\title{
Abstracts from the 53rd European Society of Human Genetics (ESHG) Conference: Interactive e-Posters
}

\author{
Volume 28 I Supplement 1 \\ Virtual Conference
}

June 6-9, 2020

(C) European Society of Human Genetics 2020. Modified from the conference website and published with permission.

Sponsorship: Publication of this supplement was sponsored by the European Society of Human Genetics. All content was reviewed and approved by the ESHG Scientific Programme Committee, which held full responsibility for the abstract selections.

Disclosure Information: In order to help readers form their own judgments of potential bias in published abstracts, authors are asked to declare any competing financial interests. Contributions of up to EUR 10 000.- (Ten thousand Euros, or equivalent value in kind) per year per company are considered "Modest". Contributions above EUR 10 000.- per year are considered "Significant".

Presenting authors are indicated with bold typeface in the contributor lists.

\section{Interactive e-Posters}

\section{P01 Reproductive Genetics/Prenatal Genetics}

\section{P01.001.A}

Prenatal diagnostics of 15q11.2 microdeletion syndrome the possibilities and challenges

K. Belemezova ${ }^{1,2}$, P. Chaveeva $^{1}$, V. Stratieva ${ }^{3,4}, M$.

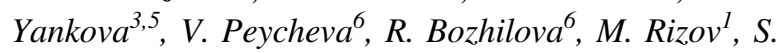
Yovinska $^{l}$, M. Hristova-Savova ${ }^{1}, R$. Kaneva ${ }^{6}$, I. Dimova $^{1,6}$

${ }^{1}$ SAGBAL „Dr Shterev“, Sofia, Bulgaria, ${ }^{2}$ University Hospital "St. Ivan Rilski", Department of Immunology, Medical University of Sofia, Sofia, Bulgaria, ${ }^{3}$ O.S.C.A.R. Clinic, Sofia, Bulgaria, ${ }^{4}$ First Specialized Hospital of Obstetrics and Gynecology „St. Sofia”, Sofia, Bulgaria, ${ }^{5}$ University Hospital "Lozenetz", Sofia, Bulgaria, ${ }^{6}$ Center of Molecular medicine, Medical University of Sofia, Sofia, Bulgaria

Microdeletion in $15 \mathrm{q} 11.2$ has been described as a distinct syndrome encompassing an area between two fragile sites in 15q (BP1 and BP2), with approximately 500 bp size, containing NIPA1, NIPA2, TUBGCP5, and CYFIP1 genes. It is a multisystemic disease affecting mostly the nervous system (intellectual deficits, delayed psychomotor development, ataxia, epilepsy, behavioral problems, etc.), followed by congenital heart defects and various dysmorphisms. This microdeletion was found in 0.57$1.27 \%$ of pediatric patients targeted for microarray analysis, mainly with developmental delay and intellectual deficits. However, not all of the deletion carriers have a clinical manifestation - it is found in $0.25 \%$ of the population of healthy controls. The penetrance of $15 \mathrm{q} 11.2$ microdelection syndrome is estimated at $10.4 \%$, which is significantly higher in de novo occurrence. We report 4 cases of prenatally established 15q11.2 microdeletion, in 3 of the cases the microdeletion was inherited from clinically healthy mothers. The indications for aCGH prenatal diagnosis were: 1) ultrasound data for unilateral cleft lip and palate in combination with an increased nuchal translucency of 3.3 $\mathrm{mm}$ - mother was the carrier; 2) second pregnancy after the first case; 3) increased risk from the biochemical screening (1:6) and tricuspid regurgitation, brother with neuropsychiatric disability - mother was a carrier; 4) Increased NT of $7.3 \mathrm{~mm}$. Our results highlight the importance of microarray analysis in the diagnostic refinement of 
pregnancies which are abnormal in early fetal screening. We emphasize the difficulties of genetic counseling in the inheritance and the incomplete penetrance of the aberrations.

K. Belemezova: None. P. Chaveeva: None. V. Stratieva: None. M. Yankova: None. V. Peycheva: None. R. Bozhilova: None. M. Rizov: None. S. Yovinska: None. M. Hristova-Savova: None. R. Kaneva: None. I. Dimova: None.

\section{P01.002.B}

Improvement of prenatal care in case of fetal anomaly of the corpus callosum using exome sequencing during the pregnancy

S. Heide ${ }^{1}$, M. Spentchian ${ }^{1}$, S. Valence ${ }^{2}, J$. Buratti $^{3}$, C.

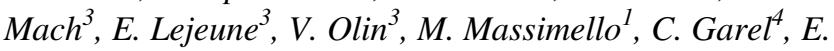
Blondiaux $^{4}$, G. Quenum-Miraillet ${ }^{5}$, S. Chantot-Bastaraud ${ }^{5}$, M. Milh ${ }^{6}$, V. des Portes ${ }^{7}$, M. Spodenkiewic ${ }^{8}, V$. Layet ${ }^{9}, R$.

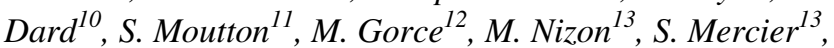

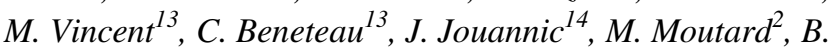
Keren $^{3}$, D. Héron ${ }^{3}$

${ }^{1} U F$ de Génétique Médicale et CRMR « Déficience intellectuelle », Département de Génétique, Groupe Hospitalier Pitié-Salpêtrière, APHP Sorbonne Université, Paris, France, ${ }^{2}$ Service de Neurologie Pédiatrique, Hôpital Armand Trousseau, APHP Sorbonne Université, Paris, France, ${ }^{3} U F$ de Génomique du Développement, Département de Génétique, Groupe Hospitalier Pitié-Salpêtrière, APHP Sorbonne Université, Paris, France, ${ }^{4}$ Service de Radiologie Pédiatrique, Hôpital Armand Trousseau, APHP Sorbonne Université, Paris, France, ${ }^{5}$ Service de Cytogénétique, Hôpital Armand Trousseau, HUEP, APHP Sorbonne Université, Paris, France, ${ }^{6}$ Service de Neurologie Pédiatrique, Hôpital La Timone, APHM, Marseille, France, ${ }^{7}$ Service de Neurologie Pédiatrique, Hospices Civils de Lyon, Bron, France, ${ }^{8}$ Service de Génétique Clinique, CHU de Reims, Reims, France, ${ }^{9}$ Service de Génétique Clinique, Hôpital du Havre, Le Havre, France, ${ }^{10}$ Service de Génétique Clinique, Hôpital de Poissy, Poissy, France, ${ }^{11}$ Centre de Référence des anomalies du développement, Service de Génétique Médicale, CHU de Dijon, Dijon, France, ${ }^{12}$ Service de génétique clinique, CHU d'Angers, Angers, France, ${ }^{13}$ Service de Génétique Clinique, CHU de Nantes, Nantes, France, ${ }^{14}$ Service de Médecine Fotale, Hôpital Armand Trousseau, HUEP, APHP Sorbonne Université, Paris, France

Purpose: Anomalies of the corpus callosum (ACC) are usually diagnosed by prenatal ultrasound. This malformation is characterized by its uncertain prognosis especially when isolated which makes the prenatal counseling difficult. Among genetic etiologies, only chromosomal anomalies are investigated during the prenatal period while $\mathrm{ACC}$ is due to single gene mutation in most cases and neurodevelopmental prognosis depends on the underlying cause.

Methods: In order to evaluate the feasibility and contribution of prenatal exome sequencing ( $\mathrm{pES}$ ) during the pregnancy, we included 66 fetuses with ACC for trio analysis. Only pathogenic variants in known ACC and/or ID genes were considered.

Results: $p E S$ results were available within a median delay of 22 days. A pathogenic SNV was identified in 12 cases (18\%). Moreover, a pathogenic CNV was identified in 4 cases. Thus, the genetic cause was determined in $22 \%$ of cases. In all cases with diagnoses, pES results enlightened the neurodevelopmental prognosis of the fetus and enabled the parents to choose to continue with or terminate the pregnancy knowingly.

Conclusions: This is the first study of pES focusing on a single malformation characterized by an uncertain prognosis. Our results demonstrate the feasibility of pES in fetuses with prenatal diagnosis of ACC. pES has an evident clinical impact. The impact of $\mathrm{pES}$ when negative remains to be evaluated

S. Heide: None. M. Spentchian: None. S. Valence: None. J. Buratti: None. C. Mach: None. E. Lejeune: None. V. Olin: None. M. Massimello: None. C. Garel: None. E. Blondiaux: None. G. Quenum-Miraillet: None. S. Chantot-Bastaraud: None. M. Milh: None. V. des Portes: None. M. Spodenkiewic: None. V. Layet: None. R. Dard: None. S. Moutton: None. M. Gorce: None. M. Nizon: None. S. Mercier: None. M. Vincent: None. C. Beneteau: None. J. Jouannic: None. M. Moutard: None. B. Keren: None. D. Héron: None.

\section{P01.003.C}

Genetic management of a small deletion in the PWS/AS imprinting center in a prenatal case

C. Pérez ${ }^{1}$, E. Pestaña ${ }^{2}$, C. Rivera ${ }^{2}$, M. Augél, L. Barranco ${ }^{1}$, A. Canellas ${ }^{1}, M$. Costa ${ }^{l}, C$. de la Iglesial, D. Fernández ${ }^{1}$, N. Palau ${ }^{l}$, B. Méndez ${ }^{1}$, M. Piqué ${ }^{1}$, M. Herrero ${ }^{1}$, D. Yeste ${ }^{l}$, L. Puig ${ }^{l}$, R. Ferreti ${ }^{1}$, M. Punzón ${ }^{l}$, S. Martín ${ }^{l}$, J. Mendoza ${ }^{l}$, N. Pinedo ${ }^{l}$, M. Rodríguez ${ }^{l}$, L. Linares ${ }^{I}$, E. Lloveras $^{l}$

${ }^{1}$ Synlab, Esplugues de Llobregat, Spain, ${ }^{2}$ Synlab, Madrid, Spain

Introduction: Imprinted genes involved in PWS and AS are under control of an imprinting center comprising two regulatory regions, PWS-SRO (around the SNRPN promoter) and the AS-SRO (35 kb upstream). A very few AS patients have small and/or atypical deletions in AS-SRO. 
Patient and Results: Pregnant patient (13 weeks) was referred because a daughter of 4 years old with development delay and peculiar phenotype and a $62 \mathrm{~Kb}$ copy number loss at 15q11.2, arr[GRCh37] 15q11.2(25119854_25177138)x1. In extended familial studies, mother (pregnant) and grandfather have both the same deletion. MS-MLPA was performed in the daughter and the pregnant. The $15 q 11.2$ deletion was confirmed in both and an altered methylation pattern in the daughter was detected correlating with an AS diagnosis. ArrayCGH analysis (CytoSure ${ }^{\mathrm{TM}}$ Constitutional v3 $8 \times 60 \mathrm{k}$, OGT, UK) was performed in chorionic villus sample and the same deletion of $62 \mathrm{~Kb}$ involving U5 exon of AS-SRO (exon 4 according NM sequence of SNRPN gene) was detected. MS-MLPA (PW/AS MS-MLPA Kit, MRC Holland) was performed in amniotic fluid with alteration of the methylation pattern, compatible with hypomethylation of the probes located in the $\mathrm{CpG}$ islands region of SNRPN gene was detected. Considering all the results, a prenatal diagnosis of AS was performed.

Comments: IC deletion have been described in a small fraction of AS patients with ID, with no disease-causing variants detected to date.Deletions involving AS-SRO are a molecular defect that must be determined as the recurrence risk in case of a familial IC deletion. Caution should be made in prenatal diagnosis and is appropriate recommending MS-MLPA analysis.

C. Pérez: None. E. Pestaña: None. C. Rivera: None. M. Augé: None. L. Barranco: None. A. Canellas: None. M. Costa: None. C. de la Iglesia: None. D. Fernández: None. N. Palau: None. B. Méndez: None. M. Piqué: None. M. Herrero: None. D. Yeste: None. L. Puig: None. R. Ferreti: None. M. Punzón: None. S. Martín: None. J. Mendoza: None. N. Pinedo: None. M. Rodríguez: None. L. Linares: None. E. Lloveras: None.

\section{P01.005.B}

\section{START: Thestudy of assistedreproductive technologies}

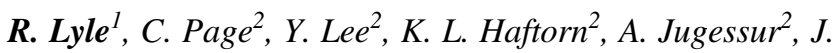

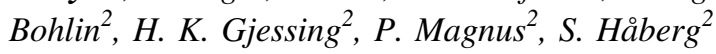

${ }^{1}$ Oslo University Hospital, Oslo, Norway, ${ }^{2}$ Norwegian Institute of Public Health, Oslo, Norway

In the last few decades there have been marked changes in patterns of fertility and family structure in rich countries. These include increasing age at childbirth, a lower number of children born to each woman or man, greater use of assisted reproductive technology (ART), higher frequency of family disruptions and increasingly complex family structures. The Centre for Fertility and Health (CeFH; www.cefh.no) consists of a multidisciplinary research team of epidemiologists, geneticists, demographers, sociologists and economists with research themes including fertility problems and intergenerational transmission of health. The START project aims to understand the causes and health consequences of subfertility and how genetic influences and epigenetic differences are associated with subfertility and Assisted Reproductive Technologies (ART). ART may introduce epigenetic changes in the embryo since the procedures are during a period of extensive epigenetic repatterning. It is unknown which genes may be affected and the potential health consequences for the children. START uses data from the Norwegian Mother, Father and Child Cohort Study (MoBa; https://www.fhi.no/en/studies/ moba/), which follows more than 110000 children and their parents. We have collected genetic and epigenetic data from 1000 trios with children born after ART, and 1000 trios from naturally conceived children analyzed with the Illumina EPIC array and the Illumina HumanCoreExome chip. Investigations are focussed on the effect of ART treatment on imprinted genes and fertility genes, and how epigenetic age acceleration interacts with fertility and ART treatment. Our results show DNA methylation changes due to both parental subfertility and ART procedures.

R. Lyle: None. C. Page: None. Y. Lee: None. K.L. Haftorn: None. A. Jugessur: None. J. Bohlin: None. H.K. Gjessing: None. P. Magnus: None. S. Håberg: None.

\section{P01.006.C}

Beckwith Wiedemann Syndrome: a prenatal case report with a novel $C D K N 1 C$ mutation

\section{P. Maia Almeida ${ }^{1,2}$, S. Moreira Ribeiro ${ }^{1}$, L. Abreu ${ }^{3}$, A. Isabel Rei ${ }^{3}$, M. Branco $^{3}$, E. Galhano ${ }^{3}$, J. Saraiva ${ }^{1,4,5}, F$. $\operatorname{Ramos}^{1,3}$}

${ }^{1}$ Medical Genetics Unit, Centro Hospitalar e Universitário de Coimbra, Coimbra, Portugal, ${ }^{2}$ Faculty of Health Sciences, University of Beira Interior, Covilhã, Portugal, ${ }^{3}$ Prenatal Diagnostic Center, Maternidade Bissaya Barreto, Centro Hospitalar e Universitário de Coimbra, Coimbra, Portugal, ${ }^{4}$ University Clinic of Pediatrics, Faculty of Medicine, University of Coimbra, Coimbra, Portugal, ${ }^{5}$ Clinical Academic Center of Coimbra, Coimbra, Portugal

Introduction: Beckwith Wiedemann Syndrome (BWS) is a genomic imprinting disorder typically diagnosed after birth. Some ultrasound features that include polyhydramnios, omphalocele, and visceromegaly may raise the clinical suspicion in prenatal. Most reported BWS cases are sporadic, but around $15 \%$ may be familial. Delineation of the molecular defects can predict familial recurrence risks and make invasive prenatal diagnosis possible. 
Case Report: We report a case of a 41-year-old pregnant woman in whom ultrasound revealed at 24-weeks corpus callosum hypoplasia, macroglossia, omphalocele, short long bones and enlarged kidneys. The pregnancy was terminated and autopsy confirmed the ultrasound findings and also enlarged ovaries and pancreas, cytomegaly of the adrenal cortex and a placental mesenchymal dysplasia. Family history was irrelevant.

Materials and Methods: Clinical and molecular characterization of a prenatal case with diagnosis of BWS. We performed array-CGH, methylation analysis at $11 \mathrm{p} 15.5$ BWS locus and Sanger sequencing of CDKN1C gene. The presence of the variant identified in the foetus was tested in the parents.

Results: Array-CGH and $11 \mathrm{p} 15.5$ methylation analysis (MS-MLPA) were normal. CDKN1C Sanger sequencing found a heterozygous novel probable pathogenic frameshift variant: NM_000076.2:c.479del p.(Pro160Argfs*112) of maternal origin.

Conclusions: This case demonstrates that search for a clear specific molecular diagnosis is key not only to confirm the diagnosis but also to provide proper genetic counselling. Although present in only $5 \%$ of the patients with no family history the identification of a CDKNIC pathogenic variant of maternal origin increases the recurrence risk to $50 \%$.

P. Maia Almeida: None. S. Moreira Ribeiro: None. L. Abreu: None. A. Isabel Rei: None. M. Branco: None. E. Galhano: None. J. Saraiva: None. F. Ramos: None.

\section{P01.007.A}

Prenatal phenotype in Beckwith-Wiedemann spectrum

D. Carli, C. Bertola, S. Cardaropoli, V. P. Ciuffreda, M. Pieretto, G. B. Ferrero, A. Mussa

Department of Public Health and Pediatrics, University of Torino, Torino, Italy

Introduction: the prenatal phenotype of BeckwithWiedemann spectrum (BWSp) is not well characterized. Here we investigate the prenatal features of large series of BWSp patients.

Methods: postnatal phenotype, genotype, prenatal ultrasound (US) features, pregnancy course and biochemical screening tests of 88 patients with BWSp (51 females) were reviewed.

Results: molecular tests had been performed in 86 patients, 80 resulted positives for BWSp-related anomalies, 6 negative with a clinical diagnosis. Seventeen (19.3\%) were conceived through artificial reproductive techniques. Twinning occurred in 9 pregnancies and resulted in 13 BWSp newborns. Pregnancy biochemical screening tests showed increased serum markers: PAPP-A at 10-13 weeks of gestational age $(n=44,1.13 \pm 0.61 \mathrm{MoM}, p<0.001)$, and $\alpha \mathrm{FP}(1.52 \pm 0.80 \mathrm{MoM}, p=0.003)$, uEstriol $(1.41 \pm 0.37 \mathrm{MoM}, p<0.001)$ and total hCG $(2.10 \pm 2.17$ MoM, $p<0.001)$ at $15-18$ weeks $(n=27)$. Morphology US scan (available in 64) revealed abdominal and head circumferences higher than normal $(1.41 \pm 1.10 \mathrm{SD}$, $p<0.001$ and $0.44 \pm 1.04, p=0.003$, respectively) with normal femur lengths. Fifty-eight patients $(66 \%)$ had a various combination of pathologic US findings, including enlarged abdominal circumference at 20 weeks' scan ( $n=17)$, macrosomia at a subsequent scan $(n=35$, of these 14 normal at 20 weeks' scan), omphalocele $(n=12)$, enlargement of abdominal organs $(n=6)$, macroglossia $(n=15)$, adrenal cysts/masses $(n=2)$, nephroureteral anomalies $(n=11)$, polyhydramnios $(n=24)$, placental enlargement $(n=2)$ or mesenchymal dysplasia $(n=4)$. Thirty cases had no detected anomaly at any US.

Conclusions: on the basis of these findings, we propose a new embryo-fetal BWSp scoring system, mirroring the post-natal one, that will implement the prenatal clinical approach of this developmental syndrome.

D. Carli: None. C. Bertola: None. S. Cardaropoli: None. V.P. Ciuffreda: None. M. Pieretto: None. G.B. Ferrero: None. A. Mussa: None.

\section{P01.008.B}

Prenatal diagnosis of a severe form of BICD2-opathy : further evidence of an extreme phenotypic spectrum

E. Marchionni $^{1}$, E. Agolini ${ }^{2}$, G. Mastromoro ${ }^{1}$, D. Guadagnolo $^{1}$, F. Di Palma', C. Pajno ${ }^{3}$, V. D’Ambrosio ${ }^{3}, M$. Roggini $^{4}$, A. Giancotti ${ }^{3}$, A. Novelli ${ }^{2}$, A. Pizzuti ${ }^{1}$

${ }^{1}$ Department of Experimental Medicine, Sapienza University of Rome, Rome, Italy, ${ }^{2}$ Laboratory of Medical Genetics, Ospedale Pediatrico Bambino Gesù, Rome, Italy, ${ }^{3}$ Department of Maternal and Child Health and Urologic Science, Sapienza University of Rome, Rome, Italy, ${ }^{4}$ Pediatrics and Child Neuropsychiatry Department, Sapienza University of Rome, Rome, Italy

Introduction: Arthrogryposis multiplex congenita (AMC) is characterized by the development of multiple joint contractures. Neurogenic and myogenic-types have been described, resulting in complete or partial movement restriction. In prenatal diagnosis decreased fetal movement is observed, the most severe forms being lethal in utero or after birth. We report a case of a male fetus at 22 gestational weeks with evidence of AMC on ultrasound, presenting fixed extended legs and flexed arms, bilateral clubfoot and absent fetal movements. Frontal oedema was also observed. After amniocentesis, karyotype and SNParray resulted normal. 
Materials and Methods: Trio-based Whole Exome Sequencing (WES) was performed on fetus genomic DNA and his unaffected parents.

Results: WES analysis disclosed a de novo heterozygous pathogenic deletion (NM_015250.3: c.1636_1638delAAT; p. Asn546del) in BICD2 gene, associated with autosomal dominant spinal muscular atrophy, lower extremitypredominant 2 (SMALED2). The couple decided to terminate the pregnancy. Babygram showed bilateral genu recurvatum, bilateral clubfoot and clubhand and multiple joints contractures.

Conclusions: BICD2 mutations are associated with severe prenatal-onset forms of spinal muscular atrophy, lower extremity-predominant 2B (SMALED2B) or milder forms with childhood-onset (SMALED2A). In literature few prenatal cases are reported, mostly with early lethal outcome. The same variant was previously described in two children with a severe postnatal phenotype but belated or milder prenatal signs. This observation expands BICD2 phenotypic spectrum, with further evidence of early-onset extreme prenatal forms. To date, the localization of the disease-causing mutations does not seem to correlate with disease severity, other molecular mechanisms should be investigated to elucidate genotype-phenotype correlations.

E. Marchionni: None. E. Agolini: None. G. Mastromoro: None. D. Guadagnolo: None. F. Di Palma: None. C. Pajno: None. V. D'Ambrosio: None. M. Roggini: None. A. Giancotti: None. A. Novelli: None. A. Pizzuti: None.

\section{P01.010.A}

Development and validation of an expanded pan-ethnic carrier screening test

S. Kyriakou, A. Achilleos, M. Georgiadou, C. Loizides, M. Nicoalou, V. Soteriou, C. Kkoufou, L. Constantinou, G. Billioud, K. Tsangaras, E. Kypri, M. Ioannides, G. Koumbaris, P. C. Patsalis

\section{NIPD Genetics, Nicosia, Cyprus}

Introduction: Carrier screening increasingly becomes more prevalent as prospective parents become more aware that the cumulative risk of having an affected child with an autosomal or X-linked condition can be higher than that for common aneuploidies. The changing admixture of populations, and the increase in gamete donation in assisted reproductive technologies have led professional organizations and professional bodies to recommend expanded carrier screening to the general population.

Materials and Methods: The test was developed using targeted in-solution hybridization enrichment followed by next generation sequencing covering all gene exons, and intron-exon boundaries, associated with 229 autosomal recessive and $\mathrm{X}$-linked conditions. The sensitivity and specificity for single nucleotide variant (SNV), indel and copy number variant (CNV) detection was ascertained in a blind validation study.

Results: SNVs and indels were detected at high sensitivity (>99.0\%) and specificity (>99.9\%). The algorithm was also designed to detect CNVs to a single or a few exons with high sensitivity and specificity. Each positive call was confirmed with an orthogonal method.

Conclusions: We have developed and validated an expanded pan-ethnic carrier screening test which includes 229 autosomal recessive and X-linked conditions. The test provides individuals and prospective parents with clinical information, helping them understand and evaluate their reproductive options.

S. Kyriakou: A. Employment (full or part-time); Significant; NIPD Genetics. A. Achilleos: A. Employment (full or part-time); Significant; NIPD Genetics. M. Georgiadou: A. Employment (full or part-time); Significant; NIPD Genetics. C. Loizides: A. Employment (full or parttime); Significant; NIPD Genetics. M. Nicoalou: A. Employment (full or part-time); Significant; NIPD Genetics. V. Soteriou: A. Employment (full or part-time); Significant; NIPD Genetics. C. Kkoufou: A. Employment (full or part-time); Significant; NIPD Genetics. L. Constantinou: A. Employment (full or part-time); Significant; NIPD Genetics. G. Billioud: A. Employment (full or part-time); Significant; NIPD Genetics. K. Tsangaras: A. Employment (full or part-time); Significant; NIPD Genetics. E. Kypri: A. Employment (full or part-time); Significant; NIPD Genetics. M. Ioannides: A. Employment (full or parttime); Significant; NIPD Genetics. G. Koumbaris: A. Employment (full or part-time); Significant; NIPD Genetics. P.C. Patsalis: A. Employment (full or part-time); Significant; NIPD Genetics.

\section{P01.014.B}

CGH-array as first-tier test in all undergoing invasive prenatal samples: an experience for a reference Centre.

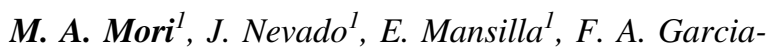 \\ Santiago ${ }^{l}$, E. Antolín ${ }^{2}, C . P e \tilde{n} a^{l}, C$. Adam ${ }^{l}$, P. D. \\ Lapunzina $^{1}$
}

${ }^{I}$ INGEMM-IdiPaz, Madrid, Spain, ${ }^{2} S^{o}$ Fisiopatología Fetal, HULP, Madrid, Spain

Introduction: In the last years microarrays (CMA) has demonstrated to be a significant tool useful in the prenatal area, mainly in fetuses with sonography anomalies. However, using array-CGH in an invasive prenatal context is still under a significant and controversial debate especially 
regarding the use of CMA in advance maternal age or maternal anxiety samples. Previous studies supported the evidence on the feasibility of introducing a-CGH as a firstline diagnostic testing routine prenatal diagnosis practice (1). Indeed, others and our laboratory have reported significant diagnostic yields also in low-risk pregnancies, between $1.6-4 \%(2,3)$.

Methods: We used customized Karyoarray ${ }^{\circledR} \mathrm{v} 3.0$ platform (4) to study consecutive invasive prenatal samples along two-year period. QF-PCR positive cases were excluded.

Results: We analyzed 800 samples and we found globally $6 \%$ of pathogenic CNVs and $2 \%$ of VUS. We segregated low-risk pregnancy samples (positive biochemical screening, soft markers, maternal anxiety, and antenatal death without sonographic anomalies). Diagnostic yield in this group was $4.5 \%$ (17/379).

Discussion: In conclusion, the use of the array-CGH in the prenatal context demonstrated a greater diagnostic capacity not only in fetuses with ultrasound abnormalities but also in low-risk pregnancies. Therefore, we definitively recommend the possibility of using the array-CGH in all those gestations that undergo invasive prenatal diagnosis, after excluding by QF-PCR the most common aneuploidies. (1)Kan AS. et al., 2014. PLoS One. 9(2):e87988. (2)Wapner RJ. et al., 2012. N Engl J Med.367:2175-2184. (3)Armengol L. et al. 2012. Hum Genet. 131:513-523. (4)Vallespin E. et al,. 2013. Am J Med Genet A.161A:1950-60.

M.A. Mori: None. J. Nevado: None. E. Mansilla: None. F.A. Garcia-Santiago: None. E. Antolín: None. C. Peña: None. C. Adam: None. P.D. Lapunzina: None.

\section{P01.016.A \\ DERIVATIVE(9)T(2;9)(P23;P22) IN HIGH RISK TWIN PREGNANCY}

\section{Kadkhoda zadeh, s. seyedhassani, f. harrazi zadeh yazdi, a. danafar, m. mirjalili, f. saeb}

yazdgenetic, yazd, Iran, Islamic Republic of

Introduction:A 39- year- old mother was referred with $\mathrm{GA}=16 \mathrm{w}+5 \mathrm{~d}$ and high risk pregnancy screening test for $\mathrm{T} 21$. The first child of this patient was a 14- years old suffering from developmental delay and mental retardation. On the other hand, this family had two spontaneous abortion. These evidences revealed enough reasons for probability of chromosomal abnormalities such as Down syndrome, monosomy $\mathrm{X}$, and other chromosomal disorder in fetus. Amniocentesis was suggested for complementary investigation.

Methods and materials: Twenty metaphases cells were studied on the basis of G-Banding technique from culture of amniocytes in two flasks. Then, karyotypig was done on the basis of G-Banding for the parents.

Result: In this study, we described a paternal chromosomal aberration of twins. Through a combination of cytogenetic assessment of fetus and parents 46, XY, der (9) $\mathrm{t}(2 ; 9)$ (p23;p22)pat was shown. Mother's karyotype was 46XX but father's karyotype revealed an abnormal chromosome complement in all cells with balanced 46, $\mathrm{XY}, \mathrm{t}(2 ; 9)(\mathrm{p} 23 ; \mathrm{p} 22)$.

Conclusions: Paternal chromosomal balanced translocation can explain repeated abortions in this family. On the other hand, unbalanced chromosomal translocation resulting from paternal inheritance lead to developmental delay in his son. This study was highly emphasized the priority of genetic counseling and attention to the family history and genetic basis of parents in evolution of repeated spontaneous abortion. Legal abortion was recommended to unbalance chromosomal abnormalities.

M. Kadkhoda zadeh: None. S. seyedhassani: None. F. harrazi zadeh yazdi: None. A. danafar: None. M. mirjalili: None. F. saeb: None.

\section{P01.017.B}

Karyotype analysis of 8531 pregnancies in prenataly identified cases with amniocentesis from south of Turkey

A. Pazarbasi ${ }^{1}$, D. Alptekin ${ }^{1}$, N. S. Ilgaz ${ }^{1}$, I. N. Uslu ${ }^{1}$, G. Ay ${ }^{l}$, S. Kocaturk-Sel', O. Demirhan ${ }^{l}$, U. Luleyap ${ }^{1}$, M. B. Yilmaz $^{1}$, N. Çetinel ${ }^{1}$, G. Evyapan ${ }^{1}$, G. Comertpay-Tufan ${ }^{1}, S$. Buyukkurt ${ }^{2}$

${ }^{1}$ Çukurova University, Faculty of Medicine, Dept. of Medical Biology, Adana, Turkey, ${ }^{2}$ Çukurova University, Faculty of Medicine, Dept. of Obstetrics and Gynecology, Adana, Turkey

Amniocentesis is a very crucial diagnostic procedure for preventing the birth of genetically defective fetuses in order to decrease the prevalence of genetic diseases in populations. A retrospective review of our amniocentesis database for the period from January 2000 to February 2020 was carried out. The karyotyping of 8531 fetuses was carried out in Department of Medical Biology from the samples of amniotic fluids which were sent from Department of Gynecology and Obstetrics of Balcali Hospital. A standart nomenclature has been developed to describe each of types of abnormality found in human chromosomes. A total of 8531 amniocentesis specimens were processed during the study period. 620 fetuses $(7.26 \%)$ had various chromosomal abnormalities. $54.51 \%$ of abnormal karyotypes (338 cases) were numerical and $43.38 \%$ (269 cases) were structural. Both numerical and structural chromosomal aberrations were observed in 13 cases $(2.09 \%)$. The ratios were as: 
trisomy $21(49.70 \%)$, trisomy $18(17.75 \%)$, monosomy X (9.46\%), trisomy $13(6.80 \%)$, Triploidy $(4.73 \%)$, Klinefelter Syndrome (3.25\%), Trisomy X (1. 18\%), XYY Syndrome $(0.88 \%)$, and the others in all numerical abnormalities. The frequent structural abnormalities were as: 46,XX/XY, inv (9) $(\mathrm{p} 11 ; \mathrm{q} 12) /(\mathrm{p} 11 ; \mathrm{q} 13)(28.25 \%), 46, \mathrm{XX} / \mathrm{XY}, 1 \mathrm{qh}(+)$ $(11.89 \%), 46, X Y, Y q h(-)(7.43 \%), 46, X X / X Y, 16 q h(+)$ (7.06\%), 46,XX/XY, 9qh(+)(4.46\%) and 46,XY, Yqh(+) $(4.08 \%)$. Balanced and unbalanced translocations, deletions and duplications were also found in less ratio. According to the literature and our results, advanced maternal age is the main cause of fetal chromosomal abnormalities. Fetal chromosomal abnormality ratio that we found was $7.26 \%$. This ratio emphasize the importance of prenatal diagnosis.

A. Pazarbasi: None. D. Alptekin: None. N.S. Ilgaz: None. I.N. Uslu: None. G. Ay: None. S. Kocaturk-Sel: None. O. Demirhan: None. U. Luleyap: None. M.B. Yilmaz: None. N. Çetinel: None. G. Evyapan: None. G. Comertpay-Tufan: None. S. Buyukkurt: None.

\section{P01.018.C}

Microarray testing in pregnancies with nuchal translucency 3-3.4 mm - time to change the cut-off.

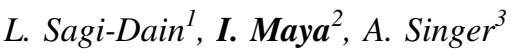 \\ ${ }^{1}$ Carmel Medical Center, Haifa, Israel, ${ }^{2}$ Rabin Medical Center, Petach Tikva, Israel, ${ }^{3}$ Sheba Medical Center, Ramat Gan, Israel}

Objective: To examine the risk for chromosomal microarray analysis (CMA) aberrations in fetuses with nuchal translucency (NT) of 3 to $3.4 \mathrm{~mm}$. Methods: The results of all CMA tests performed due to an NT of 3 to $3.4 \mathrm{~mm}$ were retrospectively retrieved from the Ministry of Health computerized database. The rates of clinically significant (pathogenic and likely pathogenic) microarray findings were compared to a previously published local control population, encompassing 5541 fetuses with normal ultrasound. Results: Overall, 619 CMA analyses were performed due to a sonographic indication of NT of 3 to $3.4 \mathrm{~mm}$. Of these, 29 (4.7\%) clinically significant copy number variants were detected, a considerably increased risk compared to control pregnancies. Fetal karyotyping would have missed $31 \%$ of the abnormal cases, while non-invasive prenatal screening (NIPS) would have overlooked $31-49 \%$ of the anomalies, depending on the method. Conclusions: The outcomes of our study, representing the largest number of CMA results in fetuses with NT of 3 to $3.4 \mathrm{~mm}$, suggest that the rate of abnormal CMA findings in these pregnancies is significantly higher compared to pregnancies with normal ultrasound. Our results support the consideration to perform invasive prenatal testing for CMA in pregnancies with NT above $3 \mathrm{~mm}$. In addition, as several of the CMA findings in these pregnancies are not associated with major sonographic anomalies, it seems that NIPS with later sonographic survey cannot adequately replace NT measurement.

L. Sagi-Dain: None. I. Maya: None. A. Singer: None.

\section{P01.020.B}

Three fetuses with achondrogenesis type 2 explained by maternal mosaicism

\author{
S. Z. Jan ${ }^{l}$, R. F. Suijkerbuijk ${ }^{l}$, Y. J. Vos ${ }^{l}$, P. M. \\ Grootscholten $^{l}$, L. Zijlstra ${ }^{l}$, K. Bouman ${ }^{1}$, M. T. M. \\ Franssen $^{2}$, M. H. Schoots ${ }^{3}$, N. Corsten-Janssen ${ }^{1}, B$. \\ Sikkema-Raddatz ${ }^{1}$
}

${ }^{1}$ Univeristy Medical Center Groningen, Department of Genetics, Groningen, Netherlands, ${ }^{2}$ Univeristy Medical Center Groningen, Department of Obstetrics, Groningen, Netherlands, ${ }^{3}$ Univeristy Medical Center Groningen, Department of Pathology and Medical Biology, Groningen, Netherlands

Background: Adding whole exome sequencing (WES) to the standard diagnostic procedure in fetuses with ultrasound anomalies improves the diagnostic yield. Identifying a diagnosis is important for parental choice and informed reproductive decision making, amongst other factors. Here we describe three fetuses in whom a diagnosis of achondrogenesis type 2 (COL2Al variant) due to maternal mosaicism was made using WES.

Materials \& Methods: A couple expecting dichorionic diamniotic twins, both with severe limb shortening and fetal hydrops, was referred to our department. A previous pregnancy had been terminated for the same reasons. QFPCR and SNP array were performed on DNA of chorionic villi from both fetuses of the current pregnancy. WES was performed on parental DNA (extracted from blood) and fetal DNA.

Results: QF-PCR and SNP-array showed no abnormalities for both fetuses. WES revealed a pathogenic, apparently de novo, COL2A1 variant c.3062_3079del p. (Pro1021_Gly1026del) in both samples, which explained the ultrasound abnormalities. Retrospective analysis confirmed the presence of the same COL2Al variant in the previous pregnancy. Re-analysis of parental WES data revealed the variant in less than $1 \%$ of maternal reads, implying an extremely low grade of mosaicism, which was confirmed by Sanger sequencing.

Conclusions: This case study highlights the added value of WES in prenatal diagnostics, including the capacity to identify extremely low-grade parental mosaicism that profoundly impact the recurrence risk of a genetic disease. It also suggests that the possibility of very low-grade 
parental mosaicism should be closely investigated when an apparently de novo variant is identified.

S.Z. Jan: None. R.F. Suijkerbuijk: None. Y.J. Vos: None. P.M. Grootscholten: None. L. Zijlstra: None. K. Bouman: None. M.T.M. Franssen: None. M.H. Schoots: None. N. Corsten-Janssen: None. B. SikkemaRaddatz: None.

\section{P01.021.C}

Prenatal diagnostics in Estonia: a 2-year prospective study assessing the performance of combined firsttrimester screening, chromosomal microarray analysis, and next-generation sequencing

K. Ridnõi ${ }^{1,2}$, K. Muru ${ }^{1,3}$, M. Keernik ${ }^{1}$, S. Pajusalu ${ }^{1,3,4}$, E. Ustav $^{5}$, P. Tammur ${ }^{1}$, T. Mölter-Väär ${ }^{1}$, T. Kahre ${ }^{1,3}, U$.

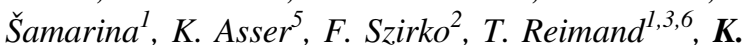
$\tilde{O}_{\text {unap }}{ }^{1,3}$

${ }^{1}$ Department of Clinical Genetics, United Laboratories, Tartu University Hospital, Tartu, Estonia, ${ }^{2}$ Centre for Perinatal Care, Women's Clinic, East-Tallinn Central Hospital, Tallinn, Estonia, ${ }^{3}$ Department of Clinical Genetics, Institute of Clinical Medicine, University of Tartu, Tartu, Estonia, ${ }^{4}$ Yale University School of Medicine, Department of Genetics, New Haven, CT, United States, ${ }^{5}$ Women's Clinic, Tartu University Hospital, Tartu, Estonia, ${ }^{6}$ Department of Biomedicine, Institute of Biomedicine and Translational Medicine, University of Tartu, Tartu, Estonia

Introduction: The implementation of cell-free fetal DNA (cff-DNA) testing in maternal blood opened possibilities to improve performance of combined first trimester screening (cFTS). On the other hand, use of new molecular methods, such as chromosomal microarray analysis (CMA) and nextgeneration sequencing (NGS), have shown benefits in diagnosis of chromosomal and genetic diseases, which are not detectable with cff-DNA screening, but require invasive procedure. We prospectively evaluated performance of cFTS in Estonia during 2 years using national guidelines with application of CMA and NGS in high-risk pregnancies.

Material and methods: We investigated 14,566 singleton pregnancies (60\% of all pregnancies). We selected 334 high-risk pregnancies for CMA diagnostic performance evaluation and 28 cases of highly dysmorphic fetuses for NGS analysis. CMA study group was divided into two groups based on the indications for testing: group A patients with high-risk for trisomies after cFTS, but normal ultrasound and group B patients who met criteria for CMA as a first-tier diagnostic test.
Results: We found the first trimester detection rate 94\%, $100 \%$ and $100 \%$ for trisomies 21,18 and 13, respectively, with false-positive rate of $3.1 \%$. The diagnostic yield of CMA was overall 3.6\% (1.7\% in Group A and 6.0\% in Group B). In NGS analysis group we report diagnostic yield of $17.9 \%$. Combining these three methods, overall detection rate of all genetic anomalies was $101(0.7 \%)$.

Conclusions: the use of CMA and NGS in high-risk pregnancies after cFTS is justified and increase diagnostic yield. Funding: Estonian Research Council grants PUT355, PRG471, and PUTJD827.

K. Ridnõi: None. K. Muru: None. M. Keernik: None. S. Pajusalu: None. E. Ustav: None. P. Tammur: None. T. Mölter-Väär: None. T. Kahre: None. U. Šamarina: None. K. Asser: None. F. Szirko: None. T. Reimand: None. K. Õunap: None.

\section{P01.023.B}

Higher incidence of congenital anomalies in children born after assisted reproduction in the Czech Republic: Population based study

A. Sipek Jr' ${ }^{1,2,3}$, V. Gregor ${ }^{2,4}$, J. Klaschka ${ }^{5,6}$, M. Maly ${ }^{5,7}$, A. Sipek $S r^{2,4,8}$

${ }^{1}$ Institute of Biology and Medical Genetics, First Faculty of Medicine, Charles University, Prague, Czech Republic, ${ }^{2}$ Department of Medical Genetics, Thomayer Hospital, Prague, Czech Republic, ${ }^{3}$ Institute of Medical Genetics, Third Faculty of Medicicne, Charles University, Prague, Czech Republic, ${ }^{4}$ Department of Medical Genetics, Pronatal Sanatorium, Prague, Czech Republic, ${ }^{5}$ Institute of Computer Science of the Czech Academy of Sciences, Prague, Czech Republic, ${ }^{6}$ Institute of Biophysics and Informatics, First Faculty of Medicine, Charles University, Prague, Czech Republic, ${ }^{7}$ National Institute of Public Health, Prague, Czech Republic, ${ }^{8}$ GENNET, Prague, Czech Republic

Introduction: The number of children conceived by assisted reproduction techniques (ART) is increasing in the Czech Republic. The main goal of this study was to evaluate the incidence of congenital anomalies in ART conceived children.

Methods: The retrospective epidemiological analysis was performed using data from the National Registry of Congenital Anomalies and National Registry of Newborns, run by the Institute of Health Information and Statistics of the Czech Republic. All diagnoses of congenital anomalies (Q00-Q99) were included. We compared the incidences of congenital anomalies in naturally conceived children and ART conceived children born in the Czech Republic during 
5 years period (2013-2017). Statistical analysis was performed by Fisher's exact test.

Results: During the selected period there were 547675 children born in the Czech Republic (531 064 were naturally conceived children and 16611 were ART conceived children). The incidence of congenital anomalies was $3.90 \%$ in naturally conceived children group and $4.35 \%$ in ART conceived children. This difference is statistically significant $(\mathrm{p}=0.003)$. Analyzing different subgroups of congenital anomalies we have found a significantly increased incidence of anomalies in two subgroups: Congenital malformations of the circulatory system (Q20Q28) and Congenital malformations of the respiratory system (Q30-Q34).

Discussion: The increased risk of congenital anomalies in ART conceived children was highlighted in many studies however the results are often diverse. In our populationbased study, we confirmed a significantly higher general incidence of congenital anomalies in ART conceived children.

The study is supported by the RVO project: "Thomayerova nemocnice - TN, 00064190".

A. Sipek Jr: B. Research Grant (principal investigator, collaborator or consultant and pending grants as well as grants already received); Modest; Research grant provided by Ministry of Health of the Czech Republic, RVO project: "Thomayerova nemocnice TN, 00064190". V. Gregor: B. Research Grant (principal investigator, collaborator or consultant and pending grants as well as grants already received); Modest; Research grant provided by Ministry of Health of the Czech Republic, RVO project: "Thomayerova nemocnice TN, 00064190". J. Klaschka: B. Research Grant (principal investigator, collaborator or consultant and pending grants as well as grants already received); Modest; Research grant provided by Ministry of Health of the Czech Republic, RVO project: "Thomayerova nemocnice TN, 00064190". M. Maly: B. Research Grant (principal investigator, collaborator or consultant and pending grants as well as grants already received); Modest; Research grant provided by Ministry of Health of the Czech Republic, RVO project: "Thomayerova nemocnice TN, 00064190". A. Sipek Sr: B. Research Grant (principal investigator, collaborator or consultant and pending grants as well as grants already received); Modest; Research grant provided by Ministry of Health of the Czech Republic, RVO project: "Thomayerova nemocnice TN, 00064190".

\section{P01.024.C}

A low-cost microsatellite marker method for quick andA low-cost microsatellite marker method for quick and reliable prenatal 22q11.2DS screening and detection in fetuses with suspected congenital heart defects
L. Torres-Juan ${ }^{l}$, M. Coll-Ferrer ${ }^{l}$, M. Prado-Farnos ${ }^{l}, R$. Martorell-Riera $^{l}$, E. Amengual-Cloquell ${ }^{1}$, A. PerezGranero $^{\prime}$, A. Tubau ${ }^{2}$, M. Juan ${ }^{2}$, J. Rosell ${ }^{1}$, I. MartinezLopez $^{l}$, D. Heine-Suñer ${ }^{l}$

${ }^{1}$ Institute of Health Research of the Balearic Islands (IDISBA) and Hospital Son Espases, Palma, Spain, ${ }^{2}$ Institute of Health Research of the Balearic Islands (IDISBA) and Hospital Son Llatzer, Palma, Spain

Congenital heart defects (CHD) are the most common form of birth defects and affect $1 \%$ of newborns and an even higher percentage of unborn fetuses. CHDs are a frequent finding during routine ultrasound prenatal screening. Once detected, it is important to determine their etiology. The $22 \mathrm{q} 11.2$ deletion syndrome (22q11.2 DS) is the most frequent known genetic cause of CHDs (1.9\% prevalence). We present a multiplex marker PCR kit to detect 22q11.2 deletions and duplications which we prospectively used in 1630 individuals and 450 fetal cases with a congenital heart defect referred by an obstetrician. Important features: 1) Low cost (there are 2 mixes of 11 pairs of primers and 2 conventional PCR reactions). 2) Fast, results can be given within hours of receiving the sample (DNA extraction -> PCR 1.5 hours -> automatic sequencer). 3) Safely rules out sample contamination by maternal DNA. 4) Uses small amounts of DNA (10 ng) and can be performed starting from amniotic fluid or non-cultured chorion biopsy or any other tissue. 5) In prenatal samples, it would be complementary to the QF-PCR (negative samples for trisomy $13,18$ or 21$)$. 6) The parental origin of the deletion/duplication is determined when the fetus is analyzed with the parents. 7) Detects atypical and typical deletions, extending the analysis far beyond that given by the FISH probe that has been historically used (TUPLE1). 8) We have developed a panel of 31 additional markers, not included in the multiplex, to be used in case of lack of informativity.

L. Torres-Juan: None. M. Coll-Ferrer: None. M. Prado-Farnos: None. R. Martorell-Riera: None. E. Amengual-Cloquell: None. A. Perez-Granero: None. A. Tubau: None. M. Juan: None. J. Rosell: None. I. Martinez-Lopez: None. D. Heine-Suñer: None.

\section{P01.026.B}

The effects of the CFTR genotype on the reproductive system in adult male patients with cystic fibrosis

\section{S. Repina ${ }^{1}$, S. Krasovskiy ${ }^{1,2}$, M. Shtaut ${ }^{l}$, G. Shmarina ${ }^{1}$, T. Adyan ${ }^{l}$, V. Chernykh ${ }^{l}$}

${ }^{1}$ Research Centre for Medical Genetics, Moscow, Russian Federation, ${ }^{2}$ Pulmonology Research Institute, Moscow, Russian Federation 
Background: Commonly male patients with Cystic Fibrosis (CF) is infertile due to obstructive azoospermia (OA), at that an influence of CFTR genotype on reproductive system is poor understood.

Materials and methods: We examined 93 Russian male CF patients (17-45 y.o., mean - $24.9 \pm 5.7$ y.o.). Physical examination, scrotal ultrasonography, hormonal, biochemical, and semen analysis were done. Standard and biochemical semen examination were done according to the WHO's (2010) recommendations. The CFTR gene variants were analyzed using by AFPL, MLPA and DNA sequencing (Sanger's method and MPS).

Results: CFTR mutations were detected in $100 \% \mathrm{CF}$ alleles. Folowing genotypes were found: F508del/F508del (20.4\%); F508del/3849+10kbC $>$ T (15.1\%); F508del/2789 $+5 \mathrm{G}>\mathrm{A}(4.3 \%) ;$ F508del/CFTRdele2.3 (4.3\%); F508del/ E92K (3.2\%); F508del/L138ins (3.2\%); F508del/327216T >A (2.2\%); F508del/W1282X (2.2\%); others (45.3\%). The spectrum of CFTR gene pathogenic variants and genotypes showed high frequency of "mild" pathogenic variants (25.8\%) and genotypes (50.5\%), as well as a relatively high frequency of $3849+10 \mathrm{kbC}>\mathrm{T}$ variant (12.4\%), which was identified in $8.2 \%$ patients with OA and $94.4 \%$ with no OA $(p<0.00001)$. Statistically significant difference was found between patients with F508del/3849+10kbC $>T$ and F508del/F508del genotypes for a prevalence of $\mathrm{OA} / \mathrm{seminal}$ ducts obstruction $(14.3 \% \mathrm{vs}$ $100 \%, p<0.05)$ and testicular hypoplasia $(26.9 \%$ vs $66.7 \%$, $p=0.03$ ).

Conclusions: CF dramatically affects reproductive organs, male fertility and semen parameters in CF patients, at that $3849+10 \mathrm{kbC}>\mathrm{T}$ mutation in the CFTR genotype is major genetic factor of male fertility.

S. Repina: None. S. Krasovskiy: None. M. Shtaut: None. G. Shmarina: None. T. Adyan: None. V. Chernykh: None.

\section{P01.029.B}

First and second trimester biochemical screening: additional value in the era of NIPS

\section{R. Michaelson-Cohen ${ }^{1,2,3}$, A. Lahad La $^{3,4}$}

${ }^{I}$ Medical Genetics Institute, Shaare Zedek Medical Center, Jerusalem, Israel, ${ }^{2}$ Department of Obstetrics and Gynecology, Shaare Zedek Medcial Center, Jerusalem, Israel, ${ }^{3}$ Hebrew University, Jerusalem, Israel, ${ }^{4}$ Clalit Health Services, Jerusalem, Israel

Background: Since implementation of NIPS, first and second trimester biochemical tests may no longer be necessary for Down Syndrome screening. However, studies suggest their ability to predict pregnancy complications. We sought to evaluate whether screening tests have value for this purpose.

Methods: Our cohort included all women who had first and/or second trimester screening from 2015-2018 and subsequently delivered in our center. Data was collected on demographics, medical history, biochemical screening and maternal-fetal pregnancy outcomes. Logistic regression was performed to examine correlations between biochemical markers and pregnancy complications using SPSS version 25.0 (SPSS, Inc).

Results: Over 3.5 years, 5400 women had screening tests and later delivered in our center. Decreased PAPP-A was associated with preeclampsia, gestational diabetes, IUGR and preterm delivery (PTD), OR=1.792, 1.364, 1.403 and 1.277 , respectively, $p<0.005$. Elevated AFP was associated with PTD, maternal blood administration, IUGR and low 5minute Apgar-score, $\mathrm{OR}=2.044,1.95,1.452$ and 1.22, respectively, $\mathrm{p}<0.033$. Decreased HCG in second trimester was correlated with IUGR, $\mathrm{OR}=1.346, \mathrm{p}<0.05$. Elevated HCG in second trimester was correlated with PTD and newborn NICU admission, $\mathrm{OR}=1.192,1.285$, respectively, $\mathrm{p}<0.015$. In both first and second trimesters, elevated HCG was associated with preeclampsia, $\mathrm{OR}=1.169,1.309$, respectively, $\mathrm{p}<0.05$. Low estriol was correlated with IUGR, OR=3.597, $\mathrm{p}<0.001$. After controlling for confounders (age, ethnicity, IVF, parity, etc.) most associations remained significant.

Discussion: Despite superiority of NIPS for Down Syndrome screening, biochemical screening is still valuable for prediction of pregnancy complications. Identifying women with high-risk pregnancies has clinical implications for prenatal care and risk-reducing management strategies.

R. Michaelson-Cohen: None. A. Lahad: None.

\section{P01.034.A}

Use of prenatal exome sequencing in fetuses with ultrasound anomalies

\section{Segura-Puimedon, B. Campos, J. Luna, C. Sintas, M. de Castro-Miró, H. Díez, S. B. Estruch, R. Garcia, L. Quintana, J. Rodríguez, L. Armengol}

Quantitative Genomic Medicine Laboratories, qGenomics, Barcelona, Spain, Esplugues de Llobregat, Spain

Introduction: Whole exome sequencing (WES) is an established diagnostic tool in postnatal settings for individuals with a suspected genetic condition. Recently, it is increasingly used as a diagnostic tool in prenatal settings as well. We present here our experience using this technology in fetuses with ultrasound anomalies. 
Material and Methods: WES was performed in 166 fetal samples with ultrasound anomalies. 104 samples were from evolutive pregnancies and 62 were from legal interruptions or stillbirths. In 61 samples, previous prenatal CGH-array was performed with negative result. Segregation studies were performed in cases with a candidate variant when possible.

Results: Common reasons for referral were skeletal anomalies, polymalformated fetuses, cerebral anomalies or specific syndrome suspicion. Pathogenic or likely pathogenic variants were identified in $n=42(25 \%)$ of samples. In $n=40(24 \%)$ cases, variants of unknown significance were identified, and in 13 of them inheritance was consistent with expected pattern, including a probable mosaicism case. In half of the cases $(50,6 \%)$ no candidate variant was identified. Diagnostic yield was higher in fetuses with skeletal anomalies, where pathogenic or likely pathogenic variants were identified in $(n=10) 31 \%$ of cases, and in fetuses with increased nuchal translucency or hidrops (Noonan syndrome suspicion), where a pathogenic variant was found in $24 \%(n=6)$ of the samples. No pathogenic variants were found in fetuses with cardiovascular anomalies $(n=9)$.

Conclusions: Exome sequencing is a valuable diagnostic tool in fetuses with ultrasound anomalies, especially when skeletal anomalies are present or when Noonan syndrome is suspected.

M. Segura-Puimedon: A. Employment (full or parttime); Significant; Quantitative Genomic Medicine Laboratories, qGenomics, Barcelona, Spain. B. Campos: A. Employment (full or part-time); Significant; Quantitative Genomic Medicine Laboratories, qGenomics, Barcelona, Spain. J. Luna: A. Employment (full or part-time); Significant; Quantitative Genomic Medicine Laboratories, qGenomics, Barcelona, Spain. C. Sintas: A. Employment (full or part-time); Significant; Quantitative Genomic Medicine Laboratories, qGenomics, Barcelona, Spain. M. de Castro-Miró: A. Employment (full or part-time); Significant; Quantitative Genomic Medicine Laboratories, qGenomics, Barcelona, Spain. H. Díez: A. Employment (full or part-time); Significant; Quantitative Genomic Medicine Laboratories, qGenomics, Barcelona, Spain. S. B. Estruch: A. Employment (full or part-time); Significant; Quantitative Genomic Medicine Laboratories, qGenomics, Barcelona, Spain. R. Garcia: A. Employment (full or parttime); Significant; Quantitative Genomic Medicine Laboratories, qGenomics, Barcelona, Spain. L. Quintana: A. Employment (full or part-time); Significant; Quantitative Genomic Medicine Laboratories, qGenomics, Barcelona, Spain. J. Rodríguez: A. Employment (full or part-time); Significant; Quantitative Genomic Medicine Laboratories, qGenomics, Barcelona, Spain. L. Armengol: A.
Employment (full or part-time); Significant; Quantitative Genomic Medicine Laboratories, qGenomics, Barcelona, Spain.

\section{P01.035.B}

AnDDI-Prenatome: The French exome sequencing study in prenatal diagnosis: causal diagnosis identified in nearly half of fetuses

F. Tran Mau Them ${ }^{1,2}$, A. Bruel ${ }^{1,2}$, A. Vitobello $^{1,2}$, S. Nambot ${ }^{3}$, S. Moutton ${ }^{3}$, A. Sorlin ${ }^{1,2,3}$, J. Delanne ${ }^{3}$, A. Ziegler ${ }^{4}$, E. Colin $^{4}$, A. Guichet ${ }^{4}$, M. Legendre ${ }^{5}$, C. Colson ${ }^{6,7}$, C. Abel ${ }^{8}$, A. Putoux 9 , J. Amiel ${ }^{10,11}$, A. Lavillaureix ${ }^{12}$, C. Quelin ${ }^{12}$, A. Guerrot ${ }^{13}$, A. Brehin ${ }^{13}$, L. Faivre ${ }^{3,2}$, C. Philippe ${ }^{1,2}$, V. Couturier ${ }^{1,2}$, Y. Duffourd ${ }^{2}$, C. Thauvin-Robinet ${ }^{1,2,14}$

${ }^{1}$ Unité Fonctionnelle d'Innovation diagnostique des maladies rares, FHU-TRANSLAD, Dijon, France, ${ }^{2}$ Inserm - UB UMR 1231 GAD «Génétique des Anomalies du Développement », FHU-TRANSLAD, Dijon, France, ${ }^{3}$ Centre de Référence Maladies rares "Anomalies du Développement et syndromes malformatifs », Centre de Génétique, FHUTRANSLAD, Dijon, France, ${ }^{4}$ Biochemistry and Genetics Department, University Hospital of Angers, Angers, France, ${ }^{5}$ Service de Génétique Médicale, CHU de Bordeaux - Hopital Pellegrin, Bordeaux, France, ${ }^{6} \mathrm{CHU}$ Lille, Clinique de Génétique Guy Fontaine, Lille, France, ${ }^{7}$ EA7364, RADEME, Université de Lille, Lille, France, ${ }^{8}$ Service de génétique et centre de diagnostic anténatal, Lyon, France, ${ }^{9}$ Service de Génétique, Centre de Référence Anomalies du Développement et Syndromes Malformatifs, Lyon, France, ${ }^{10}$ Service de Génétique Médicale et Clinique, Hôpital Necker-Enfants Malades, Paris, France, ${ }^{11}$ Equipe "Embryologie et Génétiques des malformations congénitales", Institut Imagine - INSERM U1163, Paris, France, ${ }^{12}$ Centre de Référence Maladies Rares CLAD-Ouest, CHU Hôpital Sud, Rennes, France, ${ }^{13}$ Normandie Univ, UNIROUEN, Inserm U1245 and Rouen University Hospital, Department of Genetics and Reference Center for Developmental Disorders, F 76000, Normandy Center for Genomic and Personalized Medicine, Rouen, France, ${ }^{14}$ Centre de Référence « Déficiences Intellectuelles de causes rares ", Centre de Génétique, FHU-TRANSLAD, Dijon, France

Introduction: Exome sequencing (ES) is the most powerful and current test for patients with developmental abnormalities (DA). It has considerably been deployed in postnatal indications with a diagnostic yield ranging from 30 to $40 \%$. Several prenatal studies report a diagnostic yield ranging from $15 \%$ to $35 \%$, depending on the exome strategy and the inclusion criteria. The French national network for rare 
diseases with $\mathrm{AD}$ deployed a pilot project of feasibility for rapid prenatal exome sequencing.

Materials and methods: We aimed to include 61 fetuses and both parents. The main inclusion criteria were: 2 major malformations or, 1 major and 1 minor malformation or, 1 malformation and a strong genetic suspicion. The feasibility aim was to emit a diagnostic report in 5-6 weeks. Array$\mathrm{CGH}$ and ES-trio were concomitantly performed. ES-trio was captured with the TWIST-v2 kit and sequenced on a NovaSeq6000 device with a 70X average depth.

Results: We included 23 patients. The turnaround time from receipt of samples to the diagnostic report was on average of 27 days for the negative patients and of 40 days for the positive patients. The diagnostic yield was of $44 \%$ with $7 \mathrm{SNV}$ and $3 \mathrm{CNV}$, including a tetrasomy 12p. Array$\mathrm{CGH}$ allowed a diagnosis in $13 \%$ and exome in $44 \%$. Results were concordant for CNV detection between arrayCGH and ES-trio.

Discussion: Prenatal trio-ES appears feasible in a useful time and strongly helpful for decision in $65 \%$ and genetic counseling was impactful in at least $44 \%$.

F. Tran Mau Them: None. A. Bruel: None. A. Vitobello: None. S. Nambot: None. S. Moutton: None. A. Sorlin: None. J. Delanne: None. A. Ziegler: None. E. Colin: None. A. Guichet: None. M. Legendre: None. C. Colson: None. C. Abel: None. A. Putoux: None. J. Amiel: None. A. Lavillaureix: None. C. Quelin: None. A. Guerrot: None. A. Brehin: None. L. Faivre: None. C. Philippe: None. V. Couturier: None. Y. Duffourd: None. C. Thauvin-Robinet: None.

\section{P01.036.C}

Preconception carrier screening in consanguineous couples by whole exome sequencing

A. Abulí, I. Valenzuela, M. Codina, E. Rovira, D. Palau, S. Ourani, A. Cueto-González, I. Paramonov,

E. García-Arumí, E. Tizzano, I. Cusco

\section{Hospital Vall d'Hebron, Barcelona, Spain}

Consanguineous couples have an increased risk of severe abnormalities and mortality in offspring due to autosomal recessive disorders. Exome-sequencing provides the possibility of wide preconception carrier screening (PCS) in consanguineous couples who may be at risk of rare genetic disorders. The efficacy of this strategy has been poorly explored. We retrospectively analyzed exome sequencing data from 27 affected children and 3 fetuses (after termination of pregnancy) of consanguineous couples. We explored diagnostic yield and carrier status for recessive disorders. Overall diagnostic yield was $67 \% \quad(20 / 30$ patients). We detected homozygous causative variants in 19 cases and one de novo heterozygous variant. Out of 20 causal variants, 18 were classified as pathogenic or likely pathogenic and two as unknown significance, which were reclassified as likely pathogenic because of highly specific phenotype. Based on our results and considering diagnostic yield and variant classification, causative pathogenic recessive mutations would have been detected in at least $60 \%$ of consanguineous couples by preconception exomesequencing. Most of these conditions (16/20) would not have been detected in PCS panels for most prevalent diseases considering a carrier frequency of $1 / 250$. Most of the patients were carriers of one additional heterozygous recessive mutation $(70 \%, 21 / 30)$ and a smaller number were carriers of two $(17 \%, 5 / 30)$ or more $(13 \%, 4 / 30)$. The average carrier burden of autosomal recessive conditions was of 1.7 pathogenic mutations per patient. PCS by exome-sequencing increases the detection rate of rare recessive conditions in consanguineous couples and could potentially increase their reproductive autonomy and facilitate informed decision making.

A. Abulí: None. I. Valenzuela: None. M. Codina: None. E. Rovira: None. D. Palau: None. S. Ourani: None. A. Cueto-González: None. I. Paramonov: None. E. García-Arumí: None. E. Tizzano: None. I. Cusco: None.

\section{P01.037.A}

Does maternal blood exosomal DNA effective for the fetal $R H D$ and $S R Y$ genotyping?

B. Yaşa ${ }^{l}$, O. Şahin ${ }^{2}$, S. Sözer ${ }^{1}$

${ }^{1}$ Aziz Sancar Institute of Experimental Medicine, Istanbul, Turkey, ${ }^{2}$ Clinic of Obstetrics and Gynecology, Okmeydan Training and Research Hospital, Istanbul, Turkey

Introduction: Recent advances have led to the development of important techniques in extracellular fetal DNA derived from maternal blood for prenatal diagnosis. Exosomes' DNA content is not affected structurally by nuclease activity and have a potential clinical application. Determination of fetal Rhesus $\mathrm{D}(\mathrm{RhD})$ and sex is important for prenatal diagnosis in some diseases.

Material And Method: Fetal $R H D$ and $S R Y$ genotyping was performed using exosomal DNA(exoDNA) from peripheral blood of $81 \mathrm{RhD}$ negative pregnant women with exosome isolation kit. Specific primers were applied for the $S R Y$ and $R H D$ genes amplifications by Real-Time polymerase chain reaction. The average age of the cases in the study was $28.5 \pm 5.5($ year $\pm \mathrm{SD})$. The mean gestational week of the cases was $25.81 \pm 9.02$. The exoDNA concentrations obtained were between 1.8-21.9 $\mathrm{ng} / \mu \mathrm{L}$ with an average of $11.65 \pm 8.08 \mathrm{ng} / \mu \mathrm{L}$. 
Results: The results were confirmed with the hospital's New Born Follow Up System. After analysis of the 81 cases, no amplification was observed in 16 samples, including the GAPDH gene. $100 \%$ accuracy was achieved in the RHD and $S R Y$ detection from the remaining 65 samples.

Conclusions: It was determined that fetal $R H D$ and $S R Y$ genotyping could be performed with exoDNA from maternal plasma. The isolation technique of exoDNA could be enhanced and improved for high concentration with more sensitive detection covering early pregnancy age. This technique might provide a new perspective to the prenatal diagnosis as well as to the treatment options all which require more detailed studies. The present work was supported by the Research Fund of Istanbul University. Project No. 29083

B. Yaşa: None. O. Şahin: None. S. Sözer: None.

\section{P01.038.B}

Population-based preconception expanded carrier screening for severe recessive disorders: Does a GPprovided test offer with couple results meet criteria for responsible implementation?

J. Schuurmans ${ }^{1,2}$, M. Plantinga ${ }^{l}$, E. Birnie $^{I}$, K. M. Abbott ${ }^{1}$, A. D. Diemers ${ }^{l}$, D. M. Van der Kolk ${ }^{l}$, L. M. Van den

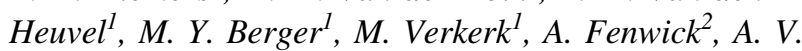

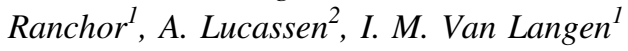

${ }^{1}$ University Medical Center Groningen, Groningen, Netherlands, ${ }^{2}$ University of Southampton, Southampton, United Kingdom

Introduction: Expanded carrier screening (ECS) enables the identification of couples who are at increased risk of having children with recessive conditions. Based on preliminary research, we conducted a pilot study of GPprovided population-based preconception ECS (2016-2018) to evaluate whether this approach would meet criteria for responsible implementation as specified by Henneman et al., EJHG, 2016. The free test-offer consisted of 50 severe early-onset childhood conditions. Only coupleresults were reported. We discuss key insights to guide large-scale implementation.

Methods: We used a mixed-methods approach. Trained GPs from nine practices invited their female patients $(n=$ 4295, aged 18-40) and partners. We addressed uptake, feasibility (e.g. pre-test counselling possible within $20 \mathrm{~min}$ utes), informed-choice, psychological outcomes (e.g. anxiety, decisional conflict) and reproductive intentions using a longitudinal survey (couples) and semi-structured interviews (GPs).
Results: 190 couples were included. 130 (approximately $15 \%$ of the eligible population) accepted the test-offer within one month and attended pre-test counselling. 117 (90\%) proceeded with testing. More than $90 \%$ of testparticipants made an informed-choice. GPs felt confident to conduct counselling, although having to make a GPappointment with both partners was a barrier. Most participants reported low anxiety and decisional conflict; this was relatively higher in test-offer decliners than acceptors.

Conclusions: Population-based preconception ECS for a specific set of severe conditions provided by motivated and trained GPs is feasible. We will discuss how responsible implementation can best be facilitated. The possibility of prenatal ECS, ECS to high-risk couples or which HCPs should offer this (e.g. midwives, fertility clinics) should now also be investigated.

J. Schuurmans: None. M. Plantinga: None. E. Birnie: None. K.M. Abbott: None. A.D. Diemers: None. D.M. Van der Kolk: None. L.M. Van den Heuvel: None. M.Y. Berger: None. M. Verkerk: None. A. Fenwick: None. A. V. Ranchor: None. A. Lucassen: None. I.M. Van Langen: None.

\section{P01.041.B}

Donor oocyte pregnancies and fetal fraction: managing patient expectations and providing accurate information

\section{Pastrick, M. K. Maisenbacher, G. Goldring, W. DiNonno, A. Ryan}

Natera, Inc, San Carlos, CA, United States

Objective: Retrospective analysis to determine if differences in fetal fraction (FF) are observed in donor oocyte pregnancies compared to the general population.

Methods: Noninvasive prenatal testing samples from singleton pregnancies were analyzed at a single reference lab. NIPT was performed using a SNP-based method with FF measured as previously described. ${ }^{1} \mathrm{FF}$ from 1611 donor oocytes was analyzed and compared to a large set of reference cases matched for maternal weight (MW) and gestational age (GA). A z-score was calculated for each donor oocyte compared to its reference data. If no impact to FF from the use of donor or in vitro fertilization (IVF), the average z-score is expected to be zero. Statistical analysis was performed using a z-test to establish if this was the case.

Results: The average $z$-score for the donor oocyte cases was -0.4. A z-test determined this deviation from normal to be significant $(p<0.00001)$, indicating lower FF in donor oocyte cases vs. corresponding reference data. 
Conclusions: Previous studies have reported lower FF in patients undergoing IVF and in donor oocyte populations. ${ }^{2,3}$ Lower FF has also been associated with increased MW, early GA, certain maternal health conditions, and abnormal fetal results (T18/T13/triploidy). ${ }^{2,3,4}$ Our results reveal statistically significant lower FF in donor oocyte pregnancies compared to matched reference data.

References 1. Zimmerman et al., Prenatal Diagnosis 2012; 32: 1-9 2. Revello R. et al., Ultrasound Obstet Gynecol. 2016 Jun;47(6):698-704. 3. Yanaihara A. et al., J Assist Reprod Genet. 2018 Mar;35(3):431-434. 4. Ebbing C. et al., PLoS One. 2013 Jul 30;8(7):e70380.

M. Pastrick: A. Employment (full or part-time); Significant; Natera, Inc. M.K. Maisenbacher: A. Employment (full or part-time); Significant; Natera, Inc. G. Goldring: A. Employment (full or part-time); Significant; Natera, Inc. W. DiNonno: A. Employment (full or parttime); Significant; Natera, Inc. A. Ryan: A. Employment (full or part-time); Significant; Natera, Inc.

\section{P01.042.C}

Repeat Associated Non-AUG (RAN)-Translation in fragile $X$ associated Premature Ovarian Insufficiency (FXPOI): FMRpolyG as predictive tool?

\section{Nguyen ${ }^{l}$, B. Messmer ${ }^{l}$, K. Hinderhofer ${ }^{2}$, T. Strowitzki ${ }^{3}$, J. Rehnitz ${ }^{1,3}$}

${ }^{1}$ Division of Reproduction Genetics, Department of Gynecological Endocrinology and Fertility Disorders, University Women's Hospital, Heidelberg, Germany, ${ }^{2}$ Institute of Human Genetics, University Heidelberg, Laboratory of Molecular Genetics, Heidelberg, Germany, ${ }^{3}$ Department of Gynecological Endocrinology and Fertility Disorders, University Women's Hospital, Heidelberg, Germany

Fragile-X-associated-primary-ovarian-insufficiency (FXPOI) is characterized by oligo/amenorrhea and hypergonadotropic hypogonadism by reason of the expansion of CGG-repeats in the $5^{\prime} \mathrm{UTR}$ of $F M R 1$, called permutation (PM) (n: 55-200). RAN-translation depending on variable CGG-repeat length is also hypothesized to cause FXPOI due to the production of a polyglycine-containing FMR1protein, FMRpolyG. FMRpolyG expression in granulosa cells (GCs) of FXPOI-model-mice leads to perturbed ovarian function. However, the expression of FMRpolyG in leukocytes and GCs of women with different ovarian response and variable CGG repeat length has not been investigated yet. We performed Western-blot (WB) to analyze the expression of FMRP and FMRpolyG in patient 's leukocytes and GCs in parallel with FMRl-geneexpressionanalysis. Firstly, we detected FMRP, both in leukocytes and GCs of women with variable ovarian reserve and different CGG-repeat-length. FMR1/FMRP was detected in a larger amount in GCs than in leukocytes, since it is a major regulator of folliculogenesis. We therefore started a pilot project investigating if variable expression-levels of FMRP and FMRpolyG detected by WB can be related to women with different ovarian reserve parameters and variable CGG repeats including "normal" and premutated ranges. Preliminary data already showed, in a PM patient undergoing oocytes cryopreservation because of her risk of developing FXPOI, a decreased level of FMRP, even though the FMRI transcription level was within the normal range, fitting to the hypothesis of kind of a perturbed protein production in this patient. We plan to further evaluate if results made in GCs can be translated to leukocytes. This would offer prospectively a predictive tool or marker of progression.

X. Nguyen: None. B. Messmer: None. K. Hinderhofer: None. T. Strowitzki: None. J. Rehnitz: None.

\section{P01.045.C}

Investigation of single nucleotide variants (SNVs) in a fertile population in association with oocyte and embryo quality

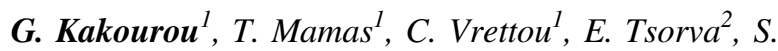

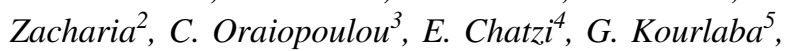
D. Christopikou ${ }^{2}$, M. Mastrominas ${ }^{2}$, M. Moisidou ${ }^{3}$, A. Chatziparasidou $^{3}$, I. Georgiou ${ }^{4}$, J. Traeger-Synodinos ${ }^{1}$

${ }^{1}$ Department of Medical Genetics, National \& Kapodistrian University of Athens, St. Sophia's Children's Hospital, Athens, Greece, ${ }^{2}$ Embryogenesis, Assisted Reproduction Unit, Athens, Greece, ${ }^{3}$ Embryolab SA, IVF Unit, Thessaloniki, Greece, ${ }^{4}$ Genetics and IVF Unit, Department of Obstetrics and Gynecology, Faculty of Medicine, University of Ioannina, Ioannina, Greece, ${ }^{5}$ Center for Clinical Epidemiology and Outcomes Research (CLEO), Athens, Greece

Introduction: Human and animal studies have provided information on a number of genes potentially impacting gamete quality and preimplantation development (with a role on meiosis, mitosis, DNA repair etc.). SNVs in these genes have been investigated in mostly infertile couples undergoing IVF, in search of potential biomarkers of IVF success. Recently, maternal SNVs in infertile women (rs1801133/rs1801131 in MTHFR, rs2305957 on chromosome 4 linked to INTU-SLC25A31-HSPA4L-PLK4MFSD8-LARP1B and PGRMC2) were associated with embryo quality or chromosomal status. We investigated the potential association of SNVs in 26 selected genes with a possible role in preimplantation development, with oocyte/embryo quality in fertile women undergoing 
Preimplantation Genetic Testing for Monogenic Disorder (PGT-M).

Materials/Methods: Maternal DNA was genotyped using exonic NGS (QiaseqTM-Targeted Custom Panel, Miseq) for 18 genes (AIRE-AMH-AURKA-AURKBAURKC-FSHR-HSPA4L-HUWE1-INTU-KHDC3LLARPIB-MFSD8-MTHFR-PGRMC2-PLK4-SENP7SLC25A31-WBP1) and 9 selected SNVs in a further 8 genes: rs175080(MLH3), rs1799963(F2), rs6025(F5), rs5918(ITGB3), rs5985(F13A1), rs1805087(MTR), rs1801394(MTRR), $\quad$ rs28756992(MLH3), $\quad$ rs2305957 (HSPA4L). The study involved 85 women and 107 PGTM cycles performed between 2013-2019. Maternal genotyping was investigated in association with number of oocytes collected/fertilized and blastocyst formation.

Results: A 20x coverage was achieved in all regions investigated, identifying 121 variants. Significant associations $(\mathrm{p}<0.05)$ were revealed for 16 SNVs in: AIRE-AMHAURKB-HSPA4L-KHDC3L-MTHFR-PLK4-SENP7-MLH3. No significant associations were revealed for rs1801131 and rs2305957.

Conclusions: Our understanding of genes that may impact the preimplantation stage and the search for genomic biomarkers predicting IVF-success may benefit by investigating a fertile population, minimizing many confounding variables. This study was supported by the State Scholarships Foundation (IKY), co-funded by national grants and the European union (ESPA 2014-2020).

G. Kakourou: None. T. Mamas: None. C. Vrettou: None. E. Tsorva: None. S. Zacharia: None. C. Oraiopoulou: None. E. Chatzi: None. G. Kourlaba: None. D. Christopikou: None. M. Mastrominas: None. M. Moisidou: None. A. Chatziparasidou: None. I. Georgiou: None. J. Traeger-Synodinos: None.

\section{P01.047.B}

Further indication of a role for de novo mutations in cases of severe idiopathic male infertility

H. E. Smith ${ }^{l}$, M. S. Oud ${ }^{2}$, P. F. de Vries $^{2}$, B. Alobaidi ${ }^{l}$, A. Virdi ${ }^{I}$, B. J. Houston ${ }^{3}$, R. M. Smits ${ }^{4}$, L. Batty ${ }^{I}$, M. K.

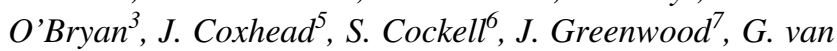
der Heijden ${ }^{2,4}$, K. McEleny ${ }^{7}$, L. Ramos ${ }^{4}$, L. E. L. M. Vissers $^{2}$, M. J. Xavier ${ }^{1}$, J. A. Veltman ${ }^{1,2}$

${ }^{1}$ Biosciences Institute, Newcastle University, Newcastle Upon Tyne, United Kingdom, ${ }^{2}$ Department of Human Genetics, Donders Institute for Brain, Cognition and Behaviour, Radboudumc, Nijmegen, Netherlands, ${ }^{3}$ School of Biological Sciences, Monash University, Melbourne, Australia, ${ }^{4}$ Department of Obstetrics and Gynaecology, Radboudumc, Nijmegen, Netherlands, ${ }^{5}$ Genomics Core Facility, Newcastle University, Newcastle Upon Tyne,
United Kingdom, ${ }^{6}$ Bioinformatics Support Unit, Newcastle University, Newcastle Upon Tyne, United Kingdom, ${ }^{7}$ Newcastle Fertility Centre, Newcastle University, Newcastle Upon Tyne, United Kingdom

Infertility affects 1 in 6 couples worldwide and in approximately $50 \%$ of these cases the infertility can be attributed to a male factor. Whilst causes such as Klinefelter's and Y chromosome microdeletions have been well distinguished, the genetic causes behind severe spermatogenic failure are largely unknown, with around $40 \%$ of all male infertility cases remaining idiopathic. De novo mutations (DNMs) arise spontaneously in the germline or postzygotically, and are known to be associated with early onset disorders with reduced reproductive fitness. These mutations, however, are vastly understudied in the field of male infertility due to the difficulty in obtaining parental samples. Here we report on the continuation and expansion of exome sequencing in our cohort of infertile patients in order to identify novel genes involved in male infertility. The role of DNMs was investigated in a total of 194 patients suffering from non-obstructive azoospermia or severe oligozoospermia and their fertile parents by adding 95 additional trios to the previously reported 99 cases. In these additional 95 trios, we have identified and validated 75 protein-altering DNMs, of which 19 are likely to affect normal gene function. Genes of particular interest are PPPIR7, RBM5 and $M S H 5$, all of which are involved in spermatogenesis and linked to infertility in mice. At this moment, none of these genes have been recognised as human male infertility genes. Our data further indicates that DNMs may play an important role in severe male infertility and emphasises the need for further replication studies in larger cohorts.

H.E. Smith: None. M.S. Oud: None. P.F. de Vries: None. B. Alobaidi: None. A. Virdi: None. B.J. Houston: None. R.M. Smits: None. L. Batty: None. M.K. O'Bryan: None. J. Coxhead: None. S. Cockell: None. J. Greenwood: None. G. van der Heijden: None. K. McEleny: None. L. Ramos: None. L.E.L.M. Vissers: None. M.J. Xavier: None. J.A. Veltman: None.

\section{P01.049.A}

A case report of a rare nonsense $Z P 1$ variant in a patient with oocyte maturation defect

E. G. Berkay, B. Karaman, G. Toksoy, B. Ozsait Selcuk, Z. O. Uyguner, S. Basaran

Istanbul Medical Faculty, Istanbul University, Istanbul, Turkey

Introduction: Oocyte maturation defect (OOMD) is a rare condition causing female infertility that can be diagnosed 
during assisted reproduction techniques (ART). OOMD related genes are $Z P 1, Z P 2, Z P 3, P A N X 1, P A T L 2, T U B B 8$, WEE2 (OMIM, 2020). We report a case of a 31-year-old woman who had four ART failures diagnosed as empty follicle syndrome and OOMD. She has short stature (-3 SD), bilateral limited extension-flexion on elbows.

Materials and Methods: Chromosome analysis and fluorescence in-situ hybridization (FISH) using X chromosome centromeric and SHOX-probe on interphase nuclei of lymphocytes and mucosal cells was investigated. Wholeexome sequencing (WES) performed via the Illumina platform. Confirmation and familial segregation analysis were performed by Sanger sequencing.

Results: Karyotyping and FISH resulted in normal, possible mosaicism was excluded. WES analysis revealed a known, rare, pathogenic homozygous variant in exon 3 (c.628C > T; p.Q210*) of $Z P 1$ gene, and her parents being first degree cousins were carriers for this variant.

Conclusions: $Z P 1$ with autosomal recessive inheritance is related to OOMD-1 (MIM_615774). Zona pellucida (ZP) is a glycoprotein structure surrounding oocytes and is essential for oocyte development. ZP contains four types of receptor proteins (ZP1-4). Our variant in $Z P 1$ is nonsense, premature stop codon causes to truncate $\mathrm{ZP1}$ receptor proteins. This is the first homozygous occurrence of this variant associated with OOMD. WES findings were also analyzed for known genes related to short stature and no pathogenic variant has been observed. WES is a valuable method to identify the genetic origin in complex, multigenic conditions like in infertility. Istanbul University Project-Number:TSA-201832135

E.G. Berkay: None. B. Karaman: None. G. Toksoy: None. B. Ozsait Selcuk: None. Z.O. Uyguner: None. S. Basaran: None.

\section{P01.051.C}

Prenatal diagnosis and molecular characterization of a jumping translocation leading to $18 p$ monosomy

J. B. Gaillard ${ }^{1}$, T. Guignard ${ }^{1}$, J. Puechberty ${ }^{2}$, A. Schneider $^{l}$, V. Gatinois ${ }^{1}$, M. Duport-Percier ${ }^{3}$, J. M. Faure ${ }^{3}$, F. Fuchs ${ }^{3}$, F. Pellestor ${ }^{1}$, P. Blanchet ${ }^{4}$, C. Wells $^{4}$

${ }^{1}$ Génétique Chromosomique, CHU, Montpellier, France, ${ }^{2}$ Maladies Génétiques de l'Enfant et de l'Adulte, CHU, Montpellier, France, ${ }^{3}$ Médecine Maternelle et Foetale, CHU, Montpellier, France, ${ }^{4}$ Génétique Prénatale et Foetopathologie, CHU, Montpellier, France

Jumping translocations are rare events in cytogenetics, characterised by a donor chromosome and multiple recipient chromosomes. To date, only ten prenatal cases have been described.

Patient: This pregnancy was a second spontaneous pregnancy of a non-consanguinous couple. A first trimester ultrasound showed an increased nuchal translucency persisting at 18 gestational weeks (GW) along with retrognatia, cleft palate and femoral shortening. Choriocentesis was performed at $14 \mathrm{GW}$.

Results: Direct examination on cytotrophoblast found a der(18)t $(18 ; 18)$ whereas the cultured karyotype on mesenchyme showed a $\operatorname{der}(18) \mathrm{t}(2 ; 18)$. Due to tissue discordance, an amiocentesis was performed at $18 \mathrm{GW}$. Surprisingly, we found, neither of the two previously identified cell lines, but a mosaic $\operatorname{der}(18) t(12 ; 18)$ with a ring 18. In addition, array $\mathrm{CGH}$ provided breakpoints for chromosomes 2 and 12 in REEPI gene (2p11.2) and TAOK3 gene (12q24.23) respectively. Whole genome sequencing on minION (Nanopore) was conducted to clarify breakpoint in chromosome 18 . Using data obtained by array $\mathrm{CGH}$, a long read with hard clipping showed a breakpoint in repeated sequence with about $90 \%$ homology with D18Z1 alpha satellite. Finally, all identified cell lines carried a 18p monosomy which could explain ultrasound abnormalities. The pregnancy was terminated. Following fetal autopsy, FISH analysis was performed on blood, thymic, lung and liver tissue and confirmed 18p monosomy in all tissues.

Conclusion: We report here a new case of jumping translocation with precise molecular characterization of breakpoints : D18Z1 as donor region and at least 3 recipient chromosomes : 2p11.2, 12q24.3 and 18q21.

J.B. Gaillard: None. T. Guignard: None. J. Puechberty: None. A. Schneider: None. V. Gatinois: None. M. Duport-Percier: None. J.M. Faure: None. F. Fuchs: None. F. Pellestor: None. P. Blanchet: None. C. Wells: None.

\section{P01.052.A}

Molecular biomarkers of ageing in men with Klinefelter syndrome

\section{E. Pohl ${ }^{1}$, S. Muschal ${ }^{2}$, S. Kliesch ${ }^{3}$, M. Zitzmann ${ }^{3}$, J. Rohayem $^{3}$, J. Gromoll ${ }^{2}$, S. Laurentino ${ }^{2}$}

${ }^{1}$ Institute of Human Genetics, University of Münster, Münster, Germany, ${ }^{2}$ Centre of Reproductive Medicine and Andrology (CeRA), Institute of Reproductive and Regenerative Biology, University Hospital Münster, 48149 Münster, Germany, Münster, Germany, ${ }^{3}$ Centre of Reproductive Medicine and Andrology (CeRA), Department of Clinical and Surgical Andrology, University Hospital 
Münster, 48149 Münster, Germany, Münster, Germany

Introduction: Males with Klinefelter syndrome (nonmosaic karyotype 47,XXY), the most common sex chromosome aneuploidy, exhibit increased rates of ageingrelated morbidities and mortality. Associated with that, a significantly reduced life span has been observed. The aim of this study was to investigate whether Klinefelter patients show molecular signs of premature ageing.

Patients and Methods: We assessed age-associated DNA methylation patterns (by pyrosequencing) and relative telomere length (TL; by quantitative polymerase chain reaction) as molecular ageing biomarkers in blood from Klinefelter patients $(n=178$ and 266 for DNA methylation and TL, respectively) aged 18-71 years. Age-matched healthy males ( $n=184$ and 196 for DNA methylation and TL, respectively) and females $(n=50)$ served as controls.

Results: Age-associated DNA methylation patterns did not indicate accelerated ageing/premature ageing in men with Klinefelter syndrome. In young Klinefelter individuals aged 18-24 years, we observed significantly longer telomeres compared to controls (mean $=1.51$ vs. 1.09 and 1.26 in female and male controls, respectively). Telomere length in subsequent age groups, however, were not significantly different to controls.

Conclusions: We did not find molecular hints for premature ageing in Klinefelter patients based on DNA methylation patterns. However, we observed higher baseline TL at adolescent age but comparable TL with progressive age in other age groups. Whether this is due to an accelerated TL attrition, putatively related to the observed co-morbidities in Klinefelter syndrome, remains to be addressed in a further longitudinal study.

Funding: German Research Foundation (DFG No. GR1547/19-1; CRU 326 No. GR1547/25-1), IZKF Münster (Grant CRA03/09)

E. Pohl: None. S. Muschal: None. S. Kliesch: None. M. Zitzmann: None. J. Rohayem: None. J. Gromoll: None. S. Laurentino: None.

\section{P01.055.A}

Maternally inherited copy-number variations may explain cases of severe idiopathic male infertility

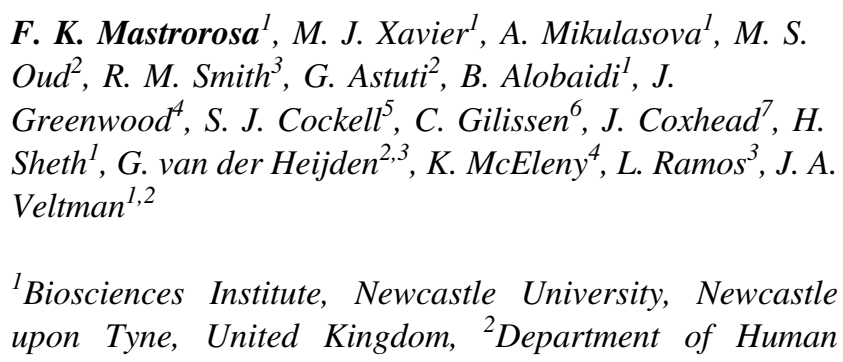

Genetics, Donders Institute for Brain, Cognition and Behaviour, Radboudumc, Nijmegen, Netherlands, ${ }^{3}$ Department of Obstetrics and Gynaecology, Radboudumc, Nijmegen, Netherlands, ${ }^{4}$ Newcastle Fertility Centre, Newcastle University, Newcastle upon Tyne, United Kingdom, ${ }^{5}$ Bioinformatics Support Unit, Newcastle University, Newcastle upon Tyne, United Kingdom, ${ }^{6}$ Department of Human Genetics, Radboud Institute for Molecular Life Sciences, Radboudumc, Nijmegen, Netherlands, ${ }^{7}$ Genomics Core Facility, Newcastle University, Newcastle upon Tyne, United Kingdom

Severe cases of infertility such as azoospermia and oligozoospermia often have genetic causes, with Klinefelter syndrome and chromosome $\mathrm{Y}$ microdeletions being the most common. However, of all infertile men, $40 \%$ have unknown aetiology after being tested for all known causes. In this study, we explored for the first time the role of maternally inherited copy-number variations $(\mathrm{CNVs})$ on a large scale, sequencing the exome of 160 patient-parents trios, a methodology primarily used to detect single nucleotide variants (SNVs) but also robust for CNV detection. Among all rare maternally inherited CNVs detected, 3 were considered of potential pathogenicity. One is a large duplication $(\sim 1.7 \mathrm{Mb})$ involving, among others, $V C X$ gene, an $\mathrm{X}$-linked multi-copy gene that leads to apoptosis and cell growth inhibition when duplicated in murine germ cells in vitro. A second case carried a $\sim 80 \mathrm{~kb}$ duplication involving two genes, one of which was $F A S$, a known regulator of testicular germ cell apoptosis. The third $\mathrm{CNV}$, instead, was a deletion of $\sim 200 \mathrm{~kb}$ affecting, among others, PSME4, a gene required for normal fertility in male mice but dispensable in females. The results of this study, although preliminary, provide a first indication that maternally inherited CNVs may explain cases of severe spermatogenic failure. Currently, additional 35 patient-parents trios and 149 patients are being screened to detect variations affecting the same genes and additional rare variations. Genome sequencing data will soon be available for all the trios, which will improve CNV detection and clarify the contribution of these variations to the disorder.

F.K. Mastrorosa: None. M.J. Xavier: None. A. Mikulasova: None. M.S. Oud: None. R.M. Smith: None. G. Astuti: None. B. Alobaidi: None. J. Greenwood: None. S.J. Cockell: None. C. Gilissen: None. J. Coxhead: None. H. Sheth: None. G. van der Heijden: None. K. McEleny: None. L. Ramos: None. J.A. Veltman: None.

\section{P01.056.B}

Improved classification of testicular histopathology to promote uniform diagnosis and discovery of genetic causes for male infertility 
G. W. van der Heijden ${ }^{l}$, M. J. Wyrwoll ${ }^{2}$, L. Ramos ${ }^{l}, J$. Veltman $^{3}, F$. Tüttelmann ${ }^{4}$

${ }^{1}$ 1. Division of Reproductive Medicine, Department of Obstetrics and Gynecology, Radboud University Medical Center, Nijmegen, Netherlands, ${ }^{2}$ Centre of Reproductive Medicine and Andrology, Department of Clinical and Surgical Andrology, University of Münster, Münster, Germany, ${ }^{3}$ Institute of Genetic Medicine, Newcastle University, Newcastle, Netherlands, ${ }^{4}$ Institute of Human Genetics, University of Münster, Münster, Germany

With the availability of Next Generation Sequencing the genetic causes of male infertility are increasingly being chartered. A genetic diagnosis combined with a clear unambiguous description of accompanying testis histology will hugely benefit interpretation of the function of identified genes. However, a universal ontology for testis histopathology is lacking. The Human Phenotype Ontology (HPO) is a formal ontology, which describes phenotypes encountered in human diseases. The HPO allows phenotype driven differential diagnostics in clinical routine and research. The current hierarchy of terms regarding azoospermia, is not a systematical one. Moreover, the majority of testicular phenotypes are not annotated yet. Building on what has been published previously and our own experience we propose a framework for testis histopathology that consists of five main categories. Four of these are subdivided to obtain information on the timing of a germ cell arrest or the severity of overall loss of spermatogenesis. To establish a lower threshold for complete spermatogenesis we determined the number of tubules containing elongating spermatids in a collection of samples of men with an obstructive azoospermia due to a previous vasectomy $(\mathrm{N}=117)$. This analysis suggested $50 \%$ to be a reasonable cut-off to distinguish hypospermatogenesis from complete spermatogenesis. The framework is easy to understand and straightforward in its application. It gathers most relevant information to study the impact of an identified mutation on spermatogenesis. We conclude that once this framework is incorporated in the HPO, the standardized vocabulary will facilitate communication and interpretation in both a clinical routine and research.

G.W. van der Heijden: None. M.J. Wyrwoll: None. L. Ramos: None. J. Veltman: None. F. Tüttelmann: None.

\section{P01.057.C}

Identification of two homozygous mutations, in the male reproductive tract specific $\beta$-defensin 126/128 genes, potentially underlie a severe sperm dysfunction.
S. Kane ${ }^{l}$, D. Lester ${ }^{\text {, S. Sameron }}{ }^{l}$, C. Barratt ${ }^{2}$, S. Martins da Silva ${ }^{2}$, S. Brown ${ }^{1}$

${ }^{1}$ School of Science, Engineering \& Technology, Abertay University, Dundee, United Kingdom, ${ }^{2}$ Reproductive and Developmental Biology, School of Medicine, Ninewells Hospital and Medical School, University of Dundee, Dundee, United Kingdom

Introduction: Idiopathic infertility affects $15 \%$ couples globally and recent evidence suggests genetic abnormalities are a causative factor in a significant number of cases. Hence, we conducted whole exome sequencing of men undergoing assisted conception to identify genetic lesions. The $\beta$-defensin 126 (DEFB126) and $\beta$-defensin 128 (DEFB128), are genes preferentially expressed in the epididymis, where these proteins may help protect the maturing sperm cells and play a vital role during sperm capacitation.

Materials and Methods: DNA extracted from the blood of 26 unrelated, sub-fertile men were sequenced using whole exome sequencing. Pathogenic rare variants were identified using bioinformatics analysis and validated using Sanger sequencing.

Results: A novel and rare homozygous pathogenic 1-bp frame-shift insertion causing a premature stop codon was identified in DEFB128 gene from one patient. Six patients showed a previously reported homozygous $2 \mathrm{bp}$ deletion, causing a frame-shift mutation in the DEFB126 gene (p. Pro106ArgfsTer?). All patients ascertained with the DEFB126/DEFB128 mutations were unable to naturally conceive and hence were sub-fertile.

Conclusions: The DEFB128 mutation (rs11396059), is rare and has not been associated with any disease to date. As the patient with the DEFB128 mutation has no other medical condition we predict that it is the cause of infertility. The DEFB126 mutation (rs140685149), is a common variant, and this homozygous variant in sub-fertile men has previously been implicated in facilitating sperm movement in the female reproductive tract. These results suggest that, $\beta$-defensin genes play a significant role in male reproduction.

This work was supported by the R-LINCS studentship, Abertay University.

S. Kane: None. D. Lester: None. S. Cameron: None. C. Barratt: None. S. Martins da Silva: None. S. Brown: None.

\section{P01.058.A}

The sperm epigenome does not display recurrent epimutations in patients with severely impaired spermatogenesis 
E. Leitao ${ }^{1}$, S. Di Persio ${ }^{2}$, S. Laurentino ${ }^{2}$, M. Wöste ${ }^{3}$, M. Dugas ${ }^{3}$, S. Kliesch ${ }^{4}$, N. Neuhaus ${ }^{2}$, B. Horsthemke ${ }^{1,5}$

${ }^{1}$ Institute of Human Genetics, University Hospital Essen, Essen, Germany, ${ }^{2}$ Centre of Reproductive Medicine and Andrology, University Hospital Münster, Münster, Germany, ${ }^{3}$ Institute of Medical Informatics, University Münster, Münster, Germany, ${ }^{4}$ Centre of Reproductive Medicine and Andrology, Department of Clinical and Surgical Andrology, University Hospital Münster, Münster, Germany, ${ }^{5}$ Institute of Human Genetics, University Hospital Münster, Münster, Germany

Introduction: Previous studies have identified aberrant DNA methylation of imprinted genes (e.g. MEST and H19) in the sperm of infertile patients, but the prevalence and genomic extent of these abnormal methylation patterns have remained unknown.

Material and Methods: We collected swim-up sperm samples from 40 normozoospermic and 93 oligoasthenoteratozoospermic patients and determined H19 and MEST methylation levels by deep bisulfite sequencing. Based on this screening, we defined three groups: normal controls (NC), abnormally methylated infertile (AMI; $n=7$ ) and normally methylated infertile (NMI; $n=86$ ), and performed whole genome bisulfite sequencing of five NC, five AMI and six NMI samples.

Results: Whole-genome comparison of NC and AMI samples revealed that each AMI sample had abnormal methylation levels of all 50 imprinting control regions and $>2,000$ germ cell-specific genes with distinct DNA methylation differences in blood and germ cells. This is consistent with the presence of somatic cell DNA in AMI samples. The comparison of NC and NMI samples revealed 19 differentially methylated regions, none of which could be validated in an independent cohort of 40 men. Analysis of the H19 CTCF-binding region 6 in samples without somatic cell DNA revealed an association between DNA methylation levels and genotype (rs2071094), but not fertility phenotype.

Conclusions: Our results suggest that somatic DNA contamination and genetic variation confound methylation studies in sperm of infertile men and that the prevalence of aberrant methylation in infertile men has been overestimated, which is reassuring for patients undergoing assisted reproduction. Funded by DFG grants HO 949/23-1 and NE 2190/3-1.

E. Leitao: None. S. Di Persio: None. S. Laurentino: None. M. Wöste: None. M. Dugas: None. S. Kliesch: None. N. Neuhaus: None. B. Horsthemke: None.
P01.059.B

miR-200 family inhibits trophoblast invasion through regulating epithelial-mesenchymal transition

M. Su ${ }^{l}, H . T$ sai $^{l}$, C. Wang $^{2}$

${ }^{I}$ Department of Obstetrics and Gynecology, National Cheng-Kung University Hospital, Tainan, Taiwan, ${ }^{2}$ Department of Cell Biology and Anatomy, National Cheng-Kung University, Tainan, Taiwan

Epithelial-to-mesenchymal transition (EMT) is a biological process that epithelial cells lose cell polarity and cellcell adhesion, and gain migratory and invasive abilities to become mesenchymal cells. In human placenta, the chorionic villi gradually transform from proliferative cytotrophoblasts and extravillous trophoblasts into a migratory and invasive trophoblast phenotype. Failure in altering the behavior and morphology of trophoblasts in placenta may result in insufficient trophoblast invasion, which leads to placental dysfunction and the diseased placenta. MiR-200 family, including miR-200a and -141 , were highly expressed in human placenta and were upregulated in patients of preeclampsia. miR-200a and miR-141 were demonstrated to inhibit the formation of primary clila in trophoblasts and therefore have a less ability for cell invasion. In this study, miR-200a and miR-141 were shown to inhibit trophoblasts (HTR-8/SVneo) invasion and migration by a transfection assay. In western blotting and qRT-PCR, miR-200a and miR-141 stimulated epithelial markers, such as E-cadherin and ZO-1, whereas suppressed mesenchymal and transcriptional factors, such as ZEB1, MMP2, MMP9 and TGF-b1. These results suggest trophoblast migration and invasion mediated by miR-200 family may through the mechanism of EMT, and may therefore predispose to develop preeclampsia in human pregnancy.

M. Su: None. H. Tsai: None. C. Wang: None.

\section{P01.060.C}

Identification of plasma miRNAs associated with endometriosis in Bulgarian patients

V. Spasova ${ }^{l}$, V. Karamisheva ${ }^{l, 2}$, O. Antonova ${ }^{l}, Z$. Hammoudeh $^{l}$, L. Koleva ${ }^{3}$, R. Staneva ${ }^{l}$, M. Ganev ${ }^{l}, D$. Nesheva $^{l}$, A. Nachev ${ }^{l, 2}$, D. Toncheva ${ }^{l}$, S. Hadzhidekova ${ }^{l}$

${ }^{1}$ Medical University of Sofia, Sofia, Bulgaria, ${ }^{2}$ University obstetrics and gynecology hospital "Maichin dom, Sofia, Bulgaria, "University Hospital "Pirogov", Sofia, Bulgaria 
Introduction: Diagnosis of endometriosis still poses a significant challenge for it usually requires surgery. The need of invasive procedure significantly contributes to late diagnosis and less effective treatment. The aim of this study is to screen for changes in miRNAs expression in peripheral blood that could be further researched as potential non-invasive diagnostic tool for endometriosis.

Materials and methods: 15 patients with surgically confirmed advanced endometriosis and 15 healthy controls were included in the study. Peripheral blood samples were collected after signed informed consent. Plasma RNA was isolated with miRNeasy-Serum/Plasma Kit in conjunction with Plasma Spike-in-Control $\quad\left(\right.$ Qiagen $\left.^{\circledR}\right)$. Equimolar amounts of RNA were used to prepare two pools: 1)late stage endometriosis and 2)healthy controls. Reverse transcription of the pool samples was performed by miScript II RT Kit $\left(\right.$ Qiagen $\left.^{\circledR}\right)$. Expression spectra of 84 miRNAs (miScript miRNA-PCR-Array-Human Serum \& Plasma, Qiagen $\left.{ }^{\circledR}\right)$ by real-time PCR quantification via miScript-SYBRGreen PCR Kit (Qiagen ${ }^{\circledR}$ ) were analyzed on Applied Biosystems ${ }^{\text {TM7500 }}$ instrument.

Results: The preliminary results revealed 65 upregulated miRNAs and no downregulated miRNAs between cases and controls. The fold change between 5 and 10 was detected in 21 miRNAs and fold change above 10 was detected in 44 miRNAs. The most significantly overexpressed miRNAs were miR-106a and miR-17-3p which are associated with cell proliferation and apoptosis.

Conclusions: MiRNA expression level differences were found in blood between endometriosis patients and healthy controls. This may lay the foundations for future in-depth research on novel non-invasive biomarker for endometriosis. Acknowledgements: Contract Д-212/12.12.2018

V. Spasova: None. V. Karamisheva: None. O. Antonova: None. Z. Hammoudeh: None. L. Koleva: None. R. Staneva: None. M. Ganev: None. D. Nesheva: None. A. Nachev: None. D. Toncheva: None. S. Hadzhidekova: None.

\section{P01.061.A}

Sequence variants in $T B X 6$ are associated with disorders of the Müllerian ducts - an Update

\author{
A. Tewes ${ }^{1}$, J. Hucke ${ }^{2}$, T. Römer ${ }^{3}$, K. Kapczuk ${ }^{4}$, C. \\ Schippert $^{5}$, P. Hillemanns ${ }^{5}$, A. Bohring ${ }^{1}$, P. Wieacker ${ }^{l}, S$. \\ Ledig $^{l}$
}

${ }^{1}$ Institute of Human Genetics, Münster, Germany, ${ }^{2}$ Department of Obstetrics and Gynecology, Wuppertal, Germany, ${ }^{3}$ Department of Obstetrics and Gynecology, Köln-Weyertal, Germany, ${ }^{4}$ Division of Gynecology, Poznan, Poland,
${ }^{5}$ Department of Obstetrics and Gynecology, Hannover, Germany

Müllerian anomalies include formation, fusion or absorption defects of the Müllerian ducts such as unicornuate, didelphys, bicornuate or septate uterus and vaginal septa as well as the Mayer-Rokitansky-Küster-Hauser syndrome (MRKHS) which is characterized by the congenital absence of uterus and vagina. The incidence of MRKHS is estimated to be 1:4.500 and 1:250 in other Müllerian anomalies. Most cases of Müllerian anomalies are sporadic; familial cases seem to be autosomal dominant inherited with reduced penetrance. Until now the knowledge about the etiology of anomalies of the Müllerian ducts is very limited. In approximately $1 \%$ of patients with Müllerian anomalies recurrent deletions in 16p11.2 encompassing TBX6 were found as causative in these patients. Moreover, in previous studies, we and others found possibly pathogenic variants in patients with Müllerian anomalies in TBX6. Since TBX6 encodes a conserved transcription factor playing an essential role in developmental processes like mesoderm formation and specification, we suggested TBX6 as strong candidate gene. Thus we performed analysis of the TBX6 gene (NM_004608) in additional 126 patients with Müllerian anomalies. We detected 4 possibly pathogenic variants $\quad(\mathrm{c} .358 \mathrm{~A}>\mathrm{G} \quad$ p. $($ Met120Val $), \quad$ c.704G $>\mathrm{A} \quad$ p. (Gly235Asp), c.815G $>$ A p.(Arg272Gln), c.1146C $>$ A p. (Tyr382*)) in TBX6 in 5/126 patients and could exclude the previously reported variant c.484G $>$ A p.(Gly162Ser) as being causative for disorders of the Müllerian ducts in the European non-Finnish population. In summary, we present increasing evidence for the association of variants in TBX6 with malformations of the Müllerian ducts.

A. Tewes: None. J. Hucke: None. T. Römer: None. K. Kapczuk: None. C. Schippert: None. P. Hillemanns: None. A. Bohring: None. P. Wieacker: None. S. Ledig: None.

\section{P01.062.B}

Circulating fetal trophoblastic cell eligible for NIPD: the exception rather than the rule

L. Cayrefourcq ${ }^{1}$, M. C. Vincent ${ }^{2}$, S. Pierredon ${ }^{2}, C$. Moutou $^{3}$, M. Imbert-Bouteille ${ }^{2}$, E. Haquet $^{4}$, J. Puechberty $^{4}$, M. Willems ${ }^{4}$, C. Liautard-Haag ${ }^{2}$, N. Molinari ${ }^{5}$, C. Zordan ${ }^{6}$, V. Dorian ${ }^{6}$, A. Chaussenot ${ }^{7}$, L. Monteil $^{8}$, C. Miry $^{9}$, Y. Petrov $^{10}$, E. Le Boette ${ }^{11}$, M. Fradin ${ }^{12}$, C. Alix-Panabiere ${ }^{1}$, C. Guissart ${ }^{2}$

${ }^{1}$ Laboratoire de cellules circulantes rares humaines_University Medical Center of Montpellier, Montpellier, France,

${ }^{2}$ Laboratoire de Genetique Moleculaire_University Medical 
Center of Montpellier, Montpellier cedex 5, France, ${ }^{3}$ Laboratoire de diagnostic pré-implantatoire_CMCO_University Medical Center of Strasbourg, Schiltigheim, France, ${ }^{4}$ Departement de Genetique Medicale_University Medical Center of Montpellier, Montpellier, France, ${ }^{5}$ DIM_University Medical Center of Montpellier, Montpellier, France, ${ }^{6}$ Service de Genetique Medicale_University Medical Center of Bordeaux, Bordeaux, France, ${ }^{7}$ Service de Genetique Medicale_University Medical Center of Nice, Nice, France, ${ }^{8}$ Service de Genetique Medicale_University Medical Center of Toulouse, Toulouse, France, ${ }^{9}$ Department of Medical Medecinee_University Medical Center of Strasbourg, Schiltigheim, France, ${ }^{10}$ Laboratoire de cytologie et cytogenetique_University Medical Center of Nimes, Nîmes, France, ${ }^{11}$ Service de Genetique Medicale_Medical Center of Saint Brieuc, Saint Brieuc, France, ${ }^{12}$ Service de Genetique Medicale_Medical Center of Saint Brieuc, Saint Brieuc, France

Introduction: Non-Invasive Prenatal Diagnosis (NIPD), based on the analysis of circulating cell-free fetal DNA (cffDNA), is successfully implemented for an increasing number of monogenic diseases. However, technical issues related to cff-DNA characteristics remain, and not all mutations can be screened with this method, particularly triplet expansion mutations that frequently concern prenatal diagnosis requests. The objective of this study was to develop an approach to isolate and analyze Circulating Trophoblastic Fetal Cells (CFTCs) for NIPD of monogenic diseases caused by triplet repeat expansion or point mutations.

Materials and Methods: We developed a method for CFTC isolation based on DEPArray ${ }^{\mathrm{TM}}$ sorting and used Huntington's disease as the clinical model for CFTC-based NIPD. Then, we investigated whether CFTC isolation and Whole Genome Amplification (WGA) could be used for NIPD in couples at risk of transmitting different monogenic diseases.

Results: Our data showed that the allele drop-out rate was 3-fold higher in CFTCs than in maternal cells processed in the same way. Moreover, we gave new insights into CFTCs by compiling data obtained by extensive molecular testing by microsatellite multiplex PCR genotyping and by WGA followed by mini-exome sequencing.

Conclusions: CFTCs appear to be often characterized by a random state of genomic degradation. This might challenge CFTC use for routine NIPD. This emphasizes the importance to have not a single diagnostic cell but multiple diagnostic cells.

This research was funded by French Ministry of Health DGOS, grant number PHRCI-16-068 (DIACCIMEX) and by Agence de la biomédecine, grant number $18 A M P 009$ (DIAFEXOME)
L. Cayrefourcq: None. M.C. Vincent: None. S. Pierredon: None. C. Moutou: None. M. Imbert-Bouteille: None. E. Haquet: None. J. Puechberty: None. M. Willems: None. C. Liautard-Haag: None. N. Molinari: None. C. Zordan: None. V. Dorian: None. A. Chaussenot: None. L. Monteil: None. C. Miry: None. Y. Petrov: None. E. Le Boette: None. M. Fradin: None. C. AlixPanabiere: None. C. Guissart: None.

P01.063.C

Development of non-invasive prenatal diagnosis for monogenic diseases: the example of Marfan syndrome and GCK diabetes

\section{T. Nouspikel ${ }^{1}$, A. Bottani ${ }^{2}$, V. Schwitzgebel ${ }^{1}$ \\ ${ }^{1}$ University Hospitals of Geneva, Geneva, Switzerland, ${ }^{2}$ Hôpital du Valais, Sion, Switzerland}

Introduction: The presence of foetal DNA in maternal plasma allows for non-invasive prenatal diagnosis of Mendelian diseases, through detection of known parental mutations. While identifying paternal mutations is straightforward, determining whether a maternal mutation is present in the foetus requires relative dosage of maternal haplotypes (RHDO), via heterozygous SNPs on either side of the mutation.

Results: A custom library of 95 SNPs spread over a $~ 1$ $\mathrm{Mb}$ region around the FBN1 gene was used for a posteriori analysis of two pregnancies in a couple with a paternal FBN1:c.5143delA mutation causing Marfan syndrome. In both cases, the mutation was detected in maternal plasma and confirmed in amniotic fluid or cord blood. We also verified that the method could determine which maternal haplotype each foetus had inherited.

In another couple with a maternal GCK:p.Gly246Arg mutation causing MODY2, a panel of 99 SNPs over $0.7 \mathrm{Mb}$ around GCK was used to analyse maternal plasma at 16 and 30 weeks gestation. In both samples, we correctly determined the maternal haplotype inherited by the foetus and diagnosed the presence of the mutation, confirmed by cord blood DNA analysis. The same results were obtained by quantifying the number of DNA molecules, obtained through molecular barcodes, rather than sequence reads. Bioinformatic reduction of foetal fractions showed that determination of maternal haplotypes remained possible down to $4.3 \%$ foetal DNA.

Conclusions: Although preliminary, our results indicate that a molecular barcode library of $\sim 100$ SNPs is adequate to achieve non-invasive prenatal diagnosis of monogenic diseases, even at low foetal fractions.

T. Nouspikel: None. A. Bottani: None. V. Schwitzgebel: None. 


\section{P01.064.A}

NIPT revealed in a pregnant woman $5 \mathrm{Mbp}-13 \mathrm{q}$ duplication, proved as a familial CNV by array CGH

I. Dimova ${ }^{1}$, I. Bradinova ${ }^{2}$, V. Peycheva ${ }^{3}, R$. Bozhilova ${ }^{3}, R$. Raynova $^{2}$, K. Kercheva ${ }^{1}$, A. Savov ${ }^{2}$, I. Kremensky ${ }^{3}, R$. Kaneva ${ }^{3}$

${ }^{1}$ Medical University Sofia, Sofia, Bulgaria, ${ }^{2}$ SBALAG "Maichin dom", Medical University Sofia, Sofia, Bulgaria,

${ }^{3}$ Molecular Medicine Center, Medical University Sofia, Sofia, Bulgaria

Subchromosomal copy number variations (CNVs), known as segmental deletions and duplications, are extensively distributed in human genome. A substantial proportion of these CNVs are associated with severe diseases, even with some newly identified microdeletion and microduplication syndromes. Recently it was showed that NIPT has a good performance in detecting fetal subchromosomal CNVs, especially for CNVs $>10 \mathrm{Mb}$, and can be incorporated into the routine NIPT chromosomal aneuploidies screening with high sensitivity and specificity. The prenatal detection of CNVs with clinical significance would benefit all the pregnant women in genetic counselling and clinical management of the pregnant outcomes. We report here a result from NIPT performed in a first trimester of a pregnant woman with third normal pregnancy. The test revealed $5 \mathrm{Mbp}-13 \mathrm{q}$ duplication and array $\mathrm{CGH}$ was indicated for confirmation of the aberration after amniocentesis. Array $\mathrm{CGH}$ confirmed the aberration in a fetus with the following size: $13 q 21.2 q 21.31(60,540,987-65,431,854) \times 3(4.89 \mathrm{Mb})$. Such duplications in $13 \mathrm{q}$ are described in the literature to be associated with the developmental delay, intellectual deficits, behavioral problems, and facial dysmorphisms. We tested all family members by array $\mathrm{CGH}$ for the presence of the aberrations - the parents and two siblings (9 years old girl and 12 years old boy). The same aberration was detected in clinically healthy mother and sister, thus determining the familial character of the aberration. In conclusion, careful evaluation should be done for each submiscroscopic aberration detected by NIPT in order to make proper classification and give an adequate genetic counselling.

I. Dimova: None. I. Bradinova: None. V. Peycheva: None. R. Bozhilova: None. R. Raynova: None. K. Kercheva: None. A. Savov: None. I. Kremensky: None. R. Kaneva: None.

\section{P01.067.A}

False positive trisomy 21 NIPT-result due to maternal inherited CNV
M. Blankenburg ${ }^{l}$, A. Weber ${ }^{l}$, S. Rauschke ${ }^{l}$, R. Becker ${ }^{2}, K$. Pötschick ${ }^{3}$, M. Stumm ${ }^{l}$

${ }^{1}$ Medicover Genetics GmbH, Berlin, Germany, ${ }^{2}$ Zentrum für Pränataldiagnostik Kudamm 199, Berlin, Germany,

${ }^{3}$ Medicover Humangenetik-Berlin-Mitte, Berlin, Germany

Introduction: Noninvasive prenatal tests (NIPT) are screening methods to detect fetal aneuploidies with high specificity and sensitivity. Nevertheless, maternal factors as copy number variations $(\mathrm{CNV})$ are reasons for discrepancies between NIPTresults and fetal karyotypes. We report a case with a conspicuous trisomy 21 result after NIPT, an unsuspicious fetal ultrasound and a normal karyotype following conventional chromosome analysis (CA) of amniotic fluid cells.

Material and Methods: Next Generation sequencing (NGS) of cfDNA, CA from peripheral blood, amniotic fluid and placental cells, Fluorescence-in-situ-Hybridization (FISH) on metaphases and interphases, array-CGH (aCGH) and quantitative PCR (qPCR).

Results: NIPT indicated a fetal trisomy 21. In contrast, ultrasound examination and amniocentesis showed normal results. Reanalysis of NIPT by WISECONDOR algorithm showed a $10 \mathrm{Mb}$ gain on chromosome 21 . No comparable cases were described in literature or databases. The parents declined further diagnostics until birth. Postpartum CA and FISH from blood and placental cells resulted in normal karyotypes. In contrast, aCGH confirmed a 1,44 Mb gain in chromosomal region $21 \mathrm{q} 21.3 \mathrm{q} 22.11$ and the aberration was classified as variant of unclear significance (VUS). Additional qPCR analyses confirmed the gain and showed it was maternal inherited. Therefore, we reclassified the aberration as a familial benign CNV.

Conclusions: To best of our knowledge, this is the first report of a small benign maternal chromosome $21 \mathrm{CNV}$ leading to a false positive trisomy 21 NIPT result. That case demonstrates again the importance of a critical reflection of genotype/phenotype correlations after NIPT.

M. Blankenburg: None. A. Weber: None. S. Rauschke: None. R. Becker: None. K. Pötschick: None. M. Stumm: None.

\section{P01.068.B}

Circulating miRNAs as biomarkers to assess fetal fraction level

G. Santoro ${ }^{1}$, S. Caporilli ${ }^{1}$, C. Lapucci ${ }^{1}$, M. Rusin ${ }^{2}, M$.

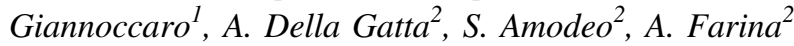

${ }^{1}$ Synlab Italia, Castenedolo, Italy, ${ }^{2}$ Università di Bologna, Dipartimento di Scienze Mediche e Chirurgiche (DIMEC), Bologna, Italy 
Non-invasive prenatal testing (NIPT) methods are widely used as screening tool for fetal aneuploidies. They are based on the isolation of cell-free DNA (cfDNA) from maternal plasma which for the majority is maternal, but a small percentage of it comes from the placenta (cell-free fetal DNA or cffDNA). cffDNA percentage or fetal fraction (FF) ranges from $<4 \%$ to $>30 \%$ and it influences NIPT outcome. According to neoBona ${ }^{\circledR}$ NIPT method used in our laboratory, invalidation occurs with a FF $<4 \%$. Recently, circulating microRNAs (miRNAs) have been investigated as potential biomarkers for screening in pregnancy. We aimed to investigate them as biomarkers in relation to FF level. Previously tested neoBona ${ }^{\circledR}$ samples were divided into one "control group" (FF $>4 \%$ ) and one "test group" (FF $<4 \%$ ). Samples were analysed in the nanostring nCounter ${ }^{\circledast}$ platform by using a pre-customised panel of 799 miRNAs. Each miRNA expression value was normalised to 1 in the "control group" and "test group" samples were analysed for expression defects. Data filtering was set using 1.5-fold of changes in signal as threshold. Ninety-three per cent of miRNAs showed no significant changes of expression whilst 4 miRNAs (named miR-579, miR-3144, miR-612 and miR-6721) were significantly over- or under-expressed. Bioinformatic analysis showed involvement of these miRNAs and their target genes in several biological pathways. Further investigations of these preliminary data could provide a new insight into the biological roles of the miRNAs in relation to FF level and in their potential use as biomarkers.

G. Santoro: None. S. Caporilli: None. C. Lapucci: None. M. Rusin: None. M. Giannoccaro: None. A. Della Gatta: None. S. Amodeo: None. A. Farina: None.

\section{P01.071.B}

An Italian public regional healthcare system experience offering non invasive aneuploidy screening test based on cfDNA (NIPT)

C. Pescucci ${ }^{1}$, F. Gerundino ${ }^{l}$, C. Giuliani ${ }^{1}$, B. Minuti ${ }^{1}, F$.

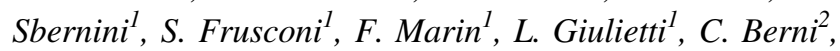

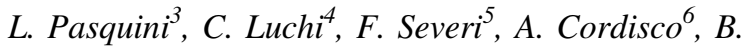

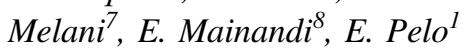

${ }^{1}$ SOD Diagnostica Genetica, Azienda Ospedaliero Universitaria Careggi, Firenze, Italy, ${ }^{2}$ P.O. Rete integrata delle malattie rare e dei difetti congeniti e sviluppo della rete dei punti nascita e della pediatria specialistica, Settore Qualità dei servizi e reti cliniche, Regione Toscana, Firenze, Italy, ${ }^{3}$ Medicina e Diagnosi Fetale, Azienda Ospedaliero Universitaria Careggi, Firenze, Italy, ${ }^{4}$ U.O. Ostericia $e$ Ginecologia, Azienda Ospedaliero-Universitaria Pisana, Pisa, Italy, ${ }^{5}$ Dipartimento di Medicina Molecolare dello sviluppo, Azienda Ospedaliero-Universitaria Senese, Siena, Italy, ${ }^{6}$ Centro Unico di Diagnosi Prenatale del P.O. Piero Palagi, Firenze, Italy, ${ }^{7}$ UO ginecologia e ostetricia - ASL DI PISTOIA, Pistoia, Italy, ${ }^{8}$ Consultori e Diagnosi Prenatale USL 4, Prato, Italy

Introduction: Non invasive prenatal testing (NIPT) to investigate the risk for fetal aneuploidies (chromosomes 13, 18 e 21 trisomies and $\mathrm{X} / \mathrm{Y}$ aneuploidies) has been demonstrated to have high sensitivity and specificity and it is worldwide accepted both as contingent screening as primary test in first trimester.

Materials and Methods: since March 2019, NIPT is offered by public healthcare system in Tuscany to pregnant women with conventional first trimester screening test (FTS) risk (nuchal translucency and biochemical measurements) ranging from 1:301-1:1000 or 1:2-1:300 if they optout invasive prenatal diagnosis. Women are counseled in different centers spread out over the regional area. Blood samples are collected in a single regional center authorized to perform the test for chromosomes 13, 18, 21, $\mathrm{X}$ and $\mathrm{Y}$ by CE-IVD VeriSeq ${ }^{\mathrm{TM}}$ NIPT Solution (Illumina Inc.).

Results: Since March to December 2019, 2199 women have been screened of which 429 with a FTS risk ranging from 1:2-1:300 and 940 from 1:301-1:1000. 396 out of 429 NIPT results were at low risk for fetal aneuploidies whereas $7 / 429$ resulted at high risk for T21 $(n=4), \mathrm{T} 18(n=1)$ and $\mathrm{XXX}(n=2)$. All these results have been confirmed by IPD. In the FTS 1:301-1:1000 risk group, 738 cases resulted at low risk for fetal aneuploidies and 5 at high risk for T21 $(n=1), \mathrm{T} 18(n=1), \mathrm{XXY}(n=2)$ and $\mathrm{XXX}(n=1)$. Only sex chromosome aneuploidy results have been confirmed by IPD. Conclusions. Preliminary data show that of the Tuscany region protocol improve the pregnancy care pathway by reducing inappropriate invasive tests.

C. Pescucci: None. F. Gerundino: None. C. Giuliani: None. B. Minuti: None. F. Sbernini: None. S. Frusconi: None. F. Marin: None. L. Giulietti: None. C. Berni: None. L. Pasquini: None. C. Luchi: None. F. Severi: None. A. Cordisco: None. B. Melani: None. E. Mainandi: None. E. Pelo: None.

\section{P01.075.C}

THE FREQUENCY OF RARE CHROMOSOMAL TRISOMIES USING GENOME WIDE NIPT

\section{M. TEKIN ${ }^{1}, H$. OZDEMIR $^{2}$, H. OZGUR ${ }^{l}, Y . S A H I N^{l}$}

${ }^{I}$ Genoks Genetic Diseases Diagnostic Center, Ankara, Turkey, ${ }^{2}$ Department of Obstetrics and Gynecology, Division of Perinatology, Gazi University Faculty of Medicine, Ankara, Turkey 
Objective: When non invasive prenatal testing (NIPT) incorporating whole genome sequencing (WGS) is performed, the autosomal aneuploidies other than trisomy 21 , 18 and 13 has the potential to be detected. We present the frequency of rare chromosomal trisomies (RATs) that were identified between 1 July 2018 and 31 December 2019

Methods: Standard centrifugation methods were used for plasma isolation. Cell free DNA (cfDNA) was isolated and library construction was prepared according to BGI's documented protocol. WGS of cfDNA was performed with BGISEQ-500 sequencer. Data was analysed using BGI's proprietary bioinformatic programme, HALOS.

Results: WGS data was generated from 13,802 NIPT cases. The mean maternal age was 33 years, fetal fraction was $10.7 \%$ and gestational age at testing was 14 weeks. Over an 18-month period, there were 30 RATs identified. The overall frequency of RATs was $0.21 \%$ in our study. Trisomies 16 and 22 were the most frequently observed RATs while other chromosomes were less frequently involved. Invasive prenatal diagnosis was performed in $37 \%$ (11/30) of cases with RATs. Three fetal aneuploidies were confirmed with amniocentesis; one was T16 and the others were $\mathrm{T} 22$

Conclusions: Rare autosomal aneuploidies are not uncommon in clinical practice when performing genomewide NIPT. The review of the published reports show a significant risk for obstetric pathology and fetal anomalies such as early miscarriage, true fetal mosaicism, intrauterine growth restriction, in utero fetal demise, and uniparental disomy. We advise genetic counseling in all cases of rare chromosomal trisomies to improve pregnancy management.

I.M. Tekin: None. H. Ozdemir: None. H. Ozgur: None. Y. Sahin: None.

\section{P01.076.A}

Next generation sequencing detects premeiotic errors in human oocytes and provides evidence of genetic influence

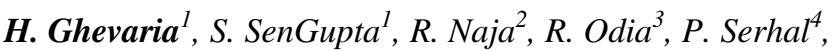
$X$. Viñals Gonzalez ${ }^{3}, X$. Sun $^{1}$, J. Delhanty ${ }^{1}$

${ }^{1}$ Preimplantation Genetics Group, University College London, London, United Kingdom, ${ }^{2}$ Molecular Genetics Laboratory, Igenomix UK Ltd, London, London, United Kingdom, ${ }^{3}$ Embryology Department, The Centre For Reproductive and Genetic Health, London, United Kingdom, ${ }^{4}$ Clinical Department, The Centre For Reproductive and Genetic Health, London, United Kingdom

As women age the risk of an aneuploid oocyte increases with most errors affecting meiosis I/II. Application of next generation sequencing (NGS) has allowed us to confirm our finding, from molecular cytogenetics, that a significant proportion of apparently meiotic aneuploidy is in fact present in the early embryo, leading to a risk of oocyte aneuploidy irrespective of age. It has also provided evidence that genetic factors influence premeiotic oocyte aneuploidy. NGS was performed on oocytes using Ion ReproSeqPGS (ThermoFisher)/VeriSeqPGS (Illumina). Immature oocytes (84) included germinal vesicles \& metaphase I oocytes. Mature oocytes (27) were metaphase II - 1st polar body complexes. Totally, 111 oocytes from 33 women, (average maternal age, 35.09 years) were tested. Overall 15/111 (13.5\%) oocytes showed premeiotic (PM) errors. Eight of 33 patients had oocytes with PM errors. Aneuploidy analysis was based on the reproductive histories of female partners donating oocytes. Interestingly the first two groups of infertility patients showed almost identical aneuploidy rates in contrast to the social oocyte freezing group. Two young women preserving oocytes prior to breast cancer treatment showed complex aneuploidies. We conclude that the application of NGS has provided accurate information regarding the frequency of aneuploidy that is due to premeiotic errors compared with that caused by errors at MI of oogenesis and that on an individual basis this is influenced by genetic factors. Grant support: CRGH

\begin{tabular}{llll}
\hline \multicolumn{3}{l}{ Classification of female partners donating oocytes } \\
\hline $\begin{array}{l}\text { Classification of female } \\
\text { partners based on } \\
\text { reproductive histories }\end{array}$ & $\begin{array}{l}\text { No. of } \\
\text { patients }\end{array}$ & $\begin{array}{l}\text { Total } \\
\text { oocytes tested }\end{array}$ & $\begin{array}{l}\text { Oocytes with } \\
\text { premeiotic } \\
\text { aneuploidy }\end{array}$ \\
\hline $\begin{array}{l}\text { 1. Primary/secondary female } \\
\text { factor infertility }\end{array}$ & 32 & $5(15.6 \%)$ \\
$\begin{array}{l}\text { 2. Females not known to be } \\
\text { infertile (probable male } \\
\text { factor infertility) }\end{array}$ & 32 & $4(12.5 \%)$ \\
$\begin{array}{l}\text { 3. Oocyte preservation due } \\
\text { to social reasons }\end{array}$ & 2 & 21 & $1(4.7 \%)$ \\
$\begin{array}{l}\text { 4. Oocyte preservation due } \\
\text { to breast cancer }\end{array}$ & 2 & 10 & $5(50 \%)$ \\
$\begin{array}{l}\text { 5. Female carriers of } \\
\text { structural rearrangements or } \\
\text { monogenic disorders (non- } \\
\text { cancer related) }\end{array}$ & 3 & 6 & 0 \\
$\begin{array}{l}\text { 6. Females at increased risk } \\
\text { of developing breast/ } \\
\text { ovarian cancer }\end{array}$ & 2 & 10 & 0 \\
\hline
\end{tabular}

H. Ghevaria: None. S. SenGupta: None. R. Naja: None. R. Odia: None. P. Serhal: None. X. Viñals Gonzalez: None. X. Sun: None. J. Delhanty: None. 
P01.080.B

Interrogating the parental and zygotic origin of de novo mutations in severe male infertility using long read sequencing

G. S. Holt ${ }^{l}$, B. Alobaidi ${ }^{l}$, L. Batty ${ }^{l}$, M. Oud ${ }^{2}$, H. Smith ${ }^{l}$, P.

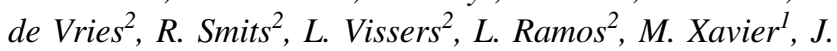
Veltman $^{l}$

${ }^{1}$ Biosciences Institute, Newcastle University, Newcastle Upon Tyne, United Kingdom, ${ }^{2}$ Department of Human Genetics, Donders Institute for Brain Cognition and Behavior, Radboud University, Nijmegen, Netherlands

Male infertility affects approximately $7 \%$ of the male population and while genetic abnormalities are predicted to make up at least $15 \%$ of the cases, only $\sim 4 \%$ are currently identified. In severe forms of male infertility, patients are found to have high numbers of genetic abnormalities and de novo mutations (DNMs). Interestingly DNMs are known to play an important role in severe genetic diseases with reduced reproductive fitness. Our research investigates the role of DNMs in severe male infertility by studying a unique cohort of azoospermic and severe oligozoospermic patients, and their fertile parents. Employing the Illumina's Novaseq6000, 100 DNMs were identified in the exomes of 99 patients. While short-read sequencing data was used to collect the location and genotypes of all informative SNPs (iSNPs) surrounding the DNMs, limitations in fragment size prevented further investigation. In-depth examination of DNMs in context with their surrounding iSNPs requires targeted capture approaches for long-read sequencing that span the entire region of interest. Using PCR amplicon sequencing on the Oxford-Nanopore MinION platform we have been able to phase $\sim 90 \%$ of the DNMs. Phasing analysis indicated that $\sim 71 \%$ of DNMs occurred in the paternal gamete pre-fertilization, which is in concordance with current literature. A link between paternal DNMs and likely causation for disease has been noted, though not all data was available for this association at this time. Investigation of allele frequency identified $91 \%$ of the DNMs occurred pre-zygotically, why they failed to impact father spermatogenesis is of particular interest for further study.

G.S. Holt: None. B. Alobaidi: None. L. Batty: None. M. Oud: None. H. Smith: None. P. de Vries: None. R. Smits: None. L. Vissers: None. L. Ramos: None. M. Xavier: None. J. Veltman: None.

\section{P01.082.A}

Polycystic ovary syndrome: Does it affect the gene expression patterns in human oocytes?

\section{P. Tulay, Z. Al-Omar, B. Ozbakir}

\section{Near East University, Nicosia, Cyprus}

Polycystic ovary syndrome is a common disorder among women of reproductive age. The aim of this study was to investigate the gene expression levels of a number of genes that is functioning in steroidogenesis and in the maintenance of telomeres.

The spare oocytes were collected from IVF patients with polycystic ovaries and routine IVF patients as controls. DNA and RNA were extracted from each oocyte. Reverse transcription of RNA was performed and real time PCR was conducted to quantify the expression levels of eleven genes, TERF1, TRF2, TINF2, TPP1, POT1, RAP1, CYP11, CYP17, CYP19, HSD17B1 and HSD3B2.

The results of this study showed that the expression levels of TERT, TRF, TINF, TPP, RAP, CYP19 and HSD3B2 were similar between the two groups. However, the expression levels of POT and CYP11Al genes were shown to be statistically different in the oocytes obtained from control patients $(\mathrm{p}<0.05)$, whereas statistically significant expression levels were observed for CYP17 in the oocytes obtained from the study group $(\mathrm{p}<0.05)$. The expression level of $H S D 17 B I$ was also shown to be statistically different in the oocytes $(\mathrm{p}<0.05)$.

The results of this study showed that different gene expression levels are observed in the oocytes obtained from females with polycystic ovaries compared to the controls. The extrapolation of the results indicates that the genes essential in maintaining DNA chromosome end integrity and steroidogenesis are crucial for the correct development of the oocytes. Any variations of the expression levels of these genes may result in anomalies of the oocyte maturation.

P. Tulay: None. Z. Al-Omar: None. B. Ozbakir: None.

\section{P01.085.A}

Regulatory variants upstream of $F L T 1$ as risk factors for preeclampsia

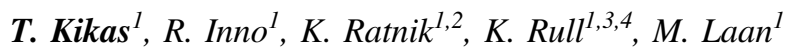

${ }^{1}$ University of Tartu, Institute of Bio- and Translational Medicine, Tartu, Estonia, ${ }^{2}$ SYNLAB Estonia OÜ, Tartu, Estonia, ${ }^{3}$ Women's Clinic, Tartu University Hospital, Tartu, Estonia, ${ }^{4}$ Department of Obstetrics and Gynecology, University of Tartu, Tartu, Estonia

Introduction: The first GWAS assessing the genetic risk to preeclampsia (PE) in placental samples by McGinnis et al., 2017 identified one statistically significant variant associated with $\mathrm{PE}$ ( $\mathrm{rs} 4769613 \mathrm{C}>\mathrm{T}$; $\left.\mathrm{OR}=1.2, \mathrm{P}=5.4 \times 10^{-11}\right)$. 
The variant is located near a well-known PE gene FLT1 encoding sFlt-1. The aim of this study was to further investigate the link between two variants highlighted in GWAS near FLTl gene (rs4769613, rs12050029; 69kb/ $158 \mathrm{~kb}$, respectively) and PE in two independent cohorts.

Materials and Methods: The study utilized placental samples from Estonian REPROMETA and HAPPY PREGNANCY (placentas $n=329 / 1768$, respectively) sample sets, including PE and non-PE controls (total $n=96 / 2001$ ). SNPs (rs4769613, rs12050029) were genotyped using Taqman assays. Association analyses and case-control testing were conducted in PLINK (linear or logistic regression, covariates gestational age, sex).

Results: Case-control testing showed an increased risk for PE with rs4769613 C allele in both Estonian sample sets, concordant with the previous study. Meta-analysis across the two cohorts improved the significance $\left(\mathrm{OR}=1.75, \quad P=2.49 \times 10^{-3}\right)$. As a novel finding, no associations between rs4769613 and other gestational complications (gestational diabetes, fetal growth, preterm birth) were detected, which suggests the variant is PEspecific. The gene expression of $F L T 1$ was upregulated only in PE placentas carrying CC- and CT genotypes (t-test, $\mathrm{P}<$ 0.05 ), whereas in maternal serum, sFlt-1 was increased in $\mathrm{PE}$ cases in all genotype groups.

Conclusions: The study emphasizes the placental rs4769613 as the only known robust and specific risk variant for PE, modulating placental FLTl expression.

Funding: IUT34-12 (Estonian Research Council); HAPPY PREGNANCY, 3.2.0701.12-0047 (European Regional Development Fund)

T. Kikas: None. R. Inno: None. K. Ratnik: A. Employment (full or part-time); Significant; SYNLAB Estonia OÜ. K. Rull: None. M. Laan: None.

\section{P01.086.B}

The role of new genetic markers and natural selection in the formation of a hereditary predisposition to preeclampsia

\section{Serebrova, E. Trifonova, V. Stepanov}

Research Institute of Medical Genetics, Tomsk National Research Medical Center of the Russian Academy of Sciences, Tomsk, Russian Federation

Introduction: Preeclampsia (PE) is a pregnancy-specific complication with the poorly understood etiopathogenesis. There is a hypothesis that the risk to the development of PE may be a consequence of natural selection affecting on placental genes responsible for trophoblast invasion and remodeling of spiral arteries. The aim of this work was to characterize the genetic components of PE by analysis of regulatory single-nucleotide polymorphisms (rSNPs) of differentially expressed genes (DEGs) in placenta and to detect the role of natural selection in its formation.

Materials and Methods: In this report we analyzed 46 rSNPs in 21 DEGs, associated with PE according to the analysis of the transcriptome in 551 patients with $\mathrm{PE}$ and 718 women with uncomplicated pregnancies of Buryat, Russian and Yakut populations using MassArray iPLEX (Sequenom). For detection of the signals of natural selection we used new INSIGHT method.

Results: We have detected significant associations of PE with 14 rSNPs in 10 genes: BHLHE40, CORO2A, INHA, LHB, NDRG1, PPPIR12C, PLIN2, RDH13, SASH1, $S Y D E 1$. The effect of weak negative selection has being shown for 4 rSNPs in 4 DEGs: rs10985257 in CORO2A gene, rs72959687 in INHA gene, rs34845949 in SASHI gene, rs2227262 in NDRG1 gene. It should be noted that these results corresponds to number of evolutionary hypotheses of the occurrence of $\mathrm{PE}$.

Conclusions: Our results demonstrate the significant role of studied rSNPs of DEGs and their adaptive changes in the hereditary predisposition to PE. This work was supported by the Russian Foundation for Basic Research (grant №1829-13045).

V. Serebrova: None. E. Trifonova: None. V. Stepanov: None.

\section{P01.087.C}

Genetic risk factors for pre-eclampsia in the Finnish population

T. Kaartokallio ${ }^{1}$, S. Ruotsalainen ${ }^{2}$, T. Palviainen ${ }^{2}$, T.

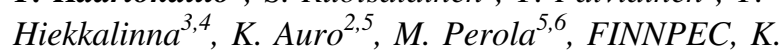
Kivinen $^{2}$, H. Laivuori ${ }^{1,2,7}$

${ }^{1}$ Medical and Clinical Genetics, University of Helsinki and Helsinki University Hospital, Helsinki, Finland, ${ }^{2}$ Institute for Molecular Medicine Finland, Helsinki Institute of Life Science, University of Helsinki, Helsinki, Finland, ${ }^{3}$ Finnish Institute for Health and Welfare, Helsinki, Finland, ${ }^{4}$ Clinical and Molecular Metabolism Research Program, University of Helsinki, Helsinki, Finland, ${ }^{5}$ National Institute for Health and Welfare, Helsinki, Finland, ${ }^{6}$ Diabetes and Obesity Research Program, University of Helsinki, Helsinki, Finland, ${ }^{7}$ Department of Obstetrics and Gynecology, Tampere University Hospital and Tampere University, Faculty of Medicine and Health Technology, Tampere, Finland

Introduction: Pre-eclampsia (PE) is a vascular pregnancy disorder with maternal and fetal genetic components. As the disease negatively affects reproductive success, the risk variants are assumed to be under purifying evolutionary 
selection. The amount of low-frequency pathogenic variants has shown to be increased in Finland due to recent bottlenecks, increasing statistical power in genetic studies. We aim to screen for genetic risk factors for PE in the families mainly originating from a northeastern Finland population isolate, and generalise the results for the whole population.

Methods: The PE family cohort consists of 15 extended families. DNA is available for 174 family members (mothers: $62 \mathrm{PE}+32$ ctrl; babies: $41 \mathrm{PE}+39$ ctrl). Using genome-wide imputed data, we have performed linkage and family-based association analyses with PSEUDOMARKER and PLINK to study segregation of loci with both maternal and fetal phenotypes. We will seek replication of the findings by association analysis in two Finnish cohorts with genome-wide imputed data available, the FINRISK population survey $(426 \mathrm{PE}+8357 \mathrm{ctrl})$ and the FINNPEC casecontrol cohort (mothers:1533 PE+995 ctrl; babies:818 PE +925 ctrl).

Results: In the linkage screen, we identified $\sim 35$ maternal and 40 fetal candidate loci when using a lenient $p$-value threshold $\mathrm{p}<5 \times 10^{-5}$. The FINRISK association analysis showed suggestive replication $\left(\mathrm{p}<5 \times 10^{-6}\right)$ of two of the loci, maternal signal in chr9 close to GASI and fetal signal in chr16 in $C D H 13$. Next steps include association analysis in FINNPEC and meta-analysis in both cohorts to verify these preliminary findings.

Funding: Academy of Finland, Erkko, Kordelin and Sohlberg Foundations, and VTR funding.

T. Kaartokallio: None. S. Ruotsalainen: None. T. Palviainen: None. T. Hiekkalinna: None. K. Auro: None. M. Perola: None. K. Kivinen: None. H. Laivuori: None.

\section{P01.089.B}

A Retrospective Study of In Vitro Fertilization and Preimplantation Genetic Testing for chromosomal aberrations (PGT-SR and PGT-A) combined with Clinical Outcome

\section{Baetens ${ }^{1}$, I. De Croo ${ }^{2}$, A. Dheedene ${ }^{1}$, K. Tilleman ${ }^{2}, B$. Menten $^{l}$}

${ }^{1}$ Center for Medical Genetics, Ghent University Hospital, Ghent, Belgium, ${ }^{2}$ Department for Reproductive Medicine, Ghent University Hospital, Ghent, Belgium

PGT for structural rearrangements (PGT-SR) can be offered to patients carrying an inversion, balanced reciprocal or Robertsonian translocation, and PGT for aneuploidy screening (PGT-A) to patients with fertility problems or recurrent miscarriages. Selecting against embryos without a chromosomal rearrangement could improve live birth rates while reducing miscarriage rates or avoiding birth of an affected child. PGT is an extended, stressful trajectory while precise outcome predictions are still hard to grasp. Counseling PGT couples remains delicate due to numerous parameters influencing outcome. Comparing success rates to (inter)national reference data is part of good clinical practice. PGT technologies evolve at high speed, going from different biopsy timepoints to more extensive genetic methodologies, making good comparisons difficult. By detailed evaluation of our in-house data, and comparison to published data from (inter)national clinics, we obtained additional insights and can provide couples with more patient-focused information. We performed a retrospective cohort study at the Department for Reproductive Medicine and the Center for Medical Genetics (Ghent University Hospital), including patients who underwent ICSI-IVF (January 2016-April 2019) followed by PGT-SR or PGTA after trophectoderm biopsy (68 and 90 oocyte retrieval cycles respectively). Making use of comprehensive chromosome screening (shallow whole genome sequencing or sWGS), we detected chromosomal abnormalities in $65 \%$ of PGT-SR embryos. In the PGT-A group, $40 \%$ of embryos showed a numerical or structural rearrangement. For translocation carriers, pregnancy rate per transfer was 58\% and live birth rate per transfer $44 \%$. For PGT-A patients, pregnancy rate and live birth rate were $39 \%$ and $25 \%$ respectively.

M. Baetens: None. I. De Croo: None. A. Dheedene: None. K. Tilleman: None. B. Menten: None.

\section{P01.090.C}

Preimplantation Genetic Testing (PGT) for cancer predisposition: A single center large cohort experience

S. Zuckerman ${ }^{1}$, O. Freireich ${ }^{l}$, T. Eldar-Geva ${ }^{2}, S$.

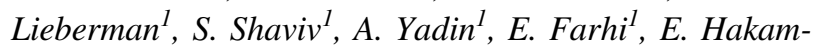
Spector ${ }^{1}$, M. Ben-Shlomo ${ }^{1}$, T. Azar ${ }^{1}$, E. Zivi ${ }^{2}$, E. Rubinstein $^{2}$, O. Schonberger ${ }^{2}$, I. Ben-Ami ${ }^{2}$, G. Altarescu ${ }^{1}$

${ }^{1}$ Medical Genetics Institute, Shaare Zedek Medical Center, Jerusalem, Israel, ${ }^{2}$ IVF unit, Shaare Zedek Medical Center, Jerusalem, Israel

In the last decade an increasing number of people carriers of cancer predisposition mutations (CCPM) are performing PGT-M. PGT-M for these late-onset and partial penetrant conditions implies that the procedure selects against embryos that might have been cancer-free. Moreover, preventive and therapeutic options for decreasing morbidity and mortality are available and ongoing cancer research might reveal treatments altering the natural history of cancer in the future. Most studies exploring perceptions of PGT-M among CCPM, were based on participants' theoretical declarative intentions. Actual use has rarely been reported and analyzed. Between 2006-2019 overall 173 CCPM were 
counseled in our center: One couple between 2006-2008 and gradual increase until 102 CCPM between 2018-2019. Six were single women (3.4\%) and 167 couples (96.5\%). Among them 145(83.8\%) were BRCA1/BRCA2 carriers (92/48 respectively; 5 BRCA1+BRCA2), 10(5.8\%) Lynch carriers, $10(5.8 \%)$ APC carriers, 4(2.3\%) TP53 carriers, 2 (1.2\%) MEN2 carriers, $1(0.6 \%)$ CHEK2 carrier and 1 $(0.6 \%)$ BMPR1A carrier. Overall, $150(86.7 \%)$ of the carriers were healthy and 23(13.2\%) were diagnosed with cancer or related condition. In 120(69.4\%) couples the carrier was the women partner; in 51(29.5\%) couples the carrier was the men partner and in $2(1.2 \%)$ couples both partners were carriers. Five) $2.8 \%$ ) couples had additional reason for PGT-M/PGT-SR. Overall Live birth rate for embryo transfer was $32 \%$. Reproductive decision-making regarding PGT-M for CCPM is a complex process. While this application for PGT-M becomes acceptable, health professionals involved in the process should be aware and address the specific issues and concerns of this group regarding medical, emotional and moral aspects.

S. Zuckerman: None. O. Freireich: None. T. EldarGeva: None. S. Lieberman: None. S. Shaviv: None. A. Yadin: None. E. Farhi: None. E. Hakam-Spector: None. M. Ben-Shlomo: None. T. Azar: None. E. Zivi: None. E. Rubinstein: None. O. Schonberger: None. I. Ben-Ami: None. G. Altarescu: None.

\section{P01.091.A}

Obstetric risks associated with preimplantation genetic testing for monogenic disorders and structural chromosomal rearrangements (PGT-M/SR) compared to regular IVF/ICSI.

M. Heijligers ${ }^{l}, Y$. Arens $^{l}$, R. van Golde ${ }^{l}$, M. van der Hoeven $^{1}$, J. Dumoulin ${ }^{1}$, I. Homminga ${ }^{2}$, C. van Tilborg ${ }^{3}$, C. de Die-Smulders ${ }^{l}$, A. van Montfoort ${ }^{1}$

${ }^{1}$ Maastricht University Medical Centre+, Maastricht, Netherlands, ${ }^{2}$ University Medical Center Groningen, Groningen, Netherlands, ${ }^{3}$ University Medical Center Utrecht, Utrecht, Netherlands

Introduction: Earlier studies on the obstetric outcome in pregnancies following PGT are inconclusive since both an increased risk and no increased risk for adverse obstetric outcome are reported. Moreover, not all of these studies distinguish between PGT for monogenic disorders or structural chromosomal rearrangements (PGT-M/SR) and PGT for aneuploidies (PGT-A).

Methods: This retrospective cohort study included all pregnancies resulting from PGT-M/SR $(n=389)$ and IVF/ ICSI treatments $(n=1667)$ conducted between 2004 and 2014. Data were collected from the medical files and from questionnaires. These data included parental characteristics, characteristics of the treatment preceding the pregnancy and obstetric and neonatal characteristics.

Results: Obstetric complications were reported in $18 \%$ and $19 \%$ of the singleton pregnancies in the PGT and regular IVF/ICSI group, respectively $(P=0.103)$. The risk for stillbirth was lower in the PGT group $(P=0.044)$. Birth weights of all singletons were converted into $\mathrm{z}$-scores and showed a comparable birth weight for the two groups $(0.09$ vs. $-0.02 ; P=0.084)$. Within the multiple pregnancies, the risk for obstetric complications $(P=0.535)$ and the incidence of fetal loss $(P=0.617)$ was comparable between the PGT and IVF/ICSI group. The mean pregnancy duration was longer $(P=0.037)$ and the mean birth weight was higher $(P=0.010)$ in the PGT group. More monozygotic pregnancies were observed in the PGT group $(P=0.009)$.

Conclusions: The more invasive nature of the PGT-M/ SR technique does not lead to an increased risk for obstetric complications as compared to a regular IVF/ICSI treatment.

M. Heijligers: None. Y. Arens: None. R. van Golde: None. M. van der Hoeven: None. J. Dumoulin: None. I. Homminga: None. C. van Tilborg: None. C. de DieSmulders: None. A. van Montfoort: None.

\section{P01.092.B}

Results gained from fetal exome sequencing inform clinical management. A report of 100 cases.

E. Dempsey ${ }^{1}$, L. Ive ${ }^{2}$, R. Dubis ${ }^{2}$, E. Serra ${ }^{2}$, H. Savage ${ }^{2}$, A.

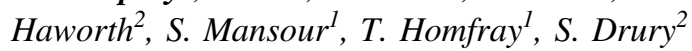

${ }^{1}$ St George's University of London, London, United Kingdom, ${ }^{2}$ Congenica Ltd, Cambridge, United Kingdom

Introduction: Exome sequencing in cases of fetal structural malformation has been shown to increase diagnostic yield (over traditional diagnostic methods) by $8-80 \%$, depending on phenotype. This technique is soon to be made available throughout England as part of a centralisation and standardisation of genetic laboratory services.

Materials and Methods: We have undertaken exome sequencing in 100 cases of fetal anomaly as part of a service improvement project at St George's Hospital. In half of cases sequencing was undertaken with the intention of informing the management of an ongoing pregnancy. In the remaining cases exome sequencing was undertaken postmortem. Exome data was analysed via the application of patient-specific phenotype panels, reducing the risk of incidental findings.

Results: Our diagnostic rate across all cases is $~ 40 \%$ and higher in cases with skeletal and oedema phenotypes. Exome results have influenced the management of pregnancy and labour. They have also directed early neonatal 
care, including enabling the treatment of a newborn with enzyme replacement therapy. The results of exome sequencing have been used for accurate assessment of recurrence risk. In addition, results can be used for prenatal testing or preimplantation genetic diagnosis in a future pregnancy.

Conclusions: Using specific case examples we will describe the way in which the results of fetal exome sequencing have influenced clinical management. We will also describe the challenges we have encountered in the implementation of a fetal exome sequencing service, including the analysis of variants of uncertain significance in phenotype-limited situations.

E. Dempsey: None. L. Ive: A. Employment (full or parttime); Significant; Congenica Ltd. R. Dubis: A. Employment (full or part-time); Significant; Congenica Ltd. E. Serra: A. Employment (full or part-time); Significant; Congenica. H. Savage: A. Employment (full or part-time); Significant; Congenica Ltd. A. Haworth: A. Employment (full or part-time); Significant; Congenica Ltd. S. Mansour: None. T. Homfray: None. S. Drury: A. Employment (full or part-time); Significant; Congenica Ltd.

\section{P01.093.C}

Whole-exome sequencing in the prenatal setting: relating fetal phenotype to genotype

\section{Leeuwen, K. Bouman, N. Corsten-Janssen}

\section{UMCG, Groningen, Netherlands}

Introduction: Whole-exome sequencing (WES) is increasingly being used in the prenatal setting. However, interpretation of WES data is complicated due to various factors including limited knowledge on the prenatal fetal phenotype of rare monogenic disorders. We therefore aimed to describe the prenatal fetal phenotype of genetic variants found by WES.

Methods: In this single-center study, we included fetuses in whom WES was indicated in a prenatal diagnostic setting between March 1, 2018 and January 1, 2020 during pregnancy or after termination of pregnancy. We performed trio WES using a phenotype-dependent filtering strategy. We documented prenatal and postnatal characteristics of fetuses and compared the phenotype with literature using PubMed.

Results: WES was performed in 93 fetuses. A pathogenic or likely pathogenic variant was found in 29 (31.2\%) fetuses ( 21 different genes). For eight genes, it was the first time a causative genetic variant was found in a prenatal setting (C2CD3,CNOT1,GATA6, PLD1,POMK,SAMD9, SMC3,THOC6). In three fetuses, some detected structural anomalies have not previously been described (PEXI-, POMK-,THOC6-variants). In three fetuses, diagnosis was filtered out in the prenatal setting due to various reasons, but could be established using additional postnatal phenotypic characteristics. In general, the prenatal phenotype on ultrasound corresponded well with postnatal phenotype although some features were not detected by the prenatal ultrasound examination.

Conclusions: Increased knowledge about the prenatal fetal phenotype is important for improving the interpretation and classification of fetal genetic variants. This will lead to improvement of parental genetic counseling as well as prenatal and neonatal care.

L. Leeuwen: None. K. Bouman: None. N. CorstenJanssen: None.

\section{P01.098.B}

Lessons learned from rapid Exome Sequencing(rES)as a standard diagnostic test in a prenatal setting for fetuses with ultrasound abnormalities.

N. Corsten-Janssen ${ }^{l}, K$. Bouman $^{l}$, A. J. Scheper ${ }^{l}$, M. T. Meems-Veldhuis ${ }^{I}$, J. B. G. M. Verheij ${ }^{1}$, R. Suijkerbuijk ${ }^{1}, L$. K. Duin ${ }^{2}$, G. T. R. Manten ${ }^{3}$, I. M. van Langen ${ }^{1}$, B. SikkemaRaddatz $^{l}$, H. Westers ${ }^{l}$, C. C. van Diemen ${ }^{l}$

${ }^{1}$ Department of Genetics, University of Groningen, University Medical Centre Groningen, Groningen, Netherlands, ${ }^{2}$ Department of Obstetrics, Gynecology and Prenatal Diagnosis, University Medical Centre Groningen, Groningen, Netherlands, ${ }^{3}$ Department of Obstetrics and Gynecology, Isala, Zwolle, Zwolle, Netherlands

Background: Adding rES to conventional genetic tests for fetuses with ultrasound abnormalities improves the diagnostic yield, but differs from performing postnatal $\mathrm{rES}$ for instance by its rapid turnaround time and the impact of a diagnosis. We will present the lessons we learned by performing $\mathrm{rES}$ as a diagnostic test in prenatal care from March 2018.

Methods: We performed trio rES analysis using a custom virtual gene panel of $\sim 4,300$ OMIM genes during pregnancy. Inclusion criteria were: 1) two or more independent major fetal anomalies, or 2) hydrops fetalis or bilateral polycystic kidneys, or 3) one major fetal anomaly and a first-degree relative with the same anomaly.

Results: We established a genetic rES-based diagnosis in 14 out of $54(26 \%)$ eligible fetuses with a mean turnaround time of 14 days. We learned the following lessons: (1) We made unexpected diagnoses using our broad virtual panel, like Zellweger syndrome in a fetus suspected of a ciliopathy. (2) We missed four diagnoses that were made postnatally using additional phenotypic information and adapted our filter strategy. 3) We were confronted with incidental unsolicited findings and changed our filter 
strategy to minimize them. (4) There is a lack of knowledge about prenatal phenotype of known genetic conditions, which hinders diagnostics. Further, we experienced the impact of time pressure to generate a diagnosis in limited time and had to deal with changing fetal phenotypes.

Conclusion: rES in the prenatal setting is feasible but challenging, and improves by adapting the process based on insights from previous experiences.

N. Corsten-Janssen: None. K. Bouman: None. A.J. Scheper: None. M.T. Meems-Veldhuis: None. J.B.G.M. Verheij: None. R. Suijkerbuijk: None. L.K. Duin: None. G.T.R. Manten: None. I.M. van Langen: None. B. Sikkema-Raddatz: None. H. Westers: None. C.C. van Diemen: None.

\section{P01.099.C}

Improved fetal sex determination in twin pregnancies in low coverage whole-genome based non-invasive prenatal testing

T. Sedlackova ${ }^{1}$, M. Hyblova ${ }^{2,3}$, J. Budis ${ }^{1,4,5}$, M. Harsanyova $^{1,6}$, M. Kucharik ${ }^{1,5}$, O. Pos ${ }^{1,6}$, M. Sekelska ${ }^{2,3}$, B. $\mathrm{Nagy}^{7}$, G. Minarik ${ }^{2,3}$, T. Szemes ${ }^{1,5,6}$

${ }^{1}$ Geneton Ltd., Bratislava, Slovakia, ${ }^{2}$ Medirex Inc., Bratislava, Slovakia, ${ }^{3}$ Trisomy Test Ltd., Bratislava, Slovakia, ${ }^{4}$ Slovak Centre of Scientific and Technical Information, Bratislava, Slovakia, ${ }^{5}$ Comenius University Science Park, Bratislava, Slovakia, ${ }^{6}$ Department of Molecular Biology, Faculty of Natural Sciences, Comenius University, Bratislava, Slovakia, ${ }^{7}$ Semmelweis University, Budapest, Hungary

Introduction: Fetal sex determination is already a common part of the standard non-invasive prenatal testing (NIPT). The current classification methods are almost entirely accurate for fetal sex prediction in singleton pregnancies, but they still show the limitations for multiple pregnancies, with 98\% reported accuracy of fetal sex prediction in twins.

Methods: We enrolled 99 maternal samples with twin pregnancies and confirmed fetus sex in this study. Since a precise estimation of the fetal fraction is a key for correct sex determination, we used a previously published improved predictor utilizing two different attributes of analyzed genomic material - length and genomic location of the sequenced DNA fragments. Subsequent fetal sex prediction was performed using an SVM classifier.

Results: Our method has shown perfect prediction accuracy in distinguishing between pregnancies with two female fetuses and pregnancies with at least one male fetus. We observed one incorrect prediction between one and two male fetuses. Overall, the accuracy of fetal sex prediction was $98.99 \%$.
Conclusions: Here we described a novel bioinformatic algorithm to determine the sex of both fetuses in twin pregnancies, by the analysis of cell-free fetal DNA from the maternal plasma. Our algorithm achieved superior accuracy in fetal sex prediction in twins compared to known bioinformatics tools. Moreover, it provides supporting graphs for visual inspection and calculation of prediction confidence.

Keywords: non-invasive prenatal testing, twin pregnancy, whole-genome sequencing, fetal sex prediction

T. Sedlackova: A. Employment (full or part-time); Modest; Geneton Ltd.. M. Hyblova: None. J. Budis: None. M. Harsanyova: None. M. Kucharik: None. O. Pos: None. M. Sekelska: None. B. Nagy: None. G. Minarik: None. T. Szemes: None.

\section{P01.100.A}

The value of prenatal array to detect submicroscopic chromosomal imbalances in cases with abnormal fetal ultrasonographic findings; a retrospective study

\section{OZER, M. Aydin, S. Aktuna, E. Unsal}

\section{MIKROGEN GENETIC DIAGNOSIS CENTER, ANKARA, Turkey}

Chromosomal microarray (CMA) is used in prenatal diagnosis for detecting CNVs which are too small to detect by karyotyping. Here we report our laboratory's array data of prenatal samples and describe phenotype-genotype correlations. Methods: 180 specimens (149 amniotic fluid, 23 chorionic villi and 8 cord blood samples) were analyzed by array CGH. Detection of genomic CNVs was performed using Agilent, 8x60K CGH Microarray. Interpretation was done according to the ACMG guidelines. Results: \%16.1 of patients (29/180), CNVs were detected. 24 of them had segmental duplication or deletion, 5 had trisomy and 1 had monosomy X. $3.3 \%$ of patients had aneuploidy, $4.4 \%$ of patients had VUS variants and \%8.8 of patients had pathogenic segmental deletions/duplications. $61.1 \%$ of the segmental pathogenic variants were deletions and most of the VUS variants were duplications $(62.5 \%) .50 \%$ of the segmental pathogenic variants were $<7 \mathrm{Mb}$ and the smallest size was $0.5 \mathrm{MB}$ (deletion). $22.2 \%$ of pathogenic variants were well-defined $\mathrm{CNV}$ syndromes. The most prevalent indications were ultrasonographic anomalies, increased triple test risk and advanced maternal age. While $30 \%$ of patients with normal results have normal ultrasound, all patients with a pathogenic variant had an ultrasound anomaly. The most defined ultrasonographic findings in cases with pathogenic variants were cardiac anomalies, increased NT and brain anomalies. Conclusion: Detection of submicroscopic imbalances by CMA increases the power 
of prenatal diagnosis. Our data emphasize the importance of CMA in the prenatal diagnosis of the fetuses with abnormal ultrasonographic findings whereas conventional karyotyping resolution is inadequate to detect submicroscopic changes.

L. Ozer: None. M. Aydin: None. S. Aktuna: None. E. Unsal: None.

P01.102.C

Targeted prenatal exome sequencing in a series of fetal structural anomalies detected by ultrasonography: first experience of the University Hospitals of Geneva

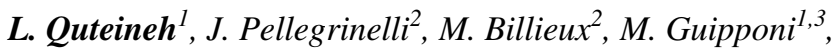
S. Laurent ${ }^{l}$, E. Hammar ${ }^{l}$, A. Murphy ${ }^{l}$, M. Carminho Amaro Rodrigues $^{l}$, T. Ait-Mouhoub ${ }^{l}$, F. Sloan-Béna ${ }^{l}$, S. Unger ${ }^{4}, F$.

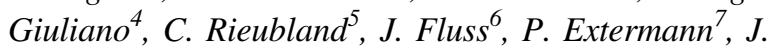
Blouin $^{1,3}$, M. Abramowicz' ${ }^{1}$ S. Fokstuen ${ }^{1}$

${ }^{1}$ Service of Genetic Medicine, Geneva, Switzerland, ${ }^{2}$ Department of gynecology and obstetrics, Geneva, Switzerland, ${ }^{3}$ Department of Genetic Medicine and Development, Geneva, Switzerland, ${ }^{4}$ Service of Genetic Medicine, Lausanne, Switzerland, ${ }^{5}$ Department of Pediatrics, Division of Human Genetics, Bern, Switzerland, ${ }^{6}$ Pediatric Neurology Unit, Geneva, Switzerland, ${ }^{7}$ Dianecho echographic center, Geneva, Switzerland

Introduction: Diagnostic genome-wide sequencing approaches to investigate prenatally detected anomalies are increasingly used in fetal medicine. We aimed to evaluate the value of targeted whole exome sequencing (WES) in fetuses with prenatal sonographic abnormalities and normal microarray and karyotype.

Materials and Methods: After informed consent was obtained, we sequenced the DNA from amniotic fluid cells of 17 fetuses by WES (Illumina NextSEQ500). We then performed bioinformatic analysis of a selected gene panel. All cases were ongoing pregnancies. The genomic results were obtained within 2 weeks.

Results: The 17 fetuses presented with the following anomalies: isolated brain malformations $(n=6)$, sustained increased nuchal translucency $(n=3)$, multiple structural anomalies $(n=3)$, polyhydramnios $(n=1)$, situs inversus $(n=1)$, renal cysts $(n=1)$, hydrops fetalis $(n=1)$ and skeletal dysplasia $(n=1)$. Causing variants were identified in 4 cases $(24 \%)$ : two with sustained increased nuchal translucency presented with a de novo variant in $R A F 1$ (p. Ser267Arg) and in BRAF (p.Thr241Arg) genes, compatible with a RASopathy, the fetus with skeletal dysplasia had a de novo variant in COL11A1 gene (p.Gln921_Pro926del) causing Stickler/Marshall syndrome and the fetus with hydrops fetalis had 2 variants in PIEZO1 gene (p.Ser182* and p.Phe1294del) causing autosomal recessive lymphatic dysplasia. Pregnancy was terminated in all 4 cases.

Conclusions: targeted WES is an adequate approach of genome-wide sequencing for ongoing pregnancies with fetal structural abnormalities suspicious for a genetic etiology and normal microarray. It has a diagnostic utility and might facilitates genetic counseling.

L. Quteineh: None. J. Pellegrinelli: None. M. Billieux: None. M. Guipponi: None. S. Laurent: None. E. Hammar: None. A. Murphy: None. M. Carminho Amaro Rodrigues: None. T. Ait-Mouhoub: None. F. Sloan-Béna: None. S. Unger: None. F. Giuliano: None. C. Rieubland: None. J. Fluss: None. P. Extermann: None. J. Blouin: None. M. Abramowicz: None. S. Fokstuen: None.

\section{P01.104.B}

Prenatal diagnosis of trisomies in CVS: are non-mosaic biallelic QF-PCR results prognostic?

\section{A. R. Pendlebury-Watt ${ }^{1}$, K. Mann ${ }^{1}$, C. Ogilvie ${ }^{2}$ \\ ${ }^{1}$ Viapath analytics, London, United Kingdom, ${ }^{2}$ Genetics Department, Guys and St. Thomas' NHS Foundation Trust, London, United Kingdom}

Placental mosaicism for chromosome aneuploidy complicates prenatal analysis of chorionic villus (CV) samples and has a reported incidence of up to $2 \%$. Quantitative fluorescence-PCR (QF-PCR) analysis of microsatellite repeats is widely used to establish chromosome copy number of uncultured CV samples; if recommended preparation methods are employed, both cytotrophoblast and mesoderm should be represented. In the case of trisomy results, the presence of three different alleles is observed in $\sim 90 \%$ of cases, confirming meiotic non-disjunction as the origin of the abnormal cell line. Where only biallelic $2: 1$ ratios are observed, the trisomy may have arisen by mitotic non-disjunction following a normal conception, in which case a normal cell line may be present; European and UK best practice guidelines recommend that non-mosaic biallelic (NMB) results should be reported more cautiously, given the increased risk of mosaicism. However, the likelihood of finding a normal cell line following an NMB result is not established. We have therefore audited 10 years of cases and compared NMB results to the follow-up karyotype and/or amniocentesis results. In total, 133 CVS showed NMB results for common trisomies, of which 131 were concordant with follow-up tests. Two trisomy 18 cases showed a normal result on amniocentesis, indicating confined placental mosaicism; neither case had ultrasound abnormalities present at referral. Therefore, in this cohort, $\sim 1.5 \%$ of NMB QF-PCR cases subsequently showed 
evidence of mosaicism, this is reduced to $0 \%$ for cases with USS abnormalities. These figures may inform the reporting and pregnancy management of NMB cases.

A.R. Pendlebury-Watt: None. K. Mann: None. C. Ogilvie: None.

\section{P01.105.C}

Parental experiences of rapid Exome Sequencing in case of serious ultrasound abnormalities during pregnancy

M. Plantinga ${ }^{l}$, L. Zwienenberg ${ }^{l}$, E. van Dijk ${ }^{I}$, H. Breet ${ }^{l}, J$. Diphoorn ${ }^{l}$, J. el Mecky ${ }^{2}$, K. Bouman ${ }^{l}$, J. Verheij ${ }^{1}$, A. Ranchor $^{3}, N$. Corsten-Janssen ${ }^{l}$, I. van Langen ${ }^{l}$

${ }^{1}$ UMCG, Clinical Genetics, Groningen, Netherlands, ${ }^{2}$ University of Southampton, CELS, Southampton, United Kingdom, ${ }^{3} U M C G, \quad$ Health Psychology, Groningen, Netherlands

Objective: Adding rapid Exome Sequencing (rES) to conventional genetic tests (QF-PCR and SNP-array) can improve the diagnostic yield of pregnancies showing ultrasound abnormalities but also includes a higher chance of unsolicited findings. rES was offered in the context of a prenatal $\mathrm{rES}$ implementation study with a turnaround time of 14 days. We evaluated how this offer was experienced in an explorative mixed-methods parental experience study.

Methods: We investigated reasons for accepting and declining $\mathrm{rES}$, the impact of rES on decision making, experiences of the counseling and test process, and understanding of test information and possible test outcomes. All couples were asked to fill two surveys, one after pre-test and one after post-test counseling. Part of the couples were approached for an interview until data saturation was reached.

Results: 44 couples participated in the survey study, 11 in the interview study. In $22 \%$ of pregnancies, a diagnosis was found, in $31 \%$ an unsolicited finding. 95\% wanted to be informed about unsolicited findings. The most important reason for accepting rES was to find a diagnosis. Most parents already decided to terminate the pregnancy on the basis of the ultrasound abnormalities, although rES results impacted decision in a few cases. The impact for future pregnancies was higher than with conventional testing and the fast turnaround was valued highly. The counseling and test process was experienced positive. Parents suggested to add information of other parents' experiences and decisional process to the test information.

Conclusion: Parents evaluate rES positively, despite the risk of unsolicited findings.

M. Plantinga: None. L. Zwienenberg: None. E. van Dijk: None. H. Breet: None. J. Diphoorn: None. J. el Mecky: None. K. Bouman: None. J. Verheij: None.
A. Ranchor: None. N. Corsten-Janssen: None. I. van Langen: None.

\section{P01.106.A}

When to test fetuses for RASopathies? Proposition from a systematic analysis of $\mathbf{3 5 3}$ multicenter cases and a postnatal cohort

A. Scott ${ }^{1}$, N. Di Giosaffatte ${ }^{2}$, V. Pinna ${ }^{2}$, P. Daniele ${ }^{2}, S$. Corno $^{3}$, V. D'Ambrosio ${ }^{3}$, E. Andreucci ${ }^{4}$, A. Marozza, F. Sirchia $^{6}$, G. Tortora ${ }^{7}$, D. Mangiameli ${ }^{8}$, C. Di Marco ${ }^{9}$, I. Donati $^{10}$, A. Zonta ${ }^{11}$, E. Grosso ${ }^{11}$, G. Mastromoro ${ }^{12}$, P.

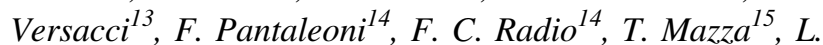
Papi $^{5}$, T. Mattina ${ }^{8}$, A. Giancotti ${ }^{3}$, A. Pizzuti ${ }^{12}$, A. Laberge ${ }^{1}$, M. Tartaglia ${ }^{14}$, M. Delrue ${ }^{1}$, A. De Luca ${ }^{2}$

${ }^{1}$ Pediatrics Department, Medical Genetics Division, CHU Sainte-Justine, University of Montreal, Montreal, QC, Canada, ${ }^{2}$ Medical Genetics Unit, Fondazione IRCCSCasa Sollievo della Sofferenza, San Giovanni Rotondo, Italy, ${ }^{3}$ Department of Maternal and Child Health and Urologic Science, "Sapienza" University, Policlinico Umberto I Hospital, Rome, Italy, ${ }^{4}$ Medical Genetics Unit, Meyer Children's University Hospital, Florence, Italy, ${ }^{5}$ Section of Medical Genetics, Department of Experimental and Clinical Biomedical Sciences, University of Florence, Florence, Italy, ${ }^{6}$ Institute for Maternal and Child Health, IRCCS "Burlo Garofolo", Trieste, Italy, ${ }^{7}$ Azienda Ospedaliero-Universitaria Ospedali Riuniti di Ancona, Ancona, Italy, ${ }^{8}$ Department of Pediatrics, Medical Genetics, University of Catania, Catania, Italy, ${ }^{9}$ Ospedale Santa Maria Nuova, Florence, Italy, ${ }^{10}$ Genetic Department, Infermi Hospital, Rimini, Italy, ${ }^{11}$ Città Della Salute e Della Scienza University Hospital, Medical Genetics Unit, Torino, Italy, ${ }^{12}$ Department of Experimental Medicine, Università Sapienza, Rome, Italy, ${ }^{13}$ Department of Pediatrics, Università Sapienza, Rome, Italy, ${ }^{14}$ Genetics and Rare Diseases Research Division, Ospedale Pediatrico Bambino Gesù, IRCCS, Rome, Italy, ${ }^{15}$ Laboratory of Bioinformatics, IRCCS Casa Sollievo della Sofferenza, San Giovanni Rotondo, Italy

Introduction: Recent studies have identified some evocative prenatal features of RASopathies e.g. lymphatic dysplasia (cystic hygroma, hydrops, effusions), congenital heart disease, polyhydramnios, and renal anomalies. The aim of this study was to better define the prenatal indications for RASopathy testing and the genotype-phenotype correlations.

Methods: 353 cases sent for prenatal RASopathy testing between 2012-2019, after a normal a-CGH, were collected. For most, 11 RASopathy genes were tested. Detailed ultrasound findings were available for 313 fetuses, whereas 
40 cases had only clinical indications for testing collected. Twenty-five RASopathy patients diagnosed postnatally were added to widen the phenotypic spectrum.

Results: See tables below for detailed results. The overall diagnostic yield was $14 \%$ (51/353). The largest contributors of mutations were PTPN11 (29\%), RITI (15\%), RAF1 $(13 \%)$ and HRAS $(12 \%)$. Severe postnatal phenotypes were in majority diagnosed prenatally and associated with mutations in HRAS, RITI and RAFl.

Conclusions: After normal a-CGH, RASopathies should be considered when any ultrasound finding of lymphatic dysplasia or suggestive congenital heart disease is found alone or in association. In the presence of persistent or associated hygroma, NT $>6 \mathrm{~mm}$, hypertrophic cardiomyopathy, or any finding combined with polyhydramnios or renal anomaly, the likelihood of RASopathy diagnosis is significantly increased.

Table 1 Prenatal ultrasound findings in 313 patients with a normal array-CGH

\begin{tabular}{|c|c|c|c|c|c|}
\hline Prenatal findings & $\begin{array}{l}\text { Total \# of } \\
\text { patients } \\
(n=313)\end{array}$ & $\begin{array}{l}\text { Prenatal } \\
\text { mutation- } \\
\text { positive } \\
\text { group }(n=48)\end{array}$ & $\begin{array}{l}\text { Mutation- } \\
\text { negative } \\
\text { group } \\
(n=265)\end{array}$ & $p$-value $e^{a}$ & $\begin{array}{l}\text { Postnatal } \\
\text { mutation- } \\
\text { positive } \\
\text { group }(n=25)\end{array}$ \\
\hline Increased NT & $\begin{array}{l}231 / \\
313(74 \%)\end{array}$ & $30 / 48(63 \%)$ & $\begin{array}{l}201 / \\
265(76 \%)\end{array}$ & 0,03 & $1 / 25(4 \%)$ \\
\hline $\mathrm{NT}>3<3,5$ & $\begin{array}{l}21 / \\
231(9 \%)\end{array}$ & $0 / 30(0 \%)$ & $\begin{array}{l}21 / \\
201(10 \%)\end{array}$ & 0,048 & \\
\hline $\mathrm{NT} \geq 3,5$ & $\begin{array}{l}145 / \\
231(63 \%)\end{array}$ & $18 / 30(60 \%)$ & $\begin{array}{l}127 / \\
201(63 \%)\end{array}$ & & - \\
\hline $\mathrm{NT}>6$ & $\begin{array}{l}60 / \\
231(26 \%)\end{array}$ & $12 / 30(40 \%)$ & $\begin{array}{l}48 / \\
201(24 \%)\end{array}$ & & \\
\hline unknown & $5 / 231(2 \%)$ & $0 / 30(0 \%)$ & $\begin{array}{l}5 / \\
201(2 \%)\end{array}$ & & \\
\hline $\begin{array}{l}\text { Increased NT } \\
\text { without } \mathrm{CH}\end{array}$ & $\begin{array}{l}86 / \\
313(27 \%)\end{array}$ & $7 / 48(15 \%)$ & $\begin{array}{l}79 / \\
265(30 \%)\end{array}$ & 0,02 & $1 / 25(4 \%)$ \\
\hline Mean NT (mm) ${ }^{\mathrm{b}}$ & $\begin{array}{l}5,01(0,7- \\
14,9)\end{array}$ & $6,59(1,25-13,5)$ & $\begin{array}{l}4,80(0,7- \\
14,9)\end{array}$ & 0,0001 & $2,58(1,0-4,1)$ \\
\hline Cystic hygroma & $\begin{array}{l}183 / \\
313(58 \%)\end{array}$ & $31 / 48(65 \%)$ & $\begin{array}{l}152 / \\
265(57 \%)\end{array}$ & 0,3 & $1 / 25(4 \%)$ \\
\hline 1st trimester $\mathrm{CH}$ & $\begin{array}{l}58 / \\
183(32 \%)\end{array}$ & $4 / 31(13 \%)$ & $\begin{array}{l}54 / \\
152(36 \%)\end{array}$ & 0,02 & - \\
\hline Persistent $\mathrm{CH}$ & $\begin{array}{l}125 / \\
183(68 \%)\end{array}$ & $27 / 31(87 \%)$ & $\begin{array}{l}98 / \\
152(64 \%)\end{array}$ & & \\
\hline Isolated* & $\begin{array}{l}89 / \\
183(49 \%)\end{array}$ & $5 / 31(16 \%)$ & $\begin{array}{l}84 / \\
152(55 \%)\end{array}$ & $<0,0001$ & - \\
\hline in association & $\begin{array}{l}94 / \\
183(51 \%)\end{array}$ & $26 / 31(84 \%)$ & $\begin{array}{l}68 / \\
152(45 \%)\end{array}$ & & \\
\hline $\mathrm{CH}$ without $\uparrow \mathrm{NT}$ & $\begin{array}{l}34 / \\
183(19 \%)\end{array}$ & $9 / 31(29 \%)$ & $\begin{array}{l}25 / \\
152(16 \%)\end{array}$ & 0,1 & $1 / 25(4 \%)$ \\
\hline $\mathrm{CH}$ with $\uparrow \mathrm{NT}$ & $\begin{array}{l}149 / \\
183(81 \%)\end{array}$ & $22 / 31(71 \%)$ & $\begin{array}{l}127 / \\
152(84 \%)\end{array}$ & & \\
\hline $\mathrm{NT}>3<3,5$ & $\begin{array}{l}17 / \\
149(11 \%)\end{array}$ & $0 / 22(0 \%)$ & $\begin{array}{l}17 / 127 \\
(13,5 \%)\end{array}$ & 0,02 & - \\
\hline $\mathrm{NT} \geq 3,5$ & $\begin{array}{l}89 / \\
149(60 \%)\end{array}$ & $11 / 22(50 \%)$ & $\begin{array}{l}78 / 127 \\
(61,5 \%)\end{array}$ & & \\
\hline NT $>6$ & $\begin{array}{l}43 / \\
149(29 \%)\end{array}$ & $11 / 22(50 \%)$ & $\begin{array}{l}32 / \\
127(25 \%)\end{array}$ & & \\
\hline Increased NF & $\begin{array}{l}68 / \\
313(22 \%)\end{array}$ & $18 / 48(38 \%)$ & $\begin{array}{l}50 / \\
265(19 \%)\end{array}$ & 0,001 & $0 / 25(0 \%)$ \\
\hline $\mathrm{NF}>6$ & $\begin{array}{l}63 / \\
68(93 \%)\end{array}$ & $15 / 18(83 \%)$ & $\begin{array}{l}48 / \\
50(96 \%)\end{array}$ & 0,08 & - \\
\hline $\mathrm{NF}>15$ & $5 / 68(7 \%)$ & $3 / 18(17 \%)$ & $2 / 50(4 \%)$ & & \\
\hline Mean NF $(\mathrm{mm})^{\mathrm{b}}$ & $\begin{array}{l}6,94(3,0- \\
28)\end{array}$ & $9,39(4,2-22)$ & $\begin{array}{l}6,28(3,0- \\
28)\end{array}$ & 0,01 & N/A \\
\hline Hydrops & $\begin{array}{l}60 / \\
313(19 \%)\end{array}$ & $20 / 48(42 \%)$ & $\begin{array}{l}40 / \\
265(15 \%)\end{array}$ & $<0,0001$ & $1 / 25(4 \%)$ \\
\hline
\end{tabular}

\begin{tabular}{|c|c|c|c|c|c|}
\hline $\begin{array}{l}\text { 1st trimester } \\
\text { hydrops }\end{array}$ & $\begin{array}{l}14 / \\
60(10 \%)\end{array}$ & $2 / 20(10 \%)$ & $\begin{array}{l}12 / \\
40(30 \%)\end{array}$ & 0,08 & - \\
\hline Persistent hydrops & $\begin{array}{l}26 / \\
60(90 \%)\end{array}$ & $18 / 20(90 \%)$ & $\begin{array}{l}28 / \\
40(70 \%)\end{array}$ & & \\
\hline $\begin{array}{l}\text { Thoracic } \\
\text { effusions or } \\
\text { ascites }\end{array}$ & $\begin{array}{l}54 / \\
313(17 \%)\end{array}$ & $22 / 48(46 \%)$ & $\begin{array}{l}32 / \\
265(12 \%)\end{array}$ & $<0,0001$ & $4 / 25(16 \%)$ \\
\hline $\begin{array}{l}\text { Congenital heart } \\
\text { disease }\end{array}$ & $\begin{array}{l}71 / \\
313(23 \%)\end{array}$ & $18 / 48(38 \%)$ & $\begin{array}{l}53 / \\
265(20 \%)\end{array}$ & 0,002 & $3 / 25(12 \%)$ \\
\hline cardiac defect & $\begin{array}{l}64 / \\
313(20 \%)\end{array}$ & $15 / 48(31 \%)$ & $\begin{array}{l}49 / \\
265(18 \%)\end{array}$ & 0,02 & $3 / 25(12 \%)$ \\
\hline $\mathrm{HCM}$ & $\begin{array}{l}13 / \\
313(4 \%)\end{array}$ & $9 / 48(19 \%)$ & $\begin{array}{l}4 / \\
265(2 \%)\end{array}$ & 0,007 & $1 / 25(4 \%)$ \\
\hline $\mathrm{HCM}$ without $\mathrm{CD}$ & $7 / 13(54 \%)$ & $3 / 9(33 \%)$ & $\begin{array}{l}4 / \\
4(100 \%)\end{array}$ & N/A & $0 / 25(0 \%)$ \\
\hline $\begin{array}{l}\text { combined } \mathrm{HCM} \\
+\mathrm{CD}\end{array}$ & $6 / 13(46 \%)$ & $6 / 9(67 \%)$ & $0 / 4(0 \%)$ & & $1 / 25(4 \%)$ \\
\hline \multicolumn{6}{|c|}{ Other associated findings } \\
\hline Polyhydramnios & $\begin{array}{l}36 / \\
313(12 \%)\end{array}$ & $14 / 48(29 \%)$ & $\begin{array}{l}22 / \\
265(8 \%)\end{array}$ & $<0,0001$ & $8 / 25(32 \%)$ \\
\hline Renal anomaly & $\begin{array}{l}46 / \\
313(15 \%)\end{array}$ & $14 / 48(29 \%)$ & $\begin{array}{l}32 / \\
265(12 \%)\end{array}$ & 0,0003 & $5 / 25(20 \%)$ \\
\hline Macrosomia & $\begin{array}{l}12 / \\
313(4 \%)\end{array}$ & $6 / 48(13 \%)$ & $\begin{array}{l}6 / \\
265(2 \%)\end{array}$ & 0,001 & $2 / 25(8 \%)$ \\
\hline $\begin{array}{l}\text { Mild } \\
\text { ventriculomegaly }\end{array}$ & $\begin{array}{l}13 / \\
313(4 \%)\end{array}$ & $7 / 48(15 \%)$ & $\begin{array}{l}6 / \\
265(2 \%)\end{array}$ & 0,0001 & $0 / 25(0 \%)$ \\
\hline $\begin{array}{l}\text { Short long } \\
\text { bones }\end{array}$ & $\begin{array}{l}18 / \\
313(6 \%)\end{array}$ & $8 / 48(17 \%)$ & $\begin{array}{l}10 / \\
265(4 \%)\end{array}$ & 0,0004 & $1 / 25(4 \%)$ \\
\hline $\begin{array}{l}\text { Only one US } \\
\text { finding }\end{array}$ & $\begin{array}{l}61 / \\
313(19 \%)\end{array}$ & $2 / 48(4 \%)$ & $\begin{array}{l}59 / \\
265(22 \%)\end{array}$ & 0,004 & $6 / 25(24 \%)$ \\
\hline $\begin{array}{l}\text { Two or more US } \\
\text { findings }\end{array}$ & $\begin{array}{l}252 / \\
313(81 \%)\end{array}$ & $46 / 48(96 \%)$ & $\begin{array}{l}254 / \\
265(78 \%)\end{array}$ & & $7 / 25(28 \%)$ \\
\hline No US finding & N/A & N/A & N/A & - & $12 / 25(48 \%)$ \\
\hline
\end{tabular}

a : chi-square statistic or Fisher exact test, as appropriate. $\alpha ; ;$; error $=$ $0,05, \mathrm{~b}: \mathrm{t}$-student test, ${ }^{*}$ without another finding, except increased NT

Table 2 Diagnostic yield of prenatal ultrasound findings

\begin{tabular}{|c|c|c|c|c|}
\hline Prenatal findings & $\begin{array}{l}\text { Diagnostic yield for NSD } \\
\text { according to indications } \\
\text { for testing (51 NSD) }\end{array}$ & $\begin{array}{l}p- \\
\text { value }^{a}\end{array}$ & $\begin{array}{l}\text { Diagnostic yield for } \\
\text { NSD according to } \\
\text { prenatal US findings } \\
\text { (48 NSD) }\end{array}$ & $p$-value ${ }^{a}$ \\
\hline & $\mathrm{n}=353$ & & $\mathrm{n}=313$ & \\
\hline $\begin{array}{l}\text { Global diagnostic } \\
\text { yield }\end{array}$ & $14 \%(51 / 353)$ & - & $15 \%(48 / 313)$ & - \\
\hline Increased NT & $11 \%(29 / 258)$ & 0,4 & $13 \%(30 / 231)$ & 0,7 \\
\hline $\mathrm{NT}>3<3,5$ & $0 \%$ & & $0 \%$ & \\
\hline $\mathrm{NT} \geq 3,5$ & $9 \%$ & 0,02 & $12 \%$ & 0,1 \\
\hline NT $>6$ & $20 \%$ & & $20 \%$ & \\
\hline alone & $1 \%$ & 0,005 & $0 \%$ & N/A \\
\hline in association & $18 \%$ & & $100 \%$ & \\
\hline $\begin{array}{l}\text { Increased NT } \\
\text { without } \mathrm{CH}\end{array}$ & $4,5 \%$ & 0,03 & $8 \%$ & 0,09 \\
\hline Cystic hygroma & $16 \%(30 / 189)$ & 0,6 & $17 \%(31 / 183)$ & 0,9 \\
\hline 1st trimester $\mathrm{CH}$ & N/A & N/A & $7 \%$ & 0,02 \\
\hline Persistent $\mathrm{CH}$ & N/A & & $22 \%$ & \\
\hline Isolated* & $9 \%$ & 0,02 & $6 \%$ & 0,0003 \\
\hline in association & $28 \%$ & & $28 \%$ & \\
\hline $\mathrm{CH}$ without $\uparrow \mathrm{NT}$ & $23 \%$ & 0,2 & $26 \%$ & 0,1 \\
\hline $\mathrm{CH}$ with $\uparrow \mathrm{NT}$ & $14 \%$ & & $15 \%$ & \\
\hline $\mathrm{NT}>3<3,5$ & - & N/A & $0 \%$ & 0,08 \\
\hline $\mathrm{NT} \geq 3,5$ & - & & $12 \%$ & \\
\hline NT $>6$ & - & & $26 \%$ & \\
\hline Increased NF & $26 \%(13 / 50)$ & 0,03 & $26 \%(18 / 68)$ & 0,02 \\
\hline$N F>6$ & $22 \%$ & 0,1 & $24 \%$ & 0,1 \\
\hline $\mathrm{NF}>15$ & $60 \%$ & & $60 \%$ & \\
\hline Hydrops & $35 \%(17 / 48)$ & 0,0001 & $33 \%(20 / 60)$ & 0,0004 \\
\hline $\begin{array}{l}\text { 1st trimester } \\
\text { hydrops }\end{array}$ & N/A & N/A & $14 \%$ & 0,1 \\
\hline Persistent hydrops & N/A & & $39 \%$ & \\
\hline
\end{tabular}




\begin{tabular}{|c|c|c|c|c|}
\hline $\begin{array}{l}\text { Thoracic } \\
\text { effusions or } \\
\text { ascites }\end{array}$ & $30 \%(12 / 40)$ & 0,01 & $41 \%(22 / 54)$ & $<0,0001$ \\
\hline $\begin{array}{l}\text { Congenital heart } \\
\text { disease }\end{array}$ & $23 \%(12 / 53)$ & 0,1 & $25 \%(18 / 71)$ & 0,03 \\
\hline cardiac defect & $23 \%(11 / 48)$ & 0,002 & $23 \%(15 / 64)$ & $<0,0001$ \\
\hline $\mathrm{HCM}$ & $60 \%(6 / 10)$ & & $69 \%(9 / 13)$ & \\
\hline $\begin{array}{l}\text { combined HCM } \\
+\mathrm{CD}\end{array}$ & $100 \%(5 / 5)$ & & $100 \%(6 / 6)$ & \\
\hline \multicolumn{5}{|c|}{ Other associated findings } \\
\hline Polyhydramnios & $50 \%(8 / 16)$ & N/A & $39 \%(14 / 36)$ & $<0,0001$ \\
\hline Renal anomaly & $29 \%(4 / 14)$ & N/A & $30 \%(14 / 46)$ & 0,01 \\
\hline Macrosomia & $57 \%(4 / 7)$ & N/A & $50 \%(6 / 12)$ & N/A \\
\hline $\begin{array}{l}\text { Mild } \\
\text { ventriculomegaly }\end{array}$ & - & N/A & $54 \%(7 / 13)$ & N/A \\
\hline $\begin{array}{l}\text { Short long } \\
\text { bones }\end{array}$ & - & N/A & $44 \%(8 / 18)$ & N/A \\
\hline \multicolumn{5}{|l|}{ General findings } \\
\hline $\begin{array}{l}\text { Only one US } \\
\text { finding }\end{array}$ & $9 \%(13 / 143)$ & 0,03 & $3 \%(2 / 61)$ & 0,002 \\
\hline $\begin{array}{l}\text { Two or more US } \\
\text { findings }\end{array}$ & $18 \%(38 / 210)$ & & $18 \%(46 / 252)$ & \\
\hline
\end{tabular}

N.B. Diagnostic yield of each major US finding was compared with the global diagnostic yield for $p$-value's calculation

A. Scott: None. N. Di Giosaffatte: None. V. Pinna: None. P. Daniele: None. S. Corno: None. V. D'Ambrosio: None. E. Andreucci: None. A. Marozza: None. F. Sirchia: None. G. Tortora: None. D. Mangiameli: None. C. Di Marco: None. I. Donati: None. A. Zonta: None. E. Grosso: None. G. Mastromoro: None. P. Versacci: None. F. Pantaleoni: None. F.C. Radio: None. T. Mazza: None. L. Papi: None. T. Mattina: None. A. Giancotti: None. A. Pizzuti: None. A. Laberge: D. Speakers Bureau/Honoraria (speakers bureau, symposia, and expert witness); Modest; Sobi. M. Tartaglia: None. M. Delrue: None. A. De Luca: None.

\section{P01.107.B}

Gene ontology enrichment analysis of congenital renal agenesis-associated genes

S. Kalantari ${ }^{1,2,3}$, I. Filges ${ }^{1,2,3}$

${ }^{1}$ Medical Genetics, Institute of Medical Genetics and Pathology, University Hospital Basel, Basel, Switzerland, ${ }^{2}$ Department of Clinical Research, University Hospital Basel, Basel, Switzerland, ${ }^{3}$ University of Basel, Basel, Switzerland

Introduction: Uni- or bilateral renal agenesis (RA) is a commonly occurring major congenital anomaly impacting fetal and neonatal outcomes. Although chromosomal abnormalities, CNVs and pathogenic variants in many genes have been associated to RA, the etiology is still poorly understood. Our aim was to better understand the biological pathways involved in RA and to identify interacting candidate pathways useful for further gene identification and molecular diagnosis in clinical care when applying genome-wide sequencing.

Materials and Methods: We used Phenolyzer to obtain all genes known as causative for syndromic and nonsyndromic uni- and bilateral RA. Using ClueGO gene enrichment analysis we classified the relationship between these genes and biological processes as defined by gene ontology.

Results: We identified 289 genes described to be involved in the pathogenesis of RA when mutated. We obtained 69 groups of enriched biological processes. About $50 \%$ included pathways directly related to the development of urogenital organ tissues (e.g. metanephros, nephric duct, ureter). Furthermore, several ciliary, axis specification, hindgut development and endocrine pathways are enriched, which may relate to different clinical presentations of RA. The results also support the hypothesis that RA is part of the ciliopathy spectrum and developmentally related to dysplastic kidneys.

Conclusions: Our gene ontology analysis shows that genes representing distinct biological pathways are significantly enriched. They may relate to different clinical presentations of RA, which will lead to an improved delineation of candidate genes and molecular diagnosis of this congenital anomaly in clinical practice.

S. Kalantari: None. I. Filges: None.

\section{P01.108.C}

Is mitochondrial DNA (mtDNA) copy number in human cleavage-stage embryos a marker of embryonic quality and development, or modified by the presence of a mtDNA mutation?

J. Steffann ${ }^{1}$, K. Chatzovoulou ${ }^{1}$, N. Gigarel ${ }^{1}$, A. Mayeur ${ }^{2}$, F. Jabot-Hanin $^{3}$, S. Monnot ${ }^{1}$, L. Hesters ${ }^{2}$, N. Frydman ${ }^{2}$, J. Bonnefont ${ }^{1}$

${ }^{1}$ Hopital Necker-Enfants Malades, Paris, France, ${ }^{2}$ Hopital Antoine-Béclère, Clamart, France, ${ }^{3}$ Université de Paris, Paris, France

Mitochondria play a critical role during the early stages of human embryo development by supplying sufficient levels of energy required for embryo survival. Because mtDNA replication is silenced during early embryonic development, the presence of a large number of mtDNA copies in the mature oocyte is mandatory. In human, several studies on blastocysts day-5 embryos have paradoxically evidenced a positive correlation between high quantities of mtDNA molecules and severe conditions, like aneuploidy, poor embryo quality, and implantation failure. However, data from day-3 embryos are much more limited and contradictory, making this point a highly controversial one. 
We have quantified the mtDNA copy number $(\mathrm{CN})$ in residual DNA of 1 or 2 cells, sampled from day-3 embryos, in a context of preimplantation genetic analysis for a nonmetabolic, non-mitochondrial DNA disorder $(n=165$, control group) and for a mtDNA mutation ( $n=16$, mitochondrial group). A great dispersion of the mtDNA CN was found, among the different cells of a given embryo, and the different embryos from a given patient. A maternal effect regulating the mtDNA $\mathrm{CN}$ was observed, but remained of unknown origin. In particular, no correlation was detected between mtDNA $\mathrm{CN}$ and maternal age or ovarian reserve. mtDNA CN did not correlate with embryonic quality and ability of an embryo to implant and give a viable pregnancy. Finally, the mtDNA CN was not influenced by the presence of a mtDNA mutation. Based on these findings, mtDNA CN at day 3 of development cannot be used to select the best embryo to transfer.

J. Steffann: None. K. Chatzovoulou: None. N. Gigarel: None. A. Mayeur: None. F. Jabot-Hanin: None. S. Monnot: None. L. Hesters: None. N. Frydman: None. J. Bonnefont: None.

P01.109.A

\section{A FAST AND NON-INVASIVE METHOD TO DETERMINE FETAL RHD GENE STATUS}

\section{B. Schreiner, E. Lindström, B. Vanherberghen, A. Hedrum}

Devyser, Hägersten, Sweden

Background: Hemolytic disease of the fetus and newborn (HDFN) is a serious complication of maternal and fetal blood group incompatibility. This occurs when a Rhesus-D negative woman, carrying a Rhesus-D positive fetus during her first pregnancy, is alloimmunized against RhD antigen and carries another Rhesus-positive fetus during a subsequent pregnancy. Nowadays, HDFN is rare since anti-D prophylaxis is provided to all $\mathrm{RhD}$ negative pregnant women. However, $40 \%$ of these women carry a Rhesusnegative fetus and are administered unnecessary treatment including all risks connected to blood-based products.

Aims: We set out to develop a fast and reliable diagnostic test assessing the fetal RHD gene status from maternal blood at the end of the first pregnancy trimester to enable targeted anti-D prophylaxis.

Methods: A real-time PCR-based assay was developed to detect cell-free fetal DNA (cffDNA) in maternal blood. The assay contains primer-probe combination specific for the RHD gene and the GAPDH house-keeping gene as internal control. CffDNA from clinical samples was automatically extracted from $1 \mathrm{~mL}$ of plasma.

Results: Devyser RHD exhibits high experiment repeatability between different reagent lots. The analytical sensitivity is down to DNA concentrations corresponding to less than 7 gene copies per reaction with $95 \%$ probability.

Summary/conclusions: Devyser RHD provides a fast, non-invasive procedure to assess the fetal RHD status in Rhesus-D negative mothers. This product is validated together with an automated DNA extraction process and reaction set-up (QIASymphony AS/SP), enabling highthroughput sample analysis with low-risk for sample mixup. Various kit sizes are available offering flexibility with sample throughput.

B. Schreiner: A. Employment (full or part-time); Significant; Devyser. E. Lindström: A. Employment (full or part-time); Significant; Devyser. B. Vanherberghen: A. Employment (full or part-time); Significant; Devyser. A. Hedrum: A. Employment (full or part-time); Significant; Devyser.

\section{P01.110.B}

Diagnosis of Rothmund Thomson Syndrome by whole genome sequencing

Q. Zhou, J. Sun, T. Wang, Y. Xing, Z. Wan, X. Yang, Z. Liu, W. Wang

\section{Genomics Institute of Beijing, Shenzhen, China}

Introduction: Rothmund-Thomson Syndrome (RTS) is a rare disorder and has been described in all ethnicities, with several identified pathogenic variants. Since there is fewer than hundreds of individuals have been described, the population prevalence is unknown. The genetic features and follow-up data of individuals with RTS are limited.

Methods: We performed a whole genome sequencing (WGS) using cord blood for an infant, who had RTS like clinical features in infancy.

Results: The infant showed a developmental delay in infancy, with a small size in both height and weight. The erythema started appearing on her face at the age of three months and then spread to the entire body. Besides, she had neither eyelashes nor eyebrows, with sparse scalp hair. After analyzing the WGS data, we found a heterozygous nonsense mutation c. $2752 \mathrm{G}>\mathrm{T}$ (p.Glu918*) and a novel frameshift-insertion c.1547_1548insC (p.Ala516fs) in the gene RECQL4, which is a known pathogenic gene of RTS. Both variants had potential effects on protein synthesis or function. Further validation indicated these were compound variants inheriting from parents.

Conclusions: Taking together with the clinical characteristics and the identification of biallelic pathogenic variants in a recessive manner, we report a diagnosed infant with RTS. Our findings provide potential application of WGS for newborn to clinical diagnosis. The follow-up study can help 
to further understand the clinical features and the genotypephenotype interactions of RTS.

Grant: The Shenzhen Municipal Government of China (NO.JCYJ20170412152854656)

Q. Zhou: None. J. Sun: None. T. Wang: None. Y. Xing: None. Z. Wan: None. X. Yang: None. Z. Liu: None. W. Wang: None.

\section{P01.111.C}

Simpson-Golabi-Behmel syndrome familial case series with an emphasis on prenatal diagnosis

N. Krasovskaja ${ }^{1,2}$, A. Matulevičienè ${ }^{1,2}, K$. Šiaurytè $\dot{e}^{2,3}, K$. ${\text { Grigalioniene } \dot{e}^{1,2}, \text { L. Ambrozaityte } \dot{e}^{1,2}, \text { A. Utkus }}^{2}$

${ }^{1}$ Vilnius University Hospital Santaros Klinikos, Vilnius, Lithuania, ${ }^{2}$ Department of Human and Medical Genetics, Institute of Biomedical Sciences, Faculty of Medicine, Vilnius University, Vilnius, Lithuania, ${ }^{3}$ Faculty of Medicine, Vilnius University, Vilnius, Lithuania

Introduction: Simpson-Golabi-Behmel syndrome (SGBS) is a rare recessively inherited $\mathrm{X}$-linked condition, with less than 300 reported cases. Characteristic features of SGBS include pre/postnatal overgrowth, distinctive craniofacial features (macrocephaly, coarse face with macroglossia), visceromegaly and increased risk of neoplasia. This condition is almost always diagnosed postnatally.

Materials and methods: A 23-y/o woman with uninformative anamnesis on the 21 weeks' gestation was referred due to abnormal fetal ultrasound findings (polyhydramnios, macrocephaly, corpus callosum agenesis, abdominal visceromegaly and abnormal genitalia). The results of fetal DNA qPCR and SNP-CGH tests from amniotic fluid revealed no chromosomal abnormalities. The precise evaluation of mother's phenotype (tall stature, coarse facial features, macroglossia) and fetal scan were key to directly sequence GPC3 gene and make the correct SGBS diagnosis prenatally.

Results: Sanger sequencing from amniotic fluid revealed hemizygous pathogenic variant NM_004484.3:c.1159C $>$ T, (NP_004475.1:p.(Arg387Ter), rs122453121, CM073110) in exon 4 of the GPC3 gene, which determines SGBS. Mother was also found to be a carrier. Despite severe polyhydramnios progression on 33 weeks' gestation, condition of the fetus was manageable and, pregnancy was carried to 38th weeks. The newborn's phenotype presented with classical SGBS features.

Conclusions: An accurate evaluation of maternal phenotype and fetal scan are essential pathognomonic signs for early suspicion of SGBS. Successful prenatal diagnosis enables to make decisions on time for family and physicians about the outcomes of pregnancy and delivery.
N. Krasovskaja: None. A. Matulevičienė: None. K. Šiaurytė: None. K. Grigalionienė: None. L. Ambrozaitytė: None. A. Utkus: None.

P01.112.A

Prenatal diagnosis of skeletal dysplasias/anomalies: high diagnostic yield using microarray and sequencing data

I. Mademont-Soler ${ }^{l}$, A. Maroto ${ }^{l}, J$. Meléndez $^{2}, A$.

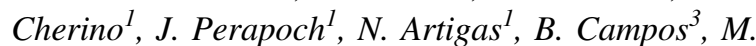
Segura $^{3}$, C. Pérez ${ }^{4}$, E. Lloveras ${ }^{4}$, J. Hernando ${ }^{1}$, X. Queralt ${ }^{1}$, M. Obón ${ }^{l}$

${ }^{1}$ Hospital Universitari de Girona Dr. Josep Trueta, Salt, Spain, ${ }^{2}$ Hospital Santa Caterina, Salt, Spain, ${ }^{3}$ Quantitative Genomic Medicine Laboratories, Barcelona, Spain, ${ }^{4}$ Synlab, Barcelona, Spain

Introduction: Skeletal dysplasias (SDs) are severe congenital disorders, mostly of genetic origin. Etiology remains unknown in $\sim 50 \%$ of cases. Materials and Methods: Nine pregnancies with ultrasound findings compatible with SDs were recruited prospectively from 2017 to 2019. A diagnostic algorithm combining microarray, Sanger and NextGeneration Sequencing was used.

Results: Definite diagnosis was achieved in 6 cases, uncertain results in 2 and negative results in 1.

\begin{tabular}{|c|c|c|c|}
\hline & Ultrasound & Genetics & Diagnosis \\
\hline $\mathrm{C} 1$ & $\begin{array}{l}\text { Skeletal anomalies, } \\
\text { oligohydramnios, } \\
\text { increased NT }\end{array}$ & $\begin{array}{l}\text { Microarray: Normal } \\
\text { WES: Normal }\end{array}$ & No \\
\hline $\mathrm{C} 2$ & $\begin{array}{l}\text { Short long bones, } \\
\text { polyhydramnios }\end{array}$ & $\begin{array}{l}\text { Microarray: Normal } \\
\text { Sanger: Pathogenic } \\
\text { variant FGFR3 }\end{array}$ & Achondroplasia \\
\hline $\mathrm{C} 3$ & $\begin{array}{l}\text { Short long bones, } \\
\text { narrow thorax }\end{array}$ & $\begin{array}{l}\text { Microarray: Normal } \\
\text { Sanger: Pathogenic } \\
\text { variant FGFR3 }\end{array}$ & $\begin{array}{l}\text { Thanatophoric } \\
\text { dysplasia }\end{array}$ \\
\hline $\mathrm{C} 4$ & $\begin{array}{l}\text { Multiple bone } \\
\text { fractures, } \\
\text { malposition of } \\
\text { extremities, skull } \\
\text { hypomineralization }\end{array}$ & $\begin{array}{l}\text { Microarray: NormalNGS } \\
\text { virtual panel: Two } \\
\text { VOUS IFT140 }\end{array}$ & Uncertain \\
\hline $\mathrm{C} 5$ & $\begin{array}{l}\text { Multiple bone } \\
\text { fractures }\end{array}$ & $\begin{array}{l}\text { Microarray: NormalNGS } \\
\text { virtual panel: Pathogenic } \\
\text { variant COL1A1 }\end{array}$ & $\begin{array}{l}\text { Osteogenesis } \\
\text { imperfecta }\end{array}$ \\
\hline C6 & $\begin{array}{l}\text { Bilateral radial } \\
\text { aplasia }\end{array}$ & $\begin{array}{l}\text { Microarray: arr } \\
\text { [GRCh37] 1q21.1 } \\
\text { (145415156_145899418) } \\
\text { x1Sanger: Pathogenic } \\
\text { variant RBM8A }\end{array}$ & TAR syndrome \\
\hline $\mathrm{C} 7$ & $\begin{array}{l}\text { Bowing of long } \\
\text { bones, } \\
\text { oligohydramnios }\end{array}$ & $\begin{array}{l}\text { Sanger: Two pathogenic } \\
\text { variants LIFR }\end{array}$ & $\begin{array}{l}\text { Stüve- } \\
\text { Wiedemann } \\
\text { syndrome }\end{array}$ \\
\hline
\end{tabular}




\begin{tabular}{|c|c|c|}
\hline C8 Arthrogryposis & $\begin{array}{l}\text { Microarray: Multiple } \\
\text { LOH regionsSanger: } \\
\text { Two pathogenic } \\
\text { variants SLC6A9 }\end{array}$ & $\begin{array}{l}\text { Glycine } \\
\text { encephalopathy } \\
\text { with normal } \\
\text { serum glycine }\end{array}$ \\
\hline C9 Arthrogryposis & $\begin{array}{l}\text { Microarray: } \\
\text { NormalWES: One } \\
\text { VOUS PIEZO2 }\end{array}$ & Uncertain \\
\hline
\end{tabular}

Conclusions: A combination of molecular cytogenetics and sequencing offers a high diagnostic yield (6/9), facilitating genetic counselling and clinical management. An individualized approach, multidisciplinary teams and the contribution of new sequencing technologies to diagnosis are recognized.

I. Mademont-Soler: None. A. Maroto: None. J. Meléndez: None. A. Cherino: None. J. Perapoch: None. N. Artigas: None. B. Campos: None. M. Segura: None. C. Pérez: None. E. Lloveras: None. J. Hernando: None. X. Queralt: None. M. Obón: None.

\section{P01.114.C}

\section{Embryo haplotype phasing based on non-invasive procedures}

L. A. Alcaraz ${ }^{1}$, C. Pérez-Pelegrín ${ }^{1}$, S. González-Reig ${ }^{1}, P$.

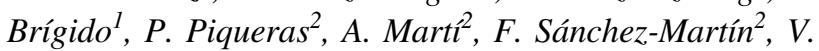
Penacho $^{l}$

\section{${ }^{1}$ Bioarray, SL, Elche, Spain, ${ }^{2}$ Ginemed, Sevilla, Spain}

Introduction: Preimplantation genetic testing (PGT) represents a widely applied genetic procedure for many IVF centers. Nowadays, for these purposes embryo material is needed. The removal of three cells in a day 5 embryo, represents an invasive procedure that could concern the embryo viability and increase the cost of a PGT. In the past few years there have been an increasing interest in the possibility of obtaining reliable DNA in the spent culture media (SCM) where the embryo is growing $(1,2)$.

Materials and Methods: For optimal PGT-M results, we aim to compare embryo biopsy and SCM. After that, we want to go further: discern whether the SCM is even a better choice to diagnose an embryo in comparison with the trophectoderm biopsy, comparing a complete embryo. All based on next generation sequencing (NGS) with PGDSeq $^{\mathrm{TM}}$ (Journey Genomics S.L.U.) technology.

Results: We can confirm a high coincidence of SCM with complete embryo and the embryo biopsy. Tis primary results are promising, even if there are less polymorphisms obtained from this kind of material, we were able to study the haplotypes and the inheritance of the embryos.

Conclusions: A non-invasive perspective on the PGT world is certainly to be the next generation of samples.
Bibliography:1. Huang, L. et al. Noninvasive preimplantation genetic testing for aneuploidy in spent medium may be more reliable than trophectoderm biopsy. Proc. Natl. Acad. Sci. 116, 14105-14112 (2019).2. Li, P. et al. Preimplantation Genetic Screening with Spent Culture Medium/ Blastocoel Fluid for in Vitro Fertilization. Sci. Rep. 8, 9275 (2018).

L.A. Alcaraz: None. C. Pérez-Pelegrín: None. S. González-Reig: None. P. Brígido: None. P. Piqueras: None. A. Martí: None. F. Sánchez-Martín: None. V. Penacho: None.

\section{P01.115.A}

Sperm protamine mRNA ratio in relation to DNA fragmentation and semen analysis

M. A. Ishchuk ${ }^{1,2}$, E. M. Komarova ${ }^{2}$, O. V. Malysheva ${ }^{2}$, I. D. Mekina', E. A. Lesik2, A. M. Gzgzyan ${ }^{2,1}$, V. S. Baranov $^{2,1}$, I. Y. Kogan ${ }^{2}$

${ }^{1}$ St. Petersburg State University, Saint-Petersburg, Russian Federation, ${ }^{2}$ The Research Institute of Obstetrics, Gynecology and Reproductology named after D.O.Ott, SaintPetersburg, Russian Federation

Introduction: Sperm contain a complex population of RNA, and the utility of sperm RNA in fertility research are currently being explored. During mammalian spermatogenesis, the chromatin structure undergoes substantial condensation. The key role in this process is played by protamines 1 and 2 (PRM1, PRM2). Abnormal condensation could lead to DNA breaks, which is linked to pregnancy loss after assisted fertilization, disturbances in embryo development and increased risk of abortions. The aim of this study was to estimate the relations between protamine mRNA ration and semen quality and further DNA fragmentation. Finding sperm-quality markers would help to understand the causes of male infertility and to improve the male reproductive health.

Materials and Methods: Human ejaculates were obtained from 33 patients and semen analyses were assessed by WHO criteria (2010). We evaluated the sperm DNA fragmentation measured by TUNEL assay. Patient's ejaculates were purified by density-gradient centrifugation, sperm cells were lysed, mRNA extracted, reverse transcribed and subjected to real-time qPCR using specific primer pairs for protamine- 1 and protamine- 2 .

Results: The sperm protamine mRNA ratio in normozoospermic men $(n=19 ; 2,86 \pm 0,67)$ differed significantly from that of patozoospermic patients $(n=14 ; 3,43 \pm 0,79$; $\mathrm{p}<0,05)$. A significant correlation was shown between sperm DNA fragmentation and the PRM2/PRM1 mRNA ratio $(r=0,33 ; p<0,05)$. In the group of patients with an 
increased sperm DNA fragmentation $(n=14 ; 3,26 \pm 0,19)$ the PRM2/PRM1 ratio was significantly higher than in the group of patients with normal rates $(n=19 ; 2,74 \pm 0,18 ; \mathrm{p}$ $<0,05)$.

Conclusions: Abnormal sperm protamine ratio associated with poor semen quality and DNA fragmentation.

M.A. Ishchuk: None. E.M. Komarova: None. O.V. Malysheva: None. I.D. Mekina: None. E.A. Lesik: None. A.M. Gzgzyan: None. V.S. Baranov: None. I.Y. Kogan: None.

\section{P01.116.B}

Investigating PHF13 induced infertility through single cell transcriptomics and non-vertebrate model organisms

\author{
E. Bosch ${ }^{l}$, S. Lukassen ${ }^{2}$, A. Gregor ${ }^{l}$, A. B. Ekici ${ }^{l}, C$. \\ Zweier $^{l}$, A. Winterpacht ${ }^{l}$
}

${ }^{1}$ Institute of Human Genetics, FAU Erlangen-Nürnberg, Erlangen, Germany, ${ }^{2}$ Digital Health Center, Berlin Institute of Health (BIH) and Charité, Berlin, Germany

Infertility is a common medical problem worldwide. Of all infertility cases, approximately $40-50 \%$ are due to "male factor" infertility, mostly caused by spermatogenic failure. Despite extensive research, there is still limited knowledge of the genetic causes and underlying molecular mechanisms. We previously showed that deficiency for the epigenetic reader PHF13 in the mouse results in testis hypoplasia due to a spermatogenic defect.

Here, we obtained single-cell RNA expression profiles from $P h f 13^{-/-}$and $P h f 13^{+-}$testis and compared them with wild type data. The results revealed a loss of meiotic sex chromosome inactivation (MSCI) in pachytene spermatocytes of PHF13 deficient mice. This is remarkable, since canonical PHF13 is only expressed in spermatogonia. In combination with ChIPseq data demonstrating an enrichment of PHF13 binding sites on the X-and Y-chromosome, we hypothesize that PHF13 mediates epigenetic changes on the sex chromosomes in spermatogonia, rendering the chromosomes competent for later inactivation.

PHF13 is evolutionarily conserved down to Bilateria including sea urchin, acorn worm and several insects like the flour beetle (Tribolium), but strikingly not in the evolutionary "young" genus Drosophila. Consequently, we ubiquitously overexpressed the Tribolium Phfl3-homolog in D. melanogaster and compared these lines with wild type lines and lines overexpressing neuronal control genes. Phf13-overexpressing strains but not the control strains show a high grade of infertility in seemingly normal flies. This strongly indicates a conserved and very specific function of this protein in germ cell development, making
PHF13 an interesting infertility factor and a fundamental player in germ cell differentiation.

E. Bosch: None. S. Lukassen: None. A. Gregor: None. A.B. Ekici: None. C. Zweier: None. A. Winterpacht: None.

\section{P01.118.A}

Telomere length in karyotypically normal and abnormal chorion in progressing pregnancies and embryonic losses

A. V. Tikhonov ${ }^{1}$, M. I. Krapivin ${ }^{l}$, A. V. PetrovskaiaKaminskaia $^{1}$, A. A. Smirnova ${ }^{2}$, Y. M. Sagurova ${ }^{3}$, O. A. Efimova $^{l}$, A. A. Pendina ${ }^{l}$, O. G. Chiryaeva ${ }^{l}$, L. I. Petrova ${ }^{l}$, V. S. Dudkina ${ }^{l}$, V. S. Baranov ${ }^{l, 3}$

${ }^{1}$ D.O. Ott Research Institute of Obstetrics, Gynecology and Reproductology, St. Petersburg, Russian Federation, ${ }^{2}$ St. Petersburg State Pediatric Medical University, St. Petersburg, Russian Federation, ${ }^{3}$ St. Petersburg State University, St. Petersburg, Russian Federation

Telomere length (TL) is considered to be an indicator of biological age. Its correct regulation during pregnancy is crucial for embryonic development. The possible contribution of TL to the developmental capacity of embryos with abnormal karyotype remains unknown so far. Here, we studied TL in karyotypically normal and abnormal chorion in progressing pregnancies and embryonic losses. The chorionic villus samples were obtained by chorion biopsy from 15 patients with progressing first trimester pregnancy referred to invasive prenatal diagnosis and by curettage from 15 patients with missed abortion. The karyotypically abnormal samples included trisomy 21 and trisomy 16 cases in both progressing pregnancies $(n=7)$ and missed abortion $(n=7)$. The telomeric regions were detected on direct metaphase preparations by qFISH. Relative TLs were measured by dividing telomeric by subtelomeric fluorescence intensity assessed in ImageJ1.49v. The results were compared by the Mann-Whitney U-test. In progressing pregnancies, the relative TL was higher in karyotypically abnormal samples compared to karyotypically normal ones ( $\mathrm{p}<0.0001$ ). In missed abortions, we observed the same tendency although the difference did not reach statistical significance $(p=0.4652)$. When comparing the relative TL between the samples with normal karyotype in progressing pregnancies and missed abortions, we revealed no difference $(p=0.8890)$. However, the relative TL was higher in the samples with abnormal karyotype in progressing pregnancies compared to those in missed abortions $(\mathrm{p}=0.0013)$. Our results suggest that in the case of karyotype abnormality, longer telomeres in chorionic villi may contribute to pregnancy success. Supported by RSF №19-75-00023. 
A.V. Tikhonov: None. M.I. Krapivin: None. A.V. Petrovskaia-Kaminskaia: None. A.A. Smirnova: None. Y.M. Sagurova: None. O.A. Efimova: None. A.A. Pendina: None. O.G. Chiryaeva: None. L.I. Petrova: None. V.S. Dudkina: None. V.S. Baranov: None.

\section{P01.120.C}

Telomere length of maternal and paternal chromosomes in human triploid zygotes

M. I. Krapivin ${ }^{l}$, A. A. Pendina ${ }^{1}$, O. A. Efimova ${ }^{1}$, I. D. Mekinal, E. M. Komarova', Y. M. Sagurova $^{2}$, I. V.

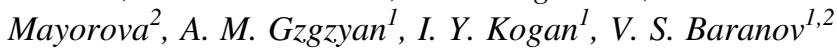

${ }^{1}$ D.O. Ott Research Institute of Obstetrics, Gynecology and Reproductology, St. Petersburg, Russian Federation, ${ }^{2}$ Saint Petersburg State University, St. Petersburg, Russian Federation

Telomeres are complex structures of tandem DNA repeats and proteins at chromosome ends. Telomere shortening due to cell divisions, oxidative stress or genetic effects may result in end-to-end chromosome joining, their degradation, and cell death. In contrast to short telomeres in oocytes, sperm telomeres become longer with age. Telomere length (TL) correlates with embryo developmental potential advocating the importance of TL analysis after fertilization. The study was performed on metaphase chromosomes of 23 triploid human zygotes from 20 couples (women aged $32.04 \pm 0.8$; men aged $34.15 \pm 1.2$ ) referred for in vitro fertilization. Paternal (sperm-derived) chromosomes were identified immunocytochemically by weak DNA methylation and strong hydroxymethylation which contrasted them with strongly methylated and poorly hydroxymethylated maternal (oocyte-derived) chromosomes. After qFISH, relative TL was measured by dividing telomeric by subtelomeric fluorescence assessed in ImageJ1.49v. The relative TL was significantly higher in the paternal compared to the maternal chromosomes (Wilcoxon signed-rank test, $\mathrm{p}<$ 0.0001). Rare zygotes demonstrated the opposite pattern: higher TL in the maternal chromosomes. However, they seem to be the exception rather than the rule suggesting that some cases of developmental arrest may be caused by altered TL pattern. Neither in sperm- nor in oocyte-derived chromosomes the relative TL correlated with paternal $(\mathrm{r}=$ $0.058 ; \mathrm{p}=0.736)$ or maternal age $(\mathrm{r}=0.155 ; \mathrm{p}=0.44)$, respectively. Thus, after fertilization, TL in paternal chromosomes is "reprogrammed". Being hypomethylated, paternal chromosomes are prone to recombination and, thus, to alternative lengthening of telomeres, which may be part of developmental program and explains the absence of correlation of TL in sperm-derived chromosomes with paternal age. Supported by RSFNo18-75-10046.
M.I. Krapivin: None. A.A. Pendina: None. O.A. Efimova: None. I.D. Mekina: None. E.M. Komarova: None. Y.M. Sagurova: None. I.V. Mayorova: None. A.M. Gzgzyan: None. I.Y. Kogan: None. V.S. Baranov: None.

P01.121.A

Transcriptomics of placental tissue: identification of potential biomarkers and biological pathways in the great obstetrical syndromes

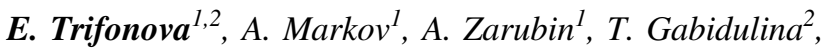 V. Stepanov ${ }^{1}$}

${ }^{1}$ Research Institute of Medical Genetics, Tomsk National Research Medical Center of the Russian Academy of Sciences, Tomsk, Russian Federation, ${ }^{2}$ Siberian State Medical University, Tomsk, Russian Federation

The great obstetrical syndromes (GOS) are common pregnancy complications that can have detrimental effects on morbidity and mortality of the mother and fetus as well as long-term health outcomes. Although they are distinct conditions, they are often considered together as they share a common etiology of inadequate placental perfusion. The aim of the work was to search for new genetic markers of GOS on the basis of integrative analysis of transcriptomic data. Genome-wide expression profiling was performed on placental tissue from preeclamptic and normal $(n=47)$ pregnancies. Thirty original datasets from patients with preterm birth, intrauterine growth restriction, preeclampsia $(n=434)$ were downloaded from Gene Expression Omnibus and were further integrated and analyzed with our data. Subsequently, 5023 differentially expressed genes (DEGs) between GOS patients and healthy women were identified. We found that the transcriptional activity of 64 DEGs changes in at least two GOS diseases. Weighted gene co-expression network analysis identified distinct gene modules associated with preterm birth, intrauterine growth restriction or preeclampsia. Using Gene Set Enrichment Analysis we identified the significant role of disturbance of intercellular interactions and regulation of proteins modification in placental tissue during the development of the GOS. We applied upstream analysis approach implemented in geneXplain platform and identified master regulators (MECOM, CDC34, UBE2E1, UBE2D4, STAT3 and $U B E 2 R 2)$ that are new therapeutic targets. These key genes may be potential biomarkers of diagnosis, therapy and prognosis for GOS. This work was supported by the Russian Foundation for Basic Research (grant No. 18-2913045, No. 18-44-700007).

E. Trifonova: None. A. Markov: None. A. Zarubin: None. T. Gabidulina: None. V. Stepanov: None. 


\section{P01.123.C}

The importance of trio WES integration into prenatal diagnosis

C. Rodriguez-Solera ${ }^{l}$, M. Martinez-Garcia ${ }^{l}$, I. Diez ${ }^{l}$, I. Sanchez-Navarro $^{l}$, E. Barrosol ${ }^{l}$, D. Rodriguez ${ }^{l}$, P. Maietta ${ }^{l}$, G. Martin-Serrano ${ }^{1}$, S. Izquierdo ${ }^{2}$, M. Miramar ${ }^{2}$, A. Rodriguez $^{2}$, F. Diaz-Flores ${ }^{3}$, M. Zeleniuc ${ }^{4}$, L. Martorell $^{5}$, J.

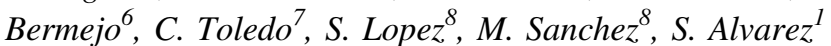

${ }^{1}$ NIMGenetics S.L, Madrid, Spain, ${ }^{2}$ Hospital Universitario Miguel Servet, Zaragoza, Spain, ${ }^{3}$ Hospital Universitario de Canarias, Tenerife, Spain, ${ }^{4}$ Personal Genetics, Bucharest, Romania, ${ }^{5}$ Hospital Sant Joan de Déu, Barcelona, Spain, ${ }^{6}$ Hospital General de Segovia, Segovia, Spain, ${ }^{7}$ Hospital Virgen de la Salud, Toledo, Spain, ${ }^{8}$ Hospital Clínico de Santiago, Santiago de Compostela, Spain

Background: Exome sequencing has proved to be a useful diagnostic tool when fetal structural anomalies suggest a genetic etiology, but other standard prenatal genetic tests did not provide a diagnosis. We describe a single laboratory experience with prenatal and fetal samples.

Material and Methods: This study includes 13 samples with ultrasound images suggesting skeletal abnormalities or rasopathies, among others. The samples origin were amniotic fluid (69\%) and fetal tissues (31\%). In six cases a whole exome sequencing with a trio analysis was performed. The genomic libraries were generated using SureSelectXT and sequenced on a Novaseq 6000. Data alignment, calling and filtering was performed using an inhouse developed pipeline. Informed consent was obtained from all participants.

Results: A molecular diagnosis was obtained on 1 amniotic fluid prenatal sample and on 1 fetal specimen (15\% global diagnostic yield). All positive cases were obtained after trio analysis. Two pathogenic variants in the ESCO2 gene associated with Roberts syndrome were identified in the prenatal sample and one homozygous variant in the $A S P M$ gene, associated with a primary microcephaly 5 , was identified in fetal tissue. Both variants were cosegregated with the disease. Ultrasound studies did not suggest the candidate genes due to the insufficient and nonspecific information about the prenatal clinical presentation of these syndromes.

Conclusion: This study highlights the importance of trio WES integration into prenatal diagnosis. Compared to ultrasound studies alone, prenatal molecular diagnoses led to a refined recurrence risk estimation, altered medical management and allows an informed reproductive planning for families.

C. Rodriguez-Solera: None. M. Martinez-Garcia: None. I. Diez: None. I. Sanchez-Navarro: None. E.
Barroso: None. D. Rodriguez: None. P. Maietta: None. G. Martin-Serrano: None. S. Izquierdo: None. M. Miramar: None. A. Rodriguez: None. F. Diaz-Flores: None. M. Zeleniuc: None. L. Martorell: None. J. Bermejo: None. C. Toledo: None. S. Lopez: None. M. Sanchez: None. S. Alvarez: None.

\section{P01.125.B}

Pregnancy outcome after failure of emergency contraception with ulipristal acetate - an observational study

\section{Hoeltzenbein, J. K. Wagner, C. Schaefer, K. Dathe}

Pharmakovigilanzzentrum Embryonaltoxikologie, Institut für Klinische Pharmakologie und Toxikologie, CharitéUniversitätsmedizin Berlin, Berlin, Germany

Introduction: Ulipristal acetate (UPA) is a selective progesterone receptor modulator used for emergency contraception (EC) after unprotected sexual intercourse. Data on pregnancy outcome after failure of EC is very limited.

Material and Methods: Observational study of prospectively ascertained pregnancies after UPA exposure for EC. In addition, retrospectively reported pregnancy outcomes were evaluated separately.

Results: Among 216 requests to the German Embryotox institute related to EC with UPA, 95 concerned prospectively ascertained pregnancies after failed EC. Follow-up data on pregnancy outcome was obtained in 56 pregnancies: 7 spontaneous abortions, 12 terminations of pregnancy (ETOP) and 37 live births. Major birth defects were not reported. Only $34 \%$ of women had taken UPA during the fertile window. Among the 4 retrospectively reported pregnancies there was one child diagnosed with Beckwith-Wiedemann-syndrome (BWS) due to hypomethylation of IC2.

Conclusions: We provide a preliminary basis for reassuring women who wish to carry their pregnancy to term after failed EC with UPA. However, due to the retrospective case report of a child with BWS after maternal UPA exposure possible epigenetic effects could not be excluded and require further evaluation. As ovarian stimulation alone (independent of artificial reproductive techniques) was previously discussed as a predisposing factor for disturbance of imprinting maintenance, UPA might have influenced preimplantation development and disturbed epigenetic processes.

This work was performed with financial support from the German Federal Institute for Drugs and Medical Devices (BfArM).

M. Hoeltzenbein: None. J.K. Wagner: None. C. Schaefer: None. K. Dathe: None. 
P01.126.C

Chromosomal microarray confirms a rare prenatal case of WAGR syndrome

M. Shetty, P. Venkatesh, S. Menezes, S. Hegde

Manipal Hospital, Bangalore, India

Introduction: WAGR syndrome is a rare genetic disorder with prevalence of 1 in 500,000 to 1 million. It affects many body systems and is named for its main features: wilms tumor, aniridia, genitourinary anomalies and range of developmental delays. Children with WAGR syndrome present with aniridia, genito-urinary defects in the new born period and are at an increased risk for developing malignancies, varying range of intellectual disability and obesity in childhood. The syndrome is a contiguous gene deletion syndrome involving chromosome $11 \mathrm{p} 13$ containing WT1 and PAX6 genes.

Materials and Methods: We report a case of 29-year-old Asian (Indian ethnicity) primigravida with nonconsanguineous marriage. Her mid trimester anomaly scan at 18 weeks gestation showed bilateral ventriculomegaly, small cavum septum pellucidum and partial agenesis of corpus callosum. Amniocentesis was performed for chromosomal microarray analysis which was performed using an Agilent $8 \times 60 \mathrm{~K}$ array. The cytogenomic microarray analysis showed 4.9MB deletion in chromosome 11 (11p13-p12).

Discussion: The prenatal diagnosis of WAGR syndrome is associated with borderline ventriculomegaly, absent corpus callosum and absent cavum septum pellucidum. In order to diagnose rare syndrome, microarray should be the first choice of investigation in the presence of any structural abnormality. This would help in understanding the prenatal presentation, potential co-morbid condition, counseling and accordingly decision making.

Conclusions: The clinical association of WAGR syndrome in postnatal period is well established but the antenatal presentation is not well-understood and reported twice in the literature earlier. Microarray study confirms these rare non-classical prenatal manifestations of WAGR syndrome.

M. Shetty: None. P. Venkatesh: None. S. Menezes: None. S. Hegde: None.

P01.127.A

Whole Exome Sequencing in 53 fetuses with abnormal ultrasound: $37 \%$ of diagnostic yield
D. Trost ${ }^{l}$, A. Boughalem ${ }^{l}$, P. Blanchet ${ }^{2}$, C. Cenni ${ }^{2}, R$. Dard $^{3}$, A. Benachi ${ }^{4}$, V. Ciorna-Monferrato ${ }^{5}$, R. Touraine ${ }^{6}$, P. Kleinfinger ${ }^{l}$, L. Lohmann ${ }^{l}$, A. Luscan ${ }^{l}$, M. Valduga ${ }^{l}, J$. Costa $^{l}$

${ }^{1}$ Laboratoire Cerba, Saint Ouen l'Aumone, France, ${ }^{2}$ Hôpital Arnaud de Villeneuve, Montpellier, France, ${ }^{3}$ Hôpital Poissy, Poissy, France, ${ }^{4}$ Hôpital Antoine Béclère, Clamart, France, ${ }^{5}$ Hôpital Metz-Thionville, Metz, France, ${ }^{6}$ Hôpital Nord, Saint Etienne, France

Introduction: Fetal structural abnormalities detected by ultrasonography, have a range of genetic causes. Chromosomal aneuploidy and structural abnormalities are explored by karyotype testing and chromosomal microarray (CGH). The utility of prenatal whole exome sequencing (WES) is discussed. Information on the interest of prenatal WES remains limited.

Methods and results: In our cohort of 53 fetuses with normal karyotype and CGH, Trio WES was performed. All fetuses had ultrasound abnormalities of poor prognosis. Most fetuses (26) were analyzed after medical termination of pregnancy, 24 analyzes were performed on ongoing pregnancies and 3 analyzes after intrauterine fetal demise. Pathogenic or likely pathogenic variants were found in 20 cases resulting in a diagnostic yield of $37 \%$. Of these, 55\% (11) were autosomal dominant with de novo variants, $45 \%$ of the diagnoses followed an autosomal recessive inheritance pattern ( 5 homozygous variants, 4 compound heterozygous variants). In urgent cases turn-around-time was 2 weeks. Reporting results for an antenatal Trio WES varies between 2-4 weeks.

Conclusions: Whole exome sequencing identified a genetic cause for the fetal anomalies in 20 of the 53 (37\%) cases analyzed. This made it possible to carry out genetic counseling for families and to offer targeted prenatal diagnosis for recessive diseases in case of a new pregnancy.

D. Trost: None. A. Boughalem: None. P. Blanchet: None. C. Cenni: None. R. Dard: None. A. Benachi: None. V. Ciorna-Monferrato: None. R. Touraine: None. P. Kleinfinger: None. L. Lohmann: None. A. Luscan: None. M. Valduga: None. J. Costa: None.

P02 Sensory Disorders (Eye, Ear, Pain)

P02.01.C

Disruption of PAX6 translation by $5^{\prime}$ UTR variants causes Congenital aniridia 
A. Filatova, T. Vasilyeva, A. Marakhonov, R. Zinchenko, M. Skoblov

Research Centre for Medical Genetics, Moscow, Russian Federation

Introduction: Congenital aniridia (AN) is a rare autosomal dominant panocular disorder caused by mutations in the PAX6 gene. Previously, we performed a molecular genetic study of a large cohort of Russian patients with AN and found six PAX6 nucleotide variants in 5'-UTR. It is known that $5^{\prime}$-UTR variants could influence the translation efficiency and cause some diseases, but for AN such a mechanism has not been previously shown. Thus, to investigate the pathogenicity of $5^{\prime}$-UTR PAX6 variants the functional studies are required.

Materials and Methods: To study 5'-UTR variants we generated luciferase constructs with full-length PAX6 5'-UTR (wt and mutants). The translation efficiency was measured by luciferase dual-assay, mRNA structure and expression level were determined by RT-PCR and qPCR respectively.

Results: Six patient-derived 5'-UTR-variants were found to lead to a significant decrease in the translation efficiency, while mRNA expression level was not significantly reduced. Three of these variants also affect splicing. Further bioinformatics analysis allows us to suggest the mechanism of 5'-UTR variants pathogenicity through disruption of an upstream ORF which possibly exists in PAX6 5'-UTR. To validate this hypothesis, we performed additional mutagenesis experiments and confirmed the essential role of uORF in the molecular pathogenesis of the disease. Moreover, we showed that this uORF normally affects the main protein translation.

Conclusions: We have identified uORF in $5^{\prime}$-UTR of the PAX6 gene and found that mutations that disrupt this uORF cause the congenital aniridia. In addition, we hypothesized that this uORF may participate in the normal regulation of PAX6 expression.

A. Filatova: None. T. Vasilyeva: None. A. Marakhonov: None. R. Zinchenko: None. M. Skoblov: None.

\section{P02.02.A}

Evaluating diagnostic challenges with $A B C A 4$-related retinal disease - experience with a 7500 IRD patient cohort sent for genetic diagnostics

J. Känsäkoski ${ }^{l}$, K. Kämpjärvi ${ }^{l}$, S. Tuupanen ${ }^{l}, K$. Wells ${ }^{l}, L$. Sarantaus $^{l}$, P. von Nandelstadh ${ }^{l}, K$. Merkkiniemi, H. Västinsalo ${ }^{l}$, E. Mårtensson ${ }^{l}$, R. Perez Carro ${ }^{l}, E$. Sankila $^{2}$, J. W. Koskenvuo ${ }^{l}$, S. Myllykangas ${ }^{1}$, T. Alastalo ${ }^{l}$

${ }^{1}$ Blueprint Genetics, Espoo, Finland, ${ }^{2}$ Helsinki University Eye Hospital, Helsinki, Finland
Introduction: $A B C A 4$ variants cause autosomal recessive Stargardt disease (STGD1), the most prevalent hereditary macular disease. Major challenges in STGD1 molecular diagnostics include (i) non-coding variants typically not targeted by available genetic tests (ii) copy number variants (CNV) missed by standard testing, and (iii) interpretation challenges with common hypomorphic variants. We evaluated the significance of challenging $A B C A 4$ variants and their contribution to STGD1 diagnostic yield in a cohort of inherited retinal disease (IRD) patients.

Materials and Methods: We included 7500 IRD patients tested at a CLIA laboratory between 2017 and 2019. Sequencing analysis, including established non-coding $A B C A 4$ variants and high resolution $\mathrm{CNV}$ detection, was done by using in-house developed and validated NGS platform.

Results: Two disease-causing ABCA4 variants were identified in 663 patients, of which $20(20 / 663 ; 3 \%)$ had an established non-coding variant and $11(11 / 663 ; 1.5 \%)$ a CNV. In 192 patients with clinically diagnosed $A B C A 4-$ related disease, only one disease-causing variant was identified. Among those, the hypomorphic allele $A B C A 4$ c.5603A $>$ T, p.(Asn1868Ile) was identified in 109 patients (109/192: 57\%), with a significantly increased allele frequency $(126 / 384=0.328)$ compared to controls in gnomAD (AF 11928/282712=0.042) (OR: 11.087, 95\% CI: $8.953-13.73, \mathrm{p}<0.001)$. The median age at genetic testing among the 109 patients was 56 years, which was significantly higher compared to 37 years among the 663 patients with two disease-causing variants.

Conclusions: Non-coding variants and CNVs contribute significantly to diagnostic yield in ABCA4-related disease. Our large dataset supports the important role of the hypomorphic allele $A B C A 4$ c.5603A $>$ T, p.(Asn1868Ile) in diagnostics of late-onset STGD1.

J. Känsäkoski: A. Employment (full or part-time); Significant; Blueprint Genetics. K. Kämpjärvi: A. Employment (full or part-time); Significant; Blueprint Genetics. S. Tuupanen: A. Employment (full or part-time); Significant; Blueprint Genetics. K. Wells: A. Employment (full or part-time); Significant; Blueprint Genetics. L. Sarantaus: A. Employment (full or part-time); Significant; Blueprint Genetics. P. von Nandelstadh: A. Employment (full or part-time); Significant; Blueprint Genetics. K. Merkkiniemi: A. Employment (full or part-time); Significant; Blueprint Genetics. H. Västinsalo: A. Employment (full or part-time); Significant; Blueprint Genetics. E. Mårtensson: A. Employment (full or part-time); Significant; Blueprint Genetics. R. Perez Carro: A. Employment (full or part-time); Significant; Blueprint Genetics. E. Sankila: A. Employment (full or part-time); Modest; Blueprint Genetics. J.W. Koskenvuo: A. Employment (full or part-time); Significant; Blueprint Genetics. 
E. Ownership Interest (stock, stock options, patent or other intellectual property); Significant; Blueprint Genetics. S. Myllykangas: A. Employment (full or part-time); Significant; Blueprint Genetics. E. Ownership Interest (stock, stock options, patent or other intellectual property); Significant; Blueprint Genetics. T. Alastalo: A. Employment (full or part-time); Significant; Blueprint Genetics. E. Ownership Interest (stock, stock options, patent or other intellectual property); Significant; Blueprint Genetics.

\section{P02.03.B}

A novel splicing variant confirms COL11A1 as a cause of autosomal dominant non-syndromic hearing loss in the DFNA37 locus

\author{
B. Vona ${ }^{l}$, A. Tropitzsch ${ }^{l}$, A. Rad ${ }^{l}$, F. Schneider ${ }^{l}, M$.
} Müller $^{l}$, T. Schade-Mann ${ }^{1}$, S. Biskup ${ }^{2}$, H. Löwenheim ${ }^{1}$

${ }^{1}$ Dept of Otolaryngology-Head \& Neck Surgery, Tuebin-
gen Hearing Research Centre, University of Tuebingen,
Tuebingen, Germany, ${ }^{2}$ CeGaT GmbH and Praxis für
Humangenetik Tuebingen, Tuebingen, Germany

Introduction: Hereditary hearing loss (HL) is a clinically and genetically heterogeneous disorder. Many genes exhibiting either non-syndromic or syndromic HL have also been identified as causing both, owing to extensive phenotypic manifestations in the form of pleiotropy. The gene COL11A1 has long been associated with autosomal dominant Marshall syndrome and Stickler syndrome type II, as well as autosomal recessive fibrochondrogenesis. Each of these syndromes have overlapping phenotypes including skeletal abnormalities, dysmorphic features, variable cleft palate, ocular and auditory phenotypes that can include mild-to-moderate $\mathrm{HL}$, and outer ear malformations. Recently, COL11A1 has been associated with autosomal dominant non-syndromic HL (DFNA37) through the genetic analysis of a large European-American family presenting a novel splice-site altering variant.

Materials and Methods: A proband from a four generation family with non-syndromic HL was ascertained. DNA from the proband was subjected to a custom-designed high-throughput sequencing panel consisting of $160 \mathrm{HL}-$ associated genes. An in vitro splice assay was performed to study the RNA-level effect of the variant.

Results: The patient was diagnosed with moderate sensorineural HL. Additional syndromic features were absent. A heterozygous variant (c.4338+2T $>$ C, p.?, NM_080629.2) was uncovered in COL11A1 that is predicted to abolish the $5^{\prime}$ splice site in exon 58 out of the 68 exons comprising COL11A1. Furthermore, this novel variant affects a highly conserved nucleotide in the alpha chain domain. In vitro splice testing confirmed abnormal splicing.

Conclusions: We report on the second DFNA37associated splice-altering variant, providing confirmatory evidence of COL11A1 as a bona fide autosomal dominant non-syndromic HL gene.

B. Vona: None. A. Tropitzsch: None. A. Rad: None. F. Schneider: None. M. Müller: None. T. Schade-Mann: None. S. Biskup: None. H. Löwenheim: None.

\section{P02.05.A}

Non-Mendelian inheritance in Bardet-Biedl syndrome: triallelism

I. Perea-Romero ${ }^{1,2}$, F. Blanco-Kelly ${ }^{1,2}$, I. Lorda-Sanchez, ${ }^{1}$, I. Sanchez-Navarro ${ }^{3}$, O. Zurita ${ }^{1,2}$, R. Riveiro-Alvarez ${ }^{1,2}, M$. Trujillo-Tiebas $^{1,2}$, A. Avila-Fernandez ${ }^{1,2}$, M. $_{\text {Corton }}{ }^{1,2}$, D. Valverde $^{4,5}$, C. Ayuso ${ }^{1,2}$

${ }^{I}$ Department of Genetics, Instituto de Investigación Sanitaria - Fundación Jiménez Díaz University Hospital Universidad Autónoma de Madrid (IIS-FJD, UAM), Madrid, Spain, ${ }^{2}$ Center for Biomedical Network Research on Rare Diseases (CIBERER), ISCIII, Madrid, Spain, ${ }^{3}$ Medical Department, NIMGenetics, Madrid, Spain, ${ }^{4}$ Department of Biochemistry, Genetics and Immunology, Biology Faculty, Vigo University, Pontevedra, Spain, ${ }^{5}$ Center for Biomedical Network Research on Rare Diseases (CIBERER), ISCIII, Pontevedra, Spain

Introduction: Bardet-Biedl syndrome (BBS) is a ciliopathy characterized by retinal dystrophy, obesity, polydactyly, cognitive impairment, renal failure and hypogonadism. Typically, it has an autosomal recessive mode of inheritance, but this heterogeneous disease has variable expressivity between and within families, which might be partially explained by oligogenic inheritance, like triallelism.

Materials and methods: A total of 61 families (78 affected cases) with an "a priori" diagnosis of BBS or BBSlike were studied using different molecular approaches (commercial genotyping microarrays, Sanger sequencing of specific genes and/or NGS technologies).

Results: The characterization rate in our cohort was $79 \%$ (48/61 families). In 5 of the families, we found a possible triallelic inheritance, involving 6 out of the 7 affected cases. Three families carried biallelic $B B S 1$ variants together with one allele in $M K K S$, one family with biallelic $M K K S$ variants and one allele in BBS5 and one additional family carried biallelic C8orf37 variants and one allele in WDPCP. The $M K K S$ (c.724G>T; p.Ala242Ser) variant was found to be part in 3 families accompanying different biallelic variants in BBS1. In one family, the triallelic case showed a more severe phenotype than the biallelic affected sibling. 
However, the phenotypic modifier effect of the triallelism could not be stablished in 4 of the families, as they were either unique cases or both affected showed triallelism.

Conclusions: Our study presents triallelism as an option in BBS diagnosis, being important to consider it in genetic counselling.

Funding: FIS (PI16/00425 and PI19/00321), University Chair UAM-IIS-FJD Genomic Medicine and FEDER (European Regional Development Fund)

I. Perea-Romero: None. F. Blanco-Kelly: None. I. Lorda-Sanchez: None. I. Sanchez-Navarro: None. O. Zurita: None. R. Riveiro-Alvarez: None. M. TrujilloTiebas: None. A. Avila-Fernandez: None. M. Corton: None. D. Valverde: None. C. Ayuso: None.

\section{P02.07.C}

Molecular genetic cause of non-syndromic congenital and juvenile cataracts in the Czech population

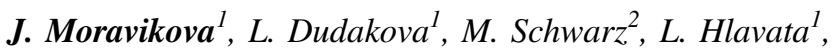
F. Malinka ${ }^{1,3}$, P. Liskova ${ }^{1,4}$

${ }^{1}$ Department of Paediatrics and Adolescent Medicine, First Faculty of Medicine, Charles University and General University Hospital in Prague, Prague, Czech Republic, ${ }^{2}$ Department of Biology and Medical Genetics, Second Faculty of Medicine, Charles University and University Hospital Motol, Prague, Czech Republic, ${ }^{3}$ Department of Computer Science, Czech Technical University in Prague, Prague, Czech Republic, ${ }^{4}$ Department of Ophthalmology, First Faculty of Medicine, Charles University and General University Hospital in Prague, Prague, Czech Republic

Introduction: Congenital and juvenile cataracts are phenotypically and genetically very heterogeneous group. In this study, we aimed to investigate the molecular genetic cause in Czech patients with bilateral congenital or juvenile cataracts not associated with other ocular or systemic clinical findings.

Materials and Methods: Whole exome sequencing was performed in 10 probands of Czech origin. Variants with minor allele frequency less than 0.005 as per GnomAD v.2 were filtered and those located in genes known to be associated with cataract development as per Cat-Map database were given a priority for further evaluation. Conventional sequencing was used to follow segregation of the presumably pathogenic variants in available first degree relatives.

Results: Out of the 10 probands 6 had a family history of congenital or infantile cataracts. In total 5 variants classified as pathogenic or likely pathogenic as per American College of Medical Genetics and Genomics guidelines were identified. Two variants were novel; c.299T>A in GJA3
(NM_021954) and c.2665G $>$ A in EPHA2 (NM_001329090). No pathogenic variants were found in 5 probands.

Conclusions: Herein we report for the first time mutational spectrum of non-syndromic cataracts in Czech patient population. The failure to identify disease-causing variants in half of the analysed cases suggests, that either the underlying mechanisms are not of genetic origin and/or that the pathogenic changes are not located in coding regions of the genome. Only two probands with family history of cataracts remained unsolved. Alternatively, larger structural variants or rearrangement could be also implicated.

Supported by AZV 17-30500A.

J. Moravikova: None. L. Dudakova: None. M. Schwarz: None. L. Hlavata: None. F. Malinka: None. P. Liskova: None.

P02.08.A

Insights into the retinal function of ciliopathy proteins gained through zebrafish models

\section{Masek, R. Bachmann-Gagescu}

University of Zurich-Medical Genetics, Zürich, Switzerland

Ciliopathies are disorders caused by dysfunction of primary cilia, ubiquitous organelles involved in signal transduction. In retinal photoreceptors (PRs), light sensation occurs in the outer segments (OSs), which represent highly specialized ciliary compartments. Consequently, retinal disease is a common manifestation in ciliopathy patients. Our efforts to elucidate the molecular functions of ciliopathy proteins rely on zebrafish mutants in genes associated with Joubert syndrome, an iconic ciliopathy associated with retinal disease in $25 \%$ of patients. We focus on genes that encode proteins participating in distinct ciliary complexes: $\mathrm{Cc} 2 \mathrm{~d} 2 \mathrm{a}$ at the transition zone, representing the gate to the cilium, or Talpid3/KIAA0586 at the basal body (BB), anchoring the cilium in the cell. While both mutants display decreased visual function, we identify distinct ultra-structural phenotypes indicating specific roles for each protein in primary cilium function. We find that $\mathrm{Cc} 2 \mathrm{~d} 2 \mathrm{a}$ organizes the vesicle fusion machinery at the periciliary margin and provides a docking point for incoming vesicles through a chain of interactions linking CC2D2A to NINL-MICAL3-RAB8. In contrast, Talpid3 is involved in BB docking and ciliogenesis upstream of Rab8a activation. Retina-specific transcriptomics identifies both shared and distinct effects on gene transcription: while transcription of phototransduction genes is decreased in both mutants, likely as a secondary effect, we observe downregulation of the BBSome, a multi- 
protein complex associated with the ciliopathy Bardet-Biedl syndrome, only in talpid3 mutants, while Nek8 is upregulated only in $c c 2 d 2 a$ mutants. In conclusion, our findings demonstrate how mutations in genes causing the same human disorder result in similar phenotypes through distinct mechanisms.

M. Masek: None. R. Bachmann-Gagescu: None.

\section{P02.09.B}

Analysis of copy number variants in a large cohort of individuals with anophthalmia, microphthalmia and coloboma

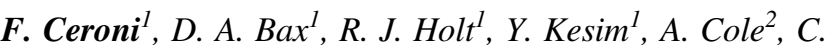
Ogilvie $^{3}$, D. Bunyan ${ }^{4,5}$, L. Cooper-Charles ${ }^{2}$, D. McMullan ${ }^{2}$, M. Griffiths ${ }^{2}$, N. K. Ragge $e^{1,2}$

${ }^{1}$ Faculty of Health and Life Sciences, Oxford Brookes University, Oxford, United Kingdom, ${ }^{2}$ West Midlands Regional Genetics Service, Birmingham Women's and Children's NHS Foundation Trust, Birmingham, United Kingdom, '3uy's and St Thomas' NHS Foundation Trust, London, United Kingdom, ${ }^{4}$ Wessex Regional Genetics Laboratory, Salisbury District Hospital, Salisbury, United Kingdom, ${ }^{5}$ Faculty of Medicine, University of Southampton, Southampton, United Kingdom

Introduction: Anophthalmia, microphthalmia and coloboma (AMC) are a genetically heterogenous collection of developmental eye disorders, with variants in $>100$ genes implicated. However, only $25-60 \%$ of patients receive genetic diagnoses. Structural variants are an important source of genetic variation, and have led to the identification of numerous eye development genes, including SOX 2 and $O T X 2$. However, few studies systematically address their contribution to AMC. Here, we investigate a large cohort of AMC families for known and novel copy number variants (CNVs).

Materials and methods: A cohort of 320 unrelated probands was screened using either diagnostic genomewide aCGH platforms $(n=156)$, including the customised OGT CytoSure 4x180K eye array, or the Illumina Infinium Global Screening Array $(n=164)$. SNP data were analysed using the algorithms QuantiSNP and PennCNV. Variants of interest were validated using $\mathrm{QPCR}$ and long-range PCR.

Results: Our analysis identified multiple variants of interest, including likely pathogenic CNVs affecting known AMC genes and rare CNVs highlighting new candidates. We describe a novel homozygous $113 \mathrm{~kb}$ deletion $19 \mathrm{~kb}$ upstream of $M A B 21 L 2$, a developmental gene involved in eye morphogenesis. This deletion removes multiple conserved cis-regulatory elements. We also identified deletions in regions with recurrent CNVs (1q21.1, 6q16, 10q11, 16p11.2, 22q11.2).

Conclusions: Our study supports the importance of CNV screening in routine diagnostic testing. It also highlights how CNVs affecting noncoding elements can provide insights into the role of regulatory regions of eye development genes in AMC. Further investigation of such variants will improve the genetic diagnostic yield and our understanding of the aetiology of these conditions.

F. Ceroni: None. D.A. Bax: None. R.J. Holt: None. Y. Kesim: None. A. Cole: None. C. Ogilvie: None. D. Bunyan: None. L. Cooper-Charles: None. D. McMullan: None. M. Griffiths: None. N.K. Ragge: None.

\section{P02.10.C}

Cataract and retinal dystrophy in $V p s 13 b^{\Delta E x 3 / \Delta E x 3}$ mice.

V. R. M. Lhussiez ${ }^{1}$, Q. Cesar ${ }^{2}$, E. Dubus ${ }^{2,3}$, M. Simonutti ${ }^{2}$, E. Lizél, S. Nguyen ${ }^{1}$, A. Geissler ${ }^{3}$, A. Bouchot ${ }^{3}$, S. Picaud ${ }^{2}$, E. F. Nandrot ${ }^{2}$, N. Acar ${ }^{4}$, L. Faivre ${ }^{1,5,6}$, C. Thauvin ${ }^{1,5,7}$, L. Duplomb $^{1,5}$, R. Da Costa ${ }^{1,5}$

${ }^{1}$ INSERM, UMR1231, Equipe GAD, Université de Bourgogne Franche Comté, Dijon, France, ${ }^{2}$ Sorbonne Université, INSERM, CNRS, Institut de la Vision, Paris, France, ${ }^{3}$ Plateforme d'Imagerie Cellulaire CellImaP/DimaCell, Inserm LNC UMR1231, Dijon, France, ${ }^{4}$ Centre des Sciences du Goût et de l'Alimentation, AgroSup Dijon, CNRS, INRAE, Université Bourgogne Franche-Comté, Dijon, France, ${ }^{5}$ FHU TRANSLAD, CHU Dijon, Dijon, France, ${ }^{6}$ Centre de Référence Anomalies du Développement et Syndromes Malformatifs, CHU Dijon, Dijon, France, ${ }^{7}$ Centre de Référence Déficiences Intellectuelles de Causes Rares, CHU Dijon, Dijon, France

Cohen syndrome (CS) is a rare genetic disorder due to variation in the VPS13B gene. It is characterized by a wide variety of clinical features that includes a typical facial dysmorphism, hypotonia, neutropenia, microcephaly, intellectual disability and severe visual impairments. In their early childhood, CS patients already suffer from myopia and a retinal dystrophy that affects both peripheral and central vision. In addition, long-term outcome studies showed that cataract reaches a high prevalence in adults with CS in their forties. To understand how VPS13B variants lead to these visual impairments and have the possibility to assess therapeutic approaches, we generated the Vps $13 b^{\Delta E x 3 / \Delta E x 3}$ mouse model. Cataract was almost systematic in 2-month-old animals. Eye fundi appeared normal until cataract development, but OCT, ERG and histological data suggest that rod homeostasis may be affected in the few $\operatorname{Vps} 13 b^{\Delta E x 3 / \Delta E x 3}$ mice without cataract after 5 months of age. Immunostaining of the lens revealed that cataract 
formation was associated with the appearance of large vacuoles in the cortical area, epithelial-mesenchymal transition and fibrosis. In later stages, cataracts became hypermature, lens capsules ruptured, and sclerotic nuclear parts dissociated. Altogether, our results show that Vps13b has a function in lens homeostasis in mice and that the $\operatorname{Vps} 13 b^{\Delta E x 3 / \Delta E x 3}$ mouse line is a useful model to study the pathomechanism leading to CS-related cataract. This work was supported with funds from the JED Fondation and from the Fondation Maladies Rares.

V.R.M. Lhussiez: None. Q. Cesar: None. E. Dubus: None. M. Simonutti: None. E. Lizé: None. S. Nguyen: None. A. Geissler: None. A. Bouchot: None. S. Picaud: None. E.F. Nandrot: None. N. Acar: None. L. Faivre: None. C. Thauvin: None. L. Duplomb: None. R. Da Costa: None.

\section{P02.12.B}

Dominant cone rod dystrophy (CORD7) previously assigned to mutation of RIMS1, is fully explained by co-inheritance of a dominant allele of PROMI

G. Arno $^{1,2}$, E. Schiff, G. Wright ${ }^{2}, N$. Waseem ${ }^{l}$, Genomics England Research Consortium, M. Michaelides ${ }^{1,2}, A . T$. Moore $^{1,2,3}$, A. R. Webster ${ }^{1,2}$

${ }^{1}$ UCL Institute of Ophthalmology, London, United Kingdom, ${ }^{2}$ Moorfields Eye Hospital, London, United Kingdom, ${ }^{3}$ University of California, San Francisco, San Francisco, CA, United States

A single missense variant (p.Arg820His) in RIMS1 has been associated with autosomal dominant cone rod dystrophy 7 (CORD-7) and enhanced cognition. This was discovered in 2003 following linkage (LOD 3.61) to a $7 \mathrm{cM}$ region on chromosome 6q, in a four generation British family in 1998. The RIMS1 gene is often included in diagnostic panels for retinal dystrophy. However, despite this, no further families with CORD7 have been reported.

Widespread availability of massively parallel sequencing has enabled detection of genomic variation at a scale not possible in the early 2000s. Access to vast datasets in gnomAD and Genomics England 100k genomes project (GE100KGP) now enables better understanding of the validity of previously accepted genetic associations.

Interrogation of the gnomAD datasets reveal 10 carriers of RIMS1 p.Arg820His (Non Finnish European allele frequency 0.0001). This would be unusual given the severity of visual impairment in adulthood and absence of the variant in further patient cohorts. Four members of the original CORD7 family were recruited to the GE100KGP, where WGS showed the two affected members to be heterozygous for a well characterised dominant allele (p.
Arg373Cys) of PROM1 located on chromosome 4p. All affected individuals subsequently were heterozygotes for this variant. Cytogenetic analysis excluded a balanced translocation that might explain co-segregation of a region on $6 \mathrm{q}$ and $4 \mathrm{p}$.

This finding suggests the rare occurrence of an alpha error despite robust linkage analysis in a large family. So far there is no evidence that perturbation of RIMS1 affects the retina in humans.

G. Arno: None. E. Schiff: None. G. Wright: None. N. Waseem: None. M. Michaelides: None. A.T. Moore: None. A.R. Webster: None.

P02.14.A

CRB1-related retinopathy superimposed on a S-adenosylhomocysteine hydrolase deficiency phenotype.

M. Di Scipio ${ }^{l}$, M. K. Grudzinska Pechhacker ${ }^{1,2}$, A. Vig ${ }^{l}, A$. Anupreet $^{l}$, E. Tavares ${ }^{l}$, A. Vincent ${ }^{1,2,3}$, E. Heon ${ }^{1,2,3}$

${ }^{1}$ Genetics and Genome Biology, The Hopsital for Sick Children, Toronto, ON, Canada, ${ }^{2}$ Department of Ophthalmology and Vision Sciences, University of Toronto, Toronto, ON, Canada, ${ }^{3}$ Department of Ophthalmology and Vision Sciences, The Hospital for Sick Children, Toronto, ON, Canada

Background: S-adenosylhomocysteine deficiency is a rare metabolic disorder for which no eye phenotype has been documented. CRB1 gene is known to cause wide spectrum of autosomal recessive retinal diseases such as Leber's congenital amaurosis, early onset cone-rod dystrophy, retinitis pigmentosa, isolated maculopathy and familial foveal retinoschisis; or autosomal dominant pigmented paravenous chorioretinal atrophy.

Materials and Methods: The present report describes a non-consanguineous family, where two children were affected with S-adenosylhomocysteine deficiency and one of them presented with reduced vision due to maculopathy. Comprehensive eye examination was performed in available family members together with color vision test, contrast sensitivity, visual fields, fundus images, OCT, full field electroretinogram, pattern electroretinogram and visual evoked potentials. Genetic testing included WES, retinal dystrophy panel and segregation analysis.

Results: Clinical whole exome sequencing revealed homozygous missense mutations in AHCY gene (c.148G $>$ A, p.Ala50Thr) as a cause of S-adenosylhomocysteine deficiency. Because of a maculopathy in the proband, retinal dystrophy gene panel sequencing revealed two heterozygous missense mutations in CRB1 gene (c.1831T $>$ C, p.Ser611Pro) and (c.3955T $>C$, p. 
Phe1319Leu) in the proband (III-1) and her mother. Mutations segregated with disease phenotype in family members.

Conclusions: Establishing an ocular genetic diagnosis may be complicated by the co-existence of a rare systemic genetic disease with previously unknown eye involvement. Extensive phenotyping and genotyping of available family members, and genotype-phenotype correlations helps to establish a diagnosis.

Grant References: Henry Brent Research Chair; Canadian Foundation Fighting Blindness; Career Development Award (Foundation fighting Blindness, USA).

M. Di Scipio: None. M.K. Grudzinska Pechhacker: None. A. Vig: None. A. Anupreet: None. E. Tavares: None. A. Vincent: None. E. Heon: None.

\section{P02.18.B}

Fundus albipunctatus in a pedigree with autosomaldominant transmission of G116R mutation in RLBPI

K. Mihova ${ }^{l}, K$. Kamenarova ${ }^{l}$, S. Cherninkova ${ }^{2}$, I. Dimova $^{l}$, F. Shakola ${ }^{1}$, V. Mitev ${ }^{l}$, R. Kaneva ${ }^{l}$

${ }^{1}$ Department of Medical Chemistry and Biochemistry, Molecular Medicine Center, Medical University of Sofia, Sofia, Bulgaria, ${ }^{2}$ Department of Neurology, University Hospital Alexandrovska, Medical University of Sofia, Sofia, Bulgaria

Introduction: Fundus albipunctatus (FA) is a rare form of congenital stationary night blindness in which white or yellow dots can be seen scattered through the fundus. Individuals complain of night blindness early in childhood without progression. By next-generation sequencing of clinical exome including all inherited retinal degeneration causative genes, we aimed to identify the disease-causing mutation in a patient with clinical signs of FA.

Materials and Methods: Using Illumina ${ }^{\circledR}$ platform and TruSight One, we identified heterozygous RLBPl-mutation in a young patient, which results in phenotype consistent with FA.

Results: A 5-years-old patient presenting ophthalmoscopic phenotype including delayed dark adaptation, aggregation of yellowish-white lesions of various sizes in the retina, and slight vascular abnormalities, was screened for mutations in all known genes previously associated with inherited retinal degeneration. We identified a heterozygous mutation, c.346G $>$ C (p.G116R), in the gene for cellular retinaldehyde-binding protein $1(R L B P 1)$, already described in a pedigree affected by autosomal-recessive FA. All other genetic variants detected in the patient's DNA were excluded after applying commonly used filtering criteria. Pedigree analysis showed the presence of p.G116R mutation in the patient's mother who was a heterozygous carrier. Mother's ophthalmological examination subsequently confirmed pre-existing stationary night blindness, prolonged dark adaptation with preserved visual acuity strongly suggesting a phenotype consistent with FA.

Conclusions: Most reported pedigrees are consistent with a recessive pattern of Mendelian inheritance of FA, although autosomal-dominant inheritance has been also reported. Our results suggest that mutation RLBP1-p. G116R may be considered as dominant-acting in pedigrees with autosomal-dominant FA.

Acknowledgements: D01-285; КП-06-Н33/12.

K. Mihova: None. K. Kamenarova: None. S. Cherninkova: None. I. Dimova: None. F. Shakola: None. V. Mitev: None. R. Kaneva: None.

P02.19.C

The G-EAR Consortium study: new candidate genes for normal hearing function (NHF) and age-related hearing loss (ARHL) in a large and deeply phenotyped cohort.

M. Brumat ${ }^{1}$, A. Morgan ${ }^{1,2}$, A. P. Nagtegaal ${ }^{3}$, B. C. Oosterloo $^{3}$, S. Seshadri, ${ }^{4,5}$, N. L. Heard-Costa ${ }^{4,6}$, G. Van

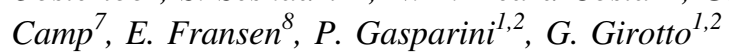

${ }^{I}$ Department of Medicine, Surgery and Health Sciences, University of Trieste, Trieste, Italy, ${ }^{2}$ Institute for Maternal and Child Health - IRCCS Burlo Garofolo, Trieste, Italy, ${ }^{3}$ Department of Otorhinolaryngology, Erasmus Medical Center, Rotterdam, Netherlands, ${ }^{4}$ Framingham Heart Study, Framingham, MA, United States, ${ }^{5}$ Glenn Biggs Institute for Alzheimer's and Neurodegenerative Diseases, University of Texas Health Sciences Center, San Antonio, TX, United States, ${ }^{6}$ Department of Neurology, Boston University School of Medicine, Boston, MA, United States, ${ }^{7}$ Center of Medical Genetics, University of Antwerp and Antwerp University Hospital, Antwerp, Belgium, ${ }^{8}$ Department of Biomedical Sciences, University of Antwerp, Antwerp, Belgium

Understanding the genetic basis of hearing traits and diseases (e.g. NHF and ARHL) is central to the development of precision medicine for the hearing impaired; to pursue this aim, fully audiometrically characterised cohorts are fundamental.

Through the international G-EAR Consortium, we united data from ten such cohorts from Europe, USA, Caucasus and Central Asia (overall $>9000$ individuals) and performed a GWAS meta-analysis on NHF and ARHL, analysing in the former case single hearing thresholds or their average value across specific frequencies, and in the latter a casecontrol phenotype (PMID:29725052). Analyses used linear 
mixed model/logistic regression; meta-analysis was performed with METAL.

Suggestive association signals were detected on chromosomes $3,8,11,16$ and 18. In particular, three genes CADM2, CALB1 and LRRC4C - result to be potentially associated with NHF and two more - $C D H 13$ and $C T I F$ with ARHL.

CADM2 encodes a protein of the nectin-like family, members of which are fundamental for the development of the inner ear; $C A L B 1$ encodes a calcium-binding protein that regulates the development of murine cochlear hair cells; LRRC4C encodes a transmembrane protein abundantly expressed in mouse inner ear. Finally, CDH13 and CTIF have been associated with NHF in previous studies, suggesting their possible role also in ARHL.

Replication is in progress in an independent cohort of $\sim 1500$ individuals, fully phenotyped; updated results will be presented. These findings provide a strong contribution to our understanding of the genetic bases of both NHF and ARHL, laying the foundation of future therapeutic targets.

M. Brumat: None. A. Morgan: None. A.P. Nagtegaal: None. B.C. Oosterloo: None. S. Seshadri: None. N.L. Heard-Costa: None. G. Van Camp: None. E. Fransen: None. P. Gasparini: None. G. Girotto: None.

\section{P02.20.A}

Further evidence of involvement of $C D C 14 A$ in autosomal recessive non-syndromic hearing loss

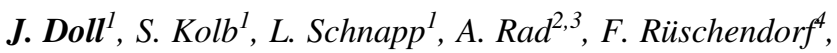
I. Khan ${ }^{5}$, A. Adli ${ }^{2}$, A. Hasanzadeh ${ }^{2}$, D. Liedtke ${ }^{1}$, S. Knaup ${ }^{l}$, M. A. H. Hofrichter ${ }^{\text {I }}$ T. Müller ${ }^{6}$, M. Dittrich ${ }^{1,6}$, I. $\mathrm{Kong}^{7}, \mathrm{H}$.

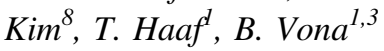

${ }^{1}$ Institute of Human Genetics, Julius Maximilians University Würzburg, Würzburg, Germany, ${ }^{2}$ Cellular and Molecular Research Center, Sabzevar University of Medical Sciences, Sabzevar, Iran, Islamic Republic of, ${ }^{3}$ Tübingen Hearing Research Centre, Department of Otorhinolaryngology, Head and Neck Surgery, Eberhard Karls University, Tübingen, Germany, ${ }^{4}$ Max Delbrück Center for Molecular Medicine in the Helmholtz Association, Berlin, Germany, ${ }^{5}$ Department of Chemistry, Bacha Khan University, Charsadda, Pakistan, ${ }^{6}$ Institute of Bioinformatics, Julius Maximilians University, Würzburg, Germany, ${ }^{7}$ Department of Animal Science, Division of Applied Life Science (BK21plus), Institute of Agriculture and Life Science, Gyeongsang National University, Jinju, Korea, Republic of, ${ }^{8}$ Neurological Disorders Research Center, Qatar Biomedical Research Institute, Hamad Bin Khalifa University, Doha, Qatar

Introduction: Hearing loss (HL) is a highly heterogeneous sensory disorder with a prevalence of 1-3 affected in 1000 newborns. Since 2016, the gene CDC14A (Cell Division Cycle 14A) is associated with autosomal recessive nonsyndromic HL (DFNB32) and hearing impairment and infertile male syndrome (HIIMS).

Materials and Methods: In order to identify likely pathogenic variants of already known and novel HL genes in Iranian and Pakistani families with sensorineural HL, exome sequencing, gene mapping approaches and bioinformatics analysis was performed. Segregation testing of variants ensued. To investigate functional consequences on the RNA-level of both loss-of-function variants and to evaluate altered expression levels, we used a minigene assay for the splice variant (c. $1421+2 \mathrm{~T}>\mathrm{C}$, p.?) and RTqPCR for the c.1041dup variant.

Results: We identified a novel homozygous CDC14A splice site variant (c.1421+2T $>C$, p.?) responsible for the disruption of a splice donor site based on in silico prediction tools. Moreover, we identified a novel homozygous frameshift variant (c.1041dup, p.Ser348Glnfs*2) in the gene $C D C 14 A$. We observed that the c. $1421+\mathrm{T}>\mathrm{C}$ variant is responsible for the activation of a cryptic splice site resulting in a truncated transcript (c.1414_1421del, p. Val472Leufs*20). Significantly reduced CDC14A mRNA levels indicated a defective transcript due to the c.1041dup variant, which is likely targeted by nonsense-mediated mRNA decay (NMD).

Conclusions: In summary, we identified and assessed the pathogenicity of two novel variants on the RNA-level and expand the spectrum of clinically relevant mutations in the gene $C D C 14 A$.

J. Doll: None. S. Kolb: None. L. Schnapp: None. A. Rad: None. F. Rüschendorf: None. I. Khan: None. A. Adli: None. A. Hasanzadeh: None. D. Liedtke: None. S. Knaup: None. M.A.H. Hofrichter: None. T. Müller: None. M. Dittrich: None. I. Kong: None. H. Kim: None. T. Haaf: None. B. Vona: None.

\section{P02.21.B}

Causes of congenital hearing loss and cochlear implant outcome

C. Landgraf ${ }^{l}$, S. von Hardenberg ${ }^{l}$, A. Lesinski-Schiedat ${ }^{2}$, A. Büchner ${ }^{2}$, B. Schlegelberger ${ }^{l}, B$. Auber ${ }^{l}$

${ }^{l}$ Department of Human Genetics, Hannover Medical School (MHH), Hannover, Germany, ${ }^{2}$ Department of Otorhinolaryngology, Hannover Medical School (MHH), Hannover, Germany

Aim: Personalize prognosis and therapy through genetic screening of potential cochlear implant patients. 
Background: Hearing loss (HL) can be divided into syndromic and non-syndromic hearing loss (NSHL, approximately $70 \%$ ). While pathogenic variants affecting the gene GJB2 account for roughly 10-30\% of NSHL, the majority of NSHL is distributed among more than 100 other genes. For many patients who do not benefit sufficiently from hearing aids, cochlear implants (CIs) are the device of choice. Performance of CI recipients is expected to depend on genetic background and a favorable outcome has been associated with pathogenic variants in genes affecting the function of the cochlear sensory organ (CSO); a negative outcome was reported for patients with variants in genes associated with spiral ganglion neuron (SGN) function.

Patients: 129 unrelated hearing impaired children and adults without pathogenic variants in $G J B 2$ and without evidence for acquired HL.

Methods: Whole exome sequencing was performed analyzing 148 genes associated with HL listed in the Deafness Variation Database and additional 681 genes listed in the Human Phenotype Ontology database based on hearing impairment.

Results: In 33 of 129 patients we identified pathogenic or likely pathogenic variants in 30 different genes, confirming the genetic heterogeneity of hereditary HL. Among these was one patient with a (homozygous) pathogenic variant in a gene proven to affect SGN function (DFNB59).

Conclusions: For meaningful results, a significantly higher number of cases is required. In addition, a further functional subdivision of patients with gene alterations influencing CSO function should be considered.

C. Landgraf: None. S. von Hardenberg: None. A. Lesinski-Schiedat: None. A. Büchner: None. B. Schlegelberger: None. B. Auber: None.

\section{P02.22.C}

In silico and in vivo analyses of novel variants identified by Whole Exome Sequencing in Argentinean deaf patients: to be or not be pathogenic

\section{P. I. Buonfiglio ${ }^{1}$, C. D. Bruque ${ }^{2}$, E. Goldschmidt ${ }^{3}, V$. Lotersztein ${ }^{4}$, S. Menazzi ${ }^{5}$, B. Paoli, P. Plazas ${ }^{7}$, A. B. Elgoyhen $^{l}$, V. K. Dalamón ${ }^{l}$}

${ }^{1}$ Instituto de Investigaciones en Ingeniería Genética y Biología Molecular "Dr. Héctor Torres" - INGEBI/ CONICET, Ciudad Autónoma de Buenos Aires, Argentina, ${ }^{2}$ Centro Nacional de Genética Médica "A.N.L.I.S. - Dr. Carlos G. Malbrán ", Ciudad Autónoma de Buenos Aires, Argentina, ${ }^{3}$ Servicio de Genética del Hospital General de Agudos "Dr. Juan A. Fernández", Ciudad Autónoma de Buenos Aires, Argentina, ${ }^{4}$ Servicio de Genética del Hospital Militar Central Cirujano Mayor "Dr. Cosme Argerich", Ciudad Autónoma de Buenos Aires, Argentina, ${ }^{5}$ Servicio de
Genética del Hospital de Clínicas "José de San Martín"., Ciudad Autónoma de Buenos Aires, Argentina, ${ }^{6}$ Servicio de Otorrinolaringología Infantil del Hospital de Clínicas "José de San Martín"., Ciudad Autónoma de Buenos Aires, Argentina, ${ }^{7}$ Tercera Cátedra de Farmacología, Facultad de Medicina, Universidad de Buenos Aires, Ciudad Autónoma de Buenos Aires, Argentina

Hereditary hearing loss (HHL) is the most common sensory disorder affecting 1 in 500 newborn children. Since HHL is related to more than 150 target genes, we designed a diagnosis strategy in order to identify pathogenic variants. A total of 1250 patients were analyzed for frequent mutations in GJB2 and GJB6 genes by Sanger Sequencing, genotyping $25 \%$ of them. From undiagnosed patients, 29 families were selected to perform Whole exome sequencing. After filtering and analysis process, $45 \%$ of patients were genotyped, identifying 23 causative mutations ( 11 novel, 12 reported) classified according to ACMG Standards. Some of the novel variants were further studied in silico by structural and stability studies of the mutated proteins. In addition, datasets from deafness and specific variant databases were correlated with different protein motifs in order to predict the theoretical pathogenicity effect of the aminoacid changes. Furthermore, knock-down phenotype rescue assays in zebrafish are underway to accomplish in vivo validation. In some cases, extensive analysis reinforced the pathogenicity prediction effect of variants and surprisingly, in one case, discouraged the deleterious effect of a genetic variant to the protein. Preliminary results in zebrafish confirmed the pathogenicity of one novel variant in the hair cell function and auditory system. This study shows that our algorithm is successful for the genetic diagnosis of deafness. Comprehensive analysis is crucial to strengthen prediction of variant pathogenicity. These findings highlight the importance of genetic studies followed by in silico and in vivo validation to better understand the genetic basis of HHL.

P.I. Buonfiglio: None. C.D. Bruque: None. E. Goldschmidt: None. V. Lotersztein: None. S. Menazzi: None. B. Paoli: None. P. Plazas: None. A.B. Elgoyhen: None. V. K. Dalamón: None.

\section{P02.23.A}

Genetic spectrum of hereditary hearing loss in multigenerational families with autosomal dominant mode of inheritance

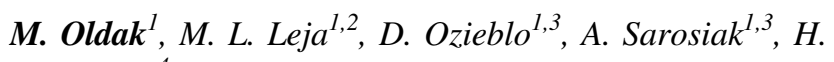
Skarzynski ${ }^{4}$

${ }^{1}$ Department of Genetics, Institute of Physiology and Pathology of Hearing, Warsaw, Poland, ${ }^{2}$ Postgraduate School of Molecular Medicine, Medical University of 
Warsaw, Warsaw, Poland, Warsaw, Poland, ${ }^{3}$ Postgraduate School of Molecular Medicine, Medical University of Warsaw, Warsaw, Poland, Warsaw, Poland, Warsaw, Poland, ${ }^{4}$ 3. Oto-Rhino-Laryngology Surgery Clinic, Institute of Physiology and Pathology of Hearing, Warsaw, Poland, Warsaw, Poland

Introduction: Hearing loss (HL) is the most common disability of human senses and genetic factors play an important role in its development. Autosomal dominant HL (ADHL) is the second most common form of inherited HL, characterized by postlingual age of onset and progression. To date 63 loci with 47 different genes were causally involved in ADHL.

Materials and Methods: Multigenerational families with ADHL were recruited for the study $(n=44)$. Genomic DNA was isolated from whole blood and buccal swabs samples. A high-throughput sequencing with custom multigene panel was performed on probands' DNA samples. Family segregation analysis of the identified variants was conducted using Sanger sequencing. All detected variants were analyzed in the context of population databases and available literature. Pathogenicity of the identified variants was predicted by different computational approaches.

Results: Genetic testing revealed probably pathogenic variants in about $52 \%$ (23/44) of the analyzed families. The majority of identified variants were novel, previously not reported and hitherto not linked to the disease. The most frequent genes with identified causative variants were MYO6, COCH, DFNA5, KCNQ4, TECTA and POU4F3.

Conclusions: Our study revealed a high involvement of novel probably pathogenic variants in the development of ADHL and confirmed a high heterogeneity of the identified genetic changes. High-throughput sequencing in $\mathrm{HL}$ patients generates large amount of data that should be interpreted carefully and confirmed by family studies. There is also a need for functional validation of the detected novel variants. Supported by: NCN Research Grant no. 2016/22/ E/NZ5/00470 SONATA BIS6

M. Oldak: None. M.L. Leja: None. D. Ozieblo: None. A. Sarosiak: None. H. Skarzynski: None.

\section{P02.24.B}

Wide spectrum of genetic hearing loss causes and large number of novel variants in cochlea implanted children

$$
\text { D. Ozieblo }{ }^{1,2}, \text { M. L. Leja }{ }^{1,2}, \text { H. Skarzynski }{ }^{3}, \text { M. Oldak }{ }^{l}
$$

${ }^{1}$ Department of Genetics, Institute of Physiology and Pathology of Hearing, Warsaw, Poland, ${ }^{2}$ Postgraduate School of Molecular Medicine, Medical University of Warsaw, Warsaw, Poland, ${ }^{3}$ Oto-Rhino-Laryngology
Surgery Clinic, Institute of Physiology and Pathology of Hearing, Warsaw, Poland

Introduction: Every year, approximately 1-6/1000 children are born with severe to profound hearing loss (HL). For this group of patients cochlear implantation (CI) is the treatment of choice. In the majority of deaf children HL is determined by DFNB1 locus pathogenic variants. Here, we aim to dissect the genetic background of HL in DFNB1-negative CI patients.

Materials and Methods: Genomic DNA was isolated from blood samples of carefully selected DFNB1-negative patients with profound prelingual $\mathrm{HL}(n=50)$ and their family members. In all probands whole exome sequencing (WES) followed by bioinformatics and expert analysis were performed. Standard Sanger sequencing was used for validation of selected variants, family segregation analysis and detection of the CEVA haplotype. Copy number variants were confirmed with qPCR.

Results: In every tested subject WES revealed at least 5 probably pathogenic variants in HL-related genes. Causative variants were identified in 33 patients and in 39\% (13/ 33 ) of them they were inherited in an autosomal dominant manner. Among the detected 53 disease-causing alleles, $55 \%(29 / 53)$ contained novel genetic alterations.

Conclusions: Obtained results show a high heterogeneity of genetic HL causes in the population of Polish DFNB1negative cochlea-implanted patients. In children with no family history of HL, the possibility of identifying causative variants inherited in both autosomal recessive and dominant manner should be taken into account. In patients with an unknown genetic cause of HL, the tested area should be expanded with whole genome analysis. Supported by NCN grant: 2017/27/N/NZ5/02369

D. Ozieblo: None. M.L. Leja: None. H. Skarzynski: None. M. Oldak: None.

\section{P02.25.C}

Diagnostic Efficiency of Clinical Exome Solution Panel in patients with Hearing loss/Hereditary Deafness by using Next Generation Sequencing

\author{
S. G. Temel $^{1,2}$, A. Alemdar ${ }^{1}$, M. Yllmaz ${ }^{3}$, L. Aliyeva $^{2}, S$. \\ Ozemri Sag ${ }^{2}$
}

${ }^{I}$ Department of Translational Medicine, Bursa Uludag University, Bursa, Turkey, ${ }^{2}$ Department of Medical Genetics, Bursa Uludag University, Bursa, Turkey, ${ }^{3}$ Faculty of Medicine, Bursa Uludag University, Bursa, Turkey

Hearing loss (HL)/Hereditary Deafness (HD) is a heterogeneous disease which multiple genes have been found to be implicated in the disease etiopathogenesis. Genetic diagnosis is highly important for HL/HD patients in order to 
ensure the etiology of the condition and counsel the patients properly. In this study, 41 patients $(22 \mathrm{M} / 19 \mathrm{~F})$ who admitted to our clinic between February 2018 and November 2019 with HL/HD were screened for 110 genes related with syndromic/non-syndromic HL/HD. NGS is considered to be the best practice for diagnosis. Large gene panels also can evaluate non-prevelant genes and candidate variants specific to populations. All exons and exon-intron boundaries were amplifed by using Clinical Exome (Sophia Genetics) Solution (CES) kit in MiSeq NGS instrument. Variant analyis (SNV, Indel and CNV) and annotations were performed by Sophia DDM platform, classified according to ACMG criteria with in-silico tools and also investigated by HGMD Professional 2019-4 and other available online bioinformatic tools. We identified $\mathrm{CNV}$ in three patients, 26 pathogenic and likely pathogenic variants in 19 of $41 \mathrm{HL} /$ HD patients (46\%) in 10 different genes. Similarly, 23 variants of uncertain significance are determined in 18 of 41 HL/HD patients (43\%) in 18 distinct genes. Besides these variations, we found 31 novel variations that are not reported before in literature or databases. Our study affirms that genetic screening of patients with HL by using CES panel not only easily diagnoses but also help counseling the patients and their family for their future risk of developing the disease.

S.G. Temel: None. A. Alemdar: None. M. Yılmaz: None. L. Aliyeva: None. S. Ozemri Sag: None.

\section{P02.26.A}

NGS approach for molecular diagnosis of hereditary hearing loss

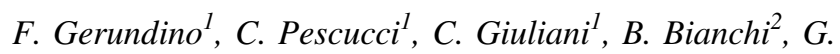
Traficante $^{3}$, S. Bargiacchi ${ }^{3}$, S. Romano ${ }^{4}, A$. Cecconi $^{5}, C . D i$ Marco $^{6}$, A. Orrico ${ }^{7,8}$, M. Mencarelli ${ }^{9}$, L. Candita ${ }^{1}, C$.

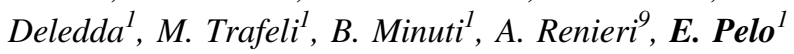

${ }^{1}$ SOD Diagnostica Genetica, AOU Careggi, Firenze, Italy, ${ }^{2}$ Audiologia SOC Otorinolaringoiatria AOU Meyer, Firenze, Italy, ${ }^{3}$ SOC Genetica Medica AOU Meyer, Firenze, Italy, ${ }^{4}$ Medicina 1, Genetica Medica AOU Pisana, Pisa, Italy, ${ }^{5}$ Ambulatorio Integrato Genetica Medica, Usl Toscana Centro, Firenze, Italy, ${ }^{6}$ Ambulatorio Integrato Genetica Medica, Usl Toscana Centro, Florence, Italy, ${ }^{7}$ Interdipartimental Program for molecular diagnosis and characterization of pathogenic mechanisms of rare genetic diseases, Siena, Italy, ${ }^{8}$ Clinical Genetics. ASL Toscana Sudest. Ospedale della Misericordia., Grosseto, Italy, ${ }^{9}$ Genetica Medica, Azienda Ospedaliera Universitaria Senese, Siena, Italy

Introduction: hearing loss (HL) is one of the most genetically heterogeneous disorders. Although pathogenic variants in GJB2 account for a large proportion of cases, many patients remain undiagnosed after DFNB1 testing. NGS analysis of HL-genes to simultaneously search for nucleotide variants and copy number variants (CNVs) greatly improve the detection rate.

Materials and methods: clinical exome sequencing followed by in silico analysis of HL-gene panel (106 genes) was carried out on 117 patients on which DFNB1 pathogenic variants were previously excluded. Eighty cases show prelingual or childhood deafness while in 37 patients HL was progressive with variable age of onset. Clinical interpretation of genetic variants was performed according to ACMG2015 by InterVar and Varsome tools and revised according to the specific guidelines for HLgenes.

Results: Forty-eight pathogenic or likely pathogenic variants and eight pathogenic CNVs (6 STRC -CATSPER2 and 2 OTOA whole gene deletions) were identified with an overall detection rate of $26.5 \%$. Specific detection rate was $32 \%(12 / 37)$ for adult cases and $24 \%$ in children. In particular, NGS identified 12 different pathogenic variants in dominant, 28 in recessive and 8 in dominant/recessive genes. The genes most involved were SLC26A4 and STRC. A single pathogenic variant in SLC26A4 gene was found in 7 cases.

Conclusions: overall detection rate of the test is in line with available data. MLPA analysis in target genes such as SLC26A4 should be performed to exclude the presence of a deletion at exonic level in cases where a single variant as been identified.

F. Gerundino: None. C. Pescucci: None. C. Giuliani: None. B. Bianchi: None. G. Traficante: None. S. Bargiacchi: None. S. Romano: None. A. Cecconi: None. C. Di Marco: None. A. Orrico: None. M. Mencarelli: None. L. Candita: None. C. Deledda: None. M. Trafeli: None. B. Minuti: None. A. Renieri: None. E. Pelo: None.

\section{P02.27.B}

Complex assessment of pathogenicity of novel variant c.516G $>C$ (p.Trp172Cys) in the $G J B 2$ gene associated with hearing loss in indigenous peoples of Southern Siberia (Russia)

\section{E. A. Maslova ${ }^{l, 2}$, M. V. Zytsar ${ }^{l}, V . Y$. Danilchenko $^{l}, K$. E. Orishchenko $^{1,2}$, O. L. Posukh ${ }^{1,2}$ \\ ${ }^{1}$ Institute of Cytology and Genetics, Novosibirsk, Russian Federation, ${ }^{2}$ Novosibirsk State University, Novosibirsk, Russian Federation}

Assessment of pathogenicity of novel variants is a primary task for molecular diagnostics of hereditary diseases. Mutations in gene GJB2 encoding transmembrane protein 
Cx26 are the common cause for hearing loss worldwide. We present a complex assessment of pathogenicity of novel non-synonymous variant c.516G $>\mathrm{C}$ (p.Trp172Cys) in GJB2 found with high frequency in deaf patients belonging to indigenous peoples of Southern Siberia (Tuvinians and Altaians).

Materials \& Methods: Genetic analysis was performed in group of deaf patients and ethnically matched controls. The GJB2 knockout HeLa cell line (obtained by CRISPR/ Cas9 system) was used to establishment of transgenic HeLa cell lines with different pathogenic GJB2-variants and GJB2-wt. Cellular Cx26 localization was investigated by immunocytochemistry (ICC). The Cx26-channels permeability was analyzed by dye (PI) loading assay.

Results: Segregation of variant c.516G $>C$ (p.Trp172Cys) with hearing loss was established in analysis of pedigrees of deaf patients and its frequency was significantly higher in patient's group than in controls. Most of in silico programs predicted a likely damaging effect of p.Trp172Cys. ICC showed predominantly cytoplasmic localization of the Cx26-p.Trp172Cys protein in contrast to Cx26-wt which represented distinct conglomerates on cell membrane. Dye (PI) loading assay revealed lower PI loading efficiencies in cells expressing mutant variant Cx26-p.Trp172Cys compared to Cx26-wt while the absence of PI accumulation was shown for other mutant Cx26-variants with known pathogenic effects.

Conclusions: Several lines of evidences support pathogenicity of novel variant c.516G >C (p.Trp172Cys) in gene GJB2 and its involving in hearing loss.

Study was supported by Project \#0324-2019-0041-C-01 and RFBR grant \#17-29-06016_ofi-m.

E.A. Maslova: None. M.V. Zytsar: None. V.Y. Danilchenko: None. K.E. Orishchenko: None. O.L. Posukh: None.

\section{P02.28.C}

\section{Genetic heterogeneity underlying hearing loss and Usher} syndrome in Saudi population

\section{K. Ramzan, F. Imtiaz, M. Al-Owain, S. Alhazzaa}

\section{King Faisal Specialist Hospital and research centre, Riyadh, Saudi Arabia}

Hearing loss is one of the most common sensory disorders in humans with both genetic and environmental etiologies. Usher syndrome (USH) is the most common cause of combined blindness and deafness. Molecular diagnosis is of great significance in revealing the molecular pathogenesis and aiding the clinical diagnosis of this disease.

Our study aims to comprehensively delineate the genetic basis of hearing loss in the individuals of Saudi Arabian origin. The identification of the causative gene in affected families with hearing loss is difficult due to extreme genetic heterogeneity and lack of phenotypic variability. Consanguineous families are a powerful resource for genetic linkage studies/homozygosity mapping for recessively inherited hearing impairment. Homozygosity mapping, linkage analysis and next generation sequencing Deafness Gene-panel and Whole Exome sequencing were conducted. Using the combined approaches, so far mutations in 32 different deafness genes including novel genes first time identified in this population, have been identified. More than 300 familial/sporadic cases were solved, including novel variants in known HL genes.

Using these innovative molecular approaches, we were able to document the most common forms of hereditary hearing loss, their incidence and distribution in the Saudi population. The overall results of this study are highly suggestive that the underlying molecular basis of hearing loss in Saudi Arabia is very genetically heterogeneous. The benefit of this study will hopefully provide the foundation for knowledge and awareness through screening of carrier status and genetic counselling, thereby having a major impact upon early intervention for and prevention of hereditary hearing loss.

K. Ramzan: None. F. Imtiaz: None. M. Al-Owain: None. S. Alhazzaa: None.

P02.29.A

ENT- Genetics Genome Boards: Over 50\% diagnostic yield

M. Carminho-Rodrigues ${ }^{1}, P$. Senn $^{2}$, M. Abramowicz ${ }^{l}$, E.

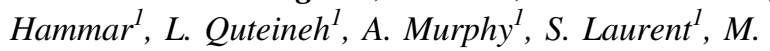
Guipponi $^{1}$, H. Cao-Van ${ }^{2}$, A. Paoloni-Giacobinol

${ }^{1}$ Medical Genetics Unit, Geneva University Hospitals, Geneva, Switzerland, ${ }^{2}$ ENT Unit, Geneva University Hospitals, Geneva, Switzerland

Introduction: Hearing impairment (HI) is the most common sensory impairment. It can be isolated or syndromic. Molecular diagnosis through whole exome sequencing (WES) can allow an improved management and surveillance of $\mathrm{HI}$ and possible associated anomalies. Here we show the results of a 3 years' experience of Ear-nose-andthroat (ENT) Genetics Genome boards.

Materials and Methods: Our cohort includes children with mild to profound, unilateral or bilateral $\mathrm{HI}$ referred by ENT clinic from 2017 to 2019. We performed WES with bioinformatics analysis of 189 genes associated with HI. The results are discussed during a genome board where geneticists and ENT specialists confront the genetic variants found by WES with the auditory phenotype of the patients. 
Results: We tested 38 children, most often with moderate, bilateral sensorineural hearing loss. The diagnostic rate was $52.6 \%$ (20/38). Among the results we found variants associated with: syndromic forms: Usher, Wolfram-like, Branchio-oto-renal, Alport, Perrault, GATA3; non-syndromic forms: Cx26, ACTG1, OTOA, ILDR1, SLC17A8, LOXHD1, TBC1D24 and STRC related HI associated or not with infertility in males.

Conclusions: The diagnostic yield of our study $(52.6 \%)$ is comparable to recent data in the literature. A genetic assessment of $\mathrm{HI}$ in young children makes the identification of the molecular cause possible, and permits to anticipate possible complications particularly in the case of syndromic forms with medical problems involving other organs or systems. Collaboration between ENT specialists and geneticists is essential to make an accurate and precise diagnosis and to personalize the follow-up and treatment of these young patients.

M. Carminho-Rodrigues: None. P. Senn: None. M. Abramowicz: None. E. Hammar: None. L. Quteineh: None. A. Murphy: None. S. Laurent: None. M. Guipponi: None. H. Cao-Van: None. A. PaoloniGiacobino: None.

\section{P02.30.B}

The utility of exome sequencing for hereditary eye diseases: single-centre experience

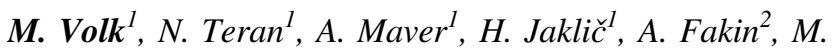
Hawlina $^{2}$, B. Peterlin ${ }^{1}$

${ }^{1}$ Institute of Genomic Medicine, UMC Ljubljana, Ljubljana, Slovenia, ${ }^{2}$ Eye hospital, UMC Ljubljana, Ljubljana, Slovenia

Introduction: Over 650 heritable conditions with ocular involvement have been described to date. These include isolated eye diseases (retinal degeneration, cataract, corneal dystrophy, optic atrophy, developmental ocular anomalies), as well as complex genetic syndromes with ocular manifestations. This group of disorders is genetically heterogeneous and diagnostically challenging. The aim of our study was to determine the clinical diagnostic utility of exome sequencing in hereditary eye diseases.

Materials and Methods: 243 Slovene unselected consecutive patients were referred to our institution from November 2013 to October 2019 because of suspected hereditary eye disease. We performed exome sequencing with the mitochondrial sequence analysis based on the offtarget exome reads. Filtered variants were analyzed according to population frequency, characterization in the ClinVar database, the putative impact of the variant, and predicted pathogenicity. Variants were classified according to ACMG standards and guidelines for variant interpretation.

Results: We found causative pathogenic or likely pathogenic variants in 102 patients. The diagnostic yield was $50 \%$ for retinal degeneration (78/156), $20 \%$ for optic atrophy (6/30), $75 \%$ for high myopia (3/4), $29 \%$ for cataract (2/7), $45 \%$ for retinoblastoma (4/9), 20\% for developmental ocular anomalies (3/15), 67\% for corneal dystrophy (2/3), and $56 \%$ for syndromic ocular disease (11/19).

Conclusions: The overall diagnostic yield for ophtalmogenetic referral in a cohort of unselected consecutive patients was $42 \%$. Therefore, the exome sequencing is a cost-effective and efficient comprehensive approach that should be considered as a first-tier investigation for diagnostics of hereditary eye diseases.

M. Volk: None. N. Teran: None. A. Maver: None. H. Jaklič: None. A. Fakin: None. M. Hawlina: None. B. Peterlin: None.

\section{P02.31.C}

Analysis of recurrent mutations in different Iranian families with hearing loss

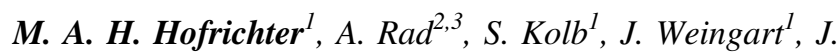

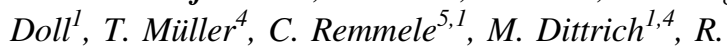

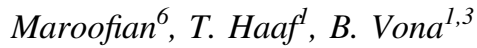

${ }^{1}$ Institute of Human Genetics, Julius Maximilians University, Würzburg, Germany, ${ }^{2}$ Cellular and Molecular Research Center, Sabzevar University of Medical Sciences, Sabzevar, Iran, Islamic Republic of, ${ }^{3}$ Department of Otorhinolaryngology, Head and Neck Surgery, Tübingen Hearing Research Centre (THRC), Eberhard Karls University, Tübingen, Germany, ${ }^{4}$ Institute of Bioinformatics, Julius Maximilians University, Würzburg, Germany, ${ }^{5}$ Center for Rare Diseases, University Hospital Würzburg, Würzburg, Germany, ${ }^{6}$ Genetics and Molecular Cell Sciences Research Centre, St George's, University of London, Cranmer Terrace, London, United Kingdom

Introduction: Hereditary hearing loss (HL) is one of the most genetically heterogeneous disorders. The genetic landscape of non-syndromic HL includes over 100 genes. In aggregate, these genes harbor several thousand causally implicated variants. The observation of recurrent variants raises the question about whether variants arose from a single ancestral allele or were the result of independent mutational events. In our study that includes 150 Iranian probands, we identified 15 families with recurrent likely pathogenic variants in the genes SMPX, SLC26A4, TECTA and TMIE.

Methods: DNA samples from index patients were exome sequenced following Illumina exome protocols. Libraries 
were sequenced with a NextSeq500 benchtop sequencer. Variant and copy number variation analysis were performed using GensearchNGS and an in-house exome analysis pipeline. Segregation analysis was performed for all families. Haplotype reconstruction allowed comparison across families.

Results: Haplotype analysis of the c.99del variant in $S M P X$ that was identified in three Iranian families suggested a common descent. Interestingly, this X-linked variant was described as a founder mutation in an isolated Newfoundland population. In addition, more than one recurrent mutation was identified in the genes SLC26A4 and TECTA in families with autosomal recessive HL that was consistent with a founder effect. In three additional families, the c. $250 \mathrm{C}>\mathrm{T}$ variant in TMIE was implicated in autosomal recessive HL without sharing further common variants, suggesting a mutational hotspot.

Conclusions: The genetic origin of recurrent mutations could be interesting for further characterization of HL genes with respect to ethnic background and susceptibility of mutational events.

M.A.H. Hofrichter: None. A. Rad: None. S. Kolb: None. J. Weingart: None. J. Doll: None. T. Müller: None. C. Remmele: None. M. Dittrich: None. R. Maroofian: None. T. Haaf: None. B. Vona: None.

\section{P02.32.A}

Genomic approaches for the analysis fo more than $\mathbf{7 0 0}$ Italian patients affected by Hereditary Hearing Loss (HHL) reveal new genes/alleles.

A. Morgan ${ }^{1,2}$, G. Pelliccione ${ }^{1}$, F. Faletra ${ }^{1}$, D. Dell'Orco ${ }^{3}$, M. Brumat ${ }^{2}$, P. Gasparini ${ }^{4,2}$, G. Girotto ${ }^{1,2}$

${ }^{1}$ Institute for Maternal and Child Health - IRCCS Burlo Garofolo, Trieste, Italy, ${ }^{2}$ Department of Medicine, Surgery and Health Sciences, University of Trieste, Trieste, Italy, ${ }^{3}$ Department of Neurosciences, Biomedicine and Movement Sciences, Section of Biological Chemistry, University of Verona, Verona, Italy, ${ }^{4}$ Institute for Maternal and Child Health - IRCCS, Burlo Garofolo, Trieste, Italy

Discerning the complex genetic basis of HHL is fundamental to facilitate a correct genetic counselling and molecular diagnosis. The use of high-throughput genome technologies has made great strides in the discovery of new alleles/genes identification. Here, 714 deeply clinically characterized patients were screened with a Targeted ReSequencing (TRS) panel of 96 deafness genes and, in negative cases, SNP-arrays were applied. Afterwards, families negative to both approaches were analysed by
Whole Exome Sequencing (WES) for new candidates identification. TRS and SNP-arrays characterized $\sim 28 \%$ of the HL cases (42\% familial and $20 \%$ sporadic cases) highlighting STRC and TECTA as the second major players in the Italian population after GJB2. Then, WES allowed the identification of five new HHL genes, two of them (SLC12A2 and USP48) just identified. As regards SLC12A2, a heterozygous missense variation (NM_001046.2, exon25, c.3431C>A, p.(T1144N)) has been identified in a three-generation HHL family. The variant is predicted as deleterious by several bioinformatic prediction tools and is not reported in any public database. Interestingly, studies in mouse and zebrafish models demonstrated Slc12a2 expression in the inner ear and its involvement in hearing loss. Moreover, to better evaluate the pathogenic effect of the identified variant, an in silico protein modelling has been performed suggesting that the substitution identified might alter the correct folding of the protein. Thanks to this approach $\sim 35 \%$ (60\% familial cases and $20 \%$ sporadic ones) of cases have been characterized confirming the large mutation's spectrum of HHL, and the importance of an accurate clinical diagnosis.

A. Morgan: None. G. Pelliccione: None. F. Faletra: None. D. Dell'Orco: None. M. Brumat: None. P. Gasparini: None. G. Girotto: None.

P02.33.B

Genetic causes of paediatric hereditary retinal diseases in Polish population

K. H. Chrzanowska ${ }^{l}$, B. Kocyła-Karczmarewicz ${ }^{2}$, A. M. Tracewska ${ }^{3}$, E. Ciara ${ }^{1}, M$. Rydzanicz ${ }^{4}$, P. Stawiński ${ }^{4}, R$. Ptoski ${ }^{4}$

${ }^{1}$ Department of Medical Genetics, The Children's Memorial Health Institute, Warsaw, Poland, ${ }^{2}$ Department of Ophthalmology, The Children's Memorial Health Institute, Warsaw, Poland, ${ }^{3}$ DNA Analysis Laboratory, PORT Polish Center for Research Technology, Wroctaw, Poland, ${ }^{4}$ Department of Medical Genetics, Medical University of Warsaw, Warsaw, Poland

Introduction: Every year, Children's Memorial Health Institute admits approximately 100 children suffering from vision impairment with suspicion of inherited retinal degeneration (IRD), which is confirmed in half of the cases. In many paediatric patients, genetic diagnosis is crucial for prognostics and future planning.

Methods: 76 probands and 22 affected family members were ascertained, and a detailed medical and family history was obtained. Ophthalmological assessment consisted, 
when feasible, of: best-corrected Snellen visual acuity (BCVA), colour vision, visual field test, dilated ophthalmoscopy, colour fundus photography, autofluorescence imaging, spectral-domain optical coherence tomography (SDOCT), fluorescein angiography and electrophysiological assessment. DNA was isolated from blood samples, and proband samples underwent targeted next-generation sequencing for 108 known IRD genes.

Results: In the vast majority of affected individuals the genetic analyses confirmed the initial diagnosis; some diagnoses, however, needed to be revised. Causal variants were found in $2 / 3$ of the families. In five cases, monoallelic pathogenic alterations were found in autosomal recessive genes. Most commonly mutated genes in this cohort were ABCA4 (23 individuals), CNGA3 (5) and CEP290 (4).

Conclusions: Genetic analysis is crucial to achieve accurate diagnosis and prognosis, which enables informed choices for the future of the affected children. Many probands were the first individual suffering from IRD in the family. An important role of genetic diagnostics is to inform the parents about their risk for having another child with the disease. Finally, emerging targeted gene therapies indicate a chance of slowing or stopping the disease progression.

Funding: National Science Centre (Poland) grant no. UMO-2015/19/D/NZ2/03193.

\section{1}

K.H. Chrzanowska: None. B. Kocyła-Karczmarewicz: None. A.M. Tracewska: None. E. Ciara: None. M. Rydzanicz: None. P. Stawiński: None. R. Płoski: None.

\section{P02.35.A}

Diagnostic exome sequencing in 50 patients with high myopia

\section{J. M. Verhoeven ${ }^{1,2}$, A. E. G. Haarman ${ }^{2,3}$, M. van Tienhoven $^{1}$, A. A. H. J. Thiadens ${ }^{2}$, S. E. Loudon ${ }^{2}$, A. de

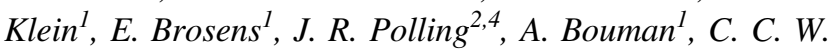 Klaver $^{5,3,2}$, A. J. A. Kievit ${ }^{1}$, L. H. Hoefsloot ${ }^{1}$}

${ }^{1}$ Dept. of Clinical Genetics, Erasmus Medical Center, Rotterdam, the Netherlands, Rotterdam, Netherlands, ${ }^{2}$ Dept. of Ophthalmology, Erasmus Medical Center, Rotterdam, the Netherlands, Rotterdam, Netherlands, ${ }^{3}$ Dept. of Epidemiology, Erasmus Medical Center, Rotterdam, the Netherlands, Rotterdam, Netherlands, ${ }^{4}$ Dept. of Orthoptics, School of Applied Science Utrecht, Utrecht, Netherlands, ${ }^{5}$ Dept. of Ophthalmology, UMC St Radboud, Nijmegen, the Netherlands, Nijmegen, Netherlands

Introduction: High myopia (HM; $\leq-6$ diopters) is a heterogeneous condition, and without clear accompanying features it can be difficult to pinpoint a genetic cause. The aim of this study was to evaluate the diagnostic yield of whole exome sequencing (WES) in patients with HM.

Materials and methods: Patients with HM were recruited by ophthalmologists and clinical geneticists. Clinical features were categorized into isolated HM, HM with other ocular features or syndromic HM with systemic involvement. WES was performed using a vision gene panel including 477 genes.

Results: WES was performed in 50 patients with HM; the majority had isolated HM (75\%). A genetic cause was identified in 11 patients (22\%; mean age at diagnosis 9.4 years (SD 7.5); mean refractive error -12.60 diopters (SD 6.22)). Four patients had mutations in known retinal dystrophy genes (GUCY2D; FAM161A; PDE6H; CAC$N A 1 F$ ); 3 female patients had a ARR3 mutation (X-linked female limited high myopia); 2 patients had homozygous or compound heterozygous COL18A1 mutations (Knobloch syndrome); 1 patient had a COL2A1 mutation (Stickler syndrome); 1 patient had a $P A X 6$ mutation. In 14 patients (28\%) a variant of unknown significance was found; followup examinations are necessary.

Conclusions: The diagnostic yield of our WES vision gene panel for $\mathrm{HM}$ was $22 \%$. It enabled us to diagnose various causes of HM, i.e. retinal dystrophies, connective tissue diseases, and non-syndromic HM, allowing appropriate follow up of systemic and ocular features and genetic counselling.

Horizon 2020 (grant 648268); NWO (grants 91815655, 91617076); Oogfonds; ODAS; Uitzicht (grant 2017-28).

V.J.M. Verhoeven: None. A.E.G. Haarman: None. M. van Tienhoven: None. A.A.H.J. Thiadens: None. S.E. Loudon: None. A. de Klein: None. E. Brosens: None. J. R. Polling: None. A. Bouman: None. C.C.W. Klaver: None. A.J.A. Kievit: None. L.H. Hoefsloot: None.

\section{P02.37.C}

Genetic architecture of inherited retinal degeneration in Germany: A large cohort study from a single diagnostic center over a 9-year period

N. Weisschuh ${ }^{l}$, C. D. Obermaier ${ }^{2,3}$, A. Bernd ${ }^{l}$, L. Kühlewein $^{1,4}$, F. Nasser ${ }^{5}$, D. Zobor ${ }^{1}$, F. Battke ${ }^{3}$, E. Zrenner $^{1,6}$, E. Weber ${ }^{l}$, B. Wissinger ${ }^{1}$, S. Biskup ${ }^{2,3}, K$. Stingl ${ }^{5}$, S. Kohl

${ }^{1}$ Institute for Ophthalmic Research, Centre for Ophthalmology, University of Tübingen, Tuebingen, Germany, ${ }^{2}$ Praxis fuer Humangenetik Tuebingen, Tuebingen, Germany, ${ }^{3} \mathrm{Cen}$ ter for Genomics and Transcriptomics, CeGaT GmbH, Tübingen, Germany, ${ }^{4}$ University Eye Hospital, Center for Ophthalmology, University of Tübingen, Tübingen, Germany, ${ }^{5}$ University Eye Hospital, Center for Ophthalmology, University of Tübingen, Tuebingen, Germany, ${ }^{6}$ Werner 
Reichardt Centre for Integrative Neuroscience (CIN), University of Tübingen, Tübingen, Germany

Introduction: The aim of this cohort study was to unravel the molecular basis of inherited retinal degeneration (IRD) in a large cohort of 2,156 affected patients diagnosed and followed-up in the largest center for inherited retinal diseases in Germany. Our study cohort accounts for approximately $7 \%$ of the estimated 30,000 IRD patients in Germany, thereby providing representative data for the prevalence and the mutation spectrum of IRD genes and mutations for the population in Germany. Presently, more than 260 'disease genes' are known to cause IRDs. The identification of the underlying disease-causing mutations has been accelerated by the introduction of next-generationsequencing technologies (NGS). Within the field of NGS platforms, the targeted capture of known 'disease genes' with its strong optimization of coverage on relevant targets has been proven superior over whole exome sequencing with respect to read depth and on-target efficiency.

Materials and Methods: DNA samples were analyzed by targeted NGS and, whenever indicated, multiplex ligation-dependent probe amplification (MLPA). Patients with single-gene disorders were analyzed by Sanger sequencing and MLPA.

Results: We achieved an overall molecular diagnostic solving rate of $70.8 \%$. A total of 1,161 distinct variants were identified, including 501 novel variants, reaffirming the known vast genetic heterogeneity of IRD in a mainly outbred European population.

Conclusions: Our study demonstrates the clinical utility of panel-based NGS in a large and highly heterogeneous cohort from an outbred population and for the first time gives a comprehensive representation of the genetic landscape of IRDs in Germany.

N. Weisschuh: None. C.D. Obermaier: None. A. Bernd: None. L. Kühlewein: None. F. Nasser: None. D. Zobor: None. F. Battke: None. E. Zrenner: None. E. Weber: None. B. Wissinger: None. S. Biskup: None. K. Stingl: None. S. Kohl: None.

\section{P02.38.A}

Retinal disease as the leading clinical manifestation in patients with unexpected inherited systemic disorders detected by whole genome sequencing

A. Liebmann ${ }^{1,2}$, P. Mazzola ${ }^{1,2}$, K. Stingl $^{3}$, T. Heinrich ${ }^{1,2}, K$. Schäferhoff $^{l, 2}$, S. Ossowski, ${ }^{1,2}$, M. Sturm ${ }^{1,2}$, S. Kohl ${ }^{4}, N$. Weisschuh $^{4}$, B. Wissinger ${ }^{4}$, O. Riess ${ }^{1,2}$, T. Haack ${ }^{1,2}$

${ }^{1}$ Institute of Medical Genetics and Applied Genomics, University of Tübingen, Tübingen, Germany, ${ }^{2}$ Centre for Rare Diseases, University of Tübingen, Tübingen,
Germany, ${ }^{3}$ University Eye Hospital, Center for Ophthalmology, University of Tübingen, Tübingen, Germany, ${ }^{4}$ Institute for Ophthalmic Research, Centre for Ophthalmology, University of Tübingen, Tübingen, Germany

Introduction: Implementation of genome-wide sequencing approaches such as exome and genome sequencing in routine diagnostics implicates the possibility of incidental findings and unexpected diagnoses. Here we present two patients, a 9-year-old girl with cone dystrophy and a 43-year old man with rod-cone dystrophy, initially referred to the Department of Ophthalmology to confirm inherited retinal disease.

Material and Methods: Genomic DNA was processed using the TruSeq DNA PCR-free Kit (Illumina) for library preparation and paired-end whole genome sequencing (WGS) was performed on a NovaSeq6000 System (Illumina). Data analysis was conducted using an in-house bioinformatics pipeline optimized for structural/copy number variant detection.

Results: WGS of the girl identified a clinically relevant homozygous deletion in $C L N 3$. The detected deletion c.461280_677+382del, p.? is the most frequent pathogenic deletion in CLN3 associated with autosomal recessive neuronal ceroid lipofuscinosis. This lysosomal storage disease is characterized by seizures, psychomotor degeneration and visual failure. Clinical course is progressive and unfavorable. WGS of the man identified a pathogenic homozygous missense variant c.635G $>\mathrm{T}$, p.Gly212Val in TMEM126B. Variants of TMEM126B are associated with autosomal recessive complex 1 deficiency. This mitochondrial disease is characterized by a variable clinical phenotype including neuromuscular, cardiovascular and metabolic symptoms and visual impairment. The detected variant has been described before in another patient with rod-cone dystrophy.

Conclusions: This study highlights the importance to inform patients about the possibility of retinal disease being the leading clinical manifestation of unexpected inherited systemic disorders. Thus, comprehensive genetic counseling and appropriate documentation of informed consent is crucial prior to performing WGS.

A. Liebmann: None. P. Mazzola: None. K. Stingl: None. T. Heinrich: None. K. Schäferhoff: None. S. Ossowski: None. M. Sturm: None. S. Kohl: None. N. Weisschuh: None. B. Wissinger: None. O. Riess: None. T. Haack: None.

P02.44.A

Shift of T-cell repertoire in cornea of keratoconus patients 
L. Skorodumova ${ }^{l}$, A. Kanygina ${ }^{l}$, A. Belodedova ${ }^{2}, E$. Sharova $^{l}$, B. Malyugin ${ }^{2}$

${ }^{1}$ Federal Research and Clinical Center of PhysicalChemical Medicine of Federal Medical Biological Agency, Moscow, Russian Federation, ${ }^{2}$ S. Fyodorov Eye Microsurgery Complex Federal State Institution, Ministry of Health of Russian Federation, Moscow, Russian Federation

Introduction: Keratoconus is a pathologic condition of the eye which results in thinning and cone-shaped deformation of cornea. While its precise cause is still unknown, keratoconus is considered to be a multifactorial disease, and immune processes are assumed to contribute to its development, yet little data is available so far on the immune microenvironment of cornea in affected patients. Here we present the assessment of $\mathrm{T}$ cell repertoire in cornea and blood samples from 3 patients with keratoconus who underwent cornea transplantation.

Materials and Methods: RNA was extracted from PBMC and cornea samples with RNeasy Micro Kit (Qiagen). RNA libraries of TCR-alpha and TCR-beta transcripts were prepared using SMARTer Human TCR a/ b Profiling Kit (Takara Bio USA Inc.) and sequenced using Illumina MiSeq platform. TRA and TRB clonotypes were assembled and quantified with MiXCR package. CDR3 sequences were annotated against VDJdb, PIRD and McPAS-TCR databases.

Results: Cornea samples for all 3 patients demonstrated significantly lower clonotype diversity than corresponding PBMC samples. We observed a shift of $\mathrm{T}$ cell repertoire in cornea compared to PBMC samples. The top abundant TCR clones in cornea were poorly represented or absent in corresponding blood samples, which indicates that they represent a tissue-specific population. For most of the CDR3 variants detected in cornea, no specificities against known antigens were found, and further research is needed to identify possible functions of these $\mathrm{T}$ cell populations.

This study was supported by RFBR as part of the research project № 17-29-06077.

L. Skorodumova: None. A. Kanygina: None. A. Belodedova: None. E. Sharova: None. B. Malyugin: None.

\section{P02.45.B}

Genomic landscape in spanish patients with ocular malformations by next generation sequencing

\author{
A. Damián ${ }^{l}$, A. Tamayo ${ }^{l}, J$. Moya $^{l}$, C. Villaverde ${ }^{l}$, \\ A. Avila-Fernández ${ }^{I}$, A. Arteche ${ }^{1}$, V. López ${ }^{2}$, M. Ballesta ${ }^{2}$,
}

E. Guillen ${ }^{2}$, S. Tahsin Swafiri ${ }^{l}$, I. Lorda ${ }^{1}$, F. Blanco-Kelly,

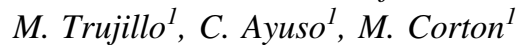

${ }^{1}$ Instituto de Investigación Sanitaria Fundación Jiménez Díaz, Madrid, Spain, ${ }^{2}$ University Hospital Virgen de la Arrixaca, Murcia, Spain

Human eye development is coordinated by complex developmental processes which involves several hundreds of genes. Any disruption in this regulation during the eye organogenesis can lead to ocular malformations such as Microphthalmia- Anophthalmia-Coloboma (MAC). These diseases are very clinically and genetically heterogeneous conditions with overlapping phenotypes; consequently the mutational load is not well known and the genotypephenotype correlations can be difficult to define. Our aim was to perform a comprehensive molecular screening in a cohort of Spanish patients with ocular malformations using different approaches of next-generation sequencing (NGS). We have findings in around 52\% of the patients. One-third carried likely causal mutations, $8 \%$ were partially characterized and $8 \%$ had VUS. Different casual mutations were found and the most prevalent in our cohort are transcription factors such as OTX2, SOX2, VSX2 and PAX6. In contrast, others variants have expanded the phenotypic spectrum of some genes classically associated with other phenotypes such as GJA8, KERA, or the lens crystallins family genes suggesting new roles of them in the early development of the eye. This results show that not only NGS is efficient for the genetic analysis of ocular malformations but also for achieving new candidate genes. Therefore, we have update our gene panels resulting in an increase in our diagnostic yield. In contrast, $34 \%$ of patients remain uncharacterized, mostly unilateral or non-syndromic cases. So that, our future purposes are to understand the mechanisms involved in this disease by identifying novel genetic causes and exploring their functional role.

A. Damián: None. A. Tamayo: None. J. Moya: None. C. Villaverde: None. A. Avila-Fernández: None. A. Arteche: None. V. López: None. M. Ballesta: None. E. Guillen: None. S. Tahsin Swafiri: None. I. Lorda: None. F. Blanco-Kelly: None. M. Trujillo: None. C. Ayuso: None. M. Corton: None.

\section{P02.46.C}

Exome-based panel testing as an efficient method to diagnose the highly heterogeneous ocular disorder spectrum Microphthalmia, Anophthalmia, Coloboma and Anterior Segment Dysgenesis (MAC-ASD)

H. Verdin ${ }^{\text {, T. T. Rosseel }}$, S. Vermeer $^{2}$, I. Balikova ${ }^{3}$, P. Kestelyn ${ }^{4}$, C. Meunier ${ }^{5}$, F. Meire ${ }^{6}$, J. Van De Velde', 
O. Vanakker ${ }^{\text {I }, ~ J . ~ V a n ~ D e n ~ E n d e ~}{ }^{7}$, M. De Rademaeker ${ }^{7}$, M. Meuwissen , M. De Vries ${ }^{8}$, Y. Sznajer ${ }^{9}$, M. Desmet ${ }^{1}, T$.

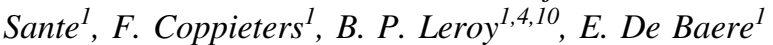

${ }^{1}$ Center for Medical Genetics, Ghent University Hospital, Ghent, Belgium, ${ }^{2}$ Center for Human Genetics, University Hospitals Leuven, Leuven, Belgium, ${ }^{3}$ Department of Ophthalmology, University Hospitals Leuven, Leuven, Belgium, ${ }^{4}$ Department of Ophthalmology, Ghent University and Ghent University Hospital, Ghent, Belgium, ${ }^{5}$ Center for Human Genetics, Institute of Pathology and Genetics, Gosselies, Belgium, ${ }^{6}$ Department of Ophthalmology, Hôpital Universitaire des Enfants Reine Fabiola, Brussels, Belgium, ${ }^{7}$ Center for Medical Genetics, University of Antwerp and Antwerp University Hospital, Antwerp, Belgium, ${ }^{8}$ Department of Ophthalmology, Antwerp University Hospital, Antwerp, Belgium, ${ }^{9}$ Center for Human Genetics, Cliniques Universitaires St-Luc, Universite Catholique de Louvain, Brussels, Belgium, ${ }^{10}$ Division of Ophthalmology and Center for Cellular \& Molecular Therapeutics, The Children's Hospital of Philadelphia, Philadelphia, PA, United States

Introduction: Microphthalmia and anophthalmia are part of a spectrum with ocular coloboma, a structural malformation resulting from incomplete fusion of the optic fissure, which likely have a shared, although heterogeneous genetic basis. They are collectively named MAC (Microphthalmia-Anophthalmia-Coloboma), characterized by a tremendous clinical heterogeneity and often associated with other ocular abnormalities such as anterior segment dysgenesis (ASD). We aimed to set up an exome-based gene panel test for MAC-ASD.

Materials and Methods: Sample preparation was done with the SureSelectXT Low Input Human All Exon V7 kit using Bravo (Agilent Technologies) and pooled libraries were sequenced on a HiSeq 3000 or NovaSeq 6000 (Illumina) aiming for a minimal coverage of $20 \mathrm{x}$ for at least $90 \%$ of the investigated regions. Data analysis was executed with a bcbio-based pipeline and variant filtering with in-house Seqplorer software. Copy number variant (CNV) analysis was done using ExomeDepth.

Results: As of May 2018, 26 patients, 12 with MAC (46.2\%) and 14 with ASD (53.8\%), underwent MACASD panel analysis. The overall diagnostic yield was 9/ $26(34.6 \%)$, in line with the reported range (11-36\%). ASD has the highest yield (50\%), compared to MAC $(16.7 \%)$. In seven out of the nine cases a (likely) pathogenic variant was identified in ALDH1A3, CPAMD8, FOXE3, MFRP, PAX6, PITX2 and PITX3. In two of the nine patients a CNV encompassing FOXCl was found.
Conclusions: Exome-based MAC-ASD panel analysis revealed a molecular diagnosis in $34.6 \%$ of a MAC-ASD cohort, highlighting the efficacy of exome-based panel testing in heterogeneous conditions.

Funding: FWO/12R3419N; FWO/1802220N; BOF15/ GOA/011.

H. Verdin: None. T. Rosseel: None. S. Vermeer: None. I. Balikova: None. P. Kestelyn: None. C. Meunier: None. F. Meire: None. J. Van De Velde: None. O. Vanakker: None. J. Van Den Ende: None. M. De Rademaeker: None. M. Meuwissen: None. M. De Vries: None. Y. Sznajer: None. M. Desmet: None. T. Sante: None. F. Coppieters: None. B.P. Leroy: None. E. De Baere: None.

\section{P02.47.A}

The enigma of Midfacial Toddler Excoriation Syndrome (MiTES), a second PRDM12 poly-alanine tract expansion disorder

\section{N. Sarveswaran ${ }^{1}$, G. Woods $^{1}$, C. Moss ${ }^{2}$}

${ }^{1}$ Cambridge Institute for Medical Research, Cambridge, United Kingdom, ${ }^{2}$ Birmingham Children's hospital, Birmingham, United Kingdom

Mid-face toddler excoriation syndrome (MiTES) is a new sensory disease first described in 2017 (PMID: 28028858). We report 12 cases in 7 families. The phenotype is of intense itch in early childhood, which causes mutilating self-injury by scratching. The intense itch is localised only to the midface and no other body parts; is not associated with altered sweating; it spontaneously subsides by 4-6 years; cognition and pain sensing is normal; there is no excess of Staph Aureus infections (on the face or elsewhere). Inheritance appeared recessive, as parents were unaffected, males and females were equally affected, and most cases were consanguineous. Older individuals "grow out" of the condition, facial injuries heal, and have normal whole-body itch and pain sensing. In six of seven families, we found the same mutation in affected individuals - a homozygous poly-Alanine repeat expansion from the normal range (7-13) to 18. Parents were always carriers. This was also found in a non-Asian, Caucasian, nonconsanguineous family. In the seventh family, we found only a heterozygous 7 base pair deletion in PRDM12, but no second mutation. What is extraordinary is that a PRDM12 homozygous 19 poly-Alanine repeat expansion causes Congenital Insensitivity to Pain; which is not associated with excess itch, leads to self-inflicted lip, tongue and corneal damage, and always cause excess S. Aureus infections. No other poly-alanine tract expansion disease has two phenotypes. We present our cellular studies to try and 
understand the disease mechanism whereby a single additional alanine can cause such different phenotypes.

N. Sarveswaran: None. G. Woods: None. C. Moss: None.

\section{P02.49.C}

Mutational analysis of 256 Russian patients with congenital aniridia and overlapping phenotypes

T. A. Vasilyeva, A. V. Marakhonov, V. V. Kadyshev, R. A. Zinchenko

Research Centre for Medical Genetics, Moscow, Russian Federation

Congenital aniridia (OMIM\#106210) is an autosomal dominant eye disorder mainly caused by heterozygous PAX6 mutations or $11 \mathrm{p} 13$ chromosome rearrangements. Pathogenic variants in some other genes could cause overlapping phenotypes, also, PAX6 missense mutations could be associated with other than aniridia phenotypes. A total of 208 unrelated families (256 patients) with clinical diagnoses of either congenital aniridia, counting WAGR syndrome, or overlapping ophthalmological diagnoses characterized by iris hypoplasia were included in the study. Familial to sporadic proportion counted for 44/164. A combination of Sanger PAX6 sequencing, whole-exome sequencing, followed by MLPA analysis of $11 \mathrm{p} 13,4 \mathrm{q} 25$, 6p25.3 loci was implemented. 204 out of 208 families (98\%) were found to have either heterozygous pathogenic nucleotide sequence variant or chromosome deletion. Disease-causing variants in PAX6 and deletions in $11 \mathrm{p} 13$ were found in $92.7 \%$. Four other genes (FOXC1, PITX2, NDP, CRYAA) explained $5.3 \%$ of the examined families. $30.3 \%$ of the cohort carried pathogenic CNVs (62 in $11 \mathrm{p} 13$ and 1 in $6 \mathrm{p} 25.3)$. A frequent $11 \mathrm{p} 13$ deletion of PAX6 3'-cis-regulatory region was determined (16/208, $7.7 \%)$. The frequency of this deletion in the cohort was higher than PAX6 hotspot c. $718 \mathrm{C}>\mathrm{T}$ rate $(9 / 208,4.3 \%)$. In total, repeated PAX6 variants were determined in 39/208 $(18,8 \%)$ families, together with $11 \mathrm{p} 13$ downstream deletion they explained 55/208 (26.4\%) of the families. PAX6 missense variants counted for $6 / 208$ (2.8\%). Mutational spectrum peculiarities in a large cohort of Russian families with congenital aniridia were established. Supported by grant RFBR 19-015-00122 and state task of the Ministry of education and science of Russia.

T.A. Vasilyeva: None. A.V. Marakhonov: None. V.V. Kadyshev: None. R.A. Zinchenko: None.

\section{P02.50.A}

\section{Revealing the role of enhancers in myopia}

J. Swierkowska ${ }^{1,2,3}$, M. S. Tedja ${ }^{1,2}$, A. E. G. Haarman ${ }^{1,2}$, M. Ghanbari $^{l}$, S. J. Erkeland ${ }^{4}$, M. Meester-Smoor ${ }^{1,2}, C . C . W$. Klaver $^{5,6,7}$, V. J. M. Verhoeven ${ }^{1,2,8}$

${ }^{I}$ Department of Epidemiology, Erasmus Medical Center, Rotterdam, Netherlands, ${ }^{2}$ Department of Ophthalmology, Erasmus Medical Center, Rotterdam, Netherlands, ${ }^{3}$ Institute of Human Genetics, Polish Academy of Sciences, Poznan, Poland, ${ }^{4}$ Department of Immunology, Erasmus Medical Center, Rotterdam, Netherlands, ${ }^{5}$ Epidemiology and Ophthalmology Departments, Erasmus Medical Center, Rotterdam, Netherlands, ${ }^{6}$ Department of Ophthalmology, Radboud University Medical Center, Nijmegen, Netherlands, ${ }^{7}$ Institute of Molecular and Clinical Ophthalmology, Basel, Switzerland, ${ }^{8}$ Department of Clinical Genetics, Erasmus Medical Center, Rotterdam, Netherlands

Introduction: Previous studies have indicated a crucial role of the non-coding genome in myopia (nearsightedness), a complex genetic trait. We aimed to identify enhancers, cisregulatory elements regulating gene expression, associated with myopia leveraging summary statistics of previous genome-wide association studies (GWAS).

Materials and Methods: GWAS on myopia related phenotypes (148,485 European, 11,935 Asian individuals) from CREAM and 23andMe were used to examine the association between enhancer-related SNPs and myopia. We obtained enhancer regions $\left(\mathrm{N}_{\text {total }}=12,244\right)$ active in eyerelated human tissues from the FANTOM5 database and performed a look-up of these regions in the GWAS. We prioritized enhancers and ANNOVAR determined target genes by ranking system based on LD, eQTLs, enhancer histone marks (HaploReg v4.1) and the association with myopia/ocular phenotype (GWAS catalog).

Results: We selected enhancers expressed in $\geq 3$ eyerelated tissue types $(\mathrm{N}=7,944)$, of which 4,610 enhancers $\left(\mathrm{N}_{\mathrm{SNPs}}=9,253\right)$ were available in the GWAS for analysis. The look-up of these regions yielded 15 significant SNPs clustering in 12 enhancers regions exceeding the Bonferroni significance threshold $\left(P=0.05 / 4,610=1.08 \times 10^{-5}\right)$. The highest ranked rs9535268 $\left(P=1.04 \times 10^{-9}\right)$ and $\mathrm{rs} 4415919$ $\left(P=3.76 \times 10^{-8}\right)$ were annotated to an intergenic enhancer region between RCBTB1 $(\sim 16 \mathrm{~kb})$ and ARL11 $(\sim 27 \mathrm{~kb})$ genes. Interestingly, SNPs within RCBTBI were associated with myopia in the GWAS.

Conclusions: We selected enhancers with putative target genes, as RCBTB1 (RCC1 and BTB domain containing protein 1), that could explain significant loci identified in GWAS and play a role in myopia pathogenesis. Support: Horizon 2020 (648268); NWO (91815655, 91617076); Uitzicht (2017-28; Oogfonds; Landelijke Stichting voor Blinden en Slechtzienden; MaculaFonds), EMBO (8529). 
J. Swierkowska: None. M.S. Tedja: None. A.E.G. Haarman: None. M. Ghanbari: None. S.J. Erkeland: None. M. Meester-Smoor: None. C.C.W. Klaver: None. V.J.M. Verhoeven: None.

\section{P02.53.A}

Functional analysis of a novel Myo6variant in hereditary hearing loss family from Qatar

M. K. Al-Kowari ${ }^{1,2}$, S. Da'as ${ }^{l}$, M. Guarch ${ }^{l}, D$.

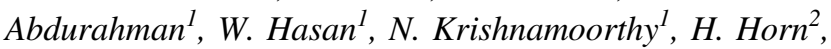
X. Estivill ${ }^{1,2}$

${ }^{1}$ Sidra Medicine, Doha, Qatar, ${ }^{2}$ Biological and Biomedical Program, Hamad Bin Khalifa University, Doha, Qatar

Introduction: Hereditary Hearing Loss (HHL) is a common genetic disorder accounting for at least $60 \%$ of prelingual deafness in children. The long tradition of consanguinity among Qatari population increases the prevalence of HHL. A novel missense variant in Myo6 was detected in two siblinges diagnosed with severe to profound HL (Alkowari et al. 2017). Prediction tools revealed that this variant is pathogenic and conserved.

Materials and methods: We functionally validate the pathogenicity of the novel variant using both cellular and animal models. HeLa cells were transiently transfected with plasmids carrying wildtype and mutated human Myo6. A zebrafish model was generated using morpholino knock down and co-injection with synthetic RNA $\left(M y o 6^{W T}\right.$ and $M y o 6^{p \cdot E 60 Q}$ ) to further characterize the phenotype.

Results: HeLa cells transfected with $M y o 6^{W T}$ and $M y o 6^{p .}$ $E 60 Q$, overexpressed the same amount of protein. Cells with $M y o 6^{W T}$ showed localization of the protein in both cytoplasm and the plasma membrane. In contrast, $M y o \sigma^{p}$. ${ }_{E 60 Q}$ is not expressed in the plasma membrane but it's restricted to the cytoplasm. Zebrafish model resulted in severe otic defects. Myo6 ${ }^{p . E 60 Q}$ RNA injection resulted in alterations in the saccule that is responsible for hearing in zebrafish ear. Additionally, we found that zebrafish with $M y o 6^{p . E 60 Q}$ displayed shorter, less abundant and disordered hair bundles in otic hair cells when compared to control and $M y o 6^{W T}$ injected zebrafish.

Conclusions: In this study we functionally characterized a novel genetic variant associated with hearing loss; in vitro studies demonstrated that c.G178C variant affects Myo6 protein trafficking. A zebrafish model confirmed the pathogenicity and identified significant otic defects.

M.K. Al-Kowari: None. S. Da'as: None. M. Guarch: None. D. Abdurahman: None. W. Hasan: None. N. Krishnamoorthy: None. H. Horn: None. X. Estivill: None.
P02.55.C

Characterizing a large Iranian cohort of non-syndromic hearing loss families using molecular inversion probe and exome sequencing

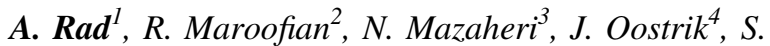
Loum $^{l}$, H. Löwenheim ${ }^{l}$, T. Haaf ${ }^{5}, H$. Galehdari ${ }^{6}, H$. Kremer $^{4}$, B. Vona ${ }^{l}$

${ }^{I}$ Department of Otorhinolaryngology, Head and Neck Surgery, Tübingen, Germany, ${ }^{2}$ Department of Neuromuscular Disorders, UCL Queen Square Institute of Neurology, London, United Kingdom, ${ }^{3}$ Department of Genetics, Faculty of Science, Shahid Chamran University of Ahvaz, Ahvaz, Iran, Islamic Republic of, ${ }^{4}$ Department of Otorhinolaryngology, Radboud University Medical Center, Nijmegen, Netherlands, ${ }^{5}$ Institute of Human Genetics, Julius Maximilians University, Würzburg, Germany, ${ }^{6}$ Department of Genetics, Faculty of Science, Shahid Chamran University of Ahvaz, Ahvaz, Iran, Islamic Republic of

Introduction: Hearing loss (HL) is the most prevalent sensory deficit. With about 120 genes implicated, nonsyndromic hearing loss (NSHL) is extremely genetically heterogeneous. In this study, we investigated extended Iranian families with hereditary NSHL to uncover the genetic landscape and characterize its genetic heterogeneity.

Materials and Methods: Using molecular inversion probes (MIPs), we targeted 89 genes involved in NSHL in 20 patients. Exome sequencing (ES) was performed in 102 GJB2-negative and unresolved MIP-screened NSHL probands. Sanger sequencing, homozygosity mapping, and in vitro functional assays were used to confirm selected variants.

Results: Our analysis resolved 50\% (10/20) of the MIPscreened patients and about 76\% (78/102) of patients subjected to ES. The overall diagnostic rate is about $78.5 \%$ (88/112). Nearly half of the diagnostic yield was attributed to the genes SLC26A4 (13.4\%), MYO15A (8.0\%), LOXHDI (7.0\%), MYO7A (6.2\%), CDH23 (5.3\%) and TMC1 (4.5\%). Our data also identified $C L R N 2$ as a novel NSHL gene in a family. A novel variant in the SPATA5 gene, known to cause epilepsy, hearing loss, and mental retardation syndrome, was implicated in a family with $\mathrm{HL}$ as the sole clinical feature.

Conclusions: Using two approaches, we could resolve roughly $78.5 \%$ of the patients undergoing genetic screening for NSHL. Our cohort also uncovered LOXHDI as a highly prevalent gene in NSHL diagnoses that was not similarly observed in large cohort screenings of Iranian HL patients. Furthermore, we introduce CLRN2 and SPATA5 as novel autosomal recessive NSHL genes. Select unresolved 
families from this cohort have been followed-up with genome sequencing.

A. Rad: None. R. Maroofian: None. N. Mazaheri: None. J. Oostrik: None. S. Loum: None. H. Löwenheim: None. T. Haaf: None. H. Galehdari: None. H. Kremer: None. B. Vona: None.

\section{P02.56.A}

Multitopographical effect of mutations in the gene PAX6 for pediatric ophthalmology

V. V. Kadyshev, T. A. Vasilyeva, A. V. Marakhonov, S. I. Kutsev, R. A. Zinchenko

FSBSI Research Centre for Medical Genetics, Moscow, Russian Federation

The expression of the PAX6 gene is realized in the formation of almost all structures of the eye - the cornea, iris, lens, optic nerve and central part of the retina. Accounting this fact, the clinical spectrum expands when there is a single pathogenetic process. Materials and methods. The sample of patients with hereditary ophthalmological pathology was 31 children (0-16 years old). The examination included clinical (ophthalmoscopy, biomicroscopy, gonyoscopy, visometry, tonometry), instrumental (electrophysiological, ultrasound, etc.), genealogical and molecular genetic (NGS, direct Sanger sequencing, MLPA) research methods. Results. Accounting the topographical principle, mutations in the PAX6 gene led to lesions in both the anterior and posterior segments of the eyeball. Structures such as the optic disc and/or macular zone (posterior segment) are affected in $100 \%$ of cases. The lesion of the anterior segment of the eye is characterized by the involvement of different structures in the process: 6 patients had corneal changes of different grades (ex.; c. 1184-2_1184delAGGinsGGA PAX6 het; c. 1184-2_1184delAGGinsGGA PAX6 het); in 5 cases pathology of the lens (NM_000280. 4(PAX6_v001):c. $765 \mathrm{G}>\mathrm{A}$ het synchronous splicing); 7 patients had different grades of iris pathology. One patient with mutation NM_000280.4(PAX6_v001):c.109dupG had a congenital malformation-colobomatous microphthalmos. Two patients had anterior segment dysgenesis (corneal, iris, anterior chamber angle, lens) (c.142-139T >C PAX6 IVS5a-3T >C het; c.718C $>\mathrm{T}, \mathrm{p} .(\operatorname{Arg} 240 *))$. Mutations in the PAX6 gene have a multitopographic effect on the eye, with the development of pathology in the anterior and posterior segments of the eyeball. Supported by RFBR grant 18-015-00090. All authors haven't conflict of interest.

V.V. Kadyshev: None. T.A. Vasilyeva: None. A.V. Marakhonov: None. S.I. Kutsev: None. R.A. Zinchenko: None.
P02.57.B

Nine Tunisian patients with typicalPeters plus syndrome

M. Guirat, S. Guidara, H. Kanoun, I. Boujelbene, N. Gharbi, I. B. Ayed, F. Abdelhedi, H. Kamoun, N. Belguith

Service de génétique médicale, CHU Hedi Chaker, Sfax, Tunisia

Peters plus syndrome is an autosomal recessive rare disorder comprising ocular anterior segment dysgenesis, short stature, hand abnormalities, distinctive facial features. It was related only to mutations in the B3GALTL gene (13q12.3), leading to the inactivation of the B1, 3glucosyltransferase. In this study, we report 9 patients with typical Peters plus syndrome. Ocular abnormality, facial dysmorphism, and rhizomelic dwarfism were present in all cases. We noted the presence of 2 new anomalies not previously described in Peters Plus syndrome, namely renal malformation and sexual ambiguity. Total sequencing of the B3GALTL gene showed the presence of the splicing mutation (c.597-2A> G) in the B3GALTL gene in 7 patients. This mutation harbours a novel splice site mutation in the B3GALTL gene that modulates the mRNA secondary structure. These data confirm a key role of the genetic study that provides diagnosis confirmation and improves genetic counseling for the families

M. Guirat: None. S. Guidara: None. H. Kanoun: None. I. Boujelbene: None. N. Gharbi: None. I.B. Ayed: None. F. Abdelhedi: None. H. Kamoun: None. N. Belguith: None.

\section{P02.58.C}

Severe forms of presbycusis are caused by very rare variants in genes underlying early-onset forms of deafness

S. boucher ${ }^{1}$, F. Wong Jun Tai ${ }^{2}$, A. Lelli ${ }^{3}$, S. Delmaghani ${ }^{3}$, A. Singh-Estivalet ${ }^{3}, T$. Dupont ${ }^{3}, V$. Michel $^{3}, N$. Wolff ${ }^{4}, A$. Bahloul $^{3}$, M. Niasme-Grare ${ }^{5}$, A. Bouaita ${ }^{3}$, D. OficjalskaPham $^{3}$, S. Chardenoux ${ }^{3}, Y$. Bouyacoub ${ }^{6}$, D. Bouccara ${ }^{7}, O$. Deguine $^{8}$, B. Fraysse ${ }^{8}$, L. Collet ${ }^{9}$, H. Thai-Van ${ }^{10}$, E. Ionescu $^{9}$, J. Kemeny ${ }^{11}$, F. Giraudet ${ }^{12}$, J. Lavieille ${ }^{13}$, A. Devèze $^{13}$, A. Roudevitch-Pujol ${ }^{14}$,V. Christophe ${ }^{15}, C$. Renard $^{16}$, V. Franco-Vidal ${ }^{17}$, C. Thibult-Apt ${ }^{17}$, V. Darrouzet $^{17}$, E. Bizaguet ${ }^{18}$, A. Coez ${ }^{19}$, A. Aubois ${ }^{14}$, G. Lefèvre $^{3}$, N. Michalski ${ }^{3}$ P. Avan ${ }^{20}$, C. Bonnet ${ }^{3}$, C. Petit $^{21}$

${ }^{1}$ INSERM UMRS1120, Institut de l'Audition, Pasteur Institute and University Hospital of Angers, Paris, France, ${ }^{2}$ INSERM UMRS1120 and Institut de l'Audition, Pasteur Institute, Paris, France, ${ }^{3}$ INSERM UMRS1120, Institut de 
l'Audition, Pasteur Institute, Paris, France, ${ }^{4}$ Institut Pasteur, Unité de RMN des Biomolécules, UMR 3528, CNRS, Paris, France, ${ }^{5}$ Service de Biochimie et Biologie Moléculaire, Hôpital d'Enfants Armand-Trousseau, APHP, Paris, France, ${ }^{6}$ INSERM UMRS1120, Pasteur Institute, Paris, France, ${ }^{7}$ Hôpital Beaujon, Hôpitaux Universitaires Paris Nord val-de-Seine, APHP, Paris, France, ${ }^{8}$ Centre Hospitalier Universitaire de Toulouse, Hôpital Larrey, Service d'Oto-Rhino-Laryngologie et Chirurgie CervicoFaciale, Toulouse, France, ${ }^{9}$ Centre Hospitalier Universitaire, Hôpital Lyon-Sud, Audiologie et Explorations Orofaciales, Lyon, France, ${ }^{10}$ Centre Hospitalier Universitaire, Hôpital Lyon-Sud, Audiologie et Explorations Orofaciales and Institut de l'Audition, Lyon and Paris, France, ${ }^{11}$ Centre Hospitalier Universitaire de Clermont Ferrand, Hôpital Gabriel Montpied, Service d'AnatomoPathologie, Clermont Ferrand, France, ${ }^{12}$ Centre Hospitalier Universitaire de Clermont Ferrand, Hôpital Gabriel Montpied, Service d'Anatomo-Pathologie, UMR 1107, INSERM and Centre Jean Perrin, Clermont Ferrand, France, ${ }^{13}$ Centre Hospitalier Universitaire de Marseille, Hôpital Nord, Service d'Oto-Rhino-Laryngologie, Marseille, France, ${ }^{14}$ Centre Hospitalier National d'Ophtalmologie des Quinze-Vingts, Centre d'Investigation Clinique, Paris, France, ${ }^{15}$ Centre Hospitalier Universitaire de Lille, Hôpital Roger Salengro, Service d'Otologie et Otoneurologie, Lille, France, ${ }^{16}$ Laboratoire d'Audiologie Renard, Lille, France, ${ }^{17}$ Centre Hospitalier Universitaire de Bordeaux, Hôpital Pellegrin, Service d'Oto-Rhino-Laryngologie et Chirurgie Cervico-Faciale, Bordeaux, France, ${ }^{18}$ Laboratoire de Correction Auditive, Paris, France, ${ }^{19}$ Laboratoire de Correction Auditive and CEA-Inserm U1000 Neuroimaging and Psychiatry and DRM, DSV, Service Hospitalier Frédéric-Joliot, Paris, France, ${ }^{20}$ INSERM UMR 1107, Laboratoire de Biophysique Neurosensorielle, Université d'Auvergne, Facultés de Médecine et de Pharmacie, Clermont Ferrand, France, ${ }^{21}$ INSERM UMRS1120, Institut de l'Audition, Pasteur Institute and Collège de France, Paris, France

Introduction: Presbycusis is a major public health issue world wide with half its variance due to genetic predisposition. The aim of this study was to identify presbycusis causative genes by whole exome sequencing (WES).

Patients and methods: We analysed 105 families, 122 simplex cases and 120 normal-hearing controls. After an in silico bioinformatic analysis to select predicted pathogenic variants, and according to the autosomal dominant mode of inheritance in $95 \%$ of the families, we studied very rare heterozygous variants (allele frequency : $\mathrm{AF} \leq 0.0001$ ).

Results: We found a significative higher ratio of pathogenic variants in autosomal dominant deafness genes carried by $24.7 \%$ of mARHL and $21,8 \%$ of sARHL (aside from three individuals carrying GJB2 biallelic variants) than in the controls $(6,7 \%)(\mathrm{p}<0,001)$. By studing a mouse model $T m c l^{N 321 I}$, we confirmed the pathogenicity of the mutation leading to congenital moderate hearing loss in homozygous mice due to a reduced mechanotransduction current amplitude, and to a progressive hearing loss beginning at 7 months and affecting the high frequencies in heterozygous carriers, validating also the mutation as responsible for a monogenic form of presbycusis.

Conclusions: WES unraveled that a large proportion of severe presbycusis is due to new mutations in autosomal dominant deafness genes arguing for a genetic continuum between early-onset deafness and presbycusis. We demonstrated also the existence of monogenic forms of presbycusis that could be targetted by new personnalized therapies like gene therapy. This study was supported by a grant from "Fondation pour l'Audition" and also by the LabEx Lifesenses (ANR-10-LABX-65).

S. boucher: None. F. Wong Jun Tai: None. A. Lelli: None. S. Delmaghani: None. A. Singh-Estivalet: None. T. Dupont: None. V. Michel: None. N. Wolff: None. A. Bahloul: None. M. Niasme-Grare: None. A. Bouaita: None. D. Oficjalska-Pham: None. S. Chardenoux: None. Y. Bouyacoub: None. D. Bouccara: None. O. Deguine: None. B. Fraysse: None. L. Collet: None. H. Thai-Van: None. E. Ionescu: None. J. Kemeny: None. F. Giraudet: None. J. Lavieille: None. A. Devèze: None. A. Roudevitch-Pujol: None. V. Christophe: None. C. Renard: None. V. Franco-Vidal: None. C. Thibult-Apt: None. V. Darrouzet: None. E. Bizaguet: None. A. Coez: None. A. Aubois: None. G. Lefèvre: None. N. Michalski: None. P. Avan: None. C. Bonnet: None. C. Petit: None.

\section{P02.59.A}

Genetic analysis of a Dutch discovery cohort suggests an association between primary open-angle glaucoma and the mitochondrial variant $\mathbf{m . 1 1 9 1 5 G / A}$ in the MT-ND4 gene

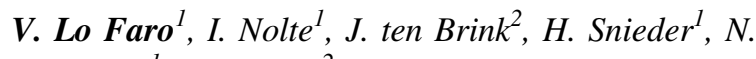
Jansonius $^{1}$, A. Bergen ${ }^{2}$

${ }^{1} U M C G$, Groningen, Netherlands, ${ }^{2} A M C$, Amsterdam, Netherlands

Introduction: To determine a possible association between mitochondrial variations and primary open-angle glaucoma (POAG) in a Dutch discovery cohort.

Materials and Methods: $140 \mathrm{mtDNA}$ single nucleotide polymorphisms (SNPs) were genotyped using the Illumina Infinium Global Screening Array-24 (GSA) 700K array set. Genetic analysis was performed in a case-control discovery 
cohort of 734 patients with POAG and 1,417 controls. The variants were classified and analyzed both as single SNPs and as haplogroups. SNP association was performed using logistic regression. The bioinformatics software HaploGrep was used to determine the haplogroup. The Chi-square test and Fisher's two-sided exact test were used for haplogroups association.

Results: For discovery, a nominal association with POAG was found for the allele A of m.11915G/A (rs2853496, $\mathrm{p}=0.016$ ), within the MT-ND4 gene. The odds ratio was 0.80 (95\% confidence interval $0.67-0.95)$ per copy of the rare allele and adjusted for gender. No haplogroup was associated with POAG in our cohort.

Conclusions: The present study reinforces the concept that mitochondrial variation contributes to POAG susceptibility. Further genetic and functional studies are required to highlight the pathophysiological mechanisms underlying primary open-angle glaucoma.

V. Lo Faro: None. I. Nolte: None. J. ten Brink: None. H. Snieder: None. N. Jansonius: None. A. Bergen: None.

P02.61.C

Whole Exome analysis versus a targeted panel for Polish patients with retinal dystrophies

E. Matczyńska ${ }^{1,2}$, P. Łyszkiewicz ${ }^{l}$, A. Wasowska ${ }^{1,2}, R$. Szymańczak ${ }^{l}$, K. Stradomska ${ }^{l}$, E. Suchecka ${ }^{l}, M$. Jędrzejowskal, S. Teper ${ }^{2}$, M. Wiacek ${ }^{3}$, A. Machalińska ${ }^{3}$, A. Boguszewska-Chachulska ${ }^{1}$

${ }^{1}$ Genomed SA, Warsaw, Poland, ${ }^{2}$ Chair and Clinical Dept. of Ophthalmology, Medical University of Silesia, Katowice, Poland, ${ }^{3}$ Pomeranian Medical University, Szczecin, Poland

A Whole Exome Sequencing (WES) approach was adopted, as a part of the recently accomplished NeuStemGen project, for identifying genetic causes of inherited retinal dystrophies (IRD) in a cohort of Polish patients. Project outcomes prompted the development of a targeted retinal panel, covering most pathogenic variants occurring in the Polish population.

WES was performed for 105 patients with clinical symptoms of retinal dystrophies. A targeted panel, consisting of 278 IRD genes (including deep intronic regions for $A B C A 4$ ), was used to sequence 29 IRD samples, including 6 samples not resolved by WES. Bioinformatic analysis was performed using standard BWA-GATK best practice pipeline and several databases, including POLGENOM, for variant frequency filtration. Copy number variants (CNVs) were analysed using the CoNVaDING program.

Over 50 pathogenic/potentially pathogenic variants were identified using the WES analysis, involving genes already known to cause IRD. The diagnostic yield of this approach, however, did not exceed $60 \%$. The pilot sequencing of the targeted retinal panel resulted in a significantly higher coverage (389x vs 78x), 98-99\% concordance of identified variants and allowed to develop a CNV analysis pipeline.

Data obtained using these two approaches were compared and support the decision of applying the large targeted panel for routine diagnostics of retinal dystrophies. This approach, focused on the complete set of retinal genes, assuring a high quality coverage and allowing for reliable analysis of CNVs and deep intronic variants, should be most efficient and cost-effective for a large cohort of Polish patients.

Partially supported by STRATEGMED1/234261/2/ NCBR/2014.

E. Matczyńska: None. P. Kyszkiewicz: None. A. Wasowska: None. R. Szymańczak: None. K. Stradomska: None. E. Suchecka: None. M. Jędrzejowska: None. S. Teper: None. M. Wiącek: None. A. Machalińska: None. A. Boguszewska-Chachulska: None.

\section{P02.63.B}

A genetic and clinical study of families with nonsyndromic retinopathy consequent upon sequence variants in HGSNAT, the gene causing Sanfilippo $\mathrm{C}$ mucopolysaccharidosis

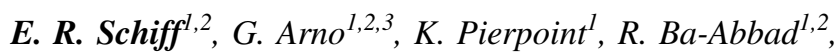
S. Nutan ${ }^{4}$, O. A. Mahroo ${ }^{1,2,5}$, M. Michaelides $^{1,2}$, K. Harvey $^{6}$, A. R. Webster ${ }^{1,2}$

${ }^{I}$ Genetics Service, Moorfields Eye Hospital, London, United Kingdom, ${ }^{2}$ UCL Institute of Ophthalmology, London, United Kingdom, ${ }^{3}$ North East Thames Regional Genetics Service, Great Ormond Street Institute of Child Health, London, United Kingdom, ${ }^{4}$ London North Genomic Laboratory Hub, Great Ormond Street NHS Foundation Trust, London, United Kingdom, ${ }^{5}$ Section of Ophthalmology, King's College London, London, United Kingdom, ${ }^{6}$ Enzyme Unit, Chemical Pathology, Paediatric Laboratory Medicine, Great Ormond Street Hospital for Children NHS Foundation Trust, London, United Kingdom

Distinct genotypes in several genes (e.g. USH2A, FLVCR1, CEP290, CLN3, MFSD8) have been shown to be associated with either syndromic or non-syndromic forms of retinitis pigmentosa (RP), a progressive degenerative condition initially affecting rod photoreceptors with subsequent cone phototreceptor loss; RP is the most prevalent form of inherited retinal disease (IRD), which is the commonest cause of blindness in the working-age population of England and Wales. HGSNAT (heparan- $\alpha$-glucosaminide $\mathrm{N}$ acetyltransferase) sequence variants cause mucopolysaccharidosis type IIIC (MPS-IIIC) or Sanfilippo C 
syndrome - a severe childhood-onset lysosomal storage disorder. Specific genotypes have, however, been associated with isolated retinal disease in a few reported individuals, thereby further extending the genes that can result in both syndromic and non-syndromic IRD. We have identified likely pathogenic variants in 13 individuals (11 families) with late onset (30s to 60s) and slowly progressing RP. None showed any other symptoms of MPS-IIIC. 7/11 families were compound heterozygotes with the allele c.1843G $>$ A, p.(Ala615Thr). We detected 6 novel sequence variants (p.(Ser296Leu); p.(Phe428Cys); p.(Gly248Ala); p. (Gly418Arg), c.1543-2A>C; c.1708delA), and 5 variants that were previously described in MPS-IIIC patients. In one family, two siblings, genetically identical for the HGSNAT locus, were discordant for retinal disease. A mild but significant reduction of HGSNAT enzyme activity in leukocytes was observed in affected individuals but urinary glucosaminoglycans were not elevated. This case series expands the phenotypic and genotypic spectrum of nonsyndromic HGSNAT-associated retinopathy, and in one family suggests the influence of trans-acting modifying factors. Funding: NIHR BRC, Moorfields Eye Hospital and UCL Institute of Ophthalmology.

E.R. Schiff: None. G. Arno: None. K. Pierpoint: None. R. Ba-Abbad: None. S. Nutan: None. O.A. Mahroo: None. M. Michaelides: None. K. Harvey: None. A.R. Webster: None.

\section{P02.64.C}

First systematic molecular genetic analysis using NGS analysis of 100 Greek patients with retinal dystrophy

\author{
S. Kamakari ${ }^{1}$, S. Koukoula ${ }^{2}$, V. Kokkinou ${ }^{1}$, L. Haer- \\ Wigman $^{3}$, I. Datseris ${ }^{4}$, M. Tsilimbaris ${ }^{5}$
}

${ }^{1}$ Ophthalmic Genetics Unit, Athens, Greece, ${ }^{2}$ Ophthalmica Institute of Ophthalmology and Microsurgery, Thessaloniki, Greece, ${ }^{3}$ Department of Human Genetics, Donders Centre for Neuroscience, Radboud University Nijmegen Medical Centre, Nijmegen, Netherlands, ${ }^{4}$ Department of Retinal Disorders, OMMA Ophthalmological Institute of Athens, Athens, Greece, ${ }^{5}$ Department of Ophthalmology, University of Crete School of Medicine, Heraklion, Greece

Purpose: Inherited Retinal Dystrophies (IRDs) are characterized by clinical variability and genetic heterogeneity. The aim of this study was to molecularly diagnose 100 Greek patients with different forms of IRDs.

Materials and Methods: 100 unrelated Greek patients were analyzed by Next Generation Sequencing (NGS), 13 and 87 of them using a 105 retinal and a 287 ophthalmic gene panel, respectively as described (Ellingford JM et al. J Med Genet 2016, Haer-Wigman L et al. Eur J Hum Genet.
2017). Additional analysis methods were used (Sanger, MLPA, array-CGH) in 6 cases.

Results: Potentially pathogenic mutations were detected in 45 retinal dystrophy genes including ABCA4, PRPF31, SPATA7, MERTK, FAM161A, CDHR1, USH2A, CNGB1, PROM1 and RP2 genes. The detection mutation rates were $46.15 \%(6 / 13)$ and $81.6 \%$ (71/87) for the 105 and 287 gene panels, respectively. These mutation rates were achieved using complementary methods in 6 cases. Final diagnoses included retinitis pigmentosa, Usher syndrome, cone-rod dystrophy and Leber congenital amaurosis and two rare cases of Knobloch and Oliver-McFarlane syndromes due to mutations in the COL18A1 and PNPLA6 genes, respectively.

Conclusions: This is the first systematic investigation of the molecular identity of 100 Greek patients with various subforms of IRDs by NGS and complementary methods leading to an overall mutation rate of $77 \%$. A plethora of novel mutations was documented further expanding the genetic heterogeneity. The molecular identification established the complete diagnosis of the patients thus contributing to family making decision, prognosis and candidacy to current and future treatments.

S. Kamakari: None. S. Koukoula: None. V. Kokkinou: None. L. Haer-Wigman: None. I. Datseris: None. M. Tsilimbaris: None.

\section{P02.65.A}

A possible epistatic interaction of two variants in autosomal dominant retinitis pigmentosa

\section{R. A. Zinchenko, T. A. Vasilyeva, V. V. Kadyshev, A. V. Marakhonov}

Research Center for Medical Genetics, Moscow, Russian Federation

Retinitis pigmentosa (RP) refers to a heterogeneous group of inherited ocular diseases that result in a progressive retinal degeneration affecting 1 in 3,000 to 5,000 people. To date, more than 80 genetic forms of RP are described. Here we perform genetic analysis of the two-generation family with 5 affected members with the terminal severe stage of RP. Whole exome sequencing (WES) of one of the patients revealed two candidate variants. The first one is pathogenic variant in the exon 16 of SNRNP200 gene (chr2:96958829G >A), leading to the missense substitution (p.Arg681Cys, NM_014014.4) in the heterozygous state. Mutations in the SNRNP200 gene in heterozygous state were described in patients with RP33 (OMIM 610359). The second variant is a novel single nucleotide variant in exon 1 of the PRPH2 gene (chr6:42689991C $>\mathrm{T}$ ) resulting in the missense substitution (p.Val28Met, NM_000322.4) in a 
heterozygous state. Mutations in the $P R P H 2$ gene in heterozygous, compound heterozygous and homozygous states were described in patients with Leber congenital amaurosis 18 (OMIM:608133), RP7 (OMIM:608133), retinitis punctata albescens (OMIM:136880), in heterozygous state - in patients with choroidal dystrophy, central areolar 2 (OMIM:613105), macular dystrophy, patterned, 1 (OMIM:169150), macular dystrophy, vitelliform, 3 (OMIM:608161). Segregation analysis revealed SNRNP200 to be co-segregated with the disease while PRPH2 variant was not found in 2 out of 5 patients. Nevertheless, it could make an impact on the clinical presentation by aggravation of clinical picture in patients bearing two variants. Supported by grant RFBR № 18-015-00090 and state task of the Ministry of education and science of Russia.

R.A. Zinchenko: None. T.A. Vasilyeva: None. V.V. Kadyshev: None. A.V. Marakhonov: None.

\section{P02.68.A}

Loss of function of RIMS2, a synaptic membrane exocytosis gene, causes a new phenotype characterized by congenital cone-rod synaptic disease associated with neurodevelopmental and pancreatic involvement

S. Mechaussier ${ }^{1}$, B. Almoallem ${ }^{2}$, C. Zeitz ${ }^{3}, K$. Van Schil ${ }^{2}, L$. Jeddawi ${ }^{4}, J$. Van Dorpe ${ }^{5}$, C. Condroyer ${ }^{3}, O$. Pelle ${ }^{6}, M$.

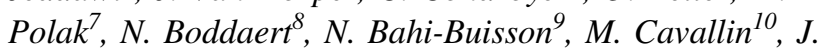
Bacquet $^{11}$, A. Mouallem-Bézière ${ }^{11}$, O. Zambrowski ${ }^{11,12}, J$. Sahe $e^{3,13,14}$, I. Audo ${ }^{3,14,15}$, J. Kaplan ${ }^{1,11}$, J. Rozet ${ }^{1}$, E. De Baere $^{2}$, I. Perrault ${ }^{l}$

${ }^{1}$ Laboratory of Genetics in Ophthalmology (LGO), INSERM UMR1163, Institute of Genetic Diseases, Imagine and Paris University, Paris, France, ${ }^{2}$ Center for Medical Genetics, Ghent University and Ghent University Hospital, Ghent, Belgium, ${ }^{3}$ Sorbonne Université, INSERM, CNRS, Institut de la Vision, Paris, France, ${ }^{4}$ Pediatric Ophthalmology Division, Dhahran Eye Specialist Hospital, Dhahran, Saudi Arabia, ${ }^{5}$ Department of Pathology, Ghent University and Ghent University Hospital, Ghent, Belgium, ${ }^{6}$ Cell Sorting Facility, INSERM UMR1163, Institute of Genetic Diseases, Imagine and Paris University, Paris, France, ${ }^{7}$ Endocrinology, Gynecology and Pediatric Diabetology Department, University Hospital Necker-Enfants Malades, Paris, France, ${ }^{8}$ Department of Pediatric Radiology, Hôpital Necker-Enfants Malades, Paris University, Paris, France, ${ }^{9}$ Pediatric Neurology Department, University Hospital Necker-Enfants Malades, Paris, France, ${ }^{10}$ Laboratory of Embryology and genetics of human malformation, INSERM UMR1163, Institute of Genetic Diseases, Imagine and Paris University, Paris, France, ${ }^{11}$ Service d'Ophtalmologie,
Centre Hospitalier Intercommunal de Créteil (CHIC), Créteil, France, ${ }^{12}$ Ophthalmology Department, University Hospital Necker-Enfants Malades, Paris, France, ${ }^{13}$ Department of Ophthalmology, The University of Pittsburgh School of Medicine, Pittsburgh, PA, United States, ${ }^{14}$ Centre Hospitalier National d'Ophtalmologie des QuinzeVingts, INSERM-DHOS CIC 1423, Paris, France, ${ }^{15}$ Institute of Ophthalmology, University College of London, London, United Kingdom

Introduction: Anomalies in pre- and post-synaptic transmission of visual information from photoreceptors to bipolar cells are responsible for stationary congenital night blindness (CSNB). Pre-synaptic disorders, characterized by congenital nystagmus and photophobia, night blindness, are known as cone-rod synaptic disorder (CRSD) can be mistaken for Leber congenital amaurosis (LCA). Full-field electroretinogram is crucial for the differential diagnosis, but it can be difficult to perform in young children. Here, we report (i) the identification of biallelic mutations in the RIMS2 gene encoding a pre-synaptic protein not yet associated with human diseases in 3/4 families initially referred for LCA and (ii) the diagnostic adjustment to CRSD with variable associated alteration including autistic behavior and insulinopathy.

Methods: Exome sequencing combined with targeted RIMS2 testing were performed in 46 prescreened patients with CRSD and 94 with LCA. Biallelic RIMS2-mutated cases underwent ophthalmologic, neurologic and metabolic assessment. RIMS2 immunostaining was performed on human retina, brain and pancreas. Nonsense variants were functionally investigated in mammalian cells.

Results: Biallelic RIMS2 sequence variants were found in four unrelated families. RIMS2, a protein regulating synaptic membrane exocytosis localizes in the human retinal outer plexiform layer, Purkinje cells and pancreatic islets. Apart from CRSD, neurodevelopmental disease was observed in all cases, and abnormal glucose homeostasis in the eldest patient. Nonsense variants lead to truncated RIMS2 and decreased insulin secretion in mammalian cells.

Conclusions: This is the first report of syndromic CRSD with neurodevelopmental or pancreatic involvement due to RIMS2 loss-of-function. The disease presentation is consistent with the localization and function of RIMS2.

S. Mechaussier: None. B. Almoallem: None. C. Zeitz: None. K. Van Schil: None. L. Jeddawi: None. J. Van Dorpe: None. C. Condroyer: None. O. Pelle: None. M. Polak: None. N. Boddaert: None. N. Bahi-Buisson: None. M. Cavallin: None. J. Bacquet: None. A. MouallemBézière: None. O. Zambrowski: None. J. Sahel: None. 
I. Audo: None. J. Kaplan: None. J. Rozet: None. E. De Baere: None. I. Perrault: None.

\section{P02.69.B}

Whole exome sequencing in Slovak patients with bilateral sensorineural hearing impairment

Z. Slobodova ${ }^{1,2}$, L. Varga ${ }^{1,2}$, S. Borecka ${ }^{2}$, I. Masindova ${ }^{1}, D$. Danis $^{1}$, L. Langova ${ }^{3}$, M. Skopkova ${ }^{l}$, M. Profant ${ }^{2}, D$. Gasperikova ${ }^{l}$

${ }^{1}$ Biomedical Research Center, Slovak Academy of Sciences, Bratislava, Slovakia, ${ }^{2}$ Department of OtorhinolaryngologyHead and Neck Surgery, Faculty of Medicine, Bratislava, Slovakia, ${ }^{3}$ Pediatric ENT Department of Medical Faculty and National Institute of Child Diseases, Bratislava, Slovakia

Background: The genetic heterogeneity of sensorineural hearing loss (SNHL) is a major hurdle to the discovery of disease-causing variants. Whole exome sequencing (WES) is a method that allows an efficient analysis of all known genes associated with hereditary hearing loss.

Aim: Using WES to identify new genetic causes of hearing impairment in the Caucasian and Roma populations in Slovakia, where standard genetic tests performed so far have not elucidated the etiology of deafness.

Patients and methods: We collected 1,369 individuals from 870 families with bilateral SNHL. The previous analysis revealed that $30 \%$ of the families have mutations in the DFNB1 locus. From the DFNB1 negative patients we selected 52 families, 42 of Caucasian and 10 of Roma ethnicity, for WES.

Results: Using WES, we identified the genetic etiology in 22 probands of Caucasian ethnicity and in 1 proband of Roma ethnicity. The detected variants were found in 18 genes (COCH, COL4A5, CREBBP, EDNRB, EYA4, ILDRI, LOXHD1, MYO6, MYO15A, OTOA, OTOG, P2RX2, SIX1, TECTA, TMC1, TMPRSS3, DIAPHI, USHIC) harboring 17 pathogenic variants and 11 likely pathogenic variants responsible for hearing impairment. 16 variants have not yet been described in the literature. In one Roma family the causal variants were found in two genes (PTPRQ and $M Y O 15 A$ ) in one individual.

Conclusion: Using WES, we detected the genetic etiology of hearing impairment in $52 \%$ probands of Caucasian ethnicity and $10 \%$ of Roma probands. WES dramatically increases the detection of pathogenic variants in known genes associated with HL. Supported by: VEGA 1/0214/16, APVV 15-067

Z. Slobodova: None. L. Varga: None. S. Borecka: None. I. Masindova: None. D. Danis: None. L. Langova:
None. M. Skopkova: None. M. Profant: None. D. Gasperikova: None.

P02.70.C

Genotype-phenotype correlations in a Spanish cohort of 506 families with bi-allelic $A B C A 4$ mutations

M. del Pozo-Valero ${ }^{l}$, R. Riveiro-Álvarez ${ }^{l}, F$. Blanco-Kelly ${ }^{l}$, J. Aguirre-Lamban ${ }^{l}$, I. Martín-Mérida ${ }^{l}$, I. Iancu ${ }^{l}, S$. Swafiri ${ }^{l}$, I. Lorda-Sanchez ${ }^{l}$, E. Rodriguez-Pinilla ${ }^{l}, M$. Trujillo-Tiebas $^{1}$, B. Jiménez-Rolando ${ }^{2}$, E. Carreño ${ }^{2}$, I. Mahillo-Fernandez ${ }^{3}$, C. Rivolta ${ }^{4}$, M. Corton ${ }^{1}$, A. AvilaFernandez $^{l}$, B. García-Sandoval ${ }^{2}$, C. Ayuso ${ }^{l}$

${ }^{1}$ Department of Genetics, Instituto de Investigación Sanitaria-Fundación Jiménez Díaz University Hospi, Madrid, Spain, ${ }^{2}$ Department of Ophthalmology, Instituto de Investigación Sanitaria-Fundación Jiménez Díaz University Hospi, Madrid, Spain, ${ }^{3}$ Department of Epidemiology, Instituto de Investigación Sanitaria-Fundación Jiménez Díaz University Hospi, Madrid, Spain, ${ }^{4}$ Institute of Molecular and Clinical Ophthalmology Basel (IOB), Basel, Switzerland, Basel, Switzerland

Introduction: $A B C A 4$ is the most mutated gene in inherited retinal dystrophies. A genotype-phenotype correlation model was described in 1999 with few cases reported. Here, we define genotype-phenotype correlations in the largest cohort study worldwide of patients: 434 with Stargardt disease (STGD1) and 72 with cone-rod dystrophy (CRD), all carrying biallelic $A B C A 4$ mutations.

Methods: Conventional genetic tools and Next Generation Sequencing technologies. Medical history and ophthalmological data were obtained for 372 patients. Genotypephenotype correlation studies were carried out for the following variables: variant type, age at onset of symptoms (AO), and clinical phenotype (STGD1 or CRD).

Results: Genotype-phenotype correlations showed that patients with biallelic truncating variants represented $41 \%$ of CRD and 6.5\% of STGD1 patients in our cohort; these cases had a significantly earlier AO than patients carrying two mutations, one of which is a missense variant. CRD patients presented earlier AO than STGD1 patients.

Conclusions: Our study, conducted in the largest $A B C A 4$-associated disease cohort reported to date, supports the genotype-phenotype model established for $A B C A 4$ variants, and broadens the mutational spectrum of the gene. $A B C A 4$ truncating variants were mostly associated with earlier $\mathrm{AO}$ and a CRD phenotype. According to our observations, $A B C A 4$ patients presenting with two truncating variants may first present features of STGD1 but eventually develop rod dysfunction, underscoring the importance of an accurate genetic diagnosis. 
Grants: Instituto de Salud Carlos III (ISCIII), CIBERER (06/07/0036), IIS-FJD Biobank PT13/0010/0012, and FIS (PI16/00425) RAREGenomics-CM (CAM, B2017/BMD3721), Conchita Rábago Foundation, Autonomous Community of Madrid (CAM, PEJ-2017-AI/BMD7256), Miguel Servet Program (CP12/03256) from ISCIII

M. del Pozo-Valero: None. R. Riveiro-Álvarez: None. F. Blanco-Kelly: None. J. Aguirre-Lamban: None. I. Martín-Mérida: None. I. Iancu: None. S. Swafiri: None. I. Lorda-Sanchez: None. E. Rodriguez-Pinilla: None. M. Trujillo-Tiebas: None. B. Jiménez-Rolando: None. E. Carreño: None. I. Mahillo-Fernandez: None. C. Rivolta: None. M. Corton: None. A. Avila-Fernandez: None. B. García-Sandoval: None. C. Ayuso: None.

P02.73.C

Functional characterization of a Xenopus tropicalis knockout and a human cellular model of RCBTB1associated inherited retinal disease shows involvement of RCBTB1 in the cellular response to oxidative stress

M. Carron $^{1,2}$, T. Naert ${ }^{l}$, G. Ascari ${ }^{2}$, S. Demuynck ${ }^{l}$, T. Van Nieuwenhuyzen ${ }^{1}$, T. Rosseel ${ }^{2}$, D. Priem ${ }^{3,4}$, A. Kremer ${ }^{4}, H$. Van Landeghem ${ }^{l}$, S. Hoogstoel ${ }^{2}$, F. Coppieters ${ }^{2}, C$. Guérin $^{5}$, K. Vleminckx ${ }^{1}$, E. De Baere

${ }^{1}$ Ghent University, Biomedical Molecular Biology, Ghent, Belgium, ${ }^{2}$ Ghent University, Center for Medical Genetics and Dept of Biomolecular Medicine, Ghent, Belgium, ${ }^{3}$ Ghent University, Ghent, Belgium, ${ }^{4}$ VIB-UGent Center for Inflammation Research, Ghent, Belgium, ${ }^{5}$ VIB Bioimaging Core, Ghent, Belgium, ${ }^{6}$ Ghent University and Ghent University Hospital, Center for Medical Genetics and Dept of Biomolecular Medicine, Ghent, Belgium

Introduction: The function of $R C B T B 1$, recently implicated in syndromic and non-syndromic inherited retinal disease (IRD), remains unknown so far. Patients with biallelic missense variants in $R C B T B 1$ display diverse IRD phenotypes such as retinitis pigmentosa. Here, we tested the hypothesis that RCBTB1 is involved in NRF2-regulated protection against reactive oxygen species in the eye, more specifically in the retinal pigment epithelium (RPE).

Materials and Methods: A Xenopus tropicalis rcbtb1 ${ }^{-1-}$ knockout (KO) was generated using CRISPR/Cas9 editing. Histology and three-dimensional electron microscopy was performed on retinas of $r c b t b 1^{-1-}$ frogs. RNA-seq analysis was performed on RCBTBI-mutated patients' lymphocytes, treated with $\mathrm{H}_{2} \mathrm{O}_{2}$, as well as on embryos from the $r c b t b 1$ ${ }^{-l-}$ KOs treated with $\mathrm{CdCl}_{2}$. An RCBTB1 knockdown cell line was generated in ARPE-19 cells and functional assays (flow cytometry, MTT-assay, cell death kinetics) assessed the consequences of RCBTB1 loss-of-function.
Results: $R c b t b 1^{-1-}$ animals showed changes in the RPE, similar to observations in human cases, including loss of apical-basal cell polarity, cuboidal cell morphology, spreading of the pigment granules and vacuolisation. NRF2 downstream targets and several metallothioneins were found to be differentially expressed, both in the KO and cellular models. Functional assays in ARPE-19 cells revealed that $R C B T B 1$ depletion affects cellular responses to external insults of oxidative stress.

Conclusions: We showed that the Xenopus tropicalis $r c b t b 1^{-1-}$ KO recapitulates the human IRD phenotype. Both in vivo and in vitro functional data show involvement of RCBTB1 in the cellular response to oxidative stress. This provides insight into the mechanism underlying RCBTB1associated IRD and uncovers potential therapeutic opportunities.

Funding: FWO/1802220N; FWO/1S52818N; BOF15/ GOA/011; BOF20/GOA/023.

M. Carron: None. T. Naert: None. G. Ascari: None. S. Demuynck: None. T. Van Nieuwenhuyzen: None. T. Rosseel: None. D. Priem: None. A. Kremer: None. H. Van Landeghem: None. S. Hoogstoel: None. F. Coppieters: None. C. Guérin: None. K. Vleminckx: None. E. De Baere: None.

P03 Internal Organs \& Endocrinology (Lung, Kidney, Liver, Gastrointestinal)

\section{P03.01.A}

A cost effective experience in diagnosis of autosomaldominant polycystic disease by custom-target NGS panel in a reference center.

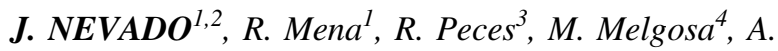

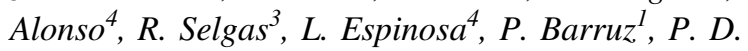
Lapunzina ${ }^{1,2}$

${ }^{1}$ INGEMM-IdiPaz, Madrid, Spain, ${ }^{2}$ CIBERER, Madrid, Spain, ${ }^{3} S^{o}$ Nefrología Adultos, HULP, Madrid, Spain, ${ }^{4} S^{\circ}$ Nefrología Infantil, HULP, Madrid, Spain

ADPKD is the most common inherited kidney disease that is caused by mutations in the $P K D 1 / P K D 2$. Most mutations take place in $P K D 1$. Its molecular diagnosis is a challenge by the high allelic heterogeneity and overlap of pseudogenes between 1-32 exons. So far, there are few data on $P K D 1 / P K D 2$ variants in the Spanish population. The main objective of this study has been to establish and know the mutations in ADPKD in our cohort. Mutational analysis of both PKD1 and PKD2 was performed on 158 unrelated families, by NGS using a custom exon-target panel from inherited nephropathies, Nephroseq ${ }^{\circledR}$. To confirm SNVs, Long-Range-PCR and Sanger sequencing were performed 
on PKD1 or PCR, and Sanger sequencing for PKD2. In addition, MLPA was used to rule out CNVs. SNVs were identified in 122 families (77.23\%): 96 families in $P K D 1$ (78.7\%) and 26 families in PKD2 (22.3\%). Globally: 64 frameshift and nonsense; 11 splicing, 36 missense, 8 in frame ins/del, and 4 large-deletions). Overall, $15 \%$ of variants were de novo and $50 \%$ were not previously described. This study demonstrates that NGS is a cost-effective experimental approach for simultaneous analysis of the PKD1 and PKD2. The analysis of mutations in the ADPKD-Spanish population expands the understanding of the genetic diversity of the different ethnic groups, enriches the databases with the new mutations, and contributes to their genetic counselling. As well as, reproductive (six pregnancies were currently successful) and/or therapeutic decision-making (a significant number of patients with highly pathogenic variants are currently under treatment with Tolvaptan).

J. Nevado: None. R. Mena: None. R. Peces: None. M. Melgosa: None. A. Alonso: None. R. Selgas: None. L. Espinosa: None. P. Barruz: None. P.D. Lapunzina: None.

\section{P03.04.A}

Clinical and molecular characteristics of young Russian patients with homo- and heterozygous AIRE mutations

E. N. Suspitsin ${ }^{1,2}$, L. V. Ditkovskaya ${ }^{1}$, T. V. Gabrusskayal, N. B. Ulanova ${ }^{l}$, D. L. Strekalov ${ }^{l}$, M. N. Guseva

${ }^{1}$ St.-Petersburg State Pediatric Medical University, St.Petersburg, Russian Federation, ${ }^{2}$ N.N. Petrov Institute of Oncology, St.-Petersburg, Russian Federation

Introduction: Hereditary defects of the AIRE gene are associated with a rare autoimmune polyendocrinopathycandidiasis-ectodermal dystrophy syndrome (APECED). The disorder has been found in many populations worldwide. The clinical manifestations are quite variable even within families, but major features include hypoparathyroidism, Addison disease, and mucocutaneous candidiasis. Although the condition usually follows autosomal-recessive inheritance there is an increasing body of evidence that some cases may be caused by monoallelic gain-of-function (GOF) mutations.

Materials and methods: 10 Russian patients (age 3-18 years) were subjected to the AIRE gene analysis. Of those, 5 had clinical signs of APECED syndrome suspected by immunologist, gastroenterologist or endocrinologist. In these patients Sanger sequencing of AIRE was performed. Another 5 patients underwent targeted sequencing of primary immunodeficiency (PID) genes including AIRE because of suspected PID condition.
Results: 6 patients had biallelic AIRE mutations with the common c.769C $>$ T (p.R257*) allele being detected in 4 individuals. One patient was homozygous for c.173C $>\mathrm{T}$ ( $\mathrm{p}$. A58V) variant recently described in Russian patients (Orlova et al, 2017). Rare c.32T>C (p.L11P) mutation was reported in Russian population for the first time. All monoallelic mutations $(n=4)$ were represented by the c.901G >A (p.V301M) allele. This variant altering functional properties of the protein was once reported in a Russian female with late-onset classical APECED (Oftedal et al, 2015).

Conclusions: Our study indicates that some GOF variants of AIRE are common in young Russian patients with manifestations of autoimmune disease.

This work was supported by RFBR grant 17-29-06069

E.N. Suspitsin: None. L.V. Ditkovskaya: None. T.V. Gabrusskaya: None. N.B. Ulanova: None. D.L. Strekalov: None. M.N. Guseva: None.

\section{P03.05.B}

Genetic and molecular analysis of urinary magnesium concentration in Scottish and Croatian populations

C. B. Joseph ${ }^{1}$, C. M. Stanton ${ }^{1}$, C. Drake ${ }^{1}$, T. S. Boutin ${ }^{1}$, J.

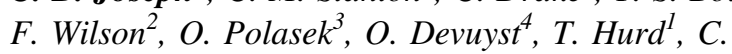
Hayward $^{1}$

${ }^{1}$ MRC Human Genetics Unit, Institute of Genetics and Molecular Medicine, University of Edinburgh, Edinburgh, United Kingdom, ${ }^{2}$ Centre for Global Health Research, Usher Institute for Population Health Sciences and Informatics, University of Edinburgh, Edinburgh, United Kingdom, ${ }^{3}$ Split Medical School, Split, Croatia, ${ }^{4}$ Institute of Physiology, University of Zurich, Zurich, Switzerland

Magnesium is the second most abundant bivalent cation in the body and is essential for many cellular processes. Renal magnesium handling plays an important role in maintaining magnesium homeostasis, however the exact biological mechanisms remain unclear. A recent genome-wide association study (GWAS) identified an association between urinary magnesium concentration $(\mathrm{uMg})$ and variants in the ARL15 gene on chromosome 5. ARL15 encodes a GTPbinding protein that regulates the magnesium transporter channel TRPM6, and other proteins involved in magnesium homeostasis in physiologically relevant cell lines. We conducted meta-analyses for urinary magnesium in 11, 617 individuals from Scottish and Croatian populations to determine possible genes involved in these traits. Mass spectrometry analysis was conducted to determine interactors of Arl15 and in-vitro knockout models of Arl15 was established using CRISPR-Cas9. The top SNP associated with $\mathrm{uMg}$ in our meta-analyses lies within a transcription 
factor binding site in an enhancer region of ARL15. Individuals homozygous for the alternate allele of rs35931 has lower urinary magnesium levels compared to individuals with the homozygous reference allele. Arl15 interacts with magnesium transporters within the distal convoluted tubule segment of the kidneys. Furthermore, we find that homozygous deletion of Arl15 is lethal at the organismal level. We hypothesise that the variant found affects the expression of ARL15, which, in turn modulates magnesium transport by regulating magnesium transporters. We are performing further functional studies to elucidate the role ARL15 plays in magnesium homeostasis both in vitro and in vivo.

C.B. Joseph: None. C.M. Stanton: None. C. Drake: None. T.S. Boutin: None. J.F. Wilson: None. O. Polasek: None. O. Devuyst: None. T. Hurd: None. C. Hayward: None.

\section{P03.08.B}

Blood chimerism in monochorionic dizygotic twins (MCDZT) identified at molecular genetic testing of permanent neonatal diabetes mellitus

\author{
B. Lorenz-Depiereux ${ }^{1}$, U. Ahting ${ }^{2}$, G. Eckstein ${ }^{1}, K$. \\ Warncke $^{3}$, T. Meitinger ${ }^{1,2}$, S. Andres ${ }^{2}$
}

${ }^{1}$ Helmholtz Zentrum München German Research Center for Environmental Health, Institute of Human Genetics, Neuherberg, Germany, ${ }^{2}$ Technical University of Munich, Institute of Human Genetics, Munich, Germany, ${ }^{3}$ Technical University of Munich School of Medicine, Kinderklinik München Schwabing, Department of Pediatrics, Munich, Germany

In most cases dizygotic (DZ) twins have a dichorionic placenta, but in some cases - especially in the context of assisted reproduction - blood chimerism in DZ twins due to placental sharing has been shown, identifying them as monochorionic dizygotic twins (MCDZT). Here we report the first case of blood chimerism in MCDZT detected as an additional finding at molecular genetic testing. Dizygotic twin brothers were born to a female couple after assisted reproduction (IVF, in vitro fertilization). One presented with permanent neonatal diabetes mellitus. Molecular genetic analysis identified a heterozygous pathogenic variant within the insulin gene (INS, dominant inheritance). Interestingly, the donor of the oocytes for IVF had been diagnosed with type 1 diabetes as an infant. Screening for the INS variant in her blood DNA was positive and altered the diagnosis to permanent neonatal diabetes mellitus. Testing of the unaffected brother for the INS variant surprisingly showed that he also carried the INS variant in blood DNA but not in DNA from buccal membrane cells. Additional microsatellite studies confirmed blood chimerism in both twins but ruled out chimerism in buccal membrane cells. Whether our findings should be classified as confined blood chimerism which is relatively common, or as an exceedingly rare reported tissue chimerism has to be elucidated by studies of other tissues.

B. Lorenz-Depiereux: None. U. Ahting: None. G. Eckstein: None. K. Warncke: None. T. Meitinger: None. S. Andres: None.

\section{P03.10.A}

Ultra-rare variants in the TRPV6 gene cause autosomal dominant chronic pancreatitis

E. Masson ${ }^{1,2}$, J. M. Chen ${ }^{l}$, V. Rebours ${ }^{3}$, M. Le Rhun ${ }^{4}, H$. Audin $^{5}$, A. Lachaux ${ }^{6}$, B. Caumont ${ }^{7}$, D. Lorenzo ${ }^{3}$, M. Fron ${ }^{8}$, K. Billiemaz ${ }^{9}$, R. Besnard ${ }^{10}$, L. Buscail ${ }^{11}$, GREPAN

(Genetic REsearch on PANcreatitis) Study Group, $\boldsymbol{C}$. Férec $^{l}$

${ }^{1}$ Univ Brest, Inserm, EFS, UMR 1078, GGB, Brest, France, ${ }^{2}$ CHRU Brest, Service de Génétique Médicale et de Biologie de la Reproduction, Brest, France, ${ }^{3}$ Department of Gastroenterology and Pancreatology, Beaujon Hospital, APHP, Clichy, and Paris-Diderot University, Paris, France, ${ }^{4}$ Service d'Hépato-Gastro-Entérologie, Cancérologie Digestive et Assistance Nutritionnelle, CHU Nantes, Nantes, France, ${ }^{5}$ Médecine 'Chauvet' à Orientation Gastro-Entérologique, CH Gabriel Martin, Saint Paul, France, ${ }^{6}$ Service de Gastroentérologie, Hépatologie et Nutrition Pédiatriques, HCL, Bron, France, ${ }^{7}$ Service de Médecine à Orientation Hépato--Gastro- Entérologique, CH Sud Gironde, Langon, France, ${ }^{8}$ Service d'Hépato-Gastro-Entérologie, CHD Vendée, La Roche-Sur-Yon, France, ${ }^{9}$ Service de Pédiatrie, CHU Saint-Etienne, Saint-Etienne, France, ${ }^{10}$ Service d'Hépato-Gastro-Entérologie et Oncologie Digestive, CHR Orléans, Orléans, France, ${ }^{11}$ Service de Gastroentérologie et Pancréatologie, CHU Toulouse, Toulouse, France

Until very recently, variants in the so far reported chronic pancreatitis (CP) genes such as PRSS1 have been found to cause/predispose to the disease through increasing trypsin activity or eliciting endoplasmic reticulum stress. In January 2020, an international collaboration reported association of functionally deficient variants in the TRPV6 gene with $\mathrm{CP}$, identifying a novel mechanism of pancreatitis due to $\mathrm{Ca}^{2+}$ dysregulation. This discovery was made essentially by analyzing young patients with idiopathic CP. Whether TRPV6 variants are responsible for autosomal dominantly inherited CP remains unknown. Herein, we analyzed the TRPV6 gene in probands of 235 such families by NGS. All families had at least two affected members and had remained genetically unexplained prior to the current analysis. We identified 11 ultra-rare variants (all were absent in 570 French controls and had an allele frequency of 0-0.000008 in gnomAD) in 11 families. Of the 11 variants, 5 were previously reported to be 
pathogenic. All the remaining six variants were novel, comprising a clear loss-of-function variant, a single amino acid deletion variant, and four missense variants. All these four missense variants were predicted to be of pathogenic relevance by Polyphen and/or SIFT and had a CADD score comparable to known pathogenic missense variants. In summary, we identified ultra-rare TRPV6 variants in $\sim 5 \%$ of the studied families. This establishes TRPV6 as a relatively frequent autosomal dominant $\mathrm{CP}$ gene for which genetic testing is warranted. Our results also suggest that haploinsufficiency of the TRPV6 gene is sufficient to cause CP. (Work supported by INSERM).

E. Masson: None. J.M. Chen: None. V. Rebours: None. M. Le Rhun: None. H. Audin: None. A. Lachaux: None. B. Caumont: None. D. Lorenzo: None. M. Fron: None. K. Billiemaz: None. R. Besnard: None. L. Buscail: None. C. Férec: None.

\section{P03.12.C}

\section{Truncating $L B X 1$ mutation in Congenital Central Hypo-} ventilation Syndrome

\section{Elbracht, M. Begemann, I. Kurth}

\section{Institute of Human Genetics, Aachen, Germany}

Undine syndrome, a congenital central hypoventilation syndrome (CCHS), derives its name from the legend of the water nymph Ondine/Undine who punished her mortal husband for his unfaithfulness with a curse of stopping breathing and dying when he fell asleep. Normally, increasing $\mathrm{CO}_{2}$ blood levels induce the hypercapnic reflex which accelerates breathing via specialized neurons of the pre-Bötzinger complex in the ventral hindbrain. Polyalanine expansions in $P H O X 2 B$ and rarely other genes have been described to cause genetic forms of CCHS by disturbing the breathing reflex. Recently, a homozygous mutation in the homeodomain transcription factor $L B X I$ was reported in two brothers with neonatal hypoventilation and complex autonomic symptoms. The mutation affects the $\mathrm{C}$-terminus of the protein and impairs cooperativity with PHOX2B, without disrupting the DNA-binding domain. We here report a female patient with severe hypoventilation who required continuous mechanical ventilation immediately after birth. During her first 11 years of life she was supplied with a tracheostomy and mask ventilation at night because of episodes of apnea. In childhood, she had severe constipation and, developed a paralytic ileus like Hirschsprung disease. Since the age of 11 she used a respiratory mask for sleeping and showed normocapnia. With consequent breathing control starting immediately after birth the now 23 years old woman developed excellently and completes a university education. Trio exome sequencing identified a homozygous frameshift-mutation in LBXI (hg19: chr10:102987166del, NM_006562.4:c.707del, p. (Val236Alafs*59)). Our patient corroborates the role of Cterminal $L B X 1$ mutations in CCHS and emphasizes the favorable clinical outcome in case of consequent treatment.

M. Elbracht: None. M. Begemann: None. I. Kurth: None.

\section{P03.13.A}

Deletion and duplication: unusual case of diagnostics for patient with cystic fibrosis

\section{S. Deryabina ${ }^{l, 2}$, O. Lagutina $^{l}, N$. Nikitina $^{l}$, E. Nikolaeva ${ }^{l}$}

${ }^{I}$ Medical Center "Health Care of Mother and Child", Yekaterinburg, Russian Federation, ${ }^{2}$ Federal State Autonomous Educational Institution of Higher Education «Ural Federal University named after the first President of Russia B.N. Yeltsin, Yekaterinburg, Russian Federation

Background: Cystic fibrosis (CF) is an autosomal recessive disease caused by a mutation in the CFTR (cystic fibrosis transmembrane conductance regulator) gene. Newborn screening for $\mathrm{CF}$ was introduced in the Russian Federation in 2006. The incidence of $\mathrm{CF}$ varies among different regions, in Sverdlovsk region it is 1:9,500. The vast majority of recurrent $C F T R$ variants are point mutations, but several populations have larger deletions and duplications, involving one or more complete exons. Here we want to report a case of $\mathrm{CF}$ with simultaneously existing deletion and duplication of different regions of 11 exon CFTR.

Case presentation: The patient (a 1-month-old girl) was born at term to non-consanguineous parents. For newborn's diagnostics CF we use a three-step IRT/IRT1个/DNA algorithm. Our patient had elevated levels of immunoreactive trypsinogen both times $(328.6 \mathrm{ng} / \mathrm{ml}$ and $299.8 \mathrm{ng} /$ $\mathrm{ml}$ respectively). Moreover, the girl had pathological sweat chlorides - $110 \mathrm{mmol} / \mathrm{L}$.

Results: The trio genetic sequencing of the CFTR gene showed heterozygous delF508 mutation in exon 11 for the child and her father. For searching another variant we were done Multiplex Ligation-mediated Probe Amplification (MLPA) of the CFTR gene. We have found heterozygous deletion of exon 9 for the girl and her mother and have proved the deletion F508 for father, but this analysis has shown also duplication of exon 11 and 2 copies F508 for proband.

Conclusions: We assume the proband has 3 copies of exon 11: two normal and one with del508F, and it is the defect copy, that is crucial for protein synthesis.

S. Deryabina: None. O. Lagutina: None. N. Nikitina: None. E. Nikolaeva: None. 


\section{P03.14.B}

Genotypic spectrum of cystic fibrosis in Cyprus with emphasis on the endemic L346P and the novel CFTRdup2 cases

A. Matthaiou ${ }^{l}$, P. Anagnostopoulou ${ }^{l, 2}$, P. Kouis ${ }^{l}$, V. Neocleous $^{3}$, T. Adamidi ${ }^{4}$, P. Ioannou ${ }^{5}$, P. Fanis ${ }^{3}$, C. Costi ${ }^{3}$, A. Georgiou ${ }^{4}$, L. A. Phylactou ${ }^{3}$, P. Yiallouros ${ }^{1,5}$

${ }^{1}$ Medical School, University of Cyprus, Nicosia, Cyprus, ${ }^{2}$ Institute of Anatomy, University of Bern, Bern, Switzerland, ${ }^{3}$ Cyprus Institute of Neurology and Genetics, Nicosia, Cyprus, ${ }^{4}$ Department of Pulmonology, Nicosia General Hospital, Nicosia, Cyprus, ${ }^{5}$ Pediatric Pulmonology Unit, Archbishop Makarios III Hospital, Nicosia, Cyprus

Introduction: The aim of this study was to describe the epidemiology of CFTR mutations in the cystic fibrosis population in Cyprus with particular emphasis on the endemic L346P (p.Leu346Pro) cases and a novel case which bears the poorly described CFTRdup2 in homozygosity.

Materials and Methods: The national cystic fibrosis patients' registry in Cyprus was searched for the demographic, diagnostic, and genotypic data of all cases whose diagnosis was genetically confirmed via the identification of two mutant CFTR alleles. In all cases, genotyping was done by Sanger sequencing for the detection of common mutations and was followed by next-generation sequencing for the identification of uncommon mutations.

Results: From an overall of 47 cases, the most common mutation was p.Phe508del with an allelic frequency of $46.9 \%$, which is compatible with its northwest-to-southeast decreasing frequency gradient in Europe. Most mutations were classified as CFTR protein processing (class II, 50\%) or conductance (class IV, 12.3\%) defects. Multiple mutations were detected in 3 cases. Seven patients with mild clinical manifestations, mostly presenting with dehydration and/or electrolyte imbalance, carried p.Leu346Pro (7.1\%), a class IV mutation detected solely in individuals of Cypriot descent. A novel case of a patient homozygous for the CFTRdup2 mutation was associated with a severe phenotype with hepatic involvement, biliary cirrhosis and eventually hepatic failure leading to death.

Conclusions: The small population of cystic fibrosis patients in Cyprus follows the regional genotypic trends, although several cases bear unique mutational profiles, which contribute to the wide genotypic and phenotypic spectrum of the disease.

A. Matthaiou: None. P. Anagnostopoulou: None. P. Kouis: None. V. Neocleous: None. T. Adamidi: None. P. Ioannou: None. P. Fanis: None. C. Costi: None. A.
Georgiou: None. L.A. Phylactou: None. P. Yiallouros: None.

P03.16.A

Distinct regulatory elements enhance $S L C 6 A 14$ expression modifying cystic fibrosis pancreas and lung phenotypes

\author{
M. Esmaeili ${ }^{1}, N$. Panjwani $^{l}$, G. He $e^{1,2}, F$. Lin $^{l}$, J. M. \\ Rommens $^{l, 3}$, L. J. Strug ${ }^{1,2}$
}

${ }^{1}$ Program in Genetics and Genome Biology, The Hospital for Sick Children, Toronto, ON, Canada, ${ }^{2}$ Division of Biostatistics, Dalla Lana School of Public Health, University of Toronto, Toronto, ON, Canada, ${ }^{3}$ Department of Molecular Genetics, University of Toronto, Toronto, ON, Canada

Introduction: Cystic Fibrosis (CF) individuals with the same CFTR mutations have variable lung and pancreatic disease severity. Genome wide association studies (GWAS) have indicated that SNP clusters in the immediate promoter region and far upstream of SLC6A14 modify meconium ileus (MI) occurrence at birth and progressive lung disease, respectively. SLC6A14 is a sodium- and chloride-dependent neutral and cationic amino acid transporter that is expressed in several tissues including lung and pancreatic duct.

Materials and Methods: We are using public data and in vitro transcriptional reporter studies to understand how the SLC6A14 promoter and upstream SNPs contribute to modification of $\mathrm{CF}$.

Results: Colocalizing SLC6A14 promoter SNPs with expression quantitative trait loci (eQTL) of multiple organs from Genotype-Tissue Expression (GTEx) suggests that the pancreas drives MI susceptibility and indicates that the eQTL effect is driven primarily by males. Reporter assays in the CFPAC-1 pancreas cell line with a $2.4 \mathrm{~kb}$ segment containing MI-associated SNPs display increased gene expression with the risk haplotypes, and exhibit transrepression with a steroid hormone receptor. The core promoter activity and haplotype effects were also evident in the lung cell lines Calu-3 and HBE. Analyses of ReMap and JASPAR databases pinpoint a series of transcription binding elements in the upstream region for ongoing investigations to understand how these segments crosstalk with the core promoter of SLC6A14 in the lung.

Conclusions: Our studies implicate differences in gene regulation of SLC6A14 in the lung versus the pancreas, with increased expression level being detrimental to $\mathrm{CF}$ phenotypes in both organs.

M. Esmaeili: None. N. Panjwani: None. G. He: None. F. Lin: None. J.M. Rommens: None. L.J. Strug: None. 


\section{P03.17.B}

Impact of next-generation sequencing on diagnosis of cystic kidney diseases

L. Obeidováa ${ }^{l}$, T. Seeman ${ }^{2}$, V. Elisakova ${ }^{l}, K$. Hirschfeldova $^{l}$, I. Zednikova ${ }^{l}$, B. Janosikova ${ }^{l}, M$. Urbanova $^{l}$, F. Liska ${ }^{l}$, O. Seda ${ }^{l}$, B. Chylikova ${ }^{l}$, J. Stekrova ${ }^{l}$

${ }^{1}$ Institute of Biology and Medical Genetics of the First Faculty of Medicine, Charles University and General University Hospital in Prague, Prague, Czech Republic, Prague, Czech Republic, ${ }^{2}$ Department of Pediatrics, 2nd Faculty of Medicine, Charles University in Prague and Motol University Hospital, Prague, Czech Republic, Prague, Czech Republic

Introduction: Cystic kidney diseases are a very heterogeneous group of chronic kidney diseases with sometimes ambiguous phenotype. Thus, the clinical diagnosis is often difficult to be made. The aim of our study was to test the ability of next-generation sequencing (NGS) to improve the diagnosis precision in a heterogeneous group of children with clinically suspected cystic kidney diseases.

Materials and Methods: Next-generation sequencing of genes responsible for the formation of cystic kidneys was performed in 32 unrelated patients with various clinically diagnosed cystic kidney diseases without a definitive genetic diagnosis.

Results: The underlying pathogenic variants were detected in $69 \%$ of patients $(n=22)$. The result of NGS correlated with the clinical diagnosis made before the NGS in $53 \%$ of patients $(n=17)$, in the remaining 15 children (47\%) the result of NGS revealed another type of cystic kidney disease than clinically suspected or did not find any causal mutation. The most common unexpected findings were variants in nephronophthisis (NPHP) genes in children with clinically suspected autosomal recessive polycystic kidney disease $(n=4)$. Overall, we found 25 (probably) pathogenic variants in the PKHD1 gene, 8 variants in the TMEM67 gene, 4 variants in the PKD1 gene, 2 variants in the HNF1B gene and 2 variants in BBS1 and NPHP1 genes, respectively.

Conclusions: NGS is a valuable tool in the diagnostics of various forms of cystic kidney diseases. Its results changed the clinically based diagnoses in $15 \%$ of the children.

Supported by the ESIF - Operational programme Prague CZ.07.1.02/0.0/0.0/17_049/0000828, GAUK 1015 and PROGRES- Q25/LF1

L. Obeidová: None. T. Seeman: None. V. Elisakova: None. K. Hirschfeldova: None. I. Zednikova: None. B. Janosikova: None. M. Urbanova: None. F. Liska:
None. O. Seda: None. B. Chylikova: None. J. Stekrova: None.

P03.18.C

Circulating miRNA as a predictive biomarker of type 2 diabetes in prediabetic patients-outcomes of 5-year prospective observational study

I. Sidorkiewicz, M. Niemira, K. Maliszewska, A. Bielska, A. Szałkowska, J. Raczkowska, G. Sokołowska, M. Górska, A. Krętowski

\section{Medical University of Bialystok, Bialystok, Poland}

Introduction: Type 2 Diabetes Mellitus (T2DM) remains the most prevalent form of diabetes. The detection of current T2DM diagnostic markers is generally late and occurs when displaying metabolic imbalance thus, novel biomarkers that could be useful as a new tool for predicting the progression from prediabetes to $\mathrm{T} 2 \mathrm{DM}$ are needed. The goal of this study was to conduct a baseline comparison of serum-circulating miRNA in prediabetic individuals with distinction between those who later progressed to T2DM and those who did not.

Materials and methods: The study group was divided patients with newly diagnosed T2DM over 5 year observational period $(n=18)$ and control group $(n=12)$ with no known history of dysglycemia. The expression level of 798 miRNAs in serum samples using the NanoString technology was examined.

Results: The study revealed that 18 miRNAs were deregulated in serum samples of patients who developed T2DM after 5 years versus non T2DM patients. Pathway analysis showed that miRNA target genes were mainly significantly enriched in homeostasis, neuronal signaling, protein complex oligomerization and core matrisome. ROC analysis demonstrated that miR-491-5p, miR-1307-3p, miR-298 and miR-1290 can be introduced as a diagnostic tool for the prediction of $\mathrm{T} 2 \mathrm{DM} \quad(\mathrm{AUC}=0.940 ; 0.894$; $0.877 ; 0.722$, respectively).

Conclusions: The results suggest that circulating miRNAs could potentially be used as predictive biomarkers of T2DM in prediabetic patients.

I. Sidorkiewicz: None. M. Niemira: None. K. Maliszewska: None. A. Bielska: None. A. Szałkowska: None. J. Raczkowska: None. G. Sokołowska: None. M. Górska: None. A. Krętowski: None.

P03.20.B

Identification of a germline interstitial deletion in14q32.13 in a child and mother with DICER1 syndrome 
I. Wieland ${ }^{I}$, I. Schanze ${ }^{1}$, A. Gnekow ${ }^{2}$, M. Kuhlen ${ }^{2}, F$. Maier $^{3}$, D. Wahl ${ }^{3}$, M. Frühwald ${ }^{2}$, M. Zenker ${ }^{1}$

${ }^{1}$ Institute of Human Genetics, University Hospital, Ottovon-Guericke University, Magdeburg, Germany, ${ }^{2}$ Swabian Children's Cancer Center, University Children's Hospital, Augsburg, Germany, ${ }^{3}$ Praxis für Humangenetik, Augsburg, Germany

Introduction: DICER1 syndrome (MIM 601200) is a rare tumor predisposition syndrome with autosomal dominant inheritance usually manifesting in childhood and adolescence. Tumor development follows Knudson's two-hit model involving the gene DICERI located on chromosome $14 \mathrm{q} 32.13$. Predisposing germline pathogenic variants (first hit) are mainly small loss-of-function mutations. Tumor-related driver mutations are specific hot-spot missense mutations in trans (second hit) located in the RNAse IIIb domain of the DICER1 protein.

Patients: We report on a female child with cystic nephroma of the right kidney and developmental delay at age 1 year. Facial features included frontal bossing, flat nasal bridge, hypertelorism and small mouth with thin lips. Body measurements were unremarkable. Her mother was reported with Sertoli-Leydig cell tumor (SLCT) and multinodular goiter at age 12 to 16 years. Clinical presentation of mother and child suggested familial inheritance of DICER1 syndrome.

Results: No typical DICERI loss-of-function germline mutation was detected in the child by standard Sanger sequencing. The child's tumor tissue of the right kidney revealed DICER1 hot-spot mutation c.5437G $>$ A p. Glu1813Lys. Subsequent molecular karyotyping of DNA from blood of the child using Affymetrix CytoScan HD SNP-Array showed a $2.31 \mathrm{MB}$ interstitial contiguous gene deletion in 14q32 (arr[hg19] 14q32.12q 32.13 (93835222_96155830)x1). The interval harbors 23 OMIM-listed genes including DICER1. The array result was finally confirmed in the blood of child and mother by MLPA.

Conclusions: Patients with DICER1 syndrome and germline $14 \mathrm{q} 32$ contiguous gene deletions infrequently have been detected. In the few patients reported to date various deletion intervals were identified suggesting no consistent chromosomal breakpoints.

I. Wieland: None. I. Schanze: None. A. Gnekow: None. M. Kuhlen: None. F. Maier: None. D. Wahl: None. M. Frühwald: None. M. Zenker: None.

\section{P03.22.A}

Two cases with central precocious puberty caused by paternally inherited novel variants in DLK1gene
V. Karaman ${ }^{1}$, E. Karakılıç Özturan ${ }^{2}$, F. Baş ${ }^{2}$, S. Başaran ${ }^{l}$, Z. Uyguner ${ }^{l}$

${ }^{1}$ Department of Medical Genetics, Istanbul Faculty of Medicine, Istanbul University, Istanbul, Turkey, ${ }^{2}$ Department of Pediatric Endocrinology, Istanbul Faculty of Medicine, Istanbul University, Istanbul, Turkey

Introduction: Gonadotropin-dependent or central precocious puberty (CPP) is caused by premature activation of the hypothalamic-pituitary-gonadal (HPG) axis. There are several genes playing roles in this pathway and two of them are maternally imprinted; 1) MKRN3 gene, located at 15q11.2, upstream suppressor of HPG axis, 2) DLK1 gene, located at $14 \mathrm{q} 32$, affecting kisspeptin signaling pathway hence important role for regulating pubertal timing.

Materials and Methods: Coding regions of the MKRN3 (NM_005664.3) and DLKI (NM_003836.6) genes were Sanger sequenced in 17 patients (15 female, 2 male). In presence of pathogenic variant, segregation analysis were performed in families.

Results: We identified two novel heterozygous variants in two unrelated cases in $D L K 1$ gene. The first variant was in exon 4 (c.357C $>\mathrm{G}$ (p.Tyr119Ter)) and the second was in intron $1(\mathrm{c} .67+78 \mathrm{C}>\mathrm{T})$, both were classified under probably pathogenic by in silico analysis. Segregation analysis presented the paternal inheritance of both, complying with the inheritance model of the genes. No pathogenic variant identified in MKRN3 gene in our cases.

Conclusions: Previously, three small and one gross deletions of $D L K 1$ were associated with CPP. Nonsense and splicing variant have first time presented in our study. The alteration identified in intron one needs further investigation on transcript level to show whether it causes splicing defect. Nevertheless, inheritance pattern complies with imprinted gene model of $D L K 1$. These results suggest that investigation of $D L K 1$ gene may contribute to the elucidation of the genetic assosiation of familial CPP. This study was supported by Scientific Research Projects Coordination Unit of Istanbul University (TDK-2018-32682)

V. Karaman: None. E. Karakılıç Özturan: None. F. Baş: None. S. Başaran: None. Z. Uyguner: None.

\section{P03.23.B}

Elucidating the molecular mechanism underlying laterality defects

D. Antony ${ }^{1,2,3}$, A. Maver ${ }^{4}$, M. A. Deardorff 5,6 , L. Slagter ${ }^{2}$, K. Wu ${ }^{2}$, Z. Bakey ${ }^{1,2}$, E. Y. Gulec ${ }^{7}$, A. Gezdirici ${ }^{7}, H$. Brunner $^{2,3}$, M. Schmidts ${ }^{1,2,3}$

${ }^{I}$ Center for Pediatrics and Adolescent Medicine, University Hospital Freiburg, Freiburg, Germany, ${ }^{2}$ Genome Research 
Division, Department of Human Genetics, Radboud UMC, Nijmegen, Netherlands, ${ }^{3}$ Radboud Institute of Molecular Life Sciences, Nijmegen, Netherlands, ${ }^{4}$ Centre for Mendelian Genomics, Clinical Institute of Medical Genetics, UMC Ljubljana, Ljubljana, Slovenia, ${ }^{5}$ Department of Pediatrics and Pathology, The Perelman School of Medicine, University of Pennsylvania, Pennsylvania, PA, United States, ${ }^{6}$ Laboratory Medicine, The Perelman School of Medicine, University of Pennsylvania, Pennsylvania, PA, United States, ${ }^{7}$ University of Health Sciences, Kanuni Sultan Suleyman training and research Hospital, Department of Medical Genetics, Istanbul, Turkey

Introduction: Laterality defects are rare developmental disorders, occurring isolated or as part of complex syndromes. Underlying cause is frequently dysfunction of motile cilia causing Primary Ciliary Dyskinesia (PCD) and rarely, non-motile cilia defects or non-ciliary causes. In addition to randomization of the left right body axis, PCD is characterized by frequent respiratory infections and infertility.

Methods: We used Whole Exome Sequencing (WES) to delineate the underlying molecular cause in 37 mainly consanguineous families with laterality defects.

Results: We identified causative variants in $32 \%$ with majority of mutations detected in genes previously associated with $\mathrm{PCD}$, including 2 small homozygous CNVs. Variants in non PCD genes, PKDIll and GJAI were also detected. We further identified homozygous null mutations in two genes not previously associated with human disease, MNS1 and DNAH9, both in cases with situs inversus but little or no respiratory symptoms. Gene matcher database revealed two additional families with biallelic DNAH9 mutation and immunofluorescence analysis revealed complete absence of DNAH9 from respiratory cilia of one affected while DNAH9 localises to the distal half of cilia in controls. Further, we found direct interaction of DNAH9 with the ODA docking complex protein CCDC114 using $\mathrm{Y} 2 \mathrm{H}$ screening also interaction with DNAH5 and DNAI2 using co-Immunoprecipitation.

Conclusions: We could only solve a third of the laterality cases genetically using WES, pointing towards non coding variations, genetic mosaicism or epigenetic effects while we were able to identify two novel human disease causing genes, MNS1 and DNAH9, both causing laterality defects with no or little respiratory disease.ERC: TreatCilia

D. Antony: None. A. Maver: None. M.A. Deardorff: None. L. Slagter: None. K. Wu: None. Z. Bakey: None. E.Y. Gulec: None. A. Gezdirici: None. H. Brunner: None. M. Schmidts: None.
P03.24.C

Insights into the proteomics of renal function: a transethnic meta-analysis and Mendelian randomization study

P. R. Matias-Garcia ${ }^{1,2,3}$, R. Wilson ${ }^{1,2}, Q$. Guo $^{4}$, S. B. Zaghlool $^{5}$, S. Sharma ${ }^{1,2}$, P. Schlosser ${ }^{6}$, A. Köttgen ${ }^{6,7}$, A. Peters $^{2,8,9}$, D. O. Mook-Kanamori ${ }^{10}$, J. Graumann ${ }^{11,12}$, W. König ${ }^{8,13,14}$, K. Hveem ${ }^{15,16}$, C. Jonasson ${ }^{15,16}$, A.

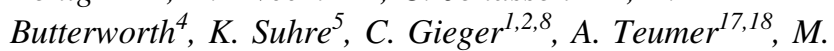
Waldenberger ${ }^{1,2,8}$

${ }^{I}$ Research Unit of Molecular Epidemiology, Helmholtz Zentrum München, German Research Center for Environmental Health, Neuherberg, Germany, ${ }^{2}$ Institute of Epidemiology, Helmholtz Zentrum München, German Research Center for Environmental Health, Neuherberg, Germany, ${ }^{3}$ School of Medicine, Technical University of Munich, Munich, Germany, ${ }^{4}$ Cardiovascular Epidemiology Unit, Department of Public Health and Primary Care, University of Cambridge, Strangeways Research Laboratory, Wort's Causeway, Cambridge, United Kingdom, ${ }^{5}$ Department of Physiology and Biophysics, Weill Cornell Medicine-Qatar, Doha, Qatar, ${ }^{6}$ Institute of Genetic Epidemiology, Faculty of Medicine and Medical Center-University of Freiburg, Freiburg, Germany, ${ }^{7}$ Department of Epidemiology, Johns Hopkins Bloomberg School of Public Health, Baltimore, MD, United States, ${ }^{8}$ German Research Center for Cardiovascular Research (DZHK), partner site Munich Heart Alliance, Munich, Germany, ${ }^{9}$ German Center for Diabetes Research (DZD), München-Neuherberg, Neuherberg, Germany, ${ }^{10}$ Department of Clinical Epidemiology, Leiden University Medical Centre, Leiden, Netherlands, ${ }^{11}$ Scientific Service Group Biomolecular Mass Spectrometry, Max Planck Institute for Heart and Lung Research, W.G. Kerckhoff Institute, Bad Nauheim, Germany, ${ }^{12}$ German Centre for Cardiovascular Research (DZHK), partner site Rhine-Main, Max Planck Institute of Heart and Lung Research, Bad Nauheim, Germany, ${ }^{13}$ Deutsches Herzzentrum München, Technical University of Munich, Munich, Germany, ${ }^{14}$ Institute of Epidemiology and Medical Biometry, University of Ulm, Ulm, Germany, ${ }^{15}$ K.G. Jebsen Center for Genetic Epidemiology, Department of Public Health, Norwegian University of Science and Technology, Trondheim, Norway, ${ }^{16}$ HUNT Research Center, Department of Public Health, Norwegian University of Science and Technology, Levanger, Norway, ${ }^{17}$ Institute for Community Medicine, University Medicine Greifswald, Greifswald, Germany, ${ }^{18}$ German Center for Cardiovascular Research (DZHK), partner site Greifswald, Greifswald, Germany 
Introduction: Renal health is reflected in the kidneys' ability to filter blood, clinically measured as the estimated glomerular filtration rate (eGFR). A number of plasma proteins have been found to be associated with renal phenotypes, but prior studies have been limited by smaller sample sizes and fewer proteins. Moreover, the causal mechanisms underlying these associations have not been investigated.

Methods: We conducted the largest observational study of plasma proteins and renal function in terms of sample size (up to $\mathrm{N}=5,285$ across four studies: KORA, INTERVAL, HUNT, QMDiab) and number of proteins $(\mathrm{k}=1,100)$. Associations with eGFR and chronic kidney disease (CKD) were tested using regression models in a trans-ethnic discovery-replication approach. We further examined the causal nature of these associations using two-sample bidirectional Mendelian randomization.

Results: 57 proteins were found to be consistently associated with eGFR in population samples of European and admixed ancestry; of these, 18 were associated with CKD. Many well-known biomarkers of renal function (e.g. cystatin-C) were identified, as were JAM-B and contactin-4 as novel biomarkers. The strongest evidence of causality was observed in the positive effect of eGFR on testican-2 $(\mathrm{p}=2.84 \mathrm{E}-04)$, a finding consistent with the biological role of testican-2 in glomeruli remodeling and kidney injury repair.

Conclusions: Our study uncovers novel plasma proteomic biomarkers for renal function and presents evidence for selected causal relationships. Plasma levels of testican-2 are reflective of mechanisms underlying progressive renal function loss, thus suggesting its applicability as a biomarker of early renal dysfunction and disease progression in the general population.

P.R. Matias-Garcia: None. R. Wilson: None. Q. Guo: None. S.B. Zaghlool: None. S. Sharma: None. P. Schlosser: None. A. Köttgen: None. A. Peters: None. D. O. Mook-Kanamori: None. J. Graumann: None. W. König: None. K. Hveem: None. C. Jonasson: None. A. Butterworth: None. K. Suhre: None. C. Gieger: None. A. Teumer: None. M. Waldenberger: None.

\section{P03.25.A}

Gain-of-function variant of the calcium-sensing receptor gene is associated with longer survival on renal replacement therapy in Caucasian patients

\author{
H. M. Rothe $\boldsymbol{e}^{1,2}$, S. Komolka ${ }^{2}$, C. Wanner $^{3}$, G. Mayer ${ }^{4}$ \\ ${ }^{1}$ Centre for Nephrology and Metabolic Disorders, Weiss- \\ wasser, Germany, ${ }^{2}$ Coburg University for Applied Sciences,
}

Coburg, Germany, ${ }^{3}$ University of Würzburg, Division of Nephrology, University Hospital, Würzburg, Germany, ${ }^{4}$ Medical University Innsbruck, Department of Internal Medicine IV, Nephrology and Hypertension, Innsbruck, Austria

Introduction: Patients with end-stage kidney disease require renal replacement therapy, i.e. dialysis or kidney transplantation, for survival. Here we report for the first time a genetic variant which is associated with longer survival on renal replacement therapy in Caucasian patients.

Materials and Methods: We conducted a survival analysis of three European end-stage kidney disease patient populations in Austria and Bavaria. 198 patients (118 male, 80 female) were genotyped for three variants of the calcium-sensing receptor gene after signing the consent form. The study started in 2005 and survival analysis was performed in 2018. During these years all patients received care according to the standards of the Austrian and German societies of nephrology.

Results: Survival on renal replacement therapy was significantly different (age and sex adjusted Cox regression $\mathrm{p}=0.04$ ) between carriers of the glycine and arginine alleles at position 990 of the calcium-sensing receptor molecule (rs1042636.) The two rs 1042636 cohorts AA (two arginine alleles) and GA/GG (at least one glycine allele) were in Hardy Weinberg equilibrium with a minor allele frequency of $7 \%$.

Conclusions: The glycine variant of rs1042636 calciumsensing receptor molecules has been shown to result in a gain-of-function, as compared with the arginine variant, in cell culture studies with transfected human embryonic kidney cells. It was also associated with lower baseline parathyroid hormone levels in the Caucasian patient cohort of the Evaluation of Cinacalcet Hydrochloride Therapy to Lower Cardiovascular Events (EVOLVE) trial. Our study shows that it is associated with longer survival on renal replacement therapy in Caucasian patients.

H.M. Rothe: None. S. Komolka: None. C. Wanner: None. G. Mayer: None.

\section{P03.26.B}

Genes regulating enteric nervous system development are impacted by Copy Number loss and modify penetrance in epistasis with $R E T$

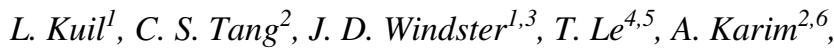
B. M. de Graaf ${ }^{l}$, R. van der Helm ${ }^{l}, Y$. van Bever ${ }^{l}$, C. E. J. Sloots $^{3}$, C. Meeussen ${ }^{3}$, D. Tibboel ${ }^{3}$, A. de Klein ${ }^{1}$, R. M. H.

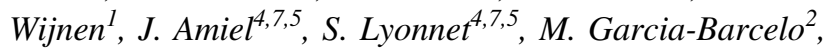


P. K. H. Tam ${ }^{2}$, M. M. Alves ${ }^{l}$, A. S. Brooks ${ }^{l}$, R. M.W. Hofstra $^{l, 8}$, E. Brosens ${ }^{l}$

${ }^{1}$ Clinical Genetics department, Erasmus Medical Centre Sophia Children's Hospital, Rotterdam, Netherlands, ${ }^{2}$ Department of Surgery, Li Ka Shing Faculty of Medicine, University of Hong Kong, Hong Kong, China, ${ }^{3}$ Paediatric Surgery department, Erasmus Medical Centre - Sophia Children's Hospital, Rotterdam, Netherlands, ${ }^{4}$ Paris Descartes - Sorbonne Paris Cité University, Imagine Institute, Paris, France, ${ }^{5}$ Laboratory of embryology and genetics of congenital malformations, INSERM UMR1163, Paris, France, ${ }^{6}$ Chittagong Research Institute for Children Surgery (CRICS), Chittagong, Bangladesh, ${ }^{7}$ Service de Génétique, Necker Enfants Malades University Hospital, APHP, Paris, France, ${ }^{8}$ Stem Cells and Regenerative Medicine, UCL Great Ormond Street Institute of Child Health, London, United Kingdom

Hirschsprung disease (HSCR) is characterized by absence of enteric ganglia, primarily in the distal colon. Approximately $18 \%$ of patients have additional anatomical malformations or associated neurodevelopmental disorders (HSCR-complex). We hypothesized that genes affected by rare Copy Number Variants contribute to disease development in "HSCR-complex" patients. Indeed, we could confirm that Copy Number $(\mathrm{CN})$ losses $(n=11)$ are enriched in these patients $(n=23)$, if no other causal variant was identified $\left(\mathrm{p}=3.64 \mathrm{E}^{-7}\right)$. At least five patients had a large de novo CNV (3 losses, 2 gains). The identified rare $\mathrm{CN}$ losses are enriched for variant intolerant genes, overexpressed in the developing mouse enteric nervous system $(\mathrm{p}=1.760 \mathrm{E}-$ 10): SLC8A1, GNL1, GABBR1, MAPK8, UFDIL, AKT3, $T B X 2, U S P 32$ and TUBB. Additionally, a loss of function variant in $T U B B$ was identified in a HSCR patient without a deleterious $\mathrm{CNV}$, and $A K T 3$ as well as UFDIL are impacted by $\mathrm{CN}$ losses in other patients. The contribution of the known HSCR predisposing risk haplotypes was comparable between patients with a pathogenic coding variant and controls $(\mathrm{P}=0.826)$. All other HSCR subgroups differed significantly with controls and between each subgroup ( $\mathrm{P}=0.02$ or lower), patients with isolated HSCR and without a RET coding variant had the highest burden, unaffected controls the lowest. Our zebrafish model reveals that disruption of six of these 9 genes - alone or via epistasis with ret- results in a HSCR phenotype. This confirms the epistatic effect of losing a copy of a gene regulating enteric nervous system development with the predisposed downregulation of RET expression.

L. Kuil: None. C.S. Tang: None. J.D. Windster: None. T. Le: None. A. Karim: None. B.M. de Graaf: None. R. van der Helm: None. Y. van Bever: None. C.E.J. Sloots: None. C. Meeussen: None. D. Tibboel: None. A. de
Klein: None. R.M.H. Wijnen: None. J. Amiel: None. S. Lyonnet: None. M. Garcia-Barcelo: None. P.K.H. Tam: None. M.M. Alves: None. A.S. Brooks: None. R.M.W. Hofstra: None. E. Brosens: None.

\section{P03.27.C}

Identification of disease-causing variants in children and adolescents with focal segmental glomerulosclerosis

S. Draut ${ }^{l}$, M. C. Braunisch ${ }^{1,2}$, K. M. Riedhammer ${ }^{1,2}$, P. M. Herr $^{l}$, R. Günthner ${ }^{1,2}$, M. Wagner ${ }^{1,3,4}$, B. Alhaddad ${ }^{l}, L$. Renders $^{2}$, U. Heemann ${ }^{2}$, C. Schmaderer ${ }^{2}$, J. Hoefele ${ }^{l}$

${ }^{1}$ Institute of Human Genetics, Klinikum rechts der Isar, Technical University of Munich, School of Medicine, Munich, Germany, ${ }^{2}$ Department of Nephrology, Klinikum rechts der Isar, Technical University of Munich, School of Medicine, Munich, Germany, ${ }^{3}$ Institute of Human Genetics, Helmholtz Zentrum München, Neuherberg, Germany, ${ }^{4}$ Institute of Neurogenomics, Helmholtz Zentrum München, Neuherberg, Germany

Introduction: A monogenic cause can be identified in approximately $30 \%$ of young patients $(<25$ years of age) with steroid-resistant nephrotic syndrome (SRNS). SRNS in children and adolescents is mostly inherited in an autosomal recessive pattern. The histological correlate of SRNS is often focal segmental glomerulosclerosis (FSGS). The aim of this study was to identify genetic causes in a cohort of children and adolescents with FSGS.

Material and Methods: 35 unrelated individuals with disease onset $<18$ years of age and suspected hereditary FSGS were analyzed using exome sequencing. Suspected hereditary FSGS was defined if at least one of the following criteria was present: absence of a secondary cause, $<18$ years of age, kidney biopsy with suspicion of a hereditary cause, extrarenal manifestations, and/or positive familial history/consanguinity. Variants were rated according to the ACMG classification. The clinical phenotype was assessed using a standardized questionnaire.

Results: In seven patients, (likely) pathogenic variants according to ACMG were identified in known diseasecausing genes (diagnostic yield: 20\%). Three variants in two different FSGS genes (INF2, TRPCO) had to be classified as VUS (Table 1).

Conclusions: The diagnostic yield of $20 \%$ highlights the importance of genetic testing in patients $<18$ years of age with nephrotic syndrome. Early genetic diagnosis could allow a personalized treatment approach with weaning of unnecessary immunosuppressive treatment, avoidance of renal biopsies and provision of well-informed genetic counselling. 
Table 1. Results of exome sequencing

\begin{tabular}{|c|c|c|c|c|c|c|}
\hline $\begin{array}{l}\text { Study- } \\
\text { ID }\end{array}$ & $\begin{array}{l}\text { Gen } \\
\text { (transcript) }\end{array}$ & $\begin{array}{l}\text { Nucleotide } \\
\text { change }\end{array}$ & $\begin{array}{l}\text { Amino } \\
\text { acid change }\end{array}$ & Segregation & Zygosity & $\begin{array}{l}\text { Solved/ } \\
\text { VUS }\end{array}$ \\
\hline $\begin{array}{l}\text { HN- } \\
\text { F19- } \\
\text { II-1 }\end{array}$ & $\begin{array}{l}\text { WT1 } \\
\text { (NM_024426.4) }\end{array}$ & c. $598 \mathrm{G}>\mathrm{T}$ & p.(Gly170Cys) & de novo & heterozygous & solved \\
\hline $\begin{array}{l}\text { HN- } \\
\text { F20- } \\
\text { II-1 }\end{array}$ & $\begin{array}{l}\text { INF2 } \\
\text { (NM_022489.3) }\end{array}$ & c.224_256del & $\begin{array}{l}\text { p. } \\
\text { (Asp75_Ser85del) }\end{array}$ & n.d. & heterozygous & VUS \\
\hline $\begin{array}{l}\text { ATS- } \\
\text { F29- } \\
\text { III-1 }\end{array}$ & $\begin{array}{l}\text { COL4A3 } \\
\text { (NM_000091.4) }\end{array}$ & $\begin{array}{l}\text { c. } 1831 \mathrm{G}>\mathrm{A} \\
\text { c. } 4421 \mathrm{~T}>\mathrm{C}\end{array}$ & $\begin{array}{l}\text { p.(Gly611Arg) } \\
\text { p.(Leu1474Pro) }\end{array}$ & $\begin{array}{l}\mathrm{p} \\
\mathrm{m}\end{array}$ & $\begin{array}{l}\text { heterozygous } \\
\text { heterozygous }\end{array}$ & solved \\
\hline $\begin{array}{l}\text { HN- } \\
\text { F201- } \\
\text { II-1 }\end{array}$ & $\begin{array}{l}\text { NPHS2 } \\
\text { (NM_014625.2) }\end{array}$ & $\begin{array}{l}\text { c. } 413 \mathrm{G}>\mathrm{A} \\
\text { c.467dup }\end{array}$ & $\begin{array}{l}\text { p.(Arg138Gln) } \\
\text { p. } \\
\text { (Leu156Phefs*11) }\end{array}$ & n.d. & $\begin{array}{l}\text { heterozygous } \\
\text { heterozygous }\end{array}$ & solved \\
\hline $\begin{array}{l}\mathrm{HN}- \\
\text { F227- } \\
\text { II-1 }\end{array}$ & $\begin{array}{l}\text { NPHSI } \\
\text { (NM_004646.3) }\end{array}$ & c.515_517del & p.(Thr172del) & $\mathrm{p}, \mathrm{m}$ & homozygous & solved \\
\hline $\begin{array}{l}\text { ATS- } \\
\text { F261- } \\
\text { II-1 }\end{array}$ & $\begin{array}{l}\text { COL4A5 } \\
\text { (NM_000945.4) }\end{array}$ & c. $645+1 \mathrm{G}>\mathrm{T}$ & p.(?) & n.d. & heterozygous & solved \\
\hline $\begin{array}{l}\text { HN- } \\
\text { F324- } \\
\text { II-1 }\end{array}$ & $\begin{array}{l}\text { TRPC6 } \\
\text { (NM_004621.5) }\end{array}$ & c. $2392 \mathrm{G}>\mathrm{C}$ & p.(Asp798His) & n.d. & heterozygous & VUS \\
\hline $\begin{array}{l}\mathrm{HN}- \\
\text { F327- } \\
\text { II-1 }\end{array}$ & $\begin{array}{l}\text { TRPC6 } \\
\text { (NM_004621.5) }\end{array}$ & c.253_264dup & $\begin{array}{l}\text { p. } \\
\text { (Phe88_Ser89ins } \\
\text { SerAspArgSer) }\end{array}$ & $\mathrm{p}$ & heterozygous & VUS \\
\hline $\begin{array}{l}\text { HN- } \\
\text { F485- } \\
\text { II-3 }\end{array}$ & $\begin{array}{l}L M X 1 B \\
\text { (NM_002316.3) }\end{array}$ & c.917_926del & $\begin{array}{l}\text { p. } \\
\text { (Met306Thrfs*50) }\end{array}$ & n.d. & heterozygous & solved \\
\hline $\begin{array}{l}\text { HN- } \\
\text { F487- } \\
\text { II-2 }\end{array}$ & $\begin{array}{l}\text { PLCE1 } \\
\text { (NM_016341.3) }\end{array}$ & c. $3346 \mathrm{C}>\mathrm{T}$ & p. $\left(\operatorname{Arg} 1116^{*}\right)$ & n.d. & homozygous & solved \\
\hline
\end{tabular}

m, maternal; n.d., not determined; p, paternal; VUS, variant of unknown significance

S. Draut: None. M.C. Braunisch: None. K.M. Riedhammer: None. P.M. Herr: None. R. Günthner: None. M. Wagner: None. B. Alhaddad: None. L. Renders: None. U. Heemann: None. C. Schmaderer: None. J. Hoefele: None.

\section{P03.29.B}

Genetic linkage analysis identifies a genomic region in $2 q 24.3$ as a candidate modulator of reduced penetrance in heritable pulmonary arterial hypertension.

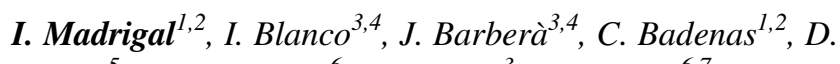

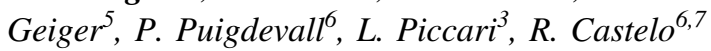

${ }^{1}$ Biochemistry and Molecular Genetics, IDIBAPS, Hospital Clinic, Barcelona, Spain, ${ }^{2}$ Centro de Investigación Biomédica en Red de Enfermedades Raras (CIBERER), Barcelona, Spain, ${ }^{3}$ Hospital Clínic de Barcelona, Institut d'Investigacions Biomèdiques August Pi i Sunyer (IDIBAPS), Barcelona, Spain, ${ }^{4}$ Centro de Investigación Biomédica en Red de Enfermedades Respiratorias (CIBERES), Madrid, Spain, ${ }^{5}$ Faculty of Computer Science, Technion Israel Institute of Technology, Haifa, Israel, ${ }^{6}$ Department of Experimental and Health Sciences, Universitat Pompeu Fabra, Barcelona, Spain, ${ }^{7}$ nstitut Hospital del Mar d'Investigacions Mèdiques (IMIM), Barcelona, Spain
Hereditary pulmonary arterial hypertension (hPAH) is an autosomal dominant disease caused, in most cases, by mutations in the BMPR2 gene. However, not all BMPR2 mutation carriers develop the disease, highlighting the presence of reduced penetrance. In order to identify genomic regions that could be contributing to incomplete penetrance, high-throughput genotyping arrays and next generation sequencing were conducted in an hPAH family. The family comprises 65 Spanish individuals spanning five different generations. One BMPR2 mutation (c.1472G> A; p.Arg491Gln) was identified in 22 members, but only 9 of them developed the disease. The SNP arrays analysis was performed in 33 family members and we identified a genomic region in $2 \mathrm{q} 24.3$, located at $38 \mathrm{Mb}$ from the $B M P R 2$ gene, which could contribute to increase the susceptibility of pulmonary hypertension in mutation carriers (Puigdevall et al., J Med Genet, 2019). Several genetic association studies have identified variants in this region associated with phenotypes involved in cardiovascular pathology, such as hypertension or ischemic cardiomyopathy, which could be related to some of the symptoms of pulmonary hypertension. In order to identify the specific variant/s that may act as genetic modifiers of $\mathrm{hPAH}$ within the candidate region, we have performed whole-genome sequencing in 16 BMPR2 mutation carriers: 6 affected individuals and 7 asymptomatic carriers. The identification of a possible genetic modifier in this family will allow us to advance in our understanding of reduced penetrance in hPAH.

Acknowledgments: ISCIIII (PI15/00483), 'fondos FEDER', Agència de Gestió d'Ajuts Universitaris i de Recerca (2017SGR1134), “CERCA Programme / Generalitat de Catalunya"

I. Madrigal: None. I. Blanco: None. J. Barberà: None. C. Badenas: None. D. Geiger: None. P. Puigdevall: None. L. Piccari: None. R. Castelo: None.

\section{P03.32.B}

HNF1B-related syndrome: genotype-phenotype correlation

C. P. Cristalli ${ }^{1,2}$, A. Mattiaccio $^{2}$, C. Graziano $^{3}$, I. Capelli $^{1}$, O. Baraldi ${ }^{1}$, G. Comai ${ }^{1}$, M. Pariali ${ }^{2,3}$, L. Gargano ${ }^{2,3}$, M. Seri ${ }^{3}$, G. La Manna ${ }^{1}$, V. Mantovani ${ }^{2,3}$

${ }^{1}$ Nephrology Dialysis and Transplantation Unit, DIMES, Bologna University, Bologna, Italy, ${ }^{2}$ Center for Applied Biomedical Reasearch (CRBA), Bologna University, Bologna, Italy, ${ }^{3}$ Medical Genetics Unit, S.Orsola-Malpighi University Hospital, Bologna, Italy

Molecular defects of HNF1B are associated with RCAD syndrome, a clinically heterogeneous disorder characterized 
by diabetes (MODY5), kidney abnormalities (cysts), cholestasis and uro-genital malformations. We performed targeted NGS of 15 genes and MLPA on 118 patients recruited by nephrology or diabetology units. 19 index cases showed molecular defects in HNF1B gene (10 pediatric and 9 adult). Six patients carried a likely pathogenic novel variant (p.G83D p.R295C, c.-67C>T, c.1207-1G>A, p.S379*, p. Q484Sfs*22) and 4 a VUS (p.G76C, p.E105K, p.A453T, p. $\mathrm{M} 532 \mathrm{~V}$ ). A functional analysis for c. $-67 \mathrm{C}>\mathrm{T}$ indicated reduced activity in $\mathrm{Caco} 2$ and Mcf7 cells. Nine cases showed the whole HNF1B gene deletion (de novo in five patients). The median age of diagnosis was 19 (SNVs) and 6 (deletion) years old, respectively. Clinical presentation included earlier onset in deleted patients (median 6 vs 19 years) and different spatial cysts localization (cortical for SNVs and medullary in at least 3 deleted cases). Diabetes was present in six patients only, possibly due to the young age of many cases involved. Two patients with urogenital malformation and 3 with cholestasis were identified. A 21year-old woman had mild developmental delay: additional array-CGH was performed and a large $(1.3 \mathrm{Mb})$ deletion was found. Contrary, a 29-years-old man homozygous for p.G83D did not show intellectual disability. In conclusion, the patients with SNVs developed a milder phenotype than those deleted, and the molecular defects were frequently of de novo origin. We confirm the wide phenotypic heterogeneity of HNF1B-related disorders and we point out that liver disease can be a major complication.

C.P. Cristalli: None. A. Mattiaccio: None. C. Graziano: None. I. Capelli: None. O. Baraldi: None. G. Comai: None. M. Pariali: None. L. Gargano: None. M. Seri: None. G. La Manna: None. V. Mantovani: None.

\section{P03.33.C}

Impact of common genetic risk variants on familial aggregation of inflammatory bowel disease

\section{H. Lee ${ }^{1,2}$, L. Hannes $^{l}$, M. Vancamelbeke $^{l}$, V. Ballet ${ }^{3}, M$. Ferrante $^{3,4}$, S. Vermeire $^{3,4}$, I. Cleynen ${ }^{1}$ \\ ${ }^{1}$ KU Leuven, Leuven, Belgium, ${ }^{2}$ University of Ulsan College of Medicine, Seoul, Korea, Republic of, ${ }^{3}$ University Hospitals Leuven, Leuven, Belgium, ${ }^{4} \mathrm{KU}$ leuven, Leuven, Belgium}

Family history is the strongest risk factor for inflammatory bowel disease (IBD). The contribution of common genetic variants to familial aggregation in multiple-affected IBD families, however, remains unclear. We included 54 multiple-affected families ( $\geq 3$ first-degree relatives affected), including 189 affected (IBD), and 133 unaffected firstdegree relatives; as well as 1768 sporadic cases and 868 unrelated non-IBD controls. For all individuals,
Immunochip genotypes were available. Weighted polygenic risk scores (PRS) were calculated using PRSice-2.0 across different $p$-value thresholds (pTs), and compared between groups. The association between PRS and familial IBD was further evaluated in age- and sex-adjusted generalized mixed regression models including family id as random effect. Using $\mathrm{pT}=0.05$, the affected relatives had a higher PRS than unaffected relatives $\left(\mathrm{P}=1.0 \times 10^{-02}\right)$, sporadic cases $\left(\mathrm{P}=4.6 \times 10^{-02}\right)$, and non-IBD controls $(\mathrm{P}<2.2 \mathrm{x}$ $\left.10^{-16}\right)$. The risk of IBD in families increased by 1.23 -fold for every incremental standard deviation in PRS. However, the proportion of the explained variance between affected and unaffected family members was smaller than that between sporadic IBD and non-IBD controls (5\% versus $17 \%$, respectively). Of note, in familial IBD, variance explained by the PRS dropped dramatically for $\mathrm{pT}>0.01$. A plausible hypothesis is that while familial cases differ from sporadic cases in low-risk (high $p$-value) variants, familial cases differ from their unaffected relatives mostly in highrisk (low $p$-value) variants. Higher PRS increases the risk for familial IBD as it does for sporadic IBD. The proportion of the explained variance in families however is limited, because of shared genetic background between family members. BIRD Grant

H. Lee: None. L. Hannes: None. M. Vancamelbeke: None. V. Ballet: None. M. Ferrante: B. Research Grant (principal investigator, collaborator or consultant and pending grants as well as grants already received); Modest; Amgen, Biogen, Janssen, Pfizer, Takeda. D. Speakers Bureau/Honoraria (speakers bureau, symposia, and expert witness); Modest; Abbvie, Amgen, Biogen, BoehringerIngelheim, Falk, Ferring, Janssen, Lamepro, MSD, Mylan, Pfizer, Takeda. F. Consultant/Advisory Board; Modest; Abbvie, Boehringer-Ingelheim, Janssen, MSD, Pfizer, Sandoz, Takeda. S. Vermeire: B. Research Grant (principal investigator, collaborator or consultant and pending grants as well as grants already received); Modest; MSD, Abbvie, Takeda, Janssen, Pfizer. D. Speakers Bureau/Honoraria (speakers bureau, symposia, and expert witness); Modest; AbbVie, MSD, Takeda, Ferring, Hospira, Pfizer, Janssen, and Tillots. F. Consultant/Advisory Board; Modest; AbbVie, MSD, Takeda, Ferring, Genentech/Roche, Shire, Pfizer Inc, Galapagos, Mundipharma, Hospira, Celgene, Second Genome, Progenity, Lilly, Arena, Gilead and Janssen. I. Cleynen: None.

\section{P03.34.A}

Combined exome/genome sequencing with in-house variant data repository mining confirm USP53 as a causal gene for intrahepatic cholestasis

S. Alawbathani ${ }^{l}, M$. Rocha ${ }^{l}, H$. Arshad Cheema ${ }^{2}, R$. Boustany $^{3}$, S. Nampoothiri ${ }^{4}$, A. Alswaid ${ }^{5}$, N. El Koofy, 
V. Karageorgou ${ }^{1}$, I. Hövel ${ }^{1}$, S. Khan ${ }^{1}$, C. Beetz ${ }^{l}$, A. Rolfs ${ }^{1,7}$, P. Bauer ${ }^{l}$, A. M. Bertoli-Avella ${ }^{l}$

${ }^{1}$ Centogene AG, Rostock, Germany, ${ }^{2}$ Children's Hospital and Institute of Child Health, Lahore, Pakistan, ${ }^{3}$ AUBMC American University of Beirut Medical Center, Beirut, Lebanon, ${ }^{4}$ Amrita Institute of Medical Sciences, Kerala, India, ${ }^{5}$ King Abdulaziz Medical City, Riyadh, Saudi Arabia, ${ }^{6}$ Abu El Reesh Children's Hospital, Cairo, Egypt, ${ }^{7}$ University of Rostock, Rostock, Germany

Cholestatic liver disease is a significant cause of morbidity and mortality in the paediatric population, starting in the early postnatal life. Severity is variable, from benign idiopathic neonatal hepatitis to intrahepatic cholestasis leading to liver failure and transplant. There are several genes described for different genetic diseases that lead to cholestasis. USP53 has been recently suggested as a candidate gene for paediatric intrahepatic cholestasis based on one family with two affected siblings. Interestingly, USP53 interacts with tight junction protein 2 (TJP2) for which pathogenic variants are known to cause progressive familial intrahepatic cholestasis. To date, the USP53 gene has not been linked to an OMIM phenotype. By combining exome/ genome sequencing and mining our in-house variant database we identified five patients from unrelated families. All affected individuals display a similar phenotype, mainly including early onset intrahepatic cholestasis. Moreover, one family showed hearing loss as an additional phenotype. All patients were homozygotes for different loss-of-function variants in the USP53 gene. USP53 (ubiquitin specific peptidase 53) is a tight junction-associated protein that is known to involve in the survival of auditory hair cells and hearing. Our results confirm USP53 as a novel gene for autosomal recessive intrahepatic cholestasis. Additionally, we demonstrate that combined exome/genome sequencing and variant database mining is an effective approach to discover and confirm novel genes related to human diseases. This accelerate the process of gene discovery and ultimately shortens the diagnostic odyssey in rare genetic disorders.

S. Alawbathani: A. Employment (full or part-time); Significant; Centogene AG, Rostock, Germany. M. Rocha: A. Employment (full or part-time); Significant; Centogene AG, Rostock, Germany. H. Arshad Cheema: None. R. Boustany: None. S. Nampoothiri: None. A. Alswaid: None. N. El Koofy: None. V. Karageorgou: A. Employment (full or part-time); Significant; Centogene AG, Rostock, Germany. I. Hövel: A. Employment (full or part-time); Significant; Centogene AG, Rostock, Germany. S. Khan: A. Employment (full or part-time); Significant; Centogene AG, Rostock, Germany. C. Beetz: A. Employment (full or part-time); Significant; Centogene AG, Rostock, Germany. A. Rolfs: A. Employment (full or part-time); Significant; Centogene AG, Rostock, Germany. P. Bauer: A. Employment (full or part-time); Significant; Centogene AG, Rostock, Germany. A. M. Bertoli-Avella: A. Employment (full or part-time); Significant; Centogene AG, Rostock, Germany.

\section{P03.35.B}

A common haplotype of the alternative serotonin transporter gene $S L C 6 A 4$ promoter P2 impacts gene expression and shows disease association in women with Irritable Bowel Syndrome

S. Mohr ${ }^{l}$, N. Hattensperger ${ }^{\text {l, C. Hammer }}{ }^{2}$, C. Martinez ${ }^{3}, S$. Berens $^{4}$, S. Schmitteckert ${ }^{l}$, L. A. Houghton ${ }^{5,6}$, M. Goebel-

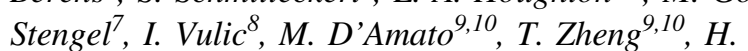
Mönnikes $^{11}$, F. Engel ${ }^{4}$, A. Gauss ${ }^{12}$, J. Tesarz ${ }^{4}$, V. Andresen ${ }^{13}$, T. Frieling ${ }^{14}$, J. Keller ${ }^{13}$, C. Pehl ${ }^{15}$, G. Clarke $^{16}$, R. Spiller ${ }^{17}$, T. G. Dinan ${ }^{16}$, E. M. Quigley ${ }^{16}$, C. Beltrán ${ }^{18}$, E. A. Mayer ${ }^{19}$, G. Sayuk ${ }^{20}$, M. Gazouli ${ }^{21}$, L. Kapur-Pojskic ${ }^{8}$, M. Bustamante ${ }^{22}$, K. Rabionet ${ }^{22}$, X. Estivil ${ }^{22}$, G. Boeckxstaens ${ }^{23}$, M. M. Wouters $^{23}$, M. Simrén ${ }^{24}$, G. A. Rappold ${ }^{1}$, M. Vicario $^{25}$, J. Santos ${ }^{25}$, R. Schäfert ${ }^{4,26}$, J. Lorenzo Bermejo ${ }^{27}$, B. Niesler ${ }^{l}$

${ }^{1}$ Department of Human Molecular Genetics, Heidelberg University, Heidelberg, Germany, ${ }^{2}$ Department of Cancer Immunology, Genentech, South San Francisco, CA, United States, ${ }^{3}$ Institut de Recerca Biomèdica de Lleida, Lleida, Spain, ${ }^{4}$ Department of General Internal Medicine and Psychosomatics, University Hospital Heidelberg, Heidelberg, Germany, ${ }^{5}$ Division of Gastroenterology \& Surgical Sciences, Leeds, United Kingdom, ${ }^{6}$ Mayo Clinic, Jacksonville, FL, United States, ${ }^{7}$ Helios Klinikum Rottweil, Rottweil, Germany, ${ }^{8}$ Institute for Genetic Engineering and Biotechnology, Sarajevo, Bosnia and Herzegovina, ${ }^{9}$ School of BioSciences, Monash University, Melbourne, Australia, ${ }^{10}$ Karolinska Institute, Solna, Sweden, ${ }^{11}$ Martin-LutherKrankenhaus, Berlin, Germany, ${ }^{12}$ Department of Gastroenterology, University Hospital Heidelberg, Heidelberg, Germany, ${ }^{13}$ Israelitisches Krankenhaus, Hamburg, Germany, ${ }^{14}$ Helios Klinik Krefeld, Krefeld, Germany, ${ }^{15}$ Krankenhaus Vilsbiburg, Vilsbiburg, Germany, ${ }^{16}$ Department of Psychiatry and Neurobehavioral Science, University College Cork, Cork, Ireland, ${ }^{17}$ Nottingham Digestive Diseases Centre, University of Nottingham, Nottingham, United Kingdom, ${ }^{18}$ Laboratory of Immuno-Gastroenterology, Universidad de Chile, Santiago de Chile, Chile, ${ }^{19}$ Oppenheimer Centre for Neurobiology of Stress, University of California, Los Angeles, CA, United States, ${ }^{20}$ Washington University School of Medicine, St. Louis, MO, United States, ${ }^{21}$ Biology National and Kapodistrian University of Athens, Medical School Athens, Athens, Greece, ${ }^{22}$ Centre for Genomic Regulation, Barcelona, Spain, ${ }^{23}$ TARGID, University 
Hospital Leuven, Leuven, Belgium, ${ }^{24}$ Institute of Medicine, University of Gothenburg, Gothenburg, Sweden, ${ }^{25}$ Institut de Recerca Vall d'Hebron, Hospital Vall d'Hebron, Barcelona, Spain, ${ }^{26}$ Universitätsspital Basel, Basel, Switzerland, ${ }^{27}$ Institute of Medical Biometry and Informatics, Heidelberg University, Heidelberg, Germany

Background: Irritable bowel syndrome (IBS) is a gut-brain disorder in which imbalances within the central and peripheral serotonergic systems contribute to disease pathophysiology and thereby shape the phenotype. The serotonin transporter gene SLC6A4 has earlier been implicated in IBS.

Material and Methods: We sequenced the alternative promoter P2 that primarily drives SLC6A4 expression in the gut and analyzed single nucleotide polymorphisms (SNP) for association with IBS. We also assessed their functional relevance by gene reporter assays, in silico analysis and comparative expression studies. In addition, we correlated detected SNPs with expression data and IBS symptoms.

Results: Detected SNPs were linked in different haplotypes and the tagging SNP rs2020938 associated with female IBS with constipation (IBS-C) in a discovery sample comprising 287 individuals. Subsequent validation and meta-analysis of rs2020938 in 2175 IBS patients and 6128 controls from eight countries confirmed the association with female IBS-C. Expression data obtained from different intestinal regions showed that the alternative promoter drives SERT expression primarily in the small intestine. Additionally, gene reporter assays pointed to a functional impact of the SNPs within this region. In silico analyses indicated differential expression regulation by transcription factors that have previously been implicated in GI disorders. Further follow up in tissue samples and clinical data from IBS patients and controls revealed that rs 2020938 correlates with differential expression in the jejunum and stool consistency.

Conclusions: Our results indicate differential expression regulation driven by a functionally relevant polymorphic promoter in female IBS-C and consolidates the importance of SLC6A4 in IBS pathogenesis.

S. Mohr: None. N. Hattensperger: None. C. Hammer: None. C. Martinez: None. S. Berens: None. S. Schmitteckert: None. L.A. Houghton: None. M. Goebel-Stengel: None. I. Vulic: None. M. D'Amato: None. T. Zheng: None. H. Mönnikes: None. F. Engel: None. A. Gauss: None. J. Tesarz: None. V. Andresen: None. T. Frieling: None. J. Keller: None. C. Pehl: None. G. Clarke: None. R. Spiller: None. T.G. Dinan: None. E.M. Quigley: None. C. Beltrán: None. E.A. Mayer: None. G. Sayuk: None. M. Gazouli: None. L. Kapur-Pojskic: None. M. Bustamante: None. K. Rabionet: None. X. Estivil: None. G. Boeckxstaens: None. M.M. Wouters: None. M. Simrén:
None. G.A. Rappold: None. M. Vicario: None. J. Santos: None. R. Schäfert: None. J. Lorenzo Bermejo: None. B. Niesler: None.

P03.36.C

Application of NGS sequencing for improved diagnosis in the pediatric nephrology setting

O. Beltcheva ${ }^{l}, K$. Kamenarova $^{l}, K$. Mihova $^{l}, G$. Zlatanova $^{2}$, F. Shakola ${ }^{1}$, D. Roussinov ${ }^{2}$, M. Gaydarova ${ }^{2}, V$. Mitev $^{l}$, R. Kaneva ${ }^{l}$

${ }^{I}$ Molecular Medicine Center, Dept. of Medical Chemistry and Biochemistry, Medical University-Sofia, Sofia, Bulgaria, ${ }^{2}$ SBAL Pediatric Diseases, Nephrology and Hemodialysis Clinic, Department of Pediatrics, Medical University - Sofia, Sofia, Bulgaria

Introduction: Renal disease first diagnosed in childhood often progresses towards chronic or end-stage kidney failure in adults. Identifying the molecular cause of the condition in each case may allow a more personalized approach to therapy and consequently increase the likelihood of preserving the renal function of the patients in adulthood.

Materials and Methods: TruSight One Sequencing Panel (Illumina) on MiSeq platform was used for mutation screening in 10 children with hematuria, proteinuria and/or compromised renal function, who did not respond well to standard treatment or presented with unusual course of the disease. Sanger sequencing was applied for confirmation and establishing the origin of the mutations. The pathogenicity of each novel variant was evaluated according to the established ACMG criteria.

Results: The NGS yielded both known and novel mutations in the sample set. In addition to well-known genes, such as WT1, GATA3, SALL1, AGXT and COL4A4/5, the analysis of the data revealed that pathogenic variants in CD96, HNF1? and TREX1 may also contribute to the etiology of renal disease in children.

Conclusions: The application of massive parallel sequencing of a large gene panel allowed us to identify the molecular cause of renal disease in a number of pediatric patients where diagnoses were hindered by atypical presentation. In several of the cases the findings allowed us to expand the clinical spectrum of the phenotypic presentation of the mutations in certain genes.

Grant references: D-73/2019; D01-285/17.12.2019

O. Beltcheva: None. K. Kamenarova: None. K. Mihova: None. G. Zlatanova: None. F. Shakola: None. D. Roussinov: None. M. Gaydarova: None. V. Mitev: None. R. Kaneva: None. 


\section{P03.37.A}

New LUTO-candidate genes identified by systematic analysis of copy number variations in 152 patients

L. M. Schierbaum ${ }^{1,2}$,J. Fabian ${ }^{2}$, S. Herms ${ }^{3}, S$. Sivalingam $^{2,3}$, S. Schneider ${ }^{2}$, S. Weber ${ }^{4}$,W. Rösch ${ }^{5}$, W. M. Merz $^{6}$, M. Feldkötter ${ }^{7}$, H. Reutter ${ }^{8,2}$, B. Hoppe ${ }^{1}$, G. Schalk ${ }^{1}$, M. Zaniew $^{9}$, A. Hilger ${ }^{1,2}$

${ }^{1}$ Division of Pediatric Nephrology, Department of Pediatrics, University Hospital Bonn, Bonn, Germany, ${ }^{2}$ Institute of Human Genetics, Children's Hospital, University of Bonn, Bonn, Germany, ${ }^{3}$ Department of Genomics, Life \& Brain Centre, Bonn, Germany, ${ }^{4}$ Department of Pediatrics, University Hospital Marburg, Marburg, Germany, ${ }^{5}$ Deparment of Pediatric Urology, St. Hedwig Krankenhaus Barmherzige Brüder, Regensburg, Germany, ${ }^{6}$ Department of Obstetrics and Prenatal Medicine, University of Bonn, Bonn, Germany, ${ }^{7}$ Division of Pediatric Nephrology, Children's Hospital, University of Zürich, Zürich, Switzerland, ${ }^{8}$ Department of Neonatology and Pediatric Intensive Care, Children's Hospital, University of Bonn, Bonn, Germany, ${ }^{9}$ Department of Pediatrics, University of Zielona Góra, Zielona Góra, Poland

Introduction: Lower urinary tract obstruction (LUTO) is caused by anatomical blockage of the bladder outflow tract or by functional impairment of urinary voiding. For anatomical blockages so far only variants in $B N C 2$ have been described as causal. Genomic copy number variations (CNVs) have been previously described as a cause of congenital anomalies of the kidneys and the urinary tract.

Used Methods: Array-based molecular karyotyping was performed in 152 LUTO patients. Raw intensity data were collected for all persons and CNVs were called with CNVPartition, QuantiSNP and PennCNV. Using PLINK version 1.07 and Excel the CNVs, identified in the patients, were compared to CNVs of 4168 controls. Overlapping CNVs between both groups were discarded. Further filtering steps were used.

Results: We identified 30 potential disease causing CNVs: 8 deletions and 22 duplications. Based on expression of the comprised genes in developing mouse urethra we prioritized $6 \mathrm{CNVs}$. One of those CNVs comprised the gene GPC5 (MIM 602446), located on chromosome $13 q 31.3$. Interestingly, of 8 patients reported in the literature with $13 \mathrm{q}$ duplications that comprise GPC5, four showed urogenital malformations. So far, GPC5 was not yet correlated with a specific clinical phenotype.

Conclusions: Systematic analysis of copy number variations in patients with LUTO is suitable for the identification of new candidate genes. We prioritized GPC5 as candidate gene. For further verification of this candidate gene, re-sequencing of LUTO patients to identify further mutation carriers and functional characterization in zebrafish is warranted.

Acknowledgment: A.C.H. is supported by BONFOR grant O-149.0123.

L.M. Schierbaum: None. J. Fabian: None. S. Herms: None. S. Sivalingam: None. S. Schneider: None. S. Weber: None. W. Rösch: None. W.M. Merz: None. M. Feldkötter: None. H. Reutter: None. B. Hoppe: None. G. Schalk: None. M. Zaniew: None. A. Hilger: None.

\section{P03.38.B}

ZSCAN12, a candidate gene for congenital lower urinary tract obstruction (LUTO)

L. Waffenschmidt ${ }^{1}$, S. Schneider ${ }^{1}$, J. Altmüller ${ }^{2,3}, H$. Thiele $^{4}$, S. Weber ${ }^{5}$, W. Rösch ${ }^{6}$, W. M. Merz ${ }^{7}$, M. Feldkötter ${ }^{8}$, B. Hoppe ${ }^{9}$, G. Schalk ${ }^{9}$, M. Zaniew ${ }^{10}$, H. Reutter ${ }^{1,11}$, M. F. Schreuder $^{12,13}$, G. M. A. Beckers ${ }^{14}$, J. A. E. van Wijk ${ }^{12}$, A. C. Hilger ${ }^{1,9}$

${ }^{1}$ Institute of Human Genetics, Children's Hospital, University of Bonn, Bonn, Germany, ${ }^{2}$ Center for Molecular Medicine Cologne (CMMC), University of Cologne, Cologne, Germany, ${ }^{3}$ Cologne Center for Genomics, University of Cologne, Cologne, Germany, ${ }^{4}$ University of Leipzig-Heart Center, Department of Internal Medicine/ Cardiology, Leipzig, Germany, ${ }^{5}$ Department of Pediatrics, University Hospital Marburg, Marburg, Germany, ${ }^{6}$ Department of Pediatric Urology, St. Hedwig Krankenhaus Barmherzige Brüder, Regensburg, Germany, ${ }^{7}$ Department of Obstetrics and Prenatal Medicine, University of Bonn, Bonn, Germany, ${ }^{8}$ Division of Pediatric Nephrology, Children's Hospital, University of Zürich, Zürich, Switzerland, ${ }^{9}$ Division of Pediatric Nephrology, Department of Pediatrics, University Hospital Bonn, Bonn, Germany, ${ }^{10}$ Department of Pediatrics, University of Zielona Góra, Zielona Góra, Poland, ${ }^{11}$ Department of Neonatology and Pediatric Intensive Care, Children's Hospital, University of Bonn, Bonn, Germany, ${ }^{12}$ Department of Pediatric Nephrology, VU University Medical Center, Pediatric Nephrourology Center, Amsterdam, Netherlands, ${ }^{13}$ Department of Pediatric Nephrology, Erasmus MC - Sophia Children's Hospital, University Medical Center, Rotterdam, Netherlands, ${ }^{14}$ Department of Pediatric Urology, VU University Medical Center, Amsterdam, Netherlands

Congenital lower urinary tract obstruction (LUTO) is usually characterised by urinary bladder outflow obstruction, it can be functional, based on a congenital dysfunction in voiding or on anatomical blockage of the urethra. LUTO has a birth prevalence of about three per 10.000 pregnancies. 
Whole-Exome-Sequencing (WES) in eight families revealed two rare variants in the ZSCAN12 gene. The first variant (c.1695-2A>C, minor allel frequency (MAF): 0,00005814 ) was identified in three affected brothers, the second variant (c.62T>C; p.Ile21Thr, MAF: 0,00002595) was identified in an affected father and his affected son. Resequencing of ZSCAN12 in 263 LUTO patients identified three further patients who all carried the same variant c.637T>C, p.Phe213Leu (MAF: 0.0002046). It has not been reported homozygous. Currently no genetic material of the parents is available for testing.

ZSCAN12 is expressed in the developing mouse urethra at embryonic day 15.5 (GUDMAP: 5824; GUDMAP Consortium) and has been found to be differentially methylated in prostate carcinoma.

Based on these preliminary data we suggest ZSCAN12 as a novel LUTO candidate gene. Further functional studies are warranted.

A.C.H. is supported by BONFOR grants O-149.0123.

L. Waffenschmidt: None. S. Schneider: None. J. Altmüller: None. H. Thiele: None. S. Weber: None. W. Rösch: None. W.M. Merz: None. M. Feldkötter: None. B. Hoppe: None. G. Schalk: None. M. Zaniew: None. H. Reutter: None. M.F. Schreuder: None. G.M.A. Beckers: None. J.A.E. van Wijk: None. A.C. Hilger: None.

\section{P03.39.C}

Biallelic mutations in the LSR gene cause a novel type of infantile intrahepatic cholestasis

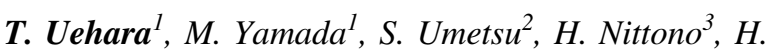
Suzuki $^{1}$, T. Fujisawa ${ }^{2}$, T. Takenouchi ${ }^{4}$, A. Inui ${ }^{2}$, K. Kosaki $^{1}$

${ }^{1}$ Center for Medical Genetics, Keio University Hospital, Tokyo, Japan, ${ }^{2}$ Department of Pediatric Hepatology and Gastroenterology, Saiseikai Yokohama-shi Tobu Hospital, Kanagawa, Japan, ${ }^{3} J u n s h i n$ Clinic BA Institute, Tokyo, Japan, ${ }^{4}$ Department of Pediatrics, Keio University Hospital, Tokyo, Japan

LSR is expressed in the liver and other organs, and represents a component protein of tricellular tight junctions. The roles of LSR in human health and disease have not been established. Only one patient with intrahepatic cholestasis with a rare variant of LSR has been reported. Herein, we report a patient who fulfilled this criterion establishing LSR deficiency as a novel cause of human liver disease. The proband was a 5-year-old female patient. At 5 months of age, the infant developed severe and uncontrollable itching. The results of blood examination at 9 months of age showed elevated serum AST, ALT and TBA. Her verbal milestones were slightly delayed. A needle biopsy of the liver at 5 years of age showed paucity of interlobular bile duct. At 6 years of age, whole-exome analysis of the samples from the patient and her parents was performed. The de novo nonsynonymous variant (c.602C $>\mathrm{T}$ ) and the maternally derived heterozygous frameshift variant (c.1396dup) were detected in the LSR gene. Immunohistochemical staining using antiLSR antibodies showed absent expression of LSR at the tricellular tight junctions in the patient. Documentation of biallelic pathogenic variants of the LSR gene and lack of LSR protein expression in the liver in our patient with infantile intrahepatic cholestasis presented in this report establishes LSR deficiency as a novel cause of infantile intrahepatic cholestasis.

T. Uehara: None. M. Yamada: None. S. Umetsu: None. H. Nittono: None. H. Suzuki: None. T. Fujisawa: None. T. Takenouchi: None. A. Inui: None. K. Kosaki: None.

P03.40.A

Neo-TAD formation on $7 q 36.3$ causes lung agenesis in three affected fetuses

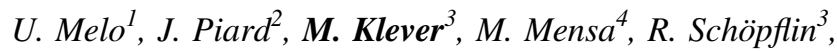
M. Holtgrewe ${ }^{3}$, F. Arbez-Gindre ${ }^{2}$, V. Guigue ${ }^{2}$, D. Gaillard ${ }^{2}$, V. Kremer ${ }^{2}$, R. Ramanah ${ }^{2}$, C. Cabrol ${ }^{2}$, D. Beule ${ }^{3}, F$. Harms $^{3}$, K. Kutsche ${ }^{3}$, M. Spielmann ${ }^{3}$, B. Fischer-Zirnsak ${ }^{3}$, L. Van Maldergem ${ }^{3}$, S. Mundlos ${ }^{3}$

${ }^{1}$ MPI, Berlin, Germany, ${ }^{2} 3$ Centre de Génétique Humaine, Université de Franche-Comté, Besançon, France, ${ }^{3} 1$ Max Planck Institute for Molecular Genetics, RG Development \& Disease, Berlin, Germany, Berlin, Germany, ${ }^{4} 1$ Max Planck Institute for Molecular Genetics, RG Development \& Disease, Berlin, Germany, Berlin, Dominican Republic

Lung development is a tightly regulated process under the control of a large number of signaling molecules. The sonic hedgehog $(\mathrm{SHH})$ signaling pathway is one of the main regulators of lung development during embryogenesis and mutations in SHH-related genes can lead to lung malformation. Here we report on three fetuses from one family with severe malformation of the respiratory system. By ultrasound screening and autopsy after termination, a complete bilateral lung agenesis and near-complete agenesis of the tracheae was observed in all fetuses. Using a combination of array-CGH, Illumina whole-genome sequencing and $\mathrm{Hi}-\mathrm{C}$ we identified a complex rearrangement at $7 \mathrm{q} 36.3$, $\sim 580 \mathrm{~kb}$ downstream the $S H H$ gene. The rearrangement consisted of a $449 \mathrm{~kb}$ triplication, a $232 \mathrm{~kb}$ duplication and a $61 \mathrm{~kb}$ deletion, involving parts of the SHH and NOM1/ MNX1/UBE3C topologically associated domains (TADs). Due to incorporation of a boundary between two TADs in the triplicated $\mathrm{CNV}$, the rearrangements lead to the formation of two neo-TADs each containing the known $\mathrm{SHH}$ enhancer MACS1 which is now placed next to MNX1, a 
transcription factor involved in caudal development. This situation can be expected to result in an ectopic activation of $M N X 1$ in the lung at a time and place where $S H H$ is normally expressed; a misexpression that likely explains the lung aplasia. The rearrangement was present in mosaic state in the clinically unaffected father, explaining the recurrence of the phenotype in his offspring's. Thus, whole-genome sequencing in combination with $\mathrm{Hi}-\mathrm{C}$ is a powerful tool to resolve and functionally interpret complex rearrangements.

U. Melo: None. J. Piard: None. M. Klever: None. M. Mensa: None. R. Schöpflin: None. M. Holtgrewe: None. F. Arbez-Gindre: None. V. Guigue: None. D. Gaillard: None. V. Kremer: None. R. Ramanah: None. C. Cabrol: None. D. Beule: None. F. Harms: None. K. Kutsche: None. M. Spielmann: None. B. Fischer-Zirnsak: None. L. Van Maldergem: None. S. Mundlos: None.

\section{P03.41.B}

Recessive and dominant patterns in Noonan Syndrome associated with $L Z T R 1$ variants

A. Cambra ${ }^{1}$, M. López-Blázquez², J. Sánchez del Pozo ${ }^{3}, J$.

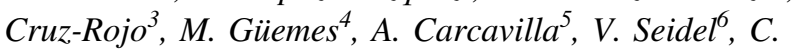
Medrano $^{2}$, B. Ezquietal

${ }^{1}$ Molecular Diagnostic Laboratory, Department of Laboratory Medicine, Hospital General Universitario Gregorio Marañón, Instituto de Investigación Sanitaria Gregorio Marañón (IiSGM), Madrid, Spain, ${ }^{2}$ Pediatric Cardiology, Department of Pediatrics, Hospital General Universitario Gregorio Marañón, Madrid, Spain, ${ }^{3}$ Pediatric Endocrinology, Department of Pediatrics, Hospital Universitario 12 de Octubre, Madrid, Spain, ${ }^{4}$ Pediatric Endocrinology, Department of Pediatrics, Hospital Universitario Niño Jesús, Madrid, Spain, ${ }^{5}$ Pediatric Endocrinology, Department of Pediatrics, Hospital Universitario La Paz, Madrid, Spain, ${ }^{6}$ Clinical Genetics, Department of Pediatrics, Hospital General Universitario Gregorio Marañón, Madrid, Spain

Noonan syndrome (NS) is an autosomal dominant disease caused by germline mutations in genes involved in the Ras/ MAPK pathway. Nevertheless, a recessive form related to biallelic variants in LZTRI gene was described in 2018. Following publications discussed phenotype and inheritance patterns associated with this gene. Here we report seven unrelated patients with clinically suspected NS and LZTRI variants. Clinical features are provided in table1. Three novel variants and four previously reported variants were identified. Two patients, NS1564 and NS960, presented a recessive form of NS. In the first patient, c.2074T $>C ; p$. (F692L) and c.2070-2A $>\mathrm{G}$ were identified and the second one was homozygous for c.2074T>C;p.(F692L), both affect the $\mathrm{BTB} / \mathrm{POZ}$ domain. In the rest of patients only one affected allele was identified: NS1414 had a stop-gain variant, c. $1084 \mathrm{C}>\mathrm{T}$;p.(R362*), located on kelch-5 domain; NS895 presented c.742G>A;p.(G248R), which lies in kelch4 domain; NS883 presented c.851G $>$ A;p.(R284H), located on kelch-5 domain; NS1199 presented a splicing variant, c. $791+1 \mathrm{G}>\mathrm{T}$; and the patient 99 was heterozygous for c. 905C $>$ T;p.(Ala302Glu), located on kelch-5 domain. Overall, these cases provide more evidence on the clinical phenotype of NS associated with LZTRI variants. These results are in accordance with the model that specifies that the location throughout the gene determines the inheritance form of NS. Our experience indicates that LZTRI is a not infrequent cause of NS, so this gene should be included in next-sequencing panels for molecular diagnosis of NS.

\begin{tabular}{|c|c|c|c|c|c|c|c|}
\hline $\begin{array}{l}\text { Patient } \\
\text { Sex }\end{array}$ & $\begin{array}{l}\text { NS1564 } \\
\text { Male }\end{array}$ & $\begin{array}{l}\text { NS960 } \\
\text { Male }\end{array}$ & $\begin{array}{l}\text { NS1414 } \\
\text { Female }\end{array}$ & $\begin{array}{l}\text { NS895 } \\
\text { Male }\end{array}$ & $\begin{array}{l}\text { NS883 } \\
\text { Female }\end{array}$ & $\begin{array}{l}\text { NS1199 } \\
\text { Female }\end{array}$ & $\begin{array}{l}\text { NS99 } \\
\text { Female }\end{array}$ \\
\hline $\begin{array}{l}\text { Face } \\
\text { dismorphology }\end{array}$ & Typical & Suggestive & Suggestive & Typical & Suggestive & Suggestive & Typical \\
\hline Short/webbed neck & Yes & Yes & No & No & Yes & No & No \\
\hline $\begin{array}{l}\text { Chest wall } \\
\text { deformity }\end{array}$ & $\begin{array}{l}\text { Broad } \\
\text { thorax }\end{array}$ & No & No & $\begin{array}{l}\text { Pectus } \\
\text { excavatum }\end{array}$ & $\begin{array}{l}\text { Pectus } \\
\text { carinatum }\end{array}$ & No & No \\
\hline Short stature & Yes & Yes & No & No & No & No & Yes \\
\hline Cardiac defect & $\mathrm{HCM}$ & $\mathrm{HCM}$ & $\mathrm{HCM}$ & PVS(mild) & $\mathrm{HCM}$ & $\mathrm{HCM}$ & $\mathrm{HCM}$ \\
\hline Cryptorchidism & Yes & Yes & & Yes & & & \\
\hline $\begin{array}{l}\text { Developmental } \\
\text { delay }\end{array}$ & Yes & No & Yes & No & No & Yes & No \\
\hline Prenatal findings & Yes & No & No & No & No & No & No \\
\hline
\end{tabular}

A. Cambra: None. M. López-Blázquez: None. J. Sánchez del Pozo: None. J. Cruz-Rojo: None. M. Güemes: None. A. Carcavilla: None. V. Seidel: None. C. Medrano: None. B. Ezquieta: None.

\section{P03.42.C}

Profiling of microRNA isoforms in ulcerative colitis

D. Nikitina ${ }^{l}$, S. Juzènas ${ }^{1,2}$, V. Šaltenien $\dot{e}^{l}$, A. Franke $^{2}, G$. Kiudelis $^{3}$, J. Kupčinskas ${ }^{1,3}$, L. Kupčinskas ${ }^{1,3}, J$. Skiecevičiene $\dot{e}^{1}$

${ }^{1}$ Lithuanian University of Health Sciences, Medical Academy, Institute for Digestive Research, Laboratory of Clinical and Molecular Gastroenterology, Kaunas, Lithuania, ${ }^{2}$ Kiel University, Institute of Clinical Molecular Biology, Kiel, Germany, ${ }^{3}$ Lithuanian University of Health Sciences, Medical Academy, Department of Gastroenterology, Kaunas, Lithuania

Introduction: Recent research has shown that so far widely studied reference miRNA sequences in miRNA databases have a large number of isoforms that can be involved in other physiological pathways compared to the reference miRNA. Ulcerative colitis (UC) is a chronic disease of the digestive system of the autoimmune origin and is attributed to the group of inflammatory bowel diseases. The aim of this study was to perform isomiRNA analysis in ulcerative colitis tissue. 
Materials and Methods: In total, 76 individuals were included in this study (32 controls, 24 patients with active $\mathrm{UC}$ and 20 patients with UC in remission phase). Small RNA sequencing was performed using the TruSeq protocol and the HiSeq2500 platform (Illumina, USA). Bioinformatic microRNA analysis was performed using the following software tools: MiRDeep2, mirAligner, Cutadapt, FASTX, RNAfold, Bowtie, BioPerl, BLASTn and R.

Results: The analysis revealed that the reference microRNA sequences make up $2.1 \%$ of all microRNA sequences and $97.9 \%$ are microRNA isoforms. Altered microRNAs and their isoforms (padj <0.01, $\log 2 \mathrm{FC}>\mid 0.9$ I) were detected in three groups: (i) active UC group compared to the HC (healthy control group) - 874; (ii) UC in the remission group compared to the $\mathrm{HC}-93$; (iii) active UC group compared to UC remission group - 632. The expression of 9 isomiRNA was strongly correlated with the intensity of the inflammation (rho> 0.7).

Conclusions: An altered profile of microRNAs and their isoforms was found in UC colon tissue, which also correlated with inflammation intensity.

D. Nikitina: None. S. Juzėnas: None. V. Šaltenienè: None. A. Franke: None. G. Kiudelis: None. J. Kupčinskas: None. L. Kupčinskas: None. J. Skiecevičienė: None.

\section{P03.43.A}

Screening for genetic polymorphisms in Bulgarian children with non-alcoholic fatty liver disease

D. Kofinova ${ }^{l}$, I. Yordanova ${ }^{2}, P$. Hadzhiyski ${ }^{1}$, R. ShentovaEneva $^{l}$, M. Baycheva ${ }^{l}$, P. Yaneva ${ }^{l}, M$. Panayotova ${ }^{3}, A$. Todorova ${ }^{2}$, D. Avdjieva-Tzavella ${ }^{l}$

${ }^{1}$ University Children's Hospital "Prof. Ivan Mitev", Sofia, Bulgaria, ${ }^{2}$ Genetic Medico-Diagnostic Laboratory Genica, Sofia, Bulgaria, ${ }^{3}$ Trakia Hospital, Stara Zagora, Bulgaria

Introduction: Non-alcoholic fatty liver disease (NAFLD) is a highly prevalent liver disease in children. NAFLD is associated with insulin resistance, obesity, and dyslipidemia. NAFLD is a multifactorial disease. Growing evidence suggest the leading role of certain gene variants, associated with rapid progression of the liver disease and dyslipidemia. The aim of this study was to investigate whether various single-nucleotide polymorphisms play any role in the development of NAFLD in Bulgarian children.

Materials and Methods: We prospectively included 22 patients with NAFLD and 10 healthy controls. Ten of the children with NAFLD underwent liver biopsy. PCR amplification with specific primers pairs for variants in the genes PNPLA3 (c.444C >G, p.I148 M), GCKR (c.1337C >T, p.P446L) and TM6SF2 (c.499C $>$ T, p.E167K) was performed followed by direct sequencing using BigDye Terminator v.3.1 Cycle Sequencing kit.

Results: The GCKR P446L gene polymorphism was found in $75.0 \%$ (24/32) of the study participants (homo- or heterozygous carriers). It was significantly higher in homozygous patients with NAFLD than in healthy controls (54.5\% vs. $10.0 \%, \mathrm{p}=0.025)$. PNPLA3 I148M was detected in 9 of the patients and 7 of the controls. TM6SF2 E167K was the rarest genetic variant, only 3 children ( 2 patients and 1 healthy child) were carriers. Two of the patients, who underwent liver biopsy had significant fibrosis F2. Patient $\mathrm{N} 1$ was homozygous for PNPLA3 I148M and heterozygous for GCKR P446L. Patient N2 was GCKR P446L heterozygous carrier.

Conclusions: GCKR P446L polymorphism is associated with development of NAFLD in Bulgarian children. Medical University Sofia, Grant "Young Investigator" Contract № D-142/02.05.2017

D. Kofinova: B. Research Grant (principal investigator, collaborator or consultant and pending grants as well as grants already received); Modest; Medical University Sofia, Grant "Young Investigator" Contract № D-142/02.05.2017. I. Yordanova: None. P. Hadzhiyski: None. R. ShentovaEneva: None. M. Baycheva: None. P. Yaneva: None. M. Panayotova: None. A. Todorova: None. D. AvdjievaTzavella: B. Research Grant (principal investigator, collaborator or consultant and pending grants as well as grants already received); Modest; Medical University Sofia, Grant "Young Investigator" Contract № D-142/02.05.2017.

\section{P03.44.B}

Molecular characterization of familial intrahepatic cholestasis by multi-gene panel analysis

A. Mattiaccio ${ }^{1,2}$, G. Vitale ${ }^{3}$, C. P. Cristalli ${ }^{1}$, M. Pariali ${ }^{1,4}, L$.

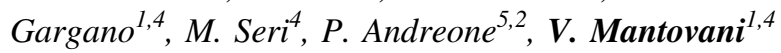

${ }^{1}$ Center for Applied Biomedical Research (CRBA), Bologna University, Bologna, Italy, ${ }^{2}$ DIMEC, Bologna University, Bologna, Italy, ${ }^{3}$ Internal Medicine for the Treatment of Severe Organ Failure, S. Orsola-Malpighi Hospital, Bologna, Italy, ${ }^{4}$ Medical Genetics Unit, S.Orsola-Malpighi University Hospital, Bologna, Italy, ${ }^{5}$ Department of Medical and Surgical Sciences, Maternal, Child and Adult Sciences, University Hospital, Modena, Italy

Introduction: Progressive Familial Intrahepatic Cholestasis (PFIC) is a group of autosomal recessive diseases that affects especially newborns and children, with progression to liver failure in the first decades of life. PFIC is classified into five types (PFIC1-5) based on the genetic defect involved in bile transport. Other benign late-onset phenotypes and non-progressive forms (BRIC, LPAC, DIC and 
ICP) are caused by heterozygous mutations in the same gene pattern.

Materials and Methods: DNA analysis of 15 candidate genes involved in PFIC and other related benign phenotypes was carried out. Amplicon-based NGS on 80 patients was performed. Bioinformatic and statistic pipelines were applied.

Results: A total of 184 different variants has been identified in our cohort: 18 pathogenic, 46 VUS, 44 likely benign and 76 benign. P/LP mutations were found in $12 \%$ of patients: 2 in $A T P 8 B 1,3$ in $A B C B 11, A B C B 4$ and TJP2 each, one in $A B C C 2, J A G 1, N O T C H 2$. Many patients had multiple variants in several genes. Patients had from 7 to 35 variants each and some SNPs were significantly associated with biochemical parameters and phenotypic features that could better explain clinics and accelerate the progression to liver failure.

Conclusions: Our detection rate is according to other studies proposing multi-gene panels. Our analysis may be useful for the molecular diagnostics of PFIC and a better characterization and understanding of the linking between molecular defects and different subtypes of the disease. The high SNPs prevalence let us to hypothesize a synergistic haplotype effect in determining different multifactorial cholestasis phenotypes and overlapping features.

A. Mattiaccio: None. G. Vitale: None. C.P. Cristalli: None. M. Pariali: None. L. Gargano: None. M. Seri: None. P. Andreone: None. V. Mantovani: None.

\section{P03.45.C}

Telomere length (TL) and oxidative stress in C57BL/6J mice

E. Kidd ${ }^{l}$, J. Pender ${ }^{l}$, M. J. Gatt ${ }^{l}$, J. Williams ${ }^{2}$, A. A. I. F. Blakemore $^{3}$, E. Meimaridou ${ }^{1}$, A. J. Walley ${ }^{4}$, U. L. Fairbrother ${ }^{1}$

${ }^{1}$ London Metropolitan University, London, United Kingdom, ${ }^{2}$ Queen Mary, London, United Kingdom, ${ }^{3}$ Brunel University, London, United Kingdom, ${ }^{4}$ St George's University of London, London, United Kingdom

High levels of oxidative stress may lead to an increased rate of telomere shortening and contribute to loss of telomere integrity. Most in vivo studies have looked for correlations between biomarkers of oxidative stress and TL, and have used leucocytes. We have used a murine model to investigate the potential impact of oxidative stress on TL in a tissue-specific manner and to assess telomere oxidation directly. Our model is a C57BL/6J mouse strain with a naturally occurring nicotinamide nucleotide transhydrogenase (NNT) deficiency. The absence of Nnt results in high levels of reactive oxygen species (ROS) in cells. DNA was extracted from healthy control mice, Nnt-deficient mice and Nnt-rescued mice (Nnt reinserted at the blastocyst stage) and their telomeres analysed. Two qPCR methods were used: $\mathrm{mmQPCR}$ to assess relative telomere length (RTL), and a formamidopyrimidine DNA glycosylase (FPG) enzyme-based qPCR method to directly determine the extent of telomere oxidation. Analysis of RTL from kidney revealed no significant difference $(\mathrm{p}>0.05)$ between the three groups. This is predictable since rapidly dividing leucocytes probably best represent early life replicative responses and tissues such as kidney, a slowly dividing tissue, represents the heritable load of TL. QPCR analysis of the DNA extracts, before and after FPG digestion showed there was no significant difference in the $\Delta \mathrm{CT}$ values between the three groups $(\mathrm{p}>0.05)$, implying there was no discernible difference in telomere oxidation levels. Further analysis will investigate tissues with variable metabolic and cellular turnover rates such as the brain, spleen, liver, heart and adipose tissue.

E. Kidd: None. J. Pender: None. M.J. Gatt: None. J. Williams: None. A.A.I.F. Blakemore: None. E. Meimaridou: None. A.J. Walley: None. U.L. Fairbrother: None.

P03.46.A

Molecular markers of severity of nonalcoholic fatty liver disease

\section{Sidorenko, V. Nazarov, S. Lapin, V. Emanuel}

Pavlov First Saint Petersburg State Medical University, Saint-Petersburg, Russian Federation

Introduction: Nonalcoholic fatty liver disease (NAFLD) is one of the most common parenchymal liver disorders. There are no reliable markers for assessment of progression of NAFLD from steatosis to steatohepatitis that leads to liver fibrosis and cirrhosis. Polymorphism I148M in PNPLA3 gene is associated with inhibition of lipid hydrolysis. Pathological variants of SERPINA1 gene promote accumulation of mutant alpha-1-antitrypsin in endoplasmic reticulum. Polymorphisms in HFE gene leads to intracellular iron accumulation and fatty degeneration of liver.

Materials and Methods: Group of 59 patients with NAFLD was selected. The assessment of structural changes in liver was carried out by transient elastometry with controlled attenuation parameter. Polymorphisms in PNPLA3, SERPINA1 and HFE genes were determined by real-time PCR.

Results: Association between homozygous carriage of I148M polymorphism and increase of ALT was found $(p=0.011)$. A connection was established between the presence of the polymorphism and more severe steatosis $(\mathrm{p}=0.045)$. AST activity was higher in PiZ carriers than in 
patients without changes in this gene $(\mathrm{p}=0.049)$. H63D and C282Y polymorphisms of the HFE gene are associated with higher ALT $(p=0.020$ and $p=0.030)$. A correlation between changes in mentioned genes and more severe fibrosis was not demonstrated.

Conclusions: Polymorphisms in the PNPLA3, SERPINA1, and HFE genes are associated with an increase in ALT and AST in patients with NAFLD. The homozygous variant of the I148M polymorphism in PNPLA3 gene and the heterozygous $\mathrm{C} 282 \mathrm{Y}$ in HFE gene are associated with more severe steatosis. However, conflicting data regarding liver fibrosis rates need further study.

D. Sidorenko: None. V. Nazarov: None. S. Lapin: None. V. Emanuel: None.

\section{P03.47.B}

Gene expression differences in non-obese, obese and previously obese subjects.

M. Tessarech ${ }^{1}$, E. Mileti ${ }^{2}, K$. Kwok $^{2}$, P. Arner ${ }^{3}$, D.

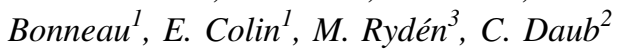

${ }^{1} \mathrm{CHU}$ Angers, Angers, France, ${ }^{2}$ Karolinska Institutet, Stockholm, Sweden, ${ }^{3}$ Karolinska University Hospital Huddinge, Stockholm, Sweden

Obesity is a worldwide public health issue associated with many comorbidities. Understanding its underlying causes and mechanisms is one of the keys to prevent and treat this condition. Besides obvious environmental causes, as physical inactivity or poor nutrition, genetics is one of the main component of obesity. Here, we perform a transcriptomic study by $5^{\prime} \mathrm{Cap}-\mathrm{mRNA}$ sequencing of subcutaneous white adipose tissue of non-obese (NO), obese (OB) and previously obese (POB) subjects, at fasting and hyperinsulinemia states. Differential gene expression analysis showed few difference in the analysis of NO compared to POB subjects, while the comparison of $\mathrm{OB}$ subjects versus $\mathrm{NO}$ and versus POB displayed many transcriptomic changes, with most of the genes in common in both comparison. Moreover, many genes were specifically involved in expression changes between $\mathrm{OB}$ and POB subjects. Gene ontology analysis revealed that those genes were involved in metabolic and cellular process pathways and in cardiovascular diseases. Fasting and hyperinsulinemia metabolic states had little influence on the results. This study demonstrates that a major gene expression difference is observed between obese and lean subjects, whether they were never obese or got rid of their fat mass after a weight loss surgery, and that after this kind of surgery, many changes in gene expression appear. Further studies are needed to identify precisely which genes are involved and if they can be a target for personalized medicine in the treatment of obesity by weight loss surgery.

M. Tessarech: None. E. Mileti: None. K. Kwok: None. P. Arner: None. D. Bonneau: None. E. Colin: None. M. Rydén: None. C. Daub: None.

P03.49.A

Autosomal Dominant Polycystic Kidney Disease in Israel: Genetic diagnosis reveals recurrent pathogenic variants

T. Paperna ${ }^{l}$, A. Mory ${ }^{l}$, A. Ofir ${ }^{l}$, N. Damouny ${ }^{l}, D$.

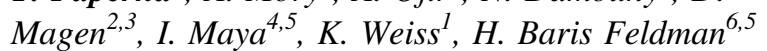

${ }^{I}$ The Genetics Institute, Rambam Health Care Campus, Haifa, Israel, ${ }^{2}$ Pediatric Nephrology Institute, Rambam Health Care Campus, Haifa, Israel, ${ }^{3}$ Faculty of Medicine, Technion, Haifa, Israel, ${ }^{4}$ The Raphael Recanati Genetic Institute, Rabin Medical Center, Beilinson Hospital, Petah Tikva, Israel, ${ }^{5}$ Sackler Faculty of Medicine, Tel Aviv University, Tel Aviv, Israel, ${ }^{6}$ The Genetics Institute, Tel Aviv Sourasky Medical Center, Haifa, Israel

Introduction: The genetic diagnosis of Autosomal Dominant Polycystic Kidney disease (ADPKD) is complicated by the genomic structure of the main causative gene $P K D 1$, due to the presence of overlapping pseudogenes. Classification of the missense variants is challenging as allelic heterogeneity is rife, and few recurrent variants are known. Data on ADPKD in Israeli populations is yet unavailable.

Materials and methods: Patient samples $(n=112)$ From Israel were referred to Rambam Genetics Institute for ADPKD gene panel testing (PKD1, PKD1, HNF1B). Next generation sequencing (NGS) was performed using either Trusight One (Illumina, $n=77$ ), or exome sequencing (ES, $n=35$ ); analysis employed the Genoox platform. NGS was supplemented by Sanger sequencing to achieve full coverage of panel. $G A N A B, D N A J B 11$ were included in ES testing.

Results: A clinically significant variant (CSV) was identified in $82 \%$ of the patients (PKD1-88\%, PKD2-7\%, HNF $1 B-5 \%)$. Three PKDl CSVs were identified only following Sanger sequencing. Truncating CSVs comprised $\sim 55 \%$ of pathogenic variants. Most CSVs (72\%) were unique; however, eight $P K D 1 \mathrm{CSVs}$ recurred in two or more patients, notably, in Jews of Persian and Ethiopian descent. HNF1B-related kidney disease was diagnosed in four individuals. Positive family history was linked with higher CSV detection rates, $81 \%$, compared with $60 \%$ when negative.

Conclusions: This first report on ADPKD genetics in Israel highlights recurrent CSVs in Israeli sub-ethnicities. $H N F 1 B$ CSVs can account for an ADPKD-like phenotype. 
Family history was a strong predictor for detection of a CSV. Our experience underscores the importance of utilizing ES as well as Sanger sequencing for variant detection.

T. Paperna: None. A. Mory: None. A. Ofir: None. N. Damouny: None. D. Magen: None. I. Maya: None. K. Weiss: None. H. Baris Feldman: None.

\section{P03.50.B}

Genome-wide association study identifies $\mathrm{CDH} 12$ as candidate gene for renal injury in patients with posterior urethral valves

L. F. M. van der Zanden ${ }^{I}$, I. A. L. M. van Rooij ${ }^{I}$, J. S. L. T. Quaedackers $^{2}$, R. Nijman ${ }^{2}$, M. Steffens ${ }^{3}$, L. L. L. de Wall, E. M. H. F. Bongers ${ }^{1}$, F. Schaefer ${ }^{4}, M$. Kirchner ${ }^{5}, R$. Behnisch $^{5}$, A. K. Bayazit ${ }^{6}$, S. Caliskan ${ }^{7}$, L. Obrycki $^{8}$, G. Montini $^{9,10}$, A. Duzova ${ }^{11}$, M. Wuttke ${ }^{12}$, R. Jennings ${ }^{13,14}, N$. A. Hanley ${ }^{13,14}$, N. J. Milmoe ${ }^{15}$, P. J. D. Winyard ${ }^{15}$, K. Y. Renkema $^{16}$, M. F. Schreuder ${ }^{l}$, N. Roeleveld ${ }^{1}$, W. F. J. Feitz ${ }^{1}$

${ }^{1}$ Radboud university medical center, Nijmegen, Netherlands, ${ }^{2}$ University Medical Center Groningen, Groningen, Netherlands, ${ }^{3}$ Isala, Zwolle, Netherlands, ${ }^{4}$ University Hospital Heidelberg, Heidelberg, Germany, ${ }^{5}$ University of Heidelberg, Heidelberg, Germany, ${ }^{6}$ Cukurova University, Adana, Turkey, ${ }^{7}$ Istanbul University-Cerrahpasa, Istanbul, Turkey, ${ }^{8}$ Children's Memorial Health Institute, Warsaw, Poland, ${ }^{9}$ Fondazione IRCCS Ca' Granda - Ospedale Maggiore Policlinico di Milano, Milan, Italy, ${ }^{10}$ University of Milan, Milan, Italy, ${ }^{11}$ Hacettepe University Faculty of Medicine, Ankara, Turkey, ${ }^{12}$ University of Freiburg, Freiburg, Germany, ${ }^{13}$ University of Manchester, Manchester, United Kingdom, ${ }^{14}$ Manchester University NHS Foundation Trust, Manchester, United Kingdom, ${ }^{15}$ UCL Great Ormond Street Institute of Child Health, London, United Kingdom, ${ }^{16}$ University Medical Center Utrecht, Utrecht, Netherlands

Introduction: Posterior urethral valves (PUV) and ureteropelvic junction obstruction (UPJO) are congenital obstructive uropathies that may impair renal development. We performed the first GWAS for renal injury in obstructive uropathy patients.

Methods: We included patients with pyeloplasty or PUV resection before age 18. Signs of renal injury were defined as dialysis, nephrectomy, kidney transplantation, estimated glomerular filtration rate $<60 \mathrm{ml} / \mathrm{min} / 1.73 \mathrm{~m}^{2}$, high blood pressure, antihypertensive medication use, proteinuria, and/ or one kidney functioning $<45 \%$. In the GWAS, we compared 141 and 216 obstructive uropathy patients with and without signs of renal injury 5 years post-surgery, respectively. For the associated SNPs, we performed stratified analyses for PUV and UPJO, and Kaplan-Meier and Cox regression analyses in the discovery and two replication samples of 102 PUV patients each. For the associated gene, we performed RNA and protein expression analyses in fetal tissues.

Results: We observed suggestive associations with six SNPs in three loci, of which rs6874819 in $\mathrm{CDH} 12$ was the strongest $\left(P=7.5 \times 10^{-7}\right)$. This SNP also seemed to be associated with time to renal injury in the PUV discovery and replication samples. RNA expression analyses showed clear expression in fetal kidneys, which was confirmed by protein immunolocalization.

Conclusions: This study identified $\mathrm{CDH} 12$ as candidate gene for renal injury in PUV. CDH12 is a target of the proinflammatory MCP-1-MCPIP pathway. We hypothesize that in PUV, MCP1 pathway upregulation due to increased urinary tract pressure is augmented in carriers of the $\mathrm{CDH} 12$ variant, exacerbating renal injury.

Grant support: Dutch Kidney Foundation (13OKJ36), Netherlands Organisation for Scientific Research (91618036).

L.F.M. van der Zanden: None. I.A.L.M. van Rooij: None. J.S.L.T. Quaedackers: None. R. Nijman: D. Speakers Bureau/Honoraria (speakers bureau, symposia, and expert witness); Modest; Astra Zeneca. M. Steffens: None. L.L.L. de Wall: None. E.M.H.F. Bongers: None. F. Schaefer: None. M. Kirchner: None. R. Behnisch: None. A.K. Bayazit: None. S. Caliskan: None. L. Obrycki: None. G. Montini: F. Consultant/Advisory Board; Modest; Merck. A. Duzova: None. M. Wuttke: None. R. Jennings: None. N.A. Hanley: F. Consultant/Advisory Board; Modest; APIS Assay Technologies. N.J. Milmoe: None. P.J.D. Winyard: None. K.Y. Renkema: None. M.F. Schreuder: None. N. Roeleveld: None. W.F.J. Feitz: None.

\section{P03.51.C}

RNA-seq approach to detect splicing alterations in primary ciliary dyskinesia and non-CF bronchiectasis

J. Legebeke ${ }^{l}$, G. Wheway ${ }^{l}, H$. Wai $^{l}$, W. Walker ${ }^{1,2}$, J. S.

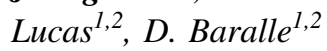

${ }^{1}$ University of Southampton, Southampton, United Kingdom, ${ }^{2}$ University Hospital Southampton, Southampton, United Kingdom

Mutations that cause PCD have been reported in $>40$ genes. Most cases are recessive, caused by biallelic changes in these genes. These account for around $70 \%$ of cases, suggesting that additional genes, and non-coding or synonymous changes in known genes, remain to be identified. Non-CF bronchiectasis is a heterogeneous group of 
disorders and adults may need to be investigated for PCD. Here we present an optimised pipeline for RNA analysis from nasal brushings taken from the inferior turbinate of patients with suspected PCD or non-CF bronchiectasis. We present analysis pipelines to investigate differences in gene expression, alternative splicing and the identification of deleterious splicing events. We developed gene-neutral and gene-specific analysis protocols, from both nasal brushings stored in RNA-later and cells from nasal brushings cultured at the air-liquid interface (ALI). We present this RNA-seq approach as a particularly useful diagnostic tool to investigate patients in whom a single pathogenic variant has been identified in a known PCD gene and where we suspect an alteration in splicing of the same gene due to a second intronic or synonymous / non-synonymous exonic variant. This represents a step towards using RNA analysis for increasing diagnostic yield in rare recessive respiratory disease. JL, DB and HT are supported by a NIHR Research Professorship to DB (RP- 2016-07-011). JL is further supported by Southampton NIHR BRC. GW is funded by a Wellcome Trust Seed Award in Science (Grant No. 204378/ Z/16/Z) and a University of Southampton Faculty of Medicine Research Management Committee research grant.

J. Legebeke: None. G. Wheway: None. H. Wai: None. W. Walker: None. J.S. Lucas: None. D. Baralle: None.

\section{P03.52.A}

Genetic testing for hereditary hyperparathyroidism in a large UK cohort

S. Mariathasan ${ }^{1}, K$. Andrews ${ }^{2}$, E. Thompson ${ }^{3}$, B. Challis ${ }^{1,4}$, S. Wilcox ${ }^{2}$, H. Pierce ${ }^{2}$, J. Hale ${ }^{1}$, S. Spiden ${ }^{3}$, G. Fuller ${ }^{5}$, H. Simpson $^{6}$, B. Fish ${ }^{7}$, P. Jani ${ }^{7}$, I. Seetho ${ }^{8}$, R. Armstrong ${ }^{2}$, L.

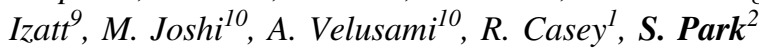

${ }^{1}$ Department of Endocrinology, Cambridge University Hospitals NHS Foundation Trust, Cambridge, United Kingdom, ${ }^{2}$ Department of Clinical Genetics, Cambridge University Hospitals NHS Foundation Trust, Cambridge, United Kingdom, ${ }^{3}$ 9.10. East Midlands and East of England NHS Genomic Laboratory Hub, Cambridge University Hospitals NHS Foundation Trust, Cambridge, United Kingdom, ${ }^{4}$ Translational Science \& Experimental Medicine, Research and Early Development, Cardiovascular, Renal and Metabolism, BioPharmaceuticals R\&D, AstraZeneca, Cambridge, United Kingdom, ${ }^{5}$ 9.10. East Midlands and East of England NHS Genomic Laboratory Hub, Cambridge University Hospitals NHS Foundation Trust, Cambridge, United Kingdom, ${ }^{6} 4$. Department of Endocrinology, University College Hospital, London, United Kingdom, ${ }^{7}$ Department of Head and Neck Surgery,
Cambridge University Hospitals NHS Foundation Trust, Cambridge, United Kingdom, ${ }^{8}$ Department of Endocrinology, Northwick Park Hospital, London North West University Hospital NHS Trust, London, United Kingdom, ${ }^{9}$ Department of Clinical Genetics, Guy's and St Thomas' NHS Foundation Trust, London, United Kingdom, ${ }^{10}$ Department of Endocrinology, Guy's and St Thomas' NHS Foundation Trust, London, United Kingdom

Primary hyperparathyroidism (PHPT) is common with a prevalence of $0.86 \%$. $10 \%$ are familial. Syndromic PHPT includes multiple endocrine neoplasia (MEN)1, MEN4, MEN2A and CDC73-related disorders. Non-syndromic causes include familial hypocalciuric hypercalcaemia (FHH), familial isolated HPT and neonatal severe PHPT. Establishing the underlying genetic cause allows for targeted, cost effective management. The 2019 National Genomic Test Directory PHPT testing criteria are PHPT and calcium creatinine clearance ratio $(\mathrm{CCCR})>0.02$ presenting $<35$ y or $<45$ y with one of a) multi-glandular disease, or b) hyperplasia, or c) ossifying fibromas of the jaw, or d) a family history (FH) of PHPT. The testing criteria for FHH is CCCR $<0.02$.

We reviewed 121 PHPT patients from the Cambridge and Guy's Endocrine Genetics clinic over a 4y period referred with multi-glandular disease, relevant $\mathrm{FH}, \mathrm{CCCR}<0.01$, age $<50 \mathrm{y}$, or $>1$ risk factor. We offered NGS panel gene testing of all known PHPT genes. Of the 121 patients, $(92 \mathrm{~F}$, mean age $41 \mathrm{y}$ ), a pathogenic variant was identified in $16 \%$ (11 CASR, 6 MEN1, 1 CDC73, 1 AP2S1) and we detected 4 VUS's. The mean CCCR was 0.0073 in those with a CASR pathogenic variant vs 0.016 in those without (p 0.003). A CCCR $<0.01$ had a sensitivity of $82 \%$ and specificity of $81 \%$ for FHH. A positive FH was a strong predictor of a pathogenic variant whereas multi-glandular disease was not.

In summary, genetic testing is recommended in PHPT at any age with i) syndromic PHPT, ii) relevant $\mathrm{FH}$ iii) a CCCR $<0.01$ and iv) multiple risk factors.

S. Mariathasan: None. K. Andrews: None. E. Thompson: None. B. Challis: None. S. Wilcox: None. H. Pierce: None. J. Hale: None. S. Spiden: None. G. Fuller: None. H. Simpson: None. B. Fish: None. P. Jani: None. I. Seetho: None. R. Armstrong: None. L. Izatt: None. M. Joshi: None. A. Velusami: None. R. Casey: None. S. Park: None.

\section{P03.55.A}

Biallelic loss of function GFRA1 variants cause bilateral renal agenesis 


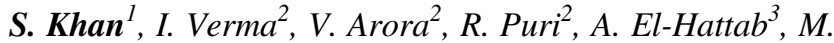

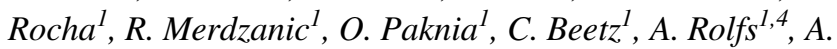
Bertoli-Avella ${ }^{l}$, P. Bauer ${ }^{l}$

${ }^{1}$ Centogene AG,, Rostock, Germany, ${ }^{2}$ Sir Ganga Ram Hospital, Institute of Genetics and Genomics, Rajinder Nagar, New Delhi, India, ${ }^{3}$ Department of Clinical Sciences, College of Medicine, University of Sharjah, Sharjah, United Arab Emirates, ${ }^{4}$ University of Rostock, Rostock, Germany

Congenital anomalies of the kidney and urinary tract comprise a broad spectrum of renal and urinary tract malformations with renal agenesis being at the most severe end of the spectrum. It usually results in death in utero or in the perinatal period. Only three genes have been identified so far as causing renal agenesis. By exome/genome sequencing within a diagnostic setting, with combined database analyses, we identified two unrelated patients presenting with congenital bilateral renal agenesis and homozygous loss of function variants $(\mathrm{LoF})$ in the GFRAl gene. Both patients had parental consanguinity and similarly affected, deceased siblings, suggesting an autosomal recessive inheritance. Patient 1 was homozygote for the nonsense variant NM_001348097.1:c.676C $>$ T, p.(Arg226*). The second patient was homozygote for a frameshift variant (NM_001348097.1:c.1294delA, p.(Thr432Profs*13)). Both variants are novel, identified for first time in our database, and presumed to lead to LoF. The GFRAl gene is not reported to be associated to any human phenotype in OMIM, but GFRA1 missense variants have been reported as associated to Hirschsprung disease in several patients, based on an oligogenic model. Gfra1-deficient mice demonstrate absence of enteric neurons and agenesis of the kidney, characteristics that are reminiscent of both Gdnf-and Retdeficient mice. The phenotype described in our patients is identical to the renal phenotype reported in Gfral null mice, however, no abnormalities of the enteric nervous system was reported in these two cases. In conclusion, these findings strongly support the causal role of GFRAl inactivating variants for an autosomal recessive form of bilateral renal agenesis.

S. Khan: A. Employment (full or part-time); Significant; Centogene AG, Rostock, Germany. I. Verma: None. V. Arora: None. R. Puri: None. A. El-Hattab: None. M. Rocha: A. Employment (full or part-time); Significant; Centogene AG, Rostock, Germany. R. Merdzanic: A. Employment (full or part-time); Significant; Centogene AG, Rostock, Germany. O. Paknia: A. Employment (full or part-time); Significant; Centogene AG, Rostock, Germany. C. Beetz: A. Employment (full or part-time); Significant; Centogene AG, Rostock, Germany. A. Rolfs: A. Employment (full or part-time); Significant; Centogene AG, Rostock, Germany. A. Bertoli-Avella: A. Employment (full or part-time); Significant; Centogene AG, Rostock, Germany. P. Bauer: A. Employment (full or part-time); Significant; Centogene AG, Rostock, Germany.

\section{P03.56.B}

WES expands the mutational spectrum in patients with Silver-Russell syndrome features

\author{
R. Meyer ${ }^{l}$, M. Begemann ${ }^{l}$, C. Hübner ${ }^{l}$, D. Dey ${ }^{l}, A$. \\ Küchler $^{2}$, M. Elgizouli ${ }^{2}$, P. Kroisel ${ }^{3}$, G. Fekete ${ }^{4}, L$. \\ Ambrozaityte $^{5}$, A. Matuleviciene ${ }^{5}$, M. Elbracht $^{1}, T$. \\ Eggermann $^{1}$
}

${ }^{I}$ Institute of Human Genetics, Medical Faculty, RWTH Aachen University, Aachen, Germany, ${ }^{2}$ Institute of Human Genetics, University Hospital Essen, Essen, Germany, ${ }^{3}$ Institute of Human Genetics, Medical University of Graz, Graz, Austria, ${ }^{4}$ II. Department of Pediatrics, Semmelweis University, Budapest, Hungary, ${ }^{5}$ Department of Human and Medical Genetics, Institute of Biomedical Sciences, Faculty of Medicine, Vilnius University, Vilnius, Lithuania

Silver-Russell syndrome (SRS) is one of the most prominent growth retardation disorders. However, both, the phenotype and underlying molecular alterations of SRS show a marked heterogeneity. SRS itself is an imprinting disorder, mainly associated with specific molecular changes at specific imprinted chromosomal regions. Disease causing point mutations are less common and vary widely in means of the affected gene. The suitability of panel-based NGS assays to identify monogenetic causes of SRS-like phenotypes has already been proven. The reasonableness of whole exome sequencing to identify point mutations in patients with Silver-Russel syndrome features however, is still the subject of ongoing discussions. We examined samples of 75 patients referred for molecular testing for SRS using different NGS-based approaches. The typical SRS-associated molecular alterations had been excluded before. The results of multi gene panel, whole exome sequencing, and triobased whole exome sequencing approaches were compared. Taking together the results of all different approaches, in 19/75 patients a disease-causing variant could be identified. Most of these variants are associated with monogenetic differential diagnoses of Silver-Russell syndrome. The diagnostic yield raised from panel to whole exome sequencing to trio-based whole exome sequencing. Broadening the molecular sequencing approach increases the mutation detection rate in patients referred for molecular testing of SRS without one of the typical molecular alterations. Some of the monogenetic disorders detected in our cohort have an important impact on the clinical management and genetic counseling of affected families mainly in terms of recurrence risks, tumor development, and mental 
development. Funding: Deutsche Forschungsgemeinschaft (EG110/15-1).

R. Meyer: None. M. Begemann: None. C. Hübner: None. D. Dey: None. A. Küchler: None. M. Elgizouli: None. P. Kroisel: None. G. Fekete: None. L. Ambrozaityte: None. A. Matuleviciene: None. M. Elbracht: None. T. Eggermann: None.

\section{P03.57.C}

'The pathogenicity analysis of mutations identified in AR gene in patients with AIS from Ukraine'

L. A. Livshits ${ }^{l}$, D. A. Sirokha ${ }^{l}$, O. V. Gorodna ${ }^{l}$, D. M. Lozhko $^{1}$, G. B. Livshyts ${ }^{1}$, N. B. Zelinska ${ }^{2}$

${ }^{1} I M B G$ NANU, Kyiv, Ukraine, ${ }^{2}$ Ukrainian Scientific and Practical Center for Endocrine Surgery, Transplantation of Endocrine Organs and Tissues of the Ministry of Health of Ukraine, Kyiv, Ukraine

Androgen Insensitivity Syndrome (AIS) is a Disorder of Sex Development (DSD) that occurs at a frequency of 1 in 20,000 live births, is the most common DSD in people with karyotype 46, XY, caused by mutations in the Androgen Receptor (AR) gene. More than 1000 AR gene mutations have been described in patients with AIS and prostate cancer. Almost 400 of them are located in ligand binding domain (LBD). The pathogenicity of mutations detected in AIS patients from Ukraine was analyzed. Directional sequencing of the PCR products of LBD-coding exons 7 and 8 was performed on DNA samples of AIS patients after informed consent. The substitution X:67722905 T>C (rs9332970), exon 7, missense mutation Ile843Thr was identified in PAIS-patient. In another PAIS-patient the substitution X: $67723745 \mathrm{C}>\mathrm{T}$ (rs137852594) samesence variant was identified. It was predicted the creation of a new enhancer motif for SRp55 splisosome protein, and as a consequence a new donor splice site at the end of the last exon 8. The substitution X:67722943 C> T (rs886041132), exon 7 - missense mutation Arg856Cys, was identified in CAIS-patient. Mutation X: $67722884 \mathrm{~T}>\mathrm{G}$, exon 7, a missense mutation Ile836Ser, was identified in CAISpatient was not annotated in Varsome, gnomAD and ARDB and identified as novel. The effect of listed missense mutations was predicted according to SIFT and PolyPhen values and classified as damaging. Analysis the substitution Ile836Ser on 3D model showed that the mutant protein does not get the correct conformation and binds dihydrotestosterone $\sim 460$ times worse than wild-type AR.

L.A. Livshits: None. D.A. Sirokha: None. O.V. Gorodna: None. D.M. Lozhko: None. G.B. Livshyts: None. N.B. Zelinska: None.
P03.59.B

Cell free DNA as potential marker of inflammatory diseases

D. Smolak ${ }^{1,2}$, G. Roman ${ }^{3}$, J. Budis ${ }^{2,4,5}$, M. Maronek ${ }^{3}$, B. Gromova $^{3}$, R. Liptak ${ }^{3}$, M. Harsanyova ${ }^{2,1}$, J. Radvanszky ${ }^{6,2}$, T. Szemes ${ }^{2,1,4}$

${ }^{I}$ Department of Molecular Biology, Faculty of Natural Sciences, Comenius University, Bratislava, Slovakia, ${ }^{2}$ Geneton Ltd., Bratislava, Slovakia, ${ }^{3}$ Institute of Molecular Biomedicine, Faculty of Medicine, Comenius University, Bratislava, Slovakia, ${ }^{4}$ Comenius University Science Park, Bratislava, Slovakia, ${ }^{5}$ Slovak Center of Scientific and Technical Information, Bratislava, Slovakia, ${ }^{6}$ Institute for Clinical and Translational Research, Biomedical Research Center, Slovak Academy of Sciences, Bratislava, Slovakia

Introduction: Ulcerative colitis is an inflammatory bowel disease (IBD) characterised by chronic inflammation of the human digestive tract. Despite efforts, the availability of effective non-invasive diagnostics assays is still limited. Cell free DNA (cfDNA) represents extracellular genetic information that already showed promises for various fields of genetic testing, including, non-invasive prenatal testing (NIPT) and cancer testing.

Materials and methods: Using a murine dextran sulfate sodium (DSS) induced colitis model we collected blood from 46 laboratory mice, grouped by stage of ulcerative colitis modeled by the duration of DSS administration. cfDNA was isolated and sequenced using the MiSeq Illumina System. Preprocessed sequenced data were mapped against mouse genome (GRCm38.p6). Statistical analyses were performed by in-house Python and R scripts.

Results: Besides the observed different fragment length distributions between control and colitis groups, we observed also a significantly increasing amount of mouse genomic material with the advancing stage of the disease, measured by the proportion of sequenced reads mappable to the mouse genome. In addition, to further investigate the pathophysiological processes behind the observed changes, we performed a sequence based differentiation of reads according to their mappability to the mouse nuclear and mitochondrial genomes, as well as of the detected bacterial communities.

Conclusions: We observed significant differences between the control group and various disease stages in sequenced data. Our results suggest the utility of cfDNA as a potential screening marker for IBD and even its specific stages and pathophysiological processes behind them.

Funded by: Slovak Research and Development Agency, grant APVV-17-0526. 
D. Smolak: A. Employment (full or part-time); Significant; Geneton Ltd.. G. Roman: None. J. Budis: A. Employment (full or part-time); Significant; Geneton Ltd.. M. Maronek: None. B. Gromova: None. R. Liptak: None. M. Harsanyova: A. Employment (full or part-time); Significant; Geneton Ltd. J. Radvanszky: A. Employment (full or part-time); Modest; Geneton Ltd. T. Szemes: A. Employment (full or part-time); Significant; Geneton Ltd..

P03.60.C

Functional characterization of variants of unknown significance in the Wilson disease-associated $A T P 7 B$ gene

A. Stalke, F. Hennig, E. Pfister, U. Baumann, M. Eilers, V. Schäffer, B. Vajen, N. Huge, T. Reinkens, B.

Schlegelberger, T. Illig, B. Skawran

\section{Hannover Medical School, Hannover, Germany}

Introduction: Wilson disease (WD) is an autosomal recessive disorder of copper excess due to pathogenic $A T P 7 B$ variants. $A T P 7 B$ codes for a copper-transporting ATPase. Sequencing of $A T P 7 B$ is an important tool to diagnose the disease. However, variants of unknown significance (VUS) are detected frequently, impeding a clear diagnosis. Functional analyses can help to unambiguously classify VUS. We aimed to functionally characterize four $A T P 7 B$ VUS, detected in patients with clinically distinct or suspected WD.

Methods: The $A T P 7 B$ open reading frame was cloned into an expression vector and the VUS were generated by site-directed mutagenesis. After HEK293T cell transfection, ATP7B expression of the mutants was quantified at mRNA and protein level by qRT-PCR and western blot. To assess the mutant's ability to export copper a copper-sensitive luciferase-based assay was used.

Results: For all VUS $A T P 7 B$ mRNA expression was significantly reduced compared to wild-type and a benign polymorphism. Three VUS also led to decreased protein expression and copper transport capacity. These VUS had been detected in clinically distinct WD patients. One VUS led to protein expression and copper transport capacity at wild-type level. It had been detected in a patient with suspected WD.

Conclusions: Our results strongly suggest a pathogenic effect for three of the VUS. For one VUS our results hint at a benign effect. On-going immunofluorescence assays will further address a possible protein mislocalization. Besides clarifying the clinical relevance of variants, functional characterization will help to understand the variant's pathomechanism contributing to personalized treatment with fewer side effects.
Supported by: DFG

A. Stalke: None. F. Hennig: None. E. Pfister: None. U. Baumann: None. M. Eilers: None. V. Schäffer: None. B. Vajen: None. N. Huge: None. T. Reinkens: None. B. Schlegelberger: None. T. Illig: None. B. Skawran: None.

P04 Skeletal, Connective Tissue, Ectodermal and Skin Disorders

P04.03.C

Geleophysic and acromicric dysplasias: clinical and molecular review of 38 patients

P. Marzin ${ }^{1,2}$, B. Thierry ${ }^{3,4}$, A. Dancasius ${ }^{1,2}$, C. Michot $^{1,2}, S$. Rondeau $^{1,2}$, G. Baujat ${ }^{1,2}$, G. Phan ${ }^{5}$, M. Le Bourgeois $^{6}$, D. Khraiche $^{7}$, C. Delacourt ${ }^{6}$, V. Cormier-Daire ${ }^{1,2}$

${ }^{I}$ Fédération de Génétique et Institut Imagine, AP-HP, Hôpital Necker-Enfants Malades, F-75015, France, ${ }^{2}$ Université de Paris, UMR1163, INSERM, F-75 015, Paris, France, ${ }^{3}$ Service d'ORL pédiatrique, AP-HP, Hôpital Necker-Enfants Malades, F-75015, France, ${ }^{4}$ Université de Paris, UMR976, INSERM, F-75 010,, Paris, France, ${ }^{5}$ Laboratoire de Cristallographie et RMN Biologiques, UMR 8015, CNRS, Université Paris Descartes, Faculté de Pharmacie, 4 Avenue de l'Observatoire, 75006, paris, France, ${ }^{6}$ Service de Pneumologie et Allergologie Pédiatriques, AP-HP, Hôpital Necker-Enfants Malades, F-75 015, Paris, France, ${ }^{7}$ Unité Médico-Chirurgicale de Cardiologie Congénital et Pédiatrique, AP-HP, Hôpital Necker-Enfants Malades, F-75 015,, Paris, France

Introduction: Geleophysic dysplasia (GD) and acromicric dysplasia (AD) are skeletal dysplasia characterized by short stature, brachydactyly and progressive joint limitation. Cardiorespiratory involvement may be responsible for a severe prognosis in GD. Dominant mutations in the FBNI and $L T B P 3$ genes are associated with $\mathrm{AD}$ and GD, whereas recessive mutations in the ADAMTSL2 gene only with GD. These three genes encode for proteins of microfibrillar network. The aim of this study is to improve understanding of natural history and genotype-phenotype correlations.

Materials and methods: This retrospective study included patients with AD and GD followed in our center, with identified mutation(s). Data from the medical records were abstracted, recorded in an Excel spreadsheet and analyzed using simple frequency analysis.

Results: We described clinical and molecular data from 38 patients (20GD, $18 \mathrm{AD}$ ) with a mean age of 17 years. Mutations in the FBN1, ADAMTSL2 and LTBP3 genes were identified in 23, 12 and 3 patients, respectively. Early death occurred in nine cases. $45 \%$ of patients presented with valvulopathy, progressive in half of cases. Respiratory 
disorders were asthma (50\%), non-cardiogenic restrictive syndrome $(30 \%)$, laryngo-tracheo-bronchial involvement $(18 \%)$ and acute non-cardiogenic pulmonary hypertension (four cases). Patients with a variant involving a cysteine in the FBN1 gene or mutations in the ADAMTSL2 gene presented a higher proportion of severe cardiorespiratory complications compared to a more benign course in patients with other mutations.

Conclusions: Genetic findings could have importance not only in the diagnosis, but also in risk stratification and clinical management of patients with suspected acromelic dysplasia.

P. Marzin: None. B. Thierry: None. A. Dancasius: None. C. Michot: None. S. Rondeau: None. G. Baujat: None. G. Phan: None. M. Le Bourgeois: None. D. Khraiche: None. C. Delacourt: None. V. CormierDaire: None.

\section{P04.04.A}

Acromesomelic dysplasia Maroteaux type, nine patients with seven novel NPR2 mutations

\section{E. Kilic ${ }^{l}$, B. Cavdarli ${ }^{2}$, G. Buyukyilmaz ${ }^{3}$, M. Kilic ${ }^{4}$}

${ }^{I}$ Division of Pediatric Genetics, Department of Pediatrics, University of Health Sciences, Ankara City Hospital, Ankara, Turkey, ${ }^{2}$ Department of Medical Genetics, Ankara City Hospital, Ankara, Turkey, ${ }^{3}$ Division of Pediatric Endocrinology, Department of Pediatrics, Ankara City Hospital, Ankara, Turkey, ${ }^{4}$ Division of Metabolism, Department of Pediatrics, Sami Ulus Children Hospital, Ankara, Turkey

Background: Acromesomelic Dysplasia, type Maroteaux (AMDM), is an autosomal recessive skeletal dysplasia caused by loss of function mutations of NPR2 gene which encodes C-type natriuretic peptid reseptor B. This rare type of dwarfizm characterized with shortening of the middle and distal segments of the limbs. Herein we reported nine patients from eight families with seven novel NPR2 mutations.

Methods: Nine patients were diagnosed AMDM in a single centre from 2015 to 2020 were enrolled in this study. Their clinical, radiological and molecular genetic findings examined retrospectively. The mutations in NPR2 gene were confirmed with direct sequencing.

Results: All nine patiens in this group were admitted to hospital with the complaint of short stature. All had short limb type dwarfism with marked shortening middle and acral segment of the limbs. All had typical dysmorphic signs, brachydactyly, significant greater toes and normal intelligence. We identified one previously reported (c.2729 C $>$ T), five novel missense (c.3029 A $>$ G, c.1738-1739 del ins TT, c.1163 G>A, c.3058 C>T, c.329 G>A) and two novel nonsense (c.1213 delT fs*, c.14 C>A fs*) mutations. All new mutations identified in this group are likely pathogenic and meaningfull with the patients typical clinical presentations.

Conclusions: Reporting new patients with novel mutations would contribute to better clinical dealination of this rare dysplasia and would help to established pathogenic mutations.

References:1. Maroteaux P, Martinelli B, Campailla E. [Acromesomelic dwarfism]. Presse Med. 1971; 79:183942.2. Khan S, Basit S, Khan MA, et al. Genetics of human isolated acromesomelic dysplasia. Eur J Med Genet. 2016;59:198-203.

E. Kilic: None. B. Cavdarli: None. G. Buyukyilmaz: None. M. Kilic: None.

\section{P04.06.C}

Severe congenital cutis laxa with generalized muscle hypotonia due to ATP6V1A mutations

G. Vogt ${ }^{1,2}, N$. El Choubassi ${ }^{1,2}$, M. Holtgrewe ${ }^{3}$, S. Krause ${ }^{4}$,

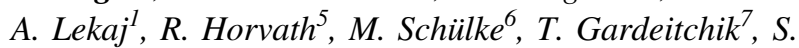
Mundlos $^{1,2}$, Á. Herczegfalvi ${ }^{8}$, H. Lochmüller ${ }^{9,10}, \mathrm{~V}$. Karcagi $^{11}$, U. Kornak ${ }^{1,2,12}$, B. Fischer-Zirnsak ${ }^{1,2}$

${ }^{1}$ Institute of Medical Genetics and Human Genetics, Charité - Universitätsmedizin, Berlin, Germany, ${ }^{2} R G$ Development \& Disease, Max Planck Institute for Molecular Genetics, Berlin, Germany, ${ }^{3}$ Core Unit Bioinformatics-CUBI, Berlin Institute of Health, Berlin, Germany, ${ }^{4}$ Department of Neurology, Friedrich-Baur-Institute, Munich, Germany, ${ }^{5}$ Department of Clinical Neurosciences, University of Cambridge School of Clinical Medicine, Cambridge, United Kingdom, ${ }^{6}$ Department of Neuropediatrics, Charité Universitätsmedizin, Berlin, Germany, ${ }^{7}$ Department of Human Genetics, Radboud University Medical Centre, Nijmegen, Netherlands, ${ }^{8}$ II. Department of Paediatric Neurology, Semmelweis Medical University, Budapest, Hungary, 'Division of Neurology, Children's Hospital of Eastern Ontario Research Institute, Ottawa, IL, Canada, ${ }^{10}$ Brain and Mind Research Institute, Ottawa, $I L$, Canada, ${ }^{11}$ Department of Molecular Genetics and Diagnostics, National Institute of Environmental Health, Budapest, Hungary, ${ }^{12}$ Institute of Human Genetics, University Medical Center, Göttingen, Germany

Introduction: Autosomal recessive cutis laxa type 2D is a severe multisystem disease due to pathogenic variants affecting ATP6V1A, encoding a component of the V-Type $\mathrm{H}^{+}$ATPase. Affected individuals show a strong cutis laxa phenotype with large skin folds at birth, hypotonia and cardiac abnormalities. 
Material and Methods: We performed single whole exome sequencing (WES) to identify the causative molecular defect in our index patient. Furthermore, Sanger sequencing, qRT-PCR and Immunoblot analyses were performed to further investigate causality of the variants identified.

Results: Here we report on two affected individuals from a non-consanguineous family from Hungary suffering from a multisystem condition with severe, generalized muscle hypotonia and and strong juvenile cutis laxa. One child died in infancy, while the other significantly improved. Using WES and co-segregation analyses we found our proband to be compound heterozygous for a novel missense and the first so far described nonsense pathogenic variant affecting ATP6V1A. Expression studies revealed a strong degradation of the allele carrying the nonsense alteration. Additionally, patients' dermal fibroblasts showed strong alteration of Cathepsin D processing indicating alterations of lysosomal function.

Conclusions: This is the second report on pathogenic variants in ATP6V1A as the cause for a severe condition with cutis laxa. Our data expand the clinical spectrum of ATP6V1A-related phenotypes and show that beside missense, also nonsense pathogenic variants are a possible cause of ATP6V1A-ARCL. Funding: The study was supported by the Deutsche Forschungsgemeinschaft (FI 2240/1-1) to BF-Z and by EURO-CDG-2 to UK.

G. Vogt: None. N. El Choubassi: None. M. Holtgrewe: None. S. Krause: None. A. Lekaj: None. R. Horvath: None. M. Schülke: None. T. Gardeitchik: None. S. Mundlos: None. Á. Herczegfalvi: None. H. Lochmüller: None. V. Karcagi: None. U. Kornak: None. B. FischerZirnsak: None.

\section{P04.07.A}

New duplication downstream of the $B M P 2$ gene in a French family with Brachydactyly type A2 (BDA2)

E. COLIN ${ }^{l}$, A. GUICHET ${ }^{l}$, F. BOUSSION ${ }^{2}, M$. TESSARECH ${ }^{l}$, A. ZIEGLER ${ }^{l}$, M. GORCE ${ }^{l}, C$. HOUDAYER ${ }^{l}$, S. MANOUVRIER ${ }^{3}$, D. BONNEAU ${ }^{1}$

${ }^{1}$ Department of Biochemistry and Genetics, Angers University Hospital, ANGERS, France, ${ }^{2}$ Department of Gynecology and Obstetrics, Angers University Hospital, ANGERS, France, ${ }^{3}$ Pole of Biology Genetic Pathology, Lille University Hospital - Center of Biology Genetic Pathology, LILLE, France

Brachydactyly type A2 (BDA2, MIM 112600) is an autosomal dominant pathology characterized by deviation and shortening of the middle phalanx of the index and / or second toe. It may be due to mutations in the BMPRIB gene
(MIM 603248) or the GDF5 gene (MIM 601146) but may also depend on structural changes in 20 p12.3 involving the $B M P 2$ gene. Indeed, microduplications downstream of the $B M P 2$ gene have been described in 5 families (2 German and 3 Chinese). Using an SNP-array technique, we identified a new duplication of $45 \mathrm{~kb}$ downstream of the $B M P 2$ gene in a French family. We describe the phenotypic and genotypic characteristics of this family and review the literature.

E. Colin: None. A. Guichet: None. F. Boussion: None. M. Tessarech: None. A. Ziegler: None. M. Gorce: None. C. Houdayer: None. S. Manouvrier: None. D. Bonneau: None.

\section{P04.11.B}

Loss-of-function variants in $\mathrm{C3ORF52}$ result in localized autosomal recessive hypotrichosis

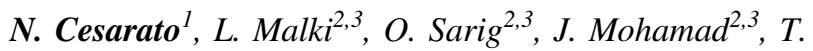
Canter $^{4}$, S. Assaf ${ }^{2,3}$, M. Pavlovsky ${ }^{2}$, D. Vodo ${ }^{2,3}$, Y. Anis ${ }^{5}, O$. Bihari $^{2}$, K. Malovitski ${ }^{2,3}$, A. Gat ${ }^{2}$, H. Thiele ${ }^{6}$, B. E. Perez White $^{4}$, L. Samuelov ${ }^{2}$, A. Nanda ${ }^{7}$, A. S. Paller ${ }^{4}$, R. C. Betz ${ }^{1}$, E. Sprecher 2,3

${ }^{1}$ Institute of Human Genetics, University of Bonn, Bonn, Germany, ${ }^{2}$ Division of Dermatology, Tel Aviv Sourasky Medical Center, Tel Aviv, Israel, ${ }^{3}$ Department of Human Molecular Genetics \& Biochemistry, Sackler Faculty of Medicine, Tel Aviv University, Ramat Aviv, Israel, ${ }^{4}$ Department of Dermatology, Northwestern University Feinberg School of Medicine, Chicago, IL, United States, ${ }^{5}$ Institute of Endocrinology, Tel Aviv Sourasky Medical Center, Tel Aviv, Israel, ${ }^{6}$ Cologne Center for Genomics, University of Cologne, Cologne, Germany, ${ }^{7}$ As'ad Al-Hamad Dermatology Center, Kuwait, Kuwait

Introduction: Localized autosomal recessive hypotrichosis has been associated with pathogenic variants in DSG4, encoding a desmosomal protein as well as in $L I P H$ and LPAR6, encoding respectively lipase $\mathrm{H}$, which catalyzes the formation of 2-acyl lysophosphatidic acid (LPA), and lysophosphatidic acid receptor 6, a receptor for LPA. LPA promotes hair growth and differentiation. In this study, we aimed at delineating the genetic basis of LAH in patients without pathogenic variants in these three genes.

Materials and Methods: Variant analysis was conducted using exome and Sanger sequencing. We then performed RT-qPCR, immunofluorescence staining, immunoblotting, enzymatic and co-immunoprecipitation assays in cell cultures and hair follicles to evaluate the consequences of the identified variants.

Results: We identified homozygous variants in C3ORF52 in four individuals from two families with LAH. C3ORF52 
was found to be co-expressed with lipase $\mathrm{H}$ in the inner root sheath of the hair follicle and the two proteins were found to directly interact. The LAH-causing variants were associated with decreased C3ORF52 expression and resulted in markedly reduced lipase H-mediated LPA biosynthesis.

Conclusions: We could connect C3ORF52 to the LPAmediated signaling pathway which, together with LPAR6 and LIPH, plays a crucial role in hair growth. LAH can be caused by abnormal function of any of these three proteins.

N. Cesarato: None. L. Malki: None. O. Sarig: None. J. Mohamad: None. T. Canter: None. S. Assaf: None. M. Pavlovsky: None. D. Vodo: None. Y. Anis: None. O. Bihari: None. K. Malovitski: None. A. Gat: None. H. Thiele: None. B.E. Perez White: None. L. Samuelov: None. A. Nanda: None. A.S. Paller: None. R.C. Betz: None. E. Sprecher: None.

P04.12.C

Evidence for a role of fibrillin-2 in de pathogenesis of carpal tunnel syndrome

S. Peeters ${ }^{1}$, A. Decramer ${ }^{2}$, S. Cain ${ }^{3}$, P. Houpt ${ }^{4}$, F. Verstreken $^{5}$, J. Noyez ${ }^{2}$, C. Hermans ${ }^{6}$, W. Jacobs ${ }^{7}$, M. Lammens $^{8}$, E. Fransen ${ }^{1}$, A. Kumar ${ }^{9}$, G. Vandeweyer ${ }^{1}$, B. Loeys $^{l}$, W. Van Hul ${ }^{1}$, C. Baldock ${ }^{3}$, E. Boudin ${ }^{1}$, G. Mortier ${ }^{1}$

${ }^{1}$ Department of Medical Genetics, University of Antwerp and Antwerp University Hospital, Antwerp, Belgium, ${ }^{2}$ Department of Orthopaedics and Traumatology, AZ Delta, Roeselare, Belgium, ${ }^{3}$ Division of Cell-Matrix Biology and Regenerative Medicine, Wellcome Centre for Cell-Matrix Research, the University of Manchester, Manchester, United Kingdom, ${ }^{4}$ Department of Plastic Surgery, Isala clinic Zwolle, Zwolle, Netherlands, ${ }^{5}$ Department of Orthopaedic surgery, AZ Monica, Deurne, Belgium, ${ }^{6}$ Center for Oncological Research Antwerp (CORE), University of Antwerp, Edegem (Belgium), Antwerp, Belgium, ${ }^{7}$ Department of Forensic Medicine and Pathology, Antwerp University Hospital and University of Antwerp, Antwerp, Belgium, ${ }^{8}$ Department of Pathological Anatomy, Antwerp University Hospital, Antwerp, Belgium, ${ }^{9}$ Department of Paediatrics, Wellcome - MRC Cambridge Stem Cell Institute Cambridge, Cambridge, United Kingdom

Introduction: Carpal tunnel syndrome (CTS) is the most common form of peripheral entrapment neuropathy. Although CTS is quite common, its pathogenesis remains largely unknown. An estimated heritability index of 0.46 and a familial occurrence in $27-39 \%$ of the cases indicate that genetic factors must play a role in the pathogenesis. We report on a peculiar family in which CTS occurred in subsequent generations at an usually young age. Additional clinical features included brachydactyly and short Achilles tendons resulting in toe walking in childhood.

Material and methods: We performed exome sequencing and genome-wide linkage analysis to identify the pathogenic variant in this family. Several functional assays were performed to further study the causality of the variant. In addition, we sequenced a cohort of 216 patients to assess the role of this new candidate gene in the pathogenesis of CTS in a larger population.

Results: We identified a heterozygous variant (c.5009T $>$ G; p.Phe1670Cys) in the fibrillin-2 (FBN2) gene that co-segregated with the phenotype in the family. Functional assays showed that the FBN2 variant impaired integrin-mediated cell adhesion and migration. Moreover, we observed an increased TGF- $\beta$ signaling and fibrosis in the carpal tissues of affected individuals. A variant burden test in a large cohort of CTS patients revealed a significantly increased frequency of rare FBN2 variants in patient alleles compared to controls.

Conclusions: Our data strongly suggest a role of FBN2 in the pathogenesis of CTS.

S. Peeters: None. A. Decramer: None. S. Cain: None. P. Houpt: None. F. Verstreken: None. J. Noyez: None. C. Hermans: None. W. Jacobs: None. M. Lammens: None. E. Fransen: None. A. Kumar: None. G. Vandeweyer: None. B. Loeys: None. W. Van Hul: None. C. Baldock: None. E. Boudin: None. G. Mortier: None.

\section{P04.14.B}

New pathways and variants in genes causing collagenopathies identified in patients with isolated cervical insufficiency

\section{Volozonoka, D. Rots, I. Kempa, A. Kornete, D.} Rezeberga, L. Gailīte, A. Miskova

\section{Riga Stradins University, Riga, Latvia}

Introduction: Cervical insufficiency is phenotypically distinguishable condition causing pregnancy loss or preterm delivery. A strong genetic component and few syndromic forms of cervical insufficiency are described. Knowledge about the genetics of human cervix remodelling during pregnancy is scarce - only 12 maternal genes and their variants are implicated in cervical insufficiency so far as identified by our systematic gene analysis before.

Material and methods: We sequenced DNA from 21 patients with clinical presentation of isolated cervical insufficiency using Illumina's TruSight One NGS kit. Genes having rare deleterious variants identified in our patients $(n=694)$ were annotated for the pathway enrichments using ConsensusPathDB. Variants in genes from enriched phenotype-relevant pathways were manually 
curated based on variant's pathogenicity, gene's expression pattern, mode of inheritance and disease mechanism, to assign likelihood for contribution to the patients' phenotype.

Results: Pathway-enrichment analysis revealed increased variant burden in 15 pathways (max $p$-value $2.47 \mathrm{E}-03$ ) playing roles in tissue mechanical and biomechanical properties, i.e. collagen biosynthesis, cell-extracellular matrix communications and previously unnoticed pathways of hemidesmosome assembly, laminins, integrins and focal adhesion. Fourteen variants of unknown significance and (likely) pathogenic variants in 11 genes - TGM2, ITGA6, COMP, COL11A1, including COL1A1, COL1A2, COL6A3, $P 3 H 1, P L O D 1, P P I B$ and TNXB causing collagenopathies in 11 patients were classified as potential to increase susceptibility to the development of non-syndromic cervical insufficiency.

Conclusions: Cervical insufficiency being an expression point in a phenotypic continuum of collagenopathies should be investigated further using multiple approaches. This might open up new opportunities to improve patient evaluation and management in the future.

L. Volozonoka: None. D. Rots: None. I. Kempa: None. A. Kornete: None. D. Rezeberga: None. L. Gailīte: None. A. Miskova: None.

\section{P04.15.C}

The phenotypic spectrum of Chitayat syndrome: Six additional affected individuals

A. Suter ${ }^{1}$, A. Abad Perez ${ }^{1}$, F. Santos-Simarro ${ }^{2}$, P. Toerring ${ }^{3}$, R. Ramos-Mejia ${ }^{4}, K$. Heath $^{2}$, V. Huckstadt ${ }^{5}, M$. Parrón-Pajares $^{6}$, M. Mensah ${ }^{1,7}$, W. Hülsemann ${ }^{8}, M$. Holtgrewe $^{1,9}$, S. Mundlos ${ }^{1,10}$, U. Kornak ${ }^{1,11}$, O. Bartsch ${ }^{12}$, N. Ehmke $e^{1,10}$

${ }^{1}$ Institute of Medical and Human Genetics, Charité Universitätsmedizin Berlin, Berlin, Germany, ${ }^{2}$ Institute of Medical and Molecular Genetics and Skeletal dysplasia multidisciplinary Unit (UMDE), Hospital Universitario La Paz and CIBERER, ISCIII, Madrid, Spain, ${ }^{3}$ Department of Clinical Genetics, Odense University Hospital, Odense, Denmark, ${ }^{4}$ Growth and Development Department, Garrahan Hospital, Buenos Aires, Argentina, ${ }^{5}$ Genetics Department, Garrahan Hospital, Buenos Aires, Argentina, ${ }^{6}$ Department of Radiology and Skeletal dysplasia multidisciplinary Unit (UMDE), Hospital Universitario la Paz, Madrid, Spain, ${ }^{7}$ Berlin Institute of Health (BIH), AnnaLouisa-Karsch-Str. 2, 10178, Berlin, Germany, ${ }^{8}$ Handchirurgie Kinderkrankenhaus Wilhelmstift, Hamburg, Germany, ${ }^{9}$ Core Unit Bioinformatics - CUBI, Berlin Institute of Health, Chariteplatz 1, Berlin, Germany, ${ }^{10} R G$ Development \& Disease, Max Planck Institute for Molecular
Genetics, Berlin, Germany, ${ }^{11}$ Institut für Humangenetik, Universität Göttingen, Göttingen, Germany, ${ }^{12}$ Institute of Human Genetics, University Medical Centre of the Johannes Gutenberg University, Mainz, Germany

Introduction: Chitayat Syndrome is characterized by hand hyperphalangism with shortened index fingers and ulnar deviation, hallux valgus, severe respiratory distress requiring assisted ventilation and mild facial dysmorphism. It is caused by the recurrent heterozygous missense variant NM_006494.2:c.266A $>$ G; p.Tyr89Cys in ERF, which encodes the ETS2 repressor factor (ERF) protein. The ETS family of transcription factors are involved in cellular proliferation and differentiation, embryological development, hematopoiesis, lymphocyte function and apoptosis. The exact pathomechanism of Chitayat syndrome remains unknown. So far, only seven individuals with molecularly confirmed Chitayat syndrome have been documented in the literature.

Methods: We performed a systematic phenotype analysis of six unpublished individuals with Chitayat syndrome.

Results: We found unreported phenotypic features and a great variability of pulmonary manifestations. Only one individual presented with a history of assisted ventilation, and one of the remaining presented with an atypical phenotype lacking any obvious pulmonary compromise.

Conclusions: Our findings extend the phenotype spectrum associated with the recurrent ERF variant c.266A $>\mathrm{G}$ and contribute to improved diagnostic strategies in individuals with hand hyperphalangism.

A. Suter: None. A. Abad Perez: None. F. SantosSimarro: None. P. Toerring: None. R. Ramos-Mejia: None. K. Heath: None. V. Huckstadt: None. M. ParrónPajares: None. M. Mensah: None. W. Hülsemann: None. M. Holtgrewe: None. S. Mundlos: None. U. Kornak: None. O. Bartsch: None. N. Ehmke: None.

\section{P04.16.A}

First family from Germany with early-onset chondrocalcinosis and osteoarthritis (CCAL1, OMIM \% 600668) caused by TNFRSF11B gene mutation

\section{A. C. Blumberg ${ }^{1}$, M. Wingendorf ${ }^{2}$, I. Spier ${ }^{3}$, V. Riehmer ${ }^{4}$, O. Bartsch}

${ }^{1}$ Institute of Human Genetics, University Medical Centre of the Johannes Gutenberg University Mainz, Mainz, Germany, ${ }^{2}$ Practice for Orthopaedics and Trauma Surgery, Kirchen/Sieg, Germany, ${ }^{3}$ Institute of Human Genetics, University Medical Centre Bonn, Bonn, Germany, ${ }^{4}$ Institute of Human Genetics, University Medical Centre Cologne, Cologne, Germany 
Chondrocalcinosis (CCAL) is a common condition caused by calcium pyrophosphate accumulation and resulting in destruction of articular cartilage, affecting 4-7\% of adults. CCAL is usually seen in polygenic disorders (hypercalcemia, gout, arthritis), but in rare cases runs in families as a dominant trait (usually CCAL2, MIM \#118600, ANKH gene). CCAL1 is extremely rare with only four families known to date: an Ashkenazy-Jewish kindred (PMIDs 2383078 and 29578045), a family from Maine (PMID 7887424), a family from the Netherlands and France (PMID 24743232), and a Long Island NY family (PMID 29578045). These families all demonstrated a specific heterozygous TNFRSF $11 B$ variant, c. $1205 \mathrm{~A}>\mathrm{T}$, modifying the stop codon (AAT>ATT) and predicting an elongated protein (p.Ter402Leuext*19). We report another 4-generation CCAL1 kindred from Rhineland-Palatinate (comprising 8 females and 3 males) also demonstrating the TNFRSF $11 B$ c. $1205 \mathrm{~A}>\mathrm{T}$ variant. All patients $(11 / 11,100 \%)$ had earlyonset osteoarthritis and chondrocalcinosis with an onset of symptoms between 16 and 30 years, and all had swelling of joints, motion restriction, and intense pain. Three probands $(3 / 10,30 \%$; no data in a deceased family member) also had digital polyarthritis. First, knee, elbow, ankle and wrist joints were affected, followed by spinal destruction. Remarkably, ball-and-socket joints (e.g. hip joints) were only minimally affected (only an affected 30-year-old male). The early onset and familial clustering led to the molecular diagnosis. This is the first CCAL1 family from Germany, providing further understanding of the clinical signs and treatment (including physical therapy, orthoses, antirheumatic drugs, experimental treatment with interleukin inhibitors, and multiple joint surgery).

A.C. Blumberg: None. M. Wingendorf: None. I. Spier: None. V. Riehmer: None. O. Bartsch: None.

\section{P04.17.B}

Three families with suspected Ehlers-Danlos syndrome and glycine substitution in COL1A1 and COL1A2 genes

\author{
S. Döhnert ${ }^{l}$, H. Reichenbach ${ }^{2}$, S. Demuth ${ }^{3}$, S. Weidensee ${ }^{3}$, \\ K. Wilhelm ${ }^{4}$, I. Schreyer ${ }^{4}$, B. Eichhorn ${ }^{1}$
}

${ }^{I}$ MVZ Mitteldeutscher Praxisverbund Humangenetik MVZ, Dresden, Germany, ${ }^{2} M V Z$ Mitteldeutscher Praxisverbund Humangenetik MVZ, Leipzig, Germany, ${ }^{3}$ MVZ Mitteldeutscher Praxisverbund Humangenetik MVZ, Erfurt, Germany, ${ }^{4}$ Zentrum für ambulante Medizin, Uniklinikum Jena gGmbH, Jena, Germany

Introduction: Glycine substitutions in the Gly-X-Y-triplet disrupt the type I collagen triple helix structure and folding, and typically result in osteogenesis imperfecta (OI) and have not yet been associated with Ehlers-Danlos syndrome
(EDS). OI is characterized by bone fragility, whereas EDS affects the connective tissue resulting in joint hypermobility, skin hyperextensibility, and tissue fragility. Although, an OI/EDS overlap" syndrome characterized by clinical hallmarks of EDS and mild or absent OI-phenotype is also described. The underlying mutation spectrum is wide, but includes glycine substitutions.

Material and Methods: Here, we report three patients mainly showing joint hypermobility and unusual skin. Panel diagnostic via sequencing by synthesis was conducted including candidate genes contributing to connective tissue disorders. Sanger sequencing was used for analyses of segregation patterns in parents.

Results: All index patients carried each an undescribed, heterozygous glycine substitution. In two cases the variant (c.1270G $>\mathrm{A} / \mathrm{p}$.(Gly424Ser) in COL1A1 gene; c. $434 \mathrm{G}>\mathrm{A} / \mathrm{p}$. (Gly145Arg) in COL1A2 gene) was maternal inherited and segregated with the symptoms in both families. In the other case the substitution occurred de novo (c.179G $>\mathrm{A} / \mathrm{p}$. (Gly60Asp) in COL1A2 gene). Comparable to our patients, glycine substitutions in the more $\mathrm{N}$-terminal region of both genes are already described with "OI/EDS overlap" syndrome. In detail, one of our families showed an EDSphenotype with additional minimal OI-symptoms (short stature, blue sclera), whereas the others were clinical classified as EDS-phenotype.

Conclusions: Despite the detection of glycine substitution, the patients could manifest a more typical EDS phenotype rather than OI. Certainly, subclinical symptoms for OI can additionally occur and should be considered.

S. Döhnert: None. H. Reichenbach: None. S. Demuth: None. S. Weidensee: None. K. Wilhelm: None. I. Schreyer: None. B. Eichhorn: None.

\section{P04.20.B}

Morphological and molecular characterization of ectopic cartilage in Crouzon Syndrome

\section{E. Candelo}

\section{Fundacion Valle del Lili, Cali, Colombia}

Background: Craniosynostosis is the premature closure of the skull sutures. Patients with these condition undergo several surgical procedures, which are not able to fully restore appearance and functionality due to insufficient bone growth and suture refusion. Most mutations disrupt genes that encode for members of the fibroblast growth factor receptor (FGFR) family.

Materials and Methods: A knock out mouse with Fgfr2c342Y/C342Y was generated for phenotypic analysis by histological staining of sections of WT, Fgfrc342y/+ heterozygous (HET) and Fgfr2c342Y/C342Y homozygous 
(HOM) mouse embryos from embryonic day (E) 14.5 and $\mathrm{E}$ 16.5 stages. Morphological characterisation of the ectopic cartilages in the mutant specimens was done against the HET and WT by calculating the surface area, perimeter, width on the parietal cartilage in each genotype and cellular characterisation by obtaining the cellular density and shape descriptors. Molecular characterisation was determined by mmunohistochemistry of Collagen I and II.

Results: We identified a significant parietal cartilage expansion in HOM embryos since E14.5 with higher perimeter, surface area, and high cellular density. Additionally, characteristic cell morphology comprised; high cellular area and perimeter and circular or rounded shape. These findings were associated with abnormal intensity and localization pattern of Collagen type I and II.

Conclusions: A possible switch off on the intramembranous ossification of the parietal and frontal primordial bone and switch on of endochondral ossification process supported by the cellular, morphological and molecular findings of the abnormal cartilage expansion lead to a novel hypothesis of coronal suture fusion that can future drug target on this condition.

\section{E. Candelo: None.}

\section{P04.22.A}

The first case of Dyssegmental Dysplasia RollandDesbuquois type with a variant in HSPG2

\section{T. Kalayci ${ }^{1}$, N. Balanda ${ }^{2}$, C. R. Ferreira ${ }^{3}$, U. Altunoglu $^{1,4}$ \\ ${ }^{1}$ Istanbul University, Istanbu Faculty of Medicine, Medical Genetics Department, Istanbul, Turkey, ${ }^{2}$ Undiagnosed Diseases Program, Common Fund, National Institutes of Health, Bethesda, MD, United States, ${ }^{3}$ Medical Genomics and Metabolic Genetics Branch, National Human Genome Research Institute, National Institutes of Health, Bethesda, MD, United States, ${ }^{4}$ Koc University, School of Medicine, Medical Genetics Department, Istanbul, Turkey}

Introduction: Dyssegmental dysplasia (DD) is a rare, autosomal recessive disorder characterized by micromelia and anisospondyly. Two distinct forms are described; Silverman-Handmaker (DDSH) characterized by severe shortening of limbs and perinatal lethality, and RollandDesbuquois (DDRD) with survival beyond infancy and milder skeletal findings. DDSH is caused by biallelic variants in HSPG2 but the aetiology of DDRD has not been explained. Here, we present the first DDRD patient reported to have an HSPG2 variant.

Materials and Methods: Detailed phenotyping and nextgeneration sequencing were performed.

Results: The patient is a 15-month-old female with short bones, flared metaphyses, camptodactyly, anisospondyly, pes equinovarus, flat face, micrognathia, cleft palate and the history of respiratory distress in infancy. She was diagnosed as DDRD given milder skeletal findings and a prolonged survival in comparison to DDSH. Whole exome analysis revealed a homozygous NM_005529.7:c.9970G $>$ A(p. Gly3324Arg) variant on HSPG2 which was predicted to be pathogenic by in silico analyses.

Conclusions: The phenotypic heterogeneity and clinical overlap between DDSH and DDRD raise the question of whether HSPG2 variants lead to distinct DD forms or represent a single disorder with variable phenotypic spectrum. In a review of the literature, we recognised three patients reported as DDSH whereas they had milder skeletal findings and survival age between 13 months and 7 years, compatible with DDRD. Strikingly, they harboured novel HSPG2 variants, one of which was detected in our case. In the light of all these findings, we conclude that DDSH and DDRD are allelic and represent a single disorder with a spectrum of severity.

T. Kalayci: None. N. Balanda: None. C.R. Ferreira: None. U. Altunoglu: None.

P04.23.B

FROM CEREBRAL HEMORRAGE AND SUSPICTION OF CHILD ABUSE TO DIAGNOSIS OF FAMILIAR EHLERS DANLOS SYNDROME DUE TO DELECION IN COL5A1 GENE

\section{P. Marin Reina ${ }^{l}$, A. Zuñiga Cabrera ${ }^{1}$, A. Parra Llorca ${ }^{l}, P$. Molina Aguilar ${ }^{2}, J_{\text {. Blasco }}^{2}$, A. Monzo Blasco ${ }^{2}$, M. Evole Buselli ${ }^{1}$}

${ }^{1}$ Hospital UyP La Fe, Valencia, Spain, ${ }^{2}$ Forensic Department, Valencia, Spain

Introduction: The misdiagnosis of Ehler Danlos syndrome (EDS) and child abuse is well known, usually in relationship to skin lesions or bone fractures. At our case, the first manifestation was an aborted lethal episode in an infant due to a cerebral hemorrhage.

Clinical Case: A 4-month-old boy was founded unconscious after sleep. He was admitted to the pediatric intensive care unit with a subdural and subarachnoid hemorrhage and severe cerebral edema. Child abuse was suspected. Familiar antecedents: Mother, 34 years, first gravida. She was followed up during infancy due to small size. She has joint hypermobility. Her brother died at sixth month due to supposed sudden infant death. One 50 years old aunt presented a cerebral hemorrhage. Healthy father, 30 years old. No consanguinity. The child presented hypermovility and skin hyperextensibility. EDS vascular type was suspected. NGS analysis was performed using Illumina (NextSeq 500) technology with a ClearSeq Inherited 
Disease panel (Agilent Thecnologies) and consisted in the analysis of the coding and intronic regions of collagen deficiency related gene. The study did not show any pathogenic variant and was completed by analysis of gene dosage by MLPA (P331; P332), resulting in patient HETEROZYGOUS for deletion of exons 5-7 of the COL5A1 gene. Presence of deletion was confirmed in affected familial members.

Conclusions: Vascular complications in nonvascular subtypes of the EDS could be life-threatening. - Misdiagnosis of child abuse should be considered in EDS - The relevance of study microdeletions in cases not diagnosed by gene sequencing.

P. Marin Reina: None. A. Zuñiga Cabrera: None. A. Parra Llorca: None. P. Molina Aguilar: None. J. Blasco: None. A. Monzo Blasco: None. M. Evole Buselli: None.

P04.25.A

Follow-up findings and genotype-phenotype correlation in 18 Turkish patients with biallelic mutation in the FKBP10 gene

A. Yüksel Ülker ${ }^{1}$, D. Uludag Alkaya ${ }^{1}$, G. Yeşil ${ }^{2}$, G. Toksoy ${ }^{3}$, Z. Uyguner ${ }^{3}$, B. Tüysüz ${ }^{1}$

${ }^{1}$ Istanbul University, Cerrahpasa Medical Faculty, Istanbul, Turkey, ${ }^{2}$ Bezmialem Vakif University, Medical Faculty, Department of Medical Genetics, Istanbul, Turkey, ${ }^{3}$ Istanbul University, Medical Faculty, Department of Medical Genetics, Istanbul, Turkey

Introduction: Osteogenesis Imperfecta (OI)-typeXI and Bruck syndrome type-I are two very rare disorders caused by biallelic FKBP10 gene mutations. While both disorders are characterized by early onset bone fractures, progressive bone deformity and scoliosis, Bruck syndrome type 1 also has congenital contractures and pterygium. FKBP10/ KRT14 mutations have been reported to be present in patients with OI-typeXI and Epidermolysis bullosa simplex (OIEBS). The aim of this study is to investigate the clinical findings and genotype-phenotype correlation in the Turkish cohort with FKBP10 mutation.

Materials and Methods: Eighteen patients from fifteen families were enrolled. FKBP10 mutations studied via sanger sequencing or next generation sequencing. The parents of all patients had consanguineous marriages. 16 patients were followed up for 6.2 years ( 7 months-15.5 years).

Results: We identified p.Gly300Ter in eleven patients and p.Gly278Argfs*95 in one patient who live same region. Ten of these patients were compatible with OI-XI, two patients had Bruck1 phenotype. One patient with Bruck1 who lives different region had a novel $(\mathrm{c} .1256+1 \mathrm{G}>\mathrm{A})$ mutation. We also found same mutation (c.321_353del) in five patient with OIEBS who lives same city. Pamidronate treatment was given to 16 follow-up patients.

Conclusions: The clinical course of the patients was progressive; bone fractures were started in the infantile period (15/18) and continued as more than 1 fracture (9/16) per year. During the follow-up period, severe limb deformity (7/16) and scoliosis (11/16) developed and no patient could walk (16/16). Although most families are not related, they had the same mutation which is attributed to the founder effect.

A. Yüksel Ülker: None. D. Uludag Alkaya: None. G. Yeşil: None. G. Toksoy: None. Z. Uyguner: None. B. Tüysüz: None.

\section{P04.26.B}

Wide sutures and fontanels, delayed speech development and a hoarse voice useful signs in diagnosing KBG syndrome, clinical, molecular and cytogenetic description of 12 patients.

A. Kutkowska-Kazmierczak ${ }^{l}, M$. Gos ${ }^{l}, J$. Castaneda ${ }^{l}, M$. Boczarl $^{l}$, A. Barczykl, A. Pietrzyk ${ }^{l}$, O. Kordowska ${ }^{l}, T$. Gambin $^{l}$, P. Wtasienko ${ }^{l}$, B. Wiśniowiecka-Kowalnik ${ }^{l}, K$. Kowalczyk ${ }^{l}$, M. Bartnik-Gtaska ${ }^{l}$, M. Smyk ${ }^{l}, M$. Paczkowska $^{l}$, I. Plaskotal, K. Niepokój' ${ }^{I}$, J. Kelly ${ }^{2}$, B. Nowakowska $^{l}$, E. Obersztyn ${ }^{l}$, J. Bal ${ }^{l}$

${ }^{1}$ Institute of the Mother and Child, Warsaw, Poland, ${ }^{2}$ Quest Diagnostics Nichols Institute, Chantilly, VA, United States

Introduction: KBG syndrome is a neurodevelopmental disorder characterized by short stature, macrodontia, developmental delay, behavioral problems as hyperactivity, velopharyngeal insufficiency causing feeding problems and speech delay and by delayed closing of sutures and fontanels. Most of the patients with KBG syndrome have a mutation of ANKRD11 gene or micro-rearrangement encompassing this gene.

Patients and methods: We present clinical evaluations of 12 patients with the clinical features of KBG syndrome at the age from 4 months to 17 years. The mutation analysis in these patients was performed using panel next generation sequencing (6pts), whole exome sequencing (WES) (2pts) and array CGH (4pts).

Results: Besides typical dysmorphic features for KBG syndrome found in all patients, the majority of patients had wide sutures and fontanels (7/12), delayed speech (9/11), feeding problems (9/12). Hoarse voice was noticed in 4 patients and short stature, below $2 \mathrm{SD}$ in two patients, between -1-2 SD in 7 patients. Abnormal behavior was noted in 4 patients, macrodontia in 3 patients. 
Conclusions: The fact that the delayed closing of sutures and fontanels was observed in more than half of our patients with KBG syndrome caused by mutations or rearrangements of ANKRD11 gene confirms the role of this gene in skull and suture formation. Speech delay is found in majority of patients with this syndrome. Rarely described sign - hoarse voice could reinforce clinical knowledge in diagnosing KBG syndrome. The studies on craniosynostoses were supported from Institute of Mother and Child in Warsaw intramural grant no. OPK-510-18-63

A. Kutkowska-Kazmierczak: None. M. Gos: None. J. Castaneda: None. M. Boczar: None. A. Barczyk: None. A. Pietrzyk: None. O. Kordowska: None. T. Gambin: None. P. Własienko: None. B. Wiśniowiecka-Kowalnik: None. K. Kowalczyk: None. M. Bartnik-Głaska: None. M. Smyk: None. M. Paczkowska: None. I. Plaskota: None. K. Niepokój: None. J. Kelly: None. B. Nowakowska: None. E. Obersztyn: None. J. Bal: None.

P04.27.C

Patients with suspected Erythrodermia variabilis of a large family carry variants in $G J B 3$ and $K R T 10$

A. Hotz ${ }^{1}$, S. Redler ${ }^{2}$, J. Kopp ${ }^{1}$, A. Zimmer ${ }^{1}$, J. Fischer ${ }^{1}$

${ }^{1}$ Institute of Human Genetics, University Medical Center Freiburg, Faculty of Medicine, Freiburg, Germany, ${ }^{2}$ Institute of Human Genetics, Heinrich-Heine-University, Medical Faculty, Düsseldorf, Germany

Introduction: Erythrokeratoderma variabilis (EKV) is a rare skin disease characterized by two features: red skin areas with sharp borderlines, which tend to shift positions; and hyperkeratosis, which can either be generalized or localized as thickened plaques. EKV is mostly inherited in an autosomal dominant manner and is mainly caused by pathogenic variants in GJB3, GJB4 or GJA1.

Results: We describe a large family with twelve patients of five generations affected by a skin disorder. The index patient shows typical features of EKV. Mutation analysis in seven affected members from three generations using Next Generation and Sanger sequencing methods revealed that all of them carry both the heterozygous unclear variant c. $783 \mathrm{G}>\mathrm{C}$, p.(Gln261His) in GJB3 and the novel heterozygous pathogenic variant c.1346A $>C$, p.(Tyr449Ser) in KRT10. Pathogenic variants in KRT10 lead to Epidermolytic ichthyosis, which is characterized by diffuse erythroderma and blistering at birth and progressive hyperkeratosis later in life and is usually inherited in an autosomal dominant manner. Furthermore, two unaffected members were analyzed, one of them carries none of the variants, the other only the GJB3 variant.
Conclusions: It is remarkable, that all seven tested affected family members from three generations carry both variants in GJB3 and KRT10. Although the KRT10 variant is pathogenic, it cannot be excluded that the GJB3 variant has an impact on the phenotype, since the initial suspected diagnosis was EKV. However, it cannot be excluded that the GJB3 variant is neutral. In this case, analysis of patients with suspected EKV should include pathogenic variants in KRT10.

A. Hotz: None. S. Redler: None. J. Kopp: None. A. Zimmer: None. J. Fischer: None.

P04.30.C

When textbooks fall short - earlier detection of Hypochondroplasia - a large UK cohort experience and systematic review.

A. Sabir ${ }^{1}$, E. Morley', J. Sheikh ${ }^{2}$, A. Singh ${ }^{2}$, A. Cocca ${ }^{1}$, M. Cheung ${ }^{1}$, M. Irving ${ }^{1}$

${ }^{I}$ Guy's and St Thomas' Hospital, London, United Kingdom, ${ }^{2}$ University of Birmingham, Birmingham, United Kingdom

Introduction: Hypochondroplasia $(\mathrm{HCH})$ is a rare autosomal dominant skeletal dysplasia caused by $F G F R 3$ mutations leading to disproportionate short stature. Classically $\mathrm{HCH}$ presents in toddlers or school-age children, as limb-to-trunk disproportion is often mild and easily overlooked during infancy. We report experiences from our UK $\mathrm{HCH}$ cohort of $\sim 30$ patients and review recent literature to explore the antenatal detection of $\mathrm{HCH}$.

Methods: We reviewed the rate of antenatal $\mathrm{HCH}$ detection in our 29 patient cohort and describe relevant case-date. We then conducted a systemic, retrospective literature review using PUBMED and MEDLINE, identifying antenatally suspected / detected $\mathrm{HCH}$ cases. Paper inclusion criteria: Antenatally suspected $\mathrm{HCH}$. Molecular confirmation of denovo HCH. Exclusion criteria: unclear antenatal scanning history.

Results: Antenatally suspected (and concurrent or deferred molecularly confirmation) $\mathrm{HCH}$ has been reported 10 times in the literature (between 2004-2019) and in 6 of our 29 patient cohort. Key sonographic markers included reduced; femur length, biparietal diameter and humeral length.

Conclusions: $\mathrm{HCH}$ is increasingly detected both antenatally and in infancy, contrary to previous descriptions. This is likely due to greater $\mathrm{HCH}$ awareness, improved imaging and easier molecular testing. Thus one should consider $\mathrm{HCH}$ outside the classical presenting period. Studying the natural history of earlier cases is important with the advent of several targeted FGFR3 therapies currently in trials for 
Achondroplasia (Biomarin's Vosorotide, Ascendis' Transchon, QED's Infagratinib and Pfizer's TA-46) as such therapies may soon be trialled in $\mathrm{HCH}$ and are likely most efficacious during earliest skeletal maturation.

A. Sabir: None. E. Morley: None. J. Sheikh: None. A. Singh: None. A. Cocca: None. M. Cheung: None. M. Irving: None.

\section{P04.31.A}

Mono-allelic deleterious variants in autosomal recessive skeletal dysplasia genes are strongly associated with idiopathic short stature

C. T. Thiel ${ }^{l}$, N. Hauer ${ }^{1}$, C. Vogl ${ }^{l}$, S. Uebe $e^{l}$, H. Sticht ${ }^{2}$, A. B. Ekici $^{l}$, C. Kraus ${ }^{l}$, H. Dörr ${ }^{3}$, A. Reis ${ }^{1}$

${ }^{1}$ Institute of Human Genetics, Friedrich-Alexander-Universität Erlangen-Nürnberg FAU, Erlangen, Germany, ${ }^{2}$ Institute of Biochemistry, Friedrich-Alexander-Universität Erlangen-Nürnberg FAU, Erlangen, Germany, ${ }^{3}$ Department of Pediatrics and Adolescent Medicine, FriedrichAlexander-Universität Erlangen-Nürnberg FAU, Erlangen, Germany

Short stature is a common concern in childhood with an incidence of $3 \%$. After exome analysis about $60 \%$ remain without diagnosis (idiopathic short stature, ISS). Recent identification of heterozygous mutations in ACAN in $1.4 \%$ of ISS individuals confirmed that single, large-effect variants strongly affect height (appr. $15 \mathrm{~cm}$ final adult height). We therefore hypothesized that heterozygous variants in other autosomal recessive skeletal dysplasia genes also contribute to ISS. We selected exome data from 368 ISS families where previous analysis showed no mutations in a known growth-associated gene and selected 114 genes known to be involved in autosomal recessive inherited skeletal dysplasia, extracted from the "Nosology and Classification of Genetic Skeletal Disorders" (Bonafe et al. 2015). From a total of 391 heterozygous variants we selected 33 variants in 26 genes which were classified as ACMG class 4/5 and compatible with segregation in the families and were thus considered strong candidates for ISS. This accounted for 31 patients (8.4\%). No class $4 / 5$ variant in any of the 26 genes was detected in 343 controls ( $\mathrm{p}<1 \mathrm{e}-$ $85)$. Frequently affected genes were ACAN (6 individuals) and NPR2 (3 individuals) representing $2.5 \%$ of the study group. In addition to missense variants in 17 genes, a nonsense variant was observed in ADAMTS10, CHST3, CYP27B1, TCIRG1, TRIP11, WDR35 and CUL7. Our findings suggest a strong association between rare monoallelic deleterious variants in genes for recessive skeletal dysplasia and idiopathic short stature and provide further evidence for the contribution of rare, large-effect variants to the etiology of this common trait.

C.T. Thiel: None. N. Hauer: None. C. Vogl: None. S. Uebe: None. H. Sticht: None. A.B. Ekici: None. C. Kraus: None. H. Dörr: None. A. Reis: None.

\section{P04.32.B}

IRAK1 gene SNPs rs1059703 and rs3027898 polymorphisms is associated with psoriasis

\author{
L. Kucinskas ${ }^{1}$, A. Bruzaite ${ }^{I}$, J. Cepaite ${ }^{l}$, V. Kucinskiene ${ }^{2}$, \\ R. Ugenskiene ${ }^{1}$, S. Valiukeviciene ${ }^{2}$
}

${ }^{1}$ Institute of Biological System and Genetic Research LUHS, Kaunas, Lithuania, ${ }^{2}$ Department of Skin and Venereal Diseases, Lithuanian University of Health Sciences (LUHS), Hospital of LUHS Kauno Klinikos, Kaunas, Lithuania

Psoriasis vulgaris and psoriatic arthritis are multifactorial diseases with an important genetic component. The clinical significance of IRAK1 genes SNPs rs1059703 ir rs3027898 are unknown for psoriasis pathogenesis. The aim of present study was to determinate IRAKI gene SNPs rs 1059703 and rs3027898 clinical importance for psoriatic arthritis and psoriasis development.Methods. 106 patients with psoriasis from Skin and Veneral disease department of Lithuanian University of Helath Sciences hospital and 52 healthy control group subjects were genotyped. The IRAKI gene SNPs rs1059703 and rs3027898 genotyping was performed using Mva1269I (BSMI) and Eco57I (AcuI) restriction enzymes. Data and statistical analysis was performed using, Microsoft Office Excel" and, IBM SPSS Statistics 20.0" programs.Results. The number of patients of IRAKI gene rs3027898 with C/A, C/C and C genotypes outnumbered control group (32 persons (30 \%) vs 5 persons $(10 \%)$. The number of patients of IRAKI rs 1059703 with $\mathrm{C} / \mathrm{T}, \mathrm{C} / \mathrm{C}$ and $\mathrm{C}$ genotype was higher compare with control group (30 persons $(30 \%)$ vs 6 persons $(11 \%)$. The statistical analysis of patients genotypes and psoriatic arthritis corelation showed no significant data of association $(\mathrm{p}<0,05)$. Odds of ratio calculation (OR) showed IRAK1 rs3027898 C/A, C/ $\mathrm{C}, \mathrm{C}$ and IRAKI rs1059703 C/T, C/C and C genotypes association with psoriasis (OR 4,06) and (OR 3,39) respectively.Conlusions. Statistically significant association of IRAKI rs3027898 and IRAKI rs3027898 SNPs with psoriatic arthritis was not determinated. IRAKI rs3027898 C/A, C/C, C genotypes and IRAKI rs1059703 C/T, C/C and $\mathrm{C}$ genotypes are associated with psoriasis development.

L. Kucinskas: None. A. Bruzaite: None. J. Cepaite: None. V. Kucinskiene: None. R. Ugenskiene: None. S. Valiukeviciene: None. 
P04.33.C

Skin cancer screening is indicated in IWC patients due to the identification of heterozygous KRT10 variants in patients with MAUIE syndrome

B. Burger ${ }^{l}$, A. Ghosh ${ }^{1,2}$, C. K. Y. $\mathrm{Ng}^{3}$, S. Piscuoglio ${ }^{1}, I$.

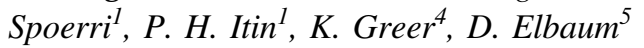

${ }^{1}$ University of Basel and University Hospital Basel, Basel, Switzerland, ${ }^{2}$ University of Zurich and ETH Zurich, Zurich, Switzerland, ${ }^{3}$ University of Bern, Bern, Switzerland, ${ }^{4}$ University of Virginia, Charlottesville, VA, United States, ${ }^{5}$ Mercy Hospitals of Bakersfield, Bakersfield, CA, United States

Ichthyosis with confetti (IWC) is an autosomal dominant skin disorder characterized by a congenital erythema and the development of numerous pale spots. About $20 \%$ of the patients are reported to develop skin cancer at young adulthood. All IWC patients are carriers of a heterozygous keratin (KRT)10 or KRT1 variant leading to a shifted reading frame and the translation of an aberrant carboxyl (C)-terminus. This results in the nuclear localization of the KRT10/KRT1 protein instead of cytoplasmic. About 20 years ago, two patients were described as MAUIE patients. They showed the typical IWC-phenotype including the development of pale spots. Both patients developed aggressive and fatal cutaneous squamous cell carcinoma (cSCC). A genetic analysis is not yet done. Our study aimed to identify an IWC-correlated frameshift variant in both patients and to clarify whether they suffered from IWC. We performed whole exome sequencing on genomic DNA from FFPE cSCC tissue of both deceased patients and screened for variants similar to IWC-associated variants. A heterozygous KRT10 variant, encoding for the aberrantly shifted $\mathrm{C}$-terminus, was identified in each patient and the nuclear localization of KRT10 was confirmed by immunofluorescence staining. Our study reclassified both MAUIE patients as patients with a specific form of IWC. The fatal course of the disease is not typical for the other IWC forms, but it emphasizes the importance of skin cancer screening in all IWC-patients. We recommend the regular intensive examination of their skin for cancers and a good education of the patients with reference to their disease.

B. Burger: None. A. Ghosh: None. C.K.Y. Ng: None. S. Piscuoglio: None. I. Spoerri: None. P.H. Itin: None. K. Greer: None. D. Elbaum: None.

\section{P04.34.A}

Kinesin family member $13 \mathrm{~A}, K I F 13 A$, is a novel gene for hypomelanosis of Ito

\section{C. $L A M$}

\section{The University of Hong Kong, Hong Kong, China}

Introduction: Hypomelanosis of Ito is a neurocutaneous syndrome, characterized by distinctive skin manifestations, with or without multisystem involvements. While the exact cause of hypomelanosis of Ito is unknown, chromosomal mosaicism as a result of sporadic gene mutation has been suggested in literature.

Materials and Methods: In our Clinics for Rare diseases Referral (CRareDr), we encountered a 3-year-old girl presenting with characteristic skin pigmentation and developmental delay, suggesting hypomelanosis of Ito This case was enrolled in our undiagnosed diseases program (UDP) for ending diagnostic odyssey. We initiated genetic analysis for neurodevelopmental impairment using whole-genome (WGS) and whole exome sequencing (WGS).

Results: A heterozygous de novo variant NM_022113.6: c.2357dupA; p.Asn786Lysfs*18 in the KIF13A gene was identified in the patient. The variant is a single nucleotide duplication causing a shift in the translational reading frame and premature termination of the protein. The variant is absent in control population in gnomAD exomes and genomes.

Conclusions: $K I F 13 A$ is a relatively new gene with a limited number of studies on its function and has not been reported to cause any hereditary conditions. To date, KIF13A is known to interact with adaptor complex AP-1, forming a complex that is responsible for the transportation of a number of proteins and receptors to plasma membrane and endosomal organelles. The role of KIF13A in melanosome biogenesis is well-demonstrated in in-vitro studies and may explain the underlying pathogenesis of skin hypopigmentation in hypomelanosis of Ito.

C. Lam: None.

\section{P04.37.A}

Functional evidence supporting the hypothesis that Neanderthals and Denisovans had a genetically specified high bone mass negatively selected in anatomically modern humans

N. Roca-Ayats ${ }^{1}$, I. Maceda ${ }^{2}$, C. Bruque ${ }^{3}, M$. Cozar $^{l}, N$. Garcia-Giralt ${ }^{4}$, L. Mellibovsky ${ }^{4}$, W. van $\mathrm{Hul}^{5}$, O. $\mathrm{Lao}^{2}, \mathrm{D}$. Grinberg $^{l}$, S. Balcells ${ }^{l}$

${ }^{1}$ University of Barcelona, CIBERER, IBUB, IRSJD, Barcelona, Spain, ${ }^{2}$ Centre Nacional d'Anàlisi Genòmica CNAGCRG, Barcelona, Spain, ${ }^{3}$ Centro Nacional de Genética Médica, ANLIS, Instituto de Biología y Medicina Experimental, CONICET, Buenos Aires, Argentina, ${ }^{4}$ Musculoskeletal Research Group, IMIM (Hospital del Mar Medical 
Research Institute), Centro de Investigación Biomédica en Red de Fragilidad y Envejecimiento Saludable (CIBERFES), ISCIII, Barcelona, Spain, ${ }^{5}$ Department of Medical Genetics, University of Antwerp, Antwerp, Belgium

Introduction: Bone density underwent gracilization through hominin evolution; while Neanderthals and Denisovans had robust and dense bones, anatomically modern humans $(\mathrm{AMH})$ show a more gracile skeleton. In this context, mutations in the first $\beta$-propeller of LRP5 result in high bone mass (HBM) in AMH, suggesting this gene as a suitable candidate for studying the hominin evolution of bone mass.

Material and Methods: Variants were looked for in the publically available Neanderthal and Denisovan genomes and functionally tested by a luciferase assay in Saos-2 cells. A molecular homology model of LRP5 was generated using MODELLER (v.9.22). Introgression was inferred in the Simons Genome Diversity Project using the CRF method. Evidences of selective pressures at LRP5 in AMH were sought using PopHuman browser.

Results: We identified five Neanderthal or Denisovan missense mutations in LRP5. Four displayed significantly greater Wnt pathway stimulation, and two also showed reduced inhibition by DKK1. Modelling suggested that these mutations either affected the 3D structure of the first $\beta$-propeller, or impinged on the interaction of LRP5 with DKK1. We observed that the LRP5 genomic region in AMH is depleted of introgression from Neanderthals and/or Denisovans and that the functional Neanderthal and Denisovan mutations are at a very low frequency in current human populations.

Conclusions: The variants identified in Neanderthal and Denisovan genomes, together with the evolutionary analyses in the LRP5 region suggest that this gene may have been involved in HBM determination in archaic hominins.

Funding: Spanish MINECO (SAF2016-75948-R; PGC2018-098574-B-I00); CIBERER (U720); AGAUR (2017 SGR 937).

N. Roca-Ayats: None. I. Maceda: None. C. Bruque: None. M. Cozar: None. N. Garcia-Giralt: None. L. Mellibovsky: None. W. van Hul: None. O. Lao: None. D. Grinberg: None. S. Balcells: None.

\section{P04.39.C}

Looking for genetic modifiers in Marfan syndrome

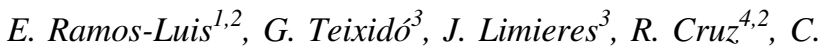
Granato $^{3}$, B. Sobrino ${ }^{5,2}$, J. Amigo ${ }^{5,2}$, A. Blanco-Verea ${ }^{1,2}$, A. Carracedo $^{5,2,4}$, A. Evangelista ${ }^{3}$, M. Brion ${ }^{1,2}$

${ }^{1} X e n e ́ t i c a$ Cardiovascular, Instituto de Investigación Sanitaria de Santiago, Santiago de Compostela, Spain, ${ }^{2}$ Grupo de Medicina Xenómica, Universidade de Santiago de Compostela, Santiago de Compostela, Spain, ${ }^{3}$ Hospital Vall d'Hebron, Servicio de Cardiología, Barcelona, Spain, ${ }^{4}$ CIBER enfermedades Raras, Santiago de Compostela, Spain, ${ }^{5}$ Fundación Pública Galega de Medicina Xenómica, Santiago de Compostela, Spain

Introduction: Marfan syndrome is an autosomal dominant disorder of connective tissue manifested in the ocular, skeletal and cardiovascular systems. It is most frequently caused by mutations in FBN1 with high clinical penetrance, but showing a high inter and intra-familial variability, that cannot be explained by the current knowledge of genotypephenotype correlation. In order to improve knowledge of the whole genetic background of this disease, this study aims to identify genetic modifiers of the phenotypic expression of Marfan syndrome, looking for the intrafamilial variability with known causal mutation in FBN1.

Material and Methods: A total of 22 Marfan syndrome patients with different severity from 8 families with known FBN1 mutation, were exome sequenced. For exome wide gene-set analyses F-SKAT (familial sequence kernell association test) was performed. At the intra-family level, non-common rare protein changing variants among firstdegree relatives with severe and mild phenotypes were selected for discriminant analysis of principal components (DAPC).

Results: F-SKAT test showed no gene with significant differences after Bonferroni test between severe and mild phenotype group of patients. Nevertheless, DAPC showed two genes of retinal homeostasis, NXNL1 gene and RP1L1 gene, as one of the principal contributors to the discrimination analysis between mild and severe phenotype.

Conclusions: The genetic architecture of Marfan syndrome is very complex, however, as it was previously described in the literature, this study point to the genes related to retinal homeostasis as possible genetic modifiers of the disease. Founding: Instituto de Salud Carlos III and Fondo Europeo de Desarrollo Regional (PI14/01062, PI16/ 00903)

E. Ramos-Luis: None. G. Teixidó: None. J. Limieres: None. R. Cruz: None. C. Granato: None. B. Sobrino: None. J. Amigo: None. A. Blanco-Verea: None. A. Carracedo: None. A. Evangelista: None. M. Brion: None.

P04.40.A

Reciprocal translocation as a possible cause of familial connective tissue disorder

B. Herr, K. Komlosi, A. Tzschach, J. Fischer, B. Gläser

Department of Human Genetics, Freiburg, Germany 
Introduction: Monogenic disorders due to apparently balanced reciprocal translocations are rarely reported. There are few reports of interstitial deletions involving $F B N 1$-gene and only one case of a patient with Marfan syndrome and a complex chromosome rearrangement. However, no disruption of $F B N 1$ due to a translocation has been reported so far.

Clinical report: We report on a mother and daughter with clinical symptoms of connective tissue disorder. The 39-year-old mother presented with congenital strabismus, muscle weakness, muscle hypotrophy of forearms and calves, reduced exercise tolerance and muscle and joint pain. Skeletal manifestations included arachnodactyly, joint laxity and foot deformity. Her father and a paternal uncle had skeletal abnormalities and died unexpectedly in young adulthood. Her 18-year-old daughter fulfilled the Ghent criteria for Marfan syndrome.

Methods and Results: Connective tissue panel diagnostics did not reveal pathogenic variants in the mother. Rbanded karyotyping of lymphocyte cultures revealed the same reciprocal translocation in mother and daughter: 46, $\mathrm{XX}, \mathrm{t}(2 ; 15)(\mathrm{q} 22 ; \mathrm{q} 21.1)$. Chromosome microarray analysis revealed no imbalances. Subsequent FisH analysis with region-specific BAC probes for $2 \mathrm{q}$ and $15 \mathrm{q}$ narrowed down the translocation break point to a region of approximately $110 \mathrm{~kb}$ from g.43663778 proximal the $3{ }^{`}$ end of $F B N 1$ to g.48772449 in intron 32-33 of FBN1.

Conclusions: In order to prove a possible disruption of $F B N 1$ we plan to perform long range PCR, next generation mapping and RNA sequencing. We emphasize the importance of a combination of cytogenetic and molecular diagnostic techniques to reveal the cause of monogenic diseases typically caused by point mutations.

B. Herr: None. K. Komlosi: None. A. Tzschach: None. J. Fischer: None. B. Gläser: None.

\section{P04.41.B}

Variant classification in Marfan syndrome: illustrating an underestimated issue in DNA-diagnostics

\section{G. Pals ${ }^{1,2,3}$, S. A. Stolk ${ }^{1}$, A. Maugeri ${ }^{1}$, D. Micha ${ }^{2}$, M. A. M.} M. Mannens ${ }^{l}$

${ }^{1}$ Amsterdam UMC, Genome Diagnostics laboratory, Amsterdam, Netherlands, ${ }^{2}$ Centre for Connective tissue disorders, Amsterdam, Netherlands, ${ }^{3}$ Prodia laboratories, Jakarta, Indonesia

Introduction: Correct classification is crucial for reliable diagnostic DNA-testing of patients. The classification of variants ranges from benign (class 1) to pathogenic (class 5). General recommendations for classification have been developed, like the American College of Medical Genetics (ACMG). Variant classification differs per method used.
We compared three different guidelines, by reanalyzing patients' data of the FBNl gene.

Materials and Methods: Variants in the FBN1 gene, found in our lab, were used to compare the outcome of three different variant classifications: the ACMG guidelines, the methods of our accredited laboratory (ISO15189-RvAM174) and recommendations from the Revised Ghent nosology of Marfan syndrome (Loeys et al. 2010).

Results: We classified, by using our guidelines, 869 variants as pathogenic. 77 of these are classified as benign according to Loeys et al. or VUS according to ACMG. A large number of variants that we classified as benign would be classified as likely benign or VUS according to the other guidelines.

Conclusions: Comparison of various variant interpretations shows that pathogenic variants were classified as VUS or even benign by using the other guidelines. This was mainly caused by using an incorrect consensus sequence for the amino acid motif of the 47 EGF domains in fibrillin-1 (Loeys et al.) or not using specific properties of the protein. Different use of frequency data causes a large difference in interpretation of benign variants. These data emphasize that knowledge of the disease and the protein involved are indispensable in correct interpretation of variants.

G. Pals: None. S.A. Stolk: None. A. Maugeri: None. D. Micha: None. M.A.M.M. Mannens: None.

\section{P04.42.C}

Identifying the molecular cause in Marfan and Marfan like phenotype in a cohort of patients from Romania - 5year single center experience

M. Puiu ${ }^{l}$, A. Chirita-Emandi ${ }^{l}$, N. Andreescu ${ }^{l}$, G. Doros $^{2}$, A. Popoiu ${ }^{2}$, A. Dumitrescu ${ }^{3}$, A. Lacatusu ${ }^{4}$, C. Zimbru ${ }^{5}$

${ }^{1}$ Genetics Discipline, Center of Genomic Medicine, "Victor Babes," University of Medicine and Pharmacy, Timisoara, Romania, ${ }^{2}$ Pediatric Department University of Medicine and Pharmacy "Victor Babes", Timisoara, Romania, ${ }^{3}$ Clinical Emergency Hospital for Children "Louis Turcanu”, Timisoara, Romania, "Victor Babes," University of Medicine and Pharmacy, Timisoara, Romania, ${ }^{5}$ Department of Automation and Applied Informatics, Politehnica University, Timisoara, Romania

Background: Connective tissue disorder represent a group of life-threatening disorders. The prevalence of these diseases is relatively low, but Marfan, Ehlers-Danlos, and Loeys-Dietz syndromes are ubiquitous, affect both genders and involve several organs, particularly the cardiovascular system, eyes, and skeleton. Mortality generally results from cardiovascular complications, mainly aortic dissection. Identifying the molecular cause can lead to a different 
therapeutic management and the possibility for prevention of reoccurrence in the family.

Aim: We aimed to retrospectively evaluate the diagnostic yield in these group o patients and present the variants identified.

Methods: Using targeted Next Generation Sequencing (NGS) with a panel for 174 genes (TruSightCardio Illumina) related to cardiologic diseases we screened 52 patients (37 males/15 females) evaluated for a Marfan/ Marfan like phenotype using Ghent nosology, between 2015-2020 at the Center for Genomic Medicine University of Medicine and Pharmacy Timisoara, Romania.

Results: Diagnosis was identified in 55.7\% (29/52) patients, most(25) pathogenic/likely pathogenic variants were found in FBN1 gene, 3 splicesite, 5 frameshift and 17 missense variants, including two variants associated with neonatal Marfan Syndrome (NM_000138.4:c.3143T>C and c. $3217 \mathrm{G}>\mathrm{A})$. Of these, 10 are previously unreported variants. Nine out of 52 patients originated from 3 families. Diagnosis in 3 other patients involved COL3A1, TGFBR1 and SLC2A10 genes.

Conclusions: This study broadens the spectrum of pathogenic variants in FBN1 gene, providing an update of the molecular basis of Marfan Syndrome in Romania.

M. Puiu: None. A. Chirita-Emandi: None. N. Andreescu: None. G. Doros: None. A. Popoiu: None. A. Dumitrescu: None. A. Lacatusu: None. C. Zimbru: None.

\section{P04.44.B}

Characterization of the pathogenetic role of POLD1 gene in Mandibular hypoplasia, Deafness, Progeroid features and Lipodystrophy (MDPL) Syndrome

C. De Masi ${ }^{1}$, P. Spitalieri ${ }^{l}$, M. Murdocca ${ }^{l}$, V. Ferradini ${ }^{1}$, M. D'Adamo', P. Sbraccia', M. Sanchez ${ }^{3}$, I. Udroiu ${ }^{4}, J$. Marinaccio $^{4}$, G. Novelli, A. Sgura ${ }^{4}$, M. D'Apice ${ }^{5}, F$. Sangiuolo ${ }^{l}$

${ }^{1}$ Dep. of Biomedicine and Prevention, Tor Vergata University, Rome, Italy, ${ }^{2}$ Dep. of Systems Medicine, Tor Vergata University, Rome, Italy, ${ }^{3}$ Core Facilities Center Section of Cytometry, Istituto Superiore di Sanità, Rome, Italy, ${ }^{4}$ Dep. of Science. "Roma Tre" University, Rome, Italy, ${ }^{5}$ Laboratory of Medical Genetics, Tor Vergata Hospital, Rome, Italy

MDPL defines a multisystem disorder identified in 22 patients worldwide and associated to mutations inPOLD1 gene, encoding the DNA polymerase $\delta$, involved in DNA replication and repair. We identified an in-frame deletion (p. Ser605del), segregating for the first time as an autosomal dominantmutation, in a MDPL patient from whom dermal fibroblasts (HDFs) have been derived. Cells displayed in vitro abnormalities of nuclear envelope morphology, presence of micronuclei, a large number of autophagosomesand a significant accumulation of prelamin A, strongly linked to altered cellular proliferation and genomicinstability. DNA damage-induced treatment in MDPLHDFs revealed a poor capacity of DNA repair, an arrestin G0/G1 phase transition, and a lower number of cells in the S phase. Moreover, Western Blot analysesshowed a nuclear reduction of POLD1 in MDPL-HDFs compared to WT in basal condition but a statisticallysignificant and persistent increase after cisplatin/1-Gy X-ray damage when compared to WT. Analysis of telomere length exhibited an increased rate of telomere shortening, accompanied by anaccumulation of unrepaired telomeric DNA damage. Furthermore, the evaluation of $\mathrm{H} 2 \mathrm{AX}$ and TelomereInduced Foci after 1-Gy $\mathrm{X}$-ray treatment enabled us to figure out that POLD1 repairs less efficiently DNAdamage and its activity is mainly associated with the homologous recombination phase. Finally, we also generated human induced pluripotent stem cells from patient's fibroblasts showing thepeculiar presence of micronuclei. Knowing the mechanistic basis for the association of DNA damage and failed DNA repair with aging wouldopen up new therapeutic possibilities for this rare disease.

C. De Masi: None. P. Spitalieri: None. M. Murdocca: None. V. Ferradini: None. M. D'Adamo: None. P. Sbraccia: None. M. Sanchez: None. I. Udroiu: None. J. Marinaccio: None. G. Novelli: None. A. Sgura: None. M. D’Apice: None. F. Sangiuolo: None.

\section{P04.45.C}

Molecular analysis of Polish patients with Mendelian Disorders of Cornification

K. Wertheim-Tysarowska ${ }^{l}, K$. Osipowicz ${ }^{2}$, A. KutkowskaKaźmierczak ${ }^{l}$, J. Sawicka ${ }^{l}$, M. Jackiewicz ${ }^{l}$, O. Kordowska ${ }^{l}$, M. Gos ${ }^{l}$, A. M. Rygiel ${ }^{l}, K$. Niepokój ${ }^{l}, K$. Woźniak ${ }^{2}, C$. Kowalewski $i^{2}$ J. Bal

${ }^{1}$ Medical Genetic Department, Institute of Mother and Child, Warsaw, Poland, ${ }^{2}$ Department of Dermatology and Immunodermatology, Warsaw Medical University, Warsaw, Poland

Introduction: Mendelian Disorders of Cornification (MeDOC) comprise syndromic or non-syndromic heterogeneous disorders manifesting by skin hyperkeratosis and/ or scaling. Mutations in approximately 40 different genes can cause MeDOCs, that can be inherited in autosomal recessive/dominant or X-linked patterns. Identification of molecular defect is essential for prognosis, proper medical interventions and genetic counseling. Nonetheless, genetic 
diversity, low incidence of majority of MeDOCs and overlapping phenotypes make diagnosis challenging. The aim of the study was to characterize molecularly Polish MeDOC patients.

Materials and methods: A group of 161 patients with clinical symptoms of MeDOC was enrolled to the study. Genetic analysis was performed using self-designed customized NGS panel. Sanger sequencing or MLPA were used for mutations verification.

Results: We identified full genotype in 125 (78\%) patients. In $17(11 \%)$ patients we didn't detect any mutation, while in 19 (12\%) we found mutation in one allele only, despite expected autosomal recessive inheritance. Overall, we detected mutations in 27 distinct genes. The 33 patients had mutations in more than one gene involved in skin barrier functioning: 13 of them had pathogenic mutations in two genes, while in remaining 20 cases variant of unknown significance was detected in at least one of the genes.

Conclusions: Our diagnostic strategy proved to be an efficient and sensitive diagnostic tool for MeDOC patients. Our data provide further information regarding molecular epidemiology of skin barrier defects and also focus on the presence of secondary mutations, which have important impact on genetic counseling and, presumably, may affect therapy. Supported by NCN:2014/13/D/NZ5/03304

K. Wertheim-Tysarowska: None. K. Osipowicz: None. A. Kutkowska-Kaźmierczak: None. J. Sawicka: None. M. Jackiewicz: None. O. Kordowska: None. M. Gos: None. A.M. Rygiel: None. K. Niepokój: None. K. Woźniak: None. C. Kowalewski: None. J. Bal: None.

\section{P04.49.A}

CRISPR/Cas9-based analysis of a missense variant in Paxip1 suggests subtle effects on palatal process distance

N. Ishorst ${ }^{1,2}$, E. Z. Kvon ${ }^{2}$, Y. Zhu ${ }^{2}$, S. Tran ${ }^{2}$, I. PlajzerFrick $^{2}$, C. S. Pickle ${ }^{2}$, S. Hölzel ${ }^{1}$, A. Harrington ${ }^{2}$, J. Godoy ${ }^{2}$, J. A. Akiyama ${ }^{2}$, L. Selleri ${ }^{3,4,5,6,7}$, I. C. Welsh ${ }^{3}$, M. M.

Nöthen ${ }^{1}$, K. U. Ludwig', E. Mangold ${ }^{1}$, D. E. Dickel ${ }^{2}$, L. A. Pennacchio $^{2,8,9}$, A. Visel $^{2,8,10}$

${ }^{1}$ Institute of Human Genetics, University of Bonn, School of Medicine \& University Hospital Bonn, Bonn, Germany, ${ }^{2}$ Environmental Genomics and Systems Biology Division, Lawrence Berkeley National Laboratory, Berkeley, CA, United States, ${ }^{3}$ Program in Craniofacial Biology, University of California at San Francisco, San Francisco, CA, United States, ${ }^{4}$ Institute of Human Genetics, University of California at San Francisco, San Francisco, CA, United States, ${ }^{5}$ Eli and Edythe Broad Center of Regeneration
Medicine and Stem Cell Research, University of California at San Francisco, San Francisco, CA, United States, ${ }^{6}$ Department of Orofacial Sciences, University of California at San Francisco, San Francisco, CA, United States, ${ }^{7}$ Department of Anatomy, University of California at San Francisco, San Francisco, CA, United States, ${ }^{8}$ U.S. Department of Energy Joint Genome Institute, Berkeley, CA, United States, ${ }^{9}$ Comparative Biochemistry Program, University of California Berkeley, Berkeley, CA, United States, ${ }^{10}$ School of Natural Sciences, University of California Merced, Merced, CA, United States

Nonsyndromic cleft lip with/without cleft palate (nsCL/P) is among the most common congenital malformations and has a multifactorial etiology. Common risk loci explain parts of the trait heritability, but a substantial fraction needs to be elucidated. Parts of the missing heritability could be explained by rare, penetrant mutations that might occur de novo in genes potentially involved in craniofacial development. A previous study by our group identified three nsCL/P susceptibility genes with rare recurrent de novo mutations (PAXIP1, MDN1, ANK1). The present study aims at assessing a contribution of the rare mutations found in these nsCL/P susceptibility genes to cleft development by using CRISPR/Cas9 generated mouse models. We created five knock-in mouse strains that carried human missense mutations in Paxipl (1 mutation), Ankl (1 mutation), and Mdnl (3 different mutations). Embryos at embryonic day E18.5 were dissected and assessed for craniofacial anomalies using skeletal preparations and microscopic assessment. No craniofacial anomalies were observed for E18.5 embryos in the $F_{0}$ generation in any of the five knock-in strains. In the $F_{1}$ generation, 2/31 E18.5 pups carrying the knocked-in Paxipl missense mutation in a heterozygous state presented with abnormal body posture, a broader snout and a subtle increase in distance between the palatal processes, suggesting a role of the missense variant in Paxipl in craniofacial development. Further follow-up studies in mice homozygous for the variants and more precise phenotyping methods are ongoing.

N. Ishorst: None. E.Z. Kvon: None. Y. Zhu: None. S. Tran: None. I. Plajzer-Frick: None. C.S. Pickle: None. S. Hölzel: None. A. Harrington: None. J. Godoy: None. J.A. Akiyama: None. L. Selleri: None. I.C. Welsh: None. M. M. Nöthen: None. K.U. Ludwig: None. E. Mangold: None. D.E. Dickel: None. L.A. Pennacchio: None. A. Visel: None.

\section{P04.50.B}

High-throughput screen for rare variants in candidate genes for orofacial clefting 


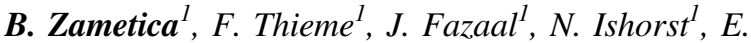
Ongkosuwito $^{2}$, C. Carels ${ }^{3}$, I. A. L. M. van Rooij ${ }^{4}$, E. Mangold $^{5}$, K. U. Ludwig ${ }^{1}$

${ }^{1}$ Institute of Human Genetics, University of Bonn, School of Medicine \& University Hospital Bonn, Bonn, Germany, Bonn, Germany, ${ }^{2}$ Department of Orthodontics and Craniofacial Biology, Radboud Institute for Molecular Life Sciences, Radboudumc, Nijmegen, Netherlands, ${ }^{3}$ Department of Human Genetics and Department of Oral Health Sciences, KU Leuven and University Hospitals KU Leuven, Leuven, Belgium, ${ }^{4}$ Department for Health Evidence, Radboud Institute for Health Sciences, Radboud university medical center (Radboudumc), Nijmegen, Netherlands, ${ }^{5}$ Institute of Human Genetics, University Hospital of Bonn, Bonn, Germany

Nonsyndromic cleft lip with/without cleft palate (nsCL/P) is a common birth defect with multifactorial etiology. Genome-wide association studies (GWAS) have revealed $>40$ risk loci that contribute to $\mathrm{nsCL} / \mathrm{P}$ risk, and it has been suggested that $\sim 30 \%$ of phenotypic variance is explained by common genetic variation. In the absence of surgery, nsCL/ $\mathrm{P}$ is a detrimental phenotype, which implies a considerable effect on reproductive fitness. Thus, rare variants are likely to also contribute to nsCL/P etiology. First studies have suggested nsCL/P candidate genes that harbor causative rare variants, or a burden thereof. These studies included the analysis of established "syndromic" genes ( $n=6$ genes), genes with established role in craniofacial development ( $n$ $=5$ ), and the identification of mutations in genes of the epithelial cadherin-p120-catenin complex segregating within multiply affected families $(n=5)$. However, independent replication is lacking. Here, we aimed at providing further genetic evidence for an involvement of these genes in $n s C L / P$ etiology, through the identification of additional evidence for rare variants from a large Central-European cohort. Using single molecule molecular inversion probes (smMIPs), we obtained high-confident sequencing results for 14 of the 16 genes in $711 \mathrm{nsCL} / \mathrm{P}$ cases, 761 populationbased controls, and 234 case-parent trios. At individual level, we identified four novel loss-of-function (LoF) variants ( 3 cases / 1 control). The variants observed in cases were LoF-mutations in $C D H 1, C T N N D 1$ and MTRR. Further statistical analyses, including analyses of missense variants, burden and pedigree analyses, are currently ongoing and will help to further understand the etiology of nsCL/P.

B. Zametica: None. F. Thieme: None. J. Fazaal: None. N. Ishorst: None. E. Ongkosuwito: None. C. Carels: None. I.A.L.M. van Rooij: None. E. Mangold: None. K. U. Ludwig: None.
P04.52.A

Phenotypic spectrum of NPR2 mutations: study of 12 patients with acromesomelic dysplasia, Maroteaux type and 8 patients with short stature.

C. Michot ${ }^{l}$, S. Prévost ${ }^{l}$, C. Haudry ${ }^{l}$, A. Tourré ${ }^{1}$, A. DieuxCoeslier ${ }^{2}$, M. Mathieu-Dramard ${ }^{3}$, M. Rossi ${ }^{4}$, A. Toutain ${ }^{5}$, G. Baujat ${ }^{l}$, S. Rondeau ${ }^{1}$, V. Cormier-Daire ${ }^{l}$

${ }^{I}$ APHP - Hôpital Necker-Enfants Malades, Paris, France, ${ }^{2} \mathrm{CHU}$ de Lille - Centre de Biologie Pathologie Génétique, Lille, France, ${ }^{3} \mathrm{CHU}$ Amiens-Picardie - Site Sud, Amiens, France, ${ }^{4} \mathrm{CHU}$ de Lyon HCL - GH Est-Hôpital Femme Mère Enfant, Bron, France, ${ }^{5} \mathrm{CHRU}$ de Tours - Hôpital Bretonneau, Tours, France

Introduction: Acromesomelic dysplasia, Maroteaux type (AMDM, MIM 602875) associates disproportionate short stature, short limbs and extremities with cone-shaped epiphyses. AMDM is caused by biallelic mutations of NPR2 (natriuretic peptide receptor B). Heterozygous NPR2 mutations are involved in idiopathic or dyschondrosteosislike short stature.

Materials and methods: Our study reviewed the clinical and molecular findings of AMDM and short stature patients with NPR2 mutations. 12 AMDM DNAs and more than 200 short stature DNAs were tested, after SHOX MLPA testing, in the molecular biology laboratory of Necker Hospital.

Results: NPR2 mutations were identified in all AMDM cases and in 8 short stature patients. 23 different mutations were identified: nonsense, frameshift, splice and missense ones in AMDM and mostly missense variants in short stature. Heterozygous parents of AMDM patients were relatively small. All AMDM patients were of short birth and final heights $(125-130 \mathrm{~cm})$. Apart small extremities, 6 had large hallux and 7 had curved radius with limited pronation. Out of 12 cases, 8 had hyperlaxity, 7 scoliosis and 8 dental misalignment and arched palate. All heterozygous patients were of normal birth size and grew between -2,5 to -1,5 SD. They had normal envergure/height ratio, even if $\mathrm{X}$-rays showed thick radius. 2/8 had IV and V brachymetacarpia.

Conclusions: our study supports AMDM genetic homogeneity. We underline the importance of specific assessments, including laxity, scoliosis and orthodontics. We confirm that NPR2 heterozygous mutations should be considered in short stature with minor skeletal changes. NPR2 function in C-natriuretic peptide signaling might lead to therapeutics options.

C. Michot: None. S. Prévost: None. C. Haudry: None. A. Tourré: None. A. Dieux-Coeslier: None. M. MathieuDramard: None. M. Rossi: None. A. Toutain: None. 
G. Baujat: None. S. Rondeau: None. V. CormierDaire: None.

\section{P04.55.A}

Non-syndromic cleft lip with/without cleft palate: Genome-wide association study in patients from The Netherlands and Belgium identifies a suggestive risk locus at 16p12.1 and supports $S H 3 P X D 2 A$ as a clefting susceptibility gene.

I. van Rooij ${ }^{1}$, K. U. Ludwig ${ }^{2}$, J. Welzenbach ${ }^{2}$, N. Ishorst ${ }^{2}$, M. Thonissen ${ }^{3}$, T. E. Galesloot ${ }^{1}$, E. Ongkosuwito ${ }^{3}$, S. J. Berge $^{4}, K$. Aldhorae ${ }^{5}$, A. Rojas-Martinez ${ }^{6}$, L. Kiemeney ${ }^{7}, J$. Vermeersch $^{8}$, H. Brunner ${ }^{9}$, N. Roeleveld ${ }^{1}, K$. Devriendt ${ }^{10}$, T. Dormaar ${ }^{11}$, G. Hens ${ }^{12}$, M. Kanpp ${ }^{13}$, C. Carels $^{14,8}, \boldsymbol{E}$. Mangold $^{2}$

${ }^{I}$ Radboud university medical center, Radboud Institute for Health Sciences, Department for Health Evidence, Nijmegen, Netherlands, ${ }^{2}$ Institute of Human Genetics, University of Bonn, School of Medicine \& University Hospital Bonn, Bonn, Bonn, Germany, ${ }^{3}$ Radboud Institute for Health Sciences, Department of Dentistry, Section of Orthodontics and Craniofacial Biology, Radboud university medical center, Nijmegen, Netherlands, ${ }^{4}$ Radboud university medical center, Department of Oral and Maxillofacial Surgery, Nijmegen, Netherlands, ${ }^{5}$ Orthodontic Department, College of Dentistry, Thamar University, Thamar, Zambia, ${ }^{6}$ Tecnologico de Monterrey, School of Medicine, and Universidad Autonoma de Nuevo Leon, Centro de Investigación y Desarrollo en Ciencias de la Salud, Monterrey, Mexico, ${ }^{7}$ Radboud university medical center, Radboud Institute for Health Sciences, Department for Health Evidence and Department of Urology, Nijmegen, Netherlands, ${ }^{8}$ Department of Human Genetics, KU Leuven, Leuven, Belgium, ${ }^{9}$ Radboud university medical center, Department of Human Genetics, and Donders Institute for Brain, Cognition and Behaviour, Nijmegen, Netherlands, ${ }^{10}$ Center for Human Genetics, University Hospitals Leuven, KU Leuven, Leuven, Belgium, ${ }^{11}$ Oral and Maxillofacial Surgery, University Hospitals Leuven, Leuven, Belgium, ${ }^{12}$ Department of Neurosciences, Experimental Otorhinolaryngology, $\mathrm{KU}$ Leuven, Leuven, Belgium, ${ }^{13}$ Institute of Medical Biometry, Informatics and Epidemiology, University of Bonn, Bonn, Germany, ${ }^{14}$ Orthodontics, University Hospitals Leuven, Leuven, Belgium

Nonsyndromic cleft lip with or without cleft palate (nsCL/P) ranks among the most common human congenital malformations. The etiology of nsCL/P is multifactorial, whereby genetic risk factors, environmental exposures, and potential gene-environment interactions all contribute to susceptibility. The present project is a genome-wide association study
(GWAS) in a medium-sized nsCL/P cohort encompassing a total of $285 \mathrm{nsCL} / \mathrm{P}$ patients and 1,212 controls from The Netherlands and Belgium. Twenty of 40 previously reported nsCL/P susceptibility loci were replicated, and at the wellestablished nsCL/P susceptibility locus at chromosome 8q24.21, the analyses identified 62 genome-wide significant SNVs. These findings underline the validity of this sample. SNV-based analysis of the data identified a yet unreported suggestive locus at chromosome 16p12.1 ( $p$-value of the lead SNV: $4.17 \times 10-07)$. This association was replicated in two of three patient/control replication series (Central European and Yemeni). The lead SNV rs56383345 maps to a $930 \mathrm{~kb}$ noncoding region at $16 \mathrm{p} 12.1$, and is not located in any currently known regulatory element. Gene-based analysis of the GWAS data prioritized SH3PXD2A at chromosome 10q24.33 as a candidate gene for nsCL/P. To date, support for this gene as a cleft gene has been restricted to data from zebrafish and a knockout mouse model. The present GWAS is the first to implicate SH3PXD2A in non-syndromic cleft formation in humans. In summary, although performed in a relatively small sample, the present GWAS generated novel insights into nsCL/P etiology, and demonstrates how medium-sized, clinically well characterized GWAS samples can improve knowledge of the genetic basis of nsCL/P.

I. van Rooij: None. K.U. Ludwig: None. J. Welzenbach: None. N. Ishorst: None. M. Thonissen: None. T.E. Galesloot: None. E. Ongkosuwito: None. S.J. Bergé: None. K. Aldhorae: None. A. Rojas-Martinez: None. L. Kiemeney: None. J. Vermeersch: None. H. Brunner: None. N. Roeleveld: None. K. Devriendt: None. T. Dormaar: None. G. Hens: None. M. Kanpp: None. C. Carels: None. E. Mangold: None.

\section{P04.56.B}

GWAS candidate genes for cleft lip with or without cleft palate: Analysis of expression data during embryonic craniofacial development.

\section{A. Siewert ${ }^{l}$, B. Reiz ${ }^{2}$, J. Welzenbach ${ }^{l}$, H. Dickten ${ }^{2}$, K. U.} Ludwig $^{1}$

${ }^{1}$ Institute of Human Genetics, University of Bonn, School of Medicine \& University Hospital Bonn, Bonn, Germany, ${ }^{2}$ FASTGenomics, Comma Soft AG, Bonn, Germany

Molecular defects during craniofacial embryonic development may result in nonsyndromic cleft lip with/without cleft palate (nsCL/P), one of the most common birth defects. Despite the possibility of surgical correction, follow-up treatment is long-term and requires an interdisciplinary approach. Also, epidemiological data indicate that individuals with nsCL/P show an increased risk for morbidities and suffer from a considerable psycho-social burden. 
The molecular pathways underlying these observations are currently unknown. We here used nsCL/P-GWAS data and array-based expression data from embryonic mice (gestational days E10.5-E12.5, Hooper et. al 2017), to identify pathways in which nsCL/P candidate genes are enriched. The 45 nsCL/P risk loci comprise $\sim 400$ genes within their topologically associated domains. Using gene-based analysis, we identified 86 genes with enrichment of common variants, suggesting their involvement in nsCL/P etiology. For each of these genes, we analyzed the expression pattern in ectodermal or mesodermal tissues of mandibular, maxillary and fronto-nasal prominences, and performed hierarchical clustering. One of the three clusters we identified comprised 33 genes, including established nsCL/P-genes like IRF6 and TFAP2a. A pathway analysis of genes within this cluster revealed a significant overrepresentation of cancer-related pathways and processes related to molecular signaling. Interestingly, affecteds with nsCL/P have been reported to be at increased risk of cancer. We will next extend our approach at single-cell level, using systematic data as recently provided (Cao et. al 2019) and the analytical ecosystem FASTGenomics (Scholz et. al 2018). This will generate novel insights into gene regulatory networks across development, and their contribution to nsCL/P.

A. Siewert: None. B. Reiz: A. Employment (full or parttime); Significant; Comma Soft AG. J. Welzenbach: None. H. Dickten: A. Employment (full or part-time); Significant; Comma Soft AG. K.U. Ludwig: None.

\section{P04.59.B}

\section{Severe Osteogenesis imperfecta with oligodontia: think} of MESD

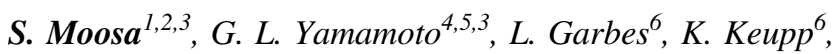
A. Beleza-Meireles ${ }^{7,8}$, C. A. Moreno ${ }^{9}$, E. R. Valadares $^{10}, S$. B. de Sousa ${ }^{7,11}$, S. Maia ${ }^{7,12}$, J. Saraiva ${ }^{7,13}$, R. S. Honjo ${ }^{14}$, C. A. Kim ${ }^{15}$, H. Cabral de Menezes ${ }^{16}$, E. Lausch ${ }^{17}$, P. V. Lorini $^{18,19}$, A. Lamounier $\mathrm{Jr}^{10}$, T. C. B. Carniero ${ }^{10}, C$. Giunta $^{20}$, M. Rohrbach ${ }^{20}$, M. Janner ${ }^{21}$, O. Semler ${ }^{22}, F$.

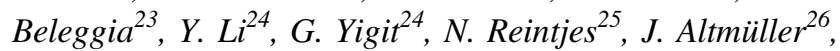
P. Nürnberg ${ }^{26}$, D. P. Cavalcanti ${ }^{9}$, B. Zabel ${ }^{17,27}, M . L$. Warman $^{28}$, D. R. Bertola ${ }^{14,5}$, B. Wollnik ${ }^{24,29}$, C. Netzer ${ }^{23,30}$

${ }^{1}$ Stellenbosch University, Tygerberg, South Africa, ${ }^{2}$ University Medical Center Göttingen, Göttingen, Germany, ${ }^{3}$ Boston Children's Hospital and Harvard Medical School, Boston, MA, United States, ${ }^{4}$ Unidade de Genética, Instituto da Criança do Hospital das Clínicas da Faculdade de Medicina da Universidade de São Paulo, São Paulo, 05403-000, Brazil;; Brazil, ${ }^{5}$ Instituto de Biociências da Universidade de São Paulo, São Paulo, Brazil, ${ }^{6}$ Institute of Human Genetics, University Hospital of Cologne and Faculty of Medicine, University of Cologne, Cologne,
Germany, ${ }^{7}$ Medical Genetics Unit, Hospital Pediátrico de Coimbra, Centro Hospitalar e Universitário de Coimbra, Coimbra, Portugal, ${ }^{8}$ Department of Clinical Genetics, St Michael's Hospital, University Hospitals Bristol, Bristol, United Kingdom, ${ }^{9}$ Skeletal Dysplasia Group, Department of Medical Genetics, Faculty of Medical Sciences, University of Campinas, Campinas, Brazil, ${ }^{10}$ Hospital das Clínicas da Faculdade de Medicina da Universidade Federal de Minas Gerais, Belo Horizonte, Belo Horizonte, Brazil, ${ }^{11}$ University Clinic of Genetics, Faculty of Medicine, University of Coimbra, Coimbra, Portugal, ${ }^{12}$ University Clinic of Genetics, Faculty of Medicine, University of Coimbra, Coimbra, Portugal, ${ }^{13}$ University Clinic of Pediatrics, Faculty of Medicine, University of Coimbra, Coimbra, Portugal, ${ }^{14}$ Unidade de Genética, Instituto da Criança do Hospital das Clínicas da Faculdade de Medicina da Universidade de São Paulo, São Paulo, Brazil, ${ }^{15}$ Instituto de Biociências da Universidade de São Paulo, São Paulo, Portugal, ${ }^{16}$ Unidade de Endocrinologia, Instituto da Criança do Hospital das Clínicas da Faculdade de Medicina da Universidade de São Paulo, São Paulo, Portugal, ${ }^{17}$ Division of Genetics, Children's Hospital, University of Freiburg, Freiburg, Germany, ${ }^{18}$ Stellenbosch UniversityDivision of Genetics, Children's Hospital, University of Freiburg, Freiburg, Germany, ${ }^{19}$ Institute of Human Genetics, University Hospital Halle, Martin Luther University Halle-Wittenberg, Halle, Germany, ${ }^{20}$ Connective Tissue Unit, Division of Metabolism, University Children's Hospital Zurich, Zurich, Switzerland, ${ }^{21}$ Division of Pediatric Endocrinology, Diabetology and Metabolism, Department of Pediatrics, Inselspital, Bern University Hospital, Bern, Switzerland, ${ }^{22}$ Faculty of Medicine, University of Cologne, 50931 Cologne, Germany; Department of Pediatrics, University Hospital Cologne, Cologne, Germany, ${ }^{23}$ Institute of Human Genetics, University Hospital of Cologne, Cologne, Germany, ${ }^{24}$ Institute of Human Genetics, University Medical Center Göttingen, Göttingen, Germany, ${ }^{25}$ Institute of Human Genetics, University Hospital of Cologne, Cologne, Ghana, ${ }^{26}$ Cologne Center for Genomics, University of Cologne, Cologne, Germany, ${ }^{27}$ Medical Faculty of the University of Magdeburg, Magdeburg, Germany, ${ }^{28}$ Orthopaedic Research Laboratories, Department of Orthopaedic Surgery, Boston Children's Hospital and Harvard Medical School, Boston, MA, United States, ${ }^{29}$ Cluster of Excellence, Multiscale Bioimaging: from Molecular Machines to Networks of Excitable Cells (MBExC), University of Göttingen, Göttingen, Germany, ${ }^{30}$ Faculty of Medicine, University of Cologne, Cologne, Germany

Osteogenesis imperfecta (OI) comprises a genetically heterogeneous group of skeletal fragility diseases. It is often clinically impossible to predict the underlying causative gene, 
thus necessitating broad multi-gene panel testing or exome sequencing. We report on five independent families with a progressively-deforming type of OI with the distinguishing feature of oligodontia. We identified four homozygous truncation or frameshift mutations in a novel gene for OI, namely MESD. MESD encodes an endoplasmic reticulum (ER) chaperone protein for the canonical Wingless-related integration site (WNT) signaling receptors LRP5 and LRP6. Because complete absence of MESD causes embryonic lethality in mice, we hypothesized that the OI-associated mutations are hypomorphic alleles since these mutations occur downstream of the chaperone activity domain but upstream of ER-retention domain. This would be consistent with the clinical phenotypes of skeletal fragility and oligodontia in persons deficient for LRP5 and LRP6, respectively. Further functional analysis showed that OI-associated MESD mutations produce hypomorphic alleles whose failure to remain within the ER significantly reduces but does not completely eliminate LRP5 and LRP6 trafficking. Since these individuals have no eye abnormalities (which occur in individuals completely lacking LRP5) and have neither limb nor brain patterning defects (both of which occur in mice completely lacking LRP6), we infer that bone mass accrual and dental patterning are more sensitive to reduced canonical WNT signaling than are other developmental processes. Multigene panels do not yet include MESD. Therefore, if an undiagnosed patient with severe OI and oligodontia presents, it may be prudent to think first of MESD.

S. Moosa: None. G.L. Yamamoto: None. L. Garbes: None. K. Keupp: None. A. Beleza-Meireles: None. C.A. Moreno: None. E.R. Valadares: None. S.B. de Sousa: None. S. Maia: None. J. Saraiva: None. R.S. Honjo: None. C.A. Kim: None. H. Cabral de Menezes: None. E. Lausch: None. P.V. Lorini: None. A. Lamounier Jr: None. T.C.B. Carniero: None. C. Giunta: None. M. Rohrbach: None. M. Janner: None. O. Semler: None. F. Beleggia: None. Y. Li: None. G. Yigit: None. N. Reintjes: None. J. Altmüller: None. P. Nürnberg: None. D.P. Cavalcanti: None. B. Zabel: None. M.L. Warman: None. D.R. Bertola: None. B. Wollnik: None. C. Netzer: None.

P04.61.A

Adult osteopetrosis with osteonecrosis of the jaws and abnormal osteoclast resorption due to a $L R R K 1$ splice site mutation

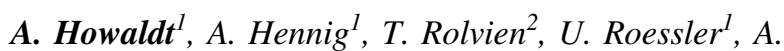

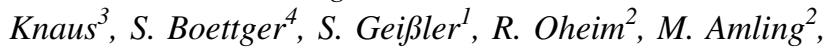
H. Howaldt ${ }^{4}$ U. Kornak ${ }^{5}$

${ }^{1}$ Charité-Universitaetsmedizin Berlin, Berlin, Germany, ${ }^{2}$ University Medical Center Hamburg-Eppendorf, Hamburg, Germany, ${ }^{3}$ School of Medicine \& University Hospital
Bonn, Bonn, Germany, ${ }^{4} J u s t u s$ Liebig Universität Gießen, Gießen, Germany, ${ }^{5}$ Universitaetsmedizin Göttingen, Göttingen, Germany

We report on a 34 year-old patient with sandwich vertebrae, platyspondyly, osteosclerosis of long bones, pathologic fractures, and anemia. In the third decade, he developed osteonecrosis of the jaws, which was progressive in spite of repeated surgical treatment. An iliac crest bone biopsy revealed an elevated trabecular density (BV/TV [\%] 32.2 vs. $21.5 \pm 14.8)$ and the presence of hypermineralized cartilage remnants leading to an overall increase in mineralization (CaMean [wt\%] 25.65 vs. 21.8). We also found large multinucleated osteoclasts with abnormal morphology and inadequate bone resorption. Although these findings were reminiscent of autosomal dominant osteopetrosis type 2, no mutations in $C L C N 7$ were detected. Subsequent whole exome sequencing identified the novel homozygous splicesite mutation c.261G $>\mathrm{A}$ in the gene LRRK1. cDNA sequencing showed nearly complete skipping of exon 3 leading to a frameshift (p.Ala34Profs*33), very likely leading to a loss of function. LRRKI encodes a protein kinase and its dysfunction can cause osteosclerotic metaphyseal dysplasia (OSMD; OMIM \# 615198). Osteoclasts differentiated in vitro from the patient's peripheral blood monocytes formed quickly and became unusually large. In resorption assays these cells formed large areas with superficial pseudo-resorption but excavated only few resorption pits (resorption pit surface surface [\%] $1.3 \pm 0.6$ vs. $15 \pm 3.6 ; \mathrm{p}<0.01$ ). Phosphorylation of L-plastin was strongly reduced in patient-derived osteoclasts, thus corroborating a LRRK1 loss of function $(0.26 \pm 0.1$ vs. $1 \pm$ $0.08 ; \mathrm{p}<0.01)$. Our analysis indicates a strong overlap of LRRK1-related OSMD with other late-onset forms of osteopetrosis, but an exceptionally leaky osteoclast resorption.

A. Howaldt: None. A. Hennig: None. T. Rolvien: None. U. Roessler: None. A. Knaus: None. S. Boettger: None. S. Geißler: None. R. Oheim: None. M. Amling: None. H. Howaldt: None. U. Kornak: None.

\section{P04.62.B}

The Irp5 knockout zebrafish as a model to study osteoporosis

P. J. Coucke, J. Bek, H. De Saffel, A. De Clercq, A. Boel, A. Willaert

Center for Medical Genetics, Gent, Belgium

Osteoporosis-pseudoglioma syndrome (OPPG) is an autosomal recessive disorder characterized by reduced bone mass and strength, caused by loss-of-function mutations in 
LRP5 (low-density lipoprotein receptor-related protein 5), a co-receptor in the WNT signaling pathway. Here we describe the first reliable genetically induced form of osteoporosis in the zebrafish in a lrp5 knockout (KO). Molecular analysis confirmed reduced lrp5 transcripts and absence of Lpr5 protein. Reduced survival was observed in lrp5 KO larvae after the onset of mineralization. The fraction of larvae that did survive, displayed delayed ossification as was shown via mineral staining. Subsequently, these fish were grown to adulthood and analyzed via $\mu \mathrm{CT}$. This revealed decreased bone volume and decreased bone mineralization in the vertebral column, which are hallmark features of osteoporosis. Next, we performed CRISPR/Cas9 mediated mutagenesis targeted at lrp 5 followed by F0 phenotype analysis (crispant screening). Miseq sequencing of the mosaic larvae revealed out-of-frame mutations in on average $90 \%$ of the reads. Consequently, we showed a similar skeletal phenotype in the F0 'crispants' to that of the stable $\operatorname{lrp5} 5^{-/}$larvae, showing that crispant screening in zebrafish is a promising approach to functionally screen a large set of osteoporosis candidate genes. In summary, we present $\operatorname{lrp} 5^{-1-}$ zebrafish as the first genetic osteoporosis zebrafish model that can be used as a screening platform for potential therapeutics. Additionally, we show that F0 crispant screening can be used for functional validation of candidate genes for osteoporosis as high rates of out-offrame mutations in the lrp5 gene are sufficient to induce phenotypes.

P.J. Coucke: None. J. Bek: None. H. De Saffel: None. A. De Clercq: None. A. Boel: None. A. Willaert: None.

\section{P04.64.A}

The p.R321C mutation in p62 protein, associated to Paget's Disease of Bone, leads to a relocation of the protein in human osteoclasts

\section{N. Gestoso-Uzal ${ }^{1,2}$, R. Usategui-Martín ${ }^{1,2}$, C. Gutiérrez- Cerrajero $^{2,1}$, A. I. Turrión Nieves ${ }^{1,3}$, J. Del Pino-Montes ${ }^{1,3}$, R. González-Sarmiento ${ }^{2,1,4}$}

${ }^{1}$ Institute of Biomedical Research of Salamanca (IBSAL), Salamanca, Spain, ${ }^{2}$ Molecular Medicine Unit, Department of Medicine, University of Salamanca, Salamanca, Spain, ${ }^{3}$ Rheumatology Service, University Hospital of Salamanca, Salamanca, Spain, ${ }^{4}$ Institute of Molecular and Cellular Biology of Cancer (IBMCC), University of SalamancaCSIC, Salamanca, Spain

Background: The most important known genetic factor predisposing to Paget's disease of bone (PDB) is mutation in SQSTM1 gene, which encodes p62 protein. We detected the c.961C>T SQSTM1 gene mutation in three PDB patients, that causes the p.R321C mutation. It has been reported that p.R321C mutation causes a blockade of autophagy and an activation of NF-kB pathway. The aim of this study was to characterize the effect of the p.R321C mutation in human osteoclasts.

Methods: Peripheral blood mononuclear cells (PBMCs) were isolated from blood samples for generating human osteoclasts. Cells were differentiated by adding M-CSF and RANKL for 3 weeks. Human osteoclasts were transiently transfected with total plasmid DNA (pCEFL-FlagSQSTM1-321C and pCEFL-Flag-SQSTM1-321R constructs). Finally, we performed a co-immunofluorescence assay to detect Flag and p62.

Results and discussion: Our results showed that, while 321R-p62 protein remained scattered though the cytoplasm, $321 \mathrm{C}$-p62 was concentrated in the periphery of osteoclasts. This relocation of $\mathrm{p} 62$ protein caused by $\mathrm{p} . \mathrm{R} 321 \mathrm{C}$ mutation could be a consequence of autophagy blockade, which has been reported that could have a crucial role in the etiology of PDB. The p.R321C mutation causes a blockade of autophagy and an activation of NF-kB pathway, therefore the alteration of the cell metabolism could be the cause of the relocation of p62 protein in human osteoclasts.

Conclusion: The p.R321C mutation in p62 protein leads to a relocation of the protein in human osteoclasts. This reinforces the hypothesis that an alteration of autophagy could be involved in PDB etiology.

This work was supported by FIS-FEDER:PI16/01920.

N. Gestoso-Uzal: None. R. Usategui-Martín: None. C. Gutiérrez-Cerrajero: None. A.I. Turrión Nieves: None. J. Del Pino-Montes: None. R. GonzálezSarmiento: None.

\section{P04.66.C}

Penttinen syndrome and infantile myofibromatosis in a patient carrying the tyrosine kinase-activating variant Asn666Ser in PDGFRB

\section{Horn ${ }^{1}$, A. T. Abad-Perez ${ }^{1}$, V. Stephan ${ }^{2}$}

${ }^{I}$ Institute of Medical Genetics and Human Genetics, Charité-Universitätsmedizin, Berlin, Germany, ${ }^{2}$ Sana Children's Hospital Lichtenberg, Berlin, Germany

Introduction: Penttinen syndrome belongs to the group of progeroid disorders and is characterized by progressive subcutaneous lipoatrophy, sclerotic skin lesions and acroosteolysis. This very rare condition is caused by a few distinct missense mutations of PDGFRB, other distinct mutations of this gene have been shown to be associated with different conditions such as infantile myofibromatosis.

Material and Methods: Here, we describe a 5-year-old male patient with infantile myofibromatosis diagnosed in his first month of life who in addition developed a 
prematurely aged appearance with lipoatrophy, scar-like skin nodules on the hands and joint contractures.

Results: Sanger sequencing revealed the heterozygous de novo mutation c. $1997 \mathrm{~A}>\mathrm{G}$; p.Asn666Ser in the PDGFRB gene. Recently, this mutation has been described in two patients affected with a severe form of Penttinen syndrome presenting a more pronounced connective tissue destruction.

Conclusions: This is the first report of an individual affected with both conditions, infantile myofibromatosis and Penttinen syndrome. This mutation lead to increased phosphorylation of downstream signalling proteins such as PTPN11. Imatinib is a strong inhibitor of phosphorylation of these targets representing a treatment option.

D. Horn: None. A.T. Abad-Perez: None. V. Stephan: None.

\section{P04.67.A}

Blood secreted miRNA hsa-miR-374b-5p inhibits osteoblast-differentiating gene UHMK1 and is upregulated in periodontitis patients R. Wiehe ${ }^{1}$, D. Bajric ${ }^{2}$ A. El Sharawy ${ }^{3}$, A. Keller ${ }^{4}, H$.
Dommisch $^{1}$, A. Schaefer

${ }^{1}$ Charité-Universitymedicine Berlin, Institute for Dental and Craniofacial Sciences, Department of Periodontology and Synoptic Dentistry, Berlin, Germany, ${ }^{2}$ University Medical Center Schleswig-Holstein, Campus Kiel, Department of Operative Dentistry and Periodontology, Kiel, Germany, ${ }^{3}$ Institute for Clinical Molecular Biology, Christian-Albrechts-University Kiel, Kiel, Germany, ${ }^{4}$ Clinical Bioinformatics, Saarland University, Saarbrücken, Saarbrücken, Germany

microRNAs interact with the 3'UTRs of target mRNAs to suppress gene expression. MicroRNAs act tissue specifically but some are secreted into blood. Recent studies reported upregulation of specific miRNAs in the inflamed oral mucosa of periodontitis patients. The regulatory targets of these miRNAs may reveal genes that are conducive to oral health. If secreted into blood, these miRNAs could serve as biomarkers to diagnose periodontitis before clinical manifestation. To identify such miRNAs, we quantified 863 miRNAs in the blood of 18 periodontitis patients and 70 periodontitis-free controls (Geniom Biochip array). miRNA hsa-miR-421 and hsa-miR-374b-5p were most significantly upregulated in blood of periodontitis cases $\left(\mathrm{P}_{\text {adjusted }}<5 \times 10\right.$ $\left.{ }^{-6}\right)$. Two previous studies that profiled microRNAs in the inflamed and healthy oral mucosa independently reported $>2$ fold upregulation of hsa-miR-374b-5p, but not hsa-miR421. Using mirVana miRNA mimics (ThermoFisher Scientific), we overexpressed hsa-miR-374b-5p in primary gingival fibroblasts ( 3 biological and 3 technical replicates) and quantified the mRNA levels in $24 \mathrm{~h}$ transfected and mock-transfected cells (Clariom D Array, ThermoFisher Scientific). The gene U2AF homology motif kinase 1 (UHMK1) was significantly downregulated in the miRNA mimic transfected cells (Fold Change $=-1.8, \mathrm{P}=0.00025$ ). Two binding sites of hsa-miR-374b-5p were predicted at the 3'UTR of UHMK1. To validate the functional mechanism, we cloned the respective 3'UTR of UHMKI into a reporter plasmid (pGL4.24, Promega). After co-transfection of the plasmid and hsa-miR-374b-5p mimic into HeLa cells for 24 hours, the reporter gene expression was significantly reduced (Fold Change $=-1.5, \mathrm{p}=0.02,3$ independent replicates). UHMK1 has a role in the coordination of osteoblast differentiation.

R. Wiehe: None. D. Bajric: None. A. El Sharawy: None. A. Keller: None. H. Dommisch: None. A. Schaefer: None.

P04.70.A

POP1-Skeletal Dysplasias : Description of two new families.

J. HARVENGT, S. ALKAN, B. FLORKIN, S. BULK, V. BOURS

\section{CHU Liège, Liège, Belgium}

Background: $P O P 1$ mutations should be considered in anauxetic dysplasia (AD) patients without $R M R P$ mutations but also in skeletal dysplasia (SD) of different severities. To date, only 5 patients were reported.

Methods: Cartilage Hair Hypoplasia $(\mathrm{CHH})$ was suspected in two siblings based on clinical and radiological evaluation. In family 1 , a 4 years boy was investigated. His older sister is also affected by SD but currently refuses to pursue genetic testing. In family 2 , two sisters presented a severe short stature : the older has $120 \mathrm{~cm}$ as final adult height. The second aged of 13 also suffered from an immune deficiency.

Results: A skeletal dysplasia gene panel revealed in the POP1 gene:Family $\mathrm{N}^{\circ} 1$ : a composite heterozygous variant c.1846A $>$ C, class3 (paternally inherited) and c.1747G $>\mathrm{T}$, class 4 (maternally inherited). Family $\mathrm{N}^{\circ} 2$ : a homozygous variant c. $632 \mathrm{G}>\mathrm{A}$, class 4 .

Discussion: $P O P 1$ patients are characterized by severe short stature of prenatal-onset and very short adult height (classically less than 1 meter, but our adult patients were $120 \mathrm{~cm}$ and near $160 \mathrm{~cm}$ ), hypodontia, midface hypoplasia, mild intellectual disability and radiological specific findings including systematic bullet-shaped middle phalanges. Notably, none of the 5 published cases were reported with immune deficiency. 
Conclusions: We describe two new families with $P O P 1$ mutations encountered in patients clinically suspected for $\mathrm{CHH}$ and achondroplasia, enhancing that the phenotypical spectrum is larger than $\mathrm{AD}$ type 2. Larger cohorts of patients are needed to improve the clinical characterization, especially the risk of having associated symptoms such as immune deficiency.

J. Harvengt: None. S. Alkan: None. B. Florkin: None. S. Bulk: None. V. Bours: None.

\section{P04.71.B}

Coding variants of the CARD14 NF-kB adaptor are associated with palmar plantar pustulosis

A. Niaouris ${ }^{1}$, S. Haddad ${ }^{l}$, A. PushpaRajah ${ }^{2}$, N. BenzianOlsson $^{1}$, P. Baum ${ }^{3}$, S. Visvanathan ${ }^{4}$, T. he APRICOT and PLUM study team ${ }^{2}$, J. Barker ${ }^{2}$, C. Smith ${ }^{2}$, F. Capon ${ }^{l}$

${ }^{1}$ King's College London, London, United Kingdom, ${ }^{2} \mathrm{St}$ John's Institute of Dermatology, Guy's and St Thomas' NHS Foundation Trust, London, United Kingdom, ${ }^{3}$ Boehringer Ingelheim Pharma GmbH \& Co. KG, Biberach, Germany, ${ }^{4}$ Boehringer Ingelheim Pharma GmbH \& Co. $K G$, Ridgefield, $C T$, United States

Caspase recruitment domain containing protein 14 (CARD14) is a soluble NF-kB adaptor protein, which is mostly expressed in keratinocytes. In the presence of inflammatory stimuli, free CARD14 molecules oligomerise, recruit a BCL10/MALT1 signalosome and activate NF-kB signalling. Gain-of-function CARD14 variants have previously been associated with inflammatory skin diseases such as psoriasis vulgaris and generalised pustular psoriasis. However, the involvement of CARD14 in palmar plantar pustulosis (PPP), a localised form of pustular psoriasis, remains unclear. Here, we analysed CARDI4 changes in 160 PPP cases (125 females, 35 males; mean age of onset: 41 years) ascertained across the UK. We identified 11 rare variants predicted to have a deleterious effect on protein function. A burden test (comparing PPP cases vs controls sequenced by the ExAC consortium) demonstrated a significant association between these changes and PPP (OR: 2.5; 95\% CI: $1.39-4.65 ; \mathrm{p}=0.0023)$. We also detected a low-frequency allele with deleterious potential (E422K) that occurred more frequently in affected individuals compared to controls (OR: $1.7 ; 95 \%$ CI: $1.07-2.69 ; \mathrm{p}=0.025$ ). To assess the effect of disease-associated variants, CARD14 FLAG-tagged plasmids were mutagenized and transfected into HEK293 cells. The depletion of free CARD14 from the cytoplasm (which is an indirect measurement of protein oligomerization) was then analysed by western blotting. This confirmed that most variants promote the formation of insoluble CARD14 oligomers. These findings implicate
CARD14 in the pathogenesis of PPP. They also suggest that the proteins encoded by disease alleles are prone to constitutive oligomerization, which will cause abnormal NF-kB activation.

A. Niaouris: None. S. Haddad: None. A. PushpaRajah: None. N. Benzian-Olsson: None. P. Baum: None. S. Visvanathan: None. T. he APRICOT and PLUM study team: None. J. Barker: None. C. Smith: None. F. Capon: B. Research Grant (principal investigator, collaborator or consultant and pending grants as well as grants already received); Significant; 3 Boehringer Ingelheim Pharma $\mathrm{GmbH} \& \mathrm{Co}$.

\section{P04.74.B}

Further delineation of Roifman syndrome: report of four new Spanish patients

I. Valenzuela ${ }^{l}$, I. Cusco ${ }^{l}$, I. Paramonov ${ }^{l}$, F. LópezGrondona $^{l}$, S. Garcia-Miñaur ${ }^{2}$, M. Palomares-Bralo ${ }^{2}$, A.

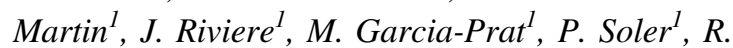
Colobran $^{l}$, E. Garcia-Arumíl, E. Tizzano ${ }^{l}$

${ }^{1}$ Hospital Vall d'Hebron, Barcelona, Spain, ${ }^{2}$ Hospital La Paz, Madrid, Spain

Roifman Syndrome (RS, OMIM 300258) is a rare disorder characterized by pre- and postnatal growth retardation, cognitive delay, spondyloepiphyseal dysplasia, antibody deficiency and facial dysmorphism. Biallelic mutations in RNU4ATAC have been reported as the molecular cause. Our series expand the phenotypic spectrum of Roifman patients. We report four patients from three unrelated families with RS. Due to the complexity of variant annotation in RNU4ATAC, molecular studies are not usually straightforward. Indeed, in families 1 and 2 exome sequencing was inconclusive. In patient 1 the diagnosis was finally uncovered by genome sequencing, in sisters 2 and 3 by Sanger sequencing upon clinical suspicion and in patient 4 by a customized gene panel. Main clinical characteristics include skeletal dysplasia (4/4), short stature (4/4), intellectual disability (4/4), antibody deficiency (3/4) and retinal dystrophy (2/4). Main dysmorphic features included markedly long philtrum and thin upper lip. Hepatomegaly was identified in 2/4 and patient 1 presented hepatic cavernoma (unreported so far in Roifamn patients). Molecular results showed variants $\mathrm{n} .8 \mathrm{C}>\mathrm{T}$ and $\mathrm{n} .50 \mathrm{G}>\mathrm{A}$ (patient 1 ); variant $\mathrm{n} .13 \mathrm{C}>\mathrm{T}$ and $\mathrm{n} .17 \mathrm{G}>\mathrm{C}$ (sisters 2 and 3 ) and two novel variants, n. $37 \mathrm{G}>\mathrm{A}$ and $\mathrm{n} .53 \mathrm{C}>\mathrm{T}$ (patient 4). Segregation analysis indicated that each variant was inherited from a healthy parent in the three families. The inclusion of new patients with Roifman increases the knowledge of mutational spectrum and it's essential to delineate the phenotype of this syndrome. The recognition of the syndrome is indispensable 
for the orientation of molecular diagnosis, prompt follow up and counseling.

I. Valenzuela: None. I. Cusco: None. I. Paramonov: None. F. López-Grondona: None. S. Garcia-Miñaur: None. M. Palomares-Bralo: None. A. Martin: None. J. Riviere: None. M. Garcia-Prat: None. P. Soler: None. R. Colobran: None. E. Garcia-Arumí: None. E. Tizzano: None.

\section{P04.77.B}

Identification of the deregulated NEK1 protein network in skeletal ciliopathies

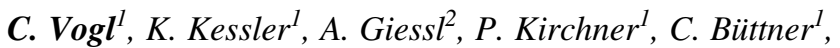

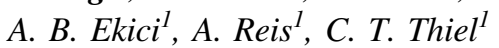

${ }^{1}$ Institute of Human Genetics, FAU Erlangen-Nürnberg, Erlangen, Germany, ${ }^{2}$ Department of Ophthalmology, FAU Erlangen-Nürnberg, Erlangen, Germany

The primary cilium is an organelle, which is present on almost all vertebrate cells. Cilia consist of the axoneme and the basal body and present various ciliary membrane receptors, which receive extracellular signals. Those signals are transmitted into the cell, where they modulate different intracellular signaling pathways. Mutations in ciliary proteins are involved in formation, maintenance and function of the primary cilium and associated with developmental defects, like brain malformations, polydactyly, kidney cysts and skeletal abnormalities. This phenotypic spectrum is present among patients with short-rib polydactyly syndromes (SRPS). This group constitutes the most frequent lethal autosomal recessive osteochondrodysplasias.

Diallelic mutations in NEK1, encoding for a basal body protein, are the underlying cause of SRPS II. To gain further insight into the pathogenic mechanism, we used two approaches: RNA sequencing of CRISPR/Cas9 mediated genomic edited HEK293T cells and scRNASeq in limb buds of E13 embryos of the Nek1 knock down mouse, and respectively compared the knock out and the wildtype in order to find differentially expressed genes. Analysis of significantly enriched pathways revealed an activation of genes in early growth response and cell cycle control and silencing of different collagens and ribosomal and histone proteins. One of the main findings was a severely reduced expression of Acan, coding for Aggrecan the most abundant cartilaginous protein, explaining the severe growth phenotype.

These results underline that the NEK1 associated defect of the primary cilium causes the deficiency in cell cycle and gene expression, mainly aggrecan, in the growth plate.
C. Vogl: None. K. Kessler: None. A. Giessl: None. P. Kirchner: None. C. Büttner: None. A.B. Ekici: None. A. Reis: None. C.T. Thiel: None.

\section{P04.79.A}

Novel variant in PLAG1 in a familiar case of SilverRussell syndrome

A. Pereda ${ }^{1}, Y$. Vado $^{2}$, I. Llano-Rivas ${ }^{3}$, N. Gorria-Redondo ${ }^{4}$, I. Díez ${ }^{5}$, G. Perez de Nanclares ${ }^{2}$

${ }^{1}$ Rare Diseases Research Group, Molecular (Epi)Genetics Laboratory, BioAraba National Health Research Institute, Vitoria-Gasteiz, Spain, ${ }^{2}$ Rare Diseases Research Group, Molecular (Epi)Genetics Laboratory, BioAraba National Health Research Institute, Vitoria-Gasteiz, Araba, Spain, ${ }^{3}$ Service of Genetics, BioCruces Health Research Institute, Hospital Universitario Cruces., Barakaldo, Bizkaia, Spain, ${ }^{4}$ Service of Paediatric Neurology, Araba University Hospital, Vitoria-Gasteiz, Araba, Spain, ${ }^{5}$ Department of Pediatric Endocrinology. BioAraba Health Research Institute, Araba University Hospital-Txagorritxu, Vitoria-Gasteiz, Araba, Spain

Introduction: Silver-Russell syndrome is a rare imprinting disorder characterized by prenatal (SGA) and postnatal growth retardation (PNGR), relative macrocephaly at birth, a prominent forehead, body asymmetry and feeding difficulties and/or low BMI. The most common underlying mechanisms are hypomethylation at H19/IGF2:IG-DMR and UPD(7)mat. Rarely, rearrangements involving 11p15, or inactivating point variants have been identified (maternal $C D K N 1 C$ or paternal IGF2 variants). Recently PLAG1 and $H M G A 2$ genes, which participate in the IGF2 pathway, have been associated with this syndrome.

Patients and Methods: We present a 9 years-old girl who was initially referred to pediatric endocrinology because of short stature. A clinical geneticist suggested the clinical diagnosis of SRS (NHC score=4: SGA, PNGR, prominent forehead and problems for feeding manifested as poor food intake). She also showed dental crowding with delayed teeth eruption, triangular face, small and bulbous nose, prominent maxillary, high ojival palate, fine voice, fine hair and learning difficulties. Her mother and maternal grandmother presented the same phenotype. After discarding alterations in $11 \mathrm{p} 15$ and chromosome 7 (MS-MLPA), a custom NGS panel for genes associated with growth was run.

Results: The study of the NGS panel revealed an heterozygous novel variant within exon 5 of PLAG1 [NM_001114634.2:c.551delA; p.(Lys184Serfs*45)], bioinformatically predicted as likely pathogenic (ACMG: PVS1; 
PM2; PP1). Familial studies by Sanger sequencing confirmed cosegregation.

Conclusions: We report the second familial case of SRS caused by a novel PLAGl frameshift alteration, and we expand the clinical spectrum to the previously described. Funding: Instituto de Salud Carlos III (PI16/00073); Basque Department of Health (GV2017/111040).

A. Pereda: None. Y. Vado: None. I. Llano-Rivas: None. N. Gorria-Redondo: None. I. Díez: None. G. Perez de Nanclares: None.

\section{P04.80.B}

Increased yield of genetic diagnoses in skeletal ciliopathies using massively parallel sequencing, structural variant and RNA analyses

A. Hammarsjo ${ }^{1}$, M. Pettersson ${ }^{1}$, D. Chitayat ${ }^{2,3}$, A. Handa ${ }^{4}$, F. Taylan ${ }^{5}$, D. Batkovskyte ${ }^{5}$, B. Anderlid ${ }^{1}$, A. E. Lin ${ }^{6}, K$.

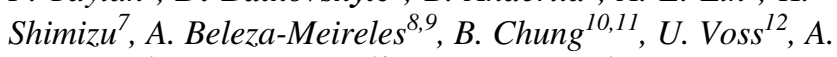

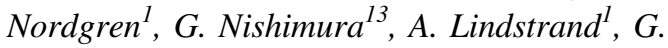
Grigelioniene ${ }^{1}$

${ }^{1}$ Department of Molecular Medicine and Surgery, Center for Molecular Medicine, Karolinska Institutet, and Clinical Genetics, Karolinska University Laboratory, Karolinska University Hospital, Stockholm, Sweden, ${ }^{2}$ Division of Clinical and Metabolic Genetics, The Hospital for Sick Children, University of Toronto, Toronto, ON, Canada, ${ }^{3}$ The Prenatal Diagnosis and Medical Genetics Program, Department of Obstetrics and Gynecology, Mount Sinai Hospital, University of Toronto, Toronto, ON, Canada, ${ }^{4}$ Department of Radiology, University of Iowa Hospitals and Clinics, Iowa City, IA, United States, ${ }^{5}$ Department of Molecular Medicine and Surgery, Center for Molecular Medicine, Karolinska Institutet, Stockholm, Sweden, ${ }^{6}$ Medical Genetics, MassGeneral Hospital for Children, Boston, MA, United States, ${ }^{7}$ Division of Medical Genetics, Saitama Children's Medical Center, Saitama, Japan, ${ }^{8}$ Department of Clinical Genetics and Genomics, Guy's Hospital, Guy's and St Thomas' NHS Foundation Trust, London, United Kingdom, ${ }^{9}$ Centre for Craniofacial and Regenerative Biology, King's College London, London, United Kingdom, ${ }^{10}$ Department of Pediatrics and Adolescent Medicine, The University of Hong Kong and Shenzhen Hospital, Futian District, Shenzhen, China, ${ }^{11}$ Department of Pediatrics and Adolescent Medicine, Queen Mary Hospital, The University of Hong Kong, Hong Kong, China, ${ }^{12}$ Department of Pediatric Radiology, Karolinska University Hospital, Stockholm, Sweden, ${ }^{13}$ Department of Pediatric Imaging, Tokyo Metropolitan Children's Medical Center, Tokyo, Japan

Introduction: Cilia are important for cell migration and signaling using multiple pathways and proteins essential for development of many body organs and systems, including the skeleton. Skeletal ciliopathies are a heterogenous group of disorders with overlapping features, caused by pathogenic variants in at least 30 genes, coding for different structural cilia proteins.

Materials and Methods: We examined 28 probands with radiographic findings of skeletal ciliopathies. For single nucleotide variant (SNV) analysis, we used whole exome and genome sequencing with in silico filtering for variants in known skeletal ciliopathy genes.

Results: We identified biallelic variants in 23 patients in DYNC2H1, KIAA0753, WDR19, C2CD3, TTC21B, EVC and $E V C 2$. Subsequent examination of four potential splice variants showed that cDNA analyses are important in facilitating molecular diagnoses in a significant proportion $(20 \%)$ of the patients and that aberrant splicing in DYNC2H1, EVC and KIAA0753 can be confirmed with RNA extracted from blood. Copy number variant (CNV) analyses was performed for the same cohort and we found one individual with an intragenic deletion of $\mathrm{DYNC} 2 \mathrm{H} 1$ and another with 1q24q25-microdeletion syndrome.

Conclusions: In conclusion, combined SNV, CNV and RNA analyses result in a high yield of genetic diagnoses $(86 \%)$ in a cohort of patients with a clinical, autopsy and radiological findings consistent with skeletal ciliopathy.

Grants: AnH - Sällskapet Barnavård and Karolinska Institutet. GG - Stiftelsen Samariten, Promobilia and Stiftelsen Frimurare. GG, AL and AN - Stockholm County Council. AN and AL - The Swedish Research Council. AN - The Swedish Childhood Cancer Foundation.

A. Hammarsjo: None. M. Pettersson: None. D. Chitayat: None. A. Handa: None. F. Taylan: None. D. Batkovskyte: None. B. Anderlid: None. A.E. Lin: None. K. Shimizu: None. A. Beleza-Meireles: None. B. Chung: None. U. Voss: None. A. Nordgren: None. G. Nishimura: None. A. Lindstrand: None. G. Grigelioniene: None.

\section{P04.82.A}

Lack of bone mineralization in a TANGO1 deficient patient

B. Guillemyn ${ }^{1}$, S. Nampoothiri ${ }^{2}$, O. Foresti ${ }^{3}$, I. Raote $^{3}$, V.

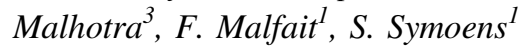

${ }^{1}$ Center for Medical Genetics Ghent, Ghent University Hospital, Department of Biomolecular Medicine, Ghent, Belgium, ${ }^{2}$ Department of Pediatric Genetics, Amrita Institute of Medical Sciences \& Research Center, Cochin, India, ${ }^{3}$ Center for Genomic Regulation, The Barcelona Institute of Science and Technology, Barcelona, Spain

Introduction: $T A N G O 1$ (Transport and Golgi organization1) encodes a transmembrane protein, located at 
Endoplasmic Reticulum (ER) exit sites. TANGO1 binds collagen in the lumen, COPII-coats in the cytoplasm and recruits membranes from the ERGIC (ER-Golgi intermediate compartment) to create an export route for bulky cargos like collagen. Recently aberrant expression of truncated TANGO1 was linked to a severe collagenopathy associated with primary obesity, diabetes mellitus and intellectual disability. We show that complete loss of TANGO1 results in human embryonic lethality with neartotal bone loss.

Material and Methods: Fetal DNA and parental blood/ fibroblast samples were obtained. Whole exome sequencing (WES) was performed. RTqPCR, Western blot, immunocytochemical staining and collagen secretion assays were conducted.

Results: Ultrasound examination of a 13-weeks-old fetus of healthy Indian consanguineous parents revealed tetramicromelia, increased nuchal translucency, absent nasal bone, pingpong appearance of the skull, shortened limb segments, and extreme skeletal hypomineralization. The pregnancy was terminated and the infantogram showed an almost boneless fetus. Fetal WES detected a homozygous 4basepair deletion in TANGO1, heterozygously present in both parents. Parental fibroblast studies demonstrated decreased TANGO1 expression and protein, and severe defects in collagen secretion.

Conclusions: Our findings underscore the importance of TANGO1 in cellular protein secretion. While absence of TANGO1 results in human embryonic lethality, aberrant expression of truncated TANGO1 results in a severe collagenopathy. This further supports the physiological role of TANGO1 in collagen export, which is necessary for bone mineralization, skin and tissue biogenesis. Moreover, the data clearly reveal the severity of human pathologies in association with levels of functional TANGO1 expression.

B. Guillemyn: None. S. Nampoothiri: None. O. Foresti: None. I. Raote: None. V. Malhotra: None. F. Malfait: None. S. Symoens: None.

\section{P04.83.B}

TAR syndrome: clinical and molecular characterization of a cohort of 26 patients and description of novel noncoding variants of $R B M 8 A$

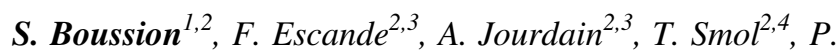

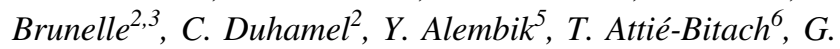

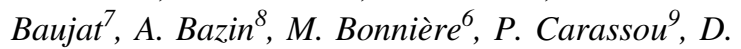
Carles $^{10}$, L. Devisme ${ }^{2,11}$, C. Goizet ${ }^{12}$, A. Goldenberg ${ }^{13}$, S. Grotto ${ }^{14}$, A. Guichet ${ }^{15}$, P. Jouk ${ }^{16}$, L. Loeuillet $^{17}$,
C. Mechler ${ }^{18}$, C. Michot ${ }^{7}$, F. Pelluard ${ }^{19}$, A. Putoux ${ }^{20,21}$, S. Whalen ${ }^{22}$, J. Ghoumid ${ }^{1,2}$, S. Manouvrier ${ }^{1,2}$, F. Petit ${ }^{1,2}$

${ }^{l} \mathrm{CHU}$ Lille, Clinique de Génétique, Centre de Référence Anomalies du Développement, Lille, France, ${ }^{2}$ Université de Lille, EA7364, Lille, France, ${ }^{3} \mathrm{CHU}$ Lille, Service de Biochimie et Oncologie Moléculaire, Lille, France, ${ }^{4} \mathrm{CHU}$ Lille, Institut de Génétique Médicale, Lille, France, ${ }^{5} \mathrm{Ser}$ vice de Génétique Médicale, CHU Strasbourg, Strasbourg, France, ${ }^{6}$ Unité d'Embryofoetopathologie, Service Histologie Embryologie et Cytogénétique, Hôpital Necker-Enfants Malades, AP-HP, Paris, France, ${ }^{7}$ Service de Génétique Clinique, Hôpital Necker - Enfants Malades, AP-HP, INSERM UMR 1163, Institut IMAGINE, Paris, France, ${ }^{8}$ Service de Diagnostic Anténatal, Hôpital René Dubois, Pontoise, France, ${ }^{9}$ Service d'Hématologie, CHR MetzThionville, Metz, France, ${ }^{10}$ Service d'Anatomo-Pathologie, CHU Bordeaux, Bordeaux, France, ${ }^{11}$ Institut d'AnatomoPathologie, CHU Lille, Lille, France, ${ }^{12}$ Service de Génétique Médicale, CHU Bordeaux, et laboratoire MRGM, INSERM U1211, Univ. Bordeaux, Bordeaux, France, ${ }^{13}$ Département de Génétique, et centre de référence anomalies du développement et syndromes malformatifs, CHU de Rouen, Centre Normand de Génomique et de Médecine Personnalisée, Rouen, France, ${ }^{14}$ Département de Génétique, Hôpital Robert Debré, AP-HP, Paris, France, ${ }^{15}$ Service de Génétique, CHU Angers, Angers, France, ${ }^{16}$ Département de Génétique, CHU Grenoble-Alpes, Grenoble, France, ${ }^{17}$ Service d'Anatomo-Cytopathologie, Hôpital Cochin, AP-HP, Paris, France, ${ }^{18}$ Unité de Foetopathologie, Hôpital Robert Debré, AP-HP, Paris, France, ${ }^{19}$ INSERM U1053-UMR BaRITOn et Unité de Foetopathologie, Service de pathologie, Hôpital Pellegrin, CHU Bordeaux, Bordeaux, France, ${ }^{20}$ Service de Génétique, Hospices Civils de Lyon, Lyon, France, ${ }^{21}$ GENDEV Team, CRNL, INSERM U1028, CNRS UMR 5292, UCBL1, Lyon, France, ${ }^{22} U F$ de Génétique Clinique, Centre de Référence Maladies Rares des Anomalies du développement et syndromes malformatifs, Hôpital Armand Trousseau, APHP, Paris, France

Thrombocytopenia-Absent Radius (TAR) syndrome is characterized by radial defect and neonatal thrombocytopenia. It is caused by bi-allelic variants of $R B M 8 A$ gene (1q21.1) with the association of a null allele and a hypomorphic non-coding variant. RBM8A encodes $\mathrm{Y} 14$, a core protein of the Exon Junction Complex involved in mRNA maturation. To date, only two hypomorphic variants have been identified. We report on a cohort of 26 patients affected with TAR syndrome. Half patients carried a 1q21.1 deletion and one of the two known hypomorphic variants. Four novel non-coding variants of $R B M 8 A$ were identified in the remaining patients. We developed experimental 
models enabling their functional characterization in vitro. Two variants, located respectively in the 5'-UTR and 3'UTR regions, are responsible for a diminished expression whereas two intronic variants alter splicing. Our results bring new insights into the molecular knowledge of TAR syndrome and enabled us to propose genetic counseling for patients' families.

S. Boussion: None. F. Escande: None. A. Jourdain: None. T. Smol: None. P. Brunelle: None. C. Duhamel: None. Y. Alembik: None. T. Attié-Bitach: None. G. Baujat: None. A. Bazin: None. M. Bonnière: None. P. Carassou: None. D. Carles: None. L. Devisme: None. C. Goizet: None. A. Goldenberg: None. S. Grotto: None. A. Guichet: None. P. Jouk: None. L. Loeuillet: None. C. Mechler: None. C. Michot: None. F. Pelluard: None. A. Putoux: None. S. Whalen: None. J. Ghoumid: None. S. Manouvrier: None. F. Petit: None.

\section{P05 Cardiovascular Disorders}

\section{P05.01.A}

Improving family screening of abdominal aorta aneurysm patients

\section{R. L. Lechner, A. S. IJpma, D. Heijsman, J. W. Roos- Hesselink, H. J. M. Verhagen, S. ten Raa, R. M. W. Hofstra, D. F. Majoor-Krakauer}

\section{Erasmus MC, Rotterdam, Netherlands}

Relatives of abdominal aneurysm patients (AAA) have an increased risk of developing aneurysms. To improve early detection and reduce risk of rupture in relatives, guidelines recommend screening of first degree relatives. Current guidelines do not specify the method or extend of aorta screening in relatives. In practice, mostly male relatives are referred for abdominal echographic screening of the abdominal aorta, possibly missing thoracic aneurysms (TAA) in relatives. To determine in which part of the aorta affected relatives of AAA patients have an aneurysm or dilatation, we reviewed the results of family history taking and family screening of families of 471 AAA patients with familial aneurysms of which medical records and/or CT images relatives were available. In total 475 affected relatives (311 male, 164 female) in 237 families had an aorta dilatation or aneurysm: $258(54,3 \%)$ had abdominal and 147 $(31 \%)$ thoracic aneurysm. Male relatives were more likely to have AAA ( $40 \%$ vs $28 \%, \mathrm{p}<0.05$ ), females to have TAA ( $32 \%$ vs $17 \%, \mathrm{p}<0.05$ ). Co-occurrence of AAA and TAA was observed in $7 \%$ of relatives. In $17 \%$ an iliac and in $2 \%$ a popliteal dilation occurred. In conclusion: relatives of AAA patients have an increased risk for dilatation of the abdominal and the thoracic aorta. To prevent underdiagnosis of aortic aneurysm in relatives and improve early detection of aortic aneurysms, a CT-scan of the total aorta and the iliac arteries should be considered as the method of choice for family screening.

Conflicts of interest: none. Funding: Lijf en Leven Foundation

R.L. Lechner: None. A.S. IJpma: None. D. Heijsman: None. J.W. Roos-Hesselink: None. H.J.M. Verhagen: None. S. ten Raa: None. R.M.W. Hofstra: None. D.F. Majoor-Krakauer: None.

\section{P05.02.B}

Yield of whole exome sequencing of aneurysm genes in 858 patients with an abdominal aorta aneurysm

\section{R. L. Lechner, A. S. IJpma, D. Heijsman, J. W. Roos- Hesselink, H. J. M. Verhagen, S. ten Raa, R. M. W. Hofstra, D. F. Majoor-Krakauer}

\section{Erasmus MC, Rotterdam, Netherlands}

A growing number of genes are associated with risk for aortic aneurysms. Similar mechanisms of remodeling of the extracellular matrix influencing the integrity of the aortic wall may play a role in abdominal and thoracic aneurysms. This helps to explain co-occurrence of AAA and TAA (25\%), relatives with AAA in familial TAA and relatives with TAA in familial AAA.

To establish the role of aneurysm genes in AAA, the yield of diagnostic whole exome sequencing (WES) of a panel of aneurysm genes in AAA patients is presented.

As part of an ongoing genetic AAA study consecutively diagnosed patients at the department of Vascular Surgery were invited for genetic counseling. In this way WES DNA testing was performed in 858 AAA patients (738 unique families, 345 with familial AAA).

A pathogenic (PV) variant occurred in 20 (3\%) AAA index cases and 7 family members and 301 AAA patients (41\%) had a variant of unknown clinical significance (VUS). PV occurred in FBN1 $(n=4)$, COL3A1 $(n=6)$, DSCH1 $(n=1), \operatorname{TGFBR2}(n=3)$, NOTCH1 $(n=1)$, PLODI $(n=1)$, MYLK $(n=1)$, MFAP5 $(n=1)$ SMAD6 $(n=1)$ and $\operatorname{SLC} 2 A 10(n=1$, heterozygous). VUS were observed in FBN2, COL5A1, MYH11, PLOD1, TGFB2, COL1A1 and COL1A2. There was no difference between familial and sporadic AAA in the WES yield. The outcome was comparable to that found in thoracic aneurysm patients.

The conclusion is that DNA diagnostics may improve stratification of familial risk for aortic aneurysms and distinguish relatives who benefit most from close surveillance.

Funding Lijf en Leven Foundation

Conflicts of interest: none 
R.L. Lechner: None. A.S. IJpma: None. D. Heijsman: None. J.W. Roos-Hesselink: None. H.J.M. Verhagen: None. S. ten Raa: None. R.M.W. Hofstra: None. D.F. Majoor-Krakauer: None.

\section{P05.03.C}

ADAMTS19 associated heart valve defects: novel genetic variants consolidating a recognizable cardiac phenotype

S. Massadeh ${ }^{1,2}$, A. AlHashem ${ }^{3}$, I. van de Laar ${ }^{4}, F$ Alhabshan $^{5}$, N. Ordonez ${ }^{6}$, S. Alawbathani ${ }^{6}$, S. Khan ${ }^{6}$, M. Kabbani ${ }^{5}$, F. Chaikhouni ${ }^{5}$, A. Sheereen ${ }^{1}, I$. Almohammed $^{1,2}$, B. Alghamdi ${ }^{1}$, S. Ahmad ${ }^{7}$, I. FrohnMulder $^{8}$, C. Beetz ${ }^{6}$, A. Rolfs ${ }^{6,9}$, P. Bauer ${ }^{6}$, M. Wessels ${ }^{4}$, M. Alaamery ${ }^{1,2}$, A. Bertoli-Avella ${ }^{6}$

${ }^{1}$ Developmental Medicine Department, King Abdullah International Medical Research Center, King Saud Bin Abdulaziz University for Health Sciences, King Abdulaziz Medical City, Ministry of National Guard Health Affairs, Riyadh, Saudi Arabia, ${ }^{2}$ The Joint Center of Excellence for Biomedicine Between King Abdulaziz City for Science and Technology (KACST) and Brigham \& Women's Hospital (BWH), Joint Centers of Excellence Program, King Abdulaziz City for Science and Technology (KACST), Riyadh, Saudi Arabia, ${ }^{3}$ Division of Pediatric Genetics, Department of Pediatrics, Prince Sultan Military Medical City, Riyadh, Saudi Arabia, ${ }^{4}$ Department of Clinical Genetics, Erasmus University Medical Center, Rotterdam, Netherlands, ${ }^{5}$ Cardiac Sciences Department, Ministry of the National Guard - Health Affairs, King Abdullah International Medical Research Center, King Saud bin Abdulaziz University for Health Sciences, Riyadh, Saudi Arabia, ${ }^{6}$ CENTOGENE AG, Rostock, Germany, ${ }^{7}$ Cardiac Center, Prince Sultan Military Medical City, Riyadh, Saudi Arabia, ${ }^{8}$ Department of Pediatric Cardiology, Erasmus University Medical Center, Rotterdam, Netherlands, ${ }^{9}$ University of Rostock, Rostock, Germany

Recently, ADAMTS19 was identified as a novel causative gene for heart valve disease (HVD), affecting mainly the aortic and pulmonary valves as described in four patients from two families and supported by a mouse knockout model.

Exome sequencing and data repository $\left(\right.$ CentoMD $\left.{ }^{\circledR}\right)$ analyses were performed to identify patients with ADAMTS19 variants (two families). A third family was recognized based on cardiac phenotypic similarities and SNP array homozygosity. Three novel loss of function $(\mathrm{LoF})$ variants were identified in six patients from three families. Clinically, all patients presented anomalies of the aortic/pulmonary valves, which included thickening of valve leaflets, stenosis and insufficiency. Three patients had (recurrent) subaortic membrane, suggesting that ADAMTS19 is the first gene identified related to discrete subaortic stenosis. In contrast to previous publication, none of these patients were reported as having a bicuspid aortic valve. However, one case presented a bi-commissural pulmonary valve. All patients displayed some degree of atrioventricular valve insufficiency. Other cardiac anomalies included atrial/ventricular septal defects, persistent ductus arteriosus, and mild dilated ascending aorta.

Our findings confirm that biallelic $\mathrm{LoF}$ variants in ADAMTS19 are causative of a specific and recognizable cardiac phenotype. We recommend considering ADAMTS19 genetic testing in all patients with multiple semilunar valve abnormalities, particularly in the presence of subaortic membrane. ADAMTS19 screening in patients with semilunar valve abnormalities is needed to estimate the frequency of the HVD related phenotype, which might be not so rare.

S. Massadeh: None. A. AlHashem: None. I. van de Laar: None. F. Alhabshan: None. N. Ordonez: A. Employment (full or part-time); Significant; CENTOGENE AG. S. Alawbathani: A. Employment (full or part-time); Significant; CENTOGENE AG. S. Khan: A. Employment (full or part-time); Significant; CENTOGENE AG. M. Kabbani: None. F. Chaikhouni: None. A. Sheereen: None. I. Almohammed: None. B. Alghamdi: None. S. Ahmad: None. I. Frohn-Mulder: None. C. Beetz: None. A. Rolfs: A. Employment (full or part-time); Significant; CENTOGENE AG. P. Bauer: A. Employment (full or parttime); Significant; CENTOGENE AG. M. Wessels: None. M. Alaamery: None. A. Bertoli-Avella: A. Employment (full or part-time); Significant; Centogene AG.

\section{P05.04.A}

Aortic aneurysm and dissection, an underestimated finding in JAG1 mutation carriers

I. Van Gucht ${ }^{1}$, J. R. Bento ${ }^{1}$, A. Van Berendoncks ${ }^{2}$, E. Van Craenenbroeck $^{2}$, J. Saenen ${ }^{3}$, S. Laga ${ }^{3}$, J. Meester ${ }^{1}$,

A. Verstraeten ${ }^{1}$, N. Boeckx ${ }^{1}$, L. Van Laer ${ }^{1}$, B. Loeys ${ }^{1}$

${ }^{1}$ Center of Medical Genetics Antwerp, University of Antwerp, University Hospital Antwerp, Edegem, Belgium, ${ }^{2}$ Department of Cardiac Surgery, University Hospital Antwerp, University of Antwerp, Edegem, Belgium, ${ }^{3}$ Department of Cardiology, University Hospital Antwerp, University of Antwerp, Edegem, Belgium

Loss-of-function Jagged-1 mutations classically lead to Alagille syndrome (AGS), a dominantly inherited disorder affecting liver, heart, eye, face and skeleton. Pulmonary artery stenosis, present in two thirds of AGS patients, is considered as a hallmark of the condition. Other vascular 
anomalies are identified in up to $10 \%$ of AGS patients. We describe a unique family with a $J A G 1$ missense variant that segregates with a wide range of cardiovascular anomalies. Whole exome sequencing in a fetus with left hypoplastic heart and left renal agenesis revealed a missense variant (c.2242T>C; p.Cys748Arg) in JAG1, which was absent from gnomAD, predicted likely pathogenic by prediction programs and maternally inherited. The mother underwent surgery for aortic coarctation at age 10. The JAGl variant was present in the mother's monozygotic twin-sister and brother but absent in another sister. Subsequent familial clinical evaluation revealed normal eye exam (no embryotoxon), normal ultrasound of kidneys and absence of hemivertebrae in all mutation carriers. Echocardiographic evaluation revealed a normal aorta in the mother, borderline aortic dilatation $(40 \mathrm{~mm})$ in her monozygotic twin-sister and a huge aortic sinus aneurysm $(58 \mathrm{~mm})$ in the brother. The brother also has scoliosis. Remarkably, in literature another JAGl variant affecting a Cys-residu has been linked to severe congenital heart disease (Tetralogy of Fallot). Aortic aneurysm was previously reported in four patients, three of which were only diagnosed on autopsy after fatal dissection. Our family data warrant regular echocardiographic followup in JAGl mutation carriers as severe aortic aneurysms remain asymptomatic and predispose to fatal aortic dissection.

I. Van Gucht: None. J.R. Bento: None. A. Van Berendoncks: None. E. Van Craenenbroeck: None. J. Saenen: None. S. Laga: None. J. Meester: None. A. Verstraeten: None. N. Boeckx: None. L. Van Laer: None. B. Loeys: None.

\section{P05.06.C}

Expanding the genetic and phenotypic spectrum of $A C T A 2$-related vasculopathies in a Dutch cohort

L. van den Bersselaar ${ }^{1}$, E. Rompen ${ }^{1}$, S. Kurul ${ }^{1}, M$. Kempers $^{2}$, A. Houweling ${ }^{3}$, E. Overwater ${ }^{3}$, Y. Hilhorst ${ }^{4}$, D. Barge-Schaapveld ${ }^{4}$, I. Krapels ${ }^{5}$, E. Dulfer ${ }^{6}$, M. Wessels $^{l}, B$. Loeys $^{2}$, J. Bekkers ${ }^{1}$, H. Bruggenwirth ${ }^{l}$, A. Maugeri ${ }^{3}$, J. Roos-Hesselink ${ }^{l}$, J. Verhagen ${ }^{l}$, I. van de Laar $^{l}$

${ }^{1}$ Erasmus Medical Center, Rotterdam, Netherlands, ${ }^{2}$ Radboud University Medical Center, Nijmegen, Netherlands, ${ }^{3}$ Amsterdam University Medical Center, Amsterdam, Netherlands, ${ }^{4}$ Leiden University Medical Center, Leiden, Netherlands, ${ }^{5}$ Maastricht University Medical Center, Maastricht, Netherlands, ${ }^{6}$ University Medical Center Groningen, Groningen, Netherlands

Introduction: Heterozygous variants in the ACTA2 gene, encoding the smooth muscle cell specific isoform of alphaactin, confer a high risk for thoracic aortic aneurysms and aortic dissections. The purpose of this retrospective multicenter study was to review the clinical and genetic data of 47 new individuals with a pathogenic or likely pathogenic ACTA2 variant and elucidate the clinical outcome of ACTA2-related vasculopathies.

Material and Methods: Index patients and relatives with a pathogenic or likely pathogenic variant in the ACTA2 gene were included in this study. Data were collected through retrospective review of available medical records using a standardized questionnaire.

Results: Forty-seven individuals from 26 families participated in our study. Mean age at last evaluation was $50.8 \pm 17.0$ years. A total of 21 different ACTA2 variants were detected in our cohort, including 19 missense variants, 1 splice site variant and 1 frameshift variant. Four variants are novel. Aortic events occurred in 30/47 (63.8\%) of patients. Mean age at first aortic event was $55.6 \pm 13.7$ years. Male gender and the presence of hypertension emerged as significant predictors of aortic events. Men were also at higher risk of aortic aneurysms compared to women. Other clinical findings included congenital heart disease in 6/47 and iris flocculi in 6/47.

Conclusions: We provide further insight into the genotype and phenotype of ACTA2-related vasculopathies. Male gender and hypertension are predisposing factors for aortic events in individuals with a pathogenic ACTA2 variant.

Supported by Dutch Heart Foundation (2014T007) and Erasmus University Rotterdam Fellowship to IvdL.

L. van den Bersselaar: None. E. Rompen: None. S. Kurul: None. M. Kempers: None. A. Houweling: None. E. Overwater: None. Y. Hilhorst: None. D. BargeSchaapveld: None. I. Krapels: None. E. Dulfer: None. M. Wessels: None. B. Loeys: None. J. Bekkers: None. H. Bruggenwirth: None. A. Maugeri: None. J. RoosHesselink: None. J. Verhagen: None. I. van de Laar: None.

\section{P05.11.B}

Prevalence of TTR mutations associated with transthyretine amyloidosis (ATTR) estimated from national exome sequencing data.

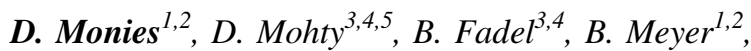 I. Alayary ${ }^{6}$, M. Abouelhoda ${ }^{1,2}$

${ }^{I}$ Department of Genetics, King Faisal Specialist Hospital and Research Centre, Riyadh, Saudi Arabia, ${ }^{2}$ Saudi Human Genome Program, King Abdulaziz City for Science and Technology, Riyadh, Saudi Arabia, ${ }^{3}$ Heart center, King Faisal Specialist Hospital \& Research Center, Riyadh, Saudi Arabia, ${ }^{4}$ Al-Faisal University, College of Medicine, Affiliate Harvard Medical School International, Riyadh, 
Saudi Arabia, ${ }^{5} \mathrm{CHU}$, Limoges, France, ${ }^{6}$ Pfizer Inc, Jeddah, Saudi Arabia

Introduction: Amyloidosis is a rare, frequently underdiagnosed systemic disease due to protein deposition that develops when a native protein misfolds into insoluble amyloid fibrils. These fibril deposits alter function of most affected organs, including the myocardium. Transthyretin amyloidosis (ATTR) is one of the two main causes of cardiac amyloidosis and occurs as a result of mutations in $T T R$. Under or misdiagnosis of affected individuals and incomplete penetrance make estimates of prevalence challenging. While worldwide prevalence is estimated to be around 50,000 individuals, with TTR V142I being the most common cardiac ATTR associated mutation, no data on TTR mutations or their frequency in Middle Eastern countries are available to date.

Materials and Methods: Blinded from clinical information, we undertook data mining of 13,906 exomes of unrelated Saudi individuals, sequenced between 2015 and 2019 as part of the Saudi Human Genome Project (SHGP). Pathogenic or highly pathogenic TTR variants were identified following filtration to remove common, synonymous, non-coding and other benign variants.

Results and conclusion: We identified seven missense variants (known or predicted pathogenic) in TTR, three of which have been previously reported in amyloidosis patients worldwide. The most frequent variants were c.424G>A:p.V142I (1:435) and c.370C >T:p.R124C (1:2,318). Prevalence of TTR variants in the general Saudi population was much higher than the actual estimated rate of ATTR cases nationally $(1: 10,000)$, which may be explained by incomplete penetrance or sub-clinical manifestation of the disease.

D. Monies: None. D. Mohty: None. B. Fadel: None. B. Meyer: None. I. Alayary: None. M. Abouelhoda: None.

\section{P05.13.A}

Investigation of NOTCH1mutation frequency in bicuspid aortic valve disease

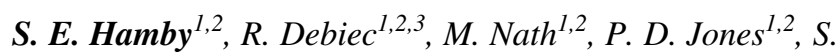
Coolman $^{1,2}$, M. Asiani ${ }^{1,2}$, S. Kharodia ${ }^{1,2}$, G. J. Skinner ${ }^{3}, N$. J. Samani ${ }^{1,2}$, A. Bolger ${ }^{1,2,3}$, T. R. Webb ${ }^{1,2}$

${ }^{1}$ University of Leicester Department of Cardiovascular Sciences, Leicester, United Kingdom, ${ }^{2}$ NIHR Leicester Biomedical Research Centre, Leicester, United Kingdom, ${ }^{3}$ East Midlands Congenital Heart Centre, Glenfield Hospital, Leicester, United Kingdom

Background: Bicuspid Aortic valve (BAV) is a common congenital heart defect present in around $1 \%$ of the population. Familial BAV has an autosomal dominant pattern of inheritance with reduced penetrance, however, the majority of BAV cases are sporadic. Pathogenic and likely pathogenic NOTCHI variants have been reported in familial, as well as up to $4 \%$ of sporadic cases.

Aim: To investigate the frequency of causative NOTCH1 mutations in a large cohort of patients of sporadic and familial BAV disease recruited to the Bicuspid aoRtic vAlVe gEnetic research (BRAVE) study.

Methods: Whole exome sequencing (WES) was performed in 12 families ( 30 affected and 25 unaffected family members). Targeted NOTCH1 sequencing was carried out in 383 sporadic BAV cases. NOTCH1 variants were filtered by gnomAD allele frequency $(<0.001)$ and potential deleterious effect (Combined Annotation Dependent Depletion (CADD) score $>20$ ). We also compared cumulative frequency of rare $\mathrm{NOTCH} 1$ variants between sporadic BAV patients and 1102 apparently healthy controls.

Results: One BAV family had a truncating NOTCHI variant (c.873C $>$ G/p.Tyr291*). There were no candidate NOTCH1 variants in any of the other 11 families. We identified seven rare and potentially deleterious NOTCHI variants in the sporadic patient group. Among which, there were no protein truncating mutations. There was no enrichment of NOTCHI variants between cases and controls (1.83\% vs 3.09\%, $p$-value 0.18 ).

Conclusions: Our data suggest that whilst occasionally responsible for familial cases of BAV, NOTCH1 mutations are an infrequent cause of sporadic forms of the disease.

S.E. Hamby: None. R. Debiec: None. M. Nath: None. P.D. Jones: None. S. Coolman: None. M. Asiani: None. S. Kharodia: None. G.J. Skinner: None. N.J. Samani: None. A. Bolger: None. T.R. Webb: None.

\section{P05.14.B}

Bicuspid aortic valve: a pilot candidate gene-based approach in a representative Czech cohort

\section{P. Votýpka ${ }^{1}$, P. Peldová ${ }^{1}$, A. Krebsováa, V. Zoubkovál, M. Balaščakovál ${ }^{1}$ M. Macek, $J r^{l}$}

${ }^{1}$ Department of Biology and Medical Genetics, 2nd Faculty of Medicine, Charles University and Motol, Prague, Czech Republic, ${ }^{2}$ Department of Cardiology, Institute for Clinical and Experimental Medicine, Prague, Czech Republic

Aim: Analysis of the genetic basis in a representative cohort of Czech patients with familiar bicuspid aortic valve (BAV) with / without aortic aneurysm (MIM\# 109730).

Materials and Methods: Altogether 100 unrelated cases were clinically examined, underwent genetic counselling and positive cases were subjected to "cascade screening" of first-degree relatives. Massively parallel sequencing (MiSeq 
platform; Illumina.com) was utilised for a custom-made panel comprising either 136, 229 or 100 cardiac/aortic conditions-related candidate genes (Sophia Genetics.com). Presence of pathogenic variants was validated by Sanger sequencing and via their segregation in respective families.

Results: Pathogenic/likely pathogenic variant (ACMG Class $\geq 4$ ) were found in $3 / 100$ patients (3\%) within $T G F B 2$, SMAD6 and FBN1. Variants of unknown significance (Class 3) were detected in $9 / 100$ patients (9\%) comprising genes FLNA, FBN1, MYH11, TAB2, TGFB2 and GATA5. A family with concurrent familial dilated cardiomyopathy, BAV and ectopia lentis was analysed as well. Likely causative DNA variants in $M Y B P C 3$ and $F B N 1$ were identified, but $F B N 1$ variants did not co-segregate with BAV.

Conclusions: The yield of genetic testing in familial forms of BAV is rather low in our cohort. Moreover, the identification of pathogenic variant in BAV-related gene is not a guarantee of their causality, hence detected variants should be carefully interpreted. The genetic basis for the familial forms of BAV will be further studied, e.g. by whole exome analysis together with improved clinical stratification of our patient cohort.

Supported by Ministry of Health of the Czech Republic, grant Nr. NV18-02-00237

P. Votýpka: None. P. Peldová: None. A. Krebsová: None. V. Zoubková: None. M. Balaščaková: None. M. Macek, Jr.: None.

\section{P05.16.A}

Electrophysiological characterization of a Brugada syndrome SCN5A Belgian founder mutation in induced pluripotent stem cell cardiomyocytes

E. Simons ${ }^{l}, A$. Nijak $^{l}$, B. Vandendriessche ${ }^{l}$, D. Van de Sande $^{2}$, E. Sieliwończyk ${ }^{1}$, A. J. Labro ${ }^{2}$, D. Snyders ${ }^{2}, D$. Schepers $^{l}$, B. Loeys ${ }^{1,3}$, M. Alaerts ${ }^{l}$

${ }^{1}$ Centre of Medical Genetics, faculty of Medicine and Health Sciences, University of Antwerp \& Antwerp University Hospital, Antwerp, Belgium, ${ }^{2}$ Laboratory of Molecular Biophysics, Physiology \& Pharmacology, Department of Biomedical Sciences, University of Antwerp, Antwerp, Belgium, ${ }^{3}$ Department of Human Genetics, Radboud University Medical Centre, Nijmegen, Netherlands

Introduction: $S C N 5 A$ encodes the $\alpha$-subunit of voltagegated cardiac sodium channel $\mathrm{Na}_{\mathrm{v}} 1.5$. Mutations in $S C N 5 A$ are identified in about $20 \%$ of patients with Brugada syndrome $(\mathrm{BrS})$, an inherited cardiac arrhythmia. We have identified an SCN5A founder mutation (c.4813+3_4813 +6dupGGGT), leading to a loss-of-function of $\mathrm{Na}_{\mathrm{v}} 1.5$ in 25 different families. Mutation carriers show variable expression of the phenotype: from asymptomatic to syncopes and $\mathrm{SCD}$. We used induced pluripotent stem cell derived cardiomyocytes (iPSC-CM) to investigate the underlying pathophysiology.

Material \& Methods: Dermal fibroblasts of three patients with different severity, and one unrelated healthy control were reprogrammed using the CytoTune ${ }^{\mathrm{TM}_{-} \mathrm{iPS}} 2.0$ Sendai Reprogramming Kit. iPSC-CMs were differentiated following a published protocol. T3 hormone addition was tested to improve maturity of the cells. We performed several differentiation rounds and investigated expression of cardiac markers using qPCR and immunocytochemistry and electrophysiological properties using patch-clamping and calcium imaging.

Results: All iPSC-CMs expressed tested markers. We observed reduction in sodium current density in patient iPSC-CMs, together with shortening of the action potential and calcium transient duration compared to the control. However, our data display variability between the differentiation batches, as well as between clones generated from one donor. Addition of T3 improved organization of the sarcomeres and decreased result variability.

Conclusions: We established iPSC-CM models for a unique Belgian SCN5A founder mutation. Despite observed variability, we could detect expected differences in electrophysiological properties of patient cells compared to controls. We prove that $\mathrm{T} 3$ addition improves the maturity of the cells, with a positive impact on the variability.

E. Simons: None. A. Nijak: None. B. Vandendriessche: None. D. Van de Sande: None. E. Sieliwończyk: None. A. J. Labro: None. D. Snyders: None. D. Schepers: None. B. Loeys: None. M. Alaerts: None.

\section{P05.18.C}

Network-based identification of novel Shox2-dependent genes in cardiac rhythm control

S. Hoffmann ${ }^{1,2}$, S. Schmitteckert ${ }^{l}$, K. Raedecke $\boldsymbol{~ R}^{1,2}, D$. Rheinert $^{l}$, S. Diebold ${ }^{3}$, R. Roeth ${ }^{1}$, B. Weiss ${ }^{1}$, M. Granzow ${ }^{4}$, B. Niesler ${ }^{1,2}$, A. Griesbeck ${ }^{l}$, V. Eckstein ${ }^{5}$, W. H. Zimmermann $^{6,7}$, S. Just ${ }^{3}$, G. A. Rappold ${ }^{1,2}$

${ }^{I}$ Department of Human Molecular Genetics, Institute of Human Genetics, University of Heidelberg, Heidelberg, Germany, ${ }^{2}$ DZHK, German Center for Cardiovascular Research, Partner Site Heidelberg/Mannheim, Heidelberg, Germany, ${ }^{3}$ Department of Internal Medicine II, University of Ulm, Ulm, Germany, ${ }^{4}$ Department of Human Genetics, Institute of Human Genetics, University of Heidelberg, Heidelberg, Germany, ${ }^{5}$ FACS Core Facility, Department of Medicine V, University Hospital Heidelberg, Heidelberg, Germany, ${ }^{6}$ Institute of Pharmacology, University Medical 
Center Goettingen, Goettingen, Germany, ${ }^{7}$ DZHK, German Centre for Cardiovascular Research, Partner Site Goettingen, Goettingen, Germany

The transcription factor SHOX2 is involved in the development and function of the sinoatrial node (SAN), which is crucial for initiating and controlling heart rhythm. SHOX2 has been associated with cardiac arrhythmias such as atrial fibrillation (AF) and sinus node dysfunction. Yet, our knowledge on co-regulatory networks associated with cardiac rhythm control in health and disease still remains limited. To dissect the role of Shox2-dependent networks, we utilized a murine embryonic stem cell (ESC) model that allowed us to investigate molecular pathways involving Shox2 in SAN-like cardiomyocytes. Differential RNA-seq-

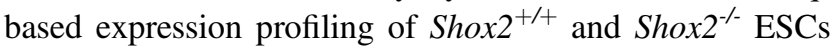
revealed 94 regulated transcripts in Shox ${ }^{-/}$SAN-like cells. Fifteen putative target genes were selected for further validation based on comparative expression analysis of our dataset with publicly available RNA-seq data of SAN and right atria enriched genes at different mouse embryonic stages. Network-based analyses integrating data from the Mouse Organogenesis Cell Atlas and the Ingenuity pathway analysis tool as well as validation in mouse and zebrafish confirmed a species conserved regulation for Shox 2 target genes in cardiac rhythm control. Our results indicate that co-regulatory networks involving SHOX2 participate in the development of conduction traits through regulatory mechanisms, thereby affecting the expression of multiple genes. This work was supported by grants from the DFG (RA 380/14-4) and DZHK (81X2500110 and 81X2500171).

S. Hoffmann: None. S. Schmitteckert: None. K. Raedecke: None. D. Rheinert: None. S. Diebold: None. R. Roeth: None. B. Weiss: None. M. Granzow: None. B. Niesler: None. A. Griesbeck: None. V. Eckstein: None. W.H. Zimmermann: None. S. Just: None. G.A. Rappold: None.

\section{P05.19.A}

Diagnostic yield of targeted versus broad next generation sequencing data analysis in inherited cardiomyopathy and arrhythmia syndromes

\author{
K. Kolokotronis ${ }^{l}$, N. Pluta ${ }^{l}$, E. Klopocki ${ }^{1}$, S. Störk ${ }^{2}, S$. \\ Rost $^{l}$, B. Gerull ${ }^{2}$
}

${ }^{1}$ Institute of Human Genetics, University of Wuerzburg, Wuerzburg, Germany, ${ }^{2}$ Comprehensive Heart Failure Center and Department of Medicine I, University Hospital Würzburg, Wuerzburg, Germany

Introduction: Although next generation sequencing has drastically changed cardiogenetics, the fact that many cases with positive family history remain unsolved after routine panel diagnostics suggests that some genetic causes remain to be discovered. In this study we examined the diagnostic yield of targeted gene panels compared to whole exome data analysis.

Methods: The cohort consisted of 61 consecutive patients with a diagnosis of cardiomyopathy or primary arrhythmia syndrome, with DCM being the most common diagnosis. The genetic analysis involved whole exome sequencing with subsequent stepwise bioinformatic analysis starting from virtual gene panels till whole exome data. Genetic variants were classified according to ACMG-criteria.

Results: Overall, in 39 out of 61 (64\%) patients a variant of interest was detected. The detection rate was highest in patients with a diagnosis of HCM $(7 / 9 ; 77 \%)$ or DCM (25/ 36; 69\%). In DCM the diagnostic yield was better compared to current literature. The majority of variants of interest were found in the disease-specific panel (46\%; 28/61), while the further analysis of an extended panel and whole exome data led to an additional diagnostic yield of $13 \%$ and $5 \%$, respectively. Whole exome data analysis also detected variants in thus far not well characterized candidate genes whose expression and function profile suggested a probable pathogenetic role.

Conclusions: Targeted gene panel analysis led to the identification of the genetic cause in the majority of solved cases. The additional diagnostic yield of whole exome data analysis was low, enabled though the detection of potentially causal variants in candidate genes.

K. Kolokotronis: None. N. Pluta: None. E. Klopocki: None. S. Störk: None. S. Rost: None. B. Gerull: None.

\section{P05.20.B}

Diagnostic yield of core panels for cardiomyopathy- and arrhythmia genes versus a larger panel of heart-disease related genes

D. M. E. I. Hellebrekers ${ }^{l}$, B. J. C. van den Bosch ${ }^{1}$, G. R. F. Claes $^{l}$, I. Roozen ${ }^{l}$, Y. E. G. Barrois ${ }^{l}, N$. Vergoossen ${ }^{l}$, A. T. M. Hendrickx ${ }^{1}$, W. van Dijk ${ }^{1}$, C. L. M. Marcelis ${ }^{2}, M$.

Kempers $^{2}$, Y. M. Hoedemaekers ${ }^{2}$, A. T. J. M. Heldermanvan den Enden ${ }^{l}$, E. K. Vanhoutte ${ }^{1}$, I. P. C. Krapels ${ }^{l}$, A. van den Wijngaard ${ }^{l}$

${ }^{1}$ Maastricht University Medical Center, Maastricht, Netherlands, ${ }^{2}$ Radboud University Medical Center, Nijmegen, Netherlands

Introduction: We evaluated the prevalence of (likely) pathogenic variants in our cardiomyopathy- and arrhythmia core panels in patients with cardiac disease. Furthermore, 
the additional diagnostic value of a larger panel of heart disease-associated genes was assessed.

Methods: This study included a large population of 2000 patients suspected of a heritable cardiac condition who underwent cardiogenetic testing at the laboratory of the Clinical Genetics Department in Maastricht. Sequencing a diagnostic panel of 46 cardiomyopathy genes and/or 27 arrhythmia genes was performed using single-molecule molecular inversion probes (smMIPs). For 347 of these patients, whole exome sequencing was performed for the analysis of a panel of 350 heart disease-associated genes.

Results: A (likely) pathogenic variant was found in $20 \%$ (336/1719) and 8\% (34/408) of patients tested for the cardiomyopathy or arrhythmia gene panel, respectively. The majority of these variants were found in the $M Y B P C 3$, MYH7, LMNA, TNNT2, MYL2 and PKP2 genes for cardiomyopathy and $S C N 5 A, K C N Q 1, R Y R 2$ and $K C N H 2$ genes for arrhythmia. Sequencing the larger panel of 350 genes in 347 patients identified an additional pathogenic variant explaining the clinical phenotype in only 3 patients.

Conclusions: Our data support parallel sequencing of core genes as the preferred strategy for genetic testing of patients suspected of having a genetic cardiomyopathy or arrhythmia. The incremental diagnostic yield of a larger panel of heart disease-associated genes is minimal. Therefore, focus should not be on analysing more genes, but on developing functional assays to predict pathogenicity of variants of unknown significance (VUS's) in the core panel genes.

D.M.E.I. Hellebrekers: None. B.J.C. van den Bosch: None. G.R.F. Claes: None. I. Roozen: None. Y.E.G. Barrois: None. N. Vergoossen: None. A.T.M. Hendrickx: None. W. van Dijk: None. C.L.M. Marcelis: None. M. Kempers: None. Y.M. Hoedemaekers: None. A.T.J.M. Helderman-van den Enden: None. E.K. Vanhoutte: None. I.P.C. Krapels: None. A. van den Wijngaard: None.

\section{P05.21.C}

Dominant mild Carvajal syndrome: arrhythmogenic cardiomyopathy caused by the heterozygous p.R1113* nonsense variant in the DSP gene.

A. T. J. M. Helderman-vd Enden ${ }^{1}$, J. G. Post ${ }^{2}$, K. J. A. F. van Kaam ${ }^{l}$, R. H. Lekanne Deprez ${ }^{3}$, D. Dooijes ${ }^{4}$, A. A. M. Wilde $^{5}$, I. P. C. Krapels ${ }^{l}$

${ }^{1}$ Department of Clinical Genetics, Maastricht University Medical Center, Maastricht, Netherlands, ${ }^{2}$ Department of Clinical Genetics, University Medical Center Utrecht, Utrecht, Netherlands, ${ }^{3}$ Department of Clinical Genetics, Amsterdam University Medical Center, Amsterdam, Netherlands, ${ }^{4}$ Department of Clinical Genetics, University
Medical Center Utrecht, Utrecht, Netherlands, ${ }^{5}$ Department of Cardiology, Amsterdam University Medical Center, Amsterdam, Netherlands

Introduction: Arrhythmogenic cardiomyopathy (ACM) is a dominant inherited cardiac disease characterized by progressive fibrofatty replacement of the myocardium that predisposes to ventricular tachycardia and sudden death. Carvajal syndrome is a rare recessive form of ACM associated with woolly hair and palmo-plantar keratoderma and pathogenic variants in the desmoplakin (DSP) gene. Some families have been described with a dominantly inherited DSP variant with features of Carvajal.

Materials and Methods: In a four generation family seggregation analysis was done for a dominantly inherited DSP p.R1113* variant. The phenotypes are described and the results are compared to literature.

Results: In the large sibship $(4 \mathrm{M}+8 \mathrm{~F})$ at least three siblings are obligate carrying the DSP pathogenic variant and have offspring with cardiac abnormalities. Five family members died suddenly, $2 / 5$ obligate carriers, $3 / 5$ the status is unknown. The curly hair is co-seggregating with the variant. Not all carriers have keratoderma. A number of individuals with the variant have no cardiac complaints so far, cardiological investigations are planned. An overview will be presented at the conference and compared to the six families described by Maruthappu et al in 2019, one of these families also has the p.R1113* variant.

Conclusion: The p.R1113* variant in the DSP gene leads to a well recognizable phenotype of mild Carvajal syndrome. Drawing the attention to this phenotype by presenting this large four generation family may help in identifying other families with this disease and in preventing sudden death.

A.T.J.M. Helderman-vd Enden: None. J.G. Post: None. K.J.A.F. van Kaam: None. R.H. Lekanne Deprez: None. D. Dooijes: None. A.A.M. Wilde: None. I.P.C. Krapels: None.

\section{P05.22.A}

Chromosome 22q11 copy number variations in paediatric and adult patients with congenital heart defects

\author{
G. K. ZODANU, D. Nagy, M. Széll, M. Oszlánczi, \\ K. Havasi, A. Kalapos, M. Katona
}

\section{UNIVERSITY OF SZEGED, SZEGED, Hungary}

Introduction: Congenital heart defect (CHD) is the most common birth defect, affects $\sim 1 \%$ of newborns. Chromosome 22q11 microdeletion syndrome is often associated with CHDs and is the most common human microdeletion, occurs in $\sim 1 / 4000$ live births in the general population and 
in $2.8-14.9 \%$ of CHD patients. 22q11 copy number variants (CNVs) are caused by non-allelic homologous recombination events in the flanking low copy repeat regions (LCRs), labeled A-H.

Materials and Methods: Our aim was to evaluate the prevalence of $22 \mathrm{q} 11 \mathrm{CNVs}$ in Hungarian CHD patients. 175 blood samples were obtained from children and adults with different isolated and combined CHDs. Samples were analyzed using multiplex ligation-dependent probe amplification (MLPA) to detect CNVs in the given chromosomal region. Positive MLPA results were confirmed with locusspecific FISH. Genotype-phenotype comparison and family screening was also performed.

Results: CNVs were detected in overall 10 cases (5.7\%). Six cases with typical microdeletion (between LCRs A and D) also known as DiGeorge syndrome, 2 cases with microduplication and 2 cases with combined microdeletion and microduplication were detected. The phenotypical appearance of patients was highly variable. CNVs were familiar in 3 cases.

Conclusions: Until present this was the largest systemic genetic screening of Hungarian CHD patients. Since the clinical diagnosis of 22q11 microdeletion/microduplication syndrome may be challenging due to its variable manifestation, systematic genetic testing may be of utmost benefit in the early diagnosis and proper management of these patients.

Funding grant: GINOP-2.3.2-15-2017-00039

G.K. Zodanu: None. D. Nagy: None. M. Széll: None. M. Oszlánczi: None. K. Havasi: None. A. Kalapos: None. M. Katona: None.

\section{P05.23.B}

Genetic variation in participants of the 100,000 Genomes Project with congenital heart disease

\author{
S. L. Baross ${ }^{1}$, S. G. Williams ${ }^{1}$, K. E. Hentges ${ }^{2}$, A. D. \\ Sharrocks $^{3}$, B. D. Keavney ${ }^{l}$
}

${ }^{1}$ Division of Cardiovascular Sciences, University of Manchester, Manchester, United Kingdom, ${ }^{2}$ Division of Evolution and Genomic Sciences, University of Manchester, Manchester, United Kingdom, ${ }^{3}$ Division of Molecular and Cellular Function, University of Manchester, Manchester, United Kingdom

Congenital heart disease is the most common birth defect, affecting approximately $1 \%$ of live births globally. Congenital heart disease shows a high degree of heritability but the genetic causes are still poorly understood.

The 100,000 Genomes Project provides whole genome sequences for patients with rare disease or cancer and their families. Over 600 participants were recruited to the project under the congenital heart disease arm of the project, almost all of whom have no known causative variant. In addition, analysis of phenotyping and clinical data identified an additional $\sim 2,500$ participants with congenital heart disease who were recruited to the project for other conditions.

We will present rare, likely pathogenic single nucleotide, short insertions/deletions and structural variants, both transmitted and de novo, from this cohort of 3,100 participants with congenital heart disease. Short variants are filtered for variants with moderate or high impact on protein function; CADD (combined annotation dependent depletion) score $\geq 20$; and absent in gnomAD v3.

This research was made possible through access to the data and findings generated by the 100,000 Genomes Project (http://www.genomicsengland.co.uk).

Grant reference: BHF FS/16/58/32734.

S.L. Baross: None. S.G. Williams: None. K.E. Hentges: None. A.D. Sharrocks: None. B.D. Keavney: None.

P05.24.C

Mutation spectrum of congenital heart disease in 73consanguineous Turkish families

\author{
W. Dong ${ }^{1}$, H. Kaymakcalan ${ }^{2}$, N. Diab ${ }^{1}$, S. Chih Jin ${ }^{1}$,

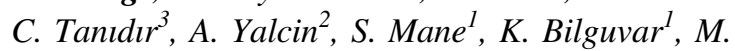 \\ Brueckner $^{l}$, R. Lifton ${ }^{l}$
}

${ }^{1}$ Yale University, New Haven, CT, United States, ${ }^{2}$ Demiroglu Bilim University, Istanbul, Turkey, ${ }^{3}$ Mehmet Akif Ersoy Hospital, istanbul, Turkey

Mutation spectrum of congenital heart disease in 73 consanguineous Turkish families

Introduction: Congenital heart disease (CHD) is the most frequent defect among live births and the leading cause of mortality from a birth defect. Nearly $90 \%$ of CHD cases have a suspected genetic etiology, but in $56 \%$ of cases the genetic etiology is unknown. Few gene discovery efforts have been made with consanguineous CHD cohorts. We recruited $74 \mathrm{CHD}$ patients from 73 consanguineous families in Turkey and used whole exome sequencing to identify affected genes in these patients. In $13.7 \%$ of the cohort, we could identify a genetic etiology and we discovered four new genes that are putatively causative for CHD. This represents an important advance in our understanding of the genetic consequences of consanguinity in CHD.

Materials and methods: 74 samples from 73 families were recruited from two pediatric cardiology clinics in Istanbul, Turkey. Cardiac phenotypes were classified into 5 major categories and whole exome sequencing was performed at the Yale Center for Genomic Analysis using Illumina. 
Result: 7 out of $73(9.6 \%)$ patients carried damaging homozygous variants in known CHD genes and 3 out of 73 $(4.1 \%)$ of them harbored other types of CHD-related genomic alterations, which explained $13.7 \%$ of the cohort.

Conclusions: Our findings is consistent with the hypothesis that high consanguinity is more likely to be associated with recessive cardiac abnormalities. Additionally, it fits the perception that the heritability for severe defects is higher than milder malformations.

Grant: Yale-National Institutes of Health Center for Mendelian Genomics

W. Dong: None. H. Kaymakcalan: None. N. Diab: None. S. Chih Jin: None. C. Tanıdır: None. A. Yalcin: None. S. Mane: None. K. Bilguvar: None. M. Brueckner: None. R. Lifton: None.

\section{P05.26.B}

Altered microRNA and target gene expression related to Tetralogy of Fallot

\section{Grunert, S. Appelt, S. Rickert-Sperling}

\section{Charité - Universitätsmedizin Berlin, Berlin, Germany}

MicroRNAs (miRNAs) play an important role in guiding development and maintaining function of the human heart. Dysregulation of miRNAs has been linked to various congenital heart diseases including Tetralogy of Fallot (TOF), which represents the most common cyanotic heart malformation in humans. Several studies have identified dysregulated miRNAs in right ventricular (RV) tissues of TOF patients. In the study presented here, we profiled genomewide the whole transcriptome and analyzed the relationship of miRNAs and mRNAs of RV tissues of a homogeneous group of 22 non-syndromic TOF patients. Observed profiles were compared to profiles obtained from right and left ventricular tissue of normal hearts. To reduce the large list of predicted target genes of dysregulated miRNAs, we applied a stringent target prediction pipeline integrating probabilities for miRNA-mRNA interaction. The final list of disease-related miRNA-mRNA pairs comprises novel as well as known miRNAs including miR-1 and miR-133, which are essential to cardiac development and function by regulating KCNJ2, FBN2, SLC38A3 and TNNI1. Overall, our study provides additional insights into posttranscriptional gene regulation of malformed TOF hearts.

M. Grunert: None. S. Appelt: None. S. RickertSperling: None.

\section{P05.28.A}

Circulating IncRNA ANRIL a potential biomarker in Tanzanian patients with coronary artery disease
G. $\boldsymbol{A K A N}^{1}$, E. Nyawawa ${ }^{2}$, B. Nyangasa ${ }^{2}$, M. K. Turkcan ${ }^{3}$, E. Mbugi ${ }^{1}$, O. Ozgen ${ }^{4}$, M. Janabi ${ }^{2}$, F. Atalar ${ }^{1}$

${ }^{I}$ MUHAS Genetics Laboratory, Biochemistry Department, School of Medicine, Muhimbili University of Health and Allied Science, Dar es Salaam, Tanzania, United Republic of, ${ }^{2}$ Muhimbili National Hospital Jakaya Kikwete Heart Institute, Dar es Salaam, Tanzania, United Republic of, ${ }^{3}$ Department of Electrical Engineering, Columbia University,, New York, NY, United States, ${ }^{4}$ Molecular Medicine Department, Aziz Sancar Experimental Medicine Research Institute, Istanbul University, Istanbul, Turkey

Background: 9p21.3 locus was identified as a hot spot locus associated with coronary artery disease $(\mathrm{CAD})$ and it contains CDKN2A and CDKN2B genes which encode the cell cycle regulators and a non-coding RNA ANRIL gene. Role of 9p21.3 locus variations in molecular mechanisms of CAD pathogenesis and the expression of neighbour-genes remain unknown. The study aims to assess of expressions of CDKN2A, CDKN2B, ANRIL and ANRIL splices variants association with CAD susceptibility, and to investigate the impact of 9p21.3 locus variations on the expression of studied genes in peripheral blood, Epicardial Adipose Tissue(EAT), Mediastinal Adipose tissue(MAT) and Subcutaneous Adipose Tissue(SAT) in Tanzanian CAD patients.

Methods\&Results: Expression of CDKN2A, CDKN2B, ANRIL, and its splice variants (circANRIL, NR003529, EU741058 and DQ485454)were measured by QRT-PCR in peripheral blood, EAT, MAT and SAT of 25 patients undergoing coronary artery bypass grafting due to CAD and 25 non-CAD patients undergoing heart valve surgery. ANRIL gene expression levels were significantly upregulated while expression levels of circANRIL were significantly down-regulated in peripheral blood of CAD patients. Moreover, increased expression levels of circANRIL were associated with less severity of CAD. ROC analysis data has also confirmed that circANRIL could act as a potential biomarker for detecting CAD.

Conclusions: Our results are the first data presenting the expressions of ANRIL and its splice variants in cardiac adipose tissues of CAD patients. We demonstrate circANRIL has a synergistic effect with ANRIL and plays a protective role in CAD. circANRIL could possibly be used as a biomarker for CAD in Tanzania.

G. Akan: None. E. Nyawawa: None. B. Nyangasa: None. M.K. Turkcan: None. E. Mbugi: None. O. Ozgen: None. M. Janabi: None. F. Atalar: None.

\section{P05.29.B}

Members of subgingival plaque bacterial complexes are present in blood of patients with coronary artery disease 
A. Suarez-Molina ${ }^{1}$, C. Fong ${ }^{2}$, L. Cifuentes- $C^{2}$, S. GuauqueOlarte $^{2}$

${ }^{1}$ Instituto Departamental de Salud de Nariño, Pasto, Colombia, ${ }^{2}$ Universidad Cooperativa de Colombia, Pasto, Colombia

Introduction: It has been hypothesized that oral bacteria can migrate, through the blood, from the mouth to the artery plaques exacerbating atherosclerosis. This study compared the bacteria present in peripheral blood of individuals with and without coronary artery disease.

Materials and Methods: RNA sequences from blood downloaded from GEO (GSE58150) correspond to 8 cases with coronary artery calcium $(\mathrm{CAC})>514$ and 8 controls $(\mathrm{CAC}=0)$. After quality controls, the sequences were aligned to the hg38 reference genome using Hisat2. The unmapped sequences were fed into Kraken to determinate bacterial taxa. Ecological indices were calculated using Vegan. The counts of bacteria between groups was compared using a Mann-Whitney U test.

Results: The mean number of species was different between cases (433.4) and controls (226.1) ( $p$-value = 0.0001 ). A total of 129 species were found only in cases and 74 were exclusive of controls. According to the Jaccard similarity index $16 \%$ of the species identified is not shared between cases and controls $(\mathrm{p}=0.001)$. The Alcaligenaceae family was more frequent in cases than in controls (FDR: 0.048). The red complex bacteria Treponema denticola was detected only in 2 cases. The mean count of bacteria belonging to subgingival plaque bacterial complexes was higher in cases (573.5) than in controls (249) $(p$-value $=$ 0.0206).

Conclusions: This study identified periodontopathogens in blood of patients with coronary artery disease. The list of microorganisms presented only in cases will be key to identify new periodontopathogens that can relate periodontitis and this cardiovascular disease. SGO received funding by CONADI-Universidad Cooperativa de Colombia, grant 1839 .

A. Suarez-Molina: None. C. Fong: None. L. CifuentesC: None. S. Guauque-Olarte: None.

P05.30.C

Molecular genetic screening in patients with spontaneous coronary artery dissection

M. Antonutti ${ }^{1}$, F. Baldan $^{2}$, L. Spedicato ${ }^{1}$, D. Zanuttini ${ }^{l}, T$.

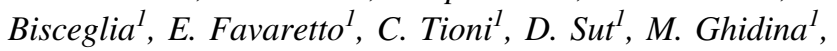

C. Lanera $^{3}$, D. Gregori ${ }^{3}$, D. Fabbro ${ }^{1}$, G. Damante ${ }^{\text {, }}$, A. Proclemer ${ }^{1}$

${ }^{1}$ ASUFC, Udine, Italy, ${ }^{2}$ University of Udine, Udine, Italy, ${ }^{3} \mathrm{DCTVPH}$, Padova, Italy

Spontaneous coronary artery dissection (SCAD) is increasingly recognized as an important cause of myocardial infarction, particularly in women often with few cardiovascular risk factors. SCAD is defined as a spontaneous tear in the coronary arterial wall that is not traumatic or iatrogenic. Studies are still underway to investigate the role of genetics in promoting SCAD; genetic diseases frequently associated with SCAD are connective tissue diseases. Considering the role of SCAD for sudden cardiac death and the increased risk for future cardiac events, an early diagnosis is crucial. We enrolled 60 patients with nonatherosclerotic angiographic diagnosis of SCAD. All patients' genomic DNA was analyzed by NGS with gene panel involved in collagenopathies and arteriopathies (ACTA2, CBS, COL3A1, COL5A1, COL5A2, ELN, FBN1, FBN2, LTBP2, MYLK, NOTCH1, MYH11, SMAD3, SMAD4, SLC2A10, TGFB2, TGFB3, TGFBR1, TGFBR2, $P L O D 1)$. Seven patients result to carry potential SCADrelated mutations. In particular, we observed a pathological COL3A1 mutation; 3 different VUS mutations in: LTBP2 (two patients), MYLK and NOTCH1. Moreover, we found a new nonsense mutation in $S M A D 3$ and a missense alteration in COL5A2; both not enlisted in public database, but that could be categorized as pathogenetic and VUS, respectively, according to the ACMG classification. These data support the use of sequencing of genes inclusive of collagenopathies and arteriopathies as an approach to evaluate genetically mediated SCAD. However, considering that only a minority of patients have clear SCAD-causative mutations, these results remark the need of further studies to define genetic mechanisms of SCAD.

M. Antonutti: None. F. Baldan: None. L. Spedicato: None. D. Zanuttini: None. T. Bisceglia: None. E. Favaretto: None. C. Tioni: None. D. Sut: None. M. Ghidina: None. C. Lanera: None. D. Gregori: None. D. Fabbro: None. G. Damante: None. A. Proclemer: None.

\section{P05.32.B}

Comparison of variant detection rate in $\mathbf{1 0 0}$ candidate genes between two representative cohorts of Czech living patients versus victims of sudden cardiac death with clinical. or post mortem diagnosis of dilated-, arrhythmogenic- and hypertrophic cardiomyopathy.

$$
\begin{aligned}
& \text { A. Krebsova }{ }^{1}, \text { P. Votýpka }{ }^{2}, \text { P. Peldová }{ }^{2}, K \text {. Ruecklova }{ }^{3} \text {, } \\
& \text { V. Zoubkova }{ }^{2} \text {, S. Pohlova-Kucerova }{ }^{4} \text {, A. Pilin }{ }^{5}, \text { M. } \\
& \text { Kulvajtova }{ }^{6}, \text { M. Kubanek }{ }^{1} \text {, M. Bilek }{ }^{5} \text {, T. Tavacova }{ }^{7} \text {, }
\end{aligned}
$$


J. Petrkova ${ }^{8}$, M. Dobias ${ }^{9}$, P. Tomasek ${ }^{10}$, M. Macek Sr. ${ }^{2}$, M. Macek Jr. ${ }^{2}$, J. Janousek ${ }^{7}$, J. Kautzner ${ }^{1}$

${ }^{1}$ Institute for Clinical and Experimental Medicine, Prague, Czech Republic, ${ }^{2}$ Department of Biology and Medical Genetics, 2nd Faculty of Medicine, Charles University and Motol University Hospital, Prague, Czech Republic, ${ }^{3}$ Paediatric Department, 3rd Faculty of Medicine, Charles University, Prague, Czech Republic, ${ }^{4}$ Department of Forensic Medicine, Faculty of Medicine in Hradec Králové, Charles University, Hradec Kralove, Czech Republic, ${ }^{5}$ Institute for forensic medicine and toxicology, 1st Faculty of Medicine, Charles University, Prague, Czech Republic, ${ }^{6}$ Institute for Forensic Medicine, 3rd Faculty of Medicine, Charles University, Prague, Czech Republic, ${ }^{7}$ Children's Heart Centre, 2nd Faculty of Medicine, Charles University and Motol University Hospital, Prague, Czech Republic, ${ }^{8}$ Department of Cardiology, Faculty Hospital Olomouc and Palacký University, Olomouc, Czech Republic, ${ }^{9}$ Institute for Forensic Medicine, Faculty Hospital Olomouc, Olomouc, Czech Republic, ${ }^{10}$ Institute for Forensic Medicine, Hospital Bulovka, 2nd Faculty of Medicine, Charles University, Prague, Czech Republic

Introduction: Hereditary cardiomyopathy is associated with risk of sudden cardiac death (SCD). Genetic aetiology of SCD in a representative Czech cohort with post mortem diagnosis of cardiomyopathy was studied and results compared to those in living cases.

Patients and Methods: Altogether 47 victims of SCD with post mortem diagnosis of hypertrophic- (HCM; 18/47), arrhythmogenic- (ACM; 19/47) and dilated cardiomyopathy (DCM; 10/47) were ascertained. Concurrently, genetic testing was performed in 114 living patients (HCM 54/ 114, ACM 22/114, DCM 38/114). NGS (MiSeq platform; Illumina.com) was utilised for a custom-made panel comprising 100 candidate genes (Sophia Genetics, Switzerland).

Results: The causative detection rate (according to ACMG.net classes 4 or 5) in SCD victims with DCM was $60 \%(6 / 10)$ and in living patients with DCM $47.4 \%$ (18/ 38). Variants in TTN, RBM20, DES and FLNC prevailed. Detection rate in ACM was $5 \%$ (1/19 in SCN5A gene) in SCD victims and $31.8 \%(7 / 22)$ [JJ1] in living patients. The detection rate in SCD victims with HCM was $16 \%(3 / 18)$ and in living patients $35 \%$ (19/54). The most prevalent gene was $M Y B P C 3$, while PRKAG2 was detected in SCD victim and in cardiac arrest survivor.

Conclusion: Post-mortem genetic analysis in DCM yields a high detection rate and allows effective primary prevention of SCD in at risk relatives. In contrast, molecular autopsy of HCM and ACM renders a much lower yield which could reflect more complex genetic aetiology in SCD. Supported by Ministry of Health of the Czech Republic, grant Nr. NV18-02-00237 and 00064203/6003

A. Krebsova: None. P. Votýpka: None. P. Peldová: None. K. Ruecklova: None. V. Zoubkova: None. S. Pohlova-Kucerova: None. A. Pilin: None. M. Kulvajtova: None. M. Kubanek: None. M. Bilek: None. T. Tavacova: None. J. Petrkova: None. M. Dobias: None. P. Tomasek: None. M. Macek Sr.: None. M. Macek Jr.: None. J. Janousek: None. J. Kautzner: None.

\section{P05.33.C}

Genome wide association analysis in dilated cardiomyopathy revealed two new susceptibility loci for systolic heart failure

S. Garnier ${ }^{1,2}$, M. Harakalova ${ }^{3,4}$,S. Weiss ${ }^{5,6}$, M. Mokry ${ }^{3,7,8}$, J. van Setten ${ }^{3}$, C. Proust ${ }^{9}$, L. Duboscq-Bidot ${ }^{1,2,10}$, A. Boland $^{11,12}$, J. Deleuze $e^{11,12,13}$, M. Dörr ${ }^{6,14}, F$. Asselbergs $^{3,15,16}$, F. Cambien ${ }^{9}$, E. Villard ${ }^{1,2,10}$, D. Trégouët ${ }^{9}$, P. Charron ${ }^{1,2,10,17}$

${ }^{1}$ Sorbonne Université, INSERM, UMR-S1166, research unit on cardiovascular disorders, metabolism and nutrition, Team Genomics \& Pathophysiology of Cardiovascular Diseases, Paris, France, ${ }^{2}$ ICAN Institute for Cardiometabolism and Nutrition, Paris, France, ${ }^{3}$ Department of Cardiology, Division Heart \& Lungs, University Medical Center Utrecht, Utrecht University, Utrecht, Netherlands, ${ }^{4}$ Regenerative Medicine Utrecht (RMU), University Medician Center Utrecht, Utrecht, Netherlands, ${ }^{5}$ Interfaculty Institute for Genetics and Functional Genomics, Department of Functional Genomics, University Medicine Greifswald, Greifswald, Germany, ${ }^{6} \mathrm{DZHK}$ (German Centre for Cardiovascular Research), partnersite Greifswald, Greifswald, Germany, ${ }^{7}$ Laboratory of Clinical Chemistry and Haematology, University Medical Center, Heidelberglaan 100, Utrecht, Netherlands, ${ }^{8}$ Laboratory for Experimental Cardiology, University Medical Center Utrecht, Heidelberglaan 100, Utrecht, Netherlands, ${ }^{9}$ INSERM UMR_S 1219, Bordeaux Population Health Research Center, University of Bordeaux, Bordeaux, France, ${ }^{10}$ APHP, Pitié-Salpêtrière Hospital, Cardiology Department, Paris, France, ${ }^{11}$ Centre National de Recherche en Génomique Humaine (CNRGH), Institut de Biologie François Jacob, CEA, Université ParisSaclay, Evry, France, ${ }^{12}$ Laboratory of Excellence GENMED (Medical Genomics), Paris, France, ${ }^{13}$ Centre d'Etude du Polymorphisme Humain, Fondation Jean Dausset, Paris, France, ${ }^{14}$ Department of Internal Medicine B, University Medicine Greifswald, Greifswald, Germany, ${ }^{15}$ Institute of Cardiovascular Science, Faculty of Population Health Sciences, University College London, London, United Kingdom, ${ }^{16}$ Health Data Research UK and Institute of Health Informatics, University College London, London, 
United Kingdom, ${ }^{17} A P-H P$, Département de génétique, Centre de Référence Maladies Cardiaques Héréditaires, Hôpital Pitié-Salpêtrière, Paris, France

We conducted the largest Genome Wide Association Study performed so far in Dilated Cardiomyopathy (DCM), a leading cause of systolic heart failure and cardiovascular death. Using a discovery phase of 2,719 cases and 4,440 controls and a replication phase of 584 independent cases and 966 controls, we identified and replicated two new DCM-associated loci one on chromosome $3 p$ (meta-analysis $\mathrm{p}=5.310^{-13}$ ) and the second on chromosome $22 \mathrm{q}$ (metaanalysis $\mathrm{p}=5.010^{-10}$ ) while confirming the two previously identified DCM loci on chromosome 10 and 1, BAG3 and HSPB7 for an estimated heritability of $31 \% \pm 8 \%$. The genetic risk score constructed from the number of lead riskalleles at these 4 loci revealed a $27 \%$ risk increased in individuals with 8 risk-alleles compared to the 5 risk alleles reference group. The two association signals were then finemapped by combining in silico and functional genomics investigations. While a few genes remain candidates at the second locus and deserve further investigations, our work clearly identified one gene as responsible for the association at the first locus whose role in the pathophysiology of DCM is supported by recent observations in human and mice. As the biological pathway in which this gene is involved is a potential target for pharmacological agents, our finding opens novel therapeutic perspectives for treating or preventing heart failure. These results provide new findings that add both on the understanding of the genetic architecture of heart failure and on potential new players involved in the pathophysiology of this devastating disease.

S. Garnier: None. M. Harakalova: None. S. Weiss: None. M. Mokry: None. J. van Setten: None. C. Proust: None. L. Duboscq-Bidot: None. A. Boland: None. J. Deleuze: None. M. Dörr: None. F. Asselbergs: None. F. Cambien: None. E. Villard: None. D. Trégouët: None. P. Charron: None.

\section{P05.34.A}

\section{Utility of genetics for risk stratification in paediatric} dilated cardiomyopathy

J. C. Herkert ${ }^{1}$, M. H. van der Meulen ${ }^{2}$, S. L. den Boer ${ }^{2}$, G. J. du Marchie Sarvaas ${ }^{3}$, N. Blom ${ }^{4,5}$, A. D. J. ten Harkel ${ }^{4}, H$. M. P. J. Breur ${ }^{6}$, L. A. J. Rammeloo ${ }^{7}$, R. Tanke ${ }^{8}$, C. Marcelis $^{9}$, I. M. B. H. van de Laar ${ }^{10}$, J. M. A. Verhagen ${ }^{10}$, A. Baas ${ }^{11}$, A. Sammani ${ }^{11}$, I. Christiaans ${ }^{1}$, D. Q. C. M. Barge-Schaapveld $^{12}$, R. H. Lekanne dit Deprez ${ }^{13}$, J. P. van Tintelen $^{11}$, M. Dalinghaus ${ }^{2}$

${ }^{1}$ University of Groningen, University Medical Center Groningen, Department of Genetics, Groningen,
Netherlands, ${ }^{2}$ Erasmus MC, University Medical Center Rotterdam, Department of Paediatric cardiology, Rotterdam, Netherlands, ${ }^{3}$ University of Groningen, University Medical Center Groningen, Department of Paediatric cardiology, Groningen, Netherlands, ${ }^{4}$ University of Leiden, Leiden University Medical Center, Department of Paediatric cardiology, Leiden, Netherlands, ${ }^{5}$ Amsterdam University Medical Center, location AMC, Department of Paediatric cardiology, Amsterdam, Netherlands, ${ }^{6}$ University of Utrecht, Wilhelmina Children's Hospital, University Medical Center Utrecht, Department of Paediatric cardiology, Utrecht, Netherlands, ${ }^{7}$ Amsterdam University Medical Center, location Free University Medical Center, Department of Paediatric cardiology, Amsterdam, Netherlands, ${ }^{8}$ Radboud University Medical Center, Department of Paediatric cardiology, Nijmegen, Netherlands, ${ }^{9}$ Radboud University Medical Center, Department of Genetics, Nijmegen, Netherlands, ${ }^{10}$ Department of Clinical Genetics, Erasmus MC, University Medical Center Rotterdam, Rotterdam, Netherlands, ${ }^{11}$ University of Utrecht, University Medical Center Utrecht, Department of Genetics, Utrecht, Netherlands, ${ }^{12}$ University of Leiden, Leiden University Medical Center, Department of Clinical Genetics, Leiden, Netherlands, ${ }^{13}$ Amsterdam University Medical Center, location AMC, Department of Clinical Genetics, Amsterdam, Netherlands

Introduction: Dilated cardiomyopathy (DCM) in children may have an underlying genetic cause in a substantial number of cases. We describe the current practice and results of genetic evaluation in children with DCM and evaluate genotype-phenotype correlations that may guide prognosis.

Methods: We performed a multicentre prospective observational study in children diagnosed with DCM from 2010-2017.

Results: One hundred forty-four patients were included. Initial diagnostic categories consisted of idiopathic DCM in 67 children (47\%), familial in 18 (13\%), neuromuscular in 7 $(5 \%)$, inborn error of metabolism in $4(3 \%)$, malformation syndrome in $2(1 \%)$, myocarditis in $23(16 \%)$ and 'other' in $23(16 \%)$. Median follow-up time was 2.1 years [IQR 1.04.3]. Hundred-seven patients (74\%) underwent genetic testing. A likely pathogenic (LP) or pathogenic (P) variant was found in 39 children (36\%); the majority in $M Y H 7$ ( $n=$ 9). Three patients had more than one LP/P variant. In at least 5/39 patients $(13 \%)$ the variant occurred de novo. During the study, 39 patients $(27 \%)$ reached a study endpoint (SE: all-cause death or heart transplantation). Transplant-free survival was significantly lower in patients with a LP/P variant $(P=0.005)$. Children who carried a LP/ $\mathrm{P}$ variant were 2.8 times more likely to reach a SE compared to children without, while clinical characteristics at 
diagnosis did not differ (hazard ratio 2.8; 95\% CI 1.3-5.8, $P=0.007$ ).

Conclusions: Genetic testing is useful to predict clinical outcome in children with DCM and should be incorporated into the initial work-up. Patients with a LP/P variant have a poorer prognosis than patients without LP/P variant.

J.C. Herkert: None. M.H. van der Meulen: None. S.L. den Boer: None. G.J. du Marchie Sarvaas: None. N. Blom: None. A.D.J. ten Harkel: None. H.M.P.J. Breur: None. L.A.J. Rammeloo: None. R. Tanke: None. C. Marcelis: None. I.M.B.H. van de Laar: None. J.M.A. Verhagen: None. A. Baas: None. A. Sammani: None. I. Christiaans: None. D.Q.C.M. Barge-Schaapveld: None. R.H. Lekanne dit Deprez: None. J.P. van Tintelen: None. M. Dalinghaus: None.

\section{P05.35.B}

Non-segregation of truncating $T T N$ variants in families with dilated cardiomyopathy (DCM)

\section{A. G. Fleming, L. Brett, S. Wilkinson, S. John, L. Briggs, M. Edwards, D. J. Morris-Rosendahl}

\section{Clinical Genomics Laboratory, London, United Kingdom}

DCM is a highly heterogeneous condition with over 30 genes implicated in inherited forms, including TTN. TTN truncating variants are found in $\sim 2 \%$ of the general population (Roberts et al. (2015) Sci Transl Med. 7(270); 270) but there is a higher association with DCM for truncating variants in cardiac isoforms of titin; therefore, only truncating variants in exons which are spliced into $>90 \%$ of TTN cardiac transcripts are reported as potentially pathogenic in DCM by our laboratory. We have identified TTN truncating variants that have been classified as either likely pathogenic or pathogenic in 82 of 613 DCM patients in our cohort. Due to the prevalence of TTN truncations in healthy individuals, predictive testing for truncating TTN variants is offered with caution, and instead segregation testing in affected relatives is first undertaken. Within our cohort we have found several variants which do not segregate with DCM. Out of a total of 36 individuals, across 25 families, we have 5 cases of non-segregation of truncating TTN variants; 3 frameshift, 1 splice site and one exonic deletion. All of these non-segregating variants are absent from populations databases and occur in exons which are spliced into $100 \%$ of cardiac TTN isoforms; and were therefore initially classified as likely pathogenic. These findings highlight the importance of a cautious approach when interpreting TTN truncating variants, as well as the risks of predictive testing; especially if this results in clinical discharge for individuals who do not carry the familial truncating $T T N$ variant.
A.G. Fleming: None. L. Brett: None. S. Wilkinson: None. S. John: None. L. Briggs: None. M. Edwards: None. D.J. Morris-Rosendahl: None.

\section{P05.37.A}

Optimization and refinement of the diagnosis of genetic dyslipidemias by targeted NGS

\section{Zuurbier, O. R. F. Mook, M. A. Haagmans, J. C. Defesche}

Amsterdam University Medical Centers, Amsterdam, Netherlands

Introduction: Genetic and acquired dyslipidemias are very frequent in the general population and strongly associated with premature atherosclerosis, myocardial infarctions, arterial complications and sudden death at young age. Genetic lipoprotein abnormalities can be explained by a limited number of genes. For optimal clinical management, risk assessment and prevention of cardiovascular disease an accurate diagnosis is an absolute requirement, but the interconnection between the metabolic pathways of different lipoprotein fractions jeopardizes this when only lipid levels are assessed.

Materials and Methods: In order to pinpoint the culprit lipoprotein fraction of clinical dyslipidemia in 4277 patients, we evaluated NGS and CNV data, including 29 dyslipidemia-associated genes that enabled to examine all separate lipoprotein components simultaneously. With this approach 10 types of dyslipidemia can be discriminated. Moreover, this panel includes a genetic risk factor for statin intolerance.

Results: A genetic cause for dyslipidemia was found in $32 \%$ of the patients. Of these, $4 \%$ carried a CNV. The specific initial clinical diagnoses of hypercholesterolemia or hypertriglyceridemia were confirmed in only 17 and $35 \%$ of patients, respectively. However, unsolicited findings causal for a second or an unexpected type of dyslipidemia, were encountered in $17 \%$ of cases, and suggest a broader role than initially anticipated for variants causing dysbetalipoproteinemia or hypertriglyceridemia.

Conclusions: The use of the NGS-Dyslipidemia gene panel accurately separated genetic from acquired dyslipidemias and within the detected genetic dyslipidemias the diagnosis was refined based on unsolicited findings in a substantial number of patients. This allows targeted and optimal treatment to reduce cardiovascular risk.

L. Zuurbier: None. O.R.F. Mook: None. M.A. Haagmans: None. J.C. Defesche: None. 


\section{P05.38.B}

Hypermethylation of NRG1 gene correlates with the presence of heart defects in Down's syndrome

\section{A. Dobosz, A. Grabowska, M. Bik-Multanowski}

Department of Medical Genetics, Jagiellonian University Medical College, Krakow, Poland

Congenital heart defects can decrease the quality of life and life expectancy in affected individuals, and constitute a major burden for the health care systems. Endocardial cushion defects are among the most prevalent heart malformations in the general population, and are extremely frequent (approximately a 100-fold higher prevalence) in children with Down syndrome. Several genes have been proposed to be involved in the pathogenesis of these malformations, but no common pathogenic DNA variants have been identified so far. Here, we focussed on constitutive, epigenetic alterations of function of selected genes, potentially important for endocardial cushion development. We used two types of microarrays, dedicated for assessment of gene promoter methylation and whole genome expression.

First, we compared the gene promoter methylation profiles between two groups of Down syndrome patients, with and without heart defects of endocardial cushion-type. Then, to determine the functional role of the detected methylation alterations, we assessed the expression of the genes of interest. We detected significant hypermethylation of the NRG1 gene promoter region in children with heart defects. NRG1 is a key factor in maturation of endocardial cushions. Supplementary gene expression assessment revealed significantly decreased activity of the ERBB3, SHC3 and SHC4 genes in children with heart defects. The above three genes are closely related to the NRG1 gene and are crucial elements of the NRG/ErbB pathway. The results of this pilot study show that hypermethylation of the NRG1 gene promoter can reflect the functional genome alteration contributing to development of congenital heart defects of endocardial cushion-type.

A. Dobosz: None. A. Grabowska: None. M. BikMultanowski: None.

P05.39.C

How Effective is Genetic Testing for Familial Hypercholesterolaemia Patients in Tayside?

\section{Leung, E. Gellatly, J. Berg}

Ninewells Hospital and Medical School, University of Dundee, Dundee, United Kingdom

Background: DNA analysis for Familial
Hypercholesterolaemia (FH), including the LDLR, PCSK 9 and $A p o E$ genes, is available to Scottish individuals with high cholesterol who fulfil Simon Broome criteria. The aim of mutation identification is to allow more effective management of the proband, as well as cascade testing and identification of at risk individuals. The ultimate aim is early statin treatment of affected individuals with prevention of cardiovascular morbidity.

Method: 60 index patients with causative $L D L R$ mutations were identified between 2010 and 2018 in NHS Tayside (Scotland). We used record linkage to assess the effect of genetic testing on cholesterol levels on probands and the number of subsequent cascade tests performed on family members. The main endpoint we used was achieving a $50 \%$ reduction of LDL cholesterol levels, as recommended by the UK National Institute of Clinical Excellence (NICE).

Results: 49/189 (25.9\%) eligible first degree relatives received cascade testing. Where data was available, 6/22 (27.3\%) patients met the 50\% reduction target in LDL-C levels. There was no demonstrable relationship between cholesterol reduction and attendance at a cardiovascular risk clinic.

Conclusions: The number of cascade tests following diagnosis in a proband is low. Even if a causative $L D L R$ mutation was identified, there was limited impact on the clinical improvement of cholesterol levels, with only a minority of patients achieving recommended cholesterol lowering following diagnosis. A better mechanism for cascade testing and management of $\mathrm{FH}$ is required to gain the maximum clinical benefit from DNA analysis in Familial Hypercholesterolaemia.

C. Leung: None. E. Gellatly: None. J. Berg: None.

\section{P05.41.A}

Mutation spectrum and polygenic score in German patients with familial hypercholesterolemia

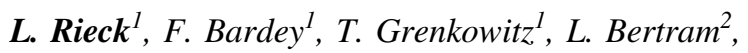

J. Helmuth ${ }^{3}$, C. Mischung ${ }^{3}$, J. Spranger ${ }^{1}$, T. Bobbert ${ }^{1}$, U. Kassner ${ }^{1}$, I. Demuth ${ }^{1}$

${ }^{l}$ Charité Universitätsmedizin Berlin, Berlin, Germany, ${ }^{2}$ Lübeck Interdisciplinary Platform for Genome Analytics, Institutes of Neurogenetics and Cardiogenetics, Lübeck, Germany, ${ }^{3}$ Department Molecular Diagnostics, Labor Berlin, Berlin, Germany

Introduction:Autosomal-dominant familial hypercholesterolemia $(\mathrm{FH})$ is a genetic disorder characterized by increased plasma concentrations of LDL-Cholesterol and a substantially elevated risk for cardiovascular disease. Mutations in three major genes are known to cause FH: the 
$L D L$ receptor gene $(L D L R)$, the apolipoprotein $B$ gene $(A P O B)$ and the proprotein convertase subtilisin/kexin 9 gene (PCSK9). Our aim is to analyze the mutation spectrum and to determine a polygenic risk score in a cohort of hypercholesterolemic patients.

Methods: We screened 336 patients who were clinically assessed by the Dutch Lipid Clinic Network (DLCN) score and suspected to have $\mathrm{FH}$ for disease causing variants and genotyped six single nucleotide polymorphisms (SNPs) to calculate the polygenic risk score for the $\mathrm{FH}$ patients and a control group of 1,946 healthy participants.

Results: We identified an FH causing variant in 117 of the 336 patients (12 novel variants) from the FH cohort. Most sequence variants were found in the LDLR gene $(84.9 \%)$ out of which $44.4 \%$ were of the missense type. The discriminatory power of the DLCN score distinguished FH mutation carriers from non-carriers with an $\mathrm{AUC}=0.76(p$ $<.001$ ) in a receiving operator characteristic (ROC) curve, similar to the value for native LDL-C (AUC $=0.77$ ( $p<$ $.001)$ ). Similar to previous studies, the mean polygenic risk score was significantly higher in $\mathrm{FH}$ mutation negative subjects than in $\mathrm{FH}$ mutation positive patients $(p<.05)$ and healthy controls $(p<.001)$.

Conclusions: This study substantiates the known clinical and genetic variability of $\mathrm{FH}$ for German hypercholesterolemic patients and examines a possible polygenic aetiology.

L. Rieck: None. F. Bardey: None. T. Grenkowitz: None. L. Bertram: None. J. Helmuth: None. C. Mischung: None. J. Spranger: None. T. Bobbert: None. U. Kassner: None. I. Demuth: None.

\section{P05.43.A}

FLNC gene in Hypertrophic Cardiomyopathy: an effort to accurate variant classification

E. Cuesta-Llavona, R. Lorca, B. Díaz-Molina, J. Lambert, B. Alonso, S. Iglesias-Álvarez, J. Reguero, E. Coto, J. Gómez

\section{Hospital Universitario Central de Asturias, Oviedo,} Spain

Hypertrophic Cardiomyopathy (HCM) is the most common inherited heart disease. Recent studies have identified the Filamin C $(F L N C)$ gene as a HCM-associated gene. The aim of this study is to assign the percentage of HCM cases which could be relationated with $F L N C$ variants.

We sequenced 924 index cases of HCM for a comprehensive cardiovascular gene panel of 194 genes, in an Ion GeneStudio S5 Sequencer. We identified 365 patients (39\%) with variants in the sarcomeric and FLNC genes. Among them, $330(90 \%)$ were carriers of sarcomeric variants, and $35(10 \%)$ harbored rare $F L N C$ variants (either not described or $\leq 10$ cases in gnomAD). Eleven patients were carriers of both sarcomeric and FLNC variant, thus their were excluded to the further analysis and variant classification since their pathogenicity is complex to measure. In this way, we finally selected 24 carriers of 20 different FLNC variants. Family studies were performed in $16 / 24$ cases. In total, we identified 55 relatives who harbor a FLNC variant, thirty nine of them have a HCM diagnosis (71\% penetrance). According to the ACMG criteria, and the performed family studies, seven variants $(35 \%)$ have been classified as pathogenic or likely pathogenic, twelve as variants of unknown significance $(60 \%)$, and a variant classified as benign or likely benign $(5 \%)$. In conclusion, at least $1.2 \%$ of HCM index cases harbor a FLNC likely pathogenic variant. However, further co-segregation and functional studies have to be performed in order to accomplish an accurate variant classification, specially in VUS variants.

E. Cuesta-Llavona: None. R. Lorca: None. B. DíazMolina: None. J. Lambert: None. B. Alonso: None. S. Iglesias-Álvarez: None. J. Reguero: None. E. Coto: None. J. Gómez: None.

\section{P05.44.B}

A founder truncating mutation in GDF1 causes autosomal-recessive right isomerism in multiplex Arab kindreds.

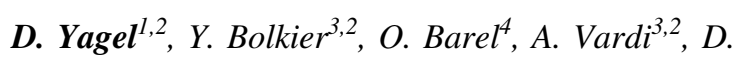
Mishali $^{3,2}$, A. Veber ${ }^{1}$, N. Shalva ${ }^{1}$,U. Katz ${ }^{3,2}$, S. Abudi ${ }^{1,2}, N$. $\mathrm{Kol}^{4}$, A. Raas-Rothschild ${ }^{5,2}$, G. Rechavi, ${ }^{4,2,6}$, B. PodeShakked $^{1,7,2}$, Y. Anikster ${ }^{1,2,6}$

${ }^{1}$ Metabolic Disease Unit, Edmond and Lily Safra Children's Hospital, Sheba Medical Center, Ramat Gan, Israel, ${ }^{2}$ Sackler Faculty of Medicine, Tel-Aviv University, Tel-Aviv, Israel, ${ }^{3}$ Department of Pediatric Cardiac Intensive Care, Edmond Safra International Congenital Heart Center, Edmond and Lily Safra Children's Hospital, Sheba Medical Center, Ramat Gan, Israel, ${ }^{4}$ Sheba Cancer Research Center, Sheba Medical Center, Ramat Gan, Israel, ${ }^{5}$ The Institute for Rare Diseases, The Danek Gertner Institute of Human Genetics, Sheba Medical Center, Ramat Gan, Israel, ${ }^{6}$ The Wohl Institute for Translational Medicine, Sheba Medical Center, Ramat Gan, Israel, ${ }^{7}$ Talpiot Medical Leadership Program, Sheba Medical Center, Ramat Gan, Israel

The genetic basis of congenital heart malformations associated with disruption of left-right (L-R) asymmetry is broad and heterogenous, with variants in over twenty five genes implicated thus far. Of these, deleterious mutations in 
the Growth/Differentiation Factor $1(G D F 1)$ gene have recently been shown to cause heterotaxy with varied complex heart malformations of left-right patterning, in 23 individuals reported to date, either in monoallelic or biallelic state. We report three unrelated individuals exhibiting heterotaxy with congenital heart defects, each originating from consanguineous kindreds of Arab-Muslim descent. Using Whole exome sequencing, a shared novel homozygous truncating c.608G $>$ A (p.W203*) mutation in the GDF1 gene, was revealed as the molecular basis of their disease. Subsequently, targeted sequencing of this variant showed full segregation with the disease in these families, with over 15 reportedly affected individuals, enabling genetic counselling, prenatal diagnosis and planning of future pregnancies. Our findings further confirm the association of GDF1 variants, heterotaxy and congenital heart defects of left-right patterning, and expand the previously described phenotypic spectrum and mutational profile. Finally, given the high rate of consanguinity and high frequency of the p.W203* mutation in the Arab-Muslim population residing near the city of Hebron, we suggest targeted screening for this variant in relevant clinical circumstances.

D. Yagel: None. Y. Bolkier: None. O. Barel: None. A. Vardi: None. D. Mishali: None. A. Veber: None. N. Shalva: None. U. Katz: None. S. Abudi: None. N. Kol: None. A. Raas-Rothschild: None. G. Rechavi: None. B. Pode-Shakked: None. Y. Anikster: None.

\section{P05.46.A}

Pathogenic variationin the copper binding domain of lysyl oxidase causes aortic dilation modified by sex and mechanical forces

\section{K. Tsang ${ }^{1}$, R. H. Knutsen ${ }^{l}$, C. J. Billington $\mathrm{Jr}^{1}$, E. Lindberg', H. Steenbock ${ }^{2}$, A. Wardlaw-Pickett ${ }^{1}$, C. K. E. Bleck $^{l}$, J. Brinckmann ${ }^{2}$, B. A. Kozel ${ }^{l}$ \\ ${ }^{l}$ National Institutes of Health, Bethesda, MD, United States, ${ }^{2}$ University of Luebeck, Luebeck, Germany}

Mutations in lysyl oxidase $(L O X)$ contribute to familial thoracic aortic aneurysm. GnomAD reveals variation throughout $L O X$, with more variants reported in the region encoding the N-terminal pro-domain. While ClinVar showed disease-associated stopgain and frameshift variants throughout LOX, the majority of pathogenic missense mutations were identified near the copper binding domain, within the relatively variant-poor C-terminal catalytic domain.

We then studied theLox ${ }^{b 2 \mathrm{~b} 370.2 \mathrm{Clo}}$ (c.G854T; p.C285F) mouse whose mutationfalls within the copper binding domain. The Lox ${ }^{\mathrm{C} 285 \mathrm{~F}}$ protein was stably secreted but exhibited decreased enzymatic activity and altered elastin crosslinks. Structural analysisby two photon and scanning electron microscopyrevealed holes in the elastic lamellar sheets that increased with age and blood pressure. As in humans, the rate of aortic dilation was higher in males, but was also amplified by pregnancy. Lox mutants showed altered transcription of matrisome genes and their aortas showed increased susceptibility to elastase.

Taken together, these findings suggest that missense variation surrounding the LOX copper binding domain increases risk for aortic aneurysm while missense variation in the region encoding the N-terminal pro-domain appears largely tolerated. Novel gene-first studies are underway to test this hypothesis in a large hospital-based cohort. Our mouse studies confirm that copper binding domain LOX variants cause disease through the production of a dysfunctional extracellular matrix that is unusually susceptible to proteolytic damage. In both humans and mice, the progressive weakening of the connective tissue leads to worsening aortic disease, modified by sex and blood pressure.

Funding: NIH HL006244, USA

K. Tsang: None. R.H. Knutsen: None. C.J. Billington Jr: None. E. Lindberg: None. H. Steenbock: None. A. Wardlaw-Pickett: None. C.K.E. Bleck: None. J. Brinckmann: None. B.A. Kozel: None.

\section{P05.47.B}

Rare variants in HCN4 identified in the general population are associated with complete atrioventricular (AV) block, 1. degree AV block and bundle branch block, results from $\mathbf{5 0 . 0 0 0}$ exomes

\author{
J. S. Jensen ${ }^{1,2}$, O. B. Vad ${ }^{1,2}$, C. Paludan-Müller ${ }^{2,1}$, P. R. \\ Lundegaard $^{\text {, }}$ J. H. Svendsen ${ }^{2,3}$, S. Hauns $\phi^{2,3}, H$. \\ Bundgaard $^{2,3}$, M. S. Olesen ${ }^{1,2}$
}

${ }^{1}$ Department of Biomedical Sciences, Faculty of Health and Medical Sciences, University of Copenhagen, Copenhagen, Denmark, ${ }^{2}$ Laboratory for Molecular Cardiology, Department of Cardiology, Centre for Cardiac, Vascular, Pulmonary and Infectious Diseases, Rigshospitalet, Copenhagen University Hospital, Copenhagen, Denmark, ${ }^{3}$ Department of Clinical Medicine, Faculty of Health and Medical Sciences, University of Copenhagen, Copenhagen, Denmark

Introduction: Cardiac conduction disorders cover disorders such as atrioventricular (AV) block and bundle branch block (BBB). Genome-wide association studies have identified more than 100 genetic loci for atrial fibrillation, including HCN4 loci associated with duration of the PR interval, a proxy for AV dysfunction. Recent candidate 
studies suggest an association between HCN4 variants and AV-block. We seek to determine the contribution of rare genetic variants in $\mathrm{HCN} 4$ to complete AV-block, 1. degree AV-block and BBB in the general population.

Materials/methods: The UK Biobank is a populationbased study of 500000 individuals including a subset with genome-wide genotyping and exome sequencing $(n=$ 45,596). In this case-control study, we included persons of genetically determined white-European ancestry and analyzed their exome data. Analyses were performed using a logistic mixed-effects model. A gene-based burden analysis and single variant test were performed to examine the relationship between $\mathrm{HCN} 4$ variants and complete $\mathrm{AV}$ block, 1. degree AV-block and $\mathrm{BBB}$ in the general population.

Results: The study included 43,371 persons. In an analysis of the HCN4 genes a significant association between rare variants (MAF < 0.01) in HCN4 gene was found (complete AV-block, $\mathrm{P}=2.5 \times 10^{-5}$, 1. degree $\mathrm{AV}$ block, $\mathrm{P}=1.3 \times 10^{-3}$ and $\mathrm{BBB}, \mathrm{P}=0.01$ ). The association to complete AV block was mostly driven by the variants Ser835Phe $\left(\mathrm{P}=2.7 \mathrm{e}^{-3}\right)$, Glu153Gly $\left(\mathrm{P}=3.5 \mathrm{e}^{-3}\right)$ and $\operatorname{Arg} 378$ Cys $\left(\mathrm{P}=6.3 \mathrm{e}^{-3}\right)$.

Conclusions: Rare $\mathrm{HCN} 4$ variants contribute to the risk of complete AV-block, 1. degree AV-block and BBB in the general population. These HCN4 variants seem to confer a substantial penetrance. Clinical screening for some of these variants seems appropriate.

J.S. Jensen: None. O.B. Vad: None. C. PaludanMüller: None. P.R. Lundegaard: None. J.H. Svendsen: None. S. Haunsø: None. H. Bundgaard: None. M.S. Olesen: None.

\section{P05.49.A}

\section{MicroRNAs as biomarkers of heart failure}

\section{A. M. Peterlin ${ }^{1}$, K. Pocivavsek ${ }^{2}$, D. Petrovic ${ }^{1}$, B. Peterlin ${ }^{3}$}

${ }^{1}$ Institute of Histology and Embriology, Faculty of Medicine, University of Ljubljana, Ljubljana, Slovenia, ${ }^{2}$ Department of Cardiovascular Surgery, University Medical Centre Ljubljana, Ljubljana, Slovenia, ${ }^{3}$ Clinical Institute of Genomic Medicine, University Medical Centre Ljubljana, Ljubljana, Slovenia

Introduction: MicroRNAs are highly investigated for their role in the pathogenesis of cardiovascular diseases. Nevertheless, evidence for clinical implementation is still lacking. In our systematic review, we evaluated the potential of microRNA as pathophysiological and diagnostic biomarkers of heart failure.

Materials and methods: The literature search was conducted in the PubMed database up to August 2019.
Newcastle-Ottawa quality assessment scale was used to assess the quality of included research papers. Using miRTarBase, we identified all known gene targets for selected miRNAs that were differentially expressed in research papers included in the systematic review. We then comprised the list of all target genes and performed gene set enrichment analysis using Enrichr. Results from KEGG, BioPlanet, and Panther databases were analyzed.

Results: We identified 72 differentially expressed microRNA molecules among groups of heart failure patients and control groups. There was no substantial overlap of differentially expressed miRNAs among different studies, only five miRNAs (miR-1228, miR-122, miR-423-5p, miR$142-3 p$, and exosomal miR-92b-5p) were differentially expressed in more than one included study. Gene set enrichment analysis, based on the gene targets of microRNAs presented in included studies, showed that gene targets of differentially expressed miRNAs were enriched in MAPK signaling pathway, TGF $\beta$ signaling pathway, PI3KAkt signaling pathway, IL-2 signaling pathway, apoptosis pathway, p53 activity regulation, angiogenesis pathway, and PDGF signaling pathway.

Conclusions: Results of our systematic review show that currently, there is insufficient support for the use of any of the presented miRNAs as a pathophysiological or prognostic biomarker in the clinical setting.

A.M. Peterlin: None. K. Pocivavsek: None. D. Petrovic: None. B. Peterlin: None.

\section{P05.51.C}

Germline variants in transcription factor $H E Y 2$ functional domains lead to congenital heart defects and thoracic aortic aneurysms

E. S. van Walree ${ }^{1,2}$, G. Dombrowsky ${ }^{3}$, I. E. Jansen ${ }^{2}$, M. Umićević Mirkov', R. Zwart ${ }^{4}$, A. Ilgun $^{4}$, D. Guo ${ }^{5}$, S. B. Clur ${ }^{6}$, A. S. Amin ${ }^{7}$, J. E. Savage ${ }^{2}$, A. C. van der Wal ${ }^{8}$, Q. Waisfisz ${ }^{9}$, A. Maugeri ${ }^{9}$, A. Wilsdon ${ }^{10}$, F. A. Bu'lock ${ }^{11}$, M. E. Hurles ${ }^{12}$, S. Dittrich ${ }^{13}$, F. Berger ${ }^{14}$, E. Audain Martinez ${ }^{3}$, M. Hitz ${ }^{3}$, D. M. Milewicz ${ }^{5}$, D. Posthuma ${ }^{2}$, H. MeijersHeijboerl $^{1}$, A. V. Postma ${ }^{1,4}$, I. B. Mathijssen ${ }^{1}$

${ }^{1}$ Department of Clinical Genetics, Amsterdam UMC, University of Amsterdam, Meibergdreef 9, Amsterdam, Netherlands, ${ }^{2}$ Department of Complex Trait Genetics, Center for Neurogenomics and Cognitive Research, Amsterdam Neuroscience, Vrije Universiteit Amsterdam, de Boelelaan 1085, Amsterdam, Netherlands, ${ }^{3}$ Department of Congenital Heart Disease and Pediatric Cardiology, Universitätsklinikum Schleswig-Holstein Kiel, Kiel, Germany, ${ }^{4}$ Department of Medical Biology, Amsterdam UMC, University of Amsterdam, Meibergdreef 9, Amsterdam, Netherlands, ${ }^{5}$ Department of Internal Medicine, McGovern 
Medical School, University of Texas Health Science Center at Houston, 6431 Fannin, Houston, TX, United States, ${ }^{6}$ Department of Pediatric Cardiology, Emma Children's Hospital, Amsterdam UMC, University of Amsterdam, Meibergdreef 9, Amsterdam, Netherlands, ${ }^{7}$ Department of Clinical and Experimental Cardiology, Amsterdam UMC, University of Amsterdam, Meibergdreef 9, Amsterdam, Netherlands, ${ }^{8}$ Department of Pathology, Amsterdam UMC, University of Amsterdam, Meibergdreef 9, Amsterdam, Netherlands, ${ }^{9}$ Department of Clinical Genetics, Amsterdam UMC, Vrije Universiteit Medisch Centrum, de Boelelaan 1118, Amsterdam, Netherlands, ${ }^{10}$ School of Life Sciences, University of Nottingham, Queen's Medical Centre, Nottingham, United Kingdom, ${ }^{11}$ East Midlands Congenital Heart Centre and University of Leicester, Glenfield Hospital, Leicester, United Kingdom, ${ }^{12}$ Wellcome Trust Sanger Institute, Wellcome Trust Genome Campus, Hinxton, United Kingdom, ${ }^{13}$ Department of Pediatric Cardiology, University of Erlangen-Nürnberg, Loschgestrasse 15, 91054, Erlangen, Germany, ${ }^{14}$ German Heart Center Berlin, Department of Congenital Heart Disease, Pediatric Cardiology, Berlin, Germany

Background: Affecting approximately $1 \%$ of all newborns, cardiovascular defects (CVDs) are the most common congenital anomaly worldwide. Here, we present a large family from a genetically isolated population presenting with CVDs, including congenital heart defects (CHD) and familial thoracic aortic aneurysms (FTAA), caused by a loss-of-function mutation in the Notch target gene $H E Y 2$, which hasn't been associated with hereditary CVDs in humans previously.

Methods: The index patient, who has severe CVD, underwent trio WES. The identified variant was subsequently screened in 30 members of this patient's family, which has a high prevalence of CVDs. Additionally, 3011 unrelated cases and 4940 controls from two datasets (one with $\mathrm{CHD}$ and one with FTAA) were screened for rare risk variants in $H E Y 2$. To investigate how the various identified variants influence gene function, the regulatory activity of mutated and wildtype HEY2 on a target gene was measured with luciferase assays and qPCR.

Results: A homozygous frameshift variant (p.G108*) in HEY2 was identified in three individuals with lifethreatening CVDs. Twenty family members were found to be heterozygous for this variant, of whom $80 \%$ had CVD. Two additional CVD datasets show enrichment for rare, likely deleterious variants in $H E Y 2$ after meta-analysis. Lastly, we demonstrate that these variants induce failure of repression of $H E Y 2$ target genes.

Conclusions: We find that rare $H E Y 2$ variants predispose to a broad spectrum of CVDs and advise clinicians to consider $H E Y 2$ as a causative gene in unsolved CVD patients. Identifying a potentially deleterious variant in HEY2 warrants clinical follow-up.

E.S. van Walree: None. G. Dombrowsky: None. I.E. Jansen: None. M. Umićević Mirkov: None. R. Zwart: None. A. Ilgun: None. D. Guo: None. S.B. Clur: None. A. S. Amin: None. J.E. Savage: None. A.C. van der Wal: None. Q. Waisfisz: None. A. Maugeri: None. A. Wilsdon: None. F.A. Bu'lock: None. M.E. Hurles: None. S. Dittrich: None. F. Berger: None. E. Audain Martinez: None. M. Hitz: None. D.M. Milewicz: None. D. Posthuma: None. H. Meijers-Heijboer: None. A.V. Postma: None. I.B. Mathijssen: None.

\section{P05.52.A}

The Unknown Genetic Architecture of Severe Hypercholesterolemia in Finland

\section{N. Junna ${ }^{1}$, S. Ruotsalainen ${ }^{1}$, S. Ripatti ${ }^{1,2}$, E. Widén $^{1}$ \\ ${ }^{1}$ FIMM, Helsinki, Finland, ${ }^{2}$ Department of Public Health, Clinicum, Faculty of Medicine, University of Helsinki, Helsinki, Finland}

While severe hypercholesterolemia is a significant risk factor for ischemic heart disease (IHD), Finnish patients remain inadequately identified and only a part of their mutation load is known. To elucidate the genetic architecture of severe hypercholesterolemia in Finland, we have screened participants of the GeneRISK-study ( $n=7342$ ) originating from South-eastern Finland for novel mutations and utilized the FinnGen-cohort ( $n=110654$ ) to assess the mutation prevalence, impact, and nationwide distribution.

Five percent ( $n=366$ ) of GeneRISK-participants had severe hypercholesterolemia (LDL $\geq 5 \mathrm{mmol} / \mathrm{l}$ ) and exomesequencing revealed 3 carriers of previously known mutations ( $L D L R$ R594Q, LDLR P309Kfs and APOB $\mathrm{R} 3527 \mathrm{Q}$, which has not previously been reported in Finland). We also identified a novel, likely pathogenic mutation, LDLR R574L. We assessed the geographical distribution of the mutation and the cardiometabolic status of mutation carriers in the nationwide FinnGen-cohort and compared them with 5 previously described hypercholesterolemia-mutations (LDLR R594Q, LDLR G844D, LDLR L401H, LDLR D579N, LDLR C352W). The novel $L D L R$ R574L-mutation was unevenly distributed in the country with a high prevalence among individuals born in the Southeast (the ceded Karjala region) and prevalence of cardiovascular disease among mutation carriers was elevated $(22 \%$ had been diagnosed with IHD and $14 \%$ with stroke). The data further showed that less than half of mutation carriers (both the novel and previously known mutations) were on statin therapy. 
Our data show that the genetic spectrum of severe hypercholesterolemia in Finland is more diverse than previously anticipated, and further highlight the urgent need for better recognition and treatment of this disease.

N. Junna: None. S. Ruotsalainen: None. S. Ripatti: None. E. Widén: None.

\section{P05.53.B}

Mendelian randomisation identifies pleiotropy between uric acid and cardiovascular diseases

E. M. Wigmore ${ }^{l}$, O. Chazara ${ }^{l}$, . FinnGen Consortium ${ }^{2}, K$. Carss $^{l}$, A. Matakidou ${ }^{l}$, C. Haefliger ${ }^{l}$

${ }^{1}$ AstraZeneca, Cambridge, United Kingdom, ${ }^{2}$ University of Helsinki, Helsinki, Finland

Elevated levels of uric acid (UA), hyperuricaemia, leads to the formation of urate crystals that cause gout. Hyperuricaemia has also been linked to cardiovascular and metabolic diseases but, unlike gout, causality has not been ascertained. Here we utilised biomarker data from the UK Biobank (UKB, $n=\sim 500,000)$ and health outcome data from a Finnish biobank, FinnGen $(n=\sim 135,000)$ to explore the causal relationship between biomarkers and disease. Genome-wide association studies (GWAS) of 48 available biomarkers were conducted in UKB and FINEMAP was applied to ascertain putative causal variants. We assessed the genetic correlation of these biomarkers with 1,485 disease endpoints in FinnGen using LD score regression. Those with a significant correlation following FDR correction were assessed for causal associations using Mendelian randomisation (MR). GWAS of UA corrected for allopurinol treatment (a UA-lowering medication) was completed on 389,114 individuals and FINEMAP identified 320 putative causal signals. Significant genetic correlations were found with multiple diseases $(n=183)$ including hypertensive diseases $\left(\mathrm{rg}=0.25, \mathrm{P}_{\mathrm{FDR}}=6.2 \times 10^{-38}\right)$, type 2 diabetes $\left(\mathrm{rg}=0.29, \mathrm{P}_{\mathrm{FDR}}=6.2 \times 10^{-38}\right)$, cardiovascular disease $\left(\mathrm{rg}=0.23, \mathrm{P}_{\mathrm{FDR}}=9.6 \times 10^{-29}\right)$ and gout $(\mathrm{rg}=0.70$, $\left.\mathrm{P}_{\mathrm{FDR}}=8.3 \times 10^{-21}\right)$. Results from MR analyses confirmed the known causal association of UA with gout (IVW estimate $=1.6, \quad \mathrm{P}_{\mathrm{FDR}}=7.8 \times 10^{-62}$ ) as well as with polyarthropathies (IVW estimate $=0.37, \mathrm{P}_{\mathrm{FDR}}=1.9 \times 10^{-12}$ ) but not with other diseases. A significant causal association was found with hypertension (IVW estimate $=0.20$, $\left.\mathrm{P}_{\mathrm{FDR}}=0.0015\right)$ but horizontal pleiotropy was also detected (MR-Egger intercept $\mathrm{P}=0.0069$ ). Our finding suggests that although MR does not support the direct association between UA and cardiovascular disease, we observe pleiotropic effects which could support therapies targeting shared pathways.
E.M. Wigmore: A. Employment (full or part-time); Significant; AstraZeneca. O. Chazara: A. Employment (full or part-time); Significant; AstraZeneca. .. FinnGen Consortium: B. Research Grant (principal investigator, collaborator or consultant and pending grants as well as grants already received); Significant; AstraZeneca, Abbvie, Biogen, Celgene, Genentech, GSK, Merck, Pfizer, Sanofi. K. Carss: A. Employment (full or part-time); Significant; AstraZeneca. A. Matakidou: A. Employment (full or parttime); Significant; AstraZeneca. C. Haefliger: A. Employment (full or part-time); Significant; AstraZeneca.

\section{P05.55.A}

Pathogenic variant in the MYBPC3 gene with founding effect in Galician population

A. Blanco-Verea ${ }^{1,2}, R$. Cruz $^{3,2}$, M. Álvarez-Barredo ${ }^{4,1,5}$, B. López-Abel ${ }^{6,1}$, R. Gil ${ }^{1,2}$, E. Ramos-Luis ${ }^{1,2}$, L. MartínezCampelo $^{1,2}$, I. Quintela ${ }^{7,2}$, J. González-Juanatey ${ }^{4,5}$, Á. Carracedo $^{8,2,3,7}$, M. Brion ${ }^{1,2,5}$

${ }^{l}$ Xenética Cardiovascular, Instituto de Investigación Sanitaria de Santiago, Santiago de Compostela, Spain, ${ }^{2}$ Grupo de Medicina Xenómica, Universidade de Santiago de Compostela, Santiago de Compostela, Spain, ${ }^{3}$ CIBER Enfermedades Raras, Santiago de Compostela, Spain, ${ }^{4}$ Complejo Hospitalario Universitario de Santiago, Servicio de Cardiología, Santiago de Compostela, Spain, ${ }^{5}$ CIBER Cardiovascular, Santiago de Compostela, Spain, ${ }^{6}$ Complejo Hospitalario Universitario de Santiago, Servicio de Pediatría, Santiago de Compostela, Spain, ${ }^{7}$ Centro Nacional de Genotipado (CEGEN-PRB3-ISCIII), Santiago de Compostela, Spain, ${ }^{8}$ Fundación Pública Galega de Medicina Xenómica, Santiago de Compostela, Spain

Introduction: The Myosin Binding Protein C3 gene (MYBPC3) is one of the main genes in which mutations involved in familial hypertrophic cardiomyopathy (HCM) appear. One of these pathogenic variants is the deletion rs397515926 that appears in the gnome AD database in only one individual of Latino origin (Allele Frequency $=0.000004913$ ). However, we have found 18 unrelated patients with HCM from Galicia with this variant, which has led us to think about a possible founding effect of this variant in the Galician population.

Materials and Methods: Trying to unmask a possible founding effect or a distant family relationship between patients, we made a genotyping with the Applied Biosystems ${ }^{\mathrm{TM}}$ Axiom ${ }^{\mathrm{TM}}$ Spain Biobank Array in 6 of these patients and in a cohort of healthy Galician and Spanish population. Principal components analysis and discriminant analysis of principal components were performed in Plink and adegenet package of $\mathrm{R}$ software. 
Results: discriminant analysis of principal components taking into account rare variants of the array and comparing our patients with the general Spanish and Galician population clearly differentiate our patients with respect to the rest.

Conclusions: Considering the results of the analysis of the genotypes between our cases and the general population, the practically null bibliographic mention and very scarce presence in databases of this variant, we could assume a possible common origin for the presence of this variant in the Galician population, highlighting a possible founding effect. Founding: Instituto de Salud Carlos III and Fondo Europeo de Desarrollo Regional (PI16/00903, CB16/ 11/00226, CB03/07/0088).

A. Blanco-Verea: None. R. Cruz: None. M. ÁlvarezBarredo: None. B. López-Abel: None. R. Gil: None. E. Ramos-Luis: None. L. Martínez-Campelo: None. I. Quintela: None. J. González-Juanatey: None. Á. Carracedo: None. M. Brion: None.

\section{P05.56.B}

The potential diagnostic value of circulating serumderived miRNAs in ischemic heart disease (IHD) as a complication of type 2 diabetes mellitus (T2DM).

A. Bielska ${ }^{1}$, W. Bauer ${ }^{1,2}$, I. Sidorkiewicz ${ }^{1}$, A. Szalkowskal, J. Raczkowskal, D. Ostrowski ${ }^{1}$, P. Prokopczuk ${ }^{3}, S$. Dobrzycki ${ }^{3}$, M. Niemira ${ }^{1}$, A. Kretowski, ${ }^{1,4}$

${ }^{1}$ Clinical Research Centre, Medical University of Bialystok, Bialystok, Poland, ${ }^{2}$ Clinical Research Centre, Medical University of Bialystok, Biatystok, Poland, ${ }^{3}$ Department of Invasive Cardiology, Medical University of Bialystok, Bialystok, Poland, ${ }^{4}$ Department of Endocrinology, Diabetology and Internal Medicine, Medical University of Bialystok, Biatystok, Poland

Introduction: Currently, IHD is the most common cause of death in patients with diabetes and it develops much earlier before clinical diagnosis. Therefore, there is an urgent need for the detection of sensitive and specific early biomarkers to predict the risk of the IHD development. We postulate that non-coding miRNAs may have higher diagnostic value for early detection of IHD than inflammatory mediators like chemokine CXCL12 and macrophage migration-inhibitory factor (MIF).

Materials and Methods: We obtained serum samples from 43 T2DM patients (24 with IHD and 19 without IHD). The levels of 798 miRNAs were analysed using NanoString nCounter Technology. Additionally, we predicted the key miRNA regulatory network and miRNAs targets and pathways that may be critical in IHD development using the Ingenuity Pathway Analysis software. The concentration of MIF and CXCL12 was estimated by enzyme-linked immunosorbent assay. Receiver operating characteristic analysis was used to assess the diagnostic value of each miRNA.

Results: Our data showed 9 miRNAs (miRNA-1224-5p, miRNA-1303, miRNA-3147, miRNA-4455, miRNA-498, miRNA-548b-3p, miRNA-548d-3p, miRNA-615-3p, miRNA-651-5p) significantly upregulated in T2DM IHD group compared to T2DM noIHD (FC>1.4 and FDR < 0.05). Furthermore, all miRNAs showed high diagnostic value (AUC>0.85). Targeted genes were identified in canonical pathways involved in the pathology of the cardiovascular system. No statistical differences were observed in the concentration of MIF and CXCL12 between tested groups.

Conclusions: Our findings suggest that circulating miRNAs might have a pivotal role in the development of IHD in T2DM patients and may be used as non-invasively biomarkers for early diagnosis.

A. Bielska: None. W. Bauer: None. I. Sidorkiewicz: None. A. Szalkowska: None. J. Raczkowska: None. D. Ostrowski: None. P. Prokopczuk: None. S. Dobrzycki: None. M. Niemira: None. A. Kretowski: None.

\section{P05.57.C}

Derivation and validation of a polygenic risk score associated with ischemic heart failure in multiethnic cohorts

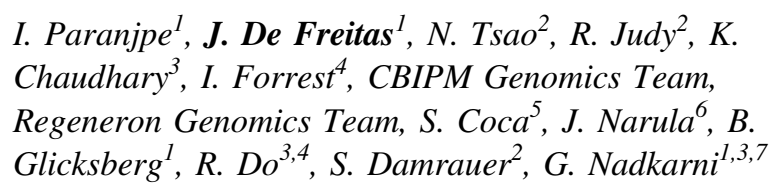

${ }^{1}$ The Hasso Plattner Institute for Digital Health at Mount Sinai, New York, NY, United States, ${ }^{2}$ Department of Surgery, Perelman School of Medicine, University of Pennsylvania, Philadelphia, PA, United States, ${ }^{3}$ The Charles Bronfman Institute for Personalized Medicine, Icahn School of Medicine at Mount Sinai, New York, NY, United States, ${ }^{4}$ Department of Genetics and Genomic Sciences, Icahn School of Medicine at Mount Sinai, New York, NY, United States, ${ }^{5}$ Division of Nephrology, Department of Medicine, Icahn School of Medicine at Mount Sinai, New York, NY, United States, ${ }^{6}$ Division of Cardiology, Department of Medicine, Icahn School of Medicine at Mount Sinai, New York, NY, United States, ${ }^{7}$ Renal Program, James J Peters VA Medical Center at Bronx, New York, NY, United States

Introduction: Mortality from heart failure (HF) secondary to coronary artery disease (CAD) is $50 \%$ and riskstratification approaches are limited. We aimed to stratify 
risk of progression to $\mathrm{HF}$ in CAD patients by developing a polygenic risk score (PRS).

Methods: Using summary statistics from a recent GWAS for $\mathrm{HF}$ secondary to $\mathrm{CAD}$ (ischemic $\mathrm{HF}$ ), we computed risk scores by varying the rho parameter in LDPred and varying the $p$-value threshold and $\mathrm{r}^{2}$. We selected the PRS that maximized the association with ischemic $\mathrm{HF}\left(\mathrm{PRS}_{\mathrm{iHF}}\right)$ from the multiethnic Mount Sinai BioMe Biobank(N=6276). We validated the association in Penn Medicine BioBank (PMBB; $\mathrm{N}=6674)$. We performed association analysis using logistic regression adjusted for age, sex, smoking history, hypertension, BMI, type 2 diabetes, and ten genetic principal components.

Results: In BioMe, one standard deviation (SD) increase in $\mathrm{PRS}_{\mathrm{iHF}}$ was significantly associated with $\mathrm{HF}(\mathrm{OR}=1.1$; $\mathrm{p}=0.001 ; 2530$ cases, 3746 controls $\mathrm{HF}$ risk increased monotonically with $\mathrm{PRS}_{\mathrm{iHF}}$ in a subset of individuals with CAD but no evidence of obstructive CAD on coronary catheterization $(\mathrm{N}=4775)$. In $\mathrm{PMBB}, \mathrm{PRS}_{\mathrm{iHF}}$ was associated with ischemic HF in European Americans (adjusted $\mathrm{OR}=1.07 ; \mathrm{p}=0.02 ; 2692$ cases, 2472 controls) but not African Africans (adjusted $\mathrm{OR}=0.94 ; \mathrm{p}=0.3 ; 921$ cases, 589 controls)

Conclusions: We derived and validated a PRS for ischemic HF in two multiethnic cohorts and demonstrated association with and without obstructive CAD. PRS $\mathrm{PHF}_{\mathrm{iHF}}$ replicated in patients of European ancestry, but not in African ancestry. Although studies on clinical utility and in more diverse populations are needed, our results suggest application in early risk stratification.

I. Paranjpe: None. J. De Freitas: None. N. Tsao: None. R. Judy: None. K. Chaudhary: None. I. Forrest: None. S. Coca: None. J. Narula: None. B. Glicksberg: None. R. Do: None. S. Damrauer: None. G. Nadkarni: None.

\section{P05.58.A}

Identifying Long QT syndrome (LQTS) patients in primary care, an approach for rare diseases.

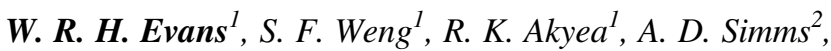 J. Kai ${ }^{l}$, N. Qureshi \\ ${ }^{1}$ University of Nottingham, Nottingham, United Kingdom, ${ }^{2}$ Department of Cardiology, Leeds Teaching Hospital NHS Trust, Leeds, United Kingdom}

Introduction: LQTS is a rare ( 1 in 2000 births) inherited cardiac arrhythmia that predisposes patients to syncope and sudden cardiac death. Identification and treatment can substantially reduce this risk. We aimed to identify the clinical features that precede the diagnosis of LQTS in primary care and use these to develop a predictive model.
Methods:1495 patients diagnosed with LQTS and 7475 propensity-score matched controls were identified in a database of primary care electronic records (CPRD). A range of clinical features that occurred at greater frequency in cases than control ( $p$ <0.05) before diagnosis were identified and incorporated into a multivariable logistic regression model.

Results:18 clinical features were incorporated into the final model, including: epilepsy (1.70 (1.12, 2.56)), palpitations $(2.22(1.80,2.74))$, collapse (1.636 (1.32, 2.03), mitral valve disease $(2.64(1.41,4.91))$, atrial fibrillation $(1.91(1.41,2.60))$ and IBS $(1.78(1.41,2.26))$ (OR $(95 \% \mathrm{CI})$ ). The discriminative accuracy of the model assessed by area under the curve (AUC) was $0.74,(95 \% \mathrm{CI}$ 0.73 to 0.75 ), with comparable performance in two more tightly phenotyped subgroups: LQTS diagnosis $<45$ years and those commenced on a betablocker \&/or fitted with an Implantable Cardioverter Defibrillator (ICD) post-diagnosis.

Conclusion: This study demonstrates that it is possible to develop a predictive model for LQTS that performs with similar accuracy to other widely adopted cardiovascular risk models. Furthermore, it shows the potential of primary care records to phenotypically identify patients that are at an increased risk of having a rare disease.

W.R.H. Evans: D. Speakers Bureau/Honoraria (speakers bureau, symposia, and expert witness); Modest; Intrabio inc. F. Consultant/Advisory Board; Modest; Mendelian.co. S.F. Weng: D. Speakers Bureau/Honoraria (speakers bureau, symposia, and expert witness); Modest; Amgen. R.K. Akyea: None. A.D. Simms: None. J. Kai: None. N. Qureshi: D. Speakers Bureau/Honoraria (speakers bureau, symposia, and expert witness); Modest; Amgen.

\section{P05.60.C}

Functional investigation of possible pathogenic EPHB4 variants associated with lymphatic related fetal hydrops and hereditary haemorrhagic telangiectasia

\section{E. Sackey, S. Martin-Almedina, K. Ogmen, D. Grigoriadis, S. Mansour, P. Ostergaard}

\section{St. George's University, London, United Kingdom}

The use of next generation sequencing (NGS) technologies has increased the capacity of rare and novel variant detection. However, this brings challenges to confirm pathogenicity of the identified variants and evaluate them functionally, both needed for precise clinical diagnostics. This study investigates nine novel possible pathogenic nonsynonymous variants in the EPHB4 (Ephrin Receptor B4) gene. EPHB4 mutations have been associated with lymphatic related fetal hydrops (LRFH) and hereditary haemorrhagic telangiectasia (HHT) both with autosomal 
dominant inheritance. Non-immune fetal hydrops is characterised by fluid accumulation in at least two extravascular fetal compartments and about $15 \%$ of the cases result from a lymphatic abnormality. HHT presents commonly with arteriovenous malformations (AVMs) in the liver and lung, in connection with cutaneous and mucosal telangiectasias. Functional analysis including immunoprecipitation, western blotting, and immunofluorescence assays were performed on nine novel variants on EPBH4 to investigate their pathogenicity. This study demonstrates the pathogenic effect of eight variants out of nine. Most of the LRFHassociated EPHB4 variants presented similar protein expression levels compared to the wildtype protein but reduced tyrosine kinase activity while HHT-associated $E P H B 4$ variants showed a reduction or total absence of protein expression. Based on these results, we suggest there could be two distinct EPHB4-associated disease phenotypes distinguished by two distinctive molecular mechanisms, but further research is necessary to elucidate this.

E. Sackey: None. S. Martin-Almedina: None. K. Ogmen: None. D. Grigoriadis: None. S. Mansour: None. P. Ostergaard: None.

\section{P05.61.A}

Identifying metabolomic fingerprints of microRNAs in cardiovascular disorders

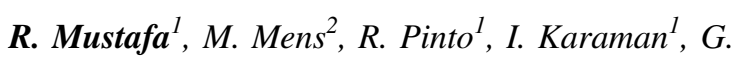

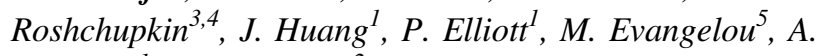
Dehghan $^{1}$, M. Ghanbari ${ }^{2}$

${ }^{1}$ Department of Epidemiology and Biostatistics, Imperial College London, London, United Kingdom, ${ }^{2}$ Department of Epidemiology, Erasmus Medical Center, Rotterdam, Netherlands, ${ }^{3}$ Department of Radiology and Nuclear Medicine, Erasmus Medical Center, Rotterdam, Netherlands, ${ }^{4}$ Department of Medical Informatics, Erasmus Medical Center, Rotterdam, Netherlands, ${ }^{5}$ Department of Mathematics, Imperial College London, London, United Kingdom

Introduction: microRNAs (miRNAs) are small non-coding RNAs that regulate gene expression. Despite an increasing evidence that supports miRNAs as potential biomarkers in cardiovascular disorders, the underlying mechanisms remain to be elucidated. We aimed to identify metabolites that mediate the effect of miRNAs on cardiovascular disorders.

Methods: We performed two steps of two-sample Mendelian Randomization (MR) to study the causal association between miRNAs and metabolites, and between the metabolites and cardiovascular disorders. We conducted genome-wide association studies (GWAS) on miRNA expression data $(\mathrm{N}=1,687)$ in the Rotterdam Study to identify genetic instruments for miRNAs. MR was performed in a hypothesis-free manner between 591 miRNAs and 886 metabolites measured by the Metabolon platform. The association of the genetic instruments with metabolites were examined in the Airwave Study $(\mathrm{N}=1,942)$. We further performed MR to investigate the causal role of identified metabolites on cardiovascular disorders using publicly available GWAS summary results.

Results: Our analysis indicated causal associations between 16 miRNAs and 21 metabolites after multiple testing correction. Among the identified metabolites, nominally significant associations were found between androsterone sulfate, cysteine sulfinic acid, and $\mathrm{N}$ palmitoylglycine with stroke, and 3-hydroxy-5cholestenoic acid with coronary artery disease. Taken together, we were able to identify metabolites that might link miR-1273h-5p, miR-3937, and miR-4753-5p with stroke, and miR-181a-2-3p with coronary artery disease.

Conclusions: Our study has added insight into understanding the role of metabolites in the link between miRNAs and cardiovascular disorders. This approach could offer a way for finding candidate biomarkers and therapeutic targets in cardiovascular disorders.

R. Mustafa: None. M. Mens: None. R. Pinto: None. I. Karaman: None. G. Roshchupkin: None. J. Huang: None. P. Elliott: None. M. Evangelou: None. A. Dehghan: None. M. Ghanbari: None.

\section{P05.62.B}

The detection of mosaic mutationsin hereditary aortic disease through next-generation sequencing

\section{Z. G. Yan, H. Yang, Y. H. Zhang, Z. Zhou}

State Key Laboratory of Cardiovascular Disease, Beijing Key Laboratory for Molecular Diagnostics of Cardiovascular Diseases, Diagnostic Laboratory Service, Fuwai Hospital, National Center for Cardiovascular Diseases, Chinese Academy of Medical Sciences, Beijing, China

Background: The mosaic carriers have been clinically normal or had only minor manifestations when the proportion of mutated cells is low, which makes it difficult to be detected. However, if the mutations were transmitted from asymptomatic carriers to their children, it will cause unexpected occurrence of diseases. So genetic tests are often required.

Methods: We choosed 563 patients with suspected marfan syndrome (MFS) or thoracic aortic aneurysm/ dissection, who had been detected by Panel. Among them, we selected 56 patients who carried likely pathogenic (pathogenic) mutations and were not de novo, their parents 
who harbored the same mutation were clinically normal. For these Trio families, we used the Life PGM ${ }^{\mathrm{TM}} \mathrm{DX}$ platform to perform deep sequencing of single amplicon (approximately 5000x) on DNA samples extracted from whole blood.

Results: We detected two mosaic mutations in these Trio families. The first patient AD700-1 who had typical MFS features carried the mutation FBN1, C1305F. After deep sequencing, $23 \%$ mutated alleles were detected in healthy father who harbored the same mutation. The second mutation $F B N 1$, c. $2167+1 \mathrm{G}>\mathrm{A}$ was identified in patient AD1833, who was suspected to be MFS. The mutation was also carried by healthy father, who was found to carry $17 \%$ of the mutant allele through deep sequencing.

Conclusions: Mosaic mutations could be a more common phenomenon in aortic diseases than has been realized so far. Therefore, whenever children are identified with harmful mutations, the parents who lack obvious phenotypic characteristics should be detected by genetic testing, which will provide more accurate guidance for genetic counseling.

Z.G. Yan: None. H. Yang: None. Y.H. Zhang: None. Z. Zhou: None.

\section{P05.63.C}

Familial aortic aneurysm syndrome caused by a +3 variant at a donor splice site of $\mathrm{MYH11}$.

B. Chesneau ${ }^{1,2}$, A. Plancke ${ }^{1}$, G. Rolland ${ }^{1}$, B. Marcheix ${ }^{3}$, Y.

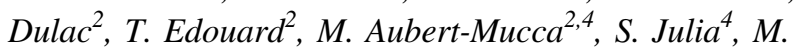
Langeois $^{2,4}$, V. Gaston ${ }^{4}$, P. Khau Van Kien ${ }^{1}$

${ }^{1}$ Unité de génétique médicale et cytogénétique, Hôpital Carémeau, CHU de Nîmes, Nîmes, France, ${ }^{2}$ Centre de Référence du syndrome de Marfan et des syndromes apparentés, Hôpital des Enfants, CHU de Toulouse, Toulouse, France, ${ }^{3}$ Département de chirurgie cardiaque, Hôpital Universitaire de Rangueil, Toulouse, France, ${ }^{4}$ Service de génétique médicale, Hôpital Universitaire de Purpan, Toulouse, France

Pathogenic variants in the myosin smooth muscle heavy chain 11 (MYH11) are associated with familial aortic aneurysm (FAA) and patent ductus arteriosus (PDA) syndrome. We report a family with two siblings who presented with type A aortic dissections. The sister made an aortic dissection at 45 years-old. The brother made an aortic dissection at 22 years-old treated with an aortic graft procedure, at 46 years-old he had revision surgery because of pseudoaneurysms complications. His daughter was diagnosed with a patent ductus arteriosus. Their father suddenly died at 28 years-old. Molecular analysis of a panel of 15 aortic aneurysm genes in the brother revealed only the variant c. $4599+3 \mathrm{~A}>\mathrm{C}$ at the 5 ' donor splice site (5'ss) of the exon 33 of MYH11 (NM_00104114.1) with a potential splicing impact. This variant was also present in the affected sister. It was absent from GnomAD and internal variant database (>1000 patients) and never reported in the literature. Interestingly, a single-nucleotide polymorphism is described at this position: c. $4599+3 \mathrm{~A}>\mathrm{G}$ (rs143288748). Interpretation of variations at the position +3 of the 5 'ss is difficult because data of the literature are conflicting with variations reported to induce aberrant splicing in some cases but not in others. We performed transcript analysis with RNA extracted from an aortic biopsy during the brother's second intervention. RT PCR revealed a skipping of the exon 33 of MYH11 predicting a truncated myosin tail and a dominant negative effect. This report confirms genotypephenotype correlations for MYHII and FAA/PDA.

B. Chesneau: None. A. Plancke: None. G. Rolland: None. B. Marcheix: None. Y. Dulac: None. T. Edouard: None. M. Aubert-Mucca: None. S. Julia: None. M. Langeois: None. V. Gaston: None. P. Khau Van Kien: None.

P05.64.A

A founder pathogenic variant in $M Y H 7$ causing hypertrophic cardiomyopathy in Catalonia

P. Fernández-Álvarez ${ }^{l}$, M. Codina ${ }^{l}, J$. Limeres $^{2}$, C. SerraJuhé $^{3}$, M. Masas Castro ${ }^{l}$, A. Cordero Lopez ${ }^{l}$, E. GarcíaArumil $^{1,4}$, E. Tizzano ${ }^{1,4}$

${ }^{1}$ Department of Clinical and Molecular Genetics and Medicine Genetics Group, VHIR, University Hospital Vall d'Hebron, Barcelona, Spain, ${ }^{2}$ Department of Cardiology, Hospital Universitari Vall d'Hebron, Barcelona, Spain, ${ }^{3}$ Department of Genetics, Hospital de la Santa Creu i Sant Pau, Barcelona, Spain, ${ }^{4}$ CIBERER, Centro de Investigación Biomédica en Red de Enfermedades Raras, ISCIII, Madrid, Spain

Pathogenic variants in $M Y H 7$ are the second most common cause of inherited hypertrophic cardiomiopathy (HCM). 262 unrelated HCM probands were screened using the Illumina TruSightCardio kit. Twenty-nine patients were heterozygous for $M Y H 7$ pathogenic variants, 6 (21\%) of them had the same variant c.2674C $>$ A p.(Gln892Lys). This variant has been previously described as a VUS in a HCM patient which harbored another pathogenic variant in $M Y H 7$ and in ClinVar, and is absent in GnomAD. In all our six patients p.(Gln892Lys) was the only probably pathogenic variant found. Patients (4 males/2 females) were of Catalan origin and had been diagnosed of HCM around 30 years-old. Familial sudden death before the fifth decade of life were reported. We extended the genetic and cardiologic study to 20 relatives of the probands. Twelve were carriers, 9 of them symptomatic and p.(Gln892Lys) cosegregated with the HCM in all the families. We studied the 
possibility of a founder effect by haplotype analysis using the SNP data from the TruSightCardio kit. We used 14 SNPs, flanking an area of 49,35 kb before $M Y H 6$ and after MYH7. A common haplotype with a calculated haplotype frequency in Iberia population of 0.0187 was shared in the 6 patients and was not found in our remaining 256 patients with HCM. Our results indicate a possible founder effect of this $M Y H 7$ pathogenic variant in HCM patients of Catalan origin.

\begin{tabular}{|c|c|c|c|c|c|c|c|c|c|c|c|c|c|}
\hline \multicolumn{14}{|c|}{ esults of habplotype analysis in the 6 patients } \\
\hline \multirow{2}{*}{$\begin{array}{l}\text { SNP } \\
\text { rs } 8006357\end{array}$} & \multirow{2}{*}{$\begin{array}{l}\begin{array}{l}\text { Position } \\
\text { (hg19) } \\
\text { chr14 } \\
\text { case 1 }\end{array} \\
23853629 \text { T }\end{array}$} & Proband & \multicolumn{2}{|c|}{1 Proband 2} & \multicolumn{2}{|c|}{2 Proband } & \multicolumn{2}{|c|}{3 Proband 4} & \multicolumn{2}{|c|}{4 Proband } & \multicolumn{2}{|c|}{5 Proband 6} & \multirow[t]{2}{*}{$\begin{array}{l}5 \text { Distance } \\
\text { from } \\
\text { Previous } \\
\text { SNP (kb) }\end{array}$} \\
\hline & & $\mathrm{C}$ & $\mathrm{T}$ & $\mathrm{C}$ & $\mathrm{T}$ & $\mathrm{C}$ & $\mathrm{T}$ & $\mathrm{C}$ & $\mathrm{C}$ & $\mathrm{C}$ & $\mathrm{T}$ & $\mathrm{C}$ & \\
\hline rs178640 & $23855569 \mathrm{~A}$ & G & A & G & G & G & A & $\mathrm{G}$ & G & $\mathrm{G}$ & A & G & 1,94 \\
\hline s2071634 & $23855849 \mathrm{C}$ & $\mathrm{T}$ & $\mathrm{C}$ & $\mathrm{T}$ & $\mathrm{C}$ & $\mathrm{T}$ & $\mathrm{C}$ & $\mathrm{T}$ & $\mathrm{T}$ & $\mathrm{T}$ & $\mathrm{C}$ & $\mathrm{T}$ & 0,28 \\
\hline s365990 & $23861811 \mathrm{~A}$ & A & $\mathrm{G}$ & A & A & A & A & A & A & A & A & A & 5,962 \\
\hline s61731179 & 23869993 & A & $\mathrm{G}$ & $\mathrm{A}$ & G & A & $\mathrm{G}$ & A & G & A & G & A & 8,182 \\
\hline rs434273 & $23872666 \mathrm{C}$ & $\mathrm{T}$ & $\mathrm{C}$ & $\mathrm{T}$ & $\mathrm{C}$ & $\mathrm{T}$ & $\mathrm{T}$ & $\mathrm{T}$ & $\mathrm{C}$ & $\mathrm{T}$ & $\mathrm{C}$ & $\mathrm{T}$ & 2,673 \\
\hline rs2331979 & $23882855 \mathrm{~A}$ & A & A & A & A & A & A & A & A & A & A & A & 10,189 \\
\hline rs2277475 & $23888665 \mathrm{~A}$ & A & $\mathrm{T}$ & A & A & A & A & $\mathrm{A}$ & $\mathrm{T}$ & A & A & A & 5,81 \\
\hline rs7157716 & $23892888 \mathrm{~A}$ & A & $\mathrm{G}$ & A & A & A & A & A & G & A & $\mathrm{A}$ & A & 4,223 \\
\hline $\begin{array}{l}\text { p. } \\
\text { (Gln892Lys) }\end{array}$ & $23893983 \mathrm{C}$ & $\mathrm{T}$ & $\mathrm{G}$ & $\mathrm{T}$ & G & $\mathrm{T}$ & $\mathrm{G}$ & $\mathrm{T}$ & G & $\mathrm{T}$ & G & $\mathrm{T}$ & 1,095 \\
\hline rs 2231126 & 23898994 & G & $\mathrm{A}$ & 0 & G & G & G & G & G & G & G & G & 0,066 \\
\hline rs 735712 & $23899060 \mathrm{C}$ & G & $\mathrm{G}$ & G & G & G & $\mathrm{G}$ & G & G & G & G & G & 0,066 \\
\hline rs2069542 & 23900794 & G & $\mathrm{A}$ & G & G & G & $\mathrm{G}$ & G & A & G & G & G & 1,734 \\
\hline s2069540 & 23902753 & A & $\mathrm{A}$ & A & G & A & A & A & A & A & G & A & 1,959 \\
\hline ss3729992 & $23902974 \mathrm{C}$ & $\mathrm{C}$ & $\mathrm{C}$ & $\mathrm{C}$ & $\mathrm{C}$ & $\mathrm{C}$ & $\mathrm{C}$ & $\mathrm{C}$ & A & $\mathrm{C}$ & $\mathrm{C}$ & $\mathrm{C}$ & 0,221 \\
\hline
\end{tabular}

P. Fernández-Álvarez: None. M. Codina: None. J. Limeres: None. C. Serra-Juhé: None. M. Masas Castro: None. A. Cordero Lopez: None. E. García-Arumí: None. E. Tizzano: None.

\section{P05.65.B}

\section{Biallelic variants in $M Y L 3$ cause autosomal recessive} cardiomyopathy

D. P. S. Osborn ${ }^{1}$, L. Emrahi ${ }^{2}$, M. T. Tabrizi ${ }^{3}$, M. Yazdchi ${ }^{3}$, N. Mazaheri, , A. Mitchell ${ }^{5}$, C. Hesse ${ }^{6}, H$. Goullée ${ }^{7}, D$. Burkardt ${ }^{5}$, N. Laing ${ }^{7}$, A. Y. B. Wan ${ }^{1}$, H. Galehdari ${ }^{4}$, R.

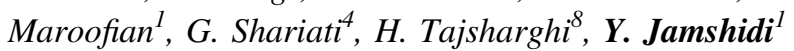

${ }^{1}$ Genetics Centre, London, United Kingdom, ${ }^{2}$ Tarbiat Modares University, Tehran, Iran, Islamic Republic of, ${ }^{3}$ Tabriz University of Medical Sciences, Tabriz, Iran, Islamic Republic of, ${ }^{4}$ Shahid Chamran University of Ahvaz, Ahvaz, Iran, Islamic Republic of, ${ }^{5}$ Case Western Reserve University, Cleveland, OH, United States, ${ }^{6}$ University of Gothenburg, Gothenburg, Sweden, ${ }^{7}$ The University of Western Australia and the Harry Perkins Institute of Medical Research, Nedlands, Australia, ${ }^{8}$ University of Skovde, Skovde, Sweden
MYL3 encodes the slow-twitch specific myosin essential light chain (ELC), and pathogenic variants in this gene are a rare cause of hypertrophic cardiomyopathy. The majority of cases reported in the literature involve MYL3 missense variants transmitted in an autosomal dominant mode of inheritance. Here, through exome sequencing of four unrelated consanguineous families we identify biallelic MYL3 sequence variants. Affected individuals present with hypertrophic or dilated cardiomyopathy of variable severity from early- to late-onset of cardiomyopathy, and sudden cardiac death in 10 individuals. We report homozygous and compound heterozygous missense variants p.Ala57Asp, p. Met173Lys and p.Glu177Gly, a novel homozygous translational stop codon variant p.Glu36Ter, and a homozygous splice variant c.482-1G>A in MYL3. G0 zebrafish larvae with CRISPR/Cas9 gene disruption or transient knockdown of the major ELC orthologue in zebrafish, cmlc1, display significantly larger ventricular chamber volume and compromised cardiac function, which cannot be rescued by reintroduction of human mutant RNA. Our genetic and functional data expand the mutational and phenotypic spectrum of MYL3 variants, identifying for the first time a homozygous loss of function variant, and highlighting the role of MYL3 in recessive hypertrophic and dilated cardiomyopathy with occurrence of sudden cardiac death.

D.P.S. Osborn: None. L. Emrahi: None. M.T. Tabrizi: None. M. Yazdchi: None. N. Mazaheri: None. A. Mitchell: None. C. Hesse: None. H. Goullée: None. D. Burkardt: None. N. Laing: None. A.Y.B. Wan: None. H. Galehdari: None. R. Maroofian: None. G. Shariati: None. H. Tajsharghi: None. Y. Jamshidi: F. Consultant/ Advisory Board; Modest; DiNAQOR AG.

\section{P05.66.C}

Impact of abdominal obesity on postprandial lipid concentrations

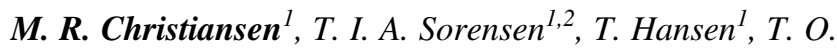 Kilpeläinen ${ }^{l}$}

${ }^{1}$ Novo Nordisk Foundation Center for Basic Metabolic Research, Faculty of Health and Medical Sciences, University of Copenhagen, Copenhagen, Denmark, ${ }^{2}$ Department of Public Health, Section of Epidemiology, University of Copenhagen, Copenhagen, Denmark

Introduction: Humans spend most of the day in a postabsorptive state, and thus prolonged and high postprandial lipemia can have major implications for the risk of cardiovascular disease. Abdominal adiposity may influence postprandial lipemia by influencing lipid metabolism after a meal. To date, $>400$ genetic loci have been discovered for association with abdominal adiposity, assessed by waist-hip 
ratio adjusted for body mass index (WHRadjBMI). Whether these variants are associated with postprandial lipid concentrations, remains unknown. We thus examined whether genetic risk score for increased waist-hip ratio adjusted for body mass index (WHRadjBMI) is associated with lipid concentrations at fasting state and after a high-fat meal.

Materials and Methods: 771 individuals of European ancestry and with obesity from the NUGENOB study consumed a liquid high-fat meal $(95 \%$ fat of the energy content). The postprandial concentrations of HDL cholesterol, LDL cholesterol, free fatty acids, glycerol and triglycerides were measured at fasting state and postprandially for 3 hours. The associations between the WHRadjBMI score and lipid concentrations were examined using linear models.

Results: At fasting state, the genetic risk score for WHRadjBMI was associated with higher fasting triglycerides $(\mathrm{P}=0.0013)$ and lower HDL cholesterol $(\mathrm{P}=0.0059)$. Postprandially, the genetic risk score was not associated with changes in triglycerides or HDL cholesterol $(\mathrm{P}>0.05)$, but was significantly associated with elevated glycerol concentrations $(\mathrm{P}=0.012)$.

Conclusions: Genetic predisposition to abdominal adiposity is associated with elevated blood glycerol concentration after a high-fat meal.

M.R. Christiansen: None. T.I.A. Sorensen: None. T. Hansen: None. T.O. Kilpeläinen: None.

\section{P05.68.B}

Familial pulmonary arterial hypertension by $K D R$ heterozygous loss of function

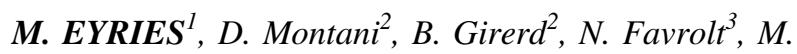

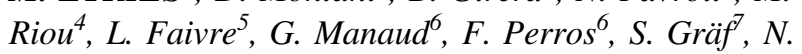
Morrell $^{8}$, M. Humbert ${ }^{2}$

${ }^{1}$ Département de génétique, Hôpitaux Universitaires Pitié Salpêtrière - Charles Foix,, Paris, France, ${ }^{2}$ Service de Pneumologie, Centre de Référence de l'Hypertension Pulmonaire, Hôpital Bicêtre, Le Kremlin Bicêtre, France, ${ }^{3}$ Service de Pneumologie et Soins Intensifs Respiratoires, Centre de référence constitutif des maladies pulmonaires rares de l'adulte, Centre de compétence de l'hypertension pulmonaire, CHU Dijon-Bourgogne, Dijon, France, ${ }^{4} \mathrm{Ser}$ vice de Pneumologie, Nouvel hôpital civil, Strasbourg, France, ${ }^{5}$ Centre de génétique, FHU TRANSLAD, Institut GIMI et UMR INSERM 1231, CHU de Dijon et Université de Bourgogne, Dijon, France, ${ }^{6} U M R \_S$ 999, Université Paris-Saclay, INSERM, Hôpital Marie Lannelongue, Le Plessis Robinson, France, ${ }^{7}$ NIHR Bioresource - Rare Diseases, Department of Medicine, Department of Haematology, Cambridge Biomedical Campus, Cambridge, United Kingdom, ${ }^{8}$ Department of Medicine, University of
Cambridge School of Clinical Medicine, Addenbrooke's and Royal Papworth Hospitals, Cambridge, United Kingdom

Introduction: Pulmonary arterial hypertension (PAH) is a rare and devastating disease, resulting from progressive obliteration of small caliber pulmonary arteries leading to cardiac failure. PAH can occur in the context of family history or genetic mutations causing heritable $\mathrm{PAH}$, or is considered as idiopathic in the absence of an identified predisposing factor. Beyond the major gene BMPR2, a gene encoding a type 2 receptor of the BMP signaling pathway several new genes predisposing to PAH have been identified during the last decade. Recently, preliminary evidence of the involvement of the KDR gene was found in a large genetic association study.

Methods: We prospectively analysed the KDR gene by targeted panel NGS sequencing in a series of $311 \mathrm{PAH}$ patients referred to a clinical molecular laboratory for genetic diagnosis of PAH.

Results: Two index cases with severe PAH were found to carry a loss-of-function mutation in the KDR gene. These two patients were clinically characterized by low diffusing capacity for carbon monoxide adjusted for hemoglobin (DLCOc) and radiological evidence of parenchymal lung disease. Segregation analysis in one family revealed that all KDR mutations carriers have a low DLCOc that is more pronounced in affected subjects compared to non-affected

Conclusions: Our study provided genetic evidence for considering KDR as a newly identified PAH causing gene since we describe the segregation of KDR mutations with $\mathrm{PAH}$ in two families. Furthermore, KDR mutations are associated with a particular form of PAH characterized by low DLCOc and parenchymal lung disease.

M. Eyries: None. D. Montani: None. B. Girerd: None. N. Favrolt: None. M. Riou: None. L. Faivre: None. G. Manaud: None. F. Perros: None. S. Gräf: None. N. Morrell: None. M. Humbert: None.

\section{P05.70.A}

PHACTR1 mRNA expression is affected by $r s 9349379$ in PBMC of patients six months after the first MI
J. Kuveljic ${ }^{1}$, T. Djuric ${ }^{1}$, M. Dekleva ${ }^{2}$, N. Markovic Nikolic ${ }^{2}$, A. Stankovic ${ }^{l}$, D. Alavantic ${ }^{l}$, M. Zivkovic ${ }^{l}$
${ }^{I}$ Vinca Institute of Nuclear Sciences, Laboratory for Radiobiology and Molecular Genetics, University of Belgrade, Belgrade, Serbia, Belgrade, Serbia, ${ }^{2}$ Department of Cardiology, University Clinical Center "Zvezdara", Belgrade, Serbia; Faculty of Medicine, University of Belgrade, Belgrade, Serbia, Belgrade, Serbia 
Aim: Phosphatase and actin regulator 1 (PHACTR1) was at first associated with early onset myocardial infarction (MI). Later on, it was associated with other vascular disorders, but still the mechanism of its effect is unknown. Rs9349379 is an eQTL for PHACTRl in coronary arteries, where A allele leads to higher expression. Also PHACTRl mRNA expression is elevated in the heart after MI, indicating its up-regulation in pathological conditions. The aim of our study was to investigate the association of $r s 9349379$ with the first $\mathrm{MI}$ in Serbian population and its effect on PHACTR1 mRNA expression in PBMC of MI patients and controls.

Methods: Blood samples from 171 controls and 156 patients with the first MI were obtained for DNA extraction. For RNA extraction, we collected 32 control samples and 65 samples of patients six months after the first MI. Genotyping and expression analysis were performed with Taqman technology. Statistical analyses were done by Statistica 8 software.

Results: The association of $r s 9349379$ with the first MI was not significant $(\mathrm{p}=0.13)$. PHACTR $1 \mathrm{mRNA}$ expression was significantly higher in MI patients compared to controls ( $\mathrm{p}=0.02$ ). We have found that $r s 9349379$ has no effect on PHACTR1 mRNA expression in control group $(\mathrm{p}=0.90)$, while AA genotype led to higher expression in patient group ( $\mathrm{p}=0.04)$.

Conclusions: Rs9349379 affects PHACTR1 mRNA expression in PBMC of MI patients, six months after the MI. In order to accurately estimate its association with MI, further analysis and larger sample size are needed. Funded by Serbian Ministry, grant III41028.

J. Kuveljic: None. T. Djuric: None. M. Dekleva: None. N. Markovic Nikolic: None. A. Stankovic: None. D. Alavantic: None. M. Zivkovic: None.

\section{P05.71.B}

Polygenic risk scores for hypertension risk stratification

O. Borisov ${ }^{1}$, E. Salvi ${ }^{2}$, L. Citterio ${ }^{3}$, C. Lanzani ${ }^{3}$, C. Barlassina $^{4}$, N. Glorioso ${ }^{5}$, P. Krawitz ${ }^{1}$, D. Cusi ${ }^{4}$, P. Manunta $^{3}$, C. Maj ${ }^{1}$

${ }^{1}$ Institute for Genomic Statistics and Bioinformatics, Bonn, Germany, ${ }^{2}$ Neuroalgology Unit, Fondazione IRCCS Istituto Neurologico "Carlo Besta", Milan, Italy, "3enomics of Renal Diseases and Hypertension Unit, Istituto di Ricovero e Cura a Carattere Scientifico (IRCCS) San Raffaele Scientific Institute; Vita-Salute San Raffaele University, Milan, Italy, ${ }^{4}$ Dept. Of Health Sciences, University of Milan, Milan, Italy, ${ }^{5}$ Hypertension and related diseases Centre Department of Clinical and Experimental Medicine University of Sassari Italy, Sassari, Italy
Introduction: Hypertension (HTN) is a major cardiovascular risk factor and responsible for $\sim 50 \%$ of cardiovascular morbidity and mortality. Blood pressure (BP) is also a complex genetic trait with heritability estimates of $30-50 \%$. Genome-wide association studies (GWAS) have identified a high number of genetic loci associated with HTN and BP traits, indicating underlying polygenic architectures. This study proposes to compute polygenic risk scores (PRS) for HTN and BP for potential genetic risk stratification.

Materials and Methods: We investigated the additive effect of common genetic variants on HTN and BP by means of regression models based on PRS. We used genotyping data of 561 cases and 731 controls from Hypergenes cohort. We computed PRS based on clumping and thresholding method using GWAS summary statistics of systolic/diastolic BP measurements from GERA cohort (99,785 health records) and the HTN case-control cohort of the UK Biobank (144,793 cases and 313,761 controls).

Results: PRS for HTN and diastolic/systolic BP are strongly associated with hypertensive status and BP measurements. The highest significance is achieved for the case-control status by considering UK Biobank effect sizes $(p=6.4 e-28, R 2=0.09)$. The area under the curve is 0.68 . The model remains significant after adjusting for sex and body mass index $(\mathrm{p}=4.1 \mathrm{e}-27)$.

Conclusions: PRS modeling shows a strong polygenic component both for HTN status and for BP levels. Despite the limited predictive accuracy expected for BP, which is largely influenced by environmental factors, the strength of associations suggests that PRS could be potentially used for genetic risk stratification.

O. Borisov: None. E. Salvi: None. L. Citterio: None. C. Lanzani: None. C. Barlassina: None. N. Glorioso: None. P. Krawitz: None. D. Cusi: None. P. Manunta: None. C. Maj: None.

\section{P05.72.C}

Biallelic variants in PPP1R13L cause paediatric dilated cardiomyopathy

H. K. Robinson ${ }^{1}$, E. Zaklyazminskaya ${ }^{2}$, I. Povolotskaya ${ }^{3}, Y$. Surikova ${ }^{4}$, L. Mallin $^{1}$, C. Armstrong ${ }^{5}$, M. R. Chrisant ${ }^{6}$, P. J.

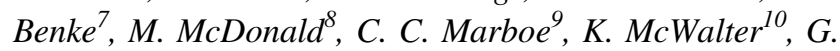
Douglas $^{10}$, K. Agre ${ }^{11}$, D. Deyle ${ }^{11}$, K. M. Balashova ${ }^{3}$, V. Kaimonov $^{3}$, N. Shirokova ${ }^{3}$, E. Pomerantseva ${ }^{3}$, C. Turner ${ }^{12}$, S. Ellard ${ }^{1,13}$

${ }^{1}$ Exeter Genomics Laboratory, Exeter, United Kingdom, ${ }^{2}$ Center of Syncope and Cardiac Arrhythmias in Children and Adolescents, Moscow, Russian Federation, ${ }^{3}$ Centre of Genetics and Reproductive Medicine "Genetico", Moscow, Russian Federation, ${ }^{4}$ B.V. Petrovsky Russian National Centre of Surgery, Moscow, Russian Federation, 
${ }^{5}$ Paediatric Cardiac Service, Bristol Royal Hospital for Children, Bristol, United Kingdom, ${ }^{6} J o e$ DiMaggio Children's Hospital, Hollywood, FL, United States, ${ }^{7}$ Charles E. Schmidt College of Medicine, Florida Atlantic University, Hollywood, FL, United States, ${ }^{8}$ Department of Pediatrics, Division of Medical Genetics, Duke University Medical Center, Durham, NC, United States, ${ }^{9}$ Department of Pathology and Cell Biology, Columbia University Medical Centre, New York, NY, United States, ${ }^{10}$ GeneDx Inc., Gaithersburg, MD, United States, ${ }^{11}$ Mayo Clinic, Department of Clinical Genomics, Rochester, MN, United States, ${ }^{12}$ Peninsula Clinical Genetics Service, Department of Clinical Genetics, Royal Devon and Exeter NHS Foundation Trust, Exeter, United Kingdom, ${ }^{13}$ Institute of Biomedical and Clinical Science, College of Medicine and Health, Exeter, United Kingdom

Introduction: Childhood DCM is a leading cause of heart failure requiring cardiac transplantation, with $\sim 5 \%$ of cases resulting in sudden death. Knowledge of the underlying genetic cause can aid prognostication and clinical management and enables accurate recurrence risk counselling. Here our aim was to identify the causative genetic variant(s) in families with children affected by severe DCM.

Materials and Methods: Exome/genome sequencing was performed on leukocyte DNA from seven children affected with severe DCM and their unaffected parents from five independent families, followed by inheritance-based gene-agnostic variant filtering. Families were brought together by an international collaborative effort facilitated by GeneMatcher.

Results: DCM presented early (range: 3 months -9 years) and was progressive, resulting in death $(n=3)$ or transplant $(n=3)$, with one child currently awaiting transplant. Biallelic variants in $P P P 1 R 13 L$ were identified in the affected children in each family. PPPIR13L encodes inhibitor of apoptosis-stimulating protein of $\mathrm{p} 53$ protein (iASPP). iASPP has roles in apoptosis, acts as a regulator of desmosomes and has been implicated in inflammatory pathways. Spontaneous biallelic loss-of-function variants in PPP1R13L orthologues have been shown to underlie bovine and murine models of DCM and biallelic variants were previously reported in a single consanguineous family with paediatric DCM.

Conclusions: Gene-agnostic exome/genome sequencing is valuable for the identification of novel and emerging candidate genes. The identification of these five families now provides sufficient evidence to support a robust genedisease association between PPPIR13L and severe paediatric DCM. PPPIR13L is therefore recommended for inclusion in panel-based genetic testing for paediatric DCM.

H.K. Robinson: None. E. Zaklyazminskaya: None. I. Povolotskaya: None. Y. Surikova: None. L. Mallin:
None. C. Armstrong: None. M.R. Chrisant: None. P.J. Benke: None. M. McDonald: None. C.C. Marboe: None. K. McWalter: A. Employment (full or part-time); Significant; GeneDx Inc. G. Douglas: A. Employment (full or part-time); Significant; GeneDx Inc.. K. Agre: None. D. Deyle: None. K.M. Balashova: None. V. Kaimonov: None. N. Shirokova: None. E. Pomerantseva: None. C. Turner: None. S. Ellard: None.

\section{P05.75.C}

Identification of ANGPT2 mutations as a novel cause of primary lymphedema

V. Leppänen ${ }^{1}$, P. Brouillard ${ }^{2}$, E. Korhonen ${ }^{1}$, N. Revencu ${ }^{3}$, G. Koh ${ }^{4}$, P. Saharinen ${ }^{l}$, K. Alitalo ${ }^{l}$, M. Vikkula ${ }^{2,5}$

${ }^{I}$ Wihuri Research Institute, Biomedicum Helsinki, Helsinki, Finland, ${ }^{2}$ Human Molecular Genetics, de Duve Institute, UClouvain, Brussels, Belgium, ${ }^{3}$ Center for Human Genetics, Cliniques universitaires Saint-Luc, UCLouvain, Brussels, Belgium, ${ }^{4}$ Center for Vascular Research, Korea Advanced Institute of Science and Technology (KAIST), Daejoen, Korea, Republic of, ${ }^{5}$ Walloon Excellence in Lifesciences and Biotechnology (WELBIO), UCLouvain, Brussels, Belgium

Purpose: Primary lymphedema (PLE) is caused by developmental and functional defects of the lymphatic system resulting in accumulation of protein-rich fluid in tissues. The 28 currently known genes causing lymphedema explain $<30 \%$ of cases. We search for additional genes to better understand pathophysiology.

Methods: We used WES to screen 543 patients within our cohort of $>900$ index patients. We filtered variants using our in-house developed Highlander software. In vitro and in vivo studies were performed to validate functional effects.

Results: We identified a complete angiopoietin-2 (ANGPT2) gene deletion in one family, and four variants of interest in four other families. All are extremely rare or absent in controls and databases, and predicted to be pathogenic by several algorithms. Functional analyses of the substitution-mutants unraveled that $3 / 4$ showed decreased ANGPT2 secretion and even inhibited secretion of the wild-type ANGPT2, suggesting that they have a dominant-negative effect. The fourth mutant, located in the dimerization interphase, showed reduced integrin- $\alpha 5$ binding and its expression in mouse skin promoted hyperplasia and dilation of cutaneous lymphatic vessels.

Conclusions: We identified five mutations in the TIE2ligand ANGPT2 as a novel cause of primary lymphedema. The mutations alter ANGPT2 structure and/or function. The data underscore loss of ANGPT2 function as the 
mechanism leading to primary lymphedema, and provide new insights into TIE2 signaling. The ANGPT2 gene is now included in the diagnostic panel used in our hospital to perform diagnostic testing for primary lymphedema. These studies were supported by Walloon Excellence in Lifesciences and Biotechnology (WELBIO).

V. Leppänen: None. P. Brouillard: None. E. Korhonen: None. N. Revencu: None. G. Koh: None. P. Saharinen: None. K. Alitalo: None. M. Vikkula: None.

\section{P05.76.A}

Novel variants in $A B I N 2$ and $T R A F 2$ implicated in pathogenesis of pulmonary arterial hypertension

N. Gallego ${ }^{1}$, D. Condon ${ }^{2}$, S. Pienkos ${ }^{2}$, V. de Jesús Pérez ${ }^{2}$, I. Hernández ${ }^{3}$, C. Pérez-Olivares ${ }^{4}, N$. Ochoa ${ }^{4}, P$. Spanish Consortium $^{5}$, P. Lapunzina ${ }^{6}$, P. Escribano ${ }^{7}$, J. Tenorio $^{6}$

${ }^{1}$ Instituto de Genética Médica y Molecular, Madrid, Spain, ${ }^{2}$ Stanford University, Standford, CA, United States, ${ }^{3}$ Hospital Universitario Fundación Jiménez Díaz, Madrid, Spain, ${ }^{4}$ Hospital Universitario Doce de Octubre, Madrid, Spain, ${ }^{5}$ Instituto de Genética Médica y Molecular, Centro de Investigación Biomédica en Red de Enfermedades Raras, Madrid, Spain, ${ }^{6}$ Instituto de Genética Médica y Molecular, Centro de Investigación Biomédica en Red de Enfermedades Raras, Madrid, Spain, ${ }^{7}$ Hospital Universitario Doce de Octubre, Centro de Investigación Biomedica en Red en Enfermedades Cardiovasculares, Madrid, Spain

Introduction: Pulmonary Arterial Hypertension (PAH) is an infrequent cardiopathy with poor prognosis despite therapeutic advances. Mutation in several known risk genes predispose to disease however, some cases remain genetically undefined. Increasing knowledge of the underlaying molecular mechanisms may lead to a better understanding of the disease and the opening of new drug targets that can improve the treatment of patients. The advance in massive paralleled sequencing technologies has allowed improving the characterization of the molecular profiling of the patients and a better clinical characterization.

Material and methods: The aim of this project is to study 20 patients and available relatives by whole exome sequencing (WES) technology in order to identify new genes and regions that can be involved in PAH. Variant prioritization was performed through bioinformatic tools and a custom algorithm. In addition, to stablish mechanistic significance, a series of in vitro studies were performed.

Results: Several candidate genes were identified after the variant prioritization. We have identified two genes (TRAF2 and TNIP2) in two unrelated families, which are related to the NFK $\beta$ pathway, associated with the progression of PAH.
In vitro studies demonstrate a possible impairment of NFK $\beta$ transcription factors localization of a modulation of NFK $\beta$-inducible genes after inhibition of ABIN2 and TRAF2.

Conclusions: Although these results have to be validated through more different functional assays, they suggested an association between disease and new genes which may also be considered for inclusion in genetic screening for $\mathrm{PAH}$ patients.

Grants: FIS-PI18/01233, Janssen unrestricted grant, FCHP.

N. Gallego: None. D. Condon: None. S. Pienkos: None. V. de Jesús Pérez: None. I. Hernández: None. C. PérezOlivares: None. N. Ochoa: None. P. Spanish Consortium: None. P. Lapunzina: None. P. Escribano: None. J. Tenorio: None.

\section{P05.77.B}

Association of two novel SCN5A intragenic duplications with epilepsy, ventricular tachycardia, fibrillation and Brugada-like Syndrome

N. Marziliano ${ }^{1}$, A. Medoro ${ }^{2}$, D. Fiscella ${ }^{3}$, A. Fiscella ${ }^{4}$, L.

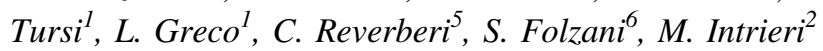

${ }^{1}$ ASL TARANTO, Taranto, Italy, ${ }^{2}$ Università degli Studi del Molise, Campobasso, Italy, ${ }^{3}$ Ospedale Garibaldi, Catania, Italy, ${ }^{4}$ Fondazione Floresta Longo, Catania, Italy, ${ }^{5}$ Centro Clinico Gemini, Parma, Italy, ${ }^{6}$ Poliambulatorio Città di Collecchio, Collecchio, Italy

In the last years, the increasing evidences about the coexistence of genetically based cardiac arrhythmias and epilepsy brings out the emerging concept of cardio-cerebral channelopathy. In this view, we describe two unrelated cases presenting cardiac and epileptic phenotypes in which two novel intragenic duplications in the SCN5A gene (c.1627_1653dup, p.Phe543_Ala551dup and c.1846_1863dup, p.Ser616_Pro621dup, respectively) were identified. The SCN5A gene encodes for the $\alpha$-subunit of the $\mathrm{Na}_{\mathrm{v}} 1.5$ channel and mutations in this gene are mainly involved in electrical channelopathies that lead to lifethreatening arrhythmias (Brugada syndrome, Long-QT syndrome, Sudden Infant Death syndrome, etc.) or structural changes in the myocardium, such as in the dilated cardiomyopathy (DCM). With the aim of characterizing the biological effects of such genetic defects, we studied the biophysical properties of the $\mathrm{Na}_{\mathrm{v}} 1.5$ channels revealing that duplication might prompt for a loss-of-function of the $\mathrm{Na}_{\mathrm{v}} 1.5$-mediated current in a zebrafish model without alterations in the heart structure and function (at least for the first mutation). Taking together, clinical and biochemical data support the idea that the intragenic in-frame 
duplications within the $S C N 5 A$ gene could be responsible for both cardiac and epileptic phenotypes.

N. Marziliano: None. A. Medoro: None. D. Fiscella: None. A. Fiscella: None. L. Tursi: None. L. Greco: None. C. Reverberi: None. S. Folzani: None. M. Intrieri: None.

\section{P05.78.C}

Clonal occurrence of the Hutchinson-Gilford progeria syndrome lamin A mutation in chronic kidney disease

G. Revechon ${ }^{1}$, H. Helgadottir ${ }^{l}$, N. Viceconte ${ }^{1}$, A. Witasp ${ }^{2}$, A. Sola Carvajal $^{1}$, D. Whisenant ${ }^{1}$, D. McGuinness ${ }^{3}, G$. Artiach $^{4}$, E. Wallen Arzt ${ }^{1}$, A. Thorell ${ }^{5}, A$. Babler $^{6}, S$.

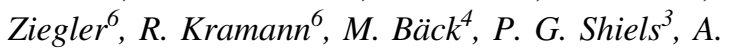
Wernerson $^{2}$, P. Stenvinkel ${ }^{2}, M$. Eriksson ${ }^{l}$

${ }^{1}$ Karolinska Institute, Dept of Biosciences and Nutrition, Huddinge, Sweden, ${ }^{2}$ Karolinska Institute, Dept of CLINTEC, Huddinge, Sweden, ${ }^{3}$ University of Glasgow, Institute for Cancer Sciences, Glasgow, United Kingdom, ${ }^{4}$ Karolinska Institute, Dept of Medicine, Stockholm, Sweden, ${ }^{5}$ Karolinska Institute, Dept of Clinical Sciences, Dept of Surgery, Ersta hospital, Stockholm, Sweden, ${ }^{6}$ University hospital RWTH Aachen, Aachen, Germany

Somatic mutagenesis occurs continuously across the life course, resulting in tissues composed of clones with different genetic makeups. While the majority of mutations have no functional significance, some can contribute to disease and aging. The nature of the arterial wall, with its media being composed of clonal smooth muscle cells, makes this tissue particularly sensitive to somatic mutagenesis. Here we analyzed progerin, the protein responsible for the premature aging disorder Hutchinson-Gilford progeria syndrome (HGPS), for its possible involvement in the accelerated vascular aging process observed in chronic kidney disease (CKD), due to similarities in vascular phenotypes. We provide evidence for expression of progerin, and the occurrence of the HGPS-causing LMNA c.1824C $>$ T mutation as a somatic mutation in CKD patient arteries. Clonal populations of vascular cells with the $1824 \mathrm{C}>\mathrm{T}$ mutation was supported by clusters of progerin positive cells in the media and a high frequency of the mutant allele in several CKD arteries. Further analysis of proliferation and DNA damage in the CKD arteries indicated regeneration and progerin-induced genomic damage. Our results implicate somatic mutations in the functional decline of the vascular wall and support that somatic mutations may become a risk factor during extensive tissue damage.

G. Revechon: None. H. Helgadottir: None. N. Viceconte: None. A. Witasp: None. A. Sola Carvajal: None. D. Whisenant: None. D. McGuinness: None. G. Artiach: None. E. Wallen Arzt: None. A. Thorell: None. A.
Babler: None. S. Ziegler: None. R. Kramann: None. M. Bäck: None. P.G. Shiels: None. A. Wernerson: None. P. Stenvinkel: None. M. Eriksson: None.

\section{P05.79.A}

Enrichment of rare variants in Loeys-Dietz syndrome genes in spontaneous coronary artery dissection but not in severe fibromuscular dysplasia

J. Meester ${ }^{l}$, A. Verstraeten ${ }^{l}$, M. Perik ${ }^{l}$, L. Van Den Heuvel $^{1}$, M. Kempers ${ }^{2}$, I. Krapels ${ }^{3}$, L. Van Laer ${ }^{1}, N$.

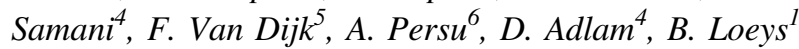

${ }^{1}$ Centre of Medical Genetics, University of Antwerp, Antwerp University Hospital, Antwerp, Belgium, ${ }^{2}$ Department of Human Genetics, Radboud University Nijmegen Medical Center, Nijmegen, Netherlands, ${ }^{3}$ Department of Clinical Genetics, Maastricht University Medical Centre, Maastricht, Netherlands, ${ }^{4}$ Department of Cardiovascular Sciences and National Institute for Health Research Leicester Biomedical Research Centre, Glenfield Hospital, Leicester, United Kingdom, ${ }^{5}$ Ehlers-Danlos Syndrome, National Diagnostic Service, Northwick Park and St. Mark's Hospitals, Harrow, United Kingdom, ${ }^{6}$ Pole of Cardiovascular Research, Institut de Recherche Expérimentale et Clinique, Université Catholique de Louvain, and Division of Cardiology, Cliniques Universitaires Saint-Luc, Université Catholique de Louvain, Brussels, Belgium

Spontaneous coronary artery dissection (SCAD) is characterized by separation of the inner intimal lining of the coronary artery from the outer vessel wall. It is the prime cause of acute myocardial infarction in women below the age of 50. Fibromuscular dysplasia (FMD) presents with condensed areas of arterial stenosis, aneurysm, tortuosity and dissection. It has become clear that FMD is prevalent in SCAD cohorts and vice versa. However, the genetic etiology of both conditions remains largely elusive. We aimed to investigate the contribution of rare genetic variants in vascular Ehlers-Danlos and the Marfan, Loeys-Dietz (LDS), Shprintzen-Goldberg syndrome genes to the etiology of SCAD and FMD in a large multi-center patient cohort. Haloplex-based gene panel sequencing of COL3A1, FBN1, TGFB2/3, SMAD2/3, TGFBRI/2 and SKI was performed in 191 SCAD patients with or without FMD and 111 isolated FMD patients. Subsequent variant filtering was performed. The gnomAD database was used as an independent control dataset and filtered identically. Case-control burden analyses were performed. Rare variants in SMAD2 are significantly enriched in SCAD patients compared to controls $(p=0.001)$. A combined LDS-gene burden analysis was performed, revealing a highly significant association (4.5\% vs $1.5 \%$; $=0.000003$ ). When performed for FMD, none of 
the analyses yielded a significant $p$-value or showed a trend. We here provide evidence for an important contribution of rare genetic variants in LDS genes to the etiology of SCAD. Our findings suggest a strong role for the TGFbeta signaling in the pathogenesis of SCAD but not FMD.

J. Meester: None. A. Verstraeten: None. M. Perik: None. L. Van Den Heuvel: None. M. Kempers: None. I. Krapels: None. L. Van Laer: None. N. Samani: None. F. Van Dijk: None. A. Persu: None. D. Adlam: None. B. Loeys: None.

\section{P05.80.B}

Genetic association study of eight steroid hormones and implications for sexual dimorphism of coronary artery disease

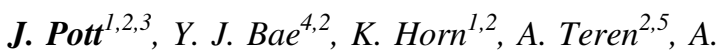
Kühnapfel $^{1,2,3}$, H. Kirsten ${ }^{1,2}$, U. Ceglarek ${ }^{4,2}$, M. Loeffler $^{1,2}$, J. Thiery ${ }^{4,2}$, J. Kratzsch ${ }^{4,2}$, M. Scholz ${ }^{1,2,3}$

${ }^{1}$ Institute of Medical Informatics, Statistics und Epidemiology, University of Leipzig, Leipzig, Germany, ${ }^{2}$ LIFE Research Center for Civilization Diseases, University of Leipzig, Leipzig, Germany, ${ }^{3}$ IFB AdiposityDiseases, University Hospital, Leipzig, Germany, ${ }^{4}$ Institute of Laboratory Medicine, Clinical Chemistry and Molecular Diagnostics, University Hospital, Leipzig, Germany, ${ }^{5}$ Heart Center Leipzig, Leipzig, Germany

Introduction: Steroid hormones act as important regulators of physiological processes in the body. Our aim is to identify genetic factors that influence steroid hormone metabolism, to detect genetic sexual dimorphisms and to investigate possible causal implications for atherosclerotic vascular disease phenotypes.

Materials and Methods: In two independent cohorts, we performed genome-wide sex-specific meta-association studies of eight steroid hormones: progesterone, 17hydroxyprogesterone (17-OHP), androstenedione, testosterone, estradiol, dehydroepiandrosterone sulfate (DHEA-S), cortisol, and aldosterone (LIFE-Adult, LIFE-Heart, max. $n$ $=7667)$. Genome-wide significant loci $\left(\mathrm{p}<5 \times 10^{-8}\right)$ were tested for sex differences in effect size. The causal relationships between hormone levels and atherosclerotic vascular diseases were investigated using sex-specific eQTL analyses and Mendelian Randomization (MR) approaches.

Results: We detected 22 genome-wide significant loci for seven steroid hormones of our panel, of which nine displayed significant sexual dimorphisms. Eight loci relate to genes coding for enzymes involved in steroid biosynthesis, almost completing the list of genes of this pathway with genetic associations. Other loci correspond, e.g., to the WNT4/ $\beta$-catenin pathway. MR revealed that cortisol, androstenedione, 17-OHP and DHEA-S had sex-specific causal effects on coronary artery disease.

Conclusions: Our study greatly improves insight into genetic regulation of steroid hormones and their dependence on sex. The significant MR estimates support the hypothesis that steroid hormones influence the development of atherosclerosis, which partly explains the higher risk for men. These results could serve as a basis for analyzing sexdimorphisms in other complex diseases.

This project was supported by the HI-MAG Project Funding for Young Scientists of the Medical Faculty of the University Leipzig.

J. Pott: None. Y.J. Bae: None. K. Horn: None. A. Teren: None. A. Kühnapfel: None. H. Kirsten: None. U. Ceglarek: None. M. Loeffler: None. J. Thiery: None. J. Kratzsch: None. M. Scholz: None.

\section{P05.81.C}

Genetic post-mortem in drug users with sudden cardiac death

\section{H. Massey, J. Dean, L. Deboys, D. O'Sullivan}

\section{Aberdeen royal infirmary, Aberdeen, United Kingdom}

Aim: To quantify our ability to make a genetic diagnosis in drug users with sudden cardiac death (SCD).

Background: While there are European recommendations for genetic testing in SCD1, these guidelines do not extend to drug users with positive toxicology at postmortem. We wanted to gain a better understanding of the genetic pick up rate in this population.

Method: We undertook a 10-year retrospective study looking at genetic diagnoses in drug related deaths discussed at the joint pathology-genetic MDT. This was compared to age and provisional aetiology matched controls.

Results: 60 drug related deaths were identified, and 40 patients underwent genetic testing. 7 variants were found of which 3 were classified as benign and 4 VUS. The most common reason for genetic testing was enlarged heart at post mortem; however the genetic pick up in this group was 0 . Patients with structurally normal hearts had the most variants detected. This would coincide with the majority of variants being found in arrhythmogenic genes. Family history did not influence genetic pick up. In the control group variants in arrhythmogenic genes again predominated correlating with the highest genetic pick up in patients with normal post-mortems. However in the control group 17 variants were detected of which 3 were pathogenic.

Conclusions: Our ability to make a genetic diagnosis in drug users with SCD is low. We need to undertake a larger 
study to characterise those drug users with a positive genetic diagnosis so testing can be rationalised.

H. Massey: None. J. Dean: None. L. Deboys: None. D. O'Sullivan: None.

\section{P05.82.A}

Insights into the genetic basis of ventricular tachycardia in Kazakhstani patients. Targeted next generation sequencing

A. R. Akilzhanova ${ }^{l}$, C. Guelly ${ }^{2}$, Z. Abilova ${ }^{l}, S$. Rakhimova $^{l}$, A. Akhmetova ${ }^{l}$, U. Kairov ${ }^{l}, S$. Trajanoski ${ }^{2}, G$. Akilzhanova $^{3}$, M. Bekbosynova ${ }^{4}$, Z. Zhumadilov $^{l}$

${ }^{1}$ Center for life sciences, National Laboratory Astana, Nazarbayev University, Nur-Sultan, Kazakhstan, ${ }^{2}$ Center for Medical Research, Medical University of Graz, Graz, Austria, ${ }^{3}$ Medical University of Semey, Pavlodar branch, Pavlodar, Kazakhstan, ${ }^{4}$ National Research Cardiac Surgery Center, Nur-Sultan, Kazakhstan

Introduction: Recent advances in genetic screening and the potential of therapeutic intervention in patents with cardiac arrhythmias have garnered this group of disorders much attention in the scientific community. The purpose of the study was to identify the genetic basis of ventricular tachycardia (VT) in Kazakhstani patients.

Material and Methods: using predeveloped a targeted panel of 96 known cardiac disease genes, associated with cardiomyopathy and arrhythmia we screened 92 individuals, diagnosed with VT and dilated cardiomyopathy (VT DCM) or idiopathic VT (iVT). Results. Targeted sequencing and stepwise filtering of the annotated variants identified 307 unique variants in 74 genes totaling up in 456 variants for the overall study group. Variants included one in/del, four splice-site and 451 single-nucleotide variants (SNV) within the coding exonic regions. Seven $(0.15 \%)$ of the SNVs were unique stop-gain variants, three of those residing in the TTN gene. 168 HGMD mutations (61 unique) were observed in 37 genes. According to ACMG all variants were classified as 9 pathogenic (KCNJ2 R218Q and TTN R5338X in iVT patients, KCNQ1 c.477 $+1 \mathrm{G}>\mathrm{A}$, LMNA Q353X, MYH7 F244L, TTN L17465X and W21011X, DSG2 c.2334+1G $>$ A, GAA W746C in VT DCM patients), 11 likely pathogenic, 97 variants with uncertain significance and rest as benign.

Conclusions: Individuals with VT either secondary to DCM or of idiopathic etiology carry multiple rare mutations and potentially pathogenic sequence variants in cardiac risk genes in a similar pattern and at a comparable frequency. Study support: grant from the Ministry Education and Science, Republic of Kazakhstan (AP05134683).
A.R. Akilzhanova: None. C. Guelly: None. Z. Abilova: None. S. Rakhimova: None. A. Akhmetova: None. U. Kairov: None. S. Trajanoski: None. G. Akilzhanova: None. M. Bekbosynova: None. Z. Zhumadilov: None.

\section{P05.83.B}

Identification of the major genetic contributors to Tetralogy of Fallot

\author{
R. M. Monaghan ${ }^{1}$, D. J. Paige ${ }^{2}$, S. G. Williams ${ }^{1}$, B. D. \\ Keavney ${ }^{l}$ \\ ${ }^{1}$ University of Manchester, Manchester, United Kingdom, \\ ${ }^{2}$ Manchester Metropolitan University, Manchester, United \\ Kingdom
}

Tetralogy of Fallot (TOF) is the most common cyanotic congenital heart defect. Rare genetic variants have been identified as important contributors to the risk of congenital heart disease, but relatively small numbers of TOF cases have been studied. We used whole exome sequencing to assess the prevalence of unique, deleterious variants in the largest cohort of nonsyndromic TOF patients reported to date. The presence of such variants was defined by their absence in the Genome Aggregation Database and bioinformatic prediction of their deleterious effect on function. The enrichment of variants in two genes, NOTCHI and FLT4, surpassed thresholds for exome-wide significance $(P$ $<5 \times 10-8)$ after correction for multiple comparisons. NOTCHI was most frequently found to harbour unique, deleterious variants $(4.5 \%$; 95\% CI, 3.2\%-6.1\%). Three $\mathrm{NOTCHI}$ variants were subjected to functional evaluation, and two showed a reduction in Jagged1-induced NOTCH signalling. FLT4 variants were found in $2.4 \%(95 \% \mathrm{CI}$, $1.6 \%-3.8 \%$ ) of our cohort. In addition, variants in the wellestablished TOF gene, TBXI, and the genes were also significantly enriched. Our study underlines the importance of sequencing large cohorts of CHD cases in order to further discover their genetic basis.

Grant reference: BHF (RG/15/12/31616)

R.M. Monaghan: None. D.J. Paige: None. S.G. Williams: None. B.D. Keavney: None.

\section{P05.84.C}

In search of genetic modifiers that explain the phenotypic variability in SMAD3-related aortopathy

J. D. Velchev ${ }^{I}$, M. Perik ${ }^{1}$, I. M. B. H. van de Laar $^{2}, J$. Richer $^{3}$, A. Verstraeten ${ }^{\text {I }}$ M. Alaerts ${ }^{1}$, B. L. Loeys ${ }^{1,4}$

${ }^{1}$ Center of Medical Genetics, University of Antwerp and Antwerp University Hospital, Antwerp, Belgium, ${ }^{2}$ Department of Clinical Genetics, Erasmus MC, University Medical Center 
Rotterdam, Rotterdam, Netherlands, ${ }^{3}$ Department of Medical Genetics, Children's Hospital of Eastern Ontario, Children's Hospital of Eastern Ontario Research Institute, Ottawa, ON, Canada, ${ }^{4}$ Department of Human Genetics, Radboud University Nijmegen Medical Center, Nijmegen, Netherlands

Introduction: Thoracic aortic aneurysm and dissection (TAAD) is a frequent cause of mortality in the Western world. Loeys-Dietz syndrome (LDS), an early-onset TAAD syndrome is caused by mutations in genes coding for components of the TGFbeta signaling, including SMAD3. Remarkably, a fraction of $S M A D 3$ mutation carriers remains cardiovascularly unaffected throughout life, suggesting the existence of genetic modifiers for aortopathy. This study implements a combination of linkage analysis and whole-genome sequencing (WGS) in order to identify genetic modifiers that explain the variability in SMAD3-related aortopathy.

Material and Methods: In two large LDS families $(n=$ 41 mutation carriers) segregating SMAD3 missense mutations and presenting variable TAAD expressivity genomewide SNP-based linkage analysis was performed. LOD scores were obtained using MERLIN and SUPERLINK and linkage was complemented with WGS of six affected and six unaffected mutation carriers.

Results: In the first family linkage analysis identified a chromosomal region (chr2:149,604,077-155,607,398) with a LOD score of 2.68, suggesting the presence of an aggravating modifier. Linkage peak was observed in the second family with a LOD score of 2.86 ( $\operatorname{chr} 18: 43,013,157$ 57,021,550). Additionally, the WGS data analysis of the second family resulted in the discovery of a rare variant in $T G F B 2$, a known TAA causative gene, exclusively present in affected mutation carriers.

Conclusions: The obtained data indicate that variants in known TAAD genes may play a role in modulating a primary causative mutation; chromosomal regions segregate with disease severity suggesting a presence of genetic modifiers and that distinct families may bear different genetic modifiers as indicated by the linkage analysis.

J.D. Velchev: None. M. Perik: None. I.M.B.H. van de Laar: None. J. Richer: None. A. Verstraeten: None. M. Alaerts: None. B.L. Loeys: None.

\section{P05.85.A}

Identification of Clinically Relevant Variants by Whole Exome Sequencing in Chinese Patients with Sporadic Non-syndromic Type A TAAD

\section{J. Guo, C. Hao, X. Hu, F. Leng, X. Liu}

Genetics and Birth Defects Control Center, Beijing Children's Hospital, Beijing, China
Introduction: Thoracic aortic aneurysm and dissection (TAAD) is a life-threatening disease, of which genetic abnormalities are considered as important risk factors. The present research aims at identifying causal variants in Chinese patients with sporadic non-syndromic type A TAAD (ATAAD).

Materials and Methods: Whole exome sequencing (WES) was performed on 73 sporadic Chinese patients with ATAAD, 30 TAAD associated genes were curated for bioinformatic analyses. Clinical differences were compared between patients with and without causal variants.

Results: 15 pathogenic/likely pathogenic variants were identified (8 novel and 7 previously described) in 4 known TAAD-causal genes (FBN1, TGFBR2, SMAD3 and ACTA2) in 15 individuals, including 11 variants in FBNI (7 missense, 3 truncating, and 1 splicing variants), 2 missense variants in TGFBR2, 1 ACTA2 frameshift variant and 1 SMAD3 frameshift variant. Significant clinical differences were found between patients with and without causal variants. Patients with TAAD-causal variants proved to have an earlier onset age, a more dilated aorta, and relatively intractable subtypes. Even without risk factor like hypertension, they might still suffer from TAAD with TAAD-causal variants.

Conclusions: The variants identified in our research might not only result in the occurrence of ATAAD, but also add complexities and difficulties to the clinical practice. Our data demonstrated that WES was an effective tool for determining genetic etiologies of non-syndromic ATAAD and could be helpful in genetic counseling for ATAAD patients and their at-risk family members.

J. Guo: None. C. Hao: None. X. Hu: None. F. Leng: None. X. Liu: None.

\section{P05.87.C}

Investigating the role of pathogenic TTN variants in heart failure by whole-exome sequencing

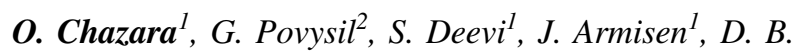
Goldstein $^{2}$, C. Haefliger ${ }^{1}$

${ }^{1}$ Astrazeneca, Melbourn, United Kingdom, ${ }^{2}$ Institute for Genomic Medicine, Columbia University Medical Center, New York, NY, United States

Heart failure (HF) affects $~ 40$ million people globally. Estimates of the genetic predisposition of developing HF is 1.69 if one parent is affected and 1.92 if both parents have HF (Framingham cohort study, Lopez et al. 2013).

Recent studies suggest higher cardiovascular genetic risk in patients with and without diagnosed heart failure if they 
carry a pathogenic loss of function variant in the gene encoding titin (TTN).

Here, we assess the role of pathogenic TTN variants by whole-exome sequencing in 5916 patients with heart failure from two clinical studies CHARM and CORONA, and 268,451 unrelated Europeans from the UK Biobank prospective cohort study, including 4634 individuals with ICD-10 codes for heart failure (HF, based on hospital inpatient diagnoses).

In both types of cohorts, we detect a significant enrichment of pathogenic TTN variants in subjects with HF diagnosis. Furthermore, after restricting the analysis to the variants located in exons that are predominantly spliced in the heart, the genetic risk was increased: from $2.54(95 \%$ CI, 1.96-3.31) to $4.52(95 \% \mathrm{CI}, 3.10-6.68)$ in the clinical studies, and from 2.95 (95\% CI, 2.42-3.57) to 4.97 (95\% CI, 3.94-6.19) in the UKBB. The enrichment observed is similar in clinical trial data and routine care health records.

As expected, individuals with a diagnosis of cardiomyopathy had the highest rate of TTN mutations, but we also observed TTN variants in up to $5 \%$ of HF patients due to other aetiologies, such as ischaemic and hypertensive disease. This result highlights the importance of TTN variants beyond cardiomyopathies.

O. Chazara: A. Employment (full or part-time); Significant; Astrazeneca. G. Povysil: None. S. Deevi: A. Employment (full or part-time); Significant; Astrazeneca. J. Armisen: A. Employment (full or part-time); Significant; Astrazeneca. D.B. Goldstein: F. Consultant/Advisory Board; Modest; Astrazeneca. C. Haefliger: A. Employment (full or part-time); Significant; Astrazeneca.

\section{P05.88.A}

\section{Non-amyloidotic transthyretin cardiomyopathy}

\section{J. Gimeno Blanes ${ }^{1}$, M. Sabater Molina ${ }^{1}$, I. San Roman ${ }^{1}$, L.} Polo $^{2}$, I. Garrido ${ }^{3}$, C. Salas ${ }^{4}$, F. Lopez Andreu ${ }^{5}$

${ }^{1}$ Inherited Cardiac Disease Unit. Universitary Hospital Virgen de la Arrixaca., Murcia, Spain, ${ }^{2}$ Hospital Universitario Virgen Arrixaca, Department of Pathological Anatomy., Murcia, Spain, ${ }^{3}$ Department of Cardiology Universitary Hospital Virgen de la Arrixaca., Murcia, Spain, ${ }^{4}$ Hospital Puerta de Hierro, Department of Pathological Anatomy, Madrid, Spain, ${ }^{5}$ Hospital General Reina Sofia, Service of Internal Medicine, Murcia, Spain

Introduction: We aim to present an unsolved case of a young patient with unclassified cardiomyopathy associated with a genetic TTR variant predicted to cause a dramatic change in protein structure. Clinical course went from asymmetric hypertrophy with restrictive phenotype to cardiac dilatation and systolic dysfunction. Pulmonary pressures were strikingly elevated.

Case: A 33 years old woman was admitted in 1997 with limiting dyspnoea. Echocardigram showed non-obstructive asymmetrical septal hypertrophy of $17 \mathrm{~mm}$ maximum at the septum and restrictive physiology. Sarcoid cardiomyopathy was ruled out with negative scintigraphy and unspecific biopsy in the absence of other extracardiac features. Atrioventricular ablation and permanent pacing were required in 2002. Clinical deterioration led to frequent heart failure admissions and one episode of syncope. Implantable cardioverter defibrillator was implanted. Emergency transplant was performed with a postoperative complicated course in 2006. Patient remained stable to date. Study of the explanted heart demonstrated macro and microscopic hypertrophy, extensive fibrosis, unspecific vacuolization and disarray. There were no signs of amyloid with usual stains and immunohistochemical typing. Sequencing of the TTR gene led to identification of a de novo frameshift mutation p.S132fs*25(g.6903_6906delinsGTAGGG) causing original stop codon lost, results in prolonged protein. Cardiac evaluation of her brother, sister and parents ruled out any sign of cardiomyopathy. All relatives were free for the TTR variant.

Conclusions: Case of cardiomyopathy associated with a TTR variant. Severe right and left heart failure clinical course with very particularly severe pulmonary hypertension ended up in heart transplant. Mechanisms of disease in this case remain unknown.

J. Gimeno Blanes: None. M. Sabater Molina: None. I. San Roman: None. L. Polo: None. I. Garrido: None. C. Salas: None. F. Lopez Andreu: None.

\section{P05.89.B}

Searching for genes associated with primary varicose veins using targeted next-generation sequencing

E. Strauss ${ }^{1,2}$, M. Zmyslowski ${ }^{3}$, B. Budny ${ }^{4}, L$. Dzieciuchowicz ${ }^{3}$, E. Wrotkowska ${ }^{4}$, M. Ruchala ${ }^{4}, K$. Ziemnicka $^{4}$, G. Oszkinis ${ }^{5}$, Z. Krasinski ${ }^{3}$

${ }^{1}$ Institute of Human Genetics of the Polish Academy of Sciences in Poznan, Poznan, Poland, ${ }^{2}$ Department of Vascular, Endovascular Surgery, Angiology and Phlebology Poznan University of Medical Sciences, Poznań, Poland, ${ }^{3}$ Department of Vascular, Endovascular Surgery, Angiology and Phlebology Poznan University of Medical Sciences, Poznan, Poland, ${ }^{4}$ Department of Endocrinology, Metabolism and Internal Medicine, Poznan University of Medical Sciences, Poznan, Poland, ${ }^{5}$ Department of General and Vascular Surgery, Poznan University of medical Sciences, Poznan, Poland 
Introduction: The aim of this study was to search for genes involved in the pathogenesis of the primary varicose veins (PVV) using targeted next-generation gene sequencing. Sporadic cases with the onset of the first symptoms of disease below or equal 40 years of age were considered.

Methods: A cohort of 66 PVV patients was collected and exome sequencing of the 35 candidates' genes was performed, which focused on the genes encoding structural elements of the vessel wall, metalloproteinases, and their protein inhibitors, proteins involved in the homeostasis of the coagulation system and regulation of sex hormones system, as well as genes previously associated with VV development.

Results: Most of the patients $(91.9 \%)$ had a positive family history of VV, considering first-, second- or thirddegree relatives. 26 potentially pathogenic changes: 12 mutations in 9 genes ( 7 known and 5 novel), 5 rare SNPs and 9 common SNPs were found. $40 \%$ of the patients had a potentially causative mutation in the candidate genes: $33 \%$ of them had 1 mutation, $3 \%$ had 2 mutations and $1.5 \%$ had 3 mutations. The association between one SNP in the MMP-9 gene and the age of the onset of PVV symptoms was also observed.

Conclusions: This report provides evidence for the heterogeneity and complexity of the genetic background of PVV in sporadic cases and shows the influence of genetic factors on the age of the onset of this disease. It also reveals attractive targets for further research in a larger group of patients and functional analyses.

E. Strauss: None. M. Zmyslowski: None. B. Budny: None. L. Dzieciuchowicz: None. E. Wrotkowska: None. M. Ruchala: None. K. Ziemnicka: None. G. Oszkinis: None. Z. Krasinski: None.

\section{P06 Metabolic and Mitochondrial Disorders}

\section{P06.01.C}

Identification of AADC patient through combined determination of 3-OMD biomarker and $D D C$ gene sequencing and copy number variation analysis

\section{Pereira ${ }^{l}$, C. Cozma ${ }^{l}$, L. Demuth ${ }^{l}$, G. Morales Torres ${ }^{l}$, M. Iurascul, P. Bauer ${ }^{l}$, A. Rolfs ${ }^{l}$, C. Lourenco ${ }^{2}$}

${ }^{1}$ Centogene AG, Rostock, Germany, ${ }^{2}$ Universidade de São Paulo, Sao Paulo, Brazil

L-amino acid decarboxylase (AADC) deficiency is caused by bi-allelic variants in the $D D C$ gene. This very rare neurometabolic disorder is clinically characterized by vegetative symptoms, oculogyric crises, dystonia, and severe neurologic dysfunction, usually beginning in infancy or childhood. Treatment has become available recently. We quantified 3-OMD (3-O-Methyldopa), a suggested AADCspecific biomarker, in dried blood spots by tandem mass spectrometry. Upon suggestive findings, we subsequently analyzed the $D D C$ gene by amplicon-based next generation sequencing (NGS) and copy number variant (CNV) screening. This two-step approach identified several patients. The ones with at least one hitherto undescribed variant are described here in detail. All three patients had pathological levels of 3-OMD. In patient 1, we identified a novel whole $D D C$ gene duplication in homozygosity. In patient 2, we identified the two missense variants p. (Ser147Ile) and p.(Val60Ala) in compound heterozygosity; interestingly, variant p.(Ser147Ile) was not only novel, but shown to have occurred de novo. In patient 3 , p. (Trp121Arg) and p.(Arg347Gln) were identified in compound heterozygosity. The former is novel, while the latter has frequently been described in AADC patients previously. Using a combined biochemical, NGS and CNV approach, a positive identification and diagnosis of AADC deficiency patients is reliably reached. This allows for an early treatment of the patients, leading to an improvement of their quality of life. Moreover, the identification of novel DDC variants and the establishment of correlations with 3-OMD levels can be expected to increase our understanding of the disease.

C. Pereira: None. C. Cozma: A. Employment (full or part-time); Significant; Centogene AG. L. Demuth: A. Employment (full or part-time); Significant; Centogene AG. G. Morales Torres: A. Employment (full or part-time); Significant; Centogene AG. M. Iurascu: A. Employment (full or part-time); Significant; Centogene AG. P. Bauer: A. Employment (full or part-time); Significant; Centogene AG. A. Rolfs: A. Employment (full or part-time); Significant; Centogene AG. C. Lourenco: None.

\section{P06.04.C}

Patients affected by autosomal recessive or dominant cutis laxa type 3 show diverse degrees of collagen alterations

N. El Choubassi ${ }^{1,2}$, L. Lugli ${ }^{3}$, C. Spada ${ }^{4}$, D. de Silva ${ }^{5}$, O.

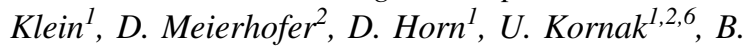
Fischer-Zirnsak ${ }^{1,2}$

${ }^{1}$ Charité Universitaetsmedizin Berlin, Berlin, Germany, ${ }^{2}$ Max Planck Institute for Molecular Genetics, Berlin, Germany, ${ }^{3}$ Division of Neonatal Intensive Care Unit, Department of Pediatrics, University Hospital, Modena, Italy, ${ }^{4}$ Department of Medical and Surgical Sciences for Mothers, Children and Adults, Post Graduate School of Paediatrics, University of Modena \& Reggio Emilia, Modena, Italy, ${ }^{5}$ Department of Physiology, Faculty of Medicine, University of Kelaniya, Ragama, Sri Lanka, 
${ }^{6}$ Institute of Human Genetics, University Medical Center Göttingen, Göttingen, Germany

Introduction: Mutations in $A L D H 18 A 1$, encoding the mitochondrial enzyme pyrroline-5 carboxylate synthase (P5CS), lead to a broad spectrum of rare genetic conditions. Besides hereditary spastic paraplegia, ALDH18A1 mutations are causative for autosomal recessive (ARCL3) and dominant (ADCL3) cutis laxa with progeroid features.

Materials and Methods: We performed whole exome (WES) and gene panel sequencing to identify pathogenic variants in our probands. These analyses were complemented by mRNA expression studies, immunofluorescence, immunoblot and mass-spectrometry approaches on patient derived fibroblasts.

Results: We report on novel pathogenic variants in three affected individuals with ARCL3/ADCL3. These variants have variable impacts on the stability of the protein and its localization within the mitochondrial network. However, targeted metabolomics revealed substantial alterations of mitochondrial proline synthesis rates. Additionally, gene expression profiling, comparative proteomics and subsequent immunoblot analyses showed alterations in expression of different collagens.

Conclusions: Our data expand the mutational spectrum of ALDH18A1-related diseases and demonstrate the importance of correct ALDH18Al localization within the mitochondria for proper functioning. Additionally, they suggest a potential connection of the mitochondrial proline synthesis pathways with the regulation of collagen synthesis.

Funding: The study was supported by the Deutsche Forschungsgemeinschaft (FI 2240/1-1) to BF-Z

N. El Choubassi: None. L. Lugli: None. C. Spada: None. D. de Silva: None. O. Klein: None. D. Meierhofer: None. D. Horn: None. U. Kornak: None. B. FischerZirnsak: None.

\section{P06.06.B}

Clinical study of the relationship between histamie intolerance and variants in the AOC1/ABP1 Gene

\author{
J. Vinas-López ${ }^{1}$, P. Ojeda ${ }^{2}$, I. Ojeda ${ }^{2}$, A. Duelo ${ }^{3}, B$. \\ García-Velasco $^{l}$, C. Lenz ${ }^{2}$, T. Perucho ${ }^{l}$, M. Berbel ${ }^{3}, A$. \\ Vásquez $^{3}$, E. Ruiz-Casares ${ }^{1,4}$
}

${ }^{1}$ Genyca, Majadahonda, Spain, ${ }^{2}$ Clínica Ojeda de Asma y Alergia Dres. Ojeda, Madrid, Spain, ${ }^{3}$ Centro de Nutrición AD Dietistas, Barcelona, Spain, ${ }^{4}$ Universidad San Pablo CEU, Madrid, Spain

Introduction: Histamine is a biogenic amine with many essential physiological activities. However, its deregulation and accumulation can cause a multitude of pathologies such as histamine intolerance. There are two enzymes involved in the metabolism of histamine: histamine N-methyltransferase (HNMT) is responsible for degrading histamine inside cells. Diamine-oxidase (DAO) is the other enzyme involved in the degradation of histamine, mainly exogenous histamine, ingested with the diet. The deficiency of DAO activity could be one of the main causes of histamine intolerance, an alteration in homeostasis that is caused by a reduced intestinal degradation of histamine, with the consequent increase in its plasma levels. Variants in AOC1/ABP1 gene, coding DAO enzyme, have been associated with a reduction in DAO activity and accumulation of histamine.

Objective: The present study aims to demonstrate the negative effect that the variants rs10156191, rs1049742 and rs 1049793 have on DAO plasma activity in Caucasian male patients with clinical manifestations associated with histamine intolerance.

Methods: We analyzed 78 white male patients with a clinical manifestation associated with histamine intolerance. Variants analyzed were genotyped by multiplex SNPE (Single Nucleotide Primer Extension).

Results: The variants rs10156191 and rs1049742 reduce significantly DAO plasma activity. Factors such as worsening symptoms after eating a diet rich in histamine or NSAID-type drugs (non-steroidal anti-inflammatory) are associated with a greater number of alternative alleles in the SNVs analyzed, and reduced DAO plasma activity.

Conclusions: These results show the predictive value of this genetic study in patients who have a clinical manifestation associated with histamine intolerance.

J. Vinas-López: C. Other Research Support (supplies, equipment, receipt of drugs or other in-kind support); Modest; Genyca. P. Ojeda: None. I. Ojeda: None. A. Duelo: None. B. García-Velasco: None. C. Lenz: None. T. Perucho: None. M. Berbel: None. A. Vásquez: None. E. Ruiz-Casares: None.

\section{P06.10.C}

Low prevalence of classic galactosemia in the Sverdlovsk Region (Russian Federation)

\section{O. Lagutina ${ }^{l}$, S. Deryabina ${ }^{1,2}$, T. Belyaeva ${ }^{l}$, N. Nikitina $^{l}$, E. Nikolaeval}

${ }^{1}$ Medical Center "Health Care of Mother and Child", Yekaterinburg, Russian Federation, ${ }^{2}$ Federal State Autonomous Educational Institution of Higher Education «Ural Federal University named after the first President of Russia B.N. Yeltsin, Yekaterinburg, Russian Federation

Introduction: Classic galactosemia is one of the five hereditary metabolic diseases, which has been included in the 
State Program of Neonatal Screening of the Russian Federation in 2006.

Aim of the study: To estimate the incidence of galactosemia in the Sverdlovsk Region based on neonatal screening data.

Patients and methods: 698550 children born in the Sverdlovsk Region from 2006 to 2019 were examined. Initially, the level of total galactose and galactose-1phosphate was determined in a dry blood spot, then an activity of the enzyme galactose phosphate-uridyl transferase (GALT) has been selected as a biochemical marker of galactosemia. The diagnosis of galactosemia should then be confirmed by genetic analysis.

Results: During the 13-year study period 657 newborns were included in the risk group and assigned for molecular genetic testing. Eight children were identified as having classical galactosemia on the basis of the enzymatic and molecular genetic analysis. Moreover, 142 babies had a galactosemia Duarte, another 134 had decreasing activity of the GALT enzyme due to non-genetic reasons. The spectrum of revealed mutations in the GALT gene: $\mathrm{K} 285 \mathrm{~N}$ (31.3\%), Q188R (25.0\%), M142K (12.5\%), E352Q (12.5\%), W316X (6.2\%), R262W (6.2\%) and one new variant P140L (6.2\%), not described earlier. Conclusion. Based on the results of newborn screening programs, the prevalence of classic galactosemia in the Ural population is 1:87 318 live birth and unlikely that it will undergo significant changes with further increase in the screening period.

O. Lagutina: None. S. Deryabina: None. T. Belyaeva: None. N. Nikitina: None. E. Nikolaeva: None.

\section{P06.12.B}

What is beyond the congenital lactic acidosis? From variants to personalized medicine

\section{Bravo-Alonso, R. Navarrete, P. Ruiz-Sala, F. Leal, M. \\ Ugarte, C. Pérez-Cerdá, B. Merinero, B. Pérez, P. \\ Rodríguez-Pombo}

Centro de Diagnóstico de Enfermedades Moleculares, Centro de Biología Molecular Severo Ochoa, UAM-CSIC, CIBERER, IDIPAZ, Madrid, Spain

Implementation of Next Generation Sequencing has dramatically increased the diagnosis success-rate of highly heterogeneous inborn-errors of metabolism such as those underlying congenital lactic acidosis. However, the lack of integrated phenotypic data, the scarce number of datasets of global metabolomics and the necessary expertise to analyze the functional effects of variants of unknown significance, are limiting the transfer of genetic data into clinical practice. Our aim in this study was to evaluate the powerful of an adequate clinical-biochemical and genetic analysis to drive a clinical response in a timely manner preventing the use of major invasive techniques. With a pipeline that combined specific datasets from metabolomics sub-sections and phenotypic information, with DNA and/or RNA analysis, we identified likely pathogenic variants in strong-candidate genes in $64 \%$ of a cohort of 39 patients with clinical suspicion of congenital lactic acidosis. These variants, 14 of which were new, were found in 19 different nuclear and two mitochondrial genes. Specific biochemical test and/or bioenergetics analysis in fibroblasts including measures of oxygen consumption rate, steady-state levels of OxPhos proteins using Blue-native geles, and changes in mitochondrial morphology, sustained the diagnosis for patients carrying novel genotypes. RNA analysis contributed to solve miss or undiagnosed patients, providing a direct insight into transcriptional perturbations caused by specific genetic changes. Finally, by rescuing normal splicing in a GFM1-patient cells carrying in heterozygous fashion the c. $689+908 \mathrm{G}>\mathrm{A}$ change with antisense oligonucleotide treatment, we corroborated the importance of molecular analysis to investigate personalized therapeutic options. LCF/PR/PR16/11110018; PI16/00573; CAM， B2017/ BMD3721

I. Bravo-Alonso: None. R. Navarrete: None. P. RuizSala: None. F. Leal: None. M. Ugarte: None. C. PérezCerdá: None. B. Merinero: None. B. Pérez: None. P. Rodríguez-Pombo: None.

\section{P06.13.C}

Hypoglycemia-some of its many reasons-a molecular update.

\section{K. Brusgaard ${ }^{1}$, Y. Alhaidan ${ }^{2}$, M. Balwi ${ }^{2}$, H. Christesen ${ }^{1}$}

${ }^{1}$ Odense University Hospital, Odense, Denmark, ${ }^{2}$ King Abdullah International Medical Research Center, Riyadh, Saudi Arabia

Hypoglycemia can be caused by numerous somatic or hereditary causes. Three major reasons are; diabetes, hyperinsulinism and ketotic/non-ketotic hyperglycemia. The brain accounts for $60 \%$ of the glucose consumption in the infants' resting state, leaving the brain vulnerable to hypoglycemia. Tight plasma glucose control is ensured by a number of complex mechanisms. In the majority of cases the underlying pathophysiology are unexplained. The goal in the presented study was to find new reasons underlying these disorders.

38 families were included for trio analyses performing exome sequencing for germline variation. Where available exome sequencing was performed on resected pancreatic tissue if no germline cause was elucidated. Data was 
analyzed using VarSeq and various filters considering recessive, $\mathrm{X}$-linked, de-novo or autosomal dominant modes of inheritance. Candidate genes was analyzed by in vitro studies using RIN-m $\beta$-cell lines and $\mathrm{KO}$ of target genes using siRNA or Crispercas9. Measurement of RNA expression levels was performed by Q-RT-PCR or microarray analysis. Insulin measurements were performed.

In $10(26 \%)$ cases the hypoglycemia was explained. In three ketotic hypoglycemia patients the reason was found in a known GSD gene, four patients was explained by novel genes. In one diabetic and one hyperinsulinism patient a novel genetic cause was found. A single hyperinsulinism patient was explained by a previously undetected mutation in a known hyperinsulinism gene. 10 additional candidate genes are currently under in vitro cellular investigation for their role in manifestation of disease in individual families. As a result of the current study the treatment was changed in three individuals

K. Brusgaard: None. Y. Alhaidan: None. M. Balwi: None. H. Christesen: None.

\section{P06.14.A}

Biochemical and molecular landscape of Fabry disease in Portugal

\section{F. E. Laranjeira ${ }^{l, 2}$, P. Rodrigues ${ }^{1,3}$, C. $_{\text {Caseiro }}$, I.} Ribeiro $^{1,2}$, H. Carmo $^{3}$, D. Quelhas ${ }^{1,2}$

${ }^{1}$ Unidade de Bioquímica Genética, Centro de Genética Médica Doutor Jacinto Magalhães, Centro Hospitalar Universitário do Porto, Porto, Portugal, ${ }^{2}$ Unidade Multidisciplinar de Investigação Biomédica, Instituto de Ciências Biomédicas Abel Salazar, Universidade do Porto, Porto, Portugal, ${ }^{3}$ UCIBIO, REQUIMTE - Laboratório de Toxicologia, Departamento de Ciências Biológicas, Faculdade de Farmácia, Universidade do Porto, Porto, Portugal

Introduction: Fabry disease is an X-linked progressive inborn error of metabolism originated by mutations in the GLA gene causing a deficiency of the lysosomal $\alpha$-galactosidase $(\alpha-\mathrm{Gal})$ hydrolase activity. The breakdown of glycolipids is impaired and the lysosomal substrate accumulation triggers the onset of this lysosomal storage disorder. Male patients usually present very low $\alpha$-Gal activity and marked substrate accumulation, and at least some of the clinical hallmarks. In females, however, there is a wide range of phenotypes leading to great controversy regarding clinical diagnosis and therapeutic decisions. Patients and methods

Data concerning leukocytes and plasma $\alpha$-Gal activity together with plasma and urine substrate accumulation for 141 males and 210 females were obtained. Correlation among biochemical parameters and with the molecular defect was evaluated.

Results: 37 genetic variants were found and the most prevalent genetic variant is c.337T $>\mathrm{C}$ p. $(\mathrm{F} 113 \mathrm{~L})$, found in $50 \%$ of males and females. Males usually present a correlation of reduced $\alpha$-Gal activity with increased substrate accumulation. In this group it was possible to identify some genetic variant-specific patterns. Heterozygous females show less pronounced effect on substrate accumulation and enzyme deficiency. The variability of patterns within carriers of the same genetic variant is also noticed.

Conclusions: Some genetic variants whose pathogenicity is controversial are indeed associated with milder biochemical phenotypes. Plasma $\alpha$-Gal activity is not recommended as only diagnosis testing because in the presence of specific variants it may give false negatives. Heterogeneity among heterozygous females should be further evaluated with relation to $\mathrm{X}$ chromosome inactivation profile.

F.E. Laranjeira: None. P. Rodrigues: None. C. Caseiro: None. I. Ribeiro: None. H. Carmo: None. D. Quelhas: None.

\section{P06.15.B}

Mutation spectrum and corresponding clinical manifestation of patients with hypertriglyceridemia in a German outpatient clinic

F. Bardey ${ }^{l}$, L. Rieck ${ }^{l}$, D. Spira ${ }^{l}$, W. März ${ }^{2}$, S. Schwab ${ }^{2}$, P. Binner $^{2}$, T. Bobbert ${ }^{l}$, J. Spranger ${ }^{1}$, E. SteinhagenThiessen $^{1}$, I. Demuth ${ }^{l}$, U. Kassner ${ }^{1}$

${ }^{I}$ Charite Universitätsmedizin Berlin, Berlin, Germany, ${ }^{2}$ SYNLAB MVZ Humangenetik GmbH, Mannheim, Germany

Introduction: A sizeable proportion of patients with severe hypertriglyceridemia (HTG) do have the monogenetic familial chylomicronemia syndrome (FCS). It remains difficult to clinically distinguish FCS from the multifactorial chylomicronemia syndrome (MCS) in the absence of genetic testing. Our aim was to characterize the genotype of our HTG patients with respect to MCS and FCS as well as to apply the clinical FCS-score proposed by Moulin and colleagues.

Methods: We studied 90 genotyped patients with severe HTG in our clinic during the last seven years. We classified the identified genetic variants (SHERLOC-criteria), followed by categorization into MCS or FCS. Clinical data were gathered from the medical records and the FCS-score was calculated for each patient.

Results: The gene sequencing revealed ten FCS patients. Four were compound heterozygous and six were 
homozygous for a pathogenic variant. Another 53 patients were categorized as MCS, of which seven were heterozygous carriers of a pathogenic variant. Altogether, we found 16 pathogenic variants of which six have not been described before. The most common was a missense variant (c. $644 \mathrm{G}>\mathrm{A}$, p.G215E) in the $L P L$ gene. In 27 (30\%) of our patients we were unable to detect any genetic alterations. In our cohort the FCS-score yielded a sensitivity of $80 \%$ and a specificity of $92.5 \%$.

Conclusions: At $11 \%$ the prevalence of FCS among our patients with severe HTG was higher than expected. The FCS score performed similar to the original study, however $20 \%$ of the FCS patients will not be detected correctly, relying on the score only.

F. Bardey: None. L. Rieck: None. D. Spira: None. W. März: None. S. Schwab: None. P. Binner: None. T. Bobbert: None. J. Spranger: None. E. SteinhagenThiessen: None. I. Demuth: None. U. Kassner: None.

\section{P06.17.A}

Gene expression and pathway analysis of omental adipose tissue from Type 2 diabetic and non diabetic Asian obese patients

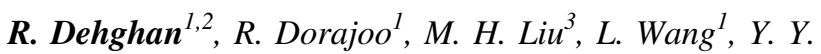

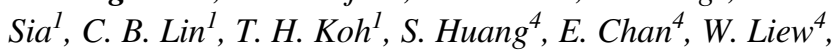

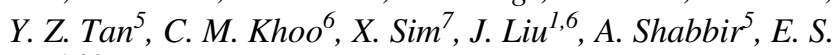
$\operatorname{Tai}^{4,6,8}$

${ }^{1}$ Genome Institute of Singapore, Agency for Science, Technology and Research, Singapore, Singapore, ${ }^{2}$ Department of Genetics and Molecular Biology, School of Medicine, Isfahan University of Medical Science, Isfahan, Iran, Islamic Republic of, ${ }^{3}$ Department of Food Science and Technology, National University of Singapore, Singapore, Singapore, ${ }^{4}$ Division of Endocrinology, Department of Medicine, National University Health System, Singapore, Singapore, ${ }^{5}$ Department of Surgery, National University Hospital, Singapore, Singapore, ${ }^{6}$ Department of Medicine, Yong Loo Lin School of Medicine, National University of Singapore, Singapore, Singapore, ${ }^{7}$ Saw Swee Hock School of Public Health, National University of Singapore, Singapore, Singapore, ${ }^{8}$ Duke-National University of Singapore Medical School, Singapore, Singapore

Introduction: Asians may have increased propensity to central obesity and related Type 2 diabetes mellitus (T2DM) risks, as compared to populations of European ancestry. Gene expression profiling of omental fat in Asians may identify additional T2DM relevant pathways.

Methods: We performed RNA-seq on 37 (16 T2DM and 21 non-T2DM) omental fats from Asian obese patients, obtained during bariatric weight-loss surgery. DESeq2 was used for differential expression analysis.

Results: 266 differentially expressed genes (DEGs) showed higher and 197 showed lower expression in T2DM vs non-T2DM samples $(P$ adj $<0.05)$. We identified $G A B R B 2$ as the top novel upregulated gene, highlighting a role for GABAergic system in omental fat with T2DM while $A C V R 1 C$ expression was most significantly downregulated and loss of function variants in $A C V R I C$ have been reported for reduced central obesity and T2DM risks. Upstream regulator analysis using our DEGs revealed OSM as the most significant regulator of up-regulated DEGs and PPARG for down-regulated DEGs. Pathway-based analyses using our DEGs connected with OSM revealed significant associations for immune-related pathways, validating recent associations of increased HMGB1 and TREM1 signaling for increased T2DM risks. Similar analyses on PPARG revealed pathways associated with adipogenesis, glucose and lipid metabolism and validated the protective association between increased Apelin signaling in omental fat and T2DM.

Conclusions: Our study on omental fat identified novel DEGs associated with T2DM. Omental fats from T2DM subjects exhibit upregulation of immune pathways probably modulated by OSM, and downregulation in genes involved in adipogenesis, glucose and lipid metabolism probably modulated by PPARG.

R. Dehghan: None. R. Dorajoo: None. M.H. Liu: None. L. Wang: None. Y.Y. Sia: None. C.B. Lin: None. T.H. Koh: None. S. Huang: None. E. Chan: None. W. Liew: None. Y.Z. Tan: None. C.M. Khoo: None. X. Sim: None. J. Liu: None. A. Shabbir: None. E.S. Tai: None.

\section{P06.18.B}

Lipodystrophy-associated PPAR $\gamma$ mutants define subclasses of target genes

M. F. Broekema ${ }^{1}$, M. Stahl Madsen ${ }^{2}$, M. Rønn Madsen ${ }^{2}$, A. Koppen $^{l}$, M. E. Kranendonk ${ }^{l}$, M. Groot Koerkamp ${ }^{l}, N$. Hamers $^{1}$, A. Borgmans ${ }^{1}$, A. M. Bonvin ${ }^{3}$, F. C. Holstege ${ }^{1}, W$. Spiering $^{1}$, H. Monajemi ${ }^{4}$, D. Cassiman ${ }^{5}$, S. Mandrup ${ }^{2}$, E. Kalkhoven ${ }^{1}$

${ }^{I}$ University Medical Center Utrecht, Utrecht, Netherlands, ${ }^{2}$ University of Southern Denmark, Odense, Denmark, ${ }^{3}$ Utrecht University, Utrecht, Netherlands, ${ }^{4}$ Rijnstate Hospital, Arnhem, Netherlands, ${ }^{5}$ Leuven University Hospitals, Leuven, Belgium

Introduction: The nuclear receptor $\operatorname{PPAR} \gamma$, encoded by the PPARG gene, is pivotal for adipocyte differentiation and function. How the different domains of PPAR $\gamma$ exactly communicate on intra- and intermolecular level to generate 
the appropriate transcriptional output in the context of chromatin is unresolved. New insights can be obtained from natural PPARG mutations, as found in patients suffering from familial partial lipodystrophy subtype 3 (FPLD3), characterized by repartitioning of adipose tissue causing insulin resistance and type 2 diabetes. We identified two FPLD3 mutations (E379K and R212Q) - located in distinct PPAR $\gamma$ domains - that impair the adipogenic capacity.

Methods and materials: We have used genome-wide sequencing-based strategies in PPARG knockout mouse embryonic fibroblasts transduced with PPAR $\gamma$ to determine how these PPARG mutations affect the ability of PPAR $\gamma$ to activate PPARG target genes in the context of native chromatin structure.

Results: Both mutations impair the ability of PPAR $\gamma$ to induce an overlapping subset of target genes, including classical PPAR $\gamma$ target genes such as $L p l$, while retaining the ability to activate other genes including Acoxl. Genome-wide DNA binding profiles indicated that regulatory regions that require PPAR $\gamma$ for chromatin remodeling are particularly sensitive to the mutations. In addition, the exact nucleotide sequence of the PPAR $\gamma$ binding sites can potentially contribute to target gene sensitivity.

Conclusions: We propose a model in which recruitment of PPAR $\gamma$ to chromatin is determined by multiple proteinprotein and protein-DNA interfaces. Our findings indicate that relatively subtle molecular defects in PPAR $\gamma$ are sufficient to cause lipodystrophy by dysregulating a subset of PPAR $\gamma$ target genes.

M.F. Broekema: None. M. Stahl Madsen: None. M. Rønn Madsen: None. A. Koppen: None. M.E. Kranendonk: None. M. Groot Koerkamp: None. N. Hamers: None. A. Borgmans: None. A.M. Bonvin: None. F.C. Holstege: None. W. Spiering: None. H. Monajemi: None. D. Cassiman: None. S. Mandrup: None. E. Kalkhoven: None.

\section{P06.21.B}

'Congenital Nicotinamide adenine dinucleotide (NAD) Deficiency Disorder treated with niacin supplementation'

A. M. Cueto-González, S. Arevalo, M. Del Toro, G. Giralt, A. Creus, M. Torres, I. Valenzuela, A. Abuli, E. Tizzano

Hospital Vall d'Hebron, Barcelona, Spain

Congenital NAD Deficiency is an extremely ultrarare disorder (OMIM 617660, ORPHA 521438) with only two previous cases reported (Shi et al. 2017). We report a new case diagnosed during pregnancy presenting cardiac malformations (Shone syndrome with high risk of aortic coarctation) and progressive intrauterine growth retardation since $20 \mathrm{w}$ with preserved head circumference and abdominal perimeter, without renal malformations or vertebral segmentation. Prenatal exome revealed a homozygous frameshift mutation in exon 1 of the HAAO gene (p.R15Gfs*99). The parents were heterozygous carriers and referred non-consanguinity. After birth the length was -3.2SD, we performed an abdominal ultrasound (normal), skeletal X-rays (normal) and cranial MRI (normal). The patient had elevated levels of 3hidroxyanthralinic acid (3HAA) and 3-OH-kynurenina and normal levels of niacin, similar to the two previously reported cases. Given that defects in Haao-null mice owing to NAD deficiency can be prevented with supplementation with nicotinic acid (Shi et al., 2017), compasive use of niacin supplementation was started at one month. Followup at 3 months shows a normal length (-1.63SD). There are only two reported patients from Australia with homozygous variants in $H A A O$ predicting loss-of-function changes in HAAO (different mutations that our patient). Cardiac, vertebral and renal malformation and hearing loss were present in both and Mondini defect and intellectual disability in one of them. To our knowledge, this is the third patient reported with NAD Deficiency and the first treated with niacin. Our patient has only three months and long term follow-up is required to determine efficacy.

A.M. Cueto-González: None. S. Arevalo: None. M. Del Toro: None. G. Giralt: None. A. Creus: None. M. Torres: None. I. Valenzuela: None. A. Abuli: None. E. Tizzano: None.

P06.29.A

Research Priorities for Primary Mitochondrial Disorders: Defined by Patients, their Carers and Clinicians.

J. Lowndes ${ }^{1}$, R. Thomas ${ }^{2}$, S. Rahman ${ }^{3}$, R. Wheeler ${ }^{4}, J$.

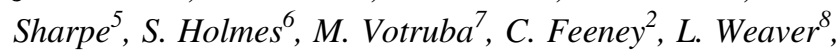

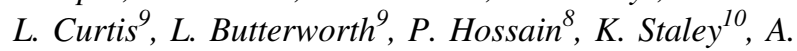
Hunter $^{10}$, S. Upadhyaya ${ }^{11}$

${ }^{1}$ Oxford University Hospitals NHS Foundation Trust, Oxford, United Kingdom, ${ }^{2}$ Wellcome Trust Centre for Mitochondrial Research, Newcastle, United Kingdom, ${ }^{3}$ UCL Great Ormond Street Institute of Child Health, London, United Kingdom, ${ }^{4}$ LHON Society, London, United Kingdom, ${ }^{5}$ Muscular Dystrophy UK, London, United Kingdom, ${ }^{6}$ National Hospital for Neurology and Neurosurgery, London, United Kingdom, ${ }^{7}$ Cardiff University and The University Hospital of Wales, Cardiff, United Kingdom, ${ }^{8}$ Metabolic Support UK, London, United Kingdom, ${ }^{9}$ The Lily Foundation, London, United Kingdom, ${ }^{10}$ Genetic Alliance UK, London, United Kingdom, ${ }^{11}$ James Lind Alliance, London, United Kingdom 
Introduction: Primary mitochondrial disorders are clinically and genetically heterogeneous, for which there is currently a lack of effective therapies. It is therefore vital to know that competitively funded research meets the needs of patients and their clinicians. The Priority Setting Partnerships (PSPs) are an established, collaborative methodology that brings patients, charity representatives and clinicians together to try to establish the most pressing and unanswered research priorities for a particular disease group.

Methods: After a scoping exercise to identify relevant partners, we chose to include questions relevant to both children and adults with primary mitochondrial disorders. A web-based questionnaire was developed and widely disseminated; asking for patients, carers and clinicians to pose their important research questions. This yielded 709 questions from 50 patients, 47 carers and 50 clinicians. These were grouped in to themes and filtered to exclude statements that were personal stories. Extensive literature searching was performed to determine which questions are unanswered based on current evidence. This process resulted in a list of 42 discrete questions. In a second web-based questionnaire, patients, carers and clinicians were invited to rank these 42 questions. This identified 24 questions that were taken forward to a workshop attended by a diverse range of patients, carers, charity representatives and clinicians. The relative merits and strengths of each question were debated to create a definitive 'top 10 of unanswered research questions'.

Results/Conclusion: Here we present the 'top 10 of unanswered research questions for primary mitochondrial disorders', as defined by the patients, their carers and clinicians.

J. Lowndes: None. R. Thomas: None. S. Rahman: None. R. Wheeler: None. J. Sharpe: None. S. Holmes: None. M. Votruba: None. C. Feeney: None. L. Weaver: None. L. Curtis: None. L. Butterworth: None. P. Hossain: None. K. Staley: None. A. Hunter: None. S. Upadhyaya: None.

P06.31.C

High coverage NGS of mtDNA proofs apparent de novo variant to be in fact not de novo

U. Ahting ${ }^{1}$, B. Lorenz-Depireux ${ }^{2}$, R. Berutti ${ }^{2}, M$.

Radivojkov-Blagojevic ${ }^{2}, F$. Radelfahr ${ }^{3}$, C. Stendel ${ }^{3}, J . U$. Walther $^{1}$

${ }^{1}$ Institut für Humangenetik. TUM, München, Germany, ${ }^{2}$ Helmholtz-Zentrum München, Institut für Humangenetik, Neuherberg, Germany, ${ }^{3}$ Friedrich-Baur-Institut, München, Germany
Introduction: Maternally inherited pathogenic mtDNA variants increase in heteroplasmy during passage from mother to affected offspring. A pathogenic variant, which is present in an affected offspring, is often not detectable in various tissues of the mother, and therefore classified as "de novo". Here we present a case, where an apparent "de novo" variant is actually not de novo, as it is detectable by high coverage NGS in the mother and other maternal relatives.

Case: The male index patient is affected by Leigh syndrome, a classical mitochondrial disease. Symptoms are psychomotor delay and generalized dystonia and MRI brain scans show bilateral symmetrical lesions in the basal ganglia and brainstem. Muscle biopsy showed ragged red fibers. Mother and grandfather are affected by Parkinson's disease.

Results: Whole exome sequencing identified pathogenic variant $\mathrm{m} .10158 \mathrm{~T}>\mathrm{C}$ in mtDNA isolated from blood. High coverage NGS revealed that the variant is present in this sample with heteroplasmy of $62 \%$ (2710 of 4371 reads). Testing of mother and healthy siblings showed that the variant is present in all investigated tissues with low heteroplasmy $(\sim 1 \%)$. Therefore the variant is inherited.

Discussion: Apparent de novo mtDNA-variants may be inherited and maternal relatives may be carriers of such variants. High coverage NGS of mtDNA can detect such cases. Increase of heteroplasmy in cases like this must be due to a strong bottleneck effect. If the bottleneck works by unequal partitioning (segregation), at least 6 cell divisions without mtDNA replication must have happened in the reported affected case.

U. Ahting: None. B. Lorenz-Depireux: None. R. Berutti: None. M. Radivojkov-Blagojevic: None. F. Radelfahr: None. C. Stendel: None. J.U. Walther: None.

\section{P06.32.A}

Molecular mitochondrial DNA analysis in patients with suspected mitochondrial disorder

\section{K. Grigalionienè, B. Burnytè, D. Vansevičiūtè, G. Žukauskaitè, L. Ambrozaitytè, A. Utkus}

Department of Human and Medical Genetics, Institute of Biomedical Sciences, Faculty of Medicine, Vilnius University, Vilnius, Lithuania

Introduction: Mitochondrial diseases represent important and common source of inherited metabolic disorders. Mitochondrial dysfunction is characterized by defects in oxidative phosphorylation and caused by pathogenic variants in either mitochondrial DNA or nuclear mitochondrial genes. A correct diagnosis is challenging due to the com- 
plexity of the clinical presentations, lack of classical diagnostic clues and genetic heterogeneity.

Patients and methods: In this study, mtDNA was examined in a cohort of 71 affected individuals (69 unrelated families) with suspected mitochondrial disorder. Comprehensive analysis of mtDNA was performed using following molecular genetic methods: Sanger sequencing of whole-length mtDNA for detection of point variants, long PCR and MLPA for detection of mtDNA deletions/ duplications and RT-PCR for mtDNA copy number analysis.

Results: Six $(8.70 \%)$ patients were identified with pathogenic mtDNA variants in the cohort of 69 unrelated patients. Common mtDNA pathogenic variant NC_012920.1:m.3243A>G with variable heteroplasmy levels was identified in three unrelated female patients with heterogeneous phenotype of MELAS. Homoplasmic mtDNA pathogenic variants NC_012920.1:m.8993T>C and NC_012920.1:m.9185T>C were identified in patients presenting with Leigh syndrome. Novel heteroplasmic 5888 bp mtDNA deletion NC_012920.1:m.6069_11956del causing Kearns-Sayre syndrome was identified using long PCR and Sanger sequencing while MLPA profile was normal. MtDNA haplogroup analysis revealed a $43.5 \%$ frequency of the most common $\mathrm{H}$ haplogroup in the patients group.

Conclusions: Benefits of mtDNA analysis manifest in the identification of pathogenic variants that could direct patients care, but limitations of the methods should be taken into account while selecting the best investigation strategy.

Supported by grant TAP LLT-02/2015

K. Grigalionienė: None. B. Burnytė: None. D. Vansevičiūtė: None. G. Žukauskaitè: None. L. Ambrozaitytè: None. A. Utkus: None.

\section{P06.34.C}

Mitochondrial disorder diagnosis; a combined genomic and transcriptomic approach

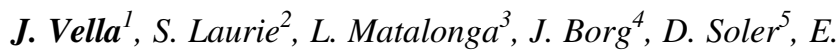

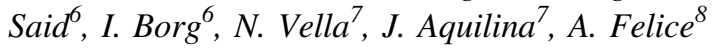

${ }^{1}$ Department of Physiology and Biochemistry and Centre for Molecular Medicine and Biobanking, University of Malta, Msida, Malta, ${ }^{2}$ Centro Nacional de Analisis Genomico (CNAG-CRG); Centre for Genomic Regulation; Barcelona Institute of Science and Technology (BIST), Universitat Pompeu Fabra (UBF), Barcelona, Spain, ${ }^{3}$ Centro Nacional de Analisis Genomico (CNAG-CRG); Centre for Genomic Regulation; Barcelona Institute of Science and Technology (BIST); Universitat Pompeu Fabra (UBF), Barcelona, Spain, ${ }^{4}$ Department of Health Sciences and Centre for Molecular Medicine and Biobanking, University of Malta, Msida, Malta, ${ }^{5}$ Department of Paediatrics, Mater Dei Hospital, Msida, Malta, ${ }^{6}$ Department of Pathology, Mater Dei Hospital, Msida, Malta, ${ }^{7}$ Department of Neurology, Mater Dei Hospital, Msida, Malta, ${ }^{8}$ Department of Physiology and Biochemistry and Centre for Molecular Medicine and Biobanking, Msida, Malta

Mitochondrial disorders are rare, genetically heterogenous diseases which affect the oxidative phosphorylation (OXPHOS) system. A combined genomic and transcriptomic approach was adopted to diagnose mitochondrial disorders in a cohort of 14 Maltese patients. Phenotypic data was recorded in the RD-Connect PhenoTips instance. The nuclear and mitochondrial genome were evaluated by whole exome sequencing. Variant filtration and prioritisation was undertaken using the RD-Connect Genome Phenome Analysis Platform (GPAP). The full transcriptome was analysed by RNA sequencing. Differential expression of candidate variants was confirmed by q-PCR. A HEK-293 cell model was used to functionally characterise the effect of a rare homozygous missense variant on mitochondrial complex 1 activity in one of the patients. Pathogenic variants were characterised according to their functional roles in OXPHOS biogenesis (see Table). The most variants were identified in Complex 1 OXPHOS subunits $(\mathrm{N}=8)$ and Complex 1 assembly factor variants were also identified in 4 of these patients. Two patients were diagnosed with MELAS. Gene expression analysis also revealed the effects of these variants.

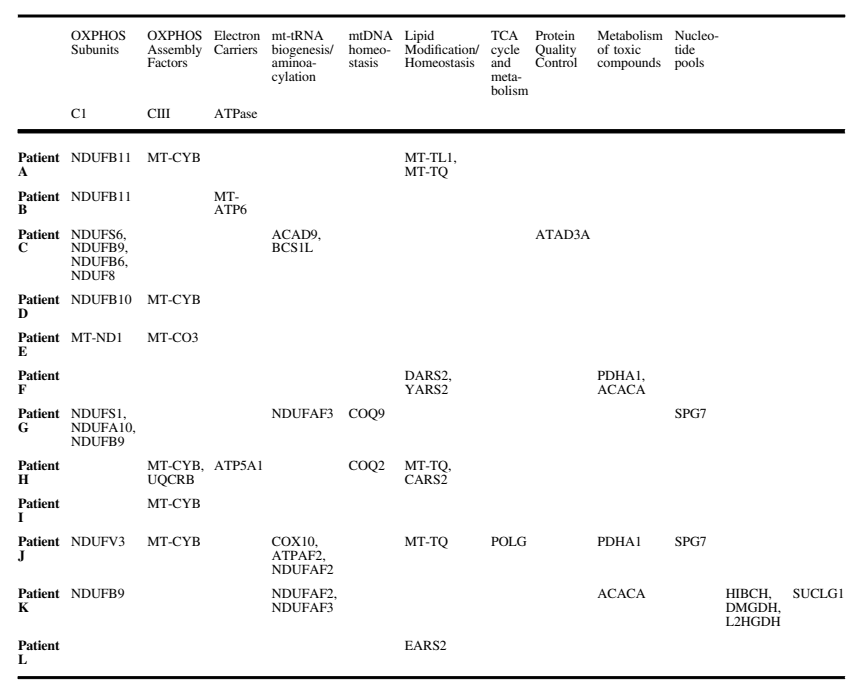

This combined genomic and transcriptomic approach could aid in establishing a diagnosis for these rare disease patients.

J. Vella: None. S. Laurie: None. L. Matalonga: None. J. Borg: None. D. Soler: None. E. Said: None. I. Borg: None. N. Vella: None. J. Aquilina: None. A. Felice: None. 
P06.35.A

Mitochondriopathy in pediatric patients with unspecific neuropediatric disease

A. van der Ven ${ }^{1}$, J. Denecke ${ }^{2}, K$. Tsiakas $^{2}, S$. Wortmann $^{3,4,5}$, D. Lessel ${ }^{1}, F$. Kortüm ${ }^{l}$, T. Bierhals ${ }^{l}, H$. Prokisch $^{3,4}$, R. Santer ${ }^{2}$, M. Hempel ${ }^{1}$

${ }^{1}$ Dept. Human Genetics, UKE Hamburg-Eppendorf, Hamburg, Germany, ${ }^{2}$ Dept. Pediatrics, UKE Hamburg-Eppendorf, Hamburg, Germany, ${ }^{3}$ Dept. Human Genetics, TU Munich, Munich, Germany, ${ }^{4}$ Dept. Human Genetics, Helmholz Centre Munich, Neuherberg, Germany, ${ }^{5}$ Dept. Pediatrics, Paracelsus Medical University, Salzburg, Austria

Introduction: Mitochondriopathies account for an important subgroup of neuropediatric conditions but often the clinical presentation may be too unspecific to hint to an a priori disease hypothesis.

Materials and Methods: We report on a deep phenotyping and trio-WES study of 492 unrelated children affected by previously undiagnosed and diverse complex neuropediatric disorders. All children underwent a standardized and comprehensive clinical work-up and trio-WES. In a subset of 31 children we had an a priori clinical suspicion of a mitochondriopathy.

Results: Within this group of patients with a clinical suspicion of an underlying mitochondriopathy, we confirmed the diagnosis by identifying a pathogenic variant in a known mitochondriopathy-associated gene in 9/31 children (29\%). In 10/31 children (32\%) we discovered a pathogenic variant in a disease gene not associated with a mitochondriopathy. For $3 / 31$ children we detected a pathogenic mutation in a candidate gene for a mitochondriopathy. In the group of 461 children with no suspicion of a mitochondriopathy, we identified pathogenic mutations in known mitochondriopathy-associated genes in 13 children (3\%). In 226/461 children (49\%) we detected pathogenic mutations in other known disease genes. For 222/461 (48\%) patients no definite genetic diagnosis could be established.

Conclusions: Overall, we molecularly established the diagnosis of a mitochondriopathy in 22 of 492 (4,5\%) unselected neuropediatric patients. This study highlights the importance of WES for the identification of children with a clinically less characteristic presentation of their underlying mitochondriopathy.

A. van der Ven: None. J. Denecke: None. K. Tsiakas: None. S. Wortmann: None. D. Lessel: None. F. Kortüm: None. T. Bierhals: None. H. Prokisch: None. R. Santer: None. M. Hempel: None.
P06.36.B

Mitochondrial DNA sequence context in the penetrance of all mt-tRNA mutations across many species: A study diagnostic implications

H. O'Keefe, R. A. Queen, J. S. Steyn, P. Lord, J. L. Elson

Newcatsle University, Newcastle, United Kingdom

Introduction: Mitochondrial disorders are heterogeneous, showing variable presentation and penetrance. Over the last three decades, our ability to recognize mitochondrial patients and diagnose these mutations, linking genotype to phenotype, has greatly improved. However, it has become increasingly clear that these strides in diagnostics have not benefited all population groups. It has been suggested that haplogroup (or lineage) context might influence the presentation and penetrance of mtDNA disease; thus, the spectrum of mutations that are associated with disease in different populations.

Methods and Materials: This paper conducted the most extensive investigation to date into the importance of haplogroup context on the pathogenicity of mtDNA mutations. We searched for proven human point mutations across 726 multiple sequence alignments derived from 33 non-human species absent of disease.

Results: 58 pathogenic point mutations arise in the sequences of these species. We assessed the sequence context and found evidence of population variants that could modulate the phenotypic expression of these point mutations masking the pathogenic effects seen in humans.

Conclusions: This supports the theory that sequence context is influential in the presentation of mtDNA disease and has implications for diagnostic practices. Our current understanding of the pathogenicity of mtDNA point mutations, primarily built on studies of individuals with haplogroups HVUKTJ, will not present a complete picture. This will impair our understanding of disease mechanisms and create a diagnostic inequality, whereby individuals who do not belonging to more commonly studied lineages will be less likely to receive a genetic diagnosis.

H. O'Keefe: None. R.A. Queen: None. J.S. Steyn: None. P. Lord: None. J.L. Elson: None.

P06.37.C

Unraveling Atypical Phenotypes in Neuronal Ceroid Lipofucinosis type 2 
A. M. Amaral ${ }^{1}$, F. Fola ${ }^{l}$, Z. A. Carneiro ${ }^{1}$, L. Vagnini ${ }^{2}$, C. Fischinger Moura De Souza ${ }^{3}$, A. Pessoa ${ }^{4}$, C. M. Lourenco ${ }^{1}$

${ }^{I}$ Faculdade de Medicina - Centro Universitario Estacio de Ribeirao Preto, Ribeirao Preto, Brazil, ${ }^{2}$ Centro Paulista de Diagnostico e Pesquisa, Ribeirao Preto, Brazil, ${ }^{3}$ Hospital de Clínicas de Porto Alegre, Porto Alegre, Brazil, ${ }^{4}$ Hospital Infantil Albert Sabin, Fortaleza, Brazil

Introduction: Late-infantile neuronal ceroid lipofuscinosis (NCL), also called neuronal ceroid lipofuscinosis type 2 (CLN2), is caused by the deficiency of lysosomal enzyme tripeptidyl peptidase-1 (TPP1) due to mutations in the CLN2 gene. The classical phenotype is late infantile presenting with language developmental delay, with its clinical signs usually appearing between the ages of 2 to 4 years with seizures and a rapid decline of psychomotor functions. Most children are bedridden at 5 years and die around the age of 10 years. Nevertheless, atypical phenotypes have been reported in the literature, creating a clinical challenge to avoid diagnosis without significant delay.

Methods: Clinical, biochemical and neuroradiological data retrospective analysis of five CLN2 patients showing atypical/protracted phenotypes

Results: All four patients ( 3 males; 2 females) showed an atypical late onset phenotype. First symptons started around 5 - 10 years of age being cerebellar ataxia the most prominent feature. Abnormal findings in brain MRI were found in all patients (cerebellar atrophy and periventricular white matter changes). Seizures were present in three patients, cognitive decline was seen in 4 patients and mild learning disabilities in one. TPP1 enzyme was decreased in all patients, although not in the typical range found in "classical" CLN2 patients.

Discussion: The protracted CLN2 phenotype was previously considered globally rare. In South America, the frequency is approximately $50 \%$ of affected individuals. Recently with the recent approval of intraventricular enzyme replacement therapy (ERT) with cerliponase alpha for CLN2 disease, early diagnosis is essential to start treatment before disease progression.

A.M. Amaral: None. F. Fola: None. Z.A. Carneiro: None. L. Vagnini: None. C. Fischinger Moura De Souza: None. A. Pessoa: None. C.M. Lourenco: None.

\section{P06.38.A}

Comprehensive characterization of a large cohort of patients with Niemann Pick disease
P. Guatibonza, C. Beetz, J. Gutierrez, S. Zafari, V. Skrahina, C. Cozma, P. Bauer, A. Rolfs

\section{Centogene AG, Rostock, Germany}

The term Niemann Pick disease (NPD) denotes a genetically and clinically heterogeneous group of lysosomal storage disorders. A deeper insight into NPD would benefit from large and uniformly characterized cohorts of patients. We queried our database for patients that had received a genetic diagnosis of NPD between December 2015 and August 2019. Information was compiled on: affected gene, age, gender, and country of origin, along with available clinical and metabolic data. A genetic diagnosis of NPD was issued for 733 patients. With $\mathrm{n}=472(64.4 \%)$, the majority had variants in $N P C 1$, while SMPD1 and NPC2 were identified as the affected genes in $255(34.8 \%)$ and 6 cases $(0.8 \%)$, respectively. Most patients were infants (median age at diagnosis 3.6 years; range 0 to 76 years). They had been referred from 52 distinct countries, with the top 4 countries (Iran, Germany, Brazil and Egypt) collectively accounting for more than $50 \%$ of referrals. Translation of the available clinical information into human phenotype ontology (HPO) terms revealed that hepato- and/ or splenomegaly was present in $76.5 \%$ of patients, intellectual disability in $27.9 \%$, and jaundice in $19.0 \%$. Further frequent HPO terms (i.e. present in $>10 \%$ ) included, abnormality of movement, ataxia, failure to thrive, and seizures. The NPD-specific biomarker LysoSM-509 was pathologically increased in $97.9 \%$ of the patients. Our cohort is amongst the largest described to date. By being well-characterized at numerous levels, it represents a valuable basis for discovering novel correlations and, thereby, for increasing our understanding of Niemann Pick disease.

P. Guatibonza: A. Employment (full or part-time); Significant; Centogene AG. C. Beetz: A. Employment (full or part-time); Significant; Centogene AG. J. Gutierrez: A. Employment (full or part-time); Significant; Centogene AG. S. Zafari: A. Employment (full or part-time); Significant; Centogene AG. V. Skrahina: A. Employment (full or parttime); Significant; Centogene AG. C. Cozma: A. Employment (full or part-time); Significant; Centogene AG. P. Bauer: A. Employment (full or part-time); Significant; Centogene AG. A. Rolfs: A. Employment (full or parttime); Significant; Centogene AG.

\section{P06.40.C}

A novel $160 \mathrm{~Kb}$ deletion identified in $P C C A$ gene using whole exome sequencing in a patient with propionic acidemia 
S. Farajzadeh Valilou ${ }^{1,2,3}$, H. Heydari ${ }^{l}$, S. Shohani ${ }^{1,4}, S$. Samadpour $^{l}$, H. Abdi ${ }^{l}$, J. Karimzad Hagh ${ }^{5}$, M. Salimi Asl ${ }^{1}$

${ }^{1}$ Division of Medical Genetics, TehranLab, Tehran, Iran, Islamic Republic of, ${ }^{2}$ Department of Medical Genetics, Kasra Hospital, Tehran, Iran, Islamic Republic of, ${ }^{3} \mathrm{Med}-$ ical Genetics Network (MeGeNe), Universal Scientific Education and Research Network (USERN), Tehran, Iran, Islamic Republic of, ${ }^{4}$ Department of Biotechnology, Faculty of Allied Medicine, Iran University of Medical Sciences, Tehran, Iran, Islamic Republic of, ${ }^{5}$ IVF Center, Cuypers and Cuypers, Hochstr. 152, Heinsberg, Germany

Background: Propionic acidemia (PA) is a severe metabolic disorder characterized by various clinical features. Herein, we present an 18-month-old male patient from consanguineous parents with early-onset of symptoms such as anemia, respiratory problem, and developmental delay. Urine examination exhibited an increased level of 3hydroxypropionic acid and methylcitric acid, led to the primary diagnosis of PA.

Methods: To identify the causative variant, DNA was extracted from the peripheral blood of the child and exons were captured and enriched to perform whole exome sequencing (WES). Data were analyzed using bioinformatics techniques and in silico predictors. Conventional PCR and Sanger sequencing were used to confirm the findings in WES. Additionally, comparative genomic hybridization (CGH)-array was carried out to study the accurate size of the copy number variation (CNV).

Results: Surprisingly, no pathogenic variants in propionic academia causing genes (PCCA and $P C C B$ ) or phenotypically related disorders' genes. Assuming the probably low coverage, we analyzed the $P C C A$ and $P C C B$ regions in the BAM file. In the $P C C A$, unlike $P C C B$, exons 12 to 21 were identified with no coverage, probably suggesting deletion of the approximately $220 \mathrm{~kb}$-size region. Not only we confirmed the novel PCCA deletion via PCR but also performing $\mathrm{CGH}$-array revealed the approximate deletion size of $160 \mathrm{~Kb}$.

Conclusions: We identified a novel homozygous deletion in the PCCA in the patient with PA using WES. Our results showed that WES can be used for identifying CNVs instead of CGH-array. However, a lot of studies should be done to improve the accuracy of this approach.

S. Farajzadeh Valilou: None. H. Heydari: None. S. Shohani: None. S. Samadpour: None. H. Abdi: None. J. Karimzad Hagh: None. M. Salimi Asl: None.

\section{P06.41.A}

A novel hemizygous loss-of-function variant in the PRPS1 gene found in a patient with features of Arts syndrome, but without hearing loss and normal levels of purine/pyrimidine metabolites

S. Puusepp ${ }^{1,2}$, K. Reinson ${ }^{1,2}$, S. Pajusalu ${ }^{1,2,3}$, A. B. P. van Kuilenburg $^{4}$, D. Dobritzsch ${ }^{5}$,W. Stenzel ${ }^{6,7}, K$. Ónap ${ }^{1,2}$

${ }^{I}$ Department of Clinical Genetics, Institute of Clinical Medicine, Faculty of Medicine, University of Tartu, Tartu, Estonia, ${ }^{2}$ Department of Clinical Genetics, United Laboratories, Tartu University Hospital, Tartu, Estonia, ${ }^{3}$ Department of Genetics, Yale University School of Medicine, New Haven, CT, United States, ${ }^{4}$ Department of Clinical Chemistry, Cancer Center Amsterdam, Amsterdam Gastroenterology \& Metabolism, Amsterdam UMC, University of Amsterdam, Amsterdam, Netherlands, ${ }^{5}$ Department of Chemistry - BMC, Uppsala University, Uppsala, Sweden, ${ }^{6}$ Department of Neuropathology, CharitéUniversitätsmedizin Berlin, corporate member of Freie Universität Berlin, Humboldt-Universität zu Berlin, and Berlin Institute of Health, Berlin, Germany, ${ }^{7}$ Leibniz Science Campus Chronic Inflammation, Berlin, Germany

Introduction: The PRPS1 gene, located on Xq22.3, encodes phosphoribosyl-pyrophosphate synthetase (PRPS), a key enzyme in purine de novo synthesis. Three clinical phenotypes are associated with loss-of-function PRPSI variants and decreased PRPS activity: Arts syndrome, Charcot-Marie-Tooth disease type 5 (CMTX5) and Xlinked non-syndromic deafness. Hearing loss is present in all of these patients. CMTX5 patients additionally present with peripheral neuropathy and optic atrophy. Arts syndrome includes developmental delay, intellectual disability, ataxia and susceptibility to infections.

Case report: We report a boy who presented with marked generalized muscular hypotonia, progressive global developmental delay, lack of speech, trunk instability, exercise intolerance, hypomimic face with opened mouth, oropharyngeal dysphagia, dysarthria, and very frequent upper respiratory tract infections. Cardiac, audiological and ophthalmological investigations were normal. A novel hemizygous variant c.130A $>$ G p.(Ile44Val) was found in the PRPS1 gene by panel sequencing. PRPS activity in erythrocytes was markedly reduced confirming the pathogenicity of the variant. In retrospect, serum uric acid levels and the first urine purine/pyrimidine analysis were normal, but analysis of the follow-up sample showed nearly absent hypoxanthine.

Conclusions: We describe a patient with features of Arts syndrome and a novel PRPS1 loss-of-function variant. However, at the current age of five years, our patient does not present with hearing loss, which has so far been reported in all of the Arts syndrome patients as congenital/ early-onset. We would also like to emphasize that the first 
purine/pyrimidine analysis was normal giving no clues towards a dysfunction in the purine-pyrimidine metabolism.

Funding: Estonian Research Council grants PUT355, PRG471, PUTJD827.

S. Puusepp: None. K. Reinson: None. S. Pajusalu: None. A.B.P. van Kuilenburg: None. D. Dobritzsch: None. W. Stenzel: None. K. Õunap: None.

P06.43.C

Infantile and early childhood onset mitochondrial myopathy due to mutations in the TK2 gene with a phenotype of spinal muscular atrophy $5 q$. The first cases in Russia.

P. Tsygankova ${ }^{l}$, S. Kurbatov ${ }^{2,3}$, I. Bychkov ${ }^{l}$, Y. Itkis ${ }^{l}$, A. Galushkin $^{l}$, K. Molewa ${ }^{4}$, V. Zabnenkova ${ }^{l}$, E. Zakharova $^{l}$

${ }^{1}$ Research centre for medical genetics, Moscow, Russian Federation, ${ }^{2}$ Voronezh Regional Clinical Consulting and Diagnostic Center, Voronezh, Russian Federation, ${ }^{3}$ Association of Neuromuscular Disorders Specialists, Medical Center "Practical Neurology", Moscow, Russian Federation, ${ }^{4}$ Department of Neurology, "Dagestan State Medical University", Machachkala, Russian Federation

Mitochondrial myopathy (MM) due to thymidine kinase 2 deficiency and spinal muscular atrophy (5q) (SMA-5q) are two potentially curable hereditary diseases with different levels of damage to the neuromuscular system and etiology. Early childhood forms have a similar phenotype and are difficult for differential diagnosis. We present the clinical characteristics and molecular defect of MM with TK2 deficiency and informing health care specialists about the possibility of optimizing differential diagnosis. Materials and methods. This study involved 9 patients with excluded SMA-5q diagnosis. TK2 mutations were detected by NGSgene panel in 3 of them. In addition, 96 DNA samples of patients with SMA-phenotype were screened for TK2 mutations by Sanger sequencing. Results. Nine patients with MM with TK2 from 6 unrelated families have been diagnosed. Three of them have been found retrospectively by analysis of 96 SMA-5q negative samples. Screening for mutations in the TK2 gene can be recommended for all children with a manifestation of the disease up to 2 years old, with rapidly progressing muscle weakness, increased CPK and/or lactate in the blood and/or detection of damage to the leg muscles on MRI. The study revealed four previously undescribed mutations in the TK2 gene: c. $169 \mathrm{G}>\mathrm{A}$ (p.Gly57Ser), c.310C $>\mathrm{T}$ (p.Arg104Cys), c.338T >A (p. Val113Glu), c.655T $>$ C (p.Trp219Arg). Early molecular diagnostic is important for the choice of experimental nucleoside bypass treatment for this group of mitochondrial diseases.
P. Tsygankova: None. S. Kurbatov: None. I. Bychkov: None. Y. Itkis: None. A. Galushkin: None. K. Molewa: None. V. Zabnenkova: None. E. Zakharova: None.

\section{P06.44.A}

TYPE 2 DIABETES AND CANCER CO-MORBIDITY: IS THERE SHARED GENETIC SUSCEPTIBILITY?

\author{
L. Zudina ${ }^{\text {, }}$ A. Demirkan ${ }^{1}$, I. Pupko ${ }^{1}$, M. J. Gunter ${ }^{2}$, M. \\ Kaakinen $^{l}$, I. Prokopenko ${ }^{l}$
}

${ }^{I}$ Department of Clinical \& Experimental Medicine, University of Surrey, Guildford, United Kingdom, ${ }^{2}$ Nutrition and Metabolism Section, International Agency for Research on Cancer, World Health Organization, Lyon, France

A plethora of epidemiological studies showed relationships between Type 2 Diabetes (T2D) and common cancers, reporting increased risks for postmenopausal breast $(\mathrm{RR}=1.20), \quad$ colorectal $\quad(\mathrm{RR}=1.30) \quad$ and pancreatic $(\mathrm{RR}=1.94)$ cancers, and decreased risk for prostate cancer $(R R=0.84)$ in $T 2 D$ patients. We aimed to dissect the genetic factors shared between T2D, pancreatic and three common cancers using genome-wide genetic variability in a large case-control and population-based context.

We evaluated the role of signals established for T2D on the susceptibility to postmenopausal breast, prostate, colorectal and pancreatic cancers in EPIC (European Prospective Investigation into Cancer and Nutrition) a combined nested case-control study of 14,553 individuals, including 4,345/ $2,120 / 426 / 499$ with postmenopausal breast/colorectal/prostate/pancreatic cancer diagnosis and 476 prevalent cases of T2D. We generated polygenic risk scores (PRS) for breast/ colorectal/prostate/pancreatic cancers and T2D using 171/ 90/154/21 and 403 established DNA variants for each disease, respectively. To perform reciprocal evaluation of PRS effects on diabetes and four cancer risks, we used the variants from original phenotype and the estimates of perallele genetic effects from the other outcome.

For all individual PRSs, we observed significant $(\mathrm{P}<$ $5 \times 10^{-5}$ ) increase in the risk of original outcomes. T2D PRS was significantly associated with risk of pancreatic cancer $\left(\mathrm{OR}=3.82[1.65-8.84], \quad \mathrm{P}=1.71 \times 10^{-3}\right)$. This effect was stronger compared to the T2D PRS effect on T2D risk $\left(\mathrm{OR}=1.53[1.35-1.75], \mathrm{P}=9.87 \times 10^{-11}\right)$. The T2D and pancreatic cancer share signals at GRP, HNF1B BCARl and LINC-PINT/KLF14 loci only.

Using the pan-European EPIC study data, we provide evidence for shared genetic factors between T2D and pancreatic cancer.

Funding: WCRF-2017/1641 
L. Zudina: None. A. Demirkan: None. I. Pupko: None. M.J. Gunter: None. M. Kaakinen: None. I. Prokopenko: None.

\section{P06.45.B}

Milder form of Vici syndrome due to novel missense variant affecting splicing

\section{Skopkova ${ }^{l}, K$. Brennerova ${ }^{2}, M$. Ostrozlikova ${ }^{3}, S$. Borecka $^{l}$, D. Gasperikova ${ }^{l}$}

${ }^{1}$ Institute of Experimental Endocrinology, Biomedical Research Center, Slovak Academy of Sciences, Bratislava, Slovakia, ${ }^{2}$ Department of Pediatrics, Medical Faculty of Commenius University and National Institute of Children's Diseases, Bratislava, Slovakia, ${ }^{3}$ Department of Laboratory Medicine, National Institute of Children's Diseases, Bratislava, Slovakia

Introduction: Vici syndrome is a rare autosomal recessive syndrome caused by mutations in the gene EPG5 encoding ectopic $\mathrm{P}$ granules protein involved in autophagy. It is a neurodevelopmental disorder with multisystem involvement including corpus callosum agenesis, cataracts, hypopigmentation, cardiomyopathy, and immunodeficiency. It has been hypothesized that the severity of phenotype is related to residual level of functional EPG5.

Patient: A 4 years old boy of Roma ethnicity with preterm birth, hypotonia, severe hypotrophy and growth retardation, developmental delay, mild brain atrophy, and urine organic acid profile suggesting mitochondrial disease was referred for whole-exome sequencing.

Results: We have found a novel variant c.4205G >A (p. Arg1402Lys) in the EPG5 gene. As it is the last base of exon 23, we performed a mini-gene assay to estimate its effect on splicing. This has shown decreased inclusion of exon 23 compared to wild-type (40\% vs. $72 \%$, respectively). RNA sequencing confirmed only presence of the correctly spliced transcript. The patient was further examined for symptoms common in Vici syndrome and hypopigmented skin areas and immunodeficiency were confirmed. Interestingly, the patient has present corpus callosum, although thin, no seizures, no cataracts and no cardiomyopathy. Additionally, he has hemodynamically important stenosis of left pulmonary artery. The typical sign - pale skin and hair were also not present.

Conclusions: Novel variant c. $4205 \mathrm{G}>\mathrm{A}$ causes aberrant splicing only in a proportion of transcripts, therefore, milder presentation of Vici syndrome in our patient is probably due to the residual presence of the EPG5 protein. Supported by VEGA 2/083/17 and APVV-17-0296.
M. Skopkova: None. K. Brennerova: None. M. Ostrozlikova: None. S. Borecka: None. D. Gasperikova: None.

P07 Immunology and Hematopoietic System

\section{P07.05.A}

A rare pair in one patient - Bernard-Soulier syndrome unraveled by 22q11.2 microdeletion syndrome

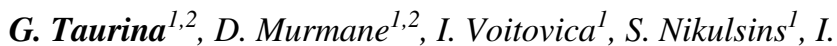 Micule $^{1,3}$ \\ ${ }^{1}$ Childrens Clinical University Hospital, Riga, Latvia, ${ }^{2}$ Riga Stradins University, Riga, Latvia, ${ }^{3}$ Biomedical Research and Study Centre, Riga, Latvia}

Introduction: The 22q11.2 deletion syndrome is one of the most common microdeletion syndromes. Bernard-Soulier syndrome, in turn, is a rare autosomal recessive bleeding disorder (prevalence <1:1 000000 ) with dysfunctional glycoprotein Ib-V-IX complex, responsible for the adhesion activity of platelets. We report a patient having both diseases.

Case report: A boy who was admitted to hospital on the third day of life due to epistaxis, petechias and umbilical cord bleeding. Brain MRI showed subdural hematoma among cerebellar lobes. Blood count revealed severe thrombocytopenia $\left(8 \times 10^{3} / \mathrm{uL}\right)$, that required several platelet infusions and did not improve with immunoglobulin treatment. An NGS analysis for Bleeding disorder gene panel was ordered, with $\mathrm{CNV}$ analysis. On the second month of life flow cytometry revealed reduced CD $42 \mathrm{~b}$ expressivity.

Results: The NGS gene panel results revealed a heterozygous pathogenic 22q11.2 microdeletion, encompassing 73 genes with $G P 1 B B$ gene included. The analysis also showed a heterozygous likely pathogenic variant in GP1BB gene c.395T $>$ A, p.(Leu132Gln). The combination of the microdeletion on one and the mutation on the other $G P I B B$ allele explains the bleeding disorder of our patient and confirms in him Bernard-Soulier syndrome. 22q11.2 microdeletion syndrome was not suspected before the DNA analysis. Afterwards at a repeated consultation the patient demonstrated signs of velopharyngeal insufficiency but as yet no other signs of the Velocardiofacial syndrome.

Conclusions: In patients with early onset bleeding disorder genetic testing with $\mathrm{CNV}$ analysis must be performed in order to not miss an autosomal recessive disease unmasked by a seemingly presymptomatic frequent microdeletion syndrome.

G. Taurina: None. D. Murmane: None. I. Voitovica: None. S. Nikulsins: None. I. Micule: None. 


\section{P07.08.A}

High intrafamilial variability in a C15orf41 associated Congenital Dyserythropoietic Anemia family indicates involvement of C2orf69 in infantile epilepsy

\section{A. Gurel ${ }^{1}$, S. Unal ${ }^{2}$, N. Yarali $^{3}$, P. Simsek Kiper ${ }^{4}$, S. Ceylaner ${ }^{5}$, O. A. Bilir ${ }^{3}$, F. Gumruk ${ }^{2}$, N. A. Akarsu ${ }^{1}, A$. Cetinkayal}

${ }^{1}$ Department of Medical Genetics, Faculty of Medicine, Hacettepe University, Ankara, Turkey, ${ }^{2}$ Pediatric Hematology Unit, Department of Pediatrics, Faculty of Medicine, Hacettepe University, Ankara, Turkey, ${ }^{3}$ Division of Pediatric Hematology, Ankara Child Health and Diseases Hematology Oncology Training and Research Hospital, Ankara, Turkey, ${ }^{4}$ Pediatric Genetics Unit, Department of Pediatrics, Faculty of Medicine, Hacettepe University, Ankara, Turkey, ${ }^{5}$ Intergen Genetic Diagnosis Center, Ankara, Turkey

Congenital dyserythropoietic anemias (CDAs) are a heterogenous group of disorders characterized by dysplastic erythroblasts, ineffective erythropoiesis, iron overload, splenomegaly and occasionally congenital limb abnormalities. Here, we report a CDA family with a novel homozygous mutation in C15orf41, one of six CDA associated genes, and unusually high intrafamilial clinical variability.

The proband, 3-month-old male born to consangineous parents, presented with transfusion-dependent anemia, malnutrition, recurrent pulmonary infections, hypotonia, refractory seizures and cerebral atrophy. His affected sibling had a milder neonatal anemia with no additional findings. Whole exome sequencing in proband revealed a homozygous missense mutation in C15orf41 (c.58C >A,p. Pro20Thr), which is not present in GnomAD and also found in the affected sibling. Another mutation in this codon (c.58C $>$ T,p.Pro20Ser) had already been associated with CDA. In order to discover a second mutation that may explain the neurological findings in the proband, we focused on twelve $>4 \mathrm{Mbp}$ runs of homozygosity and detected a nonsense mutation in C2orf69 (c.929G>A,p. Trp310Ter), which is absent in GnomAD and in-house databases. No other family member was homozygous for this variant. C2orf69 is highly uncharacterized, but we predict that absence of any homozygous truncating mutations in GnomAD indicates selective pressure on C2orf69. A high expression in brain is apparent in GTEx dataset.

In conclusion, we demonstrate that a novel mutation in C15orf41 affects a hotspot codon leading to CDA. In addition, we associate biallelic mutations in $C 20$ rf 69 with intractable infantile epilepsy. Multiple molecular diagnoses not only underlie the apparent phenotypic variability but also is useful in uncovering previously unidentified genes.

A. Gurel: None. S. Unal: None. N. Yarali: None. P. Simsek Kiper: None. S. Ceylaner: None. O.A. Bilir: None. F. Gumruk: None. N.A. Akarsu: None. A. Cetinkaya: None.

\section{P07.09.B}

A novel disorder involving dyshematopoiesis, inflammation and HLH due to aberrant $\mathrm{CDC} 42$ function

S. Coppola ${ }^{1}$, M. Lam $^{2,3,4}$, O. H. Krumbach ${ }^{5}$, G. Prencipe ${ }^{6}$, A. Insalaco ${ }^{6}$, C. Cifaldi ${ }^{7,8}$, I. Brigida ${ }^{9}$, E. Zara ${ }^{1}$, S. Scala ${ }^{9}$,

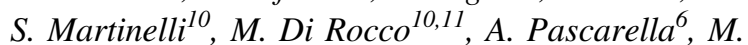
Niceta $^{12}$, F. Pantaleoni ${ }^{12}, A$. Ciolfi ${ }^{12}, F$. Carisey ${ }^{2,4}, M$. Akbarzadeh $^{5}$, L. Farina ${ }^{1,6}$, L. Pannone ${ }^{10,12}$, H. C. Erichsen $^{13}$, A. C. Horne ${ }^{14,15}$, Y. Bryceson ${ }^{16,17}$, L. TorralbaRaga $^{16}$, K. Ramme ${ }^{18}$, A. Finocchi ${ }^{19,8}$, F. Locatelli ${ }^{20}, C$. Cancrini $^{19,8}$, A. Aiuti ${ }^{9}$, M. R. Ahmadian ${ }^{5}$, J. S. Orange ${ }^{2}$, F. De Benedetti ${ }^{6}$, M. Tartaglia ${ }^{12}$

${ }^{l}$ National center for rare diseases, Istituto Superiore di Sanità, Rome, Italy, ${ }^{2}$ Department of Pediatrics, Columbia University, Irving Medical Center, New York, NY, United States, ${ }^{3}$ Translational Biology and Molecular Medicine Graduate Program and Medical ScientistTraining Program, Baylor College of Medicine, Houston, TX, United States, ${ }^{4}$ Department of Pediatrics, Baylor College of Medicine, Houston, TX, United States, ${ }^{5}$ Institute of Biochemistry and Molecular Biology II, Medical Faculty of the Heinrich-Heine University, Düsseldorf, Germany, ${ }^{6}$ Division of Rheumatology, Ospedale Pediatrico Bambino Gesù, IRCCS, Rome, Italy, ${ }^{7}$ Department of Pediatrics, Ospedale Pediatricon Bambino Gesù, IRCCS, Rome, Italy, ${ }^{8}$ Department of Systems Medicine, University of Rome Tor Vergata, Rome, Italy, ${ }^{9}$ San Raffaele Telethon Institute for Gene Therapy (SR-TIGET), IRCCS San Raffaele Scientific Institute, Milan, Italy, ${ }^{10}$ Department of Oncology and Molecular Medicine, Istituto Superiore di Sanità, Rome, Italy, ${ }^{11}$ Department of Cellular Biotechnology and Haematology, Sapienza University of Rome, Rome, Italy, ${ }^{12}$ Genetics and Rare Diseases Research Division, Ospedale Pediatrico Bambino Gesù, IRCCS, Rome, Italy, ${ }^{13}$ Department of Pediatric and Adolescent Medicine, Oslo University Hospital, Oslo, Norway, ${ }^{14}$ Pediatric Rheumatology, Astrid Lindgren Childrens Hospital, Karolinska University Hospital, Stockholm, Sweden, ${ }^{15}$ Department of Women's and Children's Health, Karolinska Institutet, Karolinska University Hospital Solna, Stockholm, Sweden, ${ }^{16}$ Department of Medicine, Karolinska Institutet, Karolinska University Hospital Huddinge, Stockholm, Sweden, ${ }^{17}$ Broegelmann Research Laboratory, Institute of Clinical Sciences, 
University of Bergen, Bergen, Norway, ${ }^{18}$ Pediatric Hematology, Immunology and HCT Section, Astrid Lindgren Children's Hospital, Karolinska University Hospital, Stockholm, Sweden, ${ }^{19}$ Department of Pediatrics, Ospedale PediatricoBambino Gesù, IRCCS, Rome, Italy, ${ }^{20}$ Department of Pediatric Hematology and Oncology, Ospedale Pediatrico Bambino Gesù, IRCCS, Rome, Italy

Introduction: The diagnosis and delineation of novel genetic syndromes is often difficult given the limited availability of patients, genetic heterogeneity, and clinical variability. Hemophagocytic lymphohistiocytosis (HLH) is characterized by hyper-inflammation driven by excessive activation and expansion of macrophages and defective Natural Killer (NK) cytotoxicity; it can be fatal if untreated. Here, we identify a previously unrecognized hematological and autoinflammatory condition in four unrelated patients with superimposable features, including neonatal-onset trilinear cytopenia with dyshematopoiesis, autoinflammation, rash, and HLH.

Methods: To delineate this new syndrome, we performed phenotype and whole-genome/exome analyses. In silico, in vitro and in vivo (C. elegans) assays allowed to characterize the impact of mutation on localization and function.

Results: All patients shared the same de novo CDC42 mutation (Chr1:22417990C >T, p.R186C) and altered hematopoietic compartment, immune dysregulation, and inflammation. We documented unique effects of the $\mathrm{p}$. $\mathrm{R} 186 \mathrm{C}$ on CDC42 localization and function. Indeed, $\mathrm{CDC} 42^{\wedge} \mathrm{R} 186 \mathrm{C}$ showed impaired interaction with known CDC42 regulators and effectors, including RHOGDI, IQGAP1 and WASP, leading to aberrant Golgi accumulation, cytoskeleton rearrangement and reduced growth and migration, which likely underlie the distinctiveness of the trait of this syndrome. Aberrant $\mathrm{CDC} 42$ function caused defective NK cytotoxicity, which likely contributes to the HLH phenotype. Timely therapeutic neutralization of IL-1 $\beta$ and IFN $\gamma$ was critical to the survival of one patient, who underwent successful bone marrow transplantation.

Conclusions: The presented data emphasize the crucial role played by CDC42 in hematopoiesis and inflammation. Early recognition of the disorder and establishment of treatment followed by bone marrow transplant are important to survival.

S. Coppola: None. M. Lam: None. O.H. Krumbach: None. G. Prencipe: None. A. Insalaco: None. C. Cifaldi: None. I. Brigida: None. E. Zara: None. S. Scala: None. S. Martinelli: None. M. Di Rocco: None. A. Pascarella: None. M. Niceta: None. F. Pantaleoni: None. A. Ciolfi: None. F. Carisey: None. M. Akbarzadeh: None. L. Farina: None. L. Pannone: None. H.C. Erichsen: None. A.C. Horne: None. Y. Bryceson: None. L. TorralbaRaga: None. K. Ramme: None. A. Finocchi: None. F. Locatelli: None. C. Cancrini: None. A. Aiuti: None. M.R.
Ahmadian: None. J.S. Orange: None. F. De Benedetti: None. M. Tartaglia: None.

\section{P07.11.A}

Studying known autoimmune risk loci in patients with CFS-ME

R. Hajdarevic, B. Lie, M. Viken

\section{University of Oslo, Oslo, Norway}

Introduction: $\mathrm{CFS} / \mathrm{ME}$ is a chronic debilitating disease of unknown origin and cause, but a heritable component is implicated, likely harbouring a multifactorial aetiology Evidence from recent studies suggest an involvement of the immune system as well as a high degree of comorbidity with other autoimmune disorders. This has led us to explore the genetic architecture of CFS/ME to further explore the immune component of this pathology. Previous genetic studies of CFS/ME were limited by heterogeneous diagnostic criteria as well as sample size $(<100$ patients), thereby lacking statistical substance.

Materials and Methods: We have genotyped 426 CFS/ ME patient (diagnosed according to the strict Canadian criteria) and 973 controls with the Illumnia immunochip. The Immunochip is designed to screen for more than 250,000 common genetic variants covering genetic regions, previously associated with autoimmune and inflammatory diseases like rheumatoid arthritis and multiple sclerosis.

Results: Our analysis has shown 16 significant and close to significant ( $\mathrm{P}$ values ranging from 6.35E-14 to 9.90E-05) genetic associations with CFS/ME scattered over several different chromosomes. The most robust finding is a block of 4 genetic variants located on chromosome 2 .

Conclusions: To the best of our knowledge, our study is the first of this magnitude in CFS/ME. The results from our discovery cohort are promising and replication studies in 2 different cohorts are in progress at the time of writing this abstract (independent Danish cohort with $>700$ samples and a UK Biobank cohort with $>1500$ samples). Grant provided by the Research Council of Norway Project number: 27268

R. Hajdarevic: None. B. Lie: None. M. Viken: None.

\section{P07.14.A}

Clonal relationship and mutational landscape of diffuse large B-cell lymphoma recurrences

M. R. Berendsen, M. van den Brand, J. Rijntjes, G. Astuti, C. J. Hess, K. M. Hebeda, W. B. C. Stevens, P. J. T. A. Groenen, J. H. van Krieken, B. Scheijen

Radboudumc, Nijmegen, Netherlands 
Introduction: Diffuse large B-cell lymphoma (DLBCL) is an aggressive disease, with a $30-40 \%$ relapse rate after firstline chemoimmunotherapy treatment. Some patients develop multiple recurrences, but the clonal relationship between the initial diagnosis and subsequent lymphomas is not routinely assessed. The molecular mechanisms underlying therapy response or failure are still poorly understood. To support diagnostics, guide therapy choices and eventually improve outcome of patients with DLBCL, we aim to define the clonal relationship and mutational landscape of DLBCL recurrences.

Materials and Methods: In a cohort of 37 matched DLBCL diagnosis and one to three relapse biopsies, clonality analyses were performed by next-generation sequencing (NGS) of immunoglobulin (IG) gene rearrangements. The clonotypes for the different IG gene rearrangements were identified by ARReST/Interrogate bioinformatics. Mutation analysis by whole exome sequencing is currently being performed on a subset of the paired diagnosis-relapse samples.

Results: IG-NGS clonality analyses on matched diagnosisrelapse(s) samples revealed identical dominant clonotype(s) in $33 / 37$ of the recurrences, even in cases with an interval period of 14 years. However, in 4/37 DLBCL patients, at least one of the recurrences was clonally unrelated.

Conclusions: DLBCL recurrences are clonally unrelated lymphomas in $\sim 10 \%$ of the cases, suggesting the occurrence of second primary lymphoma, possibly linked to a genetic predisposition or immunodeficiency. Our cohort of clonally related cases will be subjected to mutational analyses to define the patterns of clonal evolution and identify genes related to therapy resistance. These studies will help to define the genetic landscape of relapsed DLBCL.

M.R. Berendsen: None. M. van den Brand: None. J. Rijntjes: None. G. Astuti: None. C.J. Hess: None. K.M. Hebeda: None. W.B.C. Stevens: None. P.J.T.A. Groenen: None. J.H. van Krieken: None. B. Scheijen: None.

\section{P07.17.A}

Clinical and cytogenetic assessment in a cohort of Egyptian patients with Fanconi anemia: A 10 year experience

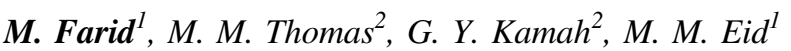 \\ ${ }^{1}$ Human Cytogenetics Department, Human Genetics and Genome Division, National Research Centre, Cairo, Egypt, \\ ${ }^{2}$ Clinical Genetics Department, Human Genetics and Genome Division, National Research centre, Cairo, Egypt}

Introduction: Fanconi anemia (FA) is a rare autosomal recessive disorder. Common clinical presentations include progressive bone marrow failure, abnormal skin pigmentations, skeletal malformations and high risk of malignancy. Due to its rarity and heterogeneous presentation, it is rather challenging to establish a definite registry on a national level. Therefore, this study describes the FA cases in the NRC 10 year registry, focusing on family history, clinical, hematological and cytogenetic findings.

Methods: This study included 225 patients. Their age ranged from 4 months to 26 years. All patients were subjected to thorough clinical examination, pedigree construction, hematological and radiological investigations. Cytogenetic assessment using diepoxybutane(DEB)induced chromosomal breakage was done to differentiate Fanconi anemia from acquired aplastic anemia.

Results: 131 patients (58\%) showed positive chromosomal breakage and were diagnosed with FA. $93 \%$ of them were of consanguineous parents and $21 \%$ showed positive family history as an affected sibling. Clinical examination showed; limb anomalies (20\%), abnormal skin pigmentation $(31 \%)$, bleeding tendency (22\%) and kidney anomalies $(10 \%)$ of the cases. Hematological investigations showed: cytopenia in one or more of blood cell types (52\%), bone marrow failure (42\%) and 34\% had received blood /blood products at least once at the time of diagnosis. Cytogenetic study showed mosaicism in $10 \%$ of the FA cases which is significant for treatment assessment.

Conclusions: This study is the first to present the clinical and cytogenetic characterizations of a cohort of Egyptian FA patients. Further large scale studies are needed to identify the prevalence of FA in the Egyptian society.

M. Farid: None. M.M. Thomas: None. G.Y. Kamah: None. M.M. Eid: None.

\section{P07.19.C}

Myeloperoxidase modulates inflammation in generalized pustular psoriasis and further rare pustular skin diseases

\section{S. Haskamp}

Institute of Human Genetics, University Hospital Erlangen, Erlangen, Germany

Generalized pustular psoriasis (GPP) is a severe multisystemic inflammatory disease characterized by neutrophilic pustulosis and triggered by pro-inflammatory IL-36 cytokines in skin. While $19-41 \%$ of patients carry bi-allelic mutations in IL36RN, the majority of patients do not carry mutations in known genes. In order to identify and characterize new pathways involved in the pathogenesis of GPP we performed whole exome sequencing in 31 GPP patients and demonstrated effects of mutations in $M P O$ encoding the neutrophilic enzyme myeloperoxidase (MPO) in myeloid 
cells. We discovered eight $M P O$ mutations resulting in MPO-deficiency in neutrophils and monocytes. Mutations were associated with GPP, primarily those resulting in complete MPO-deficiency $\quad(\mathrm{p}=3.49 \mathrm{E}-10 ; \quad \mathrm{OR}=8.63)$. Dosage of mutant $M P O$ alleles showed significant differences between 82 patients and $>3,600$ controls $(p=2.67 \mathrm{E}-$ $10)$; this effect was stronger when including IL36RN mutations $(\mathrm{p}<2.20 \mathrm{E}-16)$ and correlated with a younger age of onset ( $\mathrm{p}=0.0018)$. Activity of four proteases, previously implicated as activating enzymes of IL-36 precursors, correlated with MPO-deficiency. Formation of neutrophil extracellular traps (NETs) was reduced following phorbol myristate acetate induction $(\mathrm{p}=0.015)$. Phagocytosis assays in MPO-deficient mice and human suggested that altered neutrophil function together with impaired clearance of neutrophils by monocytes (efferocytosis) allowed prolonged neutrophil persistence in patients' skin.MPO mutations contribute significantly to GPP's pathogenesis. Our study implicates MPO as an inflammatory modulator in humans by regulating protease activity and NET formation, and modifying efferocytosis. Our findings confine using MPO inhibitors in cardiovascular diseases. MPO and affected pathways represent attractive targets for inducing resolution of inflammation in neutrophil-mediated skin diseases.

\section{S. Haskamp: None.}

\section{P07.21.B}

A rare cause of Acute Myeloid Leukemia and a delayed diagnosis of Fanconi Syndrome: Mutations in FANCE

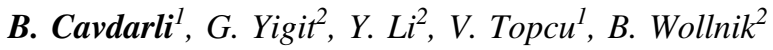

${ }^{1}$ Ankara Bilkent City Hospital, Ankara, Turkey, ${ }^{2}$ Institute of Human Genetics, University Medical Center Göttingen, Göttingen, Germany

Introduction: Genomic instability syndromes are a group of inherited disorders that include a high variety of symptoms such as congenital anomalies, bone marrow failure, and cancer susceptibility. Fanconi Syndrome(FS) is a rare syndrome belonging to this family characterized by neurodevelopmental delay, radial-ray defects, microcephaly and progressive deficiency of bone marrow production. Material and Methods: We present a 33-year-old female who was monitored for Acute Myeloid Leukemia. Clinical findings of the patient included short stature, microcephaly, neurodevelopmental delay, patchy hypopigmented dark-colored skin, signs of premature aging and chronic liver disease.

Results: Chromosomal breakage rate was detected high after mitomycin-C(MMC) treatment of cells suggesting chromosomal instability syndrome. We performed wholeexome-sequencing on the DNA of the index patient and her affected brother who had parental consanguinity. We identified an intronic variant, c.1114-8G $>$ A in FANCE that was homozygous in the index patient as well as her affected brother, and it is predicted to generate a new acceptor splice-site resulting in alternative-splicing and premature protein truncation (p.Ile372Leufs*2). So far, this variant has only been described in a single case also of Turkish origin with a similar phenotype. Besides this patient, there are currently only four additional patients carrying biallelic mutations in FANCE. All of these patients were diagnosed in the first decade of their lives.

Conclusions: We expand the clinical spectrum of patients with FANCE mutations and show that the absence of renal, cardiac and radial ray anomalies might be the cause of late diagnosis of FS caused by FANCE mutations.

B. Cavdarli: None. G. Yigit: None. Y. Li: None. V. Topcu: None. B. Wollnik: None.

\section{P07.25.C}

\section{Cell-free DNA in plasma-an essential immune system regulator}

M. Korabecna ${ }^{l}$, A. Zinkova $^{l}$, I. Brynychova ${ }^{l}, B$.

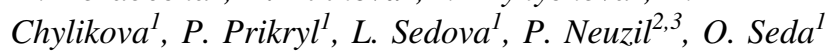

${ }^{1}$ First Faculty of Medicine, Charles University, Prague, Czech Republic, ${ }^{2}$ Northwestern Polytechnical University, Xi'an, China, ${ }^{3}$ Brno University of Technology, Brno, Czech Republic

Cell-free DNA (cfDNA) is always present in plasma, and it is utilized in prenatal diagnostics as well as in oncology and transplantology for therapy efficiency monitoring. But does the cfDNA have a physiological role? Here we show that cfDNA presence in plasma of healthy individuals plays an indispensable role in immune system regulation. We exposed THP1 cells to healthy individuals' plasma with (NP) and without (TP) cfDNA. In cells treated with NP, we found elevated expression of genes whose products maintain immune system homeostasis. Exposure of cells to TP triggered an innate immune response (IIR), documented particularly by elevated expression of pro-inflammatory interleukin 8 . The results of mass spectrometry showed a higher abundance of proteins associated with IIR activation due to the regulation of complement cascade in cells cultivated with TP. Our results provide evidence that the presence of cfDNA in plasma of healthy individuals blocks fundamental mechanisms of the inflammation process. The expression profiles show that this process could play an important role in the regulation of tissue homeostasis. This finding epitomizes one of many intriguing properties and features of the human immune system. Its detailed understanding is of crucial interest for future medical applications. Financial support: grants LTACH19005 and Progres 
Q25/LF1 of the Ministry of Education, Youth and Sport of the Czech Republic, grant RVO-VFN 64165 of the Ministry of Health of the Czech Republic. P.N. would like to acknowledge financial support from State Administration of Foreign Experts Affairs number W099109 and CEITEC Nano+ project (CZ.02.1.01/0.0/0.0/16_13/0001728).

M. Korabecna: None. A. Zinkova: None. I. Brynychova: None. B. Chylikova: None. P. Prikryl: None. L. Sedova: None. P. Neuzil: None. O. Seda: None.

P07.27.B

The impact of rare and common genetic variation in the Interleukin-1 pathway for human cytokine responses

R. C. van Deuren ${ }^{1,2,3}, P$. Arts $^{2,4}$, G. Cavalli ${ }^{1,5,6}, M$. Jaeger $^{1,3}$, M. Steehouwer ${ }^{2}$, M. van der Vorst ${ }^{2}$, C. Gilissen ${ }^{2,3}$, L. A. B. Joosten ${ }^{1,3,7}$, C. A. Dinarello ${ }^{1,6}$, M. M. Mhlanga ${ }^{8,9}$, V. Kumar ${ }^{1,10}$, M. G. Netea ${ }^{1,3,11}$, F. L. van de Veerdonk ${ }^{1,3}$, A. Hoischen $^{1,2,3}$

${ }^{1}$ Department of Internal Medicine, Radboud University Medical Center, Nijmegen, Netherlands, ${ }^{2}$ Department of Human Genetics, Radboud University Medical Center, Nijmegen, Netherlands, ${ }^{3}$ Radboud Institute of Molecular Life Sciences (RIMLS), Radboud University Medical Center, Nijmegen, Netherlands, ${ }^{4}$ Department of Genetics and Molecular Pathology, Centre for Cancer Biology, SA Pathology and the University of South Australia, Adelaide, Australia, ${ }^{5}$ Unit of Immunology, Rheumatology, Allergy and Rare Diseases, IRCCS San Raffaele Hospital and VitaSalute San Raffaele University, Milan, Italy, ${ }^{6}$ Department of Medicine, University of Colorado, Aurora, CO, United States, ${ }^{7}$ Department of Medical Genetics, Iuliu Hatieganu University of Medicine and Pharmacy, Cluj-Napoca, Romania, ${ }^{8}$ Division of Chemical Systems \& Synthetic Biology, Institute for Infectious Disease \& Molecular Medicine (IDM), Department of Integrative Biological \& Medical Sciences, University of Cape Town, Cape Town, South Africa, ${ }^{9}$ Faculty of Health Sciences, Department of Integrative Biomedical Sciences, University of Cape Town, Cape Town, South Africa, ${ }^{10}$ University of Groningen, University Medical Center Groningen, Department of Genetics, Groningen, Netherlands, ${ }^{11}$ Department for Genomics \& Immunoregulation, Life and Medical Sciences Institute (LIMES), University of Bonn, Bonn, Germany

Interleukin(IL)-1 signaling is of major importance in human innate cytokine responses. Common genetic variants in underlying genes have been linked to various inflammationmediated diseases and pathogen induced cytokine responses, but the role of rare variants remains to be elucidated. In this study, we characterize the role of rare and common genetic variation in 48 genes related to the IL-1 pathway, on in vitro stimulation-specific human cytokine responses from 463 healthy individuals. We identified strong NCF4 and CASP1 rare variant associations with IL-6 cytokine production induced by phytohaemagglutinin $\left(\mathrm{P}=7.2 \mathrm{E}^{-05}\right)$ and lipopolysacharide (LPS) $\left(\mathrm{P}=3.0 \mathrm{E}^{-05}\right)$. Additionally, common variants in IL36A and IL38 were associated to both $C$. albicans induced IL-1 $\beta$ and IL-6 (IL36A P=0.0442 and 0.0037 ; IL38 $\mathrm{P}=0.0092$ and 0.0082 ), an effect that was magnified on the respective IL-30 subpathway level (IL-1 $\beta$ $\mathrm{P}=0.0017$; IL-6 $\left.\mathrm{P}=1.8 \mathrm{E}^{-04}\right)$. The common variant signature for the immunological response to C.albicans was confirmed by an association with the anti-inflammatory phenotype group (IL-1 $\beta \mathrm{P}=1.87 \mathrm{E}^{-03}$; IL-6 $\mathrm{P}=5.75 \mathrm{E}^{-04}$ ), and we validated this for non-coding common variants. Lastly, we detected an association between rare variants in pro-inflammatory genes and LPS-induced IL-6 cytokine $\left(\mathrm{P}=2.42 \mathrm{E}^{-04}\right)$, and discovered a role for rare variants in anti-inflammatory genes on S.aureus induced IL-6 production $\left(\mathrm{P}=6.71 \mathrm{E}^{-03}\right)$. In conclusion, we show that both common and rare genetic variation in genes of the IL-1 pathway differentially influence in vitro cytokine responses to various stimuli in healthy individuals. This study therefore provides insight into potential mechanisms relevant for the characterization of genetic variant involvement in variability of immune responses and possibly inflammatorymediated diseases.

R.C. van Deuren: None. P. Arts: None. G. Cavalli: None. M. Jaeger: None. M. Steehouwer: None. M. van der Vorst: None. C. Gilissen: None. L.A.B. Joosten: None. C.A. Dinarello: None. M.M. Mhlanga: None. V. Kumar: None. M.G. Netea: None. F.L. van de Veerdonk: None. A. Hoischen: None.

\section{P07.28.C}

Correcting clinical diagnosis with targeted immundeficiency gene panel sequencing: the critical role of genetic counselling

\section{K. Arvai $^{l}$, A. Illés ${ }^{l}$, B. Kocsis-Deák ${ }^{l}$, V. Klujber ${ }^{l}$, B.}

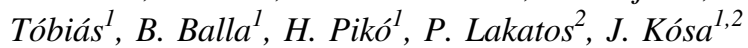

${ }^{1}$ PentacoreLab Ltd., Budapest, Hungary, ${ }^{2}$ Semmelweis University 1st Department of Internal Medicine, Budapest, Hungary

Immunodeficiency results from a failure or absence of elements of the immune system including lymphocytes, phagocytes and complement system. These immunodeficiencies can be either primary such as Bruton's disease or secondary as the one caused by HIV infectionIn our case, a 35 years old healthy female patient was referred to our lab, who lost two of her brothers with severe immunodeficiency. She is about to have her first child and 
wanted to be tested before the pregnancy. The suspected familiar diagnosis, based only clinical symptoms, was Xlinked agammaglobulinemia which is a X-linked monogenic disorder. After sequencing of the BTK gene, MLPA were carried out, both returned with negative results. After second genetic counselling it was revealed by the counsellor that the suspected phenotype is not fit entirely and targeted gene panel test was ordered, included 200 genes which are linked to immunodeficiencies. A pathogenic heterozygous deletion in the CD40LG gene (c.67dupA) was identified, which is a known cause of X-linked immunodeficiency, with hyper-IgM. HIGM is a rare immunodeficiency characterized by normal or elevated serum IgM levels associated with markedly decreased $\operatorname{IgG}, \operatorname{Ig} \mathrm{A}$, and $\operatorname{IgE}$, resulting in a profound susceptibility to bacterial infections and an increased susceptibility to opportunistic infections. Patients also tend to have neutropenia, as well as a high rate of gastrointestinal and central nervous system infections. Based on estimation only $20 \%$ of patients will reach the third decade of life. Our results emphasize the importance of multiple genetic counselling and highlights the difficulties to interpret clinical results decades ago.

K. Arvai: None. A. Illés: None. B. Kocsis-Deák: None. V. Klujber: None. B. Tóbiás: None. B. Balla: None. H. Pikó: None. P. Lakatos: None. J. Kósa: None.

\section{P07.31.C}

Hyperinflammation and secondary HLH in a patient with Interferon alpha/beta receptor 2 (IFNAR2) deficiency

C. Passarelli ${ }^{l}$, A. Civino' ${ }^{2}$, M. N. Rossi ${ }^{3}$, V. Lanari ${ }^{l}$, G. M.

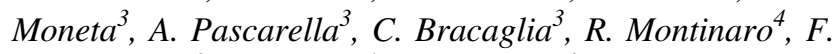
De Benedetti ${ }^{3}$, A. Novelli ${ }^{1}$, G. Prencipe ${ }^{3}$

${ }^{1}$ U.O.C. Laboratory of Medical Genetics, IRCCS Paediatric Hospital "Bambino Gesu", Rome, Italy, ${ }^{2}$ Rheumatology and Paediatric Immunology, PO "Vito Fazzi", Lecce, Italy, ${ }^{3}$ Division of Rheumatology, IRCCS Paediatric Hospital "Bambino Gesu", Rome, Italy, ${ }^{4}$ Division of Pediatry, PO "Santa Caterina Novella", Galatina, Italy

Introduction: Interferons $\alpha / \beta$ (Type I IFNs) play an important role in defense against viral infections. Here we report the case of a 22-months-old Caucasian boy presenting with high fever and lethargy five days after liveattenuated MMR (measles-mumps-rubella) vaccine. Laboratory parameters were suggestive for secondary Hemophagocityc lymphohistiocytosis (HLH), with progressive decrease in cell blood count, hyperferritinemia, elevation of liver enzymes and lactate dehydrogenase and hypofibrinogenemia.
Materials and Methods: Clinical Exome, using a custom panel including 6920 genes known as associated to genetic diseases, was sequenced on Illumina NovaSeq $6000^{\circledR}$ platform. In silico analysis was performed on the basis of the patient's clinical phenotype. Peripheral blood mononuclear cells (PBMCs) were isolated from the patient and his family and stimulated with IFN $\alpha$ or IFN $\gamma$; phosphorylation of STAT1 and Type I and II IFN signatures were analyzed by FACS analysis and RT-PCR.

Results: We identified the two novel frameshift mutations c.234delT and c.555_559delAAAAG, in a compound heterozygous status, in IFNAR2 gene (OMIM\# 602376), resulting respectively in p.Leu79Ter and p.Ile185MetfsTer12 variants. Ex vivo studies on patient's PBMCs confirmed the defect in the activation of the type I IFN signaling, but not in type II IFN response. Treatment with high dose of intravenous methylprednisolone allowed a progressive improvement of patient condition and of clinical and laboratory features.

Conclusions: Our data demonstrate that HLH episodes following administration of live-attenuated MMR vaccine could suggest the presence of a defect in the type I IFN response.

C. Passarelli: None. A. Civino: None. M.N. Rossi: None. V. Lanari: None. G.M. Moneta: None. A. Pascarella: None. C. Bracaglia: None. R. Montinaro: None. F. De Benedetti: None. A. Novelli: None. G. Prencipe: None.

P07.32.A

Genome wide association study of circulating interleukin 6 levels identifies novel loci

T. S. Ahluwalia ${ }^{1}$, N. J. Armstrong ${ }^{2}$, S. Aslibekyan ${ }^{3}, M$.

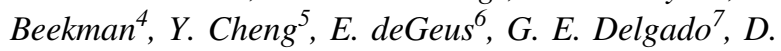
Marek $^{8}$, S. Kanoni ${ }^{9}$, I. M. Nolte ${ }^{10}$, E. Porcu ${ }^{11}$, I. Seppälä ${ }^{12}$, M. Standl ${ }^{13}$, A. Teumer ${ }^{14}$, A. Thalamuthu ${ }^{15}$, S. Trompet ${ }^{16}$, E. J. Benjamin ${ }^{17}$, M. F. Feitosa ${ }^{18}$, G. Homuth ${ }^{19}$, J. Lahti ${ }^{20}$, Y. Liu $^{21}$, N. J. Timpson ${ }^{17}$, S. Visvikis-Siest ${ }^{22}$, U. Völker ${ }^{19}$, B. T. Baune $^{23}$, D. Boomsma ${ }^{24}$, I. J. Deary ${ }^{25}$, D. M. Evans $^{17}$, M. A. Ferreira $^{26}$, T. Gaunt ${ }^{17}$, V. Gudnason ${ }^{27}$, A. Hamsten ${ }^{28}$, S. E. Humphries $^{29}$, W. Koeing ${ }^{30}$, M. Kumari ${ }^{31}$, D. A. Lawlor ${ }^{17}$, M. Nauck $^{32}$, J. F. Price ${ }^{33}$, T. I. A. Sørensen ${ }^{34}$, D. Stacey ${ }^{35}$, M. G. Stathopoulou ${ }^{22}$, T. Tanaka ${ }^{36}$, S. G. Wannamethee ${ }^{37}$, J. I. Rotter $^{38}$, A. Dehghan ${ }^{39}$, E. Boerwinkle ${ }^{40}$, H. Sneider ${ }^{10}$, B. M. Psaty ${ }^{41}$, B. P. Prins ${ }^{10}$, B. Z. Alizadeh ${ }^{10}$

${ }^{1}$ Steno Diabetes Center Copenhagen, Gentofte, Denmark, ${ }^{2}$ Murdoch university, Perth, Australia, ${ }^{3}$ University of alabama at birmingham school of public health, Birmingham, AL, United States, ${ }^{4}$ Leiden university medical center, Leiden, Netherlands, ${ }^{5}$ University of Maryland school of medicine, Baltimore, MD, United States, ${ }^{6}$ Vrije universiteit 
amsterdam, Amsterdam, Netherlands, ${ }^{7}$ Vth department of medicine university of heidelberg, Mannheim, Germany, ${ }^{8}$ SIB swiss institute of bioinformatics, Lausanne, Switzerland, ${ }^{9}$ William harvey research institute, queen mary university of london, London, United Kingdom, ${ }^{10}$ university of groningen, university medical center groningen,, Groningen, Netherlands, ${ }^{11}$ Istituto di ricerca genetica $e$ biomedica, consiglio nazionale delle ricerche, Cagliari, Italy, ${ }^{12}$ Department of clinical chemistry, tampere university, Tampere, Finland, ${ }^{13}$ Institute of epidemiology, helmholtz zentrum münchen - german research center for environmental health, Neuherberg, Germany, ${ }^{14}$ Institute for community medicine, university medicine greifswald, Greifswald, Germany, ${ }^{15}$ Neuropsychiatric institute, prince of wales hospital, Sydney, Australia, ${ }^{16}$ Department of cardiology, leiden university medical center, Leiden, Netherlands, ${ }^{17}$ MRC integrative epidemiology unit, university of bristol, Bristol, United Kingdom, ${ }^{18}$ Department of genetics, washington university school of medicine, St. Louis, MO, United States, ${ }^{19}$ Interfaculty institute for genetics and functional genomics, university medicine Greifswald, Greifswald, Germany, ${ }^{20}$ Turku institute for advanced studies, university of turku, Turku, Finland, ${ }^{21}$ Department of epidemiology and prevention, wake forest school of medicine, winston-Salem, NC, United States, ${ }^{22}$ Université de lorraine, INSERM, Nancy, France, ${ }^{23}$ Department of psychiatry and the Florey institute of neuroscience and mental health the university of Melbourne, Melbourne, Australia, ${ }^{24}$ Department of biological psychology, behaviour and movement sciences, vrije universiteit Amsterdam, Amsterdam, Netherlands, ${ }^{25}$ Centre for cognitive ageing and epidemiology, university of edinburgh, Edinburgh, United Kingdom, ${ }^{26}$ QIMR berghofer medical research institute, Brisbane, Australia, ${ }^{27}$ Faculty of medicine, university of iceland, Reykjavik, Iceland, ${ }^{28}$ Department of medicine solna, center for molecular medicine, karolinska institutet, Stockholm, Sweden, ${ }^{29}$ Institute of cardiovascular science, university college london, London, United Kingdom, ${ }^{30}$ German Heart Centre Munich, Technical University Munich, Munich, Germany, ${ }^{31}$ Department of epidemiology and public health, university college london, London, United Kingdom, ${ }^{32}$ Institute of clinical chemistry and laboratory medicine, university medicine greifswald, Greifswald, Germany, ${ }^{33}$ Usher institute of population health sciences and informatics, university of edinburgh, Edinburgh, United Kingdom, ${ }^{34}$ Department of public health, university of copenhagen, Copenhagen, Denmark, ${ }^{35}$ MRC cardiovascular epidemiology unit, university of cambridge, Cambridge, United Kingdom, ${ }^{36}$ Translational gerontology branch, national institute on aging, Baltimore, MD, United States, ${ }^{37}$ Institute of epidemiology \& health care, UCL, London, United Kingdom, ${ }^{38}$ Departments of pediatrics and medicine, harbor- ucla medical center, Los Angeles, CA, United States, ${ }^{39}$ Department of epidemiology, erasmus mc, Rotterdam, Netherlands, ${ }^{40}$ Human genetics center, university of texas, Houston, TX, United States, ${ }^{41}$ Cardiovascular health research unit, department of medicine, university of washington, Seattle, WA, United States

Interleukin-6 (IL-6) is a multifunctional cytokine with both pro and anti-inflammatory properties, synthesized by a wide range of tissues and cell types. Increased levels of circulating IL-6 in blood is associated with the pathophysiology of complex disorders like type 2 diabetes, cardiovascular and autoimmune diseases. Albeit, IL-6 levels are heritable with estimates up to $61 \%$, only a few common genetic loci associated with circulating IL-6 levels have been identified. We therefore conducted a two stage (discovery and replication) meta genome-wide association study (GWAS) of circulating serum IL-6 concentrations comprising up to 67,428 individuals of european ancestry. About 2.5 million single nucleotide polymorphisms (SNPs) were available for testing after imputation to Hap Map 2 reference panel. We conducted an inverse variance based fixed effects metaanalysis. We identified three IL- 6 associated, independent signals on chromosomes (chr) 2q14, 6p21 and 1q21, reaching genome-wide significance $\left(p<5.0 \times 10^{-8}\right)$ in the combined meta-analyses. Among the identified loci ILIF10/ ILIRN (chr 2q14, $p=1.8 \times 10^{-11}$ ), and HLA-DRB1/DRB5 (chr $6 \mathrm{p} 21, p=1.5 \times 10^{-10}$ ) were novel while IL6R $(\mathrm{chr}$ $1 \mathrm{q} 21, p=1.2 \times 10^{-122}$ ) was a known locus. Our study identifies 2 novel loci for circulating IL-6 levels uncovering new immunological and inflammatory pathways that may influence IL-6 pathobiology.

T.S. Ahluwalia: None. N.J. Armstrong: None. S. Aslibekyan: None. M. Beekman: None. Y. Cheng: None. E. deGeus: None. G.E. Delgado: None. D. Marek: None. S. Kanoni: None. I.M. Nolte: None. E. Porcu: None. I. Seppälä: None. M. Standl: None. A. Teumer: None. A. Thalamuthu: None. S. Trompet: None. E.J. Benjamin: None. M.F. Feitosa: None. G. Homuth: None. J. Lahti: None. Y. Liu: None. N.J. Timpson: None. S. VisvikisSiest: None. U. Völker: None. B.T. Baune: None. D. Boomsma: None. I.J. Deary: None. D.M. Evans: None. M.A. Ferreira: None. T. Gaunt: None. V. Gudnason: None. A. Hamsten: None. S.E. Humphries: None. W. Koeing: None. M. Kumari: None. D.A. Lawlor: None. M. Nauck: None. J.F. Price: None. T.I.A. Sørensen: None. D. Stacey: None. M.G. Stathopoulou: None. T. Tanaka: None. S.G. Wannamethee: None. J.I. Rotter: None. A. Dehghan: None. E. Boerwinkle: None. H. Sneider: None. B.M. Psaty: None. B.P. Prins: None. B.Z. Alizadeh: None.

P07.33.B 
KIR-HLA class I ligand combinations among cystic fibrosis patients

\section{K. Sosnina ${ }^{l}$, D. Zastavna ${ }^{1,2}$, O. Terpyliak ${ }^{1}, H$. Makukh $^{l}$, L. Bober $^{3}$}

${ }^{1}$ SI "Institute of Hereditary Pathology Ukrainian National Academy of Medical Sciences”, Lviv, Ukraine, ${ }^{2}$ Department of Biotechnology and Bioinformatics, Faculty of Chemistry, Rzeszow University of Technology, Al. Powstańców Warszawy 6, Rzeszow, Poland, '3Lviv Regional Children's Specialized Clinical Hospital, Lviv, Ukraine

$K I R$ and $H L A$ loci play a critical role in NK cell regulation. Different compound KIR-HLA genotypes can impart different thresholds of activation to the NK repertoire and such genotypic variation has been found to confer altered risk in a number of human diseases including infectious diseases. The aim of this study was to establish and analyze the spectrum of KIR-HLA genes in people with a confirmed diagnosis of $\mathrm{CF}$, homozygote of F508del mutation of the CFTR gene for understanding the genetic predisposition of congenital immunity key part functioning during $\mathrm{CF}$. Results: KIR and HLA class I ligands $(C 1, C 2)$ genes distribution was established in $48 \mathrm{CF}$ patients and 104 practically healthy individuals of control group. HLAC genotypes frequency did not differ in the CF group and in the control. The lower frequency of KIR2DL3, KIR2DS1 and KIR2DS4 genes $(37.5 \%, 6.25 \%$ and $0 \%)$ was found among CF patients ( $\mathrm{p}<0.05$ ) compared to control group $(67.3 \%, 20.2 \%$ and $10.5 \%)$ indicating a strong inhibitory effect on NK cells. Respectively, the frequency of KIRBB haplotype $8.34 \%$ in $\mathrm{CF}$ patients was significantly higher compared to the control $0.96 \%$ ( $\mathrm{p}<0.05)$. Analysis of combined frequencies for $i K I R+H L A(>=<) a K I R+H L A$ showed no significant difference between the study groups. $i K I R+H L A \backslash$ IKIR analysis showed significantly lower frequency $i K I R+H L A<a K I R$ combinations in the $\mathrm{CF}$ group compared to controls $(\mathrm{p}<0.05)$. Thus, the results indicate that people with cystic fibrosis have genetically predispossition of weak activation and a strong inhibition of NK functional activity, that possibly contributing to broncopulmonary infection process development.

K. Sosnina: None. D. Zastavna: None. O. Terpyliak: None. H. Makukh: None. L. Bober: None.

\section{P07.34.C}

Transcriptome-based elucidation of gender bias in systemic lupus erythematosus (SLE)
K. Varvagiannis ${ }^{1}$, D. Gerasoudis ${ }^{1}$, A. Filia ${ }^{1}$, N. Panousis ${ }^{2}$, E. Dermitzakis ${ }^{1,3,4,5}$, G. Bertsias ${ }^{6,7}$, D. Boumpas ${ }^{1,8,7,9}$, A. Banos $^{1}$, P. Makrythanasis ${ }^{1,3}$

${ }^{1}$ Biomedical Research Foundation Academy Of Athens, Athens, Greece, ${ }^{2}$ Wellcome Trust Sanger Institute, Hinxton, Cambridge, United Kingdom, ${ }^{3}$ Department of Genetic Medicine and Development, University of Geneva Medical School, Geneva, Switzerland, ${ }^{4}$ Institute of Genetics and Genomics in Geneva (iGE3), University of Geneva, Geneva, Switzerland, ${ }^{5}$ Swiss Institute of Bioinformatics, Geneva, Switzerland, ${ }^{6}$ Department of Rheumatology, Clinical Immunology and Allergy, University of Crete, Medical School, Heraklion, Greece, ${ }^{7}$ Institute of Molecular Biology and Biotechnology, Foundation for Research and Technology Hellas (FORTH), Heraklion, Greece, ${ }^{8}$ Joint Academic Rheumatology Program, National and Kapodistrian University of Athens, Medical School, Athens, Greece, ${ }^{9}$ Medical school, University of Cyprus, Nicosia, Cyprus

Background: Systemic lupus erythematosus (SLE) is a chronic autoimmune disease that can affect virtually any organ/system. Clinical features in individual patients can be highly variable, ranging from mild arthritis, skin manifestations and antinuclear antibody production to lifethreatening organ disease. SLE exhibits a striking female predominance, with ratios exceeding 9:1. The disease often displays a more aggressive clinical course in men, resulting in poorer prognosis. Several hypotheses have been put forward to explain this gender bias, including $\mathrm{X}$ chromosome inactivation, sex hormones and TLR/miRNAs altered expression.

Methods: By employing data from mRNA sequencing in whole blood samples from 142 SLE patients and 58 healthy volunteers we explore gender/disease specific patterns of gene expression and their putative functional gene networks as suggested by DAVID analysis.

Results: Genes displaying a stepwise up-/down-regulation upon comparison of their expression in the order of healthy males (HM), healthy females (HF), affected females (AF) and affected males (AM) groups, were the most overrepresented among all possible combinations. These genes appeared to belong to pathways highly relevant to SLE pathogenesis, including NOD-/Toll-like receptor signaling, ubiquitin mediated proteolysis, nucleotide excision repair and spliceosome ( $p$-values < 0.05).

Conclusions: Although preliminary, our results suggest that gender bias may - at least partially - be reflected by patterns of altered expression of genes/pathways relevant to SLE. Future analysis in lupus mouse model will reveal genetic impact on gender-specific gene expression.

K. Varvagiannis: None. D. Gerasoudis: None. A. Filia: None. N. Panousis: None. E. Dermitzakis: None. G. 
Bertsias: None. D. Boumpas: None. A. Banos: None. P. Makrythanasis: None.

\section{P07.35.A}

MicroRNAs expression may affect the lymphocyte concentration in Down syndrome

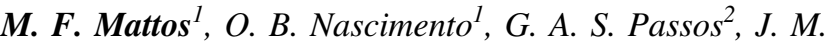 \\ Biselli $^{3}$, E. M. Goloni-Bertollo ${ }^{l}$, É. C. Pavarino ${ }^{l}$ \\ ${ }^{1}$ Unidade de Pesquisa em Genética e Biologia Molecular \\ da Faculdade de Medicina de São José do Rio Preto, São \\ José do Rio Preto, Brazil, ${ }^{2} 2$ Faculdade de Medicina de \\ Ribeirão Preto da Universidade de São Paulo - USP, \\ Ribeirão Preto, Brazil, ${ }^{3} 3$ Universidade Estadual Paulista \\ Júlio de Mesquita Filho- UNESP, São José do Rio Preto, \\ Brazil
}

Introduction: Studies have showed the importance of microRNAs in Down syndrome(DS) pathology and impaired $\mathrm{T}$ cell proliferation may result of altered microRNAexpression. Therefore, in this study we investigated the relationship between expressionpattern of hsa-miR378a-3p, hsa-miR-668-3p and hsa-miR 942-5p and lymphocytesconcentration in DS.

Material and Methods: Peripheral blood mononuclear cells were obtained from 52healthy individuals with full trisomy 21 (DS) (age 1 to 20) and 53 healthy control (HC) (age 1 to 12). MicroRNAs expression was investigated by real time polymerase chainreaction using Taqman assays. Statistical analysis was performed using OneSampleWilcoxon Signed Rank Test, in the GraphPad Prism 6 software. Bioinformatic analysiswas carried out to identify genes regulated by expressed differentially microRNAs andthe interaction between proteins using MiRTarbase v9 and STRING t-v11.0 tool.

Results: DS individuals presented over-expression of hsa-miR-378a-3p (RQ 1.22, $\mathrm{P}=0.036)$ and downexpression of hsa-miR-668-3p (RQ 0.42, $\mathrm{P}=0.018$ ). Moreover, thisindividuals presented lower lymphocytes concentration than $\mathrm{HC}$ (mean 36.70, 48.68; respectively, $\mathrm{P}=0.007$ ). Network analysis showed that genes regulated by hsa-miR-378a-3p and hsa-miR-668-3p are significantly involved in cell differentiation, celldivision, cell cycle $(\mathrm{P}=<0.001)$ and regulation of cell growth $(\mathrm{P}=0.02)$.

Conclusions: The lower lymphocytes concentration in DS may in part explain the highfrequency of immune alterations. Genes that regulates the lymphocyte concentrationsare probably affected by impaired microRNA expression. MicroRNA target genes areinvolved in biological process relevant to immune response as cell proliferation.
Grants: FAPESP (2018/09126-0 and 2018/24825-2), CNPQ (310806/2018-6), CAPES(001), FAMERP/ FUNFARME.

M.F. Mattos: None. O.B. Nascimento: None. G.A.S. Passos: None. J.M. Biselli: None. E.M. Goloni-Bertollo: None. É.C. Pavarino: None.

\section{P07.36.B}

Surprising genetic results in a case of neurofibromatosislike syndrome

M. T. Bataneant ${ }^{1,2}$, M. Puiul ${ }^{1,2}$, M. Baica ${ }^{3}$, E. Boeriu ${ }^{1,2}$, P. Urtila $^{1,2}$, C. Zaica $^{3}$, A. Beloia ${ }^{4}$, A. Chirita-Emandi ${ }^{1}$

${ }^{1}$ University of Medicine and Pharmacy "Victor Babes", Romania, Romania, ${ }^{2}$ Clinical Emergency Hospital for Children „Louis Turcanu”, Timisoara, Romania, ${ }^{3}$ Louis Turcanu Emergency Children Hospital, Timisoara, Romania, ${ }^{4}$ Louis Turcanu Emergency Children Hospital, Romania, Romania

Introduction: Neurofibromatosis is an autosomal dominant disorder consisting in cutaneous, neurologic, ophthalmologic and tumoral signs. But always other disorders that can mimic neurofibromatosis should be excluded.

Case presentation: A 10-year-old girl, from consanguineous gipsy parents, was admitted for anemia. Family history showed a sister who died due to cerebral tumors at the age of 3. The personal medical history was insignificant. The clinical examination revealed more than 6 , cafe au lait" spots larger than $0,5 \mathrm{~cm}$ and multiple axillary freckling, signs which support the diagnosis of neurofibromatosis. The clinical exam of the parents was normal. Laboratory explorations showed a normochromic, normocytic anemia, normal ferritin level, liver and kidney function, folate deficiency, but also immunological abnormalities: IgA, $\mathrm{IgG} 2$ and $\mathrm{IgG} 4$ deficiency, poor response to vaccination, low memory $\mathrm{B}$ cells and a normal number of $\mathrm{T}$ cell subpopulations. Ophthalmologic examination was normal. The cerebral MRI-scan highlighted multiple 0,5 and $4 \mathrm{~cm}$ in diameter tumors. The cerebral biopsy revealed a diffuse astrocytoma IDH mutant and she started chemotherapy. Taking in account the consanguinity, family history and the negative clinical exam of the parents we performed genetic exam - WES that revealed a pathogenic homozygous c. $2653 \mathrm{~A}>\mathrm{T}$ mutation in MSH6 gene, parents being carriers for the same mutation.

Conclusions: Homozygous mutation in MSH6 gene is a very rare type of immunodeficiency from DNA repair syndromes and it must be suspected in atypical cases of neurofibromatosis associating immunological abnormalities. A correct diagnosis allows genetic counseling and 
monitoring for the cancer development in other members of the family.

M.T. Bataneant: None. M. Puiu: None. M. Baica: None. E. Boeriu: None. P. Urtila: None. C. Zaica: None. A. Beloia: None. A. Chirita-Emandi: None.

\section{P07.37.C}

Novel candidate genes and sequence variants for susceptibility to mycobacterial infections revealed by exome sequencing

A. M. Varzari ${ }^{1,2}$, M. Dembic ${ }^{3,4,5}$, G. Hoffmann Bruun ${ }^{3}$, I. V. Deyneko $^{6}$, W. Hofmann ${ }^{7}$, V. M. Cebotari ${ }^{8}$, E. G. Privalova ${ }^{9}$, B. S. Andresen ${ }^{3}$, T. Illig ${ }^{2,7}$

${ }^{1}$ Laboratory of Human Genetics, Chiril Draganiuc Institute of Phthisiopneumology, Kishinev, Moldova, Republic of, ${ }^{2}$ Hannover Unified Biobank, Hannover Medical School, Hannover, Germany, ${ }^{3}$ Department of Biochemistry and Molecular Biology and The Villum Center for Bioanalytical Sciences, University of Southern Denmark, Odense M, Denmark, ${ }^{4}$ Department of Clinical Genetics, Odense University Hospital, Odense, Denmark, ${ }^{5}$ Department of Mathematics and Computer Science, University of Southern Denmark, Odense, Denmark, ${ }^{6}$ Group of Functional Genomics, K.A. Timiryazev Institute of Plant Physiology, Moscow, Russian Federation, ${ }^{7}$ Department of Human Genetics, Hannover Medical School, Hannover, Germany, ${ }^{8}$ Department of Paediatrics, Municipal Hospital of Phthisiopneumology, Kishinev, Moldova, Republic of, ${ }^{9}$ Laboratory of Immunology, Chiril Draganiuc Institute of Phthisiopneumology, Kishinev, Moldova, Republic of

Introduction: Mendelian Susceptibility to Mycobacterial Disease (MSMD) is a rare genetic disorder with impaired immunity against mycobacterial infections; however only half of patients with MSMD have an identified genetic etiology. Here, we attempt to detect causal mutations in three paediatric patients with possible presentations for MSMD.

Methods: Whole exome sequencing was performed on two patients with severe localized BCG infection (\#1 and \#2) and their parents, and another individual patient with disseminated TB (\#3). The functional effects of the identified variants were characterized using RT-PCR analysis of endogenous and minigene transcripts, and SPRi analysis.

Results: Six rare heterozygous variants were detected in the established MSMD genes: IL12B (case \#1, compound heterozygote), TYK2 (cases \#1 and \#2), IL12RB1 and STAT1 (case \#3). In addition, four disease-associated variants in three presumed causative genes were identified by trio-based analysis: compound missense and intronic variants in GBP2 (case \#2), a de novo heterozygous frameshift mutation in HEATR3 (case \#1), and a homozygous missense mutation in IFNW (case \#1). cDNA analysis for splice-site mutation c.373-2A $>\mathrm{C}$ in STAT1 demonstrated abnormalities in STAT1 pre-mRNA splicing. Characterization of the c. $1149+14 \mathrm{~T}>\mathrm{C}$ variant in $G B P 2$ via minigene assay revealed some exon-skipping event from the mutant construct. Additionally, SPRi analysis showed a greater affinity of silencer-bound hnRNP A1 protein to RNA oligonucleotides harboring c. $1149+14 \mathrm{~T}>\mathrm{C}$ compared to the wild type oligonucleotides, providing support to the minigene assay.

Conclusions: Our findings expand the spectrum of genetic variation possibly predisposing to mycobacterial infections in children. Funding: Alexander von Humboldt Foundation and Hanover Unified Biobank.

A.M. Varzari: None. M. Dembic: None. G. Hoffmann Bruun: None. I.V. Deyneko: None. W. Hofmann: None. V.M. Cebotari: None. E.G. Privalova: None. B.S. Andresen: None. T. Illig: None.

\section{P07.38.A}

An indel in the promoter region of NFKB1 confers protection against infection but increases risk of allergy

A. Y. Chong ${ }^{1}$, N. Brenner ${ }^{2}$, A. Jimenez Kaufmann ${ }^{3}$, A.

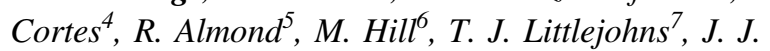

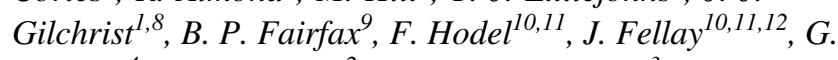
McVean $^{4}$, T. Waterboer ${ }^{2}$, A. Moreno Estrada ${ }^{3}$, A. V. S. Hill $^{1,13}$, A. J. Mentzer ${ }^{1,4}$

${ }^{l}$ Wellcome Centre for Human Genetics, University of Oxford, Oxford, United Kingdom, ${ }^{2}$ Infections and Cancer Epidemiology, German Cancer Research Center, Heidelberg, Germany, ${ }^{3}$ Human Population Genomics Lab, Laboratorio Nacional de Genómica para la Biodiversidad, Irapuato, Mexico, ${ }^{4}$ Big Data Institute, Li Ka Shing Centre for Health Information and Discovery, University of Oxford, Oxford, United Kingdom, ${ }^{5}$ UK Biobank, Stockport, United Kingdom, ${ }^{6}$ MRC-Population Health Research Unit, University of Oxford, Oxford, United Kingdom, ${ }^{7}$ Nuffield Department of Population Health, University of Oxford, Oxford, United Kingdom, ${ }^{8}$ Department of Paediatrics, University of Oxford, Oxford, United Kingdom, ${ }^{9}$ Department of Oncology, University of Oxford, Oxford, United Kingdom, ${ }^{10}$ Global Health Institute, School of Life Sciences, EPFL, Lausanne, Switzerland, ${ }^{11}$ Swiss Institute of Bioinformatics, Lausanne, Switzerland, ${ }^{12}$ Precision Medicine Unit, Lausanne University Hospital and University of Lausanne, Lausanne, Switzerland, ${ }^{13}$ Jenner Institute, University of Oxford, Oxford, United Kingdom

Introduction: Infectious agents contribute significantly to the global burden of diseases, through both acute infection 
and increasing risk of diverse chronic conditions. We leveraged the rich phenotypic data in the UK Biobank to identify genetic loci that influence response to multiple infections.

Methods: We undertook 45 genome-wide association studies of antibody responses against common pathogens, in 9,611 participants from UK Biobank, identifying NFKB1 as a common locus. Using incidence of disease in 487,409 UK Biobank participants, we investigated the association between NFKB1 variation and 91 infection and inflammation phenotypes. To better understand the impact of variation on underlying immune mechanisms, we also explored the effect of these variants on eight blood cell traits and cellular expression data.

Results: We identified an insertion-deletion variant (rs28362491), known to affect NFKB1 promoter binding, as a likely causal variant. The insertion allele has a protective effect against infection, but increases the risk of allergic disease. The same variant was associated with shifts in haematopoietic pathways, and likely impacts cell survival, antibody production and inflammation. Interestingly, this variant is relatively common in all human populations, suggesting that variation within this locus is largely maintained through balancing selection.

Conclusions: We find that disruptions to the tightly regulated immune responses and inflammatory processes can tip the balance between exacerbated immune responses and allergy, or increased risks of infection and impaired resolution of inflammation. This subtle yet demonstrable effect across the population is likely driven by modulation of NFKB1 gene expression.

A.Y. Chong: None. N. Brenner: None. A. Jimenez Kaufmann: None. A. Cortes: None. R. Almond: None. M. Hill: None. T.J. Littlejohns: None. J.J. Gilchrist: None. B.P. Fairfax: None. F. Hodel: None. J. Fellay: None. G. McVean: None. T. Waterboer: None. A. Moreno Estrada: None. A.V.S. Hill: None. A.J. Mentzer: None.

\section{P07.39.B}

CN-LOH in PIGV causes PNH, in a case of ET and nephrotic syndrome

A. Maurel-Ribes ${ }^{1}$, A. Knaus ${ }^{2}$, C. Garcia ${ }^{l}$, H. Engels ${ }^{3}$, S. Sivalingam $^{2}$, H. Hundertmark ${ }^{3}$, D. Ribes ${ }^{4}$, S. Tavitian ${ }^{5}, B$. Payrastre $^{6}$, S. Faguer ${ }^{4}$, P. Krawitz ${ }^{2}$, F. Vergez $^{7}$

${ }^{1}$ Laboratoire d'Hématologie, Centre Hospitalier Universitaire de Toulouse, Toulouse, France, ${ }^{2}$ Institute for Genomic Statistics and Bioinformatics, University of Bonn, School of Medicine \& University Hospital Bonn, Bonn, Germany, ${ }^{3}$ Institute of Human Genetics, University of Bonn, School of Medicine \& University Hospital Bonn, Bonn, Germany,
${ }^{4}$ Department of Nephrology and Organ Transplantation, University Hospital of Rangueil, Toulouse, France, ${ }^{5}$ Service D'Hématologie, Centre Hospitalier Universitaire de Toulouse, Institut Universitaire du Cancer de Toulouse, Toulouse, France, ${ }^{6}$ Inserm U1048 and Paul Sabatier University, Institute of Cardiovascular and Metabolic Diseases, Toulouse, France, ${ }^{7}$ Department of Biology Hematology, Toulouse-Oncopole University Cancer Institute (IUCT-O), Toulouse, Germany

Classical paroxysmal nocturnal hemoglobinura (PNH) is an acquired glycosylphosphatidylinositol (GPI) anchor deficiency in a hematopoietic stem cell (HSC) due to mutations in PIGA. However, the reasons for clonal expansion in an acquired loss of function of GPI biosynthesis, is still a matter of debate. Here we describe, how PNH also manifested in a patient with essential thrombocythemia (ET) after a copy number neutral loss-of-heterozygosity (CN$\mathrm{LOH})$ in $P I G V$, another gene of the GPI-anchor deficiency.

Similar to other proliferative myeloid malignancies, the clonal expansion of the HSC with ET resulted in our case from a de novo mutation in MPL (p.W515L) on chromosome 1 (1p34.2). 16.7 MB upstream of the acquired $M P L$ mutation in cis, the patient carried a heterozygous pathogenic mutation in $P I G V$ (p.R469X, 1p36.11), congenitally. A somatic CN-LOH in the HSC comprised almost the entire p-arm of chromosome 1. Hence, the $M P L$ mutation p.W5151L gained dominance and deteriorated ET and while homozygosity of p.R469X in PIGV resulted in a GPI-anchor deficiency and manifestation of PNH. In addition to ET and $\mathrm{PNH}$, the patient presented with nephrotic syndrome that ceased after treatment with eculizumab and hydroxycarbamide. We determined the origin of kidney injury as a result of podocyte instability due to an increase of non-GPI linked soluble uPAR. In this report we identified PIGV as a novel gene causing PNH and provide insights into the pathomechanism of acquired GPI anchor deficiency and expansion of PNH cells. Furthermore, we discuss the contribution of PNH to kidney injury.

A. Maurel-Ribes: None. A. Knaus: None. C. Garcia: None. H. Engels: None. S. Sivalingam: None. H. Hundertmark: None. D. Ribes: None. S. Tavitian: None. B. Payrastre: None. S. Faguer: None. P. Krawitz: None. F. Vergez: None.

P07.40.C

Human genomics of the humoral response against polyomaviruses

F. Hodel ${ }^{1,2}$, A. Y. Chong $^{3}$, P. Scepanovic ${ }^{4}$, C. W. Thorball $^{1,2}$, Z. M. Xu ${ }^{1,2}$, P. Marques-Vidal ${ }^{5}$, M. Begemann ${ }^{6}$, 
H. Ehrenreich ${ }^{6}$, N. Brenner ${ }^{7}$, N. Bender ${ }^{7}$, T. Waterboer ${ }^{7}$, A. J. Mentzer ${ }^{3,8}$, A. V. S. Hill ${ }^{3,9}$, C. Hammer ${ }^{10,11}$, J. Fellay ${ }^{1,2,12}$

${ }^{1}$ Global Health Institute, School of Life Sciences, EPFL, Lausanne, Switzerland, ${ }^{2}$ Swiss Institute of Bioinformatics, Lausanne, Switzerland, ${ }^{3}$ The Wellcome Centre for Human Genetics, University of Oxford, Oxford, United Kingdom, ${ }^{4}$ Roche Pharmaceutical Research and Early Development, F. Hoffmann-La Roche Ltd, Basel, Switzerland, ${ }^{5}$ Service of Internal Medicine, Department of Medicine, Lausanne University Hospital and University of Lausanne, Lausanne, Switzerland, ${ }^{6}$ Clinical Neuroscience, Max Planck Institute of Experimental Medicine, DFG Research Center for Nanoscale Microscopy and Molecular Physiology of the Brain, Göttingen, Germany, ${ }^{7}$ Infections and Cancer Epidemiology, German Cancer Research Center, Heidelberg, Germany, ${ }^{8}$ Big Data Institute, Li Ka Shing Centre for Health Information and Discovery, University of Oxford, Oxford, United Kingdom, ${ }^{9}$ The Jenner Institute, Oxford, United Kingdom, ${ }^{10}$ Department of Cancer Immunology, Genentech Inc., San Francisco, CA, United States, ${ }^{11}$ Department of Human Genetics, Genentech Inc., San Francisco, CA, United States, ${ }^{12}$ Precision Medicine Unit, Lausanne University Hospital and University of Lausanne, Lausanne, Switzerland

Introduction: Human polyomaviruses are widespread in human populations and some cause severe disease in immunocompromised individuals. There remains an incomplete understanding of the potential impact of human genetic variation on inter-individual responses to polyomaviruses.

Material and methods: To identify human genetic determinants of the humoral immune response against polyomaviruses, we performed genome-wide association studies and meta-analyses of immunoglobulin $\mathrm{G}$ ( $\mathrm{IgG}$ ) responses against the major capsid protein VP1 of Human polyomavirus 6 (HPyV6), BK virus (BKV), JC virus (JCV) and $\mathrm{WU}$ polyomavirus (WUPyV), using data from three independent studies grouping 15'660 individuals of European ancestry.

Results: We found evidence for an involvement of HLA class II variation in the determination of antibody responses to JCV $(\mathrm{P}=1.5 \mathrm{e}-78)$ and HPyV6 $(\mathrm{P}=3.2 \mathrm{e}-14)$. We also observed associations in $A B O$ for JCV $(\mathrm{P}=6.2 \mathrm{e}-11)$, and FUT2 for BKV, JCV and WUPyV $(\mathrm{P}=2.3 \mathrm{e}-18 ; \mathrm{P}=9.7 \mathrm{e}-26$; $\mathrm{P}=9.0 \mathrm{e}-09$, respectively), involved in blood group determination and secretor status. Lastly, we found a functional variant in $M U C 1$, previously associated with the risk of developing gastric cancer and Helicobacter pylori associated gastritis, to be associated with IgG levels against WUPyV (rs4072037, P=3.6e-33).
Conclusions: These results provide insights into the human genetic control of a family of very prevalent viruses, highlighting polymorphisms that play a modulating role on the humoral immune response against specific human polyomaviruses. Functional follow-up studies will be needed to uncover the biological mechanisms responsible for the newly discovered associations.

F. Hodel: None. A.Y. Chong: None. P. Scepanovic: None. C.W. Thorball: None. Z.M. Xu: None. P. Marques-Vidal: None. M. Begemann: None. H. Ehrenreich: None. N. Brenner: None. N. Bender: None. T. Waterboer: None. A.J. Mentzer: None. A.V.S. Hill: None. C. Hammer: None. J. Fellay: None.

\section{P07.42.B}

Increasing the diagnostic yield in patients suspected for primary immunodeficiency

A. Niskakoski, P. Kokkonen, I. Saarinen, J. Sistonen, H. Junnila, A. Kere, M. Andreevskaya, M. Muona, J. Djupsjöbacka, L. Koskinen, H. Duzkale, S. Myllykangas, J. Koskenvuo, T. Alastalo

\section{Blueprint Genetics, Espoo, Finland}

Background: Finding the genetic diagnosis for patients with suspicion of primary immunodeficiency (PID) is becoming increasingly important in the management of primary immunodeficiency and estimating the risk for family members. Simultaneous sequence and copy number variant analysis combined with customized analysis for difficult to sequence regions in specific genes such as NCF1 improves the diagnostic yield in patients suspected for PID.

Methods: A retrospective review of nearly 1700 patients suspected for PID and referred for genetic testing at Blueprint Genetics between 2018 and 2019 was performed. Patients were tested with Blueprint Genetics next generation sequencing panels related to immunology.

Results: Diagnostic yield including all immunology related panels was 14.9\% (253/1698). The diagnostic yield was highly variable between the different panels; diagnostic yield for Primary Immunodeficiency Panel was 13.3\% (138/ 1036), whereas for Severe Combined Immunodeficiency Panel it was 46.7\% (7/15). The diagnostic yield for Chronic Granulomatous Disease Panel is high (10/21, 48\%) although analysis of the $N C F 1$ gene included in this panel is complicated by two highly homologous pseudogenes $N C F 1 B$ and NCFIC. Out of the 296 reported diagnostic sequence variants, $234(79 \%)$ were unique, and only 7 diagnostic variants were reported over 3 times. Copy number variants, including deletions and duplications, were reported for 19 patients from 17 different genes. 
Conclusions: We constantly increase the diagnostic yield for PIDs by improving the sequencing technology, updating the panels with new genes discovered related to PID, and finding diagnoses from difficult to sequence regions and regions with high homology.

A. Niskakoski: A. Employment (full or part-time); Significant; Blueprint Genetics. P. Kokkonen: A. Employment (full or part-time); Significant; Blueprint Genetics. I. Saarinen: A. Employment (full or part-time); Significant; Blueprint Genetics. J. Sistonen: A. Employment (full or part-time); Significant; Blueprint Genetics. H. Junnila: A. Employment (full or part-time); Significant; Blueprint Genetics. A. Kere: A. Employment (full or part-time); Significant; Blueprint Genetics. M. Andreevskaya: A. Employment (full or part-time); Significant; Blueprint Genetics. M. Muona: A. Employment (full or part-time); Significant; Blueprint Genetics. J. Djupsjöbacka: A. Employment (full or part-time); Significant; Blueprint Genetics. L. Koskinen: A. Employment (full or part-time); Significant; Blueprint Genetics. H. Duzkale: A. Employment (full or part-time); Significant; Blueprint Genetics. S. Myllykangas: A. Employment (full or part-time); Significant; Blueprint Genetics. E. Ownership Interest (stock, stock options, patent or other intellectual property); Significant; Blueprint Genetics. J. Koskenvuo: A. Employment (full or part-time); Significant; Blueprint Genetics. E. Ownership Interest (stock, stock options, patent or other intellectual property); Significant; Blueprint Genetics. T. Alastalo: A. Employment (full or part-time); Significant; Blueprint Genetics. E. Ownership Interest (stock, stock options, patent or other intellectual property); Significant; Blueprint Genetics.

\section{P07.45.B}

Common RUNX3 missense variant contributes to psoriatic arthritis by affecting splicing and modifying signaling, activation and differentiation of T-cells

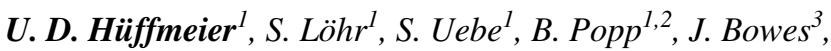
P. Kirchner ${ }^{1}$, E. Giardina ${ }^{4}$, E. Korendowych ${ }^{5}$, A. B. Ekici ${ }^{1}$, P. Ho ${ }^{3}$, F. Behrens ${ }^{6}$, M. Köhm ${ }^{6}$, G. Schett ${ }^{7}, J$. Rech $^{7}, G$. Assmann $^{8}$, A. Nimeh ${ }^{9}$, L. Padyukov ${ }^{10}$, G. Alenius ${ }^{11}$, N. J. McHugh $^{12}$, H. Sticht ${ }^{13}$, H. Burkhardt ${ }^{6}$, A. Barton ${ }^{3,14}$, A. Reis $^{l}$

${ }^{1}$ Human Genetics, University of Erlangen, Erlangen, Germany, ${ }^{2}$ Institute of Human Genetics, University of Leipzig Hospitals and Clinics, Leipzig, Germany, ${ }^{3}$ Arthritis Research UK Centre for Genetics and Genomics, University of Manchester, Manchester, United Kingdom, ${ }^{4}$ University of Rome Tor Vergata and Fondazione Policlinico Tor Vergata, Rome, Italy, ${ }^{5}$ Royal National Hospital for Rheumatic Diseases, NHS Foundation Trust, Bath, United
Kingdom, ${ }^{6}$ Division of Rheumatology and IMEFraunhofer Project Group Translational Medicine and Pharmacology, Johann Wolfgang Goethe University, Frankfurt/ Main, Germany, ${ }^{7}$ Department of Internal Medicine 3 - Rheumatology and Immunology, University of Erlangen, Erlangen, Germany, ${ }^{8}$ Department of Internal Medicine I, José-Carreras Centrum for Immuno- and Gene Therapy, University of Saarland Medical School, Homburg/ Saar, Germany, ${ }^{9}$ Department of Rheumatology, Fachklinik Bad Bentheim, Bad Bentheim, Germany, ${ }^{10}$ Karolinska Institute, Stockholm, Sweden, ${ }^{11}$ University Hospital of Umeå, Umeå, Sweden, ${ }^{12}$ University of Bath, Bath, United Kingdom, ${ }^{13}$ Bioinformatics, Institute of Biochemistry, University of Erlangen-Nürnberg, Erlangen, Germany, ${ }^{14}$ NIHR Manchester Musculoskeletal Biomedical Research Unit, Central Manchester Foundation Trust and University of Manchester, Manchester Academy of Health Sciences, Manchester, United Kingdom

Psoriatic Arthritis (PsA) is a chronic inflammatory joint disease occurring in up to $30 \%$ of patients with the most common psoriatic skin manifestation, psoriasis vulgaris (PsV). PsA is considered a T-cell mediated disease with a complex genetic basis. To date, GWAS identified $>60$ largely overlapping susceptibility loci for PsA/ PsV including $R U N X 3$, while the respective disease-contributing mechanism remained mostly uncertain. RUNX3 encodes a transcription factor expressed e.g. in immune cells, including Tcells, and skin. As genotyping arrays had insufficient coverage at this locus, we selected 32 tagging SNPs for further fine-mapping. Association analysis in 3,049 PsA patients and 6,178 controls of the Psoriatic Arthritis Genetics in Europe consortium showed significant association to an LD-wise isolated intronic SNP ( $\mathrm{p}=1.50 \mathrm{E}-11)$ and to 5 SNPs $(5.43 \mathrm{E}-12 \leq \mathrm{p} \leq 2.78 \mathrm{E}-07)$ within one intragenic LD block. Genomic annotations for 38 SNPs in high LD $\left(r^{2} \geq 0.9\right)$ with the SNPs were inconclusive, including histone modifications and DNAse-sensitive sites in immune cells. Haplotype and conditional analyses, though, pointed to diseasecontribution of the common, evolutionary conserved variant c.53T $>\mathrm{A} / \mathrm{p}$.Ile18Asn in alternative exon 1 . Targeted deep-sequencing of cDNA from CD8+ T-cells unveiled alternative splicing in homozygous wildtype carriers, but a reduced number of splicing products and lack of alternative splicing in homozygous risk-allele carriers. Comparative transcriptome analysis in CD8+ T-cells of homozygous carriers provided evidence for differentially expressed genes involved in T-cell exhaustion signaling, Th1/ Th2 activation and T-helper-cell differentiation. Our study indicates that the RUNX3 risk-allele in PsA reduces splicing variability thereby contributing to altered signaling, activation and differentiation of T-cells. 
U.D. Hüffmeier: None. S. Löhr: None. S. Uebe: None. B. Popp: None. J. Bowes: None. P. Kirchner: None. E. Giardina: None. E. Korendowych: None. A.B. Ekici: None. P. Ho: None. F. Behrens: None. M. Köhm: None. G. Schett: None. J. Rech: None. G. Assmann: None. A. Nimeh: None. L. Padyukov: None. G. Alenius: None. N. J. McHugh: None. H. Sticht: None. H. Burkhardt: None. A. Barton: None. A. Reis: None.

\section{P07.46.C}

Functional study of peptidylarginine deiminases in collagen-induced arthritis mice

\section{A. Suzuki, T. Shibuya, K. Yamamoto}

\section{RIKEN, Yokohama, Japan.}

Previously, peptidylarginine deiminase type 4 (PADI4) was identified as a susceptibility gene for Rheumatoid arthritis (RA) by genome-wide association studies in Japanese population. PADI genes can provide peptidyl citrulline which is a target antigen of anti-citrullinated peptide antibodies (ACPAs) that is highly sensitive and specific antibodies in RA. After that, PADI2 was also identified as susceptibility gene with RA. But, it is not clear how these PADI genes are involved in the development of rheumatoid arthritis. The aim of this study was to investigate the relationship between PADI4 gene and PADI2 gene in the progression of RA.

To clarify the physiological function of PADI4 and PADI2, we used collagen-induced arthritis (CIA), known as a RA model mouse. We examined that localization of PADI4 and PADI2 protein was indicated by immunohistochemistry in CIA mice. We also measured expression of PADI genes and various inflammatory cytokines in immune cells by real-time TaqMan assay and ELISA, respectively. We generated PADI4-I- and PADI2-/- mice, and performed experimental arthritis. We demonstrated that the clinical disease score was significantly decreased in PADI4-/ - mice and PADI2-/- mice.

PADI4 expression was induced by $\mathrm{CII}$ immunization. Interestingly, PADI2 expression was compensationally induced in CD11b+ cells of PADI4-/- mice. However, in PADI2-/- mice, conpensational induction of PADI4 was not obsered. It appears that PADI4 and PADI2 enhance collagen-initiated inflammatory responses.

A. Suzuki: None. T. Shibuya: None. K. Yamamoto: None.

P07.49.C

Likely pathogenic variant within zinc finger domain 4 of $B C L 11 B$ in a child with facial and dermal abnormalities and severe combined immunodeficiency
L. Olfe ${ }^{1}$, S. v. Hardenberg ${ }^{1}$, C. Klemann ${ }^{2}$, U. Baumann ${ }^{2}$, E. Tolosa $^{3}$, D. Lessel ${ }^{4}$, B. Auber ${ }^{1}$

${ }^{1}$ Department of Human Genetics, Hannover Medical School, Hanover, Germany, ${ }^{2}$ Department of Pediatric Pneumology, Allergy and Neonatology, Hannover Medical School, Hanover, Germany, ${ }^{3}$ Department of Immunology, University Medical Center Hamburg-Eppendorf, Hamburg, Germany, ${ }^{4}$ Institute of Human Genetics, University Medical Center Hamburg-Eppendorf, Hamburg, Germany

$B C L 11 B$ encodes a Cys2His2 zinc finger transcription factor involved in the development of nervous and immune systems. $B C L 11 B$ heterozygous loss-of-function variants have been associated with a neurodevelopmental disorder, alterations in the T-cell development and reduced number of type 2 innate lymphoid cells (ILC2s). Moreover, a single patient presenting with syndromic features and severe combined immunodeficiency (SCID) due to a pathogenic missense variant in $B C L 11 B$ has been reported. Here we report a newborn with developmental SCID diagnosed through neonatal SCID screening based on T-cell receptor excision circles. The child presented with microcephaly, hirsutism, loose skin folds and dysmorphic facial features, which include thin eyebrows, hypertelorism, short palpebral fissures, broad nasal bridge, anteverted, small nares, thin upper and lower lip vermillion and long philtrum. Immunophenotyping showed absence of T-cells with remaining B- and NK-cells, and in strong contrast to loss-of-function $B C L 11 B$ cases a normal amount of ILC2-cells. Wholeexome sequencing identified a likely pathogenic $B C L 11 B$ missense variant c.2428A $>$ C, p.(Asn810His), affecting one of the four specificity residues of the DNA-contacting alpha-helix within the fourth zinc finger domain. Bioinformatic prediction algorithms suggest this missense variant binds to identical alternative genomic sequences, resulting in acquisition of novel DNA-binding regions, as the previously reported one. Thereby, providing an explanation for the striking clinical similarities of the two missense cases. Our findings further suggest that only specific $B C L 11 B$ missense variants result in a severe immunodeficiency. This case further highlights the importance of neonatal SCID screening for early diagnosis and consequently the therapeutic option of hematopoietic stem cell transplantation.

L. Olfe: None. S. v. Hardenberg: None. C. Klemann: None. U. Baumann: None. E. Tolosa: None. D. Lessel: None. B. Auber: None.

P07.50.A

An atypical presentation of Immunodeficiency Centromeric Instability Facial anomalies syndrome 
C. Mehawej ${ }^{1}$, H. Khalife ${ }^{2}$, G. Dbaibo ${ }^{3}$, R. Wakim $^{4}$, C. Farra $^{l}$

${ }^{1}$ Unité de Génétique Médicale, Faculté de Médecine, SaintJoseph University, Beirut, Lebanon, ${ }^{2}$ Al Zahraa Hospital University Medical Center, Beirut, Lebanon, ${ }^{3}$ Pediatrics Department, American University of Beirut, Beirut, Lebanon, ${ }^{4}$ American University of Beirut, Beirut, Lebanon

Severe Combined Immunodeficiency Diseases (SCID) are genetic disorders characterized by profound impairment of cellular and humoral immunity. They are defined by a significant deficiency in T lymphocytes. Patients with SCID are asymptomatic at birth but infections can lead to their early death unless appropriate treatment is applied. A 10 day old girl, born to consanguineous Lebanese parents was referred to our Medical Genetics Unit for genetic evaluation, because of a family history of SCID. Lymphocytic immunophenotyping and serum immunoglobulin testing revealed a low absolute lymphocyte count, $\mathrm{T}$ cell lymphopenia with a significant decrease in naïve $\mathrm{T}$ cells and normal circulating $\mathrm{B}$ cells count including mainly immature B cells. SCID/atypical SCID was suspected in the patient. Whole Exome Sequencing (WES) was conducted and did not reveal any mutation in the SCID genes. However, reanalysis of WES data showed the presence of a homozygous missense variant, likely pathogenic, in the DNMT3B gene (NM_ 006892; c.2477G $>$ A, p.R826H). This gene is involved in the autosomal recessive ICF1 syndrome (Immunodeficiency- Centromeric instability- Facial anomalies syndrome). A reevaluation of the patient's clinical presentation and laboratory findings revealed undetectable immunoglobulin levels and facial dysmorphism (hypertelorism, micrognathia and low set ears), both characteristic of ICF1. T cell lymphopenia presenting at an early age in our proband is an unusual feature in ICF1 patients. Altogether, this case describes a severe presentation of ICF1 and highlights the heterogeneity of primary immunodeficiencies. It also stresses on the importance of early management of patients born to families with a history of SCID.

C. Mehawej: ; Research council of Saint-Joseph University (FM362). H. Khalife: None. G. Dbaibo: None. R. Wakim: None. C. Farra: None.

\section{P07.51.B}

Uncovering rare hematological entities: Shwachman Diamond syndrome in a pair of siblings with congenital neutropenia and recurrent infections

J. Trizuljak ${ }^{1,2}$, Z. Vrzalová ${ }^{1,2}$, I. Blaháková ${ }^{1,2}$, L. Radová2, Š. Pospíšilová ${ }^{1,2}$, M. Doubek ${ }^{1,2}$

${ }^{1}$ University Hospital, Brno, Czech Republic, ${ }^{2}$ Central European Institute of Technology, Brno, Czech Republic
Shwachman-Diamond syndrome (SDS) is an autosomal recessive disorder with clinical features that include pancreatic exocrine insufficiency, hematologic dysfunction, and skeletal abnormalities, Haematological malignancies occur in one third of patients. SDS is caused by homozygous or compound heterozygous mutations in SBDS gene.

We present a sibling pair with mild to moderate neutropenia, episodes of autoimmune hemolysis and recurrent infections of the oral cavity and respiratory airways. Prior investigations were inconclusive. Family history suggested AR mode of inheritance. We performed whole-exome sequencing of siblings and parents. Wholeexome libraries were prepared according to the Nimblegen SeqCap EZ Exome v3 protocol and sequencing was performed on NextSeq 500 for all of them. Furthermore, we performed in silico analysis of a virtual gene panel, focused on congenital neutropenias. Whole-exome sequencing identified a compound heterozygous genotype in SBDS gene in both siblings. We identified a likely pathogenic missense variant c.355T $>$ C; p.(Cys119Arg) in exon 3 of SBDS gene, leading to substitution of strongly conserved cysteine for arginine. This variant has been previously reported in a French family with SDS (Donadieu et al., 2012). Furthermore, we identified a rare, previously undescribed missense variant, c.536C $>\mathrm{T}$; p.(Pro179Leu), leading to prolin - leucine substitution. In-silico analysis (Align GVGD, SIFT, MutationTaster) predicts pathogenic or likely pathogenic effect. Molecular-genetic analysis of parents confirmed heterozygous carrier status. Compound heterozygous mutations in SBDS leads to disruption of SBDS gene and clinical manifestation of ShwachmanDiamond syndrome in the siblings. This study was supported by Czech Ministry of Health (grant AZV 1629447A) and Masaryk University (grant MUNI/A/1395/ 2019).

J. Trizuljak: None. Z. Vrzalová: None. I. Blaháková: None. L. Radová: None. Š. Pospíšilová: None. M. Doubek: None.

\section{P07.52.C}

Genome-to-genome analysis identifies an Asian-specific variant in the gene encoding NTCP that leads to specific HBV escape mutations during chronic infection

Z. Xü ${ }^{1}$, S. Rüeger ${ }^{1}$, P. Shea ${ }^{2}$, M. Buti ${ }^{3}$, H. L. Y. Chan ${ }^{4}$, P. Marcellin $^{5}$, O. Podlaha ${ }^{6}$, Z. Jiang ${ }^{6}$, V. Suri ${ }^{6}$, A. Gaggar ${ }^{6}$,

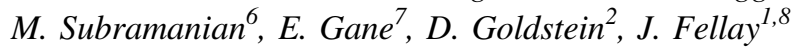

${ }^{1}$ School of Life Sciences, École Polytechnique Fédérale de Lausanne, Lausanne, Switzerland, ${ }^{2}$ Institute for Genomic Medicine, Columbia University, New York, NY, United States, ${ }^{3}$ Liver Unit, Hospital Universitario Vall d'Hebron 
and CIBEREHD del Instituto Carlos III, Barcelona, Spain, ${ }^{4}$ The Chinese University of Hong Kong, Hong Kong, China, ${ }^{5}$ Service d'Hépatologie, Hôpital Beaujon, Clichy, France, ${ }^{6}$ Gilead Sciences Inc, Foster City, CA, United States, ${ }^{7}$ Auckland Clinical Studies, Auckland, New Zealand, ${ }^{8}$ Precision Medicine Unit, Lausanne University Hospital, Lausanne, Switzerland

Introduction: Hepatitis B virus (HBV) is a major cause of chronic liver diseases, including liver cirrhosis and hepatocellular carcinoma. In chronic infections, the interplay between human and viral genetics contributes to HBV evolutionary changes. Here, we use a genome-to-genome (G2G) approach to identify sites of host-pathogen genomic interactions.

Materials and Methods: We obtained paired human exome and HBV genome data from 424 chronically infected individuals of Asian ancestry from three Phase 3 studies evaluating tenofovir-based regimens. For each variable HBV amino acid, a genome-wide association study was conducted using generalized linear mixed models and controlling for human and viral population structures.

Results: We identified strong associations $(\mathrm{p}=1.9 \mathrm{E}-12)$ between an Asian-specific missense SNP in the SLC10A1 gene encoding the HBV receptor NTCP (rs2296651) and amino acids in HBV large surface (preS1 domain) and polymerase proteins. The minor allele is present in $7 \%$ of East Asians (1KG Project) and was previously reported to associate with decreased risk of cirrhosis and hepatocellular carcinoma in chronic HBV. We also identified significant associations $(p=8.7 \mathrm{E}-13)$ between variants of HLA-A protein and amino acids in $\mathrm{HBV}$ polymerase and core proteins.

Conclusions: Our joint analysis of human and viral genomes shows that variation in NTCP exerts selective pressure on HBV during chronic infection, and confirms the impact of HLA class I variation on HBV epitopes. These results demonstrate the power of the G2G approach to identify host restriction factors and identify specific HBV amino acids likely to be involved in the interaction between $\mathrm{HBV}$ and its cellular receptor.

Z. Xu: None. S. Rüeger: None. P. Shea: None. M. Buti: None. H.L.Y. Chan: None. P. Marcellin: None. O. Podlaha: A. Employment (full or part-time); Significant; Gilead Sciences Inc. Z. Jiang: A. Employment (full or parttime); Significant; Gilead Sciences Inc. V. Suri: A. Employment (full or part-time); Significant; Gilead Sciences Inc. A. Gaggar: A. Employment (full or parttime); Significant; Gilead Sciences Inc. M. Subramanian: A. Employment (full or part-time); Significant; Gilead Sciences Inc. E. Gane: None. D. Goldstein: None. J. Fellay: None.
P07.53.A

Relationships between smoking methylation biomarkers and GPR15 + helper $\mathbf{T}$ cell levels in peripheral blood

\author{
W. Philibert ${ }^{l}$, S. R. H. Beach ${ }^{2}$, A. M. Andersen ${ }^{l}$ \\ ${ }^{1}$ University of Iowa, Iowa City, IA, United States, ${ }^{2}$ Uni- \\ versity of Georgia, Athens, GA, United States
}

Introduction: Smoking causes epigenetic changes associated with inflammatory diseases and elevated mortality. Of particular interest are changes in the level of $\mathrm{T}$ cells expressing G-protein-coupled receptor 15 (GPR15), a chemokine receptor linked with inflammatory bowel disease, multiple sclerosis and psoriasis. Accordingly, a better understanding of the mechanisms by which smoking influences variation in GPR $15^{+} \mathrm{T}$ cell subpopulations is of potential interest.

Materials and Methods: Flow cytometry and digital PCR assays were used to measure the GPR $15^{+} \mathrm{CD}^{+} \mathrm{CD} 4^{+}$ helper $\mathrm{T}\left(\mathrm{T}_{\mathrm{h}}\right)$ populations in peripheral blood from $n=62$ primarily African American young adults (aged 27-35 years) with a high rate of tobacco and cannabis use.

Results: Tobacco and cannabis smoking predicted GPR15 $^{+}\left(T_{h}\right)$ cell levels using linear regression models. Methylation of two candidate CpGs, cg19859270, located in GPR15, and cg05575921, located in Aryl Hydrocarbon Receptor Repressor (AHRR), were both significant predictors of GPR $15^{+} \mathrm{T}_{\mathrm{h}}$ cell levels, mediating the relationship between smoking and increases in GPR $15^{+} \mathrm{T}_{\mathrm{h}}$ cells. As hypothesized, the interaction between $\operatorname{cg} 05575921$ and cg19859270 was significant, indicating that low cg05575921 methylation was more strongly associated with $\mathrm{GPR}^{+} 5^{+} \mathrm{T}_{\mathrm{h}}$ cell levels for those with lower cg19859270 methylation.

Conclusions: Smoking leads changes in two CpGs, $\operatorname{cg} 05575921$ and $\operatorname{cg} 19859270$, that mediate $38.5 \%$ of the relationship between tobacco and cannabis smoking and increased GPR $15^{+} \mathrm{T}_{\mathrm{h}}$ levels in this sample. The impact of $\operatorname{cg} 19859270$ in amplifying the association between $\operatorname{cg} 05575921$ and increased GPR $15^{+} \mathrm{T}_{\mathrm{h}}$ levels is of potential theoretical interest because it may reflect a permissive interaction between different parts of the immune system.

W. Philibert: None. S.R.H. Beach: None. A.M. Andersen: None.

\section{P07.54.B}

Somatic mutations in HLA loci in patients with myeloid leukemia 
J. Neupauerová ${ }^{1}$, P. Crivello ${ }^{2}, \check{S}_{\text {. }}$ Půbalová $^{1}$, C. Šálek ${ }^{l}, S$. Nazarová ${ }^{l}, M$. Vraná $^{l}$

${ }^{1}$ Institute of hematology and blood transfusion, Prague 2, Czech Republic, ${ }^{2}$ Institute of experimental cellular therapy, University Hospital Essen, Essen, Germany

Introduction: In the HLA department of Institute of hematology and blood transfusion in Prague we deal with HLA typing of hematooncological patients and their related/ unrelated donors. The best donor compatibility is important for succesful hematopoetic stem cell transplantation (HSCT).

Materials and methods: Using NGS (Omixon, Hungary), we investigated more than 2000 patients and their HSC donors. New alleles found were confirmed by Sanger sequencing (Inno-train, Germany; CareDx, USA).

Results:. From our recent studies (2017 - 2019), in our cohort of patients, 15 new alleles were detected. Two of them were a somatic mutation in patients suffering from acute myeloid leukemia (AML); one was in the HLA-B and the other in the HLA-C locus. In both cases, the somatic mutation represents a single nucleotide polymorphism.

Conclusions: Our findings are in agreement with the known genome instability in AML. In the HLA region, somatic mutations are not so common, however, they should be given more attention since they can affect disease progression and may impact also on HSCT. Our future studies will focus on the precise identification of the new allelles and their characterization in HLA typing.

Supported by the Ministry of Health, Czech Republicconceptual development of research organization (CZ00023736, UHKT).

J. Neupauerová: None. P. Crivello: None. Š. Půbalová: None. C. Šálek: None. S. Nazarová: None. M. Vraná: None.

\section{P07.55.C}

High-throughput sequencing of T-cell receptor alpha chain clonal rearrangements in B-lineage acute lymphoblastic leukemia

\section{A. Miroshnichenkova ${ }^{l}$, A. Komkov ${ }^{1,2}$, A. Popov ${ }^{l}$, E. Volchkov $^{l}$, M. Maschan ${ }^{l}$, A. Karachunskiy ${ }^{l}, Y$. Olshanskaya $^{l}$, I. Mamedov ${ }^{2}$}

${ }^{1}$ Dmitry Rogachev National Research Center of Pediatric Hematology, Oncology and Immunology, Moscow, Russian Federation, ${ }^{2}$ Shemyakin-Ovchinnikov Institute of bioorganic chemistry, Moscow, Russian Federation

Introduction: Rearrangements of T- and B-cell receptor (TCR and BCR) genes are useful markers for clonality assessment and minimal residual disease (MRD) monitoring during the treatment of hematological malignancies. Despite their estimated lineage, B-cell leukemias manifest a remarkably high incidence of TCR gene rearrangements. According to indirect estimation, TCR alpha (TRA) incidence in B-cell precursor ALL could reach 46\%. Currently, rearrangements of all BCR and 3/4 TCR loci are used for this purpose with the exception of TRA due to the large number of multiplex primers needed to amplify all TRA segments.

Materials and Methods: We developed the first targeted sequencing-based method for identification of TRA clonal rearrangements. gDNA was extracted from a bone marrow of children with B-ALL. Amplification of TRA rearrangements was performed in multiplex PCR and subsequently sequenced on Illumina platform.

Results: TRA rearrangements were detected in 5/ 45 samples and associated with myeloid CD33+ coexpression ( $p$-value 0.0147, two-tailed Mann-Whitney Utest). Clonal rates of TRA varied from $31 \%$ to $98 \%$ and corresponded to main leukemic clones. All found TRA rearrangements were nonfunctional. TRA clones arose after leukemic transformation and have high clonal rate, which indicates these clones are most viable of all other leukemic clones.

Conclusions: The innovative study provides pathbreaking analyses of TRA occurrence in B-ALL at the DNA level and suggests that particular TRA rearrangements may be of clinical relevance in childhood B-ALL by adding a significant marker to MRD detection panel based on TCR/BCR rearrangements analysis.

The study is supported by PFBR grants 20-015-00462, 18-29-09132.

A. Miroshnichenkova: None. A. Komkov: None. A. Popov: None. E. Volchkov: None. M. Maschan: None. A. Karachunskiy: None. Y. Olshanskaya: None. I. Mamedov: None.

\section{P07.56.A}

Gene mutation spectrum of thalassemia patients from Azerbaijan

A. AGHAYEV ${ }^{l}$, V. Huseynov ${ }^{2}$, E. Aliyev ${ }^{2}$, A. Najafi ${ }^{2}, K$. Jafarova $^{2}$, A. Mammadova ${ }^{2}$, R. Bayramli ${ }^{2}$, Z. Uyguner ${ }^{3}$

${ }^{l}$ National Hematology and Transfusiology Center, Department of Genetic Diagnosis, Baku, Azerbaijan, ${ }^{2}$ National Hematology and Transfusiology Center, Baku, Azerbaijan, ${ }^{3}$ Department of Medical Genetics, Istanbul Medical Faculty, Istanbul, Turkey

Introduction: Thalassemias are defined by the absence or decrease of globin chain(s), which may lead imbalances of 
globin chains, ineffective erythropoiesis, hemolysis, and eventually to a variable degree of anemia. With the estimated carrier frequency of $\beta$-thalassemia is $4 \%-8.6 \%$ in country, the importance of premarital genetic testing, informed family counseling and preventive medical treatment cannot be overstated. Screening for traits by hemoglobin electrophoresis and biochemical analysis has become a routine application test prior to the issuance of a marriage license throughout Azerbaijan.

Methods: We aimed to evaluate prevalent mutations spectrum, the effect and their co-inheritance and/or role of polymorphisms (BCL11A, HBS1L-MYB and Xmnl SNP) on disease phenotype by using reverse dot-blot hybridization using customized strips, sequencing analysis of the complete genes and MLPA.

Results: We report here a summary finding of $H B B$ gene analysis for 265 patients and $H B A 1-H B A 2$ genes analysis for 28 patients, along with their family members totaling 570 individuals. $\beta$; thalassemia-66 were found to have homozygous, 76 possessed compound heterozygous and 80 possessed heterozygous mutations. Overall, a total of 416 alleles were found to have 34 mutations. The first 20 frequented mutations covered $87 \%$ of the entirety of all mutations. The summary range is as following: c.25_26delAA(p.Lys9Valfs) 27.9\%; c.93-21G $>$ A(IVS1 $+110 \mathrm{G}>\mathrm{A}) \quad 11.3 \%$; c. $315+1 \mathrm{G}>\mathrm{A}(\mathrm{IVS} 2+1 \mathrm{G}>\mathrm{A}) \quad 8.9 \%$. $\alpha$; thalassemia-Genotyping of 45 alleles revealed 9 mutations, the $3.7 \mathrm{~kb}$ deletion is the most frequent mutation (35.6\%), followed by $20.5 \mathrm{~kb}$ deletion (24.4\%) and $\alpha 2$ polyA2(HBA2:c.*92A > G, 13.3\%).

Conclusions: The results may help inform decisions in the design and implementation of prevention strategies and diagnostic approaches.

A. Aghayev: None. V. Huseynov: None. E. Aliyev: None. A. Najafli: None. K. Jafarova: None. A. Mammadova: None. R. Bayramli: None. Z. Uyguner: None.

\section{P07.59.A}

Genome-wide association study of susceptibility to respiratory syncytial virus infection during infancy

D. Lawless ${ }^{1}$, C. Rosas-Salazar ${ }^{2}$, T. Gebretsadik ${ }^{2}$, K. Turi ${ }^{2}$,

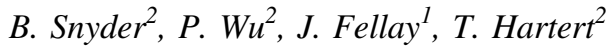

${ }^{1}$ Global Health Institute, Lausanne, Switzerland, ${ }^{2}$ Vanderbilt University School of Medicine, Nashville, TN, United States

Introduction: Human respiratory syncytial virus (RSV) infection is associated with asthma development. However, this association is confounded by shared heritability, and genetic studies have focused exclusively on severe infection. Longitudinal, population-based studies of RSV infection are thus required to assess the causal association between RSV and asthma. To determine whether there are genetic variants involved with susceptibility or resistance to infection, we performed the first GWAS on non-severe RSV infection.

Methods: We performed GWAS analyses of healthy term infants in a population-based birth cohort (INSPIRE) specifically designed to determine the effect of infant RSV infection on later child health. We compared infants who were and were not infected with RSV in the first year of life as determined by biweekly surveillance for RSV infection and serology at age 1 year.

Results: Among 1959 enrolled, 54\% of infants were RSV infected and $46 \%$ were uninfected at age 1 . There were significant differences in environmental factors associated with RSV infection, including daycare $(\mathrm{p}=0.001)$, siblings $(p=0.002)$ and black race $(p=0.002)$. GWAS analyses of a subset of 663 participants with available genotyping data, adjusted for birth month, sex, race, daycare and siblings revealed no significant associations.

Conclusions: We found no genome wide associations with risk of infant RSV infection; environmental factors were significantly associated with infection risk. These findings support using RSV infection as a means of natural randomization free from genetic confounding to study the effects of RSV infection on long-term outcomes.

Grants: NIH-U19AI095227, NIH-UL1RR024975, SNSF IZSEZ0_191968

D. Lawless: None. C. Rosas-Salazar: None. T. Gebretsadik: None. K. Turi: None. B. Snyder: None. P. Wu: None. J. Fellay: None. T. Hartert: None.

\section{P07.60.B}

The HLA and ABO genetic background impact on SARS-CoV-2 infection and COVID-19 severity

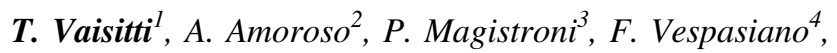

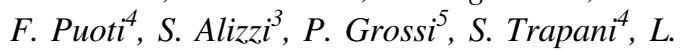

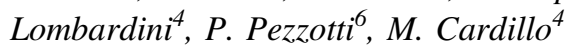

${ }^{1}$ Department of Medical Sciences, University of Turin, Turin, Italy, ${ }^{2}$ Department of Medical Sciences, University of Turin and Immunogenetics and Transplant Biology Service, Città della Salute e della Scienza University Hospital, Turin, Italy, ${ }^{3}$ Immunogenetics and Transplant Biology Service, Città della Salute e della Scienza University Hospital, Turin, Italy, ${ }^{4}$ National Transplant Center, Istituto Superiore di Sanità, Rome, Italy, ${ }^{5}$ Department of Medicine \& Surgery University of Insubria - ASST-Sette Laghi, Varese, Italy, ${ }^{6}$ Epidemiology, Biostatistics and Mathematical models, Istituto Superiore di Sanità, Rome, Italy

At the beginning of 2020, the SARS-CoV-2 outbreak has been defined by the WHO as a global health emergency. A 
peculiar feature of this infection is the extreme variability in clinical presentation, ranging from very mild sub-clinical flu-like symptoms to acute respiratory distress syndrome with high mortality. This heterogenous presentation poses the question whether individual variability of immune response to the virus can be influenced by genetic factors. Specifically, it is unknown whether HLA and AB0 can explain the different prevalence in population of SARSCoV-2 infection and its clinical evolution. To address this point, a retrospective analysis was performed on an Italian cohort composed by subjects who received organ transplantation and candidates in a waiting list in a 2002-March 2020 time frame. A total of 56,304 cases were studied with the aim of comparing HLA and ABO frequencies according to the presence $\left(\mathrm{COVID}^{+}\right)$or absence $\left(\mathrm{COVID}^{-}\right)$of SARSCoV-2 infection. The prevalence of COVID was $0.112 \%$ in the Italian population and $0.462 \%$ in waitlisted and transplanted patients. HLA-B*51 and HLA-DRB1*08 were more frequent in $\mathrm{COVID}^{+}$compared to $\mathrm{COVID}^{-}$, while HLA-DRB $1 * 07$ showed a protective effect. In transplant recipients, HLA-DRB $1 * 08$ was an indicator of mortality (1.9\% in living vs $15.2 \%$ in the deceased, OR=9.4 95\%CI [1.9-47.3]; $\mathrm{P}=.006)$. Moreover, blood group A was more frequent in $\mathrm{COVID}^{+}(45.5 \%)$ than in $\mathrm{COVID}^{-}(39.0 \%)$. Overall, this study shows for the first time that some HLA antigens influence SARS-CoV-2 infection and its clinical evolution and confirms that blood group A subjects are at greater risk of infection.

T. Vaisitti: None. A. Amoroso: None. P. Magistroni: None. F. Vespasiano: None. F. Puoti: None. S. Alizzi: None. P. Grossi: None. S. Trapani: None. L. Lombardini: None. P. Pezzotti: None. M. Cardillo: None.

\section{P08 Intellectual Disability}

\section{P08.003.C}

Angelman syndrome: a novel $U B E 3 A$ splice variant

\author{
M. G. Williams ${ }^{1,2}$, B. Hanna ${ }^{3}$, M. Freckmann ${ }^{4}, S$. \\ Bommireddipalli $^{5}$, A. Bournazos ${ }^{5}$, S. Cooper ${ }^{5}$, J.

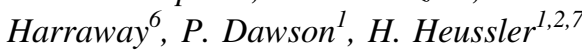

${ }^{1}$ Mater Research Institute - The University of Queensland, Woolloongabba, QLD, Australia, ${ }^{2}$ Faculty of Medicine, University of Queensland, St Lucia, QLD, Australia, ${ }^{3}$ Clinical Genetics, Westmead Hospital, Westmead, NSW, Australia, ${ }^{4}$ Clinical Genetics, Royal North Shore Hospital, St Leonards, NSW, Australia, ${ }^{5}$ Kids Neuroscience Centre, The Children's Hospital at Westmead, Westmead, NSW, Australia, ${ }^{6}$ Mater Pathology, South Brisbane, QLD, Australia, ${ }^{7}$ Child Development, The Queensland Children's Hospital, South Brisbane, QLD, Australia
Introduction: Angelman syndrome (AS) is a neurodevelopmental disorder characterised by moderate to severe developmental delay, absent or near absent speech, gait ataxia, microcephaly and seizures. Deficient expression or function of the maternally inherited $U B E 3 A$ allele results in AS. Pathogenic $U B E 3 A$ sequence variants (predominantly loss-of-function) account for $\sim 11 \%$ of Angelman syndrome cases.

A maternally inherited c.1900G $>$ C (p.(Val634Leu)) variant of uncertain significance was identified in a sevenyear-old male with clinical features consistent with Angelman syndrome (although no seizures noted). This variant affects the first nucleotide of $U B E 3 A$ exon 7 (transcript NM_130838.2) and in silico splicing tools predict weakening of the canonical 3'-splice site.

Methods: RT-PCR and Sanger sequencing of gel-purified amplicons was performed on mRNA isolated from the proband and maternal peripheral blood mononuclear cells (PBMCs). To detect aberrantly spliced transcripts targeted by nonsense-mediated mRNA decay (NMD), PBMCs were cultured in the presence of cycloheximide $(\mathrm{CHX})$ to inhibit NMD.

Results: The UBE3A:c.1900G $>$ C variant causes aberrant splicing and use of a cryptic 3'splice site (r.1899_1900ins [1900-38_1900-1]) resulting in a frameshift: p.(Val634Phefs*19). Cycloheximide inhibition of transcripts demonstrated an increase in the relative abundance of abnormally spliced transcripts, suggesting targeting by NMD. The c. $1900 \mathrm{G}>\mathrm{C}$ variant was re-classified as a pathogenic.

Conclusions: The maternally inherited UBE3A: c. $1900 \mathrm{G}>\mathrm{C}$ variant induces abnormal splicing of the predominant $U B E 3 A$ isoforms expressed in blood and brain, introducing a frameshift consistent with the pathogenesis of Angelman syndrome. Therefore, $U B E 3 A$ splicing outcomes observed in mRNA isolated from blood can inform the molecular consequences of cryptic splice variants for diagnosis and genetic counselling of Angelman syndrome.

M.G. Williams: None. B. Hanna: None. M. Freckmann: None. S. Bommireddipalli: None. A. Bournazos: None. S. Cooper: None. J. Harraway: None. P. Dawson: None. H. Heussler: None.

\section{P08.004.A}

De novo loss of function mutations in $A N K 2$ cause a neurodevelopmental disorder with autism and early onset epilepsy

M. W. A. Teunissen ${ }^{1}$, E. Lewerissa ${ }^{2}$, C. Ockeloen $^{3}, D$. Koolen $^{3}$, M. R. F. Reijnders ${ }^{4}$, R. Pfundt ${ }^{4}$, S. Seiffert ${ }^{5}, M$. Primiano $^{6}$, E. Brilstra ${ }^{7}$, J. L. Howe ${ }^{8}$, S. Scherer ${ }^{9}$, R. J. 
Vermeulen $^{l}$, R. P. W. Rouhl ${ }^{1,10}$, H. Brunner ${ }^{1,3}$, N. Nadif Kasri $^{3,11}$, M. H. Willemsen ${ }^{3}$

${ }^{1}$ Maastricht University Medical Centre, Maastricht, Netherlands, ${ }^{2}$ Cognitive Neuroscience, RadboudUMC, Nijmegen, Netherlands, ${ }^{3}$ RadboudUMC, Nijmegen, Netherlands, ${ }^{4}$ Maastricht University Medical Centre, The Netherlands, Maastricht, Netherlands, ${ }^{5}$ Hertie Institute for Clinical Brain Research, Tuebingen, Germany, ${ }^{6}$ Morgan Stanley Children's Hospital of New York-Pretbytarian, New York, NY, United States, ${ }^{7}$ Universitair Medisch Centrum Utrecht, Utrecht, Netherlands, ${ }^{8}$ Centre for Applied Genomics, The Hospital for Sick Children, Toronto, ON, Canada, ${ }^{9}$ The Hospital for Sick Children, Toronto, ON, Canada, ${ }^{10} \mathrm{Aca}$ demic Center for Epileptology Kempenhaeghe/MUMC, Maastricht, Netherlands, ${ }^{11}$ Donders Institute for Brain, Cognition, and Behaviour, Nijmegen, Netherlands

Aim: Mutations in Ankyrin 2 (ANK2), previously known to cause cardiac arrhythmia, may also cause neurodevelopmental disorders and epilepsy. We will provide insight in the spectrum of neurodevelopmental phenotypes associated with $A N K 2$ and explore the effects of $A N K 2$ mutations on neuronal function.

Methods: We describe clinical and molecular data of seven individuals with de novo loss-of-function (LoF) mutations in ANK2. These patients were collected through our database, international contacts and contacting authors of previous reports. Furthermore, we generated a heterozygous loss-of-function of ANK2 using CRISPR/Cas9 in human induced pluripotent stem cells (hIPSCs). HiPSCs were differentiated towards excitatory neurons to study neuronal function. We measured axon initial segment (AIS) plasticity, which plays an important role in regulating homeostatic plasticity in response to increased neuronal excitability. We stimulated ANK2-CRISPR neurons with $\mathrm{KCl}$ to induce chronic depolarization. We immunostained neurons for AnkG, a scaffolding protein that acts as marker for AIS.

Results: All patients had intellectual disability or developmental delay. Six patients had a formal or suspected diagnosis of autism, four patients had early onset epilepsy. Two patients had concurrent cardiac abnormalities. All patients with neurodevelopmental disorders have LoF mutations, in contrast almost all reported patients with cardiac problem had missense mutations. Functionally, heterozygous loss of ANK2 causes a more diffuse AnkG staining and showed impaired AIS plasticity upon $\mathrm{KCl}$ treatment.

Conclusions: We show that heterozygous de novo ANK2 LoF mutations cause a novel neurodevelopmental disorder with early onset epilepsy. Furthermore, we showed that heterozygous heterozygous LoF mutations in ANK2 affect neuronal function.

M.W.A. Teunissen: None. E. Lewerissa: None. C. Ockeloen: None. D. Koolen: None. M.R.F. Reijnders: None. R. Pfundt: None. S. Seiffert: None. M. Primiano: None. E. Brilstra: None. J.L. Howe: None. S. Scherer: None. R.J. Vermeulen: None. R.P.W. Rouhl: None. H. Brunner: None. N. Nadif Kasri: None. M.H. Willemsen: None.

\section{P08.005.B}

Biallelic variants in ANKRD12 cause intellectual disability, developmental delay, aphasia, hypotonia and hearing loss

M. Ansar ${ }^{1,2}$, S. A. Paracha ${ }^{3}$, E. Wohler ${ }^{4}$, M. T. Sarwar ${ }^{3}$, E. Ranza $^{1,5,6}$, F. A. Santonil ${ }^{1,7}$, J. Ahmed ${ }^{3}, H$. Goel ${ }^{8}$, D. A. Scott $^{9}$, S. E. Antonarakis ${ }^{1,5,10}$

${ }^{1}$ Department of Genetic Medicine and Development, University of Geneva, Geneva, Switzerland, ${ }^{2}$ Clinical Research Center, Institute of Molecular and Clinical Ophthalmology Basel (IOB), Basel, Switzerland, ${ }^{3}$ IInstitute of Basic Medical Sciences, Khyber Medical University, Peshawar, Pakistan, ${ }^{4}$ McKusick-Nathans Institute of Genetic Medicine, Johns Hopkins University, Baltimore, MD, United States, ${ }^{5}$ Service of Genetic Medicine, University Hospitals of Geneva, Geneva, Switzerland, ${ }^{6}$ Medigenome, The Swiss Institute of Genomic Medicine, Geneva, Switzerland, ${ }^{7}$ Department of Endocrinology Diabetes and Metabolism, University Hospital of Lausanne, Lausanne, Switzerland, ${ }^{8}$ Medicine and Public Health, University of Newcastle, Callaghan, New South Wales, Australia, ${ }^{9}$ Department of Molecular and Human Genetics, and Department of Molecular Physiology and Biophysics Baylor College of Medicine, Houston, TX, United States, ${ }^{10} i$ GE3 Institute of Genetics and Genomics of Geneva, Geneva, Switzerland

The Ankyrin repeat domain-containing protein 12 is encoded by the ANKRD12 gene. The function of this protein, and its role in human health is unclear. However, is highly expressed in the cerebellum and cerebellar hemisphere regions of brain (gtexportal.org). It is hypothesized that ANKRD12 plays a role in inhibiting the ligand dependent transactivation by recruiting histone deacetylases (HDACs) to the p160 coactivators/nuclear receptor complex. HDACs are involved in the regulation of gene expression. Pathogenic variants in another member of the Ankyrin repeat domain-containing protein, ANKRD11, cause the KBG syndrome whose phenotypes include intellectual disability, developmental delay and seizures in some patients. Here, we report six affected individuals from three unrelated 
families that carry homozygous or compound heterozygous variants in ANKRD12. Four of these individuals are from a consanguineous Pakistani family, and have a homozygous non-synonymous variant c.3425A $>$ C,p.(Lys1142Thr) in ANKRD12 (NM_015208.4). The other two are from the USA and carry compound heterozygous variants in ANKRD12; c.4511C >T,p.(Ser1504Leu)/c.3375_3377del in one, and c.5251C $>$ G, p.(Leu1751 Val)/deletion of exon 1 of the transcript NM_001204056.1 in the other. Phenotypes noted in these individuals include intellectual disability, aphasia, delayed motor milestones, hypotonia, mild hearing loss, and seizures (in one patient). Functional experiments in drosophila are in progress and will be presented in the meeting. Further investigations need to be done to understand the disease mechanism. However, the phenotypic similarity of the patients and the expression data from GTEx indicate that pathogenic ANKRD12 variants may cause a recessive disease characterized by intellectual disability, developmental delay, hypotonia and mild hearing loss.

M. Ansar: None. S.A. Paracha: None. E. Wohler: None. M.T. Sarwar: None. E. Ranza: None. F.A. Santoni: None. J. Ahmed: None. H. Goel: None. D.A. Scott: None. S.E. Antonarakis: None.

\section{P08.007.A}

Loss-of-function variants in CAPRIN1 in patients affected by autism spectrum disorder, language delay and intellectual disability with variable expressivity and incomplete penetrance

L. Pavinato $^{\text {I, J. L. Howe }}$, D. Carli ${ }^{3}$, E. Agolini ${ }^{4}$, D. A. Coviello $^{5}$, I. M. B. H. Van de Laar ${ }^{6}$, P. Y. B. Au, E. Di Gregorio $^{8}$, E. Giorgio ${ }^{1}$, E. Pozzil, M. Ferrero ${ }^{1}, S$.

Cardaropoli $^{3}$, A. Delle Vedove ${ }^{9,10,11}$, V. Salpietro ${ }^{12,13,14}, F$. Zara $^{12}$, A. Novelli ${ }^{4}$, B. Wirth ${ }^{9}$, G. B. Ferrero ${ }^{3}$, S. W. Scherer $^{15,16,2}$, A. Brusco ${ }^{1}$

${ }^{1}$ Department of Medical Sciences, University of Turin, Turin, Italy, ${ }^{2}$ The Centre for Applied Genomics, The Hospital for Sick Children, Toronto, ON, Canada, ${ }^{3}$ Department of Public Health and Pediatrics, University of Turin, Turin, Italy, ${ }^{4}$ Laboratory of Medical Genetics, Bambino Gesù Children's Hospital, Rome, Italy, ${ }^{5}$ Laboratory of Human Genetics, IRCCS Istituto Giannina Gaslini, Genova, Italy, ${ }^{6}$ Department of Clinical Genetics, Erasmus MC, University Medical Center Rotterdam, Rotterdam, Netherlands, ${ }^{7}$ Department of Medical Genetics, Alberta Children's Hospital Research Institute, University of Calgary, Calgary, AB, Canada, ${ }^{8}$ Medical Genetics Unit, Città della Salute e della Scienza University Hospital, Turin, Italy, ${ }^{9}$ Institute of Human Genetics, Center for Molecular Medicine Cologne, Cologne, Germany, ${ }^{10}$ Institute for
Genetics, University of Cologne, Cologne, Germany, ${ }^{11}$ Center for Molecular Medicine Cologne, University of Cologne, Cologne, Germany, ${ }^{12}$ Pediatric Neurology and Muscular Diseases Unit, IRCCS "G. Gaslini" Institute, Genova, Italy, ${ }^{13}$ Department of Neurosciences, Rehabilitation, Ophthalmology, Genetics, Maternal and Child Health, University of Genoa, Genova, Italy, ${ }^{14}$ Department of Neuromuscular Diseases, Queen Square Institute of Neurology, University College of London, London, United Kingdom, ${ }^{15}$ McLaughlin Centre, University of Toronto, Toronto, ON, Canada, ${ }^{16}$ Genetics \& Genome Biology Program, The Hospital for Sick Children, Toronto, ON, Canada

CAPRIN1 gene is highly expressed in brain and its product, Caprin-1 protein, is involved in the transport of mRNAs in neurons and it localizes at neuronal RNA granules, where is able to directly interact with the Fragile X Mental Retardation protein. Genome-wide studies have suggested CAPRIN1 as a new autism spectrum disorders (ASD) candidate gene. Interestingly, Caprin- $1^{+/-}$animal models show defects in the development of dendrites and dendritic spines and are characterized by a reduction in social interactions and lower response to novelty. Despite a clear involvement of Caprin-1 in neurodevelopment, the gene is still not associated with a human disease. Here we report the description of seven affected individuals with de novo and three cases with inherited loss-of-function (LoF) variants in CAPRIN1. Our cases show a broad spectrum of phenotypes, that ranges from ASD to language delay, intellectual disability, attention deficit hyperactivity disorder and epileptic seizures. Other traits, such as abnormal facial features and skeletal anomalies are observed in subgroup of cases. CAPRIN1 is predicted to be an haploinsufficient gene by computational pLI score (GnomAD). Taking advantage of the availability of patients-derived cells, we demonstrated a half-dose of CAPRIN1 mRNA levels, caused by nonsense mediated decay of the transcripts with LoF variants, that is followed by a parallel protein level half-dose. Our data lay the bases for further characterization of the involvement of CAPRIN1 in neurodevelopmental disorders, suggesting the haploinsufficiency of this gene as causative of a form of ASD with a possible incomplete penetrance and variable expressivity.

L. Pavinato: None. J.L. Howe: None. D. Carli: None. E. Agolini: None. D.A. Coviello: None. I.M.B.H. Van de Laar: None. P.Y.B. Au: None. E. Di Gregorio: None. E. Giorgio: None. E. Pozzi: None. M. Ferrero: None. S. Cardaropoli: None. A. Delle Vedove: None. V. Salpietro: None. F. Zara: None. A. Novelli: None. B. Wirth: None. G.B. Ferrero: None. S.W. Scherer: None. A. Brusco: None. 


\section{P08.008.B}

TLK2-associated intellectual disability: expanding the disease phenotype and characterizing missense variants at the molecular level

L. Pavinatol, M. Villamor-Paya ${ }^{2}$, M. Sanchiz-Calvo ${ }^{2}$, C. Andreoli $^{3}$, S. Martinelli, E. Giorgiol, D. Carli ${ }^{5}$, V. Antona $^{6}$, K. Ranguin ${ }^{7}$, C. Colson ${ }^{7}$, S. De Rubeis ${ }^{8}$, B. Alessandro $^{4}$, T. Pippucci ${ }^{9}$, P. Dimartino ${ }^{10}, M$.

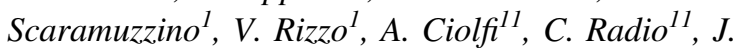
Buxbaum $^{8}$, G. B. Ferrero ${ }^{5}$, T. Marco ${ }^{11}$, T. H. Stracker ${ }^{2}$, A. Brusco $^{1}$

${ }^{1}$ Department of Medical Sciences, Turin, Italy, ${ }^{2}$ Institute for Research in Biomedicine (IRB Barcelona), The Barcelona Institute of Science and Technology, Barcelona, Spain, ${ }^{3}$ Department of Environment and Health, Istituto Superiore di Sanità, Rome, Italy, ${ }^{4}$ Department of Oncology and Molecular Medicine, Istituto Superiore di Sanità, Rome, Italy, ${ }^{5}$ Department of Pediatrics and Public Health and Pediatric Sciences, University of Turin, Turin, Italy, ${ }^{6}$ Department of Sciences for Health Promotion and Mother and Child Care "G. D'Alessandro", University of Palermo, Palermo, Italy, ${ }^{7}$ Centre de référence Maladies rares et Anomalies du développement, Service de génétique, Caen, France, ${ }^{8}$ Department of Psychiatry, Icahn School of Medicine at Mount Sinai, New York, NY, United States, ${ }^{9}$ Medical Genetics Unit, Polyclinic Sant'Orsola-Malpighi University Hospital, Bologna, Italy, ${ }^{10}$ Department of Medical and Surgical Sciences, University of Bologna, Bologna, Italy, ${ }^{11}$ Genetics and Rare Diseases Research Division, Ospedale Pediatrico Bambino Gesù, Rome, Italy

The Tousled-Like Kinase 2 (TLK2) gene was recently associated with "Mental Retardation Autosomal Dominant 57" (MIM:618050), a neurodevelopmental disorder characterized by a highly variable phenotype, including mild-tomoderate intellectual disability, behavioural abnormalities, facial dysmorphisms, microcephaly, epilepsy and skeletal anomalies. By whole-exome sequencing, we identified a novel patient with a de novo likely pathogenic variant (c.1586A>G; p.(Asp529Gly)) and a familial case with three siblings who inherited a nonsense change from an affected mother (c.1357G $>\mathrm{T}$; p.(Glu453*)). Moreover, we report a de novo 39-kb deletion encompassing TLK2 gene. Clinical phenotype included intellectual disability, facial dysmorphisms, microcephaly and anomalies of the hands as prevalent features, in accordance with previously reported cases.TLK2 is involved in many fundamental processes, including DNA replication, cell cycle checkpoint recovery and chromatin remodelling, through its regulation of the ASF1 histone chaperone. TLK2 is essential in mice for placental development but how TLK2 mutated protein affects central nervous system development remains largely unclear. Using spatial proteomics (BioID), we have investigated the proximity interaction landscape of TLK2 and analysed the effects of missense mutations on TLK2 interactions, localization and activity. These results demonstrated that TLK2 activity is strongly impaired by the p. (Asp529Gly) mutation and identified new links between TLK2 and other proteins implicated in neurological disorders. Moreover, single cell gel electrophoresis demonstrated a more relaxed state of chromatin in lymphoblastoid cells harbouring the p.(Asp529Gly) variant compared to control cells. In conclusion, our study has identified novel TLK2-patient pathogenic variants and provided new insights into the role of $T L K 2$ in intellectual disability.

L. Pavinato: None. M. Villamor-Paya: None. M. Sanchiz-Calvo: None. C. Andreoli: None. S. Martinelli: None. E. Giorgio: None. D. Carli: None. V. Antona: None. K. Ranguin: None. C. Colson: None. S. De Rubeis: None. B. Alessandro: None. T. Pippucci: None. P. Dimartino: None. M. Scaramuzzino: None. V. Rizzo: None. A. Ciolfi: None. C. Radio: None. J. Buxbaum: None. G.B. Ferrero: None. T. Marco: None. T.H. Stracker: None. A. Brusco: None.

\section{P08.009.C}

Exposure of heterozygous Tsc2 KO mice to chronic social defeat stress leads to improvement in specific behavioural domains

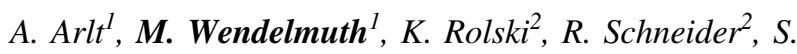 Schweiger ${ }^{1,3}$}

${ }^{1}$ Institute of Human Genetics, University Medical Centre Mainz, Mainz, Germany, ${ }^{2}$ Center of Molecular Biosciences (CMBI), Institute of Biochemistry, University of Innsbruck, Innsbruck, Austria, ${ }^{3}$ Leibniz Institute for Resilience Research (LIR), Mainz, Germany

Autism is a neurodevelopmental disorder characterized by impairment of communication and social interaction, as well as by high levels of repetitive behaviours. Due to atypical information processing within the brain, individuals with Autism highly rely on predictable procedures, e.g. daily routines. Changes of these procedures increases stress in the individuals. To investigate stress effects on autistic phenotype development, we used a Tsc2 heterozygous $\mathrm{KO}$ mouse model. Heterozygous mutations in the TSC1 or TSC2 genes, which both negatively regulated the mTOR kinase cause tuberous sclerosis, which is frequently associated with mental retardation, autism and epilepsy. We assessed sociability, anxiety, cognition and sensorimotor gating, and found significant deficits in Tsc 2 heterozygous 
KO animals. In a second step we asked, how chronic social defeat stress affects the behavior of these mice. The chronic social defeat stress (CSD) procedure used had been shown to effectively induce a depression-like phenotype in wildtype animals. Interestingly, in Tsc2 heterozygous KO mice exposure to CSD stress lead to an improvement in some behavioural domains including repetitive behavior, sensomotoric gating and working memory suggesting an inverse stress reaction in these animals. Proteomic analysis of synaptosomal fractions of the prefrontal cortex in stressed and non-stressed animals revealed a significant overlap of pathways affected in Tsc2 animals and stressed wildtype and Tsc2 KO animals. This data suggests that the mTOR kinase is involved in the response following chronic social defeat stress and surprisingly point towards a potentially beneficial effect of manageable stress in autistic children.

A. Arlt: None. M. Wendelmuth: None. K. Rolski: None. R. Schneider: None. S. Schweiger: None.

P08.010.A

\section{Germ cell mosaicism for AUTS2 exon 6 deletion}

L. Gieldon ${ }^{l}$, A. Jauch ${ }^{l}$, K. Hinderhofer ${ }^{l}, K$. Obeid $^{l}, U$. Haug $^{2}$, U. Moog ${ }^{l}$

${ }^{1}$ Institute of Human Genetics, University Hospital Heidelberg, Heidelberg, Germany, ${ }^{2}$ Center for Child Neurology and Social Pediatrics Maulbronn, Maulbronn, Germany

Introduction: Genomic rearrangements causing haploinsufficiency of AUTS2 have been associated with neurodevelopmental disorders and dysmorphic features. Over 50 patients have been described, mostly carrying de novo single exon deletions, including eight patients with exon 6 deletions. Small in-frame 5' deletions have been associated with a mild phenotype and parental inheritance.

Clinical Report and Methods: We report on two siblings, a girl and a boy aged 11 and 13 years, in whom we identified the same pathogenic $85 \mathrm{~kb}$ deletion on $7 q 11.22$ encompassing exon 6 of AUTS2 by SNP-array and subsequent confirmation with MLPA and FISH. Both children had typical symptoms of AUTS2-syndrome such as intellectual impairment and behavioral problems, but with markedly different expression. As in most children with exon 6 deletions, there were no feeding difficulties, which are otherwise common in AUTS2-syndrome. We subsequently performed targeted testing of both parents.

Results: MLPA and FISH excluded the deletion in blood samples of both parents. Conventional karyotyping did not show any structural rearrangements. Additional FISH analysis marking the flanking regions of the deletion showed normal arrangement of the signals on chromosome 7 in both parents. Parenthood was molecularly confirmed.
Conclusions: We suggest germline mosaicism as the most probable explanation for occurrence of the same deletion in these two siblings. To our knowledge this is the first report of germline mosaicism for AUTS2 syndrome. This report additionally provides further evidence of intrafamilial phenotypic variability and adds clinical information to the phenotypic spectrum of patients with AUTS2 exon 6 deletions.

L. Gieldon: None. A. Jauch: None. K. Hinderhofer: None. K. Obeid: None. U. Haug: None. U. Moog: None.

\section{P08.011.B}

Disruption of the STK24 and KLF13 genes in a female patient with a de novo balanced chromosomal translocation $t(13 ; 15)$ and a severe neurological phenotype

M. Kucharczyk ${ }^{1}$, V. Murcia Pieńkowski ${ }^{2}, K$.

Wojciechowska ${ }^{3}$, M. Lejman ${ }^{4}$, A. Pollak ${ }^{2}$, M. Rydzanicz ${ }^{2}$, M. Mtynek ${ }^{l}$, K. Pachotal, A. Madej-Pilarczyk ${ }^{l}, A$. Cieślikowska $^{l}$, D. Wicher ${ }^{l}$, M. Białecka ${ }^{l}$, A. Marczak ${ }^{l}$, D. Sielska-Rotblum ${ }^{l}$, P. Berko ${ }^{l}$, A. Gutkowska ${ }^{l}, K$. Chrzanowska ${ }^{l}$, M. Krajewska-Walasek ${ }^{l}$, R. Ptoski ${ }^{2}$

${ }^{I}$ Children's Memorial Health Institute, Department of Medical Genetics, Warsaw, Poland, ${ }^{2}$ Warsaw Medical University, Department of Medical Genetics, Warsaw, Poland, ' ${ }^{3}$ University Children's Hospital, Division of Pediatric Hematology, Oncology and Transplantology, Lublin, Poland, ${ }^{4}$ Medical University of Lublin, Department of Pediatric Hematology, Oncology and Transplantology, Lublin, Poland

Background: Mapping the breakpoints in de novo balanced chromosomal translocations (BCTs) in symptomatic individuals provides a unique opportunity to identify in an unbiased way the likely causative genetic defect and thus find novel human disease candidate genes. The aim was to fine-map breakpoints of de novo BCT in a female patient with the global developmental delay. At the age of 13 years she was referred to genetic counseling because of severe intellectual disability, macrocephaly and the lack of the speech. The autistic features and outbursts of aggression were also observed.

Methods and results: Conventional G-banding karyotyping revealed a de novo balanced chromosomal translocation 46,XX,t(13;15)(q32;q13). Microarray-based comparative genomic hybridization did not detect any imbalance. Shallow genome-wide mate pair sequencing (SGMPS) was applied to identify the exact structure of the breakpoints in the translocation. The breakpoint on chromosome 13 disrupted the STK24 gene, while the breakpoint on chromosome 15 was located in KLF13 gene. 
Discussion: The BCT identified in the presented girl disrupted genes with so far unknown (STK24) or poorly delineated impact on the phenotype ( $K L F 13$; only one previous report on mutation in this gene in the context of neurodevelopmental disorders). The function of STK24 and $K L F 13$ genes will be discussed based on available literature data. Their role as good candidates for disease loci will be suggested.

Conclusions: SGMPS in symptomatic carriers of BCTs is a powerful approach to delineate novel human gene-disease associations. This study was supported by the NCN Grant No. UMO-2016/21/B/NZ5/02541.

M. Kucharczyk: None. V. Murcia Pieńkowski: None. K. Wojciechowska: None. M. Lejman: None. A. Pollak: None. M. Rydzanicz: None. M. Młynek: None. K. Pachota: None. A. Madej-Pilarczyk: None. A. Cieślikowska: None. D. Wicher: None. M. Białecka: None. A. Marczak: None. D. Sielska-Rotblum: None. P. Berko: None. A. Gutkowska: None. K. Chrzanowska: None. M. Krajewska-Walasek: None. R. Płoski: None.

\section{P08.013.A}

Unexpected phenotypic variability in a family with epilepsy explained by independent segregation of biparental CACNA1A loss-of-function variants

\section{Kraus ${ }^{1}$, D. Mammadova ${ }^{2}$, T. Leis ${ }^{2}$, A. Ekici ${ }^{1}$, C. Thiel ${ }^{1}$,} A. Reis ${ }^{1}$, R. Trollmann ${ }^{2}$

${ }^{1}$ Institute of Human Genetics, Friedrich-Alexander Universität Erlangen-Nürnberg, Erlangen, Germany, ${ }^{2}$ Department of Pediatrics, Neuropediatrics, Friedrich-Alexander Universität Erlangen-Nürnberg, Erlangen, Germany

CACNA1A encodes a transmembrane subunit of a voltagegated calcium channel. Autosomal dominant mutations are causative for familial hemiplegic migraine, episodic ataxia type 2 , spinocerebellar ataxia type 6 , and neurodevelopmental disorders.

We now report on a family with five members affected with variable $C A C N A 1 A$-associated neurological phenotypes. The mother was diagnosed with episodic ataxia type 2 due to the heterozygous mutation p.(Ala868Profs*24). A similarly affected son was tested negative for this variant. Three further children presented with severe muscular hypotension, refractory epileptic seizures, optic atrophy and dysphagia, fatal in all within the first years. One of them was positively tested for the known maternal CACNAIA variant. However, as this was not sufficient to explain the very severe phenotype, we performed exome sequencing. Surprisingly, we identified a second heterozygous variant p.(His1826Thrfs*30) in the CACNA1A gene, which was shown to be paternally inherited. The same compoundheterozygous situation was confirmed in material from one of the other two deceased children. Furthermore, the mildly affected son was found to be heterozygous for the paternal CACNA1A variant, thus confirming his initial clinical diagnosis of episodic ataxia type 2 .

In conclusion, we report two different variants in CACNA1A segregating in either heterozygous or compound-heterozygous states in a single family with variable neurological phenotypes. To our knowledge, this is only the second observation of a severe, fatal epileptic encephalopathy associated with bi-allelic variants in CACNA1A. This case demonstrates the necessity of additional analyses to clarify unusual phenotypes even if a pathogenic variant has already been identified.

C. Kraus: None. D. Mammadova: None. T. Leis: None. A. Ekici: None. C. Thiel: None. A. Reis: None. R. Trollmann: None.

\section{P08.015.C}

Next generation candidates - Case-Level Evaluation of Candidate Genes for Neurodevelopmental Disorders

\section{Klöckner, R. A. Jamra, K. Platzer}

Institute of Human Genetics, University of Leipzig Medical Center, Leipzig, Germany

Recent work on discovery of genes to cause neurodevelopmental disorders (NDD) has indicated that probably over 1000 monogenetic causes, including those with reduced penetrance, remain to be discovered. To fulfil the aspirations of personalized medicine and power downstream research, a validation and extensive description of every candidate gene turned morbid gene is inevitable. This requires case-level evaluations to provide detailed phenotypic information and functional validation on every gene that is usually beyond the scope of large meta-analyses such as DDD.

If single exome sequencing of the index individual does not identify a genetic cause for NDD, we follow this up with parental exome sequencing on a research basis to identify novel candidate genes. In a subset of 120 individuals consisting of 60 individuals with NDD + epilepsy and 60 individuals with NDD, we identified more than 150 candidate genes using our in-house scoring system of $0-15$ points considering variant and gene attributes, literature research and inheritance. A prerequisite for all our candidate genes is a minimal expression in brain tissue derived from the GTEx database. Recurrent pathways with multiple candidate genes identified in our cohort include axon guidance, mTOR signaling, Ras signaling and 
regulation of transcription. As of now, we contributed seven patients to promising efforts validating novel NDD genes. At the time of the conference, we will present comprehensive data on our full cohort of candidate genes and progression on ongoing validation efforts.

C. Klöckner: None. R.A. Jamra: None. K. Platzer: None.

\section{P08.016.A}

\section{Core clinical features of $C E N P F$ related disorder}

M. Gensini ${ }^{1}$, G. Cappuccio ${ }^{1,2}$, S. Douzgou ${ }^{3}$, E. Bijlsma ${ }^{4}$, T. Koopmann $^{4}$, L. Hendon ${ }^{5}$, B. Isidor ${ }^{6,7}$, B. Cogné $e^{8,9}$, M.

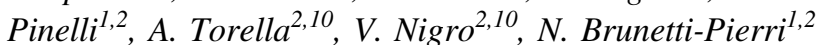

${ }^{1}$ Federico II University, Naples, Italy, ${ }^{2}$ Telethon Institute of Genetics and Medicine, Pozzuoli, Naples, Italy, ${ }^{3}$ Division of Evolution \& Genomic Sciences, School of Biological Sciences, Faculty of Biology, Medicine and Health, University of Manchester, Manchester M13 9PL, UK; Manchester Centre for Genomic Medicine, St Mary's Hospital, Manchester University NHS Found, Manchester, United Kingdom, ${ }^{4}$ Department of Clinical Genetics, Leiden University Medical Center, Leiden, Netherlands, ${ }^{5}$ Department of Pediatrics, University of Mississippi Medical Center, Jackson, MS, United States, ${ }^{6} \mathrm{CHU}$ de Nantes, Service de Génétique Médicale, Nantes 44093 Cedex 1,, Nantes, France, ${ }^{7}$ INSERM, CNRS, UNIV Nantes, l'institut du thorax, Nantes, France, ${ }^{8} \mathrm{CHU}$ de Nantes, Service de Génétique Médicale, Nantes 44093 Cedex 1, Nantes, France, ${ }^{9}$ INSERM, CNRS, UNIV Nantes, l'institut du thorax, Nantes, France, ${ }^{10}$ Department of Precision Medicine, University of Campania 'Luigi Vanvitelli', Naples, Italy

Introduction: Exome sequencing (ES) allows both expansion of the phenotype of known disorders and discovery of novel disease-causing genes. Biallelic loss-of-function (LOF) variants in CENPF gene are responsible for Stromme disease, a condition presenting with intestinal atresia, anterior ocular chamber anomalies, and microcephaly.

Cases: Through an international collaboration, 5 individuals ( 2 females and 3 males) carrying novel CENPF biallelic variants ( 1 missense and 4 LOF) were identified by ES. Four individuals were lacking the distinctive clinical abnormalities of Stromme disease, such as intestinal atresia or anterior chamber malformation of the eye. All individuals had microcephaly (average -3.2 SDS, ranging from -2 SDS to $-5.1 \mathrm{SDS}$ ) and developmental delay/intellectual disability (DD/ID). Genitourinary malformations (3/5), short stature (2/5), and epilepsy (2/5) were also observed. The five individuals shared a recognizable pattern of dysmorphic facial features with sloping forehead, flat philtrum, and anteverted nares.

Discussion: ES is rapidly changing the diagnostic process and the 'genotype-first' approach is rapidly becoming the method of choice. In the context of ES, reverse-phenotyping (i.e., refinement of clinical presentations in light of the identified genetic variants) is important for gene variant interpretation. The small case series of individuals with biallelic CENPF variants herein presented offers evidence of clinical variability of Stromme disease. Microcephaly, DD/ID and dysmorphic features are consistent even in the absence of intestinal atresia or malformation of the anterior chamber of the eye.

M. Gensini: None. G. Cappuccio: None. S. Douzgou: None. E. Bijlsma: None. T. Koopmann: None. L. Hendon: None. B. Isidor: None. B. Cogné: None. M. Pinelli: None. A. Torella: None. V. Nigro: None. N. Brunetti-Pierri: None.

\section{P08.017.B}

Further evidence to link CHD family-chromatin remodeler genes and neurodevelopment: de novo variants in CHD5 are associated with intellectual disability, epilepsy and autism

D. Lehalle ${ }^{l}$, T. Mizuguchi ${ }^{2}$, C. Nava ${ }^{l}$, N. Matsumoto ${ }^{3}, H$.

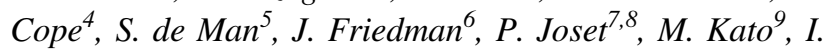

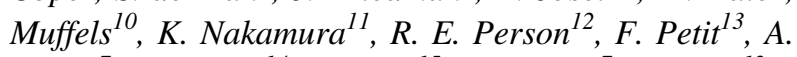
Rauch $^{7}$, V. Shashi ${ }^{14}$, T. Smol ${ }^{15}$, K. Steindl ${ }^{7}$, E. Torti ${ }^{12}, I$. Van de Laar ${ }^{16}$, S. Fuchs ${ }^{17}$, C. Depienne ${ }^{1,18}$, C. Mignot ${ }^{1}$

${ }^{1}$ Service de Génétique, Hôpital Pitié Salpêtrière, Paris, France, ${ }^{2}$ Department of Human Genetics, Yokohama City University Graduate School of Medicine, 3-9 Fukuura, Kanazawa-ku, Yokohama, Japan, ${ }^{3}$ Department of Human Genetics, Yokohama City University Graduate School of Medicine, 3-9 Fukuura, Kanazawa-ku, Yokohama, Japan, ${ }^{4}$ Department of Pediatrics, Division of Medical Genetics, Duke University School of Medicine, Durham, NC, United States, ${ }^{5}$ Department of Pediatrics, Amphia Hospital, Breda, Netherlands, ${ }^{6}$ Rady Children's Institute for Genomic Medicine, San Diego, CA, United States, ${ }^{7}$ Institute of Medical Genetics, University of Zurich, Zurich, Switzerland, ${ }^{8}$ radiz - "Rare Disease Initiative Zurich, Clinical Research Priority Program for Rare Diseases University of Zurich,", Zurich, Switzerland, ${ }^{9}$ Department of Pediatrics, Showa University School of Medicine, Tokyo, Japan, ${ }^{10}$ Department of Metabolic Diseases, University Medical Centre Utrecht, Utrecht, Netherlands, ${ }^{11}$ Department of Pediatrics, Yamagata University Faculty of Medicine, Yamagata, Japan, ${ }^{12}$ GeneDx, Inc., Gaithersburg, MD, United States, ${ }^{13} \mathrm{CHU}$ Lille, Clinique de Génétique, Lille, France, ${ }^{14}$ Program in Genetics and Genomics, Duke 
University School of Medicine, Durham, NC, United States, ${ }^{15}$ EA7364, RADEME, Université de Lille, Lille, France, ${ }^{16}$ Department of Clinical Genetics, Erasmus MC, University Medical Center Rotterdam, Rotterdam, Netherlands, ${ }^{17}$ Department ofMetabolic Diseases, University Medical Centre Utrecht, Utrecht, Netherlands, ${ }^{18}$ Institute of Human Genetics, University Hospital Essen, University DuisburgEssen, Essen, Germany

Introduction: Located in the critical region for $1 \mathrm{p} 36$ microdeletion syndrome, CHD5 encodes a member of the chromodomain helicase DNA-binding protein (CHD) family of proteins, which play a role in regulating gene transcription via modifications of histone marks and chromatin remodeling. To date, six of the nine members of the CHD family (CHD1, CHD2, CHD3, CHD4, CHD7 and CHD8) have been associated with dominant neurodevelopmental disorders. Either CHD3, CHD4 or CHD5 form the core of the nucleosome remodeling and deacetylation $(\mathrm{NuRD})$ complex, required for neural development.

Material and methods: We assembled 10 unrelated individuals with a de novo CHD5 variant and a neurodevelopmental phenotype in different centers through GeneMatcher. All patients were diagnosed through exome sequencing performed in a diagnostic setting.

Results: The patients (seven males and three females) were aged 3-18 years old. They presented with moderate to severe intellectual disability (ID) (8/10), speech impairment (7/10), epilepsy (7/10) - including suppression bursts encephalopathy $(1 / 10)$, West syndrome $(3 / 10)$, generalized tonic-clonic seizures $(2 / 10)$ and atypical absences (1/10) -, autistic features (6/10), and/or hypotonia (4/10). Brain MRI showed non-specific white matter changes in two patients and focal cortical dysplasia in one. Of the ten de novo CHD5 variants, nine were missense and one was a splice site variant. Seven were absent from the gnomAD database, while three were present at a low frequency.

Conclusions: Our findings suggest that, in line with other CHD-related disorders, heterozygous CHD5 variants are associated with a variable neurodevelopmental disorder including ID, epilepsy and autism as the main features.

D. Lehalle: None. T. Mizuguchi: None. C. Nava: None. N. Matsumoto: None. H. Cope: None. S. de Man: None. J. Friedman: None. P. Joset: None. M. Kato: None. I. Muffels: None. K. Nakamura: None. R.E. Person: A. Employment (full or part-time); Significant; GeneDx Inc.. F. Petit: None. A. Rauch: None. V. Shashi: None. T. Smol: None. K. Steindl: None. E. Torti: A. Employment (full or part-time); Significant; GeneDx Inc.. I. Van de Laar: None. S. Fuchs: None. C. Depienne: None. C. Mignot: None.

\section{P08.018.C}

CLTC mutations are also associated with familial forms of intellectual disability

C. Engel ${ }^{1}$, A. Garde ${ }^{1,2}$, B. Chalot ${ }^{2,3}$, S. Moutton ${ }^{1,2}$, C. Philippe $^{1,4}$, F. Tran Mau-Them ${ }^{1,4}$, N. Philip ${ }^{5}$, F. Riccardi ${ }^{5}$, A. Verloes ${ }^{6}$, M. Nizon ${ }^{7}$, B. Cogne ${ }^{7}$, S. Kury ${ }^{7}$, C. Thauvin ${ }^{1,2}$, P. Callier $^{1,3}$, L. Faivre ${ }^{1,2}$, A. Bruel ${ }^{1,4}$, A. Sorlin ${ }^{1,2}$

${ }^{1}$ Inserm UMR 1231 GAD, Université de Bourgogne Franche-Comté, Dijon, France, ${ }^{2}$ Centre de Génétique et Centre de Référence Anomalies du Développement et Syndromes Malformatifs de l'Interrégion Est, FHU TRANSLAD, Centre Hospitalier Universitaire Dijon Bourgogne, Dijon, France, ${ }^{3}$ Laboratoire de Génétique Chromosomique et Moléculaire, Plateau technique de biologie, Centre Hospitalier Universitaire Dijon Bourgogne, Dijon, France, ${ }^{4}$ Unité Fonctionnelle d'Innovation diagnostique des maladies rares, FHU-TRANSLAD, CHU Dijon Bourgogne, Dijon, France, ${ }^{5}$ Service de génétique médicale, $C H U$ de Marseille, Marseille, France, ${ }^{6}$ Service de génétique médicale, AP-HP Robert-Debré, Paris, France, ${ }^{7}$ Service de génétique médicale, CHU Nantes, Nantes, France

Missense and nonsense variants in $C L T C$ are responsible for a form of intellectual disability (ID) with variable nonspecific morphological features of autosomal dominant transmission (OMIM \#617854). Since 2016, 32 patients with mild to severe ID have been reported in the literature [AS1], all carrying de novo variations. We report here new patients carrying a pathogenic CLTC variant, including inherited mutations, suggesting the existence of familial forms. By a collaboration throught the French AnDDi-Rares reference centers network, we collected 10 patients (4 girls and 6 boys), from 8 families, with CLTC pathogenic variants (diagnosed by solo exome sequencing or array-CGH). They presented with mild to moderate ID, variably associated with epilepsy, stereotypies, developmental delay and autism spectrum disorders. For five patients, from three families, the variation was inherited from a parent initially considered asymptomatic, including one with a somatic mosaicism (52\%). After being questioned again about their personal history, it turned out that these parents had initially presented with learning difficulties or behavioural disorder and a favorable evolution. The identification of pathogenic CLTC variations inherited from paucisymptomatic parents allows us to envisage that this gene's phenotype is not limited to the initially described forms, exclusively caused by de novo variations. Familial forms appear to be less severe and permit to expand the phenotypic spectrum associated with CLTC mutations. This work also illustrates how difficult it is to interpret inherited variations, and that it 
is sometimes necessary to reconsider the initial clinical hypothesis with a more precise familial history.

C. Engel: None. A. Garde: None. B. Chalot: None. S. Moutton: None. C. Philippe: None. F. Tran Mau-Them: None. N. Philip: None. F. Riccardi: None. A. Verloes: None. M. Nizon: None. B. Cogne: None. S. Kury: None. C. Thauvin: None. P. Callier: None. L. Faivre: None. A. Bruel: None. A. Sorlin: None.

\section{P08.019.A}

CMIP is candidate gene for a neurodevelopmental disorder

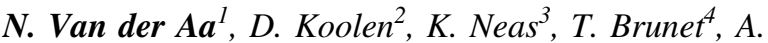

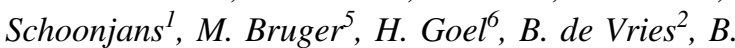 \\ Ceulemans $^{l}$, F. Kooy ${ }^{1}$
}

${ }^{1}$ University of Antwerp, Edegem, Belgium, ${ }^{2}$ Radboud Universiteit, Nijmegen, Netherlands, ${ }^{3}$ Genetic Health Service NZ, Wellington South, New Zealand, ${ }^{4}$ Institute of Human Genetics, Technical University Munich, Munchen, Germany, ${ }^{5}$ Institute of Human Genetics, Technical University Munich, Munich, Germany, ${ }^{6}$ Hunter Genetics, Newcastle, Australia

In 2012 we reported on a 16q23.2 de novo deletion in a patient with autism spectrum disorder, developmental delay and intractable epilepsy. The region contained CMIP as the only candidate gene for the clinical presentation of the patient. More recently we have gathered an additional 6 deletion cases with CMIP as the only commonly deleted gene. Three of those were reported as de novo, in two cases the deletion was inherited and in one case parental testing was not performed. One of the two inherited cases was from an affected parent. Two more de novo deletions were reported by others in the literature. In an additional patient we diagnosed a de novo stop mutation in CMIP. Common clinical features of the patients include: developmental delay and intellectual disability, autism spectrum disorder and epilepsy. Other neurological symptoms are coordination disorders and behavioural problems. CMIP encodes a protein that is part of the scaffold that links the plasma membrane to the cytoskeleton. Cytoskeletal remodelling is essential in neuronal migration and synaptic formation. Association studies found an association between CMIP and specific language impairment (SLI1). According to gnomAD, the gene is intolerant to genetic LoF variation $(\mathrm{pLi}=1)$. Thus, we report in total 9 CMIP containing deletions, the majority de novo or inherited form an affected parent and one de novo stop mutation. In combination with the predicted function of the gene our data strongly suggest that haploinsufficiency of $C M I P$ is causative of a novel neurodevelopmental disorder.
N. Van der Aa: None. D. Koolen: None. K. Neas: None. T. Brunet: None. A. Schoonjans: None. M. Bruger: None. H. Goel: None. B. de Vries: None. B. Ceulemans: None. F. Kooy: None.

\section{P08.020.B}

Whole Genome Sequence Analysis of Individuals with Cornelia de Lange Syndrome (CdLS) and CdLS-like Disorders With No Genetic Diagnosis from Targeted Sequencing

\section{K. A. Williamson ${ }^{1}$, M. Ansari ${ }^{2}$, M. R. Halachev ${ }^{3}$, A. M. Meynert ${ }^{4}$, J. Campos Parada ${ }^{4}$, D. A. Parry ${ }^{4}$, D. R. FitzPatrick}

${ }^{I}$ MRC Human Genetics Unit, Institute of Genetics and Molecular Medicine, University of Edinburgh; Simons Initiative for the Developing Brain, Simons Foundation Autism Research Initiative (SFARI), Centre for Discovery Brain Sciences, University of Edinburgh, Edinburgh, United Kingdom, ${ }^{2}$ South East Scotland Genetics Service, NHS Lothian, Edinburgh, United Kingdom, ${ }^{3}$ MRC Human Genetics Unit, Institute of Genetics and Molecular Medicine, University of Edinburgh; South East Scotland Genetics Service, NHS Lothian, Edinburgh, United Kingdom, ${ }^{4}$ MRC Human Genetics Unit, Institute of Genetics and Molecular Medicine, University of Edinburgh, Edinburgh, United Kingdom

Typical Cornelia de Lange syndrome (CdLS) is a severe, highly-characteristic multisystem neurodevelopmental disorder almost exclusively caused by heterozygous loss-offunction de novo mutations (DNM) in the gene encoding the cohesin loader, NIPBL. Atypical CdLS shows locus heterogeneity and can be caused by monoallelic DNM in genes encoding components of the cohesin system and other chromatin associated proteins. The MRC HGU cohort of CdLS individuals $\left(\mathrm{HGU}^{\mathrm{CdLS}}\right)$ has been extensively screened by arrayCGH, Sanger-, targeted gene panel-, and exome sequencing, identifying pathogenic variants in the known cohesin-related genes (NIPBL, SMC1A, SMC3, RAD21, HDAC8) and in core SFARI genes (SETD5, ANKRD11, KMT2A). We have now performed whole genome sequencing (WGS) of DNA from 107 "mutation-negative" CdLS individuals from 105 families, analysed as 42 nuclear trios, 2 quads (affected sibs and parents), and 63 singletons (197 individuals WGS in total). We have identified intragenic likely causative variants in EP300 (2), MED13L (1), EHMT1 (2), and ARID1B (1), together with previously unidentified mutations in NIPBL (9), ANKRD11 (6), KMT2A, and SETD5. We have also identified plausibly causative DNM in the 5'UTR (2) and deep intronic regions of NIPBL together with both balanced and unbalanced 
structural variants (SV) at this and other loci. In total WGS has identified a likely pathogenic variant in $30 \%$ of affected individuals providing a higher than expected diagnostic uplift from known loci. This also provides us with a cohort of genuinely undiagnosed individuals for new gene discovery.

K.A. Williamson: None. M. Ansari: None. M.R. Halachev: None. A.M. Meynert: None. J. Campos Parada: None. D.A. Parry: None. D.R. FitzPatrick: None.

P08.021.C

CTR9 heterozygous variants result in a neurodevelopmental disorder by disruption of the PAF1 complex

M. E. C. Meuwissen ${ }^{1,2}$, E. Ranza $^{3}$, A. Verstraeten ${ }^{2}, J$. Iwaszkiewicz, A. Afenjar ${ }^{5}$, M. Amaral ${ }^{6}$, D. Ballhausen ${ }^{7}, S$.

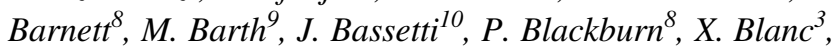

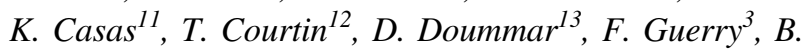
Keren $^{12}$, J. Pappas ${ }^{14}$, R. Rabin ${ }^{14}$, M. Shinawi ${ }^{15}$, A. T. VultoVan Silfhout $^{16}$, M. Wagner ${ }^{17,18}$, A. Ziegler ${ }^{9}$, V. Zoete ${ }^{19}$, S. E. Antonarakis $^{3}$, B. Loeys ${ }^{1,2}$

${ }^{1}$ Center of Medical Genetics, Antwerp University Hospital, Edegem/ Antwerp, Belgium, ${ }^{2}$ Center of Medical Genetics, University of Antwerp, Edegem/ Antwerp, Belgium, ${ }^{3}$ Medigenome, Swiss Institute of Genomic Medicine, Geneva, Switzerland, ${ }^{4}$ Molecular Modeling Group, Swiss Institute of Bioinformatics, Quartier Sorge - Bâtiment Amphipôle, Lausanne, Switzerland, ${ }^{5}$ Sorbonne Université, Centre de Référence Malformations et maladies congénitales du cervelet et déficiences intellectuelles de causes rares, département de génétique et embryologie médicale, Hôpital Trousseau, AP-HP, Paris, France, ${ }^{6}$ Hudson Alpha Institute for Biotechnology, Huntsville, AL, United States, ${ }^{7}$ Unité pédiatrique des maladies métaboliques, Service de Pédiatrie, CHUV, Lausanne, Switzerland, ${ }^{8}$ Department of Laboratory Medicine and Pathology, Mayo Clinic, Rochester, MN, United States, ${ }^{9}$ Biochemistry and Genetics Department, University Hospital of Angers, Angers, France, ${ }^{10}$ Division of Medical Genetics, Department of Pediatrics, Weill Cornell Medicine, New York, NY, United States, ${ }^{11}$ Sanford Broadway Clinic, Fargo, ND, United States, ${ }^{12}$ Sorbonne Université, Dept. of Genetics, AP-HP, La Pitié-Salpêtrière Hospital, Paris, France, ${ }^{13}$ Sorbonne Université, Neuropédiatrie, AP-HP, Hôpital d'enfants Armand Trousseau, Paris, France, ${ }^{14}$ NYU Langone Medical Center, New York, NY, United States, ${ }^{15}$ Dept. of Pediatrics, Division of Genetics and Genomic Medicine, Washington University School of Medicine, St. Louis, MO, United States, ${ }^{16}$ Department of Human Genetics, Radboud University Medical Center, Nijmegen, Netherlands, ${ }^{17}$ Institute of Human Genetics, Technical University München,
Munich, Germany, ${ }^{18}$ Institute for Neurogenomics, Helmholtz Zentrum München, Neuherberg, Germany, ${ }^{19}$ Department of Fundamental Oncology, Lausanne University, Ludwig Institute for Cancer Research, Epalinges, Switzerland

The PAF1 complex (PAF1C), consisting of several subunits including PAF1, CTR9, CDC73, RTF1 and LEO1, plays a crucial role in transcription regulation by binding of CTR9 to RNA polymerase II. It is involved in transcriptioncoupled histone modification, by promoting H3K4 and H3K36 methylation. Moreover, CTR9 also modulates the cellular trafficking of the dopamine transporter. Through GeneMatcher, molecular and clinical data of 11 probands were collected. In all probands, whole exome sequencing identified CTR 9 missense variants, encompassing $10 \mathrm{de}$ novo and 1 inherited from an affected father. Recurrent patient findings were intellectual disability, ranging from borderline to severe (10/11), speech delay (8/11), autism spectrum disorder (5/11) associated with regression and aggressive behavior during late childhood (3/11), hypotonia (6/11), joint hyperlaxity (5/11), fatigability (4/11), coordination problems (3/11) and mild dysmorphism. EM and crystal structure modelling of the activated human transcription complex Pol II-DSIF-PAF-SPT6 and the PAF1/ CTR9 complex predict a likely disruptive effect on local CTR9 structure and/or protein interaction of the different missense variants. In yeast, the role of the PAF1C in H3K4 and H3K36 methylation is exerted by regulating the activity of Set1 and Set2. Interestingly, pathogenic variants on the human orthologues SETD1A, SETD1B and SETD2 are known causes of neurodevelopmental deficits. In skin fibroblasts from four of the CTR9 mutant patients, an effect on $\mathrm{H} 3 \mathrm{~K} 4$ and $\mathrm{H} 3 \mathrm{~K} 36$ methylation is currently under investigation. In conclusion, we describe a novel dominant neurodevelopmental disorder caused by (mainly) de novo variants in $C T R 9$, likely affecting PAF1C function.

M.E.C. Meuwissen: None. E. Ranza: None. A. Verstraeten: None. J. Iwaszkiewicz: None. A. Afenjar: None. M. Amaral: None. D. Ballhausen: None. S. Barnett: None. M. Barth: None. J. Bassetti: None. P. Blackburn: None. X. Blanc: None. K. Casas: None. T. Courtin: None. D. Doummar: None. F. Guerry: None. B. Keren: None. J. Pappas: None. R. Rabin: None. M. Shinawi: None. A.T. Vulto-Van Silfhout: None. M. Wagner: None. A. Ziegler: None. V. Zoete: None. S.E. Antonarakis: None. B. Loeys: None.

\section{P08.022.A}

Expanding the clinical and molecular spectrum of the novel CYFIP2-related neurodevelopmental disorder and functional proof of aberrant WRC-mediated actin dynamics 
A. Begemann ${ }^{1}$, H. Sticht ${ }^{2}$, K. McWalter ${ }^{3}$, A. Vitobello ${ }^{4}$, L. Faivre $^{4}$, B. Alhaddad ${ }^{5}$, S. Banka ${ }^{6}$, J. Becker ${ }^{7}$, T. Bierhals ${ }^{8}$, K. Brown ${ }^{9}$, A. Bruel ${ }^{4}$, T. Brunet ${ }^{5}$, M. $_{\text {Carneiro }}{ }^{10}, K$. Cremer $^{7}$, R. Day ${ }^{11}$, A. Denommé-Pichon ${ }^{4}$, D. A. Dyment ${ }^{12}$, H. Engels ${ }^{7}$, R. Fisher ${ }^{13}$, M. Glassford ${ }^{13}$, E. S. Goh ${ }^{14}$, M. Hajianpour $^{15}$, L. R. M. Haertel ${ }^{16}$, N. Hauer ${ }^{17}$, M. Hempel He $^{8}$, T. Herget ${ }^{8}$, C. Kraus ${ }^{17}$, G. Le Guyader ${ }^{18}$, G. Lesca ${ }^{19}$, F. T. Mau-Them ${ }^{4}$, J. H. McDermott ${ }^{6}$, P. Meyer ${ }^{20}$, K. Õunap ${ }^{21}$, B. Popp $^{17,22}$, T. Reimand ${ }^{21}$, K. M. Riedhammer ${ }^{5}$, M. Russo ${ }^{1}$, L. Sadleir $^{23}$, E. Schuler ${ }^{24}$, G. Siegel ${ }^{1}$, S. Syrbe ${ }^{24}$, A. T. Van der

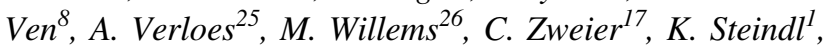
M. Zweier ${ }^{l}$, A. Rauch ${ }^{l}$

${ }^{1}$ University of Zurich, Institute of Medical Genetics, Schlieren-Zurich, Switzerland, ${ }^{2}$ Institute of Biochemistry, Emil-Fischer Center, Friedrich-Alexander-Universität Erlangen-Nürnberg, Erlangen, Germany, ${ }^{3}$ GeneDx, Gaithersburg, MD, United States, ${ }^{4}$ INSERM UMR 1231 Equipe GAD, Université de Bourgogne, Dijon, France, ${ }^{5}$ Institute of Human Genetics, Klinikum rechts der Isar, Technical University of Munich, Munich, Germany, ${ }^{6}$ Manchester Centre for Genomic Medicine, St Mary's Hospital, Manchester University NHS Foundation Trust, Health Innovation Manchester, Manchester, United Kingdom, ${ }^{7}$ Institute of Human Genetics, University of Bonn, Bonn, Germany, ${ }^{8}$ Institute of Human Genetics, Universitätsklinikum Hamburg-Eppendorf, Hamburg, Germany, ${ }^{9}$ University of Colorado Anschutz Medical Campus, Children's Hospital Colorado, Aurora, CO, United States, ${ }^{10}$ Department of Neuropediatrics, Lyon University Hospital, Lyon, France, ${ }^{11}$ Cancer Research Laboratory, Department of Biochemistry, University of Otago, Dunedin, New Zealand, ${ }^{12}$ Department of Pediatrics, University of Ottawa, Ottawa, ON, Canada, ${ }^{13}$ Division of Pediatric Genetics, Metabolism, and Genomic Medicine, Department of Pediatrics, University of Michigan, Arbor, MI, United States, ${ }^{14}$ Laboratory Medicine and Genetics, Trillium Health Partners, Mississauga, ON, Canada, ${ }^{15}$ Department of Pediatrics, Division of Medical Genetics, East Tennessee State University, Quillen College of Medicine, Mountain Home, TN, United States, ${ }^{16}$ Hospital Santa Catarina de Blumenau, Blumenau, Brazil, ${ }^{17}$ Institute of Human Genetics, Friedrich-AlexanderUniversität Erlangen-Nürnberg (FAU), Erlangen, Germany, ${ }^{18} \mathrm{CHU}$ de Poitiers, Poitiers, France, ${ }^{19}$ Department of Medical Genetics, Lyon University Hospital, Lyon, France, ${ }^{20}$ Department of Pediatric Neurology, CHU Montpellier, PhyMedExp, University of Montpellier, INSERM, CNRS, Montpellier, France, ${ }^{21}$ Department of Clinical Genetics, Institute of Clinical Medicine, University of Tartu and Tartu University Hospital, Tartu, Estonia, ${ }^{22}$ Institute of Human Genetics, University of Leipzig Hospitals and Clinics, Leipzig, Germany, ${ }^{23}$ Department of Paediatrics and Child Health, University of Otago,
Wellington, New Zealand, ${ }^{24}$ Department of Pediatrics, University Hospital Heidelberg, Heidelberg, Germany, ${ }^{25}$ UMR1141 INSERM, Université Paris Diderot, Sorbonne Paris Cité, Paris, France, ${ }^{26}$ Département Génétique Médicale, CHRU de Montpellier, Montpellier, France

We and others have recently described de novo missense variants in $C Y F I P 2$ as a novel cause of severe intellectual disability with seizures and muscular hypotonia in a total of 18 patients, with Arginine at protein position 87 recurrently substituted in the majority. CYFIP2 plays an important role in the regulation of actin polymerization as a member of the WAVE regulatory complex (WRC) and in transcription regulation via the interaction with the fragile $X$ mental retardation protein. We evidenced that the pathogenic CYFIP2 variants spatially cluster and are predicted to weaken the interaction of CYFIP2 with WASF1 or NCKAP1 of the WRC-complex. We now have identified further 18 patients harboring two previously described, one novel recurrent and 10 novel unique pathogenic missense variants, as well as the first three loss-of-function variants.

Genotype-phenotype correlation indicates a profound phenotype for substitutions at the mutational hotspot $\mathrm{p}$. Arg87 and a more variable phenotype in other recurrent missense alterations, while there is currently no evidence that haploinsufficiency leads to a similarly severe phenotype. Consistent with the role of CYFIP2 in WRC-mediated actin polymerization our functional studies demonstrate aberrant regulation of the actin cytoskeleton in all six available patient fibroblasts. Our study further delineates the clinical and molecular spectrum of this novel disease and provides evidence for WRC-mediated actin dynamics as a cellular pathomechanism.

AR was supported by the Swiss National Science Foundation (SNSF) grant 320030_179547. AB was supported by the Forschungskredit Candoc by the University of Zurich grant FK-18-025.

A. Begemann: None. H. Sticht: None. K. McWalter: A. Employment (full or part-time); Significant; GeneDx. A. Vitobello: None. L. Faivre: None. B. Alhaddad: None. S. Banka: None. J. Becker: None. T. Bierhals: None. K. Brown: None. A. Bruel: None. T. Brunet: None. M. Carneiro: None. K. Cremer: None. R. Day: None. A. Denommé-Pichon: None. D.A. Dyment: None. H. Engels: None. R. Fisher: None. M. Glassford: None. E.S. Goh: None. M. Hajianpour: None. L.R.M. Haertel: None. N. Hauer: None. M. Hempel: None. T. Herget: None. C. Kraus: None. G. Le Guyader: None. G. Lesca: None. F. T. Mau-Them: None. J.H. McDermott: None. P. Meyer: None. K. Õunap: None. B. Popp: None. T. Reimand: None. K.M. Riedhammer: None. M. Russo: None. L. Sadleir: None. E. Schuler: None. G. Siegel: None. S. Syrbe: None. A.T. Van der Ven: None. A. Verloes: None. 
M. Willems: None. C. Zweier: None. K. Steindl: None. M. Zweier: None. A. Rauch: None.

\section{P08.024.C}

Phenotypic and genotypic description of 44 patients with variants in DLG4 encoding the post-synaptic density protein PSD-95

A. Rodríguez-Palmero Seuma ${ }^{1,2}, M$. Boerrigter ${ }^{3,4}, G$. Mandrile $^{5}$, A. Pelle ${ }^{6}$, E. Giorgio ${ }^{7}$, A. Lindstrand ${ }^{8,9}, M$.

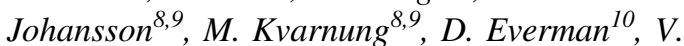
Bahrambeigi $^{10}$, A. MacKenzie ${ }^{11,12}$, J. Morton ${ }^{13}, C$. Ruivenkamp ${ }^{14}$, T. Challman ${ }^{15}$, A. Hurst ${ }^{16}$, J. Hoyer $^{17}, F$. Elmslie $^{18}$, T. Dye ${ }^{19}$, B. Isidor ${ }^{20}$, C. Haldeman-Englert ${ }^{21}$, D. Gómez-Andrés $^{22}$, A. Schlüter ${ }^{1,23}$, S. de Man ${ }^{24}$, J. Shieh ${ }^{25}$, C. Prada $^{26}$, S. Moutton ${ }^{27}$, A. Denommé-Pichon ${ }^{27}$, S. Motti ${ }^{28}$, A. Bruel $^{27}$, F. Tran Mau-Them ${ }^{27}$, S. Reiter ${ }^{29}$, C. van Ravenswaaij-Arts ${ }^{30}$, C. Shaw-Smith ${ }^{31}$, S. Parikh ${ }^{32}, K$. Aldinger $^{33}$, A. Lovgren ${ }^{34}$, A. Rauch ${ }^{35}$, M. Ross ${ }^{36}$, P. Gómez-

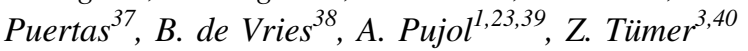

${ }^{1}$ Neurometabolic Diseases Laboratory, Bellvitge Biomedical Research Institute (IDIBELL), L'Hospitalet de Llobregat (Barcelona), Spain, ${ }^{2}$ Paediatric Neurology Unit, Hospital Universitari Germans Trias i Pujol, Badalona, Spain, ${ }^{3}$ Kennedy Center, Department of Clinical Genetics, Copenhagen University Hospital, Rigshospitalet, Copenhagen, Denmark, ${ }^{4}$ Department of Gastroenterology and Hepatology, Radboud University Medical Center, Nijmegen, Netherlands, ${ }^{5}$ Thalassemia Centre and Genetic Unit, San Luigi University Hospital, Orbassano, Italy, ${ }^{6}$ Department of Clinical and Biological Sciences, University of Turin, Torino, Italy, ${ }^{7}$ Department of Medical Sciences, University of Turin, Torino, Italy, ${ }^{8}$ Department of Clinical Genetics, Karolinska University Hospital, Stockholm, Sweden, ${ }^{9}$ Department of Molecular Medicine and Surgery, Karolinska Institutet, Stockholm, Sweden, ${ }^{10}$ Greenwood Genetic Center, Greenville, SC, United States, ${ }^{11}$ Rare Neurologic Disease Therapeutics, Department of Paediatrics, Children's Hospital of Eastern Ontario Research Institute, Ottawa, ON, Canada, ${ }^{12}$ Department of Pediatrics, Faculty of Medicine, University of Ottawa, Ottawa, ON, Canada, ${ }^{13}$ Clinical Genetics Unit Birmingham Women's Hospital, Birmingham, United Kingdom, ${ }^{14}$ Department of Clinical Genetics, Leiden University Medical Center, Leiden, Netherlands, ${ }^{15}$ Medical Director, Geisinger Autism \& Developmental Medicine Institute, Lewisburg, PA, United States, ${ }^{16}$ Department of Genetics, University of Alabama at Birmingham, Birmingham, AL, United States,

${ }^{17}$ Institute of Human Genetics, Friedrich-Alexander-Universität Erlangen-Nürnberg (FAU), Erlangen, Germany, ${ }^{18}$ South West Thames Regional Genetics Service, London, United Kingdom, ${ }^{19}$ Cincinnati Children's Hospital Medical
Center, University of Cincinnati School of Medicine, Department of Pediatrics, Cincinnati, OH, United States, ${ }^{20}$ Department of Medical Genetics, CHU Nantes, Nantes, France, ${ }^{21}$ Fullerton Genetics Center, Mission Hospital, Asheville, NC, United States, ${ }^{22}$ Child Neurology Unit. Hospital Universitari Vall d'Hebron, Vall d'Hebron Research Institute (VHIR), Barcelona, Spain, ${ }^{23}$ Center for Biomedical Research on Rare Diseases (CIBERER), Madrid, Spain, ${ }^{24}$ Department of Pediatrics, Amphia Hospital, Breda, Netherlands, ${ }^{25}$ Institute for Human Genetics, University of California San Francisco, San Francisco, CA, United States, ${ }^{26}$ Cincinnati Children's Hospital Medical Center, University of Cincinnati School of Medicine, Cincinnati, OH, United States, ${ }^{27}$ INSERM UMR1231, GAD, University of Burgundy, FHU-TRANSLAD, CHU Dijon-Bourgogne, Dijon, France, ${ }^{28}$ Bioinformatic unit, Cancer research center, Sheba Medical Center and Sackler Medical Center, Tel Aviv University, and Maccabi HMO, Tel Aviv, Israel, ${ }^{29}$ Department of Medical Genetics, Haukeland University Hospital, Bergen, Norway, ${ }^{30}$ Department of Genetics, University Medical Center Groningen, Groningen, Netherlands, ${ }^{31}$ Department of Clinical Genetics, Royal Devon and Exeter National Health Service Foundation Trust, Exeter, United Kingdom, ${ }^{32}$ Mitochondrial Medicine \& Neurogenetics, Cleveland Clinic, Cleveland, OH, United States, ${ }^{33}$ Center for Integrative Brain Research, Seattle Children's Research Institute, Seattle, WA, United States, ${ }^{34}$ Center for Mendelian Genomics, Broad Institute of MIT and Harvard, Cambridge, MA, United States, ${ }^{35}$ Institute of Medical Genetics, University of Zurich, Schlieren, Switzerland, ${ }^{36}$ Department of Pediatrics, NewYork-Presbyterian Hospital, New York, NY, United States, ${ }^{37}$ Molecular Modelling Group, Severo Ochoa Molecular Biology Centre, Madrid, Spain, ${ }^{38}$ Department of Human Genetics, Radboud University Medical Center, Nijmegen, Netherlands, ${ }^{39}$ Catalan Institution of Research and Advanced Studies (ICREA), Barcelona, Spain, ${ }^{40}$ Department of Clinical Medicine, Faculty of Health and Medical Sciences, University of Copenhagen, Copenhagen, Denmark

Introduction: Synapses are the basic functional structures that allow the transmission of information between neurons. PSD-95 protein, encoded by $D L G 4$, is located in the postsynaptic density and regulates excitatory synaptic maturation and function. Only a few patients heterozygous for pathogenic variants in $D L G 4$ have been reported to date. The characteristic features include intellectual disability (ID) (some with marfanoid habitus $(\mathrm{MH})$ ), developmental disorders including autism spectrum disorders (ASD) and schizophrenia.

Materials and Methods: A total of 44 patients (33 unpublished) were included. The unpublished cases were identified through GeneMatcher or Decipher and clinical 
investigations were carried out by the local clinicians. Pathogenic variants in DLG4 were identified through nextgeneration sequencing (NGS) based technologies.

Results: Mean age of clinical onset was 1.4 years and mean age at last evaluation was 14 years (1.5-47). The most frequent manifestations were developmental delay (25 patients) (developmental regression; 12), ID (37)(severe 9, moderate 15, mild 9), ASD (20), epilepsy (19), ADHD (16), ophthalmological manifestations (16). MH was present in 5 cases. MRI abnormalities were reported in 11 cases. Most of the DLG4 variants were truncating whereas 6 were missense, located in the functional domains of the protein. There were no differences in clinical severity between patients with the two groups of variants.

Conclusions: Clinical manifestations associated with $D L G 4$ variants are similar to those of other synaptopathies, mainly ID, ASD and epilepsy. MH is not a characteristic feature of the condition. NGS is fundamental to establish a molecular diagnosis, given the non-specific clinical spectrum here reported.

A. Rodríguez-Palmero Seuma: None. M. Boerrigter: None. G. Mandrile: None. A. Pelle: None. E. Giorgio: None. A. Lindstrand: None. M. Johansson: None. M. Kvarnung: None. D. Everman: None. V. Bahrambeigi: None. A. MacKenzie: None. J. Morton: None. C. Ruivenkamp: None. T. Challman: None. A. Hurst: None. J. Hoyer: None. F. Elmslie: None. T. Dye: None. B. Isidor: None. C. Haldeman-Englert: None. D. Gómez-Andrés: None. A. Schlüter: None. S. de Man: None. J. Shieh: None. C. Prada: None. S. Moutton: None. A. Denommé-Pichon: None. S. Motti: None. A. Bruel: None. F. Tran MauThem: None. S. Reiter: None. C. van Ravenswaaij-Arts: None. C. Shaw-Smith: None. S. Parikh: None. K. Aldinger: None. A. Lovgren: None. A. Rauch: None. M. Ross: None. P. Gómez-Puertas: None. B. de Vries: None. A. Pujol: None. Z. Tümer: None.

\section{P08.027.C}

Towards personalized treatment of Dravet syndrome using induced neurons as an ex-vivo tool

E. J. H. van Hugte ${ }^{1,2,3}$, E. Lewerissa ${ }^{1,3}$, M. Frega ${ }^{1,3}, J$. Nicolai $^{2}$, H. J. M. Majoie ${ }^{2}$, H. J. Schelhaas ${ }^{2}$, J. S.

Verhoeven $^{2}$, J. H. L. M. van Bokhoven ${ }^{1,3}$, N. Nadif Kasri ${ }^{1,3}$

${ }^{1}$ RadboudUMC, Nijmegen, Netherlands, ${ }^{2}$ Academic Centre for Epileptology Kempenhaeghe, Heeze, Netherlands, ${ }^{3}$ Donders Institute, Nijmegen, Netherlands

Dravet syndrome (DS) is a severe epileptic encephalopathy, characterized by recurrent and prolonged seizures. In addition, DS is associated with substantial cognitive and neuropsychological decline as a result of seizures during brain development. It is therefore important to timely control seizure activity. Although numerous antiepileptic drugs are available, it remains a challenge for clinicians to select effective drugs with minimal side effects for each specific patient, and a predictive tool to investigate treatment options is lacking. Moreover, Dravet syndrome is associated with both genetic and phenotypic heterogeneity, which increases the complications of finding an appropriate treatment. In $70 \%-80 \%$ of the cases, DS results from de novo mutations in the SCN1A gene, encoding the voltage gated sodium channel $\mathrm{Na}_{\mathrm{v}} 1.1$. Conversely, not every patient with a mutation in SCNIA develops DS, and also milder clinical phenotypes can arise. In addition to SCN1A, other genes have been associated with a DS phenotype. Animal models can neither recapitulate mutation-specific effects nor genetic background factors, and are unsuitable for devising personalized medicine interventions. This research proposes the development of human induced pluripotent stem cells (hiPSC), differentiated into induced neurons as a patientspecific disease model. The neurons are cultured into a neuronal network on micro-electrode arrays (MEAs), able to record network activity by embedded micro-electrodes. Here, we report a patient specific neuronal network (PNN) fingerprint of DS on both the single cell and the network level. With this PNN, we aim to provide a predictive tool to model DS and to explore treatment options in vitro.

E.J.H. van Hugte: None. E. Lewerissa: None. M. Frega: None. J. Nicolai: None. H.J.M. Majoie: None. H. J. Schelhaas: None. J.S. Verhoeven: None. J.H.L.M. van Bokhoven: None. N. Nadif Kasri: None.

\section{P08.031.A}

\section{Exome Pool-Seq Reloaded}

B. Popp ${ }^{1,2}$, G. Vasileiou ${ }^{2}$, M. Zweier ${ }^{3}$, A. B. Ekici ${ }^{2}, S$. Moortgat $^{4}$, D. Lederer ${ }^{4}$, I. Maystadt ${ }^{4}$, A. Destrée $e^{5}, S$.

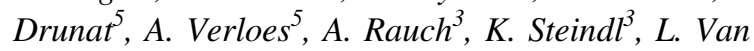

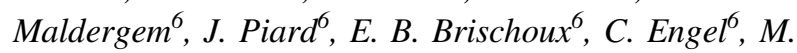
Serey-Gaut ${ }^{6}$, A. Reis $^{2}$, C. Zweier ${ }^{2}$

${ }^{1}$ Institute of Human Genetics, University of Leipzig Hospitals and Clinics, Leipzig, Germany, ${ }^{2}$ Institute of Human Genetics, Friedrich-Alexander-Universität Erlangen-Nürnberg (FAU), Erlangen, Germany, ${ }^{3}$ Institute of Medical Genetics, University of Zurich, Zurich, Switzerland, ${ }^{4}$ Centre de Génétique Humaine, Institut de Pathologie et de Génétique, Gosselies, Belgium, ${ }^{5}$ Department of Genetics, APHP-Robert DEBRE University Hospital, Denis Diderot School of Medicine, Paris University, France; INSERM UMR1141, Paris, France, ${ }^{6}$ Centre de génétique humaine CHU, Université de Franche-Comté, Besançon, France 
Falling sequencing costs has greatly advanced the identification of genetic causes for neurodevelopmental disorders (NDDs), but trio-based exome/genome sequencing remains expensive and time-consuming for large cohorts.

To screen for known and novel genetics causes of NDDs at scale we recently developed Exome Pool-Seq. This approach combines DNA pooling and capture-based exome sequencing with variant filtering in curated lists of known NDD genes and candidate genes.

We now performed Exome Pool-Seq in a cohort of 576 individuals with unsolved NDDs, collected and clinically characterized by a collaboration of five institutions from four European countries. Most individuals had previous normal microarray analysis $(93.7 \%)$ and Fragile-X testing (67.7\%). Remarkably, $48.7 \%$ already had gene panel testing without conclusive result.

We identified (likely) pathogenic truncating or previously described variants in known NDD genes in $11.5 \%$. This includes four cases with truncating $A N K R D 11$ and other commonly mutated genes like SYNGAP1, ARIDIB and $M E D 13 L$. Several pathogenic variants in autosomal dominant NDD genes (MED13, CNOT3) were inherited, highlighting a potential pitfall when filtering only for de novo variants. Analysis of missense variants absent from public databases but predicted as deleterious by novel ensemble scores increased the diagnostic yield by $5.2 \%$. Finally, we analyzed truncating variants in candidate and constrained genes and identified several candidate variants, e.g. in $K M D 2 B$ and MARK2, which have subsequently been submitted to matchmaking platforms.

This study provides new insights into the clinical and mutational spectrum of a representatively pre-analyzed European cohort of individuals with NDDs and highlights the value of curated disease gene lists.

B. Popp: None. G. Vasileiou: None. M. Zweier: None. A.B. Ekici: None. S. Moortgat: None. D. Lederer: None. I. Maystadt: None. A. Destrée: None. S. Drunat: None. A. Verloes: None. A. Rauch: None. K. Steindl: None. L. Van Maldergem: None. J. Piard: None. E.B. Brischoux: None. C. Engel: None. M. Serey-Gaut: None. A. Reis: None. C. Zweier: None.

P08.033.C

DISSEQ: Double-blind Next-Generation-Sequencing technologies (exome and gene panel) in the diagnosis of a cohort of 330 patients with an intellectual disability: concordance, discrepancies, and efficiencies.

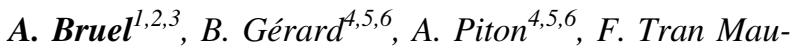 Them $^{1,2}$, A. Sorlin ${ }^{1,2,7}$, A. Sorly ${ }^{8}$, D. Lacombe L $^{9}$ S. Manouvrier $^{10}$, P. Edery ${ }^{11}$, N. Philip ${ }^{12}$, D. Geneviève ${ }^{13}$, A. Verloes $^{14}$, S. Odent ${ }^{15}$, J. Thevenon ${ }^{16}$, A. Toutain ${ }^{17}, D$. Bonneau $^{18,19}$, S. El Chehadeh ${ }^{20}$, M. Doco-Fenzy ${ }^{21}$, B.

Isidor $^{22}$, A. Goldenberg ${ }^{23}$, C. Vincent-Delorme ${ }^{24}$, O. BouteBenejean $^{10}$, L. lambert ${ }^{25}$, M. Asensio ${ }^{26}$, P. Callier ${ }^{1,2}, Y$. Duffourd $^{1,2}$, C. Lejeune $^{26}$, C. Binquet ${ }^{26}, C$. Philippe ${ }^{1,2}, L$. Faivre $^{1,2,7}$, C. Thauvin-Robinet ${ }^{1,2,3}$

${ }^{1}$ UMR1231 INSERM, GAD, Université de Bougogne, Dijon, France, ${ }^{2}$ Unité Fonctionnelle Innovation en Diagnostic génomique des maladies rares, FHU-TRANSLAD, CHU Dijon Bourgogne, Dijon, France, ${ }^{3}$ Centre de Référence maladies rares "Déficiences Intellectuelles de causes rares », CHU Dijon Bourgogne, Dijon, France, ${ }^{4}$ Institute of Genetics and Molecular and Cellular Biology, Illkirch, France; French National Center for Scientific Research, UMR7104, Illkirch, France, ${ }^{5}$ National Institute of Health and Medical Research U964, Illkirch, France, ${ }^{6}$ University of Strasbourg, Illkirch, France, ${ }^{7}$ Centre de Référence maladies rares "Anomalies du Développement et syndromes malformatifs », centre de génétique, FHU-TRANSLAD, CHU Dijon Bourgogne, Dijon, France, ${ }^{8}$ Direction Clinique de la Recherche et de l'Innovation, CHU Dijon Bourgogne, Dijon, France, ${ }^{9}$ Service de génétique médicale, CHU de Bordeaux, Centre de Référence, Anomalies du développement et Syndromes malformatifs du Sud-Ouest Occitanie Réunion, Saint-Pierre, INSERM U1211, Université de Bordeaux, Bordeaux, France, ${ }^{10}$ Centre de référence maladies rares pour les anomalies du développement NordOuest, Clinique de Génétique médicale, CHU de Lille et EA7364, Université de Lille, Lille, France, ${ }^{11}$ Department of Medical Genetics, Women Mothers and Children's Hospital, Lyon Hospices Civils, Claude Bernard Lyon 1 University, CRNL, CNRS UMR 5292, INSERM U1028, Lyon, France, ${ }^{12}$ Service de génétique médicale, CHU de Marseille, Aix Marseille Univ, INSERM, MMG, Marseille, France, ${ }^{13}$ Département de génétique médicale, Maladies rares et médecine personnalisée, CHU de Montpellier, INSERM U1183, Université de Montpellier, Montpellier, France, ${ }^{14}$ Département de Génétique, APHP-Robert DEBRE University Hospital, USPC University and INSERM UMR1141, Paris, France, ${ }^{15}$ Service de génétique clinique, CHU de Rennes, Univ. Rennes, Institute of Genetics and Development of Rennes (IGDR) UMR6290 CNRS, Rennes, France, ${ }^{16}$ Département de Génétique et Procréation, Hôpital Couple-Enfant, Université de Grenoble, CHU de Grenoble, Grenoble, France, ${ }^{17}$ Service de génétique, CHU de Tours, UMR 1253, iBrain, Université de Tours, Inserm, Tours, France, ${ }^{18}$ Centre Hospitalier Universitaire de Angers, Département de Biochimie et Génétique, Angers, France, ${ }^{19}$ Mitochondrial and Cardiovascular Pathophysiology (MITOVASC), Unité mixte de Recherche, Centre National de la Recherche Scientifique 6015, Inserm 1083, Université d'Angers, Angers, France, ${ }^{20}$ Département de Génétique Médicale, $\mathrm{CHU}$ de 
Hautepierre, Strasbourg, France, ${ }^{21}$ Service de Génétique, CHU de Reims, EA3801, SFR CAP Santé, Reims, France,

${ }^{22}$ Service de Génétique Médical, CHU Nantes, Nantes, France, ${ }^{23}$ Normandie Univ, UNIROUEN, Inserm U1245 and Rouen University Hospital, Department of Genetics and Reference Center for Developmental Disorders, Rouen, France, ${ }^{24}$ Service de Génétique Clinique Guy Fontaine, CHRU de Lille-Hôpital Jeanne de Flandre, Lille, France, ${ }^{25}$ Service de génétique médicale, CHU de Nancy, Nancy, France, ${ }^{26}$ EPICAD LNC-UMR1231, Burgundy \& Franche Comte University, Clinical Epidemiology Unit, Inserm, CIC 1432 University Hospital, Dijon, France

The introduction of Next Generation Sequencing (NGS) technologies, in combination with a broad panel of bioinformatics tools and databases, has revolutionized the field of human genetics, making it possible to have the unprecedented ability to establish molecular diagnoses in a timeand cost-effective manner. The DISSEQ study aims to assess the efficiency of different NGS strategies in the diagnosis of patients with intellectual disability from a cohort of 330 patients (205 males/127 females) without previous genetic investigation, using double-blind strategy 1 (array-CGH+FRAXA+large gene panel sequencing (459 genes)) and strategy 2 (FRAXA+exome (ES)). Preliminary results in 235/330 patients display a positive diagnosis in 85/235 (36 \%) (62 SNV, 21 CNV, 1 FRAXA, 4 doublehits): $29.7 \%$ with strategy 1 and $30 \%$ with strategy 2 . The results were concordant in $88 \%$. Gene panel and ES identified a positive diagnosis in 58/235 (25\%) and 66/235 (28\%), respectively. No SNV interpretation was missed in gene panel sequencing. Different reasons explain the defects in ES interpretation $(28 / 235 ; 12 \%)$ : atypical phenotype, double-hits, bioinformatics misalignments and difficulties to interpret missense variants within a solo strategy. But ES identified variants in new disease-causing genes, recently described and new candidate genes. For CNV diagnosis, gene panel sequencing appeared limited and required to be coupled with array-CGH, whereas ES appeared efficient. Overall, solo ES obtain slightly higher results than gene panel but a trio-based analysis should be preferred for a greater efficiency. Moreover, the possibility to reanalyze ES data confers a significant benefit due to the continuous development of knowledge.

A. Bruel: None. B. Gérard: None. A. Piton: None. F. Tran Mau-Them: None. A. Sorlin: None. A. Sorly: None. D. Lacombe: None. S. Manouvrier: None. P. Edery: None. N. Philip: None. D. Geneviève: None. A. Verloes: None. S. Odent: None. J. Thevenon: None. A. Toutain: None. D. Bonneau: None. S. El Chehadeh: None. M. Doco-Fenzy: None. B. Isidor: None. A. Goldenberg: None. C. Vincent-Delorme: None. O. Boute-Benejean: None. L. lambert: None. M. Asensio: None. P. Callier:
None. Y. Duffourd: None. C. Lejeune: None. C. Binquet: None. C. Philippe: None. L. Faivre: None. C. ThauvinRobinet: None.

\section{P08.034.A}

\section{DOES GENDER AFFECT THE FACIAL FEATURES OF FETAL ALCOHOL SYNDROME? EVIDENCE FROM A COHORT STUDY}

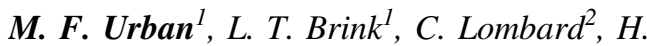 \\ Bezuidenhout $^{l}$, G. de Jong ${ }^{l}, H$. J. Odendaal ${ }^{l}$
}

${ }^{I}$ Faculty of Medicine and Health Sciences, University of Stellenbosch, Cape Town, South Africa, ${ }^{2}$ Medical Research Council of South Africa, Cape Town, South Africa

Background: By Institute of Medicine criteria, smooth philtrum and thin vermilion border of the upper lip, and short palpebral fissure length (PFL) are cardinal features of fetal alcohol syndrome (FAS). Lip assessment is by visual comparison with a photographic 5-point Likert scale (Astley score), and PFL by measurement. Astley scores of philtrum and vermilion $\geq 4$, and $P F L \leq 10^{\text {th }}$ centile define the facial features. We assessed gender differences in these features.

Methods: The Safe Passage Study is an international cohort study of prenatal alcohol exposure effects on infant outcomes. Data from 7060 pregnancies were assessed at the Cape Town site. Case report forms prospectively collected exposure information. Dysmorphologists examined oneyear-olds. Multivariate statistical analysis included gender, alcohol and smoking levels per trimester, maternal measurements, socio-economic status, and excluded drug users.

Results: After exclusions, 4399 one-year-olds were compared: 2224 (50.6\%) females and 2175 (49.4\%) males. Astley score $\geq 4$ was commoner in males than females for philtrum $(21.0 \%$ versus $13.3 \%, \mathrm{p}<0.001)$ and vermilion ( $20.6 \%$ versus $17.3 \%, \mathrm{p}=0.005$ ). On multivariate analysis males had relative risk of Astley score $\geq 4$ for philtrum of $1.53(95 \% \mathrm{CI}=1.33-1.76 ; \mathrm{p}<0.001)$ and vermilion of 1.14 $(95 \% \mathrm{CI}=1.01-1.35 ; \mathrm{p}=0.03)$. Males had wider PFLs (correlation coefficient $0.53 ; \mathrm{p}<0.001$ ).

Conclusions: Gender differences in facial features for FAS diagnosis are independent of alcohol use and may confound dysmorphic assessment. Males are more likely to score positive on lip features but less likely on PFL. Caution is warranted in reifying cut-off measurements for FAS diagnosis.

Funding support: National Institutes of Health grants U01HD055154, U01HD045935, U01HD055155, U01HD045991, and U01AA016501

M.F. Urban: None. L.T. Brink: None. C. Lombard: None. H. Bezuidenhout: None. G. de Jong: None. H.J. Odendaal: None. 


\section{P08.035.B}

Floating-Harbor Syndrome: iPSCs with atypical morphology from patient-derived cells with mutation in the SRCAP gene

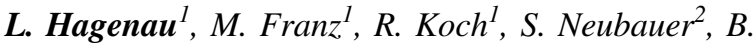 Nowack $^{2}$, L. R. Jensen ${ }^{l}$, A. W. Kuss ${ }^{l}$}

${ }^{I}$ Department of Functional Genomics, Interfaculty Institute for Genetics and Functional Genomics, University Medicine Greifswald, Greifswald, Germany, ${ }^{2}$ SensID GmbH, Rostock, Germany

Introduction: Floating-Harbor syndrome (FLHS) is a rare genetic disease characterized by growth deficiency, facial dismorphism, language delays and often mild to severe intellectual disability. It is caused by mutations in the SRCAP gene, which encodes a core component of the chromatin-remodeling complex and also acts as a transcriptional activator. We identified a de novo truncating mutation in the SRCAP gene of a patient diagnosed with FLHS and aimed to establish an induced pluripotent stem cell (iPSC) line that can serve as a model for FLHS.

Material and methods: Gingival fibroblasts from the patient and skin fibroblasts from the unaffected father were reprogrammed into iPSCs using a virus-free episomal vector system. iPSCs were tested for pluripotency marker expression (immunofluorescence and qPCR) and differentiation capability (embryoid bodies). Two and three iPSC clones were generated for the patient and the control, respectively.

Results: We were able to create iPSCs from primary fibroblasts of a FLHS patient and his father. However, the patient iPSCs displayed an atypical colony morphology with fuzzy borders and loose cell grouping. Despite the atypical appearance, the patient iPSCs were pluripotent and able to differentiate into the 3 germ-layers.

Conclusions: The patient derived iPSCs we describe can be instrumental in furthering our knowledge about the molecular mechanisms involving SRCAP and contribute to a better understanding of the aetiology of FLHS.

L. Hagenau: None. M. Franz: None. R. Koch: None. S. Neubauer: A. Employment (full or part-time); Significant; SensID GmbH. B. Nowack: A. Employment (full or parttime); Significant; SensID GmbH. E. Ownership Interest (stock, stock options, patent or other intellectual property); Significant; SensID GmbH. L.R. Jensen: None. A.W. Kuss: None.

P08.036.C

Ethnicity is an independent Risk Factor for Full Mutation Expansion in FMR1 Premutation Carriers
N. Domniz ${ }^{1}$, L. Ries Levavi ${ }^{2}$, M. Berkenstadt ${ }^{2}$, E. Pras ${ }^{2}, Y$. Cohen $^{1}$, H. Raanani ${ }^{1}$, S. Ben-Shachar ${ }^{3}$, D. Brabbing Goldstein $^{3}$, S. Elizur ${ }^{1}$

${ }^{1}$ IVF Unit, Ramat Gan, Israel, ${ }^{2}$ The Genetic Institute, Ramat Gan, Israel, ${ }^{3}$ The Genetic Institute, Tel-Aviv, Israel

Design: A cohort population-based study.

Materials and Methods: All Jewish FMR1 premutation carriers who underwent chorionic villus sampling (CVS) or amniocentesis (AC) at Sheba Medical Center and the Tel Aviv Medical center during the period of 2011-2018 were included in this study. Ethnicity was determined by selfreport as appeared in the medical records. Long range PCR for FMR1 (Asuragen, TX, USA) was performed in order to determine the number of CGG repeats and AGG interruptions in all women and fetuses. General distribution and data analysis were calculated using SPSS 18.0 and Microsoft Excel.

Results: 766 FMR1 premutation carriers underwent CVS or AC. Of them, 592 carriers had parents' concordance for ethnic background. The number of CGG repeats was significantly higher among the Ashkenazi carriers compared to Non-Ashkenazi women (median 69 vs. 64; p < 0.0001). The number of AGG interruptions was lower in Ashkenazi (35\% with no AGG's) compared to the Non-Ashkenazi carriers (20\% with 0 AGG's); $p<0.0001$.

Multivariate analysis using CGG repeats, AGG interruptions and ethnicity, revealed that women's ethnicity was an independent risk factor for a full mutation expansion $(\mathrm{p}=0.01)$.

Conclusions: Carrier's ethnicity may be an independent risk factor for a full mutation expansion among FMRI premutation carriers, suggesting the existence of further components affecting the mother-to-offspring transmission process. Further research is needed in order to identify other ethnic groups at risk and to better understand the process of CGG inter-generation expansion and the different variables that affect it. In addition, these findings may contribute for a precise genetic counselling.

N. Domniz: None. L. Ries Levavi: None. M. Berkenstadt: None. E. Pras: None. Y. Cohen: None. H. Raanani: None. S. Ben-Shachar: None. D. Brabbing Goldstein: None. S. Elizur: None.

P08.038.B

Heterozygous variants disturbing the transcriptional repressor activity of FOXP4 cause a disorder with expressive language deficits, congenital abnormalities and divergent growth parameters 
L. Snijders Blok ${ }^{1,2,3}$, A. Vino ${ }^{2}$, H. Underhill ${ }^{4}$, D. Monteil ${ }^{5}$, H. Li ${ }^{6}$, F. Reynoso Santos ${ }^{7}$, W. K. Chung ${ }^{8}$, M. Amaral ${ }^{9}, R$.

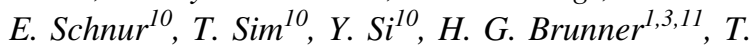
Kleefstra $^{1,3}$, S. E. Fisher ${ }^{2,3}$

${ }^{1}$ Radboud University Medical Center, Nijmegen, Netherlands, ${ }^{2}$ Max Planck Institute for Psycholinguistics, Nijmegen, Netherlands, ${ }^{3}$ Donders Institute for Brain, Cognition and Behaviour, Nijmegen, Netherlands, ${ }^{4}$ University of Utah, Salt Lake City, UT, United States, ${ }^{5}$ Naval Medical Center, Portsmouth, VA, United States, ${ }^{6}$ Emory University, Atlanta, GA, United States, 'Joe DiMaggio Children's Hospital, Hollywood, FL, United States, ${ }^{8}$ Columbia University Irving Medical Center, New York, NY, United States, ${ }^{9}$ HudsonAlpha Institute for Biotechnology, Huntsville, AL, United States, ${ }^{10}$ GeneDx, Gaithersburg, MD, United States,

${ }^{11}$ Maastricht University Medical Center, Maastricht, Netherlands

Introduction: Germline variants in FOXP4 have not yet been definitively linked to a Mendelian disorder. Although a single individual has been reported in the literature with a homozygous possibly pathogenic variant, suggesting a recessive inheritance pattern, it is currently unclear whether heterozygous FOXP4 variants can also cause a phenotype.

Methods: We assembled a cohort of eight individuals with heterozygous and mostly de novo variants in FOXP4: seven individuals with six different missense variants and one individual with a frameshift variant. We used functional assays to assess pathogenicity of the variants, and collected clinical data to delineate the associated phenotypic spectrum.

Results: Four missense variants clustered in the DNAbinding forkhead-box (FOX) domain of the encoded transcription factor. Luciferase assays showed loss-offunction effects for these four missense variants, and aberrant subcellular localization was seen for three out of four. The two missense variants located outside the functional domains of FOXP4 showed transcriptional repressor capacities similar to wild-type FOXP4, as well as normal subcellular localization patterns. Clinical data were collected for the six individuals with variants likely affecting FOXP4 functions. Recurrent features included growth abnormalities, language deficits, congenital diaphragmatic hernia, cervical spine abnormalities and ptosis.

Conclusions: Although a dominant-negative effect cannot be excluded for the missense variants, it seems that lossof-function of FOXP4 can already be sufficient to cause a phenotype. Our findings show that heterozygous variants in FOXP4 can cause an autosomal dominant disorder that is characterized by growth defects, congenital abnormalities and mild developmental delays, mainly affecting speech and language.
L. Snijders Blok: None. A. Vino: None. H. Underhill: None. D. Monteil: None. H. Li: None. F. Reynoso Santos: None. W.K. Chung: None. M. Amaral: None. R.E. Schnur: None. T. Sim: None. Y. Si: None. H.G. Brunner: None. T. Kleefstra: None. S.E. Fisher: None.

\section{P08.040.A}

Altered mitochondrial function in cells carrying a premutation or unmethylated full mutation of the FMR1 gene

V. Nobile ${ }^{1}$, F. Palumbo ${ }^{1}$, S. Lanni ${ }^{2}$, V. Ghisio ${ }^{1}$, A. Vitali ${ }^{1}$, V. Marzano ${ }^{3}$, M. Castagnola ${ }^{1}$, G. Maulucci ${ }^{1}, C$. De Angelis $^{1}$, M. De Spirito ${ }^{1}$, L. Pacinit, L. D’Andrea ${ }^{5}$, R.

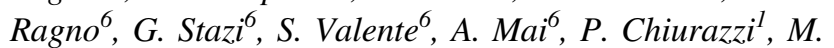
Genuardi $^{l}$, G. Neri ${ }^{l}$, E. Tabolacci ${ }^{l}$

${ }^{1}$ Catholic University, Roma, Italy, ${ }^{2}$ The Hospital for Sick Children, Toronto, ON, Canada, ${ }^{3}$ Bambino Gesù Children's Hospital IRCCS, Roma, Italy, ${ }^{4}$ Saint Camillus International University of Health and Medical Sciences, Roma, Italy, ${ }^{5}$ University of Rome Tor Vergata, Roma, Italy, ${ }^{6}$ Sapienza University, Roma, Italy

Fragile $\mathrm{X}$ related-disorders are due to a dynamic mutation of the CGG repeat at the 5' UTR of the FMRl gene, coding for the RNA-binding protein FMRP. As the CGG sequence expands from premutation (PM, 56-200 CGGs) to full mutation (>200 CGGs), FMRP synthesis decreases until it is practically abolished in fragile $\mathrm{X}$ syndrome (FXS) patients, mainly due to FMRl methylation. Cells from rare individuals with no intellectual disability and carriers of an unmethylated full mutation (UFM) produce slightly elevated levels of FMR1-mRNA and relatively low levels of FMRP, like in PM carriers. With the aim of clarifying how UFM cells differ from CTRL and FXS cells, a comparative proteomic approach was undertaken, from which emerged an overexpression of SOD2 in UFM cells, also confirmed in PM but not in FXS. The SOD2-mRNA bound to FMRP in UFM more than in the other cell types. The high SOD2 levels in UFM and PM cells correlated with lower levels of superoxide and Reactive Oxygen Species (ROS), and with morphological anomalies and depolarization of the mitochondrial membrane detected through confocal microscopy. The same effect was observed in CTRL and FXS after treatment with MC2791, causing SOD2 overexpression. These mitochondrial phenotypes reverted after knock-down with siRNA against SOD2-mRNA and FMRI-mRNA in UFM and PM. Overall, these data suggest that in PM and UFM carriers, which have high levels of FMRI transcription and may develop FXTAS, SOD2 overexpression helps to maintain low levels of both superoxide and ROS with signs of mitochondrial degradation. 
V. Nobile: None. F. Palumbo: None. S. Lanni: None. V. Ghisio: None. A. Vitali: None. V. Marzano: None. M. Castagnola: None. G. Maulucci: None. C. De Angelis: None. M. De Spirito: None. L. Pacini: None. L. D’Andrea: None. R. Ragno: None. G. Stazi: None. S. Valente: None. A. Mai: None. P. Chiurazzi: None. M. Genuardi: None. G. Neri: None. E. Tabolacci: None.

\section{P08.041.B}

A frameshift mutation in ZFHX4, located on chromosom 8q21, phenocopies the 8q21.11 microdeletion syndrome.

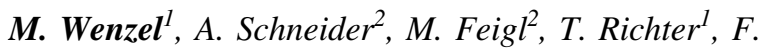
Oeffner $^{1}$, B. Zirn ${ }^{3}$

${ }^{1}$ genetikum, Neu-Ulm, Germany, ${ }^{2}$ Casa Elisa Sozialpädiatrisches Zentrum, Ravensburg, Germany, ${ }^{3}$ genetikum, Stuttgart, Germany

Introduction: Patients with 8q21.11 microdeletion syndrome including the ZHFX4 gene present with a recognizable phenotype of variable intellectual disability, behavioral abnormalities, ptosis, a round face, down-turned corners of the mouth, low-set ears, and sensorineural hearing loss.

Materials and Methods: We report a female patient and her son with the following clinical features: macrocephaly, ptosis, prominent low-set ears, a round face, down-turned corners of the mouth, developmental delay, hearing loss and behavioral abnormalities. After exclusion of the $8 \mathrm{q} 21.11$ microdeletion syndrome and other relevant chromosomal anomalies by chromosome and microarray analysis and after exclusion of fragile-X-syndrome, we applied clinical exome sequencing on an Illumina System followed by phenotype-based evaluation strategies.

Results: A heterozygous point mutation in $\mathrm{ZFHX} 4$ (c.5850_5851delAT, p.(Cys1951fs)), was identified and confirmed by sanger sequencing in both mother and son, and excluded in further unaffected family members.

Conclusions: This is the first report of a point mutation in ZFHX4 leading to the same phenotypic features as the known 8q21.11 microdeletion syndrome including ZFHX4.

M. Wenzel: None. A. Schneider: None. M. Feigl: None. T. Richter: None. F. Oeffner: None. B. Zirn: None.

\section{P08.043.A}

Human FMR1 gene expression regulation by microRNA in a cellular model
A. A. Dolskiy ${ }^{1}$, L. V. Krainikova ${ }^{1}$, A. V. Pindyurin ${ }^{2}$, L. V. Boldyreva ${ }^{2}$, D. V. Yudkin ${ }^{1}$

${ }^{I}$ FBRI SRC VB "Vector", Rospotrebnadzor, Novosibirsk Region, Russian Federation, ${ }^{2} I M C B S B$ RAS, Novosibirsk, Russian Federation

Introduction: FRAXopathies are widespread pathologies due to CGG repeat expansion in the 5'-UTR of the FMRI gene. Changes in the repeat length lead to $F M R I$ expression alteration. Previously, microRNA involvement in FMRI gene expression regulation was demonstrated in an animal model. We described nine microRNAs complementary to the 3'-UTR of FMRI with expression alterations dependent on the FMRl gene allele. Here, we present a study on the identified microRNA interactions with the 3'-UTR of FMRI in a plasmid model system.

Materials and methods: A plasmid expressing two reporter genes was designed. Both reporters were driven by constitutive promoters. One reporter was fused with the 3'UTR of the FMRl gene, while microRNA expression was driven by an inducible promoter. Measuring the protein expression of the reporter in the control and after microRNA expression induction allowed us to quantify the interaction between the microRNA and the 3'-UTR.

Results: The model plasmid was confirmed by sequencing. The activity of reporter genes was detected after transfection of HEK293 cells. The genes of nine microRNAs were cloned into a plasmid and separately transfected into the cells. The expression of reporter proteins was measured, and the interaction of every microRNA and the FMRl gene was quantified.

Conclusions: Screening of microRNA expression allowed us to study its involvement in the regulation of a specific gene. The use of a molecular model helped us to show the interaction of microRNAs and the regulatory region of the gene. The study is supported by Russian Science Foundation grant 18-15-00099.

A.A. Dolskiy: None. L.V. Krainikova: None. A.V. Pindyurin: None. L.V. Boldyreva: None. D.V. Yudkin: None.

\section{P08.044.B}

Protein abundance profiling in murine model for intellectual disability induced by FTSJ1 deficiency

L. R. Jensen ${ }^{l}$, V. Dhople ${ }^{l}$, M. Depke ${ }^{l}$, U. Völker ${ }^{l}, F$. Schmidt ${ }^{2}$, A. W. Kuss ${ }^{1}$

${ }^{1}$ Department of Functional Genomics, Interfaculty Institute for Genetics and Functional Genomics, University 
Medicine Greifswald, Greifswald, Germany, ${ }^{2}$ Proteomics Core, Weill Cornell Medicine-Qatar, Doha, Qatar

Congenital forms of intellectual disability (IQ $<50$ ) are genetically heterogeneous but affect up to $0.5 \%$ of the population. Mutations in most known intellectual disability genes often only account for a comparatively small number of cases, as exemplified by mutations in the X-linked gene FTSJ1, which are a rare cause for non-syndromic intellectual disability in affected males. FTSJI encodes a tRNA methyl-transferase that methylates tRNA phenylalanine at position 32 and 34 (wobble position) in yeast and man. We have recently established a mouse model for Ftsjl deficiency and found that in male Ftsj1-deficient mice the phenotype is not restricted to cognitive functions. In order to investigate the effects of Ftsj1 deficiency in different mammalian tissues, we generated protein extracts from brain, liver, kidney and heart from five wild type and Ftsj $1^{-/ y}$ littermate pairs, generated protein abundance profiles and compared the normalized label-free quantitation values (LFQ). The fold changes between control and Ftsj1 $1^{-/ y}$ tissues were generally at or below factor 3, but still between 54 and 131 proteins from each tissue showed a significantly different abundance $(\mathrm{p}<0.01)$. Among these we found enrichment of proteins involved in protein synthesis, energy metabolism and actin dynamics. Our results suggest that a common pathway is affected in all four different tissues but that the evolutionarily comparatively young cognitive aspects of brain function are more sensitive to changes in this functional context. It also raises the question whether additional characteristic features might not also be detectable in patients with FTSJ1 deficiency at cellular or tissue levels.

L.R. Jensen: None. V. Dhople: None. M. Depke: None. U. Völker: None. F. Schmidt: None. A.W. Kuss: None.

\section{P08.048.C}

De novo noncoding variants upstream of $M E F 2 C$ cause severe developmental disorders

N. Whiffin ${ }^{1,2,3}$, P. Danecek ${ }^{3}$, N. M. Quaife ${ }^{1,4}$, J. Kaplanis ${ }^{3}$, $K$. Samocha ${ }^{3}$, Genomics England Research Consortium, J.

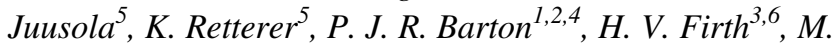
E. Hurles ${ }^{3}$, C. F. Wright

${ }^{I}$ National Heart and Lung Institute, Imperial College London, London, United Kingdom, ${ }^{2}$ Royal Brompton and Harefield NHS Foundation Trust, London, United Kingdom, ${ }^{3}$ Wellcome Sanger Institute, Hinxton, United Kingdom, ${ }^{4}$ MRC London Institute of Medical Sciences, London, United Kingdom, ${ }^{5}$ GeneDx, Gaithersburg, MD, United States, ${ }^{6}$ Clinical Genetic Department, Addenbrooke's Hospital Cambridge University Hospitals, Cambridge,
United Kingdom, ${ }^{7}$ University of Exeter Medical School, Royal Devon \& Exeter Hospital, Exeter, United Kingdom

Trio exome sequencing to profile protein-coding regions in patients from the Deciphering Developmental Disorders (DDD) study identifies a genetic diagnosis in only $~ 35 \%$ of patients. We searched 9,858 patients for de novo variants directly upstream of genes known to cause developmental disorders and identified six likely disease-causing variants upstream of $M E F 2 C$. All six probands have phenotypes that are consistent with disruptions in $M E F 2 C$, including global developmental delay (6/6) and seizures (4/6), and no other likely diagnostic variants.

These variants are predicted to cause loss-of-function through three distinct mechanisms. (1) Two 5'UTR single nucleotide variants (SNVs) create upstream open reading frames (uORFs) that overlap with the MEF2C coding sequence out-of-frame, negatively regulating translation. (2) Two further 5'UTR SNVs create upstream start codons that extend the coding sequence by three and nine amino acids, respectively. These variants are also observed de novo in four additional patients (two with each variant) sequenced by Genomics England and GeneDx. Protein structure modelling suggests that these variants disrupt DNA binding of MEF2C protein, impairing its function as a transcription factor. (3) The final two variants are large deletions $(294 \mathrm{~Kb}$ and $97 \mathrm{~Kb}$ ) that remove the N-terminal exon of the 5'UTR and the entire promoter sequence, completely ablating transcription of one allele. We will present ongoing functional assays to prove the mechanism through which these variants cause disease.

Our analyses show that non-coding variants upstream of known disease-causing genes are an important cause of severe disease and demonstrates that sequencing 5'UTRs can increase diagnostic yield.

N. Whiffin: None. P. Danecek: None. N.M. Quaife: None. J. Kaplanis: None. K. Samocha: None. J. Juusola: A. Employment (full or part-time); Significant; GeneDx, Inc. K. Retterer: A. Employment (full or part-time); Significant; GeneDx, Inc.. E. Ownership Interest (stock, stock options, patent or other intellectual property); Significant; OPKO Health, Inc.. P.J.R. Barton: None. H. V. Firth: None. M.E. Hurles: E. Ownership Interest (stock, stock options, patent or other intellectual property); Significant; Congenica Ltd. F. Consultant/Advisory Board; Significant; Congenica Ltd. C.F. Wright: None.

\section{P08.049.A}

Exploring the transcriptional landscape of hippocampus cells in homozygous Pigv c.1022C $>$ A knock-in mice by single-cell RNA-sequencing 
F. S. David ${ }^{1,2}$, M. Rodríguez de los Santos ${ }^{2,3,4}$, A. Knaus ${ }^{2}$, D. Mattei ${ }^{5}$, B. Timmermann ${ }^{6}$, P. M. Krawitz ${ }^{2}$

${ }^{1}$ Institute of Human Genetics, University of Bonn, School of Medicine \& University Hospital Bonn, Bonn, Germany, ${ }^{2}$ Institute for Genomic Statistics and Bioinformatics, University of Bonn, School of Medicine \& University Hospital Bonn, Bonn, Germany, ${ }^{3}$ Institute for Medical Genetics and Human Genetics, Charité - Universitätsmedizin Berlin, Berlin, Germany, ${ }^{4}$ Max-Planck-Institute for Molecular Genetics, FG Development and Disease, Berlin, Germany, ${ }^{5}$ Insitute of Veterinary Pharmacology and Toxicology, University of Zurich, UZH, Zürich, Switzerland, ${ }^{6}$ Sequencing Core Facility, Max Planck Institute for Molecular Genetics, Berlin, Germany

Introduction: Biallelic hypomorphic mutations in PIGV are a known cause of congenital glycosylphosphatidylinositol biosynthesis defects (GPIBD), a group of recessive syndromes with shared phenotypic features such as intellectual disability. In particular, the c.1022C $>\mathrm{A}$ transversion is frequently reported in European patients with GPIBD.

Materials and Methods: To gain further insight into the pathophysiology of neurological features observed in patients with GPIBD, we performed a single-cell RNA sequencing analysis of hippocampus samples from a recently established Pigv c.1022C $>$ A knock-in $\left(\right.$ Pigv $\left.^{341 \mathrm{E}}\right)$ mouse model.

Results: Based on the gene expression profiles of around 8,800 Pigv ${ }^{341 \mathrm{E}}$ and 7,100 wild type cells, we identified 17 cellular subgroups. Cells of both genotypes were present in all subgroups, but the distribution was skewed in Pigv $v^{341 \mathrm{E}}$ mice, e.g. with an increased fraction of subicular neurons and decreased fractions of granule cells and oligodendrocytes. The most extensive changes in transcriptional profiles due to glycosylphosphatidylinositol anchor deficiency were observed in a microglia subgroup as well as in subicular neurons. Among the affected genes, several synapse-related Gene Ontology terms were found to be enriched. Across all cellular subgroups, expression of Abll was decreased and expression of $H d c, C y p 4 x l$, and $G m 14216$ was increased in Pigv $^{341 \mathrm{E}}$ cells.

Conclusions: This single-cell RNA sequencing study of hippocampus cells in the Pigv ${ }^{341 \mathrm{E}}$ mouse model allowed us to generate new hypotheses regarding the pathophysiology of cognitive impairment in GPIBDs. In particular, differential expression of Abll suggests an involvement of the Eph/ephrin signaling pathway, which is known to play a major role in synapse formation and function.

F.S. David: None. M. Rodríguez de los Santos: None. A. Knaus: None. D. Mattei: None. B. Timmermann: None. P.M. Krawitz: None.
P08.051.C

Expanding the clinical phenotype of $\mathrm{X}$-linked mental retardation 103: new patient with deleterious mutation in KLHL15 gene

M. Ballesta-Martinez ${ }^{1,2,3}$, M. Sanchez Soler ${ }^{1,2}$, A. Serrano Anton $^{1,2}$, V. López-Gonzalez, ${ }^{1,2,3}$, L. Rodriguez Peña ${ }^{l}, L$. Armengol Dulcet ${ }^{4}$, E. Guillen Navarro ${ }^{1,2,3}$

${ }^{1}$ Hospital Clinico Universitario Virgen de la Arrixaca, Murcia, Spain, ${ }^{2}$ Insituto Murciano de Investigación Biomedica (IMIB), Murcia, Spain, ${ }^{3}$ CIBER-ER, Murcia, Spain, ${ }^{4}$ Quantitative Genomic Medicine Laboratory, qGenomics $S$. L., Barcelona, Spain

Introduction: X-linked mental retardation 103 is caused by loss of function mutations in KLHL15 gene. Only 2 families have been reported. First reported patient had intellectual disability (ID), epilepsy, brain anomalies and coarse features. Array-CGH: $22 \mathrm{~kb}$ deletion Xp22.11 involving the first three of the four exons of KLHL15 gene; mother carried the deletion with skewed XCI in lymphocyte DNA. Second report includes a large family with several affected members with mild to moderate ID in which a protein-truncating variant in KLHL15 which segregated with the phenotype was detected by exome sequencing. We report on a new case with a new causal variant in KLHL15 which further supports its implication in ID, and delineate the phenotype. Clinical description: 5 year-old boy, second child of healthy non-consanguineous parents. Mother had deceased twin brothers with ID and brain atrophy. Patient had global developmental delay, OFC $3^{\text {rd }}$ centile, coarse facial features, braquidactyly, thyroglosal cyst and ocular malformation (posterior polar cataract and choroidal coloboma of left eye). Brain MRI, abdominal and cardiac sonogram were normal. No epilepsy. Array-CGH was normal, and clinical exome sequencing detected a previously not reported variant c.417_418delGA; P. Leu139ArgfsTer2 in KLHL15 gene, of maternal origin; she presented skewed XCI in lymphocyte DNA (100:0).

Conclusions: 1- We report on a new case of X-linked MR due to loss of function mutation in KLHL15 supporting its implication in ID. 2- This case expands clinical phenotype of patients with KLHL15 mutations with ocular involvement. 4-Achieving molecular diagnosis with NGS permits adequate genetic counseling in families.

M. Ballesta-Martinez: None. M. Sanchez Soler: None. A. Serrano Anton: None. V. López-Gonzalez: None. L. Rodriguez Peña: None. L. Armengol Dulcet: None. E. Guillen Navarro: None. 


\section{P08.053.B}

Functional predictors of causative cisregulatory mutations in mendelian disease

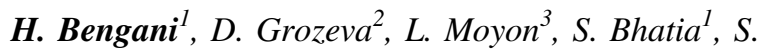
Ribeiro dos Louros ${ }^{4}, J$. Hope ${ }^{5}$, A. Jackson ${ }^{4}, J$. Prendergast $^{6}$, M. Naville ${ }^{3}$, O. Spasic-Boskovic ${ }^{7}, V . v$.

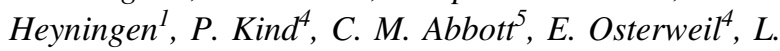
Raymond $^{7}$, H. R. Crollius ${ }^{3}$, D. R. FitzPatrick ${ }^{l}$

${ }^{1}$ MRC Institute of Genetics \& Molecular Medicine, Edinburgh, United Kingdom, ${ }^{2}$ Cambridge Institute for Medical Research, Edinburgh, United Kingdom, ${ }^{3}$ Institut de biologie de l'Ecole normale supérieure, Paris, France, ${ }^{4}$ Centre for Integrative Physiology, Patrick Wild Centre, University of Edinburgh, Edinburgh, United Kingdom, ${ }^{5}$ Centre for Genomics and Experimental Medicine, IGMM, University of Edinburgh, Edinburgh, United Kingdom, ${ }^{6}$ Roslin Institute, University of Edinburgh, Edinburgh, United Kingdom, ${ }^{7}$ Cambridge Institute for Medical Research, Cambridge, United Kingdom

Rare variants altering cis-regulatory elements (CRE) appear enriched in undiagnosed neurodevelopmental disease but few highly penetrant variants have been identified. We present a pipeline which aims to discriminate likely diagnostic CRE variants from those of neutral/low-penetrant effect. Six rare testable CRE variants were identified using targeted and whole genome sequencing in 48 unrelated males with apparent X-linked intellectual disability (XLID) but without detectable coding region variants. These variant segregated appropriately in families and altered conserved bases in CREs targeting known XLID genes. Three were unique and three were rare but too common to be plausibly causative for XLID. Cis-regulatory activity was initially tested by comparing wild-type and mutant alleles in zebrafish embryos using dual-color fluorescent enhancer reporters. Two variants showed striking changes: one plausibly causative $\left(F M R I^{\mathrm{CRE}}\right)$ and the other likely neutral/ low-penetrant $\left(T E N M 1^{\mathrm{CRE}}\right)$. These regulatory variants were then "knocked-in" to mice and both caused abnormal embryonic neural expression of their target gene. Only mice carrying the plausible $F M R I^{\mathrm{CRE}}$ showed disease-relevant behavioral defects. We consider $F M R I^{\mathrm{CRE}}$ to be plausibly disease-associated and resulting in complex misregulation of FMR1/FMRP rather than loss-of-function. This is consistent with absence of typical physical features of Fragile $\mathrm{X}$ in affected individuals and electrophysiological anomalies in the brains of post-natal $F M R I^{\mathrm{CRE}}$ mice. Thus, extreme rarity and the presence of relevant phenotypic features in knock-in mice were the only discriminative factors for causative CRE variants. Importantly, disruption of the normal in vivo pattern of target gene expression in disease- relevant tissues cannot be used as strong evidence for Mendelian disease association.

H. Bengani: None. D. Grozeva: None. L. Moyon: None. S. Bhatia: None. S. Ribeiro dos Louros: None. J. Hope: None. A. Jackson: None. J. Prendergast: None. M. Naville: None. O. Spasic-Boskovic: None. V.V. Heyningen: None. P. Kind: None. C.M. Abbott: None. E. Osterweil: None. L. Raymond: None. H.R. Crollius: None. D.R. FitzPatrick: None.

\section{P08.054.C}

Detection rate and re-analysis of exome sequencing data in a cohort of 207 individuals with neurodevelopmental disorders

M. Hebebrand, C. T. Thiel, C. Kraus, A. Ekici, A. Reis, C. Zweier

Institute of Human Genetics, Friedrich-Alexander-Universität Erlangen-Nürnberg (FAU), Erlangen, Germany

Exome sequencing is the most efficient test to diagnose patients with neurodevelopmental disorders (NDD). Given the constantly growing number of NDD associated genes, improvements in analysis tools and information on variants, regular re-analysis of negative or unclear cases is recommended. In a long-term study aiming at identifying the genetic basis of NDDs we performed exome sequencing (SureSelect_v.6; HiSeq2500) in 205 individuals from 197 families presenting with NDDs who had negative test results including CMA. Filtering against known NDD genes (SysID database) followed by identification of de novo variants in all genes resulted in a diagnostic rate of 45\% (89/ 197). Interestingly, the diagnostic yield in singletons was higher than in trios (52\% vs. $42 \%)$. We now re-analysed 105 unsolved sporadic cases regarding homozygous, compound-heterozygous, X-linked, de novo and autosomal dominant variants in all genes, considering also an updated list of 1,164 NDD genes and 1,127 candidate genes. One additional case could be diagnosed: an affected boy inherited a stop mutation in SETD5 from his healthy mother, which previously was missed due to filtering for de novo variants in a trio setting, highlighting the relevance of reduced penetrance. Furthermore, we identified 20 candidate variants in 17 genes compatible with the clinical presentation but a firm diagnosis could not be made due to lack of functional validation, including a de novo missense variant in the candidate gene NEUROD2. The relatively low re-analysis detection rate is probably due to the high initial detection rate based on a combination of diagnostic and research analysis strategy.

M. Hebebrand: None. C.T. Thiel: None. C. Kraus: None. A. Ekici: None. A. Reis: None. C. Zweier: None. 


\section{P08.055.A}

Exome-based gene panel analysis in patients with intellectual disability and epilepsy

E. D'haenens, E. Pouillie, S. Vergult, M. De Smet, T. Sante, B. Callewaert, O. Vanakker, B. Menten, A. Dheedene

Center for Medical Genetics Ghent (CMGG), Ghent, Belgium

The implementation of whole exome sequencing (WES) in the clinic has led to a steep increase in diagnostic yield in patients with (syndromic) intellectual disability (ID) and/or epilepsy. Since 2017 the Center for Medical Genetics Ghent (UZ Ghent) offers a WES-based gene panel analysis for genes related to ID and epilepsy. Here we present an overview our findings. WES was performed in $~ 1000$ probands and both parents (if available) using the SureSelect All Exon kit (Agilent technologies) enrichment followed by 2x150bp sequencing on a HiSeq3000 or NovaSeq 6000 platform (Illumina). Data analysis was done with a community developed pipeline based on the Broad Institute Best Practices (bcbio-nextgen). Virtual panel analysis was performed for 1175 genes and variant classification was based on ACMG-AMG guidelines. In $21 \%$ of cases a definitive genetic causal defect (class 4 or 5 variant) could be identified: in $60 \%$ of these patients a de novo variant was found while in $40 \%$ the causal variant was inherited. From the latter $12,5 \%$ are variants in genes related to an X-linked disorder, $41,5 \%$ to an autosomal dominant and $46 \%$ to an autosomal recessive disorder. In addition, in $25 \%$ of the patients an interesting variant of unknown significance (class 3) was reported. Currently, follow-up studies are ongoing to evaluate these class 3 variants, e.g. collecting additional phenotypic data, performing segregation and transcriptome analysis. With a diagnostic yield between $21 \%$ and $46 \%$, we can conclude that WES is a gamechanger in the diagnostic workup of patients with ID/ epilepsy.

E. D'haenens: None. E. Pouillie: None. S. Vergult: None. M. De Smet: None. T. Sante: None. B. Callewaert: None. O. Vanakker: None. B. Menten: None. A. Dheedene: None.

\section{P08.056.B}

A de novo missense mutation highlights the role of FBXO28 gene in 1q41-q42 microdeletion syndrome phenotype

N. Spataro, E. Gabau, N. Baena, M. Guitart, A. Ruiz

Corporació Sanitaria Parc Taulí, Sabadell, Spain
Introduction: Microdeletion 1q41-q42 has been associated with a distinctive neurodevelopmental syndrome. The reported clinical features include: intellectual disability, seizures, dysmorphia and brain anomalies among others. Haploinsufficiency of different genes has been proposed as the causal mechanism. Since most of the reported 1q41-q42 microdeletions encompass both FBXO28 and WDR26 genes, their causal role has been debated in recent years.

Materials and Methods: Sequencing was carried out on an Illumina platform (MiSeq) considering a panel of 460 genes related to neurodevelopmental disorders. Raw reads were mapped to the human genome using BWA. PCR duplicates were removed using Picard Tools; GATK was used for base quality score recalibration and variant discovery. Variants were annotated using Annovar and filtered according to their frequency in gnomAD database.

Results: We identify a de novo c.1043 G>T substitution not reported in gnomAD and causing a p.Arg348Leu change in FBXO28. The patient is affected by severe intellectual disability, epilepsy, brain alterations and absent speech, with a global phenotype resembling that of the 1q41-q42 microdeletion syndrome. To date, only a single de novo loss of function mutation in FBXO28 gene has been reported for a patient having similar clinical features.

Conclusions: This second case evidences the critical role of FBXO28 gene in 1q41-q42 microdeletion syndrome phenotype. FBXO28 protein is a component of the SkpCullin-F-Box complex, which mediates proteasomal degradation. Dysfunctions of the ubiquitin proteasome system have been associated to several neurodevelopmental disorders, such as: $\mathrm{X}$-linked spinal muscular atrophy, Angelman syndrome and Opitz BBB/G.

N. Spataro: None. E. Gabau: None. N. Baena: None. M. Guitart: None. A. Ruiz: None.

\section{P08.058.A}

Next generation sequencing of a cohort of individuals with syndromic intellectual disability

F. Santos-Simarro ${ }^{1,2}$, M. Pacio-Miguez ${ }^{1}$, S. GarcíaMiñaúr ${ }^{1,2}$, V. Rufo-Rabadán ${ }^{1}$, M. Solís ${ }^{1,2}, R$. Mena ${ }^{1,2}, C$. Rodríguez-Jiménez ${ }^{1,2}$, V. E. Fdez-Montaño ${ }^{1,2}$, R. MartínArenas $^{l}$, H. González-Pecellín ${ }^{l}$ Á. del Pozo ${ }^{1,2}$, E. Vallespin $^{1,2}$, M. Palomares-Bralo ${ }^{1,2}$

${ }^{1}$ INGEMM, Hospital Universitario La Paz, Madrid, Spain, ${ }^{2}$ U753, CIBERER, ISCIII, Madrid, Spain

Introduction:Global developmental delay (GDD) / intellectual disability (ID) has an estimated prevalence in the general population of 1-3\%. The genetic causes of GDD/ID are highly heterogeneous, traditionally limiting the possibility to reach a specific diagnosis. In recent years, with the 
advent of next generation sequencing (NGS), the genetic diagnosis of GDD/ID has greatly improved and expanded with currently more than a 1000 genes involved.

Methods: We present the results of NGS testing in a cohort of 456 individuals with syndromic GDD/ID (defined as GDD/ID plus additional manifestations such as congenital malformations, growth abnormalities or dysmorphic features) evaluated in our clinical genetics department over a 42-month period (Jul2016-Dec2019). All had a previous normal $60 \mathrm{~K}$ array-CGH (KaryoArray v3.0, Agilent). NGS trio analysis was performed either using a customized NGS panel containing 1253-1663 genes associated with neurodevelopmental disorders (RD-Seq® v1.0-v6.0) and/or by whole exome sequencing (WES).

Results:Causal variants were identified in 131 different genes in a total of 207 probands (45\%), the majority autosomal dominant de novo variants (66\%). Two novel GDD/ID causing genes have been identified and confirmed with further cases identified through international collaboration. Detailed results of the cohort will be presented.

Conclusions:This study reflects the vast genetic heterogeneity associated to GDD/ID. Comprehensive clinical and molecular evaluation are key to a successful etiological diagnosis, increasing our genomic understanding of GDD/ ID and ultimately allowing improved medical management and genetic counselling for these patients and their families.

F. Santos-Simarro: None. M. Pacio-Miguez: None. S. García-Miñaúr: None. V. Rufo-Rabadán: None. M. Solís: None. R. Mena: None. C. Rodríguez-Jiménez: None. V.E. Fdez-Montaño: None. R. Martín-Arenas: None. H. González-Pecellín: None. Á. del Pozo: None. E. Vallespín: None. M. Palomares-Bralo: None.

\section{P08.059.B}

Inositol monophosphatase 1 (IMPA1) mutation in intellectual disability patients impairs neurogenesis but not gliogenesis

T. Figueiredo ${ }^{1,2,3}$, A. Mendes $^{2}$, G. Kobayashi ${ }^{3}, D$. Moreira $^{3}$, D. Oliveira ${ }^{3}$, E. Goulart ${ }^{3}$, S. Stern ${ }^{2}$, F. Kok ${ }^{3}$, M.

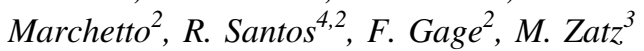

${ }^{1}$ Federal University of Alagoas, Faculty of Medicine, Maceio, Brazil, ${ }^{2}$ The Salk Institute for Biological Studies, La Jolla, CA, United States, ${ }^{3}$ Human Genome and Stem Cell Research Center, University of Sao Paulo, Sao Paulo, Brazil, ${ }^{4}$ Institute of Psychiatry and Neuroscience of Paris, Paris, France

A homozygous mutation in the inositol monophosphatase 1 (IMPA1) gene was recently identified in nine individuals with severe intellectual disability (ID) and disruptive behavior. These individuals belong to the same family from
Northeastern Brazil, which has 28 consanguineous marriages and 59 genotyped family members. IMPA1 is responsible for the generation of free inositol from de novo biosynthesis and recycling from inositol polyphosphates and participates in the phosphatidylinositol signaling pathway. To understand the role of IMPA1 deficiency in ID, we generated induced pluripotent stem cells (iPSCs) from patients and neurotypical controls and differentiated these into hippocampal dentate gyrus-like neurons and astrocytes. IMPA1-deficient neuronal progenitor cells (NPCs) revealed substantial deficits in proliferation and neurogenic potential. At low passage NPCs (P1 to P3), we observed cell cycle arrest, apoptosis, progressive change to a glial morphology and reduction in neuronal differentiation. These observations were validated by transcriptome profiling. Transcriptome analysis showed that NPCs and neurons derived from ID patients have extensive deregulation of gene expression affecting pathways necessary for neurogenesis and upregulation of gliogenic genes. IMPA1 deficiency did not affect cell cycle progression or survival in iPSCs and glial progenitor cells or astrocyte differentiation. Therefore, this study shows that the IMPAI mutation specifically affects NPC survival and neuronal differentiation.

This study was financially supported by the CEPIDFAPESP (2013/08028-1), INCT/FAPESP (2014/50931-3) and FAPESP (Process Number: 2017/19877-0 and 2016/ 09618-5).

T. Figueiredo: None. A. Mendes: None. G. Kobayashi: None. D. Moreira: None. D. Oliveira: None. E. Goulart: None. S. Stern: None. F. Kok: None. M. Marchetto: None. R. Santos: None. F. Gage: None. M. Zatz: None.

P08.060.C

Early diagnosis of NPHP1-associated developmental delay

K. Komlosi ${ }^{1}$, A. Tzschach ${ }^{1}$, K. Prückner-Engesser ${ }^{2}$, E. Komini $^{2}$, N. Hirt ${ }^{l}$, J. Fischer ${ }^{l}$, B. Gläser ${ }^{l}$

${ }^{1}$ Institute of Human Genetics, Medical Center-University of Freiburg, Faculty of Medicine, University of Freiburg, Freiburg, Germany, ${ }^{2}$ Department of Pediatrics, Schwarzwald-Baar Klinikum, Villingen-Schwenningen, Germany

Introduction: Homozygosity for a recurrent $290 \mathrm{~kb}$ deletion of NPHP1 is the most frequent cause of isolated nephronophthisis. It has also been detected in patients with Joubert syndrome (JS). JS is a rare autosomal recessive disorder characterized by a distinctive malformation of the cerebellum and brainstem, hypotonia, ataxia, developmental delay and variable additional features. 
Clinical report: We report on a 3-year-old girl born of consanguineous parents with significant psychomotor delay, expressive language disorder, muscular hypotonia, gait ataxia and increased reflexes in the lower extremities. Cranial MRI at age 8 and 33 months did not show any structural abnormality apart from a pineal cyst and lipoma of the filum terminale in spinal MRI. A detailed examination revealed bitemporal narrowing, a depressed nasal bridge, hypertelorism, convergent strabismus, long, curly eyelashes, a high-arched palate, pointed teeth and a low-set hairline.

Methods and Results: Chromosomal microarray analysis (CMA) showed a homozygous deletion of $88.97 \mathrm{~kb}$ at $2 \mathrm{q} 13$ (110879075-110968043) encompassing the entire NPHPI gene. Both parents carry the deletion in heterozygous form.

Conclusions: Homozygous NPHP1 deletion represents the first molecular defect associated with JS (JBTS4, OMIM \#609583) in a subset of mildly affected patients. Reports without the pathognomic molar tooth sign are very rare, though, subtle findings that may be missed have been reported. In our patient many features may evolve only later; there is a high risk for nephronophthisis and ophthalmologic abnormalities. Our case adds to the spectrum of JS and emphasizes the clinical utility of CMA in the elucidation of syndromic developmental delay.

K. Komlosi: None. A. Tzschach: None. K. PrücknerEngesser: None. E. Komini: None. N. Hirt: None. J. Fischer: None. B. Gläser: None.

\section{P08.061.A}

When a seemingly heterozygous CNV turns out to be compound-heterozygous - from NRXN1-associated neurodevelopmental disorder to Pitt-Hopkins-like syndrome 2

A. C. Teichmann ${ }^{1}$, M. Stiller ${ }^{l}$, S. Schubert ${ }^{l}$, S. Redler ${ }^{2}$, R. Jamra $^{1}$, K. Platzer ${ }^{1}$, N. Rahner ${ }^{3}$

${ }^{1}$ Institute of Human Genetics, University of Leipzig Medical Center, Leipzig, Germany, ${ }^{2}$ Heinrich-Heine-University, Medical Faculty, Institute of Human Genetics, Düsseldorf, Germany, ${ }^{3}$ Institute of Clinical Genetics, Bonn, Germany

Introduction: Heterozygous CNVs and SNVs of NRXNI have been associated with a wide spectrum of neurodevelopmental and neuropsychiatric disorders, including mild to moderate intellectual disability (ID), autism spectrum disorders (ASD) and schizophrenia with reduced penetrance. Furthermore homozygous and compound-heterozygous variants of NRXN1 are associated with the autosomal recessive Pitt-Hopkins-like syndrome 2, including severe ID and a spectrum of ASD, epilepsy and breathing anomalies.
Materials and Methods: We report on an 11-year old female presenting with moderate ID, no active speech development, muscular hypotonia, one febrile seizure during childhood and suspected autism. Family history includes the father with learning difficulties and a paternal aunt and cousin with learning difficulties, epilepsy and autism.

Results: SNP-Array revealed a heterozygous $0.27 \mathrm{Mb}$ deletion disrupting the exons 8-19 of NRXN1. As the phenotype of our patient was more severe than would be expected, we additionally performed NGS-panel-diagnostics, which revealed unremarkable findings in the index individual. Segregation analysis in the parents unexpectedly revealed a heterozygous deletion of exons 10-19 in the father and a heterozygous deletion of exons 8-9 in the mother. The mother is unaffected.

Conclusions: The initial diagnosis of NRXN1-associated developmental delay due to a seemingly heterozygous deletion turned into a Pitt-Hopkins-like syndrome 2 caused by a compound-heterozygous deletion in NRXN1. Further genetic testing including segregation analysis are therefore key for patients not phenotypically matching the initial diagnosis, especially in genes where multiple modes of inheritance are known.

A.C. Teichmann: None. M. Stiller: None. S. Schubert: None. S. Redler: None. R. Jamra: None. K. Platzer: None. N. Rahner: None.

\section{P08.062.B}

Comprehensive genetic analysis of intellectual disability patients from the Kashubian population using homozygosity mapping and exome sequencing

A. Charzewska ${ }^{1}$, T. Gambin ${ }^{1}, J$. Wierzba ${ }^{2}$, E. Kaczorowska ${ }^{2}$, E. Obersztyn ${ }^{1}$, B. Wojtas $\hat{s}^{3}$, B. Gielniewski ${ }^{3}$, R. Szlendak ${ }^{1}$, P. Górka-Skoczylas ${ }^{1}$, R. Tataj ${ }^{1}$, K. Kanabus ${ }^{1}$, S. Rzońca-Niewczas ${ }^{l}, J$. Bal ${ }^{l}$, D. Hoffman-Zacharskal

${ }^{1}$ Institute of Mother and Child, Warsaw, Poland, ${ }^{2}$ Medical University of Gdańsk, Gdansk, Poland, ${ }^{3}$ Nencki Institute of Experimental Biology, Warsaw, Poland

Introduction: Autosomal recessive intellectual disability (ARID) is the most common form of intellectual disability (ID) in populations with frequent parental consanguinity. However, ARID is extremely heterogeneous, and the vast majority of ARID genes are still unknown. The main research hypothesis of the study was the assumption that patients with ID belonging to the isolated Kashubian population have, because of parental consanguinity, homozygous regions in the genome where pathogenic, recessive mutations are present. 
Materials and Methods: The project included 45 families of Kashubian origin. There were 64 patients diagnosed with ID in this group. To identify regions where pathogenic, homozygous mutations may be present, an innovative, computational method combining homozygosity mapping and exome sequencing was used. In this method homozygous regions were indicated by coverage analysis involving biallele frequency calculation, transformation and segmentation.

Results: No evident homozygous regions were identified in patients of Kashubian origin. Mutations responsible for ARID were rare. It has been shown that the molecular basis of ID in this group is not ARID, but autosomal dominant ID and X-linked ID. Overall, clearly pathogenic variants have been identified in $29 \%$ of examined families.

Conclusions: This project was the first in Poland, and one of the first in Europe aiming to identify recessive risk factors involved in the pathogenesis of ID based on genomic data obtained by testing an isolated European population. The molecular basis of ID in the group of Kashubian patients proved to be not autosomal recessive, but autosomal dominant and X-linked ID.

Grant Number: NCN 014/15/D/NZ5/03426

A. Charzewska: None. T. Gambin: None. J. Wierzba: None. E. Kaczorowska: None. E. Obersztyn: None. B. Wojtaś: None. B. Gielniewski: None. R. Szlendak: None. P. Górka-Skoczylas: None. R. Tataj: None. K. Kanabus: None. S. Rzońca-Niewczas: None. J. Bal: None. D. Hoffman-Zacharska: None.

\section{P08.063.C}

Identification the role of TUB in Intellectual Disability using RNA-seq

\section{F. Peymani ${ }^{1}$, K. InanlooRahtloo ${ }^{2}$}

${ }^{1}$ Genetic Research Center, University of social welfare and Rehabilitation, Sciences, Tehran, Iran, Islamic Republic of, ${ }^{2}$ School of Biology, University College of Science, University of Tehran, Tehran, Iran, Islamic Republic of

Intellectual disability (ID) is a genetically and phenotypically heterogeneous disorder with more than 1000 candidate genes to date. Despite the accelerating rate of gene discovery for ID, the molecular mechanisms underlying the disease remain elusive. In a previous study, whole exome sequencing in a consanguineous family with five affected individuals representing ID led to the identification of a splice site variant (NM_003320:c.1281+1G>A) in TUB, a novel ID candidate gene, whose mutations have already been associated with Retinal dystrophy and obesity. TUB acts both as a membrane bound transcription regulator and an adaptor molecule in insulin signaling and GPCR trafficking in neuronal cilia. In order to investigate TUB role in the etiology of ID and identify dysregulated molecular pathways in patients, we conducted whole blood transcriptome profiling of patients and their age and sex matched healthy individuals, using RNA-seq technique. The transcription profile demonstrates deregulation of 1045 genes ( $p$-value $<0.01$, fold change $>1.5$ ), of these 155 genes were upregulated and 890 genes were downregulated. Upregulated genes show enrichment in Insulin synthesis and processing, diabetes and metabolism pathways and the molecular function of downregulated genes mainly include constituent of ribosome, transcription regulator and DNA binding. Signaling pathways involved in the regulation of translation and insulin synthesis and processing were amongst the most significant dysregulated pathways in patients. Here, we put an emphasis on the role of insulin and translation pathways in the molecular pathophysiology of ID. This study was funded by National Institute For Medical Research Development (NIMAD).

\section{F. Peymani: None. K. InanlooRahtloo: None.}

\section{P08.064.A}

Identification and functional characterization of a novel homozygous mutation in KCNMA1 encoding voltage and calcium sensitive potassium channel is associated with dyskinesia, epilepsy, intellectual disability, cerebellar and corticospinal tract atrophy

\author{
E. Yucesan ${ }^{1}$, B. Goncu ${ }^{2}$, A. Aslanger ${ }^{3}, C$. Ozgul $^{4}, S$.

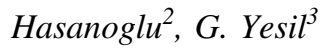

${ }^{1}$ Bezmialem Vakif University, Faculty of Medicine, Department of Medical Biology, Istanbul, Turkey, ${ }^{2}$ Bezmialem Vakif University, Experimental Research Center, Istanbul, Turkey, ${ }^{3}$ Bezmialem Vakif University, Faculty of Medicine, Department of Medical Genetics, Istanbul, Turkey, ${ }^{4}$ Medipol University, Regenerative and Restorative Medical Research Center, Istanbul, Turkey

Introduction: KCNMA1 encodes, alpha subunit of the voltage, and calcium-sensitive potassium channel, predominantly expressed in the central nervous system. Therefore abnormal function in this gene may occur neurological conditions.

Materials and Methods: We report 15 year-old patient who was born at term with healthy conditions. Motor signals were delayed, and also seizures started at the age of 18 months. EEG revealed generalized spike-wave activities. Brain MRI performed, atrophy of the cerebellum was detected. Recent clinical examination;contractures on the large joints, and dyskinetic tremor. Whole exome sequencing (WES) was performed and in-slico analyses were conducted. MCF7 and 293T cells transfected with either 
wild-type or mutant expression vectors. Cellular distribution was determined by immunofluorescence. Functional analysis was performed using electrophysiological approach based on whole-cell patch-clamp.

Results: WES revealed homozygous variation (NM_001161352.1:c.1372C>T, p.Arg458Ter). The variant was not observed in publicly available or in-house databases. Immunofluorescent staining revealed that novel variant is not interfering with synthesis of KCNMA1 however mutation exhibit dominant-negative effect on cell viability when compared to wild-type. 293T and MCF7 cells transfected with homozygous p.Arg458Ter mutation showed markedly increased KCNMA1 currents compared to controls on patch clamp recording, and these data support loss-of-function effect of all KCNMA1 mutants.

Conclusions: Herein we report a 15 -year old boy who has neurological conditions. A novel homozygous stop-gain mutation detected by WES and confirmed by conventional sequencing. Afterwards, functional characterization was conducted using two step-approach, immunostaining to detect subcellular effect of the variation and patch-clamp to detect difference between mutant vs. wild-type of the protein. Homozygous mutation was considered as causative for this clinical condition.

This study was supported by Bezmialem Vakif University, Scientific Research Projects Unit, Project No: 2.2019/7

E. Yucesan: None. B. Goncu: None. A. Aslanger: None. C. Ozgul: None. S. Hasanoglu: None. G. Yesil: None.

\section{P08.065.B}

A next generation sequencing solution to detect copy number variants, single nucleotide variants and loss of heterozygosity in intellectual disability and developmental delay samples

J. Reid, S. Kachhia, P. Dougall, J. Shovelton, D. Molha, J. Kasturiarachchi, J. Holdstock, E. Marek, V. Pullabhatla, L. Parkes, D. Hurd

\section{Oxford Gene Technology, Oxford, United Kingdom}

The detection of copy number variants (CNVs) in intellectual disability (ID) and developmental delay (DD) samples is crucial in elucidating the genetic cause of abnormality. We have developed a targeted NGS panel and analytical software to accurately detect $\mathrm{CNVs}$, as well as SNVs, indels and LOH.

The assay uses a bait capture approach, which is able to capture the exons and untranslated regions (UTRs) from over 700 genes, chosen for their relevance in ID/DD, as well as a range of backbone regions across the genome.
Combined with the CNV detection algorithm in the software, both intragenic and large 'backbone' CNVs can be confidently detected.

We implemented a web-based data analysis solution that runs OGT's NGS analysis pipeline, comprising many stateof-the-art open-source NGS software tools. These tools were carefully chosen and deployed using containers to ensure cross-platform compatibility and reproducibility. Pipeline optimisation and performance was assessed using equivalent array data and reference materials.

We will outline the results from over 200 intellectual disability and developmental research samples to demonstrate the efficiency of the CNV, SNV and LOH detection. The study demonstrated that the assay automatically called 97\% of CNVs (including small intragenic CNVs), $99 \%$ of LOHs over $5 \mathrm{Mb}$ and $100 \%$ of SNVs. The assay was able to call CNV deletions as small as $1.42 \mathrm{~kb}$ and CNVs in samples with mosaicism as low as $10 \%$. We have described an improved method to investigate ID/DD, providing critical information on not just CNVs, but SNVs and Indels as well.

J. Reid: A. Employment (full or part-time); Significant; Oxford Gene Technology. S. Kachhia: None. P. Dougall: A. Employment (full or part-time); Significant; Oxford Gene Technology. J. Shovelton: None. D. Molha: None. J. Kasturiarachchi: None. J. Holdstock: None. E. Marek: None. V. Pullabhatla: None. L. Parkes: None. D. Hurd: A. Employment (full or part-time); Significant; Oxford Gene Technology.

\section{P08.066.C}

Clinical and genetic aspects in 26 patients with KBG syndrome caused by mutation of ANKRD11.

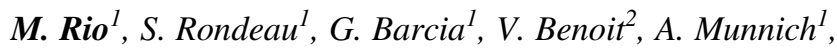 \\ C. Ormières ${ }^{l}$, A. Guimier ${ }^{l}$, S. Marlin ${ }^{l}$, J. Amiel $^{l}, S$. \\ Lyonnet $^{l}$, G. Baujat ${ }^{l}$, N. Garcelon $^{2}$, V. Cormier-Daire ${ }^{l}$ \\ ${ }^{1}$ Department of genetics, Necker Hospital, Assistance \\ Publique - Hôpitaux de Paris, Paris, France, ${ }^{2}$ Data Science \\ plateform, IMAGINE Institut, Université Paris Descartes, \\ Sorbonne Paris Cité,, Paris, France
}

Normal 021 false false false FR X-NONE X-NONE /* Style Definitions */ table.MsoNormalTable \{mso-stylename:"Tableau Normal"; mso-tstyle-rowband-size:0; msotstyle-colband-size:0; mso-style-noshow:yes; mso-stylepriority:99; mso-style-parent:""; mso-padding-alt:0cm 5.4pt $0 \mathrm{~cm} \quad 5.4 \mathrm{pt}$; mso-para-margin-top:0cm; mso-para-marginright:0cm; mso-para-margin-bottom:10.0pt; mso-para-margin-left:0cm; line-height:115\%; mso-pagination:widoworphan; font-size:11.0pt; font-family:"Calibri","sans-serif"; mso-ascii-font-family:Calibri; mso-ascii-theme-font:minor- 
latin; mso-hansi-font-family:Calibri; mso-hansi-theme-font: minor-latin; mso-fareast-language:EN-US; $\}$ KBG syndrome (OMIM 148050), first described in 1975, is a rare autosomal dominant disorder characterized by distinctive facial features, developmental delay, short stature, and skeletal anomalies. In 2011, heterozygous mutations in the ANKRD11 gene were identified in patients with KBG syndrome. Since then, 200 cases have been described expanding the clinical phenotype. Here we present 26 unrelated patients with pathogenic mutations in ANKRD11. We compare these patients with previously reported cases to further expand and update the phenotypic and mutational spectrum of this condition. The mutation occurred de novo in the 22 families where parental DNA was available and was inherited from a parent in two patients. Age at diagnosis ranged from 7 to 58 years. Developmental delay was reported in $94 \%$ of the patients. Intellectual disability was not a constant finding. Three patients attended regular school. $68 \%$ of patients have behavioral disorders, and $30 \%$ of patients have methylphenidate therapy for attention deficit hyperactivity disorder. Gastrointestinal findings were common. $56 \%$ of patients had deafness. This study allowed us to confirm clinical features already reported in the literature (deafness, feeding difficulties, cerebellar atrophy). In addition, agenesis of the olfactory bulbs, a sign not described in the literature to our knowledge, was identified in 3 patients.

M. Rio: None. S. Rondeau: None. G. Barcia: None. V. Benoit: None. A. Munnich: None. C. Ormières: None. A. Guimier: None. S. Marlin: None. J. Amiel: None. S. Lyonnet: None. G. Baujat: None. N. Garcelon: None. V. Cormier-Daire: None.

\section{P08.067.A}

Pathogenic variants in $\mathrm{KCNQ} 2$ cause intellectual deficiency without epilepsy: broadening the phenotypic spectrum of a potassium channelopathy.

E. Schaefer ${ }^{1}$, L. Mary ${ }^{2}$, E. Nourrisson ${ }^{2}$, C. Feger ${ }^{2}$, B.

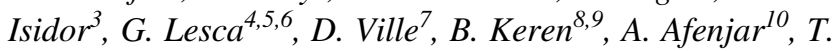
Billette $^{11}$, D. Trost ${ }^{12}$, C. Cieuta-Walti ${ }^{13}$, J. Mandel ${ }^{14,2}$, B. Gérard $^{2}$, A. Piton ${ }^{2,14}$

${ }^{1}$ Service de Génétique Médicale, Hôpitaux Universitaires de Strasbourg, Institut de Génétique Médicale d'Alsace, Strasbourg, France, ${ }^{2}$ Laboratoire de Diagnostic Génétique, Hôpitaux Universitaires de Strasbourg, Strasbourg, France, ${ }^{3}$ Service de génétique médicale, Unité de Génétique clinique, CHU de Nantes, Nantes, France, ${ }^{4}$ Service de Génétique, Hôpitaux Universitaires de Lyon, Lyon, France, ${ }^{5}$ Université Claude Bernard Lyon 1, Lyon, France, ${ }^{6}$ Centre de recherche en Neuoscience, CNRS UMRS5292, INSERM U1028, Lyon, France, ${ }^{7}$ Service de Neurologie Pédiatrique,
Hôpital Femme Mere Enfant, Centre Hospitalier Universitaire de Lyon, Lyon, France, ${ }^{8}$ Service de Génétique, Université Pierre et Marie Curie, Hôpital de la PitiéSalpêtrière, Paris, France, ${ }^{9}$ Université Pierre et Marie Curie (Université Paris 06), UMRS 1127, INSERM U 1127, CNRS UMR 7225, Institut du Cerveau et de la Moelle Épinière, Paris, France, ${ }^{10}$ Unité de Génétique Clinique, Hôpital Armand Trousseau, Groupe Hospitalier Universitaire de l'Est Parisien, Paris, France, ${ }^{11}$ Service de Neuropédiatrie, Hôpital Armand Trousseau, Groupe Hospitalier Universitaire de l'Est Parisien, Paris, France, ${ }^{12}$ Laboratoire Cerba, Saint-Ouen-l'Aumône, France, ${ }^{13}$ Institut Jérôme Lejeune, Paris, France, ${ }^{14}$ Institut de Génétique et de Biologie Moléculaire et Cellulaire, CNRS UMR-7104, Inserm U964, Université de Strasbourg, Strasbourg, France

High-throughput sequencing improved the molecular diagnosis in individuals with intellectual deficiency (ID) and, through its unbiased approach, to broaden the clinical spectra associated with each gene involved. We report herein 6 patients with isolated intellectual disability (ID), carriers of a likely pathogenic variant in $K C N Q 2$, a gene usually implicated in benign familial neonatal seizures (BFNS) or early-onset epileptic encephalopathy (EOEE). Patients were diagnosed either by targeted HTS (panel of 450-556 epilepsy- and/or ID-related genes) or exome sequencing. Pathogenicity of the variants and predictions of proteic structural changes were assessed by multiple in silico tools. All patients were children at diagnosis. Patients' ID ranged from mild to severe with predominance of speech disturbance and autistic features. All patients carried a heterozygous likely pathogenic missense variant in $K C N Q 2$, 4 of them disrupted the same amino-acid (Arg144, previously reported in EOEE). We compiled all the pathogenic variants previously reported (>130). We observed that missense variants in the voltage-sensing domain and the pore are significantly more associated to EOEE than BFNS ( $\mathrm{p}<0.01$, Fisher test). However, there was a strong overlap between variants causing EOEE, isolated ID and BFNS and an important intra-familial phenotypic variability. To conclude, pathogenic variants in $K C N Q 2$ can be associated with isolated ID. We did not highlight strong correlations between $K C N Q 2$-related phenotypes and the associated variants' localization. A second genetic hit, a burden of rare variants or other extrinsic factors may explain such a phenotypic variability. However, it is of interest to study encephalopathy genes in non-epileptic ID patients.

E. Schaefer: None. L. Mary: None. E. Nourrisson: None. C. Feger: None. B. Isidor: None. G. Lesca: None. D. Ville: None. B. Keren: None. A. Afenjar: None. T. 
Billette: None. D. Trost: None. C. Cieuta-Walti: None. J. Mandel: None. B. Gérard: None. A. Piton: None.

P08.068.B

Brain-on-a-chip: altered neuronal network development in neural cells derived from patients with intellectual disability

N. Nadif Kasri, M. Frega, B. Mossink, K. Linda, H. van Bokhoven

Radboudumc, Nijmegen, Netherlands

Great progress has been made over recent years towards the identification of intellectual disability (ID)-related genes, resulting in hundreds of genes. A remaining challenge, however, is to connect the genetic causes of ID to processes that establish and/or modify neuronal circuit function. The recent developments in induced pluripotent stem cells (iPSCs) have provided us with the ability to model patientspecific neuronal networks. We studied the development of neuronal networks of iNeurons derived from patients with Kleefstra Syndrome, Koolen-de Vries Syndrome, and Kabuki Syndrome. These are three well-described intellectual disability syndromes caused by mutations in different histone modifiers, respectively in EHMT1, KANSLland $K M T 2 D$. Little is known as to how disruption in these genes lead to altered neuronal network development. Here, we monitored the electrophysiological activity of neuronal networks coupled to multi-electrode arrays (MEAs) over time and compared their activity to healthy controls and between syndromes. Interestingly, independent clustering analysis revealed that all tested patient lines deviated from healthy control lines, which functionally clustered together. Whereas Koolen-de Vries patient lines showed a general reduced level of activity, Kleefstra syndrome and Kabuki syndrome patient lines were mainly characterized by changes in neuronal network burst activity and organisation. Interestingly, patient lines from the same syndrome phenotypically clustered together, but separately between syndromes. These data indicate that neuronal network measurement of iNeurons on MEAs is a robust and sensitive method to perform genotype-phenotype analyses for NDDs and can be a powerful platform for drug screening assays

N. Nadif Kasri: None. M. Frega: None. B. Mossink: None. K. Linda: None. H. van Bokhoven: None.

\section{P08.069.C}

Novel missense mutation of the $K M T 2 D$ gene in a girl with Kabuki syndrome
S. Riedel, M. Linné, B. Eichhorn

MVZ Mitteldeutscher Praxisverbund Humangenetik GmbH, Dresden, Germany

Introduction: Kabuki syndrome (KS) is a rare congenital disorder characterized by typical facial features, postnatal growth deficiency, intellectual disability, skeletal anomalies and cardiac defects with variable expressivity. Mutations in the KMT2D gene (lysine-specific methyltransferase 2D) and the KDM6A gene (lysine-specific demethylase 6A) have been identified as the main cause of KS. Both genes are involved in cellular differentiation processes during embryonic development. Here, we report a $4^{10 / 12}$-year old girl with the clinical diagnosis of $\mathrm{KS}$ due to typical facial dysmorphism, slightly reduced head circumference and speech delay.

Materials and Methods: We performed multi-gene panel testing via sequencing by synthesis, which includes among others the two core genes KMT2D and KDM6A associated with KS.

Results: We revealed a novel missense variation c.15869G >A / p.(Gly5290Asp) in exon 49 of the KMT2D gene in the index patient. This mutation occurred de novo, since it was absent in the clinically unaffected parents.

Conclusions: The majority of mutations (about $87 \%$ ) causing KS produce premature termination codons. However, the role of missense mutations remains weakly explored, although they may be disease-relevant dependings on the localization in the gene. The detected missense variant is situated in the functional "FY-rich" domain Cterminal (FYRC) of the protein, suggesting that it may affect function. It has previously not been annotated in GnomAD or Ensembl and is classified as "probably damaging" by several prediction data bases like PolyPhen-2. There is also a strong evidence for the disease-causing effect due to de novo occurrence and the patient's phenotype.

S. Riedel: None. M. Linné: None. B. Eichhorn: None.

\section{P08.070.A}

Refining the phenotypic \& mutational spectrum in a multinational cohort of O'Donnell-Luria-Rodan Syndrome

C. Velmans ${ }^{1}$, A. O'Donnell-Luria ${ }^{2,3}$, E. Argillit ${ }^{4}, F$. TranMau-Them ${ }^{5}$, A. Vitobello ${ }^{5}$, M. Rech ${ }^{6}$, A. Abicht ${ }^{7}$, M. Aubert-

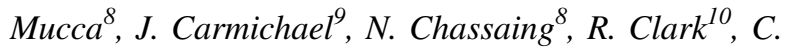
Coubes $^{11}$, K. de Dios ${ }^{12}$, B. Funalot ${ }^{13}$, M. Joseph ${ }^{9}$, C. Kenendy ${ }^{9}$, I. van de Laar ${ }^{14}$, D. Lehalle ${ }^{13}$, K. Leppig ${ }^{15}$, L. 


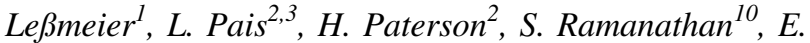

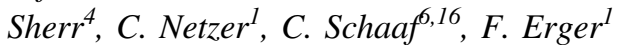

${ }^{1}$ Institute of Human Genetics, University of Cologne, Faculty of Medicine and University Hospital Cologne, Cologne, Germany, ${ }^{2}$ Manton Center for Orphan Disease Research, Boston Children's Hospital, Harvard Medical School, Boston, MA, United States, ${ }^{3}$ Program in Medical and Population Genetics, Broad Institute of MIT and Harvard, Cambridge, MA, United States, ${ }^{4}$ Brain Development Research Program, Department of Neurology, University Hospital California San Francisco, San Francisco, CA, United States, ${ }^{5}$ Inserm UMR 1231 Genetics of Developmental Disorders, FHU-TRANSLAD, University Hospital Dijon-Bourgogne, Dijon, France, ${ }^{6}$ Molecular and Human Genetics, Baylor College of Medicine, Houston, TX, United States, ${ }^{7}$ Medical Genetics Center (MGZ), Munich, Germany, ${ }^{8}$ Department of Medical Genetics, University Hospital Toulouse, Toulouse, France, ${ }^{9}$ Valley Children's Hospital, Madera, CA, United States, ${ }^{10}$ Pediatrics Specialty Clinics, Loma Linda University Medical Center, San Bernardino, CA, United States, ${ }^{11}$ Department of Medical Genetics, University Hospital Montpellier, Montpellier, France, ${ }^{12}$ Department of Medical Genetics, Dayton Children's Hospital, Dayton, OH, United States, ${ }^{13}$ Department of Clinical Genetics, University Hospital Henri Mondor, Créteil, France, ${ }^{14}$ Department of Clinical Genetics, Erasmus University Medical Center, Rotterdam, Netherlands, ${ }^{15}$ Genetic Services, Kaiser Permanente of Washington, Seattle, WA, United States, ${ }^{16}$ Institute of Human Genetics, University of Heidelberg, Faculty of Medicine and University Hospital Heidelberg, Heidelberg, Germany

Introduction: The $K M T 2 E$-associated neurodevelopmental disorder named O'Donnell-Luria-Rodan Syndrome (ODLURO) was first described by O'Donnell-Luria et al. in $2019^{1}$. This previous study included 38 patients with heterozygous, mostly truncating de novo variants in $K M T 2 E$ or microdeletions disrupting the gene. We present the second case series of patients with ODLURO, refining the mutational and phenotypic spectrum of this not-so-rare syndrome.

Results: We report 13 additional patients with ODLURO from clinical centers in Europe and the US. We detected ten heterozygous nonsense or frameshifting variants, two with predicted deleterious effects on splicing, and one microdeletion encompassing $>90 \%$ of KMT2E's coding sequence (eleven changes confirmed de novo). Eleven variants and the microdeletion are novel, one has been previously observed $^{1}$. We confirm and refine the phenotypic spectrum of the $K M T 2 E$-related neurodevelopmental disorder, especially concerning developmental delay with rather mild intellectual disability and macrocephaly with subtle facial features in most patients. In contrast to the study of O'Donnell-Luria et al. ${ }^{21}$, the rate of autism in our cohort was higher with $\sim 40 \%$, whereas seizures were reported in only a single patient with recurring febrile seizures. Furthermore, we describe additional anomalies and symptoms reported only in one or two patients, for which a potential causal relation to the disruption of $K M T 2 E$ remains to be investigated. Our study, bringing the total of known patients to more than 50 within a year of the first publication, also illustrates a surprisingly high relative frequency of ODLURO worldwide.

Footnotes $^{1}$ O'Donnell-Luria et al., Am J Hum Genet. 2019 Jun 6; 104(6): 1210-1222

C. Velmans: None. A. O'Donnell-Luria: None. E. Argilli: None. F. Tran-Mau-Them: None. A. Vitobello: None. M. Rech: None. A. Abicht: None. M. AubertMucca: None. J. Carmichael: None. N. Chassaing: None. R. Clark: None. C. Coubes: None. K. de Dios: None. B. Funalot: None. M. Joseph: None. C. Kenendy: None. I. van de Laar: None. D. Lehalle: None. K. Leppig: None. L. Leßmeier: None. L. Pais: None. H. Paterson: None. S. Ramanathan: None. E. Sherr: None. C. Netzer: None. C. Schaaf: None. F. Erger: None.

\section{P08.071.B}

Minimal 239-kb microdeletion spanning $K M T 2 E$ in a boy with neurodevelopmental disorder.

\section{K. Varvagiannis ${ }^{1,2}$, K. Kosma ${ }^{2}$, A. Mitrakos ${ }^{2}$, J. Traeger-} Synodinos $^{2}$, M. Tzetis ${ }^{2}$

${ }^{1}$ Biomedical Research Foundation Academy Of Athens, Athens, Greece, ${ }^{2}$ Department of Medical Genetics, Medical School, National and Kapodistrian University of Athens, Athens, Greece

Background: Disruptive $K M T 2 E$ variants have been recently reported to cause a neurodevelopmental disorder characterized by developmental delay (DD), intellectual disability (ID) and subtle dysmorphism. Epilepsy, autism and macrocephaly are part of the phenotype in a subset of the affected individuals.

The occurrence of truncating variants in most of these subjects and the overlapping features observed in cases with microdeletions spanning this gene suggest haploinsufficiency as the mechanism underlying this type of variants.

Clinical description and molecular findings: A male was referred for genetic evaluation at the age of 2 months in the context of prenatal-onset hydronephrosis, DD and dysmorphic features. Investigation with chromosomal microarray (CMA) revealed a 239-kb microdeletion of 7q22-q23 spanning 2 protein coding genes, namely KMT2E 
and LHFPL3. The deletion was classified as VUS at the time of initial reporting.

Re-evaluation of the CMA result upon last examination of the child at the age of 3 years and 3 months, resulted in reclassification of the variant to likely pathogenic. Similarly to other individuals harboring variants affecting $K M T 2 E$, our proband presented DD, dysmorphic features albeit without occurrence of autistic features, epilepsy or abnormal growth parameters. Review of his medical records revealed persistence of hydronephrosis without associated anomalies of the urogenital tract. The boy had extensive work-up for severe constipation, raising suspicion of possible Hirschsprung disease.

Conclusions: We provide a detailed clinical description of our proband, focusing on ill-defined previously reported issues such as bowel dysmotility while extending the phenotypic spectrum of the disorder with additional features such as hydronephrosis.

K. Varvagiannis: None. K. Kosma: None. A. Mitrakos: None. J. Traeger-Synodinos: None. M. Tzetis: None.

P08.072.C

MEA-seq for combined gene expression and neuronal network measurements in iPSC-derived neurons from Koolen-de Vries patients

A. H. A. Verboven, K. Linda, M. Hogeweg, C. A. Albers, B. B. A. de Vries, P. A. C. 't Hoen, N. Nadif Kasri

\section{Radboudumc, Nijmegen, Netherlands}

Koolen-de Vries Syndrome (KdVS) is an intellectual disability syndrome caused by haploinsufficiency of KANSL1, due to a loss-of-function mutation in KANSL1 or a microdeletion in region $17 q 21.31$. To study KdVS in vitro, we generated induced pluripotent stem cells (iPSCs) from fibroblasts of three $\mathrm{KdVs}$ patients and three healthy controls. Additionally, we introduced a loss-of-function mutation in KANSL1 in one control line using the CRISPR/ Cas9 system. For all cell lines, iPSCs were differentiated into neurons (iNeurons) by forced expression of neurogenin-2 (Ngn2) resulting in a homogeneous population of mature excitatory neurons within 30 days. MEA-seq was performed to measure neuronal activity of iNeurons using micro-electrode arrays (MEAs), followed by RNA sequencing (RNA-seq). The RNA-seq method used allows us to screen many samples at low cost by barcoding and pooling samples during the library preparation. MEA measurements revealed a decreased network burst rate and more irregular firing pattern in KdVS iNeurons. RNA-seq was performed on these iNeurons as well as on iPSCs from the same individuals. Differential expression (DE) analysis was performed to identify changes in gene expression in
KdVS patients compared to controls. Gene set enrichment analysis (GSEA) revealed cell type-specific enrichment of up- and down-regulated genes in gene sets involved in processes such as cell cycle regulation, cellular respiration and early development. These results show that MEA-seq allows for characterization of iPSC-derived neurons both on a functional and transcriptional level, and therefore is a powerful tool to study mechanisms underlying neurodevelopmental disorders.

A.H.A. Verboven: None. K. Linda: None. M. Hogeweg: None. C.A. Albers: None. B.B.A. de Vries: None. P. A.C. 't Hoen: None. N. Nadif Kasri: None.

\section{P08.073.A}

Mutations in $K P T N$, a rare cause of macrocephaly and intellectual disability

M. Pacio Miguez ${ }^{1}$, F. Santos Simarro ${ }^{1,2}$, S. GarcíaMiñaúrl, C. Rodríguez Jiménez ${ }^{1}$, Á. del Pozo ${ }^{1,2}$, M. Solís ${ }^{1}$, R. Velázquez Fragua ${ }^{3}$, V. Rufo-Rabadán ${ }^{1}$, V. Fdez Montaño ${ }^{1}$, I. Rueda Arenas ${ }^{1}$, M. Gomez del Pozo ${ }^{1}, N$. Gallego Onís ${ }^{1}$, M. Palomares Bralo ${ }^{1,2}$

${ }^{1}$ Instituto de Genética Médica y Molecular, INGEMM, Madrid, Spain, ${ }^{2}$ CIBERER, Centro de Investigación Biomédica en Red de Enfermedades Raras, ISCIII, Madrid, Spain, Spain, ${ }^{3} 3$ Servicio de Neurología Infantil. Hospital Universitario La Paz, Madrid, Spain

Introduction: Pathogenic homozygous or compound heterozygous variants in KPTN have emerged as a novel autosomal recessive neurodevelopmental disorder associated to developmental delay, intellectual disability, macrocephaly and language deficit with only 13 individuals described in the medical literature so far. We report two additional siblings with a homozygous pathogenic variant in the KPTN gene.

Materials and Methods: Variants were identified using a NGS custom panel containing 1663 genes involved in neurodevelopmental disorders (RD-Seq (C) V6.0).

Results: A homozygous dinucleotide duplication Chr19 (GRCh37):g.47984018_47984019dup; NM_007059.3: c.597_598dup; p.(Ser200Ilefs*55) in KPTN was found in a 7-year-old girl and her 3-year-old sister born to nonconsanguineous healthy parents with no family history of note. Both presented with developmental delay, macrocephaly, frontal bossing, language deficit and delayed anterior fontanel closure. Brain MRI revealed anatomical changes in one. None of them had seizures or behavioral problems. A clinical description our cases and a review of the previously reported individuals will be provided.

Conclusions: Cardinal features of KPTN-related disease observed in all 15 cases are developmental delay 
/intellectual disability, language deficit, macrocephaly (of prenatal or postnatal origin) and frontal bossing. Variable clinical features include seizures, behavioral problems, craniosynostosis, brain MRI changes and endocrine abnormalities. In summary, homozygous or compound heterozygous variants in KPTN may be a hitherto underrecognized cause of macrocephaly, developmental delay, intellectual disability and language deficit. We suggest including KPTN in gene panels for the study of patients with these features.

M. Pacio Miguez: None. F. Santos Simarro: None. S. García-Miñaúr: None. C. Rodríguez Jiménez: None. Á. del Pozo: None. M. Solís: None. R. Velázquez Fragua: None. V. Rufo-Rabadán: None. V. Fdez Montaño: None. I. Rueda Arenas: None. M. Gomez del Pozo: None. N. Gallego Onís: None. M. Palomares Bralo: None.

\section{P08.074.B}

The simultaneous presence of microcephaly, seizures, and developmental delay due to PNKP gene mutation and infantile hypophosphatasia caused by mutation in ALPL gene

\section{Sansovic, A. Bobinec, A. Measic, M. Kero, I. Barisic}

Department of Medical Genetics and Reproductive Health, Children's Hospital Zagreb, Scientific Centre of Excellence for Reproductive and Regenerative Medicine (CERRM), University of Zagreb School of Medicine, Zagreb, Croatia

Introduction: Microcephaly, seizures, and developmental delay (MCSZ; MIM \#613402) is neurodevelopmental disorder with onset in infancy caused by homozygous or compound heterozygous mutations in the PNKP gene. There is a range of phenotypic severity: some patients have early infantile epileptic encephalopathy (EIEE), whereas others have more well-controlled seizures and a protracted course. Infantile hypophosphatasia (MIM \#241500) is caused by a pathogenic mutation in the ALPL gene characterized by defective bone mineralization, a combination of typical radiological signs, hypercalcemia, hyperphosphatemia, and low alkaline phosphatase activity. We describe a family with three affected children born of allegedly nonconsanguineous parents: a twelve-year-old boy with microcephaly, craniosynostosis, EIEE, patchy/ polymicrogyria, polyneuropathy and global developmental delay (MCSZ), his eleven-year-old sister with infantile hypophosphatasia and nine-year-old sister with infantile hypophosphatasia, microcephaly, global developmental delay and paroxysmal seizures with vomiting.

Method: Clinical exome sequencing was performed in all family members using Illumina TruSight One Kit.
Results: A boy with MCSZ has a homozygous c.1253_1269dup17 pathogenic variant in the PNKP gene, the older sister has homozygous c.542C $>\mathrm{T}$ pathogenic variant in ALPL gene and the younger sister has homozygous pathogenic variants in both genes.

Conclusions: The simultaneous presence of infantile hypophosphatasia and MCSZ has never been reported before. The overlapping clinical features of infantile hypophosphatasia and MCSZ (hypotonia, craniosynostosis, seizures, short stature, failure to thrive) as well as the milder clinical course of MCSZ, resulted in delayed diagnosis of MCSZ in the female patient.

Acknowledgement: supported by CERRM and EU through the European Regional Development Fund by grant No. KK.01.1.1.01.0008.

I. Sansovic: None. A. Bobinec: None. A. Measic: None. M. Kero: None. I. Barisic: None.

\section{P08.075.C}

Updated insight into the mutational and phenotypic spectrum of $M E D 13 L$-related intellectual disability

R. Asadollahi ${ }^{1}$, P. Boonsawat ${ }^{l}$, B. Popp ${ }^{2,3}$, E. Torti ${ }^{4}$, I.

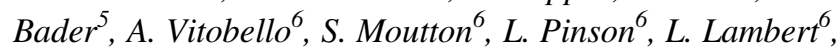
A. C. Thuresson ${ }^{7}$, M. Sobol ${ }^{7}, C$. Soussi Zander ${ }^{7}, K$.

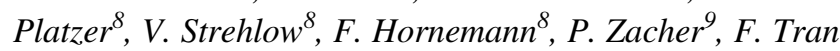
Mau-Them $^{6}$, A. L. Bruel ${ }^{6}$, M. J. Hajianpour ${ }^{10}$, R. KovacsNagy $^{11}$, G. Lay-Son ${ }^{12}$, L. Amlie-Wolf ${ }^{13}$, J. Kaplan ${ }^{14}$, A. Chassevent $^{15}$, C. Smith-Hicks ${ }^{16}$, A. Slavotinek ${ }^{17}$, M. K. Kukolich $^{18}$, K. Nugent ${ }^{19}$, E. Roeder ${ }^{19}$, Y. A. Zarate ${ }^{20}, Y$. Toshiyuki ${ }^{21}$, C. Jackel-Cram ${ }^{22}$, I. Maystadt ${ }^{23}$, S. G. Mehta ${ }^{24}$, T. A. Briggs ${ }^{25}, K$. Chandler ${ }^{25}$, A. van Haeringen ${ }^{26}, C$.

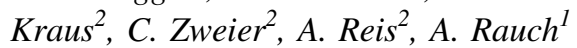

${ }^{1}$ University of Zurich, Zurich, Switzerland, ${ }^{2}$ FriedrichAlexander-Universität Erlangen-Nürnberg, Erlangen, Germany, ${ }^{3}$ University of Leipzig Hospitals and Clinics, Leipzig, Germany, ${ }^{4}$ GeneDx, Gaithersburg, MD, United States, ${ }^{5}$ Paracelsus Medical University, Salzburg, Austria, ${ }^{6}$ Inserm-Université Bourgogne-Franche Comté, Dijon, France, ${ }^{7}$ Uppsala University, Uppsala, Sweden, ${ }^{8}$ University of Leipzig Medical Center, Leipzig, Germany, ${ }^{9}$ Saxon Epilepsy Center Kleinwachau, Radeberg, Germany, ${ }^{10}$ East Tennessee State University, Tennessee, TN, United States, ${ }^{11}$ Semmelweis University, Budapest, Hungary, ${ }^{12}$ Pontificia Universidad Catolica de Chile, Santiago, Chile, ${ }^{13}$ Nemours A.I. Dupont Hospital for Children, DE, USA, Wilmington, DE, United States, ${ }^{14}$ Nemours A.I. Dupont Hospital for Children, Wilmington, DE, United States, ${ }^{15}$ Kennedy Krieger Institute, Baltimore, MD, United States, ${ }^{16}$ Johns Hopkins Medical Institutions, Baltimore, MD, United States, ${ }^{17}$ University of California, San Francisco, CA, United States, ${ }^{18}$ Cook Children's Medical Center, Fort 
Worth, TX, United States, ${ }^{19}$ Baylor College of Medicine, Houston, TX, United States, ${ }^{20}$ University of Arkansas for Medical Sciences, Little Rock, AR, United States, ${ }^{21}$ Tokyo Women's Medical University, Tokyo, Japan, ${ }^{22}$ Royal University Hospital, Saskatoon, SK, Canada, ${ }^{23}$ Centre de Génétique Humaine, Gosselies, Belgium, ${ }^{24}$ Cambridge University Hospitals NHS Foundation Trust, Cambridge, United Kingdom, ${ }^{25}$ University of Manchester, Manchester, United Kingdom, ${ }^{26}$ Leiden University Medical Center, Leiden, Netherlands

MED13L-related intellectual disability (MIM \#616789) is an autosomal dominant neurodevelopmental syndrome with $\sim 80$ cases reported so far. To further delineate its spectrum, we have studied 28 clinically well-characterized new patients (age 7 months to 31 years) harbouring pathogenic or likely pathogenic (P/LP) MED13L sequence or copy number variants. We found 21 likely gene-disrupting (LGD) de novo variants distributed throughout the gene and 7 deleterious missense variants (5 de novo, 2 unknown inheritance). We observed clustering of our new and previously reported $\mathrm{P} / \mathrm{LP}$ missense variants between the two LxxLL motifs, between Leu-Zipper and Ser-rich motifs, and within the C-terminus of the MED13L protein, which are deprived of missense variants in gnomAD. Clinically, we have been able to ascertain details of developmental milestones and growth parameters, and to find multiple novel features. Remarkably, we noticed previously unreported mild global developmental delay in 3/28 $(\sim 11 \%)$ and verbal dyspraxia in $6 / 28(\sim 21 \%)$ patients harbouring LGD variants. Moreover, we observed a higher frequency of epilepsy ( $\sim 86 \%$ vs. $\sim 29 \%$ ) and severe congenital heart defects requiring surgery ( $29 \%$ vs. $0 \%$ ) in our patients with missense compared to LGD variants. We also report the first $M E D 13 L$ patient with no obvious congenital heart defect who passed away at the age of $\sim 10$ years possibly due to respiratory insufficiency and right-heart failure. Altogether, our findings highlight new aspects of the syndrome and suggest a critical role for the MED13L C-terminus, missense defects of which may even cause a more severe phenotype than MED13L haploinsufficiency.

R. Asadollahi: None. P. Boonsawat: None. B. Popp: None. E. Torti: None. I. Bader: None. A. Vitobello: None. S. Moutton: None. L. Pinson: None. L. Lambert: None. A.C. Thuresson: None. M. Sobol: None. C. Soussi Zander: None. K. Platzer: None. V. Strehlow: None. F. Hornemann: None. P. Zacher: None. F. Tran MauThem: None. A.L. Bruel: None. M.J. Hajianpour: None. R. Kovacs-Nagy: None. G. Lay-Son: None. L. AmlieWolf: None. J. Kaplan: None. A. Chassevent: None. C. Smith-Hicks: None. A. Slavotinek: None. M.K. Kukolich: None. K. Nugent: None. E. Roeder: None. Y.A. Zarate: None. Y. Toshiyuki: None. C. Jackel-Cram:
None. I. Maystadt: None. S.G. Mehta: None. T.A. Briggs: None. K. Chandler: None. A. van Haeringen: None. C. Kraus: None. C. Zweier: None. A. Reis: None. A. Rauch: None.

\section{P08.076.A}

Investigation of the $M M P 2$ haplotype as a risk factor for the development of cerebral palsy

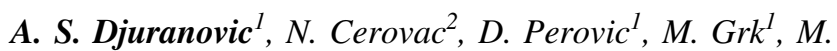
Pesic $^{l}$, T. Damnjanovic ${ }^{l}$

${ }^{1}$ Institute of Human Genetics, Belgrade, Serbia, ${ }^{2}$ Clinic of Neurology and Psychiatry for Children and Youth, Belgrade, Serbia

Introduction: Hypoxic-Ischemic Encephalopathy (HIE) is the most important cause of cerebral palsy (CP). Perinatal brain damage activates the immune system and inflammation mediators. Matrix metalloproteinases (MMPs) are necessary in inflammation and might be involved in neurodegeneration. Aim of this study was to examine possible association between $M M P 2$ promoter polymorphisms and $\mathrm{CP}$ onset in children with perinatal asphyxia.

Material and methods: Study included 96 patients aged 1 to 16 years with anamnesis of perinatal asphyxia. Detailed neurological evaluation and neuroimaging (ultrasound, computed tomography, magnetic resonance imaging) were performed for all subjects. TaqMan assays were used for genotyping rs243864 and rs243866 MMP2 gene polymorphisms. Haplotypes were calculated with Haploview software.

Results: Frequencies of rs243866 and rs243864 haplotypes in patients who developed CP were: $72.2 \%$ GT, $22.3 \% \mathrm{AG}, 2.8 \% \mathrm{GG}$ and $2.7 \% \mathrm{AT}$, while in the group of those who did not develop CP were $89.0 \%$ GT, $10.0 \%$ AG, and $1.0 \%$ GG. AG haplotype was significantly more frequent in patients with $\mathrm{CP}(\mathrm{p}=0.018)$. Also, in patients with AG haplotype, HIE and pathological neuroimaging finding were more frequently observed $(\mathrm{p}=0.012, \mathrm{p}=0.048$, respectively)

Conclusions: $M M P 2$ promoter haplotype could be a risk factor for CP in patients with perinatal asphyxia.

A.S. Djuranovic: None. N. Cerovac: None. D. Perovic: None. M. Grk: None. M. Pesic: None. T. Damnjanovic: None.

\section{P08.077.B}

Uncovering molecular mechanisms and neuronal pathways involved in SETBP1 disorder using human stem cell models 
M. M. K. Wong ${ }^{1}$, J. den Hoed ${ }^{1}$, M.Heide ${ }^{2}$, W. B. Huttner ${ }^{2}$, B. W. van Bon ${ }^{3}$, S. E. Fisher ${ }^{1,4}$

${ }^{1}$ Max Plank Institute for Psycholinguistics, Nijmegen, Netherlands, ${ }^{2}$ Max Planck Institute of Molecular Cell Biology and Genetics, Dresden, Germany, ${ }^{3}$ Radboud University Nijmegen Medical Centre, Nijmegen, Netherlands, ${ }^{4}$ Donders Institute for Brain, Cognition and Behaviour, Radboud University, Nijmegen, Netherlands

Introduction: Haploinsufficiency of the SETBPI gene causes a neurodevelopmental syndrome (SETBP1 disorder) involving expressive speech impairment and mild-moderate developmental delay. The precise functions of SETBP1, encoding the SET-binding protein 1, are yet to be discovered. Therefore, the molecular mechanisms or neuronal pathways by which rare loss-of function SETBP1 mutations cause a neurodevelopmental disorder remain largely unknown.

Materials and Methods: By employing induced pluripotent stem cell (iPSC)-derived brain organoids and transcriptomic approaches, we aim to dissect the underlying neuronal pathways. We will differentiate patient-derived iPSCs carrying de novo heterozygous SETBPI mutations and SETBPl knockout iPSCs generated with CRISPR/Cas9 gene-editing into brain organoids. Their transcriptomic profiles will be analysed at both whole organoid and single-cell levels. Morphological examination, clustering, cell-type specific differential gene expression analysis and cell lineage tracing will be performed.

Results: We have identified six truncating mutations in the SETBP1 gene in a Dutch cohort. We have generated homozygous and heterozygous SETBPl knockout iPSC lines using CRISPR/Cas9 gene-editing. Moreover, we have characterized a robust protocol for generating cerebral organoids using the isogenic control iPSC line that we used to generate SETBPl knockout iPSCs.

Conclusions: These iPSC lines are ready for cerebral organoid differentiation and subsequent downstream analyses. In the long-term, we hope that cell-culture models can provide a platform for testing drug panels with the potential to rescue aberrant molecular/cellular phenotypes that we observe. Together, this work promises to offer insights into fundamental neuronal mechanisms that go awry in SETBP1 disorder and, ultimately, to accelerate development of therapeutic applications.

M.M.K. Wong: None. J. den Hoed: None. M. Heide: None. W.B. Huttner: None. B.W. van Bon: None. S.E. Fisher: None.

\section{P08.080.B}

Smith-Kingsmore Syndrome: a case report and delineation of phenotype
D. J. Josifova ${ }^{1}$, R. Marcinkute ${ }^{1}$, C. Deshapnde ${ }^{2}, T$. Serafimova ${ }^{3}$, S. Byrne ${ }^{4}$, M. Lim $^{4}$, A. Siddiqui ${ }^{5}$

${ }^{1}$ Guy's Hospital, London, United Kingdom, ${ }^{2}$ Manchester Centre for Genomic Medicine, St. Mary's Hospital, Manchester, United Kingdom, ${ }^{3}$ University Hospital Bristol NHS Trust, Bristol, United Kingdom, ${ }^{4}$ Evelina Children's Hospital, London, United Kingdom, ${ }^{5}$ St. Thomas Hospital, London, United Kingdom

Smith-Kingsmore Syndrome (SKS) [MIM\#616638] is a rare multisystem disorder characterised by macrocephaly, dysmorphic features, malformations of cortical development (MCD) and intellectual disability, caused by heterozygous, germline variants in the mammalian target of rapamycin $(M T O R)$. At 31 months old, our patient was referred with significant global developmental delay, dysmorphic features and large head circumference (HC). Antenatally, moderate bi-ventricular dilatation and macrocephaly were observed. Ventricular dilatation was rapidly progressed in the first 6 months and stabilised by one year old. There was no history of seizures. Clinical examination revealed $\mathrm{HC}>4 \mathrm{SD}$ above the mean, curly hair, dysmorphic facial features, fleshy hands and feet with prominent fetal finger pads. She had peripheral and, more marked, core hypotonia, and was not independently mobile. Neurometabolic investigations were unremarkable. Serial MRI imaging demonstrated moderate, stable ventriculomegaly, thin corpus callosum, subtle bilateral anterior/opercular polymicrogyria and subepemdymal nodular heterotopia. Array CGH was normal. Whole exome sequencing revealed a de novo, likely pathogenic MTOR c.7217T>G p.(Val2406Gly) variant located in a highly conserved nucleotide and amino acid position in the Kinase domain. The gene encodes serine/threonine-protein kinase mTOR, member of the phosphatidylinositol 3-kinase (PI3K) family. The mTOR engages in two complexes, mTORC1 and mTORC2, and is a potent regulator of cell growth, energy metabolism, autophagy and cytoskeletal organisation. Deregulation of the mTOR pathway has been implicated in cancer, cardiovascular, autoimmune and neurodegenerative diseases, often referred to as mTORopathies. We present a new case of SKS and review the literature to further delineate the phenotype.

D.J. Josifova: None. R. Marcinkute: None. C. Deshapnde: None. T. Serafimova: None. S. Byrne: None. M. Lim: None. A. Siddiqui: None.

P08.081.C

Things you might miss in your sequencing study Reanalyzing 152 cases with neurodevelopmental disorders 


\section{T. Bartolomaeus, R. Abou Jamra, B. Popp}

Institute of Human Genetics, University of Leipzig Medical Center, Leipzig, Germany

Clinical guidelines recommend iterative re-analysis in undiagnosed cases. Re-interpretation and reporting research in light of novel data is not well investigated. Of 152 consanguineous families with NDDs we previously found a diagnosis in 56 and identified VUS or candidate genes in 53. In total 116 variants were reported in 109 families. We re-evaluated all reported variants according to updated classification guidelines or our internal candidate gene scoring system. Variants in candidate genes were uploaded to GeneMatcher and collaborations were joined/initiated. We re-processed all data using up-to-date tools, references and databases for case-level re-analysis. In 26/116 (22\%) variants the clinical relevance changed through variant classification or increased significance of candidate genes. In 4 (9\%) of the 43 undiagnosed cases a possible diagnosis was found. Of the 53 originally identified candidate genes 8 were in the meantime associated with NDDs, 2 are in submission and 3 collaborations were entered in the course of this project. Two reported variants were missed by the updated computational pipeline due to alignment (old data) or reference (ambiguous region in hg38) issues. We submitted all relevant variants to public databases and revised their previous classification. Our results support the need to re-evaluate research screening studies. We highlight potential benefits and pitfalls of computational re-analysis. Its complexity for old data should be weighed against the decreasing re-testing costs. Published variants in established and candidate disease genes are not assessable unless reported in databanks. Future studies should incorporate post-publication variant management and bio-banking of patient cells for upcoming methods.

T. Bartolomaeus: None. R. Abou Jamra: None. B. Popp: None.

\section{P08.082.A}

Missense variants in NDD-associated FBXO11 impair its localization and Fbxo11 deficiency leads to neuronal impairment in Drosophila melanogaster

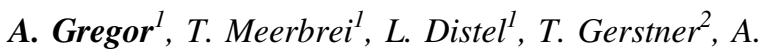 \\ Gupta ${ }^{3}$, A. Toutain ${ }^{4}$, S. A. Lynch ${ }^{5}$, C. Maxton ${ }^{6}$, J. R. \\ Lemke $^{7}$, J. A. Bernat ${ }^{8}$, H. M. Bombei ${ }^{8}$, N. Foulds ${ }^{9}$, A. \\ Kuechler $^{10}$, A. Bouman ${ }^{11}$, M. Palomares Bralo ${ }^{12}$, F. Santos \\ Simarro $^{12}$, S. Garcia-Minaur ${ }^{12}$, C. Zweier ${ }^{1}$
}

${ }^{1}$ Institute of Human Genetics, Friedrich-AlexanderUniversity Erlangen-Nürnberg, Erlangen, Germany, ${ }^{2}$ Department of Pediatrics, Sørlandet Hospital, Arendal,
Norway, ${ }^{3}$ Center for Individualized Medicine, Mayo Clinic, Rochester, MN, United States, ${ }^{4}$ Service de Génétique, CHU de Tours, Tours, France, ${ }^{5}$ Dept of Clinical Genetics, Temple Street Children's Hospital Dublin 1, Dublin, Ireland, ${ }^{6}$ Praxis für Kinderneurologie, Hamburg Altona, Hamburg, Germany, ${ }^{7}$ Institute of Human Genetics, University of Leipzig Hospitals and Clinics, Leipzig, Germany, ${ }^{8}$ Division of Medical Genetics, Department of Pediatrics, University of Iowa, Iowa City, IA, United States, ${ }^{9}$ Wessex Clinical Genetics Services, University Hospital Southampton, Southampton, United Kingdom, ${ }^{10}$ Institut für Humangenetik, Universitätsklinikum Essen, Essen, Germany, ${ }^{11}$ Department of Clinical Genetics, Erasmus MC, University Medical Center Rotterdam, Rotterdam, Netherlands, ${ }^{12}$ Institute of Medical and Molecular Genetics, University Hospital La Paz, Madrid, Spain

Recently, we identified de novo FBXO11 variants as causative for a variable neurodevelopmental disorder (NDD). We now assembled clinical and mutational information on 13 additional cases. The phenotypic spectrum remains highly variable, and the mutational spectrum includes intragenic deletions, likely gene disrupting and missense variants distributed across the protein, in line with haploinsuffiency of FBXO11 as the most likely disease mechanism.

We analyzed the effects of ten different missense variants on protein expression and localization by overexpression of mutant constructs in HEK293 and Hela cells. Variants located close to the N-terminal F-Box domain lead to altered subcellular localization and exclusion from the nucleus, and to reduced protein levels in western blot. In contrast, variants localized in the C-terminal Zn-finger UBR domain lead to an accumulation in cytoplasmatic granules and to increased protein levels in the cell. Together, these results indicate that some missense variants impair function through mislocalization or reduced expression.

Using Drosophila melanogaster as a model organism, we investigated the effects of Fbxoll deficiency on neuronal development. We found a mild impairment of basic locomotor function in the climbing assay upon neuronal knockdown and a stronger impairment upon glial knockdown. We also found decreased dendrite branching and total dendrite length upon Fbxo11 knockdown in multiple dendrite neurons of the larval body wall.

Our study further characterizes the FBXO11 associated NDD and the underlying pathomechanism and highlights the importance of Drosophila Fbxo11 for neuronal development and basic neuronal functioning.

A. Gregor: None. T. Meerbrei: None. L. Distel: None. T. Gerstner: None. A. Gupta: None. A. Toutain: None. S. A. Lynch: None. C. Maxton: None. J.R. Lemke: None. J. A. Bernat: None. H.M. Bombei: None. N. Foulds: None. 
A. Kuechler: None. A. Bouman: None. M. Palomares Bralo: None. F. Santos Simarro: None. S. GarciaMinaur: None. C. Zweier: None.

\section{P08.083.B}

Using RNA-sequencing to expand the diagnostic toolset for neurodevelopmental disorders: a pilot study

M. Radtke, D. Le Duc, R. Jamra

\section{Medical University Leipzig, Leipzig, Germany}

Introduction: Despite the inclusion of next generation sequencing in routine genetic diagnostics, around $50 \%$ of cases remain unsolved. One cause for this is the difficulty of assessing synonymous and non-coding SNVs. RNAsequencing is one way to unravel their potential causality and gain first insights in potential pathomechanisms.

Materials and Methods: We extracted RNA from PAXblood of a first cohort of 26 individuals with syndromic neurodevelopmental disorders that remained unsolved after exome sequencing. The RNA-sequencing data was analyzed with respect to gene expression and splicing alterations.

Results: A first evaluation of the sequencing data shows a strong inter-individual fluctuation, stressing the importance of selecting appropriate control groups. Differentially expressed genes were then analyzed with regard to function, interaction and disease association. In at least two individuals, we identified disruptive splicing events in genes with a known disease association. In one individual we found a significant enrichment in differentially expressed genes involved in synaptic signaling and neuronal processes. This implies a possible disturbance of neuronal pathways and offers a set of potential candidate genes for further investigations. We are still in the process of interrogating the sequencing data and will present a full report of our findings at the conference.

Conclusions: The blending of exome and transcriptomic sequencing data allows for more in depth exploration and offers the potential to identify disruptive variants which remain unnoticed by exome sequencing.

M. Radtke: None. D. Le Duc: None. R. Jamra: None.

\section{P08.084.C}

Diagnostic array-CGH and exome sequencing in a cohort of patients with neurodevelopmental disorders: genetic heterogeneity and phenotypic variability

\section{A. Pelle ${ }^{1,2}$, G. Mandrile ${ }^{3,2}$, E. Di Gregorio ${ }^{4}$, P. Dentelli ${ }^{4}$, E.

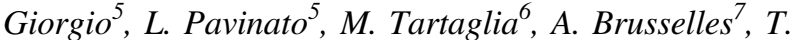

Pippucci $^{8}$, P. Dimartino ${ }^{8}$, S. De Rubeis ${ }^{9}$, G. B. Ferrero $^{10}$, A. Brusco $^{5}$, D. F. Giachino ${ }^{2,1}$

${ }^{1}$ University of Torino, Department of Clinical and Biological Sciences, Orbassano (TO), Italy, ${ }^{2}$ Service of Genetic Counseling, Department of Medicine and Oncology, AOU San Luigi Gonzaga, Orbassano (TO), Italy, ${ }^{3}$ Microcitemia, AOU San Luigi Gonzaga, Orbassano (TO), Italy, ${ }^{4} \mathrm{SC}$ Medical Genetics, AOU Città della salute e della scienza di Torino, Molinette, Torino (TO), Italy, ${ }^{5}$ Department of Medical Sciences, University of Torino, Torino (TO), Italy, ${ }^{6}$ Genetics and Rare Diseases Research Division, Ospedale Pediatrico Bambino Gesù IRCSS, Roma, Italy, ${ }^{7}$ Department of Oncology and Molecular Medicine, Istituto Superiore di Sanità, Roma, Italy, ${ }^{8}$ Department of Medical and Surgical Sciences, University of Bologna, Bologna, Italy, ${ }^{9}$ Seaver Autism Center for Research and Treatment, Icahn School of Medicine at Mount Sinai, New York, NY, United States, ${ }^{10}$ Department of Pediatrics and Public Health and Pediatric Sciences, University of Torino, Torino (TO), Italy

Neurodevelopmental disorders (NDDs) are a heterogeneous category of neuropsychiatric disorders, with onset during childhood/adolescence and a worldwide prevalence of 0,5 $2 \%$. The etiology of NDDs include both environmental and genetic causes. In this study we evaluated the genotypephenotype correlation of 201 patients with NDDs consecutively admitted to our Unit for a clinical genetic evaluation from 2006 to 2019. Based on the clinical evaluation, the appropriate genetic testing was performed for each patient: array-CGH and FMRI analysis were used as firsttier tests and specific genetic testing/WES as second-tier analysis. Array-CGH was performed in 158 cases and positive in 13 (DR 8.2\%). Two patients were carriers of a complex chromosomal rearrangements (one due to chromothripsis), a family had a recurrent $4 \mathrm{p} 15.31 \mathrm{p} 15.2$ deletion. Specific genetic tests revealed a pathogenic variant in $7.5 \%$. WES was performed in 52 patients and revealed a mutation in HNRNPH2, DLG4, IQSEC2, ATPIA3 genes (DR 16\%). Overall, 16 different genetic conditions were diagnosed and Fragile-X syndrome was the most frequent (3 cases). Detection rate of array-CGH in our cohort was roughly in line with the $10 \%$ estimated by in literature. In our cohort, the results of WES are encouraging but certainly underestimated: DR of this test would be higher if WES was employed also instead of other second-tier specific gene analysis (DR estimated: 23\%). The new knowledge and technologies would probably change the multi-step genetic testing approach, indicating WES or WGS as first step analysis after FMRl test as routine diagnostic test in all patients with NDDs. 
A. Pelle: None. G. Mandrile: None. E. Di Gregorio: None. P. Dentelli: None. E. Giorgio: None. L. Pavinato: None. M. Tartaglia: None. A. Brusselles: None. T. Pippucci: None. P. Dimartino: None. S. De Rubeis: None. G.B. Ferrero: None. A. Brusco: None. D.F. Giachino: None.

\section{P08.086.B}

Exome sequencing of Slovenian children with neurodevelopmental disorders

\section{Krgovic, S. Stangler Herodez, A. Golub, N. Kokalj Vokac}

\section{Laboratory of Medical Genetics, Maribor, Slovenia}

Introduction: Since the implementation of molecular karyotyping in diagnostics of neurodevelopmental disorders (NDDs), the understanding of the genetic causes of these diseases has significantly improved. However, with the introduction of the NGS, the setting of the genotypephenotype correlations has become even more straightforward. By determining causative mutations in individual genes, it enabled us to understand the role of individual genes in the etiology of the disease, and significantly increased the diagnostic yield for genetic diagnostic of NDDs in Slovenian children.

Methods: Clinical exome sequencing was performed by using the TruSight One Illumina Kit (4813 genes) and focusing on phenotype driven analysis on a small serie $(\mathrm{N}$ 45) of pediatric patients with various NDDs.

Results: With NGS analysis, we were able to identify the genetic origin of the disorder (pathogenic Single Nucleotide Variant - pSNV) in $29 \%$ of screened patients. In $24 \%$ of patients, a SNV classified as variant of unknown significance (VOUS) has been determined within the diseaseassociated genes.

Discussion: With phenotype-genotype driven analysis, we were able to identify rare and clinically poorly defined syndromes in previously undiagnosed patients. Among them, disorders such as Kabuki and Noonan syndrome, Menkes disease, mutations in IQSEC2 gene causing the Rett-like phenotype etc. were identified. A high percent of pSNV are also partly due to the selection of suitable patients for NGS testing based on their good clinical characterization. Our study confirmed a high efficiency of NGS in diagnostic of NDDs and enabled the diagnosis of rarely described cases which will be presented.

D. Krgovic: None. S. Stangler Herodez: None. A. Golub: None. N. Kokalj Vokac: None.
P08.087.C

Loss-of-function truncating and missense variants in NSD2 cause decreased methylation activity and are associated with a distinct neurodevelopmental phenotype

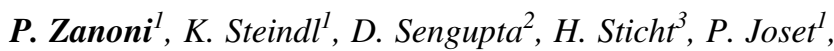
A. Baar ${ }^{l}$, C. M. A. van Ravenswaaij-Arts ${ }^{4}$, M. Shinawi ${ }^{5}, I$. Maystadt $^{6}$, N. Belnap ${ }^{7}$, V. Benoit ${ }^{6}$, B. B. A. de Vries ${ }^{8}, D$. Lacombe $^{9}$, A. Larson ${ }^{10}$, R. Pfundt ${ }^{8}$, K. Ramsey ${ }^{7}$, L. Snijders

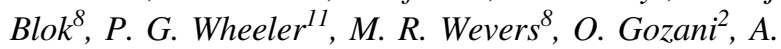
Rauch $^{1}$

${ }^{1}$ University of Zurich, Schlieren, Switzerland, ${ }^{2}$ Stanford University, Stanford, CA, United States, ${ }^{3}$ FriedrichAlexander University Erlangen-Nürnberg, Erlangen, Germany, ${ }^{4}$ University Medical Centre Groningen, Groningen, Netherlands, ${ }^{5}$ Washington University School of Medicine, St. Louis, MO, United States, ${ }^{6}$ Institut de Pathologie et de Génétique, Gosselies, Belgium, ${ }^{7}$ Translational Genomics Research Institute, Phoenix, AZ, United States, ${ }^{8}$ Radboud University Medical Center, Nijmegen, Netherlands, ${ }^{9}$ Hôpital Pellegrin CHU, Bordeaux, France, ${ }^{10}$ University of Colorado Anschutz Medical Campus, Denver, CO, United States, ${ }^{11}$ Arnold Palmer Hospital, Orlando Health, Orlando, FL, United States

Introduction: NSD2 (alias WHSC1) has been suspected to be critical for the Wolf-Hirschhorn syndrome (WHS) phenotype for decades. Recently, few case reports described the first patients harbouring heterozygous truncating NSD2 variants. In order to understand the full clinical spectrum and molecular mechanism, we investigated 15 new individuals carrying truncating or missense NSD2 variants.

Materials and Methods: Detailed phenotypic information was collected for 15 new sporadic and familial affected individuals. Clinical data were compared to the available information of 10 previously described carriers of NSD2 truncating variants or small deletions. The structural effect of missense variants was modelled in silico. Each missense variant was tested in vitro for methyltransferase activity and for the ability to reconstitute $\mathrm{H} 3 \mathrm{~K} 36 \mathrm{me} 2$ in knockout cell lines.

Results: the core phenotype associated with NSD2 pathogenic variants overlaps only partially with WHS and includes mild to moderate DD, prenatal-onset growth retardation, failure to thrive and characteristic facial features distinct from WHS. Carriers of missense variants were significantly taller and had more frequent behavioural/ psychological issues compared to carriers of truncating variants. BMI was significantly higher in older patients. All missense variants in known structures are supposed to interfere with NSD2's folding and function in silico. In vitro 
data showed that missense variants lead to a reduction of methylation activity.

Conclusions: We present the first comprehensive patient series with NSD2 pathogenic variants, providing a significant refinement of the clinical spectrum. Furthermore, we report the first missense variants and provide evidence for pathogenicity through decreased methylation activity.

Funding: SNF, NIH, Stanford-MCHRI

P. Zanoni: None. K. Steindl: None. D. Sengupta: None. H. Sticht: None. P. Joset: None. A. Baar: None. C.M.A. van Ravenswaaij-Arts: None. M. Shinawi: None. I. Maystadt: None. N. Belnap: None. V. Benoit: None. B. B.A. de Vries: None. D. Lacombe: None. A. Larson: None. R. Pfundt: None. K. Ramsey: None. L. Snijders Blok: None. P.G. Wheeler: None. M.R. Wevers: None. O. Gozani: E. Ownership Interest (stock, stock options, patent or other intellectual property); Significant; EpiCypher, Inc., Athelas Therapeutics. A. Rauch: None.

\section{P08.089.B}

\section{A novel TBL1XR1 variant in Pierpont syndrome}

\section{Stefanova ${ }^{1}$, H. Klang Åstrand ${ }^{1}$, M. Frid ${ }^{2}$, I. Hüning ${ }^{3}$}

${ }^{1}$ University Hospital Linköping, Linköping, Sweden, ${ }^{2}$ Ryhov County Hospital, Jönköping, Sweden, ${ }^{3}$ University of Lübeck, Lübeck, Germany

Pierpont syndrome (PS) (OMIM \#602342) is a rare disorder characterized by intellectual impairment, characteristic facial gestalt, microcephaly, fetal finger/toes pads and anteromedial fat pads of the heels (Pierpont et al, 1998). A recurrent de novo TBLIXRI variant [c.1337A $>\mathrm{G}$; $\mathrm{p}$. (Tyr446Cys)] is known to be disease-causing for the syndrome (Heinen et al, 2016). Additionally, other TBL1XR1 variants [c.974G > A;p.(Cys325Tyr) and c.1336 T > C;p. (Tyr446His)] have been reported in patients with a typical PS (Lemattre et al, 2018). The authors suggest comparability between the functional consequences of all these variants. TBL1XR1 haploinsufficiency, though, is reported to cause a different entity, MRD41 (OMIM\# 616944). Here we report on a four-year-old girl with a typical PS phenotype and a novel de novo heterozygous TBL1XR1 variant, c.1184A>G;p.(Tyr395Cys). We studied the threedimensional structure of TBLR1 protein and noted that the amino acid affected by this variant is located in the WD6 repeat of the WD40 domain and lies in the close vicinity of the amino acids affected by the other PS causing variants (Tyr446 and Cys325). The WD40 domain has a $\beta$-propeller-like-structure where hotspot residues at the domain's top as identified by WDSPdb (Wang et al, 2015) are likely to interact with $\beta$-catenin. Tyr395 and Tyr446 are hotspot residues and therefore predicted to be of importance for the function. In summary, a review of the clinical and molecular data of all the reported patients so far, inclusive the present patient, suggests that the TBL1XR1 variants causing PS affect most likely protein structures with comparable gene function.

M. Stefanova: None. H. Klang Åstrand: None. M. Frid: None. I. Hüning: None.

\section{P08.092.B}

PPP2R5D variants in patients with variable neurodevelopmental phenotypes

M. Aspromonte ${ }^{1,2}$, E. Leonardi ${ }^{1,2}$, R. Polli ${ }^{1,2}$, E. Bettella ${ }^{1,2}$, F. Cesca ${ }^{1,2}$, S. Sartori ${ }^{3}$, L. Turolla ${ }^{4}$, I. Donati ${ }^{5}, R$. Guerrini $^{6}$, A. Murgia ${ }^{1,2}$

${ }^{1}$ Dept. of Women's and Children's Health, University of Padova, Padova, Italy, ${ }^{2}$ Fondazione Istituto di Ricerca Pediatrica (IRP), Città della Speranza, Padova, Italy, ${ }^{3}$ Dept. of Woman's and Child's Health, University Hospital of Padova, Padova, Italy, ${ }^{4}$ U.O.S. Genetica Medica Azienda ULSS9, Treviso, Italy, ${ }^{5}$ UO Genetica Medica AUSL Romagna, Ravenna, Italy, ${ }^{6}$ Neuroscience Department, Children's Hospital A. Meyer-University of Florence, Firenze, Italy

PPP2R5D is the $\mathrm{B}$ regulatory subunit of protein phosphatase 2A (PP2A) and has been recently associated with a neurodevelopmental disorder (NDD), characterized by mild to severe Intellectual Disability (ID), Autism Spectrum Disorder (ASD), pronounced hypotonia, macrocephaly and speech impairment. To date, only 23 individuals with de novo PPP2R5D mutations have been described. Here we report five $P P P 2 R 5 D$ variants in five unrelated individuals with NDD: two novel missense, one rare intronic variants and two recurrent missense pathogenic mutations, Glu198Lys and Glu200Lys. The Glu198Lys mutations, located in the PP2A catalytic subunit interaction interface, is associated with a more severe phenotype due to its severe effects on the binding properties of the B regulatory subunit PP2A. All but one the mutations were detected by targeted NGS analysis of a ID/ASD multigenic panel in 600 NDDs subjects. Among these cases we report for the first time a dual diagnosis. A girl carrying a de novo Glu198Lys mutation, identified by whole-exome sequencing, exhibiting a very severe NDD with ID, ASD, absent speech, marked hypotonia, and typical dysmorphisms, also carry a full FMR1 gene mutation. The fragile $X$ phenotype seemed to be overshadowed by the severe $P P P 2 R 5 D$ clinical features. The other individuals carrying $P P P 2 R 5 D$ mutations (segregation analysis is currently underway) showed a rather mild ID phenotype, very variable dysmorphic features, no hypotonia and, only in case of the Glu200Lys mutation, 
absence of ASD features. These new cases confirm the phenotype correlations in recurring mutations and contribute to highlighting the variability of PPP-related NDDs.

M. Aspromonte: None. E. Leonardi: None. R. Polli: None. E. Bettella: None. F. Cesca: None. S. Sartori: None. L. Turolla: None. I. Donati: None. R. Guerrini: None. A. Murgia: None.

\section{P08.093.C}

A novel mutation in WDR62 gene in a patient who had autosomal recessive primary microcephaly with concomitant sarcoglycanopathy

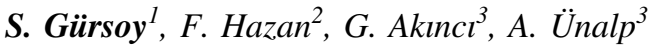

${ }^{1}$ Department of Pediatric Genetics, Dr. Behcet Uz Children's Hospital, Izmir, Turkey, ${ }^{2}$ Department of Medical Genetics, Dr. Behcet Uz Children's Hospital, Izmir, Turkey, ${ }^{3}$ Department of Pediatric Neurology, Dr. Behcet Uz Children's Hospital, Izmir, Turkey

Introduction: Autosomal recessive primary microcephaly is a rare form of primary microcephaly, characterized by a marked reduction in brain size and intellectual disability. WDR62, which is the second most frequently mutated gene, plays a significant role in neuronal progenitor cell proliferation and spindle formation.

Materials and Methods: Herein, we present a novel WDR62 gene mutation in a 11 year-old girl. The index girl patient was referred to our genetic clinic for microcephaly, epilepsy and developmental delay. Her parents had a consanguineous marriage and the patient had a 2 year-old brother who had microcephaly and developmental delay and a 10 year-old healthy brother. She had increased serum CK levels and muscle biopsy findings were compatible with sarcoglycanopathy. An autosomal recessive microcephaly panel (including 9 gene) and limb-girdle muscular dystrophy panel (including 31 gene) with MiSeq ${ }^{\circledR}$ NGS system, were performed in the patient. Sanger seguencing was used for family segregation analysis.

Results: We detected a novel homozygous frameshift mutation (c.621_622delAG; p.Glu208GlyfsTer23) in WDR62 gene. The 2 year-old brother was homozygous, whereas the healthy parents were heterozygous for this mutation. No pathogenic mutation was detected in limbgirdle muscular dystrophy panel.

Conclusions: We have identified a novel frameshift mutation in WDR62 gene and to our knowledge, this is the first case who had autosomal recessive primary microcephaly with concomitant sarcoglycanopathy. Further molecular studies for sarcoglycanopathies will allow us to better understand the genetic etiology in this patient.
S. Gürsoy: None. F. Hazan: None. G. Akıncı: None. A. Ünalp: None.

\section{P08.094.A}

Biallelic variants in PSMB1 encoding the proteasome subunit b6 cause impairment of proteasome function, microcephaly, intellectual disability, developmental delay and short stature

S. A. Paracha ${ }^{1}, M$. Ansar $^{2}, F$. Ebstein $^{3}, H . \ddot{O}_{z k o c^{4}}, J$. Iwaszkiewicz ${ }^{5}$, M. Gesemann 6 , V. Zoete ${ }^{5,7}$, E. Ranza ${ }^{2,8,9}, F$. A. Santoni ${ }^{2,10}$, M. T. Sarwar ${ }^{1}$, J. Ahmed ${ }^{1}$, E. Krüger ${ }^{3}$, R. Bachmann-Gagescu ${ }^{4,6}$, S. E. Antonarakis ${ }^{2,8,11}$

${ }^{1}$ IInstitute of Basic Medical Sciences, Khyber Medical University, Peshawar, Pakistan, ${ }^{2}$ Department of Genetic Medicine and Development, University of Geneva, Geneva, Switzerland, ${ }^{3}$ Universitätsmedizin Greifswald, Institut für Medizinische Biochemie und Molekularbiologie, Greifswald, Germany, ${ }^{4}$ Institute of Medical Genetics, University of Zurich, Zurich, Switzerland, ${ }^{5}$ Swiss Institute of Bioinformatics, Molecular Modeling Group, Lausanne, Switzerland, ${ }^{6}$ Department of Molecular Life Sciences, University of Zurich, Zurich, Switzerland, ${ }^{7}$ Department of Fundamental Oncology, Lausanne University, Ludwig Institute for Cancer Research, Lausanne, Switzerland, ${ }^{8}$ Service of Genetic Medicine, University Hospitals of Geneva, Geneva, Switzerland, ${ }^{9}$ Medigenome, The Swiss Institute of Genomic Medicine, Geneva, Switzerland, ${ }^{10}$ Department of Endocrinology Diabetes and Metabolism, University Hospital of Lausanne, Lausanne, Switzerland, ${ }^{11}$ iGE3 Institute of Genetics and Genomics of Geneva, Geneva, Switzerland

The molecular cause of the majority of rare autosomal recessive disorders remains unknown. Consanguinity due to extensive homozygosity unravels many recessive phenotypes and facilitates the detection of novel gene-disease links. Here, we report two siblings with phenotypic signs including intellectual disability, developmental delay, and microcephaly from a Pakistani consanguineous family in which we have identified homozygosity for $\mathrm{p}$ (Tyr103His) in the PSMB1 gene (Genbank NM_002793) that segregated with the disease phenotype. PSMB1 encodes a $\beta$-type proteasome subunit (i.e. $\beta 6$ ). Modeling of the $p$ (Tyr103His) variant indicates that this variant presumably weakens the interactions between PSMB1/ $\beta 6$ and PSMA5/ $\alpha 5$ proteasome subunits and thus destabilizes the 20S proteasome complex. Biochemical experiments in human SHSY5Y cells revealed that the $p$ (Tyr103His) variant affects both the processing of PSMB1//6 and its incorporation into proteasome, thus impairing proteasome activity. CRISPR/Cas9 mutagenesis or morpholino knock-down of the single psmbl zebrafish orthologue resulted in microcephaly, 
microphthalmia and reduced brain size. Genetic evidence in the family and functional experiments in human cells and zebrafish indicate that PSMB1/ $\beta 6$ pathogenic variants are the cause of a recessive disease with intellectual disability, microcephaly and developmental delay due to abnormal proteasome assembly.

S.A. Paracha: None. M. Ansar: None. F. Ebstein: None. H. Özkoç: None. J. Iwaszkiewicz: None. M. Gesemann: None. V. Zoete: None. E. Ranza: None. F. A. Santoni: None. M.T. Sarwar: None. J. Ahmed: None. E. Krüger: None. R. Bachmann-Gagescu: None. S.E. Antonarakis: None.

\section{P08.095.B}

Expanding the PTEN-associated phenotype: two patients with de novo PTEN mutations and MPS-like phenotype

\section{T. Scholz ${ }^{1}$, T. Bierhals ${ }^{1}$, J. Johannsen ${ }^{2}$, N. Muschol ${ }^{2}, M$. Hempel $^{l}$}

${ }^{1}$ UKE, Institute of Human Genetics, Hamburg, Germany, ${ }^{2}$ UKE, Department of Pediatrics, Hamburg, Germany

Introduction: Heterozygote PTEN variants are associated with different but overlapping diseases, such as PTEN hamartoma tumor syndrome, Cowden syndrome, Bannayan-Riley-Ruvalcaba syndrome and macrocephaly autism syndrome. We report on two patients with de novo PTEN mutations who presented with an MPS-like phenotype.

Case reports: Patient 1: A 3-year-old boy, who is the first child of healthy parents, was referred to hospital due to developmental delay, macrocephaly, splenomegaly and facial dysmorphism leading to suspicion of a metabolic disease. Results after extended metabolic work up were normal. The brain MRT revealed occipital white matter anomalies and enlarged perivascular spaces. Trio whole exome sequencing (WES) identified the de novo missense variant c.80A $>$ T [p.(Tyr27Phe)] in PTEN. Patient 2: A 4year-old girl, who is the second child of healthy parents, was referred to hospital due to macrocephaly, mild motor developmental delay and dysmorphic features resembling a storage disorder. Extended metabolic work up was normal. Brain MRT showed some enlarged perivascular spaces. Trio WES detected a de novo missense mutation c.103A $>\mathrm{G}$ [p.(Met35Val)] in PTEN.

Discussion: Both patients were initially referred to hospital due to suspicion of a metabolic disease, most likely MPS, which could not be verified. Instead, de novo PTEN mutations were found. The phenotype does not match the currently described PTEN-associated diseases. This leads to the assumption that there might be an additional PTEN-associated phenotype. Children who are suspected of having a lysosomal storage disease that cannot be confirmed should be considered for an extended genetic analysis including PTEN.

T. Scholz: None. T. Bierhals: None. J. Johannsen: None. N. Muschol: None. M. Hempel: None.

\section{P08.097.A}

Biallelic loss-of-function variants in $R B L 2$ in siblings with a neurodevelopmental disorder

T. Brunet ${ }^{1,2}$, M. Radivojkov-Blagojevic ${ }^{2}$, P. Lichtner $^{2}$, V. Kraus $^{3}$, T. Meitinger ${ }^{1,2}$, M. Wagner ${ }^{1,2,4}$

${ }^{1}$ Institute of Human Genetics, Technical University Munich, Munich, Germany, Munich, Germany, ${ }^{2}$ Institute of Human Genetics, Helmholtz Zentrum München, Neuherberg, Germany, Neuherberg, Germany, ${ }^{3}$ Department of Pediatrics, Klinik für Kinder- und Jugendmedizin, München Klinik Schwabing und Harlaching, Klinikum Rechts der Isar der Technischen Universität Munich, Germany, Munich, Germany, ${ }^{4}$ Institute of Neurogenomics, Helmholtz. Zentrum München, Neuherberg, Germany, Neuherberg, Germany

Introduction: The $R B L 2$ locus has been associated with intelligence but not with a monogenic disorder to date. RBL2 encodes p130, a member of the retinoblastoma protein family. Previous studies on p130 knockout mice displaying embryonic death and impaired neurogenesis highlight the importance of $R B L 2$ in brain development.

Material \& Methods: We performed Exome sequencing (ES) in two siblings with a neurodevelopmental disease and their parents. Subsequent segregation analysis was done with haplotype analysis by GSA-Array in all four (two affected and two unaffected) siblings and their parents.

Results: The two siblings share a phenotype characterized by severe intellectual disability (no ability to stand/ walk, no expressive language), stereotypies, autoaggressive behavior, dysmorphic features and supra- and infratentorial atrophy in cMRI. ES identified compound-heterozygous variants in RBL2 as the sole candidate (NM_005611.3) in both patients: A heterozygous nonsense variant c. $556 \mathrm{C}>\mathrm{T}$, p.(Arg186Ter) and heterozygous in-frame deletion of exon 13-17. Subsequent haplotype analysis showed that the two disease haplotypes segregate in the family.

Discussion: RBL2, that has not been linked to a monogenic disorder yet, is the key regulator of maintaining neuron survival among the $\mathrm{Rb}$ family members. The hypothesis of $R B L 2$ as a disease gene is underscored by the gnomAD gene constraint metrics showing a depletion of loss of function variants and p130 knockout mice exhibiting impaired neurogenesis and embryonic lethality. Our data 
establish $R B L 2$ as a candidate gene for an autosomal recessive neurodevelopmental delay.

231 words

T. Brunet: None. M. Radivojkov-Blagojevic: None. P. Lichtner: None. V. Kraus: None. T. Meitinger: None. M. Wagner: None.

\section{P08.098.B}

Cell system with inducible transcription for the study of CGG repeat instability

I. V. Grishchenko ${ }^{l}$, Y. V. Purvinsh ${ }^{l}$, I. R. Imatdinov ${ }^{l}$, S. V. Kulemzin ${ }^{2}$, D. V. Yudkin ${ }^{1}$

${ }^{I}$ FBRI SRC VB "Vector", Rospotrebnadzor, Koltsovo, Russian Federation, ${ }^{2} I M C B$ SB RAS, Novosibirsk, Russian Federation

Introduction: The molecular basis of certain hereditary neurodegenerative diseases is trinucleotide repeat expansion, which dramatically increases the number of triplet repeats. However, the expansion mechanism has yet to be defined. A failure of the DNA repair system at sites with alternative DNA structures formed by repeats can lead to expansion. This work aimed to design a cellular system for studying the instability of CGG repeats involved in FRAXopathy development during transcription and TCRNER.

Materials and Methods: CGG repeats were amplified by PCR from GC-rich templates. We transfected HEK293A cells with plasmids containing CGG repeats of different lengths. Transfected cells were selected using flow cytofluorometry or puromycin. Changes in CGG repeats were evaluated by fragment analysis. Reporter gene expression was measured by qPCR.

Results: To control transcription levels, DNA fragments of CGG repeats and the EGFP ORF were inserted into plasmids under the inducible promoter TRE. Additionally, vectors encoded DsRedExpress or puromycin-Nacetyltransferase driven by a constitutive promoter. Several types of plasmids carrying CGG repeats of various lengths were generated. HEK293A cells were transfected, and transformants were selected. In the obtained cell lines, plasmid integration sites, expression levels of fluorescent proteins and changes in (CGG)n repeat length upon induction of TRE were determined.

Conclusions: Cell lines with controlled transcription across repeats were produced. This system allows tracking changes in the repeat region. The obtained cell lines can be used as a model to study the effect of transcription and TCR-NER on repeat instability. The study is supported by Russian Science Foundation Grant 18-15-00099.
I.V. Grishchenko: None. Y.V. Purvinsh: None. I.R. Imatdinov: None. S.V. Kulemzin: None. D.V. Yudkin: None.

\section{P08.099.C}

Next Generation Sequencing for the characterization of Rett-like spectrum disorders

E. Leonardi ${ }^{l, 2}, M$. Bellini ${ }^{l, 2}, M$. C. Aspromonte ${ }^{l, 2}, M$.

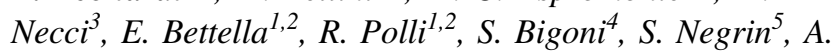

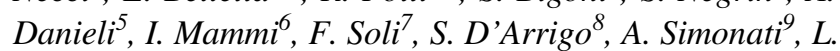

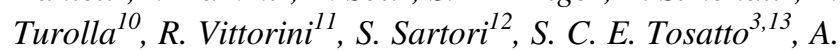
Murgial

${ }^{I}$ Dept. Woman and Child Health, University of Padua, Padova, Italy, ${ }^{2}$ Pediatric Research Institute - Città della Speranza, Padova, Italy, ${ }^{3}$ Dept. of Biomedical Sciences, University of Padua, Padova, Italy, ${ }^{4}$ 4. U. O. L. di Genetica Medica, Dip. Riproduzione e Accrescimento, Ospedale Universitario S. Anna, Ferrara, Italy, ${ }^{5}$ Child Neuropsychiatry Unit, IRCCS Eugenio Medea, Conegliano, Italy, ${ }^{6}$ Medical Genetics Unit, Dolo General Hospital, Venezia, Italy, ${ }^{7}$ Dept of Medical Genetics, Santa Chiara Hospital, Trento, Italy, ${ }^{8}$ Dept. Developmental Neurology, Fondazione IRCCS, Istituto Neurologico C. Besta, Milano, Italy, ${ }^{9}$ Dept. of Neuroscience, Biomedicine, Movement-Neurology, University of Verona, Verona, Italy, ${ }^{10}$ U.O.S. Genetica Medica, Azienda ULSS 2, Treviso, Italy, ${ }^{11}$ NPI Dept. of pediatrics, Presidio OIRM, Torino, Italy, ${ }^{12}$ Paediatric Neurology and Neurophysiology Unit, Dept. Woman and Child Health, University of Padua, Padova, Italy, ${ }^{13}$ CNR Institute of Neuroscience, Padova, Italy

The advent of next generation sequencing (NGS) allowed identifying a number of genes responsible of RTT and associated phenotypes (RTT-like). However, the etiology of the condition in a subset of RTT and RTT-like patients still remains unknown. Using the Ion Torrent platform, we developed a low-cost NGS gene panel and sequenced ca. 630 samples for early ID/ASD diagnosis. The gene panel contains 74 ID and ASD related genes, including 18 known and candidate genes associated with RTT-like disorders. We identified de novo causative mutations or likely pathogenic variants in 18 of the 90 (20\%) sequenced individuals with RTT-like disorders. Among negative cases with clinical features in the spectrum of Rett-like disorders, we performed whole exome sequencing in 31 family trios, 1 singleton, and 2 multiplex families. Pathogenic or likely pathogenic mutations were identified in 24 cases $(70 \%)$. Our findings bring additional supportive (SHANK3, GRIN2A, SYNGAP1, KCNQ2, KCNB1, PDHA1, WAC genes) or unsupportive (PTPN4) evidences for the implication of previously reported candidate genes in RTT-like 
disorders. Furthermore, we identified causative mutations in novel candidate genes and genes associated with defined syndromes, e.g. KAT6A, that have never been associated with RTT-like phenotypes. Funding: Italian Ministry of Health Young Investigator Grant GR-2011-02347754 to E. L and S.C.E.T.; Fondazione Istituto di Ricerca Pediatrica Città della Speranza, Grant 18-04 to E.L.

E. Leonardi: None. M. Bellini: None. M.C. Aspromonte: None. M. Necci: None. E. Bettella: None. R. Polli: None. S. Bigoni: None. S. Negrin: None. A. Danieli: None. I. Mammi: None. F. Soli: None. S. D'Arrigo: None. A. Simonati: None. L. Turolla: None. R. Vittorini: None. S. Sartori: None. S.C.E. Tosatto: None. A. Murgia: None.

P08.100.A

The adult phenotype of Schaaf-Yang syndrome

F. Marbach ${ }^{1}$, M. Elgizouli ${ }^{2,3}$, M. Rech ${ }^{4}, J$. Beygo $^{3}, F$. Erger $^{5}$, C. Velmans ${ }^{5}$, C. T. R. M. Stumpel ${ }^{6}$, A. P. A. Stegmann ${ }^{6}$, S. Beck-Wödl , G. Gillessen-Kaesbach ${ }^{8}, B$. Horsthemke $^{3}$, C. P. Schaaf ${ }^{1,4}$, A. Kuechler ${ }^{3}$

${ }^{1}$ Institute of Human Genetics, Heidelberg University, Heidelberg, Germany, ${ }^{2}$ Institute of Medical Genetics, University of Zurich, Zurich, Switzerland, ${ }^{3}$ Institute of Human Genetics, University Duisburg-Essen, Essen, Germany, ${ }^{4}$ Department of Molecular and Human Genetics, Baylor College of Medicine, Houston, TX, United States, ${ }^{5}$ Faculty of Medicine, University of Cologne; Institute of Human Genetics, University Hospital Cologne, Cologne, Germany, ${ }^{6}$ Department of Clinical Genetics and GROWSchool for Oncology and Developmental Biology, Maastricht University Medical Center, Maastricht, Netherlands, ${ }^{7}$ Institute of Medical Genetics and Applied Genomics, University of Tübingen, Tübingen, Germany, ${ }^{8}$ Institute of Human Genetics, University of Lübeck, Lübeck, Germany

Introduction: MAGEL2-associated Schaaf-Yang-Syndrome/SHFYNG is a rare genetic disorder, which was identified in 2013. The phenotype of SHFYNG in childhood partially overlaps with that of the well-established Prader-Willi-Syndrome/PWS. While several studies of younger individuals with SHFYNG have been recently published, the phenotype in adulthood is not well established. We recruited 7 adults (aged 18-36) with molecularly confirmed SHFYNG and collected data regarding eating habits, sleep, behavior, autonomy, psychiatric, and medical conditions, as well as information about the respective phenotypes in childhood.

Methods: Participating individuals with SHFYNG and their families received a questionnaire covering the aforementioned areas. The legal guardians of all participants provided signed consent under the IRB-approved human research protocol H-34578, Baylor College of Medicine.

Results: We identified common symptoms within our cohort, such as disturbed sleep, hypoactivity, social withdrawal, and anxiety, but noted considerable differences at the level of personal autonomy and skills. Behavioral problems were frequent, and a majority of individuals displayed weight gain, food-seeking behavior, and mild intellectual disability or borderline intellectual function. Typical symptoms of SHFYNG in childhood were reported for most individuals.

Conclusions: Our findings indicate a high variability of functional abilities and social participation of adults with SHFYNG. The prevalence of obesity was high, and lack of satiety was of concern for some caregivers. The phenotypes of PWS and SHFYNG might be more difficult to distinguish in adults than in children. Molecular genetic testing for SHFYNG should be considered in adults with the suspected diagnosis of PWS, if testing for PWS has been negative.

F. Marbach: None. M. Elgizouli: None. M. Rech: None. J. Beygo: None. F. Erger: None. C. Velmans: None. C.T.R.M. Stumpel: None. A.P.A. Stegmann: None. S. Beck-Wödl: None. G. Gillessen-Kaesbach: None. B. Horsthemke: None. C.P. Schaaf: None. A. Kuechler: None.

\section{P08.102.C}

SETD5 gene haploinsufficiency in three patients with suspected KBG syndrome

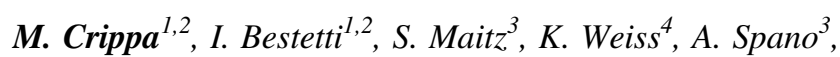
S. Smithson ${ }^{5}$, L. Larizza ${ }^{1}$, L. Cohen ${ }^{6}, K$. Low $^{5}$, P. Finelli $^{1,2}$

${ }^{1}$ IRCCS Istituto Auxologico Italiano, Cusano Milanino, Italy, ${ }^{2}$ Department of Medical Biotechnology and Translational Medicine, University of Milan, Milan, Italy, ${ }^{3}$ Clinical Pediatric Genetic Unit, Pediatric Clinic, Fondazione MBBM, San Gerardo Hospital, Monza, Italy, ${ }^{4}$ The Genetics Institute, Rambam Health Care Campus, Haifa, Israel, ${ }^{5}$ Clinical Genetics, St. Michael's Hospital, University Hospitals NHS Trust, Bristol, United Kingdom, ${ }^{6}$ Raphael Recanati Genetic Institute, Schneider Children's Medical Center of Israel, Petah Tiqva, Israel

Mendelian disorders of the epigenetic machinery (MDEMs), also called chromatin modifying disorders, are a broad group of neurodevelopmental disorders, caused by mutations in functionally related chromatin genes. KBG and the mental retardation autosomal dominant 23 (MRD23) syndromes, caused by ANKRD11 and SETD5 gene mutations respectively, belong to this broad group of disorders. We performed high resolution array CGH and trio-based 
WES on three molecularly unsolved patients with an initial KBGS diagnosis, found to carry SETD5 gene haploinsufficiency. A de novo deletion of $116 \mathrm{~kb}$ partially involving SETD5 and two de novo frameshift variants in SETD5 were identified in the patients. The clinical reevaluation of the patients was consistent with the molecular findings, however still compatible with KBGS. Phenotypic features of KBGS and MRD23 overlap to some extent, but the presence of facial features not fully consistent with KBGS can suggest differential diagnosis. Our results provide further examples that loss-of-function pathogenic variants in genes encoding factors shaping the epigenetic landscape lead to a wide phenotypic range with significant clinical overlap. To conclude we recommend clinicians to consider SETD5 gene haploinsufficiency in the differential diagnosis of KBGS, enhancing deep phenotyping and genotyping of KBGS overlapping conditions.

M. Crippa: None. I. Bestetti: None. S. Maitz: None. K. Weiss: None. A. Spano: None. S. Smithson: None. L. Larizza: None. L. Cohen: None. K. Low: None. P. Finelli: None.

\section{P08.104.B}

Expression of circadian rhythm genes in patients with neurodevelopmental syndromes displaying sleep disturbance

A. Sironi ${ }^{1,2}$, I. Bestetti ${ }^{1,2}$, S. Cipri $^{1,2}$, M. Bonati ${ }^{3}, S$.

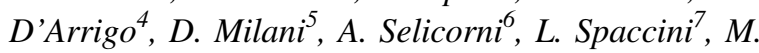
Bedeschi $^{8}$, L. Larizza ${ }^{2}$, P. Finelli ${ }^{1,2}$

${ }^{1}$ Department of Medical Biotechnology and Translational Medicine, University of Milan, Milan, Italy, ${ }^{2} L a b$. of Medical Cytogenetics and Molecular Genetics, IRCCS Istituto Auxologico Italiano, Cusano Milanino, Milan, Italy, ${ }^{3}$ Clinic of Medical Genetics, San Luca Hospital, IRCCS Istituto Auxologico Italiano, Milan, Italy, Milan, Italy, ${ }^{4}$ Developmental Neurology Division, IRCCS Fondazione Istituto Neurologico C. Besta, Milan, Italy, Milan, Italy, ${ }^{5}$ Medical Genetic Unit, Pediatric Highly Intensive Care, Fondazione IRCCS $\mathrm{Ca}$ ' Granda Ospedale Maggiore Policlinico, Milan, Italy, Milan, Italy, ${ }^{6}$ Pediatric Unit, ASST Lariana, Como, Italy, Como, Italy, ${ }^{7}$ Clinical Genetics Unit, Department of Obstetrics and Gynecology, V. Buzzi Children's Hospital, University of Milan, Milan, Italy, Milan, Italy, ${ }^{8}$ Medical Genetic Unit, IRCCS Ca'Granda Ospedale Maggiore Policlinico, Milan, Italy, Milan, Italy

Patients with neurodevelopmental disorders (NDDs) show a higher rate of sleep disturbances as compared to the general population. Sleep anomalies are a heterogeneous set of problems, including difficulties with initiating or maintaining sleep, excessive daytime sleepiness, and parasomnias.
The relationship between abnormal sleep patterns and NDDs is complex and potentially synergistic. Indeed, sleep anomalies exacerbate an already compromised neurobehavioral phenotype. Smith-Magenis Syndrome (SMS) [MIM:182290], caused by haploinsufficiency of RAIl gene, is a NDD characterized by sleep disturbances, which in $95 \%$ of the patients results from disruption of circadian rhythm with an inverted melatonin secretion. Based on the assessed role of RAI1 in the regulation of circadian rhythm, we evaluated through RT-qPCR the expression of circadian genes in peripheral blood of 18 clinically suspected SMS/ SMS-like patients, three with confirmed RAII mutation, manifesting sleep disturbance except three. Out of the 6 tested genes CLOCK, BMAL2, PER2, and NR1D1 were found deregulated in at least one patient with sleep problems: gene expression alterations were observed in 9 out 15 patients, whereas no dysregulation was identified in the 3 patients without sleep disturbance. Our data confirms a few studies suggesting a correlation between sleep disturbance in patients with molecularly defined syndromes and expands the correlation to patients with sleep disturbance who are molecularly unsolved. Our pivotal study needs to be validated on a larger cohort of patients and by testing also the melatonin receptor genes which due to their lack of expression in blood, need alternative tissues, such as buccal smears

A. Sironi: None. I. Bestetti: None. S. Cipri: None. M. Bonati: None. S. D'Arrigo: None. D. Milani: None. A. Selicorni: None. L. Spaccini: None. M. Bedeschi: None. L. Larizza: None. P. Finelli: None.

\section{P08.105.C}

Variants inSLITRK2in individuals with neurodevelopmental manifestations including intellectual disability, behavioral troubles and possible dystonia

S. EL CHEHADEH $\boldsymbol{H}^{1,2}$, S. Bakhtiari ${ }^{3,4}, J$. Wynn $^{5}$, W. K.

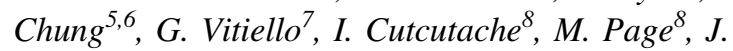
Gecz $^{9,10,11}$, K. Harper ${ }^{12,11}$, A. de Brouwer ${ }^{13}$, A. Vulto-van Silfhout $^{13}$, M. Willems ${ }^{14}$, A. Fernández Jaén ${ }^{15}$, A. Selicorni $^{16}$, S. Maitz ${ }^{17}$, E. K. Vanhoutte ${ }^{18}$, M. Armstrong ${ }^{19}$, J. Symonds ${ }^{20}$, J. Muller ${ }^{21,22}$, M. Kruer ${ }^{3,4}$, A. Piton ${ }^{23,24}$

${ }^{1}$ Service de génétique médicale, Institut de Génétique Médicale d'Alsace (IGMA), Centre de Référence des Déficiences Intellectuelles de Causes Rares, STRASBOURG, France, ${ }^{2}$ Laboratoire de Génétique Médicale, INSERM U1112, Institut de Génétique Médicale d'Alsace (IGMA), Université de Strasbourg, Strasbourg, France, ${ }^{3}$ Pediatric Movement Disorders Program, Division of Pediatric Neurology, Barrow Neurological Institute, Phoenix Children's Hospital, Phoenix, AZ, United States, ${ }^{4}$ Departments of Child Health, Neurology, Cellular \& 
Molecular Medicine and Program in Genetics, University of Arizona College of Medicine, Phoenix, AZ, United States, ${ }^{5}$ Departments of Pediatrics and Medicine, Columbia University Medical Center, New York, NY, United States, ${ }^{6}$ Department of Medicine, Columbia University, New York, NY, United States, ${ }^{7}$ Department of Translational Medicine, Federico II University, Naples, Italy, ${ }^{8}$ Translational Medicine, UCB Pharma, Slough, United Kingdom, ${ }^{9}$ Adelaide Medical School, Faculty of Health and Medical Sciences, The University of Adelaide, Adelaide, Australia,

${ }^{10}$ Robinson Research Institute, The University of Adelaide, Adelaide, Australia, ${ }^{11}$ Women and Kids, South Australian Health and Medical Research Institute, Adelaide, Australia, ${ }^{12}$ Robinson Research Institute, The University of Adelaide,, Adelaide, Australia, ${ }^{13}$ Department of Human Genetics, Radboud University Medical Center, Nijmegen, Netherlands, ${ }^{14}$ Service de Génétique Médicale, CHU de Montpellier, Montpellier, France, ${ }^{15}$ Department of Pediatrics Neurology, Quirón Hospitals, Madrid, Spain, ${ }^{16}$ Department of Pediatrics, ASST Lariana Sant'Anna Hospital, San Fermo della Battaglia, Como, Italy, ${ }^{17}$ Fondazione MBBM, Monza, Italy, ${ }^{18}$ Department of Clinical Genetics, Maastricht University Medical Centre, Maastricht, Netherlands, ${ }^{19}$ Translational Medicine, UCB Pharma, Braine-l'Alleud, Belgium, ${ }^{20}$ Paediatric Neurosciences Research Group, Royal Hospital for Children, Queen Elizabeth University Hospitals, Glasgow, United Kingdom, ${ }^{21}$ Laboratoire de Génétique Médicale, INSERM U1112, Institut de Génétique Médicale d'Alsace (IGMA), Université de Strasbourg, STRASBOURG, France, ${ }^{22}$ Laboratoires de Diagnostic Génétique, Institut de Génétique Médicale d'Alsace (IGMA), Hôpitaux Universitaires de Strasbourg, Nouvel Hôpital Civil, Strasbourg, France, ${ }^{23}$ Laboratoires de Diagnostic Génétique, Institut de Génétique Médicale d'Alsace (IGMA), Hôpitaux Universitaires de Strasbourg, Nouvel Hôpital Civil, STRASBOURG, France, ${ }^{24}$ INSERM U964, Institut de Génétique et de Biologie Moléculaire et Cellulaire, Illkirch-Graffenstaden, France

SLITRK2 is a member of the transmembrane SLITKR protein family, known to regulate neurite outgrowth and to interact with proteins involved in synapse architecture such as SHANK3. Two missense variants have been reported in SLITRK2, located on the $\mathrm{X}$ chromosome, in patients with schizophrenia (Piton et al., 2011). Through a collaborative study, we report on seven individuals (1 female and 6 males, including two siblings) carrying rare variants in SLITRK2 identified by WES. These variants include one nonsense (p.Glu461*), and five missenses changes ( $p$. Thr312Ala, p.Arg426Cys, p.Val511Met, p.Pro691Ser and p.Val201Ile), that were never reported at the hemizygous state in gnomAD populations. Three variants were de novo and three were maternally inherited. All patients had moderate to severe intellectual disability and speech delay, mostly severe. Neurological regression was observed in one patient after six years-old. Patients present behavioral issues (anxiety, aggressiveness or autism spectrum disorder) (5/6), spasticity and/or dystonia (4/7), unsteady gait (4/7), seizures (3/6), short stature (4/7), cyphoscoliosis (3/5), and variable nonspecific facial features. Nonspecific brain MRI anomalies $(3 / 6)$ included white matter reduction, ventricles dilation and thin corpus callosum. Based on these observations and the role of SLITRK2 in synapse development and organization, we believe that these variants contribute to the neurodevelopmental phenotype of our patients. We aim to gather more patients with pathogenic SLITRK2 variants and to continue with functional studies, to confirm the implication of this gene in neurodevelopmental disorder and to better delineate the clinical manifestations associated to mutations in this gene, especially at the behavioral level.

S. El chehadeh: None. S. Bakhtiari: None. J. Wynn: None. W.K. Chung: None. G. Vitiello: None. I. Cutcutache: None. M. Page: None. J. Gecz: None. K. Harper: None. A. de Brouwer: None. A. Vulto-van Silfhout: None. M. Willems: None. A. Fernández Jaén: None. A. Selicorni: None. S. Maitz: None. E.K. Vanhoutte: None. M. Armstrong: None. J. Symonds: None. J. Muller: None. M. Kruer: None. A. Piton: None.

\section{P08.106.A}

Do SMARCC2 truncating and splicing variants cause intellectual disability and developmental delay?

\section{Ö. Baysal, R. Pfundt, C. L. M. Marcelis}

Department of Human Genetics, Radboud University Medical Center, Nijmegen, Netherlands

BAFopathies are a heterogeneous group of disorders caused by variants in genes encoding the various subunits of the chromatin-remodelling complex BRG1-associated factor (BAF). De novo variants in SMARCC2, one of core subunits of the BAF complex, have been described as a cause of intellectual disability (ID) in 2019. Missense and splice variants affecting the highly conserved SMARCC2 DNAinteracting domains (SANT and SWIRM) present with a more severe phenotype overlapping with the phenotype of other BAfopathies, like Coffin-Siris and Baraitser-Winter syndrome. Truncating and splice variants affecting other regions show a much milder phenotype. According to data from the gnomAD database the SMARCC2 gene is highly intolerant to loss-of-function variants (pLI-score $=1$ ), supporting pathogenicity of this type of variants. However, we have now identified four unrelated patients harbouring predicted loss of function variants in SMARCC2 that are 
inherited from healthy (grand)parents. Whole exome sequencing was performed on these patients because of developmental delay (DD)/ID. Two unrelated patients have the same frameshift variant (c.3129del, p.Gly1044fs), inherited from their healthy father. The brother of one of these patients, who had a similar DD, did not have this variant. A third patient (c.574C $>$ T (p. (Arg 192*)) inherited a nonsense variant from his unaffected father and grandfather. The fourth patient has a predicted splice donor variant (c.317+2T>A (r.spl.?)) inherited from her healthy mother. The presence of these truncating variants in healthy individuals in four different families seems to contradict the suspected pathogenicity of this type of SMARCC2 variants. We'll discuss the possible relation between truncating SMARCC2 variants and ID.

Ö. Baysal: None. R. Pfundt: None. C.L.M. Marcelis: None.

\section{P08.107.B}

\section{SPOP: One Gene, Two Distinct Neurodevelopmental} Phenotypes

M. J. Nabais $\boldsymbol{S a ́}^{1}$, G. El Tekle Th, $^{2,3}$, A. P. M. de Brouwer ${ }^{1}, S$. L. Sawyer ${ }^{5}$, D. del Gaudio ${ }^{6}$, M. J. Parker ${ }^{7}$, F. Kanani ${ }^{7}$, M. H. van den Boogaard ${ }^{8}, K$. van Gassen ${ }^{8}$, M. I. Van Allen ${ }^{9}, K$.

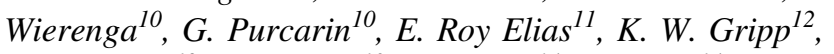
R. Procopio ${ }^{12}$, C. Mignot ${ }^{13}$, E. Argillit ${ }^{14}$, E. Sherr ${ }^{14}$, G. Cappuccio $^{15,16}$, N. Brunetti-Pierri ${ }^{15,16}$, A. Begtrup ${ }^{17}, J$. Keller-Ramey ${ }^{17}$, E. Ryan ${ }^{17}$, T. Bernasocchi ${ }^{2,3,4}$, L. van de Wiel $^{18,19}$, H. Venselaar $^{18}$, C. Gilissen ${ }^{19}$, R. Pfundt ${ }^{1}$, L. E. L. M. Vissers ${ }^{l}$, J. P. Theurillat ${ }^{2,3}$, B. B. A. de Vries $^{l}$

${ }^{1}$ Radboudumc/Donders Institute for Brain, Cognition and Behaviour, Nijmegen, Netherlands, ${ }^{2}$ Functional Cancer Genomics, Institute of Oncology Research, Oncology Institute of Southern Switzerland, Bellinzona, Switzerland, ${ }^{3}$ Faculty of Biomedical Science, Università della Svizzera Italiana, Lugano, Switzerland, ${ }^{4}$ University of Lausanne, Lausanne, Switzerland, ${ }^{5}$ Department of Genetics, Children's Hospital of Eastern Ontario and Children's Hospital of Eastern Ontario Research Institute, University of Ottawa, Ottawa, ON, Canada, ${ }^{6}$ Department of Human Genetics, University of Chicago Genetic Services Laboratory, The University of Chicago, Chicago, IL, United States, ${ }^{7}$ Department of Clinical Genetics, Sheffield Children's NHS Foundation Trust, Sheffield, United Kingdom, ${ }^{8}$ Department of Genetics, UMC Utrecht, Utrecht, Netherlands, ${ }^{9}$ Department of Medical Genetics, University of British Columbia, Vancouver, BC, Canada, ${ }^{10}$ University of Oklahoma Health Sciences Center, Oklahoma City, OK, United States, ${ }^{11}$ Special Care Clinic, Children's Hospital Colorado, and University of Colorado School of Medicine, Aurora, CO, United States, ${ }^{12}$ Division of Medical Genetics, Department of Pediatrics, A.I. duPont Hospital for Children/Nemours, Wilmington, DE, United States, ${ }^{13}$ Assistance PubliqueHôpitaux de Paris, Département de Génétique and Centre de Référence Déficiences Intellectuelles de Causes Rares, Groupe Hospitalier Pitié-Salpêtrière, Paris, France, ${ }^{14}$ Departments of Neurology and Pediatrics, Weill Institute of Neuroscience and Institute of Human Genetics, University of California, San Francisco, CA, United States, ${ }^{15}$ Telethon Institute of Genetics and Medicine, Pozzuoli, Italy, ${ }^{16}$ Department of Translational Medicine, Federico II University, Naples, Italy, ${ }^{17}$ GeneDx, Gaithersburg, MD, United States, ${ }^{18}$ Center for Molecular and Biomolecular Informatics, Radboud Institute for Molecular Life Sciences (RIMLS), Radboudumc, Nijmegen, Netherlands, ${ }^{19}$ Department of Human Genetics, RIMLS, Radboudumc, Nijmegen, Netherlands

Pathogenic variants in an increasing number of genes are associated with cancer, if somatic, and with neurodevelopmental disorders, if in the germline. SPOP (MIM: 602650) is recurrently somatically mutated in endometrial cancer and prostate cancer, resulting in gain-of-function and dominantnegative effects, respectively, towards ubiquitin ligase substrates, such as BRD2, BRD3 and BRD4 proteins (BETs). Using clinical exome sequencing, we identified eleven patients with nine different germline de novo missense SPOP variants, including two recurrent variants. These patients presented with developmental delay/intellectual disability, facial dysmorphisms and congenital anomalies. Interestingly, three individuals shared craniofacial dysmorphisms, including microcephaly, which were strikingly different from those observed in the other individuals, which included (relative) macrocephaly and hypertelorism. Based on these findings, we hypothesized that two distinct molecular pathophysiological mechanisms underlie the phenotypic differences, similarly to the observed effect of somatic SPOP variants in cancer. Indeed, measurement of BETs levels in human Ishikawa endometrial cancer cells and patient-derived cell lines showed that de novo variants identified in the microcephalic individuals result in a gain-of-function, while the other variants lead to a dominant-negative effect. These results suggest that divergent functional effects on BETs are related to distinct and clinically recognizable syndromic forms of intellectual disability with contrasting craniofacial dysmorphisms.

M.J. Nabais Sá: None. G. El Tekle: None. A.P.M. de Brouwer: None. S.L. Sawyer: None. D. del Gaudio: None. M.J. Parker: None. F. Kanani: None. M.H. van den Boogaard: None. K. van Gassen: None. M.I. Van Allen: None. K. Wierenga: None. G. Purcarin: None. E. Roy Elias: None. K.W. Gripp: None. R. Procopio: None. C. Mignot: None. E. Argilli: None. E. Sherr: None. G. 
Cappuccio: None. N. Brunetti-Pierri: None. A. Begtrup: A. Employment (full or part-time); Significant; GeneDx. J. Keller-Ramey: A. Employment (full or part-time); Significant; GeneDx. E. Ryan: A. Employment (full or parttime); Significant; GeneDx. T. Bernasocchi: None. L. van de Wiel: None. H. Venselaar: None. C. Gilissen: None. R. Pfundt: None. L.E.L.M. Vissers: None. J.P. Theurillat: None. B.B.A. de Vries: None.

\section{P08.108.C}

A new patient with a de novo $S S R 4$ variant revealed using exome sequencing contributes to the definition of phenotype and genotype of the congenital disorder of glycosylation type Iy

M. Hančárová ${ }^{l}, M$. Vlčkovál ${ }^{l} \check{S}_{\text {. Bendová }}{ }^{l}, D$. Prchalovál, V. Stránecký2 ${ }^{2}$, Z. Sedláček ${ }^{l}$

${ }^{I}$ Department Of Biology And Medical Genetics, 2nd Faculty of Medicine, Charles University, Prague, Czech Republic, ${ }^{2}$ Department of Pediatrics and Adolescent Medicine, Diagnostic and Research Unit for Rare Diseases, Charles University 1st Faculty of Medicine and General University Hospital, Prague, Czech Republic

Congenital disorders of glycosylation (CDG) are characterized by deficiency in processing of glycans. CDG type Iy (MIM300934) is a rare X-linked disorder caused by lossof-function variants in SSR4 encoding signal sequence receptor 4. CDG Iy is associated with developmental delay (DD), intellectual disability (ID), hypotonia, microcephaly, facial dysmorphism, failure to thrive and epilepsy. Carrier females are only mildly affected. SSR4 was linked with CDG Iy in 2014, and since then only 10 patients with sufficient genotype and phenotype details have been reported. We describe a 5-year-old boy with hypotonia, feeding problems, failure to thrive, DD, speech delay, microcephaly, abnormal EEG, history of seizures and facial dysmorphism (low-set dysplastic ears, preauricular pit, enophthalmos, broad nasal bridge, telecanthus, midface hypoplasia, long smooth philtrum and thin upper lip vermilion). Exome sequencing (ES) revealed a de novo hemizygous frameshift SSR4 variant NM_006280.3: c.200_2001delA, p.Y67Cfs*2 which was absent from all databases. Our patient confirms that males with CDG due to SSR4 variants show consistent phenotype with DD/ID, hypotonia, microcephaly, facial dysmorphism, feeding problems, failure to thrive and gastrointestinal and eye abnormalities. Seizures have been reported in $6 / 10$ previous patients, and our patient supports their involvement in the syndrome. On the contrary, skeletal, coagulation, cardiac and renal abnormalities mentioned among minor features were absent in our patient. A similar clinical picture is seen in many CDG types, and changes in serum transferrin levels in CDG Iy patients are easily overlooked. Therefore ES is indispensable in the deciphering of this rare disorder. Supported by 17-29423A, LM2018132 and 00064203.

M. Hančárová: None. M. Vlčková: None. Š. Bendová: None. D. Prchalová: None. V. Stránecký: None. Z. Sedláček: None.

\section{P08.109.A}

A novel SUZ12 nonsense mutation in patient with mild Cornelia de Lange-syndrome

\section{B. Hanker ${ }^{I}$, Y. Hellenbroich ${ }^{I}$, M. Wagner ${ }^{2}$, F. J. Kaiser ${ }^{3}$, I. Hüning ${ }^{l}$}

${ }^{l}$ Institut für Humangenetik, Universität zu Lübeck, Lübeck, Germany, ${ }^{2}$ Institute of Human Genetics, Technical University Munich, Munich, Germany, ${ }^{3}$ Institut für Humangenetik, Universitätsklinikum Essen, Essen, Germany

Polycomb-group proteins (PcGs) are evolutionarily conserved and are best known for their role in gene silencing. $S U Z 12$ codes for the polycomb repressive complex 2 subunit protein. Variants in genes encoding other components of PRC2 (EZH2 and EED) have been reported as causative for Weaver syndrome and Cohen-Gibson syndrome. In 2017, Imagawa et al. reported first of a patient with Weaver-like syndrome due to a mutation in SUZ12.

We report on an eight year old girl, born at term after uneventful pregnancy with body measurements within normal range. Postnatally a VSD and a persistent left vena cava were detected. Our patient has mild intellectual disability and facial features including synophrys, hypertelorism, long and deep seated ears. She showed no overgrowth, had mild hearing impairment and hypermetropia.

In suspicion of mild Cornelia de Lange-syndrome sequencing analysis of known CdLS genes on DNA isolated from blood or fibroblast were conducted but could not identify a causative variant. Karyotyping was normal and array-CGH showed a paternally inherited microduplication 16p11.2. Subsequent trio-exome sequencing revealed a de novo heterozygous nonsense variant in SUZ12 (NM_015355.2: c.1657C >T, p.Gln553Ter).

Systematic review of the literature showed only 13 patients with pathogenic variants in SUZ12 reported so far. Nine of them had overgrowth. Missense variants appear to be stronger associated with overgrowth rather than nonsense variants (Cyrus et al., 2020). Facial features suggestive for CdLS were not reported. Our findings expand the phenotypical spectrum of variants in SUZ12 and link the spectrum of CdLS-like phenotypes with another chromatin associated complex. 
B. Hanker: None. Y. Hellenbroich: None. M. Wagner: None. F.J. Kaiser: None. I. Hüning: None.

\section{P08.110.B}

Mild clinical features aid in the interpretation of syndromic gene variants in apparent non-syndromic intellectual disability patients

\section{H. Syryn, O. Vanakker}

Center for Medical Genetics, Ghent University Hospital, Gent, Belgium

Introduction: Before the common use of whole exome sequencing (WES) in routine diagnostics, hereditary syndromes were defined by distinct clinical features. However, with the increasing use of WES-based diagnostics, in particular for indications with large numbers of causal genes such as intellectual disability (ID), very mild or atypical phenotypic presentations of known syndromic causes can be observed.

Methods: We present several patients with thorough clinical description, presenting with ID in whom no syndromic diagnosis was made prior to testing. A confirmed molecular diagnosis via whole exome sequencing of a targeted panel of ID genes was made with (likely) pathogenic variants in genes such as WAC, CHD8, TBRl, and SOX5. We compare their phenotype to what has been traditionally reported in the syndromes associated with these genes and delineate mild key features useful for evaluating causality of the sequencing results.

Results: A first patient with a WAC mutation presents with a duplicated ureter and aggressive behavior. In patients with CHD8-related ID, early bone maturation is seen. Furthermore, pathogenic variants in TBRI can lead to exclusively developmental delay and absent speech. Finally, lower limbs abnormalities such as seen in LambShaffer syndrome (SOX5) contribute to determining the diagnosis.

Conclusions: We define several mild but characteristic features in patients having a variant in syndromic genes, even if an overt syndromic diagnosis in them was not clear. Taking into account these mild symptoms in patients with apparent non-syndromic ID is an important help in interpreting sequencing results, particularly in case of variants of unknown significance.

H. Syryn: None. O. Vanakker: None.

P08.111.C

Combined comprehensive approach in patients within Rett/Angelman phenotypic spectrum
A. Alexandrou ${ }^{l}$, A. Theodosiou ${ }^{l, 2}$, I. Papaevripidou ${ }^{l}$, A. Chrysanthou $^{l, 2}$, N. Salameh ${ }^{l}, P$. Evangelidou ${ }^{1,2}, V$. Christophidou-Anastasiadou ${ }^{3}$, G. A. Tanteles ${ }^{4,2}$, C. Sismani $^{1,2}$

${ }^{1}$ Cytogenetics and Genomics Department, The Cyprus Institute of Neurology and Genetics, Nicosia, Cyprus, ${ }^{2}$ The Cyprus School of Molecular Medicine, The Cyprus Institute of Neurology and Genetics, Nicosia, Cyprus, ${ }^{3}$ Clinical Genetics Department, Archbishop Makarios III Medical Centre, Nicosia, Cyprus, ${ }^{4}$ Clinical Genetics Department, The Cyprus Institute of Neurology and Genetics, Nicosia, Cyprus

Angelman and Rett syndromes are rare neurodevelopmental disorders sharing a range of phenotypic characteristics between them but also with many disorders, making accurate diagnosis challenging.

Here, we present a targeted custom NGS panel (AgilentSureDesign), developed for patients in the Rett/Angelman phenotypic spectrum. The panel includes coding regions and splice site junctions of 25 genes, causing disorders with overlapping clinical phenotypes such as Pitt-Hopkins and Mowat Wilson. Bioinformatics analysis was performed for detecting SNVs, indels and CNVs using Surecall (Agilent) along with in-house established pipelines. The panel design proved successful with a mean target depth of 550X and mean uniformity of $99 \%$.

Upon validation, 74 patients were referred for testing. Thirty patients had classic Rett or Angemann Syndrome features and were tested by Sanger sequencing and MLPA/ MS-MLPA analyses for MECP2 or UBE3A as a first tier test. Six were found positive for deletions or point mutations (one of which mosaic) and were included in the panel only as blind positive samples. All positive blind samples were successfully identified.

Out of the 68 undiagnosed patients that were analysed with the panel, 9 (6 novel) were found to have de novo pathogenic variants in UBE3A, EHMT1, CTNNB1, IQSEC2, EHMT1, WDR45 and SCN1A genes and were confirmed with Sanger sequencing. Additional 11 patients were found to have a VOUS, yielding a total detection rate of at least $13 \%$.

This custom designed panel will improve clinical detection rate and provide a comprehensive accurate testing for individuals suggestive of a disorder in Rett/Angelman spectrum.

A. Alexandrou: None. A. Theodosiou: None. I. Papaevripidou: None. A. Chrysanthou: None. N. Salameh: None. P. Evangelidou: None. V. ChristophidouAnastasiadou: None. G.A. Tanteles: None. C. Sismani: None. 


\section{P08.112.A}

Neurodevelopmental and neurologic spectrum in TECPR2-associated congenital disorder of autophagy

\author{
S. Neuser ${ }^{1}$, I. Brösse ${ }^{2}$, L. O'Grady ${ }^{3}$, D. A. Sweetser ${ }^{3}, S$. \\ Schubert ${ }^{l}$, R. Jamra ${ }^{l}$, B. Popp ${ }^{l}$
}

${ }^{1}$ Institute of Human Genetics, University of Leipzig Medical Center, Leipzig, Germany, ${ }^{2}$ Department of Pediatrics Hospital for Children and Adolescents, Heidelberg University Hospital, Heidelberg, Germany, ${ }^{3}$ Department of Medical Genetics and Metabolism, Massachusetts General Hospital, Boston, MA, United States

Autophagosomes are subcellular compartments necessary to degrade cytoplasmic content. Macro-autophagy is vital for long-lived, non-regenerating and metabolically active neurons. In 2012 a truncating founder variant in the autophagyrelated gene TECPR2 was identified in three Bukharian Jewish families. The five affected individuals were homozygous and showed spastic paraplegia complicated by syndromic intellectual disability. Subsequent reports of five unrelated individuals with this or novel truncating/missense variants confirmed autonomic involvement as a leading feature of spastic paraplegia 49 (SPG49).By trio-based exome sequencing we now identified the homozygous frameshifting TECPR2-variant c.2829del, p.(Asn944Thrfs*7) in male individual of Turkish descent with neurodevelopmental disorder (NDD) and epilepsy. RT-PCR on PAXgene stabilized blood showed normal expression of the c.2829del allele in the heterozygous parents and gel electrophoresis indicated no expression reduction in the index. Together these results suggest escape from nonsense-mediated decay. Through matchmaking we identified one additional unrelated individual with NDDs due to homozygosity for the known Ashkenazi TECPR2-variant (c.1319del, p. (Leu440Arg $\left.f s^{*} 19\right)$ ). Based on these cases and comparison with the 10 literature cases, we now provide a Human Phenotype Ontology (HPO) standardized clinical synopsis. The neurologic spectrum comprises muscular hypotonia with progression to spastic paraplegia in the first decade, abnormal reflexes and autonomic involvement. The variable neurodevelopmental disorder present in 11/12 cases can be complicated by structural brain abnormalities (7/8 cases with brain MRI) and seizures (3/12). Because NDD is a central feature in addition to the neurologic presentation, we propose to classify this disease as TECPR2-associated congenital disorder of autophagy.

S. Neuser: None. I. Brösse: None. L. O’Grady: None. D.A. Sweetser: None. S. Schubert: None. R. Jamra: None. B. Popp: None.

\section{P08.113.B}

Thauvin-Robinet-Faivre Syndrome: Report of a new patient

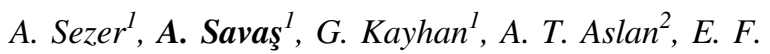
Perçin ${ }^{1}$

${ }^{1}$ Gazi University, Faculty of Medicine, Department of Medical Genetics, Ankara, Turkey, ${ }^{2}$ Gazi University Faculty of Medicine, Department of Pediatrics, Division of Pediatric Pulmonology, Ankara, Turkey

Introduction: Biallelic mutations in the acidic fibroblast growth factor intracellular-binding protein gene (FIBP) cause Thauvin-Robinet-Faivre syndrome (OMIM \#617107, TROFAS), which is characterized by overgrowth. To date, only four different patients have been reported from two unrelated families.

Patient and Methods: Whole-exome sequencing (WES) analysis was performed in a 4-year-old male patient with tall stature, macrosomia, macrocephaly, dysmorphic facial features, recurrent pulmonary infections, cryptorchidism, spontaneous erection, elbow joints laxity, prominent superficial blood vessels, developmental delay and speech delay. He had consanguineous parents.

Result: A homozygous frameshift variant c.415_416insCAGTTTG (Asp139AlafsTer3) in FIBP gene (NM_004214.5) was detected and classified as pathogenic variant. Additionally, in the Toll-Like receptor 5 gene (TLR5) (NM_003268.6), c.974A>G (p.Asn325Ser) homozygous missense variant was detected and was classified as uncertain significance.

Conclusions: Two variants in FIBP gene previously detected in patients with TROFAS have a loss-of-function effect putatively similar to the variant in our patient which caused putatively a premature protein. The patient had a history of recurrent pulmonary infections, cryptorchidism and spontaneous erection, unlike reported patients. It is not known whether our patient's history of recurrent pulmonary infections is a component of TROFAS because of the limited number of patient reports. Primary immunodeficiencies and polymorphisms in TLRs are associated with susceptibility to infections in humans and animal models and also TLR5 has protective effects against bacterial infections. Owing to the limited number of reported patients with TROFAS, new patient reports and further studies are needed to clarify the clinical properties of the disease.

A. Sezer: None. A. Savaş: None. G. Kayhan: None. A. T. Aslan: None. E.F. Perçin: None.

P08.115.A

A new case of Kaufman Oculocerebrofacial Syndrome caused by two new splicing variants in UBE3B. 
K. Uguen ${ }^{1,2,3}, C . K^{1,3}$, M. Planes $^{1,2}$, S. AudebertBellanger $^{1,2}$, S. Redon ${ }^{1,2,3}$, C. Benech ${ }^{1,3}$, S. Küry ${ }^{4,5}, S$. Peudenier $^{2}$, S. Autret ${ }^{1,3}$, I. Gourlaouen ${ }^{3}$, D. Bonneau ${ }^{6}, S$. Odent $^{7}$, S. Bézieau ${ }^{4,5}$, B. Gilbert-Dussardier ${ }^{8}$, A. Boland ${ }^{9}$, J. Deleuze $^{9}$, C. Le Maréchal ${ }^{1,2,3}$, G. Le Gac ${ }^{1,3}$, C. Ferec ${ }^{1,2,3}$

${ }^{1}$ Service de Génétique Médicale et Biologie de la Reproduction, CHRU de Brest, Brest, France, ${ }^{2}$ Centre de Référence Déficiences Intellectuelles de causes rares, Brest, France, ${ }^{3}$ Univ Brest, Inserm, EFS, UMR 1078, GGB, F29200, Brest, France, ${ }^{4} \mathrm{CHU}$ de Nantes, Service de Génétique Médicale, 9 quai Moncousu, 44093 Nantes CEDEX 1, Nantes, France, ${ }^{5}$ Université de Nantes, CNRS, INSERM, l'institut du thorax, F-44000, Nantes, France, ${ }^{6}$ Département de biochimie et génétique, CHU d'Angers, Angers, France, ${ }^{7}$ Service de Génétique Clinique, CHU de Rennes, UMR6290 CNRS, Université Rennes, Rennes, France, ${ }^{8}$ Service de Génétique, CHU de Poitiers, Poitiers, France, ${ }^{9}$ Centre National de Recherche en Génomique Humaine (CNRGH), CEA, Evry, France

Introduction: $U B E 3 B$ encodes a member of the E3 ubiquitin-conjugating enzyme family. Biallelic variants (missense, nonsense, splicing) are responsible of the Kaufman Oculocerebral Syndrome (KOS), characterized by severe intellectual disability, distinctive facial features, short stature and multiple congenital abnormalities. Here we report a patient presenting with KOS and harboring two undescribed splicing variants in $U B E 3 B$. We describe new clinical features and in vitro characterization of the two variants.

Case presentation: The propositus is an 18-year-old boy presenting with intellectual disability, microphtalmia, bilateral deafness, tetralogy of Fallot, scoliosis, hip malformation and facial dysmorphism consistent with KOS. Other clinical features not described in KOS include anal imperforation and cryptorchidism.

Results: Through a French collaborative study (HUGODIMS), trio-based whole exome sequencing identified two variants in $U B E 3 B$ : c. $1956+3 \mathrm{G}>\mathrm{C}$ and c. $1622+1 \mathrm{G}>\mathrm{C}$, each one inherited from a healthy parent. None of the variants was found in population databases. Minigene assays were performed to evaluate the effects of both variants in transiently transfected HEK293T cells. The c. $1956+3 \mathrm{G}>\mathrm{C}$ induced skipping of exon 18 with a shift in the reading frame. The c. $1622+1 \mathrm{G}>\mathrm{C}$ variant resulted in the retention of the first $61 \mathrm{bp}$ of intron 15 and the immediate insertion of a stop codon. The two variants were therefore predicted to be highly deleterious.

Conclusions: We expand the phenotype of KOS, further highlighting the interest of in vitro assays to demonstrate causality of new splicing variants.
K. Uguen: None. C. Ka: None. M. Planes: None. S. Audebert-Bellanger: None. S. Redon: None. C. Benech: None. S. Küry: None. S. Peudenier: None. S. Autret: None. I. Gourlaouen: None. D. Bonneau: None. S. Odent: None. S. Bézieau: None. B. Gilbert-Dussardier: None. A. Boland: None. J. Deleuze: None. C. Le Maréchal: None. G. Le Gac: None. C. Ferec: None.

\section{P08.116.B}

A novel loss of function mutation of the WAC gene in a subject with intellectual disability and autistic features associated with encephalopathy related to status epilepticus during slow sleep

E. Leonardi ${ }^{l, 2}, M$. Bellini ${ }^{1,2}, M$. C. Aspromonte ${ }^{l, 2}, R$.

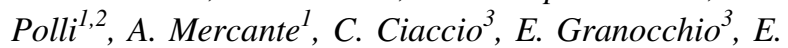
Bettella $^{1,2}$, S. Sartori ${ }^{1}$, I. Donati ${ }^{4}$, C. Pantaleoni ${ }^{3}$, C. Boniver $^{l}$, A. Murgia ${ }^{1,2}$

${ }^{I}$ Dept. Woman and Child Health, University of Padua, Padova, Italy, ${ }^{2}$ Pediatric Research Institute - Città della Speranza, Padova, Italy, ${ }^{3}$ Developmental Neurology Unit, Fondazione IRCCS Istituto Neurologico Carlo Besta, Milano, Italy, ${ }^{4}$ AUSL Romagna Infermi Hospital, Rimini, Italy

Alterations of the WAC gene have been recently associated with a neurodevelopmental disorder characterized by intellectual disability, neonatal hypotonia, behavioral problems, and mildly dysmorphic features. To date only 20 individuals have been reported with WAC mutations, which, in the majority of the cases, are de novo loss of function (LoF). Using targeted deep sequencing, we screened a cohort of 600 individuals with variable degrees of intellectual disability and identified five WAC rare variants: two variants were inherited from healthy parents; two previously reported de novo mutations, c.1661_1664del (p.Ser554*) and c. $374 \mathrm{C}>\mathrm{A}$ (p.Ser125*); and a novel c. $381+2 \mathrm{~T}>\mathrm{C}$ variant causing the skipping of exon 4 of the gene, inherited from a reportedly asymptomatic father with somatic mosaicism. A phenotypic evaluation of this individual evidenced areas of cognitive and behavioural deficits. The two individuals with previously reported WAC mutations presented phenotypic differences in comparison with reported cases. The patient carrying the novel splicing mutation had a clinical history of encephalopathy related to Status Epilepticus during slow Sleep (ESES), only recently reported in WAC individuals. This first report of a WAC somatic mosaic remarks the contribution of mosaicism in the etiology of neurodevelopmental and neuropsychiatric disorders. We summarized the clinical data of reported individuals with WAC LoF variants, which together with our findings allowed 
expanding the phenotypic spectrum of WAC-related disorders.

E. Leonardi: None. M. Bellini: None. M.C. Aspromonte: None. R. Polli: None. A. Mercante: None. C. Ciaccio: None. E. Granocchio: None. E. Bettella: None. S. Sartori: None. I. Donati: None. C. Pantaleoni: None. C. Boniver: None. A. Murgia: None.

\section{P08.118.A}

Genotype-phenotype correlation on 45 individuals with West syndrome

I. Krey ${ }^{l}$, J. Krois-Neudenberger ${ }^{2}$, J. Hentschel ${ }^{l}$, S. Syrbe ${ }^{3}$, T. Polster ${ }^{4}$ B. Hanker ${ }^{5}$, B. Fiedler ${ }^{6}$, G. Kurlemann ${ }^{7}, J . R$. Lemke $^{l}$

${ }^{1}$ Institute of Human Genetics, University of Leipzig Medical Center, Leipzig, Germany, ${ }^{2}$ Department of General Pediatrics, Division of Neuropediatrics, University Hospital, University Hospital Muenster, Germany, Germany, ${ }^{3}$ Division of Child Neurology and Metabolic Medicine, Center for Paediatrics and Adolescent Medicine, University Hospital Heidelberg, Heidelberg, Germany, ${ }^{4}$ Bethel Epilepsy Center, Mara Hospital, Bielefeld, Bielefeld, Germany, ${ }^{5}$ Institute of Human Genetics, University of Lübeck, Lübeck, Lübeck, Germany, ${ }^{6}$ Department of General Pediatrics, Division of Neuropediatrics, University Hospital Muenster, Münster, Germany, ${ }^{7}$ Hospital for Children, Bonifatius Hospital Lingen, Lingen, Germany

West syndrome is an epilepsy syndrome characterized by repetitive epileptic spasms (ES) and hypsarrhythmia, typically leading to developmental delay/intellectual disability (DD/ID). It is considered a classic epileptic encephalopathy (EE). We designed a diagnostic sequencing panel targeting 131 genes associated with epilepsy and/or EE and screened a cohort of 45 individuals with clinical diagnosis of West syndrome. We identified disease-causing single nucleotide variants in 11 out of 45 individuals affecting genes commonly associated with West syndrome (such as CDKL5, ARX) but also in genes pre- dominantly linked to other epileptic disorders (such as DEPDC5, SCN1A, WDR45, AARS). Panel analysis revealed copy number variants in two additional cases, comprising a 6,7 Mb Duplication on chromosome 2 including $\mathrm{SCN} 2 \mathrm{~A}$ and $\mathrm{SCN} 3 \mathrm{~A}$ and a supernumerary marker chromosome 15 leading to an overall diagnostic yield of $29 \%$ (13/45).

In our cohort, individuals with a disease-causing variant had significantly more severe phenotypes with respect to DD/ID, therapy resistant epilepsy and cerebral atrophy compared to genetically unclarified cases.

In addition to investigating the genotypic spectrum of West syndrome, we compared the phenotypic spectrum of clarified versus unclarified cases. Our study illustrates that West syndrome is an electro- clinical syndrome caused by various genetic disorders. Individuals without detectable genetic cause might have less encephalopathy leading to a less severe course.

(C) 2019 European Paediatric Neurology Society. Published by Elsevier Ltd. All rights reserved.

I. Krey: None. J. Krois-Neudenberger: None. J. Hentschel: None. S. Syrbe: None. T. Polster: None. B. Hanker: None. B. Fiedler: None. G. Kurlemann: None. J.R. Lemke: None.

P08.120.C

Wiedemann-Steiner Syndrome: clinical and molecular characterization of $\mathbf{1 1}$ patients from four Portuguese hospital centers

S. Fernandes ${ }^{1}$, C. S. Rosas ${ }^{1}$, M. Soeiro e Sán ${ }^{2}$ I. Carvalho ${ }^{3}$,

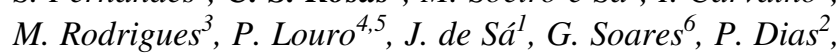
L. Ramos ${ }^{1,5}$, J. M. Saraiva ${ }^{1,7}$, S. B. Sousa ${ }^{1,8}$

${ }^{l}$ Medical Genetics Unit, Hospital Pediátrico, Centro Hospitalar e Universitário de Coimbra, Coimbra, Portugal, ${ }^{2}$ Serviço de Genética Médica, Departamento de Pediatria, Centro Hospitalar Lisboa Norte, Hospital de Santa Maria, EPE, Centro Académico de Medicina de Lisboa, Lisboa, Portugal, ${ }^{3}$ Medical Genetics Unit, Hospital Dona Estefânia, Centro Hospitalar Lisboa Central, Lisboa, Portugal, ${ }^{4}$ IPO, Lisboa, Former worker at Medical Genetics Unit, Hospital Pediátrico, Centro Hospitalar e Universitário de Coimbra, Coimbra, Portugal, ${ }^{5}$ Faculty of Health Sciences, Universidade da Beira Interior, Covilhã, Portugal, ${ }^{6}$ Serviço de Genética Médica Dr. Jacinto Magalhães, Centro Hospitalar do Porto, Porto, Portugal, ${ }^{7}$ University Clinic of Pediatrics, Faculty of Medicine, Universidade de Coimbra, Coimbra, Portugal, ${ }^{8}$ University Clinic of Genetics, Faculty of Medicine, Universidade de Coimbra, Coimbra, Portugal

Context: Wiedemann-Steiner Syndrome (WSS) is an autosomal dominant condition comprising intellectual disability, short stature, typical facial features and hypertrichosis. It is caused by loss-of-function variants in $K M T 2 A$ gene, leading to chromatin remodelling defects.

Methods: Clinical and molecular characterization of all cases with WSS diagnosis from 4 Portuguese hospital centres based on retrospective analysis of patient medical records. Clinical exome (7/11) or KMT2A targeted NGS (4/ 11) was or is being performed in all cases.

Results: We describe 8 male and 3 female unrelated patients. The age of diagnosis ranged between 2 and 18 years. The main reasons for referral were intellectual disability (8/11) and dysmorphic features (7/11). Most 
individuals had mild to moderate intellectual disability (11/ 11), behavioural problems (9/9), narrow palpebral fissures $(10 / 11)$ and downslanted (8/10), long eyelashes $(6 / 11)$, thick eyebrows (5/10) and wide nasal bridge (10/11). Hypertrichosis cubiti was present in 10/10. Short stature was present in 3/11 individuals, recurrent infections in 4/7 and sleep apnoea in 2/6 cases, one requiring non-invasive ventilation. We identified 7 novel KMT2A heterozygous variants and 3 previously described pathogenic variants. One case is awaiting for results.

Discussion: Our data are in accordance with the literature, except for short stature which seems to have lower prevalence than expected. Clinical suspicion is often challenging as the phenotypic variability is wide. In 6/10 cases, the diagnosis was not initially considered and in 4 of those only achieved by reverse phenotyping. Detailed description of national cohorts of WSS patients contributes to awareness and better-informed counselling and management.

S. Fernandes: None. C. S. Rosas: None. M. Soeiro e Sá: None. I. Carvalho: None. M. Rodrigues: None. P. Louro: None. J. de Sá: None. G. Soares: None. P. Dias: None. L. Ramos: None. J. M. Saraiva: None. S. B. Sousa: None.

\section{P08.122.B}

Biallelic ZNF407 mutation in a neurodevelopmental disorder with ID, short stature and variable microcephaly, hypotonia, ocular anomalies and facial dysmorphism

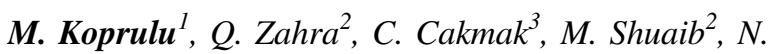
Sobreira $^{4}$, L. Kalsner ${ }^{5}$, J. Sobreira ${ }^{6}$, M. Guillen Sajoto ${ }^{7}$, S. Malik $^{2}$, A. Tolun ${ }^{1}$

${ }^{1}$ Istanbul Technical University, Istanbul, Turkey, ${ }^{2}$ Quaid-iAzam University, Islamabad, Pakistan, ${ }^{3}$ Bogazici University, Istanbul, Turkey, ${ }^{4}$ Johns Hopkins University School of Medicine, Baltimore, MD, United States, ${ }^{5}$ University of Connecticut School of Medicine and Connecticut Children's Medical Center, Farmington, CT, United States, ${ }^{6}$ Universidade Federal de Sao Paulo, Sao Paulo, Brazil, ${ }^{7}$ GeneDx, Gaithersburg, MD, United States

Background: We describe five members of a consanguineous Pakistani family (Family I) plus two unrelated patients of different ethnic origins presenting with neurodevelopmental disorders with overlapping features and biallelic ZNF407 variants. Homozygous c.5054C $>\mathrm{G}$ (p. (Ser1685Trp)) has been reported in two brothers with an intellectual disability (ID) syndrome.
Methods: SNP genotype data were used for mapping the disease locus in Family I. Gene variants were identified by exome sequencing.

Results: All of the patients we describe have ID, ranging from mild to profound, and reduced motor and cognitive skills plus variable features including short stature, microcephaly, developmental delay, hypotonia, dysarthria, deafness, visual problems, enuresis, encopresis, behavioural anomalies, delayed pubertal onset and facial dysmorphism. We first mapped the disease locus in the large family (Family I) and identified homozygous ZNF407 c.2814_2816dup (p.(Val939dup)) in the four patients of the family, and then added to the study two patients of different ethnic origins with biallelic ZNF407 variants: homozygous c.2405G $>\mathrm{T}$ (p. (Gly802Val)) in patient of Family II and compound heterozygous variants c. $2884 \mathrm{C}>\mathrm{G}$ (p. (Arg962Gly)) and c.3642G $>\mathrm{C} \quad$ (p. (Lys1214Asn)) in patient of Family III.

Conclusions: Our findings confirm that ZNF407 is a recessive neurodevelopmental disease gene, biallelic variants of which can manifest with ID and wide phenotypic variability. The observation that all four variants found in our patients are predicted as benign on canonical transcripts by computational algorithms indicates that many other variants may have escaped identification as causal in this large gene with extreme loss-of-function intolerance.

M. Koprulu: None. Q. Zahra: None. C. Cakmak: None. M. Shuaib: None. N. Sobreira: None. L. Kalsner: None. J. Sobreira: None. M. Guillen Sajoto: A. Employment (full or part-time); Modest; GeneDx. S. Malik: None. A. Tolun: None.

\section{P09 Neurogenetic and Psychiatric Disorders}

\section{P09.002.C}

Epilepsy, neuropsychogical phenotype, and neuroimaging findings in 22q11.2 deletion syndrome: a case series.

A. M. Alhashem, N. Alsaleh, H. El Ghezal, H. AlKalaf, I. B. Bouhjar, K. Tlili, B. T. Melaiki

Prince sultan military and medical city, Riyadh, Saudi Arabia

Background: The 22q11.2 deletion syndrome affects multiple organ systems, and the neurological manifestations are an important aspect of this disorder. Many are aware of cardiac anomalies associated with this uncommon genetic disorder. However, the different types of seizures, neuropsychological profile, EEG, and MRI findings seen in this condition are not well appreciated. 
Methods: Through 2010 tell 2019, Medical records of patients with $22 \mathrm{q} 11.2$ deletion syndrome were retrospectively reviewed for documentation of Neurological/neuropsychological evaluation, seizure types, EEG, and brain MRI findings.

Results: 28 patients were collected. 6 (21.4\%) patient have epilepsy, 3 have focal epilepsy and 3 have generalized epilepsy and all of them were controlled on AED. Brain MRI performed in 16 patients: normal in 6 patients $(37.5 \%)$, abnormal in 10 patients: polymicrogyri and cortical dysplasia: 2, thin corpus callosum: 3, vermis hypoplasia: 1, biliateral pallidal calcifications: 1 , developmental cerebellar veinous anomalies: 1 , cervical pilocytic astrocytoma: 1, atrophic changes: 5 Neuropsychological assessment: 3 with average intellectual ability, 9(30\%) with intellectual disabilities of variable range. 3 patients suffered of neuropsychiatric illness.

Conclusions: Patients with 22q11.2DS have a high risk of epilepsy. Patients with brain malformations should have a chromosomal microarray (CMA) performed to screen for the $22 q 11.2$ deletion syndrome. The specific genetic locus is critically linked to seizure type, and neurodevelopmental status.

A.M. Alhashem: None. N. Alsaleh: None. H. El Ghezal: None. H. AlKalaf: None. I.B. Bouhjar: None. K. Tlili: None. B.T. Melaiki: None.

\section{P09.004.B}

Confirmation of ADD3 pathogenic variants as a genetic cause of cerebral palsy

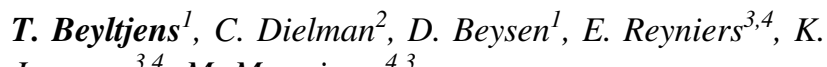
Janssens $^{3,4}$, M. Meuwissen ${ }^{4,3}$

${ }^{1}$ Department of Pediatric Neurology, Antwerp University Hospital, Edegem, Belgium, ${ }^{2}$ Department of Pediatric Neurology, Queen Paola Children's Hospital, Antwerp, Belgium, ${ }^{3}$ Center for Medical Genetics, University of Antwerp, Edegem, Belgium, ${ }^{4}$ Center for Medical Genetics, Antwerp University Hospital, Edegem, Belgium

Introduction: Cerebral palsy (CP) is a clinical descriptive term that defines a heterogeneous group of non-progressive, neurodevelopmental disorders of motor impairment, which co-occur with a wide range of medical conditions, such as intellectual disability (ID), speech/language deficits, autism, epilepsy and visual and/or hearing impairment. CP is caused by a cerebral anomaly/dysfunction that develops during pregnancy, birth or infancy. $\mathrm{CP}$ is still the most common cause of physical disability in childhood with an important impact on the quality of life of patients and, by its high prevalence of $1.5-2.5$ per 1000 live births, even on society. Recent studies demonstrate an important contribution of genetic factors.
Methods: At the Antwerp University Hospital, we therefore perform genetic testing for CP using SNP-array and a targeted whole exome sequencing-based gene panel consisting of approximately 200 genes associated with $\mathrm{CP}$ and CP-mimics.

Results: We report the findings on two siblings from a consanguineous family presenting with spastic $\mathrm{CP}$, and mild to moderate ID. Brain MRI showed hypoplastic corpus callosum and periventricular white matter abnormalities. WES demonstrated the homozygous pathogenic variant c.1100G >A (p.Gly367Asp) in ADD3 in both cases.

Conclusions: In literature, only one report describes a homozygous $A D D 3$ variant as a cause of $\mathrm{CP}$ in a consanguineous family with similar clinical findings of spastic quadriplegia or diplegia, variable ID and cerebral white matter abnormalities. Interestingly, the same variant was identified in our family, which suggests the possibility of a founder mutation. Our findings confirm the role of $A D D 3$ in $\mathrm{CP}$.

T. Beyltjens: None. C. Dielman: None. D. Beysen: None. E. Reyniers: None. K. Janssens: None. M. Meuwissen: None.

\section{P09.005.C}

Dissecting genetic factors involved in the relation between behavioural laterality and neurodevelopmental conditions

J. Schmitz ${ }^{1}$, M. Zheng ${ }^{2}$, K. F. H. Lui ${ }^{2}$, C. S. H. Ho ${ }^{3}$, C. McBride $^{2}$, S. Paracchini ${ }^{1}$

${ }^{1}$ School of Medicine, University of St Andrews, St Andrews, United Kingdom, ${ }^{2}$ Department of Psychology, Chinese University of Hong Kong, Hong Kong, Hong Kong, ${ }^{3}$ Psychology Department, The University of Hong Kong, Hong Kong, Hong Kong

Introduction: The cerebral hemispheres differ in structure and function underpinning specialisation for cognition, perception, and motor control. Atypical brain asymmetries have been implicated in psychiatric and neurodevelopmental conditions. Handedness is often used as a proxy for brain asymmetries and recent genome-wide association studies (GWAS) suggest a genetic association with schizophrenia, while other laterality measures are less explored. Here, we investigate shared genetic components between behavioural laterality phenotypes and their relevance to these conditions.

Methods and Results: We performed GWAS on hand, foot, eye and ear preference in a longitudinal British cohort (ALSPAC, $n>=5770$ ). Our results suggest an involvement of genes associated with immune response (footedness) and 
axonogenesis (eye, ear preference). In line with previous research, genes involved in body asymmetries were associated with handedness. The relevance of neurodevelopmental conditions was tested using gene set enrichment analyses and polygenic risk scores (PRS) for schizophrenia, ADHD, autism spectrum disorders and bipolar disorder. PRS for ADHD were associated with left- and more strongly with non-right-handedness, but not with other laterality phenotypes. Multivariate structural equation models (SEM) (genome-wide genetic markers in ALSPAC and classic multivariate SEM in a Chinese twin cohort $(n=$ 486)) revealed shared genetic factors for hand and foot, but not eye preference, suggesting a dissociation of motor and sensory laterality.

Conclusions: These results give further support to a shared biology between laterality and neurodevelopment, which is potentially mediated by brain asymmetry. Funding: JS is a DFG fellow (SCHM 3530/1-1). This work was funded by Royal Society grants (SP).

J. Schmitz: None. M. Zheng: None. K.F.H. Lui: None. C.S.H. Ho: None. C. McBride: None. S. Paracchini: None.

\section{P09.006.A}

Assessment of SORL1 rare variants segregation in Alzheimer disease families and in vitro models show reduced penetrance and oligogenic inheritance

G. Nicolas, C. Schramm, L. Miguel, M. Lacour, S. Rousseau, D. Wallon, C. Charbonnier, A. Zaréa, T. Frebourg, D. Campion, A. Rovelet-Lecrux, M. Lecourtois

Normandie Univ, UNIROUEN, Inserm U1245 and Rouen University Hospital, Department of Genetics and CNRMAJ, $F$ 76000, Normandy Center for Genomic and Personalized Medicine, ROUEN, France

Introduction: SORL1 rare loss-of-function (LOF) and missense predicted damaging (Mis3) variants have first been identified in early-onset Alzheimer Disease (EOAD, onset $\leq 65$ ) probands with a pedigree suggesting autosomal dominant inheritance. Then, increased burdens of rare SORL1 LOF and Mis3 variants were reported in $\mathrm{AD}$ patients with odds ratios ranging from 1.9 to 29 . Hence, using SORL1 rare variants for genetic counseling appears debatable. In addition, a functional effect of Mis3 variants cannot be inferred from bioinformatics predictions only.

Methods: We included families with a rare LOF or Mis3 variant, evaluated their segregation, and developed a functional assay.

Results: We studied 20 families with a LOF variant (mean age at onset (AAO) in probands, 55.4 years, range: [48-65]). Seventeen showed a positive family history. Mean
AAO in affected relatives was 66.3 [50-80]. One patient carried two LOF compound heterozygous variants. DNA was available in 18 relatives: $5 / 6$ affected relatives carried the familial variant $(\mathrm{AAO}=[61-78])$ while $3 / 12$ unaffected were also carriers, aged 42,71 and 95 , respectively. Strikingly, 57\% affected carriers were APOE4+, a strong $\mathrm{AD}$ risk variant, including one being homozygous, who also carried a LOF $A B C A 7$ risk variant.

We introduced one LOF variant and one Mis 3 variant using CRISPR/Cas9 in iPSCs and observed increased levels of $A \beta$ in the supernatant. The proband carrying this Mis3 variant was APOE4+ and carried a LOF rare $A B C A 7$ risk variant. We are now assessing additional missense variants.

Conclusions: SORL1 LOF variants are not fully penetrant and can be observed in an oligogenic context.

G. Nicolas: None. C. Schramm: None. L. Miguel: None. M. Lacour: None. S. Rousseau: None. D. Wallon: None. C. Charbonnier: None. A. Zaréa: None. T. Frebourg: None. D. Campion: None. A. Rovelet-Lecrux: None. M. Lecourtois: None.

\section{P09.008.C}

Albumin did not increase with Coenzyme Q10 treatment in $\mathrm{AOA1}$ patients

P. CHARLES ${ }^{1}$, V. LADEVEZE ${ }^{2}$, C. TRANCHANT ${ }^{3}, M$.

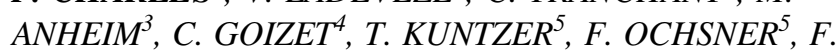

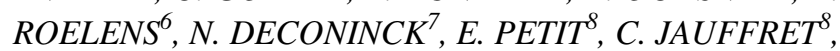
S. RIVAUD-PECHOUX', I. LEBER ${ }^{10}$, A. BRICE ${ }^{11}, S$. TEZENAS DU MONTCEL ${ }^{12}$, A. DURR ${ }^{11}$

${ }^{1}$ APHP, Genetic Department, Medical Genetic UF, Armand-Trousseau and Pitié Salpêtrière Hospitals, Centre de référence Déficiences intellectuelles de causes rares, Département de Neurologie, Hôpital de La Salpêtrière, 47 Bd de l'Hôpital 75651 Paris Cedex 13, Paris, France, ${ }^{2}$ Sorbonne University, INSERM, Institut Pierre Louis d'Epidémiologie et de Santé Publique IPLESP, 75013 Paris, France, Paris, France, ${ }^{3}$ Service de Neurologie, Hôpitaux Universitaires de Strasbourg, Strasbourg, France. Institut de Génétique et de Biologie Moléculaire et Cellulaire (IGBMC), INSERM-U964/CNRS-UMR7104/ Université de Strasbourg, Illkirch, France. Fédération de Médecine Translati, Strasbourg, France, ${ }^{4}$ CHU Bordeaux, Service de Génétique Médicale, Centre de Référence Maladies Rares «Neurogénétique», Laboratoire MRGM, INSERM U1211, Univ. Bordeaux, Bordeaux, France, Bordeaux, France, ${ }^{5}$ Unité Nerf-Muscle, Service de Neurologie, Département des neurosciences cliniques, CHU Vaudois (CHUV) et université de Lausanne, 1011 Lausanne, Suisse, Lausanne, Switzerland, ${ }^{6}$ Pediatric Neurology, AZ Delta, Roeselare, Belgium, Roeselare, Belgium, ${ }^{7}$ Neurologie pédiatrique, HUDERF av J.J. Crocq, 151020 
Bruxelles, ULB, Belgique et NMRC Gent, UZ Gent, Belgique, Bruxelles, Belgium, ${ }^{8}$ Groupe Hospitalier Pitié Salpêtrière ICM - Institut du Cerveau et de la Moelle Epinière 47, Boulevard de l'Hôpital CS21414 75646 PARIS Cedex 13, Paris, France, ${ }^{9}$ INSERM U-1127 Institut du cerveau et de la moelle épinière Hôpital de la Salpêtrière 47-91 boulevard de l'Hôpital 75651 Paris CEDEX 13 France, Paris, France, ${ }^{10}$ Sorbonne Universités, UPMC Univ Paris 06, Inserm U1127, CNRS UMR 7225, Institut du Cerveau et la Moelle épinière (ICM), Centre de référence des démences rares ou précoces, IM2A, Département de Neurologie, AP-HP - Hôpital Pitié-Salpêtrière, Paris, France, Paris, France, ${ }^{11}$ Sorbonne Université, Institut $d u$ Cerveau et de la Moelle épinière (ICM), AP-HP, Inserm, CNRS, University Hospital Pitié-Salpêtrière, Paris, France, Paris, France, ${ }^{12}$ Sorbonne Université, INSERM, Institut Pierre Louis d'Epidémiologie et de Santé Publique IPLESP, AP-HP, Hôpitaux Universitaires Pitié Salpêtrière - Charles Foix, F75013 Paris, France, Paris, France

Ataxia with oculomotor apraxia type 1 (AOA1) is a rare autosomal recessive cerebellar ataxia associated with oculomotor apraxia, hypoalbuminaemia and hypercholesterolaemia linked to Aprataxin (APTX) gene mutations. Aprataxin gene mutations are associated with decreased Coenzyme Q10 levels in muscle and plasma. Disease duration and disability are negatively correlated with albuminemia. We included 19 patients in a multicenter (six centers) controlled randomized double blind trial with Coenzyme Q10 with 2 phases, a controlled randomized phase Coenzyme Q10 versus placebo during 1 year, and an open-labelled phase with all patients treated with Coenzyme Q10 during 1 year, to estimate longer term potential side effects and tolerance. Inclusion criteria were AOA1 confirmed by genetic molecular analysis; age older than 18, hypoalbuminemia, informed consent signed. Primary endpoint was hypoalbuminemia rate evolution. Secondary endpoints were cerebellar ataxia severity (SARA and quantitative evaluations), ocular movements evaluation, quality of life (EQ5-D), depression and anxiety (PHQ-9), biological measurements (prealbuminemia, cholesterolemia, alphafoetoprotein), safety (blood counts and hepatic check) and compliance (CoEnzyme Q10 dosage). Evaluations were performed each 6 months with a phone call between each visit. Results: Albuminemia evolution was not significantly different between the 2 groups (Phase1: coQ10: -1.2 [-3.0; 1.1], Placebo: $+1.0[+0.8 ;+2.0], \mathrm{p}=0.0511)$ and no significant difference on all other evaluation criteria. Treatment was well tolerated with minor side effects: 6 during phase 1 ( 3 in each group) and 2 during phase 2. Conclusion: despite good tolerance, we failed to demonstrate an increase of albuminemia under Coenzyme Q10 treatment in AOA1 patients.
P. Charles: None. V. Ladeveze: None. C. Tranchant: None. M. Anheim: None. C. Goizet: None. T. Kuntzer: None. F. Ochsner: None. F. Roelens: None. N. Deconinck: None. E. Petit: None. C. Jauffret: None. S. Rivaud-pechoux: None. I. Leber: None. A. Brice: None. S. Tezenas du montcel: None. A. Durr: None.

\section{P09.010.B}

A nonsense variant in the $A R S D$ gene associated with young-onset Parkinson's disease

S. MIURA ${ }^{1}$, T. Shimojo ${ }^{2}$, R. Fujioka ${ }^{3}$, Y. Uchiyama ${ }^{4}, H$. Shibata $^{2}$

${ }^{I}$ Department of Neurology \& Geriatric Medicine, Ehime University Graduate School of Medicine, Toon, Japan, ${ }^{2}$ Division of Genomics, Medical Institute of Bioregulation, Kyushu University, Fukuoka, Japan, ${ }^{3}$ Department of Food and Nutrition, Beppu University Junior College, Beppu, Japan, ${ }^{4}$ Department of Radiology, Kurume University School of Medicine, Kurume, Japan

Parkinson's disease (PD) is one of the common neurodegenerative disorder defined as bradykinesia plus rest tremor or rigidity. It has been reasonable that the younger age at onset is, the higher the risk of genetic condition gets. To identify the causative nucleotide variant for the disease, we studied a Japanese pedigree consisting of a trio of a patient with sporadic young-onset PD and unaffected parents. The patient was a 35-year-old male. He had noted involuntary movement of slow torsion in his right shoulder and forearm at the age of 31 . He also noticed slow torsion in his left toe and dragged his left foot in every evening at the age of 34 . On neurological examination, he showed dystonic posture in his right wrist, mild hypertonus and bradykinesia in his right upper limb, hyperreflexia and mild spasticity in the lower extremities. Brain MRI showed no abnormality. Meta-iodobenzylguanidine (MIBG) myocardial scintigraphy showed no decrease of the heart/mediastinum ratio. Dopamine transporter (DAT) SPECT imaging showed moderately reduced dopamine uptake in the bilateral striatum. L-dopa was effective for his bradykinesia. Wholeexome sequencing of the trio combined with strict criteria revealed an X-linked nonsense variant (NM_001669: c.1556C $>\mathrm{T}$ [p.Arg498X]) in the ARSD gene encoding arylsulfatase D. Quantitative real-time PCR analysis verified that the mRNA expression level of ARSD was significantly reduced in the patient's peripheral blood cells probably due to the nonsense mediated decay. This is the first record of a disease associated with a variant of the ARSD gene.

S. Miura: None. T. Shimojo: None. R. Fujioka: None. Y. Uchiyama: None. H. Shibata: None. 


\section{P09.011.C}

Variations in genes of the mTOR-pathway link ASD and ICV

\section{Arenella ${ }^{1,2}$, N. R. Mota ${ }^{1}$, M. W. A. Teunissen ${ }^{3}$, H. G. Brunner $^{1,4}$, J. Bralten ${ }^{l}$}

${ }^{I}$ Department of Human Genetics, Radboud University Medical Center, Nijmegen, Netherlands, ${ }^{2}$ Institute of Psychiatry, Psychology \& Neuroscience, King's College London, London, United Kingdom, ${ }^{3}$ Academic Center for Epileptology Kempenhaeghe, Maastricht University Medical Center, Maastricht, Netherlands, ${ }^{4}$ Department of Human Genetics, Maastricht University Medical Center, Maastricht, Netherlands

Introduction: Large brains are commonly observed in autism spectrum disorder (ASD), but the underlying molecular basis is unclear. Brain volume is highly heritable and is partly regulated by variation in the mammalian target of rapamycin (mTOR) pathway. Rare mTOR-related variants associate with macrocephaly in syndromic intellectual disability (ID), and common mTOR-related variants are associated with intracranial volume (ICV) in the general population. We hypothesized that variations in mTORrelated genes could mediate the occurrence of enlarged brains in ASD.

Materials and methods: Looking at rare de-novo mTOR-related variants, we estimated enrichment of macrocephaly and ASD using a local ID cohort $(\mathrm{N}=2,257)$ and publicly available resources $(n=32,991)$. Looking at common variants in a unique set of mTOR-related genes, we estimated association with ASD $(\mathrm{N}=46,350)$ and ICV $(\mathrm{N}=25,974)$ using publicly available data. Using the same data, we estimated 1) genome-wide genetic correlation between ASD and ICV and 2) mTOR-restricted ASD-ICV genetic correlation.

Results: We found a significant enrichment of syndromic macrocephaly and ASD in de-novo mTOR-related variants carriers. Gene-set analyses for common mTOR-related variants revealed significant independent associations with ASD $(p=0.002)$ and ICV $(p=0.021)$. While no significant genome-wide ASD-ICV genetic correlation was present $(\mathrm{p}=0.81)$, we show a significant positive mTOR-stratified genetic correlation $(\mathrm{p}=0.027)$.

Conclusions: Our study shows that both rare and common mTOR-related genetic variations contribute to ASD and brain volume. These results are compatible with a model in which variable mTOR activation may drive both larger brain volume and ASD risk.

M. Arenella: None. N.R. Mota: None. M.W.A. Teunissen: None. H.G. Brunner: None. J. Bralten: None.
P09.013.B

Novel MAG variant in autosomal recessive cerebellar ataxia with oculomotor apraxia: molecular basis and expanded clinical phenotype

\author{
M. Santos ${ }^{1}$, J. Damásio ${ }^{1,2,3}$, C. Kun-Rodrigues ${ }^{4}, C$. \\ Barbot $^{2}$, J. Sequeiros ${ }^{1,2}$, J. Brás ${ }^{4,5}$, I. Alonso ${ }^{1,2}, R$. \\ Guerreiro $^{4,5}$
}

${ }^{1}$ UnIGENe, IBMC - Institute for Molecular and Cell Biology, i3S - Instituto de Investigação e Inovação em Saúde, Universidade do Porto, Porto, Portugal, ${ }^{2} C G P P$, IBMC - Institute for Molecular and Cell Biology, i3S Instituto de Investigação e Inovação em Saúde, Universidade do Porto, Porto, Portugal, ${ }^{3}$ Neurology Department, Centro Hospitalar do Porto, Porto, Portugal, ${ }^{4}$ Center for Neurodegenerative Science, Van Andel Institute, Grand Rapids, MI, United States, ${ }^{5}$ Division of Psychiatry and Behavioral Medicine, Michigan State University College of Human Medicine, Grand Rapids, MI, United States

Hereditary cerebellar ataxias (HCAs) are a heterogeneous group of neurodegenerative disorders, characterized by motor incoordination and unsteady gait, often associated with speech and eye movement disturbances. HCAs share clinical features and disease mechanisms with hereditary spastic paraplegias (HSPs), with several ataxia-related genes found to cause HSPs and vice-versa. In this study, we used homozygosity mapping and exome sequencing to unravel the disease-causing variant in a Portuguese family with early-onset, autosomal recessive, cerebellar ataxia with neuropathy and oculomotor apraxia. We identified a novel homozygous missense variant in $M A G$ (c.124T $>\mathrm{C}$; p. Cys42Arg), the gene encoding the myelin-associated glycoprotein (MAG). Cellular studies confirmed its detrimental effect. Our results showed that this variant reduces protein stability, impairs post-translational processing ( $\mathrm{N}$-linked glycosylation) and subcellular localization of MAG. Homozygous variants in $M A G$ have been previously associated with complicated forms of HSP. Our findings newly associate $M A G$ variants with ataxia with oculomotor apraxia. The low expression and premature degradation of the mutant protein showed the variant to cause protein lossof-function. This work has also expanded the clinical phenotype associated with disease-causing variants in MAG. Genotyping $M A G$ variants should, therefore, be considered in the diagnosis of HCAs, in addition to HSPs.

M. Santos: None. J. Damásio: None. C. Kun-Rodrigues: None. C. Barbot: None. J. Sequeiros: None. J. Brás: None. I. Alonso: None. R. Guerreiro: None. 


\section{P09.014.C}

NPTX1 mutations cause a novel form of autosomal dominant cerebellar ataxia

M. Coutelier ${ }^{1}$, M. Jacoupy ${ }^{1}$, A. Janer ${ }^{2}, F$. Renaud ${ }^{3}, G$. Saripella $^{4}, F$. Ancien ${ }^{5}, R$. Larivière $^{2}, N$. Sgarioto ${ }^{2}, R$.

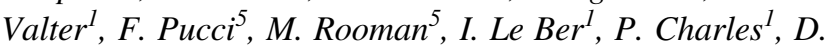
A. Tregouet ${ }^{4}$, A. Brice ${ }^{1}$, E. Shoubridge ${ }^{2}$, D. Gilis $^{5}$, A. Durr ${ }^{1}$, G. Stevanin ${ }^{l}$

${ }^{1}$ Institut du Cerveau et de la Moelle, Paris, France, ${ }^{2}$ McGill university, Montreal, QC, Canada, ${ }^{3}$ Institut Gustave Roussy, Villejuif, France, ${ }^{4}$ Institut ICAN, Paris, France, ${ }^{5}$ Universite Libre de Bruxelles, Bruxelles, Belgium

Introduction: Autosomal dominant spinocerebellar ataxias (SCA) are characterized by a marked genetic heterogeneity, with more than forty causative genes identified so far, and half the patients lacking a molecular diagnosis.

Material and Methods: In a large family with 9 symptomatic patients, we aimed at identifying a new genetic cause of SCA by performing exome sequencing combined to whole genome linkage analysis.

Results: We identified a G389R missense mutation in NPTX1, segregating in all affected cases. This mutation was recurrent in 2 independent pedigrees without a common founder effect and another missense mutation, E327G, was identified in a smaller pedigree with two affected patients. The phenotype in these 4 families is a late onset (range 3471 years) slowly progressive cerebellar ataxia, nystagmus, cognitive impairment, myoclonic tremor and mild cerebellar atrophy on MRI. Both mutations affect conserved aminoacid residues in the NP1 protein and are extremely rare or missing from public databases. We show that both mutations alter the endoplasmic reticulum morphology and induce ATF6-mediated stress, ultimately leading to increased cell cytotoxicity in COS7 cells. The E327G change also abolishes the protein secretion, as well as the formation of NP1 complexes. In silico modeling of the complex evidenced a destabilizing effect for the E327G change, located at the interface between monomers. No phenotype-genotype correlation could be established.

Conclusions: We suggest that mutations in NPTXI can lead to spinocerebellar ataxia via endoplasmic stress, following a dominant negative complex-destabilizing process or a direct alteration of the protein function.

M. Coutelier: None. M. Jacoupy: None. A. Janer: None. F. Renaud: None. G. Saripella: None. F. Ancien: None. R. Larivière: None. N. Sgarioto: None. R. Valter: None. F. Pucci: None. M. Rooman: None. I. Le Ber: None. P. Charles: None. D.A. Tregouet: None. A. Brice: None. E. Shoubridge: None. D. Gilis: None. A. Durr: None. G. Stevanin: None.

\section{P09.015.A}

Novel dominantly inherited spinocerebellar ataxia subtype caused by $S A M D 9 L$ mutation triggering mitochondrial and lysosomal dysregulation

M. Corral-Juan ${ }^{1}$, P. Casquero ${ }^{2}$, N. Giraldo Restrepo ${ }^{2}, S$. Laurie $^{3}$, A. Martinez-Piñeiro ${ }^{4}$, R. Mateo Montero ${ }^{2}, L$. Ispierto ${ }^{5}$, V. Volpini ${ }^{6}$, D. Vilas $^{5}$, R. Álvarez-Ramo ${ }^{5}, I$. Sánchez $z^{l}$, A. Matilla-Dueñas ${ }^{l}$

${ }^{1}$ Functional and Translational Neurogenetics Unit, Department of Neuroscience, Research Institute Germans Trias $i$ Pujol (IGTP), Universitat Autònoma de Barcelona-Can Ruti Campus, Badalona, Spain, ${ }^{2}$ Neurology and neurophysiology Section, Hospital Mateu Orfila, Mahón, Spain, ${ }^{3}$ CNAG-CRG, Centre for Genomic Regulation (CRG), Barcelona Institute of Science and Technology (BIST), Barcelona, Spain, ${ }^{4}$ Neuromuscular and functional studies Unit, Neurology Service, University Hospital Germans Trias $i$ Pujol (HUGTiP), Universitat Autònoma de Barcelona-Can Ruti Campus, Badalona, Spain, ${ }^{5}$ Neurodegeneration Unit, Neurology Service, Department of Neuroscience, University Hospital Germans Trias i Pujol (HUGTiP), Universitat Autònoma de Barcelona-Can Ruti Campus, Badalona, Spain, ${ }^{6} I D I B E L L$, L'Hospitalet de Llobregat, Spain

Introduction: Spinocerebellar ataxias (SCAs) consist of rare movement disorders characterised by progressive cerebellar ataxia variably associated with other neurological signs. At least 43 different SCA subtypes have been described to date evidencing the high clinical and genetic heterogeneity.

Materials and Methods: Whole-genome linkage and exome studies were performed in a Spanish five-generation family from the Balearic island of Menorca with seven affected individuals variably presenting with ataxia, nystagmus, dysarthria, polyneuropathy and pyramidal signs.

Results: Here we present a novel spinocerebellar ataxia with autosomal dominant inheritance with affected individuals presenting nystagmus as initial clinical sign and a variable age at onset ranging from 15 to 50 years old. MRI revealed cerebellar atrophy with cerebral demyelination, and EMG showed nerve conduction deficits. We identified the c.1877C $>\mathrm{T}$ (p.Ser626Leu) germline variant in the SAMD $9 L$ gene as the disease causative genetic defect with a significant LOD score $\mathrm{Z}_{\max }=3.43(\theta=0.00 ; P<3.5 \mathrm{E} 10$ $5)$. We demonstrate the mitochondrial location of human SAMD9L protein and found its levels decreased in patients' fibroblasts. The p.Ser626Leu variant is located on a predicted microtubule binding motif in SAMD9L leading to its subcellular mislocalization. Furthermore, we detected a high-copy number of the mitochondrial MT-D-LOOP 
region along with increased ATP5H, DRP1 and LAMP1 protein levels evidencing both mitochondrial and lysosomal deficits in patients' fibroblasts.

Conclusions: This study describes a new spinocerebellar ataxia subtype caused by SAMD9L, mitochondrial, and lysosomal dysregulation. This work was funded by the Spanish Health Institute Carlos III (FIS PI14/00136; PI14/ 01159; FIS PI17/00534).

M. Corral-Juan: None. P. Casquero: None. N. Giraldo Restrepo: None. S. Laurie: None. A. Martinez-Piñeiro: None. R. Mateo Montero: None. L. Ispierto: None. V. Volpini: None. D. Vilas: None. R. Álvarez-Ramo: None. I. Sánchez: None. A. Matilla-Dueñas: None.

P09.017.C

Linked-read whole genome sequencing reveals undetected variants in autism spectrum disorder

F. A. Cupaioli ${ }^{1}$, E. Mosca ${ }^{I}$, N. Di Nanni ${ }^{1}$, P. Pelucchi ${ }^{1}$, L. Milanesi $^{1}$, M. E. Raggi ${ }^{2}$, L. Villa $^{2}$, A. Mezzelani ${ }^{1}$

${ }^{1}$ National Research Council, Institute of Biomedical Technologies, Segrate, Italy, ${ }^{2}$ Scientific Institute, IRCCS Eugenio Medea, Bosisio Parini, Italy

Introduction: Autism Spectrum Disorder (ASD) is a neurodevelopmental condition with complex etiology. Although genetics play a key role in ASD, causative or predisposing genetic variants have been detected only in $30 \%$ of patients. Here, for the first time, linked-read whole genome sequencing of ASD patients is used to access disease associated regions unmappable by short-reads NGS.

Materials and Methods: 10 children with ASD, including 3 couples of affected siblings, were enrolled and HMW DNA isolated from peripheral blood. DNA was submitted to 10Xgenomics microfluidics partitioning and barcoding, library preparation and Illumina WG-NGS. Data were analyzed through 10x Long Ranger pipelines to find SNVs, in/dels and larger structural variants in comparison to 1000 genomes, genome aggregation database and NHLBI-ESP populations. Genes affected by variants were compared with those already known to be associated with ASD (SFARI database, large-scale sequencing studies, bioinformatics predictions).

Results: this approach successfully produced sequences up to $9 \mathrm{mln}$ bp in length. Among the detected variants, 405 were listed in SFARI, 43 confirmed bioinformatics predictions and 3,210 were new. Among the latter variants, 22 were homozygous in all the 3 couples of siblings.

Conclusions: this powerful approach deciphered underlying genomic heterogeneity and missing variations in ASD. Further studies will be performed to validate the new ASD variants in a large size of samples and in public data.
Data integration will be also performed to identify pathways and gene networks involved in the disorder to understand disease mechanisms and design target-driven treatment.

Acknowledgements: EU project GEMMA (grant agreement No 825033).

F.A. Cupaioli: None. E. Mosca: None. N. Di Nanni: None. P. Pelucchi: None. L. Milanesi: None. M.E. Raggi: None. L. Villa: None. A. Mezzelani: None.

\section{P09.018.A}

Changes in transcriptional activity of mitochondrial genes in patients with autism spectrum disorder

Y. Chudakova ${ }^{l}$, G. Shmarina ${ }^{1,2,3}$, A. Poletkina ${ }^{l}$, M. Konkova $^{l}$, E. Ershova ${ }^{1,2}$, N. Veiko ${ }^{1,2}$, S. Nikitina ${ }^{4}, N$. Simashkova ${ }^{4}$ S. Kostyuk ${ }^{1,2}$

${ }^{I}$ Research Center for Medical Genetics, Moscow, Russian Federation, ${ }^{2} N$. I. Pirogov Russian National Research Medical University, Moscow, Russian Federation, ${ }^{3}$ Gabrichevsky Institute of Epidemiology and Microbiology, Moscow, Russian Federation, ${ }^{4}$ Scientific Center for Mental Health, Russian Academy of Medical Sciences, Moscow, Russian Federation

Introduction: Mitochondrial dysfunction with concomitant oxidative stress is evidenced in the brains and periphery of many patients with autism spectrum disorder (ASD). This study aimed to compare the level of mitochondrial DNA (mtDNA) and transcriptional activity of mitochondrial genes in peripheral blood lymphocytes (PBL) of ASD patients (ASDP) and healthy controls (HC).

Materials and Methods: The study included 20 ASDP and $15 \mathrm{HC}$ aged 4 to 10 years. PBL DNA was isolated from plasma using phenol extraction. mtDNA content in PBL DNA samples was determined by non-radioactive quantitative dot-hybridization. PBL RNA was isolated with RNeasyPlusMiniKit('Qiagen'). Expression of 85 mitochondrial genes including those involved in regulating redox metabolism, mitochondrial response to stress, mitophagy, mitochondrial fusion, mitochondrial fission, mitochondrial transports, and those that encode for proteins of $\mathrm{I}-\mathrm{V}$ respiratory complexes, was evaluated using real-time PCR.

Results: There were no significant differences in mtDNA levels between patient and control groups. Analysis of mitochondrial gene expression revealed 3-5 fold elevation of transcript levels for UQCRFS1, SCO1, and MT-ATP6 genes (complex III, IV, and V, respectively) in PBL of ASD patients compared to those of HC. Besides, PBL of ASDP were characterized by heightened expression of PINK1 gene (tenfold) involved in mitophagy as well as FIS1 (twofold) and MFN2 (fourfold) genes regulated mitochondrial fission and fusion, respectively. 
Conclusions: Increased transcriptional activity of fusion, fission and mitophagy genes in PBL of ASDP may be associated with adaptive response maintaining functional mitochondria under disease-related metabolic or/and environmental stresses. The study was supported by RFBR grant № 17-29-06017ofi_m.

Y. Chudakova: B. Research Grant (principal investigator, collaborator or consultant and pending grants as well as grants already received); Significant; RFBR grant № 17-2906017ofi_m.. G. Shmarina: None. A. Poletkina: None. M. Konkova: None. E. Ershova: None. N. Veiko: None. S. Nikitina: None. N. Simashkova: None. S. Kostyuk: None.

P09.020.C

Exploring mechanisms of gene-environment interactions contributing to the onset of idiopathic Autism Spectrum Disorder

J. X. Santos ${ }^{1,2}$, H. Martiniano ${ }^{1,2}$, A. R. Marques ${ }^{1,2}$, C.

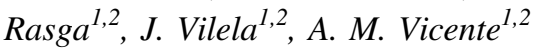

${ }^{1}$ Instituto Nacional de Saúde Doutor Ricardo Jorge, Lisbon, Portugal, ${ }^{2}$ Faculdade de Ciências, BioISI - Biosystems \& Integrative Sciences Institute, Universidade de Lisboa, Lisbon, Portugal

Sequencing studies have yielded several candidate genes for Autism Spectrum Disorder (ASD). However, the biological mechanisms underlying its onset are still unclear. Environmental factors may modulate ASD risk, with heritability estimates of 50-80\% supporting a role for gene-environment interactions in idiopathic cases. We hypothesize that ASD candidate genes interact with reported ubiquitous environmental risk factors. Thus, we interrogated the Comparative Toxicogenomics Database (CTD) for interactions between 1144 ASD candidate genes and 59 ASD-risk chemicals. A proportion analysis was performed to identify genes that selectively interact with ASD risk chemicals (and viceversa). Genetic data from ASD-individuals was inspected to identify SNVs $(n=2674)$ and CNVs $(n=3570)$. Eleven genes, including genes encoding for sex hormone receptors (AR, ESR1 and ESR2), signaling kinases (MAPK1 and $M A P K 3$ ) and xenobiotics-responding molecules (GSTM1 and SLC7A5) were found to selectively interact with ASDchemicals. Meanwhile, heavy metals, endocrine disruptors (pesticides, benzo(a)pyrene and a phthalate) and valproic acid were found to selectively target ASD-candidate genes. In ASD-cases, we found 22 loss-of-function or deleterious missense SNVs in 8/11 genes, of which 3 (ESR1, ESR2, and $M A P K 3)$ were also targeted by CNVs. External cues may dysregulate the MAPK signaling cascade, leading to neurodevelopmental problems. Hormone-mimicking toxins act as agonist/antagonist ligands to hormone receptors, while
SLC7A5 is a blood-brain barrier (BBB) transporter. Sex hormones and BBB are fundamental during early development. We highlight the need of considering genetics and environment as interacting entities. Efforts to collect earlylife exposure data from genetically susceptible patients may accelerate the implementation of health management strategies for ASD.

J.X. Santos: None. H. Martiniano: None. A.R. Marques: None. C. Rasga: None. J. Vilela: None. A.M. Vicente: None.

\section{P09.021.A}

Transcriptomic analysis unveils functional consequences of complex rearrangements associated to autism spectrum disorder

M. Costa-Roger ${ }^{l}$, M. López-Sánchez ${ }^{l}$, T. Vendrell ${ }^{2}, P$. Muñoz-Cabello ${ }^{1,3}$, B. Gener ${ }^{4}$, E. Gabau ${ }^{5}$, N. Capdevila $^{5}$, L. Pérez-Jurado ${ }^{1,6}$, I. Cuscó ${ }^{2}$

${ }^{1}$ Genetics Unit, Departament de Ciències Experimentals $i$ de la Salut, Pompeu Fabra University, Hospital del Mar Research Institute (IMIM), Parc de Salut Mar and Centro de Investigación Biomédica en Red de Enfermedades Raras (CIBERER), Barcelona, Spain, ${ }^{2}$ Department of Clinical and Molecular Genetics and Medicine Genetics Group, VHIR, University Hospital Vall d'Hebron, Barcelona, Spain, ${ }^{3}$ Unit of Clinical Genetics, Hospital del Mar, Parc de Salut Mar, Barcelona, Spain, ${ }^{4}$ Osakidetza Basque Health Service, Cruces University Hospital. Department of Genetics, Biocruces Bizkaia Health Research Institute, Bizkaia, Spain, ${ }^{5}$ Service of Clinical Genetics and Genetics Laboratory of Paediatric Unit, Parc Tauli University Hospital, Institut d'Investigació I Innovació Parc Taulí I3PT, Universitat Autònoma de Barcelona, Sabadell, Spain, ${ }^{6} \mathrm{SA}$ Clinical Genetics, Women's and Children's Hospital, South Australian Health and Medical Research Institute (SAHMRI) and University of Adelaide, South Australian Health and Medical Research Institute, Adelaide, South Australia, Australia

Introduction: Autism Spectrum Disorder (ASD) is a group of neurodevelopmental disorders characterized by socialcommunication difficulties, restricted interests and repetitive behaviors. ASD has a complex multifactorial etiology with high genetic involvement. Previous unpublished data suggested that inversions $17 \mathrm{q} 21.31$ and 8p23.1 could act as susceptibility variants. The purpose of this project is to elucidate the transcriptional consequences of these rearrangements.

Materials and Methods: We performed RNAseq analysis of peripheral blood in 13 idiopathic ASD patients and 11 controls grouped by homozygous inversion 
genotypes: non-inverted; inv8p23.1; inv17q21.31. We used HTSeq/DESeq2 to discover the differentially expressed genes (DEGs) with p.adj.value $<0.05$.

Results: We found that inversions affect the expression of genes encompassed in each inverted region and genes elsewhere in the genome. Regarding inv17q21.31, we discovered 38 DEGs, 8 from the region and some of them associated to neuronal diseases (LRRC37A,LRRC37A2, KANSL1-AS1,IL1B) or brain-related functions (ROBO1, RIN1). When exploring the interaction of phenotypegenotype, no regional genes were found deregulated. We discovered 27 DEGs for inv17q21.31 including some related to neuronal function (ATOH8,MAP1B,VSNL1), whereas for inv8p23.1 we found 73 DEGs of which 4 have been previously related to ASD (ABCA13,SLC7A8, STX1A,SLC24A2).

Conclusions: Our data indicate that inversions have a direct effect on gene expression patterns both in inverted regions and elsewhere in the genome. Moreover, we note that ASD carriers of the susceptibility alleles (inv17q21.31/ inv8p23.1) have differential expression of brain functionrelated genes. Further studies are needed to determine the involvement of these deregulated genes to the ASD phenotype. Funded by grants from the Spanish-Ministryof-Economy-and-Competiveness (FIS PI16/00369) and Ministry-of-Education-Culture-and-Sport (FPU16/06907).

M. Costa-Roger: None. M. López-Sánchez: None. T. Vendrell: None. P. Muñoz-Cabello: None. B. Gener: None. E. Gabau: None. N. Capdevila: None. L. PérezJurado: F. Consultant/Advisory Board; Modest; qGenomics. I. Cuscó: None.

\section{P09.022.B}

Systems Biology approach for the identification of neurotransmission and synaptic risk genes and disrupted biological processes in Autism Spectrum Disorder

J. Vilela ${ }^{1,2}$, H. Martiniano ${ }^{1,2}$, S. Luzi ${ }^{1,2}$, C. Rasga ${ }^{1,2}, A$. Marques $^{1,2}$, J. Santos ${ }^{1,2}$, G. Oliveira ${ }^{3,4}$, A. Vicente $^{1,2,5}$

${ }^{1}$ INSA - Instituto Nacional de Saúde Dr. Ricardo Jorge, Lisboa, Portugal, ${ }^{2}$ University of Lisboa Faculty of Sciences, BioISI - Biosystems and Integrative Science Institute, Lisboa, Portugal, ${ }^{3}$ Neurodevelopment and Autism Unit (UNDA), Serviço do Centro de Desenvolvimento da Criança, Centro de Investigação e Formação Clínica, Hospital Pediátrico, Centro Hospitalar e Universitário de Coimbra, Coimbra, Portugal, ${ }^{4}$ University Clinic of Paediatrics, Faculty of Medicine, University of Coimbra, Coimbra, Portugal, ${ }^{5}$ Instituto Gulbenkian de Ciência, Oeiras, Portugal
Autism Spectrum Disorder (ASD) is a neurodevelopmental disorder characterized by communication deficits and repetitive behavioral patterns. ASD has a strong genetic component and there is evidence supporting many putative risk genes. However, the genetics underlying the disease is unclear. Our objective was to identify neurotransmission and synaptic (NS) genes that play a role in ASD. For this purpose, we defined 1216 NS candidate genes filtering for the terms 'neurotransmitter' and 'synapse' in Gene Ontology, Reactome and KEGG, and overlapping the gene list obtained with the synaptic databases SynaptomeDB and SynSysNet, and with literature review. We then searched the Whole Exome Sequencing dataset from the Autism Sequencing Consortium (3938 cases) for ultra-rare loss of function $(\mathrm{LoF})$ Single Nucleotide Variants (MAF $<0,1 \%$; gnomAD 60146 controls) in these genes. We selected 434 LoF variants in 244 genes and identified the biological pathways enriched in these genes using Reactome. Applying the Louvain community detection algorithm to the network spanned by the selected genes and edges from the STRING database of protein-protein interactions, we identified several biological communities within the network: MAP Kinase, Calcium and $G$ alpha signaling, Interleukins and Toll-like receptors, Energy/Insulin metabolism, Phospholipids, Nicotinic acetylcholine and NMDA/AMPA receptors. Finally, we constructed a network-based gene rank through the computation of topological parameters and centrality measures in Cytoscape. The genes PRKCA, GNG13 and ANK2 involved in phosphorylation, transmembrane signaling and membrane stabilization of ion transporters and channels, which are important processes for the brain function, are the top-ranked genes.

Fundação para a Ciência e Tecnologia: (PD/BD/131390/ 2017)

J. Vilela: None. H. Martiniano: None. S. Luzi: None. C. Rasga: None. A. Marques: None. J. Santos: None. G. Oliveira: None. A. Vicente: None.

\section{P09.023.C}

Family-based whole exome sequencing allows a $25 \%$ diagnostic yield in patients with autism spectrum disorder

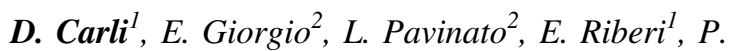
Dimartino $^{3,4}$, A. Bruselles ${ }^{5}$, S. Cardaropoli ${ }^{1}$, A. Mussa ${ }^{1}$, A. Pelle $^{6}$, G. Mandrile, V. Antona ${ }^{7}$, L. Sorasio ${ }^{8}$, E. Biamino $^{1}$, E. F. Belligni ${ }^{1}$, V. Rizzo ${ }^{2}$, F. Sirchia ${ }^{9}$, I. Bagnasco ${ }^{10}$, G. Zacchetti $^{11}$, V. G. Naretto ${ }^{11}$, G. Gai ${ }^{11}$, A. Zonta ${ }^{11}$, E. Grosso $^{11}$, C. Davico ${ }^{12}$, R. Keller ${ }^{13}$, T. Pippucci, ${ }^{3,4}$, M. Tartaglia $^{14}$, S. De Rubeis ${ }^{15}$, A. Brusco ${ }^{2,11}$, G. B. Ferrero ${ }^{1}$

${ }^{l}$ Department of Public Health and Pediatrics, University of Torino, Torino, Italy, ${ }^{2}$ Department of Medical Sciences, 
University of Torino, Torino, Italy, ${ }^{3}$ Medical Genetics Unit, Polyclinic Sant'Orsola-Malpighi University Hospital, Bologna, Italy, ${ }^{4}$ Department of Medical and Surgical Sciences, University of Bologna, Bologna, Italy, ${ }^{5}$ Department of Oncology and Molecular Medicine, Istituto Superiore di Sanità, Roma, Italy, ${ }^{6}$ Medical Genetics, San Luigi Gonzaga University Hospital, Orbassano, Italy, ${ }^{7}$ Department of Sciences for Health Promotion and Mother and Child Care "G. D'Alessandro", University of Palermo, Palermo, Italy, ${ }^{8}$ Pediatrics, Santa Croce e Carle Hospital, Cuneo, Italy, ${ }^{9}$ Institute for Maternal and Child Health IRCCS “Burlo Garofolo”, Trieste, Italy, ${ }^{10}$ Neuropsichiatria infantile Torino Sud-ospedale Martini- Asl Città di Torino, Torino, Italy, ${ }^{11}$ Medical Genetics Unit, Città della Salute e della Scienza University Hospital, Torino, Italy, ${ }^{12}$ Division of Child and Adolescent Neuropsychiatry, Department of Public Health and Pediatrics, University of Torino, Torino, Italy, ${ }^{13}$ Adult autism centre, Local Health Unit ASL City of Turin, Department of Mental Health, Torino, Italy, ${ }^{14}$ Genetics and Rare Diseases Research Division, Ospedale Pediatrico Bambino Gesù IRCSS, Roma, Italy, ${ }^{15}$ Seaver Autism Center for Research and Treatment, Icahn School of Medicine at Mount Sinai, New York, NY, United States

Introduction: autism spectrum disorders (ASD) represent a major health issue, whose genetic bases are not yet fully delineated.

Methods: we performed family-based whole exome sequencing (WES) in collaboration with the Autism Sequencing Consortium (Icahn School of Medicine at Mount Sinai) in 88 families with at least one subject presenting with ASD. Array-CGH and FMR1 analysis were negative. Patients have been clinically stratified in isolated ASD, ASD associated with other neurological signs (seizures, brain anomalies, micro/macrocephaly) and syndromic ASD (dysmorphisms, short stature/overgrowth, congenital anomalies).

Results: we found pathogenic variants in 22/88 cases (25\%). Diagnostic yield was 5/54 (9.3\%) in patients with isolated ASD (genes DSCAM, FOXP1, GRIA3, NF1, $T L K 2), 13 / 22(59.1 \%)$ in patients with associated neurologic signs (ARID1B, DEAF1, GRIN1, HNRNPH2, IRF2BPL, PHF8, PTEN, SATB2, SCN2A, SRCAP, TLK2, WDR26) and $4 / 12(33.3 \%)$ in syndromic ASD (ARIDIB, NAA15, $S H A N K 3, S E T B P 1)$. In other 9 families we identified potentially causative variants in 10 new candidate genes (ARHGAP4, ARHGAP5, CASKIN2, CTSZ, METRN, NRC2, PCDHA3, SEPT2, SLC4A2, TSPYL2). Variants of unknown significance were found in 12 families (13.6\%).

Conclusions: these data confirm that family-based WES has a high diagnostic rate in ASD. Notably, the genes found mutated in patients with isolated ASD are known to be associated with genetic syndromes (e.g. NF1), suggesting a wide phenotype variability ranging from complex syndromes to isolated ASD.

D. Carli: None. E. Giorgio: None. L. Pavinato: None. E. Riberi: None. P. Dimartino: None. A. Bruselles: None. S. Cardaropoli: None. A. Mussa: None. A. Pelle: None. G. Mandrile: None. V. Antona: None. L. Sorasio: None. E. Biamino: None. E.F. Belligni: None. V. Rizzo: None. F. Sirchia: None. I. Bagnasco: None. G. Zacchetti: None. V.G. Naretto: None. G. Gai: None. A. Zonta: None. E. Grosso: None. C. Davico: None. R. Keller: None. T. Pippucci: None. M. Tartaglia: None. S. De Rubeis: None. A. Brusco: None. G.B. Ferrero: None.

\section{P09.025.B}

Application of oligonucleotide array comparative genomic hybridization in clinical diagnostics of 500 patients with autism spectrum disorders

B. Wisniowiecka-Kowalnik ${ }^{1}$, I. Plaskotal, M. Kędzior ${ }^{l}$, J. Bernaciak $^{l}$, E. Obersztyn ${ }^{l}$, A. Kutkowska-Kaźmierczak ${ }^{l}, N$. Braun-Walickal, M. Geremek ${ }^{l}$, A. Pietrzyk ${ }^{l}$, J. Castañeda ${ }^{l}$, A. Barczyk ${ }^{l}, P$. Szyld ${ }^{l}$, J. Klapecki ${ }^{1}$, A. SobczyńskaTomaszewska ${ }^{2}$, K. Czerska ${ }^{2}$, B. Nowakowska ${ }^{1}$

${ }^{1}$ Institute of Mother and Child, Warsaw, Poland, ${ }^{2}$ MEDGEN, Warsaw, Poland

Autism Spectrum Disorders (ASDs) are a heterogeneous group of neurodevelopmental disorders with an estimated prevalence of $1.4-2 \%$ in the general population. ASDs have a complex multifactorial etiology, with genetic causes being recognized in 25-35\% of cases. Array comparative genomic hybridization studies showed that copy numer variants (CNVs), in particular rare and those encompassing brainrelated genes, are associated with idiopathic ASDs. We applied oligonucleotide microarrays (OGT) with average resolution of $30 \mathrm{kpz}$ to evaluate its usefulness for identification and characterization of CNVs in a cohort of 528 patients with ASDs. The analyses of the patients' genomes were performed using genome-wide, high-resolution (180K) array to accurate detection of copy number variation at the exon level. Probes have been designed to target 4645 genes including 227 genes relevant for etiopathogenesis of ASDs. Chromosomal microarray analysis revealed 116 nonpolymorphic $\mathrm{CNVs}$, ranging in size from $9.3 \mathrm{~kb}$ to $8.16 \mathrm{Mb}$, in $100(19 \%)$ patients. Pathogenic or likely pathogenic CNVs were identified in 42 (8\%) patients, whereas CNVs with uncertain clinical significance were identified in $68(12.8 \%)$ of cases. Due to high resolution of the selected microarray, it was possible to identify $37 \mathrm{CNVs}$ that could not have been detected using the clinical microarrays (OGT, 60k). Our study confirmed the potential 
of aCGH in elucidating the etiology of ASDs, demonstrated by the identification of one novel gene LRRTM4 as candidate for ASDs. The work was supported by projects 510-1809 and 510-18-54 from the Ministry of National Education

B. Wisniowiecka-Kowalnik: None. I. Plaskota: None. M. Kędzior: None. J. Bernaciak: None. E. Obersztyn: None. A. Kutkowska-Kaźmierczak: None. N. BraunWalicka: None. M. Geremek: None. A. Pietrzyk: None. J. Castañeda: None. A. Barczyk: None. P. Szyld: None. J. Klapecki: None. A. Sobczyńska-Tomaszewska: None. K. Czerska: None. B. Nowakowska: None.

\section{P09.026.C}

Identification of rare risk variants in 12 multiply affected families with bipolar disorder

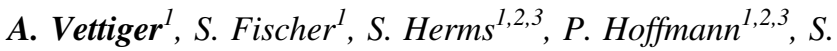
Cichon $^{4,3}$, A. Maaser ${ }^{2}$, F. Streit ${ }^{5}$, S. H. Witt ${ }^{6}$, J. Guzman-

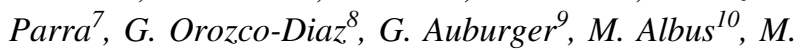
Borrmann-Hassenbach ${ }^{10}$, M. González ${ }^{7}$, S. Gil-Flores ${ }^{11}, F$. Cabaleiro-Fabeiro $^{12}, F$. Del Río Noriega ${ }^{13}$, F. Perez-

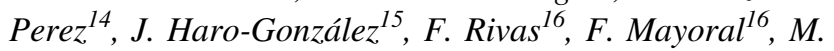
Rietschel $^{6}$, M. Nöthen ${ }^{2}$, P. Förstner ${ }^{17,1,18}$

${ }^{1}$ Human Genomics Research Group, Department of Biomedicine, University of Basel, Basel, Switzerland, ${ }^{2}$ Institute of Human Genetics, University of Bonn, School of Medicine \& University Hospital Bonn, Bonn, Germany, ${ }^{3}$ Institute of Medical Genetics and Pathology, University Hospital Basel, Basel, Switzerland Human Genomics Research Group, Department of Biomedicine, University of Basel, Basel, Switzerland, ${ }^{4}$ University of BaselHuman Genomics Research Group, Department of Biomedicine, University of Basel, Basel, Switzerland, ${ }^{5}$ Department of Genetic Epidemiology in Psychiatry, Central Institute of Mental Health, University Medical Center Mannheim/University of Heidelberg, Mannheim, Germany, Mannheim, Germany, ${ }^{6}$ Department of Genetic Epidemiology in Psychiatry, Central Institute of Mental Health, University Medical Center Mannheim/University of Heidelberg, Mannheim, Germany, ${ }^{7}$ Department of Mental Health, Institute of Biomedicine IBIMA, University Hospital of Malaga, Malaga, Spain, ${ }^{8}$ Unidad de Gestión Clínica del Dispositivo de CuidadosCríticos y Urgencias del Distrito Sanitario Málaga, Malaga, Spain, ${ }^{9}$ Department of Experimental Neurology, Division of Neurology, Goethe University Hospital, Frankfurt, Germany, ${ }^{10}$ Isar Amper Klinikum München Ost, kbo, Haar, Germany, ${ }^{11}$ Department of Mental Health, University Hospital of Reina Sofia, Cordoba, Spain, ${ }^{12}$ Department of Mental Health, University Hospital of Jaen, Jaen, Spain,

${ }^{13}$ Department of Mental Health, University Hospital of
Jerez de la Frontera, La Frontera, Spain, ${ }^{14}$ Department of Mental Health, University Hospital of Puerto Real, Department of Mental Health, Cadiz, Spain, ${ }^{15}$ Department of Mental Health, Hospital Punta de Europa, Algeciras, Spain, ${ }^{16}$ Department of Psychiatry, Carlos Haya Regional University Hospital, Malaga, Spain, ${ }^{17}$ Institute of Human Genetics, University of Bonn, School of Medicine \& University Hospital Bonn, Bonn, Bonn, Germany, ${ }^{18}$ Centre for Human Genetics, University of Marburg, Marburg, Germany

Introduction: Bipolar disorder (BD) is a common, highly debilitating neuropsychiatric disorder. It has a life-time prevalence of about $1 \%$. The disease manifests itself by reoccurring episodes of depression and mania. BD is a complex disorder caused by a combination of genetic and environmental factors. Formal genetic studies have estimated a relatively high heritability of approximately 75$80 \%$. Our actual project focuses on the identification of rare risk variants for $\mathrm{BD}$ that segregate in 12 multiply affected families from Andalusia/Spain with a total of 71 individuals, using whole exome data.

Methods: To identify risk variants that segregate within the pedigree we set up an analysis pipeline using a commercial software. The workflow includes trimming of reads, mapping to the reference genome, removal of duplicates, local realignment and variant detection. Variants that matched the segregation pattern were further analyzed using different prediction tools and for known candidate genes.

Results: We have identified 138'000 missense variants, which were familywise present in all affected and none of the healthy members. These variants were annotated using dbNSFP, including several different prediction scores. As a first approach to identify causative variants, we looked at the overlap with the 30 loci described in the BP2 GWAS from the PGC (Stahl, E.A. et al. 2019). We found 18 mutations in BD associated genes, 3 of them being rare. SYNE1 is outstanding, as 9 mutations pertain to this gene. Next steps will comprise following up of these mutations using biological pathway analysis tools and starting analysis of identified frame-shift mutations.

A. Vettiger: None. S. Fischer: None. S. Herms: None. P. Hoffmann: None. S. Cichon: None. A. Maaser: None. F. Streit: None. S.H. Witt: None. J. Guzman-Parra: None. G. Orozco-Diaz: None. G. Auburger: None. M. Albus: None. M. Borrmann-Hassenbach: None. M. González: None. S. Gil-Flores: None. F. CabaleiroFabeiro: None. F. Del Río Noriega: None. F. PerezPerez: None. J. Haro-González: None. F. Rivas: None. F. Mayoral: None. M. Rietschel: None. M. Nöthen: None. P. Förstner: None. 


\section{P09.027.A}

\section{Decrease of mitochondrial DNA copy number in C9ALS/FTD}

\author{
M. I. Alvarez-Mora ${ }^{1}$, P. Podlesniy ${ }^{2}$, T. Riazuelo ${ }^{3}, N$. \\ Serra $^{4}$, L. Rodriguez-Revenga ${ }^{5}$
}

${ }^{1}$ Hospital 12 de Octubre, IDIBAPS and CIBERER, Madrid, Spain, ${ }^{2}$ CIBERNED, BARCELONA, Spain, ${ }^{3}$ Hospital Clinic of Barcelona, BARCELONA, Spain, ${ }^{4} I I B B$ and CIBERNED, Barcelona, Spain, ${ }^{5}$ Hospital Clinic of Barcelona, IDIBAPS and CIBERER, BARCELONA, Spain

Introduction: Hexanucleotide repeat expansion in $C 9$ orf 72 gene is the most common genetic cause of amyotrophic lateral sclerosis and frontotemporal dementia (C9ALS/ FTD). Loss of C9orf72 protein function, a toxic RNA gainof-function and a gain of function by RAN translation have been proposed as triggering pathological mechanisms. In addition, mitochondrial defects have been described to be a major driver of disease initiation. MtDNA copy number has been proposed as a useful biomarker of mitochondrial dysfunction. Therefore, we investigated the role of mtDNA copy number in the pathogenesis C9ALS/FTD.

Material and Methods: Total mitochondrial and genomic DNA was extracted from postmortem human prefrontal cortex brains from 18 C 9 orf 72 samples and 11 controls. The mtDNA copy number was assessed by digital droplet PCR.

Results: A statistically significant decrease was obtained when comparing C9orf72 samples (3923 mtDNA copies/ diploid genome \pm 566 ) and controls (7017 mtDNA copies/ diploid genome \pm 909 ), with a significant decrease of $55 \%$ of mtDNA copy number in C9orf72 frontal cortex brain samples. This association was independent of age and gender.

Conclusions: A growing number of studies demonstrated the influence of mtDNA copy number reduction on neurodegeneration. Our results provide new insights into the role of mitochondrial dysfunction in the pathogenesis of C9ALS/FTD

Acknowledgements: This work was supported by the Instituto de Salud Carlos III (PI17/01067), co-financed by Fondo Europeo de Desarrollo Regional (FEDER) "una manera de hacer Europa" and AGAUR (2017 SGR1134). The CIBERER is an initiative of the Instituto de Salud Carlos III.

M.I. Alvarez-Mora: None. P. Podlesniy: None. T. Riazuelo: None. N. Serra: None. L. RodriguezRevenga: None.

\section{P09.028.B}

Study of the C9ORF72 repeat expansion in Greek patients with neurodegenerative disorders.
C. KARTANOU ${ }^{l}$, M. Breza ${ }^{l}$, C. Potagas ${ }^{2}$, E. Kapaki ${ }^{3,4}$, G. P. Paraskevas ${ }^{3,4}$, V. C. Constantinides ${ }^{3,4}, M$. Rentzos ${ }^{4}, L$. Stefanis $^{4,5,6}$, S. G. Papageorgiou ${ }^{7}$, M. Panas ${ }^{1}$, G. Koutsis ${ }^{1}$, G. Karadimal

${ }^{1}$ Neurogenetics Unit, 1st Department of Neurology, School of Medicine, Eginition Hospital, National and Kapodistrian University of Athens, Athens, Greece, ${ }^{2}$ Neuropsychology and Speech Pathology Unit, 1st Department of Neurology, School of Medicine, Eginition Hospital, National and Kapodistrian University of Athens, Athens, Greece, ${ }^{3} \mathrm{Neu}$ rochemistry and Biological Markers Unit, 1st Department of Neurology, School of Medicine, Eginition Hospital, National and Kapodistrian University of Athens, Athens, Greece, ${ }^{4} 1$ st Department of Neurology, School of Medicine, Eginition Hospital, National and Kapodistrian University of Athens, Athens, Greece, ${ }^{5}$ 2nd Department of Neurology, Attikon University Hospital, National and Kapodistrian University of Athens, Athens, Greece, ${ }^{6}$ Center of Clinical Research, Experimental Surgery and Translational Research, Biomedical Research Foundation of the Academy of Athens, Athens, Greece, ${ }^{7}$ Cognitive Disorders/Dementia Unit, 2nd Department of Neurology, Attikon University Hospital, National and Kapodistrian University of Athens, Athens, Greece

A hexanucleotide repeat expansion located in C9ORF72 gene is as an established cause of amyotrophic lateral sclerosis (ALS) and/or frontotemporal dementia (FTD). Since its discovery in 2011, many research groups have tried to assess its frequency in several neurodegenerative disorders, reporting differences among populations and phenotypic heterogeneity. The aim of the present study is to identify the C9ORF72 expansion in a broad spectrum of neurodegenerative disorders in Greece (ALS, HD-like syndromes, dementia, Parkinson's disease-PD, Hereditary Spastic Paraplegia-HSP) and healthy controls. Using molecular biological techniques including Repeat-Primed PCR (RP-PCR) 860 patients (399 with ALS, 44 with HDlike syndromes, 224 with dementia, 178 with PD and 15 with hereditary spastic paraplegia, HSP) and 321 controls were tested for the C9ORF72 repeat expansion. Thirty-eight patients with ALS (9.5\%), 2 with HD-like syndromes (4.5\%), 8 with FTD (8.5\%), 1 with AD (1.7\%), and 2 with PD $(1.1 \%)$ were expansion-positive. The expansion was not detected in the HSP or control group. The results of this study provide an update on the spectrum of C9ORF72related neurodegenerative diseases, emphasizing the importance of establishing C9ORF72 as a diagnostic test in laboratory routine for both familial and sporadic patients.

C. Kartanou: None. M. Breza: None. C. Potagas: None. E. Kapaki: None. G.P. Paraskevas: None. V.C. Constantinides: None. M. Rentzos: None. L. Stefanis: 
None. S.G. Papageorgiou: None. M. Panas: None. G. Koutsis: B. Research Grant (principal investigator, collaborator or consultant and pending grants as well as grants already received); Significant; Teva Pharmaceuticals, Genesis Pharma. Other; Significant; Novartis, Genesis Pharma, Sanofi-Genzyme, Teva. G. Karadima: B. Research Grant (principal investigator, collaborator or consultant and pending grants as well as grants already received); Modest; Pfizer. F. Consultant/Advisory Board; Modest; Roche.

P09.029.C

\section{The Cdkl5 phenotype at single cell resolution}

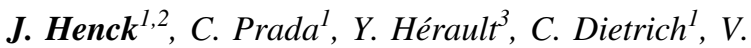
Suckow $^{l}$, V. M. Kalscheuer ${ }^{1}$, M. Spielmann ${ }^{1,2}$

${ }^{1}$ Research Group Development and Diease, Max Planck Institute for Molecular Genetics, Berlin, Germany, ${ }^{2}$ Institut für Medizinische Genetik und Humangenetik, Charité, Berlin, Germany, ${ }^{3}$ Institut de Génétique, Biologie Moléculaire et Cellulaire, IGBMC, 67404 Illkirch, France

In recent years, a multitude of single-cell sequencing technologies have been developed that now enable the study of neurodevelopmental disorders at the organismal scale and allow for detailed investigations of the roles of specific genes and regulatory sequences during development.

Here we aim to understand the underlying pathomechanism of the CDKL5 deficiency disorder (CDD), a rare genetic disorder that causes seizures, developmental delay, and severe intellectual disability, by studying the Cdk15 phenotype at single cell resolution. The CDKL5 encoded protein is a kinase responsible for normal neuronal development, synapse formation and signal transmission, but the mechanism(s) by which loss of CDKL5 expression leads to disease remains unclear.

We studied total brain isolated whole cells from hemizygous Cdk15-knockout male mouse embryos (E18.5) by single-cell RNA sequencing and were able to identify numerous cell-type-specific phenotypes. Our single-cell approach provided the necessary resolution to detect previously unidentified transcriptional changes and molecular states compared to wildtype mice. Additionally we identified altered pseudotemporal trajectories of Cdkl5 deficient brain cells during embryonic development. Our data provides new insights into the molecular pathomechanism of CDD and highlights the potential of single-cell RNA sequencing as a phenotyping tool for mouse models of neurodevelopmental disorders.

J. Henck: None. C. Prada: None. Y. Hérault: None. C. Dietrich: None. V. Suckow: None. V.M. Kalscheuer: None. M. Spielmann: None.

\section{P09.031.B}

Structural genome variations in cerebral cavernous malformations

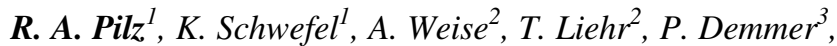
A. Spuler ${ }^{4}$, S. Spiegler ${ }^{1}$, E. Gilberg ${ }^{1}$, C. A. Hübner ${ }^{2}, U$. Felbor $^{1}$, M. Rath ${ }^{1}$

${ }^{1}$ Department of Human Genetics, University Medicine Greifswald, and Interfaculty Institute of Genetics and Functional Genomics, University of Greifswald, Greifswald, Germany, ${ }^{2}$ Institute of Human Genetics, Jena University Hospital, Friedrich Schiller University, Jena, Germany, ${ }^{3}$ Institute of Medical Diagnostics, IMD Potsdam, Potsdam, Germany, ${ }^{4}$ Department of Neurosurgery, Helios Hospital Berlin Buch, Berlin, Germany

Introduction: Autosomal dominant cerebral cavernous malformations (CCM) are leaky vascular lesions that can cause epileptic seizures and stroke-like symptoms. Germline mutations in either $C C M 1, C C M 2$ or $C C M 3$ are found in the majority of patients with multiple CCMs or a positive family history. Recently, we have identified the first copy number neutral inversion in CCM2 by whole-genome sequencing in an apparently mutation-negative CCM family.

Material and Methods: We here asked the question whether further structural genomic rearrangements can be detected within NGS gene panel data of unsolved CCM cases. Hybrid capture NGS data of eight index patients without a pathogenic single nucleotide, indel or copy number variant were analyzed using two bioinformatics pipelines.

Results: In a 58-year-old male with multiple CCMs in his brain and spinal cord, we identified a $294 \mathrm{~kb}$ insertion within the coding sequence of CCM2. Fine mapping of the breakpoints, molecular cytogenetic studies, and multiplex ligation-dependent probe amplification verified that the structural variation was an inverted unbalanced insertion that originated from 1p12-p11.2. As this rearrangement disrupts the CCM2 gene on $7 \mathrm{p} 13$, it was classified as pathogenic.

Conclusions: Our study demonstrates that efforts to detect structural variations in known disease genes increase the diagnostic sensitivity of genetic analyses for welldefined Mendelian disorders.

This study was funded by the Deutsche Forschungsgemeinschaft (DFG, German Research Foundation, RA 2876/ 2-1).

R.A. Pilz: None. K. Schwefel: None. A. Weise: None. T. Liehr: None. P. Demmer: None. A. Spuler: None. S. Spiegler: None. E. Gilberg: None. C.A. Hübner: None. U. Felbor: None. M. Rath: None. 


\section{P09.032.C}

Microcephaly-causing dynein mutations lead to severe neural stem cell proliferation defects in human cerebral organoïds

\section{S. FARCY ${ }^{1,2}$, A. Di Cicco $^{2}$, A. Baffet ${ }^{2}$, N. Bahi-Buisson ${ }^{l}$ \\ ${ }^{1}$ Institut Imagine, Paris, France, ${ }^{2}$ Institut Curie, Paris, France}

The cortex is the centre for our complexes cognitive capacities. Its development is a highly regulated process. The dynein protein is a unique molecular motor and is crucial for intra-cellular trafficking. Our team identified mutations in the gene coding for the dynein in patients with microcephaly. Interestingly, distinct mutations within this same gene lead to a different phenotype, the spinal muscular atrophy (SMA). Why some mutations affect the cortex development and some doesn't remain unclear. The project is focus on the cortex, and aim to investigate which specific neuronal dynein functions are altered, and how it can lead to microcephaly. In order to study dynein-dependant functions during cortex development, I generate cerebral organoids, 3D structures that fully recapitulate the human features of the developing cortex. I reprogrammed two cell lines from patients caring mutations leading either to microcephaly or to SMA into pluripotent stem cells (IPSC) and differentiate them into cerebral organoids. Neuronal defects have been observed only in the organoids mutated for the microcephaly. The first observation was a strong reduction in the size of the organoids, which correlates with the patient's phenotypes. In those same organoids, an alteration of the neural stem cells proliferation have been observed as well as an increase in the number of apoptotic cells. I am using the live imaging technique, a new outcome in the field. This technique allows me to investigate in real time the division of the neural stem cells as well as the newborn neurons migration to their final location.

S. Farcy: None. A. Di Cicco: None. A. Baffet: None. N. Bahi-Buisson: None.

\section{P09.033.A}

Cerebral palsy is caused by genomic variants in $27 \%$ of patients undergoing exome sequencing

F. Millan ${ }^{1}$, A. Moreno-De-Luca ${ }^{2,3,4}$, S. Lewis ${ }^{5,6}$, H. Z. Elloumi $^{1}$, D. R. Pesacreta ${ }^{2,7}$, S. Shetty ${ }^{5,6}$, C. Teigen ${ }^{l}$, M. T. Oetjens $^{2}$, J. Heim ${ }^{6}$, J. Scuffins ${ }^{1}$, K. E. Wain ${ }^{2}$, B. Norton ${ }^{5,6}$, R. I. Torene ${ }^{1}$, S. M. Myers ${ }^{2,7}$, V. Gainullin ${ }^{l}$, K. Arvai ${ }^{l}$, D. H.

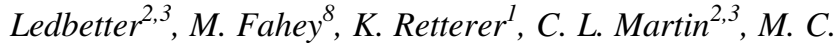
Kruer $^{5,6}$

${ }^{I}$ GeneDx, Gaithersburg, MD, United States, ${ }^{2}$ Autism \& Developmental Medicine Institute, Geisinger, Danville, PA, United States, ${ }^{3}$ Genomic Medicine Institute, Geisinger, Danville, PA, United States, ${ }^{4}$ Department of Radiology, Geisinger, Danville, PA, United States, ${ }^{5}$ Departments of Child Health, Neurology, Genetics, and Cellular \& Molecular Medicine, University of Arizona College of Medicine, Phoenix, AZ, United States, ${ }^{6}$ Cerebral Palsy \& Pediatric Movement Disorders Program, Barrow Neurological Institute at Phoenix Children's Hospital,, Phoenix, AZ, United States, ${ }^{7}$ Bucknell University, Lewisburg, PA, United States, ${ }^{8}$ Department of Paediatrics, Monash University, Melbourne, Australia

Introduction: Cerebral palsy (CP) describes a heterogeneous group of developmental brain disorders characterized by posture and movement impairments. Accumulating evidence suggests a significant genetic contribution.

Materials and Methods: Exome sequencing (ES) was performed on 1,777 patients with CP. Positive cases harbored one or two pathogenic or likely pathogenic alleles, depending on mode of inheritance, in a single gene.

Results: ES yielded a positive result in $27.5 \%$ of patients (488/1777). Causative variants were identified in genes associated with autosomal dominant $(66.0 \%)$, autosomal recessive (19.9\%), and X-linked disorders (14.1\%), encompassing 238 different genes. Trio testing revealed a high de novo rate $(71.5 \%)$. Linear modeling indicated that the likelihood of harboring a pathogenic variant was higher in patients with multiple co-morbid neurodevelopmental disorders $\left(\mathrm{OR}=1.34 ; 95 \% \mathrm{CI}=1.18-1.53 ; \mathrm{p}=7.28 \times 10^{-6}\right)$. Subset analysis from one study center $(n=208)$ showed that individuals lacking CP risk factors were 8-fold more likely to have a genetic etiology when controlling for GMFCS score (95\% CI=2.33-52.59; $\mathrm{p}=0.0052)$.

Conclusions: ES identified a genetic etiology in more than a quarter of $\mathrm{CP}$ patients, supporting genetic testing in this group. Although recurrent $\mathrm{CP}$-associated genes were elucidated, the results revealed vast genetic heterogeneity, underscoring the need for a broad genetic testing approach for CP. The high rate of de novo findings supports utilizing trio-testing. Individuals with multiple co-morbidities were more likely to have a positive finding, as were those lacking traditional CP risk factors. Genetic testing of patients with $\mathrm{CP}$ can inform an accurate recurrence risk, and provide information for prognosis, adjusted therapies, and management options.

F. Millan: A. Employment (full or part-time); Significant; GeneDx. A. Moreno-De-Luca: None. S. Lewis: None. H.Z. Elloumi: A. Employment (full or part-time); Significant; 
GeneDx. D.R. Pesacreta: None. S. Shetty: None. C. Teigen: A. Employment (full or part-time); Significant; GeneDx. M. T. Oetjens: None. J. Heim: None. J. Scuffins: A. Employment (full or part-time); Significant; GeneDx. K.E. Wain: None. B. Norton: None. R.I. Torene: A. Employment (full or part-time); Significant; GeneDx. S.M. Myers: None. V. Gainullin: A. Employment (full or part-time); Significant; GeneDx. K. Arvai: A. Employment (full or part-time); Significant; GeneDx. D.H. Ledbetter: None. M. Fahey: None. K. Retterer: A. Employment (full or part-time); Significant; GeneDx. C.L. Martin: None. M.C. Kruer: F. Consultant/Advisory Board; Modest; PTC Therapeutics.

P09.035.C

SLC12A6 gene: De novo variants cause dominantintermediate Charcot-Marie-Tooth

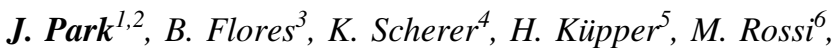
K. Rupprich ${ }^{7}$, M. Rautenberg ${ }^{1}$, N. Deininger ${ }^{1}, A$. Weichselbaum $^{5}$, A. Grimm ${ }^{2}$, M. Sturm ${ }^{1}$, U. Grasshoff ${ }^{l}$, E. Delpire $^{3}$, T. Haack ${ }^{1,8}$

${ }^{1}$ Institute of Medical Genetics and Applied Genomics, University of Tübingen, Tübingen, Germany, ${ }^{2}$ Department of Neurology and Epileptology, Hertie Institute for Clinical Brain Research, University of Tübingen, Tübingen, Germany, ${ }^{3}$ Department of Anesthesiology, Vanderbilt University School of Medicine, Nashville, TN, United States, ${ }^{4}$ Children's Clinic for Rehabilitative Services, Tucson, AZ, United States, ${ }^{5}$ Department of Neuropediatrics, University of Tübingen, Tübingen, Germany, ${ }^{6}$ Clinical Genomics, Ambry Genetics, Aliso Viejo, CA, United States, ${ }^{7}$ Department of Neuropediatrics, Essen University Hospital, Essen, Germany, ${ }^{8}$ Centre for Rare Diseases, University of Tübingen, Tübingen, Germany

Introduction: Charcot-Marie-Tooth (CMT) is the most common peripheral neuropathy. Biallelic truncating and missense variants in SLC12A6 coding for the potassium chloride cotransporter $\mathrm{KCC} 3$ have been associated with autosomal-recessive Andermann syndrome. This condition is characterized by agenesis of the corpus callosum, peripheral neuropathy and developmental delay. One heterozygous de novo missense alteration (Thr991Ala) has been reported in a patient with progressive, early-onset neuropathy with normal cognition.

Materials and Methods: We performed diagnostic trio exome sequencing on three affected individuals and their parents. We collected clinical and electrophysiological data. Wild-type and mutant KCC3 cotransporters were measured in Xenopus laevis oocytes using unidirectional ${ }^{86} \mathrm{Rb}$ uptakes.
Results: In three unrelated individuals, we identified two different de novo missense alterations (Arg207His and Tyr679Cys) in SLC12A6. Arg207His was found recurrent in two patients. Functional analyses demonstrated a significant reduction of $\mathrm{K}^{+}$influx in all mutant $\mathrm{KCC} 3$ cotransporters (Arg207His, Arg207Cys and Tyr679Cys). All patients presented early-onset sensorimotor, axonal and demyelinating neuropathy with normal cognition and brain MRI.

Conclusions: We conclude that heterozygous de novo SLC12A6 alterations can cause intermediate CMT, a milder phenotype than the autosomal-recessive disorder, Andermann syndrome. Although the patients with de novo variants in SCL12A6 had a homogenous phenotype, the biophysical properties of all mutant cotransporters were different. Furthermore, less is known about the role of histidine or cysteine on Arg207 that causes different inheritance patterns.

J. Park: None. B. Flores: None. K. Scherer: None. H. Küpper: None. M. Rossi: None. K. Rupprich: None. M. Rautenberg: None. N. Deininger: None. A. Weichselbaum: None. A. Grimm: None. M. Sturm: None. U. Grasshoff: None. E. Delpire: None. T. Haack: None.

\section{P09.036.A}

Homozygous frameshift mutation in $\mathrm{CHDH}$ associated with adult-onset Spastic Ataxia

C. Catalli ${ }^{1}$, H. Maortua Olabe ${ }^{1}$, M. Gonzalez Bouzas ${ }^{1}$, L. Garcia Naveda ${ }^{l}$, I. Llano Rivas ${ }^{l}$, B. Gener Querol ${ }^{l}$, I. Rouco Axpe ${ }^{2}$

${ }^{1}$ Osakidetza Basque Health Service, Cruces University Hospital, Department of Genetics, Barakaldo, Spain, ${ }^{2}$ Osakidetza Basque Health Service, Cruces University Hospital, Department of Neurology, Barakaldo, Spain

Introduction: Spastic ataxias (SAs) are a heterogeneous group of diseases, sharing symptoms and molecular pathways with other classes of neurodegenerative entities, like Spinocerebellar Ataxias (SCAs) and Hereditary Spastic Paraplegias (HSPs), often manifesting with complicated neurological features. Here we describe a new association with a truncating homozygous mutation in $\mathrm{CHDH}$ gene, not associated with any disease until now.

Materials and methods: A 70 years old man, son of second-cousins, presented with progressive spastic ataxia since his fifties, associated with ptosis, autonomic features, diffuse leukoencephalopathy and cortical atrophy. Routing screening for acquired forms was performed, along with common causes of ataxia caused by dynamic expansions. Exome was sequenced with xGen Exome Panel v1.0, IDT, on a NextSeq 500 by Illumina. Validated OMIM genes 
were analyzed and broad search in all candidate genes was performed in search of homozygous potentially pathologic mutations.

Results: No common SCAs nor Friedreich Ataxia expansions were detected. Exome analysis failed to find any variation amongst 33 validated genes causing autosomal recessive SA. Broad analysis extended to all OMIM and no OMIM genes led to identify a ultrarare frameshift mutation, c.386del (p.G129Vfs*89) in CHDH gene in homozygous state. No other potentially pathological variations were found.

Conclusions: CDHD is a mitochondrial enzyme involved in mitophagy and interacts with other genes like SQSTM1, PARK2 and TMEM70, which cause different neurodegenerative diseases through mitochondrial dysfunction when mutated. Functional studies are ongoing. We propose $\mathrm{CHDH}$ as candidate gene for autosomal recessive neurogenerative diseases presenting with $\mathrm{SA}$, inexplicable leukoencephalopathy or cognitive decline.

C. Catalli: None. H. Maortua Olabe: None. M. Gonzalez Bouzas: None. L. Garcia Naveda: None. I. Llano Rivas: None. B. Gener Querol: None. I. Rouco Axpe: None.

\section{P09.039.A}

Evaluation of recurrent copy number variations in patients with neurodevelopmental disorders

L. Morozin Pohovski ${ }^{1}$, A. Bobinec ${ }^{1,2}$, A. Measic ${ }^{1,2}, N$. Vidan Rogulj, I. Sansovic ${ }^{1,2}$, M. Kero ${ }^{1,2}$, L. Boban ${ }^{1,2}$, I. Barisic $^{1,2}$

${ }^{1}$ Children's Hospital Zagreb, Medical School University of Zagreb, Zagreb, Croatia, ${ }^{2}$ Department of Medical Genetics and Reproductive Health, Children's Hospital Zagreb, Scientific Centre of Excellence for Reproductive and Regenerative Medicine (CERRM), University of Zagreb School of Medicine, Zagreb, Croatia

Background: Neurodevelopmental disorders (NDDs) are a wide range of neuropsychiatric conditions caused by impaired growth and development of the central nervous system. NDDs include autism spectrum disorders, intellectual disability/developmental delay, communication disorders, attention-deficit/hyperactivity, epilepsy, specific learning problems and motor disorders. The risk of developing NDDs seems to be highly influenced by the effect of rare but recurrent copy number variations (CNVs).

Methods: We have analysed the findings of CNV studies from a cohort of 1170 patients with NDDs referred for clinical geneticist evaluation. The analysis was conducted using Agilent $60 \mathrm{~K}$ oligonucleotide array-based comparative genomic hybridization.
Results: Chromosome microarray analysis was performed in 1170 patients with NDDs and CNVs were identified in 89 children $(7,6 \%)$. We identified 45 duplications and 44 deletions involving chromosomal regions 1q21.1, 3q29, 15q11.2, 15q13.3, 16p11.2, 16p13.11 and 22q11.2. $47(52,8 \%)$ were considered to be pathogenic, 11 $(12,3 \%)$ likely pathogenic and 31 were variants of unknown significance (VUS). In 15 patients two or three copy number alterations were present.

Conclusions: A rare CNVs contribute to a significant proportion to the risk of developing NDDs. Counselling for $\mathrm{CNVs}$ remains challenging because of variable penetrance and expressivity. Further data collection is needed to fully evaluate the impact of rare CNVs on complex the etiology of NDDs. Acknowledgement: This work was supported by Scientific Center of Excellence for Reproductive and Regenerative Medicine and by the European Union through the European Regional Development Fund, under grant agreement No. KK.01.1.1.01.0008, project „Reproductive and Regenerative Medicine - Exploring New Platforms and Potentials.

L. Morozin Pohovski: None. A. Bobinec: None. A. Measic: None. N. Vidan Rogulj: None. I. Sansovic: None. M. Kero: None. L. Boban: None. I. Barisic: None.

P09.041.C

DCTN1 mutation analysis in Italian patients with PSP, MSA and DLB

R. Procopio ${ }^{1,2}$, M. Gagliardi ${ }^{1}$, M. D'Amelio ${ }^{3}$, L. Brighina ${ }^{4}$, G. Nicoletti ${ }^{1}, M$. Morelli ${ }^{2}$, G. Bonapace ${ }^{5}$, A. Quattrone ${ }^{6}$, G. $^{2}$ Annesi ${ }^{1}$

${ }^{1}$ Institute of Molecular Bioimaging and Physiology, National Research Council, Section of Germaneto, Catanzaro, Italy, ${ }^{2}$ Institute of Neurology, Department of Medical and Surgical Sciences, University Magna Graecia, Catanzaro, Italy, ${ }^{3}$ Department of Experimental Biomedicine and Clinical Neuroscience (BioNeC), University of Palermo, Palermo, Italy, ${ }^{4}$ Department of Neurology, Milan Center for Neuroscience, San Gerardo Hospital, University of Milano-Bicocca, Monza, Italy, ${ }^{5}$ University Magna Graecia, Faculty of Medicine, Pediatrics, Catanzaro, Italy, ${ }^{6} \mathrm{Neu}-$ roscience Center, University Magna Graecia, Catanzaro, Italy

Introduction: Progressive supranuclear palsy (PSP), multiple system atrophy (MSA), and dementia with Lewy bodies (DLB) are defined as atypical parkinsonisms. This term describes a syndrome characterized by typical parkinsonian features, such as bradykinesia, rigidity, postural instability and resting tremor, and other clinical signs atypical for Parkinson's disease (PD). The signs are different for each disease, but they share the pathological 
mechanisms: they presented intracellular protein aggregation in the brain of tau or $\alpha$-synuclein, which is thought to be the cause of the development of the disease. Mutations in genes encoding tau and $\alpha$-synuclein have been identified as risk factors for these diseases. DCTN1 encodes the largest subunit of dynactin complex essential in the retrograde axonal transport and cytoplasmic transport of vesicles; mutations in DCTN1 have been reported predominantly in individuals with Perry syndrome and, recently, in patients with PSP. Here we performed a mutational screening of DCTN1 in 207 patients with atypical parkinsonisms from Italy.

Material and Methods: The cohort included 79 patients with PSP, 100 patients with MSA and 28 patients with DLB. A control group of 500 healthy subjects from the same geographical area was also examined. All 32 exons and intron-exon boundaries region of DCTN1 were sequenced.

Results: Sequence analysis revealed only a synonymous variant in one of PSP case, and two intronic variants with the same frequency in patients with atypical parkinsonisms and healthy subjects cohort.

Discussion: DCTN1 mutations have not a key role in the development of atypical parkinsonisms in the Italian population.

R. Procopio: None. M. Gagliardi: None. M. D'Amelio: None. L. Brighina: None. G. Nicoletti: None. M. Morelli: None. G. Bonapace: None. A. Quattrone: None. G. Annesi: None.

\section{P09.042.A}

Genetic characterization of Hungarian patients with early onset dementia

D. Csabán ${ }^{l}$, R. Bencsik ${ }^{l}$, A. Illés ${ }^{l}, V$. Molnár $^{l}, P$. Balicza $^{l}$,

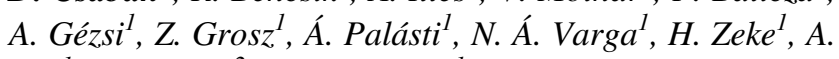
Gál $^{l}$, T. Kovács ${ }^{2}$, M. J. Molnár ${ }^{1}$

${ }^{1}$ Institute of Genomic Medicine and Rare Disorders, Semmelweis University, Budapest, Hungary, ${ }^{2}$ Department of Neurology, Semmelweis University, Budapest, Hungary

Introduction: Dementia is one of the most common neurodegenerative disorder with a previously suggested heterogeneous genetic background. In the recent years the increasing availability of Next Generation Sequencing (NGS) has made it possible to better understanding the genetic background of this disease group.

Materials and Methods: In our study 111 Hungarian patients with relatively early onset dementia (mean age of onset was $56 \pm 7.41$ years) were pre-screened for the most common $\mathrm{AD}$ or FTD associated genes by Sanger sequencing and $C 9$ orf 72 hexanucleotide repeat expansion. In subjects without positive results $(n=56)$, targeted panel sequencing was performed on the Illumina platform. The common $\mathrm{AD}$ genetic risk factors, $A P O E$ and MTHFR genotyping were performed too.

Results: In the most common dementia associated genes (PSEN1, PSEN2, APP, GRN), mutations were detected in $8.1 \%$ of the cases. Abnormal repeat number for $C 9$ orf 72 gene was identified in 6 cases. Further heterozygous possibly damaging alterations were detected by NGS in other genes such as TREM2, ABCA7, PLA2G6, C19orf12, $P A R K 7$. In some cases the coexistence of a genetic risk factors and a rare variant indicating monogenic dementia has additive effect on the phenotype.

Conclusions: In our study, variants with potential association with the early onset dementia were identified in $20.7 \%$ of the investigated cohort. With the application of NGS both rare damaging variants and genetic risk factors could be effectively identified, which could contribute to understand the complex genetic architecture of dementias and could be helpful for genetic counselling.

Disclosure: Hungarian Brain Research Program (KTIA_NAP_ 2017-1.2.1-NKP-2017-00002)

D. Csabán: None. R. Bencsik: None. A. Illés: None. V. Molnár: None. P. Balicza: None. A. Gézsi: None. Z. Grosz: None. Á. Palásti: None. N.Á. Varga: None. H. Zeke: None. A. Gál: None. T. Kovács: None. M.J. Molnár: None.

\section{P09.044.C}

Monogenic causes of developmental epileptic encephalopathies in a large cohort from Latin America

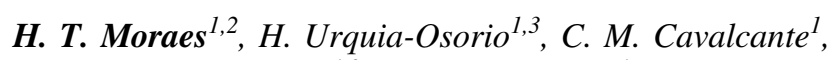
E. Almendares-Bonilla ${ }^{l, 2}$, M. M. Guerreiro ${ }^{l}, M$. Montenegro $^{l}$, B. Henning ${ }^{1,2}$, B. S. Carvalho ${ }^{4,2}$, A. Coan ${ }^{l}$, M. T. Medina ${ }^{3}$, R. Caraballo $^{5}$, I. Lopes-Cendes ${ }^{1,2}$

${ }^{1}$ School of Medical Sciences, State University of Campinas, Campinas, Brazil, ${ }^{2}$ Brazilian Institute of Neuroscience and Neurotechnology - BRAINN, Campinas, Brazil, ${ }^{3} 5$ Faculty of Medical Sciences, National Autonomous University of Honduras, Tegucigalpa, Honduras, ${ }^{4}$ Institute of Mathematics, Statistics and Computer Science, University of Campinas, Campinas, Brazil, ${ }^{5}$ Hospital JP Garrahan, Neurology, Buenos Aires, Argentina

Introduction: Recent molecular genetics techniques improved mutations detection in patients with developmental epileptic encephalopathies (DEEs). However, the current studies are performed predominantly in European populations, and information in more ethnically diverse 
groups is lacking. This study aims to search for the monogenic causes of DEEs in Latin-America.

Materials and Methods: We ascertained 316 patients from different countries: Brazil (223), Argentina (57), Chile (15), Peru (14), Uruguay (4), and Honduras (3). To date, we performed whole-exome sequencing in 100 patients. For genetic variant selection, two filters were used: i) read depth $(\mathrm{RD} \geq 10)$, allele frequency in control population $(\leq 1 \%)$ and exonic consequence; ii) 129 known genes related to DEEs. For variants of interest selection, three criteria were used: considered deleterious by variant effect predictors, impact in the protein, presence on dbSNP and CLINVAR.

Results: The mean RD was 30X, and the mean percentage of targets with at least 10x RD was $89 \%$. The mean number of genetic variants per patient was 19,5341 before the filters and 22 after the filters. We selected sixtythree variants of interest in 41 genes from 49 individuals for further studies. From the selected variants, 51 are missense, 5 nonsense, 4 frameshift indel, 2 in-frame indel, and one affects a splicing site. According to the American College of Medical Genetics classification 52 variants are of uncertain significance, 9 likely pathogenic, one likely benign, and one pathogenic.

Conclusions: The combined filters and selection criteria used identified genetic variants of potential clinical interest in $49 \%$ of Latin-American patients with DEE.

H.T. Moraes: None. H. Urquia-Osorio: None. C.M. Cavalcante: None. E. Almendares-Bonilla: None. M.M. Guerreiro: None. M. Montenegro: None. B. Henning: None. B.S. Carvalho: None. A. Coan: None. M.T. Medina: None. R. Caraballo: None. I. LopesCendes: None.

\section{P09.045.A}

Vitamin D3supplementation affects the morphology and MTHFR and caspase 3 p12 expression in the olfactory bulb of a mouse model for Down syndrome

\section{F. Campos Gomes ${ }^{1}$, J. S. Melo-Neto ${ }^{2}$, M. F. Ramires Ferrari $^{3}$, O. Y. Galvis-Alonso ${ }^{4}$, E. M. Goloni-Bertollo ${ }^{l}$, É. C. Pavarino ${ }^{1}$}

${ }^{1}$ Genetics and Molecular Biology Research Unit (UPGEM), São José do Rio Preto Medical School (FAMERP), São José do Rio Preto, Brazil, ${ }^{2}$ Institute of Health Sciences, Federal University of Pará (UFPA), Belém, Brazil, ${ }^{3}$ Department of Genetics and Evolutionary Biology, Biosciences Institute, University of São Paulo (USP), São Paulo, Brazil, ${ }^{4}$ Laboratory of Experimental Physiology, São José do Rio Preto Medical School (FAMERP), São José do Rio Preto, Brazil
Introduction: Individuals with Down syndrome (DS) have olfactory function impairment and are susceptible to develop Alzheimer's disease (AD). Olfactory dysfunction may be an early clinical symptom of AD. Recent studies show that vitamin $\mathrm{D}_{3}\left(\mathrm{VD}_{3}\right)$ has neuroprotective effects in mouse models of AD. In this study, we evaluated the effects of $\mathrm{VD}_{3}$ on the olfactory bulb (OB) in mouse model for DS.

Materials and Methods: Female mice with 14-week-old of the lineage B6EiC3Sn-Rb(12.Ts171665Dn)2Cje/CjeDnJ were distributed into experimental groups: with trisomy (Ts (CO) or $\left.\mathrm{Ts}_{(\mathrm{VD} 3)}\right)$ and wild-type $\left(\mathrm{Wt}_{(\mathrm{CO})}\right.$ or $\left.\mathrm{Wt}_{(\mathrm{VD} 3)}\right)$, and treated for 10-week with common diet (CO) or $12.500 \mathrm{IU} /$ $\mathrm{Kg}$ of $\mathrm{VD}_{3}$. At twenty-fourth week old the mice were euthanized. The OB was processed for morphological/ stereological analysis; immunochemistry/Western blotting for A $\beta-42$, Caspase-3 p12, MTHFR, NeuN and Pgp. Data were analyzed statistically $(\mathrm{P}<0.05)$. The $\mathrm{F}-(\mathrm{F})$ and $\mathrm{H}-$ statistic $(\mathrm{H})$ were used to analyze between-groups factors (trisomy; vitamin $\mathrm{D}_{3}$; interaction).

Results: Trisomy reduced the volume of the external plexiform layer $(\mathrm{F}=4.313 ; \mathrm{P}=0.046)$, number of mitral cells $(\mathrm{F}=9.941 ; \mathrm{P}=0.006)$, increased Pgp expression $(\mathrm{F}=$ 9.802; $\mathrm{P}=0.005)$, caspase-3 $\mathrm{p} 12$ in $(\mathrm{P}<0.0001)$ and $\mathrm{A} \beta-42$ $(\mathrm{H}=8.67 ; \mathrm{P}=0.003) . \mathrm{VD}_{3}$ reduced the expression of caspase-3 p12 $(\mathrm{F}=107.729 ; \mathrm{P}<0.0001)$ and MTHFR $(\mathrm{F}=13.191 ; \mathrm{P}=0.002)$ and increased the volume of the mitral cell layer $(\mathrm{F}=5.398 ; \mathrm{P}=0.034)$.

Conclusions: Ts mice show morphological and molecular changes in the $\mathrm{OB}$ and $\mathrm{VD}_{3}$ can attenuate and reverse these abnormalities indicating its neuroprotective function.

Grants: Capes (Code 001); CNPq (310806/2018-6) and FAPESP (2018/09126-0).

F. Campos Gomes: None. J.S. Melo-Neto: None. M.F. Ramires Ferrari: None. O.Y. Galvis-Alonso: None. E.M. Goloni-Bertollo: None. É.C. Pavarino: None.

\section{P09.047.C}

A clinical case of dystonia-28 childhood-onset with a previously undescribed variant in the KMT2B gene.

\section{Guseva, I. Sharkova, N. Semenova, E. Dadali}

Research Centre for Medical Genetics, Moscow, Russian Federation

Introduction: Dystonia-28 is a neurological disorder characterized by onset of progressive dystonia in the first decade of life. It is an autosomal dominant disease with incomplete penetrance. The clinical symptoms of dystonia28 are development delayed, microcephaly, focal or generalized dystonia, gait difficulties and toe walking.

Materials and methods: We present a 7-year-old girl with clinical symptoms of generalized dystonia. Motor 
development is delayed - keeps his head from a fore month, sits from a ten month, walk from a year. At the age of 2 years, she started walking on "socks" and instability was observed. At the age of 6 years, after infections, there was tension in the muscles of the left leg, involuntary movements, a turn of the left leg when walking, tiredness due to walking. The dystonia was progressive and eventually became generalized with involvement of the upper limbs, hands, neck, face, tongue, and trunk. She had microcephaly elongated face, dysarthria, generalized dystonia, and spasticity. EEG and MRI of the brain were normal.

Results: Analysis of whole genome sequencing data revealed a previously undescribed heterozygous variant c. $3626 \mathrm{G}>\mathrm{A}$ in KMT2B gene. This variant was not detected in either parent and was confirmed with Sanger sequencing.

Conclusions: We report clinical case with undescribed heterozygous variant KMT2B in the girl with a clinically recognizable form of dystonia. Verification of the genetic etiology of dystonia allowed selection of appropriate treatment strategies, determination of genetic risk and discussion of the necessary of prenatal testing.

D. Guseva: None. I. Sharkova: None. N. Semenova: None. E. Dadali: None.

\section{P09.048.A}

Understanding the mechanism of RNF216-mediated neurodegeneration: genetic, proteomic, neuropathological and cellular approach

S. Roy $^{1,2}$, P. Ervilha Pereira ${ }^{1,2}, N$. Schuermans ${ }^{1,2}$, A. Sieben $^{3,4}$, F. Thery ${ }^{5,6}$, E. Mondragon Rezola ${ }^{7}$, E.

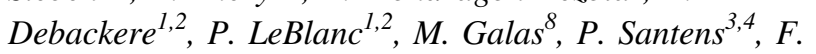
Impens $^{5,6}$, E. Bogaert ${ }^{1,2}$, B. Dermaut ${ }^{1,2}$

${ }^{1}$ Center for Medical Genetics, Ghent University Hospital, Gent, Belgium, ${ }^{2}$ Department of Biomolecular Medicine, Faculty of Medicine and Health Sciences, Ghent University, Ghent, Belgium, ${ }^{3}$ Department of Neurology, Ghent University Hospital, Gent, Belgium, ${ }^{4}$ Department of Head and Skin, Faculty of Medicine and Health Sciences, Ghent University, Ghent, Belgium, ${ }^{5}$ Department of Biomolecular Medicine, Faculty of Medicine and Health Sciences, Ghent University, Gent, Belgium, ${ }^{6}$ Center for Medical Biotechnology, VIB Ghent, Ghent, Belgium, ${ }^{7}$ Department of Neurology, University Hospital Donostia, Basque Country University, Donostia, Spain, ${ }^{8}$ Inserm, UMRS1172, JPARC, Alzheimer \& Tauopathies, Lille, France

Introduction: Neurodegenerative diseases frequently result from mutations in ubiquitin-signaling genes. Our group was among the first to report bi-allelic mutations in RNF216, an E3 ubiquitin ligase, as the genetic cause of Gordon Holmes syndrome (GHS), an adult-onset rare neurodegenerative disorder characterized by ataxia, dementia and hypogonadotropic hypogonadism or Huntington-like disorder (HDL). To date, $>10$ mutations in RNF216 have been reported not only from the catalytic active site but also from $\mathrm{N}$-terminal part of the protein.

Objective: We intend to unravel the mechanisms by which RNF216 mutations lead to GHS and HDL. We aim to identify novel RNF216 mutations for genotypephenotype correlation studies, identify protein composition of the pathological intranuclear inclusions, and understand the cellular and biochemical function of the RNF216 protein.

Methods: Frozen and fixed brain material from patient were used for generalized proteomics and neuropathological studies and patient fibroblast and Hela cells were used for cellular studies.

Results: We describe a Spanish patient with HDL having 2 novel compound heterozygous missense mutations in RNF216. The study of brain material from one of the two reported Belgian families demonstrated ubiquitin- and p62positive intranuclear inclusions, negative for Tau, $\alpha$ synuclein and TDP-43. Proteomics generated a list of candidate genes whose histopathological examination is currently ongoing. Hela cells transfected with wildtype and mutant RNF216 constructs revealed RNF216 and ubiquitin positive intranuclear speckle-like bodies.

Conclusions: The identity of intranuclear bodies should be further analyzed hoping to reveal molecular intricacies linked to this neurodegenerative disease.

Odysseus Type-I (G0H8318N) FWO grant, Starting grant (01N10319) Ghent University

S. Roy: None. P. Ervilha Pereira: None. N. Schuermans: None. A. Sieben: None. F. Thery: None. E. Mondragon Rezola: None. E. Debackere: None. P. LeBlanc: None. M. Galas: None. P. Santens: None. F. Impens: None. E. Bogaert: None. B. Dermaut: None.

\section{P09.049.B}

Identification of genes for epileptic encephalopathy: the impact of exome analysis in a pediatric cohort.

L. Musante ${ }^{\text {I, C. Zanus }}{ }^{2}$, P. Costa ${ }^{2}$, A. P. d'Adamo ${ }^{1}$, A. M. Bianco $^{l}$, G. Ragusa ${ }^{l}$, E. Athanasakis ${ }^{l}, F$. Faletra ${ }^{l}, P$. Gasparini $^{l}$, M. Carrozzi ${ }^{2}$

${ }^{1}$ SC Genetica Medica, Institute for Maternal and Child Health IRCCS Burlo Garofolo, Trieste, Italy, ${ }^{2}$ SC Neuropsichiatria Infantile, Institute for Maternal and Child Health IRCCS Burlo Garofolo, Trieste, Italy

Early onset infantile epileptic encephalopathies (EIEE) are neurodevelopmental disorders, with persistent severe electroencephalographic abnormalities, developmental delay or 
regression associated with ongoing epileptic activity and often characterized by pharmaco-resistant epilepsy. By Next-Generation Sequencing (NGS) great advances have been made in gene discovery for EIEE and several studies emphasized de novo variants as major underlying cause. Current diagnostic pathways allow molecular diagnosis to be made in about $20 \%$ of cases with EIEE and recent studies have also highlighted extreme genetic heterogeneity. Therefore, the current scenario indicates that most of the genes responsible for EIEE are probably still unknown. To this regard, our group recruited 21 patients (and their parents) with EIEE or epilepsy of unknown origin, negative for the presence of micro-arrangements, and investigated by WES, in the frame of the multicentric project EPI-IDEA, which is part of the RETE-IDEA network constituted by eight Italian pediatric IRCCS. In our study, we identified a pathogenic or very likely pathogenic variant/genotype in about $48 \%$ of the patients. Only in 2 patients a de novo mutation was identified, whereas $64 \%$ of diagnosed cases were caused by recessive genes previously associated with a seizure-related phenotype/s, including five compound heterozygosity for causative sequence and 2 homozygous. $27 \%$ of the mutations were novel variants not described before. 10 patients are currently still under investigation. Our results confirm a relevant role of mostly compound heterozygous recessive inheritance for EIEE with important implication for genetic counselling.

L. Musante: None. C. Zanus: None. P. Costa: None. A. P. d'Adamo: None. A.M. Bianco: None. G. Ragusa: None. E. Athanasakis: None. F. Faletra: None. P. Gasparini: None. M. Carrozzi: None.

\section{P09.050.C}

Clinical significance of submicroscopic copy number variants (CNVs) in the etiopathogenesis of epilepsy

\section{Plaskota, M. Bartnik-Głaska, M. Smyk, B. Wiśniowiecka- Kowalnik, K. Kowalczyk, M. Paczkowska, M. Zbiciak, J. Bernaciak, E. Szczepanik, H. Mierzewska, E. Obersztyn, B. Nowakowska}

\section{Institute of Mother and Child, Warsaw, Poland}

Epilepsy is one of the most common diseases of the central nervous system affecting about $1 \%$ of the human population. The etiology of epilepsy remains unknown in $40-50 \%$ of cases, although it is well known that approximately $30 \%$ causes is genetic. According to recent studies, the copy number variants (CNVs) are important factors in this disorder $(2-11 \%)$. We used array CGH method in a cohort of 90 patients with epilepsy, and neurodevelopmental abnormalities with or without dysmorphic features. Array $\mathrm{CGH}$ analysis was performed using genome-wide microarray with average resolution of $30 \mathrm{~kb}(180 \mathrm{~K}$, OGT) with specifically designed coverage for over 212 selected genes, known or candidate to play an important role in the pathogenesis of epilepsy. Chromosomal microarray analysis revealed 33 CNVs (17 duplications, 15 deletions, 1 triplication) in 29 out of 90 (32.2\%) patients with epilepsy. 2 pathogenic CNVs were detected in 2 patients from our cohort. 9 potentially pathogenic CNVs, and 22 CNVs with unknown clinical significance were detected in 27 individuals. All of the identified CNVs were submicroscopic in size, ranging from $1.7 \mathrm{~kb}$ to $8.85 \mathrm{Mb}$, and therefore could not have been detected by standard karyotype analysis. Additionally, 17 CNVs (51\%) could not have been detected by our clinical microarray $(60 \mathrm{~K})$. Our study highlights the role of CNVs in the etiopathogenesis of epilepsy and demonstrates that the array $\mathrm{CGH}$ is an effective tool in the genetic diagnosis of this neurodevelopmental disorder. The work was supported by projects 510-18-47, 510-18-57, 510-18-71 from the Ministry of National Education

I. Plaskota: None. M. Bartnik-Glaska: None. M. Smyk: None. B. Wiśniowiecka-Kowalnik: None. K. Kowalczyk: None. M. Paczkowska: None. M. Zbiciak: None. J. Bernaciak: None. E. Szczepanik: None. H. Mierzewska: None. E. Obersztyn: None. B. Nowakowska: None.

\section{P09.051.A}

Two novel mutations in GRIN2A and LGI1 in multigenerational Bulgarian families with different forms of epilepsy

V. Peycheva ${ }^{l}$, K. Kamenarova ${ }^{l}$, R. Tzveova ${ }^{l}$, N. Ivanova ${ }^{l}$, S. Zhelyazkova ${ }^{2}$ M. Radionova ${ }^{2}$, I. Pacheva ${ }^{3}$, I. Ivanov ${ }^{3}, I$. Litvinenko $^{4}$, V. Mitev ${ }^{l}$, I. Tournev ${ }^{2}$, A. Jordanova ${ }^{1,5}, R$. Kaneval

${ }^{1}$ Molecular Medicine Center, Department of Medical Chemistry and Biochemistry, Medical University-Sofia, Sofia, Bulgaria, ${ }^{2}$ Clinic of Neurology, University Hospital "Alexandrovska”, Department of Neurology, Medical University-Sofia, Sofia, Bulgaria, ${ }^{3}$ University Hospital "St. George”, Department of Pediatrics and Medical Genetics, Medical University- Plovdiv, Plovdiv, Bulgaria, ${ }^{4}$ University Pediatrics Hospital, Clinic of Pediatric Neurology, Medical University-Sofia, Sofia, Bulgaria, ${ }^{5}$ Molecular Neurogenenomics Group, VIB-Antwerp Center for Molecular Neurology, University of Antwerp, Antwerp, Belgium

The aim of the study was to reveal the genetic causes of the familial epilepsy in two four-generational clinically heterogeneous Bulgarian families. Both families were clinically assessed in detail and mutation analysis was performed using Clinical Exome Sequencing(CES) with 
Illumina platform. In the first family CES revealed a frameshift deletion (c.194delT,p.Leu65ArgfsTer3) affecting the GRIN2A gene. The proband presented unprovoked myoclonic and Generalized Tonic-Clonic Seizures(GTCS), motor aphasia and moderate intellectual disability (ID). In his pedigree we diagnosed a total of seven additional affected members all having GTCS with onset around 4-6 years of age. The GRIN2A gene encodes a subunit of Nmethyl-D-aspartate(NMDA) receptors found in excitatory synapses with role in neural plasticity. GRIN2A mutations are associated with focal epilepsy with speech disorder with or without cognitive disability. In the second family CES revealed a possibly pathogenic splice-acceptor variant c. $216-1 \mathrm{G}>\mathrm{A}$ in exon 2 of the LGII gene. This family consists of a total of eight affected members. The proband presented a fever-provoked GTCS, while his first-degree relatives have GTCS preceded by auditory aura and later onset. LGII gene encodes brain leucine-rich protein regulating postnatal glutamatergic synapse development. Loss of function LGII mutations are associated with partial epilepsy with auditory features. Confirmation Sanger sequencing showed that the variants segregate with disease phenotype in all available affected members from each family. Both variants are absent in public databases. Further investigations are needed to elucidate the splice-site mutation effect on RNA level. The study was supported by Grants DTK/67/2009 and DUNK/01-2/2009 of NSF, MES, Bulgaria.

V. Peycheva: None. K. Kamenarova: None. R. Tzveova: None. N. Ivanova: None. S. Zhelyazkova: None. M. Radionova: None. I. Pacheva: None. I. Ivanov: None. I. Litvinenko: None. V. Mitev: None. I. Tournev: None. A. Jordanova: None. R. Kaneva: None.

\section{P09.053.C}

$A D G R V 1$ is a susceptibility gene for familial forms of Genetic Generalized Epilepsy

\section{Dahawi ${ }^{1,2}$, M. S Elmagzoub ${ }^{3}$, E. A Ahmed ${ }^{4}, F$. A Elmugadam $^{5}$,W. A Abdelgadir ${ }^{6}$, O. Abdalla ${ }^{7}, J$. Buratti $^{8}, E$. Noé ${ }^{9}$, M. Alzubeir ${ }^{5}$, R. Abubakr ${ }^{10}$, S. Baldassari ${ }^{9}, S$. Baulac $^{9}$, L. Elsayed ${ }^{11}$, A. E Ahmed ${ }^{5}$, E. Leguern ${ }^{9,12}$}

${ }^{1}$ University of Khartoum, Faculty of medicine, department of physiology, Khartoum, Sudan, ${ }^{2}$ niversity of Khartoum, Faculty of medicine, department of physiology, Khartoum, Sudan, ${ }^{3}$ National Ribat University Faculty of Medicine, khartoum, Sudan, ${ }^{4}$ UNESCO Chair on Bioethics, University of Khartoum, Faculty of Medicine, Sudan, khartoum, Sudan, ${ }^{5}$ University of Khartoum, Faculty of medicine, Sudan, khartoum, Sudan, ${ }^{6}$ Al-Neelain University, Faculty of science and technology, department of biochemistry and molecular biology, Khartoum, Sudan., khartoum, Sudan,
${ }^{7}$ University of Khartoum, Faculty of medicine, department of physiology, Sudan, khartoum, Sudan, ${ }^{8}$ Department of genetics, La Pitié-Salpêtrière Hospital, AP-HP Sorbonne Université, paris, France, ${ }^{9}$ Institut du Cerveau et de la Moelle épinière, INSERM U1127, CNRS UMR7225, paris, France, ${ }^{10}$ University of Khartoum, Faculty of medicine, Sudan, Khartoum, Sudan, ${ }^{11}$ University of Khartoum, Faculty of medicine, department of biochemistry, Sudan, khartoum, Sudan, ${ }^{12}$ Department of genetics, La PitiéSalpêtrière Hospital, AP-HP Sorbonne Université, Paris, France

Objective:Genetic generalized epilepsies (GGE) including childhood absence epilepsy (CAE), juvenile absence epilepsy (JAE), juvenile myoclonic epilepsy (JME), and GGE with tonic-clonic seizures (TCS) alone (GGE-TCS), are mainly determined by genetic factors with a likely poly/ oligogenic mode of inheritance. Recent studies on large American or European cohorts showed that susceptibility genes for GGE are numerous and their variants rare, challenging their identification. In this study, we aimed to assess the GGE genetic etiology in a Sudanese population.

Methods:We collected 13 large consanguineous families with GGE from Sudan including 34 patients to search for GGE susceptibility genes. We performed whole exome sequencing (WES) in DNA samples of probands from 13 families. We filtered non-silent ultrarare/rare variants identified in each family and compared them with publicly available data and ethnicity-match controls.

Results: We identified in a family of 3 siblings with GGETCS one ultra-rare missense variant in ADGRVI, a gene recently involved in the pathogenesis of sporadic myoclonic epilepsies. Five additional $A D G R V 1$ ultra rare/rare missense variants segregated with GGE in three of the remaining families. The frequency of this class of $A D G R V 1$ variants was significantly higher in our cohort compared to gnomAD African controls $(\mathrm{P}=0.0018)$. A homozygous patient for an $A D G R V 1$ variant presented a more severe phenotype than her heterozygous brother, suggesting a dosage effect.

Significance:Our study showed for the first time the segregation of ADGRVI variants with familial GGE, extending the phenotype spectrum of ADGRVI-related epilepsies to CAE/JAE and GTC epilepsy. This work was supported by INSERM (France), IHU-A-ICM (France), the Sudanese Ministry of Higher Education.

M. Dahawi: None. M. S Elmagzoub: None. E. A Ahmed: None. F. A Elmugadam: None. W. A Abdelgadir: None. O. Abdalla: None. J. Buratti: None. E. Noé: None. M. Alzubeir: None. R. Abubakr: None. S. Baldassari: None. S. Baulac: None. L. Elsayed: None. A. E Ahmed: None. E. Leguern: None. 


\section{P09.054.A}

\section{EFHC2 variants in juvenile myoclonic epilepsy}

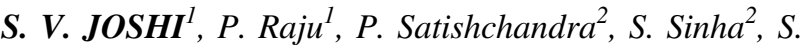
Nayak $^{l}$, A. Anand ${ }^{l}$

${ }^{1}$ Jawaharlal Nehru Centre for Advanced Scientific Research, Bengaluru, India, ${ }^{2}$ National Institute of Mental Health and Neurosciences, Bengaluru, India

Juvenile myoclonic epilepsy (JME) is a brain disorder characterized by frequent myoclonic seizures often accompanied by generalized tonic-clonic seizures during adolescence. Here, we present, our work on contribution of $E F H C 2$ in predisposition to JME. EFHC2 encodes a protein containing three DM10 domains and a putative EF-hand motif. EFHC2 is expressed in the mammalian brain regions: cerebral cortex, hippocampus, hypothalamus and cerebellum. In cultured mammalian cells, it localizes to the spindle poles and midbody. We have identified seven potentially pathogenic $E F H C 2$ variants present almost exclusively among $500 \mathrm{JME}$ patients examined. On overexpression of EFHC2 carrying these variants, significant levels of spindle abnormalities, multi-polarity and chromosome segregation defects are observed. We analyze the contribution of the DM10 domains to subcellular localization and function of the protein. EFHC2 is a homolog of EFHC1, a known JME causing gene. Cellular defects caused by EFHC2 variants in cultured mammalian cells are partially rescued by EFHC1, and vice versa. EFHC2 coprecipitates with $\alpha$-tubulin and $\gamma$-tubulin suggesting that EFHC2 is a microtubule-associated protein. EFHC2's role in microtubule organization during cell cycle are being currently studied.

We would like to acknowledge the grant BMS/TF/TransNeuro-2014-3681/Jul-2015/47/KA/Govt.

S.V. Joshi: None. P. Raju: None. P. Satishchandra: None. S. Sinha: None. S. Nayak: None. A. Anand: None.

\section{P09.055.B}

Delineating the phenotypic spectrum of STX1B associated epilepsy

M. Schlögl ${ }^{1}$, M. Krenn ${ }^{1,2}$, E. Pataraia ${ }^{2}$, S. Schröder ${ }^{3}$, J. A. Mayr $^{4}$, C. Rauscher ${ }^{4}$, D. Kotzot ${ }^{5}$, F. Zimprich ${ }^{2}, T$.

Meitinger ${ }^{l}$, M. Wagner ${ }^{1,6}$

${ }^{1}$ Institute of Human Genetics, Munich, Germany, ${ }^{2}$ Department of Neurology, Medical University of Vienna, Vienna, Austria, ${ }^{3}$ Dr. von Hauner Children's Hospital, LudwigMaximilians-University Munich, Munich, Germany, ${ }^{4}$ University Children's Hospital, Salzburger Landeskliniken (SALK), Salzburg, Austria, ${ }^{5}$ Unit of Clinical Genetics,
Salzburg, Austria, ${ }^{6}$ Institute for Neurogenomics, Helmholtz Zentrum München, Neuherberg, Germany

Introduction: $S T X 1 B$ (Syntaxin 1B) encodes a presynaptic protein which is part of the SNARE complex. De novo, mostly loss of function variants cause a spectrum of epileptic disorders. These have recently been divided into four groups: (1) Genetic epilepsies with febrile seizures plus (GEFS+), (2) Genetic generalized epilepsy (GGE), (3) Developmental and epileptic encephalopathy (DEE) and (4) Focal epilepsy. Most patients with $S T X 1 B$-associated epilepsy can be classified as GEFS+, whereas those with GGE or focal epilepsy are less common.

Materials and Methods: We screened our diagnostic inhouse exome sequencing database comprising 4000 datasets for cases with de novo and/or loss of function variants in $S T X 1 B$ and reviewed the clinical information of the respective patients.

Results: We identified four patients with pathogenic or likely pathogenic variants in STX1B: c.293G>A, p.(Ser98Asn); c.165dup, p.(Gln56ThrfsTer3); p.(Phe215CysfsTer9) and c.537+1G>A, p.(?). All patients had epilepsy and the clinical spectrum ranged from febrile and afebrile seizures to developmental and epileptic encephalopathy with cerebral malformations.

Conclusions: Our findings confirm the broad clinical spectrum of STXIB associated epilepsies. We identified three loss of function variants in differently affected individuals implying that a one-to-one genotypephenotype correlation cannot be established.

M. Schlögl: None. M. Krenn: None. E. Pataraia: None. S. Schröder: None. J.A. Mayr: None. C. Rauscher: None. D. Kotzot: None. F. Zimprich: None. T. Meitinger: None. M. Wagner: None.

\section{P09.057.A}

Whole-exome sequencing is effective for clarification of unsolved causes of epileptic encephalopathies

\section{Sedlackova ${ }^{l}$, P. Lassuthova ${ }^{1}, K$. Sterbova ${ }^{2}$, M. Vlckova $^{3}$,} D. Stanek ${ }^{l}$, P. Seeman ${ }^{l}$

${ }^{1}$ DNA laboratory, Department of Pediatric Neurology, 2nd Faculty of Medicine, Charles University and University Hospital Motol, Prague, Czech Republic, ${ }^{2}$ Department of Pediatric Neurology, 2nd Faculty of Medicine, Charles University and University Hospital Motol, Prague, Czech Republic, ${ }^{3}$ Department of Biology and Medical Genetics, 2nd Faculty of Medicine, Charles University and University Hospital Motol, Prague, Czech Republic

Introduction: Epileptic encephalopathies (EE) are severe epilepsies of childhood with early onset of usually drug- 
resistant seizures and developmental delay or regression. Their etiology is extremely heterogeneous. Whole-exome sequencing (WES) was used in patients previously unsolved using standard diagnostic methods and clinical experience.

Patients and Methods: Here we present WES results of 48 EE patients. These patients were selected based on previous experience by the expert in epilepsy genetics from the cohort of patients unsolved after targeted NGS gene panel.

Results: To date, causative variants were found in ten patients $(20.8 \%)$ in genes explaining the cause of EE (HUWE1, UBTF, SERPINI1, SLC1A4, GABRB2, DHDDS, PPP2R5D, NARS2, YWHAG, KMT2A). In other six patients $(12.5 \%)$, seven suspected variants of unknown causality were found and wait to be validated and interpreted by family segregation or phenotype confirmation by the physician (CACNA1A, SNAP25, NLGN3, CBL, DONSON, $S P A T A 5, P A K 3)$. In further three patients, we found variants in genes not associated with EE or any other human disease (KCNJ4, TULP4, PHYHIP). Five variants out of twenty presented were already published before (by different authors) and the rest of them are novel.

Conclusions: WES represents efficient tool for clarification of various EE types. It has an important potential for novel candidate genes detection, however, international collaboration is required for searching other patients for genotype-phenotype confirmation. WES may help in selected patients to obtain genetic diagnosis in those who remained unclarified after targeted NGS gene panel (up to $40 \%$ of patients). Supported by: MH CR AZV 15-33041

L. Sedlackova: None. P. Lassuthova: None. K. Sterbova: None. M. Vlckova: None. D. Stanek: None. P. Seeman: None.

\section{P09.059.C}

A novel Italian family with Familial Adult Myoclonic Epilepsy (FAME) broadens the phenotypic and molecular spectrum of FAME2 caused by a pentanucleotide expansion in STARD7

\author{
L. Veneziano ${ }^{1}$, A. Coppola ${ }^{2}$, L. Fontana ${ }^{3}$, S. Rossi ${ }^{l}$, L. \\ Camerota $^{4,3}$, E. Mantuano ${ }^{1}$, A. Suppa ${ }^{5,6}$, L. Bilo $^{2}$, M.

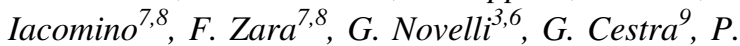 \\ Striano $^{7,8}$, A. Botta ${ }^{3}$, M. Cozzolino ${ }^{1}$, F. Brancati ${ }^{1,4,10}$
}

${ }^{1}$ Institute of Translational Pharmacology, National Research Council, Rome, Italy, ${ }^{2}$ Department of Neuroscience, Reproductive and Odontostomatological Sciences, Federico II University of Naples, Naples, Italy, ${ }^{3}$ Department of Biomedicine and Prevention, Tor Vergata University of Rome, Rome, Italy, ${ }^{4}$ Institute of Medical Genetics, Department of Life, Health and Environmental Sciences, University of L'Aquila, L'Aquila, Italy,
${ }^{5}$ Department of Human Neurosciences, Sapienza University of Rome, Rome, Italy, ${ }^{6}$ IRCCS Neuromed Institute, Pozzilli, Italy, ${ }^{7}$ Pediatric Neurology and Muscular Diseases Unit, IRCCS Giannina Gaslini, Genova, Italy, ${ }^{8}$ Department of Neurosciences, Rehabilitation, Ophthalmology, Genetics, Maternal and Child Health, University of Genoa, Genova, Italy, ${ }^{9}$ Institute of Molecular Biology and Pathology, National Research Council, Rome, Italy, ${ }^{10}$ Laboratory of Molecular and Cell Biology, IRCCS Istituto Dermopatico dell'Immacolata, Rome, Italy

Familial Adult Myoclonic Epilepsy (FAME; OMIM Phenotypic Series - PS601068) is an autosomal dominant adultonset disorder characterized by cortical myoclonic tremor and epilepsy. FAME is genetically heterogeneous with 6 distinct disease-causative genes identified over the last two years. Notably, all share an emerging common pathomolecular mechanism consisting in the expansion of non-coding (intronic) pentanucleotide repeats at distinct loci. We report a novel three generation FAME pedigree with 3 affected subjects and a healthy carrier. Two sibs had full-blown FAME phenotype, while a 32-year-old girl manifested only myoclonus starting at age 25 . Molecular genetics investigation by means of repeat primed PCR (RP-PCR) detected the pathogenic ATTTC pentanucleotide expansion in STARD7, diagnostic of FAME2. The ATTTT repeat reference sequence was not identified in the expansion. Sanger sequencing confirmed an apparent loss of heterozygosity in affected members and, more interestingly, depicted a complex structure of wild-type (not expanded) alleles. Of note, ATTTC-STARD7 expansion was confirmed in the unaffected 89-year-old mother of affected sibs. To our knowledge this is the first report of reduced penetrance in a molecularly confirmed STARD7 expansion carrier individual. In addition, the identification of atypical wild-type alleles broadens the repeat structure repertoire suggesting further complexity at the STARD7/FAME2 locus.

L. Veneziano: None. A. Coppola: None. L. Fontana: None. S. Rossi: None. L. Camerota: None. E. Mantuano: None. A. Suppa: None. L. Bilo: None. M. Iacomino: None. F. Zara: None. G. Novelli: None. G. Cestra: None. P. Striano: None. A. Botta: None. M. Cozzolino: None. F. Brancati: None.

P09.062.C

Mitochondrial dysfunction and oxidative stress contribute to the phenotype of FOXP1 deficiency

J. Wang, H. Fröhlich, G. A. Rappold

Institut für Humangenetik, Heidelberg, Germany 
Patients with FOXP1 haploinsufficiency present intellectual disability, autism spectrum disorder, severe speech and language impairments as well as motor dysfunction. To unravel the pathological mechanism underlying FOXP1 syndrome, we analysed $F$ oxp $^{+/-}$mice, which closely reflect FOXP1 haploinsufficiency. We can show that Foxp1 $^{+/-}$animals exhibit behavioural deficits such as reduced neonatal ultrasonic vocalization, hyperactivity, anxiety and alterations in spatial navigation and memory. Furthermore, they manifest decreased exercise capacity and endurance. We also found that $F o x p 1^{+/}$animals display a reduced striatal expression of proteins involved in mitochondrial biogenesis and dynamics (Pgc-1 $\alpha$, Tfam, Opa1 and Drp1), suggesting that mitochondrial dysfunction may contribute to the symptoms. To address this issue in more detail, we investigated mitochondria in WT and Foxpl ${ }^{+/-}$ animals. We demonstrate that Foxpl $^{+/-}$striatal neurons display a disrupted mitochondrial membrane potential. In addition, we detected an increased abundance of reactive oxygen species, increased cytochrome $\mathrm{C}$ release as well as lipid peroxidation in the Foxpl ${ }^{+/}$striatum. In summary, our results indicate mitochondrial dysfunction in murine Foxp1 deficiency, suggesting that mitochondrial dysfunction and increased oxidative stress may also contribute to the deficits in FOXP1 Syndrome.

J. Wang: None. H. Fröhlich: None. G.A. Rappold: None.

\section{P09.063.A}

Fragile $X$ premutation mouse embryonic stem cells as an in vitro model for CGG repeat expansion and disease pathology

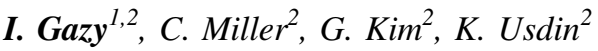

${ }^{1}$ KwaZulu-Natal Research Innovation and Sequencing Platform (KRISP), University of KwaZulu-Natal, Durban, South Africa, ${ }^{2}$ Laboratory of Cell and Molecular Biology, NIDDK, National Institutes of Health, Bethesda, MD, United States

The Fragile-X related disorders (FXDs) are Repeat Expansion Disorders (REDs) that result from expansion of a CGG-repeat tract in the FMRI gene. While expansion affects both disease risk and severity in humans, the underlying molecular mechanism responsible is unknown. Despite a high frequency of expansions in vivo, existing patient-derived cell models do not show significant repeat instability. Here, we describe the generation of mouse embryonic stem cells (mESCs) carrying an expanded CGGrepeat tract in the endogenous murine Fmrl gene. We show that these cells show high frequency repeat expansions, visible in a matter of days in culture. Moreover, using the CRISPR-Cas9 gene editing system, we show that these expansions are dependent on MSH2, a factor essential for expansions in vivo. Altogether, our data has implications for the timing of expansion and allows these cells to be useful for modeling the expansion mutation. Additionally, we demonstrate that these cells have abnormalities consistent with human disease including elevated mitochondrial copy numbers and Fmrl hyperexpression, that we show is $\mathrm{O}_{2}$-sensitive. Thus, this new stem cell model should facilitate studies of both expansion and the consequences of expansion during early embryonic development.

I. Gazy: None. C. Miller: None. G. Kim: None. K. Usdin: None.

P09.065.C

Autophagic alterations in skin fibroblasts from Fragile $\mathrm{X}$-associated tremor/ataxia syndrome patients

L. Rodriguez-Revenga ${ }^{l}$, M. Alvarez-Mora ${ }^{l}$, I. GonzalezCasacuberta $^{2}$, T. Barcos ${ }^{1}$, G. Garrabou ${ }^{2}$

${ }^{1}$ Hospital Cinic, Barcelona, Spain, ${ }^{2}$ CIBERER, Barcelona, Spain

Introduction: Fragile X-associated tremor/ataxia syndrome (FXTAS) is a late-onset neurodegenerative disorder with reduced penetrance that appears in adult FMRI premutation carriers (55-200 CGGs). The neuropathological hallmark of FXTAS consists of presence of ubiquitin-positive nuclear inclusions that are broadly distributed throughout the brain. Additionally, bioenergetic deficits and mitochondrial dysfunction have been also reported. Since autophagy promotes cell survival by elimination of damaged organelles and proteins aggregates, we aimed to study its potential role in the pathogenesis of FXTAS.

Material and Methods: Skin fibroblasts cultures from FXTAS patients $(n=4)$ were used to quantify autophagic flux (time-course bafilomycin a1-mediated autophagic inhibition) by measuring by western blot autophagic substrates (p62 levels), autophagosome formation (LC3BII content) and mitochondrial amount (VDAC1 expression).

Results: The analysis of autophagic flux in skin fibroblasts cultures evidenced enhanced autophagic pathway activation and increased mitochondrial amount in FXTAS patients compared to controls. Increased autophagosome number was also confirmed by confocal microscopy.

Conclusions: These findings suggest that despite the increase in autophagic flux, abnormal cell waste may contribute to the pathology of FXTAS fibroblasts by causing the accumulation of incomplete degraded mitochondria, among other subtracts. As a result, we postulate 
that FXTAS fibroblasts suffer from a bioenergetic collapse, leading to cell stress that triggers cell death.

Acknowledgements: This work was supported by the Instituto de Salud Carlos III (PI17/01067 and PI18/00498), co-financed by Fondo Europeo de Desarrollo Regional (FEDER) "una manera de hacer Europa" and AGAUR (2017 SGR1134). The CIBERER is an initiative of the Instituto de Salud Carlos III.

L. Rodriguez-Revenga: None. M. Alvarez-Mora: None. I. Gonzalez-Casacuberta: None. T. Barcos: None. G. Garrabou: None.

\section{P09.068.C}

Contribution of lysosomal gene variants to Parkinson's Disease penetrance in GBA mutation carriers

L. Straniero ${ }^{l}$, V. Rimoldi ${ }^{1,2}$, S. Bonvegna ${ }^{3}$, G. Melistaccio ${ }^{l}$, G. Soldà ${ }^{l, 2}$, M. Aurelì, A. Di Fonzo ${ }^{5}$, A. Zecchinelli ${ }^{3}, G$.

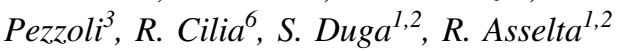

${ }^{1}$ Humanitas University, Pieve Emanuele (Milano), Italy, ${ }^{2}$ Humanitas Clinical and Research Center, IRCCS, Rozzano (Milano), Italy, ${ }^{3}$ Parkinson Institute, ASST "Gaetano PiniCTO”, Milano, Italy, ${ }^{4}$ Department of Medical Biotechnology and Translational Medicine, University of Milan, Milano, Italy, ${ }^{5}$ Foundation IRCCS Ca' Granda Ospedale Maggiore Policlinico, Neurology Unit; Dino Ferrari Center, Neuroscience Section, Department of Pathophysiology and Transplantation, University of Milan, Milano, Italy, ${ }^{6}$ Fondazione IRCCS Istituto Neurologico 'Carlo Besta', Milano, Italy

Introduction: Parkinson's disease (PD) is the second most common neurodegenerative disorder affecting more than $1 \%$ of the over-65 population. To date, mutations in the GBA gene, coding for the lysosomal glucocerebrosidase, represent the most frequent cause of genetic PD. Besides $G B A$, other lysosomal genes have also been implicated in PD pathogenesis, such as SMPD1, NPCl, and SCARB2. The reason why patients with the same mutation may or may not develop neurodegeneration and PD is still unclear. To this end, we evaluated the impact of the burden of deleterious mutations in lysosomal genes in patients heterozygous for pathogenic $G B A$ mutations vs asymptomatic $G B A$-mutation carriers.

Materials and Methods: This analysis was performed using a custom NGS panel by exploiting a DNA pooling strategy. The panel included 50 genes responsible for lysosomal storage disorders (LSD) and all previously reported PD-genes to evaluate if they contribute to PD penetrance in $G B A$-mutation carriers.

Results and Conclusions: Statistical analysis revealed a significantly increased burden of deleterious variants in
LSD genes in $\mathrm{PD}$ cases $(\mathrm{P}=0.003)$. In particular, the $36 \%$ of patients was carrier of at least one mutation in an LDS gene compared to the $23 \%$ of controls. Moreover, performing single-gene analysis, we observed that a second mutation in $G B A$ represents the strongest modulator of GBA itself (5\% vs $1 \%, \mathrm{P}=0.012$ ). The identification of genetic lesions in lysosomal genes increasing the risk to develop PD may contribute to classify patients in subgroups most likely to benefit from existing and upcoming targeted therapies.

L. Straniero: None. V. Rimoldi: None. S. Bonvegna: None. G. Melistaccio: None. G. Soldà: None. M. Aureli: None. A. Di Fonzo: None. A. Zecchinelli: None. G. Pezzoli: None. R. Cilia: None. S. Duga: None. R. Asselta: None.

\section{P09.069.A}

Modelling motor-neurons in a dish to study the effects of a homozygous codon-stop mutation in GDAP1 responsible for Charcot-Marie-Tooth disease

F. Miressi ${ }^{l}$, N. Benslimane ${ }^{l}$, L. Richard ${ }^{1,2}, S$. Bourthoumieu $^{1,3}$, M. Rassat $^{l}$, C. Laroche $^{4}$, L. Magy ${ }^{1,2}$, C.

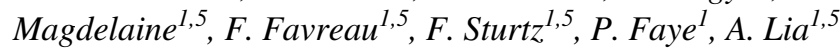

${ }^{1}$ EA6309 - University of Limoges, Limoges, France, ${ }^{2} \mathrm{CHU}$ Limoges - Service de Neurologie, Limoges, France, ${ }^{3} \mathrm{CHU}$ Limoges - Service de Cytogénétique, Limoges, France, ${ }^{4}$ CHU Limoges - Service de Pédiatrie, Limoges, France, ${ }^{5}$ CHU Limoges - Service de Biochimie et Génétique Moléculaire, Limoges, France

Introduction: Charcot-Marie-Tooth (CMT) disease, the most common inherited peripheral neuropathy in humans, is caused by mutations in more than 90 genes. Among these, GDAP1 encodes for a mitochondrial protein important for mitochondrial dynamic and protection from ROS. However, motor neurons (MNs), the mainly involved cells in this neuropathy, cannot be obtained from patients. Our goal was to generate MNs derived from iPSc (induced-pluripotentstem-cells) in order to investigate the effects of GDAP1mutations.

Materials and Methods: We created iPSc from fibroblasts of two unaffected subjects and a CMT-patient carrying a GDAPl homozygous codon-stop mutation. iPSc were differentiated in neural progenitors (NP), then in motor neurons (MN). To study GDAP1 expression, we realized RNA-extraction, qRT-PCR and immunocytochemistry. Electronic microscopy and functional tests were also achieved.

Results: By studying GDAPl mRNA expression in Fibroblasts, iPSc, NPs and MNs, on controls' cells, we observed that GDAP1 is mostly expressed in neural cells (NPs and MNs), showing the importance of generating 
MNs to study GDAPl mutations effects. Interestingly, in all patient's cell types, GDAP1 expression was low, suggesting that GDAPl mRNA, containing a premature termination codon (PTC), is probably degraded by the nonsensemediated-decay (NMD) system. These results have been confirmed by immunocytochemistry. Then, we analyzed morphological and functional properties of our cellular models.

Conclusions: Cellular models are useful tools to investigate the impact of genomic mutations on functional level. Our study highlighted the importance of employing the good cellular model (MNs), to study the altered molecular mechanisms associated to GDAP1-mutations in CMT disease.

F. Miressi: None. N. Benslimane: None. L. Richard: None. S. Bourthoumieu: None. M. Rassat: None. C. Laroche: None. L. Magy: None. C. Magdelaine: None. F. Favreau: None. F. Sturtz: None. P. Faye: None. A. Lia: None.

\section{P09.074.C}

Telomere length and mitochondrial DNA copy number changes in Huntington's Disease

\section{B. A. Fekete, A. Illés, D. Csabán, H. Zeke, V. Molnár, M. J. Molnár}

Institute of Genomic Medicine and Rare Disorders, Semmelweis University, Budapest, Hungary

Introduction: Telomere length was previously shown to be decreased in several neurodegenerative disorders including Huntington's Disease (HD) while mitochondrial DNA copy number changes were reported to show conflicting results. Simultaneous measurements and detailed data on disease severity is lacking.

Materials and methods: HD patients and preclinical patients (pathologic CAG expansion carriers) were staged ( $n=142)$ based on the Shoulson-Fahn clinical staging system (Stage I-V. and preclinical). A healthy control group was used $(n=71)$. mtDNA copy number and telomere lengths were measured with real-time PCR technique.

Results: For preclinical HD patients the average telomere length is $133.8( \pm 88.2)(\mathrm{kb} / \mathrm{cel})$ and mtDNA copy number is $387.5( \pm 167.4)$ (pcs/cel). Stage I HD patients have an average telomere length of $138.7( \pm 101)$, mtDNA copy number of 393.7 ( \pm 117.4$)$, while stage II telomere length is $124.5( \pm 74.8)$ and mtDNA copy number is $382.5( \pm 119.8)$ and stage III telomer lengths is $94.1( \pm 65.8)$ while mtDNA copy number is $326.5( \pm 127)$. We did not have any stage IV and V HD patients. Average telomere length for the control patients is $167.3( \pm 84.7)$ while mtDNA copy number is $201.5( \pm 103.7)$
Conclusions: HD patients have a significantly $(\mathrm{p}<0,05)$ lower telomer length and higher mtDNA copy number than healthy controls. Both telomere length and mitochondrial copy number decreased with HD disease severity (with a small spike in Stage I patients) but the changes were not statistically significant in that regard with the given sample sizes.

B.A. Fekete: None. A. Illés: None. D. Csabán: None. H. Zeke: None. V. Molnár: None. M.J. Molnár: None.

\section{P09.077.C}

Redefining the spectrum of disorders with white matter abnormalities in the genomic era

P. Kaur ${ }^{1}$, N. Kausthubham ${ }^{1}$, A. Shrikiran ${ }^{2}$, R. Bhat ${ }^{2}, L$. Edward Lewis ${ }^{2}$, S. Nampoothiri ${ }^{3}$, S. J. Patil ${ }^{4}$, S. Sharma ${ }^{5}$, S. Bijarnia Mahay ${ }^{6}$, S. Pai ${ }^{7}$, A. Kaur ${ }^{8}$, K. C. Rakshith ${ }^{7}, N$. Kamath $^{9}$, S. Bajaj ${ }^{10}$, R. Shetty ${ }^{11}$, R. Shenoy ${ }^{12}$, B. Banerjee ${ }^{13}$, M. Kamate ${ }^{14}$, B. Baliga ${ }^{7}$, K. M. Girisha ${ }^{1}$, A. Shukla ${ }^{1}$

${ }^{1}$ Department of Medical Genetics, Kasturba Medical College, Manipal, Manipal Academy of Higher Education, Manipal, India, ${ }^{2}$ Department of Paediatrics, Kasturba Medical College, Manipal, Manipal Academy of Higher Education, Manipal, India, ${ }^{3}$ Department of Paediatric Genetics, Amrita Institute of Medical Sciences and Research Centre, Kochi, India, ${ }^{4}$ Division of Genetics, Mazumdar Shaw Medical Center, Narayana Health City, Bangalore, India, ${ }^{5}$ Department of Paediatrics, Lady Hardinge Medical College, New Delhi, India, ${ }^{6}$ Institute of Medical Genetics and Genomics, Sir Ganga Ram Hospital, New Delhi, India, ${ }^{7}$ Department of Neurology, Kasturba Medical College, Mangalore, Manipal Academy of Higher Education, Manipal, India, ${ }^{8}$ Department of Paediatrics, Postgraduate Institute of Medical Education and Research, Chandigarh, India, ${ }^{9}$ Department of Paediatrics, Kasturba Medical College, Mangalore, Manipal Academy of Higher Education, Manipal, India, ${ }^{10}$ Jaslok Hospital and Research Centre, Mumbai, India, ${ }^{11}$ Department of Paediatrics, Indiana Hospital and Heart Institute, Mangalore, India, ${ }^{12}$ Department of Paediatrics, K.S. Hegde Medical Academy, NITTE University, Mangalore, India, ${ }^{13}$ Department of Paediatrics, Manipal Hospitals, Bangalore, India, ${ }^{14}$ Department of Paediatrics, Jawaharlal Nehru Medical College, Belgaum, India

Genetic disorders with central nervous system white matter abnormalities, also referred to as leukodystrophies and/or genetic leukoencephalopathies (LD/gLE), are a heterogeneous group of neurodegenerative conditions. Till date, 316 monogenic and 2 chromosomal disorders are designated as LD/gLE. Though neuroimaging was the key to diagnosis over last two decades, broad-spectrum genetic 
testing has now taken a central role in diagnosis. Methods: We reviewed literature to retract a list of genetic disorders with white matter abnormalities. We also evaluated 109 individuals from 101 families with white matter abnormalities. Exome sequencing (ES) was performed for genomic evaluation. Results: Literature review rendered 445 conditions with white matter abnormalities. One-hundred-andtwo conditions present with hypomyelination and 343 conditions have de/dysmyelination. Of the total, 129 disorders have not yet been designated as LD/gLE but have predominant white matter abnormalities. Diagnostic yield of ES in heterogenous cohorts of individuals with white matter abnormalities has been noted to be in the range of $40-50 \%$. ES has led to discovery of 63 novel conditions with white matter abnormalities in the past decade. In our cohort of 109 families, 14 had hypomyelination and 95 had de/dysmyelination. Molecular diagnosis was established in 67 families (66.33\%). Thirty-eight (59.37\%) of 64 disease-causing variants were novel. Four novel conditions with pathogenic variants in ISCA1, AIMP2, EXOSC1 and ANKRD17 were identified. Conclusions: The spectrum of genetic disorders with white matter abnormalities is ever-increasing and ES has emerged as a highly efficient modality for diagnosis of known conditions as well as elucidation of novel conditions.

P. Kaur: None. N. Kausthubham: None. A. Shrikiran: None. R. Bhat: None. L. Edward Lewis: None. S. Nampoothiri: None. S.J. Patil: None. S. Sharma: None. S. Bijarnia Mahay: None. S. Pai: None. A. Kaur: None. K.C. Rakshith: None. N. Kamath: None. S. Bajaj: None. R. Shetty: None. R. Shenoy: None. B. Banerjee: None. M. Kamate: None. B. Baliga: None. K.M. Girisha: None. A. Shukla: None.

\section{P09.079.B}

De novo KIF1A mutations play an important role in the development of cerebral palsy

\section{Beysen ${ }^{1}$, F. Roelens ${ }^{2}$, S. Kenis ${ }^{1}$, E. Reyniers ${ }^{3}, K$. Janssens $^{3}$, M. Meuwissen ${ }^{3,4}$}

${ }^{1}$ Dept. of Pediatric Neurology, Antwerp University Hospital, Edegem, Belgium, ${ }^{2}$ Dept. of Pediatrics, AZ Delta, Roeselare, Belgium, ${ }^{3}$ Center for Medical Genetics, University of Antwerp, Antwerp, Belgium, ${ }^{4}$ Center for Medical Genetics, Antwerp University Hospital, Edegem, Belgium

Introduction: Cerebral palsy $(\mathrm{CP})$ is a clinical descriptive term defining a heterogeneous group of non-progressive, neurodevelopmental disorders of motor impairment, which co-occur with a wide range of medical conditions, including intellectual disability, speech and language deficits, autism, epilepsy and visual and/or hearing impairment. Recent studies demonstrate an important contribution of genetic factors.

Materials and methods: We performed diagnostic genetic studies in $100 \mathrm{CP}$-patients that are followed at the Pediatric Neurology department using a whole exome sequencing (WES) based gene panel consisting of 200 genes associated with $\mathrm{CP}$ and CP-mimics.

Results: Here, we report the presence of de novo KIF1A variants in a relatively large proportion of our $\mathrm{CP}$ population (7/100 patients tested, 7\%). These variants were located in the KIFIA motor domain, absent in control databases, predicted pathogenic by in silico prediction programs and reported as likely pathogenic (class $4 / 5$ ) or pathogenic (class 5/5). Patients presented with developmental delay and mild to moderate ID (7/7), spastic paraplegia $(7 / 7)$, periventricular white matter abnormalities (4/7), corpus callosum hypoplasia (2/7), cerebellar atrophy (1/7), behavioural problems (ADHD/autism) (4/7) and seizures (1/7). These findings were already reported in KIF1A variants.

Conclusions: Variants in KIFlA, which encodes a kinesin axonal motor protein, have been described to cause variable neurological disorders. This is the first report in which de novo KIFIA mutations are demonstrated to be an important cause of $\mathrm{CP}$ associated with early onset spasticity and a frequent finding of periventricular white matter abnormalities.

D. Beysen: None. F. Roelens: None. S. Kenis: None. E. Reyniers: None. K. Janssens: None. M. Meuwissen: None.

\section{P09.080.C}

Complex social behaviour deficiencies in a mouse model for Kleefstra Syndrome

\section{M. van der Werf ${ }^{l, 2}, M$. Negwer ${ }^{1}$, P. Decuzzi $i^{2}$, H. van Bokhoven $^{l}$, N. Nadif Kasri ${ }^{1}$ \\ ${ }^{1}$ Radboudumc, Nijmegen, Netherlands, ${ }^{2}$ Italian Institute of Technology, Genoa, Italy}

Haploinsufficiency of the EHMT1 gene is the cause of Kleefstra Syndrome (KS), a disorder characterized by intellectual disability, autism spectrum disorders and specific facial features. EHMTI encodes the Euchromatic Histone Lysine Methyltransferase 1 (EHMT1) protein, which in complex with EHMT2 can deposit methyl groups on histone 3 lysine 9 (H3K9me1/H3K9me2), important epigenetic marks for gene repression. The Ehmt1 ${ }^{+/-}$mouse model, with heterozygous knockout of Ehmtl, shows phenotypic resemblance of the human disorder with developmental delay, learning and memory deficits, craniofacial abnormalities and diminished response to social novelty. To 
evaluate social behaviour in the $E h m t 1^{+/}$mouse model in more depth, we used the recently developed Live Mouse Tracker system (de Chaumont et al. 2019). This system can automatically track and score behaviour of a group of mice in a home cage-like setting. We recorded $\mathrm{Ehmt}^{+/-}$mice and their wildtype littermates over time periods of 23 hours and analyzed general activity, isolated behaviour, dyadic interactions and group dynamics in familiar social contexts, thereby excluding social novelty. Our results indicate that Ehmt1 ${ }^{+/}$mice are generally hypoactive when compared to wildtype littermates and display specific social behaviour deficits, in particular in group dynamics. These phenotypes will be further investigated as endpoint measures for phenotypic rescue in drug studies.

This work was supported by the K.I.D.S.IQ Project and the IIT MINDED project (European Union's Horizon 2020 research and innovation programme, Marie SktodowskaCurie grant agreement No 754490)

I.M. van der Werf: None. M. Negwer: None. P. Decuzzi: None. H. van Bokhoven: None. N. Nadif Kasri: None.

\section{P09.082.B}

Clinical and genetic findings in 14 patients with Wiedemann Steiner syndrome caused by mutation in the KMT2A gene

C. ORMIERES ${ }^{1,2}$, S. Rondeau ${ }^{l}$, G. Barcia ${ }^{l}$, S. Lyonnet ${ }^{1,3}$, A. Guimier ${ }^{l}$, C. Michot $^{l}$, G. Baujat ${ }^{l}$, V. Cormier-Daire ${ }^{1,2}$, M. Rio $^{l}$

${ }^{1}$ Fédération de Génétique et Institut Imagine, AP-HP, Hôpital Necker-Enfants Malades, F-75015 Paris, France, Paris, France, ${ }^{2}$ Université de Paris, UMR1163, INSERM, F-75 015, Paris, France, Paris, France, ${ }^{3}$ Université de Paris, UMR1163, INSERM, F-75 015, Paris, France, Paris, France, Paris, France

Introduction: Wiedemann Steiner syndrome (WSS) (605130 OMIM), is a rare autosomal dominant disorder characterized by distinctive facial features, variable developmental delay, short stature, and hypertrichosis especially of the elbows; related to heterozygous mutations in the KMT2A gene. To date, more than 100 cases have been described expanding the clinical phenotype. The aim of this study is to improve the clinical description of patients with WSS.

Materials and methods: This retrospective study included patients followed in our center with confirmed WSS. Data from the medical records were abstracted, recorded in an Excel spreadsheet and analyzed using simple frequency analysis. We compare these patients with previously reported cases.
Results: We described 14 unrelated patients with pathogenic mutations in KMT2A. All the mutation occurred de novo. Age at diagnosis ranged from 2 to 19 years, sex ratio 1. All patients presented with global developmental delay with a wide range of severity, only $14 \%$ with severe intellectual disability. $86 \%$ of the patients have language disorders. Gastrointestinal findings were common (feeding difficulties $100 \%$, constipation $22 \%$ ). $94 \%$ of the patients had an hypertrichosis whose more than half with hairy elbow. Abnormalities of craniocervical junction were reported in 4 patients (29\%). Two patients developed conductive deafness.

Conclusions: This study improved the description of natural history of WSS, especially about neurological development. Moreover, our cohort emphases the importance of early detection of craniocervical junction abnormalities and deafness.

C. Ormieres: None. S. Rondeau: None. G. Barcia: None. S. Lyonnet: None. A. Guimier: None. C. Michot: None. G. Baujat: None. V. Cormier-Daire: None. M. Rio: None.

\section{P09.083.C}

A rare missense variant in the ATP2C2 gene is associated with developmental language disorder (DLD)

A. Martinelli, M. Rice ${ }^{2}$, S. Smith ${ }^{3}$, J. Talcott ${ }^{4}$, J. Stein ${ }^{5}$, L. Kent $^{1}$, M. Hayiou-Thomas ${ }^{6}$, D. Newbury ${ }^{7}$, C. Hulme ${ }^{5}, S$. Pitt $^{l}$, S. Paracchini ${ }^{l}$

${ }^{1}$ University of St Andrews, St Andrews, United Kingdom, ${ }^{2}$ University of Kansas, Lawrence, KS, United States, ${ }^{3}$ University of Nebraska, Lincoln, NE, United States, ${ }^{4}$ Aston University, Birmingham, United Kingdom, ${ }^{5}$ University of Oxford, Oxford, United Kingdom, ${ }^{6}$ University of York, York, United Kingdom, ${ }^{7}$ Oxford Brookes University, Oxford, United Kingdom

Developmental Language Disorder (DLD) is a common condition presenting unexpected difficulties in expressing and understanding spoken language. DLD presents extensive comorbidity with other disorders like dyslexia and ADHD. DLD is highly heritable but few genes have been identified so far. Through an exome sequencing analysis we identified a rare missense mutation $(\operatorname{chr} 16: 84405221 \mathrm{G}>\mathrm{A}$ (GRCh38.p12)) in the ATP2C2 gene. Exactly the same variant was reported previously in a different exome sequencing study for DLD, and ATP2C2 was previously proposed as susceptibility gene for language disorders by linkage and association studies. We followed this finding up by genotyping the mutation in cohorts selected for DLD and comorbid disorders. We found that the variant had a higher frequency in DLD cases $(1.8 \%, \mathrm{~N}=360)$ compared to 
cohorts selected for dyslexia $(0.8 \%, \mathrm{~N}=520)$ and ADHD $(0.7 \%, \mathrm{~N}=150)$, which presented frequencies comparable to reference databases $(0.9 \%, \mathrm{~N}=24,046$ gnomAD controls). Analysis of quantitative phenotypes in the ALSPAC birth cohort showed that carriers $(\mathrm{N}=42)$ of the variant had, as a group, lower mean scores on a range of reading and language-related measures compared to controls $(\mathrm{N}=1825)$, e.g. carriers had a mean score 0.4 SD lower than noncarriers on a phoneme awareness task. We are currently investigating the potential effects of this variant on protein function with a range of biochemical and electrophysiology assays. This work is supported by an Action Medical Research Action/The Chief Scientist (CSO) Office, Scotland grant (GN2614) and a Cunningham Trust grant.

A. Martinelli: None. M. Rice: None. S. Smith: None. J. Talcott: None. J. Stein: None. L. Kent: None. M. HayiouThomas: None. D. Newbury: None. C. Hulme: None. S. Pitt: None. S. Paracchini: None.

\section{P09.085.B}

Contribution and consequences of LIS1 dosage in physiopathological mechanism of LIS1 mutationsrelated lissencephaly

C. MAILLARD ${ }^{1,2}$, M. Philbert ${ }^{3}$, M. Cavallin ${ }^{4}$, S. Auvin ${ }^{5}, M$.

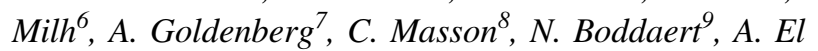

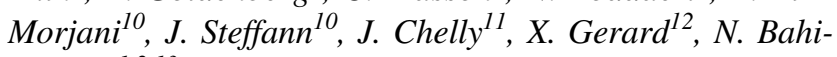
Buisson $^{1,2,13}$

${ }^{1}$ Genetics and Development of the Cerebral Cortex, Imagine Institute, Paris, France, ${ }^{2}$ Sorbonne Paris Cite University, Paris, France, ${ }^{3}$ University Hospital of Martinique, Fort-de-France, Martinique, ${ }^{4}$ Meyer Pediatric Hospital, Florence, Italy, ${ }^{5}$ Pediatric Neurology and Metabolic Diseases, Robert Debré Hospital, Paris, France, ${ }^{6}$ Pediatric Neurology, Aix-Marseille University, Marseille, France, ${ }^{7}$ Unit of Clinical Genetics, University of Rouen, Rouen, France, ${ }^{8}$ Plateform of Bioinformatic, Imagine Institute, Paris, France, ${ }^{9}$ Pediatric Radiology, Necker-Enfants Malades Hospital, Paris, France, ${ }^{10}$ Unit of Clinical Genetics, Necker-Enfants Malades Hospital, Paris, France, ${ }^{11}$ Genetics and Physiopathology of Neurodevelopmental and Epileptogenic Diseases, IGBMC, Illkirch, France, ${ }^{12}$ Laboratory of Genetics in Ophthalmology, Imagine Institute, Paris, France, ${ }^{13}$ Pediatric Neurology, NeckerEnfants Malades Hospital, Paris, France

Introduction: LIS1 is an ubiquitous protein of which loss of function mutations lead to lissencephalies, whereas LIS1 overexpression causes microcephaly. This suggests cerebral development is very sensitive to LIS1 dosage. The main goal of this project is to evaluate the dose-effect impact of LIS1 on the different degrees of lissencephalies' severity.
Material and methods: Four lines of fibroblast from patients with LISI de novo heterozygous mutations were used. Two of them displaying a severe phenotype carry an intronic mutation $(\mathrm{c} .1002+1 \mathrm{G}>\mathrm{T})$ or a missense (c. $445 \mathrm{C}>\mathrm{T}$ ) while the two other patients presenting a less severe phenotype display a splice site mutation (c.569$10 \mathrm{~T}>\mathrm{C})$. LIS1 transcripts and proteins were quantified and the subcellular distribution of the protein was visualized by immunofluorescence.

Results: Analysis of LIS1 mRNA showed that the c.569$10 \mathrm{~T}>\mathrm{C}$ mutant transcripts were degraded while both c. $445 \mathrm{C}>\mathrm{T}$ et $\mathrm{c} .1002+1 \mathrm{G}>\mathrm{T}$ mutant transcripts, associated to the severe phenotypes, were not. Yet, no difference of LIS1 protein level was detected between patient and control lines, by Western Blot. But, immunofluorescence targeting LIS1 after proteasome inhibition allowed the detection of clusters in fibroblasts associated to the severe phenotypes (c. $1002+1 \mathrm{G}>\mathrm{T} ;$ c. $445 \mathrm{C}>\mathrm{T}$ ).

Conclusions: In LIS1 lissencephalies, our data suggest that more severe forms would be associated to an abnormal repartition of the LIS1 protein forming clusters after proteasome inhibition, which might be toxic for the cell. Recent studies indicate the clusters might be stress granules, membraneless organelles involved in post-transcriptional regulation and translational control, formed by RNA-RNA, protein-protein and RNA-protein interactions, in which LIS1 would bind RNA-binding proteins.

C. Maillard: None. M. Philbert: None. M. Cavallin: None. S. Auvin: None. M. Milh: None. A. Goldenberg: None. C. Masson: None. N. Boddaert: None. A. El Morjani: None. J. Steffann: None. J. Chelly: None. X. Gerard: None. N. Bahi-Buisson: None.

\section{P09.087.A}

The involvement of MAN2C1 in the development of cortical malformations

L. Kerkhove ${ }^{l}$, H. Yildirim $^{l}$, S. Seneca ${ }^{1,2}$, A. Gheldof ${ }^{1,2}, D$.

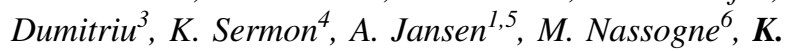
Stouffs $^{1,2}$

${ }^{1}$ Neurogenetics Research Group, Reproduction - Genetics and Regenerative Medicine Research Cluster, Vrije Universiteit Brussel, Brussels, Belgium, ${ }^{2}$ Centre for Medical Genetics, Universitair Ziekenhuis Brussel (UZ Brussel), Brussels, Belgium, ${ }^{3}$ Pediatric Radiology Unit, Cliniques universitaires Saint-Luc, UCLouvain, Brussels, Belgium, ${ }^{4}$ Reproduction and Genetics Research Group, Reproduction - Genetics and Regenerative Medicine Research Cluster, Vrije Universiteit Brussel, Brussels, Belgium, ${ }^{5}$ Pediatric Neurology Unit, Department of Pediatrics, UZ Brussel, Brussels, Belgium, ${ }^{6}$ Pediatric Neurology Unit, Cliniques universitaires Saint-Luc, UCLouvain, Brussels, Belgium 
The development of the cerebral cortex is a complex process and pathogenic variants in $>200$ genes have been associated with malformations of cortical development (MCD). Yet, the etiology still remains to be elucidated in a large group of MCD patients. The proband of this study, a 17 -year old boy presented with a complex brain malformation including severe cortical dysplasia, agenesis of the corpus callosum, interhemispheric cysts and pontocerebellar hypoplasia. Parents are non-consanguineous. The father of the proband has a simple arachnoid cyst. Whole exome sequencing showed a novel homozygous variant (c.607G $>$ A, p.(Gly203Arg)) in the MAN2C1 (NM_006715.3) gene. This variant was not reported earlier and classified as variant of uncertain significance (VUS 3). The $\mathrm{MAN2Cl}$ gene encodes a cytosolic mannosidase and is involved in the degradation of misfolded glycoproteins. Possibly, as a consequence of the accumulation of proteins, the organization and maturation of neurons is altered during fetogenesis. Preliminary RT-PCR results showed that the expression level of $\mathrm{MAN} 2 \mathrm{Cl}$ is downregulated in fibroblasts compared to controls, although this downregulation was not significant. The variant in MAN2Cl causes a $\pm 50 \%$ decrease in cytosolic alpha-mannosidase enzymatic activity in the patient compared to controls. RNA sequencing analysis in patient and control fibroblasts showed up/down regulation of multiple glycosylated proteins. The functional consequences of the detected variant and the exact role of the MAN2C gene in the development of MCD need further investigation.

L. Kerkhove: None. H. Yildirim: None. S. Seneca: None. A. Gheldof: None. D. Dumitriu: None. K. Sermon: None. A. Jansen: None. M. Nassogne: None. K. Stouffs: None.

\section{P09.089.C}

The association of CTDP1 mutation withmalformations of cortical development

H. Yildirim ${ }^{1}$, L. Kerkhove ${ }^{1}$, B. I. Dimitrov ${ }^{2}$, E. Cannaerts $^{2}$, K. Sermon ${ }^{3}$, K. Stouffs ${ }^{1}$, A. C. Jansen ${ }^{4}$

${ }^{I}$ Neurogenetics Research Group, Research Cluster Reproduction - Genetics and Regenerative Medicine, Vrije Universiteit Brussel, Jette, Belgium, ${ }^{2}$ Center for Medical Genetics, UZ Brussel, Jette, Belgium, ${ }^{3}$ Reproduction and Genetics Research Group, Research Cluster Reproduction Genetics and Regenerative Medicine, Vrije Universiteit Brussel, Jette, Belgium, ${ }^{4}$ Pediatric Neurology Unit, Department of Pediatrics, UZ Brussel, Jette, Belgium

Malformations of cortical development (MCD) represent a major cause of developmental disabilities and severe epilepsy. Some of the MCD causative genes have been identified, but part remain unknown. Here, we describe a patient with heterozygous variants in the $\mathrm{C}$-terminal domain phosphatase 1 (CTDPl) gene, a gene potentially involved in MCD. CTDP1 functions as a phosphatase which dephosphorylates the C-terminus of RNA polymerase II, making it available for initiation of gene expression.

A 2.5-year old girl presented with congenital arthrogryposis, periventricular gray matter heterotopia, optic atrophy, absent optic chiasma and unilateral auditory neuropathy. Trio exome analysis revealed that the index is compound heterozygous for two novel variants in CTDP1: c. $609 \mathrm{G}>\mathrm{C}$, p. (Gln203His) and c.2665C $>$ T, p. (Arg889Trp). To assess the gene expression levels of $C T D P 1$, real-time polymerase chain reaction (RT-PCR) is performed. The phosphatase activity of CTDP1 was assessed by performing western blotting (WB) of its target RNA polymerase II.

RT-PCR data showed a non-significant downregulation of CTDP1 expression in the patient versus controls. By performing WB, more phosphorylated RNA polymerase II was detected in patient cells.

Our preliminary analyses show that the phosphatase activity of CTDP1 is altered in the patient compared to controls, supporting its role in MCD. CTDP1 is involved in the regulation of RNA polymerase II and forms a complex with INTS1 and INTS8. Variants in the latter genes result in a phenotype similar to that of in our index patient, further suggesting a role for CTDP1 in brain development.

H. Yildirim: None. L. Kerkhove: None. B.I. Dimitrov: None. E. Cannaerts: None. K. Sermon: None. K. Stouffs: None. A.C. Jansen: None.

\section{P09.090.A}

Malformations of cortical development: international consensus recommendations on diagnostic work-up

R. Oegema ${ }^{1}$, T. Barakat ${ }^{2}$, N. Bahi -Buisson ${ }^{3}$, E. Aronica $^{4,5}$, V. Conti ${ }^{6}$, M. Zaki ${ }^{7}$, W. Dobyns ${ }^{8,9}$, A. Fry ${ }^{10,11}$, T. Geis ${ }^{12}$, D. Gomez Andres $^{13}$, R. Guerrini ${ }^{6}$, U. Hehr ${ }^{14}$, A. Jansen ${ }^{15,16}$, R. Leventer $^{17}$, D. Amron ${ }^{18,19}$, G. Mirzaa ${ }^{8,9}$, E. Parrini ${ }^{6}, D$. Pilz $^{20}$, E. Said ${ }^{21,22}$, D. Soler ${ }^{23}$, K. Stouffs ${ }^{24}$, L. M. Valor ${ }^{25}$, O. Reiner ${ }^{26}$, I. Pogledic ${ }^{27}$, M. Wilke ${ }^{28}$, G. M. S. Mancini ${ }^{28}$, N. Di Donato ${ }^{29}$

${ }^{1}$ UMC Utrecht - WKZ, Utrecht, Netherlands, ${ }^{2}$ Department of Clinical Genetics, Erasmus MC University Medical Center, Rotterdam, Netherlands, ${ }^{3}$ Pediatric Neurology Necker Enfants Malades UniversityHospital Imagine Institute Paris France, Paris, France, ${ }^{4}$ Amsterdam UMC, University of Amsterdam, Dept (Neuro)pathology, Amsterdam, Netherlands, ${ }^{5}$ Stichting Epilepsie Instellingen Nederland (SEIN), Hoofddorp, Netherlands, ${ }^{6}$ Pediatric Neurology, Neurogenetics and Neurobiology Unit and 
Laboratories, Department of Neuroscience, A. Meyer Children's Hospital, University of Florence, Florence, Italy, ${ }^{7}$ Clinical Genetics Department, Human Genetics and Genome Research Division, National Research Centre, Cairo, Egypt, ${ }^{8}$ Center for Integrative Brain Research, Seattle Children's Research Institute, Seattle, WA, United States, ${ }^{9}$ Department of Pediatrics, University of Washington, Seattle, WA, United States, ${ }^{10}$ Institute of Medical Genetics, University Hospital of Wales, Cardiff, United Kingdom, ${ }^{11}$ Division of Cancer and Genetics, School of Medicine, Cardiff University, Cardiff, United Kingdom, ${ }^{12}$ Department of Pediatric Neurology, Klinik St. Hedwig, University Children's Hospital Regensburg (KUNO), Regensburg, Germany, ${ }^{13}$ Child Neurology. Hospital Universitari Vall d'Hebron, Barcelona, Spain, ${ }^{14}$ Center for and Institute of Human Genetics, University of Regensburg, Regensburg, Germany, ${ }^{15}$ Pediatric Neurology Unit, Department of Pediatrics, UZ Brussel, Brussels, Belgium, ${ }^{16} \mathrm{Neu}$ rogenetics Research Group, Vrije Universiteit Brussel, Brussels, Belgium, ${ }^{17}$ Department of Neurology, Royal Children's Hospital, Murdoch Children's Research Institute and University of Melbourne Department of Pediatrics, Melbourne, Australia, ${ }^{18}$ Kannerklinik, Centre Hospitalier de Luxembourg, Luxembourg, Luxembourg, ${ }^{19}$ Hôpital Universitaire des Enfants Reine Fabiola, Université Libre de Bruxelles, Brussels, Belgium, ${ }^{20}$ West of Scotland Clinical Genetics Service, Queen Elizabeth University Hospital, Glasgow, United Kingdom, ${ }^{21}$ Section of Medical Genetics, Mater dei Hospital, Msida, Malta, ${ }^{22}$ Department of Anatomy and Cell Biology, University of Malta, Msida, Malta, ${ }^{23}$ Department of Paediatrics, Mater dei Hospital, Msida, Malta, ${ }^{24}$ Centre for Medical Genetics, UZ Brussel; Neurogenetics Research Group, Vrije Universiteit Brussel, Brussels, Belgium, ${ }^{25}$ Hospital Universitario Puerta del Mar, INiBICA, Cádiz, Spain, ${ }^{26}$ Department of Molecular Genetics, Weizmann Institute of Science, Rehovot, Israel, ${ }^{27}$ Department of Biomedical Imaging and Image Guided Therapy, Medical University of Vienna, Vienna, Austria, ${ }^{28}$ Department of Clinical Genetics, Erasmus MC University Medical Center, Rotterdam, Netherlands, ${ }^{29}$ Institute for Clinical Genetics, TU Dresden, Dresden, Germany

Introduction: Malformations of cortical development (MCD) are a group of predominantly genetic disorders due to abnormal formation of cerebral cortex during fetal brain development. Main examples of MCD are lissencephaly, heterotopia, and polymicrogyria. MCD place a significant burden on affected individuals, their families and society, as patients often suffer from lifelong symptoms including drug-resistant epilepsy, cerebral palsy, and intellectual disability. Although next-generation sequencing has become broadly available worldwide, marked differences in diagnostic yield remain due to broad genetic and clinical heterogeneity and lack of guidelines in the recognition and classification of these rare disorders.

Methods: The international network on brain malformations Neuro-MIG formed a multidisciplinary task force. Through expert meetings, literature search, and the Delphi consensus method a diagnostic strategy for individuals with MCD was developed.

Results: We present consensus recommendations on the optimal work flow applicable to any individual with MCD. This includes a flow chart for the clinical work-up, laboratory standards, and a curated MCD gene panel list. In addition, we discuss the differential diagnosis, possible diagnostic pitfalls and special recommendations for specific MCD subtypes.

Conclusions: These recommendations will aid clinicians in providing the optimal diagnostic strategy, which will improve genetic and prognostic counseling and patient care. Financial support: Neuro-MIG is supported by COST Action CA16118 (https://www.neuro-mig.org/).

R. Oegema: None. T. Barakat: None. N. Bahi -Buisson: None. E. Aronica: None. V. Conti: None. M. Zaki: None. W. Dobyns: None. A. Fry: None. T. Geis: None. D. Gomez Andres: None. R. Guerrini: None. U. Hehr: None. A. Jansen: None. R. Leventer: None. D. Amron: None. G. Mirzaa: None. E. Parrini: None. D. Pilz: None. E. Said: None. D. Soler: None. K. Stouffs: None. L.M. Valor: None. O. Reiner: None. I. Pogledic: None. M. Wilke: None. G.M.S. Mancini: None. N. Di Donato: None.

\section{P09.091.B}

Mutations of $\mathrm{PCDHGC4}$ encoding protocadherin gamma-C4 cause primary microcephaly and intellectual disability

M. Iqbal ${ }^{1,2,3}$, S. M. Baig ${ }^{3}$,U. Abdullah ${ }^{4}$, E. U. H.

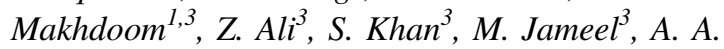

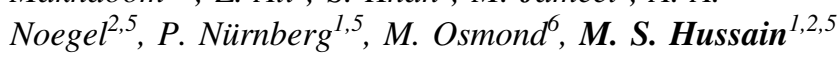

${ }^{1}$ Cologne center for Genomics, University of Cologne, Cologne, Germany, ${ }^{2}$ Institute of Biochemistry I, Medical Faculty, University of Cologne, Cologne, Germany, ${ }^{3}$ Human Molecular Genetics Laboratory, Health Biotechnology Division, National Institute for Biotechnology and Genetic Engineering (NIBGE) College, PIEAS, Faisalabad, Pakistan, ${ }^{4}$ University Institute of Biochemistry and Biotechnology (UIBB), PMAS-ARID Agriculture University, Rawalpindi, Pakistan, ${ }^{5}$ Center for Molecular Medicine Cologne (CMMC), University of Cologne, Cologne, Germany, ${ }^{6}$ Children's Hospital of Eastern Ontario Research Institute, University of Ottawa, Ottawa, ON, Canada 


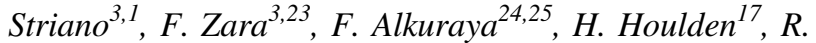
Maroofian $^{15}$, D. Silver ${ }^{4}$

${ }^{1}$ Pediatric Neurology and Muscular Diseases Unit, IRCCS Istituto Giannina Gaslini, Genoa, Italy, ${ }^{2}$ Department of Neuromuscular Disorders, Institute of Neurology, University College London, London, United Kingdom, ${ }^{3}$ Department of Neurosciences, Rehabilitation, Ophthalmology, Genetics, Maternal and Child Health, University of Genoa, Genoa, Italy, ${ }^{4}$ Signature Research Program in Cardiovascular and Metabolic Disorders, Duke-NUS Medical School, Singapore, Singapore, ${ }^{5}$ Department of Genetics, King Faisal Specialist Hospital and Research Centre, Riyadh, Saudi Arabia, ${ }^{6}$ Research Centre for Medical Genetics, Moscow, Russian Federation, ${ }^{7}$ Department of Otorhinolaryngology Head \& Neck Surgery, School of Medicine, University of Maryland, Baltimore, MD, United States, ${ }^{8}$ Center for Genetic Diseases, Shaheed Zulfiqar Ali Bhutto Medical University, Pakistan Institute of Medical Sciences, Islamabad, Pakistan, ${ }^{9}$ National Centre of Excellence in Molecular Biology, University of the Punjab, Lahore, Pakistan, ${ }^{10}$ Department of Neuroscience and Cell Biology and Child Health Institute of New Jersey, Rutgers Robert Wood Johnson Medical School, New Brunswick, NJ, United States, ${ }^{11}$ Neuroradiology Unit, IRCCS Istituto Giannina Gaslini, Genova, Italy, ${ }^{12}$ Bioinformatics Institute, Agency for Science, Technology and Research (A*STAR), 30 Biopolis St., Matrix No. 07-01, Singapore, Singapore, ${ }^{13}$ Department of Biological Sciences, National University of Singapore, Singapore, Singapore, ${ }^{14}$ Centre for Computational Biology, DUKE-NUS Medical School, Singapore, Singapore, ${ }^{15}$ Genetics Research Centre, Molecular and Clinical Sciences Institute, St George's, University of London, Cranmer Terrace, London, United Kingdom, ${ }^{16}$ Department of pediatric diseases, Faculty of medicine, Mashhad University of Medical Sciences, Mashhad, Iran, Islamic Republic of, ${ }^{17}$ Department of Neuromuscular Disorders, Institute of Neurology, University College London, London, United Kingdom, ${ }^{18}$ Independent Clinical Bioinformatics Laboratory, Moscow, Russian Federation, ${ }^{19}$ Genomed Ltd., Moscow, Russian Federation, ${ }^{20}$ Department of Obstetrics and Gynecology, King Saudi University, Riyadh, Saudi Arabia, ${ }^{21}$ Pediatrics Department, Security Forces Hospital, Riyadh, Saudi Arabia, ${ }^{22}$ Department of Pediatric Diseases, Mashhad University of Medical Sciences, Mashhad, Iran, Islamic Republic of, ${ }^{23}$ Unit of Medical Genetics, IRCCS Istituto Giannina Gaslini, Genoa, Italy, ${ }^{24}$ Department of Genetics, King Faisal Specialist Hospital and Research Center, Riyadh, Saudi Arabia, ${ }^{25}$ Department of Anatomy and Cell Biology, College of Medicine, Alfaisal University, Riyadh, Saudi Arabia
Introduction: Major Facilitator Superfamily Domain containing 2a $(M F S D 2 A)$ is an essential endothelial lipid transporter at the blood-brain barrier. Biallelic MFSD2A pathogenic variants cause autosomal recessive primary microcephaly 15 (MCPH15, OMIM\# 616486). We sought to expand our knowledge of the phenotypic spectrum of MCPH15 and demonstrate the underlying mechanism of inactivation of the MFSD2A transporter.

Materials and Methods: We carried out detailed analysis of the clinical and neuroradiological features of eight individuals from seven consanguineous families with severe microcephaly. Genetic investigation was performed through exome sequencing. Structural insights on the human Mfsd2a model and in vitro biochemical assays were used to investigate the functional impact of the identified variants.

Results: All patients had primary microcephaly and severe developmental delay. Brain MRI showed variable degrees of white matter reduction, ventricular enlargement, callosal hypodysgenesis, and pontine and vermian hypoplasia. ES led to the identification of the novel biallelic MFSD2A variants p.(Pro493Leu), p.(Pro164Thr), p. (Arg326His), p.(Val250Phe), p.(Gln462HisfsTer17) and p. (Cys251SerFsTer3), and the recurrent variants $p$. (Thr198Met) and p.(Thr159Met), all resulting in either reduced MFSD2A expression and/or transport activity.

Conclusions: Our study further delineates the phenotypic spectrum of MCPH15, refining its clinical and neuroradiological characterization and supporting that MFSD2A deficiency causes early prenatal brain developmental disruption. We also show that poor MFSD2A expression despite normal transporter activity is a relevant pathomechanism in MCPH15.

Funding: NRF Singapore (NRF2016NRF-NRFI001-15); BMRC A*STAR; MRC (MR/S01165X/1, MR/S005021/1, G0601943); UCLH/UCL Biomedical Research Centre; Rosetree Trust; Ataxia UK; MSA Trust; Brain Research UK; Sparks GOSH Charity; Muscular Dystrophy UK-USA; March of Dimes USA; NINDS/NIH (R01 RNS107428A).

M. Scala: None. G. Chua: None. C. Chin: None. H. Alsaif: None. B. Artem: None. S. Riazuddin: None. S. Riazuddin: None. M. Manzini: B. Research Grant (principal investigator, collaborator or consultant and pending grants as well as grants already received); Significant; March of Dimes USA. M. Severino: None. A. Kuk: None. H. Fan: B. Research Grant (principal investigator, collaborator or consultant and pending grants as well as grants already received); Significant; Biomedical Research Council of A*STAR. Y. Jamshidi: None. M. Beiraghi Toosi: None. M. Doosti: None. E. Karimiani: None. V. Salpietro: None. E. Dadali: None. G. Baydakova: None. F. Konovalov: None. E. Lozier: None. E. O'Connor: None. Y. Sabr: None. A. Alfaifi: None. F. 
Ashrafzadeh: None. P. Striano: None. F. Zara: None. F. Alkuraya: None. H. Houlden: None. R. Maroofian: None. D. Silver: B. Research Grant (principal investigator, collaborator or consultant and pending grants as well as grants already received); Significant; National Research Foundation grants, Singapore NRF2016NRF-NRFI001-15.

\section{P09.096.A}

Prevalence of Machado-Joseph disease (MJD/SCA3) explained by migration and multiple founder effects

S. Martins ${ }^{1,2}$, I. P. D. Costa ${ }^{1,2}$, P. Giunti ${ }^{3}$, M. Watanabe W $^{4}$ H.

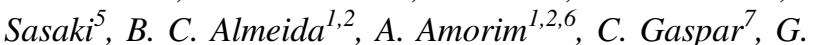
Nicholson ${ }^{8}$, M. L. Saraiva-Pereira ${ }^{9,10}$, S. Tsuji ${ }^{11}$, Q. Li ${ }^{12}, S$. Chen $^{12}$, G. Landouré ${ }^{13,14,15}$, P. Maciel ${ }^{16}$, R. Zaltzman ${ }^{17,18}$, R. Sharony ${ }^{17,18}, Y$. Zhou ${ }^{19}$, J. Barros ${ }^{20}$, J. L. Loureiro ${ }^{21,22}$, V. T. Cruz $^{23,24}$, L. Ruano ${ }^{22,24}$, E. R. P. Brunt ${ }^{25}$, M. Marc Corral-Juan $^{26}$, M. Hsieh ${ }^{27}$, L. Tranebjarg ${ }^{28}$, O. Olajumoke $^{29}$, S. A. Ogun ${ }^{29}$, M. F. Finkel ${ }^{30}$, C. Gordon ${ }^{17,18}$, M. Cornejo-Olivas ${ }^{31,32}$, K. Fischbeck ${ }^{15}$, A. MatillaDueñas $^{26}$, V. Volpini ${ }^{33}$, O. Riess ${ }^{34}, Z$. Wu ${ }^{12}$, G. A. Rouleau ${ }^{7}$,

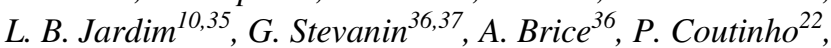
B. Soong ${ }^{38,39}$, L. P. Ranum ${ }^{40}$, A. Durr ${ }^{36}$,J. Sequeiros ${ }^{1,21}$

${ }^{1}$ i3S, Univ Porto, Porto, Portugal, ${ }^{2}$ IPATIMUP, Porto, Portugal, ${ }^{3}$ Univ Coll London Inst Neurol, Univ London, London, United Kingdom, ${ }^{4}$ NBI TA-CNS/Immunol, Tokyo, Japan, ${ }^{5}$ Fac Med Grad Sch Medicine, Hokkaido Univ, Sapporo, Japan, ${ }^{6}$ Fac Ciências, Univ Porto, Porto, Portugal, ${ }^{7}$ Montreal Neurol Inst; McGill Univ, Montréal, QC, Canada, ${ }^{8}$ Concord Hosp; Univ Sydney, Sidney, Australia, ${ }^{9}$ ICBS, UFRGS, Porto Alegre, Brazil, ${ }^{10}$ Hosp Clin Porto Alegre, Porto Alegre, Brazil, "'Univ Tokyo, Tokyo, Japan, ${ }^{12}$ Res Ce Neurol Sec Affil Hosp; Zhejiang Univ Sch Med, Hangzhou, China, ${ }^{13}$ Fac Méd d'Odonto, USTTB, Bamako, Mali, ${ }^{14}$ Cen Hosp Univ Point " $G$ ", Bamako, Mali, ${ }^{15} \mathrm{Nt}$ Inst Neurol Dis Stroke, Bethesda, MD, United States, ${ }^{16}$ Life Health Sci Res Inst, Sch Medicine, Univ Minho, Braga, Portugal, ${ }^{17}$ Sackler Fac Medicine, Tel Aviv Univ, Tel Aviv, Israel, ${ }^{18}$ Meir Med Cen, Kfar Saba, Israel, ${ }^{19}$ MedStar Med Gr, MedStar St Mary's Hosp MedStar Georgetown Univ Hosp, Leonardtown, MD, United States, ${ }^{20}$ Hosp Sto António, CHP - Cen Hosp Univ Porto, Porto, Portugal, ${ }^{21}$ IBMC, Univ Porto, Porto, Portugal, ${ }^{22}$ CHEDV, Feira, Portugal, ${ }^{23}$ Hosp Pedro Hispano, Matosinhos, Portugal, ${ }^{24}$ ISPUP, Porto, Portugal, ${ }^{25}$ Univ Hosp Groningen, Groningen, Netherlands, ${ }^{26}$ Health Sci Res Inst Germans Trias i Pujol (IGTP); Univ Autòn Barcelona, Badalona, Spain, ${ }^{27}$ Tunghai Univ, Taichung, Taiwan, ${ }^{28}$ Rigshospit /Kennedy Cen; Inst Clin Med, Univ Copenhagen, Copenhagen, Denmark, ${ }^{29}$ Lagos St Univ Coll Med (LASUCOM) /Teach Hosp (LASUTH), Ikeja, Nigeria,

${ }^{30}$ Physic Reg Med Cen, Naples, FL, United States, ${ }^{31}$ Inst
Nac Cienc Neurol, Lima, Peru, ${ }^{32}$ Univ Per Cayetano Heredia, Lima, Peru, ${ }^{33}$ Bellvitge Biomed Res Inst (IDIBELL), L'Hosp Llobregat, Barcelona, Spain, ${ }^{34}$ Inst Med Genet Appl Genom, Univ Tübingen, Tübingen, Germany, ${ }^{35}$ UFRGS, Porto Alegre, Brazil, ${ }^{36}$ Inst Cerveau Moelle Épinière, Pitié-Salpetrière Hosp, Paris, France, ${ }^{37}$ EPHE, PSL Res Univ, Paris, France, ${ }^{38}$ Shuang Ho Hosp; Taipei Neurosc Inst, Taipei Med Univ, Taipei, Taiwan, ${ }^{39}$ Taipei Vet Gen Hosp; Ntl Yang-Ming Univ, Taipei, Taiwan, ${ }^{40}$ Coll Med, Univ Florida, Gainesville, FL, United States

Machado-Joseph disease (MJD/SCA3) is a neurological disorder caused by an unstable $(\mathrm{CAG})_{\mathrm{n}}$ in $A T X N 3$, ranging usually 61-87 repeats in patients. Although MJD is the most frequent dominant ataxia worldwide, our previous studies suggest that de novo expansions would not explain its worldwide prevalence. To date, only two major MJD mutational origins have been identified: the Machado lineage, more recent, most frequent in parts of Portugal; and the ancient Joseph lineage, of Asian origin, spread worldwide. We extended our previous studies and tested the potential of additional markers for higher-resolution MJD haplotypes. We analysed a total of 370 families, from 30 world populations (5 continents); assessed a set of 30 SNPs in cis with the $(\mathrm{CAG})_{\mathrm{n}}$ expansion, to establish lineages; and genotyped 7 STRs flanking $A T X N 3$, to construct phylogenetic networks. In addition to the Joseph $(n=210)$ and Machado ( $n$ $=90$ families) pure lineages, we observed 7 sublineages, differing from those two main haplotype backgrounds at 1-3 variants. Based on extended STR-haplotypes, the highest gene diversity was observed in Joseph-lineage families from the USA $(0.91 \pm 0.05)$ and Asia $(0.88 \pm 0.03)$, followed by central European populations (around 0.50), with Portuguese families showing the lowest diversity $(0.18 \pm 0.08)$. Besides its epidemiological and historical interest, these variants could be of great value to study phenotypic variation and genetic modifiers linked to these haplotypes; to study genetic instability of the $(\mathrm{CAG})_{\mathrm{n}}$; and for therapeutical interventions, including gene-based targeting, namely in the best design for allele-specific ATXN3 silencing, that might be applicable to most MJD patients. SM is funded by FCT:CEECIND/00684/2017

S. Martins: None. I.P.D. Costa: None. P. Giunti: None. M. Watanabe: None. H. Sasaki: None. B.C. Almeida: None. A. Amorim: None. C. Gaspar: None. G. Nicholson: None. M.L. Saraiva-Pereira: None. S. Tsuji: None. Q. Li: None. S. Chen: None. G. Landouré: None. P. Maciel: None. R. Zaltzman: None. R. Sharony: None. Y. Zhou: None. J. Barros: None. J.L. Loureiro: None. V.T. Cruz: None. L. Ruano: None. E.R.P. Brunt: None. M. Marc Corral-Juan: None. M. Hsieh: None. L. Tranebjærg: None. O. Olajumoke: None. S.A. Ogun: None. M.F. Finkel: None. C. Gordon: None. M. Cornejo- 
Olivas: None. K. Fischbeck: None. A. Matilla-Dueñas: None. V. Volpini: None. O. Riess: None. Z. Wu: None. G. A. Rouleau: None. L.B. Jardim: None. G. Stevanin: None. A. Brice: None. P. Coutinho: None. B. Soong: None. L.P. Ranum: None. A. Durr: None. J. Sequeiros: None.

\section{P09.097.B}

Conserved methyltransferase NSUN5: a novel genetic modifier of Machado-Joseph disease (MJD/SCA3)?

\section{Huang ${ }^{1}$, E. Nibbeling ${ }^{2}$, J. d. Vries ${ }^{3}$, E. Brunt ${ }^{3}$, C. Verschuuren-Bemelmans $^{1}$, T. Schmidt ${ }^{4}$, D. S. Verbeek ${ }^{1}$}

${ }^{1}$ Department of Genetics, University of Groningen, University Medical Center Groningen, Groningen, Netherlands, ${ }^{2}$ Department of Clinical Genetics, Leiden University Medical Centre, Leiden, Netherlands, ${ }^{3}$ Department of Neurology, University of Groningen, University Medical Center Groningen, Groningen, Netherlands, ${ }^{4}$ Institute of Medical Genetics \& Applied Genomics, University of Tuebingen, Tuebingen, Germany

Machado-Joseph disease (MJD) or spinocerebellar ataxia (SCA3) is caused by a coding CAG repeat expansion in Ataxin-3 (ATXN3). The CAG repeat length is inversely correlated with the age of onset (AO) but only explains up to $60-70 \%$ of $\mathrm{AO}$ variation. Thus, additional modifiers of AO need to be identified. Whole exome sequencing was performed in 4 early $\mathrm{AO}$ and 5 late $\mathrm{AO}( \pm 10$ years deviation) patients of a large Dutch MJD/SCA3 family ( $n_{\text {affec- }}$ ted_total=32) via Illumina HiSeq. Sequencing data were analyzed via an in-house bioinformatics pipeline and Alissa (Agilent). ATXN3 protein aggregates were quantified using a filter-trap assay. We identified a rare non-synonymous variant c.472G $>$ C, p.Gly158Arg in the highly conserved methyltransferase domain of NSUN5 in the late AO cases but not in the cases with early AO. NSUN5 was previously shown to regulate lifespan and stress resistance in yeast, worms, and flies. Upon screening of all 32 affected family members, we identified 8 cases carrying the p.Gly158Arg variant, who showed an average delay in $\mathrm{AO}(7.9 \pm 4.8$ years) compared to non-carriers. Upon silencing of NSUN5 in HEK293T cells transiently expressing ATXN3-148Q increased protein aggregates were observed compared to control.

Our preliminary results point to NSUN5 as likely modifier gene of $\mathrm{AO}$ in MJD/SCA3. Further studies are needed to confirm if the p.Gly158Arg variant changes NSUN5's modifying role on ATXN3 protein aggregation and toxicity. China Scholarship Council (CSC) \#201608440359 and de Jan Kornelis de Cock Stichting grant (MH), JPND eRARE grant and Rosalind Franklin Fellowship of the University of Groningen (DSV).

M. Huang: None. E. Nibbeling: None. J.D. Vries: None. E. Brunt: None. C. Verschuuren-Bemelmans: None. T. Schmidt: None. D.S. Verbeek: None.

P09.098.C

Clinical and molecular characterization of a large MPAN cohort

P. Sparber ${ }^{l}$, T. Krylova ${ }^{l}$, S. Repina ${ }^{l}$, N. Demina ${ }^{l}, G$. Rudenskayal, I. Sharkoval, A. Sharkov ${ }^{2,3,4}$, V. Kadishev ${ }^{I}$, I. Kanivets $^{3}$, S. Korostelev ${ }^{3}$, E. Pomerantseva ${ }^{5}$, M. Ivanova ${ }^{6}$, S. Mikhailova ${ }^{7}$, E. Zaharova $^{1}$, A. Filatova $^{1}$, M. Skoblov $^{1}$

${ }^{1}$ Research center of medical genetics, Moscow, Russia, Moscow, Russian Federation, ${ }^{2}$ Veltischev Research and Clinical Institute for Pediatrics Pirogov RNRMU, Moscow, Russian Federation, ${ }^{3}$ Genomed ltd., Moscow, Russian Federation, ${ }^{4}$ Institute of Gene Biology RAS, Moscow, Russian Federation, ${ }^{5}$ Genetico ltd., Moscow, Russian Federation, ${ }^{6}$ Oftalmic ltd., Moscow, Russian Federation, ${ }^{7}$ Russian Children's Clinical Hospital, Moscow, Russian Federation

Introduction: Mitochondrial membrane protein-associated neurodegeneration (MPAN) is a rare neurological disorder with an autosomal recessive mode of inheritance and characterized by spasticity, optic nerve atrophy, neuropsychiatric abnormalities and most important iron accumulation in the basal ganglia. MPAN is caused by biallelic pathogenic variants in the C19orf12 gene and is considered the third most common clinical form of neurodegeneration with brain iron accumulation. Nevertheless, only few patient cohorts were described and MPAN clinical and genetic diversity is of great interest.

Material and methods: For cohort formation, we analyzed the internal database in Research Centre for Medical Genetics in Moscow and several commercial genetic laboratories. Patients were examined by a clinical geneticist and gave their inform consent to participate in the study. Mutation analysis was performed by whole exome sequencing or by Sanger sequencing of C19orf12 gene. Functional analysis of c. $193+5 \mathrm{G}>\mathrm{A}$ variant was performed using a minigene assay. Disease prevalence was calculated with the Hardy-Weinberg equilibrium. Allelic imbalance was detected using RT-PCR.

Results: We gathered a cohort of 17 confirmed MPAN patients with detailed clinical information. The common c.210_214del variant accounted for $73,5 \%$ of all the pathogenic alleles. We detected four previously undescribed variants and several patient with an unusual clinical presentation. The estimated MPAN prevalence was 
1:600000. In one heterozygous carrier, a pronounced allelic imbalance with lower expression levels of the wild-type allele was detected.

Conclusion: We describe the third largest cohort of MPAN patients to date with novel clinical findings. Our work expand the clinical and mutational spectrum of MPAN.

P. Sparber: None. T. Krylova: None. S. Repina: None. N. Demina: None. G. Rudenskaya: None. I. Sharkova: None. A. Sharkov: None. V. Kadishev: None. I. Kanivets: None. S. Korostelev: None. E. Pomerantseva: None. M. Ivanova: None. S. Mikhailova: None. E. Zaharova: None. A. Filatova: None. M. Skoblov: None.

P09.100.B

A recurrent de novo CAPRIN1 mutation causes a novel progressive early onset neurodegenerative disorder

A. Delle Vedove ${ }^{1,2,3}$, G. Zanni ${ }^{4}$, M. Eckenweiler ${ }^{5}, M$. Storbeck $^{1,2,3}$, S. Barresi ${ }^{6}$, S. Pizzi ${ }^{6}$, S. Hosseinibarkooie ${ }^{1,2,3}$, N. Mendoza-Ferreira ${ }^{1,2,3}$, I. Hölker ${ }^{1,2,3}$, F. Körber ${ }^{7}, M$. Kye $^{1,2}$, E. Bertini ${ }^{4}$, J. Kirschner ${ }^{8}$, M. Tartaglia ${ }^{4}, B$. Wirth $^{1,2,3}$

${ }^{1}$ Institute of Human Genetics, Cologne, Germany, ${ }^{2}$ Center for Molecular Medicine Cologne, Cologne, Germany, ${ }^{3}$ Institute for Genetics, Cologne, Germany, ${ }^{4}$ Department of Neurosciences, Ospedale Pediatrico Bambino Gesù, Rome, Italy, ${ }^{5}$ Clinic for Neuropediatrics and Muscular Disorders, Freiburg, Germany, ${ }^{6}$ Genetics and Rare Diseases Research Division, Ospedale Pediatrico Bambino Gesù, Rome, Italy, ${ }^{7}$ Institute of Diagnostic and Interventional Radiology, Cologne, Germany, ${ }^{8}$ Department of Neuropediatrics, University Hospital Bonn, Bonn, Germany

Introduction: Neurodegenerative disorders (NDs) represent increasingly prevalent and severely debilitating diseases. In several cases, their molecular diagnosis remains elusive.

Materials and methods: Trio-based WES and Sanger sequencing led to the identification of the disease-causative variant. Its functional impact is being investigated in HEK293T cells and iPSCs.

Results: We describe two individuals from two unrelated non-consanguineous families with an identical de novo mutation in CAPRIN1 (Cell Cycle Associated Protein 1): an 8 year-old girl with progressive muscular atrophy, who developed ataxia and cognitive decline at 11 years, and a 7 year-old boy with spinocerebellar ataxia and learning difficulties. Both carry an identical de novo c.1535C $>\mathrm{T}$ variant (p.Pro512Leu) which is not reported in gnomAD and affects a highly conserved residue. CAPRIN1 is abundant in the brain, where it regulates the transport and translation of mRNAs of genes involved in synaptic plasticity. As other ND-related proteins, it is a component of stress granules and harbours a prion-like domain (PRD). In accordance with the in silico predictions suggesting that the p.P512L mutation increases its aggregation propensity, CAPRIN1 ${ }^{\text {P512L }}$ overexpression causes the formation of insoluble aggregates in transfected HEK293T cells. To investigate the mutation effects in neurons, we successfully introduced the missense mutation in human iPSCs using the CRISPR/Cas9 system.

Conclusions: Our results suggest that CAPRIN1 ${ }^{\mathrm{P} 512 \mathrm{~L}}$ is prone to aggregation. As CAPRIN1 deficiency is linked to autism-spectrum disorders (ASD) in humans, we identify CAPRIN1 as a novel gene implicated in NDs, and a novel class of mutations perturbing CAPRIN1 function in a dominant manner.

A. Delle Vedove: None. G. Zanni: None. M. Eckenweiler: None. M. Storbeck: None. S. Barresi: None. S. Pizzi: None. S. Hosseinibarkooie: None. N. MendozaFerreira: None. I. Hölker: None. F. Körber: None. M. Kye: None. E. Bertini: None. J. Kirschner: None. M. Tartaglia: None. B. Wirth: None.

P09.101.C

Implementation of WES-based gene panel testing in patients with adult-onset neurological disorders at the University Hospital of Ghent: a retrospective observational analysis of 176 patients

N. Schuermans ${ }^{1,2}$, E. Debackere ${ }^{1,2}$, S. Symoens $^{1,2}$, B. Poppe $^{1,2}$, P. Santens ${ }^{3,4}$, J. De Bleecker ${ }^{3,4}$, D. Hemelsoet ${ }^{3,4}$, B. Dermaut ${ }^{1,2}$

${ }^{1}$ Center for Medical Genetics, Ghent University Hospital, Ghent, Belgium, ${ }^{2}$ Department of Biomolecular Medicine, Faculty of Medicine and Health Sciences, Ghent University, Ghent, Belgium, ${ }^{3}$ Department of Neurology, Ghent University Hospital, Ghent, Belgium, ${ }^{4}$ Department of Head and Skin, Faculty of Medicine and Health Sciences, Ghent University, Ghent, Belgium

Introduction: Neurodegenerative inherited diseases are an important cause of morbidity and mortality. A molecular diagnosis is elementary for genetic counseling and potential therapeutic strategies. In January 20196 novel WES-based gene panels were introduced at the Ghent University Hospital to facilitate diagnostics in patients with neurodegenerative diseases. In this study we want to establish the added value of these panels and define patient- and diseasespecific characteristics associated with a high diagnostic yield. 
Methods: 6 novel gene panels were introduced in diagnostic setting at the Centre for Medical Genetics Ghent: 1. Ataxia/spasticity 2. Leukodystrophy 3. Paroxysmal/ episodic disorders 4. Movement disorders 5. Neurodegeneration with brain iron accumulation 6. Progressive myoclonic epilepsy

Results: From January the $1^{\text {st }}$ up till December $31^{\text {st }} 2019$ 176 cases have been analyzed. The mean age of the patients was 50 years with a majority of the patients being adults (98\%). The leukodystrophy panel was most popular (43\%), followed by the ataxia/spasticity panel (26\%) and the movement disorders panel $(20 \%)$. The overall diagnostic yield was $15 \%$. In another $15 \%$ variants of unknown significance were found. The diagnostic yield was highest among patients presenting with spinocerebellar ataxia $(30 \%)$. The causal mutations were missense $(62 \%)$, nonsense $(25 \%)$, splice-site $(10 \%)$ and deletion $(3 \%)$ mutations.

Conclusions: Gene panel testing is a successful tool to diagnose inherited neurological diseases. The overall diagnostic yield after analysis of 176 cases was $15 \%$, with another $15 \%$ of potential but unsure diagnoses. Patients suffering from ataxia and/or spastic paraplegia were most likely to obtain a molecular diagnosis $(30 \%)$.

N. Schuermans: None. E. Debackere: None. S. Symoens: None. B. Poppe: None. P. Santens: None. J. De Bleecker: None. D. Hemelsoet: None. B. Dermaut: None.

\section{P09.102.A}

NMIHBA causing PRUNE1 variants result in loss of exopolyphosphatase activity.

H. Nistala ${ }^{1}$, J. Dronzek ${ }^{l}$, C. Gonzaga-Jauregui ${ }^{1}$, S. Chim ${ }^{l}$, S. Rajamani ${ }^{2}$, S. Nuwayhid ${ }^{2}$, D. Delgado ${ }^{2}$, E. Burke ${ }^{3}$, E.

Karaca $^{4}$, M. C. Franklin ${ }^{2}$, P. Sarangapani ${ }^{2}$, M. Podgorski ${ }^{2}$, Y. Tang ${ }^{2}$, M. G. Dominguez ${ }^{2}$, M. Withers ${ }^{5}$, R. A.

Deckelbaum $^{2}$, C. J. Scheonherr ${ }^{1}$, W. A. Gahl ${ }^{3}$, M. C.

Malicdan $^{3}$, B. Zambrowicz ${ }^{2}$, N. W. Gale ${ }^{2}$, R. A. Gibbs ${ }^{5}$, W. K. Chung $^{6}$, J. R. Lupski ${ }^{5}$, A. N. Economides ${ }^{1}$

${ }^{1}$ Regeneron Genetics Center, Tarrytown, NY, United States, ${ }^{2}$ Regeneron Pharmaceuticals, Inc., Tarrytown, NY, United States, ${ }^{3}$ Undiagnosed Diseases Program Translational Laboratory, NHGRI, Bethesda, MD, United States, ${ }^{4}$ University of Alabama, Birmingham, AL, United States, ${ }^{5}$ Baylor College of Medicine, Houston, TX, United States, ${ }^{6}$ Columbia University Medical Center, New York, NY, United States

Neurodevelopmental disorder with microcephaly, hypotonia, and variable brain anomalies (NMIHBA) is an autosomal recessive neurodevelopmental and neurodegenerative disorder characterized by global developmental delay and severe intellectual disability. Microcephaly, progressive cortical atrophy, cerebellar hypoplasia and delayed myelination are neurological hallmarks in affected individuals. NMIHBA is caused by biallelic variants in PRUNE1 encoding prune exopolyphosphatase 1 . We provide in-depth clinical description of two affected siblings harboring compound heterozygous variant alleles, c.383G $>\mathrm{A}$ (p. Arg128Gln), c.520G>T (p.Gly174*) in PRUNE1. To gain insights into disease biology, we biochemically characterized missense variants within the conserved N-terminal aspartic acid-histidine-histidine (DHH) motif and provide evidence that they result in the destabilization of protein structure and/or loss of exopolyphosphatase activity. Genetic ablation of Prunel results in midgestational lethality in mice, associated with perturbations to embryonic growth and vascular development. Our findings suggest that NMIHBA results from hypomorphic variant alleles in humans and underscore the potential key role of PRUNE1 exopolyphoshatase activity in neurodevelopment.

H. Nistala: A. Employment (full or part-time); Significant; Regeneron Pharmaceuticals, Inc.. E. Ownership Interest (stock, stock options, patent or other intellectual property); Modest; Regeneron Pharmaceuticals, Inc.. J. Dronzek: None. C. Gonzaga-Jauregui: A. Employment (full or part-time); Significant; Regeneron Pharmaceuticals, Inc.. E. Ownership Interest (stock, stock options, patent or other intellectual property); Modest; Regeneron Pharmaceuticals, Inc. S. Chim: A. Employment (full or part-time); Significant; Regeneron Pharmaceuticals, Inc. E. Ownership Interest (stock, stock options, patent or other intellectual property); Modest; Regeneron Pharmaceuticals, Inc. S. Rajamani: A. Employment (full or part-time); Significant; Regeneron Pharmaceuticals, Inc.. E. Ownership Interest (stock, stock options, patent or other intellectual property); Modest; Regeneron Pharmaceuticals, Inc. S. Nuwayhid: A. Employment (full or part-time); Significant; Regeneron Pharmaceuticals, Inc. E. Ownership Interest (stock, stock options, patent or other intellectual property); Modest; Regeneron Pharmaceuticals, Inc. D. Delgado: A. Employment (full or part-time); Significant; Regeneron Pharmaceuticals, Inc.. E. Ownership Interest (stock, stock options, patent or other intellectual property); Modest; Regeneron Pharmaceuticals, Inc.. E. Burke: None. E. Karaca: None. M.C. Franklin: A. Employment (full or part-time); Significant; Regeneron Pharmaceuticals, Inc.. E. Ownership Interest (stock, stock options, patent or other intellectual property); Modest; Regeneron Pharmaceuticals, Inc. P. Sarangapani: A. Employment (full or part-time); Significant; Regeneron Pharmaceuticals, Inc. E. Ownership Interest (stock, stock options, patent or other intellectual property); Modest; Regeneron Pharmaceuticals, Inc. M. Podgorski: A. Employment (full or part-time); Significant; 
Regeneron Pharmaceuticals, Inc.. E. Ownership Interest (stock, stock options, patent or other intellectual property); Modest; Regeneron Pharmaceuticals, Inc. Y. Tang: A. Employment (full or part-time); Significant; Regeneron Pharmaceuticals, Inc.. E. Ownership Interest (stock, stock options, patent or other intellectual property); Modest; Regeneron Pharmaceuticals, Inc. M.G. Dominguez: A. Employment (full or part-time); Significant; Regeneron Pharmaceuticals, Inc.. D. Speakers Bureau/Honoraria (speakers bureau, symposia, and expert witness); Significant; Regeneron Pharmaceuticals, Inc.. M. Withers: None. R.A. Deckelbaum: A. Employment (full or part-time); Significant; Regeneron Pharmaceuticals, Inc.. E. Ownership Interest (stock, stock options, patent or other intellectual property); Significant; Regeneron Pharmaceuticals, Inc. C. J. Scheonherr: A. Employment (full or part-time); Significant; Regeneron Pharmaceuticals, Inc.. E. Ownership Interest (stock, stock options, patent or other intellectual property); Significant; Regeneron Pharmaceuticals, Inc.. W. A. Gahl: None. M.C. Malicdan: None. B. Zambrowicz: A. Employment (full or part-time); Significant; Regeneron Pharmaceuticals, Inc.. E. Ownership Interest (stock, stock options, patent or other intellectual property); Significant; Regeneron Pharmaceuticals, Inc. N.W. Gale: A. Employment (full or part-time); Significant; Regeneron Pharmaceuticals, Inc.. E. Ownership Interest (stock, stock options, patent or other intellectual property); Significant; Regeneron Pharmaceuticals, Inc.. R.A. Gibbs: None. W.K. Chung: None. J.R. Lupski: E. Ownership Interest (stock, stock options, patent or other intellectual property); Significant; 23andMe, Lasergen Inc.. F. Consultant/Advisory Board; Significant; Regeneron Pharmaceuticals Inc, Novartis International AG,. A.N. Economides: A. Employment (full or part-time); Significant; Regeneron Pharmaceuticals, Inc.. E. Ownership Interest (stock, stock options, patent or other intellectual property); Significant; Regeneron Pharmaceuticals, Inc..

\section{P09.103.B}

Exome Sequencing in Bangladeshi families with neurodevelopmental disorders

H. Morsy ${ }^{1,2}$, V. Salpietro ${ }^{l}$, S. Banu ${ }^{3}$, M. Zaman ${ }^{3}, S$. Efthymiou $^{l}$, R. Maaroofian ${ }^{l}$, A. Fragkouli ${ }^{l}$, T. Bourinaris ${ }^{l}$, SYNaPS Study Group, J. vandrovcova ${ }^{l}$, H. Houlden ${ }^{l}$

${ }^{1}$ Institute of Neurology, London, United Kingdom, ${ }^{2}$ Medical Research Institute, Alexandria, Egypt, ${ }^{3} \mathrm{ICH}$ and SSF Hospital Mirpur, Dhaka, Bangladesh

Background: Neurodevelopmental disorders are characterized by high clinical and genetic heterogeneity. Their molecular basis had not been well characterized in
Bangladesh. Through the Synaptopathies and Paroxysmal Syndromes (SYNaPS) Study Group, we aimed to elucidate the genetic basis of neurodevelopmental disorders affecting the Bangladeshi patients.

Methods: We applied whole exome sequencing (WES) in a cohort of 50 Bangladeshi families with primarily Mendelian neurodevelopmental diseases. In families without likely pathogenic variants in known disease causing genes; novel candidate genes were prioritized according to functional impact, rarity in multiple databases especially in Southeast Asia population, presence in multiple families with overlapping phenotypes, mutational tolerance of the gene, animal studies, predicted deleteriousness by several in silico tools and expression of the protein in affected tissues.

Results: We found known pathogenic or novel variants in known disease genes with phenotypic expansion in 18 families and 12 novel candidates in 10 families including RTN4R, ITGB8 and ACBD6. Overall, molecular diagnosis was provided by variants in known genes in 36 families (72\%) and by novel candidate genes in additional 10 families, resulting in a potential molecular diagnostic rate of $92 \%$. Homozygous variants were causative in only $34.7 \%$, despite high rate of consanguinity in our cohort.

Conclusions: Our study shows the benefits of WES analysis in neurodevelopmental disorders in non-European populations and highlights ongoing difficulties in variant interpretation. In our cohort, only six of identified variants were previously reported, the remaining were novel variants and hence interpretation of pathogenicity was challenging especially in cases with atypical phenotypes.

H. Morsy: None. V. Salpietro: None. S. Banu: None. M. Zaman: None. S. Efthymiou: None. R. Maaroofian: None. A. Fragkouli: None. T. Bourinaris: None. J. vandrovcova: None. H. Houlden: None.

\section{P09.104.C}

Update on a sponsored no-cost epilepsy gene panel for seizure onset between 2-4 years of age: Results from 682 tests

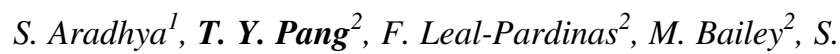

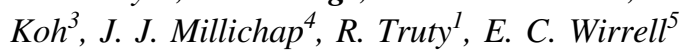

${ }^{1}$ Invitae, San Francisco, CA, United States, ${ }^{2}$ BioMarin Pharmaceutical Inc., Novato, CA, United States, ${ }^{3}$ Emory University School of Medicine, Atlanta, GA, United States, ${ }^{4}$ Ann \& Robert H. Lurie Children's Hospital of Chicago, Chicago, IL, United States, ${ }^{5}$ Mayo Clinic, Novato, CA, United States

Neuronal Ceroid Lipofuscinosis Type 2 (CLN2) disease often presents with epilepsy at approximately three years of age accompanied by a history of language delay. Behind the 
Seizure $^{\circledR}$ (BTS) is a US-based, sponsored, no-cost, targeted, next-generation sequencing epilepsy gene panel for children suspected to have genetic epilepsy. The goal is to utilize BTS to lower the age of CLN2 disease diagnosis.

Eligibility criteria were: age-at-test-order between 24-60 months and unprovoked seizure onset $>24$ months. BTS uses an epilepsy gene panel of $180+$ genes with the option to include preliminarily associated genes.

Non-BTS epilepsy gene panel testing, which includes no limitation for age-at-test-order and age-of-seizure-onset, has shown a $15.5 \%(n=1234 / 7939)$ molecular diagnostic yield (MDx) overall and $0.05 \%$ specifically involving TPP1 gene (CLN2 disease). Within the BTS program, the overall MDx was $6.74 \%$ ( $n=46 / 682)$. However, the yield of TPP1 positives was $0.88 \%$, representing a 17.6 -fold increase in comparison to non-BTS cohort. Age of CLN2 disease diagnosis was earlier than as described in the literature (3.89-year versus 5-year). CLN2 disease was not a suspected diagnosis for 5 out of 6 individuals tested using BTS. Other genes that contributed to positive molecular diagnoses in the BTS program included CACNA1A, CHD2, EHMT1, FOXG1, FRRS1L, GRIN2A, IQSEC2, KANSL1, KIAA2022, MECP2, PACS1, PCDH19, PPT1, PURA, SCN1A, SCN2A, SLC6A1, STX1B, SYNGAP1, TSC1 and ZEB2.

The BTS program has facilitated earlier CLN2 disease identification. These findings indicate that broad epilepsy gene panel tests can increase diagnostic yield for CLN2 disease and simultaneously identify other genetic causes of epilepsy.

S. Aradhya: A. Employment (full or part-time); Significant; Invitae. E. Ownership Interest (stock, stock options, patent or other intellectual property); Significant; Invitae. T.Y. Pang: A. Employment (full or part-time); Significant; BioMarin Pharmaceutical Inc.. E. Ownership Interest (stock, stock options, patent or other intellectual property); Significant; BioMarin Pharmaceutical Inc. F. Leal-Pardinas: A. Employment (full or part-time); Significant; BioMarin Pharmaceutical Inc.. E. Ownership Interest (stock, stock options, patent or other intellectual property); Significant; BioMarin Pharmaceutical Inc. M. Bailey: A. Employment (full or part-time); Significant; BioMarin Pharmaceutical Inc.. E. Ownership Interest (stock, stock options, patent or other intellectual property); Significant; BioMarin Pharmaceutical Inc.. S. Koh: None. J.J. Millichap: None. R. Truty: A. Employment (full or part-time); Significant; Invitae. E. Ownership Interest (stock, stock options, patent or other intellectual property); Significant; Invitae. E.C. Wirrell: F. Consultant/Advisory Board; Modest; BioMarin Pharmaceutical Inc., Biocodex.
P09.105.A

A copy number variation map of pediatric neuropsychiatric traits

M. Zarrei ${ }^{l}$, D. Merico ${ }^{1,2}$, C. L. Burton ${ }^{3}$, W. Engchuan ${ }^{l}$, E. J. Higginbotham ${ }^{l}, J$. Wei ${ }^{l}$, J. R. MacDonald ${ }^{l}, B$. Thiruvahindrapuram ${ }^{l}$, A. J. S. Chan ${ }^{l}$, T. Nalpathamkalam ${ }^{l}$, L. J. Strug ${ }^{1,4}$, R. F. Wintle ${ }^{l}$, R. K. C. Yuen ${ }^{l}$, M. WoodburySmith $^{1,5}$, C. R. Marshall ${ }^{1,6,7}$, P. D. Arnold ${ }^{8,9,10}, J$.

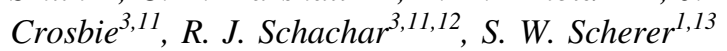

${ }^{I}$ The Centre for Applied Genomics, Program in Genetics and Genome Biology, The Hospital for Sick Children, Toronto, ON, Canada, ${ }^{2}$ Deep Genomics Inc., Toronto, ON, Canada, ${ }^{3}$ Neurosciences and Mental Health Program, The Hospital for Sick Children, Toronto, ON, Canada, ${ }^{4}$ Division of Biostatistics, Dalla Lana School of Public Health, University of Toronto, Toronto, ON, Canada, ${ }^{5}$ Institute of Neuroscience, Newcastle University, Newcastle upon Tyne, United Kingdom, ${ }^{6}$ Genome Diagnostics, Department of Paediatric Laboratory Medicine, The Hospital for Sick Children, Toronto, ON, Canada, ${ }^{7}$ Laboratory Medicine and Pathobiology, University of Toronto, Toronto, ON, Canada, ${ }^{8}$ Program in Genetics and Genome Biology, The Hospital for Sick Children, Toronto, ON, Canada, ${ }^{9}$ Mathison Centre for Mental Health Research and Education, University of Calgary, Calgary, AB, Canada, ${ }^{10}$ Departments of Psychiatry and Medical Genetics, Hotchkiss Brain Institute, Cumming School of Medicine, University of Calgary, Calgary, AB, Canada, ${ }^{11}$ Department of Psychiatry, University of Toronto, Toronto, ON, Canada, ${ }^{12}$ Institute of Medical Science, University of Toronto, Toronto, ON, Canada, ${ }^{13}$ Department of Molecular Genetics and McLaughlin Centre, University of Toronto, Toronto, ON, Canada

'Spit for Science' is a cohort of over 17,000 youth (6-17 years of age) with associated psychiatric phenotype measurements and saliva DNA collection. Quantitative phenotype data were collected for attention deficit hyperactivity disorder (ADHD), obsessive compulsive disorder (OCD), and response inhibition using Stop-Signal Reaction Time (SSRT). We genotyped unrelated Caucasians $(n=4,817)$ using Illumina HumanCoreExome beadchips. We performed global and functional gene-set burden analyses, and locus association test on rare CNVs using linear regression against different traits.

We identified CNVs linked with neuropsychiatric disorders in 191 (4.0\%) participants with elevated standardized z-scores for all traits. These CNVs included i) sexchromosome and autosomal aneuploidies in $12(0.25 \%)$ individuals, ii) 13 unique genomic loci previously implicated in NDD in $52(1.1 \%)$ different participants, and iii) 
CNVs affecting genes with known functions in the human brain such as ASTN2, DLGAP2, MACROD2, NRXN1, and others. ADHD traits were associated with increased deletion burden ( $p=0.014)$, whereas there was an increased burden of duplication for SSRT ( $p=0.034)$. We found no global CNV burden for OCD traits. ADHD traits showed significant enrichment for deletions in genes associated with mouse neurophenotypes $(p=0.009)$ and with human brain gene expression $(p=0.04)$. OCD and SSRT traits showed enrichment for duplications in genes expressed in human brain ( $p=0.004$ and 0.016 , respectively). SSRT traits showed enrichment for duplications in eight loci: $1 q 21.1,1 q 42.3,2 q 37.3,9 q 22.2,12 q 13.13,18 p 11.21$, and $22 \mathrm{q} 11.21$.

These results highlight the importance of mapping the prevalence and impact of rare variants in community samples to improve understanding of pediatric mental health.

M. Zarrei: None. D. Merico: None. C.L. Burton: None. W. Engchuan: None. E.J. Higginbotham: None. J. Wei: None. J.R. MacDonald: None. B. Thiruvahindrapuram: None. A.J.S. Chan: None. T. Nalpathamkalam: None. L. J. Strug: None. R.F. Wintle: None. R.K.C. Yuen: None. M. Woodbury-Smith: None. C.R. Marshall: None. P.D. Arnold: None. J. Crosbie: None. R.J. Schachar: None. S. W. Scherer: None.

\section{P09.106.B}

Genetic epidemiology analysis of theNOTCH3gene mutations in Hungary

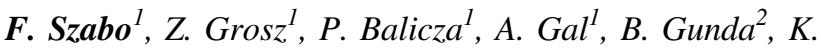
Sas $^{3}$, M. Molnár ${ }^{1}$

${ }^{1}$ Semmelweis University, Institute of Genomic Medicine and Rare Disorders, Budapest, Hungary, ${ }^{2}$ Semmelweis University, Department of Neurology, Budapest, Hungary, ${ }^{3}$ Albert Szent-Györgyi Clinical Center, University of Szeged, Szeged, Hungary

Background: Cerebral autosomal dominant arteriopathy with subcortical infarcts and leukoencephalopathy (CADASIL) is a hereditary, small vessel disease associated with resurrent stroke syndrome, migraine, progressive cognitive decline, and psychiatric disturbances. The worldwide prevalence of CADASIL has been estimated at approximately 5:100,000.

Methods: Total coding region of the NOTCH3 gene was analyzed with Sanger or NGS sequencing in 650 patients (359 male, 291 female; mean age $45.27 \pm 15.95$ years) with the MRI sign of small vessel disease. In our cohort non genetic risk factors for cerebovascular disorders have been excluded.
Results: Damaging variants has ben in found in $7.84 \%$ of the cases, in 51 patiens 16 missense substitutions were found in the NOTCH3 gene. Twelve have been previously described as pathogenic variants (p. R90C, C146R, C208R, C440R C448R, C75R, R110C R141C, R153C, R169C, $\mathrm{R} 182 \mathrm{C}$, R1076C), while 4 were novel, likely pathogenic alterations (p. C222S, G420C, R133C, R207C). These variants were not present in controls and were predicted as disease causing by in silico analysis.

Conclusions: In the Hungarian cohort the most common rare variant was the $\mathrm{R} 90 \mathrm{C} \mathrm{NOTCH3}$ gene, the mutation frequency was, $2.5 \%$. In the Caucasian population this variant is the most frequent pathogenic variant. We detected rare variants characteristic of Asian population as well (R133C). We can conclude that the henetic epidemiological data of the Hungarian pateints with CADASIL can be explained by the Hungarian history. This study was supported by the Hungarian Brain Research Program (KTIA_NAP_2017-1.2.1-NKP-2017-00002).

F. Szabo: None. Z. Grosz: None. P. Balicza: None. A. Gal: None. B. Gunda: None. K. Sas: None. M. Molnár: None.

\section{P09.107.C}

Biallelic loss-of-function NRROS variants impairing active TGF- $\beta 1$ delivery cause a severe infantile onset neurodegenerative condition with intracranial calcification

X. Dong ${ }^{1,2}$, N. B. Tan $^{1,2,3}$, K. B. Howell ${ }^{1,2,4}$, S. Barresi ${ }^{5}$, J. L. Freeman ${ }^{1,4}$, D. Vecchio ${ }^{5}$, M. Piccione ${ }^{6}, F$. Clementina Radio $^{5}$, D. Calame ${ }^{7,8}$, S. Zong ${ }^{1}$, S. Eggers ${ }^{1,3}$, I. E. Scheffer ${ }^{1,2,9}$, T. Y. Tan ${ }^{1,2,3}$, N. J. Van Bergen ${ }^{1,2}$, M. Tartaglia $^{5}$, J. Christodoulou ${ }^{1,2,3}$, S. M. White Wh, $^{1,2}$

${ }^{I}$ Murdoch Children's Research Institute, Parkville, Victoria, Australia, ${ }^{2}$ Department of Paediatrics, University of Melbourne, Parkville, Victoria, Australia, ${ }^{3}$ Victorian Clinical Genetics Services, Parkville, Victoria, Australia, ${ }^{4}$ Department of Neurology, Royal Children's Hospital, Parkville, Victoria, Australia, ${ }^{5}$ Genetics and Rare Diseases Research Division, Ospedale Pediatrico Bambino Gesù, Rome, Italy, ${ }^{6}$ Department of Science for Health Promotion and Mother and Child Care, Università degli Studi di Palermo, Palermo, Italy, ${ }^{7}$ Baylor College of Medicine, Houston, TX, United States, ${ }^{8}$ Texas Children's Hospital, Houston, TX, United States, ${ }^{9}$ Department of Medicine, University of Melbourne, Austin Health, Heidelberg, Victoria, Australia

Introduction: NRROS is a leucine-rich repeat-containing protein that associates with latent TGF- $\beta 1$ and anchors it on the cell surface, a requirement for activation of TGF- $\beta 1$ in 
microglia. We report six individuals from four families with biallelic variants in NRROS. All affected individuals had refractory epilepsy, developmental regression, and reduced white matter volume with delayed myelination. Intracranial calcification was detected in three individuals. These features are consistent with those observed in the Nrros knockout mouse.

Methods: Confocal microscopy in HEK293T cells was used to investigate the localisation of wild-type and mutant NRROS proteins. Flow cytometry was performed to analyse the influence of mutant NRROS proteins in anchoring latent TGF- $\beta 1$ to the cell surface.

Results: Wild-type and mutant NRROS proteins were found to co-localise with latent TGF- $\beta 1$ intracellularly. However, flow cytometry showed that the mutant NRROS proteins failed to anchor latent TGF- $\beta 1$ on the cell surface, as compared to wild-type NRROS. Moreover, wild-type NRROS rescued the defect of the disease-associated mutants in presenting latent TGF- $\beta 1$ to the cell surface.

Conclusion: Our findings suggest that loss of NRROS function causes a severe childhood-onset neurodegenerative condition with features suggestive of a disordered response to inflammation.

Funding: Research at Murdoch Children's Research Institute is supported by the Victorian Government's Operational Infrastructure Support Program and the Harbig Foundation. NBT acknowledges the Australian NHMRC CRE in Neurocognitive Disorders for their support. $\mathrm{KBH}$ is supported by funding from the Australian NHMRC. MT acknowledges the Italian Ministry of Health (Ricerca Corrente 2019) and Fondazione Bambino Gesù (Vite Coraggiose).

X. Dong: None. N.B. Tan: None. K.B. Howell: None. S. Barresi: None. J.L. Freeman: None. D. Vecchio: None. M. Piccione: None. F. Clementina Radio: None. D. Calame: None. S. Zong: None. S. Eggers: None. I.E. Scheffer: None. T.Y. Tan: None. N.J. Van Bergen: None. M. Tartaglia: None. J. Christodoulou: None. S.M. White: None.

\section{P09.108.A}

De novo NSF mutations cause early infantile epileptic encephalopathy

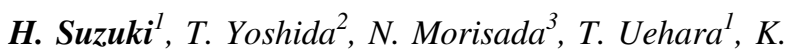
Kosaki $^{l}$, T. Takano-Shimizu ${ }^{4}$, T. Takenouchi ${ }^{1}$

${ }^{1}$ Keio University, Tokyo, Japan, ${ }^{2}$ Kyoto University, Kyoto, Japan, ${ }^{3}$ Kobe Children's Hospital, Hyogo, Japan, ${ }^{4}$ Kyoto Institute of Technology, Kyoto, Japan

Introduction: N-ethylmaleimide-sensitive factor (NSF) plays a critical role in intracellular vesicle transport, which is essential for neurotransmitter release. Dysregulation of neurotransmitter releasing process has been recognized as the mechanistic basis for neurological disorders. Here, we describe two patients with de novo pathogenic variants in $N S F$ with early infantile epileptic encephalopathy.

Clinical Report: Patient 1 was a girl with no significant family medical history. She was born at 37 weeks of gestation by uneventful delivery after an uneventful pregnancy. Immediately after birth, she developed continuous tonic seizures. Electroencephalography showed burstsuppression patterns. Patient 2 was a 1-year-old girl with no significant family medical history. In her prenatal period, she exhibited failure to thrive, fetal hydrops, and anemia. After birth, she had no spontaneous respiration, which required mechanical ventilation, and had frequent myoclonic seizures. Her electroencephalography showed burstsuppression patterns.

Results: Trio-based exome sequencing revealed a de novo heterozygous variant in the NSF gene (NM_006178.3) in both the probands: c. $1375 \mathrm{G}>\mathrm{A}$ chr17:44782125G $>\mathrm{A}$ (GRCh37) p.Ala459Thr in patient 1 and c.1688C $>\mathrm{T}$ chr17:44791279C>T (GRCh37) p.Pro563Leu in patient 2. Functional analysis: When ectopically expressed in the developing eye of Drosophila in the presence of normal fly protein, the mutant human NSF protein severely affected eye development, while the wild-type human protein had no detectable effect under the same conditions. This finding suggested that the two variants had a dominant negative effect.

Conclusions: De novo heterozygous mutations in the NSF cause early infantile epileptic encephalopathy. Research grant: The Japan Agency for Medical Research and Development (JP18ek0109301, JP19gk0110038, and JP18ek0109288h0002).

H. Suzuki: None. T. Yoshida: None. N. Morisada: None. T. Uehara: None. K. Kosaki: None. T. TakanoShimizu: None. T. Takenouchi: None.

\section{P09.109.B}

Homozygous variants of PACS1cause an autosomal recessive developmental and epileptic encephalopathy

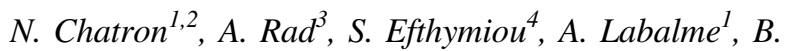

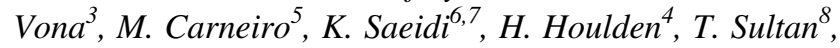 \\ R. Maroofian $^{4}$, G. LESCA ${ }^{1,9}$
}

${ }^{I}$ Department of Medical Genetics, Hospices Civils de Lyon, Lyon, France, ${ }^{2}$ Institut NeuroMyoGène, CNRS UMR 5310 INSERM U1217, Université de Lyon Université Claude Bernard Lyon 1, Lyon, France, Lyon, France, ${ }^{3}$ Department of Otorhinolaryngology, Head and Neck Surgery, Tuebingen Hearing Research Centre (THRC), Eberhard Karls University, Tuebingen, Germany, ${ }^{4}$ Department of 
Neuromuscular Disorders Institute of Neurology, University College London, Queen Square, London, United Kingdom, ${ }^{5}$ Department of Pediatric Neurology, Hospices Civils de Lyon, Lyon, France, ${ }^{6}$ Neuroscience Research Center, Institute of Neuropharmacology, Kerman University of Medical Sciences, Kerman, Iran, Islamic Republic of, ${ }^{7}$ Department of Medical Genetics, Kerman University of Medical Sciences, Kerman, Iran, Islamic Republic of, ${ }^{8}$ Department of Pediatric Neurology, Institute of Child Health, The Children's Hospital Lahore, Lahore, Pakistan, ${ }^{9}$ Institut NeuroMyoGène, CNRS UMR 5310 - INSERM U1217, Université de Lyon Université Claude Bernard Lyon 1, Lyon, France

PACS1 (OMIM 607492) encodes a trans-Golgi-membrane traffic regulator that directs protein cargo and several viral envelope proteins. It is upregulated during human embryonic brain development and has low expression after birth. Homozygous KO mice are lethal. A recurrent missense variant was reported in patients with SchuursHoeijmakers syndrome (OMIM 615009), an autosomal dominant disorder characterized by intellectual disability, distinct craniofacial features, and variable additional congenital abnormalities. Seizures of various types are frequent and usually respond well to antiepileptic drugs. This variant was suggested to act via a dominant-negative or a gain-offunction mechanism. No other pathogenic variant has been reported to date. We report on four patients from three unrelated families, with homozygous variants of PACS1. They presented with severe developmental delay, poor eye contact, absent language. Seizures started in infancy. They were of various types (focal seizures in one patient, spams in another one and generalized seizures in three patients) and drug resistant. EEG showed multifocal spikes or generalized sharp waves. Brain MRI showed variable patterns in patients, including delayed myelination or cerebral atrophy. Patients did not show the dysmorphic features reminiscent of Schuurs-Hoeijmakers syndrome. One family carried a homozygous stop variant and the two others different unreported homozygous missense variants, affecting highly conserved amino acids. These variants were absent or with a very low occurrence in the gnomAD v2.1 database. These observations suggest that biallelic variants of PACS1 can cause a phenotype of developmental epileptic encephalopathy. Functional analyses will be performed to characterize the consequences of these variants.

N. Chatron: None. A. Rad: None. S. Efthymiou: None. A. Labalme: None. B. Vona: None. M. Carneiro: None. K. Saeidi: None. H. Houlden: None. T. Sultan: None. R. Maroofian: None. G. Lesca: None.

\section{P09.117.A}

Mitochondrial-specific polygenic risk score is associated with increased Parkinson's disease risk

\section{Z. Landoulsi ${ }^{1}$, D. Bobbilit ${ }^{1,2}$, S. Pachcheck ${ }^{l}$, G. Arena ${ }^{3}$, NCER-PD Consortium, R. Krüger ${ }^{3,4,5}$, P. May ${ }^{l}$}

${ }^{1}$ Bioinformatics Core, Luxembourg Centre for Systems Biomedicine, University of Luxembourg, Belvaux, Luxembourg, ${ }^{2}$ Megeno S.A., Esch-sur-Alzette, Luxembourg, ${ }^{3}$ Clinical and Experimental Neuroscience, Luxembourg Centre for Systems Biomedicine, University of Luxembourg, Belvaux, Luxembourg, ${ }^{4}$ Parkinson Research Clinic, Centre Hospitalier de Luxembourg, Luxembourg, Luxembourg, ${ }^{5}$ Transversal Translational Medicine, Luxembourg Institute of Health, Strassen, Luxembourg

Introduction: Strong evidence indicates that mitochondrial dysfunction is implicated in Parkinson's disease (PD) pathophysiology. The underlying mechanism of mitochondrial processes in the sporadic form of PD is still largely unknown. Polygenic risk scores (PRS) gather the total risk for disease in an individual based on common variants, where the risk alleles and their effects are defined by previous genome-wide association studies (GWAS). Here we evaluate whether an additive genetic risk of variants from gene-sets is associated with increased PD risk.

Material and Methods: A Luxembourgish cohort of 656 controls and 633 PD patients were genotyped on the Neurochip array. PRS for each individual were calculated with PRSice-2, using the GWAS data from Nalls et al., 2018. The PRS was tested for association with PD status with mitochondrial and other pathway-related gene-sets. The Parkinson's Progression Markers Initiative (PPMI) dataset included 363 PD cases and 165 controls genotyped with NeuroX chip.

Results: The results show a significantly higher common variant burden in PD cases compared to controls in mitochondrial gene-sets (odds ratio: 1.31 [1.18, 1.48] per one standard deviation; empirical-P $=8.9$ e-04). The PRS was also associated with PD status within the known PD genes, lysosomal and autophagy gene-sets. The same result was observed with the (PPMI) data.

Conclusions: Our study suggests that cumulative effect of common variants in mitochondrial genes significantly contribute to PD. Association tests targeting specific mitochondrial pathways and functional studies could determine the level of involvement of each mitochondrial process in the disease onset and progression.

Z. Landoulsi: None. D. Bobbili: None. S. Pachcheck: None. G. Arena: None. R. Krüger: None. P. May: None. 
P09.120.A

Genetic and clinical report of ten additional patients with PNKP-related ataxia (AOA4)

S. Sousa ${ }^{l, 2}$, J. Oliveira ${ }^{l, 2}$, J. Parente-Freixo ${ }^{l, 2}$, I. Alonso ${ }^{l, 2}$,

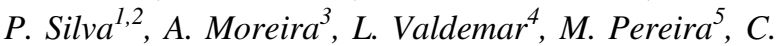

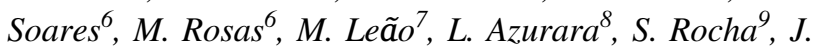
Ferreira $^{8}$, J. Damásio ${ }^{1,2,10}$, J. Sequeiros ${ }^{1,2}$

${ }^{l}$ CGPP-IBMC, Universidade do Porto, Porto, Portugal, ${ }^{2} i 3 S$ - Instituto de Investigação e Inovação em Saúde, Universidade do Porto, Porto, Portugal, ${ }^{3}$ Serviço de Neurologia, Hospital de Dona Estefânia, Centro Hospitalar de Lisboa Central, EPE, Lisboa, Portugal, ${ }^{4}$ Serviço de Neurologia, Hospital do Divino Espírito Santo de Ponta Delgada, EPE, Ponta Delgada, Portugal, ${ }^{5}$ Serviço de Neurologia, Centro Hospitalar e Universitário de Coimbra, EPE, Coimbra, Portugal, ${ }^{6}$ Consulta de Neurologia Geral, Centro Hospitalar Universitário de São João, EPE, Porto, Portugal, ${ }^{7}$ Serviço de Genética Médica, Centro Hospitalar Universitário de São João / Faculdade de Medicina da Universidade do Porto, Porto, Portugal, ${ }^{8}$ Serviço de Neurologia Pediátrica, Hospital de São Francisco Xavier, Centro Hospitalar de Lisboa Ocidental, EPE, Lisboa, Portugal, ${ }^{9}$ Serviço de Neurologia, Hospital de Braga, Braga, Portugal, ${ }^{10}$ Centro Hospitalar Universitário do Porto, Hospital de Santo António, Porto, Portugal

Ataxia with oculomotor apraxia (AOA) is the second most frequent recessive ataxia in Portugal. It is characterized by childhood-onset cerebellar ataxia, sensorimotor axonal neuropathy, oculomotor apraxia and dystonia; with a variable degree of cognitive impairment. Diallelic variants in the polynucleotide kinase 3'-phosphatase $(P N K P)$ gene were shown to be the cause of AOA4, by our group, in 2015. We now reviewed genetic and clinical data from a sequential series with pathogenic variants in $P N K P, 2015$ through 2019, to update mutational spectrum and reassess phenotypes; we report 10 additional AOA4 patients. We used Sanger sequencing of PNKP or a NGS multigene (whole-exome based) panel for recessive ataxias, according to clinical request; 10 Portuguese AOA patients ( 7 families), were found to be homozygotes ( 2 families) or compound heterozygotes (5 families), bearing 4 known PNKP pathogenic variants: c.1221_1223del (p.Thr408del) and c.1123G $>\mathrm{T}$ (p.Gly375Trp) (each present in 4 families), c.1315_1330delinsGGGGACG (p.Arg439_Pro444delinsGlyAspAla) and c. $1029+2 \mathrm{~T}>\mathrm{C}$ (r.spl) (each present in 1 family); and 2 novel variants: c.1510del (p.Arg504Glyfs*) and c.1282_1283insACAAACCCAGAC (p.Ala428delinsAspLysProArgPro), for which pathogenicity is being confirmed. Incidentally, through multigene panels, six other patients with ataxia (or other differential diagnoses) carried a single pathogenic or likely- pathogenic variant in $P N K P$. This study expands considerably the number of AOA4 patients genetically characterized by our group, from 11 reported in 2015 to a total of 21 . Further, confirms AOA4 as among the most frequent subtypes of recessive ataxia in Portugal. Considering the limited number of patients reported in the literature so far $(n=8)$, studies in other populations are much needed.

S. Sousa: None. J. Oliveira: None. J. Parente-Freixo: None. I. Alonso: None. P. Silva: None. A. Moreira: None. L. Valdemar: None. M. Pereira: None. C. Soares: None. M. Rosas: None. M. Leão: None. L. Azurara: None. S. Rocha: None. J. Ferreira: None. J. Damásio: None. J. Sequeiros: None.

\section{P09.121.B}

Genome-Wide Association Study of Postoperative Cognitive Dysfunction in older surgical patients

N. Göttel ${ }^{1,2}$, M. Rickenbacher ${ }^{l}$, C. S. Reinbold ${ }^{3}, S$.

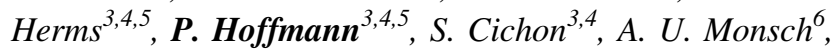
L. A. Steiner ${ }^{1,2}$

${ }^{I}$ Department of Anesthesia, Prehospital Emergency Medicine and Pain Therapy, University Hospital Basel, University of Basel, Basel, Switzerland, ${ }^{2}$ Department of Clinical Research, University of Basel, Basel, Switzerland, ${ }^{3}$ Human Genomics Research Group, Department of Biomedicine, University of Basel, Basel, Switzerland, ${ }^{4}$ Institute of Medical Genetics and Pathology, University Hospital Basel, Basel, Switzerland, ${ }^{5}$ Institute of Human Genetics, University of Bonn, School of Medicine \& University Hospital Bonn, Bonn, Germany, ${ }^{6}$ Memory Clinic, University Center for Medicine of Aging Basel, Felix Platter Hospital, Basel, Switzerland

Introduction: Postoperative cognitive dysfunction (POCD) is a common neurocognitive complication after surgery, particularly in elderly patients. Various studies suggest genetic risk factors for POCD. This prospective cohort study aimed to detect genome-wide associations with POCD in older patients.

Methods: Study participants aged $\geq 65$ years completed a battery of neuropsychological tests consisting of the Consortium to Establish a Registry for Alzheimer's Disease-Neuropsychological Assessment Battery (CERAD-NAB), Trail Making Tests $\mathrm{A}$ and $\mathrm{B}$, and the Phonemic Fluency Test (S-words) before, and at 1 week and 3 months after major noncardiac surgery. Test variables were converted into standard scores (z-scores) based on demographic variables. POCD was diagnosed if the decline was $>1$ standard deviation in 2 or more of the 15 variables in the test battery. A genome-wide association study 
(GWAS) was performed to determine potential alleles that are linked to the phenotype POCD.

Results: Sixty-three patients with blood samples were included in the study. POCD was diagnosed in $47.6 \%$ of patients at 1 week and in $34.2 \%$ of patients at 3 months after surgery. Insufficient sample quality led to exclusion of 26 patients. In the remaining 37 patients, a GWAS was performed, but no association with POCD was found. A subsequent gene set analysis on 36 genes using VEGAS2 did not reveal any significant associations.

Conclusions: In this patient cohort, a GWAS did not reveal an association between specific genetic alleles and POCD at 1 week and 3 months after surgery. Future genetic analysis should focus on specific candidate genes for POCD.

N. Göttel: None. M. Rickenbacher: None. C.S. Reinbold: None. S. Herms: None. P. Hoffmann: None. S. Cichon: None. A.U. Monsch: None. L.A. Steiner: None.

P09.122.C

Functional characterization of a novel variant in $P O L G 2$ shows disrupted mitochondrial integrity

M. Dulovic Mahlow ${ }^{l}$, M. Borsche $e^{1,2}$, H. Baumann ${ }^{l}, S$. Tunc $^{2}$, E. Knappe ${ }^{l}$, S. Özcakir ${ }^{1}, J$. Trinh $^{l}, N$. Brüggemann $^{1,2}$, K. Lohmann ${ }^{1}$

${ }^{1}$ Institute of Neurogenetics, Luebeck, Germany, ${ }^{2}$ Department of Neurology, UKSH, Luebeck, Germany

Introduction: Pathogenic variants in the DNA polymerasegamma2 (POLG2) cause a rare mitochondrial disease with variable phenotypic presentations.

Material and methods: We performed exome sequencing in a family with ataxia. To interpret pathogenicity of a POLG2 variant, we used deep mitochondrial DNA (mtDNA) sequencing, tested for mitochondrial deletions; and established primary fibroblast lines from two mutation carriers to investigate mitochondrial integrity by immunocytochemistry, immunoblotting, and flow cytometry.

Results: The index patient, a 55-year-old woman, presented with signs of vertigo at 30 years, followed by the development of cerebellar ataxia and progressive ophthalmoplegia. Her brother and mother had onset of ataxia at ages of 40 and 50 years. By exome sequencing, we identified a segregating, heterozygous, novel missense variant (c.1270T $>$ C [p.Ser424Pro], NM_007215.3) in POLG 2 in all three affected family members. Initially, this was a variant of uncertain significance (VUS). We found a higher number of mtDNA sequence changes in blood cells from the mutation carriers compared to matched healthy controls. However, large mtDNA deletions were not detected in the blood samples. In POLG2-mutant fibroblasts, we demonstrated an impaired mitochondrial network, a decreased mitochondrial membrane potential, and decreased TOMM20 protein levels in comparison to healthy controls. A muscular biopsy was not available.

Conclusions: We provide multiple lines of evidence that this POLG2 variant is associated with impaired mitochondrial integrity suggesting its pathogenicity in respect to the mitochondrial phenotype (ataxia and progressive external ophthalmoplegia) in this family. Our work highlights the importance of functional characterization of VUS to enable meaningful genetic counselling.

M. Dulovic Mahlow: None. M. Borsche: None. H. Baumann: None. S. Tunc: None. E. Knappe: None. S. Özcakir: None. J. Trinh: None. N. Brüggemann: None. K. Lohmann: None.

P09.123.A

Extending the phenotypic spectrum of NEDHIB (neurodevelopmental disorder with hypotonia and variable intellectual and behavioral impairment) in a girl with multiple anomalies and a de novo frameshift mutation in POLR2A.

\section{J. Behunova, F. Laccone}

Institute of Medical Genetics, Medical University of Vienna, Vienna, Austria

The gene $P O L R 2 A$ is coding polymerase II, RNA, subunit $A$ and its heterozygous mutations have been described to cause global developmental delay with hypotonia, abnormal behaviour, feeding and visual problems, strabismus, variable craniofacial dysmorphic features, frequent infections, and, eventually, structural brain anomalies. To date only 16 patients with $P O L R 2 A$ mutations have been described in a single publication, three of them carrying a truncating mutation. Many features in the published patients are overlapping with those of our patient. However, none of the patients reported presented a polydactyly (in our girl preaxial with a double thumb on the left), a thumb hypoplasia (in our girl just a ,soft tissue thumb“, which had been removed, on the right), anal anteposition and stenosis, and a heart defect (ASDII). There was significant neonatal hypotonia. Unsupported walking was possible only at the age of 4 years. Developmental delay now at the age of 14 years is severe. The girl does not speak at all. After the first decade the tendon-reflexes of the legs decreased and bilateral pes cavus developed, possibly manifesting a peripheral neuropathy. There are craniofacial dysmorphic features, but no microcephaly. Whole exome sequencing (incl. CNV) identified a 2 bp deletion in POLR2A which is supposed to cause a frameshift and a premature stop of protein synthesis. 
No other DNA variants were identified that could explain the symptoms unusual for NEDHIB. The healthy unrelated parents do not carry the mutation, two sisters are healthy.

J. Behunova: None. F. Laccone: None.

\section{P09.124.B}

Polygenic-wide analysis to predict genetic risk profiles on brain morphometry over phenome-wide diseases

N. Vilor-Tejedor ${ }^{1,2,3,4}$, R. Guigo ${ }^{1,4}$, G. Operto ${ }^{2,5,6}, M$.

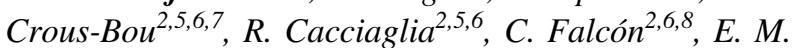

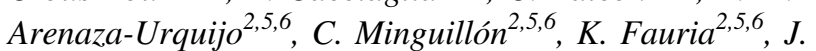
Molinuevo $^{2,4,5,6}$, J. Gispert ${ }^{2,4,6,8}$

${ }^{1}$ Centre for Genomic Regulation (CRG), The Barcelona Institute for Science and Technology., Barcelona, Spain, ${ }^{2}$ Barcelonaßeta Brain Research Center (BBRC), Pasqual Maragall Foundation., Barcelona, Spain, ${ }^{3}$ Department of Clinical Genetics, ERASMUS Medical Center., Rotterdam, Netherlands, ${ }^{4}$ Universitat Pompeu Fabra, Barcelona, Spain, ${ }^{5}$ Centro de Investigación Biomédica en Red de Fragilidad y Envejecimiento Saludable (CIBERFES), Madrid, Spain, ${ }^{6}$ IMIM (Hospital del Mar Medical Research Institute), Barcelona, Spain, ${ }^{7}$ Department of Epidemiology, Harvard TH Chan School of Public Health, Boston, MA, United States, ${ }^{8}$ Centro de Investigación Biomédica en Red Bioingeniería, Biomateriales y Nanomedicina, Madrid, Spain

Neurodegenerative diseases are associated with progressive neuronal damage along with an accelerated brain atrophy, which is influenced by multiple genetic factors. Polygenic risk scores (PRS) are used for identifying the genetic architecture of complex diseases in order to predict disease genetic risk, as well as testing their shared genetic architecture. In this study, we investigated the association between PRS related to neurodegenerative and complex diseases and brain morphometry. A total of 479 cognitively unimpaired middle-age participants at increased risk of $\mathrm{AD}$ (ALFA cohort) with available genotyping and neuroimaging data were included. PRS were calculated from the most recent genome-wide association meta-analyses for Alzheimer's disease (AD), Parkinson disease, stroke, hypertension, hypercholesterolemia, diabetes, depression, cerebrovascular disease, and CSF core AD biomarkers. Association analyses included brain regional volumes as outcomes. Associations were estimated using linear regression models adjusted for age, sex, education, APOE$€ 4$ status, and total intracranial volume. We found increased PRS average of AD, p-tau and A $\beta 42$ levels among ALFA participants. Furthermore, results suggested evidence of an association between increased risk of cholesterolemia and reduced volumes of left accumbens area, increased risk of cardiovascular disease and reduced volumes of thalamus proper, as well as increased risk of having higher p-tau levels and reduced volumes of right thalamus proper and right cerebellum cortex. We provide strong evidence that genetic liability of cardiovascular diseases and core $\mathrm{AD}$ biomarkers influences brain morphology in ALFA participants. In addition, across all diseases, ALFA participants showed increased genetic risk of $\mathrm{AD}$ and altered CSF biomarker.

N. Vilor-Tejedor: None. R. Guigo: None. G. Operto: None. M. Crous-Bou: None. R. Cacciaglia: None. C. Falcón: None. E.M. Arenaza-Urquijo: None. C. Minguillón: None. K. Fauria: None. J. Molinuevo: F. Consultant/Advisory Board; Modest; Roche Diagnostics, Genentech, Novartis, Lundbeck, Oryzon, Biogen, Lilly, Janssen, Green Valley, MSD, Eisai, Alector, BioCross, GE Healthcare, ProMIS Neurosciences. J. Gispert: None.

\section{P09.125.C}

Biallelic loss of function mutations in JAM2 cause primary familial brain calcification

Z. Cen, Y. Chen, S. Chen, D. Yang, L. Wang, H. Wang, X. Chen, P. Liu, B. Zhang, W. Luo

The Second Affiliated Hospital, Zhejiang University School of Medicine, Hangzhou, China

Introduction: Primary familial brain calcificationis a monogenic disease characterized by bilateral calcification in the basal ganglia and other brain regions, and commonly presents motor, psychiatric, and cognitive symptoms. Currently, four autosomal dominant (SLC20A2, PDGFRB, $P D G F B, X P R 1)$ and one autosomal recessive (MYORG) causative genes have been identified.

Materials and Methods: Homozygosity mapping and whole genome sequencingwere performed in a consanguineous family with two affected siblings diagnosed with primary familial brain calcificationto detect novel causative gene. Further genetic screening was performed in a cohort of 398 probands with primary familial brain calcification.

Results: A homozygous frameshiftmutation (c.140delT, p.L48*) in the JAM2gene in theconsanguineous family. Further more, a homozygous start codon mutation (c.1A $>\mathrm{G}$, p.M1?) and compound heterozygous mutations [c.504G $>$ C, p.W168C and c.(67+1_68-1)_(394+1_395-1), p. Y23_V131delinsL], respectively, in two unrelated familieswere detected. In Chinese hamster ovary cells, truncated His-tagged JAM2 proteins were detected by western blot following transfection of p.Y23_V131delinsLmutant plasmid, while no protein was detected following transfection of 
p.L48* or p.1M? mutant plasmids. In immunofluorescence experiments, the p.W168C mutant JAM2 protein failed to translocate to the plasma membrane.

Conclusions: Our findings offer a novel causative gene for primary familial brain calcification, whose vital function and high expression in the neurovascular unitfurther supports impairment of the neurovascular unit as the root of primary familial brain calcificationpathogenesis.

Z. Cen: None. Y. Chen: None. S. Chen: None. D. Yang: None. L. Wang: None. H. Wang: None. X. Chen: None. P. Liu: None. B. Zhang: None. W. Luo: None.

P09.126.A

Novel progressive myoclonus epilepsy syndrome caused by a recurrent homozygous variant of $S L C 7 A 6 O S$

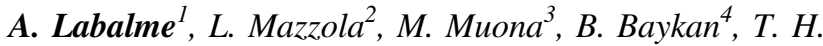

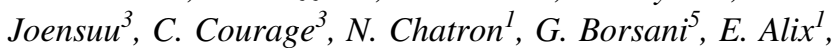
F. Ramond ${ }^{6}$, R. Touraine ${ }^{6}$, S. F. Berkovic ${ }^{7}, N$. Bebek ${ }^{4}, A$. Lehesjoki ${ }^{3}$, G. Lescal

${ }^{1}$ University Hospital of Lyon, Lyon, France, ${ }^{2}$ University Hospital of Saint Etienne, Saint Etienne, France, ${ }^{3}$ Folkhälsan Research Center, Helsinki, Finland, ${ }^{4}$ Istanbul Faculty of Medicine, Istanbul, Turkey, ${ }^{5}$ University of Brescia, Brescia, Italy, ${ }^{6}$ University Hospital of SaintEtienne, Saint-Etienne, France, ${ }^{7}$ Epilepsy Research Centre, University of Melbourne, Victoria, Australia

Purpose: Progressive myoclonus epilepsies are a genetically heterogeneous group of rare Mendelian disorders defined by the combination of action and reflex myoclonus, other types of epileptic seizures, and progressive neurocognitive impairment. In addition to the classical diseases, namely Unverricht-Lundborg and Lafora, several other genetic forms have been identified during the past years. Despite recent advances, a substantial proportion of cases remain without a molecular diagnosis and additional genes still remain to be identified.

Methods: Individuals from two families from different ethnic backgrounds with several individuals affected with a similar presentation of progressive myoclonus epilepsy resembling Unverricht-Lundborg disease were analyzed by exome sequencing.

Results: Affected individuals from both families were homozygous for the same p.(Gln64Arg) variant affecting a splice site in $S L C 7 A 6 O S$. It occurs 7 times in gnomAD as a heterozygote, and is absent as a homozygote. Haplotype analysis suggests the variant arose independently in the two families. Analysis of cDNA from lymphoblastoid cells of a patient showed a partial abolition of the splice site and the creation of an abnormal isoform with the retention of intron 1 (NM_032178.2:r.191_193ins[ag;192+1_193-1]). RT-
qPCR and western blot showed $\mathrm{a} \approx 80 \%$ reduction of cDNA and $60 \%$ of protein. Gene knockdown experiments in zebrafish indicate that slc7a6os plays a critical role in defined areas of the developing CNS.

Conclusions: Our results suggest that $S L C 7 A 6 O S$ may be a novel gene causing progressive myoclonus epilepsy phenotype resembling Unverricht-Lundborg disease.

A. Labalme: None. L. Mazzola: None. M. Muona: None. B. Baykan: None. T.H. Joensuu: None. C. Courage: None. N. Chatron: None. G. Borsani: None. E. Alix: None. F. Ramond: None. R. Touraine: None. S. F. Berkovic: None. N. Bebek: None. A. Lehesjoki: None. G. Lesca: None.

\section{P09.128.C}

The genotype-phenotype correlation of intragenic RBFOX1 deletions.

\section{A. Paczkowska, M. Geremek, B. Nowakowska}

\section{Institute of Mother and Child, Warsaw, Poland}

The $R B F O X 1$ gene encodes a protein that regulates premRNA splicing in developmental genes expressed in muscle, heart and neuronal cells. Intragenic deletions of RBFOX1 gene have been identified as susceptibility loci and reported in patients with aggressive behavior, conduct disorder, epilepsy and autism spectrum disorder. Despite of the documented correlation with neurodevelopmental disorders, the deletions of $R B F O X 1$ gene are often inherited from the healthy parents, therefore the clinical interpretation of CNVs within this gene is still unclear. We applied oligonucleotide microarrays (OGT) in a cohort of 9430 patients with neurodevelopment disorders and their healthy relatives. The analyses of genomes were performed using genome-wide array to accurate detection of copy number variation at the exon level in $R B F O X 1$ gene. Chromosomal analysis revealed intragenic $R B F O X 1$ deletions in 36 patients. The differential distribution across the gene between patients with neurodevelopmental disorders and the control group allowed to distinguished evidently pathogenic areas within the gene. Deletions of exones 1-2 and 13-14 have high penetrance, and are mainly associated with conduct disorder and autism spectrum disorder. While the middle part of the gene and all intronic CNVS were observed with higher frequency in the control group, suggesting their benign impact on the phenotype.

The work was supported by MINDDS - CA COST Action CA16210 - Maximising Impact of research in NeuroDevelopmental DisorderS 
M.A. Paczkowska: None. M. Geremek: None. B. Nowakowska: None.

\section{P09.130.B}

Deep targeted-NGS identifies RLS genes by differential burden with low-frequency variants and differential target sequencing depth

E. Tilch ${ }^{1}$, B. Schormair ${ }^{1}$, C. Zhao ${ }^{1}$, A. Salminen ${ }^{1}$, A. Antic Nikolic $^{l}$, E. Holzknecht ${ }^{2}$, B. Högl ${ }^{2}$, W. Poewe ${ }^{2}$, C.

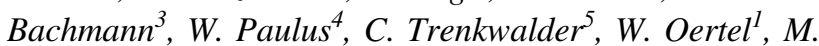
Hornyak $^{6}$, I. Fietze ${ }^{7}$, K. Berger ${ }^{8}$, P. Lichtner ${ }^{9}$, C. Gieger ${ }^{10}$, A. Peters ${ }^{10}$, B. Müller-Myhsok ${ }^{11,12}$, A. Hoischen ${ }^{13}$, J. Winkelmann $^{1,11,14}$, K. Oexle ${ }^{1}$

${ }^{1}$ Institute of Neurogenomics, Helmholtz Zentrum München GmbH, German Research Center for Environmental Health, Neuherberg, Germany, ${ }^{2}$ Department of Neurology, Medical University of Innsbruck, Innsbruck, Austria, ${ }^{3}$ Department of Neurology, Paracelsus Clinic Osnabrück, Osnabrück, Germany, ${ }^{4}$ Department of Clinical Neurophysiology, Georg August University, Göttingen, Germany, ${ }^{5}$ Center of Parkinsonism and Movement Disorders, Paracelsus-Elena Hospital, Kassel, Germany, ${ }^{6}$ Neuropsychiatry Center Erding/Munich, Erding, Germany, ${ }^{7}$ Department of Cardiology and Angiology, Center of Sleep Medicine, Charité-Universitätsmedizin Berlin, Berlin, Germany, ${ }^{8}$ Institute of Epidemiology and Social Medicine, University of Münster, Münster, Germany, ${ }^{9}$ Institute of Human Genetics, Helmholtz Zentrum München GmbH, German Research Center for Environmental Health, Neuherberg, Germany, ${ }^{10}$ Institute of Epidemiology, Helmholtz Zentrum München GmbH, German Research Center for Environmental Health, Neuherberg, Germany, ${ }^{11}$ MunichCluster for Systems Neurology, Munich, Germany, ${ }^{12}$ Max Planck Institute of Psychiatry, Munich, Germany, ${ }^{13}$ Department of Human Genetics, Radboud University Medical Center, Nijmegen, Netherlands, ${ }^{14}$ Department of Neurogenetics and Institute of Human Genetics, Technical University of Munich, Munich, Germany

Introduction: Restless legs syndrome (RLS) is one of the most common and burdensome neurological disorders. Patients typically present with an urge to move the legs at rest at night which severely impairs sleep and quality of life. Based on previous GWAS results, we performed mutational load analysis of putatively causal candidate genes.

Materials and Methods: We sequenced exons and promotors of 84 genes in 4,649 RLS patients and 4,982 population-based controls from Germany in a targeted NGS approach with 11,214 molecular inversion probes (MIPs). We compared the variant load (MAF $<=0.05$ ) by gene between cases and controls using standard burden testing. As variants in a MIP's binding regions affect its sequencing yield, we also analyzed target sequencing depths as an integrative and independent assessment of the differential variant burden.

Results: Differential variant burden results of both types of analysis overlapped significantly. Therefore, 14 genes were significantly associated and validated. Both types of analysis also converged significantly in fine-mapping potentially causative domains within these genes.

Conclusions: By assessing differential burden with intragenic low-frequency variants we identified at least 14 putatively causative genes in RLS. In keeping with other polygenic diseases, the variant burden was higher in patients. Methodologically, we demonstrate that target depth analysis is useful for independent validation of conventional variant-based burden tests.

Acknowledgements: DFG (project 310572679), RLS e. V., and SRLS.

E. Tilch: None. B. Schormair: None. C. Zhao: None. A. Salminen: None. A. Antic Nikolic: None. E. Holzknecht: None. B. Högl: None. W. Poewe: None. C. Bachmann: None. W. Paulus: None. C. Trenkwalder: None. W. Oertel: None. M. Hornyak: None. I. Fietze: None. K. Berger: None. P. Lichtner: None. C. Gieger: None. A. Peters: None. B. Müller-Myhsok: None. A. Hoischen: None. J. Winkelmann: None. K. Oexle: None.

\section{P09.131.C}

Extension of the phenotypes caused by $R N F 13$ variants to include a choreo-dystonic movement disorder

\section{E. Ranza ${ }^{l}$, X. Blanc ${ }^{l}, F$.Santoni ${ }^{l}, F$. Guerry $^{l}, S$.} Antonarakis $^{1}$, C. Korff

${ }^{I}$ Medigenome, Swiss Institute of Genomic Medicine, Geneva, Switzerland, ${ }^{2}$ Pediatric Neurology, Geneva University Hospitals, Geneva, Switzerland

The substantial recent advances in genetic diagnosis, have identified numerous pathogenic variants on genes involved in neurodevelopment in patients with developmental delay and epilepsy. Among those, heterozygous missense gain-offunction variants in $R N F 13$ leading to altered signaling of the endoplasmic reticulum stress response, have been recently reported in three children with congenital microcephaly, encephalopathy with epilepsy, blindness, and failure to thrive (PMID 30595371). None of these patients presented with movement disorders. We here report a child with early-onset global developmental delay, acquired microcephaly, failure-to-thrive, infantile spasms, focal seizures and gastrointestinal dysfunction, who also presented at 3 years with a choreo-dystonic movement disorder. An 
extended metabolic work-up was normal. Brain MRI did not reveal significant abnormalities. A trio whole-exome sequencing revealed a heterozygous de novo c.932delT:(p. Leu311TyrfsTer2) variant in the RNF13 gene. The variant results in a frameshift near the carboxyl terminus of the predicted protein sequence (codon 311 of 380), producing an abnormal protein with a postulated gain of function effect. Interestingly the previously described pathogenic variants were in the same location of the primary protein sequence p.(Leu311Ser) and p.(Leu312Pro).To our knowledge, our patient is the fourth described so far. In addition to previously reported symptoms, the phenotype of this child also included a choreo-dystonic movement disorder; thus the phenotypes related to $R N F 13$ pathogenic variants are extended to include a movement disorder. The RNF13 should be added to the panel of candidate genes in patients with abnormal movements, in addition to those involved in encephalopathy and early-onset epilepsy.

E. Ranza: None. X. Blanc: None. F. Santoni: None. F. Guerry: None. S. Antonarakis: None. C. Korff: None.

\section{P09.136.B}

DNMT1 overexpression results in dysregulation of multiple genes associated with neurological disorders and abnormal neurogenesis.

\section{S. Saxena ${ }^{I}$, S. Choudhury ${ }^{2}$, A. Sampath ${ }^{I}$, K. Naga Mohan ${ }^{1}$}

${ }^{1}$ BITS PILANI HYDERABAD CAMPUS, hyderabad, India, ${ }^{2}$ BITS PILANI HYDERABAD CAMPUS, Hyderabad, India

In the context of DNMT1 overexpression as one of the etiological factors for schizophrenia (SZ), and abnormal neurogenesis associated with Dnmt1 ${ }^{\text {Tet/Tet }}($ Tet/Tet) mouse embryonic stem cells, we studied the effects of DNMT1 overexpression on neurogenesis. Transcriptome analysis of Tet/Tet neurons showed dysregulation of a significant number of genes associated with neurological disorders. Downregulation of Shh was the main event associated with dysregulation of genes involved in multiple pathways such as Wntl and Wnt3a signaling, extracellular matrix synthesis, homeobox family and other transcription factors required for brain development. Transcriptome analysis of Tet/Tet ES cells to understand earlier events also showed dysregulation of a significant number of genes associated with neurodevelopment and neurological disorders. Specifically, Bmp4, an antagonistic molecule to Shh, emerged with the highest number of interacting partners. In ES cells, high Bmp4 transcript levels were associated with low transcript levels of Lrp2, Htral and Bmper (negative regulators of Bmp4) and Dpysl2 (negatively regulated by BMP4). Consequently, as in case of some SZ-patient derived iPS cells, Tet/Tet ES cells possessed a decreased potential to produce neurons and oligodendrocytes but an increased potential in forming glial cells. RRBS data on ESCs and neurons did not reveal any significant difference in the level of DNA methylation of the promoters of the affected genes. DNMT1 affects on these genes therefore, appear to be independent of catalytic activity. Taken together, increased DNMT1 levels results in abnormal neurogenesis by affecting multiple SZ-associated genes and pathways in a DNA-methylation independent manner.

S. Saxena: None. S. Choudhury: None. A. Sampath: None. K. Naga Mohan: None.

P09.140.C

Two intronic variants in cis in both alleles of the POLR3A gene cause progressive spastic ataxia with hypodontia

A. Felner ${ }^{1,2}$, A. Lossos ${ }^{3}$, E. Kogan ${ }^{2}$, Z. Argov ${ }^{3}$, C. GonzagaJauregui ${ }^{4}$, A. R. Shuldiner ${ }^{4}$, N. Lev-El Halabi ${ }^{l}$, I.

Lagovcky ${ }^{l}$, S. Naftaly Nathan ${ }^{l}$, L. Bazak ${ }^{l}$, G. Lidzbarsky ${ }^{l}$, L. Basel-Salmon ${ }^{1,5,6}$, Y. GOLDBERG ${ }^{l}$

${ }^{1}$ Raphael Recanati Genetics Institute, Rabin Medical Center, Petah Tikva, Israel, ${ }^{2}$ 2. Department of Neurology, Rabin Medical Center, Beilinson Hospital, Petah Tikva, Israel, ${ }^{3}$ Department of Neurology, Hadassah-Hebrew University Medical Center, Jerusalem, Israel, ${ }^{4}$ Regeneron Genetics Center, Tarrytown, NY, United States, ${ }^{5}$ Sackler Faculty of Medicine, Tel-Aviv University, Tel Aviv, Israel, ${ }^{6}$ Felsenstein Medical Research Center, Petah Tikva, Israel

Background: POLR3A encodes the largest subunit of the DNA-dependent RNA polymerase-III. Mutations in this gene are associated with dysregulation of tRNA production and other non-coding RNAs. Biallelic POLR3A pathogenic variants are associated with several phenotypes, including Hypomyelinating leukodystrophy, hypogonadism, hypodontia, progressive spastic ataxia, and the progeroid disorder Wiedemann-Rautenstrauch syndrome (WRS). Genotypephenotype correlation is unclear, though some variants have been associated mainly with spastic ataxia and others with WRS.

Methods: Phenotypic analysis and exome sequencing were performed in 4 affected siblings, two healthy siblings and their mother.

Results: All affected siblings (two men, two women; ages 45-58) had early childhood-onset hypodontia and adolescent-onset progressive spastic ataxia. None had 
dysmorphism or hypogonadism. They all had biallelic POLR3A pathogenic variants. The mother was heterozygotous and both healthy siblings had wild-type alleles. Each mutated allele had two intronic splicing-altering variants in cis, leading to skipping of exons 14 and 26. This allele was reported previously in compound heterozygous combinations in patients with severe progeroid WRS.

Conclusions: This is the first report addressing the clinical consequence associated with a unique homozygous pathogenic allele in the POLR3A gene. Our findings indicate that homozygosity for this pathogenic allele causes spastic ataxia with hypodontia, rather than a progeroid syndrome, dysmorphism or hypogonadism previously attributed to this allele when found in a compound heterozygous state with other alleles. Our findings contribute to the characterization of genotype-phenotype correlation in POLR3A-related disorders.

A. Felner: None. A. Lossos: None. E. Kogan: None. Z. Argov: None. C. Gonzaga-Jauregui: A. Employment (full or part-time); Modest; Regeneron. A.R. Shuldiner: A. Employment (full or part-time); Modest; Regeneron. N. Lev-El Halabi: None. I. Lagovcky: None. S. Naftaly Nathan: None. L. Bazak: None. G. Lidzbarsky: None. L. Basel-Salmon: None. Y. Goldberg: None.

\section{P09.141.A}

Increased gene expression ofGINS2in patients with Specific Language Impairment

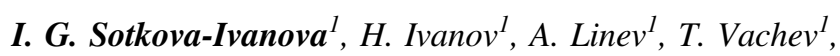
N. Popov ${ }^{2}$, I. Ivanov ${ }^{l}$, I. Pacheval, R. Yordanova ${ }^{l}$, V. Stoyanova ${ }^{l}$

${ }^{1}$ Medical Univercity Plovdiv, Department of Pediatrics and Medical Genetics, Medical University Plovdiv, Plovdiv, Bulgaria, ${ }^{2}$ Psychiatric ward for active treatment, State Phsychiatry Hospital Pazardzhik, Plovdiv, Bulgaria

Introduction: Specific Language Impairment (SLI) is a disorder characterized by slow, abnormal language development, despite normal intelligence, adequate social environment and in the absence of any explanatory medical conditions. Most children with this disorder do not present any other cognitive or neurological deficits and have normal non-verbal IQ. This condition is highly heritable and affects between $5 \%$ and $8 \%$ of pre-school children. Lately investigations have begun to uncover genetic factors that may contribute to susceptibility to language impairment. In our previous study we conducted a pool expression analysis and GINS2 was the most up regulated gene.

Materials and Methods: The study aimed to examine the individual expression profile of GINS2 gene by ddPCR in the peripheral blood of 48 individuals divided into two groups: children with SLI and healthy controls.

Results: Mean values of 15,4 copies/20mcl was found in the SLI group and 8,8 - in the control one. The difference was statistically significant $-\mathrm{P}=0.03$, proving that the GINS2 gene is up regulated in the SLI group. GINS2, located in 16q21 is part of the GINS complex which plays an essential role in the initiation of DNA replication, and progression of DNA replication forks. There are a couple of case reports that report speech delay in patients with $16 \mathrm{q} 21$ duplications. A recent study found a connection with GINS2 expression and neuroinflamation.

Conclusions: Comparative gene expression analysis is useful in discovering novel genetic markers associated with SLI. Elevated gene expression of GINS2 might contribute to the elucidation of the pathogenesis of SLI.

I.G. Sotkova-Ivanova: None. H. Ivanov: None. A. Linev: None. T. Vachev: None. N. Popov: None. I. Ivanov: None. I. Pacheva: None. R. Yordanova: None. V. Stoyanova: None.

P09.142.B

A $357 \mathrm{~kb}$ duplication encompassing the ATP2B3 gene in $\mathrm{Xq} 28$ is associated with $\mathrm{X}$-linked spinocerebellar ataxia (SCAX1) in two males within one family

Y. Stratis, F. Stock, C. Müller-Hofstede, S. Ledig, J. Horvath, P. Wieacker, A. Bohring, A. Röpke

\section{Institute of Human Genetics, Münster, Germany}

$\mathrm{X}$-linked transmission of spinocerebellar ataxia (SCAX) is relatively rare. One missense mutation in $A T P 2 B 3$ resulting in spinocerebellar ataxia 1 (SCAX1) is reported twice in the literature as the cause of SCAX1 in two families. The $A T P 2 B 3$ gene encodes for a member of the calmodulin-activated plasma membrane $\mathrm{Ca}^{2+}$ ATPase family (PMCA3) which extrudes excess calcium from the cell to maintain neuronal $\mathrm{Ca}^{2+}$-homeostasis, especially in the cerebellum. The 46 year old index patient was referred to our institute because of early onset cerebellar ataxia. $\mathrm{He}$ showed a delayed motor development as an infant, impaired gross and fine motor skills, dysarthria, and hypoplasia of the cerebellum. Intellectual development was normal. Kennedy's disease was excluded and a NGS panel for ataxia related genes showed no pathogenic variants. Array-CGH revealed a duplication of $357 \mathrm{~kb}$ in $\mathrm{Xq} 28$. This duplication spans eight genes, including ATP2B3 and two more OMIM genes (BGN, FAM38A). Further analysis of female family members confirmed this duplication in the patient's mother, her sister and the sister's daughter. Her three year old son also displayed 
ataxia and a delayed motor development. He was proved to carry the duplication as well. Based on the family constellation and the known literature for the $A T P 2 B 3$ gene we highly assume that the duplication in Xq28 comprising $A T P 2 B 3$ is causative for the spinocerebellar ataxia within this family, implicating a dysregulation of the calcium homeostasis in neuronal cells by the duplication of the $A T P 2 B 3$ gene.

Y. Stratis: None. F. Stock: None. C. Müller-Hofstede: None. S. Ledig: None. J. Horvath: None. P. Wieacker: None. A. Bohring: None. A. Röpke: None.

P09.144.A

Genetic risk factors for tendency to suicide detected in a cohort GWAS study

\section{A. Haghighatfard ${ }^{l}$, F. Mohammadpour ${ }^{2}$}

${ }^{1}$ Islamic Azad university-Tehran medical sciences branch, Tehran, Iran, Islamic Republic of, ${ }^{2}$ University of Guilan, Rasht, Iran, Rasht, Iran, Islamic Republic of

Suicide is a major public health problem, with almost one million people victims' world-wide each year. Psychological risk factors for suicidal behaviors are including, psychiatric and medical illness, impulsivity, aggression, alcohol and drug abuse specially stimulants, and low stress resilience. Genetic risk factors of suicide are not completely clarified. Our study aimed to detect genetic risk factors that increasing the vulnerability to committing suicide. DNA was extracted from blood samples of 8240 saved suicide victims with at least one attempt to suicide in last 12 months that were suffering from psychosis and 5000 healthy subjects. Genotyping for the subjects was performed using the Affymetrix Genome-Wide Human SNP Array 6.0 (Affymetrix, Santa Clara, CA) according to the manufacturer's protocol. Several SNPs were detected with genome-wide significance. The significant association found in 77 SNPs involved in dopaminergic pathway and neurodevelopment of CNS. 56 of significantly related SNPs were found as risk factors of psychiatric disorders specially schizophrenia; other 21 SNPs were related to the mitochondrial complexes, transcription factors and growth factor genes. The study showed strong genetic bases of suicidal thoughts and could be used for providing genetic prognostic markers for prediction of suicide risk especially in subjects with psychosis. Also importance of mitochondrial complexes and growth factors in neuorobilogical functions and decision making processes of brain were showed.

A. Haghighatfard: None. F. Mohammadpour: None.
P09.145.B

Expanding phenotypic spectrum of TAF1 variants: Report of two novel families

J. Pozojevic ${ }^{1}$, M. G. Pauly ${ }^{l}$, N. Schell ${ }^{1}$, H. Pawlack ${ }^{l}$, C. Klein $^{l}$, A. Westenberger ${ }^{l}$, N. Brüggemann ${ }^{1,2}$

${ }^{1}$ Institute of Neurogenetics, Lübeck, Germany, ${ }^{2}$ Department of Neurology, Lübeck, Germany

Introduction: Pathogenic variants affecting the TAF1 (TATA-box binding protein associated factor 1) coding region have been shown to cause a complex X-linked neurodevelopmental syndrome that includes intellectual disability, dysmorphic features, and neurological manifestations. The changes described to date are predominantly missense and distributed along the entire gene. A noncoding TAFl variant causes X-linked dystonia-parkinsonism (XDP), an adult-onset neurodegenerative movement disorder.

Patients and Methods: By whole-exome sequencing, the genetic cause of $\mathrm{X}$-linked intellectual disability was investigated in male patients from two families. In other available family members, the presence of relevant $T A F 1$ variants was investigated by targeted Sanger sequencing. Where appropriate, a standard X-chromosome inactivation assay was used.

Results: The proband from Family 1 clinically manifested with global developmental delay including motor, speech and cognitive abilities and carried the c.1606G $>\mathrm{A}$ (p.(Asp536Asn)) TAF1 variant and the MT-COI m.6054G $>$ A (p.(Asp51Asn)) mitochondrial variant in the heteroplasmic state. In Family 2, the affected male patients presented with early onset spastic paraplegia and mild cognitive impairment, all carrying a missense (c.5418G $>$ C; p.(Glu1806Asp)) variant in TAF1. Their mother was a heterozygous carrier of the same change and developed adult-onset parkinsonism, spasticity, and dementia. Her Xchromosome inactivation (XCI) was random.

Conclusions: Two novel $T A F 1$ variants were identified as the likely disease causes in our patients. The affected members of Family 2 presented with pyramidal signs, suggesting that the TAF1 variants other than those related to XDP may cause an affection of additional neurological systems.

J. Pozojevic: None. M.G. Pauly: None. N. Schell: None. H. Pawlack: None. C. Klein: None. A. Westenberger: None. N. Brüggemann: None.

P09.146.C

Brain lipid profile of early onset Tay-Sachs disease mouse model 
T. Sengul ${ }^{1}$, M. Can ${ }^{l}$, S. Akyildiz Demir ${ }^{l}$, C. Klose ${ }^{2}$, M. Surma $^{2}$, V. Seyrantepe ${ }^{l}$

${ }^{1}$ Izmir Institute of Technology, Izmir, Turkey, ${ }^{2}$ Lipotype GmbH, Dresden, Germany

Introduction: Tay-Sachs disease is one of the lysosomal disorders caused by mutation in Hexa gene. HEXA gene deficiency affects the central nervous system owing to GM2 accumulation in lysosomes resulting in progressive neurodegeneration in patient. Previoulsy generated Hexa-/Neu3-/- mice mimic neuropathological and clinical abnormalities of early onset Tay-Sachs patients. Lipids are important players in the central nervous system functionality. Several studies showed that neurodegenerative diseases indicate alterations in lipid metabolism. Here, we aimed to clarify the secondary accumulation of lipids and presence of unregulated lipid metabolism in brain of early onset Tay-Sachs disease mice model by lipidome profiling.

Materials and methods: Cortex and hippocampus of 5month-old of WT, Hexa-/-, Neu3-/- and Hexa-/-Neu3-/mice were analyzed by mass spectrometry and lipid levels belongs to glycerolipids, glycerophospholipids, sphingolipids, sterol lipids were evaluated by using shotgun lipidomics approach.

Results: In glycerophospholipid class, high level of LPC, LPE and PG lipids while low level of PC, PS lipids were detected in cortex and hippocampus of Hexa-/-Neu3-/- mice compared to age matched control group. Significantly high levels ceramide and sterolipid were also measured in cortex. Alterations in glycerolipid class was detected in both regions.

Conclusion: Here we report that in addition to abnormal GM2 accumulation in lysosomes, secondary but distinct lipid alterations may also contribute to pathophysiology of early onset Tay-Sachs disease suggesting the possible dysfunctionality of cellular pathways such as autophagy.

Acknowledgement: Research was funded by TUBITAK $215 Z 083$.

T. Sengul: None. M. Can: None. S. Akyildiz Demir: None. C. Klose: None. M. Surma: None. V. Seyrantepe: None.

\section{P09.147.A}

Role of oxidative stress in the pathogenesis of Tay-Sachs disease mouse model

N. Ates, H. Basirli, T. Caliskan, A. Nalbant, V. Seyrantepe

Izmir Institute of Technology, Izmir, Turkey

Introduction: Tay-Sachs disease is one of the lethal lysosomal storage disorders caused by mutations on the HEXA gene leading to GM2 accumulation which causes neurodegeneration. Hexa-/-Neu3-/- mice mimic neuropathologic phenotype of the disease. Oxidative stress has been demonstrated to be involved in the pathogenesis of many neurodegenerative diseases. In this research we investigated whether the oxidative stress has an effect on the neuropathology mice.

Material and Methods: Brain sections and fibroblasts from WT, Hexa-/-, Neu3-/- and Hexa-/-Neu3-/- mice as well as neuroglia were studied. Oxidative stress markers were analysed by RT-PCR, western blot and protein carbonylation assay. The intracellular ROS level was measured by using $\mathrm{H}_{2}$ DCFDA and analysed by FACScan.

Results: Significant increase in protein carbonyl levels was determined particularly in cerebellum of Hexa-/ Neu3-/- mice. Similarly, we determined significant change in neuroglia but not in Hexa-/-Neu3-/- fibroblast. However, we found significantly high level of catalase and Ttase 1 mRNA in Hexa-/-Neu3-/- fibroblasts. In addition, oxidized DCFDA was also increased in Hexa-/-Neu3-/- fibroblast indicating high level of ROS but surprisingly we detected the low level of ROS in neuroglia. Significant increase in the level of APE1/Ref-1 protein was observed in cerebellum and thalamus of Hexa-/-Neu3-/-; however, in fibroblast there was no significant difference among samples. Significantly decreased level of catalase and increased Ttase 1 mRNA were detected in neuroglia compared to control.

Conclusions: We suggest that abnormal GM2 accumulation in lysosomes trigger oxidative stress leading alterations in redox homeostasis in early-onset Tay-Sachs disease pathology. The research was supported by TUBITAK$215 Z 083$

N. Ates: None. H. Basirli: None. T. Caliskan: None. A. Nalbant: None. V. Seyrantepe: None.

\section{P09.149.C}

Neurodegenerative-like phenotype of a TTR mutation deciphered by bioinformatic tools

\author{
A. Lunati $^{l}$, C. Delamarche ${ }^{2}$, P. Diraison ${ }^{3}$, I. Isabelle \\ Quintin-Roue $^{4}$, B. Funalot ${ }^{1}$, P. Fanen ${ }^{1}$
}

${ }^{I}$ Département de génétique; CHU Henri Mondor, Créteil, France, ${ }^{2}$ Professor - Retired from University of Rennes 1 , Rennes, France, ${ }^{3}$ Service de neurologie, $\mathrm{CH}$ de Cornouaille, Quimper, France, ${ }^{4}$ Service d'anatomie et cytologie pathologiques, CHRU, Brest, France

Hereditary transthyretin amyloidosis (ATTR) leads to a variable phenotype and involve many organs. Here we report one patient presenting with central neurological and ophthalmological signs, amyloid deposits on subarachnoid 
strongly linked to neuronal differentiation. VASH1 and VASH2, in complex with SVBP, have recently been identified as responsible for intracellular tubulin detyrosination activity. Biallelic loss-of-function mutations in SVBP have much recently been described in patients showing intellectual disability, microcephaly, ataxia and hypotonia.

Methods: All patients underwent accurate clinical evaluation and provided informed consent. Whole-exome sequencing (WES) was performed using patients' DNA samples, and segregation in relatives was confirmed by Sanger sequencing. Primary fibroblasts were generated from patients' skin biopsies, and cultured cells were incubated with paclitaxel to analyze detyrosinated tubulin levels by immunofluorescence and western blot. cDNA of VASHI and wild-type/mutated forms of SVBP were cloned into a pCMV-Tag4A vector, and then cotransfected into HeLa cells for Western Blot analysis.

Results: We report two families with multiple patients harboring an homozygous missense variant in $S V B P$ (p. Leu49Pro). Affected individuals show spastic paraplegia, peripheral neuropathy, verbal apraxia and intellectual disability, in absence of microcephaly, broadening the previously reported phenotype. In vitro experiments in patients' fibroblasts demonstrate a significant loss of VASH1/2 detyrosination activity and decreased SVBP levels. Cotransfection studies show that SVBP p.Leu49Pro variant reduces both VASH1 and SVBP soluble quantities or their stability.

Conclusions: Our results expand the phenotypic spectrum of SVBP mutations to complex spastic paraplegia without microcephaly, and confirm for the first time that a detyrosination defect is observed in patients' cell lines, enabling functional testing of $S V B P$ variants and extended pathogenetic studies.

E. Verdura: None. N. Launay: None. G. Fernandez Garcia de Eulate: None. J. Ondaro: None. A. Schlüter: None. M. Ruiz: None. S. Fourcade: None. A. López de Munain: None. A. Pujol: None.

\section{P09.152.C}

Another case with childhood-onset Neurodegeneration due to a UBTF variant identified by whole-exome sequencing (WES).

\section{Zengeler ${ }^{1}$, H. Gabriel ${ }^{1}$, S. Biskup ${ }^{1}$, C. Schell-Apacik ${ }^{2}$ \\ ${ }^{1}$ Praxis für Humangenetik, Tuebingen, Germany, ${ }^{2}$ Medi- cover Human Genetics Berlin-Westend MVZ, Berlin, Germany}

Recently, Edvardson and collegues identified the de novo variant c.628G $>\mathrm{A}$; p.Glu210Lys in the gene $U B F T$ in cases with childhood-onset Neurodegeneration. In these patients, regression of skills occurred at 2.5-7 years. Initially motor regression followed by language regression was found. Brain MRI revealed diffuse cortical atrophy of the subcortical white matter. UBFT encodes a transcription factor, which facilitate the assembly of the transcription initiation complex of the RNA polymerase I (PolI), which is crucial for rRNA synthesis. Functional studies showed that the UBTF variant leads to a stronger binding affinity to the rDNA promotor and increased 18s rRNA expression. As a result the chromatin structure at the rDNA locus was decondensed. In rats enhanced PolI activity promotes neurite outgrowth, which can lead to neurodegenerative processes (Edvardson et al., 2017). Here we report on another patient carrying the de novo p.Glu210Val UBTF variant identified by WES. The psychomotoric development of the boy was slightly delayed at first. He started walking on his own with $1 \frac{1}{2} 2$ year, and ataxic gait was noted. At the age of 5 years, he was unable to walk without assistance, and was wheelchair-bound at the age of 7. Subsequently, the boy developed tetraparesis, global retardation, and severe scolio-kyphosis. Around the age of $1 \frac{1 / 2}{2}$ the boy started to use identifiable words but his speech deteriorated and by the age of 7 he presented with slurred speech, and subsequently stopped using words. MRI of the brain performed at the age of 14 years revealed supratentorial atrophy.

D. Zengeler: None. H. Gabriel: None. S. Biskup: None. C. Schell-Apacik: None.

\section{P09.154.B}

Expanding the spectrum of WDR62 mutations : description of new cases

L. Ruaud ${ }^{1,2}$, S. Drunat ${ }^{3,2}$, A. Ernault ${ }^{l}$, Y. Capri $^{l}$, L. Van Maldergem $^{4,5}$, C. Engel ${ }^{4,5}$, C. Altuzarra ${ }^{6}$, A. Bayat $^{7}, S$. Moortgart $^{8}$, I. Maystadt ${ }^{8}$, M. Abramowicz ${ }^{9}$, C. Zweier ${ }^{10}$, I. Lorenz $^{11}$, D. Haye ${ }^{12}$, F. Giuliano ${ }^{12}$, S. Vaessen ${ }^{13}$, L. Servais $^{14}$, E. Di Maria ${ }^{15,16}$, F. Faravelli ${ }^{15}$, J. Kohlhase ${ }^{17}, T$. Bast $^{18}$, N. Miladi ${ }^{19,20}$, A. Agadr ${ }^{21,22}$, S. Auvin ${ }^{23}$, A. Verloes $^{1,2}$, S. Passemard ${ }^{1,2}$

${ }^{1}$ Service de génétique clinique, AP-HP, Hôpital Robert Debré, Paris, France, ${ }^{2}$ Université de Paris, Neurodiderot, Inserm, Paris, France, ${ }^{3}$ Service de génétique moléculaire, AP-HP, Hôpital Robert Debré, Paris, France, ${ }^{4}$ Centre de génétique humaine, CHRU de Besançon, Besançon, France, ${ }^{5}$ Université de Bourgogne-Franche Comté, Besançon, France, ${ }^{6}$ Service de pédiatrie, CHRU Besançon, Besançon, France, ${ }^{7}$ Danish Epilepsy Centre, Filadelfia, Denmark, ${ }^{8}$ Centre de Génétique Humaine, Institut de Pathologie et Génétique, Charleroi, Belgium, ${ }^{9}$ Laboratoire de Diagnostic Moléculaire et Génomique, Hôpitaux universitaires de Genève HUG, Genève, Switzerland, 
${ }^{10}$ Intitute of Human Genetics, Friedrich-Alexander-Universität Erlanger-Nürnberg, Erlangen, Germany, ${ }^{11}$ Department of Pediatrics, Division of Neuropediatrics, FriedrichAlexander-Universität Erlangen-Nürnberg, Erlangen, Germany, ${ }^{12}$ Service de génétique, CHRU Nice, Nice, France, ${ }^{13} 12$ Centre de génétique humaine, Hôpital de La Citadelle, CHU de Liège, Liège, Belgium, ${ }^{14}$ Neuromuscular Reference Center, Hôpital de La Citadelle, CHU de Liège, Liège, Belgium, ${ }^{15}$ Unit of Medical Genetics, Galliera Hospital, Genova, Italy, ${ }^{16}$ Department of Health Sciences, University of Genova, Genova, Italy, ${ }^{17}$ SYNLAB Center for Human Genetics, Freiburg, Germany, ${ }^{18}$ Children's Hospital, Epilepsy Center Kork, Kehl, Germany, ${ }^{19}$ Service de neurologie pédiatrique, Magreb Médical, Tunis, Tunisia, ${ }^{20}$ Université de Tunis El Manar, Tunis, Tunisia, ${ }^{21}$ Service de pédiatrie, Hopital militaire Mohamed V, Rabat, Morocco, ${ }^{22}$ Faculté de médecine Mohamed V, Rabat, Morocco, ${ }^{23}$ Service de neurologie pédiatrique, APHP, Hôpital Robert Debré, Paris, France

MCPH2 (MicroCephaly Primary Hereditary 2), caused by WDR62 mutations, is the second most common form of MCPH. WDR62, located on chr19q13.12, encodes WD repeat-containing protein 62 , playing a role for mitotic division, proliferation of neuronal progenitor and neuronal migration. To date, more 100 patients with pathogenic homozygous or compound heterozygous variants in WDR62 were reported. They have developmental delay with constant speech impairment, moderate to severe intellectual disability (ID) and multiple brain malformations including hypoplasia of corpus callosum and migration disorders (polymicrogyria, pachygyria, lissencephaly and schizencephaly). Nevertheless, no patient have been properly assessed by appropriated neuropsychological tests. Using multi-gene panel or exome sequencing, we identified 18 cases with WDR62 variants and report clinical, neuropsychological assessment and neuroradiological phenotype. Phenotypic spectrum of our patients is very large ranging from severe antenatal presentation leading to profound motor handicap, to mild-moderate ID, learning abilities and autonomy in daily life. We were surprised to discover that some patients had a VCI at 50 and able to read and write, similar to what is described with ASPM. Some patients had normal brain structure on MRI except microcephaly. Interestingly, some patients develop ataxia with age, which was never reported. Epilepsy is not constant but pharmaco-sensitive for all patients unless one. We report here the largest series of WDR62 patients with a very precise clinical, neuropsychological and neuroradiological characterization. We demonstrate that WRD62 mutations do not always cause severe ID/motor handicap and cortical malformation. However, we have concerns regarding the long term neurological outcome.

L. Ruaud: None. S. Drunat: None. A. Ernault: None. Y. Capri: None. L. Van Maldergem: None. C. Engel: None. C. Altuzarra: None. A. Bayat: None. S. Moortgart: None. I. Maystadt: None. M. Abramowicz: None. C. Zweier: None. I. Lorenz: None. D. Haye: None. F. Giuliano: None. S. Vaessen: None. L. Servais: None. E. Di Maria: None. F. Faravelli: None. J. Kohlhase: None. T. Bast: None. N. Miladi: None. A. Agadr: None. S. Auvin: None. A. Verloes: None. S. Passemard: None.

\section{P09.155.C}

Identification of new variants in amyotrophic lateral sclerosis patients by whole genome sequencing data

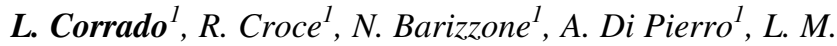

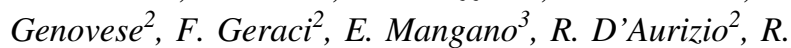
Bordoni $^{3}$, D. Corà ${ }^{l}$, F. Favero ${ }^{1}$, F. De Marchi ${ }^{4}$, G. De Bellis $^{3}$, G. Manzini ${ }^{2,5}$, M. Severgnini ${ }^{3}$, M. Pellegrini ${ }^{2}, L$. Mazzinit $^{4}$, S. D'Alfonsol

${ }^{1}$ University of Eastern Piedmont UPO, NOvara, Italy, ${ }^{2}$ Institute of Informatics and Telematics of CNR, Pisa, Italy, ${ }^{3}$ National Research Council of Italy, Institute for Biomedical Technologies, Segrate (MILANO), Italy, ${ }^{4} A L S$ Center AOU Maggiore della Carità, NOvara, Italy, ${ }^{5}$ University of eastern Piedmont, UPO, Alessandria, Italy

Amyotrophic lateral sclerosis (ALS) is a fatal motor neuron disorder with adult onset. Mutation in over 30 genes account for less than $65 \%$ of ALS familial cases. To explore ALS missing heritability, we performed WGS of 70 ALS/Frontotemporal Dementia (FTD) patients negative for mutations in main known causative genes, focusing on a panel of 699 genes implicated in 5 neurodegenerative disorders (NDDs, ALS, Parkinson's disease, FTD, Ataxia and Spastic Paraparesis). We analysed and prioritized mutations in the coding sequence, repeats expansions, and structural variants by standard bioinformatic tools and we implemented a novel pipeline to prioritize non coding-variants with a possible pathogenetic effect. We identified 11 pathogenic variants. Four of them map in genes causative of rare forms of ALS (KIF5A, NEK1, TBK1 OPTN). The remaining 7 variants map in genes associated with different NDDs and never reported in ALS. Among them, ABCA7, TGM6 and CCDC88C genes associated respectively to Alzheimer disease and autosomal dominant spinocerebellar ataxia. In addition, we found the most common causative mutation of PD in LRRK2, in an ALS patient with a PD positive family history. Among the non-coding variants, we prioritized 5 deep intronic variants predicting an effect on 
splicing mechanism whose functional impact is under investigation with in vitro studies. We found pathogenetic variants in ALS patients in genes involved in other NDDs, reinforcing the idea of a shared genetic cause and a possible overlapping condition between these disorders. We identified for the first time non-coding variants with a possible pathogenic role in ALS

L. Corrado: None. R. Croce: None. N. Barizzone: None. A. Di Pierro: None. L.M. Genovese: None. F. Geraci: None. E. Mangano: None. R. D'Aurizio: None. R. Bordoni: None. D. Corà: None. F. Favero: None. F. De Marchi: None. G. De Bellis: None. G. Manzini: None. M. Severgnini: None. M. Pellegrini: None. L. Mazzini: None. S. D'Alfonso: None.

\section{P09.156.A}

A novel bioinformatic approach for the family-based genetic analysis of whole-genome sequencing data from 8 multigenerational Spanish families with bipolar disorder

S. B. Fischer ${ }^{1,2}$, M. Fink ${ }^{3}$, C. K. Y. $\mathrm{Ng}^{4,5}$, C. S. Reinbold ${ }^{6,1,2}$, A. Maaser-Hecker ${ }^{7}$, F. Streit ${ }^{8}$, S. H. Witt $^{8}, J$. GuzmanParra $^{9}$, G. Orozco-Diaz ${ }^{10}$, G. Auburger ${ }^{11}$, M. Albus ${ }^{12}, M$. Borrmann-Hassenbach ${ }^{12}$, M. J. González ${ }^{9}$, S. Gil-Flores ${ }^{13}$, F. J. Cabaleiro Fabeiro ${ }^{14}$, F. del Río Noriega ${ }^{15}$, F. PerezPerez $^{16}$, J. Haro-González ${ }^{17}, F$. Rivas $^{18}, F$. Mayoral ${ }^{18}, S$. Herms $^{1,2,7}$, M. Rietschel ${ }^{8}$, M. M. Nöthen ${ }^{7}$, P. Hoffmann ${ }^{1,2,7}$, A. J. Forstner ${ }^{19,7,1}$, S. Cichon ${ }^{1,2,7,20}$

${ }^{1}$ Human Genomics Research Group, Department of Biomedicine, University of Basel, Basel, Switzerland, Basel, Switzerland, ${ }^{2}$ Institute of Medical Genetics and Pathology, University Hospital Basel, Basel, Switzerland, Basel, Switzerland, ${ }^{3}$ Novartis Pharma AG, Basel, Switzerland, Basel, Switzerland, ${ }^{4}$ Institute of Pathology, University Hospital Basel, Basel, Switzerland, Basel, Switzerland, ${ }^{5}$ Department of Biomedicine, Hepatology Laboratory, University of Basel, Basel, Switzerland, Basel, Switzerland, ${ }^{6}$ Center for Lifespan Changes in Brain and Cognition (LCBC), Department of Psychology, University of Oslo, Oslo, Norway, Oslo, Norway, ${ }^{7}$ Institute of Human Genetics, University of Bonn, School of Medicine \& University Hospital Bonn, Bonn, Germany, Bonn, Germany, ${ }^{8}$ Department of Genetic Epidemiology in Psychiatry, Central Institute of Mental Health, University Medical Center Mannheim/University of Heidelberg, Mannheim, Germany, Mannheim, Germany, ${ }^{9}$ Department of Mental Health, Institute of Biomedicine IBIMA, University Hospital of Malaga, Malaga, Spain, Malaga, Spain, ${ }^{10}$ Unidad de Gestión Clínica del Dispositivo de Cuidados Críticos y
Urgencias del Distrito Sanitario Málaga - Coin-Gudalhorceedicine, Malaga, Spain, Malaga, Spain, ${ }^{11}$ Department of Experimental Neurology, Division of Neurology, Goethe University Hospital, Frankfurt, Germany, Frankfurt, Germany, ${ }^{12}$ Isar Amper Klinikum München Ost, kbo, Haar, Germany, Haar, Germany, ${ }^{13}$ Department of Mental Health, University Hospital of Reina Sofia, Cordoba, Spain, Cordoba, Spain, ${ }^{14}$ Department of Mental Health, University Hospital of Jaen, Jaen, Spain, Jaen, Spain, ${ }^{15}$ Department of Mental Health, University Hospital of Jerez de la Frontera, La Frontera, Spain, La Frontera, Spain, ${ }^{16}$ Department of Mental Health, University Hospital of Puerto Real, Department of Mental Health, Cádiz, Spain, Cádiz, Spain, ${ }^{17}$ Department of Mental Health, Hospital Punta de Europa, Algeciras, Spain, Algeciras, Spain, ${ }^{18}$ Department of Psychiatry, Carlos Haya Regional University Hospital, Malaga, Spain, Malaga, Spain, ${ }^{19}$ Centre for Human Genetics, University of Marburg, Marburg, Germany, Marburg, Germany, ${ }^{20}$ Institute of Neuroscience and Medicine INM-1, Research Center Jülich, Jülich, Germany, Jülich, Germany

Introduction: Bipolar Disorder (BD) is a complex neuropsychiatric disorder, with an estimated heritability of 60$80 \%$. Common genetic risk factors identified by GWAS explain $25-38 \%$ of the phenotypic variance. It is assumed that rare variants may also play a substantial role and explain part of the hidden heritability. One strategy to identify such variants is Whole Genome Sequencing (WGS) of large multiply affected families with BD. In the present study we conducted WGS of 108 individuals in 8 multigenerational affected families of Spanish origin.

Methods: Sequencing was performed with 30x mean coverage on an Illumina HiSeq2500 and variant calling was performed using the GATK best practice workflow. For downstream analysis we developed a variant based scoring model. We combined information on family structure and the proportion of allele sharing amongst the affected individuals plus the biological impact for each variant based on different prediction classifiers such as CADD or SNPeff.

Results: We focused on the identification of rare variants following two analyses pathways for the WGS data: an "extended exome" analysis, focusing on the high impact and protein-coding variants only. Secondly we applied the model to the full WGS dataset, including also the noncoding part of the genome. On average we identified 4.4 million variants per individual. In two families, we identified stop mutations as the top scored variants in the genes TAS2R19 and PPEF2. Additional top scoring variants were identified in the non-coding/intronic regions of the genome. 
S.B. Fischer: None. M. Fink: None. C.K.Y. Ng: None. C.S. Reinbold: None. A. Maaser-Hecker: None. F. Streit: None. S.H. Witt: None. J. Guzman-Parra: None. G. Orozco-Diaz: None. G. Auburger: None. M. Albus: None. M. Borrmann-Hassenbach: None. M.J. González: None. S. Gil-Flores: None. F.J. Cabaleiro Fabeiro: None. F. del Río Noriega: None. F. Perez-Perez: None. J. HaroGonzález: None. F. Rivas: None. F. Mayoral: None. S. Herms: None. M. Rietschel: None. M.M. Nöthen: None. P. Hoffmann: None. A.J. Forstner: None. S. Cichon: None.

\section{P10 Neuromuscular Disorders}

\section{P10.01.B}

A case of fetal arthrogryposis due to $A C T A 1$-associated myopathy inherited from an affected mosaic mother

U. Grasshoff ${ }^{l}$, K. Schoner ${ }^{2}$, T. Haack ${ }^{l}$, K. Schaeferhoff ${ }^{l}, P$. Mazzolal, S. Beck-Woedl ${ }^{l}$, D. Rating ${ }^{3}$, H. Goebel ${ }^{4}, S$. Hentze $e^{5}$

${ }^{1}$ Medical Genetics and Applied Genomics, Tübingen, Germany, ${ }^{2}$ Institute of Pathology, Philipps University Marburg, Marburg, Germany, ${ }^{3}$ Pediatric Neurology, University Children's Hospital, Heidelberg, Germany, ${ }^{4}$ Department of Neuropathology, Charitè University Hospital, Berlin, Germany, ${ }^{5}$ Human Genetical Practice, Heidelberg, Germany

Introduction: Pathogenic variants in ACTA1 (OMIM *102610) have been associated with a spectrum of rare myopathies. Inheritance is described as autosomal-recessive as well as autosomal-dominant. The clinical phenotypes can be highly variable regarding age at onset and severity. The severe form of the disorder is characterized by the absence of spontaneous movement, arthrogryposis, and respiratory failure at birth.

Case report: We report on a young woman who presented with a congenital non-progressive muscular disease with unilateral paresis of $\mathrm{N}$. facialis, muscular hypotonia and non-symmetrical muscular atrophy. A phenotype-based exome analysis showed no causative mutation. During her first pregnancy a sonogram at 14 weeks of gestation diagnosed severe arthrogryposis of the fetus with akinesia of the hips and ulnar deviation of the hands. The pregnancy was terminated. Fetal autopsy documented arthrogryposis multiplex as well as facial dysmorphism, bilateral talipes equinovarus and extreme atrophy of muscle fibres. Histological examination was concordant with a severe structural (actin) myopathy. The subsequent trio exome analysis prioritized an apparently de novo heterozygous ACTAl missense variant (c.226G>C,p. Val118Met) in the fetus. A re-examination of the maternal data revealed a low mosaicism $(15 \%)$ in the maternal blood sample for the same variant.

Conclusions: We postulate that the asymmetric maternal myopathy is caused by a postzygotic mosaic mutation in ACTA1. The fetus inherited the ACTAl variant in a then non-mosaic state and developed the severe lethal phenotype. An analysis of the ACTAI variant in maternal muscle tissue is intended.

U. Grasshoff: None. K. Schoner: None. T. Haack: None. K. Schaeferhoff: None. P. Mazzola: None. S. BeckWoedl: None. D. Rating: None. H. Goebel: None. S. Hentze: None.

P10.02.C

Whole exome sequencing reveals a novel homozygous mutation in the $A D A M 22$ gene, underpinning its association with severe neurologic morbidity.

L. Sagi-Dain ${ }^{1}$, S. Sagi ${ }^{2}$, J. Haddad-Halloun ${ }^{1}$, V. Adir ${ }^{l}$, O. Sadeh $^{l}$, A. Peleg ${ }^{1}$

${ }^{1}$ Carmel Medical Center, Haifa, Israel, ${ }^{2}$ Bnei Zion Medical Center, Haifa, Israel

Background: Numerous genes have been implicated in congenital arthrogryposis and epilepsy. In this report we describe a second reported case of ADAM22 mutations, associated with severe neurologic manifestations.

Case presentation: We describe a female infant with congenital arthrogryposis and severe hypotonia including diaphragmatic weakness. Intractable seizures appeared in the age of several days, and the patient passed away at the age of one year. Family history evaluation revealed similar symptoms in daughter of paternal cousin, who passed away at the age of 6 months. This patient had hypotonia, seizures, respiratory difficulty, and her brain MRI showed progressive atrophy of the white matter. Consanguinity was noted in parents of both patients.

Results: Whole exome sequencing of the patient revealed a novel homozygous variant c.2077-2A $>C$ in intron 23 of ADAM22 gene. The variant was not found in large exome databases, and several in-silico prediction programs classified the mutation as "pathogenic" vs no benign predictions. Family segregation analysis demonstrated heterozygous carrier state in both parents, their healthy daughter, as well as in parents of additional familial case with similar symptoms. Literature search yielded one description of compound heterozygous mutations in ADAM22 gene, 
causing rapidly progressing cerebral atrophy, intractable seizures, and intellectual disability. Previous research showed that knockout mice for ADAM22 manifest with lethal seizures during the first postnatal weeks.

Conclusions: Our case provides valuable information to the scarce literature evidence describing the association between mutations in ADAM22 gene to severe neurologic morbidity.

L. Sagi-Dain: None. S. Sagi: None. J. HaddadHalloun: None. V. Adir: None. O. Sadeh: None. A. Peleg: None.

\section{P10.03.A}

The genetic architecture of Amyotrophic lateral sclerosis and the role of comorbidity in Italian population

I. Palmieri ${ }^{1,2}$, S. Conti ${ }^{3}$, O. Pansarasa ${ }^{1}$, L. Diamanti ${ }^{4}, S$. Gagliardi $^{l}$, M. Filosto $^{5}$, C. Cereda ${ }^{1}$

${ }^{1}$ Genomic and post-genomic Center, IRCCS Mondino Foundation, Pavia, Italy, ${ }^{2}$ Department of Molecular Medicine, University of Pavia, Pavia, Italy, ${ }^{3}$ Department of Biology and Biotechnology "L. Spallanzani", University of Pavia, Pavia, Italy, ${ }^{4}$ General Neurology, IRCCS Mondino Foundation, Pavia, Italy, ${ }^{5}$ Center for Neuromuscular Diseases and Neuropathies, Unit of Neurology, ASST 'Spedali Civili', University of Brescia, Brescia, Italy

Introduction: Amyotrophic Lateral Sclerosis (ALS) is a neurodegenerative disease (ND) characterized by heterogeneous clinical manifestations due to both genetic and phenotypic overlapping with other NDs, such as Frontotemporal dementia (FTD), Parkinson's disease (PD) and Hereditary spastic paraplegia (HSP) (Elert-Dobkowska et al., 2019; Abramzon et al., 2020). Here, we screened an Italian population of 210 sporadic ALS patients for 95 genes related to ALS, FTD, PD and HSP to enlighten the genetic burden in ALS clinical spectra.

Materials and Methods: Next Generation Sequencing (NGS) was done using a customized panel of 175 genes (SureSelect, Agilent). The eVai software (https://evai. engenome.com) was used for variant calling and classification. Pathogenic, likely pathogenic and uncertain variants were retained and validated by Sanger sequencing. C9orf72 repeat expansion was assessed using the AmplideX ${ }^{\circledR}$ PCR/ CE C9orf72 Kit (Asuragen).

Results: 81 ALS patients carry one or more variants in ALS/FTD associated genes. Interestingly, there are also ALS patients that carry variants in PD (19) or in HSP (9) linked genes and patients that carry variants in genes related either to ALS and other NDs. A summary is listed in the table below:

\begin{tabular}{|c|c|c|c|c|c|}
\hline $\begin{array}{l}\text { Variants } \\
\text { linked to }\end{array}$ & $\begin{array}{l}\mathrm{N}^{\circ} \\
\text { patients (\%) }\end{array}$ & $\begin{array}{l}\mathrm{N}^{\circ} \text { patients } \\
\text { with } \\
\text { 1 variant }\end{array}$ & $\begin{array}{l}\mathrm{N}^{\circ} \text { patients } \\
\text { with } 2 \\
\text { variants }\end{array}$ & $\begin{array}{l}\mathrm{N}^{\circ} \text { patients } \\
\text { with } 3 \\
\text { variants }\end{array}$ & $\begin{array}{l}\mathrm{N}^{\circ} \text { patients } \\
\text { with }>3 \\
\text { variants }\end{array}$ \\
\hline ALS/FTD & $\begin{array}{l}81 \\
(38,57 \%)\end{array}$ & 56 & 17 & 5 & 3 \\
\hline PD & $19(9,05 \%)$ & 14 & 7 & 0 & 0 \\
\hline HSP & $9(4,29 \%)$ & 7 & 2 & 0 & 0 \\
\hline $\begin{array}{l}\text { ALS/FTD } \\
+ \text { PD }\end{array}$ & $\begin{array}{l}30 \\
(14,29 \%)\end{array}$ & -- & 9 & 15 & 6 \\
\hline $\begin{array}{l}\text { ALS/FTD } \\
+ \text { HSP }\end{array}$ & $16(7,62 \%)$ & -- & 10 & 4 & 2 \\
\hline $\mathrm{PD}+\mathrm{HSP}$ & $4(1,90 \%)$ & -- & 2 & 1 & 1 \\
\hline $\begin{array}{l}\text { ALS/FTD } \\
+ \text { PD } \\
+ \text { HSP }\end{array}$ & $16(7,62 \%)$ & -- & -- & 5 & 11 \\
\hline $\begin{array}{l}\text { No } \\
\text { Variants }\end{array}$ & $\begin{array}{l}35 \\
(16,67 \%)\end{array}$ & -- & -- & -- & -- \\
\hline
\end{tabular}

Conclusions: Our study confirmed that the genetic architecture of ALS is extremely complex, highlighting the importance to screen also genes related to other NDs. Considering a broader genetic spectrum will help in understanding also atypical clinical presentations.

I. Palmieri: None. S. Conti: None. O. Pansarasa: None. L. Diamanti: None. S. Gagliardi: None. M. Filosto: None. C. Cereda: None.

\section{P10.04.B}

Different levels of soluble nuclear SOD1 activate specific pathways in Peripheral Blood Mononuclear Cells of ALS patients

S. Gagliardi ${ }^{1}$, M. Garofalo ${ }^{1,2}$, C. Pandini ${ }^{1,2}$, M. Bordoni $^{1}$, L. Diamanti ${ }^{3}$ D. Sproviero ${ }^{1}$, O. Pansarasa ${ }^{l}$, C. Cereda ${ }^{l}$

${ }^{1}$ Genomic and post-genomic Center, IRCCS Mondino Foundation, Pavia, Italy, ${ }^{2}$ Department of Biology and Biotechnology "L. Spallanzani", University of Pavia, Pavia, Italy, ${ }^{3}$ General Neurology, IRCCS Mondino Foundation, Pavia, Italy

Introduction: Amyotrophic Lateral Sclerosis (ALS) is a fatal neurodegenerative disease caused by loss of motorneurons. SOD1 may have a toxic role in the pathogenesis when the protein aggregates in the cytoplasm; increased accumulation of nuclear SOD1 (nSOD1) represents a protective cellular reaction (Cereda et al., 2013; Bordoni et al., 2019). We investigated pathways activated by nSOD1 in Peripheral Blood Mononuclear Cells (PBMCs) of ALS patients by dividing them depending on the "high" or "low" concentration of nSOD1.

Methods: Subcellular fractionation from PBMCs of ALS patients (18) and healthy controls (12) was performed and proteins were quantified by Western blotting. RNA libraries were prepared by Illumina TruSeq Stranded RNA Library 
and run on NextSeq (Illumina). Differential expression analysis was performed using $\mathrm{R}$ package EBSeq.

Results: We obtained two gene expression patterns for high and low nSOD patients. Differentially expressed genes in patients with high nSOD1 form a cluster similar to controls compared to low nSOD1 group. Pathways activated in high nSOD1 patients are related to the upregulation of SEMA6B, a semaphorin involved in axon guidance, and HSP70, molecular chaperones ensuring the correct protein folding. In low nSOD1 group the upregulation of KDM4C and $\mathrm{S} 100 \mathrm{~B}$ may be responsible for hyper-demethylation of histone H3 impaired in ALS.

Conclusions: Our findings highlight the importance of subcellular localization of soluble nSOD1 in ALS patients. We observed a different behavior of RNA regulation in the two groups of patients, leading to pathways conferring "protection" where nSOD1 was high, and a general "perturbations" where nSOD1 was low.

S. Gagliardi: None. M. Garofalo: None. C. Pandini: None. M. Bordoni: None. L. Diamanti: None. D. Sproviero: None. O. Pansarasa: None. C. Cereda: None.

\section{P10.06.A}

Efficiency of high-throughput sequencing in the diagnosis of genetic causes of cerebral palsy

V. Udalova ${ }^{l}$, I. Kanivets ${ }^{l}$, D. Pyankov ${ }^{l}$, I. Komarkov ${ }^{l}, K$. Gorgishely $^{l}$, S. Korostelev ${ }^{1,2}$

${ }^{1}$ LLC GENOMED, Moscow, Russian Federation, ${ }^{2}$ Federal State Autonomous Educational Institution of Higher Education I.M. Sechenov First Moscow State Medical University of the Ministry of Health of the Russian Federation (Sechenov University), Moscow, Russian Federation

Introduction: Cerebral Palsy (CP) is a group of clinically heterogeneous conditions, data on the genetic causes of which are currently underrepresented. The aim of our work is to determine the effectiveness of high-throughput sequencing in patients with $\mathrm{CP}$.

Materials and methods: 604 patients, in whose clinical signs was CP, were examined using NGS-based tests (gene panels, containing up to 2000 genes, whole exome and genome sequencing). Data analysis was performed using inhouse pipeline.

Results: Among the provided clinical data, spastic manifestations were described in 256 patients $(39.07 \%)$, atonic-astatic - in 111 patients $(18.38 \%)$, mixed form - 45 patients $(7.45 \%)$. Intellectual developmental disorder, speech delay were observed in 380 patients $(62.91 \%)$, seizures - in 267 patients $(44.21 \%)$. After testing definitive diagnosis can be made in $31.8 \%$ cases, a possible cause was detected in $17.5 \%$, a negative result was obtained in $50.6 \%$. Mutations in the LAMA2, ATM, MECP2, CAPN3, KCNQ2, RYR1, SPAST, SCO2, SLC16A2, SLC2A1, SPG11, STXBP1 genes were most often found in the investigated group. Copy number variations (CNVs) were determined by coverage analysis in 31 patients $(10.4 \%)$.

Conclusions: In our study NGS-based tests detected $31.8 \%$ clinically significant variants in patients with $\mathrm{CP}$. The results of our work show that $\mathrm{CP}$ should not be considered as an independent nosology, but as a key symptom in many hereditary diseases, including genetically heterogeneous.

V. Udalova: None. I. Kanivets: None. D. Pyankov: None. I. Komarkov: None. K. Gorgishely: None. S. Korostelev: None.

\section{P10.08.C}

Unraveling the genetic architecture of autosomal recessive Charcot-Marie-Tooth neuropathies

C. Armirola ${ }^{l}$, K. Peeters ${ }^{l}$, E. de Vriendt ${ }^{l}$, S. Amor-Barris ${ }^{l}$, A. Jordanova ${ }^{1,2}$

${ }^{I}$ VIB-UAntwerp Center of Molecular Neurology, Antwerp, Belgium, ${ }^{2}$ Department of Medical Chemistry and Biochemistry, Medical University-Sofia, Sofia, Bulgaria

Introduction: Even though autosomal recessive CharcotMarie-Tooth neuropathy (ARCMT) represents less than $10 \%$ of CMT patients in Europe, in endogamous populations it accounts for $30-50 \%$ of all CMT cases. The pathogenesis of ARCMT is poorly understood. Over 30 ARCMT genes have been identified so far, yet they only explain a small portion of cases. The aim of this project is to identify novel causative ARCMT genes.

Methods: Based on whole exome sequencing data of a cohort of 135 ARCMT families highly enriched with consanguineous pedigrees, we perform homozygosity mapping and focus on the resulting autozygous regions to do variant filtering and prioritization. The functional effect of the variants in candidate ARCMT genes is further characterized in vitro and in vivo, using Drosophila melanogaster as our main model of choice.

Results: In 2 siblings with severe axonal CMT we have found a homozygous splice-site variant in a mitochondrial nuclear-encoded gene, that works as an assembly factor for cytochrome c oxidase. Screening for additional cases identified 5 additional families with 9 different variants. The splice variant leads to frame recovery and expression of an alternative transcript which lacks one exon. Immunoblotting showed that the new isoform is effectively translated into protein. The functional characterization of 
the mutant protein in patient-derived lymphoblasts and in yeast models is currently ongoing.

Conclusions: We have established a gene discovery pipeline for ARCMT that combines exome sequencing with homozygosity mapping and functional experiments in cellular or in vivo models. The characterization of the latest candidate is underway.

C. Armirola: None. K. Peeters: None. E. de Vriendt: None. S. Amor-Barris: None. A. Jordanova: None.

\section{P10.10.B}

\section{CRISPR interference as therapy strategy for COL VI-} CMD

\section{F. Haarich, Z. Aherrahrou, J. Erdmann}

Institute for Cardiogenetics, University of Lübeck, Lübeck, Germany

Introduction: The spectrum disorder Collagen VI congenital muscular dystrophy (COL VI-CMD) is caused by mutations in COL6A1, COL6A2 and COL6A3. The phenotypic spectrum consists of the mildest and late onset form Bethlem Myopathy (BM, OMIM \#158810), intermediate phenotypes and the most severe and early onset form Ullrich congenital muscular dystrophy (UCMD, OMIM \#254090). There is still no treatment for COL VI-CMD. Considering that haploinsufficiency is tolerated for COL6A2, specific knockdown of the allele including a pathogenic dominant-negative variant is under investigation as a treatment strategy for COL VI-CMD. The only approach for COL6A2 so far targeted a variant in intron 9 (c.954+17_954+22del28). We focus on a group of pathogenic variants that lead to substitutions of essential Glycines in triple helical repeats (Gly-X-Y).

Materials and Methods: The aim is to utilize CRISPR interference to repress the transcription of the mutated allele and to use common variants to differentiate between alleles. After the identification of common heterozygous variants in potential target sites (promotors, enhancers) via Sanger sequencing, the target region was enriched using Cas9 enrichment and sequenced with an Oxford Nanopore MinION. The data analysis was performed with a script containing several command line tools.

Results: A 48kb region including enhancers, promotors and 24 exons of COL6A2 was successfully enriched, sequenced and the found variants were phased with the pathogenic variant.

Outlook: Allele-specific guideRNAs will be used to repress the transfection of the pathogenic allele.

The project is supported by a grant of the German Society of Muscular Diseases (DGM).
F. Haarich: None. Z. Aherrahrou: None. J. Erdmann: None.

P10.12.A

Molecular diagnosis of merosin-deficient congenital muscular dystrophy in Moroccan patients by NGS and identification of a novel mutation

\author{
Y. El Kadiri ${ }^{1,2}$, I. Ratbi ${ }^{1}$, A. Zrhidri ${ }^{1,2}$, N. Birouk ${ }^{3}, Y$.
} Kriouile $^{4}$, A. Sefiani ${ }^{1,2}$, J. Lyahyai ${ }^{l}$

${ }^{\text {I}}$ Centre de Recherche en Génomique des Pathologies Humaines (GENOPATH), Faculté de Médecine et de pharmacie, Mohammed V University, Rabat, Morocco, ${ }^{2}$ Département de Génétique Médicale, Institut National d'Hygiène, Rabat, Morocco, ${ }^{3}$ Service de Neurophysiologie clinique, Hôpital des Spécialités, Rabat, Morocco, ${ }^{4}$ Unité de Neuropédiatrie et maladies neurométaboliques, Service de Pédiatrie PII- Hôpital d'enfant de Rabat, Faculté de Médecine et de pharmacie, Mohammed $V$ University, Rabat, Morocco

Introduction: Congenital muscular dystrophies (CMDs) are a clinically and genetically heterogeneous group of neuromuscular disorders. The most common CMD subtype is laminin- $\alpha 2$ or merosin deficient congenital muscular dystrophy (also known as MDC1A), accounting for 30-40\% of CMD cases. It is characterized by muscle weakness at birth or within the first months of life, delay in motor milestones, elevated creatine kinase (CK) and white matter abnormalities in brain MRI. MDC1A is an autosomal recessive condition caused by LAMA2 gene mutations.

Patients and methods: We investigated six unrelated Moroccan patients with clinical and paraclinical signs of congenital muscular dystrophy. We performed a molecular analysis of a customized panel of 24 genes involved in different forms of muscular dystrophies by targeted next generation sequencing. Mutations were confirmed by Sanger sequencing.

Results: Five known homozygous mutations: c.32T $>$ C (p.Leu11Pro), c.1377delC (p.Tyr460ThrfsTer15), c.6993$2 \mathrm{~A}>\mathrm{C}, \mathrm{c} .8244+1 \mathrm{G}>\mathrm{A}, \mathrm{c} .8244+3 \_8244+6 \operatorname{del} \mathrm{AAGT}$, and a novel homozygous nonsense variant c.2217G $>$ A (p. Trp739Ter) were identified in the LAMA2 gene.

Conclusions: Next generation sequencing has improved genetic diagnosis pathway for heterogeneous diseases like muscular dystrophies. Our results allowed to provide appropriate management of patients and genetic counseling to families, and to expand mutational spectrum of LAMA2 gene in Moroccans.

Y. El Kadiri was supported by an excellence research grant from the National Center for Scientific and Technical Research, Rabat-Morocco. 
Y. El Kadiri: None. I. Ratbi: None. A. Zrhidri: None. N. Birouk: None. Y. Kriouile: None. A. Sefiani: None. J. Lyahyai: None.

\section{P10.13.B}

The importance of targeted sequencing in the diagnosis of congenital muscular dystrophy type $1 \mathrm{~A}$

V. Antsupova ${ }^{1}$, I. Lastivka ${ }^{2}$, L. Khlunovska ${ }^{2}$, M. Ryznychuk $^{2}$, L. Hodovaniuk ${ }^{3}$

${ }^{1}$ Bohomolets National medical university, Kyiv, Ukraine, ${ }^{2}$ HSEE of Ukraine, "Bukovinian State Medical University", Chernivtsi, Ukraine, ${ }^{3}$ Bukovynian Center for Rehabilitation and Integrated Rehabilitation of Children, Chernivtsi, Ukraine

Introduction: Type 1A Merosindeficient congenital muscular dystrophy (CMD1A) is an autosomal recessive neuromuscular disease caused by a mutation of the LAMA2 gene encoding the laminin $\alpha 2$-chain (merosine). Although CMD1A in the European population takes the first place $(50 \%)$ in the structure of all CMD, there are difficulties in diagnosing this pathology.

Materials and Methods: Case CMD1A. Used clinical and genealogical, molecular-genetic, laboratory and instrumental methods.

Results: A 3-year-old boy with a diagnosis of WerdnigHoffmann spinal amyotrophy complicated by arthrogryposis was sent to consult a geneticist. At birth, the child had diffuse hypotension with the involvement of facial muscles, a weak cry, a decrease in reflexes. At the age of 5 months, the child did not roll over; when verticalized, it did not rest on its legs. In 1 year appeared flexion contracture of large joints, a delay in motor development. Examination of genetics: the child has severe diffuse muscular hypotension, weakness of the axial muscles, proximal muscles of the limbs, face. Keeled chest deformity, contracture of the knee and ankle-foot joints. Blood CPK is 10 times higher than normal; ENMG - signs of primary muscle damage; MRI: damage to the white matter of the brain. Targeted sequencing in the LAMA2 gene revealed mutations 2049_2050del(pArg683Serfs*21) and c.7732C $>\mathrm{T}(\mathrm{p}$. Arg2578*) in a compound heterozygous state. Final diagnosis: Merosindeficient congenital muscular dystrophy type $1 \mathrm{~A}$.

Conclusions: The knowledge of the key sign CMD1A allows you to immediately scrape the patient for moleculargenetic diagnostics. Core diagnosis, more important in predicting seizure; adequate therapy and rehabilitation of the patient.
V. Antsupova: None. I. Lastivka: None. L. Khlunovska: None. M. Ryznychuk: None. L. Hodovaniuk: None.

P10.14.C

Further delineation of clinical and molecular characteristics of congenital myasthenic syndromes in Indian families

\section{S. Mishra, K. Girisha, A. Shukla}

Kasturba Medical College, Manipal Academy of Higher Education, Manipal, Karnataka, India

Introduction: Congenital myasthenic syndrome (CMS) is a clinically and genetically heterogeneous group of disorders characterized by a neuromuscular transmission defect. They are rare and often treatable genetic disorders depending on the underlying molecular defect. This study expands the mutational spectrum of CMS in India.

Materials and Methods: We performed detailed clinical evaluation in seven patients with CMS from five families and singleton exome sequencing in five index patients. Validation of the identified variant and segregation analysis was done by Sanger sequencing.

Results: All patients had autosomal recessive pattern of inheritance. Clinically significant variants were identified in $4 \mathrm{CMS}$ disease-causing genes in five families: $\operatorname{COLQ}(2 / 5)$, DOK7 (1/5), RAPSN (1/5) and CHRNE (1/5). We identified 2 novel variants (c.930_933delCATG in DOK7 and c.1016_1032+2dup in CHRNE) and 2 known variants (c. $955-2 \mathrm{~A}>\mathrm{C}$ in $C O L Q$ and $\mathrm{c} .490 \mathrm{C}>\mathrm{T}$ in $R A P S N$ ) in homozygous state. $C O L Q$ variant c.955-2A $>\mathrm{C}$ was identified in two unrelated families. Patient with RAPSN variant had a later onset of disease while all others had an earlyonset of disease. All patients with COLQ variant had generalized muscle weakness, those with $D O K 7$ and RAPSN variants had limb-girdle weakness, and with CHRNE variant had predominant ocular weakness. Patients with $C O L Q$ and $D O K 7$ variants showed improvement with salbutamol and CHRNE with pyridostigmine therapy.

Conclusions: In CMS, genetic diagnosis is essential to guide pharmacotherapy and avoid adverse reactions to acetylcholinesterase inhibitors in $C O L Q, D O K 7$, and slow channel CMS (AChR subunit genes). This study adds a small but significant cohort of patients with CMS from India.

S. Mishra: None. K. Girisha: None. A. Shukla: None.

\section{P10.15.A}

Rare heterozygous DHTKD1 variants in patients with amyotrophic lateral sclerosis 
I. Gogol ${ }^{1,2}$, A. Osmanovic ${ }^{2}$,H. Martens ${ }^{1}$, M. Widjaja $^{1,2}, K$. Müller $^{3}$, O. Schreiber-Katz ${ }^{2}$, G. Schmidt ${ }^{l}$, B. Auber ${ }^{l}$, P. Andersen ${ }^{4}$, A. Ludolph ${ }^{3}, J$. Weishaupt ${ }^{3}, F$. Brand $^{l}$, S. Petri ${ }^{2}$, R. G. Weber ${ }^{l}$

${ }^{1}$ Department of Human Genetics, Hannover Medical School, Hannover, Germany, ${ }^{2}$ Department of Neurology, Hannover Medical School, Hannover, Germany, ${ }^{3}$ Department of Neurology, Ulm University, Ulm, Germany, ${ }^{4}$ Department of Neurosciences, Umeå University, Umeå, Sweden

Introduction: Amyotrophic lateral sclerosis (ALS) is a neurodegenerative disorder characterized by upper and lower motor neuron loss. Numerous genes regulating mitochondrial function have been associated with neurodegeneration. In a pilot whole-exome sequencing study of 27 ALS patients, rare heterozygous variants in the DHTKD1 gene were recurrently identified, including one nonsense variant. DHTKDl encodes a dehydrogenase subunit acting in L-lysine degradation in mitochondria. Pathogenic variants in DHTKD1 have been described causative of autosomal recessive 2-aminoadipic and 2-oxoadipic aciduria and autosomal dominant Charcot-Marie-Tooth disease type 2. Here, we aimed to investigate the frequency and clinical implications of rare DHTKDl variants in an ALS cohort.

Methods: We performed whole-exome $(n=46)$ and targeted $(n=179)$ sequencing of 225 unrelated ALS patients of central European ancestry. All patients were diagnosed according to the El Escorial criteria by a specialized neurologist. Clinical (e.g. age and region of onset, ALS subtype, and disease progression) and diagnostic (e.g. electrophysiological, biochemical) characteristics are being analyzed to discover genotype-phenotype relations.

Results: Five missense and one nonsense variant, all rare (MAF $<0.5 \%$ ) or novel and predicted to be pathogenic, were identified in 10 of 225 patients. Two of these variants were previously described in patients with 2-aminoadipic and 2-oxoadipic aciduria. Preliminary clinical correlations indicate a slightly later ALS onset in DHTKDI variant carriers (median: 71 years) compared to non-carriers (median: 63 years).

Conclusions: We identified rare heterozygous DHTKD1 variants in $4.4 \%$ of central European ALS patients. Potential phenotype-genotype correlations and frequency of rare DHTKDl variants in other ALS cohorts are currently being assessed.

I. Gogol: None. A. Osmanovic: None. H. Martens: None. M. Widjaja: None. K. Müller: None. O. SchreiberKatz: None. G. Schmidt: None. B. Auber: None. P. Andersen: None. A. Ludolph: None. J. Weishaupt: None. F. Brand: None. S. Petri: None. R.G. Weber: None.
P10.16.B

Dystrophinopathy patients with non-contiguous molecular alterations: diagnosis and characterization of the genetic mechanisms involved

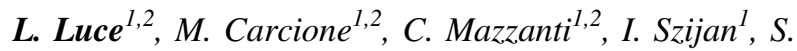
Menazzi $^{3}$, L. Francipane ${ }^{3}$, J. Nevado $^{4,5}$, P. Lapunzina ${ }^{4,5}$, L.

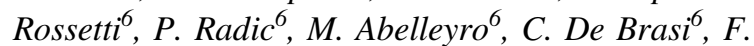
Giliberto $^{1,2}$

${ }^{1}$ Universidad de Buenos Aires; Departamento de Microbiología, Inmunología, Biotecnología y Genética; Cátedra de Genética; Laboratorio de Distrofinopatías, Buenos Aires, Argentina, ${ }^{2}$ CONICET - Universidad de Buenos Aires; Instituto de Inmunología, Genética y Metabolismo (INIGEM), Buenos Aires, Argentina, ${ }^{3}$ Universidad de Buenos Aires, Hospital de Clínicas "José de San Martín", División de Genética, Buenos Aires, Argentina, ${ }^{4}$ Hospital Universitario La Paz, IdiPAZ, Instituto de Genética Médica y Molecular (INGEMM), Madrid, Spain, ${ }^{5}$ Centro de Investigaciones Biomédicas en Red para Enfermedades Raras (CIBERER), Madrid, Spain, ${ }^{6}$ CONICET - Academia Nacional de Medicina, Instituto de Medicina Experimental (IMEX), Buenos Aires, Argentina

Introduction: Dystrophinopathies are neuromuscular Xlinked recessive diseases caused by $D M D$ mutations. Molecular alterations in this gene are large deletions/ duplications in $80 \%$ of cases and small mutations in the remaining. Several authors reported the occurrence of noncontiguous rearrangements within the same $D M D$ allele, with frequencies up to $4 \%$. The present work aims to characterize the incidence of complex rearrangements in an Argentinian dystrophinopathy cohort and unravel the causing molecular mechanisms.

Materials and Methods: We analyzed 437 boys with clinical diagnosis of Dystrophinopathy. The following techniques were implemented: MLPA, WES, WGS, PCRSanger Sequencing, CGH Array and HUMARA assay. In 2 cases, breakpoints were precisely determined, so we performed a bioinformatic screening of microhomologies, interspersed repeats, secondary structures and recombinogenic motifs $50 \mathrm{pb}$ surrounding each breakpoint.

Results: We detected 6 patients carrying complex rearrangements in DMD: 2 deletions-duplications, 3 noncontiguous duplications and 1 large deletion plus a $20 \mathrm{pb}$ insertion. These accounted for $1.4 \%$ of our cohort. In a deletion-duplication case, familial segregation and bioinformatics analysis suggested that the duplication was the first mutagenic event caused by Fork Stalling and Template Switching (FoSTeS), while the deletion occurred secondly by Non-homologous end joining. Furthermore, bioinformatic screening of the deletion plus insertion propose that 
the deletion was due to Microhomology-mediated end joining, while the insertion arose by FoSTeS.

Conclusions: Our findings widen the understanding of the molecular events that may take place in $D M D$ and characterize the occurrence of complex rearrangements in our dystrophinopathy cohort. This study was supported by PTC Therapeutics and University of Buenos Aires.

L. Luce: None. M. Carcione: None. C. Mazzanti: None. I. Szijan: None. S. Menazzi: None. L. Francipane: None. J. Nevado: None. P. Lapunzina: None. L. Rossetti: None. P. Radic: None. M. Abelleyro: None. C. De Brasi: None. F. Giliberto: None.

\section{P10.17.C}

Elucidating myopathies with high creatine-kinase- from unsolved cases to common diagnosis

C. L. Serban ${ }^{1,2}$, N. Andreescu ${ }^{1,2}$, I. Jurca-Simina ${ }^{1,2}, A$. Corches $^{1,2}$, A. Chiriţă Emandi ${ }^{1,2}$, M. Puiu ${ }^{1,2}$

${ }^{1}$ Victor Babes University of Medicine and Pharmacy, Timisoara, Romania, ${ }^{2}$ Louis Turcanu Clinical Emergency Hospital for Children, Timişoara, Romania

Background: One of the most common causes for myopathies are related to X-linked DMD gene, including Duchene Muscular Dystrophy(DMD) and Becker Muscular Dystrophy(BMD). Other autosomal recessive myopathies affect males(M) and females(F) consisting of limb-girdle myopathies.

Method: 42 patients with high levels of creatine-kinase addressed the Regional Center of Medical Genetics Timis County, Romania (October 2017-September 2019), either having suggestive symptoms of myodystrophy (23M and $1 \mathrm{~F})$ or for verifying carrier $(6 \mathrm{M}$ and $10 \mathrm{~F})$.

Results: DMD was diagnosed in 13M, with hemizygous variants in $D M D$ gene (using MPLA) with following exons deletion: $10-11 ; 13-17 ; 22-41 ; 39-43 ; 46-53 ; 51 ; 58$,exon $3-7$ duplication and variants (using NGS): c.7174dupG; c.8608C >T; c.8688_8689delAG; c.10738delG. BMD was diagnosed by MLPA in 6M: deletions in exons 45-47 and 45-51. Two male cousins had a previously unreported splice site NM_004009.3:c.175-1G>T pathogenic variant in DMD gene. After negative testing for DMD gene, limb-girdle muscular dystrophy was confirmed using NGS in compound heterozygosity in FKRP gene in 3 patients, of which 2 were siblings(M/F): c. $148 \mathrm{~A}>\mathrm{G} / \mathrm{c} .985 \mathrm{G}>\mathrm{A}$, and one unrelated (c.826C $>\mathrm{A} / \mathrm{c} .935 \mathrm{G}>\mathrm{C}$ ). Pompe disease was diagnosed in two brothers showing pathogenic variants in $G A A$ gene (exon 18 deletion) and in one unrelated patient with c.784G $>A /$ ? . Carrier status for DMD was identified in 6 out of 10F. Using MLPA for DMD and a NGS panel of
4813 genes, no pathogenic variants were detected in $4 \mathrm{M}$ and $1 \mathrm{~F}$ (persistent extremely elevated CK levels).

Conclusions: This study shows a diagnostic yield of almost $80 \%$ and lists the variants associated with myodystrophies, underling the limitation in establishing the diagnosis in patients with increased CK levels.

C.L. Serban: None. N. Andreescu: None. I. JurcaSimina: None. A. Corches: None. A. Chiriță Emandi: None. M. Puiu: None.

\section{P10.21.A}

The floppy child syndrome is not only caused by mutations in genes related to neuromuscular disorders.

M. Gos ${ }^{I}$, E. Dębek ${ }^{1}$, A. Madej-Pilarczyk ${ }^{2}$, A. PotulskaChromik $^{3}$, J. Pilch ${ }^{4}$, R. Śmigiel ${ }^{5}$, J. Wierzba ${ }^{6,7}$, R. Posmyk ${ }^{8}$, J. Fijak-Moskal ${ }^{9}$, A. Kutkowska-Kaźmierczak ${ }^{l}$, A. JakubiukTomaszuk ${ }^{10}$, B. Wojtaś $^{11}$, B. Gielniewskil ${ }^{11}$, A. KosteraPruszczyk ${ }^{3}, J$. Bal ${ }^{1}$, M. Jędrzejowska ${ }^{12,2}$

${ }^{I}$ Department of Medical Genetics, Institute of Mother and Child, Warsaw, Poland, ${ }^{2}$ Department of Medical Genetics, The Children's Memorial Health Institute, Warsaw, Poland, ${ }^{3}$ Department of Neurology, Medical University of Warsaw, Warsaw, Poland, ${ }^{4}$ Department of Pediatrics and Neurology for Children and Adolescents, Medical University of Silesia, Katowice, Poland, ${ }^{5}$ Department of Paediatrics and Rare Disorders, Wroclaw Medical University, Wroctaw, Poland, ${ }^{6}$ Department of General Nursery, Medical University of Gdańsk, Gdańsk, Poland, ${ }^{7}$ Department of Pediatrics, Hematology and Oncology, Medical University of Gdansk, Gdańsk, Poland, ${ }^{8}$ Department of Clinical Genetics, Medical University of Biatystok, Biatystok, Poland, ${ }^{9}$ Department of Medical Genetics, Polish-Amercian Institute of Pediatrcs, Jagiellonian University, Krakow, Poland, ${ }^{10}$ Department of Pediatric Neurology and Rehabilitation, Medical University of Biatystok, Biatystok, Poland, ${ }^{11}$ Laboratory of Molecular Neurobiology, Neurobiology Center, Nencki Institute of Experimental Biology, Polish Academy of Sciences, Warsaw, Poland, ${ }^{12}$ Neuromuscular Department, Mossakowski Medical Research Centre, Polish Academy of Sciences, Warsaw, Poland

Introduction: The, floppy child syndrome" is one of the most unambiguous clinical manifestations. Neonatal or childhood hypotonia can present with other symptoms, like decreased muscle tone, arthrogryposis or developmental delay making a definite clinical diagnosis challenging. We aimed to assess the genetic causes of this condition, with interest in neuromuscular disorders (NMD).

Patients and methods: For 124 patients with excluded common genetic causes of early hypotonia (e.g. SMA, PWS, MTMI and ACTA1 mutations), an exome sequencing 
have been performed. The first-tier analysis of exome data has been done towards the identification of pathogenic variants in NMD-, arthrogryposis- or connective tissue disorder-related genes.

Results: Mutations in genes related to neuromuscular disorders (NMD) were identified in 42 patients. In 21 (32\%) and $18(27 \%)$ probands, we have found mutations in genes related to congenital myopathies or muscular dystrophies, respectively. The commonly affected genes included LMNA, RYRI and TTN with 5, 4 and 3 patients with pathogenic variants, respectively. In 13 patients, mutations in non-NMD genes were found, including 2 cases with mutation in FKBP14 (Ehlers-Danlos syndrome), PIEZO2 (distal arthrogryposis) and $N A L C N$ (hypotonia with psychomotor delay). Mutations in DDX3X, COL9A3, NEXMIF, PPP2R1A, CTBP1, SLC16A2 and GRIA3 were found in single cases.

Conclusions: The genetic cause of floppy child syndrome was identified in 55/124 (44.3\%) patients. Although the most commonly mutated genes are related to NMD, other genes involved in etiology of connective tissue disorders and syndromic hypotonia should be also considered.

The study was supported from National Science Centre grant no. UMO-2015/17/B/NZ5/01368

M. Gos: None. E. Dębek: None. A. Madej-Pilarczyk: None. A. Potulska-Chromik: None. J. Pilch: None. R. Śmigiel: None. J. Wierzba: None. R. Posmyk: None. J. Fijak-Moskal: None. A. Kutkowska-Kaźmierczak: None. A. Jakubiuk-Tomaszuk: None. B. Wojtaś: None. B. Gielniewski: None. A. Kostera-Pruszczyk: None. J. Bal: None. M. Jędrzejowska: None.

\section{P10.22.B}

Diagnostic of facioscapulohumeral muscular dystrophy in Russian patients based on newly developed qPCR approach

\section{N. Zernov, M. Skoblov, A. Guskova}

Research centre for medical genetics, Moscow, Russian Federation

Introduction: Facioscapulohumeral muscular dystrophy (FSHD) is one of the most prevalent congenital myodystrophies. FSHD molecular-genetic diagnostics requires expensive and laborious methods. Here we present a newly developed simplified qPCR-based diagnostic approach and obtained results.

Materials and Methods: Blood-derived DNA samples from 64 FSHD patients and 39 their phenotypically healthy relatives were examined by Southern blotting, molecular combing, and a newly developed qPCR-based approach.
Results: We validated the qPCR-based approach on 26 individuals with at least one allele of chromosome 4 D4Z4 array contracted up to 10-1 repeats, and on 19 individuals without the contraction. In $96 \%$ of the cases, qPCR-based results were concordant with the Southern blotting and molecular combing references. Remaining 60 individuals were analyzed only by qPCR-based approach. In a group of 64 affected individuals, FSHD diagnosis was confirmed for $36(56.3 \%)$ patients. In $69 \%$ of the confirmed cases, pathogenic alleles had number of D4Z4 repeats in range between 3-6. We defined 13 carriers in a group of 39 healthy relatives $(33.3 \%)$. Among them, $76.9 \%$ of cases had the permissive allele with number of the D4Z4 repeats from 6 to 8 . There were no significant prevalence between sex in any observed groups.

Conclusions: In this work we present newly developed FSHD diagnostics approach based on qPCR and first study of the FSHD genotyping in Russian patients. We observed enrichment of the permissive alleles in the range of 3-6 D4Z4 units among affected individuals, whereas among carriers permissive alleles were enriched in the range 6-8 D4Z4 units.

N. Zernov: None. M. Skoblov: None. A. Guskova: None.

P10.24.A

Molecular -genetic study of hereditary spastic paraplegia in Russian patients

V. A. Kadnikova, O. P. Ryzhkova, G. E. Rudenskaya, A. V. Polyakov

Research Centre for Medical Genetics, Moscow, Russian Federation

Introduction: Hereditary spastic paraplegia (HSP) is highly heterogeneous group of neurodegenerative diseases that is characterized by progressive spasticity and weakness of the lower limbs and mild sensory dysfunction, due to the axonal degeneration in the pyramidal tract. Mutations in about 80 different loci and 60 genes have been associated with HSP pathogenesis. Thus the application of NGS technologies can enable the enhanced molecular diagnosis of HSP because multiple loci can be analyzed simultaneously. Herein we characterize a cohort of Russian HSP patients using NGS techniques along with the routine diagnostics.

Materials and Methods: We screened DNA samples from 198 unrelated probands with HSP. Sanger sequencing of SPAST gene was performed in 60 DNA samples, and ATL1 was analyzed in 24 of partially overlapping samples. DNA with unidentified mutations and non-tested samples 
were examined by MLPA-analysis and by target gene panel containing 63 HSP related genes.

Results: We diagnosed the genetic type of HSP for 97 (49\%) patients (SPG4 - 54.6\%, SPG3A - 14.4\%, SPG30 $6.2 \%$, SPG1 $1-6.2 \%$, SPG8 - 3.1\%, SPG17 - 3.1\%, and 9 more forms less than in $3 \%$ each). We find 88 mutations across diagnosed patient, whereas 39 mutations were new. Remarkably, $85.2 \%$ of mutations were discovered by NGS and Sanger sequencing; and $14.8 \%$ of larger genomic rearrangements - by MLPA-analysis.

Conclusions: Our data show that combination of NGS and routine methods allows for effective HSP diagnostics. This is the first overview of the HSP mutations frequencies in Russian population that have to be further expanded.

V.A. Kadnikova: None. O.P. Ryzhkova: None. G.E. Rudenskaya: None. A.V. Polyakov: None.

P10.25.B

Genetic analysis of 50 unrelated cases affected with hereditary spastic paraplegia (HSP): a novel candidate gene

R. Hajati ${ }^{l}$, A. Davarzani ${ }^{1}$, S. Hashemi ${ }^{l}$, M. Rahimi Bidgoli $^{l}$, L. Javan Parast ${ }^{1}$, M. Pashaei ${ }^{1}$, F. DanaeeFard ${ }^{l}$, H. Najmabadi ${ }^{l}$, M. Rohani ${ }^{2}$,F. Fatehi ${ }^{3}$, S. Nafissi ${ }^{3}$, A. Alavi ${ }^{1}$

${ }^{1}$ Genetics research center, University of Social Welfare and Rehabilitation Sciences, Tehran, Iran, Islamic Republic of, ${ }^{2}$ Department of Neurology, Iran University of Medical Sciences, Hazrat Rasool Hospital, Tehran, Iran, Islamic Republic of, ${ }^{3}$ Department of Neurology, Tehran University of Medical Sciences, Tehran, Iran, Islamic Republic of

Introduction: Hereditary spastic paraplegia (HSP) is a genetically heterogeneous neurodegenerative disorder is characterized by lower-limb spasticity and weakness. To date, more than 70 genes have been identified that contribute to the cause of HSP. Despite the use of nextgeneration sequencing-based methods, genetic-analysis has failed in the finding of causative-genes in $~ 50 \%$ of HSP patients, indicating a significant genetic heterogeneity in the disease and emphasizing that the majority of HSP-genes have remained unknown.

Materials and Methods: To identification of HSPcausing genes, whole-exome sequencing (WES) was performed in 50 unrelated-Iranian probands. Candidate variants were confirmed and co-segregated in the family members. Functional studies including RNAseq for a family harboring a variation in a novel candidate-gene are currently in progress.

Results: Variations in 18 known HSP-causing genes including SPEG, ERLIN1, ERLIN2, ATAD3A, SPAST (four families), ENTPD1, SPG7 (two families), KIF5A, CYP7B1,
ZFYVE26, GJC2, CAPN1, ZFYVE26, KIF1B, SPG11 (four families), ATL1 (two families), MFN2, C19orf12, and one novel candidate-HSP gene were identified.

Discussion: HSP-causing genes were detected in 26 probands(52\%). Also, a novel candidate-gene that is exclusively expressed in the brain and directly involved in axonogenesis was identified. Given that the HSP is a lengthdependent, "dying-back" axonopathy, the candidate-gene can be an important HSP-causative gene. The research presented the powers of WES for facilitating genediscovery and identification of causative genes for diseases with genetic heterogeneity. The precise mechanisms underlying the HSP are unknown. Identification of novel genes and molecular-pathways will enhance our knowledge of the cellular pathways that are involved in the disease pathogenesis.

Grant-Reference:no.963846(NIMAD)

R. Hajati: None. A. Davarzani: None. S. Hashemi: None. M. Rahimi Bidgoli: None. L. Javan Parast: None. M. Pashaei: None. F. DanaeeFard: None. H. Najmabadi: None. M. Rohani: None. F. Fatehi: None. S. Nafissi: None. A. Alavi: None.

P10.26.C

GWAS on hand grip strength: new insights into the genetics of muscular strength support a strong genetic component at different ages.

\section{F. Abbondanza, S. Paracchini}

\section{University of St Andrews, St Andrews, United Kingdom}

Introduction: Hand grip strength (HGS) is a widespread proxy for muscular strength and a predictor of cardiovascular disease and poor health outcomes. Genetic studies in adult cohorts (e.g. UK Biobank) found multiple genes associated with HGS and showed a modest heritability $\left(\mathrm{h}^{2} \sim 24 \%\right)$. However, the genetics of HGS remains largely elusive and has never been explored in a cohort of young individuals.

Materials and Methods: We performed GWAS analyses on three measures of HGS in the ALSPAC dataset $(\mathrm{N} \sim 5,400$, mean age $=11.8$ years $)$, namely HGS for the right and left hand and grip strength maximal, i.e. best result regardless of the hand used. Analyses were performed in PLINK and functional mapping with FUMA. Genetic correlations and SNP-heritability were computed with LD Score Regression using summary statistics from UK Biobank.

Results: We reported a novel genome-wide significant hit for HGS (rs2968991, p < 1.75E-08) with the right hand and we replicated a common association in UK Biobank for the three traits $(\mathrm{p}<1 \mathrm{E}-06)$. We reported a higher SNP- 
heritability than previous studies and highlighted a moderate-to-high genetic correlation between HGS in the current study and in the UK Biobank.

Conclusions: In addition to a novel association, our analysis replicated some of the associations previously reported both at pathway (skeletal muscle fibres and neurological disorders pathways) and gene-specific (HOXB3) level. This, together with the modest-to-high genetic correlation with HGS in the UK Biobank suggests a stable genetic component of HGS at different ages. This work was funded by the Royal Society.

F. Abbondanza: None. S. Paracchini: None.

\section{P10.27.A}

new mutation causing hypokalemic periodic paralysis in a young child, a practical example for helpfulness of genotype first approach

\section{Shariati, R. Jafarzadeh Esfehani, R. Boostani, M. Soudyab, A. Sadr-Nabavi}

Mashhad University of Medical Sciences, Mashhad, Iran, Islamic Republic of

Introduction: Hypokalemic periodic paralysis(HOKPP) is a rare disorder usually manifesting by episodes of muscle weakness or paralysis. However in some cases, clinical presentation may be atypical and may be misdiagnosed with other possible progressive neurological diseases. The present report describes a case of HOKPP which was first misdiagnosed as a case of distal spinal muscular atrophy (DSMA).

Material and methods: A 5 years old male child referred because of variable distal limb weakness. The patient had a history of hypotonia at birth, evidence of irritable myopathy on electro-diagnostic study and elevated creatinephosphokinase. The whole exome sequencing(WES) revealed a variant with unknown significant(VUS) in IGHMBP2 gene $(\mathrm{c} .403 \mathrm{~A}>\mathrm{G})$ causing DSMA. However, the VUS was not confirmed in segregation analysis and the patient's WES data was reanalyzed with a genotype first approach.

Results: The FASTQ files were reanalyzed by Seamless ${ }^{\mathrm{TM}}$ software and the pathogenicity of 112 variants which were pathogenic or likely pathogenic were checked in Varsome $^{\mathrm{TM}}$ and Sixteen pathogen and likely pathogen variants were remained checked in $\mathrm{OMIM}^{\mathrm{TM}}$ database. Only 1 candidate variant in CACNA1S gene(c.3724A $>\mathrm{G})$ causing HOKPP was remained. After a brief history taking, prolonged episodes of muscle weakness after exercises in school was reported. Our segregation study on patient's family confirmed the diagnosis of HOKPP and the patient's father also reported milder and similar weakness in his childhood and carried the same mutation.

Conclusions: The present experience demonstrates that a genotype first approach can be helpful in discovering novel mutation in rare diseases even with atypical clinical presentations or negative WES results.

M. Shariati: None. R. Jafarzadeh Esfehani: None. R. Boostani: None. M. Soudyab: None. A. SadrNabavi: None.

P10.29.C

A case of Neuromyotonia associated to a C9ORF72 CCCGG expansion

G. Bonapace ${ }^{l}$, R. Gullace ${ }^{l}$, R. Procopio ${ }^{2,3}$, M. Gaglardi ${ }^{3}$, F. Fortunato $^{2}$, A. Gambardella ${ }^{2,3}$, P. Valentino $^{2}$, G. Annesi ${ }^{3}$

${ }^{1}$ University Magna Graecia, Faculty of Medicine, Pediatrics, Catanzaro, Italy, ${ }^{2}$ Institute of Neurology, University "Magna Graecia" of Catanzaro, Department of Medical Sciences, Catanzaro, Italy, ${ }^{3}$ Neuroimaging Research Unit, IBFM/CNR; Institute of Neurology, Catanzaro, Italy

Introduction: Neuromyotonia is a peripheral nerve hyperexcitability (PNH) syndrome that presents as continuous motor activity. Mutations in the KCNAl gene, which encodes the voltage-gated potassium channel, Kv1.1 subunit, have been found to be associated with this syndrome.

Materials and Methods: The patient was a 56-year man presenting cramps painful stiffness of calves, undulating wave-like rippling of left thigh muscles. The neurological examination showed visible myokymias of left thigh. Electromyography revealed spontaneous continuous single motor unit discharges firing at a high intraburst frequency $(>150 \mathrm{~Hz}$ )compatible with neuromyotonic discharges, on vastus medialis of the left tight. Single needle electromyography and nerve conduction studies were unremarkable. Brain MRI and laboratory test excluded autoimmune or paraneoplastic conditions. The Molecular analysis revealed no point mutation on the KCANAl gene. Based on the recently identified role for $C 9 O R F 72$ protein, in modulating the membrane channel subunits maturation, an Ampliconlength analysis on the C9ORF72 gene, was performed.

Results: Capillary electrophoresis analysis of the patient amplicon revealed the presence of a 94 pathological CCCGG expansion.

Conclusions: The Kv1.1 subunit recycling processes is driven by specific Rab proteins and regulated by the C9ORF72 protein acting both as Rab effector and component of the GTP ase exchange factor GEF. We hypothesize that the loss of function of the $c 9$ orf 72 protein, due to the CCCGG expansion can affect the trafficking of 
the Kv1.1 monomers leading to a reduction in the channel subunits correctly allocated into the plasma membrane, altering the normal flows of potassium during the action potential generation.

G. Bonapace: None. R. Gullace: None. R. Procopio: None. M. Gaglardi: None. F. Fortunato: None. A. Gambardella: None. P. Valentino: None. G. Annesi: None.

\section{P10.30.A}

Identification of two rare homozygous frameshift variants in $L B X 1$ and PAPD5/TENT4B genes in siblings with chronic respiratory insufficiency

D. Musalkova ${ }^{l}$, L. Noskova ${ }^{l}$, V. Stranecky ${ }^{l}, P$. Melenovska $^{l}$, R. Dobrovolny ${ }^{l}, H$. Treslova ${ }^{l}, K$. Hodanova ${ }^{l}$, H. Hartmannoval ${ }^{1}$ A. Gregorova ${ }^{2}$, T. Honzik ${ }^{l}$, S. Kmoch ${ }^{l}$

${ }^{1}$ Research Unit for Rare Diseases, Department of Pediatrics and Adolescent Medicine, First Faculty of Medicine, Charles University, Prague, Czech Republic, ${ }^{2}$ Department of Medical Genetics, University Hospital Ostrava, Ostrava, Czech Republic

We report a case of two siblings with chronic respiratory insufficiency, psychomotor retardation, and suspected congenital myopathy. The children were born to consanguineous parents of Roma ethnicity.

Exome sequencing identified two rare homozygous frameshift variants in $L B X 1$ and PAPD5 genes. These variants were present in heterozygous state in the parents.

LBX1 is a transcription factor that affects migration of muscle precursor cells and it has been associated with adult idiopatic scoliosis. Identical frameshift variant (p.Val236AlafsTer59) was described in a recent publication (Hernandez-Miranda et al., 2018) as a variant that interferes only with a subset of Lbx1 functions, specifically, with the ability to cooperate with Phox $2 b$. This disturbance leads to congenital central hypoventilation. The variant was present in one individual of Roma ethnicity in our internal database but is absent from GnomAD database. Ethnicity-specific genomic databases would be useful tools to estimate population frequency of candidate variants.

The effect of the homozygous frameshift variant in PAPD5/TENT4B gene (p.Ser610ArgfsTer56) is not clear. The gene is classified as intolerant to the loss of function $(\mathrm{pLI}=0,999)$ but it is not associated with any disorder. The TENT4B enzyme was recently described as a noncanonical poly(A) polymerase that generates mixed poly(A) tails with intermittent guanyl residues that shield mRNA from degradation (Lim et al., 2018).

Two homozygous candidate variants in siblings born to consanguineous parents substantially complicate a potential prenatal or preimplantation genetic testing in the family. At the same time, it underscores the importance of wholeexome sequencing.

D. Musalkova: None. L. Noskova: None. V. Stranecky: None. P. Melenovska: None. R. Dobrovolny: None. H. Treslova: None. K. Hodanova: None. H. Hartmannova: None. A. Gregorova: None. T. Honzik: None. S. Kmoch: None.

\section{P10.31.B}

Investigating the pathogenicity of CAPN3 c.1746-20C $>$ G splicing variant and its association with LGMD R1 calpain3-related

M. Mroczek ${ }^{l}$, J. Hudson ${ }^{2}$, A. Töpf $f^{l}$, R. Barresi ${ }^{l}$, J. Duff ${ }^{\prime}$, E. England $^{3}$, K. Chao ${ }^{3}$, M. Sobalska-Kwapis ${ }^{4}$, D. Strapagiel ${ }^{4}$, D. MacArthur ${ }^{3}$, V. Straub ${ }^{1}$

${ }^{1}$ Translational and Clinical Research Institute, Newcastle upon Tyne, United Kingdom, ${ }^{2}$ Highly Specialized Service (HSS) for LGMDs, Newcastle upon Tyne, United Kingdom, ${ }^{3}$ Analytic and Translational Genetics Unit, Massachusetts General Hospital, Boston, MA, United States, ${ }^{4}$ The Biobank Lab, Department of Molecular Biophysics, Lodz, Poland

Limb girdle muscular dystrophy (LGMD) R1 calpain3related is the most common autosomal recessive form of LGMD. The CAPN3 c. 1746-20C $>$ G variant has a population frequency of above $>1 \%$ and there is conflicting information regarding its pathogenicity in the literature. In approximately 2.000 exome sequenced patients included in the MYO-SEQ project we identified c.1746-20C $>$ G 26 times in heterozygosity and 1 time in homozygosity. We recruited further centres from Poland, Estonia, Latvia and UK. We identified the c.1746-20C $>$ G together with CAPN3 c.598_612del, c.550delA, p.Arg39Cys, p.Gly234Arg and other pathogenic heterozygous CAPN3 variants. One individual affected with LGMD was homozygous for c.174620C $>$ G. Most of the patients were of Polish or Eastern European origins. In all these cases there was reduced calpain3 expression on Western Blots and muscle MRI in available cases was suggestive for LGMD R1 calpain3related. The phenotype of these patients was keeping up with mild calpainopathy with characteristic hypertrophies. Population studies suggest allele frequency of 0.017 in Poland and 0.015 in Estonia. No homozygous healthy individuals were identified so far in our populational studies and according to gnomAD there are 3 homozygous individuals (out of 282.874). In some populations the variant was rare or nearly absent. Functional studies are ongoing. Population frequency data suggest that c.1746-20C $>$ G may be a hypomorphic variant. Preliminary haplotype analysis of a patient homozygous for c. 1746-20C $>\mathrm{G}$ revealed a 
haplotype block, indicating this might be a founder allele in Polish population. CAPN3 c. 1746-20C $>$ G variant may be often missed as many tests include only 10bp of flanking intronic region.

M. Mroczek: None. J. Hudson: None. A. Töpf: None. R. Barresi: None. J. Duff: None. E. England: None. K. Chao: None. M. Sobalska-Kwapis: None. D. Strapagiel: None. D. MacArthur: None. V. Straub: None.

\section{P10.33.A}

Genetic variability and clinical spectrum of limb-girdle muscular dystrophy in a cohort of Turkish patients

D. Mortazavi ${ }^{1}$, E. Vanl Yavuz ${ }^{2}$, E. Borklu Yucel ${ }^{3}$, S. Avcl ${ }^{3}$, S. Eraslan ${ }^{3}$, P. Oflazer ${ }^{2}$, H. Kayserili ${ }^{3}$

${ }^{1}$ Koç University, Graduate School of Health Science, Istanbul, Turkey, ${ }^{2}$ Department of Neurology, Koç University School of Medicine, Istanbul, Turkey, Istanbul, Turkey, ${ }^{3}$ Department of Medical Genetics, Koç University School of Medicine, Genetic Diagnostics Center Istanbul, Turkey, Istanbul, Turkey

Background: Limb-Girdle Muscular Dystrophies (LGMDs) are clinically and genetically heterogeneous group of neuromuscular disorders affecting hip and shoulder muscles. Features include hypertrophy of calf muscles and elevated level of serum CK. Age of onset, progression and distribution of weakness vary according to genetic subtypes which are related to more than 30 genes. We aimed to correlate the clinical manifestations and clinical exome sequencing results of 29 patients with LGMD from Turkey.

Method: 29 patients with clinical diagnosis of LGMD were recruited to NGS-based clinical exome screening of 4811 genes, we performed during 2017-2019, at tertiary health center, Istanbul/Turkey.

Results: In 28 patients, 34 variants were identified, seven of variants are novel. In 28 patients, the most common subtypes were LGMD R1 calpain3-related in eleven (39.28\%), LGMD R2 dysferlin-related in nine (32.14\%), Sarcoglycanopathies in four [14.28\%; LGMD R3 $\alpha$-sarcoglycan-related in three (10.71\%), LGMD R5 $\gamma$-sarcoglycan-related in one(3.57\%)], LGMD R14 POMT2-related, LGMD R20 ISPD-related, LGMD R7 telethonin-related and LGMD R10 titin-related, each in one patient (3.57\%) and one patient with no variant related to the phenotype. Rare clinical presentations were observed, one LGMD R10 titin-related patient with cognitive/psychomotor retardation and a LGMD R14 POMT2-related patient with distal weakness. Increased liver aminotransferases (ALT/AST) led to diagnosis of neuromuscular diseases in seven patients.
Conclusions: The result of the cohort led to better understanding of the relative prevalence of different LGMD subtypes and genotype-phenotype correlation in Turkish population.

D. Mortazavi: None. E. Vanlı Yavuz: None. E. Borklu Yucel: None. S. Avcı: None. S. Eraslan: None. P. Oflazer: None. H. Kayserili: None.

\section{P10.34.B}

Expanding of mutation spectrum in Muscular Dystrophies: A Turkish Cohort

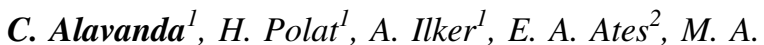

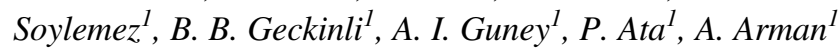

${ }^{1}$ Marmara University School of Medicine, Medical Genetics Department, Istanbul, Turkey, ${ }^{2}$ Marmara University Pendik Research and Training Hospital, Medical Genetics Department, Istanbul, Turkey

Muscular dystrophies are heterogeneous group of disorder. More than 40 genes were identified and genotype-phenotype correlation is not clear for most of cases. The aim of this study is to improve the genotypic features of muscular dystrophies other than Duchenne-Becker Muscular Dystrophy with novel mutations. Thirty-three patients who were suffering from muscular dystrophy were referred to our clinic. Sixteen patients were male and seventeen patients were female. Mean age was 22,06 years and mean age of disease onset was 11,48 years. Creatinine kinase levels were between 27.000 and 90 U/L(mean 2.964). Next-Generation Sequencing(NGS) panel which has 33 muscular dystrophy related genes were sequenced for all patients. Reported pathogenic mutations in fourteen patients and novel mutations in twelve patients were identified. One patient's biopsy had shown megaconial changes, NGS panel revealed homozygous c.1031G>A(p. Arg344Gln) known mutation in CHKB gene. Another mutation in $\mathrm{CHKB}$ gene which was novel homozygous c.1130G $>$ T(p.Arg377Leu) mutation was detected in elevenyear-old patient. Novel homozygous c. $195+3 \mathrm{~A}>\mathrm{T}$ mutation in SGCG gene was identified in patient whose biopsy showed pathogenic staining with gamma-sarcoglycan. In thirty-fouryear-old patient and her sibling who had same clinic novel homozygous c.3666delA(p.Asp1223Thefs*24) mutation in DYSF gene were identified. In CAPN3 gene two novel homozygous mutations were detected, which were c.691T $>C$ (p.Phe231Leu) and c.632+2T>G. In one patient, CNV analyses revealed SYNE2 gene exon 65-66 deletion. Homozygous SGCA c.850C $>\mathrm{T}$ (p.Arg284Cys) mutation in two unrelated patients were detected, which indicates this mutation may have founder effect. Herein we present 33 patients' genetic test results with recurrent and novel mutations which contribute the literature. 
C. Alavanda: None. H. Polat: None. A. Ilker: None. E. A. Ates: None. M.A. Soylemez: None. B.B. Geckinli: None. A.I. Guney: None. P. Ata: None. A. Arman: None.

\section{P10.35.C}

Genetic analysis of 309 Iranian patients with muscular dystrophy and congenital myopathy by next generation sequencing

M. Asadollahi ${ }^{1}$, A. Karamzade ${ }^{I}$, M. Taghizadeh ${ }^{2}, Z$. Golchehre $^{I}$, P. Nourmohammadi ${ }^{2,3}, N$. Nourpour ${ }^{2,3}, M$. Jamshidifar $^{2,3}, \quad R$. Taheri ${ }^{2,3}$, A. Keramatipour ${ }^{3}, \quad M$. Keramatipour $^{1,2,3}$

${ }^{1}$ Department of Medical Genetics, School of Medicine, Tehran University of Medical Sciences, Tehran, Iran, Islamic Republic of, ${ }^{2}$ Watson Genetic Laboratory, North Kargar Street, Tehran, Iran, Islamic Republic of, ${ }^{3} N G S$ Department, Pishgam Biotech Company, North Kargar Street, Tehran, Iran, Islamic Republic of

Muscular dystrophies and congenital myopathies are a large group of heterogeneous muscle disorders. Here, next generation sequencing (NGS) was used to investigate genomic variants including copy number variants (CNV) to determine causative variants behind clinical presentations in patients. DNA samples were extracted from 309 patients with presentation of muscular dystrophy and congenital myopathy who were referred to our laboratory. NGS was performed to investigate genes associated with muscular disorders. NGS findings were validated using other molecular methods such as MLPA, QPCR, long PCR, and PCR-Sanger sequencing. In 275 out of 309 patients, variants with pathogenic or likely pathogenic classifications $(88.9 \%)$ were detected. Based on genetic finding and other clinical and para-clinical data, diagnosis was confirmed in these 275 patients. Total number of known or novel detected pathogenic and likely pathogenic variants is 303 variants in 51 genes. The most frequent genes causing the disease in this study were DMD $(68 / 309,22 \%)$, LAMA2 (36/309, 11\%), and SGCB (31/309, 10\%). Moreover, the most common mutations detected in our patients were gross deletion/duplication variants in DMD gene, SGCB deletions and deletion of exon 7 in SMN1 gene. Detailed analysis of data will be presented in the meeting. In addition to obtaining valuable data regarding genetic epidemiology of hereditary muscular diseases in Iran, this study showed great value of NGS for defining causative variants in patients with muscular diseases. High frequency of $\mathrm{CNV}$ s detected as the causative variants in patients shows the necessity of developing efficient tools for CNV analysis on NGS data.

M. Asadollahi: None. A. Karamzade: None. M. Taghizadeh: None. Z. Golchehre: None. P. Nourmohammadi: None. N. Nourpour: None. M. Jamshidifar:
None. R. Taheri: None. A. Keramatipour: None. M. Keramatipour: None.

\section{P10.36.A}

Usefulness of studying myopathies and muscular dystrophies through Exome Sequencing: an experience of 73 cases

Y. Moreno-Saez ${ }^{l}$, A. Romera-López ${ }^{l}$, A. Andújar ${ }^{l}$, A. López $^{2}$, T. Otero ${ }^{1}$, M. D. Ruíz', S. Valverde ${ }^{l}$, C. CasañMartínez $^{l}$, N. Serrano ${ }^{1}$, J. L. Díaz ${ }^{l}$, J. García ${ }^{l}$, A. Gómez ${ }^{l}$, C. Collado ${ }^{1}$, A. Arilla ${ }^{l}$, C. M. Moya ${ }^{l}$, A. Matilla-Dueñas ${ }^{3}$, S. Santillán ${ }^{1}$

${ }^{1}$ Sistemas Genómicos, Paterna (Valencia), Spain, ${ }^{2}$ SIGENFundación Santa $\mathrm{Fe}$ de Bogotá, Bogotá (Colombia), Colombia, ${ }^{3}$ Health Sciences Research Institute Germans Trias i Pujol, Badalona (Barcelona), Spain

Muscle weakness and hypotonia usually suggest a clinical diagnosis of myopathy or muscular dystrophy. However, these pathologies include more than 50 subtypes and are associated with more than 250 genes, making an accurate diagnosis challenging.

Genetic testing through NGS has a huge impact on diagnosis due to its ability to analyze, in parallel, large number of genes and due to the possibility of reanalyzing non-conclusive cases including new disease-causing genes. Such potential has been proven to be even useful for the diagnosis of families with a strong history of hereditary disease when no affected members are available and healthy relatives must be tested.

Here, we applied NGS to a cohort of 73 patients with clinical or familial suspicion of myopathy or muscular dystrophy. Samples were analyzed using SureSelect XT Human All Exon kit (Agilent) and a HiSeq sequencer (Illumina). An in-house pipeline was used for the bioinformatic analysis. Fifty-four out of 73 individuals were analyzed using a whole clinical exome approach while the others were analyzed through virtual panels. Variant filtering was performed based on variant effect, familial pattern of inheritance, allele frequency and information available in databases.

Genetic diagnosis was achieved in 23 out of 73 patients $(31,5 \%)$ including a patient with a complex phenotype of tricuspid insufficiency with structural heart disease and fibromyalgia. In 39 patients only VOUS were detected $(53,4 \%)$. In 11 out of 73 patients $(15,1 \%)$ no candidate variants were found. These results confirm exome sequencing as an effective strategy to study myopathies and muscular dystrophies.

Y. Moreno-Saez: None. A. Romera-López: None. A. Andújar: None. A. López: None. T. Otero: None. M.D. 
Ruíz: None. S. Valverde: None. C. Casañ-Martínez: None. N. Serrano: None. J.L. Díaz: None. J. García: None. A. Gómez: None. C. Collado: None. A. Arilla: None. C.M. Moya: None. A. Matilla-Dueñas: None. S. Santillán: None.

\section{P10.37.B}

NGS-based testing in diagnostics of hereditary neuromuscular disorders: observations on a large cohort from a clinical bioinformatician's perspective

M. Ampleeval, E. Tolmacheval, I. Komar'kov' ${ }^{2}, N$. Shcherbakova ${ }^{3}$, D. Khmelkova ${ }^{4}$, E. Surkova $^{2}$, O. Ryzhkova ${ }^{5}$, V. Kaimonov ${ }^{4}$, I. Mironova ${ }^{4}$, E. Pomerantseva ${ }^{4}$, A. Isaev ${ }^{4}$, E. Dadali ${ }^{5}$, D. Pyankov ${ }^{2}$, A. Antonets ${ }^{2}$, I. Kanivets ${ }^{2}$, S. Korostelev $^{2}, \boldsymbol{F}$. Konovalov ${ }^{1}$

${ }^{1}$ Independent Clinical Bioinformatics Laboratory, Moscow, Russian Federation, ${ }^{2}$ Genomed Ltd., Moscow, Russian Federation, ${ }^{3}$ Veltischev Research and Clinical Institute for Pediatrics, Moscow, Russian Federation, ${ }^{4}$ Center for Genetics and Reproductive Medicine "Genetico”, Moscow, Russian Federation, ${ }^{5}$ Research Centre for Medical Genetics, Moscow, Russian Federation

Next generation sequencing (NGS) is a powerful tool for accurate diagnostics in genetically heterogenous hereditary disorders. From 2015 till 2020 our variant analysis team interpreted NGS data for 837 patients with myopathy or myasthenia, mostly from Russia and other Russianspeaking countries. The set included 271 clinical exomes, $176 \mathrm{WES}, 16 \mathrm{WGS}$, and 374 custom panels of several types comprising in most cases $>2000$ genes. After processing via an automated pipeline, the variants were uploaded into a custom database-driven software and assessed by clinical bioinformaticians, with subsequent review of the reports by medical geneticists, when possible. As a result, 336 patients $(40 \%)$ had definitive molecular diagnostic findings explaining the phenotype (mostly mutations in LAMA2, CAPN3, DMD, RYR1, NEB, SEPN1, $D Y S F, C H R N E, T T N)$ and 123 patients (15\%) had a possible diagnosis based on variants of unknown significance with a need for additional evidence. Maximum success rate was in infants who were less than 1 year old at the time of test (59\%; 53/90 cases) and minimum success rate was in adults 18 years and older (33\%; 56/170 cases). In 59 successful cases $(18 \%$ of $336 ; 7 \%$ of total) the result was due to detection of CNVs, and this was not limited to $D M D$ deletions/duplications. The CNV ratio here is higher than in our other patient cohorts, indicating high importance of $\mathrm{CNV}$ analysis step in myopathy/myasthenia cases. Molecular diagnostics of neuromuscular disorders is complex due to their clinical variability and genetic heterogeneity, requiring a combination of exome-scale NGS and thorough data analysis.

M. Ampleeva: None. E. Tolmacheva: None. I. Komar'kov: None. N. Shcherbakova: None. D. Khmelkova: None. E. Surkova: None. O. Ryzhkova: None. V. Kaimonov: None. I. Mironova: None. E. Pomerantseva: None. A. Isaev: None. E. Dadali: None. D. Pyankov: None. A. Antonets: None. I. Kanivets: None. S. Korostelev: None. F. Konovalov: None.

P10.38.C

Myotonia congenita: a series of familial cases with causative pathogenic variants in both CLCN1 and SCN4A

K. Šiauryt $\dot{\boldsymbol{e}}^{1,2}$, A. Matulevičien $\dot{e}^{3,2}$, L. Cimbalistiene $\dot{e}^{3,2}$, A. Morkūniene $\dot{e}^{3,2}$, A. Vaitkevičius ${ }^{4}$, A. Utkus ${ }^{3}$

${ }^{1}$ Faculty of Medicine, Vilnius University, Vilnius, Lithuania, ${ }^{2}$ Centre for Medical Genetics at Vilnius University Hospital Santaros Klinikos, Vilnius, Lithuania, ${ }^{3}$ Department of Human and Medical Genetics, Institute of Biomedical Sciences, Faculty of Medicine, Vilnius University, Vilnius, Lithuania, ${ }^{4}$ Clinic of Neurology and Neurosurgery, Institute of Clinical Medicine, Faculty of Medicine, Vilnius University, Vilnius, Lithuania

Introduction: In cases of non-dystrophic myotonias (NDM) phenotype-genotype correlations are difficult to discern due to the overlap of nosologic entities caused by mutations in CLCN1 (17q23.3) and SCN4A (7q34) genes. Patients may exhibit phenotypes including dominant and recessive myotonia congenita (MC), paramyotonia congenita (PC) and sodium channel myotonia (SCM). Here we report on a familial case series where members with $\mathrm{MC}$ of varying severity harbour different combinations of pathogenic variants in these two genes.

Materials and methods: Five out of six family members display the classical spectrum of MC features. Proband (aged 57), her two daughters (aged 37 and 24), her grandson (aged 17), and a cousin (aged 45) have similar complaints of muscle rigidity in the extremities and face, provoked by cold or potentially elicited by percussion of muscles. The youngest grandson (aged 8) does not exhibit symptoms representative of his age. EMG of three individuals (mother, older daughter, cousin) showed classical excessive myotonic discharges.

Results: Sanger sequencing revealed heterozygous previously reported missense mutation NM_000083.3: c.2680C $>$ T (NP_000074.3: p.(Arg894Ter)) in exon 23 of the CLCN1 gene of every family member excluding the 
cousin. Another heterozygous previously reported missense mutation NM_000334.4:c.4343G >A (NP_000325.4:p. (Arg1448His)) in exon 24 of the SCN4A gene was found in every family member except for the youngest grandson.

Conclusions: In cases when NDM phenotype severity is varied in members of the same family, molecular genetic analysis of both CLCNI and SCN4A genes should be considered.

K. Šiaurytė: None. A. Matulevičienè: None. L. Cimbalistienè: None. A. Morkūnienè: None. A. Vaitkevičius: None. A. Utkus: None.

P10.41.C

Molecularly confirmed pontocerebellar hypoplasia in an extensive family with four affected children

Z. Kubiritova ${ }^{1,2,3}$, E. Radvanska ${ }^{4}, F$. Bauer $^{4}$, J. Barosova $^{5}$, M. Hyblova ${ }^{6,7}$, T. Szemes ${ }^{2,3,8}$, L. Kadasi ${ }^{1,2}$, J. Radvanszky ${ }^{1,3}$

${ }^{1}$ Slovak Academy of Sciences, Biomedical Research Center, Bratislava, Slovakia, ${ }^{2}$ Department of Molecular Biology, Faculty of Natural Sciences, Comenius University, Bratislava, Slovakia, ${ }^{3}$ Geneton Ltd., Bratislava, Slovakia, ${ }^{4}$ Department of Neonatology, Faculty Hospital Nove Zamky, Nove Zamky, Slovakia, ${ }^{5}$ Genet Ltd., Nitra, Slovakia, ${ }^{6}$ Trisomy Test Ltd., Bratislava, Slovakia, ${ }^{7}$ Medirex Inc., Bratislava, Slovakia, ${ }^{8}$ Comenius University Science Park, Bratislava, Slovakia

Introduction: Pontocerebellar hypoplasia $(\mathrm{PCH})$ is a rare and heterogenous group of neurodegenerative disorders having typically prenatal onset. Patients present with hypotonia and severe atrophy of various parts of their brain, resulting in slow development, movement problems and intellectual disability, even death.

Material and Methods: In our family, we identified the causative mutation by massively parallel sequencing, using a clinical-exome kit (Illumina) in three affected patients (post-mortem, from archived DNA samples). Following the initial finding, we screened the respective genomic position in the remaining family members using Sanger sequencing.

Results: In the affected family members we identified a rare homozygous pathogenic variant c. $92 \mathrm{G}>\mathrm{C}$ in the EXOSC 3 gene, causing an autosomal recessive $\mathrm{PCH}$ type 1B. Unaffected parents and four siblings are heterozygous carriers, one healthy sibling has a wild type genotype and three affected siblings were homozygous and died soon after birth (2-6 months of age). Two other siblings were not analyzed, however, one of them also died soon after birth and suffered most probably from the same disorder.

Conclusions: We report detailed clinical findings of the largest $\mathrm{PCH}$ type 1B family published up to date, with four affected children. The causative variant was reported to be more frequent in the Roma population of the Czech Republic (considered a founder mutation). Based on the close connection of the populations of Slovakia and the Czech Republic, we hypothesize, these two alleles may have a common origin. Therefore, we are still investigating their haplotype background. Funding: APVV-18-0319; MZSR_2018/46-SAV-5; VEGA_2/0167/20.

Z. Kubiritova: None. E. Radvanska: None. F. Bauer: None. J. Barosova: None. M. Hyblova: None. T. Szemes: None. L. Kadasi: None. J. Radvanszky: None.

P10.42.A

Biallelic mutations in neurofascin cause neurodevelopmental impairment and peripheral demyelination

S. Efthymiou ${ }^{l}$, V. Salpietro ${ }^{l}$, N. Malintan ${ }^{l}$, M. $_{\text {Poncelet }}{ }^{2}, Y$. Kriouile $^{3}$, S. Fortuna ${ }^{4}$, R. de Zorzi ${ }^{4}$, K. Payne ${ }^{5}$, L. Henderson $^{6}$, A. Cortese ${ }^{l}$, S. Maddirevula ${ }^{7}$, N. Alhashmi ${ }^{8}, S$.

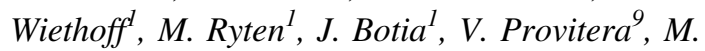
Schuelke ${ }^{10}, J$. Vandrovcova ${ }^{1}$, L. Walsh ${ }^{5}$, E. Torti ${ }^{6}$, V. Iodice $^{l}$, M. Najafi ${ }^{11}$, E. Karimiani ${ }^{12}$, R. Maroofian ${ }^{l}, K$. Siquier-Pernet ${ }^{13}$, N. Boddaert ${ }^{13}$, P. De Lonlay ${ }^{13}, V$. Cantagrel $^{13}, M$. Aguennouz ${ }^{14}, M$. El Khorassani ${ }^{3}, M$. Schmidts $^{11}$, F. S. Alkuraya ${ }^{15}$, S. Edvardson ${ }^{16}$, M. Nolano ${ }^{9}$, J. Devaux ${ }^{2}$, H. Houlden ${ }^{1}$

${ }^{1}$ UCL Institute of Neurology, London, United Kingdom, ${ }^{2}$ INSERM U1051, Institut de Neurosciences de Montpellier (INM), Montpellier, France, ${ }^{3}$ University Mohammed V of Rabat, Rabat, Morocco, ${ }^{4}$ University of Trieste, Trieste, Italy, ${ }^{5}$ Riley Hospital for Children, Indianapolis, IN, United States, ${ }^{6}$ GeneDx, Gaithensburg, MD, United States, ${ }^{7}$ King Faisal Specialist Hospital and Research Centre, Riyadh, Saudi Arabia, ${ }^{8}$ College of Medicine, Sultan Qaboos University, Oman, Oman, ${ }^{9}$ Istituti Clinici Scientifici Maugeri IRCCS, Naples, Italy, ${ }^{10}$ Charite' Universita" tsmedizin, Berlin, Germany, ${ }^{11}$ Radboud University Medical Center, Nijmegen, Netherlands, ${ }^{12}$ St George's University of London, London, United Kingdom, ${ }^{13}$ Paris Descartes Sorbonne Paris, Paris, France, ${ }^{14}$ University of Messina, Sicily, Italy, ${ }^{15}$ Alfaisal University, Riyadh, Saudi Arabia, ${ }^{16}$ Hadassah Medical Center, Jerusalem, Israel

Axon pathfinding and synapse formation are essential processes for nervous system development and function. The assembly of myelinated fibres and nodes of Ranvier is mediated by a number of cell adhesion molecules of the immunoglobulin superfamily including neurofascin, encoded by the NFASC gene, and its alternative isoforms Nfasc186, Nfasc140 and Nfasc155. We identified 10 individuals from six unrelated families, exhibiting a neurodevelopmental disorder characterized with a 
spectrum of central and peripheral neurological involvement, who were found by NGS to carry frameshift and non-synonymous variants. Expression studies using immunostaining-based techniques identified absent expression of the Nfasc155 isoform as a consequence of the frameshift variant and a significant reduction of expression was also observed in association with two nonsynonymous variants affecting the fibronectin type III domain. Cell aggregation studies revealed a severely impaired Nfasc155-CNTN1/CASPR1 complex interaction as a result of the identified variants. Immunofluorescence staining of myelinated fibres from two affected individuals showed a severe loss of myelinated fibres and abnormalities in the paranodal junction morphology. Our results establish that recessive variants affecting the Nfasc155 isoform can affect the formation of paranodal axoglial junctions at the nodes of Ranvier. The genetic disease caused by biallelic NFASC variants includes neurodevelopmental impairment and a spectrum of central and peripheral demyelination as part of its core clinical phenotype. Our findings support possible overlapping molecular mechanisms of paranodal damage at peripheral nerves in both the immune-mediated and the genetic disease. Wellcome Trust strategic award (Synaptopathies WT093205 MA and WT104033AIA)

S. Efthymiou: None. V. Salpietro: None. N. Malintan: None. M. Poncelet: None. Y. Kriouile: None. S. Fortuna: None. R. de Zorzi: None. K. Payne: None. L. Henderson: None. A. Cortese: None. S. Maddirevula: None. N. Alhashmi: None. S. Wiethoff: None. M. Ryten: None. J. Botia: None. V. Provitera: None. M. Schuelke: None. J. Vandrovcova: None. L. Walsh: None. E. Torti: None. V. Iodice: None. M. Najafi: None. E. Karimiani: None. R. Maroofian: None. K. Siquier-Pernet: None. N. Boddaert: None. P. De Lonlay: None. V. Cantagrel: None. M. Aguennouz: None. M. El Khorassani: None. M. Schmidts: None. F. S. Alkuraya: None. S. Edvardson: None. M. Nolano: None. J. Devaux: None. H. Houlden: None.

\section{P10.43.B}

Detection and interpretation of variants in dystroglycanopathy-causing genes in a cohort of 1,803 patients with unexplained limb-girdle muscle weakness

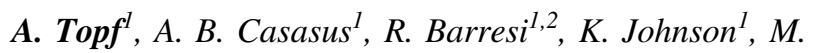

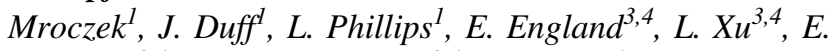
Valkanas $^{3,4}$, D. G. MacArthur ${ }^{3,4}$, V. Straub ${ }^{1}$

${ }^{1}$ John Walton Muscular Dystrophy Research Centre, Translational and Clinical Research Institute, Newcastle University, Newcastle, United Kingdom, ${ }^{2}$ Muscle Immunoanalysis Unit, Newcastle upon Tyne, United Kingdom,
${ }^{3}$ Analytic and Translational Genetics Unit, Massachusetts General Hospital, Boston, MA, United States, ${ }^{4}$ Program in Medical and Population Genetics, Broad Institute of MIT and Harvard, Boston, MA, United States

Background: Dystroglycanopathies are a clinically and genetically heterogeneous group of disorders characterised by limb-girdle muscle weakness (LGMW) often in combination with brain abnormalities. To date, 18 disease-causing genes have been identified, all encoding for proteins involved in the glycosylation of $\alpha$-dystroglycan and thus affecting its binding to the extracellular matrix.

Methods: As part of the MYO-SEQ project, detailed clinical and histological information was collected for 1,803 patients with unexplained LGMW. Whole-exome sequencing was carried out and genes known to be associated with dystroglycanopathies were analysed to identify likely pathogenic variants.

Results: Suspected disease-causing variants in 8 genes were detected in 47 patients: DPM3 and ISPD in one patient each, FKTN, POMK and POMT1 in two patients, GMPPB in four patients, POMT2 in 14 patients and FKRP in 21 patients. The overall frequency for the disease group was $2.6 \%$. The phenotypes were highly variable, generally presenting with progressive proximal muscle weakness and high CK, yet cognitive impairment was not always observed. Muscle biopsies were available for 21 cases; reported results of $\alpha$-dystroglycan labelling were inconsistent, indicating that standardised immunohistochemical protocol and training in its interpretation are crucial. In addition, we looked at undiagnosed patients carrying two or more heterozygous pathogenic variants in two different dystroglycanopathy genes, however we did not find evidence of digenic inheritance in this pathway.

Conclusions: Given their genetic and phenotypic heterogeneity, here we highlight the advantage of using nextgeneration sequencing in combination with traditional histopathological analysis to improve the diagnosis of dystroglycanopathies as a rare disease group.

A. Topf: None. A.B. Casasus: None. R. Barresi: None. K. Johnson: None. M. Mroczek: None. J. Duff: None. L. Phillips: None. E. England: None. L. Xu: None. E. Valkanas: None. D.G. MacArthur: None. V. Straub: None.

P10.44.C

A treatable hereditary polyneuropathy due to impaired Vitamin B6 metabolism

P. N. Torbati ${ }^{1}$, E. G. Karimiani ${ }^{2,3}$, N. M. Ferreira ${ }^{4}$, R.

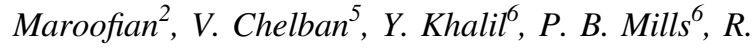




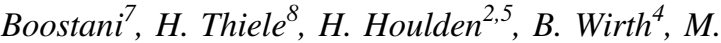
Karakaya $^{4}$

${ }^{1}$ Department of Molecular Genetics, Next Generation Genetic Polyclinic, Mashhad, Iran, Mashhad, Iran, Islamic Republic of, ${ }^{2}$ Molecular and Clinical Sciences Institute, St. George's University of London, London, United Kingdom, ${ }^{3}$ Department of Molecular Genetics, Next Generation Genetic Polyclinic, Mashhad, Iran, Islamic Republic of, ${ }^{4}$ Institute of Human Genetics, Center for Molecular Medicine Cologne (CMMC), Institute of Genetics, and Center for Rare Diseases Cologne, University of Cologne, Cologne, Germany, ${ }^{5}$ Department of Neuromuscular Diseases, UCL Queen Square Institute of Neurology, University College London, London, United Kingdom, ${ }^{6}$ Genetics and Genomic Medicine, Great Ormond Street Institute of Child Health, University College London, London, United Kingdom, ${ }^{7}$ Neurology Dept., Ghaem Hospital, Medical School, Mashhad University of Medical Sciences, Mashhad, Iran, Islamic Republic of, ${ }^{8}$ Cologne Center for Genomics (CCG), University of Cologne, Cologne, Cologne, Germany

Introduction: PDXK encodes for a pyridoxal kinase, which converts inactive B6 vitamers to the active cofactor pyridoxal 5'-phosphate (PLP), which is an essential co-factor involved in vital metabolic pathways, including neurotransmitter production and amino acid biosynthesis. Recently, biallelic pathogenic variants in PDXK were shown to cause axonal Charcot-Marie-Tooth disease with optic atrophy that responds to PLP supplementation.

Methods: We performed whole exome sequencing and segregation analysis using Sanger sequencing in order to uncover the pathogenic variant(s) in a family affected by an undiagnosed autosomal recessive peripheral neuropathy. Pathogenicity of the variant was confirmed via enzymatic assays and mass spectroscopy on dried blood-spot samples, derived from all members of the family.

Results: Here, we report two affected individuals, aged 17 and 14 years old, from a consanguineous Iranian family presenting with a childhood-onset sensorimotor axonal neuropathy and first signs of optic atrophy. Genetic analysis identified a segregated novel homozygous missense variant in PDXK, (NM_003681.4); c.225T>A, p.(Asn75Lys). We show that the variant leads to reduced PDXK enzymatic activity with low PLP. The relatively early diagnosis and PLP replacement restored the PLP plasma levels with observable clinical improvement.

Conclusions: We recommend that patients presenting with autosomal recessive childhood-onset axonal neuropathy and optic atrophy should be screened for PDXK mutations, as this is a rare opportunity for treatment.
P.N. Torbati: None. E.G. Karimiani: None. N.M. Ferreira: None. R. Maroofian: None. V. Chelban: None. Y. Khalil: None. P. B. Mills: None. R. Boostani: None. H. Thiele: None. H. Houlden: None. B. Wirth: None. M. Karakaya: None.

\section{P10.45.A}

Novel HINT1 variant identified in Lithuanian patients with autosomal recessive axonal neuropathy with neuromyotonia

B. Burnyte ${ }^{1,2}$, A. Morkuniene $e^{1,2}$, K. Grigalioniene G, $^{1,2}$, L. Ambrozaityte ${ }^{1,2}, R$. Buneviciute ${ }^{3,2}$, A. Vaitkevicius $^{3,2}$, E. Preiksaitiene $e^{1,2}$, A. Matuleviciene ${ }^{1,2}$, L. Cimbalistiene Ci,2, $^{1,2}$ A. Utkus ${ }^{1,2}$

${ }^{1}$ Department of Human and Medical Genetics, Institute of Biomedical Sciences, Faculty of Medicine, Vilnius University, Vilnius, Lithuania, ${ }^{2}$ Vilnius University Hospital Santaros Klinikos, Vilnius, Lithuania, ${ }^{3}$ Institute of Clinical Medicine, Faculty of Medicine, Vilnius University, Vilnius, Lithuania

Aim: Autosomal recessive axonal neuropathy with neuromyotonia (ARAN-NM) is a rare subtype of hereditary neuropathy. HINT1 gene c.110G>C, (p.Arg37Pro) variant already reported in the literature as the most common pathogenic variant [P. Laššuthová, 2016]. Here, we describe genetic and phenotypic characteristics of patients with ARAN-NM in Lithuania.

Materials and methods: By using multigene NGS panel, we identified 8 patients with recessive variants in HINTI gene from 2014 to 2019.

Results: Eight patients showed homozygous or compound heterozygous variants in HINT1 gene, including previously reported pathogenic variant c.110G >C p.(Arg37Pro) and a novel variant c.299A $>$ G p.(Glu100Gly): i) five patients harboured a homozygous pathogenic variant c. $110 \mathrm{G}>\mathrm{C}$, ii) two patients had compound heterozygous variants (c. $110 \mathrm{G}>\mathrm{C}$ and c.299A $>\mathrm{G}$ ), and iii) one patient had a homozygous novel variant, which was also identified in affected brother. Co-segregation of these variants in affected sibs or healthy relatives confirmed a recessive inheritance. Patients with homozygous novel and previously reported HINTI variants shared pure axonal motor neuropathy.

Conclusions: Novel variant in HINT1 has been identified from three unrelated patients. Our data expand the spectrum of pathogenic variants in HINT1 and its significance in molecular diagnosis. Additionally, our findings support that previously reported variant $\mathrm{c} .110 \mathrm{G}>\mathrm{C}$ may result from a founder mutation in European populations. 
B. Burnyte: None. A. Morkuniene: None. K. Grigalioniene: None. L. Ambrozaityte: None. R. Buneviciute: None. A. Vaitkevicius: None. E. Preiksaitiene: None. A. Matuleviciene: None. L. Cimbalistiene: None. A. Utkus: None.

\section{P10.46.B}

The result of NGS disease specific panel for practical genetic diagnostics of neuromuscular disorders.

I. Haiboniuk ${ }^{l}, H$. Makukh ${ }^{l}$, O. Semeriak ${ }^{2}$, M. Dushar ${ }^{l}, H$. Akopyan $^{l}$

${ }^{1}$ Institute of Hereditary Pathology NAMS of Ukraine, Lviv, Ukraine, ${ }^{2}$ Lviv Regional Clinical Hospital, Lviv, Ukraine

Introduction:The genetic determined neuromuscular disease are characterized by broad spectrum of clinical disorders and clinical polymorphism.

Materials and Methods:We analyze the results of using NGS for practical genetic diagnostics. Genetic testing was performed among 65 person (55\% male), 1 to 56 y.o with clinicaly unexplained progressive neuromuscular pathology. DNA samples was extracted from blood leucocytes and common mutilations for DMD and SMA were excluded by target testing. The Illumina technology for 289 genes associated with muscular dystrophies, myopathies, neuropathies was performed at INVITAE laboratory, USA. All clinically significant observations were additionally confirmed.

Results:The positive results - identification of at least one pathogenic variant in the gene panel, were received for 35/ $65(54 \%)$ of cases. The conformation of CAPN3 autosomal recessive limb-girdle muscular dystrophy was the most frequent, 9.2\%: three patients c.550del homozygous and two compound with other mutations. Charcot-Marie-Tooth disease was diagnosed in association with mutations in PMP22, MFN2, GJB1, GDAP1 genes in 6.2\%. The other than deletion DMD genetic variants were identified in 4 males. The interesting finding of this study: TCF4 deletion of entire coding sequence associated with Pitt-Hopkins syndrome; copy number gains for X-chromosome genes and after cytogenetic testing 47, XXY karyotype in boy with SMA- like clinical phenotype. In $40 \%$ of patients the diagnosis of monogenic disorder was confirmed with use of NGS disease specific panel. The clinical treatment of VUS is challenging.

Conclusions:The collaboration between molecular geneticist, clinical geneticist and neurologist guarantee the correct diagnosis for patients with orphan neuromuscular disease.

I. Haiboniuk: None. H. Makukh: None. O. Semeriak: None. M. Dushar: None. H. Akopyan: None.
P10.47.C

Identification of a novel PRUNE1 gene mutation in a patient with severe neurodevelopmental disorder characterized by hypotonia and epileptic encephalopathy

L. Lazaros ${ }^{1}$, D. Palaiologou ${ }^{1}$, A. Romito ${ }^{2}$, A. Pantou ${ }^{I}$, A. Marais $^{2}$, E. Kanavakis ${ }^{l}$

${ }^{1}$ Genesis Genoma Lab, Genetic Diagnosis, Clinical Genetics \& Research, Athens, Greece, ${ }^{2}$ Centogene AG, Rostock, Germany

Introduction: The application of whole exome sequencing (WES) has revealed the genetic basis of an autosomal recessive neurodevelopmental disorder characterized by microcephaly, hypotonia and variable brain anomalies (NMIHBA). In the current study, we describe a severe case of NMIHBA due to the compound heterozygosity of a missense and a novel frameshift variant in the PRUNE1 gene.

Materials and Methods: The affected proband is a 3.5year-old girl characterized by generalized muscular hypotonia, drug-resistant epilepsy (10-15 myoclonic seizures daily) and severe swallowing problems. Serious developmental and speech delay is also present. The clinical examination of the patient has detected scoliosis and kyphosis, high myopia, frequent respiratory tract infections, tooth enamel loss and severe constipation. WES was performed in three family members (proband \& parents) using SureSelect Human All Exon V6 kit in a HiSeq4000 instrument. The detected variants were confirmed by Sanger sequencing analysis.

Results: WES analysis detected two heterozygous PRUNE1 gene variants in the affected proband, c.316G $>$ A (p.Asp106Asn) and c.181_185delTCTGA (p. Ser61Thrfs*6), the first of paternal and the second of maternal origin, confirming the compound heterozygosity in the index patient.

Conclusions: Given that PRUNE1 gene has recently been associated with neurodevelopmental disorders and consequently it is not included in older custom NGS gene panels, WES application constituted the most efficient approach for the molecular investigation of the specific case. These results highlight the utility of WES in order to reveal the genetic etiology of complex phenotypes, allowing the better classification of the patients and the design of personalized therapeutic strategies.

L. Lazaros: None. D. Palaiologou: None. A. Romito: None. A. Pantou: None. A. Marais: None. E. Kanavakis: None. 


\section{P10.48.A}

Variants causing mitochondrial dysfunction are not rare in non-5q SMA: Re-evaluation of thirty families by exome sequencing

M. Karakaya ${ }^{1}$, N. Keller ${ }^{1}$, J. Altmueller ${ }^{2}$, S. Motameny ${ }^{2}, H$. Thiele $^{2}$, G. Wunderlich ${ }^{3}$, J. Kirschner ${ }^{4}$, B. Schrank ${ }^{5}, R$.

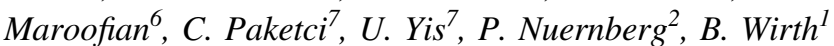

${ }^{1}$ University Hospital Cologne, Institute of Human Genetics, Cologne, Germany, ${ }^{2}$ Cologne Center for Genomics, Cologne, Germany, ${ }^{3}$ University Hospital Cologne, Department of Neurology, Cologne, Germany, ${ }^{4}$ University Hospital Bonn, Department of Neuropediatrics, Bonn, Germany, ${ }^{5}$ DKD HELIOS Kliniken, Department of Neurology, Wiesbaden, Germany, ${ }^{6}$ Molecular and Clinical Sciences Institute, St. George's University of London, London, United Kingdom, ${ }^{7}$ Dokuz Eylül University, Department of Pediatric Neurology, Izmir, Turkey

Introduction: Spinal muscular atrophy (SMA) without deletions/mutations in SMN1 (non-5q-SMA) is genetically very heterogeneous. Due to the strongly overlapping phenotypes, the differential diagnosis of non-5q-SMAs is challenging.

Materials/Methods: 52 patients analysed by a large gene panel with 479 neuromuscular disease-causing genes received no conclusive results. We re-evaluated 21 unsolved cases by trio whole-exome sequencing (trioWES), in addition to nine families without previous panel analysis.

Results: In 10/21 individuals with negative panel results, trio-WES revealed the pathogenic variant (48\%). Of these ten identified genes, five $(50 \%)$ were associated with genes involved in mitochondrial function and stability (FDXR, NDUFS6, ECHS1, MPV17, VPS13D). The remaining genes encoded for proteins of diverse functions, with one variant in the lysosomal enzyme-encoding gene $A R S A$, one in a gene required for amino acid synthesis (SEPSECS) and one in the muscle protein-coding TTN. Additionally, we identified a variant in a gene relevant for cellular stress response $(A D P R S)$ and one encoding an enzyme essential for vitamin B6 metabolism $(P D X K)$. Among nine patients with no prior gene panel testing, we diagnosed four patients (4/9) with pathogenic variants in known neuromuscular disease genes.

Discussion: Inborn errors of organelle metabolism might primarily manifest with neuropathic features in childhood. Our preliminary analysis shows that mutations in mitochondrial proteins are the leading cause in this spectrum. Due to abundance and high number of the associated genes, and high clinical variability, we recommend performing WES instead of gene panels, in order to address the extreme heterogeneity of the causal pathomechanisms.

Grant reference: Deutsche Forschungsgemeinschaft (Wi945/19-1)

M. Karakaya: None. N. Keller: None. J. Altmueller: None. S. Motameny: None. H. Thiele: None. G. Wunderlich: None. J. Kirschner: None. B. Schrank: None. R. Maroofian: None. C. Paketci: None. U. Yis: None. P. Nuernberg: None. B. Wirth: None.

\section{P10.49.B}

Comparison of gene panel and alpha-glucosidase activity on dried blood spot in patients with suspected muscle disease in order to screen and diagnose Pompe disease

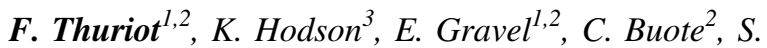
Gravel $^{1,2}$, S. Lévesque L,2 $^{1,2}$

${ }^{I}$ Université de Sherbrooke, Sherbrooke, QC, Canada, ${ }^{2}$ Sherbrooke Genomic Medicine, Sherbrooke, QC, Canada, ${ }^{3}$ Dynacare, Laval, QC, Canada

Pompe disease is an autosomal recessive disorder caused by alpha-glucosidase (GAA) deficiency, leading to a progressive limb-girdle weakness and respiratory insufficiency. Limb-girdle weakness is the most common form of muscle weakness and Pompe disease is hard to differentiate, delaying diagnosis and administration of treatment. Enzymatic activity of GAA is wildly used to screen patients but sequencing of $G A A$ is required to confirm the diagnosis. In order to investigate if potential Pompe cases could be missed by using a gene panel approach alone, we screened 876 patients with a suspected muscle disease, and compared GAA enzymatic activity on dried blood spot with an 89gene panel. Eight patients were diagnosed with Pompe disease; enzymatic activity and sequencing results were concordant. Of the 36 patients carrying a single heterozygous $G A A$ variant, only one patient harboring a pathogenic heterozygous variant had low enzyme activity overlapping with levels seen in Pompe cases suggesting that a second variant could have been missed. RNA studies are pending. Finally, nine patients had low or borderline enzyme activity, but no variant was detected. These patients have a low risk of having Pompe disease since enzymatic activity is close to the threshold and does not overlap with confirmed Pompe cases. With gene panels being increasingly performed as a first-tier test for patients with suspected muscle disorders, our study emphasizes the need to perform reflex enzymatic activity in patients with a single $G A A$ variant. RNA sequencing should be considered if it the enzymatic result is compatible with Pompe. *Supported by Sanofi-Genzyme. 
F. Thuriot: None. K. Hodson: A. Employment (full or part-time); Significant; Dynacare. E. Gravel: None. C. Buote: None. S. Gravel: D. Speakers Bureau/Honoraria (speakers bureau, symposia, and expert witness); Modest; Sanofi-Genzyme. S. Lévesque: B. Research Grant (principal investigator, collaborator or consultant and pending grants as well as grants already received); Significant; Sanofi-Genzyme. D. Speakers Bureau/Honoraria (speakers bureau, symposia, and expert witness); Modest; SanofiGenzyme.

\section{P10.50.C}

\section{A homozygous frameshift mutation in PTRH2 gene}

\author{
S. Seneca ${ }^{1,2}$, L. Régal ${ }^{3}$, M. Becker ${ }^{4}$, K. Stouffs ${ }^{1,2}$, A. \\ Gheldof $^{1,2}$, E. Scalais ${ }^{5}$
}

${ }^{1} U Z$ Brussel, Center for Medical Genetics, Brussels, Belgium, ${ }^{2}$ Neurogenetics Research Group, Reproduction Genetics and Regenerative Medicine Research Cluster, Vrije Universiteit Brussel (VUB), Brussels, Belgium, ${ }^{3}$ Division of Pediatric Neurology, UZ Brussel, Brussels, Belgium, ${ }^{4}$ Pediatric Department, Centre Hospitalier de Luxembourg, Luxembourg City, Luxembourg, ${ }^{5}$ Division of Pediatric Neurology, Centre Hospitalier de Luxembourg, Luxembourg City, Luxembourg

The identification of the genetic defect of a neurological disease is complex, and many patients are still without a molecular diagnosis. With massive parallel sequencing and a laboratory developed Mendeliome gene panel, we identified a truncation mutation in the PTRH2 gene in two affected siblings. This multifunctional and evolutionary well conserved enzyme encodes the mitochondrial peptidyltRNA hydrolase 2 protein (Ptrh2), and prevents the accumulation of peptidyl-tRNA. It is also a key determinant in cell survival, growth and apoptosis processes. The precise role of the protein in muscle disease has still to be delineated. Our index patients are the 15- and 13-year-old son and daughter of non-consanguineous healthy Nepalese parents. Both patients had multiple neurological symptoms including sensorineural deafness, psychomotor and language delay, epilepsy and behavioral disturbances, subsequently motor regression with ataxia, peripheral neuropathy and later, in one of them, diabetes mellitus. In both patients, pancreatic echography disclosed hyper-echogenicity. A homozygous pathogenic 1 base pair insertion c.127dupA was revealed in the PTRH2 gene of both siblings, and confirmed with Sanger sequencing. This frameshift causes a premature translation stop p.(Ser43Lysfs*11), and is present in heterozygosity in both parents. Originally, a homozygous deletion variant in the PTRH 2 gene was identified as the molecular cause of global developmental delay, hearing loss and ataxia. Since 2014, the identification of additional patients has documented a clinical heterogeneity, and expanded the clinical spectrum of pathogenic PTRH2 variants. They are now known as the cause of infantile multisystem neurologic, endocrine and pancreatic disease (IMNEPD).

S. Seneca: None. L. Régal: None. M. Becker: None. K. Stouffs: None. A. Gheldof: None. E. Scalais: None.

\section{P10.51.A}

Genetic analysis of recessive rhabdomyolysis - mutations in LPIN1 (Lipin-1)

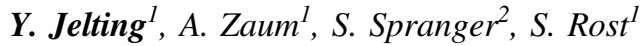 \\ ${ }^{1}$ University of Würzburg, Institute for Human Genetics, \\ Würzburg, Germany, ${ }^{2}$ Praxis für Humangenetik, Bremen, \\ Germany
}

Lipin-1, a protein coded by the LPIN1 gene, plays an important role in the human triglyceride synthesis. Mutations in LPIN1 lead to recurrent rhabdomyolysis with myoglobinuria, myalgia and generalized weakness in childhood. We investigated two independent cases with rhabdomyolysis. Patient 1 is a six-year-old Turkish boy with healthy consanguineous parents. His sister died of a respiratory infection at the age of 3 . He showed massively increased creatinine kinase (CK) levels (up to $400.000 \mathrm{U} / \mathrm{l}$ ) and myoglobinuria. Patient 2 is a German 36-year-old women with myalgia, increased CK values and a history of kidney failure after gastrointestinal infection in early childhood. The DNA was processed by next-generation sequencing (NGS) after enrichment of a custom-made myopathy panel (65 genes). Data analysis was done by GensearchNGS. Three different variants in LPIN1 could be identified, a large recurrent deletion including exon 18, a nonsense variant and a single base pair duplication. Patient 1 carries the deletion and the duplication, each in homozygous state. The duplication has not been described before, is not present in gnomAD and leads to a frameshift with a premature stop codon at position 769 . Patient 2 carries the same deletion of exon 18 and a known nonsense variant leading to a stop codon at position 19 . The breaking points of the deletion could be identified exactly by NGS. Our findings revealed a novel and already known mutations in LPIN1 and verified the breakpoints of the recurrent deletion. This confirms the causative role of LPINI mutations in patients with muscular disorders.

Y. Jelting: None. A. Zaum: None. S. Spranger: None. S. Rost: None.

P10.52.B

Autosomal recessive spinocerebellar ataxia 20: a novel insertion in the SNX14 gene 
A. Harari-shaham ${ }^{l}$, L. Shemer ${ }^{l}$, V. Adir ${ }^{l}$, O. Sadeh ${ }^{l}, H$. Baris-Feldman ${ }^{2}$, A. Mory ${ }^{3}$, L. Sagi-Dain', A. Peleg ${ }^{1}$

${ }^{1}$ Carmel medical center, Haifa, Israel, ${ }^{2}$ Rambam medical center, Haifa, Israel, ${ }^{3}$ Rambam Medical Center, Haifa, Israel

Background: Autosomal recessive spinocerebellar ataxia 20 (SCAR20) is a rare disorder, mainly characterized by intellectual disability, ataxia, coarse facial features, progressive loss of Purkinje cells in the cerebellum and often hearing loss and skeletal abnormalities.

Case report: We describe a 3.5 years old boy, fourth child to consanguineous parents, double first cousins from Druze origin.

Prenatal sonographic examination demonstrated periventricular pseudo-cyst, mild unilateral ventriculomegaly, small and dysmorphic corpus callosum, dysmorphic small cave of septum pellucidum and shortened long bones of the legs $\left(5^{\text {th }}\right.$ percentile). Amniocentesis for chromosomal microarray analysis yielded normal results. Magnetic resonance imaging (MRI) demonstrated hypogenesis of corpus callosum and delayed myelination of the white matter. Examination of the 3.5 year old boy at our genetics clinic demonstrated global severe developmental delay, vision impairment, severe hypotonia, lower motor neuron lesion characteristics and some dysmorphic signs.

Results: Two bioinformatics analyses of exome sequencing did not reveal any pathogenic findings. However, the third exome re-evaluation raised suspicion of about 200bp deletion in the SNX14 gene, including part of exon 13 and intron 14. The approximated boundaries were Chr6 (GRCh37) g.86252986_86253202del.

Sanger sequencing and a PCR fragment analysis revealed a homozygote 142bp insertion. Chr6 (GRCh37) g.86252986_86252987ins[142], the insertion interrupted the coding region of the SNX14 gene, NM_153816.5; c.1320_1321ins[142].

Conclusions: We report a novel SNX14 gene mutation, causing a severe phenotype of a recently described SCAR20. Our findings emphasize the limitation of the exome analysis and the importance of the use of different analysis platforms.

A. Harari-shaham: None. L. Shemer: None. V. Adir: None. O. Sadeh: None. H. Baris-Feldman: None. A. Mory: None. L. Sagi-Dain: None. A. Peleg: None.

P10.53.C

SMN2genes study by NGS in spinal muscular atrophy: towards identification of new SMA modifiers

L. Blasco-Pérez ${ }^{l}$, I. Paramonov ${ }^{I}$, L. Iranzo ${ }^{1}$, D. Martinez-

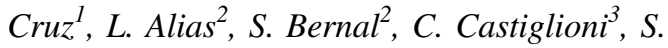

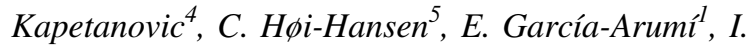
Cuscó $^{1}$, E. F. Tizzano ${ }^{1}$

${ }^{I}$ Department of Clinical and Molecular Genetics and Medicine Genetics Group, VHIR, University Hospital Vall d'Hebron, Barcelona, Spain, ${ }^{2}$ Servei de Genètica, Hospital de la Santa Creu i Sant Pau, Barcelona, Spain, ${ }^{3}$ Clínica Las Condes, Santiago de Chile, Chile, ${ }^{4}$ Hospital Universitario de Basurto, Bilbao, Spain, ${ }^{5}$ Copenhagen University Hospital, Copenhagen, Denmark

Introduction: Spinal muscular atrophy (SMA) is a neuromuscular disorder caused by loss of function of both copies of SMN1. SMA patients are classified into different clinical groups. Copy number of SMN2, the highly homologous copy of $S M N 1$, is considered the most important phenotypic modifier of the disease but this correlation is not absolute. In the present therapeutic scenario with SMN2 splicing modifiers patients receive expensive treatments and there are different degrees of responses (responders, slow responders, non-responders). All SMN2 copies are not always equivalent. Thus, in deep analysis of the SMN2 genotype could provide useful information to further characterize SMA patients.

Materials and Methods: We study 53 SMA patients with homozygous SMN1-deletion to determine their SMN2 sequences. DNA samples were sequenced by Long-PCR and NGS. The amplified region includes the complete SMN2 gene (promoter, 5'UTR and 3'UTR regions). Variant analysis was performed using a bioinformatic pipeline adapted to the complexity of the region.

Results: We found previously described modifier variants c. $859 \mathrm{G}>\mathrm{C}$, c.835-44A $>\mathrm{G}$, c.835-1897C $>\mathrm{T}$ in 5 patients, and 10 new candidates, being two of them recurrent. Additionally we have detected SMN1-SMN2 hybrid in two patients.

Conclusions: our technique was useful to accurately characterize the SMN2 copies, identifying described and new modifier variants. Further functional analysis of those candidates should be essential for categorization. In the current framework of new therapies it is extremely important to characterize historical and new patients that receive further advanced treatments. Funded by Biogen and FIS PI18/00687

L. Blasco-Pérez: None. I. Paramonov: None. L. Iranzo: None. D. Martinez-Cruz: None. L. Alias: None. S. Bernal: None. C. Castiglioni: None. S. Kapetanovic: None. C. Høi-Hansen: None. E. García-Arumí: None. I. Cuscó: None. E.F. Tizzano: None.

P10.54.A

Distinguishing SMN1 and SMN2: nanopore sequencing as a powerful tool to assign variants 
K. Eggermann, T. Pfister, I. Kurth, F. Kraft

Institute of Human Genetics RWTH University Hospital, Aachen, Germany

Spinal Muscular Atrophy (SMA)-5q is an autosomalrecessive neuromuscular disorder with a frequency of app. $1: 4,900$ in the European population. Mutations in the Survival Motor Neuron (SMN) 1 gene are responsible for the disease while the copy number of SMN2 modifies the patient's phenotype. Both genes are located in a highly homologous, duplicated and inverted region on chromosome 5q. The majority of patients carry a homozygous deletion of SMN1 but $3-4 \%$ of patients are compound heterozygous for the deletion on one and single nucleotide variants (SNV) on the other allele. There are not more than five unique sequence differences between SMN1 and SMN2, only one of those, c.840C/T, in the coding sequence and the others in intronic or non-coding regions. When a patient suspected of having SMA carries a heterozygous deletion of SMN1, direct sequencing of the SMN1 gene to detect a second pathogenic SNV is necessary. Due to the nearly $100 \%$ sequence identity it is almost impossible to distinguish SMN1 and SMN2 sequences with standard sequencing approaches and a SNV cannot be assigned unequivocally to one of the genes. Several sequencing strategies to overcome this problem have been introduced, all of them being rather laborious. We have established a long-range Nanopore sequencing protocol that allows unequivocal assignment of variants to SMN1 or SMN2, and thereby improves the diagnostics for the patient and genetic counselling for the family. In times of promising therapeutic approaches for SMA-5q, it is crucial to make a fast and unambiguous diagnosis of the disease.

K. Eggermann: None. T. Pfister: None. I. Kurth: None. F. Kraft: None.

\section{P10.55.B}

Update on SMN1 mutations in Spanish SMA patients: description of new rare missense, nonsense and splicing mutations

L. Alías ${ }^{1}$, S. Bernal ${ }^{1}$, F. March ${ }^{2}$, I. Cuscó ${ }^{3}$, L. Blasco $^{3}$, S. Quero $^{2}$, M. Simón ${ }^{2}$, E. Martínez-Sánchez ${ }^{2}$, P. Fuentes-

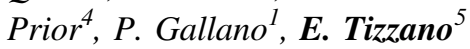

${ }^{1}$ Genetics Department. Hospital Sant Pau, CIBERER U705, Barcelona, Spain, ${ }^{2}$ Genetics Department. Hospital Sant Pau, Barcelona, Spain, ${ }^{3}$ Medical Genetics Group, VHIR, Barcelona, Spain, ${ }^{4}$ Molecular Bases of Disease, IIBSant Pau, Barcelona, Spain, ${ }^{5}$ Dept. Of Clinical and
Molecular Genetics and Rare Diseases Unit, Hospital Vall d'Hebrón, Barcelona, Spain

Background: Homozygous absence of SMN1 is the major genetic cause of SMA $(90 \%)$, the remaining cases are hybrid SMN2-SMN1 genes $(4.6 \%)$ and rare point mutations (5\%). When a patient has no homozygous deletion, SMA molecular diagnostic is of utmost importance to include him in a specific advanced treatment considering the current SMA landscape.

Materials and Methods: The $S M N$ genes were quantified by MLPA in 931 unrelated Spanish SMA patients. Sanger sequencing was usefully to detect point mutations in the SMN1 gene. C212 and C272 molecular markers were analysed to establish the familial haplotype.

Results: From our SMA cohort: $834(89.6 \%)$ showed homozygous absence of the SMN1; 42 (4.5\%) showed homozygous absence of only exon 7 , harbouring at least one SMN2-SMN1 hybrid gene; 46 (4.9\%) showed a point mutation in the SMN1 gene, being the c.399_402delAGAG in exon 3 and the c.770_780dup11 in exon 6 responsible of the $65.2 \%$ of the cases (20 and 10 respectively), other 11 known and 3 novel (missense:c.808A $>\mathrm{C}$, nonsense: c.188C $>\mathrm{A}$ and splicing:c.724-1G $>$ C) point mutations were detected. Interestingly, just one SMN1 deleted allele was found in 9 symptomatic patients but any other pathogenic variant in the other allele has been currently identified.

Conclusions: Although the vast majority of Spanish SMA cases present an homozygous deletion of the SMN1 gene, a minority of cases can be explained due to other rare mutations that are necessary to discover by Sanger or Next Generation Sequencing. Genetic confirmation of these patients is crucial in order to include them in therapeutic protocols. FIS-PI18/00687

L. Alías: None. S. Bernal: None. F. March: None. I. Cuscó: None. L. Blasco: None. S. Quero: None. M. Simón: None. E. Martínez-Sánchez: None. P. FuentesPrior: None. P. Gallano: None. E. Tizzano: None.

\section{P10.56.C}

Analytical validation of a multiplex PCR/CE assay for simultaneous determination of SMN1/SMN2 exon 7 copy number and variant status

W. Laosinchai-Wolf, J. L. Larson, J. Janovsky, K. Jefferson, D. Patel, M. Church, H. Zhu, G. J. Latham, J. N. Milligan

Asuragen, Austin, TX, United States

Introduction: Spinal Muscular Atrophy (SMA) is an autosomal recessive neuromuscular disease commonly caused by homozygous loss of function deletions in SMN1 
exon7, frequently resulting in infant mortality. SMN2, a paralog of SMN1, modulates SMA severity. Recent breakthrough therapies rely on quantification of SMN1 and SMN2 copies to inform treatment, and early detection is critical. Additionally, detection of c.859G $>$ C in SMN2 is linked to reduced disease severity and prognosis while gene duplication variants c. $* 3+80 \mathrm{~T}>\mathrm{G}$ and c. $* 211$ - 212 del in SMN1 can inform carrier risk in several populations.

Methods: We validated the AmplideX ${ }^{\oplus}$ PCR/CE SMA Plus Kit, which quantifies 0 to $\geq 4$ SMN1 and SMN2 exon7 copy numbers and the three informative variants. Automated analysis with AmplideX PCR/CE Reporter software incorporates batch and sample QC checks to qualify results. We assessed single-site precision and accuracy of SMN1 and SMN2 copy number and variant detection against reference methods using DNA samples isolated from whole blood with over five purification methods. We also assessed analytical specificity, reagent stability, and other performance factors.

Results and Conclusion: SMN1 and SMN2 copy number and variant measurements were concordant with reference methods for all isolation methods. Further, results were precise across 200 sample measurements generated with three reagent lots and two operators over multiple runs/ days. The assay was specific for all genes and variants. The AmplideX PCR/CE SMA Plus Kit is in development for in vitro diagnostic use for carrier screening and disease diagnosis, and provides accurate and reliable results in under four hours.

W. Laosinchai-Wolf: A. Employment (full or part-time); Significant; Asuragen. J.L. Larson: A. Employment (full or part-time); Significant; Asuragen. J. Janovsky: A. Employment (full or part-time); Significant; Asuragen. K. Jefferson: A. Employment (full or part-time); Significant; Asuragen. D. Patel: A. Employment (full or part-time); Significant; Asuragen. M. Church: A. Employment (full or part-time); Significant; Asuragen. H. Zhu: A. Employment (full or part-time); Significant; Asuragen. G.J. Latham: A. Employment (full or part-time); Significant; Asuragen. J.N. Milligan: A. Employment (full or part-time); Significant; Asuragen.

\section{P10.57.A}

The <"fortunate" > couple

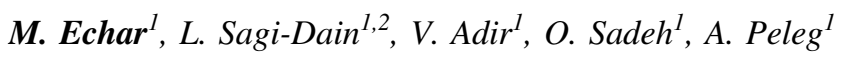

${ }^{1}$ Carmel Medical Center, Haifa, Israel, ${ }^{2}$ The Ruth and Bruce Rappaport Faculty of Medicine, Technion - Israel Institute of Technology, Hifa, Israel

Introduction: Spinal Muscular Atrophy (SMA) is an autosomal recessive neuromuscular disorder caused by loss of lower motor neurons. All types of SMA are caused by homozygous loss of the SMN1 gene, resulting in low levels of the survival motor neuron protein, insufficient to protect motor neurons. Homozygous deletions of exon 7 in SMN1 gene in clinically asymptomatic individuals are extremely rare, involving less than 30 described cases. These patients usually have several copies of the SMN2 gene, which compensates for the loss of SMN1 product.

Case presentation: An asymptomatic 31-years old woman was referred for pregestational genetic testing within the Israeli national genetic carrier-screening program for reproductive purposes. Carrier screening was performed using Multiplex ligase-dependent probe amplification (MLPA) Probemix P060.

Results: The patient was diagnosed with homozygous absence of exon 7 in the SMN1 gene. The deletion was validated using MLPA Probemix P021 and Real TimePolymerase Chain Reaction. MLPA testing revealed three copies of SMN2 exon 7 and 8. Genetic testing of the spouse revealed three copies of exon 7 in SMN1.

Discussion: Carrier testing for reproductive partner of SMA requires SMN1 dosage analysis. If an individual is found to have at least two SMN1 copies, the probability of being a carrier is approximately 1/670 (mainly taking into consideration the $2 \%$ frequency of two SMN1 copies on the same chromosome). However, in our unique and pretty fortunate couple the risk for SMA disease in the progeny is very low due to the spouses' extra copy.

M. Echar: None. L. Sagi-Dain: None. V. Adir: None. O. Sadeh: None. A. Peleg: None.

\section{P10.58.B}

Profiling of the oculomotor nucleus and spinal cord in amyotrophic lateral sclerosis using single-nuclei RNA sequencing (snRNA-seq)

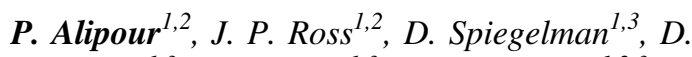

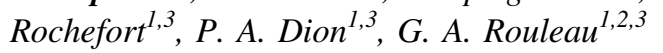

${ }^{1}$ Montreal Neurological Institute, and Hospital, McGill University, Montréal, QC, Canada, Montreal, QC, Canada, ${ }^{2}$ Department of Human Genetics, McGill University, Montréal, QC, Canada, Montreal, QC, Canada, ${ }^{3}$ Department of Neurology and Neurosurgery, McGill University, Montréal, QC, Canada, Montreal, QC, Canada

Introduction: Amyotrophic lateral sclerosis (ALS) is a progressive neurodegenerative disorder which involves loss of upper and lower motor neurons (MNs) in the central nervous system (CNS). While both upper and lower MNs are affected, some subtypes of lower MNs are resistant to degeneration such as oculomotor nuclei located in the midbrain. The molecular mechanism underlying this differential vulnerability among MNs is currently unknown. 
Using snRNA-seq to profile expression in ALS tissues will explain the changes in cell type proportions associated with neurodegeneration.

Materials \& Methods: Postmortem brain tissues have been collected from 30 tissue samples from oculomotor nucleus, medulla, and spinal cord of ALS cases and healthy controls. Nuclei isolation and library preparation using the Chromium Single Cell $3^{\prime}$ Reagent Kits v2 (10X Genomics) has been performed aiming for 6,000 nuclei per sample. The average sequencing depth per sample was $360 \mathrm{M}$ reads using the NovaSeq 6000 (Illumina). Cellranger software was used for alignment and generation of feature-barcode matrices. The R package Seurat was applied for downstream analysis such as differential gene expression and cell clustering.

Preliminary Results: We have identified differences in surviving cell types between severely affected and spared regions of the CNS. Further, we identified cell population differences and cell-specific dysregulation of genes between ALS patients and controls, as well as between different regions of the CNS.

Conclusions: Knowing the mechanisms underlying tissuespecific neurodegeneration could help understand why some cell types are resistant to degeneration and how it could be possible to simulate this resistance in vulnerable regions.

P. Alipour: None. J. P. Ross: None. D. Spiegelman: None. D. Rochefort: None. P. A. Dion: None. G. A. Rouleau: None.

\section{P10.59.C}

New splice site mutation in SPG7

\section{A. Zaum, F. Zimmer, N. Pluta, E. Kunstmann, S. Rost}

\section{Institute of Human Genetics, Wuerzburg, Germany}

Introduction: $S P G 7$ encodes paraplegin, a protein that is part of a metalloprotease of the inner mitochondrial membrane. Mutations in SPG7 cause an autosomal recessive form of hereditary spastic paraplegia (SPG), a clinically and genetically heterogeneous group of neurological diseases. Due to the heterogeneity of SPGs and other neuropathies, genetic diagnostics by whole exome sequencing (WES) is beneficial and performed successfully in our laboratory for different (virtual) gene panels.

Methods: We present the case of a patient with clinical suspicion of SPG. For molecular diagnosis, we used WES and analysed a (virtual) SPG-panel consistent of 13 genes. To validate one variant's effect on splicing, we isolated RNA from whole blood and retranscriped it into cDNA, followed by Sanger sequencing.

Results: We identified two heterozygous variants in SPG7: one recurrent pathogenic variant (p.(Ala510Val)) and a variant of uncertain significance $($ c. $1779+5 G>C)$. Prediction tools calculated a decreased probability for the usage of the donor splice site adjacent to the second variant. However, RNA analysis confirmed the usage of a cryptic splice site (c.1779+42) that leads to the inclusion of 14 amino acids in the protein. The insertion is in frame, but occurs in a functionally important peptidase domain of the protein.

Conclusions: In summary, we present the case of a new SPG7 splice site variant, with confirmed effect on RNA splicing. Together with the second variant, it confirms and specifies the clinical diagnosis. Therefore, WES is a powerful tool to diagnose heterogeneous neurological disorders.

A. Zaum: None. F. Zimmer: None. N. Pluta: None. E. Kunstmann: None. S. Rost: None.

P10.60.A

Detection of cis duplication of SMN1 in healthy carrier, a droplet digital PCR method to improve genetic counselling

\section{VEREBI, C. Vignal, S. Drunat}

\section{Robert Debre University Hospital, Paris, France}

Introduction: The c. $* 3+80 \mathrm{~T}>\mathrm{G}$ and c. $* 211 * 212 \mathrm{del}$ SMN1 variants had been initially associated with SMN1 cis duplication in the Ashkenazi population (Luo et al. Genet Med. 2014). Our study falls within improvement of genetic counseling for carriers of SMA as already done for Spanish population (Alías et al. EJHG. 2018).

Materials and methods: We used a previously published ddPCR design for the variant c. $* 3+80 T>G$ detection (Vidal-Folch et al. Clin Chem. 2018) and a custom made design for the variant c.*211_*212del. Carrier status was established after familial studies around SMA cases.

Results: See Table ; SMA for Spinal Muscular Atrophy ; *Corresponding to the same individuals ; ${ }^{\&} 27$ individuals carried the two variants, the last individual only carried the c. $* 3+80 \mathrm{~T}>\mathrm{G}$ variant.

Conclusions: The variants were absent in $1 / 0$ healthy carriers which corroborate the high association with multiple and furthermore cis duplicate SMN1 genes. We can assume that the percentage of variants carriers in $2 / 0$ carriers individual is about $43 \%$ in our population which place itself between the average $20 \%$ and $46 \%$ in Spanish and Ashkenazi population respectively(Luo et al. Genet Med. 2014, Alías et al. EJHG. 2018). The variant study leaded to the estimation of $11.48 \%$ of cis duplication carrier in our general population, allowing us to increase the sensitivity of our SMA carrier detection test. 
Cis duplication SMN1 variants in SMA patients, in SMA carriers and in general population

\begin{tabular}{|c|c|c|c|c|c|c|c|c|}
\hline & $\begin{array}{l}\text { SMA patients with } \\
\text { homozygous deletion of } \\
S M N I(n=48)\end{array}$ & $\begin{array}{l}\text { SMA } \\
\text { carriers } \\
=76)\end{array}$ & & & $\begin{array}{l}\text { General } \\
\text { population } \\
(n=191)\end{array}$ & & & \\
\hline Genotype & $0 S M N 1$ & $\begin{array}{l}1 S M N 1 \\
(0 / 1)\end{array}$ & $\begin{array}{l}2 \\
S M N 1 \\
(0 / 2)\end{array}$ & $\begin{array}{l}\stackrel{3}{S M N I} \\
(0 / 3)\end{array}$ & $2 S M N 1$ & $\stackrel{3}{S} M N 1$ & $\stackrel{4}{S} M N 1$ & $\stackrel{5}{S M N 1}$ \\
\hline c. $3^{*}+80 \mathrm{~T}>\mathrm{G}$ & 0 & 0 & $\begin{array}{l}16^{*} \\
(43.2 \%)\end{array}$ & $\begin{array}{l}2^{*} \\
(100 \%)\end{array}$ & $1^{*}(0.8 \%)$ & $\begin{array}{l}28^{\&} \\
(43.8 \%)\end{array}$ & $\begin{array}{l}5^{*} \\
(83.3 \%)\end{array}$ & $\begin{array}{l}1^{*} \\
(100 \%)\end{array}$ \\
\hline$\underset{* 211 \_* 212 \mathrm{del}}{\mathrm{c}}$ & $1(2.1 \%)$ & 0 & $\begin{array}{l}16^{*} \\
(43.2 \%)\end{array}$ & $\begin{array}{l}2^{2 *} \\
(100 \%)\end{array}$ & $1 *(0.8 \%)$ & $\begin{array}{l}27 \\
(42.1 \%)\end{array}$ & $\begin{array}{l}5 * \\
(83.3 \%)\end{array}$ & $\begin{array}{l}1_{(100 \%}^{*} \\
\text { (100) }\end{array}$ \\
\hline $\begin{array}{l}\text { Total studied } \\
(n=315)\end{array}$ & 48 & 37 & 37 & 2 & 120 & 64 & 6 & 1 \\
\hline
\end{tabular}

C. Verebi: None. C. Vignal: None. S. Drunat: None.

P10.62.C

Long-read amplicon sequencing detects a bi-allelic $S M N 1>S M N 2$ gene conversion allele that does not guarantee a spinal muscular atrophy (SMA) phenotype

H. Muallem ${ }^{\text {, J. Harting }}{ }^{2}$, C. Heiner ${ }^{2}$, S. Y. Scher ${ }^{3}, J$. Ekstein $^{3}$, D. A. Zeevi ${ }^{l}$

${ }^{I}$ Dor Yeshorim Committee for Prevention of Jewish Genetic Diseases, Jerusalem, Israel, ${ }^{2}$ Pacific Biosciences, Menlo Park, CA, United States, ${ }^{3}$ Dor Yeshorim Committee for Prevention of Jewish Genetic Diseases, New York, NY, United States

Introduction: Spinal muscular atrophy (SMA) is caused primarily by bi-allelic loss of SMNI exon 7 via deletion and/or gene conversion to $S M N 2$. Here we show that biallelic $S M N 1>S M N 2$ gene conversion presents with mild, or completely absent, disease phenotype.

Materials and Methods: A consanguineous couple and 4 children were selected for investigation. Two children were asymptomatic and the other two children were diagnosed with mild Type 3 SMA (at ages 13 and 28, respectively). For each sample, SMN1 exon7 dosage was measured by qPCR and $S M N 1 / 2$ alleles were determined by long read amplicon Single Molecule, Real-Time (SMRT) sequencing on the Sequel System (Pacific Biosciences) followed by segregation analysis.

Results: The qPCR assay identified a carrier state in both parents, non-carrier state in one unaffected child, and complete absence of SMN1 exon 7 in 2 affected children and 1 unaffected child. Long-read amplicon analysis identified identical genotypes in the parents, including one normal SMN chromosome (bearing 1 copy of SMN1 and 1 copy of SMN2) and one gene conversion SMN chromosome (bearing one normal copy of SMN2 and one SMN1>SMN2 gene converted copy). The non-carrier unaffected child was homozygous for the normal SMN chromosome, while the 2 affected children and 1 unaffected child were each homozygous for the gene conversion SMN chromosome.

Conclusions: $S M N 1>S M N 2$ gene conversion, which is fairly common in the general population, was readily detected with qPCR and long amplicon sequencing. Our results suggest that bi-allelic loss of SMN1 by gene conversion is not necessarily indicative of disease.

H. Muallem: None. J. Harting: A. Employment (full or part-time); Significant; Pacific Biosciences. C. Heiner: None. S.Y. Scher: None. J. Ekstein: None. D.A. Zeevi: None.

\section{P10.64.B}

Possible X-linked genetic and epigenetic modifiers of proximal spinal muscular atrophy

\section{A. Yurchenko ${ }^{1}$, E. Patskun ${ }^{2}$, S. Kravchenko ${ }^{3}$, N. Hryshchenko $^{3}$}

${ }^{I}$ Educational and Scientific Center "Institute of Biology and Medicine" of Taras Shevchenko National University of Kyiv, Kyiv, Ukraine, ${ }^{2}$ Municipal Non-Profit Enterprise "Transkarpathian Clinical Regional Hospital named after Andrii Novak", Uzhgorod, Ukraine, ${ }^{3}$ Institute of Molecular Biology and Genetics NAS of Ukraine, Kyiv, Ukraine

Introduction: Proximal spinal muscular atrophy (SMA) is a neuromuscular autosomal recessive disease, mostly caused by a homozygous deletion in SMN1 gene. Despite SMA is a classic monogenic disease, the clinical phenotype of patients varies from the most acute infantile type to a relatively mild chronic juvenile type. Some genetic modifiers of SMA are already known, such as a type of SMN1 mutation and SMN2 copy number variation. It was shown that the female SMA phenotype was significantly differing from the phenotype of the male patients. The aim of our investigation is to study an association of SMA phenotype with X-linked genetic and epigenetic factors.

Materials and Methods: The study groups consisted of female $(n=103)$ and male $(n=75)$ patients with determined SMN1 and SMN2 genotypes. The analysis of $\mathrm{X}$-chromosome inactivation (XCI) was done by methylspecific CAG-genotyping of AR gene. The rs2070169 and rs4339766 of UBA1 were analyzed by RFLP-method. Spearman's correlation, regression analysis and MannWhitney test were used for the statistical analysis.

Results: The correlation between the patients' SMN1 and SMN2 genotypes and SMA phenotypes was stronger in the male group. Skewed XCI was detected in $45.5 \%$ of females, these patients significantly more often $(\mathrm{p}<0.05)$ had differ phenotype than expected. It was demonstrated that rs2070169 and rs4339766 had no significant effect on SMA phenotype.

Conclusions: It was confirmed that a XCI skewing may influence on SMA phenotype. The skewed XCI in SMA patients lead both to milder and severer phenotypes. 
A. Yurchenko: None. E. Patskun: None. S. Kravchenko: None. N. Hryshchenko: None.

\section{P10.66.A}

Loss of costameric supervillin causes myopathy with myofibrillar disorganization and autophagic vacuoles

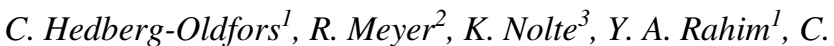

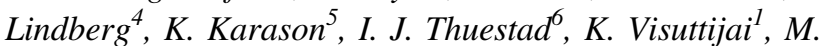
Geijer $^{7,8}$, M. Begemann ${ }^{2}, F$. Kraft ${ }^{2}$, E. Lausberg ${ }^{2}$, L. Hitpa $^{9}$, R. Götzl ${ }^{10}$, E. J. Luna ${ }^{11}$, H. Lochmüller ${ }^{12,13}, S$. Koschmieder ${ }^{14}$, M. Gramlich ${ }^{15}$, B. Gess ${ }^{16}$, M. Elbracht ${ }^{2}, J$. Weis $^{3}$, I. Kurth ${ }^{2}$, A. Oldfors ${ }^{1}$, C. Knopp ${ }^{2}$

${ }^{1}$ Department of Pathology and Genetics, Sahlgrenska Academy, University of Gothenburg, Gothenburg, Sweden, ${ }^{2}$ Institute of Human Genetics, Medical Faculty, RWTH Aachen University, Aachen, Germany, ${ }^{3}$ Institute of Neuropathology, Medical Faculty, RWTH Aachen University, Aachen, Germany, ${ }^{4}$ Department of Neurology, Neuromuscular Centre, Sahlgrenska University Hospital, Gothenburg, Sweden, ${ }^{5}$ Department of Cardiology and Transplant Institute, Sahlgrenska University Hospital, Gothenburg, Sweden, ${ }^{6}$ Department of Pediatrics, Skane University Hospital, Malmo, Sweden, ${ }^{7}$ Department of Radiology, Sahlgrenska Academy, University of Gothenburg, Gothenburg, Sweden, ${ }^{8}$ Department of Clinical Sciences, Lund University, Lund, Sweden, ${ }^{9}$ Department of Diagnostic and Interventional Radiology, Medical Faculty, RWTH Aachen University, Aachen, Germany, ${ }^{10}$ Department of Plastic Surgery, Hand and Burn Surgery, Medical Faculty, RWTH Aachen University, Aachen, Germany, ${ }^{11}$ Division of Cell Biology and Imaging, Department of Radiology, University of Massachusetts Medical School, Worcester, MA, United States, ${ }^{12}$ Children's Hospital of Eastern Ontario Research Institute, Division of Neurology, Department of Medicine, The Ottawa Hospital, Ottawa, ON, Canada, ${ }^{13}$ Brain and Mind Research Institute, Ottawa, ON, Canada, ${ }^{14}$ Department of Hematology, Onkology, Hemostaseology and Stem Cell Transplantation, Medical Faculty, RWTH Aachen University, Aachen, Germany, ${ }^{15}$ Department of Invasive Electrophysiology, Medical Faculty, RWTH Aachen University, Aachen, Germany, ${ }^{16}$ Department of Neurology, Medical Faculty, RWTH Aachen University, Aachen, Germany

Introduction: Archvillin, the muscle specific isoform of supervillin encoded by the SVIL gene, is a large sarcolemmal protein. Despite an important role of supervillin in muscle cell physiology has been suggested, no muscular disease associated with SVIL mutations has been described. We here report a new structural myopathy in four individuals of two unrelated, consanguineous families caused by biallelic loss-of-function mutations in SVIL.

Material and Methods: Patients were comprehensively investigated clinically, and whole exome sequencing on genomic DNA was performed in all affected individuals. Skeletal muscle biopsies were morphological analyzed by enzyme and immunohistochemical techniques and electron microscopy.

Results: Affected patients share a distinctive phenotype that includes a wide neck, anteverted shoulders, and a prominent trapezius muscle together with variable contractures besides further skeletal and mild heart muscle involvement. Muscle biopsies of both families demonstrated complete loss of supervillin in muscle tissue by western blot and immunohistochemical analyses. Comprehensive light and electron microscopical investigations of muscle biopsies showed striking similarities between patients and revealed a structural myopathy with prominent lobulated type 1 fibers, myofibrillar disintegration and signs of altered proteostasis and impaired autophagy.

Conclusions: The results underline the importance of supervillin for the structural integrity of muscle fibers in humans and show that recessive loss-of-function mutations in SVIL cause a novel myopathy with distinct histological, ultrastructural and clinical findings. Funding: This study was supported by the Swedish Research Council to AO (grant number 2018-02821) and by the German Research Foundation (DFG) to IK (KU1587/4-1).

C. Hedberg-Oldfors: None. R. Meyer: None. K. Nolte: None. Y.A. Rahim: None. C. Lindberg: None. K. Karason: None. I.J. Thuestad: None. K. Visuttijai: None. M. Geijer: None. M. Begemann: None. F. Kraft: None. E. Lausberg: None. L. Hitpaß: None. R. Götzl: None. E.J. Luna: None. H. Lochmüller: None. S. Koschmieder: None. M. Gramlich: None. B. Gess: None. M. Elbracht: None. J. Weis: None. I. Kurth: None. A. Oldfors: None. C. Knopp: None.

\section{P10.68.C}

Unexpected identification of a novel 11 bp deletion mutation in $T A R D B P$ causing a slowly progressive autosomal dominant myopathy

\section{P. Ervilha Pereira ${ }^{1,2}$, N. Schuermans ${ }^{1,2}$, A. Meylemans ${ }^{3,4}$, S. Roy ${ }^{1,2}$, P. Leblanc ${ }^{1,2}$, J. Aalders ${ }^{5}$, E. Debackere ${ }^{1,2}$, O. Vanakker $^{1,2}$, J. Van Hengel ${ }^{5}$, S. Symoens ${ }^{1,2}$, J. De Bleecker $^{3,4}$, B. Dermaut ${ }^{1,2}$, E. Bogaert ${ }^{1,2}$}

${ }^{I}$ Department of Biomolecular Medicine, Faculty of Medicine and Health Sciences, Ghent University, Gent, Belgium, ${ }^{2}$ Center for Medical Genetics, Gent University Hospital, Gent, Belgium, ${ }^{3}$ Department of Neurology, Ghent University Hospital, Gent, Belgium, ${ }^{4}$ Department of Head and 
Skin, Faculty of Medicine and Health Sciences, Ghent University, Gent, Belgium, ${ }^{5}$ Department of Human Structure and Repair, Faculty of Medicine and Health Sciences, Ghent University, Gent, Belgium

Background: In Amyotrophic Lateral Sclerosis (ALS) and Frontotemporal Dementia (FTD), TDP-43 proteinopathy occurs in $97 \%$ of ALS cases and $45 \%$ of FTD patients. Furthermore, missense mutations in the C-terminus of TDP43 are a genetic cause of ALS, emphasizing the crucial role of this protein in the disease's pathogenesis.

Objective: To identify and characterize the genetic cause of an autosomal dominant slowly progressive neurogenic myopathy in a large Belgian family.

Methods: Whole exome sequencing and segregation analysis of candidate causal variants.

Results: We identified a novel causal $11 \mathrm{bp}$ deletion in the TARDBP gene (c.1152_1162del) segregating with the disease (2-point LOD-score of 2.4). The disease presented as a slowly progressive neurogenic myopathy with an average age-of-onset of 32 years (range: 18-40) and a disease duration of $>45$ years. No family members showed classical ALS or FTD pathology. Unlike previously described classical ALS-causing mutations in TARDBP, this mutation causes a frameshift that leads to an entirely novel C-terminus. This region of the protein is key for the formation of TDP-43 aggregates, therefore this novel mutation provides a unique opportunity to dissect the pathogenic role of TDP-43.

Conclusions: Since the molecular mechanisms responsible for TDP-43 pathology remain fairly unknown, unravelling the biophysical properties of TDP-43 aggregation is of utmost importance. Functional analysis this novel and unusual TARDBP 11 bp deletion (c.1152_1162del) using patient iPSC-derived neurons and Drosophila models are ongoing.

Funding was obtained through following grants: Odysseus Type I grant (G0H8318N), Research Foundation Flanders (FWO) and Starting grant (01N10319), Ghent University Special Research Fund

P. Ervilha Pereira: None. N. Schuermans: None. A. Meylemans: None. S. Roy: None. P. Leblanc: None. J. Aalders: None. E. Debackere: None. O. Vanakker: None. J. Van Hengel: None. S. Symoens: None. J. De Bleecker: None. B. Dermaut: None. E. Bogaert: None.

P10.69.A

TTN gene analysis in UK patients with congenital myopathies

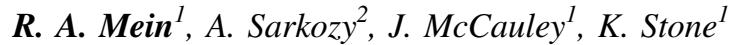

${ }^{I}$ Genetics Department, Viapath, London, United Kingdom,

${ }^{2}$ The Dubowitz Neuromuscular Centre, London, United Kingdom

Recessive TTN gene variants cause congenital myopathy (CM) with variable presentation, progression and pathological features. Implementation of Next generation sequencing (NGS) has enabled screening of the TTN gene in routine diagnostic testing and is highlighting the considerable prevalence of this myopathy. The UK Highly Specialised Service for CM started TTN analysis in 2016 as part of a panel of genes associated with congenital myopathy. We present results from 99 patients with $T T N$ sequence variants. In most cases other potentially relevant CM genes had been excluded either prior to or at the same time as TTN analysis. 23 patient have homozygous/compound heterozygous (likely) pathogenic variants which were confirmed to be in trans in 13 patients. 21 patients had one or more missense variants of uncertain significance in addition to a (likely) pathogenic TTN variant, confirmed to be in trans in 16 patients. A further 13 patients had a heterozygous pathogenic variant but a second causative variant was not identified. The remaining 42 patients had one $(n=18)$, two (12), or more than three (4) variants of uncertain significance. The high number of missense variants with insufficient evidence to classify as likely pathogenic, even in patients with strong clinical, pathological and MRI features, means that functional characterisation of missense variants is required and further investigation such as transcriptome analysis is necessary to identify additional pathogenic variants in in those patients with a single heterozygous pathogenic variant.

R.A. Mein: None. A. Sarkozy: None. J. McCauley: None. K. Stone: None.

\section{P10.70.B}

Preferential $\mathrm{X}$ chromosome inactivation in women with myasthenia gravis

V. Nicoli ${ }^{1,2}, S$. Tabano $^{3}$, P. Colapietro ${ }^{3}, M$. Miozzo $^{3}, R$.

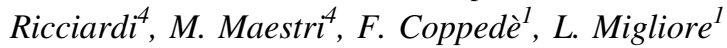

${ }^{I}$ Department of Translational Research and of New Surgical and Medical Technologies, University of Pisa, Pisa, Italy, ${ }^{2}$ Doctoral School in Genetics, Oncology and Clinical Medicine, Department of Medical Biotechnologies, University of Siena, Siena, Italy, ${ }^{3}$ Medical Genetics, Department of Pathophysiology \& Transplantation, 
University of Milan, Milan, Italy, ${ }^{4}$ Department of Clinical and Experimental Medicine, Neurology Unit, University of Pisa, Pisa, Italy

Introduction: Myasthenia gravis (MG) is an autoimmune disease characterised by female prevalence (3:1). Several mechanisms were proposed as explanation of gender bias in autoimmune diseases, including skewed $\mathrm{X}$ chromosome inactivation (XCI) and X chromosome dosage, sex hormones, and variants of genes involved in autoimmunity development. Normally, XCI is a biological event that occurs randomly, and females are thus functional mosaics for the X-linked genes. A skewed XCI pattern can lead to an unbalanced expression of certain X-linked alleles, for this reason it has been hypothesized that females may be more prone to certain diseases. Indeed, a high frequency of skewed XCI pattern has been observed in several autoimmune disorders characterized by female predominance. No data are yet available concerning myasthenia gravis, so that we investigated the XCI pattern in MG females with this autoimmune disease.

Materials and Methods: XCI analysis was performed on blood samples of 84 myasthenic women and 111 healthy age-matched female controls. XCI was analysed by human androgen receptor (HUMARA) assay.

Results: In $72 \mathrm{MG}$ informative patients the frequency of skewed XCI pattern $(\geq 75 \%)$ was $35,0 \%$, which was higher than in healthy controls $(14,4 \% ; p=0.002)$. Furthermore, the prevalence of skewed pattern was observed mostly in youngest patients ( $<50$ years; $p=0.008)$, suggesting an age-independent effect.

Conclusions: In this study, we observed preferential XCI in MG female patients, this could suggest a potential role of this mechanism in the disease etiology, and in the increased prevalence of autoimmune disorders in females.

V. Nicolì: None. S. Tabano: None. P. Colapietro: None. M. Miozzo: None. R. Ricciardi: None. M. Maestri: None. F. Coppedè: None. L. Migliore: None.

\section{P10.71.C}

Truncating mutations in $Y I F 1 B$ cause a progressive encephalopathy with various degrees of mixed movement disorder, microcephaly, and epilepsy

N. Kaya ${ }^{l}$, M. AlMuhaizeal, R. AlMass ${ }^{l}$, A. AlHargan ${ }^{l}$, A. AlBader $^{l}$, E. Medico Salsench ${ }^{2}$, J. Howaidi ${ }^{3}$, J. Ihinger $^{4}$, P. Karachunski $^{5}$, A. Begtrup ${ }^{6}$, M. Castell ${ }^{7}$, P. Bauer $^{7}$, A. Bertoli-Avella ${ }^{7}$, I. Kaya ${ }^{1}$, J. AlSufayan ${ }^{3}$, L. AlQuait ${ }^{3}$, A.

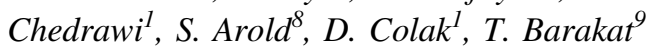

${ }^{1}$ King Faisal Specialist Hospital and Research Centre, Riyadh, Saudi Arabia, ${ }^{2}$, Erasmus MC University Medical Center, Rotterdam, Netherlands, ${ }^{3}$ AlFaisal University,
Riyadh, Saudi Arabia, ${ }^{4}$ University of Minnesota Medical Center, Minneapolis, MN, United States, ${ }^{5}$ Department of Neurology, University of Minnesota, Minneapolis, MN, United States, ${ }^{6}$ GeneDx, Gaithersburg, MD, United States, ${ }^{7}$ CENTOGENE AG, Rostock, Germany, ${ }^{8}$ King Abdullah University of Science and Technology, Riyadh, Saudi Arabia, ${ }^{9}$ Erasmus MC University Medical Center, Rotterdam, Netherlands

YIF1B, an intracellular transmembrane protein, involves in trafficking of molecules from the endoplasmic reticulum to the Golgi apparatus in eukaryotic cells. Errors in such transportation between the organelles can lead to human diseases and implicated in nervous system disorders. Here, we describe 6 patients from 5 unrelated families presenting with progressive encephalopathy with various degrees of mixed movement disorder, dysphagia, hypotonia, microcephaly, and epilepsy. Common genetic errors found in all these patients are homozygous truncating variants (c.186dupT:p.Ala64fs; c.360_361insACAT:p.Gly121fs; c.598G>T:p.Glu200*) in YIF1B. The genetic analysis was carried out using whole exome sequencing. All affected individuals shared a similar phenotypic features. The residues in the variants are highly conserved among different species and were absent in publicly available databases such as GnomAD. YIF $1 B$ is ubiquitously expressed molecule in various tissues including neuronal cells and specifically in raphe neurons. We performed computational structural analysis of the variants. The modeling predicted that the variants lead to unstructured molecules with loss of function. Our network and functional analyses revealed coregulation of 171 genes with $Y I F 1 B$ in over 46,000 human RNAseq samples $(r \geq 0.9)$. Our data indicates that disease causing variants in $Y I F 1 B$ in humans lead to a novel neurogenetic disorder. Additional studies are required to disentangle the precise pathobiological mechanisms of this autosomal recessive novel disease entity.

We are grateful to the patient families for their participation. This research received intramural funds from KFSHRC, grants from NSTIP/KACST (NK, DC), KSCDR (NK), KAUST (STA), NARSAD (TSB). We extend our special thanks to the funding agencies.

N. Kaya: None. M. AlMuhaizea: None. R. AlMass: None. A. AlHargan: None. A. AlBader: None. E. Medico Salsench: None. J. Howaidi: None. J. Ihinger: None. P. Karachunski: None. A. Begtrup: None. M. Castell: Other; Modest; CENTOGENE. P. Bauer: Other; Modest; CENTOGENE. A. Bertoli-Avella: Other; Modest; CENTOGENE. I. Kaya: None. J. AlSufayan: None. L. AlQuait: None. A. Chedrawi: None. S. Arold: None. D. Colak: None. T. Barakat: None. 


\section{P11 Multiple Malformation/Anomalies Syndromes}

\section{P11.01.A}

\section{1q21.1 CNVs}

A. Grangeia ${ }^{1,2}$, J. Pinto ${ }^{3,2}$, M. Leão $o^{1,3}$, S. Guimarães ${ }^{4}, C$. Ramalho $^{5,6,2}$, R. Oliveira ${ }^{1}$, S. Dória ${ }^{3,2}$

${ }^{1}$ Medical Genetics Service, Centro Hospitalar e Universitário São João (CHUSJ), Porto, Portugal, ${ }^{2}$ I3S-Instituto de Investigação e Inovação em Saúde, University of Porto, Porto, Portugal, ${ }^{3}$ Department of Genetics, Faculty of Medicine (FMUP), University of Porto, Porto, Portugal, ${ }^{4}$ Pathological Anatomy Service, Centro Hospitalar $e$ Universitário São João (CHUSJ)/Faculty of Medicine University of Porto (FMUP), Porto, Portugal, ${ }^{5}$ Department of Obstetrics, Centro Hospitalar e Universitário São João (CHUSJ), Porto, Portugal, ${ }^{6}$ Department of Obstetrics, Gynecology and Pediatrics, Faculty of Medicine (FMUP), Porto, Portugal

Introduction: The 1q21.1 deletion/duplication syndrome is associated to a variable clinical phenotype, characterized by neurodevelopmental disorder, craniofacial dysmorphisms and heart defects. The Thrombocytopenia Absent Radius (TAR) syndrome is characterized by hypo-megakaryocytic thrombocytopenia, radius agenesis with thumbs. This study describes ten patients with 1q21.1 CNV including one clinical TAR syndrome.

Material and Methods: An array-CGH (Agilent $4 \times 180 \mathrm{~K}$ ) was performed in 1508 patients with different indications.

Results: Ten CNVs in 1q21.1 region were identified, 6 pathogenic and 4 probably pathogenic. Nine patients showed a variable clinical phenotype, autism spectrum disorders (ASD), intellectual disability (ID), developmental delay and craniofacial dysmorphisms, and a fetus with radius agenesis; three CNVs included the deletion of RBM8A gene, but only the fetus had clinical TAR.

Discussion/Conclusion: The presence of a CNV in the 1q21.1 region could represent a contiguous gene deletion/ duplication syndrome (autosomal dominant inheritance) or TAR syndrome (autosomal recessive inheritance).In the fetus with absent radius, the $R B M 8 A$ gene sequencing revealed no pathogenic coding variants, so further studies will be performed to identify a second variant (intronic or promotor regions) that could explain the TAR phenotype.In 1q21.1deletion patients the most common psychiatric manifestation includes internalizing disorders. In 1q21.1duplications, ASD, attention deficit-hyperactivity disorder and ID are the most common features. Our patients' clinical characterization and a comparison of the deletion versus duplication phenotypes will be done in detail. This study highlights the clinical usefulness of aCGH as a first-tier test for evaluation of patients with neurodevelopmental disorders and malformation syndromes.

A. Grangeia: None. J. Pinto: None. M. Leão: None. S. Guimarães: None. C. Ramalho: None. R. Oliveira: None. S. Dória: None.

\section{P11.02.B}

Phenotypic heterogeneity in 22q11.2 deletion syndrome: CNVs as genetic modifiers for congenital heart disease in a Brazilian cohort

M. Zamariolli ${ }^{1}$, A. G. Dantas ${ }^{1}$, N. Nunes ${ }^{1}$, M. MoysésOliveira $^{1}$, I. C. Sgardioli ${ }^{2}$, D. C. Q. Soares ${ }^{3}$, V. L. Gil-DaSilva-Lopes $^{2}$, C. A. Kim ${ }^{3}$, M. I. Melaragno ${ }^{1}$;

${ }^{1}$ Universidade Federal de São Paulo, São Paulo, Brazil, ${ }^{2}$ Universidade Estadual de Campinas, Campinas, Brazil, ${ }^{3}$ Universidade de São Paulo, São Paulo, Brazil.

Introduction: The clinical heterogeneity in $22 \mathrm{q} 11.2$ deletion syndrome (22q11.2DS) might be caused by complex mechanisms including variants in other regions of the genome, known as genetic modifiers. Congenital heart disease (CHD) is one of the most relevant phenotypes and previous studies have shown that copy number variants (CNVs) outside the $22 q 11.2$ region could play a role in its variable expressivity. However, those described loci account for a small proportion of the variability. Therefore, CNV analysis in new cohorts from different ancestry-based population are a valuable resource to identify a wider range of modifiers.

Materials and Methods: We performed SNP-array (CytoScanHD) in 77 Brazilian patients with 22q11.2DS (55 with CHD and 22 with normal cardiac phenotype). Genome-wide CNV analysis was performed by two algorithms (PennCNV and Rawcopy) and the overlapping CNVs were considered for downstream analysis. The CNVs content was annotated using AnnotSV and compared between patients with and without CHD using Gene Set Enrichment Analysis (GSEA).

Results: We identified 407 CNVs (308 deletions and 99 duplications). CNV-affected genes in patients with CHD were enriched for pathways related to transition metal ion binding, cytoskeleton and stabilization of membrane potential. Some of these genes have been previously associated to cardiac alterations (OTUD7A, DNAH5, KIF6, EVC, KCNK17).

Conclusions: Further exploration of the genes in these pathways could reveal new modifiers to the cardiac phenotype in 22q11.2DS patients. This study will leverage new insights to the understanding of genetic modifiers involved in the expressivity of cardiac alterations in 22q11.2DS. Financial support: CAPES, FAPESP, Brazil. 
M. Zamariolli: None. A.G. Dantas: None. N. Nunes: None. M. Moysés-Oliveira: None. I.C. Sgardioli: None. D.C.Q. Soares: None. V.L. Gil-Da-Silva-Lopes: None. C. A. Kim: None. M.I. Melaragno: None.

\section{P11.05.B}

Modelling Rho GTPase dysregulation in Adams-Oliver syndrome: a disorder of vasculogenesis?

\section{E. Benson, D. P. Osborn, G. S. Whitley, L. Southgate \\ St George's University of London, London, United King- dom}

Introduction: Adams-Oliver syndrome (AOS) is a debilitating disorder of limb, scalp and vascular birth defects, providing a unique window into the regulation of human embryogenesis. AOS is considered to be a disorder of vasculogenesis; however, this remains to be determined. We have previously identified pathogenic mutations of the Rho GTPase regulators ARHGAP31 and DOCK6, implicating Rho dysregulation in AOS aetiology. Gain-of-function ARHGAP31 mutations and DOCK6 loss-of-function impact cytoskeletal dynamics through abnormal inactivation of Cdc42 and Rac1. Nonetheless, their role in human blood vessel formation requires further exploration. Here, we sought to generate in vitro and in vivo models of Rho dysregulation to investigate the mechanism of vascular disruption in AOS.

Materials and Methods: Rho GTPase dysregulation was modelled in zebrafish embryos (Danio rerio) and human vascular smooth muscle cells. Gene expression patterns were assessed by quantitative PCR and whole-mount in situ hybridisation. Gene knockdown was conducted using splice-blocking morpholino oligonucleotides.

Results: Both genes were expressed in human vascular smooth muscle cells and early stages of vascular development in zebrafish. Whole-mount in situ hybridisation in zebrafish embryos indicated arhgap 31 and dock6 are localised to the vasculature, ocular and cranial regions, highlighting a correlation with the human phenotype. Furthermore, dock6 morphant embryos displayed vascular defects and microphthalmia, analogous to the clinical features observed in DOCK6-related AOS.

Conclusions: Our preliminary results support a role for ARHGAP31 and DOCK6 during vascular development. Further interrogation of these models will broaden our understanding of Rho GTPase biology in the vasculature and the mechanisms driving AOS pathogenesis.

C.E. Benson: None. D.P. Osborn: None. G.S. Whitley: None. L. Southgate: None.
P11.06.C

Expanding the phenotypic spectrum of AlkurayaKucinskas syndrome: defining the mildest end

E. Çepni ${ }^{1}$, U. Altunoğlu ${ }^{2,3,4}, S$. Avcl $^{3}$, S. Eraslan ${ }^{3}, H$. Kayserili $^{1,2,3}$

${ }^{1}$ Institute of Health Sciences, Koç University School of Medicine, Istanbul, Turkey, ${ }^{2}$ Medical Genetics Department, Koç University School of Medicine, Istanbul, Turkey, ${ }^{3}$ Diagnostic Center for Genetic Diseases, Koç University Hospital, Istanbul, Turkey, ${ }^{4}$ Medical Genetics Department, Istanbul Faculty of Medicine, Istanbul, Turkey

Alkuraya-Kucinskas syndrome (ALKKUCS, OMIM \#617822) is a recently described, ultra-rare autosomal recessive neurodevelopmental disorder characterized by structural, cortical and parenchymal brain abnormalities, global developmental delay/intellectual deficit and joint contractures. The phenotypic spectrum of 15 previously reported cases range from mild-to-moderate intellectual deficit with microcephaly to a phenotype characterized by severe ventriculomegaly and/or brainstem dysgenesis with intrauterine or neonatal death. Only three children survived till childhood. We here report two new ALKKUCS cases from two unrelated consanguineous families. The first patient was presented with antenatal ultrasound findings of severe hydrocephaly, interhemispheric cyst, hydropic changes with cystic hygroma and joint contractures. Pedigree analysis showed two similarly affected siblings and four affected cousins. Postmortem examination was compatible with a lethal contracture phenotype. Whole exome sequencing (WES) revealed a 'likely pathogenic' homozygous variant in the KIAAl109 gene. The second patient was consulted at 9 years of age. She had a history of NICU care due to poor sucking/weak swallowing reflex and cardiac arrest in early neonatal period. Clinical findings included mild myopathy of the neck muscles, pes equinovarus, scapula alata and camptodactyly. Identification of a homozygous variant in the KIAA1109 gene, segregating with the phenotype, made the diagnosis of ALKKUCS possible, placing the case to the mildest end of the phenotypic spectrum. Cases we here report highlights the power of WES in identifying genetic etiopathogenesis of rare disorders; and expand the phenotypic spectrum of ALKKUCS.

E. Çepni: None. U. Altunoğlu: None. S. Avcı: None. S. Eraslan: None. H. Kayserili: None.

\section{P11.11.B}

Severe scoliosis in the patient with a novel variant $p$. Pro237ArgfsTer7 in the WDR45 gene 


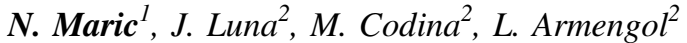

${ }^{1}$ University Clinical Centre of the Republic of Srpska, Banjaluka, Bosnia and Herzegovina, ${ }^{2}$ Quantitative Genomic Medicine Laboratories SL, Barcelona, Spain

Introduction: Mutations in the WDR45 gene have been described as the cause of beta-propeller protein-associated neurodegeneration (BPAN), an X-linked dominant disorder with brain iron accumulation. It typically presents in early childhood with global developmental delay, seizures and autistic-like behavior. Following an initial static phase, these symptoms progress to dystonia, parkinsonism and dementia in early adulthood. We report a case with a novel pathogenic variant in the WDR45 gene that exhibited an early progression of symptoms and severe scoliosis which, to our knowledge, has not been previously reported in BPAN.

Case report: A 10-year-old girl is the second child of healthy unrelated parents. At 14 months, developmental delay was noted. She had autistic-like behavior, didn't walk until the age of 3-year and was mute. Seizures were never noticed but EEG was abnormal. Her condition was stable until the age of 7-year when her cognitive skills further declined. In that time, scoliosis was noted together with bradykinesia, rigidity and postural instability. Scoliosis was severely progressive and needed surgical treatment. At the age of 9-year she lost the ability to walk. Whole exome sequencing revealed a novel heterozygous frameshift pathogenic variant in the WDR45 gene p.Pro237ArgfsTer7, verified by Sanger sequencing, that wasn't present in either parent. No other variants of studied genes associated with scoliosis were identified.

Conclusions: Our case is a very rare example of BPAN with severe scoliosis that might be due to a novel variant in the WDR45 gene and thus expands the knowledge of the phenotype-genotype correlation of BPAN.

N. Maric: None. J. Luna: None. M. Codina: None. L. Armengol: None.

\section{P11.14.B}

\section{CC2D1A AS A NOVEL CILIOPATHY GENE}

I. SAKIN ${ }^{1}$, G. TUNCEL ${ }^{2}$, S. OZEMRI SAG ${ }^{3}$, O. I. KAPLAN ${ }^{4}$, M. K. KHOKHA ${ }^{5}$, M. C. ERGOREN ${ }^{2}$, E. DENIZ ${ }^{6}$, S. G. Temel ${ }^{3}$, MarmaRare Group

${ }^{1}$ Medical Student, School of Medicine, Acibadem Mehmet Ali Aydinlar University, Istanbul, Turkey, Istanbul, Turkey, ${ }^{2}$ Department of Medical Biology, Faculty of Medicine, Near East University, 99138 Nicosia, Cyprus, Nicosia, Cyprus, ${ }^{3}$ Bursa Uludag University, Faculty of Medicine, Department of Medical Genetics, Bursa, Turkey., Bursa, Turkey,
${ }^{4}$ Abdullah Gul Universitesi, Doga Bilimleri Fakultesi, Sumer Kampusu, 38090, Kocasinan, Kayseri, Turkey., Kayseri, Turkey, ${ }^{5}$ Pediatric Genomics Discovery Program, Department of Pediatrics and Genetics, Yale University School of Medicine, New Haven, Connecticut, USA., New Haven, CT, United States, ${ }^{6}$ Pediatric Genomics Discovery Program, Departments of Pediatrics and Genetics, Yale University School of Medicine, 333 Cedar Street, New Haven, Connecticut 06510, USA., New HAVEN, CT, United States

Normal 0 false false false EN-US JA X-NONE /* Style Definitions */ table.MsoNormalTable \{mso-style-name:"Table Normal"; mso-tstyle-rowband-size:0; mso-tstyle-colband-size:0; mso-style-noshow:yes; mso-style-priority:99; mso-style-parent:""; mso-padding-alt:0in 5.4pt 0in 5.4pt; mso-para-margin:0in; mso-para-margin-bottom:.0001pt; mso-pagination:widow-orphan; font-size:12.0pt; fontfamily:Cambria; mso-ascii-font-family:Cambria; mso-asciitheme-font:minor-latin; mso-hansi-font-family:Cambria; mso-hansi-theme-font:minor-latin;\} Cilia are organelles that protrude from the apical aspect of the cells. They can be either motile or immotile-sensory and play many distinct roles in human development. Defective cilia lead to a group of inherited, monogenic disorders referred as ciliopathies, including disorders such as Bardet-Biedl syndrome, polycystic kidney disease, Joubert syndrome, and nephronophthisis. Here, we present homozygous CC2D1A c. $1186 \mathrm{C}>\mathrm{T}$ (p.Arg396*) variant identified in our proband with left renal cysts, autistic features and Mental Motor Retardation (MMR), his sibling has also austictic features, obesity and MMR, as a candidate ciliopathy gene. To analyze the effect of CC2D1A in ciliogenesis, we utilized the frog Xenopus and C.Elegans model systems. Xenopus tadpole epidermis harbor numerous multiciliated cells that generate head-to-tail polarized fluid flow. When we knocked down CC2D1A in frog Xenopus tropicalis using CRISPR/CAS9 system, and analyzed epidermal flow using Optical Coherence Tomography Imaging, we observed loss of epidermal flow in F0 mutant tadpoles. When we further examined the epidermal surface in these tadpoles using immunofluorescence, we detected the loss of multiciliated cells. In parallel, we also showed the loss of cilia in C. Elegans CC2D1A knock-out model utilizing the CRISPR/ Cas9 system. Together, this work demonstrates that CC2D1A is required for proper ciliogenesis in multiciliated cells, providing a plausible mechanism for its role in our patient phenotype. <!--EndFragment-->

I. Sakin: None. G. Tuncel: None. S. Ozemri sag: None. O.I. Kaplan: None. M.K. Khokha: None. M.C. Ergoren: None. E. Deniz: None. S.G. Temel: None. 


\section{P11.15.C}

Chitayat syndrome: a rare but potentially underdiagnosed condition ?

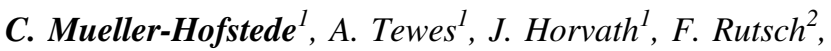
A. Bohring ${ }^{l}$

${ }^{1}$ Institut für Humangenetik, University Hospital, Muenster, Germany, ${ }^{2}$ Klinik für Kinder- und Jugendmedizin, Allgemeine Pädiatrie, University Hospital, Muenster, Germany

We report on a ten months old boy, third child of nonconsanguineous parents. The pregnancy was uncomplicated without ultrasound abnormalities. Body measurements were in the normal range. Bowed hands and feet with bilateral shortened and ulnar deviated index fingers, additional bone nuclei of digits II / III of both hands and clinodactyly of digiti II, III, IV were noticed. The feet showed bilateral hallux valgus. Neonatal respiratory distress required initially high-flow oxygen therapy.

At presentation the boy showed a pectus excavatum and mild facial dysmorphism (hypertelorism, broad nasal bridge, short columella) in addition to the abovementioned abnormalities. The general development was age-appropriate.

Due to the combination of the symptoms Chitayat syndrome has been considered. Sequence analysis identified the de novo heterozygous variant c.266A $>\mathrm{G}$ (p.(Tyr89Cys)) in the $E R F$-gene.

Chitayat et al. first described 1993 a patient with hyperphalangism, brachydactyly, bronchomalacia and facial anomalies. Since then, only 6 other patients have been described in literature. In 2016 the recurrent variant c. $266 \mathrm{~A}>\mathrm{G}$ (p.(Tyr89Cys)) in the $E R F$-gene was identified as the underlying molecular cause.

Heterozygous mutations (predominantely loss-of-function) in the $E R F$-gene are described in syndromal craniosynostosis 4 . The above-mentioned variant has not yet been described in this context. This could indicate, that the variant may have a different influence on protein function and potentially leads to a gain-of-function.

We suggest that the rare condition of Chitayat syndrome should always be considered in children with the combination of characteristic skeletal abnormalities of the hands and feet especially hyperphalangism with respiratory distress.

C. Mueller-Hofstede: None. A. Tewes: None. J. Horvath: None. F. Rutsch: None. A. Bohring: None.

\section{P11.16.A}

Isolated familial choanal atresia: a new entity in the phenotypic spectrum of $K M T 2 D$ gene
A. Garde ${ }^{1,2}$, F. Tran Mau-Them ${ }^{2}$, S. Nambot ${ }^{1,2}$, M. Fradin ${ }^{3}$, S. Odent ${ }^{3}, Y$. Duffour ${ }^{2}$, S. Banka ${ }^{4}$, C. Philippe ${ }^{2}$, C. ThauvinRobinet $^{1,2}$, L. Faivre F,2 $^{1,2}$

${ }^{1}$ Centre de Référence Anomalies du Développement et Syndromes Malformatifs, FHU TRANSLAD, CHU de Dijon, Dijon, France, ${ }^{2}$ Inserm UMR1231 GAD, Génétique des Anomalies du Développement, Université de Bourgogne, Dijon, France, ${ }^{3}$ Service de Génétique Clinique, IGDR (Institut de Génétique et développement de Rennes) (S.O.), CHU de Rennes, Rennes, France, ${ }^{4}$ Division of Evolution and Genomic Sciences, School of Biological Sciences, Faculty of Biology, Medicine, and Health, The University of Manchester, Manchester, United Kingdom

Introduction: Loss of function variants in $K M T 2 D$ can be responsible of Kabuki syndrome type 1 (KS1). Recently, missense variants in exon 38 or 39 in KMT2D have been found in patients presenting a new phenotype with multiple malformations distinct from KS1, including choanal atresia and absence of intellectual disability. Choanal atresia (CA) is a congenital disorder characterized by a bony or membranous septum between the nose and nasopharynx. CA can occur as an isolated form or in syndromic presentations.

Patients and Method: A newborn girl was referred to our medical department because of choanal atresia. Given the fact that 8 members of the family spreading across 3 generations had non-syndromic choanal atresia, we decided to perform exome sequencing (ES) in our patient and one cousin, the most distant affected relative.

Results: In 2014, ES was first concluded as negative. Following the publication entitled "A restricted spectrum of missense KMT2D variants cause a multiple malformations disorder distinct from Kabuki syndrome" (Cuvertino et al., 2020), we interrogated our database for missense variants in exons 38-39 of KMT2D. We identified a p.Glu3569Gly, in exon 38 , segregating with choanal atresia in the family. One obligate carrier did not present choanal atresia, suggesting incomplete penetrance. Prediction scores are in favor of the pathogenicity and functional analyses have been proposed.

Conclusions: Considering these results and the recent literature, we suggest that missense variants in exon 38 of $K M T 2 D$ can be responsible of even milder phenotypes such as isolated choanal atresia.

A. Garde: None. F. Tran Mau-Them: None. S. Nambot: None. M. Fradin: None. S. Odent: None. Y. Duffour: None. S. Banka: None. C. Philippe: None. C. Thauvin-Robinet: None. L. Faivre: None.

P11.17.B

Chromatinopathies: clinical hypothesis versus molecular diagnosis 
C. Cesario, M. Gnazzo, E. Pisaneschi, R. Capolino, L. Sinibaldi, M. Dentici, M. Digilio, A. Novelli

Laboratory of Medical Genetics, Medical Genetics, Bambino Gesù Pediatric Hospital, Rome, Italy

Introduction: Defects of the structural or regulatory components of cohesin lead to various multisystem malformation syndromes described as "cohesinopathies", that share clinical findings such as distinctive facial features, growth retardation, intellectual disability and limb abnormalities. Moreover, in patients presenting classical and non-classical features of cohesinopathies were identified pathogenic variations in key chromatin-associated factors, genetically different from cohesin, but with overlapping functions, such as transcriptional regulation, chromatin remodeling and epigenetic modifications; these conditions could be considered part of a larger disease family categorized as "chromatinopathies".

Materials and Methods: Between 2017 and 2018, 40 cohesinopathies/chromatinopathies patients carrying heterozygous pathogenic variants have been diagnosed at the Bambino Gesù Children Hospital, with initial clinical hypothesis of KBG syndrome, CHARGE association, Kabuki syndrome, Cornelia de Lange syndrome and Coffin-Siris syndrome. A custom Chromatinopathies NGS panel was used to analyze genes associated with these syndromes.

Results: In $22.5 \%$ of cases molecular defects causing phenotypic conditions different from the initial clinical hypothesis were identified in other genes coding for cohesin, transcriptional regulators, chromatin-associated protein complex and its interactors, such as MBD5 (OMIM:611472), BRD4 (OMIM:608749), SOX11 (OMIM:600898), SMARCA2 (OMIM:600014), KDM5C (OMIM:314690), KMT2A (OMIM:159555) and MECP2 (OMIM:300005).

Conclusions: Because of phenotypic overlap among chromatin disorders, clinical diagnosis of these patients is often challenging or rather impossible without genetic evidence. In our cohort, the high rate of positive cases not matching with the first clinical suspect suggests that broad spectrum molecular analysis is crucial to reach accurate diagnosis and will probably led to the identification of additional genotype-phenotype associations.

C. Cesario: None. M. Gnazzo: None. E. Pisaneschi: None. R. Capolino: None. L. Sinibaldi: None. M. Dentici: None. M. Digilio: None. A. Novelli: None.

\section{P11.18.C}

Phenotype-genotype analysis in a large cohort of 250 individuals with a chromosome $6 q$ deletion
A. Engwerda ${ }^{l}$, S. E. Meijer ${ }^{l}$, P. Bouman ${ }^{2}$, N. F. Simoes de Souza $^{1}$, B. Frentz ${ }^{3}$, B. C. T. Flapper ${ }^{4}$, N. Corsten-Janssen ${ }^{1}$, E. H. Gerkes ${ }^{l}$, M. A. Swertz ${ }^{l}$, M. Plantinga ${ }^{l}$, T. Dijkhuizen ${ }^{l}$, W. S. Kerstjens-Frederikse ${ }^{I}$, C. M. A. van RavenswaaijArts $^{l}$

${ }^{1}$ University of Groningen, University Medical Centre Groningen, Department of Genetics, Groningen, Netherlands, ${ }^{2}$ Facebook contact parent, Chromosome 6 Facebook group, Utrecht, Netherlands, ${ }^{3}$ Vanboeijen, Assen, Netherlands, ${ }^{4}$ University of Groningen, University Medical Centre Groningen, Department of Paediatrics, Groningen, Netherlands

Chromosome 6q deletions are rare and little is known about their phenotypical consequences. Parents of children with such a deletion search the internet and unite in international social media platforms. A successful collaboration with the Chromosome 6 Facebook group allowed us to collect data of 109 newly identified individuals.

Families can sign up for the study via the secured project's website by uploading the proband's array report. Phenotype data is collected directly from the individuals or parents via an online multilingual questionnaire. Literature case reports are added to the database using the same questionnaire. Up till January 2020 this resulted in a total of 250 individuals.

Subgroups are made based on the predicted haploinsufficiency genes within the deletions, leading to detailed phenotype descriptions for 18 subgroups thus far.

Gastroesophageal reflux, trachea/laryngo/bronchomalacia, congenital heart defects, cerebral defects, seizures and vision and respiratory problems are predominant in 6q14.2q15 deletions. In contrast, 6q11q14.1 deletions present less severe clinical characteristics.

Deletions including the gene TAB2 (6q25.1) are characterized by facial dysmorphisms, short stature, heart defects and connective tissue problems and resemble a Noonan-like syndrome.

Within the $6 \mathrm{q} 25.2 \mathrm{q} 25.3$ region $A R I D 1 B$ plays a prominent role, resulting in a Coffin-Siris syndrome phenotype when deleted.

Terminal 6q26q27 deletions are characterised by microcephaly, hypotonia, balance problems, vision problems, seizures and brain abnormalities. Deletions including PARK2 presented with more severe developmental delay.

In conclusion, social media are instrumental in collecting large numbers of detailed data on rare chromosome aberrations, resulting in a more precise description of the phenotypic spectrum.

A. Engwerda: None. S.E. Meijer: None. P. Bouman: None. N.F. Simoes de Souza: None. B. Frentz: None. B. C.T. Flapper: None. N. Corsten-Janssen: None. E.H. 
Gerkes: None. M.A. Swertz: None. M. Plantinga: None. T. Dijkhuizen: None. W.S. Kerstjens-Frederikse: None. C.M.A. van Ravenswaaij-Arts: None.

\section{P11.19.A}

\section{Cilium Phenotyping using patient-derived cells to} accurately diagnose ciliopathies

M. M. Oud ${ }^{1,2}$, R. van Beek ${ }^{1,2}$, C. van Manen ${ }^{1,3}, D$. Lugtenberg $^{l}$, E. M. H. F. Bongers ${ }^{l}$, R. Roepman ${ }^{1,3}, H$. Yntema $^{l}$, L. E. L. M. Vissers ${ }^{1,2}$

${ }^{1}$ Department of Human Genetics, Radboud University Medical Centre, Nijmegen, Netherlands, ${ }^{2}$ Donders Institute for Brain, Cognition and Behavior, Radboud University Medical Centre, Nijmegen, Netherlands, ${ }^{3}$ Radboud Institute for Molecular Life Sciences, Radboud University Medical Centre, Nijmegen, Netherlands

Introduction: Cilia are small signaling organelles protruding from the cell membrane of nearly every vertebrate cell. Ciliary dysfunction leads to rare recessive multiorgan disorders, collectively termed ciliopathies, which have overlapping genotype-phenotype correlations, making accurate diagnosis challenging. Our aim is to develop a novel cell-based functional assay to improve accurate diagnosis of ciliopathies on the basis of the underlying mechanism of disease.

Materials and Methods: Skin-fibroblasts from 10 ciliopathy patients (from 3 different subgroups) and 8 controls were cultured in vitro and immunofluorescence was applied to determine cilium phenotype parameters; 1) ciliogenesis, based on presence or absence of cilium markers, 2) cilium length, measured by the combined signal of an axonemal and a cilium membrane marker, and 3) intraflagellar transport (IFT), quantified by the area of the ciliary tip using the IFT88 signal.

Results: Cilium phenotypic data from skin-fibroblasts of eight controls yielded comparable and reproducible results: $88 \%$ cells were ciliated, the cilium length was $3.30 \pm 0.251 \mu \mathrm{m}$, and the IFT88 ciliary tip area was $0.13 \pm 0.02 \mu \mathrm{m}^{2}$. Analysis of the cilium phenotype in the 10 patients for three clinical subgroups not only showed significant differences in cilium length and IFT results, but also allowed to distinguish the three ciliopathy subgroups from one another.

Conclusions: In summary, standardized analysis of the cilium phenotype can lead to identifiable clusters for different ciliopathies. Therefore, we believe that standardized tests based on cilium phenotypic data can provide additional proof for conclusive diagnosis of ciliopathies, which is essential in routine diagnostic care.
M.M. Oud: None. R. van Beek: None. C. van Manen: None. D. Lugtenberg: None. E.M.H.F. Bongers: None. R. Roepman: None. H. Yntema: None. L.E.L.M. Vissers: None.

\section{P11.20.B}

Clinical and diagnostic implications of whole-genome sequencing (WGS) of 42 patients affected by rare diseases: the first Italian experience on a diagnostic basis.

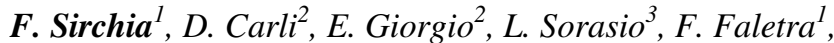
A. Fabretto ${ }^{1}$, F. Guidolin ${ }^{4}$, V. Antona ${ }^{5}$, J. Belmont ${ }^{6}, J$.

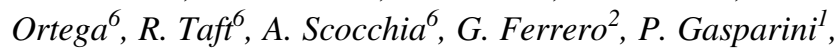
A. Brusco $^{2}$

${ }^{1}$ IRCCS Burlo Garofalo di Trieste, Trieste, Italy, ${ }^{2}$ University of Turin, Torino, Italy, ${ }^{3}$ Santa Croce e Carle Hospital, Cuneo, Italy, ${ }^{4}$ University of Padova, Padova, Italy, ${ }^{5}$ University of Palermo, Palermo, Italy, ${ }^{6}$ Illumina Inc., San Diego, CA, United States

We report the collaboration between the Medical Genetics Units in Torino and Trieste with the Illumina Company in a diagnostic setting by trio-WGS. We selected 42 complex pediatric patients $<16$ years with normal CGH/SNP-arrays without recognizable genetic disorders. We found a certain molecular diagnosis in 26 (62\%; ultrarare genes in the literature were $A S X L 1, A S X L 3, C S N K 2 A 1, D D X 3 X, D Y R K 1 A$, FLNA, HIPEV2, LMNA, NFIX, PACS1, PDE4D, PDHA1, PIGA, PIEZO2, PIGO, RRM2B, SATB2, SETBP1, SRCAP and $T U B B 3)$. Three cases $(3 / 26,11.5 \%)$ had two diseases, and 3 further could potentially be double diagnoses. A mosaic chr 9 trisomy (not previously identified by array$\mathrm{CGH}$ ) and a mat-UPD1 were also identified. In 21/26 (81\%) the disease was associated with a de novo, highlighting the importance of trio analysis; in 1 patient the disease was inherited from the affected mother (ANKRD11, KBG syndrome). One or more VUSs were found in 5/43 (11.5\%) patients. SNVs were in the coding region/nearby intron suggesting our high diagnostic rate may be related to a more complete coverage of WGS versus exome, and/or our intertwined bioinformatics and clinical prioritization of variants. The diagnosis changed the management of all positive cases, including referral to palliative cures (RRM2B; mtDNA depletion syndrome), referral for a specific follow-up, or cancer surveillance program (ASXL1). We provided a correct recurrence risk for future pregnancies for parents, including a consanguineous couple with a de novo causative variant in their child. In conclusion, we show how WGS can dramatically increase our ability to reach a genetic diagnosis in undiagnosed cases. 
F. Sirchia: None. D. Carli: None. E. Giorgio: None. L. Sorasio: None. F. Faletra: None. A. Fabretto: None. F. Guidolin: None. V. Antona: None. J. Belmont: None. J. Ortega: None. R. Taft: None. A. Scocchia: None. G. Ferrero: None. P. Gasparini: None. A. Brusco: None.

P11.21.C

A case with complex small supernumerary marker chromosome consisting $19 p$ and $22 q$

\section{S. Altiner, N. Yürür Kutlay}

Ankara University School of Medicine, Department of Medical Genetics, Ankara, Turkey

Introduction: Complex small supernumerary marker chromosomes (sSMC) consist of chromosomal material derived from more than one chromosome (Liehr et al., 2013). Here, we describe a complex sSMC derived from a maternal translocation between chromosomes $19 \mathrm{p}$ and $22 \mathrm{q}$ in a patient with intellectual disability and short stature.

Materials and Methods: G-banding, FISH, and microarray were performed in order to define sSMC.

Results: The patient presented with a complex sSMC derived from a 3:1 segregation of a maternal balanced $\mathrm{t}$ $(19 ; 22)(\mathrm{p} 13 ; \mathrm{q} 11)$ reciprocal translocation and resulting in tertiary trisomy of $19 \mathrm{p} 13.3$ and $22 \mathrm{q} 11.1 \mathrm{q} 11.21$.

Conclusions: Genotype-phenotype correlation and assigning a specific feature to an aberration are often difficult in cases with combined partial trisomies. Besides, numerical and structural abnormalities involving chromosome 19 is relatively rare compared to other chromosomes. In comparison to patients with similar aberrations, our patient showed a phenotype similar to $19 \mathrm{p} 13.3$ duplication, regarding intellectual disability and growth retardation (Orellana et al., 2015). This is the first complex sSMC originating from chromosome 19 and 22. Functional analysis of the genes in these regions may provide a better explanation about the gene-dosage effects on the phenotype.

References Liehr, T., Cirkovic, S., Lalic, T., Guc-Scekic, M., de Almeida, C., Weimer, J., et al. (2013). Complex small supernumerary marker chromosomes - an update. Mol. Cytogenet. 6, 46. Orellana C, Roselló M, Monfort S, Mayo S, Oltra S, Martínez F. (2015). Pure duplication of 19 p13.3 in three members of a familyintellectual disability and literature review. Definition of a new microduplication syndrome. Am J Med Genet A. 167:1614-20.

S. Altiner: None. N. Yürür Kutlay: None.

\section{P11.23.B}

Clinical and molecular description of 202 cases of Cornelia de Lange Syndrome and differential diagnoses
G. VERA, A. Goldenberg, F. Lecoquierre, N. Drouot, K. Cassinari, F. Charbonnier, S. Coutant, O. Quenez, T. Frebourg, G. Nicolas, P. Saugier-Veber

Rouen University Hospital, Department of Genetics, $F$ 76000, Normandy Center for Genomic and Personalized Medicine, Reference Center for Developmental Disorders, and Normandie Univ, UNIROUEN, Inserm U1245, Rouen, France

Introduction: Cornelia de Lange Syndrome (CdLS) is a multisystem disorder characterised by growth retardation, microcephaly, dysmorphism, hypertrichosis, limb and organ malformations, feeding difficulties, and neurodevelopmental disorder. Clinical heterogeneity is illustrated by variable severity and atypical cases overlapping with some other syndromes. Mutations in 6 genes from the cohesin complex can cause CdLS: NIPBL, SMC1A, HDAC8, SMC3, $R A D 21$ and $B R D 4$. We present results from a retrospective study of 202 cases referred between 2011 and 2019.

Methods: Clinical data were reviewed by an expert clinician before molecular testing. Phenotypes were classified as typical/mild/atypical CdLS or another diagnosis. We applied a clinical score for all suspected CdLS cases, and compared values per molecular outcome and phenotype. A gene panel sequencing assessed 5/6 CdLS and 17 differential diagnoses genes $(\sim 700 \mathrm{x}$ depth of coverage on average).

Results: Differential diagnoses were suspected for 23 individuals. CdLS was suspected for 179 individuals: 66 typical, 40 mild and 73 atypical. Molecular testing identified a (likely) pathogenic variant for 47/66 typical (71\%), 18/40 mild (45\%), and 14/73 atypical (19\%) individuals confirming CdLS for 73 of them. A differential diagnosis was identified for 6 atypical individuals. We identified 11 mosaic mutations with variable phenotypes; 9 were detected in saliva and were undetectable in blood. Clinical scores were significantly higher for typical individuals and for pathogenic variants carriers.

Conclusions: Applying deep gene panel sequencing from other tissues than blood is an effective strategy for typical CdLS given the number of mosaics, while atypical phenotypes could be resolved by first-tier exome/genome sequencing.

G. Vera: None. A. Goldenberg: None. F. Lecoquierre: None. N. Drouot: None. K. Cassinari: None. F. Charbonnier: None. S. Coutant: None. O. Quenez: None. T. Frebourg: None. G. Nicolas: None. P. Saugier-Veber: None.

P11.24.C

Identifying Cornelia de Lange Syndrome by facial phenotypes using Face2Gene 
A. Latorre-Pellicer ${ }^{1}$, Á. Ascaso ${ }^{2}$, L. Trujillano ${ }^{2}$, M. GilSalvador $^{l}$, M. Arnedo ${ }^{l}$, C. Lucia-Campos ${ }^{l}$, R. AntoñanzasPerez $^{1}$, I. Marcos-Alcalde ${ }^{3,4}$, I. Parenti ${ }^{5,6}$, G. Bueno-

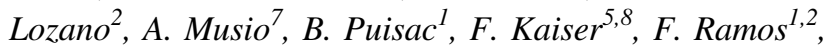
P. Gómez-Puertas ${ }^{3}$, J. Piél

${ }^{1}$ Unit of Clinical Genetics and Functional Genomics, Department of Pharmacology-Physiology, School of Medicine, University of Zaragoza, CIBERER-GCVO2 and IISAragon, Zaragoza, Spain, ${ }^{2}$ Department of Paediatrics, Hospital Clínico Universitario "Lozano Blesa", Zaragoza, Spain, ${ }^{3}$ Molecular Modelling Group, Centro de Biología Molecular Severo Ochoa, CBMSO (CSIC-UAM), Madrid, Spain, ${ }^{4}$ Bioscience Research Institute, School of Experimental Sciences, Universidad Francisco de Vitoria, UFV, Pozuelo de Alarcón, Spain, ${ }^{5}$ Section for Functional Genetics, Institute of Human Genetics, University of Lübeck, Lübeck, Germany, ${ }^{6}$ Institute of Science and Technology (IST) Austria, Klosterneuburg, Austria, ${ }^{7}$ Istituto di Ricerca Genetica e Biomedica, Consiglio Nazionale delle Ricerche, Pisa, Italy, ${ }^{8}$ Institute for Human Genetics, University Hospital Essen, University of Duisburg-Essen, Essen, Germany

Introduction: DeepGestalt technology, and its app Face2Gene, is having a growing impact on the diagnosis and management of genetic diseases by analysing the features of affected individuals. This is of particular interest for Cornelia de Lange syndrome (CdLS), in which heterogeneity in clinical presentation and phenotype overlap with different syndromes, and the still widely unknown molecular pathomechanisms makes its diagnosis challenging for paediatricians. Here, we performed a phenotypic study on a cohort of 49 individuals harbouring causative variants in known CdLS genes in order to evaluate Face2Gene utility and sensitivity in the clinical diagnosis of CdLS.

Material and Methods: We analysed the frontal images of all individuals using Face2Gene technology. All individuals with CdLS were subjected to molecular analysis by next generation sequencing. The CdLS clinical score was computed by trained physicians according to the international consensus criteria of CdLS.

Results: Based on the profile images of patients, a diagnosis of CdLS was within the top five predicted syndromes for $97.9 \%$ of our cases and even listed as first prediction for $83.7 \%$. The age of patients did not seem to affect the prediction accuracy, whereas our results indicate a correlation between the clinical score and affected genes. Furthermore, each gene presents a different pattern recognition that may be used to develop new neural networks with the goal of separating different genetic subtypes in CdLS.
Conclusions: Overall, we conclude that computerassisted image analysis based on deep learning could support the clinical diagnosis of CdLS.

A. Latorre-Pellicer: None. Á. Ascaso: None. L. Trujillano: None. M. Gil-Salvador: None. M. Arnedo: None. C. Lucia-Campos: None. R. Antoñanzas-Perez: None. I. Marcos-Alcalde: None. I. Parenti: None. G. Bueno-Lozano: None. A. Musio: None. B. Puisac: None. F. Kaiser: None. F. Ramos: None. P. Gómez-Puertas: None. J. Pié: None.

\section{P11.25.A}

HDAC8-related Cornelia de Lange Syndrome - cases report

M. S. Santos ${ }^{l}$, S. Fernandes ${ }^{1}$, M. Lopes-de-Almeida ${ }^{2}$, P. M. Almeida $^{l}$, S. Maia ${ }^{1,3}$, C. F. Reis ${ }^{4}, J . S a^{1,3}$

${ }^{1}$ Medical Genetics Unit, Centro Hospitalar e Universitário de Coimbra, Coimbra, Portugal, ${ }^{2}$ Medical Genetics Unit Hospital de Braga (former worker of Medical Genetics Unit, Centro Hospitalar e Universitário de Coimbra), Braga, Portugal, ${ }^{3}$ University Clinic of Genetics, Faculty of Medicine - Universidade de Coimbra, Coimbra, Portugal, ${ }^{4}$ Medical Genetics Unit, Centro Hospitalar e Universitário do Porto (former worker of Medical Genetics Unit, Centro Hospitalar e Universitário de Coimbra), Porto, Portugal

Introduction: Cornelia de Lange Syndrome (CdLS) is a heterogeneous genetic disorder best known for its distinctive craniofacial features of microbrachycephaly, highly-arched eyebrows, synophrys and anteverted nares, as well as upper-limb defects, growth retardation, intellectual disability and hirsutism. Five genes have been identified but only $4 \%$ of cases are associated with $H D A C 8$, in contrast to $60 \%$ associated with NIPBL gene. Variants in HDAC8 present themselves with phenotypical differences from the classical-CdLS, therefore having no evident clinical diagnose. In the past 3 years, with whole exome sequencing (WES), we identified three cases of HDAC8 CdLS, including a long-term accompanied, yet undiagnosed, 20 year old girl.

Cases report: Three girls were studied due to development disorder and multiple dysmorphologies not resembling of any particular syndrome. After initial inconclusive analysis, further testing with WES found that each one presented a variant in the $H D A C 8$ gene, one already known and classified as pathogenic, and two unknown classified as likely pathogenic. The next table lists some alterations usually linked to HDCA8-CdLS and found in our patients. 


\begin{tabular}{|c|c|c|c|}
\hline & Patient 1 & Patient 2 & Patient 3 \\
\hline $\begin{array}{l}\text { Age of first } \\
\text { evaluation }\end{array}$ & 10 years & 5 months & 3 years \\
\hline Age of diagnosis & 20 years & 35 months & 10 years \\
\hline Variant $H D A C 8$ & $\begin{array}{l}\text { c. } 467 \mathrm{~A}>\mathrm{G} p \\
\text { (Asn156Ser) }\end{array}$ & $\begin{array}{l}\text { c. } 706 \mathrm{C}>\mathrm{T} p \\
\left(\mathrm{Gln} 236^{*}\right)\end{array}$ & $\begin{array}{l}\text { c.653G }>\text { A p. } \\
\text { (Gly218Asp) }\end{array}$ \\
\hline $\begin{array}{l}\text { Distinctive facial } \\
\text { features of CdLS }\end{array}$ & No & $\begin{array}{l}\text { No, but low } \\
\text { anterior hairline }\end{array}$ & $\begin{array}{l}\text { No, but } \\
\text { synophrys and } \\
\text { arched } \\
\text { eyebrows }\end{array}$ \\
\hline $\begin{array}{l}\text { Other facial } \\
\text { features described } \\
\text { in } H D A C 8 \\
\text { variants }\end{array}$ & $\begin{array}{l}\text { Hooding of } \\
\text { lids and Ptosis }\end{array}$ & Telecanthus & $\begin{array}{l}\text { Thin upper lip } \\
\text { and Widely } \\
\text { spaced teeth }\end{array}$ \\
\hline $\begin{array}{l}\text { Upper-limb } \\
\text { defects }\end{array}$ & $\begin{array}{l}\text { Short } 5^{\text {th }} \text { finger } \\
\text { and Proximally } \\
\text { set thumbs }\end{array}$ & None & $\begin{array}{l}\text { Short } 5^{\text {th }} \text { finger } \\
\text { and Proximally } \\
\text { set thumbs }\end{array}$ \\
\hline $\begin{array}{l}\text { Growth } \\
\text { retardation }\end{array}$ & No & $\begin{array}{l}\text { Yes (Height and } \\
\text { Weight }=\mathrm{P}<1 \text {, } \\
>-3.0 \mathrm{SD} \text { ) }\end{array}$ & No \\
\hline $\begin{array}{l}\text { Intellectual } \\
\text { Disability }\end{array}$ & Severe & Moderate & Moderate \\
\hline $\begin{array}{l}\text { Motor } \\
\text { development } \\
\text { (walking) }\end{array}$ & 36 months & 27 months & 24 months \\
\hline $\begin{array}{l}\text { Verbal } \\
\text { development } \\
\text { (first words) }\end{array}$ & 30 months & 18 months & 24 months \\
\hline Hirsutism & Yes & No & No \\
\hline
\end{tabular}

Conclusions: These cases enlighten the heterogeneity of phenotypes and the clinical diagnostic challenge regarding syndromes in the spectrum of a very well-known pathology. It also shows how the evolution of genetical tests now allows us to finally attribute a "name" and a better followup to some of our long-term undiagnosed patients.

M.S. Santos: A. Employment (full or part-time); Significant; Centro Hospitalar e Universitário de Coimbra. S. Fernandes: A. Employment (full or part-time); Significant; Centro Hospitalar e Universitário de Coimbra. M. Lopes-de-Almeida: A. Employment (full or part-time); Significant; Hospital de Braga. P.M. Almeida: A. Employment (full or part-time); Significant; Centro Hospitalar e Universitário de Coimbra. S. Maia: A. Employment (full or part-time); Significant; Centro Hospitalar e Universitário de Coimbra. C.F. Reis: A. Employment (full or part-time); Significant; Centro Hospitalar e Universitário do Porto. J. Sá: A. Employment (full or part-time); Significant; Centro Hospitalar e Universitário de Coimbra.

\section{P11.26.B}

A homozygous missense variant inTUBGCP2alter the gtubulin ring complex leading to abnormal cortical development, pontocerebellar atrophy and altered myelination

Y. Oktay ${ }^{1,2,3}$, S. Gungor ${ }^{4}$, S. Hiz ${ }^{5}$, A. Yaramis ${ }^{6}$, Á. Aranguren-Ibáñez ${ }^{7}, U$. Yis ${ }^{5}$, E. Sonmezler ${ }^{2}$, E. Yilmaz ${ }^{2}, B$. Ekinci $^{2}$, M. Aslan ${ }^{4}$, S. Balaraju ${ }^{8}$, N. Szabo ${ }^{9}$, S. Laurie ${ }^{10}, S$. Beltran $^{10}$, D. Hathazi ${ }^{9}$, D. MacArthur ${ }^{11}$, A. Roos ${ }^{12}, H$. Lochmuller $^{13}$, I. Vernos $^{14}$, R. Horvath ${ }^{9}$

${ }^{1}$ Izmir Biomedicine and Genome Center (IBG), Izmir, Turkey, ${ }^{2}$ Izmir International Biomedicine and Genome Institute, IBG-izmir, Dokuz Eylul University, Izmir, Turkey, ${ }^{3}$ Dept. of Medical Biology, Faculty of Medicine, Dokuz Eylul University, Izmir, Turkey, ${ }^{4}$ Inonu University, Faculty of Medicine, Turgut Ozal Research Center, Department of Paediatric Neurology, Malatya, Turkey, ${ }^{5}$ Dokuz Eylul University, Faculty of Medicine, Department of Pediatric Neurology, Izmir, Turkey, ${ }^{6}$ Pediatric Neurology Clinic, Diyarbakir Memorial Hospital, Diyarbakir, Turkey, ${ }^{7}$ Centre for Genomic Regulation (CRG), The Barcelona Institute of Science and Technolog, Barcelona, Spain, ${ }^{8}$ John Walton Muscular Dystrophy Research Centre, Institute of Translational and Clinical Research, Newcastle University, Newcastle upon Tyne, United Kingdom, ${ }^{9}$ Department of Clinical Neurosciences, John Van Geest Cambridge Centre for Brain Repair, University of Cambridge School of Clinical Medicine, Cambridge, United Kingdom, ${ }^{10} C N A G-C R G$, Centre for Genomic Regulation, Barcelona Institute of Science and Technology, Barcelona, Spain, ${ }^{11}$ Analytic and Translational Genetics Unit, Massachusetts General Hospital, Boston, MA, United States, ${ }^{12}$ Leibniz Institut für Analytische Wissenschaften, ISAS, Dortmund, Germany \& Pediatric Neurology, University Hospital, University of Duisburg-Essen, Faculty of Medicine, Essen, Germany, ${ }^{13}$ Children's Hospital of Eastern Ontario Research Institute; Division of Neurology, Department of Medicine, The Ottawa Hospital; and Brain and Mind Research Institute, University of Ottawa, Ottawa, ON, Canada, ${ }^{14}$ Centre for Genomic Regulation (CRG), The Barcelona Institute of Science and Technology, Barcelona, Spain

Microtubules are long fibers made of protofilaments of $\alpha$ and $\beta$-tubulin heterodimers. Microtubule organizing centers are protein complexes composed of several proteins forming the $\gamma$-tubulin ring complex or $\gamma$-TuRC that directly drives MT nucleation. By whole exome sequencing we identified a novel homozygous variant in exon 8 of TUBGCP2(NM_001256617.1:c.1015G>A, p.Glu339Lys), which encodes $\gamma$-TuRC component GCP2 protein, in two siblings (10 yo female and 8 yo male) from a consanguineous Turkish family. Although TUBGCP2 mutations were recently reported in families with developmental delay, dysmorphic features, hypotonia, epilepsy, 
microcephaly and lissencephaly spectrum changes, we for the first time investigated the cellular defects caused by GCP2 mutations in patient fibroblasts by immunocytochemical, proteomic and in silico analyses. We studied the localization of some $\gamma$-TuRC components and associated proteins in control and patient fibroblasts in interphase and in mitosis by immunofluorescence, and the levels of the GCP2 protein along the cell cycle. Our data suggested that the localization of $\gamma$-TuRC complex was perturbed in mitosis but not in interphase. Label-free LC-MS/MS analysis of patient-derived fibroblasts identified 26 upregulated and 24 down-regulated proteins, which were enriched for tubulin and cytoskeletal proteins that play role in neurodevelopment. In summary, we describe two siblings carrying a novel homozygous TUBGCP2 variant with dysmorphic signs, severe developmental delay and epilepsy, and show that, in addition to a neuronal migration defect, brainstem atrophy and disturbed myelination are also associated with TUBGCP2 mutations, explaining the variable clinical and imaging findings. *TUBITAK research grant 216S771, Newton Fund (UK/Turkey, MR/N027302/1), AFM \#21644.

Y. Oktay: None. S. Gungor: None. S. Hiz: None. A. Yaramis: None. Á. Aranguren-Ibáñez: None. U. Yis: None. E. Sonmezler: None. E. Yilmaz: None. B. Ekinci: None. M. Aslan: None. S. Balaraju: None. N. Szabo: None. S. Laurie: None. S. Beltran: None. D. Hathazi: None. D. MacArthur: None. A. Roos: None. H. Lochmuller: None. I. Vernos: None. R. Horvath: None.

\section{P11.28.A}

Clinical \& genetic characteristics of syndromes associated with craniosynostosis

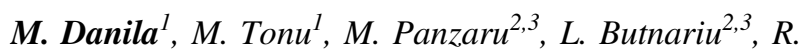
Popescu $^{2,3}$, C. Banescu ${ }^{4}$, I. Resmerita ${ }^{3}$, E. Gavril $^{3}$, E. V. Gorduza $^{1,3}$, C. Rusu ${ }^{2,3}$

1. "Cuza-Voda" Obstetrics and Gynecology Hospital, Iasi, Romania, ${ }^{2} 2$. "Saint Mary” Emergency Children's Hospital - Regional Medical Genetics Centre, Iasi, Romania, ${ }^{3} 3$. "Grigore T. Popa" University of Medicine and Pharmacy Department of Medical Genetics, Iasi, Romania, ${ }^{4} 4$. Clinical County Emergency Hospital, Targu Mures, Romania

Craniosynostosis represents a premature fusion of one or more cranial sutures leading to secondary distortion of skull shape resulting in skull deformities with a variable presentation. Craniosynostosis may occur in an isolated setting or as part of a syndrome. FGFR2, FGFR3, FGFR1, TWIST1 and $E F N B$ genes are major causative genes of genetic syndromes with craniosynostosis. Our aim was to investigate the clinical and genetic aspects for the patients recorded with craniosynostosis in the files of the Iasi Regional Medical Genetics Center for the last 15 years. For each case we have studied clinical features to highlight the evocative signs for diagnosis and to establish the correlation with the laboratory results. In all patients we applied imagistic investigations (RX or CT) that was concordant with craniosynostosis types. We identified 99 patients suspected for craniosynostosis. From these, 84 presented an isolated form of craniosynostosis, while 15 patients had a syndromic form (Apert, Saethre-Chotzen, Crouzon, Carpenter syndromes). We investigated 15 patients with syndromic forms of craniosynostosis by MLPA analysis using kit P064 and P080 (MRC Holland ${ }^{\circledR}$ ). We identified the mutation in TWIST1 gene associated with Saethre-Chotzen syndrome in 4 patients. Another mutation was detected in FGFR2 gene, being discovered in a case of Apert syndrome. In familial cases we accorded genetic counselling in concordance with results of molecular analyses and we discussed the possibility of surgical therapy. Our cases illustrate the genetic heterogeneity among cranyosinostosis syndromes. Molecular diagnosis is very important for an adequate genetic counseling and guidance for patients with syndromic craniosynostosis.

M. Danila: None. M. Tonu: None. M. Panzaru: None. L. Butnariu: None. R. Popescu: None. C. Banescu: None. I. Resmerita: None. E. Gavril: None. E.V. Gorduza: None. C. Rusu: None.

\section{P11.29.B}

The clinical features of individuals of Menke-Hennekam syndrome

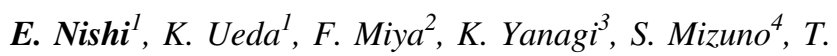
Kaname $^{3}$, K. Kosaki $^{5}$, N. Okamotol

${ }^{1}$ Department of Medical Genetics, Osaka Women's and Children's Hospital, Osaka, Japan, ${ }^{2}$ Department of Medical Science Mathematics, Medical Research Institute, Tokyo Medical and Dental University, Tokyo, Japan, ${ }^{3}$ Department of Genome Medicine, National Research Institute for Child Health and Development, Tokyo, Japan, ${ }^{4}$ Department of Clinical Genetics, Aichi Developmental Disability Center Central Hospital, Kasugai, Japan, ${ }^{5}$ Center for Medical Genetics, Keio University School of Medicine, Tokyo, Japan

Menke-Hennekam syndrome-1 (MKHK1) is a congenital disorder characterized by variable impairment of intellectual development, feeding difficulties, autistic behavior, hearing impairment, short stature, microcephaly, and facial dysmorphisms [Menke et al., 2016; Menke et al., 2018]. MKHK1 is caused by heterozygous mutation in exon 30 or 31 of CREBBP (OMIM 600140). CREBBP mapped 
16 13.3 encodes CREB-binding protein, the heterozygous loss of function variants are well-known causes of Rubinstein-Taybi syndrome Type 1 (RSTS1, OMIM 180849) which is characterized by developmental delay, mild to moderate intellectual disability, short stature, microcephaly, broad thumbs, broad halluces, and facial dysmorphism including highly arched eyebrows, long eyelashes, downslanting palpebral fissures, broad nasal bridge, beaked nose with the nasal septum, highly arched palate, mild micrognathia [Hennekam, 2006; Petrij et al., 1995]. The clinical features included facial characteristics of individuals of MKHK1 are developmental delay or intellectual disability, short stature, microcephaly, short palpebral fissures, telecanthus, depressed nasal bridge, short nose, anteverted nares, short columella, and long philtrum. Although both syndromes have variants in the same gene, $C R E B B P$, the phenotype of individuals with MKHK1 are differed substantially from phenotype of RSTS1[Menke et al., 2016; Menke et al., 2018]. Here we report four further individuals with de novo pathogenic variants, affecting the last amino acids of exon 31 of CREBBP. One has a recurrent variant, and the othes have novel variants including two nonsense variants. All of them did not recognize the characteristics of RSTS like broad thumbs, but rather shared the clinical features of MKHK1.

E. Nishi: None. K. Ueda: None. F. Miya: None. K. Yanagi: None. S. Mizuno: None. T. Kaname: None. K. Kosaki: None. N. Okamoto: None.

\section{P11.31.A}

Two different de novo mutations causing syndromic developmental delay in two patients from a consanguineous Turkish family

L. Castilla-Vallmanya ${ }^{1}$,S. Acar ${ }^{2}$, Ö. Giray-Bozkaya ${ }^{3}$, A.

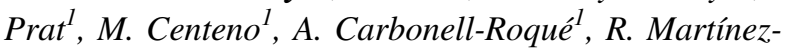
Cabrera $^{l}$, R. Rabionet ${ }^{1}$, D. Grinberg ${ }^{l}$, S. Balcells ${ }^{l}, R$. Urreizti $^{1}$

${ }^{1}$ Department of Genetics, Microbiology and Statistics, Faculty of Biology, University of Barcelona, IBUB, IRSJD, CIBERER, Barcelona, Spain, ${ }^{2}$ Department of Pediatric Genetics, Dr. Behcet Uz Children's Hospital, Izmir, Turkey, ${ }^{3}$ Department of Pediatric Genetics, Dokuz Eylül University, Izmir, Turkey

Introduction: We present a Turkish family with two cousins (OC15 and OC15b) affected with syndromic developmental delay, microcephaly and trigonocephaly but with some phenotypical traits distinct between them. OC15 showed asymmetrical skeletal defects and syndactyly, while OC $15 \mathrm{~b}$ presented with a more severe microcephaly and semilobal holoprosencephaly. All four progenitors were related (the two mothers being sisters and the two fathers being cousins once removed). Additionally, OC15 parents were consanguineous (again cousins once removed).

Material and Methods: WES analysis of patient OC15 was performed as a singleton inside the URD-Cat project. The OC15b trio was sequenced at the National Center of Genomic Analyses (CNAG; Barcelona, Spain). Selected variants were validated by Sanger sequencing.

Results: We did not identify any shared variant that could be associated with the disease. Instead, each patient presented a de novo heterozygous variant in a different gene. OC15 carried a nonsense mutation in PORCN (p. Arg95*), a gene responsible for Goltz-Gorlin syndrome, while OC15b carried an indel mutation in ZIC2 leading to the substitution of 3 residues by a proline (p.His404_Ser406delinsPro). Autosomal dominant mutations in ZIC2 have been associated with holoprosencephaly 5. Both variants are absent in the general population and are predicted to be pathogenic.

Conclusions: These two de novo heterozygous variants identified in the two patients seem to explain the major phenotypic alterations of each particular case, instead of a homozygous variant that would be expected by the underlying consanguinity.

Funding: Associació Síndrome Opitz C, Spain; Spanish MINECO (SAF2016-75948-R, FECYT-PRECIPITA); Catalan Government (PERIS SLT002/16/00174); CIBERER (U720).

L. Castilla-Vallmanya: None. S. Acar: None. Ö. GirayBozkaya: None. A. Prat: None. M. Centeno: None. A. Carbonell-Roqué: None. R. Martínez-Cabrera: None. R. Rabionet: None. D. Grinberg: None. S. Balcells: None. R. Urreizti: None.

\section{P11.32.B}

Non-mosaic germline cancer hotspot mutation p. Ser1344Leu in the RNase IIIa domain of DICERI causes a GLOW syndrome-like phenotype

\author{
S. A. Frisk ${ }^{1,2}$, E. Pontén ${ }^{l}$, K. Lagerstedt-Robinson ${ }^{1,2}, R$.

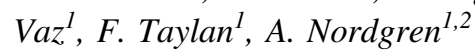 \\ ${ }^{1}$ Karolinska Institutet, Solna, Sweden, ${ }^{2}$ Karolinska Uni- \\ versity Hospital, Stockholm, Sweden
}

DICER 1 is an endoribonuclease central to the generation of microRNAs (miRNAs), important for embryogenesis and early somatic development. Germline DICERI mutations cause DICER1 syndrome - a cancer predisposition syndrome with an increased risk of a variety of tumors, most commonly pleuropulmonary blastomas, cystic nephromas, Sertoli-Leydig cell tumors and multinodular goiter. 
Recently, biallelic mutations of DICERl involving the RNase III domain were reported in $1 \%$ of Wilms tumor (WT).

Here, we present an 18-year old male with macrocephaly, lung and renal cysts, polydactyly, autism, intellectual disability, scoliosis, multiple nevi, ear pits and WT. Whole genome sequencing identified a de novo heterozygous c.4031C > T, p.Ser1344Leu variant located in the RNase IIIa domain of DICER1. The phenotype in our patient resembles two previously reported patients with GLOW (Global developmental delay, Lung cysts, Overgrowth and Wilms tumor)syndrome harboring mosaic hotspot mutations in the RNase IIIb domain. His symptoms support novel results from integrated genetic analysis and in vitro cell experiments where RNase IIIa p.Ser1344 site mutations cause miRNA-strain imbalance, analogous to RNase IIIb mutations involving the catalytic core, causing phenotype commonalities with tissue specific tumors. In addition, his symptoms with macrocephaly, polydactyly, multiple nevi, and intellectual disability resemble phenotypes seen in other overgrowth syndromes that activate PI3K/AKT/mTOR signaling.

In conclusion, we show that non-mosaic germline mutation p.Ser1344Leu in the RNase IIIa domain of DICER1 is compatible with life and causes a GLOW syndrome-like phenotype through a novel pathogenic mechanism in the RNase IIIa domain.

Grant references: Swedish Childhood Cancer Foundation

S.A. Frisk: None. E. Pontén: None. K. LagerstedtRobinson: None. R. Vaz: None. F. Taylan: None. A. Nordgren: None.

\section{P11.33.C}

Whole Exome Sequencing in Brazilian individuals with intellectual disability, Neurodevelopmental Delay and (or) Multiple Congenital Anomalies with Copy Number Variants of Uncertain Clinical Significance detected by Chromosomal Microarray Analysis

S. Spineli-Silva ${ }^{1}, N$. de Leeuw ${ }^{2}$, A. P. dos Santos ${ }^{l}, N$. Leijsten $^{2}$, M. H. A. Ruiterkamp-Versteeg ${ }^{2}$, J. R. M. Prota ${ }^{1}$, A. T. Maciel-Guerra ${ }^{l}$, A. Marques-de-Farial, C. E. Steiner $^{l}$, V. L. Gil-da-Silva-Lopes ${ }^{1}$, T. P. Vieira ${ }^{l}$

${ }^{1}$ Department of Medical Genetics and Genomic Medicine, School of Medical Sciences, State University of Campinas, Campinas, São Paulo, Brazil, ${ }^{2}$ Department of Human Genetics, Radboud University Medical Center, Nijmegen, Netherlands

Introduction: The identification of variants of unknown clinical significance (VOUS) is a constant challenge during the interpretation of copy number variants (CNVs) detected in individuals with multiple congenital anomalies (MCA) and (or) intellectual disability (ID)/ developmental delay (DD). The aim of this study is to perform whole exome sequencing (WES) in these individuals to search for single nucleotide variants (SNVs) related to the phenotype.

Methods: We selected 36 individuals with CNVs classified as VOUS, from a group of 228 individuals presenting MCA and (or) ID/DD previously investigated by Chromosomal Micorarray Analysis. To date, WES was performed for 18 individuals using the Agilent SureSelect Target Enrichment V5 (Agilent Technologies, Santa Clara, CA, USA) sequenced on the Illumina HiSeq platform (Illumina, San Diego, CA, USA).

Results: WES revealed pathogenic nucleotide variants in 4/18 (22,2\%) individuals (ANKRD11; JAG1; KMTD2; $E B F 3)$. In all of them the clinical phenotype was compatible with the mutations found. In addition, a class IV, likely pathogenic SNV was found in one individual (FOXL2), and SNVs classified as VOUS were found in an additional five individuals (ARID2; GNAS; SOX11 and NSDHL; ASXL2; $T G F B R$ 1), one of them also with a pathogenic variant in another gene. None of the SNVs found matched the CNVs classified as VOUS.

Conclusions: Since the (likely) pathogenic SNVs found in 5/18 (28\%) individuals explain the clinical phenotype, we conclude that the CNVs classified as VOUS were rare findings, with no clinical relevance.

Financial support: CAPES, FAPESP (\#2018/08890-9) and FAEPEX-Unicamp.

S. Spineli-Silva: None. N. de Leeuw: None. A.P. dos Santos: None. N. Leijsten: None. M.H.A. RuiterkampVersteeg: None. J.R.M. Prota: None. A.T. MacielGuerra: None. A. Marques-de-Faria: None. C.E. Steiner: None. V.L. Gil-da-Silva-Lopes: None. T.P. Vieira: None.

\section{P11.35.B}

EFTUD2 and Mandibulofacial Dysostosis with Microcephaly - An Australian case series

\section{S. O'Sullivan', J. McGaughran ${ }^{1}$, L. McGregor ${ }^{2}$}

${ }^{1}$ Genetic Health Queensland, Royal Brisbane and Women's Hospital, Brisbane, QLD, Australia, ${ }^{2}$ SA Clinical Genetics Service, Women and Children's Hospital, Adelaide, Australia

Introduction: Mandibulofacial dysostosis with microcephaly (MFDM) was recognised as a distinct clinical entity in 2006, with the genetic basis later attributed to haploinsufficiency of the EFTUD2 gene. EFTUD2 pathogenic variants cause mis-splicing of pre mRNAs, disrupted gene expression and cell apoptosis. MFDM is characterised by 
first and second pharyngeal arch anomalies, hearing loss, microcephaly, developmental delay, intellectual impairment, dysmorphism and systemic malformations. The prevalence is currently unknown, with 110 affected individuals reported in the literature. MFDM is an important differential for other multiple congenital malformation syndromes such as CHARGE syndrome.

Materials and methods: A systematic review of the literature was undertaken using Pubmed. A proforma was devised to capture the demographic, clinical and genetic variables of identified patients. Other clinical genetic services in Australia were contacted to contribute.

Results: Four patients with EFTUD2 related MFDM were identified at two Australian centres. Two patients were monochorionic monoamniotic (MCMA) twins. One twin died secondary to complications of MFDM in the first year of life, his surviving twin was recently reviewed at age 7 years. Two patients were last reviewed aged 15 and 30 years respectively.

Conclusions: This case series contributes further to the literature, allowing for greater expansion of the known phenotype for this rare congenital multiple malformation syndrome. In particular, our report of MCMA twins of varying clinical manifestations, supports the view that while MFDM is highly penetrant, it is variably expressive. Our case series may further guide surveillance and management of this small, but highly diverse, patient group.

S. O'Sullivan: None. J. McGaughran: None. L. McGregor: None.

\section{P11.37.A}

Genome wide methylation profiling, EpiSign, as a diagnostic test

\section{Alders ${ }^{l}$, P. Henneman ${ }^{l}$, E. Aref-Eshghi ${ }^{2}$, A. Venema ${ }^{l}, J$. Kerkhof $^{2}$, B. Sadikovic ${ }^{2,3}$, M. Mannens ${ }^{1}$}

${ }^{1}$ Amsterdam UMC, Amsterdam, Netherlands, ${ }^{2}$ London Health Sciences Centre, London, ON, Canada, ${ }^{3}$ Western University, London, ON, Canada

A growing number of neurodevelopmental syndromes are shown to have unique genomic DNA methylation profiles. These episignatures can be used to diagnose patients with such syndromes. We have recently implemented genome wide methylation profiling as a diagnostic test in our laboratory. This test, named EpiSign(v1), is able to recognize 22 syndromes, 14 of which are syndromes with specific episignatures, (for example for Kabuki syndrome, BAFopathies and Cornelia de Lange syndrome), 6 are imprinting disorders and 2 are repeat expansion disorders. Validation of the test was based on 38 positive samples (with known pathogenic variant) and 24 negative controls. All samples were called correctly provided that the DNA was extracted from peripheral blood and there was no low mosaicism for the pathogenic variant. Reasons for requesting an EpiSign clinical test were: the presence of a variant of unknown clinical significance, clinical diagnosis without molecular confirmation, or as a test complementary to WES/WGS, without a prior (suspected) diagnosis. In approximately $40 \%$ of cases with a suspected (VUS present) or clinical diagnosis, EpiSign was able to confirm this diagnosis. This shows that EpiSign is a powerful new diagnostic tool, in particular for patients for whom conventional genetic testing was inconclusive. In March 2020 an updated version of EpiSign will be available (EpiSignv2), allowing diagnosis of an additional 23 syndromes (45 in total). For a current list of syndromes detected by EpiSign see https://genomedia gnostics.amsterdamumc.nl/

M. Alders: None. P. Henneman: None. E. Aref-Eshghi: None. A. Venema: None. J. Kerkhof: None. B. Sadikovic: None. M. Mannens: None.

\section{P11.39.C}

FOXG1 variants can be associated with milder phenotypes than congenital Rett syndrome

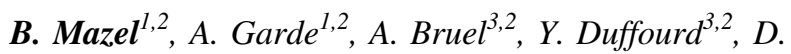
Lopergolo $^{4,5}$, A. M. Pinto ${ }^{5}$, M. A. Mencarelli ${ }^{5}$, R. Canitano $^{6}$, F. Valentino ${ }^{4}$, C. Fallerini ${ }^{4}$, F. Papa ${ }^{4}$, F. Mari ${ }^{4,5}$, N. BahiBuisson $^{7,8}$, A. Munnich ${ }^{9}$, R. Pfundt ${ }^{10}$, D. Fitzpatrick ${ }^{11}, C$.

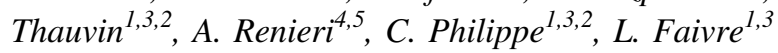

${ }^{I}$ Centre de Référence Anomalies du Développement et Syndromes Malformatifs, FHU TRANSLAD - Centre Hospitalier Universitaire de Dijon, Dijon, France, ${ }^{2}$ Laboratoire de Génétique chromosomique et moléculaire, UF Innovation en diagnostic génomique des maladies rares, Centre Hospitalier Universitaire de Dijon, Dijon, France, ${ }^{3}$ Inserm UMR1231 GAD, Génétique des Anomalies du Développement, Université de Bourgogne, Dijon, France, ${ }^{4}$ Medical Genetics, University of Siena, Siena, Italy, ${ }^{5}$ Genetica Medica, Azienda Ospedaliera Universitaria Senese, Siena, Italy, ${ }^{6}$ Division of Child and Adolescent Neuropsychiatry, University Hospital of Siena, Siena, Italy, ${ }^{7}$ Service de Neurologie Pédiatrique, Hôpital Necker Enfants Malades, Paris, France, ${ }^{8}$ Inserm, Equipe "Embryologie et Génétiques des malformations congénitales" Institut des Maladies Génétiques, Institut Imagine, Paris, France, ${ }^{9}$ Service de Génétique Médicale et Clinique, Hôpital Necker Enfants Malades, Paris, France, ${ }^{10}$ Department of Human Genetics, Radboud University Medical Center, Nijmegen, Netherlands, ${ }^{11}$ MRC Human Genetics Unit, MRC Institute of Genetics and Molecular Medicine at the University of Edinburgh, Edinburgh, United Kingdom 
Introduction : Forkhead box G1 (FOXG1) haploinsufficiency has been associated in 2008 with a severe neurodevelopmental phenotype with features of Rett syndrome, but earlier onset in the first months of life. Most patients did not achieve unassisted sitting or walking, had no speech and were not able to use their hands purposefully. For years, targeted sequencing of FOXG1 gene has only been prescribed in such severe cases and did not permit to describe the full clinical spectrum associated with FOXG1 variants. The advent of exome/genome sequencing now permits to diagnose unbiased clinical presentations.

Methods : A collaborative call was sent through the ERN-ITHACA, in order to gather detailed clinical cases with a heterozygous FOXGl variant associated with a mild phenotype, defined as a patients who could walk unassisted and/or acquire speech.

Results : Eight patients were collected from different European countries, all carrying a pathogenic or likely pathogenic de novo missense $F O X G 1$ variant, the majority being located in the forkhead conserved site 1. Patients usually presented with non-specific ID, distinct from the congenital Rett variant, microcephaly and epilepsy being rarely associated.

Conclusion : These findings corroborate with the recent genotype-phenotype analysis (Mitter et al., 2017) proposing to delineate 5 different FOXG1 genotype groups. Milder phenotypes were associated with FOXG1 missense variants in the forkhead conserved site. This information is valuable for better prognosis after the identification of a FOXG1 variant in young children but also for the interpretation of new FOXG1 variants identified after exome/genome sequencing.

B. Mazel: None. A. Garde: None. A. Bruel: None. Y. Duffourd: None. D. Lopergolo: None. A.M. Pinto: None. M.A. Mencarelli: None. R. Canitano: None. F. Valentino: None. C. Fallerini: None. F. Papa: None. F. Mari: None. N. Bahi-Buisson: None. A. Munnich: None. R. Pfundt: None. D. Fitzpatrick: None. C. Thauvin: None. A. Renieri: None. C. Philippe: None. L. Faivre: None.

\section{P11.41.B}

Trio genome sequencing reveals a high proportion of ultra-rare genetic disorders due to de novo mutations in children with medical complexity

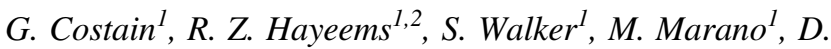
Veenma $^{l}$, M. Snell ${ }^{l}$, M. Curtis ${ }^{l}$, S. Luca ${ }^{l}$, j. Buera ${ }^{l}$, D.

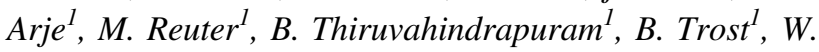

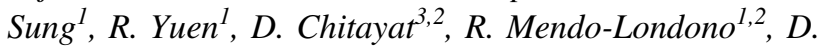

Stavropoulos $^{1,2}$, S. W. Scherer ${ }^{1,2}$, C. W. Marshall ${ }^{1,2}, R . R$.

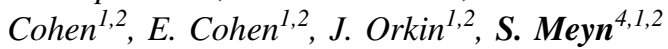

${ }^{I}$ The Hospital for Sick Children, Toronto, ON, Canada, ${ }^{2}$ The University of Toronto, Toronto, ON, Canada, ${ }^{3}$ Mount Sinai Hospital, Toronto, ON, Canada, ${ }^{4}$ University of Wisconsin - Madison, Madison, WI, United States

Children with medical complexity have $\geq 1$ chronic condition(s), multiple subspecialist involvement, and high healthcare utilization. We hypothesized that genome sequencing (GS) has high potential to diagnose children with medical complexity, and that cohorts of these children are enriched for novel genetic disorders.

Screening 545 children with medical complexity from a Complex Care Program yielded 143 children suspected of an undiagnosed genetic condition, despite having undergone an average of four genetic tests per proband. 49/143 were evaluated through clinical genetic assessments and found to be phenotypically complex, with a median of 24 Human Phenotype Ontology terms/patient. Short read GS was performed on these 49 probands, including 2 quartets, 40 trios, and 3 dyads.

Despite prior genetic testing, GS yielded a new molecular diagnosis for $15 / 49$ probands $(31 \%)$. The majority of diagnostic variants were sequence level variants, although an exonic duplication causing Kabuki syndrome found by GS escaped detection by CMA, ES, or MLPA analysis. Of note, only three autosomal recessive disorders were identified and $>90 \%(11 / 12)$ of the dominant and $\mathrm{X}$ linked disorders identified by GS were caused by de novo mutations. The majority of disorders (9/15) were ultra rare ( $<25$ reported cases), including three associated with novel disease genes FBXW7, H3F3B, and RAC3. Importantly, using DECIPHER, Matchmaker Exchange, and ClinVar, similarly affected individuals were rapidly identified around the globe.

Our results suggest that trio-based genome sequencing is a high yield testing strategy for this patient population, which appears to be enriched for de novo mutations, ultrarare disorders and novel genetic syndromes.

G. Costain: None. R.Z. Hayeems: None. S. Walker: None. M. Marano: None. D. Veenma: None. M. Snell: None. M. Curtis: None. S. Luca: None. J. Buera: None. D. Arje: None. M. Reuter: None. B. Thiruvahindrapuram: None. B. Trost: None. W. Sung: None. R. Yuen: None. D. Chitayat: None. R. Mendo-Londono: None. D. Stavropoulos: None. S.W. Scherer: None. C.W. Marshall: None. R.R. Cohen: None. E. Cohen: None. J. Orkin: None. S. Meyn: E. Ownership Interest (stock, stock options, patent or other intellectual property); Modest; Gene42. 
P11.42.C

The first patient with mesomelic dysplasia and urogenital abnormalities associated with a de novo heterozygous variant in $\mathrm{HOXA11}$ gene

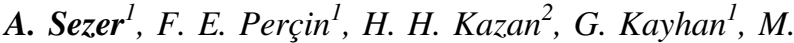 Aktürk ${ }^{3}$}

${ }^{1}$ Gazi University, Faculty of Medicine, Department of Medical Genetics, Ankara, Turkey, ${ }^{2}$ Middle East Technical University, Department of Biological Sciences, Ankara, Turkey, ${ }^{3}$ Gazi University, Faculty of Medicine, Department of Endocrinology and Metabolism, Ankara, Turkey

Introduction: Hox genes encode transcription factors playing critical roles during animal embryonic development and many of these genes have been shown to related to developmental disorders in animal models and patients. Hoxall mutant mice exhibit homeotic transformations in thoracic and sacral vertebrae, zeugopodal phenotype in forelimb and hindlimb, and urogenital abnormalities. A certain heterozygous variant in HOXA11 gene has been associated with radioulnar synostosis and amegakaryocytic thrombocytopenia 1 (RUSAT1, OMIM \#605432). Here we present the first patient with mesomelic dysplasia and urogenital abnormalities putatively related to a de novo variant in HOXA11 gene.

Materials and Methods: An 18-year-old female patient with normal intelligence was presented with disproportionate short stature, scoliosis, mesomelic limb shortening, radial and ulnar bowing, fusions in carpal bones, operated pes equinovarus, short $4^{\text {th }}-5^{\text {th }}$ metatarsals, primary amenorrhea due to hypoplasia of uterus, and vesicoureteral reflux. Whole-exome sequencing (WES) analysis was applied to investigate the genetic etiology.

Results: WES analysis revealed heterozygous c.881T $>\mathrm{G}$ (p.Met294Arg) variant in HOXA11 (NM_005523.6) gene. The variant was determined as de novo by family study.

Conclusions: To date, only a heterozygous premature stop-codon variant in HOXA11 gene has been associated with RUSAT1 in two families. Some skeletal problems without mesomelic dysplasia and urogenital abnormalities have been reported in this condition. However, our patient did not have RUSAT. This may be due to the different functional effects of these variants, although they are located in the same protein domain (homeodomain). In vitro functional studies are ongoing to evaluate the effect of the variant, c. $881 \mathrm{~T}>\mathrm{G}$.

A. Sezer: None. F.E. Perçin: None. H.H. Kazan: None. G. Kayhan: None. M. Aktürk: None.

\section{P11.44.B}

SEVERE RESPIRATORY DISTURBANCE IN NEONATAL INTENSIVE CARE UNIT WITH A RECURRENT NONSENSE VARIANT IN MAGEL2 GENE

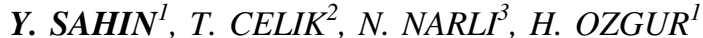

${ }^{I}$ Genoks Genetic Diseases Diagnostic Center, Ankara, Turkey, ${ }^{2}$ Department of Child Neurology, Private Practice, Adana, Turkey, ${ }^{3}$ Department of Pediatrics, Division of Neonatology, Cukurova University Faculty of Medicine,, Adana, Turkey

Schaaf-Yang syndrome (SYS) is characterized by neonatal hypotonia, feeding difficulties, developmental delay/intellectual disability, hypogonadism and short stature. SYS is caused by pathogenic mutations in the paternal allele of the MAGEL2 gene located in the maternally imprinted region of Prader-Willi syndrome. A 4-months-old male was referred to our genetic laboratory because of respiratory distress, hypotonia and small hands, camptodactyly of the third and fourth fingers. And the other sibling of the family died after 90 days of aggravated apnea. Whole exome sequencing (WES) performed on DNBSEQ-G400 sequencer and Sanger sequencing were used to validate the findings. WES was revealed a novel truncating pathogenic variant in MAGEL2 gene inherited from the unaffected father and confirmed by Sanger sequencing. We report 2 newborns affected with SYS in one family with severe respiratory distress one of them died. Considering neonatal intensive care conditions where time is overvalued, WES is an important diagnostic method in rare cases with atypical courses.

Y. Sahin: None. T. Celik: None. N. Narli: None. H. Ozgur: None.

\section{P11.46.A}

The genetic landscape of Joubert syndrome in Italy

V. Serpieri ${ }^{l}$, S. Nuovo ${ }^{2}$, A. Micalizzi ${ }^{3}$, F. D'Abrusco ${ }^{1}, P$. Accorsi $^{4}$, R. Battini ${ }^{5,6}$, F. Benedicenti ${ }^{7}$, E. Bertini ${ }^{8}, S$. Bigoni $^{9}$, M. Bonati ${ }^{10}$, R. Borgatti ${ }^{11}$, M. Briguglio ${ }^{12}$, A. $^{2}$. Casella $^{l}$, R. Ciccone $^{l}$, S. D'Arrigo ${ }^{13}$, M. Dotti $^{14}, F$. Emma $^{15}$, E. Fazzil ${ }^{16,17}$, M. Ginevrino ${ }^{18}$, L. Giordano ${ }^{16}$, I. Moroni $^{19}$, M. Romani ${ }^{20}$, R. Romaniello ${ }^{21}$, S. Signorini ${ }^{11}, F$. Stanzial $^{7}$, N. Vanacore $^{22}$, G. Zanni ${ }^{8}$, E. Valente $^{1,23}$

${ }^{I}$ Dept. of Molecular Medicine, University of Pavia, Pavia, Italy, ${ }^{2}$ Dept. of Paediatrics, Child Neurology ad Psychiatry, Sapienza University of Rome, Rome, Italy, ${ }^{3}$ Lab. of Medical Genetics, IRCCS Bambin Gesù Children's Hospital, Rome, Italy, ${ }^{4}$ Child Neuropsychiatry Unit, Spedali Civili di Brescia, Brescia, Italy, ${ }^{5}$ Dept. of Developmental 
Neuroscience, IRCCS Stella Maris Foundation, Pisa, Italy, ${ }^{6}$ Dept. of Clinical and Experimental Medicine, University of Pisa, Pisa, Italy, ${ }^{7}$ Genetic Counseling Service, Dept. of Pediatrics, Regional Hospital of Bolzano, Bolzano, Italy, ${ }^{8}$ Lab. of Molecular Medicine, Unit of Neuromuscolar and Neurodegenerative Disorders, Dept. of Neurosciences, IRCCS Bambin Gesù Children's Hospital, Rome, Italy, ${ }^{9}$ Medical Genetics Unit, Ospedale Universitario S. Anna, Ferrara, Italy, ${ }^{10}$ Clinic of Medical Genetics, IRCCS Istituto Auxologico Italiano, Milan, Italy, ${ }^{11}$ Child and Adolescent Neuropsychiatry Unit, IRCCS Mondino Foundation, Pavia, Italy, ${ }^{12}$ Child and Adolescent Neuropsychiatry, University of Messina, Messina, Italy, ${ }^{13}$ Dept. of Developmental Neurology, IRCCS Foundation, Istituto Neurologico C. Besta, Milan, Italy, ${ }^{14}$ Dept. of Medicine, Surgery and Neuroscience, University of Siena, Siena, Italy, ${ }^{15}$ Dept. of Nephrology and Urology, Unit of Nephrology and Dialysis, IRCCS Bambin Gesù Children's Hospital, Rome, Italy, ${ }^{16}$ Child and Adolescent Neurology and Psychiatry Unit, Children's Hospital, Spedali Civili di Brescia, Brescia, Italy, ${ }^{17}$ Dept. of Human Neuroscience, SapienzaUniversity of Rome, Rome, Italy, ${ }^{18}$ Unit of Genetics, Università Cattolica del Sacro Cuore, Rome, Italy, ${ }^{19}$ Dept. of Paediatric Neuroscience, IRCCS Foundation, Istituto Neurologico C. Besta, Milan, Italy, ${ }^{20}$ Molecular Genetics Laboratory, Eurofins GENOMA Group, Rome, Italy, ${ }^{21}$ Neuropsychiatry and Neurorehabilitation Unit, Scientific Institute IRCCS Eugenio Medea, Bosisio Parini, Lecco, Italy, ${ }^{22}$ National Center for Disease Prevention and Health Promotion, National Institute of Health, Rome, Italy, ${ }^{23}$ IRCCS Mondino Foundation, Pavia, Italy

Joubert syndrome (JS) is a rare genetically heterogeneous disorder, with recessive inheritance, characterized by a unique cerebellar and brainstem malformation (the "molar tooth sign") and variable organ involvement, mainly eyes, kidneys and liver. JS is related to dysfunction of the primary cilium and, up to now, about 40 causative genes are known, overall responsible for about $60 \%$ cases. Over 15 years, we recruited 595 JS families (mostly Italian), of whom 507 underwent genetic screening either by Sanger sequencing of selected JS genes, targeted sequencing or WES. With these strategies, we identified biallelic pathogenic variants in 284 probands (56\%). Six major genes accounted for two-thirds of mutated cases (CEP290 14.8\%; CPLANE1 14.4\%; CC2D2A 12.3\%; TMEM67 10.9\%; AHI1 9.5\%; KIAA0586 5.3\%). Additional 86 probands (17\%) carried a single pathogenetic variant in a JS gene: to date, 42 of them underwent high-resolution custom array, which disclosed large heterozygous deletions in 5 probands (1 AHI1, 2 CPLANE1, 2 KIAA0586). Array-negative single heterozygous carriers are currently undergoing RNA analysis to investigate the presence of cryptic variants affecting splicing. In 137 probands, no pathogenic variants were identified. Yet, most of them have been tested only for a subset of known genes, and WES is in progress. Clinical evaluation of JS-mutated cases confirmed strong genephenotype correlates for some major genes (CEP290 with JS+retina+kidney; CPLANE1 with pure JS or OFDVI syndrome; TMEM67 with JS+liver; AHI1 with JS+retina), while no obvious correlates emerge for CC2D2A and KIAA0586. These findings have major implications for prognosis and counselling of JS families.

V. Serpieri: None. S. Nuovo: None. A. Micalizzi: None. F. D'Abrusco: None. P. Accorsi: None. R. Battini: None. F. Benedicenti: None. E. Bertini: None. S. Bigoni: None. M. Bonati: None. R. Borgatti: None. M. Briguglio: None. A. Casella: None. R. Ciccone: None. S. D'Arrigo: None. M. Dotti: None. F. Emma: None. E. Fazzi: None. M. Ginevrino: None. L. Giordano: None. I. Moroni: None. M. Romani: None. R. Romaniello: None. S. Signorini: None. F. Stanzial: None. N. Vanacore: None. G. Zanni: None. E. Valente: None.

\section{P11.47.B}

Description of the molecular and phenotypic spectrum of Kabuki syndrome in $\mathbf{7 3}$ Chinese patients

\section{Y. Wang, Y. Shen, J. Wang, X. Wang}

\section{Shanghai Children's Medical Center, Shanghai,} China, China.

$<$ META NAME $=$ "author" $\mathrm{CONTENT}=$ "月巴月半小夜 曲"> Introduction: To retrospective analysis the clinical phenotypes and genotypes of Kabuki syndrome patients diagnosed in a single center. Meanwhile, the clinical dataof Chinese KS patients reported in the previous literature were summarized. To explore the characteristics of phenotype and genotype of Chinese KS patients. Materials and Method: The objects who were referred to Shanghai Children's Medical Center from July 2017 to December 2019 diagnosed with KS were included, and Chinese KS patients reported in the database were collected. The clinical phenotypes Chinese KS patients were compared with nonChinese KS patients to further analyze and summarize the characteristics of their phenotype and genotype. Results: A total of Chinese KS patients include 32 cases diagnosed by our hospital and 41 reported cases in the database. By comparing the clinical phenotypes of Chinese KS patients with non-Chinese KS patients, we found that the incidence of some facial features was higher in Chinese KS patients, but congenital heart disease, cleft palate, infant finger pad and vision abnormality was lower. The difference of the above phenotypic incidence was statistically significant. Sixty-five pathogenic mutations of KMT2D gene, 7 
pathogenic mutations of KDM6A gene and 1 copy number mutation of KDM6A gene were found. Conclusions: Through this study, we found that the incidence of several phenotypes in Chinese KS patients vs non-Chinese KS patients was statistically significant. This study expanded the phenotype and genotype of KS in China, and provided a theoretical basis for its diagnosis, treatment and prognosis.

Y. Wang: None. Y. Shen: None. J. Wang: None. X. Wang: None.

\section{P11.49.A}

Clinical features and identification of mutation spectrum in 23 Turkish children with Kabuki syndrome

\section{E. Usluer ${ }^{l}$, G. Yeşil $l^{2}$, N. Güneş ${ }^{1}$, D. Uludag Alkaya ${ }^{l}, B$. Tüysüz ${ }^{l}$}

${ }^{1}$ Istanbul University, Cerrahpasa Medical Faculty, Istanbul, Turkey, ${ }^{2}$ Bezmialem Vakif University, Medical Faculty, Department of Medical Genetics, Istanbul, Turkey

Introduction: Kabuki syndrome (KS) is a rare genetic disorder characterized by distinct facies (long palpebral fissures with eversion of the lateral portion of the lower eyelid and large ears), persistent fetal finger pads and intellectual disability (ID). It is caused by heterozygous mutations in KMT2D (56-75\%) or KDM6A (3-8\%) genes. We investigated clinical and molecular findings of 23 patients diagnosed with KS.

Methods: Twenty-three patients clinically were enrolled. Exons and exon-intron boundaries of KMT2D and KDM6A were studied by Sanger sequencing method.

Results: We detected different pathogenic mutations (five missense, four nonsense, five small deletion, one small insertion, one splicing) in KMT2D gene in 16 patients (69.6\%), seven of them were novel. None of patients had a mutation in KDM6A gene. All of the patients had typical facial features, fetal finger pads and ID (6 severe, 17 mild). The patients also had short stature (11/23), renal anomalies (11/23), congenital heart defects (CHD) (11/23), cleft palate (5/23), epilepsy (8/23), abnormal brain MRI findings (6/23), congenital hypothyroidism (6/23) and premature thelarche (2/23).

Conclusions: Up to date 829 different mutations in $K M T 2 D$ gene have been reported. This study also revealed that novel mutations are common in KMT2D gene. The absence of mutations in $30.4 \%$ of patients points genetic heterogeneity and the need to investigate indels and new candidate genes. In addition, while patients with KMT2D gene mutations have CHD and renal abnormalities, the absence of those without mutations supports the effect of KMT2D gene on heart and kidney development.

E. Usluer: None. G. Yeşil: None. N. Güneş: None. D. Uludag Alkaya: None. B. Tüysüz: None.

\section{P11.50.B}

KBG syndrome: common and uncommon clinical features based on 31 new patients

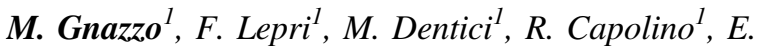

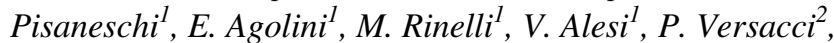
S. Genovese ${ }^{l}$, C. Cesario ${ }^{l}$, L. Sinibaldi ${ }^{l}$, A. Baban $^{l}, A$.

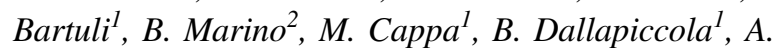
Novelli $^{l}$, M. Digilio ${ }^{l}$

${ }^{1}$ Laboratory of Medical Genetics, Medical Genetics, Rare Diseases, Pediatric Cardiology, Endocrinology Units and Scientific Rectorate, Bambino Gesù Pediatric Hospital, Rome, Italy, ${ }^{2}$ Pediatric Cardiology, Department of Pediatrics, Sapienza University, Rome, Italy

Introduction: KBG syndrome (MIM \#148050) is an autosomal dominant disorder characterized by developmental delay, intellectual disability, distinct craniofacial anomalies, macrodontia of permanent upper central incisors, skeletal abnormalities and short stature. Molecular defect results from loss of function mutations or deletions of the ANKRD11 gene, encoding for an inhibitor of liganddependent transcriptional activation.

Materials and Methods: KBG patients were analized with NGS Cohesinopathies gene panel while clinical exome sequencing was performed to study patients with a likely association of neurofibromatosis. Common clinical features have been reviewed together with unusual findings, clinical expression in the first years of life and distinctive associations.

Results: This single-center study reports the clinical features of $31 \mathrm{KBG}$ patients of Bambino Gesù Children Hospital: 28 with ANKRD11 pathogenic variants and 3 with 16q24 deletions. In addition to ANKRD11 causative mutation, pathogenic $N F 1$ variants have been found in two patients. Unusual manifestations emerging from present series include juvenile idiopathic arthritis, dysfunctional dysphonia, multiple dental agenesis, idiopathic precocious telarche, oral frenula, motor tics, lipoma of corpus callosum, pilomatrixoma and endothelial corneal polymorphic dystrophy. Facial clinical markers suggesting KBG syndrome before 6 years of age include ocular and mouth conformation, wide eyebrows, synophrys, long black eyelashes, long philtrum and thin upper lip.

Conclusions: The spectrum of clinical features in KBG is wide. It is likely that uncommon clinical presentation and overlapping features in patients with multiple variants are responsible for underdiagnosis in KBG syndrome. Improved knowledge about typical and atypical symptoms is useful for clinical management of this disorder.

M. Gnazzo: None. F. Lepri: None. M. Dentici: None. R. Capolino: None. E. Pisaneschi: None. E. Agolini: 
None. M. Rinelli: None. V. Alesi: None. P. Versacci: None. S. Genovese: None. C. Cesario: None. L. Sinibaldi: None. A. Baban: None. A. Bartuli: None. B. Marino: None. M. Cappa: None. B. Dallapiccola: None. A. Novelli: None. M. Digilio: None.

\section{P11.52.A}

Pathogenic variant in $K L H L 11$ in six individuals from a family with lichen planus, ungueal hypoplasia and cancer. ¿A new entity with novel gene?

J. Tenorio ${ }^{1}$, J. Nevado ${ }^{1}$, J. Fort ${ }^{2}$, M. Feito ${ }^{3}$, V. MartínezGlez ${ }^{1}$, R. De Lucas ${ }^{3}$, A. Fernández ${ }^{4}$, V. Ruíz-Pérez ${ }^{5}$, L. Montoliú $^{4}$, M. Palacín ${ }^{2}$, P. Lapunzina ${ }^{1}$

${ }^{1}$ INGEMM, Madrid, Spain, ${ }^{2}$ Institute for Research in Biomedicine, Barcelona, Spain, ${ }^{3}$ Dermatology Department - Hospital Universitario La Paz, Madrid, Spain, ${ }^{4}$ Centro Nacional de Biotecnología, Madrid, Spain, ${ }^{5}$ Instituto de Investigaciones Biomédicas Alberto Sols, Madrid, Spain

Lichen planus (LP) is a chronic inflammatory disorder that affects the skin, oral mucosa and hair. The lesions of the LP tend to chronify and sometimes can lead to malignancy neoplasias. LP can be associated with several systemic disorders such as metabolic syndrome, diabetes mellitus, hypertension, etc. We here present a 2-generation family with six affected members with LP, nail dystrophy and in two of them, carcinoma of the tongue and other localizations, with several members of the families developing cancer. Clinical features segregates along the family, suggesting an autosomal dominant pattern of inheritance. Thus, whole exome sequencing was performed in all available members of the family (including healthy and affected members), allowing to identify a heterozygous missense variant in KLHL11 that segregates with the phenotype. This variant is not present in the control population databases. In addition, there is no disease associated with KLHL11 yet. KLHL11 belongs to the KELCH superfamily -42 genes described -, and encoded for a protein that have a BTB/ POZ, BACK five KELCH domains. The BTB/POZ domain is involved in protein-protein binding and dimerization. The BACK domain does not have a known function and the KELCH motifs create a tertiary structure of $\beta$-helix which is involved in extracellular functions, cellular integrity and protein binding. Kelch proteins acts as homodimer, and the variant detected it is suspected to impair this dimerization. It's the first time that a KLHL11 is associated with lichen planus and neoplasia. Functional assays are necessary to confirm the pathogenic mechanism.

J. Tenorio: None. J. Nevado: None. J. Fort: None. M. Feito: None. V. Martínez-Glez: None. R. De Lucas:
None. A. Fernández: None. V. Ruíz-Pérez: None. L. Montoliú: None. M. Palacín: None. P. Lapunzina: None.

\section{P11.53.B}

Mabry syndrome: A novel recurrent PGAP3 variant in an African population

H. Bezuidenhout ${ }^{1}$, S. Bayley ${ }^{1}$, K. Fieggan ${ }^{2}$, N. Makubalo $^{3}$, C. Spencer ${ }^{2}$, S. Moosa ${ }^{1}$, L. Smit ${ }^{1}$, C. Uren ${ }^{l}$, M. Moller ${ }^{l}$, C. Kinnear $^{l}$, M. Urban ${ }^{l}$

${ }^{1}$ Division of Molecular Biology and Human Genetics, University of Stellenbosch and Tygerberg Hospital, Cape Town, South Africa, ${ }^{2}$ Division of Medical Genetics, Groote Schuur Hospital and University of Cape Town, Cape Town, South Africa, ${ }^{3}$ Dora Nginza Hospital, Port Elizabeth, South Africa

Background: Mabry syndrome (MS) is a rare autosomal recessive disorder caused by pathogenic variants in one of several genes involved in glycosylphosphatidylinositol metabolism. The phenotype includes severe intellectual disability, distinctive but subtle facial features, hypotonia, variable congenital malformations and persistently elevated alkaline phosphatase (ALP).

Methods: The search for an unidentified syndrome causing the above clinical features led to the clinical identification of a case of MS at Tygerberg Academic Hospital. This was assisted by Face2Gene phenotype matching and confirmed by elevated ALP. Whole exome sequencing detected a homozygous candidate variant (PGAP3:c.557G>C, p.Arg186Thr). A case series was assembled by retrospective and prospective case finding at three South African sites using database searches, Face2Gene phenotype matching, and/or ALP levels and Sanger sequencing for the candidate variant.

Results: Nine clinical cases were identified and Sanger sequencing could be performed on 7, all proved homozygous for the candidate variant. The likely pathogenic variant is absent from population databases, affects a highly conserved amino acid and is predicted damaging/deleterious. Observed clinical features in all included severe intellectual disability, absent speech, hypotonia and cleft palate in (5/9) (56\%). ALP levels were significantly raised. Most discriminating facial features include hypertelorism, long palpebral fissures, broad nasal tip and tented upper lip.

Conclusion: A previously undiagnosed syndrome was identified in an indigenous South African population, with computer-assisted syndromology being instrumental. We describe 9 cases of MS and their genetic basis. The common homozygous variant in $P G A P 3$, in the absence of consanguinity, suggests genetic drift and investigation is ongoing. 
H. Bezuidenhout: None. S. Bayley: None. K. Fieggan: None. N. Makubalo: None. C. Spencer: None. S. Moosa: None. L. Smit: None. C. Uren: None. M. Moller: None. C. Kinnear: None. M. Urban: None.

\section{P11.54.C}

De novo variants in $M C F 2 L$ are associated with congenital blindness, hearing loss, microcephaly, ID and autism

D. J. Smits, L. Donker Kaat, R. Schot, M. Wilke, M. van Slegtenhorst, G. M. S. Mancini

Erasmus University Medical Centre, Rotterdam, Netherlands

Introduction: $M C F 2 L$ encodes for the Dbs protein which is a Rho-specific guanine nucleotide exchange factor (RhoGEF). Rho-GEFs accelerate the exchange activity of Rho GTPases and thereby regulate several growth related processes. MCF2L interacts with RAC1 via its plekstrin homology domain, and thereby in turn activates RHOA. RAC1 activating mutations have been associated with megalencephaly and RHOA mosaic postzygotic inactivating variants have been recently associated with a neuroectodermal syndrome with asymptomatic leukoencephalopathy, microphthalmia or other ocular, dental and skeletal anomalies. Previously, GWAS studies pointed to $M C F 2 L$ as locus for osteoarthritis.

Results: We identified heterozygous de novo missense variants in the spectrin-like repeat domain of $M C F 2 L$ in two unrelated boys. They presented with nearly identical and recognizable phenotypic features including mild facial dysmorphisms, microphthalmia with persistent hyperplastic primary vitreous, congenital retinal detachment, mild hearing loss, microcephaly, cognitive impairment, autism, epilepsy, pyramidal signs and congenital thyroid insufficiency. Brain MRI in one child (10 years of age) showed focal cortical dysplasia of the insula, one periventricular nodular heterotopia and calcifications. In the other child, the MRI showed neonatal cerebellar subdural hygroma, modest intraparenchymal parietal and vitreous bleeding.

Conclusions: These phenotypic similarities suggest a causal implication for the $M C F 2 L$ variants, which enlarge the broad clinical spectrum of mutations in proteins involved in regulation of Rho-protein signalling and emphasize the importance of this pathway in neurodevelopmental disorders.

D.J. Smits: None. L. Donker Kaat: None. R. Schot: None. M. Wilke: None. M. van Slegtenhorst: None. G.M. S. Mancini: None.

\section{P11.55.A}

The Qatari Mendelian program - A premium resource for gene discovery and data sharing for rare diseases from the greater Middle East

W. Aamer ${ }^{l}$, A. Almaraghi ${ }^{l}$, A. Al-Kurbi ${ }^{1,2}$, S. Padmajeya ${ }^{l}$, A. Al-Shakaki ${ }^{3}$, the Qatari Mendelian Program, A. Robay ${ }^{3}$, M. Kamal $^{1,4}$, A. Akil ${ }^{1}$, T. Ben Omran ${ }^{1,4,3}$, K. A. Fakhro ${ }^{1,3,2}$

${ }^{I}$ Sidra Medicine, Doha, Qatar, ${ }^{2}$ Hamad Bin Khalifa University, Doha, Qatar, ${ }^{3}$ Weill Cornell Medical College, Doha, Qatar, ${ }^{4}$ Hamad Medical Corporation, Doha, Qatar

The recent investment in biomedical research and the launch of a large-scale national genome program for Qatar have motivated the establishment of the Qatari Mendelian Program - a multi-stakeholder initiative offering WGS to patients with idiopathic disorders in an area of the world where consanguinity levels are high $(>50 \%)$ and clinical care is largely centralized. Following our recent pilot of 30 families (PMID:31625567), we have now sequenced the whole genomes of a further 336 individuals (including 115 patients) from 87 families with various conditions, including: Autism $(n=23)$, neurodevelopmental and neuromuscular $(n=16)$, metabolic and endocrine $(n=14)$, craniofacial $(n=10)$, sensory $(n=7)$, immune $(n=7)$ and other $(n=10)$ disorders. We identified novel causative mutations in known disease genes in 34 families (40.2\%), including $29 \mathrm{SNV}$ s/indels and 5 structural variants. Notably, we discovered a complex rearrangement (tandem duplication and deletion) affecting exons 1-6 of IRF6 in three siblings with Popliteal pterygium syndrome, confirmed by de novo local re-assembly of WGS reads and PCR. For another 15 families, we identified putatively damaging variants in novel candidate genes, e.g. a missense variant in the CPSG4 shared by two siblings with severe hypotonia, dysmorphic features and myoclonic encephalopathy with seizures. Homozygous zebrafish we generated by CRISPR displayed highly similar phenotypes, prompting deeper functional studies to elucidate gene mechanism. The Qatari Mendelian program continues to enroll 2-4 families per week, consented for sample/data sharing, thus creating an international genomic and phenotypic resource for mutation matching and gene discovery from the globally under-represented greater Middle Eastern populations.

W. Aamer: None. A. Almaraghi: None. A. Al-Kurbi: None. S. Padmajeya: None. A. Al-Shakaki: None. A. Robay: None. M. Kamal: None. A. Akil: None. T. Ben Omran: None. K.A. Fakhro: None. 
P11.57.C

Menke-Hennekam syndrome subtypes caused by variants in the Zinc finger domains $\mathrm{ZZ}$ and TAZ2 and the fourth intrinsically disordered linker of CBP and p300

L. A. Menke ${ }^{1,2}$, Z. A. Jenkins ${ }^{2}$, E. Williams ${ }^{2}$, G. Gimenez, A. C. O'Neill' ${ }^{2}$ R. C. Hennekam ${ }^{1}$, S. P. Robertson ${ }^{2}$

${ }^{1}$ Department of Paediatrics, Amsterdam UMC, University of Amsterdam, Amsterdam, Netherlands, ${ }^{2}$ Department of Women's and Children's Health, Dunedin School of Medicine, University of Otago, Dunedin, New Zealand, ${ }^{3}$ Department of Pathology, Dunedin School of Medicine, University of Otago, Dunedin, New Zealand

Introduction: CREB-binding protein (CBP, encoded by $C R E B B P)$ and its paralog E1A-associated protein p300 (EP300) are histone acetylators and important cofactors for transcription. Variants that produce a null allele or destroy their catalytic function cause Rubinstein-Taybi syndrome, while clustered pathogenic variants in exon 30/31 cause phenotypes now bundled under the umbrella term MenkeHennekam syndrome (MKHK, OMIM 618332 and 618333).

Materials and Methods: To distinguish MKHK subtypes and define their characteristics, molecular and extended clinical data on 53 individuals (25 unpublished) with a variant in CBP/p300 (hg19; residues 1710-1872 and 1673-1835, respectively) were systematically gathered. Morphological characteristics were assessed by an expert (RCH) blinded to the molecular data. Variants were plotted on 3D protein domains using iCn3D software.

Results: Variants clustered closely around the Zinc binding residues of two Zinc finger domains ( $\mathrm{ZZ}$ and TAZ2) and within the first $\alpha$-helix of the fourth intrinsically disordered linker (ID4) of CBP/p300. All variants were predicted to disturb local protein structure and therefore likely the function of $\mathrm{CBP} / \mathrm{p} 300$. By evaluating morphological and physical features, we discerned one clearly delineated and three likely subtypes.

Conclusions: Variants in the first $\alpha$-helix of ID4 cause a separate, recognisable MKHK subtype. Although numbers are still small, three other possible subtypes can be discerned in $\mathrm{ZZ}$ and two regions within TAZ2. LAM was funded by the Janivo Foundation and Emma Children's Hospital Foundation.

L.A. Menke: None. Z.A. Jenkins: None. E. Williams: None. G. Gimenez: None. A.C. O'Neill: None. R.C. Hennekam: None. S.P. Robertson: None.
P11.60.C

Microcephalic osteodysplastic primordial dwarfism type II and pachygyria: morphometric analysis in a 2 yearsold girl

M. Dentici, M. Rossi-Espagnet, L. Pasquini, C. Carducci, M. Lucignani, D. Longo, E. Agolini, A. Novelli, M. V. Gonfiantini, M. Digilio, A. Bartuli

Bambino Gesú Pediatric Hospital, IRCCS, Rome, Italy., Rome, Italy

Microcephalic osteodysplastic primordial dwarfism (MOPD) type II is a rare disorder characterized by skeletal dysplasia, severe proportionate short stature, insulin resistance and cerebrovascular abnormalities including cerebral aneurysms and moyamoya disease. MOPD type II is caused by mutations in the pericentrin (PCNT) gene, which encodes a protein involved in centrosomes function.

We report a 2 year old girl affected by MOPD type II caused by two compound heterozygous loss of-function variants in PCNT gene, of which one is a novel variant (c.5304delT; p.Gly1769AlafsTer34). The patient presented atypical brain MRI findings consistent with pachygyria. This was confirmed by morphometric analysis of cortical thickness and gyrification index by comparing MRI data of the patient with a group of 8 age-matched healthy controls. The statistical analysis revealed a significant and diffuse increase of cortical thickness with an anterior predominant pattern and diffuse reduced gyrification $(\mathrm{p}<0.05)$.

These findings provide new evidences to the emergent concept that malformations of cortical development are complex disorders and that new genetic findings contribute to the fading of classification borders.

M. Dentici: None. M. Rossi-Espagnet: None. L. Pasquini: None. C. Carducci: None. M. Lucignani: None. D. Longo: None. E. Agolini: None. A. Novelli: None. M.V. Gonfiantini: None. M. Digilio: None. A. Bartuli: None.

\section{P11.61.A}

Mowat-Wilson syndrome: growth charts
I. Ivanovski ${ }^{1}$, O. Djuric ${ }^{2}$, S. Broccoli ${ }^{2}$, S. Caraffi $^{1}, P$.

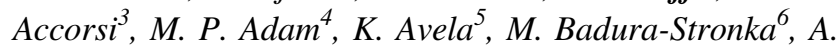 Bayat $^{7,8}$, J. Clayton-Smith ${ }^{9,10}$, D. Cordellit ${ }^{11}$, G. Cuturilo ${ }^{12}$, V. Di Pisa ${ }^{13}$, J. Dupont Garcia ${ }^{14}$, R. Gastaldi ${ }^{15}$, L. Giordano $^{3}$, A. Gualal ${ }^{16}$, C. Hoei-Hansen ${ }^{17}$, M. Inaba ${ }^{18}$, A. Iodice $^{19}$, J. Nielsen ${ }^{20}$, V. Kuburovic ${ }^{21}$, B. Lazalde-Medina ${ }^{22}$, B. Malbora ${ }^{23}$, S. Mizuno ${ }^{18}$, O. Moldovan ${ }^{14}$, R. Møller ${ }^{24,25}$, P. Muschke ${ }^{26}$, C. Pantaleoni ${ }^{27}$, C. Piscopo ${ }^{28}$, M. Poch- Olive $^{29}$, I. Prpic $^{30}$, M. Purificacion ${ }^{31}, F$. Raviglione $e^{32}, E$.

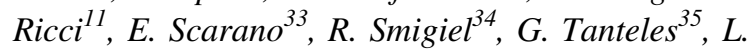




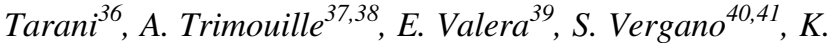
Writzl $^{42}$, B. Callewaert ${ }^{43}$, S. Savasta ${ }^{44}$, M. Street ${ }^{45}$, L. Iughetti $^{46,47}$, S. Bernasconi ${ }^{48}$, P. Giorgi Rossi ${ }^{49}$, L. Garavelli ${ }^{1}$

${ }^{1}$ Medical Genetics Unit AUSL IRCCS RE, Reggio Emilia, Italy, ${ }^{2}$ Epidemiology Unit, Azienda Unità Sanitaria Locale - IRCCS di Reggio Emilia, Italy, Reggio Emilia, Italy, ${ }^{3}$ Neuropsychiatric Department, Spedali Civili Brescia, Brescia, Italy, ${ }^{4}$ Division of Genetic Medicine, University of Washington School of Medicine, Seattle, WA, United States, ${ }^{5}$ Department of Clinical Genetics, Helsinki University Hospital, Helsinki, Finland, ${ }^{6}$ Department of Medical Genetics, Poznan University of Medical Sciences, Poznań, Poznan', Poland, 'Department of Pediatrics, University Hospital of Copenhagen/Hvidovre, Copenhagen, Denmark, ${ }^{8}$ Department of Clinical Genetics, Rigshospitalet, University Hospital of Copenhagen, Copenhagen, Denmark, ${ }^{9}$ Manchester Centre for Genomic Medicine, University of Manchester, St Mary's Hospital, Manchester, United Kingdom, ${ }^{10}$ Division of Evolution and Genomic Sciences, School of Biological Sciences, Faculty of Biology, Medicine and Health, University of Manchester, Manchester, United Kingdom, ${ }^{11}$ Child Neurology and Psychiatry Unit, Pediatric Department, S. Orsola Hospital in Bologna, University of Bologna, Bologna, Italy, ${ }^{12}$ Faculty of Medicine, University of Belgrade, Belgrade, Serbia, ${ }^{13}$ Manchester Centre for Genomic Medicine, St Mary's Hospital, Manchester University NHS Foundation Trust, Health Innovation Manchester, Manchester, United Kingdom, ${ }^{14}$ Serviço de Genética Médica, Departamento de Pediatria, Hospital de Santa Maria, Centro Hospitalar Lisboa Norte, Centro Académico de Medicina de Lisboa, Lisbon, Portugal, ${ }^{15}$ UOC Clinica Pediatrica, Istituto Giannina Gaslini, Genova, Italy, ${ }^{16}$ SOC Pediatria, Ospedale Castelli, Verbania, Italy, ${ }^{17}$ Department of Paediatrics, Copenhagen University Hospital, Rigshospitalet, Copenhagen, Denmark, ${ }^{18}$ Department of Pediatrics, Central Hospital, Aichi Human Service Center, Kasugai, Japan, ${ }^{19}$ Child Neurology and Psychiatry Unit, AUSLIRCCS, Reggio Emilia, Italy, ${ }^{20}$ Department of Pediatrics, Zealand University Hospital Roskilde, Roskilde, Denmark, ${ }^{21}$ Department of Cardiology, Mother and Child Health Care Institute, Belgrade, Serbia, ${ }^{22}$ Biomedical Research Unit, Mexican Institute of Social Security, Durango, Mexico, ${ }^{23}$ Department of Pediatric Hematology \& Oncology, Tepecik Training and Research Hospital, Izmir, Turkey, ${ }^{24}$ Danish Epilepsy Centre, Dianalund, Denmark, ${ }^{25}$ Institute for Regional Health Services, University of Southern Denmark, Odense, Denmark, ${ }^{26}$ Institute for Human Genetics, University Hospital Magdeburg, Magdeburg, Germany, ${ }^{27}$ Developmental Neurology Unit, Fondazione IRCCS Istituto Neurologico Carlo Besta, Milano, Italy, ${ }^{28}$ U.O.S.C. Medical Genetics, A.O.R.N. "A.
Cardarelli", Naples, Italy, ${ }^{29}$ Department of Pediatrics, $H$. San Pedro, La Rioja, Logrono, Spain, ${ }^{30}$ Department of Pediatrics-Child Neurology Service, University Hospital Rijeka, Medical Faculty, University of Rijeka, Rijeka, Croatia, ${ }^{31}$ Dismorphology and Reproductive Genetics, Neonatal Research Group, Health Research Institute Hospital La Fe, University \& Polytechnic Hospital La Fe, Valencia, Spain, ${ }^{32}$ Child Neuropsychiatry Unit, U.O.N.P.I. A ASST-Rhodense, Rho, Milano, Italy, ${ }^{33}$ Unit of Pediatrics, Department of Medical and Surgical Sciences, Policlinico St. Orsola-Malpighi Hospital, University of Bologna, Bologna, Italy, ${ }^{34}$ Department of Genetics, Wroclaw Medical University, Wroclaw, Wroclaw, Poland, ${ }^{35}$ Clinical Genetics Clinic, Cyprus Institute of Neurology and Genetics, Nicosia, Cyprus, ${ }^{36}$ Department of Pediatrics, University "La Sapienza”, Rome, Italy, ${ }^{37} \mathrm{CHU}$ de Bordeaux, Service de Génétique Médicale, Bordeaux, France, ${ }^{38}$ INSERM U1211, Univ. Bordeaux, Bordeaux, France, ${ }^{39}$ Department of Pediatrics, Ribeirão Preto Medical School, University of São Paulo, São Paulo, Brazil, ${ }^{40}$ Department of Pediatrics, Eastern Virginia Medical School, Norfolk, VA, United States, ${ }^{41}$ Division of Medical Genetics and Metabolism, Children's Hospital of The King's Daughters, Norfolk, VA, United States, ${ }^{42}$ Clinical Institute of Medical Genetics, University Medical Centre Ljubljana, Ljubljana, Slovenia, ${ }^{43}$ Ghent University, 26656, Center for Medical Genetics Ghent, Ghent, Belgium, ${ }^{44}$ Pediatric Clinic, IRCCS Policlinico "S. Matteo" Foundation, University of Pavia, Pavia, Italy, ${ }^{45}$ Division of Pediatric Endocrinology and Diabetology, Department of Mother and Child, Azienda Unità Sanitaria Locale - IRCCS di Reggio Emilia, Reggio Emilia, Italy, ${ }^{46}$ Post-graduate School of Pediatrics, University of Modena and Reggio Emilia, Modena, Italy, ${ }^{47}$ Department of Medical and Surgical Sciences of Mother, Children and Adults, Pediatric Unit, University of Modena and Reggio Emilia, Modena, Italy, ${ }^{48}$ Microbiome Research Hub, University of Parma, Parma, Italy, ${ }^{49}$ Epidemiology Unit, Azienda Unità Sanitaria Locale - IRCCS di Reggio Emilia, Reggio Emilia, Italy

Mowat-Wilson syndrome (MWS) is a genetic condition caused by heterozygous mutations or deletions of the ZEB2 gene, and characterized by typical face, intellectual disability, epilepsy, Hirschsprung disease, and congenital anomalies. The charts for males and females aged from 0 to 16 years were constructed using a total of 2,865 measurements of 99 MWS patients, of different ancestries. The data were collected through collaboration with the Italian MWS association (AIMW) and the MWS Foundation. To model the growth charts, the GAMLSS package for the R statistical program was used. Height, weight, body mass index (BMI) and occipito-frontal circumference (OFC) were compared to healthy children using $\mathrm{CDC}$ growth charts. In 
newborns, weight and length were distributed as in the general population, while head circumference was slighlty smaller. Weight and height distribution was slightly shifted to lower values than the general population until the age of 7 , when the difference increased. BMI distribution was almost similar to that of the non- affected children until the age of 7 , then values in the general population increase rapidly and in MWS children increase with a less steep slope. Microcephaly develops gradually in infancy, not all children were microcephalic and the head circumference was often between the $3^{\text {rd }}$ and the $10^{\text {th }}$ centile. These charts contribute to the understanding of the natural history of MWS and should assist pediatricians and other caregivers in providing optimal care to MWS individuals with problems related to physical growth. This is the first study on growth in patients with MWS.

I. Ivanovski: None. O. Djuric: None. S. Broccoli: None. S. Caraffi: None. P. Accorsi: None. M.P. Adam: None. K. Avela: None. M. Badura-Stronka: None. A. Bayat: None. J. Clayton-Smith: None. D. Cordelli: None. G. Cuturilo: None. V. Di Pisa: None. J. Dupont Garcia: None. R. Gastaldi: None. L. Giordano: None. A. Guala: None. C. Hoei-Hansen: None. M. Inaba: None. A. Iodice: None. J. Nielsen: None. V. Kuburovic: None. B. Lazalde-Medina: None. B. Malbora: None. S. Mizuno: None. O. Moldovan: None. R. Møller: None. P. Muschke: None. C. Pantaleoni: None. C. Piscopo: None. M. Poch-Olive: None. I. Prpic: None. M. Purificacion: None. F. Raviglione: None. E. Ricci: None. E. Scarano: None. R. Smigiel: None. G. Tanteles: None. L. Tarani: None. A. Trimouille: None. E. Valera: None. S. Vergano: None. K. Writzl: None. B. Callewaert: None. S. Savasta: None. M. Street: None. L. Iughetti: None. S. Bernasconi: None. P. Giorgi Rossi: None. L. Garavelli: None.

\section{P11.62.B}

A novel GREB1L c.705G $>$ T missense variant identified by whole-exome sequencing in a three-generation family with Müllerian and renal agenesis: a new candidate gene in Mayer-Rokitansky-Küster-Hauser

(MRKH) syndrome

M. K. Herlin ${ }^{1,2}$, A. T. Højland ${ }^{1,3}$, V. Q. Le $e^{l}$, A. Ernst ${ }^{l}, H$.

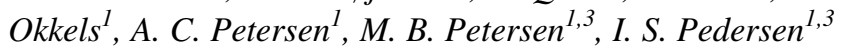

${ }^{1}$ Aalborg University Hospital, Aalborg, Denmark, ${ }^{2}$ Aarhus University Hospital, Aarhus, Denmark, ${ }^{3}$ Aalborg University, Aalborg, Denmark

Introduction: Mayer-Rokitansky-Küster-Hauser (MRKH) syndrome, also referred to as Müllerian aplasia, is a congenital anomaly characterised by uterovaginal agenesis in females with a normal 46,XX karyotype. Prevalence is 1 in
5,000 females. MRKH syndrome is often associated with extragenital malformations (type 2), predominantly kidney anomalies such as renal agenesis. The aetiology remains unknown, however rare reports of familial occurrence suggest a genetic cause following autosomal dominant inheritance with incomplete penetrance.

Material and Methods: We investigated a unique threegeneration family with two female cousins with type 2 MRKH syndrome including unilateral renal agenesis and two deceased male relatives with renal agenesis. We performed whole-exome sequencing (WES) of genomic DNA from eight family members.

Results: We identified a novel likely pathogenic (CADD = 33) c.705G $>\mathrm{T}$ missense variant in GREB1L - a gene recently identified as a novel cause of bilateral renal agenesis. Previous literature include several cases of bilateral renal agenesis in female foetuses also having uterus agenesis, which supports GREBIL as an important gene in both kidney and female genital tract development. The missense variant was confirmed in all affected family members, however, the variant was vertically transmitted through two unaffected women suggesting incomplete penetrance following a parent-origin-specific manner.

Conclusions: We suggest GREBIL as a novel and promising candidate gene in MRKH syndrome aetiology, especially in patients with type $2 \mathrm{MRKH}$ syndrome also having kidney abnormalities. The incomplete penetrance observed in our family could be explained by genomic imprinting and is currently subject to further investigation.

M.K. Herlin: None. A.T. Højland: None. V.Q. Le: None. A. Ernst: None. H. Okkels: None. A.C. Petersen: None. M.B. Petersen: None. I.S. Pedersen: None.

\section{P11.64.A}

Singleton clinical exom sequencing in fetuses with multiple anomalies

K. Gahle, M. Shoukier, C. Daumer-Haas, C. Bagowski, N. Hirschberger, S. Minderer, T. Schramm, K. P. Gloning

Prenatal Medicine Munich, Munich, Germany

Introduction: Identifying the genetic cause of fetal structural anomalies is a diagnostic challenge. We present here the results of singleton clinical exome sequencing (SCES) of 60 fetuses with complex malformations.

Materials and Methods: From January 2019 to January 2020 , in total 60 prenatal cases with complex structural anomalies, not fitting any specific syndrome, were investigated by targeted NGS of the coding regions of 5230 selected genes (Custom Constitutional Panel $17 \mathrm{Mb}$, Agilent, Santa Clara, CA, USA). Variant pathogenicity assessment by familial co-segregation analysis for was 
carried out when necessary. According to the ACMG-AMP guidelines (2015), variants were classified as classes 1 to 5 . Fetal karyotypes were normal.

Results: Likely pathogenic (class 4) and pathogenic (class 5) variants were identified in 14 (23\%) out of the 60 cases. The variants were found in 13 different genes: DHCR7 (2 cases), AFF4, ARSE, CNTNAP1, ERCC5, FAT4, KIF7, MAGED2, MYH3, NIPBL, RAF1, SHH and TSEN54. In addition variants of uncertain significance (class 3) were detected in 6 cases $(10 \%)$. No variants (class $3-5)$ were identified in a total of 40 fetuses (67\%). Our findings show a strong correlation between the detection rate of causative mutations and the complexity of the fetal phenotype.

Conclusions: SCES facilitates the identification of genetic causes with high diagnostic yield and should be considered for molecular genetic diagnosis in cases of complex fetal malformations. This unique challenge of exome-based prenatal diagnostics requires a team of prenatal and human genetic specialists.

K. Gahle: None. M. Shoukier: None. C. Daumer-Haas: None. C. Bagowski: None. N. Hirschberger: None. S. Minderer: None. T. Schramm: None. K.P. Gloning: None.

\section{P11.67.A}

Spectrum of Noonan Syndrome: Is genotype predicted by phenotype?

\section{E. Tsoutsou, N. Marinakis, K. Kosma, S. Psoni, J. Traeger- Synodinos}

National \& Kapodistrian University of Athens, Athens, Greece

Introduction: Noonan syndrome (NS) is an autosomal dominant disorder belonging to a group of developmental disorders called RASopathies with overlapping features and multiple causative genes. The variable clinical phenotype associated with NS and Noonan-like disorders is partly due to genetic heterogeneity, whereby mutations in many genes may result in different phenotypic expression. In this study we present the genotypic and phenotypic spectrum of NS illustrated by the analysis of 15 patients.

Materials and Methods: Fifteen patients with phenotypic characteristics of NS were referred to the Laboratory of Medical Genetics, following clinical evaluation, pre-test counselling and signed consent. Clinicians requested whole exome sequencing (WES, 19,000 genes). Library preparation used Sophia Genetics kits and Nextera Rapid Capture Exome (Illumina), run on a NextSeq-500 (Illumina). Bioinformatics analysis used SOPHiA DDM ${ }^{\circledR}$ and VarAFT. Variants were categorised according to ACMG guidelines; only "Pathogenic" or "Likely pathogenic" variants were reported.

Results: Heterozygous missense variants were identified in 10/15 patients, achieving a diagnostic yield of $67 \%$. Causative alterations included known pathogenic variants in the PTPN11 gene (5 variants), 2 in RIT1 gene, 1 in KRAS gene, 1 in SOS1 gene and 1 in SHOC2 gene.

Discussion: In most cases, the NS genotype cannot be predicted by phenotype. Even typical cases with characteristic morphological features, pulmonary valve stenosis and short stature, as well as atypical cases, were positive for pathogenic variants in any currently known Noonan causative genes. WES supports marked improvement in diagnostic-yield for NS, including unusual phenotypes and sometimes revealing unusual genotypes.

E. Tsoutsou: None. N. Marinakis: None. K. Kosma: None. S. Psoni: None. J. Traeger-Synodinos: None.

\section{P11.68.B}

MEK inhibitor treatment in a newborn with Noonan syndrome and severe obstructive hypertrophic cardiomyopathy

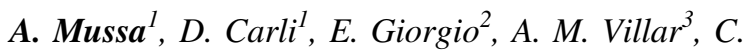 Carbonara $^{4}$, G. Paolo, G. Botta ${ }^{5}$, G. Andelfinger ${ }^{6}, M$.

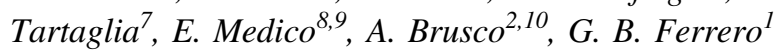

${ }^{1}$ Department of Public Health and Pediatric Sciences, University of Torino, Torino, Italy, ${ }^{2}$ Department of Medical Sciences, University of Torino, Torino, Italy, ${ }^{3}$ Pediatric Cardiology, Regina Margherita Children Hospital, Città della Salute e della Scienza, Torino, Italy, ${ }^{4}$ Neonatal Intensive Care Unit, S.Anna Hospital, Città della Salute e della Scienza, Torino, Italy, ${ }^{5}$ Department of Pathology, S. Anna Hospital, Città della Salute e della Scienza, Torino, Italy, ${ }^{6}$ Cardiovascular Genetics, CHU Sainte Justine, Department of Pediatrics, Université de Montréal, 3175, Chemin Côte Sainte Catherine,, Montréal, QC, Canada, ${ }^{7}$ Genetics and Rare Diseases Research Division, Ospedale Pediatrico Bambino Gesù, IRCCS, Roma, Italy, ${ }^{8}$ Laboratory of Oncogenomics, Candiolo Cancer Institute, FPOIRCCS, Candiolo, Italy, ${ }^{9}$ Department of Oncology, University of Torino, Torino, Italy, ${ }^{10}$ Medical Genetics Unit, Città della Salute e della Scienza, Torino, Italy

We report a preterm female newborn, with a gestation characterized by polyhydramnios and fetal hypertrophic cardiomyopathy (HCM), presenting facial dysmorphisms consistent with Noonan syndrome (NS), who required resuscitation and mechanical ventilation at birth. Echocardiography confirmed severe obstructive HCM and dysplastic pulmonary valve. At the $2^{\text {nd }}$ day of life, she developed a cerebral ventricular haemorrhage. A 
heterozygous de novo c.770C $>$ T p.Ser257Leu variant in RAF1 (NM_002880), typically associated with severe HCM and pulmonary hypertension, confirmed the clinical diagnosis. She developed severe congestive heart failure (CHF) requiring amine administration and mechanical ventilation. Given the near-terminal CHF, Trametinib - a selective inhibitor of MEK1/2 approved for the treatment of cancers with RAS/MAPK hypersignaling - was introduced $(0.022$ $\mathrm{mg} / \mathrm{kg} / \mathrm{day}$ ). There was prompt improvement in clinical condition, allowing inotropes and mechanical ventilation withdrawal; consistent with HCM stabilization, nt-pro-BNP decreased from 30,805 to $2,355 \mathrm{pg} / \mathrm{ml}$. During the next month, treatment was continued without side effects, allowing a progressive reduction of respiratory support. Cardiac ultrasound showed a tendency to HCM improvement but revealed the sudden appearance of pulmonary artery dilation. At 3 months of age, she underwent ventriculoperitoneal shunt device placement for post haemorrhagic hydrocephalus: after surgery she showed a rapidly worsening of $\mathrm{CHF}$ and died at $3 \frac{1}{2} 2$ months of age from untreatable CHF. Autopsy showed an obstructive HCM, severe dilation of pulmonary artery trunk and branches with complete disarray of both gross and fine pulmonary vascular anatomy. Whole RNA sequencing from white blood cells before and during treatment was performed to define transcriptional effects of MEK inhibition.

A. Mussa: None. D. Carli: None. E. Giorgio: None. A. M. Villar: None. C. Carbonara: None. G. Paolo: None. G. Botta: None. G. Andelfinger: None. M. Tartaglia: None. E. Medico: None. A. Brusco: None. G.B. Ferrero: None.

\section{P11.69.C}

Research on a pathogenesis of cognitive and neurofunctioning impairments in patients with Noonan syndrome. A role of RAS/MAPK signaling pathway gene disturbances

\section{N. Braun-Walicka ${ }^{1}$, A. Pluta ${ }^{2,3}$, T. Wolak ${ }^{2}, E . M a j^{2}, A$.

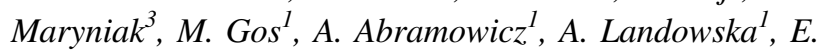 Obersztyn $^{l}, \mathrm{~J} . \mathrm{Bal}^{1}$}

${ }^{I}$ Department of Medical Genetics, Institute of Mother and Child, Warsaw, Poland, ${ }^{2}$ Bioimaging Research Center, World Hearing Center at the Institute of Physiology and Pathology of Hearing, Kajetany, Poland, ${ }^{3}$ Department of Child and Family Clinical Psychology of the University of Warsaw, Warsaw, Poland

Introduction: Noonan syndrome (NS) is one of the most common genetic conditions caused by mutations in particular genes of the RAS/MAPK signaling pathway. Patients with NS might have cognitive problems in language skills, memory, attention, executive functioning and decreased overall intelligence level. The resting-state functional connectivity (rsFC) analysis could be particularly useful for characterizing functional brain network differences in populations with intellectual disabilities.

Materials and Methods: According to our knowledge this is first study on functional connectivity in Noonan syndrome. 28 subjects with Noonan syndrome and 23 healthy took part in rs-fMRI data acquisition using a $3 \mathrm{~T}$ Siemens PRISMA scanner. All subsequent analysis of rsfMRI findings was performed in CONN-fMRI Functional Connectivity (FC) toolbox ver. 18b. We tested for group differences in between- and within-network rsFC connectivity for key seeds of salience and default mode network. In addition,, subjects underwent a complex assessment of cognitive abilities with the use of Stanford-Binet Intelligence Scales.

Results: Overall, both hypo- and hyper- connectivity within salience and DMN networks were revealed in subjects with Noonan syndrome. For example, patients exhibit increased connectivity between DMN network MPFC seed and superior frontal gyrus bilaterally and PCC and cerebellum. Simultaneously, they demonstrated decreased connectivity within precuneus which is a key region of DMN. In respect of IQ, subjects with Noonan syndrome showed decreased Verbal and Nonverbal IQ compared to healthy controls.

Conclusions: These findings imply that the functional architecture of rsFC might be altered in subjects with Noonan syndrome.

Supported from NCN research projects no. 2016/21/N/ HS6/02891

N. Braun-Walicka: None. A. Pluta: None. T. Wolak: None. E. Maj: None. A. Maryniak: None. M. Gos: None. A. Abramowicz: None. A. Landowska: None. E. Obersztyn: None. J. Bal: None.

\section{P11.70.A}

Genotype and phenotype in patients with Noonan syndrome caused by SOS 2 variants

C. Lissewski ${ }^{1}$, V. Chune ${ }^{2}$, F. Pantaleoni ${ }^{3}$, A. De Luca ${ }^{4}, Y$. Capri $^{2}$, J. Brinkmann ${ }^{1}$, F. Lepri ${ }^{3}$, P. Daniele ${ }^{4}$, E. Leenders ${ }^{5}$, L. Mazzanti ${ }^{6}$, K. Kutsche ${ }^{7}$, A. Küchler ${ }^{8}$, M. Gérard ${ }^{2}, J$. Amiel $^{2}, Y$. Vial ${ }^{2}$, I. van der Burgt ${ }^{5}$, E. Andreucci ${ }^{9}, G$. Mastromoro $^{10}$, M. C. Digilio ${ }^{3}$, H. Cavé $e^{2,11}$, M. Tartaglia ${ }^{3}$, M. Zenker ${ }^{I}$

${ }^{1}$ Institute of Human Genetics, University Hospital Magdeburg, Magdeburg, Germany, ${ }^{2}$ Département de Génétique, Hopital Robert Debré, Assistance Publique des Hopitaux de Paris (AP-HP), Paris, France, ${ }^{3}$ Genetics and Rare Diseases Research Division, Ospedale Pediatrico Bambino Gesù, 
IRCCS, Rome, Italy, ${ }^{4}$ Medical Genetics Unit, IRCCS Casa Sollievo della Sofferenza, San Giovanni Rotondo, Italy, ${ }^{5}$ Department of Human Genetics, Radboud University Medical Center, Nijmegen, Netherlands, ${ }^{6}$ Pediatric Rare Diseases Unit, Department of Pediatrics, S.Orsola-Malpighi University Hospital, University of Bologna, Bologna, Italy, ${ }^{7}$ Institute of Human Genetics, University Medical Center Hamburg-Eppendorf, Hamburg, Germany, ${ }^{8}$ Institut für Humangenetik, Universitätsklinikum Essen, Universität Duisburg-Essen, Essen, Germany, ${ }^{9}$ Medical Genetics Unit, Meyer Children's University Hospital, Florence, Italy, ${ }^{10}$ Department of Experimental Medicine, Sapienza University, Policlinico Umberto I Hospital, Rome, Italy, ${ }^{11}$ Inserm U1131, Institut de Recherche Saint-Louis, Université de Paris, Paris, France

Introduction: RASopathies are caused by pathogenic variants in genes encoding members or modulators of the RAS/ MAPK signalling pathway. Noonan syndrome is the most common entity among this group of disorders and is characterized by heart defects, short stature, variable developmental delay and typical facial features. Pathogenic variants in $S O S 2$, encoding a guanine nucleotide exchange factor for RAS, have recently been identified in patients with Noonan syndrome. The number of published cases with pathogenic SOS2 variants is still limited and little is known about genotype-phenotype correlation.

Materials and Methods: We collected clinical and genotype data from 17 previously unpublished individuals carrying a disease-causing SOS2 variant. Results: Most individuals had one of the previously reported pathogenic variants; only three had novel changes at the established mutation hotspots, which were classified as likely pathogenic. The phenotype of the 17 patients fits well into the spectrum of Noonan syndrome and is most similar to the phenotype observed in patients with SOS1-related Noonan syndrome, with ectodermal anomalies as common and short stature and learning disabilities as infrequent findings. The spectrum of heart defects in SOS2-related Noonan syndrome was consistent with the known spectrum of cardiac anomalies in RASopathies, but no specific heart defect was particularly predominating. Notably, lymphatic anomalies were extraordinarily frequent in our cohort, affecting more than half of the patients.

Conclusions: SOS2-related Noonan syndrome is phenotypically similar to SOS1-related Noonan syndrome and associated with a particularly high risk of lymphatic complications that may have a significant impact on morbidity and quality of life.

C. Lissewski: None. V. Chune: None. F. Pantaleoni: None. A. De Luca: None. Y. Capri: None. J. Brinkmann: None. F. Lepri: None. P. Daniele: None. E. Leenders: None. L. Mazzanti: None. K. Kutsche: None. A. Küchler:
None. M. Gérard: None. J. Amiel: None. Y. Vial: None. I. van der Burgt: None. E. Andreucci: None. G. Mastromoro: None. M.C. Digilio: None. H. Cavé: None. M. Tartaglia: None. M. Zenker: None.

\section{P11.71.B}

Investigating the genetic intersection of orofacial clefting and normal facial variation

K. Indencleef ${ }^{l}, H$. Hoskens $^{l}$, M. Lee ${ }^{2}, J . D$. White $^{3}$, R. J.

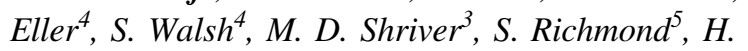
Peeters $^{1}$, M. L. Marazita ${ }^{2}$, J. R. Shaffer ${ }^{2}$, S. M. Weinberg ${ }^{2}$, G. Hens ${ }^{I}$, P. Claes ${ }^{I}$

${ }^{1}$ KULeuven, Leuven, Belgium, ${ }^{2}$ University of Pittsburgh, Pittsburgh, PA, United States, ${ }^{3}$ Pennsylvania State University, State College, PA, United States, ${ }^{4}$ Indiana University Purdue University Indianapolis, Indianapolis, IN, United States, ${ }^{5}$ Cardiff University, Cardiff, United Kingdom

Orofacial clefting is the most common craniofacial malformation in humans. Previous research has demonstrated that unaffected relatives of patients with non-syndromic cleft lip with/without cleft palate (NSCL/P) show distinctive facial features, which can be considered an endophenotype. The presence of this facial endophenotype is potentially an expression of underlying genetic susceptibility to NSCL/P. To investigate this, we applied partial least squares regression (PLSR) to define a set of endophenotypic traits in a dataset of 3D facial images from 264 unaffected parents of patients with NSCL/P and 3,171 controls. The PLSR provides a $p$-value and direction of effect (or trait) for each endophenotype. We observed nominally significant $(\mathrm{p}<$ 0.05 ) differences between parents and controls involving 59 endophenotypic traits. Next, we quantified these discovered endophenotypic traits in another dataset of 8,246 unaffected European individuals and then subjected these traits to GWAS. We identified 29 independent lead SNPs that were associated with at least one of the tested endophenotypic risk phenotypes at the genome-wide level $(\mathrm{p}<5 \mathrm{e}-8)$. Of the 29 lead SNPs, 22 are in the proximity of SNPs previously associated with normal facial variation and 15 are near genes that show evidence of roles in orofacial clefting. This study supports the existence of an overlap between the genetic pathways involved in normal facial development and orofacial clefting.

Grants: National Institutes of Health (1-RO1DE027023), the Research Fund KU Leuven (BOF-C1, C14/15/081) and the Research Program of the The Research Foundation-Flanders (Belgium) (FWO, G078518N) 
K. Indencleef: None. H. Hoskens: None. M. Lee: None. J.D. White: None. R.J. Eller: None. S. Walsh: None. M. D. Shriver: None. S. Richmond: None. H. Peeters: None. M.L. Marazita: None. J.R. Shaffer: None. S.M. Weinberg: None. G. Hens: None. P. Claes: None.

\section{P11.76.A}

Further definition of the proximal 19p13.3 microdeletion/microduplication syndrome and implication of PIAS4 as the major contributor

P. Lapunzina ${ }^{l}$, J. Tenorio ${ }^{l}$, J. Nevado ${ }^{l}$, A. GonzálezMeneses $^{2}$, P. Arias ${ }^{l}$, I. Dapía ${ }^{l}, C$. Venegas-Vega ${ }^{3}, M$. Calvente $^{4}$, A. Hernández ${ }^{1}$, L. Landera ${ }^{5}$, S. Ramos-Sorigué ${ }^{1}$, T. Consortium ${ }^{1}$, J. Cigudosa ${ }^{4}$, L. Pérez-Jurado ${ }^{6}$

${ }^{1}$ Medical and Molecular Genetics Institute (INGEMM), Madrid, Spain, ${ }^{2}$ Dysmorphology and Metabolism unit, Hospital Universitario Virgen del Rocío, Sevilla, Spain, ${ }^{3}$ Unidad de Genética, Hospital General de México, México, Spain, ${ }^{4}$ NIMGENETICS, Madrid, Spain, ${ }^{5}$ Congenital malformations laboratory, Federal University of Rio de Janeiro, Madrid, Spain, ${ }^{6}$ Women's and Children's Hospital, South Australian Health and Medical Research Institute (SAHMRI), Adelaide, Australia

The proximal 19p13.3 microdeletion/microduplication (prox19p13.3del/dup) syndrome is a recently described disorder with common clinical features including developmental delay, intellectual disability, speech delay, facial dysmorphic features with ear defects, anomalies of the hands and feet, umbilical hernia and hypotonia. While deletions are associated with macrocephaly, patients with duplications have microcephaly. The SRO in multiple patients $(113.5 \mathrm{~Kb})$ included three genes and one pseudogene, with a suggested major role of PIAS4 in determination of the phenotype and head size in these patients. Here we refine the prox 19p13.3del/dup with four additional patients; two with microdeletions, one with microduplication and for the first time as far as we know one family with singlenucleotide nonsense variant in PIAS4:NM_015897.4: exon9:c.1063C>T:p.Gln355* and concordant phenotype. The variant was detected by NGS and is not present in her healthy parents. PIAS4 is an E3 ubiquitin ligase encoded by PIAS4 and acts as a small ubiquitin-like modifier (SUMO) ligase that regulates the interaction between E2 enzymes such as UBE2I and the substrateThe patient with the PIAS4 LoF variant displayed a phenotype quite similar to deletion patients -including the macrocephaly and many other core features of the syndrome. Patient's SNV was inherited from her mother who is similarly affected. Thus, our data indicate that PIAS4 is a major contributor to the proximal $19 \mathrm{p} 13.3 \mathrm{del} / \mathrm{dup}$ syndrome phenotype. In summary, we report the first patient with a pathogenic variant in PIAS4and three additional rearrangements at the proximal 19p13.3 locus. These observations add further evidence about the molecular basis of this microdeletion/microduplication syndrome.

Grants: FIS PI15-01481

P. Lapunzina: None. J. Tenorio: None. J. Nevado: None. A. González-Meneses: None. P. Arias: None. I. Dapía: None. C. Venegas-Vega: None. M. Calvente: None. A. Hernández: None. L. Landera: None. S. Ramos-Sorigué: None. T. Consortium: None. J. Cigudosa: None. L. Pérez-Jurado: None.

\section{P11.77.B}

Expanding the spectrum of PPP2R3C-related syndromic phenotypes to $\mathrm{XX}$ gonadal dysgenesis without ocular or muscular involvement

U. Altunoglu ${ }^{l}$, E. Börklü-Yücel ${ }^{l}$, A. Shukla ${ }^{2}$, N. Escande-

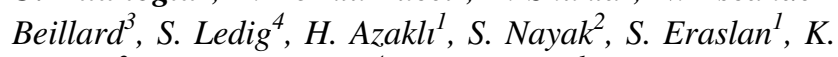
Girisha $^{2}$, I. Kennerknecht ${ }^{4}$, H. Kayserili ${ }^{1}$

${ }^{1}$ Koc University School of Medicine, Medical Genetics Department, Istanbul, Turkey, ${ }^{2}$ Department of Medical Genetics, Kasturba Medical College, Manipal Academy of Higher Education, Manipal, India, ${ }^{3}$ Institute of Medical Biology, A*STAR, Singapore, Singapore, ${ }^{4}$ Institut für Humangenetik, Westfaelische Wilhelms - Universitaet Muenster, Muenster, Germany

Homozygous mutations in $P P P 2 R 3 C$ have been reported to cause a syndromic 46,XY complete gonadal dysgenesis phenotype with extragonadal manifestations (Gonadal dysgenesis, dysmorphic facies, retinal dystrophy and myopathy, GDRM, MIM\# 615902) in patients from four unrelated families, whereas heterozygous mutations have been linked to reduced fertility with teratozoospermia (SPGF36, MIM\# 618420) in male carriers. We present eight patients from four unrelated families of Turkish and Indian descent with three different germline homozygous PPP2R3C mutations including a novel in-frame duplication (c.639_647dupTTTCTACTC, p.Ser213_Tyr215dup). All patients exhibited recognizable facial dysmorphisms with broad eyebrows, underdeveloped alae nasi, thin vermilion of the lips and low-set ears, allowing for gestalt diagnosis. 46,XX patients had hypergonadotropic hypogonadism and nonvisualized gonads, and were diagnosed due to primary amenorrhea along with absence of secondary sexual characteristics and/or unique facial gestalt. 46,XY affected individuals displayed a spectrum of genital phenotype from ambiguous to complete female, with Müllerian remnants confirmed by imaging and/or histopathology. Four of eight patients were evaluated by fundoscopy, optical coherence 
tomography, and electromyogram, and had no evidence of retinal dystrophy or a myopathy. We expand the spectrum of PPP2R3C-related syndromic phenotypes to XX and incomplete XY gonadal dysgenesis without ocular involvement or myopathy. We speculate a possible role for $P P P 2 R 3 C$ in the early signalling cascade controlling sex determination in humans, since it causes gonadal dysgenesis in both sexes.

U. Altunoglu: None. E. Börklü-Yücel: None. A. Shukla: None. N. Escande-Beillard: None. S. Ledig: None. H. Azaklı: None. S. Nayak: None. S. Eraslan: None. K. Girisha: None. I. Kennerknecht: None. H. Kayserili: None.

\section{P11.79.A}

The diverse pleiotropic effects of spliceosomal protein PUF60: a case series of Verheij syndrome

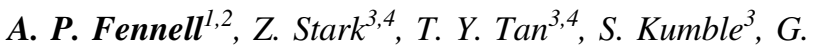
Poke $^{5}$, C. McKeown ${ }^{5}$, M. F. Hunter ${ }^{1,2}$

${ }^{1}$ Monash Genetics, Melbourne, Australia, ${ }^{2}$ Monash University, Melbourne, Australia, ${ }^{3}$ Victorian Clinical Genetics Services, Melbourne, Australia, ${ }^{4}$ The University of Melbourne, Melbourne, Australia, ${ }^{5}$ Genetic Health Service New Zealand, Wellington, New Zealand

Introduction: Verheij syndrome (VRJS) is a neurodevelopmental and multiple congenital anomaly syndrome caused by heterozygous SNVs in PUF60 or interstitial deletions of the 8q24.3 region, where PUF60 is located. PUF60 encodes a splicing factor which facilitates assembly of the spliceosome and preferentially activates specific exons enriched for uridine upstream of their 3' splice sites. We undertook a literature review of VRJS caused by PUF60 SNVs to ascertain the known mutational and phenotypic spectrum. This identified 25 individuals exhibiting marked phenotypic heterogeneity.

Case reports: We report four additional patients, including the first described patients of Khmer and Indian ethnicities, with previously unreported heterozygous PUF60 variants (3 confirmed de novo, 1 undetermined) identified through clinical exome sequencing. These included two novel frameshift variants (p.(Pro385Leufs*12) and p.(Gly268Alafs*18)), a novel missense change (p. (Arg146Cys)) and one inframe deletion variant (p. (Ala150_Phe152del)). All four patients had global developmental delay (GDD) and underwent thorough phenotypic assessment identifying variable dysmorphism, growth delay, and congenital anomalies. Features unique to our Khmer and Indian patients were talon cusps and proximal insertion of the right hallux, and craniosynostosis, respectively.
Discussion: In combining this detailed phenotypic data with the previously reported, and variably phenotyped, individuals we further refine the known frequencies of features associated with VRJS. These include GDD (100\%), short stature (68\%), limb anomalies (64\%), axial skeletal anomalies $(57 \%)$, cardiac anomalies $(62 \%)$, auditory impairment $(50 \%)$, colobomata $(31 \%)$ and other ocular anomalies (61\%). This case series, incorporating two patients from previously unreported ethnicities, further delineates the mutational spectrum and pleiotropy of PUF60 variants.

A.P. Fennell: None. Z. Stark: None. T.Y. Tan: None. S. Kumble: None. G. Poke: None. C. McKeown: None. M. F. Hunter: None.

\section{P11.80.B}

\section{Perinatal mortality in Rasopathies}

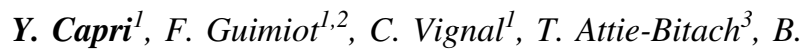
Bessiere $^{3}$, J. Martinovic ${ }^{4}$, P. Letard ${ }^{5}$, L. Loeuillet ${ }^{6}, S$. Khung-Savatovsky', M. Bonniere ${ }^{3}$, N. Pouvreaul, C.

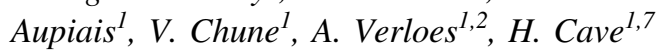

${ }^{1}$ CHU Robert Debre, Paris, France, ${ }^{2}$ INSERM UMR 1141, Paris, France, ${ }^{3} \mathrm{CHU}$ Necker, Paris, France, ${ }^{4} \mathrm{CHU}$ Antoine Béclère, Paris, France, ${ }^{5}$ Hôpital Jean Verdier, Paris, France, ${ }^{6} \mathrm{CHU}$ Cochin, Paris, France, ${ }^{7}$ INSERM UMR 1131, Paris, France

Rasopathies are a group of multiple congenital anomalies syndromes clinically and biologically similar including Noonan, Cardio-Facio-Cutaneous and Costello syndromes. Rasopathies occur in a dominant or sporadic manner and are caused by abnormality of ubiquitous proteins belonging to the cell signalling pathway RAS-MAPKinase. Prenatal symptoms including abnormal nuchal translucency, serosis effusion or polyhydramnios lead to suspect Rasopathies during pregnancy but little is known about the prognosis of foetuses with prenatal symptoms. By studying the clinical presentation and the distribution of molecular anomalies in affected subjects who died in perinatal period, we aimed to find prognostic elements which could help in counseling. Among more than 1500 positives patients for a mutation in one of the 18 known genes involved in Rasopathies from the French cohort, we selected subjects who died in perinatale period (during pregnancy or within the 28 days after birth). We collected 65 subjects with severe clinical presentation leading to pregnancy termination (34), stillbirth (15) or death within the 28 days after birth (16). Mutations occurred de novo in 43, was inherited in 14. PTPN11 was involved in 33 cases $(50,7 \%), R A F 1$ in $12(18,5 \%), R I T 1$ in $10(15,4 \%)$, SOS1 in $3(4,6 \%), B R A F$ in 3 , and HRAS, NRAS, LZTRI and NF1 in 1 for each gene. If PTPN11 
remains the major gene, RITI and RAFI are more represented in this lethal population compared to the post natal distribution of Rasopathie genes. Whereas SOS1, which is usually the $2^{\text {nd }}$ gene in Noonan syndrome, is underrepresented.

Y. Capri: B. Research Grant (principal investigator, collaborator or consultant and pending grants as well as grants already received); Modest; Agence de la Biomédecine. F. Guimiot: None. C. Vignal: None. T. Attie-Bitach: None. B. Bessiere: None. J. Martinovic: None. P. Letard: None. L. Loeuillet: None. S. Khung-Savatovsky: None. M. Bonniere: None. N. Pouvreau: None. C. Aupiais: None. V. Chune: None. A. Verloes: None. H. Cave: None.

\section{P11.81.C}

The role of $A 2 M L 1$ variants in Noonan syndrome remains unverified

\section{J. Brinkmann ${ }^{1}$, C. Lissewski ${ }^{1}, V$. Pinna $^{2}, Y$. Vial $^{3,4}, F$.

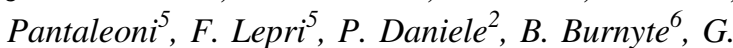 Cuturilo $^{7,8}$, C. Fauth ${ }^{9}$, D. Kotzot ${ }^{9}$, E. Y. Güleç ${ }^{10}$, V. Iotova $^{11}$, G. Forzano ${ }^{12}$, D. Schanze ${ }^{1}, F$. Ramond ${ }^{13}, M$. Havlovicová $^{14}$, G. E. Utine ${ }^{15}$, P. O. Simsek-Kiper ${ }^{15}$, A.

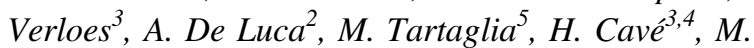 Zenker $^{l}$}

${ }^{1}$ Institute of Human Genetics, University Hospital Magdeburg, Magdeburg, Germany, ${ }^{2}$ Medical Genetics Section, IRCCS Fondazione Casa Sollievo della Sofferenza, San Giovanni Rotondo, Italy, ${ }^{3}$ Department of Genetics, Hopital Robert Debré, Assistance Publique des Hopitaux de Paris (AP-HP), Paris, France, ${ }^{4}$ Inserm U1131, Institut de Recherche Saint-Louis, Université de Paris, Paris, France, ${ }^{5}$ Genetics and Rare Diseases Research Division, Ospedale Pediatrico Bambino Gesù, Rome, Italy, ${ }^{6}$ Institute of Biomedical Sciences, Faculty of Medicine, Vilnius University, Vilnius, Lithuania, ${ }^{7}$ Faculty of Medicine, University of Belgrade, Belgrade, Serbia, ${ }^{8}$ University Children's Hospital, Belgrade, Serbia, ${ }^{9}$ Institute of Human Genetics, Medizinische Universität Innsbruck, Innsbruck, Austria, ${ }^{10}$ Medical Genetics Section, Health Sciences University, Kanuni Sultan Suleyman Training and Research Hospital, Istanbul, Turkey, ${ }^{11}$ Department of Pediatrics, Varna Medical University, Varna, Bulgaria, ${ }^{12}$ Department of Biomedical, Experimental and Clinical Sciences, University of Florence, Florence, Italy, ${ }^{13}$ Department of Genetics, Hôpital Nord, Saint Etienne University Hospital, SaintEtienne, France, ${ }^{14}$ Department of Biology and Medical Genetics, Charles University 2nd Faculty of Medicine and University Hospital Motol, Prague, Czech Republic, ${ }^{15}$ Department of Pediatric Genetics, Hacettepe University, Ankara, Turkey
Introduction: The RASopathies are a group of clinically and genetically heterogeneous developmental disorders caused by dysregulation of the RAS/MAPK signalling pathway. Mutations in several components and regulators of this pathway have been identified as pathogenetic cause. In 2015, missense variants in $A 2 M L 1$ were reported in three unrelated families with clinically suspected Noonan syndrome (NS). Two subsequent publications reported three additional A2ML1 variants in NS patients. However, an association of $A 2 M L 1$ variants with NS has not been replicated since. Herein, we report our experience with A2ML1 mutational screening in a large cohort of patients with clinically suspected NS or another RASopathy.

Materials and Methods: Individuals included in this study belong to a cohort of 1029 patients with clinically suspected NS/RASopathy that underwent multigene panel sequencing of RASopathy-related genes including A2ML1.

Results: We report on 15 individuals carrying rare variants in $A 2 M L 1$, including previously published variants. The respective $A 2 M L 1$ variant was inherited in all cases where parental DNA was available. Seven individuals had an alternate disease-causing genetic aberration. In cases with A2ML1 mutations only, careful re-analysis of the phenotype indicated that clinical features were insufficient to meet criteria for definite clinical diagnosis of NS in most. None of the A2ML1 variants evaluated herein reach a classification above class 3 (VUS) with strict application of published classification criteria.

Conclusions: Our findings underscore that current evidence is insufficient to support a causal relation of variants in A2ML1 and NS. Inclusion of A2ML1 mutation screening in diagnostic RASopathy testing cannot be recommended.

J. Brinkmann: None. C. Lissewski: None. V. Pinna: None. Y. Vial: None. F. Pantaleoni: None. F. Lepri: None. P. Daniele: None. B. Burnyte: None. G. Cuturilo: None. C. Fauth: None. D. Kotzot: None. E.Y. Güleç: None. V. Iotova: None. G. Forzano: None. D. Schanze: None. F. Ramond: None. M. Havlovicová: None. G.E. Utine: None. P.O. Simsek-Kiper: None. A. Verloes: None. A. De Luca: None. M. Tartaglia: None. H. Cavé: None. M. Zenker: None.

\section{P11.82.A}

Characterization of a unique karyotype resulting from a complex rearrangement involving the chromosomes 3 and 4 using chromosome analysis, FISH and arrayCGH in a girl with facial dysmorphism 


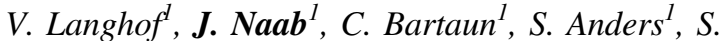
Demuth $^{2}$, T. Liehr ${ }^{3}$, M. Klaus ${ }^{1}$

${ }^{1}$ MVZ Mitteldeutscher Praxisverbund Humangenetik GmbH, Dresden, Germany, ${ }^{2} M V Z$ Mitteldeutscher Praxisverbund Humangenetik GmbH, Erfurt, Germany, ${ }^{3}$ Universitätsklinikum Jena, Institut für Humangenetik, Jena, Germany

Genomic rearrangements can result in losses, gains, translocations and insertions of DNA fragments thereby modifying genome architecture, and potentially having clinical consequences. We report on a four-month-old girl with facial dysmorphism. Physical and motoric development was normal. Karyotype analysis presented mosaicism, showing one partially deleted chromosome 4 in all analysed cells and an additional ring chromosome of unknown origin in only part of the cells. Array CGH detected different gains 4p15.33p15.31, 4p15.1p12 and 4p12q12 and one deletion in $4 q 13.3 q 22.1$. Two gains showed mosaicism of $76 \%$. The deletion was detected in a mosaic level of $35 \%$. Therefore, we suggested that the additional ring chromosome consisted of the detected aberrations showing mosaicism. To prove this presumption, we performed FISH. The results confirmed our hypothesis concerning the ring chromosome and surprisingly identified an insertion of material of chromosome 4 in the short arm of one chromosome 3 in all of the analysed metaphase spreads. In relation to the array result, this insertion might represent the gain of $4 \mathrm{p} 15.33 \mathrm{p} 15.31$, detected without mosaicism. As well as for the gains as for the deletion, different severe clinical effects were described including developmental delay, behavioural problems, epileptic seizures and facial dysmorphisms. The clinical features could differ due to size of the copy number changes as well as to mosaic level and distribution in different tissues. Our results suggest that the mosaicism of the $4 \mathrm{q}$ deletion could explain the mild clinical features of the little girl. Analysis of the parents showed that the chromosome abnormalities occurred de novo.

V. Langhof: None. J. Naab: None. C. Bartaun: None. S. Anders: None. S. Demuth: None. T. Liehr: None. M. Klaus: None.

\section{P11.85.A}

Fetal phenotype of Rubinstein-Taybi syndrome caused by $C R E B B P$ mutations

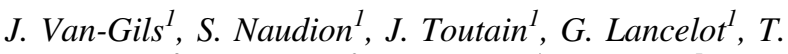
Attié-Bitach ${ }^{2}$, S. Blesson ${ }^{3}$, B. Demeer ${ }^{4}$, B. Doray ${ }^{5}$, M.
Gonzales $^{6}$, J. Martinovic ${ }^{2}$, S. Whalen $^{7}$, B. Arveiler ${ }^{l}, P$. Fergelot $^{l}$, D. LACOMBE ${ }^{l}$

${ }^{1}$ Medical Genetics, BORDEAUX, France, ${ }^{2}$ Medical Genetics, Paris Necker, France, ${ }^{3}$ Medical Genetics, Tours, France, ${ }^{4}$ Medical Genetics, Amiens, France, ${ }^{5}$ Medical Genetics, Saint Denis, La Réunion, France, ${ }^{6}$ Medical Genetics, Paris Trousseau, France, ${ }^{7}$ Medical Genetics, Paris Pitié, France

Rubinstein-Taybi syndrome (RSTS; OMIM 180849) is an autosomal dominant developmental disorder characterized by facial dysmorphism, broad thumbs and halluces associated with intellectual disability. RSTS is caused by alterations in CREBBP (about 60\%) and EP300 genes (8\%). RSTS is often diagnosed at birth or during early childhood but generally not suspected during antenatal period. We report nine cases of well-documented fetal RSTS. Two cases were examined after death in utero at 18 and 35 weeks of gestation and seven cases after identification of ultrasound abnormalities and termination of pregnancy. On prenatal sonography, a large gallbladder was detected in two cases, and brain malformations were noted in four cases, especially cerebellar hypoplasia. However, the diagnosis of RSTS has not been suggested during pregnancy. Fetal autopsy showed that all fetuses had large thumbs and/ or suggestive facial dysmorphism. A CREBBP gene anomaly was identified in all cases. Alterations were similar to those found in typical RSTS children. This report will contribute to a better knowledge of the fetal phenotype to consider the hypothesis of RSTS during pregnancy. Genotyping allows reassuring genetic counseling.

J. Van-Gils: None. S. Naudion: None. J. Toutain: None. G. Lancelot: None. T. Attié-Bitach: None. S. Blesson: None. B. Demeer: None. B. Doray: None. M. Gonzales: None. J. Martinovic: None. S. Whalen: None. B. Arveiler: None. P. Fergelot: None. D. Lacombe: None.

\section{P11.87.C}

SETBP1 gene variants: from Schinzel-Giedion syndrome to mild neurodevelopmental disorder, a challenge for the clinician.

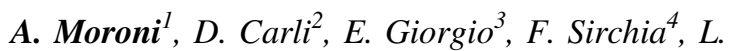
Pavinato $^{3}$, S. Cardaropoli ${ }^{2}$, P. Di Martino, ${ }^{5,6}$, A. Mussa ${ }^{2}, T$. Pipucci $^{6,5}$, S. De Rubeis ${ }^{7}$, A. Brusco ${ }^{1,3}$, G. B. Ferrero $^{2}$

${ }^{I}$ Medical Genetics Unit, Città della Salute e della Scienza University Hospital, Turin, Italy, ${ }^{2}$ Division of Pediatrics, Dep. of Public Health and Pediatric Sciences, University of Turin, Turin, Italy, ${ }^{3}$ Department of Medical Sciences, University of Turin, Turin, Italy, ${ }^{4}$ Institute for Maternal and Child Health - IRCCS "Burlo Garofolo", Trieste, Italy, 
${ }^{5}$ Medical Genetics Unit, Polyclinic Sant'Orsola-Malpighi University Hospital, Bologna, Italy, ${ }^{6}$ Department of Medical and Surgical Sciences, University of Bologna, Bologna, Italy, ${ }^{7}$ Seaver Autism Center for Research and Treatment, Icahn School of Medicine at Mount Sinai, New York, NY, United States

Introduction: Genotype-phenotype correlations for SETBP1 gene are extremely complex and clinically relevant. Nonsense variants and whole gene deletions cause intellectual disability with marked speech delay, seizures and facial dysmorphisms. Recurrent missense variants at codons 868-871, forming the critical consensus sequence of the degradation signal, have been associated with classic Schinzel-Giedion syndrome (SGS; MIM269150) which is characterized by severe developmental delay, facial dysmorphisms, epilepsy, hydronephrosis, cardiac and brain anomalies and progressive neurodegeneration, malignancies and frequent early death. Patients with missense mutation near the degradation sequence (codons 862, 867, and 873) exhibit a milder SGS phenotype. Here we report two patients with novel de novo missense heterozygous SETBP1 variants, presenting with mild and atypical phenotypes.

Case report: Patient 1 is a 3 years old female with developmental delay, stunted growth, typical SGS facial dysmorphisms, neutropenia, and joint hypermobility, carrying a p.Ser854Tyr variant. Patient 2 is a 15 years old male, with speech delay and borderline intellectual disability with autistic traits, carrying the p.Pro995Leu variant, not associated with other sign of SGS.

Conclusions: Phenotype severity in patients carrying SETBP1 missense variants depends on localization and biological effect of the variants, providing a likely explanation to the mild phenotypes we have observed in these patients. Clinical evaluation and prognosis definition in SETBP1 patients are challenging. Further functional and genotype-phenotype correlation studies are essential to establish their natural history and management indications.

A. Moroni: None. D. Carli: None. E. Giorgio: None. F. Sirchia: None. L. Pavinato: None. S. Cardaropoli: None. P. Di Martino: None. A. Mussa: None. T. Pipucci: None. S. De Rubeis: None. A. Brusco: None. G.B. Ferrero: None.

\section{P11.88.A}

Maternal uniparental disomy of chromosome 20 (UPD (20)mat) and advanced maternal age in Silver Russell Syndrome: identification of three new cases.

S. Russo ${ }^{l}$, D. Minervino ${ }^{l}$, P. Tannorella ${ }^{l}$, S. Guzzetti ${ }^{l}$, L. Calzari $^{\prime}$, G. Patti ${ }^{2}$, M. Maghnie ${ }^{2}$, A. E. M. Allegri ${ }^{2}, D$.

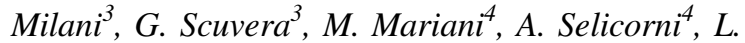
Larizza $^{l}$

${ }^{1}$ Istituto Auxologico Italiano, Cusano Milanino (MI), Italy, ${ }^{2}$ UOC Clinica Pediatrica, Istituto Giannina Gaslini, Genova, Italy, ${ }^{3}$ Unità di Pediatria ad alta Intensità di Cura, Fondazione IRCSS Ca' Granda Ospedale Maggiore Policlinico, MILANO, Italy, ${ }^{4}$ UOC Pediatria, ASST Lariana, Como, Italy

Silver Russell Syndrome (SRS, MIM \#180860) is a rare (1:30,000-1:100,000) growth retardation disorder, whose clinical diagnosis is based on the Netchine-Harbison clinical scoring system (NH-CSS), combining six features: pre- and postnatal growth failure, relative macrocephaly, prominent forehead, body asymmetry and feeding difficulties. The molecular mechanisms underlying SRS are highly heterogeneous, including (epi)genetic deregulation at multiple loci. Over $50 \%$ of SRS show loss of methylation (LOM) at the paternal H19/IGF2:IG-DMR (chr11p15.5), about 10\% maternal uniparental disomy of chromosome 7 (UPD(7) mat), while pathogenic CNVs, $14 \mathrm{q} 32$ abnormalities, rare CDKN1C and IGF2 mutations, UPD(16)mat and UPD(20) mat have been reported in a minority of cases. Our study was aimed to evaluate the frequency of the rarest known molecular defects in a cohort of SRS patients with a NH$\mathrm{CSS} \geq 3$. Within a selected cohort of $176 \mathrm{SRS}, 55$ patients had LoM at H19/IGF2:IG-DMR (31.3\%) and 17 cases showed UPD(7)mat (9.7\%). Further investigations on the 104 remaining patients identified 3 cases sharing UPD20mat $(1,7 \%)$, the most frequent out of the rare alterations. The patients, with a clinical score $>3$, shared prominent forehead, feeding difficulties and postnatal growth delay, while one case had relative macrocephaly, and another body asymmetry. Interestingly maternal age in these three UPD(20)mat cases is $>40$ years and literature survey pointed out on a total of 16 cases a mean of 38 years, likely due to its origin by trisomy rescue. Our study identifies UPD(20)mat as a subclass among patients with $\mathrm{NH}-$ CSS $\geq 3$ and elevated maternal age suggesting to introduce this pathomechanism in the SRS diagnostic flow-chart.

S. Russo: None. D. Minervino: None. P. Tannorella: None. S. Guzzetti: None. L. Calzari: None. G. Patti: None. M. Maghnie: None. A.E.M. Allegri: None. D. Milani: None. G. Scuvera: None. M. Mariani: None. A. Selicorni: None. L. Larizza: None.

\section{P11.89.B}

First record of homozygous loss of function variants in the $S L F 2$ gene in patients with chromosome instability syndromes 
N. Nahavandi ${ }^{l}$, Z. Yüksel ${ }^{l}$, M. Drasdo ${ }^{l}$, A. Kron ${ }^{l}$, P. Vogt ${ }^{l}$, A. Jonasson ${ }^{l}$, S. Ahmed Seth ${ }^{2}$, M. Tareg Mustafa ${ }^{2}, K$. Danish $^{2}$

${ }^{1}$ Bioscientia Institute for Medical Diagnostics, Human Genetics, Ingelheim, Germany, ${ }^{2}$ King Fahad Military Medical Complex, Dhahran, Saudi Arabia

The chromosome instability syndromes are a group of predominantly recessively inherited conditions associated with defects in DNA repair mechanisms that lead to chromosomal instability and chromosomal breakage. The so far known Double Strand Break Repair (DSBR) associated inherited genetic disorders share cancer predisposition, immunodeficiency, neurological disease and aplastic anemia as main clinical phenotypes. Here we present two patients with the shared clinical symptoms of severe intrauterine growth restriction, short stature, hypotonia, respiratory insufficiency and dysmorphic features. Chromosomal analysis for both patients revealed increased amount of numerical and structural chromosome aberrations, indicative for a genetic instability syndrome. Whole exome sequencing analysis revealed novel homozygous loss of function variants in SLF2 [p. (Arg190*) and p. (Asp783Serfs*53)]. SLF2 (SMC5-SMC6 complex localization factor 2) plays a role in the DNA damage response (DDR) pathway by regulating post-replication repair of UVdamaged DNA and genomic stability maintenance. The SLF1-SLF2 complex acts to link RAD18 with the SMC5SMC6 complex at replication-coupled interstrand crosslinks (ICL) and DNA double-strand breaks (DSBs) sites on chromatin during DNA repair. To the best of our knowledge we are presenting here the first human phenotype of a chromosome instability syndrome in association with $S L F 2$. Our findings underline the importance of $S L F 2$ in the DDR pathway and introduce the first human chromosomal and phenotypic presentation associated with a defect in this gene. In addition to diagnostic analysis, reporting scientific findings in disease candidate genes seems to be a crucial task of the diagnostic laboratory settings.

N. Nahavandi: None. Z. Yüksel: None. M. Drasdo: None. A. Kron: None. P. Vogt: None. A. Jonasson: None. S. Ahmed Seth: None. M. Tareg Mustafa: None. K. Danish: None.

\section{P11.90.C}

Prevalence of NF1 missense mutations and candidate modifier genes in 12 familial and 15 sporadic spinal neurofibromatosis patients

\author{
P. Riva ${ }^{l}$, E. Mangano ${ }^{2}$, C. Cesaretti ${ }^{3}$, P. Bettinaglio ${ }^{l}, R$.
}

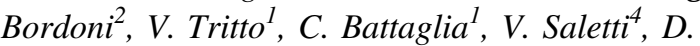

Bianchessi $^{4}$, M. Marina Melone ${ }^{5}$, C. Schettino ${ }^{5}, F$. Natacci $^{3}$, M. Eoli $^{4}$

${ }^{1}$ Univrsità degli Studi di Milano, Milano, Italy, ${ }^{2} \mathrm{CNR}$, Milano, Italy, ${ }^{3}$ Fondazione IRCCS Cà Granda Ospedale Maggiore Policlinico, Milano, Italy, ${ }^{4}$ Fondazione IRCCS Istituto Neurologico Carlo Besta, Milano, Italy, ${ }^{5}$ Università della Campania Luigi Vanvitelli, Napoli, Italy

Spinal Neurofibromatosis (SNF) is a specific form of Neurofibromatosis type 1 (NF1) characterized by bilateral neurofibromas involving all spinal roots. This feature allows to specifically distinguish SNF from Neurofibromatosis type 1 (NF1), and Multiple Neurofibromas Few Spinal Root (MNFSR), presenting single/few isolated spinal neurofibromas. While the clinical phenotype of SNF, MNSFR and classical NF1 can be revealed by spinal MRI, little is known on the molecular basis underlying these conditions. We investigated 12 NF1 families with at least one SNF patient, 15 sporadic SNF/MNSFR patients, diagnosed by Spinal MRI. We applied Targeted NGS using a panel consisting of 139 genes encoding RAS pathway effectors, neurofibromin interactors and genes mapping at $17 \mathrm{q} 11.2$ region. On identified $26 \mathrm{NF} 1$ variants, $36,6 \%$ were missense mutations, indicating an increased prevalence in our cohort versus 27,7\% missense/nonsense mutations reported in Italian (PMID: 26740943) and in a British (PMID:28637487) NF1 population. Furthermore, we evaluated rare variants with damaging predictors in genes of RAS pathway and neurofibromin interactors. In more than one sporadic case possible pathogenic variants were found in LIMK2, RASAL1, RASAL3, SOS1, A2ML1, MAP3K1, while in more than one SNF family were detected RASAL1, RASAL3, MAP3K1 genes variations. Our results confirm the high frequency of missense mutations in SNF as reported (PMID: 26740943, PMID:25211147), indicating neurofibromin gain-of-function variants as associated to SNF. In some patients, the co-occurrence of potential pathogenic variants in NF1 related genes are detected with severe phenotypes, suggesting that they might be modifier genes and promising pharmacological targets. Italian Ministry of Health RF-2016-02361293.

P. Riva: None. E. Mangano: None. C. Cesaretti: None. P. Bettinaglio: None. R. Bordoni: None. V. Tritto: None. C. Battaglia: None. V. Saletti: None. D. Bianchessi: None. M. Marina Melone: None. C. Schettino: None. F. Natacci: None. M. Eoli: None.

\section{P11.91.A}

New cohort of patients with CEDNIK syndrome expands the phenotypic and genotypic spectra 
A. Y. Mah-Som ${ }^{l}$, C. Skrypnyk ${ }^{2}$, R. Hamad ${ }^{3}$, V. Nivrutti ${ }^{3}$, A. Guerin $^{4}$, M. Shinawi ${ }^{1}$

${ }^{1}$ Washington University School of Medicine, Saint Louis, MO, United States, ${ }^{2}$ Department of Molecular Medicine, Al Jawhara Center for Molecular Medicine, Genetics and Inherited Diseases, College of Medicine and Medical Sciences, Manama, Bahrain, ${ }^{3}$ Division of Medical Genetics, Department of Pediatrics, Bahrain Defense Force Hospital, Manama, Bahrain, ${ }^{4}$ Department of Pediatrics, Queen's University, Kingston, ON, Canada

Introduction: CEDNIK (cerebral dysgenesis, neuropathy, ichthyosis, and keratoderma) syndrome (MIM\# 609528) is a rare condition (15 published cases) caused by biallelic lossof-function variants in SNAP29, which encodes a protein involved in membrane trafficking processes. Clinical manifestations include developmental delay (DD)/ intellectual disability (ID), brain malformations including cortical migration and corpus callosum (CC) defects, failure to thrive, and skin abnormalities.

Materials and Methods: Clinical trio exome or targeted sequencing was performed for each case to elucidate the cause of DD/ID and of other specific findings.

Results: We report five patients with homozygous predicted loss-of-function variants in $S N A P 29$, four of which are from consanguineous marriages. All patients exhibit DD/ID, ichthyosis and/or palmoplantar keratoderma, and hypotonia with spasticity. 3/5 subjects had seizures and $4 / 5$ had ophthalmological findings. Other phenotypes were variable between cases, including facial features, poor growth and feeding difficulties, and recurrent respiratory infections. The cohort includes 2 siblings with a c.2T>C variant who have a milder neurological phenotype, a patient with the most C-terminal variant yet described (c.622G $>\mathrm{T}$ ) with a more severe phenotype, and two patients with previously described variants (c.354dupG, c.487dupA).

Conclusions: We describe five additional patients with CEDNIK syndrome, including novel SNAP29 variants and the oldest known surviving individual. Our cohort expands the genotypic and phenotypic (seizures and strabismus) spectrum of CEDNIK syndrome.

Table 1. Phenotypic and genotypic data of patients with CEDNIK syndrome

\begin{tabular}{|c|c|c|c|c|}
\hline Patient & $1 \& 2$ & 3 & 4 & 5 \\
\hline Age (yrs) & 19,12 & 8 & 4 & 7 \\
\hline Sex & $\mathrm{F}, \mathrm{M}$ & $\mathrm{F}$ & $\mathrm{F}$ & M \\
\hline $\begin{array}{l}\text { SNAP29 } \\
\text { variant }\end{array}$ & c. $2 \mathrm{~T}>\mathrm{C}(\mathrm{p}$. Met $1 ?)$ & $\begin{array}{l}\text { c.354dupG(p. } \\
\text { Leu119Alafs*15) }\end{array}$ & $\begin{array}{l}\text { c. } 622 \mathrm{G}>\mathrm{T}(\mathrm{p} . \\
\text { Glu208*) }\end{array}$ & $\begin{array}{l}\text { c.487dupA(p. } \\
\text { Ser163Lysfs*6) }\end{array}$ \\
\hline Ethnicity & $\begin{array}{l}\text { Amish, } \\
\text { Mennonite }\end{array}$ & Mexican & Tibetan, Indian & Arab \\
\hline Consanguin. & Yes & Yes & No & Yes \\
\hline $\begin{array}{l}\text { Neurologic } \\
\text { phenotype }\end{array}$ & Leukodystrophy & Polymicrogyria (PMG) & $\begin{array}{l}\text { PMG, } \\
\text { hypoplastic CC }\end{array}$ & $\begin{array}{l}\text { Lissencephaly, } \\
\text { PMG, cerebellar }\end{array}$ \\
\hline
\end{tabular}

\begin{tabular}{|c|c|c|c|c|}
\hline & Seizures & No seizures & No seizures & $\begin{array}{l}\text { hypoplasia, } \\
\text { agenesis of CC } \\
\text { Seizures }\end{array}$ \\
\hline & $\begin{array}{l}\text { Hypotonia, } \\
\text { spasticity } \\
\text { +/-clonus }\end{array}$ & $\begin{array}{l}\text { Cerebral palsy, spastic } \\
\text { diplegia }\end{array}$ & $\begin{array}{l}\text { Profound } \\
\text { hypotonia }\end{array}$ & $\begin{array}{l}\text { Profound } \\
\text { hypotonia, } \\
\text { scoliosis }\end{array}$ \\
\hline $\mathrm{DD} / \mathrm{ID}$ & Mild-moderate & Moderate-severe & Severe & Severe \\
\hline Eye findings & Strabismus & $\begin{array}{l}\text { Strabismus, foveal } \\
\text { hypoplasia, roving eye } \\
\text { movements }\end{array}$ & $\begin{array}{l}\text { Cortical visual } \\
\text { impairment, } \\
\text { nystagmus }\end{array}$ & $\begin{array}{l}\text { Strabismus, } \\
\text { microphthalmia, } \\
\text { nystagmus }\end{array}$ \\
\hline Skinphenotype & $\begin{array}{l}\text { Ichythyosis, } \\
\text { plantar } \\
\text { keratoderma }\end{array}$ & $\begin{array}{l}\text { Ichthyosis, } \\
\text { palmoplantar } \\
\text { keratoderma }\end{array}$ & Ichthyosis & $\begin{array}{l}\text { Ichthyosis, } \\
\text { palmoplantar } \\
\text { keratoderma }\end{array}$ \\
\hline Miscellaneous & $\begin{array}{l}\text { No significant } \\
\text { dysmorphism, no } \\
\text { recurrent } \\
\text { respiratory } \\
\text { infections }\end{array}$ & $\begin{array}{l}\text { Deaf, recurrent } \\
\text { respiratory infections }\end{array}$ & $\begin{array}{l}\text { Deaf, G-tube, } \\
\text { recurrent } \\
\text { respiratory } \\
\text { infections, } \\
\text { plagiocephaly }\end{array}$ & $\begin{array}{l}\text { Recurrent } \\
\text { respiratory } \\
\text { infections, } \\
\text { microcephaly, } \\
\text { micrognathia }\end{array}$ \\
\hline $\begin{array}{l}\text { Previous } \\
\text { report of } \\
\text { SNAP29 } \\
\text { variant }\end{array}$ & PMID: 31748968 & $\begin{array}{l}\text { Tiwana } 2019 \text { AAN } \\
\text { abstract }\end{array}$ & Novel & PMID: 21073448 \\
\hline
\end{tabular}

A.Y. Mah-Som: None. C. Skrypnyk: None. R. Hamad: None. V. Nivrutti: None. A. Guerin: None. M. Shinawi: None.

\section{P11.93.C}

Loss-of-function mutations of TMEM260 cause autosomal recessive polycystic kidney, cerebral atrophy and cardiac malformation

T. M. Keszthelyi ${ }^{1,2}$, M. Varga $^{3}$, D. Czimer ${ }^{3}$, D. Ralbovszki ${ }^{3}$, L. Ablonczy ${ }^{4}$, C. Bole ${ }^{5}$, C. Antignac ${ }^{5}, K$. Tory ${ }^{1,2}$

${ }^{1}$ Semmelweis University, Ist Department of Pediatrics, Budapest, Hungary, ${ }^{2}$ MTA-SE Lendület Nephrogenetic Laboratory, Budapest, Hungary, ${ }^{3}$ Department of Genetics, Eötvös Loránd University, Budapest, Hungary, ${ }^{4}$ Gottsegen György National Institute of Cardiology, Budapest, Hungary, ${ }^{5}$ INSERM, UMR 1163, Imagine Institute, Paris, France

Introduction: Association of polycystic kidney, cerebral atrophy and truncus arteriosus had not been reported in the medical literature until recently. We aimed to identify the causal gene and to explore the function of the encoded protein.

Materials and methods: Exome sequencing was performed in a 5-year-old male. The localization of the protein was studied by immunofluorescence staining in transiently transfected IMCD3 cells after the induction of ciliogenesis. Mutant zebrafish lines were generated by the CRISPR/ Cas9 system.

Results: We identified compound heterozygous truncating mutations in the TMEM260 gene: c.592_593delTT, p. Leu198Valfs44* (paternal) and c.1854C >A (p.Tyr618*) (maternal). Out of the two isoforms (ENST00000261556.11, long"; ENST00000538838.5, short"), only the long is affected by the p.Tyr618* mutation, allowing the short isoform to be properly translated. We 
successfully generated tmem 260 biallelic loss-of-function mutant zebra danio lines. Homozygous mutants with different LOF mutations show no obvious phenotype. Interestingly, in contrast to the proteins implicated in ciliary kidney disorders, TMEM260 long isoform showed a moderate cytoplasmic and a strong nuclear membrane localization. The short isoform located in the cytoplasm and strongly in the nucleus. Neither isoforms were detectable in the primary cilium. Mutations of TMEM260 cause autosomal recessive polycystic kidney, cerebral atrophy and truncus arteriosus.

Conclusions: Our results are strongly supported by one publication indicating the role of TMEM260 in this syndrome. The preserved translation of the short isoform in all three affected families strongly suggest that the dysfunction of the short isoform is intrauterine lethal.

T.M. Keszthelyi: None. M. Varga: None. D. Czimer: None. D. Ralbovszki: None. L. Ablonczy: None. C. Bole: None. C. Antignac: None. K. Tory: None.

P11.96.C

Possible improvement in phenotype definition of Weidemann-Steiner syndrome by Whole Exome Sequencing

\section{E. Fiorentini, G. Traficante, R. Artuso, G. Gori, G.} Forzano, S. Giglio

Medical Genetics Unit, Department of Clinical and Experimental Biomedical Sciences 'Mario Serio', University of Florence, I-50139 Florence, Italy., Florence, Italy

We report the case of a boy evaluated mainly for trigonocephaly and generalized seizures. His critical events were poorly controlled by antiepileptic multi-drug therapy. His phenotype with some facial features (thick eyebrows, epicanthus, long filtering, thin upper lip), growth and psychomotor development delay, renal ectopia, complete hip dislocation, thin corpus callosum with scarcity of white matter, did not suggest any specific condition. Karyotype and CGH-array analysis were normal. Whole exome sequencing (WES) revealed a heterozygous frameshift variant in $K M T 2 A$, resulting in a premature stop codon. Variants in KMT2A have been already described in patients with Wiedeman-Steiner Syndrome (WSS). This gene encodes a histone methyltransferase involved in the regulation of chromatin-mediated transcription and it is widely expressed in most human tissues. The majority of WSSassociated variants reported so far are de novo loss-offunction, predicted to result in premature termination of translation and nonsense-mediated decay of the corresponding transcripts. WSS is a rare autosomal dominant disorder, characterized by short stature, developmental delay and intellectual disability, hypertrichosis (cubiti-hairy elbow-or generalized) and facial dysmorphism. Concerning the incidence of bone anomalies, to date trigonocephaly (a feature at our first evaluation) has been reported only in one case, while craniovertebral junction anomalies have been described in two other patients; both of these signs were not included in the features associated with WSS. Further data will be needed to evaluate the exact incidence of these features in subjects with WSS associated with haploinsufficiency of a gene involved in histone-mediated chromatin remodeling.

E. Fiorentini: None. G. Traficante: None. R. Artuso: None. G. Gori: None. G. Forzano: None. S. Giglio: None.

P11.97.A

Phenotypic spectrum of Xp22 duplication; A tertiary center experience N. Alsaleh ${ }^{1}$, I. Ben Abdallah
Ghezal

${ }^{I}$ Division of Genetic and Metabolic Medicine, Department of Pediatrics, Prince Sultan Military Medical City, Riyadh, Saudi Arabia, ${ }^{2}$ Cytogenetics and Molecular Genetics Division, Prince Sultan Military Medical City, Riyadh, Saudi Arabia, ${ }^{3}$ Department of Pediatrics, College of Medicine, AlFaisal University, Riyadh, Saudi Arabia

Introduction: Evidence strongly supports the use of chromosomal microarray analysis as a first-tier cytogenetic diagnostic tool in patients with developmental delay/intellectual disability and multiple congenital anomalies. Copy number variants $(\mathrm{CNV})$ involving the $\mathrm{X}$ chromosome has been previously described, mainly deletions (e.g. Leri-Weill dyschondrostsosis). However, little is known about phenotypes arising from duplications. In this study, we reviewed CNVs involving the short arm of the $\mathrm{X}$ chromosome, specifically duplications involving band P22. This region contains 194 genes, only 107 have an OMIM description (e.g. SHOX and STS).

Materials and Methods: Cases that underwent array comparative genomic hybridization between years (20162019) were reviewed. Indication for testing was variable, ranging from developmental delay, seizures to multiple congenital anomalies. We focused on cases with duplication involving Xp22

Results: 69 cases reported with CNVs involving the X chromosome. (33/69) with Xp22 duplication. (17/33) Females and (16/33) males. (1/33) involving Xp22.2, (1/ 33) involving Xp22.3, (1/33) involving Xp22.32, (12/33) involving Xp22.31 and (18/33) involving Xp22.33. The size of the duplication ranged from $147 \mathrm{~Kb}$ to $2.3 \mathrm{Mb}$. The detected CNVs ranged from benign or likely benign (12/ 
$33)$, pathogenic or likely pathogenic (9/33) and variants of unknown significance (12/33). Some cases were de novo, others were inherited from a healthy parent. Variable expressivity and low penetrance were observed in this cohort.

Conclusions: Deletions affecting the $\mathrm{X}$ chromosome is usually associated with a known clinical syndrome that could be male lethal. However, duplications tend to be milder, showing variable expressivity and penetrance, which makes it hard to confirm pathogenicity.

N. Alsaleh: None. I. Ben Abdallah: None. A. Al Hashem: None. H. El Ghezal: None.

\section{P11.98.B}

Deleterious de novo variants of $\mathrm{X}$-linked $\mathrm{ZC4H} 2$ in females cause a variable phenotype with neurogenic arthrogryposis multiplex congenita

S. G. M. Frints ${ }^{1,2}$, F. Hennig ${ }^{3}$, R. Colombo ${ }^{4,5}, S$.

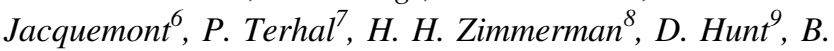
A. Mendelsohn ${ }^{10}$, U. Korda $\beta^{11}$, R. Webster ${ }^{12}, M$. Sinnema ${ }^{1,2}$, O. Abdul-Rahman $^{13}$, V. Suckow ${ }^{3}$, A. Fernández$J_{\text {Jaén }}{ }^{14}$, Deciphering Developmental Disorders (DDD) Study, S. J. C. Stevens ${ }^{1,2}$, M. V. E. Macville ${ }^{1,2}$, K. van Roozendaal $^{1}$, S. Al-Nasiry ${ }^{15}$, K. van Gassen ${ }^{7}$, N. Utzig ${ }^{16}$, S. M. Koudijs ${ }^{17}$, L. McGregor ${ }^{18}$, S. M. Maas ${ }^{19}$, D. Baralle ${ }^{9,20}$, A. Dixit ${ }^{21}$, P. Wieacker ${ }^{22}$, M. Lee ${ }^{23}$, A. S. Lee ${ }^{24,25}$, E. C. Engle $^{24,25,26}$, G. Houge ${ }^{27}$, A. G. L. Douglas ${ }^{9,28}$, C. Longman $^{29}$, S. Joss ${ }^{29}$, G. A. Gradek ${ }^{27}$, D. Velasco ${ }^{30}$, R. C. Hennekam ${ }^{31}$, H. Hirata ${ }^{32}$, V. M. Kalscheuer ${ }^{33}$

${ }^{I}$ Department of Clinical Genetics, Maastricht University Medical Center+, azM, Maastricht, Netherlands, ${ }^{2}$ Department of Genetics and Cell Biology, Faculty of Health Medicine Life Sciences, Maastricht University Medical Center+, Maastricht University, Maastricht, Netherlands, ${ }^{3}$ Max-Planck-Institute for Molecular Genetics, Berlin, Germany, ${ }^{4}$ Catholic University of the Sacred Heart, Rome, Italy, ${ }^{5}$ Center for the Study of Rare Inherited Diseases (CeSMER), Niguarda Ca' Granda Metropolitan Hospital, Milan, Italy, ${ }^{6}$ Service de Génétique Médicale, CHUV, Lausanne, Switzerland, ${ }^{7}$ Laboratories, Pharmacy and Biomedical Genetics Division, University Medical Centre Utrecht, Utrecht, Netherlands, ${ }^{8}$ Department of Pediatrics, Division of Medical Genetics, University of Mississippi Medical Center, Mississippi, MS, United States, ${ }^{9}$ Wessex Clinical Genetics Service, University Hospital Southampton NHS Foundation Trust, Southampton, United Kingdom, ${ }^{10}$ Division of Genetics, Department of Pediatrics, University of California, San Francisco, CA, United States, ${ }^{11} \mathrm{MVZ}$ für Humangenetik und Molekularpathologie GmbH, Zweigstelle Greifswald, Greifswald, Germany, ${ }^{12}$ The Department of Neurology and Neurosurgery, The Children's Hospital at
Westmead, Westmead, Australia, ${ }^{13}$ Munroe-Meyer Institute for Genetics \& Rehabilitation, University of Nebraska Medical Center, Omaha, NE, United States, ${ }^{14}$ Hospital Universitario Quirón de Madrid, Madrid, Spain, ${ }^{15}$ Department of Obstetrics and Gynecology, Prenatal Diagnostics \& Therapy, Maastricht University Medical Center+, azM, Maastricht, Netherlands, ${ }^{16}$ Universitätsmedizin Greifswald, Klinik für Kinder- und Jugendmedizin, Greifswald, Germany, ${ }^{17}$ Department of Neurology, Maastricht University Medical Center+, azM, Maastricht, Netherlands, ${ }^{18}$ SA Clinical Genetics Service, Women's and Children's Hospital, Adelaide, Australia, ${ }^{19}$ Department of Clinical Genetics, Amsterdam UMC location AMC, Amsterdam, Netherlands, ${ }^{20}$ Faculty of Medicine, University of Southampton, Southampton, United Kingdom, ${ }^{21}$ Nottingham University Hospitals NHS Trust, City Hospital Campus,, Nottingham, United Kingdom, ${ }^{22}$ Institute of Human Genetics, Westfälische Wilhelms Universität Münster, Münster, Germany, ${ }^{23}$ Department of Pediatrics, Division of Pediatric Neurology, University of Mississippi Medical Center, Mississippi, MS, United States, ${ }^{24}$ Department of Neurology, Boston Children's Hospital and Harvard Medical School, Boston, MA, United States, ${ }^{25}$ Broad Institute of MIT and Harvard, Cambridge, MA, United States, ${ }^{26}$ Department of Ophthalmology, Boston Children's Hospital and Harvard Medical School, Boston, MA, United States, ${ }^{27}$ Department of Medical Genetics, Haukeland University Hospital, Bergen, Norway, ${ }^{28}$ Human Development and Health, Faculty of Medicine, University of Southampton, Southampton, United Kingdom, ${ }^{29}$ West of Scotland Regional Genetic Centre, Queen Elizabeth University Hospital, Glasgow, United Kingdom, ${ }^{30}$ Department of Pediatrics, Munroe-Meyer Institute for Genetics \& Rehabilitation, University of Nebraska Medical Center, Omaha, NE, United States, ${ }^{31}$ Department of Pediatrics, Amsterdam UMC, Amsterdam, Netherlands, ${ }^{32}$ Department of Chemistry and Biological Science, College of Science and Engineering, Aoyama Gakuin University, Sagamihara, Japan, ${ }^{33}$ Research Group Development and Disease, Max-Planck-Institute for Molecular Genetics, Berlin, Germany

Introduction: Pathogenic variants in the $\mathrm{X}$-linked gene $\mathrm{ZC} 4 \mathrm{H} 2$ cause an infrequently described syndromic form of arthrogryposis multiplex congenita (AMC) with central and peripheral nervous system involvement (ZC4H2-Associated Rare Disorders, ZARD).

Materials and Methods: We performed exome sequencing and array-CGH and tested $\mathrm{ZC} 4 \mathrm{H} 2$ missense variants identified in affected patients in zebrafish.

Results: We present genetic and detailed phenotypic information on 23 newly identified families and simplex cases that include 19 affected females from 18 families and 14 affected males from nine families. The genetic spectrum 
of $\mathrm{ZC} 4 \mathrm{H} 2$ defects comprises novel and recurrent mostly inherited missense variants in affected males, and de novo splicing, frameshift, nonsense, and partial $\mathrm{ZC} 4 \mathrm{H} 2$ deletions in affected females. Two newly identified missense variants were investigated by studies in zebrafish and the results supported pathogenicity. Of note, the 15 females with deleterious de novo $\mathrm{ZC} 4 \mathrm{H} 2$ variants presented with phenotypes ranging from mild to severe, and their clinical features overlapped with those seen in affected males. By contrast, of the nine carrier females with inherited $\mathrm{ZC} 4 \mathrm{H} 2$ missense variants that were deleterious in affected male relatives, four were symptomatic. We also compared clinical phenotypes with previously published cases of both sexes and provide an overview on 48 males and 57 females from 42 families.

Conclusions: We propose $\mathrm{ZC} 4 \mathrm{H} 2$ as a good candidate for early genetic testing of males and females with a clinical suspicion of fetal hypo-/akinesia and/or (neurogenic) AMC. Grants: DDD study HICF-1009-003 and WT098051, Dutch NWO VENI OND1312421 (to SGM), NEI R01EY027421 and NHLBI X01HL132377 (to ECE).

S.G.M. Frints: None. F. Hennig: None. R. Colombo: None. S. Jacquemont: None. P. Terhal: None. H.H. Zimmerman: None. D. Hunt: None. B.A. Mendelsohn: None. U. Kordaß: None. R. Webster: None. M. Sinnema: None. O. Abdul-Rahman: None. V. Suckow: None. A. Fernández-Jaén: None. S.J.C. Stevens: None. M.V.E. Macville: None. K. van Roozendaal: None. S. Al-Nasiry: None. K. van Gassen: None. N. Utzig: None. S.M. Koudijs: None. L. McGregor: None. S.M. Maas: None. D. Baralle: None. A. Dixit: None. P. Wieacker: None. M. Lee: None. A.S. Lee: None. E.C. Engle: None. G. Houge: None. A.G.L. Douglas: None. C. Longman: None. S. Joss: None. G.A. Gradek: None. D. Velasco: None. R.C. Hennekam: None. H. Hirata: None. V.M. Kalscheuer: None.

\section{P12 Cancer Genetics}

\section{P12.001.C}

Functional annotation of 5p15 risk locus for Barrett's esophagus and esophageal adenocarcinoma

\section{J. Schröder ${ }^{l}$, R. Hollstein ${ }^{1}$, I. Gockel ${ }^{2}$, R. Thieme ${ }^{2}$, K. U. Ludiwg $^{l}$, J. Schumacher ${ }^{3}$, A. C. Böhmer ${ }^{l}$}

${ }^{1}$ Institute of Human Genetics, University of Bonn, School of Medicine \& University Hospital Bonn, Bonn, Germany, ${ }^{2}$ Department of Visceral, Transplant, Thoracic and Vascular Surgery, University Hospital of Leipzig, Leipzig, Germany, ${ }^{3}$ Center for Human Genetics, University Hospital of Marburg, Marburg, Germany
Esophageal adenocarcinoma (EA) and its precancerous condition Barrett's esophagus (BE) are multifactorial diseases with rising prevalence rates in Western populations. Our recent integrative analysis of GWAS and GTEx eQTL data showed that the risk allele of the sentinel SNP (rs147462972) at the genome-wide significant locus 5p15 is associated with increased SLC9A3 expression in esophageal mucosa tissue. Interestingly, upregulation of this gene has been linked to gastro-esophageal reflux disorder, a major risk factor for BE development. Therefore, we hypothesize that rs 147462972 maps to a regulatory element influencing SLC9A3 expression. To study the regulatory mechanisms at $5 \mathrm{p} 15$, chromatin interactions are analyzed using circular chromosome conformation capture (4C) technology from two distinct viewpoints, the promoter of SLC9A3 and the region around rs147462972. The method was first established in induced pluripotent stem cells (iPSC), where numerous interactions at the locus and beyond were observed. Further analyses will be performed in different cell lines representative of the course of BE/EA development: healthy epithelium (EPC1), Barrett's esophagus (CPA), intraepithelial neoplasia (CP-B), and EA (OE19).

No interaction between the SLC9A3 promoter and the sentinel SNP was detected at 5p15 in iPSC. A lack of this interaction was assumed, since these cells do not express SLC9A3. Based on these results, the experiments in esophageal cell lines are currently ongoing and the results will be presented at the conference. Our work will help to understand the contribution of the risk locus $5 \mathrm{p} 15$ to the etiology of BE and EA, and the role of SLC9A3 in their pathogenesis.

J. Schröder: None. R. Hollstein: None. I. Gockel: None. R. Thieme: None. K.U. Ludiwg: None. J. Schumacher: None. A.C. Böhmer: None.

\section{P12.002.A}

Discovery of novel tumor-associated exonic incertions of polymorphic retroelements in human genome

\section{A. Y. Komkov ${ }^{1,2}$, M. Saliutina ${ }^{1,3}$, M. Kulikov ${ }^{3}$, E. Volchkov ${ }^{2}$, S. Urazbakhtin ${ }^{1,3}$, A. Popov ${ }^{2}$, M. Maschan ${ }^{2}$, Y. Lebedev ${ }^{1}, Y$. Olshanskaya $^{2}$, I. Mamedov $^{1,2}$}

${ }^{1}$ Shemyakin-Ovchinnikov Institute of Bioorganic Chemistry of the Russian Academy of Sciences, Moscow, Russian Federation, ${ }^{2}$ Dmitry Rogachev National Medical Research Center Of Pediatric Hematology, Oncology and Immunology, Moscow, Russian Federation, ${ }^{3}$ Lomonosov Moscow State University, Moscow, Russian Federation

Introduction: Retroelements (RE) make a significant contribution to many mono- and polygenic disorders, including different types of cancer. New RE insertion can lead to exon 
deletion, exon disruption and alternative splicing, including formation of RE-containing exon. Most tumor-related insertions are individual-specific owing to germline or somatic retroposition events. Here we investigate exonic polymorphic RE insertions (PREI) distributed in population.

Materials and methods: Two cohorts of 200 healthy donors and 120 pediatric lymphoblastic leukemia patients were used for this study. Whole genome identification of $\mathrm{RE}$ insertions was conducted using target high-throughput sequencing of genome fragments adjusted to insertions of active RE. Novel detected PREIs were validated using capillary sequencing. PREI allele status in donors was identified using locus-specific PCR.

Results: We identified five exonic PREIs among all detected polymorphic insertions. Frequency of bi-allelic PREI (AluYa5) in PRAMEF4 gene exon was significantly higher in healthy female cohort compared to healthy males ( $p$-value $=0.05$, two-tailed Fisher's exact test) and in Tlineage leukemia male cohort compared to healthy male donors $(p$-value $=0.01)$. Leukemia samples with bi-allelic PREI in PRAMEF4 had specific ( $p$-value $=0.008)$ immunophenotype: CD33-CD13-CD34-CD117-CD56-CD1a $+\mathrm{CD} 3+\mathrm{CD} 4+\mathrm{CD} 8+\mathrm{CD} 10+$ inherent to more mature lymphoblasts.

Discussion: The results demonstrate that PREI in PRAMEF4 exon could participate in leukemogenesis in gender-dependent manner affecting male patients despite AluYa5-containing exons present in population. We hypothesize that bi-allelic insertion in PRAMEF4 exon is insufficient for leukemogenesis and requires additional mutations in sex chromosomes because of gender-specific nature of the observed phenomenon. The study was supported by RFBR grant 18-315-20038 and RSF grant 18-14-00244.

A.Y. Komkov: None. M. Saliutina: None. M. Kulikov: None. E. Volchkov: None. S. Urazbakhtin: None. A. Popov: None. M. Maschan: None. Y. Lebedev: None. Y. Olshanskaya: None. I. Mamedov: None.

\section{P12.003.B}

Driver mutations in pediatric core binding factor acute myeloid leukemia

\section{T. Nasedkina ${ }^{1}$, L. Ghukasyan ${ }^{1}$, G. Krasnov ${ }^{1}$, L. Baidun ${ }^{2}, S$. Ibragimova ${ }^{3}$}

${ }^{1}$ Engelhardt Institute of Molecular Biology of the Russian Academy of Sciences, Moscow, Russian Federation, ${ }^{2}$ Russian Children's Clinical Hospital, Moscow, Russian Federation, ${ }^{3}$ Scientific Research Institute of Hematology and Blood Transfusion, Tashkent, Uzbekistan
Introduction: Core binding factor acute myeloid leukemia (CBF-AML) combines two entities, which differ significantly in their clinical and biological features, AML with $\mathrm{t}(8 ; 21)$ and AML with inv(16). High-throughput sequencing methods reveal characteristic mutation spectra in two leukemia subtypes; however, the role of different mutation events in tumorigenesis remains unclear.

Patients and methods: In total, 9 patients with $t(8 ; 21)$ and 16 patients with inv(16) were investigated (13 boys and 12 girls, mean age 7.2 years). For 18 patients, paired samples at diagnosis and remission were analyzed. Coding regions of 84 genes, involved in the pathogenesis of AML, were captured with SeqCap EZ Target Enrichment System (NimbleGen, Roche) and sequenced using NextSeq500 Illumina platform.

Results: In both AML subtypes, somatic mutations in the genes KIT (40\%), NRAS (24\%), KRAS (16\%), FLT3 (12\%) were found. The KIT mutations were more common for inv (16) compared to $\mathrm{t}(8 ; 21)(56 \%$ vs. $11 \%, \mathrm{p}=0.04)$. In the group with inv(16), KIT mutations in exon $8(66 \%)$ prevailed comparing with exon 17 (33\%); two patients $(12.5 \%)$ had biallelic KIT mutations. In group with $\mathrm{t}(8 ; 21)$, mutations in the SMC1A (cohesin complex), CBL, CBLB, $A S X L 1, T E T 1, E Z H 2$ genes were revealed. In patients with inv(16), mutations in the KDM6A and KMT2A genes, involved in chromatin modification, were found.

Conclusions: Mutational profiling revealed characteristic differences in spectrum and frequency of somatic mutations in patients with $\mathrm{t}(8 ; 21)$ and inv(16). Novel mutations, which may be involved in leukemia development, were identified. The work was supported by the Russian Science Foundation (grant \# 18-15-00398).

T. Nasedkina: None. L. Ghukasyan: None. G. Krasnov: None. L. Baidun: None. S. Ibragimova: None.

\section{P12.004.C}

Molecular studies for gain of cytarabine-resistance in AML cells with FLT3-ITD mutations

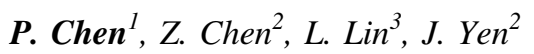

${ }^{1}$ Center of Medical Genetics, Hualien Tzu Chi Hospital, Hualien, Taiwan, ${ }^{2}$ Department of Molecular Biology and Human Genetics, Tzu Chi University, Hualien, Taiwan, ${ }^{3}$ Department of Clinical Laboratory Sciences and Medical Biotechnology, National Taiwan University, Taipei, Taiwan

Introduction: Although cytarabine (ara-C) has remained an important cornerstone for treatment of acute myeloid leukemia (AML) for decades, chemo-resistance is common and the overall survival of adult AML patients is less than $40 \%$. Internal tandem duplication mutations in the FLT3 
tyrosine kinase receptor (FLT3-ITD) account for up to $30 \%$ of cases and are associated with a poor outcome. To understand the role of FLT3-ITD in the development of drug resistance. An ara-C resistant cell line (MV-4-11-CR) was established from parent MV-4-11 cells which harbors FLT3-ITD. Here, we compared MV-4-11-CR with MV-411 cells, and explored the underling mechanisms for developing resistance in FLT3-ITD AML cells.

Materials and Methods: MV-4-11-CR and MV-4-11 cells were performed tests including MTT assay, RTqPCR, Western blot, G-banding and SKY.

Results: MV-4-11-CR cells were highly resistant to ara-C by their high IC50 value, and enhanced cell growth rate. This cell line was confirmed to retain the homologous FLT3-ITD mutations. However, a significant increase of FLT3-ITD mRNA and protein was observed. To test whether FLT3-ITD activation is crucial for resistance, we measured the effects of combined treatment of cells with ara-C and FLT3 inhibitors. Inhibition of FLT3 was notably effective in cell viability, but not effective in drug sensitivity. We further compared genomic alterations and found multiple chromosomal changes and complex karyotypes. Moreover, down-regulation of deoxycytidine kinase (DCK), a cytarabine metabolic enzyme, was observed in MV-4-11-CR.

Conclusions: We suggest that gain the resistance to ara-C may be associated with chromosomal aberrations and DCK deficiency in FLT3-ITD+ AML cells.

P. Chen: None. Z. Chen: None. L. Lin: None. J. Yen: None.

\section{P12.007.C}

Nanopore-sequencing-based high-resolution karyotyping of $\mathrm{AML}$ patients

\author{
F. Kraft ${ }^{l}$, P. Krüger ${ }^{l}$, S. Koschmieder ${ }^{2}$, E. Jost ${ }^{2}, M$. \\ Crysandt $^{2}$, I. Kurth ${ }^{I}$
}

${ }^{1}$ Institute of Human Genetics, University Hospital RWTH Aachen, Aachen, Germany, ${ }^{2}$ Department of Medicine (Hematology Oncology, Hemostaseology, and Stem Cell Transplantation), University Hospital RWTH Aachen, Aachen, Germany

Introduction: The current diagnostic scheme for classification of acute myeloid leukemia (AML) patients is usually based on cytogenetic and molecular biology methods. The turnaround time for karyotype analysis is up to two weeks, due to the indispensable cultivation of hematopoietic cells. Although initial PCR or FISH-based testing for recurrent structural variations (SV) can be carried out within few hours, the AML classification is demanding and requires accurate karyotyping.
Methods: We utilized nanopore-sequencing to overcome the time-consuming karyotyping and further increase resolution and accelerate SV analysis for AML classification. In detail, low-coverage whole genome sequencing (LC-WGS) was carried out in combination with a modified SMURFseq protocol on AML bone marrow or blood samples ( $\geq 20 \%$ blasts). LC-WGS data was used to identify classification-relevant SV in AML. Genome-wide CNV analysis was done from SMURFSeq data.

Results: For all 16 samples the results from nanoporesequencing confirmed the SV identified by conventional cytogenetic and FISH analyses. Translocations, inversions, tandem duplications and CNVs were identified at single base resolution. We could detect additional small CNVs in growth regulating genes which may modify the aggressiveness of the leukemia. Moreover, the procedure from DNA isolation to obtaining results requires less than $48 \mathrm{~h}$ and is not operator dependent. The method can also generate karyotype data from samples for which the cell cultivation failed.

Conclusions: We show that nanopore-sequencing can speed-up and potentially improve the comprehensive analysis of SV in AML. Rapid classification according to ELN risk stratification will immediately impact on targeted and risk adapted treatment of AML patients.

F. Kraft: None. P. Krüger: None. S. Koschmieder: None. E. Jost: None. M. Crysandt: None. I. Kurth: None.

\section{P12.008.A}

Classification of $A T M$ variants identified in Spanish patients with suspicion of hereditary cancer

L. Feliubadaló ${ }^{1,2,3}$, M. Santamariña Peña ${ }^{4,5,6}$, L. Porras ${ }^{7,8}$, A. Moles-Fernández ${ }^{9}$, A. Sánchez ${ }^{10,11}$, A. Blanco ${ }^{12,13,5}$, O. Diez $^{9,14}$, S. Gutiérrez-Enríquez ${ }^{9}$, M. de la Hoya ${ }^{15,16}$, A. Lopez-Novo $^{17}$, A. Osorio ${ }^{18,5}$, M. Pineda ${ }^{1,2,3}$, D. Rueda ${ }^{19,20}$, C. Ruiz-Ponte ${ }^{21,5}$, A. Vega $a^{12,13,5}$, I. J. Molina ${ }^{22}$, X. de la Cruz $^{7,8,23}$, C. Lázaro $^{1,2,3}$

${ }^{1}$ Hereditary Cancer Program, Catalan Institute of Oncology, IDIBELL, Hospitalet de Llobregat, Spain, ${ }^{2}$ Program in Molecular Mechanisms and Experimental Therapy in Oncology (Oncobell), IDIBELL, Hospitalet de Llobregat, Spain, ${ }^{3}$ Centro de Investigación Biomédica en Red de Cáncer (CIBERONC), Madrid, Spain, ${ }^{4}$ Grupo de Medicina Xenómica. Instituto de Investigación Sanitaria de Santiago de Compostela, Santiago de Compostela, Spain, ${ }^{5}$ CIBERER, Madrid, Spain, ${ }^{6}$ Universidad de Santiago de Compostela, Santiago de Compostela, Spain, ${ }^{7}$ Research Unit in Clinical and Translational Bioinformatics, Vall d'Hebron Institute of Research (VHIR), Barcelona, Spain, ${ }^{8}$ Universitat Autònoma de Barcelona, Barcelona, Spain, ${ }^{9}$ Hereditary Cancer Genetics Group, Vall d'Hebron 
Institute of Oncology (VHIO), Barcelona, Spain, ${ }^{10} \mathrm{Heredi-}$ tary Cancer Program, Catalan Institute of Oncology, Barcelona, Spain, ${ }^{11}$ Aberystwyth University, Aberystwyth, United Kingdom, ${ }^{12}$ Fundación Pública Galega Medicina Xenómica-SERGAS, Santiago de Compostela, Spain, ${ }^{13}$ Instituto de Investigación Sanitaria de Santiago de Compostela (IDIS), Santiago de Compostela, Spain, ${ }^{14}$ Area of Clinical and Molecular Genetics, University Hospital of Vall d'Hebron, Barcelona, Spain, ${ }^{15}$ CIBER-ONC, Instituto de Salud Carlos III, Madrid, Spain, ${ }^{16}$ Molecular Oncology Laboratory, Hospital Clinico San Carlos, IdISSC (Instituto de Investigación Sanitaria del Hospital Clínico San Carlos), Madrid, Spain, ${ }^{17}$ Fundacion Publica Galega de Medicina Xenómica, Grupo de Medicina XenomicaUniversidade de Santiago de Compostela, Instituto de Investigacion Santiago de Compostela (E035), Santiago de Compostela, Spain, ${ }^{18}$ Human Genetics Group, Spanish National Cancer Research Centre (CNIO), Madrid, Spain, ${ }^{19}$ Digestive Cancer Research Group, 12 de Octubre Research Institute, Madrid, Spain, ${ }^{20}$ Molecular Biology Laboratory, 12 de Octubre University Hospital, Madrid, Spain, ${ }^{21}$ Fundacion Publica Galega de Medicina Xenomica, Grupo de Medicina Xenomica-Universidade de Santiago de Compostela, Instituto de Investigacion Sanitaria de Santiago de Compostela (E035), Santiago de Compostela, Spain, ${ }^{22}$ Institute of Biopathology and Regenerative Medicine, University of Granada, Granada, Spain, ${ }^{23}$ Institució Catalana de Recerca i Estudis Avançats (ICREA), Barcelona, Spain

The use of NGS gene panels for clinical testing has increased the diagnostic yield, but also the frequency of variants of unknown significance (VUS) per patient. With the aim of improving and standardizing variant classification, we'll create a national registry of genetic variants in cancer predisposing-genes. To that end, seven Spanish molecular laboratory groups currently using NGS panels for hereditary cancer diagnostics created a variant database. Our current database gathers all variants with less than $1 \%$ allele frequency in all GnomAD outbred populations, starting with the ATM gene, study because of its moderate increase in breast cancer risk and its remarkable number of VUSes. The methodology consisted of: 1) selection and curation of 50 representative variants from our cohort; 2) evaluation of each by three members; and 3) discussion of evidences for classification in monthly teleconferences. As $A T M$-specific guidelines have not been published, we have previously carried out our own ACMG/AMP criterion adjustment. So far, 842 variants belonging to 730 tested patients with different hereditary cancer conditions. Among the 265 unique variants, 97 appeared more than once, and 35 had differences in classification among groups. Our new
ATM-specific criteria have allowed us to improve and standardize the classification of the 50 pilot variants. We'll next submit our classifications to international databases and extend our database to other Spanish participants and hereditary cancer genes. Grant support: Carlos III National Health Institute - FEDER funds [PI19/00553; PI16/00563; PI16/01898; SAF2015-68016-R and CIBERONC]; the Government of Catalonia [PERIS_MedPerCan and URDCat projects, 2017SGR1282 and 2017SGR496].

L. Feliubadaló: None. M. Santamariña Peña: None. L. Porras: None. A. Moles-Fernández: None. A. Sánchez: None. A. Blanco: None. O. Diez: None. S. GutiérrezEnríquez: None. M. de la Hoya: None. A. Lopez-Novo: None. A. Osorio: None. M. Pineda: None. D. Rueda: None. C. Ruiz-Ponte: None. A. Vega: None. I. J. Molina: None. X. de la Cruz: None. C. Lázaro: None.

\section{P12.009.B}

Establishing a scalable automation approach with the epMotion and Ampliseq for Illumina

\author{
M. Hahn ${ }^{1}$, V. Montel ${ }^{2}$, N. Mouttham ${ }^{2}$, E. Gancarek ${ }^{3}, S$. \\ Hamels $^{3}$, U. Wilkening ${ }^{1}$, J. Tsai ${ }^{2}$ \\ ${ }^{I}$ Eppendorf AG, Hamburg, Germany, ${ }^{2}$ Illumina Inc, San \\ Diego, CA, United States, ${ }^{3}$ Eppendorf Application Tech- \\ nologies SA, Namur, Belgium
}

Due to the growing number of relevant genes and variants, next generation sequencing (NGS) has become a standard technique in the field of cancer research and molecular pathology. AmpliSeq ${ }^{\mathrm{TM}}$ panels for Illumina ${ }^{\circledR}$ have been introduced to study the growing number of interesting variants in an increasing number of relevant genes. In theory, library preparation with such panels promise the ability query many interesting loci in DNA or RNA isolated from a broad range of specimen, including blood, cell culture, and formalin-fixed paraffin-embedded (FFPE) tissue at low efforts. However, especially working with the latter materials can represent practical challenges that require robust and established workflows for analytical studies. Here we sought to establish a workflow for this purpose using automated liquid handling for library preparation on the epMotion system that can accommodate both RNA and DNA samples. We show exemplary NGS sequencing data achieved with the AmpliSeq for Illumina Focus panel assembled from individual DNA and RNA reference sample sets with known mutations and quality generated in a single automated setup. We found high run-to-run reproducibility and repeatability $\left(\mathrm{R}^{2}>0.98\right)$ with $100 \%$ success rate for library preparation (DNA $n=$; RNA $n=$ preparations). Call rates of known variants $\left(R^{2}>0.99\right)$ and fusion transcripts were consistent over several independent experimental replicates. Furthermore 
variant calling and index representation were comparable to manual preparations performed on the same samples. The flexibility and scalability of this approach may aid in the routine study of different samples and throughputs, while reducing the hands-on time.

M. Hahn: A. Employment (full or part-time); Significant; Eppendorf AG. V. Montel: A. Employment (full or parttime); Significant; Illumina Inc. N. Mouttham: A. Employment (full or part-time); Significant; Illumina Inc. E. Gancarek: A. Employment (full or part-time); Significant; Eppendorf Application Technologies SA. S. Hamels: A. Employment (full or part-time); Significant; Eppendorf Application Technologies SA. U. Wilkening: A. Employment (full or part-time); Significant; Eppendorf AG. J. Tsai: A. Employment (full or part-time); Significant; Illumina Inc.

\section{P12.010.C}

Family with mesothelioma and variant of unknown significance in the BAP1 gene

B. W. van Paassen, N. M. van Poppelen, R. Cornelissen, J. von der Thüsen, A. de Klein, E. Kilic

\section{Erasmus MC, Rotterdam, Netherlands}

The BAP1 tumor predisposition syndrome (BAP1-TPDS) is a hereditary tumor syndrome caused by germline pathogenic variants in BAP1. It is associated with increased risks for uveal melanoma, mesothelioma, cutaneous melanoma, renal cell carcinoma, and cutaneous BAP1-inactivated nevi. A 50 year old female patient presented with pneumothorax, caused by bilateral mesothelioma, with no history of asbestos exposure. Molecular analysis of the tumor showed a pathogenic variant and a variant of unknown significance (the c.535C $>\mathrm{T}$, p.(Arg179Trp) variant) in the right tumor. The left tumor showed the same variant of unknown significance (VUS) as found in the other tumor, but no second mutation. Both tumors showed no staining for BAP1 by immunohistochemistry. DNA analysis of a blood sample confirmed that the VUS is present in the germline. RNA analysis showed expression of both the wild type and the variant allele in the same amount. Clinical work up of the patient showed no signs of uveal melanoma. Testing of healthy parents showed the VUS is present in the 80 year old mother of the proband. In the meantime the mother also developed mesothelioma, with no history of asbestos exposure. Molecular analysis of the tumor of the mother showed the VUS that is present in germline and another somatic VUS in the other BAP1 allele. The tumor was positive for staining of the BAP1 protein. Family history is positive for basal cell carcinoma, leiomyosarcoma of the kidney and lung cancer. At the moment further testing of affected family members is conducted.

B.W. van Paassen: None. N.M. van Poppelen: None. R. Cornelissen: None. J. von der Thüsen: None. A. de Klein: None. E. Kilic: None.

\section{P12.012.B}

Clinically important variants in Slovene bilateral breast cancer patients tested between 2014 and 2019

G. Klancar ${ }^{l}$, P. Skrel ${ }^{l}$, V. Setrajcic Dragos ${ }^{l}$, V. Stegel ${ }^{l}$, A. Blatnik $^{2}$, K. Strojnik ${ }^{2}$, M. Banjac ${ }^{2}$, M. Krajc ${ }^{2}$, S. Novakovic ${ }^{l}$

${ }^{1}$ Institute of Oncology, Division of Diagnostics, Department of Molecular Diagnostics, SI-1000 Ljubljana, Slovenia, ${ }^{2}$ Cancer Genetics Clinic, Institute of Oncology Ljubljana, Ljubljana, Slovenia, SI-1000 Ljubljana, Slovenia

Introduction: Bilateral breast cancer accounts for 2-11\% of all breast cancer cases. By the literature, $5-30 \%$ of patients with bilateral breast cancer carry a pathogenic variant in the high-penetrant $B R C A$ genes. Our aim was to determine the spectrum of pathogenic variants in HBOC genes among Slovene bilateral breast cancer patients.

Materials and Methods: Among 2483 HBOC patients tested between years 2014 and 2019, 205 patients with bilateral breast cancer were included in the study. gDNA was extracted from peripheral blood and NGS sequencing was performed using Nextera_DNA_Library_Preparation_Kit in combination with Illumina's TruSight_Cancer_Panel or TruSight_Hereditary_Panel. All variants were classified according to ACMG_guidelines.

Results: Among 205 patients with bilateral breast cancer, $124(60.5 \%)$ had positive HBOC family history and 81 (39.5\%) had no HBOC family history. A pathogenic variant in one of HBOC genes listed in the NCCN guidelines was detected in 58 patients, which represents $28.3 \%$ of all patients. Most of them (67.2\%) were patients with positive HBOC family history. Majority of patients with pathogenic variant harbored variant in BRCA genes: $50.0 \%$ in BRCAl and $27.6 \%$ in $B R C A 2$. Other pathogenic variants were discovered also in other genes CHEK2 (6.9\%), PALB2 (5.2\%), TP53 (3.4\%), CDKN2A (3.4\%), PTEN (1.7\%) and PMS2 (1.7\%).

Conclusions: Majority of bilateral breast cancer patients with positive family history had pathogenic variant in HBOC genes: $68.9 \%$ of cases in BRCA genes and $61.5 \%$ cases in other genes. The frequency of BRCA pathogenic variants in Slovene patients with bilateral breast cancer is similar to frequencies reported in the literature.

G. Klancar: None. P. Skrel: None. V. Setrajcic Dragos: None. V. Stegel: None. A. Blatnik: None. K. Strojnik: 
None. M. Banjac: None. M. Krajc: None. S. Novaković: None.

P12.013.C

Comprehensive analysis of prominent molecular biomarkers for TGCT diagnostics

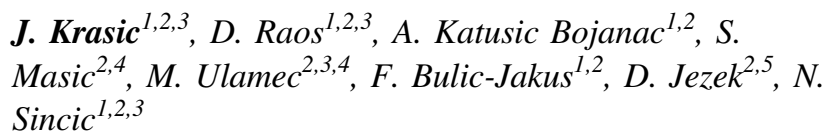

${ }^{1}$ Department of Medical Biology, School of Medicine University of Zagreb, Zagreb, Croatia, ${ }^{2}$ Centre of Excellence for Reproductive and Regenerative Medicine, School of Medicine University of Zagreb, Zagreb, Croatia, ${ }^{3}$ EpiMark, Group for Research on Epigenetic Biomarkers, Zagreb, Croatia, ${ }^{4}$ University Clinical Hospital Center Sestre milosrdnice, Ljudevit Jurak Clinical Department of Pathology and Cytology, Zagreb, Croatia, ${ }^{5}$ Department of Histology and Embryology, School of Medicine University of Zagreb, Zagreb, Croatia

Introduction: Testicular germ cell tumours (TGCTs) are classified into seminoma (SE) and non-seminoma (NSE) of various types. They originate from germ cell neoplasia in situ (GCNIS) caused by genetic and epigenetic incidents. With currently used TGCT biomarkers lacking specificity and sensitivity, stronger discriminatory biomarkers for various forms of TGCTs are needed. Our goal was to evaluate the diagnostic potential of POU5F1, NANOG, KIT, SALL4, HOXA9 and MGMT assessed at the protein level (current clinical use) alongside mRNA expression, DNA alteration and DNA methylation using prominent databases.

Materials and Methods: For immunohistochemical detection, 108 FFPE TGCTs and 48 tumour-free testes were analysed semi-quantitatively. TGCT patient's data were retrieved from TCGA while healthy adult testis data from GTEx datasets, and analysed using XENA, UALCAN and cBioPortal platforms.

Results: Our results suggest that TGCT could be discriminated from healthy tissue by difference in mRNA levels (all investigated genes), while SE from NSE by increased mRNA level (KIT) and DNA hypomethylation (MGMT and HOXA9). Identification of individual NSE types and GCNIS was possible only by analysing gene expression at the protein level (HOXA9, KIT, SALL4 and $P O U 5 F 1)$, since databases present NSE as a single entity and no data on GCNIS.

Conclusions: There is discriminatory value in gene expression at the mRNA level and DNA methylation of analysed genes in TGCT diagnostics. Promoting the inclusion of further molecular analyses, rather than just at the protein level, enhances the development of TGCT diagnostics as has recently been done for other types of tumours.

J. Krasic: None. D. Raos: None. A. Katusic Bojanac: None. S. Masic: None. M. Ulamec: None. F. Bulic-Jakus: None. D. Jezek: None. N. Sincic: None.

\section{P12.014.A}

BIRC5 gene polymorphisms and gene expression of eight different isoforms in breast cancer

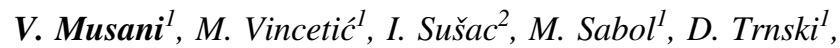
P. Ozretic ${ }^{1}$

${ }^{1}$ Rudjer Boškovic Institute, 10000 Zagreb, Croatia, ${ }^{2}$ Eljuga Polyclinic, 10000 Zagreb, Croatia

Introduction: Survivin (encoded by BIRC5 - baculoviral IAP repeat containing 5) gene is the smallest member od the family of inhibitors of apoptosis (IAP) proteins. Breast cancer (BC) is one of the most common cancers and is one of the leading causes of cancer related deaths in women in the world.

Materials and Methods: The whole coding region, including alternate exons $\mathrm{S}-2 \alpha, \mathrm{S}-2 \mathrm{~B}$ and $\mathrm{S}-3 \mathrm{~B}$, as well as 5' and 3'UTR regions of BIRC5 gene was genotyped in eighteen $\mathrm{BC}$ patients. Eight different BIRC5 gene isoforms (S-WT, S- $2 \alpha, \mathrm{S}-2 \mathrm{~B}, \mathrm{~S}-3 \alpha, \mathrm{S}-3 \mathrm{~B}, \mathrm{~S}-\Delta \mathrm{ex} 3, \mathrm{~S}-3 \gamma \mathrm{M}$ and S$3 \gamma \mathrm{V}$ ) were analyzed at the mRNA expression level in $\mathrm{BC}$ samples and matching healthy tissues. TBP gene was used as housekeeping control.

Results: Eleven different polymorphisms were found in the breast cancer samples. All eight isoforms were detected in BC samples, but isoforms $\mathrm{S}-3 \gamma \mathrm{M}$ and $\mathrm{S}-3 \gamma \mathrm{V}$ were not expressed in any of the healthy matching tissues. S-WT and $\mathrm{S}-2 \alpha$ isoforms had the highest expression, followed by $\mathrm{S}$ $\Delta$ ex3, S-3B, S-2B, S-3 $\alpha$, and $\mathrm{S}-3 \gamma \mathrm{M}$ and $\mathrm{S}-3 \gamma \mathrm{V}$ had the lowest. All isoforms, except for $\mathrm{S}-2 \mathrm{~B}, \mathrm{~S}-3 \gamma \mathrm{M}$ and $\mathrm{S}-3 \gamma \mathrm{V}$, had significantly higher expression in cancer tissue in comparison to healthy tissue. Expression of three isoforms, S-3 $\alpha$, S-3 $\gamma \mathrm{M}$ and S-3 $\gamma \mathrm{V}$ showed significant association with polymorphisms c.-644T $>$ C, c.9386T $>C$, c.9809T $>C$ and c. $10611 \mathrm{C}>\mathrm{A}$.

Conclusions: At least eight different BIRC5 isoforms are expressed in breast cancer. The isoforms showed significant positive association in BC tissues, and less so in healthy tissue. The isoforms showed significant association with different polymorphisms. 
V. Musani: None. M. Vincetić: None. I. Sušac: None. M. Sabol: None. D. Trnski: None. P. Ozretić: None.

P12.016.C

Rare congenital disease: Bloom syndrome. Complex cytogenetic and molecular genetic analysis

R. Michalovská ${ }^{l}$, V. Krulišovál, T. Píšll L. Hruškovál ${ }^{l}, H$. Paszekovál, M. Gančarčíková ${ }^{2}, Z$. Vlčkovál

${ }^{1}$ GHC Genetics, Praha, Czech Republic, ${ }^{2} L L G$, Pardubice, Czech Republic

Introduction: Bloom syndrome (BS) is a very rare autosomal recessive disease characterized by prenatal and postnatal growth retardation, photosensitive skin changes, immune deficiency, insulin resistance, and significantly increased risk of cancer in very young age. BS is caused by pathogenic variants in the $B L M$ gene. Absence of functional BLM protein causes chromosome instability, excessive homologous recombination, and increased numbers of sister chromatids exchanges.

Materials and Methods: We performed massive parallel sequencing on NextSeq (Illumina) using Sequence Capture (ROCHE) panel of 55 genes. Examination of spontaneous chromosome aberrations (ZCA) and classical conventional cytogenetic analysis ( $\mathrm{G}$ band) were used as standard cytogenetic procedures.

Results: The 12 year old boy born from second physiological pregnancy $(\mathrm{sp} 2760 \mathrm{~g}$, PD $46 \mathrm{~cm}$ ) was genetically examined for a number of non-specific clinical problems and due to the occurrence of cancer in the family, respectively. We detected variants in $B L M$ gene in the compound heterozygous state c.1642C $>\mathrm{T}$ (p.Gln548Ter, rs200389141, inherited maternally, class 5) and c.2015A $>\mathrm{G}$ (p.Gln672Arg, rs747281324, inherited paternally, class 4), which confirmed clinical diagnosis of Bloom syndrome. Subsequent cytogenetic analysis of SCE and ZCA supports the outcome of molecular genetic testing and presents complex examination in the rare chromosome instability syndrome of the genome

Conclusions: Given the anamnestic data, a very rare disease called Bloom syndrome was predicted as a possible genetic background of the patient's phenotype.

R. Michalovská: None. V. Krulišová: None. T. Píss: None. L. Hrušková: None. H. Paszeková: None. M. Gančarčíková: None. Z. Vlčková: None.

P12.017.A

Differential expressed genes in BRAF-positive versus BRAF-negative papillary thyroid carcinomas
S. Schipor, D. Manda, C. Poalelungi, I. Nedelcu, A. Muresan

"C. I. Parhon" National Institute of Endocrinology, Bucharest, Romania

Introduction: BRAFV600E is the most prevalent mutation in patients with papillary thyroid carcinoma (PTC). The successful targeted therapies against this mutation explain the importance of the analysis of concomitant molecular transformations in this pathology.

Materials and methods: In order to evaluate the differential gene expression profile between BRAF-positive and BRAFnegative samples in PTC patients we used DNA Agilent microarray. There were included 24 patients ( 8 classical PTC, 14 follicular variant of PTC, 2 insular and/or sclerosing variant of PTC), with 2 samples per patient (tumor and normal samples) obtained shortly after surgery. Patients gave their informed consent for study before surgery. Microarray analysis was performed following Agilent One-Color Microarray-Based Gene Expression protocol, ver 6.6, using SurePrint G3 Human Gene Expression arrays $8 \times 60 \mathrm{Kv} 2$. BRAF status was determined by Sanger sequencing.

Results: The list of differential expressed genes (DEGs) between $\mathrm{BRAF}(+)$ and $\mathrm{BRAF}(-)$ samples was obtained by statistical analysis using GeneSpring GX-PA 14.5 software. Moderated T-Test with $\mathrm{p}<0.05$ identified 541 DEGs. Applying a fold-change $>2$ the list restricted to 536 DEGs (366 up-regulated, 170 down-regulated). A subsequent analysis (one-way ANOVA, $p$-value with Benjamini-Hochberg correction) gave a final list of 125 DEGs, 91 genes upregulated and 34 down-regulated between cPTC and the other types of tumors. The Kegg pathway analysis suggested "pathways in cancer" as the most significant signal pathway, followed by $\mathrm{N}$ glycan biosynthesis and glycolysis/gluconeogenesis.

Conclusions: Differential gene expression analysis could be a valuable source of information regarding the molecular changes and pathways involved in BRAF-related papillary thyroid carcinomas (CNCS-UEFISCDI grant PCCA-20113.2-1337)

S. Schipor: None. D. Manda: None. C. Poalelungi: None. I. Nedelcu: None. A. Muresan: None.

\section{P12.018.B}

Identification of BRCA1/2 large deletions in DNA isolated from FFPE tumor tissues and blood.

A. Podgorska ${ }^{l}$, A. Stachurska ${ }^{l}$, E. Kwiatkowska ${ }^{l}$, A. JaniecJankowskal, A. Grzelak ${ }^{l}$, D. Nowakowska ${ }^{2}$, M. ProchorecSobieszek ${ }^{l}$, A. Tysarowski ${ }^{l}$

${ }^{1}$ Department of Pathology and Laboratory Diagnostics, Maria Sklodowska-Curie National Research Institute of 
Oncology, Warsaw, Poland, ${ }^{2}$ Genetic Outpatient Clinic, Maria Sklodowska-Curie National Research Institute of Oncology, Warsaw, Poland

Introduction: Pathogenic germline mutations in $B R C A 1$ and $B R C A 2$ can be identified in more than $10 \%$ of hereditary breast and ovarian cancers. With the introduction of targeted therapy for BRCA-deficient recurrrent ovarian cancer and breast cancer, testing for somatic/germline mutations in BRCAl/2 genes in tumor tissue became essential for treatment decisions. Identification of pathogenic $B R C A 1 / 2$ variants, especially large rearrangements in DNA isolated form FFPE, is challenging due to the high fragmentation grade of formalin-treated DNA and chemical modifications that lower the DNA quality. Therefore, the aim of the study was to verify the usefulness of NGS analysis of BRCA1/2 large rearrangements detection in DNA isolated from blood and FFPE samples.

Materials and Methods: 920 blood samples from patients with ovarian/breast cancer and 233 FFPE tissue samples from ovarian cancer patients were enrolled. FFPE tumor cell content was determined by a pathologist and tumor tissue was micro-dissected prior to DNA isolation. The NGS technology was used to detect BRCAl/2 large rearrangements in all the samples. All detected BRCA1/2deletions were confirmed by MLPA method.

Results: In total we detected 18 large deletions: 12 in 920 blood-derived DNA and 6 in 233 FFPE isolated DNA. In 15 cases deletions were detected in BRCA1 gene and 3 in BRCA2.

Conclusions: The detection rate of large rearrangements was similar in both FFPE samples and blood derived DNA reaching $1 \%$ and $3 \%$, respectively. The results show that BRCA1/2 large rearrangements can be reliably detected in both FFPE and blood derived DNA by next-generation sequencing (NGS).

A. Podgorska: None. A. Stachurska: None. E. Kwiatkowska: None. A. Janiec-Jankowska: None. A. Grzelak: None. D. Nowakowska: None. M. Prochorec-Sobieszek: None. A. Tysarowski: None.

\section{P12.019.C}

Identification and characterization of regulatory variants in BRCA1/2 causing hereditary breast and ovarian cancer

J. Bublitz, J. L. van Luttikhuizen, S. Schubert, W. Hofmann, G. Schmidt, B. Schlegelberger, D. Steinemann

\section{Department of Human Genetics, Hannover, Germany}

Introduction: The genetic predisposition causing hereditary breast and ovarian cancer (HBOC) remains in the majority of patients unknown. We hypothesize that a subset of HBOC is caused by regulatory variants located outside of coding regions of the highly penetrant genes $B R C A l$ and BRCA2 (BRCA1/2).

Materials and Methods: We selected 96 high-risk HBOC patients tested negative for (likely) pathogenic variants in 94 cancer-associated genes using the TruSight Cancer panel and high resolution CGH-arrays. Their genomic BRCA1/2 loci including introns and flanking intergenic regions, comprising $140 \mathrm{~kb}$ each, were first enriched using a SureSelect custom panel and subsequently sequenced on Illumina NextSeq 500.

Results: Of 629 and 663 detected variants in $B R C A l$ and $B R C A 2$, respectively, 100 variants per gene had a MAF $\leq 0.1 \%$ in the general population (gnomAD) and $<5 \%$ in our cohort. Using in silico analyses, such as CADD, ncER, and Alamut, remaining variants were further prioritized. Among others, one BRCAl variant (c.5333-469T>A, intron 21 of 23 ) is predicted to create a novel acceptor splice site, which appears stronger than the canonical site. The variant might lead to intron retention, which is currently tested on transcription level to prove the predicted splicing effect.

Conclusions: As the field of genomic sequencing and variant interpretation rapidly progresses, regulatory variants should be included in the search for cancer predisposing variants. The identification and characterization of genetic predispositions outside the coding genome cannot only provide HBOC patients with superior treatment and prevention plans, but may also offer new insights into transcriptional regulation of $B R C A 1 / 2$.

J. Bublitz: None. J.L. van Luttikhuizen: None. S. Schubert: None. W. Hofmann: None. G. Schmidt: None. B. Schlegelberger: None. D. Steinemann: None.

\section{P12.020.A}

Is HRT safe for BRCA mutation carriers after risk reduction salpingo-oophorectomy? A 7.5 year cohort study

S. Gabizon-Peretz ${ }^{1}$, S. Paluch-Shimon ${ }^{1,2}$, S. Armon ${ }^{1,3}$, E. Levy-Lahad $^{1,4}$, R. Michaelson-Cohen ${ }^{1,3,4}$

${ }^{1}$ Hebrew University of Jerusalem, Jerusalem, Israel, ${ }^{2}$ Division of Oncology, Shaare Zedek Medical Centre, Jerusalem, Israel, ${ }^{3}$ Department of Obstetrics \& Gynecology, Shaare Zedek Medical Center, Jerusalem, Israel, ${ }^{4}$ Medical Genetics Institute, Shaare Zedek Medical Center, Jerusalem, Israel

Introduction: Hormonal replacement treatment (HRT) after Risk-Reducing-Salpingo-oophorectomy (RRSO) in BRCA1/2 mutation carriers is controversial. In the general population, HRT slightly raises breast cancer (BC) risk $(\mathrm{HR}=1.34)$. However, it is unclear whether this applies to mutation carriers. Our hypothesis was that short-term use of 
HRT from RRSO till age 50 would not elevate BC risk significantly more than baseline risk of healthy carriers.

Methods: A retrospective cohort of 306 healthy BRCA1/ 2 mutation carriers who had undergone RRSO was followed-up for a mean duration of 7.26 years. We compared BC incidence over time in carriers who received HRT vs. those who didn't.

Results: During follow-up, BC incidence was 22/150 $(14.7 \%)$ in HRT group vs. $16 / 156$ women $(10.2 \%)$ in nonHRT group $(\mathrm{OR}=1.371, \mathrm{NS})$, when controlled for age, the association appeared stronger $(\mathrm{OR}=1.996$, NS). However, survival analysis didn't demonstrate a significant difference in $\mathrm{BC}$ incidence $(\mathrm{HR}=1.777, \mathrm{NS})$, possibly due to shorter followup in HRT group (6.2 vs. 8.3 years, $p<0.001)$. Median age at $\mathrm{BC}$ diagnosis appeared earlier ( 43.5 vs. 49 years, respectively), and latency period to BC diagnosis seemed shorter in HRT group vs. non-HRT group (invasive BC 1.58 vs. 3.45 years, respectively, DCIS 1.92 vs. 6.52 years, respectively), however, neither of these differences was significant.

Conclusions: In our BRCA carrier cohort, followed for $\sim 7.5$ years post-RRSO, there is no evidence that HRT elevates breast cancer incidence, or significantly shortens the latency period between RRSO and cancer diagnosis. However, caution in HRT administration is warranted until larger, prospective studies show similar results.

S. Gabizon-Peretz: None. S. Paluch-Shimon: None. S. Armon: None. E. Levy-Lahad: None. R. MichaelsonCohen: None.

\section{P12.021.B}

Recurrent BRCA2 Pathogenic Exon 5-11 Duplication in the Christian Arab Population in Northern Israel

\section{N. Ekhilevitch}

rambam, HAIFA, Israel

Most of the genetic research of BRCA1/2 genes germline mutations in Israel have been focused on the Jewish Israeli Population. The Christian Arab population in Israel accounts for about $2 \%$ of the population. Unlike in the Jewish population founder $B R C A 1 / 2$ mutations have not yet been reported in the Christian Arab population.

Here we describe a recurrent $B R C A 2$ pathogenic rearrangement in four patients from different families with a personal and/ or familial history of Hereditary Breast and Ovarian Cancer (HBOC). During the years 2015-2019, 20 Christian Arab patients with a personal or familial history of breast (BC) and/ or ovarian (OC) cancer were referred to $B R C A 1 / 2$ testing (Sequencing and Copy Number Variants (CNV's) analysis). A recurrent gross duplication of the genomic region encompassing exons 5-10 and the 5' portion of exon 11 of the BRCA2 gene was found in 4 out of 20
(20\%) patients from different Christian Arab families. This duplicated is expected to result in an absent or disrupted protein product. A similar duplication has been reported in the literature in two individuals affected with BC. The Exon 5-11 BRCA2 Duplication is a recurrent rearrangement, possibly founder in the Christian Arab population in Northern Israel. It is important to include CNV's analysis in all Christian Arab patients suspected for HBOC.

Farther research is needed to evaluate the carrier frequency of this rearrangement in the Christian Arab population and the degree of its penetrance. These results should help guide the BRCA1/2 genes testing strategy in this population.

\section{N. Ekhilevitch: None.}

P12.022.C

Hereditary breast and ovarian cancer: two cases of double heterozigosity for pathogenic variants in the $B R C A 1$ or BRCA2 and ATM genes

P. Theisen ${ }^{l}$, P. Rodrigues ${ }^{l}$, C. Silva ${ }^{2,3}$, D. Carpinteiro $^{2}, L$. Ribeiro $^{4}$, H. Carreiro ${ }^{5}, H$. Gervásio ${ }^{6}$, J. Leal da Silva ${ }^{7}, L$. Vieira $^{2,3}$, J. Gonçalves ${ }^{3}$

${ }^{1}$ Molecular Genetics Unit, Human Genetics Department, National Institute of Health Dr. Ricardo Jorge, Lisbon, Portugal, ${ }^{2}$ Technology and Innovation Unit, Human Genetics Department, National Institute of Health Dr. Ricardo Jorge, Lisbon, Portugal, ${ }^{3}$ Centre for Toxicogenomics and Human Health (ToxOmics) Nova Medical School, Faculty of Medical Sciences, New University of Lisbon, Lisbon, Portugal, ${ }^{4}$ Hematology-Oncology Service, CUF Infante Santo Hospital, Lisbon, Portugal, Lisbon, Portugal, ${ }^{5}$ Oncological Risk Consultation, CUF Infante Santo Hospital, Lisbon, Portugal, Lisbon, Portugal, ${ }^{6}$ Hematology-Oncology Service, CUF Viseu Hospital, Viseu, Portugal, ${ }^{7}$ Medical Oncology Service, Luz Arrábida Hospital, Vila Nova de Gaia, Portugal

Introduction: Hereditary breast and ovarian cancer (HBOC) is estimated to represent 5-10\% of all breast and ovarian cancer cases. Pathogenic germline variants in BRCA1 and BRCA2 account for $25 \%$ of familial cases. The identification of genetic defects in HBOC patients allows detection of carriers that can benefit from cancer risk management protocols, and predictive genetic testing to atrisk family members, after appropriate genetic counseling. Two female patients with a personal and family history of cancer were studied by next-generation sequencing (NGS).

Methods: NGS using TruSight Cancer Panel (Illumina) followed by bioinformatic analysis of 18 genes associated with HBOC was performed. Pathogenic variants were confirmed by Sanger sequencing. 
Results: A rare event of double heterozigosity for pathogenic variants was identified in both patients: patient A was heterozygous for BRCA1:c.2037delinsCC, p. (Lys679Asn*4) and ATM:c.3802delG, p.(Val1268*) and patient B carried both BRCA2:c.6001dupT, p.(Ser2001Phefs*2) and ATM:3435_3436delTGinsA, p. (Asp1145Glufs*11). After genetic counseling, three relatives of patient A were analyzed: while one of her two healthy sons was heterozygous for the ATM variant, the other was a double heterozygote for BRCA1: c.2037delinsCC and ATM:c.3802delG; a female cousin, recently diagnosed with breast cancer, was a carrier of ATM:c.3802delG only.

Conclusions: The identification of these two rare cases of double heterozigosity for pathogenic variants in BRCAl/ $B R C A 2$ and $A T M$ genes, highlights the importance of using NGS-gene panel testing in HBOC. If molecular analysis had been restricted to $B R C A$ genes only, the pathogenic ATM variants would have been missed in both families, depriving them of appropriate genetic counseling and cancer risk management.

P. Theisen: None. P. Rodrigues: None. C. Silva: None. D. Carpinteiro: None. L. Ribeiro: None. H. Carreiro: None. H. Gervásio: None. J. Leal da Silva: None. L. Vieira: None. J. Gonçalves: None.

P12.023.A

The landscape of $B R C A 1$ and $B R C A 2$ variants from 300,000 individuals in the UK Biobank population sampling

\section{Z. Ghazoui}

AstraZeneca, Cambridge, United Kingdom.

Prior prevalence estimates of $B R C A 1$ and $B R C A 2$ proteintruncating variants (PTVs) have been generally limited to studies that have ascertained for cancer patients or individuals with a family history of cancer. The aim of this study is to utilise one of the largest sequenced Biobank populations to investigate the prevalence of BRCA1 and BRCA2 PTVs and ClinVar pathogenic/likely pathogenic classified missense variants. We analysed whole-exome sequence data from 300,000 UK Biobank participants (https://www.ukbiobank. ac.uk/), which is a prospective cohort study linked to deep phenotypic data. Among the 300,000 exomes, we identified 1332 carriers of high confidence rare PTVs in BRCA1/2 and another set of 62 carriers of rare ClinVar pathogenic/likely pathogenic classified missense variants in BRCA1/2. Of the 1394 BRCA1/2 putatively pathogenic variant carriers, 529 $(38.0 \%)$ have been diagnosed with cancer, 455 (32.6\%) have a reported family history of cancer but are not diagnosed with cancer and $410(29.4 \%)$ have no reported history (or family history) of cancer. For the 529 individuals with a cancer diagnosis we further evaluated the contribution that they have to 100 distinct ICD10 primary cancer phenotypes and identified that ovarian ( $5.3 \%$ of reported), breast ( $2 \%$ of reported), prostate (1\% of reported), pancreas (1.5\% of reported) and lung $(1.1 \%$ of reported) cancers are amongst the cancers that are significantly enriched for these BRCA1/2 putatively pathogenic variants. This study provides an unbiased summary of germline $B R C A 1 / 2$ contribution to various primary cancers and highlights the value of careful variant interpretation.

Z. Ghazoui: A. Employment (full or part-time); Significant; AstraZeneca.

P12.024.B

Mutational spectrum of tumor susceptibility genes in Bulgarian BRCA1/2 negative patients with ovarian cancer

M. Atanasoska ${ }^{1,2}$, R. Vazharova ${ }^{1,3}$, L. Balabanski ${ }^{1}, S$. Ivanov $^{l}$, S. Yaneva-Staykova ${ }^{1}$, D. Toncheva ${ }^{1,4}$

${ }^{1}$ Gynecology and assisted reproduction hospital "Malinov MD”, Sofia, Bulgaria, ${ }^{2}$ Sofia University St. Kliment Ohridski, Faculty of Biology, Department of Genetics, Sofia, Bulgaria, ${ }^{3}$ Sofia University St. Kliment Ohridski, Faculty of Medicine, Department of Biology, Medical genetics and Microbiology, Sofia, Bulgaria, ${ }^{4}$ Department of Medical Genetics, Medical University of Sofia, Sofia, Bulgaria

Introduction: There are more than 840 ovarian cancer cases in Bulgaria and more than 240000 worldwide, every year. OC represents $3.7 \%$ of all female cancers and it is the main cause for cancer deaths with gynecologic malignancy. Fifteen to thirty five percent of the familial cases are carriers of pathogenic variants in tumor suppressor genes. There are more than 16 known genes associated with hereditary ovarian carcinogenesis. Here we present a retrospective review of rare genetic variants in different tumor suppressor genes in BRCA1/2 negative Bulgarian patients with diagnosed ovarian cancer.

Materials and Methods: Our cohort includes 91 patients diagnosed with ovarian cancer. Their DNA samples were investigated for the presence of pathogenic variants in 94 cancer-predisposing genes. Sequence analysis was performed on an Illumina platform.

Results: We identified germline pathogenic variants in $24 \%(21 / 91)$ of the patients. The pathogenic variants in the 
BRCA1/2 genes were detected in $17 \%(16 / 91)$ of the patients. In $6.6 \%(5 / 75)$ of BRCA1/2 negative patients pathogenic loss-of-function variants were observed in $A T M$, CHEK2, FANCA, FANCL and BLM genes.

Conclusions: The risk of ovarian cancer increases parallel with the modernization and the social economic development. The precise and fast identification of pathogenic variants in the ovarian cancer-predisposing genes is a key in the decision-making process for therapy strategies. Our results gain a better understanding of the genetic complexity of the disease and may result in a more personalized approach for cancer risk management in a preventive settings, early diagnosis, prognosis and treatment of ovarian cancer.

M. Atanasoska: None. R. Vazharova: None. L. Balabanski: None. S. Ivanov: None. S. Yaneva-Staykova: None. D. Toncheva: None.

P12.026.A

Relevance of RNA analysis for the classification of $B R C A 1$ intronic variants and clinical management of family members

S. Sousa ${ }^{1,2}$, M. Ferreira ${ }^{1,2}$, S. Maia ${ }^{3,4}, F$. Ramos $^{3}, M$.

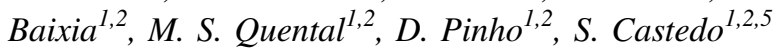

${ }^{1}$ Institute of Molecular Pathology and Immunology of the University of Porto (Ipatimup), Porto, Portugal, ${ }^{2}$ Institute for Research and Innovation in Health (i3S), University of Porto, Porto, Portugal, ${ }^{3}$ Medical Genetics Unit, Hospital Pediátrico, Centro Hospitalar e Universitário de Coimbra, Coimbra, Portugal, ${ }^{4}$ University Clinic of Genetics, Faculty of Medicine, University of Coimbra, Coimbra, Portugal, ${ }^{5}$ Faculty of Medicine, University of Porto, Porto, Portugal

Introduction: Carriers of $B R C A 1$ or $B R C A 2$ pathogenic variants have a high lifetime risk of developing breast and/or ovarian cancer. The most common pathogenic variants in BRCAl gene are nonsense and frameshift variants, however there are well documented pathogenic missense and splice site variants. Classification of intronic variants can be challenging and frequently demands further molecular studies. We identified a breast cancer (BC) family with six family members affected with BC. In the proband c. $4675+3 \mathrm{~A}>\mathrm{C} B R C A 1$ variant was identified, which was later found to be present in two more affected relatives (of the remaining patients, two were deceased and one awaits genetic counselling). Due to lack of further evidence, this variant was classified as variant of unknown significance, according to the ACMG guidelines. To clarify the clinical significance of this variant, we decided to evaluate its splicing impact.

Material and Methods: We performed RT-PCR in cDNA synthetized from a RNA sample of the proband, followed by Sanger sequencing, to evaluate the splicing impact of the c. $4675+3 \mathrm{~A}>\mathrm{C}$ BRCA1.

Results: We were able to identify an aberrant transcript, as a result of the creation of a new splice site, located 5upstream from the canonical site that is translated into a truncated protein, p.Gln1556Glyfs*14.

Conclusions: Based on these findings and the absence of this variant in the population, we were able to reclassify the c. $4675+3 \mathrm{~A}>\mathrm{C}$ BRCA1 variant as pathogenic, thereby warranting the genetic study of at risk relatives, and making carriers eligible for surveillance and, when clinically applicable, treatment with PARPi.

S. Sousa: None. M. Ferreira: None. S. Maia: None. F. Ramos: None. M. Baixia: None. M.S. Quental: None. D. Pinho: None. S. Castedo: None.

P12.028.C

Investigating of genetic variants, associated with breast cancer in Russian women

T. Lisitsa ${ }^{l}$, A. Danishevich ${ }^{l}$, M. Litvinova ${ }^{l}$, N. Bodunova ${ }^{l}$, E. Shagimardanova ${ }^{2}$, L. Shigapova ${ }^{2}$, O. Gusev v $^{2,3}$

${ }^{1}$ Moscow Clinical Scientific Center n.a. Loginov, Moscow Healthcare Department, Moscow, Russian Federation, ${ }^{2}$ Institute of Fundamental Medicine and Biology, Kazan (Volga Region) Federal University, Kazan, Russian Federation, ${ }^{3}$ Cluster for Science, Technology and Innovation Hub, RIKEN, Wako, Japan

Introduction: Breast cancer is the most common cancer in women. Genetic testing is used to identify hereditary breast cancer. Hereditary breast cancer is a polygenic disease, and next-generation sequencing allows the most comprehensive genetic examination of patients.

Materials and Methods: We performed the analysis of the genomic DNA of 62 patients with a diagnosis of breast cancer, who were treated in Moscow Clinical Scientific Center n.a. Loginov. The average age at diagnosis were $43 \pm 6.5$ years. Three patients were diagnosed with bilateral breast cancer, and another 4 had a combination of breast cancer and other malignancy. A family history of 31 patients was burdened with malignant tumors. All patients had negative test results for 8 frequent mutations in the BRCA1/2 genes. DNA was isolated from leukocytes of peripheral blood. For sample preparation, enrichment by hybridization with NimbleGen probes (Roche) was used.

Results: We identified 140 variants in 49 genes in 48 patients with a minor allele frequency $<1 \% .57$ variants were annotated as variants with uncertain clinical significance. In 12 patients, pathogenic and likely pathogenic variants were found in the genes BRCA1, BRCA2, MSH6, PALB2, TP53, CTNNA1, FANCE. 
Conclusions: It is necessary to conduct prospective monitoring of patients, as well as segregation analysis in families, to estimate clinical significance of the identified genetic variants. Investigation the effect of cancerassociated genes variants can help to understand the processes of carcinogenesis and individualize the treatment and further observation and provide genetic counseling for patients and their relatives.

T. Lisitsa: None. A. Danishevich: None. M. Litvinova: None. N. Bodunova: None. E. Shagimardanova: None. L. Shigapova: None. O. Gusev: None.

P12.030.B

Study of relative mRNA expression of TRAIL-induced apoptotic pathway components in breast carcinogenesis

E. Roupou ${ }^{l}$, M. Michelli ${ }^{l}$, I. Chatziandreou ${ }^{l}, N$. Michalopoulos $^{2}$, P. Karathanasis ${ }^{3}$, A. Lazaris ${ }^{1}$, A. Saetta ${ }^{l}$

${ }^{1} 1$ st Department of Pathology, School of Medicine, National and Kapodistrian University of Athens, Athens, Greece, ${ }^{2}$ Department of Surgery Attikon Hospital, School of Medicine, National and Kapodistrian University of Athens, Athens, Greece, ${ }^{3} 1$ st Department of Propaedeutic Surgery Hippokrateion Hospital, School of Medicine, National and Kapodistrian University of Athens, Athens, Greece

Introduction: Exploitation of TRAIL apoptotic pathway as a therapeutic target led in promising results in vivo, and although the efficacy of such monotherapies/combination therapies in clinical trials did not meet the expectations, TRAIL pathway still remains a promising target due to its high selectivity towards cancer cells. TRAIL pathway components' expression patterns could possibly serve as future predictive biomarkers for patient assortment regarding TRAIL targeting therapies.

Materials and Methods: Relative mRNA expression of TRAIL pathway genes (TRAIL, DR4, DR5, DcR1, DcR2, cFLIP, BCL2) was evaluated in 90 breast cancer tissues, using the RT-PCR/ $\Delta \Delta \mathrm{Ct}$ method. The SPSSv22 package was used for statistical analysis.

Results: The aforementioned genes presented elevated mRNA levels ranging from $8 \%$ to $17 \%$ of the cases and reduced mRNA levels between $31 \%-65 \%$ of the cases. Linear correlations were observed, indicating the simultaneous gene expression, with the strongest found between cFLIP/DR4 $(\mathrm{R}=0,709, \mathrm{p}<0,001)$ and cFLIP/DR5 $(\mathrm{R}=0,793, \mathrm{p}<0,001)$. Several significant statistical correlations with clinicopathological characteristics emerged: a) T-category with TRAIL, DcRI, cFLIP mRNA expression, b) $\mathrm{N}$ metastasis status with $D R 4, D c R 1, c F L I P$, c) p-Stage category with $c F L I P$, receptors' genes, and d) pPrognostic stage with $c F L I P, B C L 2$, receptors' genes.
Conclusions: In our study of different components of TRAIL apoptotic pathway, relative mRNA levels correlated with clinicopathological characteristics and multiple simultaneous expression patterns emerged. Taking into consideration these findings, it is of significant importance to stratify breast cancer patients using predictive biomarkers in order to maximize the efficacy of TRAIL targeting therapies.

E. Roupou: None. M. Michelli: None. I. Chatziandreou: None. N. Michalopoulos: None. P. Karathanasis: None. A. Lazaris: None. A. Saetta: None.

P12.031.C

Elucidating the role of microRNAs in cholesterol mediated drug resistance in breast cancer

\section{B. A. Monchusi, G. Palma, M. Kaur}

University of the Witwatersrand, Braamfontein, Johannesburg, South Africa

Introduction: Breast cancer is the most commonly diagnosed and leading cause of cancer-related deaths amongst women globally. TAM resistance is the major challenge faced during endocrine therapy in ER+ breast cancer. Several TAM resistance mechanisms have been proposed. Recently lipid reprogramming was reported to be involved in TAM resistance. It has emerged that certain miRNAs are involved in cholesterol metabolism and cancer drug resistance pathways. We hypothesise that miRNAs controlling cholesterol metabolism and homeostasis are potential targets to be investigated in cholesterol-mediated drug resistance in breast cancer.

Materials and methods: To investigate the role of miRNAs in cholesterol-mediated drug resistance, bioinformatic tools and in vitro cell based assays were utilised. Estrogen positive $(\mathrm{ER}+)$ breast cancer was of interest as drug resistance is a major problem in patients with ER+ breast cancer.

Results: We find that inhibition of hsa-miR-128-3p with $\mathrm{AP}+\mathrm{TAM}$ treatment induced cell death and decreased cholesterol levels in MCF7 cells. Through focused pathway study using the RT2 profiler microarray, we find that genes that were differentially expressed promoted cholesterol efflux and drug metabolism while genes that mediate drug resistance were reduced. The work also highlights another potential mechanism of AP in ER+ breast cancer, where it acts as a negative regulator of hsa-miR-128.

Conclusions: The findings demonstrate that targeting these miRNAs presents an opportunity for developing new strategies to combat anticancer drug resistance.

Acknowledgements: This research has been funded by grant \#113442 from the NRF. BM has been funded by 
Innovation Postdoctoral Scholarship \#11319414 from the NRF.

B.A. Monchusi: None. G. Palma: None. M. Kaur: None.

\section{P12.034.C}

Comparative analysis of MLPA and "CNV by NGS" for detecting copy number variations in hereditary cancer patients

N. B. Agaoglu ${ }^{1,2}$, G. Alkurt ${ }^{2,3}$, E. Celik ${ }^{2,3}$, I. M. Akcay ${ }^{2,3}$, B. Unal $^{1,2}$,J. Yildiz ${ }^{2,3}$, G. Dinler-Doganay ${ }^{2,3}$, L. Doganay ${ }^{2,4}$

${ }^{1}$ Department of Clinical Genetics, Umraniye Teaching and Research Hospital, University of Health Sciences, Umraniye, Istanbul, Turkey, ${ }^{2}$ GLAB (Genomic Laboratory), Health Directorate of Istanbul, Umraniye, Istanbul, Turkey, ${ }^{3}$ Department of Molecular Biology and Genetics, Istanbul Technical University, Maslak, Istanbul, Turkey, ${ }^{4}$ Department of Gastroenterology and Hepatology, Umraniye Teaching and Research Hospital, University of Health Sciences, Umraniye, Istanbul, Turkey

Copy number variations (CNVs) are the dosage difference of the genes and are among disease-causing mutations in hereditary cancer syndrome. MLPA (Multiplex Ligationdependent Probe Amplification) is widely used to detect CNVs. Recent advancements in bioinformatics made deletion/duplication analysis possible within the NGS data without any further experimentation. Here, with this study, we compared the results of MLPA with CNV detection algorithm (CNV by NGS) in colorectal and breast cancer patients.

Our study included 755 (683 breast-ovarian and 72 colorectal cancer) patients. MLPA test was performed using exon specific primers for BRCA1/2, MLH1, MSH2, MSH6, $P M S 2$ and EPCAM. NGS libraries were prepared by using targeted multi-gene capture panel (coding regions and splice junctions of aforementioned genes) and sequenced by NextSeq using paired-end reads. CNV by NGS analysis was performed with Sophia DDM bioinformatics software.

In 724 patients (96\%) no deletion/duplication was detected in CNV by NGS, consistent with the results of MLPA. In 21 patients (2.8\%), the same germline pathogenic variation was detected in both CNV by NGS and MLPA analyses. CNV by NGS gave a false positive result in 12 patients $(1.6 \%)$ and a false negative result in only 1 patient $(0.13 \%)$, in which MSH6 e1 deletion was only detected with MLPA.

Our experience reveals that the sensitivity of CNV by NGS is comparable to MLPA. Yet its considerably high false positive rates necessitate confirmation of the positive findings with an alternative method.

N.B. Agaoglu: None. G. Alkurt: None. E. Celik: None. I.M. Akcay: None. B. Unal: None. J. Yildiz: None. G. Dinler-Doganay: None. L. Doganay: None.

\section{P12.035.A}

RGS7 is recurrently mutated in melanoma and promotes migration and invasion of human cancer cells

n. qutob $^{1}$, I. Masuho $o^{2}$, r. emmanuel ${ }^{3}$, M. Alon $^{3}$, i. cohen $^{l}$, A. Dipizio $^{4}$, J. Madore ${ }^{5}$, A. Kahloum ${ }^{6}$, T. Ziv ${ }^{7}$, R. Levi ${ }^{3}, J$. Gartner $^{6}$, V. Hill ${ }^{6}$, J. Lin $^{8}$, Y. Hevroni ${ }^{3}$, P. Greenberg ${ }^{1}$, A. Brodezki $^{3}$, S. Rosenberg ${ }^{6}$, M. Kosloff, , N. Hayward ${ }^{10}$, A.

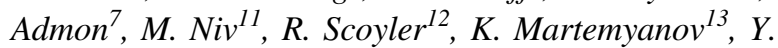
samuels ${ }^{3}$

${ }^{1}$ weizmann Institute of Science, rehovot, Israel, ${ }^{2}$ The Scripps Research Institute, florida, FL, United States, ${ }^{3}$ weizmann Institute of Science, Rehovot, Israel, ${ }^{4}$ hebrew University, rehovot, Israel, ${ }^{5}$ Royal Prince Alfred Hospital, ramallah, Australia, ${ }^{6}$ US National Institutes of Health, Bethesda, MD, United States, ${ }^{7}$ Technion-Israel Institute of Technology, haifa, Israel, ${ }^{8}$ US National Institutes of Health, Marylant, MD, United States, ${ }^{9}$ University of Haifa, Haifa, Israel, ${ }^{10}$ University of Sydney, Sydney, Australia, ${ }^{11}$ hebrew University, Rehovot, Israel, ${ }^{12}$ Royal Prince Alfred Hospital, Sydney, Australia, ${ }^{13}$ The Scripps Research Institute, Florida, FL, United States

Analysis of 501 melanoma exomes revealed RGS7, which encodes a GTPase-accelerating protein (GAP), to be a tumorsuppressor gene. RGS7was mutated in $11 \%$ of melanomas and was found to harbor three recurrent mutations (p.R44C, p. E383K and p.R416Q). Structural modeling of the most common recurrent mutation of the three (p.R44C) predicted that it destabilizes the protein due to the loss of an H-bond and salt bridge network between the mutated position and the serine and aspartic acid residues at positions 58 as 61, respectively. We experimentally confirmed this prediction showing that the p.R44C mutant protein is indeed destabilized. We further show RGS7 p.R44C has weaker catalytic activity for its substrate $\mathrm{G} \alpha_{0}$, thus providing a dual mechanism for its loss of function. Both of these effects are expected to contribute to loss of function of RGS7 resulting in increased anchorage-independent growth, migration and invasion of melanoma cells. By mutating position 56 in the R44C mutant from valine to cysteine, thereby enabling the formation of a disulfide bridge between the two mutated positions, we slightly increased the catalytic activity and reinstated protein stability, leading to the rescue of RGS7's function as a tumor suppressor. Our findings identify RGS7 as a novel melanoma driver and point to the clinical relevance of 
using strategies to stabilize the protein and, thereby, restore its function.

N. qutob: None. I. Masuho: None. R. emmanuel: None. M. Alon: None. I. cohen: None. A. Dipizio: None. J. Madore: None. A. Kahloum: None. T. Ziv: None. R. Levi: None. J. Gartner: None. V. Hill: None. J. Lin: None. Y. Hevroni: None. P. Greenberg: None. A. Brodezki: None. S. Rosenberg: None. M. Kosloff: None. N. Hayward: None. A. Admon: None. M. Niv: None. R. Scoyler: None. K. Martemyanov: None. Y. samuels: None.

P12.036.B

Unsettling NGS findings related to 94 cancer genes in pediatric patients- is there benefit or harm in reporting?

\section{A. Chirita Emandi ${ }^{l}$, N. Andreescu ${ }^{l}$, C. G. Zimbru ${ }^{1,2}$, M. Puiu ${ }^{l}$}

${ }^{1}$ Center of Genomic Medicine, Medical Genetics Discipline, University of Medicine and Pharmacy "Victor Babes", Timisoara, Romania, ${ }^{2}$ Department of Automation and Applied Informatics, Politehnica University Timisoara, Timisoara, Romania

Large panels of genes - also called the "clinical exome" are increasingly used in medical practice, where "incidental" or "unsolicited" findings are common. There is a lack of consistency regarding protocols for addressing such findings between different laboratories. The benefit of reporting secondary findings in NGS related to cancer genes in children may have implications for family members, nevertheless, could also cause distress.

Aim: to retrospectively investigate germline variants in 94 genes implicated in oncogenesis, in patients referred to NGS testing for various rare genetic diseases and reevaluate the utility of reporting different classes of pathogenicity.

Methods: We used in silico prediction software to classify variants and conducted manual review to further examine secondary findings frequencies in 229 children with rare diseases, that underwent sequencing using a 4813 gene panel.

Results: The reanalysis revealed 27907 variants, of which 176 were considered after filtering. Nine pathogenic expected variants (in $B R C A 1, B M P R 1 A, F A N C A, F A N C B$, $F A N C C, N B N, S T K 11, M S H 6)$ and four unexpected variants (in $B R C A 2, P A L B 2, P M S 2, R A D 50$ ) were reported to patients. Five secondary, probably pathogenic variants in $A T M, A T R, B L M$ (in two patients), SUFU and $S D H B$ genes, presented potential cancer susceptibility, raised ethical debate in reporting them.
Conclusions: In retrospect, $4.4 \%$ (10/229) of individuals in our cohort had unexpected NGS findings related to cancer. More efforts are needed to create an updatable consensus in reporting variants in cancer predisposing genes, especially for children. Strategies are required to ensure that consent process informs of both value and risk of additional genetic information.

A. Chirita Emandi: None. N. Andreescu: None. C.G. Zimbru: None. M. Puiu: None.

\section{P12.040.C}

Somatic mutation analysis of cell-free DNA in the plasma of gastric cancer patients

\section{G. Streleckiene ${ }^{1}$, M. Forster ${ }^{2}$, L. Kupcinskas ${ }^{1}$, J.} Skieceviciene ${ }^{1}$

${ }^{1}$ Lithuanian University of Health Sciences, Kaunas, Lithuania, ${ }^{2}$ Institute of Clinical Molecular Biology, Kiel, Germany

Introduction: Since the discovery of the circulating plasma cell-free DNA (cfDNA) the origin and characteristics of cfDNA were extensively studied. It was shown that cfDNA could harbor genetic aberrations from malignant tissue. However, there is a lack of comprehensive studies conducted in gastric cancer (GC) comparing tumor tissue DNA and plasma cfDNA mutational profiles. The aim of this study was to analyze cfDNA yield and to compare tumor tissue DNA and cfDNA mutational profiles for GC patients.

Materials and Methods: GC tissue and blood were collected from 30 patients who were recruited at the Department of Gastroenterology, LUHS Hospital. Tumor tissue was obtained from the primary lesion and peripheral blood was drawn before the surgery. cfDNA amount was quantified by laser-induced fluorescence-based microcapillary electrophoresis. Whole exome sequencing was performed for the tissue samples and targeted sequencing for the plasma cfDNA samples.

Results: Significantly higher yield of cfDNA was determined for GC patients. Mutations in GC related genes were detected for $76.7 \%$ of the patients. Top 10 mutated genes: KMT2C, TP53, TTN, FAT4, SYNE1, EPHB1, GLI3, PIK3CA, TRRAP, and APC. A custom gene panel was created for plasma cfDNA sequencing and analysis. Finally, correlation of mutant allele frequencies between tissue and plasma cfDNA samples was determined.

Conclusions: Our results demonstrated that cfDNA yield is significantly higher in GC patients and reflects mutation profile in GC tissue, therefore may enable cfDNA analysis for monitoring of the GC patients' disease state. Supported by the grant from Research Council of Lithuania No. LMTK-712-01-0130_MULTIOMICS. 
G. Streleckiene: None. M. Forster: None. L. Kupcinskas: None. J. Skieceviciene: None.

P12.041.A

BCR-ABL1 fusion gene acquisition of endothelial cells from K562 spent serum

\section{C. Ç. Koçana, S. F. Toprak, S. Sözer}

Aziz Sancar Institute of Experimental Medicine, Istanbul, Turkey

Introduction: Chronic Myeloid Leukaemia(CML) is a hematopoietic stem cell originated slow-growing myeloproliferative neoplasm. CML is associated with $B C R-A B L 1$ located on Philadelphia chromosome[t(9;22)(q34;q11)]. Recent studies revealed that some leukemic cells might release $B C R-A B L 1$. Our hypothesis is; the cell-free $B C R$ $A B L 1$ might transfect the susceptible cells and may transform them into leukemic cells. This study aims to understand whether endothelial cells EC could acquire cell-free $B C R-A B L 1$ in vitro.

Materials-Methods: $B C R-A B L 1$ positive CML cell line (K562) is cultivated and the spent media was collected every 96 hours, some saved for DNA isolation and rest was aliquot and stored in $-80^{\circ} \mathrm{C}$. $B C R$ - $A B L 1^{-}$EC line(HUVEC) was cultured with $1 \times 10^{5} / \mathrm{ml}$ density in 96-well plates for transfection. HUVEC culture media was mixed with $40 \mu \mathrm{l}$ of K562 spent media without any nucleases and incubated for $48 \mathrm{~h}$ at $37^{\circ} \mathrm{C}$. After $48 \mathrm{~h}$, HUVEC was washed and detached from the plate. Following gDNA isolation, PCR analysis were performed for $B C R-A B L 1$. BCR-ABL1 product of $418 \mathrm{bp}$ was analysed by electrophoresis in $2 \%$ gel.

Results: $B C R-A B L 1^{-}$HUVEC was transfected easily with $B C R-A B L 1^{+}$K562 spent media. BCR-ABL1 insertion into the HUVEC genome was confirmed by PCR analysis.

Conclusions: Metastasis of the haematological malignancies usually referred to the cancer cells migration and insertion into the different distant organs. Some studies revealed the theory of genometastasis; transfection of healthy cells by cell-free DNA without any induction. This study provides evidence for $B C R-A B L 1$ insertion into the different type of cells from their origin including EC. This process might also provide some answers to other cancer prognosis as well. Project No:TDB-2019-28744

C.Ç. Koçana: None. S.F. Toprak: None. S. Sözer: None.

P12.042.B

EGFR mutation testing in cf-DNA from NSCLC patients using Cobas $^{\circledR}$ EGFR mutation test v2
M. Michelli, I. Giannopoulou, I. Chatziandreou, A. Zougros, E. Roupou, A. A. Saetta

1st Department of Pathology, School of Medicine, National and Kapodistrian University of Athens, Athens, Greece

Introduction: EGFR mutation analysis in circulating cellfree tumor DNA (cf-DNA) from plasma constitutes a convenient and less invasive alternative method for NSCLC patients without adequate tumor material and for disease monitoring.

Materials and Methods: We examined 420 blood samples from patients with NSCLC of which 102 had also FFPE tissue tested. Circulating cell-free tumor DNA (cfDNA) isolation from plasma and detection of EGFR mutations was performed using Cobas ${ }^{\circledR}$ EGFR mutation v2.

Results: EGFR mutations in cf-DNA were detected in $26 \%$ of the samples. The most common mutations were deletions in exon $19(62 \%)$, followed by point mutations in exon 21 (31\%). A resistance mutation, p.Thr790Met was displayed in $24 \%$ of the mutant cases. The presence of mutations in primary samples reached $12 \%$ whereas for follow up samples the percentage of mutations was $40 \%$. Three out of fifteen tissue samples with Invalid cobas result, showed presence of EGFR mutation in cfDNA. The presence of EGFR mutations in plasma and tumor tissues was correlated with gender $(\mathrm{p}<0.001, \mathrm{p}=0.031)$ as were more common in female patients. The overall concordance of EGFR mutation status in plasma and tumor was $80 \%$, specificity $100 \%$, PPV $100 \%$, NPV $72 \%$.

Conclusions: The above analysis adds to current data indicating that cf-DNA EGFR mutation testing has a very high PPV. The PPV indicates that EGFR mutations could be reported with certainty, whereas the sensitivity of the test indicates that a biopsy should be obtained for patients with an EGFR mutation-negative cfDNA test.

M. Michelli: None. I. Giannopoulou: None. I. Chatziandreou: None. A. Zougros: None. E. Roupou: None. A.A. Saetta: None.

P12.043.C

Selection criteria for assembling a pediatric cancer predisposition gene panel for use in routine diagnostics

A. Byrjalsen ${ }^{\text {, I. J. Diets }}{ }^{2}$, J. Bakhuizen ${ }^{3,4}$, T. van Overeem Hansen $^{l}$, K. Schmiegelow ${ }^{5}$, A. Gerdes ${ }^{l}$, R. P. Kuiper ${ }^{3}, H$. Merks $^{3}$, K. Wadt ${ }^{l}$, M. C. J. Jongmans ${ }^{3,4}$

${ }^{1}$ Department of Clinical Genetics, Rigshospitalet, Copenhagen, Denmark, ${ }^{2}$ Department of Human Genetics, Radboud university medical center, Nijmegen, Netherlands, ${ }^{3}$ Princess Máxima Center for pediatric oncology, Utrecht, 
Netherlands, ${ }^{4}$ Department of Genetics, University Medical Center Utrecht, Utrecht, Netherlands, ${ }^{5}$ Department of Paediatrics and Adolescent Medicine, Rigshospitalet, Copenhagen, Denmark

Introduction: Genomic sequencing technologies are used increasingly in childhood cancer diagnostics and research. This provides opportunities for standardized screening of both known and putative cancer predisposition syndromes (CPS) in children with cancer. This confers a challenge regarding what gene panel to use in a diagnostic setting.

Methods: Criteria were developed to select genes eligible for a childhood CPS gene panel. Genes were divided into two categories: 1) genes associated with conditions that can only be caused by this specific gene, and 2) grouped genes that give rise to the same CPS. The criteria were applied to multiple sources of in total 392 candidate genes.

Results: Single genes were included in the panel when a minimum of five children with cancer and a pathogenic variant in the gene were reported in literature. In addition, evidence must be present supporting a causal relation with the malignancy (i.e. studies describing somatic second hits in the tumor or segregation in families). Genes were grouped if they are associated with a heterogenous CPS and if they share the same cellular pathway or mechanism. At least two genes in the group must fulfill the criteria for individual genes for the group to be included in the panel. In total, we included 139 genes in the childhood cancer gene panel.

Conclusions: We have made criteria for the development of a childhood cancer gene panel. Currently, 139 genes are included in the gene panel. Regular updates in the future are necessary for novel genes.

A. Byrjalsen: None. I.J. Diets: None. J. Bakhuizen: None. T. van Overeem Hansen: None. K. Schmiegelow: None. A. Gerdes: None. R.P. Kuiper: None. H. Merks: None. K. Wadt: None. M.C.J. Jongmans: None.

\section{P12.044.A}

Combination of Panobinostat and Chloroquine as a therapeutic strategy against ovarian cancer

M. Ovejero-Sánchez ${ }^{1,2,3}$, D. Iglesias-Corral ${ }^{2,1,3}$, P. MartínBejarano Soto ${ }^{4,2}$, M. A. Pérez-Nieto ${ }^{1,2}$, P. García-Vallés $^{2}$, R. González-Sarmiento ${ }^{1,2,3}$, A. B. Herrero ${ }^{1,2,3}$

${ }^{1}$ Institute of Biomedical Research of Salamanca (IBSAL), Salamanca, Spain, ${ }^{2}$ Molecular Medicine Unit, Department of Medicine, University of Salamanca, Salamanca, Spain, ${ }^{3}$ Institute of Molecular and Cellular Biology of Cancer (IBMCC), Salamanca, Spain, ${ }^{4}$ Institute of Molecular and
Cellular Biology of Cancer (IBMCC), University of Salamanca-CSIC, Salamanca, Spain

Introduction: Ovarian cancer (OC) is the most lethal gynecologic malignancy, therefore new and potent treatments are needed. Chloroquine is known to generate reactive oxygen species (ROS) causing DNA double strand breaks (DSBs). Some HDACi have been reported to inhibit DNA repair by homologous recombination (HR). This study aims to investigate the effect of Chloroquine, Panobinostat and their combination in the survival of different OC cell lines.

Material and methods: OC cell lines OVCAR-8, SKOV-3, IGROV-1 and A2780 were used. MTT, cell cycle and apoptosis assays were performed to test chloroquine and Panobinostat anticancer activity. Synergistic interaction between these two drugs was assessed using the median dose effect analysis of Chou and Talalay, and combination indexes were calculated. DCFH-DA staining was used to determine ROS generation. $\gamma$-H2AX and Rad51 foci were detected by immunofluorescence. To analyze HR, a reporter GFP cassette was integrated into the genome of SK-OV-3 and a unique DSB was introduced by transfection with an endonuclease encoding plasmid. HR-efficiency in the presence or absence of Panobinostat was calculated by quantifying the number of GFP + cells by flow cytometry.

Results: Chloroquine and Panobinostat decreased cell viability and induced G0/G1 and G2/M cell cycle arrest, respectively. The combination of both drugs caused a synergistic effect in all cell lines that was reverted by the addition of an antioxidant. Treatment with Panobinostat decreased the efficiency of HR due to the inhibition of Rad51 recruitment to DSBs.

Conclusion: Our study suggests that the ChloroquinePanobinostat combination could be a therapeutic strategy against OC.

M. Ovejero-Sánchez: None. D. Iglesias-Corral: None. P. Martín-Bejarano Soto: None. M.A. Pérez-Nieto: None. P. García-Vallés: None. R. González-Sarmiento: None. A.B. Herrero: None.

P12.045.B

Tumorsphere derived chordoma cancer stem cells have low migration and invasion capacities

N. EKIMCI GURCAN ${ }^{1,2}$, U. OZBEY $Y^{1,2}$, M. BAYINDIR

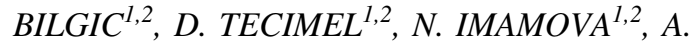
KUSKUCU ${ }^{l}$, O. F. BAYRAK

${ }^{1}$ Yeditepe University, School of Medicine, Department of Medical Genetics, Istanbul, Turkey, ${ }^{2}$ Yeditepe University, 
Institute of Natural and Applied Sciences, Department of Biotechnology, Istanbul, Turkey

Introduction: Tumorsphere formation is a widely used cancer stem cell (CSC) enrichment method. The protocol is based on cultivating cancer cells on a non-attachment surface with specific growth factors and absence of serum for enrichment of cancer cells with higher stem-like properties. Studies suggest that invasion and metastasis capabilities of cancer cells are driven by increased epithelial mesenchymal transition (EMT) status and associated with CSC phenotype. However, EMT characteristics of tumorspherederived CSCs are poorly defined. In this study, migration/ invasion capabilities of tumorsphere-derived chordoma CSCs, and their EMT related gene expression levels are investigated.

Materials and Methods: U-CH1 and MUG-Chor 1 Chordoma cell line derived tumorspheres were cultured on ultra-low attachment plate in serum free culture medium with specific supplements. Pluripotency and EMT related gene expression profiles were evaluated using qPCR. Migration capacity and invasiveness were assessed via Boyden chamber assay.

Results: Elevated SOX2, KLF4 and NANOG expressions in chordoma tumorspheres comparing parental cell lines indicates an enriched population with high pluripotency. Additionally, downregulation of CK19 and upregulation of Twist, Snail and ZEB1 in tumorspheres offers an epithelial-mesenchymal transition phenotype. In contrast migration/invasion capacities of tumorsphere derived cells are decreased, a signature EMT marker, CDH1 expression is increased.

Conclusions: Our results demonstrates that tumorsphere formation assay may enrich cancer stem-like cell populations with high stemness potential, but these tumorspherederived cells are losing their capacity to migrate, probably due to the high $\mathrm{CDH} 1$ expression. Suggesting, $\mathrm{CDH} 1$ has a therapeutic potential for chordomas with high migrative capacity.(TUBITAK project number: 118S691)

N. Ekimci gurcan: None. U. Ozbey: None. M. Bayindir bilgic: None. D. Tecimel: None. N. Imamova: None. A. Kuskucu: None. O.F. Bayrak: None.

\section{P12.046.C}

Genetic profiling of chronic lymphocytic leukemia patients in Estonia during 2018-2019

\section{Tooming ${ }^{l}$, P. Tammur ${ }^{l}$, A. Auser ${ }^{l}$, Ü. Murumets ${ }^{l}, U$. Šamarina ${ }^{1}$, T. Kahre ${ }^{1,2}$}

${ }^{1}$ Tartu University Hospital United Laboratories Department of Clinical Genetics, Tartu, Estonia, ${ }^{2}$ University of Tartu, Institute of Clinical Medicine, Department of

\section{Clinical Genetics, Tartu, Estonia}

Introduction: Chronic lymphocytic leukemia (CLL) is the most common type of leukemia in adults. Laboratory methods such as next-generation sequencing (NGS), fluorescence in situ hybridization (FISH) and chromosomal microarray (CMA) are used to investigate CLL patients. The aim of this study was to genetically profile and to get an overview of genetic alterations of the CLL patients in Estonia.

Patients and methods: 378 patient samples were referred to the Tartu University Hospital Clinical Genetics Center during 2018-2019 from 3 major tertiary referral hospitals. Patients were tested with Illumina TruSight Myeloid panel (NGS-54 genes), FISH (TP53, trisomy12, ATM deletion, DLEU1 deletion) and CMA (HumanCytoSNP-12). FISH or CMA was carried out on 343 patients. NGS was performed in 210 patients. Both NGS and FISH or CMA was performed in 175 patients.

Results: Altogether 50/378 patients (13,2\%) tested positive for TP53 mutations or del17p. TP53 pathogenic mutations were detected in $40(19 \%)$ patients. FISH/CMA revealed $23(6,7 \%)$ patients with del17p. $13(3,4 \%)$ patients harboured both TP53 mutations and del17p. Additionally, pathogenic mutations were found in $A B L 1, A S X L 1, B C O R$, BRAF, DNMT3A, GATA2, IDH2, KRAS, MYD88, NOTCH1, NRAS, RAD21, SF3B1, SRSF2 genes by NGS panel and by FISH trisomy12, ATM and DLEU1 deletions. Unfortunately, in 160 FISH/CMA-negative cases NGS was not performed and in 22 TP53-negative NGS cases FISH/ CMA was not analysed.

Conclusions: Both NGS and FISH/CMA testing should be considered to establish a status of TP53 mutation/del17p to improve patient stratification and optimize therapeutic decisions. Funding: Estonian Research Council grant PRG471.

M. Tooming: None. P. Tammur: None. A. Auser: None. Ü. Murumets: None. U. Šamarina: None. T. Kahre: None.

P12.047.A

Variants in the ASXL1 and \&gt; for DMNT3A genes may provide a personalized approach to targeted CML therapy

E. P. Adilgereeva ${ }^{1}$, A. G. Nikitin ${ }^{2}$, D. G. Zheglo ${ }^{1}$, O. A. Shukhov ${ }^{3}$, S. A. Smirnikhinal, E. Y. Chelysheva ${ }^{3}, A . V$. Lavrov $^{l}$, A. G. Turkina ${ }^{3}$, S. I. Kutsev ${ }^{l}$

${ }^{1}$ Research Centre for Medical Genetics, Moscow, Russian Federation, ${ }^{2}$ Pulmonology Research Institute, Federal Medical-Biological Agancy of Russia, Moscow, Russian Federation, ${ }^{3}$ National Research Center for Hematology, 
Moscow, Russian Federation

Introduction: Chronic myeloid leukemia (CML) accounts for about $15 \%$ of all leukemias in adults. Great success has been achieved in the treatment of CML due to the development of tyrosine kinase inhibitors (TKI), but $20-40 \%$ of patients are resistant to targeted therapy. The aim of the study is to detect exome variants predicting effectiveness of CML therapy.

Materials and methods: Blood was sampled from 60 CML patients at the time of diagnosis, before starting the TKI therapy. Later, 30 of the patients matched the ELN2013 criteria of optimal response, 18 were included in warning group, and 12 were resistant to therapy. Exome sequencing was performed on the Illumina NextSeq 550 Sequencing System. Bioinformatic analysis included SnpEff (analysis of all transcripts), ANNOVAR (analysis of allele frequencies in gnomAD, 1000G, and ESP6500, algorithms in silico prediction of pathogenity of SIFT, PolyPhen2, MutationTaster, FATMM, CADD, DANN, REVEL, M-CAP), and Alamut Batch (influence on splicing, dbsnp, ClinVar, COSMIC, and HGMD).

Results: Loss-of-function variants were revealed in the ASXL1 and DNMT3A genes in 33\% of patients in the group of resistant to therapy TKI, but not in other groups. $25 \%$ of patients in the treatment-resistant group had variants only in $A S X L 1$, while $8 \%$ had variants in both ASXL1 and DNMT3A.

Conclusions: The identified variants in ASXL1 and DNMT3A may be associated with resistance to TKI therapy and serve as prognostic markers of the TKI therapy effectiveness at the stage of CML diagnosis, and thus provide a personalized approach to the targeted CML therapy.

E.P. Adilgereeva: None. A.G. Nikitin: None. D.G. Zheglo: None. O.A. Shukhov: None. S.A. Smirnikhina: None. E.Y. Chelysheva: None. A.V. Lavrov: None. A.G. Turkina: None. S.I. Kutsev: None.

\section{P12.049.C}

Detection of Androgen Receptor in plasma circular RNAs in Prostate Cancer

\author{
P. Vázquez-Cárdenas ${ }^{1,2,3}$, A. Martel-Martel ${ }^{1,2,4}$, M. A. \\ Pérez-Nieto ${ }^{l}$, J. L. García-Hernández ${ }^{1,3}$, I. Misiewicz- \\ Krzeminska ${ }^{1,3}$, Á. J. Virseda-Rodríguez, S. Marcos- \\ Asensio $^{5}$, F. Gómez-Veiga ${ }^{5}$, J. J. Cruz-Hernández ${ }^{1,2,4}$, A. B. \\ Herrero $^{2,3}, R$. González-Sarmiento ${ }^{1,2,3}$
}

${ }^{1}$ Biomedical Research Institute of Salamanca (IBSAL), Salamanca, Spain, ${ }^{2}$ Molecular Medicine Unit, Department of Medicine, University of Salamanca, Salamanca, Spain, ${ }^{3}$ Institute of Molecular and Cellular Biology of Cancer (IBMCC), University of Salamanca-CSIC, Salamanca, Spain, ${ }^{4}$ Medical Oncology Service, University Hospital of Salamanca, Salamanca, Spain, ${ }^{5}$ Translational Research Group of Urology GITUR-IBSAL, University Hospital of Salamanca, Salamanca, Spain

Introduction: Androgen receptor (AR) plays a crucial role in prostate cancer $(\mathrm{PCa})$ and the presence of the receptor in blood cancer is being used as a biomarker. Circular RNAs (circRNAs) are a newly discovered type of endogenous non-coding RNAs, characterized by a closed continuous loop, which can regulate gene expression by acting as microRNA or RNA-binding protein sponges. In this work we wanted to test whether circRNAs derived from the AR gene (circ-AR) could also be detected in blood samples and used as PCa biomarker.

Methods: Peripheral blood was drawn from 35 patients with diagnostic of metastasic PCa. Whole blood $(10 \mathrm{~mL})$ was collected in EDTA tubes. Plasma was isolated from the supernatant by centrifugation at RT, 20 minutes and 1500 $\mathrm{rpm}$. Total RNA was extracted from patient serum/plasma and VCAP cell line using the miRNeasy and miRNeasy Mini kits respectively. RNA samples were treated with RNase $\mathrm{R}$ for 30 minutes at $37^{\circ} \mathrm{C}$ to digest linear RNA and circRNAs were isolated again. Circ-AR expression was analysed by qPCR using GAPDH mRNA for normalization. Divergent primers for circ-AR were designed using the CircInteractome webtool and the convergent primers of GAPDH were designed by Primer-BLAST.

Results: Circ-AR was detected by RT-qPCR in plasma PCa patients and in VCAP cell line. To validate the existence of a circ-AR, Sanger sequencing was performed on the PCR product amplified with the divergent primers.

Conclusions: Circ-AR was detected by RT-qPCR in all plasma samples, indicating that these molecules could be used as novel biomarkers in PCa.

P. Vázquez-Cárdenas: None. A. Martel-Martel: None. M.A. Pérez-Nieto: None. J.L. García-Hernández: None. I. Misiewicz-Krzeminska: None. Á.J. Virseda-Rodríguez: None. S. Marcos-Asensio: None. F. Gómez-Veiga: None. J.J. Cruz-Hernández: None. A.B. Herrero: None. R. González-Sarmiento: None.

P12.050.A

Analytical validation of the Droplet Digital PCR system for the detection of the most frequent clinically relevant EGFR, BRAF and KRAS hotspot tumor variants 
A. Hallermayr ${ }^{l}$, J. Romic-Pickl ${ }^{l}$, A. Benet-Pagès ${ }^{1,2}$, V. Steinke-Lange ${ }^{l}$, E. Holinski-Feder ${ }^{l}$

${ }^{1}$ MGZ - Medical Genetics Center, Munich, Germany, ${ }^{2}$ Genomics Institute, University of California Santa Cruz, Santa Cruz, CA, United States

Introduction: Precision medicine targeting specific genetic variants in cancer can be used for prediction and prognosis of disease progression and outcome. This requires accurate molecular profiling of the tumor, which is traditionally performed using DNA extracted from tumor tissue. Liquid biopsies can overcome some of the challenges regarding tissue biopsies and offer several advantages like real-time monitoring of driver hotspot variants, although highly sensitive methods are required to identify these variants in ctDNA.

Materials and Methods: The Droplet Digital ${ }^{\mathrm{TM}}$ PCR (ddPCR) technology requires low amounts of input DNA and enables quantification of low variant allele frequencies (VAF) of targeted variants, yet accuracy varies between assays and laboratory protocols.

Results: We conducted the analytical validation of five ddPCR assays, for the detection of the most frequent clinically relevant EGFR, BRAF and KRAS hotspot tumor variants and established precise thresholds between negative and positive results as well as VAF quantification. All assays showed very low limits of blank and detection ranging from $0 \%$ to $0.11 \%$ fractional abundance and $0.08 \%$ to $0.4 \% \mathrm{VAF}$ of the targeted variants, respectively. However, striking differences were observed between ddPCR assays for the detection of low VAFs of the targeted variants.

Conclusions: In particular, the observed difference in performance in low VAF variant detection and quantification demonstrates the importance of specific analytical validation for each assay to obtain reliable results of ctDNA analysis.

A. Hallermayr: None. J. Romic-Pickl: None. A. BenetPagès: None. V. Steinke-Lange: None. E. HolinskiFeder: None.

\section{P12.052.C}

\section{A case of $M S H 2$-related constitutional mismatch repair} deficiency syndrome

\section{E. M. Leter ${ }^{\text {I }}$ M. J. L. Ligtenberg ${ }^{2,3}$, R. S. van der Post ${ }^{3}$, N. Hoogerbrugge $^{2}$, A. R. Mensenkamp ${ }^{2}$}

${ }^{1}$ Department of Clinical Genetics, Maastricht University Medical Center, Maastricht, Netherlands, ${ }^{2}$ Department of Human Genetics, Radboud University Medical Center, Nijmegen, Netherlands, ${ }^{3}$ Department of Pathology, Radboud University Medical Center, Nijmegen, Netherlands
Constitutional mismatch repair deficiency (CMMRD) syndrome is a rare autosomal recessive hereditary cancer condition, characterized by early onset colorectal cancer, leukemia and lymphoma, brain tumors and other malignancies. We report a 24-year-old man referred to our outpatient genetic clinic because of rectal cancer. His sister had died of an astrocytoma at age 14. Their father was diagnosed with prostate, bladder and renal cancer at age 47 and 58. A paternal uncle died of esophageal cancer at age 50, a paternal aunt was diagnosed with breast cancer at age 70 and the paternal grandfather died of liver cancer at age 70 . The maternal grandfather died of bladder cancer at age 77 . The family history did not include additional cancer cases amongst first or second degree relatives. Immunohistochemistry of the rectal cancer showed mismatch repair deficiency with loss of MSH2, atypical diminished staining of MSH6 and presence of MLH1 and PMS2. Genetic analyses revealed two pathogenic heterozygous germline variants in $\mathrm{MSH} 2$, c.1759 $+3 \mathrm{~A}>\mathrm{G}$ (p.(Ser554fs)) and a deletion of the first exon, c. (?_-1)_(211+1_212-1)del. RNA analyses showed that the splice site variant results in a loss of exon 11 from the transcript. Parental testing showed that both variants were inherited from a different parent. The latter finding confirmed CMMRD due to bi-allelic pathogenic $M S H 2$ variants in our patient. Formalin-fixed paraffin-embedded tissue analysis revealed that his deceased sister also carried both $\mathrm{MSH} 2$ variants. This case again highlights how an index case of CMMRD may lack a clear family history of Lynch syndrome associated tumors.

E.M. Leter: None. M.J.L. Ligtenberg: None. R.S. van der Post: None. N. Hoogerbrugge: None. A.R. Mensenkamp: None.

\section{P12.053.A}

Colorectal Cancer in Adolescents and Young Adults: Comprehensive Analyses of the Clinical and Molecular Features

R. M. de Voer, I. Diets, R. van der Post, R. Weren, E. Kamping, T. de Bitter, L. Elze, R. Verhoeven, E. VinkBörger, A. Eijkelenboom, A. Mensenkamp, I. Nagtegaal, M. Jongmans, M. Ligtenberg

\section{Radboudumc, Nijmegen, Netherlands}

Colorectal cancer (CRC) at adolescent and young adult age (AYA; $\leq 25$ years) is a rare condition. Here we analyzed the clinicopathological and molecular features of CRC at AYA age to improve genetic counselling, surveillance and, ultimately, treatment and disease outcome. We retrospectively collected clinical information and tumor tissues from AYAs with CRC between 2000 and 2017 in the Netherlands. 
Tumor DNAs were analyzed for microsatellite instability, mutations in 56 hotspot regions and/or driver genes, and genome-wide somatic copy number aberrations. A total of 139 patients (58\% male) were diagnosed with $\mathrm{CRC} \leq 25$ years of age (mean age 22) between 2000 and 2017. Mucinous and/or signet-ring-cell components were observed in $33 \%$ of cancers. A genetic tumor risk syndrome was confirmed in $39 \%$ of cases. Negative predictors of outcome were a younger age at diagnosis, signet-ring-cell carcinoma, the absence of a genetic tumor risk syndrome, and a diagnosis at advanced stage of disease. Compared to CRCs $\geq 60$ years of age from The Cancer Genome Atlas, microsatellite stable (MSS) CRCs at AYA age more frequently harbor somatic mutations in TP53 and POLE, less frequently have $A P C$ mutations and are enriched for nearlydiploid MSS tumor genomes. Our data suggest that CRCs at AYA age have a biology and etiology different from sporadic CRCs at older age. Therefore, treatment of AYAs with CRC should be optimized based on their molecular genetic phenotypes. Performing comprehensive sequencing of germline and tumor DNA in every individual diagnosed with $\mathrm{CRC} \leq 25$ years of age would aid in this optimization.

R.M. de Voer: None. I. Diets: None. R. van der Post: None. R. Weren: None. E. Kamping: None. T. de Bitter: None. L. Elze: None. R. Verhoeven: None. E. VinkBörger: None. A. Eijkelenboom: None. A. Mensenkamp: None. I. Nagtegaal: None. M. Jongmans: None. M. Ligtenberg: None.

\section{P12.054.B}

Detection of germline genetic variants in hereditary colorectal cancer

\section{F. Eiengård ${ }^{1,2}$, A. Rohlin ${ }^{1,2}$, E. Mårtensson ${ }^{1}, T$. Olausson $^{1,2}$, T. Zagoras ${ }^{1,2}$, U. Lundstam ${ }^{3}$, S. Gebre- Medhin $^{4,5}$, M. Nordling ${ }^{1,2}$}

${ }^{1}$ Department of Laboratory Medicine, Institute of Biomedicine, Sahlgrenska Academy at University of Gothenburg, Gothenburg, Sweden, ${ }^{2}$ Department of Clinical Genetics and Genomics, Sahlgrenska University Hospital, Gothenburg, Sweden, ${ }^{3}$ Department of Surgery, Sahlgrenska Academy at University of Gothenburg, Sahlgrenska University Hospital/ Östra, Gothenburg, Sweden, ${ }^{4}$ Division of Clinical Genetics, Department of Laboratory Medicine, Lund University, Lund, Sweden, ${ }^{5}$ Department of Clinical Genetics and Pathology, Skåne University Hospital, Lund, Sweden

Introduction: Germline pathogenic variants in genes established to be associated with hereditary colorectal cancer (CRC) explain the disease in approximately 5-6\% patients. Due to technical limitations and variant interpretation challenges, high penetrant pathogenic variants are frequently reported only in the exonic regions of the genes.

Materials and methods: The study included 206 patients with suspected hereditary CRC referred to the Cancer Genetics Counselling Clinic at Sahlgrenska University Hospital in Gothenburg, Sweden. Variant screening was performed using a comprehensive genomic panel including established and also putative CRC predisposition genes (covering $50 \mathrm{~kb}$ upstream and downstream of the genes and intron regions). Variants, including CNVs (copy-number variants), were evaluated regarding pathogenicity. In addition, 40 out of the 206 patients were further investigated through whole-genome sequencing (WGS).

Results: The spectrum of pathogenic variants was determined. The findings include identification of previously unreported variants and putative causative variants in genetically unexplained patients with hereditary CRC.

Conclusions: The combination of high-throughput panelsequencing and WGS identifies a broader set of pathogenic variants in patients with hereditary $\mathrm{CRC}$, which is crucial for clinical follow up of patients and predictive testing of relatives. The study was supported by grants from the Swedish state under the agreement between the Swedish government and the county councils, the ALF-agreement (ALF-725011) and the Swedish Cancer Society (Grant no 18 0357).

F. Eiengård: None. A. Rohlin: None. E. Mårtensson: None. T. Olausson: None. T. Zagoras: None. U. Lundstam: None. S. Gebre-Medhin: None. M. Nordling: None.

\section{P12.056.A}

A new approach for the identification of novel candidate genes of susceptibility to colorectal cancer

A. Lopez-Novo' ${ }^{1}$, A. Dacal ${ }^{2}$, D. Remedios ${ }^{3}$, J. Cubiella ${ }^{3}$, V. Alvarez-Sanchez, M. J. Ladra-Gonzalez, , F. FernandezLopez $^{5}$, A. Alvarez-Castro ${ }^{5}$, J. M. Cameselle-Teijeiro ${ }^{6}$, M. Cuatrecasas $^{7}$, F. Balaguer ${ }^{8}$, S. Castellvi-Bel ${ }^{9}, C$. Fernandez-Rozadilla ${ }^{1}$, C. Ruiz-Ponte ${ }^{1,10}$

${ }^{1}$ Fundacion Publica Galega de Medicina Xenomica, Grupo de Medicina Xenomica-Universidade de Santiago de Compostela, Instituto de Investigacion Sanitaria de Santiago de Compostela (E035), Santiago de Compostela, Spain, ${ }^{2}$ Servicio de Digestivo, Hospital Universitario Lucus Augusti, Instituto de Investigacion Sanitaria de Santiago de Compostela (E035), Lugo, Spain, ${ }^{3}$ Servicio de Digestivo, Complexo Hospitalario Universitario de Ourense, Centro de Investigacion Biomedica en Red de Enfermedades Hepaticas y Digestivas (CIBERehd), Ourense, Spain, ${ }^{4}$ Servicio de Digestivo, Complexo Hospitalario de Pontevedra, 
Pontevedra, Spain, ${ }^{5}$ Servicio de Digestivo, Complexo Hospitalario Universitario de Santiago de Compostela, Santiago de Compostela, Spain, ${ }^{6}$ Servicio de Anatomia Patologica, Complexo Hospitalario Universitario de Santiago de Compostela, Santiago de Compostela, Spain, ${ }^{7}$ Servicio de Anatomia Patologica, Institut D'Investigacions Biomediques August Pi i Sunyer, Centro de Investigacion Biomedica en Red de Enfermedades Hepaticas y Digestivas (CIBERehd) and Tumor Bank-Biobank, Hospital Clinic, Barcelona, Spain, ${ }^{8}$ Servicio de Digestivo, Institut D'Investigacions Biomediques August Pi i Sunyer, Centro de Investigacion Biomedica en Red de Enfermedades Hepaticas y Digestivas (CIBERehd), Hospital Clinic, Universitat de Barcelona, Barcelona, Spain, ${ }^{9}$ Grupo de Oncologia Gastrointestinal y Pancreatica, Institut D'Investigacions Biomediques August Pi i Sunyer, Centro de Investigacion Biomedica en Red de Enfermedades Hepaticas y Digestivas (CIBERehd), Hospital Clinic, Universitat de Barcelona, Barcelona, Spain, ${ }^{10}$ Centro de Investigacion Biomedica en Red de Enfermedades Raras (CIBERER), Santiago de Compostela, Spain

Introduction: Whole-exome sequencing (WES) was expected to identify genetic variation that explained the missing heritability in colorectal cancer (CRC). The strategies mainly relied on prioritization of recurrent loss-offunction rare germline variants in genes previously involved in cancer pathways. However, not only a very small number of candidate genes with strong scientific evidence were identified, but a large number were proposed whose role in colorectal tumorigenesis is not yet established. The failure to identify the real causative genes could be due to the genetic heterogeneity underlying CRC. Our aim is to molecular characterize early-onset MMR-proficient CRC at somatic level to further identify novel candidate genes by integrating germline and tumor omic data.

Materials and Methods: WES and RNA-seq were performed on paired normal-tumor tissues from a phenotypically homogeneous cohort of 20 early-onset $(<50 \mathrm{y})$ MMR-proficient CRC patients. Differential expression analysis (DESeq2), functional enrichment analysis (G: Profiler), consensus molecular subtyping (CMSCaller) and signature profile (MuSiCa) were carried out.

Results: According to consensus molecular subtyping, colorectal tumors were classified as: CMS1 (3), CMS2 (2), CMS3 (4), CMS4 (6), the remaining were still not classified.

Conclusions: These data show the molecular heterogeneity underlying this phenotypically homogeneous cohort of early onset MMR-proficient CRC. Thus, a more comprehensive prioritization of germline variants according to the molecular subtype of each tumor will be followed increasing the probabilities to identify the causative germline CRC risk variants. As a result a more personalized genetic diagnostic could be achieved.

Grant support: ISCIII and FEDER funds PI17/00509; Predoctoral Fellowship (GAIN, Xunta de Galicia)

A. Lopez-Novo: None. A. Dacal: None. D. Remedios: None. J. Cubiella: None. V. Alvarez-Sanchez: None. M.J. Ladra-Gonzalez: None. F. Fernandez-Lopez: None. A. Alvarez-Castro: None. J.M. Cameselle-Teijeiro: None. M. Cuatrecasas: None. F. Balaguer: None. S. CastellviBel: None. C. Fernandez-Rozadilla: None. C. RuizPonte: None.

\section{P12.057.B}

GNMTpolymorphisms are associated with perineural invasion and age of disease onset among colorectal cancer patients

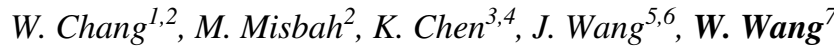

${ }^{I}$ Master Program for Clinical Pharmacogenomics and Pharmacoproteomics, School of Pharmacy, Taipei Medical University, Taipei, Taiwan, ${ }^{2}$ Department of Clinical Pharmacy, School of Pharmacy, Taipei Medical University, Taipei, Taiwan, ${ }^{3}$ Graduate Institute of Medical Sciences, College of Medicine, Taipei Medical University, Taipei, Taiwan, ${ }^{4}$ Department of Biochemistry and Molecular Cell Biology, School of Medicine, College of Medicine, Taipei Medical University, Taipei, Taiwan, ${ }^{5}$ Graduate Institute of Clinical Medicine, College of Medicine, Kaohsiung Medical University, Kaohsiung, Taiwan, ${ }^{6}$ Center for Biomarkers and Biotech Drugs, Kaohsiung Medical University, Kaohsiung, Taiwan, ${ }^{7}$ The Ph.D. Program for Translational Medicine, College of Medical Science and Technology, Taipei Medical University, Taipei, Taiwan

Introduction: Glycine N-methyl transferase (GNMT) is known to play an important role in the pathogenesis of cancer. However, the effects of GNMT polymorphisms on colorectal cancer (CRC) are still unclear. Herein, we investigated the role of GNMT in CRC.

Materials and Methods: The study sample consists of 352 CRC patients. Clinical variables including parameters of staging, vascular invasion, perineural invasion, and age of CRC onset were collected. Five single nucleotide polymorphisms (SNPs) including rs9471976, rs9462856, rs11752813, rs10948059, and rs2274517 were genotyped. The associations of SNPs with clinical variables were examined by a two-stage approach: chi-square test and logistic regression were used for categorical variables; Kaplan-Meier method and Cox proportional hazard model were used for age of onset.

Results: The minor alleles of three SNPs were associated with a higher risk of perineural invasion: $T$ of rs9471976 
$(\mathrm{OR}=1.70,95 \% \mathrm{CI}=(1.13-2.55)), C$ of $\mathrm{rs} 9462856(\mathrm{OR}=$ $1.66,95 \% \mathrm{CI}=(1.11-2.49))$, and $T$ of $\mathrm{rs} 2274517(\mathrm{OR}=$ $1.64,95 \% \mathrm{CI}=(1.10-2.45))$. Furthermore, subjects carrying $G G$ genotype of rs11752813 have a younger age of onset compared with other subjects $(\mathrm{HR}=3.02,95 \% \mathrm{CI}=(1.09$, 8.36)). According to GTEx database, these four SNPs may influence expression levels of PEX6 and GNMT in transverse and sigmoid colon tissues.

Conclusions: GNMT is associated with risk of perineural invasion and age of disease onset among CRC patients.

This study was supported by the Ministry of Science and Technology, Taiwan (MOST105-2628-B-038-001-MY4 and MOST 106-2314-B-038-052-MY3) and the International Office of Taipei Medical University (TMU105-5803003-111 and TMU105-5803-010-111).

W. Chang: None. M. Misbah: None. K. Chen: None. J. Wang: None. W. Wang: None.

\section{P12.059.A}

Assessing prevalence of microsatellite instability and Lynch syndrome amongst colorectal cancer patients in India

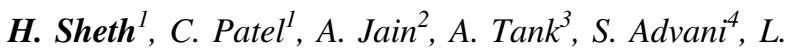

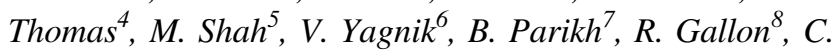
Hayes $^{8}$, G. M. Borthwick ${ }^{8}$, M. S. Jackson ${ }^{8}$, M. SantibanezKoref $^{8}$, J. Burn ${ }^{8}$, F. Sheth ${ }^{l}$, J. Sheth ${ }^{l}$, S. Trivedi ${ }^{1}$

${ }^{1}$ FRIGE's Institute of Human Genetics, Ahmedabad, India, ${ }^{2}$ CIMS Hospital, Ahmedabad, India, ${ }^{3}$ Dwarika Clinic, Ahmedabad, India, ${ }^{4}$ Sushrut Hospital, Mumbai, India, ${ }^{5}$ Zydus Cancer Hospital, Ahmedabad, India, ${ }^{6}$ Nishtha Surgical Hospital and Research Centre, Patan, India, ${ }^{7}$ HOC Vedanta, Ahmedabad, India, ${ }^{8}$ Newcastle University, Newcastle upon Tyne, United Kingdom

Microsatellite instability (MSI) and Lynch syndrome (LS) prevalence in colorectal cancer (CRC) patients is estimated to be $15 \%$ and $3 \%$ in European populations. However, prevalence estimates from India are not available. We aim to systematically assess the prevalence of MSI and LS in CRC patient population from India. Venous blood and primary tumour biopsies were prospectively collected and analysed for MSI using Promega fragment length analysis assay and BRAF V600E using Q-PCR. Patients with MSIhigh $B R A F$-negative tumour biopsies were assessed for LS using germline DNA sequencing of the mismatch repair genes (MLH1, MSH2, MSH6, PMS2 and EPCAM). Within 9 months of a 3 year study, 38 patients have been recruited with CRC. MSI testing in these patients showed MSI-high phenotype in 10 patients $(26.3 \%)$. Of these, 9 patients (90\%) were negative for BRAF V600E mutation. Germline testing in 6 of these patients have shown known and novel mutations in the MLHI gene. Ad hoc analysis of the prospective study cohort suggests higher prevalence of MSI and LS in CRC patient population in India. Analysis of the entire patient cohort $(n=0)$ in 2022 will provide a robust estimate of the prevalence of MSI and LS in CRC patients in India. These estimates would help in formulation of national guidelines for MSI testing, LS diagnosis and chemoprevention strategies of CRC in India. Study is funded by Gujarat State Biotech Mission (GSBTM/JDR\&D/604/ 2019/297).

H. Sheth: B. Research Grant (principal investigator, collaborator or consultant and pending grants as well as grants already received); Modest; HS is a PI of the study which is funded by the Gujarat State Biotech Mission (GSBTM/JDR\&D/604/2019/297). E. Ownership Interest (stock, stock options, patent or other intellectual property); Modest; HS is a co-inventor on a filed international patent describing methods of identifying microsatellite instability (PCT/GB2019/052148). C. Patel: None. A. Jain: None. A. Tank: None. S. Advani: None. L. Thomas: None. M. Shah: None. V. Yagnik: None. B. Parikh: None. R. Gallon: E. Ownership Interest (stock, stock options, patent or other intellectual property); Modest; RG is a co-inventor on a filed international patent describing methods of identifying microsatellite instability (PCT/GB2019/ 052148). C. Hayes: None. G.M. Borthwick: None. M.S. Jackson: E. Ownership Interest (stock, stock options, patent or other intellectual property); Modest; MSJ s a coinventor on a filed international patent describing methods of identifying microsatellite instability (PCT/GB2019/ 052148). M. Santibanez-Koref: E. Ownership Interest (stock, stock options, patent or other intellectual property); Modest; MSK is a co-inventor on a filed international patent describing methods of identifying microsatellite instability (PCT/GB2019/052148). J. Burn: E. Ownership Interest (stock, stock options, patent or other intellectual property); Modest; JB is a co-inventor on a filed international patent describing methods of identifying microsatellite instability (PCT/GB2019/052148). F. Sheth: None. J. Sheth: None. S. Trivedi: None.

\section{P12.060.B}

Role of miR-1246 in colorectal carcinogenesis

R. Lukosevicius ${ }^{l}$, V. Salteniene ${ }^{l}$, S. Juzenas ${ }^{1,2}, R$. Inciuraite $^{l}$, K. Balciute ${ }^{l}$, P. Ruzgys ${ }^{3}$, J. Skieceviciene ${ }^{l}, J$. Kupcinskas ${ }^{l}$

${ }^{1}$ Institute for Digestive Research, Lithuanian University of Health Sciences, Kaunas, Lithuania, ${ }^{2}$ Institute of Clinical Molecular Biology, Christian-Albrechts-University, Kiel, Germany, ${ }^{3}$ Biophysical Research Group, Vytautas Magnus University, Kaunas, Lithuania 
Introduction: Colorectal cancer (CRC) is one of the most prevalent cancers worldwide and is the second leading cause of cancer-related mortality. CRC detection rates have improved; however, disease-related mortality remains very high. Comprehensive miRNA analysis showed deregulated miRNA profiles in CRC. In order to understand functional relevance of miRNA deregulation, studies analyzing their target genes are of major importance.

Materials and methods: MTT, colonogenic, wound healing, dual-light luciferase reporter gene, gene and protein expression assays were performed in SW620, HCT-116 and Caco-2 cell lines after cell transfection with hsa-miR-1246 mimic and inhibitor to determine miR-1246 impact in cell function changes and to confirm its target genes.

Results: Reduced cell viability was observed in Caco-2 and SW620 cell lines 72 hours after transfection with miR1246 inhibitor. Colonogenic test results demonstrated that inhibition of miR-1246 decreased colony number only in Caco-2 cell line. Inhibition of miR-1246 decreased covered gap area in wound healing assay in Caco-2 and HCT-116 cells. CFTR, AXIN2 CRC-associated putative target genes of miR-1246 were confirmed by dual light luciferase assay. Overexpression of miR-1246 decreased the expression of CFTR and AXIN2 genes in SW620 cell line 48 hours after transfection. Tendency of higher CFTR and AXIN2 expression in protein level was observed after inhibition of miR-1246 in Caco-2 and SW620 cell lines.

Conclusions: AXIN2 and CFTR are direct targets of miR1246. Overexpression or inhibition of miR-1246 affected cell viability, colony formation and migration in CRC cell lines.

Funding: Funded by the Research Council of Lithuania (grant. no. S-MIP-17-22)

R. Lukosevicius: None. V. Salteniene: None. S. Juzenas: None. R. Inciuraite: None. K. Balciute: None. P. Ruzgys: None. J. Skieceviciene: None. J. Kupcinskas: None.

P12.061.C

Molecular profile of tumor DNA from patients with metachronous colorectal cancer

S. Tapial $^{1,2}$, D. Rueda ${ }^{1,2}$, D. Gómez-Sánchez ${ }^{3}, N$. Carrizo $^{3}$, Y. Rodríguez-Gil ${ }^{4}$ I. Rey ${ }^{2}$, J. Perea ${ }^{5}$

${ }^{1}$ Digestive Cancer Research Unit, 12 de Octubre Research Institute, Madrid, Spain, ${ }^{2}$ Hereditary Cancer Laboratory, 12 de Octubre University Hospital, Madrid, Spain, ${ }^{3}$ Lung Cancer Research Unit, 12 de Octubre Research Institute, Madrid, Spain, ${ }^{4}$ Pathology Department, 12 de Octubre University Hospital, Madrid, Spain, ${ }^{5}$ Surgery Department, Fundación Jiménez Díaz University Hospital, Madrid, Spain
Introduction: Colorectal cancer (CRC) is one of the most common malignant tumors. Patients with $\mathrm{CRC}$ have an increased risk for developing metachronous CRC (MCRC) during follow-up evaluation. It is still unknown which patients are prone to this condition, despite genomics studies have enhanced our ability to identify molecular changes associated with CRC. The clinician's challenge is to propose a tight follow-up and control the possible return of cancer. This study is focusing on the characterization of mutational profile of MCRC that could inform in regards to prognosis and therapy decision.

Materials and Methods: A total of 90 formalin-fixed, paraffin-embedded (FFPE) tumor DNA from 45 patients diagnosed with MCRC were selected to perform next generation sequencing (NGS) using Ion Torrent platform and Ampliseq cancer hotspot panel v2 (Thermofisher). Data analysis was performed using Torrent Suite and Ion Reporter software (Thermofisher). The annotated variants were visualized using IGV (Broad Institute).

Results: The most frequent mutations in initial tumors were located in APC (48,9\%), KRAS $(42,2 \%), T P 53$ $(28,3 \%)$ and PIK3CA $(8,9 \%)$, while in metachronous tumors the most common mutations were identified in KRAS (50,0\%), APC (42,2\%), TP53 (15,6\%), FBXW7 $(13,3 \%)$ and $C T N N B 1(8,9 \%)$. Remarkably, three $(6,7 \%)$ patients showed the same mutations in both tumors. Moreover, those patients with $A P C$ mutation in their inicial tumor showed a tendency to develop later their metachronous tumors $(\mathrm{p}=0,05)$.

Conclusions: These results highlight the genetic features of MCRC that could influence for tailored surveillance programs. Additionally, data showed the mutational profile differences between both tumors in MCRC patients.

S. Tapial: None. D. Rueda: None. D. Gómez-Sánchez: None. N. Carrizo: None. Y. Rodríguez-Gil: None. I. Rey: None. J. Perea: None.

P12.062.A

Constitutional mismatch repair deficiency is the differential diagnosis in $0.41 \%$ of NF1/SPRED1-mutation negative children suspected of sporadic neurofibromatosis type 1

J. A. Perez-Valencia ${ }^{1}$, R. Gallon ${ }^{2}$, Y. Chen ${ }^{3}, J . K_{\text {Koch }}^{4}$, M. Keller $^{4}$, K. Oberhuber ${ }^{4}$, A. Gomes ${ }^{3}$, J. Zschocke ${ }^{4}$, J. Burn $^{2}$, M. Jackson ${ }^{2}$, M. Santibanez-Koref ${ }^{2}$, L. Messiaen ${ }^{3}, \boldsymbol{K}$.

Wimmer $^{4}$

${ }^{1}$ Institute of Human Genetics, Medical University Innsbruck, Innsbruck, Austria, ${ }^{2}$ Faculty of Medical Sciences, Newcastle University, Newcastle, United Kingdom, ${ }^{3}$ Department of Genetics, University of Alabama at 
Birmingham, Birmingham, AL, United States, ${ }^{4}$ Institute of Human Genetics, Medical University Innsbruck, Innsbruck, Austria

Introduction: Biallelic germline mismatch repair (MMR) gene mutations cause constitutional MMR deficiency (CMMRD), a highly penetrant childhood cancer syndrome phenotypically overlapping with neurofibromatosis type 1 (NF1). CMMRD testing in suspected NF1 children without NFI/SPRED1 mutations enables inclusion of CMMRD positives into monitoring programs prior to tumor onset. However, testing is associated with potential harms. The prevalence of CMMRD among these children, although unknown, is estimated to be as low as $0.39 \%$.

Methods: We have previously developed a simple and scalable microsatellite instability (MSI) assay of nonneoplastic leukocyte DNA to reliably detect CMMRD. Here we used this assay to retrospectively screen a cohort of $>700$ children suspected of sporadic NF1, but lacking NF1/ SPRED1 germline mutations after highly sensitive mutation analysis.

Results: For three MSI-positive patients, identification of MMR gene germline mutations confirmed the diagnosis of CMMRD. Founder mutations NM_000535.5(PMS2): c.736_741delinsTGTGTGTGAAG, prevalent in Europe and North America, and NM_000179.2(MSH6):c.10C $>$ G, affecting 1:400 French Canadians, represented two of five mutations. CMMRD prevalence was $3 / 735(0.41 \%$, $95 \%$ CI: $0.08-1.19 \%)$.

Conclusions: Our empirical data provide reliable numbers urgently needed for genetic counseling, and confirm previous prevalence estimations on which the Care for CMMRD (C4CMMRD) consortium guidelines are based. These advocate CMMRD testing of preselected patients rather than offering reflex testing to all suspected sporadic NF1 children lacking NF1/SPRED1 mutations. The MMR mutations in the CMMRD patients identified here suggest that the possibility of founder effects should be considered, in addition to C4CMMRD guidelines, when counseling NF1/SPRED1 mutation negative children.

This work was funded by Austrian Science Funds.

J.A. Perez-Valencia: None. R. Gallon: None. Y. Chen: None. J. Koch: None. M. Keller: None. K. Oberhuber: None. A. Gomes: None. J. Zschocke: None. J. Burn: None. M. Jackson: None. M. Santibanez-Koref: None. L. Messiaen: None. K. Wimmer: None.

\section{P12.063.B}

Profiling the mutational spectrum of hereditary colorectal cancers in Turkey by using multi-gene panel tests
E. Celik ${ }^{1,2}$, I. M. Akcay ${ }^{1,2}$, G. Alkurt ${ }^{1,2}$, J. Yildiz ${ }^{1,2}$, N. B.

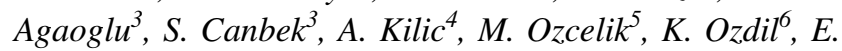
Zemheri $^{7}$, G. Dinler-Doganay ${ }^{1,2}$, L. Doganay ${ }^{1,6}$

${ }^{I}$ GLAB (Genomic Laboratory), Health Directorate of Istanbul, Istanbul, Turkey, ${ }^{2}$ Department of Molecular Biology and Genetics, Istanbul Technical University, Istanbul, Turkey, ${ }^{3}$ Department of Clinical Genetics, Umraniye Teaching and Research Hospital, University of Health Sciences, Istanbul, Turkey, ${ }^{4}$ Department of General Surgery, Umraniye Teaching and Research Hospital, University of Health Sciences, Istanbul, Turkey, ${ }^{5}$ Department of Oncology, Umraniye Teaching and Research Hospital, University of Health Sciences, Istanbul, Turkey, ${ }^{6}$ Department of Gastroenterology and Hepatology, Umraniye Teaching and Research Hospital, University of Health Sciences, Istanbul, Turkey, ${ }^{7}$ Department of Pathology, Umraniye Teaching and Research Hospital, Umraniye, University of Health Sciences, Istanbul, Turkey

Introduction: Colorectal cancers (CRC) are the third most common cancers and leading causes of death in Turkey. Up to $10 \%$ of all CRC cases are considered hereditary. Here, we aimed to characterize the spectrum of pathogenic variants in Turkish CRC patients and investigated the phenotype-genotype relationships of these variants.

Materials and Methods: We sequenced the coding regions and splice junctions of 25 cancer susceptibility genes in 174 unrelated CRC patients and 457 elderly controls with no personal and familial cancer history using multi-gene panel testing. We also performed MLPA analyses for mismatch repair genes (MLH1, MSH2, MSH6, PMS2) and EPCAM 3'UTR to detect gross deletions.

Results: We detected pathogenic germline variants in $24.1 \%$ (19/79) of high-risk CRC patients, 6.3\% (6/95) of low-risk CRC patients, and 2.6\% (12/457) of healthy controls. $54.2 \%(n=24)$ of all pathogenic variants were in mismatch repair genes $M L H 1(n=7), M S H 2(n=4)$ and MSH6 $(n=2) .2$ of these variants were gross deletions. MUTYH c.1437_1439delGGA variant was the most prevalent pathogenic variant. Monoallelic MUTYH mutations were not significantly associated with colorectal cancers $(\mathrm{p}=0.2849) .47 \%$ of patients with MMR gene defects had loss of MMR gene expression in their tumors. 4\% (7/174) of all patients had mutations in well-known breast cancer genes and other cancer susceptibility genes. Variants of uncertain significance (VUS) were detected in more than $34 \%$ of all subjects.

Conclusions: This study provides important information to estimate the contribution of cancer susceptibility genes to CRC in Turkish population, and to improve the interpretation of VUS. 
E. Celik: None. I.M. Akcay: None. G. Alkurt: None. J. Yildiz: None. N.B. Agaoglu: None. S. Canbek: None. A. Kilic: None. M. Ozcelik: None. K. Ozdil: None. E. Zemheri: None. G. Dinler-Doganay: None. L. Doganay: None.

\section{P12.065.A}

Atypical deletion of $\mathbf{5 q}$ in myelodysplastic syndromes (MDS)

Z. Zemanova ${ }^{l}, J$. Brezinova $^{2}, K$. Svobodoval ${ }^{l}, H$. Lhotska ${ }^{l}$, S. Izakova ${ }^{1}$, L. Lizcova ${ }^{l}$, D. Vesela ${ }^{l}$, S. Ransdorfova ${ }^{2}$, I. Mendlikova $^{2}$, K. Michalova ${ }^{1}, J$. Cermak $^{2}$, A. Jonasova ${ }^{3}$

${ }^{1}$ Center of Oncocytogenomics, Institute of Medical Biochemistry and Laboratory Diagnostics, General University Hospital and First Faculty of Medicine, Charles University, Prague, Czech Republic, ${ }^{2}$ Institute of Hematology and Blood Transfusion, Prague, Czech Republic, ${ }^{3} 1$ st Medical Department, General University Hospital and First Faculty of Medicine, Charles University, Prague, Czech Republic

Introduction: The interstitial deletion $\operatorname{del}(5 q)$ is a recurrent cytogenetic aberration in bone marrow of patients with myelodysplastic syndromes (MDS). The extent of the del (5q) varies in individual cases. Two different commonly deleted regions (CDRs) have been published: the proximal $5 q 31.2$ region associated with a high-risk MDS, and the distal CDR 5q32-5q33 involved in the pathogenesis of MDS with isolated del $(5 q)$. However, rare cases of atypical deletions not including defined CDRs have also been reported.

Materials and methods: During 1993-2019 we examined bone marrow cells of 3714 MDS patients by conventional G-banding and I-FISH (Abbott). Extent of del(5q) was analyzed using multicolor banding (mBAND; MetaSystems) and/or aCGH (Illumina, Agilent).

Results: del(5q) was detected in 920/3714 patients $(24,8 \%)$. Most of them had large deletion spanning both defined CDRs. Atypical deletions with retained CDRs were identified in $9 / 920$ cases $(1 \%$; 4M/5F; median age, 73 years). In five of them del(5q) was a sole aberration, in four cases it was detected together with other chromosomal rearrangements. In all cases, deletion was localized proximally to the $5 \mathrm{q} 31$ region. The size of the deleted segment ranged from 25.46 to $53.19 \mathrm{Mb}$ (median $38.51 \mathrm{Mb}$ ).

Conclusions: Our results suggest that del(5q) may occur outside of defined CDRs. Although these findings are extremely rare, they show that also genes located outside known CDRs may contribute to the malignant progression of MDS. The identification of these genes will lead to better understanding of the MDS pathogenesis and may contribute to identify new therapeutic targets. Supported by RVOVFN64165, IHBT-00023736.

Z. Zemanova: None. J. Brezinova: None. K. Svobodova: None. H. Lhotska: None. S. Izakova: None. L. Lizcova: None. D. Vesela: None. S. Ransdorfova: None. I. Mendlikova: None. K. Michalova: None. J. Cermak: None. A. Jonasova: None.

\section{P12.066.B}

Genomic heterogeneity of tumor evolution in primary and recurrent diffuse gliomas

L. Lizcova ${ }^{l}$, D. Vesela ${ }^{l}, K$. Svobodova ${ }^{l}, H$. Cechova ${ }^{2}, H$. Lhotska $^{l}$, L. Pavlistova ${ }^{l}$, L. Hodanova ${ }^{l}$, Z. Krejcik ${ }^{2}, S$.

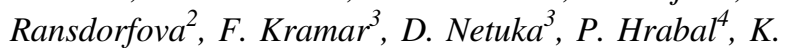
Michalova $^{l}$, Z. Zemanova ${ }^{l}$

${ }^{1}$ Center of Oncocytogenomics, General University Hospital and 1st Faculty of Medicine, Charles University in Prague, Prague, Czech Republic, ${ }^{2}$ Institute of Hematology and Blood Transfusion, Prague, Czech Republic, ${ }^{3}$ The Neurosurgery and Neurooncology Department, Military University Hospital and 1st Faculty of Medicine, Charles University in Prague, Prague, Czech Republic, ${ }^{4}$ Department of Pathology, Military University Hospital, Prague, Czech Republic

Introduction: Diffuse gliomas are highly heterogenous tumors with occurrence of recurrent lesions in majority of patients. During disease progression gliomas undergo cellular and genomic evolution. However, the mechanism of this complicated process associated with treatment failure is poorly understood.

Materials and Methods: We compared genomic profiles of 28 primary and recurrent gliomas. To assess copy number variations, $I D H 1 / / D H 2$ mutation status and hypermethylation of $M G M T$ and $M L H 3$ promoters we used array CGH/SNP (Illumina, Agilent), I-FISH (Abbott Molecular, MetaSystems) and MLPA/MS-MLPA (MRC Holland).

Results: In 27/28 cases, the genomic profiles of recurrent tumors were different from their primaries and shared the genetic/epigenetic alterations depending on the evolutionary pattern, i.e. linear or divergent. Except typical aberrations, recurrent lesions in all these patients included additional gains/losses of various parts of different chromosomes resulting in complex rearrangements. Repeatedly affected regions were detected in bands $2 \mathrm{q} 36.3,3 \mathrm{p} 11.1,8 \mathrm{q} 24.13$, 13q14, and 22q11.1. Besides copy number variations, cnLOH were proved in $25 / 28$ cases, predominantly located 
at $7 p, 9 p, 12 p$, and $17 p$. In one case, newly acquired chromothripsis of chromosomes 1, 9, 11 and 17 was observed. Hypermethylation of MGMT and/or $M L H 3$ promoter was detectedin 10/28 recurrent tumors.

Conclusions: Our study proved heterogenous pattern of clonal evolution in gliomas which is characterized by acquisition of new genomic aberrations and leads to high genome instability. We proved, that recurrences are genetically/epigenetically different from their primaries and may arise from one major tumor clone or by one or more subclones presented within primary tumors. Supported by RVO-VFN64165

L. Lizcova: None. D. Vesela: None. K. Svobodova: None. H. Cechova: None. H. Lhotska: None. L. Pavlistova: None. L. Hodanova: None. Z. Krejcik: None. S. Ransdorfova: None. F. Kramar: None. D. Netuka: None. P. Hrabal: None. K. Michalova: None. Z. Zemanova: None.

\section{P12.067.C}

Development of a novel, online training platform for genomics education in cancer care; preparing healthcare professionals for "Generation Genome"

V. Tripathi ${ }^{1}$, A. E. Coulson ${ }^{1}$, E. Cox ${ }^{2}$, Z. Beydoun ${ }^{3}$, V. Reeve $^{3}$, J. Malawana ${ }^{2}$, A. Kulkarni ${ }^{1}$

${ }^{1}$ South East Thames Regional Genetics Service, Guy's and St Thomas' NHS Foundation Trust, London, United Kingdom, ${ }^{2}$ Medics.Academy, London, United Kingdom, ${ }^{3}$ Guy's and St Thomas' NHS Foundation Trust, London, United Kingdom

Introduction: Genomic testing in the UK is changing, with more clinicians ordering genomic tests for patient care. The UK Government has called for improved education to appropriately order and interpret tests. Existing evidence highlights the benefits of online learning, as well as those of blended delivery approaches, and initiatives need to be scalable to the wider workforce. We developed an online platform (Nucleus) to address this need across for cancer healthcare professionals in our region.

Materials and Methods: Collaborating with healthcare technology company, Medics.Academy, Nucleus was developed based on face-to-face education delivered by Guy's Hospital. Scoping exercises, literature reviews and recommendations from national healthcare and governmental bodies identified target audiences, training needs and the utility of online training. Content was developed and reviewed by genetics clinicians. User testing data of an initial Nucleus module was collected.
Results: Modules in basics of cancer genomics, cancer genomics susceptibility, and precision oncology (technological and clinical applications) have been developed. Podcasts and blogs are currently in development. Initial user testing has been very positive with users rating the platform as relevant to practice $(\bar{x}=4.57 / 5)$, with appropriate length $(\overline{\mathrm{x}}=4.29 / 5)$ and pace $(\overline{\mathrm{x}}=4.14 / 5)$.

Conclusions: Nucleus can facilitate scalable cancer genomics education and is well-received by clinicians. Modules will be combined with face-to-face teaching in a blended learning course to be piloted in June 2020 . Subsequent expansion of further modules is planned over the next 18 months.

V. Tripathi: None. A.E. Coulson: None. E. Cox: None. Z. Beydoun: None. V. Reeve: None. J. Malawana: None. A. Kulkarni: None.

\section{P12.068.A}

Using genetics to understand the relationship between non-cancerous gynaecological diseases and endometrial cancer risk

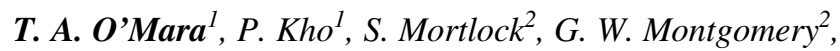
A. B. Spurdle ${ }^{l}$ D. M. Glubb ${ }^{l}$

${ }^{\text {I}}$ QIMR Berghofer Medical Research Institute, Brisbane, Australia, ${ }^{2}$ The Institute for Molecular Bioscience, University of Queensland, Brisbane, Australia

Endometriosis, polycystic ovary syndrome (PCOS) and uterine fibroids are three prevalent non-cancerous gynaecological diseases, affecting women of reproductive age, with unclear effects on endometrial cancer risk. Using GWAS summary statistics for each of these diseases (n cases: 14,949 endometriosis; 4,890 PCOS; 20,101 uterine fibroid; 12,906 endometrial cancer), we performed genetic correlation, genetic causal inference (latent causal variable and Mendelian randomization analysis) and gene-based analyses (fastBAT analysis) to understand the relationship between these three non-cancerous gynaecological diseases and risk of endometrial cancer. We found positive genetic correlation between endometrial cancer and PCOS $\left(r_{\mathrm{g}}=0.37, \mathrm{P}=2 \times 10^{-3}\right)$ and endometrial cancer and uterine fibroids $\left(r_{\mathrm{g}}=0.24, \mathrm{P}=7 \times 10^{-3}\right)$. There was no evidence of genetic correlation between endometrial cancer and endometriosis. As body mass index (BMI) is a shared risk factor for PCOS, uterine fibroids and endometrial cancer we adjusted genetic correlation results for BMI. Upon this adjustment, the significant correlation between uterine fibroids and endometrial cancer remained; however, the genetic correlation between PCOS and endometrial cancer 
was attenuated. Despite the significant genetic correlation observed, we did not find evidence supporting a causal relationship between any of the three non-cancerous gynaecological diseases and endometrial cancer development. Gene-based analyses prioritized seven endometriosis and five uterine fibroids-associated genes that overlapped with endometrial cancer-associated genes, highlighting potential shared pathways affecting risk of these diseases. No PCOS-associated genes overlapped with endometrial cancer-associated genes. Our study provides insights into the shared relationship between PCOS, uterine fibroids and endometrial cancer, and avenues for future research. Funded by NHMRC grants to TOM, ABS, GWM

T.A. O'Mara: None. P. Kho: None. S. Mortlock: None. G.W. Montgomery: None. A.B. Spurdle: None. D.M. Glubb: None.

P12.074.A

Proofing the concept of a circulating tumor DNA-based clinically scalable workflow focused on ESR1 epigenetic modification in hormone receptor positive Metastatic Breast Cancer

A. Franzoni ${ }^{1}$, D. Basile ${ }^{2}$, L. Allegri ${ }^{3}$, D. Viotto ${ }^{4}$, C. Corvaja $^{2}$, L. Bortot ${ }^{2}$, E. Bertoli ${ }^{5}$, S. Buriolla ${ }^{2}$, G. Targato ${ }^{5}$, L. Da Ros ${ }^{6}$, S. Russo, , M. Bonotto , L. Gerratana ${ }^{2}, B$. Belletti $^{4}$, G. Baldassarre ${ }^{4}$, F. Puglisi ${ }^{2}$, G. Damante $^{8}$

${ }^{1}$ Institute of Human Genetics-ASUFC Udine, Udine, Italy, ${ }^{2}$ DAME-University of Udine, Department of Medical Oncology-IRCCS CRO Aviano, Udine/Aviano, Italy, ${ }^{3}$ DAME-University of Udine, Udine, Italy, ${ }^{4}$ Department of Cancer Research and Advanced Diagnostics-IRCCS CRO Aviano, Aviano, Italy, ${ }^{5}$ DAME-University of Udine, Department of Oncology-ASUFC Udine, Udine, Italy, ${ }^{6}$ Department of Medical Oncology-IRCCS CRO Aviano, Aviano, Italy, ${ }^{7}$ Department of Oncology-ASUFC Udine, Udine, Italy, ${ }^{8}$ Institute of Human Genetics-ASUFC Udine; DAMEUniversity of Udine, Udine, Italy

Background: ESRl genomic alterations have been largely explored in Metastatic Breast Cancer (MBC) but little is known about their epigenetics. Aim of the study was to test the feasibility of a circulating tumor DNA (ctDNA)-based workflow focused on ESRI mutations and promoter methylation in MBC patients (pts) treated with endocrine therapy (ET).

Methods: overall, 49 pts eligible for first line ET were prospectively enrolled. Mutations were evaluated through NGS, while the methylation of ESRI promoter A and B (met_promA/B) was assessed through methylation-specific
ddPCR. Associations between prom $\mathrm{A} / \mathrm{B}$, pathological characteristics and mutations were explored through Fisher's exact test and Mann-Whitney test.

Results: Aromatase inhibitors were the main ET backbone $(70.83 \%)$. Median met_promA was $38.5 \%$, met_promB was $33 \%$. Lower met_promA was observed in pts with liver metastases $(\mathrm{P}=0.0211)$ while for met_promB no differences were observed among sites, with the exception of numerically higher levels in pts with soft tissue involvement. Met_promA was significantly lower in pts with ESR1 mutations (median 41 vs 25 for ESR1 wild-type and mutated; $\mathrm{P}=0.0091$ ) and numerically lower in pts with a PIK3CA mutation (median 40.5 vs 24 for PIK3CA wildtype and mutated). Three clusters were defined by combining met_promA and $\mathrm{B}$, with a significant association between PIK3CA mutations and Cluster1 $(\mathrm{P}=0.0025)$.

Conclusions: The study showed the feasibility of a ctDNA-based epigenetic evaluation. Intriguingly, differential methylation levels were observed across baseline features, suggesting a broader impact of epigenetic modulation of ESRI in breast cancer biology.

A. Franzoni: None. D. Basile: None. L. Allegri: None. D. Viotto: None. C. Corvaja: None. L. Bortot: None. E. Bertoli: None. S. Buriolla: None. G. Targato: None. L. Da Ros: None. S. Russo: None. M. Bonotto: None. L. Gerratana: None. B. Belletti: None. G. Baldassarre: None. F. Puglisi: None. G. Damante: None.

\section{P12.075.B}

Identification of modifier and dependency genes for human hereditary cancer syndromes using CRISPR/ Cas9 mediated genome editing in Xenopus tropicalis

\section{T. Naert ${ }^{l}$, D. Dimitrakopoulou ${ }^{l}$, D. Tulkens ${ }^{l}, S$.} Demuynck $^{l}$, K. Vleminckx ${ }^{1,2,3}$

${ }^{1}$ Department of Biomedical Molecular Biology, Ghent, Belgium, ${ }^{2}$ Department of Biomolecular Medicine, Ghent, Belgium, ${ }^{3}$ Cancer Research Institute Ghent, Ghent, Belgium

CRISPR/Cas9 mediated genome editing allows gene disruption experiments in a multitude of organisms. Xenopus tropicalis shares with zebrafish an aquatic habitat and easy manipulations associated with its external development. However, unique features make it a favorable and powerful organism for human disease modeling. Unlike zebrafish, Xenopus tropicalis has a true diploid genome. Hence, gene disruption studies are not suffering from redundancy. Its genome shows high synteny with humans, assisting identification orthologs of human genes. Targeted injection of 
individual blastomeres allows manipulations of specific tissues and organs. We generated the first genetic cancer models in Xenopus tropicalis. Upon mosaic targeting of the tumor suppressor gene $A p c$ using CRISPR/Cas9, tadpoles rapidly ( $<1.5$ months) and efficiently developed a range of neoplasia characterizing Familial Adenomatous Polyposis (FAP), including intestinal neoplasia, desmoid tumors and medulloblastomas. Similarly, Rbl/Rbll double mosaic mutant tadpoles rapidly develop retinoblastoma. Most recently we documented the generation of Tp53 heterozygous and homozygous mutant animals that develop several neoplasms characterizing Li-Fraumeni Syndrome, including leukemia and sarcoma. The rapid kinetics of tumor development in the tadpoles/froglets pave the way for their use as pre-clinical models. These cancer models provide unique possibilities for identification of modifier and dependency genes using multiplexed CRISPR/Cas9 gRNA injections (e.g. tumor suppressor gene + candidate dependency gene). We present our results with multiplexed gene targeting in a FAP associated desmoid tumor model identifying EZH2 as a druggable dependency factor. These genetic Xenopus tropicalis models offer a unique experimental platform in the field of human cancer research and medical genetics.

T. Naert: None. D. Dimitrakopoulou: None. D. Tulkens: None. S. Demuynck: None. K. Vleminckx: None.

\section{P12.077.A}

Diagnostic yield of a comprehensive panel for cancer predisposition genes

K. De Leeneer ${ }^{1,2}$, T. Rosseel ${ }^{l}$, R. De Putter ${ }^{1,2}$, I. Vandernoot ${ }^{3}$, D. t'Kint de Roodenbeke ${ }^{4}$, L. Wilderoo-Van Wouwe $^{l}$, C. Kumps ${ }^{l}$, B. Poppe $e^{1,2}$, K. B. M. Claes $^{1,2}$

${ }^{1}$ CMGG Ghent University Hospital, Ghent, Belgium, ${ }^{2}$ Cancer Research Institute Ghent (CRIG), Ghent, Belgium, ${ }^{3}$ Centre de génétique humaine - ULB, Brussels, Belgium, ${ }^{4}$ Jules Bordet Institute, Brussels, Belgium

We validated and implemented a custom panel of 66 genes associated with familial cancer syndromes. Target enrichment was done by SeQCap EZ Hypercap (Roche) followed by sequencing on a Miseq or a Novaseq (Illumina) instrument. Data analysis was performed with an in house developed pipeline, allowing to limit the analysis to subpanels specified by the clinician. In total, 1522 patients referred to our hospital for clinical testing were investigated. We analyzed the following subpanels: melanoma $(n=113)$, colon cancer $(n=437)$, HBOC $(n=1026)$. Combinations of panels were analyzed for some patients, depending on personal and/or family history. In 270 patients (18\%) a variant (Class V, Class IV) was detected in the requested panel. Our diagnostic yield increased with $3 \%(n=47)$ compared to what we would have obtained if we had applied our previous panels for these indications. This increase is mainly attributable to the expansion of the panels with moderate risk genes, like ATM (19 pathogenic variants detected), BRIP1 (7 pathogenic variants) and CHEK2 (6 mutations other than c. 1100delC) in our HBOC panel. Interestingly, we also identified several variants in genes associated with rare syndromes - like NTHL1 (homozygous), BAP1, POT1, MITF, RAD51D, CDH1, HOXB13, STK11 and BMPR1A. The technique also allowed to detect several mosaic patients (TP53) or patients displaying clonal hematopoiesis in their lymphocytes (TP53, ATM). Further validations include $\mathrm{CNV}$ detection and expansion with additional genes allowing to analyze all patients with a presumed predisposition for cancer in a uniform workflow.

K. De Leeneer: None. T. Rosseel: None. R. De Putter: None. I. Vandernoot: None. D. t'Kint de Roodenbeke: None. L. Wilderoo-Van Wouwe: None. C. Kumps: None. B. Poppe: None. K.B.M. Claes: None.

\section{P12.078.B}

\section{Family HealthLink:A web-based family history triage tool}

\section{K. M. Sweet}

\section{Ohio State University, Columbus, OH, United States}

Although family history remains an essential tool for assessment of risk, diagnostics and prevention, it is often not systematically collected or assessed in clinical settings. Thus, individuals at increased risk for common diseases such as cancer and/or coronary heart disease (CHD) might not be identified or referred appropriately. A web-based tool known as Family HealthLink which assesses familial cancer and CHD risk was made available for the general public (2010-2018) and as part of patient registration (2014-2018) with over 200,000 users. A one-page anonymous user survey was made available on the website during a one-month period (Jan-Feb 2018). Of 502 survey participants, 460 (91.6\%) found FHL easy to use; 476 (94.8\%) felt the assessment was easy to understand; and $365(72.7 \%)$ found the assessment accurate and complete. $337(67.1 \%)$ felt the program helped the user to learn more about cancer and CHD disease risk; 421 (83.8\%) would encourage a family member to use the program, and $377(75.1 \%)$ planned to talk to their healthcare provider about their risk. Qualitative 
research on patient users showed that certain aspects of FHL could be executed more effectively, to include increased opportunity for personalization of the web interface and risk messaging. FHL version 3.0 is under development and will allow for 1) automatic integration and updating of professional guidelines (e.g. NCCN guidelines for hereditary cancer); 2) expansion as a mobile application and for EPIC electronic health record integration; and 3) capacity to deliver tailored, evidence-based risk messages.

K.M. Sweet: None.

P12.080.A

Two novel APC deletions resulting in a hybrid APCSRP19 transcript with high sequence homology to U2AF35 splicing factor

M. Staninova Stojovska ${ }^{l}$, N. Matevska Geshkovska ${ }^{l}, B$. Angelovska $^{2}$, N. Mitreski ${ }^{2}$, M. Panovski ${ }^{3}$, A. J. Dimovski ${ }^{1}$

${ }^{1}$ Faculty of Pharmacy, Skopje, Macedonia, The Former Yugoslav Republic of, ${ }^{2}$ University Clinic for Radiotherapy and Oncology, UKIM-Faculty of Medicine, Skopje, Macedonia, The Former Yugoslav Republic of, ${ }^{3}$ University Clinic for Digestive Surgery, UKIM-Faculty of Medicine, Universtity "Ss Cyril and Methodius, Skopje, Macedonia, The Former Yugoslav Republic of

Germline mutations in the APC gene are the underlying cause of familial adenomatous polyposis (FAP), an autosomal dominant syndrome characterized by the presence of multiple colorectal (CRC) adenomatous polyps. Although truncating mutations in the APC gene are the most common defects in FAP patients, recent papers indicate that in a significant number of cases the disease develops due to large deletions that cannot be detected with routine testing. Here we describe two novel germline large deletions of the APC gene detected in 4 unrelated families presenting a severe FAP phenotype. The deletions were characterized using MLPA, high-resolution array-CGH and bridging-PCR followed by Sanger sequencing methodologies. Both deletions (123.466bp and $139.889 \mathrm{bp}$ ) had a 5' breakpoint in intron 4 of the APC gene and a 3' breakpoint in intron 4 of the SRP19 gene, indicating that these regions are prone to recombination errors and might also be frequently affected by somatic rearrangements in sporadic CRC. RT-PCR analysis showed that the deletions resulted in the production of a hybrid APC-SRP19 transcript with an ORF of 550 AA, with $\sim 92 \%$ sequence homology with the ZRSR2 gene. The ZRSR2 gene codes for the U2 small nuclear ribonucleoprotein auxiliary factor $35 \mathrm{kD}$ subunit-related protein-2, which plays an important role in RNA splicing. Mutations in this gene have been found in various hematological malignancies associated with poor overall survival. We describe the detection of a potentially novel biomarker that can be used both as a marker for early detection and a target for individualized therapy in CRC patients.

M. Staninova Stojovska: None. N. Matevska Geshkovska: None. B. Angelovska: None. N. Mitreski: None. M. Panovski: None. A.J. Dimovski: None.

\section{P12.084.B}

Investigating the role of $C D 44 v 6$ in Gastric Cancer: development of exon-v6 skipping models by CRISPR/ Cas9

\section{S. Lobo $^{1,2}$, C. Pereira ${ }^{1,2,3}$, C. Oliveira ${ }^{1,2,3}$, G. Almeida ${ }^{1,2,3}$ \\ ${ }^{1}$ Instituto de Investigação e Inovação em Saúde (I3s), Porto, Portugal, ${ }^{2}$ Instituto de Patologia e Imunologia da Universidade do Porto (IPATIMUP), Porto, Portugal, ${ }^{3}$ Faculdade de Medicina da Universidade do Porto (FMUP), Porto, Portugal}

Introduction: Gastric cancer (GC) is the $5^{\text {th }}$ most common cancer and $3^{\text {rd }}$ with highest mortality. Standard of care for advanced disease is conventional chemotherapy. CD44v6containing isoforms are de novo expressed in GC, are often correlated with cancer aggressiveness, and may predict chemotherapy response in vitro. Whether expression of CD44v6-containing variants, or presence of exon-v6 per se in these isoforms, determine tumor behavior and therapy response is unknown. This is relevant for therapeutic design and constitutes our main aim.

Materials and Methods: Using CRISPR/Cas9, we specifically deleted exon-v6, whilst maintaining the reading frame, from two GC cell lines endogenously expressing CD44v6. Edited cell lines were characterized and treated with cisplatin and 5-fluorouracil. Cell survival was compared to $w t$ controls.

Results: We obtained homozygous edited cell lines lacking exon-v6 that maintained expression of remaining CD44 variant portions. Edited cells' transcripts resulted in frame v5-v7 splicing, mimicking complete exon-v6 skipping. Drug treatments' results demonstrate that removing specifically exon-v6 does not affects GC cells chemotherapy response, however, skipping exon-v6 is sufficient to impair GC cells self-renewing and decrease proliferation levels. Moreover, CD44v6-containing isoforms knockdown modulates cell survival in GC cells.

Conclusions: We successfully designed exon-v6 skipping models in GC cells lines. These exon skipping models are extremely important to disclose exon-v6 specific role in GC chemotherapy response. We conclude that CD44v6containing isoforms modulate cell survival in GC cells and, furthermore, exon-v6 is capable of, by itself, altering one of 
cancer hallmarks by decreasing the proliferation capacity of GC cells.

Funding: $\quad$ FEDER/COMPETE:2020/POCI/Portugal;2020/FCTproject-POCI-01-0145-FEDER-007274); NORTE2020/PORTUGAL2020/ERD project-NORTE-070124-FEDER-000029;TDC/CTM-AN/120958/2010;SFRH/ BD/113031/2015(CP);iFCT/POPH-QRENType4.2,EDF, MCTES(IF/00615/2013;GMA)

S. Lobo: None. C. Pereira: None. C. Oliveira: None. G. Almeida: None.

\section{P12.085.C}

$A R I D 1 A$ and FAT4 genes are frequently mutated and deleted ina cohort of Omani patients with gastric adenocarcinoma

Y. Tamimi ${ }^{1}$, A. Al-Abri ${ }^{1}$, F. A-Kindi ${ }^{1}$, M. Al-Riyami ${ }^{2}$, M. AlMoundhri ${ }^{2}$

${ }^{1} \mathrm{SQU}$, Muscat, Oman, ${ }^{2} \mathrm{SQUH}$, Muscat, Oman

Background: Gastric cancer (GC) is one of the most common diseases worldwide. The composition of the molecular profiles of this disease is still not fully understood. ARIDIA and FAT4 were identified as novel driving genes with a pivotal role in GC pathogenesis. In this study, we used Sanger and gene panel exome sequencing (GPES) to assess the incidence rate of somatic mutations in FAT4 and ARIDIA in a cohort of Omani patients with GC and compared results to the TCGA and GEPIA databases.

Methods: DNA from 75 samples were subjected to GPES as well as Sanger sequencing to screen for alterations in ARIDIA and FAT4. In addition, we used different bioinformatics tools to retrieve TCGA data for 117 and 119 cases mutated in FAT4 and ARIDIA, respectively. Mutations and deletions were subsequently correlated with clinical-pathological features and survival data.

Results: GPES data revealed that $44.2 \%$ of patients displayed deletions within the ARIDIA gene, and 27.9\% carried mutations. Samples from $20.9 \%$ of patients were found to have deletions in FAT4, and $37.2 \%$ displayed variations. Sanger sequencing analysis of FAT4 identified novel missense mutations in $8 \%$ of cases and genomic deletions in exon-17 in $21.3 \%$ of samples. In silico analysis of tertiary protein structures, predicted alterations in two domains that contained mutations.

Conclusions: A markedly higher frequency of mutations/ deletions in ARIDIA and FAT4 genes were found in GC patients from the Middle East region, reflecting the importance of genetic background component variation among different ethnic groups.

Y. Tamimi: None. A. Al-Abri: None. F. A-Kindi: None. M. Al-Riyami: None. M. Al-Moundhri: None.
P12.087.B

Microsatellite instability and Epstein-Barr Virus status in Russian gastric cancer patients.

A. Danishevich ${ }^{l, 2}$, N. Pospekhova ${ }^{l}$, A. Stroganoval ${ }^{l}, D$. Golovina $^{l}$

${ }^{l}$ Federal State Budgetary Institution «N.N. Blokhin National Medical Research Center of Oncology», Moscow, Russian Federation, ${ }^{2}$ Moscow Clinical Research center n.a. A.S. Loginova, Moskow Helthcare Department, Moscow, Russian Federation

Introduction: According to the The Cancer Genome Atlas (TCGA) Research Network classification the Epstein-Barr Virus-associated $(\mathrm{EBV}+)$ and Microsatellite instabilityassociated (MSI) tumors are the molecular subtypes of gastric cancer. These markers are used as prognostic factors and prediction of chemotherapy and immunotherapy efficacy.

Materials and Methods: FFPE tumor samples and clinical information from 150 gastric cancer patients treated in N.N. Blokhin NMRCO in 2010-2019 have been investigated. Five quasimonomorphic mononucleotide markers were used for the detection of MSI status. EBV status has been determined by the quantitative Real-time-PCR.

Results:The clinicopathological characteristics of the gastric cancer patients and their association with the presence of EBV and MSI status are shown in Table 1. The majority of EBV-positive $(n=12,92,31 \%, \mathrm{p}<0,05)$ and MSI-High tumor samples $(n=6,54,55 \%, \mathrm{p}>0,05)$ were detected in males. EBV-associated $(61,54 \%)$ and MSIHigh $(63,64 \%)$ gastric cancer cases were more often diagnosed among patients over 55 years old. Half of malignancies $(n=7,53,85 \%$ of EBV+ and $n=5,45,45 \%$ of MSI-High) were located in the gastric body. Diffuse or mixed subtypes were frequently $(61,54 \%)$ revealed in EBV + samples, whereas $77,00 \%$ MSI-High were intestinal. There were no samples with both MSI-high and EBV+ in our research.

Table 1.

\begin{tabular}{llllll}
\hline & & $\begin{array}{l}\text { EBV- } \\
\text { positive }\end{array}$ & $\begin{array}{l}\text { EBV- } \\
\text { negative }\end{array}$ & $\begin{array}{l}\text { MSI- } \\
\text { High }\end{array}$ & MSI-stable \\
\hline & $\mathrm{n}(\%)$ & $13(8,67)$ & $137(91,33)$ & $11(7,33)$ & $\begin{array}{l}139 \\
(92,67)\end{array}$ \\
$\begin{array}{l}\text { Gender } \\
\text { Male }\end{array}$ & $74(49,33)$ & $12(92,31)$ & $62(45,26)$ & $6(54,55)$ & $68(48,92)$ \\
Female & 76 & $1(7,69)$ & $75(54,74)$ & $5(45,45)$ & $71(51,08)$ \\
Age $(\mathrm{yr})$ & $(50,67)$ & & & & \\
$\geq 55$ & 94 & $8(61,54)$ & $85(62,04)$ & $7(63,64)$ & $88(63,31)$ \\
$<55$ & $(62,67)$ & & & & \\
& 56 & $5(38,46)$ & $52(37,96)$ & $4(36,36)$ & $51(36,69)$ \\
& $(37,33)$ & & & &
\end{tabular}




\begin{tabular}{|c|c|c|c|c|c|}
\hline median & 55,05 & 54,46 & 63,31 & 57,50 & 64,18 \\
\hline \multicolumn{6}{|l|}{ Localization } \\
\hline Cardia/ fundus & $\begin{array}{l}42 \\
(28,00)\end{array}$ & $5(38,46)$ & $37(27,00)$ & $2(18,18)$ & $40(28,78)$ \\
\hline Body & $\begin{array}{l}58 \\
(38,67)\end{array}$ & $7(53,85)$ & $51(37,23)$ & $5(45,45)$ & $53(38,13)$ \\
\hline Pylorus/antrum & $\begin{array}{l}20 \\
(13,33)\end{array}$ & $1(7,69)$ & $19(13,87)$ & 0 & $20(14,38)$ \\
\hline $\begin{array}{l}>1 \\
\text { topographic area }\end{array}$ & $\begin{array}{l}30 \\
(20,00)\end{array}$ & 0 & $30(21,90)$ & $4(36,36)$ & $26(18,70)$ \\
\hline \multicolumn{6}{|l|}{ Subtype } \\
\hline Intestinal & $\begin{array}{l}70 \\
(46,67)\end{array}$ & $5(38,46)$ & $65(47,45)$ & $7(77,00)$ & $67(48,20)$ \\
\hline Diffuse/ Mixed & $80(53,33)$ & $8(61,54)$ & $72(52,55)$ & $4(44,00)$ & $72(51,8)$ \\
\hline \multicolumn{6}{|l|}{ Stage } \\
\hline I & $\begin{array}{l}20 \\
(13,33)\end{array}$ & $2(15,38)$ & $18(13,14)$ & 0 & $20(14,38)$ \\
\hline II & $\begin{array}{l}28 \\
(18,67)\end{array}$ & $4(14,28)$ & $24(17,52)$ & $2(18,18)$ & $26(18,70)$ \\
\hline III & $\begin{array}{l}55 \\
(36,67)\end{array}$ & $5(38,46)$ & $50(36,49)$ & $6(54,54)$ & $49(35,25)$ \\
\hline IV & $\begin{array}{l}47 \\
(31,33)\end{array}$ & $2(15,38)$ & $45(32,84)$ & $3(27,27)$ & $44(31,65)$ \\
\hline
\end{tabular}

Conclusions: The received results correspond to the worldwide data and provide new possibilities for the treatment of Russian patients with gastric cancer.

A. Danishevich: None. N. Pospekhova: None. A. Stroganova: None. D. Golovina: None.

P12.089.A

Oncology research applications on the GenapSys high accuracy sequencing platform

\author{
M. Fallahi, S. Paliwal, A. Nabi, S. Bagchi, M. Jouzi, A. \\ Thomas, M. Barmi, S. Sankar, H. Esfandyarpour
}

\section{GenapSys Inc, Redwood City, CA, United States}

Next Generation Sequencing (NGS) technologies have revolutionized basic biological and clinical research, especially in oncology. NGS cancer assays are used to determine cancer predisposition, identify tumor mutations, monitor treatment response and develop personalized therapies. Here, GenapSys presents a scalable, low cost, and high accuracy sequencing platform based on electrical impedance detection. We demonstrate that Chips with $1 \mathrm{M}, 16 \mathrm{M}$ and 144M sensors can be run on the same GenapSys instrument, with greater than $99 \%$ raw accuracy and an average read length of about $150 \mathrm{bp}$. We highlight its applications for oncology research, using diverse cancer panels on reference and clinical samples.

We tested hybrid-capture and multiplex PCR-based cancer panels on a range of DNA sources, including oncology reference standards derived from cell line DNA, cfDNA standards, clinical samples: FFPE, fresh frozen tumor tissue, and blood. For hybrid-capture libraries, we used the IDT xGen Pan Cancer and Exome Research panels. We detected low frequency mutations in the range of $1 \%-24.5 \%$ across multiple reference standards with the cancer panel, and observed high correlation in allele frequency with expected values $(\mathrm{R} 2>0.99)$. Similar results were obtained with the Ion AmpliSeq Cancer Hotspot Panel v2. Whole exome sequencing and pan cancer sequencing of clinical FFPE, fresh frozen and blood samples showed high concordance (F1-score 95\% - 99\%) of SNV mutation calling with industry standard technology. Thus, we demonstrate that the GenapSys Sequencing Platform is an accurate, scalable, and low cost solution for oncology research on a wide range of sample types and NGS assays.

M. Fallahi: A. Employment (full or part-time); Significant; GenapSys. S. Paliwal: A. Employment (full or part-time); Significant; GenapSys Inc. A. Nabi: A. Employment (full or part-time); Significant; GenapSys Inc. S. Bagchi: A. Employment (full or part-time); Significant; GenapSys Inc. M. Jouzi: A. Employment (full or parttime); Significant; GenapSys Inc. A. Thomas: A. Employment (full or part-time); Significant; GenapSys Inc. M. Barmi: A. Employment (full or part-time); Significant; GenapSys Inc. S. Sankar: A. Employment (full or parttime); Significant; GenapSys Inc. H. Esfandyarpour: A. Employment (full or part-time); Significant; GenapSys Inc.

\section{P12.091.C}

The role of miR-375 in the pathogenesis of gastrointestinal stromal tumors

U. Gyvyte $^{l}$, J. Bekampyte ${ }^{2}$, G. Gudoityte ${ }^{2}$, S. Juzėnas ${ }^{l, 3}$, G. Streleckiene $^{l}$, R. Lukosevicius $^{l}$, R. Inciuraite ${ }^{l}$, P. Ruzgys ${ }^{4}$, L. Kupcinskas ${ }^{I}$, J. Skieceviciene ${ }^{I}$

${ }^{1}$ Institute for Digestive Research, Lithuanian University of Health Sciences, Kaunas, Lithuania, ${ }^{2}$ Institute of Oncology, Lithuanian University of Health Sciences, Kaunas, Lithuania, ${ }^{3}$ Institute of Clinical Molecular Biology, ChristianAlbrechts University, Kiel, Germany, ${ }^{4}$ Biophysical Research Group, Vytautas Magnus University, Kaunas, Lithuania

Introduction: Deregulated miRNA profiles and their contribution to carcinogenesis have been observed in virtually all types of cancer. However, their contribution to the pathogenesis of rare gastrointestinal stromal tumors (GISTs) is not well defined yet. In this study, we aimed to investigate the role of miR-375 - a well-known tumor-suppressive miRNA - in the pathogenesis of GIST.

Materials and Methods: MirVana miRNA mimic and negative control were transfected to the GIST-T1 cell line using the lipofection technique. Target gene and protein expression were detected by RT-qPCR and Western Blot, respectively. Alterations in cell viability, migration rate and 
apoptotic cell counts, as well as miRNA-target gene interaction, were evaluated by MTT, Wound Healing, Annexin V FITC and Dual-Light Luciferase assays, respectively. Statistical analysis was performed using the computing environment R.

Results: Overexpression of miR-375 reduced levels of KIT gene mRNA (1.9-fold, $\mathrm{p}<0.05)$ and protein $(1.4$-fold, $\mathrm{p}<0.05)$ in GIST-T1 cell line. Further functional analysis revealed a significant effect of miR-375 on cell viability (reduced by $47 \%, \mathrm{p}<0.05$ ) and migration rates (reduced by up to $28 \%$ in different time points, $\mathrm{p}<0.05)$. GIST-T1 cells with KIT 3'UTR construct (pos855-861) showed decreased luciferase activity down to $76 \%$ when miR-375 was overexpressed.

Conclusions: Our findings indicate that miR-375 could suppress GIST by targeting KIT and contribute to the pathogenesis of GIST. This study was funded by the Research Council of Lithuania (Grant No: MIP-006/2014).

U. Gyvyte: None. J. Bekampyte: None. G. Gudoityte: None. S. Juzènas: None. G. Streleckiene: None. R. Lukosevicius: None. R. Inciuraite: None. P. Ruzgys: None. L. Kupcinskas: None. J. Skieceviciene: None.

P12.092.A

Massive GIST as first manifestation of neurofibromatosis type I

B. S. Küchler ${ }^{1}$, W. Heinritz ${ }^{2}$, A. Bier $^{2}$, S. Reif ${ }^{2}$, C. Jänecke ${ }^{2}$, M. Großmann ${ }^{2}$, J. Plaschke ${ }^{1}$, S. Krüger ${ }^{2}$

${ }^{I}$ MEWIGEN, Dresden, Germany, ${ }^{2} \ddot{U B A G}$ für Humangenetik Oberelbe/Spree, Dresden/Cottbus, Germany

Gastrointestinal stromal tumours (GISTs) are the most common mesenchymal tumours in the gastrointestinal tract. The incidence is estimated at 4.3 - 22 cases per million. GISTs are primarily sporadic (85-90\%) caused by somatic activating mutations in the KIT or PDGFRA genes, or less commonly $B R A F$ or $R A S$. SDH gene inactivation is involved in about $2 \%$. Mutations in the Neurofibromin gene (NF1) were found in $6-9 \%$. Clinicians should always consider a germline condition, as our illustrative case demonstrates.

Here we report a patient (55y) who suffered from weight loss, intermittent sweating at night and postprandial abdominal pain. Computer tomography revealed a massive duodenal tumour $(14 \times 9 \times 9 \mathrm{~cm})$. The excised GIST was tested negative for mutations in KIT, PDGFRA and BRAF. No pathogenic germline mutations were revealed in $S D H B$, SDHC, SDHD, FLCN and PRKARAIA. However, the pathogenic mutation c.6006+1G>T (NM_001042492.2) was identified in the NF1 gene. Subsequent clinical examinations revealed multiple nodular skin alterations most likely representing neurofibromas and several pale café-au-lait spots.

It is remarkable that the syndromic condition of this patient was missed until the genetic diagnosis was made. The diagnosis in such patients is complicated by the high de novo mutation rate of about $50 \%$. Therefore it is important, to consider neurofibromatosis type I also upon the apparently sole appearance of rare manifestations. Finally, patients with a subtle cutaneous phenotype are still at high risk of developing severe tumours.

B.S. Küchler: None. W. Heinritz: None. A. Bier: None. S. Reif: None. C. Jänecke: None. M. Großmann: None. J. Plaschke: None. S. Krüger: None.

P12.095.A

Identification of predisposing germline variants in familial glioma

R. Nurminen ${ }^{* 1,2}$, E. Afyounian ${ }^{* 1,2}$, J. Tuominen ${ }^{1,2}, M$.

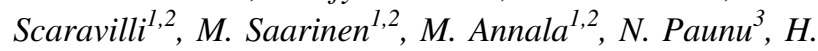
Haapasalo $^{4,5}$, M. Nykter ${ }^{1,2,6}$, K. J. Granberg ${ }^{1,2,6}$, * contributed equally

${ }^{1}$ BioMediTech, Faculty of Medicine and Health Technology, Tampere University, Tampere, Finland, ${ }^{2}$ Tays Cancer Center, Tampere University Hospital, Tampere, Finland, ${ }^{3}$ Department of Oncology, Tampere University Hospital, Tampere, Finland, ${ }^{4}$ Fimlab Laboratories Ltd, Tampere, Finland, ${ }^{5}$ Department of Pathology, Tampere University, Tampere, Finland, ${ }^{6}$ Science Center, Tampere University Hospital, Tampere, Finland

Introduction: Although gliomas are generally sporadic, there are families with several members affected by these tumors. With exception of Li-Fraumeni syndrome, little is known about predisposing factors underlying familial aggregation of glioma cases. In this study, we aim to identify rare glioma predisposing variants for familial glioma.

Materials and methods: We performed exome sequencing of 13 individuals (8 affected and 5 unaffected family members) from four Finnish glioma families with 50X coverage using blood-derived DNA. We called germline variants by using an in-house developed and GATK pipeline. Variants were filtered based on population allele frequency and functional annotation. Variants, which were shared by all genotyped affected members in at least one family, were validated by targeted sequencing in 15 additional glioma families. A total of 115 known gliomarelated variants were also covered with targeted sequencing. We are currently analysing targeted sequencing data.

Results: A total of 470 variants were identified in four glioma families after exome sequencing. Majority of 
variants were private mutations within one family (86\%). A total of 8 variants were shared between affected cases and present in two to four families. No familial gliomaassociated DNA copy number variants were detected. Preliminary analysis of targeted sequencing revealed that $24 \%$ of variants were shared between two or more families and $41 \%$ were not detected in any family.

Conclusions: Our results indicate that Finnish glioma families carry both private mutations and mutations which are shared between families. The role of variants as predisposing factors of glioma will be further evaluated.

R. Nurminen*: None. E. Afyounian*: None. J. Tuominen: None. M. Scaravilli: None. M. Saarinen: None. M. Annala: None. N. Paunu: None. H. Haapasalo: None. M. Nykter: None. K.J. Granberg: None.

\section{P12.096.B}

Meta-GWAS of multiple myeloma patients fromthe Nordic Region identifies a new risk locus in chromosome 13

L. Duran-Lozano ${ }^{1}$, G. Thorleifsson ${ }^{2}$, A. Lopez de Lapuente Portilla $^{1}$, E. Johnsson ${ }^{1}$, I. Turesson ${ }^{3}$, N. Abildgaard ${ }^{4}, U$. Mellqvist $^{5}$, N. Andersen ${ }^{6}$, A. Waage $^{7}$, A. Vangsted $^{8}$, M. Hansson $^{3,9}$, U. Thorsteinsdóttir ${ }^{2}$, T. Rafnar ${ }^{2}, K$. Stefánsson ${ }^{2}$, B. Nilsson ${ }^{1,10}$

${ }^{1}$ Hematogenomics Department of Laboratory Medicine, Lund University, Lund, Sweden, ${ }^{2}$ deCODE Genetics, Reykjavik, Iceland, ${ }^{3}$ Lund University Hospital, Lund, Sweden, ${ }^{4}$ Department of Haematology, AgeCare, Odense University Hospital, Odense, Denmark, ${ }^{5}$ Sodra Alvsborgs Sjukhus Borås, Borås, Sweden, ${ }^{6}$ Department of Haematology, Århus University Hospital, Aarhus, Denmark, ${ }^{7} \mathrm{St}$ Olavs Hospital and Norwegian University of Science, Trondheim, Norway, ${ }^{8}$ Department of Haematology, University Hospital of Copenhagen at Rigshospitalet, Copenhagen, Denmark, ${ }^{9}$ Myeloma research group, Department of Laboratory Medicine, Lund University, Lund, Sweden, ${ }^{10}$ Broad Institute, Cambridge, MA, United States

Introduction: Multiple myeloma (MM) is a common hematological malignancy, characterized by an uncontrolled expansion of plasma cells in the bone marrow. First-degree relatives of $\mathrm{MM}$ patients have 2 to 4 times higher risk of MM. Previous studies have identified 24 genomic loci associated with MM risk. However, these loci only explain 15 to $20 \%$ of the heritability, indicating that the molecular basis of MM predisposition is incompletely understood.

Materials and Methods: To identify MM risk loci, we performed a genome-wide association study in the Nordic region totalling 5,320 cases and 422,289 controls from Denmark, Iceland, Norway and Sweden.
Results and conclusions: We found a new MM risk associated locus at chromosome 13. Further analysis using blood and plasma cell eQTL data identified several potential candidate genes at the locus, as well as a likely causal variant mapping to a regulatory element that is selectively open in plasma cells. Our study increases the number of known MM risk loci to 25.

Grants and fellowships: Swedish Research Council (2018-00424), Swedish Cancer Society (2017/265), Nordic Cancer Union (R217-A13329), Canfaster (EU-H2020MSCA-COFUND-2016-754299).

L. Duran-Lozano: None. G. Thorleifsson: A. Employment (full or part-time); Modest; Amgen Genetics. A. Lopez de Lapuente Portilla: None. E. Johnsson: None. I. Turesson: None. N. Abildgaard: None. U. Mellqvist: None. N. Andersen: None. A. Waage: None. A. Vangsted: None. M. Hansson: None. U. Thorsteinsdóttir: A. Employment (full or part-time); Modest; Amgen Genetics. T. Rafnar: A. Employment (full or part-time); Modest; Amgen Genetics. K. Stefánsson: A. Employment (full or part-time); Modest; Amgen Genetics. B. Nilsson: None.

\section{P12.097.C}

Impact of NGS in management of haematological malignancies: experience after one year of routine in Eurofins-Biomnis laboratory

V. Geromel ${ }^{1}$, P. Mouty ${ }^{1}$, C. Chal ${ }^{1}$, J. delaunay ${ }^{2}$, S. Sadot Labouvier $^{2}$, R. Kaphan ${ }^{3}$, B. Rossignol ${ }^{4}$, K. Le D $\hat{u}^{5}$, J.

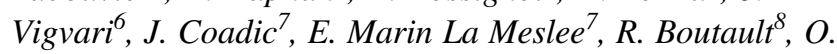
Roualdes $^{9}$, C. Bourdin' ${ }^{9}$, A. Petit ${ }^{9}, 1$, M. Roumiguières ${ }^{9}$, L. Raymond $^{l}$, B. Quilichini ${ }^{9,1}$

${ }^{1}$ Molecular genetics department of Eurofins-Biomnis laboratory, Lyon, France, ${ }^{2}$ Haematological clinical department, L'hôpital Privé du confluent, Nantes, France, ${ }^{3}$ Internal-oncology medicine department, Hospital center of Cannes, Cannes, France, ${ }^{4}$ Haematological clinical department, la Dracénie hospital center, Draguignan, France, ${ }^{5}$ Haematological department, Jean Bernard Le Mans center, Le Mans, France, ${ }^{6}$ Internal medicin department, Sémur en Auxois Hospital Center, Semur-en-Auxois, France, ${ }^{7}$ Labm, Bioloire laboratory, Nantes, France, ${ }^{8}$ Labm, Labomaine Laboratory,, Le Mans, France, ${ }^{9}$ Cellular haematology/cytogenetics department, EurofinsBiomnis laboratory, LYON, France

The biological management of haematological malignancies requires close collaboration between cellular hematology, cytogenetics and molecular biology. In contrast to traditional single-gene methods, next-generation sequencing (NGS) is a molecular diagnostic methodology that can study multiple 
regions of genomic tumor DNA in a single assay. Since 2019, over 500 analyses were routinely performed with a commercial panel allowing the analysis of 30 genes (Myeloid solution $^{\mathrm{TM}}$ by SOPHiA GENETICS). Our experience is presented here. Some clinical cases were selected, for which the results of the NGS analysis have contributed either to an improvement in patient management, or to call into question the diagnostic or prognostic value of the molecular anomalies observed. In particular, the diagnostic value of CHIPs (Clonal Hematopoiesis of Indeterminate Potential) and the impact of double mutations JAK2/CALR and JAK2/MPL in the progression of myeloproliferative neoplasia are reviewed. In addition, the CNV information provided by NGS is useful for the characterization of these diseases and complements the cytogenetic result. In chronic myeloid malignancies, the latest WHO classification includes only mutations of the JAK2, CALR, MPL, CSF3R and SF3B1 genes in strict diagnostic criteria. However, the current OMS classification of these haematological malignancies will certainly be redefined by a more exhaustive molecular approach based on recent NGS contributions.

V. Geromel: None. P. Mouty: None. C. Chal: None. J. delaunay: None. S. Sadot Labouvier: None. R. Kaphan: None. B. Rossignol: None. K. Le Dû: None. J. Vigvari: None. J. Coadic: None. E. Marin La Meslee: None. R. Boutault: None. O. Roualdes: None. C. Bourdin: None. A. Petit: None. M. Roumiguières: None. L. Raymond: None. B. Quilichini: None.

\section{P12.098.A}

Epigenetically regulated miR-129-5p functions as tumor suppressor in liver cancer by directly targeting $H D G F$

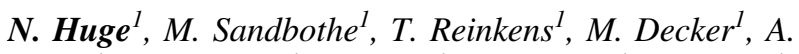

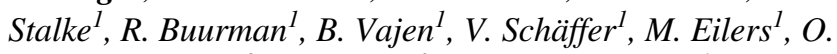 \\ Dittrich-Breiholz ${ }^{2}, F$. Kühnel ${ }^{3}$, B. Schlegelberger ${ }^{1}, T$. \\ Illig $^{1,4}$, B. Skawran ${ }^{1}$
}

${ }^{1}$ Department of Human Genetics, Hannover Medical School, Hannover, Germany, ${ }^{2}$ Research Core Unit Genomics, Hannover Medical School, Hannover, Germany, ${ }^{3}$ Department of Gastroenterology, Hepatology and Endocrinology,, Hannover Medical School, Hannover, Germany, ${ }^{4}$ Hannover Unified Biobank, Hannover Medical School, Hannover, Germany

Introduction: In hepatocellular carcinoma (HCC) an overexpression of histone deacetylases (HDACs) has become evident. This results in chromatin compaction and silencing of microRNAs (miRNAs). To identify epigenetically regulated miRNAs we analyzed global miRNA expression after HDAC inhibition in HCC cell lines. Amongst others, we identified miR-129-5p to be re- expressed after histone acetylation. MiR-129-5p has been shown to act tumor suppressive in several tumor entities and we aimed to investigate its role in HCC tumorigenesis.

Materials and Methods: After transfection of HCC cell lines (HLE, HLF, Huh7, HepG2) with miR-129-5p, tumorrelevant functional effects were analyzed using in vitro assays and a xenograft mouse model. Luciferase reporter assays were performed to validate hepatoma-derived growth factor $(H D G F)$ as a direct target of miR-129-5p. $H D G F$ expression and survival data of public HCC datasets were analyzed. Cancer-related functions of HDGF were examined after siRNA-mediated knockdown.

Results: MiR-129-5p increased apoptosis and decreased proliferation and migration in vitro and tumor growth in vivo. Direct binding of miR-129-5p to the HDGF-3'UTR via a noncanonical binding site was validated. $H D G F$ knockdown increased apoptosis and attenuated cell viability, migration, and $\mathrm{Wnt} / \beta$-catenin signaling activity of $\mathrm{HCC}$ cells. Overexpression of $H D G F$ correlated with poor overall survival, especially in Wnt-inactive HCCs.

Conclusions: HDAC inhibition induces expression of the tumor suppressor miR-129-5p which plays an important role in hepatocarcinogenesis by regulating $H D G F$. In the future, direct transfer of miR-129-5p or inhibition of its target genes may be considered for HCC therapy. In this context, inhibition of HDGF may be particularly beneficial in Wnt-inactive HCCs.

N. Huge: None. M. Sandbothe: None. T. Reinkens: None. M. Decker: None. A. Stalke: None. R. Buurman: None. B. Vajen: None. V. Schäffer: None. M. Eilers: None. O. Dittrich-Breiholz: None. F. Kühnel: None. B. Schlegelberger: None. T. Illig: None. B. Skawran: None.

\section{P12.099.B}

Characterization of somatic copy number variations in head and neck squamous cell carcinomas

N. Gestoso-Uzal ${ }^{1,2,3}$, J. L. García ${ }^{3,1}$, J. FernándezMateos $^{1,2}, \boldsymbol{P}$. García-Vallés ${ }^{2}$, D. Iglesias-Corral ${ }^{3,1,2}$, A. Martel-Martel $^{1,2}$, E. Del Barco ${ }^{4,1}$, M. A. Gómez Muñoz ${ }^{5,1}$, A. B. Herrero ${ }^{2,1,3}$, R. González-Sarmiento ${ }^{2,1,3}$, J. J. CruzHernández ${ }^{4,1,2,3}$

${ }^{1}$ Institute of Biomedical Research of Salamanca (IBSAL), Salamanca, Spain, ${ }^{2}$ Molecular Medicine Unit, Department of Medicine, University of Salamanca, Salamanca, Spain, ${ }^{3}$ Institute of Molecular and Cellular Biology of Cancer (IBMCC), University of Salamanca-CSIC, Salamanca, Spain, ${ }^{4}$ Medical Oncology Service, University Hospital of Salamanca-IBSAL, Salamanca, Spain, ${ }^{5}$ Service of Pathology, University Hospital of Salamanca, Salamanca, Spain 
Background: Most Head and Neck Squamous Cell Carcinomas (HNSCC) are diagnosed in locally advanced stages and, despite advances in treatment, survival rates are still very low. The aim of this study was to identify recurrent somatic alterations in HNSCC that could be relevant in cancer initiation, progression or stratification.

Patients and methods: Tumour samples from 98 patients included in clinical trial TTCC-2007-01 were used. All patients presented locally advanced epidermoid carcinomas (non-metastatic stages III and IV) at different locations; oral cavity, oropharynx, hypopharynx and larynx. Tumours were analysed by OncoScan ${ }^{\circledR}$ assay and bioinformatic tools (Bioconductor package, GISTIC and WebGestalt software), to detect genome-wide copy number variations and loss of heterozygosity.

Results and discussion: We identified genomic regions recurrently amplified (1q, 3q, 5p, 8q and 12p) and deleted ( $3 p, 9 p$ and 11q) in HNSCC. We considered the amplification in 1q21.3, identified in 58\% of the samples to be particularly interesting. Gene enrichment analysis in this region revealed a significant association with processes related to epithelial development. Region 1q21.3 contains FLG, IVL and LOR genes, that codify proteins highly expressed in the epithelium. Germinal mutations in these genes have been associated with genodermatosis suggesting that their somatic alteration could also be involved in the pathogenesis of HNSCC.

Conclusion: The chromosomic region 1q21.3, that contains FLG, IVL and LOR genes, is recurrently amplified in HNSCC. The present study brings new data to HNSCC research widening the spectrum of changes that could help to identify critical regions for tumorigenesis.

Funded by FIS-FEDER:PI18/01476.

N. Gestoso-Uzal: None. J.L. García: None. J. Fernández-Mateos: None. P. García-Vallés: None. D. IglesiasCorral: None. A. Martel-Martel: None. E. Del Barco: None. M.A. Gómez Muñoz: None. A.B. Herrero: None. R. González-Sarmiento: None. J.J. CruzHernández: None.

P12.100.C

Identification of a novel regulator of the hepatic leukemia factor HLF ortholog

\section{Silva Afonso ${ }^{1,2,3}$, O. Akpoghiran ${ }^{3}, K . K$ Ah $^{3}$}

${ }^{1}$ Centro Hospitalar Universitario de Coimbra, Coimbra, Portugal, ${ }^{2} I C V S / 3 B$ 's - PT Government Associate Laboratory, Braga, Portugal, ${ }^{3}$ Department of Neuroscience and the Farber Institute for Neurosciences, Thomas Jefferson University, Philadelphia, PA, United States

Introduction: HLF is associated with precursor B-cell acute lymphoblastic leukemia as part of a fusion gene. The functional regulation of HLF is poorly understood. From previous studies, we were led to hypothesize that taranis (tara) regulates HLF ortholog. tara has four orthologs: CDCA4 (Cell Division Cycle Associated 4), SERTAD2 (SERTA Domain Containing 2), SERTAD3 (SERTA Domain Containing 3), and SERTAD1 (SERTA Domain Containing 1). The first three tara orthologs are associated with diseases.

Materials and Methods: Genomic editing with CRISPR/ Cas9, protein quantification through western blotting, protein co-immunoprecipitation, transcriptional activity assays in heterologous cell lines, and statistical analysis.

Results: TARA enhances the transcriptional activity of HLF ortholog. In the nucleus, HLF ortholog levels are affected by tara. TARA forms a physical complex with HLF ortholog. More specifically, of the four conserved domains present in all four tara orthologs, the SERTA domain is the only domain required for the transcriptional activity of HLF ortholog.

Conclusions: In this work, we shed new light into the molecular mechanisms that regulate the transcriptional activity of HLF ortholog. Plausibly, CDCA4, SERTAD2, SERTAD3, and/or SERTAD 1 may regulate HLF trough an identical molecular mechanism.

D. Silva Afonso: None. O. Akpoghiran: None. K. Koh: None.

\section{P12.101.A}

Argonaute-RIP-Sequencing identifies new microRNA449a target genes in hepatocellular carcinoma

T. Reinkens ${ }^{1}$, N. Huge ${ }^{1}$, A. Stalke ${ }^{l}$, B. Vajen ${ }^{1}$, O. Dittrich-

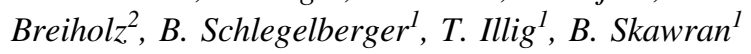

${ }^{1}$ Department of Human Genetics, Hannover Medical School, Hannover, Germany, ${ }^{2}$ Research Core Unit Genomics, Hannover Medical School, Hannover, Germany

Introduction: MicroRNAs are important transcriptional regulators that target multiple molecules affecting disease development. The regulatory potential of tumor suppressor miRNAs represents an interesting option for cancer therapeutics. It has been shown that overexpression of miR449a reduces tumor growth of hepatocellular carcinoma in vivo and in vitro. However, only few direct target genes of miR-449a have been validated to date. Therefore, we analyzed the targetome of miR-449a to decipher the large network of target genes and involved signaling pathways in order to understand its role in hepatocellular carcinoma.

Methods: HLE cells transfected with miR-449a were used for Argonaute-RNA immunoprecipitation (RIP) and subsequent high-throughput sequencing. Luciferase reporter 
assays were performed to validate PEA15, PPP1CA and TUFT1 as direct targets of miR-449a. Expression of these genes in correlation with survival data in public HCC datasets was analyzed. Cancer-related functional effects were examined after siRNA-mediated knockdown of PEA15.

Results: Ago-RIP-Seq identified 183 genes as direct targets of miR-449a. Seven genes were selected for further investigation, as they are potential tumor suppressors according to manual literature search. Reduced mRNA expression following miR-449a overexpression was confirmed in all of these genes. Direct binding of miR-449a to the 3'UTR of PEA15, PPPICA and TUFT was validated. In HCC datasets overexpression of PEA15, PPPICA or TUFT1 correlated with poor overall survival.

Conclusions: Ago-RIP-Seq is a promising method to decipher the network of micro-RNA target genes. Here, we identified several new miR-449a targets providing potential targets for cancer therapy.

This project is supported by the German Cancer Aid funding.

T. Reinkens: None. N. Huge: None. A. Stalke: None. B. Vajen: None. O. Dittrich-Breiholz: None. B. Schlegelberger: None. T. Illig: None. B. Skawran: None.

\section{P12.103.C}

A categorised approach for women with a diagnosis of breast cancer and a negative breast cancer susceptibility gene test requesting risk-reducing mastectomy

A. Kerr ${ }^{l}$, A. Kulkarni ${ }^{1}$, L. Izatt ${ }^{l}$, A. Shaw ${ }^{l}$, B. DeSouza ${ }^{l}$, D.

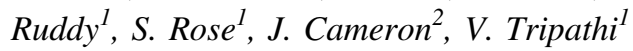

${ }^{1}$ Clinical Genetics Service, Guy's and St Thomas' NHS Foundation Trust, London, United Kingdom, ${ }^{2}$ School of Medicine, Cardiff University, Cardiff, United Kingdom

Introduction: Making an evidence based decision about risk-reducing mastectomy (RRM) is complex. We developed and audited an algorithm for women with a diagnosis of breast cancer, a negative breast cancer susceptibility gene test and a high-risk breast cancer family history requesting RRM. This incorporates current National Institute for Health and Care Excellence (NICE) Familial Breast Cancer Clinical guideline [CG164] (2013) and the findings from Reiner et al. (2013 and 2018) who found that women diagnosed $<45$ years had a high risk of developing a second primary breast cancer when they had a first degree relative (FDR) with breast cancer.

Methods and materials: 39 women with breast cancer diagnosed $<45$ years old, a negative breast cancer susceptibility gene test and a FDR with breast cancer were identified out of 213 women who had genetic testing between 2017-2019. A BOADICEA risk score was calculated to determine the risk of a second breast cancer in these women.

Results: We present findings that highlight a lack of correlation between the used risk stratification sources, including BOADICEA and Reiner et al. (2013 and 2018).

Conclusions: We conclude that clinicians can utilise scenario-based guidance to assist in making decisions about RRM in affected individuals for specific high-risk family histories, which is derived from the use of validated tools such as BOADICEA and also prospective data from the literature.

Outcome: We propose using an audited categorised approach to surgical RRM decision-making in women with unilateral breast cancer, which incorporates personal and family history based guidance.

A. Kerr: None. A. Kulkarni: None. L. Izatt: None. A. Shaw: None. B. DeSouza: None. D. Ruddy: None. S. Rose: None. J. Cameron: None. V. Tripathi: None.

P12.104.A

CanVaS: A national registry of Greek cancer patient genetic variation

D. Kalfakakou ${ }^{l}$, F. Fostira ${ }^{l}$, P. Apostolou ${ }^{l}$, A. Papathanasiou $^{l}$, I. E. Gavra', V. Dellatola ${ }^{l}$, I. S. Vlachos $^{2,3}$, Z. G. Scouras ${ }^{4}$, E. Drosopoulou ${ }^{4}$, D. Yannoukakos $^{l}$, I. Konstantopoulou ${ }^{1}$

${ }^{1}$ Molecular Diagnostics Laboratory, INRaSTES, National Center for Scientific Research "Demokritos", Athens, Greece, ${ }^{2}$ Cancer Research Institute, Department of Pathology, Beth Israel Deaconess Medical Center / Harvard Medical School, Boston, MA, United States, ${ }^{3}$ Broad Institute of MIT and Harvard, Cambridge, MA, United States, ${ }^{4}$ Department of Genetics, Development \& Molecular Biology, School of Biology, Aristotle University of Thessaloniki, Thessaloniki, Greece

Introduction: Recent advances in sequencing technologies have allowed the integration of large genetic variation datasets in public resources, providing the foundation for precision medicine. They have also introduced the necessity for inter- and intra-population genetic/genomic data recording, in order to capture variation between and within populations. National genetic variation registries could serve both ends by vastly increasing the level of detail for the relevant population, while directly affecting local patient management.

Methods: We report CanVaS, a Cancer Variation reSource aiming to document the genetic variation of cancer patients in Greece. Currently, CanVaS comprises germline genetic data 
from $\sim 11,000$ Greek cancer patients, analyzed for the presence of variants in 1-94 cancer susceptibility genes.

Results: The dataset incorporates $\sim 3,700$ functionally annotated coding variants and $\sim 3,000$ variants in noncoding loci. For each variant, allele frequency for the Greek population, clinical significance interpretation and anonymized family, segregation information, and phenotypic traits of the carriers are included. Moreover, it provides information on the geographic distribution of the variants across the country, enabling the study of Greek population isolates. Direct comparisons between Greek (sub)populations with relevant genetic resources is supported, allowing fine-grain localized adjustment of guidelines and clinical decision-making. Most importantly, anonymized data are available for download, while our first priority is integration/interconnection with other resources, such as the Leiden Open Variation Database.

Conclusions: CanVaS can become a stepping-stone for a countrywide effort to characterize the cancer mutation landscape, concurrently supporting national and international cancer research.

Acknowledgments: Supported by Stavros Niarchos Foundation Industrial Research Fellowship Program.

D. Kalfakakou: None. F. Fostira: None. P. Apostolou: None. A. Papathanasiou: None. I.E. Gavra: None. V. Dellatola: None. I.S. Vlachos: None. Z.G. Scouras: None. E. Drosopoulou: None. D. Yannoukakos: None. I. Konstantopoulou: None.

\section{P12.105.B}

Non-Lynch familial nonpolyposis colorectal cancer is partially explained by accumulation of CRC-associated low risk variants

L. Valle ${ }^{1,2}, P$. Mur $^{1,2}, N$. Bonifaci ${ }^{1}$, A. Díez-Villanueva ${ }^{3,4}$, E. $^{3,4}$ Muntél, M. Alonso $^{3,4}$, G. Aizal, M. Navarro ${ }^{1,2}$, J. Brunet $^{1,5,2}$, V. Moreno ${ }^{3,4}$, G. Capellá ${ }^{1,2}$

${ }^{1}$ Hereditary Cancer Program, Catalan Institute of Oncology; Oncobell Program, IDIBELL, Hospitalet de Llobregat, Barcelona, Spain, ${ }^{2}$ CIBERONC, Madrid, Spain, ${ }^{3}$ Unit of Biomarkers and Susceptibility, Cancer Prevention and Control Program, Catalan Institute of Oncology; Oncobell Program, IDIBELL, Hospitalet de Llobregat, Barcelona, Spain, ${ }^{4}$ CIBERESP, Madrid, Spain, ${ }^{5} I D I B G i$, Girona, Spain

Introduction: Much of the genetic predisposition to colorectal cancer (CRC) cannot be explained by pathogenic variants in known high/moderate-penetrance cancerpredisposing genes. Here we aim to investigate whether part of the genetically unexplained familial/early-onset
CRC cases may be attributed to the accumulation of low risk alleles for $\mathrm{CRC}$.

Material and methods: A weighted polygenic risk score (wPRS) based on $95 \mathrm{CRC}$ risk alleles identified and validated through genome-wide association studies (Huyghe et al. Nature genetics 2018), was determined in 421 familial/early-onset unrelated CRC patients (fCRC), 1,077 sporadic CRC patients, and 1,642 cancer-free controls. Logistic regression was used to model the risk. Two-sided t-test was applied to compare means among cohorts. Additionally, we grouped individuals (fCRC and controls) into 20 quantiles. Odds ratios (OR) were estimated taking the quantile 10 as reference.

Results: The average wPRS was statistically higher in fCRC patients compared to either controls $\left(\mathrm{P}_{\mathrm{t} \text {-test }}=2.2 \mathrm{e}-16\right)$, with an OR of 22.8, or sporadic CRC patients $\left(\mathrm{P}_{\mathrm{t} \text {-test }}=0.004\right)$. Familial CRC patients in the highest $\left(20^{\text {th }}\right)$ wPRS quantile were at a four-fold increased CRC risk than those in the middle (10th) quantile. The frequency of high-wPRS fCRC patients who had developed multiple (synchronous or metachronous) colorectal tumors and that had familial CRC history was higher than in low-wPRS patients. The heritability explained by the studied alleles was of $37.48 \%$ for fCRC.

Conclusions: The accumulation of low risk $\mathrm{CRC}$ alleles may explain a relevant proportion of familial/early-onset CRC cases without germline pathogenic variants in known hereditary cancer genes.

L. Valle: None. P. Mur: None. N. Bonifaci: None. A. Díez-Villanueva: None. E. Munté: None. M. Alonso: None. G. Aiza: None. M. Navarro: None. J. Brunet: None. V. Moreno: None. G. Capellá: None.

\section{P12.106.C}

Assessment of the causal association of recently proposed candidate genes with colorectal cancer and polyposis predisposition

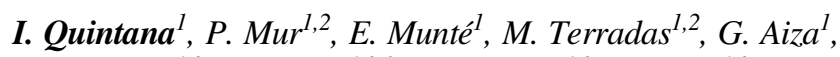

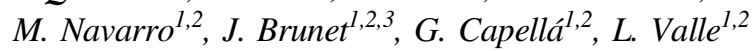

${ }^{1}$ Hereditary Cancer Program, Catalan Institute of Oncology; Oncobell Program, IDIBELL, Hospitalet de Llobregat, Barcelona, Spain, ${ }^{2}$ CIBERONC, Madrid, Spain, ${ }^{3} I D I B G i$, Girona, Spain

Introduction: A previous in silico study identified 49 genes as the most-frequently enriched in rare damaging germline variants in samples with putative somatic loss of heterozygosity ( $\mathrm{LOH})$ in cancer patients, thus presenting as good candidates for cancer predisposition. Based on their function, involvement in CRC carcinogenesis and expression in colonic mucosa, we selected six of those genes: NSD1, 
HDAC10, KRT24, BBS1, ACACA and TP63, to evaluate their actual involvement in colorectal cancer predisposition.

Materials and methods: Germline mutational screening of the six genes was performed in 738 patients (465 familial/early onset CRC patients, 177 (attenuated) familial adenomatous polyposis and 96 serrated polyposis patients) by using pooled DNA amplification followed by targeted next generation sequencing. The impact of the identified missense variants was analyzed by using 13 pathogenicity prediction tools. Co-segregation studies and second hit analysis in tumors or polyps are currently being performed.

Results and conclusions: Twenty-four carriers of disruptive or predicted deleterious variants in the studied genes were identified. Assessment of allele frequencies in controls compared to familial/early-onset CRC patients showed an association of NSDl disruptive variants with increased $\mathrm{CRC}$ risk, and no association was detected for $A C A C A$. No conclusive evidence was found for $H D A C 10$, KRT24, BBS1 and TP63. Results from co-segregation and second hit analyses will provide further evidence in favor or against the association of those genes with CRC predisposition.

Grant: BES-2017-079701 (Co-funded by European Social Fund. ESF investing in your future)

I. Quintana: None. P. Mur: None. E. Munté: None. M. Terradas: None. G. Aiza: None. M. Navarro: None. J. Brunet: None. G. Capellá: None. L. Valle: None.

\section{P12.108.B}

Clinical audit of multigene panel testing at the Royal Melbourne Hospital

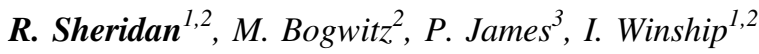 \\ ${ }^{1}$ University of Melbourne, Parkville, Australia, ${ }^{2}$ Royal Melbourne Hospital, Parkville, Australia, ${ }^{3}$ Peter MacCal- lum Cancer Centre, Parkville, Australia}

Introduction: In 2016 the Parkville Familial Cancer Centre at the Royal Melbourne Hospital introduced panel based genetic testing to screen for hereditary cancer syndromes. Multigene panel tests allow simultaneous screening for pathogenic variants in multiple cancer predisposition genes and replaced iterative single gene tests, which proved to be slow, inefficient and expensive. This study seeks to validate the clinical testing criteria used by the Familial Cancer Centre and to identify areas of improvement to increase overall clinical utility.

Materials and Methods: This study used a tailored database extraction to collate panel testing results over a 28month period, and these results were analysed in conjunction with relevant patient data, such as primary cancer type, age of diagnosis, family history, and relevant phenotypic features.

Results: A total of 757 patients met testing criteria for state funding, and were offered panel testing relevant to their cancer diagnosis and/or family history. Pathogenic variants were detected in $13 \%(n=101)$. Individual panel yield varied, with a trend suggesting that clear concomitant phenotypic features, such as skin signs with renal cancer, increased the likelihood of a panel yielding a positive result. Positive results also varied by specific cancer pathology. Of patients presenting with a primary breast cancer $(n=335), 12 \%$ had a pathogenic mutation. The mutation pickup rate for a triple hormone-receptor negative breast cancer $n=$ was $18 \%$, but only $10 \%$ for breast cancers with at least one hormone-receptor positive pathology result ( $n=237)$.

Conclusions: This project demonstrates that ongoing review of publicly funded testing is warranted.

R. Sheridan: None. M. Bogwitz: None. P. James: None. I. Winship: None.

P12.110.A

Oncopanel screening for hereditary cancers: a retrospective review of a hospital-based experience

E. Yilmaz ${ }^{1,2}$, E. Borklu Yucel ${ }^{2}$, S. Avci ${ }^{2}, H$. Azakli $^{2}$, P.

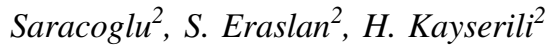

${ }^{1}$ Research Center for Translational Medicine (KUTTAM), Koç University, Istanbul, Turkey, ${ }^{2}$ Department of Medical Genetics, School of Medicine, Koç University, Istanbul, Turkey

In the last decade, cancer, as a large group of diseases described as an uncontrollable abnormal cell growth that can start in almost any organ or tissue of the body, becomes the major health problem and the second leading cause of death globally. The leading types are lung, breast, colorectal, prostate and stomach cancers. 18.1 million new cases were diagnosed globally in 2019 and only 5-10\% of them are attributed to hereditary factors. Genetic testing can be offered not only to identify the individuals' cancer risk, but also to enable the possibility of molecular targeted therapies. Here, we report the retrospective analysis of a single institution's 3-year experience of oncopanel sequencing, which targets 94 genes and 284 SNPs, carried out by using peripheral blood samples. 125 unrelated patients with a male:female ratio of $1: 4$ (mean age 40 (sd 6,51)) were screened. Our studies showed that $72 \%$ of the cases have at least one variant classified as pathogenic, possibly pathogenic or of unknown significance in targeted regions. Pathogenic variants were detected in $12 \%$, and possible pathogenic variants were detected in $18 \%$ of the cases. 
CHEK2 and ATM were detected as most common genes in patients with pathogenic mutations. Although pathogenic variant detection rate is relatively high, variants of unknown significance (VUS), which were found in $42 \%$ of the cases, are also needed to be evaluated carefully, especially in hereditary forms of cancer. It is obvious that more research on VUS and assessment of their effect on cancer development is essential to shed light to underlying pathogenicity and medical management.

E. Yilmaz: None. E. Borklu Yucel: None. S. Avci: None. H. Azakli: None. P. Saracoglu: None. S. Eraslan: None. H. Kayserili: None.

\section{P12.111.B}

TP53 pathogenic variants in colorectal cancer patients in absence of Li-Fraumeni-associated phenotypes

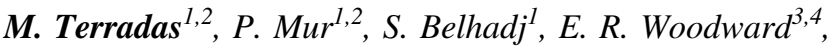
G. J. Burghel ${ }^{3}$, P. M. Munoz-Torres ${ }^{1}$, I. Quintana ${ }^{1}$, M. Navarro $^{l}$, J. Brunet ${ }^{1,5}$, C. Lázaro ${ }^{1}$, M. Pineda ${ }^{l}$, V.

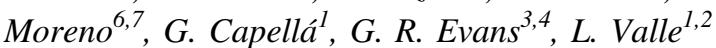

${ }^{1}$ Hereditary Cancer Program, Catalan Institute of Oncology; Oncobell Program, IDIBELL, Hospitalet de Llobregat, Spain, ${ }^{2}$ CIBERONC, Madrid, Spain, ${ }^{3}$ Manchester Centre for Genomic Medicine, St Mary's Hospital, Manchester University NHS Foundation Trust, Manchester, United Kingdom, ${ }^{4}$ Division of Evolution and Genomic Sciences, Faculty of Biology, Medicine and Health, University of Manchester, Manchester, United Kingdom, ${ }^{5}$ Catalan Institute of Oncology, IDIBGi, Girona, Spain, ${ }^{6}$ Unit of Biomarkers and Susceptibility, Cancer Prevention and Control Program, Catalan Institute of Oncology, IDIBELL, Hospitalet de Llobregat, Spain, ${ }^{7}$ CIBERESP, Madrid, Spain

Introduction: Germline TP53 pathogenic variants cause Li-Fraumeni syndrome (LFS), an aggressive multi-tumorpredisposing condition. Due to the implementation of multigene panel testing, TP53 variants have been detected in individuals without LFS suspicion, e.g. colorectal cancer (CRC) patients. We aim to decipher whether these findings are the result of detecting background population prevalence or the etiologic basis of CRC.

Materials and Methods: TP53 mutational screening was performed in 473 familial/early-onset CRC cases. Our findings were analyzed together with results from five additional studies that evaluated the prevalence of TP53 germline variants in CRC patients (total $n=6,200$ ). Data from control population and LFS cases were obtained from gnomAD and the IARC TP53 database, respectively. All variants were reclassified according to ACMG/AMP guidelines (both applying the TP53 rule specifications (v.1) provided by the ClinGen TP53 Expert Panel, and automatically using PathoMan).

Results: (Likely) pathogenic variants were identified in $0.03 \%$ of controls and $\sim 0.21 \%$ of CRC patients ( $\mathrm{p}<0.0001)$, none of whom fulfilled the clinical criteria established for TP53 testing. Loss-of-function/splice-site variants were significantly associated with $\mathrm{CRC}$ as compared with controls $(\mathrm{OR}=19 ; \mathrm{p}<0.001)$. Compared to LFS, enrichment of this type of variants and of variants with no dominant negative effect was observed in CRC patients.

Conclusions: TP53 pathogenic variants should not be unequivocally associated with LFS. The association with $\mathrm{CRC}$ risk may be explained by the absence of dominant negative effects and/or the loss-of-function nature of the variants.

M. Terradas: None. P. Mur: None. S. Belhadj: None. E.R. Woodward: None. G.J. Burghel: None. P.M. Munoz-Torres: None. I. Quintana: None. M. Navarro: None. J. Brunet: None. C. Lázaro: None. M. Pineda: None. V. Moreno: None. G. Capellá: None. G.R. Evans: None. L. Valle: None.

\section{P12.112.C}

Clinical significance of gene mutations and polymorphic variants and their association with prostate cancer risk in Polish men.

M. Heise ${ }^{l}$, P. Jarzemski ${ }^{2}$, A. Junkiert-Czarneckal, A. Bak ${ }^{l}$, M. Pilarska-Deltow ${ }^{l}$, M. Borysiak ${ }^{2}$,O. Haus ${ }^{1}$

${ }^{1}$ Department of Clinical Genetics, Faculty of Medicine, Collegium Medicum, Nicolaus Copernicus University, Bydgoszcz, Poland, ${ }^{2}$ Department of Urology, Jan Biziel University Hospital, Bydgoszcz, Poland

Introduction: We tested the association of $B R C A 1$, BRCA2, CHEK2, CDKN2A, HOXB13, MLH1, NBS1, NOD2, PALB2, CYP1B1, PON1, 8q24 mutations and polymorphisms with prostate cancer (PC) risk and analyzed the impact changes on disease clinical course, including overall survival time.

Material: DNA from 110 men with PC from all over Poland and from 110 men - healthy volunteers.

Methods: Sequencing, AS-PCR, RFLP-PCR, multiplexPCR.

Results: The percentage of men with $\geq 1$ change was higher in PC group $(61,8 \%)$ than in healthy men $(52,3 \%)(p$ $=0,15)$. The presence of at least two changes was associated with shorter patient survival than the presence of one or no change ( $p=0,14)$. The G84E of HOXB13 was detected in $2,9 \%$ of PC men and in no healthy man 
$(p=0,19$, trend, $\mathrm{OR}=7,21)$. The average age at $\mathrm{PC}$ onset of men with CYP1B1 432GG and PON1 55AA originated from Hereditary Prostate Cancer Families (HPC) was significantly younger than the age of men with these variants from families without HPC (respectively: $p=0,0077, p=0,0350$ ).

Conclusions: The presence of at least one genetic change is an unfavorable factor in PC. The presence of $\geq 2$ changes may be associated with shorter survival. The HOXB13 (G84E) are probably associated with PC development and with hereditary form of the disease. The CYP1B1 (432GG) and PON1 (55AA) are associated with younger age at $\mathrm{PC}$ onset in men from HPC families. The HOXB13 (G84E), NOD2 (3020insC) and PON1 (55AA) are assiciated with shorter overall survival.

This study was supported by the found of the Collegium Medicum.

M. Heise: None. P. Jarzemski: None. A. JunkiertCzarnecka: None. A. Bąk: None. M. Pilarska-Deltow: None. M. Borysiak: None. O. Haus: None.

P12.113.A

Evaluation of the clinical impact of a questionnairebased self-assessment family history of colorectal cancer compared to a professional pedigree survey

\section{S. I. Steiner ${ }^{1,2}$, B. Denecke ${ }^{2}$, U. Berger ${ }^{2}$, V. J. Scheble ${ }^{3}$, A. Dufke ${ }^{l}$}

${ }^{1}$ Institute of Medical Genetics and Applied Genomics, University Hospital Tübingen, Tübingen, Germany, ${ }^{2} \mathrm{Com}-$ prehensive Cancer Center Tübingen-Stuttgart, University Hospital Tübingen, Tübingen, Germany, ${ }^{3}$ Department of Internal Medicine I, University Hospital Tübingen, Tübingen, Germany

Introduction: The "German Cancer Society" is providing a questionnaire-based risk-score to delineate persons at risk for familial colorectal cancer, intended for easy use by nongeneticists in daily routine with colon cancer patients. The intention is to support therapists in detecting patients that should undergo a genetic counselling regarding hereditary tumour predisposition syndrome. The monocentric study aimed to analyse and evaluate the value of this score compared with a standard three-generation pedigree analysis.

Material and Methods: Starting in June 2018, 100 colorectal cancer patients treated at the University Tübingen were randomly picked over a 14 months period. After patients completed the questionnaire, a pedigree and a survey on the comprehensibility of the questionnaire were conducted. Pedigrees and available histopathological data were then evaluated according to the Bethesda and
Amsterdam guidelines and analysed for other familial tumour syndromes. The results were compared to the selfassessment risk-score.

Results: In $41 / 100$ patients the questionary screening approach indicated a familial colorectal cancer risk compared to 22 patients by pedigree analysis. Into the positive questionary group fell $16 / 22$ of these patients. In 9 cases the family trees identified criteria for other familial cancer syndromes.

Conclusions: The questionnaire is a valuable screening tool for identifying family risk of colorectal cancer. In order to exploit the full potential of the questionnaire, it is advisable to use it with a medical or professional perspective and assistance, because sensitivity and specificity is significantly lower compared to pedigree analysis.

S.I. Steiner: None. B. Denecke: None. U. Berger: None. V.J. Scheble: None. A. Dufke: None.

\section{P12.115.C}

Immune checkpoint genes expression vary among paediatric an adult glioblastoma models

R. Mehrian-Shai ${ }^{1}$, D. Rogachevsky ${ }^{1}$, P. A. Stempor ${ }^{2,3}$, A. Toren $^{4,1}$, P. Dobosz $z^{5,1}$

${ }^{1}$ Paediatric Haemato-Oncology, Oncology Department, Cancer Research Centre, Sheba Medical Centre Hospital, Tel Hashomer, Ramat Gan, Israel, ${ }^{2}$ School of Life Sciences, Gurdon Institute, Department of Genetics, Tennis Court Rd, Cambridge CB2 1QN, Cambridge, United Kingdom, ${ }^{3}$ The Wellcome Trust/CRUK Gurdon Institute, University of Cambridge, Tennis Court Rd,, Cambridge, United Kingdom, ${ }^{4}$ Oncology Department, Sackler Faculty of Medicine, Tel Aviv University, 6997801, Tel Aviv, Israel, ${ }^{5}$ Department of Haematology, Oncology and Internal Diseases, Medical University of Warsaw, Warsaw, Poland

Introduction: Glioblastoma (GB) is the most aggressive malignant primary brain tumour in both children and adults. Regardless of age, GB is incurable and associated with poor prognosis. Because of its highly invasive nature, it is impossible to remove all tumour cells during surgical resection, making relapse inevitable, thus, GB treatment presents one of the greatest challenges. Molecular data reveal significant differences between the paediatric and adult GBs, e.g. recurrent somatic mutations in some histone variants. The aim of the study was to determine differences in gene expression among paediatric and adult GB, suggesting the possible reason for failures of immunotherapy in GB.

Materials and methods: GB cell lines: paediatric (KNS42, SF188, SF8628), adult (U87, U251, T98G), and 
normal human astrocytes were used as a reference. RNA was harvested and the gene expression level was determined using RT-PCR. Selected genes with proven involvement for immunotherapy response have been analysed.

Results: Of all 27 analysed genes, only a small fraction seems to be expressed in GB, some of which were not analysed in GB literature so far. Expression of those seem to be higher in paediatric GB, e.g. BACH2 and IL-6R.

Conclusions: Most of the genes involved in the formation of immunological synapse are significantly down-regulated or not expressed at all in GB, providing the potential explanation for many failures of checkpoint inhibitors immunotherapy in GB. However, differences in gene expression might favour paediatric GB over adult GB as a candidate for future research leading to the successful immunotherapy.

R. Mehrian-Shai: None. D. Rogachevsky: None. P.A. Stempor: E. Ownership Interest (stock, stock options, patent or other intellectual property); Modest; Smart Immune Ltd.. A. Toren: None. P. Dobosz: E. Ownership Interest (stock, stock options, patent or other intellectual property); Modest; Smart Immune Ltd.

P12.119.A

TP53 with in cis RAD51C pathogenic variants modulates the TP53-dependent tumorigenesis to sebaceous gland carcinomas

J. Hentschel ${ }^{1}$, D. Le Duc ${ }^{l}$, S. Neuser ${ }^{l}$, M. Stiller ${ }^{1,2}$, C. Meier $^{l}$, E. Jäger ${ }^{3}$, R. Abou Jamra ${ }^{l}$, K. Platzer ${ }^{l}, A$. Monecke $^{2}$, M. Ziemer ${ }^{4}$, A. Markovic ${ }^{4}, H$. Bläker ${ }^{2}, J . R$. Lemke $^{l}$

${ }^{1}$ Institute of Human Genetics, University Medical Center Leipzig, Leipzig, Germany, ${ }^{2}$ Institute of Pathology, University Medical Center Leipzig, Leipzig, Germany, ${ }^{3}$ Department of Endocrinology, Nephrology, and Rheumatology, University Medical Center Leipzig, Leipzig, Germany, ${ }^{4}$ Department of Dermatology, Venereology and Allergology, University of Leipzig Medical Center, Leipzig, Germany

Introduction: Pathogenic variants in the tumor suppressor TP53 cause Li-Fraumeni syndrome, a cancer predisposition syndrome with high risks for childhood- and adult-onset malignancies e.g. breast cancer, soft tissue or bone sarcoma, brain tumors and adrenocortical carcinoma.

Case description: We describe a 43-year-old male with germline pathogenic variants in TP53 and RAD51C located in cis on chromosome 17, who has so far not developed any tumors of the LFS-spectrum. Instead, he developed a sebaceous gland carcinoma (SGC), without detection of mismatch repair deficiency, which is the only known cause of SGC to date. These findings are replicated by a Trp53Rad51c-double-mutant cis mouse-model, which develops SGCs, but suppresses the characteristic Trp53-associated tumor spectrum.

Methods: Variants were identified using tumor panel next generation sequencing. Phasing of the two variants was done using short tandem repeat analysis.

Conclusions: In line with the observations in Trp53Rad51c-double mutant cis mice, we propose that the human in cis co-occurrence of pathogenic TP53 and RAD51C variants substantially transforms the Li-Fraumeni phenotype to a predisposition to SGC.

J. Hentschel: None. D. Le Duc: None. S. Neuser: None. M. Stiller: None. C. Meier: None. E. Jäger: None. R. Abou Jamra: None. K. Platzer: None. A. Monecke: None. M. Ziemer: None. A. Markovic: None. H. Bläker: None. J.R. Lemke: None.

P12.121.C

Cellfree tumour DNA analysis detects copy number alterations in gastrooesophageal cancer patients.

K. Wallander ${ }^{l, 2}$, J. Eisfeldt ${ }^{l}$, M. Lindblad $^{l, 2}$, D. Nilsson ${ }^{1,2}$, K. Billiau ${ }^{l}$, H. Foroughi ${ }^{l}$, M. Nordenskjöld ${ }^{l}$, A. Leiden ${ }^{1,2}$, E. Tham $^{1,2}$

${ }^{1}$ Karolinska Institutet, Stockholm, Sweden, ${ }^{2}$ Karolinska University Hospital, Stockholm, Sweden

Background:Analysis of cell-free tumour DNA, a liquid biopsy, is a promising biomarker for cancer. We have performed a proof-of principle study to test the applicability in the clinical setting, analysing copy number alterations (CNAs) in plasma and tumour tissue from 44 patients with gastro-oesophageal cancer.

Methods:DNA was isolated from blood plasma and a tissue sample from each patient. Array-CGH was applied to the tissue DNA. The cell-free plasma DNA was sequenced by low-coverage whole-genome sequencing using a clinical pipeline for non-invasive prenatal testing. WISECONDOR and ichorCNA, two bioinformatic tools, were used to process the output data and were compared to each other.

Results:Cancer-associated CNAs could be seen in 59\% (26/44) of the tissue biopsies. In the plasma samples, a targeted approach analysing 61 regions of special interest in gastro-oesophageal cancer detected cancer-associated CNAs with a z-score $>5$ in 11 patients. Broadening the analysis to a whole-genome view, 17/44 patients (39\%) had cancer-associated CNAs using WISECONDOR and 13 (30\%) using ichorCNA. Of the 26 patients with tissueverified cancer-associated CNAs, 14 (54\%) had corresponding CNAs in plasma. Potentially clinically actionable 
amplifications overlapping the genes $V E G F A, E G F R$ and $F G F R 2$ were detected in the plasma from three patients.

Conclusions:We conclude that low-coverage wholegenome sequencing without prior knowledge of the tumour alterations could become a useful tool for cell-free tumour DNA analysis of total CNAs in plasma from patients with gastro-oesophageal cancer.

K. Wallander: None. J. Eisfeldt: None. M. Lindblad: None. D. Nilsson: None. K. Billiau: None. H. Foroughi: None. M. Nordenskjöld: None. A. Leiden: None. E. Tham: None.

\section{P12.123.B}

A novel long non coding RNA FGF10AS and sense transcript are correlated with poor prognosis in colorectal cancer

L. Rejali ${ }^{1,2}$, M. Hashemi ${ }^{3}$, H. Asadzadeh Aghdaei ${ }^{4}$, E. Nazemalhosseini $^{2}$, S. Seyednal

${ }^{1}$ Department of Biology, Faculty of Biological Sciences, Islamic Azad University, North Tehran Branch, Tehran, Iran, Islamic Republic of, ${ }^{2}$ Gastroenterology and Liver Diseases Research Center, Research Institute for Gastroenterology and Liver Diseases, Shahid Beheshti University of Medical Sciences, Tehran, Iran, Islamic Republic of, ${ }^{3}$ Department of New Sciences, Tehran Medical Branch, Islamic Azad University, Tehran, Iran, Islamic Republic of, ${ }^{4}$ Basic and Molecular Epidemiology of Gastrointestinal Disorders Research Center, Research Institute for Gastroenterology and Liver Diseases, Shahid Beheshti University of Medical Sciences, Tehran, Iran, Islamic Republic of

Introduction: Natural antisense transcripts (NATs) are inclusively predicted to play a leading role in cancer progression. Growth factors contribute to cell growth, differentiation, angiogenesis, and most significantly tumor formation in many types of cancers. The present study aimed to evaluate the relationship of fibroblast growth factor 10 (FGF10) and FGF10AS expression with clinicopathological features in CRC advancement.

Methods \& Materials: This study was navigated on 100 CRC tumoral and parallel adjacent normal tissues. The expression levels of FGF10 and FGF10AS were evaluated by the q-RT PCR method. For findings validation, expression levels were measured in HCT116 and SW480 cell lines. Immunohistochemistry analysis was performed systematically for FGF10 expression appraisal.

Results: A substantial increase and decrease in expression levels were perceived in FGF10 $(P<0.001)$ and FGF10AS $(P>0.02)$ in tumoral tissues, in comparison with the adjacent normal tissues. Besides, upregulation of sense and downregulation of antisense were strongly correlated with the TNM stage $(P<0.007$ and $P<0.004)$, vascular invasion $(P<0.03$ and $P<0.01)$, lymph invasion $(P<0.02$ and $P<$ $0.04)$ and differentiation $(P<0.01$ and $P<0.02)$ respectively. The area under the ROC curve for the prognostic value of FGF10 was about 0.84 (95\% confidence interval, 0.7710.912 ). The results of linear regression analysis confirmed a negative correlation between FGF10AS expression and sense transcript. ( $r=-0.02$ )

Conclusion: The relationship between the expression levels of FGF10 and FGF10AS in tumoral and adjacent normal tissues indicated that sense and antisense $F G F$ RNAs could be remarkable prognostic biomarkers for achieving effective treatment and higher survival rates.

L. Rejali: None. M. Hashemi: None. H. Asadzadeh Aghdaei: None. E. Nazemalhosseini: None. S. Seyedna: None.

P12.124.C

Intragenic deletions within the putative tumor suppressor $L S A M P$ are rare in primary neuroblastomas but common in cancer cell lines from diverse origin

\section{Mösle ${ }^{1}$, H. Christiansen ${ }^{2}$, S. Ackermann ${ }^{3}$, A. Weber ${ }^{l}$}

${ }^{I}$ Institute of Human Genetics, Justus-Liebig-Universität, Giessen, Germany, '2Department of Pediatric Oncology, Hematology and Hemostaseology, University Children's Hospital, Leipzig, Germany, ${ }^{3}$ Experimental Pediatric Oncology University Children's Hospital, Cologne, Germany

Introduction: Intragenic deletions within the LSAMP-Gene on human chromosome 3 have been described in osteosarcomas and osteosarcoma cell lines before. We asked for the frequency of intragenic $L S A M P$ copy number variations (CNVs) in primary neuroblastomas, neuroblastoma cell lines and cell lines from other cancer origin.

Materials and Methods: Specific real time PCRs were established for genomic regions of the seven exons of the LSAMP-gene (NM_002338.4) and six genomic control regions. Genomic copy number of the seven LSAMP-exons was calculated according to the $2^{-\Delta \Delta \mathrm{Ct}}$ method, using $n=$ 20 control DNAs for calibration. LSAMP-exon CNVs were defined with a $2^{-\Delta \Delta \mathrm{Ct}}$ value below (deletion) or above (duplication) the $2^{\text {nd }}$ standard deviation (SD) of the mean of the controls. In total we investigated genomic DNA from $n$ $=54$ primary neuroblastomas, $n=12$ neuroblastoma cell lines and $n=17$ cell lines of other malignancies.

Results: We found CNVs in LSAMP in $n=14$ (26\%) primary neuroblastomas. The findings did not correlate with tumor stage, age at diagnosis or survival. Furthermore, we found distinct CNVs of one or multiple LSAMP-exons in $n$ $=16$ out of the $n=29$ cell lines investigated (55\%). 
Conclusions: In primary neuroblastomas LSAMP CNVs were found only in a minority of cases. The clinical relevance for primary neuroblastoma development remains unclear. The common finding of LSAMP intragenic CNVs in cell lines of neuroblastoma and other cancer origin gives further evidence of a tumor suppressor function of LSAMP and makes us assume, that a loss of LSAMP function might be relevant for growth progression of tumor cells.

L. Mösle: None. H. Christiansen: None. S. Ackermann: None. A. Weber: None.

\section{P12.126. B}

Distinct Landscapes of Genomic Alterations between Lung Carcinoids and Non-Small Cell Lung Cancers

C. Domingo-Sabugo ${ }^{l}$, E. Starren ${ }^{l}$, A. Mandal ${ }^{l}, A$. Nastase $^{1}$, L. Hoang, M. Edwards ${ }^{1,2}$, D. Morris-

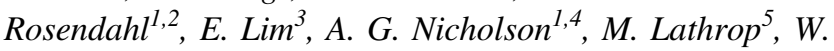
Cookson $^{1}$, M. Moffatt ${ }^{1}$

${ }^{1}$ National Centre for Mesothelioma Research, Imperial College London, London, United Kingdom, ${ }^{2}$ Clinical Genetics and Genomics Royal Brompton and Harefield NHS Foundation Trust, London, United Kingdom, ${ }^{3}$ Department of Medicine, Royal Marsden Hospital, London, United Kingdom, ${ }^{4}$ Department of Pathology, Royal Brompton and Harefield NHS Trust, London, United Kingdom, ${ }^{5}$ Department of Human Genetics, McGill University-Génome Québec Innovation Centre, Québec, QC, Canada

Introduction: Genomic profiling of tumours has become a crucial component of precision cancer medicine. A comparison of the molecular alterations between Non-Small Cell Lung Cancer (NSCLC) and Lung Carcinoid (LC) subtypes however is missing and might further improve patient stratification.

Methods: We sequenced 216 paired tumour-normal samples using a combination of whole-exome and targeted capture sequencing. Additionally, all samples underwent Illumina OmniExpressExome (v1.6) SNP genotyping.

Results: Out of 108 lung cancer patients, 22 had LC and 86 NSCLC. Those with NSCLCs showed lower survival time compared to LCs $(P=0.0148)$. Recurrent mutations were found in TP53 (81\%), KRAS (34\%), STK11 (23\%) and KEAP1 $(21 \%)$ in NSCLC but none of these genes were mutated in the LC subtype. Similarly, Copy Number (CN) burden was also higher in NSCLC than in LCs, with $10 \%$ and $5 \%$ of genome respectively harbouring $\mathrm{CN}$ alterations. Recurrent amplifications in MYC (35\%), SOX2 (20\%) and MDM2 (11\%) were found in NSCLC but only $18 \%$ of LC patients showed $M Y C$ amplifications and none were detected in SOX2 and MDM2. Furthermore, MTAP and
CDKN2A were co-deleted at frequencies of $\sim 30 \%$ in NSCLC but at $4.5 \%$ only in LCs.

Conclusions: Molecular profiles in NSCLC and LC are substantially different and this may have direct consequences in the therapy decision process. For example, preferential loss of the $C D K N 2 A$ locus in NSCLC could impact the response to immunotherapy drugs. In contrast, as $M D M 2$ is a negative regulator of TP53, MDM2 amplification in NSCLC could indicate therapeutic efficacy of MDM2 inhibitors in p53 mutant tumours.

C. Domingo-Sabugo: None. E. Starren: None. A. Mandal: None. A. Nastase: None. L. Hoang: None. M. Edwards: None. D. Morris-Rosendahl: None. E. Lim: None. A. G. Nicholson: None. M. Lathrop: None. W. Cookson: None. M. Moffatt: None.

P12.127.C

A Novel High Multiplex and $\operatorname{ddPCR}^{\mathrm{TM}}$ Based Technology for Lung Cancer MutationScreening

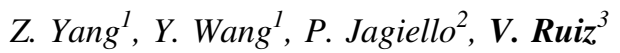

${ }^{1}$ Atila BioSystems, Inc, Mountain View, CA, United States, ${ }^{2}$ Fujirebio Europe N.V., Zwijnaarde, Belgium, ${ }^{3}$ Fujirebio Europe NV, Ghent, Belgium

Introduction: Genetic mutations have been found in many different genes, including EGFR, KRAS, HER2, MET and BRAF in the cancer cells of NSCLC patients. Testing for these mutations and tailoring therapy accordingly are widely accepted as standard practice. Recently a novel technology that enables high multiplex amplification reactions was developed by Atila Biosystems. With the droplet digital (ddPCR ${ }^{\mathrm{TM}}$ ) technology, the Atila iCARE (RUO) Lung cancer mutation screening kit identifies and quantifies 99 possible mutation sites in two reaction wells in a rapid and cost-effective manner.

Materials and Methods: Artificially prepared DNA samples were used to evaluate analytical sensitivity and specificity. We used enzymatically digested normal human genomic DNA as negative DNA samples and mutation bearing plasmid DNA as spike in positive DNA. A serial dilution of mutations at $0 \%, 0.1 \%, 0.2 \%, 0.5 \%, 1 \%$, and $10 \%$ were used to evaluate assay sensitivity.

Results: The iCARE Lung cancer screening assay targets 42 mutations in EGFR, 41 mutations in KRAS, 1 mutation in BRAF, 13 mutations in HER2, and 1 mutation in MET. The assay differentiates mutations from wildtype targets based on cluster locations in the $\mathrm{ddPCR}^{\mathrm{TM}}$ two-dimensional graphs. All mutations were successfully detected in serially diluted samples containing from $8 \%$ to $0.1 \%$ spiked mutations. 
Conclusions: The iCARE Lung Cancer Mutation Screening Assay kit allows quantification and screening for 99 NSCLC mutations of multiple samples in a rapid and costeffective manner. The assay has reached $0.1 \%$ sensitivity for mutation detection in all 5 genes and showed no false positive in wildtype DNA samples.

Z. Yang: A. Employment (full or part-time); Significant; Atila BioSystems, Inc. Y. Wang: A. Employment (full or part-time); Significant; Atila BioSystems, Inc. P. Jagiello: A. Employment (full or part-time); Significant; Fujirebio Europe N.V.. V. Ruiz: None.

\section{P12.130.C}

Design and implementation of the first one-stop multidisciplinary clinic for Lynch syndrome in the United Kingdom (UK)

B. DeSouza, V. Tripathi, A. Shaw, L. Izatt, S. Demartino, G. Tritto, P. Ross, G. Mehra, A. Schizas, M. George, C. Firth, K. Hilario, C. Reinholtz, A. Kulkarni

Guy's and St Thomas' NHS Foundation Trust, London, United Kingdom

Introduction: The clinical benefit of a multidisciplinary clinic (MDC) model has been well documented for a variety of medical conditions. We designed and implemented a novel MDC for Lynch syndrome (LS) patients, which aims to improve treatment outcomes, participation in research trials, and patient satisfaction.

Methods: From January 2019, LS patients in our region were invited to attend a bimonthly MDC offering genespecific evidence-based cancer risk management. Patients could choose to see the gastroenterology, colorectal, gynaecology, medical oncology, research, and clinical psychology teams. Clinical outcomes and patient satisfaction were evaluated over 12 months.

Results: Thirty-eight LS patients were seen (8 MLH1, 18 MSH2, 8 MSH6, 4 PMS2); mean age was 46y (range: 20$69 y) ; 15$ patients had colorectal cancer $(39.5 \%)$. Twenty-six $(68.4 \%)$ patients were female; $6(23.1 \%)$ had undergone prior risk-reducing gynaecological surgery, 7 (27\%) underwent surgery at our institution $(n=4)$ or locally $(n=3)$, and $13(50 \%)$ were too young for surgery. Eight patients $(30.8 \%)$ transferred their colonoscopic surveillance to our care due to concerns about local provision. Nineteen patients $(73.1 \%)$ accessed psychological support and 10 patients $(38.5 \%)$ were recruited to research studies. Twentysix patients $(68.4 \%)$ completed a post-clinic satisfaction questionnaire; $96.2 \%(n=25)$ rated their clinic experience as excellent or very good.

Conclusions: We have designed and implemented an effective multidisciplinary model of care for LS, which addresses unmet needs in this patient group. Other institutions are encouraged to adopt a coordinated MDC service for LS. We will continue to evaluate the MDC's impact on disease-specific outcomes in future reports.

B. DeSouza: None. V. Tripathi: None. A. Shaw: None. L. Izatt: None. S. Demartino: None. G. Tritto: None. P. Ross: None. G. Mehra: None. A. Schizas: None. M. George: None. C. Firth: None. K. Hilario: None. C. Reinholtz: None. A. Kulkarni: None.

\section{P12.131.A}

Lynch syndrome: gene variants in 35 patients and cascade screening of individuals at-risk

H. P. Saraçoğlu ${ }^{1}$, E. Börklü Yücel ${ }^{2}$, S. Eraslan ${ }^{2}$, E. Yllmaz, ${ }^{3,4}$, U. Altunoğlu ${ }^{2,3}$, H. Kayserili ${ }^{2,3}$

${ }^{1}$ Cellular and Molecular Medicine, Institute of Health Sciences, Koç University, Istanbul, Turkey, ${ }^{2}$ Diagnostic Center for Genetic Diseases, Koç University Hospital, Istanbul, Turkey, ${ }^{3}$ Medical Genetics Department, Koç University School of Medicine (KUSOM), Istanbul, Turkey, ${ }^{4}$ Research Center for Translational Medicine (KUTTAM), Koç University, Istanbul, Turkey

Lynch syndrome (LS), or hereditary nonpolyposis colorectal cancer (HNPCC), is a disorder caused by heterozygous mutations in mismatch repair (MMR) genes; which are MLH1, MSH2, MSH6, PMS2, and EPCAM. Pathogenic variants in $M L H 1$ and $M S H 2$ are linked to $50 \%$ and $40 \%$ of the cases, respectively. It is estimated that 1 in 300-440 individuals have LS. Besides colon cancer, patients have increased risks of rectum, endometrium and ovarian cancers. Genetic diagnosis relies on panel screening and MLPA of MMR genes. The aim of the study is to identify LS causal variants in a small cohort of 35 patients from Turkey. In 35 patients tested for LS by sequence panel comprising 94 genes associated with hereditary cancers/cancer susceptibility, $24(68.6 \%)$ had a variant. Out of 24 , nine variants $(37.5 \%)$ were pathogenic or likely pathogenic. While 4/9 (44.4\%) of the variants resided in $M L H 1$, two $(22.2 \%)$ in MSH2; and one each MSH2, MSH6 and PMS2. VUS distribution of the cohort was, four $(26.7 \%)$ in $M L H 1$, one $(6.7 \%)$ in $M S H 2$, five $(33.3 \%)$ in $M S H 6$, four $(26.7 \%)$ in PMS2 and one $(6.7 \%)$ in EPCAM. The results of germline panel screening for LS prompted genetic counselling. Screening at-risk individuals in LS families, cascade screening, provides opportunity for cancer prevention and early detection, through follow-up protocols, intensive surveillance, and/or prophylactic surgeries.

H.P. Saraçoğlu: None. E. Börklü Yücel: None. S. Eraslan: None. E. Yılmaz: None. U. Altunoğlu: None. H. Kayserili: None. 


\section{P12.132.B}

The prevalence of MMR gene mutations in Estonian general population

\section{Roht ${ }^{1,2}$, S. Pajusalu ${ }^{2,3}$, U. Šamarina ${ }^{3}$, T. Kahre $e^{2,3}, K$. Önap ${ }^{2,3}$}

${ }^{1}$ Tartu University Hospital, United Laboratories, Department of Clinical Genetics, Tallinn, Estonia, ${ }^{2}$ University of Tartu, Institute of Clinical Medicine, Department of Clinical Genetics, Tartu, Estonia, ${ }^{3}$ Tartu University Hospital, United Laboratories, Department of Clinical Genetics, Tartu, Estonia

Introduction: Lynch syndrome is an autosomal-dominant cancer predisposition syndrome caused by germline mutations of mismatch-repair (MMR) genes (MLH1, MSH2, MSH6, PMS2) and EPCAM gene. At the moment, there are 76 Lynch syndrome patients in Estonia.

Aim: To determine the prevalence of MMR gene mutations in Estonian general population.

Material and methods: Data from Estonian Genome Center, 2420 genomes and 2356 exomes, was analyzed to calculate the estimated prevalence of MMR and EPCAM gene variants.

Results: Genome sequencing detected disease-causing variants in following genes: one in $M S H 2$, two in PMS2, three in MSH6 and none in MLHI and EPCAM genes. Exome sequencing revealed one disease-causing variant in $M L H 1, M S H 6$ and EPCAM genes and none in MSH2 and $P M S 2$ genes. Altogether eight mutations were found and based on that the estimated prevalence of MMR gene mutations in Estonian general population is 1:597 (Poisson 95\% CI: 1:1384 - 1:303). In Estonian patient cohort MLHI was the most prevalent gene mutated (34 patients) followed by MSH2 (24), MSH6 (10), PMS2 (3) and EPCAM (2) genes. From MMR genes MSH6 gene variants were detected most frequently in our general population. The prevalence of MSH6 mutations was also higher than described in the literature in our patient cohort.

Conclusions: We present Estonian general population prevalence of Lynch syndrome associated MMR gene variants based on Estonian Genome Center data. According to literature, MMR gene variants prevalence in the world is 1:370-450, which compared to Estonian data is $~ 1.5$ times higher.

Funding: Estonian Research Council grants PRG471, PUTJD827.

L. Roht: None. S. Pajusalu: None. U. Šamarina: None. T. Kahre: None. K. Õunap: None.
P12.133.C

Whole Exome Sequencing approach identified candidate genetic modifiers of Lynch syndrome penetrance

E. Dámaso ${ }^{l}$, A. Castillejo ${ }^{l}$, A. Sánchez-Heras ${ }^{2}, M$. Castillejo $^{1}$, J. Soto ${ }^{2}$

${ }^{1}$ Elche University Hospital - FISABIO, Elche, Spain, ${ }^{2}$ Elche University Hospital, Elche, Spain

-Introduction:Lynch syndrome (LS) is an autosomaldominant cancer-predisposing syndrome caused by mutations in mismatch repair genes (MMR). Germline alterations in MMR genes shows a substantial level of cancer risk heterogeneity. Identifying genetic modifiers of risk could help to achieve a more precise cancer risk prediction and more efficient personalized surveillance programs. We aimed to identify new potential genetic modifiers of LS penetrance using Whole Exome Sequencing (WES).

-Materials and Methods: Twenty-three individuals from 15 families carrying MMR pathogenic variants in $M L H 1$ or MSH2 were sequenced by WES. Fifteen patients were diagnosed of LS-spectrum cancers before age 45 and 8 were healthy carrier individuals aged over 55. WES was performed using SureSelect-Human-AllExonV6+UTR (AgilentTechnologies) libraries and sequencing on Illumina-NovaSeq6000 at 100X. Alignment and variant calling was performed by SureCall and variant filtering by a custom pipeline for cohort analyses on AlissaInterpret software (AgilentTechnologies).

-Results: Among 254.316 total called variants, we only considered those present in over $80 \%$ of cancer patients and less than $20 \%$ of healthy carriers, and found 20 genetic variants in 18 genes $[\mathrm{RR}=2.571$; 95\%CI: 1.05-6.15; $p=0.023]$. Four variants were located in coding regions and 16 in UTRs or intronic sequences. Population frequency of all variants is over 20\% (GnomAD). Intriguingly, 17 of these have been also reported as somatic mutations in tumors, 9 of them in colorectal adenocarcinoma (ICGC).

-Conclusions: We identified 20 new variants as possible risk modifier for LS. Further studies in larger cohorts of LS patients are needed to confirm their cancer risk impact.

-Grant_ID: PI17/01082(ISCIII_AES)

E. Dámaso: None. A. Castillejo: None. A. SánchezHeras: None. M. Castillejo: None. J. Soto: None.

P12.134.A

GERMLINE BIALLELIC MUTATIONS IN MCM8 ARE ASSOCIATED WITH EARLY-ONSET LYNCHLIKE SYNDROME 
M. Golubicki ${ }^{1}$, L. Bonjoch ${ }^{2}$, J. G. Acuña-Ochoa ${ }^{2}$, M. Díaz-

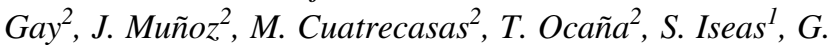
Mendez ${ }^{1}$, D. Cisterna ${ }^{1}$, S. A. Schubert ${ }^{3}$, M. Nielsen ${ }^{3}$, T. van

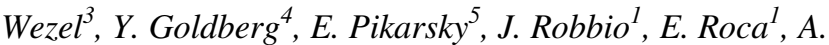
Castells $^{2}$, F. Balaguer ${ }^{2}$, M. Antelo ${ }^{1}$, S. Castellvi-Bel ${ }^{2}$

${ }^{1}$ Hospital of Gastroenterology "Dr. C. B. Udaondo", Buenos Aires, Argentina, ${ }^{2} I D I B A P S$, Barcelona, Spain, ${ }^{3}$ Leiden University Medical Center, Leiden, Netherlands, ${ }^{4}$ Rabin Medical Center, Petah Tikva, Israel, ${ }^{5}$ Hebrew University-Hadassah Medical School, Jerusalem, Israel

Introduction: Lynch-like syndrome (LLS) refers to individuals with mismatch repair (MMR)-deficient tumors, with no pathogenic germline mutation identified in a MMR gene. Biallelic somatic inactivation or cryptic MMR germline mutations not detected during genetic testing have been proposed to be involved, although germline pathogenic genetic variants in highly penetrant genes other than MMR genes could also be causing this phenotype.

Material and Methods: Sixteen patients with nonpolyposis LLS colorectal cancer (CRC) diagnosed before the age of 40 were selected for germline and tumor wholeexome sequencing. A knockout cellular model for a candidate recessive gene was generated by CRISPR/Cas9 and genetic variants were produced by mutagenesis and ectopically expressed. Subsequently, DNA damage, microsatellite instability and mutational signatures were monitored.

Results: Two potentially pathogenic recessive MCM8 germline variants were detected in an early-onset CRC LLS male patient with infertility problems. Two somatic $M L H 1$ pathogenic genetic variants were also detected. DNA damage was evident for $M C M 8^{K O}$ cells and the analyzed genetic variants. Microsatellite instability and mutational signatures in $M C M 8^{K O}$ cells were compatible with an involvement of MCM8 in MMR.

Conclusions:Some LLS CRC cases may be caused by underlying germline mutations in genes different than MMR genes, especially in patients with an early onset. We suggest $M C M 8$ as a new gene involved in germline CRC predisposition and infertility with a recessive inheritance pattern.

Funding: Foundation Nelia et Amadeo Barletta, FJCI2017-32593, CIBEREHD, 2019FI_B2_00203, Argentinian Cancer Institute, FIS/FEDER (17/00878), CERCA Program, GRPRE 2017SGR21, GRC 2017SGR653, Dutch Digestive Foundation (MLDS FP13-13), COST Action CA17118.

M. Golubicki: None. L. Bonjoch: None. J.G. AcuñaOchoa: None. M. Díaz-Gay: None. J. Muñoz: None. M. Cuatrecasas: None. T. Ocaña: None. S. Iseas: None. G.
Mendez: None. D. Cisterna: None. S.A. Schubert: None. M. Nielsen: None. T. van Wezel: None. Y. Goldberg: None. E. Pikarsky: None. J. Robbio: None. E. Roca: None. A. Castells: None. F. Balaguer: None. M. Antelo: None. S. Castellvi-Bel: None.

\section{P12.135.B}

Implementing mainstreaming genetic testing of $B R C A 1 /$ 2 for cancer patients in a Portuguese tertiary hospital: preliminary results

\section{Soeiro e Sá, J. Dupont, A. B. Sousa}

Serviço de Genética Médica, Hospital de Santa Maria, Centro Hospitalar e Universitário Lisboa Norte, Centro Académico de Medicina de Lisboa, Lisboa, Portugal, Lisbon, Portugal

Aim: To implement a similar programme to the original "Mainstreaming Cancer Genetics" of BRCA1/2 testing in our Hospital, extending the criteria to include prostate cancer.

Methods: We defined patient selection criteria and designed a protocol for patient referral, informed consent, sample collection, and genetic testing. We centralized the process through Genetics, making sure we had knowledge of all patients included and their results. We promoted educational sessions for oncologists, prepared informative brochures for patients, and produced standard clinical reports to accompany laboratory test results. Statistical analysis was done after codification.

Results: Since September 2018, 106 patients were referred, of whom 94 were included. The most frequent inclusion criteria was breast cancer $(\mathrm{BC})<40$ years $(n=32)$, followed by ovarian cancer $(n=18)$, and triple negative BC $(n=15)$. Of 86 available results, we identified 9 (10.5\%) pathogenic variants ( 8 germinal/1 somatic) and 6 VUS (5 germinal/ 1 somatic). All patients in whom a variant was identified had a genetics appointment within a mean time of $17.9 \pm 15.8$ days. The mean response time of genetic testing was $51.7 \pm 9.8$ days. The mean time from patient referral to final results (sent to the oncologist) was $67.7 \pm 18.8$ days.

Conclusions: We decreased our waiting time for urgent patients and lessened the burden of extra appointments in our clinic, while providing faster results that might influence patients' treatment. In the future we will assess impact on patients and their families, degree of satisfaction with the protocol (both from patients and oncologists), and review the classification of previously identified VUS.

M. Soeiro e Sá: None. J. Dupont: None. A.B. Sousa: None. 


\section{P12.137.A}

The single nucleotide polymorphism rs2235503 $\mathrm{C}>\mathrm{A}$ leads to increased $M S L N$ gene transcription and it is strongly associated with the levels of SMRP in vivo

\section{R. Silvestri ${ }^{1}$, P. Pucci ${ }^{2}$, C. De Santi ${ }^{3}$, I. Dell'Anno ${ }^{1}, S$} Miglietta $^{4}$, A. Corrado ${ }^{5}$, V. Nicoli ${ }^{6}$, D. Marolda ${ }^{l}$, M.

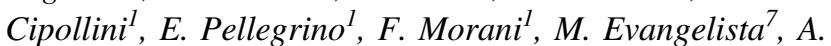

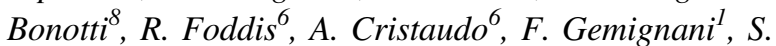
Landi $^{l}$

${ }^{1}$ University of Pisa, Department of Biology, Pisa, Italy, ${ }^{2}$ The Open University, School of Life Health and Chemical Sciences, Milton Keynes, United Kingdom, ${ }^{3}$ Royal College of Surgeons in Ireland, Department of Molecular and Cellular Therapeutics, Dublin, Ireland, ${ }^{4}$ IRCCS San Raffaele Scientific Institute, San Raffaele Telethon Institute for Gene Therapy (SR-Tiget), Milano, Italy, ${ }^{5}$ University of Milan, Department of Bioscience, Milano, Italy, ${ }^{6}$ University of Pisa, Department of Translational Research and of New Surgical and Medical Technologies, Pisa, Italy, ${ }^{7} \mathrm{CNR}$, Institute of Clinical Physiology (IFC), Pisa, Italy, ${ }^{8}$ University Hospital of Pisa, Preventive and Occupational Medicine, Pisa, Italy

Introduction: The "soluble mesothelin-related peptide" (SMRP) is one of the most promising diagnostic biomarkers for malignant pleural mesothelioma (MPM). However, several factors can affect SMRP levels, hampering its accuracy and limiting its usage in the clinical practice. In the present work, we explored the hypothesis that genetic variants within the MSLN promoter could represent a confounding factor able to affect SMRP levels.

Methods: Blood samples from a cohort of 70 MPM and 689 non-MPM subjects were analyzed to assess the association between the SMRP levels and four haplotypic variants of $M S L N$ promoter: haplotype \#1 (reference), \#2, \#3, \#4. Functional studies were carried out in Met-5A and Mero14 cell lines using a fluorescence reporter assay to assess the differences between the activity of the four variants of the $M S L N$ promoter and to identify the SNPs responsible for the observed differences.

Results: Carriers of haplotype \#2 showed a significantly higher level of SMRP when compared with the reference group ( $p$-value $<10^{-5}$ ). The functional study confirmed the role of haplotype \#2, which caused a $41 \%$ increase in the reporter fluorescence intensity $\left(p\right.$-value $\left.=6 \times 10^{-4}\right)$. The in vitro analysis also led to the identification of rs $2235503 \mathrm{C}>\mathrm{A}$ as the SNP responsible for the increased activity of haplotype \#2 (35\% increase in the reporter intensity; $p<10^{-5}$ ).

Conclusions: The rare variant of rs $2235503 \mathrm{C}>\mathrm{A}$ leads to an increase of SMRP levels through the enhancement of $M S L N$ expression. The establishment of a personalized cut- off value based on this SNP could increase the accuracy of SMRP in the diagnosis of MPM.

R. Silvestri: None. P. Pucci: None. C. De Santi: None. I. Dell'Anno: None. S. Miglietta: None. A. Corrado: None. V. Nicolì: None. D. Marolda: None. M. Cipollini: None. E. Pellegrino: None. F. Morani: None. M. Evangelista: None. A. Bonotti: None. R. Foddis: None. A. Cristaudo: None. F. Gemignani: None. S. Landi: None.

\section{P12.138.B}

Pathogenic variants in cancer predisposition genes in patients with stage II melanoma

S. Armeanu-Ebinger ${ }^{1}$, T. Amaral ${ }^{2}$, T. Sinnberg ${ }^{2}, C$. Roggia $^{l}$, F. Hilke ${ }^{1,3}$, H. Niessner ${ }^{2}$, I. Bonzheim ${ }^{4}$, T. Haack ${ }^{l}$, O. Rieß $\beta^{I}$, C. Garbe ${ }^{2}$, C. Schroeder ${ }^{l}$

${ }^{1}$ Institute of Medical Genetics and Applied Genomics, Tübingen, Germany, ${ }^{2}$ Department of Dermatology, Tübingen, Germany, ${ }^{3}$ Department of Dermatology, Venereology and Allergology, Berlin, Germany, ${ }^{4}$ Institute of Pathology and Neuropathology, Tübingen, Germany

Introduction: Melanoma is an aggressive cutaneous tumor with an increasing incidence. Sun exposure is one of the major risk factors. Recent studies indicate that 6-7\% of all melanoma patients carry a pathogenic germline variant in a cancer predisposition gene (CPG) while the association with melanoma is often unknown. We screened a large cohort of stage II melanoma patients for pathogenic germline variants in CPGs.

Materials and Methods: DNA from paired tumor and normal FFPE tissue of 196 patients with stage II melanoma was sequenced with a NGS custom enrichment panel covering 693 genes, selected promotor and intronic regions. We used megSAP (https://github.com/imgag/megSAP) for NGS data analysis and germline variants in 152 CPGs were filtered for population allele frequency $(\mathrm{AF}<1 \%)$ and classified according to ACMG guidelines.

Results: We detected pathogenic or likely pathogenic variants in recessive and dominant CPGs in 18 out of 196 patients $(9.2 \%$, including ATM, BRCA1, CHEK2, MSH2, PALB2, SDHA). A second hit in tumor tissue was detected in one patient carrying an ATM mutation. Tumors with germline pathogenic variants in MSH2 did not reveal microsatellite instability. Besides relevance for surveillance and families, five pathogenic variants affected DNA repair genes with potential relevance for therapy.

Conclusions: Pathogenic germline variants in CPGs in stage II melanoma patients are frequent and of relevance for surveillance, families and potentially therapy. Further studies are needed to investigate whether pathogenic 
variants in CPGs that are not known to be related to melanoma contribute to melanoma development.

S. Armeanu-Ebinger: None. T. Amaral: B. Research Grant (principal investigator, collaborator or consultant and pending grants as well as grants already received); Significant; Novartis. T. Sinnberg: B. Research Grant (principal investigator, collaborator or consultant and pending grants as well as grants already received); Significant; Novartis. C. Roggia: None. F. Hilke: None. H. Niessner: None. I. Bonzheim: None. T. Haack: None. O. Rieß: B. Research Grant (principal investigator, collaborator or consultant and pending grants as well as grants already received); Significant; Novartis. C. Garbe: B. Research Grant (principal investigator, collaborator or consultant and pending grants as well as grants already received); Significant; Novartis. C. Schroeder: B. Research Grant (principal investigator, collaborator or consultant and pending grants as well as grants already received); Significant; Novartis.

\section{P12.139.C}

Whole exome sequencing of patients with early onset melanoma (age $<40$ years)

E. Chatziioannou ${ }^{1}$, K. Kypraiou ${ }^{2}$, G. Vatsellas ${ }^{1}$, M. Plaka ${ }^{2}$, T. Loupis ${ }^{1}$, D. M. Vrachnos ${ }^{1}$, D. Polydorou ${ }^{2}$, D.

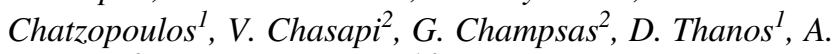
Stratigos $^{2}$, P. Makrythanasis ${ }^{1,3}$

${ }^{1}$ Biomedical Research Foundation of the Academy of Athens, Athens, Greece, ${ }^{2}$ First Department of Dermatology and Venereology, National and Kapodistrian University of Athens, Medical School, Andreas Syggros Hospital for Skin and Venereal Diseases, Athens, Greece, ${ }^{3}$ Department of Genetic Medicine and Development, Medical School, University of Geneva, Geneva, Switzerland

Introduction: Cutaneous melanoma develops frequently in people over the age of 50 and can arise sporadically or in the setting of a germline predisposition in approximately $10 \%$ of cases. During the last years high penetrance genes have been discovered and genes related to nevus count and pigmentation have been linked to melanoma. However only $50 \%$ of the patients that demonstrate positive family history carry germline mutation in the above-mentioned genes, suggesting that other genes or an interplay of genes may be implicated in its carcinogenesis. Additionally, the prevalence of pathogenic variants in these genes in cases of early onset of melanoma is poorly studied.

Materials and Methods: We performwhole exome sequencing of DNA extracted from blood of 80 patients with early onset melanoma (diagnosis occurring before 40 years of age). We first assess a panel of genes containing genes hitherto associated with familial melanoma, genodermatoses,
DNA repair, telomere maintenance, pigmentation, nevus count and hereditary predisposition to other cancers and subsequently expand our analysis to the whole exome. The family history isre-examined according to results.

Results: We have analyzed data from 10 patients. In 1 patient we have identified one novel pathogenic variant in $C D K N 2 A$. Aggregate data per gene, family of genes and pathways are catalogued and studied.

Conclusions: The present study is ongoing, and we aim to define the prevalence of known high penetrance genes and to identify potential candidate variants/genes implicated in cases of early onset of melanoma.

E. Chatziioannou: None. K. Kypraiou: None. G. Vatsellas: None. M. Plaka: None. T. Loupis: None. D.M. Vrachnos: None. D. Polydorou: None. D. Chatzopoulos: None. V. Chasapi: None. G. Champsas: None. D. Thanos: None. A. Stratigos: None. P. Makrythanasis: None.

P12.140.A

Patients with germline pathogenic variants in DNA repair genes show increased susceptibility to asbestosinduced malignant pleural mesothelioma

M. Sculco ${ }^{1}$, M. La Vecchia ${ }^{1}$, E. Casalone ${ }^{2}$, D. Ferrante ${ }^{3}$, A.

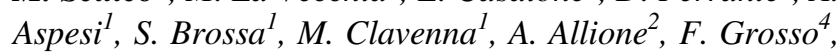
R. Libener ${ }^{5}$, A. Muzio $^{6}$, O. Rena ${ }^{7}$, C. $_{\text {Casadio }}{ }^{7}$, G. Baietto $^{7}$,

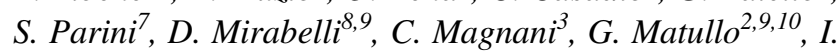
Dianzani $^{1,9}$

${ }^{1}$ Department of Health Sciences, Università del Piemonte Orientale, Novara, Italy, ${ }^{2}$ Department of Medical Sciences, Università di Torino, Torino, Italy, ${ }^{3} \mathrm{CPO}$-Piemonte e Unit of Medical Statistics and Epidemiology, Università del Piemonte Orientale, Novara, Italy, ${ }^{4}$ Mesothelioma Unit, AO SS Antonio e Biagio e Cesare Arrigo, Alessandria, Italy, ${ }^{5}$ Pathology Unit, AO SS Antonio e Biagio e Cesare Arrigo, Alessandria, Italy, ${ }^{6}$ Division of Medical Oncology, Ospedale Santo Spirito, Casale Monferrato (AL), Italy, ${ }^{7}$ Thoracic Surgery Unit, AOU Maggiore della Carità, Novara, Italy, ${ }^{8}$ Unit of Cancer Epidemiology, CPO-Piemonte $e$ Università di Torino, Torino, Italy, ${ }^{9}$ Interdepartmental Center for Studies on Asbestos and other Toxic Particulates “G. Scansetti”, Università di Torino, Torino, Italy, ${ }^{10}$ Medical Genetics Unit, AOU Città della Salute e della Scienza di Torino, Torino, Italy

Introduction: Malignant pleural mesothelioma (MPM) is a rare and aggressive cancer caused by exposure to asbestos. A role for inherited predisposition was hypothesized since the 10$17 \%$ of individuals exposed to high levels of asbestos develop MPM and some families present multiple MPM cases.

Materials and Methods: We investigated 107 cancerpredisposing genes in 144 MPM patients (16 familiar, 
128 sporadic) by NGS. Lifelong asbestos exposure was quantified in 131 patients. Moreover, we performed WES in two families, each with three MPM cases.

Results: We identified 17 pathogenic truncating variants (PTVs, in PALB2, BRCA1, FANCI, ATM, SLX4, BRCA2, FANCC, FANCF, PMS1, XPC, SBDS, CDKN2A, VHL, $B R I P 1$ ) and 27 rare missense variants (evaluated as pathogenic by 6 in silico prediction tools), mostly in genes involved in homologous recombination repair that repairs double strand breaks in DNA caused by asbestos. PTVs carriers represent $11 \%$ of the patients and show a statistically significant lower asbestos exposure in comparison to non-mutated patients $(p=0.0003)$. Only one of the six patients analyzed by WES, showed a PTV in PMS2, a gene involved in mismatch repair, suggesting that asbestos exposure may have a predominant role in some families.

Conclusions: Overall, these data suggest that patients with germline variants in DNA repair genes are less proficient to repair the DNA damage induced by asbestos and show increased susceptibility to asbestos-induced MPM. These patients carrying predisposing variants may respond to drugs that induce synthetic lethality.

Grants: HeRMes project (compensation to MPM patients of Casale Monferrato) (ID, CM); AIRC2018IG21390 (GM)

M. Sculco: None. M. La Vecchia: None. E. Casalone: None. D. Ferrante: None. A. Aspesi: None. S. Brossa: None. M. Clavenna: None. A. Allione: None. F. Grosso: None. R. Libener: None. A. Muzio: None. O. Rena: None. C. Casadio: None. G. Baietto: None. S. Parini: None. D. Mirabelli: None. C. Magnani: None. G. Matullo: None. I. Dianzani: None.

\section{P12.141.B}

Functional variants of the Methylenetetrahydrofolate reductase in childhood acute lymphoblastic leukemia

\section{R. Frikha}

Medical Genetic department, Hedi Chaker Hospital and Faculty of Medicine, Sfax, Tunisia

Introduction: Functional variants of the Methylenetetrahydrofolate reductase (MTHFR) gene result in disturbance in folate metabolism and may affect susceptibility to childhood acute lymphoblastic leukemia (ALL) [1]. In addition, a potential impact of such variants in the efficacy of methotrexate has been reported but the results are conflicting [2]. The aim of this study was to investigate the possible role of MTHFR C677T (rs1801133; C>T) and A1298C (rs1801131; A >C) polymorphism as a risk factor for the development of ALL in a cohort of Tunisian children.

Methods: This study was conducted in 28 children with ALL treated with the EORTC 58951. Clinical and biological data were recorded. Genomic DNA was extracted from whole venous blood and genotyping were carried out with restriction fragment length polymorphism (RFLP).

Results: The allelic frequency of MTHFR $677 \mathrm{~T}$ and A1298C was $14.3 \%$ and $42.8 \%$ respectively. A particular history of relapse and toxicity during methotrexate therapy was reported in ALL patients with the MTHFR 677T variant.

Conclusions: Despite the limit of this study; our finding highlights the impact of functional variants of MTHFR on leukemogenesis. Awareness of folate metabolism is mandatory to prevent childhood leukemia. 1. Yang, M., Association between MTHFR C677T Polymorphism and Risk of Acute Lymphoblastic Leukemia: A Meta-Analysis Based on 51 Case-Control Studies. Medical Science Monitor, 2015. 21: p. 740-748. 2.Lopez-Lopez, E., et al., A systematic review and meta-analysis of MTHFR polymorphisms in methotrexate toxicity prediction in pediatric acute lymphoblastic leukemia. The Pharmacogenomics Journal, 2012. 13(6): p. 498-506.

\section{R. Frikha: None.}

\section{P12.144.B}

miR-20b and miR-451a Are Involved in Gastric Carcinogenesis through the PI3K/AKT/mTOR Signaling Pathway: Data from Gastric Cancer Patients, Cell Lines and INS-GAS Mouse Model

G. Streleckiene ${ }^{l}, \boldsymbol{R}$. Inciuraite ${ }^{l}, S$. Juzenas $^{1,2}, V$. Salteniene $^{l}$, R. Steponaitiene ${ }^{1}$, U. Gyvyte ${ }^{1}$, P. Ruzgys ${ }^{3}$, A. Link $^{4}$, J. Kupcinskas ${ }^{1,5}$, J. Skieceviciene ${ }^{1}$

${ }^{1}$ Institute for Digestive Research, Lithuanian University of Health Sciences, Kaunas, Lithuania, ${ }^{2}$ Institute of Clinical Molecular Biology, Christian-Albrechts-University of Kiel, Kiel, Germany, ${ }^{3}$ Biophysical Research Group, Vytautas Magnus University, Kaunas, Lithuania, ${ }^{4}$ Department of Gastroenterology, Hepatology and Infectious Diseases, Otto-von-Guericke University, Magdeburg, Germany, ${ }^{5}$ Department of Gastroenterology, Lithuanian University of Health Sciences, Kaunas, Lithuania

Introduction: Gastric cancer (GC) is one of the most prevalent and lethal cancer worldwide. Many studies have shown that development of malignancies is mainly driven by alterations of cellular signaling pathways. MicroRNAs are small noncoding molecules playing an essential role in various biological processes. The aim of this study was to 
evaluate the functional role of miR-20b and miR-451a and their target genes in GC.

Materials and methods: To determine the expression level of miR-20b and miR-451a in vivo and in vitro, biopsy samples of GC patients, healthy controls, INS-GAS mice, AGS and MKN28 cell lines samples were analysed using qRT-PCR. To confirm putative microRNA target-genes and determine miR-20b and miR-451a impact on cell function, luciferase reporter, protein expression, MTT, clonogenic, apoptosis, wound healing assays were performed in GC cell cultures.

Results: Deregulated expression of miR-20b and miR451a was determined in GC cell lines and the INS-GAS mouse model. Western blot and luciferase reporter assay have also confirmed that miR-20b directly regulates expression of PTEN and TXNIP, and miR-451a - CAVI and TSC1. Loss-of-function experiments revealed that down-regulation of miR-20b and upregulation of miR451a expression exhibits an anti-tumor effect in vitro (miR20b: reduced viability, colony formation, increased apoptosis rate, and miR-451a: reduced colony forming ability).

Conclusions: This study identified that expression of miR-20b and miR-451a are deregulated in vitro and in vivo and have a tumor suppressive role in GC through regulation of the PI3K/AKT/mTOR signaling pathway. Funding: This research was funded by Research Council of Lithuania (Grant number MIP-007/2014).

G. Streleckiene: None. R. Inciuraite: None. S. Juzenas: None. V. Salteniene: None. R. Steponaitiene: None. U. Gyvyte: None. P. Ruzgys: None. A. Link: None. J. Kupcinskas: None. J. Skieceviciene: None.

\section{P12.145.C}

The importance of diagnostic and prognostic utility of miRNAs analysis in bladder cancer.

\section{E. M. Borkowska ${ }^{1}$, T. Konecki ${ }^{2}$, M. Pietrusinski ${ }^{1}, M$. Borowiec $^{l}$, Z. Jablonowski ${ }^{2}$}

${ }^{1}$ Medical University of Lodz Department of Clinical Genetics, Lodz, Poland, ${ }^{2}$ Medical University of Lodz I Clinic of Urology, Lodz, Poland

Introduction: Bladder cancer (BC) is still characterized by a very high death rate in patients with this disease. One of the reasons for this is the lack of adequate markers which could help determine the biological potential of the tumor to develop into its invasive stage. Some microRNAs (miRNAs) correlate with disease progression. The purpose of this study was to identify which miRNAs can accurately predict the presence of $\mathrm{BC}$ and can differentiate low grade (LG) tumors from high grade (HG) tumors.
Materials and methods:The study included 55 patients with diagnosed bladder cancer and 30 persons belonging to the control group. The expression of seven selected miRNAs was estimated with the real-time PCR technique according to miR-103-5p (for the normalization of the results). Receiver operating characteristics (ROC) curves and the area under the curve (AUC) were used to evaluate the feasibility of using selected markers as biomarkers for detecting $\mathrm{BC}$ and discriminating non-muscle invasive $\mathrm{BC}$ (NMIBC) from muscle invasive BC (MIBC).

Results: For HG tumors, the relevant classifiers are miR205-5p and miR-20a-5p, whereas miR-205-5p and miR$182-5 \mathrm{p}$ are for $\mathrm{LG}(\mathrm{AUC}=0.964$ and $\mathrm{AUC}=0.992$, respectively). NMIBC patients with LG disease are characterized by significantly higher miR-130b-3p expression values compared to patients in HG tumors.

Conclusions: Our findings could be of clinical importance. The implementation of miR-205-5p, miR-20a-5p and miR-130b-3p into routine practice can be an alternative to screening or the follow up of treatment effects. This work was supported by Medical University Grant 502-03/5-13802/502-54-146, Grant 502-03/5-138-02/502-54-133.

E.M. Borkowska: None. T. Konecki: None. M. Pietrusinski: None. M. Borowiec: None. Z. Jablonowski: None.

\section{P12.149.A}

Evidence of cancer predisposition gene pathogenic variant mosaicism identified through concurrent sequencing of tumour samples and blood from individuals with multiple primary tumours

J. Whitworth, J. Martin, O. Giger, F. Nice, G. Clark, M. Maranian, F. Rodger, NIHR BioResource Rare Diseases Consortium, E. Maher

\section{University of Cambridge, Cambridge, United Kingdom}

Tumours are often conceptualised as occurring due to germline variants in cancer predisposition genes (CPGs) or solely somatic mutations but mosaicism for a CPG variant has also been widely described. Mosaic individuals are at increased risk of neoplasms and are not readily detected clinically. Multiple primary tumours in the same individual (MPT) represent an opportunity to detect mosaicism as variants may be present in both tumours but not blood. We sequenced separate primaries from three MPT individuals with a panel assay incorporating 350 cancer genes on DNA from FFPE tissue, and corresponding blood samples with whole-genome sequencing. Variants in tumour samples were filtered to include non-synonymous or splice variants present in both tumours from each individual and exclude likely false positives. $2 / 3$ individuals had truncating CPG 
variants in both of their tumours that were undetected in their blood sample or in other tumours in the same sequencing run $(n=23)$. One had a $V H L$ frameshift variant detected in bilateral clear cell renal cell carcinomas diagnosed at age 40 and 47 years. Variant allele fractions (VAF) were $41 \%$ and $49 \%$ respectively and $0 / 37$ germline reads showed evidence of the variant. Another case had a TP53 nonsense variant in bilateral breast carcinomas diagnosed at age 48 and 52 years. VAFs were $22 \%$ and $15 \%$ and $0 / 34$ germline reads showed variant base calls. Neither individual had a family history of tumours in first degree relatives. These results suggest appreciable frequency of CPG pathogenic variant mosaicism in MPT cases without a prominent family history cancer.

J. Whitworth: None. J. Martin: None. O. Giger: None. F. Nice: None. G. Clark: None. M. Maranian: None. F. Rodger: None. E. Maher: None.

\section{P12.151.C}

NGS analysis of germline mutations in patients with multiple primary neoplasia

A. Mitkova ${ }^{1}$, R. Dodova ${ }^{1}$, M. Koleva ${ }^{2}$, A. Andreeva ${ }^{3}$, V. Petkoval $^{1}$ D. Pencheval, M. Tzekova-Chernopolska ${ }^{4}, I$.

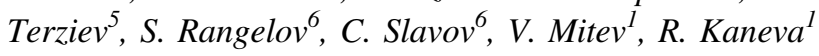

${ }^{1}$ Molecular Medicine Center, Department of Medical Chemistry and Biochemistry, Faculty of Medicine, Medical University of Sofia, Sofia, Bulgaria, ${ }^{2}$ Department of Medical Oncology, Multifunctional Hospital for Active Treatment "Serdica", Sofia, Bulgaria, ${ }^{3}$ Department of Medical Oncology, University Hospital "Queen Joanna ISUL", Sofia, Bulgaria, " Endoscopy Ward, Clinic of ENT Diseases, University Hospital "Queen Joanna - ISUL”, Sofia, Bulgaria, "Department of General and Clinical Pathology, Medical Faculty/ Department of Pathological Anatomy, University Hospital "Queen Joanna - ISUL”, Medical University of Sofia, Sofia, Bulgaria, ${ }^{6}$ Department of Urology, Medical Faculty/ Clinic of Urology with Specific Activity in Andrology, University Hospital "Queen Joanna - ISUL”, Medical University of Sofia, Sofia, Bulgaria

Introduction: Multiple primary neoplasia (MPN) has been defined as more than one primary malignancy found in one and the same individual. The main factors implicated in MPN development are genetic factors (hereditary predisposition to cancer); hormonal factors; lifestyle (smoking, alcohol consumption), unhealthy diet and obesity; environmental factors etc. The aim of the current study was to explore the role of genetic predisposition for MPN development.

Materials and Methods: The study included 36 MPN patients, selected according to recognized IACR/IARC guidelines, of which 20 females (56\%) and 16 males (44\%). Fifty five $\%$ of the patients had family history of cancer and $30.5 \%$ had an early onset $(<50 y)$. The analysis of germline mutations was performed by NGS of a panel of 94 tumorassociated genes on a MiSeq platform (Illumina).

Results: Around $18 \%$ of the total variants were evaluated as clinically significant. Among selected variants $33.4 \%$ were pathogenic, $13.3 \%$ likely pathogenic and $53.3 \%$ variants of uncertain significance (VUSs). Pathogenic/ likely pathogenic variants were detected mainly in the genes BRCA1, BRCA2, MMR genes and $C D H 1$ while VUSs in GPC3, DIS3L2, PRF1, STK11, DICER1, RET etc.

Conclusions: Overall, the genetic cause of MPN was found in $42.7 \%$ of the patients. Further research is needed to evaluate the functional effect of all VUSs. Thus clinical multi-gene panel testing is recommended for detection of germline mutations in high-risk MPN individuals.

Acknowledgments: Grants KP-06-OPR03/17/ 19.12.2018, National Science Fond; D01-285/17.12.2019, Ministry of Education and Science, Bulgaria

A. Mitkova: None. R. Dodova: None. M. Koleva: None. A. Andreeva: None. V. Petkova: None. D. Pencheva: None. M. Tzekova-Chernopolska: None. I. Terziev: None. S. Rangelov: None. C. Slavov: None. V. Mitev: None. R. Kaneva: None.

\section{P12.152.A}

A substantial proportion of adults with multiple primary tumors carry pathogenic variants in cancerpredisposition genes

F. Brugnoletti ${ }^{1,2}$, J. Vos $^{3}$, J. Schuurs-Hoeijmakers ${ }^{l}$, B. Hilhorst $^{1}$, A. Mensenkamp ${ }^{1}$, M. Ligtenberg ${ }^{1,4}$, L. Remondini $^{5}$, M. Pomponi ${ }^{5}$, E. Lucci Cordisco ${ }^{2,5}$, M. Genuardi $^{2,5}$, R. de Voer ${ }^{1}$, N. Hoogerbrugge ${ }^{1}$

${ }^{1}$ Department of Human Genetics, Radboud Institute for Molecular Life Sciences, Radboud university medical center, Nijmegen, Netherlands, ${ }^{2}$ Istituto di Medicina Genomica, Università Cattolica del Sacro Cuore, Fondazione Policlinico Universitario A. Gemelli, Rome, Italy, ${ }^{3}$ Department of Human Genetics, Radboud Institute for Health Sciences, Radboud university medical center, Nijmegen, Netherlands, ${ }^{4}$ Department of Pathology, Radboud Institute for Molecular Life Sciences, Radboud university medical center, Nijmegen, Netherlands, ${ }^{5}$ UOC Genetica Medica, Fondazione Policlinico Universitario A. Gemelli IRCCS, Rome, Italy

Multiple primary tumors and young age at diagnosis are hallmarks of inherited cancer predisposition. However, the identification of a genetic cause in individuals with multiple primary malignancies is still challenging due to phenotypic 
variability, presence of phenocopies and mostly used candidate gene approaches. To unravel the presence of an inherited cancer predisposition, we performed whole-exome sequencing (WES) on germline DNA from 44 individuals selected for the presence of either: i) $\geq 2$ primary tumors $<65$ years; ii) one tumor and one additional feature (e.g. macrocephaly, benign tumors) $<65$; or, iii) an adult type of cancer $<25$. We analyzed sequencing data for germline variants in 211 known cancer predisposition genes (CPGs) and classified each variant based on the AMCG/AMP guidelines. Pathogenic or likely pathogenic variants in CPGs were identified in 10/44 individuals (23\%). Two individuals carried one pathogenic variant that explains their tumor phenotype (PTEN;NF1). In five individuals pathogenic/likely pathogenic variants in CHEK2 $(n=3)$, $\operatorname{HOXB13}(n=1)$ and FANCM $(n=1)$ that may have contributed to their malignancies were identified. In three individuals, the analysis revealed heterozygous pathogenic variants in genes associated with recessive disorders (POLH, $n=2$; RAD50, $n=1$ ). In our highly selected cohort of adults with multiple primary tumors we identified pathogenic/likely pathogenic variants in CPGs in $23 \%$ of patients. This analysis resulted in a molecular diagnosis for two individuals and for eight individuals we identified alterations that may have cooperated in cancer development, giving cues for further genome-wide analysis on the germline and tumor tissues that are now ongoing.

F. Brugnoletti: None. J. Vos: None. J. SchuursHoeijmakers: None. B. Hilhorst: None. A. Mensenkamp: None. M. Ligtenberg: None. L. Remondini: None. M. Pomponi: None. E. Lucci Cordisco: None. M. Genuardi: None. R. de Voer: None. N. Hoogerbrugge: None.

\section{P12.153.B}

\section{MYD88 p.L265P mutation in DLBCL cases with CNS} relapse

\section{E. Voropaeva ${ }^{1}$, T. Pospelova ${ }^{2}$, M. Voevoda ${ }^{l}$, V. Maximov ${ }^{l}$,} V. Stupak ${ }^{3}$, I. Avzalov ${ }^{3}$

${ }^{1}$ Institute of Therapy and Preventive Medicine - branch of ICG SB RAS, Novosibirsk, Russian Federation, ${ }^{2}$ Novosibirsk State Medical University, Novosibirsk, Russian Federation, ${ }^{3}$ Research Institute of Traumatology and Orthopedics, Novosibirsk, Russian Federation

Previously reported about detection of the MYD88 p.L265P mutation in the cerebrospinal fluid of a patient with secondary CNS lymphomas. The aim of the study was to identify the mutational features associated with high risk of secondary CNS involvement in DLBCL.

Methods: Diagnostic FFPE tissue blocks of patients with DLBCL, who subsequently developed a CNS relapse, were taken. Whole exome sequencing of 7 tumor samples was performed. Additionally DLBCL whole-exome sequencing of dates presented in the cBioPortal for Cancer Genomics were analyzed: cases with $(n=48)$ and without $(n=355)$ proven CNS relapse were selected.

Results: In the tumor tissue of patients with DLBCL the pathogenic aberrations in the MYD88, TP53, PIM1, CD79B, MSH2, CARD11, NOTCH1, SMARCA4, KMT2D and EP300 genes were detected. The MYD88 p. L265P recurrent mutation was detected in 3/7 of samples. According to the cBioPortal for Cancer Genomics database, in DLBCL patients with CNS relapses mutations in the MYD88 gene were detected in $14 / 48,29.2 \%$ of cases. In the sample of patients without secondary CNS lesions, the central nervous system mutations in the MYD88 gene were $54 / 355,15.2 \% \quad(p=0.016)$. The mutation p.L265P compose $12 / 14(85.7 \%)$ and $32 / 54(59.3 \%)$ cases of MYD88 aberrations, accordingly. Thus, CNS relapse developed in 12/44 (27.3\%) MYD88 p.L265P carriers and only in $36 / 359(10.0 \%)$ patients without this mutation $(\mathrm{p}<0.001)$. The CNS relapse risk in mutation MYD88 $\mathrm{p}$. L265P carriers was $\mathrm{OR}=3.365,95 \%$ CI $(1.59 ; 7.11)$.

Conclusions: It is shown that testing DLBCL for MYD88 mutations is a potentially approach to diagnosing patients with high CNS relapse risk.

E. Voropaeva: None. T. Pospelova: None. M. Voevoda: None. V. Maximov: None. V. Stupak: None. I. Avzalov: None.

\section{P12.154.C}

Quantification of JAK2V617F mutation burden by droplet digital PCR in patients with myeloproliferative neoplasms

H. Y. Ivanov ${ }^{l}$, A. Linev ${ }^{l}$, I. Zheliazkov ${ }^{l}$, V. Graklanov ${ }^{2}$, E. Beleva $^{3}$, V. Goranova-Marinova ${ }^{2}$, Z. Grudeva-Popova ${ }^{3}$, V. Stoyanova ${ }^{1}$

${ }^{1}$ Medical University Plovdiv, Departament of Pediatrics and Medical Genetics, Plovdiv, Bulgaria, ${ }^{2}$ Medical University Plovdiv, First Department of Internal Medicine, Clinic of Hematology,, Plovdiv, Bulgaria, ${ }^{3}$ Medical University Plovdiv, Department of Clinical Oncology, Plovdiv, Bulgaria

Philadelphia-negative chronic myeloproliferative neoplasms (MPNs) are characterized by excessive production of terminally differentiated blood cells that are fully functional. Classical MPNs have been classified into 3 entities: polycythemia vera (PV), essential thrombocythemia (ET), and primary myelofibrosis (PMF). The JAK2 V617F mutation is detected in nearly $90 \%$ of PV patients, and in around a half of all ET and MF patients The aim of this 
study was to verify the effectiveness of digital droplet PCR (ddPCR) in the diagnosis of JAK2 V617F-mutated MPN and to investigate how the mutated allele burden correlate with the clinic.

Materials and methods: the JAK2 V617F mutation have been evaluated/quantified by ddPCR in 21 patients with MPN of whom 15 had the mutation.

Results: an increase of mutant allele burden from ET to PV and PMF was observed. A moderate negative correlation between the mutation burden and the platelet counts was found. A correlation between splenomegaly and the mutation burden was also observed. In conclusion, our study showed that quantification of the JAK2 V617F mutational burden by ddPCR could represent a new and promising technology for detection of JAK2 mutation in MPNs and the follow up of the patients may reflect the clinical response to treatment.

The study was funded by Project BG05M2OP001-1.0020005-C 01, Center for Competence "Personalized Innovative Medicine (PERIMED)", funded by the Operational Program "Science and Education for Intelligent Growth" 2014-2020, co-funded by the European Union for regional development.

H.Y. Ivanov: None. A. Linev: None. I. Zheliazkov: None. V. Graklanov: None. E. Beleva: None. V. Goranova-Marinova: None. Z. Grudeva-Popova: None. V. Stoyanova: None.

\section{P12.156.B}

Three novel splicing mutations in the NF1 gene cause exon skipping

\section{Kyprianou ${ }^{l}$, V. Anastasiadou ${ }^{2}$, P. Gerasimou ${ }^{l}, A$.}

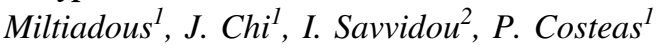

${ }^{1}$ Molecular Hematology-oncology, Karaiskakio Foundation, Nicosia, Cyprus, ${ }^{2}$ Department of Clinical Genetics, Archbishop Makarios III Medical Centre, Nicosia, Cyprus

Introduction: Neurofibromatosis type 1 is a condition characterized by changes in skin coloring (café-au-lait spots) and the growth of tumors (neurofibromas) along nerves in the skin, brain, and other parts of the body. The signs and symptoms of this condition vary widely among affected people.

Case Description: Three patients with clinical diagnosis of Neurofibromatosis type 1 were investigated for molecular evidence to support.

Methodology: A custom Haloplex/ Sureselect (Agilent) NGS panel was used to investigate mutations in Neurofibromatosis associated genes as per manufacturer's instructions. RNA investigation for exon skipping was employed.
Interpretation: Three heterozygous novel splice-site mutations in the NF1 gene were detected. The c.1642$2 \mathrm{~A}>\mathrm{G}$, c. $1722-1 \mathrm{G}>\mathrm{A}, \mathrm{c} .6085-3 \mathrm{C}>\mathrm{A}$ variants are all classified as pathogenic according to $\mathrm{ACMG}$ recommendations and Bioinformatics tools predicted that this novel mutations have pathogenic effects on splicing machinery. RNA investigation revealed that these variants lead in exon 15, exon 16, and exon 41 skipping of the NF1 gene, respectively.

Conclusions: To date, more than 1,000 NF1 mutations that cause neurofibromatosis type 1 have been identified. Most of these mutations are unique within families. Many NF1 mutations result in the production of an extremely short or abnormal version of the protein neurofibromin. This study highlights the presence of three novel splicing mutations in the NF1 gene that lead in exon skipping, causing the production of aberrant protein isoforms.

I. Kyprianou: None. V. Anastasiadou: None. P. Gerasimou: None. A. Miltiadous: None. J. Chi: None. I. Savvidou: None. P. Costeas: None.

P12.158.A

Splicing variants in hereditary cancer genes: clinical utility of functional RNA analysis

K. Agiannitopoulos ${ }^{l}$, G. Pepe ${ }^{l}$, E. Papadopoulou ${ }^{l}$, G. Tsaousis $^{1}$, S. Kampouri ${ }^{1}$, S. Maravelaki ${ }^{1}$, A. Fassas ${ }^{2}$, C. Christodoulou $^{3}$, R. Iosifidou 4 , S. Karageorgopoulou ${ }^{5}, C$. Markopoulos $^{6}$, I. Natsiopoulos ${ }^{7}$, K. Papazisis ${ }^{8}$, M. Vasilaki-

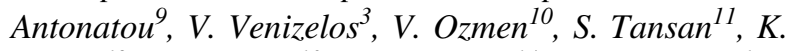
Kaban $^{12}$, D. T. Eniu ${ }^{13}$, A. Chiorean ${ }^{14}$, G. Nasioulas ${ }^{1}$

${ }^{1}$ Genekor MSA, Athens, Greece, ${ }^{2}$ St. Luke's Hospital, Thessaloniki, Greece, ${ }^{3}$ Metropolitan Hospital, Athens, Greece, ${ }^{4}$ Theagenio Anticancer Hospital, Thessaloniki, Greece, ${ }^{5}$ IASO Hospital, Athens, Greece, ${ }^{6}$ Athens Medical Center, Athens, Greece, ${ }^{7}$ Interbalkan Medical Center of Thessaloniki, Thessaloniki, Greece, ${ }^{8}$ Euromedica General Clinic, Thessaloniki, Greece, ${ }^{9}$ Metropolitan General Hospital, Athens, Greece, ${ }^{10}$ Faculty of Medicine Istanbul University, Istanbul, Turkey, ${ }^{11}$ Tansan Oncology, Istanbul, Turkey, ${ }^{12}$ American Hospital, Istanbul, Turkey, ${ }^{13}$ Institutul Oncologic Prof. Dr. I. Chiricuta, Cluj, Romania, ${ }^{14}$ University of Medicine and Pharmacy "Iuliu Haţieganu", Cluj, Romania

Introduction: Next generation sequencing (NGS) approaches have simplified multi-gene testing; yet the interpretation of variants remains a challenge. Most splicing variants (SVs) in genes associated with hereditary cancer are often classified as variants of uncertain significance (VUS) due to the lack of functional RNA analysis. The aim of this study 
was to investigate the occurrence of SVs in hereditary cancer genes the clinical utility of RNA analysis.

Material and Methods: We performed a retrospective analysis of 887 individuals referred to our laboratory for genetic testing using a NGS panel of 36 hereditary cancer genes. Functional RNA analysis for SVs was performed with RNA extracted from peripheral blood lymphocytes following RT-PCR and Sanger Sequencing.

Results: A SV was identified in $22 / 887$ individuals $(2.5 \%)$ and $7 / 22(31.8 \%)$ were classified as pathogenic and $5 / 22(22.7 \%)$ as likely pathogenic. The remaining 10 variants were initially classified as VUS (45.5\%) and RNA analysis could be performed for 2 variants. RNA analysis of a variant in ATM (c.2125-1G $>\mathrm{T}$ ) revealed the deletion of 11 bases (r.2125del), thus resulting in a frameshift (p. (Ile709Phefs*22)). Moreover, for a $M S H 2$ variant (c.1510 $+1 \_1510+2$ dupGT), it was shown that the insertion of 2 bases (r.1510dup) causes a frameshift, p.(Gly5040Glyfs*39). The two novel variants were reclassified from VUS to Pathogenic.

Conclusions: In our cohort, SVs accounted for $8.6 \%$ of pathogenic and $2.5 \%$ of VUS results. RNA analysis assisted in the reclassification of $20.0 \%$ of splicing VUS. RNA analysis is essential in the case of uncharacterized SVs, for proper classification and personalized management of patients.

K. Agiannitopoulos: None. G. Pepe: None. E. Papadopoulou: None. G. Tsaousis: None. S. Kampouri: None. S. Maravelaki: None. A. Fassas: None. C. Christodoulou: None. R. Iosifidou: None. S. Karageorgopoulou: None. C. Markopoulos: None. I. Natsiopoulos: None. K. Papazisis: None. M. Vasilaki-Antonatou: None. V. Venizelos: None. V. Ozmen: None. S. Tansan: None. K. Kaban: None. D.T. Eniu: None. A. Chiorean: None. G. Nasioulas: None.

\section{P12.160.C}

Evaluating the performance of the NF2 Genetic Severity Score and the potential impact of using functional assays to improve prognosis prediction in a cohort of Spanish patients

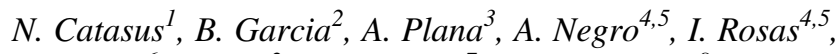

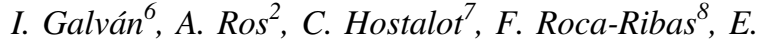
Amilibia $^{8}$, R. de Cid 6 , I. Bielsa ${ }^{3}$, E. Serra ${ }^{5}$, I. Blanco ${ }^{2}, \boldsymbol{E}$. Castellanos $^{4,5}$

${ }^{1}$ Hereditary Cancer Group, Germans Trias i Pujol Research Institute (IGTP-PMPPC);, Badalona, Spain, ${ }^{2}$ Clinical Genetics and Genetic Counseling Program, Germans Trias i Pujol University Hospital (HUGTiP),
Badalona, Spain, ${ }^{3}$ Dermatology Department, Germans Trias i Pujol University Hospital (HUGTiP), Badalona, Spain, ${ }^{4}$ Clinical Genomics Unit, Germans Trias i Pujol University Hospital (HUGTiP), Badalona, Spain, ${ }^{5}$ Hereditary Cancer Group, Germans Trias i Pujol Research Institute (IGTP-PMPPC), Badalona, Spain, ${ }^{6}$ Genomes for Life-GCAT Lab Group, Germans Trias i Pujol Research Institute (IGTP-PMPPC), Badalona, Spain, ${ }^{7}$ Neurosurgery Department, Germans Trias i Pujol University Hospital (HUGTiP), Badalona, Spain, ${ }^{8}$ Otorhinolaryngology Department, Germans Trias i Pujol University Hospital (HUGTiP), Badalona, Spain

Introduction: Neurofibromatosis Type 2 (NF2) is an autosomal dominant disorder characterized by the development of multiple schwannomas, particularly at vestibular nerves, and meningiomas ${ }^{1}$. A genotype-phenotype association is well established in NF2. A Genetic Severity Score (GSS) for NF2 mutations was developed to help predicting the disease course ${ }^{2}$. In the present study, we evaluated the performance of the GSS in our Spanish NF2 cohort and analyzed the potential added value of including functional molecular information.

Methods: Patients: GSS was validated using the NF2 Spanish Reference Center HUGTiP-ICO cohort. Functional assays: Merlin, ERK and their phosphorylated forms were measured by Western Blot using patient-derived fibroblasts. Association analysis: Mann-Whitney U test and regression model analysis.

Results: The application of the GSS to the Spanish NF2 cohort confirmed the identification of significant correlations across patient's phenotype and NF2 mutation: Spanish patients that harbor a truncating mutation in the $N F 2$ gene were associated with a most severe disease. However, for mutations in mosaicism and mutations associated to moderate phenotypes, GSS did not predict their clinical outcome as expected. We identified a correlation between the phenotype and the degree of activation of ERK pathway. We developed a lineal regression model to predict NF2 prognosis from functional and molecular data.

Conclusions: We validated the GSS in our cohort although a significant phenotypic variability intragroup was identified. To improve the performance of GSS, we evaluated the additional use of functional assays that allowed a better classification patients with mild and moderated phenotypes by applying a new statistical model.

N. Catasus: None. B. Garcia: None. A. Plana: None. A. Negro: None. I. Rosas: None. I. Galván: None. A. Ros: None. C. Hostalot: None. F. Roca-Ribas: None. E. Amilibia: None. R. de Cid: None. I. Bielsa: None. E. Serra: None. I. Blanco: None. E. Castellanos: None. 
P12.161.A

NF2 low grade mosaicism detected by deep sequencing of multiple tumor and normal tissue samples - a case report

\author{
C. Roggial, M. Schadeck ${ }^{2,1}$, U. Faust ${ }^{l}$, S. Armeanu- \\ Ebinger ${ }^{l}$, A. Dufke ${ }^{l}$, G. Tabatabai ${ }^{3}$, O. Riess ${ }^{l}, C$. \\ Schroeder ${ }^{l}$
}

${ }^{1}$ Institute of Medical Genetics and Applied Genomics, University Hospital Tübingen, Tübingen, Germany, ${ }^{2} S Y N$ $L A B$ Humangenetik Freiburg GmbH, Freiburg i. Br., Germany, ${ }^{3}$ Department of Neurosurgery, University Hospital Tübingen, Tübingen, Germany

Neurofibromatosis 2 (NF2, MIM\#101000) is a rare autosomal dominant disorder characterized by multiple schwannomas and mostly bilateral vestibular schwannoma (VS). Mutation carriers may develop meningiomas, ependymomas and retinal hamartomas. Mosaicism is a known phenomenon which is difficult to detect by conventional sequencing and is therefore impeding cascade screening and adequate surveillance for offspring-carriers. We describe a 27-year-old woman reaching out for preconception advice while presenting with singular VS, a facial nerve schwannoma, multiple cerebral bilateral meningiomas and medullar schwannomas, first diagnosed at the age of 26 years. Her parents or other family members were not affected, suggesting a de novo mutation in the NF2-gene. Previously external performed Sanger sequencing did not detect any pathogenic mutation in NF2 in the patient's blood. We analyzed two different tumors of the patient (facial nerve schwannoma and medullar schwannoma) by nextgeneration sequencing (NGS) and found the same pathogenic clonal NF2 variant (c. 362dup:p.Val122Glyfs*8) together with a second hit ( $\mathrm{LOH})$ in both tumors. The same pathogenic frameshift NF2-Variant was subsequently confirmed by NGS in blood and in skin (with an allele frequency of $0.5 \%$ and $1 \%$, respectively), while no mutation was detected in genes associated with schwannomatosis (LZTRI, SMARCBI and PRKARIA), ruling out the main differential diagnosis of NF2. Deep sequencing of blood through modern sequencing techniques such as NGS can improve the sensitivity of detection of mosaicism compared to traditional sequencing allowing therefore adequate genetic counseling concerning the risk for offsprings to inherit NF2 and specific surveillance for children carrying the mutation.

C. Roggia: None. M. Schadeck: None. U. Faust: None. S. Armeanu-Ebinger: None. A. Dufke: None. G. Tabatabai: None. O. Riess: None. C. Schroeder: None.
P12.163.C

Hematological phenotypes. Validation of a custom NGS panel including telomere and other leukemiapredisposing genes

C. Matteucci, F. Pellanera, V. Nofrini, M. Quintini, P. Gorello, S. Arniani, C. Camerini, E. Mavridou, M. Moretti, T. Pierini, C. Nardelli, A. G. Lema Fernandez, V. Bardelli, D. Di Giacomo, C. Mecucci

University of Perugia, Perugia, Italy

A growing number of hereditary hematological malignancies have been reported since the extended use of NGS that greatly improved our knowledge on germline mutations underlying myeloid neoplasms. We developed a customized diagnostic NGS panel (CHHD_A_v1 NGS panel, SOPHiA Genetics) including 21 established leukemia predisposing and 35 telomere biology genes. Cases with an hematological phenotype (cytopenia(s), myelodysplastic syndromes, acute myeloid leukemia) were selected on personal and/or familial history. Libraries were pooled and sequenced on Illumina MiSeq. Fastq files were analyzed with SOPHiA DDM software. Coding variants with variant fraction between 40 and 60 or $>90$, and minor allele frequency $<0.01$ in most common databases (g1000, ExAC, Gno$\mathrm{mAD}$, esp5400) were classified according to the ACMG guidelines. From 46 diagnostic samples 209 rare variants emerged; 47 were non-coding, 160 coding (84 missense, 4 frameshift, 3 inframe indel, 3 nonsense, 1 splice site, 65 synonymous) and 2 involved TERC gene; 78/160 were benign/likely benign, 73 VUS, 3 likely pathogenic and 6 pathogenic. In this group two novel variants affected $R A D 50$ gene. Undescribed, likely pathogenetic variant affected $C E B P A$ in one familial leukemia and RUNXI in one post-transplant donor leukemia. A novel TERC VUS g.303G $>$ A was associated with frank telomere pathology. Moreover, the DDX41 G92D missense VUS (rs371460149), very rare in GnomAD genome database (frequency $=0.000021$,) was found in $3 / 46$ cases $(6.5 \%)$ of this highly selected series, suggesting it plays a role in MDS/AML. Sharing of NGS results will deeply improve our knowledge on the occurrence and role of rare variants at candidate genes in hematological phenotypes.

C. Matteucci: None. F. Pellanera: None. V. Nofrini: None. M. Quintini: None. P. Gorello: None. S. Arniani: None. C. Camerini: None. E. Mavridou: None. M. Moretti: None. T. Pierini: None. C. Nardelli: None. A.G. Lema Fernandez: None. V. Bardelli: None. D. Di Giacomo: None. C. Mecucci: None. 
P12.164.A

Functional characterization of non-coding regulatory drivers in Chronic Lymphocytic Leukemia

A. Réal, H. Ongen, N. M. R. Lykoskoufis, C. Borel, G. Puga Yung, J. D. Seebach, E. T. Dermitzakis

\section{University of Geneva, Geneva, Switzerland}

Most cancers are associated with coding mutations. However, the effects of non-coding regulatory drivers in cancer development remains poorly understood. In a previous study (BioRxiv 174219), we identified 32 local modules of coordinated non-coding regulatory elements (CRDs, FDR $5 \%$ ) that accumulated an excess of somatic mutations in 150 patients with chronic lymphocytic leukemia (CLL). We hypothesized that CRDs with excess of somatic mutations (after controlling for mutational confounders) can have a driver role in CLL development. We aimed to functionally characterize the effect of significant CRDs on their candidate target genes.

We developed an in vitro model in which 317 geneticallydifferent lymphoblastoid cell lines (LCLs) are evaluated for variations in cancer-like phenotypes such as cell migration (chemotaxis), proliferation (mitotic index) and apoptosis (Fas-induced). In a preliminary screening on 42 LCLs, we found 50 significant genome-wide SNPs associated with these phenotypes $\left(\mathrm{P}<=1 \mathrm{e}^{-06}\right)$. Among them, we identified a SNP correlating with proliferation $\left(\mathrm{p}<=4.762 \mathrm{e}^{-07}\right)$ that is an eQTL (expression quantitative trait loci) associated with the GSDMB (gasdermin B) gene ( $\mathrm{P}<=0.0001$ ), which has been linked with apoptosis and cancer regulation.

We are currently in the process to investigate the expression profile of all 317 LCLs. These data together with phenotypic data will allow us to functionally characterize LCLs with differential expression of candidate driver genes. This could provide an experimental framework to explore and dissect the contribution of the noncoding cancer genome in CLL, helping to understand the biological complexity of these effects. Experiments are ongoing and updated results will be presented.

A. Réal: None. H. Ongen: None. N.M.R. Lykoskoufis: None. C. Borel: None. G. Puga Yung: None. J.D. Seebach: None. E.T. Dermitzakis: None.

\section{P12.165.B}

Molecular predictors of non-muscle invasive bladder cancer progression
M. Smal ${ }^{1}$, A. Rolevich ${ }^{2}$, N. Nikitchenko ${ }^{1}$, S. Krasny ${ }^{2}$, R. Goncharova $^{l}$

${ }^{1}$ Institute of Genetics and Cytology NASB, Minsk, Belarus, ${ }^{2}$ N.N. Alexandrov National Cancer Center of Belarus, Lesnoy, Minsk District, Belarus

Non-muscle invasive bladder cancer (NMIBC) comprises approximately $75-85 \%$ of primary bladder cancer cases, has low mortality rate, but is burdened by high rates of recurrence and progression. The risk of the latter is defined by the complex of clinicopathologic features which, however, provide low accuracy in predicting clinical outcomes. The aim of our study was to determine the prognostic value of FGFR3, HRAS, KRAS, PIK3CA, TP53 gene mutations, $R U N X 3$, p16 abnormal methylation, as well as $C D K N 2 A$ and $R B 1$ loss of heterozygosity ( $\mathrm{LOH}$ ) with regard to progression in a prospective cohort of 249 NMIBC patients. The multivariate Cox regression analysis, after adjusting for clinical parameters, revealed an independent effect of previous recurrence episodes (HR 25.1; 95\%CI 4.6-135.3; $\mathrm{p}<$ 0.001), TP53 mutation (HR 135.4; 95\%CI 11.6-1558.3; $\mathrm{p}<$ 0.001 ), RUNX3 (HR 20.7; 95\%CI 2.8-152.9; $\mathrm{p}=0.003$ ) and pl6 methylation (HR 19.1; 95\%CI 2.9-127.1; $\mathrm{p}=0.002$ ), as well as $R B 1$ LOH (HR 20.5; 95\%CI 3.7-114.5; $\mathrm{p}=0.001$ ) on NMIBC progression. Simultaneous presence of at least 3 established predictors resulted in significantly decreased 5year progression-free survival $(14.3 \pm 13.2 \%)$ as compared with a lesser number of indicated molecular alterations $(91.9 \pm 2.8 \%)(\mathrm{p}<0.001$, log-rank test $)$. Using logistic regression, we built a prediction model, comprising all five prognostic factors. The 5-marker panel was able to discriminate between patients with and without progression with $84.6 \%$ sensitivity, $91.7 \%$ specificity, and 0.88 AUC (p $<0.001$ ). The data obtained demonstrate that the use of molecular markers in addition to clinicopathologic ones may help in stratifying NMIBC patients into groups with different prognosis for the selection of optimal treatment regimens.

M. Smal: None. A. Rolevich: None. N. Nikitchenko: None. S. Krasny: None. R. Goncharova: None.

\section{P12.166.C}

Prevalence and clinical outcomes of non-small cell lung cancer (NSCLC) patients (pts) with potential pathogenic germline variants (pPGVs) in British Columbia (BC), Canada

S. Kuang, A. Karsan, S. Yip, I. Bosdet, S. Young, T. Tucker, K. A. Schrader, S. Sun

BC Cancer, Vancouver, BC, Canada 
Background: Multi-gene panel tumour testing (TT) has been available in BC since 2016 for pts with metastatic NSCLC. BRCA+ and mismatch repair deficient cancers may respond favourably to platinum chemotherapy and immunotherapy, respectively. We performed a review of NSCLC pts with potential pathogenic germline variants (pPGVs) identified by TT.

Methods: NSCLC pts with pPGVs from 2016-2019 were identified. TT was by next-generation sequencing of tissue for: $\quad A K T 1, A L K, B R C A 1, B R C A 2, C C N D 1, C C N D 3, C I C$, EGFR,ERBB2, ERBB3,FUBP1,HRAS,IDH1,IDH2,KIT, KRAS,MAP2K1,MET,NRAS, PDGFRA,PIK3CA,PTEN, ROS1,SDHA,SDHB,SDHC,SDHD, with APC,BMPRIA, CDH1,CDK4,CDKN2A,MLH1,MSH2, MSH6,MUTYH, NF1,PALB2,PMS2,POLD1,POLE,PTEN,SMAD4,STK11,

TP53 added Dec 2018. Clinical and treatment data were obtained.

Results: Of 2074 pts, pPGVs were found in 83(4\%), commonly BRCAl $(n=23), B R C A 2(n=29), \operatorname{MSH} 6(n=8)$, $\operatorname{MSH} 2(n=5) .2 \mathrm{pts}$ had pPGVs in both BRCAl and BRCA2. $33 / 83$ pts(40\%) were referred to genetics, 10 confirmed germline and 16 somatic. 23/83(28\%) pts had other driver mutations: 19 EGFR, $2 A L K$ and $2 B R A F$ V600E. 16/38 BRCA+ pts received platinum therapy with median time to progression of 29 weeks(range 6-64 weeks). 1 pt with $M S H 2$ and KRAS G12C variants received immunotherapy with progression at 4 months.

Conclusions: pPGVs were identified in $4 \%$ of NSCLCs, commonly BRCA1/BRCA2 and MSH2/MSH6, with other targetable driver mutations present in $28 \%$. Concomitant KRAS variant may be a mechanism of resistance to immunotherapy. These pts may benefit from clinical trials with combined targeted therapy +/- immunotherapy. Referral rates for germline testing were low, and future directions include reflex genetics referral and testing for pts with pPGVs.

Patient Demographics and Disease Characteristics

\begin{tabular}{ll}
\hline Characteristic & $\frac{\mathrm{pPGVs}(n=83)}{68(43-86)}$ \\
$\begin{array}{l}\text { Median (range) age, } \mathbf{y} \\
\text { Gender, n (\%) }\end{array}$ & $39(47 \%)$ \\
Male & $44(53 \%)$ \\
Female & \\
Ethnicity, n (\%) & $73(88 \%)$ \\
Non-Asian & $10(12 \%)$ \\
Asian & \\
Histology, n (\%) & $3(4 \%)$ \\
Squamous & $80(96 \%)$ \\
Non-squamous & \\
Smoking history, n (\%) & $25(30 \%)$ \\
Current &
\end{tabular}

SPRINGER NATURE

\begin{tabular}{ll} 
Former & $40(48 \%)$ \\
Never & $17(20 \%)$ \\
Driver mutations, n (\%) & \\
$E G F R$ & $19(23 \%)$ \\
$A L K$ & $2(2 \%)$ \\
$B R A F$ V600E & $2(2 \%)$ \\
Multiple primaries, n (\%) & \\
Yes & $16(19 \%)$ \\
No & $63(76 \%)$ \\
Site of metastasis, n (\%) & \\
Visceral & $43(52 \%)$ \\
Brain & $11(13 \%)$ \\
\hline
\end{tabular}

S. Kuang: None. A. Karsan: None. S. Yip: None. I. Bosdet: None. S. Young: None. T. Tucker: None. K.A. Schrader: None. S. Sun: F. Consultant/Advisory Board; Modest; Pfizer, AstraZeneca, BMS, Novartis.

P12.167.A

Mutation analysis in liquid biopsy from Lithuanian nonsmall cell lung cancer patients

\author{
S. Jarmalaite $^{l, 2}$, A. Sestokaite ${ }^{1,2}$, V. Gedvilaite ${ }^{l}, R$. \\ Sabaliauskaite $^{1}$, S. Cicenas ${ }^{l}$ \\ ${ }^{1}$ National Cancer Institute, Vilnius, Lithuania, ${ }^{2}$ Institute of \\ Biosciences, Life Sciences Center, Vilniu University, \\ Vilnius, Lithuania
}

Introduction \& Objectives: Non-small cell lung cancer (NSCLC) is a common and rapidly progressing cancer with poor survival rates. Blood cell-free DNA (cfDNA) has been proven to have prognostic potential as well as being useful for molecular profiling and monitoring disease burden. Next-generation sequencing (NGS) is the most efficient and sensitive method to detect mutations from cfDNA. The aim of this study was to screen Lithuanian NSCLC samples for common lung cancer-related mutations by targeted NGS and to determine their associations with clinical features.

Materials \& Methods: Analysis was conducted in NSCLC patient plasma samples before treatment and after clinical progression. Sequencing libraries were prepared from cfDNA using Oncomine Lung panel targeting 11 gene 180 hotspot mutation regions.

Results: Out of all 39 analysed plasma samples, in 24 samples taken before treatment largely from patients with advanced NSCLC, we identified 34 protein-coding mutations. Of them - 2 mutations were INDELS. Most common pathogenic variants in Lithuanian population were $K R A S$ and PIK3CA. Moreover, higher mutation load was detected in patient samples after clinical progression $v s$ samples before treatment. Specifically, in a group of 
patient's overall mutation count was found 4-fold higher in samples after immediate progression diagnosed during routine check-up at end of the treatment as compared to patient samples before treatment. In conclusion, plasma cfDNA is useful for molecular profiling of NSCLC patients to capture clinically relevant somatic alterations in advanced stage patients and could be used as prognostic biomarker.

S. Jarmalaite: None. A. Sestokaite: None. V. Gedvilaite: None. R. Sabaliauskaite: None. S. Cicenas: None.

\section{P12.168.B}

Further delineation of the NTHL1 associated syndrome: a report from the French oncogenetic consortium

F. Boulouard ${ }^{1,2}$, E. Kasper ${ }^{1}$, M. Buisine ${ }^{3}$, G. Lienard $^{l}, S$.

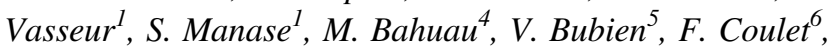
V. Cusin', M. Dhooge ${ }^{8}$, L. Golmard 9 , V. Goussot ${ }^{10}, N$. Hamzaouil $^{11}$, E. Lacaze LI $^{12}$ S. Lejeune ${ }^{13}$, J. Mauillon ${ }^{1}$, S. Pinson $^{14}$, C. Tlemsani ${ }^{15}$, C. Toulas ${ }^{16}$, J. Rey ${ }^{17}$, N. Uhrhammer $^{18}$, G. Bougeard-Denoyelle ${ }^{1}$, T. Frebourg ${ }^{l}, C$. Houdayer $^{\text {I, S. Baert-Desurmont }}{ }^{1}$

${ }^{1}$ Département de Génétique et Inserm U1245, UNIROUEN, Normandie Univ, CHU de Rouen, Centre Normand de Génomique et de Médecine Personnalisée, Rouen, France, ${ }^{2}$ Laboratoire de génétique moléculaire Centre François Baclesse, Caen, France, ${ }^{3}$ Institut de Biochimie et Biologie Moléculaire, Oncogénétique Moléculaire, CHU de Lille, Inserm UMR-S 1172, Université de Lille, Lille, France, ${ }^{4}$ Departement de Biochimie-biologie moléculaire, Pharmacologie, génétique médicale CHU Henri Mondor, Créteil, France, ${ }^{5}$ Département de BioPathologie, Unité d'Oncogénétique - Laboratoire de génétique moléculaire CLCC Institut Bergonié, Bordeaux, France, ${ }^{6}$ Département de Génétique, UF d'oncogénétique et d'angiogénétique moléculaire CHU Paris-GH La Pitié Salpêtrière-Charles FoixHôpital Pitié-Salpêtrière, Paris, France, ${ }^{7}$ Département de Génétique - CHU Paris-GH La Pitié Salpêtrière-Charles Foix - Hôpital Pitié-Salpêtrière, Paris, France, ${ }^{8}$ Service de gastro-entérologie CHU Paris Centre - Hôpital Cochin, Site Cochin, Paris, France, ${ }^{9}$ Service de Génétique - Unité de Génétique constitutionnelle - CLCC Institut Curie, PSL Research University, Paris, France, ${ }^{10}$ Département de biologie et pathologie des tumeurs - CLCC GeorgesFrançois Leclerc, Dijon, France, ${ }^{11}$ Laboratoire d'oncogénétique, CHU Paris Centre - Hôpital Cochin, Site Cochin, Paris, France, ${ }^{12}$ Service de génétique médicale GH du Havre - Hôpital Jacques Monod, Le Havre, France, ${ }^{13}$ Clinique de Génétique "Guy Fontaine" - Pôle de Biologie Pathologie Génétique - CHU de Lille - Hôpital Jeanne de Flandre, Lille, France, ${ }^{14}$ Service de Génétique Moléculaire et Clinique - Unité Médicale de Génétique des Cancers et des Maladies Multifactorielles - CHU de Lyon HCL - GH Est, Lyon, France, ${ }^{15}$ Service de cancérologie - CHU Paris Centre - Hôpital Cochin, Site Cochin, Paris, France, ${ }^{16}$ Laboratoire d'oncogénétique - Institut Universitaire $d u$ Cancer Toulouse - Oncopole, Toulouse, France, ${ }^{17}$ Laboratoire de Biologie des Tumeurs Solides - CHU de Montpellier - Hôpital Arnaud de Villeneuve, Montpellier, France, ${ }^{18}$ Laboratoire de Biologie Médicale OncoGènAuvergne - CLCC Jean Perrin, Clermont-Ferrand, France

Biallelic mutations in the NTHL1 (Nth Like DNA Glycosylase 1) gene cause a novel autosomal recessive hereditary cancer syndrome predisposing to adenomatous polyposis and colorectal cancer. Half of the biallelic carriers also display multiple colonic or extra-colonic primary tumors, mainly breast, endometrium, urothelium and brain tumors. Published data designates NTHLI as an important contributor to hereditary cancers but also underlines the scarcity of available information. Thanks to the French oncogenetic consortium (Groupe Génétique et Cancer), a well-organized network of laboratories and family cancer clinics, we collected NTHL1 variants from 6432 patients attending a visit for hereditary colon cancer $(n=3529)$ or other hereditary cancers $(n=2903)$. We found 7 patients with pathogenic, loss of function, biallelic NTHL1 germline variations. All carriers were from the "colorectal cancer or polyposis" collection and no variant was identified in the "extra digestive" collection of patients. Among the 7 biallelic carriers, 6 underwent colonoscopy and all of them presented adenomatous polyps. For digestive cancers, average age at diagnosis was 59 and we reported colorectal, duodenal, caecal and pancreatic cancer. None of these tumors showed microsatellite instability. Extra-digestive malignancies included sarcoma, basal cell carcinoma, breast cancer, urothelial carcinoma, and scalp melanoma. We reported the second largest NTHL1 collection. NTHL1 is not yet considered for diagnostic purposes in France. Despite tumor risks to be precisely defined, these novel data may favor NTHL1 inclusion in diagnostic panel testing. Colonic surveillance should be based on MUTYH recommendations and extra-colonic surveillance has to be defined.

F. Boulouard: None. E. Kasper: None. M. Buisine: None. G. Lienard: None. S. Vasseur: None. S. Manase: None. M. Bahuau: None. V. Bubien: None. F. Coulet: None. V. Cusin: None. M. Dhooge: None. L. Golmard: None. V. Goussot: None. N. Hamzaoui: None. E. Lacaze: None. S. Lejeune: None. J. Mauillon: None. S. Pinson: None. C. Tlemsani: None. C. Toulas: None. J. Rey: None. N. Uhrhammer: None. G. Bougeard-Denoyelle: None. T. Frebourg: None. C. Houdayer: None. S. BaertDesurmont: None. 


\section{P12.169.C}

Characterization of BRCA deficiency in ovarian cancer: implications for treatment and outcome

\section{G. Barbero ${ }^{1}$, R. Zuntini ${ }^{2}$, A. Perrone ${ }^{3}$, D. Rubino ${ }^{4}, M$.} Grippa $^{l}$, G. Innella ${ }^{l}$, A. De Leo ${ }^{5}$, C. Ceccarelli ${ }^{5}, L$. Godino $^{1}$, S. Miccoli ${ }^{1}$, S. Ferrari ${ }^{1}$, D. Santini ${ }^{5}$, P. De Iaco ${ }^{3}$, C. Zamagni ${ }^{4}$, D. Turchetti ${ }^{2,1}$

${ }^{1}$ Unit of Medical Genetics, Academic Hospital S.OrsolaMalpighi, Bologna, Italy, ${ }^{2}$ Unit of Medical Genetics, Department of Medical and Surgical Sciences, University of Bologna, Bologna, Italy, ${ }^{3}$ Unit of Oncological Gynecology, Department of Medical and Surgical Sciences, University of Bologna, Bologna, Italy, ${ }^{4}$ Unit of Medical Oncology, Academic Hospital S.Orsola-Malpighi, Bologna, Italy, ${ }^{5}$ Unit of Pathology, Academic Hospital S.OrsolaMalpighi, Bologna, Italy

Impairment of BRCA function in Ovarian Cancer (OC) has proven to predict response to platinum-based chemotherapy and PARP-inhibitors; consequently, BRCA1/2 sequencing is being routinely used to inform medical treatment of $\mathrm{OC}$ patients. A comprehensive assessment of BRCA defects was performed in 30 clinically characterized OC patients to obtain a picture of BRCA alterations and to test the accuracy of standard diagnostic approaches. BRCA1/2 sequence, copy number, and promoter methylation were analyzed in freshfrozen OC tissue. Twelve patients (40\%) showed clear evidence of BRCA deficit (BD): $6(20 \%)$ carried germline pathogenic variants (3 in BRCA1, 3 in BRCA2) and one (3.3\%) a somatic BRCA2 mutation, all displaying Loss-OfHeterozygosity; 5 (16.7\%) had one BRCA1 copy deleted and the other methylated; in the remaining 18 cases, BRCA deficit was undetected/unclear (BU). 29 patients received platinumbased chemotherapy, with baseline clinical features not significantly differing by BRCA status: after a median follow-up of 44.2 months, 15 had experienced progression: mean PFS was 43.5 months (95\%CI: 35.6-51.3) in the BD and 28 months (95\%CI: $21.1-35.0)$ in the BU group $(p=0.018)$. BRCA sequence analysis in respective Formalin-FixedParaffin-Embedded (FPPE) samples detected all the variants found in fresh-frozen tissue, but also multiple additional somatic variants, including pathogenic variants (allelic load $20-40 \%$ ) in 2 tumours previously classified as BU. Our findings suggest that BRCA sequencing in FFPE OC tissue may involve a risk of false positive results, while missing a substantial fraction of BRCA defective (methylated) tumours. Supported by LOTO Onlus, AstraZeneca, Fondazione Del Monte

G. Barbero: None. R. Zuntini: None. A. Perrone: None. D. Rubino: None. M. Grippa: None. G. Innella:
None. A. De Leo: None. C. Ceccarelli: None. L. Godino: None. S. Miccoli: None. S. Ferrari: None. D. Santini: None. P. De Iaco: None. C. Zamagni: None. D. Turchetti: B. Research Grant (principal investigator, collaborator or consultant and pending grants as well as grants already received); Modest; AstraZeneca. D. Speakers Bureau/Honoraria (speakers bureau, symposia, and expert witness); Modest; MSD.

\section{P12.173.A}

Mutation analysis of uterine lavage fluid from Lithuanian ovarian cancer patients

R. Sabaliauskaite ${ }^{l}$, I. Vaicekauskaite ${ }^{l, 2}$, D. Zilovic $^{l, 2}, R$. Ciurliene $^{I}$, S. Jarmalaite ${ }^{1,2}$

${ }^{1}$ National Cancer Institute, Vilnius, Lithuania, ${ }^{2}$ Institute of Biosciences, Life Sciences Center, Vilniu University, Vilnius, Lithuania

Introduction \& Objectives: Ovarian cancer (OC) is a leading cause of death in gynecological cancers with more than 250000 new cases diagnosed each year, $60 \%$ of these at an advanced stage. OC has only $29 \%$ 5-year survival, causing high mortality rates. However, currently there is no effective screening methodology or accurate early diagnostic test for OC. A few proof-of-principle studies demonstrated the use of next-generation sequencing (NGS) of DNA of uterine lavage, to identify mutations in patients with known gynecologic cancers. The aim of this study was to screen uterine lavage and ovarian tissue samples form Lithuanian OC patients for common cancer-related mutations by targeted NGS and to determine their associations with clinical features.

Materials \& Methods: Cell-free DNA from 36 uterine lavage fluid and DNA from 18 ovarian tissue samples were analysed using NGS. The sequencing libraries were prepared using Ion AmpliSeq ${ }^{\mathrm{TM}}$ On-Demand Panel targeting 10 most common OC related genes.

Results: DNA mutations were detected in: 13 of 36 (36\%) uterine lavage samples ( 21 of 36 mutations known as pathogenic) and 10 of 18 (55.56\%) tissue samples (18 of 30 mutations known to be pathogenic). 19 mutations (11 pathogenic) were detected in both cell-free and tissue samples from the same patients in 11 of $17(64.71 \%)$ sample pairs. The most often mutated genes were TP53, $B R A C 1$ and ARIDIA. In conclusion, cell-free DNA samples obtained from uterine lavage could be useful for molecular profiling of OC patients.

R. Sabaliauskaite: None. I. Vaicekauskaite: None. D. Zilovic: None. R. Ciurliene: None. S. Jarmalaite: None. 
P12.174.B

Mutations in the ATM gene are a frequent cause of apparently sporadic pancreatic carcinoma

\section{E. Kunstmann, S. Rost, A. Gehrig}

\section{Department of Human Genetics, Würzburg, Germany}

Introduction: Pancreatic cancer is an either inherited or acquired rare type of abdominal cancer. Smoking, alcohol consumption as well as diet and obesity are well known risk factors of this acquired disease. Germline mutations in BRCA2 are the most common cause of familial pancreatic cancer. In addition, the prevalence of ATM mutations has been found to be significantly higher in familial pancreatic cancer cases than in controls. Here we present data that mutations in the ATM gene may be a frequent cause of apparently sporadic pancreatic cancer.

Materials and Methods: We analyzed pedigree information and genes associated with pancreatic cancer (BRCA1/ 2, ATM, CDKN2A, CHEK2, NBN and PALB2) in 8 families.

Results: Three families showed a pathogenic mutation in BRCA2, four families had pathogenic mutations in ATM. The index patients were all men and diagnosed with pancreatic carcinoma at the age $<60$ years. One patient had no further family member with a history of cancer. The father of one index patient was diagnosed with tongue cancer. Two families showed several family members suffering from cancer like breast cancer, sarcoma, colorectal cancer and brain tumours. No mutation was detected in one patient.

Conclusions: None of these presented pedigrees fulfilled the criteria of any hereditary tumor syndrome. Nevertheless, pathogenic germline mutations in the ATM gene were detected in half of patients leading to the possibility of predictive testing in healthy family members. Therefore, genetic testing including ATM should be offered to patients with pancreatic carcinoma despite a negative family history of cancer.

E. Kunstmann: None. S. Rost: None. A. Gehrig: None.

\section{P12.180.B}

\section{Molecular characterization of metastatic eccrine porocarcinoma}

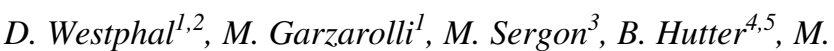

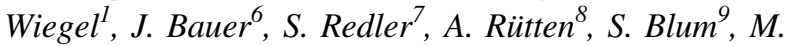

Laniado $^{9}$, S. Ugurel $^{10}$, J. Maschke ${ }^{1}$, S. Beissert ${ }^{1,2}, S$.

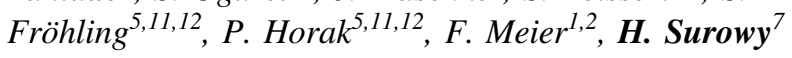

${ }^{1}$ Department of Dermatology, Faculty of Medicine and University Hospital Carl Gustav Carus, Technische
Universität Dresden, Dresden, Germany, ${ }^{2}$ National Center for Tumor Diseases (NCT) Dresden, Dresden, Germany, ${ }^{3}$ Department of Pathology, Faculty of Medicine and University Hospital Carl Gustav Carus, Technische Universität Dresden, Dresden, Germany, ${ }^{4}$ Division of Applied Bioinformatics, National Center for Tumor Diseases (NCT) Heidelberg, Heidelberg, Germany, ${ }^{5}$ German Cancer Consortium (DKTK) Heidelberg, Heidelberg, Germany, ${ }^{6}$ Department of Dermatology, University Medical Center Tübingen, Tübingen, Germany, ${ }^{7}$ Institute of Human Genetics, University Clinic Düsseldorf (UKD), Heinirch-Heine University, Düsseldorf, Germany, ${ }^{8}$ Institute of Dermatopathology, Friedrichshafen, Germany, ${ }^{9}$ Department of Radiology, Faculty of Medicine and University Hospital Carl Gustav Carus, Technische Universität Dresden, Dresden, Germany, ${ }^{10}$ Department of Dermatology, University of Essen, Essen, Germany, ${ }^{11}$ Department of Translational Medical Oncology, National Center for Tumor Diseases (NCT) Heidelberg and German Cancer Research Center (DKFZ) Heidelberg, Heidelberg, Germany, ${ }^{12}$ DKFZ-Heidelberg Center for Personalized Oncology (HIPO), Heidelberg, Germany

Background: Eccrine Porocarcinoma (EPC) arise from distal portions of sweat ducts and constitute one of the rarest forms of malignant skin neoplasias, with high recurrence and metastasis rates. Due to their largely unknown etiology and molecular pathology, no effective therapeutic recommendations exist, especially in metastatic EPC.

Methods: We performed whole exome sequencing (WES) in normal and metastatic tissue of an EPC case, supplemented by RNA-Sequencing, array-CGH and immunohistochemistry, and functional analyses.

Results: The metastatic EPC tissue exhibits a high somatic point mutation burden of 21 tumor-specific alterations per $\mathrm{MB}$. The dominance of $\mathrm{C}>\mathrm{T}$ transitions and double-substitutions point towards the UV-light exposure related tumor mutation signatures (Cosmic-Version3) $7 \mathrm{a} / 7 \mathrm{~b}$. Known cancer driver mutations are present in $A P C, P T E N$ and TP53. In addition, multiple gene families related to cell proliferation, migration, adhesion and connectivity are notably enriched among genes harbouring missense or truncating mutations or exhibiting dysregulated gene expression, for example PI3K subunits and protein kinases. In addition, wide-spread copy-number changes are present throughout the genome, including gain of chr7p $(E G F R)$ and loss of chr9p $(C D K 2 N A)$. A strongly CNValtered region on chr11q contains a highly expressed CTNND1:PAK1 in-frame fusion gene that likely retains Wnt-signalling as well as p21-binding and protein kinase properties. Presence of the altered region in an early metastasis suggests this fusion as a potentially dominant driver in tumor development. 
Conclusions: Our results demonstrate the involvement of multiple cellular signalling pathways in EPC tumor development, many of which are involved in other cancer types, and point towards potential treatment targets.

D. Westphal: None. M. Garzarolli: None. M. Sergon: None. B. Hutter: None. M. Wiegel: None. J. Bauer: None. S. Redler: None. A. Rütten: None. S. Blum: None. M. Laniado: None. S. Ugurel: None. J. Maschke: None. S. Beissert: None. S. Fröhling: None. P. Horak: None. F. Meier: None. H. Surowy: None.

P12.181.C

Genetic and in vitro analysis in a large family with a $P R D M 10$ variant and a phenotype partly resembling Birt-Hogg-Dubé syndrome

I. van de Beek ${ }^{1}$, I. E. Glykofridis ${ }^{1}$, Q. Waisfisz ${ }^{1}$, H. MeijersHeijboerl $^{l}$, M. A. M. van Steensel ${ }^{2,3}$, R. M. F. Wolthuis ${ }^{l}$, A. C. Houweling ${ }^{1}$

${ }^{1}$ AmsterdamUMC, Vrije Universiteit Amsterdam, Amsterdam, Netherlands, ${ }^{2}$ Lee Kong Chian School of Medicine, Nanyang Technological University, Singapore, Singapore, ${ }^{3}$ Skin Research Institute of Singapore, Agency for Science, Technology and Research, Singapore, Singapore

Introduction: Birt-Hogg-Dubé syndrome (BHD) is characterized by fibrofolliculomas, lung cysts, pneumothorax and renal cell carcinoma (RCC), and is caused by heterozygous germline mutations in the FLCN gene. Fibrofolliculomas are considered pathognomonic for BHD. We here present a family of which the proband has a histologically confirmed fibrofolliculoma, more extensive skin lesions not completely consistent with BHD and over 50 lipomas. He has no identifiable lung cysts on chest CT. At least 15 family members were reported to be affected by multiple lipomas, skin lesions and/or RCC. No mutation was detected in FLCN.

Materials and Methods: We performed whole exome sequencing (WES) and segregation analysis in four affected family members.

Results: The Cys677Tyr missense variant in PRDM10 was the only potentially causative variant present in the DNA of all four affected relatives. We hypothesized a gainof- function effect based on limited data of PRDM10 knockout mice and the absence of $\mathrm{LOH}$ of PRDM10 in both lipoma and RCC tissues derived from the family. To assess the effect of the variant, we overexpressed PRDM10 wildtype and mutant protein in Renal Proximal Tubule Epithelial Cells (RPTEC). Preliminary data show that overexpression of PRDM10 in RPTEC results in decreased expression of FLCN. GPMNB expression increased upon both FLCN deficiency and PRDMIO overexpression.

Conclusions: We identified a PRDMIO variant as the most likely cause in a family with multiple lipomas, RCC and skin lesions partly resembling BHD.

I. van de Beek: None. I.E. Glykofridis: None. Q. Waisfisz: None. H. Meijers-Heijboer: None. M.A.M. van Steensel: None. R.M.F. Wolthuis: None. A.C. Houweling: None.

\section{P12.182.A}

Prevalence of pathogenic variants in cancer predisposition genes in patients with pancreatic cancer: A multicentre clinical study

A. Eliades ${ }^{1}$, E. Fountzila ${ }^{2}$, C. Loizides $^{l}$, A. Achilleos $^{l}$, C. Marinou $^{l}$, C. Kkoufou ${ }^{l}$, L. Constantinou ${ }^{l}, K$. Tsangaras ${ }^{l}$, E. Kypri ${ }^{1}$, M. Ioannides ${ }^{1}$, G. Fountzilas ${ }^{3}$, G. Koumbaris ${ }^{1}$, P. C. Patsalis ${ }^{1}$

${ }^{1}$ NIPD Genetics Public Company Ltd, Nicosia, Cyprus, ${ }^{2}$ Euromedica General Clinic of Thessaloniki, Thessaloniki, Greece, ${ }^{3}$ Laboratory of Molecular Oncology, Hellenic Foundation for Cancer Research/Aristotle University of Thessaloniki, Thessaloniki, Greece

Introduction: Pancreatic cancer is one of the top leading causes of cancer-related deaths with more than 432,000 patients were predicted to die worldwide from the disease in 2018 according to the World Health Organisation. Most patients have a one-year survival rate of $20 \%$ and a fiveyear survival rate of $7 \%$. These low survival rates are mainly because in most cases at the time of diagnosis malignancy has already progressed to advanced stages. It is estimated that Identification of high-risk individuals via genetic screening can improve survival rates.

Materials and methods: This multicentre retrospective study enrolled 572 patients diagnosed with pancreatic adenocarcinoma (PDAC). Germline DNA was extracted from these patients and subjected to targeted in solution hybridisation-based next generation sequencing using the PreSENTIA pan-cancer panel covering 62 genes associated with cancer predisposition. Results were validated by an orthogonal method. Clinical and family history data were collected from the participants.

Results: We have identified pathogenic variants in 18 genes associated with hereditary cancer predisposition including genes that were previously associated with increased risk for pancreatic cancer including PALB2, ATM, BRCA1, BRCA2, MSH6 and APC. We have additionally identified pathogenic variants in genes not 
previously associated with pancreatic adenocarcinoma such as SDHB, FANCM and RET.

Conclusions: The results suggest that a substantial proportion of patients with PDAC are carriers of pathogenic germline variants in predisposition genes and highlight the clinical utility of germline multigene testing. Importantly relatives of patients that are carriers of pathogenic variants could benefit from germline testing.

A. Eliades: A. Employment (full or part-time); Significant; NIPD Genetics Public Company Ltd. E. Fountzila: None. C. Loizides: A. Employment (full or part-time); Significant; NIPD Genetics Public Company Ltd. A. Achilleos: A. Employment (full or part-time); Significant; NIPD Genetics Public Company Ltd. C. Marinou: A. Employment (full or part-time); Significant; NIPD Genetics Public Company Ltd. C. Kkoufou: A. Employment (full or part-time); Significant; NIPD Genetics Public Company Ltd. L. Constantinou: A. Employment (full or part-time); Significant; NIPD Genetics Public Company Ltd. K. Tsangaras: A. Employment (full or part-time); Significant; NIPD Genetics Public Company Ltd. E. Kypri: A. Employment (full or part-time); Significant; NIPD Genetics Public Company Ltd. M. Ioannides: A. Employment (full or part-time); Significant; NIPD Genetics Public Company Ltd. G. Fountzilas: None. G. Koumbaris: A. Employment (full or part-time); Significant; NIPD Genetics Public Company Ltd. P.C. Patsalis: A. Employment (full or part-time); Significant; NIPD Genetics Public Company Ltd.

\section{P12.183.B}

Post-biopsy cell-free DNA in blood: an open window on primary prostate cancer genetics and biology

M. Corbetta ${ }^{1}$, M. Lazzeri ${ }^{2}$, C. Chiereghin ${ }^{l}$, I. De Simone ${ }^{l}$, M. Zuradelli ${ }^{1}$, G. Soldà ${ }^{l}$, R. Asselta ${ }^{l}$, N. M. Buffi ${ }^{l}, P$. Casale $^{2}$, R. Hurle ${ }^{2}$, G. Lughezzani ${ }^{1}$, A. Saita ${ }^{2}$, G. Guazzoni ${ }^{1}$, S. Duga ${ }^{1}$

${ }^{1}$ Humanitas University, Pieve Emanuele - Milan, Italy, ${ }^{2}$ Humanitas Clinical and Research Center, IRCCS, Rozzano - Milan, Italy

Introduction: The analysis of circulating cell-free DNA (ccfDNA) represents a promising strategy for non-invasive cancer diagnosis. However, the fraction of cancer-derived ccfDNA (ctDNA) is variable, and particularly low in primary disease in capsulated organs like prostate. Most studies explored ctDNA in advanced metastatic prostate cancer (PCa), conversely, detection of ctDNA in localized disease is challenging. Inflammation, exercise, or tissue injury, substantially increase ccfDNA; we therefore hypothesized that prostate biopsies (each typically picking 14-18 samples from the gland) might release prostate-derived ccfDNA/ ctDNA, which could be exploited for molecular tests.

Materials and Methods: We collected tissue/blood samples (before and after biopsy at different timepoints) from 34 patients undergoing prostate biopsy: 26 with $\mathrm{PCa}, 8$ negative. ccfDNA was extracted with the Maxwell-RSC ccfDNA Plasma Kit, quantified by Qubit; size-distribution analysis was performed by Tape-station. Somatic mutations, identified by RNAseq on biopsies using the TruSight RNA Pan-Cancer Panel, were searched in ccfDNA (before/after biopsy) by targeted NGS (NEBNext Ultra II DNA Library Prep; mean depth 280,000x).

Results: We demonstrated a significant transient increase in plasma ccfDNA after biopsy (4.23.6 $\pm 3.39 \mathrm{ng}$ vs. $2.72 \pm 1.02 \mathrm{ng}$ per $\mathrm{mL}$ plasma; $\mathrm{P}$-value $=0.0098$ after $1 \mathrm{~h}$; $9.32 \pm 5.08 \mathrm{ng}$ vs. $6.74 \pm 4.35 \mathrm{ng}, \mathrm{P}=0.0052$ after $2 \mathrm{~h}$ ). ccfDNA molecules extracted after biopsy were also significantly longer. Finally, targeted NGS on pre/postbiopsy ccfDNA confirmed a significant enrichment (2.15164 fold) in the ctDNA fraction.

Conclusions: Plasma post-biopsy ccfDNA represents a source of pan-prostate DNA, a major advantage over the analysis of single biopsies. This opens the possibility to analyze somatic mutations/epigenetics markers with prognostic value in primary PCa.

M. Corbetta: None. M. Lazzeri: None. C. Chiereghin: None. I. De Simone: None. M. Zuradelli: None. G. Soldà: None. R. Asselta: None. N.M. Buffi: None. P. Casale: None. R. Hurle: None. G. Lughezzani: None. A. Saita: None. G. Guazzoni: None. S. Duga: None.

\section{P12.184.C}

Study of Androgen Receptor and variant 7 in TumorEducated Platelets in Metastasic Prostate Cancer

P. Vázquez-Cárdenas ${ }^{1,2,3}$, M. C. Moreno-Samaniego ${ }^{l}$, J. L. García-Hernández ${ }^{2,3}$, I. Misiewicz-Krzeminska ${ }^{2,3}$, Á. J. Virseda-Rodríguez, S. Marcos-Asensio ${ }^{4}$, A. B. Herrero ${ }^{1,2}$, F. Gómez-Veiga ${ }^{4}$, J. J. Cruz-Hernández ${ }^{1,3,5}$, R. GonzálezSarmiento $^{1,2,3}$

${ }^{1}$ Molecular Medicine Unit, Department of Medicine, University of Salamanca, Salamanca, Spain, ${ }^{2}$ Institute of Molecular and Cellular Biology of Cancer (IBMCC), University-CSIC, Salamanca, Spain, ${ }^{3}$ Institute of Biomedical Research of Salamanca (IBSAL), Salamanca, Spain, ${ }^{4}$ Translational Research Group of Urology GITUR-IBSAL, University Hospital of Salamanca, Salamanca, Spain, ${ }^{5}$ Oncology Service, University Hospital of Salamanca, Salamanca, Spain

Introduction: Liquid biopsies is a non-invasive method to detect tumor-associated biomarkers. This study aimed to detect 
the presence of androgen receptor (AR) and the androgen receptor variant 7 (AR-V7) a biomarker associated with worse prognosis, in blood samples of prostate cancer (PCa) patients by two methods: RT-qPCR from platelet-isolated RNA and determination of $\mathrm{AR}$ and $\mathrm{AR}-\mathrm{V} 7$ protein levels in plasma using capillary nano-immunoassay.

Methods: The study included 35 patients with $\mathrm{PCa}$ and 16 healthy donors. Whole blood $(10 \mathrm{~mL})$ was collected and platelets were isolated from the supernatant by centrifugation at room temperature for 20 minutes at $1500 \mathrm{rpm}$. RNA was extracted and RT-qPCR for GAPDH, ITGA2B, AR and AR-V7 was performed. Proteins were extracted to detect AR and AR-V7 using Simple West Protein analysis.

Results: Both AR and AR-V7 expression were detected by RT-qPCR. Five patients exhibited a high expression of the 2 receptors, 8 showed low levels and 22 patients showed expression levels similar to those of the VCAP PCa cell line, that was used as a positive control. These biomarkers were absent in the 16 healthy donors, as expected. AR protein was detected by Simple West in all plasma patients. However, AR-V7 protein was only detected in 11 patients.

Conclusions: The results demonstrate that platelets contain tumor-associated RNA and could be used for detection of AR and AR-V7 expression in blood samples in $\mathrm{PCa}$ patients. Moreover, determination of AR-V7 protein by nano-immunoassay represents a novel methodology and may serve to detect this biomarker associated with bad prognosis.

P. Vázquez-Cárdenas: None. M.C. Moreno-Samaniego: None. J.L. García-Hernández: None. I. Misiewicz-Krzeminska: None. Á.J. Virseda-Rodríguez: None. S. Marcos-Asensio: None. A.B. Herrero: None. F. Gómez-Veiga: None. J.J. Cruz-Hernández: None. R. González-Sarmiento: None.

\section{P12.185.A}

First results of germline genetic testing in Slovenian patients with prostate cancer

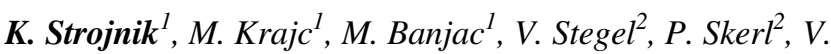
Setrajcic Dragos ${ }^{2}$, G. Klancar $^{2}$, S. Novakovic ${ }^{2}$, A. Blatnik ${ }^{1}$

${ }^{1}$ Cancer Genetics Clinic, Institute of Oncology, Ljubljana, Slovenia, ${ }^{2}$ Department of Molecular Diagnostics, Institute of Oncology, Ljubljana, Slovenia

Introduction: Metastatic prostate cancer (PC) has recently been introduced as an independent criterion for germline $B R C A 1 / 2$ genetic testing. Clinical studies indicate that variants in other DNA-repair genes might also have therapeutic implications. The prevalence of germline pathogenic variants/likely pathogenic variants (PV/LPVs) in
BRCA1/2 and other DNA-repair genes in the Slovenian population of PC patients is unknown.

Methods: We performed a retrospective analysis of all PC patients referred to our institution for germline genetic testing since 2015. In 2018, we implemented genetic testing for systemic therapy decision making in metastatic PC patients. The majority (53/57) were tested using a multigene panel. In four cases Sanger sequencing was performed for known familial variants.

Results: Overall, we identified 40 patients with metastatic and 17 patients with localized PC. We detected a significant increase in referral rates with 8 patients $(14.0 \%)$ referred between 2015 and 2017, 11 (19.3\%) in 2018 and 38 $(66.7 \%)$ in 2019. PV/LPVs in 4 different DNA-repair genes were found in $17.5 \%(7 / 40)$ of metastatic PC patients (three in BRCA2, two in CHEK2, one in RAD51C, one in ATM). Two (11.8\%) patients with localized PC had PV/LPVs in two DNA-repair genes (one in BRCA1, one in MSH2).

Conclusions: Our preliminary results show a high prevalence of germline PV/LPVs in DNA-repair genes in Slovenian metastatic PC patients. Although not all detected variants are in known prostate-susceptibility genes, they might prove important in the future as new targeted therapy options become available, as well as for identifying other family members at risk.

K. Strojnik: None. M. Krajc: None. M. Banjac: None. V. Stegel: None. P. Skerl: None. V. Setrajcic Dragos: None. G. Klancar: None. S. Novakovic: None. A. Blatnik: None.

\section{P12.186.B}

Wide range of phenotypes in a family with PTEN germline mutation

\section{Henke, F. Stock, A. Bohring, U. Siebers-Renelt}

Institut für Humangenetik, Universitätsklinikum Münster, Münster, Germany

We report on a family with clustering of premenopausal breast cancer and other tumors. The first woman appearing in our outpatient clinic was diagnosed with breast cancer (bilateral DCIS) at the age of 26. Five more family members displaying breast cancer were identified (age spectrum 27 to 75 years at diagnosis). Criteria for hereditary breast and ovarian cancer were therefore fulfilled. A complete record of family history revealed several other diseases (clear cell renal cell carcinoma, melanoma, bilateral thyroid cancer, mucoepidermoid carcinoma of trachea, ovarian cancer, endometrium cancer, benign lesion of glandula parotis, gastrointestinal polyps (including hamartomas), polypoid lesion of pancreas, benign thyroid lesions and vascular malformations). Therefore, we included PTEN in the 
genetic testing in addition to the core genes associated with hereditary breast and ovarian cancer (e.g. BRCA1, BRCA2, $P A L B 2, C H E K 2, R A D 51 C$, RAD51D, ATM; true risk panel $\left.{ }^{\circledR}\right)$. Sequencing detected a germline deleterious variant (c.1003C $>$ T, p. $\left.\left(\operatorname{Arg} 335^{*}\right)\right)$ in PTEN which could also be found in three other family members. Molecular analysis in four further individuals is pending. Germline mutations in the PTEN tumor suppressor gene cause PTEN hamartoma tumor syndrome (PHTS) which is characterised by frequent occurrence of benign and malign lesions, especially an elevated lifetime risk for developing breast and thyroid cancer. This case underlines the variability of phenotypes associated with PHTS even within one family. It highlights the importance of a detailed family history and stresses the necessity of profound knowledge about rare tumor predisposition syndromes in breast cancer families.

C. Henke: None. F. Stock: None. A. Bohring: None. U. Siebers-Renelt: None.

\section{P12.187.C}

\section{Recognizing PTEN Hamartoma Tumour Syndrome by} only 3 simple criteria.

\section{N. Hoogerbrugge, J. Meek, J. Schieving, J. Schuurs, J. R. Vos}

Radboud university Medical Center, Nijmegen, Netherlands

Introduction: Patients with PTEN Hamartoma Tumour Syndrome (PHTS) have $80-100 \%$ risk to develop cancer, often in multiple organs and at early age. The majority $(80 \%)$ of PHTS patients is not yet identified as such and does not receive proper cancer risk management. We aim for easy-to-apply criteria - usable for almost every healthcare professional - to identify those at high risk of carrying a PTEN mutation in a phase prior to cancer development.

Methods: In a cohort of 176 paediatric and adult PHTS patients phenotypical characteristics were assessed by one of two expert clinicians. Prevalence of early-onset characteristics and their (combined) sensitivity to identify PHTS was determined for all patients and by age group $(0-12,12-$ $25,25+$ years)

Results: Macrocephaly is an early-onset characteristic that was present in $82 \%$ of all patients and in $100 \%$ of those below age $12(n=58)$. Multinodular Struma (MNS) development was detected in $97 \%$ of patients age $25+$. Multiple oral features (gingival hypertrophy, tongue and/or mucosal papillomas, or high palate) were detected in $94 \%$ of patients above age 25 .

Simplified criteria were formulated as the presence of two out of three of the following features: macrocephaly; MNS; and multiple oral features. In adult patients this has a sensitivity of $95 \%$ (95\% CI: 90-98\%).

Conclusions: Our simplified criteria, specifically the oral phenotype, could serve as an early trigger initiating the more elaborate assessment of patients suspected of a PTEN mutation. They can be assessed by most clinical specialists that see complex patients unknowingly presenting with PHTS.

N. Hoogerbrugge: None. J. Meek: None. J. Schieving: None. J. Schuurs: None. J.R. Vos: None.

\section{P12.188.A}

Renal cell carcinoma (RCC) predisposition genes: Who should be tested?

A. Andreou ${ }^{1,2}$, B. Yngvadottir ${ }^{1}$, L. Bassaganyas ${ }^{1}$, P. S. Smith $^{l}$, R. Barber ${ }^{3}$, K. Ong ${ }^{3}$, D. H. Lim ${ }^{3}$, Genomics England Research Consortium, RCC GeCIP Domain members, D. R. Mole $^{4}$, S. Turajilic ${ }^{5}, K$. Litchfield ${ }^{5}$, A. J.

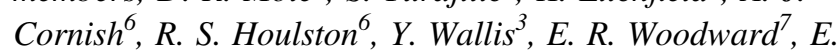
R. Maher ${ }^{1}$

${ }^{I}$ Department of Medical Genetics, University of Cambridge, Cambridge, United Kingdom, ${ }^{2}$ Cancer Research UK Cambridge Institute, Cambridge, United Kingdom, ${ }^{3}$ West Midlands Regional Genetics service, Birmingham Women's and Children's NHS Trust, Birmingham, United Kingdom, ${ }^{4}$ University of Oxford, Oxford, United Kingdom, ${ }^{5}$ Francis Crick Institute, London, United Kingdom, ${ }^{6}$ Institute of Cancer Research, London, United Kingdom, ${ }^{7}$ Manchester Centre for Genomic Medicine, St Mary's Hospital, Manchester University NHS Foundation Trust, Manchester, United Kingdom

Approximately 3\% of renal cell carcinoma (RCC) is thought to be inherited. Syndromic and non-syndromic forms have been described. It has been recommended that RCC patients with young age at onset ( $\leq 45$ years), multicentric/bilateral tumours, family history or with presence of syndromic clinical features should be referred for genetic assessment and testing (1).

Our aim was to determine the detection rate of a clinical 5 gene panel (FLCN, FH, VHL, MET, SDHB) of RCC predisposition genes. We retrospectively evaluated a referral-based cohort of non-syndromic RCC patients $(n=$ 262) and found a diagnostic yield of 2.7\% [7/262 pathogenic/likely pathogenic variants (P/LP)]. We then interrogated the unselected RCC cohort $(n=862)$ recruited to the 100,000 Genomes Project for germline P/LP variants in these genes and found a detection rate of $0.9 \%$ (8 P/LP variants). Five out of the $8 \mathrm{P} / \mathrm{LP}$ variants were seen in participants with early onset disease ( $\leq 45$ years). 
We then investigated whether P/LP variants might be detected in cancer predisposition genes (CPGs) less frequently tested using a virtual panel of 137 CPGs. Analysis is ongoing but to date additional P/LP variants have been identified in SDHA, MITF and CHEK2. The availability of comprehensive genomic analysis can better inform the genetic architecture of inherited RCC and refine clinical recommendations for diagnostic testing.

Reference:1. Reaume MN, Graham GE, Tomiak E, Kamel-Reid S, Jewett MA, Bjarnason GA, et al. Canadian guideline on genetic screening for hereditary renal cell cancers. Can Urol Assoc J. 2013;7(9-10):319-23.

A. Andreou: None. B. Yngvadottir: None. L. Bassaganyas: None. P.S. Smith: None. R. Barber: None. K. Ong: None. D.H. Lim: None. D.R. Mole: None. S. Turajilic: None. K. Litchfield: None. A.J. Cornish: None. R.S. Houlston: None. Y. Wallis: None. E.R. Woodward: None. E.R. Maher: None.

\section{P12.190.C}

The influence of the type of predisposing $R B 1$ variants on the incidence of malignancies

\section{P. Ketteler ${ }^{1,2}$, I. Hülsenbeck ${ }^{1}$, M. Frank ${ }^{3}$, B. Schmidt ${ }^{3}, K$. Jöckel ${ }^{3}$, D. R. Lohmann ${ }^{2}$}

${ }^{1}$ Department of Pediatrics 3, Essen, Germany, ${ }^{2}$ Institute of Human Genetics, Essen, Germany, ${ }^{3}$ Institute of Medical Informatics, Biometry and Epidemiology, Essen, Germany

Survivors of heritable retinoblastoma have a high risk to develop other malignancies later in life. The incidence of these second malignancies (SPMs) is significantly higher after external beam radiotherapy. We have tested if the incidence of SPM with and without previous irradiation is distinct depending on the type of the predisposing $R B 1$ gene variant as indicated by retinoblastoma penetrance. From 1940 to 2008, 655 national patients were treated for heritable retinoblastoma at the German referral center. Complete information on SPMs until 2012 and data on constitutional $R B 1$ variant was available for 317 patients (48.3\%). With respect to the type, predisposing $R B 1$ gene variants were classified by observed penetrance for retinoblastoma (incomplete and complete penetrance type variants). SPMs occurred in 51 of 317 survivors of heritable retinoblastoma. In non-irradiated retinoblastoma survivors, the incidence rate per 1,000 person years (IR) of SPMs was lower compared to the irradiated patients (IR $11.1[95 \%$ confidence interval 7.9-15.1] vs. IR 4.3 [2.1 -7.9]; $p<0.01$ ). The IR of SPMs was higher in patients with complete penetrance type variants compared to incomplete type variants $(p<0.05)$. Without previous irradiation, SPM were only observed in patients with complete penetrance type $R B 1$ variants. The association between the type of $R B 1$ variant and penetrance of retinoblastoma in childhood is also present with respect to the risk for SPMs later in life. This finding will help to develop screening programs tailored to the individual risk of survivors with heritable retinoblastoma. The Deutsche Kinderkrebsstiftung and the Wilhelm-Sander Stiftung financed this study.

P. Ketteler: None. I. Hülsenbeck: None. M. Frank: None. B. Schmidt: None. K. Jöckel: None. D.R. Lohmann: None.

\section{P12.191.A}

Comprehensive investigation of factors defining retroelement activity in colorectal cancer.

M. V. Saliutina, A. Y. Komkov, S. Z. Urazbakhtin, A. O. Smirnova, G. A. Nurmanov, Y. B. Lebedev, I. Z. Mamedov

Shemyakin-Ovchinnikov Institute of Bioorganic Chemistry of Russian Academy of Science, Moscow, Russian Federation

Introduction: Retroelement (RE) activity is one of the most important causes of human genome instability. Somatic insertions of L1 and Alu were detected in some cancer types (colorectal, lung, breast). Despite the abundance of data about the regulation of RE activity, the exact mechanisms of $\mathrm{RE}$ activation in particular cancer types are still unclear. In this study we investigate the correlation between RE insertional activity in colorectal cancer and other factors, such as gene expression, L1 transcription and methylation profiles of young L1 copies.

Materials and Methods: sequencing libraries of L1 and Alu flanking sequences were prepared from DNA isolated from paired normal and tumor tissue samples. Digested DNA was ligated to adapters containing unique molecular identifiers (UMI) and amplified with primers, specific to young RE subfamilies. The same DNA samples were used for investigation of methylation profile of young L1-copies by enzymatic based conversion of 5-mC with subsequent amplification and sequencing of L1Hs promoters. Total RNA was extracted from the same tissue samples and used for RNA-seq library preparation.

Results: Tumor-specific insertions were identified in 18 tumors from 55 analyzed normal/tumor paired samples. In these paired samples we also identified L1HS-copies with demethylated promoters. We characterized the differential expression of genes that influence RE activity and transcription activity of individual L1 copies.

Conclusions: In this work, we conducted a first systematic study of multiple factors that can influence RE 
transpositional activity in colorectal cancer. Funding: this work was supported by RSF18-14-00244 grant.

M.V. Saliutina: None. A.Y. Komkov: None. S.Z. Urazbakhtin: None. A.O. Smirnova: None. G.A. Nurmanov: None. Y.B. Lebedev: None. I.Z. Mamedov: None.

\section{P12.193.C}

Sporadic vestibular schwannoma: a molecular testing summary

K. V. Sadler ${ }^{l}$, N. L. Bowers ${ }^{l}$, C. Hartley ${ }^{I}$, P. T. Smith ${ }^{l}, S$. Tobi ${ }^{l}$, A. J. Wallace ${ }^{l}$, A. T. King ${ }^{2}$, S. K. W. Lloyd ${ }^{3}$, S. A. Rutherford $^{2}$, O. N. Pathmanaban ${ }^{2}$, C. Hammerbeck-Ward ${ }^{2}$, S. Freeman ${ }^{3}$, E. Stapleton ${ }^{3}$, A. Taylor ${ }^{4}$, A. Shaw ${ }^{5}$, D. Halliday $^{6}$, M. J. Smith ${ }^{l}$, D. G. Evans ${ }^{l}$

${ }^{1}$ Department of Genomic Medicine, St Mary's Hospital, Manchester Academic Health Sciences Centre (MAHSC), Division of Evolution and Genomic Science, University of Manchester, Manchester, United Kingdom, ${ }^{2}$ Department of Neurosurgery, and Neuroradiology Manchester Centre for Clinical Neurosciences, Salford Royal NHS Foundation Trust, Manchester Academic Health Sciences Centre (MAHSC), Manchester, United Kingdom, ${ }^{3}$ Department of Otolaryngology, Manchester Royal Infirmary, Manchester Academic Health Sciences Centre (MAHSC), University of Manchester, Manchester, United Kingdom, ${ }^{4}$ East Anglian Medical Genetics Service, Cambridge University Hospitals NHS Trust, Cambridge, United Kingdom, ${ }^{5}$ Department of Medical and Molecular Genetics, Guy's and St Thomas' NHS Foundation Trust, London, United Kingdom, ${ }^{6}$ Oxford Centre for Genomic Medicine, Nuffield Orthopaedic Centre, Oxford University Hospitals NHS Trust, Oxford, United Kingdom

Objectives: Cases of sporadic vestibular schwannoma (sVS) have a low rate of association with germline pathogenic variants. However, some individuals with sVS can represent undetected cases of neurofibromatosis type 2 (NF2) or schwannomatosis. Earlier identification of patients with these syndromes can facilitate more accurate familial risk prediction and prognosis.

Methods: Cases of sVS were ascertained from a local register at the Manchester Centre for Genomic Medicine. Genetic analysis was conducted in NF2 on blood samples for all patients, and tumour DNA samples when available. LZTRI and SMARCBI screening was also performed in patient subgroups.

Results: Age at genetic testing for VS presentation was young in comparison to previous literature, a bias resulting from updated genetic testing recommendations. Mosaic or constitutional germline NF2 variants were confirmed in $2 \%$ of patients. Pathogenic germline variants in LZTRl were found in $3 \%$ of all tested patients, with a higher rate of $5 \%$ in patients $<30$ years. No pathogenic SMARCBI variants were identified within the cohort. Considering all individuals who received tumour DNA analysis, $69 \%$ of patients were found to possess two somatic pathogenic NF2 variants, including those with germline LZTRI pathogenic variants, supporting the multi-hit mechanism of LZTRIassociated VS tumourigenesis.

Conclusions: Undiagnosed schwannoma predisposition may account for a significant proportion of apparently sVS cases, especially at lower presentation ages. Loss of NF2 function is a common event in VS tumours and may represent a targetable common pathway in VS tumourigenesis.

K.V. Sadler: None. N.L. Bowers: None. C. Hartley: None. P.T. Smith: None. S. Tobi: None. A.J. Wallace: None. A.T. King: None. S.K.W. Lloyd: None. S.A. Rutherford: None. O.N. Pathmanaban: None. C. Hammerbeck-Ward: None. S. Freeman: None. E. Stapleton: None. A. Taylor: None. A. Shaw: None. D. Halliday: None. M.J. Smith: None. D.G. Evans: None.

\section{P12.195.B}

POLE, POLD1 and RNF43 screening in serrated polyposis syndrome

Y. Soares de Lima ${ }^{l}$, C. Arnau-Collell ${ }^{1}$, M. Díaz-Gay ${ }^{1}, J$. Muñoz ${ }^{l}$, S. Carballal ${ }^{1}$, L. Bonjoch ${ }^{l}$, L. Moreira $^{l}, J$. Lozano $^{2}$, T. Ocaña ${ }^{1}$, M. Cuatrecasas ${ }^{3}$, A. Díaz de Bustamante $^{4}$, A. Castells ${ }^{1}$, L. Bujanda ${ }^{5}, J$. Cubiella ${ }^{6}$, D. Rodríguez-Alcalde $^{7}$, F. Balaguer ${ }^{1}$, C. Ruiz-Ponte ${ }^{8}, L$. Valle $^{9}$, V. Moreno ${ }^{10}$, S. Castellví-Bel ${ }^{l}$

${ }^{1}$ Gastroenterology Department, Institut d'Investigacions Biomèdiques August Pi i Sunyer (IDIBAPS), Centro de Investigación Biomédica en Red de Enfermedades Hepáticas y Digestivas (CIBERehd), Hospital Clínic, Barcelona, Spain, ${ }^{2}$ Bioinformatics Platform, Centro de Investigación Biomédica en Red de Enfermedades Hepáticas y Digestivas (CIBERehd), Barcelona, Spain, ${ }^{3}$ Pathology Department, Institut d'Investigacions Biomèdiques August Pi i Sunyer (IDIBAPS), Centro de Investigación Biomédica en Red de Enfermedades Hepáticas y Digestivas (CIBERehd) and Tumor Bank-Biobank, Hospital Clínic, Barcelona, Spain, ${ }^{4}$ Genetics Unit, Hospital Universitario de Móstoles, Madrid, Spain, ${ }^{5}$ Gastroenterology Department, Hospital Donostia-Instituto Biodonostia, Centro de Investigación Biomédica en Red de Enfermedades Hepáticas y Digestivas (CIBERehd), Basque Country University (UPV/EHU), San Sebastián, Spain, ${ }^{6}$ Gastroenterology Department, Complexo Hospitalario Universitario de Ourense, Instituto de Investigación Sanitaria Galicia Sur, Centro de 
Investigación Biomédica en Red de Enfermedades Hepáticas y Digestivas (CIBERehd), Ourense, Spain, ${ }^{7}$ Digestive Disease Section, Hospital Universitario de Móstoles, Madrid, Spain, ${ }^{8}$ Fundación Pública Galega de Medicina Xenómica, Grupo de Medicina Xenómica_USC, Instituto de Investigación Sanitaria de Santiago (IDIS), Centro de Investigación Biomédica en Red de Enfermedades Raras (CIBERER), Santiago de Compostela, Spain, ${ }^{9}$ Hereditary Cancer Program, Catalan Institute of Oncology, Oncobell, Institut d'Investigació Biomèdica de Bellvitge (IDIBELL), Centro de Investigación Biomédica en Red de Cáncer (CIBERONC), Barcelona, Spain, ${ }^{10}$ Unit of Biomarkers and Susceptibility, Cancer Prevention and Control Program, ICO, Institut d'Investigació Biomèdica de Bellvitge (IDIBELL), Centro de Investigación Biomédica en Red de Epidemiologia y Salud Pública (CIBERESP), University of Barcelona, Barcelona, Spain

Introduction: Serrated polyposis syndrome (SPS) is a condition characterized by large and multiple polyps and colorectal cancer (CRC) predisposition. RNF43 has been recently related to SPS, and POLE and POLDI to multiple polyposis predisposition. We aimed at screening for potentially pathogenic mutations in these genes in a SPS cohort.

Materials and methods: Initially, 39 SPS cases underwent whole-exome sequencing. Subsequently, a replication cohort of 211 SPS patients were analyzed in a custom panel containing POLE, POLDI and RNF43. Rare, potentially pathogenic missense and truncation variants were prioritized with an in-house bioinformatics pipeline.

Results: Ten missense variants were identified in POLE. Truncation variants were identified in POLE and POLDI and RNF43. To date, all variants are considered as of uncertain significance except for the one in RNF43 (p. $\operatorname{Arg} 132 *)$. We have started functional studies using CRISPR/Cas9 gene editing in cellular models for the frameshift $P O L D 1$ candidate variant (p.Lys648fs).

Conclusions: RNF43 variants seem to be a very rare contributor to germline SPS predisposition. POLE and POLDl variants may predispose in some cases to SPS although further functional assessment of truncating variants and missense variants outside the exonuclease domain of POLE and POLDI is compulsory to evaluate their impact in germline SPS predisposition.

Grant support: LCF/BQ/DI18/11660058(ID100010434) funded by Marie Skłodowska-Curie grant agreement no. 713673, FJCI-2017-32593, 2019FI_B2_00203, Fondo de Investigación Sanitaria/FEDER (16/01292, 17/00878), AECC (GCB13131592CAST), Spanish Ministry of Science (SAF2016-80888-R, BIO2017-89754-C2-2R), PERIS (SLT002/16/00398, SLT002/16/0037), Generalitat de Catalunya (GRPRE 2017SGR21, GRC 2017SGR653,
2017SGR723， 2017SGR861， 2017SGR1282), COST Action CA17118, CIBEREHD, CIBERONC and CIBERER.

Y. Soares de Lima: None. C. Arnau-Collell: None. M. Díaz-Gay: None. J. Muñoz: None. S. Carballal: None. L. Bonjoch: None. L. Moreira: None. J. Lozano: None. T. Ocaña: None. M. Cuatrecasas: None. A. Díaz de Bustamante: None. A. Castells: None. L. Bujanda: None. J. Cubiella: None. D. Rodríguez-Alcalde: None. F. Balaguer: None. C. Ruiz-Ponte: None. L. Valle: None. V. Moreno: None. S. Castellví-Bel: None.

\section{P12.196.C}

Colorectal cancer genetic variants are also associated with Serrated polyposis syndrome susceptibility

C. Arnau-Collell ${ }^{l}$, Y. Soares de Lima ${ }^{l}$, M. Díaz-Gay ${ }^{l}, J$. Muñoz $^{l}$, S. Carballal ${ }^{l}$, L. Bonjoch ${ }^{l}$, L. Moreira ${ }^{l}, J$. Lozano $^{2}$, T. Ocaña ${ }^{l}$, M. Cuatrecasas ${ }^{3}$, A. Díaz de Bustamante $^{4}$, A. Castells $^{1}$, G. Capellă ${ }^{5}$, L. Bujando ${ }^{6}, J$. Cubiella $^{7}$, D. Rodríguez-Alcalde ${ }^{8}, F$. Balaguer ${ }^{1}$, C. Ruiz-

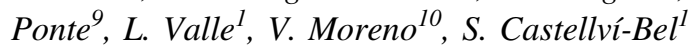

${ }^{I}$ Gastroenterology Department, Institut d'Investigacions Biomèdiques August Pi i Sunyer (IDIBAPS), Centro de Investigación Biomédica en Red de Enfermedades Hepáticas y Digestivas (CIBEREHD), Hospital Clínic, Barcelona, Spain, ${ }^{2}$ Bioinformatics Platform, Centro de Investigación Biomédica en Red de Enfermedades Hepáticas y Digestivas (CIBEREHD), Barcelona, Spain, ${ }^{3}$ Pathology Department, Institut d'Investigacions Biomèdiques August Pi i Sunyer (IDIBAPS), Centro de Investigación Biomédica en Red de Enfermedades Hepáticas y Digestivas (CIBEREHD) and Tumor Bank-Biobank, Hospital Clínic, Barcelona, Spain, ${ }^{4}$ Genetics Unit, Hospital Universitario de Móstoles, Madrid, Spain, ${ }^{5}$ Hereditary Cancer Program, Catalan Institute of Oncology, Oncobell, Institut d'Investigació Biomèdica de Bellvitge (IDIBELL), Centro de Investigación Biomédica en Red de Cáncer (CIBERONC), Barcelona, Spain, ${ }^{6}$ Gastroenterology Department, Hospital DonostiaInstituto Biodonostia, Centro de Investigación Biomédica en Red de Enfermedades Hepáticas y Digestivas (CIBEREHD), Basque Country University (UPV/EHU), San Sebastiàn, Spain, ${ }^{7}$ Gastroenterology Department, Complexo Hospitalario Universitario de Ourense, Instituto de Investigación Sanitaria Galicia Sur, Centro de Investigación Biomédica en Red de Enfermedades Hepáticas y Digestivas (CIBEREHD), Ourense, Spain, ${ }^{8}$ Digestive Disease Section, Hospital Universitario de Móstoles, Móstoles, Spain, ${ }^{9}$ Fundación Pública Galega de Medicina Xenómica, Grupo de Medicina Xenómica_USC, Instituto de Investigación Sanitaria de Santiago (IDIS), Centro de Investigación Biomédica en Red de Enfermedades Raras (CIBERER), 
Santiago de Compostela, Spain, ${ }^{10}$ Unit of Biomarkers and Susceptibility, Oncology Data Analytics Program, Catalan Institute of Oncology (ICO); Colorectal Cancer Group, ONCOBELL Program, Bellvitge Biomedical Research Institute (IDIBELL); CIBERESP, Barcelona, Spain

Background: Serrated polyposis syndrome (SPS) is a clinical entity characterized by multiple serrated polyps throughout the colon and increased risk for colorectal cancer (CRC). The basis for SPS genetic predisposition is largely unknown. Common, low-penetrance genetic variants have been consistently associated with CRC susceptibility, however, their role in SPS genetic predisposition has not been yet explored.

Objective: The aim of this study was to evaluate if common low-penetrance genetic variants for CRC risk are also implicated in SPS genetic susceptibility.

Methods: A case-control study was performed in 219 SPS patients and 548 asymptomatic controls analyzing 65 CRC susceptibility variants. A risk prediction model for SPS predisposition was developed.

Results: Statistically significant associations with SPS were found for 7 genetic variants (rs4779584-GREM1, rs16892766-EIF3H, rs3217810-CCND2, rs992157PNKD1/TMBIM1, rs704017-ZMIZ1, rs11196172-TCF7L2, rs6061231-LAMA5). The GREM1 risk allele was remarkably overrepresented in SPS cases compared to controls $(\mathrm{OR}=1.573,1.21-2.04, \quad P$-value $=0.0006) . \mathrm{A} 4$-fold increase in SPS risk was observed when comparing subjects within the highest decile of variants $(\geq 65)$ with those in the first decile $(\leq 50)$.

Conclusions: Genetic variants for CRC risk are also involved in SPS susceptibility, being the most relevant ones rs4779584-GREM1, rs16892766-EIF3H and rs3217810CCND2.

Grant support: FJCI-2017-32593, 2019FI_B2_00203, LCF/BQ/DI18/11660058, Fondo de Investigación Sanitaria/ FEDER (16/01292, 17/00878), AECC (GCB13131592CAST), Spanish Ministry of Science (SAF2016-80888-R, BIO2017-89754-C2-2R), PERIS (SLT002/16/00398, SLT002/16/0037), Generalitat de Catalunya (GRPRE 2017SGR21, GRC 2017SGR653, 2017SGR723, 2017SGR861, 2017SGR1282), COST Action CA17118, CIBEREHD, CIBERONC and CIBERER.

C. Arnau-Collell: None. Y. Soares de Lima: None. M. Díaz-Gay: None. J. Muñoz: None. S. Carballal: None. L. Bonjoch: None. L. Moreira: None. J. Lozano: None. T. Ocaña: None. M. Cuatrecasas: None. A. Díaz de Bustamante: None. A. Castells: None. G. Capellà: None. L. Bujando: None. J. Cubiella: None. D. RodríguezAlcalde: None. F. Balaguer: None. C. Ruiz-Ponte: None. L. Valle: None. V. Moreno: None. S. Castellví-Bel: None.
P12.199.C

Fluorescence in situ hybridization FISH for an accurate diagnosis of soft tissue sarcomas : single center experience

R. El koubaiti ${ }^{l}, A$. Mazti $^{2}$, M. Maaroufi ${ }^{3}$, M. El Idrissi ${ }^{4}, A$. El Ibrahimi ${ }^{4}$, A. El Mrini ${ }^{4}$, T. Bouhafa ${ }^{5}$, S. El Fakir ${ }^{6}, S$. Arifi $^{7}$, L. Chbani ${ }^{2}$

${ }^{I}$ Faculty of Medicine and Pharmacy, Sidi Mohamed Ben Abdallah University, Fez, Morocco, ${ }^{2}$ Pathological Anatomy and Molecular Pathology Department; Hassan II University Hospital, Fez, Morocco, ${ }^{3}$ Radiology department, Hassan II University Hospital, Fez, Morocco, ${ }^{4}$ TraumaOrthopedics 2 service, Hassan II University Hospital, Fez, Morocco, ${ }^{5}$ Radiotherapy department, Hassan II University Hospital, Fez, Morocco, ${ }^{6}$ Department of Epidemiology and Public Health, Hassan II University Hospital, Fez, Morocco, ${ }^{7}$ Medical oncology departement. Hassan II university Hospital. Medical school of University Sidi Mohamed Ben Abdallah, Fez, Morocco

Introduction: Soft tissue sarcomas (STS) are heterogeneous group of tumors. Pathological diagnosis relies on morphology and immunohistochemistry. It should be complemented by molecular technique, when the diagnosis is doubtful, and when it may have prognostic and/or predictive relevance. This study aims to assess the clinical effect of implementation of FISH to improve sarcoma misdiagnosis.

Materials and Methods: It is a retrospective study conducted in the pathology department of Hassan II university hospital between 2010 and 2018. 110 cases of mesenchymal tumors were included. HES coloration was performed in all cases followed by immunohistochemistry. FISH was performed in all cases. Probes used were Vysis break apart; EWSR1, MDM2, and SS18.

Results: The median age is 38 years [1-82]. On the basis of histology and immunohistochemical stains. There were 33 ewing sarcoma, 21 synovial sarcomas, 28 lipomas, 5 liposarcomas, and 23 unclassified sarcomas. FISH was successful in 97\%. EWSR1 gene was rearranged in $72 \%$ of cases. For synovial sarcomas the rearrangement of SS18 was detected in 71\%. MDM2 was amplified in $40 \%$ of atypical lipomatous tumors and dedifferentiated liposarcomas, whereas $93 \%$ of lipomas were negative, with 2 cases labelled as liposarcomas after FISH MDM2.

Conclusions: This is the first and largest moroccan series for molecular diagnosis of STS by FISH. It is important to implement this technique in a local laboratory to make it available at routine for moroccan patients. Our study shows that FISH improves sarcomas misdiagnosis, and it is very 
useful when histology and immunohistochemistry can't accurately classify tumors.

R. El koubaiti: None. A. Mazti: None. M. Maaroufi: None. M. El Idrissi: None. A. El Ibrahimi: None. A. El Mrini: None. T. Bouhafa: None. S. El Fakir: None. S. Arifi: None. L. Chbani: None.

\section{P12.201.B}

Elucidating the spliceogenic effect of variants in hereditary cancer genes: tools to prioritize RNA studies and challenges in applying ACMG guidelines

P. Rofes ${ }^{l}$, M. Menéndez ${ }^{l}, S$. González $z^{l}, E$. Tornero ${ }^{l}, C$. Gómez $^{2}$, G. Vargas ${ }^{l}$, E. Montes ${ }^{2}$, M. Salinas ${ }^{1}$, A. Solanes ${ }^{2}$, J. Brunet ${ }^{l}, A^{\prime}$. Teulél, G. Capellál, L. Feliubadalól, J. del Valle $^{l}$, M. Pineda ${ }^{l}$, C. Lázaro ${ }^{l}$

${ }^{1}$ Hereditary Cancer, Catalan Institute of Oncology ONCOBELL-IDIBELL, CIBERONC, Barcelona, Spain, ${ }^{2}$ Hereditary Cancer, Catalan Institute of Oncology ONCOBELLIDIBELL, Barcelona, Spain

Purpose: RNA analyses are a potent tool to identify spliceogenic effects of DNA variants, although they are timeconsuming and cannot always be performed. Here, we present splicing assays of 20 variants representing a variety of mutation types in ten hereditary cancer genes, together with an attempt to incorporate these results into ACMG classification guidelines.

Methods: We selected 16 point mutations, 3 exon duplications and one single-exon deletion prioritized by in silico algorithms. RNA was extracted from short-term lymphocyte cultures to perform RT-PCR and Sanger sequencing. Whenever possible, allele-specific expression was assessed by $\mathrm{SNaPshot}$.

Results: We detected aberrant transcripts in 14 variants (70\%). We faced the difficulty of variant interpretation comparing old classification standards to generic ACMG guidelines and devised a proposal to weight functional analyses at RNA level. According to ACMG guidelines only 12 were reclassified as pathogenic/likely pathogenic, since the remaining two did not gather enough evidence.

Conclusions: Our study highlights the importance of RNA studies to improve variant classification. However, it also evidences the challenge of incorporating these results into generic ACMG guidelines and the need to refine these criteria gene-specifically. Nevertheless, $60 \%$ of variants were reclassified, thus improving genetic counseling and surveillance for carriers of these variants.

Grant support Contract grant sponsor: Supported by the Carlos III National Health Institute funded by FEDER funds - a way to build Europe - [PI19/00553; PI16/00563; PI16/ 01898; SAF2015-68016-R and CIBERONC]; the
Government of Catalonia [Pla estratègic de recerca i innovació en salut (PERIS_MedPerCan and URDCat projects), 2017SGR1282 and 2017SGR496].

P. Rofes: None. M. Menéndez: None. S. González: None. E. Tornero: None. C. Gómez: None. G. Vargas: None. E. Montes: None. M. Salinas: None. A. Solanes: None. J. Brunet: None. Á. Teulé: None. G. Capellá: None. L. Feliubadaló: None. J. del Valle: None. M. Pineda: None. C. Lázaro: None.

\section{P12.202.C}

Transcript analysis in cancer predisposition genes for variants reinterpretation

A. Scandurra ${ }^{l}$, R. Pietrobono ${ }^{l}$, M. Pomponi ${ }^{2}, L$. Remondini $^{2}$, M. Colangelo ${ }^{2}$, A. Vaisfeld ${ }^{I}, A$. Panfili ${ }^{l}, F$.

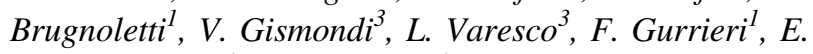
Lucci Cordisco ${ }^{I}$, M. Genuardi ${ }^{l}$

${ }^{I}$ Fondazione Policlinico Universitario A. Gemelli, IRCSS Università Cattolica del Sacro Cuore, Roma, Italy, ${ }^{2}$ Fondazione Policlinico Universitario A. Gemelli, IRCSS, Roma, Italy, ${ }^{3}$ Ospedale Policlinico San Martino, Genova, Italy

Introduction: Genetic testing has a pivotal role in the diagnosis of hereditary cancer predisposition syndromes. However, variant interpretation is still a challenging step and often the lack of functional studies leads clinicians to classify them as variants of uncertain significance (VUS). RNA functional studies can improve the characterization of variants thus allowing the implementation of personalized strategies in cancer patient care.

Materials and Methods: VUSs were analysed using the Human Splicing Finder (HSF) software, to predict the effect of intronic and exonic variants on splicing. Thereafter, RNA was extracted from short-term lymphocyte cultures (96h) from peripheral blood and /or from lymphoblastoid lines, both in presence and absence of puromycin to inhibit Nonsense Mediated Decay. cDNA was obtained and amplification products were analyzed by gel electrophoresis and Sanger sequencing to detect any abnormal splicing patterns.

Results and Discussion: A total of fourteen VUS, twelve intronic and two exonic, in the following genes have been analyzed: MSH2, MLH1, MSH6, APC, PMS2, MUTYH, PTEN, TP53, NF1, SPRED1. Ten of them affected exon incorporation as a consequence of intronic sequence changes. In the remaining four no differences were observed in transcript sequences in both untreated and puromycin-treated cultures.

Conclusions: Our study shows that $71 \%$ of the splicing variants analyzed by RNA sequencing can be reclassified as pathogenetic or likely pathogenetic. These preliminary data 
suggest that RNA analysis should be a part of the diagnostic workflow in patients with VUS in hereditary cancer predisposition genes in order to improve genetic testing accuracy and patients managment.

A. Scandurra: None. R. Pietrobono: None. M. Pomponi: None. L. Remondini: None. M. Colangelo: None. A. Vaisfeld: None. A. Panfili: None. F. Brugnoletti: None. V. Gismondi: None. L. Varesco: None. F. Gurrieri: None. E. Lucci Cordisco: None. M. Genuardi: None.

\section{P12.204.B}

Facile genome-wide detection of genomic fusions and other somatic abnormalities through Bionano Genome Imaging

\section{A. Hastie}

\section{Bionano Genomics, San Diego, CA, United States}

Genome instability is a key hallmark of cancer as it allows the genome to acquire growth advantage by amplifying or creating oncogenes by creating gene fusions and by deleting/inactivating tumor suppressor genes. Several gene fusions have been discovered and exploited for therapies as they produce fusion proteins that can be targeted by chemotherapeutics such as tyrosine-kinase inhibitors targeting BCR-ABL used to treat CML. In addition, other genomic fusions serve as diagnostic or prognostic indicators important for cancer management. Whole genome approaches to detect fusions have innate limitations, for example, karyotyping is low resolution and whole genome sequencing is blind in the $\sim 66 \%$ of the genome containing repeats. Bionano genome imaging is able fill the gap, it provides high resolution and ability to span genomic repeats.

Here we demonstrate an easy, fluid, largely automated workflow for whole genome detection of fusions and other somatic variants. The Bionano workflow involves a novel rapid isolation protocol for extremely high molecular weight DNA, labeling the DNA to create a barcode across the genome and automated data collection using the Bionano Saphyr instrument. This complete assay can be conducted within 4 days from tissue to variant calls. We detected numerous fusions that have not previously been detected. In a standard assay, whole genome imaging can detect variants at 5\% allele fraction and with increased coverage fusions down to $1 \%$ allele fraction can be efficiently detected! Whole genome optical mapping for fusion detection is surely going to play an increasing role in cancer research going forward.

A. Hastie: A. Employment (full or part-time); Significant; Bionano Genomics.
P12.206.A

Targeted RNA sequencing enables detection of relevant translocations, single nucleotide variants and automatic leukemia classification

B. Sikkema-Raddatz ${ }^{l}$, K. de Lange ${ }^{l}$, E. N. de Boer ${ }^{l}$, A. Bosga $^{l}$, M. Z. Alimohamed ${ }^{l}$, L. F. Johansson ${ }^{1,2}$, A. B.

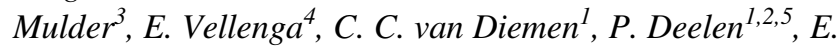
van den Berg ${ }^{1}$

${ }^{1}$ Department of Genetics, University Medical Center, Groningen, Netherlands, ${ }^{2}$ Genomics Coordination Center, University Medical Center, Groningen, Netherlands, ${ }^{3}$ Department of Laboratory Medicine, University Medical Center, Groningen, Netherlands, ${ }^{4}$ Department of Hematology, University Medical Center, Groningen, Netherlands, ${ }^{5}$ Department of Genetics, University Medical Centre, Utrecht, Netherlands

Background: Leukemia patients carry a wide range of chromosomal and molecular abnormalities which affect their prognosis and treatment options. Since no simple technique is available that detects all relevant abnormalities, the choice of techniques is depending on the referral reason. Therefore, we tested targeted transcriptome sequencing as a single platform to detect all relevant variants compared to current techniques.

Material and Methods: In a cohort of 136 primary diagnosed leukemia patients we performed RNA sequencing of 1,385 genes (TruSight RNA Pan-Cancer, Illumina). We used a decision tree based on principle component analysis of expression profiles to guide leukemia classification. Gene fusions were detected, and overexpression for the genes EVI1, CCND1 and BCL2 was quantified. SNV/ indels were analyzed in a virtual myeloid and lymphoid panel of 54 and 72 genes, respectively. Results were compared to current techniques.

Results: Compared to current techniques specificity for detecting relevant variants was $100 \%$. Two variants were missed:c.2447A $>\mathrm{T}$ in $K I T$ [qPCR at $10^{-4}$ ], and BCL2 overexpression with $\mathrm{t}(14 ; 18)(\mathrm{q} 32 ; \mathrm{q} 21)$ in $5 \%$ of the cells. With RNA sequencing six additional fusion genes and in two samples overexpression of $C C N D 1$, due to a $\mathrm{t}(11 ; 14)$ (q13;q32), were detected. We predicted leukemia classification correctly in $81 \%$ of patients and improved classification in three patients.

Conclusions: Targeted RNA sequencing combined with our workflow for data analysis is feasible and improves detection of relevant variants. Expression patterns can assist to establish the leukemia subtype. The diagnostic value of our novel approach will be tested in a large prognostic cohort. 
B. Sikkema-Raddatz: None. K. de Lange: None. E.N. de Boer: None. A. Bosga: None. M.Z. Alimohamed: None. L.F. Johansson: None. A.B. Mulder: None. E. Vellenga: None. C.C. van Diemen: None. P. Deelen: None. E. van den Berg: None.

\section{P12.208.C}

OGG1 maintains telomere stability under oxidative stress conditions

J. M. Baquero ${ }^{1}$, C. Benítez-Buelga ${ }^{2}$, R. Torres-Ruiz ${ }^{3,4}, S$. Rodríguez-Perales $^{3}$, T. Helleday ${ }^{2,5}$, J. Benítez ${ }^{1,6,7}$, A. Osorio $^{1,6}$

${ }^{1}$ Human Genetics Group, Human Cancer Genetics Programme, Spanish National Cancer Research Centre, Madrid, Spain, ${ }^{2}$ Science for Life Laboratory, Department of Oncology-Pathology, Karolinska Institutet, Solna, Sweden, ${ }^{3}$ Molecular Cytogenetics Group, Human Cancer Genetics Programme, Spanish National Cancer Research Centre, Madrid, Spain, ${ }^{4}$ Josep Carreras Leukemia Research Institute, Barcelona, Spain, ${ }^{5}$ Weston Park Cancer Centre, Sheffield, United Kingdom, ${ }^{6}$ Spanish Network on Rare Diseases, Madrid, Spain, ${ }^{7}$ Human GenotypingCEGEN Unit, Human Cancer Genetics Programme, Spanish National Cancer Research Centre, Madrid, Spain

Introduction: Oxidative stress disrupts telomere homeostasis triggering genome instability. The most common oxidative DNA lesion at the telomeres is 8-oxoguanine which is mainly removed by OGG1, initiating base excision repair pathway. Given that telomere maintenance is crucial for cancer progression, OGG1 inhibition could represent an attractive and unexplored opportunity for compromising telomere integrity in cancer cells.

Materials and Methods: We characterized OGG1 repair dynamics at telomeres in the U20S cancer cell line and we investigated the consequences at telomeres of either CRISPR-Cas9 mediated OGG1 depletion or pharmacological OGG1 inactivation with the recently developed OGG1 inhibitor TH5487.

Results: we found that OGG1 is recruited to the telomeres in response to oxidative stress. Besides, Upon induction of oxidative DNA damage, lack of OGG1 activity impairs BER at telomeres causing accumulation of oxidative DNA damage, telomere losses and consequently inducing mitotic and proliferation defects.

Conclusions: Our findings demonstrate that OGG1 is required to preserve telomere homeostasis under oxidative stress conditions in cancer cells and, present OGG1 inhibitors as a tool to induce oxidative DNA damage at telomeres, with potential implications in cancer and aging research.

Grants: J.M.B. is supported by grant FPU15/01978 from the Spanish Ministry of Education, Culture and Sport. A.O. is supported by grant FIS PI19/00640 supported by FEDER funds. JB's laboratory is partially funded by FIS PI16/ 00440 supported by FEDER funds, H2020 BRIDGES project and the Spanish Network on Rare Diseases (CIBERER).

J.M. Baquero: None. C. Benítez-Buelga: None. R. Torres-Ruiz: None. S. Rodríguez-Perales: None. T. Helleday: None. J. Benítez: None. A. Osorio: None.

P12.211.C

Identification of genetic events that cooperated with $R A S$ or PAX8-PPARG in the genesis of thyroid carcinoma

T. N. R. Carneiro ${ }^{1}$, L. Bim ${ }^{1}$, V. C. Buzzato ${ }^{2}$, V. Galdeno ${ }^{2}$, E. A. Lee $^{3}$, P. Galante ${ }^{2}$, J. Cerutti ${ }^{1}$

${ }^{1}$ Federal University of São Paulo, Department of Morphology and Genetics, São Paulo, Brazil, ${ }^{2}$ Centro de Oncologia Molecular, Hospital Sírio-Libanês, São Paulo, Brazil, ${ }^{3}$ Division of Genetics and Genomics, Boston Children's Hospital and Harvard Medical School, Boston, MA, United States

Introduction: $R A S$ point mutations and $P A X 8 / P P A R G$ fusion are the most frequent genetic alterations in benign and malignant thyroid tumours. The presence of these genetic alterations across a large spectrum of thyroid lesions suggests that they might be an early mutational event in a subset of indolent thyroid lesions that are prone to develop metastases and dedifferentiate. However, it is still not clear whether additional genetic events are needed to progress from a benign in situ to an invasive and more aggressive tumor phenotype.

Material and Methods: To identify new point mutations, fusions or alteration of gene expression that could cooperate with $R A S$ or $P A X 8 / P P A R G$, we performed and analyzed RNA-seq from 14 thyroid tumors samples harboring $R A S$ point mutation or PAX8/PPARG fusion. GRCh38 was used as reference genome. We additionally analyzed RNA-seq from 65 thyroid carcinomas positive for RAS or PAX8/ $P P A R G$ rearrangement available through The Cancer Genome Atlas Program (TCGA).

Results: Forty five percent of $R A S$ positive thyroid carcinomas showed exclusive mutations in genes that belong to the same pathway, which regulates important cellular processes as growth and division and recently has been associated with tumor progression. Additionally, the expression levels of coding genes that belong to this 
pathway were also altered in both our own and TCGA carcinoma samples evaluated in this study.

Conclusions: We here identified somatic mutations in genes that belong to the same pathway in a large fraction of $R A S$ positive cancer samples suggesting that they may cooperate with $R A S$ to drive thyroid tumor progression.

T.N.R. Carneiro: None. L. Bim: None. V.C. Buzzato: None. V. Galdeno: None. E.A. Lee: None. P. Galante: None. J. Cerutti: None.

\section{P12.212.A}

\section{Germline truncating variants in TINF2 cause cancer} through telomere length elongation

I. Schmutz ${ }^{1}$, A. Mensenkamp ${ }^{2}$, M. Haadsma ${ }^{2}$, L. Spruijt ${ }^{2}$, R. de Voer $^{2}$, T. de Lange ${ }^{1}$, M. Jongmans ${ }^{2,3,4}$

${ }^{1}$ Rockefeller University, New York, NY, United States, ${ }^{2}$ Radboud UMC, Nijmegen, Netherlands, ${ }^{3} U M C$ Utrecht, Utrecht, Netherlands, ${ }^{4}$ Princess Máxima Center for Pediatric oncology, Utrecht, Netherlands

Shelterin protects chromosome ends from the DNA damage response and controls telomere length homeostasis. Shelterin is composed of TRF1, TRF2, TIN2, Rap1, TPP1 and POT1. Within shelterin, TIN2 interacts with TRF1, TRF2, and TPP1. TIN2 contributes to telomere protection and is critical for limiting telomerase-mediated telomere elongation. We identified novel germline variants in exon 5 of TINF2 (encoding TIN2) in four probands affected by multiple malignancies. Three probands shared c. $604 \mathrm{G}>\mathrm{C}$, which results in alternative splicing causing early truncation of the protein. This variant was maternally inherited in two probands. The second variant, c.557del (p.(Ser186fs), causes a premature stop codon. These six individuals in this study developed 13 malignancies, including three papillary thyroid cancers and three breast cancers. No LOH or second hits in TINF 2 were observed in three tumors tested. The truncated TIN2 proteins encoded by c.604G $>C$ and c.557del do not interact with TRF1, a main factor in telomere length control. Consistent with loss of TRF1 binding, three patients had lymphocyte telomere lengths in the $99^{\text {th }}$ percentile. CRISPR/Cas9 edited RPE1 cells heterozygous for the TINF2 variants showed that the truncated TIN2 proteins do not cause telomere damage or genome instability but result in telomere elongation. The data establish that the TINF2 variants predispose to cancer through inappropriate telomere elongation and suggest that telomere shortening is a potent tumor suppressor pathway in multiple cancer types. We suggest that previously described cancer-predisposing POT1 variants also act through inducing telomere elongation rather than by generating genome instability.
I. Schmutz: None. A. Mensenkamp: None. M. Haadsma: None. L. Spruijt: None. R. de Voer: None. T. de Lange: None. M. Jongmans: None.

\section{P12.214.C}

ERN GENTURIS guidelines for the Li-Fraumeni and Heritable TP53-Related Cancer syndromes

\author{
T. Frebourg ${ }^{1}$, S. B. Lagercrantz ${ }^{2}$, C. Oliveira ${ }^{3}, R$. \\ Magenheim ${ }^{4}, D$. Evans $^{5}$, the European Reference Network \\ GENTURIS
}

${ }^{I}$ Department of Genetics, Rouen University Hospital and Inserm U1245, Normandie University, UNIROUEN, Normandy Centre for Genomic and Personalized Medicine, Rouen, France, ${ }^{2}$ Hereditary Cancer Unit, Department of Clinical Genetics, Karolinska University Hospital, Stockholm, Sweden, ${ }^{3} i 3 S$ Instituto de Investigação e Inovação em Saúde \& Institute of Molecular Pathology and Immunology of the University of Porto, and Porto Comprehensive Cancer Center, Porto, Portugal, ${ }^{4}$ Community Representative, Berlin, Germany, ${ }^{5}$ Manchester Centre for Genomic Medicine, Division of Evolution and Genomic Sciences, University of Manchester, MAHSC, St Mary's Hospital, Manchester University Hospitals NHS Foundation Trust, Manchester, United Kingdom

Fifty years after the recognition of the Li-Fraumeni syndrome (LFS), our perception of cancers related to germline alterations of TP53 has drastically changed: (i) germline TP53 alterations are often identified among children with cancers, in particular soft-tissue sarcomas, adrenocortical carcinomas, central nervous system tumours or among adult females with early breast cancers, without familial history. This justifies the expansion of the LFS concept to a wider cancer predisposition syndrome designated heritable TP53related cancer (hTP53rc) syndrome; (ii) the interpretation of germline TP53 variants remains challenging and should integrate epidemiological, phenotypical, bioinformatics prediction and functional data; (iii) the penetrance of germline disease-causing TP53 variants is variable, depending both on the type of variant (dominant-negative variants being associated with a higher cancer risk) and on modifying factors; (iv) whole-body MRI (WBMRI) allows early detection of tumours in variant carriers and (v) in cancer patients with germline disease-causing TP53 variants, radiotherapy and conventional genotoxic chemotherapy contribute to the development of subsequent primary tumours. It is critical to perform TP53 testing before the initiation of treatment in order to avoid in carriers, if possible, radiotherapy and genotoxic chemotherapies. In children, the recommendations are to perform clinical examination and abdominal ultrasound every 6 months, 
annual WBMRI and brain MRI from the first year of life, if the TP53 variant is known to be associated with childhood cancers. In adults, the surveillance should include every year clinical examination, WBMRI, breast MRI in females from 20 until 65 years and brain MRI until 50 years.

T. Frebourg: None. S.B. Lagercrantz: None. C. Oliveira: None. R. Magenheim: None. D. Evans: None.

\section{P12.215.A}

Higher TP53 somatic mutation prevalence from liquid biopsy analysis in smoker non-small-cell lung cancer patients

\section{H. Erdem, T. Bahsi}

Department of Medical Genetics, University of Health Sciences, Dr. Abdurrahman Yurtaslan Ankara Oncology Training and Research Hospital, Ankara, Turkey

Introduction: Non-small-cell lung cancer constitutes $80 \%$ part of all lung cancers. TP53 is a tumor suppressor gene in the DNA damage pathway, and also is the most frequently mutated gene in various malignancies. According to the AACR Project GENIE Consortium, TP53 mutations present in $41.88 \%$ of all non-small-cell lung carcinoma patients. Due to the many carcinogens in tobacco smoke, smokingrelated cancers have a high genome-wide burden of mutations. To support this theory, TP53 mutation prevalence of smoker and non-smoker non-small-cell lung cancer patients were compared with liquid biopsy.

Materials and Methods: Totally, circulating tumor DNA of 82 smoker non-small-cell lung cancer patients and 51 never-smoker non-small-cell lung cancer patients were performed using commercially available liquid biopsy platforms. TP53 gene hotspot regions were analyzed. Variants were evaluated according to AMP/ASCO/CAP classification guidelines. Only Tier I and Tier II variants were reported.

Results: TP53 mutations were described in 41/133 $(30.8 \%)$ of the samples. In never-smokers $12 / 51$ (23.5\%) patients and in smokers, 30/82 (36.5\%) patients carry a TP53 mutation. Moreover, the prevalence of truncating mutations was higher in smokers $8 / 30(26.6 \%)$ compared to non-smokers $2 / 16(16.6 \%)$.

Conclusions: Tobacco exposure causes a heavy burden of genomic alterations in TP53 at somatic level in nonsmall-cell lung cancer. The higher incidence of truncating mutations in smokers shows that the damaging effect of the resulting mutations is higher in smokers than neversmokers. In future research, clinical outcomes and detection of drug sensitivity/resistance status of this study group in different histological subtypes will contribute to personalized therapy studies.
H. Erdem: None. T. Bahsi: None.

P12.216.B

PTEN transcript analysis in 10 unrelated patients with suspected but unexplained Cowden syndrome

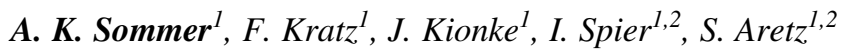

${ }^{1}$ Institute of Human Genetics, University Hospital Bonn, Bonn, Germany, ${ }^{2}$ Center for Hereditary Tumor Syndromes, University Hospital Bonn, Bonn, Germany

Introduction: Cowden syndrome (CS) is the adult onset subtype of the PTEN hamartoma tumor syndrome and a cancer predisposition syndrome, primarily linked to germline PTEN mutations. However, in $30-40 \%$ of patients with suspected CS, no causative PTEN mutation can be identified in routine diagnostics. In particular, pathogenic variants located outside the coding regions remain undetected.

Material and Methods: To uncover deep intronic PTEN mutations, ten clinically well characterized, unrelated patients with suspected CS and missing PTEN mutation were tested for aberrant splicing by cDNA analysis. In addition, three patients with suspected splice variants (PTEN:c.209+5G>A, PTEN:c.634+5G>A, PTEN:c.210$12 \mathrm{C}>\mathrm{G})$ found in routine diagnostic were analyzed. The coding exons 1-9 of PTEN were amplified in three overlapping fragments using specific primers. Amplification products were separated by gel-electrophoresis and sequenced in both directions. Deviant patterns were excised, eluted, reamplified, and sequenced.

Results: In the three patients with an intronic variant, the pathogenicity could be confirmed: In two patients, an exon deletion was found, and in one patient an insertion of $11 \mathrm{bp}$ could be revealed. All unexplained patients did not show aberrant patterns on the gel-image. Currently, sequencing of the PCR products is being performed to uncover cryptic splice variants resulting in short aberrations.

Conclusions: Rare, pathogenic deep intronic germline mutations have already been described in several hereditary tumor syndromes. However, systematic screening of a small cohort of unexplained CS patients did not detect aberrant splicing so far. In contrast, suspected splice variants in three patients could be confirmed by the transcript analysis.

A.K. Sommer: None. F. Kratz: None. J. Kionke: None. I. Spier: None. S. Aretz: None.

P12.217.C

Translocation $t(14 ; 16)(q 32 ; q 23)$ in patients with multiple myeloma (MM) 
L. Pavlistova ${ }^{l}$, A. Berkova ${ }^{l}, K$. Svobodova $^{I}$, S. Izakova ${ }^{l}$, I.

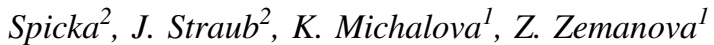

${ }^{1}$ Center of Oncocytogenomics, General University Hospital and First Faculty of Medicine, Charles University in Prague, Prague 2, Czech Republic, ${ }^{2} 1$ st Medical Department-Department of Hematology, General University Hospital and First Faculty of Medicine, Charles University in Prague, Prague 2, Czech Republic

Introduction: Translocation $\mathrm{t}(14 ; 16)(\mathrm{q} 32 ; \mathrm{q} 23)$ is rare chromosomal aberration (CA) detected in $2-4 \%$ patients with multiple myeloma (MM). Currently, it is included in prognostic scoring system as high-risk aberration. However, the prognostic value of $t(14 ; 16)$ remains controversial as it was not confirmed in several MM series. Thus, further studies are needed to verify its true clinical relevance. The aim of this study was to assess the frequency and prognostic impact of $t(14 ; 16)$ in our cohort of patients.

Material and Methods: During 2004-2019 we examined bone marrow samples of $1100 \mathrm{MM}$ patients using FISH on immunofluorescently labeled plasma cells (cIg FISH). t $(14 ; 16)$ was verified with dual fusion probe (Abbott). Kaplan-Maier analysis was performed to evaluate the overal (OS) and event-free (EFS) survival of IGH/MAF positive cases.

Results: $\mathrm{t}(14 ; 16)$ was detected in 26/1100 patients $(2,4 \%)$. The atypical FISH pattern of fusion signals and numerical changes of $14 \mathrm{q} 32$ and $16 \mathrm{q} 23$ regions were detected in 8 and 10 cases, respectively. In 17 patients (65\%), two or more additional CA were observed. $\mathrm{t}(14 ; 16)$ with another high-risk CA - deletion of TP53 - was found in four patients, three of them died within the short period from the diagnosis (9-15 months). We proved the significantly shorter OS in $\mathrm{t}(14 ; 16)$ positive cases $(p=0,1)$, for EFS the difference was not significant.

Conclusions: In this study, the frequency of $t(14 ; 16)$ was consistent with published data and our results support its negative prognostic impact. Combination with other CA and variability of FISH pattern will be discussed. Supported by RVO-64165 and Progres Q28.

L. Pavlistova: None. A. Berkova: None. K. Svobodova: None. S. Izakova: None. I. Spicka: None. J. Straub: None. K. Michalova: None. Z. Zemanova: None.

\section{P12.218.A}

Multigene panel testing of triple negative breast cancer patients from Russia
I. Abramov ${ }^{1}$, A. Ikonnikova ${ }^{1}, Y$. Korneva $^{2}$, O. Shisterova ${ }^{3}$, M. Emelyanova ${ }^{l}$, T. Nasedkina ${ }^{l}$

${ }^{I}$ Engelhardt Institute of Molecular Biology RAS, Moscow, Russian Federation, ${ }^{2}$ Smolensk State Medical University, Smolensk, Russian Federation, ${ }^{3}$ Smolensk regional clinical oncologic dispensary, Smolensk, Russian Federation

Introduction: Triple-negative breast cancer is characterized by lack of expression of estrogen, progesterone, and HER2 receptors. It is highly aggressive disease, more common occurring in younger women and is associated with hereditary forms of breast cancer caused by pathogenic mutations, mostly in the BRCA1/2 genes.

Methods: In the present study, we used next-generation sequencing (NGS) to analyze mutations in FFPE (formalin fixed embedded) tissues samples of 12 patients with triplenegative breast cancer (mean agewas 50,8 years). Target enrichment with coding sequences of APC, ATM, BRCA1, BRCA2, CDH1, CDH3, CDK4, CHEK2, MET, MLH1, MSH2, MSH6, MUTYH, NBN, PALB2, PMS2, PTEN, RET, STK11, TP53, VHL genes was done using Nimblegen (Roche) custom panel of oligonucleotide probes. The sequencing was performed on MiSeq Illumina platform with coverage up to1000x.

Results: Both germline and somatic mutations in tumor cells have been identified comparing sequencing results from paired tumor and normal tissue samples. Two pathogenic germline mutations were found in the BRCA1 gene (185delAG,3347delAG), mutations in the BRCA2 (886delGT) and TP53(c.733G $>$ A) genes. Pathogenic somatic mutations were found in the MUTYH (c.734G > A), PTEN (c.640C >T) and TP53 (c.637C > T) genes. The genotyping results were compared with clinical data.

Conclusion: Triple-negative breast cancer is a heterogeneous group of diseases. Knowledge of genetic profiles and identification of predictive biomarkersis important for further improvement of therapy results. The work was supported by the Russian Ministry of Science and High Education (grant\# 05.604.21.0234, unique number RFMEFI60419X0234).

I. Abramov: None. A. Ikonnikova: None. Y. Korneva: None. O. Shisterova: None. M. Emelyanova: None. T. Nasedkina: None.

P12.219.B

Rapid isolation of high quality ultra-high molecular weight (UHMW) genomic DNA (gDNA) from blood, bone marrow aspirates (BMAs)and fresh frozen tumors 
H. B. Sadowski, Y. Zhang, K. Pham, H. Way, M. Oldakowski

\section{Bionano Genomics, San Diego, CA, United States}

Optical mapping with Bionano Genome imaging is a powerful tool for comprehensive detection of structural variations (SVs) in cancer genomes. A crucial step in this workflow is isolating intact UHMW gDNA. For routine study of leukemic samples, we developed Bionano Prep SP kits for rapid, solution phase, isolation of UHMW gDNA from blood and BMAs. We also developed a rare variant bioinformatic pipeline that detects SVs at $5 \%$ allele fraction. Together, these can potentially replace a suite of "classical" cytogenetic tests for leukemia analysis (karyotyping, FISH, CNV microarrays) with a single Bionano Genome Imaging analysis. For solid tumors, we developed a novel protocol to isolate UHMW gDNA from small amounts $(\leq 10 \mathrm{mg})$ of fresh frozen tissues. Homogenized tissues are processed with the Bionano Prep SP kit, where lysis is coupled with purification steps to bind, wash and elute UHMW gDNA. With this protocol, a batch of 8 samples can be processed in $<6 \mathrm{~h}$. Eluted UHMW gDNA (3-19 $\mu$ g DNA/10 mg) is compatible with Direct Label and Stain protocol. When run on a Saphyr Chip, the purified gDNA gives high throughputs $(>1.3 \mathrm{Tbp} /$ sample in 24h) with excellent single molecule metrics of DNA size (N50>150 kbp of 260-300 kbp) and mapping rate (80-92\%), sufficient for use in SV analysis of cancer genomes from solid tumors. This protocol has been tested on fresh frozen human tumors including liver, lung, kidney, bladder, brain, colon, thyroid, prostate, breast, and ovary. A comprehensive analysis of SVs in these tumors will be presented.

H.B. Sadowski: A. Employment (full or part-time); Significant; BioNano Genomics. Y. Zhang: A. Employment (full or part-time); Significant; BioNano Genomics. K. Pham: A. Employment (full or part-time); Significant; BioNano Genomics. H. Way: A. Employment (full or parttime); Significant; BioNano Genomics. M. Oldakowski: A. Employment (full or part-time); Significant; BioNano Genomics.

\section{P12.221.A}

Germline loss-of-function variants in $M B D 4$ are rare in Finnish patients with uveal melanoma

\section{P. E. Repo ${ }^{1,2}$, J. E. Jänttil ${ }^{1}$, R. Järvinen ${ }^{1,2}$, M. Täll ${ }^{2}, V$. Raivio $^{2}$, T. T. Kivelä ${ }^{2}$, J. A. Turunen ${ }^{2,1}$}

${ }^{1}$ Folkhälsan Research Center, Helsinki, Finland, ${ }^{2}$ Department of Ophthalmology, University of Helsinki and Helsinki University Hospital, Helsinki, Finland
Introduction: Uveal melanoma (UM) is the most common primary intraocular cancer in adults. Metastases develop in approximately $50 \%$ of patients with poor prognosis regardless of treatment. UM tumors generally acquire unusually few somatic mutations. Eight genes are recognized as drivers of its tumorigenesis, typically consisting of one mutually exclusive mutation in GNAQ, GNA11, PLCB4 or CYSLTR2 followed by one mutually exclusive mutation in EIFIAX, SF3B1, $S R S F 2$ or $B A P 1$. Germline variants that predispose to UM have been described in $B A P 1$. Recently, germline loss-offunction (LOF) variants in the methyl-CpG binding domain-4 (MBD4) gene were found in four patients with UM. Loss of functional MBD4 induces a hypermutated profile, making tumors susceptible to immune checkpoint inhibitors. We conducted a population based screening of MBD4 in Finnish patients with UM to identify pathogenic variants.

Materials and Methods: Sanger sequencing of MBD4 in 440 Finnish patients with UM.

Results: We identified eight exonic missense variants in 51 (12\%) patients. Only one is likely to alter MBD4 function. We found no LOF variants (95\% confidence interval [CI], $0.0-0.8 \%$ ). Frequency of LOF variants in the general Finnish population is $0.052 \%$ in gnomAD, suggesting one might occur by chance in one in 2000 patients with UM.

Conclusions: Germline variants altering MBD4 function do not seem to predispose to UM in Finland. Somatic biallelic loss of MBD4 might modify the mutational burden in UM and change its response to immune checkpoint inhibitors.

Funding: The Helsinki University Hospital Research Fund; the Cancer Foundation; the Sigrid Jusélius Foundation; the Eye Foundation.

P.E. Repo: None. J.E. Jäntti: None. R. Järvinen: None. M. Täll: None. V. Raivio: None. T.T. Kivelä: D. Speakers Bureau/Honoraria (speakers bureau, symposia, and expert witness); Modest; Santen Finland. J.A. Turunen: D. Speakers Bureau/Honoraria (speakers bureau, symposia, and expert witness); Modest; Thea, Finland, Blueprint Genetics, Finland. F. Consultant/Advisory Board; Modest; Novartis, Finland.

P12.223.C

Ocular phenotype of retinal haemangioblastomas correlated to von Hippel-Lindau tumor suppressor genotype: mutations and functional domains

A. Hajjaj ${ }^{l}$, K. A. van Overdam ${ }^{2}$, R. A. Oldenburg ${ }^{l}$, E. Brosens $^{l}$, A. M. W. van den Ouweland ${ }^{l}$, A. de Klein ${ }^{l}$, E. Kilic $^{l}$

${ }^{1}$ Erasmus MC, Rotterdam, Netherlands, ${ }^{2}$ The Rotterdam Eye Hospital, Rotterdam, Netherlands 
Introduction: von Hippel-Lindau (VHL) disease is a familial disorder characterized by a predisposition to develop retinal haemangioblastomas $(\mathrm{RH})$ among other tumours. Phenotypic heterogeneity of this ocular tumours has led to a difficulty in the management of RH. We aimed to correlate the different ocular phenotypes in VHL patients with structural VHL variants in different functional domains.

Materials and Methods: Retrospective analysis of a longitudinal cohort of $33 \mathrm{VHL}$ germline mutation carriers and RH. Clinical and genetic data was obtained to analyse the correlation of genotype with phenotype.

Results: VHL patients were categorised in genotypic categories, based on the presumed effect on the VHL protein. Interface A is important for VCB-complex formation, interface B forms the HIF- $\alpha$ binding site, and interface $\mathrm{C}$ plays a role in the Cullin-2 interaction. Twelve patients had a mutation that corresponds with interface $\mathrm{C}, 11$ patients in interface $\mathrm{A}$, and 9 patients in interface $\mathrm{B}$. One patient had a whole gene deletion. An aggressive ocular phenotype was most observed in patients with mutations in interface C (75\%), followed by interface B (44.4\%) and interface $\mathrm{A}(18.2 \%)$. The highest number of amino acid substitutions were present in the interface $\mathrm{C}$ domain, where $50 \%$ of the variants were missense mutations.

Conclusions: Variants in the domain that corresponds with the interaction with Cullin-2 are more often missense mutations and had a higher prevalence of an aggressive ocular phenotype of $\mathrm{RH}$ in this study. The scaffold protein Cullin-2 seems to play a more important role in the progression of $\mathrm{RH}$.

A. Hajjaj: None. K.A. van Overdam: None. R.A. Oldenburg: None. E. Brosens: None. A.M.W. van den Ouweland: None. A. de Klein: None. E. Kilic: None.

\section{P12.225.B}

Whole genome sequencing reveals high variability of genetic risk variants associated with hereditary cancer

N. Danial-Farran, M. Khayat, C. Gafni, L. Peretz Peled, E. Mamlook, E. Chervinsky, S. A. Shalev

Genetics, Ha'Emek Medical Center, Afula, Israel

While germ-line mutations in BRCA1 and BRCA2 genes account for the major risk factors for breast cancer in the Israeli populations due to high frequencies of founder variants in the Ashkenazi families, many Ashkenazi and nonAshkenazi families from Northern Israel with strong family history remain undiagnosed. We used Whole genome sequencing (WGS) data generated from 15 blood samples to analyze 15 such families. The genetic variants were detected and analyzed using Variantyx Genomic Intelligence platform, version 2.8.0.0. We identified the first $\mathrm{CNV}$ in HMMR gene (chr5:163,475,593-163,476,336) that includes a partial deletion of exon and intron 11. In addition, we identified an SNV located in mir-153 seed side of the 3, UTR region of $A K T 1$ gene, c.*437c $>\mathrm{T}$, which was previously found to associate with lung cancer. In other four families, we detected novel nonsynonymous variants with high potential of pathogenicity due to their low frequencies in the general population and conserved residue in the protein levels: c. $1205 \mathrm{~A}>\mathrm{G}$ in $C H E K 2$ gene, c. $1283 \mathrm{C}>\mathrm{G}$ in STK11 gene, c. $3683 \mathrm{~A}>\mathrm{G}$ in $B R C A 1$ gene and c. $128 \mathrm{G}>\mathrm{A}$ in $P H B$ gene. In other 9 families, no suspected variants were found. Further studies are needed to confirm the pathogenicity of the detected variants, but our study demonstrates the vast variability of genetic causes for hereditary cancer in our population, the complexity of the mechanisms in familial cancer, and the challenge of detecting those mutations for better clinical approach and genetic counseling purposes. Li Ka Shing Foundation Grant for Joint Research Program between Shantou University and 2023476Technion-Israel Institute of Technology

N. Danial-Farran: None. M. Khayat: None. C. Gafni: None. L. Peretz Peled: None. E. Mamlook: None. E. Chervinsky: None. S. A. Shalev: None.

P12.227.A

"Mapping of Wnt-Frizzled interactions through mRNA coexpression patterns in breast and colorectal cancer"

A. Zougros ${ }^{1}$, M. Michelli ${ }^{1}$, I. Chatziandreou ${ }^{1}, N$. Michalopoulos $^{2}$, A. C. Lazaris $^{1}$, A. A. Saetta ${ }^{1}$

${ }^{1} 1$ st Department of Pathology, School of Medicine, National and Kapodistrian University of Athens, Athens, Greece, ${ }^{2}$ Department of Surgery, Attikon Hospital, School of Medicine, National and Kapodistrian University of Athens, Athens, Greece

Introduction: Wnt pathway regulates important cell functions and is frequently deregulated in breast and colorectal cancer. Wnt signaling is mediated through the interaction of 19 Wnt ligands and 10 Frizzled receptors; however the specificity of their interaction and their clinical significance remains largely unknown.

Materials and Methods: WNT2, WNT3, WNT5A, FZD4, FZD6 and FZD7 mRNA expression levels in 102 colorectal and 82 breast tumours were determined by RT-PCR and the $\Delta \Delta \mathrm{Ct}$ method. Possible correlations of Wnt pathway components' expression levels with clinicopathological data were investigated.

Results: In breast carcinomas, decreased relative mRNA expression levels of WNT2, WNT5A, FZD4, FZD6 and FZD7 were found in 54\%, 57\%, 80\%, $48 \%$ and $82 \%$ of 
carcinomas, respectively. Furthermore, increased relative mRNA expression levels were observed in WNT3 in 54\% of the cases. Expression levels of WNT3 and FZD6 were significantly correlated with tumour grade $(p=0,035)$ and pT category of tumours $(p=0,011)$, respectively. Regarding colorectal carcinomas, increased relative mRNA expression levels of FZD7, WNT5A, FZD4 and FZD6 ranged from $25-40 \%$ of the samples and WNT2, WNT3 levels were determined at $59 \%$ and $60 \%$ respectively. No clinicopathological correlation was found in colorectal cancer. Positive linear correlations were established among several of the investigated genes in both types of cancer.

Conclusions: Deregulated expression of the investigated genes was found in both breast and colorectal cancer. The linear correlations determined among Wnts and Frizzleds are indicative of their functional interaction and could point out the complexity that characterizes the Wnt-Frizzled interactions in breast and colon carcinogenesis.

A. Zougros: None. M. Michelli: None. I. Chatziandreou: None. N. Michalopoulos: None. A.C. Lazaris: None. A.A. Saetta: None.

\section{P13 Genome Variation and Architecture}

\section{P13.02.A}

22q11.2 copy number variations in patients with orofacial cleft defects with or without congenital heart disease

\section{M. Cardenas-Nieto ${ }^{\text {I }}$ J. Martinez-Lozano ${ }^{2}$, I. Briceño ${ }^{2}$, M. Forero-Castrol}

${ }^{1}$ Escuela de Ciencias Biológicas, Grupo de investigación en Ciencias Biomédicas (GICBUPTC), Universidad Pedagógica y Tecnológica de Colombia, Tunja, Colombia, Tunja, Colombia, ${ }^{2}$ Facultad de Medicina, Grupo de investigación en Genética Humana, Universidad de La Sabana, Chía, Colombia, Bogota, Colombia

Introduction: Cleft lip and/or palate (CL/P) and congenital heart disease (CHD) are the most common malformations observed in newborns. $15-20 \%$ of patients with CL/P and CHD can carry some type of chromosome abnormality. Between 5 to $8 \%$ of CL/P cases and 75 to $80 \%$ of CHD cases present the cryptic chromosomal microdeletion 22q11.2. Herein, we identified the genetic abnormalities in patients with CL/P and CHD using cytogenetic and MLPA analysis.

Materials and Methods: 14 patients with CL/P and CHD from Operation Smile Foundation (Bogotá-Colombia) were included in this study. G-banded chromosome analysis and 22q11 region was studied using MLPA (Kit P250-B2) and/or FISH (TUPLE1-HIRA LSR ARSA) techniques. Clinical parameters were evaluated in all patients.

Results: Patients' age ranged 2-20 years (median 7 years). $28.6 \%$ of the patients showed CP and $71.4 \%$ CLP, the main CHD observed were: tricuspid valve insufficiency $(42.9 \%)$, atrial septum defects $(42.9 \%)$ and ventricular septum defects $(35.7 \%)$. Other phenotypic traits included abnormalities in male genital organs $(66.6 \%)$, alterations in bone tissue $(57.2 \%)$, abnormalities in the teeth $(50 \%)$, delayed language development (42.9\%), and mental disorders $(100 \%)$. All patients had normal karyotype. One male patient (20 years) carried the 22q11.2 microdeletion, and another female patient (19 years) carried the microduplication of TOP3B gene located on 22q11.2 region. Both cases had CL/P and CHD including persistent arterial ductus, and congenital mitral valve abnormalities and bivalve aorta, respectively.

Conclusions: Screening of 22q11.2 alterations in patients with CL/P and CHD would be very helpful in their early diagnosis, treatment, and genetic counseling.

D.M. Cardenas-Nieto: None. J. Martinez-Lozano: None. I. Briceño: None. M. Forero-Castro: None.

\section{P13.03.B}

Investigation of indel variants in candidate-gene panel for 22q11.2 deletion syndrome

N. Nunes ${ }^{l}$, A. G. Dantas ${ }^{l}$, M. Zamariolli, ${ }^{l}$ D. C. Q.

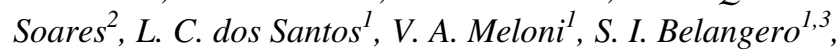
V. L. Gil-Da-Silva-Lopes ${ }^{4}$, C. A. Kim ${ }^{2}$, M. I. Melaragno ${ }^{1}$

${ }^{1}$ Universidade Federal de São Paulo - UNIFESP, São Paulo, Brazil, ${ }^{2}$ Instituto da Criança, Universidade de São Paulo - USP, São Paulo, Brazil, ${ }^{3}$ Laboratory of Integrative Neuroscience, UNIFESP, São Paulo, Brazil, ${ }^{4}$ Faculdade de Ciências Médicas, Universidade Estadual de Campinas, Campinas, Brazil

Introduction: The 22q11.2 deletion syndrome (22q11.2DS) results from hemizygous deletions of chromosome 22 segments and is the most frequent microdeletion syndrome observed in humans. The 22q11.2 region involved in the deletion is quite complex and contains highly repetitive low copy repeats (LCR), DNA sequences that make the region susceptible to recurrent genomic rearrangements. Our studies, as well as others in the literature, indicate that $22 \mathrm{q} 11.2$ deletion syndrome results in a wide variety of heterogeneous clinical signs, such as congenital cardiac malformations, characteristic facial features, immune deficiency, and psychiatric disorders. One of the hypotheses lies in the fact that allelic variation, such as indels, within the non-deleted 22q11.2 allele or in other 
genes outside the $22 \mathrm{q} 11.2$ region could influence the phenotype outcome of the 22q11.2DS.

Materials and Methods: We performed Ion Ampliseq in peripheral blood from $6022 \mathrm{q} 11.2 \mathrm{DS}$ patients to sequence seven candidate genes (CRKL, MAPK1, HIRA, TANGO2, PI4KA, ZFPM2, and JAM3), located in and outside the 22q11.2 hemizygous region. To predict indels that may have a role as modifiers, we used genomic variants filtering by deep learning models in NGS (GARFIELD-NGS), which rely on machine learning models to efficiently distinguish clinically pathogenic from neutral indels.

Results: We identified indel variants that may have a role as modifiers in the 22q11.2DS.

Conclusions: These results show how the application of new methodologies for predicting variants can guide to new findings with great exploration potential. Financial support: CAPES, FAPESP, Brazil.

N. Nunes: None. A.G. Dantas: None. M. Zamariolli: None. D.C.Q. Soares: None. L.C. dos Santos: None. V.A. Meloni: None. S.I. Belangero: None. V.L. Gil-Da-SilvaLopes: None. C.A. Kim: None. M.I. Melaragno: None.

\section{P13.04.C}

\section{LCR22q11.2 hypervariability is human specific}

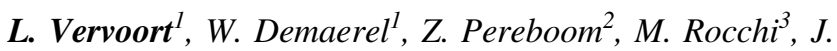
R. Vermeesch ${ }^{l}$

${ }^{1}$ Laboratory for Cytogenetics and Genome Research, $K U$ Leuven, Leuven, Belgium, ${ }^{2}$ Centre for Research and Conservation, Royal Zoological Society of Antwerp, Antwerp, Belgium, ${ }^{3}$ Department of Biology, University of Bari, Bari, Italy

Introduction: Low copy repeats (LCRs) are amongst the most complex regions in the human genome. Scrutinizing these structures unraveled their importance during evolution. LCRs play an important role in the disease mechanism of genomic disorders as well. Non-allelic homologous recombination between chromosome 22 LCRs (LCR22s) cause the 22q11.2 deletion syndrome. LCR22-A hypervariability was uncovered in human. However, it remains unknown whether such variability is human specific or is also a feature of other primates.

Materials and Methods: The LCR22 variability in the Great Apes was charted using an LCR22-specific fiberFISH. The structures of five chimpanzees, one bonobo, two gorillas, and six orangutans were de novo assembled to assess the level of inter- and intraspecies variation. In addition, we used Bionano optical mapping to chart the overall $22 q 11.2$ structure of the orangutans.

Results: The chimpanzee and bonobo harbored the smallest LCR22-A allele observed in humans, containing core duplicons, without intra-species variation. The gorilla haplotype had small expansions compared to the chimpanzee. In contrast, the overall 22q11.2 region is different in the orangutan, including rearrangements in the sequence between the LCR22s, intra-individual structural variation, and LCR22-A haplotypes not resembling the human ones.

Conclusions: For the first time, the LCR22s subunits were evolutionary charted and uncovered lineage-specific differences. The LCR22-A hypervariability is human specific, since no structural variation was observed in chimpanzee or bonobo. This suggests an important role of the locus in human adaptation and evolution.

Funding: FWO GOE1117N, Jerome Lejeune Project 1665

L. Vervoort: None. W. Demaerel: None. Z. Pereboom: None. M. Rocchi: None. J.R. Vermeesch: None.

P13.05.A

Extensive (epi)genetic profiling of pre- and post-natal tissues from female monozygotic twins discordant for Beckwith-Wiedemann syndrome

L. Fontana ${ }^{1,2}$, M. Bedeschi ${ }^{3}$, G. A. Cagnoli ${ }^{3}$, J. Costanza ${ }^{2}$, G. Moresco $^{2}$, C. Faré , N. Persico $^{4,5}$, S. Gangi ${ }^{6}$, M. Porro $^{7}$, P. F. Ajmone $^{8}$, P. Colapietro $^{1,2}$, S. M. Sirchia ${ }^{9}$, M. R. Miozzo $^{1,2}$, S. Tabano ${ }^{1,10}$

${ }^{1}$ Medical Genetics, Department of Pathophysiology \& Transplantation, Università degli Studi di Milano, Milan, Italy, ${ }^{2}$ Research Laboratories Coordination Unit, Fondazione IRCCS Ca' Granda Ospedale Maggiore Policlinico, Milan, Italy, ${ }^{3}$ Medical Genetics Unit, Fondazione IRCCS $\mathrm{Ca}$ ' Granda Ospedale Maggiore Policlinico, Milan, Italy, ${ }^{4}$ Obstetrics and Gynecology Unit, Fondazione IRCCS Ca' Granda Ospedale Maggiore Policlinico, Milan, Italy, ${ }^{5}$ Department of Clinical Sciences and Community Health, Università degli Studi di Milano, Milan, Italy, ${ }^{6}$ Neonatal Intensive Care Unit, Fondazione IRCCS Ca' Granda Ospedale Maggiore Policlinico, Milan, Italy, ${ }^{7}$ Pediatric Physical Medicine \& Rehabilitation Unit, Fondazione IRCCS Ca' Granda Ospedale Maggiore Policlinico, Milan, Italy, ${ }^{8}$ Child and Adolescent Neuropsychiatric Unit, Fondazione IRCCS Ca' Granda Ospedale Maggiore Policlinico, Milan, Italy, ${ }^{9}$ Medical Genetics, Department of Health Sciences, Università degli Studi di Milano, Milan, Italy, ${ }^{10}$ Medical Genetics Unit, Fondazione IRCCS Ca' Granda, Ospedale Maggiore Policlinico, Milan, Italy

Background: Beckwith-Wiedemann syndrome (BWS) is an overgrowth disorder caused by defects at the $11 \mathrm{p} 15.5$ imprinted region. Many cases of female monozygotic (MZ) twins discordant for BWS have been reported, but no definitive conclusions have been drawn regarding the link 
between epigenetic defects, twinning process, and gender. Here, we report a comprehensive characterization and follow-up of female MZ twins discordant for BWS.

Methods: 11p15.5 methylation pattern and multilocus methylation defects (MLID) profiling were performed by pyrosequencing and MassARRAY in pre- and post-natal tissues. Whole-exome sequencing (WES) was carried out to identify MLID causative mutations. X-chromosome inactivation (XCI) status was defined by HUMARA test.

Results: Both twins share ICR2 LOM and MLID in blood and the epigenetic defect in the healthy twin remained stable over time. ICR2 LOM appeared non-homogenously distributed in the placental samples. Twins shared the same severe XCI skewing pattern. No MLID causative mutations were identified by WES.

Conclusion: This is the first reported case in which methylation analyses was extended to extraembryonic tissues, and results suggest that caution is required when attempting prenatal diagnosis in similar cases. Although the causative mechanism of LOM remains unsolved, the XCI pattern, along with mosaic LOM, suggests that both twinning and LOM/MLID occurred after XCI commitment. This study was supported by the Italian Ministry of Health (Grant RF-2013-02359454) and Fondazione IRCCS Ca' Granda Ospedale Maggiore Policlinico (Grant 5X1000-519 02 and Ricerca Corrente-519 03 to MM).

L. Fontana: None. M. Bedeschi: None. G.A. Cagnoli: None. J. Costanza: None. G. Moresco: None. C. Faré: None. N. Persico: None. S. Gangi: None. M. Porro: None. P.F. Ajmone: None. P. Colapietro: None. S.M. Sirchia: None. M.R. Miozzo: None. S. Tabano: None.

\section{P13.07.C}

New loci associated with carotenoid blood concentrations in the Lithuanian population cohort

\section{Domarkiene ${ }^{1}$, A. Mazeikiene ${ }^{2}$, G. Petrauskaite ${ }^{1}$, Z. A. Kucinskiene $^{2}$, V. Kucinskas ${ }^{1}$}

${ }^{I}$ Department of Human and Medical Genetics, Institute of Biomedical Sciences, Faculty of Medicine, Vilnius University, Vilnius, Lithuania, ${ }^{2}$ Department of Physiology, Biochemistry, Microbiology and Laboratory Medicine, Institute of Biomedical Sciences, Faculty of Medicine, Vilnius University, Vilnius, Lithuania

Introduction: Variation in carotenoid bioavailability at an individual and population levels might depend on hostrelated factors where genetic variation has a part. It manifests through the proteins involved in carotenoid intestinal absorption and metabolism, blood lipoprotein transport or tissue uptake. This study aims to identify novel SNPs which could be associated with carotenoid serum concentrations.
Materials and Methods: 265 self-reported healthy individuals of Lithuanian ethnicity were genotyped (Illumina HumanOmniExpress-12v1.0 or v1.1 and Infinium OmniExpress-24v1.2 arrays) and blood serum concentrations of $\beta$ - and $\alpha$-carotene, $\beta$-cryptoxanthin, lycopene, lutein and zeaxanthin were measured (Shimadzu Prominence HPLC system). According to the carotenoid concentrations individuals were subdivided in to the quartiles. Q1 and Q4 were used for the following association analysis. The set of 2868 SNPs in 150 potential candidate genes (assumed for direct or indirect role in the carotenoid bioavailability) was analysed using PLINK v1.90b software $(\alpha=0.05 / \mathrm{n})$.

Results: SNPs $\operatorname{rs} 2279238(p=2.129 \times 10-5)$ and rs11039155 $(p=2.984 \times 10-5)$ in NR1H3 and rs550619 $(p=4.844 \times 10-5)$ in APOB genes were associated with higher zeaxanthin concentration. SNP rs756473 ( $p=$ $7.422 \times 10-5)$ in RDH12 gene was associated with higher lycopene concentration. Twenty-one SNPs in locus 10q23.33 including CYP2C9, CYP2C18 and CYP2C19 genes were significantly associated with higher $\beta$-carotene concentration.

Conclusions: Four novel genomic loci were found to be associated with carotenoid serum levels. Zeaxanthin, lycopene and $\beta$-carotene serum concentrations might depend on genetic variation in NR1H3, APOB, RDH12 and CYP2C9 genes.

This research is part of the LITGEN project (VP1-3.1ŠMM-07-K-01-013) and was funded by the European Social Fund under the Global Grant measure.

I. Domarkiene: None. A. Mazeikiene: None. G. Petrauskaite: None. Z.A. Kucinskiene: None. V. Kucinskas: None.

\section{P13.08.A}

Transriptome analysis reveals distinct responses to structural variations at the $C D H 1$ gene

\section{R. Barbosa-Matos ${ }^{l}$, M. Ferreira ${ }^{l}$, A. Ferro $^{l}$, B. Mesquita ${ }^{l}$, H. Pinheiro ${ }^{l}, S$. Valente $^{l}$, P. Oliveira ${ }^{l}$, C. Oliveira ${ }^{1,2}$ \\ ${ }^{1}$ Instituto de Investigação e Inovação em Saúde, Porto, Portugal, ${ }^{2}$ Department of Pathology, Faculty of Medicine of the University of Porto, Porto, Portugal}

Introduction: $C D H 1$ encodes E-Cadherin which is critical for epithelial tissue architecture and cell-cell adhesion. $\mathrm{CDH1}$ impairment is associated with metastatic spread in most epithelial cancers (somatic), early-onset of gastric and breast cancer and cleft-lip/palate (germline), however the germline and somatic mechanisms described so far can't justify all $\mathrm{CDH} 1$-associated phenotypes. We explored expression profile changes induced by structural variations 
across the $C D H 1$ gene body to infer the role of coding and non-coding sequences.

Material and Methods: We CRISPR/Cas9 deleted an out-of-frame exon (Exon_deletion), an intronic cis-regulatory element (iCRE) and an intronic portion not assigned to regulatory function (Partial_intron). Obtained clones were characterized by qRT-PCR, western-blotting and immunocytochemistry and further studied through RNA-Seq followed by Differential Expression (DE) analysis using the DESeq2 package.

Conclusions: Compared to parental cells, Exon_deletion triggers $\mathrm{CDH1}$ loss, with 19-downregulated and 34upregulated genes, being the Top-5 pathways associated with inflammatory-responses; iCRE deletion also triggers CDH1 loss, having 1106-downregulated and 1238upregulated genes and its Top-5 pathways are associated with shifting from cadherin- to protocadherin-mediated adhesion, cell motility and migration; Partial_intron deletion didn't impact $\mathrm{CDH} 1$ expression and showed 153downregulated and 220-upregulated genes, the Top-5 pathways are associated with vasoconstriction and bloodvessel size. Both clones harbouring $\mathrm{CDH} 1 \mathrm{LoF}$ share $35 \mathrm{DE}$ genes, and only 10 genes are DE in all clones. Our CRISPR/ Cas9 assay reveals unanticipated transcriptomic consequences of $\mathrm{CDH} 1$-locus associated structural variation and demonstrates that different portions of the locus play different regulatory functions.

Funding: Solve-RD project received funding from European Union's Horizon2020 research and innovation programme, grant agreement $\left.\mathrm{N}^{\circ}: 779257 ; 2\right)$ FEDER/COMPETE, “POCI-01-0145-FEDER-030164".

R. Barbosa-Matos: None. M. Ferreira: None. A. Ferro: None. B. Mesquita: None. H. Pinheiro: None. S. Valente: None. P. Oliveira: None. C. Oliveira: None.

\section{P13.09.B}

New pathogenic structural variants responsible for Charcot-Marie-Tooth disease

I. Pyromali ${ }^{1}$, A. Perrani ${ }^{2}$, A. Nizou ${ }^{1}$, C. Magdelaine ${ }^{2,1}$, P. Derouault $^{3}$, S. Bourthoumieu ${ }^{4}$, F. Sturtz ${ }^{1,2}$, A. S. Lia $^{1,2}$

${ }^{1}$ EA6309-Neuropathies Périphériques et Maintenance Myélinique, University of Limoges, Limoges, France, ${ }^{2}$ Laboratoire de Biochimie et de génétique moléculaire, CHU Dupuytren, Limoges, France, ${ }^{3} \mathrm{CHU}$ Limoges, Unité Fonctionnelle de Bioinformatique, F-87000 Limoges, Limoges, France, ${ }^{4}$ Service de cytologie, cytogénétique et de génétique médicale, $\mathrm{CHU}$ de Limoges, Limoges, France

Introduction: Charcot-Marie-Tooth (CMT) is the most common hereditary neuropathy characterized by damages of both motor and sensory peripheral nerves. CMT transmission modes can be various (dominant, recessive, autosomal or X-linked) and there are more than 90 genes involved. PMP22 duplication was the first described mutation in this disease, explaining around $15 \%$ of CMT patients. However, to date, the majority of the reported mutations in CMT patients are single nucleotide variants detected by targeted Next Generation Sequencing (NGS) and Structural Variants (SVs) have rarely been described. The aim of our study was to identify new SVs involved in CMT disease.

Materials and Methods: DNA from 695 CMT patients were analysed by NGS using an amplicon targeted sequencing panel and we performed a specific bioinformatic analysis of NGS data by Cov'Cop and CovCopCan softwares looking for new pathogenic SVs involved in CMT.

Results: Thank to this approach, we found new SVs in 107 CMT patients, highlighting the importance of SVs analysis during CMT patients' examination. 28 were small deletions (3-6 amplicons), 10 were large deletions, 49 were small duplications and 20 were large duplications. We will focus here on two of these large deletions, we proved then to be responsible for our patients' disease.

Conclusions: These SVs would not have been detected using the typical NGS analyses. We believe our strategy using Cov'Cop and CovCopCan could increase the diagnosis rate of patients suffering from neuropathies and could be used by molecular biologists and geneticists to improve diagnosis of all inherited diseases.

I. Pyromali: None. A. Perrani: None. A. Nizou: None. C. Magdelaine: None. P. Derouault: None. S. Bourthoumieu: None. F. Sturtz: None. A.S. Lia: None.

\section{P13.10.C}

Chromosome conformation capture (4C-seq and UMI4C) in human retina combined with whole genome sequencing for the detection and functional interpretation of noncoding genetic defects in North Carolina Macular Dystrophy, a regulatory disease

S. Van de Sompele ${ }^{l}$, E. D'haene ${ }^{l}$, T. Van der Snickt ${ }^{l}, P$. Liskova $^{2}$, S. Vergult ${ }^{1}, J$. van den Ende ${ }^{3}$, A. A. Bergen ${ }^{4}$, I. Balikova $^{5}$, J. De Zaeytijd ${ }^{6}$, F. S. Shaya ${ }^{7}$, C. Rivolta ${ }^{8}, K$.W. Small $^{7}$, E. De Baere ${ }^{1}$

${ }^{I}$ Center for Medical Genetics, Ghent University and Ghent University Hospital, Ghent, Belgium, ${ }^{2}$ Research Unit for Rare Diseases, Charles University and General University Hospital, Prague, Czech Republic, ${ }^{3}$ Center for Medical Genetics, Antwerp University Hospital, Antwerp, Belgium, ${ }^{4}$ Department of Ophthalmology, Amsterdam UMC, University of Amsterdam, Amsterdam, Netherlands, 
${ }^{5}$ Department of Ophthalmology, Queen Fabiola Children's University Hospital, Brussels, Belgium, ${ }^{6}$ Department of Ophthalmology, Ghent University and Ghent University Hospital, Ghent, Belgium, ${ }^{7}$ Macula and Retina Institute, Los Angeles and Glendale, Los Angeles, CA, United States, ${ }^{8}$ Institute of Molecular and Clinical Ophthalmology, Basel University Hospital, Basel, Switzerland

Introduction: Integrated -omics increasingly reveal noncoding mutations in Mendelian disorders, mostly deepintronic splicing mutations, while noncoding mutations in cis-regulatory elements (CREs) are more scarce. An example of these are regulatory changes near PRDM13 or IRXI in autosomal dominant North Carolina Macular Dystrophy (NCMD). NCMD has a recognizable phenotype of the central retina, making it interesting for regulatory variants. We aimed at dissecting the regulatory landscapes of PRDMI3 and IRXI to find novel causes of NCMD and to understand disease mechanisms.

Methods: Chromosome conformation capture (4C-seq and UMI-4C) was applied on postmortem adult human retinas to fine-map interactions of retinal CREs with the $P R D M 13$ and IRXI promoters. Interaction profiles were integrated with additional retinal epigenomic data in UCSC. Twenty-five NCMD families underwent assessment of known defects followed by whole genome sequencing.

Results: 4C-seq and UMI-4C profiles, together with other retinal epigenomic profiles, revealed noncoding putative CREs interacting with the PRDMI3 and IRXI promoters. One of them overlaps with a novel noncoding sequence variant located upstream of $P R D M 13$, and is active in developmental central retina. Interestingly, another novel and known noncoding variant at the same position and a novel duplication overlap with a noncoding putative CRE upstream of PRDM13 containing a DNase hypersensitive site.

Conclusions: Retinal interaction profiles of the PRDM13 and $I R X I$ regions advance the interpretation of noncoding variants and provide insight into the cis-regulatory mechanisms underlying NCMD. The genetic architecture of NCMD was expanded with novel noncoding variants with a likely effect on PRDM13 regulation.

Funding: FWO/1145719N; FWO/1802220N; EJPRD19234 Solve-RET.

S. Van de Sompele: None. E. D'haene: None. T. Van der Snickt: None. P. Liskova: None. S. Vergult: None. J. van den Ende: None. A.A. Bergen: None. I. Balikova: None. J. De Zaeytijd: None. F.S. Shaya: None. C. Rivolta: None. K.W. Small: None. E. De Baere: None.

\section{P13.14.A}

Identification of Structural Variants on Whole Genome Sequencing data for clinical purposes
C. Marconi, F. Masclaux, F. Mattioli, S. Laurent, S. Gimelli, E. Stathaki, A. Vannier, L. Lemmens, V. Kremer, S. Fokstuen, A. Giacobino, T. Ait Mouhoub, M. Carminho Amaro Rodrigues, L. Quteineh, T. Nouspikel, M. Guipponi, J. Blouin, F. Sloan-Béna, M. Abramowicz

\section{Hopitaux Universitaires de Genève, Geneva, Switzerland}

While WGS is being largely used in scientific research, its application in the diagnostic routine is still lagging behind. In order to develop a pipeline for the identification of SVs from short-reads WGS data that can be translated into transferred to clinical purposes we tested 7 previously published (publically available) algorithms for SV identification and evaluated their Sensitivity and False-DiscoveryRate. We observed variable level of sensitivity (36-87\%) depending on the tool and SV type and size. Read-depth approaches worked better for large deletions (79-86\%), while combined paired/split-reads approaches gave better identification of small deletions (81-94\%). High sensitivity was found for insertions (93\%) and inversions (96-100\%). As described by others, we report a high number of False Positives (FP) $\left(10^{2}-10^{3}\right)$ as main issue. To reduce FP, we decided to use one to two tools for each SV type and selected the one with highest sensitivity. After filtering based on quality parameters, SVs were annotated using annotSV and candidate SVs were selected based on frequency, involved genes and inheritance pattern. We applied the approach to a first set of 8 patients for whom no genetic cause was identified using aCGH and panel targeted whole exome sequencing a few years ago. The first results include identification of an homozygous $22.6 \mathrm{~kb}$ deletion encompassing 5'UTR and exon 1 of ERGICl in a patient with arthrogryposis. Biallelic mutations of ERGICl were previously associated to arthrogryposis in a single family. Of note, this gene was initially not included in our WES gene panel for this pathology.

C. Marconi: None. F. Masclaux: None. F. Mattioli: None. S. Laurent: None. S. Gimelli: None. E. Stathaki: None. A. Vannier: None. L. Lemmens: None. V. Kremer: None. S. Fokstuen: None. A. Giacobino: None. T. Ait Mouhoub: None. M. Carminho Amaro Rodrigues: None. L. Quteineh: None. T. Nouspikel: None. M. Guipponi: None. J. Blouin: None. F. Sloan-Béna: None. M. Abramowicz: None.

\section{P13.17.A}

Germline chromothripsis: Integration of $\mathrm{Hi}-\mathrm{C}$ and longread sequencing reveals the structure of highly rearranged chromosomes

$$
\begin{aligned}
& \text { R. Schöpflin }{ }^{1,2}, \text { U. Souto Melo } \\
& \text { Jungnitsch }^{1,2}, \text { D. Heller }
\end{aligned}
$$


Heinrich $^{3}$, J. Herztberg ${ }^{1,2,3}$, R. Acuna-Hidalgo ${ }^{1,2}, S$. Türkmen $^{1}$, M. Bugge ${ }^{6}$, I. Vogel ${ }^{7}$, V. Beensen ${ }^{8}$, G. Barbi ${ }^{9}$, B. Prager $^{10}$, A. Latos-Bieleńska ${ }^{11,12}$, N. Tommerup ${ }^{6}$, V. M. Kalscheuer $^{2}$, M. Spielmann ${ }^{1,2}$, M. Vingron $^{3}$, S. Mundlos ${ }^{1,2}$

${ }^{1}$ Institute for Medical and Human Genetics, Charité Universitätsmedizin Berlin, Berlin, Germany, ${ }^{2}$ Max Planck Institute for Molecular Genetics, $R G$ Development \& Disease, Berlin, Germany, ${ }^{3}$ Max Planck Institute for Molecular Genetics, Department of Computational Molecular Biology, Berlin, Germany, ${ }^{4}$ Core Unit Bioinformatics, Berlin Institute of Health, Berlin, Germany, ${ }^{5}$ Charité Universitätsmedizin Berlin, Berlin, Germany, ${ }^{6}$ Wilhelm Johannsen Center for Functional Genome Research, Department of Cellular and Molecular Medicine, University of Copenhagen, Copenhagen, Denmark, ${ }^{7}$ Department of Clinical Genetics, Aarhus University Hospital, Aarhus, Denmark, ${ }^{8}$ Institute of Human Genetics and Anthropology, Friedrich-Schiller University, Jena, Germany, ${ }^{9}$ Institut für Humangenetik, Universitätsklinikum Ulm, Ulm, Germany, ${ }^{10}$ Praxis für Humangenetik, Kinderzentrum Dresden-Friedrichstadt, Dresden, Germany, ${ }^{11}$ Department of Medical Genetics, Poznan University of Medical Sciences, Poznan, Poland, ${ }^{12}$ Center for Medical Genetics GENESIS, Poznan, Poland

Chromothripsis is characterized by the shattering of one or a few chromosomal segments followed by a chaotic reassembly of the chromosomal fragments. Commonly observed in cancer, chromothripsis-like rearrangements also occur as a rare event in the germline. We investigated 10 cases with congenital complex or even chromothripsis-like rearrangements and surprisingly mild phenotypes, despite massive large-scale rearrangements. To understand and predict the pathogenic effects of rearrangements, a reconstruction of the shattered chromosomes with precise mapping of the breakpoints is needed. However, due to the high error rate in breakpoint detection by short-read whole-genome sequencing this remains challenging.

Here, we address these issues by combining Hi-C with PacBio long-read sequencing for the reconstruction of patient genomes. Hi-C was very efficient and sensitive in detecting large scale rearrangements and provided a global coarse-grained atlas of the fusion events and information on their layout. In contrast, PacBio reads provided fine-grained local information of breakpoints, due to long (10-50 kb) reads and the reduced sensitivity to repetitive regions. Hi-C was used to evaluate the breakpoint calls obtained from PacBio sequencing thereby reducing the candidate list to 550 rearrangements $>5 \mathrm{Mb}$ per individual. In a next step, the derivative chromosomes were reconstructed using both technologies jointly. The potentially pathogenic effects of these highly rearranged genomes were assessed by mapping the breakpoints in the context of 3D chromatin structure and topologically associated domains (TADs). Thus, we present an efficient method for the reconstruction and evaluation of shattered chromosomes in a cohort of individuals with chromothripsis-like rearrangements.

R. Schöpflin: None. U. Souto Melo: None. D. Heller: None. J. Jungnitsch: None. M. Klever: None. M. Holtgrewe: None. E. Comak: None. V. Heinrich: None. J. Herztberg: None. R. Acuna-Hidalgo: None. S. Türkmen: None. M. Bugge: None. I. Vogel: None. V. Beensen: None. G. Barbi: None. B. Prager: None. A. Latos-Bieleńska: None. N. Tommerup: None. V.M. Kalscheuer: None. M. Spielmann: None. M. Vingron: None. S. Mundlos: None.

\section{P13.18.B}

Radiation-induced alterations in the genome and exome of human gingiva fibroblasts

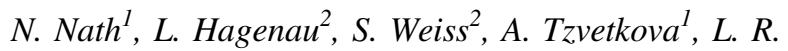

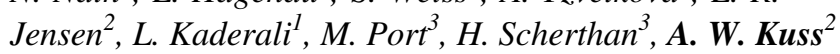

${ }^{1}$ Institute of Bioinformatics, University Medicine Greifswald, Greifswald, Germany, ${ }^{2}$ Department of Functional Genomics, Interfaculty Institute for Genetics and Functional Genomics, University Medicine Greifswald, Greifswald, Germany, ${ }^{3}$ Bundeswehr Institute for Radiobiology affil. to the University of Ulm, München, Germany

Little is known about the mutational impact of ionizing radiation (IR) exposure on a genome-wide level. Recent advancements in sequencing technology, however, have opened up new avenues for studying and characterizing global genomic IR-induced effects. Here, we present our investigation of putative IR-induced mutation signatures in whole genome and exome sequencing datasets of human gingiva fibroblasts (HGFs), which were exposed to increasing doses of X-radiation in combination with different post-exposure repair intervals. For our analyses we developed a bioinformatics workflow incorporating three stringent filtering steps for the removal of non-IR induced variants, to eliminate sequencing errors and to remove variants with low sequencing coverage. We studied the accumulation of single nucleotide variants (SNVs) as well as insertions and deletions (Indels) and statistically analyzed the accumulation of variants with respect to different genomic features such as cytogenetic bands and topologically associating domains (TAD). SNVs showed low transition/transversion (Ti/Tv) mutation ratios, while the number of deletions exceeded the number of insertions. SNVs in gene sets related to stress response and DNArepair, were situated mostly in intronic, up- or downstream regions and sequence ontology analyses showed that they 
were mostly neutral without effects on protein function. Taken together, our results provide evidence for a characteristic genomic meta-signature of variants after IR exposure. Furthermore, effects related to structural and/or functional chromatin domains of particular chromosomal areas were noted, which may reflect DNA repair pathway choice.

N. Nath: None. L. Hagenau: None. S. Weiss: None. A. Tzvetkova: None. L.R. Jensen: None. L. Kaderali: None. M. Port: None. H. Scherthan: None. A.W. Kuss: None.

\section{P13.20.A}

\section{Current Human Reference Genome and Missing Information}

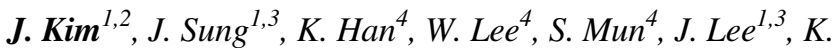
$\mathrm{Bahk}^{l}$, I. Yang ${ }^{5}$, Y. Bae, C. $\mathrm{Kim}^{6}$, J. Kim ${ }^{7}, \mathrm{~J} . \mathrm{Seo}^{8}$

${ }^{I}$ Genome \& Health Big Data Branch, Department of Health Science, School of Public Health, Seoul National University, Seoul, Korea, Republic of, ${ }^{2}$ Interdisciplinary program in Bioinformatics, Seoul National University, Seoul, Korea, Republic of, ${ }^{3}$ Institute of Health \& Environment, Seoul National University, Seoul, Korea, Republic of, ${ }^{4}$ Department of Nanobiomedical Science, BK21 PLUS NBM Global Research Center for Regenerative Medicine, Dankook University, Cheonan, Korea, Republic of, ${ }^{5}$ Korea Research Institute of Standards and Science, Daejeon, Korea, Republic of, ${ }^{6}$ Bioinformatics Institute, Macrogen Inc., Seoul, Korea, Republic of, ${ }^{7}$ Genomic Medicine Institute, Medical Research Center, Seoul National University, Seoul, Korea, Republic of, ${ }^{8}$ Precision Medicine Center, Seoul National University Bundang Hospital, Seongnam, Korea, Republic of

The human reference genome (GRCh38) serves as a template for sequencing analysis, but it might lack some ethnicspecific sequences particularly for Asians. To understand the extent and characteristics of missing information, we juxtaposed GRCh38 with high-contiguity genome assembly (AK1) of a Korean, to show that $\sim 53.4 \mathrm{Mbp}$ of AK1 sequences $(\sim 1.8 \%$ of genome) were missing in GRCh38, which harbors $\sim 1,390$ putative coding elements. To validate the possible information loss, we analyzed the "unmapped" (to GRCh38) reads of fourteen individuals' deep sequencing data (5 East-Asians, 4 Europeans, and 5 Africans), to find that $\sim 5.3 \mathrm{Mbp}$ of previously deserted unmapped sequences were recovered by using $\mathrm{AK} 1$ as reference. The recovered regions included 38 globally shared ( $\geq 7$ individuals out of 14 ) regions with $>25$ candidate coding elements. We also verified that many of these common missing regions in GRCh38 existed in multiple populations and a chimpanzee by PCR analysis. Our study illuminates the usefulness of precise ethnic genomes, and supports the rationale for building a pan-genome reference.

J. Kim: None. J. Sung: None. K. Han: None. W. Lee: None. S. Mun: None. J. Lee: None. K. Bahk: None. I. Yang: None. Y. Bae: None. C. Kim: None. J. Kim: None. J. Seo: None.

\section{P13.22.C}

Genetic aetiology of early onset severe obesity revealed in half of the affected cases from a consanguineous population of Pakistan

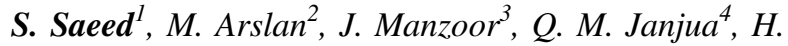
Ayesha $^{5}$, E. Durand ${ }^{6}$, W. I. Khan ${ }^{7}$, T. A. Butt ${ }^{8}$, A. Bonnefond ${ }^{6}$, P. Froguel ${ }^{1}$

${ }^{1}$ Faculty of Medicine, Imperial College London, London, United Kingdom, ${ }^{2}$ School of Life Sciences, Forman Christian College (A Chartered University), Lahore, Pakistan, ${ }^{3}$ Department of Paediatric Endocrinology, Children's Hospital, Lahore, Pakistan, ${ }^{4}$ Department of Physiology, University College of Medicine and Dentistry, University of Lahore, Lahore, Pakistan, ${ }^{5}$ Department of Paediatrics, Punjab Medical College, Faisalabad, Pakistan, ${ }^{6}$ EGID - UMR 1283 - 8199, Lille, France, ${ }^{7}$ Children Hospital and Institute of Child Health, Multan, Multan, Pakistan, ${ }^{8}$ Department of Pediatrics, Fatima Memorial Hospital, Lahore, Pakistan

Introduction: Single gene pathogenic mutations account for only $5-7 \%$ of obese cases in European populations. No assessment of their prevalence has yet been made in consanguineous populations.

Materials and Methods: DNA from 225 severely obese probands (BMI-SDS>3.5), were first screened for the mutations in $L E P$ and $M C 4 R$ genes by Sanger sequencing. This was then followed by an in-house developed augmented whole-exome sequencing (CoDE-seq) enabling direct and simultaneous detection of CNVs and point mutations in coding regions. The variants associated with obesity were graded according to ACMG criteria to predict pathogenicity.

Results: We report 110 cases (49\%) with 55 different pathogenic variations (49 point mutations; $6 \mathrm{CNVs}$ ), in known monogenic obesity genes: Point mutations were identified in $L E P, L E P R, M C 4 R$ and $A D C Y 3$ (47\%, 14\%, $11 \%$ and $3.5 \%$ of cases, respectively). Mutations causing syndromic obesity were identified in BBSs and ALMS1 (15.5\% and $3.5 \%$ of cases, respectively). CNVs affecting obesity associated genes (LEPR, PWS $16 \mathrm{p} 11.2)$ accounted for $5.5 \%$ of patients. In addition, CoDE-seq also identified 28 rare or novel CNVs in $22(10 \%)$ obese subjects, in regions associated with intellectual disability. Lastly we 
highlighted variants in 4 potential candidate genes for obesity warranting further investigation.

Conclusions: Here, we demonstrate a remarkably high frequency (49\% plus potentially 10\%) of monogenic obesity in a consanguineous population. Large inbred populations provide a genetically enriched material to elucidate the missing heritability of obesity and for the discovery of novel mechanisms influencing energy balance.

Acknowledgements: The study was funded by French National Research Agency (ANR-10-LABX-46, ANR-10EQPX-07-01).

S. Saeed: None. M. Arslan: None. J. Manzoor: None. Q.M. Janjua: None. H. Ayesha: None. E. Durand: None. W.I. Khan: None. T.A. Butt: None. A. Bonnefond: None. P. Froguel: None.

\section{P13.23.A}

Molecular characterisation of NF1 in Cypriot neurofibromatosis type 1 patients

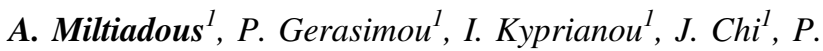
Costeas $^{l}$, V. Anastasiadou ${ }^{2}$

${ }^{1}$ Karaiskakio Foundation, Nicosia, Cyprus, ${ }^{2} 2$ Department of Clinical Genetics, Archbishop Makarios III Medical Centre, Nicosia, Cyprus

Introduction: NF1 is a large gene containing at least 57 exons and encoding a protein sized 2818 aminoacids. NF1 mutations are generally associated to Neurofibromatosis type 1, an autosomal dominant disease characterised by café-au-lait spots and neurofibromas. Studying the NF1 gene has been challenging over the years due to its large size and the similarity it shares with over seven NF1 pseudogenes.

Materials and Methods: Forty patients with suspected or confirmed clinical diagnosis of neurofibromatosis type 1, were screened with NGS (Haloplex and Sureselect panels from Agilent), Sanger sequencing and MLPA (MRCHolland) for NF1 copy number detection.

Results: Twenty-nine patients (73\%) harboured pathogenic or likely pathogenic variants in NF1, of which 11 $(37 \%)$ variants were novel. Eight $(28 \%)$ of all variants were nonsense, 8 (28\%) were missense, $6(21 \%)$ were located on splice sites (3 were investigated and resulted in exon skipping), 1 (3\%) was an Indel and $6(21 \%)$ were all novel frameshift variants. Two (5\% of all patients), harboured an NF1 multi-exon deletion, and a whole NF1 gene deletion, respectively. More than half of all variants detected, lie in exons that share $>90 \%$ similarity with NF1 pseudogenes. The identified variants were equally distributed along the gene.
Conclusions: We present the first study done in neurofibromatosis type 1 Cypriot patients, in which nonsense and missense changes are the most common type of variants observed. We highlight the importance of copy number testing in neurofibromatosis patients as well as distinguishing true pathogenic variants from variants occurring in NF1 pseudogenes.

A. Miltiadous: None. P. Gerasimou: None. I. Kyprianou: None. J. Chi: None. P. Costeas: None. V. Anastasiadou: None.

\section{P13.24.B}

Unraveling genomic interactions at nsCL/P associated GWAS loci using Circular Chromosome Conformation Capture (4C)

R. Hollstein ${ }^{l}$, J. Schröder ${ }^{l}$, J. Welzenbach ${ }^{l}$, M. Bartusel ${ }^{2}$, A. Rada-Iglesias ${ }^{2,3}$, K. U. Ludwig ${ }^{1}$

${ }^{1}$ Institute of Human Genetics, University of Bonn, School of Medicine \& University Hospital Bonn, Bonn, Germany, ${ }^{2}$ Center for Molecular Medicine Cologne (CMMC), University of Cologne, Cologne, Germany, ${ }^{3}$ Institute of Biomedicine and Biotechnology of Cantabria (IBBTEC), University of Cantabria, Cantabria, Spain

Non-syndromic cleft lip with or without cleft palate (nsCL/ $\mathrm{P}$ ) is among the most common birth defects worldwide. Its etiology is multifactorial with a strong genetic component, which is supported by previous identification of 45 risk loci through GWAS. As the majority of risk loci map to noncoding regions, molecular effects on gene regulation or chromatin organization have been suggested. However, the translation of genetic findings into pathomechanisms is challenging, due to the early embryonic time point of facial development and thus, the lack of accessible relevant human tissues for functional analyses. Our group has previously shown that associated risk variants for nsCL/P are strongly enriched in active regulatory elements of human neural crest cells (hNCCs). To unravel genomic interactions and possible target genes of associated variants, we are performing circular chromosome conformation capture (4C) in hNCCs. We selected one novel risk locus on chr.20q13 to establish the method. According to GTEx, the lead SNP is an eQTL for EYA2 in multiple tissues. Notably, EYA2 is a developmental gene expressed in hNCCs that is involved in cellular mechanisms such as migration and apoptosis. Our preliminary data based on the viewpoint around the sentinel SNP suggest an interaction between its region and the promoter region of EYA2. Analyses using the promoter region of $E Y A 2$ as a second viewpoint and allele-specific investigations are currently on the way, to validate the interaction. Overall, our approach will help to identify target 
genes that are involved in the molecular pathology of nsCL/ $P$ at GWAS loci.

R. Hollstein: None. J. Schröder: None. J. Welzenbach: None. M. Bartusel: None. A. Rada-Iglesias: None. K.U. Ludwig: None.

\section{P13.27.B}

A new genetic variants in DNAI2 detected by target sequencing in a newborn with Primary Ciliary Dyskinesia

M. Rocca ${ }^{1}$, C. Vinanzi ${ }^{1}$, A. Michelucci ${ }^{2}$, M. Caligo ${ }^{2}, A$. Valetto $^{3}$, M. De Santi ${ }^{4}$, V. Bertini ${ }^{3}$, G. Piatti ${ }^{5}$, C. Foresta ${ }^{l}$

${ }^{1}$ University of Padova, Padova, Italy, ${ }^{2}$ University Hospital of Pisa, Pisa, Italy, ${ }^{3}$ IRCSS Eugenio Medea, Pisa, Italy, ${ }^{4}$ University of Siena, Siena, Italy, ${ }^{5}$ University of Milano, Milano, Italy

Introduction: Primary Ciliary Dyskinesia (PCD) is a rare recessive disease characterized by motile cilia dysfunction. Clinical manifestations of this disease include upper and lower respiratory tract infections, laterality defects and infertility. To date, mutations in approximately 40 different genes have been found to be causative of PCD. Here, we report the case of a new-born with PCD carrying a new homozygous deletion in DNAI2 gene.

Materials and Methods: Transmission electron microscopy was carried out for diagnosis of PCD. To identify genetic cause of disorder, DNA sequencing by a custom multigene next generation sequencing panel and aCGH were performed.

Results: Transmission electron microscopy showed the absence of outer dynein arms in all analysed axonemes and abnormal inner dynein arm in more than $90 \%$ of axonemes. Target sequencing and microarray analysis detected a homozygous $6.9 \mathrm{~Kb}$ deletion including exons $7-8-9$ of DNAI2 gene.

Conclusions: In this study, we describe a novel homozygous deletion of exons 7-9 of DNAI2 found in a 4-month old girl with respiratory distress, ventriculomegaly, situs inversus, patent foramen ovale, absent outer dynein arms and abnormal inner dynein arms. Although most of the PCD cases are the result of mutations in gene encoding axonemal proteins or protein related to dynein arm assembly, variants in DNAI2 gene are rarely found in PCD patients. The application of high-throughput technologies allowed us, hence, to identify a new intragenic deletion of DNAI2 gene that could likely cause the loss of function of the protein resulting in a severe PCD phenotype.

M. Rocca: None. C. Vinanzi: None. A. Michelucci: None. M. Caligo: None. A. Valetto: None. M. De Santi:
None. V. Bertini: None. G. Piatti: None. C. Foresta: None.

\section{P13.29.A}

Haploinsufficiency of the Primary Familial Brain Calcification gene $S L C 20 A 2$ mediated by disruption of a regulatory element

K. CASSINARI ${ }^{1}$, A. Rovelet-Lecrux ${ }^{1}$, S. Tury ${ }^{2}$, O. Quenez ${ }^{1}$, A. Richard ${ }^{l}$, C. Charbonnier ${ }^{1}, R$. Olaso ${ }^{3}$, A. Boland $^{3}, J$. Deleuze $^{3}$, J. Besancenot ${ }^{4}$, B. Delpont ${ }^{4}$, D. Pouliquen ${ }^{5}$, F. Lecoquierre $^{l}$, P. Chambon ${ }^{1}$, C. Thauvin-Robinet ${ }^{6}, D$. Campion $^{1}$, T. Frebourg ${ }^{1}$, J. Battini ${ }^{7}$, G. Nicolas ${ }^{1}$

${ }^{I}$ Normandie Univ, UNIROUEN, Inserm U1245 and Rouen University Hospital, Department of Genetics and CNRMAJ, $F$ 76000, Normandy Center for Genomic and Personalized Medicine, Rouen, France, ${ }^{2}$ Institut de Recherche en Infectiologie de Montpellier (IRIM), Université de Montpellier, CNRS, Rouen, France, ${ }^{3}$ Centre National de Recherche en Génomique Humaine (CNRGH), Institut de Biologie François Jacob, CEA, Université ParisSaclay, F-91057, Evry, France, ${ }^{4}$ Department of internal medicine and systemic diseases, Dijon University Hospital, Dijon, France, ${ }^{5}$ Normandie Univ, UNIROUEN, Inserm U1245 and Rouen University Hospital, Department of Neurology and CNR-MAJ, F 76000, Normandy Center for Genomic and Personalized Medicine, Rouen, France, ${ }^{6}$ Inserm UMR 1231 GAD, Genetics of Developmental disorders, Université de Bourgogne-Franche Comté, FHU TRANSLAD, Dijon, France, ${ }^{7}$ Institut de Recherche en Infectiologie de Montpellier (IRIM), Université de Montpellier, CNRS, Montpellier, France

Objective: Primary Familial Brain Calcification (PFBC) is a rare cerebral microvascular calcifying disorder with diverse neuropsychiatric expression. Four autosomaldominant PFBC genes have been reported. Haploinsufficiency of SLC2OA2, which encodes an inorganic phosphate (Pi) importer, is a major cause of PFBC. During the analysis of exome sequencing data of 71 genetically unexplained unrelated PFBC patients, we detected an 8p11.21 deletion, mapping $35 \mathrm{~kb}$ upstream of SLC2OA2 and segregating with PFBC in 3 patients, and assessed its consequences on gene function.

Methods: We explored SLC20A2 blood expression by RT-ddPCR, performed an ex vivo Pi uptake assay and we deleted a putative SLC2OA2 enhancer mapping to this region in HEK293 cells by CRISPR/Cas9.

Results: The deletion carriers/normal controls ratio of relative $S L C 20 A 2$ mRNA levels was $60.2 \%(p<0.001)$. This was comparable to that of patients carrying an $S L C 20 A 2$ premature stop codon $(63.4 \%, p<0.001)$. The 
proband exhibited a $39.3 \%$ decreased Pi uptake in blood ( $p$ $=0.015)$. In HEK293 cells, we observed a $39.8 \%$ decrease in relative $S L C 20 A 2 \mathrm{mRNA}$ levels after normalization on DNA copy number $(p<0.001)$ in a bulk analysis.

Discussion: We identified a deletion encompassing an enhancer of SLC20A2 expression, with carriers showing haploinsufficiency in similar ranges than loss-of-function allele carriers. Deleting this element in a cellular model was associated with similarly reduced mRNA levels. Here, we used a simplified CRISPR/Cas9 assay associated to (RT-) ddPCR which allows a rapid study of DNA/RNA extracted from the cellular bulk. We propose a 3-step strategy to identify and easily assess the effect of such non-coding events.

K. Cassinari: None. A. Rovelet-Lecrux: None. S. Tury: None. O. Quenez: None. A. Richard: None. C. Charbonnier: None. R. Olaso: None. A. Boland: None. J. Deleuze: None. J. Besancenot: None. B. Delpont: None. D. Pouliquen: None. F. Lecoquierre: None. P. Chambon: None. C. Thauvin-Robinet: None. D. Campion: None. T. Frebourg: None. J. Battini: None. G. Nicolas: None.

\section{P13.30.B}

Cleft palate and left hydronephrosis in a fetus with a non-contiguous $6,7 \mathrm{Mb}$ gain and $2,1 \mathrm{Mb}$ loss in chromosome 22: unfolding an atypical ring chromosome from array to karyotype

S. Serafim ${ }^{l}$, B. Marques ${ }^{l}$, S. Pedrol, C. Alves ${ }^{l}$, A. R. Tarelho $^{l}$, L. Simao ${ }^{1}$, J. Furtado ${ }^{1}$, S. Rangel ${ }^{1}$, R. Peliano ${ }^{1}$, C. Ferreira ${ }^{l}$, N. Silva ${ }^{1}$, M. Silva ${ }^{l}, F$. Brito $^{l}$, A. Bernardo $^{2}$, I.

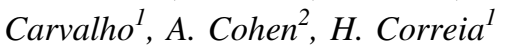

${ }^{1}$ Instituto Nacional de Saúde Dr. Ricardo Jorge, Departamento de Genética, Unidade de Citogenética, Lisboa, Portugal, ${ }^{2}$ Maternidade Alfredo da Costa, Lisboa, Portugal

Chromosomal microarray analysis (CMA) is the recommended genetic test in pregnancies with ultrasound abnormalities allowing the identification of small pathogenic copy number variations (CNV). Nevertheless karyotype may be needed to clarify the underlying mechanism of complex rearrangements. Here we report a fetus referred for prenatal diagnosis due to cleft palate and left hydronephrosis uncovered during second trimester ultrasound. Affymetrix Cytoscan 750 CMA revealed a $6,7 \mathrm{Mb}$ interstitial gain at 22q12.2q13.1 and a 2,1 Mb terminal loss at $22 q 13.32 q 13.33$, in a male fetus. The deletion includes SHANK3 whose haploinsufficiency causes PhelanMcDermid syndrome (PMS) and hence considered as a pathogenic CNV. Patients with PMS mainly exhibit neurological and behavioural symptoms, not detectable in a prenatal ultrasound, although hydronephrosis, as described in the fetus, is one of the few congenital abnormalities occasionally reported. To exclude a complex rearrangement and evaluate recurrence risk karyotype was performed both in the fetus and the parents revealing a de novo ring chromosome 22 in all cells analyzed. While terminal deletions in ring chromosomes are expected, the non-contiguous gain/ loss identified here questions whether this ring could have been generated by a mechanism of chromothripsis/chromoanagenesis, as recently described in a child with PMS and a complex ring 22. Although the duplicated region was considered of unknown clinical significance, the cleft palate observed in the fetus has not been described in PMS patients and may be a consequence of this more complex rearrangement. After genetic counseling the parents opted to terminate the pregnancy.

S. Serafim: None. B. Marques: None. S. Pedro: None. C. Alves: None. A.R. Tarelho: None. L. Simao: None. J. Furtado: None. S. Rangel: None. R. Peliano: None. C. Ferreira: None. N. Silva: None. M. Silva: None. F. Brito: None. A. Bernardo: None. I. Carvalho: None. A. Cohen: None. H. Correia: None.

P13.32.A

The intronic pathogenic variant in $S C N 2 A$ associated with neurodevelopmental disorder and epileptic encephalopathy.

\section{A. A. Sharkov ${ }^{1,2}$, P. Sparber ${ }^{3}$, A. Filatova $^{3}$, M. Skoblov ${ }^{3}, I$. Kanivets $^{2,4}$, D. Pyankov ${ }^{2}$, S. Korostelev ${ }^{2}$ \\ ${ }^{1}$ Veltischev Research and Clinical Institute for Pediatrics of the Pirogov RNRMU, Moscow, Russian Federation, ${ }^{2}$ Gen- omed LLC, Moscow, Russian Federation, ${ }^{3}$ Research Centre for Medical Genetics, Moscow, Russian Federation, ${ }^{4}$ Rus- sian Medical Academy of Continuous Professional Educa- tion, Moscow, Russian Federation}

We present a clinical case of 4-year-old girl with epileptic encephalopathy related to de novo intronic variant in $S C N 2 A$ gene. The pregnancy, delivery and family history were unremarkable. Psychomotor development was considered normal until 2 y.o., but slightly autistic features were recognised at 1 y.o. Seizures onset was at 18 months like as clusters of generalised tonic-clonic seizures, she had also daily generalised tonic seizures, myoclonic and myoclonic-atonic seizures, sometimes febrile provoked. Multiple antiepileptic drugs (AEDs) and hormonal treatment were not effective, the best effect was on the combination Carbamazepine with Levetiracetam. Severe developmental and motor regress, autistic traits, muscle hypotonia with high tendon reflexes, ataxia were reported at 2 y.o. EEG at 4 y.o. showed slow background, a bifrontal 
and generalized discharges, low-average index in the sleep. MRI was unremarkable. Gene panel showed a variant in SCN2A gene (chr2:166170123A $>$ G), confirmed de novo by Sanger. To investigate the potential impact of the NG_008143.1 (NM_021007.2): c.1035-7A $>$ G variant on splicing and due to the fact that $S C N 2 A$ gene expression is brain restricted, splicing minigene assay was performed. Wild-type and mutant alleles containing 9 and 10 exons with the corresponding intronic sequences were cloned into pSpl3-Flu2 plasmid. Minigene assay showed that c.1035$7 \mathrm{~A}$ variant activate a cryptic intronic acceptor site which leads to 6 nucleotide extension of exon 9 (NP_066287.2:p. (Gly345_Gln346insTyrSer). This insertion affects the extracellular domain of SCN2A protein where several pathogenic missense variants were previously described. To our knowledge, this is the first functional analysis of a splicing variant in $S C N 2 A$ gene.

A.A. Sharkov: A. Employment (full or part-time); Modest; Genomed LLC. B. Research Grant (principal investigator, collaborator or consultant and pending grants as well as grants already received); Modest; Marinus Pharmaceuticals. D. Speakers Bureau/Honoraria (speakers bureau, symposia, and expert witness); Modest; Eisai, Alkaloid AD Skopje, BioMarin. P. Sparber: None. A. Filatova: None. M. Skoblov: None. I. Kanivets: A. Employment (full or part-time); Modest; Genomed LLC. D. Pyankov: A. Employment (full or part-time); Modest; Genomed LLC. S. Korostelev: A. Employment (full or part-time); Modest; Genomed LLC.

\section{P13.33.B}

Cis-acting variants modify the behavior of PiS allele from moderately deficient to null phenotype in Alpha-1 Antitrypsin Deficiency

N. Matamalal, G. Gomez-Mariano ${ }^{1}$, J. Perez ${ }^{2}$, B. Baladrón $^{1}$, M. Torres-Durán ${ }^{3}, F$. Michel ${ }^{4}$, R. Saez ${ }^{4}, J$. Hernández-Pérez ${ }^{5}$, I. Belmonte ${ }^{6}, F$. Rodriguez-Frias ${ }^{6}, I$. Blanco $^{7}$, P. Strnad ${ }^{8}$, S. Janciauskiene ${ }^{9}$, B. MartinezDelgado $^{1,10}$

${ }^{1}$ Instituto de Salud Carlos III, Majadahonda, Madrid, Spain, ${ }^{2}$ Instituto Universitario de Enfermedades Tropicales y Salud Pública de Canarias, Universidad de La Laguna, Tenerife, Spain, ${ }^{3}$ Hospital Álvaro Cunqueiro. EOXI Vigo. Pneumovigo I+i. IIS Galicia Sur, Vigo, Spain, ${ }^{4}$ Hospital Universitario Donostia, Donostia, Spain, ${ }^{5}$ Hospital General de la Palma, Canarias, Spain, ${ }^{6}$ Hospital Vall d'Hebron, Barcelona, Spain, ${ }^{7}$ Registro Español de pacientes con déficit de alfa-1 antitripsina (REDAAT), Barcelona, Spain, ${ }^{8}$ University Hospital Aachen, Aachen, Germany, ${ }^{9}$ Hannover Medical School, Hannover, Germany, ${ }^{10}$ CIBER de

\section{Enfermedades Raras (CIBERER), Madrid, Spain}

Introduction: Alpha-1 Antitrypsin deficiency (AATD) is an inherited condition characterized by reduced levels of serum AAT due to mutations in SERPINA1 gene. The PiS (Glu264Val) is one of the most frequent deficient alleles of AATD which reduce by $40 \%$ the protein level. This allele is the most frequent in Caucasians.

Material and Methods: After genotyping the most frequent variants PiZ and PiS in AATD patients, there are deficient individuals with lower serum levels of AAT than expected for the initial genotype that should be further analyzed by sequencing coding exons of SERPINA1 gene, and protein phenotyped by isoelectric focusing. Novel variants were found and were functionally characterized by expressing them in a cellular model.

Results: We identify and characterize two new alleles carrying $\mathrm{S}$ mutation, but producing null phenotype. We describe two previously unknown missense variants confirmed to be in-cis configuration each of them with the $\mathrm{S}$ mutation, and demonstrated that they modify the properties of the PiS allele in terms of higher AAT intracellular retention and no extracellular secretion. Plasma samples from patients carrying these combinations confirmed nullphenotype behavior. These represent a novel type of AATD alleles that we named 'PiS-plus' alleles, as they have more pathogenic properties comparing to typical deficient PiS alleles, and modify clinical consequences.

Conclusions: This finding highlights that phasing genetic variants in AATD is an important issue to establish a definitive diagnosis. The discovery of different PiS-plus alleles could contribute to explain the phenotypical variability observed among patients. (Grant: AESI PI17CIII/00042).

N. Matamala: None. G. Gomez-Mariano: None. J. Perez: None. B. Baladrón: None. M. Torres-Durán: None. F. Michel: None. R. Saez: None. J. HernándezPérez: None. I. Belmonte: None. F. Rodriguez-Frias: None. I. Blanco: None. P. Strnad: None. S. Janciauskiene: None. B. Martinez-Delgado: None.

\section{P13.34.C}

Combined involvement of the human-specific duplications at 16p11.2 in iron metabolism and immune defense

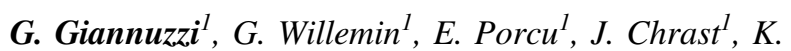
Hoekzema $^{2}$, 16p11.2 Consortium, Z. Kutalik ${ }^{1}$, E. Eichler ${ }^{2}$, A. Reymond ${ }^{l}$

${ }^{1}$ University of Lausanne, Lausanne, Switzerland, ${ }^{2}$ University of Washington, Seattle, WA, United States

Human-specific duplications at chromosome 16p11.2 
mediate recurrent pathogenic copy-number variations through non-allelic homologous recombination. These copy-number polymorphic duplications (3-8 copies) are under positive selection and include BOLA2, whose expansion might have an adaptive role in protecting against iron deficiency. It is unknown whether other genes mapping to this duplicate segment, like $S L X 1$ that takes part in the SLX1-SLX4 complex involved in the control of genome stability, might have a role too.

As SLX4 mutations are associated with the inability to produce blood cells, we assessed leukocyte differential counts in 379,385 controls and individuals who have lost or gained copies of BOLA2-SLX1: 89 chromosome 16p11.2 deletion and 56 reciprocal duplication carriers from the UK Biobank. We found that the deletion is associated with higher neutrophil $(P=7 \mathrm{e}-6)$ and lower lymphocyte $(P=3 \mathrm{e}-3)$ counts. Upon stratification of the deletion carriers of our clinical cohort by BOLA2-SLX1 copy number, we found an association between low BOLA2-SLX1 dosage and lymphocyte count $<1.5 \mathrm{M} / \mathrm{ml}$ of blood, as $4 / 7$ carriers with three copies had this phenotype compared to $0 / 12$ carriers with more than three copies $(P=9 \mathrm{e}-3)$. We are currently measuring leucocyte differential counts in $S l x l b$-knockout mice.

Our results show that the BOLA2-SLX1 copy number in humans is positively associated with lymphocyte counts and its increase might improve our immune system. They pinpoint one locus potentially involved in the lymphocyte count difference between humans and apes and highlight a potential dual adaptive role of BOLA2-SLX1 expansion in improving both iron metabolism and immune defense.

G. Giannuzzi: None. G. Willemin: None. E. Porcu: None. J. Chrast: None. K. Hoekzema: None. Z. Kutalik: None. E. Eichler: None. A. Reymond: None.

\section{P13.35.A}

\section{A new family with spastic paraplegia type 51 and novel mutations in AP4E1}

I. Winkler ${ }^{1}$, P. Miotla ${ }^{2}$, M. Lejman ${ }^{3}$, A. Pietrzyk ${ }^{4}$, M. Kacprzak ${ }^{4}$, M. Kubiak ${ }^{1}$, M. Sobczyńska-Tomaszewska,$I$. Jaszczuk ${ }^{3,5}$

${ }^{1}$ St'Johns Center Oncology, Lublin, Poland, ${ }^{2}$ II Department of Gynecology, Medical University of Lublin, Lublin, Poland, ${ }^{3}$ Department of Paediatric Haematology, Oncology and Transplantology, Children Clinical Hospital, II Dept. of Paediatrics, Lublin, Poland, ${ }^{4}$ MedGen Medical Centre, Warszawa, Poland, ${ }^{5}$ Departament of Cancer Genetics with Cytogenetic Laboratory, Medical University of Lublin, Lublin, Poland

Autosomal recessive mutations in AP-4 complex subunit €-
1 (AP-4E1) gene on chromosome 15q21.2 are known to cause spastic paraplegia 51 (SPG51). However, since only a few families affected by this mutation have been reported, the exact phenotype of SPG51 remains poorly characterized. In addition, a previous study identified an autosomal dominant mutation in AP4E1 gene to be associated with persistent stuttering. The aim of the present study was to characterize the phenotype of a pediatric patient displaying significant psychomotor retardation, intellectual disability and paraplegia. This phenotype was identified to be associated with a novel mutation in the AP4E1 gene, c.942_943 +3 delinsCC. The pediatric patient showed severe delayed psychomotor development, impaired global physical development and general illness. Movement disorders were evident during the neonatal period. Moreover, magnetic resonance imaging identified hypoplasia of the corpus callosum. The proband DNA was tested by multiplex ligationdependent probe amplification and array comparative genomic hybridization. Subsequently, next generating sequencing (NGS) was performed in the patient, and Sanger sequencing was performed in his family members. The present study identified a previously unknown diseaseinducing AP4E1 gene mutation via NGS.

\begin{tabular}{|c|c|c|c|c|c|c|c|}
\hline \multicolumn{8}{|c|}{ Clinical features of genetically confirmed APE1 patients } \\
\hline Identifier & Gender & $\begin{array}{l}\text { Age } \\
\text { at } \\
\text { onset }\end{array}$ & $\begin{array}{l}\text { Normal } \\
\text { speech }\end{array}$ & Spasticity & Epilepsy & $\begin{array}{l}\text { Other signs/ } \\
\text { symptoms } \\
\text { present }\end{array}$ & $\begin{array}{l}\text { Severe } \\
\text { Intellectual } \\
\text { disability }\end{array}$ \\
\hline $\begin{array}{l}\text { Reported } \\
\text { by } \\
\text { Moreno- de } \\
\text { Luca et al. }\end{array}$ & $\mathrm{F}$ & 23 & - & + & + & $\begin{array}{l}\text { Stereotypic } \\
\text { laughter,, } \\
\text { Babinski sign, } \\
\text { drooling, } \\
\text { hyperreflexia }\end{array}$ & + \\
\hline $\begin{array}{l}\text { Reported } \\
\text { by } \\
\text { Moreno- de } \\
\text { Luca et al. }\end{array}$ & M & 22 & - & + & + & $\begin{array}{l}\text { Stereotypic } \\
\text { laughter, } \\
\text { Babinski sign, } \\
\text { drooling, } \\
\text { hyperreflexia }\end{array}$ & + \\
\hline $\begin{array}{l}\text { Reported } \\
\text { by Abou- } \\
\text { Jamra et al. }\end{array}$ & M & 11 & - & + & - & $\begin{array}{l}\text { Foot deformity, } \\
\text { neonatal } \\
\text { hypotonia }\end{array}$ & + \\
\hline $\begin{array}{l}\text { Reported } \\
\text { by Abou- } \\
\text { Jamra et al. }\end{array}$ & $\mathrm{F}$ & 6 & - & + & + & $\begin{array}{l}\text { Stereotypic } \\
\text { laughter, foot } \\
\text { deformity, } \\
\text { neonatal } \\
\text { hypotonia }\end{array}$ & + \\
\hline $\begin{array}{l}\text { This report } \\
\text { (proband) }\end{array}$ & M & 12 & - & + & + & $\begin{array}{l}\text { Neonatal } \\
\text { hipotonia, } \\
\text { ambulation- } \\
\text { wheelchair }\end{array}$ & + \\
\hline $\begin{array}{l}\text { This report } \\
\text { (brother) }\end{array}$ & M & 16 & - & + & + & $\begin{array}{l}\text { Neonatal } \\
\text { hipotonia, } \\
\text { ambulation- } \\
\text { wheelchair }\end{array}$ & + \\
\hline
\end{tabular}

\begin{tabular}{|c|c|c|c|}
\hline \multicolumn{4}{|c|}{ Clinical features of proband and his family } \\
\hline & Proband & Brother & Father \\
\hline Intelligence & Severe intellectual disability & Deep intellectual disability & Normal \\
\hline $\begin{array}{l}\text { Speech } \\
\text { development }\end{array}$ & $\begin{array}{l}\text { A few words (He understands } \\
\text { simple commands) }\end{array}$ & $\begin{array}{l}\text { No speech development (He } \\
\text { doesn't understand } \\
\text { commands) }\end{array}$ & Normal \\
\hline Heigh & Short stature $(<3 \mathrm{pc})$ & Short stature $(<3 \mathrm{pc})$ & $\begin{array}{l}\text { Medium } \\
\text { stature }(10 \mathrm{pc})\end{array}$ \\
\hline Microcephaly & Present & Present & No \\
\hline Neurologic & Yes & Yes & No \\
\hline
\end{tabular}


spastic

Movement Crawling, standing only with Crawling, standing and Total suport. He doesn't walk. walking only with support Contractures. Abduction Increasing contractures contracture of the fingers. despite rehabilitation. Knee valgus. Hypoplas of Abduction contracture of the corpus callosum. Several the fingers. Knee valgus. diabetes episodes of increased

drowsiness, increased ptosis,

decrease in muscle tone.

Metabolic and mitochondria

diseases were excluded.

I. Winkler: None. P. Miotla: None. M. Lejman: None. A. Pietrzyk: None. M. Kacprzak: None. M. Kubiak: None. M. Sobczyńska-Tomaszewska: None. I. Jaszczuk: None.

\section{P13.37.C}

Genetic effects on splicing and expression in normal colon tissue gives insights into the genetic architecture of complex traits and diseases

V. Diez-Obrero ${ }^{1,2,3,4}$, M. Devall ${ }^{5,6}$, F. Moratalla-

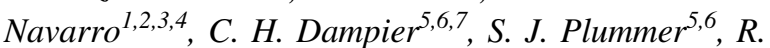

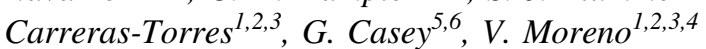

${ }^{1}$ Oncology Data Analytics Program, Catalan Institute of Oncology, L'Hospitalet de Llobregat (Barcelona), Spain, ${ }^{2}$ ONCOBELL Program, Bellvitge Biomedical Research Institute (IDIBELL), L'Hospitalet de Llobregat (Barcelona), Spain, ${ }^{3}$ Consortium for Biomedical Research in Epidemiology and Public Health (CIBERESP), Madrid, Spain, ${ }^{4}$ Department of Clinical Sciences, Faculty of Medicine, University of Barcelona, Barcelona, Spain, ${ }^{5}$ Center for Public Health Genomics, University of Virginia, Charlottesville, VA, United States, ${ }^{6}$ Department of Public Health Sciences, University of Virginia, Charlottesville, VA, United States, ${ }^{7}$ Department of Surgery, University of Virginia, Charlottesville, VA, United States

Associations of genetic variation with tissue-specific alternative splicing and gene expression can help characterize the molecular basis of gene regulation, complex trait risk loci, and disease mechanisms. Therefore, cataloguing quantitative trait loci (QTLs) across tissues is a priority in complex disease genetics.

In this study, we profiled alternative splicing and gene expression with bulk RNA sequencing (RNA-seq) in colon tissue biopsies from a novel dataset of 445 healthy individuals.

We found transcriptomic differences between colon anatomic locations and identified 1,817 splicing QTLs (sQTLs) and 9,831 expression QTLs (eQTLs). These QTLs were enriched at regulatory elements and explained a proportion of genetic heritability not only for colorectal cancer and inflammatory bowel disease risk, but also for cognitive traits and psychiatric and neurodegenerative disease risk. We replicated the results using GTEx transverse colon data and made an interactive web resource to explore the results (http://barcuvaseq.org/).

Overall, our findings provide evidence of genetic regulation of alternative splicing and gene expression in colon tissue at risk loci for complex traits and diseases.

Grant references: NIH/NCI CA143237; NIH/NCI CA204279; NIH/NCI CA201407; ISC III - FEDER PI1400613; CIBERESP CB07/02/2005; Catalan Government DURSI 2014SGR647; EU H2020 - MSC grant No 796216; FPU16/00599.

V. Diez-Obrero: None. M. Devall: None. F. MoratallaNavarro: None. C.H. Dampier: None. S.J. Plummer: None. R. Carreras-Torres: None. G. Casey: None. V. Moreno: None.

P13.38.A

PopDel identifies medium to large deletions jointly in tens of thousands of genomes

S. Niehus ${ }^{1,2}$, J. Schönberger ${ }^{1,2}$, H. Jónsson ${ }^{3}$, E. Björnsson ${ }^{3}$, D. Beyter ${ }^{3}$, H. P. Eggertsson ${ }^{3}$, P. Sulem ${ }^{3}$, K. Stefánsson ${ }^{3,4}$, B. V. Halldórsson ${ }^{3,5}$, B. Kehr ${ }^{1,2}$

${ }^{1}$ Berlin Institute of Health (BIH), Berlin, Germany, ${ }^{2}$ Charité - Universitätsmedizin Berlin, corporate member of Freie Universität Berlin, Humboldt-Universität zu Berlin, and Berlin Institute of Health, Berlin, Germany, ${ }^{3}$ deCODE genetics/Amgen Inc, Reykjavik, Iceland, ${ }^{4}$ Faculty of Medicine, School of Heath Sciences, University of Iceland, Reykjavik, Iceland, ${ }^{5}$ School of Science and Engineering, Reykjavik University, Reykjavik, Iceland

Catalogs of genetic variation for large numbers of individuals are a foundation for modern research on human diversity and disease. Creating such catalogs for small variants from whole-genome sequencing (WGS) data is now commonly done for thousands of individuals collectively. We have transferred this joint calling idea from SNPs and indels to larger deletions and developed the first joint calling tool, PopDel, that can detect and genotype deletions in WGS data of tens of thousands of individuals simultaneously as demonstrated by our evaluation on data of up to 49,962 human genomes. Good sensitivity, precision and the correctness of genotypes are demonstrated by extensive tests on simulated and real data and comparison to other state-of-the-art SV-callers. PopDel detects deletions in 
HG002 and NA12878 with high sensitivity as shown by the large overlap with different high-confidence reference call sets while maintaining a low false positive rate. On data of up to 6,794 trios, inheritance patterns are in concordance with Mendelian inheritance rules and exhibit a close to ideal transmission rate of $49.6 \%$. PopDel reliably reports common, rare as well as de novo deletions and the deletions reflect reported population structure. Meaningful quality measures and metrics of the variants like genotype quality, variant likelihood ratio and allele frequency within the cohort are reported along with the variants, enabling further downstream analysis. Therefore, PopDel enables routine scans for deletions in large-scale sequencing studies and we are currently in the process of implementing the detection of other SV-types. BMBF Grant \#031L0180

S. Niehus: None. J. Schönberger: None. H. Jónsson: None. E. Björnsson: None. D. Beyter: None. H.P. Eggertsson: None. P. Sulem: None. K. Stefánsson: None. B.V. Halldórsson: None. B. Kehr: None.

\section{P13.42.B}

The chromatin 3D structure reorganization in Trisomy 21

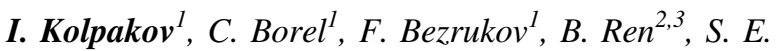 Antonarakis ${ }^{l}$}

${ }^{1}$ University of Geneva, Geneva, Switzerland, ${ }^{2}$ Ludwig Cancer Research Institute, San Diego, CA, United States, ${ }^{3}$ School of Medicine, University of California, San Diego, CA, United States

The pathogenetic mechanisms of human trisomy 21 (T21) include the dosage effect of functional genomic elements on chr21, global dysregulation of gene expression and the individual genomic variability. In this study, we hypothesize that the extra-copy of chr21 may change the chromatin $3 \mathrm{D}$ structure, which in turn may affect gene expression. We have used $\mathrm{HiC}$ experiments in 3 primary fibroblast cell lines from a pair of monozygotic twins discordant for T21 (to eliminate the noise of the genome sequence variation). We have also used transcriptome, Lamin-associated domains (LAD from PMID, and 24740065) H3K4me3 datasets from fibroblasts. No differences between T21 and euploid cischromatin interactions (TADs) were detected. We have, however, observed significant differences in higher-order chromatin contacts, largely explained by differences in A/B compartmentalization. Remarkably, we have detected 83 genomic regions $(>1 \mathrm{Mb})$ that change the compartment in $\mathrm{T} 21$ (from $\mathrm{B}$ to $\mathrm{A}$, or $\mathrm{A}$ to $\mathrm{B}$ ). These reorganized regions
$(\Delta \mathrm{ABt} 21)$ comprise a total of $\sim 250 \mathrm{Mb}$ of the genome. Genes within $\Delta \mathrm{ABt} 21$ regions display a dysregulated expression in T21: upregulated in the $\mathrm{A}$, and downregulated in the B compartment. A substantial number of the $\Delta \mathrm{ABt} 21$ regions are constitutive annotated as LADs suggesting a partial repositioning of sequences at the nuclear envelope components to the transcriptionally active compartment in T21. A fraction of the observed differences were also observed in comparisons between unrelated T21 and euploid fibroblasts. In conclusion, we have identified measurable chromatin structure differences in T21 versus an euploid nucleus, that likely result in gene expression differences in $\mathrm{T} 21$.

I. Kolpakov: None. C. Borel: None. F. Bezrukov: None. B. Ren: None. S.E. Antonarakis: None.

P13.43.C

Topological Assessment of the $K C N Q 1$ variants: a genespecific approach for variant interpretation

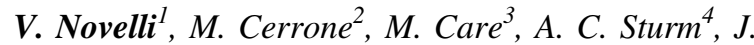

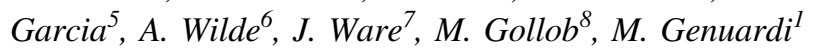

${ }^{1}$ Fondazione Policlinico A. Gemelli, IRCCS; Catholic University of the Sacred Heart, Rome, Italy, ${ }^{2}$ New York University, NYC, NY, United States, ${ }^{3}$ Toronto General Hospital and University of Toronto, Toronto, ON, Canada, ${ }^{4}$ Geisinger Genomic Medicine Institute, Danville, CA, United States, ${ }^{5}$ Invitae Corp., San Francisco, CA, United States, ${ }^{6}$ Heart Center, Department of Clinical and Experimental Cardiology, Amsterdam, Italy, ${ }^{7}$ National Heart and Lung Institute and Medical Research Council London Institute of Medical Sciences, Imperial College London, London, United Kingdom, ${ }^{8}$ Division of Cardiology, Toronto General Hospital and University of Toronto,, Toronto, ON, Canada

Background: Variant interpretation is becoming the most challenging step of genetic testing. The introduction of the ACMG guidelines set more stringent criteria, trying to standardize variant assessment in the clinical settings. However, this approach has universal applicability and therefore requires significant expertise to be applied correctly for specific genes and diseases.

Purpose: we highlight the potential role of the location of variants within the protein topology for ion channels genes, supporting variant interpretation process.

Methods and Results: Data from 3580 probands, referred for LQTS genetic testing, was analyzed for $K C N Q 1$ gene variants. In particular, the N-Terminal, 
Transmembrane linker pore region (TLP) and C-Terminal regions were used to assess the variant position. Subsequently, four conservative helical regions, within the Cterminal, were incorporated in the analysis. The GnomAD population was used as a control. Our analysis revealed that missense variants localized in the TLP have the highest probability for pathogenicity (expected predictive value $(E P V)=98,4$. Within the TLP, the pore and interlink domains showed the highest EPV, 100\%, and $99.8 \%$ respectively. Hence results confirmed the critical role of the four helical regions within the C- terminal. In particular, helix B and helix C provide an EPV of $100 \%$, identifying a critical conserved region between 509-562 aa.

Conclusions: Topological location of variants in the $K C N Q 1$ gene can be used as a gene-specific criterion for variant interpretation. Further studies stratifying the risk based on the variant location also in other ion channel genes could open new frontiers for the clinical application of genetic testing results.

V. Novelli: None. M. Cerrone: None. M. Care: None. A.C. Sturm: None. J. Garcia: None. A. Wilde: None. J. Ware: None. M. Gollob: None. M. Genuardi: None.

\section{P13.44.A}

\section{ENIGMA - Etalon of National Interpreted Genome Map of the Czech population}

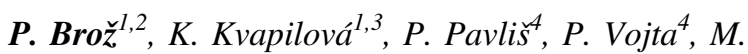
Bendová $^{4}, J$. Drábek ${ }^{4}$, J. Srovnal ${ }^{4}$ L. Daniel ${ }^{1}$, P. Kvapil ${ }^{1}$, M. Hajdúch ${ }^{4}$

${ }^{1}$ Institute of Applied Biotechnologies a.s., Prague, Czech Republic, ${ }^{2}$ Second Faculty of Medicine Charles University, Prague, Czech Republic, ${ }^{3}$ Faculty of Science Charles University, Prague, Czech Republic, ${ }^{4}$ Institute of Molecular and Translational Medicine, Faculty of Medicine and Dentistry, Palacky University, Olomouc, Czech Republic

Introduction: Due to the variability of the human genome the proper interpretation of genomic data is fundamentally dependent on the appropriate bioinformatics pipeline and reference database. Local human genome standards serve as a reference, furthermore, the general ones help clinical geneticists to discern pathogenic mutations from benign polymorphisms.

Materials and Methods: Samples were selected following strict criteria from Czech healthy blood donors. Nextgeneration sequencing was performed using the PCR-free whole genome kit followed by sequencing on Illumina NovaSeq 6000 (WGS). The genome variant calling format was generated by the Dragen Bio-IT platform. Variants were annotated using the Variant Effect Predictor version 99 and the bioinformatic pipeline was validated using the Genome in a Bottle standard. RSIDs were obtained from dbSNP build 153. Clinical relevance annotations were generated using ClinVar version 20200210.

Results: 100 samples were sequenced with average read depth $>30 X$. The median of the total number of variants was $4,736,609$, the median number of: heterozygous 2,904,376, homozygous 1,930,728, SNPs 3,823,298, INDELs 902,433 and MNPs 11,187, biallelic 4,236,049 and multiallelic 501,887. The median number of transitions $3,568,436$ and transversions $1,841,354$ with $\mathrm{Ti} / \mathrm{Tv}$ ratio 1.9 .

Conclusions: WGS protocol including the bioinformatics analysis was created and validated to abide the EN ISO 15189 requirements. The variant analysis pipeline has been verified and validated using the genome standard. The draft of the digital genome of the Czech population was created and will be expanded to up to 1,000 samples. CZ.01.1.02/ 0.0/0.0/16_084/0010360, LM2015064

P. Brož: None. K. Kvapilová: None. P. Pavliš: None. P. Vojta: None. M. Bendová: None. J. Drábek: None. J. Srovnal: None. L. Daniel: None. P. Kvapil: None. M. Hajdúch: None.

\section{P13.46.C}

Costitutional chromoanasynthesis: a familiar chromosome explosion

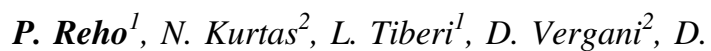
Formicola $^{2}, F$. Vanderwert ${ }^{l}$, A. Provenzano ${ }^{1}$, A. Pagliazzi ${ }^{2}$, S. Guarducci ${ }^{2}$, O. Zuffardi ${ }^{3}$, S. Giglio ${ }^{1,2}$

${ }^{1}$ Dpt. of Biomedical Experimental and Clinical Sciences "Mario Serio", University of Florence, Florence, Italy, ${ }^{2}$ Medical Genetics Unit, Meyer Children's University Hospital, Florence, Italy, ${ }^{3}$ Dpt. of Molecular Medicine, University of Pavia, Pavia, Italy

Chromoanasynthesis is characterized by extensive genomic rearrangements including variable DNA copy-number changes and several breakpoints, clustered into one or few chromosomes. Here, we studied a family case with a multiple abortion history and four affected members showing neurological defects. The index case is a three-year-old girl characterized by corpus callosum hypoplasia, bilateral cleft palate, speech delay and facial dysmorphisms. The father, paternal aunt and grandmother showed partial corpus callosum hypoplasia, psychiatric disorder and short stature, respectively. By conventional cytogenetics, we detected an unbalanced reciprocal translocation $\mathrm{t}(4 ; 8)(\mathrm{p} 15.2 ; \mathrm{q} 12)$ segregating in the four individuals through three-generation. 
Array-CGH showed a 5,8 Mb duplication at 4p15.32-p15.2 in all the translocation carriers. In order to better characterize the chromosomal rearrangement, we performed a whole genome sequencing (WGS) 50x of the proband, father and grandmother. As a result, we revealed the hidden complexity of the translocation event classified as chromoanasynthesis. Taking advantage from discordant reads and coverage depth, we detected six breakpoints clustered within $12,6 \mathrm{Mb}$ on $4 \mathrm{p}$ and $8 \mathrm{q}$ arms, duplications, deletions and inversions, defining the intricate genomic architecture of the translocation. All the breakpoints were confirmed by sanger sequencing, showing that only NKAIN3 is disrupted in its intron. NKAIN3 has not been associated with any known human disease, but its high expression in corpus callosum highlights the gene as a potential candidate for the neurological findings. Our data call for WGS as first tier genomic analysis in order to properly delineate the complexity of chromosomal rearrangements and shed light on the genotype-phenotype association.

P. Reho: None. N. Kurtas: None. L. Tiberi: None. D. Vergani: None. D. Formicola: None. F. Vanderwert: None. A. Provenzano: None. A. Pagliazzi: None. S. Guarducci: None. O. Zuffardi: None. S. Giglio: None.

\section{P13.47.A}

Whole genome sequencing of unexplained inherited retinal disease cases

Z. Fadaie ${ }^{1}$, L. Whelan', A. Dockery', T. Ben-Yosef ${ }^{3}, J$.

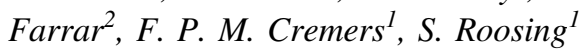

${ }^{1}$ Radboud University Medical Center, Nijmegen, Netherlands, ${ }^{2}$ The School of Genetics \& Microbiology, Smurfit Institute of Genetics, Trinity College Dublin, Dublin, Ireland, ${ }^{3}$ The Rappaport Faculty of Medicine, TechnionIsrael Institute of Technology, Haifa, Israel

Purpose: Inherited retinal diseases are a major cause of visual impairment. This clinically heterogeneous disorders are caused by mutations in many genes. Nevertheless, 30$40 \%$ of cases remain genetically unexplained. Therefore, we aimed to identify the genetic cause in unresolved cases through whole genome sequencing (WGS).

Methods: After gene-panel or exome sequencing prescreening methods, we performed WGS to identify the causative variants in 100 unresolved cases. After initial prioritization, a focused in-depth prioritization took place of all deep-intronic variants and structural variants in genes with candidate variants. In addition, functional analysis of the putative splice-site altering variants was performed using a midigene-based in-vitro splice assay.

Results: We identified a genetic cause in 21 patients. Among others, causative coding variants were observed in genes such ATXN7, CEP78, EYS, FAM161A, PEX5, and $R P G R I P 1$, . Gene disrupting structural variants were also observed in $P D E 6 B$ and EYS. Moreover, in 49 monoallelic cases, we identified candidate variants based on the possible introduction of a cryptic splice site leading to a pseudoexon. For variants with a good genotype-phenotype correlation invitro splice assays were performed.

Conclusions: We confirmed that WGS is a powerful tool to identify causative variants, when pre-screening methods remain negative. This was most efficient in cases with a distinct clinical diagnosis. We highlight the importance of the analysis of non-coding regions beyond the noncanonical splice-sites. We suggest that WGS provides the optimal detection method to enable a genetic diagnosis for unresolved cases.

Funding: Foundation Fighting Blindness USA Project Program Award, Uitzicht, Fighting Blindness Ireland.

Z. Fadaie: None. L. Whelan: None. A. Dockery: None. T. Ben-Yosef: None. J. Farrar: None. F.P.M. Cremers: None. S. Roosing: None.

\section{P14 Cytogenetics}

\section{P14.01.C}

$1 p 36$ deletion syndrome - Iasi Regional Medical Genetics Centre's Experience

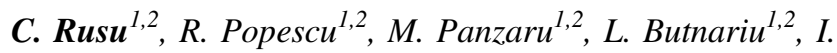 \\ Resmerita $^{l}$, E. Gavril ${ }^{1}$
}

I"Grigore T Popa" University of Medicine and Pharmacy, Iasi, Romania, "2f Maria" Children's Hospital, Regional Medical Genetics Centre, Iasi, Romania

1 p36 deletion syndrome is a common subtelomeric microdeletion (1/5,000 newborns), characterized by distinctive facial features, hypotonia, intellectual disability, seizures, heart defects, hearing impairment, and prenatal onset growth deficiency. Although usually clinically recognizable, the syndrome has a considerable phenotypic variation. We present six cases identified using MLPA and/or Array$\mathrm{CGH}$ in our center in order to illustrate particularities found in our patients and correlate them with genotype. We identified 3 de novo interstitial $1 \mathrm{p} 36$ microdeletions of different sizes (2.48 $\mathrm{Mb}$ to $5.90 \mathrm{Mb}$ ) and 3 with reciprocal duplication (one patient with 4q35.1dup and two brothers with 14q32.33dup). All cases had a wide phenotypic spectrum. Most cases had perinatal distress and hypotonia. Constant features were severe intellectual disability and seizures. Even if straight eyebrows, deep-set eyes and pointed chin are considered highly suggestive for the diagnosis, they are present only in some of the cases. The particularities found in the "pure" 1p36 microdeletions 
include: familial hypocalciuric hypercalcemia, pilonidal sinus, tall stature, obesity, rare eyebrows in external 1/3 (2 cases). The additional chromosomal anomalies present in 3 of the patients contribute to the phenotype severity, and additionally modify the clinical manifestations of $1 \mathrm{p} 36$ deletion. Analyzing the familial cases, we noticed that the same chromosomal anomaly generates distinct phenotypes in the two brothers. In conclusion, we are presenting six cases with $1 \mathrm{p} 36$ microdeletion to discuss the frequency of definitory features and illustrate particularities.

C. Rusu: None. R. Popescu: None. M. Panzaru: None. L. Butnariu: None. I. Resmerita: None. E. Gavril: None.

\section{P14.03.B}

\section{The utility of FISH for post-array CGH investigations}

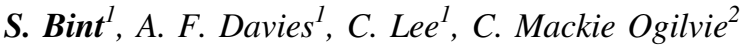

${ }^{1}$ Genetics Laboratories, Guy's Hospital, London, United Kingdom, ${ }^{2}$ King's College London, London, United Kingdom

Array CGH is widely used for the detection of chromosome imbalance in patients with congenital anomalies or neurodevelopmental disorders. The significance of unique interstitial copy number imbalances detected by this technique is largely informed by the carrier status of the parents, who are generally tested by targeted array CGH. If neither parent carries the imbalance, further studies may be carried out using fluorescence in situ hybridisation (FISH) to establish if either parent carries a balanced chromosome rearrangement. In the absence of such a rearrangement, their recurrence risk approaches zero; however, a balanced rearrangement results in a 50\% risk of imbalance in future pregnancies. Since 2008, our centre has tested approximately 50,000 patients, with imbalances detected in $\sim 22 \%$; after parental array CGH testing 1,083 imbalances were not detected in either parent. Parents and probands of 203 nonLCR-mediated cases were tested using FISH probes targeted at the regions of imbalance. Deletions represented 175 of these cases, one of which was inherited from a parent with a balanced rearrangement (ins $(1 ; 11)$ ). There were 28 duplications, 27 of which were tandem, and the other inherited from a parent with a balanced rearrangement (ins $(6 ; 4))$. The overall prevalence of parental balanced rearrangements in these cases is therefore $2 / 203(\sim 1 \%)$. This figure is lower than that previously published, and may indicate that testing for these rearrangements may not be financially justified. Interestingly, we found no cases of de novo duplications caused by insertion of the duplicated region into a distant locus, indicating that this mutational event is rare.
S. Bint: None. A.F. Davies: None. C. Lee: None. C. Mackie Ogilvie: None.

P14.04.C

Retrospective investigation of array-CGH prenatal diagnosis at Geneva Hospital: a cohort of 626 pregnancies

S. Gimelli ${ }^{l}$, E. Stathaki ${ }^{l}$, V. Kremer ${ }^{l}$, B. Martinez de Tejada Weber $^{l}$, P. Extermann ${ }^{2}$, J. Pellegrinelli ${ }^{l}$, M. Billieux ${ }^{l}, I$. Eperon $^{1}$, V. Mirlesse ${ }^{1}$, B. Heggel-Hort ${ }^{3}$, B. Plaschy ${ }^{4}$, T. Von Kanel $^{4}$, S. Fokstuen ${ }^{1}$, R. Robyr ${ }^{5}$, E. Ranza ${ }^{6,1}, S$. Antonarakis $^{6,7}$, M. Abramowicz ${ }^{l}, F$. Sloan-Béna ${ }^{l}$

${ }^{1} H U G$, Geneva, Switzerland, ${ }^{2}$ Dianecho, Geneva, Switzerland, ${ }^{3}$ Valais Hospital, Sion, Switzerland, ${ }^{4}$ Valais Hospitals, Sion, Switzerland, ${ }^{5}$ Echofemme, Geneva, Switzerland, ${ }^{6}$ Medigenome, Geneva, Switzerland, ${ }^{7}$ University of Geneva, Geneva, Switzerland

Chromosomal microarray (CMA) is recommended prenatally as a first-tier investigation in case of abnormal fotal ultrasound. Following the identification of fœetal anomalies, molecular diagnostic investigations using CMA will diagnose a clinically significant subchromosomal copy number variation $(\mathrm{CNV})$ in approximately $6 \%$ of pregnancies. We performed a retrospective study including 626 fetuses referred to Genetic Medicine in Geneva Hospitals, Switzerland, between 2013 and 2019. The cohort consisted of 458 amniotic fluids and 168 chorionic villi. Referrals for abnormal ultrasound findings were the most frequent indication. Other indications were: high risk for trisomy in maternal serum screening or NIPT, advanced maternal age and family history of chromosomal abnormalities. Cytogenetic investigations included Agilent 44K oligonucleotide array-CGH and karyotyping analysis. Array-CGH identified pathological numerical or large structural rearrangements in a total of 32 cases $(5 \%)$ and microdeletions/duplications associated to known syndromes in 16 foetuses $(2,5 \%)$. Variants of unknown significance were identified in 10 pregnancies $(1,5 \%)$.Conventional karyotyping identified 2 additional aberrations with one de novo balanced translocation and one case of low trisomy 16 mosaicism. After years of experience, our center has developed a strong technical and clinical experience of CMA testing in prenatal diagnosis. This approach improves the yield of chromosome aberration detection for fetuses with abnormal ultrasound scans. In addition, karyotyping remains useful for the mechanistic explanation of chromosome rearrangement and prevention of recurrence in some cases.

S. Gimelli: None. E. Stathaki: None. V. Kremer: None. B. Martinez de Tejada Weber: None. P. Extermann: None. J. Pellegrinelli: None. M. Billieux: None. I. 
Eperon: None. V. Mirlesse: None. B. Heggel-Hort: None. B. Plaschy: None. T. Von Kanel: None. S. Fokstuen: None. R. Robyr: None. E. Ranza: None. S. Antonarakis: None. M. Abramowicz: None. F. Sloan-Béna: None.

\section{P14.06.B}

De novo deletion of the $B C L 11 A$ gene in 2 p16.1

\section{A. Röpke, Y. Stratis, C. Müller-Hofstede, A. Bohring, F. Tüttelmann}

\section{University Hospital Münster, Münster, Germany}

We report on a 17-month-old girl of nonconsanguineous parents. The pregnancy had occurred after intracytoplasmic sperm injection. The girl presented with difficulties of feeding, speech and motor delay, microcephaly, brachycephaly, and unspecific craniofacial dysmorphism. The cranial MRI was unremarkable. Array analysis discovered a heterozygous deletion of approximately $156 \mathrm{~kb}$ in $2 \mathrm{p} 16.1$, confirmed in the child and excluded in parents by quantitative PCR. This deletion encompasses exclusively the entire BCL11A gene. Sequence variations in BCL11A (MIM 606557) are associated with the autosomal-dominant Dias-Logan syndrome (MIM 617101). Larger deletions including the BCL11A and neighboring genes were found in patients with the 2p16.1-p15 deletion syndrome (MIM 612513). Both syndromes are characterized by developmental delay, intellectual disability, microcephaly, dysmorphic features and persistent fetal hemoglobin. Deletions in 2p16.1 comprising only the BCL11A gene are rarely reported. Until now, only two patients with a BCL11A deletion were described. The patient described by Balci et al. (2015) showed brain malformations, developmental delay and distinctive facial features. Peter at al. (2014) reported a patient with hypotonia, delays in gross motor skills and a severe speech disorder. However, this patient had no cranial and skeletal anomalies, microcephaly or growth retardation. In summary, this is the third patient described with entire but exclusively deletions of BCL11A. These patients demonstrate features of Dias-Logan syndrome which broadly overlap with 2p16.1-p15 deletion syndrome and support the assumption that BCL11A is the candidate gene of most findings seen in patients with 2p16.1-p15 deletion.

A. Röpke: None. Y. Stratis: None. C. Müller-Hofstede: None. A. Bohring: None. F. Tüttelmann: None.

\section{P14.07.C}

A high detection rate for chromosomal microarray testing in 837 microcephaly cases.
R. Sukenik-Halevy, L. Basel-Salmon, R. Tomashov Matar, S. Kahanaa, S. Yacobson, I. Agmon-Fishman, I. Maya

Recanati Genetic Institute, Rabin Medical Center, Petah Tikva, Israel

Introduction: Microcephaly is a deviation from normal head growth that may be the first indication of an underlying genetic syndrome. We aimed to assess the yield of chromosomal microarray (CMA) testing for prenatally detected microcephaly as well as for postnatal cases.

Materials and Methods: CMA testing was performed for 88 prenatally detected microcephaly cases and for 749 postnatal cases at a single lab. We assessed the yield of CMA in relation to specific clinical characteristics.

Results: Pathogenic and likely pathogenic (LP) results were detected in $4.6 \%$ of prenatal cases, significantly higher than the rate reported in low risk pregnancies $(1.4 \%)$. The male to female ratio in this cohort was 3 . The yield was not affected by gender nor by any other clinical parameter. The yield for postnatal cases was $15 \%$ with a significantly higher detection rate for cases in which dysmorphism, hypotonia, epilepsy, congenital heart defects (CHD), learning disabilities (LD) and a history of intrauterine growth restriction (IUGR) were reported. There were no specific recurrent copy number variations (CNVs). The rate of variants of unknown significance was $3.94 \%$.

Conclusions: The yield of CMA testing is significant both for prenatal as well as for postnatal microcephaly cases. Microcephaly combined with dysmorphism, hypotonia, epilepsy, CHD, LD and IUGR significantly increases the likelihood of an abnormal CMA result.

\begin{tabular}{llllll}
\hline & $\begin{array}{l}\text { pathogenic } \\
\text { and } \\
\text { LP CNVs }\end{array}$ & $\begin{array}{l}\text { Karyotype } \\
\text { detectable }\end{array}$ & $\begin{array}{l}\text { Known } \\
\text { syndrome }\end{array}$ & $\begin{array}{l}\text { Involvement of a } \\
\text { known } \\
\text { pathogenic gene }\end{array}$ & $\begin{array}{l}\text { Additional } \\
\text { CNVs }\end{array}$ \\
\hline Prenatal (88) & $4.6 \%$ & $25 \%$ & $75 \%$ & $0 \%$ & $25 \%$ \\
Postnatal (749) & $15 \%$ & $17.9 \%$ & $60.1 \%$ & $18.75 \%$ & $17 \%$ \\
Total (837) & $13.9 \%$ & $18.1 \%$ & $61.2 \%$ & $18.1 \%$ & $17.2 \%$ \\
\hline
\end{tabular}

R. Sukenik-Halevy: None. L. Basel-Salmon: None. R. Tomashov Matar: None. S. Kahanaa: None. S. Yacobson: None. I. Agmon-Fishman: None. I. Maya: None.

P14.08.A

The chromosomal microarray analysis of products of conception in pregnancy loss. 
E. G. Panchenko ${ }^{I}$, I. V. Kanivets ${ }^{1,2}$, I. I. Romanova ${ }^{1,3}$, Y. K. Kievskayal, E. V. Kudryavtseva, ${ }^{1,4}$ D. V. Pyankov ${ }^{1}$, S. A. Korostelev $^{I}$

${ }^{1}$ Genetic Center «Genomed», Moscow, Russian Federation, ${ }^{2}$ Russian medical Academy of continuing professional education of the Ministry of health of the Russian Federation, Moscow, Russian Federation, ${ }^{3}$ Federal University of Kazan, Kazan, Russian Federation, ${ }^{4}$ Ural State Medical University, Yekaterinburg, Russian Federation

Introduction: The miscarriage is an actual problem, that frequency is from $15-25 \%$ to $55 \%$, reaching $80 \%$ in the first trimester. The reccurent pregnancy loss (RPL) is observed in $2-5 \%$ of cases. Chromosomal abnormalities (CAs) are a common cause of miscarriages and RPL. In 2014-2019 we performed 2201 analyses of POC to evaluate the prevalence and structure of chromosomal abnormalities.

Materials and Methods: 2201 samples of DNA from POC were studied by the chromosomal microarray analysis (CMA), using CytoScan Optima array (ThermoFisher Scientific, USA).

Results: CAs were detected in $49.57 \%$ of cases, of which aneuploidy of one chromosome, including mosaic forms, were detected in $72.69 \%$, aneuploidy more, then one chromosome, including mosaic forms $-6.97 \%$, triploidy $-10.72 \%$, other CAs with possible clinical significance $-8.62 \%$ (of these $-15.95 \%$ were terminal deletions and duplications of non-homologous chromosomes, indicating a high probability of unbalanced translocation), tetraploidy - $1.01 \%$. In $5.7 \%$ samples the size of CAs was less, than the theoretical resolution of the karyotype analysis (10Mb), of which in $48.48 \%$ of cases, such CAs were found as part of complex CAs.

Conclusions: Chromosomal abnormalities in POC can be cause of miscarriages and RPL and some complex rearrangements may present an increased risk for next pregnancies. In our study CAs were detected in $49,57 \%$ POC samples.

E.G. Panchenko: None. I.V. Kanivets: None. I.I. Romanova: None. Y.K. Kievskaya: None. E.V. Kudryavtseva: None. D.V. Pyankov: None. S.A. Korostelev: None.

\section{P14.11.A}

Incidence of constitutional chromosome aberrations in patients with hematological diseases

M. Valerianova ${ }^{l}$, S. Ransdorfova ${ }^{1,2}$, I. Sarova $^{1,2}, Z$. Zemanova $^{1,2}$, M. Onderkova ${ }^{1}$, S. Izakova ${ }^{2}$, A. Jonasova $^{3}, J$. Cermak $^{l}$, J. Brezinova ${ }^{l}$

${ }^{1}$ Institute of Hematology and Blood Transfusion, Prague, Czech Republic, ${ }^{2}$ Center of Oncocytogenomics, Institute of
Medical Biochemistry and Laboratory Diagnostics, General University Hospital and First Faculty of Medicine, Charles University, Prague, Czech Republic, ${ }^{3}$ 1st Medical Department, General University Hospital and First Faculty of Medicine, Charles University, Prague, Czech Republic

Introduction: Constitutional chromosome aberrations are usually a random finding (except of trisomy 21) in cytogenetics examination of patients with hematological diseases. In retrospective study over the last 21 years, we followed the incidence of constitutional chromosome aberrations detected in connection with the diagnosis of hematological malignancy and/or other hematological disorder. We compared our data with the incidence of specific chromosome aberrations in previously published studies.

Materials and Methods: We analyzed karyotypes of bone marrow or peripheral blood cells in 23201 patients with myeloid or lymphoid disorders diagnosed in 19982019. Subsequently, we verified suspected constitutional chromosome abnormalities by examination of PHA stimulated peripheral blood lymphocytes. For karyotyping, we used conventional G-banding completed with molecular cytogenomics techniques. Patients with Down syndrome were evaluated as a special group.

Results: In our cohort, we established constitutional chromosome aberrations in 92/23201 patients (0.4\%). Autosomal aberrations were more frequent than sex chromosomal abnormalities. Trisomy $21(n=18)$, balanced reciprocal translocations $(n=18)$ and Robertsonian translocations $(n=$ 17 ) occurred most often in a group of patients with autosomal anomalies. Sex chromosome aneuploidies were more frequent in patients with male phenotype $(n=23)$ than female phenotype $(n=12)$, mosaic variants of karyotype $(n=10)$ were present in both phenotypes.

Conclusions: The incidence of constitutional chromosome aberrations in our cohort of patients with hematological diseases is corresponding to their frequency in population. Our results, similarly as other studies, indicate that the connection of hematological disorders and constitutional chromosome aberrations seems to be random, except of acute leukemia and constitutional trisomy 21 . Supported by MH-DRO(IHBT,00023736), RVOVFN64165.

M. Valerianova: None. S. Ransdorfova: None. I. Sarova: None. Z. Zemanova: None. M. Onderkova: None. S. Izakova: None. A. Jonasova: None. J. Cermak: None. J. Brezinova: None.

P14.12.B

Detailed cytogenetic analysis of a preconception adult male sample revealed clonal evolution and (probably) changed his life 
N. Oliva-Teles ${ }^{1,2}$, S. Pires $^{1}$, M. Mota-Freitas ${ }^{1,2}, C$. Candeias $^{1,2}$, E. Lopes ${ }^{l}, P$. Oliveira ${ }^{l}$, A. Gavina ${ }^{l}, C$. Azevedo Soares $^{3,2}$, A. Fortuna ${ }^{3,2}$, N. Tkachenko ${ }^{3}$

${ }^{1}$ Centro Hospitalar Universitário do Porto/Centro de Genética Médica Jacinto Magalhães, Unidade de Citogenética, Porto, Portugal, Porto, Portugal, ${ }^{2}$ Unit for Multidisciplinary Research in Biomedicine/Institute of Biomedical Sciences Abel Salazar of University of Porto (UMIB/ICBAS, UP), Porto, Portugal, Porto, Portugal, ${ }^{3}$ Centro Hospitalar Universitário do Porto/Centro de Genética Médica Jacinto Magalhães, Serviço de Genética Médica, Porto, Portugal, Porto, Portugal

Genetic services that specialise in preconception analysis have traditionally included karyotyping among their routine genetic studies. If there is any particular clinical suspicion of a specific disorder, other cytogenomic analyses may be offered. The authors present the case of an asymptomatic 32 year-old male with non-consanguineous parents from European and African ancestry, who received preconception genetic counselling at a medical genetics appointment. After having given his written informed consent for genetic testing, the patient was screened for carrier status for the most common variants of cystic fibrosis (CF) and spinal muscular atrophy (SMA), sickle cell anemia (SCA) and thalassemia, and balanced chromosomal anomalies by karyotype. No pathogenic variants were found on the $\mathrm{CF}$ and SMA screening and no anomalous results were found for SCA and thalassemia. However, in three independent cultures, the patient's blood karyotype revealed a 46, XY line and another line with a translocation between the long arms of chromosomes 9 and 22, designated as Philadelphia chromosome, well-known to be associated with hematologic malignancy. The karyotype was thus: 46,XY,t $(9 ; 22)$ (q34;q11.2)[6]/46,XY[33]. He was counselled for the test results and a complete blood count was performed immediately showing an elevated white blood count with $2 \%$ blasts, thrombocytosis, and hemoglobin values on the lower limit of normal. He was diagnosed with Chronic Myeloid Leukemia and initiated treatment with imatinib with a good clinical evolution. The authors enhance the fact that disclosure of incidental findings related with cancer in preconception genetic counselling was very relevant to clinical care in the case described.

N. Oliva-Teles: None. S. Pires: None. M. Mota-Freitas: None. C. Candeias: None. E. Lopes: None. P. Oliveira: None. A. Gavina: None. C. Azevedo Soares: None. A. Fortuna: None. N. Tkachenko: None.

P14.13.C

When one CNV is not enough and array needs karyotype and to go FISHing

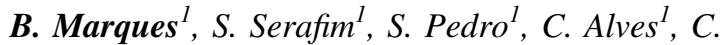
Ferreira $^{l}$, A. R. Tarelho ${ }^{l}$, R. Peliano $^{l}$, L. Simão $o^{l}$, M. Silva ${ }^{l}$, N. Silva ${ }^{1}$, M. Viegas ${ }^{1}$, F. Brito ${ }^{1}$, A. Medeira ${ }^{2}$, H. Correia ${ }^{l}$

${ }^{1}$ Instituto Nacional Saúde Doutor Ricardo Jorge, Lisboa, Portugal, ${ }^{2}$ Diferenças - Centro de Desenvolvimento Infantil, Lisboa, Portugal

Chromosomal microarray analysis (CMA) allows the detection of microdeletions/microduplications at a higher resolution but nonetheless karyotype and FISH studies may be required to clarify the underlying mechanism and recurrence risk of complex rearrangements. Here we present a 3-year-old girl with attention deficit/hyperactivity disorder (ADHD), speech disorder (SD), developmental delay (DD), and strabismus with a $3.6 \mathrm{Mb}$ terminal loss at 9p24.3p24.2, a $433 \mathrm{~Kb}$ interstitial gain at 15q13.3, and a $3.3 \mathrm{Mb}$ terminal gain at Xp22.33, uncovered by Affymetrix Cytoscan HD CMA. The 15q13.3 gain, inherited from the unaffected father, includes part of CHRNA7 and it's a known region with incomplete penetrance and variable expressivity associated to $\mathrm{DD}, \mathrm{SD}$, and ADHD, apparently fitting to the patient phenotype. However, to assess a predicted unbalanced $\mathrm{t}(\mathrm{X} ; 9)$ karyotype and FISH were performed, both in the patient and the parents, revealing a de novo submicroscopic unbalanced translocation - 46,XX.ish $\operatorname{der}(9) \mathrm{t}(\mathrm{X} ; 9)$ (p22.33;p24.3p24.2)(305J7-T7-;DXYS129+).arr[GRCh37] 9p24.3p24.2(203861_3824004)x1,15q13.3

(32003537_32436519)x3 pat,Xp22.33(168546_3489129) $x 3$. The few reported patients with $9 p$ deletion show a variable phenotype which may include DD, SD, and strabismus, among others, features also described in our patient. The Xp gain has an unknown contribution for the overall phenotype. Trio SNP-array showed the $\operatorname{der}(9)$ to be of maternal origin and therefore a balanced $t(X ; 9)$ with low level/gonadal mosaicism in the mother may exist and thus recurrence risk is not completely excluded. This case shows of how CNVs may contribute cumulative to a phenotype and the importance of keeping both cytogenetics and cytogenomics present in a diagnostic setting as CMA may need clarification by karyotype/FISH.

B. Marques: None. S. Serafim: None. S. Pedro: None. C. Alves: None. C. Ferreira: None. A. R. Tarelho: None. R. Peliano: None. L. Simão: None. M. Silva: None. N. Silva: None. M. Viegas: None. F. Brito: None. A. Medeira: None. H. Correia: None.

P14.14.A

Diploid/triploid mosaicism in a child with normal development 
M. S. Andersen ${ }^{1}$, K. Pallesen ${ }^{2}$, M. A. Kabel ${ }^{3}$, M. B. Engelund $^{l}$, C. Fagerberg ${ }^{l}$

${ }^{I}$ Department of Clinical Genetics, Odense University Hospital, Odense, Denmark, ${ }^{2}$ Department of Dermatology and Allergy Centre, Odense University Hospital, Odense, Denmark, ${ }^{3}$ H.C. Andersen Children's Hospital, Odense University Hospital, Odense, Denmark

Introduction: Diploid/triploid mosaicism is a rare entity, where a triploid cell line is present with varying tissue distribution. Cognitive deficit of varying degree is reported in most published cases. Other frequently reported features are growth retardation, syndactyly or clinodactyly, hypotonia, truncal obesity, body or facial asymmetry and pigmentary changes of the skin in the distribution of Blaschko's lines. We present a case with diploid/triploid mosaicism in a girl with normal development and review reported cases of diploid/triploid mosaicism.

Materials and Methods: Affymetrix Cytoscan HD chromosomal micro array (CMA), and G-band karyotyping.

Results: A 3,5-year old girl was referred for evaluation of irregular skin pigmentation following Blaschko's lines. She was otherwise healthy with normal development. Karyotyping of fibroblasts from hyperpigmented and normal skin, revealed mosaicism with a triploid cell line $(69, \mathrm{XXX})$ in $39 \%$ and $24 \%$ respectively. CMA verified mosaicism in the hyperpigmented skin biopsy but failed to detect the lowgrade mosaic in normal skin. A normal karyotype 46,XX was seen in blood.

Conclusions: This case confirms, that diploid/triploid mosaicism can be seen in persons with normal development, representing one end of a broad phenotypic spectrum. Genetic testing is biased towards individuals with cognitive deficit, and it is therefore likely that diploid/triploid mosaicism is underdiagnosed in patients with normal development. While triploid mosaicism was seen by karyotyping in hyperpigmented as well as normal skin, CMA failed to detect the triploid cell line in normal skin, confirming that karyotyping is still a valid and useful diagnostic method for pigmentary anomalies.

M.S. Andersen: None. K. Pallesen: None. M.A. Kabel: None. M.B. Engelund: None. C. Fagerberg: None.

\section{P14.15.B}

Genotoxicity induced in vitro by water soluble indoor $\mathbf{P M}_{2.5}$ fractions in relation to eight heavy metals concentrations

\author{
E. Kogianni ${ }^{l}$, C. Samara ${ }^{l}$, T. S. Lialiaris $^{2}$ \\ ${ }^{1}$ Aristotle University of Thessaloniki, Dept of Chemistry, \\ Thessaloniki, Greece, ${ }^{2}$ Dekokritus University of Thrace,
}

Medical School, Dept of Genetics, Alexandroupolis, Greece

Introduction: Airborne particulate matter (PM) generally contains a large number of genotoxic substances. Heavy metals concentrations in water-soluble outdoor PM fractions have been found consistent with genotoxicity to a significant degree, when no sufficient evidence exists for the indoor PM corresponding fractions.

Materials and Methods: The genotoxicity induced by water extractable particulate matter $\mathrm{PM}_{2.5}$ selected from indoor air of 20 workplaces in Alexandroupolis (Northeastern Greece) was examined in relation to the concentrations of the heavy metals $\mathrm{Cu}, \mathrm{Pb}, \mathrm{Mn}, \mathrm{Ni}, \mathrm{Co}, \mathrm{Zn}, \mathrm{Cr}$ and $\mathrm{Cd}$. For the determination of genotoxicity, the Sister Chromatid Exchange (SCE) induction assay was applied in human peripheral lymphocytes from healthy volunteer donors, whereas metals concentrations were determined using atomic absorption spectroscopy. Measurements took place in three water-soluble fractions: the total fraction $\mathrm{WS}_{\mathrm{A}}$ (filtered through $0.45 \mu \mathrm{m}$ ), the dissolved fraction $\mathrm{WS}_{\mathrm{B}}$ (filtered through $0.22 \mu \mathrm{m}$ ) and the non-chelexed dissolved fraction $\mathrm{WS}_{\mathrm{C}}$ (filtered through Chelex-100 resin).

Results: Statistically significant genotoxicity has been induced by all three fractions in relation to the control. When the results have been air volume- and $\mathrm{PM}_{2.5}$ massnormalized, the number of SCE's decreases from $\mathrm{WS}_{\mathrm{A}}$ to $\mathrm{WS}_{\mathrm{C}}$, while the difference of SCE's between $\mathrm{WS}_{\mathrm{A}}$ and $\mathrm{WS}_{\mathrm{C}}$ is statistically significant in the air volume- normalized approach. Also, significant correlations between a number of metals and the induced genotoxicity resulted in all three fractions, which are weaker in the air volume- normalized approach.

Conclusions: Heavy metal concentrations seem to play an important role to genotoxicity induced by water-soluble indoor $\mathrm{PM}_{2.5}$ fractions.

E. Kogianni: None. C. Samara: None. T.S. Lialiaris: None.

\section{P14.16.C}

Assessment of genotoxicity at low Doxorubicin doses in mice using chromosomal aberration assay

\section{R. M. Sionel ${ }^{\text {, L. L. Berca }}{ }^{2}$, S. Spandole Dinu ${ }^{1}$, P. Apostol ${ }^{3}$, M. Toma ${ }^{4}$, D. Cimponeriu ${ }^{1}$, R. I. Nica ${ }^{4}$}

${ }^{I}$ Faculty of Biology, University of Bucharest, Bucharest, Romania, ${ }^{2}$ National Institute of Research and Development for Food Bioresources - IBA Bucharest, Bucharest, Romania, ${ }^{3}$ Clinica Sante, Bucharest, Romania, ${ }^{4}$ Central Military Emergency Clinical Hospital, Bucharest, Romania 
Our aim is to investigate if high fat diet can interfere with genotoxicity of low doxorubicin (DOX) doses on bone marrow cells. We investigated four groups of adult BALBc mice that received normal diet (groups NFI, NFII) or pork greaves (groups PGI, PGII) for 7 days. At the beginning of the $8^{\text {th }}$ day mice from each group received a single intraperitoneal injection with DOX (doses range: $1.7-7.7 \mathrm{mg} / \mathrm{kg}$ body weight) or isotonic saline solution. All doses were tested in quadruplicate. In the $10^{\text {th }}$ (groups NFI, PGI) and in the $14^{\text {th }}$ (groups NFII, PGII) days from the beginning of the experiment the mice were euthanized and the chromosome aberrations were tested in bone marrow cells. We initialy assumed that the brutal shift of diet from normal food to pork greaves will have a big impact on mice weight, but the results have shown that DOX had a stronger impact cousing ambrupt weight loss. After 3- or 7-days post-injection with low DOX doses the number of chromosomal aberrations was significantly increased when compared to control group $(p<0.05)$. From observed aberrations the frequencies of polyploidy and deletion were significantly higher in mice who received 5.7-7.1 $\mathrm{mg} / \mathrm{kg}$ when compared to those treated with lower doses. The clastogenic impact of DOX in bone marrow cells seem to not be influenced by alternative diet.

R.M. Sionel: None. L. Berca: A. Employment (full or part-time); Modest; National Institute of Research and Development for Food Bioresources - IBA Bucharest. S. Spandole Dinu: None. P. Apostol: None. M. Toma: A. Employment (full or part-time); Modest; Central Military Emergency Clinical Hospital. D. Cimponeriu: A. Employment (full or part-time); Modest; Faculty of Biology, University of Bucharest. R.I. Nica: A. Employment (full or part-time); Modest; Central Military Emergency Clinical Hospital.

\section{P14.17.A}

Environmental impact on genotoxicity measured with the micronucleus test

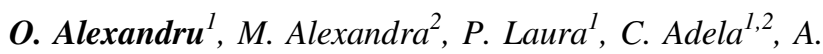
Nicoleta $^{1,2}$, P. Maria ${ }^{1,2}$

${ }^{1}$ Clinical Emergency Hospital for Children "Louis Turcanu", Timisoara, Romania, ${ }^{2}$ Genetics Department, Genomic Medicine Timisoara University of Medicine and Pharmacy "Victor Babes", Timisoara, Romania

Introduction: Genotoxicity represents the damage done to DNA by ionizing radiation. This damage can lead to the formation of random mutations in somatic cells and subsequently to the formation of different types of neoplasia. We aim to: Study the association of environmental factors with the genotoxic effect of ionizing radiation.
Material and Methods: We tested 64 doctors and nurses working in the Radiology and Radiotherapy departments using the micronucleus test. Along with the test, the medical personnel received a questionnaire regarding personal lifestyle choices as well as additional sources of exposure to genotoxic agents. Using the data received from the questionnaire we attempted to establish correlations between specific lifestyle choices and the results of the micronucleus test.

Results: We looked at correlations between age, overall number of risk factors, individual risk factors, employment length and the number of micronuclei per 1000 lymphocytes. Our results showed no significant correlation between the variables measured. Participants with risk factors such as smoking (40\%), alcohol consumption (19\%) and X-ray exposure in the last 5 years $(58 \%)$ showed no measurable increase in the average number of micronuclei. Only participants that had undergone CT-scans in the last 5 years (26\%) showed an increased average of their micronucleus test $(8.375 \%$ o compared to the overall average of $5.7 \%$ ).

Conclusions: The environment seems to play a negligible role in number of micronuclei, as a proxy for genotoxicity, however increased number of participants could bring additional information. Exposure to CT scans was shown to be associated with genotoxicity.

O. Alexandru: None. M. Alexandra: None. P. Laura: None. C. Adela: None. A. Nicoleta: None. P. Maria: None.

\section{P14.18.B}

Interstitial 11q12.1q13.1 duplication in patients with intellectual disability

B. Gläser ${ }^{1}$, K. Komlosi ${ }^{1}$, T. Haack ${ }^{2}$, S. Waldmüller ${ }^{2}$, A. Gazou $^{2}$, A. Müller ${ }^{2}$, T. Liehr ${ }^{3}$, J. Fischer ${ }^{1}$, A. Tzschach ${ }^{1}$

${ }^{1}$ Institute of Human Genetics, Freiburg, Germany, ${ }^{2}$ Institute of Medical Genetics and Applied Genomics, Tübingen, Germany, ${ }^{3}$ Institute of Human Genetics, Jena, Germany

Interstitial duplications of chromosome region $11 q 12.1 q 13.1$ are rare. We report on two siblings with intellectual disability in whom an interstitial de-novoduplication of chromosome region $11 \mathrm{q} 12.1 \mathrm{q} 13.1$ was detected. The 35-year-old female index patient had mild intellectual disability, but no malformations or other major health problems. Her 28-year-brother had moderate intellectual disability. Array-CGH analyses revealed an identical $7.83 \mathrm{Mb}$ duplication in chromosome region $11 \mathrm{q} 12.1 \mathrm{q} 13.1$ (genomic position according to hg19: chr11:57453530$65234091)$ in both patients. The duplication was confirmed by FISH analyses and whole-exome sequencing. The 
duplication was present neither in the healthy parents nor in the healthy sister, suggesting germline mosaicism in one of the parents. The findings in the family reported here indicate that duplications of chromosome region $11 \mathrm{q} 12.1 \mathrm{q} 13.1$ are associated with mild to moderate intellectual disability, but not with major malformations or other severe health problems. Reports of additional patients with overlapping duplications will be needed to elucidate the contribution of individual genes to the clinical phenotype.

B. Gläser: None. K. Komlosi: None. T. Haack: None. S. Waldmüller: None. A. Gazou: None. A. Müller: None. T. Liehr: None. J. Fischer: None. A. Tzschach: None.

\section{P14.21.B}

A case report of a possible novel deletion syndrome involving the KCTD16 gene

G. Gori ${ }^{1}$, F. Di Giovanni ${ }^{1}$, A. Pagliazzi ${ }^{1}$, S. Bargiacchi ${ }^{2}, S$. Guarducci $^{2}$, F. Pochiero $^{3}$, S. Giglio $^{l}$

${ }^{1}$ Department of Biomedical Experimental and Clinical Sciences "Mario Serio", University of Florenc, Florence, Italy, ${ }^{2}$ Medical Genetics Unit, Meyer Children's University Hospital, Florence, Italy, ${ }^{3}$ Metabolic and Muscular Unit, Neuroscience Department, Meyer Children's Hospital, Florence, Italy

Here we describe the case of a male newborn of non consanguineous parents, born by cesarean section for intrauterine growth retardation. In occasion of genetic evaluation, performed at age two years, we have ascertained hypospadias, microcephaly, dysmorphic facial features and developmental delay, in association with hyperCK-emia and high levels of transaminases. Array-CGH analysis showed a de novo interstitial deletion of approximately 194 kilobases in the $5 \mathrm{q} 31.3$ region, which includes part of the KCTD16 gene, to date not associated with any specific human disease. KCTD16 is one of the auxillary subunits of the GABA-B receptor and has been demonstrated to influence neuronal excitability by regulating GABA-B receptormediated gating of postsynaptic ion channels. This subunit is highly expressed in many brain regions, including amygdala and hippocampus, and regulates GABA-B receptors' action on postsynaptic ion channels. KCTD16, essentially, influences GABA-B modulation of neuronal excitability, oscillatory network activity and cognitive functions. Our report is the first finding of an intragenic deletion of KCTD16 and we could speculate that haploinsufficiency of this gene may have played a role in determining our patient's neurodevelopmental phenotype. We plan to perform further molecular analyses (whole exome sequencing) since we cannot rule out the neurological phenotype may be determined by biallelic variants of this gene. Furthermore, our patient's phenotype could also be caused by other known disease-genes. We will then proceed with functional studies to verify the real association of this gene with a new syndrome.

G. Gori: None. F. Di Giovanni: None. A. Pagliazzi: None. S. Bargiacchi: None. S. Guarducci: None. F. Pochiero: None. S. Giglio: None.

\section{P14.23.A}

Mosaic loss of chromosome $\mathrm{Y}$ is more prevalent in patients with prostate cancer

T. Kobayashi ${ }^{1}$, T. Hachiya ${ }^{2}, K$. Hino $^{3}$, T. Ando $o^{3}, M$.

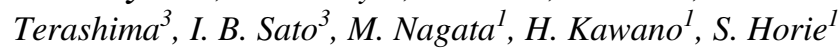

${ }^{I}$ Department of Urology, Juntendo University, Graduate School of Medicine, Hongo, Bunkyo-ku, Tokyo, Japan, ${ }^{2}$ Department of Advanced Informatics for Genetic Disease, Juntendo University, Graduate School of Medicine, Hongo, Bunkyo-ku, Tokyo, Japan, ${ }^{3}$ Genesis Healthcare Co., Ebisu, Shibuya-ku, Tokyo, Japan

Introduction: Clinical utility of mosaic loss of chromosome Y (mLOY) for cancer screening or for predicting cancer prognosis is controversial. We examined the prevalence of $\mathrm{mLOY}$ in patients with prostate cancer.

Materials and Methods: DNA microarray analysis was performed on saliva samples from 176 prostate cancer patients and 5402 control subjects. From the fluorescence intensity data of 2464 probes located at male-specific region of chromosome $\mathrm{Y}$, the median of $\log \mathrm{R}$ ratio (mLRR-Y) was calculated, and subjects with mLRR-Y $\leq-0.15$ was classified into the mLOY group. Age distribution was matched in prostate cancer and control groups, and the frequency of mLOY was compared. Furthermore, we investigated the association of mLOY with age at diagnosis, PSA value at diagnosis (iPSA), progression to castrationresistant prostate cancer (CRPC), and treatment methods among prostate cancer patients.

Results: Of the 165 cases in each of the age-matched groups, the number of subjects with mLOY was significantly higher in prostate cancer compared to control (9 in prostate cancer and 0 in control; $P$-value $=0.003)$. Among the prostate cancer patients, there were no significant differences in age at diagnosis, iPSA, and the proportion of the cases that progressed to CRPC. Regarding treatment methods, mLOY was not associated with surgery, but was significantly associated with radiotherapy $(P$-value $=$ 0.021).

Conclusions: $\mathrm{mLOY}$ occurs more frequently in patients with prostate cancer than in the control group. Among prostate cancer patients, $\mathrm{mLOY}$ is more likely to occur in cases who have been treated with radiation. 
T. Kobayashi: None. T. Hachiya: A. Employment (full or part-time); Significant; Genome Analytics Japan Inc. K. Hino: A. Employment (full or part-time); Significant; Genesis Healthcare Co. T. Ando: A. Employment (full or part-time); Significant; Genesis Healthcare Co. M. Terashima: A. Employment (full or part-time); Significant; Genesis Healthcare Co. I.B. Sato: A. Employment (full or part-time); Significant; Genesis Healthcare Co.. E. Ownership Interest (stock, stock options, patent or other intellectual property); Significant; Genesis Healthcare Co.. M. Nagata: None. H. Kawano: None. S. Horie: B. Research Grant (principal investigator, collaborator or consultant and pending grants as well as grants already received); Significant; Genesis Healthcare Co..

\section{P14.25.C}

Mosaic Turner syndrome of maternal origin in a child resulting from a consanguineous marriage: evaluation of the folate metabolism

O. Borghi Nascimento ${ }^{1}$, B. Pavarino Bertelli ${ }^{2}$, M. Fraga Mattos $^{1}$, A. L. Galbiatti Dias da Silval ${ }^{1}$, E. M. GoloniBertollo $^{1}$, E. Medina-Acosta ${ }^{3}$, T. Louvain de Souza ${ }^{3}$, É. C. Pavarino $^{l}$

${ }^{1}$ Unidade de Pesquisa em Genética e Biologia Molecular da Faculdade de Medicina de São José do Rio Preto FAMERP, São José do Rio Preto, Brazil, ${ }^{2}$ União das Faculdades dos Grandes Lagos- UNILAGO, São José do Rio Preto, SP, São José do Rio Preto, Brazil, ${ }^{3}$ Universidade Estadual do Norte Fluminense Darcy Ribeiro - UENF, Campos dos Goytacazes, Brazil

Introduction: Turner syndrome affects approximately 1:2000 female live births. 45,X/47, XXX mosaicism is seen in only $1 \%$ of cases; often caused by early post-zygotic chromosomal non-disjunction in disomic cells. Abnormal folate metabolism, as a result of genetic polymorphisms, can result in chromosomal non-disjunction. The present study evaluated the parental origin of non-disjunction and polymorphisms in genes involved in the folate metabolism in $45, \mathrm{X} / 47, \mathrm{XXX}$ mosaic patient and their parents.

Materials and Methods: Allelic segregation analysis for the STR marker located in the Retinitis pigmentosa 2 (RP2) gene was performed using quantitative fluorescent polymerase chain reaction (QF-PCR). The RFC1A80G, MTRA66G, MTHFRC667T, MTHFRA1298C, CBS844ins68 and DHFRdel19pb polymorphisms were investigated by PCR and enzymes restriction.

Results: The amniocentesis karyotype, revealed 45,X/47, XXX mosaicism. Postnatal karyotype confirmed mosaicism and the genealogy revealed consanguineous union, first cousins. The patient's DNA sample exhibited a triallelic profile which two are of maternal origin (alleles $315 \mathrm{bp}$ and $319 \mathrm{bp}$ ) and one of paternal origin (309bp allele). The analysis also indicated that $45, \mathrm{X}$ cells maintain maternal $\mathrm{X}$ represented by the $319 \mathrm{bp}$ allele. In the patient, the allelic variants RFC1A80G, MTRA66G, MTHFRC667T, MTHFRA1298C and DHFRdel19pb were observed in heterozygosity. The presence of heterozygosity also was observed in her mother (MTHFRC667T, DHFRdel19pb and MTHFRA1298C) and in her father (MTRA66G, RFC1A80G and MTHFRA1298C). CBS844ins68 alleles were homozygous in the family trio.

Conclusions: It is possible that the presence of allelic variants involved in the folate metabolism might have favored errors in chromosomal disjunction during maternal gametogenesis, resulting in Turner syndrome mosaic.

Grants: FAPESP(2018/09126-0), CNPq(310806/20186), CAPES(001), FAMERP/FUNFARME.

O. Borghi Nascimento: None. B. Pavarino Bertelli: None. M. Fraga Mattos: None. A.L. Galbiatti Dias da Silva: None. E.M. Goloni-Bertollo: None. E. MedinaAcosta: None. T. Louvain de Souza: None. É.C. Pavarino: None.

P14.26.A

A Case With Turner-Down Syndrome

S. Canbek ${ }^{1}$, H. C. Emeksiz', A. Ozturk Kaymak ${ }^{3}$, A. Tukun ${ }^{3}$

${ }^{1}$ Medical Genetics Department, Umraniye Training and Research Hospital, Istanbul, Turkey, ${ }^{2}$ Pediatric Endocrinology Department, Medeniyet University Goztepe Training and Research Hospital, Istanbul, Turkey, ${ }^{3}$ Medical Genetics Department, Duzen Laboratories, Ankara, Turkey

Introduction: We reported a patient with the mosaic form of Turner Syndrome and Down Syndrome, which is extremely rare. A 16-year-old girl was evaluated for her short stature and minor dysmorphic features. She has mild mental retardation, short stature and minor dysmorphic features with mild hypotelorism, mild epicanthic folds, long philtrum, micrognathy, retrognathy, mild low set ears, mild low posterior hairline, short neck, short fifth fingers, increased distance between the first and second toes, skin syndactyly of second to third toes, bilateral proximal outlet of fourth toe. She has no gonadal dysgenesia and has regular menstrual periods. The patient's pelvic and renal ultrasound and echocardiographic examinations were normal. She is the seventh child of 53 years old mother and 55 years old father.

Materials and Methods: Cytogenetic analysis of peripheral blood samples by using G-banding revealed mosaicism with 2 cell lines $(47, X X+21[16] / 45, X[14])$. FISH analysis revealed that $78.5 \%$ of the cells had 
monosomy X karyotype and $21.5 \%$ of the cells had XX karyotype; trisomy 21 was detected in $7.4 \%$ of the cells.

Results: The cytogenetic analysis of the patient revealed mosaicism of Turner and Down syndrome. Her FISH analyses from urine and buccal swab confirmed that result. Her parental chromosome analyses were normal.

Conclusions: The incidence of Turner / Down Syndrome is very rare. To date, less than 100 patients have been identified. We present this case to emphasize that classical cytogenetic analysis is still very important for the diagnosis of chromosomal abnormalities

S. Canbek: None. H.C. Emeksiz: None. A. Ozturk Kaymak: None. A. Tukun: None.

\section{P14.30.B}

Verification of balanced and unbalanced structural variants by optical genome mapping

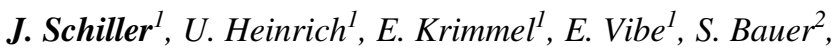

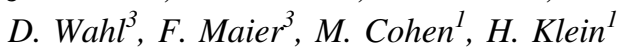

${ }^{1}$ MVZ Martinsried, Martinsried, Germany, ${ }^{2}$ Bionano Genomics, San Diego, CA, United States, ${ }^{3}$ Medical Practice for Genetic Counseling and Psychotherapy, Augsburg, Germany

Conventional chromosome analysis is capable of detecting both copy number (CNV's) and balanced structural variants (SV's), but only at a low resolution. Microarray analysis can detect CNV's at a high resolution level, but is unable to detect copy neutral SV's. To get a deeper insight in such variants and to narrow down the breakpoint regions, we started a verification study using 20 samples with known chromosome and microarray analysis results using Bionano Genomics' optical mapping technology. Bionano Genomics offers a complete workflow solution from sample preparation, DNA imaging and genomic data analysis enabling the identification of balanced and unbalanced SV's. Ultrahighmolecular-weight DNA (size 50kb to $\geq 1 \mathrm{Mb}$ ) was extracted from fresh human blood followed by a single direct-labeling enzymatic reaction to incorporate fluorophores at a specific 6-basepair sequence motif, yielding approximately 15 labels per $100 \mathrm{~kb}$. The SAPHYR-Instrument loads samples into nanochannels linearizing the labeled sample DNA and visualizing the label pattern. An algorithm converts the images into molecules and aligns molecules 'de novo' to construct consensus genome maps. Cross-Mapping to a reference sequence enables an automated SV detection. In a runtime of about $24 \mathrm{~h}$, an effective coverage of approx. 318-381x (minimum required 80x for non-mosaic samples) was achieved with a total DNA ( $>=150 \mathrm{~kb})$ output of 1300
Gb and a map rate of $78-89 \%$. We will discuss our results and demonstrate that this technology can be applied in a clinical diagnostic setting having the potential to replace conventional cytogenetics and microarray analysis.

J. Schiller: None. U. Heinrich: None. E. Krimmel: None. E. Vibe: None. S. Bauer: A. Employment (full or part-time); Modest; Bionano Genomics. D. Wahl: None. F. Maier: None. M. Cohen: None. H. Klein: None.

P14.32.A

Molecular manifestations of Phelan-McDermid syndrome. Assotiation to ring chromosome

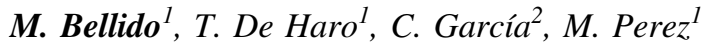

${ }^{1}$ Hospital Universitario Virgen de las Nieves, Granada, Spain, ${ }^{2}$ Hospital Universitario Juan Ramon Jjimenez, Huelva, Spain

Background Phelan-McDermid syndrome (PHMDS) is typically characterized by autism spectrum disorder, absent to severely delayed speech, and neonatal hypotonia. The PHMDS wide phenotypic variation is attributed partly to the type and size of $22 \mathrm{q} 13$ genomic deletion, partly to additional undefined factors. This deletion encompasses the SHANK3 gene which is thought to be the critical gene for the neurodevelopmental features. We report the cytogenetic and molecular characterization of a constitutional de novo ring chromosome $22(\mathrm{r}(22))$ in a patient with autism and language disorders Methods GTG-banding karyotype was performed according to standard protocols. Twenty metaphases chromosomes were examined with a 400-550 bands of resolution. Array-CGH analysis was performed by a $\mathrm{CGX}^{\mathrm{TM}} \mathrm{HD}$ v1,1 4-plex array $180 \mathrm{k}$ (PerkinElmer), with an average resolution of $40 \mathrm{~kb}$ in the backbone and $20 \mathrm{~kb}$ in the regions of interest. Results Karyotype results were 46,XY,r (22)(p13q13.33). Array-CGH revealed a terminal deletion on the long arm of chromosome 22, involving region 22q13.33 with an estimated size of $734.84 \mathrm{~Kb}$, including SHANK3 gene; arr (GRCh19) 22q13.33(50,443,314$51,178,149) \times 1$. Conclusion The determination of the size of the deletion by aCGH is very useful for the association with described syndromes associated with deletions or duplications. However, the possibility that this deletion leads the formation of a ring chromosome can only be evaluated by performing a karyotype which is also necessary to analyse the presence or absence of mosaicism respect to ring chromosome, and therefore, the clinical symptoms and evolution of the patient could have implications for genetic counselling. 
M. Bellido: None. T. De Haro: None. C. García: None. M. Perez: None.

\section{P14.33.B}

De novo microdeletions at $6 q 14.1$ encompassing the $P H I P$ gene in two girls with developmental delay, intellectual disability, behavioral problems obesity, and dysmorphic features

A. Boughalem ${ }^{\text {, }}$ D. Trost ${ }^{l}$, A. Bazin ${ }^{2}$, C. Allioux ${ }^{3}$, A. Lantres $^{2}$, P. Kleinfinger ${ }^{l}$, S. Zimmer ${ }^{1}$, L. Lohmann ${ }^{1}$, A. Luscan $^{1}$, M. Valduga ${ }^{1}$, J. Costa ${ }^{l}$

${ }^{1}$ Laboratoire Cerba, Saint Ouen l'Aumone, France, ${ }^{2}$ Hôpital René Dubos, Pontoise, France, ${ }^{3}$ Hôpital De Saint Denis, Saint Denis, France

Heterozygous deleterious variants in the pleckstrin homology domain-interacting protein (PHIP) located on chromosome 6q14.1, have been associated with Chung-Jansen syndrome MIM \# 617991. Only 9 patients with microdeletions spanning the 6q14.1 region including the PHIP gene have been reported with a phenotype similar to ChungJansen syndrome. However, these patients have a number of additional clinical findings, likely due to the deletion of adjacent genes in 6q14. Here, we report two interstitial deletions at 6q14.1 involving the PHIP gene, in two unrelated patients with a common phenotype of developmental delay, intellectual disability, behavioral problems, obesity and dysmorphic features. Methods and results: SNP-CGH analysis using Affymetrix Cytoscan HD microarray has been performed. A $642 \mathrm{~kb}$ microdeletion at $6 \mathrm{q} 14.1$ $(79,326,616$ 79,968,863 hg 19) has been detected in the first female patient presenting with obesity, developmental delay, behavioral and facial abnormalities. A smaller $54 \mathrm{~kb}$ intragenic microdeletion at 6q14.1, spanning part of exon 8exon 24 of the PHIP gene (79687488_79741515 hg19) has been detected in the second female patient presenting with behavioral problems, developmental delay, dysmorphic features. Her endocrine problems (other than obesity) included hypothyroidism, generalized hirsutism and precocious puberty. Conclusions: To our knowledge the $54 \mathrm{~kb}$ deletion is the smallest microdeletion described in this region by the use of SNP-CGH analysis. The patients we report here show clinical overlap with the phenotypic spectrum of previously reported in individuals with $P H I P$ variants, which confirms that haploinsufficiency of PHIP is sufficient to cause Chung-Jansen syndrome. This is the first report of endocrine problems eg. Hirsutism, in ChungJansen syndrome.

A. Boughalem: None. D. Trost: None. A. Bazin: None. C. Allioux: None. A. Lantres: None. P. Kleinfinger:
None. S. Zimmer: None. L. Lohmann: None. A. Luscan: None. M. Valduga: None. J. Costa: None.

\section{P14.35.A}

Rescue of the karyotype with ring chromosome 8 in human induced pluripotent stem cells

T. V. Nikitina ${ }^{l}$, M. M. Gridina ${ }^{2}, Y$. M. Minina ${ }^{2}$, A. A. Kashevarova $^{l}$, Y. S. Yakovleva ${ }^{l, 3}$, D. A. Fedotov ${ }^{3}$, M. E.

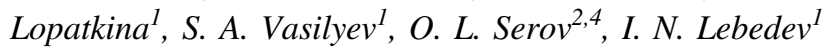

${ }^{1}$ Research Institute of Medical Genetics, Tomsk NRMC, Tomsk, Russian Federation, ${ }^{2}$ Institute of Cytology and Genetics SB RAS, Novosibirsk, Russian Federation, ${ }^{3}$ Siberian State Medical University, Tomsk, Russian Federation, ${ }^{4}$ Novosibirsk State University, Novosibirsk, Russian Federation

Introduction: Ring chromosomes are usually found as a part of mosaic karyotype and dynamic mosaicism is a frequent event in the patient's cells. Induced pluripotent stem cells (iPSCs) proved to be a valuable tool for the modelling of the dynamics of ring chromosome mosaicism through multiple cell divisions.

Materials and Methods: We obtained 6 iPSC lines from skin fibroblasts of patient with 46,XY,r(8) and compared karyotypes of fibroblasts and iPSCs at different passages using G-banding, FISH and SNP-arrays.

Results: Molecular karyotyping revealed that ring chromosome originated from an inverted duplication 8p23.1-p11.22 with a terminal deletion at 8p23.3-p23.1. We found $85 \%$ fibroblasts with $\mathrm{r}(8)$ and $15 \%$ with monosomy 8 , but later a clone of the cells with $\mathrm{t}(7 ; 8)$ arose, that have gained a proliferative advantage with more than $80 \%$ of the cells after P20. iPSC karyotypes were similar with the initial fibroblasts in the early passages $(90 \%$ of the cells had $\mathrm{r}(8)$ at $\mathrm{P} 4)$, but later changes occurred in the line iTAF11-4: only $2 \%$ of the cells had the ring at P11 (100 cells were counted per passage). SNP-arrays revealed signs of chromothripsis in early passage and 46,XY karyotype at P11 with an UPD(8)pat.

Conclusions: Molecular and cytogenetic analyses allow to track the mitotic behavior of the ring chromosome in iPSCs culture. Spontaneous correction of the 46,XY,r(8) karyotype via uniparental disomy origin has been registered in one of the 6 iPSC lines. This study was supported by Russian Science Foundation, grant 16-15-10231.

T.V. Nikitina: None. M.M. Gridina: None. Y.M. Minina: None. A.A. Kashevarova: None. Y.S. Yakovleva: None. D.A. Fedotov: None. M.E. Lopatkina: None. S.A. Vasilyev: None. O.L. Serov: None. I.N. Lebedev: None. 


\section{P14.36.B}

A case of ring chromosome 21 with hemifacial microsomia, developmental and speech delay analysed with WGS and patient-derived iPS

\section{J. Schuy ${ }^{1}$,J. Eisfeldt ${ }^{1,2,3}$, M. Pettersson ${ }^{1,2}, N$. Shahrokhshahi ${ }^{2}$, M. Shahsavani ${ }^{1}$, D. Nilsson ${ }^{1,2,3}$, A. Falk ${ }^{4}$, A. Lindstrand ${ }^{1,2}$}

${ }^{1}$ Department of Molecular Medicine and Surgery and Center for Molecular Medicine, Karolinska Institutet, Stockholm, Sweden, ${ }^{2}$ Department of Clinical Genetics, Karolinska University Hospital, Stockholm, Sweden, ${ }^{3}$ Science for Life Laboratory, Karolinska Institutet Science Park, Stockholm, Sweden, ${ }^{4}$ Department of Neuroscience, Karolinska Institutet, Stockholm, Sweden

Introduction: Ring chromosomes are rarely occurring circularized chromatin which can arise during mitosis if the telomeric sequences are lost. Structural variants (e.g. deletions) often accompany the formation of ring chromosomes, leading to neurodevelopmental disorders which severity strongly depends on the affected genes. However, the exact mechanism and biological effect of ring chromosomes is still unknown. Here, we present RD_P26, a 6-year old girl presenting hemifacial microsomia, developmental and speech delay, and a ring chromosome 21, 46,XX,r(21) (p11.2q22.3).

Methods: Characterization with chromosomal microarray, whole genome sequencing and breakpoint junction (BPJ) PCR unravelled three alternating deletions and two inversions with templated and non-templated insertions in the BPJs. Remarkably, this loss of genetic material, affecting 29\% (70/242) of the protein-coding genes on chromosome 21, is not lethal. Moreover, the ring chromosome is stable through multiple passages of neuroepithelial stem cells (NES), differentiated from patient-derived induced pluripotent stem cells. Furthermore, we analysed the transcriptome of NES from RD-P26 and compared the expression profile to two trisomy 21 patients and three healthy controls.

Results: $11.6 \%$ genes (2080/17831) were differentially expressed. Of the 171 genes located on chromosome 21, $24 \%(41 / 171)$ were differentially expressed. Within the three deleted segments, 19 genes were significantly downregulated and there were no significant upregulations, consistent with haploinsufficiency. Moreover, the downregulated gene expression was mirrored by an elevated gene dosage in the trisomy 21 patients.

Conclusions: The hemizygous genes located within the deletions are considered as likely causing the patient's phenotype, also other chromosomal rearrangements were found. Funding: Swedish science council 201702936.

J. Schuy: None. J. Eisfeldt: None. M. Pettersson: None. N. Shahrokhshahi: None. M. Shahsavani: None. D. Nilsson: None. A. Falk: None. A. Lindstrand: None.

\section{P14.38.A}

Tetrasomy of SCN2A associated with refractory neonatal epileptic encephalopathy

M. Krumbiegel ${ }^{1}$, R. Trollmann ${ }^{2}$, D. Mammadova ${ }^{2}$, A. Schnell ${ }^{2}$, C. Kraus ${ }^{1}$, A. B. Ekici ${ }^{1}$, A. Reis ${ }^{1}$, C. Zweier ${ }^{1}$

${ }^{1}$ Institute of Human Genetics, Friedrich-Alexander-Universität Erlangen-Nürnberg, Erlangen, Germany, ${ }^{2}$ Department of Pediatrics, University Hospital for Children and Adolescents, Friedrich-Alexander-Universität ErlangenNürnberg, Erlangen, Germany

Autosomal dominant missense or truncating mutations or deletions of sodium channel genes such as SCN1A, SCN2A or SCN9A cause a broad spectrum of neonatal epileptic encephalopathies with variable clinical phenotypes. The sodium channel gene family clusters in $2 \mathrm{q} 24.3$, and duplications of this region have been rarely described. All reported patients presented with severe, early-onset seizures and variable developmental and behavioral anomalies.

We now report on a female newborn who presented from first day of life with severe muscular hypotonia and prolonged focal cerebral seizures. Seizures were initially therapy refractory, but a high dose parenteral therapy with Phenytoin suppressed seizures almost completely, indicating a mutation in SCN2A gene as possible cause. Trio exome sequencing, however, did not reveal a causative variant in SCN2A or other epilepsy-related genes. Chromosomal microarray analysis with an Affymetrix CytoScan HD Array revealed a copy number gain of 5,516 kb in $2 q 24.3 q 31.1$ affecting 34 genes including the sodium channel gene cluster on $2 \mathrm{q} 24.3$. The proximal part of 0.5 $\mathrm{Mb}$ containing SCN2A was present in four copies and the distal part of $5 \mathrm{Mb}$ (containing SCN1A and SCN9A) in three copies. The aberration was confirmed, and a parental cryptic translocation was excluded by FISH analyses.

We therefore consider this copy number gain in $2 q 24.3 q 31.1$ to be disease-causing in our patient. To our knowledge, this is the first copy number gain in this region that includes a triplication of SCN2A. Tetrasomy of SCN2A might therefore contribute to the particularly severe phenotype.

M. Krumbiegel: None. R. Trollmann: None. D. Mammadova: None. A. Schnell: None. C. Kraus: None. A.B. Ekici: None. A. Reis: None. C. Zweier: None. 


\section{P14.39.B}

Profound body asymmetry in a girl with a developmental delay carrying germline partial trisomy $15 q$ and somatic duplication 11 p15

\section{K. Bilska ${ }^{l}$, U. Heinrich ${ }^{l}$, E. Krimmel ${ }^{l}$, D. Wahl $^{2}$, I. Rost ${ }^{l}$, T. Eggermann $^{3}$, F. Maier $^{2}$}

${ }^{1}$ MVZ Martinsried, Martinsried, Germany, ${ }^{2}$ Medical Practice for Genetic Counseling and Psychotherapy, Augsburg, Germany, ${ }^{3}$ Institute of Human Genetics, Medical Faculty, RWTH Aachen University, Aachen, Germany

We present a 3 1/2 year old Chechen girl with severe body asymmetry, developmental delay and dysmorphic features. Chromosome analysis from cultured peripheral lymphocytes was performed, which showed an additional chromosomal material on the short arm of one chromosomes 22. For further characterization of the chromosomal aberration, we performed array CGH analysis and detected an almost $25 \mathrm{Mb}$ large terminal duplication $15 \mathrm{q} 24.3 \mathrm{q} 26.3$. The combination of these two methods showed a de-novo pure partial trisomy $15 \mathrm{q}$ with the karyotype 46,XX,der(22)t(15;22)(q24.3;p12). The Region 15q24.3-qter contains 151 proteins and is associated with overgrowth and developmental delay. In order to clarify the cause of the asymmetry, methylation-sensitive MLPA and qPCR was performed on mucosal cells of the left and right side. Triplicated copy number variation of $11 \mathrm{p} 15.5$ with unsuspicious methylation status was found in mucosal cells of the left side and is known for the growth retardation syndrome Silver-Russel and IMAGe. Subsequent quantitative PCR showed at least 20.8 $\mathrm{Mb}$ de-novo duplication 11p15.5p15.1. No mosaicism was found in mucosal cells of the right side. This case shows a complex phenotype-genotype-correlation with coexistence of a germline and a somatic variant leading to hemihypertrophy and hemihypotrophy and may provide new insights of the precise human embryonic and fetal growth network.

K. Bilska: None. U. Heinrich: None. E. Krimmel: None. D. Wahl: None. I. Rost: None. T. Eggermann: None. F. Maier: None.

\section{P14.42.B}

Hi-C enables the 3D characterization of small supernumerary marker chromosomes

\author{
J. Jungnitsch ${ }^{1,2}$, M. Klever ${ }^{1,2}$, U. Melo ${ }^{2,1}$, R. Schöpflin ${ }^{2,1}$, \\ C. A. Prada-Medina ${ }^{2}$, T. Liehr ${ }^{3}$, S. Mundlos ${ }^{1,2}$, M. \\ Spielmann ${ }^{1,2}$ \\ ${ }^{1}$ Institute for Medical Genetics and Human Genetics, \\ Charité - University Medicine Berlin, Berlin, Germany, \\ ${ }^{2}$ Max Planck Institute for Molecular Genetics, Research \\ Group Development and Disease, Berlin, Germany,
}

${ }^{3}$ Institute of Human Genetics, Jena University Hospital, Friedrich Schiller University, Jena, Germany

For the last five decades, small supernumerary marker chromosomes (sSMCs) have been known to exist and are estimated to be present in $\sim 3.3$ million human individuals, while approximately a third of them are clinically affected. sSMCs comprise chromosomal parts, which occur additionally to the physiological 46 chromosomes. These extra chromosomes appear in various morphological shapes, e.g. ring, inverted duplicated or centric minute chromosomes. Chromosome conformation capture technologies have the potential to detect structural variants and advanced the interpretation of altered gene expression caused by them. Applying these novel technologies could give new insights into the emergence of sSMCs and their genotype-phenotype correlation. In this study we applied $\mathrm{Hi}-\mathrm{C}$ in lymphoblastoid cell lines from four samples of individuals presenting developmental delay carrying different sSMCs (1 ring, 1 inverted duplicated, 2 centric minute chromosomes). These samples were previously screened by FISH and aCGH. In addition we also performed RNA-seq to investigate dysregulation of genes included in the sSMC sequence, which when combined with Hi-C could uncover yet undiscovered mechanisms (e.g. enhancer adoption). Strikingly, Hi-C was able to detect the sSMCs and the breakpoints at a higher resolution compared to karyotype and aCGH. Furthermore, Hi-C helped to reconstruct the 3D genome architecture of sSMCs compared to their physiological counterparts. We show that the Hi-C technology is able to contribute substantially to the detailed characterization of sSMCs. Future studies will elaborate the cytogenetic and morphological characteristics further and eventually be able to explain and hopefully treat syndromes and phenotypes resulting from these chromosomal aberrations.

J. Jungnitsch: None. M. Klever: None. U. Melo: None. R. Schöpflin: None. C.A. Prada-Medina: None. T. Liehr: None. S. Mundlos: None. M. Spielmann: None.

\section{P14.43.C}

Complex small supernumerary marker chromosome sSMC originates from 6 different parts of 5 chromosomes

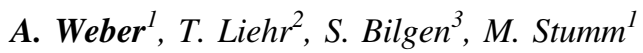

${ }^{I}$ Medicover Genetics GmbH, Berlin, Germany, ${ }^{2}$ Zentrum für ambulante Medizin, Praxis für Humangenetik, Jena, Germany, ${ }^{3}$ Medicover Bochum MVZ, Bochum, Germany

Introduction: The risk for phenotypic abnormalities of patients carrying an sSMC depends on several factors as tissue specific mosaicism, the number of additional dosage 
sensitive genes, and/or the possibility of impaired imprinting due to the origin of the supernumerary chromosomal material. Especially, a valid genotype/phenotype correlation is very difficult in patients with sSMCs originating from different parts of multiple chromosomes, because complex sSMCs can multiply all these effects. We report on a 39year-old patient with hypogonadotropic hypogonadism, infertility, small testicles, obesity, gynecomastia, hyperthyroidism, rheumatism and psychiatric problems, carrying a unique highly complex sSMC.

Methods: The patient's lymphocytes and DNA from peripheral blood cells were analyzed by conventional chromosome analysis (GTG banding) and array comparative genomic hybridization (aCGH). Additionally, the sSMC was microdissected, amplified, labeled and hybridized as fluorescence in situ hybridization (FISH) probe.

Results: aCGH detected copy number gains in chromosomal regions 8q22.3q23.1, 9q33.3q34.11, 14q21.1, $14 \mathrm{q} 21.1 \mathrm{q} 21.2,15 \mathrm{q} 21.2$ and $21 \mathrm{q} 21.1$ with evidence of mosaicism. Therefore, the sSMC originates at least from 6 different parts of 5 chromosomes. After microdissection of the sSMC and reverse FISH, the localization of the DNA gains on the respective chromosomes could be confirmed. Around 50\% of peripheral blood (mesoderm) and buccal mucosa cells (ectoderm) contained the sSMC.

Conclusions: Complex sSMCs are very rare and the formation mechanisms of such complex structural constellations are still unclear, but chromothripsis-like processes could be considered. To best of our knowledge, our patient is the first one reported, carrying a sSMC with such a high complexity.

A. Weber: None. T. Liehr: None. S. Bilgen: None. M. Stumm: None.

\section{P14.44.A}

An unusual case of CLL with cytogenetical $t(11 ; 14)$ but two different IGH/CCND1 rearrangements

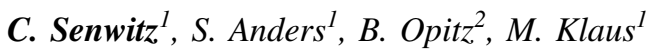 \\ ${ }^{1}$ Mitteldeutscher Praxisverbund Humangenetik, Dresden, Germany, ${ }^{2}$ Onkologisches Zentrum St. Elisabeth und St. Barbara, Halle, Germany}

The translocation $\mathrm{t}(11 ; 14)(\mathrm{q} 13 ; \mathrm{q} 32)$ occurs mainly in MCL, but is also found in a wide range of other B-cell lymphoma. We report about a case of a 58 year old male patient with suspected CLL and $\mathrm{t}(11 ; 14)(\mathrm{q} 13 ; \mathrm{q} 32)$, detected by chromosome banding analysis (CBA). Additional fluorescence in situ hybridization (FISH) of the IGH and CCND1 loci (dual fusion probe) unexpectedly revealed two independent clones: One clone $(1 ; 60 \%)$ harbored the fusion signal constellation for the standard translocation $\mathrm{t}(11 ; 14)(\mathrm{q} 13$; q32) with corresponding IGH/CCND1 colocalization on derivative chromosomes (der) 11 and 14, respectively. The other clone $(2 ; 40 \%)$ showed a variant translocation $t$ $(11 ; 14)$, including the pathognomonically relevant IGH/ CCND1 colocalization on der(11), but no fusion signal on der(14). Instead, the 3'CCND1 signal was found on an Fgroup-chromosome. Further FISH-analysis revealed the involvement of one chromosome 19 with isolated translocation of the 3'CCND1 region from 11q to 19p. Both clones 1 and 2 cannot be distinguished by CBA at 300 band levels. We suspect that the variant IGH/CCND1 rearrangement led to a fusion of 3'CCND1 and FSTL3 (follistatin-like-3) on chromosome 19, but due to the lack of commercially available FISH probes for this region this assumption could not be verified. The respective translocation $\mathrm{t}(11 ; 19)(\mathrm{q} 13$; p13) [CCND1/FSTL3] constitutes a very rare event with to date only one CLL case described. Impact on prognosis is unclear. As a conclusion, we recommend additional FISH analysis of the IGH and CCND1 loci in every case of cytogenetically assured $t(11 ; 14)$, in order to preclude cryptic variant rearrangements and to ensure proper disease monitoring.

C. Senwitz: None. S. Anders: None. B. Opitz: None. M. Klaus: None.

\section{P14.47.A}

A case of maternal UPD20 detected by routine SNP array diagnostics

\section{E. Blom, J. J. Saris, L. J. C. M. van Zutven, L. H. Hoefsloot}

\section{Erasmus Medical Center, Rotterdam, Netherlands}

In our laboratory we perform routine SNP array analysis for several clinical phenotypes, including intellectual disability, congenital abnormalities, and prenatal indications. Although this technique is not primarily aimed for UPD (Uniparental Disomy) diagnostics, a patient with a maternal UPD of chromosome 20 was discovered. UPD as a consequence of heterodisomy is not detectable by SNP array analysis. However, the presence of (large) homozygous regions on a single chromosome can be suggestive for an isodisomy UPD, which can be further tested for with another technique. Here, we present a case of an infant with severe feeding difficulties needing gastric-tube feeding. Routine SNP array analysis did not show any particularities, except two terminal regions of homozygosity, at 20p and 20q, which is suggestive for UPD. To confirm the possible presence of UPD, we requested parental samples and 
performed a SNP trio-analysis. SNP trio-analysis indeed confirmed the presence of UPD20, which showed of a maternal origin. Maternal UPD20, or upd(20)mat syndrome, is a very rare condition for which only a very limited number of cases are described in literature. The main symptoms of this disorder are prenatal and postnatal growth retardation and (severe) feeding difficulties requiring gastric-tube feeding in the first years of life. Literature of the upd(20)mat syndrome and mechanisms leading to UPD formation are reviewed.

E. Blom: None. J.J. Saris: None. L.J.C.M. van Zutven: None. L.H. Hoefsloot: None.

\section{P14.50.A}

Trisomy 14 Mosaicism in form of an Robertson Translocation $\operatorname{der}(14 ; 14)$ in a child with skeletal abnormalities

\section{Wenzel ${ }^{l}$, I. Dietze-Armana ${ }^{1}$, W. Hütter ${ }^{2}$, K. Mehnert $^{l}$ \\ Igenetikum, Neu-Ulm, Germany, ${ }^{2}$ Praxisklinik Frauenstraße, Ulm, Germany}

Introduction: Mosaic trisomy 14 is a rare chromosomal disorder. Full trisomy 14 is often reported in abortion. Features in affected individuals with mosaic trisomy 14 can vary. Symptoms that have been most commonly reported include intrauterine growth restriction, failure to thrive, developmental delay, asymmetric growth, abnormal skin pigmentation, distinctive facial characteristics and structural malformations.

Case Report: A 6-month-old female was referred for genetic counselling because of short femora and humeri. The girl showed additionally a prominent forehead but no developmental delay and a normal skin at this point. In pregnancy intrauterine growth retardation was noted. Noninvasive prenatal testing for chromosome 13, 18, 21 and gonosomes showed no abnormalities. No further examinations from parents were desired.

Results: Because of described skeletal anomalies and facial dysmorphism molecular diagnostic of FGFR3 was performed and no mutation was found. Chromosome analysis of peripheral blood revealed a mosaic trisomy 14 in form of a Robertson translocation 46,XX,+14,der(14;14) in $12 \%$ of cells. Fluorescence in situ hybridization of 37 nuclei from buccal mucosa showed three copies in $5 \%$ of cells. To exclude an uniparental disomy 14 we performed an STR marker analysis. No upd(14) was detected. Chromosome analysis of both parents were normal.

Conclusions: To our knowledge, this is the first report of trisomy 14 mosaicism in form of a Robertson translocation.
Our patient with intrauterine growth retardation matched with many patients described in literature.

M. Wenzel: None. I. Dietze-Armana: None. W. Hütter: None. K. Mehnert: None.

\section{P15 New Technologies and Approaches}

\section{P15.05.C}

Creating artificial human genomes using generative neural networks

B. Yelmen ${ }^{1,2}$, A. Decelle ${ }^{3}$, L. Ongaro ${ }^{1,2}$, D. Marnetto ${ }^{1}, C$.

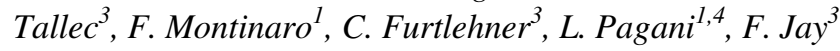

${ }^{I}$ Institute of Genomics, University of Tartu, Tartu, Estonia, ${ }^{2}$ Institute of Molecular and Cell Biology, University of Tartu, Tartu, Estonia, ${ }^{3}$ Laboratoire de Recherche en Informatique, Paris, France, ${ }^{4}$ APE Lab, Department of Biology, University of Padova, Padua, Italy

Generative models have shown breakthroughs in a wide spectrum of domains due to recent advancements in machine learning algorithms and increased computational power. Despite these impressive achievements, ability of generative models to create realistic synthetic data is still under-exploited in genetics and absent from population genetics. Yet a known limitation of this field is the reduced access to many genetic databases due to concerns about violations of individual privacy, although they would provide a rich resource for data mining and integration towards advancing genetic studies. In this study, we demonstrated that deep generative adversarial networks (GANs) and restricted Boltzmann machines (RBMs) can be trained to learn the high dimensional distributions of real genomic datasets and generate novel high-quality artificial genomes (AGs) with none to little privacy loss.

We show that our generated AGs replicate characteristics of the source dataset such as allele frequencies, linkage disequilibrium, pairwise haplotype distances and population structure. Moreover, they can also inherit complex features such as signals of selection and genotype-phenotype associations. To illustrate the promising outcomes of our method, we showed that imputation quality for low frequency alleles can be improved by augmenting reference panels with AGs and that the RBM latent space provides a relevant encoding of the data, hence allowing further exploration of the reference dataset and features for solving supervised tasks.

Generative models and AGs have the potential to become valuable assets in genetic studies by providing a rich yet compact representation of existing genomes and highquality anonymous substitutes for private databases. 
B. Yelmen: None. A. Decelle: None. L. Ongaro: None. D. Marnetto: None. C. Tallec: None. F. Montinaro: None. C. Furtlehner: None. L. Pagani: None. F. Jay: None.

\section{P15.06.A}

Reconstruction of complex genomic rearrangements with 3rd generation sequencing in rare diseases: two chromothripsis cases

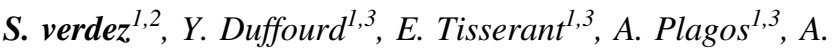
Bruel $^{1,3}$, F. Tran Mau-Them ${ }^{1,2,3}$, C. Philippe ${ }^{1,2,3}$, C. Thauvin $^{1,2,3,4}$, L. Faivre ${ }^{1,2,3,4}$, N. Chatron ${ }^{5,6}$, C. SchluthBolard $^{5,6}$, P. Callier ${ }^{1,2,3}$, D. Sanlaville ${ }^{5,6}$, A. Vitobello ${ }^{1,3}$

${ }^{1}$ UMR1231 GAD, Inserm - University of BurgundyFranche Comté, Dijon, France, ${ }^{2} U F$ Innovation en diagnostic génomique des maladies rares, Dijon, France, ${ }^{3}$ Unité Fonctionnelle Innovation en Diagnostic génomique des maladies rares, FHU-TRANSLAD, Dijon, France, ${ }^{4}$ Centre de Référence maladies rares " Anomalies du développement et syndromes malformatifs », centre de génétique, FHU-TRANSLAD, Dijon University Hospital, Dijon, France, ${ }^{5}$ Hospices Civils de Lyon, Service de Génétique, CHU de Lyon, Lyon, France, ${ }^{6}$ Equipe GENDEV INSERM U1028, CNRS, UMR5292, Lyon, France

Long-read genome sequencing (LRGS) is revealing its utility to identify complex chromosomal rearrangement involving multiple breakpoints or low-complexity regions. Chromothripsis, as an example, results from rearrangement of multiple chromosomal double-stand breaks, obtained from a single catastrophic event, giving rise to complex derivative chromosomes. In 2 chromothripsis patients presenting with syndromic intellectual disability, and without candidate gene accounting for their clinical presentation after short-read genome sequencing (30X srGS) analysis, we performed de novo assembly using 20X SMRT (Sequel 1 system - Pacific Bioscience) with the aim to reconstruct the map of derivative chromosomes.

We utilized two assembly tools (Falcon, Canu) and took advantage of available short-read Illumina reads to correct sequencing errors originated from long reads (polishing steps). Contigs containing at least one breakpoint were mapped against the Genome Reference Consortium Human Build 37 (GRCh37/hg19) using Blast in order to calculate the percentage of identity.

On average, Falcon allowed to obtain 111 contigs with $98,84 \%$ (SD 0.070) identity whereas 81 contigs with 98,92\% (SD 0.001) identity were obtained with Canu. The average size of the chromosomal blocks contained in these contigs were $206 \mathrm{~kb}$ (SD 104kb) for Falcon and $185 \mathrm{~kb}$ (SD $11 \mathrm{~kb})$ for Canu.

In conclusion our bioinformatic method allowed us to precisely reconstruct the sequence of their derived chromosomes, highlighting genomic events concerning chromosomes $4,11,13,14,15,21$ for the first patient and 3,10, 12 for the second patient. Overall the combination of srGS and lrGS permitted to resolve complex chromosomal rearrangements, not otherwise achievable by srGS alone.

S. verdez: None. Y. Duffourd: None. E. Tisserant: None. A. Plagos: None. A. Bruel: None. F. Tran MauThem: None. C. Philippe: None. C. Thauvin: None. L. Faivre: None. N. Chatron: None. C. Schluth-Bolard: None. P. Callier: None. D. Sanlaville: None. A. Vitobello: None.

\section{P15.07.B}

Targeted and genome-wide discovery of genomic interactions, structural variants, and gene regulatory mechanisms using the Arima-HiC platform

\section{J. Belton, D. Reid, X. Zhou, S. Mac, S. Tin, C. Caneda, J.} Kirkland, A. Schmitt

\section{Arima Genomics, Inc., San Diego, CA, United States}

Three-dimensional genome organization is critical for genome function. Hi-C technology has enabled high throughput analyses of chromatin conformation, providing valuable insights into the mechanisms of chromatin folding and gene regulation. However, conventional $\mathrm{Hi}-\mathrm{C}$ methods can suffer from laborious workflows, sub-optimal analytical sensitivity and reproducibility, and prohibitively expensive sequencing requirements. Realizing these challenges, Arima developed a 6-hour Hi-C protocol that improves analytical sensitivity through rapid, reproducible, multiple restriction enzyme Hi$\mathrm{C}$ chemistry. This improved Hi-C chemistry detects more loops from significantly reduced sequencing depth. For example, Arima-HiC identified >12,500 loops in GM12878 cells from just 600 million raw reads.

New technologies are being built on the Arima-HiC platform. For instance, Arima developed an optimized lowinput protocol proven to detect $>10,000$ loops from 50,000 cells. Additionally, targeted genome conformation assays such as HiChIP and Capture-HiC have emerged. Our $\mathrm{H} 3 \mathrm{~K} 27 \mathrm{ac}$ and H3K4me3 HiChIP data demonstrate reproducible detection of long-range gene regulatory interactions at a fraction of the cost as genome-wide Hi-C. Moreover, Capture-HiC enables discovery of chromatin loops around oncogenes and tumor suppressors at 500bp resolution, from only $\sim 10 \mathrm{M}$ reads per genetic locus. Lastly, Arima-HiC data is emerging as powerful tool for genome-wide discovery of 
structural variants (SVs) in cancer genomes. Analysis of a human GIST revealed $>70 \mathrm{SVs}$, with several of these forming "neo-TADs", bringing genes (or fusion genes) into new regulatory environments potentiating mis-regulation and enhancer hijacking. Overall, Arima-HiC aims to be a flexible protocol for multiple "C" assays (Hi-C, HiChIP, Capture-HiC) that will accelerate research across human genetics and epigenetics.

J. Belton: A. Employment (full or part-time); Significant; Arima Genomics, Inc. D. Reid: A. Employment (full or part-time); Significant; Arima Genomics, Inc. X. Zhou: A. Employment (full or part-time); Significant; Arima Genomics, Inc. S. Mac: A. Employment (full or part-time); Significant; Arima Genomics, Inc. S. Tin: A. Employment (full or part-time); Significant; Arima Genomics, Inc. C. Caneda: A. Employment (full or part-time); Significant; Arima Genomics, Inc. J. Kirkland: A. Employment (full or part-time); Significant; Arima Genomics, Inc. A. Schmitt: A. Employment (full or part-time); Significant; Arima Genomics, Inc.. E. Ownership Interest (stock, stock options, patent or other intellectual property); Significant; Arima Genomics, Inc..

\section{P15.08.C}

Comprehensive characterization of cell-free coding and non-coding RNAs using polyadenylation ligationmediated sequencing (PALM-Seq)

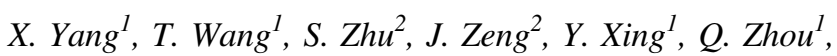

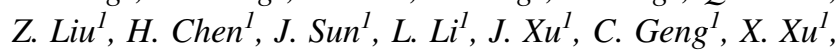

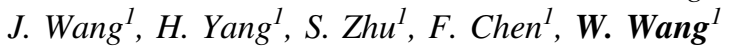

${ }^{1}$ BGI-Shenzhen, Shenzhen, China, ${ }^{2}$ Shenzhen Maternity and Child Healthcare Hospital, Shenzhen, China

Introduction: Both cell-free coding and non-coding RNAs serve as promising biomarkers for disease prediction, diagnosis and prognosis, but it remains challenging to characterize cell-free RNAs (cfRNAs) comprehensively.

Methods: We developed polyadenylation ligationmediated sequencing (PALM-Seq) to sequence cell-free mRNA, long non-coding RNA (lncRNA), and small RNAs simultaneously, and validated it by applying it to plasma and amniotic fluid samples.

Results: By comparing the involvement and noninvolvement of the steps of T4 PNK treatment and targeted RNA depletion, we showed that PALM-Seq could simultaneously capture cell-free coding RNA, lncRNA and various small RNAs, and efficiently deplete redundant RNAs, with comparable performances of existing cell-free mRNA or small RNA sequencing methods. Describing cfRNAs in plasma and amniotic fluid with PALM-Seq revealed distinct RNA composition and similarly featured 3' ends of mRNA and IncRNA. Furthermore, we identified a group of mRNAs, lncRNAs and microRNAs (miRNAs) in plasma that could perfectly distinguish pregnant and nonpregnant women.

Conclusions: Altogether, PALM-Seq enables comprehensive characterization of cell-free coding and non-coding RNAs, which could stimulate further progress in biomarker discovery, health status monitoring, and intercellular communication research.

Grant: Shenzhen Municipal Government of China (No. JCYJ20170412152854656).

X. Yang: None. T. Wang: None. S. Zhu: None. J. Zeng: None. Y. Xing: None. Q. Zhou: None. Z. Liu: None. H. Chen: None. J. Sun: None. L. Li: None. J. Xu: None. C. Geng: None. X. Xu: None. J. Wang: None. H. Yang: None. S. Zhu: None. F. Chen: None. W. Wang: None.

P15.09.A

Integration of genome-wide methylation analysis with transcription analysis and genome sequencing enables molecular confirmation in a patient with CHARGE syndrome

J. L. Granadillo, D. J. Wegner, M. Willing, K. Sisco, J. Wambach, D. Baldridge, F. S. Cole

Washington University School of Medicine, Saint Louis, MO, United States

Introduction: $C H D 7$ pathogenic variants cause more than 90\% of CHARGE syndrome cases, an autosomal dominant condition characterized by coloboma of the iris, retina, and/ or optic disk; choanal atresia, cranial nerve dysfunction, characteristic CHARGE syndrome ear malformations, developmental delay, congenital cardiac defects, and poor growth. Approximately $10 \%$ of cases have no known genetic cause.

Materials and Methods: We report the case of a patient with clinical features of CHARGE syndrome and nondiagnostic genetic testing, including chromosome microarray, $C H D 7$ sequencing and deletion/duplication analysis, SEMA3E sequencing, and trio Whole Exome Sequencing. After enrollment in the NIH-funded Undiagnosed Diseases Network (UDN), a comprehensive clinical assessment, genome-wide methylation analysis (GMA), Whole Genome Sequencing (WGS), and CHD7 RNA studies were performed.

Results: The proband is a Hispanic 5-year-old male with global developmental delay, bilateral optic nerve coloboma, strabismus, nystagmus, bilateral hearing loss, repaired persistent ductus arteriosus, bicuspid aortic valve, micropenis, and cryptorchidism. Physical exam was notable for a 
squared face and CHARGE-like ears. CT scan of the temporal bones revealed cochlear and vestibular malformations, hypoplastic facial nerves, and hypoplastic left internal auditory canal. GMA revealed a $C H D 7$-associated epigenetic signature. WGS with focused bioinformatic analysis of CHD7 and removal of all variant filters detected a de novo 15 bp deletion in intron 4 of $C H D 7$, denoted c.223920_2239-6delGTCTTGGGTTTTTGT (NM_017780.3). RNA studies confirmed that this deletion causes disruption of the canonical 3' splice site, and introduction of a premature stop codon.

Conclusions: Integrating genomic, epigenomic, and transcriptomic methods may increase the diagnostic yield for CHARGE and other genetic syndromes.

J.L. Granadillo: None. D.J. Wegner: None. M. Willing: None. K. Sisco: None. J. Wambach: None. D. Baldridge: None. F.S. Cole: None.

\section{P15.10.B}

A novel generation of digital PCR allows fast, convenient and accurate chimerism monitoring after hematopoietic stem cell transplantation

\section{E. Gourri, B. M. Frey, S. Meyer}

Department of Molecular Diagnostics and Cytometry, Blood Transfusion Service Zurich, Swiss Red Cross, Schlieren, Switzerland

Introduction: Monitoring of chimerism in patients after stem-cell transplantation is part of the surveillance of impending clinical relapse. We previously established a method for monitoring based on detection of SNVs by Taqman $^{\mathrm{TM}}$ assays on a chip-digital PCR (chdPCR) platform (Thermofisher). This method enabled reliable monitoring on nearly 2,000 samples. However, substantial hands-on-time renders this platform less suitable for high sample throughput. We aimed to establish an alternative system to our approved chdPCR platform providing higher sample throughput and minimal hands-on-time.

Material and Methods: The Opal ${ }^{\mathrm{TM}}$ dPCR chip represents the larger unit of the Naica ${ }^{\mathrm{TM}}$ crystal $\mathrm{dPCR}$ system (Stilla) and allows SNV detection in approximately 20,000 droplets. Crystal dPCR combines automated droplet formation and thermocycling on a single device (Naica ${ }^{\mathrm{TM}}$ Geode). Fluorescence signal of each droplet is measured in the Naica ${ }^{\mathrm{TM}}$ Prism instrument. With 48 reactions per run, the system represents a fast and economical alternative to other platforms.

Results: Artificial DNA mixes provided reproducible quantification of the minor allele, even below $0.25 \%$. Notably, the clinically required $0.5 \%$ minor allele sensitivity was achieved with only $5 \mathrm{ng}$ of input DNA. Additionally, the reliability of the crystal dPCR platform was confirmed on previously monitored patient's samples and numerous EQA samples.

Conclusions: This new generation of dPCR is fully compatible with our established SNV-based chimerism monitoring and has been proven to be fast, convenient and highly accurate. In addition to minimal hands-on-time, and consumable use, the low amount of input DNA per reaction should be emphasized, opening the option of lineage specific chimerism monitoring.

E. Gourri: None. B.M. Frey: None. S. Meyer: None.

\section{P15.13.B}

CNV analysis based on whole genome sequencing for distant relatedness determination M. Korabecna ${ }^{l}$, A. Zinkova
Pazourkova $^{l}$, I. Brynychova
, Simkova

${ }^{I}$ First Faculty of Medicine, Charles University, Prague, Czech Republic, ${ }^{2}$ Second Faculty of Medicine, Charles University, Prague, Czech Republic

The samples from 100 healthy non-related individuals from Czech population were used for whole genome sequencing (WGS) on NovaSeq 6000 (Illumina) to prepare the database of biallelic structural variants (CNV, SVs) existing in this population. After careful data filtering, the sets of well characterized individual variants were used for, fingerprinting" and relatedness determination. $35000 \mathrm{SVs}$ were detected in the Czech population, 8969 structural variants (SVs) per individual on average (median 9389). The number of detected SVs per individual is highly dependent on the mean coverage value. The sufficient coverage to capture all SVs in the sample should be significantly higher than the conventionally used value equal to 30 . To test the genome wide analysis of SVs for relatedness determination, we used WGS data from 10 individuals belonging to a threegeneration family. For the relationship category prediction, the following coefficients were computed by using package Demerelate in R: Bxy, Sxy, Mxy, rxy, Lxy, Li, Ioiselle, wang, wang.fin. The distributions of values of all these coefficients for different relationships were computed first using all detected variants as markers. Then the variants not having exactly identical genomic positions within all samples were removed. We demonstrated that WGS in association with appropriate bioinformatic tools is highly effective for SVs detection, the determination of their extent and population frequency. Our approach may lead to the successful determination of coefficients of relationships also for pairs with $\mathrm{r}=0.125$. Supported by the Ministry of Interior of the Czech Republic grant no. VI20172020102. 
M. Korabecna: None. A. Zinkova: None. I. Brynychova: None. E. Pazourkova: None. H. Simkova: None. J. Geryk: None.

\section{P15.14.C}

Practical applications of CRISPR knock in: generation of cacnalc mutant zebrafish

E. Sieliwonczyk, B. Vandendriessche, M. Alaerts, D. Knapen, L. Vergauwen, C. Moers, D. Snyders, B. Loeys, D. Schepers

University of Antwerp, Antwerpen, Belgium

Introduction: The similarity between the human and the zebrafish cardiac action potentials makes zebrafish an attractive model for cardiogenetic disorders. However, introducing base pair substitutions (knock in, KI) by the CRISPR/Cas9 technique in zebrafish remains challenging. In our efforts to generate a KI zebrafish model of the human CACNAlc c. $2570 \mathrm{C}>\mathrm{G}$ long $\mathrm{QT}$ syndrome mutation, we have combined early genotyping with next-generation sequencing (NGS) to improve the KI efficiency.

Methods: Zebrafish eggs were injected with Cas9 mRNA or protein, 1-2 gRNA and four conformations of ssODN: identical ("target") or complementary ("non-target") to the gRNA binding sequence, symmetric or asymmetric. KI detection was performed by NGS on a MiSeq instrument (Illumina). To select embryos for breeding, DNA was extracted from live embryos with the Zebrafish Embryo Genotyping device.

Results: Variable KI rates were observed, ranging from 0.1 to $1.58 \%$. The highest KI rate was observed with Cas9 mRNA with 1 gRNA and the right asymmetric target ssODN. Embryos from the Cas9 mRNA injection group containing $>0.2 \% \mathrm{KI}$ reads were raised to maturity and crossed out to assess germline transmission. One out of 10 fish passed the edited allele on to $25 \%$ of its offspring.

Conclusions: KI experiments in zebrafish show a high degree of variability. The KI efficiency tends to be lower in disease modeling than in methodological studies as the experiments are more restrictive. We intend to overcome these limitations by combining early genotyping with an NGS detection technique.

Grants: This research is supported by the Fund for Scientific Research, Flanders.

E. Sieliwonczyk: None. B. Vandendriessche: None. M. Alaerts: None. D. Knapen: None. L. Vergauwen: None. C. Moers: None. D. Snyders: None. B. Loeys: None. D. Schepers: None.

P15.16.B
Validation of INNO-LiPA CFTRiage for prenatal diagnostic

P. Reichgelt ${ }^{l}$, R. van de Graaf ${ }^{l}$, W. Deelen ${ }^{l}$, J. Vanden Broecke $^{2}$, L. Tremmerie $^{3}$, M. Wilke ${ }^{l}$

${ }^{I}$ Department of Clinical Genetics, Rotterdam, Netherlands, ${ }^{2}$ Fujirebio Europe NV, Ghent, Belgium, ${ }^{3}$ Fujirebio Eurpe NV, Ghent, Belgium

Cystic fibrosis (CF) is a genetic disorder caused by aberrant variants in the $\mathrm{CF}$ transmembrane conductance regulator (CFTR) gene. For a full diagnosis of CF multiple tests are needed, among which genetic testing is one of the most important. During the first round of genetic testing different tests are performed covering most frequent pathogenic variants $(>1 \%)$. Fujirebio Europe N.V.'s newly available commercial test INNO-LiPA CFTRiage was launched for identification of 88 known pathogenic variants in the CFTR gene in whole blood, dried blood spots and buccal brushes. Here we describe the validation of the INNO-LiPA CFTRiage for prenatal samples. Earlier INNO-LiPA CFTR19/CFTR17+Tn Update/CFTR Deletions + 6/CFTR Italian Regional tests were used for detection of variants in a prenatal setting. 34 (prenatal) samples were investigated for the validation of INNO-LiPA CFTRiage. The test set consisted of earlier identified pathogenic variants derived from four chorionic villi samples (CVS, one normal, three mutated), 28 amniotic fluid samples (AF, 25 normal, 3 mutated), one Intra Uterine Foetal Death (IUFD) sample (normal) and one non-prenatal control. The experiments were performed at the department of Clinical Genetics (Erasmus MC, Rotterdam, NL). The automatic read out was performed by Fujirebio Europe N.V. Results were compared with earlier obtained data. 34/34 were identical (one sample needed to be diluted 1/10).We tested a limited number of prenatal samples. All gave the expected result. Therefore we think that INNO-LiPA CFTRiage is suitable for detection of pathogenic variants in a prenatal setting using DNA derived from CVS and AF.

P. Reichgelt: None. R. van de Graaf: None. W. Deelen: None. J. Vanden Broecke: C. Other Research Support (supplies, equipment, receipt of drugs or other in-kind support); Modest; Fujirebio Europe NV. L. Tremmerie: C. Other Research Support (supplies, equipment, receipt of drugs or other in-kind support); Modest; Fujirebio Europe NV. M. Wilke: None.

\section{P15.17.C}

An analytical comparison of commonly used arrays vs. shallow whole genome sequencing for cytogenetic testing 
S. Verma, R. Keshavan, S. Shams

\section{BioDiscovery, El Segundo, CA, United States}

High-density SNP array, with its ability to detect regions of homozygosity and copy number variants (CNVs), is now the most commonly used platform for cytogenetic testing. Nextgeneration sequencing (NGS), traditionally used for detection of small base-level genomic changes, is now gaining wider acceptance as a platform for detecting CNVs in addition to single nucleotide variants (SNVs). With the reduction in cost of whole-genome sequencing (WGS), many labs are considering using "shallow" WGS to replace microarrays for cytogenetic testing. To assess content and design of microarrays, we performed analytical analyses on ten commonly used SNP array platforms for cytogenetic testing offered by Illumina and Thermo Fisher/Affymetrix. The set of arrays included the Global Screening Array (GSA) and the Global Diversity Array (GDA) platforms from Illumina since they offer a very low-cost option. The analyses included number and coverage statistics on heterozygous probe distribution for detection of $\mathrm{AOH}$ events, probes spanning exons/genes, and distribution of probes genome-wide covering regions with clinical significance from sources such as ClinGen, OMIM, DDG2P, and including a number of additional genes strongly associated with constitutional and oncology testing. Here we will share the results of the array comparison project as they compare with each other and with shallow WGS, both in terms of coverage as well as cost.

S. Verma: A. Employment (full or part-time); Significant; BioDiscovery. E. Ownership Interest (stock, stock options, patent or other intellectual property); Modest; BioDiscovery. R. Keshavan: A. Employment (full or part-time); Significant; BioDiscovery. E. Ownership Interest (stock, stock options, patent or other intellectual property); Modest; BioDiscovery. S. Shams: A. Employment (full or part-time); Significant; BioDiscovery. E. Ownership Interest (stock, stock options, patent or other intellectual property); Significant; BioDiscovery.

\section{P15.19.B}

Enabling variant calling in challenging FFPE samples by coupling a novel library preparation chemistry with exome sequencing

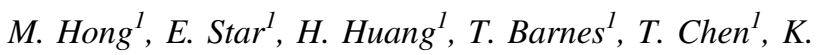

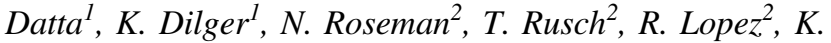
Scott $^{2}$, L. A. Lewis ${ }^{2}$, S. Groenewold ${ }^{2}$, L. Ren ${ }^{2}$, J. Zhou ${ }^{1}$, K.

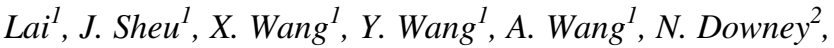
A. Potts ${ }^{l}$, U. Das Chakravarty ${ }^{l}$, B. Min ${ }^{l}$, P. J. Lau ${ }^{l}, S$. Henck $^{l}$

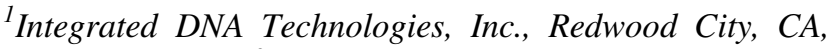
United States, ${ }^{2}$ Integrated DNA Technologies, Inc.,

\section{Coralville, IA, United States}

Comprehensive tumor profiling using NGS is fundamentally transforming oncology research. However, converting archival tissue samples into libraries is often challenging due to the low quantity and quality of DNA. Here we present accurate detection of variants in the human exome using the novel chemistry of the xGen Prism DNA library preparation kit, optimized for low-input and degraded samples, with xGen Research Exome v2.0 hybrid-capture enrichment. The IDT Exome v2 panel was used to carry out targeted sequencing of Prism DNA libraries generated from archival FFPE samples. The unique library preparation is enabled by an engineered mutant ligase and proprietary adapters that prevent chimeras and suppress dimer-formation, thereby maximizing the conversion of input DNA to sequencing libraries. We achieved high yields of library (300-400 ng) from input amounts as low as $25 \mathrm{ng}$ for severely damaged FFPE samples (DIN 1-3), $>90 \%$ ontarget rates and uniform depth of coverage ( $>96 \%$ bases covered at $>20 \mathrm{X}$ and $>98 \%$ bases covered at $>10 \mathrm{X}$ ) for FFPE samples across a wide range in quality. We also observed minimal exon drop-outs in difficult-to-target genes for severely damaged FFPE material. To validate the variant calling performance of the Prism-Exome workflow, we used the Horizon OncoSpan FFPE reference control which contains 1-92\% AF SNVs and Indels and achieved $>98 \%$ sensitivity across $\sim 250$ SNVs and Indels. This study demonstrates that the xGen Exome Research v2, when combined with xGen Prism DNA library preparation, provides researchers with a complete human exome FFPEsequencing solution with robust performance across FFPE samples of varying quality.

M. Hong: A. Employment (full or part-time); Significant; IDT. E. Star: A. Employment (full or part-time); Significant; IDT. H. Huang: A. Employment (full or parttime); Significant; IDT. T. Barnes: A. Employment (full or part-time); Significant; IDT. T. Chen: A. Employment (full or part-time); Significant; IDT. K. Datta: A. Employment (full or part-time); Significant; IDT. K. Dilger: A. Employment (full or part-time); Significant; IDT. N. Roseman: A. Employment (full or part-time); Significant; IDT. T. Rusch: A. Employment (full or part-time); Significant; IDT. R. Lopez: A. Employment (full or part-time); Significant; IDT. K. Scott: A. Employment (full or part-time); Significant; IDT. L.A. Lewis: A. Employment (full or part-time); Significant; IDT. S. Groenewold: A. Employment (full or part-time); Significant; IDT. L. Ren: A. Employment (full or part-time); Significant; IDT. J. Zhou: A. Employment (full or part-time); Significant; IDT. K. Lai: A. Employment (full or part-time); Significant; IDT. J. Sheu: A. Employment (full or part-time); Significant; IDT. X. Wang: A. Employment (full or part-time); Significant; IDT. Y. 
Wang: A. Employment (full or part-time); Significant; IDT. A. Wang: A. Employment (full or part-time); Significant; IDT. N. Downey: A. Employment (full or part-time); Significant; IDT. A. Potts: A. Employment (full or parttime); Significant; IDT. U. Das Chakravarty: A. Employment (full or part-time); Significant; IDT. B. Min: None. P. J. Lau: A. Employment (full or part-time); Significant; IDT. S. Henck: A. Employment (full or part-time); Significant; IDT.

\section{P15.21.A}

Quantitative facial phenotyping for Koolen-de Vries and 22q11.2 deletion syndrome

\section{A. J. M. Dingemans ${ }^{1}$, D. E. Stremmelaar ${ }^{1}$, R. Van der} Donk $^{1}$, L. E. L. M. Vissers ${ }^{1}$, D. A. Koolen ${ }^{1}$, P. Rump ${ }^{2}$, J. Y. Hehir-Kwa ${ }^{3}$, B. B. A. De Vries ${ }^{1}$

${ }^{1}$ Radboudumc, Nijmegen, Netherlands, ${ }^{2} U M C G$, Groningen, Netherlands, ${ }^{3}$ Princess Máxima Center for Pediatric Oncology, Bilthoven, Netherlands

\begin{tabular}{|c|c|c|c|c|}
\hline \\
\hline \multicolumn{5}{|c|}{$\begin{array}{l}\text { Introduction: The Koolen-de Vries syndrome (KdVS) } \\
\text { is a multisystem syndrome with variable facial features } \\
\text { caused by a } 17 \mathrm{q} 21.31 \text { microdeletion or KANSL1 truncat- } \\
\text { ing mutation. As the facial gestalt of KdVS is similar to } \\
\text { the gestalt of the } 22 \mathrm{q} 11.2 \text { deletion syndrome } \\
\text { (22q11.2DS), we assessed whether our previously } \\
\text { described hybrid quantitative facial phenotyping algo- } \\
\text { rithm could distinguish between these two syndromes, } \\
\text { and, in addition, whether there is a facial difference } \\
\text { between the molecular KdVS subtypes. } \\
\text { Materials and Methods: We applied our algorithm to } 2 \mathrm{D} \\
\text { photographs of } 97 \text { patients with KdVS ( } 78 \text { microdeletions, } \\
19 \text { truncating mutations) and } 48 \text { patients with } 22 \mathrm{q} 11.2 \mathrm{DS} \text { as } \\
\text { well as age, gender and ethnicity matched controls with } \\
\text { intellectual disability ( } n=145 \text { ). } \\
\text { Results: The facial gestalts of KdVS and } 22 \mathrm{q} 11.2 \mathrm{DS} \\
\text { are both recognizable, yet different from one another ( } p= \\
7.5 * 10^{-10} \text { and } p=0.0052 \text {, respectively). }\end{array}$} \\
\hline $\begin{array}{l}\mathrm{N} \text { Observed } \\
\text { matches }\end{array}$ & & & & P-value \\
\hline $2 q 11.2 D S \quad 48 \quad 25$ & 23 & 15.0 & 33.0 & \\
\hline & & & & \\
\hline
\end{tabular}

Furthermore, the facial gestalts of KdVS caused by a $17 q 21.31$ microdeletion and KANSL1 truncating mutation are indistinguishable $(p=0.981$ and $p=0.130)$. Further application to 3 patients with a variant of unknown clinical significance in KANSL1 showed that these do not match a clinical diagnosis of KdVS.

Conclusions: Our data highlight quantitative facial phenotyping not only as a powerful tool to distinguish syndromes with overlapping facial dysmorphisms but also to establish pathogenicity of variants of unknown clinical significance.

A.J.M. Dingemans: None. D.E. Stremmelaar: None. R. Van der Donk: None. L.E.L.M. Vissers: None. D.A. Koolen: None. P. Rump: None. J.Y. Hehir-Kwa: None. B.B.A. De Vries: None.

P15.22.B

High throughput analysis of disease repeat expansions and contractions by optical mapping

E. T. Lam, J. Wang, D. Zhang, T. Wang, A. W. C. Pang, H. B. Sadowski, A. R. Hastie, M. Oldakowski

\section{Bionano Genomics, San Diego, CA, United States}

Repeat expansions and contractions are associated with degenerative disorders such as facioscapulohumeral muscular dystrophy (FSHD). The phenotype severity is often correlated with the extent of pathogenic expansion or contraction. Inherently, these repeats are unstable. The repeat size in somatic cells can vary over time and be heterogeneous, which impacts the age of onset in both the current and successive generations. Most repeat disorders have no direct treatments.

Southern blotting is the current gold standard for analyzing pathogenic repeats. While sequencing-based methods are being developed, these efforts are hampered by limitations in read lengths and the repetitive and polymorphic nature of these regions. Optical mapping with Bionano Genome Imaging enables analysis of ultra-high molecular weight DNA molecules that can span large repeats. In addition, a comprehensive streamlined workflow inclusive of sample preparation, DNA imaging, and genomic data analysis has been developed for highthroughput analysis of repeats of interest.

We recently demonstrated the utility of the platform by focusing on the D4Z4 repeat contraction on chromosome $4 q 35$, which is a marker of FSHD when present on a permissive haplotype. The analysis had high sensitivity, specificity, and reproducibility. We analyzed 30 FSHDpositive samples. 28 of them had repeat contractions on the $4 \mathrm{qA}$ haplotype. The two samples that did not show repeat contractions were run twice; the results were consistent. We 
lso analyzed 58 FSHD-negative samples; none had repeat counts in the clear pathogenic range. With the method described above, we have analyzed other repeat expansions with high sensitivity.

E.T. Lam: A. Employment (full or part-time); Significant; Bionano Genomics. E. Ownership Interest (stock, stock options, patent or other intellectual property); Modest; Bionano Genomics. J. Wang: A. Employment (full or parttime); Significant; Bionano Genomics. E. Ownership Interest (stock, stock options, patent or other intellectual property); Modest; Bionano Genomics. D. Zhang: A. Employment (full or part-time); Significant; Bionano Genomics. E. Ownership Interest (stock, stock options, patent or other intellectual property); Modest; Bionano Genomics. T. Wang: A. Employment (full or part-time); Significant; Bionano Genomics. E. Ownership Interest (stock, stock options, patent or other intellectual property); Modest; Bionano Genomics. A.W.C. Pang: A. Employment (full or part-time); Significant; Bionano Genomics. E. Ownership Interest (stock, stock options, patent or other intellectual property); Modest; Bionano Genomics. H.B. Sadowski: A. Employment (full or part-time); Significant; Bionano Genomics. E. Ownership Interest (stock, stock options, patent or other intellectual property); Modest; Bionano Genomics. A.R. Hastie: A. Employment (full or part-time); Significant; Bionano Genomics. E. Ownership Interest (stock, stock options, patent or other intellectual property); Modest; Bionano Genomics. M. Oldakowski: A. Employment (full or part-time); Significant; Bionano Genomics. E. Ownership Interest (stock, stock options, patent or other intellectual property); Modest; Bionano Genomics.

\section{P15.23.C}

Global Screening Array as a First Line Test for Mutation Detection in Familial Hypercholesterolaemia

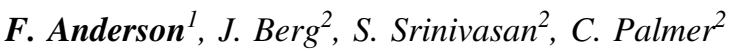 \\ ${ }^{1}$ Dundee School of Medical, Dundee, United Kingdom, ${ }^{2}$ Dundee School of Medicine, Dundee, United Kingdom}

Background: Familial Hypercholesterolaemia (FH) is a common autosomal dominant disorder caused by mutations in $L D L R, P C S K 9$ or $A P O B$. Mutation analysis allows early detection and treatment of at risk individuals, preventing cardiovascular morbidity. In Scotland, despite availability of testing to anyone fulfilling Simon Broome Criteria, it is estimated that $80 \%$ of individuals remain undiagnosed. Use of a screening array of known mutations has the potential to detect actionable mutations more cheaply, allowing testing of many more individuals and improving detection of pathogenic variants in the population.
Method and Results: We evaluated the potential clinical utility of an array based approach to mutation analysis for FH. In NHS Tayside, between 2010 and 2018, 37 pathogenic variants and 8 variants of uncertain significance were identified in $L D L R$ in 75 individuals. We compared these to the manifest of the 2016 Illumina Infinium Global Screening Array-24 V2.0, containing 1077 mutations implicated in FH. All 26 of the pathogenic point mutations were present on the array, and 10/11 of the deletions had overlapping SNVs present- giving a potential coverage of actionable variants of $97 \%$. 6/8 $(75 \%)$ of variants of uncertain significance were also represented.

Conclusions: We conclude that array technology for DNA mutation analysis has potential for screening for $\mathrm{FH}$ mutations in clinical practice. with the possibility of designing different clinical models for case identification. With careful optimisation of this low cost test option, a much greater proportion of mutation carriers could be identified and treated, with significant impact on cardiovascular disease in the population.

F. Anderson: None. J. Berg: None. S. Srinivasan: None. C. Palmer: None.

P15.24.A

A flexible workflow solution including error correction by molecular barcoding for low inputffpe tissue biopsy and circulating tumor DNA samples

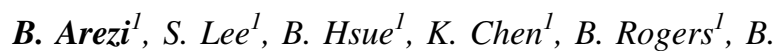

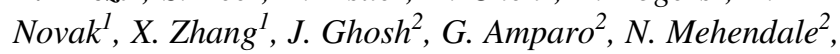 \\ C. Pabon $^{2}$, J. Ku ${ }^{2}$
}

${ }^{1}$ Agilent Technologies, la jolla, CA, United States, ${ }^{2}$ Agilent Technologies, Santa Clara, CA, United States

Accurate low allele frequency variant calling by next generation sequencing (NGS) can be challenging with FFPE specimens and circulating tumor DNA due to limited DNA availability, poor DNA quality, and variation in tumor fraction from sample to sample. Other practical considerations such as turnaround time, multiplexing capability and workflow simplicity or technological limitations such as index cross-contamination and index hopping with newer Illumina sequencing chemistries can also influence how readily NGS is applied to clinical tumor samples. Here we address these challenges by developing a complete but flexible workflow solution which is optimized for a wide range of DNA input (10-200 ng), various sample types (intact or FFPE samples), different shearing methods (mechanical vs. enzymatic), sequencing chemistries $(2 \times$ $100 \mathrm{bp}$ or $2 \times 150 \mathrm{bp}$ ), 90-minute or overnight bait hybridization, and up to 384 unique dual sample indices to eliminate index hopping. Furthermore, we incorporate 
duplex molecular barcodes at the ligation step to filter out PCR errors by making consensus calls using MBC information from both strands (duplex MBC), hybrid (duplex MBC where info is available), single strand (single MBC) or discard $\mathrm{MBC}$ information (no $\mathrm{MBC}$ ), based on the application. While both duplex and single MBC improve the accuracy of low VAF detection compared to no MBC, duplex MBC enables the most effective error correction. As we will demonstrate, the number of false positive calls of low VAF $(\leq 4 \%)$ for duplex MBC were reduced significantly by $74 \%$ and $93 \%$ compared to single MBC and no MBC, respectively.

B. Arezi: A. Employment (full or part-time); Significant; Agilent Technologies. S. Lee: A. Employment (full or parttime); Significant; Agilent Technologies. B. Hsue: A. Employment (full or part-time); Significant; Agilent Technologies. K. Chen: A. Employment (full or part-time); Significant; Agilent Technologies. B. Rogers: A. Employment (full or part-time); Significant; Agilent Technologies. B. Novak: A. Employment (full or part-time); Significant; Agilent Technologies. X. Zhang: A. Employment (full or part-time); Significant; Agilent Technologies. J. Ghosh: A. Employment (full or part-time); Significant; Agilent Technologies. G. Amparo: A. Employment (full or parttime); Significant; Agilent Technologies. N. Mehendale: A. Employment (full or part-time); Significant; Agilent Technologies. C. Pabon: A. Employment (full or parttime); Significant; Agilent Technologies. J. Ku: A. Employment (full or part-time); Significant; Agilent Technologies.

\section{P15.26.C}

An NGS-based approach for the detection of $G B A$ variants including recombinations with the pseudogene GBAP

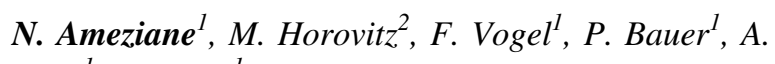

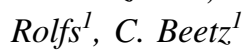

${ }^{1}$ Centogene AG, Rostock, Germany, ${ }^{2}$ Tel Aviv University, Tel Aviv, Israel

Pathogenic biallelic alterations in GBA cause Gaucher disease, while monoallelic alterations represent a risk factor for Parkinson's disease. The mutational spectrum consists of single nucleotide variants and small insertions/deletions as well as of recombination-mediated rearrangements with the nearby pseudogene GBAP. The latter type of alteration has been difficult to detect and characterize by the standard $G B A$ diagnostic approaches due to the application of an initial $G B A$-specific long-range PCR. We developed a capture-based NGS protocol and a dedicated bioinformatics pipeline that exploits the relative number of aligned reads to
$G B A$ and $G B A P$ to detect recombinational events. We applied our tool to a set of samples with known and suspected GBA alterations, which had been comprehensively analyzed by various methods including Southern blotting and cDNA cloning. We detected all known SNVs and small insertions/deletions. For cases with suspected rearrangements, we observed altered relative $G B A$ and $G B A P$ read ratios. The findings were of two kinds: 1) suggestive of recombination-mediated conversions, 2) suggestive of recombination-mediated large deletions. Our observations are consistent with theoretical considerations put forward previously. In conclusion, we have developed a method that can reliably detect all types of disease-relevant alterations in $G B A$. This will allow for accurate molecular classification of Gaucher patients and of individuals at higher risk for Parkinson's disease.

N. Ameziane: A. Employment (full or part-time); Significant; Centogene AG. M. Horovitz: None. F. Vogel: A. Employment (full or part-time); Significant; Centogene AG. P. Bauer: A. Employment (full or part-time); Significant; Centogene AG. A. Rolfs: A. Employment (full or part-time); Significant; Centogene AG. C. Beetz: A. Employment (full or part-time); Significant; Centogene AG.

\section{P15.34.B}

Genetic aberration detection through whole exome sequencing in comparison with microarray-based methods in children with global developmental delay and intellectual disability

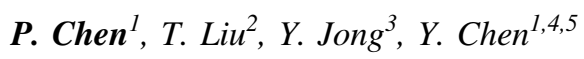

${ }^{1}$ Department of Life Sciences and Institute of Genome Sciences, Taipei, Taiwan, ${ }^{2}$ Genome Research Center, National Yang-Ming University, Taipei, Taiwan, ${ }^{3}$ Kaohsiung Medical University, Kaohsiung, Taiwan, ${ }^{4}$ Division of Genetics and Metabolism, Department of Pediatrics, Taipei Veterans General Hospital, Taipei, Taiwan, ${ }^{5}$ Department of Education and Research, Taipei City Hospital, Taipei, Taiwan

Developmental delay (DD) is a common pediatric condition, with copy number variations (CNVs) considered one of its major causes. This study aims to determine the efficiency of whole exome sequencing (WES) as a first-tier diagnostic test to detect aberrations including $\mathrm{CNVs}$ and single nucleotide variants (SNVs) in comparison with array comparative genomic hybridization (aCGH) for DD. Subjects (541) diagnosed with global developmental delay (GDD) or intellectual disability (ID) of unknown cause have been enrolled, and aCGH tests have a positive rate of $23.7 \%$ (111/468). Fragile X syndrome or Rett-like syndrome tests and treatable targeted NGS-based panel were also utilized 
for assessment. Furthermore, WES was performed on 34 cases with Agilent SureSelect target enrichment system using Illumina HiSeq2000. WES data were analyzed using Varseq v2.1.0 and Illumina BaseSpace Variant Interpreter. Trio analyses were performed on 24 aCGH-negative cases, thus far revealing 4 known rare pathogenic variants associated with GDD/ID. To determine the consistency in CNV detection between aCGH and WES tests, 10 aCGH-positive singletons were included. CNVs were detected in 11 out of 13 regions using WES. On average, $115 \mathrm{CNVs}$ with $p$ values $<0.01$ were called per case. These CNVs ranged from $119 \mathrm{bp}$ to $15 \mathrm{Mbp}$ in span, with duplications accounting for $65.0 \%$ of total calls. Approximately $1 \%$ (30/2764) of CNVs were drawn for qPCR validation, and $40 \%$ (12/30) of the tested CNVs have relative quantities suggestive or indicative of their CNV types. WES provides comprehensive and high-throughput information with the potential to cut medical expenditures, elevate diagnostic yield, and reduce diagnostic odyssey.

P. Chen: None. T. Liu: None. Y. Jong: None. Y. Chen: None.

\section{P15.35.C}

Comprehensive single-nucleotide, indel, structural, and copy-number variant detection in human genomes with PacBio HiFi reads

\section{A. M. Wenger, W. J. Rowell, A. Töpfer, L. Hickey \\ Pacific Biosciences, Menlo Park, CA, United States}

Introduction: Long-read sequencing has revealed more than 20,000 structural variants spanning over $12 \mathrm{Mb}$ in a healthy human genome. Short-read sequencing fails to detect most structural variants but has remained the more effective approach for small variants, due to $10-15 \%$ error rates in long reads, and copy-number variants ( $\mathrm{CNVs})$, due to lack of effective long-read variant callers. The development of PacBio highly accurate long reads (HiFi reads) with read lengths of $10-25 \mathrm{~kb}$ and quality $>99 \%$ presents the opportunity to capture all classes of variation with one approach.

Methods: We sequence the Genome in a Bottle benchmark sample $\mathrm{HGO02}$ and an individual with a presumed Mendelian disease with HiFi reads. We call SNVs and indels with DeepVariant and extend the structural variant caller pbsv to call CNVs using read depth and clipping signatures.

Results: For 18-fold coverage with $13 \mathrm{~kb}$ HiFi reads, variant calling in HG002 achieves an F1 score of $99.7 \%$ for SNVs, $96.6 \%$ for indels, and $96.4 \%$ for structural variants. Additionally, we detect more than $300 \mathrm{CNVs}$ spanning around $10 \mathrm{Mb}$. For the Mendelian disease case, HiFi reads reveal thousands of variants that were overlooked by shortread sequencing, including a candidate causative structural variant.

Conclusions: These results illustrate the ability of HiFi reads to comprehensively detect variants, including those associated with human disease.

A.M. Wenger: A. Employment (full or part-time); Significant; Pacific Biosciences. W.J. Rowell: A. Employment (full or part-time); Significant; Pacific Biosciences. A. Töpfer: A. Employment (full or part-time); Significant; Pacific Biosciences. L. Hickey: A. Employment (full or part-time); Significant; Pacific Biosciences.

\section{P15.37.B}

Targeting clinically significant dark regions of the human genome with high accuracy long read sequencing

\section{A. Aro, C. Heiner, Y. Tsai, J. Harding, J. Ziegle}

\section{Pacific Biosciences, Menlo Park, CA, United States}

Introduction: There are many clinically important genes in "dark" regions of the human genome, with poor coverage due to low amplification or difficulty mapping with other technologies. These dark regions often contain highly homologous genes and pseudo genes, repeat expansions, and areas of biased base composition. Ambiguous mapping due to the limitations of short-read sequencing prevents accurate assessment of structural rearrangements and importantly, short-reads limit the ability to phase across gene regions.

Materials and Methods: We investigated several important dark region genes using PacBio SMRT Sequencing using long-range PCR and/or direct sequencing using a CRISPR/cas9 mediated No-Amp for target enrichment approach. Genes included HTT, FMR1, CYP21A2 and $H B B$. Sample prep and sequencing methods are described in detail.

Results: Here we show results from successfully sequencing a key portion of the HTT gene using both NoAmp and long-range PCR. This comparison includes a set of samples with moderate to extensive repeat expansions, demonstrating highly accurate sequencing of the repeat region with both approaches. General advantages and disadvantages of each method are discussed.

Conclusion: We demonstrate that accurate, long read sequencing provides new opportunities for sequencing clinically relevant but difficult regions of the human genome that are underrepresented in short-read sequencing. The preferred method of targeting depends on the gene of interest and project goals. SMRT Sequencing of these regions enables a better understanding of the relationship between genetic factors and personal health. 
L.A. Aro: A. Employment (full or part-time); Significant; Pacific Biosciences of California. E. Ownership Interest (stock, stock options, patent or other intellectual property); Modest; Pacific Biosciences of California. C. Heiner: A. Employment (full or part-time); Significant; Pacific Biosciences. E. Ownership Interest (stock, stock options, patent or other intellectual property); Modest; Pacific Biosciences of California. Y. Tsai: A. Employment (full or part-time); Significant; Pacific Biosciences. E. Ownership Interest (stock, stock options, patent or other intellectual property); Modest; Pacific Biosciences of California. J. Harding: A. Employment (full or part-time); Significant; Pacific Biosciences. E. Ownership Interest (stock, stock options, patent or other intellectual property); Modest; Pacific Biosciences of California. J. Ziegle: A. Employment (full or part-time); Significant; Pacific Biosciences. E. Ownership Interest (stock, stock options, patent or other intellectual property); Modest; Pacific Biosciences of California.

\section{P15.38.C}

Genetic profiling and early cancer detection using NGSbased Liquid Biopsy

\section{A. Wasowska ${ }^{1,2}$, E. Matczyńska ${ }^{1,2}$, M. Krawczyk ${ }^{1}, P$. Eyszkiewicz ${ }^{1}$, A. Kowalik ${ }^{3}$, M. Chtopek ${ }^{3}, J$. Kosakowski ${ }^{1}, M$. Jurkowskal, A. Boguszewska-Chachulska ${ }^{1}$}

${ }^{1}$ Genomed SA, Warsaw, Poland, ${ }^{2}$ Medical University of Silesia, Katowice, Poland, ${ }^{3}$ Holy Cross Cancer Centre, Kielce, Poland

There is an urgent need for early and efficient cancer detection, risk stratification, and therapy monitoring. Circulating cell-free tumor DNA (ctDNA) may provide sensitive and representative marker for that purpose. The project aimed at developing non-invasive genetic diagnostics and cancer monitoring, based on the liquid biopsy (LB) for cancer patients and people at risk. Cell-free DNA (cfDNA) of 82 breast, colorectal or lung cancer cases was isolated from plasma. Additionally, breast and colorectal frozen tumours from 12 patients were collected together with the corresponding 9 blood samples. A deep coverage WGS of the tumour DNA and a standard coverage WGS for the corresponding germline DNA were performed to assess mutational burden and somatic CNVs in 10 patients. A shallow WGS (sWGS) and targeted breast cancer-related gene panel sequencing was performed for $80 \mathrm{LB}$ samples. For 6 tumour samples most common PIK3CA somatic variants in breast cancer were identified (H1047R and E545K). None of these variants was uncovered in the LB samples, while somatic variants identified in ctDNA were missing in the tumour DNA. As expected, the CNV analysis performed for tumour samples allowed to distinguish subclonal tumour variations for the same patient. A method allowing breast cancer detection at a relatively early stage, based on sWGS and CNV analysis, was developed. Discrepancies observed between the tumour and the LB results may be partially due to clonal variants in the heterogenous tumour tissues, but it also suggests the need for a further improvement regarding sensitivity of liquid biopsy methods.

Funding: RPMA.01.02.00-14-6209/16.

A. Wassowska: None. E. Matczyńska: None. M. Krawczyk: None. P. Łyszkiewicz: None. A. Kowalik: None. M. Chłopek: None. J. Kosakowski: None. M. Jurkowska: None. A. Boguszewska-Chachulska: None.

P15.39.A

Utilize of liquid biopsy in monitoring circulating tumor DNA in a patent with colorectal cancer

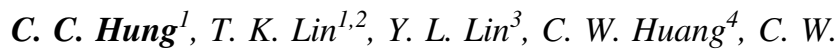

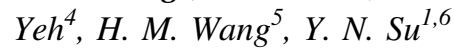

${ }^{1}$ Sofiva Genomics Co., Ltd., Taipei, Taiwan, ${ }^{2}$ Graduate Institute of Clinical Medicine, College of Medicine, National Taiwan University, Taipei, Taiwan, ${ }^{3}$ Division of Hematology and Oncology, Department of Internal Medicine, Cardinal Tien Hospital, Taipei, Taiwan, ${ }^{4}$ National Center for High Performance Computing, National Applied Research Laboratories, Hsinchu, Taiwan, ${ }^{5}$ Division of Colorectal Surgery, China Medical University Hospital, Taichung, Taiwan, ${ }^{6}$ Dianthus Maternal Fetal Medicine Clinic, Taipei, Taiwan

Background: Liquid biopsy is a minimal invasive examining circulating tumor DNA (ctDNA) in the blood, compared with invasive tissue biopsy. Frequency detecting of molecular alterations in ctDNA should be an alternative tool for monitoring disease progression.

Method: We used next-generation sequencing (NGS) based ctDNA panel assay containing 197 targeted genes and sequenced on an Illumina NextSeq 550. A 41-year old patient with stage IV colorectal cancer was examined in this study.

Results: We found 8 mutations in this patient in 2018 when he found the cancer and the mutation sites were APC c.3189 delT (50.81\%), TP53 c.339 delC (55.46\%), KRAS c.38 G>A (39.38\%), EYS c. 157 T $>$ G (0.38\%), KIT c.147 $\mathrm{C}>\mathrm{T}(0.31 \%)$, PHACTR1 c.760 C>G (0.20\%), CSMD3 c.837 T>G (0.08\%) and SLITRK4 c.329 A $>C$ (0.05\%). Physician chose neoadjuvant therapy, CCRT (concurrent chemoradiotherapy) and Avastin for this patient. Two months later, we focused on monitoring APC c.3189 delT, TP53 c.339 delC and KRAS c.38 G>A mutation sites, and the mutation rates were $0.69 \%, 0.72 \%$ and $0.34 \%$, respectively. After given the same treatment, the mutation 
rates were increased to $16.08 \%, 14.02 \%$ and $10.25 \%$, respectively. The cancer therapist decided to change to immunotherapy combined with targeted therapy, and the mutation rates decreased to $5.92 \%, 5.59 \%$ and $4.35 \%$, respectively.

Conclusions: The earlier determination of ctDNA can help track tumor response and resistance to therapy, and may also help prevent recurrence. The more clinical validation and utility of liquid biopsy would improvet of quality of life in cancer patients.

C.C. Hung: A. Employment (full or part-time); Significant; Sofiva Genomics Co., Ltd. T.K. Lin: A. Employment (full or part-time); Significant; Sofiva Genomics Co., Ltd.. Y.L. Lin: None. C.W. Huang: None. C.W. Yeh: None. H.M. Wang: None. Y.N. Su: A. Employment (full or part-time); Significant; Sofiva Genomics Co., Ltd..

\section{P15.40.B}

New revolutionary approach for Klippel-Trenaunay Syndrome: cfDNA NGS Liquid Biopsy

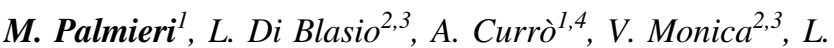
Di Sarno ${ }^{l}$, G. Doddato ${ }^{1,4}$, E. Frullanti ${ }^{1}$, G. Chaiverina ${ }^{2,3}$, A. Giliberti ${ }^{1,4}$, B. Mussolin ${ }^{2}$, C. Fallerini ${ }^{1,4}$, F. Molinaro ${ }^{5}$, M. Vaghi ${ }^{6}$, L. Primo ${ }^{2,3}$, A. Renieri ${ }^{1,4}$, A. Pinto ${ }^{4}$

${ }^{1}$ Medical Genetics, University of Siena, Siena, Italy, ${ }^{2}$ Candiolo Cancer Institute FPO-IRCCS, Candiolo, Italy, ${ }^{3}$ Deparment of Oncology, University of Turin, Turin, Italy, ${ }^{4}$ Genetica Medica, Azienda Ospedaliera Universitaria Senese, Siena, Italy, ${ }^{5}$ Chirurgia Pediatrica, Azienda Ospedaliera Universitaria Senese, Siena, Italy, ${ }^{6}$ Chirurgia Vascolare, Ospedale Maggiore di Crema, Largo Ugo Dossena, Crema, Italy

NGS-liquid biopsy using cell-free DNA (cfDNA) has emerged as an innovative non-invasive approach for early detection and monitoring of cancer. This approach overcoming the space-time profile constraint of tissue biopsies opens a new scenario also for vascular malformations due to somatic mosaicism. We propose here this approach as a fast non-invasive reliable tool for Klippel-Trenaunay syndrome (KTS) diagnosis. Six patients group suffering from KTS were selected. Blood samples from both peripheral and efferent vein from vascular malformation were collected and cfDNA extracted. Tissue biopsies from vascular lesions were also collected. CfDNA libraries were performed using Oncomine ${ }^{\mathrm{TM}}$ Pan-Cancer Cell-Free Assay. Ion Proton for sequencing and Ion Reporter Software for analysis were used. Different mutations on PIK3CA gene were detected in all patients including p.Glu542Lys and p.Glu545Gly. The same mutation detected in tissue was detected in liquid biopsy with a variant allele frequency (VAF) ranging from
$0,1-0,2 \%$ in cfDNA from peripheral vein to $1-2 \%$ in cfDNA from efferent vein. We demonstrate that cfDNA-NGSLiquid Biopsy is able to identify the PIK3CA mutation detected in affected tissues. Moreover, we have shown that blood sample withdrawal at the lesion site increases VAF rate with an order of magnitude above the limit of detection (usually $0,05 \%$ ) lowering the risk of false negative. Finally, the non-invasiveness of the method avoids any risk of bleeding, being easily performed also in children. We propose this method as method of choice for KTS diagnosis and subsequent therapy tailored to the genetic defect. We acknowledge ILA association.

M. Palmieri: None. L. Di Blasio: None. A. Currò: None. V. Monica: None. L. Di Sarno: None. G. Doddato: None. E. Frullanti: None. G. Chaiverina: None. A. Giliberti: None. B. Mussolin: None. C. Fallerini: None. F. Molinaro: None. M. Vaghi: None. L. Primo: None. A. Renieri: None. A. Pinto: None.

P15.41.C

Optimising long-range PCR methodology and long-read DNA sequencing

L. E. Batty ${ }^{l}$, B. Alobaidi ${ }^{1}$, G. S. Holt ${ }^{1}$, M. S. Oud ${ }^{2}, H$. E.

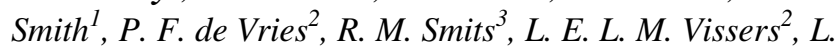
Ramos $^{3}$, M. J. Xavier ${ }^{1}$, J. A. Veltman ${ }^{l}$

${ }^{1}$ Biosciences Institute, Newcastle University, Newcastle upon Tyne, United Kingdom, ${ }^{2}$ Department of Human Genetics, Donders Institute for Brain, Cognition and Behaviour, Radboudumc, Nijmegen, Netherlands, ${ }^{3}$ Department of Obstetrics and Gynaecology, Radboudumc, Nijmegen, Netherlands

Long range PCR for targeted long-read sequencing has many practical uses, however the inherent sequencing error ( 10\%) of Oxford Nanopore Technologies (ONT) necessitates significant sequencing depths to achieve acceptable levels of confidence. Several methods exist for target capturing, most can be broadly categorised under PCR and CRISPR-Cas enrichment approaches. Here we report on the usage of long-range PCR to target capture large regions spanning rare de novo mutations linked to male infertility for sequencing with ONT for phasing analysis. Long-range PCR was used on $5-10 \mathrm{~kb}$ regions to achieve minimum sequencing depths of $>2,000 \mathrm{x}$ coverage, $>10$ fold that of CRISPR enrichments and $>90 \%$ less off-target sequencing. Individually unique target regions removed the necessity for barcodes, saving time, cost and yield. This approach allowed bringing costs to as little as $€ 15$ per sample in runs of 100 samples. The optimised method involved specialised primer design with our bespoke primer tool and improved PCR conditions, followed by sequencing on ONT MinION 
with adapted library preparation. Using input concentrations twice the standard recommendation achieved $>2$-fold increased to previous yields. "Refuelling" flowcells mid-run with ATP-containing sequencing buffer restored nanopores from $<1 \%$ to $>80 \%$, a further 6.8 fold increase in yield. More than 30 billion base calls are consistently obtained following this procedure, matching best-in field flowcell performance. Currently available methods for long-read sequencing and target capture can be expensive, time consuming and overly off target. Our proposed methodology provides a combination of common approaches that have been optimised to achieve greater results more efficiently.

L.E. Batty: None. B. Alobaidi: None. G.S. Holt: None. M.S. Oud: None. H.E. Smith: None. P.F. de Vries: None. R.M. Smits: None. L.E.L.M. Vissers: None. L. Ramos: None. M.J. Xavier: None. J.A. Veltman: None.

\section{P15.42.A}

Challenges in identifying a large complex chromosomal rearrangement by long reads sequencing

\section{B. Jenko Bizjan ${ }^{1}$, T. Katsila ${ }^{2}$, T. Tesovnik ${ }^{l}$, R. $\check{S}_{k e t}{ }^{1}, M$. Debeljak $^{l}$, J. Kovačl}

${ }^{1}$ University Children's Hospital Ljubljana, Ljubljana, Slovenia, ${ }^{2}$ Institute of Chemical Biology, National Hellenic Research Centre, Athens, Greece

Background: The success in the identification of genomic structural variations (SVs) in routine clinical protocols mainly depends on the complexity and size of SVs. Short and/or simple SV are being successfully identified by cytogenetic techniques or short read sequencing, while large nested and complex rearrangements demand case-specific investigation via the application of novel emerging technologies. For a clinical case with developmental delay cytogenetic analysis revealed a large complex de novo chromosomal rearrangement. We aimed to evaluate the potential of whole genome long-reads sequencing to resolve the precise variant configuration and its underlying mechanism.

Methods: Long-reads sequencing was performed by MinION Oxford Nanopore Technologies. Bioinformatic pipeline of reference-based alignment with SVs calling in combination with De novo assembly was applied. Reads were aligned to the reference using NGMLR or minimap2, following by detection of SVs with SVIM and Sniffles. Canu was used for De novo assembly.

Results: We have performed 6 effective runs using MinION long-read sequencing and reached 13,2x theoretical coverage of the human genome. Median read quality was 12.44 , with an average N50 read length of $10.2 \mathrm{~kb}$. Read-depth analysis identified the gain for triplication and duplication, yet the precise breakpoints cannot be defined (reads coverage variation). NGMLR alignment in combination with Canu-trimming, followed by SVIM variant calling, resulted in a few probable inversions and one tandem duplication.

Conclusions: For determination of precise breakpoints of a large nested SV, which is much larger than the average read, long-reads sequencing needs to be combined with additional long-range technologies.

B. Jenko Bizjan: None. T. Katsila: None. T. Tesovnik: None. R. Šket: None. M. Debeljak: None. J. Kovač: None.

\section{P15.43.B}

Advanced Whole Genome Sequencing Using an Etirely PCR-free Massively Workflow

\section{Zhao}

\section{MGI, ShenZhen, China}

$<$ META NAME ="author" CONTENT ="赵霞 $(X i a$ Zhao)">Introduction: Systematic errors can be introduced from DNA amplification during massively parallel sequencing library preparation and sequencing array formation. PCR-free genomic library preparation methods were previously shown to improve whole genome sequencing (WGS) quality on the Illumina platform, especially in calling InDels. We hypothesized that substantial InDel errors continue to be introduced by the remaining PCR step of DNA cluster generation.

Materials and Methods: Here, we sequenced PCR-free libraries as paired-end 150-base reads on MGI's PCR-free DNBSEQ $^{\mathrm{TM}}$ arrays to accomplish the first true PCR-free WGS. We used MGI's PCR-free WGS library preparation kits as recommended or with some modifications to prepare several NA12878 libraries. We also assessed the process data performances with three variant calling pipelines.

Conclusions: Reproducibly high-quality libraries were obtained with low coverage bias and less than $1 \%$ read duplication. Using machine learning (ML) methods (DeepVariant or DNAscope), variant calling performance (SNPs F-measure $>99.94 \%$, InDels F-measure $>99.6 \%$ ) exceeded widely accepted standards. Furthermore, PCR-free WGS libraries sequenced on the PCR-free DNBSEQ ${ }^{\mathrm{TM}}$ platform have up to $55 \%$ less InDel errors compared to the NovaSeq platform, confirming that DNA clusters contain PCRgenerated errors. We also found some clinical relevant gene locis can only be detected by PCR-free DNBSEQ ${ }^{\mathrm{TM}}$. Therefore, true PCR-free DNBSEQ ${ }^{\mathrm{TM}}$ WGS provides a powerful solution for improving WGS accuracy while reducing cost and analysis time, thus facilitating future 
precision medicine, cohort studies, and large population genome projects.

X. Zhao: None.

\section{P15.45.A}

Nanopore sequencing of differentiating murine neuronal precursor cells

\section{Hewel, C. Vennin, B. Lutz, S. Gerber}

Johannes Gutenberg University of Mainz, Mainz, Germany

Nanopore sequencing has emerged as a promising approach to overcome some of the limitations of classical NextGeneration-Sequencing. Among the benefits of this technique are direct and PCR-free sequencing of RNA or DNA, native detection of basepair-modifications and full-length read sequencing. This has led to a number of interesting applications, also for clinical purposes. Here, we were especially interested in the detection of transcript isoforms during neuronal differentiation, thus making use of the capability of a nanopore sequencer to provide full-length transcript sequences. Previous studies have shown that during neuronal differentiation the 3 'ends of transcripts can extend by several kilo-basepairs and the presence or absence of this feature conveys critical function. Moreover, key players in neuronal differentiation, such as the BDNFgene have been shown to have several distinct isoforms, that specifically trigger different receptors, evoking varying signalling cascades. To investigate transcript isoforms during neuronal differentiation, we employed a differentiation protocol in a cell line of murine neuronal precursor cells. During differentiation, the cells were harvested on 4 timepoints. The RNA was converted to cDNA and library preparation for a nanopore sequencing device was done as per manufacturers specifications. Subsequently sequencing on a nanopore device and basecalling using the MinKNOW software followed. Conclusively, we were able to test the feasibility of nanopore sequencing in our lab and to investigate complex splice isoforms during neuronal differentiation. Results will be presented and discussed.

C. Hewel: None. C. Vennin: None. B. Lutz: None. S. Gerber: None.

\section{P15.46.B}

High-throughput genome sequencing facility: Implementing NovaSeq(s)

Z. Gerber, J. Tassin, F. Jobard, F. Sandron, D. Delafoy, S. Meslage, G. Septier, C. Baulard, J. Perrier, D. Derbala, E. Menard, C. Besse, D. Lechner, J. Garnier, B. Fin, E.
Jakoby, F. Gavory, D. Bacq-Daian, V. Meyer, A. Boland, J. Deleuze, R. Olaso

CEA / National Center for Research in Human Genomics, Evry, France

We have optimised next-generation sequencing on the NovaSeq 6000 platform for our major sequencing applications: whole genome (PCR free), whole exome, and RNAseq. Our optimal loading concentration for each application is $2.0 \mathrm{nM}, 1.5 \mathrm{nM}$ and $2.25 \mathrm{nM}$ in standard workflow, respectively, resulting in $77-85 \%$ of clusters passing filter and low level of optical duplicates. We observed index-dependent differences in sequencing performance in the NovaSeq: libraries with certain indexes are consistently under- or overrepresented (27\% less or $32 \%$ more than the average number of reads per library, calculated across 68 runs). In contrast, we observed little effect of insert size on clustering efficiency (for insert sizes ranging $265-476 \mathrm{bp}$ in the same flowcell). The end-of-read quality scores are higher in the NovaSeq compared to HiSeq $\mathrm{X}$ Five, resulting in less sequencing errors. In contrast, we observed slightly increased systematic bias in $\mathrm{C}>\mathrm{A}$ and $\mathrm{G}$ $>\mathrm{T}$ errors at the end of NovaSeq reads, possibly due to the dual channel technology. The reduction in run time is an important advantage over the HiSeq platform, for example in our emergency neonatal genome sequencing for Fastgenomics. In conclusion, NovaSeq produces more output in shorter time, with sequencing quality similar to previous generation of sequencers. However, the unprecedented plexity allowed by the large sequencing capacity of NovaSeq presents new challenges in pooling certain indexes.

Z. Gerber: None. J. Tassin: None. F. Jobard: None. F. Sandron: None. D. Delafoy: None. S. Meslage: None. G. Septier: None. C. Baulard: None. J. Perrier: None. D. Derbala: None. E. Menard: None. C. Besse: None. D. Lechner: None. J. Garnier: None. B. Fin: None. E. Jakoby: None. F. Gavory: None. D. Bacq-Daian: None. V. Meyer: None. A. Boland: None. J. Deleuze: None. R. Olaso: None.

\section{P15.47.C}

High-throughput NGS Library Normalization Method

\section{P. Mielinis, A. Stašytè, A. Lubys}

\section{Thermo Fisher Scientific, Vilnius, Lithuania}

Introduction: With the advances in the next-generation sequencing and capacity of sequencers growing, more and more sample can be analyzed in a single run. To achieve efficient, well balanced run, all libraries must be carefully quantified and pooled. Current strategies rely on qPCR, 
ddPCR, fluorometry (Qubit) or capillary electrophoresis, however, they are either time consuming, costly or inaccurate, leading to unbalanced ratios between pooled libraries, especially when handling many samples. Underrepresented libraries may require re-sequencing, further increasing the cost of the experiment.

Materials and Methods: Here we describe a quantification-free NGS library normalization method, based on library tagging via PCR. Tagged libraries are then bound by a capturing reagent and purified using magnetic beads. Multiple libraries can be pooled early in the normalization procedure yielding excellent sample distribution within a pool and significantly shortening the whole normalization procedure.

Results: We show that the normalization method produces well balanced library pool with coefficient of variation $(\mathrm{CV})$ being controlled under $<10 \%$ with no observed effect on GC bias or genomic content, making it very convenient to use with samples within an extreme range of GC content.

Conclusions: Although the technology is compatible with diverse library types, including whole human genome, exome or small genome, the method is going to be the most beneficial when working with plentiful of samples and various inputs of genomic material. Due to the simplicity of materials and a workflow, the normalization has a potential to be automated, simplifying the normalization and pooling procedures even further.

P. Mielinis: A. Employment (full or part-time); Modest; Thermo Fisher Scientific. A. Stašytė: None. A. Lubys: A. Employment (full or part-time); Modest; Thermo Fisher Scientific.

\section{P15.48.A}

When Whole Exome Sequencing fails: Lessons learned from Whole Genome Sequencing

A. Eliyahu ${ }^{1,2,3}$, D. Marek-Yagel ${ }^{1,3}$, B. Pode-Shakked ${ }^{1,4,3}$, A.

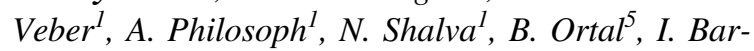
Joseph $^{5}$, O. Nayshool ${ }^{5}$, B. Ben-Zeev ${ }^{6,3}$, G. Heimer ${ }^{6,7}$, O. Staretz-Chacham ${ }^{8}$, D. Oz-Levi ${ }^{9}$, N. Pode-Shakked ${ }^{10,11,3}, Y$. Anikster $^{1,3,12}$

${ }^{1}$ Metabolic Disease Unit, Edmond and Lily Safra Children's Hospital, Sheba Medical Center, Tel-Hashomer, Israel, ${ }^{2}$ The Danek Gertner Institute of Human Genetics, Sheba Medical Center, Tel-Hashomer, Israel, ${ }^{3}$ Sackler Faculty of Medicine, Tel-Aviv University, Tel Aviv, Israel, ${ }^{4}$ The Talpiot Medical Leadership Program, Sheba Medical Center, Tel Hashomer, Israel, ${ }^{5}$ The Genomic Unit, Sheba Cancer Research Center, Sheba Medical Center, TelHashomer, Israel, ${ }^{6}$ Pediatric Neurology Unit, Edmond and Lily Safra Childrens Hospital, Tel-Hashomer, Israel,
${ }^{7}$ Sackler Faculty of Medicine, Tel-Aviv University, Tel Aviv, Italy, ${ }^{8}$ Metabolic Clinic, Pediatric Division, Soroka Medical Center, Ben-Gurion University, Beer Sheva, Israel, ${ }^{9}$ Department of Molecular Genetics, Weizmann Institute of Science, Rehovot, Israel, ${ }^{10}$ The Talpiot Medical Leadership Program, Sheba Medical Center, Tel-Hashomer, Israel, ${ }^{11}$ Pediatric Stem Cell Institute, Edmond and Lily Safra Childrens Hospital, Sheba Medical Center, Tel-Hashomer, Israel, ${ }^{12}$ Wohl Institute for Translational Medicine, Sheba Medical Center, Tel-Hashomer, Israel

Exome sequencing is an extremely efficient way to to identify disease-causing variations and increasingly used in healthcare and research. However, in certain cases, either due to the complexity or the location of the aberration, whole exome sequencing (WES) might not yield a pathogenic variant, and whole genome sequencing (WGS) may be required. We report herein four recent cases diagnosed in our lab, which were all initially missed by WES and later resolved only by sequencing the entire genome:1-Promoter mutation: Four patients of two unrelated families, with portal vein thrombosis and macrocephaly. WGS revealed the $-270 \mathrm{C}>\mathrm{G}$ homozygous mutation, located in the promoter of the PIGM gene.2-Large deletions: Eight patients from 7 different families of Iraqi-Jewish origin with autosomal recessive intractable diarrhea of infancy. Linkage analysis with WGS enabled the detection of two overlapping deletions on chromosome 16.3-Intronic mutation: Two siblings with PS6 syndrome without mutation in the coding sequence of the candidate gene, ARSB. WGS, revealed deep intronic mutation in the gene, which leads to insertion of a pseudo-exon.4-Large duplication: Three female siblings of Iraqi-Jewish descent, presented with cerebellar ataxia, encephalopathy, seizures and cognitive disability. WGS with Bam file analysis identified a novel biallelic duplications in the COQ5 gene. It is clear that WGS is a powerful tool, which can improve the rate of discovery of disease-causing mutations, especially in such challenging cases. With the falling cost of WGS, more individuals are pursuing this option, which enable to enrich the databases, and lead to better diagnosis.

A. Eliyahu: None. D. Marek-Yagel: None. B. PodeShakked: None. A. Veber: None. A. Philosoph: None. N. Shalva: None. B. Ortal: None. I. Bar-Joseph: None. O. Nayshool: None. B. Ben-Zeev: None. G. Heimer: None. O. Staretz-Chacham: None. D. Oz-Levi: None. N. PodeShakked: None. Y. Anikster: None.

P15.51.A

Phased WGS by direct library preparation from 240 FACS sorted cells for comprehensive genetics in multiple myeloma 
C. Gran ${ }^{1,2}$, L. Peña-Pérez ${ }^{l}, J$. Eisfeldt ${ }^{l}$, C. Gustafsson ${ }^{l}, J$. Borg Bruchfeld ${ }^{l, 2}$, J. Hauenstein ${ }^{l}$, N. Frengen ${ }^{l}, F$.

Taborsak-Lines ${ }^{3}$, R. Olsen ${ }^{4}, P$. Ewels ${ }^{4}, A$. Lindstrand ${ }^{1,2}, H$. Nahi $^{1,2}$, R. Månsson ${ }^{l}$

${ }^{1}$ Karolinska Institutet, Stockholm, Sweden, ${ }^{2}$ Karolinska University Hospital, Stockholm, Sweden, ${ }^{3}$ Royal Institute of Technology, Stockholm, Sweden, ${ }^{4}$ Stockholm University, Stockholm, Sweden

Introduction: multiple myeloma (MM) is an incurable and aggressive plasma cell malignancy characterized by genetic heterogeneity and complexity. In addition to single nucleotide variants (SNV), tumor cells often carry multiple complex structural variants (SV) and copy number variations (CNV). Nonetheless, only limited genetic characterization is performed by fluorescence in situ hybridization (FISH) in standard care. Linked reads whole genome sequencing (WGS) - or phased WGS - has shown great promise for providing improved calling of structural aberrations. However, its implementation has been hampered by the difficulty of high molecular weight (HMW) DNA preparation.

Materials and Methods: here we demonstrate that DNA preparation can be circumvented by direct loading of 240 FACS sorted cells into the linked read library preparation. Besides providing a simplified workflow, this procedure results in longer DNA molecules (median 190kbp) and longer phase blocks (median N50 length $16.9 \mathrm{Mbp}$ ) compared to standard methods.

Results: in the ongoing initial benchmarking, all CNVs and SVs previously found by FISH were identified through linked read sequencing. Additionally, several aberrations not detected by an expanded FISH panel were identified. The latter remain to be verified by FISH.

Conclusions: the use of linked read technologies combined with our direct cell loading protocol and diagnostic flow cytometry can feasibly establish a more comprehensive genetic characterization for $\mathrm{MM}$ - and other diseases - in clinical routine. This will contribute to improved diagnostics and risk stratification in MM.

C. Gran: None. L. Peña-Pérez: None. J. Eisfeldt: None. C. Gustafsson: None. J. Borg Bruchfeld: None. J. Hauenstein: None. N. Frengen: None. F. TaborsakLines: None. R. Olsen: None. P. Ewels: None. A. Lindstrand: None. H. Nahi: None. R. Månsson: None.

P15.52.B

Precise correction of heterozygous $\mathrm{SHOX} 2$ mutations in iPSCs derived from patients with atrial fibrillation via genome editing and sib-selection
S. A. Sumer ${ }^{1,2}$, S. Hoffmann ${ }^{1,2}$, S. Laue $^{3,4}$, B. Campbell ${ }^{3}$, K. Raedecke $^{l, 2}$, V. Frajs ${ }^{1}$, S. Clauss ${ }^{5,4}$, S. Kääb ${ }^{5,4}$, H. Janssen ${ }^{6}$,

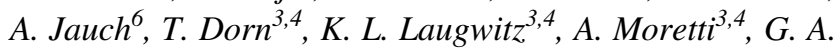
Rappold $^{1,2}$

${ }^{I}$ Department of Human Molecular Genetics, Institute of Human Genetics, University of Heidelberg, Heidelberg, Germany, ${ }^{2}$ DZHK (German Center for Cardiovascular Research), Partner Site Heidelberg/Mannheim, Heidelberg, Germany, ${ }^{3}$ First Department of Medicine, Cardiology, Klinikum rechts der Isar - Technical University of Munich, Munich, Germany, ${ }^{4}$ DZHK (German Center for Cardiovascular Research), Partner Site Munich, Munich, Germany, ${ }^{5}$ Department of Medicine I, Klinikum Grosshadern, University of Munich (LMU), Munich, Germany, ${ }^{6}$ Department of Human Genetics, Institute of Human Genetics, University of Heidelberg, Heidelberg, Germany

Patient-specific iPSCs offer unprecedented opportunities for the investigation of multigenic disease, personalized medicine and stem cell therapy. For complex diseases, such as atrial fibrillation $(\mathrm{AF})$, precise correction of the associated mutation is crucial. Here, we generated and corrected iPSC lines from two AF patients carrying different heterozygous SHOX2 mutations. In this context, we developed a novel strategy for the scarless correction of heterozygous mutations. It is based on a stochastic enrichment by sib-selection (subdivision of edited cells into small cell pools), followed by allele quantification via digital PCR and next generation sequencing to detect isogenic subpopulations. This method does not require the use of antibiotic selection or cell sorting and can be easily combined with existing improvements for gene editing such as modified Cas9 enzymes and base editors. By avoiding the insertion of silent mutations that are used to prevent re-cutting by Cas9, this method is suited for the correction of intronic or intergenic variants. The presented strategy helps to overcome the low efficiency of homology-dependent repair in iPSCs and therefore facilitates the generation of isogenic control lines that represent the gold standard for modeling complex cardiac diseases in vitro. In summary, we generated the first AF model using patient-specific and gene-corrected iPSCs and developed a novel method for the correction of heterozygous mutations. This work has been funded by DFG RA380/14-4 (to GA.R), DZHK 81X2500133 and 81X2500113 (to S.H.), 81X2600603 and 81X2600607 (to A.M.) and German Research Foundation Transregio (to A.M., K-L.L.).

S.A. Sumer: None. S. Hoffmann: None. S. Laue: None. B. Campbell: None. K. Raedecke: None. V. Frajs: None. S. Clauss: None. S. Kääb: None. H. Janssen: None. A. Jauch: None. T. Dorn: None. K.L. Laugwitz: None. A. Moretti: None. G.A. Rappold: None. 


\section{P15.53.C}

Fast genome sequencing in less than 40 days for newborn patients in intensive care unit: a feasibility study allowing diagnosis in $56 \%$ of cases in a French hospital network

A. Denommé-Pichon ${ }^{1,2,3}$, A. Vitobello ${ }^{1,2,3}, R$. Olaso ${ }^{4}, A$. Ziegler $^{5}$, B. Isidor ${ }^{6,7}$, M. Jeanne ${ }^{8,9}$, C. Racine ${ }^{1,2,3}, F$. Tran Mau-Them ${ }^{1,2,3}$, V. Couturierr, C. Pö̈ ${ }^{l}$, T. Jouan ${ }^{l}$, A.

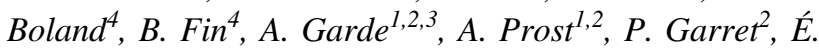

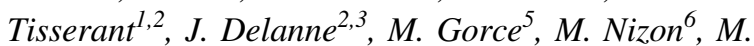

Vincent $^{6}$, S. Moutton ${ }^{2,3}$, S. Nambot ${ }^{2,3}$, Y. Capri ${ }^{10}$, J. Van Gils $^{11}$, T. Busa ${ }^{12}$, S. Sigaudy ${ }^{12}$, M. Fradin ${ }^{13,14}$, A. Lavillaureix $^{13}$, P. Rollier ${ }^{13}$, L. Pasquier ${ }^{13}$, M. Barth ${ }^{5}$, A.

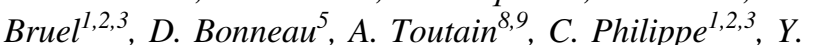
Duffourd $^{1,2,3}$, P. Callier $^{2,15}$, J. Deleuze , L. Faivre $^{1,2,3}, C$. Thauvin-Robinet ${ }^{1,2,3}$, A. Sorlin ${ }^{1,2,3}$

${ }^{1}$ Unité Fonctionnelle Innovation en Diagnostic génomique des maladies rares, FHU-TRANSLAD, CHU Dijon Bourgogne, Dijon, France, ${ }^{2}$ UMR1231 GAD, Inserm - Université Bourgogne-Franche Comté, Dijon, France, ${ }^{3}$ Centre de Référence Maladies Rares "Anomalies du développement et syndromes malformatifs », centre de génétique, FHU-TRANSLAD, CHU Dijon Bourgogne, Dijon, France, ${ }^{4}$ Centre National de Recherche en Génomique Humaine (CNRGH), Institut de Biologie François Jacob, CEA, Université Paris-Saclay and LabEx GENMED, Évry, France, ${ }^{5}$ Department of Biochemistry and Genetics, Angers University Hospital and UMR CNRS 6015-INSERM 1083, Angers, France, ${ }^{6}$ Service de génétique médicale, CHU de Nantes, Nantes, France, ${ }^{7}$ Inserm, CNRS, Université de Nantes, l'institut du thorax, Nantes, France, ${ }^{8}$ Service de Génétique, Centre Hospitalier Universitaire de Tours, Tours, France, ${ }^{9}$ UMR 1253, iBrain, Université de Tours, Inserm, Tours, France, ${ }^{10}$ Service de Génétique Clinique, CHU Robert Debré, Paris, France, ${ }^{11}$ Service de Génétique Médicale, CHU de Bordeaux, Bordeaux, France, ${ }^{12}$ Département de génétique médicale, CHU Timone enfants, AP-HM, Marseille, France, ${ }^{13}$ Service de Génétique Médicale, Centre Référence Anomalies du Développement, CHU Rennes, Rennes, France, ${ }^{14}$ Service de Génétique Médicale, CH Saint Brieuc, Saint Brieuc, France, ${ }^{15}$ Laboratoire de génétique chromosomique et moléculaire, FHUTRANSLAD, CHU Dijon Bourgogne, Dijon, France

Introduction: Fast etiological diagnosis for infants in intensive care unit (ICU) remains a major challenge for rare diseases with early onset and rapid evolution, whose genetic causes are very heterogeneous. We tried to assess the feasibility of fast genetic diagnosis by trio-GS (genome sequencing) in newborns and children requiring an urgent diagnosis for their care in a French hospital network. This study was designed to identify pitfalls and evaluate our capacity to deliver GS in emergency.

Materials and Methods: A French prospective multicentric pilot study included newborns or infants in neonatal or pediatric ICU, affected with severe phenotype that made suspect a genetic disease without obvious clinical diagnosis and required etiological diagnosis in emergency for guiding medical care. Pitfalls and duration of each biological step were noted. Every time the duration was above the expected limit, the causes were analysed and blockers handled.

Results: For the 34 newborns and infant included, the positive molecular diagnosis rate (IV and $\mathrm{V}$ classes variations) was 56\% (19/34) and suggested care management changes for $47 \%$ (9/19) of the positive cases. The rapid trio-GS strategy allowed to obtain a median duration of 45 days (27 to 92 ) from blood sampling to results communication to the clinician. Especially during the first three months, we identified several pitfalls, causing steps longer than planned. Corrective measures or protocol adaptations enabled a clear decrease of such difficulties.

Conclusions: Usefulness of rapid trio-GS is undeniable for patients in ICU, but requires adapting all stages of the classic circuit.

A. Denommé-Pichon: None. A. Vitobello: None. R. Olaso: None. A. Ziegler: None. B. Isidor: None. M. Jeanne: None. C. Racine: None. F. Tran Mau-Them: None. V. Couturier: None. C. Poë: None. T. Jouan: None. A. Boland: None. B. Fin: None. A. Garde: None. A. Prost: None. P. Garret: None. É. Tisserant: None. J. Delanne: None. M. Gorce: None. M. Nizon: None. M. Vincent: None. S. Moutton: None. S. Nambot: None. Y. Capri: None. J. Van Gils: None. T. Busa: None. S. Sigaudy: None. M. Fradin: None. A. Lavillaureix: None. P. Rollier: None. L. Pasquier: None. M. Barth: None. A. Bruel: None. D. Bonneau: None. A. Toutain: None. C. Philippe: None. Y. Duffourd: None. P. Callier: None. J. Deleuze: None. L. Faivre: None. C. Thauvin-Robinet: None. A. Sorlin: None.

\section{P15.54.A}

A three-year follow up study on 104 exome-sequenced patients with paediatric-onset diseases: Exome reanalysis and the impacts on clinical management and outcome

J. L. F. Fung ${ }^{\text {I, S. Huang }}{ }^{2}$, M. H. C. Yu ${ }^{l}$, C. C. Y. Chung ${ }^{1}$, M. C. Y. Chan ${ }^{1}$, S. Pajusalu', C. C. Y. Mak', M. Lek ${ }^{2}$, B. H. Y. Chung $^{I}$

${ }^{1}$ Department of Paediatrics and Adolescent Medicine, LKS Faculty of Medicine, University of Hong Kong, Hong Kong, Hong Kong, ${ }^{2}$ Department of Genetics, Yale School of 


\section{Medicine, Yale University, New Haven, CT, United States}

Introduction: Whole exome sequencing (WES) has become one of the important diagnostic tools in clinical genetics with mean diagnostic rate of $36 \%$. Many studies have illustrated the diagnostic and immediate clinical impact of WES. However, a vast majority of patients remain undiagnosed and there is little documentation of the longterm(>1year) clinical utility.

Methods: This is a three-year follow-up analysis to our previous publication(PMID:30109123). The diagnostic yield was $41 \%$ (43/104). 46 non-diagnosed patients consented for the reanalysis. Depending on the availability, either raw sequencing data were reanalyzed, or stored DNA were re-sequenced. The long-term outcome of 36 diagnosed patients were measured by clinical follow-up and review of medical records.

Results: WES reanalysis gave rise to twelve additional diagnoses (table), boosting the overall diagnostic yield from $41 \%$ to $53 \%$. The reanalysis of one patient had lead the discovery of MN1 C-Terminal Truncation (MCTT) syndrome caused by mutations in MNl(PMID:31834374). Currently, we are prospectively collecting and sharing more clinical information on the disease. If you have a patient with MCTT syndrome or would like to learn more, please visit https://humandiseasegenes.nl/mn1/. After three years of follow-up, change in clinical management was observed in $72.2 \%$ of the families $(26 / 36)$, leading to positive change in outcome in four patients(11\%).

Conclusions: WES reanalysis provides promising additional diagnoses to patients. Longitudinal follow-up showed that a genetic diagnosis gave rise to positive management and outcomes for patients.

\begin{tabular}{|c|c|c|c|c|c|c|}
\hline ID & Sample Type & Gene & Variant(s) & Segregation & Diagnosis (OMIM) & Inheritance \\
\hline U018 & DNA & ATPIA3 & $\begin{array}{l}\text { c. } 954 \mathrm{C}>\mathrm{G}, \\
\text { (p.Ile318Met) }\end{array}$ & De novo & $\begin{array}{l}\text { Dystonia-12 (128235); } \\
\text { Alternating hemiplegia of } \\
\text { childhood } 2(614820)\end{array}$ & $\mathrm{AD}$ \\
\hline U022 & DNA & PACS1 & $\begin{array}{l}\text { c. } 607 \mathrm{C}>\mathrm{T} \text {, } \\
\text { p.(Arg203Trp) }\end{array}$ & Unknown & $\begin{array}{l}\text { Schuurs-Hoeijmakers syndrome } \\
\text { (615009) }\end{array}$ & $\mathrm{AD}$ \\
\hline U036 & DNA & $M N 1$ & $\begin{array}{l}\text { c.3870_3879dup } \\
\text { TGACGCCAAG, } \\
\text { p.(Ala1294Ter) }\end{array}$ & De novo & $\begin{array}{l}\text { MNI C-Terminal Truncation } \\
\text { (MCTT) Syndrome (no } \\
\text { OMIM\# yet) }\end{array}$ & $\mathrm{AD}$ \\
\hline U043 & DNA & PTPN11 & $\begin{array}{l}\text { c. } 5 \mathrm{C}>\mathrm{T} \text {, } \\
\text { p.(Thr2lle) }\end{array}$ & De novo & Noonan Syndrome (163950) & $\mathrm{AD}$ \\
\hline U045 & DNA & SKIV $2 L$ & $\begin{array}{l}\text { c. } 1404-2 \mathrm{~A}>\mathrm{G} \\
\text { c. } 1647+1 \mathrm{G}>\mathrm{A}\end{array}$ & $\begin{array}{l}\text { Compound } \\
\text { heterozygous }\end{array}$ & $\begin{array}{l}\text { Trichohepatoenteric syndrome } \\
2(614602)\end{array}$ & AR \\
\hline U057 & DNA & PRFI & $\begin{array}{l}\text { c.1018G }>A, \\
\text { p.(Asp340Asn) }\end{array}$ & Homozygous & $\begin{array}{l}\text { Hemophagocytic } \\
\text { lymphohistiocytosis, familial, } 2 \\
(603553)\end{array}$ & AR \\
\hline U066 & DNA & $M F N 2$ & $\begin{array}{l}\text { c. } 707 \mathrm{C}>\mathrm{T} \text {, } \\
\text { p. }(\mathrm{Thr} 236 \mathrm{Met})\end{array}$ & Unknown & $\begin{array}{l}\text { Charcot-Marie-Tooth disease, } \\
\text { axonal, type 2A2A (609260); } \\
\text { Hereditary motor and sensory } \\
\text { neuropathy VIA(601152) }\end{array}$ & $\mathrm{AD}$ \\
\hline U071 & DNA & COL11A1 & $\begin{array}{l}\text { c. } 3115 \mathrm{G}>\mathrm{A}, \\
\text { p.(Gly1039Ser) }\end{array}$ & Unknown & $\begin{array}{l}\text { Stickler Syndrome, type II } \\
\text { (604841); Marshall Syndrome } \\
(154780)\end{array}$ & $\mathrm{AD}$ \\
\hline U075 & DNA & SPTANI & $\begin{array}{l}\text { exon10-12 } \\
\text { deletion }\end{array}$ & Unknown & $\begin{array}{l}\text { Epileptic encephalopathy, early } \\
\text { infantile, } 5 \text { (613477) }\end{array}$ & $\mathrm{AD}$ \\
\hline U077 & Data & SPTAN1 & $\begin{array}{l}\text { c. } 4828 \mathrm{C}>\mathrm{T}, \\
\text { p.(Arg1610Trp) }\end{array}$ & De novo & $\begin{array}{l}\text { Epileptic encephalopathy, early } \\
\text { infantile, } 5 \text { (613477) }\end{array}$ & $\mathrm{AD}$ \\
\hline U086 & Data & GNBI & $\begin{array}{l}\text { c.239T>C, } \\
\text { p.(lle80Thr) }\end{array}$ & Unknown & $\begin{array}{l}\text { Mental retardation, autosomal } \\
\text { dominant } 42(616973)\end{array}$ & $\mathrm{AD}$ \\
\hline U094 & Data & $\operatorname{COQ7}$ & $\begin{array}{l}\text { c.599_600delins } \\
\text { TAATGCATC, p. } \\
\text { (Lys200Ilefs*56); } \\
\text { c.319C }>\text { T, p. } \\
\text { (Arg107Trp) }\end{array}$ & $\begin{array}{l}\text { Compound } \\
\text { heterozygous }\end{array}$ & $\begin{array}{l}\text { Coenzyme Q10 deficiency, } \\
\text { primary, } 8 \text { (616733) }\end{array}$ & AR \\
\hline
\end{tabular}

J.L.F. Fung: None. S. Huang: None. M.H.C. Yu: None. C.C.Y. Chung: None. M.C.Y. Chan: None. S. Pajusalu: None. C.C.Y. Mak: None. M. Lek: None. B.H.Y. Chung: None.

\section{P15.55.B}

Sequencing the previously unsequencable using Amplification-free Targeted Enrichment powered by CRSIPR/Cas9

\section{J. Ekholm, N. Piganeau, Y. Tsai, I. McLaughlin, J. Ziegle}

\section{Pacbio, Menlo Park, CA, United States}

Genomic regions with extreme base composition bias and repetitive sequences have long proven challenging for targeted enrichment methods, as they rely upon some form of amplification. Similarly, most DNA sequencing technologies struggle to faithfully sequence regions of low complexity. This has especially been trying for repeat expansion disorders such as Fragile X disease, Huntington disease and various Ataxias, where the repetitive elements range from several hundreds of bases to tens of kilobases. We have developed a robust, amplification-free targeted enrichment technique, called No-Amp Targeted Sequencing, that employs the CRISPR/Cas9 system. In conjunction with SMRT Sequencing, which delivers long reads spanning the entire repeat expansion, high consensus accuracy, and uniform coverage, these previously inaccessible regions are now accessible. This method is completely amplificationfree, therefore removing any PCR errors and biases from the experiment. Furthermore, this technique also preserves native DNA molecules, allowing for direct detection and characterization of epigenetic signatures. The No-Amp method is a two-day protocol that is compatible with multiplexing of multiple targets and multiple samples in a single reaction, using as little as $1 \mu \mathrm{g}$ of genomic DNA input per sample. We have successfully targeted a number of repeat expansion disorder loci (HTT, FMR1, ATXN10, TCF4, C9orf72) with alleles as long as $>2700$ repeat unites ( $>13$ $\mathrm{kb}$ ). Using the No-Amp method we have isolated hundreds of individual on-target molecules, allowing for reliable repeat size estimation, mosaicism detection and identification of interruption sequences - all aspects of repeat expansion disorders important for better understanding the underlying disease mechanisms.

J. Ekholm: None. N. Piganeau: None. Y. Tsai: None. I. McLaughlin: None. J. Ziegle: None.

\section{P15.56.C}

Innovative approach for digital gene expression and alternative polyadenylation profiling 
Z. Kapustina ${ }^{1,2}$, J. Sulgaite ${ }^{1,2}$, S. Zeimyte ${ }^{2}$, A. Lubys $^{2}$

${ }^{1}$ Vilnius University, Vilnius, Lithuania, ${ }^{2}$ Thermo Fisher Scientific Baltics UAB, Vilnius, Lithuania

Introduction: High-throughput RNA sequencing offers a comprehensive analysis of transcriptome complexity originated from regulatory events such as differential gene expression, alternative polyadenylation and others, and allows the increase in diagnostic capacity and precision.

Here, we report TAS-seq (Terminator-Assisted Synthesisbased sequencing) - a novel RNA-seq library preparation method directed towards mRNA 3' termini. We demonstrate specific enrichment for 3'UTR/polyA tail junctions by a simplest possible one-step protocol which takes under 2 hours to complete. To achieve that, we synthesized modified terminators which allow to generate cDNA fragments labeled by sequencing adapters at both termini at the reverse transcription step.

Results: To demonstrate proof-of-principle and evaluate dose-response in resulting libraries, we have utilized Universal Human Reference RNA, Human Brain Reference RNA and ERCC spike-in controls. We obtained highquality stranded libraries from $20 \mathrm{pg}-1$ ug of total RNA which implies the utility of our approach for single-cell sequencing. This notion was confirmed upon successful integration of TAS-seq into the droplet-based scRNA-seq protocol. Moreover, modified terminators allow for easy introduction of unique molecular identifiers to correct for PCR bias.

Conclusions: We conclude that for high-throughput gene expression profiling applications which do not specifically require information on alternative splicing events, 3' mRNA termini counting approach is a cost-effective alternative to whole transcriptome sequencing. TAS-seq workflow proposed in this work makes it one of the simplest RNA-seq library preparation methods reported in the field.

Z. Kapustina: None. J. Sulgaite: None. S. Zeimyte: None. A. Lubys: None.

\section{P15.57.A}

The RNA-sequencing approach recapitulates the molecular pathogenesis of $\mathrm{PHOX} 2 \mathrm{~B}$ polyalanine expansion mutations in Congenital Central Hypoventilation Syndrome

T. Bachetti ${ }^{1}$, E. Di Zanni ${ }^{2}$, F. Giacopelli ${ }^{2}$, R. Cusano ${ }^{3}$, P. $U v a^{3}$, I. Ceccherini ${ }^{2}$

${ }^{1}$ Laboratory of Developmental Neurobiology, DISTAV, University of Genova, Genova, Italy, ${ }^{2}$ UOSD Laboratory of Genetics and Genomics of Rare Diseases, Istituto
Giannina Gaslini, Genova, Italy, ${ }^{3}$ Centre for Advanced Studies, Research and Development in Sardinia (CRS4), Science and Technology Park Polaris, Pula, CA, Italy

Introduction: Autonomic control of breathing is defective in congenital central hypoventilation syndrome (CCHS) patients, who thus require lifelong ventilatory supports. Heterozygous +4 to +13 residue expansions of a poly-Ala stretch in the PHOX2B gene do cause CCHS. In vitro studies have shown formation of toxic intracytoplasmic aggregates and impaired PHOX2B mediated transactivation of target genes, that can be reversed by treatments inducing heat shock response and autophagy (i.e. 17AAG).

Material and Methods: RNA-sequencing analysis was performed in SK-N-BE cells transfected with WT and $+13 \mathrm{Ala}$ PHOX $2 B$ and relative controls, with/without 17AAG treatment. Data were analyzed through a standard pipeline and lists of differentially expressed genes subjected to Gene Set Enrichment Analysis (GSEA).

Results: Gene expression profiles of transfectant cell lines allowed us to 1) rank genes and pathways resulting to drive the most deleterious effects of the +13 Ala PHOX $2 B$ mutation (i.e. apoptosis, proteasome, autophagy, etc); 2) confirm the effect of 17AAG in stimulating pathways already reported to be beneficial to CCHS (i.e. rapamycin, progesterone); 3) validate a number of differentially expressed genes and verified the effect of newly identified pathogenic mechanisms.

Conclusions: we have recapitulated i) the presence of pathways-hallmarks-perturbations already known to play a role in polyAla dependent CCHS pathogenesis and ii) the beneficial effect of drugs like 17AAG, in reverting deleterious polyAla consequences, thus providing a "proof of concept" of the feasibility of the RNA-seq approach to disclose molecular mechanisms of orphan diseases.

Major Funding: This study was supported by The CCHS Network and the Italian CCHS association (AISICC).

T. Bachetti: None. E. Di Zanni: None. F. Giacopelli: None. R. Cusano: None. P. Uva: None. I. Ceccherini: None.

P15.62.C

Understanding the inconsistencies of the smallRNAseq results by exploring different library preparation technologies

M. González-López ${ }^{1}$, E. Aparicio-Puerta ${ }^{2}$, N. Macias-

Cámara $^{l}$, L. Bárcena ${ }^{l}$, J. L. Lavín ${ }^{l}$, D. Fernández-

Ramos $^{1,3}$, P. Fernández-Tussy ${ }^{l}$, M. Reig ${ }^{4}$, L. Boix ${ }^{4}, J$. 


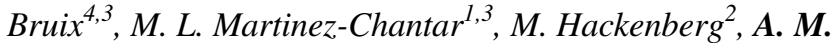
Aransay $^{1,3}$

${ }^{1}$ CIC bioGUNE, Derio, Spain, ${ }^{2}$ Department of Genetics, University of Granada, Granada, Spain, ${ }^{3}$ CIBERehd, Madrid, Spain, ${ }^{4}$ Fundació Clínic per a la Recerca Biomédica (FCRB), IDIBAPS, University of Barcelona, Barcelona, Spain

ABSTRACT The discovery of an important role of microRNAs in the disease pathogenesis along with the finding of these molecules in bodily fluids, has positioned microRNAs as one of the most promising biomarkers categories. However, despite the considerable amount of research conducted in this field, the medical use of microRNAs as biomarkers and the translation of microRNA profiling into meaningful clinical data remain a work in progress. This scenario might be caused by the low reproducibility among studies and the low disease-specificity of the microRNAs reported as biomarkers. Here, we aimed to demonstrate that the selection of kit and protocol for smallRNAseq library preparation, the method of choice for microRNA profiling, can have a great impact in the inconsistency of the results. Starting from the same samples, we prepared smallRNAseq libraries using: three commercially available kits based on fixed adapter ligation, two based on degenerated adapters ligation, one based on template switch technology, and one based on monoadapter ligation and circularization, exploiting all current smallRNAseq library preparation methods. We identified strengths and weaknesses of each protocol regarding sensitivity, accuracy and reproducibility. In addition, we evaluated the use of unique molecular identifier sequences in two of these kits. Remarkably, Illumina's kit seemed to be the outlier when compared to the rest of protocols in spite of being the most widespread library preparation kit.

FINANCIAL SUPPORTElkartek-Programs 2017-2020, Bizkaia-County and Severo-Ochoa (SEV-2016-0644), to MG, NM-C, LB, JLL and AMA; Spanish-Government (AGL2017-88702-C2-2-R) to M.H; MINECO (IFI16/ 00041) to E.A-P; MINECO to D.F-R., P.F-T. and M.L.MC.

M. González-López: None. E. Aparicio-Puerta: None. N. Macias-Cámara: None. L. Bárcena: None. J.L. Lavín: None. D. Fernández-Ramos: None. P. Fernández-Tussy: None. M. Reig: None. L. Boix: None. J. Bruix: None. M. L. Martinez-Chantar: None. M. Hackenberg: None. A. M. Aransay: None.

P15.65.C

A simple next generation sequencing method to detect sequence variants causing alpha and beta thalassemia
B. Vanherberghen, A. Hedrum

Devyser, Hägersten, Sweden

Background: Thalassemia's are inherited blood disorders characterized by abnormal hemoglobin production. Today, several methods are used sequentially for variant analysis of thalassemia patients, adding costs and increasing turnaround times. These methods include, but are not limited to, GAP-PCR, Sanger sequencing and MLPA. Often, if a variant or deletion is found in one gene, analysis of the other gene is not performed.

Method: We developed an amplicon-based NGS method on the Illumina sequencers using a single oligo-mix to detect virtually all known variants for alpha and beta thalassemia. The method includes sequencing of $\mathrm{HBB}$, HBA1 and HBA2 genes as well as direct detection of the most common deletions in alpha and beta thalassemia (GAP-PCR). Regions upstream and downstream of the above genes are sequenced to detect deletions by $\mathrm{CNV}$ analysis. The protocol is rapid and user-friendly, requiring less than one hour of hands-on time.

Results: Evaluation of 125 clinical samples revealed that all expected single nucleotide variants (SNVs) and deletions were detected. Additional variants were found in 15\% (19/ $125)$ of samples and occurred primarily in the other gene ( $i$. $e$. if the original variant was in the alpha gene, the additional variant was found in the beta gene and vice-versa).

Conclusions: We report the successful development of a rapid, NGS-based assay to detect variants involved in both alpha and beta thalassemia which identified all confirmed variants, and picked up previously undetected variants. These findings demonstrate the need for a single method that can simultaneously detect variants in both alpha and beta thalassemia's.

B. Vanherberghen: A. Employment (full or part-time); Significant; Devyser. A. Hedrum: A. Employment (full or part-time); Significant; Devyser.

\section{P15.66.A}

Knockdown of TIRR and MAD2L2 for enhancing of genome editing efficacy

\section{A. Anuchina, M. Zaynitdinova, L. Belova, A. Lavrov, S. Smirnikhina}

Research Centre for Medical Genetics(RCMG), Moscow, Russian Federation

Introduction: Enhancing of genome editing method through manipulation with repair key factors is a widespread technique nowadays. Here we performed a knockdown of two factors involved in homology directed repair 
process - TIRR and MAD2L2. These proteins are linked with non-homologous end joining pathway, which inhibition is considered to improve target-editing efficacy by homology-directed repair (HDR).

Materials and Methods: siRNA was designed with siRNAfit program. Transfection of HEK-293T cells was performed with the use of Turbofect transfection reagent. Transfection efficacy was measured with flow cytometer Flomax. Expression analysis was performed with real-time PCR; expression was normalized on TFRC, TBP, GAPDH housekeeping genes.

Results: It was shown that one of two designed siRNAs for TIRR and all three designed siRNAs for MAD2L2 significantly suppress the expression of corresponding genes. Knockdown for siTIRR was $52 \%$ (p 0,03), maximal knockdown for siMAD2L2 was 93.8\% $(p=0,02)$. Additionally, enhancing of TIRR expression as a response on three non-targeting siRNA was described in comparison with non-transfected cells and cells with transfection reagent only (maximal enhancing was in 2,4 times, $\mathrm{p} \sim 0,02)$. We are running now experiments to estimate the impact of developed knockdown on the HDR efficacy and anticipate to finalize them for ESHG'20 presentation.

Conclusions: we developed efficient knockdown of two key factors involved in DNA repair pathways - TIRR and MAD2L2. We also discovered a unique reaction of TIRR transcript expression in response to any targeted and nontargeted artificial siRNA, which should be discovered in more details and can possibly used to further control DNA repair process.

A. Anuchina: None. M. Zaynitdinova: None. L. Belova: None. A. Lavrov: None. S. Smirnikhina: None.

\section{P15.67.B}

Effectiveness and sustainability of a multidimensional approach based on tele-health and multi-OMIC testing: the Italian Undiagnosed Patients Network.

\section{F. Radio, A. Bartuli, A. Novelli, Italian Undiagnosed Patients Network, M. Tartaglia, B. Dallapiccola}

\section{Ospedale Pediatrico Bambino Gesù, IRCCS, Rome, Italy}

Introduction: Recent advances in genomic sequencing and its implementation in clinical practice are widely recognised as cost-effective diagnostic milestones. However, availability of these technologies in the clinical setting is still limited and not widely accessible. The diagnostic odyssey of undiagnosed subjects and their families is still a major constrain preventing appropriate management and care. We report on a two-year experience of the Italian Undiagnosed Patients Network of experts based on an e-health approach combined with a multi-OMIC workflow.
Materials and Methods: Since January 2018, 312 patients from 24 Italian rare diseases reference centers have been evaluated in the frame of 35 multidisciplinary sessions involving 20 experienced clinicians selected on a case-tocase basis. About $40 \%$ of patients underwent a multi-OMIC analysis directed by clinical presentation.

Results: The effectiveness (evidence-based) and sustainability (cost-effectiveness) of this multidimensional approach was proven by a significant reduction of diagnostic costs $(>25 \%)$ and an incremental rate of diagnoses $(>60 \%)$.

Conclusions: The proposed approach has produced a multiplying effect, allowing the improvement of care of undiagnosed patients greater than it would be expected by simply adding the single experts' abilities. Sharing competence among network members fosters the development of new knowledge and creates a critical mass of patients. In the case of ultra-rare and unknown disorders, pooling of a few patients only triggers new opportunities for research.

F. Radio: None. A. Bartuli: None. A. Novelli: None. M. Tartaglia: None. B. Dallapiccola: None.

\section{P15.68.C}

Improving transcriptome analysis by incorporating unique molecular identifiers into RNA-sequencing

\section{Posfai, K. Krishnan, C. Song, P. Liu, G. Naishadham, B. W. Langhorst, E. T. Dimalanta, T. B. Davis}

New England Biolabs, Ipswich, MA, United States

RNA-sequencing is a powerful tool for the study of gene regulation and function. Applications of RNA-sequencing have vastly expanded with improvements to low RNA input library preparation methods. Although analyzing low-input samples is necessary to answer certain biological questions, generating these libraries introduces greater biases with additional PCR cycles that amplify transcripts disproportionately. To better gauge true transcript abundance levels, unique molecular identifiers (UMIs) can be used to distinguish PCR duplicates from distinct original molecules. Accurate identification of such artifacts can significantly impact quantification of transcript abundance. To address this need in RNA-seq, we have developed barcoded adaptors incorporating UMIs.

We have developed 96 UMI-containing barcoded adaptors optimized across various RNA inputs (10 $\mathrm{ng}$ to $1 \mu \mathrm{g}$ ). Ligation of barcoded adaptors followed by PCR enrichment produced high-quality library and sequencing metrics (e.g. yields and coverage across transcript length). However, duplication rates significantly differed when utilizing traditional computational approaches to identify duplicates 
based on mapping position compared to analysis incorporating UMIs. As many as $90 \%$ of reads identified as duplicates using read position alone were determined to in fact originate from unique molecules, increasing the total number of reads available for further analysis.

In this study we show that incorporation of UMIs into RNA-sequencing analysis allows for a more accurate calculation of transcript abundance. Use of UMIs allows for identification of true read duplicates in RNA-seq, thereby increasing transcript detection accuracy and improving sensitivity of differential expression analysis. Furthermore, our adaptors incorporate barcodes during adaptor ligation allowing for PCR-free library pooling.

D. Posfai: A. Employment (full or part-time); Significant; New England Biolabs. K. Krishnan: A. Employment (full or part-time); Significant; New England Biolabs. C. Song: A. Employment (full or part-time); Significant; New England Biolabs. P. Liu: A. Employment (full or parttime); Significant; New England Biolabs. G. Naishadham: A. Employment (full or part-time); Significant; New England Biolabs. B.W. Langhorst: A. Employment (full or part-time); Significant; New England Biolabs. E.T. Dimalanta: A. Employment (full or part-time); Significant; New England Biolabs. T.B. Davis: A. Employment (full or part-time); Significant; New England Biolabs.

\section{P15.70.B}

Next-Generation Sequencing approach for a wide range of genetic disorders: the experience of a Portuguese Academic Hospital

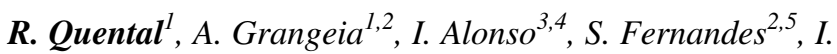
Miguel $^{3}$, C. Moura ${ }^{1,2,5}$, J. P. Freixo ${ }^{4}$, M. Amorim ${ }^{3}$, J. P. Oliveira $^{1,2,5}$, R. Oliveira ${ }^{1}, M$. Lea a $o^{1,5}$, *The first two authors contributed equally to thework

${ }^{1}$ Medical Genetics Service, São João University Hospital Centre, Porto, Portugal, ${ }^{2} i 3 S$ - Institute for Research and Innovation in Health, University of Porto, Porto, Portugal, ${ }^{3}$ Genetyca-ICM Laboratory, Porto, Portugal, ${ }^{4}$ CGPP Centre for Predictive and Preventive Genetics, IBMC Institute for Molecular and Cell Biology, i3S - Institute for Research and Innovation in Health, University of Porto, Porto, Portugal, ${ }^{5}$ Department of Genetics, Faculty of Medicine of the University of Porto, Porto, Portugal

Next-generation sequencing (NGS) has been increasingly used in clinical practice for the diagnosis of genetic disorders. Clinical exome (CE) and whole-exome sequencing (WES) have improved the diagnostic yield in neurodevelopmental disorders (NDD), with diagnostic rates of around $36 \%$. We evaluated the diagnostic yield of $\mathrm{CE}$ and in trio WES in 324 and 117 patients, respectively, referred to our
Medical Genetics outpatient clinic for diagnostic assessment. Patients have been grouped based on their clinical features: isolated NDD (developmental delay, intellectual disability, autism spectrum disorder and epilepsy); NDD plus (any of the previous plus an additional neurological, systemic or dysmorphic manifestation); NMD (neuromuscular disorders); NDG (neurodegenerative disorders); MCA (multiple congenital anomalies); and "others".A definite diagnosis was achieved in 27,8\% (90/324) and 26,5\% (31/ 117) patients by CE and WES, respectively. The NDD and NDD plus groups represented 55\% (243/441) of our patients, with a diagnostic yield ranging from $28.2 \%$ to $31.1 \%$ (WES and CE, respectively). Among the different clinical categories, diagnostic yield was highest in the NDG $(15 / 38,39.5 \%$ by CE) and MCA $(6 / 18,33.3 \%$ by WES $)$ and lowest in the "others" $(1 / 14,7.1 \%$ by WES). Achieving a diagnosis is important for patients and their families, as it can alter clinical management and the provision of accurate genetic counseling. Our results are globally consistent with those of comparable studies, highlighting CE and WES as efficient strategies for the diagnosis of a wide range of suspected genetic disorders, particularly of patients with NDG, NDD and MCA.

R. Quental: None. A. Grangeia: None. I. Alonso: None. S. Fernandes: None. I. Miguel: None. C. Moura: None. J. P. Freixo: None. M. Amorim: None. J.P. Oliveira: None. R. Oliveira: None. M. Leão: None.

P15.72.A

Whole exome sequencing reduce diagnostic odyssey of patients with epilepsy and intellectual disability

R. Minardi ${ }^{l}$, L. Laura ${ }^{1,2}$, T. Pippucci ${ }^{3}$, M. C. Baroni ${ }^{4}$, C. Stipa $^{l}$, B. Mostacci ${ }^{l}$, G. Severi ${ }^{5}$, V. Carelli ${ }^{1,2}$, M. Seri $^{5}$, P. Tinuper $^{1,2}$, F. Bisulli ${ }^{1,2}$

${ }^{1}$ IRCCS, Istituto delle Scienze Neurologiche di Bologna, UOC Neurological Clinic, Bologna, Italy, ${ }^{2}$ Department of Biomedical and Neuromotor Sciences, University of Bologna, Bologna, Italy, ${ }^{3}$ Medical Genetics Unit, Polyclinic Sant'Orsola-Malpighi University Hospital, Bologna, Italy, ${ }^{4}$ Department of Biomedical and Surgical Sciences (DIMEC) University of Bologna, Bologna, Italy, ${ }^{5}$ Medical Genetics Unit Polyclinic Sant'Orsola-Malpighi University Hospital, Bologna, Italy

Introduction: Patients with epileptic encephalopathy (EE) and developmental encephalopathy (DE) usually undergo multiple clinical evaluations and several genetics test before reaching the final diagnosis. The purpose of this study was to assess the utility of Whole-Exome Sequencing (WES) in patients with EE/DE with unknown aetiology. 
Material and Methods: We analysed the exomes of a cohort of 71 adult unrelated patients with EE/DE, which had already undergone single or multiple prior genetic tests (array-CGH, single gene or gene-panel screening) resulted negative. Variants were classified according to the American College of Medical Genetics and Genomics (ACMG) guidelines. Significant variants were validated and verified for inheritance by Sanger sequencing, when possible.

Results: In 18 patients WES analysis disclosed a total of 24 causative variants in epilepsy associated genes with either autosomal dominant (DYNC1H1; ZBTB20; CACNA1E; DYRK1A; ANKRD11; GABRG2; KCNB1; KCNH5; SCN1A; GABRB2; YWHAG; STXBP1), recessive (PRODH; $L A M B 1$; PNKP; APC2; RARS2) or X-linked (KIAA2022; $S M C 1 A)$ inheritance. Eleven out of 24 variants were novel. In $4(22 \%)$ patients the new genetic diagnosis had therapeutic implications. We estimated a diagnostic delay of 38.5 years (18-64.9).

Conclusions: In our cohort of undiagnosed adult EE/DE patients, WES analysis allowed us to obtain a diagnostic yield of $25.3 \%$. Despite the considerable diagnostic delay, genetic diagnosis had a significant impact on patients' management, allowing in selected cases for a targeted therapy. Concluding, early WES analysis in EE/DE patients would prevent "diagnostic odyssey" and unnecessary costs.

R. Minardi: None. L. Laura: None. T. Pippucci: None. M.C. Baroni: None. C. Stipa: None. B. Mostacci: None. G. Severi: None. V. Carelli: None. M. Seri: None. P. Tinuper: None. F. Bisulli: None.

\section{P15.73.B}

Validation of a new technology for whole genome amplification (WGA) and NGS sequencing in preimplantation genetic testing (PGT)

\section{S. Popov ${ }^{1}$, S. Ladygin', V. Zavarin ${ }^{1}$, S. Avdeychik', M.} Tofilo' ${ }^{1}$, D. Fomin ${ }^{2}$, A. Belyaev ${ }^{3}$

${ }^{1}$ Medical Genomics, Tver, Russian Federation, ${ }^{2}$ The Net-
work of Medical Centers «Fomin Clinic», Moscow, Russian
Federation, ${ }^{3}$ LLC AB-Vector, San Diego, CA, United
States

We have validated new WGA technology, AB-WGA (AB Vector, USA) for aneuploidy detection (PGT-A). The advantage of this technology is exhaustive random priming of the template DNA, which is crucial for minimizing amplification bias and improving genome coverage. Unlike most other WGA methods that either sacrifice specificity of copy number variation (CNV) detection for high genome coverage, or genome coverage for increased accuracy of CNV detection, AB-WGA provides nearly complete genome coverage while maintaining high accuracy of $\mathrm{CNV}$ detection. Genomic DNA from the biopsy material (3-10 cells) was extracted and amplified using AB-WGA kit. Libraries were sequenced using either Miseq reagent kit V3 (150 cycles) or NextSeq 500 reagent kit v2.5 (75 cycles). CNV-charts generation was performed using software developed by AB Vector. For comparison, we sequenced several trophectoderm biopsies (TE) samples using Illumina VeriSeq-PGS kit. CNV-charts showed 100\% karyotype matching between Illumina VeriSeq-PGS and AB-WGA kits. We also analyzed CNV-charts of TE and the inner cell mass of embryos (ICM). We have observed high concordance between TE and ICM samples using the AB Vector technology. About $10 \%$ of samples have shown different karyotypes between TE and ICM, similar to the recent study (Victor et al. 2018). Performance of the new kit was quite satisfactory, featuring a simplified protocol, better throughput and improved CNV resolution. Further studies are required for validation of this technology in PGD for detection of monogenic disorders as well as for registration of the kit as an IVD medical product for use in reproductive genetics.

S. Popov: None. S. Ladygin: None. V. Zavarin: None. S. Avdeychik: None. M. Tofilo: None. D. Fomin: None. A. Belyaev: None.

\section{P15.76.B}

Partner independent fusion gene detection by multiplexed CRISPR Cas9 enrichment and long read Nanopore sequencing

C. Stangl ${ }^{1,2,3}$, S. de Blank ${ }^{1}$, I. Renkens ${ }^{1}$, L. Westera ${ }^{4,5}, T$. Verbeek $^{l}$, J. Espejo Valle-Inclan ${ }^{1,3}$, R. Chamorro

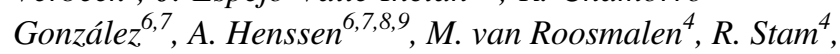
E. Voest ${ }^{2,3}$, W. Kloosterman ${ }^{1,10,11}$, G. van Haaften ${ }^{1}, G$. Monroe $^{1}$

${ }^{1}$ UMC Utrecht, Utrecht, Netherlands, ${ }^{2}$ Netherlands Cancer Institute, Amsterdam, Netherlands, ${ }^{3}$ Oncode Institute, Amsterdam, Netherlands, ${ }^{4}$ Princess Máxima Center for Pediatric Oncology, Utrecht, Netherlands, ${ }^{5}$ Dutch Childhood Oncology Group, Utrecht, Netherlands, ${ }^{6}$ CharitéUniversitätsmedizin Berlin, Berlin, Germany, ${ }^{7}$ Experimental and Clinical Research Center (ECRC) of the MDC and Charité Berlin, Berlin, Germany, ${ }^{8}$ German Cancer Consortium (DKTK), Partner Site Berlin, and German Cancer Research Center (DKFZ), Heidelberg, Germany, ${ }^{9}$ Berlin Institute of Health, Berlin, Germany, ${ }^{10}$ Cyclomics, Utrecht, Netherlands, ${ }^{11}$ Frame Cancer Therapeutics, Amsterdam, Netherlands

Fusion genes are hallmarks of various cancer types and important determinants for diagnosis, prognosis and treatment. Fusion gene partner choice and breakpoint-position 
promiscuity restricts diagnostic detection, even for known and recurrent configurations. To accurately and impartially identify fusions, we here developed FUDGE: FUsion Detection from Gene Enrichment. FUDGE couples targetselected and strand-specific CRISPR/Cas9 activity for fusion gene driver enrichment - without prior knowledge of fusion partner or breakpoint-location - to long-read Nanopore sequencing with the bioinformatics pipeline NanoFG. FUDGE has flexible target-loci choices and enables multiplexed enrichment for simultaneous analysis of several genes in multiple samples in one sequencing run. We observe on-average 665 fold breakpoint-site enrichment and identify nucleotide resolution fusion breakpoints - within two days. The assay identifies cancer cell line and tumor sample fusions irrespective of partner gene or breakpointposition. FUDGE is a rapid and versatile fusion detection assay, providing unparalleled opportunity for diagnostic pan-cancer fusion detection. Furthermore, this method paves the way for other applications of targeted long-read sequencing studies in genetics.

C. Stangl: None. S. de Blank: None. I. Renkens: None. L. Westera: None. T. Verbeek: None. J. Espejo ValleInclan: None. R. Chamorro González: None. A. Henssen: None. M. van Roosmalen: None. R. Stam: None. E. Voest: None. W. Kloosterman: None. G. van Haaften: None. G. Monroe: None.

\section{P16 Diagnostic Improvements and Quality Control}

\section{P16.01.A}

Evaluation of SpliceAI in predicting abnormal splicing in a cohort of 964 patients with rare and undiagnosed diseases

\section{Yamada, T. Uehara, H. Suzuki, K. Kosaki}

Center for medical genetics, Keio University School of Medicine, Tokyo, Japan

Background: Prediction of variants associated with abnormal splicing from exome or whole genome sequencing data without transcriptome data is difficult and unreliable for non-canonical splicing variants that occur outside of the canonical donor/ acceptor consensus sites (gt/ag). Recently, an algorithm SpliceAI based on machine learning theory was developed to quantify variant's potential to cause abnormal splicing as the delta score that ranges from zero to one. Clinical utility of SpliceAI has not been established.

Methods: Using SpliceAI, we scored all the de novo variants obtained from 964 trio exome data which derived from Japan's national clinical sequencing project Initiative on Rare and Undiagnosed diseases (IRUD). The delta score above 0.2 was considered positive.

Results: Among 964 patients, 255 patients had one or more de novo variants with positive delta score $(>=0.2)$. A total of 332 variants was detected including 264 noncanonical variants. The list of genes to which these 264 variants belonged included 63 known disease causative genes. Thirty seven of the 63 genes could account for de novo autosomal dominant or X-linked conditions. Among 37 patients with de novo non-canonical variants in these genes, 15 patients had phenotype compatible with the genotype (41\%). Analysis of 964 patients unraveled de novo non-canonical splicing variants with positive delta score among 31 loss-of-function-intolerant genes of which loss-of-function mutations are likely to be pathogenic.

Discussions: Positive predictive value of Splice AI at non-canonical sites exceeded $40 \%$ and 31 loss-of-functionintolerant genes were demonstrated to be fair candidates of novel disease-causative genes. (grant number JP19K17342)

M. Yamada: None. T. Uehara: None. H. Suzuki: None. K. Kosaki: None.

\section{P16.03.C}

\section{B-allele frequency distribution in PCR-free Whole} Genome Sequencing

E. Souche, L. Dehaspe, J. Allemeersch, J. Van Bouwel, S. Deman, W. Bossuyt

Department of Human Genetics, KU Leuven, Leuven, Belgium

Whole Genome Sequencing (WGS) analysis provides information on Single Nucleotide Variants (SNVs), indels and Copy Number Variants (CNVs). As such, WGS may replace SNP arrays and (clinical) Whole Exome Sequencing (WES) for the detection of CNVs and SNVs respectively. WGS is thought to outperform WES as it suffers from limited biases due to the PCR-free library preparation and the absence of capture. However, the B-allele frequency distribution (BAF) of WGS heterozygous variants deviates from the expected binomial normal distribution. An asymmetric enrichment of variants with low BAF $(<0.25)$ is observed in both Genome In A Bottle (GIAB) cell lines and patients, excluding cell culture artefacts as a source. No similar enrichment is observed for heterozygous variants with a high BAF (>0.75). Applying more stringent quality thresholds to the data, such as extracting GIAB confident regions or increasing depth, diminishes the width of the distribution but does not remove it. GIAB data shows that filtering out variants with low BAF successfully diminishes the number of false positives but at the price of removing true positives. Such classical filtering strategies should thus 
be applied cautiously on real data sets. These findings also have implications in the use of B-allele frequencies for $\mathrm{CNV}$ calling and haplotyping.

E. Souche: None. L. Dehaspe: None. J. Allemeersch: None. J. Van Bouwel: None. S. Deman: None. W. Bossuyt: None.

P16.04.A

Shallow whole-genome sequencing of cell-free DNA for B-cell lymphoma diagnosis and disease monitoring: a standardized approach with underappreciated potential

\section{Raman}

\section{Ghent University, 9000, Belgium}

B-cell lymphoma (BCL) tumors are physically related to the cardiovascular system and generally have a large cell turnover. As a collective, they are therefore excellent candidates for liquid biopsy (LB)-based research. The omnipresent, cheap and standardized shallow whole-genome sequencing (sWGS) of cell-free DNA for copy number profiling, is not sufficiently explored. To resolve this, a prospective cohort of 38 Hodgkin (HL) and 86 nonHodgkin lymphoma (nHL) patients has been recruited, following sWGS of 255 samples.

At diagnosis, LBs had detectable abnormalities in $84.2 \%$ of $\mathrm{HL}$ and $74.4 \%$ of nHL patients. Profiles from paired tissue biopsies showed high correlation within nHL $(r=0.815 \pm 0.043)$; and, compared to tissue, LBs had alterations with mostly higher amplitudes for both $\mathrm{HL}$ $(P=.005)$ and $\mathrm{nHL}(P=.026)$, implying tumor DNA is more abundant in blood samples. In addition, sequencing reads were mapped to viral reference genomes, which indicated that $15 \mathrm{HL}$ and $13 \mathrm{nHL}$ cases had significantly elevated titers of Epstein-Barr virus (EBV) at diagnosis. All patients that tested positive during routine EBV testing, had increased plasmatic viral read fractions.

Longitudinal analysis indicated that, when detectable, copy number patterns were similar across restaging moments in refractory/relapsed patients. Moreover, the overall anomaly of copy number profiles correlated with disease status.

Finally, predictive modeling demonstrates that LBs might aid in case of doubt between, e.g., HL versus nHL: receiver operating characteristic analysis produced an area under the curve of 0.972 . This could be helpfull when the disease is located in sites that are difficult to reach.

\section{Raman: None.}

\section{P16.06.C}

Blended phenotypes: an unusual expression of genetic heterogeneity
J. Parente-Freixo ${ }^{1,2}$, J. Oliveira ${ }^{1,2}$, R. Quental $^{3}$, P. Silva ${ }^{1,2}$, S. Sousa ${ }^{1,2}$, D. Gonçalves ${ }^{4}$, C. Moura ${ }^{2,3}$, M. Leão ${ }^{3}, J$. Sequeiros $^{1,2}$

${ }^{1}$ CGPP-IBMC, Universidade do Porto, Porto, Portugal, ${ }^{2} i 3 S$ - Instituto de Investigação e Inovação em Saúde, Universidade do Porto, Porto, Portugal, ${ }^{3}$ Serviço de Genética Médica, Centro Hospitalar Universitário de São João, Universidade do Porto, Porto, Portugal, ${ }^{4}$ Serviço de Pediatria, Centro Hospitalar Universitário de São João, Universidade do Porto, Porto, Portugal

During recent years, DNA sequencing capacity has considerably improved genetic diagnostic rate, contributed to identification of new genetic causes of rare monogenic diseases, and challenged our perception about the extension and complexity of some phenotypes. Comprehensive genomic approaches, clinical exome and whole-exome sequencing (WES) have shown that, in a small subset, the presented phenotype is the product of concomitant monogenic disorders in a single patient, giving rise to so-called "blended phenotypes". We used a WES approach in 4,027 individuals referred for genetic diagnostic study at CGPP (2016-2019), for targeted disease-specific multigene panels $(n=3,284)$; or for clinical exome or analysis of WES in a trio $(n=743)$, in clinically heterogeneous presentations. We systematically reviewed the genetic data of these 743 cases, either (singleton) clinical exome $(n=629)$ or WES in trio (patient and both parents) $(n=114)$. Patients showing variants classified as pathogenic or likely pathogenic, in at least two different genes, and data (at least partly) compatible with phenotype and known inheritance pattern for their disease, were reassessed. Five patients had two genetic conditions: two dominant diseases were found in 3 cases; 2 patients had an autosomal recessive and a dominant disease. Though parental studies were not possible for all cases, of note is that 2 patients had de novo variants occurring in two different gene-pairs: HUWE1 and CHAMP1, and SETD5 and KIF21A. Frequency of the blended (overlapping or combined) phenotypes was $\sim 0.67 \%$ in our cohort ( $n=743$ ). This work further expands knowledge and need for awareness about such conditions in a diagnostic setting.

J. Parente-Freixo: None. J. Oliveira: None. R. Quental: None. P. Silva: None. S. Sousa: None. D. Gonçalves: None. C. Moura: None. M. Leão: None. J. Sequeiros: None.

\section{P16.08.B}

Transcriptomic changes of cell-free RNA in plasma with different blood storage times and temperatures 


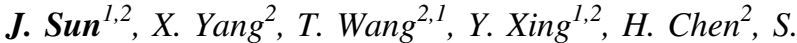

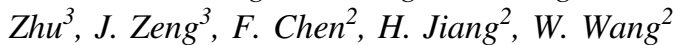

${ }^{1}$ BGI Education Center, University of Chinese Academy of Sciences, Shenzhen 518083, China, ${ }^{2}$ BGI-Shenzhen, Shenzhen 518083, China, ${ }^{3}$ Obstetrics Department, Shenzhen Maternity and Child Healthcare Hospital, Shenzhen, China

Introduction: Cell-free RNA (cfRNA) in plasma can serve as potential biomarkers for detecting and monitoring diseases. However, the transcriptomic profile of cfRNA is impacted by the delay before blood fractionation. Yet no study has evaluated systematically the influence of blood storage condition on the expression of cfRNA in plasma.

Materials and Methods: We collected fresh blood samples from four healthy subjects. After allowing blood samples to stand at room temperature (RT) and $4^{\circ} \mathrm{C}$ for various times (0h, 2h, 6h and 24h), plasma was separated and cfRNA was extracted and sequenced by PALM-seq, an integrated sequencing method for cell-free long and small RNA. Then, the expressions of cfRNA were quantitated and compared with the expression at $0 \mathrm{~h}$.

Results: Differential expression analysis reveals that the storage time influenced the transcriptomic profile with increasing significantly changed genes. The effects were noted after blood stored for $24 \mathrm{~h}$ at $4{ }^{\circ} \mathrm{C}$ and for $6 \mathrm{~h}$ at RT. Moreover, blood stored at RT has a greater impact on the expression of cfRNA than stored at $4^{\circ} \mathrm{C}$. Most of the upregulated genes of $24 \mathrm{~h}$ are monotonically increasing over time, while down-regulated genes of $24 \mathrm{~h}$ are fluctuating. Compared with lncRNA and mRNA, miRNA is more stable and has a higher Pearson correlation with the expression at $\mathrm{Oh}$.

Conclusions: Our study depicts the changes of cfRNA transcriptomic profile in different blood storage times and storage temperatures, and provides relevant research with a list of genes whose expressions are sensitive to blood storage conditions.

Grant: Shenzhen Municipal Government of China (No. JCYJ20170412152854656).

J. Sun: None. X. Yang: None. T. Wang: None. Y. Xing: None. H. Chen: None. S. Zhu: None. J. Zeng: None. F. Chen: None. H. Jiang: None. W. Wang: None.

\section{P16.09.C}

Monitoring the impact of extraction methods on cfDNA quality

\author{
E. Graf ${ }^{l}$, C. Pocernich ${ }^{2}$ \\ ${ }^{1}$ Agilent Technologies, Waldbronn, Germany, ${ }^{2}$ Agilent \\ Technologies, Ankeny, IA, United States
}

Introduction: Cell-free DNA (cfDNA) plays an emerging role as source for potential new biomarkers. However, cfDNA still represents a demanding sample type due to restricted abundance and complex fragment size distribution. Standardizing and monitoring pre-analytical parameters, including cfDNA extraction, is inevitable to achieve best possible sample quality and yield.

Materials and Methods: CfDNA was extracted with nine different kits and then analyzed with an electrophoretic assay tailored for cfDNA analysis. The assay provides quantitative results for total DNA as well as cfDNA separated from high molecular weight (HMW) DNA. The quality score \%cfDNA is provided as additional quality parameter, determining the percent cfDNA of total DNA in presence of HWM DNA. This set of quality parameters adjusted to the needs of cfDNA characterization was applied to compare the extraction kits.

Results: The nine cfDNA extraction kits varied in rate of yield and their size selection capacity to remove HMW DNA. In general, each kit afforded either superior yield or high selectivity towards cfDNA.

Conclusions: High yield may be crucial for plasma with low cfDNA content, yet if the plasma is expected to contain considerable amounts of HMW DNA, the selectivity towards cfDNA might be favored. Even if no preference for a single kit could be established, the results present a straightforward way to monitor the impact of pre-analytical parameters on cfDNA yield and quality. Automatic evaluation of a quality score for cfDNA allows to define a threshold for objective sample quality assessment.

E. Graf: A. Employment (full or part-time); Significant; Agilent Technologies. C. Pocernich: A. Employment (full or part-time); Significant; Agilent Technologies.

\section{P16.13.A}

Comparative evaluation of Exome-CNV calling with Chromosomal Microarray Analysis for the identification of large copy number variations

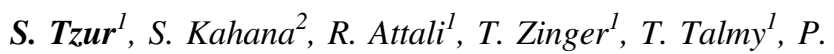
Smirin-Yosef ${ }^{1}$, R. Matar ${ }^{2}$, L. Bazak ${ }^{2,3}$, N. Ruhrman-Shahar ${ }^{2}$, L. Basel-Salmon ${ }^{2,3,4,5}$

${ }^{1}$ Emedgene Technologies, Tel Aviv, Israel, ${ }^{2}$ Recanati Genetics Institute, Beilinson Hospital, Rabin Medical Center, Petach Tikva, Israel, ${ }^{3}$ Sackler Faculty of Medicine, Tel Aviv University, Tel Aviv, Israel, ${ }^{4}$ Pediatric Genetics Clinic, Schneider Children's Medical Center of Israel, Petach Tikva, Israel, ${ }^{5}$ Felsenstein Medical Research Center, Petach Tikva, Israel 
In this study, we evaluated the potential use of Exome CopyNumber Variations calling (Exome-CNV) with that of chromosomal microarray analysis (CMA) for the identification of large structural variations. For that aim, we compared exome $\mathrm{CNV}$ calling results of 45 individuals with results of CMA analysis. The samples belong to the patients with developmental delay/intellectual disability with or without additional abnormalities for whom no pathogenic or likely pathogenic CNVs were reported by CMA and hence, they underwent exome trio testing. The CMA analysis identified 64 regions overlapping with coding regions, but 27 of them were designated as CMA artifacts. The remaining 37 CMA regions (in 27 samples), included 11 deletions (average 537Kbp), and 26 duplications (average size $665 \mathrm{Kbp})$. Exome sequencing was performed following enrichment with TWIST Human Core Exome plus kits (average coverage $>250 x$ ). Exome-CNV calling was executed using default parameters of DRAGEN Ver. 3.4.14 based on read-depth method. In the current methodology, 33 out of 37 (89\%) regions reported by CMA were successfully identified by the Exome-CNV calling. The remaining 4 regions were duplications in size ranging between 256Kbp$890 \mathrm{Kbp}$. In addition, Exome-CNV calling identified at least 150 more high-quality CNV regions not reported by CMA, and also encountered a large number of false positives. We concluded that default parameters of DRAGEN CNV calling for exome samples should be optimized to increase the accuracy and reduce the number of false positives in order to make it comparable with CMA analysis.

S. Tzur: E. Ownership Interest (stock, stock options, patent or other intellectual property); Significant; Emedgene. S. Kahana: None. R. Attali: None. T. Zinger: None. T. Talmy: None. P. Smirin-Yosef: None. R. Matar: None. L. Bazak: None. N. Ruhrman-Shahar: None. L. Basel-Salmon: None.

\section{P16.14.B}

New genomic rearrangements identified in genes associated to inborn errors of metabolism

N. Castejon-Fernandez, I. Bravo-Alonso, R. Navarrete, F. Leal, M. Ugarte, P. Rodríguez-Pombo, B. Pérez

Centro de Diagnóstico de Enfermedades Moleculares. Universidad Autónoma de Madrid, Madrid, Spain

Recent developments in high-throughput sequence capture have made next generation sequencing (NGS) of routine use when making genetic diagnoses. Improvement of the depth of coverage allows for the detection of large genomic deletions in addition to single nucleotide variations ( $\mathrm{SNVs}$ ), although further validations are needed for an accurate diagnosis. The present study proposes a bioinformatic pipeline to exploit the potential use of the NGS to detect $\mathrm{CNV}$ in addition to SNVs.

This work includes genetic analysis of 21 unsolved cases with suspicion of metabolic disorders. We have used two NGS panels for genetic studies: a targeted exome panel (panel1) and the Clinical-Exome Sequencing TruSightTM One [Illumina] (panel2). Bioinformatic pipeline consist on a first step of annotation of SNVs and a second step -if necessary- of CNVs analysis using an algorithm based on the DeCON R package modified in-house, combined with post-computational filter-selection to reduce false positives.

The bioinformatic pipeline allowed the identification of eighteen large deletions in 16 different genes, 7 of which were novel. The results of the SNPs arrays studies and/or long range PCR analysis showed that a high number of genomic rearrangements detected involved $A l u$ sequences. Two cases showed Alu insertion that was not detected by either panel due to the incorrect alignment.

These results show that the NGS can detect genomic rearrangements plus SNVs with a well-defined pipeline what reduces the response time in the genetic diagnosis, but other molecular assays are needed for the correct characterization of them.

N. Castejon-Fernandez: None. I. Bravo-Alonso: None. R. Navarrete: None. F. Leal: None. M. Ugarte: None. P. Rodríguez-Pombo: None. B. Pérez: None.

\section{P16.15.C}

Fast, scalable validation of NGS diagnostics using hundreds of clinical samples

T. Medina, M. Beukers, P. B. T. Neerincx, K. M. Abbott, B. Sikkema-Raddatz, M. A. Swertz

University Medical Center Groningen, Groningen, Netherlands

Introduction: High-confidence variant call sets, such as those offered by the Genome in a Bottle Consortium, are the current gold standard for evaluating NGS analysis pipelines. Individual samples do not, however, include significant numbers of rare or clinically relevant variants. Reanalysis of in-house samples known to contain such variants complements this deficiency, but is limited by its lack of scalability. We present a method for incorporating variantrelevant NGS reads from a multitude of clinical samples into a single hybrid control for efficient pipeline validation.

Materials and Methods: NIST reference sample NA24385 was whole-exome sequenced using a custom GATK-based clinical diagnostic pipeline. Using our developed VaSeBuilder tool (https://github.com/molgenis/Va SeBuilder), sequencing reads aligning in a continuous region around patient variant loci were added to the 
NA24385 read set. NA24385 reads in the same alignment window were removed. The resulting hybrid set of reads was then routinely added to diagnostic analysis batches as an in silico spike-in control for continuous validation.

Results: NA24385 sequencing reads were exchanged with reads representing 1283 clinically relevant autosomal variant calls from 656 patient WES samples. Of the 1081 SNVs and 202 indels added, 98.8\% are recalled in concordance with their original samples.

Conclusions: The compilation of an artificial genome enriched with clinically relevant variants allows for much more efficient and relevant validation of NGS pipelines than by using high-confidence call sets alone. In addition, this method can be automated and used continuously for routine diagnostic validation with every analysis run.

Grant: Work supported by NWO Vidi Grant 917.16.455

T. Medina: None. M. Beukers: None. P.B.T. Neerincx: None. K.M. Abbott: None. B. Sikkema-Raddatz: None. M.A. Swertz: None.

\section{P16.17.B}

A rapid multiplex PCR assay and software system with broad coverage of pathogenic CFTR mutations for diverse populations

G. Latham, P. Rao, S. Gokul, E. Hallmark, C. Parker, D. Patel, B. Hall

\section{Asuragen, Inc., Austin, TX, United States}

Introduction: ECFS practice guidelines recommend detection of at least one abnormal CFTR mutation in $>96 \%$ Cystic Fibrosis (CF) patients. ACMG and ACOG guidelines in the US recommend a targeted CFTR panel of 23 variants. We addressed both European and US recommendations with a panel derived from common mutations indexed in CFTR2 and reflected in the US population using an integrated system of PCR reagents and analysis software.

Methods: We developed a prototype, two-tube panel using AmplideX ${ }^{\circledast}$ PCR that genotypes 65 mutations, including SNPs, INDELs, CNVs and the IVS8 poly-T/TG modifier, within five hours. Amplicons were resolved by capillary electrophoresis (CE) on a 3500 Genetic Analyzer (Thermo Fisher). Evaluated samples included synthetic DNA $(n=43)$ and genomic DNA from cell lines $(n=79)$, whole blood $(n=300)$, and residual clinical samples with known pathogenic variants $(n=35)$. Genotypes were assigned using a prototype AmplideX Reporter software module. Sanger sequencing confirmed all variants.

Results: The CFTR mutation panel targeted at least one pathogenic mutation in $>99 \%$ of CF patients, and represented $92.6 \%$ of variant alleles in the US population. Mutations and zygosity agreed with reference calls for
$>98 \%$ of variants across different samples. Automated software calls agreed with manual calls yet required $<100$ fold analysis time. Carrier rates for presumed healthy blood donors were compared with published values for the relevant groups.

Conclusions: We developed and evaluated a prototype multiplex, allele-specific PCR/CE system with up-to-date, evidence-based mutation content, a streamlined workflow, and automated software. This system can genotype diverse populations for $\mathrm{CF}$ carrier screening and diagnostics.

G. Latham: A. Employment (full or part-time); Significant; Asuragen, Inc.. E. Ownership Interest (stock, stock options, patent or other intellectual property); Significant; Asuragen, Inc. P. Rao: A. Employment (full or part-time); Significant; Asuragen, Inc.. S. Gokul: None. E. Hallmark: A. Employment (full or part-time); Significant; Asuragen, Inc. C. Parker: A. Employment (full or part-time); Significant; Asuragen, Inc. D. Patel: A. Employment (full or part-time); Significant; Asuragen, Inc. B. Hall: A. Employment (full or part-time); Significant; Asuragen, Inc.. E. Ownership Interest (stock, stock options, patent or other intellectual property); Significant; Asuragen, Inc.

\section{P16.18.C}

A cost-effective solution for the genetic analysis of cystic fibrosis combining next generation sequencing and fragment analysis methods

\section{Zaouter}

Devyser AB, Stockholm, Sweden

Introduction: Cystic fibrosis (CF) is a severe genetic disease caused by mutations in the CFTR gene. The identification of mutations is a key step in the process of diagnosis and carrier screening. However, the high mutation variability between different geographical and ethnic populations makes the analysis of the CFTR gene challenging and has a direct bearing on the strategy of molecular analysis of CF.

Methods: We developed two assays for a multi-level testing strategy that maximizes mutation detection while minimizing costs. A first multiplex allele-specific PCR assay followed by fragment analysis was used to allow the simultaneous detection of the 68 most common European mutations in only two PCR reactions per sample. A second, amplicon-based NGS assay was employed to detect all mutations in exons and flanking intronic sequences of CFTR using one single PCR mix per sample. The performance of these two assays was evaluated by analyzing samples carrying pathogenic mutations that were previously characterized using commercially available kits. 
Preliminary results: The allele-specific PCR analysis of 41 samples from Spain carrying a total of 60 mutations revealed that 57 of these mutations were included in our mutation panel and identified, providing a high detection rate of $95 \%$. The NGS analysis of 32 samples carrying 116 mutations showed a sensitivity and specificity of $>99 \%$, combined with an excellent coverage uniformity of $>99.8 \%$.

Conclusions: The combination of a fragment analysis assay enabling high mutation detection rate with a comprehensive NGS assay is a cost-effective strategy that is well suited for routine laboratories.

C. Zaouter: A. Employment (full or part-time); Significant; Devyser AB.

\section{P16.19.A}

\section{ISCN 2020: What's new and how can we use it?}

\section{J. McGowan-Jordan ${ }^{1}$, R. J. Hastings ${ }^{2}, J$. T. den Dunnen ${ }^{3}$}

${ }^{1}$ CHEO/University of Ottawa, Ottawa, ON, Canada, ${ }^{2} \mathrm{John}$ Radcliffe Hospital, Oxford University Hospitals NHS Foundation Trust, Oxford, United Kingdom, ${ }^{3}$ Leiden University Medical Center, Leiden, Netherlands

Clear descriptions of clinical and experimental findings are essential for consistent communication amongst individual scientists, clinicians, and groups. In laboratory genetic studies this has happened through the International System for Human Cytogenomic Nomenclature (ISCN) and Human Genome Variation Society (HVGS). The Standing Committee of the ISCN has established a standard system for the description of numerical and structural chromosome abnormalities characterized by various methods. The next revision of these standards will be released in 2020, and will address issues raised from members of the cytogenomics community and vetted through the Standing Committee. These include clarity on general principles with more specific definitions of terms. Improvements to oncology, FISH, microarray and region specific assay nomenclatures will be emphasized. ISCN 2020 will align the rules governing the description of all chromosome abnormalities regardless of technology used. The "view" of chromosomes will move from centromere-focused to one based on genomic sequence from pter to qter; sex chromosomes abnormalities will be presented first followed by those affecting autosomes for all techniques. Continued improvements and refinements to guidelines first released in 2016 in collaboration with HGVS will also be included. This new standard combines aspects of both HGVS and ISCN and will meet the needs of both the cytogenomic and molecular communities, making information on both the chromosomal and base-pair level readily evident and providing a consistent description of large intra- and inter-chromosomal rearrangements. Elements and examples of these improvements to ISCN and the combined standard will be presented to illustrate their utility.

J. McGowan-Jordan: None. R.J. Hastings: None. J.T. den Dunnen: None.

P16.21.C

Exome sequencing as the first-line investigation for developmental disorders in South Africa: an evaluation of current practice, the patient cohort and potential challenges

\section{E. K. Wiener, Z. Lombard, A. Krause, the DDD-Africa Study}

Division of Human Genetics, National Health Laboratory Service and School of Pathology, Faculty of Health Sciences, University of the Witwatersrand, Johannesburg, South Africa

Introduction: Latest guidelines for the diagnosis of developmental disorders (DD) suggests exome sequencing as the first-line investigation, however, the applicability of such guidelines in an African setting is uncertain. Due to the dearth of research into DD in Africa, there is little knowledge of the characteristics of the patient cohort with DD and current diagnostic processes. This knowledge is essential before considering implementation of such guidelines.

Methods: A clinical file audit was performed on 934 patients presenting to Medical Genetics clinics in Johannesburg South Africa in 2017.

Results: $83 \%$ of the patient cohort presented with features of DD with $37 \%$ having global developmental delay. Three main groups of patients were identified within the cohort according to their different diagnostic processes. The first group (18\%) with genetically confirmed aneuploidies were often diagnosed by general clinicians before referral. The second group (25\%) presented with conditions that were clinically recognisable, by medical geneticists, and so received clinical diagnoses with $39 \%$ confirmed genetically. The third and largest group (57\%) presented with nonspecific, unrecognisable conditions. Of these, $92 \%$ remain undiagnosed, even after all appropriate available testing.

Conclusions: Patients with DD are the largest group of patients seen at this Medical Genetics clinic. Current diagnostic testing approaches are limited, so where conditions are unclear or unrecognisable, diagnostic yield is very low. Therefore, there is a large cohort of patients who would benefit from implementation of first-line exome sequencing, from a time, diagnosis and cost perspective. NIH funding award: U01MH115483.

E.K. Wiener: None. Z. Lombard: None. A. Krause: None. 


\section{P16.23.B}

Genomic testing in more than 1,000 individuals from Pakistani families results in high diagnostic yield and remarkable clinical impact

A. Bertoli-Avella ${ }^{1}, H$. Cheema ${ }^{2}$, V. Skrahina ${ }^{1}$, M. Nadeem Anjum $^{2}$, N. Salman ${ }^{2}$, A. Saeed ${ }^{2}$, C. Beetz ${ }^{1}$, J. Perez-Lopez ${ }^{1}$, M. Rocha ${ }^{l}$, S. Alawbathani ${ }^{1}$, C. Pereira ${ }^{1}$, M. Hovakimyan ${ }^{l}$, I. Patric ${ }^{l}$, O. Paknial, N. Ameziane ${ }^{l}$, C. Cozmal, P. Bauer ${ }^{l}$, A. Rolfs $s^{1,3}$

${ }^{1}$ CENTOGENE AG, Rostock, Germany, ${ }^{2}$ Pediatric Department of Gastroenterology. Children's Hospital of Lahore Hospital, Lahore, Pakistan, ${ }^{3}$ University of Rostock, Rostock, Germany

To establish the genetic diagnosis, and to asses diagnostic yield and clinical impact in patients with no previous access to genetic testing.

We implemented a collaborative diagnostic program in Lahore, Pakistan. Local physicians carefully ascertained pediatric patients at their departments. More than 1,586 genetic tests were performed in 1,019 individuals consisting of 349 index cases and 670 relatives. Most performed tests were exome/genome sequencing (ES/GS) and specific gene panels.

In $61.3 \%$ of the patients $(n=214)$ a genetic diagnosis was established based on pathogenic and likely pathogenic variants. In $19.8 \%$ (69 cases), variants of unknown clinical significance were reported. Diagnostic yield was higher in consanguineous families. Most identified disorders were metabolic diseases corresponding to the main referral clinical department. Furthermore, in 28 patients, genetic diagnosis relied on additional biochemical testing, which allowed for rapid assessment of the functional effect of the relevant variants. Remarkably, the genetic diagnosis had a direct impact on clinical management in $79.4 \%$ of the patients (170/ 214). Most relevant consequences were therapy related decisions such as initiation of the appropriated treatment in a timely manner (e.g. biotin). Others included referral to other medical specialties and possibility of prenatal/predictive testing. Finally, we report 12 candidate genes among 66 cases with no clear genetic diagnosis.

Our results emphasize the importance of careful clinical assessment, patient selection and physician-to-lab communication as crucial steps of the diagnostic process. The high diagnostic yield, clinical impact, and relevant research findings demonstrate the utility of genomic testing, especially when used as first-line genetic test.

A. Bertoli-Avella: A. Employment (full or part-time); Significant; CEnTOGENE AG. H. Cheema: None.
V. Skrahina: A. Employment (full or part-time); Significant; CENTOGENE AG. M. Nadeem Anjum: None. N. Salman: None. A. Saeed: None. C. Beetz: A. Employment (full or part-time); Significant; CENTOGENE AG. J. Perez-Lopez: A. Employment (full or part-time); Significant; CENTOGENE AG. M. Rocha: A. Employment (full or part-time); Significant; CENTOGENE AG. S. Alawbathani: A. Employment (full or part-time); Significant; CENTOGEnE AG. C. Pereira: A. Employment (full or part-time); Significant; CENTOGENE AG. M. Hovakimyan: A. Employment (full or part-time); Significant; CEntogene AG. I. Patric: A. Employment (full or part-time); Significant; CENTOGENE AG. O. Paknia: A. Employment (full or part-time); Significant; CENTOGENE AG. N. Ameziane: None. C. Cozma: A. Employment (full or part-time); Significant; CENTOGENE AG. P. Bauer: A. Employment (full or part-time); Significant; CENTOGENE AG. A. Rolfs: A. Employment (full or part-time); Significant; CENTOGENE AG.

P16.24.C

A simple and cost-effective method for CNV confirmation and segregation analysis by EvaGreen digital PCR

E. Sanchez ${ }^{1}$, A. Fabre ${ }^{1}$, M. Rama ${ }^{2}$, A. Schneider ${ }^{1}$, V. Gatinois $^{l}$, F. Pellestor ${ }^{l}$, J. B. Gaillard ${ }^{l}$

${ }^{1}$ Génétique Chromosomique, CHU, Montpellier, France, ${ }^{2}$ Cytogénomique des déficiences intellectuelles et anomalies du développement, $\mathrm{CHU}$, Lille, France

Introduction: The use of array comparative genomic hybridization $(\mathrm{aCGH})$ as a diagnostic tool in molecular genetics has facilitated the identification of copy number variations (CNVs). In this study, we present a simple and highly accurate method for CNV confirmation and segregation analysis using an EvaGreen single colour, digital PCR (dPCR).

Methods: In an EvaGreen multiplex assay (Biorad), the fluorescence amplitude varies with amplicon size, PCR efficiency and primer concentrations allowing amplicon separation. A gene ratio can then be calculated by absolute quantification of events for the target and reference genes with no need of standard curves. For each CNV, we developed a duplex assay with RPP30 as reference gene and designed primers for the target gene with an amplicon size between 100 and $250 \mathrm{bp}$. In order to more easily separate amplicons, we used 50nM and 150nM for target and reference primers respectively. We compared results obtained from dPCR (DQ) to aCGH ( $\log _{2}$ ratio) and qPCR (normalized ratio) from 137 samples (blood, amniotic fluid, chorionic villi). 
Results: Our experiments showed that dPCR is a reproducible method with a low variation coefficient $(5 \%)$. Correlations are equivalent between both molecular method, i.e. $\mathrm{dPCR}$ and $\mathrm{qPCR}$, compared to results obtained by aCGH $\left(r^{2}=0.964, p=1\right)$. Same analytical sensitivity was obtained for experiments from 1.25 to $10 \mathrm{ng}$ DNA by well, thus allowing sample saving in particular for prenatal diagnosis.

Conclusions:This dPCR assay in a single well is a simple, reliable and cost-efficient method which could be an interesting alternative to relative quantification assay as qPCR.

E. Sanchez: None. A. Fabre: None. M. Rama: None. A. Schneider: None. V. Gatinois: None. F. Pellestor: None. J.B. Gaillard: None.

\section{P16.25.A}

Best practice guidelines for genetic testing in dystrophinopathies

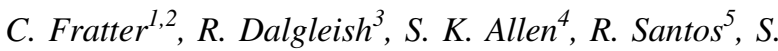
Abbs $^{6}$, S. Tuffery-Giraud ${ }^{7}$, A. Ferlini ${ }^{8,9}$

${ }^{1}$ Oxford Genetics Laboratories, Oxford, United Kingdom, ${ }^{2}$ Genomics Quality Assessment (GenQA), Edinburgh, United Kingdom, ${ }^{3}$ Department of Genetics and Genome Biology, Leicester, United Kingdom, ${ }^{4}$ West Midlands Regional Genetics Laboratory, Birmingham, United Kingdom, ${ }^{5}$ Centro Hospitalar Universitário do Porto, Porto, Portugal, ${ }^{6}$ Cambridge University Hospitals NHS Foundation Trust, Cambridge, United Kingdom, ${ }^{7}$ Laboratory of Genetics of Rare Diseases (LGMR), University of Montpellier, Montpellier, France, ${ }^{8}$ Department of Medical Sciences, Ferrara, Italy, ${ }^{9}$ Dubowitz Neuromuscular Unit, University College London, London, United Kingdom

Dystrophinopathies are X-linked genetic diseases resulting from dystrophin gene (DMD) sequence variants. Genetic testing is recommended as part of routine clinical practice since a genetic diagnosis allows confirmation of the clinical diagnosis, ensuring appropriate care and follow up access to personalised treatments, carrier identification, and family planning. The advent of next generation sequencing-based diagnostics has prompted the update of testing guidelines, previously published in 2010. The compilation of these revised guidelines was facilitated by the European Molecular Genetics Quality Network (EMQN).We have considered several issues. Technical aspects include variant detection (including small variants and copy number variations); RNA analysis and its use in DMD genetic testing; genotype-phenotype correlation as an implication of genetic testing. Male and female carrier diagnostic flowcharts have been revised with specific recommendation about known or unknown familial pathogenic variant identification. The entire cohort of prenatal diagnostic workflows, including non-invasive foetal sexing and diagnosis (NIPD) and preimplantation genetic diagnosis are discussed. A section is dedicated to variant annotation, which is of paramount importance for accurate variant reporting. We also provide recommendations on results reporting (in males and carriers), and interpretation of atypical variants or genomic configurations. We discuss and delineate possible modalities of reporting incidental findings related to the DMD gene analysis. The impact of genetic testing in personalized therapies and clinical trials enrollment procedures is also discussed. Finally we provide recommendations on laboratory settings, including accreditation, ERN network (Euro-NMD) participation, Orphanet platform registration and participation in quality-assessment schemes.

C. Fratter: None. R. Dalgleish: None. S.K. Allen: None. R. Santos: None. S. Abbs: None. S. TufferyGiraud: None. A. Ferlini: B. Research Grant (principal investigator, collaborator or consultant and pending grants as well as grants already received); Significant; Sarepta Ther, PTC Therapeutics. D. Speakers Bureau/Honoraria (speakers bureau, symposia, and expert witness); Modest; Sarepta, PTC. F. Consultant/Advisory Board; Modest; Sarepta, PTC.

\section{P16.26.B}

Targeted next-generation-sequencing analysis of 93 patients with suspected Ellis Van Creveld syndrome: a clinically relevant approach with improved diagnostic yield

F. Piceci-Sparascio ${ }^{l}$, I. Torrente ${ }^{l}$, M. D'Asdia ${ }^{l}$, V. Guida ${ }^{l}$, F. Consoli ${ }^{1}$, B. Torres ${ }^{1}$, L. Bernardini ${ }^{1}$, T. Mazza ${ }^{2}$, M.

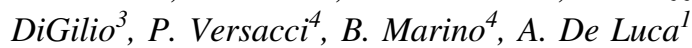

${ }^{1}$ Medical Genetics Unit, Fondazione Casa Sollievo della Sofferenza, IRCCS, San Giovanni Rotondo, Italy, ${ }^{2}$ Laboratory of Bioinformatics, Fondazione Casa Sollievo della Sofferenza, IRCCS, San Giovanni Rotondo, Italy, ${ }^{3}$ Medical Genetics Unit, Bambino Gesù Children's Hospital and Research Institute, IRCCS, Rome, Italy, ${ }^{4}$ Department of Pediatrics, Sapienza University, Rome, Italy

Ellis-Van Creveld (EvC) syndrome is a rare autosomal recessive skeletal ciliopathy presenting with postaxial polydactyly, ectodermal dysplasia and congenital heart disease. EvC is caused by mutations in EVC or EVC2 genes in approximately $85 \%$ of cases. From year 2003 to 2019 we collected and analyzed for mutations by DHPLC and/or Sanger sequencing the $E V C$ and $E V C 2$ genes in a cohort of 93 patients suspected of having EvC syndrome. After this screening, 42 patients remained mutation-negative. In this 
study, we used a targeted next-generation-sequencing (NGS) skeletal ciliopathy panel to reanalyze this EVC/ $E V C 2$ mutation-negative cohort. NGS analysis identified compound heterozygous or homozygous genotypes in 15 out of the 42 cases analyzed (36\%). Of these, 5 subjects harbored mutations in either $E V C$ or $E V C 2$, and 10 were found to be mutated in other ciliary genes. Of these, $6 / 10$ carried mutations in the $D Y N C 2 H 1$ gene associated with Jeune syndrome, comprising one compound heterozygous for a $\mathrm{DYNC} 2 \mathrm{H} 1$ splice site change and a $3.6 \mathrm{Mb}$ de novo deletion encompassing the entire $D Y N C 2 H 1$ gene, $3 / 12$ were mutated in DYNC2LII, a gene associated with a wider clinical spectrum including EvC-like phenotypes, and a fetus was mutated in NEKI, a gene associated with type II short rib-polydactyly syndrome (Majewski type). In conclusion, NGS technology represents a powerful approach in the field of skeletal ciliopaties, where the application of a small NGS targeted panel can be successfully applied to revaluate based on molecular findings, the diagnosis of individuals with clinically-overlapping phenotypes.

F. Piceci-Sparascio: None. I. Torrente: None. M. D'Asdia: None. V. Guida: None. F. Consoli: None. B. Torres: None. L. Bernardini: None. T. Mazza: None. M. DiGilio: None. P. Versacci: None. B. Marino: None. A. De Luca: None.

\section{P16.27.C}

Chromosomes in the DNA world - preserving cytogenomic competence of diagnostic medical genetic laboratories

\section{R. Hochstenbach ${ }^{1}$, T. Liehr ${ }^{2}$, R. Hastings ${ }^{3}$}

${ }^{1}$ Department of Clinical Genetics, Amsterdam UMC, Amsterdam, Netherlands, ${ }^{2}$ Institute of Human Genetics, University Clinic Jena, Jena, Germany, ${ }^{3}$ GenQA, Oxford University Hospitals Foundation Trust, Oxford, United Kingdom

Introduction: In a future where Whole Genome Sequencing (WGS) will predominate, clinical laboratory geneticists (CLGs) with knowledge about chromosomes will be needed because not all clinically relevant chromosomal aberrations can be revealed by WGS. CLGs who specialize in cytogenomics are able to recognize when there is a need for cytogenetic follow-up studies after WGS. Here, we present evidence from recent External Quality Assessments (EQAs) that competence in clinical cytogenomics is at risk.

Methods: Recent EQAs organized by GenQA were evaluated in which identical critical errors had been made by at least five participants. We also used public sources about age distributions of CLGs, job opportunities and training programs.
Results: We identified critical errors at both the analytical and interpretative level that would adversely affect patient management, revealing that, in many laboratories, there is a deficit in CLGs with cytogenomic expertise. This may deteriorate further as $25-30 \%$ of EBMG-registered CLGs with experience in cytogenetics will retire in the next decade. At the same time, trainees entering the field have a preference for molecular genetics. We show that job opportunities in molecular genetics are in excess over those in cytogenomics, and that current CLG training programs tend to accommodate less time for cytogenomics.

Discussions: Here, we define the minimal knowledge a CLG should have about cytogenomics in a world predominated by WGS. We argue that laboratory directors and boards of professional organizations in clinical genetics can preserve cytogenomic competence by providing adequate CLG training programs and attracting sufficient numbers of capable trainees.

R. Hochstenbach: None. T. Liehr: None. R. Hastings: None.

\section{P16.28.A}

Testing and improving DeepGestalt's discriminatory power on photographs of individuals with and without a genetic syndrome

J. T. Pantel ${ }^{1,2}$, N. Hajjir ${ }^{1,3}$, M. Danyel ${ }^{1,4}$, J. Elsner $^{1}$, A. Abad-Perez ${ }^{l}$, P. Hansen ${ }^{1,5}$, M. Spielmann ${ }^{1,6}$, D. Horn ${ }^{1}$, C. E. Ott ${ }^{1}$, M. A. Mensah ${ }^{1,7}$

${ }^{1}$ Institute of Medical Genetics and Human Genetics, Charité - Universitätsmedizin Berlin, corporate member of Freie Universität Berlin, Humboldt-Universität zu Berlin and Berlin Institute of Health, Berlin, Germany, ${ }^{2}$ Institute for Genomic Statistics and Bioinformatics, University Hospital Bonn, Rheinische Friedrich-Wilhelms-Universität Bonn, Bonn, Germany, ${ }^{3}$ Klinik für Pädiatrie mit Schwerpunkt Gastroenterologie, Nephrologie und Stoffwechselmedizin, Charité - Universitätsmedizin Berlin, corporate member of Freie Universität Berlin, Humboldt-Universität zu Berlin and Berlin Institute of Health, Berlin, Germany, ${ }^{4}$ Berlin Center for Rare Diseases, Charité - Universitätsmedizin Berlin, corporate member of Freie Universität Berlin, Humboldt-Universität zu Berlin and Berlin Institute of Health, Berlin, Germany, ${ }^{5}$ The Jackson Laboratory for Genomic Medicine, Farmington, CT, United States, ${ }^{6}$ Max Planck Institute for Molecular Genetics, RG Development $\&$ Disease, Berlin, Germany, ${ }^{7}$ Berlin Institute of Health (BIH), Berlin, Germany

Introduction: Facial recognition methodologies such as DeepGestalt (Face2Gene) are increasingly used in the 
diagnostic process in clinical genetics. Previous studies on DeepGestalt's quality focused on its sensitivity in patients with facial dysmorphisms. However, any diagnostic procedure also requires negative testing to determine its accuracy. We present these systematic tests of DeepGestalt on photographs of healthy individuals and suggest a machinelearning driven approach to infer presence/absence of a syndromic facies from DeepGestalt's results.

Materials and Methods: We collected 19 images for each of 17 distinct syndromes. 323 images of healthy individuals were matched to these regarding age, sex and ethnicity. Altogether, we analyzed 646 frontal photographs. We accessed DeepGestalt (v. 19.1.7) using Face2Gene CLINIC and evaluated results for the suggested diagnoses' frequencies, ranks and scores. We trained and tested a linear support vector machine (SVM) using a leave-one-out scheme to distinguish the two classes of images based on DeepGestalt's output.

Results: We confirm DeepGestalt's high true positive rate (top-10-sensitivity 91\%). Furthermore, false positives showed an interesting distribution. DeepGestalt suggested 171 distinct differential diagnoses in the top-10-results lists of the healthy control cohort. Frequencies differed: For example - although known to the system - it never falsely suggested Apert syndrome, while it proposed Fragile X syndrome in $50 \%$ of healthy controls.

DeepGestalt's top-1-scores do fairly predict presence/ absence of a syndromic facies (AUROC: 0.71). Using a linear SVM on DeepGestalt's output enhances this prediction (AUROC: 0.89).

Conclusions: Our data facilitate the interpretation of DeepGestalt's results and may help improve it and similar systems.

J.T. Pantel: None. N. Hajjir: None. M. Danyel: None. J. Elsner: None. A. Abad-Perez: None. P. Hansen: None. M. Spielmann: None. D. Horn: None. C.E. Ott: None. M. A. Mensah: None.

\section{P16.29.B}

Benchmarking SNP microarray analysis of compromised DNA and impact on forensic genetic genealogy

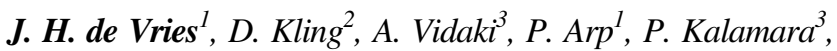 M. M. P. J. Verbiest ${ }^{l}, A$. G. Uitterlinden ${ }^{l}, M$. Kayser ${ }^{3}$}

${ }^{l}$ Department of Internal Medicine, Erasmus MC University Medical Center, Rotterdam, Netherlands, ${ }^{2}$ National Board of Forensic Medicine, Linköping, Sweden, ${ }^{3}$ Department of Genetic Identification, Erasmus MC University Medical Center, Rotterdam, Netherlands

Introduction: SNP microarrays have recently demonstrated its first successes in forensic genetic genealogy and more forensic applications are foreseen. However, array technology was not developed for low quantity and quality forensic DNA. Here we systematically tested how DNA quantity and quality impacts array-based genotyping accuracy and consequent success in identification of relatives.

Methods: We used the Illumina GSA-v2 array for blood DNA with varying degrees of DNA concentration and degradation. We established high-quality reference data, which allowed quantifying genotyping error rate and investigating its impact on the success of identifying relatives, simulated from these reference data.

Results: Stepwise decrease of DNA quantity from 200 nanogram down to 6.25 picogram led to a gradual decrease in the SNP call rate from $100 \%$ to $43 \%$, an increase in heterozygote discordance from $0 \%$ to $73 \%$ while the homozygote discordance remained zero down to 250 picogram and then increased to $21 \%$. However, the identification rate for relatives remained stable at $100 \%$ down to 250 picogram for siblings and $1^{\text {st }}$ cousins and down to 1 nanogram for $2^{\text {nd }}$ cousins and $3^{\text {rd }}$ cousins, before the identification rate decreased to zero at 25 picogram and below. Analyses on artificially degraded DNA are ongoing and not yet completed.

Conclusions: Our study provides the first empirical evidence on SNP microarray genotyping accuracy in compromised DNA samples and illustrates the consequence of genotyping errors for genetic genealogy. Our results are relevant for forensic genetic genealogy and any other forensic microarray applications such as appearance prediction and biogeographic ancestry inference.

J.H. de Vries: None. D. Kling: None. A. Vidaki: None. P. Arp: None. P. Kalamara: None. M.M.P.J. Verbiest: None. A.G. Uitterlinden: None. M. Kayser: None.

\section{P16.30.C}

CADA: network-based gene prioritization with phenotypic features

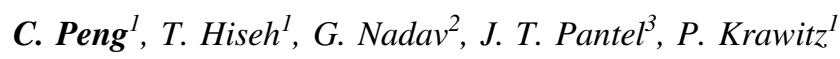

${ }^{1}$ Institute for Genomic Statistics and Bioinformatics University Hospital Bonn, Bonn, Germany, ${ }^{2}$ FDNA Inc., Boston, MA, United States, ${ }^{3}$ Charité - Universitätsmedizin Berlin, Berlin, Germany

Introduction: Human Phenotype Ontology (HPO) is widely used for deep phenotyping of patients with Mendelian disorders. Lots of existing tools have been developed to search the semantic similarity in the ontology and achieved high accuracy in the task of gene prioritization by patients' phenotypic features. However, since the similarity search is biased among diseases of varying prevalences, 
these tools usually perform poorly on patients with rare diseases. Therefore, we propose Case-Annotation plus Disease-Annotation (CADA), a network-based gene prioritization method to improve the gene prioritization for rare diseases patients.

Materials and Methods: The diagnosed disease, diseasecausing gene and phenotypic features of 11,000 patients from the Clinvar and our hospitals are collected. Along with the phenotypic features and their annotated diseases and genes from HPO, A knowledge graph, incorporating nodes of diseases, genes, patients and phenotypic features as well as their relationships as edges, is prepared. Then node2vec is used for node embeddings generation. With node embeddings, the gene prioritization is achieved by similarity-based node link prediction.

Results: The patients with the same disease and diseasecausing gene were located in close proximity in node embeddings. Clustering visualization shows that diseases with similar phenotypic features tend to cluster together as well. The prioritization of disease-causing gene improved on more than $60 \%$ of rare disease patients.

Conclusions: By integrating real-world case annotations and existing disease annotations from HPO, CADA provides a new way to address the semantic search bias problem in existing tools for the gene prioritization of rare disease patients with phenotypic features.

C. Peng: None. T. Hiseh: None. G. Nadav: None. J.T. Pantel: None. P. Krawitz: None.

\section{P16.31.A}

Need for a precise molecular diagnosis in BeckwithWiedemann and Silver-Russell syndrome. What has to be considered and why it is important

\section{T. Eggermann, J. Brück, K. Eggermann, I. Kurth, M. Begemann, M. Elbracht}

\section{Institute of Human Genetics, Aachen, Germany}

Molecular diagnostic testing of imprinting disorders (ImpDis) is challenging due to the broad spectrum of molecular defects and their mosaic occurrence. Clinically, these entities are heterogeneous as well, and they show a notable phenotypic overlap. However, the precise identification of the type of defect is a prerequisite for the clinical management and genetic counselling. In fact, the contribution of the four major alterations (UPDs, CNVs SNVs, epimutations) varies considerably in the currently known ImpDis. Due to their formation mechanism, epimutations are particular prone to mosaicism, as well as upd(11)pat in Beckwith-Wiedemann syndrome (BWS). It is therefore not surprising that ImpDis are un- and misdiagnosed. We will report on our experience of testing in $>1000$ patients referred for diagnostic testing of BWS and Silver-Russell syndrome (SRS). Though the majority of patients carried the expected molecular results, we identified a considerable number of unexpected alterations, new changes as well as undiagnosed epimutations / UPDs due to extremely discrepant mosaic distributions. Additionally, the rate of multilocus imprinting disturbances (MLID) and genomewide uniparental disomies is further specified. Altogether, these cases show that comprehensive tests targeting different tissues and including different methods have to be applied, combined with a thorough clinical examination. The precise molecular diagnosis is required as the basis for a targeted management (e.g. ECG and tumor surveillance in BWS, growth treatment in SRS). For genetic counselling it is needed for recurrence risk estimation which is hindered by the impact of the spatial arrangements of imprinting elements and their interactions on the phenotype.

T. Eggermann: None. J. Brück: None. K. Eggermann: None. I. Kurth: None. M. Begemann: None. M. Elbracht: None.

P16.33.C

A cost-effective model for the pathway of care of $\mathrm{CDH} 1$ related hereditary diffuse gastric cancer syndrome (HDGC)

L. Garrido ${ }^{* 1,2}$, L. Sousa ${ }^{* 2,3,4}$, T. Nércio ${ }^{l}$, L. Castro ${ }^{l}, R$. Leal $^{3}$, S. Costa ${ }^{1,5}$, A. Magalhães ${ }^{1,5}$, A. F. Pereira ${ }^{1}, R$. Guimarães $^{I}$, L. Ferro ${ }^{1}$, L. Vilarinho ${ }^{I}$, I. Gullo ${ }^{1,3,5}, H$. Pinheiro $^{3}$, S. Sousa ${ }^{2,3}$, A. P. Neto ${ }^{3,5}$, L. Capela ${ }^{3,5}$, C. Caeiro $^{l}$, I. Augusto ${ }^{l}$, D. Almeida ${ }^{l}$, G. Pinto ${ }^{l}$, C. Teixeira ${ }^{l}$, V. Devezas ${ }^{1,5}$, G. Macedo ${ }^{1}$, J. L. Fougo ${ }^{1,5}$, J. Preto ${ }^{1,5}$, J. Barbosa $^{1,5}$, J. Costa-Maia ${ }^{1}$, M. Baptistal, ${ }^{1,5}$, S. Fernandes $^{2,3,5}$, F. Carneiro ${ }^{1,2,3}$, S. Castedo ${ }^{1,2,3}, C$. Oliveira ${ }^{2,3,5}$

${ }^{1}$ Centro Hospitalar e Universitário São João (CHUSJ), Porto, Portugal, ${ }^{2}$ European Reference Network on Genetic Tumour Risk Syndromes (GENTURIS) \& SolveRD, Solving the unsolved in rare diseases, Grant No 779257 \& Porto. Comprehensive Cancer Centre (P.CCC), Porto, Portugal, ${ }^{3}$ Instituto de Investigação e Inovação em Saúde (i3s) \& Institute of Molecular Pathology and Immunology of the University of Porto (Ipatimup), Porto, Portugal, ${ }^{4}$ Department of Economics and Economic Policies Research Unit (NIPE), University of Minho, Braga, Portugal, ${ }^{5}$ Faculty of Medicine of the University of Porto (FMUP), Porto, Portugal

Introduction: $\mathrm{CDH} 1$-germline mutations cause HereditaryDiffuse-Gastric-Cancer(HDGC) syndrome. Penetrance is 60\%-80\% in both genders for diffuse-gastric-cancer(DGC), and $40 \%$ in females for lobular-breast-cancer(LBC). In 
asymptomatic-carriers, risk-reduction gastrectomy (RRG) and mastectomy (RRM), complemented by intensive surveillance, prevents disease. We present a cost-model specific for HDGC care-pathway demonstrating that enrolment in disease-specific care-pathways at Expert Centres and adoption of risk-reduction measures is cost-effective and life-saving.

Material and Methods: We calculated costs for optimal HDGC care-pathway in the public Portuguese Health System(PHS); and analysed clinical and financial records (2008-2018) from structured oncogenetics/high-risk consultations from 115 at risk individuals from 7 HDGC-CDH1 families, at a GENTURIS Healthcare Provider Centre.

Results: This model predicts that a non-carrier costs 350 $€$, being discharged from further follow-up. An asymptomatic-carrier (male/female) undergoing RRG costs $14 \mathrm{~K} €$ the $1^{\text {st }}$ year, $160 € /$ year the 2 following years and 125 $€ /$ year thereafter. Hospital expenses with female-carriers undergoing RRM is $9 \mathrm{~K} €$ the $1^{\text {st }}$ year, $200 €$ the following year and $100 € /$ year thereafter. Asymptomatic-carriers deciding for surveillance cost $700 € /$ year. However, if developing disease (early-detected) during surveillance, cost increases to $15 \mathrm{~K} €$ for DGC and $10 \mathrm{~K} €$ for LBC in the $1^{\text {st }}$ year, with overall good prognosis. If late-detected during surveillance, prognosis is poor and cost raises the $1^{\text {st }}$ year to $32 \mathrm{~K} €$ if DGC, $28 \mathrm{~K} €$ if LBC and 60K€ if both.

Conclusions: This study, the first on cost impact of preventive multidisciplinary-care applied to rare-cancersyndromes performed at a European Reference Centre, shows that prevention/early diagnosis in $C D H 1$-mutationcarriers is life-saving and considerably less-costly for the PHS than treating patients with expressed disease. *Equal contribution

L. Garrido*: None. L. Sousa*: None. T. Nércio: None. L. Castro: None. R. Leal: None. S. Costa: None. A. Magalhães: None. A.F. Pereira: None. R. Guimarães: None. L. Ferro: None. L. Vilarinho: None. I. Gullo: None. H. Pinheiro: None. S. Sousa: None. A.P. Neto: None. L. Capela: None. C. Caeiro: None. I. Augusto: None. D. Almeida: None. G. Pinto: None. C. Teixeira: None. V. Devezas: None. G. Macedo: None. J.L. Fougo: None. J. Preto: None. J. Barbosa: None. J. Costa-Maia: None. M. Baptista: None. S. Fernandes: None. F. Carneiro: None. S. Castedo: None. C. Oliveira: None.

P16.34.A

Identifying the genetic causes of hereditary angioedema through whole exome sequencing in the Canary Islands, Spain.

A. Mendoza-Alvarez ${ }^{I}$, A. Muñoz-Barrera ${ }^{2}$, I. MarcelinoRodriguez $^{l}$, L. Rubio-Rodríguez ${ }^{2}$, E. Tosco-Herrera ${ }^{l}, B$. Guillen-Guio $^{l}$, A. Corrales ${ }^{I}$, A. Iñigo-Campos ${ }^{2}$, A.
Callero $^{3}$, J. Garcia-Robaina ${ }^{3}$, R. González-Montelongo ${ }^{2}, J$. Lorenzo-Salazar $^{2}$, C. Flores ${ }^{1,2,4}$

${ }^{1}$ Research Unit, Hospital Universitario Nuestra Señora de Candelaria, Santa Cruz de Tenerife, Canary Islands, Spain, ${ }^{2}$ Genomics Division, Instituto Tecnológico y de Energías Renovables (ITER), Granadilla de Abona, Canary Islands, Spain, ${ }^{3}$ Deparment of Allergy, Hospital Universitario Nuestra Señora de Candelaria, Santa Cruz de Tenerife, Canary Islands, Spain, ${ }^{4}$ CIBER de Enfermedades Respiratorias, Instituto de Salud Carlos III, Madrid, Spain

Introduction: Hereditary angioedema (HAE) is a rare disease caused by $\mathrm{C} 1$ inhibitor dysfunction and the dysregulation of the kinin cascade. The updated HAE management guidelines recommend performing genetic tests to reach a precise diagnosis. Unfortunately, genetic tests are still uncommon in the diagnosis routine. Here we characterized for the first time the genetic causes of HAE in individuals from Canary Islands (Spain).

Material and Methods: Whole-exome sequencing data was obtained from 35 enrolled patients ( 21 families) and the results were analyzed with the Hereditary Angioedema Database Annotation (HADA) tool for ACMG pathogenicity classification and causal variant prioritization. Manual reclassification of prioritized variants according to ACMG guidelines was assessed in those families without known causal variants.

Results: Three known causal variants were detected in 4 families: rs38940870, classified as likely pathogenic, and rs35788383 and rs118204456, classified as of uncertain significance. In two unrelated patients without any described causal variant within SERPING1 or F12, we detected two novel variants in SERPING1 of uncertain significance that were manually reclassified as likely pathogenic. A total of 17 families $(81 \%)$ remained without a genetic diagnosis of HAE based on the current knowledge.

Conclusions: All described or novel causal variants found affected SERPING1 and F12 genes. However, further in-depth sequence analysis is required to fully describe the genetic variation causing HAE.

Funding: Ministerio de Ciencia, Innovación y Universidades (RTC-2017-6471-1; AEI/FEDER, UE); ITER agreement OA17/008; SEAIC Foundation (18_A01); Fundación Canaria Instituto de Investigación Sanitaria de Canarias (FPIFIIS19/48); CajaSiete-ULL (AMA); Instituto de Salud Carlos III (CD19/00231; IMR).

A. Mendoza-Alvarez: D. Speakers Bureau/Honoraria (speakers bureau, symposia, and expert witness); Modest; Takeda Pharmaceutical Company. A. Muñoz-Barrera: None. I. Marcelino-Rodriguez: None. L. Rubio-Rodríguez: None. E. Tosco-Herrera: None. B. Guillen-Guio: 
None. A. Corrales: None. A. Iñigo-Campos: None. A. Callero: None. J. Garcia-Robaina: None. R. GonzálezMontelongo: None. J. Lorenzo-Salazar: None. C. Flores: None.

\section{P16.35.B}

Thrombophilic gene mutations as risk factors for recurrent pregnancy loss

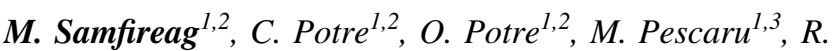
Tudor $^{1,4}$, I. Ionita ${ }^{1,2}$, H. Ionita $^{l, 2}$, A. Anghel $^{l}$

${ }^{1}$ University of Medicine and Pharmacy "Victor Babes", Timisoara, Romania, ${ }^{2}$ Hematology Clinic, Municipality Emergency Hospital, Timisoara, Romania, ${ }^{3}$ Hematology Clinic, Varnamo, Sweden, ${ }^{4}$ Neurology Clinic, Timisoara County Emergency Clinical Hospital, Timisoara, Romania

Introduction: Hereditary thrombophilia is a genetic disposition related to hypercoagulable states generated by acquired conditions of haemostasis; pregnancy changes the haemostatic system into a hypercoagulable state, which increases throughout pregnancy and is utmost around delivery ${ }^{[1]}$. Genetic testing for thrombophilia mutations implies the study through different methodologies of realtime polymerase chain reaction and DNA microarrays of specific genes: the polymorphic methylenetetrahydrofolate reductase (MTHFR) mutations - C677T and A1298C, Factor V Leiden (FVL), and two polymorphisms - the coagulation factor XIII (FXIII) Val34Leu and plasminogen activator inhibitor- 1 (PAI-1) 4G/5G.

Materials and Methods: We performed a retrospective study on a group of 40 pregnant caucasian women, diagnosed with hereditary thrombophilia during 20172019, in the west part of Romania, investigated by analyzing the main thrombofilic gene mutations involved as risk factors in the process of coagulation and of the nucleic acids metabolism.

Resuls: The overall relative risk for early pregnancy loss was significant increased for the homozygosity of PAI-1 and for the MTHFR mutations $(\mathrm{p}<0,05)$. We noticed no statistically relevant association of any of the other tested mutations with early pregnancy loss.

Conclusions: The genetic testing for thrombophilic gene mutations as risk factors for recurrent pregnancy loss revealed that the main genes involved in mechanism of hereditary thrombophilia were PAI-1 and MTHFR.

M. Samfireag: None. C. Potre: None. O. Potre: None. M. Pescaru: None. R. Tudor: None. I. Ionita: None. H. Ionita: None. A. Anghel: None.
P16.38.B

Assessing performance of pathogenicity predictors using clinically-relevant variant datasets.

A. C. Gunning, V. Fryer, J. Fasham, A. H. Crosby, S. Ellard, E. Baple, C. F. Wright

\section{University of Exeter, Exeter, United Kingdom}

Purpose: Pathogenicity predictors are integral to genomic variant interpretation but, despite their widespread usage, an independent validation of performance using a clinicallyrelevant dataset has not been undertaken.

Methods: We derive two validation datasets: an "open" dataset containing variants extracted from publiclyavailable databases, similar to those commonly applied in previous benchmarking exercises, and a "clinically-representative" dataset containing variants identified through research/diagnostic exome and panel sequencing. Using these datasets, we evaluate the performance of three recent meta-predictors, REVEL, GAVIN and ClinPred, and compare their performance against two commonly used in silico tools, SIFT and PolyPhen-2.

Results: Although the newer meta-predictors outperform the older tools, the performance of all pathogenicity predictors is substantially lower in the clinicallyrepresentative dataset. Using our clinically-relevant dataset, REVEL performed best with an area under the ROC of 0.81 . Using a concordance-based approach based on a consensus of multiple tools reduces the performance due to both discordance between tools and false concordance where tools make common misclassification. Analysis of tool feature usage may give an insight into the tool performance and misclassification.

Conclusions: Our results support the adoption of metapredictors over traditional in silico tools, but do not support a consensus-based approach as recommended by current variant classification guidelines. We acknowledge funding from Wellcome [200990].

A.C. Gunning: None. V. Fryer: None. J. Fasham: None. A.H. Crosby: None. S. Ellard: None. E. Baple: None. C.F. Wright: None.

P16.40.A

NGS custom panel assessment for sequencing circulating tumor DNA samples in patients with colorectal cancer

R. Tebar-Martinez ${ }^{1,2}$, J. Martín-Arana ${ }^{2,3,4}$,E. Seda ${ }^{l}, S$. Blesa $^{l}$, A. Ferrer-Martínez ${ }^{2,4}$, S. Zuñiga ${ }^{1,3}$, N. Tarazona ${ }^{2,4}$, 
S. Roselló ${ }^{2}$, D. Roda ${ }^{2}$, M. Huerta ${ }^{2}$, P. Rentero-Garrido ${ }^{1}, A$. Cervantes $^{2,4}$

${ }^{1}$ Personalize Medicine Unit, Biomedical Research Institute INCLIVA, Valencia, Spain, ${ }^{2}$ Department of Medical Oncology, Biomedical Research Institute INCLIVA, Valencia, Spain, ${ }^{3}$ Bioinformatics and Biostatistics Unit, Biomedical Research Institute INCLIVA, Valencia, Spain, ${ }^{4}$ CIBERONC, ISCIII Institute of Health Carlos III, Madrid, Spain

Introduction: In colorectal cancer (CRC), liquid biopsies suppose a non-invasive alternative for biomarker identification and minimal residual disease detection. Our aim is to evaluate the potential of a custom gene panel that includes unique molecular identifier technology (UMI) for analysis of plasma cell-free DNA (cfDNA).

Materials and Methods: Horizon Discovery's standards HD780 and HD816 simulating cfDNA and plasma patient samples, respectively, were selected for evaluation of 8 mutations at different variant allele frequencies (VAF). $2 \mathrm{ml}$ of each HD816 standards were purified using QIAamp Circulating Nucleic Acid Kit (QIAgen). A custom gene panel of 29 frequently mutated CRC genes was designed for QIAseq technology (QIAgen). cfDNA library preparation input was 10ng, 20ng, 40ng and 80ng from HD780 standards and 40ng of purified HD816. HD780 libraries were generated according manufacturer's instructions, whereas HD816 included modifications. All libraries were sequenced on the NextSeq platform at $>30,000 \mathrm{X}$ and processed with an in-house pipeline for UMI processing.

Results: Coverage with non-duplicated reads (NDR) were proportional to the cfDNA input, up to $80 \mathrm{ng}$. Panel sensitivity at $1 \%$ VAF was $87.5 \%$ with $20 \mathrm{ng}$ input and $100 \%$ with $40 \mathrm{ng}$ or higher input. 3 out of 8 mutations were lost at $10 \mathrm{ng}$. Sensitivity at $0.1 \%$ was $25 \%$ in $20 \mathrm{ng}$ and $40 \mathrm{ng}$ cfDNA inputs. Similar sensitivity and non-significant variations $(\mathrm{P}=0.35)$ in NDR coverage were found between HD816 and HD780.

Conclusions: Our panel reaches $100 \%$ sensitivity at $1 \%$ VAF with cfDNA expected input. Adjustments are needed to reach higher sensitivity.

Tébar-Martínez's PhD contract is funded by Spanish Association Against Cancer (AECC).

R. Tebar-Martinez: None. J. Martín-Arana: None. E. Seda: None. S. Blesa: None. A. Ferrer-Martínez: None. S. Zuñiga: None. N. Tarazona: None. S. Roselló: None. D. Roda: None. M. Huerta: None. P. Rentero-Garrido: None. A. Cervantes: B. Research Grant (principal investigator, collaborator or consultant and pending grants as well as grants already received); Modest; Roche, Merck Serono, SERVIER, Novartis, Lilly, Tesaro, Johnson \&
Johnson, MedImmune, Theradex, Astellas Pharma, Array BioPharma. D. Speakers Bureau/Honoraria (speakers bureau, symposia, and expert witness); Modest; Merck Serono, Roche, SERVIER. F. Consultant/Advisory Board; Modest; Bayer, Pierre Fabre.

\section{P16.41.B}

detecting down $\&$ fragile $\mathrm{X}$ syndromes by using deep machine learning from facial images

R. Jafarzadeh-Esfehani ${ }^{l}$, M. Mirzaei Fard ${ }^{2}$, F. HabibiHatam-Ghale $^{2}$, M. Beiraghi-Toosi ${ }^{l}$, S. Eslami ${ }^{l}$, A. SadrNabavi $^{1}$

${ }^{I}$ Mashhad University of Medical Sciences, Mashhad, Iran, Islamic Republic of, ${ }^{2}$ Ferdowsi University of Mashhad, Mashhad, Iran, Islamic Republic of

Introduction: Delayed diagnosis of genetic syndromes is an important challenge for health care providers. Among various attempts towards reducing the rate of delayed diagnosis, using machine learning is considered as a new and rapidly developing solution. In the present study, validation of a new diagnostic algorithm for diagnosis of two common genetic syndromes with unique facial phenotype is discussed.

Material and methods: The frontal and lateral picture datasets were gathered from Iranian patients who were diagnosed to have down or fragile $\mathrm{X}$ syndromes as well as healthy patients without any genetic syndrome with prominent facial appearance. After processing the pictures, landmark detection, feature extraction, feature selection, and classification were performed. Five support vector machine(SVM) Classifier designed for the present study. Each classifier took features of frontal and side view images as input and presented its decision from one of the states as output. After implementing all of the classifiers on the input data, true parameters for each category were considered and the input image matched with the category with higher count of true statements.

Results: Among 100 syndromic and 200 healthy patients, the accuracy, sensitivity and specificity of the software was calculated as $0.986,1.000$ and 0.961 respectively which was greater than human inelegance for similar pictures.

Conclusion: We have demonstrated that our proposed algorithm using both frontal and lateral picture of syndromic patients can achieve considerable accuracy and can be used as a screening program helping the physicians in order to detect the genetic syndrome with facial features.

R. Jafarzadeh-Esfehani: None. M. Mirzaei Fard: None. F. Habibi-Hatam-Ghale: None. M. BeiraghiToosi: None. S. Eslami: None. A. Sadr-Nabavi: None. 


\section{P16.43.A}

A standardized framework for the control and estimation of measurement uncertainty for clinical genomic tests

I. Delon ${ }^{1}$, A. S. Devonshire ${ }^{2}$, C. Mattocks ${ }^{3}$, R. Hawkins ${ }^{4}, S$. Tobi ${ }^{5}$, S. Patton ${ }^{5}$, S. L. R. Ellison ${ }^{2}$

${ }^{1}$ East Midlands and East of England Genomic Laboratory Hub, Cambridge, United Kingdom, ${ }^{2}$ National Measurement Laboratory at LGC, Teddington, United Kingdom, ${ }^{3}$ West Midlands, Oxford and Wessex Genomic Laboratory Hub, Salisbury, United Kingdom, ${ }^{4}$ NIBSC, Ridge, United Kingdom, ${ }^{5}$ EMQN, Manchester, United Kingdom

The quality and comparability of laboratory medicine results underpins accurate diagnosis and disease monitoring in patient care pathways. Measurement uncertainty is the quality metric that informs the testing laboratory and its users about test fitness for purpose, whether a result is above or below a treatment threshold and its degree of comparability with other laboratories. An ISO15189 laboratory medicine accreditation requirement, the measurement uncertainty of a test is intended to encompass all random and systematic effects on a measurement result. However current models to assess measurement uncertainty have been developed in the field of physical measurement or analytical chemistry and do not apply well to clinical genomic tests. Here we describe two new principles to standardise measurement uncertainty evaluation for genomic tests: 1) the 'examinand' -a general concept that applies to both qualitative and quantitative properties- is defined by the genomic target sequence and the source of the sample, 2) the combination of type of data output and reported result, which may be qualitative or quantitative, defines the requirement for the type of analysis. We propose the use of a structured cause-effect diagram for mapping sources of measurement uncertainty in the pre-analytical, analytical and post-analytical phases, along with standard templates for recording sources of uncertainty and their mitigation, in addition to measurement uncertainty calculation when applicable. We propose that using this standardised framework to guide laboratories through measurement uncertainty evaluation will contribute to improving the quality of genomic results and increasing patient's safety and equity.

I. Delon: None. A.S. Devonshire: None. C. Mattocks: None. R. Hawkins: None. S. Tobi: None. S. Patton: None. S.L.R. Ellison: None.

\section{P16.44.B}

Clinical utility of Mendeliome sequencing with respect to whole exome sequencing
M. Zollino ${ }^{l}$, S. Frangella ${ }^{l}$, P. N. Doronzio ${ }^{l}$, S. Maietta ${ }^{l}$, D. Orteschi $^{l}$, S. Amenta ${ }^{\text {I }}$ S. Ricciardi ${ }^{l}$, L. Masini ${ }^{2}$, M. De Santis $^{2}$, G. Vento ${ }^{3}$, R. Manfredi ${ }^{4}$, V. Nigro ${ }^{5}$, M. Pinelli, TUDP Consortium, G. Marangi ${ }^{I}$

${ }^{1}$ Institute of Genomic Medicine, Università Cattolica del Sacro Cuore, Fondazione Policlinico A. Gemelli IRCCS, Roma, Italy, ${ }^{2}$ Department of Obstetrics and Gynecology, Università Cattolica del Sacro Cuore, Fondazione Policlinico A. Gemelli IRCCS, Roma, Italy, ${ }^{3}$ Department of Pediatrics, Università Cattolica del Sacro Cuore, Fondazione Policlinico A. Gemelli IRCCS, Roma, Italy, ${ }^{4}$ Department of Radiological Sciences, Università Cattolica del Sacro Cuore, Fondazione Policlinico A. Gemelli IRCCS, Roma, Italy, ${ }^{5}$ TIGEM (Telethon Institute of Genetics and Medicine), Napoli, Italy

Whole exome sequencing (WES) can be considered the test of choice to search for intragenic variants in unexplained monogenic diseases not caused by CNVs. To verify whether mendeliome (or clinical exome) sequencing can be still proven to be useful, we critically evaluated our experience with 64 patients affected by syndromic conditions that were likely monogenic in origin. Relevant for this analysis were also the following aspects: 1) planning of new pregnancies; 2) early use of mendeliome sequencing in the diagnostic procedure; 3 ) parental consent. By mendeliome sequencing, we were able to reach the genetic diagnosis in $15(23,4 \%)$ cases, of which $8(53.3 \%)$ had a de novo autosomal heterozygous variant; $5(33.3 \%)$ were compound heterozygous or homozygous for an autosomal recessive condition; 2 (13.3 $\%)$ had an X-linked condition. Variants were mostly in classes IV and V. Clinical re-evaluation of patients was crucial for result validation. We could expand the phenotypic spectrum of known conditions in $30 \%$ of cases. Prenatal diagnosis in subsequent pregnancies was consistently performed in several occasions. By WES, precision diagnosis was expanded to three genes not included in the mendeliome. By contrast, a high number of VUS was observed, for which additional research activities were required and planned. The use of mendeliome sequencing as an early step of the diagnostic workflow, critically oriented on the basis of the clinical phenotype, has proven efficient also in terms of cost/benefit. Strategies for the use of mendeliome sequencing, mainly in silico, are here suggested.

M. Zollino: None. S. Frangella: None. P.N. Doronzio: None. S. Maietta: None. D. Orteschi: None. S. Amenta: None. S. Ricciardi: None. L. Masini: None. M. De Santis: None. G. Vento: None. R. Manfredi: None. V. Nigro: None. M. Pinelli: None. G. Marangi: None. 


\section{P16.47.B}

Clinical utility of the functional mRNA evaluation of rare genetic variants in diagnostic practice

C. R. A. Duff-Farrier ${ }^{l}$, R. Whittington ${ }^{l}, J$. Honeychurch ${ }^{l}$, J. Evans ${ }^{l}, K$. Low ${ }^{2}$, J. Kennedy ${ }^{2}$, S. Smithson ${ }^{2}$, M. Williams ${ }^{1}$

${ }^{1}$ Bristol Genetics Laboratory, Bristol, United Kingdom, ${ }^{2}$ Clinical Genetics, University Hospitals Bristol, Bristol, United Kingdom

High throughput sequencing in clinical diagnostics generates many genetic variants that require interpretation. A proportion of these affect mRNA splicing, and a range of in silico tools are employed to predict their effect, however these are limited in scope and may be unreliable. Evaluation of splicing variants using targeted RT-PCR is useful to demonstrate functionality. The implementation of a functional splicing service at Bristol Genetic Laboratory has demonstrated high clinical utility as complementary approach to support ACMG classification (code PS3), to deliver additional positive diagnoses, and to provide further information into underlying disease mechanisms. RT-PCR investigation was utilised in a cohort of ten index patients from different disease backgrounds, using peripheral blood or skin fibroblasts. In eight patients $(80 \%)$ the investigated variant demonstrated a splicing effect. Notable examples include: (i) ANKRD11 c.744G >A, p.(Trp202Glyfs*99) resulting in exon 7 skipping and causing loss of a critical Dbox sequence, compatible with a diagnosis of KBG syndrome. (ii) KAT6A c.1364-2A>T, p.(Asp455Glyfs*10) at a dinucleotide consensus splice site resulting in activation of a cryptic acceptor site in exon 8, causing KAT6A syndrome and demonstrating a novel disease mechanism. (iii) $T A Z$ c.646+1del, p.(Ile196_Gly216del) at a dinucleotide consensus splice site resulting in exon 8 skipping and premature protein truncation. The first report of Barth syndrome in a heterozygous female. (iv) WDR45 c.236$18 \mathrm{~A}>\mathrm{G}$, p.(Asp44Alafs*9) resulting in activation of a cryptic acceptor site in intron 5 , intron inclusion, and a frameshift. The first report of a 'deep intronic' WDR45 variant casual in Neurodegeneration with Brain Iron Accumulation.

C.R.A. Duff-Farrier: None. R. Whittington: None. J. Honeychurch: None. J. Evans: None. K. Low: None. J. Kennedy: None. S. Smithson: None. M. Williams: None.

\section{P16.48.C}

How SMRT sequencing can improve the prognosis and genetic counseling in DM1 patients
S. Tom $\hat{\boldsymbol{e}}^{1}$, J. Ziegle ${ }^{2}$, Y. Tsai ${ }^{2}$, J. Harting ${ }^{2}$, J. Bonnefont ${ }^{3}$, G.

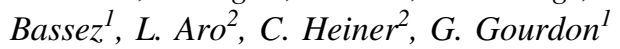

${ }^{I}$ INSERM UMR974, Sorbonne Université, Centre de Recherche en Myologie, Paris, France, ${ }^{2}$ Pacific Biosciences, Menlo Park, CA, United States, ${ }^{3}$ Molecular genetic laboratory, Necker Hospital, Paris, France

Myotonic dystrophy type 1 (DM1) results from the expansion of an unstable CTG repeat that usually increases across generations and over time in somatic tissues. CTG repeat instability and DM1 clinical manifestations depend on the length of the repeat itself and the purity of the repeated sequence. The genetic counseling in DM1 is very complex, due to the highly variable clinical presentation and technical difficulties in determining the size and purity of the CTG expansion. We used Single Molecule Real-Time sequencing (SMRT) developed by Pacific Biosciences (PacBio) to precisely measure large CTG repeat size and identify sequence interruptions of expanded allele to understand clinical and genetic variability in DM1 patients. We sequenced 10 DM1 patients with CTG repeat expansion ranging from 130 to > 1000 CTG repeats on the Sequel System. We amplified expanded alleles by PCR and pooled samples equi-mass for library construction, using $10 \mathrm{ng}$ per sample. Selective AMPure purification was done to enrich for expanded alleles. We obtained more than $77 \%$ full DM1 reads per sample, with $>70 \%$ of the reads from expanded alleles. The data includes long reads in the expected size range for all samples, including DM1 patients with more than 1000 CTG repeats. SMRT sequencing is very promising to sequence large triplet repeat expansions and also to identify CTG repeat interruptions and estimate somatic mosaicism in DM1 patients. This method can improve the prognosis and counseling offered to patients. This work was supported by Pac bio and the Association Institut de Myologie.

S. Tomé: None. J. Ziegle: None. Y. Tsai: None. J. Harting: None. J. Bonnefont: None. G. Bassez: None. L. Aro: None. C. Heiner: None. G. Gourdon: None.

P16.50.B

Pathogenic Neurofibromatosis type 1 (NF1) RNAsplicing resolved by targeted RNAseq

R. Koster ${ }^{1}$, D. Tserpelis ${ }^{1}$, K. B. M. Claes $^{2}$, A. D. C. Paulussen $^{l}$, M. Sinnema ${ }^{l}$, R. Szklarczyk ${ }^{l}, K$. Derks ${ }^{l}$, M. van Geel $^{l}$, M. J. Blok ${ }^{l}$

${ }^{I}$ Department of Clinical Genetics, Maastricht University Medical Center+, Maastricht, Netherlands, ${ }^{2}$ Centre for Medical Genetics, Ghent University Hospital, Ghent, Belgium 
Introduction: Neurofibromatosis type 1 (NF1) is caused by loss-of-function variants in the NF1 gene, about $30 \%$ effect RNA-splicing. Approximately $10 \%$ are missed by conventional DNA-diagnostics, because they are located outside the region of interest, or are misinterpreted by in silico splicing predictions. We designed a targeted RNAseq-based approach to detect pathogenic RNA-splicing and thereby causative DNA-variants.

Methods: RNA was extracted from lymphocytes followed by targeted NF1 RNA-seq. An in-house developed tool (QURNAS) was used to calculate an enrichment score (ERS) for each splicing event. A high enrichment score indicates high abundance of a potentially pathogenic splicing event.

Results: A validation was first performed with nine known pathogenic NF1 splice-variants and two wildtype NF1 samples. All normal reference exon-exon splice junctions, several novel and 16 out of 17 previously reported naturally occurring splice events were detected. Furthermore, all expected pathogenic splice events were detected based on high ERS. Next, we blindly replicated the molecular diagnosis (based on RT-PCR) of ten patients from Ghent. Since using our approach all pathogenic splicing events and corresponding splice-variants were detected. Additionally, using RNAseq we identified a de novo deep intronic pathogenic splice variant in a patient with a clear NF1 phenotype, whom lacked a molecular diagnosis.

Conclusions: Targeted NF1 RNAseq can be successfully used to characterize variants of unknown significance (VUS) and identify undetected pathogenic RNA-splicing variants, additionally to DNA-based diagnostics.

R. Koster: None. D. Tserpelis: None. K.B.M. Claes: None. A.D.C. Paulussen: None. M. Sinnema: None. R. Szklarczyk: None. K. Derks: None. M. van Geel: None. M.J. Blok: None.

\section{P16.51.C}

Sample identity quality control in Next Generation Sequencing (NGS) routine diagnostics using KASP

\section{F. Scharf, J. Romic-Pickl, T. Wohlfrom, A. Benet-Pagès, B. Neitzel, A. Abicht, E. Holinski-Feder}

\section{Medical Genetics Center MGZ, Munich, Germany}

The parallel processing of multiple samples in NGS applications requires the exclusion of possible sample swaps. We present a QC approach based on the genotyping of single nucleotide polymorphisms (SNPs) with Kompetitive Allele Specific PCR (KASP) technology and the comparison to NGS data for the verification of sample identities. 16 SNPs covered in our NGS capture kit (Agilent SureSelectXT) were selected. To reliably detect sample swaps, 1) haplotype overlaps between samples need to be excluded, i.e. no sample has been pooled twice (uniqueness) and 2) the identity of each sample needs to be confirmed by comparing KASP and NGS data. Due to possible dropouts or erroneous KASP results, some comparisons might not always be possible. The minimal number of successful comparisons and maximum number of mismatches for reliable conclusions was calculated. Concerning uniqueness, the probability of encountering two samples with the same haplotype over a certain number of SNPs in a certain number of samples was calculated; the cutoffs for identity confirmation are based on the probability of a sample showing one specific haplotype over a certain number of SNPs. Results showed that at least two differing SNPs in all pairwise comparisons of the samples exclude overlaps with $>99 \%$ certainty. With a cutoff of maximally four dropouts or three dropouts and one mismatch the correct sample assignment can be verified with a probability of $>99 \%$. The identity confirmation is integrated in our NGS analysis pipeline. Samples failing the criteria are automatically identified and held back from further processing.

F. Scharf: None. J. Romic-Pickl: None. T. Wohlfrom: None. A. Benet-Pagès: None. B. Neitzel: None. A. Abicht: None. E. Holinski-Feder: None.

\section{P16.53.B}

Evaluation and comparison of novel amplicon-based NGS library preparation protocols across multiple sequencing platforms

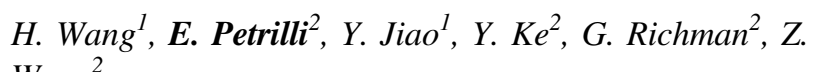
Wang $^{2}$

${ }^{1}$ Pillar Shanghai Zhengu Biotech Co. Ltd, Shanghai, China, ${ }^{2}$ Pillar Biosciences, Natick, MA, United States

Introduction: SLIMamp allows multiplex-PCR of tiled amplicons in a single tube, which enables targeting of large exons for NGS analysis with a streamlined process. This technology was previously demonstrated to be a sensitive and robust NGS library prep method for Illumina instruments. To benefit the largest number of patients and researchers, SLIMamp library prep was made compatible with multiple sequencing platforms that are common worldwide including the MGISEQ.

Materials and Methods: Five ONCO/Reveal assays for MGI were evaluated (multi-cancer, multi-cancer with CNV, multi-cancer cfDNA, lung fusions, and lung and colon cancer) on the MGISEQ-2000. Libraries were prepared and sequenced by two laboratories (Zhengu and MGI). 14 CRC samples were tested and compared on the MGISEQ-2000 
and NextSeq CN500 platforms. Automated library preparation by the MGISP-100 was also evaluated.

Results: Across the five assays, mapping and on-target rates and coverage uniformity at $0.2 \mathrm{x}$ of the mean demonstrated high performance (generally $>95 \%$ ). Variant calls from 14 CRC samples for the ONCO/Reveal MultiCancer Panel sequenced on the MGISEQ-2000 and NextSeq CN500 demonstrated an $\mathrm{R}^{2}$ value of $97 \%$. Variants were concordantly called from $2 \%$ to $65 \%$ VAF. The MGISP-100 reduced hands-on-time to 30 minutes.

Conclusions: All ONCO/Reveal assays demonstrated high performance when sequenced on MGISEQ-2000 and Illumina platforms. Automated library preparation utilizing the MGISP-100 reduced hands-on time while providing sensitive and robust performance. Concordance was demonstrated across platforms, specifically the NextSeq CN500 and MGISEQ-2000. ONCO/Reveal assays are sensitive and robust solutions across multiple sequencing platforms that can be used by researchers across the globe.

H. Wang: A. Employment (full or part-time); Significant; Pillar Shanghai Zhengu Biotech Co. Ltd. E. Petrilli: A. Employment (full or part-time); Significant; Pillar Biosciences. E. Ownership Interest (stock, stock options, patent or other intellectual property); Significant; Pillar Biosciences. Y. Jiao: A. Employment (full or part-time); Significant; Pillar Shanghai Zhengu Biotech Co. Ltd. Y. Ke: A. Employment (full or part-time); Significant; Pillar Biosciences. E. Ownership Interest (stock, stock options, patent or other intellectual property); Significant; Pillar Biosciences. G. Richman: A. Employment (full or parttime); Significant; Pillar Biosciecnes. E. Ownership Interest (stock, stock options, patent or other intellectual property); Significant; Pillar Biosciences. Z. Wang: A. Employment (full or part-time); Significant; Pillar Biosciences. E. Ownership Interest (stock, stock options, patent or other intellectual property); Significant; Pillar Biosciences.

\section{P16.54.C}

\section{Robustness and accuracy validation of fetal fraction} estimates in NIPT

\section{Miceikaite, C. Brasch-Andersen, C. Fagerberg, M. J. Larsen}

\section{Department of Clinical Genetics, Odense University} Hospital, Odense, Denmark

Introduction: Fetal fraction (FF) is the proportion of total cell-free DNA present in the maternal plasma that is of fetal origin. Sufficient amount of fetal DNA is crucial to avoid false negative NIPT results. Accurate and robust methods to estimate fetal fraction are therefore essential to ensure a valid NIPT result. To what extent the total number of sequencing reads influence the fetal fraction estimate is unexplored. In this study, we tested the robustness of the widely used SeqFF method to provide recommendations on the optimal number of reads needed to obtain accurate fetal fraction estimates.

Materials and Methods: Retrospective data from WGS NIPT samples $(n=516)$ obtained as a part of our local prenatal screening program (Department of Clinical Genetics, OUH, Denmark) were included. For all samples $>30 \mathrm{M}$ (million) reads had been obtained. Data were randomly down-sampled before estimating the fetal fraction and results were compared with SNP-based and chrY-based FF estimates.

Results: Overall, SeqFF estimates highly correlated with both the SNP-based (R-squared=0.88) and chrY-based (Rsquared=0.92) methods. By down-sampling, we observed that the SeqFF estimates were highly affected by the total number of reads used. Accurate SeqFF estimates were achieved using $>10 \mathrm{M}$ reads. By increasing the number of reads further, additional gain in accuracy was observed. Including more than $20 \mathrm{M}$ reads did not result in further significant improvement.

Conclusions: FF estimation is highly affected by the total number of reads used. It is recommended to use no less than $10 \mathrm{M}$ reads to obtain accurate FF estimates for NIPT.

I. Miceikaite: None. C. Brasch-Andersen: None. C. Fagerberg: None. M.J. Larsen: None.

\section{P16.55.A}

Repeatability assessment of a prenatal cell-free DNA test

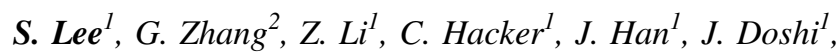
K. Kirkconnell ${ }^{1}$, R. Stokowski ${ }^{1}$, E. Martin ${ }^{1}$

${ }^{1}$ Roche Sequencing Solutions, Inc., San Jose, CA, United States, ${ }^{2}$ Roche Sequencing Solutions, Inc., Pleasanton, CA, United States

Introduction: Clinical implementation of a complex molecular test such as cell-free DNA (cfDNA) testing for fetal trisomy requires extensive validation; however, for most commercially available cfDNA tests there is little published performance data beyond the establishment of sensitivity and specificity. The objective of this study was to assess the repeatability of results for a prenatal cfDNA test.

Materials and Methods: The Harmony ${ }^{\circledR}$ prenatal test on the Ariosa Cell-Free DNA System (CE-IVD) was assessed across multiple lots of reagents and operators. Panels were contrived from genomic DNA to generate sufficient replicates to assess repeatability across multiple factors. This resulted in a robust sample size of 1,710 samples with fetal fractions reflecting those observed in a general pregnancy population. 
Results: Overall $97 \%$ of samples met the test's quality control thresholds. Sensitivity was 100\% for trisomy 21 (142/ $142), 100 \%$ for trisomy 18 (122/122), 100\% for trisomy 13 (114/114), $97.6 \%$ for the $22 \mathrm{q} 11.2$ deletion (120/123), and $100 \%$ for monosomy X (123/123). Specificity was $100 \%$ $(1,034 / 1,034)$. Sensitivity $(100 \%)$ was in complete agreement between the lots for trisomy 21 , trisomy 18 , trisomy 13 , and monosomy X. Sensitivity for 22q11.2 deletion in the 3 lots $[100 \%(41 / 41), 95 \%(40 / 41)$, and 97.5\%(39/40)] was not significantly different $(p=0.25)$.

Conclusions: The study demonstrated high sensitivity and specificity with repeatable results over different lots, with operators, runs, and days. There was no significant lotto-lot or inter-operator variability in sensitivity and specificity.

S. Lee: A. Employment (full or part-time); Significant; Roche Sequencing Solutions, Inc. G. Zhang: A. Employment (full or part-time); Significant; Roche Sequencing Solutions, Inc. Z. Li: A. Employment (full or part-time); Significant; Roche Sequencing Solutions, Inc. C. Hacker: A. Employment (full or part-time); Significant; Roche Sequencing Solutions, Inc. J. Han: A. Employment (full or part-time); Significant; Roche Sequencing Solutions, Inc. J. Doshi: A. Employment (full or part-time); Significant; Roche Sequencing Solutions, Inc. K. Kirkconnell: A. Employment (full or part-time); Significant; Roche Sequencing Solutions, Inc. R. Stokowski: A. Employment (full or part-time); Significant; Roche Sequencing Solutions, Inc. E. Martin: A. Employment (full or part-time); Significant; Roche Sequencing Solutions, Inc..

\section{P16.56.B}

Development of matched maternal-fetal non-invasive prenatal testing (NIPT) reference materials compatible with a broad range of assay methods

\section{A. M. Caruso ${ }^{\text {I }}$, F. L. Tomson ${ }^{2}$, Y. Konigshofer ${ }^{2}, R$.

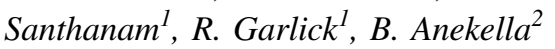 \\ ${ }^{1}$ SeraCare LifeSciences, Milford, MA, United States, ${ }^{2}$ Ser- aCare LifeSciences, Gaithersburg, MA, United States}

Introduction: The availability of appropriate reference materials that mimic the characteristics of clinical samples for proficiency testing, assay validations, and run controls is very limited. Seracare has developed a new technology and formulation to provide a sustainable source of patient-like NIPT reference materials. These materials are compatible with a broad range of NIPT assay methods and should enable clinical labs to validate and monitor NIPT assays.

Methods: Antepartum and postpartum samples were obtained from patients with high-risk pregnancies under IRB approved protocols. ccfDNA was isolated from ante- and post-partum samples. This isolated ccfDNA was amplified, encapsulated by a proprietary lipophilic procedure and blended into a synthetic plasma to create patientlike samples.

Results: These new NIPT reference materials demonstrated patient-like fragment distribution (for both maternal and fetal sizing) with a major peak of around 160-170 bp and minor peaks above $250 \mathrm{bp}$. The materials have been extensively tested by multiple commercial NIPT assays, such as Harmony (Ariosa), VeriSeq (Illumina) or Panorama (Natera). The trisomy 21 and 18 were called accurately as male or female trisomy 21 and female trisomy 18 at fetal fractions of $11 \%, 13 \%$, and $18 \%$ respectively. We also created an aneuploidy negative control - female and male euploid material with fetal fractions at $16.6 \%$ and $9.4 \%$.

Conclusions: The SeraCare portfolio of NIPT materials with patient-like sample attributes are suitable for a broad range of currently available NIPT methods. The availability of matched fetal-maternal reference materials is particularly important for the transition of NIPT into mainstream clinical practice.

A.M. Caruso: A. Employment (full or part-time); Significant; SeraCare LifeSciences. F.L. Tomson: A. Employment (full or part-time); Significant; Seracare. Y. Konigshofer: A. Employment (full or part-time); Significant; Seracare. R. Santhanam: A. Employment (full or part-time); Significant; Seracare. R. Garlick: A. Employment (full or part-time); Significant; Seracare. B. Anekella: A. Employment (full or part-time); Significant; Seracare.

\section{P16.57.C}

a novel NIR spectroscopic qualitative method to monitor the diet compliance in PKU patients

\author{
M. F. M. Vismara ${ }^{1}, A$. Valentini ${ }^{2}$, G. Bonapace ${ }^{3}, O$. \\ Marasco $^{4}$, G. Scozzafava ${ }^{4}$, M. Ashour ${ }^{5}$, M. Pittelli $^{4}, T$.

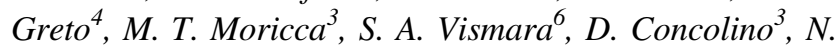 \\ Perrotti $^{7}$
}

${ }^{1}$ Department of Histology, University "Magna Gracia", Catanzaro, Italy, ${ }^{2}$ CARAVAGGIO medical center, Roma, Italy, ${ }^{3}$ Department of Medical and Surgical Science, Pediatric Unit, University "Magna Graecia", Catanzaro, Italy, ${ }^{4}$ Centro Regionale di Screening Neonatale, A.O.U. "Mater Domini", Catanzaro, Italy, ${ }^{5}$ Department of Health Sciences, Nephrology Unit, "Magna Graecia" University, Catanzaro, Italy, ${ }^{6}$ Ente Ospedaliero Cantonale del Ticino, Istituto pediatrico della Svizzera italiana, Lugano, Switzerland, ${ }^{7}$ Department of Health Sciences, University "Magna Graecia", Catanzaro, Italy

Introduction: Phenylketonuria (PKU MIM 261600) is a human metabolic disease, caused by mutations in the 
phenylalanine hydroxylase gene. Children born with PKU, thanks to the newborn screening and early dietary interventions, can have relatively normal lives. The diet allows them to keep Phe level in range. The best indicator of dietary compliance is regular monitoring of Phe concentrations by using a Liquid Chromatography (LC) based procedure. We developed a qualitative chemometric model able to separate PKU patients based on their response to the diet in three different categories: Low $(<2 \mathrm{mg} / \mathrm{dl})$, Compensate $(2,5-6 \mathrm{mg} / \mathrm{dl})$ and High $(6,5-20 \mathrm{mg} / \mathrm{dl})$ with NIR spectroscopy.

Methods and Results: A qualitative chemometric model was elaborated using 3000 NIR spectra, from 562 DBS PKU samples from the three different groups, based on their Phe levels assessed by LC. Using a sensor calibrated in the Near infra-Red spectrum, 20 scans per sample in duplicate were acquired. Principal component analysis (PCA) was conducted. The chemometric model was validated by blind external procedure. Our model is able to correctly assign unknown sample to the Low, Compensate, or High group with a linearity of 0,91 and a $\sigma=0,078$, showing a sensitivity ratio between the number of the correctly classified samples (CCS) and the number of the known samples belonging to each class $(\mathrm{KS}): \mathrm{Sn}=\mathrm{CCS} \cdot 100 \mathrm{KS}$ close to $100 \%$.

Conclusions:We demonstrated that it is possible to use NIR spectroscopy to quickly monitor the diet compliance in PKU patients.

M.F.M. Vismara: None. A. Valentini: None. G. Bonapace: None. O. Marasco: None. G. Scozzafava: None. M. Ashour: None. M. Pittelli: None. T. Greto: None. M.T. Moricca: None. S.A. Vismara: None. D. Concolino: None. N. Perrotti: None.

\section{P16.58.A}

\section{Analysis of 15q11.2-q13 locus reorganizations}

\section{S. Chernushyn ${ }^{l}$, N. Hryshchenko ${ }^{l}$, S. Kravchenko ${ }^{1}, E$. Patskun $^{2}$}

${ }^{1}$ Institute of Molecular Biology and Genetics, Kiev, Ukraine, ${ }^{2}$ Municipal Non-Profit Enterprise "Transkarpathian Clinical Regional Hospital named after Andrii Novak", Uzhgorod, Ukraine

Introduction: $15 \mathrm{q} 11-\mathrm{q} 13$ is a region harboring several genes regulated by genomic imprinting, as a result, genes subject to regulation by genomic imprinting are functionally haploid, having only a single functional copy. Prader-Willi syndrome (PWS), Angelman syndromes (AS) and 15q11q13.1 duplication syndrome (dup15q) are 3 clinically distinct disorders associated with multiple anomalies and intellectual disability typically resulting from $\mathrm{CNV}$ of imprinted genes.

Materials and Methods: Analysis of CNVs in 15q11q13 and UPD15 was performed using STR markers D15S817, D15S1513, D15S822, D15S1002 genotyping. The STR markers were selected according to their high heterozygosity and location in the region adjacent of PWS and AS loci.

Results: The STR markers genotyping in the analyzed groups revealed 4 PWS patients with maternal UPD15 (isoand heterodisomic). Three PWS and one AS had the 15q11q13 deletions: paternal del 15q in PWS and maternal del15q in AS patients. The maternal age of UPD15 patients was significantly higher (40,3years) than in mothers of del15q patients $(28,4$ years). In two AS-like patients with seizures 15q11-q13 duplication was detected, one dup15q was inherited from the effected mother and another one was a de novo maternal mutation.

Conclusion: The selected set of STR markers allows detecting all types of molecular genetic disorders of 15q11q13 chromosomal region as well as to differentiate the mutation type and its origin.

S. Chernushyn: None. N. Hryshchenko: None. S. Kravchenko: None. E. Patskun: None.

\section{P16.59.B}

Update of the EMQN / ACGS best practice Guidelines for Molecular Analysis of Prader-Willi and Angelman Syndromes

\author{
J. Beygo ${ }^{l}$, K. Buiting ${ }^{l}$, S. Ramsden ${ }^{2}$, R. Ellis ${ }^{3}$, J. Clayton- \\ Smith $^{2,4}$, D. Kanber ${ }^{l}$
}

${ }^{1}$ Institut für Humangenetik, Universitätsklinikum Essen, Universität Duisburg-Essen, Essen, Germany, ${ }^{2}$ Manchester Centre for Genomic Medicine, Manchester University Hospitals NHS Foundation Trust, Manchester Academic Health Sciences Centre, Manchester, United Kingdom, ${ }^{3}$ Department of Medical Genetics, Yorkhill NHS Trust, Yorkhill Hospital Glasgow, Glasgow, United Kingdom, ${ }^{4}$ Division of Evolution and Genomic Sciences School of Biological Sciences University of Manchester, Manchester, United Kingdom

Prader-Willi (PWS, \#176270) and Angelman syndrome (AS, \#105830) are rare neurogenetic imprinting disorders caused by genetic and epigenetic disturbances affecting the chromosomal region $15 \mathrm{q} 11 \mathrm{q} 13$ which harbours a cluster of imprinted genes. The parent-of-origin specific expression of these genes is regulated by an imprinting centre. The molecular defects include large deletions of 5-7 Mb, uniparental disomies, imprinting defects (ID) with or without imprinting centre deletions, mosaic IDs, point mutations 
and deletions of the UBE3A gene as well as rare chromosomal rearrangements. Given the broad nature of the underlying molecular causes and the different techniques needed to establish the molecular genetic diagnosis and hence the recurrence risk, diagnostic of PWS and AS is challenging. Furthermore, there is increasing need for proper, universally understandable and standardised diagnostic reports. In the light of improvements and new developments regarding techniques and differential diagnoses together with reporting standards we updated the best practice guidelines for the PWS and AS diagnostics. The guidelines were open for consultation and amendment by the broad community of labs participating in the annual European Molecular Genetics Quality Network (EMQN) schemes for PWS and AS diagnostic and were approved by the EMQN and the Association for Clinical Genomic Science (ASCG).

J. Beygo: None. K. Buiting: None. S. Ramsden: None. R. Ellis: None. J. Clayton-Smith: None. D. Kanber: None.

\section{P16.64.A}

Mainstreaming genomic reporting in Australia- recommendations from an Australian Genomics working group

J. M. Fletcher ${ }^{1}$, K. Bloggs ${ }^{2}$, L. Gilkes ${ }^{3}$, J. Harraway ${ }^{4}$, S.

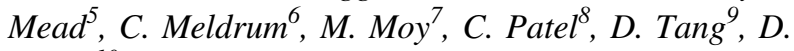
Walsh $^{10}$

${ }^{1}$ Genetics and Molecular Pathology,, North Adelaide, Australia, ${ }^{2}$ Australian Genomics Health Alliance, Murdoch Children's Research Institute, Melbourne, Australia, ${ }^{3}$ University of Notre Dame, Fremantle, Australia, ${ }^{4}$ Sullivan Nicolaides Pathology, Brisbane, Australia, ${ }^{5}$ NSW Health Pathology, Sydney, Australia, ${ }^{6}$ New South Wales Health Pathology, Newcastle, Australia, ${ }^{7}$ Partridge General Practice, Adelaide, Australia, ${ }^{8}$ Genetic Health Queensland, Brisbane, Australia, ${ }^{9}$ Murdoch Children's Research Institute, Melbourne, Australia, ${ }^{10}$ Queen Elizabeth Hospital, Adelaide, Australia

Introduction: A recent national audit of genetic testing dispelled the myth that most tests are ordered by genetic health professionals. Genetic pathology reports can be difficult to understand, and need to be easily understood by healthcare providers so they can be used to improve patient outcomes. Additionally, a new, national health e-resource now allows patients to independently access their own pathology results, including genetic results in some jurisdictions.

Methods: A multi-disciplinary working party was convened. Example reports and literature were reviewed through different lenses. Membership comprised general practitioners, a surgeon, clinical geneticist, genetic counsellor, genomic scientist and genetic pathologists. FleshKincaid and SMOG were used to analyse report readability. The Australian Genomics Community Advisory Group reviewed the model factsheet and recommendations.

Results: The level of information that requestors wanted in reports was different, with more detail requested by genetic professionals. All wanted a clear summary of the findings, with a standardised, consistent order of report elements. Readability was identified as a major issue. Plain language is important, but accuracy of genetic information must be retained in line with accreditation guidelines. Recommendations were formulated and included a model laboratory report template and model report interpretation fact sheet for primary referrers.

Conclusions: Laboratories reporting genomic tests should simplify their reports as much as possible. Synoptic reporting for genomic tests should be encouraged. The matter has been referred to the Royal College of Pathologists of Australasia for implementation. Australian Genomics Health Alliance is funded by NHMRC grant 1113531 and the Medical Research Future Fund

J.M. Fletcher: None. K. Bloggs: None. L. Gilkes: None. J. Harraway: None. S. Mead: None. C. Meldrum: None. M. Moy: None. C. Patel: None. D. Tang: None. D. Walsh: None.

\section{P16.65.B}

Sample identity confirmation in MPS workflows using pxlence's Human Sample ID Kit

\section{Dunon ${ }^{1}$, F. Coppieters $^{2,1}$, J. Vandesompele ${ }^{2,1}, S$. Lefever $^{2,1}$ \\ ${ }^{l}$ pxlence bvba, Dendermonde, Belgium, ${ }^{2}$ Ghent University, Gent, Belgium}

Background: Gene panel, whole exome (WES) and genome sequencing (WGS) are routinely performed. However, library preparations are complex and laborious, and prone to sample mix-ups. Here, we developed and validated an easy and cost-effective sample identity test for independent genotyping of 44 SNPs and 6 gender markers.

Methods: Target SNPs were selected based on their minor allele frequency, exonic location and overlap with the capture region of various WES enrichment kits. For compatibility with FFPE DNA and cfDNA samples, short 60-100 bp amplicons were designed. Library preparation is achieved by a limited-cycle indexing PCR step on universal tails incorporated in the target-specific primers. We applied this novel Human Sample ID Kit on 3 FFPE samples, 1 cellfree reference DNA and $6 \mathrm{GiB}$ reference DNA samples with 
input amounts ranging from 1 upto $20 \mathrm{ng}$. Performance was evaluated by paired-end sequencing on a MiSeq instrument.

Results: Excellent homogeneous coverage and on-target specificity was achieved. For all DNA samples, all SNPs displayed a coverage higher than $20 \%$ of the mean and a mean on-target rate of $94.3 \%$. More impressively, $93.2 \%$ of the SNPs had a uniform coverage within 2-fold of the mean. Based on the resulting genotypes, DNA samples could be unambiguously discriminated and linked to the corresponding exomes.

Conclusions: We developed and validated a novel sample tracking test for WES and WGS, involving a ready-to-sequence 2-step multiplex PCR reaction. The Human Sample ID Kit has a discrimination power of over 1:85,000 and enabled unambiguous discrimination of each DNA sample.

V. Dunon: A. Employment (full or part-time); Significant; pxlence bvba. F. Coppieters: E. Ownership Interest (stock, stock options, patent or other intellectual property); Significant; pxlence bvba. J. Vandesompele: E. Ownership Interest (stock, stock options, patent or other intellectual property); Significant; pxlence bvba. S. Lefever: E. Ownership Interest (stock, stock options, patent or other intellectual property); Significant; pxlence bvba.

\section{P16.66.C}

Sanger validation of NGS variants: is still always necessary?

A. Arteche-López ${ }^{1}$, A. Ávila Fernández ${ }^{2}$, R. Riveiro Álvarez', M. López Martínez ${ }^{2}$, A. Giménez Pardo ${ }^{2}$ C. Vélez $^{2}$, J. Gallego Merlo ${ }^{2}$, I. García Varal ${ }^{1}$ B. Almoguera ${ }^{2}$, A. Bustamante Aragonés ${ }^{2}$, F. Blanco Kelly ${ }^{2}$, S. Tahsin Swafiri ${ }^{2}$, E. Rodríguez Pinilla ${ }^{2}$, I. Lorda Sánchez ${ }^{2}, M$. Trujillo Tiebas ${ }^{1}$, C. Ayuso García ${ }^{2}$

${ }^{1}$ University Hospital 12 de Octubre. Fundación Jiménez Díaz University Hospital, Madrid, Spain, MADRID, Spain, ${ }^{2}$ Fundación Jiménez Díaz University Hospital, Madrid, Spain, MADRID, Spain

Introduction: As it has already been reported, Sanger sequencing validation appears to have limited utility for good quality single nucleotide variants (SNVs) NGSdetected. However, more studies are needed for indels variants and/or copy number variations (CNVs). We present the largest single-center Sanger validation study, with a subset data of clinical exomes in 825 characterized cases, in order to confirm the high concordance expected of our clinical exome diagnostic tool. We also aim to expand the validations of our small deletion/duplication variants and CNVs detected by NGS.
Materials and Methods: Clinical exomes were analysed from peripheral blood samples using the TruSight One Sequencing panel (Illumina) or the Clinical Exome Solution (Sophia Genetics). A total of 1099 good quality NGSdetected variants $(>20 \mathrm{X}$ coverage, $>20 \%$ heterozygosity and Quality $=$ PASS $)$ and $30 \mathrm{CNVs}$ were further confirmed by Sanger sequencing and MLPA or array-CGH according to convenience, respectively. Sequencing primers were designed manually or with the ExonPrimer tool, and checked in the SNPchecker program.

Results: Though 11 Sanger discrepancies that required new primers designs were found, we had $100 \%$ concordance for the 858 SNVs $(80.16 \%)$ and 321 (19.64\%) indel variants analysed $(n=1099)$. However, we only had a concordance of $96 \%$ for CNVs $(n=30)$, as we had a false positive deletion in homozygosis.

Conclusions: NGS is a robust technique for the detection for good quality SNVs/Indel variants, but validation for CNVs is still necessary. Limitations of Sanger sequencing should always be considered. Each laboratory might stablish their own thresholds before deciding to discontinue Sanger confirmation studies.

A. Arteche-López: None. A. Ávila Fernández: None. R. Riveiro Álvarez: None. M. López Martínez: None. A. Giménez Pardo: None. C. Vélez: None. J. Gallego Merlo: None. I. García Vara: None. B. Almoguera: None. A. Bustamante Aragonés: None. F. Blanco Kelly: None. S. Tahsin Swafiri: None. E. Rodríguez Pinilla: None. I. Lorda Sánchez: None. M. Trujillo Tiebas: None. C. Ayuso García: None.

\section{P16.68.B}

Multisite evaluation of a single-tube SMN1/2 PCR/CE assay system that assesses copy number and expanded content for spinal muscular atrophy

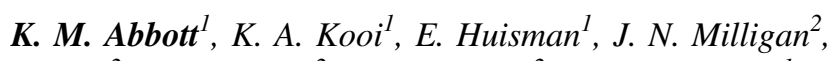
H. Zhu' ${ }^{2}$, J. L. Larson ${ }^{2}$, G. J. Latham ${ }^{2}$, H. H. Lemmink ${ }^{1}$

${ }^{1}$ University Medical Center Groningen, Groningen, Netherlands, ${ }^{2}$ Asuragen, Inc., Austin, TX, United States

Spinal Muscular Atrophy (SMA) is an autosomal recessive neuromuscular disease that is often fatal before age two. Approximately $95 \%$ of cases are caused by homozygous deletion of SMN1 exon7. SMN2 is a homolog of SMN1 whose copy number is inversely correlated with disease severity, making quantification of both genes relevant to recent breakthrough treatments. Additionally, detection of three variants - c.*3+80T>G, c.*211_*212del in SMN1 associated with gene duplication, and c.859G $>\mathrm{C}$ in $S M N 2$ linked to reduced disease severity - can improve carrier detection and inform therapy decisions. We evaluated an 
assay that quantifies SMN1 and SMN2 exon7 copy numbers and the three variants in a single PCR reaction. We used the AmplideX ${ }^{\oplus}$ PCR/CE SMN1/2 Plus kit to test over 100 whole blood or cell-line DNA samples. PCR products were separated using Applied Biosystems ${ }^{\mathrm{TM}}$ 3730/3730xl Genetic Analyzers and analyzed with AmplideX Reporter software. We also compared the included kit calibrator to calibration using samples from each purification method. Copy number calls for SMN1 and SMN2 were over 95\% concordant with reference method results over multiple runs. Variant calls were $100 \%$ concordant for all samples with known genotypes. Performance was similar regardless of calibration method although calibration with purified samples reduced variability. The assay accurately and precisely quantified exon7 for both SMN1 and SMN2 at or above four copies and detected the three clinically-significant variants in under four hours from DNA to genotype. The expanded content of the assay may provide an all-in-one option for laboratories interested in SMA carrier screening and diagnostics.

K.M. Abbott: None. K.A. Kooi: None. E. Huisman: None. J.N. Milligan: A. Employment (full or part-time); Significant; Asuragen, Inc. H. Zhu: A. Employment (full or part-time); Significant; Asuragen, Inc. J.L. Larson: A. Employment (full or part-time); Significant; Asuragen, Inc. G.J. Latham: A. Employment (full or part-time); Significant; Asuragen, Inc.. H.H. Lemmink: None.

\section{P16.70.A}

Towards the integration of RNA-seq into diagnostic pipelines for the identification of pathogenic splicing variants in Mendelian disorders

\section{F. Rowlands ${ }^{1}$, D. Baralle ${ }^{2}$, A. G. L. Douglas ${ }^{2}, R$. O'Keefe $^{l}$, S. Hubbard ${ }^{l}$, T. A. Briggs ${ }^{l}$, J. M. Ellingford ${ }^{l}$ \\ ${ }^{1}$ University of Manchester, Manchester, United Kingdom, ${ }^{2}$ University of Southampton, Southampton, United King- dom}

Background: RNA-seq shows promise as a diagnostic tool to elucidate the effects of genomic variants impacting premRNA splicing. However, it remains difficult to speculate in which disease and tissue types it may be most beneficial in improving diagnostic yield. We thus aimed to develop a bioinformatics approach for predicting cross-tissue RNAseq suitability for given disease genes of interest.

Methods: To identify characteristics of pathogenic variation, we compared RNA-seq data from patients with known splicing defects with control individuals from the GTEx dataset across whole blood, lymphoblastoid cell line (LCL) and skeletal muscle. We then devised a gene-specific approach to predict the RNA sequencing depth required to achieve sufficient coverage of transcripts for given disease genes. This was applied to all 285 gene panels listed in the Genomics England PanelApp repository.

Results: Between 22 and 84 panels were predicted to be amenable to RNA-seq analysis dependent on tissue choice. Diseases such as mucopolysaccharidosis showed particular promise as candidates for RNA-seq-based diagnostics, as 37 $M$ sequencing reads in LCL-based RNA-seq was predicted sufficient to cover $90 \%$ of splice junctions with at least 8 reads in 16 of the 17 panel genes.

Conclusions: We present here a bioinformatics framework for the prediction of RNA-seq suitability across disease and tissue contexts, which may ultimately facilitate the appropriate integration of RNA-seq into clinical diagnostic pipelines.

Funding: Medical Research Council (MRC; 1926882) CASE PhD studentship with QIAGEN

C.F. Rowlands: B. Research Grant (principal investigator, collaborator or consultant and pending grants as well as grants already received); Modest; QIAGEN. D. Baralle: None. A.G.L. Douglas: None. R. O'Keefe: None. S. Hubbard: None. T.A. Briggs: None. J.M. Ellingford: None.

P16.72.C

Splicing analysis of blood RNA can increase diagnostic rate and resolve variants of uncertain significance

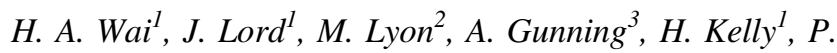
Cibin $^{l}$, E. G. Seaby ${ }^{1,4}$, K. Spiers-Fitzgerald ${ }^{1}$, J. Lye ${ }^{l}, S$. Ellard $^{3}$, N. S. Thomas ${ }^{1,2}$, D. J. Bunyan ${ }^{1,2}$, A. G. L. Douglas $^{1,5}$, D. Baralle ${ }^{1,5}$

${ }^{1}$ University of Southampton, Southampton, United Kingdom, ${ }^{2}$ Wessex Regional Genetics Laboratory, Salisbury, United Kingdom, ${ }^{3}$ Exeter Genomics Laboratory, Exeter, United Kingdom, ${ }^{4}$ Broad Institute of MIT and Harvard, Cambridge, MA, United States, ${ }^{5}$ Wessex Clinical Genetics Service, Southampton, United Kingdom

Introduction: In the setting of single-gene disorders, the high prevalence of variants of uncertain significance limits the clinical diagnostic rate of DNA-based sequencing methodologies. A significant proportion of such variants may cause disease through disruption of RNA splicing. We examined the effects on splicing of a large set of variants of uncertain significance clinically ascertained through diagnostic genetic testing. We also compared the experimentally determined RNA splicing data to bioinformatic predictions made by several commonly used splicing tools.

Materials and Methods: 257 variants of uncertain significance were analysed from across three separate laboratories. Blood RNA was collected in PAXgene tubes and targeted RT-PCR was undertaken with agarose gel 
electrophoresis and Sanger sequencing analysis of products. 17 samples also underwent RNA sequencing of total RNA with depletion of globin mRNA and rRNA. Targeted analysis of splicing was performed on RNA-seq data using Sashimi plot visualisation. Alamut, HSF 3.1 and SpliceAI software were used for splicing predictions.

Results: 85/257 (33\%) of analysed variants were found to affect splicing. Skipping of the upstream exon was the most frequent abnormality (39/85) and this was most often seen in association with variants within the splice donor region. SpliceAI had an accuracy of 0.91 and outperformed other prediction tools in terms of sensitivity and specificity.

Conclusions: Analysis of splicing from blood RNA is useful in identifying splicing abnormalities that cannot reliably be predicted from DNA sequence alone. This provides functional evidence of effect in a significant proportion of uncertain variants, helping in classification of pathogenicity. NIHR_RP-2016-07-011.

H.A. Wai: None. J. Lord: None. M. Lyon: None. A. Gunning: None. H. Kelly: None. P. Cibin: None. E.G. Seaby: None. K. Spiers-Fitzgerald: None. J. Lye: None. S. Ellard: None. N.S. Thomas: None. D.J. Bunyan: None. A.G.L. Douglas: None. D. Baralle: None.

\section{P16.74.B}

Apparently isolated trigonocephaly: diagnostic yield thanks to Whole Exome Sequencing

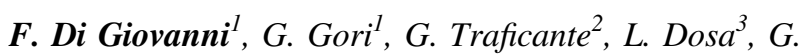

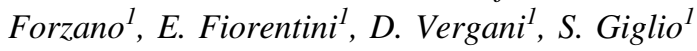

${ }^{1}$ Department of Biomedical Experimental and Clinical Sciences "Mario Serio", University of Florence, F, Florence, Italy, ${ }^{2}$ Medical Genetics Unit, Meyer Children's University Hospital, Florence, Italy, ${ }^{3}$ Integrated Unit of Medical Genetics, USL Toscana Centro, Florence, Italy

The recent availability of whole exome/whole genome sequencing has led to the characterization of the molecular basis of an increasing number of craniosynostosis, although there is still little information about isolated trigonocephaly. It usually occurs sporadically and results from the premature closure of the metopic sutures with consequent deformation of the anterior calvarium. We describe two patients (a female and a male) with isolated trigonocephaly. WES disclosed in both cases a mutation in the PTCHI gene: a de novo heterozygous frameshift variant in the female and a heterozygous stop (inherited from the father later discovered to be affected) in the male. Mutations in PTCHI have been found to cause Gorlin syndrome, an autosomal dominant condition characterized by multiple basal cell carcinomas, odontogenic keratocysts, skeletal anomalies, macrocephaly, dysmorphisms and an increased risk of tumors in early infancy. Skeletal anomalies with defects in the shape of the skull, ribs and spine are often described, but clinical data do not refer specifically to the presence of trigonocephaly which instead is frequently reported in patients with $9 \mathrm{q} 22.3$ deletion. $P T C H 1$, included in the region, is the only gene for which the deletion is known to represent most of the features of patients with this rearrangement. Our report reinforces the clinical utility of WES even in apparently non-syndromic trigonocephaly: in fact, the early diagnosis of PTCH1 variants allowed us the early diagnosis of Gorlin syndrome with consequent prompt surveillance, since approximately $5 \%$ of all children with variants in this gene develop medulloblastoma.

F. Di Giovanni: None. G. Gori: None. G. Traficante: None. L. Dosa: None. G. Forzano: None. E. Fiorentini: None. D. Vergani: None. S. Giglio: None.

\section{P16.75.C}

Genome-wide association study on glycation and its implication on the diagnosis of type II diabetes mellitus

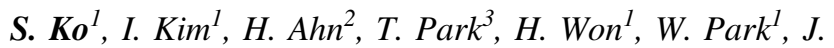 $\mathrm{Kim}^{4}$}

${ }^{1}$ Samsung Advanced Institute for Health Sciences \& Technology, Sungkyunkwan University, Seoul, Korea, Republic of, ${ }^{2}$ Department of Life Sciences, Pohang University of Science and Technology, Pohang, Korea, Republic of, ${ }^{3}$ Division of Cardiology, Department of Medicine, Heart, Stroke and Vascular Institute, Samsung Medical Center, Sungkyunkwan University School of Medicine, Seoul, Korea, Republic of, ${ }^{4}$ Samsung Genome Institute, Biomedical Research Institute, Samsung Medical Center, Sungkyunkwan University School of Medicine, Seoul, Korea, Republic of

For the detection of diabetes, screening for the level of glucose and glycated hemoglobin (HbAlc) in blood had been widely used. Several reports showed that HbA1c was more stable indicator of diabetes than blood glucose, since HbA1c level depicts the average of blood glucose in 2-3 months. However, it has not been critically tested whether genetic predisposition to glycation differs among individuals, which would alter the HbA1c levels given the same glucose levels. To answer this question, we here investigated $\sim 270 \mathrm{~K}$ individuals with 1,892 cases of type 2 diabetes (T2D) in UK Biobank for the blood glucose and HbA1c, and their associated single nucleotide polymorphisms (SNPs). We discovered that 2,349 SNPs were associated only with HbA1c level (dubbed as glycation SNPs), while 2,407 and 720 lead SNPs were associated with HbA1c and glucose, respectively. These glycation SNPs were related to genes such as SPTA1, SPTB, and ANK1, indicating their 
functional implication on glycation. We further built polygenic risk score (PRS) from the glycation SNPs, which performed as an indicator of the deviated $\mathrm{HbAlc}$ levels given the same glucose level across different individuals. In addition, we categorized individuals into several groups along with the PRS, and showed that those groups should have different criteria of $\mathrm{HbA1c}$ level to maintain equivalent sensitivity and precision of diabetes diagnosis. We believe that our approach provides insights into personalized diagnosis of complex diseases.

S. Ko: None. I. Kim: None. H. Ahn: None. T. Park: None. H. Won: None. W. Park: None. J. Kim: None.

P16.77.B

Applying PP1 to variants where internal cosegregation data exists at CHEOGenetics Diagnostic Laboratory

L. Little, L. Bronicki, O. Jarinova, A. Smith, L. Huang, J. McGowan-Jordan

\section{CHEO, Ottawa, ON, Canada}

Introduction: The "PP1" variant classification criteria created by the American College of Medical Genetics and Genomics (ACMG) and Association of Molecular Pathology (AMP) can be used as supporting, moderate or strong level of evidence towards pathogenicity if a variant cosegregates with disease in multiple affected family members in a gene definitively known to cause the disease. Application of PP1 has improved the quality of variant interpretation if evidence towards cosegregation exists in the literature. However, the CHEO Genetics Diagnostic Laboratory does not currently apply PP1 when cosegregation of a variant is observed in a family tested internally. Several obstacles were identified in implementing the use of PP1 for internal data, including the statistical evaluation of cosegregation, which is not easily computable by most laboratory personnel. Therefore, a protocol was designed to address these obstacles and create a standardized process.

Materials and Methods: Specific cutoffs for pathogenicity were chosen, as informed by Jarvik et al. (2016). A retroactive chart review was performed on variants where $\geq 2$ family members were tested internally. PP1 was applied when appropriate.

Preliminary Results: PP1 was applied to $3 / 3$ likely pathogenic (LP) variants and 7/11 variants of uncertain clinical significance (VUS). Of these, all LP variants were upgraded to pathogenic and 5 VUS's were upgraded to likely pathogenic.

Conclusions: Using a standardized protocol for applying PP1 when evidence of cosegregation exists internally has improved the overall quality of variant interpretation at
CHEO Genetics Diagnostic Laboratory, and can alter clinical management for patients and their families.

L. Little: None. L. Bronicki: None. O. Jarinova: None. A. Smith: None. L. Huang: None. J. McGowanJordan: None.

P16.78.C

PanelApp: accelerating international consensus on virtual gene panels through collaboration on a federated open platform

Z. Stark ${ }^{1,2,3}$, R. E. Foulger ${ }^{4,5}$, E. Williams ${ }^{4,5}$, B. Thompson ${ }^{6}$, C. Patel ${ }^{7}$, I. U. S. Leong ${ }^{4}$, L. C. Daugherty ${ }^{4,5}$, S. Leigh $^{4}$, C.

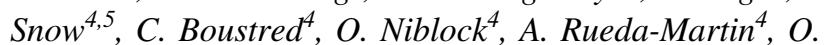
Gerasimenko $^{4}$, E. Ivanov ${ }^{4}$, K. Savage ${ }^{4}$, W. Bellamy ${ }^{4}$, V. San

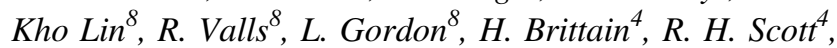
E. R. A. Thomas ${ }^{4}$, A. de Burca ${ }^{4}$, A. L. T. Tavares ${ }^{4}, Z$. Hyder $^{4}$, M. McEntagart ${ }^{4}$, C. Turnbull ${ }^{4}$, S. M. White ${ }^{1,3}, T . Y$. Tan $^{1,3}$, A. Yeung ${ }^{1}$, L. Downie ${ }^{1,3}$, S. Lunke ${ }^{1,3}$, J. Deller ${ }^{9}$, Z. C. Deans $^{9,10}$, S. L. Hill', M. J. Caulfield ${ }^{4}$, K. N. North ${ }^{11,2,3}$, A. Rendon $^{4,5}$, O. Hofmann ${ }^{8,2}$, E. M. McDonagh ${ }^{4}$

${ }^{I}$ Victorian Clinical Genetics Services, Melbourne, Australia, ${ }^{2}$ Australian Genomics Health Alliance, Melbourne, Australia, ${ }^{3}$ University of Melbourne, Melbourne, Australia, ${ }^{4}$ Genomics England, London, United Kingdom, ${ }^{5}$ Genomics England Biodata Innovation Centre, Cambridge, United Kingdom, ${ }^{6}$ Royal Melbourne Hospital, Melbourne, Australia, ${ }^{7}$ Genetic Health Queensland, Brisbane, Australia, ${ }^{8}$ Centre for Cancer Research, University of Melbourne, Victorian Comprehensive Cancer Centre, Melbourne, Australia, ${ }^{9}$ National Health Service England, London, United Kingdom, ${ }^{10}$ National Health Service Improvement, London, United Kingdom, ${ }^{11}$ Murdoch Children's Research Institute, Melbourne, Australia

Introduction: Robust gene-disease validity assessments are the foundation of accurate variant interpretation in diagnostic genomics. Virtual gene panels for specific clinical indications improve the efficiency of genomic analysis, yet there is wide variability in the processes for panel creation and maintenance, and in panel content.

Methods: PanelApp is an open platform for virtual gene panel curation that captures structured gene-disease validity assessments, with evidence thresholds governing gene inclusion in panels. Developed by Genomics England for the 100,000 Genomes Project, it is now used by the NHS Genomic Medicine Service, and has been deployed by Australian Genomics for use by Australian diagnostic laboratories. We have established a collaboration to enable technical co-development and systematic comparison of virtual panels between the two PanelApps, sharing expert reviews to accelerate discordance resolution. 
Results: Of the 511 virtual gene panels in the two PanelApps, 79 panel pairs cover directly comparable clinical indications; 182 panels are partially comparable; 171 panels are unique. Of the first 20 panels compared, 709 discrepant genes were identified. Sharing expert reviews has resulted in inclusion of 285 new genes for diagnostic use, and removal of 186, with curation ongoing. Common reasons for discrepant gene ratings include differences in panel scope definition; differential weighting of functional evidence; and challenges in systematic timely identification of new evidence establishing or refuting gene-disease associations.

Conclusions: We have accelerated improvements in evidence-based diagnostic genomics in two healthcare systems through a federated open platform that supports shared expert curation of virtual gene panels, while retaining separate governance.

Z. Stark: None. R.E. Foulger: None. E. Williams: None. B. Thompson: None. C. Patel: None. I.U.S. Leong: None. L.C. Daugherty: None. S. Leigh: None. C. Snow: None. C. Boustred: None. O. Niblock: None. A. RuedaMartin: None. O. Gerasimenko: None. E. Ivanov: None. K. Savage: None. W. Bellamy: None. V. San Kho Lin: None. R. Valls: None. L. Gordon: None. H. Brittain: None. R.H. Scott: None. E.R.A. Thomas: None. A. de Burca: None. A.L.T. Tavares: None. Z. Hyder: None. M. McEntagart: None. C. Turnbull: None. S.M. White: None. T.Y. Tan: None. A. Yeung: None. L. Downie: None. S. Lunke: None. J. Deller: None. Z.C. Deans: None. S.L. Hill: None. M.J. Caulfield: None. K.N. North: None. A. Rendon: None. O. Hofmann: None. E.M. McDonagh: None.

\section{P16.79.A}

The influence of storage conditions on WBCs transcriptome profiles

X. Yanru ${ }^{1,2}, Y . X i^{2}$, C. Haixiao ${ }^{2}$, Z. Sujun ${ }^{3}$, X. Jinjin ${ }^{2}$, C.

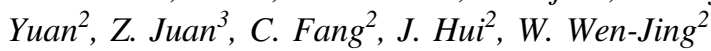

${ }^{I}$ School of Future Technology, University of Chinese Academy of Sciences, Beijing, China, ${ }^{2}$ BGI-Shenzhen, Shenzhen, China, ${ }^{3}$ Obstetrics Department, Shenzhen Maternity and Child Healthcare Hospital, Shenzhen, China

Introduction: Transcriptome levels of white blood cells (WBCs) play an important role in disease diagnosis, prognosis and biomarker discovery research. Peripheral blood is usually collected and treated for further experiments, but the effect of storage conditions on WBCs transcriptome remains largely unknown.

Methods: Blood samples from healthy donors were drawn into EDTA tubes and stored at both $4^{\circ} \mathrm{C}$ and room temperature
(RT, $18-22^{\circ} \mathrm{C}$ ) for $0 \mathrm{~h}, 2 \mathrm{~h}, 6 \mathrm{~h}$ and $24 \mathrm{~h}$. Total cellular RNA was extracted from WBCs after red blood cells lysis, and transcriptome profiles were assessed by RNA sequencing.

Results: Compared with samples treated immediately, the proportion of neutrophils decreased, but that of $\mathrm{T}$ cells increased in the samples stored at RT for 24h. Samples maintained at $4^{\circ} \mathrm{C}$ were less influenced than that at RT within $24 \mathrm{~h}$. We identified 1428 up-regulated and 86 downregulated genes at $4^{\circ} \mathrm{C}$, while there were 4493 genes upregulated and 5982 genes down-regulated at RT. Upregulated genes at $4^{\circ} \mathrm{C}$ were mostly related to HDACs deacetylate histones, and that at RT were mostly related to mRNA processing. Genes down-regulated at RT were mostly due to leukocyte activation involved in immune response. Reference genes such as CHMP2A and PSMB4 were relatively stable, while the expression of IL1RN, which was similar in both men and women, increased more in women than men after $24 \mathrm{~h}$ stored at RT.

Conclusions: We provide a full-scale ex vivo change of WBCs expression profiles, and suggest that these influences should not be ignored in relative studies.

Funding: Shenzhen Municipal Government of China (No.JCYJ20170412152854656).

X. Yanru: None. Y. Xi: None. C. Haixiao: None. Z. Sujun: None. X. Jinjin: None. C. Yuan: None. Z. Juan: None. C. Fang: None. J. Hui: None. W. Wen-Jing: None.

\section{P16.80.B}

Modelling diagnostic yield in the 100,000 Genomes Rare Disease Project using panel-based analysis and complementary approaches

E. R. A. Thomas ${ }^{1,2,3}$, D. Pasko ${ }^{l}$, C. Boustred ${ }^{l}$, D. Kasperaviciute $^{1,2}$, M. Bleda ${ }^{1}$, A. Rueda Martin ${ }^{1}$, D. Smedley $^{1,2}$, E. McDonagh ${ }^{1}$, H. Brittain ${ }^{1,4}$, A. Tucci, ${ }^{1,5}, Z$. Deans $^{6}$, S. L. Hill ${ }^{6}$, T. Fowler ${ }^{1,2}$, A. Rendon ${ }^{1,7}$, M. J. Caulfield $^{1,2}$, R. H. Scott ${ }^{1,2,8}$, Genomics England Research Consortium

${ }^{1}$ Genomics England, London, United Kingdom, ${ }^{2}$ Queen Mary University of London, London, United Kingdom, ${ }^{3}$ Imperial College Healthcare NHS Trust, London, United Kingdom, 'Birmingham Women's and Children's Hospital, Birmingham, United Kingdom, ${ }^{5}$ University College London, London, United Kingdom, ${ }^{6}$ NHS England Genomics Unit, London, United Kingdom, ${ }^{7}$ University of Cambridge, Cambridge, United Kingdom, ${ }^{8}$ Great Ormond Street Hospital, London, United Kingdom

Background: The 100,000 Genomes Project Rare Disease programme developed a gene panel-based analysis strategy aiming to maximise specificity and sensitivity of automated variant prioritisation before manual review by NHS clinical 
scientists. HPO terms were collected prior to analysis. Over 35,000 families have been analysed using this approach.

Methods: Using $>950$ confirmed genetic diagnoses in patients with intellectual disability, we modelled the outcome of different strategies for automated prioritisation and manual review, focused on diagnostic sensitivity.

Results: $87 \%$ of genetic diagnoses were prioritised using a single gene panel selected for the original diagnostic hypothesis. The primary reason for non-prioritisation was that the causative gene was in a different panel; sensitivity increased to $92 \%$ using automated HPO-based rules to apply additional panels.

Neuromuscular disorders (analysed with smaller phenotype-specific panels) gave a panel-based yield of $38 \%$, increasing to $71 \%$ with one broad panel.

Diagnoses of metabolic and mitochondrial conditions, some treatable, were made where these conditions were compatible with the phenotype data but outside the gene panel for the primary diagnostic hypothesis.

Of the $13 \%$ with non-prioritised diagnoses, $72 \%$ were trios or larger family structures; we will discuss the impact of family structure on diagnostic yield.

For families where trio testing is not possible, phenotypebased variant ranking algorithms such as Exomiser contribute.

Conclusions: No single analysis strategy can identify all diagnoses. Collecting detailed phenotype data at the time of test initiation permits a multi-faceted strategy combining broad gene panels, bespoke panel assignment, phenotypebased prioritisation, and gene agnostic analysis, to maximise diagnostic yield.

E.R.A. Thomas: A. Employment (full or part-time); Significant; Genomics England. D. Pasko: A. Employment (full or part-time); Significant; Genomics England. C. Boustred: A. Employment (full or part-time); Significant; Genomics England. D. Kasperaviciute: A. Employment (full or part-time); Significant; Genomics England. M. Bleda: A. Employment (full or part-time); Significant; Gen. A. Rueda Martin: A. Employment (full or part-time); Significant; Genomics England. D. Smedley: A. Employment (full or part-time); Significant; Genomics England. E. McDonagh: A. Employment (full or part-time); Significant; Genomics England. H. Brittain: A. Employment (full or part-time); Significant; Genomics England. A. Tucci: None. Z. Deans: None. S.L. Hill: None. T. Fowler: A. Employment (full or part-time); Significant; Genomics England. A. Rendon: A. Employment (full or part-time); Significant; Genomics England. M.J. Caulfield: A. Employment (full or part-time); Significant; Genomics England. R.H. Scott: A. Employment (full or part-time); Significant; Genomics England.
P16.81.C

Validation of Clinically Relevant Variant Detection from Whole Genome Sequencing for NHS England's Genomic Medicine Service

D. Kasperaviciute ${ }^{l, 2}$, K. R. Smith ${ }^{1,2}$, K. Ibanez ${ }^{l, 2}$, D. Pasko $^{l}$, M. Bleda ${ }^{l}$, S. Walker ${ }^{l}$, D. Polychronopoulos ${ }^{l}$, A. Rueda Martin $^{l}$, M. Kayikci ${ }^{l}$, J. Lopez ${ }^{l}$, E. Thomas ${ }^{1,2,3}$, E. Baple ${ }^{l}$, A. Tucci ${ }^{1,2}$, R. H. Scott ${ }^{1,4}$, J. Polke ${ }^{5}$, R. Labrum ${ }^{5}$, L. Santos ${ }^{5}$, K. Eggleton ${ }^{5}$, D. Mcmullan ${ }^{6}$, D. Morrogh ${ }^{7}, J . W . A h n^{8}, Z$. Deans $^{9}$, S. Hill', M. J. Caulfield ${ }^{1,2}$, A. Rendon ${ }^{1,10}$, Genomics England Research Consortium

${ }^{1}$ Genomics England, London, United Kingdom, ${ }^{2}$ Queen Mary University of London, London, United Kingdom, ${ }^{3}$ Imperial College Healthcare NHS Trust, London, United Kingdom, ${ }^{4}$ Great Ormond Street Hospital, London, United Kingdom, ${ }^{5}$ Neurogenetics Unit UCL Institute of Neurology, London, United Kingdom, ${ }^{6}$ West Midlands Regional Genetics Laboratory, Birmingham Women's and Children's Foundation Trust, Birmingham, United Kingdom, ${ }^{7}$ North East Thames Regional Genetics Service, Great Ormond Street Hospital for Children, NHS Foundation Trust, London, United Kingdom, ${ }^{8}$ East Midlands and East of England NHS Genomic Laboratory Hub, Cambridge University Hospitals NHS Foundation Trust, Cambridge, United Kingdom, ${ }^{9}$ NHS England, London, United Kingdom, ${ }^{10}$ Department of Haematology, University of Cambridge, Cambridge, United Kingdom

Introduction: Standard benchmarking approaches for whole genome sequencing (WGS) variant detection are not sufficiently informative for rare pathogenic variants that fall outside "high confidence" regions or represent classes of variation absent from the truth sets. While developing the new WGS pipeline as part of a new genomic medicine service for England's National Health Service, we validated the variant detection of other diagnostically important classes of variants.

Materials and Methods: The detection of diagnostic variants was assessed using genome data from 100,000 Genomes project participants with diagnostic variants detected and/or confirmed by orthogonal methods.

Results: The sensitivity of diagnostic variants detection was as following: de novo small variants $99.4 \%$ (CI 98.43$99.96 \%, N=354$ ), mitochondrial small variants $100 \%$ (CI, $82.92-100 \%, N=10$ ), pathogenic CNVs $>10 \mathrm{~kb}$ size $97.1 \%$ (CI 93.71-99.28\%, $N=136$ ). The STR detection of full expansions in 13 loci reached sensitivity and precision of $100 \%$ (CI $88.8-100 \%, 16$ positive and 428 negative controls), however, the sensitivity was tested in controls identified by the earlier version of the WGS pipeline and confirmed by orthogonal methods, and therefore may be 
overestimated. We further show high reproducibility of CNV and STR calling using 80 monozygotic twin pairs.

Conclusions: We show high sensitivity, and, where possible, precision, of our rare disease pipeline to identify clinically relevant variants in families with rare disease. We also present a framework for validation of WGS pipelines for clinical service, and highlight the challenges posed by the lack of unbiased truth sets of diagnostically relevant variants.

D. Kasperaviciute: A. Employment (full or part-time); Significant; Genomics England. K.R. Smith: A. Employment (full or part-time); Significant; Genomics England. K. Ibanez: A. Employment (full or part-time); Significant; Genomics England. D. Pasko: A. Employment (full or parttime); Significant; Genomics England. M. Bleda: A. Employment (full or part-time); Significant; Genomics England. S. Walker: A. Employment (full or part-time); Significant; Genomics England. D. Polychronopoulos: A. Employment (full or part-time); Significant; Genomics England. A. Rueda Martin: A. Employment (full or parttime); Significant; Genomics England. M. Kayikci: A. Employment (full or part-time); Significant; Genomics England. J. Lopez: A. Employment (full or part-time); Significant; Genomics England. E. Thomas: A. Employment (full or part-time); Significant; Genomics England. E. Baple: A. Employment (full or part-time); Significant; Genomics England. A. Tucci: A. Employment (full or part-time); Significant; Genomics England. R.H. Scott: A. Employment (full or part-time); Significant; Genomics England. J. Polke: None. R. Labrum: None. L. Santos: None. K. Eggleton: None. D. Mcmullan: None. D. Morrogh: None. J.W. Ahn: None. Z. Deans: None. S. Hill: None. M.J. Caulfield: A. Employment (full or part-time); Significant; Genomics England. A. Rendon: A. Employment (full or part-time); Significant; Genomics England.

\section{P16.82.A}

The contribution of whole genome sequencing in establishment of genetic diagnosis in undiagnosed patients with rare genetic disorders

\section{A. Maver, G. Bergant, B. Peterlin}

Clinical institute for Medical Genomics, Ljubljana, Slovenia

A significant proportion of patients with rare genetic disorders remain undiagnosed despite comprehensive genetic testing using exome sequencing (ES). It is known that pathogenic genetic variants may reside in non-coding regions or may arise due to mutational mechanisms not detectable using ES. We therefore aimed to characterize the utility of whole genome sequencing (WGS) in diagnosing exome-negative patients. We performed WGS in a 30 patients with negative ES result and high suspicion of genetic etiology based on one of the following inclusion criterions: (1) observation of multiple affected family members, (2) a specific diagnosis with high probability of genetic etiology, (3) presence of significant blocks of homozygosity, or (4) observation a single pathogenic variant in a gene associated with recessive inheritance. We developed an in-house pipeline for analysis of sequence variation in WGS and have extended it with approaches for analysing structural variation (including translocations and retrotransposition events), polynucleotide expansions, mitochondrial variation and artificial intelligence-based splice variant effect prediction approaches. Among 30 selected patients, we identified the molecular cause in 9 (30.0\%) using WGS. We detected deep intronic pathogenic variants in 2 patients, a pathogenic promoter variant in 1 patient, a small structural variant in 1 patient, a complex rearrangement in 1 patient, a translocation in 1 patient, polynucleotide expansions in 2 patients and a retrotransposition event in 1 patient. The results of our study indicate that WGS may represent a valuable approach to establish a diagnosis in a carefully selected subset of patients with a negative ES result.

A. Maver: None. G. Bergant: None. B. Peterlin: None.

\section{P17 Bioinformatics and Statistical Methods}

\section{P17.002.C}

A convolutional neural network based model to predict allele-specific expression by regulatory sequence

\section{Z. Zhang ${ }^{1,2}$, J. van der Velde ${ }^{1,2}$, GoNL consortium, BIOS} consortium, R. Sinke ${ }^{l}$, M. Swterz ${ }^{1,2}$

${ }^{1}$ University Medical Center Groningen, Groningen, Netherlands, ${ }^{2}$ Genomics Coordination Centre, Groningen, Netherlands

Allele-specific expression (ASE) refers to the imbalanced abundance of allelic copies from one transcript. It is the consequence of many regulation events such as cisregulatory variants. Recent studies imply its potential roles in diseases and practical value in genome diagnostics. However, routine genome diagnostics heavily depend on DNA sequencing and usually ignore the regulation of gene expression which can be indicated by ASE. Therefore, a well-developed prediction model is capable to attribute the value of ASE to the successful genome diagnosis.

We here report on a deep learning model built to understand how the ASE effects of a transcript associated 
with the adjacent regulatory elements using NGS DNA haplotypes obtained from the Genome of the Netherlands project and RNA sequencing results from Biobank-based Integrative Omics Studies. Then, given a transcript, for paternal and maternal alleles, the ASE effects are quantified from the RNA sequencing results and potential regulatory sequences are acquired from whole-genome sequencing results. Finally, we are piloting a convolutional neural network, which values the objects in an image-like twodimension matrix (in our case, it is the regulatory sequence) to distinguish one from the other. We also plan to apply the model on diseases-related genes or transcripts to evaluate its added value to the diagnosis. Ideally, the model can contribute to the genome diagnostics and general biological studies by supplying functional interpretation that exploits expression evidence. This study is supported by the Chinese Scholarship Consortium and NWO-VIDI grant.

Z. Zhang: None. J. van der Velde: None. R. Sinke: None. M. Swterz: None.

\section{P17.005.C}

Conditional estimation of the contribution of genomic annotations to common complex diseases

\author{
M. Patxot ${ }^{l}$, A. Kousathanas ${ }^{\text {, S. E. Ojavee }}{ }^{I}$, D. Trejo \\ Banos $^{l}$, M. R. Robinson ${ }^{2}$ \\ ${ }^{1}$ University of Lausanne, Lausanne, Switzerland, ${ }^{2}$ Institute \\ of Science and Techonology Austria, Vienna, Austria
}

Despite efforts to estimate the contribution of genomic regions to common complex trait variation, the distribution of effect sizes across functional annotations remains unknown. Previous studies estimate enrichment across annotations in a series of follow-up analyses rather than utilizing functional information to assess enrichment conditional on the rest of the genome.

Here, we introduce a scalable Bayesian model that utilizes genomic annotations and individual-level data to jointly estimate marker effects while accounting for LD. SNP effects for each annotation group are modelled as a series of normal distributions providing inference on the genetic architecture of genomic enrichment. Effect size distributions can be compared across annotations and the genetic variance for each group simultaneously quantified.

In simulations we demonstrate our method gives unbiased estimates of annotation enrichment under extreme LD scenarios. We apply the model to height, body mass index (BMI), type-2-diabetes (T2D) and coronary artery disease (CAD) in the UK Biobank. We find a stark contrast between BMI and height: BMI being highly polygenic with tiny effect sizes located 10 to $1000 \mathrm{~kb}$ from exons whereas height shows a variety of effect sizes in coding and non- coding regions. T2D and CAD are highly polygenic and we also observe that promoter regions contribute less than $5 \%$ to the phenotypic variance of all four traits.

This method provides a full quantification of genetic architectures underlying complex traits and determines which genomic regions are influential, improving disease risk prediction.

This work was funded by an SNSF Eccellenza Grant (PCEGP3_181181) to M.R.R.

M. Patxot: None. A. Kousathanas: None. S.E. Ojavee: None. D. Trejo Banos: None. M.R. Robinson: None.

\section{P17.006.A}

Automated re-analysis of over 7,500 exomes and genomes within Solve-RD demonstrates its power to deliver diagnosis for rare disease patients

L. Matalonga ${ }^{1}$, D. Danis ${ }^{2}, S . L^{3}$, D. Piscia ${ }^{l}, C$. Hernandez $^{l}$, R. Tonda ${ }^{l}$, S. Laurie ${ }^{l}$, I. Gut ${ }^{4}$, K. van der Velde $^{3}$, A. Topf ${ }^{5}$, R. Schüle ${ }^{6}$, M. Synofzik ${ }^{6}$, L. Vissers ${ }^{7}$, R. de

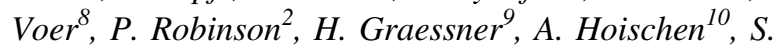
Beltran $^{4}$

${ }^{1}$ Centro Nacional de Análisis Genómico (CNAG-CRG), Center for Genomic Regulation, Barcelona Institute of Science and Technology (BIST), Barcelona, Spain, Barcelona, Spain, ${ }^{2}$ The Jackson Laboratory for Genomic Medicine, Farmington, CT 06032, USA, Farmington, CT, United States, ${ }^{3}$ Genomics Coordination Center, University Medical Centre Groningen, Groningen, The Netherlands, Groningen, Netherlands, ${ }^{4}$ Centro Nacional de Análisis Genómico (CNAG-CRG), Center for Genomic Regulation, Barcelona Institute of Science and Technology (BIST), Barcelona, Spain. Universitat Pompeu Fabra (UPF), Barcelona, Spain, Barcelona, Spain, ${ }^{5}$ John Walton Muscular Dystrophy Research Centre, Translational and Clinical Research Institute, Newcastle University, Newcastle upon Tyne, UK, Newcastle, United Kingdom, ${ }^{6}$ Department of Neurodegenerative Diseases, Hertie-Institute for Clinical Brain Research and Center of Neurology, University of Tübingen, Tübingen, Germany; German Center for Neurodegenerative Diseases (DZNE), University of Tübingen, Tübingen, Germany, Tübingen, Germany, ${ }^{7}$ Department of Human Genetics, Donders Centre for Neuroscience, Radboud University Medical Center, Nijmegen, The Netherlands, Nijmegen, Netherlands, ${ }^{8}$ Department of Human Genetics, Radboud Institute for Molecular Life Sciences, Radboud University Medical Center, Nijmegen, The Netherlands, Nijmegen, Netherlands, ${ }^{9}$ Institute for Medical Genetics and Applied Genomics, University of Tübingen, Tübingen, Germany; Centre for Rare Diseases, University Hospital of Tübingen, Tübingen, Germany; European Reference Network for Rare Neurological Diseases, 
Tübingen, Germany, ${ }^{10}$ Department of Human Genetics, Radboud University Medical Center, Nijmegen, The Netherlands. Radboud Expertise Center for Immunodeficiency and Autoinflammation, Department of Internal Medicine, Radboud University Medical Center, Nijmegen, The Netherlands., Nijmegen, Netherlands

Exome and genome sequencing is a cost-effective approach to shorten the time to diagnosis for rare disease patients. Data analysis and interpretation is one of the main challenges and still many cases remain unsolved. However, scientific knowledge evolves rapidly and periodic data reanalysis has been shown to increase the diagnostic yield by up to $5-10 \%$.We have reanalysed 7,495 genomic datasets from unsolved rare disease patients and relatives submitted in the context of the "Solve-RD - solving the unsolved rare diseases" project. Data was processed through the RD-Connect Genome-Phenome Analysis platform (GPAP: https://platform.rd-connect.eu/), and reanalysed using different innovative bioinformatics approaches that enable variant filtering and prioritisation combining genomic and clinical data: i) VFPS, a programmatic approach integrated with the GPAP ii) Exomiser, a tool for phenotypedriven prioritisation of disease-causing variants and iii) CAPICE, a computational method for consequence-agnostic pathogenicity estimation. A consensus output with a minimal set of variant annotations has been defined for all approaches to further facilitate data interpretation and results feedback. Candidate variants are made available in a secured cloud to clinical researchers of the four participating European Reference Networks (euro-NMD, ITHACA, RND and GENTURIS) for data interpretation. Preliminary results on the VFPS approach, where more than 2500 candidate variants from 1700 patients have been returned, already provided diagnosis for $3-8 \%$ of patients. Solved cases are being used to train and improve the tools' algorithms. Solve-RD (H2020-779257)

L. Matalonga: None. D. Danis: None. S. Li: None. D. Piscia: None. C. Hernandez: None. R. Tonda: None. S. Laurie: None. I. Gut: None. K. van der Velde: None. A. Topf: None. R. Schüle: None. M. Synofzik: None. L. Vissers: None. R. de Voer: None. P. Robinson: None. H. Graessner: None. A. Hoischen: None. S. Beltran: None.

\section{P17.007.B}

GenerAVI: Variant Interpreter and Genetic Analysis Summary Generator

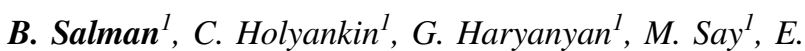 \\ Celik $^{1}$, D. Bozkulak ${ }^{1}$, S. A. U. Iseri ${ }^{2}$
}

${ }^{I}$ Gen Era Diagnostics, Bioinformatics Department, Istanbul, Turkey, ${ }^{2}$ Istanbul University, Aziz Sancar DETAE, Istanbul, Turkey
One of the most challenging tasks in the area of clinical genetics is to determine the pathogenicity of genetic variations and provide standardized clinical evaluation for the genetic data. Therefore, we have developed the GenerAVI. v2 tool for the automatic interpretation of bulk variants by American College of Genetics and Genomics (ACMG) criteria. The GeneraAVI.v2 also allows users to make their own interpretation and stores them in a disease-specific manner. The GenerAVI.v2 is developed using $\mathrm{C}++$ and Python. The platform uses vcf files as the file input. After preliminary filtering based on quality and previously known false-positive calls, all variants are annotated using Ensembl Variant Effect Predictor. Disease-specific allele frequency, relative position on the gene, in silico pathogenicity tool metrics, ClinVar records, and more parameters are used for automated ACMG classifications. GeneraAVI.v2 tool can automatically evaluate and report all variants detected in terms of 15 known ACMG criteria. It also analyzes by modified PVS1 criteria which are based on the nonmediated-decay pathway and alternative splicing events. It also allows users to make their own assessments for criteria that cannot be automated. The tool keeps track of each curation made and uses these evaluations in future analysis. The GenerAVI.v2 tool helps researchers and clinicians to easily create genetic analysis reports with automated ACMG classifications and generate dynamic internal frequency and clinical interpretation database.

B. Salman: None. C. Holyankin: None. G. Haryanyan: None. M. Say: None. E. Celik: None. D. Bozkulak: None. S.A.U. Iseri: None.

\section{P17.010.B}

Mobile elements identification in genes from exome sequencing data of patients affected with developmental and/or neurological abnormalities

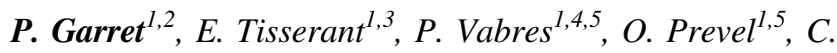 Philippe $^{1,3}$, A. Boughalem ${ }^{2}, J$. Costa ${ }^{2}$, D. Trost ${ }^{2}$, A. Vitobello $^{1,3}$, L. Faivre ${ }^{1,6}$, C. Thauvin-Robinet ${ }^{1,3,7}$, Y. Duffourd $^{1,3}$

${ }^{1}$ UMR1231 GAD, Inserm, 21070 Dijon, France, ${ }^{2}$ Laboratoire CERBA, Saint-Ouen l'Aumône, France, ${ }^{3}$ Unité Fonctionnelle Innovation en Diagnostic génomique des maladies rares - FHU-TRANSLAD - Dijon University Hospital, Dijon, France, ${ }^{4}$ Centre de Référence maladies rares «maladies dermatologiques en mosaïque »-service de dermatologie - FHU-TRANSLAD - Dijon University Hospital, Dijon, France, ${ }^{5}$ Service Dermatologie - Dijon University Hospital, Dijon, France, ${ }^{6}$ Centre de Référence maladies rares "Anomalies du développement et syndromes malformatifs »- centre de génétique - FHU- 
TRANSLAD - Dijon University Hospital, Dijon, France, ${ }^{7}$ Centre de Référence maladies rares "déficiences intellectuelles de cause rare »- centre de génétique - FHUTRANSLAD - Dijon University Hospital, Dijon, France

Introduction: About $27 \%$ of the human genome is composed of mobile elements (MEs), which can create genomic instability leading to genetics diseases. The mechanism of LINE1, Alu or SVA insertion has been described in several pathologies. It has been estimated that about $0.3 \%$ of all mutations are due to de novo insertion. Nowadays, the massive development of high-throughput sequencing provides the opportunity to identify new MEs insertions on large scale.

Methodology: Exome sequencing (ES) data from patients affected with developmental and/or neurological abnormalities has been analyzed by MELT, a tool identifying MEs insertions. The results were filtered by frequency, impacted region and gene function.

Results: ES data from 3,322 individuals (2,500 probands) were analyzed. 496,312 possible MEs were detected. After filtering, 516 probands with 1,070 rare MEs in non-intronic regions were obtained. Following phenotype comparison and PCR validation, 3 candidates were kept. The most convincing was found in a suspected consanguineous patient referred for poikilodermia. An homozygous insertion was identified in FERMT1 gene involved in Kindler syndrome, a condition belonging to the class of poikilodermia. In two other patients, an exonic and a 3'UTR insertions were identified in 2 genes involved respectively in mental retardation/epileptic encephalopathy and epilepsy. RNA-Seq analyzes are ongoing to study transcriptional consequences.

Conclusions: This project aims to identify new insertions in genes and highlight the interest to include MEs detection in ES pipeline to reduce diagnostic wavering. This work on preexisting ES data represents an additional argument in favor of ES suitability as an unique exam.

P. Garret: None. E. Tisserant: None. P. Vabres: None. O. Prevel: None. C. Philippe: None. A. Boughalem: None. J. Costa: None. D. Trost: None. A. Vitobello: None. L. Faivre: None. C. Thauvin-Robinet: None. Y. Duffourd: None.

P17.011.C

Whole-genome sequencing and bioinformatics analysis of M.tuberculosis multi-drug resistant strains from Kazakhstan

\section{U. Kairov}

Lab of Bioinformatics and Systems Biology, CLS, NLA, Nur-Sultan, Kazakhstan

1 Laboratory of Bioinformatics and Systems Biology, CLS,
NLA, Nazarbayev University, Nur-Sultan, Kazakhstan.2 Laboratory of Plants Genomics and Bioinformatics, NCB, Nur-Sultan, Kazakhstan.3 Laboratory of Genomic and Personalized Medicine, CLS, NLA, Nazarbayev University, Nur-Sultan, Kazakhstan

Introduction: Tuberculosis (TB) is an infectious disease of bacterial etiology, remains a significant public health problem worldwide. Drug-resistant TB is a public health problem in the Kazakhstan and drug susceptibility testing and genotyping methods of M.tuberculosis (MTB) play important role in tuberculosis treatment and control for TB transmission. It is extremely important to determinate susceptible and resistant strains with different mutations in genes encoding drug metabolism of MTB strains in Kazakhstan.

Materials and Methods: The whole genome sequencing data from 8 multidrug-resistant (MDR) clinical isolates of MTB were examined.

Results: Known pathogenic and novel variants were found. Genomic variants (SNPs and InDels) in de novo assembled and annotated whole-genomes and specific/novel variants in drug-resistant genes of MDR strains from Kazakhstan were observed. MDR clinical isolates of the Beijing family and their complete genomes were studied for the first time in Kazakhstan.

Conclusions: These findings may provide a basis for expansion of the reference MTB database, and further investigation of virulence and transmissibility patterns in MDR strains. This study may provide a basis for creation of the reference database, the subsequent study, and comparison with the different drug-resistant isolates circulating in Kazakhstan. This work highlights the importance of expanded targets for drug resistance detection in MTB isolates.

Grant references: AP05134737; AP05136106 of MES RK.

U. Kairov: None.

\section{P17.012.A}

Multi-dimensional biomarker machine learning tool for immune-oncology: combinatorial gene expression predict patient's survivability

\section{P. Stempor ${ }^{1,2}$, P. Dobosz $z^{3,4,2}$}

${ }^{I}$ The Gurdon Institute, University of Cambridge, Cambridge, United Kingdom, ${ }^{2}$ SmartImmune LTD, Cambridge, United Kingdom, ${ }^{3}$ Department of Haematology, Oncology and Internal Diseases, Medical University of Warsaw, Warsaw, Poland, ${ }^{4}$ Paediatric Haemato-Oncology, Oncology Department, Cancer Research Centre, Sheba Medical Centre Hospital, Ramat Gan, Israel 
Introduction: Checkpoint blockade immunotherapy, which utilizes immune system to selectively destroy tumour cells, becomes an effective treatment for cancer. However, success rate is low: many patients do not respond, whereas others suffer from adverse effects. Immune response is mediated by immunological synapse - checkpoint proteins interface between lymphocytes and antigen presenting cells. Understanding complex interactions within immunological synapse will yield new biomarkers, with application to clinical trials optimization, companion diagnostics to existing drugs and better combination therapies.

Materials and Methods: We developed a computational method that infers gene interaction networks from expression profiles in large cohorts of patients using unsupervised Bayesian machine learning model. Our method also reduces the dimensionality of data producing factors that represent differences between cancer sub-types and individual patients. We apply this method to bladder and kidney carcinoma TCGA cohorts.

Results and Conclusions: We used the panel of 21 genes expressed on cancer side of immunological synapse and 3 transcription factors that regulate them. We found that a combinatorial markers have significantly better predictive power for patient survivability rate in comparison to single gene expression: $p$-value of 0.0002 for KIRC and 0.0076 BLCA cohorts. Further, we build de-novo gene interactions networks from expression data and analysed influence (loadings) of genes on our combinatorial model in context of other co-expressed genes. Combinations containing NFE2L2, ICOSLG and PD-L1 are particularly predictive in KIRC, and NFE2L2 and VISTA in BLCA. Hence, we show utility of machine learning tools to generate combinatorial, multi-dimensional biomarkers from gene expression measurements.

GrantRefs: https://orcid.org/0000-0002-9464-7475

P. Stempor: E. Ownership Interest (stock, stock options, patent or other intellectual property); Modest; SmartImmune LTD. P. Dobosz: E. Ownership Interest (stock, stock options, patent or other intellectual property); Modest; SmartImmune LTD.

\section{P17.013.B}

Softclipping of gene specific primers after primer extension enrichment to remove artificial sequences from downstream analysis

\section{J. Rehker, M. Ball, C. Heydt, R. Büttner, S. Merkelbach- Bruse}

\section{University Hospital Cologne, Cologne, Germany}

Primer based amplification of genomic target regions suffers from the fact that the primer sequence is initially indistinguishable from amplified captured sequence of the specimen. This might result in artifacts and undetected variants when amplicons overlap each other as variant callers take both into account, thereby reporting either incorrect allelic fractions or not calling the variant at all. Multiplex PCR as well as primer extension enrichment suffer from this drawback. Multiple tools are available that trim adapters from raw sequence. However there is an advantage to soft clip primers after alignment as it allows for more accurate indel detection at the ends of a captured fragment. Multiple open source tools are available for soft clipping of PCR primers based on the alignment start/end position, but they do not seem applicable for primer extension enrichment. We therefore developed PESC (Primer Extension Soft Clipper). It matches the starting position and length of the primer to the alignment of read one. The primer is softclipped. The tool then continues to check the end of read two of the same readpair for overlap with the identified primer and soft clips it as well. PESC is written in Python and can remove primers at a speed of $\sim 1.2$ million reads / minute on a regular desktop computer given a primer file for detection of tumor mutational burden with $\sim 19000$ entries. With a memory footprint of $13.7 \mathrm{MB}$ in this setup it should integrate well into sequencing pipelines.

J. Rehker: None. M. Ball: None. C. Heydt: None. R. Büttner: None. S. Merkelbach-Bruse: None.

\section{P17.014.C}

Biological constraints on GWAS SNPs at suggestive significance thresholds reveals additional BMI loci

\section{R. Hammond, A. D. Wells, S. F. A. Grant}

Children's Hospital of Philadelphia, Philadelphia, PA, United States

GWAS requires increasingly larger sample sizes to overcome extensive correction for multiple testing in order to uncover additional signals. As an alternative, we aimed at salvaging suggestive association signals in a series of increasingly powered BMI GWAS efforts by intersecting our ATAC-seq and high-resolution promoter-focused Capture $\mathrm{C}$ datasets. The biological constraint of insisting each SNP both coincide with open chromatin and be contacted directly by a gene promoter at sub- $1 \mathrm{~kb}$ resolution triaged the vast majority of SNPs, leaving a manageable residual number for further interpretation. Conducting retrospective analyses utilizing three published GIANT BMI GWAS efforts of increasing sample size from 2010, 2015 and 2018, we characterized promoter contacts with open SNPs at varying suggestive sub-threshold $P$-values from $5 \times 10^{-7}$ to $5 \times 10^{-4}$ in cell types relevant to neuronal, endocrine and immune function. We identified sub-threshold signals in the 
2010 dataset corresponding to 14 BMI loci that only achieved genome-wide significance in 2015, including NAVI, SCG3, PRKDI and NLRC3. Similarly, extending our 2010-based observations to the 2018 dataset we observed 64 loci. Furthermore, analyzing the 2015 dataset, we identified 160 sub-threshold loci that went on to achieve genome-wide significance in 2018. The proportion of implicated SNPs, of course, varied with the given thresholds, with the most notable outcome being $90 \%$ of 2010 biologically-constrained independent $P<5 \times 10^{-5}$ signals going on to be genome-wide significant in 2018. Finally, applying this approach to the latest 2018 dataset implicated multiple independent novel loci. In conclusion, placing biological constraints on sub-threshold signals revealed additional loci in existing GWAS datasets.

R. Hammond: None. A.D. Wells: None. S.F.A. Grant: None.

\section{P17.015.A}

Genomics analyses on the cloud versus on-premise: comparison of performance, costs and accessibility

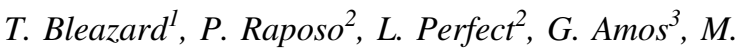

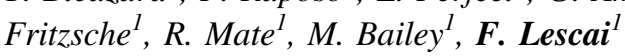

${ }^{I}$ Division of Analytical Biological Sciences, National Institute of Biological Standards and Control (NIBSC), Medicines and Healthcare products Regulatory Agency (MHRA), South Mimms, London, UK, Potters Bar, United Kingdom, ${ }^{2}$ UK Stem Cell Bank, National Institute of Biological Standards and Control (NIBSC), Medicines and Healthcare products Regulatory Agency (MHRA), South Mimms, London, UK, Potters Bar, United Kingdom, ${ }^{3}$ Division of Bacteriology, National Institute of Biological Standards and Control (NIBSC), Medicines and Healthcare products Regulatory Agency (MHRA), South Mimms, London, UK, Potters Bar, United Kingdom

When planning future infrastructure for genomics analyses, most institutions face the difficult choice of whether to expand an on-premise cluster, switch entirely to the cloud or build a hybrid setup. Despite data in the literature about the performance of cloud solutions, and the portability of individual applications, we could not identify an exhaustive, use case based comparison between the available options, which was specific for genomics and providing metrics useful for decision making at institutional level. We have therefore designed three use cases, representing some of the most demanding applications in terms of computing and storage footprint: Human Whole Genome Sequencing, Single Cell RNA Sequencing, Shotgun Metagenome Sequencing. The use of large-sized datasets, for a total of 7.5TB, also allowed us to evaluate the effect of data compression solutions. We have designed the analysis pipelines using Nextflow on a Slurm scheduler, with Singularity containers to maximise portability and reproducibility. By employing widely used applications, like GATK, Cell Ranger and Seurat, Metaphlan and Humann2, we ensured the relevance of our results for the community. This allowed us to collect and compare metrics in six dimensions consistently across different environments: performance, scheduling, cost, running features, data transfer as well as risk/benefit. We performed the comparison on a highperformance computing (HPC) cluster on-premise and on a similarly designed Cyclecloud HPC on Azure. Our results will help life science organisations to map their computing needs to the use cases and develop their computing infrastructure roadmaps.

T. Bleazard: None. P. Raposo: None. L. Perfect: None. G. Amos: None. M. Fritzsche: None. R. Mate: None. M. Bailey: None. F. Lescai: None.

P17.021.A

TAD-aware prioritization of putative pathogenic copy number variants

\section{J. Hertzberg ${ }^{1,2}$, M. Vingron $^{l}$, S. Mundlos ${ }^{1,2}$, G. Gallone $^{l}$}

${ }^{I}$ Max Planck Institute for Molecular Genetics, Berlin, Germany, ${ }^{2}$ Institute for Medical and Human Genetics, Charité Universitätsmedizin, Berlin, Germany

Introduction: The prioritization of genetic mutations is of fundamental importance for the genomics, genetics and clinical communities. Current methods prioritizing larger chromosomal alterations such as Copy Number Variants (CNVs) based on their pathogenicity are limited by the lack of publicly available variant calls. We combined pathogenic and non-pathogenic CNVs from multiple data sources to analyze the utility of functional annotation, such as regulatory domains defined by TAD boundaries, in the prioritization of pathogenic CNVs.

Methods: We matched pathogenic and non-pathogenic sets by size and variant count and conducted a series of enrichment tests to investigate any correlations between pathogenicity and overlap with genomic annotations. Finally, we implemented a novel method to annotate CNVs for manual and automated prioritization.

Results: We confirm a correlation between the pathogenicity of a CNV and its tendency to overlap with genecentric and regulatory elements. Additionally, we present the TAD annotation (TADA) tool, a method to annotate $\mathrm{CNVs}$ with respect to their regulatory environment and a proof-of-concept classification of pathogenic variants with high accuracy $(\mathrm{AUC}=0.8911)$. 
Conclusions: Considering recent advances in sequencing and variant calling, the focus on larger genomic alterations in diagnostics will likely increase, raising the need for manual and automated prioritization methods. Our results show that the prioritization of pathogenic CNVs based on functional annotation is a promising approach. With an increasing number of publicly available variant catalogues we aim to improve the classifier's predictive power and adapt our approach to all types of structural variants.

J. Hertzberg: None. M. Vingron: None. S. Mundlos: None. G. Gallone: None.

P17.023.C

Critical comparison of colocalization algorithms on large datasets

V. Rallo ${ }^{1,2}$, A. Angius ${ }^{2,1}$, M. Steri ${ }^{2}$, C. Sidore ${ }^{2}$, F. Cucca $^{1,2}$

${ }^{1}$ University of Sassari, Sassari, Italy, ${ }^{2}$ Institute for Genetic and Biomedical Research (IRGB - CNR), Monserrato (CA), Italy

Introduction: Great efforts have focused on identifying causal genetic variants shared between quantitative traits (QTs) and diseases. Several algorithms integrate association data and provide insights on the role of QTs in disease predisposition, such as Mendelian randomization, coincident associations and colocalization, all of them having benefits and drawbacks. Among these three, the colocalization aims at identifying shared haplotypes influencing both QTs and disease to find common molecular mechanisms. Here, we carry out a comparison of three colocalization algorithms (coloc, eCAVIAR and gwas-pw) reviewing their strengths and weaknesses.

Materials and Methods: We tested three colocalization software using GWAS summary statistics of 272 immune QTs measured in up to 2,870 individuals from SardiNIA cohort and public data GWAS from a case-control metaanalysis on rheumatoid arthritis (RA) (>100,000 individuals). The three tools use different approaches based on Bayes factors to evaluate colocalization signals.

Results: We considered as true positive the 270 traitlocus associations colocalizing (YC) or not-colocalizing (NC) with RA for at least 2 of the 3 tools, resulting in a total of $14 \mathrm{YC}$ and $256 \mathrm{NC}$. Coloc and gwas-pw showed the highest concordance (100\%), followed by eCAVIAR (98\%).

Conclusions: The comparison showed a high concordance among the colocalization algorithms. This suggests that the choice of tool does not have a strong impact on the colocalization results and the identification of links between QTs or diseases. We can assert that the colocalization is a robust approach to investigate connections between QTs and to advance understanding of biological mechanisms behind disease predisposition.

V. Rallo: None. A. Angius: None. M. Steri: None. C. Sidore: None. F. Cucca: None.

\section{P17.024.A}

Allelic heterogeneity and complex patterns of genetic effects across traits and cell types

K. Tayeb, M. Arvanitis, B. J. Strober, A. Battle

Johns Hopkins University, Baltimore, MD, United States

Association studies have identified thousands of genetic variants associated with phenotypes ranging from molecular traits to disease. These data offer the opportunity to compare genetic effects across traits and tissues. For example, by intersecting disease loci with expression quantitative trait loci (eQTL) we can help identify the regulatory mechanisms and target genes influencing disease. However, it remains challenging to identify which associations observed in multiple traits are driven by shared causal variants as opposed to distinct mechanisms confounded by linkage disequilibrium (LD). The problem is particularly challenging in the presence of allelic heterogeneity, where multiple causal alleles occur in the same genomic region. In fact, the same region may harbor some shared and some distinct causal effects across different tissues, conditions, or traits.

We introduce a probabilistic model, CAFEH, which leverages multiple association studies to perform colocalization and fine-mapping across studies in the presence of allelic heterogeneity. CAFEH jointly models summary statistics from each study, using a factor analysis approach to identify patterns of association driven by shared and distinct causal variants. CAFEH outperforms competing colocalization methods, specifically when multiple studies are provided. We apply CAFEH to eQTLs from 49 GTEx tissues, demonstrating much more extensive tissuespecificity than previously reported, and numerous cases of allelic heterogeneity with different causal components affecting different tissues. Applying CAFEH to UK Biobank data along with GTEx, we connect these patterns to shared causal factors with disease. Overall, our method improves understanding of allelic heterogeneity and shared genetic effects across traits and tissues.

K. Tayeb: None. M. Arvanitis: None. B.J. Strober: None. A. Battle: None.

P17.027.A

Comprehensive CNV re-analysis of over 5,000 exomes from undiagnosed Rare Disease patients collated by Solve-RD 
S. Laurie $^{1,2}$, G. Bullich ${ }^{1,2}$, G. Demidov ${ }^{3}$, L. Johansson ${ }^{4}, F$. Musacchia $^{5}$, M. Pauper ${ }^{6}$, K. Sablauskas $^{6}$, L. Matalonga $^{1}$, I.

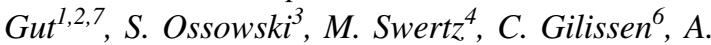

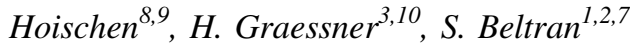

${ }^{1}$ Centro Nacional de Análisis Genómico (CNAG-CRG), Center for Genomic Regulation, Barcelona, Spain, ${ }^{2}$ Barcelona Institute of Science and Technology (BIST), Barcelona, Spain, ${ }^{3}$ Institute of Medical Genetics and Applied Genomics, University of Tübingen, Tübingen, Germany, ${ }^{4}$ University Medical Center Groningen (UMCG), Groningen, Netherlands, ${ }^{5}$ Telethon Institute of Genetics and Medicine (TIGEM), Pozzuoli, Italy, ${ }^{6}$ Department of Human Genetics, Radboud Institute for Molecular Life Sciences, Radboud University Medical Center, Nijmegen, Netherlands, ${ }^{7}$ Universitat Pompeu Fabra (UPF), Barcelona, Spain, ${ }^{8}$ Radboud Expertise Center for Immunodeficiency and Autoinflammation, Department of Internal Medicine, Radboud University Medical Center, Nijmegen, Spain, ${ }^{9}$ Department of Human Genetics and Department of Internal Medicine, Radboud University Medical Center, Nijmegen, Netherlands, ${ }^{10} \mathrm{Cen}-$ tre for Rare Diseases, University of Tübingen, Tübingen, Germany

One of the key objectives of the H2020 Solve-RD project (http://solve-rd.eu/) is the detailed reanalysis of 19,000 unsolved exome sequencing datasets from rare disease cases, through the application of state-of-the-art tools and methods, in order to attempt to solve them. Raw sequencing data is processed, and genetic variants uploaded to the RDConnect Genome-Phenome Analysis Platform (https://pla tform.rd-connect.eu/). While a percentage of cases are being solved by automated reanalysis of short variants and examination by domain experts, many datasets remain unsolved. For an important proportion of these, the detection of copy number variants may help resolve the case, as it may not have been attempted previously or our approach is more comprehensive.

The accurate detection of CNVs from exome sequencing data is known to be a very challenging task due to the inherent stochasticity in the capture enrichment process. The challenge within SolveRD is amplified due to the submitted data being extremely heterogeneous in nature, having been sequenced in different places, at different times, using different machines and protocols. Of note, the data submitted includes samples from more than 20 different commercial exome sequencing kits.

To date we have generated CNV calls from over 5,000 exomes, using three CNV calling algorithms, ExomeDepth, Conifer, and ClinCNV, with tailored settings, and a clustering algorithm, ClusterWES to define which samples should be batched together. Here we detail our workflow, report on the outcome of these analyses, and provide some recommendations for others who wish to undertake similar analyses.

S. Laurie: None. G. Bullich: None. G. Demidov: None. L. Johansson: None. F. Musacchia: None. M. Pauper: None. K. Sablauskas: None. L. Matalonga: None. I. Gut: None. S. Ossowski: None. M. Swertz: None. C. Gilissen: None. A. Hoischen: None. H. Graessner: None. S. Beltran: None.

P17.029.C

Optimized strategy for copy-number calling in targeted next-generation sequencing panels

M. Vyverman, R. De Smet, G. Vinterhalter, B. Slabbinck, J. Deceukeleire, K. Bettens, J. Crappe

Agilent Technologies, Gent, Belgium

Introduction: Copy number variations (CNVs) represent a significant fraction of pathogenic germline mutations found in human conditions. Reliable identification of CNVs from next-generation sequencing (NGS) data is challenging as it is impacted by many different factors, such as sample quality, sequencing depth, library preparation, ... Here we present a CNV calling algorithm developed and optimized for Agilent's SureMASTR (amplicon based) NGS panels.

Materials and methods: The algorithm is developed for the specific needs of the enrichment strategies of Agilent's SureMASTR panels and consists of the following: (1) Normalization by using a pooled reference from the same sequencing run, eliminating the need for matched normal samples. (2)Bias correction to control for GC-, length- and other technology-specific biases in the sequencing data. (3) Quality filtering to remove low-quality samples, PCR plexes or genomic regions. (4)Hidden Markov Models for calling. We also optimized the algorithm for dosage quotient (DQ) calculation and DQ-visualization of FFPE and FFT samples. This algorithm is incorporated in the MASTR Reporter, the analysis and quality control software for (Sure)MASTR assays. MASTR Reporter CNV calling is for Research Use Only.

Results: We tested the germline CNV calling algorithm on >500 clinical samples analyzed with 5 different SureMASTR panels and sequenced on Illumina MiSeq, MiniSeq and NextSeq platforms. Analyzed samples included 28 experimentally confirmed exon- and genelevel CNVs. Across the different panels we observed a specificity $>=98 \%$ and sensitivity of $100 \%$.

Conclusions: We present an algorithm for reliable CNV detection, that is specifically optimized for NGS targeted sequencing panels.

M. Vyverman: A. Employment (full or part-time); Significant; Agilent Technologies. R. De Smet: A. 
Employment (full or part-time); Significant; Agilent Technologies. G. Vinterhalter: A. Employment (full or part-time); Significant; Agilent Technologies. B. Slabbinck: A. Employment (full or part-time); Significant; Agilent Technologies. J. Deceukeleire: A. Employment (full or part-time); Significant; Agilent Technologies. K. Bettens: A. Employment (full or part-time); Significant; Agilent Technologies. J. Crappe: A. Employment (full or part-time); Significant; Agilent Technologies.

\section{P17.033.A}

\section{FlowCat: Automated classification of B-cell lymphoma} subtypes

N. Mallesh ${ }^{1}$, M. Zhao ${ }^{1}$, A. Höllein, ${ }^{2}$, R. Schabath ${ }^{2}, T$.

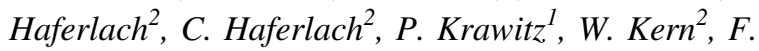
Elsner $^{3}$, H. Lüling ${ }^{3}$

${ }^{1}$ Institute for Genomics Statistics and Bioinformatics, Bonn, Germany, ${ }^{2}$ Munich Leukemia Laboratory, Munich, Germany, ${ }^{3}$ Res Mechanica GmbH, Munich, Germany

Leukemia and lymphoma are hematological disorders involving malignant neoplasms of leukocytes. Multichannel flow cytometry (MFC) is an integral diagnostic method for diagnosis and monitoring of those disorders. MFC data analysis includes manual gating to interpret cell populations. Although several computational approaches are available to automate the process of gating, these methods require expert supervision to adjust the gate definitions. We created an automated tool to directly classify MFC data to diagnosis labels without any human processing. MFC data (FCS files) were obtained from routine diagnostic of 20,622 patients. The dataset includes 8 B-cell lymphoma subtypes and healthy controls. Three 9-color MFC tubes were used to measure up to 50,000 cells per case. Compensated and transformed flow cytometry data from a single case is used to train a self-organizing map, which is used as input to a convolutional neural network generating class predictions. We achieved an overall weighted F1 score of 0.92 .We use transfer learning to classify data acquired with a different flow cytometry panel. We were able to increase the unweighted F1 score from 0.33 to 0.42 of a second network trained with 10,079 samples from a 5-color panel by transferring the knowledge from our base network described above. We demonstrate an automated classification of FCS data into multiclass labels with high accuracy. We also show that the transfer of knowledge between networks trained with data from different flow cytometry protocols can improve the classification performance of such networks, especially for the rare subtypes with very little data available.
N. Mallesh: None. M. Zhao: None. A. Höllein,: None. R. Schabath: None. T. Haferlach: None. C. Haferlach: None. P. Krawitz: None. W. Kern: None. F. Elsner: None. H. Lüling: None.

\section{P17.034.B}

Genetic intolerance across homologous protein domains identifies $T T N$ missense variants with a role in dilated cardiomyopathy

\section{Zhang $^{1,2}, P$. Theotokis ${ }^{1,2}, N$. Whiffin ${ }^{1,2}, J$. Ware $^{1,2}$ \\ ${ }^{1}$ National Heart and Lung Institute, Imperial College London, London, United Kingdom, ${ }^{2}$ Cardiovascular Research Centre, Royal Brompton and Harefield NHS, Foundation Trust London, London, United Kingdom}

Introduction: The interpretation of TTN missense variants in dilated cardiomyopathy (DCM) presents a profound challenge in variant interpretation since rare missense variants in TTN are prevalent in the population, and indistinguishable from those in patients with DCM. Previous studies assessing the relevance of TTN missense variants, using sequence conservation across species or structural features of proteins have been inconclusive.

Methods: Since $\sim 70 \%$ of the titin protein sequence is composed of $\sim 300$ Fibronectin type-III (fn3) and Immunoglobin I-set (I-set) domains, we quantified and aggregated the degree of depletion of neutral missense variation within the human genome at each domain position of the two domain families over homologous amino acids, by comparing the number of neutral variations based on a sequence-context model and variations observed among 141,456 humans from the Genome Aggregation Database (gnomAD).

Results: Six domain positions from fn 3 and I-set domains are significantly depleted of missense variants within gnomAD populations (z-test $P<0.01$ ). These constrained domain sites are enriched for ClinVar pathogenic variants in TTN and other proteins containing fn 3 or I-set domains. We combined our constraint scores with existing variant effect prediction tools to prioritise TTN missense variants. The predicted pathogenic TTN missense variants have shown with an increased association with DCM $(n=1,343)$ compared with health controls $(n=2,313)$ in a metaanalysis across three populations of different ancestries.

Conclusions: The aggregation of constraint within the human population across homologous domains represents a complementary and effective approach to prioritize missense TTN variants with single amino acid resolution of large deleterious impact.

X. Zhang: None. P. Theotokis: None. N. Whiffin: None. J. Ware: None. 


\section{P17.035.C}

The Gasdermin E Gene Has Potential as a Pan-Cancer Biomarker, While Discriminating between Different Tumor Types

J. Ibrahim ${ }^{1,2}$, K. Op de Beeck ${ }^{1,2}$, E. Fransen ${ }^{1,3}$, M. Peeters $^{2,4}$, G. Van Camp ${ }^{1,2}$

${ }^{1}$ Center of Medical Genetics, University of Antwerp and Antwerp University Hospital, Antwerp, Belgium, ${ }^{2}$ Center for Oncological Research, University of Antwerp and Antwerp University Hospital, Antwerp, Belgium, ${ }^{3}$ StatUa Centre for Statistics, University of Antwerp, Antwerp, Belgium, ${ }^{4}$ Department of Medical Oncology, Antwerp University Hospital, Antwerp, Belgium

Introduction: Due to the elevated rates of incidence and mortality of cancer, early and accurate detection is crucial for achieving optimal treatment. Molecular biomarkers remain important screening and detection tools, especially in light of novel blood-based assays. DNA methylation in cancer has been linked to tumorigenesis, but its value as a biomarker has not been fully explored. In this study, we have investigated the methylation patterns of the Gasdermin $\mathrm{E}$ gene across 14 different tumor types using The Cancer Genome Atlas (TCGA) methylation dataset $(N=6502)$.

Methods: Differential methylation was tested through linear mixed models. Binary logistic regression and partial-least squares discriminant analysis prediction models were used for identifying pan-caner and tumorspecific markers. We were able to identify six $\mathrm{CpG}$ sites that could effectively distinguish tumors from normal samples in a pan-cancer setting (AUC $=0.86$ ). This combination of pan-cancer biomarkers was validated in six independent datasets (AUC $=0.84-0.97)$. Moreover, we tested 74,613 different combinations of six $\mathrm{CpG}$ probes, where we identified tumor-specific signatures that could differentiate one tumor type versus all the others (AUC $=0.79-0.98)$. In all, methylation patterns exhibited great variation between cancer and normal tissues, but were also tumor specific.

Conclusions: Our analyses highlight that a Gasdermin E methylation biomarker assay, not only has the potential for being a methylation-specific pan-cancer detection marker, but can also discriminate between different tumor types. Funding: Research Foundation Flanders (FWO), Kom op tegen Kanker (KOTK) and the Research Fund of the University of Antwerp (GEOMED).

J. Ibrahim: None. K. Op de Beeck: None. E. Fransen: None. M. Peeters: None. G. Van Camp: None.

\section{P17.036.A}

Longitudinal changes in blood metabolite levels from young- to middle adulthood are related to epigenetic variability genome-wide

\author{
I. Pupko ${ }^{l}$, H. Draisma ${ }^{l}$, L. Zudina ${ }^{l}$, Z. Balkhiyarova ${ }^{l}$, M. \\ Wielscher ${ }^{2}$, M. Ala-Korpela ${ }^{3}$, S. Sebert ${ }^{3}$, M. Järvelin ${ }^{2,3}$, M. \\ Kaakinen $^{l}$, I. Prokopenko ${ }^{l}$
}

${ }^{1}$ University of Surrey, Guildford, United Kingdom, ${ }^{2}$ Imperial College London, London, United Kingdom, ${ }^{3}$ University of Oulu, Oulu, Finland

Associations between metabolite levels and DNA methylation (DNAm) have been reported. However, the relationship between longitudinal changes in metabolite levels and differential DNAm is underexplored. We assessed associations between epigenome-wide blood DNAm and changes in the levels of blood proton nuclear magnetic resonance $\left({ }^{1} \mathrm{H}-\mathrm{NMR}\right)$ metabolomics-based traits. For 595 non-diabetic individuals from the Northern Finland Birth Cohort 1966 with metabolomics data at ages 31 (T1) and 46 years (T2) as well as concurrent DNAm data (T2), we calculated the average change in level per year between $\mathrm{T} 1$ and $\mathrm{T} 2$ for each of the 228 metabolomics-based variables. We used our methylSCOPA software for fast singlepredictor longitudinal epigenome-wide association study (EWAS). We regressed out participant sex from the change in metabolomic variable level and used the resulting residuals as predictors of DNAm in EWAS for 832,569 markers on the Illumina (San Diego, CA, USA) MethylationEPIC BeadChip. We quality-controlled, residualized, and normalized the DNAm data, mapped genomic locations to CGCh37/hg19, and adopted the Bonferronicorrected $P$-value $=4.4 \times 10^{-10}$ threshold to denote epigenome- and metabolome-wide statistical significance of association between predictor and epigenetic mark. We detected an epigenome- and metabolome-wide significant association between DNAm at $\operatorname{cg} 16904859$ (chr1:206, 849,$\left.995 ; \beta=2.31 ; \mathrm{SE}=0.36 ; P=3.2 \times 10^{-10}\right)$ and change over time in the level of triglycerides in extremely large very low-density lipoprotein particles and potentially also chylomicrons. Additionally, we identified 67 epigenomewide significant $\left(P<1 \times 10^{-7}\right)$ predictor-epigenetic mark associations, involving 28 unique epigenetic markers and 53 unique metabolomics-based variables. Using methylSCOPA, we detected associations between longitudinal changes in blood metabolite levels and DNAm.

Funding: H2020-SC1-2019-874739, WCRF-2017/1641. 
I. Pupko: None. H. Draisma: None. L. Zudina: None. Z. Balkhiyarova: None. M. Wielscher: None. M. Ala-Korpela: None. S. Sebert: None. M. Järvelin: None. M. Kaakinen: None. I. Prokopenko: None.

\section{P17.037.B}

Towards a more efficient genetic diagnosis of epilepsy: switching between a mendelian genes space and a clinical phenotypes space

\section{A. Cisterna ${ }^{1}$, A. Sabater ${ }^{1}$, D. Zhang ${ }^{2}$, J. A. Hardy ${ }^{2}$, M. Ryten $^{2,3}$, I. Díez ${ }^{4}$, P. Maietta ${ }^{4}$, S. Álvarez ${ }^{4}$, J. A. Botía ${ }^{1,2}$ \\ ${ }^{I}$ Departamento de Ingeniería de la Información y las Comunicaciones, Universidad de Murcia, Murcia, Spain, ${ }^{2}$ Department of Molecular Neuroscience, UCL Institute of Neurology, London, United Kingdom, ${ }^{3}$ Department of Medical and Molecular Genetics, King's College London School of Medicine, Guy's Hospital, London, United Kingdom, ${ }^{4}$ NIM- Genetics Genómica y Medicina S.L, Madrid, Spain}

Introduction: Epilepsy is a group of neurological disorders characterized by recurrent epileptic seizures. 35\% of cases have a genetic basis. For those, roughly the failed differential diagnoses are about $15 \%$. It is difficult to diagnose epilepsy, in many cases, a genetic study of the patient is required, and these may lead to false negatives. For those cases, we estimate similarity between epilepsy and other close disorders by transforming gene sets into phenotype sets to provide a clinical similarity between genetic diseases. The tool is called PhenoExam.

Methods: PhenoExam uses a score that evaluates how many relevant phenotypes the reference panel shares with the test panel and how similar is the representation of the shared phenotypes in relative terms. It works on HPO and MGI ontologies.

Results: We have created a phenotypic space for 120 diseases whose gene-based definitions where obtained from Genomics England PanelApp. Panels closest to epilepsy are hereditary ataxia, inherited white matter disease and hereditary neuropathy. Through PhenoExam we have refined the epilepsy panel predicting new epilepsy genes. They have a higher PhenoExam score of phenotype similarity than random genes $\left(\mathrm{P}<10^{-5}\right)$. Amongst those genes are KIF1A, NFASC, RYR2, CELF2.

Conclusions: PhenoExam allows us to situate diseases, defined by a set of genes into a metric space based on phenotypical descriptions emerging from individual genes. We confirmed that new genes likely to cause epilepsy recapitulate and correlate with relevant phenotypes extracted from NIMGenetics epilepsy panel. PhenoExam is an R package available at https://github.com/alexcis95/PhenoExam.

Fundación Séneca and NIMGenetics.
A. Cisterna: B. Research Grant (principal investigator, collaborator or consultant and pending grants as well as grants already received); Modest; NIMGenetics. A. Sabater: None. D. Zhang: None. J.A. Hardy: None. M. Ryten: None. I. Díez: A. Employment (full or part-time); Significant; NIMGenetics. P. Maietta: A. Employment (full or part-time); Significant; NIMGenetics. S. Álvarez: A. Employment (full or part-time); Significant; NIMGenetics. J.A. Botía: None.

P17.038.C

Differentially expressed gene analysis from wholetranscriptome sequencing of patients with esophageal squamous cell carcinoma from Kazakhstan

A. Sharip ${ }^{I}$, S. Rakhimova ${ }^{l}$, A. Molkenov ${ }^{l}, U$. Kozhamkulov $^{l}, Y$. Zhukov ${ }^{2}$, M. Omarov ${ }^{2}$, A. Akilzhanova ${ }^{l}$, U. Kairov $^{l}$

${ }^{I}$ National Laboratory Astana, Nur-Sultan, Kazakhstan, ${ }^{2}$ Oncology Center, Nur-Sultan, Kazakhstan

Introduction: Esophageal cancer is the eighth most common cancer worldwide and sixth in Kazakhstan. Esophageal squamous cell carcinoma (ESCC) is the predominant histological subtype of esophageal cancer and diagnosed at late stage. The aim of the project was to identify genetic basis of ESCC by analysing differentially expressed genes (DEGs) from whole-transcriptome sequencing of Kazakhstani patients.

Materials and Methods: Tissue samples were obtained from 25 ESCC-affected individuals immediately after IvorLewis esophagectomy from Oncology Center in NurSultan. Whole transcriptome sequencing was performed following the TruSeq RNA Protocol. STAR software and DESeq2 package have been used for mapping and defining differentially expressed genes. Functional analysis of DEGs was performed using various $\mathrm{R}$ packages.

Results: The study sized 14 men and 11 women, average age of patient $65.5 \pm 7.7$ years $88 \%$ of the patients were diagnosed with advanced stages T3-T4. Analysis of tumor and normal esophageal tissues identified 1197 DEGs, comprising 883 upregulated and 314 downregulated genes (with adjusted $p$-value $<0.05$ ). We found significant 4 upregulated and 6 down-regulated KEGG pathways ( $p$ value $<0.05$ ). Top 300 DEGs were mapped to PPI network and functional enrichment analysis was performed on identified three modules of closely connected nodes (genes).

Conclusions: ESCC with moderate dysplasia is the most common histologic subtype of esophageal cancer in our patients and is characterized by a poor prognosis. Highthroughput sequencing approach allows identifying 
molecular pathways involved in esophageal carcinogenesis that could improve diagnosis and treatment strategies.

Acknowledgments: Work was supported by grant of the Ministry of education and science \#AP05134722, \#AP05135430 and \#AP05136106.

A. Sharip: None. S. Rakhimova: None. A. Molkenov: None. U. Kozhamkulov: None. Y. Zhukov: None. M. Omarov: None. A. Akilzhanova: None. U. Kairov: None.

P17.039.A

MLI-score: a machine learning tool generating an integrated score for pathogenic variant prediction

E. Benetti ${ }^{1}$, D. Rosati ${ }^{1}$, C. Fallerini ${ }^{2}, F$. Mari $^{2,3}, A$. Renieri $^{2,3}$, S. Furini ${ }^{1}$

${ }^{1}$ Department of Medical Biotechnologies, University of Siena, Siena, Italy, ${ }^{2}$ Medical Genetics, University of Siena, Siena, Italy, ${ }^{3}$ Medical Genetics, University of Siena, Siena, Italy Genetica Medica, Azienda Ospedaliera Universitaria Senese, Siena, Italy

Exome sequencing is gaining importance in the clinical practice thanks to its high accuracy and the decreasing costs. However, a great challenge in the analysis of exome sequencing experiments is how to identify variants that are clinically relevant among an overwhelming number of variants with no functional consequences. Many bioinformatic scores exist for predicting the pathogenicity of genetic variants. In this work, we compared their performances, and we tested if it was possible to improve the accuracy of pathogenicity predictions by combining the available bioinformatic scores into a unified Machine Learning model using Logistic Regression. This integrated strategy improves the performance by almost $5 \%$ with respect to the best algorithm available in the literature and interestingly reduces by $7 \%$ the rate of benign variants that are wrongly classified as pathogenic. By integrating additional features, among which Minor Allele Frequency (MAF) and the number of Pubmed articles related to the rs code (pubmed_rss), the Machine Learning model further improves the performance of almost $10 \%$ with respect to the best single algorithm alone and further decreases the rate of false positive up to $11 \%$. This novel integrated score for pathogenic variant prediction, that incorporates multiple sources of information into a single score, can therefore facilitate the analysis of exome sequencing experiments and speed up the identification of pathogenic variants by performing a more stringent and accurate selection of variants.

E. Benetti: None. D. Rosati: None. C. Fallerini: None. F. Mari: None. A. Renieri: None. S. Furini: None.
P17.041.C

Refining reference human protein-coding gene annotation

\section{A. Frankish, The GENCODE Consortium}

\section{EMBL-EBI, Hinxton, United Kingdom}

The GENCODE consortium continues to produce foundational genome annotation for the human and mouse reference genomes. GENCODE identify and classify, with high accuracy, all protein-coding genes, pseudogenes, long noncoding RNAs and small RNAs based on defined biological evidence and make this annotation freely available for the benefit of biomedical research and genome interpretation. We will describe the ongoing extension of annotation workflows to use long transcriptomic data from PacBio and ONT in combination with RNAseq data to produce accurate full-length transcript structures, CAGE, RAMPAGE and 3'enriched RNAseq to identify transcript ends, and targeted proteogenomics and phylogenetic assessment to support decisions regarding protein-coding potential. Alongside the efforts to identify genes and alternatively-spliced (AS) transcripts we continue to work closely with our colleagues at other resources to resolve conflicts. We have undertaken an extensive exercise to compare GENCODE and Uniprot annotation. The GIFTS (Genome Integration with FuncTion and Sequence) project has created a common framework for UniProtKB-Ensemb1/GENCODE mapping. Using GIFTS, GENCODE annotators have investigated 716 Uniprot entries that do not map to a protein-coding gene in the Ensembl/GENCODE human geneset. The review process has led to updates to Ensembl/GENCODE gene annotation, discussions with Uniprot curators regarding entries that do not display high protein-coding potential in the genomic context, and identification of a set of loci where resourcespecific criteria preclude agreement.

This work was supported in part by the National Human Genome Research Institute (NHGRI) (2U41HG007234), Wellcome Trust (WT108749/Z/15/Z) and the European Molecular Biology Laboratory.

\section{A. Frankish: None.}

\section{P17.043.B}

Deciphering non-coding variants with GeneHancer regulatory regions

S. Fishilevich, R. Barshir, T. Iny Stein, M. Safran, D. Lancet

Weizmann Institute of Science, Rehovot, Israel 
Whole genome sequencing (WGS) variant analysis and interpretation requires an exploration of non-genic functional genomic elements such as promoters and enhancers, involved in development and implicated in disease. The comprehensive identification of regulatory elements and their gene targets is a significant challenge. We created GeneHancer (PMID:28605766), a regulatory element database within the GeneCards Suite (https://www.geneca rds.org/), with 400,000 enhancers and promoters. Information is amalgamated from ENCODE, Ensembl, FANTOM5, VISTA, dbSUPER, EPDnew, UCNEbase, CraniofacialAtlas and GTEx. GeneHancer creates a unique non-redundant and comprehensive view of regulatory elements, including their target gene associations, transcription factor binding sites, tissue specificity and super-enhancer mapping. We recently focus on phenotype/disease annotation of such elements, based on variant-trait mappings from the GWAS Catalog, and literature-curated evidence from DiseaseEnhancer and NCBI Entrez Gene. GeneHancer is now used by a considerable fraction of GeneCards' 4 million user base to annotate non-coding variants, and is included as a native regulation track at the UCSC genome browser, where it is the only source for explicit regulatory regions and gene associations. GeneHancer provides an indispensable augmentation for the GeneCards' NGS disease interpretation tools: VarElect, a phenotype interpreter (PMID:27357693), and TGex, a VCF-to-report analyzer (PMID:31888639), used by major clinical sequencing centers. Non-coding variants are mapped to regulatory regions, and then prioritized with respect to diseases and phenotype keywords via direct and target gene-mediated links. Such capacities provide a comprehensive route to deciphering the clinical significance of non-coding single nucleotide and structural variations, thus helping to elucidate unsolved disease cases. Support: LifeMap Sciences grant

S. Fishilevich: None. R. Barshir: None. T. Iny Stein: None. M. Safran: None. D. Lancet: B. Research Grant (principal investigator, collaborator or consultant and pending grants as well as grants already received); Significant; LifeMap Sciences.

\section{P17.049.B}

Towards a joint standardised "default" transcript set: Matched Annotation from the NCBI and EMBL-EBI (MANE)

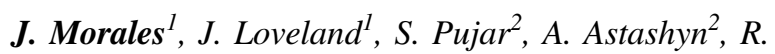
Bennett $^{l}$, C. Davidson ${ }^{l}$, O. Ermolaeva ${ }^{2}$, C. Farrell ${ }^{2}$, L. Gil $^{l}$, J. M. Gonzalez', V. Joardar ${ }^{2}, M . K^{1}{ }^{l}, V . K^{2}$ dali $^{2}, K$.
McGarvey $^{2}$, A. McMahon ${ }^{l}$, S. Rangwala ${ }^{2}$, G. Threadgold ${ }^{1}$, F. Cunningham ${ }^{1}$, A. Frankish ${ }^{1}$, T. Murphy ${ }^{2}$

${ }^{I}$ European Bioinformatics Institute (EMBL-EBI), Hinxton, United Kingdom, ${ }^{2}$ National Center for Biotechnology Information, National Library of Medicine, Bethesda, MD, United States

The accurate description of transcripts is foundational for clinical genomics. GENCODE (annotation produced by EMBL-EBI) and RefSeq (NCBI) produce comprehensive and complementary human gene annotation. The large number of alternatively spliced transcripts, together with unstandardised use of canonical transcripts across resources, can present challenges, especially in the clinical context. To address this, EMBL-EBI and NCBI are working together on the Matched Annotation from the NCBI and EMBL-EBI (MANE) initiative. The aim is to generate a genome-wide transcript set that: 1) aligns to GRCh38, 2) includes pairs of $100 \%$ identical Ensembl/GENCODE (ENST) and RefSeq (NM) transcripts and 3) is well-supported. The output, the MANE Select set, will include one transcript that is representative of biology at each protein-coding locus across the genome. Select transcripts are identified using independent computational pipelines complemented by manual review and discussion. The pipelines utilise evidence of functional potential such as expression levels, evolutionary conservation, and clinical significance. Currently, we have released MANE Select transcripts for $75 \%$ of protein-coding loci across the genome, including $83 \%$ of validated clinical genes and $90 \%$ of ACMG59 genes. We aim to achieve genome-wide coverage in 2020 and are working closely with partners to encourage adoption of the MANE set on browsers and in clinical workflows. We envision this dataset serving as a unified highvalue reference set for use in comparative genomics, clinical reporting and basic research. Released transcripts are available on all genome browsers and on the NCBI's FTP site. This work is supported by: Wellcome Trust-WT200990/Z/16/ Z; EMBL-Core-Funds; NIH-U41HG007234 and NIHIntramural-Research-program.

J. Morales: None. J. Loveland: None. S. Pujar: None. A. Astashyn: None. R. Bennett: None. C. Davidson: None. O. Ermolaeva: None. C. Farrell: None. L. Gil: None. J.M. Gonzalez: None. V. Joardar: None. M. Kay: None. V. Kodali: None. K. McGarvey: None. A. McMahon: None. S. Rangwala: None. G. Threadgold: None. F. Cunningham: None. A. Frankish: None. T. Murphy: None.

P17.051.A

Leveraging correlated risks to increase power in Genome-Wide Association Studies 


\section{N. Mounier ${ }^{1,2}$, Z. Kutalik ${ }^{1,2}$}

${ }^{1}$ University Center for Primary Care and Public Health, Lausanne, Switzerland, ${ }^{2}$ Swiss Institute of Bioinformatics, Lausanne, Switzerland

Introduction: Genome-Wide Association Studies (GWASs) are nowadays often conducted in more than 1 million samples. Improved discovery, by further increasing study sizes is not the only strategy. Leveraging information from published studies of related traits can improve inference. To this end, we developed a Bayesian GWAS approach that builds informative priors for each single nucleotide variant (SNV) using GWASs of related risk factors (RFs).

Methods: Mendelian Randomisation (MR) is used to derive multivariate causal effects of a set of iteratively selected RFs on the target outcome. These causal effects of the RFs on the outcome are then combined with the SNV's effect on the identified RFs to build a Bayesian prior. Bayes Factors (BFs) are used to quantify the evidence in favour of the prior. P-values are assigned to these BFs to allow the identification of associated SNVs.

Results: We found a set of universally optimal parameters (instrument strength, clumping stringency and shrinkage intensity) that maximises the out-of-sample squared correlation between prior and observed effects (up to $40 \%$ ). We also derived an analytical formula for the null BFs distribution, leading to a 6-fold reduced runtime and a more accurate estimation of BFs P-values, compared to permutation-based approaches. The method now also provides posterior and direct (part of observed effect not mediated through the RFs) effect estimates that can be used for downstream analyses.

Conclusions: The method, as well as functions facilitating results visualisation, have been implemented in an $\mathrm{R}$ package (https://github.com/n-mounier/bGWAS) and can be applied to any GWAS summary statistics.

N. Mounier: None. Z. Kutalik: None.

P17.052.B

An online platform for GWAS/EWAS summary statistics harmonisation and data imputation

\section{A. Demirkan ${ }^{1,2,3}$, K. Rudometkin ${ }^{1}$, L. Zudina ${ }^{l}, Z$. Balkhiyarova $^{3,1}$, M. Kaakinen ${ }^{1,3}$, I. Prokopenko ${ }^{I}$}

${ }^{1}$ Department of Clinical \& Experimental Medicine, University of Surrey, Guildford, United Kingdom, ${ }^{2}$ Department of Genetics, University Medical Center Groningen, Groningen, Netherlands, ${ }^{3}$ Department of Medicine, Section Genomics of Common Disease, Imperial College London, London, United Kingdom
With the increasing utility of data driven methods in science, ownership of complete datasets enabling use of multivariate statistical methods has turned out to be of major value. As an outcome of this trend, approaches that tackle missingness in epidemiological and omic datasets are of increasing demand. We developed a user-friendly webbased portal and an analytical platform ANGLIA: "An online platform for imputing and aNalyzinG epidemioLogical -omIcs dAta" for the users with (epi)genomic and other omic data, to address the issues raising from data missingness and to enable data harmonisation at the summary statistics level. With ANGLIA we aim to address the challenges by integrating approaches to imputation and multi-variable analysis of omics data both at the individual and summary statistics levels by implementing a framework of existing and novel analytical tools. These include refining existing and developing new high-performance tools for missing data imputation, performing multi-phenotype (epi) genome-wide association studies (EWAS/GWAS) and performing EWAS/GWAS of composite measurements, as well as upcycling GWAS summary statistics to more recent genotype imputation panels. The showcase of the developed platform is located at http://www.anglia.prokopenkogroup. org/ and will serve for the very immediate needs of the scientists working in the fields of genetics, epidemiology and statistical omics, and eventually to different branches of medicine through more accurate research of the omics data. Our proposed framework has potential to enhance statistical omics association studies of any kind, and enable new insilico discoveries to advance further our understating of complex human conditions. Funding: H2020-SC1-2019874739, WCRF-2017/1641.

A. Demirkan: None. K. Rudometkin: None. L. Zudina: None. Z. Balkhiyarova: None. M. Kaakinen: None. I. Prokopenko: None.

\section{P17.053.C}

Accounting for age-of-onset, disease prevalence, and family information in Genome-wide Association Studies.

\author{
E. M. Pedersen, J. W. Dreier, K. Musliner, ISPYCH-Broad \\ Consortium, A. Børglum, J. McGrath, S. Dalsgaard, J. \\ Christensen, P. B. Mortensen, E. Agerbo, F. Privé, B. J. \\ Vilhjalmsson
}

\section{Aarhus University, Aarhus, Denmark}

Most genome-wide association studies (GWAS) account only for information from the genotyped individuals. However, in epidemiological studies additional family and temporal information, e.g. family history, disease prevalence and age-of-onset, are routinely accounted for. 
The recently proposed methods; GWAX: genome-wide association study by proxy (Liu et al., Nat Genet 2017), LTFH: liability threshold model, conditional on family history (Hujoel et al., BioRxiv 2019) and Cox regression GWAS (Staley et al., EJHG 2017) incorporate either family history or temporal information, but not both. Here we present a new method that extends LT-FH to account for both family and temporal information.

We compared our approach to both standard GWAS, GWAX, and LT-FH using simulated polygenic liabilities and case-control phenotypes. When only accounting for sex of family members, we observed a modest improvement (3\% increased power) over LT-FH and large improvement over GWAX (53\% increased power) in simulations assuming a disease prevalence of $4 \%$ in males and $1 \%$ in females. Accounting for changes in disease prevalence over time, age-of-onset, and oversampling cases (a common strategy in genetic studies) further increases statistical power. We applied our approach to several psychiatric and neurological disorders in the iPSYCH study of five serious mental disorders: ADHD, Autism, Bipolar disorder, Depression, and Schizophrenia with more than 80,000 psychiatric cases and 50,000 controls.

In summary, the liability-threshold model is a promising approach to increase power in GWAS when family history, disease prevalence over time, and temporal information is available.

E.M. Pedersen: None. J.W. Dreier: None. K. Musliner: None. A. Børglum: None. J. McGrath: None. S. Dalsgaard: None. J. Christensen: None. P.B. Mortensen: None. E. Agerbo: None. F. Privé: None. B.J. Vilhjalmsson: None.

\section{P17.054.A}

Engaging the GWAS community to increase Summary Statistics data sharing in the GWAS Catalog

\section{Cerezo ${ }^{1}$, J. A. L. MacArthur ${ }^{1}$, A. Buniello ${ }^{1,2}, J$.

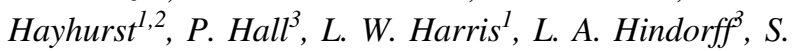 Jupp $^{l}$, E. Lewis ${ }^{l}$, C. Malangone ${ }^{l}$, A. McMahon ${ }^{l}, J$. Morales $^{l}$, E. Sollis ${ }^{l}$, J. Stewart ${ }^{l}$, D. Suveges ${ }^{I}$, P. L. Whetzel $^{1}$, T. Burdett ${ }^{1}$, F. Cunningham ${ }^{1}$, P. Flicek ${ }^{1}, H$. Parkinson $^{l}$}

${ }^{1}$ EMBL-EBI, Cambridge, United Kingdom, ${ }^{2}$ Open Targets, Cambridge, United Kingdom, ${ }^{3}$ Division of Genomic Medicine, National Human Genome Research Institute, National Institutes of Health, Bethesda, MD, United States

In recent years, summary statistics (SumStats) from genome-wide association studies (GWAS) have been increasingly used to provide insights into the mechanisms of complex disease, predict disease risk, prioritise candidate loci, and discover drug targets. The availability of SumStats has also proven beneficial for the development of new statistical methods, conditional associations, and integration with other omics. The NHGRI-EBI GWAS Catalog (www. ebi.ac.uk/gwas) expanded its scope to include SumStats in 2017 and is now the largest and most updated freely available SumStats resource. Thanks to the increase of SumStats links in research articles, along with direct submission to the Catalog, the percentage of new publications with available SumStats in the Catalog has increased from 6\% in 2017 to $44 \%$ in 2019 and now includes over 10,000 datasets. Despite an increase in author submissions, the percentage of publications with available SumStats remains relatively low, with $86 \%$ of Catalog publications not having available SumStats. In an aim to facilitate the data sharing, we have developed a web-based deposition interface to support author submission of SumStats and meta-data from publications. We are also planning a workshop to address the standards, infrastructure and incentives required to promote and enable sharing of SumStats. The workshop will bring together participants from the diverse stakeholder space, including researchers, journal editors, funding bodies, and biobank representatives. The aim is to reach a consensus and publish an agreed action plan for SumStats sharing and standardization which will have a positive impact on the GWAS community.

M. Cerezo: None. J.A.L. MacArthur: None. A. Buniello: None. J. Hayhurst: None. P. Hall: None. L. W. Harris: None. L.A. Hindorff: None. S. Jupp: None. E. Lewis: None. C. Malangone: None. A. McMahon: None. J. Morales: None. E. Sollis: None. J. Stewart: None. D. Suveges: None. P.L. Whetzel: None. T. Burdett: None. F. Cunningham: None. P. Flicek: None. H. Parkinson: None.

\section{P17.055.B}

ITHANET: An information and database community portal for haemoglobinopathies

\section{Stephanou, A. Minaidou, S. Tamana, M. Xenophontos, C. W. Lederer, P. Kountouris, M. Kleanthous}

The Cyprus Institute of Neurology and Genetics, Nicosia, Cyprus

Introduction: The ITHANET portal (www.ithanet.eu) is an expanding, publicly available biomedical resource dedicated to haemoglobinopathies. It provides a manually curated, literature-derived collection of published genetic and epidemiological data, also integrating the latest updates on news, events, publications, clinical trials, funding opportunities, and many more. 
Materials and Methods: A team of expert biocurators is involved in the collection, validation and annotation of information with weekly updates on scientific literature collected from PubMed, also incorporating new and updated information from existing public databases. Contributions are welcome and clearly acknowledged.

Results: ITHANET offers a range of curated databases; IthaGenes currently stores information on over 2900 fully annotated variations in more than 350 globin-related loci and genes. Most variations are assigned at least one of 60 phenotypic annotations in IthaGenes, taken from the Human Phenotype Ontology. IthaMaps stores epidemiological information as documented in published literature for a total of over 185 countries and illustrates this information on a dynamic global to regional map. IthaChrom provides digitised reports (as kindly provided by Bio-Rad Laboratories Inc.) of standard diagnostic HPLC analyses on more than 600 haemoglobin variants.

Conclusions: ITHANET is the most comprehensive knowledgebase on haemoglobinopathies and the official partner of the Human Variome Project's Global Globin Network for data storing, curation and sharing within and between countries. Also, this partnership fosters the development of a genotype-phenotype database for better health care delivery and forms the basis of an Expert Panel application for haemoglobinopathy-related variant classification under the Clinical Genome Resource.

Funded project: EXCELLENCE/1216/0256.

C. Stephanou: None. A. Minaidou: None. S. Tamana: None. M. Xenophontos: None. C.W. Lederer: None. P. Kountouris: None. M. Kleanthous: None.

\section{P17.056.C}

Inter-omics analysis in hepatocellular carcinoma

\section{Jihad, I. Yet}

Department of Bioinformatics, Graduate School of Health Sciences, Hacettepe University, Ankara, Turkey

Introduction: The main aim of this study is to understand the biological mechanisms involved in disease susceptibility and progression, by linking CNV, DNA methylation and gene expression to Hepatocellular carcinoma (HCC) using Bayesian networks (BN).

Methods and Results: To infer causality in HCC by fitting $\mathrm{BN}$ we used $\mathrm{HCC}$ single-cell sequencing dataset contained 25 samples. We built three BN structures representing the alternative hypotheses of the causal relations between $\mathrm{CNV}$, gene expression and methylation. The parameters of these networks were estimated by using MLE. Afterwards, we examined the compatibility of these structures with the data by using the AIC score. After fitting all the models, there were 17 models where the best model was at least ten times more likely than the second best model.

Discussion and Conclusion: The availability of different levels of "-omics" data allowed us to explore the relationship between disease-associated "-omics" profiles, specifically for genetic variation, methylation and gene expression. We build three BN structure for the alternative causal relations and detected several cases of three-way associations where either genetically driven DNA methylation levels impact gene expression profiles, or genetically driven gene expression traits impact DNA methylation levels. Overall, these results suggest that the effects of DNA methylation can be both active on gene expression levels, and passive, by being a consequence for gene expression levels. This study shows the potential of integrating other "-omics" data for complex diseases.

M. Jihad: None. I. Yet: None.

\section{P17.057.A}

A novel machine learning-based approach for screening Individuals at risk of hereditary haemochromatosis

\author{
P. Martins Conde ${ }^{1,2}$, T. Sauter ${ }^{1}$, T. P. Nguyen ${ }^{1,2}$ \\ ${ }^{1}$ University of Luxembourg, LSRU, Esch-sur-Alzette, Lux- \\ embourg, ${ }^{2}$ Megeno S.A., Esch-sur-Alzette, Luxembourg
}

Hereditary haemochromatosis $(\mathrm{HH})$ is an autosomal recessive disease, where homozygosity for $\mathrm{C} 282 \mathrm{Y}$ in the HFE gene accounts for $80 \%-85 \%$ of clinical cases among the Caucasian population. $\mathrm{HH}$ is characterised by the accumulation of iron, which, if untreated, can lead to the development of liver cirrhosis and liver cancer. An iron overload screening tool (IRON), employing traditional statistical methods on self-reported and non-laboratory data, has been proposed. This tool obtained an area under the curve (AUC) of $68.5 \%$, demonstrating great potential of integrating different types of self-reported and clinical data into computational screening tools. However, the utility of such tools is limited by challenges associated with efficiently handling and combining large amounts of structured and unstructured data from different sources. We have developed a novel computational model to identify individuals at risk of $\mathrm{HH}$, using the family study data of the HEIRS cohort. This dataset, consisting of 254 cases and 701 controls, contains variables extracted from questionnaires and biomarker values. These variables were ranked using statistical and recursive feature elimination methods. The model was trained on an XGBoost classifier using the most significant risk factors: HFE $\mathrm{C} 282 \mathrm{Y}$ homozygosity, age, mean corpuscular volume, iron level, serum ferritin level, transferrin saturation, and unsaturated iron-binding capacity. Parameter 
optimisation was carried out with multiple runs, resulting in an AUC of $87.03 \% \pm 5.43$ for 10 -fold stratified crossvalidation. Since iron overload is preventable and treatable if diagnosed early, individuals at high risk can benefit from efficient screening strategies employing artificial intelligence-based approaches.

P. Martins Conde: A. Employment (full or part-time); Significant; Megeno S.A.. T. Sauter: None. T.P. Nguyen: A. Employment (full or part-time); Significant; Megeno S.A.

P17.059.C

AutoMap: High performance homozygosity mapping using sequencing data

M. Quinodoz ${ }^{1,2,3}$, V. Peter ${ }^{1,3,4}$, N. Bedoni ${ }^{2}$, B. Royer

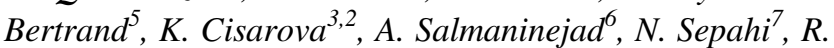

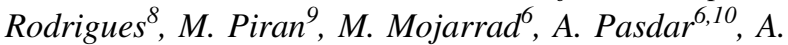
Asad $^{7}$, A. Sousa ${ }^{8,11}$, L. Coutinho-Santos ${ }^{12}$, A. SupertiFurga $^{5}$, C. Rivolta ${ }^{1,3,13}$

${ }^{1}$ Insitute of Molecular and Clinical Ophtalmology Basel, Basel, Switzerland, ${ }^{2}$ Department of Computational Biology, Unit of Medical Genetics, University of Lausanne, Lausanne, Switzerland, ${ }^{3}$ Department of Genetics and Genome Biology, University of Leicester, Leicester, United Kingdom, ${ }^{4}$ Institute of Experimental Pathology, Lausanne University Hospital (CHUV), Lausanne, Switzerland, ${ }^{5}$ Service of Medical Genetics, Lausanne University Hospital (CHUV), Lausanne, Switzerland, ${ }^{6}$ Department of Medical Genetics, Faculty of Medicine, Mashhad University of Medical Sciences, Masshad, Iran, Islamic Republic of, ${ }^{7}$ Noncommunicable Diseases Research Center, Fasa University of Sciences, Fasa, Iran, Islamic Republic of, ${ }^{8}$ Department of Medical Genetics, Hospital Santa Maria, Centro Hospitalar Universitário Lisboa Norte (CHULN), Lisbon Academic Medical Center (CAML), Lisbon, Portugal, ${ }^{9}$ Bioinformatics and Computational Biology Research Center, Shiraz University of Medical Sciences, Shiran, Iran, Islamic Republic of, ${ }^{10}$ Division of Applied Medicine, Medical School, University of Aberdeen, Aberdeen, United Kingdom, ${ }^{11}$ Medical Faculty, Lisbon University, Lisbon, Portugal, ${ }^{12}$ Instituto de Oftalmologia Dr Gama Pinto, Lisbon, Portugal, ${ }^{13}$ University of Basel, Basel, Switzerland

Through the identification of genomic runs of homozygosity (ROHs), homozygosity (autozygosity) mapping is a powerful approach to find autosomal recessive mutations, especially in consanguineous families. Many tools exists for $\mathrm{ROH}$ discovery that rely on data from genotyping arrays or exome sequencing (ES). Since most of these tools had bad performances or were difficult to use, we have developed a new tool: AutoMap (Autozygosity Mapper). AutoMap directly uses standard VCF files as primary source of data. A careful variant filtering step is implemented prior to the identification of ROHs by a sliding-window approach and subsequent filtering. The tool was first trained on ES data from 26 samples from consanguineous families, and further validated in 26 additional samples. AutoMap displayed similar performance for both training and validation cohorts, with sensitivity of $90.5 \%$ and $92.4 \%$ respectively and showed the best sensitivity/specificity combination with respect to 8 existing tools. When applied to unpublished ES data, AutoMap allowed the discovery of ROHs containing homozygous non-coding variants that led to the identification of disease genes for five novel conditions including the Liberfarb syndrome. AutoMap is a reliable tool that can predict, in less than a minute, ROHs with high specificity and sensitivity by using a single VCF file. Our tool is available both via a web-based interface, for a quick analysis with default parameters, as well as a command-line package, allowing assessment of a large numbers of samples, the customization of many parameters and analysis of VCFs from whole-genome sequencing (WGS) experiments.

M. Quinodoz: None. V. Peter: None. N. Bedoni: None. B. Royer Bertrand: None. K. Cisarova: None. A. Salmaninejad: None. N. Sepahi: None. R. Rodrigues: None. M. Piran: None. M. Mojarrad: None. A. Pasdar: None. A. Asad: None. A. Sousa: None. L. CoutinhoSantos: None. A. Superti-Furga: None. C. Rivolta: None.

\section{P17.060.A}

How reliable is HPO-based phenotyping?

F. Boschann ${ }^{1}$, M. Schuelke ${ }^{1}$, E. Seelow ${ }^{1}$, R. Steinhaus ${ }^{2,1}$, D. Hombach $^{2,1}$, P. N. Robinson ${ }^{3}$, D. Seelow ${ }^{2,1}$

${ }^{1}$ Charité - Universitätsmedizin Berlin, Berlin, Germany, ${ }^{2}$ Berliner Institut für Gesundheitsforschung, Berlin, Germany, ${ }^{3}$ The Jackson Laboratory for Genomic Medicine, Farmington, CT, United States

The Human Phenotype Ontology has become a widely used tool for the precise 'deep' phenotyping of patients. However, it remains unclear how accurate and reproducible HPO-based phenotyping is. Different physicians put emphasis on different features, provide different detail levels, and have different opinions on the inclusion of noncardinal symptoms. The complex structure of the ontology and the sheer amount of terms may also add some uncertainty, especially for novel users. 
With The Reliable HPO, we strive to address these issues. We encourage physicians and biomedical researchers to 'phenotype' simulated patients according to their health records. By comparing different HPO phenotype profiles of the same patient, we aim to determine the normal divergence in the HPO-based description of a patient's symptoms and to identify missing or misleading terms.

This knowledge will help us and other groups to better estimate a realistic 'fuzziness' of symptoms when developing software that utilises the HPO, for instance for the prioritisation of mutations in clearly described diseases. In addition, the results can and will be used for an optimisation of the names, synonyms, and descriptions of HPO terms. We include functions to suggest more appropriate names of the symptoms and new synonyms and to point out missing symptoms.

We will of course share all our findings with the community.

Please contribute to The Reliable HPO project at http://www.human-phenotype-ontology.org/reliableHPO/

F. Boschann: None. M. Schuelke: None. E. Seelow: None. R. Steinhaus: None. D. Hombach: None. P.N. Robinson: None. D. Seelow: None.

\section{P17.062.C}

Phenotypic data architecture in peer-reviewed literature relating to genetically-determined developmental disorders

\section{T. M. Yates ${ }^{1}$, J. Campbell ${ }^{1}$, T. I. Simpson ${ }^{2}$, D. R. FitzPatrick ${ }^{I}$}

${ }^{1}$ Institute of Genetic and Molecular Medicine, University of Edinburgh, Edinburgh, United Kingdom, ${ }^{2}$ Institute for Adaptive and Neural Computation, School of Informatics, University of Edinburgh, Edinburgh, United Kingdom

Introduction: Informatic approaches to phenotypic data extraction from the peer-reviewed literature are needed, to maximise speed and accuracy of diagnosis in geneticallydetermined developmental disorders (GDD). We present analysis of phenotypic data in full-text papers published over one year in the American Journal of Human Genetics (AJHG). This will form the basis for development of an automated extraction pipeline.

Materials and Methods: AJHG abstracts from 20172018 describing childhood onset disease were selected. Phenotypic descriptors were annotated in full-text manuscripts and supplemental files (if these contained case reports) using hypothes.is (https://hypothes.is). Annotations were mapped to HPO (Human Phenotype Ontology) terms by computing term frequency (TF) and inverse document frequency (IDF), splitting spans into character trigrams, and calculating cosine similarity values.

\section{Results:}

\begin{tabular}{|c|c|c|c|c|c|}
\hline $\begin{array}{l}\text { Manuscript } \\
\text { type }\end{array}$ & $\begin{array}{l}\text { Manuscript } \\
\text { count }\end{array}$ & $\begin{array}{l}\text { Relevant (to } \\
\text { genotype in } \\
\text { paper)/total } \\
\text { number of } \\
\text { phenotype } \\
\text { annotations }\end{array}$ & $\begin{array}{l}\text { Relevant } \\
\text { annotations } \\
\text { per } \\
\text { document } \\
\text { (median) }\end{array}$ & $\begin{array}{l}\text { Relevant } \\
\text { annotations } \\
\text { per } \\
\text { document } \\
\text { with exact } \\
\text { match to } \\
\text { HPO (mean) }\end{array}$ & $\begin{array}{l}\text { Relevant } \\
\text { annotations per } \\
\text { document with } \\
\text { match to HPO } \\
\text { above cosine } \\
\text { similarity } \\
\text { threshold } \\
0.8 \text { (mean) }\end{array}$ \\
\hline Article & 25 & $\begin{array}{l}1277 / \\
2052(62 \%)\end{array}$ & 67 & 39.9 & 48.6 \\
\hline Report & 43 & $\begin{array}{l}1796 / \\
2623(68 \%)\end{array}$ & 57 & 33.6 & 38.6 \\
\hline Supplemental & 33 & $\begin{array}{l}4726 / \\
5027(94 \%)\end{array}$ & 124 & 50.6 & 97.4 \\
\hline Total & 101 & $\begin{array}{l}7799 / \\
9702(80 \%)\end{array}$ & 67 & 40.7 & 60.3 \\
\hline
\end{tabular}

Table 1. Proportion of phenotype terms in corpus, mapped back to HPO using tf-idf and cosine similarity.

Conclusions: We demonstrate a significant volume of extractable phenotypic data contained within full-text papers describing GDD, most of which can be mapped to HPO terms using closest string match methods. The design of an automated system to extract phenotypes from the scientific literature will be informed by the data architecture presented here, with substantially more information in supplementary files. Ultimately, genotype-phenotype correlation using literature-derived disease models derived from this system will significantly increase the rapidity and accuracy of diagnosis in GDD.

Grant reference: Wellcome Strategic Award 200990/Z/ $16 / Z$

T.M. Yates: None. J. Campbell: None. T.I. Simpson: None. D.R. FitzPatrick: None.

\section{P17.064.B}

Efficient phasing and imputation of low-coverage sequencing data using large reference panels

\section{S. Rubinacci, D. Ribeiro, R. Hofmeister, O. Delaneau}

\section{University of Lausanne, Lausanne, Switzerland}

Low-coverage whole genome sequencing followed by imputation has been proposed as a cost-effective genotyping approach. However, its competitiveness against SNP arrays is undermined as imputation methods are computationally expensive.

Here we describe a method for imputation of lowcoverage sequencing datasets called GLIMPSE. The efficiency our method is achieved by combining advanced 
data structures (PBWT) with a new powerful linear-time sampling algorithm. As a consequence, the computational time to impute a single variant decreases as the size of the reference panel increases, which is an important property since larger panels are constantly made available.

We compared the performance of GLIMPSE to other lowcoverage imputation methods: BEAGLE, GENEIMP and STITCH. GLIMPSE is more accurate for the full allelefrequency range and for rare variants the difference is more pronounced, as GLIMPSE gets an increased accuracy of more than $20 \%$. Notably, these improvements are obtained in running times that are several orders of magnitude shorter of other methods, archiving imputation of a full genome for less than $1 \$$.

Our results show that $1 \mathrm{x}$ coverage enables effective association studies in standard eQTL settings and is better suited than SNP arrays to access the impact of rare variations. We also show that this increased accuracy is more evident for populations not well-represented on SNP arrays. Finally, we demonstrate the potential of $4 \mathrm{x}$ coverage outperforming every other setting tested.

Overall, we demonstrate the potential of low-coverage imputation and suggest a paradigm shift in the design of future genomic studies.

Swiss National Science Foundation project grant (PP00P3_176977).

S. Rubinacci: None. D. Ribeiro: None. R. Hofmeister: None. O. Delaneau: None.

\section{P17.065.C}

Machine learning to distinguish true somatic variants from noise in tumour only next generation sequencing data

\section{Jaramillo Oquendo, H. Parker, J. C. Strefford, J. Gibson, S. Ennis}

University of Southampton, Southampton, United Kingdom

Introduction: Discovery of driver mutations in cancer play a key role in developing staging systems and targeted drugs to fight this disease. Identification of somatic mutations is complex, often aided by concurrent analysis of germline tissue, which is not always available. However, detecting true somatic mutations without germline tissue may leave numerous false positives in the data, requiring validation or time-consuming manual curation. Our aim was to develop an unsupervised machine learning model to distinguish true somatic variants from noise in tumour-only data.

Methods: Haloplex ${ }^{\mathrm{HS}}$ libraries were prepared from splenic marginal zone lymphoma tumour samples $(n=62)$, sequenced, and variants were called with an in-house pipeline that populated numerous quality metrics. Variants were filtered to enrich for somatic mutations and manually curated by two independent investigators, labelling each variant true or false. Ten features were selected after evaluation of all quality metrics for their value in the model. Clustering was performed using $\mathrm{k}$ means.

Results: Variants $(n=873)$ clustered into five groups, true variants in one group and false into four, with $92 \%$ agreement to manual curation labels. Our approach accurately predicted 210/217 (97\%) and 637/656 (97\%) of the variants labelled true and false respectively. Principal component analysis identified mutant allele frequency and quality as the features with the greatest effect on variance between true and false clusters.

Conclusions: This approach successfully identified the majority of true variants with high sensitivity and specificity, allowing for a faster and more accurate method of filtering out noise in tumour-only samples.

Acknowledgements: C.J.O. funded by COLCIENCAS.

C. Jaramillo Oquendo: None. H. Parker: None. J.C. Strefford: None. J. Gibson: None. S. Ennis: None.

P17.066.A

GenNet framework: interpretable neural networks for predicting phenotype from genotype

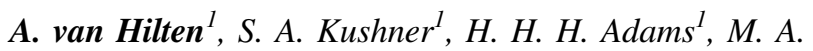
Ikram $^{l}$, W. J. Niessen ${ }^{1,2}$, G. V. Roshchupkin ${ }^{I}$

${ }^{1}$ Erasmus MC, Rotterdam, Netherlands, ${ }^{2} T U$ Delft, Delft, Netherlands

Introduction: Neural networks are currently the state of the art in many domains but seldom applied for genetic risk prediction. Reliable genetic risk prediction demands a network that is able to handle a large number of genetic variants, as well as produce interpretable results that provide insights into the underlying biological mechanisms.

Methods: We propose GenNet, a framework with novel interpretable genetic neural network architectures. Prior biological knowledge (such as DNA annotations, pathways, and gene expression) is used to create layers limited to functionally meaningful connections, thereby constructing computational feasible and interpretable network architectures. Each node represents a biological entity (e.g. gene, pathway). Learned weights represent the contribution of these entities to the overall prediction providing the important entities without any secondary analysis.

Results: The framework's effectiveness is demonstrated by predicting various diseases or traits across a wide spectrum of polygenicity. Table 1 shows the performance and the genes with the largest predictive contributions per phenotype. The framework is not limited to gene-based results. It also includes architectures based on genomic, tissue, cell type, and other functional annotations. 
Conclusion: We developed GenNet, a framework for interpretable neural networks to predict phenotype from genotype data by incorporating prior biological knowledge. Given the interpretability of the network, we anticipate this approach as having the potential for uncovering novel insights into the genetic architecture of complex diseases.

\begin{tabular}{lllllll}
\hline Overview of the results on the held-out test set & & & & \\
\hline Study & Dataset & Phenotype & Heritability & $\begin{array}{l}\text { AUC } \\
\text { Lasso }\end{array}$ & $\begin{array}{l}\text { AUC } \\
\text { GenNet }\end{array}$ & $\begin{array}{l}\text { Three most } \\
\text { important genes }\end{array}$ \\
\hline Eye color & $\begin{array}{l}\text { Rotterdam } \\
\text { (genotype array) }\end{array}$ & $\begin{array}{l}\text { Blue vs. } \\
\text { non-blue }\end{array}$ & $0.80-0.98$ & 0.68 & 0.75 & $\begin{array}{l}\text { HERC2, } \\
\text { OCA2, LAMC1 }\end{array}$ \\
Skin color & $\begin{array}{l}\text { UK biobank } \\
\text { (exome) }\end{array}$ & $\begin{array}{l}\text { Brown / } \\
\text { black } \\
\text { vs. fair }\end{array}$ & $0.99-1$ & 0.97 & 0.98 & $\begin{array}{l}\text { RPL23AP87, } \\
\text { SMARCAD1, }\end{array}$ \\
Hair color & $\begin{array}{l}\text { UK biobank } \\
\text { (exome) }\end{array}$ & $\begin{array}{l}\text { Red } \\
\text { vs. blond }\end{array}$ & $0.70-0.97$ & 0.78 & 0.83 & $\begin{array}{l}\text { MLP1R } \\
\text { OCA2, TC2N }\end{array}$ \\
Hair color & $\begin{array}{l}\text { UK biobank } \\
\text { (exome) }\end{array}$ & $\begin{array}{l}\text { Dark } \\
\text { brown } \\
\text { vs. red }\end{array}$ & $0.70-0.97$ & 0.79 & 0.88 & $\begin{array}{l}\text { MC1R, } \\
\text { OCA2, ZCCHC4 }\end{array}$ \\
Hair color & $\begin{array}{l}\text { UK biobank } \\
\text { (exome) }\end{array}$ & $\begin{array}{l}\text { Blond vs. } \\
\text { dark brown }\end{array}$ & $0.70-0.97$ & 0.64 & 0.75 & $\begin{array}{l}\text { OCA2, } \\
\text { TC2N, EXOC2 }\end{array}$ \\
Balding & $\begin{array}{l}\text { UK biobank } \\
\text { (exome) }\end{array}$ & $\begin{array}{l}\text { Pattern 1 } \\
\text { vs. } \\
\text { pattern } 4\end{array}$ & $0.60-0.70$ & 0.57 & 0.57 & $\begin{array}{l}\text { NGEF, } \\
\text { ANKR18B, }\end{array}$ \\
Case vs. & $0.73-0.93$ & 0.59 & 0.60 & $\begin{array}{l}\text { LINC00266-1, } \\
\text { CSMD1, }\end{array}$ \\
Bipolar & $\begin{array}{l}\text { UK biobank } \\
\text { (exome) }\end{array}$ & $\begin{array}{l}\text { control } \\
\text { TCERG1L }\end{array}$ \\
Schizophrenia & $\begin{array}{l}\text { Sweden } \\
\text { (exome) }\end{array}$ & $\begin{array}{l}\text { Case vs. } \\
\text { control }\end{array}$ & $0.80-0.85$ & 0.65 & 0.74 & $\begin{array}{l}\text { ZNF773, } \\
\text { PCNT, DYSF }\end{array}$ \\
\hline
\end{tabular}

A. van Hilten: None. S.A. Kushner: None. H.H.H. Adams: None. M.A. Ikram: None. W.J. Niessen: A. Employment (full or part-time); Significant; Quantib BV.. G.V. Roshchupkin: None.

\section{P17.067.B}

The added value of RNA sequencing on top of WES for variant interpretation and diagnosis of patients with Mendelian disorders

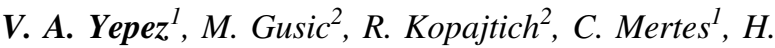 Prokisch $^{2}$, J. Gagneur ${ }^{1}$ \\ ${ }^{1}$ Technical University of Munich, Garching, Germany, ${ }^{2}$ Helmholtz Zentrum Munich, Munich, Germany}

Introduction: RNA sequencing (RNA-seq) is a promising technology to complement Whole Exome Sequencing (WES) for pinpointing the genetic cause of Mendelian diseases. However, with ever-growing and improving databases of WES samples, and better variant effect predictors, the relative advantage of RNA-seq may be questioned.

Materials and Methods: We performed RNA-seq on fibroblasts from 245 individuals affected with a mitochondrial disease who had previously undergone WES, which represents the largest RNA-seq compendium for rare disease diagnostics. We built a computational pipeline to prioritize genes harbouring either mono-allelic expression of a rare allele, aberrant expression level using OUTRIDER, or aberrant splicing using a novel algorithm called FRASER. Moreover, we pinpointed rare variants associated with the prioritized genes either by re-annotating WES variants in the light of the RNA-seq results or by calling variants from the RNA-seq data.

Results: We provided a diagnosis for $15 \%$ (24 out of 165) of WES unsolved cases. We also showed that the majority of OMIM diseases genes are expressed in each of the following clinically accessible tissues: whole blood, muscle, and fibroblasts, suggesting that RNA-seq of accessible, yet not affected, tissues may be suitable for a broad range of rare diseases. We share our computational workflow, called DROP (Detection of RNA Outliers Pipeline), that integrates all analysis steps, can leverage parallel computing infrastructures, and generates browsable web page reports.

Conclusions: Collectively, these results further establish RNA-seq as an effective strategy for validating or invalidating variants of unknown significance and for the discovery of pathogenic gene regulatory events.

V.A. Yepez: None. M. Gusic: None. R. Kopajtich: None. C. Mertes: None. H. Prokisch: None. J. Gagneur: None.

\section{P17.068.C}

Genotyping rare variants with high accuracy using microarray technology

O. Mizrahi Man, M. H. Woehrmann, T. Webster, J. Gollub, A. Roter, J. Schmidt

Thermo Fisher Scientific, Santa Clara, CA, United States

Introduction: UK Biobank is a health resource that follows the health and well-being of 500,000 participants, all genotyped with very high accuracy on Thermo Fisher Scientific's Applied Biosystems ${ }^{\mathrm{TM}}$ Axiom $^{\mathrm{TM}}$ microarrays. A recent pre-publication based on this data set claimed that microarrays may not be the technology of choice for calling very rare variants, defined as having minor allele frequency below $0.01 \%$. We show that a genotyping algorithm modification tailored to very rare variants achieves excellent accuracy for these variants.

Materials and Methods: We have optimized the genotyping algorithms for very rare variants. For common variants the location of the heterozygous cluster provides powerful evidence for heterozygous calls. Rare variants usually have a single sample in the het cluster, making the call less robust. An in-depth analysis of the distribution of replicate probe signal on Axiom microarrays revealed this distribution can be used to significantly improve the accuracy of heterozygous calls.

Results: Eight separate data sets with 1000 Genomes Project truth originally achieved positive predictive values 
(PPVs) ranging from $60 \%$ to $93 \%$ for very rare variant calls. The new algorithms increased all PPVs to near $90 \%$ while losing on average only $0.5 \%$ of correct calls. We will report the results of applying the new algorithms to rare variant genotyping in the UK Biobank cohort.

Conclusions: Improved algorithms for genotype calls for Axiom microarrays improve the PPV of very rare variants while minimally affecting sensitivity.

Analysis of UK Biobank data is conducted using UK Biobank Resource under Application Number 55681.

O. Mizrahi Man: None. M.H. Woehrmann: A. Employment (full or part-time); Significant; Thermo Fisher Scientific. T. Webster: A. Employment (full or part-time); Significant; Thermo Fisher Scientific. J. Gollub: A. Employment (full or part-time); Significant; Thermo Fisher Scientific. A. Roter: A. Employment (full or part-time); Significant; Thermo Fisher Scientific. J. Schmidt: A. Employment (full or part-time); Significant; Thermo Fisher Scientific.

\section{P17.070.B}

AdapMiR: a new tool for trimming adapters and counts calculation in miRNA sequencing

\section{A. Zarubin, A. Markov, M. Nazarenko}

Research Institute of Medical Genetics, Tomsk National Research Medical Center, Tomsk, Russian Federation

Introduction: Trimming of adapter sequences from miRNA-seq data is a common preprocessing step during NGS data analysis. Overtrimmed reads and adapter contamination can be challenging for downstream analysis. We have proposed an algorithm called AdapMiR for the trimming adapters and further analysis of miRNA expression that turns these drawbacks into advantages. Our algorithm can achieve high accuracy of adapter trimming and discard non-miRNA reads.

Material and Methods: AdapMiR was implemented in $\mathrm{R}$ language. miRNA-seq data of atherosclerotic plaques $(n=5)$ and healthy vessels $(n=5)$ was used as a test dataset. Mature miRNAs sequences were obtained from miRBase and used for construction of artificial reference including adapter sequences. BWA and edgeR were used for read alignment and differential expression analysis. We compared developed algorithm with miARma-Seq and Trimmomatic in high sensitivity mode. AdapMiR is freely available (https:/github.com/alekseizarubin/AdapMiR).

Results and discussion: Results of adapMiR and Trimmomatic differed for $55 \%$ of miRNA reads. Moreover, we noticed a peculiarity in Trimmomatic output: the lesser read length the more probable was overtrimming, but longer read sequences ( $\geq 20 \mathrm{bp}$ ) could contain adapter fragments. In contrast, AdapMiR did not produced excessively trimmed reads while filtered out erroneously read nucleotides. Only $2 \%$ of total counts per sample differed between algorithms. The number of differentially expressed miRNAs between atherosclerotic plaques and healthy vessels were equal ( $n=$ 151), but resulting lists of both algorithms was different by 18 miRNA.

Conclusions: AdapMiR is comparable to miARma-Seq approach. Our algorithm improves the accuracy of singleend read trimming by adding adapter sequences to the reference.

A. Zarubin: None. A. Markov: None. M. Nazarenko: None.

P17.073.B

Multisite de novo mutations after paternal exposure to ionizing radiation

\author{
F. Brand ${ }^{1}$, M. Holtgrewe ${ }^{2}$, A. Knaus ${ }^{1}$, D. Beule ${ }^{2}$, P. M. \\ Krawitz
}

${ }^{1}$ Institut for genomics statistics and bioinformatics, Bonn, Germany, ${ }^{2}$ Berlin Institute of Health, Berlin, Germany

In our ongoing study we evaluate the effects of ionizing radiation on the offspring of exposed soldiers.

We sequenced the entire genomes of 56 families with 87 children on an Illumina NovaSeq 6000. Eighteen offspring of twelve families have earlier been sequenced on a HiSeq $\mathrm{X}$, three of which have now been resequenced. In our comparison we found a sequencing pattern with an increased rate of $\mathrm{A}>\mathrm{C}$ and $\mathrm{T}>\mathrm{G}$ transversions on the NovaSeq, most probably due to its novel two-color chemistry.

Our focus lies on specific mutational patterns such as MSDNs (at least two de novo mutations within $20 \mathrm{bp}$ ), SVs and CNVs, which could be a result of DNA damage from exposure to ionizing radiation before conception of the children.

From our large cohort we are able to reliably estimate age effects and compare the performance of different variant calling applications. We confirmed the origin of all MSDNs using long-read and Sanger sequencing and validated all complex de novo variants. We found that MSDNs occurred only on the paternal allele. Since only fathers were exposed to ionizing radiation, this finding strengthens our hypothesis that MSDNs could serve as a biomarker to identify previous exposure for exposure to ionizing radiation.

In our cohort we detected 1.125 MSDNs/offspring of exposed fathers compared to 0.3 MSDNs/offspring in unexposed controls. These numbers are corrected for age effects and platform specific mutational signatures. The 
increased rate of MSDNs could serve as a biomarker for DNA damage caused by prolonged exposure to irradiation.

F. Brand: None. M. Holtgrewe: None. A. Knaus: None. D. Beule: None. P.M. Krawitz: None.

\section{P17.077.C}

unCOVERApp: an interactive graphical webapplication for clinical assessment of sequence coverage at the base-pair level

\section{E. Iovino ${ }^{l}$, T. Pippucci $i^{2}$}

${ }^{1}$ University of Bologna, Department of Medical and Surgical Sciences, Bologna, Italy, ${ }^{2}$ Medical Genetics Unit, Sant'Orsola-Malpighi University Hospital, Bologna, Italy

Introduction: The rapid spread of Next Generation Sequencing technology has been coupled since its beginning with development of bioinformatic tools for data analysis and interpretation. However, despite increasing accuracy of available approaches, the need to assess sequencing quality of the analysis targets at the base-pair resolution poses growing challenges, especially in the clinical settings. In diagnostics indeed, meticulous investigation of every single target base is often required to exclude that pathogenic events across the gene of interest may be missed due to uneven sequence coverage. We present unCOVERApp an application both for graphical inspection both dbNSFP-based annotations of sequence coverage at base-pairs levels.

Method: unCOVERApp is a freely available application written in $\mathrm{R}$ and developed with Shiny packages (https:// github.com/Manuelaio/unCOVERApp).

Results: unCOVERApp is a suite of graphical and statistical tools to support the clinical assessment and annotation of low-sequence coverage positions in genomic data, providing interactive plots displaying gene sequence coverage, functional and clinical annotations of base-pair positions within user-defined coverage thresholds to support user's judgement about their possible clinical relevance. Importantly, the binomial calculator expresses the probability that a variant is missed given its expected allelic fraction and sequence coverage. The maximum credible allele frequency calculator allows to label existing variants within low coverage regions based on allele frequency calculated according to the disease model instead of using general cut-offs (e.g. 0.05).

Conclusions: uncoverApp is a graphical interface tool designed to help especially clinically-oriented users in easy inspection of coverage profiles of the gene of interest at the base-pair level.

E. Iovino: None. T. Pippucci: None.

\section{P17.079.B}

Prioritisation of non-coding regions based on human genomic constraint and primary sequence context with deep learning

\section{Vitsios, S. Petrovski}

\section{AstraZeneca, Cambridge, United Kingdom}

Elucidating functionality in human non-coding sequence is a key genomics challenge. It has been shown that intolerance to variation of coding and proximal non-coding sequence is a strong predictor of human disease relevance. Here, we expand on published methods by applying a tiled genome-wide Residual Variation Intolerance Score (gwRVIS) to Whole Genome Sequencing (WGS) data from 62,784 individuals with lower gwRVIS corresponding to greater intolerance to genetic variation. We verified that gwRVIS distinguishes sequence occupied by human Mendelian disease genes from intergenic sequence (median gwRVIS -0.54 versus $-0.011 ; p=2.4 \times 10^{-195}$ ). Remarkably, despite being focused solely on human lineage purifying selection, gwRVIS identified sequence occupied by known ultra-conserved non-coding elements as the most intolerant category (median gwRVIS $=-0.85 ; p<$ $\left.1 \times 10^{-320}\right)$. gwRVIS also boosts predictive performance in pathogenic variant detection from ClinVar in intergenic regions, lincRNAs and UTRs. We then employ gwRVIS along with functional genomic annotation (including methylation and chromatin accessibility) and primary genomic sequence to build "Junk Annotation" RVIS (JARVIS): a comprehensive deep learning model to prioritise non-coding variants using only human lineage-specific information. Despite not incorporating any conservation information, JARVIS outperforms every comparable score on pathogenicity inference of non-coding variants and performs comparably or better in some cases over current state-of-the-art methods that do employ conservation information. Both gwRVIS and JARVIS capture previously inaccessible human-lineage constraint information as derived from 62,784 WGS to help prioritise genetic variants found in the human non-coding regulatory sequence and further enable the pursuit of novel drug targets and biomarkers in the non-coding genome.

D. Vitsios: A. Employment (full or part-time); Significant; AstraZeneca. S. Petrovski: A. Employment (full or part-time); Significant; AstraZeneca.

\section{P17.080.C}

Analysis of mutation burden in Parkinson's disease using machine learning, a case-control study 
G. Bergant, A. Maver, A. Kovanda, B. Peterlin

Clinical Institute of Genomic Medicine, Ljubljana, Slovenia

Introduction: Despite known risk factors and monogenic causes most sporadic and familial cases of Parkinson's disease (PD) remain idiopathic. In our study we hypothesize that polygenic causes explain a proportion of idiopathic cases and use machine learning on exome sequencing data to identify individuals at risk for PD.

Materials and Methods: We analyzed genetic variants detected by clinical exome sequencing of 68 early onset or familial PD patients with no discovered monogenic cause and 323 control individuals. All single nucleotide coding variants with genotype quality $\geq 100$ have been selected and the number of germline variants per gene has been calculated for all individuals. A single dataset containing the calculations, information on all analyzed genes and the individual's disease status has been prepared as an input to the ML analysis. We employed a supervised ML algorithm, gradient boosted trees with regularization (eXtreme Gradient Boosting), which used the observed patterns to determine, first, the possibility to predict the disease status on the test group and, second, the subset of genes with the highest contribution to the prediction.

Results: ML approach yielded the accuracy of $80.5 \%$, and area under the receiver-operator characteristic curve of $74.3 \%$ (sensitivity: $30.8 \%$, specificity: $90.6 \%$ ). The genes contributing highly to the algorithms prediction were analyzed and a pathway enrichment analysis was performed.

Conclusions: The results show that the argument for a significant polygenic risk on the development of PD is promising, however, larger studies are necessary to further determine its impact.

G. Bergant: None. A. Maver: None. A. Kovanda: None. B. Peterlin: None.

\section{P17.082.B}

Phenotype-based search using free online databases is ineffective in diagnostics of mendelian disorders

\author{
A. Fellner ${ }^{1,2}$, N. Ruhrman-Shahar ${ }^{1,3}$, N. Orenstein $^{3,4}, G$. \\ Lidzbarsky $^{l}$, O. Hagari ${ }^{l}$, H. Brown-Shalev ${ }^{l}$, L. Bazak $^{l}$, L. \\ Basel-Salmon ${ }^{1,3,5}$
}

${ }^{1}$ Raphael Recanati Genetics Institute, Rabin Medical Center, Beilinson Hospital, Petah Tikva, Israel, ${ }^{2}$ Department of Neurology, Rabin Medical Center, Beilinson Hospital, Petah Tikva, Israel, ${ }^{3}$ Sackler Faculty of Medicine, Tel-Aviv University, Tel-Aviv, Israel, ${ }^{4}$ Pediatric Genetics
Clinic, Schneider Children's Medical Center of Israel, Petah Tikva, Israel, ${ }^{5}$ Laboratory of Immunology and Genetics, Felsenstein Medical Research Center, Petah Tikva, Israel

Introduction: Searching online databases for a genetic diagnosis that fits a clinical phenotype is used routinely in clinical genetics. We investigated the diagnostic yield of phenotype-based search using free online databases.

Materials and methods: We included consecutive solved cases from our whole-exome database. For each case, the combination of Human Phenotype Ontology (HPO) terms reported by the clinician was used to search three commonly used databases: Online Mendelian Inheritance in Man (OMIM), Phenolyzer and Mendelian. We examined whether the actual molecular diagnosis (AMD) found by whole-exome sequencing was included in the search results (First 300 in OMIM and Phenolyzer; All 100 in Mendelian).

Results: One hundred consecutive cases were included (43 females; mean age: 10 years). Search results included the AMD in none of the databases in $33.0 \%$ of cases, and in one, two or three databases in $30.0 \%, 20.0 \%$ and $17.0 \%$ of cases, respectively. The first 5 search results included the AMD in only 9.0\%, 6.0\% and 7.0\%, in OMIM, Phenolyzer and Mendelian, respectively. When included, the AMD's mean rank in the results' list was 97 (range: 1-295), 56 (1270) and 37 (1-92) in these databases, respectively.

Conclusions: This study demonstrates that phenotypebased search using free online databases is ineffective in finding a probable diagnosis of mendelian conditions. It emphasizes the need for better search tools and for a more structured approach in HPO terms selection, and may suggest that, at least in some cases, a genotype-first approach with backward phenotyping may be more appropriate.

A. Fellner: None. N. Ruhrman-Shahar: None. N. Orenstein: None. G. Lidzbarsky: None. O. Hagari: None. H. Brown-Shalev: None. L. Bazak: None. L. Basel-Salmon: None.

\section{P17.084.A}

Developing genetic risk model for quantitative traits considering epistasis

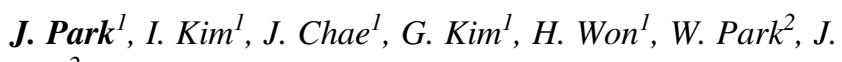
$\operatorname{Kim}^{2}$

${ }^{1}$ Samsung Advanced Institute for Health Sciences and Technology, Sungkyunkwan University, Samsung Medical Center, Seoul, Korea, Republic of, ${ }^{2}$ Samsung Genome Institute, Samsung Medical Center, Seoul, Korea, Republic of 
As massive resources of genotype and phenotype data become available, genetic risk score holds great potential to improve precision medicine. Unfortunately, the problem of missing heritability still prevails, despite several previous studies had improved the conventional polygenic risk score, for which risk alleles were weighted by the effect size of their association with given trait and summed up. To alleviate the problem here we systematically explore epistasis between genes that may modulate polygenic risk score for greater accuracy. Specifically, we developed a model assuming multiplicative interactions between different loci and applied the model to blood assay quantitative traits in UK Biobank. To avoid overfitting emerged from enumerated variables depicting many interactions between loci, we employed a shrinkage method. We found that the epistasis model indeed improved polygenic risk score for several traits, including total bilirubin and Lipoprotein A. We further investigated the functional relationships between genes that may contribute to the discovered epistatic effects.

J. Park: None. I. Kim: None. J. Chae: None. G. Kim: None. H. Won: None. W. Park: None. J. Kim: None.

\section{P17.085.B}

Differential positive selection in complex traits: interpopulation study of local adaptation in loci associated with hair colour

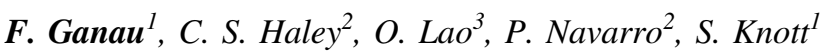 \\ ${ }^{1}$ Institute of Evolutionary Biology, University of Edinburgh, Edinburgh, United Kingdom, ${ }^{2}$ MRC Human Genetics Unit, University of Edinburgh, Edinburgh, United Kingdom, ${ }^{3}$ CNAG-CRG, Barcelona, Spain}

When humans left Africa and dispersed around the world, they met novel environments to which their phenotypes were not adapted. The resulting local selective pressures in each of these geographically distinct populations varied in direction and strength, and led to a plethora of divergent phenotypic adaptations. This research studies the genomic fingerprint left by these processes for hair colour, a trait with substantial inter and intra-population phenotypic variation, that we use as a proof-of-concept for differential adaptation. Genomic regions strongly associated with hair colour are defined based on a published GWAS of the white British population. Evidence for positive selection in these regions is evaluated with a single, composite statistic. This statistic is produced by merging five different tests of positive selection with the De-correlated Composite of Multiple Signals method, used for multivariate analyses. By applying conservative P-rank transformations, we integrate signal information from both of haplotype homozygosity and Site-Frequency Spectrum (SFS) into a single, normally distributed parameter of adaptation. Association between hair colour and selection is tested using permutation-based methodologies. Significant regions from the British haircolour GWAS are tested for selection across all 26 populations present in the 1000 Genomes database (https://www. internationalgenome.org/), comparing how positive selection shaped each of them. As selective sweeps often lead to extreme allele frequencies (MAF, it is possible that intermediate size alleles undergoing recent adaptation are nearly fixed and thus undetectable by GWAS. To evaluate this effect the analysis is repeated selecting GWAS regions based on effect sizes instead of $p$-values.

F. Ganau: None. C.S. Haley: None. O. Lao: None. P. Navarro: None. S. Knott: None.

P17.086.C

GenomeMixer and NiPTUNE: novel bioinformatics tools to improve Non-Invasive Prenatal Testing for fetal aneuploidies

V. Duboc ${ }^{1}$, D. Pratella ${ }^{2}$, M. Milanesio ${ }^{2,3}$, J. Boudjarane ${ }^{4}, S$. Descombes $^{2}$, V. Paquis-Flucklinger ${ }^{1,2}$, S. Bottini ${ }^{2}$

${ }^{1}$ Université Côte d'Azur, CHU, Inserm U1081, CNRS UMR7284, IRCAN, Nice, France, ${ }^{2}$ MDLab - MSI Université Cote d'Azur, Nice, France, ${ }^{3}$ Inria Sophia Antipolis, Epione Research Project, Sophia Antipolis, France, ${ }^{4}$ Département de génétique médicale, CHU la Timone, Marseille, France

Introduction: The discovery of free circulating fetal DNA in maternal blood has led to the development of NonInvasive Prenatal Testing (NIPT) techniques for detection of fetal aberrations such as trisomy 21. NIPT is based on the analysis of small fragments of DNA, circulating freely in the maternal blood, by massively parallel sequencing. Its reliability relies heavily on both the presence in the maternal blood of a sufficient amount of fetal DNA (ff for fetal fraction) and a sufficient sequencing depth (sd). Bioinformatics tools have been developed to determine the ff but there is currently no reference method in clinical practice.

Materials and Methods: We developed GenomeMixer, an approach to estimate confidence intervals for aneuploidies prediction, usable by all diagnostic laboratories and NiPTUNE, a package to automate NIPT analysis. GenomeMixer creates synthetic sequencing data mixing reads from pregnant women with fetal aneuploidies and from non-pregnant women in order to determine the threshold of both minimum ff and sd necessary to detect aneuploidies reliably. Niptune pipeline is composed by several modules including quality control, ff estimation by different methods and aneuploidies prediction.

Results: We tested our tools on 2 different cohorts composed by 377 and 1055 samples including respectively 
10 and 24 aneuploidies. GenomeMixer allowed to determine minimal threshold of both ff and sd. NiPTUNE was validated thanks to the retrospective study performed on the cohorts.

Conclusions: We have developed bioinformatics tools to empower each diagnostic laboratory to determine the confidence intervals of both ff and sd necessary to a reliable NIPT.

V. Duboc: None. D. Pratella: None. M. Milanesio: None. J. Boudjarane: None. S. Descombes: None. V. Paquis-Flucklinger: None. S. Bottini: None.

P17.087.A

Classifying Rare Genetic Variants by Enriching and Clustering on 3D Protein Structure Domains

\section{J. D. Stephenson ${ }^{1}$, R. A. Laskowski ${ }^{1}$, M. E. Hurles ${ }^{2}$, J. M.} Thornton ${ }^{1}$

${ }^{1}$ EMBL-EBI, Hinxton, United Kingdom, ${ }^{2}$ Wellcome Sanger Institute, Hinxton, United Kingdom

Introduction: Variant classification is an important step in reducing the diagnostic odyssey for patients with rare genetic disorders. For missense variants their direct effect is largely determined by their local environment as dictated by their position in the protein structure. This means that other variants in the local environment may lead to a similar protein consequence. Our method classifies variants of unknown significance by considering their positions in structure relative to known pathogenic or neutral variants.

Materials and Methods: We map neutral and known pathogenic variants to the most relevant protein 3D structures and then enrich the data to aid pattern detection by combining variant data across structurally conserved regions of proteins. Following this we discover clusters of known pathogenic and neutral variants on the protein structure domain assemblage with parameters optimised using machine learning to capture variant effects common across proteins with shared structure. Single VUS or entire cohorts are then compared with our pre-calculated clusters across all conserved structural domains containing over 100,000 protein structures.

Results: Our method classifies individual VUS based on their proximity to pre-calculated neutral/pathogenic regions with confidence values based on domain specific model classification precision. Using this method we have analyzed a set of 30,000 missense VUS from patients with rare developmental disorders and uncovered several previously unreported gene associations.

Conclusions: Our method offers a complementary approach to other pathogenicity predictors by combining evolutionary structure conservation with previously annotated variant locations.

J.D. Stephenson: None. R.A. Laskowski: None. M.E. Hurles: None. J.M. Thornton: None.

\section{P17.089.C}

Making the most of Clumping and Thresholding for polygenic scores

\section{F. Privé ${ }^{1,2}$, B. J. Vilhjálmsson ${ }^{1}$, H. Aschard ${ }^{3}$, M. G. B. Blum ${ }^{2}$}

${ }^{1}$ Aarhus University, Aarhus, Denmark, ${ }^{2}$ Univ. Grenoble Alpes, Grenoble, France, ${ }^{3}$ Institut Pasteur, Paris, France

Polygenic prediction has the potential to contribute to precision medicine. Clumping and Thresholding $(\mathrm{C}+\mathrm{T})$ is a widely used method to derive polygenic scores. We implement an efficient way to derive thousands of different $\mathrm{C}+\mathrm{T}$ polygenic scores corresponding to a grid over several hyper-parameters. We find that optimizing over the proposed four hyper-parameters improves the predictive performance of $\mathrm{C}+\mathrm{T}$ in both simulations and real data applications, as compared to tuning only the $p$-value threshold of $\mathrm{C}+\mathrm{T}$. A particularly large increase can be noted when predicting depression status, from an AUC of 0.557 (95\% CI: [0.544-0.569]) when tuning only the $p$ value threshold in $\mathrm{C}+\mathrm{T}$ to an AUC of 0.592 (95\% CI: [0.580-0.604]) when tuning all four hyper-parameters of $\mathrm{C}$ $+\mathrm{T}$. Overall, by optimizing $\mathrm{C}+\mathrm{T}$ polygenic scores, one can achieve prediction accuracies on par with more sophisticated approaches such as LDpred and lassosum (Vilhjálmsson 2015, Mak 2017).

We further propose Stacked Clumping and Thresholding (SCT). Instead of choosing one set of hyper-parameters that maximizes prediction in some training set, SCT learns an optimal linear combination of all $\mathrm{C}+\mathrm{T}$ scores (stacking). We apply SCT to 8 different case-control diseases in the UK biobank data and find that SCT significantly improves prediction accuracy compared to $\mathrm{C}+\mathrm{T}$, resulting in an average AUC increase of 0.035 over standard $\mathrm{C}+\mathrm{T}$. We conclude with extensions of SCT.

F. Privé: None. B.J. Vilhjálmsson: None. H. Aschard: None. M.G.B. Blum: None.

\section{P17.090.A}

Identifying single nucleotide polymorphisms modulating gene expression by transcription factors. A novel approach for regulatory QTLs. 
F. Moratalla-Navarro ${ }^{1,2,3}, V$. Diez-Obrero ${ }^{1,2,3}, R$.

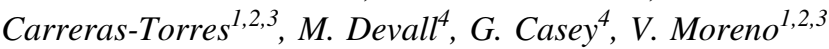

${ }^{1}$ Oncology Data Analytitcs Program, Catalan Institute of Oncology (ICO), L'Hospitalet de Llobregat, Spain, ${ }^{2}$ Colorectal Cancer Group, ONCOBELL program, Bellvitge Biomedical Research Institute (IDIBELL), L'Hospitalet de Llobregat, Spain, ${ }^{3}$ Consortium for Biomedical Research in Epidemiology and Public Health (CIBERESP), Madrid, Spain, ${ }^{4}$ Public Health Sciences, University of Virginia, Charlottesville, VA, United States

Introduction: The functional role of expression quantitative trait loci (eQTLs) often remains to be explained. Most of the single nucleotide polymorphisms (SNPs) involved in are located in regulatory regions such as promoters or enhancers, and could interact with transcription factors (TFs) to regulate gene expression (regQTLs). We hypothesize a main role of TFs mediating the association between SNPs and gene expression.

Materials and Methods: Colon tissue biopsies and blood samples were obtained from 191 healthy donors, from which both gene expression and genotype data were generated. Using expression data, a consensus Gene Regulatory Network (GRN) was constructed by merging three different GRN inference methods (ARACNE-ap, BC3, and GENIE3), to get only robust interactions. Finally, regression models were applied to detect regQTLs, using co-expression of TF - gene pairs learned by GRN and SNPs located in regulatory sites.

Results: We found a total of 1,427 different interactions between regQTLs, TFs and target genes, comprising 73 unique target genes of 109 TFs and 1,212 unique regQTLs (FDR < 0,01). We plan to enlarge the existing dataset to nearly 500 samples during the next months.

Conclusions: In this study, we present a novel in-silico method that identified 1,212 unique regQTLs, and could help to explain regulatory interactions and genome-wide disease associations. Preliminary results still need to be validated.

Grant references:NIH/NCI CA143237; NIH/NCI CA204279; NIH/NCI CA201407

F. Moratalla-Navarro: None. V. Diez-Obrero: None. R. Carreras-Torres: None. M. Devall: None. G. Casey: None. V. Moreno: None.

\section{P17.092.C}

INFRAFRONTIER services for rare disease research

A. Ali Khan, R. Michna, M. Raess, S. Fessele, M. Hrabĕ de Angelis, INFRAFRONTIER Consortium

INFRAFRONTIER GmbH, Munich, Germany
According to the EU, a rare disease is defined as a disease afflicting fewer than 1 in 2000 individuals. It is estimated that 30 million people in Europe suffer from a rare disease. There are currently about 7000 rare diseases known with more being discovered continually. The field of rare diseases suffers from a deficit of medical and scientific knowledge. These diseases reside in the 'unchartered space' of biomedical research with their treatment marred by a dearth of medical and biochemical knowledge. This has led to development and implementation of several strategies to tackle the treatment of rare and debilitating diseases. For example, the EU supports research into rare diseases through Horizon 2020, the EU Framework Programme for Research and Innovation. Via Horizon 2020, about €900 million is available to more than 160 collaborative projects related to rare diseases. The core services of INFRAFRONTIER (the European Research Infrastructure for Mouse Models) comprise of the systemic phenotyping of mouse mutants in the participating mouse clinics, and the archiving and distribution of mouse mutant lines by the European Mouse Mutant Archive (EMMA) which also include lines important for rare disease research. To consolidate and present the INFRAFRONTIER / EMMA resources related to rare diseases in an easily accessible location, the INFRAFRONTIER and Rare Diseases web page was created, providing access to a) EMMA strains related to rare diseases b) EMMA publications related to rare diseases and c) information on INFRAFRONTIER activities at rare disease conferences. This webpage can be found at: https://www.infrafrontier.eu/infrafrontier-and-ra re-diseases.

A. Ali Khan: None. R. Michna: None. M. Raess: None. S. Fessele: None. M. Hrabĕ de Angelis: None.

\section{P17.093.A}

Exome-wide identification of pathogenic missense variants by Paralogue Annotation

N. Li, E. Mazaika, R. Walsh, P. Theotokis, M. Ahmed, M. Jang, X. Zhang, N. Whiffin, P. Barton, J. Ware

Imperial College London, London, United Kingdom

Introduction: Distinguishing novel pathogenic from rare but benign variants is a key challenge in clinical genetics. Machine learning tools that predict the consequence of missense variants typically perform unreliably on novel variants since there is little information to be used in training sets. We previously developed Paralogue Annotation (PA); a method that does not require training, but instead exploits an under-utilised source of information. We look for functionally equivalent variants of known consequence at equivalent positions across paralogous protein 
families and use their information to predict pathogenicity. This method was validated in a restricted set of arrhythmia syndrome genes. Here, we extend it exome-wide.

Materials and Methods: Paralogue relationships as defined by Ensembl were examined in 7,016 proteincoding genes associated with human diseases, that harbor 48,258 (Likely) Pathogenic/Benign missense variants from ClinVar. PA and current established prediction tools were applied to this and multiple independent test sets.

Results: We identify 3,621 genes that have at least 1 paralogue and show that equivalent pathogenic variants from other related proteins provides strong supporting evidence for pathogenicity at $98 \%$ precision for ClinVar variants. Other tools have a lower precision (mean 57.3\% less) and higher false-positive rate (mean $98.4 \%$ more) across all variants tested. With current variants in ClinVar, we identify 178,378 potentially informative annotations exome-wide.

Conclusions: PA represents an under-used source of information with an extremely high positive predictive value for identifying novel and rare disease-causing variants. As more variants arise, the utility and power of PA will increase over time.

N. Li: None. E. Mazaika: None. R. Walsh: None. P. Theotokis: None. M. Ahmed: None. M. Jang: None. X. Zhang: None. N. Whiffin: None. P. Barton: None. J. Ware: None.

\section{P17.094.B}

A population-specific reference genome built by integrating three de novo Japanese genome assemblies

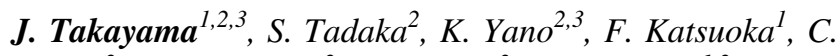
Gocho $^{2}$, T. Funayama ${ }^{2}$, S. Makino ${ }^{2}$, Y. Okamura ${ }^{1,2}$, A.

Kikuchi $^{4}$, J. Kawashima ${ }^{2}$, A. Otsuki ${ }^{2}$, J. Yasuda ${ }^{2}$, S. Kure ${ }^{2,4}$, K. Kinoshita ${ }^{1,2,5}$, M. Yamamoto $^{1,2}$, G. Tamiya ${ }^{1,2,3}$

${ }^{1}$ Advanced Research Center for Innovations in NextGeneration Medicine, Tohoku University, Sendai, Japan,

${ }^{2}$ Tohoku Medical Megabank Organization, Tohoku University, Sendai, Japan, ${ }^{3}$ RIKEN Center for Advanced Intelligence Project, Tokyo, Japan, ${ }^{4}$ Department of Pediatrics, Tohoku University School of Medicine, Sendai, Japan,

${ }^{5}$ Graduate School of Information Sciences, Tohoku University, Sendai, Japan

The human reference genome is an invaluable resource both for basic and clinical genetic research. However, the reference genome lacks some population-specific sequences, is mainly composed of genomes from African or European origins, and has $>90,000$ rare reference alleles. Here we report JG1, a population-specific reference genome built by integrating three de novo Japanese genome assemblies. We performed three de novo hybrid assemblies of $>100 \mathrm{X}$ PacBio long reads and $>100 \mathrm{X}$ Bionano optical maps per individual. After errorcorrection with the long and $>50 \mathrm{X}$ short reads, the three assemblies were meta-assembled by using mate-pair information. We then replaced minor alleles in the meta-assembled genome by major alleles defined among the three individual assemblies. Finally, we built pseudo-chromosomes by anchoring each scaffold to each chromosome on the basis of marker positional information from genetic and radiation hybrid maps. The resulting genome JG1 was highly contiguous (Scaffold NG50 $=141 \mathrm{Mb}$ ), concordant with GRCh38 (only 1,654 mis-assemblies), and replaced minor reference allele in $96 \%$ of $650,718 \mathrm{SNV}$ sites (non-reference allele frequency $>90 \%$ in Japanese population). Moreover, we performed confirmatory exome re-analysis of rare diseases using JG1 as a reference, and found that the number of total variant calls was smaller in JG1 than in the reference hs $37 \mathrm{~d} 5$, while the variant call set retained the causal variants. These results suggest that integration of multiple assemblies is an effective way to build a high-quality population-specific reference genome. JG1 is publicly available and can be browsed in the jMorp website (https://jmorp.megabank. tohoku.ac.jp/).

J. Takayama: None. S. Tadaka: None. K. Yano: None. F. Katsuoka: None. C. Gocho: None. T. Funayama: None. S. Makino: None. Y. Okamura: None. A. Kikuchi: None. J. Kawashima: None. A. Otsuki: None. J. Yasuda: None. S. Kure: None. K. Kinoshita: None. M. Yamamoto: None. G. Tamiya: None.

\section{P17.095.C}

Accurate and simultaneous identification of differential expression and splicing using hierarchical Bayesian analysis

P. N. Robinson ${ }^{1}$, G. Karlebach
, P. Hansen

${ }^{1}$ The Jackson Laboratory for Genomic Medicine, Farmington, CT, United States, ${ }^{2}$ Charité Universitätsmedizin, Berlin, Germany

The regulation of mRNA controls both overall gene expression as well as the distribution of mRNA isoforms encoded by the gene. Current algorithmic approaches focus on characterization of significant differential expression or alternative splicing without integrating both events. Here, we present Hierarchical Bayesian Analysis of Differential Expression and ALternative Splicing (HBA-DEALS), which simultaneously characterizes differential expression and splicing in cohorts. The statistical model of HBADEALS characterizes genes as having differential gene expression (DGE), differential alternative splicing (DAST), 
both, or neither. We analyzed 50 simulated datasets for DGE with HBA-DEALS and five other top-performing DGE analysis tools: voom, DESeq2, edgeR, baySeq, and NOISeq. We analyzed the same datasets for DAST with rMATS. HBA-DEALS displayed a larger area under the precision-recall curve than the other approaches did for both DGE and DAST. To assess the accuracy of HBA-DEALS using real data, we ran HBA-DEALS on estimated isoform levels from the Genotype-Tissue Expression (GTEx) project. We used HBA-DEALS to identify differentially expressed and differentially spliced genes in 20 different pairs of tissues and found a high degree of overlap of genes identified as differentially expressed or spliced $(\mathrm{p}<2.23 \times$ $10^{-308}$ for all comparisons, hypergeometric test). Based on an analysis of the GTEx data, we demonstrate the existence of sets of genes that show predominant DGE or DAST across a comparison of 20 tissue pairs, and show that these sets have pervasive differences with respect to gene structure, function, membership in protein complexes, and promoter architecture.

P.N. Robinson: None. G. Karlebach: None. P. Hansen: None. D.F.T. Veiga: None. R. Steinhaus: None. D. Danis: None. S. Li: None. O. Anczukow: None.

P17.096.A

ROHMM, a flexible HMM approach for detecting homozygosity using next generation sequencing data

G. Çelik ${ }^{1}$, T. Tuncali ${ }^{2}$

${ }^{1}$ Health Sciences Institute, Ankara Yildirim Beyazit University, Ankara, Turkey, ${ }^{2}$ Department of Medical Genetics, Ankara University School of Medicine, Ankara, Turkey

Introduction: Runs of long homozygous stretches (ROH) are considered to be the result of consanguinity and usually contain recessive deleterious disease causing mutations. Several algorithms have been developed to detect ROHs. Here, we developed a simple, alternative strategy by examining $\mathrm{X}$ chromosome non-pseudoautosomal region (Non-PAR) to detect the ROHs from NGS data utilizing the genotype probabilities and the hidden Markov model (HMM) algorithm as a tool, namely ROHMM. It is implemented purely in java and contains both command line and GUI. ROHMM can operate in a range from single sample to population scale data alike.

Materials and Methods: NGS genotype data is from $1000 \mathrm{G}$ phase3. 25 deep sequenced whole genome data (10 males, 15 females) were processed to collect genotyping results from the $\mathrm{X}$ chromosome Non-PAR. Genotype probabilities were integrated into HMM algorithm along with dynamic transition probabilities calculated from distances between each locus.
Results: Simulation results demonstrated that ROHMM can detect ROHs of varying lengths with great precision and accuracy compared to PLINK, BCFtools, and H3M2. On real population data, ROHMM proved to be as robust as using allele frequencies or sliding window methods. Comparisons between heterozygosity ratio and ROHMM's inferences have shown a significant correlation which supports the integrity of the ROHMM for the population studies.

Conclusions: We present a novel approach for the detection of ROHs. ROHMM's strength comes from the emission parameters deduced from NGS genotype topology rather than allele frequencies that may contain potential sampling bias, and flexibility for application to any size of data.

G. Çelik: None. T. Tuncalı: None.

P17.098.C

'Deep dive' disease gene re-annotation in GENCODE: identifying and reporting new variant interpretations of likely clinical relevance.

\section{J. M. Mudge, T. Hunt, J. M. Gonzalez, A. Frankish}

European Molecular Biology Laboratory, Hinxton, United Kingdom

The accuracy of variant interpretation directly relates to the 'trueness' of gene annotation. However, current annotations are limited in scope and quality, and these deficiencies foster an opaque view of disease biology. We present a drive by Ensembl-GENCODE to radically improve our understanding of disease gene structure and function, deploying a fully integrated, manually supervised workflow incorporating state-of-the-art transcriptomics datasets with enormous tissue coverage alongside targeted proteogenomics and in-depth phylogenetic assessment. Crucially, we can specifically identify 'missing' gene features that appear most likely to be biologically relevant, and thus of potential clinical importance. Previously, our re-annotation of 191 epilepsy genes led to the identification of 3 novel de novo SCN1A mutations within a panel of 122 patients with Dravet's syndrome. We are expanding this work in collaboration with clinical partners to query additional genes and experimental datasets, and our efforts have now progressed into gene panels for other diseases. We will present potentially crucial new understandings of genes such as $P O L G$, which is linked to mitochondrial disease. Nonetheless, genic insights provided by Ensembl-GENCODE do not efficiently propagate into the clinic, for two major reasons: firstly, genomics projects - both small and large scale typically utilise older annotations; secondly, investigative work and diagnostics often incorporate a single transcript 
model per gene. We will therefore outline our proposed strategy for the targeted dissemination of new annotation information into different parts of the scientific community and would welcome feedback on these ideas. Supported by NHGRI Award U41HG007234.

J.M. Mudge: None. T. Hunt: None. J.M. Gonzalez: None. A. Frankish: None.

\section{P17.099.A}

Determining the role of gene expression on human sexual dimorphism

\section{E. Porcu ${ }^{1,2}$, A. Claringbould ${ }^{3}$, K. Lepik $^{4,5}$, BIOS Consortium, L. Franke ${ }^{3}$, F. A. Santoni ${ }^{6}$, A. Reymond ${ }^{l}, Z$. Kutalik $^{5,2}$}

${ }^{1}$ Center for Integrative Genomics, University of Lausanne, Lausanne, Switzerland, ${ }^{2}$ Swiss Institute of Bioinformatics, Lausanne, Switzerland, ${ }^{3}$ University Medical Centre Groningen, Groningen, Netherlands, ${ }^{4}$ Institute of Computer Science, University of Tartu, Tartu, Estonia, ${ }^{5}$ University Center for Primary Care and Public Health, Lausanne, Switzerland, ${ }^{6}$ Endocrine, Diabetes, and Metabolism Service, Centre Hospitalier Universitaire Vaudois (CHUV), Lausanne, Switzerland

Despite the prevalence of many diseases differs between women and men, only few published genome-wide association studies (GWAS) have been performed in a sexstratified manner. Nowadays the molecular bases of sex associated differences in complex traits remain poorly understood. We hypothesized that given the marked causal involvement of gene expression levels in complex traits, sex-biased trait associations might be driven by sex-biased eQTLs.To challenge this assumption, we performed a genome-wide analysis of sex-specific whole blood RNAseq eQTLs from 3,447 individuals. Amongst the preselected 9 million SNP-gene pairs (based on sex-combined association), we identified 18 genes with significantly (FDR $5 \%$ ) different eQTL effects in men and women. PheWAS analyses for these 18 eGenes on $>700$ traits revealed that sex-biased eQTLs in CDIP1 and PSMD5 translate into sexspecific trait-associations for trunk predicted mass. However, such examples are sporadic and sex-specific expression regulation does not systematically propagate to highlevel traits. Next, we applied a sex-specific transcriptomewide Mendelian Randomization approach (TWMR) by combining sex-specific summary statistics for both eQTLs and complex traits and observed the presence of a compensatory effect downstream of gene expression, e.g. genes with stronger eQTLs in women have weaker womanspecific causal effects on complex traits. Finally, we show that the sex-specific GWAS associations are not driven by sex-biased eQTLs. Our findings suggest that sex-specific trait associations can rarely occur as a consequence of sexspecific gene expression regulation in whole blood, hence other omics data are necessary to better understand the genetic basis of sexual dimorphism.

E. Porcu: None. A. Claringbould: None. K. Lepik: None. L. Franke: None. F.A. Santoni: None. A. Reymond: None. Z. Kutalik: None.

\section{P17.100.B}

SCelVis: exploratory single cell data analysis on the desktop and in the cloud B. Obermayer ${ }^{1,2}$, M. Holtgrewe
Messerschmidt $^{1,2}$, D. Beule
, Nieminen

${ }^{1}$ Berlin Institute of Health, Berlin, Germany, ${ }^{2}$ Charite Universitaetsmedizin, Berlin, Germany, ${ }^{3}$ Max-DelbrueckCenter for Molecular Medicine in the Helmholtz Association, Berlin, Germany

Introduction: Single cell omics technologies present unique opportunities for biomedical and life sciences from lab to clinic, but the high dimensional nature of such data poses challenges for computational analysis and interpretation. Furthermore, FAIR data management as well as data privacy and security become crucial when working with clinical data, especially in cross-institutional and translational settings. To facilitate analysis and interpretation of single-cell data by users without bioinformatics expertise, we present SCelVis, a flexible, interactive and user-friendly app for web-based visualization of preprocessed single-cell data.

Methods: SCelVis is implemented in Python using Dash by Plotly and available as a Python package, via Conda/ Bioconda and as a Docker image under the permissive MIT license. All components are based on open standards and interfaces, enabling further development and integration with third party pipelines and analysis components. The GitHub repository is https://github.com/bihealth/scelvis.

Results: In SCelVis, users can survey multiple interactive visualizations of their single cell expression data and cell annotation, define cell groups by filtering or manual selection and perform differential gene expression, and download raw or processed data for further offline analysis. SCelVis can be run both on the desktop and cloud systems and accepts input from local and various remote/cloud sources using open protocols. We use SCelVis in connection with an iRODS based omics data management system for interdisciplinary single cell data analysis in translational research and clinical applications. Other users found it useful to easily create standalone websites that allow efficient exploration of their published data sets. 
B. Obermayer: None. M. Holtgrewe: None. M. Nieminen: None. C. Messerschmidt: None. D. Beule: None.

P17.102.A

Unveiling Early Differentiation induced by Histone Deacetylase inhibitors in Neuroblastoma cell lines: a Single-Cell Transcriptomics Approach

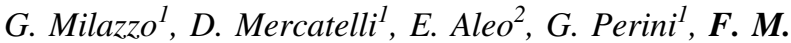 Giorgi $^{1}$}

${ }^{1}$ University of Bologna, Bologna, Italy, ${ }^{2} I G A$ Technology Services, Udine, Italy

The process of tumorigenesis cannot be fully explained solely by genetic alterations. Epigenetic regulation is an underinvestigated basal cause initiating and sustaining cancer cells development. In this context, Histone deacetylases (HDACs) play a fundamental role in remodeling chromatin and thus regulating gene expression towards cell proliferation and de-differentiation (PMID 21416059). Currently, there are more than 20 HDAC inhibitor drugs approved for cancer treatment or in clinical trials. These inhibitors can promote cell differentiation and apoptosis and ultimately reduce cancer cell growth, infiltration and angiogenesis. However, little is known on the early transcriptional response induced by HDAC inhibitors, particularly in Neuroblastoma. Current transcriptomics techniques (microarrays and bulk RNA-Seq) lack the ability to understand short-term transcriptional responses at a single cell resolution. Here, we measure transcript expression from 2000 single cells from Neuroblastoma cell lines treated with an HDAC pan-inhibitor using 10x Genomics with Cell Hashing and Illumina sequencing. Our analysis of singlecell gene expression profiles detected an increased proportion of cells in G1 phase in inhibitor-treated Neuroblastoma cells. Amongst these we were able to identify progressively differentiated and apoptotic cell populations. We also detect a high heterogeneity in individual cells transcriptional response upon drug delivery, linked with short-term rewiring and/or the insurgence of resistance mechanisms. This can help understanding how HDAC inhibitors can benefit from the combination with other drugs, in order to further guide the tumor's molecular machinery towards differentiation and apoptosis.

G. Milazzo: None. D. Mercatelli: None. E. Aleo: None. G. Perini: None. F.M. Giorgi: None.

\section{P17.104.C}

Integrative somatic variant calling in paired FFPE tumor and peripheral blood samples to identify candidate mutations to follow-up in circulating tumor DNA for tracking minimal residual disease in localized colorectal cancer patients

J. Martín Arana ${ }^{1,2,3}$, R. Tébar-Martínez ${ }^{1,4}$, N. Tarazona ${ }^{1,2}$, P. Rentero-Garrido ${ }^{4}$, J. Carbonell-Asins ${ }^{3}$, C. MartínezCiarpaglini $^{5}$, C. Alfaro-Cervello ${ }^{5}$, M. Huerta ${ }^{1}$, S. Rosello ${ }^{1,2}$, A. Ferrer-Martínez ${ }^{1,2}$, D. Roda ${ }^{1,2}$, S. Zúñiga ${ }^{3,4}$, A. Cervantes $^{1,2}$

${ }^{1}$ Department of Medical Oncology, INCLIVA Biomedical Research Institute, Valencia, Spain, ${ }^{2}$ CIBERONC, Instituto de Salud Carlos III, Madrid, Spain, ${ }^{3}$ Bioinformatics and Biostatistics Unit, INCLIVA Biomedical Research Institute, Valencia, Spain, ${ }^{4}$ Precision Medicine Unit, INCLIVA Biomedical Research Institute, Valencia, Spain, ${ }^{5}$ Department of Pathology, INCLIVA Biomedical Research Institute, Valencia, Spain

Introduction: Mutation tracking in circulating tumour DNA (ctDNA) is used to detect minimal residual disease (MRD) in localized colorectal cancer (CRC) patients. Sequencing of tumor-only samples restricts the selection of tracking somatic mutations to those already known to be pathogenic. To increase the number of candidate mutations to monitor patients, sequencing of paired tumor-normal samples is required.

Validation and optimization of different variant calling tools are essential to maximize sensitivity scores. The aim of this work is developing an integrative bioinformatics pipeline to compare the use of only-tumor versus paired normal-tumor samples to identify candidate somatic mutations to follow-up in ctDNA to detect MRD.

Materials and Methods: Tumor Formalin Fixed Paraffin Embedded (FFPE) and peripheral blood samples of 150 CRC patients were sequenced using a 29-gene custom panel including Unique Molecular Identifiers technology. For pipeline development, five different algorithms were tested on a mixture of HapMap cell lines and 2 commercial standards.

Results: Smcounter2, LoFreq and Mutect2 reported best sensitivity values. $11 \%$ of patients could not be monitored because no pathogenic variants were detected using tumoronly samples, whereas all 150 patients presented at least two somatic variants when adding normal sample data. In addition, paired samples analysis identified somatic variants in 16 genes where no pathogenic variants had been previously detected using tumor-only samples.

Conclusions: A combination of different variant calling tools is necessary to achieve best sensitivity ranges. Paired tumor-normal sample analysis increases the percentage of patients to be monitored to detect MRD.

J.MA contract is founded by CIBERONC. 
J. Martín Arana: None. R. Tébar-Martínez: None. N. Tarazona: None. P. Rentero-Garrido: None. J. Carbonell-Asins: None. C. Martínez-Ciarpaglini: None. C. Alfaro-Cervello: None. M. Huerta: None. S. Roselló: None. A. Ferrer-Martínez: None. D. Roda: None. S. Zúñiga: None. A. Cervantes: B. Research Grant (principal investigator, collaborator or consultant and pending grants as well as grants already received); Modest; Roche, Merk Serono, SERVIER, Novartis, Lilly, Tesaro, Johnson \& Johnson, Medlmmune, Theradex, Astellas Pharma, Array BioPharma. D. Speakers Bureau/Honoraria (speakers bureau, symposia, and expert witness); Modest; Merk Serono, Roche, SERVIER. F. Consultant/Advisory Board; Modest; Bayer, Pierre Fabre.

\section{P17.105.A}

Performance evaluation of deleteriousness prediction methods for intronic SNVs in next generation sequences

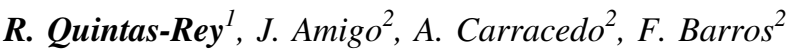

${ }^{1}$ Grupo de Medicina Xenómica, USC, Santiago de Compostela, Spain, ${ }^{2}$ Fundacion Publica Galega de Medicina Xenomica - Sergas, Grupo de Medicina Xenomica CIBERER, Universidad de Santiago de Compostela, Santiago de Compostela, Spain

Introduction: Alterations in splicing sites (ss) are estimated to explain approximately $10 \%$ of human disease causal variants. Mutations outside the ss but affecting "regulatory elements" can be up to $25 \%$. Accurate deleteriousness prediction for intronic variants is crucial for diagnostic purposes. Many deleteriousness prediction methods have been developed, but their relative values are still unclear in practical applications. We comprehensively evaluated the predictive performance of two complementary deleteriousness-scoring methods using information from real patients.

Material and Methods: We selected the dbscSNV (both ADA and RF scores) and SPIDEX algorithms, that study variants in splicing consensus regions or in regulatory regions respectively. The tools, either alone or in combination, were tested on 29294 gene intronic SNVs that have previously been characterised by ClinVar as either "pathogenic" (430) or "benign" (28864). The sensitivity, specificity and positive and negative predictive values were calculated. Moreover, we applied the algorithms to WES data from undiagnosed patients, and we analysed the mRNA sequence from genes that fitted the patient's phenotype.

Results: The highest sensitivity corresponds to dbscSNV with $96.55 \%$ while the best specificity is for SPIDEX with $95.78 \%$. When considering the 3 scores (SPIDEX,
dbscSNV ADA and RF Score), the sensitivity and specificity values were $60.7 \%$ and $94.6 \%$. The Positive and Negative Predictive Value were $14.45 \%$ and $99.39 \%$. The results for 20 undiagnosed cases are presented.

Conclusions: Besides the low positive predictive value, the combination of both algorithms leads less than $1 \%$ of false negatives, so their routine use can be recommended for diagnostic purposes.

R. Quintas-Rey: None. J. Amigo: None. A. Carracedo: None. F. Barros: None.

\section{P17.106.B}

Functional and in-silico interrogation of rare genomic variants impacting RNA splicing for the diagnosis of genomic disorders

J. M. Ellingford ${ }^{1}$, H. B. Thomas ${ }^{1}$, C. Rowlands ${ }^{1}$, G. Arno ${ }^{2}$, G. Beaman ${ }^{1}$, B. Gomes-Silval, C. Campbell ${ }^{3}$, N. Gossan',

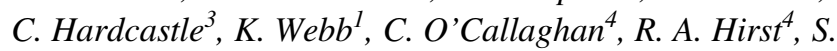
Ramsden $^{3}$, E. Jones ${ }^{3}$, J. Clayton-Smith ${ }^{1}$, A. R. Webster ${ }^{2}$, Genomics England Research Consortium, R. T. O'Keefe', W. G. Newman' ${ }^{\text {, G. C. M. Black }}{ }^{1}$

${ }^{I}$ University of Manchester, Manchester, United Kingdom, ${ }^{2}$ University College London, London, United Kingdom, ${ }^{3}$ Manchester Universities NHS Foundation Trust, Manchester, United Kingdom, ${ }^{4}$ University of Leicester, Leicester, United Kingdom

Purpose: To develop a comprehensive analysis framework to identify pre-messenger RNA splicing pathogenic variants in the context of rare disease.

Methods: We ascertained variants of uncertain significance (VUS) through clinical multi-disciplinary team meetings, and defined an accurate in-silico strategy for the prioritization of variants impacting splicing through comparison to functional analyses. We retrospectively applied these prioritization strategies to a large cohort of 2783 individuals who had previously received genomic testing for rare genomic disorders, and assessed clinical impact.

Results: We identified 21 VUS that potentially impacted splicing, and used cell based splicing assays to identify those variants which disrupted normal splicing. We established the comparative utility of 9 in-silico strategies for variant prioritization. We analysed 1,346,744 variants identified through diagnostic testing for 2783 individuals, and observed that clinical impact is highly dependent on the specific incorporated in-silico prioritization strategy. We show that incorporation of one of these strategies into diagnostic testing would improve clarity in clinical analysis for $15 \%$ of the individuals surveyed. Prioritized variants could provide new molecular diagnoses or provide 
additional support for molecular diagnosis for up to 81 individuals within our cohort.

Conclusions: Incorporation of an in-silico and functional analysis framework for splicing pathogenic variants improves clarity in diagnostic reporting, increases diagnostic yield and, with the advent of targeted treatment strategies, can directly alter patient clinical management.

J.M. Ellingford: None. H.B. Thomas: None. C. Rowlands: None. G. Arno: None. G. Beaman: None. B. Gomes-Silva: None. C. Campbell: None. N. Gossan: None. C. Hardcastle: None. K. Webb: None. C. O'Callaghan: None. R.A. Hirst: None. S. Ramsden: None. E. Jones: None. J. Clayton-Smith: None. A.R. Webster: None. R.T. O'Keefe: None. W.G. Newman: None. G.C.M. Black: None.

\section{P17.107.C}

A comprehensive in silico analysis of the functional, structural and stability impact of the PALB2 p. Leu1143His variant of uncertain clinical significance.

M. Zanti, M. A. Loizidou, K. Michailidou, C. Machattou, P. Pirpa, G. M. Spyrou, K. Kyriacou, A. Hadjisavvas

The Cyprus Institute of Neurology and Genetics, Nicosia, Cyprus

Introduction: Germline genetic testing can identify women with pathogenic mutations who are at increased risk of BC. However, genetic testing may reveal a variant of uncertain clinical significance (VUS). These variants pose significant challenges for clinicians since, their ambiguous association with disease interferes with effective genetic counseling. Although, in-vitro characterization of these variants should be performed, it is often not feasible to carry out functional interpretation for every single VUS. To this end, we conducted a comprehensive in silico analysis of the structural, functional and stability impact of the PALB2 p.Leu1143His VUS which was identified in 9 Cypriot BC patients with extensive family history of the disease.

Materials and Methods: A combination of structural bioinformatics tools were used for the in silico classification of the PALB2 p.Leu1143His VUS.

Results: Stability analyses based on $\Delta \Delta \mathrm{G}$ calculations (10 prediction tools) predicted a destabilization of the mutant PALB2 protein. The substitution from the nonpolar, hydrophobic Leucine to the positively charged, hydrophilic Histidine, results in a minor change of the surface charge and conformation. Both amino acids are exposed to the surface (cut-off value: Relative Solvent Accessibility -RSA $>25 \%$ ) since RSA demonstrated similar values $(71.3 \%$ and $83.1 \%$ ) upon mutagenesis. Results on protein stability and structure end up being contradicting.
Conclusions: Although in silico tools are valuable for the interpretation of VUS, functional studies are needed to draw more definite conclusions on variant classification.

Grant: EU H2020; 669026; Establishment of the Bioinformatics Chair at the CING (BIORISE).

M. Zanti: None. M.A. Loizidou: None. K. Michailidou: None. C. Machattou: None. P. Pirpa: None. G.M. Spyrou: None. K. Kyriacou: None. A. Hadjisavvas: None.

\section{P17.108.A}

Towards a better understanding of the low discovery rate of short-read based insertion variant callers

\section{W. Delage ${ }^{I}, J$. Thevenon $^{2}$, C. Lemaitre ${ }^{I}$}

${ }^{1}$ Univ Rennes, CNRS, Inria, IRISA - UMR 6074, F-35000, Rennes, France, ${ }^{2}$ Unité de Génétique Clinique, Pôle Couple Enfant, CHU de Grenoble Site Nord-Hôpital CoupleEnfant, 38043, Grenoble, France

Since 2009, numerous tools have been developed to detect structural variants using short read technologies. Insertions are one of the hardest type to discover and are drastically underrepresented in gold standard variant call sets. The advent of long read technologies has completely changed the situation. In 2019, two independent cross technologies studies have published the most complete variant call sets with sequence resolved insertions in human individuals. Among the reported insertions, only $15 \%$ could be discovered with short-read based tools. In this work, we performed an in-depth analysis of these unprecedented insertion call sets, in order to investigate the causes of such failures. We have first established a precise classification of insertion variants according to three different layers of characterization: the nature of the inserted sequence, the genomics context of the insertion site and the breakpoints junction complexity. Because these levels are intertwined, we used simulations to characterize the impact of each complexity factor. Most reported insertions exhibited characteristics that may interfere with their discovery: 50\% were tandem repeat expansions, $40 \%$ contained homologies larger than $20 \mathrm{bp}$ within their breakpoints junctions and $70 \%$ were located in simple repeats. Consequently, the recall of short-read based variant callers was significantly lower for such insertions (7\% vs 54\% for mobile element and novel insertions). Simulations showed that the most impacting factor on the discovery rate was the insertion type rather than the genomics context, and that the different factors of insertion complexities were handled differently depending on the chosen tool. 
W. Delage: None. J. Thevenon: None. C. Lemaitre: None.

P17.109.B

Vaquita-LR: A new bioinformatics tool for identifying structural variants using long and short reads

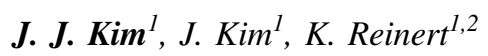

${ }^{I}$ Max Planck Institute for Molecular Genetics, Berlin, Germany, ${ }^{2}$ Freie Universität Berlin, Berlin, Germany

Introduction: The identification of structural variation in the genome is difficult due to the lack of a "perfect" sequencing technology. By combining short and long read sequencing data, Vaquita-LR is a novel bioinformatics approach to identify more variants with higher accuracy by utilizing the strengths of one method to overcome the weaknesses of the other.

Materials and Methods: Vaquita-LR is an extension of Vaquita, a short read variant caller, which has been modified to identify potential variants in long reads in addition to short reads and merge them to provide a final set of variants. Vaquita-LR takes into consideration the read depth, read length, and other characteristics of the sequencing data to add weight to more confident calls. Additionally, it adapts techniques from Pilon to improve the accuracy of the long read data using relevant short read information.

Results: By combining short and long reads, Vaquita-LR is able to outperform other structural variant callers, including meta callers which combine results from other callers. Importantly, supplying long reads is effective at sequencing depths as shallow as $1 \mathrm{x}$. Using this combination allows Vaquita-LR to better filter out false positives while retaining true positives, providing a better list of possible causal variants for further investigation.

Conclusions: With the abundance of data available today, it is important to consider how the data can be effectively merged to better serve our needs. Vaquita-LR is an initial step in showing the usefulness of integrating different sequencing data types when identifying structural variants.

J.J. Kim: None. J. Kim: None. K. Reinert: None.

P17.110.C

Identification of subnetwork markers for diagnosis and prognosis of Middle-Eastern patients with colorectal cancer using integrative genomic and network-based analysis
O. Al-Harazi ${ }^{1,2}$, I. H. Kaya ${ }^{1,3}$, A. El Allali ${ }^{2}$, N. Kaya ${ }^{4}$, D. Colak $^{l}$

${ }^{I}$ Department of Biostatistics, Epidemiology and Scientific Computing, King Faisal Specialist Hospital and Research Centre, Riyadh, Saudi Arabia, ${ }^{2}$ College of Computer and Information Sciences, Computer Science Department, King Saud University, Riyadh, Saudi Arabia, ${ }^{3}$ College of Medicine, Alfaisal University, Riyadh, Saudi Arabia, ${ }^{4}$ Department of Genetics, King Faisal Specialist Hospital and Research Centre, Riyadh, Saudi Arabia

Introduction: The identification of gene signatures for disease prognosis and diagnosis is considered a major step. Artificial intelligence and machine learning methodologies have been used to investigate the molecular characteristics of diseases. It has been reported that subnetwork markers are reliable with high accuracy in disease classification.

Materials and Methods: We developed and used a subnetwork-based method to identify network markers for Saudi colorectal cancer (CRC) patients. Whole-genome gene expression and copy number alterations (CNAs) datasets were gathered from publically available databases. We then performed an integrated analysis of mRNA and CNA datasets followed by a gene interaction network analysis using several publically available databases. We clustered the identified gene network into subnetworks and calculated an activity score for each significant subnetwork. The scores were then used as feature values to build a support vector machine (SVM) classification model.

Results: The integrated genomic analysis revealed 24 differentially expressed genes with CNAs. Network analysis revealed 15 significant subnetwork markers associated with CRC. The SVM classifier using individual marker genes showed $94.7 \%$ accuracy, whereas the classifier with the subnetwork markers achieved $98.4 \%$ accuracy in identifying CRC patients. Furthermore, we validated the prognostic potential of our subnetwork markers on independent CRC datasets with detailed clinical data.

Conclusions: Subnetwork markers that are based on integrated genomic and network analyses yield more robust and accurate classification results compared to gene markers.

Acknowledgement: This study is funded by the Research Grant (RAC\#2110006 to DC). We would like to thank Ms. Sukina Qanbar for her administrative support.

O. Al-Harazi: None. I.H. Kaya: None. A. El Allali: None. N. Kaya: None. D. Colak: None.

P17.111.A

ePOSSUM2: A web app for predicting the impact of DNA variants on transcription factor binding 
S. Proft ${ }^{1}$, R. Steinhaus ${ }^{1}$, P. N. Robinson ${ }^{2}$, D. Seelow ${ }^{1}$

${ }^{1}$ Charité, Berlin, Germany, ${ }^{2}$ The Jackson Laboratory, Farmington, CT, United States

Modelling gene regulation is a major challenge in biomedical research. Mutations in transcription factor binding sites (TFBS) may cause the misregulation of genes, eventually leading to disease.

In 2016, we published a study that compared the ability of 179 binding models for 82 human transcription factors to identify experimentally verified "real" in vivo binding sites derived from ENCODE ChIP-seq data. Our results showed that several models do not reliably detect TFBSs. Using these results, we created ePOSSUM, a web application for predicting the impact of DNA variants on transcription factor binding.

Here we present an update of the application. ePOSSUM2 has been rebuild from the ground up with a modern $\mathrm{C}++$ back-end that runs many computations in milliseconds.

In ePOSSUM2, web users can easily upload VCF files to identify and rank variants that most likely affect gene expression. ePOSSUM2 supports more than 4,000 different binding models, including transcription factor flexible models. It features an improved log-weighted matching algorithm that takes into account the wild-type dinucleotide distribution surrounding matching sites. For each variant, ePOSSUM2 predicts the effect on transcription factor binding. To account for the poor performance of several binding models, it also reports a negative or positive predictive value.

ePOSSUM2 is freely available at: http://www.muta tiondistiller.org/ePOSSUM2

S. Proft: None. R. Steinhaus: None. P.N. Robinson: None. D. Seelow: None.

\section{P17.113.C}

TaPAS db: A centralised storage solution for casemanagement and variant interpretation for diagnostic targeted next generation sequencing data

\section{J. Adams ${ }^{1,2}$, M. Owens ${ }^{1}$, M. Sloman ${ }^{1}$, A. Moleirinho ${ }^{l}, K$.

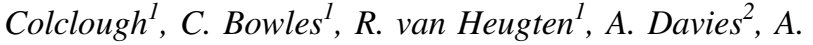 Parrish $^{l}$}

${ }^{1}$ Exeter Genomics Laboratory, Royal Devon and Exeter NHS Foundation Trust, Exeter, United Kingdom, ${ }^{2}$ University of Manchester, Manchester, United Kingdom

Analysis of panels of genes associated with particular clinical indications by targeted Next Generation Sequencing (tNGS) is offered routinely in diagnostic laboratories to maximise the likelihood of finding a genetic diagnosis. In
2019, Exeter Genomics Laboratory (EGL) performed tNGS testing for approximately 2000 patients, generating hundreds of variants per patient requiring secure storage and interpretation. Efficient variant prioritisation and interpretation is essential for delivering a timely diagnosis which can be facilitated by cross-referencing results from previous patients and applying filtering strategies based on ACMG variant classification guidelines. Historically, data for each patient has been stored independently, limiting the ability to detect trends across patients and utilise information from previous analyses.

Here we present TaPAS, a variant interpretation interface and centralised data store for variants identified using EGL's in-house tNGS bioinformatics pipeline. Built using the Django framework based around an SQL database, TaPAS dynamically filters and displays variants based on the intrinsic characteristics of the variants and ACMG variant classification criteria, with JavaScript elements used to access external databases and manipulate local programs such as Alamut $^{\circledR}$ Visual and IGV to visualise variants. It maintains a historical record of previous analyses, displaying variant frequency in the patient cohort, previous variant classifications and allows the application of additional panels to the data as required. TaPAS streamlines the process of variant classification and genomic diagnosis, optimises interpretation and reanalysis of tNGS data and reduces turnaround times.

J. Adams: None. M. Owens: None. M. Sloman: None. A. Moleirinho: None. K. Colclough: None. C. Bowles: None. R. van Heugten: None. A. Davies: None. A. Parrish: None.

\section{P17.114.A}

Using transcription factor sequence information to interpret rare variants in non-coding regions

\section{H. K. Zieger, F. Thieme, A. Schmidt, J. Fazaal, E. Mangold, J. Welzenbach, K. Ludwig}

Institute of Human Genetics, University of Bonn, School of Medicine \& University Hospital Bonn, Bonn, Germany

Common diseases are characterized by variants from different parts of the allelic spectrum. While common risk variants are identified by GWAS, the conclusive identification of causal private/rare variants remains challenging. Collapsing rare variants into groups, as performed in gene burden analyses, is difficult for non-coding regions, due to the lack of functional annotations. Here we combine resequencing data of non-coding regions with information of transcription factor binding sites (TFBS), to identify novel etiological variants and molecular players in non-syndromic cleft lip with/without cleft palate (nsCL/P). We used data of 
691 European cases and 854 European controls, in which functional candidate regions at three GWAS risk loci (total $56.7 \mathrm{~kb}$ ) had been resequenced using molecular inversion probes. Based on 624 rare variants $(\mathrm{MAF}<1 \%)$ and position weight matrix information, we predicted TFBS for 810 human transcription factors from JASPAR2020. Of the 370 motifs that were hit by at least one rare variant (total number: 4,013), 21 motifs were hit significantly more often in cases compared to controls. When integrating the predicted allelic effect on TF binding capacity, we identified an additional set of nine TFs where in aggregate the variants' effects on the motif were stronger in cases. Across both approaches, consistent effects were observed for TF motifs of FOXO6 and ZEB1, suggesting them as molecular players in $\mathrm{nsCL} / \mathrm{P}$ at these loci. This analysis framework will improve systematic evaluation of rare variant data and can be extended to large data sets, such as from whole genome sequencing studies.

H.K. Zieger: None. F. Thieme: None. A. Schmidt: None. J. Fazaal: None. E. Mangold: None. J. Welzenbach: None. K. Ludwig: None.

\section{P17.115.B}

Meta-analysis of cancer transcriptomes using Independent Component Analysis

\section{A. Seisenova, A. Sharip, A. Molkenov, D. Karabayev, U. Kairov}

Laboratory of Bioinformatics and Systems Biology, Center for Life Sciences, National Laboratory Astana, Nazarbayev University, Nur-Sultan, Kazakhstan

Introduction: Independent Component Analysis (ICA) is a matrix factorization method for data dimension reduction. ICA has been widely applied for the analysis of transcriptomic data for blind separation of biological, environmental and technical factors affecting gene expression. This study aimed to analyze cancer data using the ICA for identification and comprehensive analysis of reproducible signaling pathways and molecular signatures in cancer.

Materials and Methods: In this study, four independent cancer transcriptomic datasets GSE26886, GSE69925, GSE32701 and GSE21293 (Affymetrix) from GEO databases were used. R Bioconductor and Matlab have been used for normalization. A bioinformatics tool «BiODICA Independent Component Analysis of Big Omics Data» was applied to compute independent components (ICs). Gene Set Enrichment Analysis (GSEA) and ToppGene uncovered the most significantly enriched pathways. Construction and visualization of gene networks and graphs were performed using the OFTEN method, Cytoscape and HPRD database.
Results: The correlation graph between decompositions into 30 ICs was built with absolute correlation values exceeding 0.15. Clusters of components - pseudocliques were observed in the structure of the correlation graph. Top 500 most contributing genes of each ICs in pseudocliques were mapped to the PPI network to construct signaling pathways for gene interaction. Some cliques were composed of densely interconnected nodes and included components common to most cancer types, while others were common to some of them.

Conclusions: The results of this investigation may reveal potential biomarkers of carcinogenesis, functional subsystems in the tumor cells, and helpful in predicting the early development of a tumor.

A. Seisenova: None. A. Sharip: None. A. Molkenov: None. D. Karabayev: None. U. Kairov: None.

P17.116.C

Single and cross-tissue conditional transcriptome wide analyses reveal independent and shared susceptibility genes for psychiatric traits and inflammatory bowel diseases

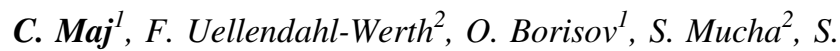

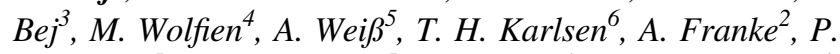

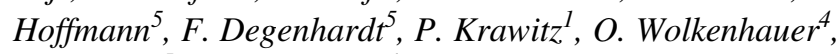
M. Nöthen ${ }^{5}$ D. Ellinghaus ${ }^{1}$

${ }^{1}$ Institute for Genomic Statistics and Bioinformatics (IGSB), Bonn, Germany, ${ }^{2}$ Institute of Clinical Molecular Biology, Christian Albrechts University of Kiel, Kiel, Germany, ${ }^{3}$ Institute of Computer Science, Department of Systems Biology and Bioinformatics, University of Rostock, Kiel, Germany, ${ }^{4}$ Institute of Computer Science, Department of Systems Biology and Bioinformatics, University of Rostock, Germany, Rostock, Germany, ${ }^{5}$ Institute of Human Genetics, University of Bonn, School of Medicine \& University Hospital, Bonn, Germany, ${ }^{6}$ Norwegian PSC Research Center, Department of Transplantation Medicine, Division of Cancer Medicine, Surgery and Transplantation, Oslo University Hospital, Rikshospitalet, Oslo, Norway

Introduction: Genome-wide association studies (GWAS) have identified hundreds of significantly associated loci for different psychiatric and inflammatory phenotypes. Noteworthy, genetic correlations have been reported for these traits indicating the presence of potential shared molecular mechanisms.

Materials and Methods: We performed transcriptomewide association study (TWAS) based on the largest available GWAS results for a number of psychiatric and inflammatory traits using the unified test for molecular signature (UTMOST). We conducted multi-phenotype 
TWASs by considering single and cross-tissue expression signals based on cis-eQTL. We selected 23 relevant tissues for brain and gut traits from GTEx, STARNET, and BLUEPRINT databases. Single tissue associations were combined by means of the Generalized Berk-Jones (GBJ) test and we performed genome-wide conditional analysis to detect independent TWAS signals at loci with multiple TWAS hits.

Results:A relatively high number of genes were found to be associated with the analyzed traits. We observed strong inflation of TWAS signals most likely due to correlated predicted expression among same-locus genes. Gene prioritization based on genome-wide conditional analyses strongly reduced the number of associated genes and highlighted specific associations including known risk genes (e.g., SMAD3 for inflammatory bowel disease). Interestingly some genes are associated both with brain and gut related phenotypes suggesting common regulation mechanisms of the gut-brain axis.

Conclusions: TWAS was able to prioritize geneassociations in multiple psychiatric and inflammatory traits supporting commonly regulated genes of the gut-brain axis. Noteworthy, Conditional analysis at loci with multiple TWAS hits can discriminate between spurious associations due to correlation and true-association signals.

C. Maj: None. F. Uellendahl-Werth: None. O. Borisov: None. S. Mucha: None. S. Bej: None. M. Wolfien: None. A. Weiß: None. T.H. Karlsen: None. A. Franke: None. P. Hoffmann: None. F. Degenhardt: None. P. Krawitz: None. O. Wolkenhauer: None. M. Nöthen: None. D. Ellinghaus: None.

\section{P17.118.B}

VarFish - Collaborative Fishing for Causative Variants

M. Holtgrewe ${ }^{1}$, O. Stolpe ${ }^{1}$, M. Nieminen ${ }^{1}$, S. Mundlos ${ }^{2}$, A. Knaus $^{3}$, U. Kornak ${ }^{2}$, D. Seelow ${ }^{4}$, L. Segebrecht ${ }^{4}, M$.

Spielmann $^{4}$, B. Fischer-Zirnsak ${ }^{4}$, F. Boschann ${ }^{4}$,U. Scholl ${ }^{4}$, N. Ehmke ${ }^{4}$ D. Beule ${ }^{1}$

${ }^{1}$ CUBI - Core Unit Bioinformatics, Berlin Institute of Health, Berlin, Germany, ${ }^{2}$ Charité - Universitätsmedizin Berlin, Berlin, Germany, ${ }^{3}$ Institute for Genomic Statistics and Bioinformatics, University of Bonn, Bonn, Germany, ${ }^{4}$ Charité - Universitätsmedizin Berlin, Berlin, Germany

VarFish is an easy-to-use web-based database system designed for empowering geneticists in the analysis of clinical and whole exome sequencing variant data sets for individuals and families. It provides a set of tools for supporting the full workflow from (i) variant data quality control, (ii) variant filtration and (iii) efficient assessment of variants based on visual alignment inspection and annotation data such as functional and frequency annotation. The system allows to organize data into a folder structure of projects with access control. Variant quality metrics can be displayed project- (and thus cohort-) wise or for single cases/families. The variants themselves can be filtered based on genotype, population frequency, variant effect, quality metrics, and annotation such as membership in ClinVar or HGMD Public. A special ClinVar-centric view allows for the easy screening of variants based on pathogenicity annotation in ClinVar. After filtration of the variants, their quick and efficient assessment is supported by various tools: color flags and commenting allows for simple yet effective note-taking, remote-controlling the genome viewer IGV to display a variant's locus, and some important database excerpts are available directly within the system. Assessment documentation according to ACMGAMP is also supported. Further, link-out to external databases (e.g., ENSEMBL or NCBI), allow the assessment of variants using MutationTaster, SIFT, or various splice site assessment tools. Filtered data sets can be downloaded as VCF or Excel files (including comments and flags) or submitted to external tools such as MutationDistiller. VarFish also allows for querying the GA4GH beacon network for individual variants.

M. Holtgrewe: None. O. Stolpe: None. M. Nieminen: None. S. Mundlos: None. A. Knaus: None. U. Kornak: None. D. Seelow: None. L. Segebrecht: None. M. Spielmann: None. B. Fischer-Zirnsak: None. F. Boschann: None. U. Scholl: None. N. Ehmke: None. D. Beule: None.

\section{P17.120.A}

Variant Record Fusion: a bioinformatics pipeline for Tumor-only NGS testing in clinical routine

S. Lehnert ${ }^{1}$, H. Vranckx ${ }^{2}$, S. Vander Borght ${ }^{2}, C$. Helsmoortel $^{2}$, I. Vanden Bempt ${ }^{1}$,W. Bossuyt ${ }^{1}$

${ }^{1}$ KULeuven, Leuven, Belgium, ${ }^{2}$ UZ Leuven, Leuven, Belgium

Here we present a novel approach for the detection and filtering of variants for NGS cancer diagnostic tests without tumor matched normal samples. A combination of different variant callers allows a wider set of variant types to be detected. Current approaches that make use of different variant callers rely on merging or concatenating individual sets of variant calls that have high confidence for a specific caller. This approach however, needs to tradeoff between low sensitivity or specificity for variants that have lower confidence calls in individual variant callers, but are still true variants. Our approach obviates this problem by fusing normalized and decomposed variant-records of all different 
callers to allow filtering of individual variants based on the information from all different callers. Variants in the fused dataset are subsequently filtered using parameters specific to the individual caller, to a combination of different callers and to the combined variant calls of the entire run. The pooled likelihood matrixes of different callers in fused variant entries proved to be especially useful for categorizing low frequent variant calls $(\mathrm{AF}<=5 \%)$. This pipeline was brought in clinical routine for a solid tumor gene panel and a hematological malignancy gene panel. We used 3 variant callers (unified genotyper $(2 \mathrm{x})$ and MuTect) and defined over 20 filter rules. Only 2 from 25 cutoffs required a slight adaptation between the two different sample tests with different sample types, FF and FFPE, indicating a general applicability of this approach.

S. Lehnert: None. H. Vranckx: None. S. Vander Borght: None. C. Helsmoortel: None. I. Vanden Bempt: None. W. Bossuyt: None.

\section{P17.121.B}

CADD-Splice - Improving genome-wide variant effect prediction using deep learning derived splice scores

\section{P. Rentzsch ${ }^{1,2}$, M. Schubach ${ }^{1,2}$, J. Shendure ${ }^{3,4}$, M. Kircher ${ }^{1,2}$}

${ }^{1}$ Berlin Institute of Health, Berlin, Germany, ${ }^{2}$ Charité Universitätsmedizin Berlin, Berlin, Germany, ${ }^{3}$ Brotman Baty Institute for Precision Medicine, Seattle, WA, United States, ${ }^{4}$ Department of Genome Sciences, Seattle, WA, United States

The assembly of genomic exons into mRNAs via splicing is of critical importance for the correct synthesis of proteins. As such, genomic variants in splicing regions have been linked to various genetic diseases. In order to assist the identification of splice altering variants from genetic data, a number of methods aim to predict splicing effects. Recently, deep neural networks (DNNs) have been shown to achieve better results on predicting splice variants genome-wide. However, these methods have been barely adopted in genome-wide variant effect prediction.

We use the Multiplexed Functional Assay of Splicing using Sort-seq (MFASS) dataset to assess several new machine learning methods that score variant splicing effects. To build a general variant effect model, we integrate two of those approaches into our genome-wide variant prioritization tool Combined Annotation Dependent Depletion (CADD, cadd.gs.washington.edu), that weights and integrates a diverse collection of other annotations.

We show that the new DNN scores improve prediction across multiple variant categories. While splice effect scores show superior performance on splice variants, the specialized predictors cannot compete with other variant scores in general variant interpretation, as these also account for nonsense and missense effects that do not alter splicing. This suggests that by integrating domain-specific information from for example splice scores, variant prioritization can generally be improved. We believe that this effect is universal and outlines the importance of developing domain specific scores for regulatory sequences, UTRs or noncoding RNA species.

P. Rentzsch: None. M. Schubach: None. J. Shendure: E. Ownership Interest (stock, stock options, patent or other intellectual property); Modest; Patent application on basis of CADD. M. Kircher: E. Ownership Interest (stock, stock options, patent or other intellectual property); Modest; Patent application on basis of CADD.

P17.123.A

Quantifying sequencing performance for clinical WGS

T. U. Dincer ${ }^{1}$, N. Johnson ${ }^{2}$, C. Colombo ${ }^{3}$, M. A. Bekritsky ${ }^{2}$, M. A. Eberle $^{2}$, D. R. Bentley ${ }^{3}$

${ }^{1}$ UCLA, Biological chemistry, Los Angeles, CA, United States, ${ }^{2}$ Illumina, Inc, San Diego, CA, United States, ${ }^{3}$ Illumina UK, Cambridge, United Kingdom

Clinical whole genome sequencing (WGS) pipelines must deliver quantifiably accurate and reproducible variant calls. Publicly available truthsets allow labs to measure variant calling performance in curated sets of samples. Truthset generation methods seek to exclude any problematic genomic regions that may contain both stochastic and systematic errors from the source samples. In clinical variant calling, complementary standards to identify high- and lowconfidence sites are needed in order to detect systematic effects that generalize to any sample.

With increased sequencing throughput, we can query population-level sequence data to obtain 1000s of independent per-site measures. To test this approach, we collected metrics (e.g. base quality, depth and mapping quality) from $>30$ samples and trained a logistic regression model to learn the relationship of quality metrics to small variant calling accuracy.

When applying the model to independent samples, we found that it can effectively discriminate regions of high, medium and low predicted accuracy, as well as identify common deletions regions. Of the non-N autosomal genome, we predict $\sim 91 \%, \sim 2 \%$, and $\sim 5.4 \%$ has high, medium, and low accuracy, while $\sim 1.6 \%$ contains common deletions. SNP concordance is $>99.95 \%,>86.5 \%,>62.1 \%$ and $>80.5 \%$ respectively, demonstrating the validity of the approach, which is further supported by performance assessment using the Platinum Genomes truthset. 
Profiling systematic error proves a promising approach to predict per-site variant calling accuracy and annotate variant and invariant bases. This will reduce the need for orthogonal validation of calls and the curation burden, making clinical WGS quicker, less expensive, and more reliable.

T.U. Dincer: A. Employment (full or part-time); Significant; Illumina, Inc. N. Johnson: A. Employment (full or part-time); Significant; Illumina, Inc. E. Ownership Interest (stock, stock options, patent or other intellectual property); Significant; Illumina, Inc. C. Colombo: A. Employment (full or part-time); Significant; Illumina UK. E. Ownership Interest (stock, stock options, patent or other intellectual property); Significant; Illumina, Inc. M.A. Bekritsky: A. Employment (full or part-time); Significant; Illumina, Inc. E. Ownership Interest (stock, stock options, patent or other intellectual property); Significant; Illumina, Inc. M.A. Eberle: A. Employment (full or part-time); Significant; Illumina, Inc. E. Ownership Interest (stock, stock options, patent or other intellectual property); Significant; Illumina, Inc. D.R. Bentley: A. Employment (full or part-time); Significant; Illumina UK. E. Ownership Interest (stock, stock options, patent or other intellectual property); Significant; Illumina, Inc.

\section{P17.124.B}

Reproduce easily: analysis of matching tumor-normal NGS data with the Sarek workflow

\section{S. Juhos ${ }^{1}$, M. Garcia ${ }^{1}$, T. Díaz De Ståhl ${ }^{1}$, M. Mayrhofer ${ }^{2}, J$. Sandgren $^{l}$, M. Nistér ${ }^{1}$}

${ }^{1}$ Karolinska Institutet, Solna, Sweden, ${ }^{2}$ NBIS, Science for Life Laboratory, Uppsala, Sweden

Introduction: High throughput sequencing for precision medicine is now a routine method. Numerous tools have to be used, and analysis is time consuming. We propose Sarek, an open-source container based bioinformatics workflow for germline or matching tumor-normal pairs, written in Nextflow, to process WGS, whole-exome or gene-panel samples.

Materials and methods: Sarek is part of nf-core, a collection of peer-reviewed workflows; supported environments are Conda, Docker and Singularity. It is systemagnostic: can be used on single machines, clusters (HPC) or in a cloud such as AWS, with little difference between setups. Additional software can be included as new modules. Several model organism references are available (including Human GRCh37 and GRCh38). The pipeline reports germline and somatic SNVs and SVs (by HaplotypeCaller, Strelka, Mutect2, Manta and TIDDIT). CNVs, purity and ploidy is estimated by ASCAT and
Control-FREEC. Furthermore, a broad set of QC metrics is reported at the end of the workflow with MultiQC.

Results: From FASTQs to annotated VCFs it takes three days for a $90 \mathrm{X} / 90 \mathrm{X}$ sample on a 48 cores node. Sarek is used in production at the National Genomics Infrastructure Sweden for germline and cancer samples for the Swedish Childhood Tumor Biobank and other research groups.

Conclusions: Sarek is an easy-to-use tool for germline or cancer NGS samples, to be downloaded from https://nf-co. re/sarek under MIT license.

Supporting grants: Swedish Research Council (201700630, 2017-00656), the Swedish Childhood Cancer Fund (BTB: BB2017-0001; BB2018-0001; BB2019-0001), and the Knut and Alice Wallenberg Foundation (KAW 2014.0278).

S. Juhos: None. M. Garcia: None. T. Díaz De Ståhl: None. M. Mayrhofer: None. J. Sandgren: None. M. Nistér: None.

\section{P18 Personalized Medicine and Pharmacogenomics}

\section{P18.01.B}

Reduced penetrance of pathogenic ACMG variants in a deeply phenotyped cohort study and evaluation of ClinVar classification over time

\section{J. G. J. van Rooij, L. Broer, J. Verlouw, F. J. A. van Rooij, R. Kraaij, A. G. Uitterlinden, A. J. M. H. Verkerk}

\section{Erasmus MC, Rotterdam, Netherlands}

Introduction: We studied the penetrance of pathogenic classified variants in an elderly Dutch population from the Rotterdam study for which deep phenotyping is available. We screened the 59 actionable genes for which reporting of "known" pathogenic variants was recommended by the ACMG, and demonstrate that determining what constitutes as a "known" pathogenic variant can be quite challenging.

Methods: We defined known pathogenic as classified pathogenic by both ClinVar and HGMD. In 2,628 individuals, we performed exome sequencing and identified known pathogenic variants. We investigated the clinical records of carriers and evaluated clinical events during 25 years of follow-up for evidence of variant pathogenicity.

Results: Out of 3,815 variants detected in the 59 ACMG genes, 17 variants were considered known pathogenic by both ClinVar and HGMD. For 14/17 variants the ClinVar classification had changed over time. We identified 24 carriers of these variants, which were confirmed by Sanger sequencing. In only three participants (13\%) we could observe at least one clinical event possibly caused by the variant. 
Conclusions: We show that the definition of "known pathogenic" is often unclear and should be approached carefully. Additionally variants marked as known pathogenic do not always have clinical impact on their carriers. Definition and classification of true (individual) expected pathogenic impact should be defined carefully.

J.G.J. van Rooij: None. L. Broer: None. J. Verlouw: None. F.J.A. van Rooij: None. R. Kraaij: None. A.G. Uitterlinden: None. A.J.M.H. Verkerk: None.

\section{P18.03.A}

A complex pharmacogenomic landscape of ADME genes in sub-Saharan Africa, revealed by genetic characterisation of high coverage whole-genome data

H. Othman ${ }^{1}$, J. da Rocha ${ }^{1}$, G. Botha ${ }^{2}$, D. Twesigomwe ${ }^{l}, S$. Ahmed $^{3}$, L. Cottino ${ }^{I}$, B. Drögemöller ${ }^{4}, F$. M. Faisal ${ }^{3}, P$. Machanick $^{5}$, M. Mbiyavanga ${ }^{2}, S$. Panji ${ }^{2}$, G. Wright ${ }^{4}$, M. C. Simuunza $^{6}$, S. Baldwin ${ }^{7}$, M. Chiano ${ }^{7}, C$. Cox $^{7}, A$. Gross $^{8}, P$. Thomas $^{7}$, F. J. Gamo ${ }^{9}$, S. Hazelhurst ${ }^{1}$, as members of the H3A Consortium

${ }^{1}$ Sydney Brenner Institute for Molecular Bioscience, Wits University, Johannesburg, South Africa, ${ }^{2}$ Computational Biology Division and H3ABioNet, Department of Integrative Biomedical Sciences, University of Cape Town, Cape Town, South Africa, ${ }^{3}$ Centre for Bioinformatics and Systems Biology, Faculty of Science, University of Khartoum, Khartoum, Sudan, ${ }^{4}$ University of British Columbia, Vancouver, BC, Canada, ${ }^{5}$ Department of Computer Science, Rhodes University, Grahamstown, South Africa, ${ }^{6}$ Department of Disease Control, School of Veterinary Medicine, University of Zambia, Lusaka, Zambia, ${ }^{7}$ GlaxoSmithKline, Stevenage, United Kingdom, ${ }^{8}$ GlaxoSmithKline, Sydney, Australia, ${ }^{9}$ Diseases of the Developing World, Tres Cantos Medicines Development Campus, GlaxoSmithKline, Tres Cantos, Spain

Introduction: Investigating the pharmacogenomics landscape of ADME (Absorption, Distribution, Metabolism, and Excretion) genes is important to understand their implications on drug response. ADME variation has been characterised across European and Asian populations, but data relative to African populations are available only for a few ADME genes.

Materials and Methods: We present the first study aiming to characterise the pharmacogenomics landscape of all ADME genes in sub-Saharan Africa using 458 high coverage whole genomes and other publicly accessible datasets.

Results: Our study shows a significant level of variability in ADME genes. We identified 930 potential high impact coding variants, most of which are rare and discrete to a single African subgroup. Significant frequency differences (i.e. $>10 \%$ ) are seen in common high impact variants between subgroups. For some of the novel variants, we show that their impact could be deleterious at the protein structure level. Copy number variation was detected in 116 ADME genes, with equal ratios of duplications/deletions. For variants of known clinical outcome, we note that most of these are rare in Africa compared to European populations, potentially reflecting the underrepresentation of these groups in past research.

Conclusions: Our work highlights that the ADME landscape in African populations is diverse and shows the importance of rare variation held within individual population clusters. The "genetic diversity bottleneck" in precision medicine might increase the burden in developing targeted therapies at sub-population levels. Moreover, current arraybased genotyping technologies have severe limitations to be applied as the main method in precision medicine applications.

H. Othman: None. J. da Rocha: None. G. Botha: None. D. Twesigomwe: None. S. Ahmed: None. L. Cottino: None. B. Drögemöller: None. F.M. Faisal: None. P. Machanick: None. M. Mbiyavanga: None. S. Panji: None. G. Wright: None. M.C. Simuunza: None. S. Baldwin: A. Employment (full or part-time); Significant; GlaxoSmithKline. M. Chiano: A. Employment (full or part-time); Significant; GlaxoSmithKline. C. Cox: A. Employment (full or part-time); Significant; GlaxoSmithKline. A. Gross: A. Employment (full or part-time); Significant; GlaxoSmithKline. P. Thomas: A. Employment (full or part-time); Significant; GlaxoSmithKline. F.J. Gamo: A. Employment (full or part-time); Significant; GlaxoSmithKline. S. Hazelhurst: B. Research Grant (principal investigator, collaborator or consultant and pending grants as well as grants already received); Significant; GlaxoSmithKline.

\section{P18.05.C}

GLCCI1, ORMDL-GSDMB and GNGT2 variants increase exacerbation risk in adults with asthma

A. Edris ${ }^{1,2}$, E. W. De Roos ${ }^{2}$, M. J. McGeachie ${ }^{3}$, K. M. C.

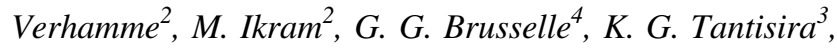
C. Iribarren ${ }^{5}, M$. Lu $u^{6}, A . C$. Wu $u^{7}$ B. H. Stricker ${ }^{2}, L$. Lahousse $^{1,2}$

${ }^{1}$ Ghent University, Ghent, Belgium, ${ }^{2}$ Erasmus MC, Rotterdam, Netherlands, ${ }^{3}$ Channing Division of Network Medicine, Brigham and Women's Hospital, Boston, MA, United States, ${ }^{4}$ Ghent University Hospital, Ghent, Belgium, ${ }^{5}$ Division of Research, Kaiser Permanente Northern California, Oakland, CA, United States, ${ }^{6}$ Division of Research, Kaiser Permanente Northern California, Oakland, CA, United 
States, ${ }^{7}$ Precision Medicine Translational Research (PROMoTeR) Center, Department of Population Medicine, Harvard Pilgrim Health Care Institute and Harvard Medical School, Boston, MA, United States

Introduction: Asthma exacerbations may be associated with genetic variants affecting the disease's underlying inflammatory pathways. We investigated the association of single nucleotide polymorphisms (SNPs) on the risk of exacerbations in adult patients with asthma, adjusted for anti-inflammatory treatment effects.

Methods: Ten SNPs which previously associated with asthma, change in lung function or exacerbations in children, were investigated within adults with asthma from the population-based Dutch Rotterdam study $(n=588)$ with replication in the American GERA cohort $(n=9,842)$. Hurdle regression models were used in the analysis to account for both the incidence and the frequency of exacerbations. All models were adjusted for age, sex, BMI, inhaled corticosteroid (ICS) use, smoking, treatment step according to GINA guidelines and the interaction between ICS use and the genotypes. The Logarithm of the follow-up time was used as an offset variable. $\mathrm{P}$ values less than 0.005 were considered Bonferroni significant.

Results: Rs7216389 (ORMDL-GSDMB, previously associated with risk of exacerbations in children) and rs37973 (GLCCII, previously associated with $\mathrm{FEV}_{1}$ change) were significantly associated with an increased subsequent exacerbation risk in count models in both adult asthmatic cohorts.(rs7216389 Discovery Rate Ratio(RR): 1.67; $\mathrm{p}<0.005$, Replication: 1.10; $\mathrm{p}<0.005$, rs37973 Discovery RR: $1.31 ; \mathrm{p}<0.005$, Replication: 1.08; $\mathrm{p}<0.005$ ) Additionally, rs17637472 (GNGT2) was nominally associated with exacerbations in the Dutch cohort, and significantly in the American cohort.

Conclusions: GLCCI1, ORMDL-GSDMB and GNGT2 variants may affect exacerbation risk in adults with asthma regardless of ICS treatment.

A. Edris: None. E.W. De Roos: None. M.J. McGeachie: None. K.M.C. Verhamme: None. M. Ikram: None. G.G. Brusselle: None. K.G. Tantisira: None. C. Iribarren: None. M. Lu: None. A.C. Wu: None. B.H. Stricker: None. L. Lahousse: None.

\section{P18.06.A}

Genetic and epigenetic factors of glucocorticoid sensitivity in asthma patients

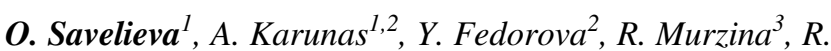
Gatiyatullin $^{3}$, E. Etkina ${ }^{3}$, E. Khusnutdinova ${ }^{2,3}$

${ }^{1}$ Bashkir state university, Ufa, Russian Federation, ${ }^{2}$ Institute of Biochemistry and Genetics - Subdivision of Ufa
Federal Research Centre, Russian Academy of Sciences, Ufa, Russian Federation, ${ }^{3}$ Bashkir State Medical University, Ufa, Russian Federation

Glucocorticoids are the most effective drugs for asthma. Up to $50-60 \%$ of differences in sensitivity to therapy in asthma patients is due to genetics. We studied polymorphisms in genes involved in the metabolism of glucocorticoids (GLCCI1, TBXT, ALLC, FBXL7, MAGI2) and the methylation status of GLCCII gene in asthma patients and healthy individuals. The study was conducted in 350 children with asthma and 363 non-atopic children of different ethnicity from the Volga-Ural region of Russia. Genotyping was carried out by RT-PCR. The methylation status of the promoter region of the GLCCII gene was determined based on bisulfite conversion of DNA, the methylation-sensitive melt curve analysis and sequencing of samples with different levels of methylation. The association of the $G L C C I 1 * \operatorname{rs} 37973 G$ allele with uncontrolled asthma $(p=$ $0,02, \mathrm{OR}=1,74)$ in Tatars was defined. We found the association of the $T B X T^{*} \mathrm{rs} 2305089 T$ allele with asthma in Bashkirs $(p=0,02, \mathrm{OR}=2,16)$. The association of the $F B X L 7 *$ rs $10044254 A A$ genotype with significantly reduced MEF25 in Russians $(p=0,03, \mathrm{OR}=2,75)$ and association of the MAGI2* rs $2691529 T C$ genotype with normal spirometry values in Tatars were established $(p<0,05)$. Hypermethylation of the promoter region of the GLCCII gene was detected only in asthma patients $(p=0,02$, $\mathrm{OR}=15,1)$. Patients with an increased level of the GLCCII gene methylation showed reduced FEV1 $(p=0,04)$, MEF25 $(p=0,006)$ MEF50 $(p=0,04)$ compared with asthma patients with low level of the GLCCII gene methylation. These results suggest that GLCCI1, TBXT, FBXL7, MAGI2 genes play an important role in the glucocorticoids pathway and pathogenesis of asthma. The study supported by the RFBR grants №17-04-02195 and №19-31-590055.

O. Savelieva: None. A. Karunas: None. Y. Fedorova: None. R. Murzina: None. R. Gatiyatullin: None. E. Etkina: None. E. Khusnutdinova: None.

\section{P18.07.B}

An admixture mapping analysis of asthma exacerbations in African Americans identifies the association of local ancestry at $3 q 27.3-28$

\section{E. Herrera-Luis ${ }^{l}$, F. Lorenzo-Diaz ${ }^{1}, A$. Mak $^{2}$, E. G. Burchard $^{2,3}$, M. Pino-Yanes ${ }^{4,5,6}$}

${ }^{1}$ Genomics and Health Group, Department of Biochemistry, Microbiology, Cell Biology and Genetics, Universidad de La Laguna, San Cristobal de La Laguna, Spain, ${ }^{2}$ Department of Medicine, University of California San Francisco, 
San Francisco, CA, United States, ${ }^{3}$ Department of Bioengineering and Therapeutic Sciences, University of California San Francisco, San Francisco, CA, United States, ${ }^{4}$ Genomics and Health Group, Department of Biochemistry, Microbiology, Cell Biology and Genetics, Universidad de La Laguna, San Cristobal de La Laguna, Spain, ${ }^{5}$ CIBER de Enfermedades Respiratorias, Instituto de Salud Carlos III, Madrid, Spain, ${ }^{6}$ Instituto de Tecnologías Biomédicas (ITB), Universidad de La Laguna, San Cristobal de La Laguna, Spain

Introduction: African ancestry has been consistently associated with asthma risk and exacerbations in African Americans. However, the genetic variation involved in severe asthma exacerbations in this population remains scarcely explored.

Aim: To perform an admixture mapping and fine mapping of severe asthma exacerbations in African Americans.

Methods: A total of 970 African American asthma patients (448 exacerbators and 522 non-exacerbators) genotyped with the Axiom LAT1 array (Affymetrix) were analyzed. Local ancestry was assessed with ELAI for 412,603 SNPs using data from Yorubans as reference panel. Association of local ancestry with asthma exacerbations was assessed with logistic regression models. A $p \leq 1.63 \times 10^{-4}$ was used to declare significance based on the number of independent ancestry blocks. Fine mapping was performed with data imputed to the, 1000 Genome Project reference panel. Association of polymorphisms and exacerbation status in the last 12 months was assessed by binary logistic regression with correction for age, sex, asthma severity and global African ancestry.

Results: African ancestry at chromosome 3q27.3-28 was associated with exacerbations. The strongest association $\left(p=3.79 \times 10^{-6}\right.$, odds ratio: $1.84,95 \%$ confidence interval: 1.42-2.39) was located in the region containing the $L P P$ gene, which has been previously associated with asthma. Additionally, fine mapping identified three common polymorphisms that remained significant after multilocus correction for the number of effective tests.

Conclusions: African ancestry and three polymorphisms at 3q27.3-q28 are associated with asthma exacerbations. Future work will include evaluation of rare variants and replication in independent studies.

Supported by grant SAF2017-83417R \& fellowship PRE2018-083837 MINECO/AEI/FEDER, UE.

E. Herrera-Luis: None. F. Lorenzo-Diaz: None. A. Mak: None. E. G. Burchard: None. M. PinoYanes: None.

\section{P18.09.A}

Genetic variability of antioxidant mechanisms and late adverse events of breast cancer radiotherapy
K. Goricar ${ }^{l}$, J. T. Stojanov Konda ${ }^{2}$, V. Dolzan ${ }^{l}, T$. Marinko $^{3,2}$

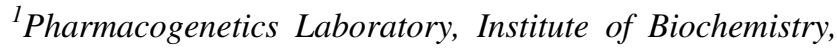
Faculty of Medicine, University of Ljubljana, Ljubljana, Slovenia, ${ }^{2}$ Faculty of Medicine, University of Ljubljana, Ljubljana, Slovenia, ${ }^{3}$ Institute of Oncology Ljubljana, Ljubljana, Slovenia

Introduction: Radiotherapy enables good long-term local control and survival in early breast cancer patients. However, some patients experience acute or late adverse events that can affect treatment protocol and decrease patient's quality of life. Genetic factors can contribute to the interindividual variability in occurrence of adverse events. As radiation leads to increased production of reactive oxygen species, contributing to oxidative stress, our aim was to evaluate the association of common polymorphisms in antioxidant genes with late adverse events of radiotherapy in breast cancer patients.

Materials and Methods: Our study included 101 HER2positive early breast cancer patients treated with adjuvant radiotherapy. We isolated DNA from buccal swabs and genotyped all patients for CAT rs1001179, GSTP1 rs1138272, GSTP1 rs1695, SOD2 rs4880, PON1 rs854560 and $P O N 1$ rs662 polymorphisms using competitive allele-specific PCR. Association of polymorphisms with adverse events was evaluated using logistic regression.

Results: Median follow-up after radiotherapy was 4.0 (2.6-5.4) years. In total, 33 (32.7\%) patients reported grade $\geq 2$ late adverse events according to LENT-SOMA criteria, and $12(11.9 \%)$ experienced grade 2 skin toxicity according to CTC criteria. Late adverse events were significantly more common in carriers of at least one polymorphic PONI rs854560 allele $(\mathrm{OR}=3.03 ; 95 \% \mathrm{CI}=1.07-8.53, P=$ 0.036 ) and carriers of at least one polymorphic $C A T$ rs 1001179 allele $(\mathrm{OR}=2.71 ; 95 \% \mathrm{CI}=1.00-7.33, P=$ 0.049) after adjustment for clinical variables.

Conclusions: Polymorphisms in antioxidant genes were associated with adverse events of radiotherapy and could serve as biomarkers contributing to the personalization of radiotherapy in breast cancer patients.

Research grants: ARRS J3-1753, P1-0170 and P3-0321.

K. Goricar: None. J.T. Stojanov Konda: None. V. Dolzan: None. T. Marinko: None.

P18.12.A

Development of a Point of Care Test for CYP2C19AIlowing Genotype Guided Antiplatelet Prescribing to Prevent Recurrent Ischaemic Strokes 
J. H. McDermott ${ }^{1}$, S. Ainsworth ${ }^{2}$, S. Wright ${ }^{3}$, D. Sen ${ }^{4}$, G. Miele $^{2}$, C. J. Smith ${ }^{4}$, K. Payne ${ }^{3}$, W. G. Newman ${ }^{1}$

${ }^{1}$ Manchester Centre for Genomic Medicine, Manchester, United Kingdom, ${ }^{2}$ Genedrive PLC, Manchester, United Kingdom, ${ }^{3}$ Manchester Centre for Health Economics, Manchester, United Kingdom, ${ }^{4}$ Greater Manchester Comprehensive Stroke Centre, Manchester, United Kingdom

Background: Clopidogrel is an antiplatelet medication recommended for secondary prevention of ischemic strokes and transient ischemic attacks in the UK. Activation is required via the $\mathrm{P} 450$ cytochrome system, of which CYP2C19 is a major component. CYP2C19 is highly polymorphic, with over 30 loss of function (LOF) alleles. Evidence suggests that outcomes could be improved if individuals with LOF alleles were prescribed alternative antiplatelets. We aimed to develop a point of care test (POCT) to identify CYP2C19 status in a clinically relevant timeframe, model the effect of implementation and capture patient views on acceptability.

Methods: An asymmetric PCR was designed to amplify CYP2C19 alleles prior to melt curve analysis using fluorescent hybridisation probes. The assay was developed on the Genedrive ${ }^{\circledR}$ rapid thermocycler using synthetic DNA target templates before validation with genomic DNA. A model-based cost-effectiveness analysis was used to assess the indicative incremental costs and consequences of genotype-guided prescribing, compared with current practice. A focus-group composed of stroke survivors explored acceptability of genotype-guided prescribing.

Results: The assay detects $* 1, * 2, * 3, * 4, * 4 \mathrm{~b}, * 10$ and $* 17$ CYP2C19 alleles in diplotype, accounting for over $96 \%$ of global variation. This POCT identifies metaboliser status in $<60$ minutes via buccal swab. Qualitative evidence suggested genotype-guided prescribing was acceptable to patients. Indicative economic analysis suggested genotypeguided prescribing has the potential to improve health outcomes and save resource, but a definitive trial is required.

Conclusions: This assay identifies $C Y P 2 C 19$ metaboliser status in a clinically relevant timeframe. The assay is portable, non-invasive and facilitates tailored antiplatelet prescribing in the acute care setting.

J.H. McDermott: None. S. Ainsworth: A. Employment (full or part-time); Significant; Genedrive PLC. S. Wright: None. D. Sen: None. G. Miele: A. Employment (full or part-time); Significant; Genedrive PLC. C.J. Smith: None. K. Payne: None. W.G. Newman: None.

\section{P18.14.C}

CYP2D6 predicted phenotype frequencies and major ethnic differences among 8457 cases.
B. Esquivel, G. Elnashar, V. Tam, J. Sheard, J. England, J. Sevlie, M. Donaldson, B. Metzger, B. Hartung

\section{OneOme, Minneapolis, MN, United States}

Introduction: Throughout world populations, different allelic frequencies of CYP2D6 have been observed. CYP2D6 is a prominent phase I metabolizing pathway that mediates up to $25 \%$ of clinically used drugs. The goal of this study was to identify CYP2D6 allelic frequencies in an ethnically diverse patient population.

Materials and Methods: DNA samples were collected from 8457 participants who consented to receive the RightMed Comprehensive test (OneOme, USA). The RightMed Comprehensive test report provides pharmacogenomic interpretation for 27 genes (111 alleles) and guidance on $300+$ medications. CYP2D6 genotypes and their respective predictive phenotype interpretation were analyzed.

Results: Out of a total of 8457 participants, $68.9 \%$ selfidentified as White or Caucasian, $4.4 \%$ as Hispanic or Latino, $3.4 \%$ as Asian, and $2.6 \%$ as Black or African American. The remaining $19.1 \%$ of participants selected Unknown or did not provide ethnicity. Poor metabolizers (PMs) with an activity score (AS) of 0 were identified in $7 \%$ of individuals identifying as White, $5 \%$ of those identified as Hispanic or Latino, $1 \%$ of Asian individuals, and $1 \%$ of Black or African Americans. Overall, 6\% of the total participants were classified as PMs.

Conclusions: Pharmacogenomics has become a valuable clinical tool for more effective prescribing by diminishing the risk of adverse drug reactions for several medications resulting in increased success. CYP2D6 plays an essential role, and with more than 110 allele variants, the frequencies fluctuate among populations. Ultimately, for a better understanding and proper implementation, broader allele coverage and accurate collection of sub-ethnicities is needed.

B. Esquivel: A. Employment (full or part-time); Significant; OneOme. E. Ownership Interest (stock, stock options, patent or other intellectual property); Modest; OneOme. F. Consultant/Advisory Board; Modest; PWN Health. Other; Significant; Genetest. G. Elnashar: None. V. Tam: A. Employment (full or part-time); Significant; OneOme. J. Sheard: A. Employment (full or part-time); Significant; OneOme. J. England: A. Employment (full or part-time); Significant; OneOme. J. Sevlie: A. Employment (full or part-time); Significant; OneOme. M. Donaldson: A. Employment (full or part-time); Significant; OneOme. B. Metzger: A. Employment (full or part-time); Significant; OneOme. B. Hartung: A. Employment (full or part-time); Significant; OneOme. 


\section{P18.16.B}

Imputation of CYP2D6 copy-number variation and frequency of key pharmacogenetic variants in Finnish population

K. Häkkinen ${ }^{1,2}$, M. Lähteenvuo ${ }^{1,2}$, T. Jukuri ${ }^{2,3}, K$. Suokas $^{2,4}$, J. Niemi-Pynttäri ${ }^{2,5}$, T. Kieseppä ${ }^{2,6,7}, T$. Männynsalo ${ }^{2,5}$, A. Wegelius ${ }^{2,6,7}$, W. Haaki ${ }^{2,8}, K$. Lahdensuo $^{2,9}$, R. Kajanne ${ }^{10}$, M. Kaunisto ${ }^{10}$, A. Tuulio-

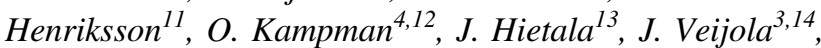
J. Lönnqvist ${ }^{7,15}$, E. Isometsä ${ }^{6}$, T. Paunio ${ }^{6,7,15}$, J. Suvisaari ${ }^{7}$, E. Kalso ${ }^{16}$, M. Niemi ${ }^{17,18}$, J. Tiihonen ${ }^{1,19,20}$, A. Ahola-

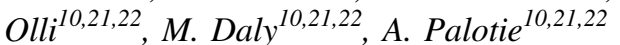

${ }^{1}$ Department of Forensic Psychiatry, Niuvanniemi Hospital, University of Eastern Finland, Kuopio, Finland, ${ }^{2}$ Institute for Molecular Medicine Finland (FIMM), University of Helsinki, years 2016-2018, Helsinki, Finland, ${ }^{3}$ Department of Psychiatry, University of Oulu, Oulu, Finland, ${ }^{4}$ Tampere University, Tampere, Finland, ${ }^{5}$ Social Services and Health Care Sector, City of Helsinki, Helsinki, Finland, ${ }^{6}$ University of Helsinki, Helsinki University Hospital, Psychiatry, Helsinki, Finland, ${ }^{7}$ Mental Health Unit, Finnish Institute for Health and Welfare, Helsinki, Finland, ${ }^{8}$ University of Turku, Turku, Finland, ${ }^{9}$ Mehiläinen, Helsinki, Finland, ${ }^{10}$ Institute for Molecular Medicine Finland (FIMM), University of Helsinki, Helsinki, Finland, ${ }^{11}$ Department of Psychology and Logopedics, Faculty of Medicine, University of Helsinki, Helsinki, Finland, ${ }^{12}$ Department of Psychiatry, Pirkanmaa Hospital District, Tampere, Finland, ${ }^{13}$ Department of Psychiatry, University of Turku and Turku University Hospital, Turku, Finland, ${ }^{14}$ Department of Psychiatry, Oulu University Hospital, Oulu, Finland, ${ }^{15}$ Department of Psychiatry, University of Helsinki, Helsinki, Finland, ${ }^{16}$ Pain Clinic, Department of Anesthesiology, Intensive Care and Pain Medicine, University of Helsinki and Helsinki University Hospital, Helsinki, Finland, ${ }^{17}$ Department of Clinical Pharmacology, University of Helsinki and Helsinki University Hospital, Helsinki, Finland, ${ }^{18}$ Individualized Drug Therapy Research Program, Faculty of Medicine, University of Helsinki, Helsinki, Finland, ${ }^{19}$ Department of Clinical Neuroscience, Karolinska Institutet, Stockholm, Sweden, ${ }^{20}$ Center for Psychiatry Research, Stockholm City Council, Stockholm, Sweden, ${ }^{21}$ Stanley Center for Psychiatric Research, The Broad Institute of MIT (Massachusetts Institute of Technology) and Harvard, Cambridge, MA, United States, ${ }^{22}$ Analytical and Translational Genetics Unit, Massachusetts General Hospital, Boston, MA, United States

Introduction: Pharmacogenetic studies have been limited by small sample size mainly through complexity of $C Y P 2 D 6$-locus. Here, we demonstrate that CYP2D6 gene duplication can be imputed accurately using existing imputation algorithms and report the frequencies of pharmacogenetic variants in a population isolate of Finland.

Materials and Methods: Genotyping of 10,075 psychosis cases from SUPER-Finland was performed with Illumina Global Screening Array. 9,381 samples have been whole-exome sequenced and 897 whole-genome sequenced. We used Finnish BrePainGen sample $(n=$ 926 with GWAS-chip and CNV data) to construct the imputation panel for CYP2D6 CNV. To validate the imputation approach, we masked the CNV in a subset of BrePainGen samples and imputed it back and correlated against genotyped CNV.

Results: In the imputation of CNV duplication 20 out of 22 duplications $(90.9 \%)$ in validation data set were imputed correctly. CYP2D6 metabolizer statuses were distributed as follows: Ultrarapid (UM) 5.4\%, intermediate (IM) $0.9 \%$, poor (PM) $2.8 \%$ and normal (NM) $90.9 \%$. Correspondingly, for CYP2C19 UM 28.1\%, IM 28.7\%, PM 3.5\%, and NM $39.7 \%$.

Conclusions: The $C Y P 2 D 6$ can be imputed with high accuracy allowing large pharmacogenetic studies. We hereby confirm the 2-3 fold enrichment of CYP2D6 UMs in Finland compared to other European populations.

Funding: The Stanley Center for Psychiatric Research at the Broad Institute of MIT and Harvard, Academy of Finland, Governmental Research Funds to the Helsinki University Hospital, The Finnish Cultural Foundation, The Finnish Foundation for Psychiatric Research, The Ministry of Social Affairs and Health Finland, through the developmental fund for Niuvanniemi Hospital, Kuopio, Finland

K. Häkkinen: None. M. Lähteenvuo: None. T. Jukuri: None. K. Suokas: None. J. Niemi-Pynttäri: None. T. Kieseppä: None. T. Männynsalo: None. A. Wegelius: None. W. Haaki: None. K. Lahdensuo: None. R. Kajanne: None. M. Kaunisto: None. A. Tuulio-Henriksson: None. O. Kampman: None. J. Hietala: None. J. Veijola: None. J. Lönnqvist: None. E. Isometsä: None. T. Paunio: None. J. Suvisaari: None. E. Kalso: None. M. Niemi: None. J. Tiihonen: None. A. Ahola-Olli: A. Employment (full or part-time); Significant; Abomics. M. Daly: None. A. Palotie: None.

\section{P18.17.C}

Monogenic diabetes gene screening in a transnational multicenter study from eleven countries of the Mediterranean area

M. Vaxillaire ${ }^{1}$, A. Bonnefond ${ }^{1,2}$, S. Liatis ${ }^{3}$, L. Ben Salem Hachmi $^{4}$, A. Jotic ${ }^{5}$, M. Boissel ${ }^{1}$, S. Gaget ${ }^{1}$, E. Durand ${ }^{l}$, E. Vaillant $^{l}$, M. Derhourhi ${ }^{1}$, N. Larcher ${ }^{l}$, F. Allegaert ${ }^{1}, R$. Medlej ${ }^{6}$, A. Chadli, Z. Arbouche ${ }^{7}$, A. Belhadj, M. 
Chaieb $^{10}$, J. Raposo ${ }^{11}$, H. Ilkova ${ }^{12}$, D. Loizou ${ }^{13}$, N. Lalic ${ }^{5}, J$.

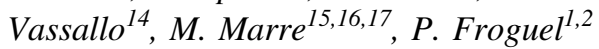

${ }^{1}$ UMR 1283, European Genomic Institute for Diabetes, University of Lille and Pasteur Institute of Lille, Lille, France, ${ }^{2}$ Department of Metabolism, Section of Genomics of Common Disease, Imperial College London, London, United Kingdom, ${ }^{3}$ Diabetes Center, Laiko General Hospital, University of Athens, Athens, Greece, ${ }^{4}$ Department of Endocrinology and Metabolic Diseases, National Institut of Nutrition, Tunis, Tunisia, ${ }^{5}$ Department of Endocrinology, Diabetes and Metabolic Diseases, Faculty of Medicine, University of Belgrade, Belgrade, Serbia, ${ }^{6}$ Department of Endocrinology, Chronic Care Centre, Saint Joseph University, Beirut, Lebanon, ${ }^{7}$ Department of Endocrinology, Ibn Rochd University Hospital, Casablanca, Morocco, ${ }^{8}$ Department of Endocrinology, Beni Messous University Hospital, Alger, Algeria, ${ }^{9}$ Department of Internal Medicine, Dr Ben Badis University Hospital, Constantine, Algeria, ${ }^{10}$ Department of Endocrinology and Diabetology, University Hospital of Farhat Hached, Sousse, Tunisia, ${ }^{11}$ Diabetology Department, Diabetes Portugal, Lisbon, Portugal, ${ }^{12}$ Department of Internal Medicine, Cerrahpasa Medical Faculty, Istanbul University, Istanbul, Turkey, ${ }^{13}$ IATRODIAGNOSIS Medical Center, Nicosia, Cyprus, ${ }^{14}$ Centre for Molecular Medicine and Biobanking, University of Malta Medical School, Msida, Malta, ${ }^{15}$ Department of Diabetology-Endocrinology-Nutrition, Hôpital Bichat, Paris, France, ${ }^{16}$ Research Unit 1138, Cordeliers Research Centre, Paris, France, ${ }^{17}$ University Paris Diderot, Sorbonne Paris Cité, Paris, France.

Introduction: Diagnosis of monogenic diabetes has important implications for diabetes precision medicine and health expenditure. Its prevalence in many countries remains unclear, although young-adult non-autoimmune diabetes is increasing. Here, we conducted a transnational multicenter study to identify rare pathogenic mutations causing monogenic diabetes in young-adults, and to assess the diagnosis rates, genetic subtypes and specificities between countries.

Materials and Methods: We performed exome sequencing in 204 unrelated diabetic cases (age-at-diagnosis: $26.1 \pm 9.1$ years) clinically selected by 30 medical centers in eleven Mediterranean countries. Rare coding variants in 35 targeted genes were evaluated for mutation pathogenicity using American College of Medical Genetics and Genomics guidelines. One-way ANOVA, chi-squared test, principal component analysis and factor analysis of mixed data were used for data analyses.

Results: Fourty pathogenic (P) or likely pathogenic (LP) mutations were identified in 36 patients yielding an overall genetic diagnosis in $17.6 \%$ of the participants. $G C K$ (37.5\%), HNFlA (27.5\%), ABCC8 (12.5\%) and HNF4A
(7.5\%) mutations accounted for the majority of cases. We found country wise highly variable diagnosis rates (up to $40 \%$ in Greece) and interestingly a higher mutation rate in European ancestry participants. Lower body mass index and HbA1c level at study inclusion, and less insulin treatment at diagnosis and inclusion are hallmarks of the mutation carriers.

Conclusions: In practice, our findings impacted patient long-term monitoring and therapeutic regimen. They also highlight broad clinical and genetic spectrum of youngonset diabetes in Mediterranean countries.

Funded by the French National Research Agency and the Mediterranean Group for Study of Diabetes.

M. Vaxillaire: None. A. Bonnefond: None. S. Liatis: None. L. Ben Salem Hachmi: None. A. Jotic: None. M. Boissel: None. S. Gaget: None. E. Durand: None. E. Vaillant: None. M. Derhourhi: None. N. Larcher: None. F. Allegaert: None. R. Medlej: None. A. Chadli: None. Z. Arbouche: None. A. Belhadj: None. M. Chaieb: None. J. Raposo: None. H. Ilkova: None. D. Loizou: None. N. Lalic: None. J. Vassallo: None. M. Marre: None. P. Froguel: None.

\section{P18.19.B}

Comparison of EGFR mutation detection among FFPET, bronchoalveolar lavage (BAL) and blood samples in patients with non-small cell lung Cancer

\section{Dimitrova ${ }^{1}$, N. Yanev ${ }^{2}$, S. Bichev ${ }^{l}$, D. Kostadinov ${ }^{2}$, A.} Savov ${ }^{1}$

${ }^{1}$ National Genetics Laboratory, Sofia, Bulgaria, ${ }^{2}$ Medical University of Sofia, Department of Pulmonary Diseases University Hospital for Pulmonary Diseases "St. Sofia", Sofia, Bulgaria

Background: FFPET samples remain the golden standard for EGFR genotyping. In many cases the initial material is insufficient. In those cases EGFR genotyping could be done on BAL or blood samples. In the last few years liquid biopsies prove to be reliable method for EGFR characterization, on the other hand cytology samples are not used that often. In this study we compare the detection rate of EGFR mutations in BAL and blood samples compared to FFPET samples.

Materials and Method: For a period of 9 months 10 patients with EGFR activating mutations have been selected. DNA extraction was performed with QiaAmp Circulating Nucleic Acid kit for plasma and BAL samples. For FFPET samples we used QIAamp DNA FFPE Tissue Kit. EGFR genotyping was performed with therascreen EGFR Plasma or tissue RGQ PCR Kits. 
Results and Discussion: Activating mutations were confirmed based on EGFR genotyping in FFPET samples. 6 patients had deletions in exon 19, 3 had L858R substitution in exon 21 and one patient had G719x in exon 18. In 9 cases mutations were confirmed in corresponding BAL samples and they were detected in only 4 plasma samples. In 1 case, in both liquid biopsy samples- BAL and plasma, we found T790M mutation along with deletion in exon 19, but not in the corresponding FFPET sample.

Conclusions: This study shows that BAL samples are superior to plasma samples and almost equal to FFPET samples in detecting EGFR mutations, therefore cytology samples should be preferred before blood samples when possible.

I. Dimitrova: None. N. Yanev: None. S. Bichev: None. D. Kostadinov: None. A. Savov: None.

P18.20.C

Genetic test utilization in a large US healthcare database of patients with indications of rare genetic disease

B. Schroeder ${ }^{l}$, N. Gonzaludo ${ }^{1}$, K. Everson ${ }^{2}$, J. Sullivan ${ }^{2}, R$. J. Taft ${ }^{l}$, J. Belmont ${ }^{l}$

${ }^{1}$ Illumina, Inc, San Diego, CA, United States, ${ }^{2}$ Precision Health Economics, Los Angeles, CA, United States

Introduction: Our understanding of the genetic etiology of many rare diseases has grown substantially in recent years. However, there has been little real-world evidence published regarding the epidemiology and care delivery of patients with suspected rare genetic diseases at the population level. The objective of this analysis was to characterize the population of patients with indications of rare genetic diseases in a large US healthcare claims database, with a focus on utilization of genetic testing.

Materials and Methods: This retrospective administrative claims analysis used the Optum Clinformatics database. A total of 12,023,329 patients met inclusion criteria, including continuous enrollment. Curated lists of ICD-9 and ICD-10, CPT codes, and other billing codes were generated to identify patients with indications of suspected genetic diseases. Combinations of variables were used to define patient cohorts for analysis, with different levels of stringency used to define wider vs more conservative cohorts. Separate analyses were completed on critically-ill infants and other pediatric patients.

Results: Up to $12 \%$ of pediatric patients $(1,450,702)$ and up to $53 \%$ of critically ill infants $(26,322)$ had indicators of genetic disease. Across all cohorts, only a small minority of patients received any genetic testing-between 3 to $9 \%$ based on different cohort criteria. Mean time to first genetic test was $>350$ days for pediatric patients and $>140$ days for critically-ill infants.

Conclusions: The results of this study highlight the substantial patient population with indicators of suspected genetic diseases, but notably, a significant underutilization of genetic testing.

B. Schroeder: A. Employment (full or part-time); Significant; Illumina, Inc. E. Ownership Interest (stock, stock options, patent or other intellectual property); Significant; Illumina, Inc. N. Gonzaludo: A. Employment (full or part-time); Significant; Illumina, Inc. E. Ownership Interest (stock, stock options, patent or other intellectual property); Significant; Illumina, Inc. K. Everson: F. Consultant/Advisory Board; Significant; Illumina, Inc. J. Sullivan: F. Consultant/Advisory Board; Significant; Illumina, Inc. R.J. Taft: A. Employment (full or part-time); Significant; Illumina, Inc. E. Ownership Interest (stock, stock options, patent or other intellectual property); Significant; Illumina, Inc. J. Belmont: A. Employment (full or part-time); Significant; Illumina, Inc. E. Ownership Interest (stock, stock options, patent or other intellectual property); Significant; Illumina, Inc.

\section{P18.22.B}

Polygenic risk score improves the prediction ability in knee and hip osteoarthritis

B. Sedaghati-khayat ${ }^{1}$, J. G. J. van Rooij ${ }^{l}$, C. G. Boer ${ }^{l}$, L. Broer $^{1}$, A. J. M. H. Verkerk' ${ }^{1}$ E. Zeggini ${ }^{2}$,.. GO consortium $^{1,2}$, A. G. Uitterlinden ${ }^{1,3}$, J. B. J. van Meurs ${ }^{1,3}$

${ }^{I}$ Department of Internal medicine, Erasmus MC, Rotterdam, Netherlands, ${ }^{2}$ Institute of Translational Genomics, Helmholtz, Zentrum München, German Research Center for Environmental Health, Neuherberg, Germany, ${ }^{3}$ Department of Epidemiology, Erasmus MC, Rotterdam, Netherlands

Introduction: Osteoarthritis (OA) is the most common joint disorder worldwide, with mostly unknown etiology but with substantial heritability. We estimated an individual's genetic risk of OA using a polygenic risk score (PRS) calculated from the latest GWAS of the Genetics of Osteoarthritis (GO)-consortium. This project is part of "Genotyping On All Patients(GOALL)" of Erasmus MC to return genetic information to the patient and clinician.

Methods: We analyzed all 6,291 Rotterdam Study (RS-I base line cohort) Caucasian participants with 25 years follow-up, containing 1,177 knee and 561 incident hip OA cases. PRS were constructed for HipOA (43-SNPs) and KneeOA (23-SNPs) with 11-SNPs shared. Standardized PRSs (zPRS) were constructed to estimate the odds ratios per standard deviation (SD) of zPRS for each subject, weighted by effect sizes and adjusted for age/sex. External 
validation was performed in the clinical Cohort Hip and Cohort Knee (CHECK), and RS-II and RS-III cohorts.

Results: The zPRS for HipOA predicted prevalent HipOA $\left(p=6.7 \times 10^{-4} ; \mathrm{OR}=1.1\right)$. Moreover, the incident HipOA was predicted $\left(p=6.2 \times 10^{-4} ; \mathrm{OR}=1.4\right)$ with the area under the curve (AUC) of 0.59 . When the zPRS were added to the predictive AUC of 0.68 for age/sex, it increased to 0.70 . The $\mathrm{zPRS}$ for KneeOA only predicted prevalent KneeOA ( $\left.p=1.5 \times 10^{-6} ; \mathrm{OR}=1.2\right)$. The top $10 \%$ of the zPRS compared to the rest of the population had a 2 times higher risk for HipOA incidence $\left(p=7.7 \times 10^{-4}\right)$ and $50 \%$ higher risk for KneeOA incidence $\left(p=3.7 \times 10^{-2}\right)$.

Conclusions: Our work shows the potential of PRS as a clinical prediction tool for prevalent and incident knee and hip OA.

B. Sedaghati-khayat: None. J.G.J. van Rooij: None. C. G. Boer: None. L. Broer: None. A.J.M.H. Verkerk: None. E. Zeggini: None. .. GO consortium: None. A.G. Uitterlinden: None. J.B.J. van Meurs: None.

\section{P18.24.A}

\section{International mapping of national genomics initiatives}

\author{
T. Leonardo Alves ${ }^{1}$, M. E. Jansen ${ }^{1}$, B. Hoebee ${ }^{2}$, W. \\ Rodenburg ${ }^{1}$
}

${ }^{1}$ National Institute for Public Health and the Environment, Centre for Health Protection, Bilthoven, Netherlands, ${ }^{2}$ National Institute for Public Health and the Environment, Centre for Population Screening, Bilthoven, Netherlands

Introduction: The potential benefits of population-based genomics are considered substantial, but little is known about the type, nature and scale of national genomic plans. We aimed to describe ongoing genomics initiatives identified in middle and high-income settings.

Methods: A convenience sample of countries was selected based on expert referral and google searches. Data was extracted and collated covering diverse items of interest: country, type and scope of the initiative, funding, participants, aims, activities, inception and conclusion date, drivers and actors involved.

Results: A sample of 25 countries was included and a total of 30 initiatives were found. Most have a national scope $(N=27)$ and focus on Prevention and Screening as well as on Diagnostics and Treatment $(N=10)$. If the initiative is national, then it is often population-based. Models are varied, from funding platforms to governmentlead initiatives, as well as individual research projects. In some countries, there is full public funding while others opt for public-private-partnerships. The scale of financing varies largely, depending on the span of activities: research, infrastructure, development of genomic testing and analysis, as well as capacity-building and education. Drivers and actors involved also differ depending on the nature of the initiative, but universities and health research centres often have a leading role within consortia or networks.

Conclusions: There is not a one-size fits all approach to genomic plans. Countries seem to design initiatives according to their priorities. However, lessons learned and arguments for a certain model can hold valuable information for individual countries and international genomic plans.

T. Leonardo Alves: None. M.E. Jansen: None. W. Rodenburg: None.

\section{P18.25.B}

No relationship was found between IL28B rs12979860 polymorphism and spontaneous clearance of $\mathrm{HDV}$ in Mongolian population

Z. Batsuuri, ${ }^{1,2}$, P. Bat-Ulzii ${ }^{3}$, A. Enkhbat $^{3}$, K. Batbold ${ }^{1}$, B. Munkhjin $^{4}$, G. Chimed ${ }^{2}$, B. Dendev ${ }^{3}$, N. Dashdorj ${ }^{5}$, E. Perenlei $^{6}$, O. Oidovsambuu ${ }^{4}$

${ }^{I}$ Global Communication LLC, Ulaanbaatar, Mongolia, ${ }^{2}$ New Mongol Institute of Technology, Ulaanbaatar, Mongolia, ${ }^{3}$ Liver Center, Ulaanbaatar, Mongolia, ${ }^{4}$ National University of Mongolia, Ulaanbaatar, Mongolia, ${ }^{5}$ Onom Foundation, Ulaanbaatar, Mongolia, ${ }^{6}$ University of Texas Medical Branch, Galvestone, TX, United States

Introduction: Host genetic factors play an important role for viral infection. Numerous studies showed that the SNP rs12979860 of IL28B gene is clearly associated with spontaneous clearance of $\mathrm{HCV}$, but not that of $\mathrm{HBV}$. However, only few studies were done in very small number of people to reveal the possible effect of SNP rs12979860 genotypes in case of HDV infection. In this study, we did comprehensive statistical analysis to show whether there is a relationship between SNP rs12979860 and spontaneous clearance of HDV using relatively large number of people.

Methods: Total of 820 participants including of 3 groups (HDV spontaneously cleared $n=215$, HDV chronic $n=$ 304 and healthy $n=301$ ) were participated in this study according to their serological and molecular biological diagnostic results. For each participant, SNP rs12979860 was genotyped by RFLP and Sanger sequencing. Pearson's chi square test and odds ratios were applied for assessing the association between SNP rs12979860 and spontaneous clearance of HDV.

Results: Our results are shown as following table. 


\begin{tabular}{|c|c|c|c|c|c|c|c|c|c|}
\hline \multirow[t]{2}{*}{ Genotype } & \multirow[t]{2}{*}{$\begin{array}{l}\text { HDV } \\
\text { cleared }\end{array}$} & \multirow[t]{2}{*}{$\begin{array}{l}\text { HDV } \\
\text { chronic }\end{array}$} & \multirow[t]{2}{*}{ Healthy } & \multicolumn{2}{|c|}{$\begin{array}{l}\text { HDV cleared } \\
\text { vs. chronic }\end{array}$} & \multicolumn{2}{|c|}{$\begin{array}{l}\text { HDV cleared } \\
\text { vs. healthy }\end{array}$} & \multicolumn{2}{|c|}{$\begin{array}{l}\text { HDV chronic } \\
\text { vs. healthy }\end{array}$} \\
\hline & & & & OR & $95 \% \mathrm{CI}$ & OR & $95 \%$ CI & OR & $95 \% \mathrm{Cl}$ \\
\hline $\mathrm{CC}$ & 184 & 257 & 269 & 1.09 & $\begin{array}{l}0.66- \\
1.77\end{array}$ & 0.71 & $\begin{array}{l}0.42- \\
1.2\end{array}$ & 0.65 & $\begin{array}{l}0.4- \\
1.05\end{array}$ \\
\hline $\mathrm{CT}+\mathrm{TT}$ & 31 & 47 & 32 & & & & & & \\
\hline
\end{tabular}

Conclusions: Our analysis showed that spontaneous clearance of HDV is not dependent on the genotypes of rs12979860 polymorphism. This project was funded by CRDF Global (agreement number: DAA2-17-62984-1).

Z. Batsuuri: A. Employment (full or part-time); Significant; Global Communication LLC. B. Research Grant (principal investigator, collaborator or consultant and pending grants as well as grants already received); Significant; New Mongol Institute of Technology. C. Other Research Support (supplies, equipment, receipt of drugs or other in-kind support); Significant; National University of Mongolia. P. Bat-Ulzii: None. A. Enkhbat: None. K. Batbold: None. B. Munkhjin: None. G. Chimed: None. B. Dendev: None. N. Dashdorj: None. E. Perenlei: None. O. Oidovsambuu: None.

\section{P18.26.C}

Identification of novel genetic risk factors in the conserved haplotype region surrounding the LCT locus on chromosome 2 q21.

\section{A. Cairo ${ }^{1}$, S. Spena ${ }^{2}$, E. Pappalardo ${ }^{2}$, M. Mancuso ${ }^{1}$, F. Peyvandi, ${ }^{1,2}$}

${ }^{1}$ Angelo Bianchi Bonomi Haemophilia and Thrombosis center, Fondazione IRCCS Ca' Granda Ospedale Maggiore Policlinico and Fondazione Luigi Villa, Milan, Italy, ${ }^{2}$ Università degli studi di Milano, Department of Pathophysiology and Trasplantation, Milan, Italy

Introduction: Inhibitors development affects about $30 \%$ of patients with severe hemophilia A (HA). Different environmental and genetic risk factors are involved in this process.[1,2] Recently, we identified a SNV (rs3754689) in the LCT gene potentially linked with this predisposition.[3] Since this variant is benign [4] and is located in a conserved haplotype block, we hypothesized that the association signal captured by this variant is probably located in coinherited, neighboring genes like R3HDM1, UBXN4 and miRNA-1281 , previously linked with autoimmune disorders and therefore potential clinically relevant targets. The aim of this study is to identify novel genetic risk factors associated with inhibitor development in R3HDM1, UBXN4, CXCR4, MCM6, miRNA-128-1 genes in patients with severe HA.

Materials and Methods: 246 Italian patients with severe HA with (72) or without (174) inhibitor was sequenced by
TruSeq Custom Amplicon (Illumina). Data were analyzed according to the guidelines reported by the Broadinstitute (https://software.broadinstitute.org/gatk/best-practices/).

Statistical analysis was performed by Plink, PlikSeq.

Results: 228 variants passed the quality controls: 56 common $(\mathrm{MAF} \geq 1 \%)$ and 172 rare $(\mathrm{MAF}<1 \%)$. Logistic regression of common variants confirmed the protective role of the rs 3754689 missense variant. Other 4 variants resulted significantly associated. Two are localized in $L C T$, a genomic region involved in the regulation of the $U B X N 4$ expression. Rare variants resulted not associated.

Conclusions: This study confirmed the association of the chromosomal region around the $L C T$ locus with inhibitor development in patients with severe HA. Further investigations are necessary to evaluate the expression of target genes and miR-128-1.

A. Cairo: None. S. Spena: None. E. Pappalardo: None. M. Mancuso: None. F. Peyvandi: D. Speakers Bureau/ Honoraria (speakers bureau, symposia, and expert witness); Modest; Alnylam, Grifols, Roche, Takeda. D. Speakers Bureau/Honoraria (speakers bureau, symposia, and expert witness); Significant; Kedrion. F. Consultant/Advisory Board; Modest; Bioverativ, Roche, Sanofi, Takeda.

P18.27.A

Identification of genomic signature as diagnostic and prognostic blood biomarker for early hepatocellular carcinoma using integrated cross-species genomic and network analyses

I. H. Kaya ${ }^{1}$, O. Al-Harazi $i^{2}$ M. Al-Eid ${ }^{2}$, L. Alfantoukh ${ }^{2}$, M. Al-Sabayel ${ }^{2,3}$, N. Kaya ${ }^{2}$, D. Colak $^{2}$

${ }^{1}$ AlFaisal University, Riyadh, Saudi Arabia, ${ }^{2}$ King Faisal Specialist Hospital and Research Centre, Riyadh, Saudi Arabia, ${ }^{3}$ AlMaarefa University, College of Medicine, Riyadh, Saudi Arabia

Introduction: Hepatocellular carcinoma (HCC) is the most common primary cancer originating in the liver and thirdleading cause of cancer-related deaths worldwide. The disease is mostly diagnosed at advanced stages, hence has poor prognosis. Robust biomarkers are therefore urgently needed for early diagnosis and prognostic estimation.

Materials and Methods: We performed integrated genomic and network-based analyses of early HCC (eHCC) using genome-wide gene expression profiling datasets from human eHCC samples and eHCC rat model. The validation of diagnostic and prognostic value of the identified gene signature was done using independent datasets of gene expression profiling of tissue samples as well as peripheral blood mononuclear cells from patients with eHCC. 
Results: We identified 41-gene signature that is conserved across species and expressed in blood. We validated the diagnostic and prognostic potential of the gene signature on independent datasets with detailed clinical data. We developed a prognostic model based on our gene signature that demonstrated that the high expression was significantly associated with poor disease outcome. Multivariate regression analysis revealed that our gene set predicted the disease outcome independent of other clinical factors.

Conclusions: Our results suggest that integrated crossspecies genomic and network analysis may provide a robust methodology to identify key biological programs associated with eHCC and may lead to improved diagnosis, prognosis, and therapeutic options.

Acknowledgement: This study is funded by the Research Grant (RAC\#2110006, 2180030 to DC). We thank our individual sponsors who generously donated to this research. We also would like thank Ms. Sukina Qanbar for her administrative support.

I.H. Kaya: None. O. Al-Harazi: None. M. Al-Eid: None. L. Alfantoukh: None. M. Al-Sabayel: None. N. Kaya: None. D. Colak: None.

\section{P18.31.B}

Reports of incidental findings in a cohort of 500 exomes of Brazilian patients were common and show potential impact in life-expectancy

C. R. D. C. Quaio ${ }^{1,2}$, C. M. Moreira ${ }^{l}$, P. R. SacramentoBobotis $^{l}$, G. M. Novo-Filhol, M. G. Penna ${ }^{l}$, R. R. F. Sousa ${ }^{l}$, A. C. G. Trindade ${ }^{l}$, C. R. Furuzawal ${ }^{1}$ D. P.

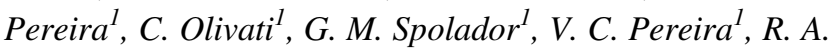

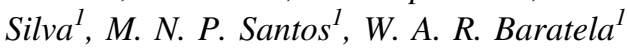

${ }^{1}$ Fleury Medicina e Saúde, Sao Paulo - SP, Brazil, ${ }^{2}$ Faculdade de Medicina da Universidade de São Paulo, São Paulo - SP, Brazil

Incidental findings in next-generation sequencing-based exams correspond to deleterious variants in clinicallyactionable conditions discovered unintentionally in genes that were not the primary objective of analysis. American College of Medical Genetics and Genomics (ACMG) has published recommendations for reporting incidental findings in selective genes.

We report incidental findings from 500 whole exomes performed for clinical/diagnostic purposes at a private laboratory in Brazil and discuss potential impact in life expectancy. Information about potential life expectancy impact of early diagnosis of genetic conditions was reviewed from cost-effectiveness studies available in the literature: we considered a potential increase of 21 years in life expectancy for variants in congenital cardiomyopathy genes, 7.6 years for long QT and 6.0 years in highly penetrant breast cancer genes.

Incidental findings in genes enlisted by ACMG were found in eight patients $(1.6 \%)$, including four variants in genes associated with cardiac conditions (KCNH2, $M Y B P C 3, D S C 2, M Y H 7)$ and four variants in cancerpredisposing genes (TP53, BRCA1, PMS2, BRCA2). We additionally reported findings whose impact in lifeexpectancy was not calculated: 15 (3\%) pathogenic heterozygous variants in autosomal recessive conditions of ACMG list (LDLR, MUTYH, ATP7B, APOB genes) and three variants $(0.6 \%)$ for autosomal dominant cancerpredisposing conditions not in ACMG list (CHEK2, RAD51C, BRIP1).

We estimate that the reports of incidental findings may have increased life expectancy in our cohort by 88.6 years, or 0.18 year per cohort patient. Since general accessibility to genomic testing is increasing, analysis of clinicallyactionable conditions show potential benefit for public health policies in the future.

C.R.D.C. Quaio: A. Employment (full or part-time); Significant; Fleury Medicina e Saude. C.M. Moreira: A. Employment (full or part-time); Significant; Fleury Medicina e Saude. P.R. Sacramento-Bobotis: A. Employment (full or part-time); Significant; Fleury Medicina e Saude. G. M. Novo-Filho: A. Employment (full or part-time); Significant; Fleury Medicina e Saude. M.G. Penna: A. Employment (full or part-time); Significant; Fleury Medicina e Saude. R.R.F. Sousa: A. Employment (full or parttime); Significant; Fleury Medicina e Saude. A.C.G. Trindade: A. Employment (full or part-time); Significant; Fleury Medicina e Saude. C.R. Furuzawa: A. Employment (full or part-time); Significant; Fleury Medicina e Saude. D. P. Pereira: A. Employment (full or part-time); Significant; Fleury Medicina e Saude. C. Olivati: A. Employment (full or part-time); Significant; Fleury Medicina e Saude. G.M. Spolador: A. Employment (full or part-time); Significant; Fleury Medicina e Saude. V.C. Pereira: A. Employment (full or part-time); Significant; Fleury Medicina e Saude. R. A. Silva: A. Employment (full or part-time); Significant; Fleury Medicina e Saude. M.N.P. Santos: A. Employment (full or part-time); Significant; Fleury Medicina e Saude. W. A.R. Baratela: A. Employment (full or part-time); Significant; Fleury Medicina e Saude.

\section{P18.33.A}

inflammation status modulates the effect of host genetic variation on intestinal gene expression in inflammatory bowel disease

S. $\boldsymbol{H u}^{l}$, W. T. Uniken Venema ${ }^{l}, H$. Westra ${ }^{l}$, A. Vich Vila ${ }^{l}$, R. Barbieri ${ }^{l}$, M. D. Voskuill ${ }^{l}$, T. Blokzijl ${ }^{l}$, B. H. Jansen ${ }^{l}$, Y. 
Li $^{l}$, M. J. Daly ${ }^{2}$, R. J. Xavier ${ }^{2}$, E. A. Festen ${ }^{l}$, G. Dijkstra ${ }^{l}$, R. K. Weersma ${ }^{l}$

${ }^{1}$ UMCG, Groningen, Netherlands, ${ }^{2}$ Broad Institute of Harvard and MIT, Boston, MA, United States

Introduction: More than 240 genetic loci have been associated with Inflammatory bowel disease (IBD), but little is known about how they contribute to disease development in involved tissue. We hypothesized that host genetic variation affects gene expression in an inflammation-dependent way.

Materials and Methods: We investigated 299 snapfrozen intestinal biopsies from inflamed and non-inflamed mucosa from 171 IBD patients. RNA-sequencing was performed, and genotypes were determined using whole exome sequencing and genome wide genotyping. In total, 28,746 genes and 6,894,979 SNPs were included. Linear mixed models identified 7,667 independent intestinal cisexpression quantitative trait loci (cis-eQTLs) $(\mathrm{FDR}<0.05)$ and interaction analysis revealed 122 inflammationdependent intestinal cis-eQTLs $(\mathrm{FDR}<0.05)$, including several for known IBD-risk genes and genes encoding immune-cell receptors and antibodies. The inflammationdependent $c i s$-eQTL SNPs mainly interact with prevalence of immune cell types.

Conclusions: Inflammation-dependent intestinal ciseQTLs reveal genetic susceptibility under inflammatory conditions that can help identify the cell types involved and the underlying pathways, knowledge that may guide future drug development and profile patients for precision medicine in IBD.

S. Hu: None. W.T. Uniken Venema: None. H. Westra: None. A. Vich Vila: None. R. Barbieri: None. M.D. Voskuil: None. T. Blokzijl: None. B.H. Jansen: None. Y. Li: None. M.J. Daly: None. R.J. Xavier: None. E.A. Festen: None. G. Dijkstra: None. R.K. Weersma: None.

\section{P18.34.B}

Integrated analysis of transcriptomic and genomic data reveals blood markers with diagnostic and prognostic potential in non-small cell lung cancer

\section{T. Kaya ${ }^{1}$, O. Al-Harazi ${ }^{2}$, M. Aldosary ${ }^{2}$, A. Al-Bakheet ${ }^{3}$,

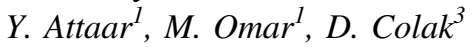

${ }^{1}$ King Faisal School, Riyadh, Saudi Arabia, ${ }^{2}$ King Faisal Specialist Hospital and Research Centre, Riyadh, Saudi Arabia, ${ }^{3}$ King Faisal Specialist Hospital and Research Center, Riyadh, Saudi Arabia

Introduction: Lung cancer is the second most common cancer and the main leading cause of cancer-associated death worldwide. Non-small cell lung cancer (NSCLC) accounts for about $85 \%$ of lung cancer diagnoses, which enforces the importance of diagnosing NSCLC patients reliably and as early as possible in order to reduce the risk of mortality.

Materials and Methods: We identified blood-based gene markers for NSCLC by utilizing an integrated analysis of global gene expression and copy number alterations of NSCLC patients using array-based techniques. The gene signature was then validated using independent datasets of gene expression profiling of over 1000 NSCLC patients. We also validated the classification performance and the prognostic potential of the gene signature using independent datasets with detailed clinical information.

Results: The high expression of our gene signature is associated with a poor disease outcome. Significantly altered functions, pathways, and gene networks in NSCLC-associated genes revealed alterations in several cancer-related pathways that may have importance for NSCLC transformation, further validating our gene signature's diagnostic and prognostic potential.

Conclusions: Our findings suggest that integrated genomic and network analysis is likely to provide a reliable approach to identify genes that are associated with NSCLC and may lead to improved diagnosis and therapeutic options, without any invasive procedures required.

Acknowledgement: This study is funded by the Research Grant (RAC\#2110006 to DC). We would like to thank Ms. Sukina Qanbar for her administrative support.

M.T. Kaya: None. O. Al-Harazi: None. M. Aldosary: None. A. Al-Bakheet: None. Y. Attaar: None. M. Omar: None. D. Colak: None.

\section{P18.38.C}

Decomposing the genetic burden of chronic diseases into multitrait signatures to support drug development

H. Julienne ${ }^{l}$, V. Laville ${ }^{l}$, Z. R. McCaw ${ }^{2}, Z . H e^{3}$, V. Guillemot $^{l}$, C. Lasry ${ }^{l}$, A. Ziyatdinov ${ }^{4}$, A. Vaysse ${ }^{l}, P$. Lechat $^{1}$, H. Ménager ${ }^{1}$, W. Le Goff ${ }^{5}$, P. Kraft ${ }^{2,4}$, I. IonitaLaza $^{6}$, B. J. Vilhjálmsson ${ }^{7}, H$. Aschard ${ }^{1,4}$

${ }^{1}$ Department of Computational Biology - USR 3756 CNRS, Institut Pasteur, Paris, France, ${ }^{2}$ Department of Biostatistics, Harvard TH Chan School of Public Health, Boston, MA, United States, ${ }^{3}$ Department of Neurology and Neurological Sciences, Stanford University School of Medicine, Stanford, CA, United States, ${ }^{4}$ Department of Epidemiology, Harvard TH Chan School of Public Health, Boston, MA, United States, ${ }^{5}$ Sorbonne Université, INSERM, UMR_S 1166 ICAN, Paris, France, ${ }^{6}$ Department of Biostatistics, Columbia University, New York, NY, United States, ${ }^{7}$ National Centre for Register-based Research, Aarhus University, Aarhus, Denmark 
Only a small fraction of molecules entering the drug development process will actually be approved for market use. Leveraging genetic information could improve the success rate of drug clinical trial. Previous work showed that molecules with genetic evidence of association with the disease have greater chance of being eventually approved, and that adverse effects can be partially predicted from genetic associations. Here, we argue that multitrait genomewide association studies (GWAS) is a powerful framework for harnessing genetics to inform drug targeting by: 1) increasing power to identify new drug targets; 2 ) associating drug targets with biological pathways through clustering; and 3) suggesting drug targets with no indication of likely adverse effects. We conducted a multitrait GWAS of metabolic (lipid and glycemic) traits using our recently developed JASS package (http://jass.pasteur.fr/index.html). We identified 405 significant associations, detected six multitrait association signatures (i.e. clusters of genetic variants with similar association pattern) and associated each signature with metabolic pathways. We investigated the links between the identified multitrait association signatures and coronary heart diseases (CHD). Signatures associated with an increased risk of type 2 diabetes, low density lipoprotein or triglycerides were also significantly associated to CHD. Conversely, signatures associated to high density lipoprotein did not exhibit protective effect on CHD. These conclusions were concordant with past or ongoing random control trials on the following molecules: probucol, cetrapid, alirocumab, ezetimibe, and volanesorsen. We provide a list, for signatures associated with CHD, of druggable genes with their associated metabolic pathways that have not been investigated yet.

H. Julienne: None. V. Laville: None. Z.R. McCaw: None. Z. He: None. V. Guillemot: None. C. Lasry: None. A. Ziyatdinov: None. A. Vaysse: None. P. Lechat: None. H. Ménager: None. W. Le Goff: None. P. Kraft: None. I. Ionita-Laza: None. B.J. Vilhjálmsson: None. H. Aschard: None.

P18.41.C

Comprehensive Genomic analysis of Parkinson 's disease Cases of an Arab Descent

\section{B. R. Mubarak}

King Faisal specialist hospital and Research center, Riyadh, Saudi Arabia

Despite over 2 decades of Parkinson's disease (PD) research, the genetic picture remains unclear. Until now, no universal risk variant(s) have been found. At least 40 different loci have been implicated in PD from genome-wide association studies and an equal number of candidate genes have been revealed by whole exome sequencing-based studies. Thus, highlighting the genetic heterogeneity of PD. Moreover, most of the large scale studies have been conducted in European or Asian cohorts and their findings rarely replicated across populations. Therefore, inclusion of non-European data sets is necessary not only for validating previous findings but also for variant/gene discovery. Genetic studies on complex neurodegenerative disorders in Arabs are lacking. Here we report on the first comprehensive genomic study on 60 PD cases of Arab descent. A three-stage analysis strategy was tailored to detect rare variants in PD. By applying this strategy, 125 new variants affecting 117 genes were identified in $85 \%$ of the cases. All of the genes have not be previously linked to PD except two (EIF4G1 and ATP13A2). Further prioritization of our data generated a list of high confidence variants in 20 genes involved in neuronal function and/or having mouse models with relevant phenotypes.

B.R. Mubarak: None.

\section{P18.43.B}

How can we capture clinical variation and progression in deeply phenotyped patient cohorts?

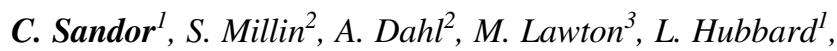
B. Bojovic ${ }^{2}$, M. Peyret-Guzzon ${ }^{2}$, H. Matten ${ }^{2}$, C. Blancher ${ }^{2}$, N. Williams ${ }^{1}, Y$. Ben-Shlomo ${ }^{3}, M . \mathrm{Hu}^{2}$, D. Grosset ${ }^{4}, J$. Marchini $^{2}$, C. Webber ${ }^{1}$

${ }^{1}$ Cardiff University, Cardiff, United Kingdom, ${ }^{2}$ University of Oxford, Oxford, United Kingdom, ${ }^{3}$ University of Bristol, Bristol, United Kingdom, ${ }^{4}$ Queen Elizabeth University Hospital, Glasgow, United Kingdom

Introduction: There is large variation in the clinical presentations and progression between patients with the same disorder. The generation of deeply and longitudinally phenotyped patient cohorts offers an enormous potential to identify disease subtypes with prognostic and therapeutic utility.

Material and Methods: Using three large cohorts of Parkinson's disease (PD) patients with extensive clinical observational data repeatedly collected over many years, we developed a Bayesian multiple phenotype mixed model incorporating the genetic relationships between individuals that was able to reduce a large number of diverse clinical measurements into a smaller number of continuous underlying factors ("phenotypic axes"). This approach overcomes many limitations associated with clustering methods and better reflects the more continuous phenotypic variation observed amongst patients. 
Results: We identify three principal axes of PD patient phenotypic variation which are reproducibly found across three independent, deeply and diversely phenotyped cohorts. Together they explain over $75 \%$ of the observed clinical variation and remain robustly captured with a fraction of the clinically-recorded features. The most influential axis was associated with the genetic risk of Alzheimer's disease (AD) suggesting PD patients with a high genetic risk for Alzheimer's disease are more likely to develop a more aggressive form of PD including dementia symptoms.

Conclusions: Our approach has the potential to accelerate our understanding of how disease manifests and progresses. This approach provides universal disease benchmarks through which patients may be appropriately compared, through which the underlying disease-modifying mechanisms can be understood and appropriately stratified, and personalised therapeutic strategies and treatments can be developed.

C. Sandor: None. S. Millin: None. A. Dahl: None. M. Lawton: None. L. Hubbard: None. B. Bojovic: None. M. Peyret-Guzzon: None. H. Matten: None. C. Blancher: None. N. Williams: None. Y. Ben-Shlomo: None. M. Hu: None. D. Grosset: None. J. Marchini: None. C. Webber: None.

\section{P18.46.B}

Post-operative toxic reaction reversed through pharmacogenetics screening: a case report

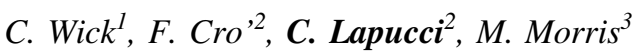

${ }^{1}$ Praxis am Lindspitz, Winterthur, Switzerland, ${ }^{2}$ Synlab Italia Srl, Castenedolo, Italy, ${ }^{3}$ Synlab Switzerland, Lausanne, Switzerland

We report the case of 78-year-old woman with a $3^{\text {rd }}$ episode of a toxic icteric hepatitis (liver parameters 4-7x). Each occurrence was after surgical intervention and postoperative infections, necessitating antibiotics and analgesics (paracetamol, amoxicillin-clavulanic acid).

Personal history: coronary heart disease with coronary stent 3 years previously. Drug history: atorvastatin, acetylsalicylic-acid and valsartan. Stopping atorvastatin, a "commonly poorly-tolerated" drug, led to no improvement; the patient continued to feel weak and remained icteric even after 4 weeks.

She was screened for variants in 33 pharmacologicallyrelevant genes (MyPGx). Results predicted Poor Metabolizer/Low Function phenotypes in some PharmGKB Very Important Pharmacogenes including CYP3A5, NAT2 and SLC- and ABC-Transporters, plus Intermediate Metabolizer phenotypes in CYP2C9, CYP2D6, UGT1A1 and others.
Based on the patient's haplotypes, the MyPGx algorithm predicted possible increased toxicity/risk of side effects for both valsartan and atorvastatin. Valsartan is principally excreted unchanged $(80 \%)$ but is also metabolized by CYP2C9, which showed a decreased function in the patient $(* 1 / * 2)$.

Taking into account these results, valsartan was replaced by olmesartan and atorvastatin by rosuvastatin; in both cases the alternative drug belonged to the same class but the key pharmacogene is SLCO1B1 (normal function, *1A/ *1B). After only two days the patient felt "as good as before"; within 4 weeks, liver transaminases sank to quasinormal values. The complex genetic findings suggest several interactions initially caused by post-operative drugs but knowing the predicted activity of key pharmacogenes facilitates selection of the best treatment leading, in this patient, to reversing the toxic reaction she was experiencing.

C. Wick: None. F. Cro': None. C. Lapucci: None. M. Morris: None.

\section{P18.47.C}

Quantifying Known and Novel Clinically Actionable Pharmacogenetic Variations in Whole-exome Sequencing(WES) Data of 1,132 Chinese Subjects

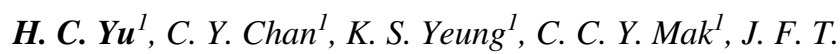

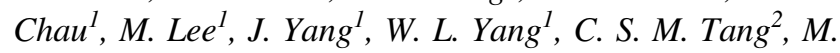

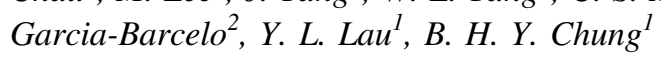

${ }^{1}$ Department of Paediatrics \& Adolescent Medicine, LKS Faculty of Medicine, The University of Hong Kong, Pokfulam, Hong Kong, ${ }^{2}$ Department of Surgery, LKS Faculty of Medicine, The University of Hong Kong, Pokfulam, Hong Kong

Introduction: Pharmacogenetics (PGx) is an important area of precision medicine. Currently, lack of large-scale exome/ genome-wide data precludes clinical application of preemptive PGx testing in Chinese. In this study, we analyze known and novel variants in clinically actionable pharmacogenes on the HKU Pediatric Exome Cohort in Hong Kong.

Methods: WES data of 1,132 unrelated Chinese subjects were analyzed using a two-tiered approach. The 1st-tier analysis targeted known PGx variants based on a list of 135 curated actionable variants. The 2nd-tier analysis focused on discovery of rare, predicted deleterious variants in 108 genes with PharmGKB Level 1/2 evidence based on 4 bioinformatic tools on missense (CADD, REVEL and PREDICT) and loss-of-function (CADD and LOFTEE) variants.

Results: We identified 29 known PGx variants (21.5\%) in the curated variant list, comprising of 57 drug-gene 
combinations. The most prevalent variants are rs1065852 in CYP2D6 (60.7\%), followed by rs12777823 in CYP2C8CYP2C9 region $(31.5 \%)$ and rs4244285 in CYP2C19 (30.6\%). Of the 1,132 Chinese subjects, $98.9 \%$ harbored at least 1 known actionable PGx variant $($ median $=3$ ). Additionally, we discovered 561 rare variants in 108 PharmGKB Level 1/2 genes with diversified functional effects such as non-synonymous nucleotide substitution ( $n$ $=520)$, premature gain of stop codon $(n=33)$ and disruption of reading frame $(n=8)$.

Conclusions: We have shown the abundance of known and novel functional PGx variants that are potentially clinically actionable. This study addresses the research gap of inadequate Chinese samples by large-scale PGx studies and also demonstrates the feasibility of secondary usage of WES data on PGx.

H.C. Yu: None. C.Y. Chan: None. K.S. Yeung: None. C.C.Y. Mak: None. J.F.T. Chau: None. M. Lee: None. J. Yang: None. W.L. Yang: None. C.S.M. Tang: None. M. Garcia-Barcelo: None. Y.L. Lau: None. B.H.Y. Chung: None.

\section{P18.50.C}

Aripiprazole and olanzapine may induce dysregulation of the glucose utilization through the alteration of the Myc signalling as detected by RNA-seq expression analysis of treated healthy volunteers

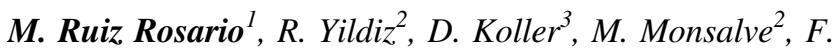
Abad-Santos $^{3,4,5}$, J. C. Cigudosa 6

${ }^{1}$ Scientific \& Innovation Department, NIMGenetics, Madrid, Spain, ${ }^{2}$ Instituto de Investigaciones Biomédicas "Alberto Sols" (CSIC-UAM), Madrid, Spain, ${ }^{3}$ Clinical Pharmacology Department, Hospital Universitario de la Princesa, Instituto Teófilo Hernando, Universidad Autónoma de Madrid (UAM), Instituto de Investigación Sanitaria la Princesa (IP), Madrid, Spain, ${ }^{4}$ UICEC Hospital Universitario de la Princesa, Plataforma SCReN (Spanish Clinical Reseach Network), Instituto de Investigación Sanitaria la Princesa (IP), Madrid, Spain, ${ }^{5}$ Pharmacology Department, Facultad de Medicina, Universidad Autónoma de Madrid, Madrid, Spain, ${ }^{6}$ Departamento de Universidad, Innovación y Transformación Digital. Gobierno de Navarra, Navarra, Spain

Introduction: Aripiprazole and olanzapine are atypical antipsychotics used mainly for schizophrenia treatment. Being schizophrenia a chronic disorder, it requires lifelong medication, that subsequently triggers metabolic Adverse Drug Reactions (ADRs).
Objective: Identify biomarkers that may contribute to the short-term prediction of metabolic dysfunctions associated with antipsychotic treatment.

Materials and Methods: An open, controlled, randomised, crossover clinical trial in healthy volunteers was carried out to evaluate the short-term effects (5 days) of olanzapine and aripiprazole in healthy volunteers. Blood samples were collected before and after each treatment. A total of 48 samples were analysed with Illumina total RNASeq technology, followed by differential expression analyses.

Results: A euclidean distance heatmap grouped the 4 samples from 4 volunteers, suggesting a lack of effect due to the treatments while separating the samples of the other 8 volunteers. A Gene Set Enrichment Analysis (GSEA) showed the opposite effect of aripiprazole and olanzapine on the expression of Oxidative phosphorylation and Myc targets gene sets $(\mathrm{p}<0.001)$. While aripiprazole produced a Normalized Enrichment Score (NES) of -2.9 and -2.4 respectively, olanzapine produced a NES of 4.9 and 5.7 respectively. The Myc network coordinates the cellular utilization of glucose by regulating the mitochondrial mass and activity.

Conclusions: Our results suggest that the volunteers could be stratified according to their response to the treatments. Both aripiprazole and olanzapine may induce dysregulation of the glucose utilization due to the alteration of the Myc signalling. Further validation is ongoing. This project was funded by the EU Framework Horizon 2020 under GA 721236.

M. Ruiz Rosario: None. R. Yildiz: None. D. Koller: None. M. Monsalve: None. F. Abad-Santos: None. J.C. Cigudosa: None.

\section{P18.53.C}

Polygenic risk score utilization in bone transcriptome differential expression analyses

V. Prijatelj ${ }^{\text {, S. Seppe }}{ }^{2}$, C. M. Medina-Gomez ${ }^{1}$, M. Dietz ${ }^{3}$, J. A. M. Verlouw ${ }^{1}$, C. G. Boer ${ }^{l}$, J. B. J. van Meurs ${ }^{1}$, A. J. van Wijnen ${ }^{3}$, K. M. Gautvik ${ }^{2}$, E. B. Wolvius ${ }^{1}$, F. Rivadeneiral

${ }^{I}$ Erasmus MC, University Medical Center Rotterdam, Rotterdam, Netherlands, ${ }^{2}$ Institute of Basic Medical Sciences, University of Oslo, Oslo, Norway, ${ }^{3}$ Department of Orthopedic Surgery, Mayo Clinic, Rochester, MA, United States

Introduction: Clinically defined phenotypes are prone to confounding which may obscure classification of cases and controls. We aimed at using recall-by-genotype (RbG) to leverage polygenic risk scores (PRS) for low bone mineral 
density (BMD) and osteoarthritis (OA) seeking to enhance transcriptome differential expression analyses (DEA).

Materials: Whole-tissue bone samples were obtained from either trans-iliacal biopsies or femoral head fragments as part of hip replacement surgery in 121 participants. BMD was measured at the Total Hip (TH-BMD) and Total Body (TB-BMD) using DXA. Transcriptome profiling was done on all samples using RNA-sequencing. Iliac samples $(n=$ 71) were genome-wide genotyped. DEA was done by grouping participants into cases and controls based on either on TH-BMD T-values (<-2.5 and >-1.0 respectively) or OA status. Weighted PRS were constructed for TB-BMD and OA. DEA was repeated on transcriptome profiles using RbG targeting the upper and lower PRS quintiles.

Results: Higher expression of SOST and DKK1 was observed in patients with higher BMD replicated by both approaches (phenotypic and recall-by-genotype grouping). The latter approach also identified differential expression of $T P B G$ and MEPE. Differential expression of OA identified known (SLC39A8, CTSK, MTF1) and novel OA genes.

Conclusions: Our findings illustrate PRS can enhance transcriptome DEA when used in a RbG framework. Further, the approach allows triangulation of functional genomics evidence implicating shared and independent pathways underlying osteoporosis and osteoarthritis.

\begin{tabular}{|c|c|c|c|c|}
\hline Name & Effect Size & Standard Error & P-value & FDR \\
\hline$S Y T$ & 0.084 & 0.017 & $2.02 \mathrm{E}-06$ & $7.05 \mathrm{E}-05$ \\
\hline WIFI & 0.053 & 0.014 & $2.66 \mathrm{E}-04$ & $2.34 \mathrm{E}-03$ \\
\hline ANGPT4 & 0.055 & 0.015 & 2.67E-04 & $2.34 \mathrm{E}-03$ \\
\hline DKKI & 0.048 & 0.013 & 4.37E-04 & $2.78 \mathrm{E}-03$ \\
\hline SCIN & 0.059 & 0.017 & 7.03E-04 & $3.51 \mathrm{E}-03$ \\
\hline$A R M H I$ & 0.108 & 0.032 & $1.16 \mathrm{E}-03$ & $4.52 \mathrm{E}-03$ \\
\hline COL8A2 & 0.095 & 0.028 & $1.08 \mathrm{E}-03$ & $4.52 \mathrm{E}-03$ \\
\hline MMP14 & 0.101 & 0.032 & $2.13 \mathrm{E}-03$ & $6.79 \mathrm{E}-03$ \\
\hline PLCDI & 0.132 & 0.043 & $2.86 \mathrm{E}-03$ & $8.35 \mathrm{E}-03$ \\
\hline SPACA9 & 0.103 & 0.035 & 4.13E-03 & $1.08 \mathrm{E}-02$ \\
\hline$F G F 1$ & 0.040 & 0.014 & $4.31 \mathrm{E}-03$ & $1.08 \mathrm{E}-02$ \\
\hline SOST & 0.024 & 0.009 & $1.18 \mathrm{E}-02$ & $2.44 \mathrm{E}-02$ \\
\hline SFRP4 & 0.041 & 0.016 & $1.19 \mathrm{E}-02$ & $2.44 \mathrm{E}-02$ \\
\hline CHITI & 0.029 & 0.013 & $2.19 \mathrm{E}-02$ & 4.03E-02 \\
\hline IRX5 & 0.047 & 0.021 & $2.80 \mathrm{E}-02$ & 4.46E-02 \\
\hline$K L K 4$ & 0.038 & 0.017 & $2.62 \mathrm{E}-02$ & 4.46E-02 \\
\hline$T P B G$ & 0.068 & 0.030 & $2.80 \mathrm{E}-02$ & 4.46E-02 \\
\hline COLIIA1 & 0.028 & 0.013 & $3.15 \mathrm{E}-02$ & $4.59 \mathrm{E}-02$ \\
\hline MEPE & 0.027 & 0.012 & $3.28 \mathrm{E}-02$ & $4.60 \mathrm{E}-02$ \\
\hline$N E X N$ & -0.103 & 0.025 & $2.34 \mathrm{E}-05$ & 4.10E-04 \\
\hline PDE5A & -0.112 & 0.031 & 4.77E-04 & $2.78 \mathrm{E}-03$ \\
\hline PRKGI & -0.085 & 0.026 & $1.30 \mathrm{E}-03$ & $4.55 \mathrm{E}-03$ \\
\hline$R A B 27 B$ & -0.055 & 0.021 & $9.28 \mathrm{E}-03$ & $2.17 \mathrm{E}-02$ \\
\hline ATP13A4 & -0.041 & 0.017 & $1.98 \mathrm{E}-02$ & $3.84 \mathrm{E}-02$ \\
\hline
\end{tabular}

V. Prijatelj: None. S. Reppe: None. C.M. MedinaGomez: None. M. Dietz: None. J.A.M. Verlouw: None. C.G. Boer: None. J.B.J. van Meurs: None. A.J. van
Wijnen: None. K.M. Gautvik: None. E.B. Wolvius: None. F. Rivadeneira: None.

\section{P18.55.B}

Biobank-based genotype-first model for return of actionable findings: follow-up and extended impact by cascade screening of $B R C A 1 / 2$ carriers

M. Palover ${ }^{1,2}$, L. Leitsalu ${ }^{l}$, T. Sikka ${ }^{l, 2}$, A. Reigo $^{l}$, T. Nikopensius $^{1}$, T. Esko ${ }^{l}$, A. Metspalu ${ }^{1,2}$, P. Padrik ${ }^{3,4}, N$. Tõnisson ${ }^{1,5}$

${ }^{I}$ Estonian Genome Centre, Institute of Genomics, Tartu, Estonia, ${ }^{2}$ Institute of Molecular and Cell Biology, University of Tartu, Tartu, Estonia, ${ }^{3}$ Hematology and Oncology Clinic, Tartu University Hospital, Tartu, Estonia, ${ }^{4}$ Institute of Clinical Medicine, University of Tartu, Tartu, Estonia, ${ }^{5}$ Dept. of Clinical Genetics, United Laboratories, Tartu University Hospital, Tartu, Estonia

Introduction: accumulating data from population studies intensifies the need to update best practices on how clinically significant genetic findings are handled in research cohorts and clinical practice.

Objective: We propose a model on how to distinguish, contact and return high-risk genetic findings to biobank participants.

Methods: Genotype-first approach was applied to identify carriers of pathogenic variants in BRCAl/2 genes and offer return of results with genetic counselling to the Estonian biobank participants. We evaluated the long-term effect of reporting actionable genetic findings.

Results: 22 of 40 carriers contacted opted for receiving results. 18 of 21 appreciated being contacted; three were unsure. 10 had relatives attending cascade screening. Within one year, five of 16 female carriers opted for risk-reducing surgery. Over follow-up of 30 months, 10 adhered to surveillance recommendations. Only eight of 21 qualified for high-risk assessment based on clinical criteria.

Discussion: This study highlights the significance of population genomic studies for personal early risk evaluation. It provides evidence for the genotype-first approach to improve long-term outcomes for individuals, unaware of their high genetic risk. We demonstrate that biobank participants as well as their family members appreciate receiving results that exemplifies how individuals from general population would respond. Our results indicate that age and family history are poor discriminators of risk alone. Our study is a basis of ongoing national pilot study on genetics of female breast cancer. This research was supported by the PUT PRG555, IUT20-60 and RITA1/0142-01 grants from the Estonian Research Council and European Regional Development Fund. 
M. Palover: None. L. Leitsalu: None. T. Sikka: None. A. Reigo: None. T. Nikopensius: None. T. Esko: None. A. Metspalu: None. P. Padrik: None. N. Tõnisson: None.

\section{P18.57.A}

\section{Treatabolome: a rare diseases treatment awareness} project

A. Atalaia ${ }^{1}$, R. Thompson ${ }^{2}$, A. Corvo $^{3}$, L. Carmody $^{4}$, D. Piscia $^{3}$, L. Matalonga ${ }^{3}$, A. Macaya ${ }^{5}$, A. Lochmuller ${ }^{6}$, B. Fontaine $^{7}$, B. Zurek ${ }^{8}$, C. Hernandez-Ferrer ${ }^{3}$, C. Reinhard ${ }^{9}$, D. Gómez-Andrés ${ }^{5}$, J. F. Desaphy ${ }^{10}$, K. Schon ${ }^{6}, K$. Lohmann $^{11}$, M. Jennings ${ }^{6}$, M. Synofzik ${ }^{12,13}$, O. Riess ${ }^{9}, R$. Ben Yaou ${ }^{14}$, T. Evangelista ${ }^{15}$, T. Ratnaike ${ }^{16}$, V. Bros-

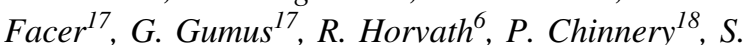

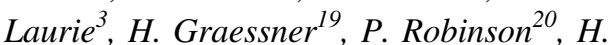
Lochmuller $^{21,22,23}$, S. Beltran ${ }^{3}$, G. Bonne ${ }^{24}$

${ }^{1}$ Soborne Universite, Inserm U974, institut de Myologie, Hopital Pitie-Salpetriere, Paris, Paris, France, ${ }^{2}$ Children's Hospital of Eastern Ontario Research Institute, Ottawa, ON, Canada, ${ }^{3}$ CNAG-CRG, Centre for Genomic Regulation (CRG), Barcelona Institute of Science and Technology (BIST), Barcelona, Spain, ${ }^{4}$ The Jackson Laboratory For Genomic Medicine, Farmington, CT, United States, ${ }^{5}$ Paediatric Neurology, Vall d'Hebron University Hospital and VHIR (Euro-NMD, ERN-RND), Barcelona, Spain, ${ }^{6}$ Department of Clinical Neurosciences, University of Cambridge School of Clinical Medicine, Cambridge Biomedical Campus, Cambridge, United Kingdom, ${ }^{7}$ Soborne Universite, Inserm U974, Hopital Pitie-Salpetriere, Paris, Paris, France, ${ }^{8} 7$ Institute of Medical Genetics and Applied Genomics, University of Tuebingen, Tuebingen, Germany, ${ }^{9}$ Institute of Medical Genetics and Applied Genomics, University of Tuebingen, Tuebingen, Germany, ${ }^{10}$ Department of Biomedical Sciences and Human Oncology, School of Medicine, University of Bari Aldo Moro, Bari, Italy, ${ }^{11}$ Institute of Neurogenetics, University of Lübeck, Lübeck, Germany, ${ }^{12}$ Department of Neurodegenerative Diseases, Hertie-Institute for Clinical Brain Research and Center of Neurology, University of Tübingen, Tübingen, Germany, ${ }^{13}$ German Center for Neurodegenerative Diseases (DZNE), University of Tübingen, Tübingen, Germany, ${ }^{14}$ Soborne Universite, Inserm U974, Institut de Myologie, Hopital Pitie-Salpetriere, Paris, Paris, France, ${ }^{15}$ Unité de Morphologie Neuromusculaire, Institut de Myologie, GHU PitiéSalpêtrière; Sorbonne Université, AP-HP, INSERM, Centre de référence Des Maladies Neuromusculaires Nord/Est, Ile de France, Paris, France, ${ }^{16}$ Department of Paediatrics, Cambridge University Hospitals NHS Foundation Trust; Department of Clinical Neurosciences, University of Cambridge, Cambridge, United Kingdom, ${ }^{17}$ EURORDIS - Rare Diseases Europe, Paris, France, ${ }^{18}$ Department of Clinical
Neurosciences, School of Clinical Medicine, University of Cambridge and MRC Mitochondrial Biology Unit, Cambridge Biomedical Campus, Cambridge, United Kingdom, ${ }^{19}$ Institute of Medical Genetics and Applied Genomics, University of Tuebingen, Germany, Tuebingen, Germany, ${ }^{20} 4$ The Jackson Laboratory For Genomic Medicine, Farmington, CT, United States, ${ }^{21}$ Department of Neuropediatrics and Muscle Disorders, Medical Center - University of Freiburg, Faculty of Medicine, University of Freiburg, Freiburg, Germany, ${ }^{22}$ Children's Hospital of Eastern Ontario Research Institute; Division of Neurology, Department of Medicine, The Ottawa Hospital, Ottawa, ON, Canada, ${ }^{23}$ Brain and Mind Research Institute, University of Ottawa, Ottawa, ON, Canada, ${ }^{24}$ Sorbonne Université Inserm UMRS 974, Center of Research in Myology, Institut de Myologie, G.H. Pitié-Salpêtrière, Paris, France

Rare diseases are individually rare but globally affect about $6 \% \%$ of the population according to the latest calculations. Recent estimates point to over 10000 rare diseases, of which 6172 disorders are listed in Orphanet, $71.9 \%$ of which being genetic and $69.9 \%$ having paediatric-onset. These diseases' rarity is contextual, varying with the population considered and also geographically. Nonetheless, disease rarity uniformly translates into a delayed diagnosis, with $25 \%$ of patients having to wait for 5 to 30 years to have one. It is essential, at the time of diagnosis, to be able to relay information about potential gene and variant-specific therapies to patients. "Solve-RD - solving the unsolved rare diseases" is a research project funded by the European Commission for five years(2018-2022) aiming at improving the diagnostic and therapeutic management of rare diseases. It will deliver seven implementation steps, including one dedicated to treatment as part of the"genetic knowledge web". With this in mind, we propose a method of allowing clinicians to access this information promptly to benefit patients affected by rare disease and their families. The"Treatabolome" is an evidence-appraised database of rare diseases treatments for specific genes and variants to be made available through genetic diagnosis and support tools, for example, the RD Connect Genome-Phenome Analysis Platform. The Treatabolome aims at contributing to raising awareness and increasing the visibility of existing rare diseases' treatments to clinicians.

Grants: European Reference Networks ERN Euro-NMD and ERN-RND (project ID 739510), Solve-RD project EU's Horizon 2020 grant 779257.

A. Atalaia: None. R. Thompson: None. A. Corvo: None. L. Carmody: None. D. Piscia: None. L. Matalonga: None. A. Macaya: None. A. Lochmuller: None. B. Fontaine: None. B. Zurek: None. C. Hernandez-Ferrer: None. C. Reinhard: None. D. Gómez-Andrés: None. J.F. Desaphy: None. K. Schon: None. K. Lohmann: None. 
M. Jennings: None. M. Synofzik: D. Speakers Bureau/ Honoraria (speakers bureau, symposia, and expert witness); Modest; Movements Disorder Society. F. Consultant/ Advisory Board; Modest; Actelion Pharmaceuticals. O. Riess: F. Consultant/Advisory Board; Modest; Illumina. R. Ben Yaou: None. T. Evangelista: None. T. Ratnaike: None. V. Bros-Facer: None. G. Gumus: None. R. Horvath: None. P. Chinnery: None. S. Laurie: None. H. Graessner: F. Consultant/Advisory Board; Modest; Roche. P. Robinson: None. H. Lochmuller: None. S. Beltran: None. G. Bonne: None.

\section{P18.58.B}

C3435T $A B C B 1$ polymorphism predicts therapeutic response to cyclosporine in psoriasis patients.

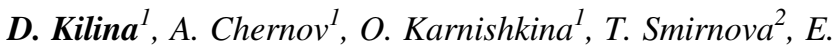
Galimova $^{l, 3}$

${ }^{1}$ Center for Preclinical and Translational Research of Federal State Budgetary Institution "Almazov National Medical Research Centre" of the Ministry of Health of the Russian Federation, St. Petersburg, Russian Federation, ${ }^{2}$ City dispensary of Dermatology and Venerology, St. Petersburg, Russian Federation, ${ }^{3}$ Sechenov Institute of Evolutionary Physiology and Biochemistry Russian Academy of Sciences, St. Petersburg, Russian Federation

Introduction: Psoriasis is a chronic immune-mediated skin disease affecting 2-3\% of the Caucasian population. Cyclosporine, a potent calcineurin inhibitor acting selectively on T-cells, is used for the treatment of severe psoriasis, show significant variability in efficacy, which associated with varying degrees of toxicity. The detection of pharmacogenetic markers of treatment response may be useful in predicting clinical response to psoriasis therapies and would help in the improvement of personalized therapy. The aim of the present study was to assess the association rs3213619, rs2229109, rs1128503, rs2032582 singlenucleotide polymorphisms (SNPs) in the $A B C B 1$ gene with response to cyclosporine treatment in Russian psoriasis patients.

Materials and Methods: DNA samples were collected from 168 patients with psoriasis. Genotyping of SNPs was performed by real-time polymerase chain reaction using the CFX 96 ${ }^{\mathrm{TM}}$ Real-Time Cycler (BioRad, USA). For casecontrol association study we used $\chi^{2}$ tests with Pearson contingency tables were implemented in PLINK version 1.06 to compute the $\mathrm{P}$-values and corresponding odds ratios (OR) with $95 \%$ confidential intervals (CI) for allelic association.

Results: The variant rs1045642 in the $A B C B 1$ gene showed an association with a negative response to cyclosporine therapy $(\mathrm{OR}=2.58,95 \% \mathrm{CI}=1.64-4.06)$ in Russians. Carriers of the dominant model (TT + CTvsCC) have 1.6-fold higher risk $(\mathrm{OR}=4.01,95 \% \mathrm{CI}=1.82-8.98)$ of no response to cyclosporine treatment than recessive $(\mathrm{TTvsCC}+\mathrm{CT})$ model $(\mathrm{OR}=2.50,95 \% \mathrm{CI}=1.14-5.50)$.

Conclusions: Further, larger studies are required to confirm our findings and replicate them in various populations before implementation in the clinical practice.

D. Kilina: None. A. Chernov: None. O. Karnishkina: None. T. Smirnova: None. E. Galimova: None.

\section{P18.59.C}

Prevalence of actionable germline variants within a 53gene inherited cancer risk gene panel in a cohort of 2,642 cancer patients classified by an AI inference engine

\section{F. M. De La Vega ${ }^{l}$, T. Friedrich ${ }^{l}$, S. Nohzadeh-

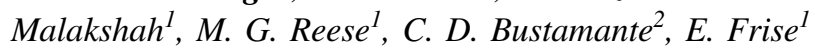

${ }^{1}$ Fabric Genomics, Oakland, CA, United States, ${ }^{2}$ Stanford University School of Medicine, Stanford, CA, United States

Implementation of cancer risk gene tests continues to grow driven by new reimbursement pathways and technological advances. However, disagreement exists on what genes and populations should be screened, their clinical utility and the cost/benefit of such tests. This debate should be informed by an understanding of the prevalence of cancer risk variants in different population cohorts. Here we analyzed germline variants from a cohort of 2,658 patients with 38 different types of cancer, whose tumor and matching normal genomes were sequenced as part of the ICGC PanCancer Analysis of Whole Genomes project. We identified 377,025 non-synonymous germline variants within the regions of an expanded 53-gene hereditary cancer risk panel, which includes genes associated with breast, ovarian, colon, prostate, lung, and other cancers. For expedited and consistent assessment of variant pathogenicity, we utilized a recently developed artificial intelligence-based forwardchaining inference engine, ACE, which implements the ACMG-AMP criteria leveraging variant annotations and codified gene-condition curations. ACE classified automatically $97 \%$ of variants, leaving only 1,085 unique variants annotated with a subset of the ACMG criteria for manual review. Overall $5 \%$ of cancer patients were carriers of pathogenic/likely pathogenic (P/LP) variants, with prevalence higher in patients of renal cell, pancreas adenocarcinoma, ovarian, breast, and liver cancers. A higher frequency of the P/LP variants was observed in the genes ATM, KCHN2, BRCA1/2, and PMS2. As population screening for cancer risk is being actively discussed by 
healthcare stakeholders, our results illustrate that previously established guidelines could miss people with potentially actionable variants.

F.M. De La Vega: A. Employment (full or part-time); Significant; Fabric Genomics, Inc.. E. Ownership Interest (stock, stock options, patent or other intellectual property); Significant; Fabric Genomics, Inc. T. Friedrich: A. Employment (full or part-time); Significant; Fabric Genomics, Inc. S. Nohzadeh-Malakshah: A. Employment (full or part-time); Modest; Fabric Genomics, Inc. M.G. Reese: A. Employment (full or part-time); Significant; Fabric Genomics. E. Ownership Interest (stock, stock options, patent or other intellectual property); Significant; Fabric Genomics. C.D. Bustamante: None. E. Frise: A. Employment (full or part-time); Significant; Fabric Genomics. E. Ownership Interest (stock, stock options, patent or other intellectual property); Significant; Fabric Genomics.

\section{P18.60.A}

Exploring secondary findings in a Spanish cohort of 641 patients undergoing whole exome sequencing

\section{Codina Solà, E. Rovira Moreno, A. Abulí Vidal, I. Valenzuela Palafoll, A. Cueto Gonzalez, F. López Grondona, I. Paramonov, S. Ourani, C. Serra Juhé, E. García Arumí, I. Cuscó Martí, E. Tizzano Ferrari}

\section{Hospital Vall Hebron, Barcelona, Spain}

Introduction: Exome-sequencing is being used across diverse clinical settings allowing primary diagnosis but also screening for unrelated disorders. The ACMG (2017) recommends offering the analysis of 59 medically actionable genes (secondary findings, SFs). However, little is known about acceptance rates and penetrance of SFs in patients without family history.

Materials and Methods: We retrospectively reviewed patients $(n=641)$ who underwent exome-sequencing in a Spanish tertiary center from May 2017 to September 2019. During pretest genetic counseling all patients were offered to know SFs or to opt out. We analyzed acceptance and detection rate. In addition, we reviewed type of findings and family history.

Results: The great majority of patients opted to receive SFs. A secondary finding was identified in 11 patients $(1,7 \%)$. Pathogenic variants were found in genes related to cancer (SDHB, BRCA2, PMS2, STK11), cardiomyopathies (MYBPC3, BAG3, TNNT2, DSC2, DSP) and channelopathies $(R Y R 2, S C N 5 A)$. Segregation studies indicated that all variants were inherited with exception of one de novo variant in $R Y R 2$. Only one family (BAG3) met criteria for an a priori genetic testing. In the rest of cases, carriers evaluated so far had no personal/family history and showed no signs of the disease.

Conclusions: Our results show a high acceptance for the return of SFs. Virtually none of the variants would have been a priori identified, since most patients did not have family history to justify specific genetic testing. Further clinical evaluation is being performed to assess penetrance of SFs in the absence of family history.

M. Codina Solà: None. E. Rovira Moreno: None. A. Abulí Vidal: None. I. Valenzuela Palafoll: None. A. Cueto Gonzalez: None. F. López Grondona: None. I. Paramonov: None. S. Ourani: None. C. Serra Juhé: None. E. García Arumí: None. I. Cuscó Martí: None. E. Tizzano Ferrari: None.

\section{P18.61.B}

Impairments of smell perception: the role of sex, age and genes

M. Concas $^{1}$, T. Battistuzzi $i^{2}$, M. Cocca ${ }^{l}$, B. Spedicati ${ }^{3}$, G. Pelliccione $^{l}$, P. Gasparini ${ }^{1,3}$, G. Girotto ${ }^{1,3}$

${ }^{I}$ Institute for Maternal and Child Health - IRCCS "Burlo Garofolo", Trieste, Italy, ${ }^{2}$ Department of Life Sciences, University of Trieste, Trieste, Italy, ${ }^{3}$ Department of Medicine, Surgery and Health Sciences, University of Trieste, Trieste, Italy

Smell deficits affect quality of life leading to loneliness and depression and represent an early symptom of neurodegenerative diseases. Here, we investigated the role of age, sex and genetics factors in smell perception in a large Italian cohort (1155 individuals). Smell ability was assessed using the SNIFFIN' sticks test. Number of errors (NoE) was counted and a linear mixed model applied to evaluate the influence of age and sex. A mean NoE of $1.76 \pm 1.65$ was detected. Male sex and increased age were associated with a greater $\operatorname{NoE}(p<0.05)$. A subset of subjects $(802$ out of 1155) was analysed by WGS to detect human Loss of Function variants (LoF). The number of biallelic LoF mutations (NoM) on 35 olfactory receptors (OR) genes (27 of which clearly expressed in the olfactory epithelium) was counted and subjects (228) grouped accordingly. The NoM was $4.19 \pm 1.99$, while NoE was $2.54 \pm 1.95$. Age accounts for $22 \%$ of the variability in NoE, while NoM has no impact. Excluding elderly people ( $\geq 70 \mathrm{y}, n=75$ ) from the analysis, we found that NoE increases with NoM, suggesting a strong role of genetics factors. NoM explains $6 \%$ of NoE variability, while age only $4.9 \%(p<0.05)$.

Our findings confirm age as a strong factor influencing smell deficits, and, for the first time, highlight the role of genetics factors (i.e. mutations in OR genes) in predisposing to smell deficits in the general population. Moreover, we 
showed that at a given time, the aging process has such strong impact that exceeds the individual's genetic predispositions.

M. Concas: None. T. Battistuzzi: None. M. Cocca: None. B. Spedicati: None. G. Pelliccione: None. P. Gasparini: None. G. Girotto: None.

\section{P18.62.C}

Pharmacogenomics of varenicline using linked primary care data in the UK

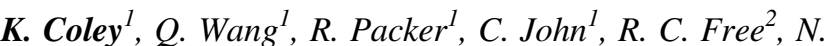

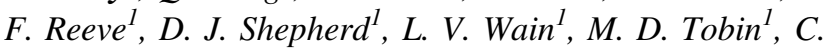
Batini $^{1}$

${ }^{1}$ Department of Health Sciences, University of Leicester, Leicester, United Kingdom, ${ }^{2}$ Leicester NIHR BRC, Institute for Lung Health, Department of Infection, Immunity \& Inflammation, University of Leicester, Leicester, United Kingdom

Background: Varenicline is a partial agonist to the $\alpha_{4} \beta_{2}$ nicotinic acetylcholine receptors (nAChRs), which are involved in nicotine-induced reward pathways, dependence and withdrawal. Varenicline is the second most commonly prescribed smoking cessation medication after nicotine replacement therapy, and has the greatest therapeutic efficacy. We present the first case-control candidate gene study of varenicline effectiveness using EXCEED, a cohort of $\sim 10,000$ individuals based in the UK.

Methods: An electronic phenotyping algorithm using prescription Read Codes in primary care data linked to EXCEED participants was used to identify individuals with varenicline prescriptions $(N=185)$. Cases and controls were defined as smokers at $\geq 1$ year follow up and nonsmokers at $\geq 1$ year follow up, respectively. We tested 198 tag SNPs, spanning 12 biologically relevant candidate genes encoding proteins involved in varenicline activity and metabolism.

Results: Seven SNPs were associated with varenicline effectiveness at nominal significance $(\mathrm{P}<0.05)$, but none passed the Bonferroni corrected threshold. These SNPs were located in nAChR subunit genes CHRNA4, CHRNB4 and $C H R N A 7$, and have not been previously reported as associated with varenicline effectiveness or smoking behaviour traits.

Future work: This study was limited by statistical power due to low sample size. Therefore, we intend to use the same approach to ascertain case-control status for individuals with varenicline prescriptions using primary care data linked to 230,000 UK Biobank participants. This will form the foundations for a genome-wide association study to identify unbiased genetic associations with varenicline effectiveness.

K. Coley: None. Q. Wang: None. R. Packer: None. C. John: None. R.C. Free: None. N.F. Reeve: None. D.J. Shepherd: None. L.V. Wain: B. Research Grant (principal investigator, collaborator or consultant and pending grants as well as grants already received); Significant; GSK. M.D. Tobin: B. Research Grant (principal investigator, collaborator or consultant and pending grants as well as grants already received); Significant; GSK, Orion. C. Batini: None.

\section{P18.64.B}

Impact of BRCA1 mutation status on the therapeutic efficacy of CDK4/6 inhibitors in triple negative breast cancer

\section{G. Guney Eskiler ${ }^{1}$, Z. Ozman ${ }^{2}$}

${ }^{1}$ Sakarya University, Faculty of Medicine, Department of Medical Biology, Sakarya, Turkey, ${ }^{2}$ Sakarya University, Institute of Health Science, Department of Medical Biochemistry, Sakarya, Turkey

Introduction: The cyclin-dependent kinases (CDK)-retinoblastoma (RB) pathway plays a vital role in the progression of breast cancer (BC). Therefore, CDK4/6 inhibitors have great attention as a new therapeutic strategy for the treatment of BC. On the basis of genetic feature, there is a strong associations between TN phenotype and BRCAl mutation status. However, the effectiveness of CDK4/6 inhibitors in triple negative breast cancer (TNBC) has not been fully examined in terms of BRCAl mutation status. Therefore, we explored the therapeutic effects of abemaciclib on two different TNBC cells and identified the underlying mechanisms of apoptotic cell death.

Materials and Methods: The cytotoxicity, Annexin V, cell cycle and RT-PCR analysis were performed to determine anti-cancer effects of abemaciclib on HCC1937 (BRCA1 mutant) and MDA-MB-231 cells (BRCA1 wildtype).

Results: Abemaciclib significantly inhibited the proliferation of TNBC cells in a dose and time dependent manner ( $\mathrm{p}<0.05$ ). However, HCC1937 cells were more sensitive to abemaciclib than MDA-MB-231 cells. After 24 hours treatment with $1.5 \mu \mathrm{M}$ abemaciclib, the percentage of total apoptotic cells was $63.5 \%$ and $11.7 \%$ in $\mathrm{HCC} 1937$ and MDA-MB-231 cells, respectively. We observed that more cells accumulated in G0/G1 phase through down-regulation of CCDN1 (1.7-fold; $p=0.001$ ) and RB1 (0.7-fold; $p=$ $0.015)$ in HCC1937 cells.

Conclusions: Abemaciclib exerted potential anti-cancer effects on TNBC cells and BRCAl mutation status increased 
therapeutic efficacy of abemaciclib. Our results suggest a greater benefit for the future clinical trials of abemaciclib in the treatment of BRCAl mutation carriers with TNBC.

G. Guney Eskiler: None. Z. Ozman: None.

\section{P18.65.C}

Polygenic risk scores could improve venous trombosis risk estimation in Factor $\mathrm{V}$ Leiden mutation carriers

\section{T. Paajanen ${ }^{l}$, H. Marjonen ${ }^{l}$, M. Marttila ${ }^{l}$, A. Haukkala ${ }^{2}$, H. Kääriäinen ${ }^{l}$, M. Perola $^{l, 3}$, K. Kristiansson ${ }^{l}$}

${ }^{1}$ Finnish Institute for Health and Welfare, Helsinki, Finland, ${ }^{2}$ University of Helsinki, Helsinki, Finland, ${ }^{3}$ Research Program for Clinical and Molecular Metabolism, Faculty of Medicine, University of Helsinki, Helsinki, Finland

Introduction: In P5 FinHealth study we have 3449 participants from a biobanked population-based study cohort FinHealth. We estimated participants' polygenic risk scores (PRSes) and ten years total risks for venous thromboembolism (VTE) and returned the information to participants via internet portal. In the process we studied how the known VTE predisposing variant Factor V Leiden affects the risk of VTE in the presence of PRS.

Materials and Methods: Our VTE PRS, containing 6.5 million genomic variants, was based on UK Biobank GWAS summary statistics and adjusted for the effects of linkage disequilibrium (LD) with Python package LDpred. We generated PRSes also separately for Factor V Leiden carriers and non-carriers. We used Cox regression to estimate the effects of genetic (PRS) and traditional (e.g. gender, age, smoking, BMI, prevalent $\mathrm{CHD}$ or T2D) risk factors on future risk of VTE event in a large Finnish follow-up cohort $(N=28.000)$.

Results: PRS associates significantly with a 10-year VTE risk (Factor V Leiden carriers: $\mathrm{HR}=1.8, p=7 * 10^{4}$ and non-carriers: $\mathrm{HR}=1.4, p=2 * 10^{6}$ ). Moreover, Net reclassification improvement (NRI) analysis showed that the model with PRS correctly re-classifies cases in both groups: Factor V Leiden carriers $(p=0.009)$ and non-carriers $(p=0.013)$, compared to a model without PRS.

Conclusions: In our study we show that VTE PRS offers additional value to VTE risk estimation even in the presence of the known VTE predisposing variant Factor V Leiden. We have returned personal genetic and absolute 10-year risk estimates and instructions for their health care for the P5 participants in February 2020.

T. Paajanen: None. H. Marjonen: None. M. Marttila: None. A. Haukkala: None. H. Kääriäinen: None. M. Perola: None. K. Kristiansson: None.
P18.66.A

Added value of clinical whole-genome sequencing: Cooccurring rare diseases and pharmacogenetic profiling

S. M. Caspar ${ }^{l, 2}$, A. Najafi ${ }^{l}$, J. Meienberg $^{l}$, T. Schneider ${ }^{l}$, C. Henggeler $^{l}$, G. Matyas ${ }^{l}$

${ }^{1}$ Center for Cardiovascular Genetics and Gene Diagnostics, Schlieren-Zurich, Switzerland, ${ }^{2}$ Laboratory of Translational Nutrition Biology, ETH Zurich, Schwerzenbach, Zurich, Switzerland

Introduction: In the current genomics era, interpretation of high-throughput sequencing data constitutes the main bottleneck on the path to accurate diagnosis of Mendelian disorders. Large-scale reference cohorts such as ExAC/ gnomAD are valuable for the population-frequency-based filtering of the myriad of detected sequence variants. Using the example of fibrillinopathies, such as FBN1-related Marfan syndrome (MFS) and FBN2-related congenital contractural arachnodactyly (CCA), we assessed the frequency and co-occurrence of pathogenic FBN1/ $F B N 2$ sequence variants in ExAC/gnomAD and the largest Swiss database of Marfan genomes, respectively.

Methods: By focusing on a priori (likely) pathogenic sequence variants in ExAC/gnomAD, we calculated prevalence estimates for MFS and CCA. Furthermore, we screened whole genomes (60× WGS) of $\sim 550$ patients with rare (aortic) disorders for (likely) pathogenic and functional sequence variants in $F B N 1 / F B N 2$, and 12 pharmacogenes, respectively.

Results: We show the presence of pathogenic FBN1/2 variants in the apparently healthy reference cohort ExAC/ gnomAD, providing prevalence estimates for MFS and CCA. In our Swiss cohort, we identified two families with dual FBN1/FBN2 mutations, explaining the variable phenotype within these families including clinical features of MFS and CCA (PMID:31506931). In one family, we also detected a pharmacogenetically actionable variant in a drug metabolizing enzyme.

Conclusions: Our results not only demonstrate that apparently healthy reference data sets may include individuals with late-onset or unrecognized disease, but also show that fibrillinopathies occur more frequently than expected and may co-occur. Furthermore, we emphasize the importance and increasing possibility of detecting digenic and, as an added value, pharmacogenetically relevant sequence variants using WGS.

S.M. Caspar: None. A. Najafi: None. J. Meienberg: None. T. Schneider: None. C. Henggeler: None. G. Matyas: None. 


\section{P18.67.B}

Effectual delivery of Nef-MPER-V3 fusion protein using LDP12 cell penetrating peptide as a HIV-1 vaccine candidate

\section{S. Sabaghzadeh ${ }^{1}$, S. Sadat ${ }^{2}$, A. Bolhassani $^{2}$}

${ }^{1}$ Islamic azad university of tehran medical branch, faculty of advanced sciences \& technology, Tehran, Iran, Islamic Republic of, ${ }^{2}$ Pasteur institute of iran, department of hepatitis and AIDS, Tehran, Iran, Islamic Republic of

Introduction: Human immunodeficiency virus (HIV) which causing acquired immune deficiency syndrome (AIDS) is one of the main universal health concern. In spite of using antiretroviral treatments, there are no authorized HIV vaccines. The development in using therapeutic proteins is affected by the limitation on permeability and selectivity of cell membranes. Thus, utilizing cellpenetrating peptides (CPPs) as protein transfection reagents or protein transduction domains (PTDs) has been expanded. In addition, heat shock proteins suchlike Hsp27 as intracellular chaperones motivate efficient immune responses.

Material and Methods: Following by the confirmation of the plasmid construct HIV-1 Nef-MPER-V3-LDP12pET-28a by NdeI/XhoI restriction enzymes digestion, applying prokaryotic expression, SDS-PAGE and westernblotting were utilized. Next, the target protein was purified on Ni-NTA agarose column, dialyzed and evaluated by spectrophotometry. For transfection, $4 \times 10^{4}$ HEK-293T cells/well were used and supplied by complete DMEM medium. Then LDP12-Nef-MPER-V3, Nef-MPER-V3, Hsp27-GFP-turbofect and GFP-turbofect were applied to each well and assessed by western-blotting.

Results: The gene's presence was confirmed by digestion as 825 bp band and sequencing. Moreover, western-blot analysis indicated a desired expression of Hsp27-GFP, GFP, LDP12-Nef-MPER-V3, and Nef-MPER-V3 as 54, $\sim 27, \sim 37$, and $\sim 35 \mathrm{kDa}$ bands, respectively. Our data showed the significantly expression of fusion constructs in Rosetta strain and also, was accurately purified under denaturing condition.

Conclusions: Briefly, this stable construct (LDP12-NefMPER-V3) with high cellular penetrance and low toxicity could be used as a favorable procedure in HIV-1 vaccine strategy, that is sufficiently capable of inducing specific strong immune responses.

Grant: Seyed mehdi sadat, Pasteur institute of Iran

S. Sabaghzadeh: None. S. Sadat: None. Bolhassani: None.
P19 Genetic Epidemiology, Population Genetics and Evolutionary Genetics

\section{P19.001.B}

12q24 locus affects BMI through alcohol drinking and dietary behaviors

M. Igarashi ${ }^{1}$, S. Nogawa ${ }^{2}$, K. Kawafune ${ }^{2}$, T. Hachiya ${ }^{2,3}, H$.

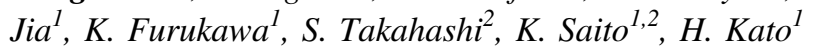

${ }^{1}$ The University of Tokyo, Tokyo, Japan, ${ }^{2}$ Genequest Inc, Tokyo, Japan, ${ }^{3}$ Genome Analytics Japan Inc, Tokyo, Japan

Introduction: The 12q24 locus, East Asian-specific linkage disequilibrium (LD) region, includes $A L D H 2$ gene which involves in alcohol metabolism. It has been reported that a single nucleotide polymorphism (SNP) in $A L D H 2$, rs671, is associated with various dietary behaviors (DBs), susceptibility to diseases and clinical measurements including BMI. The purpose of this study was to investigate how the pleiotropic effects of 12q24 affect human health through DBs.

Materials and Methods: Genomic DNA was extracted from saliva samples of 12,315 Japanese subjects and genotyped by SNP array. We collected data about residential area, sex, age, and 56 DBs by Web questionnaire survey. The sex-stratified phenome-wide association study (PheWAS) for rs671 was performed by PLINK with multiple testing correction $(P<0.05 / 56)$.

Results: The PheWAS analysis revealed that 11 and 7 DBs were significantly associated with rs671 in male and female, respectively. After adjusting for alcohol intake frequency, $6 \mathrm{DBs}$ in male and $2 \mathrm{DBs}$ in female were significantly associated with rs671. Almost all associations with DBs, except for coffee intake, were attenuated by adjustment of alcohol intake frequency. The mediation analysis revealed that $5 \mathrm{DBs}$ in male and $3 \mathrm{DBs}$ in female were mediated between alcohol drinking and BMI.

Conclusions: This study showed that the $12 \mathrm{q} 24$ locus affects BMI through complicated pathways with and without the mediation of alcohol drinking. Furthermore, it was suggested that sex-specific DBs was involved in the pathway. Our findings can contribute to personalized nutrition using rs671 in East Asians. This work was supported by Grants-in-Aid (19K11709) from JSPS.

M. Igarashi: None. S. Nogawa: A. Employment (full or part-time); Modest; Genequest Inc. K. Kawafune: A. Employment (full or part-time); Modest; Genequest Inc. T. Hachiya: A. Employment (full or part-time); Modest; Genequest Inc. H. Jia: None. K. Furukawa: None. S. A. Takahashi: A. Employment (full or part-time); Modest; 
Genequest Inc. K. Saito: A. Employment (full or part-time); Modest; Genequest Inc. H. Kato: None.

\section{P19.005.C}

Variants associated with Alzheimer's disease development revealed by analyzing genome data of healthy individuals and Bulgarian supercentenarians

D. N. Nikolova ${ }^{l}$, D. Serbezov ${ }^{l}$, L. Balabanski ${ }^{l, 2}, S$. Karachanak-Yankova $^{l, 3}$, M. Mihailova $^{l}$, V. Damyanova $^{l}, R$. Staneva $^{l}$, D. Nesheva ${ }^{l}$, Z. Hammoudeh ${ }^{l}$, S. Hadjidekova ${ }^{l}$, D. Toncheva ${ }^{1,2}$

${ }^{1}$ Medical University Sofia, Sofia, Bulgaria, ${ }^{2}$ Hospital "Malinov", Sofia, Bulgaria, ${ }^{3}$ Sofia University "St. Kliment Ohridski", Sofia, Bulgaria

Introduction: Alzheimer's disease (AD) is predominantly a disease of the elderly and is a major cause of dementia, slowly progressing towards disability and death. Genetic studies are mainly related to the search for single mutations with a pathogenic effect, as well as separate variants of genes that would modify the risk of developing symptoms earlier or later in life.

Materials and methods: DNAs from 32 centenarians (100 to 106 years) and 61 healthy individuals aged 18-30 years have been exome sequenced in two separate pools. 1930 variants from DisGeNet database known to be associated with $\mathrm{AD}$ have been searched.

Results: Five pathogenic variants have been found in young healthy control group: rs63750264 in APP gene is associated with early onset AD, while rs 145999145 in PLD3, rs72470545 in HTRA2, rs2070045 in SORL1, rs429358 in APOE are associated with late onset AD.

Conclusion: Centenarians are a model of a "good quality of life" and most of them do not show any manifestations of dementia. They are "gold standard" for revealing protective genetic variants or absence of pathogenic/risky ones, the latter considered a triggering mechanism to unlock AD. Our analysis shows that young healthy individuals carry risk variants for predisposition to $\mathrm{AD}$. This suggests that the exome screening is useful for early diagnosis and prevention of neurodegenerative disorders.

Acknowledgements: Contract DN 03/7 from 18.12.2016 and KP-06-N33/5 from 13.12.2019 of the National Science Fund of Bulgaria.

D.N. Nikolova: None. D. Serbezov: None. L. Balabanski: None. S. Karachanak-Yankova: None. M. Mihailova: None. V. Damyanova: None. R. Staneva: None. D. Nesheva: None. Z. Hammoudeh: None. S. Hadjidekova: None. D. Toncheva: None.

\section{P19.006.A}

The first Y-STR haplotype of the Medieval Russia Individual from the Tver Region.

G. D. Stepanov
Mustafin $^{2}$

${ }^{1}$ Research Centre for Medical Genetics, Moscow, Russian Federation, ${ }^{2}$ Moscow Institute of Physics and Technology, Moscow, Russian Federation

Introduction: The study of ancient human DNA is rapidly developing, although research on ancient samples at the level of paleo-populations is almost not conducted. One of the difficult regions of Eastern Europe's ethnogenesis is medieval Russia. The aim of this research is to obtain the Ychromosomal STR-haplotype of an individual buried in the XII century on the territory of the Tver Kremlin and compare it with the haplotypes of modern populations.

Materials and Methods: We used bone fragments and teeth from individual found in the burial of the early necropolis, wherein 1285 the construction of the Transfiguration Cathedral in the Tver Kremlin took place. The burial was formed before the construction of the cathedral, which is confirmed by radiocarbon analysis: date from the 12th13th centuries. The methods were used in the work: DNA was extracted on a Silica Spin column; capillary electrophoresis and the $\mathrm{Yfiler}^{\circledR}$ Plus kit were used to determine the lengths of polymorphic STR-loci of Y-chromosome.

Results: A Y-chromosomal STR-haplotype was obtained. Of the 25 loci included in the Yfiler ${ }^{\circledR}$ Plus kit, 23 loci are identified. Analysis using predictors shows that this haplotype belongs to the haplogroup R1a1a1b1a1a (M458). An analysis of publications revealed 3 identical haplotypes by 16 common loci in populations: the Russian of Ivanovo and Penza regions, the southern Germans.

Conclusions: This study confirms the possibility of studying Medieval Russia samples. The haplotype is widespread in the modern Russian population. Further expansion of the sample will allow making a complete picture of medieval Tver.

G.D. Stepanov: None. I. Alborova: None. R.A. Zinchenko: None. K.K. Mustafin: None.

\section{P19.007.B}

Genome-wide association study meta-analysis identifies three novel loci for circulating anti-Müllerian hormone levels in women

R. M. G. Verdiesen ${ }^{l}$, Y. T. van der Schouw ${ }^{l}$, C. H. van Gils $^{l}$, W. M. M. Verschuren ${ }^{1,2}$, F. J. M. Broekmans ${ }^{3}$, M. C. Borges $^{4,5}$, A. L. G. Soares ${ }^{4,5}$, D. A. Lawlor ${ }^{4,5,6}$, A. H. Eliassen $^{7,8}$, D. P. Sandler ${ }^{9}$, S. D. Harlow ${ }^{10}$, J. A. Smith ${ }^{10}$, N. 
Santoro $^{11}$, A. S. Karlamanga ${ }^{12}$, M. J. Schoemaker ${ }^{13}$, A. J. Swerdlow $^{13,14}$, A. Murray ${ }^{15}$, K. S. Ruth ${ }^{15}$, N. C. OnlandMoret $^{1}$

${ }^{1}$ Julius Center for Health Sciences and Primary Care, University Medical Center Utrecht, Utrecht, Netherlands, ${ }^{2}$ National Institute for Public Health and the Environment (RIVM), Bilthoven, Netherlands, ${ }^{3}$ Department of Reproductive Medicine and Gynaecology, University Medical Center Utrecht, Utrecht, Netherlands, ${ }^{4}$ MRC Integrative Epidemiology Unit at the University of Bristol, Bristol, United Kingdom, ${ }^{5}$ Population Health Sciences, Bristol Medical School, University of Bristol, Bristol, United Kingdom, ${ }^{6}$ Bristol NIHR Biomedical Research Centre, Bristol, United Kingdom, ${ }^{7}$ Channing Division of Network Medicine, Department of Medicine, Brigham and Women's Hospital and Harvard Medical School, Boston, MA, United States, ${ }^{8}$ Department of Epidemiology, Harvard TH Chan School of Public Health, Boston, MA, United States, ${ }^{9}$ Epidemiology Branch, National Institute of Environmental Health Sciences, Durham, NC, United States, ${ }^{10}$ Department of Epidemiology, University of Michigan, Ann Arbor, MI, United States, ${ }^{11}$ Department of Obstetrics and Gynecology, University of Colorado School of Medicine, Denver, CO, United States, ${ }^{12}$ Division of Geriatrics, Department of Medicine, David Geffen School of Medicine, University of California at Los Angeles, Los Angeles, CA, United States, ${ }^{13}$ Division of Genetics and Epidemiology, The Institute of Cancer Research, London, United Kingdom, ${ }^{14}$ Division of Breast Cancer Research, The Institute of Cancer Research, London, United Kingdom, ${ }^{15}$ University of Exeter Medical School, University of Exeter, Exeter, United Kingdom

Introduction: Anti-Müllerian hormone (AMH) is expressed by primary ovarian follicles in premenopausal women; circulating AMH levels can be measured until menopause. Variation in age-specific AMH levels has been associated with breast cancer and polycystic ovary syndrome (PCOS), amongst other diseases. Identification of genetic variants underlying variation in AMH levels could elucidate these AMH-disease associations.

Methods: We performed a meta-analysis of genome-wide association studies including data from 7,049 premenopausal female participants of European ancestry, to identify SNPs associated with circulating AMH levels. Studyspecific analyses were adjusted for age at blood collection and population stratification, and summary statistics were meta-analysed using a standard error-weighted approach.

Results: We identified four loci for circulating AMH. We confirmed the previously reported association with the MCM8 locus and identified three novel signals in or near $A M H, T E X 41$, and CDCA7. The strongest signal was a nonsynonymous missense variant located in exon 5 of the
$A M H$ gene (rs10417628, $\beta(\mathrm{SE})=-0.34(0.05), p=1.2 \mathrm{x}$ $\left.10^{-11}\right)$. LD score regression analyses indicated a strong positive genetic correlation among SNPs for AMH levels and for age at menopause $\left(r_{\mathrm{g}}=0.82, \mathrm{FDR}=0.003\right)$. Twosample Mendelian Randomization analyses did not support a causal effect of circulating $\mathrm{AMH}$ on breast cancer or PCOS.

Conclusions: We identified three novel loci for AMH levels in premenopausal women. Whilst this study almost doubled the sample size of the most recent GWAS, we may still lack power to determine causal effects of AMH on for example breast cancer. RMGV was supported by The Netherlands Organization for Scientific Research (Grant number: 022.005.021).

R.M.G. Verdiesen: None. Y.T. van der Schouw: None. C.H. van Gils: None. W.M.M. Verschuren: None. F.J.M. Broekmans: B. Research Grant (principal investigator, collaborator or consultant and pending grants as well as grants already received); Modest; Merck Serono, Gedeon Richter, Ferring BV, Roche. M.C. Borges: None. A.L.G. Soares: None. D.A. Lawlor: B. Research Grant (principal investigator, collaborator or consultant and pending grants as well as grants already received); Modest; Medtronic Ltd. B. Research Grant (principal investigator, collaborator or consultant and pending grants as well as grants already received); Significant; Roche Diagnostics. A.H. Eliassen: None. D.P. Sandler: None. S.D. Harlow: None. J.A. Smith: None. N. Santoro: F. Consultant/Advisory Board; Modest; Ansh Laboratories. A.S. Karlamanga: None. M.J. Schoemaker: None. A.J. Swerdlow: None. A. Murray: None. K.S. Ruth: None. N.C. Onland-Moret: None.

\section{P19.008.C}

Founder effect in the French-Canadian population of the Saguenay-Lac-St-Jean region in Quebec: screening strategies for rare diseases and local initiatives under development

T. Cruz Marino, M. Desmeules, J. Leblanc, A. Pratte, J. Tardif, R. Guerin, L. Bouchard

\section{CIUSSS Saguenay-Lac-St-Jean, Chicoutimi, QC, Canada}

Introduction: French-Canadian settlers arriving from the $17^{\text {th }}$ century onward provided the genetic background of Saguenay-Lac-St-Jean's population. A founder effect has been well described in this Quebec region. A pilot program started in 2010 for carrier screening of ARSACS, tyrosinemia type I, Leigh syndrome French-Canadian type, and hereditary sensory-motor neuropathy with/without agenesis of the corpus callosum. In 2018, this program became province-wide through a web-based strategy that 
reached previously underserved regions. Simultaneously, new founder conditions have been identified.

Methods: We review the particularities and the results of the web-based test; we present a new group of founder conditions, as well as the strategies under development for their accurate diagnosis.

Results: During the first two years, more than 3000 individuals from 17 regions of Quebec province opted for taking the web-based carrier test, the majority of them were females, and preconceptional predominated over prenatal requests. A project for neonatal screening of vitamin Ddependent rickets is about to begin on a research basis as an initial step. Other initiatives are being discussed for the screening or early diagnosis of mucolipidoses type II, Zellweger disease, junctional epidermolysis bullosa, MPICDG, Naxos disease, APECED, autosomal recessive deafness type 57, and familial hypertrophic cardiomyopathy type 1.

Conclusions: The web-based carrier testing allows equitable access to all individuals coming from SaguenayLac-St-Jean and living in any region of Quebec province. This data highlights the importance of developing local strategies that suit the needs of founder populations.

T. Cruz Marino: None. M. Desmeules: None. J. Leblanc: None. A. Pratte: None. J. Tardif: None. R. Guerin: None. L. Bouchard: None.

\section{P19.009.A}

Arsenic efficient metabolism in Mexican Indigenous reinforces the arsenical hypothesis

R. I. Montero-Delgado ${ }^{1}$, M. López-González ${ }^{l}$, G. Noris ${ }^{2}$, C.

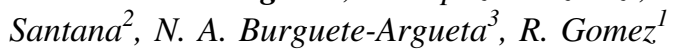

${ }^{I}$ Toxicology Department - CINVESTAV-IPN, Ciudad de Mexico, Mexico, ${ }^{2}$ BIMODI, Santiago de Queretaro, Queretaro, Mexico, ${ }^{3}$ Instituto de Ciencias Biológicas, Universidad de Ciencias y Artes de Chiapas, Tuxtla Gutiérrez, Chiapas, Mexico

Introduction: Mexico is an arsenic-endemic country with more than two million individuals exposed, chronically, to elevated concentrations of this metalloid via drinking water. Arsenic-3-methyltransferase, encoded by AS3MT gene, is a key enzyme in the metabolism of inorganic arsenic, methylating it and eliminating in the urine. Dimethylarsinic acid (DMA) has been associated with more efficiently arsenic metabolism a reduced sensitivity to this metalloid; monomethylarsonic acid (MMA) with elevated toxicity. Varying MMA and DMA proportions present interpopulation and inter-ethnic variations. Native Americans groups, whose ancestors arrived at the Americas $>12,000$ ybp seem to metabolize arsenic more efficiently than
European-derived populations who have exhibited high toxicity risk. Thus, arsenic tolerance could be related to long-term exposition. The contemporary Native Americans, descendants of the ancient Mesoamerican cultures, along with a prominent admixed population between European and Indigenous (Mestizos), constitute a suitable scenario to delineate the genetic variation of AS3MT polymorphisms, which have been hardly studied.

Methods and Results: Using 28 Ancestry Informative Markers and genotyping four AS3MT polymorphism (rs11191439, rs3740400, rs7085104 and rs10748835) we found that individuals with predominant Native American ancestry exhibited higher frequencies of the "efficient" haplotype TGGA (0.47) than Mestizos (0.31). Crs11191439 allele related to higher toxicity risk was also absent in the tested Native Americans samples; the frequencies in Mestizos and European derived populations were $5 \%$ and $12 \%$, respectively.

Conclusions: Our findings could reinforce the arsenical hypothesis about genetic adaptation related to long-term exposition. This study was financed by Conacyt, grants 261268, 178239 (to RG) and 728535 (to RM).

R.I. Montero-Delgado: None. M. López-González: None. G. Noris: None. C. Santana: None. N.A. Burguete-Argueta: None. R. Gomez: None.

\section{P19.010.B}

Novel asthma risks within loci enriched in African ancestry in Southwestern Europeans

C. Díaz-García ${ }^{l}$, E. Suarez-Pajes ${ }^{l}$, H. Rodríguez-Pérez ${ }^{l}, J$. M. Lorenzo-Salazar ${ }^{2}$, I. Marcelino-Rodríguez ${ }^{I}$, A. Corrales $^{1,3}$, X. Zheng ${ }^{4}$, A. Callero ${ }^{5}$, E. Perez-Rodriguez, ${ }^{5}$ J. C. Garcia-Robaina ${ }^{5}, R$. González-Montelongo ${ }^{2}, C$. Flores $^{1,2,3}$, B. Guillen-Guio ${ }^{l}$

${ }^{1}$ Research Unit, Hospital Universitario N.S. de Candelaria, Universidad de La Laguna, Santa Cruz de Tenerife, Spain, ${ }^{2}$ Genomics Division, Instituto Tecnológico y de Energías Renovables (ITER), Santa Cruz de Tenerife, Spain, ${ }^{3}$ CIBER de Enfermedades Respiratorias, Instituto de Salud Carlos III, Madrid, Spain, ${ }^{4}$ Department of Biostatistics, University of Washington, Seattle, WA, United States, ${ }^{5}$ Allergy Unit, Hospital Universitario N.S. de Candelaria, Universidad de La Laguna, Santa Cruz de Tenerife, Spain

Introduction: An important proportion of asthma genetic risks remain unknown, especially in populations with African genetic influences, which associate with greater burden of asthma. Since the population of the Canary Islands has a high North African genetic ancestry among Southwestern Europeans and presents the highest asthma prevalence in Spain, here we examined asthma associations 
in six loci showing an excess of African ancestry in this population.

Methods: A two-stage study was conducted in 564 asthma cases and 923 controls from the Canary Islands genotyped on the Axiom Genome-Wide CEU1 array (Affymetrix). Imputation was performed using the HRC reference panel and logistic regressions were used to test association. Classic alleles from seven HLA genes were imputed and association testing was assessed with HIBAG.

Results: Statistical significance was reached for a 3' UTR variant of HLA-DQB1 (meta-analysis $\mathrm{OR}=1.74 ; 95 \%$ CI:1.42-2.14; $p=1.30 \mathrm{e}-7)$ that colocalizes with an eQTL for several HLA and complement genes. This was associated with asthma, lung traits and peanut allergy. The most prominent classic HLA allele associations were HLA-DQB $1 * 02: 01$ and HLA-DQA1*05:01. These cluster in the DQ2.5 haplotype that is frequent in North Africa and associates with a high-risk of celiac disease and type-1 diabetes.

Conclusions: Targeted analyses of six regions enriched in African ancestry in the Canary Islanders revealed novel asthma risks linked to classic HLA alleles known to confer a high-risk for autoimmune diseases.

Funding: Instituto de Salud Carlos III (PI14/00844, PI17/ 00610, FI18/00230, CD19/00231), co-funded by ERD Funds, "A way of making Europe" from the EU; ITER agreement OA17/008.

C. Díaz-García: None. E. Suarez-Pajes: None. H. Rodríguez-Pérez: None. J.M. Lorenzo-Salazar: None. I. Marcelino-Rodríguez: None. A. Corrales: None. X. Zheng: None. A. Callero: None. E. Perez-Rodriguez: None. J.C. Garcia-Robaina: None. R. González-Montelongo: None. C. Flores: None. B. Guillen-Guio: None.

\section{P19.011.C}

Genome-wide association study of asthma-COPD overlap syndrome identifies new signals of association

C. John ${ }^{1}$, A. L. Guyatt ${ }^{1}$, N. Shrine ${ }^{1}$, T. Olafsdottir ${ }^{2,3}, J$.

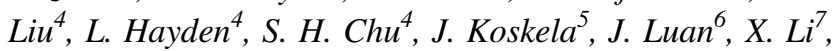
N. Terzikhan ${ }^{8}, H . X u^{9}$, T. M. Bartz ${ }^{10}$, H. Petersen ${ }^{11}, S$. Leng $^{11}$, G. Thorleifsson ${ }^{2,3}$, D. A. Meyers ${ }^{7}$, E. R. Bleecker ${ }^{7}$, L. C. Sakoda ${ }^{12}$, C. Iribarren ${ }^{12}$, Y. Tesfaigzi ${ }^{4}$, S. A. Gharib ${ }^{10}$, J. Dupuis ${ }^{9}$, L. Lahousse ${ }^{8,13}$, V. E. Ortega ${ }^{14}$, K. Stefansson ${ }^{2,3}$, I. Sayers ${ }^{15,16}$, I. P. Hall ${ }^{15,16}$, C. Langenberg ${ }^{6}$, S. Ripatti ${ }^{5,17}$, T. Laitinen ${ }^{18,19}$, A. C. Wu ${ }^{20}$, J. Lasky-Su ${ }^{4}$, C. Hayward ${ }^{21}$, B. Brumpton $^{22}$, A. Langhammer ${ }^{22}$, I. Jonsdottir ${ }^{2,3}$, M. H. Cho ${ }^{4}$, L. V. Wain $^{1,23}$, M. D. Tobin ${ }^{1,23}$

${ }^{1}$ University of Leicester, Leicester, United Kingdom, ${ }^{2}$ deCODE genetics/Amgen, Reykjavik, Iceland, ${ }^{3}$ University of Iceland, Reykjavik, Iceland, ${ }^{4}$ Brigham and Women's Hospital and Harvard Medical School, Boston, MA, United
States, ${ }^{5}$ Institute for Molecular Medicine Finland, University of Helsinki, Helsinki, Finland, ${ }^{6}$ MRC Epidemiology Unit, University of Cambridge School of Clinical Medicine, Cambridge, United Kingdom, ${ }^{7}$ University of Arizona, Tucson, AZ, United States, ${ }^{8}$ Erasmus Medical Center, Rotterdam, Netherlands, ${ }^{9}$ Boston University School of Public Health, Boston, MA, United States, ${ }^{10}$ University of Washington, Seattle, WA, United States, ${ }^{11}$ Lovelace Respiratory Research Institute, Albuquerque, NM, United States, ${ }^{12}$ Division of Research, Kaiser Permanente of Northern California, Oakland, CA, United States, ${ }^{13}$ Ghent University, Ghent, Belgium, ${ }^{14}$ Wake Forest School of Medicine, Winston-Salem, NC, United States, ${ }^{15}$ University of Nottingham, Nottingham, United Kingdom, ${ }^{16}$ NIHR Nottingham Biomedical Research Centre, Nottingham, United Kingdom, ${ }^{17}$ Broad Institute of MIT and Harvard, Cambridge, MA, United States, ${ }^{18}$ Turku University Hospital, Turku, Finland, ${ }^{19}$ University of Turku, Turku, Finland, ${ }^{20}$ Harvard Pilgrim Health Care Institute and Harvard Medical School, Boston, MA, United States, ${ }^{21}$ MRC Human Genetics Unit, Institute of Genetics and Molecular Medicine, University of Edinburgh, Edinburgh, United Kingdom, ${ }^{22}$ Norwegian University of Science and Technology (NTNU), Levanger, Norway, ${ }^{23}$ NIHR Leicester Biomedical Research Centre, Leicester, United Kingdom

Asthma and COPD are common causes of morbidity and mortality worldwide. Some individuals have characteristics of both conditions ("asthma-COPD overlap syndrome", ACOS), and evidence suggests they experience significantly worse outcomes than those with asthma or COPD alone. Although hundreds of genetic associations with asthma, COPD, and lung function have been identified, the genetics of ACOS have been little studied and no genetic variants have specifically been associated.

We tested 7,693,381 genetic variants for association with ACOS (defined as self-reported asthma and spirometric airflow limitation) in 8068 cases and 40,360 healthy controls from UK Biobank (stage 1). Signals with $P<5 \times 10^{-6}$ in this analysis, and $\mathrm{P}<0.01$ in two sensitivity analyses in UK Biobank using (i) controls with asthma but no COPD and (ii) controls with COPD but no asthma, were meta-analysed among the results from UK Biobank and 15 independent cohorts with an additional 4301 cases and 48609 controls (stage 2). All cases/controls were of European ancestry.

Thirty-one genetic variants met criteria for inclusion in stage 2. Of these, eight were associated at genome-wide significance $\left(P<5 \times 10^{-8}\right)$ and showed stronger evidence of association in the stage 2 meta-analysis than in UK Biobank alone, including signals near $H L A-D Q B 1$, C5orf56, GLB1, FAM105A, PHB, TSLP and IL17RD, and 
an intergenic signal on chromosome 5 not previously associated with asthma, COPD or lung function.

Whilst our results suggest that many genetic variants associated with ACOS are shared with asthma and/or COPD, we identify a new signal which is distinct and may implicate cellular pathways characteristic of ACOS.

C. John: None. A.L. Guyatt: None. N. Shrine: None. T. Olafsdottir: A. Employment (full or part-time); Significant; deCODE genetics/Amgen. J. Liu: None. L. Hayden: None. S.H. Chu: None. J. Koskela: None. J. Luan: None. X. Li: None. N. Terzikhan: None. H. Xu: None. T. M. Bartz: None. H. Petersen: None. S. Leng: None. G. Thorleifsson: A. Employment (full or part-time); Significant; deCODE genetics/Amgen. D.A. Meyers: None. E.R. Bleecker: F. Consultant/Advisory Board; Significant; ALK-Abello, AztraZeneca, Glaxo Smith Kline, MedImmune, Novartis, Regeneron, Sanofi Genzyme, TEVA. Other; Significant; AstraZeneca, MedImmune, Boehringer Ingelheim, Genentech, Novartis, Regeneron, Sanofi Genzyme. L.C. Sakoda: None. C. Iribarren: None. Y. Tesfaigzi: None. S.A. Gharib: None. J. Dupuis: None. L. Lahousse: None. V.E. Ortega: None. K. Stefansson: A. Employment (full or part-time); Significant; deCODE genetics/Amgen. I. Sayers: None. I.P. Hall: B. Research Grant (principal investigator, collaborator or consultant and pending grants as well as grants already received); Significant; GSK, Boehringer Ingelheim, Orion. C. Langenberg: None. S. Ripatti: None. T. Laitinen: None. A.C. Wu: None. J. Lasky-Su: None. C. Hayward: None. B. Brumpton: None. A. Langhammer: None. I. Jonsdottir: A. Employment (full or part-time); Significant; deCODE genetics/Amgen. M.H. Cho: B. Research Grant (principal investigator, collaborator or consultant and pending grants as well as grants already received); Significant; GSK, Bayer. D. Speakers Bureau/Honoraria (speakers bureau, symposia, and expert witness); Significant; Illumina, AstraZeneca. L.V. Wain: B. Research Grant (principal investigator, collaborator or consultant and pending grants as well as grants already received); Significant; GSK. M.D. Tobin: B. Research Grant (principal investigator, collaborator or consultant and pending grants as well as grants already received); Significant; GSK, Orion.

\section{P19.014.C}

The landscape of autosomal-recessive pathogenic variants in the European population

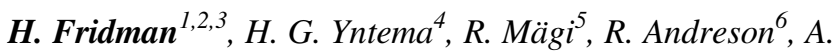
Metspalu ${ }^{5}$, S. Carmi ${ }^{2}$, E. Levy-Lahad ${ }^{1,3}$, C. Gilissen ${ }^{4}, H$. G. Brunner ${ }^{4,7}$

${ }^{1}$ Medical Genetics Institute, Shaare Zedek Medical Center, Jerusalem, Israel, ${ }^{2}$ Braun School of Public Health and
Community Medicine, The Hebrew University of Jerusalem, Jerusalem, Israel, ${ }^{3}$ Faculty of Medicine, The Hebrew University of Jerusalem, Jerusalem, Israel, ${ }^{4}$ Department of Human Genetics, Radboud University Medical Centre, Nijmegen, Netherlands, ${ }^{5}$ Estonian Genome Centre, Institute of Genomics, University of Tartu, Tartu, Estonia, ${ }^{6}$ Estonian Biocentre, Institute of Genomics, University of Tartu, Tartu, Estonia, ${ }^{7}$ Department of Clinical Genetics, Maastricht University Medical Center, Maastricht, Netherlands

Background: A major public health goal is to detect at-risk couples (ARCs) for various autosomal-recessive (AR) diseases. Currently, the number and distribution of recessive alleles in the population is not known at genomic-scale. Existing estimates are extrapolations from specific phenotypes and gene-sets.

Methods: We used 4120 exome-sequences of genetically unrelated Dutch samples and an independent replication cohort of 2330 exome-sequences of genetically unrelated Estonian samples, representing two distinct European populations. We analyzed 1758 genes classified as ARonly by OMIM and extracted all exonic and flanking intronic variants. We created a list of pathogenic/likelypathogenic variants $(\mathrm{P} / \mathrm{LPs})$, based on automated analysis and databases of classified variants. We simulated all possible matings within the Dutch and Estonian cohorts (8.5M/2.7M theoretical couples, respectively).

Results: We estimate that there are 2.0 recessive heterozygous P/LPs per individual in the Dutch population giving rise to $1.3 \%$ ARCs. We replicate these results in the Estonian cohort where we find 1.6 recessive heterozygous P/LPs per individual, resulting in $1.5 \%$ ARCs. The robustness of our approach was confirmed by the fact that $>97 \%$ of variants had a carrier frequency of at most $0.5 \%$ (in both populations), and the remaining $\sim 3 \%$ were wellknown population-specific founder mutations.

Conclusions: In two distinct European populations, we find similar estimates for carrier frequency per person and for the proportion of ARCs for a recessive disease. This is the first overall estimate of AR carriership in European populations and will inform schemes for introducing preconception carrier screening for AR diseases.

H. Fridman: None. H.G. Yntema: None. R. Mägi: None. R. Andreson: None. A. Metspalu: None. S. Carmi: F. Consultant/Advisory Board; Modest; MyHeritage. E. Levy-Lahad: None. C. Gilissen: None. H.G. Brunner: None.

P19.015.A

THL Biobank, a multi-omics infrastructure for systems biology 
A. Joensuu ${ }^{1,2}$, K. Silander ${ }^{1}$, M. Perola ${ }^{1,2}$, S. Soini ${ }^{1}$

${ }^{I}$ THL Biobank, Finnish Institute for Health and Welfare (THL), Helsinki, Finland, ${ }^{2}$ Research Program for Clinical and Molecular Metabolism, Faculty of Medicine, University of Helsinki, Helsinki, Finland

Background: Large genomics projects have pinpointed specific genomic variants that associate with disease endpoints. Studying the biological pathways of these associations often require a systems biology approach. Since 1992, Finnish Institute for Health and Welfare (THL) has collected DNA samples from all participants of its health examination surveys. Today, THL enables access to vast amounts of genomic and other omics data through its biobank.

Materials \& Methods: THL Biobank currently hosts omics data from 11 Finnish study cohorts. The collections include: GWAS chip data $\mathrm{N}>109,000$, ( $>90,000$ imputed in FinnGen project to a population-specific reference panel); WES $\mathrm{N}>12,500$; WGS $\mathrm{N}>2,000$; methylation data $\mathrm{N}>500$; gene expression data $\mathrm{N}>550$ and telomere data $\mathrm{N}>7,000$. NMR metabolomics is available for $\mathrm{N}>40,000$. The omics data can be combined with a wide range of questionnaire, clinical examination and laboratory variables, and study participants can be followed up (for up to 27 years) from national registers for disease endpoints and drug purchases. THL Biobank's most diverse dataset, DILGOM (a subset of FINRISK 2007), includes data from all of the above listed data types. Besides data, cells are available for $>10,000$ and RNA for $>3,000$.

Results \& Conclusions: THL Biobank is accessible on FAIR-principles for researchers and companies worldwide. Since 2015, 130 biobank projects have been initiated, including interesting projects in pharmacogenomics, evolutionary genetics, return of genetic information to biobank participants and cardiovascular and cancer research. Genomic data requests are included in $94 \%$ of the biobank applications (122/130). In 2019, a marked increase was seen in applications on metabolomic data and recall studies.

A. Joensuu: A. Employment (full or part-time); Modest; Negen Ltd. K. Silander: None. M. Perola: None. S. Soini: None.

\section{P19.016.B}

Genetic associations with birth weight and bivariate genetic correlations with birth weight in an Indigenous American population

L. E. Wedekind ${ }^{1,2}$, W. Hsueh ${ }^{2}$, S. Kobes ${ }^{2}$, W. C. Knowler ${ }^{2}$, L. J. Baier ${ }^{2}$, R. L. Hanson ${ }^{2}$

${ }^{1}$ University of Oxford, Nuffield Department of Medicine, Oxford, United Kingdom, ${ }^{2}$ National Institute of Diabetes and Digestive and Kidney Diseases (NIDDK), Phoenix Epidemiology \& Clinical Research Branch, Phoenix, AZ, United States

Birth weight has high heritability $\left(h^{2}=0.54\right)$ and substantial genetic correlations with metabolic traits in adulthood. This GWAS of birth weight was conducted in an Indigenous population from the southwestern US. 3700 individuals had genotypes available from a population-specific Axiom array (Affymetrix; Santa Clara, CA; 512,319 SNPs) and birth weight data. Genotypes for another $4.6 \mathrm{M}$ variants were imputed using WGS data from 266 community members. Birth weight was ascertained from birth and medical records, and normalised separately by sex via rank-based transformation.

Gestational age data were available for $60 \%$ of participants; to address missingness, multiple imputation of gestational age was performed. Genetic associations were analyzed using a mixed model in SOLAR-Eclipse, accounting for genetic relationships (inferred from marker data), adjusting for birth year, gestational age and the $1^{\text {st }} 5$ genetic PCs.180 SNPs had suggestive significance $(p<0.0001)$ with birth weight; the 10 strongest associations are listed by chromosome ( $\mathrm{Chr}$ ) and position (Position37):

\begin{tabular}{|c|c|c|c|c|c|c|c|c|c|c|}
\hline Gene & rsID & $\mathrm{Chr}$ & Position37 & $\mathrm{A} 1$ & A2 & $\mathrm{A} 1 \mathrm{~F}$ & $\mathrm{GT}^{*}$ & $\begin{array}{l}\beta \text { (SD birth } \\
\text { weight per } \\
\text { A1 copy) }\end{array}$ & SE & $\mathrm{p}$ \\
\hline$U B E 4 B$ & rs2180184 & 1 & 10235946 & $\mathrm{~T}$ & $\mathrm{C}$ & 0.68 & G & 0.12 & 0.03 & $6.24 \times 10^{-6}$ \\
\hline$A B C C 8$ & - & 11 & 17417205 & $\mathrm{~T}$ & $\mathrm{C}$ & 0.02 & G & 0.46 & 0.09 & $1.14 \times 10^{-6}$ \\
\hline - & rs 2324532 & 13 & 40830879 & $\mathrm{~T}$ & G & 0.11 & I & 0.18 & 0.04 & $8.95 \times 10^{-6}$ \\
\hline - & - & 13 & 40838524 & $\mathrm{~T}$ & G & 0.10 & G & 0.18 & 0.04 & $1.02 \times 10^{-5}$ \\
\hline - & rs35219516 & 13 & 40843260 & $\mathrm{C}$ & $\mathrm{T}$ & 0.90 & I & -0.18 & 0.04 & $6.23 \times 10^{-6}$ \\
\hline - & - & 13 & 40848849 & $\mathrm{C}$ & $\mathrm{T}$ & 0.90 & I & -0.18 & 0.04 & $6.57 \times 10^{-6}$ \\
\hline - & rs61515650 & 19 & 1729004 & $\mathrm{~T}$ & $\mathrm{C}$ & 0.81 & I & 0.15 & 0.03 & $3.53 \times 10^{-6}$ \\
\hline - & rs10589986 & 19 & 1729070 & CGT & $\mathrm{C}$ & 0.82 & I & 0.15 & 0.03 & $4.34 \times 10^{-6}$ \\
\hline$D M D$ & rs17341316 & $\mathrm{X}$ & 33349779 & G & A & 0.68 & I & -0.09 & 0.02 & $1.11 \times 10^{-5}$ \\
\hline$D M D$ & rs5928238 & $\mathrm{X}$ & 33350078 & $\mathrm{~T}$ & A & 0.71 & I & -0.10 & 0.02 & $9.80 \times 10^{-6}$ \\
\hline
\end{tabular}

${ }^{\ddagger} \mathrm{G}=$ directly-genotyped; $\mathrm{I}=$ imputed

Missense variant $A B C C 8 \mathrm{R} 1420 \mathrm{H}$ (chr11:17417205)— whose activating mutations cause hyperinsulinemia of infancy - had the strongest association with birth weight $\left(\beta=0.46 ; p=1.14 \times 10^{-6}\right)$. Among 180 SNPs with suggestive evidence, results for one SNP (LOC105378114 rs73030292, chr6:166190683; $p=4.83 \times 10^{-5}$ ) directionally replicated a previously reported association at genome-wide significance $\left(p=1.15 \times 10^{-10}\right.$; Early Growth Genetics Consortium). This study identifies novel genetic associations with birth weight and demonstrates $A B C C 8 \mathrm{R} 1420 \mathrm{H}$ is a strong determinant of birth weight in this population. 
L.E. Wedekind: None. W. Hsueh: None. S. Kobes: None. W.C. Knowler: None. L.J. Baier: None. R.L. Hanson: None.

\section{P19.018.A}

Racial and age-related differences in breast cancer: Comprehensive comparison of epidemiological, clinicopathological features and genomic signatures

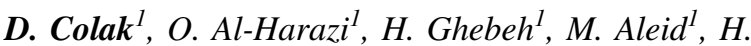
Khalil $^{l}$, E. Aljarba ${ }^{l}$, N. Bawyan ${ }^{l}, H$. Alassiry ${ }^{l}, M$. Almarzouqi ${ }^{1}$, D. S. Ajarim ${ }^{1}$, B. H. Park ${ }^{2}$, J. Quackenbush ${ }^{3}$, A. S. Al-Zahrani ${ }^{l}$, N. Kaya ${ }^{l}$

${ }^{1}$ King Faisal Specialist Hospital and Research Center, Riyadh, Saudi Arabia, Riyadh, Saudi Arabia, ${ }^{2}$ VanderbiltIngram Cancer Center, Vanderbilt University, Nashville, $T N$, United States, ${ }^{3}$ Dana-Farber Cancer Institute, Boston, MA, United States

Introduction: Age and ethnicity are important factors in breast cancer (BC) disparities and outcome. The underlying biology creating these discrepancies is not well understood. We aimed to identify ethnic/racial differences in epidemiological, clinicopathological, and genomic characteristics of breast cancer in young women (BCYW) as compared to elderly women across different populations.

Materials and Methods: We used data from Cancer Incidence in Five Continents Volume X reports, Gulf Centre for Cancer Registration, including clinical and survival data from 11,244 Saudi/Arab women, 107,938 African-American and 882,242 non-Hispanic White women (US NCI SEER database), and transcriptomic profile of breast tumors in young and elderly women cohorts from four different ethnic/racial origins.

Results: Our results indicated distinct epidemiological and biological features in BCYW as compared to the elderly patients across different populations. Notably higher immune related processes in young Arab and UK women, to a certain extent in African-American women. Moreover, we identified genes and upstream regulators that are distinct or common in early- vs late-onset BC among four different populations.

Conclusions: To our knowledge, this is the first study to examine the molecular, epidemiological, genomic signatures, and survival of women with BC patients in Arab and Western populations in age-specific cohorts. The population-specific molecular alterations and distinct clinicopathological characteristics may explain the racial and age-related discrepancies in disease outcome and may lead to more effective treatment strategies.
Acknowledgement: This study is funded by the Research Grant (RAC\#2110006 to DC). We would like to thank Ms. Sukina Qanbar for her administrative support.

D. Colak: None. O. Al-Harazi: None. H. Ghebeh: None. M. Aleid: None. H. Khalil: None. E. Aljarba: None. N. Bawyan: None. H. Alassiry: None. M. Almarzouqi: None. D.S. Ajarim: None. B.H. Park: None. J. Quackenbush: None. A.S. Al-Zahrani: None. N. Kaya: None.

\section{P19.019.B}

Evidencing the genetic differences of Canary Islanders in the Spanish diversity landscape

I. Marcelino-Rodriguez ${ }^{l}$, M. Bolaños-Mendoza ${ }^{l}, H$. Rodríguez-Pérez ${ }^{l}$, J. M. Lorenzo-Salazar ${ }^{2}$, L. Ciuffreda ${ }^{l}, B$. Guillen-Guio $^{1}$, R. González-Montelongo ${ }^{2}$, I. Quintela ${ }^{3}$, Á. Carracedo $^{4}$, C. Flores ${ }^{1,2,5}$

${ }^{I}$ Research Unit, HUNSC, Universidad de La Laguna, Santa Cruz de Tenerife, Spain, ${ }^{2}$ Genomics Division, Instituto Tecnológico y de Energías Renovables (ITER), Santa Cruz de Tenerife, Spain, ${ }^{3}$ Grupo de Medicina Xenomica, CEGEN-ISCIII-Universidade de Santiago de Compostela, Santiago de Compostela, Spain, ${ }^{4}$ Grupo de Medicina Xenomica, CIBERER-Universidade de Santiago de Compostela-Fundacion Galega de Medicina Xenomica (SERGAS), Santiago de Compostela, Spain, ${ }^{5}$ CIBER de Enfermedades Respiratorias, Instituto de Salud Carlos III, Madrid, Spain

Introduction: The Spanish DNA National Bank (NDNAB) was created to promote genomic research in the country. Recent efforts have characterized the genetic substructure of mainland populations and their recent genetic influences. Leveraging the NDNAB population controls, here we aimed to analyze the genetic structure of Spain with a particular focus on their relationships with Canary Islanders, a geographically isolated population with a sizeable proportion of African alleles.

Methods: Genotypes from 758,740 variants in 3,526 NDNAB controls, represented by donors from 19 autonomous regions including the Canary Islands, were obtained with the Axiom Spain Biobank Array (Affymetrix). Population structure was assessed using a combination of principal component analyses, complementary highdimensional reduction techniques (UMAP), and modelbased ancestry estimates.

Results: Approximate population-aggregated clusters were identified using grandparental geographical origin, language and ancestry strata. Evident groups were observed 
in major axes of differentiation according to autonomous regions, most obviously represented by Catalans, Basques and Galicians as has been described before. However, another clear axis of differentiation was due to the Canary Islanders.

Conclusions: These results will aid in selecting NDNAB controls and guide association testing in genomic studies in the Spanish population.

Funding: Instituto de Salud Carlos III (CD19/00231), Ministerio de Ciencia, Innovación y Universidades (RTC2017-6471-1; AEI/FEDER, UE), and agreement OA17/008 with ITER. The genotyping service was carried out at CEGEN-PRB3-ISCIII, supported by grant PT17/0019, of the PE I+D+i 2013-2016, funded by ISCIII and ERDF.

I. Marcelino-Rodriguez: None. M. Bolaños-Mendoza: None. H. Rodríguez-Pérez: None. J.M. Lorenzo-Salazar: None. L. Ciuffreda: None. B. Guillen-Guio: None. R. González-Montelongo: None. I. Quintela: None. Á. Carracedo: None. C. Flores: None.

P19.021.A

Causal effects of gut microbiota on celiac disease: a twosample mendelian randomization approach

I. Garcia-Santisteban ${ }^{l}$, A. Cilleros-Portet ${ }^{l}$, E. Moyua ${ }^{l}$, A. $K_{\text {Kurilshikov }}^{2}$, A. Zhernakova ${ }^{2}, K$. Garcia-Etxebarria ${ }^{3}, N$. Fernandez-Jimenez ${ }^{1}$, J. Bilbao ${ }^{1,4}$

${ }^{1}$ University of the Basque Country (UPV/EHU) \& BioCruces-Bizkaia Health Research Institute, Leioa, Spain, ${ }^{2}$ University of Groningen and University Medical Center Groningen, Groningen, Netherlands, ${ }^{3}$ Biodonostia Health Research Institute, Donostia, Spain, ${ }^{4}$ Spanish Biomedical Research Center in Diabetes and associated Metabolic Disorders (CIBERDEM), Madrid, Spain

Introduction: Celiac disease (CeD), the most common food intolerance, is a complex immune-mediated inflammatory condition triggered by ingestion of gluten in genetically predisposed individuals. Literature suggests that alterations in the gut microbiota composition and function precede the onset of $\mathrm{CeD}$. Considering that microbiota characteristics are determined by host genetics, we speculate that the genetic makeup of $\mathrm{CeD}$ patients could elicit disease manifestation through alterations in the intestinal microbiota.

Materials and Methods: To evaluate the causal relationship of gut microbiota with the risk of developing CeD, we performed a Two-Sample Mendelian Randomization (2SMR) analysis. Exposure data was obtained from three raw datasets of a previous Genome Wide Association Study (GWAS) of gut microbiota composition and function, and outcome data from summary statistics of the most relevant GWAS on CeD.

Results: We have identified a number of putative causal associations between gut microbiota composition and function with SNPs associated with CeD. Regarding bacterial composition, most of the associated SNPs are related to Firmicutes phylum, whose relative abundance has been reported to be altered in $\mathrm{CeD}$ patients. In terms of functional units, we have linked SNPs located in intronic regions of known $\mathrm{CeD}$-associated genes, such as $L P P$ or PUS10, to a variety of bacterial metabolic pathways.

Conclusions: This study represents the first 2SMR approach to elucidate the causal relationships between microbiome and CeD from GWASs. Next steps should focus on deciphering the underlying molecular mechanisms from a basic and clinical perspective.

Funding: ISCIII 16/00258 and GVSAN2018/111086 to JRB and GVSAN2019111085 to NFJ.

I. Garcia-Santisteban: None. A. Cilleros-Portet: None. E. Moyua: None. A. Kurilshikov: None. A. Zhernakova: None. K. Garcia-Etxebarria: None. N. FernandezJimenez: None. J. Bilbao: None.

\section{P19.022.B}

Assessing the association of common genetic variants in $E P H B 4$ and $R A S A 1$ with severity phenotypes in cerebral cavernous malformation

F. M. Choksi ${ }^{1}$, S. Weinsheimer ${ }^{1}$, J. Nelson ${ }^{1}$, L. Pawlikowska ${ }^{1}$, C. Fox ${ }^{1}$, A. Zafar ${ }^{2}$, M. Mabray ${ }^{2}, J$. Zabramski ${ }^{3}$, A. Akers ${ }^{4}$, B. Hart ${ }^{2}$, L. Morrison ${ }^{2}$, C. E. McCulloch ${ }^{1}$, H. Kim ${ }^{l}$

${ }^{1}$ University of California San Francisco, San Francisco, CA, United States, ${ }^{2}$ University of New Mexico, Albquerque, NM, United States, ${ }^{3}$ Barrow Neurological Institute, Phoenix, AZ, United States, ${ }^{4}$ Angioma Alliance, Durham, NC, United States

Introduction: Familial cerebral cavernous malformation (CCM) is an autosomal dominant disease caused by mutations in KRIT1, CCM2 and PDCD10. KRIT1 shares pathways with $R A S A 1$, as suggested by their interaction with the Rap1A protein. EPHB4 and RASA1 mutations cause capillary malformation-arteriovenous malformation (CM-AVM), which resemble lesions seen in familial CCM. We thus investigated whether common variants in EPHB4 and RASAl are modifiers of CCM disease severity, including intracerebral hemorrhage $(\mathrm{ICH})$, total and large lesion count.

Methods: Familial CCM cases enrolled in the Brain Vascular Malformation Consortium were included $(n=$ $354)$. Total lesions and large lesions $(>5 \mathrm{~mm})$ were counted 
on MRI; ICH history at enrollment was assessed by medical records/self-report. Samples were genotyped on the Affymetrix Axiom Genome-Wide LAT1 Human Array. We tested 7 variants with minor allele frequency (MAF) $\geq 5 \%$ (3 in $E P H B 4,4$ in $R A S A 1)$ using multivariable regression, adjusting for age, sex, and 3 principal components (to account for population stratification). Significance was based on Bonferroni adjustment for multiple comparisons $(0.05 / 7=0.007)$.

Results: At baseline, $30.1 \%$ of cases had $\mathrm{ICH}$, with median total lesion count of 12 (IQR: 5-44) and large lesion count of 3 (IQR: $1-5$ ); mean age was $39 \pm 21$ years. EPHB4 variants were not associated with CCM severity phenotypes $(P>0.007)$. One RASAl intronic variant (rs72783711 A>C) was significantly associated with $\mathrm{ICH}(\mathrm{OR}=2.33, \mathrm{P}<$ $0.001)$, but not with total $(P=0.728)$ or large lesion count $(P=0.015)$.

Conclusion: RASA1 variants may be associated with $\mathrm{ICH}$ in CCM. Further investigations with longitudinal and larger studies are needed to confirm these findings.

Grant support: NIH U54NS065705.

F.M. Choksi: None. S. Weinsheimer: None. J. Nelson: None. L. Pawlikowska: None. C. Fox: None. A. Zafar: None. M. Mabray: None. J. Zabramski: None. A. Akers: None. B. Hart: None. L. Morrison: None. C.E. McCulloch: None. H. Kim: None.

\section{P19.023.C}

CFTR variant $\mathrm{W} 1282 \mathrm{R}$ is a novel pathogenic cystic fibrosis mutation

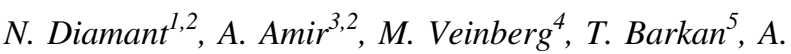

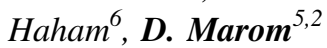

${ }^{1}$ Pedaitric Pulmonary Unit, Dana-Dwek Children's Hospital, Tel Aviv Sourasky Medical Center, Tel Aviv, Israel, ${ }^{2}$ Sackler School of Medicine, Tel Aviv University, Tel Aviv, Israel, ${ }^{3}$ Pediatric Gastroentorology, Liver and Nutrition Clinic, Dana-Dwek Children's Hospital, Tel Aviv Sourasky Medical Center, Tel Aviv, Israel, ${ }^{4}$ Pediatric Surgery, DanaDwek Children's Hospital, Tel Aviv Sourasky Medical Center, Tel Aviv, Israel, ${ }^{5}$ Genetics Institute, Tel Aviv Sourasky Medical Center, Tel Aviv, Israel, ${ }^{6}$ Neonatal Intensive Care Unit, Dana-Dwek Children's Hospital, Tel Aviv Sourasky Medical Center, Tel Aviv, Israel

Introduction: Bi-allelic CFTR variants, causing dysfunction of the cystic fibrosis transmembrane regulator protein, result in cystic fibrosis. Controversy exists regarding pathogenicity of the W1282R CFTR variant, classified as a variant of unknown significance in scant case reports. $\mathrm{W} 1282 \mathrm{R}$ is not registered in the CFTR2 database and is not included in expanded population carrier screen panels.
Methods: we report on two male siblings with cystic fibrosis and compound heterozygosity for W1282X and W1282R CFTR variants.

Results: a second born male to unrelated parents of mixed Ukraine and Ashkenazi Jewish descent presented prenatally with meconium ileus requiring emergency postnatal surgical intestinal resection followed by cholestasis and malabsorption with undetected stool elastase levels. Parental prenatal ethnic based common mutation carrier screen was positive for paternal heterozygosity for the known pathogenic CFTR variant W1282X. Maternal screen was negative. Sequencing based expanded carrier screen showed similar results. Next-generation sequencing of a multi-gene monogenic neonatal cholestasis panel revealed compound heterozygosity for $\mathrm{W} 1282 \mathrm{X}$ and $\mathrm{W} 1282 \mathrm{R}$. The latter classified as a variant of unknown significance. Retrospectively, his 19-months old brother was diagnosed with failure to thrive, abdominal distention and steatorrhea (fecal fat 12.6 gram/24 hours). Two separate sweat chloride levels were 101 and $103 \mathrm{mmol} / \mathrm{L} \mathrm{Eq.} \mathrm{NaCL}$, respectively. Stool elastase was undetectable. The genetic diagnosis prompted pancreatic enzyme replacement therapy that resulted in significant clinical improvement. Both siblings lack respiratory symptoms.

Conclusions: W1282R is a pathogenic CFTR variant. It should be included and reported in population genetic carrier screening panels. Further research will establish allele frequency.

N. Diamant: None. A. Amir: None. M. Veinberg: None. T. Barkan: None. A. Haham: None. D. Marom: None.

\section{P19.024.A}

Interrogating and correcting fine-scale genetic structure in large $(>36,000$ samples $)$ GWAS datasets using scalable haplotype sharing methods

\section{R. P. Byrne ${ }^{1}$, W. van Rheenen ${ }^{2}$, J. H. Veldink ${ }^{2}$, R. L. McLaughlin ${ }^{1}$}

${ }^{I}$ Smurfit institute of genetics, Trinity College Dublin, Dublin, Ireland, ${ }^{2}$ Department of Neurology, Brain Center Rudolf Magnus, University Medical Center Utrecht, Utrecht, Netherlands

Introduction: We characterised finescale population structure in an Amyotrophic Lateral Sclerosis (ALS) GWAS dataset of 36,052 individuals of European descent using the scalable haplotype painting algorithm PBWTpaint, and evaluated its application in correcting subtle population stratification undetectable using single marker PCA. 
Methods: We implemented the PBWT-paint algorithm to generate a haplotype sharing matrix describing coancestry between 36,052 individuals. To evaluate the effectiveness of this approach for correcting subtle population stratification we fit principal components (PCs) calculated from the coancestry matrix as covariates in a logistic regression model GWAS and compared metrics of statistical inflation ( $\lambda_{\mathrm{GC}}$ and LD-score regression intercept) to a model using single marker PCs as covariates. Additionally we explored the extent of structure captured by PBWT-paint using tdistributed stochastic neighbour embedding and identified genetic clusters using Louvain network clustering.

Results: The coancestry matrix revealed both striking broadscale genetic structure between countries and subtle genetic structure within countries, making it an appealing option for correcting overt and cryptic GWAS confounding. We observed that both the $\lambda_{\mathrm{GC}}$ and $\mathrm{LD}$-score regression intercept were significantly closer to 1 when using PCs of the haplotype sharing matrix, signifying lower inflation and confounding from population structure. Notably, the GWAS analysis corrected using haplotype sharing PCs as covariates retained the power to detect all significant loci from the original meta analysis of the data, alongside an additional hit at the established ALS locus TBK1, suggesting that it has improved power over traditional approaches.

Grants: MND Association (957-799); Science Foundation Ireland (17/CDA/4737); European Research Council (772376-EScORIAL).

R.P. Byrne: None. W. van Rheenen: None. J.H. Veldink: None. R.L. McLaughlin: None.

\section{P19.025.B}

CEMARA database in the AnDDI-Rares network: 10 years of epidemiology in developmental disorders in France

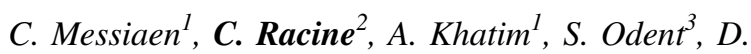
Lacombe $^{4}$, S. Manouvrier ${ }^{5}$, P. Edery ${ }^{6}$, N. Philip ${ }^{7}, D$. Geneviève $^{8}$, C. Thauvin-Robinet ${ }^{2,9,10}$, L. Pasquier ${ }^{3}, F$.

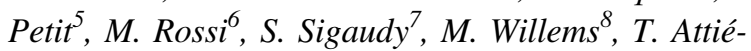
Bitach $^{11}$, P. Roux-Levy ${ }^{10}$, L. Demougeot ${ }^{9}$, L. Ben Slama ${ }^{9,11}$, the AnDDI-Rares network, C. Binquet ${ }^{12}$, A. Sandrin ${ }^{1}, A$. Verloes $^{13}$, L. Faivre $2,9,10$

${ }^{1}$ Banque Nationale de Données Maladies Rares, DSIWIND, APHP, Paris, France, ${ }^{2}$ Centre de Référence Anomalies du Développement et Syndromes Malformatifs, Dijon Bourgogne University Hospital, Dijon, France, ${ }^{3}$ Centre de Référence Anomalies du Développement et Syndromes Malformatifs, Hôpital Sud, CHU de Rennes, Rennes, France, ${ }^{4}$ Centre de Référence Anomalies du Développement et Syndromes Malformatifs, CHU Bordeaux et INSERM U1211, Bordeaux, France, ${ }^{5}$ Centre de
Référence Anomalies du Développement et Syndromes Malformatifs, CHRU de Lille, Lille, France, ${ }^{6}$ Centre de Référence Anomalies du Développement et Syndromes Malformatifs, Hôpital Femme-Mère-Enfant, Hospices Civils de Lyon, Lyon, France, ${ }^{7}$ Centre de Référence Anomalies du Développement et Syndromes Malformatifs, Département de génétique médicale, $\mathrm{CHU}$ de Marseille Hôpital de la Timone, Marseille, France, ${ }^{8}$ Centre de Référence Anomalies du Développement et Syndromes Malformatifs, CHU Montpellier, Montpellier, France, ${ }^{9}$ Filière AnDDI-Rares, CHU Dijon, Dijon, France, ${ }^{10}$ INSERM UMR1231 et FHU Translad, Université de Bourgogne, Dijon, France, ${ }^{11}$ Foetopathologie, Hôpital Necker-Enfants Malades, Paris, France, ${ }^{12}$ Centre d'Investigation Clinique - Epidémiologie Clinique, CHU de Dijon, Dijon, France, ${ }^{13}$ Centre de Référence Anomalies $d u$ Développement et Syndromes Malformatifs, AP-HP-NordUniversité de Paris, Hôpital Robert Debré, Department of Medical Genetics and INSERM UMR1141, Paris, France

Introduction: The French Ministry of Health has implemented a vast epidemiological program on rare diseases (RD), in parallel of the establishment of reference and expertise centres for RD in France. A majority of these centres have adopted the CEMARA web-portal, set up by the national data repository for all RD, which allows the collation of data for patients seen within these centres. Since 2007, a minimal data set (MDS) was collected prospectively for all these patients.

Methods: After a 10-year period, we analyzed anonymously and retrospectively administrative, demographic, care organization and diagnoses data.

Results: 228,243 individuals were enrolled from 2007 to 2017 through the AnDDI Rares network, including 167,361 affected individuals (median age 11 years, 54\% children, $46 \%$ adults) with a balanced sex-ratio. Among the 2,872 $\mathrm{RD}, 1,907(66,5 \%)$ were recorded in less than 10 patients. $45.6 \%$ of patients had no diagnosis. Given the centres' geographical coverage, patients have an average distance to travel of $25.1 \mathrm{~km}$, suggesting an efficiency of the health organization for RD. Focus can be done by disease groups such as chromosomal abnormalities (enabling an extensive overview) or by diseases; leading to operable large cohorts of patients with diverse data within the MDS (demographic, clinical, medical care) and more if filled out.

Conclusions: The main objective of CEMARA is, in the field of RD, to provide support for epidemiological, clinical or therapeutic studies, for which a collective and cooperative effort between centres is necessary, and to provide qualitative/quantitative indications for the establishment of national health policies.

C. Messiaen: None. C. Racine: None. A. Khatim: None. S. Odent: None. D. Lacombe: None. S. 
Manouvrier: None. P. Edery: None. N. Philip: None. D. Geneviève: None. C. Thauvin-Robinet: None. L. Pasquier: None. F. Petit: None. M. Rossi: None. S. Sigaudy: None. M. Willems: None. T. Attié-Bitach: None. P. Roux-Levy: None. L. Demougeot: None. L. Ben Slama: None. C. Binquet: None. A. Sandrin: None. A. Verloes: None. L. Faivre: None.

P19.026.C

Phylostratigraphic analysis of co-expression network reveals evolutionary compositions of preeclampsia

A. A. Babovskaya ${ }^{l}$, E. A. Trifonova ${ }^{l}$, A. A. Zarubin ${ }^{l}$, Z. S.

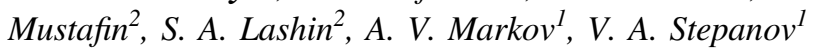

${ }^{1}$ Research Institute of Medical Genetics, Tomsk, Russian Federation, ${ }^{2}$ Institute of Cytology and Genetics SB RAS, Novosibirsk, Russian Federation

Preeclampsia (PE) is a complication of pregnancy characterized by new-onset hypertension and proteinuria of gestation, with serious consequences for mother and infant. However, the evolutionary machineries of PE are still largely unknown. Here, we used a method that combines phylostratigraphy information with gene co-expression networks to extensively study the evolutionary compositions of PE. The present co-expression network construction yielded 3987 nodes based on $129 \mathrm{PE}$ and normal pregnancy expression samples obtained from the GEO database. A total of 31 modules were identified as PE related clusters and 34 modules were identified in control group. Using WGCNA, we found 6 clusters containing 86 genes associated only with PE. We identified 9 hub genes (RAD21, YY1, CXCL9, CXCL10, IFI44, IFI44L, GYPA, GYPB and IFIH1) in these clusters using cytoHubba (MCC, rank < 5) and STRING. As part of evolutionary analysis, we calculated the phylostratigraphic age index (PAI) and the divergence index (DI) using Orthoscape. We note that $70,9 \%$ genes in network have PAI $\leq 3$, but interesting that most of hub genes (GYPA, GYPB, IFI44, IFI44L, CXCL9, CXCL10) have PAI'5 (Vertebrata); more susceptible to evolutionary processes were genes GYPA, GYPB, IFI44, IFI44L (DI 3,918-0,834), only YY1 and RAD21 have PAI $\leq 1$ (Eukaryota) and low DI $(0,054-0,186)$. These results demonstrate that most hub genes for this network diverged from their ancestors. The integrated analysis of the topology of PE modules and the phylogenetic data revealed an evolutionary pattern of PE in human and identified new potential biomarkers PE.

A.A. Babovskaya: None. E.A. Trifonova: None. A.A. Zarubin: None. Z.S. Mustafin: None. S.A. Lashin: None. A.V. Markov: None. V.A. Stepanov: None.

\section{P19.028.B}

A polygenic architecture of medication-use across medical conditions

P. D. Rohde ${ }^{1}$, M. Nyegaard ${ }^{2}$

${ }^{1}$ Aalborg University, Aalborg, Denmark, ${ }^{2}$ Aarhus University, Aarhus, Denmark

Introduction: Genomics has been forecasted to revolutionise human health by improving medical treatment through a better understanding of the genetic epidemiology of human diseases. Despite great successes of the last decade's genome-wide association studies (GWAS), the results have to a limited extent been translated to genomic medicine. We propose, that one route to get closer to improved medical treatment is by understanding the genetics of medicationuse. We hypothesise that, irrespectively of medical condition, medication-use per se has a unique genetic heritable architecture.

Methods: Medication-use from 335.744 individuals from the UK Biobank was obtained, and we conducted a GWAS on 9 million imputed genetic variants. We estimated the heritability of medication-use and partitioned the genetic variance across different categories. Using five-fold cross validation we performed within sample prediction of medication-use.

Results: We identified 57 independent loci associated with variation in medication-use and estimated that $14 \%$ of the total variation was attributable to common genetic variants. The largest fraction of genetic variance was captured by variants with low to medium minor allele frequency. In particular coding and conserved regions, as well as transcription start sites, displayed significantly enrichment of heritability. The mean prediction accuracy was 0.14 .

Conclusions: These results demonstrate that medicationuse per se is a complex trait irrespectively of medical condition. Thus, if the goal is to improve medical treatment it might be insufficient to solely rely on analyses of complex diseases as medication-use per se has its own genetic architecture.

Funding: PDR has received funding from the Lundbeck Foundation (R287-2018-735).

P.D. Rohde: B. Research Grant (principal investigator, collaborator or consultant and pending grants as well as grants already received); Significant; Lundbeck Foundation (R287-2018-735). M. Nyegaard: None.

P19.029.C

Carnitine and risk of complex disorders, a Mendelian Randomisation study 


\section{ABAR, V. ZUBER, A. DEHGHAN}

IMPERIAL COLLEGE LONDON, LONDON, United Kingdom

Background: Carnitine, a water-soluble quaternary amine, is essential for the normal functioning of all tissues and has an important role in energy metabolism by transferring long-chain fatty acids (acyl groups) to the mitochondrial matrix for $\beta$ oxidation and depleting the acyl groups out of the mitochondria. Accumulating evidence from animal and human studies indicates that carnitine and its acylderivatives may have potential beneficiary effect in lipid profile modification, glycaemic control, and modification of vascular cognitive impairment in dementia patients.

Objective: The overarching aim of the study is to investigate whether carnitine levels have causal effects on risk of complex disorders including coronary artery disease (CAD), type 2 diabetes (T2D) and Alzheimer's disease (AD) by applying Two-sample Mendelian Randomisation (MR) analysis.

Methods: A meta-analysis of genome-wide association studies (GWASs) of the Airwave cohort participants and publicly available results from a meta-analysis of the KORA and TwinsUK studies was used to identify instruments for carnitine. Summary statistics data for $\mathrm{CAD}, \mathrm{T} 2 \mathrm{D}$ and $\mathrm{AD}$ were obtained from publicly available GWAS meta-analyses conducted by the CARDIoGRAMplusC4D, DIAGRAM and IGAP consortiums. MR analyses were performed using the inverse-variance-weighted (IVW), weighted-median, MR Pleiotropy RESidual Sum and Outlier (PRESSO) test and MR-Egger regression.

Results: There was evidence of a significant causal inverse association between carnitine and coronary artery disease. No further evidence for a causal effect observed between carnitine and other outcomes including T2D and AD.

Conclusions: The study findings suggest that carnitine has a protective effect on the risk of coronary artery disease.

L. Abar: None. V. Zuber: None. A. Dehghan: None.

\section{P19.030.A}

The results of the program of DNA diagnostics in patients with cystic fibrosis in the RF in 2018-2019

E. Zhekaite, E. Kondratyeva, N. Petrova, R. Zinchenko, O. Pylaeva, S. Kutsev, A. Voronkova, V. Sherman, $R$. Budzinsky

Research Centre for Medical Genetics, Moscow, Russian Federation

Introduction: According to the national cystic fibrosis (CF) registry 2017 , a genetic study was performed in $92.4 \%(n=$
2816) of patients with CF in RF, the frequency of identified pathogenic variant (PV) was $88.3 \%(80.2 \%$ had $2 \mathrm{PV}$, in $16.1 \%$ one, $3.7 \%$ - none $)$. A total of $7.6 \%(n=231) \mathrm{CF}$ patients need genetic diagnostics.

Materials and Methods: within realization the program of DNA diagnostics in patients with CF in 2018-2019, 350 patients (265 patients from the national registry and 85 patients with $\mathrm{CF}$ unspecified) were examined for frequent PV in CFTR. DNA was isolated from leukocytes using standard procedures, sequencing was performed in 52 patients, and MLPA was performed 20 patients.

Results: We were able to increase the number of diagnosed patients, and the percent of identified PV in 18 distant RF regions. As a result of the work carried out, 2 PV were identified in 168 patients, one in 34 , none in 84 (the diagnosis is not confirmed). 64 blood samples are still being sequenced.

Conclusion: Due to the improvements in CF diagnostics, an increased number of regions is added to the national registry. A larger number of newly diagnosed patients still need DNA diagnostics. The program will continue in 20202021. The work was carried out with the support of the "Ostrova" charitable Foundation.

E. Zhekaite: None. E. Kondratyeva: None. N. Petrova: None. R. Zinchenko: None. O. Pylaeva: None. S. Kutsev: None. A. Voronkova: None. V. Sherman: None. R. Budzinsky: None.

\section{P19.031.B}

Stochastic variation explains differences in the number of de novo mutations between families

J. E. Hampstead ${ }^{1}$, J. M. Goldmann ${ }^{1}$, W. S. W. Wong ${ }^{2}$, T. Turner $^{3}$, A. Wilfert ${ }^{3}$, M. A. Jonker ${ }^{4}$, R. Bernier ${ }^{5}$, M. A. Huynen $^{6}$, L. Gomez, R. Baker ${ }^{7}$, D. Ascher ${ }^{7}$, S. Hazrati ${ }^{7}$, T.

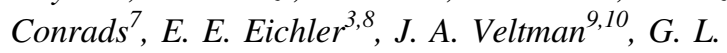
Maxwell $^{11,7}$, C. Gilissen ${ }^{1}$

${ }^{1}$ Department of Human Genetics, Radboud Institute for Molecular Life Sciences, Radboud University Medical Center, Nijmegen, Netherlands, ${ }^{2}$ Coherent Logic Limited, McLean, VA, United States, ${ }^{3}$ Department of Genome Sciences, University of Washington School of Medicine, Seattle, WA, United States, ${ }^{4}$ Department for Health Evidence, Radboud University Medical Center, Nijmegen, Netherlands, ${ }^{5}$ Department of Psychiatry and Behavioral Sciences, University of Washington, Seattle, WA, United States, ${ }^{6}$ Center for Molecular and Biomolecular Informatics, Radboud University Medical Center, Nijmegen, Netherlands, ${ }^{7}$ Inova Translational Medicine Institute (ITMI), Inova Health Systems, Falls Church, VA, United States, ${ }^{8}$ Howard Hughes Medical Institute, University of Washington, Seattle, WA, United States, ${ }^{9}$ Biosciences 
Institute, Faculty of Medical Sciences, Newcastle University, Newcastle Upon Tyne, United Kingdom, ${ }^{10}$ Department of Human Genetics, Donders Centre for Neuroscience, Radboud University Medical Center, Nijmegen, Netherlands, ${ }^{11}$ Department of Obstetrics and Gynecology, Inova Health Systems, Falls Church, VA, United States

Human germline de novo mutations (DNMs) are both a driver of evolution and an important cause of genetic disease. The number of DNMs in the human germline is correlated with parental age at conception, but parental age explains only part of the observed variation in the number of DNMs between individuals. Here, we investigated whether there is a family-specific contribution to the number of DNMs in offspring. We analyzed DNMs from four large whole genome sequencing cohorts of diverse ancestry. First, we compared the difference in the number of DNMs in 111 dizygotic twin pairs to those from 578 pairs of parental age-matched unrelated children, but we did not observe a significant familyspecific contribution. Subsequently we compared differences in DNMs from siblings to those of randomly matched individuals with correction for parental age, and again observed no significant differences. Finally, we used a random effects model to directly estimate the potential impact of familyspecific effects on variation in DNM number using a cohort of 1590 families with two offspring and a second cohort of 45 families with an average of 9.3 offspring. We estimated a familial variance component of only $5.2 \%$. Additionally, we found almost no difference between the observed number of DNMs in these offspring and those expected based on a stochastic Poisson model. We therefore conclude that familyspecific contribution to DNM number in offspring is small and that stochasticity explains a large proportion of the observed variation.

J.E. Hampstead: None. J.M. Goldmann: None. W.S. W. Wong: None. T. Turner: None. A. Wilfert: None. M. A. Jonker: None. R. Bernier: None. M.A. Huynen: None. L. Gomez: None. R. Baker: None. D. Ascher: None. S. Hazrati: None. T. Conrads: None. E.E. Eichler: None. J. A. Veltman: None. G.L. Maxwell: None. C. Gilissen: None.

\section{P19.032.C}

Identifying and characterising germline hypermutators

J. Kaplanis ${ }^{l}$, P. Danecek ${ }^{l}$, E. Prigmore ${ }^{l}$, R. Rahbari ${ }^{l}$, P. Short ${ }^{l}$, J. Korbel ${ }^{2}$, Genomics England Research

Consortium, M. Hurles ${ }^{1}$

${ }^{I}$ Wellcome Sanger Institute, Cambridge, United Kingdom, ${ }^{2}$ European Molecular Biology Laboratory, Heidelberg, Germany
The human germline mutation rate is known to vary between individuals. Most of the variation in the population is explained by parental age, but little is known about rare outliers with extreme mutation rates. We have tackled this problem using both phenotype and genotype driven approaches.

For our phenotype driven approach, we identified germline hypermutators and sought genetic causes for this trait. We identified five children with an excessive number of de novo mutations (DNMs) across 20,000 individuals from the Deciphering Developmental Disorders study and Genomics England Limited dataset. They all have $>3.5$ times DNMs as expected ranging from 257-426 DNMs per individual. In four children, the excess DNMs all phased paternally implicating the father as a germline hypermutator. We identified a potentially causal variant in two of the fathers: a rare homozygous missense mutation in the gene MPG and a rare homozygous nonsense mutation in XPC. For the remaining child, the mutations appear to have occurred post-zygotically indicating this is unlikely attributable to a parental hypermutator. The genotype-driven approach focussed on whether variants in DNA repair genes impact germline mutation rates. First, we interrogated variants in an established cancer mutator gene, MBD4, and found they did not have the same effect in the germline. Second, we performed analyses of the impact of rare damaging variants in DNA repair genes on individual germline mutation rate.

Our analyses provide new insights into the role of genetic variation on the human germline mutation rate and uncover possible genetic causes of germline hypermutation.

J. Kaplanis: None. P. Danecek: None. E. Prigmore: None. R. Rahbari: None. P. Short: None. J. Korbel: None. M. Hurles: E. Ownership Interest (stock, stock options, patent or other intellectual property); Significant; Congenica Ltd. F. Consultant/Advisory Board; Significant; Congenica Ltd.

\section{P19.034.B}

Gene-based rare variant analysis of whole-exome sequencing in relation to eosinophil counts

\section{J. R. Höglund, W. E. Ek, T. Karlsson, Å. Johansson}

Uppsala University, Uppsala, Sweden

During the last decade, thousands of associations between common variants and complex traits and diseases have been identified. With rapid advancement in high-throughput sequencing technology, progress in sequencing rare and low-frequency variants is constantly being made. For rare variants, region-based analyses have been suggested to be 
more powerful as compared to standard variant association testing. In this study, we used the sequence kernel association test (SKAT) to test for association with eosinophil counts, performed in the initial release of the UK Biobank whole-exome sequencing dataset $(N=49,960)$. We tested for five different scenarios, with various single nucleotide variant (SNV)-weighting functions. The primary SKAT analyses resulted in 51 significant transcripts throughout the five scenarios, all colocalizing with at least one GWAS signal. When adjusting for all significant, primary and conditional, top GWAS hits, five transcripts mapping to three regions (CLC, IL-33 and ST20-MTHFS) remained significant in the SKAT tests. The association with $C L C$ seems to be driven by two common variants, not previously associated with eosinophil counts. In contrast, variants with a minor allele frequency below $0.5 \%$ seem to drive the association with $I L-33$. For most of the SKAT signals, a single-marker GWAS seems to capture the majority of variation in eosinophil count. However, it is plausible that a larger sample size is needed to fully resolve the effect of rare variants. This project is funded by SciLifeLab, the Swedish Research Council (VR), the $\AA$ Wiberg, M Borgström, A, M Rudbergs, and the Swedish Heart and Lung foundation.

J.R. Höglund: None. W.E. Ek: None. T. Karlsson: None. Å. Johansson: None.

\section{P19.038.C}

Clinical application of whole exome and clinical exome sequencing as a diagnostic tool in Colombian population

\section{A. J. Muñoz-Bolaños, S. J. Maradei, I. T. Bernal, Y. M. Gómez}

\section{BIOTECGEN S.A., Bogotá, Colombia}

Introduction: Whole and clinical exome sequencing (WES, CES) has been used increasingly in clinical diagnostics for a variety of indications to identify the underlying genetic cause of disease. As a clinical diagnostic laboratory, we sought to review our experience analyzing 124 WES and CES cases over the past 4 years to better understand the clinical contexts in which WES/CES can provide a plausible explanation for the presenting symptoms.

Materials and Methods: We reviewed 124 consecutive cases referred to our laboratory for WES/CES from 2016 to 2019 and performed clinical categorization.

Results: We analyzed 124 cases, with 97 (78.2\%) submitted as proband only, $2(1.6 \%)$ with one additional family member, and 25 (20.2\%) with two additional family members. A definitive result (Pathogenic and Likely pathogenic) was provided in $41.1 \%$, a possible/probable (VOUS) result was provided in $38.7 \%$, a candidate gene result as the only finding was provided in $3.2 \%$, and a negative result was provided in $16.9 \%$. Secondary findings were offered to all participants, of whom 120 opted out (96.7\%), but were found in only 2 cases $(1.7 \%)$.

Conclusions: Positive results impacted patient care, regardless of whether the established diagnosis was treatable. Cases that remained unsolved (VOUS) should be carefully considered, and variant reclassification must be followed up. Reanalysis of data generated by WES and CES may eventually yield a diagnosis for patients with negative or uncertain results. Even when the results were noninformative, WES/CES allowed the medical team to streamline diagnostic evaluations and/or focus on managing known issues.

A.J. Muñoz-Bolaños: None. S.J. Maradei: None. I.T. Bernal: None. Y.M. Gómez: None.

\section{P19.039.A}

\section{Genetic basis if falling risk susceptibility}

K. Trajanoska ${ }^{1}$, L. J. Seppala ${ }^{2}$, C. Medina-Gomez ${ }^{l}, Y$. $\mathrm{Hsu}^{3}$, S. Zhou ${ }^{4}$, N. M. van Schoor ${ }^{5}$, L. C. de Groot ${ }^{6}$, D.

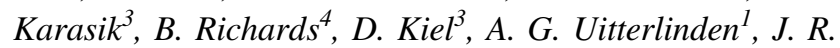
Perry $^{7}$, N. van der Velde ${ }^{2}, F$. Day ${ }^{7}, F$. Rivadeneira ${ }^{1}$

${ }^{I}$ Department of Internal Medicine, Erasmus MC University Medical Center, Rotterdam, Netherlands, ${ }^{2}$ Department of Internal Medicine, Section of Geriatric Medicine, Academic Medical Center, University of Amsterdam, Amsterdam, Netherlands, ${ }^{3}$ Hinda and Arthur Marcus Institute for Aging Research, Hebrew SeniorLife, Roslindale, MA, United States, ${ }^{4}$ Lady Davis Institute, Jewish General Hospital, McGill University, Montreal, QC, Canada, ${ }^{5}$ Department of Epidemiology and Biostatistics, Amsterdam Public Health research institute, VU University Medical Center, Amsterdam, Netherlands, ${ }^{6}$ Wageningen University, Division of Human Nutrition, Wageningen, Netherlands, ${ }^{7}$ MRC Epidemiology Unit, University of Cambridge School of Clinical Medicine, Cambridge, United Kingdom

Introduction: Both extrinsic and intrinsic factors predispose older people to fall. We performed a genome-wide association analysis (GWAS) to investigate how much of an individual's fall susceptibility can be attributed to genetics in 89,076 cases and 362,103 controls from the UK Biobank Study.

Material and Methods: Individuals were genotyped with the Afymetrix UKBiobank chip. The GWAS model included age and sex analysed with BOLT-LMM v.2.3.1. We used LD score regression to: 1). Estimate falling risk heritability 2). Test for genetic correlation with other traits and 3). Identify tissues where expression of genes related to fall-associated variants are enriched. Finally, we tested for 
causal relationships between several risk factors and falls using Mendelian Randomization (MR).

Results: We identified three novel fall loci mapping to RP11-6N13.1 on 5q21.3 (rs2431108, OR=1.03, $p=$ $4.20 \times 10^{-08}$ ), near PER4 on $7 \mathrm{p} 21.3$ (rs2709062, OR $=1.03$, $P=3.4 \times 10^{-08}$ ) and near TSHZ3 on $19 \mathrm{q} 12$ (rs2111530, $\left.\mathrm{OR}=1.03, p=1.2 \times 10^{-08}\right)$. Falling risk heritability was modest $(2.5 \%)$. Falls had positive genetic correlation with fracture risk $(\mathrm{rg}=0.35, \mathrm{se}=0.05)$, insomnia $(\mathrm{rg}=0.42$, $\mathrm{se}=0.05)$, neuroticism $(\mathrm{rg}=0.26, \mathrm{se}=0.08)$ and $\mathrm{ADHD}$ $(\mathrm{rg}=0.44$, se $=0.14)$; and negative genetic correlation with muscle strength $(\mathrm{rg}=-0.24, \mathrm{se}=0.04)$, intelligence $(\mathrm{rg}=$ $-0.12, \quad \mathrm{se}=0.04)$ and social well-being $(\mathrm{rg}=-0.29$, $\mathrm{se}=0.05)$. Brain and in particular cerebellum tissue showed the highest gene expression enrichment (FDR $<5 \%$ ) for fallassociated variants. MR analyses found a causal effect of high BMI and low relative handgrip on increased falling risk.

Conclusions: Falling risk constitutes a heritable, heterogeneous and polygenic trait genetically correlated with fracture risk and grip strength. The cerebellum tissue enrichment of falls-associated variants corroborates the mediation of postural balance in the etiology of falls.

K. Trajanoska: None. L.J. Seppala: None. C. MedinaGomez: None. Y. Hsu: None. S. Zhou: None. N.M. van Schoor: None. L.C. de Groot: None. D. Karasik: None. B. Richards: None. D. Kiel: None. A.G. Uitterlinden: None. J.R. Perry: None. N. van der Velde: None. F. Day: None. F. Rivadeneira: None.

\section{P19.041.C}

Reduced male reproductive success drives selective constraint on human genes

E. J. Gardner ${ }^{1}$, M. D. C. Neville ${ }^{1}$, K. E. Samocha ${ }^{1}$, M. E. K.

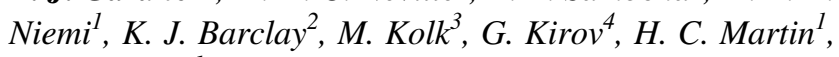
M. E. Hurles ${ }^{1}$

${ }^{1}$ Wellcome Sanger Institute, Hinxton, United Kingdom, ${ }^{2}$ Max Planck Institute for Demographic Research, Rostock, Germany, ${ }^{3}$ Stockholm University, Stockholm, Sweden, ${ }^{4}$ School of Medicine, Cardiff University, Cardiff, United Kingdom

Sequencing of human populations has revealed highly variable patterns of natural selection acting on human genes, with a minority of genes appearing to be under strong selection against heterozygous variants that abolish the function of the encoded protein. Although these selectively constrained genes are strongly enriched for dominant single gene disorders, for most of these genes the nature of the selective constraint is unknown. We called large deletions from SNP genotyping data on 500K UK Biobank participants and analysed protein truncating variants from exome data on $50 \mathrm{~K}$ UK Biobank participants. We determined the cumulative burden of likely loss-of-function variants in each UK Biobank participant, weighted according to the degree of selective constraint of the impacted gene(s), which we term their $S_{\text {het }}$ burden. We found that increasing $S_{\text {het }}$ burden results in substantially reduced male reproductive success, driven by an increased risk of childlessness $\left(p=4 \times 10^{-15}\right)$. This reduction in reproductive success is much weaker in females $(p=$ $0.0026) . S_{\text {het }}$ burden is also associated with reduced fluid intelligence, educational attainment and household income, but without strong sex-bias. We present evidence that this reduced male reproductive success is most likely mediated by cognitive and behavioural traits, which, due to female preferences for mating partners likely to invest resources in their offspring, renders male carriers of such variants less able to find mating partners. Our findings support a substantial role for sexual selection due to mate choice preferences shaping the recent evolution of human genes.

E.J. Gardner: None. M.D.C. Neville: None. K.E. Samocha: None. M.E.K. Niemi: None. K.J. Barclay: None. M. Kolk: None. G. Kirov: None. H.C. Martin: None. M.E. Hurles: E. Ownership Interest (stock, stock options, patent or other intellectual property); Modest; Congenica.

\section{P19.042.A}

Modification of Heritability for Educational Attainment and Fluid Intelligence by Socioeconomic Deprivation in the UK Biobank

\section{Rask-Andersen, T. Karlsson, W. Ek, A. Johansson}

\section{Uppsala University, Uppsala, Sweden}

Educational attainment, measured either in years of education or whether an individual has attended college or university, constitutes a complex genetic trait that correlates with cognitive traits, such as intelligence. It is strongly linked to quality-of-life measurements such as subjective well-being, paid employment, overall health, mortality and obesity. Socioeconomic factors have been suggested to influence the effect of education- and intelligenceassociated genetic variants. However, results from previous studies on the interaction between socioeconomic status and education or intelligence have been inconsistent. We therefore assessed the effect of socioeconomic deprivation on education- and intelligence-associated genetic variants by estimating the SNP heritability for fluid intelligence, educational attainment and years of education, in subsets of the UK Biobank with different degrees of social deprivation using LD score regression (LDSC). We also generated polygenic scores with LDpred and tested for 
interactions with social deprivation. The SNP heritability for all phenotypes were found to increase with socioeconomic deprivation. Polygenic scores for all phenotypes were also found to interact with socioeconomic deprivation, where the effects of polygenic scores increase with increasing deprivation. These results imply that genetics have a larger influence on educational and cognitive outcomes in more socioeconomically deprived UK citizens, which has serious implications for equality of opportunity.

This project is funded by grants from the Swedish Society for Medical Research (SSMF), and the Swedish Medical Research Council (Project Number 2015-03327).

M. Rask-Andersen: None. T. Karlsson: None. W. Ek: None. A. Johansson: None.

\section{P19.043.B}

\section{Genetic structure of the Catalan Pyrenean population}

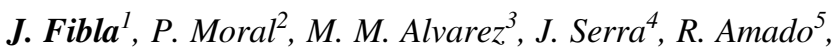
M. Feixes ${ }^{6}$, F. Soldevilla ${ }^{7}$ I. Maceda ${ }^{8}$, O. Lao $^{8}$

${ }^{1}$ Unitat de Genètica Humana - Genètica de Malalties Complexes, Universitat de Lleida-IRBLleida, Lleida, Spain, ${ }^{2}$ Departament de Biologia Evolutiva, Ecologia i Ciències Ambientals, Universitat de Barcelona, Barcelona, Spain, ${ }^{3}$ Universitat de Barcelona, Barcelona, Spain, ${ }^{4}$ Laboratori d'Anàlisis Clíniques, Hospital Comarcal del Pallars, Lleida, Spain, ${ }^{5}$ Hospital Sant Bernabé, Lleida, Spain, ${ }^{6} 4$ Servei d'Atenció Primària, Lleida, Lleida, Spain, ${ }^{7}$ Fundació Privada Hospital Sant Jaume D'Olot, Girona, Spain, ${ }^{8}$ Population Genomics Group, Centre Nacional d'Anàlisi Genòmica, Centre de Regulació Genòmica (CRG-CNAG), Parc Científic de Barcelona, Barcelona, Spain

Introduction: Until now, most of the populations considered in population genetics studies are of urban origin, representing large geographical or even continental regions. The study of genetic variation of rural populations such as the Pyrenean one, can be of great interest. In addition, the existence of natural barriers and the well characterized demographic history, make the genetic study of this population as an important piece of the puzzle on genetic variation in Europe.

Materials and Methods: We selected for this study a sample of 435 subjects with their eight grandparents born in the Catalan Pyrenean counties. DNA was extracted from blood. Genotyping data was obtained from each subject by using the Axiom PMDA genotyping array at the CEGEN facilities. Genotypes were merged with 1000 Genome Phase III data resulting in a final data set of 2939 individuals and 552.000 common SNPs.

Results: A PCA analysis shows the distinctive European genetic constitution of the Pyrenean population, as compared to other continents. Within Europe, the Pyrenean population could be clearly distinguished from other European populations. Within Pyrenean a west-east gradient in genetic variability was noticed. STRUCTURE analysis reveals a west-east gradient on Norden European ancestry as well as an inverse gradient on South European ancestry.

Conclusions: The genetic diversity of the populations of the Catalan Pyrenees is macro-scale within the European genetic diversity, following the same macro-spatial patterns that are observed within Europe and, in particular, with the populations of the South of Europe.

J. Fibla: None. P. Moral: None. M.M. Alvarez: None. J. Serra: None. R. Amado: None. M. Feixes: None. F. Soldevilla: None. I. Maceda: None. O. Lao: None.

P19.045.A

Genome-wide association meta-analysis of individuals of European ancestry identifies new loci explaining a substantial fraction of dietary intake and heritability

O. Mompeo ${ }^{l}$, P. Hysi ${ }^{l}$, A. M. Valdes ${ }^{2}$, S. E. Berry ${ }^{l}$, R.

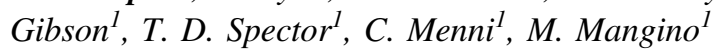

${ }^{1}$ King's College London, London, United Kingdom, ${ }^{2}$ NIHR Nottingham Biomedical Research Centre, Nottingham, United Kingdom

Introduction: Dietary intake is highly heritable and has an impact on cardiovascular disease. However, genetic variants influencing overall diet intake at a genome-wide significant level have not been identified. The aim of this study is to identify genetic loci for the Dietary Approaches to Stop Hypertension (DASH) diet, a diet quality index previously associated to cardiovascular disease.

Materials and Methods: We firstly calculated DASH heritability in TwinsUK (DZ $=706$; $\mathrm{MZ}=778)$. We then conducted a genome-wide analysis (GWA) in both the UKBiobank cohort $(n=173,456)$ and TwinsUK ( $n=$ 2968) using PLINK 2.0 and GEMMA respectively, correcting for sex, age, energy intake, smoking and socioeconomic status. Finally we conducted a GWA meta-analysis using METAL.

Results: DASH heritability was $40 \%$ [95\%C.I. 0.260.46], with the remaining 60\% [95\%C.I. 0.53-0.73] due to non-shared environmental factors. We identified five novel independent genomic risk loci: Chr1 (rs66495454, Beta $(\mathrm{SE})=-0.029$ (0.0034), $P=3.22 \times 10-17) ; \mathrm{Chr} 3$ (rs56331918, Beta(SE) $=0.022(0.0036), P=2.76 \times 10$ 9); Chr5 (rs550997965, $\operatorname{Beta}(\mathrm{SE})=-0.020 \quad$ (0.0036), $P=1.46 \mathrm{e}-08)$; Chr8 (rs73195303, Beta(SE) $=-0.025$ (0.0044), $P=9.85 \times 10-9)$ and Chr12 (rs1054442, Beta $(\mathrm{SE})=-0.019(0.0034) ; P=3.70 \times 10-8)$. These loci have 
been respectively associated with NEGR1, ARPP21, ZSWIM6, MSRA and DDN genes all previously linked to BMI or psychiatric traits. We also identified genetic correlations between DASH and anthropometric traits, health-related traits, and educational attainment.

Conclusions: These results confirm the polygenic nature of the dietary phenotypes and provide important new insights into the genetic aetiology of diet quality intake.

Grant: MRC AimHy (MR/M016560/1) project by the Chronic Disease Research and the Denise Coates Foundation.

O. Mompeo: None. P. Hysi: None. A.M. Valdes: None. S.E. Berry: None. R. Gibson: None. T.D. Spector: None. C. Menni: None. M. Mangino: None.

P19.048.A

Hereditary haemochromatosis: Lifetime risks of primary hepatic carcinoma and mortality in the UK Biobank

J. Atkins ${ }^{1}$, L. C. Pilling ${ }^{1}$, J. A. H. Masoli ${ }^{l}$, C. Kuo ${ }^{2}$, J. D. Shearman ${ }^{3}$, P. C. Adams ${ }^{4}$, D. Melzer ${ }^{1}$

${ }^{1}$ University of Exeter Medical School, Exeter, United Kingdom, ${ }^{2}$ Center on Aging, University of Connecticut Health Center, Farmington, CT, United States, ${ }^{3}$ South Warwickshire NHS Foundation Trust, Warwickshire, United Kingdom, ${ }^{4}$ Department of Medicine, University of Western Ontario, London, ON, Canada

Background:Hereditary haemochromatosis $(\mathrm{HH})$ is predominantly caused by the HFE p.C282Y homozygous mutation. Hepatic carcinomas and mortality risks are raised in clinically diagnosed $\mathrm{HH}$, especially with liver cirrhosis. However, outcomes are unclear in mutation carriers in the community setting without clinically diagnosed $\mathrm{HH}$.

Methods:UK Biobank European descent participants ( $n=451,186,40-70$ years) including 2,890 p.C282Y homozygotes, followed via hospitalization records, national cancer registry, primary care records and death certificates. Cox regression models adjusted for age, assessment center, genotyping array and genetic principal components. Kaplan Meier lifetable probabilities of incident diagnoses were estimated from age 40 to 75 .

Results:Incidence of hepatic carcinomas was higher in male p.C282Y homozygotes compared to those without mutations (HR: 14.1, 95\% CI: 7.6-26.1). However, no associations were observed in female p.C282Y homozygotes, p.C282Y heterozygotes or p.C282Y/p.H63D compound heterozygotes. Lifetables estimate project that by age $75,7.2 \%$ (95\% CI: $3.9-13.1$ ) of male p.C282Y homozygotes develop hepatic carcinoma and $19.5 \%$ (95\% CI 15.8-24.0) of homozygote men would die, compared to
$15.1 \%$ (95\% CI:14.7-15.5) in those without mutations. Female p.C282Y homozygotes also had an increased risk of death from hepatic carcinomas (HR: 3.36, 95\% CI:1.0610.67).

Conclusions:In a large sample of community volunteers, p.C282Y homozygosity was associated with substantial risks of hepatic cancer and excess mortality in males. As haemochromatosis iron overload is preventable and treatable by phlebotomy, early case ascertainment is needed for hereditary haemochromatosis, including at the community level. Our findings support the case that p.C282Y homozygous individuals identified incidentally during DNA sequencing should be notified.

Funding: MRC MR/S009892/1.

J. Atkins: None. L.C. Pilling: None. J.A.H. Masoli: None. C. Kuo: None. J.D. Shearman: None. P.C. Adams: None. D. Melzer: None.

\section{P19.049.B}

Genetic loci that increase obesity risk but protect against cardiometabolic comorbidities are embedded in regulatory regions of adipocyte function

L. O. Huang ${ }^{\text {, A. Aauch }}{ }^{2,3}$, E. Mazzaferro ${ }^{4}$, C. S. Bayrak, S. Carribio ${ }^{6}$, M. Preuss ${ }^{5}$, N. Chami ${ }^{5}$, Z. Wang ${ }^{5}$, U. Schnick, N. Yang ${ }^{5}$, Y. Itan ${ }^{5}$, A. Vidal-Puig ${ }^{6,7}$, M. den Hoed ${ }^{4}, S$. Mandrup $^{3}$, T. O. Kilpeläinen ${ }^{1,8}$, R. J. F. Loos Lo, $^{5,90}$

${ }^{1}$ Novo Nordisk Foundation Center for Basic Metabolic Research, University of Copenhagen, Copenhagen N, Denmark, ${ }^{2}$ Molecular Endocrinology \& Stem Cell Research Unit, Department of Endocrinology and Metabolism, University of Southern Denmark, Odense, Denmark, ${ }^{3}$ Functional Genomics \& Metabolism Research Unit, Department of Biochemistry and Molecular Biology, University of Southern Denmark, Odense, Denmark, ${ }^{4}$ The Beijer Laboratory and Department of Immunology, Genetics and Pathology, Uppsala University and SciLifeLab, Uppsala, Sweden, ${ }^{5}$ The Charles Bronfman Institute for Personalized Medicine, Icahn School of Medicine at Mount Sinai, New York, NY, United States, ${ }^{6}$ Wellcome Trust Sanger Institute, Wellcome Trust Genome Campus, Hinxton, United Kingdom, ${ }^{7}$ Metabolic Research Laboratories, Addenbrooke's Treatment Centre, Institute of Metabolic Science, Addenbrooke's Hospital, University of Cambridge, Cambridge, United Kingdom, ${ }^{8}$ Department of Environmental Medicine and Public Health, Icahn School of Medicine at Mount Sinai, New York, NY, United States, ${ }^{9}$ The Genetics of Obesity and Related Metabolic Traits Program, The Icahn School of Medicine at Mount Sinai, New York, NY, United States, ${ }^{10}$ The Mindich Child Health and Development Institute, Icahn School of Medicine at Mount Sinai, New York, NY, United States 
Introduction: Obesity is typically associated with higher risk of cardiometabolic complications. However, some obese individuals, the metabolically healthy obese (MHO), do not suffer from cardiometabolic comorbidities. Recent evidence suggests that genetic factors may contribute to this paradoxical phenotype. We aim to 1) ascertain the genetic variants that predispose $\mathrm{MHO}, 2$ ) identify enriched tissues and gene sets of these variants, 3) examine regulatory roles of neighbouring eQTL and DNase1 hypersensitive sites of these variants, and 4) prioritize causal genes.

Materials \& Methods: We 1) used publicly available GWAS statistics and identified loci associated with pairwise combinations of three adiposity and eight cardiometabolic traits, 2) conducted DEPICT tissue and gene set enrichment analyses on identified loci, 3) examined nearby enhancer dynamics in DNase1 hypersensitive sites during in vitro adipogenesis, and 4) performed ingenuity pathway analysis to prioritize causal genes.

Results: We 1) identified 62 independent loci reaching genome-wide significance, 2) highlighted adipose tissue and liver as the most significantly enriched tissues, and the protein-protein interaction partners of $O A T$ gene as the most significantly enriched pathway, involved in glutamaterelated energy metabolism, 3) confirmed the 62 loci more likely overlap with adipose tissue eQTLs and DNase1 hypersensitive sites that increase accessibility during adipogenesis, 4) prioritized 61 putative causal genes may identify novel biomarkers and future drug targets.

Conclusions: Combining GWAS meta-analysis with follow-up studies, our findings provide unequivocal insights into the biological pathways that underlie MHO. Funding: Danish Council for Independent Research (DFF611000183), Novo Nordisk Foundation (NNF18CC0034900)

L.O. Huang: None. A. Rauch: None. E. Mazzaferro: None. C.S. Bayrak: None. S. Carribio: None. M. Preuss: None. N. Chami: None. Z. Wang: None. U. Schnick: None. N. Yang: None. Y. Itan: None. A. Vidal-Puig: None. M. den Hoed: None. S. Mandrup: None. T.O. Kilpeläinen: None. R.J.F. Loos: None.

\section{P19.051.A}

The importance of Human Knockouts in a deeper characterization of Mendelian disorders

\author{
B. Spedicati ${ }^{l}$, F. Faletra ${ }^{2}$, R. Palmisano ${ }^{1}$, C. Barbieri ${ }^{3}$, G.

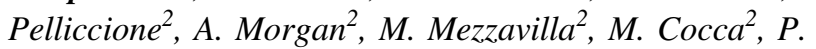 \\ Gasparini $^{1,2}$, G. Girotto $^{1,2}$
}

${ }^{1}$ Department of Medicine, Surgery and Health Sciences, University of Trieste, Trieste, Italy, ${ }^{2}$ Medical Genetics, Institute for Maternal and Child Health - I.R.C.C.S. "Burlo Garofolo", Trieste, Italy, ${ }^{3}$ Division of Genetics and Cell
Biology, San Raffaele Scientific Institute, Milan, Italy

Rare biallelic Loss of Function (LoF) variants leading to Human Knockout (HKO) might be efficiently characterized studying inbred populations, specifically genetic isolates. WGS data of 947 samples from three Italian isolated cohorts have been analysed to select biallelic LoF variants; after filtering, 28 variants in "autosomal recessive" genes were retained. We identified two groups of HKO: a) individuals showing clinical features of Mendelian recessive disorders but without a previous genetic diagnosis and b) subjects who do not show any clinical characteristic signs/symptoms. In group a) interesting examples involve three genes and three individuals respectively: Patient-1, KO for GPR68 gene and showing amelogenesis imperfecta clinical features, Patient-2 KO for F12 gene and presenting increased APTT values with normal wound healing, Patient-3 KO for $C 7$ gene and displaying an increased susceptibility to systemic infections. In group b) Patient-4, KO for FANCL gene, despite a positive chromosome fragility assay, does not display Fanconi-Anemia hematological features but only a clinical history of head/neck carcinoma and a short stature. We are currently expanding our collection of KO individuals detecting subjects who are compound heterozygous carriers of LoF mutations in genes involved in autosomal recessive disorders and, so far, 54 individuals have been detected. Functional studies to evaluate the potential phenotypic consequences of this variants will be performed. In conclusion, thanks to the study of HKO we lay the foundation for preventive strategies (as in the case of group a) but also for searching new protective variants/genes and therapeutic approaches for the study of Mendelian disorders.

B. Spedicati: None. F. Faletra: None. R. Palmisano: None. C. Barbieri: None. G. Pelliccione: None. A. Morgan: None. M. Mezzavilla: None. M. Cocca: None. P. Gasparini: None. G. Girotto: None.

\section{P19.052.B}

The genetic landscape of hypertension - results from a 5690 Romanian individuals
R. Ursu ${ }^{1,2,3}$, P. Iordache ${ }^{4}$, V. Radoi ${ }^{1,2,5}$, G. Ursu ${ }^{6}$, N. Cucu ${ }^{7}$, V. Chirica ${ }^{8}$, D. Iacob ${ }^{9}$, C. Sima ${ }^{10}$, O. Dragoi ${ }^{11}$, O. Croitoru $^{12}$, E. Poenaru ${ }^{12}$, L. Bohiltea ${ }^{1,5}$, A. Manolescu $^{13}$, V. Jing ${ }^{12,10}$
1"Carol Davila" University of Medicine and Pharmacy, Department of Medical Genetics, Bucharest, Romania, ${ }^{2}$ Synevo Romania, Central Reference Laboratory, Depart- ment of Medical Genetics, Bucharest, Romania, ${ }^{3}$ National Institute for Mother and Child Health "Alessandrescu- Rusescu", Bucharest, Romania, "Carol Davila" 
University of Medicine and Pharmacy, Department of Epidemiology, Bucharest, Romania, ${ }^{5}$ National Institute for Mother and Child Health "Alessandrescu-Rusescu", Bucharest, Romania, ${ }^{6}$ National Authority of Quality Management in Health, Bucharest, Romania, ${ }^{7}$ University of Bucharest, Faculty of Biology, Department of Genetics, Bucharest, Romania, " Mina Minovici" National Institute of Legal Medicine, Bucharest, Romania, ${ }^{9}$ The National Institute for Infectious Diseases "Matei Bals", Bucharest, Romania, "10"Prof. Dr. Theodor Burghele" Clinical Hospital, Department of Urology, Bucharest, Romania, ${ }^{11}$ Fundeni Clinical Institute, Department of Haematology, Bucharest, Romania, "12"Carol Davila" University of Medicine and Pharmacy, Bucharest, Romania, ${ }^{13}$ Reykjavik University, School of Science and Engineering, Reykjavik, Iceland

Background: The purpose of this study is to idetify highrisk variants associated with hypertension (HTA) in the Romanian population.

The current presentation asseses the final results of a 3 part study comprising the first and the largest GWAS on hypertension in Romanians.

Material and Methods: The total cohort includes a number of 5690 individuals, of which 2190 with hypertension and 3500 heathy controls. Genetic testing was performed at DeCode Genetics, Reykjavik, Iceland. A multiple GWAS assay has been performed for the identification of variants associated with hypertension, HTA risk factors (smoking, alcohol, coffee consumption) and HTA comorbidities (obesity, diabetes mellitus).

Results: Tissue-specific protein expression, gene function and gene-gene interactions have been analyzed for assessing a possible biological explanation for the association between the identified variants and HTA. Expression quantitative trait loci (eQTL) were assessed for variants in the reported locations for a better understanding of their involvement in HTA. The results revealed over 5000 genetic variants correlated with HTA, some well documented and in genes known to be involved in HTA (clusters on chromosomes 1p,1q,3q,4p,5q,7q,12p,15q,17q,20q,a.o. or CRNKL1,C19Orf12,CCDC51,C20Orf26,ZNF420,

ZNF571, a.o. intragenic variants). Approx. 4100 SNVs were identified in correlation with diabetes mellitus (DM) and obesity. Variants correlated with both HTA and DM were identified (TBX20, ANK2, a.o. genes). Two other clusters on chromosomes $19 q 12$ and 20p11.21 revealed associations with both HTA and obesity.

Conclusions: The results revealed several gene clusters correlated with HTA. The validity of these results for the Romanian population need to be confirmed by replication studies. The research is part of EU ProMark, ROMCAN and AppGenEdu projects.
R. Ursu: None. P. Iordache: None. V. Radoi: None. G. Ursu: None. N. Cucu: None. V. Chirica: None. D. Iacob: None. C. Sima: None. O. Dragoi: None. O. Croitoru: None. E. Poenaru: None. L. Bohiltea: None. A. Manolescu: None. V. Jinga: None.

\section{P19.053.C}

Human leukocyte antigen-wide association metaanalysis of idiopathic pulmonary fibrosis susceptibility

M. L. Paynton ${ }^{1}$, R. J. Allen ${ }^{1}$, J. Oldham ${ }^{2}, S . M a^{3}, R$. Braybrooke $^{4,5}$, UK ILD Consortium, I. Sayers ${ }^{5,6}$, I. P.

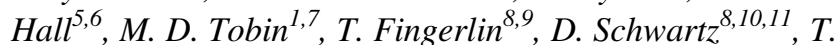
M. Maher ${ }^{12,13}$, P. Molyneaux ${ }^{12,13}$, Y. Zhang ${ }^{14}, N$.

Kaminski $^{15}$, B. Guillen-Guio ${ }^{16}$, C. Flores ${ }^{16,17,18}$, I. Noth $^{3}$, R. G. Jenkins ${ }^{6}$, E. J. Hollox ${ }^{19}$, L. V. Wain ${ }^{1,7}$

${ }^{1}$ Department of Health Sciences, University of Leicester, Leicester, United Kingdom, ${ }^{2}$ Department of Internal Medicine, University of California Davis, Davis, CA, United States, ${ }^{3}$ Division of Pulmonary \& Critical Care Medicine, University of Virginia, Charlottesville, VA, United States, ${ }^{4}$ Division of Epidemiology and Public Health, University of Nottingham, Nottingham, United Kingdom, ${ }^{5}$ National Institute for Health Research, Nottingham Biomedical Research Centre, Nottingham University Hospitals, Nottingham, United Kingdom, ${ }^{6}$ Division of Respiratory Medicine, University of Nottingham, Nottingham, United Kingdom, ${ }^{7}$ National Institute for Health Research, Leicester Respiratory Biomedical Research Centre, Glenfield Hospital, Leicester, United Kingdom, ${ }^{8}$ Center for Genes, Environment and Health, National Jewish Health, Denver, CO, United States, ${ }^{9}$ Department of Biostatistics and Informatics, University of Colorado, Denver, CO, United States, ${ }^{10}$ Department of Medicine, University of Colorado, Denver, CO, United States, ${ }^{11}$ Department of Immunology, University of Colorado, Denver, CO, United States, ${ }^{12}$ National Institute for Health Research, Respiratory Clinical Research Facility, Royal Brompton Hospital, London, United Kingdom, ${ }^{13}$ National Heart and Lung Institute, Imperial College, London, United Kingdom, ${ }^{14}$ Division of Pulmonary, Allergy and Critical Care Medicine and Simmons Center for Interstitial Lung Diseases, University of Pittsburgh School of Medicine, Pittsburgh, PA, United States, ${ }^{15}$ Section of Pulmonary, Critical Care and Sleep Medicine, Yale School of Medicine, New Haven, CT, United States, ${ }^{16}$ Research Unit, Hospital Universitario N.S. de Candelaria, Universidad de La Laguna, Santa Cruz de Tenerife, Spain, ${ }^{17}$ CIBER de Enfermedades Respiratorias, Instituto de Salud Carlos III, Madrid, Spain, ${ }^{18}$ Genomics Division, Instituto Tecnológico y de Energías Renovables, Santa Cruz de Tenerife, Spain, 
${ }^{19}$ Department of Genetics and Genome Biology, University of Leicester, Leicester, United Kingdom

Idiopathic pulmonary fibrosis (IPF) is a progressive interstitial lung disease with poor prognosis and unknown cause. The human leukocyte antigen (HLA) region is one of the most complex regions in the genome and influences the risk of inflammatory and respiratory conditions. The extreme genetic diversity of the HLA challenges the interpretation of association studies, therefore specific imputation strategies exist to capture HLA alleles and amino acid changes. The $H L A-D Q B 1 * 06: 02$ allele has been previously associated with fibrotic idiopathic interstitial pneumonias.

We combined bespoke HLA imputation with the latest SNP imputation (using the Haplotype Reference Consortium panel). We tested the association of 39,570 SNPs, HLA alleles and amino acid changes across the HLA region with IPF susceptibility in 1,905 IPF cases and 13,876 controls from three datasets (UK [612 cases, 3,366 controls], Chicago [500 cases, 510 controls] and UUS [793 cases and 10,000 controls]). Sex and 10 principal components were included as covariates. The results were meta-analysed using a fixed-effects weighted analysis.

An intronic variant near ZNRDIASP (rs3132684, $\mathrm{OR}=1.24(1.14-1.35), \quad P=2.41 \times 10^{-7}$ ) passed a Bonferroni corrected significance threshold $\left(\mathrm{P}<2.8 \times 10^{-6}\right)$ in this meta-analysis. rs3132684 was consistently significant $(\mathrm{P}<0.05)$ across all three datasets and is possibly an eQTL for several classical and non-classical HLA genes. The HLA-DQB1*06:02 was not associated with IPF $(P=0.57)$.

We present the largest study of HLA variation in IPF susceptibility to date, using a combination of SNP imputation and HLA imputation. One variant was consistently associated with IPF susceptibility in our data, further analysis is required to determine the functional effect.

M.L. Paynton: None. R.J. Allen: None. J. Oldham: B. Research Grant (principal investigator, collaborator or consultant and pending grants as well as grants already received); Significant; NHLBI. C. Other Research Support (supplies, equipment, receipt of drugs or other in-kind support); Modest; Boehringer Ingelheim, Genentech. S. Ma: None. R. Braybrooke: None. I. Sayers: B. Research Grant (principal investigator, collaborator or consultant and pending grants as well as grants already received); Significant; GlaxoSmithKline, Anaptysbio Inc., Boehringer Ingelheim. I.P. Hall: B. Research Grant (principal investigator, collaborator or consultant and pending grants as well as grants already received); Significant; Medical Research Council, GlaxoSmithKline, Boehringer Ingelheim. M.D. Tobin: B. Research Grant (principal investigator, collaborator or consultant and pending grants as well as grants already received); Significant; GlaxoSmithKline,
Orion Pharma. T. Fingerlin: None. D. Schwartz: A. Employment (full or part-time); Significant; Eleven P15, Inc.. B. Research Grant (principal investigator, collaborator or consultant and pending grants as well as grants already received); Significant; NIH-NHLBI, DOD Focused Program Grant. E. Ownership Interest (stock, stock options, patent or other intellectual property); Significant; US Patent serial no: 8,673,565;. T.M. Maher: B. Research Grant (principal investigator, collaborator or consultant and pending grants as well as grants already received); Significant; GlaxoSmithKline, UCB, Astra Zeneca. C. Other Research Support (supplies, equipment, receipt of drugs or other in-kind support); Modest; Boehringer Ingelheim, Roche, Bayer, Prometic, Apellis, Samumed, Galapagos, Celgene, Indalo, Pliant, Blade Therapeutics, Respivant, Novartis, Bristol-Myers Squibb. P. Molyneaux: C. Other Research Support (supplies, equipment, receipt of drugs or other in-kind support); Modest; Boehringer Ingelheim, Hoffman-La Roche, AstraZeneca. Y. Zhang: None. N. Kaminski: C. Other Research Support (supplies, equipment, receipt of drugs or other in-kind support); Modest; Biogen Idec, Boehringer Ingelheim, Third Rock, Miragen, Pliant, Samumed, NuMedii, Indaloo, Theravance, LifeMax, Three Lake Partners, Optikira. E. Ownership Interest (stock, stock options, patent or other intellectual property); Significant; New Threapies in Pulmonary Fibrosis. B. Guillen-Guio: None. C. Flores: None. I. Noth: B. Research Grant (principal investigator, collaborator or consultant and pending grants as well as grants already received); Significant; NIH. C. Other Research Support (supplies, equipment, receipt of drugs or other inkind support); Modest; Genentech, Boehringer Ingelheim. R.G. Jenkins: B. Research Grant (principal investigator, collaborator or consultant and pending grants as well as grants already received); Significant; GlaxoSmithKline, Astra Zeneca, Biogen, Galecto. C. Other Research Support (supplies, equipment, receipt of drugs or other in-kind support); Modest; Boehringer Ingelheim, Galapagos, Heptares, Numedii, Pliant, Promedior, Redex, Roche. E.J. Hollox: None. L.V. Wain: B. Research Grant (principal investigator, collaborator or consultant and pending grants as well as grants already received); Significant; GlaxoSmithKline.

\section{P19.055.B}

Influence of the genetic liability on the association between infections and autism

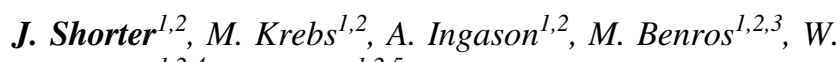
Thompson $^{1,2,4}$, T. Werge $e^{1,2,5}$

${ }^{1}$ Institute of Biological Psychiatry, Copenhagen, Denmark, ${ }^{2}$ iPSYCH, The Lundbeck Foundation Initiative for 
Integrative Psychiatric Research, Copenhagen, Denmark, ${ }^{3}$ Psykiatrisk Center Kobenhavn, Mental Health Services in the Capital Region of Denmark, Copenhagen, Denmark, ${ }^{4}$ University of California, San Diego, San Diego, CA, United States, ${ }^{5}$ Department of Clinical Medicine, University of Copenhagen, Copenhagen, Denmark

Several recent studies have suggested a role of infections in the development of mental health disorders. A genetic liability, measured through Polygenic Risk Score (PRS), for infections and mental health disorders could also drive this association. Here, we studied the genetic liability on the association between several different types of infections that led to hospital visits, with the risk of being diagnosed with autism spectrum disorder (ASD). To study this association, we used the Danish-based iPSYCH case-control study population, as well as a cohort of randomly sampled individuals from the Danish population. We observe that having certain types of infection, but not all types, can increase the lifetime risk of autism for those with a genetic liability. This risk for ASD diagnosis following a severe infection was the highest for those with the highest PRS for ASD

J. Shorter: None. M. Krebs: None. A. Ingason: None. M. Benros: None. W. Thompson: None. T. Werge: None.

\section{P19.056.C}

Evaluating the causal effects of protein biomarkers on inflammatory diseases

\section{W. E. Ek, T. Karlsson, J. Höglund, M. Rask-Andersen, Å. Johansson}

Department of Immunology, Genetics and Pathology, Uppsala, Sweden

Protein biomarkers are indicators, often measured in blood, that are used to estimate severity or presence of disease. A number of inflammatory protein biomarkers have been identified to be associated with development of inflammatory diseases or disease outcomes. These proteins might therefore serve as potential drug targets, or shed light into the pathogenesis of the diseases. However, if the biomarker itself increase the risk (is causal) of getting the disease is usually not known. Here we applied a two sample Mendelian randomization (MR) approach, using data from two cohorts (NSPHS and UK Biobank), to investigate the causal effect of 20 inflammatory protein biomarkers, known or suggested, on 18 inflammatory diseases. We identified five statistically significant biomarkers (IL-12B, IL-15RA, TNFB, TWEAK, VEGF-A), having a tentative causal effect on eight different inflammatory diseases (FDR $\leq 0.05$ ). IL12B showed a causal effect on psoriatic arthritis, IL-15RA on eczema, TNFB on Type 1 diabetes, rheumatoid arthritis and inflammatory polyarthropathies. We also identified a causal effect of TWEAK on asthma and a causal effect of VEGF-A on both inflammatory bowel disease and its sub phenotype ulcerative colitis. The causal effects on disease progress investigated here, may be used to further evaluate the potential of these proteins as drug targets

This project is funded by SciLifeLab, the Swedish Research Council (VR), the Åke Wiberg, M Borgström, A, $M$ Rudbergs, and the Swedish Heart and Lung foundation.

W.E. Ek: None. T. Karlsson: None. J. Höglund: None. M. Rask-Andersen: None. A. Johansson: None.

\section{P19.057.A}

\section{Inheritance patterns of polygenic risk scores}

M. J. Knol, E. A. Loehrer, R. A. Oldenburg, R. J. H. Galjaard, J. G. J. van Rooij, M. F. van Dooren, A. G. Uitterlinden, R. M. W. Hofstra, S. Lamballais, H. H. H. Adams

Erasmus MC University Medical Center, Rotterdam, Netherlands

Introduction: Genome-wide association studies have identified genetic variants that, together in polygenic risk scores (PRS), confer substantial risk for many complex traits. While clinical implementation of PRS is underway, it remains unclear how such scores propagate in pedigrees. Here, we determine the inheritance patterns of PRS to aid clinical counselling of genetic liability for complex traits.

Methods: We simulated polygenic risk for six complex diseases in 10,000 multigenerational families, modelling both random and consanguineous mating. We determined inheritance patterns for up to ten generations. Furthermore, we examined biparental inheritance patterns.

Results: Correlations of polygenic risk ranged from $47.1 \%$ to $49.0 \%$ for between first-degree relatives and from $23.1 \%$ to $24.3 \%$ for second-degree relatives (Table 1). When both parents' PRS is known, the ranges become narrower for their offspring, especially for the extremes (Table 2). In high-risk ( $>95 \%)$ individuals, median polygenic risk for first- and second-degree offspring was respectively $85.2 \%$ and $69.7 \%$, and approximated population average in the fifth generation. Results were similar across traits, and for random and consanguineous mating.

Discussion: Extreme polygenic risk propagates for multiple generations and is particularly informative in biparental inheritance. Future steps include validation in real multigenerational datasets and extension to larger number of genetic variants.

Table 1. Correlations between the polygenic risk of the $1^{\text {st }}$ and both the $2^{\text {nd }}$ and $3^{\text {rd }}$ generation. 


\begin{tabular}{|c|c|c|c|c|}
\hline Trait & $\begin{array}{l}\text { SNP-based } \\
\text { heritability }\end{array}$ & $\begin{array}{l}\text { Number of genetic } \\
\text { variants }\end{array}$ & $\begin{array}{l}\text { Correlation } 1^{\text {st }} \text { and } 2^{\text {nd }} \\
\text { generation }\end{array}$ & $\begin{array}{l}\text { Correlation } 1 \text { st and } 3^{\text {rt }} \\
\text { generation }\end{array}$ \\
\hline Atrial fibrillation & $21.8 \%$ & 94 & $47.5 \%$ & $23.5 \%$ \\
\hline $\begin{array}{l}\text { Age-related macular } \\
\text { degeneration }\end{array}$ & $11.6 \%$ & 34 & $48.4 \%$ & $24.2 \%$ \\
\hline Breast cancer & $30.4 \%$ & 172 & $48.9 \%$ & $24.2 \%$ \\
\hline Coronary artery disease & $14.8 \%$ & 86 & $49.0 \%$ & $24.2 \%$ \\
\hline $\begin{array}{l}\text { Inflammatory bowel } \\
\text { disease }\end{array}$ & $11.1 \%$ & 232 & $47.1 \%$ & $23.1 \%$ \\
\hline Type 2 diabetes & $44.8 \%$ & 403 & $48.7 \%$ & $24.3 \%$ \\
\hline
\end{tabular}

Table 2. Multigenerational inheritance of breast cancer* polygenic risk, showing median (interquartile range) percentile scores.**

\begin{tabular}{|c|c|c|c|c|c|c|}
\hline \multicolumn{2}{|l|}{$\begin{array}{l}\text { First } \\
\text { generation } \\
\text { polygenic } \\
\text { risk }\end{array}$} & $0-5 \%$ & $25-30 \%$ & $50-55 \%$ & $75-80 \%$ & $95-100 \%$ \\
\hline \multicolumn{7}{|c|}{ Multigenerational polygenic risk of offspring, given random mating } \\
\hline \multirow[t]{9}{*}{ Generation } & 2 & $15.6(5.1-32.2)$ & $38.1(17.5-61.1)$ & $52.6(30.4-74.0)$ & $65.1(44.2-82.8)$ & $85.2(67.7-95.0)$ \\
\hline & 3 & $29.1(11.9-54.5)$ & $41.7(18.3-68.2)$ & $52.5(27.2-76.8)$ & $57.0(31.4-78.8)$ & $69.7(44.3-88.2)$ \\
\hline & 4 & $38.9(17.2-64.9)$ & $46.0(21.9-71.1)$ & $50.7(24.9-74.6)$ & $53.5(28.5-78.0)$ & $59.6(32.4-81.8)$ \\
\hline & 5 & $44.8(21.1-71.5)$ & $48.2(23.5-74.0)$ & $50.8(25.4-75.6)$ & $52.6(27.5-76.5)$ & $54.6(28.1-78.4)$ \\
\hline & 6 & $47.5(23.0-72.8)$ & $49.2(24.4-74.6)$ & $50.2(24.9-74.6)$ & $51.7(25.9-76.4)$ & $52.1(26.7-76.5)$ \\
\hline & 7 & $49.0(24.4-74.2)$ & $49.3(24.5-74.6)$ & $49.9(25.1-75.1)$ & $50.8(25.6-75.5)$ & $51.1(25.7-75.6)$ \\
\hline & 8 & $49.8(24.9-75.0)$ & $49.9(25.0-74.6)$ & $50.3(25.0-75.1)$ & $50.6(25.5-75.4)$ & $50.8(25.3-75.6)$ \\
\hline & 9 & $49.9(24.9-74.8)$ & $49.7(24.7-74.8)$ & $50.3(25.0-75.2)$ & $50.3(25.2-75.2)$ & $50.3(25.3-75.4)$ \\
\hline & 10 & $50.0(25.0-75.0)$ & $49.9(24.9-74.8)$ & $50.1(24.9-75.2)$ & $50.0(24.9-75.0)$ & $50.2(25.0-75.1)$ \\
\hline \multicolumn{7}{|c|}{ Polygenic risk for second generation, given first-generation consanguineous parents } \\
\hline \multirow[t]{2}{*}{ Consanguinity } & $\begin{array}{l}\text { Second- } \\
\text { degree }\end{array}$ & $12.5(4.0-28.9)$ & $36.6(18.2-59.4)$ & $51.3(29.0-72.9)$ & $66.7(43.9-84.4)$ & $87.7(71.1-96.0)$ \\
\hline & $\begin{array}{l}\text { Third- } \\
\text { degree }\end{array}$ & $14.7(4.9-32.5)$ & $38.0(18.6-60.9)$ & $51.3(29.0-73.0)$ & $65.2(42.3-83.6)$ & $85.8(68.3-95.2)$ \\
\hline \multicolumn{7}{|c|}{ Polygenic risk for second generation, given polygenic risk of second } \\
\hline \multirow[t]{5}{*}{$\begin{array}{l}\text { Polygenic risk } \\
\text { second parent }\end{array}$} & $0-5 \%$ & $1.9(0.5-5.7)$ & $9.4(3.4-20.5)$ & $15.9(6.6-30.6)$ & $26.6(13.4-44.4)$ & $51.7(31.5-70.1)$ \\
\hline & $25-30 \%$ & $9.4(3.4-20.5)$ & $28.2(14.2-45.7)$ & $39.7(23.1-58.3)$ & $53.7(34.9-71.0)$ & $77.3(59.5-89.5)$ \\
\hline & $50-55 \%$ & $15.9(6.6-30.6)$ & $39.7(23.1-58.3)$ & $51.6(33.1-69.5)$ & $65.9(47.4-81.1)$ & $85.8(71.8-94.3)$ \\
\hline & $75-80 \%$ & $26.6(13.4-44.4)$ & $53.7(34.9-71.0)$ & ) $65.9(47.4-81.1)$ & $77.9(61.2-89.1)$ & $91.9(82.4-97.2)$ \\
\hline & $95-100 \%$ & $51.7(31.5-70.1)$ & $77.3(59.5-89.5)$ & 85.8 (71.8-94.3) & 91.9 (82.4-97.2) & $98.0(93.7-99.4)$ \\
\hline
\end{tabular}

* Similar patterns were observed for the other traits.

**The top part of the table assumes knowledge of one parent's polygenic risk (with both random and consanguineous mating), whereas the bottom part assumes knowledge of both parents' polygenic risk.

M.J. Knol: None. E.A. Loehrer: None. R.A. Oldenburg: None. R.J.H. Galjaard: None. J.G.J. van Rooij: None. M.F. van Dooren: None. A.G. Uitterlinden: None. R.M.W. Hofstra: None. S. Lamballais: None. H.H.H. Adams: None.

\section{P19.059.C}

Comparative analysis of WGS data from Bulgarian centenarians and findings from meta-GWA studies reveals genetic loci associated with longevity

\section{T. Balabanski ${ }^{1,2}$, D. Serbezov ${ }^{l}$, R. Vazharova ${ }^{2,3}, S$.} Karachanak-Yankova ${ }^{l}$, R. Staneva ${ }^{l}$, M. Mihaylova ${ }^{l}, V$. Damyanova $^{l}$, D. Nesheva ${ }^{l}$, Z. Hammoudeh ${ }^{l}$, M. Atanasoska $^{2}$, M. Ganev ${ }^{l}$, O. Antonova ${ }^{l}$, V. Spasova ${ }^{l}, D$. Nikolova ${ }^{l}$, S. Hadjidekova ${ }^{l}$, D. Toncheva ${ }^{1,2}$

${ }^{1}$ Department of Medical Genetics, Medical University of Sofia, Sofia, Bulgaria, ${ }^{2}$ Genomic Laboratory, Malinov Clinic, Sofia, Bulgaria, ${ }^{3}$ Medical Faculty, Sofia University "St. Kliment Ohridski”, Sofia, Bulgaria
Human lifespan is a complex, multifactorial trait resulting from the interplay of many genetic variants with small effect and a wide range of environmental factors. It is estimated that the heritability of longevity in humans could be up to $25 \%$. To identify potential factors that contribute to this phenotype we performed whole-genome sequencing on DNA samples from 16 centenarians from the Bulgarian population. Their genotypes were extracted at more than 20 genetic loci associated with high parental lifespan - an appropriate measure for heritable longevity, from two large GWAS meta-analyses (AncestryDNA and LifeGen) utilising the UK Biobank and their own cohorts. We established that 5 genetic variants were significantly enriched within our centenarian cohort compared to individuals of nonFinnish European ancestry in the gnomAD population database. Only one of them was exonic - the wellrecognised rs429358-T in the $A P O E$ gene, while the other ones: rs10455872-A in LPA, rs6224-G in FURIN, rs599839-G between CELSR2 and PSRC1 and rs9872864$\mathrm{G}$ in $I P 6 K 1$, were non-coding suggesting that mechanisms regulating gene expression might play a role in longevity. Interestingly, all these genes are involved in different cardiometabolic conditions. Combinations of these variants, as well as with other factors, could therefore be protective against cardiovascular risk factors - a common cause of death in many older people, or could potentially slow down the process of biological ageing. A larger cohort of precious samples from centenarians could validate these findings and provide one more piece of the complex puzzle of human longevity.

Grant reference: DN03/7(18.12.2016), NSF, Bulgaria

L.T. Balabanski: None. D. Serbezov: None. R. Vazharova: None. S. Karachanak-Yankova: None. R. Staneva: None. M. Mihaylova: None. V. Damyanova: None. D. Nesheva: None. Z. Hammoudeh: None. M. Atanasoska: None. M. Ganev: None. O. Antonova: None. V. Spasova: None. D. Nikolova: None. S. Hadjidekova: None. D. Toncheva: None.

\section{P19.060.A}

Analysis of population-specific protein-truncating variants in gnomAD suggests novel criteria for annotation of loss-of-function alleles

\section{Y. A. Barbitoff ${ }^{1,2,3}$, R. K. Skitchenko ${ }^{1,2,4}$, J. S. Kornienko ${ }^{l}$, E. M. Maksiutenko ${ }^{3}$, A. V. Predeus ${ }^{1}$}

${ }^{1}$ Bioinformatics Institute, St. Petersburg, Russian Federation, ${ }^{2}$ Dpt. of Genomic Medicine, D.O.Ott Research Institute of Obstetrics, Gynecology, and Reproduction, St. Petersburg, Russian Federation, ${ }^{3}$ Dpt. of Genetics and Biotechnology, St. Petersburg State University, St. 
Petersburg, Russian Federation, ${ }^{4}$ ITMO University, St. Petersburg, Russian Federation

Putative loss-of-function (pLoF) variants are of ultimate relevance to Mendelian disease pathology. As such variants in disease-related genes are under strong negative natural selection, their frequency across major ancestral groups is expected to be highly similar. In this study, we tested this assumption by systematically assessing the presence of highly prevalent population-specific PTVs in human genes using gnomAD data. We discovered unusually high incidence of population-specific $\mathrm{pLoF}$ variants in all major ancestral groups, with a total of 1,972 genes harboring such population-specific sites. Such prevalence of PTVs does not conform to a deterministic statistical model developed by Cassa et al. in 2017, indicating either systematic differences in disease penetrance in different human populations or the failure of current annotation criteria to accurately predict the loss-of-function potential of PTVs. We demonstrate that high-frequency population-specific PTVs tend to reside in exons that have low conservation scores and belong to transcripts with low expression levels, suggesting that these variants should not be treated as genuine LoF alleles. As such, additional data resources should be used during interpretation of genotypes in both population genetics and clinical genetics studies.

Y.A. Barbitoff: None. R.K. Skitchenko: None. J.S. Kornienko: None. E.M. Maksiutenko: None. A.V. Predeus: None.

\section{P19.061.B}

Causal relationship between adiponectin and diabetic retinopathy: a Mendelian randomization study in an Asian population

Y. C. Huang ${ }^{1,2}$, F. J. Tsai ${ }^{2,3}$, Y. W. Chang ${ }^{2}$, C. M. Wu ${ }^{l}$, W. L. Liao $^{4,5}$

${ }^{1}$ China Medical University, Taichung, Taiwan, ${ }^{2}$ Human Genetic Center, Department of Medical Research, China Medical University Hospital, Taichung, Taiwan, ${ }^{3}$ Department of Health and Nutrition Biotechnology, Asia University, Taichung, Taiwan, ${ }^{4}$ Graduate Institute of Integrated Medicine, China Medical University, Taichung, Taiwan, ${ }^{5}$ Center for Personalized Medicine, China Medical University Hospital, Taichung, Taiwan

Introduction: Adiponectin (APN), a cytokine released by adipocytes, is involved in different pathways, such as insulin sensitivity, inflammation, and atherogenesis. However, the association between APN and diabetic retinopathy (DR) has been inconsistent from observational studies. To clarify the causal relationship, we used a Mendelian randomization (MR) analysis to test the hypothesis that elevated circulating APN levels causally results in DR.

Materials and Methods: A GWAS database-catalog of 47 single nucleotide polymorphisms (SNPs) reaching significance in East-Asians was utilized in 1251 diabetic patients in Taiwan. APN-SNPs that were genetically independent, and without pleiotropic effect were selected as instrumental variables (IV). Including 3 different genetic risk scores (GRS): GRS $_{\text {All }}$ combined all 47 SNPs, GRS $_{\text {APN }}$ combined 5 SNPs significantly associated with APN level, and $\mathrm{GRS}_{\text {Limited }}$ comprised $16 \mathrm{GRS}_{\mathrm{All}}$-SNPs with a threshold $\left(\mathrm{P}<5 \times 10^{-8}\right.$ for GWAS).

Results: The MR-inverse-variance weighted method analysis showed that for each 1-SD increase in genetically induced increase in plasma APN, the OR of having DR was $\beta=0.20$ (95\% CI: $-0.46-0.85, P=0.553$ ) for $\mathrm{GRS}_{\mathrm{APN}}, 0.61$ (95\% CI: $0.10-1.13, P=0.020)$ for $\mathrm{GRS}_{\mathrm{All}}$, and 0.57 (95\% CI: -0.06 to $1.20, P=0.078$ ) for $\mathrm{GRS}_{\text {Limited }}$. Sensitivity analysis, including MR-egger regression and the weightedmedian approach, did not provide evidence of the pleiotropic effect of IVs.

Conclusions: Limited evidence for the causal role of APN in DR risk among diabetic patients was shown based on MR analysis in the present study. This work was supported by research grants from Ministry of Science and Technology of Taiwan.

Y.C. Huang: None. F.J. Tsai: None. Y.W. Chang: None. C.M. Wu: None. W.L. Liao: None.

\section{P19.064.B}

Investigating causal relations between sleep duration and risks of adverse obstetric outcomes: a Mendelian randomization study

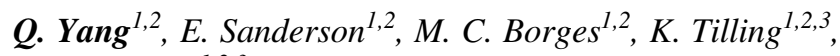
D. A. Lawlor ${ }^{1,2,3}$

${ }^{I}$ MRC Integrative Epidemiology Unit, University of Bristol, Bristol, United Kingdom, ${ }^{2}$ Population Health Sciences, Bristol Medical School, University of Bristol, Bristol, United Kingdom, ${ }^{3}$ NIHR Bristol Biomedical Centre, University Hospitals Bristol NHS Foundation Trust and University of Bristol, Bristol, United Kingdom

Introduction: Shorter sleep duration has been linked with higher risks of adverse obstetric outcomes, but it remains unclear whether those associations are causal.

Materials and Methods: We conducted a two-sample Mendelian randomization (MR) study with 78 single nucleotide polymorphisms for sleep duration using individual-participant data from UK biobank (208171 women), Avon longitudinal study of parents and children 
(7635 women) and born in Bradford (2977 women) to investigate effects of sleep duration on obstetric outcomes.

Results: Using inverse variance weighted method, there was little evidence to support an effect of sleep duration on the risk of pregnancy loss (odds ratio [OR] per additional hour/day $1.02,95 \%$ confidence interval [CI]: 0.92-1.14), stillbirth (OR 1.19, 95\% CI: 0.87-1.64), miscarriage (OR 0.99, 95\% CI: 0.88-1.12), gestational diabetes (OR 0.69, 95\% CI: 0.32-1.49), hypertensive disorders in pregnancy (OR 1.45, 95\% CI: 0.90-2.34), perinatal depression (OR $0.85,95 \%$ CI: $0.57-1.28)$, preterm birth (OR 0.80, 95\% CI: 0.37-1.70), low offspring birthweight (OR 0.93, 95\% CI: 0.63-1.37) or high offspring birthweight (OR 0.82, 95\% CI: 0.52-1.29) Sensitivity analyses using MR-Egger, weighted median and weighted mode methods provided similar estimates.

Conclusions: We did not find consistent evidence supporting linear effects of sleep duration on obstetric outcomes. However, even with large sample sizes some estimates are imprecise, and some point estimates suggest a potentially important clinical effect (e.g. $45 \%$ increased odds of hypertensive disorders. In two-sample MR we cannot explore the possibility of non-linear effects. All authors work in a Unit supported by UK Medical Research Council (MC_UU_00011/3, MC_UU_00011/6).

Q. Yang: None. E. Sanderson: None. M.C. Borges: None. K. Tilling: None. D.A. Lawlor: B. Research Grant (principal investigator, collaborator or consultant and pending grants as well as grants already received); Modest; Medtronic, Roche Diagnostics.

\section{P19.065.C}

Clostridium shows a higher abundance in less neurovascular and neurodegenerative aspects: a microbiomewide association study

H. Karamujić-Čomić ${ }^{l}$, S. Ahmad ${ }^{l}$, D. Radjabzadeh ${ }^{l}, B$. Bonnechere $^{2}$, R. Kaddurah-Daouk ${ }^{3}$, A. G. Uitterlinden ${ }^{1}, R$.

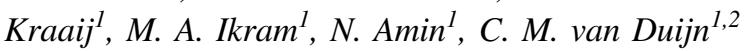

${ }^{1}$ Erasmus University Medical Center, Rotterdam, Netherlands, ${ }^{2}$ University of Oxford, Oxford, United Kingdom, ${ }^{3}$ Duke University, Durham, NC, United States

Introduction: There is an important interplay between the intestinal microbiome and brain, commonly known as the gut-brain-axis. Several studies addressed the role of the microbiome in neurologic diseases. Here, we studied the role of stool microbiota in cognitive function, neurovascular aspects, and neurodegenerative aspects in the general population.

Methods: In the population-based Rotterdam Study, we studied the association between stool microbial taxa and cognitive function, and neurovascular and neurodegenerative traits, measured on brain magnetic resonance imaging, in $\sim 1,200$ participants. Stool microbiota were profiled using $16 \mathrm{~s}$ rRNA gene sequencing. We used linear regression analysis, accounted for multiple testing, and adjusted for major confounders, i.e., age, sex, technical covariates, medication use, alcohol, smoking, body mass index, and hypertension.

Results: The genus Clostridium sensu stricto 1 showed a higher abundance in participants with better cognitive function $(\operatorname{beta}(\beta)$ (standard error(SE)) 0.023(0.01), $p=$ $\left.3.22 \times 10^{-2}\right)$, larger global brain volume $(\beta(\mathrm{SE}) 0.0019$ $\left.(0.0006), p=1.83 \times 10^{-3}\right)$, less white matter hyperintensities $\left(\beta(\mathrm{SE})-0.024(0.007), p=5.38 \times 10^{-4}\right)$, and smaller lateral ventricular volume $(\beta(\mathrm{SE})-0.013(0.005), p=$ $\left.1.81 \times 10^{-2}\right)$. The family Clostridiaceae 1 also showed a higher abundance in these traits, with the same direction of effect and very similar effect estimates. The class Clostridia, order Clostridiales, family Christensenellaceae, and genus Christensenellaceae $R 7$ group showed a higher abundance in better cognition. Of note is that the agreement of our findings with those of studies of Alzheimer's disease is high.

Conclusions: Clostridium shows a higher abundance in participants with better cognitive function, less neurovascular aspects, and less neurodegenerative aspects. We find that different microbiota are associated to neurovascular and neurodegenerative pathology.

H. Karamujić-Čomić: None. S. Ahmad: None. D. Radjabzadeh: None. B. Bonnechere: None. R. Kaddurah-Daouk: None. A.G. Uitterlinden: None. R. Kraaij: None. M.A. Ikram: None. N. Amin: None. C.M. van Duijn: None.

\section{P19.066.A}

The mitochondrial DNA landscape of Thracians from Bronze Age Bulgaria

D. V. Nesheva ${ }^{1}$, A. Modi ${ }^{2}$, S. Sarno ${ }^{3}, S . V^{2} i^{2}, S$. Karachanak-Yankova ${ }^{1}$, D. Luiselli ${ }^{4}$, E. Pilli ${ }^{2}$, M. Lari ${ }^{2}, C$. Vergata $^{2}, Y$. Yordanov ${ }^{5}$ D. Dimitrova 6 , P. Kalcev ${ }^{7}, R$.

Staneva ${ }^{l}$, O. Antonoval, S. Hadjidekova ${ }^{l}$, A. Galabov $^{8}, D$. Toncheva $^{l}$, D. Caramelli ${ }^{2}$

${ }^{1}$ Department of Medical genetics, Medical Faculty, Medical University - Sofia, Sofia, Bulgaria, ${ }^{2}$ Department of Biology, University of Florence, Florence, Italy, ${ }^{3}$ Department of Biological, Geological and Environmental Sciences, University of Bologna, Bologna, Italy, ${ }^{4}$ Department of Cultural Heritage, University of Bologna, Ravenna, Italy, ${ }^{5}$ Institute of Experimental Morphology, Pathology and Anthropology with Museum, Bulgarian Academy of Sciences, Sofia, Bulgaria, ${ }^{6}$ The Regional Historical Museum of Sliven, 
Sliven, Bulgaria, ${ }^{7}$ The Regional Historical Museum of Stara Zagora, Stara Zagora, Bulgaria, ${ }^{8}$ The Stephan Angeloff Institute of Microbiology, Bulgarian Academy of Sciences, Sofia, Bulgaria

Introduction: Thracians are one of the best documented civilizations that inhabited the Bulgarian lands during the Bronze Age. They left evidence in the rich cultural and historical heritage. By conducting molecular anthropological studies on Thracians mtDNA samples we aim to reveal their genetic characteristics and contribution to the gene pool of present day populations.

Materials and methods: We have reconstructed and analyzed complete mitochondrial genomes from 25 Bronze Age individuals sampled in three different Bulgarian necropolises. The molecular phylogenetic analyses were performed following strict guidelines and standards. The Thracian mitogenomes were compared with different modern and ancient populations for understanding possible genetic relationships.

Results: The obtained mtDNA sequences were assigned to 21 different haplogroups, representative of the mitochondrial variability of Western Eurasia. The presented haplogroups in our samples $\mathrm{H}, \mathrm{K}, \mathrm{J}$ and $\mathrm{T}$ arose in frequency in different European populations during the Neolithic. Sublineages of the haplogroup $U$ mark the genetic pool of European pre-LGM hunter-gatherers. The results support the hypothesis of Thracians origin from the local people with a low percentage of gene flow from the Steppe.

Conclusions: The Thracian maternal gene pool is mostly represented by Western Eurasian haplogroups. After comparing between the complete mitochondrial sequences of Thracians and Eurasian (ancient and contemporary) populations we observed that Thracians genetic profiles reflect their nexus geographical position between east and west. This study has an important implication for understanding the origins of the peopling in this part of Europe and for enlarging the knowledge on the ancient Bronze Age civilizations.

D.V. Nesheva: None. A. Modi: None. S. Sarno: None. S. Vai: None. S. Karachanak- Yankova: None. D. Luiselli: None. E. Pilli: None. M. Lari: None. C. Vergata: None. Y. Yordanov: None. D. Dimitrova: None. P. Kalcev: None. R. Staneva: None. O. Antonova: None. S. Hadjidekova: None. A. Galabov: None. D. Toncheva: None. D. Caramelli: None.

\section{P19.067.B}

Human mitochondrial eQTLs and the $m^{\mathbf{1}} \mathrm{A}$ 16S rRNA modification explain tissue-specific mitochondrial gene expression patterns
T. Cohen, C. Mordechai, A. Eran, D. Mishmar

\section{Ben Gurion University of the Negev, Beer Sheva, Israel}

Expression quantitative trait loci (eQTLs) are instrumental in genome-wide identification of regulatory elements, yet were overlooked in the mitochondrial DNA (mtDNA). By analyzing 5079 RNA-seq samples from 23 tissues we identified association of ancient mtDNA SNPs (haplogroups T2, L2, J2 and V) with tissue-dependent mtDNA gene-expression. Secondly, the discovery of mtDNA eQTLs in both coding and non-coding mtDNA regions, propose the identification of novel mtDNA regulatory elements. Third, we identified mostly negative correlation between $\mathrm{m}^{1} \mathrm{~A} 947$ 16S rRNA modification levels and mtDNA gene-expression in twelve body sites. Such association was compromised in skin which was exposed to sun, as compared to sun-unexposed skin from the same individuals, thus suggesting an impact of UV on mtDNA gene expression. Taken together, our findings reveal that both mtDNA SNPs and mt-rRNA modification affect mtDNA gene expression in a tissue-dependent manner.

T. Cohen: None. C. Mordechai: None. A. Eran: None. D. Mishmar: None.

\section{P19.071.C}

The incidence of phacomatoses in the Rostov region

A. V. Antonets ${ }^{l}$, S. S. Amelina ${ }^{l}$, E. V. Degtereva ${ }^{l}, S . V$. Mordanov $^{l}$, R. A. Zinchenko ${ }^{2}$

${ }^{1}$ Rostov Medical State University, Rostov-on-Don, Russian Federation, ${ }^{2}$ Medical Genetic Research Center named after Academician N.P. Bochkov, Moscow, Russian Federation

Introduction: Phacomatoses, also known as Neurocutaneous syndromes (NCS), include a heterogeneous group of disorders characterized by abnormalities of both the integument and central nervous system. Disorders classified as NCS include neurofibromatosis 1 (NF1), tuberous sclerosis (TS), von Hippel-Lindau (VHL) disease, PHACE syndrome, ataxia telangiectasia (AT), linear nevus syndrome (LNS), Sturge-Weber Syndrome (SWS), hypomelanosis of Ito (HOI), and incontinentia pigmenti (IP). Most disorders are inherited as autosomal dominant conditions with variable penetrance or can be caused by somatic mosaic mutation (SWS).

Materials and Methods: The purpose of the present research was studying of prevalence of phakomatosis in Rostov region (12 districts). The total number of investigated population was 497460 persons. The research was conducted under the original protocol providing for 
detection of more than 2500 various hereditary diseases (OMIM) and syndromes including all phacomatoses.

Results: The most frequent observed phacomatoses were NF1, TS, VHL, SWS accounted for 55, 15, 1, 7 cases correspondingly. The overall prevalence of phacomatoses in Rostov region was 1:6378, namely NF1 was 1:9045, TS 1:33164, VHL - 1:497460, SWS 1:71066. For some patients additional molecular testing was available. Conclusion. We estimated the incidence of phacomatoses in the Rostov region that could allow to plan genetic support. Phacomatoses should be considered by doctors of different specialties.

A.V. Antonets: None. S.S. Amelina: None. E.V. Degtereva: None. S.V. Mordanov: None. R.A. Zinchenko: None.

\section{P19.072.A}

\section{Genetic correlation between pigmentation traits in} european populations

\section{E. pairo-castineira ${ }^{l}, J$. Cornelissen $^{I}$, O. Canela-Xandri, K. Rawlik ${ }^{2}$, A. Tenesa ${ }^{1}$, I. Jackson ${ }^{1}$}

${ }^{I}$ MRC - Human genetics unit, Edinburgh, United Kingdom, ${ }^{2}$ Roslin Institute, Edinburgh, United Kingdom

Many of the biology of human pigmentation traits is shared between them, but there are some specific genes and pathways for each trait, and the similiarities and differences between them still need still to be understood. Recently we published a GWAS for hair colour variation in Europeans showing 8 genes related to red hair and more than 100 genes associated to blonde to black hair colour. Another GWAS found 20 loci related to UV sensitivity, and the largest study of skin colour variation in Europeans found 9 genes. In this study, we performed a GWAS of 452,264 UKBiobank European individuals for hair colour, skin colour, and ease of tanning, using DISSECT, calculated the genetic correlations and studied the commonalities and differences. We identified 655 independent SNPs related to red hair in 128 genes, many of them within $3 \mathrm{MB}$ of MC1R, but we find new associations in regions related to pigmentation such MITF, a transcription factor controlling the pigmentation, and MFSD12, a gene related to darker skin in African populations. Using Whole exome sequencing for 50,000 UKBiobank individuals, we identified $44 \mathrm{MC} 1 \mathrm{R}$ protein coding variants in red hair individuals, of whom 96.4\% had $2 \mathrm{MC} 1 \mathrm{R}$ variants. We found 519 genes related to hair colour, 338 to skin colour and 293 to ease of tanning, of which 161 were common, with enriched function for melanin synthesis (pval 10-7) and lipid-bidning proteins (pval 10-9). Hair colour specific genes (288) are enriched for keratinocyte-expressed genes $(\mathrm{pval}=)$ as we expected from the previous analysis

E. pairo-castineira: None. J. Cornelissen: None. 0. Canela-Xandri: None. K. Rawlik: None. A. Tenesa: None. I. Jackson: None.

\section{P19.073.B}

Irish demographic insights utilising rare variants captured by whole-genome-sequencing

\section{E. H. Gilbert ${ }^{1,2}$, A. Corvin $^{3}$, A. Helgason ${ }^{4,5}$, G. Cavalleri $^{1,2}$}

${ }^{1}$ Royal College of Surgeons in Ireland, Dublin, Ireland, ${ }^{2}$ FutureNeuro SFI Research Centre, RCSI, Dublin, Ireland, ${ }^{3}$ Trinity College Dublin, Dublin, Ireland, ${ }^{4}$ deCODE Genetics, Reykjavik, Iceland, ${ }^{5}$ University of Iceland, Reykjavik, Iceland

Genetic structure within Ireland has recently been demonstrated using common genetic variants. Irish genetic structure inferred by rarer variants is less well understood. A population's demographic history impacts the whole variant frequency spectrum, including rare variants. These variants are usually younger than common variation, and often have a functional impact. Understanding the distribution of rare variants in Ireland will therefore elucidate genetic history and disease risk in Ireland.

In this context, we generated 30x short-read wholegenome-sequencing and microarray genotype data on 880 Irish individuals, 280 of whom had genealogies mapped to specific regions of Ireland. We filtered variants by standard quality control thresholds and generated 'genotype counts' matrices which recorded the frequencies of each variant in each Irish region. In parallel we estimated the functional impact of each variant, and generated haplotype data from the microarray data on these individuals.

We investigated the impact of the demographic history of Ireland on the full spectrum of Irish genetic variation using this data. We report the different site frequency spectra for the four Irish provinces alongside analysis of haplotype data that estimates Irish population size for the last 40 generations, detecting evidence of a modest population size compared to England. Using rare variation to detect population structure, we calculated $\mathrm{F}_{\mathrm{ST}}$ distances and proportion of $f_{2}$ variants between Irish regions, revealing the structure of rare variant sharing in Ireland. Our results have implications for the research of rare disease variants within Ireland and populations of Irish ancestry and inform on Irish history.

E.H. Gilbert: None. A. Corvin: None. A. Helgason: None. G. Cavalleri: None. 


\section{P19.075.A}

The fine-scale genetic structure $\&$ adaptive positive selection in the Lithuanian population from high-density SNP data

\author{
A. Urnikyte ${ }^{1}$, A. Flores Bello ${ }^{2}$, M. Mondal ${ }^{3}$, A. Molyte $\dot{e}^{1}, D$. \\ Comas $^{2}$, F. Calafell ${ }^{2}$, E. Bosch ${ }^{2}$, V. Kucinskas ${ }^{1}$ \\ ${ }^{1}$ Vilnius University, Vilnius, Lithuania, ${ }^{2}$ Universitat Pom- \\ peu Fabra, Barcelona, Spain, ${ }^{3}$ Estonian Biocentre, Tartu, \\ Estonia
}

The analysis of geographically specific regions and the characterization of fine-scale patterns of genetic diversity may facilitate a much better understanding of the microevolutionary processes affecting local human populations. Here we generated genome-wide high-density SNP genotype data in 425 individuals from six geographical regions in Lithuania and combined our dataset with available ancient and modern data to explore genetic population structure, ancestry components and signatures of natural positive selection in the Lithuanian population. Our results show that Lithuanians are a homogenous population, genetically differentiated from neighbouring populations but within the general expected European context. Moreover, we not only confirm that Lithuanians preserve one of the highest proportions of western, Scandinavian and eastern hunter-gather ancestry components found in European populations but also that of an steppe Early to Middle Bronze Age pastoralists, which together configure the genetic distinctiveness of the Lithuanian population. Finally, among the top signatures of positive selection detected in Lithuanians, we identified several candidate genes related with diet $(P N L I P, P P A R D)$, pigmentation (SLC24A5, TYRP1 and PPARD) and the immune response (BRD2, HLA-DOA, IL26 and IL22).

This study was supported by LITGEN project, which was approved by the Vilnius Regional Research Ethics Committee No. 158200-05-329-79, date: 2011-05-03, and by Direcció General de Recerca, Generalitat de Catalunya (2017SGR702), Agencia Estatal de Investigación (AEI) and Fondo Europeo de Desarrollo Regional (FEDER) with project grants BFU2016-77961-P and CGL2016-75389-P, and the Unidad de Excelencia María de Maeztu", funded by the MINECO (ref: MDM-2014-0370).

A. Urnikyte: None. A. Flores Bello: None. M. Mondal: None. A. Molytè: None. D. Comas: None. F. Calafell: None. E. Bosch: None. V. Kucinskas: None.

\section{P19.076.B}

Positively selected TNKS haplotype: from evolutionary advantage to the health risk in an Arabian Peninsula population

\author{
E. Muthukrishnan ${ }^{\text {, }, ~ A . ~ L . ~ C . ~ d . ~ S a n t o s ~}{ }^{2,3}$, O. Gokcumen ${ }^{3}$, \\ F. Al-Mulla ${ }^{l}$, T. A. Thanaraj ${ }^{l}$ \\ ${ }^{1}$ Dasman Diabetes Institute, Dasman, Kuwait, ${ }^{2}$ Federal \\ University of Pernambuco, Recife, Brazil, ${ }^{3}$ State University \\ of New York at Buffalo, Buffalo, NY, United States
}

Introduction: Following the out-of-Africa diaspora, the Arabian Peninsula has experienced several waves of human migrations despite the prevailing extreme and varying environmental conditions. The potential adaptation that shaped the extant human populations of the hot and dry environment of the Arabian Peninsula have been scarcely studied.

Materials and Methods: We explored natural selection in the Arabian Peninsula region by analyzing 662,750 single nucleotide variants in 583 Kuwaiti and 96 Saudi Arabian individuals conducting multiple statistical tests, including integrated Haplotype Score (iHS), Cross Population Extended Haplotype Homozygosity (XP-EHH), Population Branch Statistics (PBS), and log-likelihood ratio scores (LLRS).

Results: Our integrative approach identified several genomic regions with strong signals of positive selection in both the populations, many of which contain loci associated with metabolic traits, asthma and blood-related phenotypes. We highlight a haplotype overlapping TNKS region in chromosome 8 , associated with metabolic traits and hypertension. The TNKS haplotype exemplifies a general trend in which a more rapid metabolism rate and hypertension have been selected in the Kuwaiti population, and potentially conferred some degree of fitness advantage to the Kuwaiti ancestors for surviving in the extremely dry and hot ecological environments, while posing a considerable health risk to present-day Kuwaitis.

Conclusions: Our results suggest that past adaptive trends have further predisposed Kuwaiti populations to the health risks at the genetic level. Overall, the mechanisms through which the TNKS haplotype conferred a fitness advantage and how the same haplotype predisposes the population to metabolic diseases remain fascinating and could be explored in future research. 
E. Muthukrishnan: None. A.L.C.D. Santos: None. O. Gokcumen: None. F. Al-Mulla: None. T.A. Thanaraj: None.

\section{P19.077.C}

Association of PPARGC1A gene polymorphism with strength\&power athlete status in two cohorts of European elite athletes

\section{Gineviciene ${ }^{1}$, A. Utkus ${ }^{1}$, L. Andruschenko ${ }^{2}$, M. Aksenov ${ }^{2}$ \\ ${ }^{1}$ Vilnius University, Vilnius, Lithuania, ${ }^{2}$ Plekhanov Russian University of Economics, Moscow, Russian Federation}

Introduction: The peroxisome proliferator-activated receptors $\gamma$ coactivator- $1 \alpha$ (encoded by PPARGC1A gene) is crucial regulator of energy homeostasis, adaptive thermogenesis, mitochondrial biogenesis and metabolism. The aim of this case-control study was to investigate the association of PPARGC1A (G>A, Gly482Ser, rs8192678) polymorphism with athletes' status in two European Caucasians cohorts from Lithuania (LT) and Russia (RU).

Materials and Methods: The study involved 160 professional strength/power athletes (47 LT and $113 \mathrm{RU}$ (86 weightlifters, 60 powerlifters, 14 throwers)) and 1,202 controls (255 LT and 947 RU, healthy unrelated nonathletes). Genotyping of PPARGC1A polymorphism was performed by polymerase chain reaction and restriction fragment length polymorphism analysis method.

Results: Statistically significant differences in genotype distribution were observed in all (LT+RU) powerlifters group (PPARGC1A: GG 62.3\%, GA 18\%, AA 19.7\%) compared with weightlifters (GG 47.7\%, GA 45.3\%, AA $7 \% ; P=0.0009$ ) as well as with throwers (GG 50\%, GA $50 \%$, AA $0 \% ; P=0.019$ ) and with controls (GG 46.3\%, GA $43.4 \%$, AA $10.3 \%, P=0.0002$ ). Odds ratio (OR) of PPARGC1A AA genotype 2.11 (95\% CI: 1.09-4.09, $P=0.026)$ was larger compared to $\mathrm{G} / \mathrm{G}$ genotype $\mathrm{OR}=1.92$ (95\% CI: 1.13-3.26, $P=0.016$ ) for powerlifters. Calculated OR indicate that the AA genotype is more favourable for powerlifter athletes.

Conclusions: Our results indicate that PPARGC1A AA genotype is beneficial to sports disciplines characterized by a predominantly anaerobic energy production and is related to greater power performance (such as powerlifters). We conclude that PPARGC1A (rs8192678) polymorphism is associated with strength/power athlete status in two European populations.

V. Gineviciene: None. A. Utkus: None. L. Andruschenko: None. M. Aksenov: None. P19.078.A
DNA methylation at five CpG sites of nasal brushings can predict childhood allergic disease

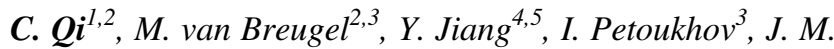

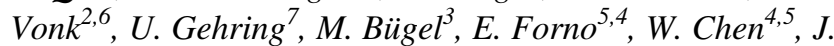
C. Celedón ${ }^{4,5}$, G. H. Koppelman ${ }^{1,2}$, C. X $u^{8,9,10}$

${ }^{1}$ Dept. of Pediatric Pulmonology and Pediatric Allergy, Beatrix Children's Hospital, University Medical Center Groningen, University of Groningen, Groningen, Netherlands, ${ }^{2}$ University of Groningen, University Medical Center Groningen, GRIAC Research Institute, Groningen, Netherlands, ${ }^{3}$ MI company, Amsterdam, Netherlands, ${ }^{4}$ Dept. of Pediatrics, University of Pittsburgh School of Medicine, Pittsburgh, PA, United States, ${ }^{5}$ Division of Pulmonary Medicine, UPMC Children's Hospital of Pittsburgh, Pittsburgh, PA, United States, ${ }^{6}$ University of Groningen, University Medical Center Groningen Dept. of Epidemiology, Groningen, Netherlands, ${ }^{7}$ Institute for Risk Assessment Sciences, Utrecht University, Utrecht, Netherlands, ${ }^{8}$, Centre for Individualised Infection Medicine, CiiM, a joint venture between the Hannover Medical School and the Helmholtz Centre for Infection Research, Hannover, Germany, ${ }^{9}$ Department of Internal Medicine, Radboud University Medical Center, Nijmegen, Netherlands, ${ }^{10}$ Department of Gastroenterology, Hepatology and Endocrinology, TWINCORE, Centre for Experimental and Clinical Infection Research, a joint venture between the Hannover Medical School and the Helmholtz Centre for Infection Research, Hannover, Germany

Background: The development of allergic, IgE mediated disease, such as asthma, rhinitis, and eczema, has a strong genetic and epigenetic contribution ${ }^{1,2}$. However, diagnosing allergic disease in childhood remains challenging, especially in young children.

Methods: We aimed to predict the presence of allergic disease (i.e. either asthma, rhinitis or eczema in combination with $\geq 1$ positive allergen-specific $\operatorname{IgE}$ ) in children. The model was built using data including lifestyle, environmental factors, genetic variation, and blood and nasal DNA methylation $(450 \mathrm{~K})$, collected from 348 subjects at 16 years old from a birth cohort, with 67 children having allergic disease. Elastic net regulation was used to train the model. The model performance was validated by 10 -fold cross validation and was replicated in an independent cohort with 481 children.

Results: We observed that most predict power can be attributed to nasal DNA methylation. Other factors did not improve the prediction of allergic disease. The final model was based on nasal DNA methylation levels at only $5 \mathrm{CpGs}$ with the prediction performance of AUC 0.86 in the 
discovery cohort and AUC 0.83 in the replication cohort. Matched nasal gene expression data was used to annotate these CpGs. Genes that were significantly correlated to the 5 CpGs were enriched in immune pathways including Interleukin-4 and Interleukin-13 signaling, neutrophil degranulation and leukocyte transendothelial migration.

Conclusions: We present a prediction model for allergic disease based on only $5 \mathrm{CpGs}$ from nasal epithelial cells. This model shows great potential to diagnose the presence of allergic disease in children.

C. Qi: None. M. van Breugel: A. Employment (full or part-time); Modest; MI company. Y. Jiang: None. I. Petoukhov: A. Employment (full or part-time); Modest; MI company. J.M. Vonk: None. U. Gehring: None. M. Bügel: A. Employment (full or part-time); Modest; MI company. E. Forno: None. W. Chen: None. J.C. Celedón: None. G.H. Koppelman: None. C. Xu: None.

\section{P19.080.C}

Frequency analysis of potentially protective genome variants in the Lithuanian population

\section{G. Zukauskaite, L. Ambrozaityte, I. Domarkiene, T. Rancelis, V. Kucinskas}

Vilnius University, Faculty of Medicine, Department of Human and Medical Genetics, Vilnius, Lithuania

The aim of this study is to determine potential protective genome variants (PGVs) in the general Lithuanian population using the data of the project "LITGEN". Exome sequencing and high-throughput beadchip genotyping data analysis was performed for 98 and 466 subjects of the Lithuanian descent, respectively. A catalog of 144 known PGVs was compiled using databases and publications. The catalog was set as a reference of PGVs for the LITGEN data. Allele frequencies were calculated and compared to the general European population as well as individual European populations based on $1000 \mathrm{G}$ project data. Statistical analysis (Hardy-Weinberg equilibrium, $\chi 2$ or F-test, $\mathrm{p} \leq 0.05$ ) was performed. Six missense variants were significantly more frequent in the study group and may have protection against alcohol dependence $(A D H 1 C$, rs698, $p=0.05)$, type 2 diabetes $(P P A R G, \operatorname{rs} 1801282, p=0.05$; SLC30A8, rs13266634, $p=0.03$ (FIN), $p=0.03$ (GBR), $p=0.04(\mathrm{CEU}))$, coronary heart disease (ZC3HCl, rs11556924, $p=0.04$ (GBR), $\mathrm{p}<0.01$ (FIN)), obesity $(S H 2 B 1$, rs 7498665, $p=0.03)$, esophageal cancer (PLCE1, rs2274223, $p=0.04)$. Although PGVs may reduce the risk of disease, there are additional genetic mechanisms that control this process. Not only the effects of single genomic variants are important, but their interactions and interactions between regulatory elements as well.
Detection of PGVs would not only provide a better understanding of the etiopathogenesis mechanisms and microevolutionary processes, but could also be used for the analysis for the development of novel therapies.

This research is part of the LITGEN project (VP1-3.1ŠMM-07-K-01-013) and was funded by the European Social Fund under the Global Grant measure.

G. Zukauskaite: None. L. Ambrozaityte: None. I. Domarkiene: None. T. Rancelis: None. V. Kucinskas: None.

\section{P19.081.A}

Identification of urinary metabolite quantitative trait loci in children and their interaction with dietary factors

B. Calvo ${ }^{1,2,3}$, C. H. Lau ${ }^{4}$, A. Siskos ${ }^{4}$, L. Maitre ${ }^{1,2,3}$, K. B.

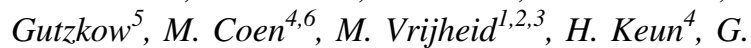
Escaramís $^{7}$, M. Bustamante ${ }^{1,2,3}$, Human Early Life Exposome (HELIX)

${ }^{1}$ ISGlobal, Barcelona, Spain, ${ }^{2}$ Universitat Pompeu Fabra (UPF), Barcelona, Spain, ${ }^{3}$ CIBER Epidemiología y Salud Pública (CIBERESP), Madrid, Spain, ${ }^{4}$ Imperial College London, London, United Kingdom, ${ }^{5}$ Norwegian Institute of Public Health, Oslo, Norway, ${ }^{6}$ AstraZeneca, Cambridge, United Kingdom, ${ }^{7}$ Universitat de Barcelona (UB), Barcelona, Spain

Introduction: Human metabolism is influenced by genetic and environmental factors. Previous studies have identified $>23$ single nucleotide polymorphisms (SNPs) associated with $>28$ urine metabolites levels (metabQTLs) in adults, but there are no publications in children. The aim of the present study is the identification of urinary metabQTLs in children.

Material and Methods: Genome-wide association studies (GWAS) of 44 urine metabolite levels measured with ${ }^{1} \mathrm{H}$ NMR were conducted in 996 children from the Human Early Life Exposome (HELIX) project.

Results: Twelve statistically significant urine metabQTLs were identified, involving 11 unique loci and 10 different metabolites. Comparison with findings in adults revealed that 6 metabQTLs were already known, 1 had been described in serum and 2 involved the same locus but different urinary metabolites. The remaining 2 metabQTLs represent novel associations: (i) rs2274870 for 3hydroxyisobutyrate annotated to Nipsnap Homolog $3 A$ (NIPSNAP3A), that codes for a protein highly expressed in the kidneys that participates in the vesicular transport; and (ii) rs12575496 for taurine, which is in a gene desert region. We also found that urinary taurine levels were affected by the combined action of rs12575496 and dietary patterns of meat and beverages. 
Conclusions: Overall, we identified 12 urinary metabQTLs in children, being 2 of them novel. A substantial part of the identified loci affected similarly urinary metabolite levels in children and in adults. The metabQTL for taurine seemed to be specific to children and interacted with dietary patterns.

$\begin{array}{lrr}\text { Funding: } & \text { ISCIII } & \text { PI17/01225; }\end{array}$

ENVIRONMENT 308333

B. Calvo: None. C.H. Lau: None. A. Siskos: None. L. Maitre: None. K.B. Gutzkow: None. M. Coen: None. M. Vrijheid: None. H. Keun: None. G. Escaramís: None. M. Bustamante: None.

P19.082.B

Population-scale comprehensive variant discovery with PacBio HiFi reads

\section{R. Vogelsang, S. B. Kingan, A. M. Wenger}

\section{Pacific Biosciences, Menlo Park, CA, United States}

Introduction: Catalogs of human variation continue to increase in scale, but they remain incomplete by relying on short-read sequencing and reference-based variant calling. Short reads have low sensitivity for structural variants (SVs, differences $\geq 50 \mathrm{bp}$ ), miss variants in segmental duplications that comprise $5-10 \%$ of human genome, and do not phase haplotypes. Further, large sequences absent in the reference genome are ignored. Highly accurate long-read ( $\mathrm{HiFi})$ sequencing overcomes these limitations.

Results: Long-read sequencing has been used successfully to create ethnicity-specific reference genomes of Asian, African, and European populations. Each genome adds up to $10 \mathrm{Mb}$ of unique sequence beyond GRCh38 and provides a superior target against which to map short reads. We demonstrate that de novo assembly with $\mathrm{HiFi}$ reads reliably produces genomes that approach GRCh38 in contiguity and accuracy (contig N50 >30 Mb, accuracy $>$ Q55).

$\mathrm{HiFi}$ reads also provide high precision and recall for variant detection at 15 -fold coverage. For the Genome in a Bottle benchmark samples, DeepVariant and pbsv achieve an F1 score of $99.7 \%$ for SNVs, 96.6\% for indels, and $96.4 \%$ for SVs. WhatsHap produces a phase block N50 >100 kb. HiFi reads resolve 152 of 193 medically relevant genes noted to be difficult for short reads.

Conclusions: We propose to improve catalogs of human variation using HiFi reads through: 1) de novo assembly of a representative male and female from each subpopulation to discover novel sequences; and 2) whole-genome sequencing of thousands of individuals to discover small variants in difficult regions and SVs genome-wide.
R. Vogelsang: A. Employment (full or part-time); Significant; Pacific Biosciences. S.B. Kingan: A. Employment (full or part-time); Significant; Pacific Biosciences. A. M. Wenger: A. Employment (full or part-time); Significant; Pacific Biosciences.

\section{P19.083.C}

The dynamics of incidence rates for recessive disorders in growing populations under different mating schemes

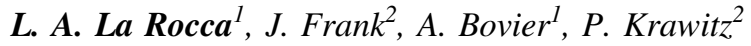 \\ ${ }^{1}$ Institute for Applied Mathematics, Bonn, Germany, ${ }^{2}$ Insti- \\ tute for Genomic Statistics and Bioinformatics, Bonn, \\ Germany
}

Despite increasing data from population-wide sequencing studies, the risk for recessive disorders in consanguineous partnerships is still heavily debated. An important aspect that has not sufficiently been investigated theoretically, is the influence of inbreeding on mutation load and incidence rates when the population sizes change. We therefore developed an individual-based population model to study these dynamics for a wide range of growth and mating conditions. In the phase of population expansion and shortly afterwards, our simulations show that there is a drop of diseased individuals at the expense of an increasing mutation load for random mating. On the other hand both parameters remain almost constant in highly consanguineous partnerships. This explains the empirical observation in present times that a high degree of consanguinity is associated with an increased risk of autosomal recessive disorders. However, the simulations also state that the higher frequency of severe recessive disorders such as intellectual disability in inbred populations is a transient phenomenon before a mutation-selection balance is reached again. Mathematic simulations can help to gain new insights in processes, where several factors interact in a complex manner.

L.A. La Rocca: None. J. Frank: None. A. Bovier: None. P. Krawitz: None.

\section{P19.085.B}

Temporal variation of regulatory organization based on longitudinal RNA-seq data in twins
A. Ramisch Ri,2, D. Ribeiro $^{3}$, J. S. El-Sayed Moustafa ${ }^{4}$, G.

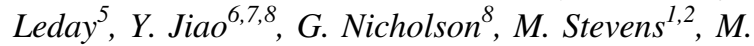

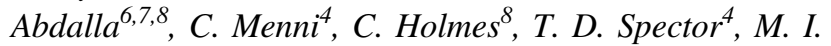


McCarthy ${ }^{6,7,9}$, S. Richardson ${ }^{5}$, O. Delaneau ${ }^{3}$, K. S. Small ${ }^{4}$, E. T. Dermitzakis ${ }^{1,2}$

${ }^{1}$ Department of Genetic Medicine and Development, Faculty of Medicine, University of Geneva, Geneva, Switzerland, ${ }^{2}$ Institute of Genetics and Genomics of Geneva, Geneva, Switzerland, ${ }^{3}$ Systems and Population Genetics Group, Department of Computational Biology, University of Lausanne, Lausanne, Switzerland, ${ }^{4}$ Department of Twin Research and Genetic Epidemiology, King's College London, London, United Kingdom, ${ }^{5}$ Medical Research Council, Biostatistics Unit, Cambridge, United Kingdom, ${ }^{6}$ Wellcome Centre Human Genetics, Nuffield Department of Medicine, University of Oxford, Oxford, United Kingdom, ${ }^{7}$ Oxford Centre for Diabetes, Endocrinology and Metabolism, Radcliffe Department of Medicine, University of Oxford, Oxford, United Kingdom, ${ }^{8}$ Department of Statistics, University of Oxford, Oxford, United Kingdom, ${ }^{9}$ Oxford NIHR Biomedical Research Centre, Oxford University Hospitals Trust, Oxford, United Kingdom

The human genome can be partitioned into domains of regulations that are necessary to foster gene expression in development, homeostasis and disease. A study by Delaneau et al. (Science 364, eaat8266, 2019) used populationbased chromatin data to identify cis-regulatory domains (CRDs), which show cell type-specific coordinated chromatin activity, follow the local chromatin organization into topologically associated domains, and were observed to control coexpression of nearby genes. Since CRDs are also under strong genetic control, they offer a great insight into the regulatory organization of the human genome. However, chromatin data are not available for most human genetics' studies, rendering the identification of CRDs expensive on a large scale. Therefore, we extend our previous work to characterize regulatory organization of the genome using only gene expression data from RNA-seq studies and genotypes. Specifically, we identify coexpression domains (CODs) as groups of significantly correlated proximal genes with inter-individual coordinated expression patterns, and assess their temporal dynamics exploiting a longitudinal dataset from the MultiMuTHER study, including 336 individuals with whole-blood RNA-seq data collected at three time-points over seven years, and imputed genotypes from the TwinsUK cohort. We identify genetic variants associated to the coordinated expression regulation of the CODs, as well as to their age-associated changes. Using the unique properties of twin studies, we perform a variance decomposition analysis to estimate CODheritability. Finally, we perform association studies to clinically relevant phenotypes in an attempt to identify
CODs involved in the regulation of cardiometabolic traits such as cardiovascular diseases and diabetes.

A. Ramisch: None. D. Ribeiro: None. J.S. El-Sayed Moustafa: None. G. Leday: None. Y. Jiao: None. G. Nicholson: None. M. Stevens: None. M. Abdalla: None. C. Menni: None. C. Holmes: None. T.D. Spector: None. M.I. McCarthy: None. S. Richardson: None. O. Delaneau: None. K.S. Small: None. E.T. Dermitzakis: None.

\section{P19.087.A}

Whole-exome sequencing provides insights into monogenic disease prevalence in Northwest Russia

R. K. Skitchenko ${ }^{1,2,3}$, Y. A. Barbitoff ${ }^{2,3}$, M. Vashisth ${ }^{1,2}$, O. I. Poleshchuk $^{2,3}$, A. E. Shikov ${ }^{2,4}$, E. A. Serebryakova', Y. A. Nasykhova $^{3}$, D. E. Polev ${ }^{3}$, A. R. Shuvalova , I. V. Shcherbakova ${ }^{3}$, M. A. Fedyakov ${ }^{4}$, O. S. Glotov ${ }^{4,3}$, A. S. Glotov $^{4,3}$, A. V. Predeus ${ }^{2}$

${ }^{1}$ ITMO, St. Petersburg, Russian Federation, ${ }^{2}$ Bioinformatics Institute, St. Petersburg, Russian Federation, ${ }^{3}$ Department of Genomic Medicine, D.O. Ott Research Institute of Obstetrics, Gynaecology and Reproduction, St. Petersburg, Russian Federation, ${ }^{4}$ City Hospital No. 40, St. Petersburg, Russian Federation

Allele frequency data from large exome and genome aggregation projects such as the Genome Aggregation Database (gnomAD) are of ultimate importance to the interpretation of medical resequencing data. However, allele frequencies might significantly differ in poorly studied populations that are underrepresented in large-scale projects, such as the Russian population. In this work, we leveraged our access to a large dataset of 1,021 exome samples to analyze genetic variation in the Northwest Russia. We compared the spectrum of genetic variants to the dbSNP build 151, and made estimates of ClinVar-based autosomal recessive (AR) disease allele prevalence as compared to gnomAD r. 2.1. In total, around $10 \%$ of discovered variants were not present in dbSNP. We report statistically significant overrepresentation of pathogenic variants for several Mendelian disorders, including phenylketonuria (PAH, rs5030858), Wilson's disease (ATP7B, rs76151636), factor VII deficiency ( $F 7$, rs36209567), kyphoscoliosis type of Ehlers-Danlos syndrome (FKBP14, rs542489955), and several other recessive pathologies. We also make primary estimates of monogenic disease incidence in the population, with retinal dystrophy, cystic fibrosis, and phenylketonuria being the most frequent AR pathologies. Our observations demonstrate the utility of population-specific allele frequency data to the diagnosis of monogenic disorders. 
This work was supported by the alpha-Endo program (the CAF Foundation), RFBR grant 18-415-130008, and D.O. Ott Research Institute of Obstetrics, Gynaecology and Reproductology, project 558-2019-0012 of FSBSI. Equipment of the Resource Center «Biobank» and "Computational Centre" of SPBU was used.

R.K. Skitchenko: None. Y.A. Barbitoff: None. M. Vashisth: None. O.I. Poleshchuk: None. A.E. Shikov: None. E.A. Serebryakova: None. Y.A. Nasykhova: None. D.E. Polev: None. A.R. Shuvalova: None. I.V. Shcherbakova: None. M.A. Fedyakov: None. O.S. Glotov: None. A.S. Glotov: None. A.V. Predeus: None.

\section{P19.088.B}

Autosomal diversity of Russian old settlers living in the shores of the Arctic Ocean in the Sakha Republic (Eastern Siberia)

A. V. Solovyev ${ }^{1,2}$, T. V. Borisova ${ }^{2}$, A. I. Kushniarevich ${ }^{3}$, E. Metspalu $^{3}$, F. M. Teryutin ${ }^{2,4}$, V. G. Pshennikova, ${ }^{2,4}$, G. P. Romanov $^{2,4}$, A. M. Cherdonova ${ }^{2}$, A. N. Alekseev ${ }^{1}$, T. E. Burtseva $^{2,4}$, E. K. Khusnutdinova ${ }^{5,6}$, M. Metspalu ${ }^{3}, R$. Villems $^{3}$, N. A. Barashkov ${ }^{2,4}$, S. A. Fedorova ${ }^{2,4}$

${ }^{1}$ The Institute for Humanities Research and Indigenous Studies of the North, Federal Research Center "Yakut Science Center of Siberian Branch of Russian Academy of Science”, Yakutsk, Russian Federation, ${ }^{2}$ M.K. Ammosov North-Eastern Federal University, Yakutsk, Russian Federation, ${ }^{3}$ Estonian Biocentre, Institute of Genomics, Tartu, Estonia, ${ }^{4}$ Yakut Scientific Center for Complex Medical Problems, Yakutsk, Russian Federation, ${ }^{5}$ Institute of Biochemistry and Genetics, Ufa Federal Research Center of the Russian Academy of Sciences, Ufa, Russian Federation, ${ }^{6}$ Bashkir State University, Ufa, Russian Federation

The "Russkoe Ust'e" is a small village located on the shores of the Arctic Ocean in Northeast Siberia $\left(71^{\circ}\right.$ $\left.08^{\prime} 02.2^{\prime \prime} \mathrm{N} 149^{\circ} 16^{\prime} 17.4^{\prime \prime} \mathrm{E}\right)$, with a total population of 128 people. The main population is Russian old settlers - ruskoustintsy. The ruskoustintsy have preserved old Russian traditions and dialect typical for Russians of the 16th century, despite living 400 years neighboring with indigenous Siberian populations (Paleo-Asiatic, Uralic, Tungusic and Turkic people) which have expanded in this territory in various historical period. In our days it's amazing to see a Slavic population among the indigenous people of this land. The aim of this study is to analyze the autosomal diversity and to reconstruct genetic history of Russian old settlers living in the Arctic region. DNA samples of 30 unrelated residents of "Russkoe Ust'e" were collected and genotyped using the Illumina OmniExpress $730 \mathrm{~K}$. The samples were analyzed using ADMIXTURE approach (Alexander et al., 2009). Final dataset included 192776 SNP's presented in 5458 individuals from 365 different worldwide populations. The ADMIXTURE $(\mathrm{K}=8)$ analysis showed that all 30 individuals in average have two major components - European $(42.4 \%)$ and Siberian $(41.3 \%)$ and minor different components (Middle-Eastern, East Asian, American-15\% and $1.3 \%$ others). The results do not refute the hypothesis that the first settlers of "Russkoe Ust'e" were the Russian Pomors, that fled from the persecution of the Ivan-IV the Terrible from the old territories of the Velikiy Novgorod ( 1570 year). This study was supported by Ministry of Science and Higher Education of Russia (basic_part_to_NEFU) and RFBR_grants (\#18-05-600035_Arctica, \#1934-60023_Perspektiva).

A.V. Solovyev: None. T.V. Borisova: None. A.I. Kushniarevich: None. E. Metspalu: None. F.M. Teryutin: None. V.G. Pshennikova: None. G.P. Romanov: None. A.M. Cherdonova: None. A.N. Alekseev: None. T. E. Burtseva: None. E.K. Khusnutdinova: None. M. Metspalu: None. R. Villems: None. N.A. Barashkov: None. S.A. Fedorova: None.

\section{P19.089.C}

Exploring the genetic architecture of the human neurological proteome using whole genome sequencing
G. Png ${ }^{1}$, A. Barysenka ${ }^{2}$, L. Repetto ${ }^{3}$, X. Shen Sh, $^{3,5}$, E.

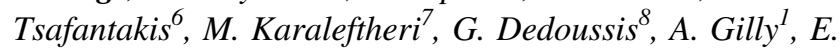 Zeggini $^{1}$

${ }^{1}$ Institute of Translational Genomics, Helmholtz Zentrum Munich - German Research Center for Environmental Health, Neuherberg, Germany, ${ }^{2}$ Helmholtz Zentrum Munich, Neuherberg, Germany, ${ }^{3}$ Centre for Global Health Research, Usher Institute, University of Edinburgh, Edinburgh, United Kingdom, ${ }^{4}$ State Key Laboratory of Biocontrol, School of Life Sciences, Sun Yat-sen University, Guangzhou, China, ${ }^{5}$ Department of Medical Epidemiology and Biostatistics, Karolinska Institute, Stockholm, Sweden, ${ }^{6}$ Anogia Medical Centre,, Anogia, Greece, ${ }^{7}$ Echinos Medical Centre, Echinos, Greece, ${ }^{8}$ Department of Nutrition and Dietetics, School of Health Science and Education, Harokopio University of Athens, Athens, Greece

The human proteome has a stronger genetic component compared to many complex diseases, making it a valuable resource of potential disease biomarkers and drug targets. This is especially so for highly polygenic neurological disorders whose mechanisms remain elusive. Here, we present the first sequence-based protein quantitative trait loci (pQTL) analysis of 92 neurological proteins. We perform a meta-analysis using deep whole-genome sequencing 
(WGS) data from two isolated Greek cohorts, MANOLIS (22.5x WGS; $N=1,356)$ and Pomak (18.4x WGS; $N=$ 1,537). A total of 123 independently-associated variants in 84 loci reach study-wide significance $\left(P<1.14 \times 10^{-10}\right)$ for 63 proteins, all of which are at least nominally significant $\left(P<3.78 \times 10^{-4}\right)$ and have the same direction of effect in both cohorts. To further elucidate the genetic architecture, independent variants were classified into 89 (72\%) cis- and $34(28 \%)$ trans-acting pQTLs. Ten variants have consequences equal to or more severe than missense, and 33 overlap regulatory regions. We also discover variants that have previously been linked to psychiatric disorders. For example, an intronic trans-pQTL in the ITIH4 gene is associated with increased NEP levels ( $r$ 2239547; $P=1.19 \times 10^{-129}$; BETA $=0.637983$; $\left.\mathrm{SE}=0.026328\right)$, and is an established risk variant for schizophrenia and bipolar disorder. This analysis represents the largest and only WGS-based pQTL study of neurological proteins to date, delivering insight into the rare and common genetic variant landscape underlying the human neurological proteome and its connection to neurological diseases.

G. Png: None. A. Barysenka: None. L. Repetto: None. X. Shen: None. E. Tsafantakis: None. M. Karaleftheri: None. G. Dedoussis: None. A. Gilly: None. E. Zeggini: None.

\section{P19.090.A}

\section{Widespread sexual differences in genetic architecture in} UK Biobank

E. Bernabeu ${ }^{1}$, O. Canela-Xandri ${ }^{2}$, K. Rawlik ${ }^{l}$, A. Talenti ${ }^{l}$, J. Prendergast ${ }^{1}$, A. Tenesa ${ }^{1,2}$

${ }^{1}$ The Roslin Institute, University of Edinburgh, Edinburgh, United Kingdom, ${ }^{2}$ MRC Human Genetics Unit, University of Edinburgh, Edinburgh, United Kingdom

Despite males and females sharing nearly identical genomes, there are differences between the sexes in complex traits and in the risk, incidence, and prevalence of a wide array of diseases. Gene by sex interactions $(\mathrm{GxS})$ are thought to underlie some of this sexual dimorphism. However, the extent and basis of these interactions are poorly understood.

Here we provide insights into both the scope and mechanism of GxS across the genome of circa 450,000 individuals of European ancestry and 530 complex traits in UK Biobank. We report sex differences in heritability for 71 traits, through genetic correlations for 69 traits, and in associations of genetic variants located in the autosome and $\mathrm{X}$-chromosome for 103 traits. We also show that sex specific polygenic risk scores may lead to a modest improvement of phenotypic prediction and highlight how sex agnostic analyses could be missing loci of interest. Finally, we studied the potential functional role of this dimorphism through sex-biased eQTL and gene-level analyses.

This study marks the largest examination of the genetics of sexual dimorphism to date. Our findings parallel previous reports, proving the widespread presence of sexual genetic heterogeneity across complex traits, though be it of generally modest magnitude. This warrants the need for future studies to consider sex-stratifying analyses, in order to both shed light into possible sex-specific molecular mechanisms as well as improve prediction of high-level phenotypes.

E. Bernabeu: None. O. Canela-Xandri: None. K. Rawlik: None. A. Talenti: None. J. Prendergast: None. A. Tenesa: None.

\section{P19.092.C}

Experimental system to enhance the discovery and translatability of causal links between host genetics and RSV-ALRI susceptibility

\section{A. Destouni}

Louisiana State University, Baton Rouge, LA, United States

Introduction: Respiratory syncytial virus (RSV) is the most significant cause of acute lower respiratory tract infections (ALRI) and ALRI caused deaths in children $<5$ years of age globally. Heritability of RSV-ALRI is $20 \%$ indicating that extensive genotype-by-environment interactions determine phenotypic variation. In this context, RSVALRI GWA studies are underpowered, because adequately sized infant cohorts have been unattainable. Integrating GTEx eQTLs from adult lungs is not informative due to the age-dependent pulmonary/immune RSV induced responses.

Materials and Methods: We developed an experimental system combining the BXD family of recombinant inbred strains with the neonatal mouse model of infant RSVimmunopathology. We infected 28 BXD strains and measured levels of viral NS1 gene transcription in lungs to obtain quantitative measure of the host-virus interaction. Heritability of NS1 transcription was 67\%. We mapped QTLs with Haley-Knott regression (5000 permutations and 2000 bootstraps). The analysis yielded 2 QTL intervals in chromosomes 14 (LRS=18.8, $p=0.05)$ and 13 $(\mathrm{LRS}=11.34, \quad p=0.63)$. Two recent genome-wide association studies (GWAS) of human bronchiolitis associated SNPs in VSTM4 and DRGX and JARID2, all three of which map to homologous regions in our RSV intervals. Thus, quantifying the viral NS1 transcript level in the neonatal mouse model captures host variants in conserved 
sequences with associations between host SNPs and bronchiolitis in human infants.

Conclusions: With the neonatal BXD isogenic panel, we effectively boost heritability by reducing the environmental variance in an age-appropriate model. This resource is poised to expedite the discovery and translatability of causal links between host genetics and RSV-ALRI susceptibility.

A. Destouni: None.

\section{P19.094.B}

Periconceptional environment predicts leukocyte telomere length in a cross-sectional study of 7-9 year old rural Gambian children

K. Maasen ${ }^{1,2,3}$, P. T. James ${ }^{4}$, A. M. Prentice ${ }^{3}$, S. E.

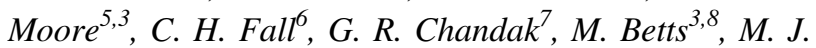
Silver $^{3}$, J. L. Buxton ${ }^{9,10}$

${ }^{1}$ Wageningen University, Wageningen, Netherlands, ${ }^{2}$ Maastricht University Medical Centre, Maastricht, Netherlands, ${ }^{3}$ Medical Research Council Unit The Gambia at the London School of Hygiene and Tropical Medicine, Fajara, Gambia, ${ }^{4}$ London School of Hygiene and Tropical Medicine, London, United Kingdom, ${ }^{5}$ King's College London, London, United Kingdom, ${ }^{6}$ University of Southampton, Southampton, United Kingdom, ${ }^{7}$ CSIR-Centre for Cellular and Molecular Biology, Hyderabad, India, ${ }^{8}$ University College London, London, United Kingdom, ${ }^{9}$ Kingston University, London, United Kingdom, ${ }^{10}$ UCL Great Ormond Street Institute of Child Health, London, United Kingdom

Introduction: Early life exposures are important predictors of adult disease risk. Although the underlying mechanisms are largely unknown, maintenance of telomeres may be involved. We investigated the relationship between seasonal differences in parental environmental exposures at the time of conception in rural Gambia and leukocyte telomere length (LTL) in their offspring.

Materials and Methods: LTL was measured in two cohorts of children aged 2 yrs $(N=487)$ and 7-9 yrs $(N=$ 218), using multiplex quantitative PCR. The association between date of conception and LTL was examined using Fourier regression models with age, sex, leukocyte cell composition, maternal periconceptional BMI, child birth weight, maternal and infant supplementation, and maternal folate considered as potential confounders.

Results: In the older children, we found evidence for an effect of season in a crude, seasonality only model [likelihood ratio test (LRT) $\left.\chi^{22}=8.27, p=0.016\right]$. LTL was greatest in children conceived in September (in the rainy season), and smallest in those conceived in March (in the dry season), with an effect size (LTL peak - nadir) of
$0.60 \mathrm{z}$-scores. This association remained after adjustment for multiple potential confounders. No effect of season was evident in the younger children (LRT $\chi^{2} 2=4.24, p=0.12$ ).

Conclusions: Our results suggest that conception during the dry season in The Gambia could adversely influence telomere maintenance in postnatal tissues, resulting in a detectable effect on mean LTL by mid-childhood but not before 2 years of age.

Funders: Bill and Melinda Gates Foundation (0PP1135346), MRC (MR/N006208/1 \& MC-A7605QX00), DFID \& the DBT, India (BT/IN/DBT-MRC/, DFID/24/GRC/2015-16).

K. Maasen: None. P.T. James: None. A.M. Prentice: None. S.E. Moore: None. C.H. Fall: None. G.R. Chandak: None. M. Betts: None. M.J. Silver: None. J. L. Buxton: None.

\section{P19.095.C}

Associations between telomere length shortening and cardio-metabolic components: a longitudinal study

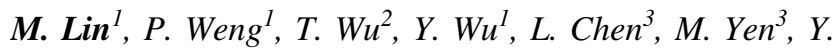

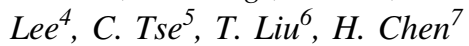

${ }^{I}$ Institute of Public Health, National Yang-Ming University, Taipei, Taiwan, ${ }^{2}$ Department of Public Health, Chung-Shan Medical University, Taichung, Taiwan, ${ }^{3}$ School of Oral Hygiene, College of Oral Medicine, Taipei Medical University, Taipei, Taiwan, ${ }^{4}$ Department of Internal Medicine, National Taiwan University Hospital, Taipei, Taiwan, ${ }^{5}$ Bureau of Health and Welfare, Lienchiang County Government, Matsu, Taiwan, ${ }^{6}$ Lienchiang County Government, Matsu, Taiwan, ${ }^{7}$ Institute of Epidemiology and Preventative Medicine, College of Public Health, National Taiwan University, Taipei, Taiwan

Introduction: Telomeres are the tips of chromosomes and play a critical role in maintaining chromosomal stability by protecting chromosome ends from damage and degradation. Telomere length (TL) is shortened progressively with age. Many studies revealed that age, sex, ethnicity, smoking, obesity, lack of exercise could result in shorter telomere length. Several studies also reported significant associations between shorter TL and deregulated metabolic syndrome components. The study was aimed to evaluate if the telomere length and shorting are associated with cardiometabolic components.

Methods: A total of 2698 adults were recruited from the Matsu community-based integrated health screening project. Relative telomere length was determined at baseline and after ten years. Multiple regression analyses and GEE adjusting for other covariates were performed to examine 
the relationship between relative telomere length and cardio-metabolic components.

Results: We found the metabolic syndrome group had significantly longer TL attrition $(p=0.028)$, and higher TL attrition rate over 10 years $(p=0.014)$. We further found statistically significant associations between relative telomere length at baseline and elevation of body mass index $(p=0.017)$, diastolic blood pressure $(p=0.043)$ at baseline as well as with the systolic blood pressure $(p=0.043)$ at the year 2015 after adjusting for age and gender. We also observed that the longer TL attrition was significantly associated with increased levels of fasting glucose $(p=0.006), \quad \operatorname{HbA1C}(p=0.014)$, triglycerides $(p=0.015)$, and total cholesterol $(p=0.034)$ at the year 2015 .

Conclusions: Our results confirmed the previous knowledge of the associations between telomere length and cardio-metabolic components.

Grant No: MOST 106-2314-B-010-020-MY3

M. Lin: None. P. Weng: None. T. Wu: None. Y. Wu: None. L. Chen: None. M. Yen: None. Y. Lee: None. C. Tse: None. T. Liu: None. H. Chen: None.

\section{P19.096.A}

Significant associations between telomere length related genetic loci and cardio-metabolic components

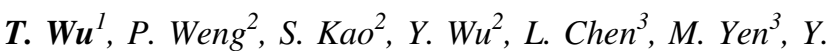

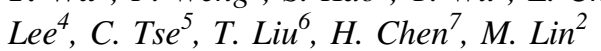

${ }^{l}$ Department of Public Health, Chung-Shan Medical University, Taichung, Taiwan, ${ }^{2}$ Institute of Public Health, National Yang-Ming University, Taipei, Taiwan, ${ }^{3}$ School of Oral Hygiene, College of Oral Medicine, Taipei Medical University, Taipei, Taiwan, ${ }^{4}$ Department of Internal Medicine, National Taiwan University Hospital, Taipei, Taiwan, ${ }^{5}$ Bureau of Health and Welfare, Lienchiang County Government, Matsu, Taiwan, ${ }^{6}$ Lienchiang County Government, Matsu, Taiwan, ${ }^{7}$ Institute of Epidemiology and Preventative Medicine, College of Public Health, National Taiwan University, Taipei, Taiwan

Introduction:Telomeres are at the end of chromosomes with the repeat sequences of TTAGGG. Telomere length (TL) would be shortened during DNA replication. Many studies revealed that age, sex, ethnicity, smoking, obesity, and lack of exercise were the factors linked with shorter TL length. Several studies reported significant associations between shorter TL and deregulated metabolic syndrome (MetS) components. Moreover, a few genetic loci associated with telomere length have been identified. The study was aimed to evaluate if the TL related genetic loci are associated with cardio-metabolic components.
Methods: A total of 2543 adults were recruited from the Matsu community-based integrated health screening project. A total of 43 SNPs on $17 \mathrm{TL}$ related genes were genotyped. Multiple regression analyses adjusting for other confounding covariates were performed to examine the relationship between TL related genetic loci and cardiometabolic components.

Results: We examined 43 SNPs in 17 genes and found these 43 SNPs had no significant associations with MetS, but some SNPs were related to MetS components. The results of multiple logistic regression showed that rs78975791, rs622961, rs11191853, rs2287641, and rs41269537 were associated with lower HDL; rs820152, rs622961 and rs1684149 were associated with increased levels of blood sugar; rs4125537 was associated with elevated TG; rs13265931 and rs6062302 were associated with wider waist circumference; rs13265931, rs4841210, and rs75877904 were associated with higher blood pressure (all with $P$-value $<0.05$ ).

Conclusions: Our study identified several TL related gene variations were associated with cardio-metabolic components.

Grant No: MOST 106-2314-B-010-020-MY3

T. Wu: None. P. Weng: None. S. Kao: None. Y. Wu: None. L. Chen: None. M. Yen: None. Y. Lee: None. C. Tse: None. T. Liu: None. H. Chen: None. M. Lin: None.

\section{P19.097.B}

Leukocyte telomere length is related to brain parenchymal fraction and attention/speed in the elderly: Results of the Austrian Stroke Prevention Study

\section{P. Gampawar, R. Schmidt, H. Schmidt}

\section{Medical University of Graz, Graz, Austria}

The role of leukocyte telomere length (LTL) in brain aging is controversial. Here we explored the association between LTL and 1) global MRI correlates of brain aging including brain parenchymal fraction (BPF), white matter hyperintensities (WMH) as well as 2) global (g-factor) cognition and the cognitive domains of attention/speed, conceptualization, memory, and visuopractical skills in 909 participants of the Austrian Stroke Prevention Study. There were $388(42.7 \%)$ men, and the mean age was $65.9 \pm 8.0$ years. Longer LTL was significantly associated with larger BPF $(\beta=0.43, p<0.001)$ and larger WMH load $(\beta=0.03$, $p=0.04$ ) after adjusting for age, sex, vascular risk factors, and ApoE4 carrier status. The effect on BPF was more significant in the subgroups of women $(\beta=0.51$, $p=0.001)$, age $>65$ years $(\beta=0.58, p=0.002), \mathrm{BMI} \geq 25$ $(\beta=0.40, p=0.004)$, education $\leq 10$ years $(\beta=0.42$, $p=0.002)$, hypertensives $(\beta=0.51, p=0.001)$, 
cardiovascular disease (CVD) $(\beta=0.58, p=0.005)$, nondiabetics $(\beta=0.42, \mathrm{p}<0.001)$ and Apoe4 non-carriers $(\beta=0.49, p<0.001)$. The effect on WMH was significant within hypertensives $(\beta=0.04, p=0.02)$, non-diabetics $(\beta=0.03, p=0.01)$, and in those with education $\leq 10$ years $(\beta=0.03, p=0.04)$, and ApoE4 allele $(\beta=0.03$, $p=0.02)$. There was a significant association between LTL and the cognitive domain of attention/speed, which was confined to the subgroups of $\mathrm{BMI} \geq 25(\beta=0.04, p=0.05)$ and education $\leq 10$ years $(\beta=0.04, p=0.05)$. The effect of LTL on attention/speed was partly mediated in both subgroups by BPF $(\beta=0.02,95 \% \mathrm{CI}=0.01: 0.03)$ when tested by bootstrapping. Our results support a strong protective role of longer LTL on global brain volume, which in turn may contribute to better cognitive functions, especially in the attention/speed domain in the elderly.

P. Gampawar: None. R. Schmidt: None. H. Schmidt: None.

\section{P19.098.C}

Thyroid cancer, obesity and blood glucose: a Mendelian Randomization study

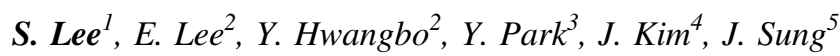

${ }^{1}$ Institute of Health \& Environment, Seoul National University, Seoul, Korea, Republic of, ${ }^{2}$ Center for Thyroid Cancer, National Cancer Center, Goyang, Korea, Republic of, ${ }^{3}$ Department of Internal Medicine, Seoul National University College of Medicine, Seoul, Korea, Republic of, ${ }^{4}$ Department of Cancer Biomedical Science, Graduate School of Cancer Science and Policy, National Cancer Center, Goyang, Korea, Republic of, ${ }^{5}$ Department of Public Health, Graduate School of Public Health, Seoul National University, Seoul, Korea, Republic of

Background: Glucometabolic traits such as obesity, hyperglycemia and height have been associated with an increased risk of thyroid cancer (TC). It is, however, largely unknown whether the risk factors are causal and bear potential significance for intervention. We attempted to investigate the causal relationship between thyroid cancer risk and selected epidemiologic risk factors applying Mendelian Randomization (MR) approaches

Methods: A total of 17,935 healthy individuals from the Korean Genome Epidemiology Study and 1,214 thyroid cancer cases from 2 hospitals were selected with epidemiologic, clinical and genome information. Genetic risk scores of BMI, fasting plasma glucose (FPG) and height were constructed and used as instrumental variables (IV) to analyze the causal association in the two-sample IV analyses. Additionally inverse-weighted variance, weighted median methods were performed to estimate the causal effect size of TC risk. Further, MR-Egger regression was used to assess potential pleiotropic effects as well.

Results: IV analyses demonstrated that BMI, FPG, and height GRS and risk of overall TC (BMI: OR $=1.05$, $p=0.363$; FPG: $\mathrm{OR}=1.03, p=0.396$; height: $\mathrm{OR}=1.01$, $p=0.379)$ as well as subgroups of TC did not show significant associations. Pleiotropic effects were not found, and statistical power ranged from 0.67 to 0.79 .

Conclusion: Our findings suggest that obesity and FPG may not be the potential intervention target to prevent TC risk, despite the findings from observational studies.

S. Lee: None. E. Lee: None. Y. Hwangbo: None. Y. Park: None. J. Kim: None. J. Sung: None.

P19.100.B

Differential genetic and environmental effects on immune parameters and functions in type 1 diabetes and health

X. Chu $^{1,2,3}$, A. W. M. Janssen ${ }^{4}$, H. Koenen ${ }^{5}$, L. Chang ${ }^{1}$, X.

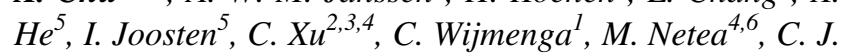
$\operatorname{Tack}^{7}, Y . \mathrm{Li}^{1,2,3,4}$

${ }^{1}$ Department of Genetics, University of Groningen, Groningen, Netherlands, ${ }^{2}$ Centre for Individualised Infection Medicine, CiiM, a joint venture between the Hannover Medical School and the Helmholtz Centre for Infection Research, Hannover, Germany, ${ }^{3}$ TWINCORE, Centre for Experimental and Clinical Infection Research, a joint venture between the Hannover Medical School and the Helmholtz Centre for Infection Research, Hannover, Germany, ${ }^{4}$ Department of Internal Medicine and Radboud Institute for Center for Infectious Diseases, Radboud University Medical Center, Nijmegen, Netherlands, ${ }^{5}$ Department of Laboratory Medicine, Laboratory Medical Immunology, Radboud University Medical Center, Nijmegen, Netherlands, ${ }^{6}$ Department for Genomics \& Immunoregulation, Life and Medical Sciences Institute (LIMES), University of Bonn, Bonn, Germany, ${ }^{7}$ Department of Internal Medicine and Radboud Institute for Molecular Life Sciences, Radboud University Medical Center, Nijmegen, Netherlands

Background: Inter-individual variability in immune parameters and functions determines the susceptibility to immune-mediated diseases. Recent studies from the Human Functional Genomics Project have successfully characterized the impact of genetic and environmental factors on immune response in health. However, how this variability relates to the risk of developing an autoimmune disease, such as type 1 diabetes (T1D), is largely unknown.

Methods: Blood samples were collected from 243 T1D and 534 healthy volunteers of Western European descent. 
$>200$ immune cell populations and >100 cytokine-stimulus pairs were measured in both cohorts. We used a systems approach to comprehensively assess the impact of environmental and genetic factors on immune phenotypes. The results were compared between T1D patients and healthy controls.

Results: Age and seasons show impact on both immune cell abundance and cytokine production upon stimulation, e.g. resting $\mathrm{T}$ regs show higher levels in summer. The T1D associated genetic factors show stronger impact on $\mathrm{T}$ cell than $\mathrm{B}$ cell subpopulations in peripheral blood $\left(P=4.17 \times 10^{-5}\right.$, K.S. test $)$, highlighting the importance of $\mathrm{T}$ cell immunity in T1D. Rs35092096 of the CCR region associated with CCR5 $+\mathrm{CD} 4+$ regulatory $\mathrm{T}$ cells $\left(P=1.64 \times 10^{-12}\right)$ showed co-localization $(\mathrm{H} 3=0.95)$ with T1D, suggesting a shared regulation of this locus. Genome-wide quantitative trait loci (QTL) mapping revealed 16 significant loci in T1D. $75 \%$ of the identified QTLs are specific in T1D, implying a distinct regulatory mechanism of immune parameters and functions between auto-immune disease and health.

Conclusions: Our approach provides novel insights into immune trait variability in T1D.

X. Chu: None. A.W.M. Janssen: None. H. Koenen: None. L. Chang: None. X. He: None. I. Joosten: None. C. Xu: None. C. Wijmenga: None. M. Netea: None. C.J. Tack: None. Y. Li: None.

\section{P19.101.C}

Evaluation of the causal effect of desaturase activity on the risk of type 2 diabetes and coronary artery disease

\section{S. Jäger ${ }^{1,2}$, R. Cuadrat ${ }^{1,2}$, P. Hoffmann ${ }^{3,4}, C$. Wittenbecher ${ }^{1,2,5}$, M. B. Schulze $e^{I, 2,6}$}

${ }^{1}$ Department of Molecular Epidemiology, German Institute of Human Nutrition Potsdam-Rehbruecke (DIfE), Nuthetal, Germany, ${ }^{2}$ German Center for Diabetes Research (DZD), Neuherberg, Germany, ${ }^{3}$ Human Genomics Research Group, Department of Biomedicine, University of Basel, Basel, Switzerland, ${ }^{4}$ Institute of Human Genetics, University of Bonn, School of Medicine \& University Hospital Bonn, Bonn, Germany, ${ }^{5}$ Department of Nutrition, Harvard T.H. Chan School of Public Health, Boston, MA, United States, ${ }^{6}$ Institute of Nutritional Science, University of Potsdam, Potsdam, Germany

Background: Delta 5-desaturase (D5D) and delta 6desaturase (D6D) (estimated from fatty acids measured in blood fractions) were associated with cardiometabolic risk, however, causality needs to be clarified.

Material and Methods: We investigated causal effects of D5D and D6D activities on the risk of type 2 diabetes
(T2DM) and coronary artery disease (CAD) in two sample Mendelian randomization (MR) studies. Selection of genetic instruments for D5D (20:4n-6/20:3n-6 ratio) and D6D activity (18:3n-6/18:2n-6 ratio) relied on genome-wide association studies (GWAS) and gene-wide screens (restricted to the $F A D S$-gene region that encodes for both desaturases) in a representative sub-cohort $(n=1853)$ of the European Prospective Investigation into Cancer and Nutrition (EPIC)-Potsdam Study. Outcome associations were drawn from DIAGRAM and CARDIoGRAM GWAS. We applied univariable MRs (single desaturases) and multivariable MR (combining both) and addressed confounding by linkage disequilibrium (LD) as all instruments from FADS1 (D5D) are in LD with FADS2 (D6D) variants.

Results: Univariable MRs revealed risk-increasing total effects of both, D6D (odds ratio (OR) [95\% confidence interval $(\mathrm{CI})]=1.08[1.06-1.09])$ and $\mathrm{D} 5 \mathrm{D}(\mathrm{OR}=1.03$ [1.01-1.04]) on T2DM; and risk-increasing total effect of $\mathrm{D} 6 \mathrm{D}(\mathrm{OR}=1.06$ [1.02-1.11] $)$ on CAD. The multivariable MR could not unambiguously allocate a direct causal effect to either of the individual desaturases.

Conclusions: Our results suggest that D6D is causally linked to cardiometabolic risk, which is likely due to downstream production of fatty acids and products resulting from high D6D activity. For D5D, higher T2DM risk reflects the genetic linkage with D6D rather than an independent effect.

S. Jäger: None. R. Cuadrat: None. P. Hoffmann: None. C. Wittenbecher: None. M.B. Schulze: None.

\section{P19.102.A}

Association of tyrosine kinase 2 polymorphisms with baseline viral load and advanced fibrosis in chronic hepatitis $\mathrm{C}$ patients

A. M. V. Silva ${ }^{l}$, L. Alvarado-Arnez ${ }^{2}$, O. C. L. Bezerra ${ }^{l}, T$. Azamor $^{l}$, M. R. Alves ${ }^{l}$, T. L. Calvo ${ }^{1}$, C. Bayma ${ }^{l}$, J. Silva ${ }^{l}$, A. F. Souza ${ }^{l}$, A. M. Andrade ${ }^{l}$, J. R. Xavier ${ }^{l}$, M. S. Maia ${ }^{l}$, P. D. Picon , A. D. Ano Bom ${ }^{l}$, D. S. Matos ${ }^{1}$, M. O. Moraes ${ }^{1}$

${ }^{I}$ Fundação Oswaldo Cruz (FIOCRUZ), RIO DE JANEIRO, Brazil, ${ }^{2}$ Universidad Privada Franz Tamayo - UNIFRANZ, Bolivia, Cochabamba, Bolivia, Plurinational State of, ${ }^{3}$ Hospital de Clínicas de Porto Alegre, Porto alegre, Brazil

Introduction: Single nucleotide polymorphisms (SNPs) in interferon lambda 3 and 4 (IFNL3/4) are well-established prognostic markers for Pegylated-Interferon-alpha/ribavirin (Peg-IFN- $\alpha / \mathrm{RBV}$ ) treatment in Chronic Hepatitis C (CHC). In other viral infections, an antagonistic effect between IFN$\lambda$ and IFN- $\alpha$ levels was described. We aimed to evaluate both a functional and genetic association of SNPs in the 
interferon-alpha 1 receptor (IFNARI) and tyrosine kinase 2 (TYK2) over clinical parameters in CHC patients.

Materials and Methods: Three IFNARI and two TYK2 SNPs were genotyped in $525 \mathrm{CHC}$ patients treated with Peg-IFN- $\alpha /$ RBV, using TaqMan assays for allelic discrimination. Gene expression was evaluated for IFNL3, IFNA1, and IFNARI genes in 24 patients stratified by genotypes, considering different time points (0-1-3-12 weeks of treatment), using Biomark-HD System. Mann-Whitney, Kruskal-Wallis and Dunn's post-test was used for comparisons.

Results: Heterozygous CHC patients at TYK2 rs12720356 presented an association with a high baseline viral load $(\mathrm{OR}=2.0 / \mathrm{CI} 1.1-3.7 / p=0.03)$. Also, heterozygous at $T Y K 2$ rs3456443 $(\mathrm{OR}=2.6 / \mathrm{CI} 1.1-5.9 / p=0.02)$ were associated with advanced fibrosis. IFNAR1 SNPs were not associated with any of the outcomes tested. No differences were observed when comparing IFNA1, IFNARI and IFNL3 levels between the different treatment time points. Stratifying according to the different SNP genotypes, only higher IFNL3 levels were significantly different in IFNAR1 rs2843710-G carriers. Due to low sample size gene expression levels were not stratified for TYK2 genotypes.

Conclusions: High baseline viral load and advanced fibrosis in CHC patients were associated with TYK2 polymorphisms, which is consistent with the role of this SNP in TYK2 function controlling viral replication.

A.M.V. Silva: None. L. Alvarado-Arnez: None. O.C.L. Bezerra: None. T. Azamor: None. M.R. Alves: None. T. L. Calvo: None. C. Bayma: None. J. Silva: None. A.F. Souza: None. A.M. Andrade: None. J.R. Xavier: None. M.S. Maia: None. P.D. Picon: None. A.D. Ano Bom: None. D.S. Matos: None. M.O. Moraes: None.

\section{P19.103.B}

Comprehensive analysis of circulating viral DNA in maternal plasma at population-scale

V. Eldem ${ }^{1}$, E. Akdeniz ${ }^{2}$, B. Nevruz ${ }^{2}$, I. M. Tekin ${ }^{2}$, H. Özgür ${ }^{2}$, G. Serim ${ }^{2}$, D. Aydın', M. Aslan ${ }^{2}$, M. Durdu ${ }^{2}, \ddot{O}$. Alan $^{2}, G$. $\ddot{O} z d o \breve{g} a n^{2}, V$. Oflas $^{2}$

${ }^{1}$ Department of Biology, Faculty of Science, Istanbul University, Istanbul, Turkey, ${ }^{2}$ Genoks Genetic Disease Diagnostic Center, Ankara, Turkey

Introduction: In recent years, low-coverage whole-genome sequencing has become the method of choice for noninvasive prenatal testing (NIPT) to evaluate the most common chromosome aberrations. Although nearly all WGSgenerated NIPT data analysis is limited to detect common trisomies, the possible re-use of NIPT data increasingly becomes popular and expanding. Here, we systematically analyzed NIPT data at the population-scale to characterize the virome in maternal plasma.

Materials and Methods: A total of 13,802 NIPT data were generated from Turkish pregnant women during the period of 2018-2019 at Genoks Diagnostic Center. Sequencing was performed on BGI SEQ-500, producing 35-bp single-end reads. Following quality filtering, clean reads that did not map to human reference genome were blasted against the NCBI viral reference sequence database (release 97). Only matching reads with e-value $<1 \mathrm{e}-10$, a percent identity $\geq 97$ and alignment length $\geq 32$ bp were kept for further analysis. After removing alignments to bacteriophages, remaining alignments were used for virus abundance estimation.

Results: About 110 billion clean reads from 13,802 samples were used for profiling plasma virome, 3947 samples $(28.6 \%)$ have at least one viral hit and 607 samples $(4.4 \%)$ have at least two significant alignment hits. Human herpesvirus $6 \mathrm{~A}$ and $6 \mathrm{~B}$, hepatitis $\mathrm{B}$ virus and human endogenous retrovirus $\mathrm{K}$ were enriched in terms of prevalence and abundance. The maternal blood in the Turkish population was appeared to have a different viral distribution compared to pregnant Europeans and Chinese.

Conclusions: WGS-generated NIPT data can be a powerful approach for detecting circulating viral DNAs.

V. Eldem: None. E. Akdeniz: None. B. Nevruz: None. I.M. Tekin: None. H. Özgür: None. G. Serim: None. D. Aydın: None. M. Aslan: None. M. Durdu: None. Ö. Alan: None. G. Özdoğan: None. V. Oflas: None.

\section{P20 Functional Genomics and Epigenomics}

\section{P20.05.B}

Whole genome methylation analysis in families with ALS-linked SOD1 mutations

\author{
F. Coppedè ${ }^{1}$, D. Licastro ${ }^{2}$, S. Dal Monego ${ }^{2}$, L. Mosca $^{3}, U$.

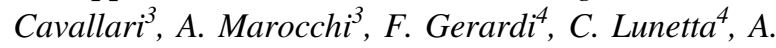 \\ Stoccoro $^{1}$, L. Migliore ${ }^{1}$ \\ ${ }^{1}$ Dept of Translational Research and of New Surgical and \\ Medical Technologies, University of Pisa, Pisa, Italy, \\ ${ }^{2}$ AREA Science Park, Basovizza, Trieste, Italy, ${ }^{3}$ Medical \\ Genetics Unit, Department of Laboratory Medicine, ASST \\ Grande Ospedale Metropolitano Niguarda, Milan, Italy, \\ ${ }^{4}$ NEuromuscular Omnicentre (NEMO), Fondazione Serena \\ Onlus, Milan, Italy
}

Introduction: Our previous investigation of the global levels of 5-methylcytosine (5-mC) in blood DNA samples from families with $S O D 1$ mutations revealed increased 5$\mathrm{mC}$ levels in patients with amyotrophic lateral sclerosis 
(ALS) with respect to their unaffected relatives, including presymptomatic/paucisymptomatic carriers and non-carrier family members. In the present study we performed a genome-wide methylation analysis in order to characterize the methylation differences among groups.

Materials and Methods: A total of 18 blood DNA samples from 3 different ALS families were collected for the study, including 4 ALS patients, 10 presymptomatic/ paucisymptomatic carriers, and 4 non-carrier relatives. All the samples were processed with reduced representation bisulfite sequencing (RRBS) and analyzed using RnBeads pipeline.

Results: A preliminary multidimensional scaling analysis of the dataset suggests that, despite coming from different families, the 4 ALS patients share a similar methylation pattern across more than 23.000 investigated $\mathrm{CpG}$ islands, that can be distinguished from the patterns observed in noncarriers of ALS-linked SOD1 mutations. By contrast, presymptomatic/paucisymptomatic carriers show a more heterogeneous $\mathrm{CpG}$ island methylation profile. We are now cleaning the dataset from confounding factors in order to reveal the gene promoters and the pathways that are differentially methylated among groups.

Conclusions: The present investigation takes advantage of well characterized families enriched in presymptomatic/ paucisymptomatic carriers of ALS-linked SOD1 mutations and could help revealing preclinical methylation signatures of the disease.

F. Coppedè: None. D. Licastro: None. S. Dal Monego: None. L. Mosca: None. U. Cavallari: None. A. Marocchi: None. F. Gerardi: None. C. Lunetta: None. A. Stoccoro: None. L. Migliore: None.

\section{P20.07.A}

Association between asbestos exposure and epigenetic age acceleration

${\text { G. } \text { Cugliari }^{1,2}, \text { A. Allione }}^{1,2}$, S. Guarrera ${ }^{1,2}$, C. Viberti $^{1,2}, F$. Grosso $^{3}$, E. Casalone ${ }^{1,2}$, M. Betti ${ }^{3}$, D. Ferrante ${ }^{4,5}, A$.

Aspesi $^{6}$, C. Casadio ${ }^{7}$, R. Libener ${ }^{8}$, E. Piccolini ${ }^{9}$, D.

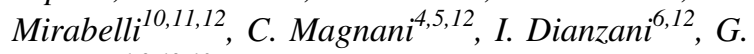
Matullo $^{1,2,12,13}$

${ }^{1}$ Department of Medical Sciences, University of Turin, Turin, Italy, ${ }^{2}$ Italian Institute for Genomic Medicine, IIGM, Turin, Italy, ${ }^{3}$ Division of Medical Oncology, SS. Antonio e Biagio General Hospital, Alessandria, Italy, ${ }^{4}$ Medical Statistics and Cancer Epidemiology Unit, Department of Translational Medicine, University of Piemonte Orientale, Novara, Italy, ${ }^{5}$ Cancer Epidemiology Unit, CPO-Piemonte, Novara, Italy, ${ }^{6}$ Department of Health Sciences, University of Piemonte Orientale, Novara, Italy, ${ }^{7}$ Thoracic Surgery Unit, AOU Maggiore Della Carità, Novara, Italy, ${ }^{8}$ Pathology Unit, SS. Antonio e Biagio General Hospital, Alessandria, Italy, ${ }^{9}$ Pneumology Unit, Santo Spirito Hospital, Casale Monferrato, Italy, ${ }^{10}$ Cancer Epidemiology Unit, Department of Medical Sciences, University of Turin, Turin, Italy, ${ }^{11}$ Cancer Epidemiology Unit, CPO Piemonte, Turin, Italy, ${ }^{12}$ Interdepartmental Center for Studies on Asbestos and Other Toxic Particulates "G. Scansetti", University of Turin, Turin, Italy, ${ }^{13}$ Medical Genetics Unit, AOU Città della Salute e della Scienza, Turin, Italy

Introduction: Although the mechanisms of carcinogenesis in Malignant Pleural Mesothelioma (MPM) are incompletely understood, chronic inflammation is critically involved in the pathogenesis as a possible result of asbestos exposure. Age is one of the strongest predictors of chronic disease and mortality, but biological responses to aging differ among people and can have an important role in the consideration of the relationship with disease-related covariates. Aging eludes precise definition at the systemic level and denotes a multitude of processes at the cellular level.

Material and Methods: We investigated a cohort of 300 subjects exposed between 2000 and 2010. Using methylation-based "clock" (Horvath), namely extrinsic epigenetic age acceleration (EEAA), we defined biological age acceleration for each of three hundred asbestos-exposed subjects. EEAA is defined as the weighted average of DNAm age and imputed proportions of naïve CD8+ $\mathrm{T}$ cells, memory $\mathrm{CD} 8+\mathrm{T}$ cells and plasmablasts. Asbestos exposure-related variation in age acceleration measures and 95\% confidence intervals (CIs) were estimated using multiple regression models.

Results: EEAA showed that biological age acceleration was statistically significantly associated with increased asbestos exposure (Horvath's clock: Estimate $=0.704$, 95\% CI: $0.067,1.475, P=0.043)$.

Conclusions: DNAm profiles have been used as indices of biological age and the roles of epigenetic age acceleration measures were investigated. Our results suggest the potential application of DNAm profiles, considering age acceleration measures, in blood to develop non-invasive tests for asbestos-exposed subjects. In detail, epigenetic age acceleration may play a role as a proxy of internal dose of asbestos exposure.

G. Cugliari: None. A. Allione: None. S. Guarrera: None. C. Viberti: None. F. Grosso: None. E. Casalone: None. M. Betti: None. D. Ferrante: None. A. Aspesi: None. C. Casadio: None. R. Libener: None. E. Piccolini: None. D. Mirabelli: None. C. Magnani: None. I. Dianzani: None. G. Matullo: None. 


\section{P20.08.B}

Autism spectrum disorder-associated CHD8 Suppression impacts on histone $\mathrm{H} 3$ lysine 36 trimethylation and alters RNA alternative splicing.

E. Kerschbamer ${ }^{1}$, T. Tripathi ${ }^{1}$, S. Erdin ${ }^{2,3}$, E. Salviato ${ }^{4}, F$. Di Leva ${ }^{l}$, E. Sebestyen ${ }^{4}$, M. Arnoldi ${ }^{l}$, M. Benelli ${ }^{5}, J$.

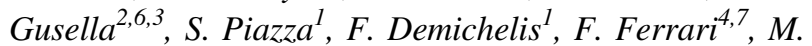
Talkowski $^{2,6,3}$, M. Biagioli $^{l}$

${ }^{1}$ University of Trento, Trento, Italy, ${ }^{2}$ Massachusetts General Hospital, Boston, MA, United States, ${ }^{3}$ Program in Medical and Population Genetics, Broad Institute, Boston, MA, United States, ${ }^{4}$ IFOM, the FIRC Institute of Molecular Oncology, Milano, Italy, ${ }^{5}$ Bioinformatics Unit, Hospital of Prato, Istituto Toscano Tumori, Prato, Italy, ${ }^{6}$ Department of Neurology, Harvard Medical School, Boston, MA, United States, ${ }^{7}$ CNR Institute of Molecular Genetics "Luigi Luca Cavalli-Sforza”, Pavia, Italy

Disruptive mutations in the chromodomain helicase DNA binding protein 8 ( $C H D 8$ ) have been recurrently and independently associated to Autism Spectrum Disorders (ASD). In normal cellular physiology, CHD8 co-purifies with MLL1 and MOF transcriptional activation complex, with elongating RNAPII and directly binds to DNA promoters and enhancers regions, thus a regulatory role in transcriptional initiation and elongation could be postulated. Here we investigated how chromatin landscape reacts to $C H D 8$ suppression by analyzing a panel of histone modifications in induced pluripotent stem cells-derived neural progenitors. We interrogated transcriptionally active and repressed regions as well as active and poised enhancers. CHD8 suppression leads to a significant reduction $(47.82 \%)$ in histone H3K36me3 at gene bodies, particularly impacting on transcriptional elongation chromatin states. Highly expressed, CHD8-bound genes present a stronger and more significant H3K36me3 reduction compared to CHD8 unbound genomic regions. Genes presenting histone H3K36me3 reduction are implicated in "macromolecule catabolic", "cell cycle" and "mRNA processing" biological processes and enriched for "essential and constrained genes", 'FMRP targets in brain', 'post synaptic genes', whose expression peaks early during nervous system. Significantly, CHD8-suppression associated histone H3K36me3 reduction does not functionally impact on global transcriptional levels, but significantly correlates with altered alternative splicing pattern of $\sim 2000$ protein coding genes implicated in "RNA splicing", "mitotic cell cycle phase transition" and "mRNA processing", especially affecting alternative first exon and exon skipping events. In summary, our results point toward broader molecular consequences of CHD8 suppression, implicating altered histone deposition/maintenance and RNA processing regulation as important regulatory processes in ASD.

E. Kerschbamer: None. T. Tripathi: None. S. Erdin: None. E. Salviato: None. F. Di Leva: None. E. Sebestyen: None. M. Arnoldi: None. M. Benelli: None. J. Gusella: None. S. Piazza: None. F. Demichelis: None. F. Ferrari: None. M. Talkowski: None. M. Biagioli: None.

P20.10.A

Exposure of bisphenol A (BPA) in relation to idiopathic central precocious puberty (ICPP) in girls and the epigenetic mechanism

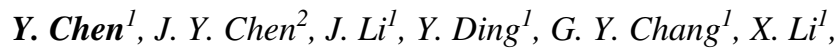

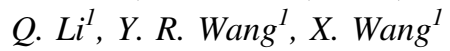

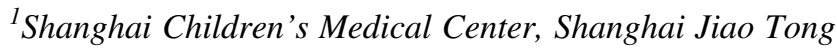
University School of Medicine, Shanghai, China, ${ }^{2}$ Xin Hua Hosipital, Shanghai Jiao Tong University School of Medicine, Shanghai, China

Background: The estrogenic effect of BPA has been widely demonstrated. The relationship between BPA exposure and ICPP in girls is still undefined. Changing of methylation levels of target genes of DNA methyltransferases (DNMTs) and HPG axis may be epigenetic regulation mechanism of BPA exposure induced ICPP in girls Objectives: To investigate the relationship between BPA exposure and ICPP in girls and the epigenetic regulation mechanism.

Methods: 157 6-9 years old girls diagnosed as precocious puberty were recruited from Shanghai Children's Medical Center from July 2017 to August 2018. After detailed laboratory examinations, 56 girls of them were diagnosed as ICPP. At the same time, 56 healthy 6-9 years girls were recruited as control group. All of the participants' urinary BPA concentrations were detected. Methylation levels of $\mathrm{CpG}$ islands of target genes from peripheral blood samples were detected for 56 ICPP girls and 56 healthy girls.

Results: Cases in higher urinary BPA concentration groups were associated with higher risk of ICPP [specific gravity (SG) adjusted $(\mathrm{OR}=2.09,95 \% \mathrm{CI}: 1.05-4.17$, $p=0.037)$; creatinine adjusted $(\mathrm{OR}=1.98,95 \%$ CI: 1.01 $3.89, p=0.046)$ ]. Higher urinary BPA concentration (SG adjusted) group was associated with higher level of methylation of $\mathrm{CpG}$ islands of DNMT3B $(\mathrm{Z}=-1.792$, $p=0.037$ ).

Conclusions: BPA exposure may increase the risk of ICPP in girls. Higher methylation level of $\mathrm{CpG}$ islands of DNMT3B may be associated with higher level of BPA exposure. 
Y. Chen: None. J.Y. Chen: None. J. Li: None. Y. Ding: None. G.Y. Chang: None. X. Li: None. Q. Li: None. Y.R. Wang: None. X. Wang: None.

\section{P20.12.C}

The association between cardiometabolic risk factors and DNA methylation aging across adult lifespan in the Rhineland study

D. Liu $^{l}$, N. Aziz ${ }^{1,2}$, M. M. B. Breteler ${ }^{1,3}$

${ }^{1}$ German Center for Neurodegenerative Diseases (DZNE), Bonn, Germany, ${ }^{2}$ Department of Neurology, University of Bonn, Bonn, Germany, ${ }^{3}$ Institute for Medical Biometry, Informatics and Epidemiology (IMBIE), Faculty of Medicine, University of Bonn, Bonn, Germany

Introduction: DNA methylation-based biomarkers of aging (DNAm aging) are associated with cardiometabolic diseases. Although this may be due to their relation with cardiometabolic risk factors, limited data are available on the association between cardiometabolic risk factors and DNAm aging in the general population.

Methods: We performed a cross-sectional analysis of data from the Rhineland Study, a population-based prospective cohort study in Bonn, Germany. Four types of DNAm aging acceleration estimates (including AgeAccel. Horvath, AgeAccel.Hannum, AgeAccelPheno, and AgeAccelGrim), defined as within individual differences between the epigenetic and chronological age, were calculated. The effects of 20 cardiometabolic risk factors on DNAm aging acceleration were assessed using multiple linear regressions.

Results: Among 3327 participants (mean age 55.0 years (range 30 - 95), 56.0\% women), 14 risk factors were significantly associated with AgeAccelPheno [change in years $(95 \% \mathrm{CI})$ per standard deviation increase]: HDL -0.47 $(-0.81,-0.12)$, triglycerides $0.28(0.04,0.51)$, total cholesterol $0.40(0.09,0.70)$, glucose $0.44(0.21,0.67)$, insulin $0.68(0.45,0.91)$, insulin resistance $0.62(0.39,0.85)$, diastolic blood pressure $0.35(0.11,0.58)$, systolic blood pressure $0.29(0.06,0.53), \mathrm{C}$-reactive protein $0.92(0.70$, 1.14), cystatin $C 0.77(0.53,1.01)$, glomerular filtration rate $-0.67(-0.91,-0.44)$, body mass index $0.99(0.68,1.31)$, waist circumference $1.03(0.78,1.28)$ and percentage body fat $0.90(0.64,1.15)$. Comparable results were obtained when using AgeAccelGrim, AgeAccel.Horvath or AgeAccel.Hannum as outcomes (data not shown).

Conclusions: Multiple cardiometabolic risk factors were consistently associated with various DNAm aging acceleration, which may underlie the association between DNAm aging and cardiometabolic morbidity and mortality.
D. Liu: None. N. Aziz: None. M.M.B. Breteler: None.

P20.13.A

Large scale chromatin accessibility fine mapping of cardiovascular disease associated loci in the human liver

C. D. Brown, B. Wenz, S. Ramdas, M. Caliskan, D. Xin, K. Olthoff, A. Shaked

University of Pennsylvania, Philadelphia, PA, United States

Genome-wide association studies (GWAS) have identified thousands of loci associated with human traits. However, the causal variant, gene, cell type, and mechanism underlying these associations remain unclear. Most GWAS variants are in linkage disequilibrium (LD) with many other non-coding variants, complicating fine-mapping and mechanistic characterization. Cell-type specific gene regulation plays a causal role at many complex trait-associated loci. Changes in chromatin accessibility are key mechanisms leading to gene expression differences. To understand the mechanisms that lead to cardiovascular disease (CVD), the leading cause of mortality worldwide, we quantified chromatin accessibility in more than 200 human liver tissue samples. We identify more than 500,000 open chromatin regions, many of which exhibit tissue-specific activity, and identify transcription factor binding sites at nucleotide resolution. Integration with epigenomic profiles identified specific cis-regulatory elements such as promoters and enhancers. We identifiy thousands of cis-regulatory elements whose chromatin accessibility is associated with local genetic variants, called chromatin accessibility quantitative trait loci (caQTLs). At dozens of CVD GWAS loci, chromatin interactions and colocalization of molecular QTL and GWAS signals generate specific hypotheses about causal variants, affected genes, and cell types. With a combination of experimental approaches in multiple model systems, we fine-map complex trait-associated loci to single-nucleotide resolution. These findings highlight the benefits of integrating multiple cellular traits for the identification and characterization of disease-causing variants and contribute to basic understanding of genetic and epigenetic regulation of gene expression.

C.D. Brown: None. B. Wenz: None. S. Ramdas: None. M. Caliskan: None. D. Xin: None. K. Olthoff: None. A. Shaked: None.

\section{P20.14.B}

DNA methylation profile in Cystic Fibrosis patients with C677T variant of MTHFR gene 
M. Ghughunishvili, S. Surmava, T. Tkemaladze, M. Gagua, E. Abzianidze, E. Kvaratskhelia

\section{Tbilisi State Medical University, Tbilisi, Georgia}

Introduction: Cystic fibrosis (CF) is the most common lethal monogenetic disease in the Caucasian population. Genetic and epigenetic modifiers may play a considerable role in the phenotypic heterogeneity of CF disease. Methylenetetrahydrofolate reductase (MTHFR) is a key regulatory enzyme in the metabolism of folate. A common variant of the MTHFR gene, C677T, is associated with variety of disorders, including cancer, chronic inflammation and etc. In this study we investigated the DNA methylation level of the LINE-1 and Alu interspersed repetitive sequences (IRS) in CF patients with the C677T variant of the MTHFR gene.

Materials and methods: The study was approved by the ethics committee of Tbilisi State Medical University. All patients were genotyped for the CFTR gene. Genomic DNA was isolated from peripheral blood mononuclear cells (PBMCs) of CF patients using QIAamp DNA Mini Kit (Qiagen, US). For Bisulfite treatment of DNA we used EpiTect Bisulfite Conversion Kit (Qiagen, US). The MTHFR C677T polymorphism was detected by PCRRELP method. Methylation levels of LINE-1 and Alu were examined by the combined bisulfite restriction analysisinterspersed repetitive sequences (COBRA-IRS).

Results: Significant differences were observed in methylation levels of both LINE-1 and Alu sequences between CF patients with $\mathrm{CC}$ and TT genotypes. In patients with TT genotypes, frequency of ${ }^{\mathrm{u}} \mathrm{C}{ }^{\mathrm{u}} \mathrm{C}$ (unmethylated Cytosine) Alleles was lower than in patients with $\mathrm{CC}$ genotypes.

Conclusions: Our results indicated that identification of CF patients with the MTHFR C677T variant and study of DNA methylation levels may be useful for personalized treatment approaches.

M. Ghughunishvili: None. S. Surmava: None. T. Tkemaladze: None. M. Gagua: None. E. Abzianidze: None. E. Kvaratskhelia: None.

\section{P20.15.C}

Identification of CFTR cis-regulatory variants

M. COLLOBERT T , K. Rouault ${ }^{2,1}$, C. L'Hostis ${ }^{3,1}$, M. Audrézetet, C. Férec ${ }^{2,1}$, S. Moisan ${ }^{2,1}$

${ }^{1}$ Univ Brest, Inserm, EFS, UMR 1078, GGB, Brest, France,

${ }^{2}$ Laboratory of Molecular Genetics and Histocompatibility, CHRU Brest, Brest, France, ${ }^{3}$ Gaétan Saleün association, Brest, France

Although, more than 2075 variants have been discovered in Cystic Fibrosis Transmembrane conductance Regulator
(CFTR) gene, responsible of Cystic Fibrosis (CF) or one of CFTR-related disorders, the congenital bilateral absence of vas deferens (CBAVD), some patients have an incomplete genotype or present extremes phenotypes. Development of chromatin conformation study techniques has identified several long-range regulatory elements of CFTR gene. 17 cis-regulatory regions of CFTR gene have been sequenced in 63 CBAVD patients. By comparing to European population, some variants display a frequency significantly different. In particular, one variant located in cis-regulatory region of intron 21, is 40 times more frequent in this group. Activity tests in Caco-2 intestinal cells have shown strong cooperative effects of the intron 21 enhancer with several strong enhancers specific to intestinal cells (Intron 1, 11 and at $+15.6 \mathrm{~kb}$ of the CFTR gene) on CFTR promoter activity. Insertion of the interest variant into intron 21 shows a strong decrease of CFTR promoter activity and the loss of enhancers cooperation. Chromatin immunoprecipitation (ChIP) analyses of HNF1a and EP300 transcription factors have shown their fixations in intron 1, 11 and 21 enhancers of CFTR gene. This variant could affect the recruitment of regulatory elements. In conclusion, some significantly variants are identified in CBAVD patients with incomplete genotype, notably one regulatory variant localised in the intron 21 enhancer of $C F T R$ gene. This region plays an important role in regulation of CFTR locus in combining with 3 others intestinal specific enhancers.

M. Collobert: None. K. Rouault: None. C. L'Hostis: None. M. Audrézet: None. C. Férec: None. S. Moisan: None.

\section{P20.17.B}

From man to mouse: The discovery and validation of CYR61 as a regulator of body composition

\section{S. Metz, T. J. Grevengoed, T. O. Kilpeläinen, M. P. Gillum}

Novo Nordisk Foundation Center for Basic Metabolic Research, Copenhagen, Denmark

Obesity is a major contributor to global burden of chronic disease, in particular type 2 diabetes and cardiovascular disease. There is currently a great unmet need for developing effective and safe anti-obesity treatments. Genomic studies provide novel molecular targets for the treatment of obesity by discovering genes and pathways involved in obesity. We have recently identified an association between the rare variant Ser316Cys in CYR61, implicated in angiogenesis, and increased body fat percentage $\left(P=1.1 \times 10^{-9}\right)$, in particular trunk fat percentage $\left(3.8 \times 10^{-11}\right)$. The Cys316 minor allele was associated with a $0.4 \%$ higher body fat percentage and $0.5 \%$ higher trunk fat percentage. 
In a mouse model overexpressing human Cyr61 (hCyr61) in the fat tissue, we show that the hCyr61-overexpression leads to increased body fat percentage under high fat diet, due to a switch in body composition, while body weight did not differ from wild type mice. The endogenous Cyr61 remained unaffected by hCyr61, but is abundantly expressed in all fat depots, clearly supporting our hypothesis of CYR61 being involved in fat tissue development.

Our results suggest a critical role of CYR61 in the regulation of body composition and adipocyte development, which may be mediated by adipogenesis. Ongoing in vitro and in vivo experiments will provide further insights into the mechanistic role of Cyr61 in the development of obesity and metabolic dysfunction. Ultimately, understanding the fundamental mechanisms of adipogenesis and CYR61 may enable new therapeutic options for the treatment of obesity by lowering fat mass and retaining lean mass.

S. Metz: None. T.J. Grevengoed: None. T.O. Kilpeläinen: None. M.P. Gillum: None.

\section{P20.19.A}

The penetrance of aortic arch defects in 22q11DS can be modulated by dietary vitamin A levels and is dependent on the maternal genotype

\section{E. Amengual-Cladera ${ }^{l}$, D. Medina Chavez ${ }^{1}$, E. Lynton Pons $^{l}$, P. Sureda Horrach ${ }^{1}$, V. J. Asensio Landa ${ }^{l}$, J. Rocha ${ }^{2}$, J. Ruiz Rodriguez ${ }^{1}$, A. Tubau ${ }^{3}$, M. Juan ${ }^{3}$, G. Lania $^{4}$, M. Bilio ${ }^{4}$, A. Baldini ${ }^{4}$, L. Ruiz Guerra ${ }^{5}$, C. Vives Bauza $^{5}$, M. Logotheti ${ }^{6}$, E. Pilalis ${ }^{6}$, A. Chatziioannou ${ }^{6}, D$. Heine Suñerl}

${ }^{1}$ Hospital Universitari Son Espases/Institut d'Investigació Sanitaria de Palma (IDISBA), Palma, Spain, ${ }^{2}$ Grup de Biologia Computacional $i$ Bioinformàtica (BIOCOM), Universitat de les Illes Balears, Palma, Spain, ${ }^{3} 4$ Servicio de Ginecología y Obstetricia, Hospital Universitario Son Llàtzer, Palma, Spain, ${ }^{4}$ Institute of genetics and Biophysics Adriano Buzzati-Traverso, Palma, Spain, ${ }^{5}$ Grupo Neurobiología, Fundació Institut d'Investigació Sanitària de les Illes Balears (IdISBa, Palma, Spain, ${ }^{6}$ Institute of Biology, Medicinal Chemistry and Biotechnology, NHRF, 11635 Athens, Greece; e-NIOS Applications PC, Palma, Spain

$22 \mathrm{q} 11 \mathrm{DS}$ is caused by a recurrent $3 \mathrm{Mb}$ deletion present in $90 \%$ of patients. Despite this genomic homogeneity there is a great phenotypic variability. This indicates the existence of genetic and/or epigenetic modifiers. Which are exactly these modifiers and, in particular, how they act is still poorly defined, but diet could be one of the candidates. Our hypothesis is that altered vitamin A (vitA) levels in the diet of pregnant mothers could modify the penetrance of one of the phenotypic traits (Aortic Arch Defects; AADs). We used a mouse model (Df1) with a hemizygous deletion equivalent to the human 22q11.2DS. Our experimental setup consisted of 3 diet groups (vitA-supplemented, -control and vitA-deficient) and 2 types of crosses between Df1 mothers with WT fathers and WT mothers with Df1 fathers. Embryos were collected at gestational day E18.5 and phenotyped. Serum retinol levels were determined in the blood of the pregnant females. AADs are more frequent in the Df1 embryos of WT mothers fed a vitA supplemented diet $(47 \%)$ than in those fed with a vitA deficient diet (29\%). On the contrary, when the mother is Df1 we observe more AADs in Df1 embryos of mothers fed a vitA deficient $\operatorname{diet}(45 \%)$ than in those fed a supplemented diet $(27 \%)$. In general, independently of the diet, we find a correlation of higher frequencies of AADs with lower expression of mitochondrial genes, and lower expression of key heart development genes. Ongoing studies on genome differential methylation will also be presented.

E. Amengual-Cladera: None. D. Medina Chavez: None. E. Lynton Pons: None. P. Sureda Horrach: None. V.J. Asensio Landa: None. J. Rocha: None. J. Ruiz Rodriguez: None. A. Tubau: None. M. Juan: None. G. Lania: None. M. Bilio: None. A. Baldini: None. L. Ruiz Guerra: None. C. Vives Bauza: None. M. Logotheti: None. E. Pilalis: None. A. Chatziioannou: None. D. Heine Suñer: None.

\section{P20.20.B}

The Reversion Curve of cg05575921 Methylation in Response to Smoking Cessation: A New Dynamic Epigenetic Tool for Monitoring and Incentivizing Smoking Cessation.

\section{R. A. Philibert, K. Dawes, M. Dogan, W. Philibert, B. Hundley, J. Mills, J. Long, A. Gerke, J. Wilson, P. Nagpal, A. Comellas, E. Hoffman}

\section{University of Iowa, Iowa City, IA, United States}

Current methods for assessing and monitoring the effectiveness of smoking cessation are either unreliable (i.e. $\mathrm{CO}$ monitors) or confounded by use of nicotine replacement (i.e. cotinine assays). This prevents the use of highly effective, socially responsive, incentive-based reinforcement smoking cessation therapies. Recent developments in epigenetics may address this shortcoming. Previously, we have shown that a methylation sensitive digital PCR (MSdPCR) assay of DNA methylation at cg05575921 can precisely quantitate current cigarette consumption using DNA from saliva or whole blood. However, whether changes in that DNA methylation can be used to monitor the effectiveness of smoking cessation was uncertain. To determine this, we monitored serum cotinine and 
determined DNA methylation levels for ninety days in 63 subjects participating in a nicotine replacement-free incentive based smoking cessation program. (NIH R01 HL130883) Overall, 18 of 63 (29\%) subjects had undetectable levels of cotinine at the 30 day visit and maintained undetectable levels through the 90 day exit visit. Correspondingly, cg05575921 levels showed a marked time and dose-dependent reversion of methylation with $\operatorname{cg} 05575921$ levels increasing over $10 \%$ with 3 months of cessation in the heaviest smokers (initial cg05575921<50\%), while levels in more moderate smokers increasing only $7 \%$ after 3 months. We conclude that MSdPCR cg05575921 assessments are a sensitive, rapid method of monitoring the effectiveness of smoking cessation. Because these DNA methylation levels are not affected by nicotine replacement and are quantitative in nature, they can be used in socially responsible, incentive-based programming for the quantitative reduction of smoking induced harms.

R.A. Philibert: A. Employment (full or part-time); Significant; Behavioral Diagnostics. E. Ownership Interest (stock, stock options, patent or other intellectual property); Significant; Behavioral Diagnostics. K. Dawes: None. M. Dogan: None. W. Philibert: None. B. Hundley: None. J. Mills: None. J. Long: None. A. Gerke: None. J. Wilson: None. P. Nagpal: None. A. Comellas: None. E. Hoffman: None.

\section{P20.21.C}

Leveraging a co-methylation network to predict regulatory effects of DNA methylation sites and human phenotypes

\section{S. Li, C. Qi, P. Deelen, F. Boulogne, N. de Klein, J. van der Velde, G. Koppelman, L. Franke, M. Swertz, H. Westra}

\section{University Medical Centre Groningen, Groningen, Nether- lands}

DNA methylation is an important epigenetic signal with potential regulatory effects on gene expression, and aberrant methylation patterns have been previously used as biomarkers for disease. While we have shown recently that methylated DNA sites are correlated with gene expression levels in blood, the function of many other DNA methylation sites and co-methylation, however, is unclear. Previous studies have used gene co-expression networks to predict links between genes and Human Phenotype Ontology (HPO) terms. Here, we compare co-methylation and coregulation patterns, and investigate the functions of DNA methylation sites to improve gene-symptom predictions.

For this purpose, we have created a co-methylation network using 33,000 public 450k Illumina methylation assays from various tissues and cell types. We identified
6,067 significant correlations between DNA methylation and genes through a meta-analysis using blood, nasal and brain tissues. These correlations link methylation sites with genes, and consequently to HPO terms associated with those genes. Using this data, we will compare the comethylation network with our previously published coexpression network consisting of 32,000 samples in terms of their power to predict HPO term membership per gene and methylation site. We expect that this comparison will assign methylation sites to human phenotypes, and provide functional interpretation for many others.

Our preliminary results comparing networks in 2905 blood samples show that the co-methylation and coexpression networks have comparable predictive power. In the future, we will combine the two networks to improve prioritization of disease-causing genes.

S. Li: None. C. Qi: None. P. Deelen: None. F. Boulogne: None. N. de Klein: None. J. van der Velde: None. G. Koppelman: None. L. Franke: None. M. Swertz: None. H. Westra: None.

P20.25.A

Difference of significant methylation change by heavy metal concentration in atopic dermatitis

S. Yu, E. Koh, S. Kim, S. Lee, J. Lee, S. Hwang

Hanyang University, Ansan, Korea, Republic of

As environmental pollution is getting worse, many environmental diseases are caused by various factors. For this reason, the importance of research between environmentally harmful substances and diseases is increasing. In particular, exposure to harmful substances during pregnancy affects the fetus and can lead to disease. Therefore, epigenome was studied to confirm the change caused by environmental factors. The effect of heavy metals on atopic dermatitis, which is representative of environmental diseases, was examined using DNA methylation experiments, one of the methods for identifying epigenomes. The samples used in this study were cord blood DNA from some MOCEH cohort samples. The degree of methylation according to the concentration of heavy metals was confirmed in 84 children diagnosed with atopic dermatitis. For methylation profiles, an experiment was performed using an Infinium Human Methylation EPIC kit (Illumina, CA, USA) and analyzed using GenomeStudio (version 2011.1). As a result, 37 genes in mercury and 58 in lead were found in regions with significant methylation differences when exposed to high concentrations compared to low concentrations. Pathway analysis was performed using genes selected from each heavy metal group, and it was confirmed that specific genes were involved in the cytokine signaling in Immune system. 
In conclusions, the effect of heavy metals on atopic dermatitis was confirmed. In other words, it was confirmed that environmental diseases may be caused by harmful environmental substances. This study was supported by Korea Environment Industry \& Technology Institute(KEITI) through "the Environmental Health Action Program", funded by Korea Ministry of Environment(MOE) (2017001360005).

S. Yu: None. E. Koh: None. S. Kim: None. S. Lee: None. J. Lee: None. S. Hwang: None.

\section{P20.27.C}

Dive into Epigenetics and Gene Regulation - Navigation using the ENCODE Portal

\section{J. N. Au, I. Gabdank, Y. Luo, M. Kagda, B. Lam, I.}

Youngworth, P. Adenekan, U. K. Baymuradov, S. Miyasato, M. Simison, K. Graham, O. Jolanki, J. P. Jou, J. Lee, C. Litton, K. Z. Lin, E. O'Neill, P. Sud, F. Tanaka, J. S. Strattan, B. C. Hitz, J. M. Cherry

Department of Genetics, School of Medicine, Stanford University, Stanford, CA, United States

The Encyclopedia of DNA Elements (ENCODE) project is an international collaboration of research groups funded by the NHGRI, whose main goal is to identify all functional elements of the human genome. The ENCODE portal (https://www.encodeproject.org/), established and maintained by the ENCODE Data Coordination Center (DCC), provides unrestricted access to data generated by the members of the ENCODE consortium and other related projects such as Model organism Encyclopedia of Regulatory Networks (modERN), Genomics of Gene Regulation (GGR), Roadmap Epigenomics Project (Roadmap), and ENCODE G-TEx (EN-Tex). The DCC develops and updates the data model and uniform processing pipelines that allow transparency and reproducibility of the experimentally collected and computationally generated data. With a constant influx of data, the DCC consistently implements new ways for the users of the ENCODE portal to search and identify related experiments, understand how assays were performed, and track the provenance of the data. Currently, the ENCODE portal hosts more than 15 thousand datasets, representing experimental results for over 75 distinct tissue and cell types using more than 40 distinct high-throughput genomic assays.

Richness, diversity, and structure of the data on the ENCODE portal make it an invaluable resource for the scientific community. The presence of curated and standardized experimental results from assays like RNA-seq, ChIPseq, ATAC-seq, and Hi-C allow for convenient accessibility to any genomics investigator. Here we show how the
ENCODE portal can be used as a multifaceted lens to investigate various biological and regulatory properties of chromatin state and gene expression.

U24HG009397-01

J.N. Au: None. I. Gabdank: None. Y. Luo: None. M. Kagda: None. B. Lam: None. I. Youngworth: None. P. Adenekan: None. U.K. Baymuradov: None. S. Miyasato: None. M. Simison: None. K. Graham: None. O. Jolanki: None. J.P. Jou: None. J. Lee: None. C. Litton: None. K. Z. Lin: None. E. O'Neill: None. P. Sud: None. F. Tanaka: None. J.S. Strattan: None. B.C. Hitz: None. J. M. Cherry: None.

P20.28.A

Impact of natural compounds in the DNA methylation of RASSF1A in triple-negative breast cancer cell lines and epi-drug discovery

C. A. RAINHO, J. H. M. Assumpção, F. A. S. France, D. P. Callegari, B. M. Barbosa, A. A. S. Takeda, J. M. Sforcin

SAO PAULO STATE UNIVERSITY - UNESP, Botucatu, Brazil

Introduction: Triple-negative breast cancer (TNBC) is an aggressive subtype of breast cancer frequently associated with resistance to chemotherapy, a higher risk of recurrence and metastasis. Combined epi-drugs targeting DNA methyltransferases (DNMTs) and histone deacetylases (HDACs) are a promising alternative to reverse acquired therapy resistance. Since natural origin products are considered a promising source of epi-drugs, the present study aimed to identify propolis-derived small molecules able to interact with human DNMT1 or HDAC2.

Materials and Methods: The effects of propolis treatment were evaluated in four TNBC cell lines (BT-20, BT-549, MDA-MB-231, and MDA-MB-436). Cell viability was accessed by the MTT test and the DNA methylation analysis was performed by Methylation-Specific Polymerase Chain Reaction (MS-PCR). A set of 3,146 propolisderived compounds were retrieved from scientific literature and used in the molecular screening strategy.

Results: Propolis reduces the cell viability of TNBC cells and partly demethylates the promoter region of the tumor suppressor RASSF1A as well as modulates the RASSF1 protein expression levels in BT-549 cells. After filtering and curating 1,002 propolis-derived chemicals, $58.5 \%$ of the molecules were grouped into five most representative clusters according to the components' atomic positions. After hierarchical clustering, the in silico screening analysis indicated catechin 3'-O-gallate and propolin-B as possible new DNMT and HDAC inhibitors, respectively. 
Conclusions: Our data indicate that propolis can modulate the expression of the RASSF1 gene via epigenetic mechanisms. Also, the computational approach predicted that propolis-derived compounds can interact with DNMT1 and HDAC2 and should considered in future studies of new epi-drugs identification.

C.A. Rainho: None. J.H.M. Assumpção: None. F.A.S. France: None. D.P. Callegari: None. B.M. Barbosa: None. A.A.S. Takeda: None. J.M. Sforcin: None.

\section{P20.29.B}

Line 1 methylation patterns in placenta and cord blood at birth reveal associations with neonatal weight and maternal methylation

O. Rondinone ${ }^{l}$, S. Motta ${ }^{2}$, P. Colapietro ${ }^{3}, J$. Costanza $^{l}, M$. Camanni $^{l}$, E. Beretta ${ }^{2}$, L. Fontana ${ }^{1}$, A. Morotti ${ }^{1}, S$.

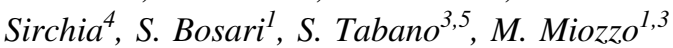

${ }^{1}$ Scientific Direction, Fondazione IRCCS Ca' Granda Ospedale Maggiore Policlinico, Milan, Italy, ${ }^{2} 1$ Medical Genetics, Department of Pathophysiology \& Transplantation, Università degli Studi di Milano, Milan, Italy, ${ }^{3}$ Medical Genetics, Department of Pathophysiology \& Transplantation, Università degli Studi di Milano, Milan, Italy, ${ }^{4} 3$ Medical Genetics, Department of Health Sciences, Università degli Studi di Milano, Milan, Italy, ${ }^{5}$ Medical Genetics Unit, Fondazione IRCCS Ca' Granda Ospedale Maggiore Policlinico, Milan, Italy

During intrauterine life, environmental perturbations can influence newborn outcome by means of DNA methylation modifications with long-term consequences. L-1 (Long Interspersed Nucleotide Elements) sequences are transposable elements accounting for $17 \%$ of the human genome, considered a surrogate for global methylation. To investigate the global methylation at birth in association with neonatal birth, we recruited at the delivery 195 women with singleton, spontaneous and uncomplicated pregnancies and we investigated L-1 methylation by pyrosequencing in cord and maternal blood and in placental samples. We found a significant severe L-1 hypomethylation in placenta from SGA (Small for Gestational Age, $\leq 10$ percentile) compared with normal and LGA babies (Large for Gestational Age). This finding suggests a compensatory epigenetic mechanism in response to a reduced growth; or a placental insufficiency responsible for SGA. This association was not present in cord blood. A strong correlation (Pearson $r=0,79$, pvalue $<0,01)$ of L-1 methylation between maternal $(62,7 \%$ +/- 5,3) and cord (mean+/- StDev: 63,3\% +/- 5,4) blood was found. This similarity seems to be lost after birth, based on the weak correlation ( $r=0,26, p$ value $>0,05$ ) observed in the L-1 methylation levels of 31 mothers and children
( $>4$ years of age). In order to explore also the methylation profiling of gene promoters in these cases, MethylSeq experiments are ongoing.

O. Rondinone: None. S. Motta: None. P. Colapietro: None. J. Costanza: None. M. Camanni: None. E. Beretta: None. L. Fontana: None. A. Morotti: None. S. Sirchia: None. S. Bosari: None. S. Tabano: None. M. Miozzo: None.

\section{P20.31.A}

Frameshift mutations at the C-terminus of HIST1H1E result in a specific DNA hypomethylation signature

A. Ciolfi, ${ }^{1}$ E. Aref-Eshghi ${ }^{2,3}$, S. Pizzi ${ }^{1}$, L. Pedace ${ }^{1}$, E. Miele $^{1}$, J. Kerkhof ${ }^{2,3}$, E. Flex ${ }^{4}$, S. Martinelli ${ }^{4}$, F. C. Radio ${ }^{\text {, }}$ C. A. L. Ruivenkamp ${ }^{5}$, G. W. E. Santen ${ }^{5}$, E. K. Bijlsma ${ }^{5}$, D. Q. C. M. Barge-Schaapveld ${ }^{5}$, K. Ounap ${ }^{6,7}$, V. Mok Siu $^{3}, F$. F. Kooy ${ }^{8}$, B. Dallapiccola ${ }^{1}$, B. Sadikovic ${ }^{2,3}$, M. Tartaglia ${ }^{1}$

${ }^{1}$ Ospedale Pediatrico Bambino Gesù, Rome, Italy, ${ }^{2}$ Western University, London, ON, Canada, ${ }^{3}$ London Health Sciences Centre, London, ON, Canada, ${ }^{4}$ Istituto Superiore di Sanità, Rome, Italy, ${ }^{5}$ Leiden University Medical Center, Leiden, Netherlands, ${ }^{6}$ Tartu University Hospital, Tartu, Estonia, ${ }^{7}$ University of Tartu, Tartu, Estonia, ${ }^{8}$ University of Antwerp, Antwerp, Belgium

Introduction: Histones regulate dynamic packaging of nuclear DNA in chromatin to achieve efficient compaction of the genome as well as to regulate and coordinate DNA replication, transcription, and repair. Recently, a narrow spectrum of functionally equivalent frameshift mutations at the C-terminal tail of HIST1H1E, a member of the linker histone family, has been causally linked to Rahman syndrome. Previous work demonstrated that these mutations disrupt proper compaction of DNA and affected multiple cellular processes, resulting in accelerated replicative senescence, and are associated with a specific methylation pattern.

Results: Methylome analysis from peripheral blood samples of six affected subjects led us to identify $\sim 9,000$ $\mathrm{CpG}$ probes located in $\sim 600$ differentially methylated regions, defining a specific and predominantly hypomethylated pattern. This "episignature" was enriched for genes involved in neuronal system development and function, in particular at the synaptic level. A computational classifier trained on these methylation profiles yielded full sensitivity and specificity in detecting subjects carrying dominantacting HIST1H1E frameshift mutations among healthy controls or individuals carrying pathogenic lesions in other proteins of the epigenetic machinery associated with human disease. Applying this model to a cohort of probands 
without clinical diagnosis allowed us to reach diagnosis for a previously undiagnosed patient.

Conclusions: We demonstrate an epigenetic signature in subjects with a defined class of HISTIHIE mutations. We show that this signature is specific and can be used to reach molecular diagnosis. These findings further demonstrate the utility of DNA methylation episignatures as a tool to favor diagnosis and genetic variant classification.

A. Ciolfi: None. E. Aref-Eshghi: None. S. Pizzi: None. L. Pedace: None. E. Miele: None. J. Kerkhof: None. E. Flex: None. S. Martinelli: None. F.C. Radio: None. C.A. L. Ruivenkamp: None. G.W.E. Santen: None. E.K. Bijlsma: None. D.Q.C.M. Barge-Schaapveld: None. K. Ounap: None. V. Mok Siu: None. F.F. Kooy: None. B. Dallapiccola: None. B. Sadikovic: None. M. Tartaglia: None.

P20.32.B

Germline mutations in $F A F 1$ are associated with hereditary colorectal cancer

L. Bonjoch $^{1}$, S. Franch-Expósito ${ }^{2}$, P. Garre ${ }^{3}$, S. Belhadj ${ }^{4}$, J. Muñoz ${ }^{2}$, C. Arnau-Collell ${ }^{2}$, M. Díaz-Gay ${ }^{2}$, A. GratacósMulleras $^{2}$, G. Raimondi ${ }^{5}$, C. Esteban-Jurado ${ }^{2}$, Y. Soares de Lima $^{2}$, C. Herrera-Pariente ${ }^{2}$, M. Cuatrecasas ${ }^{6}$, T. Ocaña ${ }^{2}$, A. Castells ${ }^{2}$, C. Fillat ${ }^{5}$, G. Capellá ${ }^{4}$, F. Balaguer ${ }^{2}, T$. Caldés $^{3}$, L. Valle ${ }^{4}$, S. Castellvi-Bel ${ }^{2}$

${ }^{1}$ IDIBAPS-Hospital Clínic-CIBERehd, Barcelona, Spain, ${ }^{2}$ Gastroenterology Department, Centro de Investigación Biomédica en Red de Enfermedades Hepáticas y Digestivas (CIBEREHD), Hospital Clínic, IDIBAPS, Barcelona, Spain, ${ }^{3}$ Molecular Oncology Laboratory, Hospital Clínico San Carlos, Instituto de Investigación Sanitaria del Hospital Clínico San Carlos (IdISSC), Madrid, Spain, ${ }^{4}$ Hereditary Cancer Program, Catalan Institute of Oncology, IDIBELL, Hospitalet de Llobregat, Spain, ${ }^{5}$ Centro de Investigación Biomédica en Red de Enfermedades Raras (CIBERER), Gastrointestinal and Pancreatic Oncology, IDIBAPS, Barcelona, Spain, ${ }^{6}$ Department of Pathology, Hospital Clínic, IDIBAPS, Barcelona, Spain

Introduction: A significant proportion of colorectal cancer (CRC) cases present familial aggregation but the underlying inherited cause is unknown. The aim of this study is to identify new genes involved in the germline predisposition to CRC, by means of an exhaustive functional characterization of genetic variants previously prioritized by our research group in familial $\mathrm{CRC}$ cases.

Materials and Methods: We analyzed 75 patients with familial CRC with an unknown germline basis from 40 families by whole-exome sequencing. An external replication cohort of 473 families was also examined by targeted- gene sequencing. We developed a FAF1 knockout cellular model by CRISPR-Cas gene editing, and the identified genetic variants were produced by site-directed mutagenesis and expressed by transfection. Apoptosis, proliferation and protein-specific functional assays were carried out to characterize $F A F 1$ role in cancer predisposition.

Results: A predicted pathogenic FAF1 variant (c.1111G >A; p.Asp371Asn) was detected in the initial cohort segregating in four patients of the same family. A second genetic variant was detected in the replication cohort (c.254G $>$ C; p.Arg85Pro). Both variants showed protein instability and, once transfected, produced an abnormal cellular phenotype by promoting apoptosis resistance and sustaining both NF- $\mathrm{BB}$ and Wnt signaling pathways.

Conclusions: Our findings suggest that FAF1 may be involved in inherited predisposition to $\mathrm{CRC}$, being resistance to apoptosis the plausible pathogenic mechanism.

Grant support: FJCI-2017-32593, 2019FI_B2_00203, LCF/BQ/DI18/11660058, Fondo de Investigación Sanitaria/ FEDER (16/01292, 17/00878), AECC (GCB13131592CAST), Spanish Ministry of Science (SAF2016-80888-R, BIO2017-89754-C2-2R), PERIS (SLT002/16/00398, SLT002/16/0037), Generalitat de Catalunya (GRPRE 2017SGR21, GRC 2017SGR653, 2017SGR723, 2017SGR861, 2017SGR1282), COST Action CA17118, CIBEREHD, CIBERONC, CIBERER.

L. Bonjoch: None. S. Franch-Expósito: None. P. Garre: None. S. Belhadj: None. J. Muñoz: None. C. Arnau-Collell: None. M. Díaz-Gay: None. A. GratacósMulleras: None. G. Raimondi: None. C. EstebanJurado: None. Y. Soares de Lima: None. C. HerreraPariente: None. M. Cuatrecasas: None. T. Ocaña: None. A. Castells: None. C. Fillat: None. G. Capellá: None. F. Balaguer: None. T. Caldés: None. L. Valle: None. S. Castellvi-Bel: None.

\section{P20.34.A}

Androgenetic alopecia: First functional evidence for an interaction between two GWAS loci

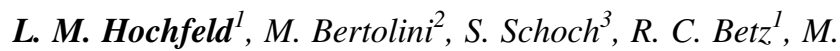
M. Nöthen ${ }^{l}$, S. Heilmann-Heimbach ${ }^{l}$

${ }^{1}$ Institute of Human Genetics; University of Bonn, School of Medicine \& University Hospital Bonn, Bonn, Germany, ${ }^{2}$ Monasterium Laboratory, Skin and Hair Research Solutions, Münster, Germany, ${ }^{3}$ Department of Neuropathology; University of Bonn Medical Center, Bonn, Germany

One of the strongest genetic risk loci for androgenetic alopecia (AGA) is located in WNT10A (2q35). Here, a significant regulatory effect of the lead variants on the expression of the hair cycle regulator WNT10A has been 
reported. Interestingly, one of these variants is located within a binding site for the transcription factor EBF1, whose gene is located at another AGA risk locus (5q33.3). We hypothesized that changes in EBF1 mediated regulation of WNT10A expression may contribute to the deregulated hair cycle dynamics observed in AGA. To analyze a potential interaction between EBF1 and WNT10A, we performed luciferase reporter assays in HEK cells. Our experiments confirmed that EBF1 activates the WNT10A promoter and that the WNT10A/EBF1 interaction was decreased with the AGA risk allele. To further investigate this potential interaction in AGA-relevant tissue and identify the relevant cell population we performed immunofluorescence co-staining in human scalp hair follicle. The strongest co-expression of EBF1 and WNT10A was observed in hair follicle keratinocytes of the inner and outer root sheath. A major challenge in complex genetics is to bridge the gap between association and function. To our knowledge, our study is the first functional follow-up study on AGA that (i) together with previous mRNA data enabled identification of a regulatory mechanism at an AGA risk locus and (ii) proves functional interaction between two risk loci that is likely to contribute to the pathophysiological changes in hair cycle dynamics.

L.M. Hochfeld: None. M. Bertolini: None. S. Schoch: None. R.C. Betz: None. M.M. Nöthen: None. S. Heilmann-Heimbach: None.

\section{P20.38.B}

Integrated analysis of transcriptional regulation in hypertrophic cardiomyopathy cased by truncating MYBPC3 mutations

J. Pei ${ }^{l}$, M. Schuldt ${ }^{2}$ E. Nagyova ${ }^{1}$, Z. Gu ${ }^{l}$, S. E. Bouhaddani $^{1}$, J. Calis ${ }^{1}$, L. M. Dorsch ${ }^{2}$, N. A. M. van den Dungen ${ }^{1}$, N. Lansu', B. J. Boukens ${ }^{3}$, I. R. Efimov ${ }^{4}$, M. Michels $^{5}$, M. C. Verhaar ${ }^{1}$, R. de Wegerl, A. Vink ${ }^{1}, H$. W. Uh $^{1}$, C. Cheng ${ }^{1}$, D. W. Kuster ${ }^{2}$, M. Mokry' ${ }^{1}$, J. van der Velden $^{2}, F$. W. Asselbergs ${ }^{1,6,7}$

${ }^{1}$ Universitair Medisch Centrum Utrecht, Utrecht, Netherlands, ${ }^{2}$ Amsterdam UMC, Vrije Universiteit Amsterdam, Amsterdam, Netherlands, ${ }^{3}$ Amsterdam UMC, Amsterdam, Netherlands, ${ }^{4}$ The George Washington University, Washington, WA, United States, ${ }^{5}$ Erasmus Medical Centre, Rotterdam, Netherlands, ${ }^{6}$ Netherlands Heart Institute, Utrecht, Netherlands, ${ }^{7}$ University College London, London, United Kingdom

Introduction: Transcription factors (TFs) bind to regulatory DNA sequences, such as promoters and enhancers, to control the level of RNA transcription and protein translation as a reaction to a disease stimulus. However, little is known about how the patient's heart epigenetically responds to pathogenic truncating variants (tv) in the MYBPC3 gene that cause hypertrophic cardiomyopathy (HCM), a devastating cardiac disease with high morbidity and mortality.

Methods and Results: To address the interplay between the chromatin activity and the subsequent RNA and protein products in patients, we performed H3K27ac ChIP-seq, RNA-seq, and proteomics on human cardiac tissues from 13 tvMYBPC3 HCM patients and 10 healthy donors. We identified 9,310 differentially acetylated regions and 2,033 differentially expressed genes between patients and controls using Deseq2. Gene enrichment analysis showed biological processes mostly in extracellular matrix formation and lipid metabolism. Besides, we identified a list of TFs that showed altered mRNA expression levels and the acetylation levels of their binding regions were altered. We also obtained top 2,000 regions and genes that were driving the major variation among all samples using O2PLS. Allelic imbalance of wild type and mutated MYBPC3 mRNA expression was observed. After combining results from both approaches in patients vs. controls, we further detected the expression levels of 45 proteins significantly changed in the same direction as changes in their encoding genes. Notably, several identified proteins have not been linked to HCM previously.

Conclusions: Our integrated omics analysis identified novel candidates involved in cellular remodeling and dysfunction in tvMYBPC3 HCM.

J. Pei: None. M. Schuldt: None. E. Nagyova: None. Z. Gu: None. S.E. Bouhaddani: None. J. Calis: None. L.M. Dorsch: None. N.A.M. van den Dungen: None. N. Lansu: None. B.J. Boukens: None. I.R. Efimov: None. M. Michels: None. M.C. Verhaar: None. R. de Weger: None. A. Vink: None. H.W. Uh: None. C. Cheng: None. D.W. Kuster: None. M. Mokry: None. J. van der Velden: None. F.W. Asselbergs: None.

\section{P20.39.C}

ImprintSeq: A new sequencing panel to detect methylation alterations in human imprinted regions

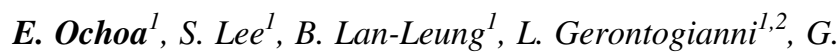
Pérez de Nanclares ${ }^{3}$, R. Martinez ${ }^{3}$, G. Clark ${ }^{1,4}$, E. Martin $^{4}$, L. A. Castano ${ }^{3}$, L. Bottolo ${ }^{1,2,5}$, E. R. Maher ${ }^{1}$

${ }^{1}$ Department of Medical Genetics, Cambridge, United Kingdom, ${ }^{2}$ The Alan Turing Institute, London, United Kingdom, ${ }^{3}$ Biocruces-Bizkaia Health Research Institute, Barakaldo, Spain, ${ }^{4}$ Cambridge NIHR BRC Stratified Medicine Core Laboratory NGS Hub, Cambridge, United Kingdom, ${ }^{5}$ MRC Biostatistics Unit, Forvie Site, Cambridge, United Kingdom 
Congenital imprinting disorders (CIDs) are a group of developmental disorders with overlapping clinical and molecular features that result from genetic and epigenetic alterations in imprinted genes and imprinting control regions. Though specific CIDs are associated with locusspecific imprinting defects, a subset of cases showed multiple imprinting defects across the genome (multi-locus imprinting disturbances (MLIDs)). MLID has been described in a broad ranfge of CIDS but most frequently Beckwith-Wiedemann syndrome (BWS), Silver-Russell syndrome (SRS), Transient neonatal diabetes (TNDM) and Pseudohypoparathyroidism type 1B (PHP1b). The specific impact of MLIDs on clinical phenotype is not well defined though some patients with MLID will have a discordant epigenotype-phenotype (e.g. clinical features of SRS and molecular findings associated with SRS). The availability of an accurate, quantitative and scalable highthroughput method to interrogate many imprinted regions simultaneously would be advantageous for MLID research and clinical diagnostics. We have designed a custom targeted methylation sequencing panel that covered the most relevant imprinting DMRs. We tested ImprintSeq in healthy controls $(n=70)$ and CIDs $(n=142)$ including BWS $(n=$ 59), SRS $(n=26)$, TNDM $(n=15)$ and PHP1b $(n=42)$. ImprintSeq allowed the identification of MLIDs and locus specific methylation defects, as well as provides a useful tool to explore the significance of MLID in CIDs.EO is funded by/supported by/receives funding from the National Institute for Health Research [Cambridge Biomedical Research Centre at the Cambridge University Hospitals NHS Foundation Trust]. This research was supported by the Cambridge NIHR BRC Stratified Medicine Core Laboratory NGS Hub and a MRC Clinical Infrastructure Award.

E. Ochoa: None. S. Lee: None. B. Lan-Leung: None. L. Gerontogianni: None. G. Pérez de Nanclares: None. R. Martinez: None. G. Clark: None. E. Martin: None. L.A. Castano: None. L. Bottolo: None. E.R. Maher: None.

\section{P20.42.C}

Identification and characterization of functional BMIassociated CpGs at the LGALS3BP locus

M. Gomez-Alonso ${ }^{\text {, I. Dahlman }}{ }^{2}$, C. Gieger ${ }^{1}$, A. Peters ${ }^{3,4}$, J. Winkelmann ${ }^{5}$, H. Laumen $^{6}$, A. Kretschmer ${ }^{1}$, M. Waldenberger ${ }^{l, 4}$

${ }^{1}$ Research Unit Molecular Epidemiology, Institute of Epidemiology, Helmholtz Zentrum München, German Research Center for Environmental Health, Neuherberg, Germany, ${ }^{2}$ Department of Medicine, Karolinska Institutet, Stockholm, Sweden, ${ }^{3}$ Institute of Epidemiology, Helmholtz
Zentrum München, German Research Center for Environmental Health, Neuherberg, Germany, ${ }^{4}$ German Center for Cardiovascular Research (DZHK), Partner Site Munich Heart Alliance, Munich, Germany, ${ }^{5}$ Institute of Neurogenomics, Helmholtz Zentrum München, German Research Center for Environmental Health, Neuherberg, Germany, ${ }^{6}$ Research Center for Nutrition and Food Sciences, School of Life Sciences Weihenstephan, Technische Universität München, Freising, Germany

Methylation of the LGALS3BP locus (galactoside-binding soluble 3 binding protein), which encodes an extracellular matrix lectin, has been linked to body mass index (BMI), type 2 diabetes and related adverse outcomes of adiposity through epigenome wide association studies (EWAS). Revealing the functional role of methylation at these $\mathrm{CpG}$ sites ( $\mathrm{CpGs}$ ) is essential to understand gene regulation and ultimately metabolic disease pathophysiology.

First, we used statistical analyses to prioritize these CpGs. An EWAS on BMI (blood, $N=1888$; IlluminaMethylationEPIC array) confirmed four $L G A L S 3 B P$ associated $\mathrm{CpGs}\left(\mathrm{P}<8 \times 10^{-8}\right)$. We then applied transcription wide analyses (Illumina-HumanHT-12v3 array) and assessed methylation profile correlation in all $23 \mathrm{CpGs}$ at the locus using samples form blood $(N=1727)$, subcutaneous adipocytes $(N=127)$, peripheral blood mononuclear cells, omental and subcutaneous adipose tissue $(N=80)$. Five CpGs were associated with only the LGALS3BP transcript $\left(\mathrm{P}<4.9 \times 10^{-08}\right)$, three of which overlap BMI hits. All associated CpGs are contained in a main cluster region.

Based on our results, we selected BMI-associated CpGs for functional analyses. Electrophoretic mobility shift assays (THP-1, Jurkat and HepG2 cells) demonstrated methylation-specific differential protein binding for all assessed CpGs (cell-type- and CpG-site-specific). Preliminary results for dual-luciferase reporter gene assays (THP-1 and Jurkat cells) suggest methylation-dependent transcriptional activity and for liquid chromatography-mass spectrometry (HepG2 cells) allow identification of differential binding proteins.

Our findings support specific molecular mechanisms involved at each of the $\mathrm{CpGs}$, providing new insights into the underlying functional basis influenced by BMIassociated methylation at the $L G A L S 3 B P$ locus.

Monica Gomez-Alonso holds a CONACyT-Mexico fellowship for doctoral studies.

M. Gomez-Alonso: None. I. Dahlman: None. C. Gieger: None. A. Peters: None. J. Winkelmann: None. H. Laumen: None. A. Kretschmer: None. M. Waldenberger: None. 


\section{P20.43.A}

Characterization of the modulatory role of LZTR1 in the control of RAS-MAPK signaling

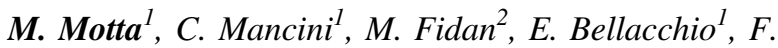
Pantaleoni $^{l}$, K. Schneider-Heieck ${ }^{2}$, S. Coppola ${ }^{3}$, G. Borck ${ }^{4}$, L. Salviati ${ }^{5}$, M. Zenker ${ }^{6}$, I. C. Cirstea ${ }^{2}$, M. Tartaglia ${ }^{1}$

${ }^{1}$ Genetics and Rare Diseases Research Division, Ospedale Pediatrico Bambino Gesù, Rome, Italy, ${ }^{2}$ Institute of Comparative Molecular Endocrinology, Ulm University, Ulm, Germany, ${ }^{3}$ National Centre for Rare Diseases, Istituto Superiore di Sanità, Rome, Italy, ${ }^{4}$ Institute of Human Genetics, Ulm University, Ulm, Germany, ${ }^{5}$ Department of Pediatrics, Università degli Studi di Padova, Padova, Italy, ${ }^{6}$ Institute of Human Genetics, University Hospital Magdeburg, Magdeburg, Germany

Noonan syndrome (NS), the most common RASopathy, is caused by mutations affecting RAS-MAPK signaling. Recently, genome scanning has allowed the discovery of novel NS-related genes, representing signal transducers or modulators not belonging to the "classical" RAS pathway. Among them, the leucine zipper-like transcriptional regulator 1 (LZTR1), encoding a member of the BTB/POZ protein superfamily, was identified in patients carrying both dominant or recessive form of NS. Furthermore, constitutional monoallelic mutations in the same gene cause schwannomatosis, a cancer-prone disorder clinically distinct from NS. LZTR1 has been suggested to act as substrate receptor for the CUL3-RING ubiquitin ligase (CRL3) complex, a multi-subunit RING-class E3 ligase implicated in protein mono- and poly-ubiquitination. Here we demonstrate that dominant NS-causing mutations do not influence protein stability and subcellular localization nor perturb binding of LZTR1 to CUL3, but specifically affect the surface of LZTR1 Kelch domain, mediating substrate recognition. On the contrary, recessive NS-causing variants behave as loss-of-function mutations, affecting LZTR1 synthesis, stability, and/or binding to CUL3. Collectively, our data suggest a model in which LZTR1 contributes to the ubiquitination of proteins functioning as positive modulators of the RAS-MAPK pathway. LZTRI mutations variably impair binding of these substrates to the multi-component ligase complex and their efficient ubiquitination and degradation, resulting in MAPK signaling upregulation.

M. Motta: None. C. Mancini: None. M. Fidan: None. E. Bellacchio: None. F. Pantaleoni: None. K. SchneiderHeieck: None. S. Coppola: None. G. Borck: None. L. Salviati: None. M. Zenker: None. I.C. Cirstea: None. M. Tartaglia: None.

\section{P20.45.C}

Pre-pregnancy maternal body mass index (BMI) and placental methylation: a joint meta-analysis of 2,951 mother-child pairs

N. Fernandez-Jimenez ${ }^{1}$, R. Fore ${ }^{2}$, M. Bustamante ${ }^{3}, C$. Lesseur ${ }^{4}$, T. Kvist ${ }^{5}$, A. Binder ${ }^{6}$, F. Tian ${ }^{7}$, J. MartorellMarugán $^{8}, J$. Loke ${ }^{9}$, L. Santa Marina ${ }^{10}$, M. Lacasaña ${ }^{11}, K$. Bakulski $^{12}$, J. Lepeule ${ }^{13}$, J. M. Craig ${ }^{9}$, C. J. Marsit ${ }^{14}$, K. B. Michels $^{15}$, K. Räikkönen ${ }^{5}$, M. R. Karagas ${ }^{16}$, B. Heude ${ }^{17}$, M. F. Hivert ${ }^{2}$, J. R. Bilbaol

${ }^{I}$ University of the Basque Country (UPV/EHU), Leioa, Spain, ${ }^{2}$ Harvard Medical School, Boston, MA, United States, ${ }^{3}$ ISGlobal, Institute for Global Health, Barcelona, Spain, ${ }^{4}$ Icahn School of Medicine at Mount Sinai, New York, NY, United States, ${ }^{5}$ University of Helsinki, Helsinki, Finland, ${ }^{6}$ University of Hawaii Cancer Center, Honolulu, HI, United States, ${ }^{7}$ Emory University, Atlanta, GA, United States, ${ }^{8}$ GENYO Centre for Genomics and Oncological Research: Pfizer/University of Granada/Andalusian Regional Government, Granada, Spain, ${ }^{9}$ Centre for Molecular and Medical Research, School of Medicine, Faculty of Health Deakin University, Geelong, Australia, ${ }^{10}$ Biodonostia Health Research Institute, Donostia, Spain, ${ }^{11}$ Andalusian School of Public Health (EASP), Granada, Spain, ${ }^{12}$ University of Michigan, Ann Arbour, MI, United States, ${ }^{13}$ Univ. Grenoble Alpes, Inserm, CNRS, Grenoble, France, ${ }^{14}$ Rollins School of Public Health, Emory University, Atlanta, GA, United States, ${ }^{15}$ Fielding School of Public Health, University of California, Los Angeles, Los Angeles, CA, United States, ${ }^{16}$ Geisel School of Medicine, Dartmouth College, Lebanon, NH, United States, ${ }^{17}$ Universite de Paris, CRESS, Inserm, INRAE, Paris, France

Introduction: Maternal obesity during pregnancy increases the future risk for obesity, diabetes and heart disease in offspring but the molecular mechanisms are still unclear. Placenta is a key organ in fetal development and enables the mother-child interplay. The placental methylome could be a marker of this interaction and reflect the functional changes derived from in utero exposures.

Methods: To assess whether pre-pregnancy maternal BMI is associated with DNA methylation in placenta we combined DNA methylation data from 11 cohorts of the PACE consortium (AQUA, EARLI, EDEN, Gen3G, GENEIDA, HEBC, INMA, ITU, MARBLES, NHBCS and RICHS) analyzed with EPIC $(n=996)$ and $450 \mathrm{~K}$ $(n=1,955)$ arrays. We modelled the associations with and without adjustment for cellular heterogeneity, estimated with a reference-free deconvolution algorithm. We adjusted all models for maternal age, parity, education and smoking 
status. Finally, we meta-analyzed the estimates of 2,951 placental methylomes using the GWAMA software (fixed effects).

Preliminary results: We identified 51 and 39 BMIassociated $\mathrm{CpGs}$ passing Bonferroni correction with and without cell-type adjustment, respectively. Among the genes closest to the significant $\mathrm{CpGs}$, we found obesityrelated genes such as $G P X 1, B B S 9, E B F 2$ or PPP2R2A; type 2 diabetes genes such as DDAH1, CMIP and SGSM2, and a number of developmental players, suggesting that maternal BMI impacts the epigenetic regulation of healthrelated genes in placenta. In the near future, we will ascertain the functional impact of these signatures in placental transcription, and their interaction with the genomic variation of children and mothers.

Funding: GVSAN2018/111086 to JRB and PI18/01142FEDER to LSM.

N. Fernandez-Jimenez: None. R. Fore: None. M. Bustamante: None. C. Lesseur: None. T. Kvist: None. A. Binder: None. F. Tian: None. J. Martorell-Marugán: None. J. Loke: None. L. Santa Marina: None. M. Lacasaña: None. K. Bakulski: None. J. Lepeule: None. J.M. Craig: None. C.J. Marsit: None. K.B. Michels: None. K. Räikkönen: None. M.R. Karagas: None. B. Heude: None. M.F. Hivert: None. J.R. Bilbao: None.

P20.46.A

DNA methylation status of interspersed repetitive sequences and pro-inflammatory cytokines in migraine patients with MTHFR C667T variant

\section{N. Kraveishvili, ${ }^{l, 2}$, M. Kvintradze ${ }^{l}$, S. Surmava ${ }^{l}, M$. Zarandia $^{l}$, T. Gorgiladze ${ }^{3}$, E. Kvaratskhelia ${ }^{l}, E$. Abzianidze ${ }^{l}$}

${ }^{1}$ Tbilisi State Medical University, Tbilisi, Georgia, ${ }^{2}$ Tbilisi Institute of Medicine, Tbilisi, Georgia, ${ }^{3}$ Batumi Shota Rustaveli State University, Batumi, Georgia

Introduction: Epigenetic alterations may have a role in the pathogenesis of complex diseases such as Migraine. This study aimed to determine the methylation level of the Line1 and Alu interspersed repetitive sequences in migraine patients with C677T variant of MTHFR gene as well as methylation status of the TNF- $\alpha$ and IL- 8 gene promoters.

Materials and methods: The study was approved by the ethics committee of Tbilisi State Medical University. Genomic DNA was isolated from peripheral blood mononuclear cells (PBMCs) of migraine patients and healthy controls using QIAamp DNA Mini Kit (Qiagen, US). For Bisulfite treatment of DNA, we used EpiTect Bisulfite Conversion Kit (Qiagen, US). The MTHFR C677T polymorphism was detected by PCR-RELP method.
Methylation levels of Line-1 and Alu in were examined by the combined bisulfite restriction analysis-interspersed repetitive sequences (COBRA-IRS). Methylation levels of TNF- $\alpha$ and IL- 8 in patients and controls were examined by methylation-specific real-time qPCR.

Results: For Alu sequences, significant differences were observed in the frequency of unmethylated Cytosines between PBMCs of patients with MTHFR C677T polymorphism and healthy controls. However, no statistically significant differences for LINE-1 were detected. In addition, our results showed significant low level of methylation in TNF- $\alpha$ and IL- 8 promoters in the migraine patients than those in healthy controls $(\mathrm{p} \leq 0.05)$.

Conclusions: We demonstrated that changes in the DNA methylation of IRS elements and genes involved in inflammation might contribute to the pathogenesis of migraine in patients with MTHFR C677T variant. Such research could lead to the discovery of diagnostic biomarkers and therapeutic targets.

N. Kraveishvili: None. M. Kvintradze: None. S. Surmava: None. M. Zarandia: None. T. Gorgiladze: None. E. Kvaratskhelia: None. E. Abzianidze: None.

\section{P20.47.B}

Circulating miRNAs as potential biomarkers to classify patients with the different grades of glioma

M. Niemira ${ }^{l}$, A. Erol ${ }^{l}$, I. Sidorkiewicz ${ }^{l}$, A. Bielska $^{l}, A$. Szalkowska $^{l}$, G. Sokolowska ${ }^{l}, J$. Raczkowska ${ }^{l}$, E. Siewiec ${ }^{l}$, M. Moniuszko ${ }^{2}$, J. Reszec ${ }^{3}$, A. Kretowski ${ }^{1}$

${ }^{1}$ Clinical Research Centre, Medical University of Bialystok, Bialystok, Poland, ${ }^{2}$ Department of Regenerative Medicine and Immune Regulation, Medical University of Bialystok, Bialystok, Poland, ${ }^{3}$ Department of Medical Pathomorphology, Medical University of Bialystok, Bialystok, Poland

Introduction: MicroRNA is a single-stranded non-coding small RNA, which is considered as a new type of 'oncomiRs' or 'tumour suppressors'. Abnormal expression of miRNAs contributes to glioma initiation and progression. In this study, we aimed to determine differentially expressed miRNAs in the serum between glioblastoma (GBM) and low-grade (LGG) patients.

Materials and Methods: Serum miRNAs of 40 glioblastomas and 12 of low-grade glioma patients $(n=52)$ have been isolated. Using the NanoString nCounter Analysis System expression of 798 miRNAs has been analysed. Quantitative RT-PCR was further performed to verify the expression of selected miRNAs with an increased number of samples. Gene ontology, Ingenuity Pathway Analysis Software, and Cytoscape with STRING plug-in tool were employed to the prediction of putative target 
genes and their role in glioma development. Receiver operating characteristic (ROC) curves were constructed to determine miRNA sensitivity and specificity.

Results: According to the cut-off criteria $(\mathrm{FC}>2$, FDR $<0.05$ ), a total of $125 \mathrm{DE}$ miRNAs were obtained including 7 upregulated and 118 downregulated miRNAs. The analysis predicted 9929 target genes related to RAS/ MAPK signalling pathway, ephrin signalling pathway, nNOS signalling in neurons, and glioma signalling. ROC analyses demonstrated that all miRNAs had significant potential diagnostic value (AUC $>0.75$ ). The highest AUC values (AUC $>0.95$ ) were observed for five miRNAs: miR324-3p, miR-301b-3p, miR-1271-3p, miR-103a-3p, and miR-491-3p.

Conclusions: This study highlights the potential for serum-derived miRNAs as biomarkers for the progression of glioma and predicting the prognosis of affected patients. Data were generated by the Centre for Artificial Intelligence of the Medical University of Bialystok.

M. Niemira: None. A. Erol: None. I. Sidorkiewicz: None. A. Bielska: None. A. Szalkowska: None. G. Sokolowska: None. J. Raczkowska: None. E. Siewiec: None. M. Moniuszko: None. J. Reszec: None. A. Kretowski: None.

\section{P20.48.C}

Gene-centric compilation of the human ncRNA repertoire: A new vista for disease interpretation

\section{R. Barshir, S. Fishilevich, T. Iny Stein, M. Safran, D. Lancet}

\section{Weizmann Institute of Science, Rehovot, Israel}

Non-coding RNA (ncRNA) genes, amounting to $>20 \%$ of the non-protein-coding genome, assume increasing importance, with accumulating evidence of involvement in disease mechanisms. A comprehensive non-redundant genecentric view of human ncRNA genes and their annotations is critical for understanding their involvement in health and disease, and for whole genome sequencing (WGS) interpretation. GeneCards, the widely used human gene database, is integrating data of ncRNA records and annotations from EBI's RNAcentral, and its 15 primary sources, as well as from Ensembl, NCBI Entrez Gene and HGNC. Many ncRNA sources are transcript-centric, and for WGS variant analysis it is essential to transform this information into a unique and all-encompassing compendium of ncRNA genes. Utilizing the capacities of GeneCards, being genecentric, we clustered overlapping transcript entries from all sources using an algorithm based on genomic coordinates. This strategy eliminates redundancies and allows for full coverage of genomic gene positions. Adhering to
GeneCards strategy, each RNA gene, with affiliated transcripts, appears with annotations from all relevant sources. The unified compilation includes more than 200,000 ncRNA gene entries, including $\sim 100,000$ Piwi-interactingRNAs.VarElect, GeneCards' NGS interpretation tool [PMID 27357693], allows disease interpretation of variants mapped to ncRNAs by two routes. First, direct ncRNAdisease associations are extracted from our knowledgebase, now augmented by novel ncRNA-disease connections. Second, our strengthened capacity to associate ncRNAs with protein-coding genes now enables interpretation of poorly characterized ncRNAs. The combination of an extensive ncRNA knowledgebase with gene interpretation algorithms allows fathoming the disease-related significance of non-coding variants identified by WGS. Support: LifeMap Sciences grant

R. Barshir: None. S. Fishilevich: None. T. Iny Stein: None. M. Safran: None. D. Lancet: B. Research Grant (principal investigator, collaborator or consultant and pending grants as well as grants already received); Significant; LifeMap Sciences.

\section{P20.53.B}

Epigenome-wide association study in knee osteoarthritis generates novel insights into disease mechanisms

P. Kreitmaier ${ }^{1}$, M. Suderman ${ }^{2}$, L. Southam ${ }^{1}$, J. Steinberg ${ }^{1,3}$, J. M. Wilkinson ${ }^{4}$, E. Zeggini ${ }^{1}$

${ }^{1}$ Institute of Translational Genomics, Helmholtz Zentrum München, Neuherberg, Germany, ${ }^{2}$ MRC Integrative Epidemiology Unit, Bristol Medical School, University of Bristol, Bristol, United Kingdom, ${ }^{3}$ Cancer Research Division, Cancer Council NSW, Sydney, Australia, ${ }^{4}$ Department of Oncology \& Metabolism, The University of Sheffield, Sheffield, United Kingdom

Osteoarthritis (OA) is a common degenerative joint disease. To promote the development of efficient therapy approaches, it is first necessary to unravel the genomic architecture of OA. We investigated DNA methylation in cartilage and synovium tissue from 97 patients, the largest epigenetic data set for knee OA to date. Genome-wide DNA methylation data from primary chondrocytes from macroscopically intact (low-grade) and degraded (high-grade) cartilage as well as synovium samples were generated from patients that underwent total knee replacement for OA. We conducted an epigenome-wide association study (EWAS) to identify methylation marks of cartilage degeneration. Genotypedependent methylation changes (mQTL effects) were identified in synovium, low-grade and high-grade cartilage across the genome. We used Mendelian randomization (MR) to investigate putative causal effects between 
methylation state and $\mathrm{OA}$, and colocalisation analysis to identify causal loci. The EWAS discovered 15,328 differentially methylated positions exceeding the genome-wide significance threshold $\left(P<1.24 e^{-7}\right)$ between low-grade and high-grade cartilage. We identified 2,477 differentially methylated regions linked to cartilage degeneration. The mQTL analysis revealed widespread associations between genetic variants and methylation in all three examined tissues, with partial overlap between tissue types. Two of these mQTLs colocalised with OA-associated variants, thus helping resolve GWAS signals. The application of MR identified methylation loci with a putative causal influence on OA. In this study, we identify widespread epigenomic profile changes related to cartilage degeneration. We find likely effector genes for methylation and GWAS signals, enhancing our understanding of the mechanisms underpinning disease development and progression in OA.

P. Kreitmaier: None. M. Suderman: None. L. Southam: None. J. Steinberg: None. J.M. Wilkinson: None. E. Zeggini: None.

\section{P20.54.C}

\section{A comprehensive transcriptional map of knee osteoarthritis}

G. Katsoula ${ }^{1}$, L. Southam ${ }^{1}$, J. Steinberg ${ }^{2}$, J. M. Wilkinson ${ }^{3}$, E. Zeggini ${ }^{1}$

${ }^{1}$ Helmholtz Zentrum München, Neuherberg, Germany, ${ }^{2}$ Cancer Research Division, Cancer Council NSW, Sydney, Australia, ${ }^{3}$ Department of Oncology \& Metabolism, The University of Sheffield, Sheffield, United Kingdom

Osteoarthritis is a degenerative joint disease with huge public health burden and no curative therapy. Therefore, it is urgent to translate insights from genomics into disease mechanisms. We molecularly endotype joint tissues from 200 patients undergoing total knee replacement.

We generated RNA-seq data from patient knee tissues (primary chondrocytes from low-grade and high-grade cartilage, synoviocytes, and adipocytes) and genotype data from peripheral blood. We: (1) investigate gene expression differences between low and high-grade cartilage (2) examine alterations in chondrocyte population markers and (3) determine expression quantitative loci (eQTL) effects in all the aforementioned tissues.

Differential expression analysis identified 4,512 and 4,365 significantly upregulated and downregulated genes in high-grade cartilage respectively. Strong effects were observed for genes associated with osteoarthritis pathology including CRLF1 ( $\mathrm{P}=1.54 \mathrm{e}^{-86}$, $\left.\mathrm{LFC}>2\right)$ and CHRDL2, $\left(P=9.19 \mathrm{e}^{-64}, \mathrm{LFC}<-2\right)$. Functional analyses pointed significantly enriched terms including skeletal system development $\left(\mathrm{P}=3.49 \mathrm{e}^{-06}\right)$, ossification $\left(P=1.48 \mathrm{e}^{-05}\right)$ and activation of ECM-receptor interaction pathway $\left(P=4.14 \mathrm{e}^{-06}\right)$. We detected significant downregulation in the expression of effector chondrocytes markers in highgrade cartilage, a chondrocyte population associated with high metabolic activity in early-stage osteoarthritis. The eQTL analysis revealed widespread significant associations between genetic variants and gene expression in all tissues.

We have identified large-scale transcriptional signatures of disease grade in key tissues of the knee joint in the largest osteoarthritis transcriptomic analysis to date. The identified genes and eQTL forward the understanding of molecular mechanisms in osteoarthritis. The impacted pathways serve as potential targets for novel therapeutics.

G. Katsoula: None. L. Southam: None. J. Steinberg: None. J.M. Wilkinson: None. E. Zeggini: None.

P20.55.A

MethylSeq Direct workflow: a fast method for DNA methylation analysis

\section{E. Schreiber, S. Jackson}

ThermoFisher Scientific, South San Francisco, CA, United States

Introduction: Methylation at carbon 5 in the cytosine (5$\mathrm{meC}$ ) of the $\mathrm{CpG}$ dinucleotide is a key epigenetic regulator of developmental processes in higher eukaryotes. NGS analysis of DNA methylation associated with cancer and other pathologies revealed specific loci where $\mathrm{CpG}$ methylation influences the cell physiology. These discoveries can be expanded by analyzing discrete methylation changes in the large number of archived samples. However, retrospective studies using the rich resource of FFPE samples are hampered by the degraded nature of DNA extracted.

Materials methods and Results: We present a workflow for DNA methylation analysis method that is compatible with FFPE tissues. We demonstrate the use of a method for bisulfite sequencing, termed the MethylSeq Direct workflow, using PCR and sequencing reagents of the Applied Biosystems $^{\mathrm{TM}}$ BigDye $^{\mathrm{TM}}$ Direct Cycle Sequencing Kit to determine the methylation status in the p16 and MGMT gene promoters as examples. FFPE extracted DNA is bisulfite converted using the Thermo Scientific ${ }^{\mathrm{TM}}$ EpiJET ${ }^{\mathrm{TM}}$ Bisulfite Conversion Kit. PCR primers specific for bisulfite converted sequences are designed using Applied Biosystems ${ }^{\mathrm{TM}}$ MethylPrimer Express ${ }^{\mathrm{TM}}$ Software. PCR using these M13-tagged primers is used to amplify the locus of interest. The amplicon is sequenced using BigDye Direct Sequencing reagent and purified using the Applied Biosystems ${ }^{\mathrm{TM}}$ BigDye XTerminator ${ }^{\mathrm{TM}}$ Kit. The sequencing reaction can 
be analyzed on the Applied Biosystems ${ }^{\mathrm{TM}}$ SeqStudio ${ }^{\mathrm{TM}}$ Genetic Analyzer, or another model.

Conclusions: The entire workflow for bisulfite conversion, PCR, and DNA sequencing, takes 7 hours, including an hour of hands-on time enabling the use of archived FFPE samples for retrospective studies.

E. Schreiber: A. Employment (full or part-time); Significant; Thermo Fisher Scientific. S. Jackson: A. Employment (full or part-time); Significant; Thermo Fisher Scientific.

\section{P20.56.B}

DNA methylome- and transcriptome-wide profiling in peripheral blood mononuclear cells identifies potential predictors of desensitization to peanut allergy through oral immunotherapy

A. Arnau Soler ${ }^{1,2}$, S. Ashley ${ }^{1,2}$, K. Blümchen ${ }^{3}$, K. Beyer ${ }^{4}, Y$. Lee $e^{1,2}$

${ }^{1}$ Max Delbrück Center for Molecular Medicine, Berlin, Germany, ${ }^{2}$ Clinic for Pediatric Allergy, Experimental and Clinical Research Center, Charité University Medical Center, Berlin, Germany, ${ }^{3}$ Department of Children and Adolescent Medicine, Division of Pneumology, Allergology and Cystic Fibrosis, University Hospital Frankfurt, Frankfurt am Main, Germany, ${ }^{4}$ Department of Pediatric Pulmonology, Immunology and Intensive Care Medicine, Charité University Medical Center, Berlin, Germany

Peanut allergy is a common disease and the most common cause of anaphylaxis in childhood. Peanut oral immunotherapy (OIT) can successfully desensitise many peanutallergic children. Although such treatment appears effective and safe for the majority of patients, life-threatening allergic reactions can still occur in a minority of children. Therefore, predictors of positive OIT response are still required. Blood samples were collected at baseline from 38 peanut-allergic children who successfully completed an OIT trial. Methylation and RNA sequencing data was obtained from isolated peripheral blood mononuclear cells (PBMCs) following in vitro cultivation both in medium and peanut antigen stimulation conditions over 48 hours. We conducted DNA methylome- and transcriptome-wide profiling of both positive response to treatment and complete desensitisation, accurately phenotyped by an oral food challenge before and after the OIT trial. We developed a random forest classifier using PBMC multi-omics data. Several significant differentially methylated $\mathrm{CpGs}$, differentially methylated regions and differentially expressed genes (DEGs) were identified. A few differentially methylated $\mathrm{CpGs}$ identified were associated with gene expression levels of significant DEGs. Gene set enrichment analysis revealed many pathways enriched by DEGs. Our random forest classifier showed accuracies of over $70 \%$ and $90 \%$ in distinguishing positive treatment responders from non-responders and completely desensitised from non-completely desensitised children, respectively. Our results identify potential biomarkers of positive peanut OIT response. If validated, such omics markers may serve to predict OIT response, to better select children that could benefit from OIT, and to identify targetable pathways underpinning successful response to OIT in peanut-allergic children.

A. Arnau Soler: None. S. Ashley: None. K. Blümchen: None. K. Beyer: None. Y. Lee: None.

\section{P20.57.C}

Pharmacotranscriptome effects of melanocortin derivative in acute stress

I. B. Filippenkov ${ }^{l}$, V. V. Stavchansky ${ }^{l}$, L. V. Dergunova ${ }^{l}$, N. Y. Glazova ${ }^{l}$, N. G. Levitskaya ${ }^{2}$, E. A. Sebentsova ${ }^{l}$, L. V. Valieval $^{l}$, D. D. Khukhareva ${ }^{2}$, S. A. Limborska ${ }^{l}$, N. F. Myasoedov ${ }^{l}$

${ }^{1}$ Institute of Molecular Genetics, Russian Academy of Sciences, Moscow, Russian Federation, ${ }^{2}$ Lomonosov Moscow State University, Moscow, Russian Federation

Introduction: The pharmacotranscriptome studies of antistress drugs are an actual for the translation medicine. The synthetic analogue of melanocortin (MEHFPGP, Semax) effectively improves cognitive function under acute stress (AS). The work is devoted to the genome-wide expression profiling in the rat hippocampus under the action of Semax after AS.

Materials and Methods: rat model of AS, highthroughput RNA sequencing (RNA-Seq), real-time RTPCR, bioinformatics.

Results: Using RNA-Seq we revealed 1529 differential expression genes (DEGs) with cut-off $>1.5$ and padj $<0.05$ at $4.5 \mathrm{~h}$ after AS. Semax administration of $100 \mu \mathrm{g} / \mathrm{kg}$ at $30 \mathrm{~min}$ before AS, led to 944 DEGs at $4.5 \mathrm{~h}$ after AS. Bioinformaticaly, DEGs after AS were associated with a wide range of catalytic and binding molecular functions of proteins encoding by them. Simultaneously, Semax significantly modulated the expression of DEGs associated with catalytic (Dpp3, Dhcr 7, Gpx8), transmembrane transporter (Slc13a5, Slco2b1, Slc7a15), transcription regulator (Stat2, Etv5, Irf5), G-protein receptor (Gpr17, Adoral), cytokine receptor (Il3ra, Csf3r) activity and has the effect of compensating for the effects of AS. Moreover, Semax has an additional effect on protein metabolism, increasing the activity of genes involved in protein synthesis (Rpl15, Rps16, Rps27l) and decreasing the activity of genes involved in protein degradation ( $G b a, C l n 5, C t s h)$. 
Conclusions: The data obtained revealed the antiinflammatory and immunomodulatory transcriptional effects of the melanocortin analog Semax after AS. We believe that further genome-wide non-coding RNAs profiling can reveal novel insight to the peptide regulation in rat brain after AS. This work was supported by grant from the Russian Science Foundation 19-14-00268.

I.B. Filippenkov: None. V.V. Stavchansky: None. L.V. Dergunova: None. N.Y. Glazova: None. N.G. Levitskaya: None. E.A. Sebentsova: None. L.V. Valieva: None. D.D. Khukhareva: None. S.A. Limborska: B. Research Grant (principal investigator, collaborator or consultant and pending grants as well as grants already received); Significant; Grant from the Russian Science Foundation 19-14-00268. N.F. Myasoedov: None.

\section{P20.61.A}

Association between prenatal stress and infant DNA methylation

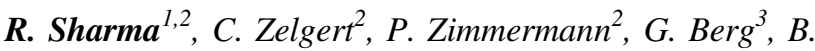

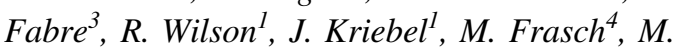
Waldenberger $^{1}$, S. Lobmaier ${ }^{2}$, M. Antonelli ${ }^{2,3}$

${ }^{1}$ Helmholtz Zentrum Munich, Munich, Germany, ${ }^{2}$ Dept. of OBGYN, Klinikum rechts der Isar, Technical Univ of Munich, Munich, Germany, ${ }^{3} I B C N$, Facultad de Medicina, Universidad de Buenos Aires, Buenos Aires, Argentina, ${ }^{4}$ Dept. of OBGYN \& CHDD, Univ of Washington, Seattle, WA, Seattle, WA, United States

Introduction: Maternal stress before and during pregnancy can profoundly affect the in-utero and postnatal child development. We hypothesize that children affected by prenatal stress and thus epigenetic reprogramming might show alterations of the fetal autonomic nervous system and Hypothalamic- Pituitary axis.

Methods: We evaluated epigenome-wide methylation sites in the saliva of infants born to stressed and nonstressed mothers. In a prospective study of expecting mothers, we screened women for chronic stress exposure at the $28^{\text {th }}$ week using the Cohen Perceived Stress Scale (PSS) and Prenatal Distress questionnaires (PDQ). Expectant mothers were classified into the stressed group (SG, PSS$10 \geq 19, N=55$ ); the control group (CG, PSS-10 < 19, $N=55$ ) matched $1: 1$ for parity, maternal age and gestational age. On the day of delivery, maternal blood and maternal head hair were collected for cortisol measurements. DNA methylation was measured in saliva samples of the newborn using the EPIC Bead Chip array (758132 $\mathrm{CpG}$ ). In order to identify associations between cortisol and methylation, linear regression models adjusting for confounders (sex, age and smoking) were run.
Results: Cortisol measurements were 63\% higher in SG versus CG. The top hit of the regression analysis is $\operatorname{cg} 15652683$ ( $\mathrm{p}=2.16 \mathrm{e}-06$ ), a $\mathrm{CpG}$ annotated to VIPR2 gene (Chr 7q36.3) that function as neurotransmitters and neuroendocrine hormones.

Conclusions: Our work is ongoing and aims to see if the prenatal stress can be employed as a predictive biomarker of child neurodevelopmental outcome.

R. Sharma: None. C. Zelgert: None. P. Zimmermann: None. G. Berg: None. B. Fabre: None. R. Wilson: None. J. Kriebel: None. M. Frasch: None. M. Waldenberger: None. S. Lobmaier: None. M. Antonelli: None.

\section{P20.62.B}

Genetic and epigenetic regulation of chaperones in the pathogenesis of pseudoexfoliation syndrome and glaucoma

\section{R. S. Kapuganti, B. Padhy, B. Hayat, P. P. Mohanty, D. P.} Alone

National Institute of Science Education and Research, Bhubaneswar, Khurda, India

Introduction: Pseudoexfoliation (PEX) is a systemic agerelated disorder of protein aggregation with prominent ocular manifestation. The etiology of PEX is multifactorial involving both genetic and epigenetic factors. This study aims to understand the genetic and epigenetic regulation of molecular chaperones, clusterin (CLU) and heat shock protein 70 (HSP70), respectively, in PEX pathology.

Materials and Methods: Fluorescence based capillary electrophoresis was employed for genotyping variants in $C L U$ in an Indian cohort comprising of 136 PEX patients and 89 controls. mRNA and proteins levels were checked through qRT-PCR and western blotting. DNA methylation of HSP70 was assessed through bisulfite sequencing. Functional and molecular analysis were done by luciferase reporter assays, chromatin immunoprecipitation, electrophoretic mobility shift assays, CRISPR deletions in HEK293 and human lens epithelial (HLE-B3) cells.

Results: We found deregulated expression of CLU and HSP70 in the lens capsule of PEX affected individuals. Two variants in $C L U$, rs2279590 and rs3087554 showed a significant association with PEX as risk factors. Further, rs2279590 was found to be a functional variant that regulates $C L U$ expression through interaction with HSF1. Further, bisulfite sequencing revealed DNA hypermethylation of HSP7O in PEX patients leading to its transcriptional silencing which was further validated by its restoration by DNA methyltransferase inhibitor in HLE-B3 cells.

Conclusions: This study shows that the molecular chaperones are deregulated in PEX affected individuals, 
contributing to the severity of the disease. CLU and HSP70 expression is regulated through different mechanisms, i.e., genetic and epigenetic, implying a complex interlink between genetic and environmental effects in PEX pathophysiology.

R.S. Kapuganti: None. B. Padhy: None. B. Hayat: None. P.P. Mohanty: None. D.P. Alone: None.

P20.63.C

EpiWE project, the molecular signature of violence in women's victim genome

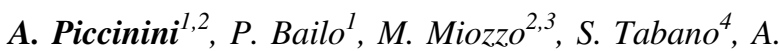
Kustermann $^{5}$, S. F. Gaudi ${ }^{6}$

${ }^{1}$ Department of Biomedical Sciences for Health - Università degli Studi di Milano, Milan, Italy, ${ }^{2}$ Department of Pathophysiology and Transplantation, Medical Genetics, Università degli Studi di Milano, Milan, Italy, ${ }^{3}$ Fondazione IRCCS Ca' Granda Ospedale Maggiore Policlinico,, Milan, Italy, ${ }^{4}$ Fondazione IRCCS Ca' Granda Ospedale Maggiore Policlinico, Milano, Università degli Studi di Milano, Milan, Italy, ${ }^{5}$ Department of Women's and Children's Health and Service for Sexual and Domestic Violence (SVSeD), Fondazione IRCCS Ca' Granda Ospedale Maggiore Policlinico,, Milan, Italy, ${ }^{6}$ Italian National Institute of Health, Roma, Italy

Introduction: Violence against women represents a chronic and global health plague and, due to its serious consequences on women's health, needs to be approached at multisciplinary level. The short-term effects of violence are more evident, while the exhaustive evaluation of long-term effects still remains a challenge. It has been demonstrated that the signs ofviolence interfere with genome plasticity and gene expression through epigenetic mechanisms.

Materials and Methods: Cases were enrolled by systematic sampling of victims of violence screened at the Emergency Department in all the italian regions. The psychological evaluation was performed in a cohort of 700 women. Biological samples from women's victims were analysed for differential methylation in a panel of genes involved in post traumatic stress disorder.

Results: Quantitative methylation evaluation of a panel of genes already reported as associated with trauma/stressrelated disorders was performed by MassARRAY (Agena, Bioscience) in 63 victims and 80 controls. The evaluation of LINE1 methylation, for global methylation assessment is in progress, (pyrosquencing, Qiagen).

Conclusions: Early detection of post-traumatic stress disorder cases represent a priority area of research, which is focused not only on the susceptibility but also on the resilience or not resilience to PTSD. The interaction between genome and environmental factors, such as violence, represents one of the major challenges in precision and preventive medicine. By knowing the mechanisms responsible for resilience, we can derive clues about the best treatment and the best public policy.

A. Piccinini: None. P. Bailo: None. M. Miozzo: None. S. Tabano: None. A. Kustermann: None. S.F. Gaudi: None.

P20.64.A

trans-acting sequence variants affecting protein abundances of the genes encoding human ribosomal proteins

J. Ryu, C. Lee

Soongsil University, Seoul, Korea, Republic of

Introduction: Ribosomal protein is the essential component of ribosome which is a molecular machinery to translate the genetic information from the mRNA templates into proteins. Their expression dysregulation is known to be involved in the survival and growth of human cells, and pathogenesis of human disease such as cancer. Nevertheless, little is known for how genetic factor regulated their gene expression across expression stage from mRNA transcription to translation.

Material and Methods: We analyzed the genetic associations of the expression level at three different type expression stages of 27 small ribosomal proteins and 43 large ribosomal proteins using mixed model.

Results: Eighteen quantitative trait loci (QTL) were found out for protein abundance of 21 ribosomal proteins, but not for the expression level for mRNA transcript and ribosomal occupancy of their corresponding genes. Among these QTLs, three pQTL were associated with three different long non-coding RNA expression, which target genes may produce ribosomal components or may control the metabolic cues for ribosome synthesis, which are involved in apoptosis, cell division, and cancer.

Conclusions: The results implied that the variants could be identified through the different translation of target gene by affecting expression level of lncRNA. Further studies were needed to understand their underlying mechanism for regulating gene expression of the ribosomal proteins.

Grant Reference: This work was supported by National Research Foundation of Korea (NRF) grants funded by the Ministry of Education (NRF-2018R1A6A3A11049586) and by the Ministry of Science and ICT (NRF2018R1A2B6004867).

J. Ryu: None. C. Lee: None.

P20.65.B 
Methylation profile of locally advanced rectal carcinomas and neoadjuvant chemoradiotherapy response

L. Matos do Canto ${ }^{1}$, M. C. Barros-Filho ${ }^{2}$, D. Marinho ${ }^{3}$, B. E. C. Kupper ${ }^{2}$, M. F. S. Begnami ${ }^{4}$, C. Scapulatempo-Neto ${ }^{5}$, B. M. Havelund ${ }^{l}$, J. Lindebjerg ${ }^{l}$, F. A. Marchi ${ }^{2}, J$. Baumbach $^{6}$, S. Aguiar-Jr ${ }^{2}$, S. R. Rogatto ${ }^{1,7}$

${ }^{1}$ Vejle University Hospital, Vejle, Denmark, ${ }^{2}$ A.C.Camargo Cancer Center, Sao Paulo, Brazil, ${ }^{3}$ Psykiatrisk Center Sct Hans, Roskilde, Denmark, ${ }^{4}$ Sirio-Libanes Hospital, Sao Paulo, Brazil, ${ }^{5}$ Barretos Cancer Hospital, Barretos, Brazil, ${ }^{6}$ Technical University of Munich, Munich, Germany, ${ }^{7}$ Danish Colorectal Cancer Center South, Vejle, Denmark

Introduction: Locally advanced rectal cancer (LARC) is treated with pre-operative chemoradiotherapy (nCRT) and surgery. Patients achieving complete pathological response (pCR, $15 \%$ to $30 \%$ ) present better overall survival and lower rates of recurrence compared to incomplete responders (pIR). Predicting treatment response markers are needed in these cases.

Methods: The methylation status of 32 pre-treatment LARC biopsies $(\mathrm{pCR}=11, \mathrm{pIR}=21)$ and 5 normal rectal (NT) tissues was assessed (Infinium_EPIC_platform-Illumina). Differentially methylated (DM) probes (false discovery rate $<5 \%$ and $|\Delta \beta|>0.15$ ) were identified using limma. A predictive classifier was developed using the recursive feature elimination method and Diagonal Linear Discriminant Analysis. The classifier performance was calculated using the leave-one-out cross-validation test (BRB array tools v.4.4.0). The methylation status of these CpGs was evaluated in 77 LARC cases (32 array-dependent and 45 independent) using bisulfite-pyrosequencing.

Results: The comparison between pCR and pIR revealed 24,428 and 75,398 DM CpGs, respectively. The CpGs exclusively altered in each group $(3,470$ in pCR vs. NT and 54,440 in pIR vs. NT) were most frequently hypermethylated in pCR (44\% vs. $18 \%$ in pIR). pCR cases presented more DM CpGs in promoter regions (49\% vs. $37 \%$ in pIR) and $\mathrm{CpG}$ islands (66\% vs. $36 \%$ in pIR). The classifier, based on 3CpGs, could discriminate cases with $100 \%$ sensitivity and $90 \%$ specificity. Pyrosequencing confirmed the DM status of the 3CpGs in pCR vs. pIR ( $p$-values: CpGA: 0.0107, CpG-B: 0.0023, CpG-C: 0.0138). Conclusions: The methylation profile of $\mathrm{pCR}$ cases diverges from pIR. Three CpGs could predict nCRT response in LARC cases.

L. Matos do Canto: None. M.C. Barros-Filho: None. D. Marinho: None. B.E.C. Kupper: None. M.F.S. Begnami: None. C. Scapulatempo-Neto: None. B.M. Havelund: None. J. Lindebjerg: None. F.A. Marchi: None. J. Baumbach: None. S. Aguiar-Jr: None. S.R. Rogatto: None.

\section{P20.66.C}

Predicting the effects of regulatory variants by deep learning

\author{
M. Schubach ${ }^{1,2}$, L. Krützfeldt ${ }^{1,2}$, S. Röner ${ }^{1,2}$, M. Kircher $^{1,2}$ \\ ${ }^{1}$ Charité - Universitätsmedizin Berlin, Berlin, Germany, \\ ${ }^{2}$ Berlin Institute of Health (BIH), Berlin, Germany
}

Machine learning methods are routinely applied in medicine to prioritize and implicate deleterious genetic variants. However, their focus has been on coding sequence. Here, we address regulatory changes, jointly referring to changes in promoter and distal regulatory elements covering $~ 5-20 \%$ of the non-coding genome. Pathogenic regulatory variants still correspond to a minority of known variants and quantitative readouts are not commonly available. Further, larger reporter assay datasets are limited to specific loci or readouts of standing variation. While inherently biased and unsuited for machine learning, these datasets provide important validation and show that although myriad annotations and scores exist, none consistently predicts experimental regulatory variant effects.

Here, we develop a deep neural network (DNN), trained on active and non-active regulatory regions from multiple cell-types. We selected open chromatin peaks from publicly available DNase data (e.g. HepG2, HEK293T, K562, iPSCs) and use a multi-task convolutional network based only on genomic sequence. The multi-task approach provides critical insights into regulatory mechanisms and our model outperforms others' in predicting experimental variant effects. This method inherently learns sequence features of epigenetic factors and DNA binders, and enables us to study sequence correlates of chromatin marks and transcription factor binding. Using recent methods for DNN interpretation, we extract important regulatory mechanisms from our model, identifying motifs of various key players in cell-type specific transcriptional regulation. We conclude that DNNs have a high potential in predicting regulatory effects and tissue-specific regulation, a critical step forward in the interpretation of genome-wide variants in common and rare diseases.

M. Schubach: None. L. Krützfeldt: None. S. Röner: None. M. Kircher: None.

\section{P20.67.A}

Epigenome wide association study in Restless-Legs Syndrome

N. Mirza-Schreiber ${ }^{l}$, S. Naher ${ }^{l}$, B. Schormair ${ }^{l}$, C. Zhao ${ }^{l}$, R. Wilson ${ }^{2}$, M. Waldenberger ${ }^{2}, C$. Gieger ${ }^{2}$, M. MüllerNurasyid ${ }^{3}$, A. Peters ${ }^{4}$, C. Bachmann ${ }^{5}$, W. Paulus ${ }^{6}$, C. 


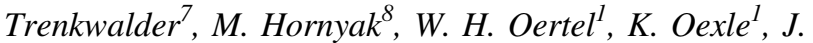
Winkelmann ${ }^{1,9,10}$

${ }^{1}$ Institute of Neurogenomics, Helmholtz Zentrum München GmbH, German Research Center for Environmental Health, Neuherberg, Germany, ${ }^{2}$ Institute of Molecular Epidemiology, Helmholtz Zentrum München GmbH, German Research Center for Environmental Health, Neuherberg, Germany, ${ }^{3}$ Institute of Genetic Epidemiology, Helmholtz Zentrum München GmbH, German Research Center for Environmental Health, Neuherberg, Germany, ${ }^{4}$ Institute of Epidemiology, Helmholtz, Zentrum München GmbH, German Research Center for Environmental Health, Neuherberg, Germany, ${ }^{5}$ Department of Neurology, Paracelsus Clinic, Osnabrück, Germany, ${ }^{6}$ Department of Clinical Neurophysiology, Georg August University Göttingen, Göttingen, Germany, ${ }^{7}$ Center of Parkinsonism and Movement Disorders, Paracelsus-Elena Hospital, Kassel, Germany, ${ }^{8}$ Neuropsychiatry Center Erding/Munich, Erding/München, Germany, ${ }^{9}$ MunichCluster for Systems Neurology, Munich, Germany, ${ }^{10}$ Institute of Human Genetics, Technical University of Munich, Munich, Germany

"Epigenetics" refers to the regulation of genetic information processing. DNA methylation is an important epigenetic mechanism which impacts gene expression in reaction to genetic, developmental or environmental conditions. Conventional epigenome-wide associations studies (EWAS) inquire the relation of phenotypes to the genomic DNA methylation pattern. We performed an EWAS of blood cell DNA from Restless-Legs Syndrome (RLS) patients and age- and sex-matched controls, using the Illumina EPIC (850K) chip. EWAS results were generated by applying mixed models in two datasets with 1000 and 2000 subjects, respectively, submitted to rigorous quality control and inflation correction, and then combined by meta-analysis. We obtained three genome-wide significant methylation sites. The strongest signal ( $p$-value $\leq 1.6 e-22)$ was located within MLLT6. This site is annotated to be promotor associated, suggesting promotor inactivation and consequent down regulation of MLLT6 expression in RLS patients. Gene products of MLLT6 and other MLL genes act as transcriptional regulators of MEISI, a gene highly associated with RLS. Our study is currently in the replication stage, where we intend to verify our findings. Because DNA methylation and gene expression are tissue-specific, we also generate EWAS and other omics results in post-mortem brain tissue samples of 25 RLS patients and 15 controls across four different brain regions. We thus aim for a comprehensive understanding of RLS pathogenesis and potential entry points for therapy.

Acknowledgments: International EU-RLS-GENE Consortium
N. Mirza-Schreiber: None. S. Naher: None. B. Schormair: None. C. Zhao: None. R. Wilson: None. M. Waldenberger: None. C. Gieger: None. M. MüllerNurasyid: None. A. Peters: None. C. Bachmann: None. W. Paulus: None. C. Trenkwalder: None. M. Hornyak: None. W.H. Oertel: None. K. Oexle: None. J. Winkelmann: None.

\section{P20.68.B}

SNP rs3087898 located next to m6A motif influences gluten-induced RNA methylation changes that regulate intestinal inflammation via allele-specific XPO1 translation

A. Olazagoitia-Garmendia ${ }^{l}$, L. Zhang ${ }^{2}$, P. Mera ${ }^{3}, I$.

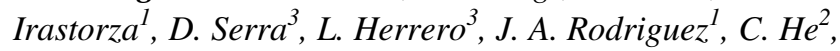
J. R. Bilbao ${ }^{1}$, A. Castellanos-Rubio ${ }^{l}$

${ }^{1}$ University of the Basque Country (UPV/EHU), Leioa, Spain, ${ }^{2}$ Howard Hugues Medical Institute, University of Chicago, Chicago, IL, United States, ${ }^{3}$ Institut de Biomedicina de la Universitat de Barcelona (IBUB), Universitat de Barcelona, Barcelona, Spain

Celiac disease (CD) is an inflammatory intestinal disorder that develops in genetically susceptible individuals upon gluten ingestion. Gluten triggers a proinflammatory response but the underlying mechanisms remain unclear. CD-associated SNPs map mainly to noncoding regions, and their effects are difficult to assess. Additionally, posttranscriptional RNA modifications, including m6A, have been shown to play key roles in different autoimmune diseases. We hypothesize that noncoding $\mathrm{CD}$-associated SNPs can alter m6A levels in adjacent motifs, affecting different cell processes and increase $\mathrm{CD}$ predisposition. SNP rs3087898, on the 5' UTR of XPO1 gene, is close to an m6A motif. We confirmed methylation in this region, and observed that the RNA carrying the CD-risk allele is more methylated and binds YTHDF1 reader more efficiently, promoting higher XPOI translation. m6A-related machinery KO produced lower XPO1 protein levels; while gluten stimulations provoked increased XPO1, METTL3 and YTHDF1 expression and an enhanced XPO1-YTHDF1 interaction, both in vitro and in vivo. Finally, we confirmed that the genotype of the associated SNP influences XPO1 protein levels in human intestinal biopsy samples. In addition, biopsies from $\mathrm{CD}$ patients stimulated with gluten also presented XPO1, METTL3 and YTHDF1 induction. We demonstrate that intergenic SNPs associated to autoimmune diseases can alter m6A levels and contribute to pathogenesis. This is the first approach linking gluten with alterations in m6A-related mechanisms. We observe that gluten induces the m6A machinery for an allele-specific increase of 
m6A levels on the 5'UTR of XPO1, leading to augmented XPO1 and NFkB activation in patients harboring the risk allele.

A. Olazagoitia-Garmendia: None. L. Zhang: None. P. Mera: None. I. Irastorza: None. D. Serra: None. L. Herrero: None. J.A. Rodriguez: None. C. He: None. J.R. Bilbao: None. A. Castellanos-Rubio: None.

\section{P20.69.C}

Implementation of functional assays to determine the clinical impact of Runt-related transcription factor 1 (RUNX1) variants in familial platelet disorder with associated myeloid malignancies (FPDMM)

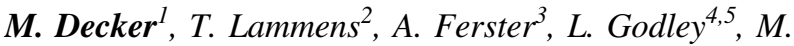
Erlacher $^{6}$, A. Yoshimi ${ }^{6}$, C. Niemeyer ${ }^{6}$, D. Steinemann ${ }^{1}$, B. Schlegelberger $^{1}$, T. Illig ${ }^{1,7}$, T. Ripperger ${ }^{l}$

${ }^{1}$ Department of Human Genetics, Hannover Medical School, Hannover, Germany, ${ }^{2}$ Department of Pediatric Hematology-Oncology, Ghent University Hospital, Ghent, Belgium, ${ }^{3}$ Department of Pediatric Hematology-Oncology, Children's University Hospital Queen Fabiola, Brussels, Belgium, ${ }^{4}$ Department of Human Genetics, The University of Chicago, Chicago, IL, United States, ${ }^{5}$ Section of Hematology/Oncology, Department of Medicine, The University of Chicago, Chicago, IL, United States, ${ }^{6}$ Division of Pediatric Hematology and Oncology, Department of Pediatrics and Adolescent Medicine, University of Freiburg, Freiburg, Germany, ${ }^{7}$ Hannover Unified Biobank, Hannover Medical School, Hannover, Germany

Introduction: RUNX1 bound to core binding factor $\beta$ $(\mathrm{CBF} \beta)$ functions as an essential transcriptional regulator in hematopoiesis. Pathogenic germline loss-of-function RUNX1 variants cause autosomal-dominantly inherited FPDMM, which is associated with thrombocytopenia, thrombocytopathy, and predisposition to hematological malignancies. To functionally characterize RUNX1 variants of uncertain significance (VUS) and, thus, determine their clinical impact, we established and applied a set of functional assays.

Methods: To address heterodimerization with $\mathrm{CBF} \beta$, expression and phosphorylation, and transcriptional activation, we applied a flow cytometry-based fluorescence energy transfer (FRET) assay, western blotting, and luciferase reporter assays in nephrogenic HEK293T and hematopoietic HEL cells, respectively. In comparison to RUNX1 wild-type and six known pathogenic RUNX1 variants, we analyzed nine previously reported germline missense VUS.

Results: We observed significant impairments in our functional assays for all known pathogenic variants. Three out of nine VUS displayed significantly reduced activity resembling known pathogenic variants. For two VUS, there was clear evidence for a significant functional impairment, while two other VUS showed slightly reduced functionality, and the remaining two displayed activity comparable to wild-type RUNX1.

Conclusions: We have successfully established functional assays and proven their clinical applicability in FPDMM families. Our data supports classification of three RUNX1 VUS as (likely) pathogenic and two RUNX1 VUS as (likely) benign. For two additional variants, there is clear evidence for their functional relevance, but further investigations are required to prove their pathogenicity and to facilitate conclusive classifications of the remaining two VUS.

This project has been supported by the European Hematology Association (EHA).

M. Decker: None. T. Lammens: None. A. Ferster: None. L. Godley: None. M. Erlacher: None. A. Yoshimi: None. C. Niemeyer: None. D. Steinemann: None. B. Schlegelberger: None. T. Illig: None. T. Ripperger: None.

\section{P20.70.A}

Profiling the chromatin landscape of early neurodevelopment using scATAC-seq

\section{C. A. Mannens, L. Hu, E. P. Braun, P. Lönnerberg, S. Linnarsson}

\section{Karolinska institutet, Solna, Sweden}

Understanding the molecular processes underlying healthy development of the human brain is crucial in the modelling and investigation of neurodevelopmental disorders. While many evolutionarily conserved developmental processes are well studied in mouse models, our understanding of humanspecific neurodevelopmental processes is limited. Our lab aims to provide transcriptomic and epigenomic reference atlases with single-cell resolution. In this ongoing project, we are characterizing chromatin accessibility of cell types in the human brain during early development (5 - 13 p.c.w.) using single-cell ATAC-sequencing.So far, we have sequenced a few tens of thousands of cells and will profile up to one million individual human cells, identifying both region- and cell lineage-specific enhancers as well as enriched motifs and gene accessibility. We use a two-step approach to feature identification, first performing superficial decomposition and clustering on binned genomic ranges to stratify peak calling and then performing Hierarchical Poisson Factorization on more detailed PeakxCell data to identify lineages and cell types. We have identified 29 clusters and 325.484 unique peaks. 115.048 of these 
peaks are intergenic and 28.622 of these peaks (25\%) don't align to any known element in the GENCODE GRCh38 reference, indicating the presence of unannotated cell lineage specific enhancers. To validate the cluster identities and lineages, our scATAC-seq data will be integrated with the scRNA-seq data that has been acquired as part of the Human Developmental Cell Atlas initiative. This combined resource may serve as a reference for future studies relating to development of the human nervous system.

European Union - H2020-SC1-BHC-2018-2020 BRAINTIME

C.C.A. Mannens: None. L. Hu: None. E.P. Braun: None. P. Lönnerberg: None. S. Linnarsson: None.

\section{P20.71.B}

Monocyte heterogeneity in health and inflammatory disease

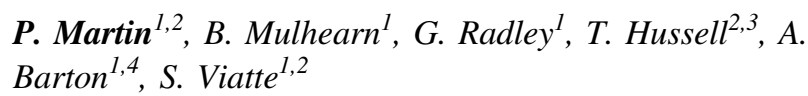

${ }^{1}$ Centre for Genetics and Genomics Versus Arthritis, Centre for Musculoskeletal Research, Manchester Academic Health Science Centre, University of Manchester, Manchester, United Kingdom, ${ }^{2}$ The Lydia Becker Institute of Immunology and Inflammation, Faculty of Biology, Medicine and Health, University of Manchester, Manchester, United Kingdom, ${ }^{3}$ Manchester Collaborative Centre for Inflammation Research, The Lydia Becker Institute of Immunology and Inflammation, University of Manchester, Manchester, United Kingdom, ${ }^{4}$ Manchester University NHS Foundation Trust, Manchester Academic Health Science Centre, NIHR Manchester Biomedical Research Centre, Manchester, United Kingdom

Rheumatoid arthritis (RA) is a common chronic inflammatory disease affecting $1 \%$ of the population. T-cells play a key role in initiating and/or maintaining the disease and antigen presentation is important in mediating this. Monocytes, precursors of antigen presenting cells, are typically characterised by expression of two cell surface proteins (CD14 and CD16). However, recent studies have shown further heterogeneity, some associated with RA. Understanding monocyte heterogeneity is therefore crucial in understanding the mechanisms leading to autoimmunity. We therefore sought to investigate peripheral blood monocyte heterogeneity, using single cell RNA-seq (scRNA-seq), from three healthy volunteers and three patients with RA. Overall, we analysed 17,831 profiles and identified fourteen clusters. There was a clear separation of classical (CD14 $\left.{ }^{+} \mathrm{CD} 16^{-}\right)$and non-classical $\left(\mathrm{CD} 14^{-} \mathrm{CD} 16^{+}\right)$monocyte subtypes, as well as intermediate $\left(\mathrm{CD} 14^{+} \mathrm{CD} 16^{+}\right)$and "natural killer" $(0.38 \%)$ identities. Interestingly, we identified a cluster defined by expression of the pro-inflammatory cytokine interleukin 1 beta (IL-1 $\beta$ ) in both healthy and RA samples (3.62\%), which recently has been shown to be expanded in RA synovia. These findings show that monocytes are indeed more diverse than previously indicated, highlighting the power of scRNA-seq to uncover this heterogeneity. Further work will be required to validate these findings and functionally define a role for this IL- $1 \beta$ monocyte subset. However, the presence of proinflammatory monocytes in the blood of healthy individuals would suggest that they are not a feature of RA specifically, but may interact with other cell types to influence or cause disease. Supported by the MRC (MC_PC_15072) and Versus Arthritis $(21745,20385)$.

P. Martin: None. B. Mulhearn: None. G. Radley: None. T. Hussell: None. A. Barton: None. S. Viatte: None.

P20.73.A

DNA methylation analysis of multiple imprinted DMRs in Sotos syndrome reveals IGF2-DMR0 as a DNA methylation-dependent, $P 0$ promoter-specific enhancer

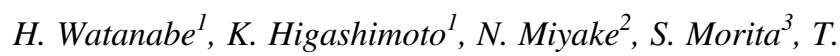
Horii ${ }^{3}$, M. Kimura ${ }^{3}$, T. Suzuki ${ }^{4}$, T. Maeda ${ }^{1}$, H. Hidaka ${ }^{1}$, S. Aoki $^{l}$, H. Yatsuki ${ }^{l}$, N. Okamoto ${ }^{5}$, T. Uemura ${ }^{l}$, I. Hatada ${ }^{3}$, N. Matsumoto ${ }^{2}$, H. Soejima ${ }^{l}$

${ }^{1}$ Saga University, Saga, Japan, ${ }^{2}$ Yokohama City University Graduate School of Medicine, Yokohama, Japan, ${ }^{3}$ Gunma University, Maebashi, Japan, ${ }^{4}$ Nagoya University, Nagoya, Japan, ${ }^{5}$ Osaka Women's and Children's Hospital, Osaka, Japan

Haploinsufficiency of NSD1, which dimethylates histone H3 lysine 36 (H3K36), causes Sotos syndrome (SoS), an overgrowth syndrome. DNMT3A and DNMT3B recognizes H3K36me3 through PWWP domain to exert de novo DNA methyltransferase activity and establish imprinted differentially methylated regions (DMRs). Since decrease of H3K36me3 and genome-wide DNA hypomethylation in SoS were observed, hypomethylation of imprinted DMRs in SoS was suggested. We explored DNA methylation status of 28 imprinted DMRs in $31 \mathrm{SoS}$ patients with NSDI defect and found that hypomethylation of IGF2-DMR0 and IGDMR in a substantial proportion of SoS patients. Luciferase assay revealed that IGF2-DMR0 enhanced transcription from the IGF2 $\mathrm{P} 0$ promoter but not the $\mathrm{P} 3$ and $\mathrm{P} 4$ promoters. ChIP-qPCR revealed active enhancer histone modifications at IGF2-DMR0, with high enrichment of H3K4me1 and H3K27ac. CRIPSR-Cas9 epigenome editing revealed that specifically induced hypomethylation at IGF2DMR0 increased transcription from the $\mathrm{P} 0$ promoter but not 
the P3 and P4 promoters. NSD1 knockdown suggested that NSD1 targeted IGF2-DMR0; however, IGF2-DMR0 DNA methylation and $I G F 2$ expression were unaltered. This study could elucidate the function of $I G F 2$-DMR0 as a DNA methylation dependent, P0 promoter-specific enhancer. NSD1 may play a role in the establishment or maintenance of IGF2-DMR0 methylation during the postimplantation period.

H. Watanabe: None. K. Higashimoto: None. N. Miyake: None. S. Morita: None. T. Horii: None. M. Kimura: None. T. Suzuki: None. T. Maeda: None. H. Hidaka: None. S. Aoki: None. H. Yatsuki: None. N. Okamoto: None. T. Uemura: None. I. Hatada: None. N. Matsumoto: None. H. Soejima: None.

\section{P20.74.B}

Methylation profile at $14 q 32$ in a family with $D L K 1$ small deletion associated with Temple syndrome

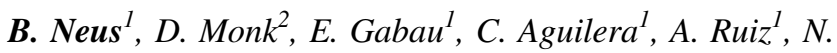

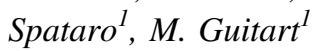

${ }^{1}$ Corporació sanitària Parc taulí, SABADELL, Spain, ${ }^{2} I D I-$ BELL Institut d'Investigació Biomèdica de Bellvitge, Hospitalet de Llobregat, Spain

Introduction:Temple syndrome (TS) is a rare condition caused by an abnormal expression of genes at the imprinted locus $14 \mathrm{q} 32$ that harbors a cluster of imprinted genes. The parental origin dependent expression pattern of imprinted genes is regulated by the sperm-derived imprinting control region, the IG-DMR, that regulates the somatic establishment of the MEG3-DMR on the paternal allele, as well as the maternally methylated MEG8-DMR and DLK1-DMR intervals.

Methodology:A female two years old with mild psychomotor retardation and hypotonia presents a $69 \mathrm{~kb}$ deletion of DLK1 identified by aCGH (8x60K ISCA, Agilent technologies). The MS-MLPA (MS-MLPA probemix ME032-A1, MRC Holland) technique was used to determine the inheritance, methylation pattern and dose analysis. Bisulphite PCR and pyrosequencing was used to assess methylation at imprinted DMRs not included in the MS-MLPA assay.

Results:The deletion is inherited from her father and from her grandmother, also her uncle is a carrier. The methylation pattern at the IG-DMR and MEG3-DMR was normal in carriers by MS-MLPA. It was confirmed by pyrosequencing, which also revealed carriers were hypomethylated at the DLK1 and MEG8-DMRs consistent with maternal transmission, whilst the proband presented with mild hypermethylation at the DLK1-DMR.
Conclusions:The loss of expression of $D L K 1$ on the paternal allele is responsible for the clinical features of psychomotor retardation and mild hypotonia suggestive of Temple syndrome. This small deletion narrowed the minimal region and methylation results supports that $D L K 1$ loss of function is sufficient to cause the main features of Temple syndrome.

B. Neus: None. D. Monk: None. E. Gabau: None. C. Aguilera: None. A. Ruiz: None. N. Spataro: None. M. Guitart: None.

\section{P20.75.C}

Pervasive and CpG-dependent promoter-like characteristics of transcribed enhancers

R. Steinhaus ${ }^{1,2}$, T. Gonzalez ${ }^{3,4}$, D. Seelow ${ }^{1,2}$, P. N. Robinson ${ }^{3,5}$

${ }^{1}$ Berlin Institute of Health, Berlin, Germany, ${ }^{2}$ Charité Universitätsmedizin Berlin, Berlin, Germany, ${ }^{3}$ The Jackson Laboratory for Genomic Medicine, Farmington, CT, United States, ${ }^{4}$ Harvey Mudd College, Claremont, CA, United States, ${ }^{5}$ Institute for Systems Genomics, University of Connecticut, Farmington, CT, United States

Introduction: The temporal and spatial expression of genes is controlled by promoters and enhancers. Findings obtained over the last decade that not only promoters but also enhancers are characterized by local transcription have challenged the traditional notion that promoters and enhancers represent distinct classes of regulatory elements. Over half of human promoters are associated with $\mathrm{CpG}$ islands (CGIs), relatively $\mathrm{CpG}$-rich stretches of generally several hundred nucleotides that are often associated with housekeeping genes. Only about $6 \%$ of transcribed enhancers defined by CAGE-tag analysis are associated with CGIs.

Results: Here we present a detailed analysis of the relation between enhancer and promoter characteristics and the presence or absence of CGIs. We show that transcribed enhancers share a number of CGI-dependent characteristics with promoters, including statistically significant local overrepresentation of core promoter elements. CGI-associated enhancers are longer, display higher directionality of transcription, greater expression, and a lesser degree of tissue specificity, as well as a higher frequency of transcription-factor binding events than enhancers not associated with CGIs. Genes putatively regulated by CGIassociated enhancers are enriched for transcription regulator activity.

Conclusions: Our findings show that CGI-associated enhancers display a series of characteristics related to 
sequence, expression, and function that distinguish them from transcribed enhancers not associated with CGIs.

R. Steinhaus: None. T. Gonzalez: None. D. Seelow: None. P.N. Robinson: None.

\section{P20.76.A}

Characterisation of $c i s$-regulatory elements of $G J B 2$ and whole genome sequencing of heterozygous patients with NSHL

\section{A. Le Nabec, A. Quillévéré, C. Le Maréchal, C. Férec, S. Moisan}

\section{Inserm UMR 1078, Brest, France}

Three-dimensional chromatin organization plays a key role on gene expression. Gene regulation depends on cis-regulatory elements which can interact with gene promoter by chromatin loop. Alteration of chromatin architecture and/or cis-acting elements can lead to cis-ruption disorder. Numerous unelucidated nonsyndromic hearing loss and deafness 1 (DFNB1) cases carrying out only one heterozygous pathogenic mutation on Gap Junction Beta 2 (GJB2) gene, led to strongly suggest the presence of distant cisregulation. Thanks to chromatin conformation study we previously identified several GJB2 cis-regulatory elements and purposed a DFNB1 3D looping model. Now, to more characterize these cis-acting elements, we analysed cooperative effect of these regions, we combined few regions together and showed that association of two enhancers increased the enhancer activity. Moreover, we focused on 10 patients with incomplete genotype. We realized a whole genome sequencing with HiSeq 4000 by IntegraGen Genomics. The results are currently being analysed. Our results allow to confirm an enhancer activity of these cisregulatory regions and their cooperative effects. Then, we expect that whole genome sequencing will allow to identify other mutations with loss / gain function or CNV which can explain pathology of our patients.

A. Le Nabec: None. A. Quillévéré: None. C. Le Maréchal: None. C. Férec: None. S. Moisan: None.

\section{P20.77.B}

Identification of Mowat-Wilson Syndrome (MOWS) ZEB2 gene-desert located enhancers active during neural differentiation of human induced pluripotent stem cells

J. C. Birkhoff ${ }^{l}$, R. W. W. Brouwer ${ }^{1}$, P. Kolovos ${ }^{2}$, A. L.

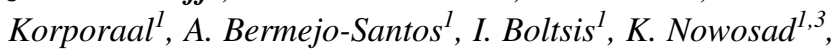

M. C. G. N. van den Hout ${ }^{l}$, F. G. Grosveld ${ }^{l}$, W. F. J. van IJcken $^{l}$, D. Huylebroeck ${ }^{1,4}$, A. Conidi ${ }^{l}$

${ }^{1}$ Erasmus Medical Center, Rotterdam, Netherlands, ${ }^{2}$ Democritus University of Thrace, Alexandroupolis, Greece, ${ }^{3}$ Medical University of Lublin, Lublin, Poland, ${ }^{4} \mathrm{KU}$ Leuven, Leuven, Belgium

Introduction: The transcription factor ZEB2 exerts multiple functions in vivo and uses different mechanisms of action and protein partners for controlling embryonic and adult cell fate decisions and maturation. As shown in various cell type specific knockout mouse models, some of these mechanisms explain major aspects of human MOWS (OMIM\#235730). ZEB2 is located downstream of a $\sim 3,3$ Mb-long gene desert, which is evolutionary conserved in other vertebrates. We mapped chromatin conformation changes here over $7.4 \mathrm{Mb}$ (chr2:143270465-150642631; GRCh37/hg19) to identify regulatory elements (REs) of ZEB2.

Material and Methods: Temporal Targeted Chromatin Capture (T2C) was used in human induced Pluripotent Stem Cells (iPSCs) during neural differentiation. Candidate REs, and their co-operations, were characterized by luciferase assays in transiently transfected cells, including neuroprogenitor cells (NPCs).

Results: We identified three novel, co-operative enhancers $\sim 500 \mathrm{~kb}$ upstream the ZEB2 transcription start site. They display strongest proximity (in T2C) with the ZEB2 promoter at early NPC/rosette cell stage, where ZEB2 mRNA levels increase significantly.

Conclusions: This work illustrates the value of $\mathrm{T} 2 \mathrm{C}$ in terms of high-resolution mapping novel REs at affordable sequencing cost. We are the first to document the dynamics of far-distant neural-active REs for ZEB2 located in the upstream gene desert. T2C can thus be used to identify and characterize REs that might be affected in those MOWS patients where no ZEB2 protein-encoding mutation could be identified.

J.C. Birkhoff: None. R.W.W. Brouwer: None. P. Kolovos: None. A.L. Korporaal: None. A. BermejoSantos: None. I. Boltsis: None. K. Nowosad: None. M.C. G.N. van den Hout: None. F.G. Grosveld: None. W.F.J. van IJcken: None. D. Huylebroeck: None. A. Conidi: None.

P20.78.C

Loss of imprinting of the human-specific imprinted gene ZNF597 causes prenatal growth retardation and dysmorphic features reminiscent of Silver-Russell syndrome 
K. Yamazawa ${ }^{1}$, T. Inoue ${ }^{2}$, Y. Sakemi ${ }^{3}$, T. Nakashima ${ }^{3}, H$. Yamashita $^{3}$, K. Khono ${ }^{4}$, H. Fujita ${ }^{5}$, K. Enomoto ${ }^{6}, K$. Nakabayashi ${ }^{7}$, K. Hata $^{7}$, M. Nakashima ${ }^{1}$, T. Matsunaga ${ }^{1}$, A.

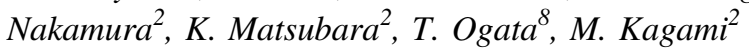

${ }^{1}$ Medical Genetics Center, National Hospital Organization Tokyo Medical Center, Tokyo, Japan, ${ }^{2}$ Department of Molecular Endocrinology, National Research Institute for Child Health and Development, Tokyo, Japan, ${ }^{3}$ Department of Pediatrics, National Hospital Organization Kokura Medical Center, Fukuoka, Japan, ${ }^{4}$ Navitas Clinic Kawasaki, Kawasaki, Japan, ${ }^{5}$ Meguro Avenue Kid's Clinic, Tokyo, Japan, ${ }^{6}$ Enomoto Children's Clinic, Moriya, Japan, ${ }^{7}$ Department of Maternal-Fetal Biology, National Research Institute for Child Health and Development, Tokyo, Japan, ${ }^{8}$ Department of Pediatrics, Hamamatsu University School of Medicine, Hamamatsu, Japan

Introduction: ZNF597, encoding a zinc finger protein, is the human-specific maternally expressed imprinted gene located on $16 \mathrm{p} 13.3$. The parent-of-origin expression of ZNF597 is regulated by the ZNF597:TSS-DMR of which only paternal allele acquires methylation during postimplantation period. Overexpression of ZNF597 may contribute to some of the phenotypes associated with maternal uniparental disomy of chromosome 16 (UPD(16)mat), and some UPD(16)mat patients presenting with Silver-Russell syndrome (SRS) phenotype have recently been reported.

Materials and Methods: A 6-year-old boy partially presented with SRS-like features such as prenatal growth restriction, macrocephaly at birth, forehead protrusion in infancy, and clinodactyly of the right fifth finger. Methylation, expression, microsatellite marker, single nucleotide polymorphism array, and trio whole-exome sequencing analyses were conducted.

Results: Isolated hypomethylation of the ZNF597:TSSDMR and subsequent loss of imprinting and overexpression of ZNF597 were confirmed in the patient. Epigenetic alterations such as UPD and other methylation defects were excluded. Pathogenic sequence or copy number variants affecting his phenotypes were not identified, indicating that primary epimutation occurred postzygotically.

Conclusions: This is the first case of isolated ZNF597 imprinting defect, who showed prenatal growth retardation and some dysmorphic features reminiscent of SRS. ZNF597 overexpression may comprise the underlying molecular mechanism of developing SRS phenotype in UPD(16)mat. Further investigations and case series are needed to establish a new imprinting disorder entity involving the ZNF597 domain.

Grants: This work was supported by a Grant-in-Aid for Scientific Research (B) from the Japan Society for the Promotion of Science (16H05362).
K. Yamazawa: None. T. Inoue: None. Y. Sakemi: None. T. Nakashima: None. H. Yamashita: None. K. Khono: None. H. Fujita: None. K. Enomoto: None. K. Nakabayashi: None. K. Hata: None. M. Nakashima: None. T. Matsunaga: None. A. Nakamura: None. K. Matsubara: None. T. Ogata: None. M. Kagami: None.

\section{P21 New Treatments for Genetic Disorders}

\section{P21.1.A}

CLOVES syndrome as a consequence of a PIK3CA variant the" long and far " way to confirm the diagnosis and therapeutic options

S. Morlot, B. Auber, G. Schmidt, S. von Hardenberg, B. Schlegelberger

\section{Medical School Hanover, Hannover, Germany}

We report on an 8-year-old Iranian boy who was diagnosed with CLOVES syndrome, a disease within the PIK3CArelated overgrowth spectrum (PROS) caused by mosaic pathogenic PIK3CA variants, characterized by congenital lipomatous asymmetric overgrowth of the trunk as well as lymphatic, capillary, venous and combined vascular malformations, epidermal nevi, skeletal and spinal anomalies. The patient presented with a large "portwine", multiple superficial small hemangiomas, macrodactylia of fingers and toes, syndactylies and swelling on the right breast. The patient reported pain in the upper and lower extremities. Motor and cognitive development was age-appropriate. Exome analysis from peripheral blood revealed no pathogenic variant in the genes PTPN11, PIK3CA, AKT3, PIC3R2, PTEN, SDHB, KLLN, SDHD, GNAQ and AKT1 (Macrogen, Korea)).

We suspected mosaic CLOVES syndrome due to a postzygotic PIK3CA variants gained in the early embryonic period. We performed parallel panel sequencing (IDT custom panel) from different tissues. No pathogenic variant was detected in the PIK3CA gene in blood and oral mucosa. In a skin punch (hypertrophic hand) the pathogenic PIK3CA variant c.328_330del p.(Glu110del) was detected with an allele frequency of $4 \%$. A fibroblast culture of this punch reveled the pathogenic $P I K 3 C A$ variant with an allele frequency of $44 \%$.

There is no curative therapy available. However in a clinical trial with the PIK3CA inhibitor BYL719 (Alpelisib) carried out in 19 patients with CLOVES syndrome improved symptoms. (Venot et al. 2018; PMID: 29899452)). In Germany Alpesilib is not yet approved for the treatment of CLOVES syndrome, but its application for off-label use is aspired. 
S. Morlot: None. B. Auber: None. G. Schmidt: None. S. von Hardenberg: None. B. Schlegelberger: None.

\section{P21.5.B}

Stability and safety of induced pluripotent stem cells for cartilage regeneration

M. Poulou ${ }^{1}$, I. Papathanasiou ${ }^{2}$, E. Mourmoura ${ }^{2}, T$. Brantzos $^{1}$, I. Sfougataki ${ }^{3}$, A. Mertzanian $^{3}$, E. Goussetis $^{3}$, V. Trachana $^{4}$, A. Tsezou ${ }^{2,4}$, M. Tzetis ${ }^{1}$

${ }^{I}$ National and Kapodistrian University of Athens, Faculty of Medicine, Laboratory of Medical Genetics, Athens, Greece, ${ }^{2}$ University of Thessaly, Faculty of Medicine, Laboratory of Cytogenetics and Molecular Genetics, Larissa, Greece, ${ }^{3}$ Aghia Sofia Children's Hospital, Bone Marrow Transplantation Unit, Athens, Greece, ${ }^{4}$ University of Thessaly, Faculty of Medicine, Dept. of Biology, Larissa, Greece

Introduction: Osteoarthritis (OA), the most common form of degenerative joint disease of adult population worldwide is expected to increase in incidence due to aging population. Pharmacological treatments aim at pain relief, while surgical joint replacement is costly and requires a long recovery period. The limited ability of articular cartilage to regenerate make approaches for cartilage regeneration a reasonable approach. Patient-specific induced pluripotent stem-cells (iPSCs) and production of derived mesenchymal stem-cells (iMSCs) from mesenchymal bone marrow stem-cells (BMMSCs) were tested and evaluated regarding their proliferative capacity and stability for potential use for cartilage regeneration.

Material and Methods: BM-MSCs cells isolated from the iliac crest of OA patients and normal controls, were reprogrammed to produce iPSCs and derived iMSCs. Reprogramming to generate iPSCs, was done using a modified synthetic mRNA method. Different clones of each undifferentiated iPSC line were tested for their genetic stability, pluripotency, teratoma formation and for their patient-specific origin as previously described. After mesenchymal differentiation and expansion each iPSCMSC line was examined for specific MSC markers. CNV detection was done using aCGH.

Results: The clinical safety of the produced cells was proven even in higher passages, by analysis of their genetic stability through array-CGH of various clones of iPSCs and iMSCs cells, in comparison to the original MSCs.

Conclusions: Array-CGH revealed a chromosomal pattern exhibiting mostly polymorphic CNVs thus proving genetic stability. Some clones acquired CNV deletions after continuous passaging, a phenomenon extensively observed and related to culture adaptation. Work sponsored under the
Operational Program "Competitiveness, Entrepreneurship \& Innovation" (EPAnEK)

M. Poulou: None. I. Papathanasiou: None. E. Mourmoura: None. T. Brantzos: None. I. Sfougataki: None. A. Mertzanian: None. E. Goussetis: None. V. Trachana: None. A. Tsezou: None. M. Tzetis: None.

P21.7.A

Efficient and concise protocol for neural conversion of human fibroblasts as an autologous cell source for an early cyto-gene therapy of genetic based neurodegenerative diseases

\section{S. S. Hosseini Farahabadi ${ }^{1,2}, K$. Ghaedi ${ }^{1,2}$, M. Nasr- Esfahani ${ }^{2}$}

${ }^{1}$ Department of Cell and Molecular Biology and Microbiology, Faculty of Biological Science and Technology, Isfahan, Iran, Islamic Republic of, ${ }^{2}$ Department of Cellular Biotechnology at Cell Science Research Center, Royan Institute for Biotechnology, ACECR, Isfahan, Iran, Islamic Republic of

Introduction: A revolutionary new approach to produce cells is to induce transdifferentiation to make it conventional in therapeutic strategies. In this project, we describe a brief cocktail of small molecules including Dorsomorphin (DSM) and Trichostatin A (TSA) to produce safe induced neuroectodermal cells (iNCs). These iNCs can be used for a combinatorial cell-gene therapy strategy to treat or cure of genetic-based neurodegenerative diseases such as Amyotrophic Lateral Sclerosis (ALS) in newborns.

Materials and Methods: Human fibroblast cells were treated with DSM, in presence of TSA and a cocktail of neurotrophic factors including brain-derived neurotrophic factor, glial cell line-derived neurotrophic factor and ciliary neurotrophic factor, in neural induction medium. On day 6, cells were seeded on culture dishes coated with poly-Lornithine + Laminin + Fibronectin. Cell characterization was carried out by morphological tracking, gene expression profiling of markers including Vimentin, PAX6 and Nestin by real time PCR, immunofluorescence staining and flow cytometry. Statistical analysis was performed by SPSS in three independent replicate. Comparisons were analyzed by independent-sample $t$-test and One-Way ANOVA, $\mathrm{p}<0.05$.

Results: This protocol mediated neuroectodermal conversion of human fibroblasts within 12 days with an average efficiency of $24 \%$, promises a fast strategy to produce human iNCs for cyto-gene therapy of nervous system disorders.

Conclusions: Our protocol is a safe conversion protocol which shortens path of clinical application of iNCs. Benefiting from these important features, it makes it 
possible for us to edit genetic mutations of newborns with neural disorders such as ALS with an early cell-gene therapy strategy.

S.S. Hosseini Farahabadi: None. K. Ghaedi: None. M. Nasr-Esfahani: None.

\section{P21.8.B}

The pharmacological chaperone N-n-Butyldeoxygalactonojirimycin enhances $\beta$-galactosidase processing and activity in the fibroblasts of a patient with infantile GM1-gangliosidosis

F. Mohamed ${ }^{l}$, M. Al-Sorkhy ${ }^{2}$, M. Ghattas ${ }^{2}$, L. Al-Gazali ${ }^{1}$, O. Al-Dirbashi ${ }^{1}$, F. Al-Jasmi ${ }^{1}$, B. R. Ali ${ }^{l}$

${ }^{1}$ United Arab Emirates University, Al-Ain, United Arab Emirates, ${ }^{2}$ Al-Ain University, Al-Ain, United Arab Emirates

Introduction: Variants affecting the conformations of secretory and membrane proteins are usually retained in the ER then degraded. However, some partially misfolded ERretained proteins may retain some biological activity and are therefore good drug targets. GM1-Gangliosidosis, a lysosomal storage disorder, is associated with mutations in GLB1 leading to $\beta$-galactosidase ( $\beta$-Gal) deficiency. Deoxygalactonojirimycin (DGJ) and its derivatives are pharmaceutical chaperones that could bind to mutated $\beta-G a l$ in the ER promoting its folding and trafficking to lysosomes.

Patient and Methodology: An Emirati child has been diagnosed with infantile GM1-Gangliosidosis with GLB1 mutations (p.D151Y). Confocal microscopy and biochemical analysis were used to establish the subcellular localization of $\beta$-Gal and its enzymatic activity was measured using $0.8 \mathrm{mM} \quad$ 4-Methylumbelliferyl $\beta$-Dgalactopyranoside.

Results: We show that p.D151Y $\beta$-Gal in patient's fibroblasts retained $<1 \%$ residual activity due to impaired cellular processing and trafficking. Molecular simulation analysis revealed that the amino acid substitution significantly affected the enzyme conformation. However, p. D151Y $\beta$-Gal was amenable for partial rescue by culturing the fibroblasts in the presence of glycerol or at low temperature. The DGJ derivatives butyl (NB-DGJ) and nonyl (NN-DGJ) were examined as potential pharmacological chaperones for the mutant enzyme. Although NN-DGJ showed higher affinity to $\beta$-Gal, it didn't show a significant enhancement in p.D151Y $\beta$-Gal activity. However, NBDGJ promoted p.D151Y $\beta$-Gal maturation and enhanced its activity up to $\sim 4.5 \%$ of normal control activity within $24 \mathrm{hrs}$ which significantly increased to $\sim 10 \%$ over a period of six days.
Conclusions: NB-DGJ is a promising pharmacological chaperone in infantile GM1 missense variants.

Grant number: UAEU31R134.

F. Mohamed: None. M. Al-Sorkhy: None. M. Ghattas: None. L. Al-Gazali: None. O. Al-Dirbashi: None. F. AlJasmi: None. B.R. Ali: None.

P21.9.C

Medical therapy of vascular Ehlers-Danlos syndrome: Challenging the paradigm of interchangeable antihypertensive drugs

N. Dubacher ${ }^{1,2}$, S. M. Caspar ${ }^{1,3}$, J. Meienberg ${ }^{1}$, G. Matyas ${ }^{1}$

${ }^{1}$ Center for Cardiovascular Genetics and Gene Diagnostics, Schlieren-Zurich, Switzerland, ${ }^{2}$ University Hospital Balgrist, University of Zurich, Switzerland; Institute for Biomechanics, ETH Zurich, Zurich, Switzerland, ${ }^{3}$ Laboratory of Translational Nutrition Biology, ETH Zurich, Schwerzenbach, Zurich, Switzerland

Introduction: Patients with the rare connective tissue disorder vascular Ehlers-Danlos syndrome (vEDS) are at increased risk for fatal aortic ruptures. Using a mouse vEDS model, we established an objective read-out system for the assessment of the clinically highly relevant biomechanical integrity of the murine thoracic aorta. By means of our novel read-out system, we aimed to assess the effects of antihypertensive drugs on the biomechanical integrity of the weakened murine vEDS thoracic aorta as potential medical therapy in vEDS.

Method: Mice modelling vEDS were treated with the beta-blockers celiprolol $\left(\right.$ Selectol $\left.^{\circledR}\right)$ or bisoprolol $\left(\right.$ Bilol $\left.^{\circledR}\right)$ or the ARB losartan (losartan Actavis ${ }^{\circledR}$ ) for 4 weeks. 1.5-mmlong sections of the ascending and descending murine thoracic aorta were mounted on a tissue puller and uniaxially stretched until rupture while recording the tensile force (in $\mathrm{mN}$ ).

Results: The rupture force was significantly lower in untreated heterozygous compared to wild-type mice and decreased with increasing distance from the heart for both heterozygotes and wild-types. We showed that celiprolol but neither bisoprolol nor losartan increased the thoracic aortic rupture force in heterozygous mice (PMID:31056650 and 31693161).

Conclusions: Our novel and objective read-out system is suitable for detecting significant differences in the rupture force of the murine thoracic aorta and allows the assessment of the effect of candidate drugs on the biomechanical integrity of the aorta. Although the added value of other antihypertensive drugs in vEDS, if any, is unknown, 
celiprolol, but not losartan and bisoprolol, is currently the medical therapy of choice for vEDS, until further evidence emerges.

N. Dubacher: None. S.M. Caspar: None. J. Meienberg: None. G. Matyas: None.

\section{P22 Genetic Counselling / Services / Education}

\section{P22.04.A}

The story of BRCA1:c.4096 $+3 \mathrm{~A}>\mathrm{G}-$ is it pathogenic?

\section{Stefansdottir ${ }^{l}$, J. J. Jonsson ${ }^{1,2,3,4}$}

${ }^{1}$ Department of Genetics and Molecular Medicine, Landspitali National University Hospital, Reykjavik, Iceland, ${ }^{2}$ Dept. of Biochemistry and Molecular Biology, Univ. of Iceland, Reykjavik, Iceland, ${ }^{3}$ Faculty of Medicine, Univ. of Iceland, Reykjavik, Iceland, ${ }^{4}$ Genetical Committee of the University of Iceland and Landspitali, Reykjavik, Iceland

The founder pathogenic variants (PV), BRCA1:c.5074G >A and the BRCA2:c.767_771delCAAAT were for many years thought to be the only BRCA PVs in the Icelandic population. Of the two, the c.767_771delCAAAT is more prevalent, with $0.8 \%$ carrier rate. Therefore, genetic testing was mostly limited to these two variants. The extensive electronic pedigrees used in genetic counselling in Iceland, show clearly the hereditary breast and ovarian (HBOC) patterns in families, aiding decision on genetic testing. However, in one part of the country, the Westfjords, hardly any invidual tested postive for either of the founder PVs despite many HBOC families with classic BRCA1 cancer pattern. By sequencing the BRCA genes, the first patient tested positive for the BRCA1 c. $4096+3 \mathrm{~A}>\mathrm{G}$ in 2010 , and in 2014, another one. The two patients were related within $6^{\circ}$ of relatedness. But, the variant had conflicting pathogenity in ClinVar, mostly of unknown significance. In 2017, a Danish study classified the variant as VUS (1), based mainly on one homozygous healthy individual. We sought information from deCode genetics (personal communication), which established $0.2 \%$ population frequency and pathogenity, with 16 fold risk of ovarian cancer and 3.5 fold risk of breast cancer. In 2019, an Icelandic study found their data to accord with the variant being pathogenic (2). Of the 467 tested for this variant, 87 are postive (19\%). All positive individuals trace their ancestry to the Westfjords and no other significant HBOC PV has been found there. The variant is now routinely tested for, in HBOC families with roots in the Westfjords.

V. Stefansdottir: None. J.J. Jonsson: None. P22.05.B
Evaluating cancer genomics education for mainstream clinicians in South East London, Kent, Surrey and East Sussex; can technology bridge the gaps?

A. E. Coulson ${ }^{1}$, E. Cox², J. Malawana ${ }^{2}$, Z. Beydoun ${ }^{3}$, V. Reeve $^{3}$, A. Kulkarni ${ }^{l}$, V. Tripathi ${ }^{l}$

${ }^{1}$ South East Thames Regional Genetics Service, Guy's and St Thomas' NHS Foundation Trust, London, United Kingdom, ${ }^{2}$ Medics. Academy, London, United Kingdom, ${ }^{3}$ Guy's and St Thomas' NHS Foundation Trust, London, United Kingdom

Introduction: Genomic testing in the UK is changing, and cancer clinicians are increasingly utilising genomics to inform treatment, prognosis and future risks. The UK Government has called for improved education in appropriately ordering and interpreting genomic tests. We aimed to evaluate existing local cancer genomics training to understand training needs and barriers, contributing to the development of a new online platform (Nucleus) for futureproof, scalable training.

Materials and Methods: Delegates from Guy's Hospital Cancer Genetics course completed questionnaires about cancer genomics education. This evidence contributed to development of Nucleus, an online education platform. User testing of an initial Nucleus module was assessed to determine the utility of a change in delivery of education.

Results: Delegates identified time (56\%) and funding (44\%) as barriers to accessing training. Prior to Nucleus development, $55 \%$ of delegates felt online training was an acceptable mode for education, though face-to-face was popular $(77 \%)$. Following implementation, Nucleus user testing was highly positive. Users rated it as relevant to practice $(\overline{\mathrm{x}}=4.57 / 5)$, with appropriate length $(\overline{\mathrm{x}}=4.29 / 5)$ and pace $(\bar{x}=4.14 / 5)$, and felt it was easy to navigate.

Conclusions: Education is a priority for the implementation of new genomic testing services. Face-to-face teaching is valued but barriers and scalability of training for larger clinician groups must be considered. Online training can overcome barriers by providing instant access to relevant and appropriate education, as demonstrated by Nucleus. Future work will develop further modules and blendedlearning approaches to education.

A.E. Coulson: None. E. Cox: None. J. Malawana: None. Z. Beydoun: None. V. Reeve: None. A. Kulkarni: None. V. Tripathi: None.

P22.07.A

Governance of Cancer Genetic Counseling: A Systematic Literature Review 
L. Abu Jamous, H. Abdelrazeq, A. Tbakhi, M. Odeh

\section{King Hussain Cancer Center, Amman, Jordan}

Cancer Genetic Counselling (CGC) involves trained persons to help individuals or family members comprehending genetic disorders, heredity and risk factors, with the aim to choose the appropriate course of action in the affected family members. This research is aimed at informing (using the systematic literature review method) the existence and effectiveness of automated CGC governance frameworks. The gap in the literature revealed the absence of a formalised approach to linking CGC processes which are currently highly dependent on the person involved in counseling sessions. Although professional guidelines are used to identify patients at risk, they are not in a standardised form. Few resources exist in CGC service assessment which are mainly the National Cancer Institute Community Cancer Centers (NCCCP) and the CGC Assessment Tool (CGCAT) tool. Also, four core bodies are mainly responsible for generating policies for CGC services with no major differences between their policy documents. CGC Quality requirements include accountability, value, safety, equity, sustainability, effectiveness, etc. Seven core CGC processes are mainly followed from patient identification to postcounseling. However, there is no consensus on metrics to inform the satisfaction of quality requirements of CGC programmes. Moreover, no comprehensive CGC frameworks exit that reflect the interaction between standards, quality requirements, policies, and processes. In conclusion, without such interactions, automatic governance for each CGC case is not possible which has led the researchers to embark on developing a CGC process architectural framework to inform governance of CGC processes, and their effectiveness in reducing incidence and mortality in relation to hereditary.

L. Abu Jamous: None. H. Abdelrazeq: None. A. Tbakhi: None. M. Odeh: None.

P22.09.C

Communication between specialist genetic services, CCGs and GPs

J. Gordon ${ }^{1}$, J. Adlard ${ }^{2}$, R. Jewell ${ }^{2}$, J. Hayward ${ }^{2}$

${ }^{1}$ Hull Teaching Hospitals NHS Trust, Hull, United Kingdom, ${ }^{2}$ Yorkshire Regional Genetics Service, Leeds, United Kingdom

Background: Genomics services are advancing rapidly. Development of these services must allow for effective information dissemination to, and communication with, primary care.
Methods: We developed an e-survey to assess Clinical Commissioning Groups' (CCGs) mechanisms of information dissemination and General Practitioners' (GPs) access to specialist advice. All 17 local CCGs were invited to participate. A semi-structured interview script was developed for face-to-face interviews.

Results: $82 \%$ of surveys were completed. CCGs disseminated information to GPs mostly by emails to practice managers $(100 \%)$ and GPs $(93 \%)$, educational events (86\%), and CCG newsletters (86\%). Overall, educational events were identified as the most effective and emails to GPs relatively ineffective. The most common methods for GPs to access specialist advice were phone (79\%) and electronic 'advice and guidance' (A\&G) systems (79\%). Phone and electronic advice were the preferred options for a new genetics advice service.

Three respondents consented to interview. All felt that short, practical updates on genetics (rather than a full dedicated session) would be useful at their educational events. All had website portals for referral information, on which they felt genetics content would be useful. Simple updates could also be emailed to CCGs for dissemination via newsletter. Electronic $A \& G$ was the favoured specialist advice method.

Conclusions: Short, practical educational sessions could be used to share genetics updates. Simple updates could be emailed to CCGs (for inclusion in newsletters/emails to practice managers) or uploaded onto CCG portals. Electronic advice methods, such as $A \& G$ and email, should be considered in genomic medicine service development.

J. Gordon: None. J. Adlard: None. R. Jewell: None. J. Hayward: None.

\section{P22.10.A}

A service evaluation: exploring the communication of microarray-comparative genome hybridisation copy number variant results of uncertain significance in a paediatric setting

\section{Jacobs-Pearson ${ }^{l, 2}, J_{\text {Rankin }}{ }^{1}$}

${ }^{1}$ Peninsula Department of Clinical Genetics, Exeter, United Kingdom, ${ }^{2}$ School of Biological Sciences, Univeristy of Manchester, Manchester, United Kingdom

Chromosomal microarray-comparative genome hybridisation (array-CGH) is a first line diagnostic test for children presenting in paediatric departments. The clinical significance of array-CGH results is not always understood, introducing uncertainties for interpretation and challenges for clinicians and patients. The aim of this study was to look at the experiences of paediatricians in providing uncertain array-CGH results to their patients; ascertaining their 
confidence in interpretation and communication of uncertain information and reviewing the current support in place. An online mix-methods questionnaire was used to capture the data. Descriptive statistical and thematic qualitative analysis was carried out on the data. Ten paediatricians across three hospitals took part. All paediatricians had varied experience in communicating uncertain array-CGH results and a mixed knowledge of genomic testing and its possible implications for the patient and their family. This study demonstrated a general understanding of array-CGH results and their implications alongside a willingness to communicate uncertainty to patients. The importance of ongoing education and support for paediatricians was highlighted, along with the need for additional patient information. These findings are important as genomics becomes embedded throughout clinical practice; gaining insight into experiences of those on the frontline helps to improve the support for clinicians and patients alike to ensure high quality patient care.

\section{Jacobs-Pearson: None. J. Rankin: None.}

\section{P22.11.B}

A nationally agreed cross-professional competency framework to facilitate genomic testing

A. Pichini ${ }^{1,2}$, A. Seller ${ }^{2}$, M. Bishop ${ }^{2}$

${ }^{1}$ University Hospitals Bristol NHS Foundation Trust, Bristol, United Kingdom, ${ }^{2}$ Health Education England Genomics Education Programme, Birmingham, United Kingdom

Building on current medical practice, genomic medicine highlights unique considerations with regards to consent including addressing needs of the wider family, the complexity and uncertainty of genomic information, data sharing protocols, and returning results. England is implementing a national Genomic Medicine Service, leading to genomic testing being utilised across an increasing number of specialties and resulting in a requirement for workforce development around the consent conversation. In response to this, The Genomics Education Programme has developed a nationally agreed cross-professional competency framework outlining the knowledge, skills and behaviours required to facilitate genomic testing. Using the principles of the nominal group technique, a one-day expert consensus meeting reviewed clinical scenarios in iterative rounds, mapped themes to an initial framework and voted on areas of inconsistency. A revised framework was open for consultation with key stakeholders including patient communities and Royal Medical Colleges before being finalised. A short-term external evaluation has been conducted, assessing how early adopters are using or intending to use the framework. Evidence-based competencies are an important basis to support responsible delivery of genomic medicine. This framework can be used as a guide for best practice by clinicians involved in requesting genomic tests. For those delivering education, the framework provides a foundation to identify learning needs and structure training, such that consent conversations can be delivered in a consistent manner across specialties. In addition, these competencies can be used as a reference to evaluate how consent is facilitated in different specialty areas to enhance the delivery of genomic medicine.

A. Pichini: None. A. Seller: None. M. Bishop: None.

P22.12.C

Minds made up? The scope for deliberation in predictive genetic testing

\section{S. D. Doheny, A. Clarke}

Division of Cancer and Genetics, Cardiff, United Kingdom

Certain tests are offered to patients in the expectation that the results of the test will be of more use to the patient than to clinicians managing their care. In genetics, the paradigmatic example is the predictive genetic test for Huntington's Disease where patients may want to plan for a future with or without the disease. Similarly, genetic testing for BRCA 1/2 and some other familial cancers may be offered to patients before they may want to engage in surveillance or preventative treatment.

Following established guidelines, counseling for these investigations proceeds by first providing patients with information about the test while counseling on the aspects of the test that the patient might want to consider. This is to prompt reflection by the patient, before proceeding with the test. The question we raise is: to what extent do patients use this time to deliberate on the decision to take the test.

To explore the deliberations of patients, we gathered ethnographic observations of clinic consultations and post decision interviews with patients, and asked patients to keep a record of their thoughts during this period. In this paper, we explore the extent to which patients engage in deliberation by focusing on the patient's record of their reflections and deliberations. In our analysis, we explore how the temporal span of the patients' own deliberations extended beyond that of the clinical setting, and how various aspects of the decision could prompt a more or less deliberative engagement on the part of the patient.

S.D. Doheny: None. A. Clarke: None. 


\section{P22.14.B}

The use of eConsultation to improve delivery of clinical genetics to primary care: Preliminary findings of geneticist data

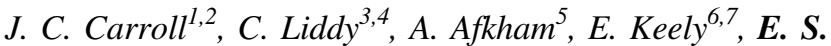
Goh $^{8,9}$, G. E. Graham ${ }^{10,11}$, J. A. Permaul ${ }^{1}$, J. Allanson ${ }^{10,11}$, G. Farrell ${ }^{12}$, R. Heisey ${ }^{2,13}$, T. Makuwaza ${ }^{1}$, D. P. Manca ${ }^{14}$, M. A. O'Brien ${ }^{2}, R$. Segal ${ }^{15}$, E. Grunfeld ${ }^{2,16}$, on behalf of CanIMPACT

${ }^{1}$ Ray D. Wolfe Department of Family Medicine, Mount Sinai Hospital, Sinai Health System, Toronto, ON, Canada, ${ }^{2}$ Department of Family \& Community Medicine, University of Toronto, Toronto, ON, Canada, ${ }^{3}$ Bruyere Research Institute, Ottawa, ON, Canada, ${ }^{4}$ Department of Family Medicine, University of Ottawa, Ottawa, ON, Canada, ${ }^{5}$ Champlain Local Health Integration Network, Ottawa, Ottawa, ON, Canada, ${ }^{6}$ Division of Endocrinology and Metabolism, Ottawa Hospital, Ottawa, ON, Canada, ${ }^{7}$ Department of Medicine, University of Ottawa, Ottawa, ON, Canada, ${ }^{8}$ Laboratory Medicine and Genetics, Trillium Health Partners, Mississauga, ON, Canada, ${ }^{9}$ Department of Laboratory Medicine \& Pathobiology, University of Toronto, Toronto, ON, Canada, ${ }^{10}$ Department of Pediatrics, University of Ottawa, Ottawa, ON, Canada, ${ }^{11}$ Department of Genetics, Children's Hospital of Eastern Ontario, Ottawa, ON, Canada, ${ }^{12}$ Memorial University of Newfoundland, St. John's, NL, Canada, ${ }^{13}$ Department of Family and Community Medicine, Women's College Hospital, Toronto, ON, Canada, ${ }^{14}$ Department of Family Medicine, University of Alberta, Edmonton, AB, Canada, ${ }^{15}$ Division of Medical Oncology, University of Ottawa, Ottawa, ON, Canada, ${ }^{16}$ Ontario Institute for Cancer Research, Toronto, ON, Canada

Introduction: Electronic consultation (eConsult) is a secure online platform connecting primary care providers and specialists that may help improve wait times for genetics consultations and educate primary care providers (PCPs) about genetic conditions. Our objective is to determine if eConsult is an effective method to improve delivery of clinical genetics in primary care. Specifically, the perceptions of PCPs and geneticists about the effectiveness of genetics eConsults and their implementation into practice are examined.

Materials and Methods: A mixed methods implementation study using data collection forms and interviews was undertaken with PCPs and 7 geneticists in 2 regions of Ontario, Canada. PCPs complete a questionnaire evaluating value and utility of each eConsult. Geneticists track the type and appropriateness of eConsults. Descriptive thematic analysis was used for the semi-structured interviews with geneticists.

Results: In 2019, 112 genetics eConsults were analyzed (94 adult, 18 pediatric). Cancer eConsults were most common $(56,50 \%)$, with possible genetic syndromes $(9$, $8 \%$ ) being next most common. Geneticists did not expect PCPs to know the answer for $46 \%$ (52) of eConsult questions posed. Interview findings at 3 months show that geneticists are positive about eConsult and see value in educating PCPs through eConsults, but challenges include underuse of the service by PCPs.

Conclusions: Geneticist data without PCPs' assessment of utility of eConsults are presented. Results show that hereditary cancer eConsults are common, PCPs appear to be asking appropriate questions, and geneticists are generally positive about eConsult. This study is funded by the Canadian Institutes of Health Research.

J.C. Carroll: None. C. Liddy: None. A. Afkham: None. E. Keely: None. E.S. Goh: None. G.E. Graham: None. J. A. Permaul: None. J. Allanson: None. G. Farrell: None. R. Heisey: None. T. Makuwaza: None. D.P. Manca: None. M.A. O'Brien: None. R. Segal: None. E. Grunfeld: None.

\section{P22.16.A}

Creating an Master of Science in Genetic counselling in Sweden that fulfils EBMG-criteria

\section{R. Pestoff ${ }^{1,2}$, P. Söderkvist ${ }^{2}$, C. Ingvoldstad Malmgren ${ }^{3}$}

${ }^{I}$ Department of Clinical Genetics, Linköping, Sweden, ${ }^{2}$ Department of of Biomedical and Clinical Sciences, Linköping University, Linköping, Sweden, ${ }^{3}$ Department of Molecular medicine, surgery, rare diseases; Karolinska Institute, Solna, Sweden

There is a sharp increase in demand for high quality genetic counselling, however there is no MSc education in genetic counselling in Sweden. This emerging issue led to publications on the need for higher education for genetic counsellors in Sweden, and discussions with several Universities regarding this possibility. Subsequently, a process of creating the first MSc in Genetic counselling in Sweden that fulfils the eligibility criteria for EBMG certification (European Board of Medical Genetics) as genetic counsellor has been started at the University of Linköping. Multidisciplinary workshops, discussions with the EBMG-board, compiling research and higher educational programs for genetic counselling have resulted in a preliminary curricula adapted to the Swedish context. A 2-year MSc in Genetic counselling (120 ECTS) with first student admissions in the fall 2021 is proposed. The responses from stakeholders at the University of Linköping and the clinical genetics 
community in Sweden have been positive. The proposed MSc in genetic counselling programme will allow graduates from Sweden to apply for EBMG professional registration and lead to strengthening the role and competences of genetic counsellors as a pivotal member of multidisciplinary teams regarding genetic disorders and support to families in Swedish health care.

R. Pestoff: None. P. Söderkvist: None. C. Ingvoldstad Malmgren: None.

\section{P22.17.B}

Rolling out Whole Exome and Whole Genome Sequencing to mainstream services. The experience of the Oxford Centre for Genomic Medicine.

\author{
H. Stewart, E. Blair, C. Campbell, J. Craft, U. Kini, J. \\ Lowndes
}

\section{OUH, Oxford, United Kingdom}

With the approaching completion of the 100,000 Genomes Project and the reconfiguration of genetic laboratories, Clinical Genetic Services have been instrumental in rolling out Whole Exome/Genome Sequencing for Rare Diseases to mainstream clinical services. In April 2019, we began planning, assigning priorities and contacting then meeting individual lead clinicians and contributors to 100,000 Genomes Project. We held teaching sessions for clinical teams, taught at regional speciality meetings and hosted meetings for regional early adopters. Subjects included testing techniques, use of the National Genomic Test Directory, Record of Discussion, test request forms, NGIS and giving genomic results. Teams of educators comprised clinical scientists, SpRs, genetic counsellors and clinical geneticists, reaching out to peripheral hospitals and specialist colleagues. Mainstream clinical teams were encouraged to identify 'Genomic Champions and Practitioners' to educate their teams and work together in networks. We used tools including role-play, interactive discussions, recording frequently-asked-questions and development of slide-decks to ensure consistency and avoid duplication of effort. We worked with Genomic Champions to develop care-pathways, using a 'Do Once and Share' model. We worked with colleagues in Wessex and Birmingham to share good practice and Health Education England developing documents and competency frameworks. By December 2019 we had started rapid Whole Exome Sequencing for NICU/ PICU, made ready services in Phase 1 of the WGS roll-out and are readying those in Phase 2. The Oxford Genomic Medicine Centre and Genetic Laboratories embedded all this engagement, education and support in Genomic Academies, over-arching, virtual structures that received widespread support from mainstream clinicians.
H. Stewart: None. E. Blair: None. C. Campbell: None. J. Craft: None. U. Kini: None. J. Lowndes: None.

P22.18.C

The efficacy of genetic counselling for familial colorectal cancer. Initial findings from a randomised controlled trial

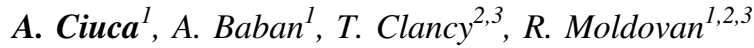

${ }^{1}$ Babes-Bolyai University, Department of Psychology, ClujNapoca, Romania, ${ }^{2}$ Manchester Centre for Genomic Medicine, St Mary's Hospital, Manchester University Hospitals NHS Foundation Trust, Manchester Academic Health Science Centre, Manchester, United Kingdom, ${ }^{3}$ Division of Evolution and Genomic Sciences, School of Biological Science, University of Manchester, Manchester, United Kingdom

Introduction: Genetic counselling (GC) for familial colorectal cancer (fCRC) has been shown to improve outcomes such as emotional distress and screening adherence. This is the first randomised clinical trial to evaluate the efficacy of $\mathrm{GC}$ for fCRC.

Method: We included individuals affected or at risk for fCRC (Lynch syndrome, APC-associated polyposis, MUTYH-associated polyposis or clinically defined fCRC). Participants are randomised to (1) genetic counselling and standard care or (2) standard care alone (control). Measures include: Genetic Counselling Outcome Scale (GCOS), knowledge, risk perception, emotional distress, screening/ surveillance behaviours, perceived social support, decisional conflict and quality of life.

Results: We currently recruited 24 participants with an average age of 42 years old, $80 \%$ females, $40 \%$ at risk and $60 \%$ individuals diagnosed various types of fCRC cancer. Non-parametric Mann-Whitney-U Test was used to compare differences at post-test between the two groups: delta change for GCOS $(p=0.06)$, level of knowledge $(p=0.03)$ and trust in knowledge $(p=0.01)$ are indicative of improvement. Additionally, Wilcoxon-Signed-Rank Test was used to compare pre-post scores for the genetic counselling group: GCOS $(p=0.04)$, level of knowledge $(p=0.02)$, trust and utility of knowledge $(p=0.07)$, anxiety $(p=0.05)$, depression $(p=0.06)$, intention of screening $(p=0.15)$, decisional conflict $(p=0.11)$.

Conclusions: Our initial data suggest improvement for the primary endpoint (GCOS) and secondary endpoints (knowledge, emotional distress, screening intention, and decisional conflict). We hypothesize that these trends will be maintained and our findings will be further supported by the sample $(n=68)$ we intend to recruit in order to ensure power for statistical and clinical significance. 
A. Ciuca: None. A. Baban: None. T. Clancy: None. R. Moldovan: None.

\section{P22.19.A}

Optimizing Familial Hypercholesterolaemia care in the Amsterdam region: the role of GPs

\section{A. Haasnoot, C. G. van El, M. C. Cornel, P. Slottje}

Amsterdam UMC, location VUMC, Amsterdam, Netherlands

Introduction: In the Netherlands in 2013 the national Familial Hypercholesterolaemia screening programme ended. While general practitioners (GPs) were always involved in finding index cases, after 2013 especially follow-up and active cascade screening was no longer systematically pursued by an organization. The number of family screenings dropped. Aim of this study was to explore views on the role of GPs in FH screening and care, and identify barriers and facilitators for optimizing care.

Materials and Methods: Two focus groups (17 GPs) and eight interviews (GPs, vascular specialists, a clinical geneticist, health law expert and consultants of the Dutch Expertise Centre for Inheritance Testing of Cardiovascular Disease (LEEFH) coordinating FH screening) were conducted. Descriptive analyses of data from anonymized electronic medical records of 26988 patients registered in 50 general practices in Amsterdam, the Netherlands addressed registration of International Classification of Primary Care (ICPC-1) codes and free text.

Results: Registration using ICPC code T93.04 (having FH) was suboptimal and varied between practices. GPs had specific knowledge gaps. Although GPs acknowledged their role in discussing with patients the relevance of informing first degree relatives, active support and checking whether patients actually had informed the family was welcomed by some stakeholders, but regarded unfeasible by several GPs.

Conclusions: For optimising $\mathrm{FH}$ care more adequate information should be available for GPs, patients and family. Knowledge of GPs should be increased. Attunement between primary and secondary care and LEEFH should be strengthened. Adequate registration in primary care should be stimulated.

Funded via APH research institute Innovation grant.

D.A. Haasnoot: None. C.G. van El: None. M.C. Cornel: None. P. Slottje: None.

P22.21.C

Gamete Donors Retrospective Diagnosis of Genetic Conditions, Exploration of Referral Pathways for appropriate genetic counselling
V. Shaikly ${ }^{1}$, K. A. Sage S $^{2}$

${ }^{1}$ Fertility Genetics, London, United Kingdom, ${ }^{2}$ CARE Fertility UK, Nottingham, United Kingdom

Aim: To gain understanding of the implications for patients and regional genetic services after receiving notification of a genetic condition associated with a donor used for assisted conception treatment.

Methods: This study reports on the experience across 2 private assisted conception centres between January 2018 January 2020 for alerts issued by donor banks. The conditions reported were reviewed and considered in the context of the patients' current assisted conception situation. The implications for patients using the specific donor were categorised to look for common referral pathways and how and if these can be met by local genetic services.

Conclusion and recommendations: Potential referrals to genetic services could arise for patients who: have had a live birth, have embryos in storage or have donor samples in storage for future/sibling use. Alternatively access to genetic counselling services could be provided by the fertility clinic to reduce the impact on local and regional genetic services. Patients should be supported by both genetic services and their fertility clinic when receiving genetic information about a donor so they can make empowered and informed decisions about their next steps. Clear pathways of communication between the fertility clinic and genetic services are required to provide a streamlined patient service when a donor is retrospectively found to have or be at increased risk of passing on a genetic condition.

V. Shaikly: None. K.A. Sage: None.

\section{P22.22.A}

Genetic counsellor, patient and carers' views on an Australian clinical genetics service information system

\section{Speechly ${ }^{l}$, R. Stenhouse ${ }^{l}, Y$. Berman ${ }^{2}, K$. Barlow-} Stewart $^{1}$, J. Fleming ${ }^{1}$, D. Petrie ${ }^{3}$, B. Culling ${ }^{4}$

${ }^{1}$ University of Sydney, Northern Clinical School, St Leonards, Sydney, Australia, ${ }^{2}$ Department of Clinical Genetics, Royal North Shore Hospital, St Leonards, Sydney, Australia, ${ }^{3}$ Williams Syndrome Australia, Coogee, Sydney, Australia, ${ }^{4}$ Service Delivery, eHealth NSW, Chatswood, Sydney, Australia

Introduction: The Genetic Information System (GIS) is a database of familial genetic information, which was introduced into the Australian state of New South Wales and the Australian Capital Territory in 2010 and 2013; creating a virtual state-wide service for 31 genetics services. As the 
use and storage of family genetic information in the GIS impacts clinicians, patients and carers, the aim of this study was to explore their views about this database.

Materials and Methods: Semi-structured telephone interviews were conducted with genetic counsellors experienced with the GIS. Findings from these interviews were used to inform focus groups with past patients/carers from public clinical genetics services. Interviews and focus groups were audio-recorded, transcribed and analysed using thematic analysis.

Results: Clinicians $(n=12)$ perceived that sharing of information via the GIS was valuable, although they identified challenges in achieving consistent data entry, keeping data current and aligned with other health systems, and they raised concerns regarding future challenges. Patients and carers $(n=14)$ also supported the sharing of family genetic information; but were cautious about the use of information for relatives' healthcare; and the stewardship of family information. Overall, the majority of participants identified similar advantages and privacy concerns regarding the sharing of family genetic information and wanted patients/carers to be better informed about the GIS early in the genetic counselling process - especially about privacy of family genetic information.

Discussion: We hope these findings may inform further development of the GIS; leading to increased patient/carer knowledge through education and development of patient resources.

C. Speechly: None. R. Stenhouse: None. Y. Berman: None. K. Barlow-Stewart: None. J. Fleming: None. D. Petrie: None. B. Culling: None.

\section{P22.23.B}

Adopting a gene through Human Disease Genes website series facilitates a clinical diagnosis for rare genetic disorders

A. J. M. Dingemans ${ }^{1}$, D. E. Stremmelaar ${ }^{1}$, A. Remortele ${ }^{l}, J$.

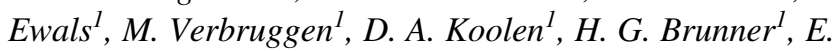
E. Eichler ${ }^{2}, J$. Gecz $^{3}$, B. B. A. de Vries ${ }^{1}$

${ }^{1}$ Radboudumc, Nijmegen, Netherlands, ${ }^{2}$ University of Washington School of Medicine, Seattle, WA, United States,

${ }^{3}$ University of Adelaide, Adelaide, Australia

The Human Disease Genes website series (HDG) is an international library of websites for professional information on the clinical consequences of novel variants in the human genome (https://humandiseasegenes.com/). The HDG is an initiative of the Human Genetics Department of the Radboud University Medical Center, Nijmegen, in collaboration with the University of Washington and the University of Adelaide. Each gene website is moderated by a dedicated team of clinicians and molecular biologists, and provides up-to-date - mostly unpublished - clinical information on one specific gene or copy number variant. HDG aims to fill the gap between first publication of several cases and consecutive publication of a large review paper. Professionals can use the information of the disorder for genetic counseling and will have the opportunity to share clinical data. Patients and parents/caregivers will find useful information on the disease and have the opportunity to share detailed clinical information through the website of our partner GenIDA. HDG is also a platform where researchers can collect and share (functional) data. Recently a new version of the websites has been launched which not only allows collection of phenotypic data using HPO, but provides an up-to-date graphical overview of the clinical data that have been collected. These tools will be highly valuable for the clinical practice. Currently, over 400 genes have been adopted by more than 300 moderators world-wide. If you wish to adopt a gene and join the team of moderators please, contact us at the poster or via e-mail: info@humandiseasegenes.com.

A.J.M. Dingemans: None. D.E. Stremmelaar: None. A. Remortele: None. J. Ewals: None. M. Verbruggen: None. D.A. Koolen: None. H.G. Brunner: None. E.E. Eichler: None. J. Gecz: None. B.B.A. de Vries: None.

\section{P22.24.C}

Introducing the genetic counselling profession in Austria

\author{
G. F. Schwaninger ${ }^{1,2}$, C. M. Benjamin ${ }^{2}$, S. Rudnik- \\ Schöneborn ${ }^{l}$, J. Zschoke ${ }^{l}$
}

${ }^{1}$ Institut for Human Genetics, Medical University, Innsbruck, Austria, ${ }^{2}$ Center for Medical Education, School of Medicine, Cardiff University, Cardiff, United Kingdom

Different to most European countries, the genetic counselling profession has yet to be established in Germany, Austria and German-speaking Switzerland. In 2019 a Masters level course was established in Genetic and Genomic Counselling at the Medical University Innsbruck, Austria, the first in a German speaking country. The primary aim of this research study was to help the course director, who is also the first author, explore the perspectives of key stakeholders in Austrian genetic services regarding prerequisites, opportunities and challenges of implementing MSc trained genetic counsellors in Austria.

A qualitative approach using semi-structured interviews with open-ended questions and thematic analysis was performed. The nine participants were medical geneticists at different hierarchy levels, policy makers at universities, and members of professional committees. Participants were recruited from human genetics departments and universities 
in three geographic regions of Austria, to elicit their opinions and attitudes on the subject.

Emerging factors for the successful integration of the newly educated genetic counsellors into the Austrian healthcare system are: Acceptance by medical geneticists, awareness of the genetic counselling profession amongst the medical community, a solid legal basis for the profession, and a precise definition of their professional role within the medical genetic services.

This study provides evidence to better understand catalysts and obstacles of the implementation of genetic counselling education and profession in Austria. It outlines the challenges of introducing this new healthcare profession in the German-speaking healthcare systems.

G.F. Schwaninger: None. C.M. Benjamin: None. S. Rudnik-Schöneborn: None. J. Zschoke: None.

\section{P22.25.A}

Demand for genetic counselling services in Germany: past, present and future

I. Nippert ${ }^{1}$, J. Schmidtke ${ }^{2}$, J. Epplen ${ }^{3}$, R. Glaubitz ${ }^{4}$, P. R. Nippert $^{5}$, H. Tönnies ${ }^{6}$

${ }^{1}$ Institut für Humangenetk, Universitätsklinikum Münster, Münster, Germany, ${ }^{2}$ Institut für Humangenetik, Medizinische Hochschule Hannover, Hannover, Germany, ${ }^{3}$ RuhrUniversität Bochum, Bochum, Germany, ${ }^{4}$ amedes-genetics, Hannover, Germany, ${ }^{5}$ Westfälische Wilhelms-Universität, Münster, Germany, ${ }^{6}$ Geschäftsstelle Gendiagnostik-Kommission, Robert Koch-Institut, Berlin, Germany

Introduction: Medical geneticists are providing the majority of genetic counselling services in Germany. With the Act on Genetic Testing (GenDG) the German legislator has issued far reaching regulations for genetic services, including genetic counselling.

Objectives: To assess demand for genetic counselling services before and after the enactment of GenDG.

Material and Methods: Data on genetic counselling services (2005-2017)provided within the framework of the German statutory health insurance (GKV) were obtained via a database query at the Central Institute of the National Association of Statutory Health Physicians (ZIV-KV) and via regional (state) associations of statutory health physicians. Additional data on supply and demand were obtained from the GenBIn database.

Results: Demand for genetic counselling has steadily increased at an average rate of $6 \%$ per year since 2009 . The increase has started well before the enactment of GenDG. Change in demand is characterized by increased selfreferrals and by increased referrals by other specialists than obstetricians/gynecologists. Waiting times have increased.
Conclusions: Increased demand may be attributed to a multiplicity of factors, such as advances in technology, increased public awareness, increased awareness among nongeneticist physicians and the GenDG. While demand is steadily growing, the number of medical geneticists remains almost constant. Capacity limits may be reached if both trends continue. GenBIn funded by Robert Koch Institute (Berlin) on behalf of the Federal Ministry of Health, Germany

I. Nippert: None. J. Schmidtke: None. J. Epplen: None. R. Glaubitz: None. P.R. Nippert: None. H. Tönnies: None.

\section{P22.26.B}

\section{Mapping counselling supervision at European level}

M. Paneque ${ }^{1}$, L. Guimarães ${ }^{2}$, J. Bengoa $^{3}$, S. Pasalodos ${ }^{4}$, C.

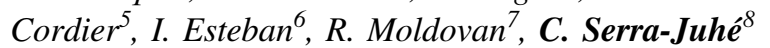

${ }^{1} i 3 S$ - Instituto de Investigação e Inovação em Saúde, Universidade do Porto; IBMC - Institute for Molecular and Cell Biology, Universidade do Porto; Centre for Predictive and Preventive Genetics (CGPP), Universidade do Porto, Porto, Portugal, ${ }^{2} i 3 S$ - Instituto de Investigação e Inovação em Saúde, Universidade do Porto; IBMC - Institute for Molecular and Cell Biology, Universidade do Porto; Centre for Predictive and Preventive Genetics (CGPP), Universidade do Porto; Associação de Apoio à Juventud, Porto, Portugal, ${ }^{3}$ Hôpital Necker Enfants Malades, Paris, France, ${ }^{4}$ Genomic Medicine Unit, Navarrabiomed, Biomedical Research Centre, Navarra, Spain, ${ }^{5}$ Synlab Genetics, Lausanne, Switzerland, ${ }^{6}$ Ninewells Hospitals, Dundee, United Kingdom, ${ }^{7}$ Babeş-Bolyai University, Cluj-Napoca, Romania, ${ }^{8}$ Hospital de la Santa Creu $i$ Sant Pau, Barcelona, Spain

Genetic testing is becoming more commonplace in general and specialist health care, and should always be accompanied by genetic counselling, according to legislation in many European countries and recommendations by professional bodies.

Personal and professional competence is necessary to be able to provide safe and effective genetic counselling. Clinical and counselling supervision of practitioners plays a key role in quality assurance of practice and providing a safety environment for patients and professionals. However, in many European countries, genetic counsellors are still an emerging professional group and supervision is not routinely offered. The relevance of counselling supervision for the improvement of genetics healthcare services is not yet widely discussed or established.

This study aimed to explore the current status of genetic counseling supervision provision across Europe and to 
ascertain factors that might be relevant for the successful implementation of counselling supervision.

A total of 100 practitioners responded to the online survey; respondents were from 18 countries, with the majority working in France (27\%) and Spain (17\%). Only 34 participants reported having access to genetic counselling supervision; country of origin, the existence of a regulation system and years of experience were factors identified as relevant influencing access and characteristics of counselling supervision.

Although there is a growing number of genetic counsellors trained at European level, just a few countries have implemented and required as mandatory the access to genetic counselling supervision, which is essential to ensure a safe and effective genetic counselling.

M. Paneque: None. L. Guimarães: None. J. Bengoa: None. S. Pasalodos: None. C. Cordier: None. I. Esteban: None. R. Moldovan: None. C. Serra-Juhé: None.

P22.27.C

An e-learning course "virtual genetic counselling in hereditary cancer" for undergraduate education in Human Genetics

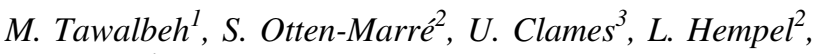 H. Rieder ${ }^{l}$}

${ }^{1}$ Institute of Human Genetics, University Clinic Duesseldorf, Duesseldorf, Germany, ${ }^{2}$ Communication in Medical Education Duesseldorf (CoMED), Institute of Psychosomatics and Psychotherapy, University Clinic, Duesseldorf, Germany, ${ }^{3}$ Multi Media Center, Heinrich-Heine-University, Duesseldorf, Germany

The identification and adequate care of individuals, which are at a high risk to develop cancer, is the major benefit of genetic counselling in families with a suspected family history of a cancer predisposition syndrome. The process from the initial suspicion to the final genetic risk assessment in an individual includes several steps, in which affected and unaffected family members have to be taken care of. To demonstrate the psychological impact as well as the interaction between the physician and the respective family members, we set up a video based online accessible virtual genetic counselling session in a cancer family. A family with Lynch syndrome was created. Simulation patients were instructed to act as affected and unaffected family members according to predefined roles. A specialist in human genetics was advised to conduct a counselling session according to a routine situation. Videos were taken from different camera angles and cut according to the counselling process. An e-learning module was developed by using an e-learning course development software (Articulate $360^{\circledR}$ ).
Each video sequence was limited to 2 minutes duration. The e-learning course was intergrated as a sharable object reference module (SCORM) in an elearning content managment system (ILIAS ${ }^{\circledast}$ ). In the course each step of five different consultation-hours of the genetic counselling was visualized by the respective video sequences. Only the most relevant sections were included so that the students were able to accomplish the course within $40 \mathrm{~min}$. The course is available under https://ilias.hhu.de/ilias/goto.php?target $=\mathrm{ca}$ t_663694\&client_id=UniRZ.

Funded by the eLearning Foerderfonds, Heinrich-HeineUniversity, Duesseldorf, Germany

M. Tawalbeh: None. S. Otten-Marré: None. U. Clames: None. L. Hempel: None. H. Rieder: None.

P22.28.A

The impact of children affected by psychiatric conditions: The caregivers' perspective

M. M. Radu ${ }^{l}$, A. Ciucă $\breve{a}^{l}$, S. Pintea ${ }^{l}$, A. Baban ${ }^{l}$, E.

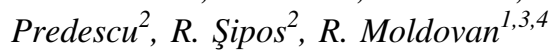

${ }^{1}$ Department of Psychology, Babeş-Bolyai University, ClujNapoca, Romania, ${ }^{2}$ Emergencies Clinical Hospital for Children, Cluj-Napoca, Romania, ${ }^{3}$ Division of Evolution and Genomic Sciences, School of Biological Science, University of Manchester, Manchester, United Kingdom, ${ }^{4}$ Manchester Centre for Genomic Medicine, St Mary's Hospital, Manchester University Hospitals NHS Foundation Trust, Manchester Academic Health Science Centre, Manchester, United Kingdom

Introduction: Families of children and adolescents diagnosed with psychiatric disorders often find it difficult to deal with daily responsibilities, distress, stigma or shame. Yet only few studies investigated the psychological wellbeing and challenges amongst the caregivers of young people diagnosed with psychiatric diagnosis.

Methods: A cross sectional study was conducted from October 2018 to October 2019 in an outpatient pediatric psychiatry clinic. Family members (e.g. parents, grandparents) of young people diagnosed with psychiatric conditions (e.g. affective disorders, autism spectrum disorders, attention deficit hyperactivity disorder, obsessive-compulsive disorder) were invited to participate. We explored their knowledge about psychiatric disorders, illness perception, burden of care, stigma, wellbeing and quality of life.

Results: 70 caregivers (60 females, 10 males, $40.5 \pm 8.4$ average age) participated in the study. The burden of care is positively associated with caregivers' knowledge and perceptions of the negative consequences the disorder can have for the patient $(\mathrm{r}=0.52, \mathrm{p}<0.01)$ and relatives 
$(\mathrm{r}=0.25, \mathrm{p}<0.05)$ as well as with the personal blame towards the patient $(r=0.30, p<0.01)$. Both stigma and burden of care were shown to significantly impact caregivers' wellbeing $(r=-0.29, p<0.05 ; r=-0.42, p<$ $0.01)$ and quality of life $(r=-0.39, p<0.01 ; r=-0.42$, $\mathrm{p}<0.01$ ).

Conclusions: Caregivers experience burden of care, low wellbeing and quality of life and increased levels of stigma. Insight into the impact of psychiatric disorders on caregivers can help professionals develop and adapt psychosocial interventions for families impacted by psychiatric disorders such as psychiatric genetic counselling.

M.M. Radu: None. A. Ciucă: None. S. Pintea: None. A. Baban: None. E. Predescu: None. R. Şipos: None. R. Moldovan: None.

\section{P22.29.B}

\section{New technology in genetic counselling}

\section{G. Turbin ${ }^{1,2}$}

${ }^{1}$ Addenbrooke's Hospital, Cambridge, United Kingdom, ${ }^{2}$ University of Manchester, Manchester, United Kingdom

Background: Genetic counselling is in a transitional phase. There is a radical shift as genetic testing becomes a routine part of healthcare. With the volume of genetic testing increasing, and resources stretched, technological solutions have been developed to help people understand the genetic testing they are being offered. These include decision aids, 'chatbots' and online learning materials.

In this context, the role of new technology and genetic counsellors remains uncertain. I argue that research is needed to look at how patients can benefit from both genetic counsellors and new technology in the genomic age.

Methods: Six films were created by the genetic counselling team at Addenbrooke's Hospital and the Society and Ethics Research Group from Wellcome Genome Campus. The films use the metaphor of music to communicate genomic information.

Six genetic consultations in which the films were used were observed, and the same patients were interviewed. Ethnographic field notes from the observations and interviews were analysed thematically.

Results: Themes emerged around technology, shared space, body language, emotion, information giving and personalisation. The qualitative data also allowed for an exploratory analysis into the relationship between technology and power in genetic counselling consultations.

Implications: This research provides insight into the role of technology in genetics; how it can both hinder and enhance genetic counselling practice. I also discuss the results in the context of evidence-based genetic counselling. I argue that even with technological innovations, time is still a key element if patients are to benefit from both new technologies and genetic counselling.

\section{I.G. Turbin: None.}

\section{P22.31.A}

Survey of the competences of hospital based doctors in providing care to patients with genetic diseases

\section{Curtisova ${ }^{1,2}, H$. Adamová $^{2}$}

${ }^{1}$ Department of Human Genetics, Fakultni nemocnice Olomouc, Olomouc, Czech Republic, ${ }^{2}$ Palacky University, Olomouc, Czech Republic

Aim: to assess the knowledge and skills related to clinical genetics in a group of Czech hospital non-genetic specialists, compare the results with those obtained during an assessment of the international e-learning project GenEquip and to see if they correlate with their medical speciality, years from graduation, previous referral of patients for genetic consultation or testing and self-perceived knowledge of the management of patients with heritable conditions.

Methods: the anonymous modified two-part questionnaire developed by the Gen-Equip project was randomly distributed to physicians of a Czech teaching hospital.

Results: scores of the participants $(n=46)$ were significantly lower than those in the Gen-Equip group $(n=75)-53.2$ vs. 60.5 for part 1 of the test and 48.4 vs 75.3 for part 2 of the test. The scores were significantly higher in participants who previously referred patients for genetic consultation $(53.8 \%$ vs. $43.5 \%)$ or for genetic laboratory testing (53.9\% vs. $44.8 \%$ ) than those who had made no referrals. There was no significant dependence of the results of the test and the years from graduation, the specialisation of the participants or their self-perceived knowledge of the management of patients with heritable conditions.

Conclusions: the level of knowledge and skills related to clinical genetics may affect the readiness of physicians to refer their patients for appropriate genetic care. In our sample the knowledge and skills were not influenced by the years from graduation, medical speciality or self-perceived knowledge of the management of patients with heritable condition.

V. Curtisova: None. H. Adamová: None.

\section{P22.33.C}

A new measure for genetic counselling quality assessment: patients' perspectives 
M. Carvalho $^{1,2}$, F. Rodrigues ${ }^{3}$, J. Saraiva ${ }^{3,4,5}$, A. Leonardo $^{6}$, A. Sousa ${ }^{6}$, V. Machado ${ }^{7}$, M. Gonçalves-Rocha ${ }^{8}$, J. Sequeiros ${ }^{l}$, M. Serra de Lemos $^{2,9}$, M. Paneque ${ }^{l}$

${ }^{1}$ IBMC-CGPP, i3S, Porto, Portugal, ${ }^{2}$ Faculdade de Psicologia e de Ciências da Educação, Universidade do Porto, Porto, Portugal, ${ }^{3}$ CHUC - Medical Genetics Unit, Hospital Pediátrico, Centro Hospitalar e Universitário de Coimbra, Coimbra, Portugal, ${ }^{4}$ University Clinic of Pediatrics, Faculty of Medicine, University of Coimbra, Coimbra, Portugal, ${ }^{5}$ Clinical Academic Center of Coimbra, Coimbra, Portugal, ${ }^{6}$ CHULN-HSM - Serviço de Genética Médica, Centro Hospitalar Universitário de Lisboa Norte - Hospital de Santa Maria, Lisboa, Portugal, ${ }^{7}$ EPER - Hospital de Santo Espírito da Ilha Terceira, Açores, Portugal, ${ }^{8}$ Unidade de Genética Médica, Hospital de Braga, Braga, Portugal, ${ }^{9}$ Centro de Psicologia, Universidade do Porto, Porto, Portugal

International genetic testing guidelines recommend appropriate genetic counselling provision before and after genetic testing is undertaken. In spite of that, literature on quality assessment of genetic counselling is scarce. Measures available are insufficient, poorly disseminated, and focused mainly on genetic counselling effects. A theoretically-underpinning scale for quality assessment by healthcare professionals was recently published; however, the need for a measure for patients' assessment persisted. This study describes the development and initial validation of a theoretically-informed scale for genetic counselling quality assessment by patients. Scale items were developed based on the pre-existing quality assessment scale for professionals, the reciprocal-engagement model of genetic counselling practice, and literature on genetic counselling patients' perceptions. Items were refined through cognitive interviews. Patients from five Portuguese clinical genetics services $(n=123)$ completed the scale. Principal component analysis using direct oblimin was conducted to establish the initial construct validity of the scale and reduce the number of items. Cronbach's alpha was calculated as a measure of internal reliability. Final scale consisted of 51 items scale organised into six dimensions. The scale presented adequate internal reliability. Initial evidence of construct validity was also demonstrated. This scale is the first theoretically-underpinned measure designed for patient's assessment of genetic counselling quality. Genetic counselling professionals and researchers can use this scale to identify areas for quality improvement of genetic healthcare services and design interventions. We discuss practical implications and clinical utility of this new tool as well as challenges of the validation process at genetic services.

M. Carvalho: None. F. Rodrigues: None. J. Saraiva: None. A. Leonardo: None. A. Sousa: None. V. Machado:
None. M. Gonçalves-Rocha: None. J. Sequeiros: None. M. Serra de Lemos: None. M. Paneque: None.

\section{P22.34.A}

Patients' knowledge about genetics and genetic testing on the example of neoplasm diagnostics

\section{A. Tupaj, O. Dusza, M. Smerdzyński, M. Malarska}

\section{Medical University of Lodz, Lodz, Poland}

Introduction: Commercial genetic tests are getting more and more available. As some of them may don't have a real diagnostic value, it is important to help patients in choosing the right one. We've decided to examine peoples' knowledge about genetics and genetic testing by using the example of neoplasm diagnostics.

Materials and Methods: We've conducted a survey on a group of 1161 people about their knowledge and opinion about the genetic testing in neoplasm diagnostics. The survey was posted on the Internet, the mean age of our patients was 24.84 years old, $80.4 \%$ of them were female, $19.6 \%$ - male.

Results: The early results of our study show that $61.6 \%$ of patients would perform a genetic test without a specialist consultation and that the majority of them would trust in the commercial testing results. At the same time, $48.8 \%$ of respondents were unable to give a correct answer to the question about the genetic background of neoplasms. Some respondents were also unable to give an example of a genetic test, mentioning mammography, cytology or medical ultrasound.

Conclusions: Our study clearly shows that patients don't have sufficient knowledge about genetics but at the same time they perform or would perform a genetic test without a specialist's consultation. As this may lead to the misinterpretation of the results, we consider it essential to find a way to educate patients about diagnostic value of genetic tests. In our further work we want to consider the role of General Practitioners in this process.

A. Tupaj: None. O. Dusza: None. M. Smerdzyński: None. M. Malarska: None.

\section{P22.35.B}

Challenges and achievements of genetic education in Republic of Georgia among medical professionals

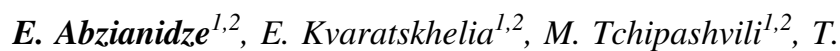 \\ Gigineishvili $^{1,2}$, E. Imnadze $e^{1,2}$, T. Tkemaladze $e^{1,2}$ \\ ${ }^{1}$ Tbilisi State Medical University, Dept. of Molecular and \\ Medical Genetics, Tbilisi, Georgia, ${ }^{2}$ Georgian Society of \\ Medical Genetics and Epigenetics, Tbilisi, Georgia
}


In mid-90's, teaching of molecular and medical genetics gained priority at the Tbilisi State Medical University (TSMU). The University has been able to incorporate ASHG/APHMG Medical School Core Curriculums (1995, 2001, 2013) into an undergraduate medical program. Students' Scientific Debate Club "FBG - Future Belongs to Genetics" was established in 1995, which held 25 students' conferences and events. In 2006 a department of Molecular $\&$ Medical Genetics was established in our university for the first time in Georgia. We have embedded problem-based learning (PBL) within the integrated course, centered on clinical case studies; High quality scientific and educational literature, including textbooks: Thompson\&Thompson Genetics in Medicine (7th and 8th editions) have been translated and published by the department. Several local and international conferences, including "Genomics and Epigenomics in Personalized Medicine" (2016, 2017), "Human Genome and Heath" $(2018,2019)$ have been organized by the department and the Georgian Society of Medical Genetics and Epigenetics (GSMGE). In addition, since 2014 we have been running a Georgian DNA Day Essay Contest for Georgian and international students. Our department and GSMGE, in collaboration with ESHG, encourage young scientists to participate in the annual conferences organized by ESHG. In 2014 the research and diagnostic laboratory was founded at the department, where $\mathrm{PhD}$ programs, CME courses and educational courses for students in genetics, genomics and epigenomics were designed and developed. As a result, nowadays, many medical professionals in Georgia have sound understanding of genetics and can successfully deploy their knowledge in their clinical practice, when needed.

E. Abzianidze: None. E. Kvaratskhelia: None. M. Tchipashvili: None. T. Gigineishvili: None. E. Imnadze: None. T. Tkemaladze: None.

\section{P22.36.C}

Measuring physician practice and preparedness for genomic medicine: a national survey

\section{E. King ${ }^{1,2}$, A. Nisselle ${ }^{1,2,3}$, B. Mc Claren ${ }^{1,2,3}$, S. Metcalfe ${ }^{1,2,3}$, C. L. Gaff ${ }^{1,2,3}$, on behalf of the Australian Genomics Workforce \& Education Working Group}

\section{${ }^{1}$ Australian Genomics Health Alliance, Parkville, Australia, ${ }^{2}$ Murdoch Children's Research Institute, Parkville, Austra- lia, ${ }^{3}$ The University of Melbourne, Parkville, Australia}

Even as genomic medicine is implemented globally, there remains a lack of rigorous, national assessments of physicians' current genomic practice and continuing genomic education (CGE) needs. We therefore developed an expert-reviewed survey based in theory (behavioural change) and empirical qualitative data (McClaren et al., Front Genet 2019). Domains included: personal characteristics (demographics, specialty, years of practice); current genomic medicine practice; perception of how proximal genomic medicine is to practice; preparedness to practice genomics (competence and confidence); and preferences for future roles and models of care in genomic medicine and for CGE. The survey was deployed nationally through medical colleges, societies, hospitals, social media and snowballing in 2019, with 409 analysable responses from physicians across Australia. Respondents represented 30 specialties (majority paediatricians, 20\%), from mainly public hospitals $(70 \%)$ in metropolitan areas $(75 \%)$. Only $13 \%$ of respondents ordered whole exome/genome sequencing tests in their practice, with the main reason for not ordering tests being relevance to practice (39\%). A third (32\%) referred patients to a clinical genetics service for testing and this was largely unchanged (34\%) when reporting future preferences. Two-thirds of respondents felt genomics will soon impact their practice and, while a third recently completed CGE, only a quarter felt prepared. Respondents lacked confidence in genomic knowledge and ability to explain concepts to patients and incorporate test results in patient management. These data can inform genetic services (re test referral patterns) and support genomic education providers to best meet learner needs and develop a competent, genomic literate workforce.

\section{Grant reference: GNT1113531}

E. King: None. A. Nisselle: None. B. McClaren: None. S. Metcalfe: None. C.L. Gaff: None.

\section{P22.37.A}

The Young Geneticists Network (YGN): using social media to unite trainees in Human Genetics around the world

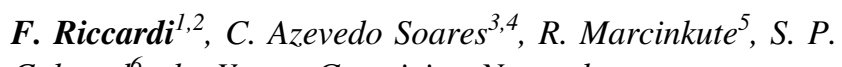
Calapod $^{6}$, the Young Geneticists Network

${ }^{1}$ Department of Medical Genetics, La Timone children Hospital, AP-HM, France, Marseille, France, ${ }^{2}$ Marseille Medical Genetics, INSERM UMR 1251, Aix-Marseille Université, Marseille, France, ${ }^{3}$ Serviço de Genética Médica, Centro de Genética Médica Jacinto Magalhães, Centro Hospitalar Universitário do Porto, Porto, Portugal, ${ }^{4}$ Unit for Multidisciplinary Research in Biomedicine, Instituto de Ciências Biomédicas Abel Salazar/Universidade do Porto, Porto, Portugal, ${ }^{5}$ Department of Clinical Genetics, Guy's and St Thomas' NHS Foundation Trust, London, United Kingdom, ${ }^{6}$ Medical Genetics Centre, St. Mary Children's Hospital, Iasi, Romania 
Human Genetics is a popular field for postgraduate students. However, training is very heterogeneous and partitioned with isolated trainees learning highly-specific expertise areas. There was a need for an international group easily accessible online to connect young human geneticists around the world. We aim to present how this group operates and develops. Based on the Facebook social media, we created the "Young Geneticists Network" private group in February 2018 (https://www.facebook.com/groups/ young.geneticist/). All people registered on Facebook can request to join the YGN group. Requests are reviewed by administrators. All members can post and share text, photos, and multimedia related to Human Genetics topics with other members who receive notifications. We present anonymous group insights data obtained until November 2019.We gathered 529 members from 57 different countries and selected 14 volunteer moderators. The majority of the members were under 34 years old $(74.9 \%)$ and female (74.6\%). Six European countries gathered most of the members (55\%): France (108), Italy (71), Romania (30), The Netherlands (28), Portugal (27) and the United Kingdom (27). We counted 37 posts with 74 comments and 408 reactions from the community. The YGN is a collaborative group that allows members to share training opportunities (online courses, funding resources,...) and queries related to Human Genetics. We are able to engage a global audience of peers in a very short period. We believe this group will expand and allow better international collaboration to standardize training and facilitate international large-scale genetics projects.

F. Riccardi: None. C. Azevedo Soares: None. R. Marcinkute: None. S.P. Calapod: None.

\section{P22.39.C}

Exploring the genomic education needs within the NHS workforce: a cardiac genetic nurse perspective.

\section{A. Braddel, M. Watson}

Wessex Clinical Genetics Service, Southampton, United Kingdom

Introduction: Nurses represent the largest professional group within the National Health Service (NHS) and are central to the integration of genomics into routine healthcare. Cardiac Genetic Nurse (CGN) roles have been developed in recent years to streamline the care for patients and families affected by an inherited cardiac condition. Like many nurses, CGNs prior exposure to genomics and the implications surrounding inherited conditions is limited. This study aimed to explore the education needs and support required for CGNs to fulfil their role.
Materials and Methods: CGNs were given the opportunity to express their interest in participating, during an Association of Inherited Cardiac Conditions conference and a British Heart Foundation study day. A purposive sampling approach was adopted to invite CGNs working within various NHS Trusts across the United Kingdom to participate. Semi-structured interviews were conducted with CGNs $(n=7)$, which were recorded, transcribed and independently coded by two researchers.

Results: Through thematic analysis, it emerged that CGNs have a hybrid role requiring a broad range of skills and, despite some overlap, are distinct from the role of a genetic counsellor. A key theme identified was the skills associated with managing the uncertainty of genetic information. The interviews highlighted that effective learning requires immersion in the clinical environment and the need for a CGN-specific competency framework to be developed.

Conclusions: This study demonstrates the need to define the competencies required for the role of a CGN, to standardise practice nationally and ensure that nursing and genetic counselling resources are applied effectively.

A. Braddel: None. M. Watson: None.

\section{P22.40.A}

We need to talk about the Fragile $X$ Syndrome: a genetic short film (episode one)

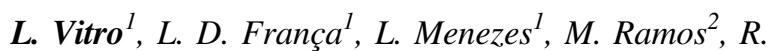
Mello $^{3}$, D. Gongora ${ }^{4}$, L. Videira ${ }^{4}$, E. Leite ${ }^{5}$, S. M.

Vasconcelos $^{6}$, A. Malinverni ${ }^{7}$, G. Carvalheira ${ }^{2}$

${ }^{1}$ Genetics Division, Department of Morphology and Genetics, EPM/EPE, Unifesp, Sao Paulo, Brazil, ${ }^{2}$ Genetics Division, Department of Morphology and Genetics, EPM, Unifesp, Sao Paulo, Brazil, ${ }^{3}$ Department of Speech Therapy, EPM, Unifesp, Sao Paulo, Brazil, ${ }^{4}$ Department of Institutional Communication, Unifesp, Sao Paulo, Brazil, ${ }^{5}$ Administration of Escola Paulista de Enfermagem, EPE, Unifesp, Sao Paulo, Brazil, ${ }^{6}$ Department of Health Information Technology, EPM, Unifesp, Sao Paulo, Brazil, ${ }^{7}$ Pathology Laboratory, Department of Pathology, EPM, Unifesp, Sao Paulo, Brazil

Introduction: Fragile X Syndrome (FXS) is a dominant Xlinked disease. It is considered the second most common cause of intellectual disability (ID), with an incidence of $5: 10,000$, being prevalent in males. The majority of FXS is caused by FMR1 mutations. As it is a X-linked disorder, females with premutation (range from 55 to 200 CGG repeats) may present early ovarian failure, while males may present tremors and ataxia. On the other hand, individuals with complete mutation (greater than 200 CGG repeats) 
could present moderate to severe ID (30\% females and $90 \%$ males), due to FMRI silencing. As this disorder presents complex etiology, FXS education documentary had been prepared, containing the main syndrome's themes: historical data, genetic causes and their phenotypic consequences.

Materials and Methods: This documentary is a short film divided in three episodes, which the first one will be available on the website http://ucgenetica.sites.unifesp.br/ index.php/pt/. In this episode, the molecular mechanisms of FXS genetic explain how the symptoms are manifested and their clinical consequences. To promote accessibility, the LIBRAS language and English subtitles were used.

Results: This documentary had been presented to 83 undergraduate nursing students. The evaluation revealed that $98 \%$ of the students assimilated the FXS genetic content.

Conclusions: This documentary proved to be an efficient strategy to disseminate FXS information, using a simple and objective language. The next step is to assess whether this short film will have the same applicability, in a playful way, to inform family members, students in public schools, and the general population.

L. Vitro: None. L.D. França: None. L. Menezes: None. M. Ramos: None. R. Mello: None. D. Gongora: None. L. Videira: None. E. Leite: None. S.M. Vasconcelos: None.

\section{A. Malinverni: None. G. Carvalheira: None.}

\section{P22.41.B}

Development of the course in laboratory genetics and genomics for medical students in the Republic of Georgia

\section{E. Kvaratskhelia, M. Zarandia, T. Tkemaladze, E. Ekaladze, N. Tevzadze, E. Abzianidze}

\section{Tbilisi State Medical University, Tbilisi, Georgia}

Introduction: A new elective course "Principles of Laboratory Genetics and Genomics" has been developed for undergraduate medical students at Tbilisi State Medical University in the United State Medical Doctor Program. The aim of this course is to offer students an opportunity to conduct experiments based on molecular genetics to understand the role of genetic analysis in the diagnosis of human diseases.

Methods: In this course, third year medical students who have passed molecular biology and medical genetics enrolled. Since October 2019 this course has been run twice with 21 participants. Course components include basic genetic concepts, current molecular genetics methodologies (hands-on labs), and interpretation of results (total 20hr.). In addition, the course includes a scientific paper discussion session and an essay writing task. Laboratory activities include DNA extraction, PCR of repetitive elements, gene mutation detection, and Real Time PCRbased DNA profiling. We used kits and reagents developed for educational purposes, e.g. Crime Scene Investigation Kit (BioRad).

Results: Students were motivated and enthusiastic to perform well in the lab; they were able to develop their collaborative skills when working together on inquiry-based lab lessons. Post-course evaluation showed that $75 \%$ of learners agreed or strongly agreed that the course had increased their understanding in the methods of laboratory genetics and the sessions had educational value.

Conclusions: Our pilot course is effective in improving the level of genetic knowledge and practical genetic skills among medical students. In addition, using lab activities, students will be inspired and encouraged to participate in biomedical research projects.

E. Kvaratskhelia: None. M. Zarandia: None. T. Tkemaladze: None. E. Ekaladze: None. N. Tevzadze: None. E. Abzianidze: None.

P22.43.A

Experiences with mainstreaming of rapid gene panel DNA diagnostics in breast cancer patients

K. J. A. F. van Kaam ${ }^{1}$, B. A. H. Caanen ${ }^{l}$, M. L. Haadsma', A. M. van Remortele ${ }^{2}$, A. R. Mensenkamp ${ }^{2}$, R. J. Blok ${ }^{1}, M$. van Geel ${ }^{1}$, E. M. Leter ${ }^{1}$, W. A. G. van Zelst-Stams ${ }^{2}$, M. J. L. Ligtenberg $^{2}, N$. Hoogerbrugge ${ }^{2}$

${ }^{1}$ MUMC+, Maastricht, Netherlands, ${ }^{2}$ Radboud University Medical Center, Nijmegen, Netherlands

Introduction: Genetic test results can now be available within 2-3 weeks and are therefore increasingly considered for determining treatment options in breast cancer patients. To facilitate timely test results, we aimed to shift counselling and requests for rapid DNA diagnostics for these patients from clinical geneticists to treating physicians and evaluate this transition towards mainstreaming.

Methods: Nine regional hospitals were included from July 2018 onwards. Treating physicians were asked for their needs and barriers to participate. DNA diagnostics consisted of gene panel analysis for BRCA1, BRCA2, PALB2, CHEK2 and ATM.

Results: The website www.DNAfirst.nl was developed to provide treating physicians with hands-on information. Barriers included time investment during consultation and perceived incompetence of providing breast cancer screening advice for family members. Up to December 2019, 351 requests for DNA diagnostics were made and 42 pathogenic variants were found $(12 \%)$. All patients with a pathogenic variant were subsequently referred to a clinical geneticist. 
32/42 pathogenic variants were found in patients meeting the Dutch criteria for DNA testing for hereditary breast cancer $(n=271)$, whereas $10 / 42$ of pathogenic variants were found in patients who did not meet these criteria $(n=$ $57)$ or for whom this was unknown $(n=23)$. Structured evaluation of quality of care and experiences of doctors and patients is ongoing and results will be available shortly.

Conclusions: Mainstreaming of rapid gene panel DNA diagnostics in breast cancer patients appears to be feasible.

K.J.A.F. van Kaam: None. B.A.H. Caanen: None. M. L. Haadsma: None. A.M. van Remortele: None. A.R. Mensenkamp: None. R.J. Blok: None. M. van Geel: None. E.M. Leter: None. W.A.G. van Zelst-Stams: None. M.J.L. Ligtenberg: None. N. Hoogerbrugge: None.

\section{P22.44.B}

Implementing BRCA mainstreaming in Australia: a qualitative study

R. O Shea ${ }^{l}$, N. Rankin ${ }^{1}$, M. Kentwell ${ }^{2}$, M. Gleeson ${ }^{3}$, K. M.

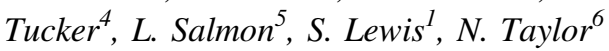

${ }^{1}$ Faculty of Medicine and Health, University of Sydney, Sydney, Australia, ${ }^{2}$ Parkville Familial Cancer Centre, Peter MacCallum Cancer Centre and Royal Melbourne Hospital, Parkville, Victoria, Melbourne, Australia, ${ }^{3}$ Hunter Family Cancer Service, Newcastle, Australia, ${ }^{4}$ Hereditary Cancer Clinic, Prince of Wales Hospital and Clinical School, University of New South Wales, Sydney, Australia, ${ }^{5}$ Department of Clinical Genetics, Austin Health, Melbourne, Australia, ${ }^{6}$ Faculty of Medicine and Health, University of Sydney and Cancer Research Division, Cancer Council NSW, Sydney, Australia

Background: Sub-optimal referrals and identification of hereditary cancers are evident internationally. Mainstreaming programs for $B R C A$ gene testing for epithelial ovarian cancer have been introduced to address this problem. However, the successes and challenges of integrating genetics into the Australian oncology health system are unknown.

Methods: Study invitations were emailed through the heads of departments in oncology, genetics and the Mainstreaming Working Party in Australia. Semistructured telephone interviews were designed using the Consolidated Framework for Implementation Research (CFIR). Interviews were transcribed and inductive content analysis was conducted.

Results: Twenty-two participants from 8 health organisations participated and the following themes were found: Theme 1: Embracing the shift to mainstream genetic testing: clinical utility of testing, preferences on the mainstreaming process, considerations for future adaptation and a patient centred process, Theme 2: Role delineation and responsibilities for mainstream genetic testing: communication networks, genetics point of contact, role delineation, collaboration. Theme 3: Influencing factors on sustaining mainstreaming: ongoing training needs, resources and funding, real world adaptation, private and public system complexity, champions and engagement. Theme 4: Interventions for mainstreaming: embedded genetic counsellor in oncology, results follow up and tracking system, flowchart of mainstreaming processes.

Conclusions: Our findings identify that participants recognise the need for integration of genetic testing in oncology, but that further adaptation of interventions and processes is essential to sustain a feasible model. An understanding of individual and organisational factors that influence mainstreaming programs will help to prepare for future integration of genetic testing in other cancer types.

R. O Shea: None. N. Rankin: None. M. Kentwell: Other; Modest; Astrazeneca. M. Gleeson: Other; Modest; Astrazeneca. K.M. Tucker: Other; Modest; Astrazeneca. L. Salmon: None. S. Lewis: None. N. Taylor: None.

P22.47.B

Diagnostic yield and recognized barriers of an adult Neurogenetics clinic

\section{A. Peleg ${ }^{1}$, L. Sagie-Dain ${ }^{1,2}$, D. Golan ${ }^{3,2}$}

${ }^{1}$ Human Genetics Institute, Lady Davis Carmel Medical Center, Haifa, Israel, ${ }^{2}$ Ruth and Bruce Rappaport Faculty of Medicine, Technion - Israel Institute of Technology, Haifa, Israel, ${ }^{3}$ Department of neurology, Lady Davis Carmel Medical Center, Haifa, Israel

Background: The advent of molecular genetic technologies paved a path for the diagnosis of many neurological disorders. Joint evaluation by a neurologist and a medical genetics specialist can potentially increase diagnostic effectiveness by ensuring the exclusion of non-genetic mimics and by rationally selecting appropriate genetic diagnostic tools. Therefore, a monthly adult Neurogenetics clinic was established.

Methods: A retrospective review of medical records.

Results: 85 patients were evaluated. Age: $47.1 \pm 15.7$, Male: $40(47 \%), 44(51 \%)$ had a positive family history. Disease duration was typically long $(11.4 \pm 0.9$ years $)$. Futile use of expensive diagnostic modalities was very common [45(52\%) had repeated MRI, 28(32\%) hospitalized for observation in neurologic departments, 12(14\%) had a normal metabolic workup, 4 (4\%) with a non-conclusive muscle biopsy, 1 with a normal cerebral angiography]. Following clinical evaluation molecular genetic testing was offered to $70(82 \%)$ patients. In the other $15(18 \%)$ routine 
workup for the exclusion of non-genetic mimics was not complete, obtainable information regarding family members was missing or that a neurogenetic disorder seemed improbable. 29 (34\%) patients received a definitive diagnosis, either a genetic (29\%) or non-genetic (5\%). The adherence to genetic testing recommendations was $62 \%$. The reasons for non-adherence were lack of public funding for the required test $(56 \%)$ and patient decision not to proceed (44\%).

Conclusions: Given the frequent futile use of expensive modalities due to diagnostic uncertainty and the complexity of clinical genomic data analysis, a comprehensive multidisciplinary neurogenetic clinics seems reimbursement and cost effective for the patients and medical health institutions.

A. Peleg: None. L. Sagie-Dain: None. D. Golan: None.

\section{P22.48.C}

A survey of the pregnant women about their preferences for the prenatal tests with different characteristics in Russia

E. Baranova ${ }^{l}$, E. Zayaeva ${ }^{2}$, L. Zhuchenko ${ }^{2}, S$. Shchelykalina ${ }^{3}, V$. Izhevskaya ${ }^{4}$

${ }^{I}$ Federal State Budgetary Educational Institution of Further Professional Education "Russian Medical Academy of Continuous Professional Education" of the Ministry of Healthcare of the Russian Federation, Moscow, Russian Federation, ${ }^{2}$ Federal State Budgetary Educational Institution of Further Professional Education Russian Medical Academy of Continuous Professional Education of the Ministry of Healthcare of the Russian Federation, Moscow, Russia, Moscow, Russian Federation, ${ }^{3}$ Pirogov Russian National Research Medical University, Moscow, Russia, Moscow, Russian Federation, ${ }^{4}$ Research Centre for Medical Genetics, Moscow, Russia, Moscow, Russian Federation

The aim of this study was to assess the opinions of pregnant women about prenatal tests in Russia. Materials and methods: Totally 800 questionnaires were collected from pregnant women living in 16 regions of Russia. Results: The statement "Every pregnant woman must undergo combined screening in the first trimester" was agreed by the majority of respondents $-90.16 \% .63 .38 \%$ of the respondents did not know before our survey about NIPT. After explaining its capabilities, $74.74 \%$ believe that it should be used. It was revealed that despite the high compliance to the early prenatal screening between pregnant women in general, only a little more than half of the women with an increased risk of chromosome abnormalities were ready to undergo invasive diagnosis in the fetus and only a quarter of the pregnant women were ready to terminate the pregnancy with chromosome abnormalities in the fetus. Women who were ready to terminate the pregnancy were statistically significantly older, had more pregnancies, were at a later gestational age. It was more important for them to receive the test results as early as possible with as much additional information as possible and have the state/insurance type of payment for the test (Mann-Whitney, $\mathrm{p}<0.002$ ). Conclusion: The survey will allow comparing the results with other countries where NIPT is used in early prenatal screening and to develop recommendations for our country. This work was supported by the RFBR grant No. 18-013-01175.

E. Baranova: None. E. Zayaeva: None. L. Zhuchenko: None. S. Shchelykalina: None. V. Izhevskaya: None.

P22.49.A

The contribution of an oncogenetics consultation while offering a theranostic approach of genome-wide analyses

G. BERTOLONE ${ }^{1,2}$, E. COSSET ${ }^{l}$, A. BAURAND ${ }^{2,1}$, C. SAWKA ${ }^{2,1}$, M. ROBERT ${ }^{2}$, A. LANCON ${ }^{1,2}, M . R E D A^{3}, C$.

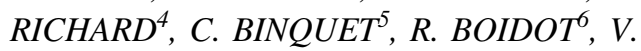
DERANGERE ${ }^{7}$, V. GOUSSOT ${ }^{8}, J$. ALBUISSON $^{7}, L$. FAVIER $^{3}$, F. GHIRINGHELLI $I^{3}, S . N^{2}$ AMBOT ${ }^{2,1}, L$. FAIVRE $^{2,1}$

${ }^{I}$ Service des consultations d'oncogénétique, Centre Georges François Leclerc, 1 rue du Pr Marion, BP 77980, Dijon, France, ${ }^{2}$ Centre de génétique, CHU de Dijon, Hôpital d'enfants, Dijon, France, ${ }^{3}$ Service d'oncologie médicale, Centre Georges François Leclerc, 1 rue du Pr Marion, BP 77980, Dijon, France, ${ }^{4}$ Plateforme de transfert en biologie cancérologique, Centre Georges François Leclerc, 1 rue du Pr Marion, BP 77980, Dijon, France, ${ }^{5}$ Inserm 1231, Université de Bourgogne FrancheComté Centre d'investigation clinique et d 'épidémiologie clinique 1432, CHU de Dijon, Hôpital d'enfants, Dijon, France, ${ }^{6}$ Unité de biologie moléculaire Centre Georges François Leclerc, 1 rue du Pr Marion, BP 77980, Dijon, France, ${ }^{7}$ Biologie et pathologie des tumeurs, Centre Georges François Leclerc, 1 rue du Pr Marion, BP 77980, Dijon, France, ${ }^{8}$ Unité de biologie clinique, Centre Georges François Leclerc, 1 rue du Pr Marion, BP 77980, Dijon, France

Introduction: Targeted therapies have emerged, requiring a better delineation of the tumor genetic characteristics. In this context, the EXOMA study (2016-2019) proposed somatic/constitutional exome for patients with advanced cancer. Considering the risk for identifying genetic predisposition, with genetic counseling and management 
implications, a genetic consultation was proposed; which value needed to be assessed.

Methods: A 20 minutes consultation, with a prior collection of family history by the patient, was carried out within 15 days of inclusion in EXOMA. Tumoral and constitutional analyses were limited to 317 genes involved in oncogenesis.

Results: In 36/716 patients (5\%), a constitutional pathogenic variant was identified in 13 actionable genes, but half were already identified in the care circuit. Among 18 cases identified by the exome, 10/18 did not meet the predisposition search criteria, and in $2 / 10$, the variation can be considered as incidental (unrelated to the presented cancer). In 3/18 cases, a genetic consultation had been previously performed, but the analyses were limited to $B R C A / M M R$. In $5 / 18$ cases, the criteria for the search of predisposition were present but not identified by the oncologist. In 18 additional patients, a variant was identified in genes that do not lead to a specific surveillance protocol in France, such as ATM/CHECK2.

Conclusions: In $2.5 \%$ of patients, a constitutional genetic predisposition, related or not to the clinical presentation, was identified, making it important to inform the patient beforehand. This low percentage should be balanced with the burden of proposing such urgent circuits. Other modes of information are being explored.

G. Bertolone: None. E. Cosset: None. A. Baurand: None. C. Sawka: None. M. Robert: None. A. Lancon: None. M. Reda: None. C. Richard: None. C. Binquet: None. R. Boidot: None. V. Derangere: None. V. Goussot: None. J. Albuisson: None. L. Favier: None. F. Ghiringhelli: None. S. Nambot: None. L. Faivre: None.

\section{P22.52.A}

The impact of a "Psychiatric Genetics for Genetic Counselors" workshop on genetic counsellor attendees: an exploratory study

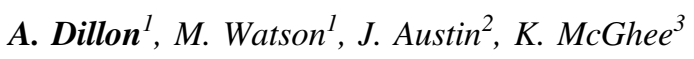

${ }^{1}$ Wessex Clinical Genetics Service, University Hospital Southampton NHS Foundation Trust, Southampton, United Kingdom, ${ }^{2}$ BC Mental Health and Addictions Research Institute, Vancouver, BC, Canada, ${ }^{3}$ Bournemouth University, Bournemouth, United Kingdom

Introduction: Genetic counselling is the process of supporting patients' and families' adaption to genetic information. Psychiatric genetic counseling has been proven to be effective in improving patients' empowerment, selfefficacy, and knowledge even in the absence of genetic testing. Despite this, only one specialist psychiatric genetic counselling clinic currently exists. In order to engage and empower genetic counsellors in providing psychiatric genetic counselling worldwide, a two-day workshop: "Psychiatric Genetic Counseling for Genetic Counselors", was developed and implemented. The aim of the present study was to qualitatively explore the impact of the workshop.

Materials and Methods: Semi-structured interviews were carried out with twelve genetic counsellors who attended the workshop between 2015 and 2018. Interviews were then transcribed, and thematic analysis was used to identify themes from the data. Constant comparative method was employed on the qualitative data themes throughout analysis.

Results: $100 \%$ of participants reported an increase in empowerment in offering psychiatric genetic counselling to patients. Participants also reflected how the workshop normalised mental illness and aided the counsellors in identifying sources of their personal stigma. The workshop also provided participants with a practical tool to describe multifactorial inheritance, the Jar Model.

Conclusions: The "Psychiatric Genetic Counseling for Genetic Counselors" workshop empowered genetic counsellors to feel confident and comfortable offering psychiatric genetic counselling to patients.

A. Dillon: None. M. Watson: None. J. Austin: None. K. McGhee: None.

\section{P22.54.C}

What happens during and after the disclosure of ACMG 59 secondary genomic findings? A systematic review of the literature

\section{F. M. Facio, J. C. Sapp, K. L. Lewis, L. G. Biesecker}

National Human Genome Research Institute, Bethesda, MD, United States

The American College of Medical Genetics and Genomics (ACMG) released its initial guidelines for the return of secondary genomic findings (SF) in 2013. The main reason for returning SF include the opportunity to tailor medical care to the individual and his/her at-risk relatives. We conducted a systematic review to summarize the published data supporting the communication of SF and downstream actions taken by individual recipients.

We searched six major biomedical databases (CINAHL, Embase, PubMed, Scopus, Web of Science, and PsychInfo) to identify peer-reviewed publications between 01/Jan/2012 and 18/Jun/2019 describing how SF findings are communicated, and/or any outcomes associated with disclosure, including psychosocial impact, healthcare behaviors, family communication/cascade testing. 
A total of 1751 citations were retrieved. After deduplication, 990 citations were screened and 847 rejected. The remaining 143 citations were reviewed, and 20 met inclusion criteria. Most studies $(n=10)$ investigated both process and outcomes, and a few of these reported similar rates of compliance with post-disclosure recommendations $(\sim 70 \%)$; rates of family communication were as high as $90 \%$. Other studies focused on the disclosure process $(n=4)$ or post-disclosure outcomes $(n=6)$ only. Across studies the psychosocial impact of receiving SF was minimal.

Since the release of the ACMG guidelines, only twenty studies have examined the disclosure process or evaluated clinical utility. Our review suggests that not all SF recipients communicate results to their doctors or relatives, and a substantial fraction may not engage in the recommended healthcare behaviors. These early published experiences may help shape research priorities as we move towards personalized medicine.

F.M. Facio: None. J.C. Sapp: None. K.L. Lewis: None. L.G. Biesecker: None.

\section{P22.56.B}

Single-gene pathology in pediatric patients referred for genetic counseling for a period of five years

\section{Stoyanova ${ }^{1,2}$, L. Angelova ${ }^{l}$, M. Hachmeriyan ${ }^{1,2}, M$.} Levkova $^{1,2}$, V. Miteva ${ }^{1,2}$

${ }^{1}$ Department of Medical Genetics, Medical University, Varna, Bulgaria, ${ }^{2}$ Laboratory of Medical Genetics, University Hospital Saint Marina, Varna, Bulgaria

Introduction: As a whole, the group of single-gene disorders is often considered to be primarily affecting childhood - almost $90 \%$ of this pathology is manifested by puberty. Our aim is to evaluate the activity of the Genetics Counselling Unit in diagnosis of children with clinically suspected monogenic pathology.

Materials and methods: The study includes pediatric patients referred to the Genetics Unit of the University Hospital Saint Marina, Varna for a period of five years (2015-2019), who were genetically consulted for suspected or confirmed single-gene disorders Clinical phenotyping, imaging examinations, literature review and specialized computer programs/dysmorphology databases were applied. Appropriate molecular genetic and metabolic investigations were offered.

Results: 343 out of $706(48,5 \%)$ consulted children, including 94(27,4\%) infants, were clinically suspective for single-gene disorders. The great majority - 276(80,5\%) were referred by Pediatric clinics of the hospital. Molecular genetic testing (in Bulgarian Genetic laboratories or abroad) was performed to 212 children $(61,8 \%)$. In $83 \%$ it was financed completely privately. Based on these analyses, 105 $(49,5 \%)$ patients were detected with single-gene conditions, mainly autosomal recessive entities in $83(79 \%)$ of them. The spectrum of all genetic diagnoses is presented in details and analyzed. The identified genetic defects in some patients led to the detection of carriers in close relatives and prenatal diagnosis.

Conclusions: Although individually rare, single gene disorders totally are responsible for a significant proportion of childhood pathology. Genetic counseling is an integral part of medical care for patients and their families. Reimbursement of genetic testing by National Health Insurance Fund would increase detection rate.

M. Stoyanova: None. L. Angelova: None. M. Hachmeriyan: None. M. Levkova: None. V. Miteva: None.

P22.58.A

Completely new set of free educational inheritance quiz apps for students: their use and international uptake.

A. P. Tobias ${ }^{1}$, E. S. Tobias ${ }^{2}$

${ }^{1}$ University of Edinburgh, Edinburgh, United Kingdom, ${ }^{2}$ University of Glasgow, Glasgow, United Kingdom

Introduction: Undergraduate medical and science students frequently search for self-assessment quizzes to help them learn the inheritance modes of well-known genetic disorders. Addressing this, a new set of quiz apps has recently been created by the authors and released on both app stores for undergraduate students. These apps are separate from the already-popular Clinical Genomics Guide and Clinical Genomics Quiz apps that the authors previously created for post-graduates and professionals.

Methods: Three new multi-platform, self-contained apps were created using the authors' Apple \& Google developer licences. Each app contains 15 multiple-choice questions, testing knowledge of conditions' inheritance-modes (autosomal dominant, recessive, X-linked or mitochondrial). The apps award points for questions answered correctly at first attempt. Upon quiz completion, total and percentage scores are displayed and the app teaches students the correct answers to all incorrectly-answered questions. The apps are compatible with all currently used iPhone (iOS) and Android smartphones and tablets.

Results: Having passed strict, extensive testing by Apple and Google, the Genetic Inheritance Quiz A, B and C apps (progressing in difficulty) are available, free, on both official App Stores. They have been installed $>750$ times worldwide in 4 months. Feedback comments for the apps, rated 5-stars overall, have been highly positive, commending the layout, corrected-answer-list, usability, "massive" helpfulness and "fun". Links to the apps are provided from 
the ESHG Education page and from www.genomicsapps. org.

Conclusions: Although time-consuming to create, they have been found useful to many individuals internationally. Feedback and suggestions for future additions would be welcomed at: edward.tobias@glasgow.ac.uk

A.P. Tobias: None. E.S. Tobias: None.

\section{P22.59.B}

Support groups for brca carriers and their relatives: motivations and satisfaction of participants

\section{B. Bertonazzi, L. Godino, L. Polpatelli, S. Miccoli, D. Turchetti}

\section{Sant'Orsola-Malpighi Hospital, Bologna, Italy}

To assess helpfulness and satisfaction about support or focus groups and psychoeducational interventions in BRCA carriers, plus recurrent themes expressed by participants, we have conducted a systematic review and a prospective explorative cohort study. Peer-reviewed papers published between January 1995 and May 2019 were searched using five databases. Of 1505 studies identified, 27 were reviewed in full-text, 9 met the inclusion criteria. Satisfaction and usefulness of support groups was analyzed by three studies, which reported high level of satisfaction, appreciation of psychological support and interest for future psychoeducational groups. Major themes emerged were distress and feelings of guilt about transmission to offspring, when to disclose information to relatives, anxiety and depression related. Between September 2018 and January 2020 we arranged six support groups for BRCA carriers and their families. Of 43 individuals participating to at least one group, $23(53 \%)$ participated to more than one. Main reasons reported for participating were: desire to meet other BRCA carriers $(17 ; 40 \%)$ and the need for further information (14; 33\%). 95\% felt comfortable and 90\% considered the group very useful. Twenty-seven participants $(62,7 \%)$ declared their expectations had been completely satisfied; almost all declared they would participate to future groups. Major themes emerged were: how and when to disclose the information to offspring (particularly sons), why minors cannot be tested, differences between BRCA1 and BRCA2, prophylactic surgery, diet and hormone therapy. Our findings support the usefulness of support groups for longitudinal follow-up of BRCA families.

Supported by GOforLife and Susan G. Komen Italia Onlus.

1

B. Bertonazzi: None. L. Godino: None. L. Polpatelli: None. S. Miccoli: None. D. Turchetti: None.
P22.60.C

Patients' understanding of routine genetic testing

M. Levkova ${ }^{1,2}$, M. Hachmeriyan ${ }^{1,2}$, V. Miteva ${ }^{1,2}$, M. Stoyanova $^{1,2}$, L. Angelova ${ }^{1}$

${ }^{1}$ Department of Medical Genetics, Medical University Varna, Varna, Bulgaria, ${ }^{2}$ Laboratory of Medical Genetics, University Hospital St Marina, Varna, Bulgaria

Introduction: Over the past decade, there has been a major increase in the number of genetic tests available. However, it is still not clear whether the patients could interpret the result themselves even for a routine genetic testing such as thrombophilia.

Materials and Methods: We present a survey among 111 randomly selected patients, carried out at the Laboratory of Medical genetics, Varna. All of the participants underwent a genetic testing for hereditary predisposition to thrombophilia and their knowledge was evaluated before and after the test using Likert scale. Correlation analysis and t-tests were applied.

Results: Mean age of the participants was $33.77 \pm 5.79$ years. $74.77 \%$ of them had a masters degree. Most of the questionned $(70.27 \%)$ were referred to genetic testing by a medical specialist, $29.73 \%$ decided on their own to test themselves due to family predisposition. There was a positive correlation between receiving information from a medical doctor $(p=0.014)$ and the degree of understanding about the test. A negative correlation existed $(p=0.001)$ between the level of knowledge and informing on your own about the test. There was no statistically significant difference $(p=0.891)$ for the correlation between the source of information and awareness of the result. However, the degree of understanding was higher $(p=0.003)$, when the result was explained by a medical specialist. The education level did not correlate with a better pre or post test knowledge ( $p=0.949)$.

Conclusions: Our findings demonstrate the importance of pre and post test counseling of the patient and its crucial role in providing information about genetic testing to the patients.

M. Levkova: None. M. Hachmeriyan: None. V. Miteva: None. M. Stoyanova: None. L. Angelova: None.

\section{P22.61.A}

Mapping of resources for undiagnosed and newly diagnosed ultra-rare disease networks

G. H. Gumus ${ }^{1}$, S. Broley ${ }^{2}$, C. Patch ${ }^{3,4}$, M. Montolio del Olmo $^{5}$, K. Boycott ${ }^{6}$, L. Faivre ${ }^{7}$, B. Zurek ${ }^{8}$, M. Macek ${ }^{9}$, H. Graessner $^{10,11}$, S. Bellagambi ${ }^{12}$, D. Stemkens ${ }^{13}$, C. van 
Doorne $^{14}$, A. Metcalfe ${ }^{15}$, A. Costa ${ }^{16}$, G. Robert ${ }^{16}$, L. Roberts $^{17}$, M. Pritchard ${ }^{17}$, H. Scheffer ${ }^{18}$, V. Boulanger ${ }^{19}, \mathrm{~J}$. Lewis $^{20}$, H. Cederroth ${ }^{21}$, M. Cederroth ${ }^{21}$, P. Arias ${ }^{22}, V$. Bros-Facer ${ }^{l}$

${ }^{1}$ EURORDIS-Rare Diseases Europe, Paris, France, ${ }^{2}$ Genetic Services of Western Australia, Perth, Australia, ${ }^{3}$ Genomics England, Queen Mary University, London, United Kingdom, ${ }^{4}$ Society and Ethics Research, Connecting Science, Wellcome Genome Campus, Cambridge, United Kingdom, ${ }^{5}$ Duchenne Parent Project, Madrid, Spain, ${ }^{6}$ Children's Hospital of Eastern Ontario Research Institute, University of Ottawa, Ottawa, ON, Canada, ${ }^{7}$ Centre de Géntétique et FHU TRANSLAD, CHU de Dijon et Université de Bourgogne, Dijon, France, ${ }^{8}$ University of Tübingen, Tubingen, Germany, ${ }^{9}$ Charles University and Motol University Hospital, Prague, Czech Republic, ${ }^{10} \mathrm{Cen}$ tre for Rare Diseases, University and University Hospital of Tübingen, Tubingen, Germany, ${ }^{11}$ European Reference Network for Rare Neurological Diseases (ERN-RND), Tübingen, Germany, ${ }^{12}$ UNIAMO FIMR Italian Alliance for Rare Diseases, Rome, Italy, ${ }^{13}$ VSOP, Dutch Patient Alliance for Rare and Genetic Diseases, Soest, Netherlands, ${ }^{14}$ European Federation of Hereditary Ataxias, United Kingdom, London, United Kingdom, ${ }^{15}$ Faculty of Health and Wellbeing, Sheffield Hallam University, Sheffield, United Kingdom, ${ }^{16}$ Florence Nightingale Faculty of Nursing \& Midwifery, King's College London, London, United Kingdom, ${ }^{17}$ Genetic Alliance UK (SWAN UK), London, United Kingdom, ${ }^{18}$ Radboud University Nijmegen Medical Centre, Nijmegen, Netherlands, ${ }^{19}$ National Organization for Rare Disorders, Danbury, CT, United States, ${ }^{20}$ Genetic and Rare Diseases Information Center, ICF International,, Rockville, $M D$, United States, ${ }^{21}$ Wilhelm Foundation, Sandbacken, Brottby, Sweden, ${ }^{22}$ Spanish Federation of Rare Diseases (FEDER), Madrid, Spain

Introduction: Every year, 65000 children across Europe are born with an undiagnosed disease (1). The diagnostic odyssey lasts 5 years on average for a rare disease patient (2). This delay in diagnosis prevents patients from accessing proper care and treatment. Several initiatives have been established to improve the lives of people with undiagnosed rare diseases. However, currently, there is not a comprehensive mapping of resources for undiagnosed or newly diagnosed Ultra-Rare diseases. Within the EU funded project, Solve-RD, EURORDIS initiated the Community Engagement Task Force (CETF), a multi-stakeholder community of patients, scientists, and clinicians, to support the needs of the undiagnosed and recently diagnosed patients and leave a legacy of a strengthened undiagnosed community.
Methodology: Key stakeholders in the field of diagnosis were invited to become members of CETF. These include Undiagnosed Diseases Network International (UDNI), SWAN (Syndrome Without A Name) Europe, Patient Advocacy Groups within the European Reference Networks and The Global Commission to End the Diagnostic Odyssey for Children.

Results: Members gathered through 3 teleconferences and a face-to-face meeting over 15 months where they discussed issues addressing specific needs of undiagnosed rare disease patients and their families such as lack of information for medical professionals and the need for standardization when receiving a diagnosis. Resources (toolkits, surveys, factsheets, illustrations) were compiled to develop a roadmap.

Conclusions: Regular and meaningful engagement of representatives across key initiatives represents the start of an active undiagnosed community. Importantly, patients as equal and valued partners are driving forward active collaborations with different stakeholders.

G.H. Gumus: None. S. Broley: None. C. Patch: None. M. Montolio del Olmo: None. K. Boycott: None. L. Faivre: None. B. Zurek: None. M. Macek: None. H. Graessner: None. S. Bellagambi: None. D. Stemkens: None. C. van Doorne: None. A. Metcalfe: None. A. Costa: None. G. Robert: None. L. Roberts: None. M. Pritchard: None. H. Scheffer: None. V. Boulanger: None. J. Lewis: None. H. Cederroth: None. M. Cederroth: None. P. Arias: None. V. Bros-Facer: None.

\section{P22.62.B}

Dying to see you; Deaths on a Clinical genetics waiting list; what are the consequences?

\section{Bradley, S. Lynch}

National Centre for Medical Genetics, Dublin 12, Ireland

Introduction: The value of a Clinical Genetics consultation is difficult to quantify. In order to estimate negative effects and by default the value of a genetics consultation; we sought to identify the consequences to the patient and families who died on the waiting-list. Methods Audit records of 40 referrals who died prior to appointment.

Results: Age range at referral: 11 days to $82 \mathrm{yrs}$. Thirty five of the 40 had never been seen; 5/40 died awaiting a follow up appointment. The majority (26/40) were cancer referrals. Ten had developmental delay/dysmorphism; 3 were cardiac genetic referrals, \& a dementia referral. We determined that $15 / 40$ deaths posed a significant risk to genetic health in their relatives [proband not seen, no family contact, no genetic testing \& no DNA stored]; 10/40 posed a moderate risk [DNA stored or predictive test available but 
no family contact]; $13 / 40$ posed a minimal risk [proband never counselled] and in $2 / 40$ cases the families were fully counselled. From the 25 probands where the death posed a moderate or significant risk, we identified a minimum of 118 ( $\sim 5$ individuals per proband) first degree living relatives. DNA was stored on only $5 / 25$ precluding ability to risk stratify in 20 families unless another relative is subsequently genetically diagnosed. Most families did not avail of offers of appointments posthumously raising concern that relatives remain unaware of their genetic risks.

Conclusions: Our study reveals that the relatives of $65 \%$ $(25 / 40)$ of probands are substantially disenfranchised if their affected relative dies whilst awaiting an appointment.

L. Bradley: None. S. Lynch: None.

P22.63.C

Impact of terminating the pregnancy after receiving a diagnosis of Down syndrome from an South African perspective

\section{J. Scott, M. F. Urban, D. Skinner}

\section{Stellenbosch University, Cape Town, South Africa}

Introduction: Advances in prenatal testing technologies lead to more women receiving a prenatal diagnosis of Down syndrome (DS). As this is an incurable disabling condition, they are confronted with the decision of having a termination of pregnancy (TOP). This has long-term psychosocial implications which are poorly understood. The aim of this study was to gain an understanding of the experiences of South African women who have had a TOP for DS and the psychosocial impact thereof.

Methods: A phenomenological qualitative approach was used. Eight participants were purposively selected from a prenatal clinic at a state sector hospital in South Africa (SA). They were interviewed shortly after being admitted to hospital for the TOP and again 6 to 12 months afterwards. The audio-recordings were transcribed and thematic analysis was used.

Results: Prenatal testing services are not widely accessible in SA and therefore the participants all had a secondtrimester TOP. The themes identified with regards to the psychological implications were: grief, impact of seeing stillborn, guilt, isolation, finding meaning, decision satisfaction and finding peace. The social impact included change in workplace, TOP stigma, moving house and change in relationships. The participants valued support from religious groups, partners, family, friends, health care workers and employers.

Conclusions: The major long-term psychosocial impact of a TOP made it difficult for the women to return to their normal daily routine. Understanding the impact of this event will deepen knowledge on how to best care for these women and may subsequently improve prenatal testing and counselling practices.

C.J. Scott: None. M.F. Urban: None. D. Skinner: None.

P23 Ethical, Legal and Psychosocial Aspects in Genetics

P23.02.B

Managing actionable secondary findings beyond highpenetrant genes through personalised health surveillance

R. Koesters, T. Hasenberg, K. U. Beiser, E. Felber

Megeno S.A., Esch-sur-Alzette, Luxembourg

Genome-informed prevention holds the promise of improving clinical outcomes among individuals at significant risk for developing a disease with a strong genetic component, by enabling personalised phenotypic surveillance for monitoring predictive biomarkers and detecting early disease symptoms. However, in the context of lowpenetrance diseases, gathering reliable phenotypic information to facilitate such early detection is challenging, since many at-risk individuals will never develop the disease. In particular, there is a risk of overdiagnosis and overtreatment, leading to an inefficient use of scarce healthcare resources. Furthermore, it is crucial that clinically healthy individuals are not prematurely designated as patients. To address these issues, we have developed a rigorous, 100point-scale scoring scheme for prioritising low-penetrance diseases to facilitate long-term risk monitoring and early disease detection. A key variable to our scoring scheme is "at-home phenotyping", that is, the ease and accuracy with which an at-risk individual can regularly sample and monitor relevant phenotypic markers over time, without recourse to their medical professional. We have applied the scoring scheme to evaluate and rank 23 monogenic disorders characterised by reduced penetrance and variable expressivity. The scores for the 23 disorders ranged from 40.0 to 88.3 , with a mean of 65.3 (median of 65.8 ). Disorders with the highest scores were hereditary hemochromatosis (88.3), familial hypercholesterolemia (86.7), and hereditary hyperparathyroidism (85.0). By systematically evaluating and ranking medically relevant gene-disease associations, it may be possible to prioritise low-penetrance disorders for facilitating long-term personalised health surveillance in an efficient and responsible manner.

R. Koesters: A. Employment (full or part-time); Significant; Megeno S.A. T. Hasenberg: A. Employment (full or part-time); Significant; Megeno S.A. K.U. Beiser: A. Employment (full or part-time); Significant; Megeno S. 
A. E. Felber: A. Employment (full or part-time); Significant; Megeno S.A..

P23.03.C

Parental communication of BRCA1/2 mutation status to children:links between gender norms and health practices

F. Gauna ${ }^{1}$, C. Noguèsl ${ }^{1,2}$, E. Mouret-Fourme ${ }^{3}$, I. Coupier ${ }^{4}$, V. Mari ${ }^{5}$, J. Moretta-Serra ${ }^{6}$, A. Bouhnik ${ }^{l}$, S. Carof ${ }^{7,1}$

${ }^{1}$ Aix-Marseille Univ, INSERM, IRD, SESSTIM, Sciences Economiques \& Sociales de la Santé \& Traitement de l'Information Médicale, Marseille, France, ${ }^{2}$ Département d'Anticipation et de Suivi des Cancers, Oncogénétique clinique, Institut Paoli-Calmettes, Marseille, France, ${ }^{3}$ Department of Genetics, Institut Curie, Paris, France, ${ }^{4}$ Gastroenterology and Genetic Department, Montpellier Hospital, Montpellier, France, ${ }^{5}$ Unité d'Oncogénétique, Centre Antoine Lacassagne, Nice, France, ${ }^{6}$ Institut Paoli Calmette, Department d'Anticipation et de Suivi du Cancer, Pôle Clinique Consultations d'Oncologie Genétique, Marseille, France, ${ }^{7}$ Institut Gustave Roussy (IGR), Villejuif, France

Objective: Our study aimed to determine psychosocial factors associated with the gendered of uptake of genetic counseling among children of BRCA1/2 mutation carriers.

Methods: We conducted a qualitative study using semistructured interviews from a comprehensive approach. Data were analyzed using thematic analysis.

Results: Thirty mutation carriers (23 women, 7 men) aged from 40 to 60 years took part in the interviews. The participants included are parents of an overall of 72 children (31 minors including 17 girls and 41 adults including 24 women). The themes included: mutation discovery, body and risk management and communication to their children from disclosure to genetic test up-taking. We explored the role of both parents in the transmission of cancer prevention practices, considering their own risk management, the disclosure of the mutation to their children and their role on the genetic test up-taking of their children. We found that gender differences are explained both by different social roles, but also by different relationship to cancers linked to BRCA and perceived as "feminine" and also by different health practices.

Conclusions: Cancer prevention is shaped by the complex links between gender norms and health practices. This has practical implications since the genetic risk communication and genetic test up-taking could be better framed taking this information into account
F. Gauna: None. C. Noguès: None. E. Mouret-Fourme: None. I. Coupier: None. V. Mari: None. J. MorettaSerra: None. A. Bouhnik: None. S. Carof: None.

P23.06.C

Cancer worry and willingness to undergo colonoscopy at different risk levels; results from a population-based survey in Sweden

\author{
C. Hawranek ${ }^{l}$, J. Maxon ${ }^{1}$, A. Andersson ${ }^{1}$, B. Van \\ Guelpen $^{1,2}$, B. Numan ${ }^{l}$, A. Rosén ${ }^{l}$
}

${ }^{l}$ Department of Radiation sciences, Oncology, Umeå university, Umeå, Sweden, ${ }^{2}$ Wallenberg Centre for Molecular Medicine, Umeå University, Umeå, Sweden

Introduction: Inherited genetic risk factors are known to induce cancer worry in at-risk individuals, affecting risk perception and screening uptake. The Cancer Worry Scale (CWS) has been used to evaluate cancer worry in these populations. This is the first population-based study describing the distribution of cancer worry and willingness to partake in screening for colorectal cancer (CRC).

Materials and Methods: Data was sourced through a probability-based citizen panel. In total, 1800 individuals between ages 18-74 were invited. 943 respondents (52\% response rate) completed an 6-item CWS (min 6, max 24) and questions on willingness to undergo colonoscopy in hypothetical situations of having a 5\%,10\% or $70 \%$ lifetime risk of developing CRC.

Results: Cancer worry scores were significantly higher among women [mean 10.10 (SD 3.09)] than men [mean 9.17 (SD 2.59)], $p<0.01$. Personal cancer history was associated with higher worry scores [11.00 (SD 3.63)] vs [9.46 (SD 2.72)], $p<0.05$, but age, educational level and country of birth was not, $p>0.05$. Women's higher scores persisted regardless of previous cancer or not. In the CRCscreening scenarios, the proportion willing to undergo colonoscopy increased with increasing levels of hypothetical risk; 58\% (5\% lifetime risk), 80\% (10\% lifetime risk) and $92 \%$ (70\% lifetime risk).

Conclusions: Gender and cancer history was related to increased cancer worry in this population-based sample. Willingness to undergo colonoscopy increased significantly with the presented increased risk of CRC. Interestingly, our population-based results overlapped with previously reported CSW-scores in several different cancer populations.

Grants: Cancer research foundation in Norrland and Forte.

C. Hawranek: None. J. Maxon: None. A. Andersson: None. B. Van Guelpen: None. B. Numan: None. A. Rosén: None. 


\section{P23.08.B}

Clinical utility of genomic sequencing: A measurement toolkit

R. Z. Hayeems ${ }^{1,2,3}$, D. Dimmock ${ }^{4}$, D. Bick ${ }^{5}$, J. Belmont ${ }^{6}$, R. Green $^{7}$, B. Lanpher ${ }^{8}$, R. Mendoza ${ }^{3}$, V. Jobanputra ${ }^{9}, S$. Taylor $^{10}$, S. Kulkarni ${ }^{11}$, M. Grove ${ }^{12}$, E. Ashley ${ }^{12}$,.. On Behalf of The Medical Genome Initiative ${ }^{13}$

${ }^{1}$ Program in Child Health Evaluative Sciences, The Hospital for Sick Children, Toronto, ON, Canada, ${ }^{2}$ Institute of Health Policy Management and Evaluation, University of Toronto, Toronto, ON, Canada, ${ }^{3}$ Centre for Genetic Medicine, The Hospital for Sick Children, Toronto, ON, Canada, ${ }^{4}$ Rady Children's Hospital Institute for Genomic Medicine, San Diego, CA, United States, ${ }^{5} \mathrm{Hud}-$ sonAlpha Institute for Biotechnology, Huntsville, $A L$, United States, ${ }^{6}$ Illumina Inc., Houston, TX, United States, ${ }^{7}$ Broad Institute of MIT and Harvard, Cambridge, MA, United States, ${ }^{8}$ Mayo Clinic, Rochester, MN, United States, ${ }^{9}$ New York Genome Center, New York, NY, United States, ${ }^{10}$ Illumina Inc., San Diego, CA, United States, ${ }^{11}$ Baylor Genetics and Baylor College of Medicine, Houston, TX, United States, ${ }^{12}$ Stanford Medicine, Stanford, CA, United States, ${ }^{13}$ The Medical Genome Initiative, San Diego, CA, United States

Background: Whole genome sequencing (WGS) is positioned to become the most robust strategy for achieving timely diagnosis of rare diseases. Despite its favorable diagnostic performance compared to conventional testing strategies, routine use of WGS is hampered by insufficient evidence of clinical utility. Largely, this evidence gap stems from complexities and inconsistencies in how this concept is defined and measured. For example, what constitutes clinical utility for WGS varies by stakeholder perspective, clinical context, and test application. A rapidly evolving technology landscape and challenges associated with executing robust comparative study designs in the context of rare disease further impede progress in this area.

Methods: An expert working group, representing a range of clinical settings in which WGS is in translation, was formed to provide practical guidance to practitioners and researchers to address the challenge of measuring clinical utility.

Results: Following a consensus-based process and aligned with a broad definition of clinical utility, we propose a conceptually-grounded and empirically-guided measurement toolkit focused on four domains of clinical utility: diagnostic thinking efficacy, therapeutic efficacy, patient outcome efficacy and societal efficacy. For each domain, we offer specific indicators and measurement strategies that can be integrated into new and ongoing studies.

Conclusions: While we focus on diagnostic applications of WGS for rare germline diseases, this toolkit offers a flexible framework for best practices related to measuring clinical utility for a range of WGS applications. As such, it provides a resource for laboratories, clinicians, and researchers looking to characterize the value of WGS beyond the laboratory.

R.Z. Hayeems: None. D. Dimmock: None. D. Bick: None. J. Belmont: A. Employment (full or part-time); Significant; Illumina Inc.. E. Ownership Interest (stock, stock options, patent or other intellectual property); Significant; Illumina Inc.. R. Green: None. B. Lanpher: None. R. Mendoza: None. V. Jobanputra: None. S. Taylor: A. Employment (full or part-time); Significant; Illumina Inc.. E. Ownership Interest (stock, stock options, patent or other intellectual property); Significant; Illumina Inc.. S. Kulkarni: None. M. Grove: None. E. Ashley: None. .. On Behalf of The Medical Genome Initiative: None.

\section{P23.09.C}

The relationship between COMT polymorphism, parenting behaviour and problem behaviors: a prospective study

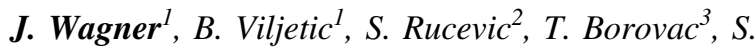
Vučković ${ }^{2}$, D. Krupić ${ }^{2}$

${ }^{1}$ Medical faculty, University of Osijek, Osijek, Croatia, ${ }^{2}$ Faculty of humanities and social sciences, University of Osijek, Osijek, Croatia, ${ }^{3}$ Faculty of education, University of Osijek, Osijek, Croatia

Introduction: Both genetic (i.e., Catechol-Omethyltransferase Val158Met/COMT-rs4680) and environmental factors (e.g., negative parenting practices including authoritarian and permissive parenting styles) have been implicated as vulnerability factors for future problem behaviors. The present study examined the susceptibility properties of COMT genotype to adverse and favorable parenting styles in relation to childhood problem behaviors.

Materials and Methods: The sample was composed of community children $\left(N=175 ; 80\right.$ boys; $\mathrm{T} 1_{\text {age }}=6.98$; $\left.\mathrm{T} 2_{\text {age }}=9.78\right)$ and their parents chosen through a multistage stage random sampling procedure. At $\mathrm{T} 1$, children were genotyped for COMT-rs4680, whereas parents rated their parenting styles. DNA was extracted from buccal swabs and genotyped using Taqman $^{\mathrm{TM}}$ SNP genotyping assay on Applied Biosystems ${ }^{\mathrm{TM}} 7500$ Real-Time PCR System. At $\mathrm{T} 2$, teachers rated children's problem behaviors. As in previous studies, we divided the sample into those with the 
high-activity Val/Val genotype (20.9\%) and those with 1 or more Met alleles (79.1\%).

Results: Using a regression analysis, a significant interactive effect was observed only for the authoritarian parenting and COMT genotype. Specifically, children who had faced authoritarian parents and were Val homozygotes exhibited more future problem behaviors $(p=.041)$ than did their Met-carrying counterparts. Notably, in the absence of authoritarian parenting practices, Val homozygotes displayed significantly lower aggression scores than did Met carriers $(p=.039)$.

Conclusions: These results suggest that Val homozygotes are more malleable to the effect of negative home environment on problem behaviors.

Grant: Presented study is a part of the ECLAT longitudinal study 2017-2021 funded by the Croatian science foundation (HRZZ-IP-2016-06-3917-ECLAT).

J. Wagner: None. B. Viljetic: None. S. Rucevic: None. T. Borovac: None. S. Vučković: None. D. Krupić: None.

\section{P23.10.A}

\section{Addressing genetic findings with familial implications in} clinical consent

\section{A. M. Phillips ${ }^{1}$, E. Niemiec ${ }^{2}$, H. C. Howard ${ }^{2}$, K. Kagkelari $^{3}$,} P. Borry ${ }^{l}$, D. F. Vears ${ }^{4}$

${ }^{1}$ University of Leuven, Leuven, Belgium, ${ }^{2}$ Uppsala University, Uppsala, Sweden, ${ }^{3}$ University of Crete, Heraklion, Greece, ${ }^{4}$ University of Melbourne, Melbourne, Australia

When a disease-causing genetic variant is identified in an individual, communicating this information to family members can play an essential role in the early diagnosis of relatives. Yet, many patients struggle with communicating these results and it is unclear to what extent this is discussed during the consent process. To better understand if and how the communication of genetic information to family members is addressed in the clinical context, we systematically searched for consent forms for diagnostic genomic sequencing online and used content analysis to analyze the forms. We identified 68 consent forms from 11 countries. The forms indicated that genetic findings were often presented as familial in nature, yet varied in how findings with familial implications were discussed. A minority of providers addressed communication of genetic information with family members, with differences in whether the health professional or the patient would be responsible for this process. Several forms offered patients options regarding communication that contradicted national guidelines and legislation regarding the disclosure of results in the absence of patient consent. This is concerning because it shows how patients and clinicians could be misled about whether or not communication is permissible in cases where the patient does not consent. Our research questions whether providers and health professionals receive adequate education and training, especially with regards to the ethical problems raised by genetic findings. Based on our analysis, we make recommendations for how to address genetic findings with familial implications in the clinical consent process.

A.M. Phillips: None. E. Niemiec: None. H.C. Howard: None. K. Kagkelari: None. P. Borry: None. D.F. Vears: None.

\section{P23.14.B}

Attitudes of Costa Rican individuals towards donation of personal genetic data for research

G. Chavarria-Soley ${ }^{1,2}, M$. Francis ${ }^{1,2}, F$. Jimenez $^{1,2}, A$. Avila $^{2}$, M. Castro-Gomez ${ }^{2}$, H. Raventos ${ }^{1,2}$

${ }^{1}$ Escuela de Biologia, Universidad de Costa Rica, San Pedro, Costa Rica, ${ }^{2}$ Centro de Investigacion en Biologia Celular y Molecular, Universidad de Costa Rica, San Pedro, Costa Rica

Introduction: Technological advances in molecular genetics have resulted in the generation of vast amounts of genomic data. Sharing study data within the research community raises important issues such as trust, privacy, and potential consequences. The goal of this study is to explore public attitudes and concerns in a sample of Costa Rican individuals regarding the donation of personal genetic data for research purposes.

Materials and Methods: A total of 224 Costa Rican individuals answered the anonymous online survey "Your DNA, Your Say". The survey covers basic demographic information, attitudes, trust in research professionals, and concerns regarding donation of medical and genetic data.

Results: A majority of individuals (93\%) are willing to donate their DNA and medical information to at least one kind of research professional (from three categories: medical doctor, non-profit researcher, for-profit researcher). Willingness to donate differs significantly according to the potential use of the data and is lowest in the case of forprofit research. The top concerns about sharing of genetic data are discrimination by health or life insurance companies and employers. When asked about levels of trust in different professionals and the government, the top ranked categories were the person's own medical doctor and researchers at a university in the person's country.

Conclusions: This is the first study dealing with the attitude toward sharing of genetic and medical information in Costa Rica. In general, we found a higher willingness to share genetic information for research purposes than what has been reported for other non- Latin American countries. 
G. Chavarria-Soley: None. M. Francis: None. F. Jimenez: None. A. Avila: None. M. Castro-Gomez: None. H. Raventos: None.

\section{P23.19.A}

Human genome and gene-editing: opinions of National Ethics/Bioethics Committees in Europe.

\section{G. Floridia $^{\text {, C. Mannelli }}{ }^{2}$, C. Petrini ${ }^{1}$}

${ }^{1}$ Istituto Superiore di Sanità, Rome, Italy, ${ }^{2}$ Candiolo Cancer Istitute, FPO-IRCCS, Italian Ministry of Health, University of Turin, Candiolo, Rome, Turin, Italy

Introduction: Advanced genome editing (GE) technologies, as the CRISPR/Cas9 system, are very promising for prevention and treatment of human diseases. Ethical concerns arise from the use of GE on human germ-cell lines and embryos, whose debate has been amplified by the birth of two Chinese gene-edited twin girls. In the last few years National Ethics/Bioethics Committees (NC) in Europe have been releasing documents about the use of GE, in particular on human genome.

Materials and Methods: In this work we summarise and analyse the following documents released by European NC: a) "Statement from the Danish Council on Ethics on genetic modification of future humans In response to advances in the CRISPR technology", 2016; b) "GE: an ethical review", Nuffield Council on Bioethics" (NuCoB), 2016; c) Opinion N25/2016, Swiss National Advisory Commission on Biomedical Ethics, 2016 ; d) "Recent developments in human genome modification: GE”, Hellenic NC, 2016; e) "Ethical issues in GE using CRISPR/CAS9", Italian National Bioethics Committee, 2017; f) "GE and human reproduction", $\mathrm{NuCoB} 2018$; g) "Intervening in the Human Germline", German Ethics Council, 2019.

Results: Different ethical opinions, even inside a same Committee, are expressed about genetic modifications concerning early embryos not finalised to reproduction and germ cells/fertilised cells edited for therapeutic purposes, e.g. to remove disease susceptibility in the offspring.

Conclusions: Bioethical viewpoints across European NC vary indeed in a considerable way, from those focused mainly on the potential benefits of these methodologies to those more cautious.

G. Floridia: None. C. Mannelli: None. C. Petrini: None.

\section{P23.20.B}

What public concerns exist regarding genetic information: Japanese experiences and beyond

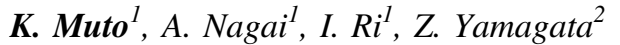

${ }^{1}$ The University of Tokyo, Tokyo, Japan, ${ }^{2}$ University of Yamanashi, Yamanashi, Japan

In Japan, direct-to-consumer genetic testing has been also freely accessible without any regulations since 1990s. The Japanese government finally launched a comprehensive policy for promoting cancer genomic medicine and storing anonymized patients' clinical and genomic data to the National Data Repository in 2017. However, discussions regarding the prevention of genetic discrimination have not yet been active. The objectives of this survey were to determine the extent of genetic literacy and attitudes of the Japanese public toward genomic medicine and to estimate the prevalence of genetic discrimination. Cross-sectional and anonymous online surveys were distributed to adults in Japan in February 2017 and again in January 2020. The findings of the surveys conducted in $2017(N=10,881)$ and $2020(N=10,245)$ were compared, referring to our dataset since 2005. The recognition rate of "genome" became higher $(69.7 \%$ in $2005,73.2 \%$ in $2017,85.1 \%$ in 2020 respectively). Respondents' experiences of being required to submit personal genetic information were "life insurance for medical benefits or death benefits" (2.1\% in $2017,1.5 \%$ in 2020 respectively) and "from my partner or his/her relatives" ( $0.3 \%$ in both years). Overall benefits of using genetic information were recognized higher than concerns. As genome medicine becomes more familiar to people in the near future, their concerns about the utilization of genetic information may increase. The policy to prevent genetic discrimination should be considered urgently.

K. Muto: None. A. Nagai: None. I. Ri: None. Z. Yamagata: None.

\section{P23.21.C}

Public's awareness of and attitudes towards research biobanks in Latvia: concerns regarding genetic and medical data donation for research

\section{Rovite ${ }^{1}$, S. Mezinska ${ }^{2}$, L. Tzivian $^{2}$, J. Kaleja $^{3}$, I. Mileiko $^{3}$, D. Santare ${ }^{2}$}

${ }^{1}$ Latvian Biomedical Research and Study centre, Riga, Latvia, ${ }^{2}$ University of Latvia, Institute of Clinical and Preventive Medicine, Riga, Latvia, ${ }^{3}$ University of Latvia, Riga, Latvia

Introduction: In 2018 Latvia signed the declaration 'Towards access to at least 1 million sequenced genomes in the European Union by 2022', which aims to link genomic health data throughout the EU. The genetic research in Latvia is currently regulated by several national regulations, 
but the most have been developed more than 15 years ago and do not meet current international standards preventing the successful functioning of biobanks and genetic research data obtainment in Latvia. The aim of the study was to analyze the public's attitudes, concerns and trust in research biobanking and genetic research in Latvia.

Materials and Methods: The questionnaire was based on 2010 Eurobarometer questionnaire (questions number B12B18 in the original questionnaire). Five nationally relevant questions were added. The survey was conducted in March 2019 and used multi-stage stratified random sampling to obtain a representative sample of the general population of Latvia (aged 18 to 75 years). Statistical analysis was performed using SPSS software, v. 23.

Results: 1017 responders participated in the survey. $35.6 \%$ expressed concerns donating their medical history data and $27.5 \%$ their genetic data to the research, respondents were less concerned for donating their lifestyle information (25.8\%), blood (25.5\%) or tissue (26.7\%). $59.6 \%$ supported the data and biological sample exchange between EU member states for research purposes. $25.6 \%$ of respondents were familiar with term "biobank", 19.2\% have heard about national biobank.

Conclusions: The survey participants had higher concerns for donating their genetic and medical data to research than biological samples or lifestyle data.

V. Rovite: None. S. Mezinska: None. L. Tzivian: None. J. Kaleja: None. I. Mileiko: None. D. Santare: None.

\section{P23.23.B}

How much do cancer patients value whole genome sequencing? A cross-sectional survey using the willingness-to-pay technique

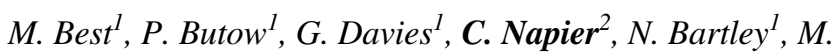
Ballinger $^{3}$, B. B. Biesecker ${ }^{4}$, I. Juraskova $^{l}$, B. Meiser ${ }^{5}$, T. Schlub ${ }^{6}$, D. Thomas ${ }^{3}$, D. Goldstein ${ }^{7}$, Members of the

PiGeOn Project

${ }^{1}$ School of Psychology, University of Sydney, Australia, ${ }^{2}$ University of Sydney, School of Psychology, Sydney, Australia, ${ }^{3}$ Garvan Institute of Medical Research, Sydney, Australia, ${ }^{4}$ RTI International, Washington, DC, United States, ${ }^{5}$ Psychosocial Research Group, University of NSW, Australia, ${ }^{6}$ School of Public Health, University of Sydney, Australia, ${ }^{7}$ Prince of Wales Hospital, Sydney, Australia

Introduction: Genomic Sequencing (GS) to identify high cancer risk will soon enter clinical practice at significant cost to the health system. This study aimed to quantify perceived value of GS to cancer patients and their relatives, and factors associated with value.
Materials and Methods: Participants were recruited on consent to a genomics study. Eligible participants (with cancer of likely genetic aetiology, or a first-degree relative) completed a questionnaire prior to GS. Willingness-to-pay was assessed via a hypothetical trade-off scenario of actionable result return-rates of $1 \%, 10 \%, 20 \%, 30 \%$, $40 \%$ or $50 \%$ respectively.

Results: Of 348 probands and 213 relatives (92\% response rate), $81 \%$ would have GS for as little as a $1 \%$ actionable return-rate. Participants would pay a median of $\$ 1,000$ for return-rates of at least $20 \%$ (probands) or $30 \%$ (relatives), and $\$ 300$ for lower return-rates. 114 (35\%) probands and $64(31 \%)$ relatives would pay $\$ 1,000$ for a $1 \%$ actionable return rate, while $108(33 \%)$ of probands and 76 (36\%) of relatives would not pay $\$ 1,000$ even for a $50 \%$ return rate. Probands with common cancers and negative attitudes to uncertainty were more likely to have GS; those with higher education were more willing to pay $\$ 1,000$ and $\$ 3,000$ at lower return-rates.

Conclusions: This study found high interest in, but lower willingness-to-pay for GS in patients with cancers of likely genetic aetiology and their first-degree relatives, possibly due to inability to pay. To avoid inequity, the health system should consider universal coverage of GS. Funded by NHMRC and CINSW.

M. Best: None. P. Butow: None. G. Davies: None. C. Napier: None. N. Bartley: None. M. Ballinger: None. B. B. Biesecker: None. I. Juraskova: None. B. Meiser: None. T. Schlub: None. D. Thomas: None. D. Goldstein: None.

\section{P23.26.B}

Genetic testing decision-making: deciding between control and fate

\author{
B. M. Zimmermann ${ }^{1}$, D. Shaw ${ }^{1}, K$. Heinimann ${ }^{2}, L$. \\ Knabben $^{3}$, B. Elger ${ }^{l}$, I. Koné
}

${ }^{1}$ University of Basel, Institute of Biomedical Ethics, Basel, Switzerland, ${ }^{2}$ University Hospital Basel, Institute for Medical Genetics and Pathology, Basel, Switzerland, ${ }^{3}$ University Hospital of Bern, Department of Obstetrics and Gynecology, Bern, Switzerland

Individuals considering genetic testing for cancer predisposition must reflect on factual knowledge, relational considerations and emotions. Genetic counselling supports their decision-making process. However, factors influencing the genetic testing decision-making process have not been studied in Switzerland so far. This study thus aims to investigate the decision-making process of people undergoing genetic counselling for cancer predisposition in Switzerland. Grounded theory is the methodological 
approach of this qualitative interview study. Eighteen individuals were retrospectively asked about their decisionmaking process, including both acceptors and decliners of genetic testing. Additionally, participants were asked in hypothetical vignettes whether they would undergo genetic testing for nonactionable genetic variants. Two healthcare professionals providing genetic counselling were interviewed for data triangulation. We found that the underlying life philosophy of either leaving life to fate or preferring control influences genetic testing decision-making importantly. However, other factors might shape this decision: People look for certainty; anticipate consequences; are socially influenced; simplify risks; decide intuitively or reflectively, and trust in science to different degrees. Our results visualise a theory of genetic testing decision-making that comprises both acceptors and decliners. It might help healthcare professionals and carriers informing family members to anticipate differing life philosophies, and, thus, differing attitudes towards genetic testing for both actionable and non-actionable genetic variants.

B.M. Zimmermann: None. D. Shaw: None. K. Heinimann: A. Employment (full or part-time); Modest; University Hospital Basel. L. Knabben: A. Employment (full or part-time); Modest; University Hospital Bern. B. Elger: None. I. Koné: None.

\section{P23.27.C}

The impact of incidental findings in clinical exome sequencing, a qualitative interview study

V. van der Schoot ${ }^{l}$, S. J. Viellevoije ${ }^{2}$, F. Tammer ${ }^{2}, H$. G.

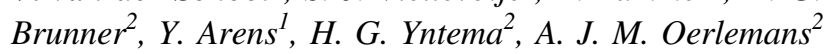

${ }^{1}$ MUMC+, Maastricht, Netherlands, ${ }^{2}$ Radboudumc, Nijmegen, Netherlands

Introduction: Incidental findings (IFs) in clinical exome sequencing are variants unrelated to the initial clinical question, that could be of medical relevance to patients and/ or their families. The management and disclosure of IFs is a topic of debate. Little is known about the impact of IFs on patients' lives.

Materials and Methods: We conducted 20 semistructured face-to-face interviews with patients and/or their relatives who had an oncological $(n=10)$ or cardiological $(n=10)$ IF disclosed.

Results: Almost all patients indicated they would undergo genetic testing again. For participants, the impact of an IF consists of the psychological, physical and the financial impact. These dimensions are interrelated and appear to be influenced by the actionability, understanding the implications of the IF, the pre-test health of the patient and the social context.
Conclusions: Disclosure of IFs in exome sequencing does not seem to greatly affect the lives of patients and their family if patients are enabled to realize the actionability of the IF and get a grip on the consequences of the finding. We observe a difference in perceived impact of oncological and cardiological IFs. We propose a coordinating role for the clinical geneticist to facilitate the empowerment of patients with an IF.

V. van der Schoot: None. S.J. Viellevoije: None. F. Tammer: None. H.G. Brunner: None. Y. Arens: None. H.G. Yntema: None. A.J.M. Oerlemans: None.

\section{P23.28.A}

GenIDA, a participatory international database to collect and analyze medically relevant information on genetic forms of intellectual disability or autism: novel findings on Koolen de Vries syndrome and comparative analysis of behavioral and language features in KdVS, Kleefstra and KBG syndromes

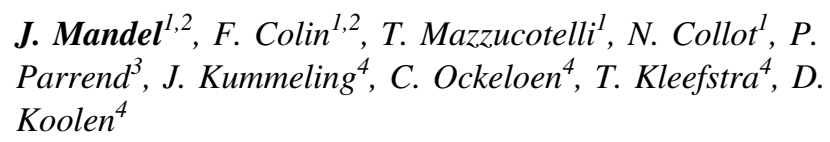
Koolen $^{4}$

${ }^{1} I G B M C$, Illkirch, Eurometropole Strasbourg, France, ${ }^{2}$ University of Strasbourg, Strasbourg, France, ${ }^{3}$ iCube lab CNRS UMR7357 Université de Strasbourg, Strasbourg, France, ${ }^{4}$ Dept of Human Genetics, Radboud University Medical Cantre, Nijmegen, Netherlands

Introduction: GenIDA was launched in 2017 as a participatory and international study of specific genetic forms of intellectual disability (ID) with or without autism or epilepsy. The extreme heterogeneity of these neurodevelopmental disorders and the rarity of identified cases for many of the implicated genes render the goal of defining the clinical spectrum, comorbidities and natural history a daunting task. We reasoned that a participatory approach would be a relevant alternative whereby clinical information is entered and updated by the family of the proband using a structured online questionnaire in 5 languages at https:// genida.unistra.fr We used the Koolen-de Vries syndrome (KdvS 17q21.31del / KANSL1mut) for a proof-of-concept of the approach.

Methods and Results: The current questionnaire has 41 multiple choice questions and 92 sub-questions analyzed automatically, and 5 open text qualitative questions. Currently, the questionnaire has been filled for 808 patients with a known genetic diagnosis. The KdVS cohort is the largest, with 202 probands. Results obtained for the frequency of epilepsy or other previously reported comorbidities are consistent with published data. Inclusion of probands from a wide age range generates data on natural 
history. A major finding was the identification of recurrent respiratory problems in $40 \%$ of patients, including asthma (39 cases) and pneumonia (31 cases, mostly before 10y). We could study use, perceived efficacy and reported adverse effects of antiepileptic drugs. We compared behavioral and language aspects for KdVS, Kleefstra syndrome $(n=114)$ and $\mathrm{KBG}$ syndrome $(n=38)$, showing major differences.

Conclusion: The data validate our participatory approach.

J. Mandel: None. F. Colin: None. T. Mazzucotelli: None. N. Collot: None. P. Parrend: None. J. Kummeling: None. C. Ockeloen: None. T. Kleefstra: None. D. Koolen: None.

\section{P23.31.A}

The social shaping of a diagnosis in Next Generation Sequencing

\section{J. M. L. Kuiper ${ }^{1}$, P. Borry ${ }^{2}$, D. Vears ${ }^{2}$, I. Van Hoyweghen ${ }^{1}$ \\ ${ }^{1}$ Centre for Sociological Research, KU Leuven, Leuven, Belgium, ${ }^{2}$ Centre for Biomedical Ethics and Law, KU Leuven, Leuven, Belgium}

Although Next Generation Sequencing (NGS) has increased our ability to test and diagnose, the type of data that is generated by NGS techniques and their interpretation leaves room for a considerable amount of uncertainty. NGS not only generates a vast amount of data, the results are often not very clear-cut. In many instances, analysis does not reveal a straightforward diagnostic cause for the symptoms, requiring a more complex interpretation and negotiation process. The type of delineation work this lack of clarity necessitates is not uncommon in medicine: diagnostic definitions and criteria change over time, which allows new diagnoses to arise and others to be lumped together, split or dissolved. In NGS, the boundaries of what counts as 'evidence' for variant interpretation, what is relevant to report to patients, and what constitutes a diagnosis is continuously reshaped in everyday practice. In this early implementation phase of NGS, in which standardization efforts are not yet solidified, the boundary struggles in laboratory and clinical practice are particularly visible. To explore the complexities in the social shaping of diagnosis in NGS settings, we critically review the literature on the sociology of health and illness, sociology of diagnosis, and science and technology studies on the meaning and social construction of diagnosis. We then assess the current state of NGS practice through this lens. We discuss what it entails to give and receive a diagnosis using NGS, how this differs from diagnoses identified through more 'traditional' diagnostic technologies, and what role uncertainty plays in this.
J.M.L. Kuiper: None. P. Borry: None. D. Vears: None. I. Van Hoyweghen: None.

\section{P23.32.B}

The psychological impact of receiving an incidental finding with Non-Invasive Prenatal Testing (NIPT)

I. M. Bakkeren ${ }^{1}$, L. Henneman ${ }^{2}$, M. N. Bekker ${ }^{3}$, E. H. van Vliet-Lachotzki ${ }^{4}$, R. H. Galjaard ${ }^{l}$

${ }^{1}$ Erasmus Medical Centre, Rotterdam, Netherlands, ${ }^{2}$ Amsterdam UMC, Amsterdam, Netherlands, ${ }^{3}$ Utrecht University Medical Center, Utrecht, Netherlands, ${ }^{4}$ Dutch Genetic Alliance (VSOP), Soest, Netherlands

Since April 2017, the non-invasive prenatal test (NIPT) is offered to all pregnant women in the Netherlands as a first trimester prenatal screening test. Findings other than trisomy 21,18 or 13 are reported on request of the pregnant woman, $78 \%$ of women chose to have these reported. The first year, a total of 207 incidental findings (IF) other than the three common trisomies were detected. This survey study examined women's experiences of being informed of an IF and its psychological impact. One-hundred thirteen $(54.6 \%)$ women completed the questionnaire. Most women (59.3\%) chose for IF because they wanted to know as much as possible about the health of their unborn child. Slightly more than half $(57.5 \%)$ did not find deciding for IF difficult, however only $25.6 \%$ felt they had an idea of what IF could be before having NIPT. Almost all (98.2\%) women met with a clinical geneticist to discuss their IF, for $82.3 \%$ of them this helped in understanding their result. Sixteen women $(14.2 \%)$ ended their pregnancy because of abnormalities resulting from the IF, whereas $85(75.2 \%)$ gave birth to a child without anomalies. For most women $(85.9 \%)$ the IF caused them great worry. However, a vast majority $(89.3 \%)$ would opt for NIPT again in a future pregnancy, of whom $71 \%$ would opt for IF again. This study gives an insight in how pregnant women experience receiving an IF from NIPT. For most women the IF caused worry, however most of them would opt for NIPT with IF again.

I.M. Bakkeren: None. L. Henneman: None. M.N. Bekker: None. E.H. van Vliet-Lachotzki: None. R.H. Galjaard: None.

\section{P23.33.C}

Opportunistic screening for actionable pharmacogenetic variants: why not apply knowledge to patient-data that is already available? 
T. Rigter ${ }^{l}$, M. E. Jansen ${ }^{l}$, G. M. W. R. de Wert ${ }^{2}$, M. C. Cornel $^{1}$

${ }^{1}$ AmsterdamUMC (location VUmc), Amsterdam Public Health Institute, Dept. Clinical Genetics, section Community Genetics, Amsterdam, Netherlands, ${ }^{2}$ Maastricht University, Department of Health, Ethics and Society, Maastricht, Netherlands

Analyzing clinical exome and genome sequences beyond the primary indication (opportunistic screening) has been subject to international debate. Arguments evolved around the 2013 American College of Medical Genetics recommendations to analyze and disclose a minimum list of actionable variants. Reasons to support opportunistic screening include, utilizing available data as an opportunity to identify and manage risks for the selected highly penetrant genetic disorders. Experts, however, have also questioned the clinical validity and utility of these findings and posed serious ethical and social issues around informed consent, equity, potential psychosocial harm of receiving results and impact on health care budgets. Pharmacogenetic variants have received little attention in these debates, while guidelines with actionable gene-drug prescriptions are available. The "Dutch Consortium for Ethical, Legal, and Social Issues of Personalized Medicine (ELSI-PM)" organized a workshop on opportunistic screening for pharmacogenetic variants, for relevant Dutch stakeholders. We aimed to elucidate the arguments for and against offering opportunistic pharmacogenetic screening to patients undergoing sequencing with an overview of variants relevant for future drug prescriptions; their personal "myDNAmedicationcard". Participants agreed that such practice would pertain screening, should therefore meet corresponding screening criteria, and does not automatically fall under the duty of the physician offering the initial sequencing. Although many argued that it could be beneficial to have your personal pharmacogenetic information readily available when requiring drugs, many uncertainties emerged. Pilot studies could provide more insight in consequences of opportunistic pharmacogenetic screening. ELSI-PM is funded by ZonMw (Netherlands Organisation for Health Research \& Development; grantno. 846003102)

T. Rigter: None. M.E. Jansen: None. G.M.W.R. de Wert: None. M.C. Cornel: None.

\section{P23.34.A}

Extent of knowledge about prenatal diagnostic testing in Poland

\section{L. Podstawka, M. Malarska}

\section{Medical University of Lodz, Lodz, Poland}

Aim: The aim was to assess the extent of knowledge of prenatal diagnostic testing among Polish people.

Methods: This study was conducted via an online anonymous survey published on social media, targeted at Polish citizens of different age, sex, residence and educational background. 1034 respondents answered a series of questions.

Results: The results revealed that among respondents there are still many false beliefs about the antenatal examination, as well as many concerns regarding health service in general. Among other results, it was observed that $53 \%$ do not know what amniocentesis is and of those who know $25 \%$ do not understand the risks associated with the procedure, $38.2 \%$ do not know what indications for CVS are, only $30 \%$ know what a free-DNA test is, $9 \%$ think that an ultrasound scan is harmful to the fetus and $26.4 \%$ of the respondents with children did not have any prenatal tests done during pregnancy.

Conclusions: Currently in Poland, the extent of knowledge and understanding of prenatal diagnostic testing is rather pooras survey answers showed that people do not understand the procedures used in prenatal tests, their effectiveness and possible risks. Additionally, some of the respondents left remarks which suggested that they have a problem with access to prenatal diagnostics due to lack of information about the antenatal examinations. What became clear is that there is a need to raise awareness about this issue, as well as educate patients and improve health service in Poland.

\section{M.L. Podstawka: None. M. Malarska: None.}

\section{P23.35.B}

Family factors in a prenatal clinic; the extended trajectory of a single decision

\author{
A. J. Clarke ${ }^{1}$, S. Doheny ${ }^{1}$, O. Uzun ${ }^{2}$ \\ ${ }^{1}$ Cardiff University, Cardiff, United Kingdom, ${ }^{2}$ Cardiff and \\ Vale University Health Board, Cardiff, United Kingdom
}

Decisions about whether to continue or terminate a pregnancy, when the fetus is affected by a genetic condition and/ or a malformation, are often difficult. Research into such decisions is also difficult and sensitive. These decisions can raise many issues: ethical, social and practical.

We report on the multiple factors considered by one family in the course of making their decision about a pregnancy in which the fetus was found to be affected by Down syndrome and a cardiac malformation. 
We used multiple data sources to achieve this, collecting audio recordings of fetal cardiology clinics, a diary of thoughts and feelings kept by the pregnant woman until after the pregnancy, an audio recording of one family conversation and one interview at follow-up after the end of the study. We concentrate on the diary and the recorded family discussion as such material is not often accessed.

The topics discussed include the contrasting feelings of the couple about a possible termination of the pregnancy, the perceived opinions of friends and family members, the efforts of the pregnant woman's mother to be supportive, and the family's efforts to 'read' the opinions of antenatal clinic professionals. We also note the family's understandings of the research process and its implications for "informed consent".

This approach to research - recruiting patients as active participants in research into their experiences and decisions - adds substantially to our appreciation of their situation and their decisions and will be of value for reflective practice and training.

ESRC Grant ES/R003092/1

A.J. Clarke: None. S. Doheny: None. O. Uzun: None.

\section{P23.36.C}

\section{Medical Genetics in Preventive Healthcare in Europe:} Current Status and Future Directions

\author{
A. Blazkova ${ }^{1,2}$, M. Zapp ${ }^{1}$, G. Lenzini ${ }^{1}$ \\ ${ }^{1}$ University of Luxembourg, Esch-sur-Alzette, Luxembourg, \\ ${ }^{2}$ Megeno S.A., Esch-sur-Alzette, Luxembourg
}

The potential of predictive and presymptomatic genetic testing to foster preventive healthcare has been widely recognised. However, the extent to which genetic tests are currently utilised for prevention purposes remains unknown. In this study, we explore the practices of a large sample of European prevention centres (PCs) - i.e. medical institutions specialised in health maintenance and disease prevention - regarding their use of medical genetics. We identified PCs located within the European Economic Area based on their membership of relevant professional organisations, participation in academic and business events related to preventive medicine, and a comprehensive online search. We reviewed PCs' websites and information materials, such as marketing brochures and videos, to gain insights into the availability of genomics-guided preventive services. In cases where provided information was deemed insufficient, PCs were contacted with additional questions via email. Those PCs who did not reply to our email inquiries within two weeks were subsequently contacted via a phone call. Our search identified 74 PCs located in 20 European countries. The highest number of PCs were based in Germany $(17 / 74 ; 23 \%)$, followed by Spain $(9 / 74 ; 12 \%)$ and the Czech Republic (7/74; $9 \%)$. Of these, only six $(8 \%)$ PCs fully incorporate genetic testing into their services to personalise preventive healthcare pathways for their customers. These PCs also employ qualified genetics professionals who support customers through genetic counseling. The paucity of genome-informed services among PCs indicates a slow rate of adoption of genomics in preventive healthcare.

A. Blazkova: A. Employment (full or part-time); Modest; Megeno S.A.. M. Zapp: None. G. Lenzini: B. Research Grant (principal investigator, collaborator or consultant and pending grants as well as grants already received); Significant; Megeno S.A..

\section{P23.37.A}

The latent class analysis to clarify the factors associated with the structure of the public attitude toward the genome research at the survey in Japan.

\section{Z. Yamagata ${ }^{1}$, K. Muto ${ }^{2}$}

${ }^{1}$ University of Yamanashi, Chuo, Japan, ${ }^{2}$ The Institute of Medical Science, The University of Tokyo,, Tokyo, Japan

Aim: The aim of this study was to clarify the structure of the public attitude toward the genome research by the latent class analysis.

Methods: The nationwide surveys about the attitude toward the genome research were conducted in 2016 in Japan. The participants were comprised of 2,000 people (age, 20-69), selected from the Japanese general population by using the two-step stratified random sampling method.

Results: Five clusters were assumed as an explanation model of six variables related to the knowledge of genome and attitudes toward genomic research promotion about three themes; basic genome research, genome research related to agriculture and medicine at the survey in 2016. They were able to be named "Group of aggressive promotion" (41.5\%), "Group of passive support”(20.8\%), "Group not making judgment" (19.2\%), "Group making prudent judgment" (18.5\%), and "Group not interested in genome". The results in 2008 and 2009 were almost the same as that in2005. It is possible to forecast to which cluster to belong according to respondent's attribute, and we can forecast the reaction to other questions by using a cluster oppositely. For examples, "Group of aggressive promotion" is the layer of a high academic background, and is positive to donate their blood for the genome research, "Group making prudent judgment" is high academic back ground persons as same as "Group of aggressive promotion", and is interesting in the science and technology, but is negative to the blood donation for the research. 
Z. Yamagata: None. K. Muto: None.

\section{P23.38.B}

Exploring responsibility about reproduction in the accounts of individuals at risk for and af-fected by late onset neurological diseases: a qualitative study

\section{Á. Mendes $^{1}$, J. Sequeiros ${ }^{1}$, A. Clarke $^{2}$}

${ }^{1}$ UnIGENe and CGPP, IBMC - i3S, Universidade do Porto, Porto, Portugal, ${ }^{2}$ Institute of Medical Genetics, School of Medicine, Cardiff University, Cardiff, United Kingdom

People with a family history of hereditary disease commonly face difficult questions about reproduction. This study reports accounts of their reproductive decisions from people at-risk for or affected by the incurable, late-onset neurological diseases, familial amyloid polyneuropathy ATTRVal30Met and Machado-Joseph disease. We draw on individual and family semi-structured interviews with participants recruited through the respective national patients' associations ( $n=35,19$ women; mean age: $39.2 y$, range 17-68). Data were analysed thematically. Findings revealed that participants drew on various - sometimes ambivalent and competing - relations to their awareness of genetic risk and their wish to have children. The elimination of genetic risk was perceived as responsible behaviour by some participants. For others, however, responsibility entailed accepting risks, because they prioritized values such as parenthood, family relationships and the value of life, above any concern about genetic disease. Some accounts were fraught with ambivalence, repentance and guilt, especially when children were born before participants knew of their or their partner's risk. Hope for future scientific advances was often emphasized by those participants who already had children at-risk. We discuss the findings in the context of the participants' negotiations between reproductive risks and their sense of responsibility to self and others (including those not yet born), and how the accumulated multigenerational experience with the disease influence participants' decisions. We conclude that 'genetic responsibility' is present not only in the accounts of those who chose not to have children but also in those who knew their situation beforehand and still chose to have at-risk children.

Á. Mendes: None. J. Sequeiros: None. A. Clarke: None.

P23.39.C

ELSI challenges raised by sequencing technology in French pediatric oncology services
S. Le Tirant ${ }^{1}$, E. Rial-Sebbag ${ }^{l}$, S. Julia $^{1,2}$, L. Hervouet $^{3}, S$. de Montgolfier ${ }^{3,4}$

${ }^{I}$ LEASP, UMR 1027 Inserm / Université Paul Sabatier, Toulouse, France, ${ }^{2} \mathrm{CHU}$ de Toulouse, Service de Génétique médicale, Hôpital Purpan, Toulouse, France, ${ }^{3}$ Institut de Recherche Interdisciplinaire sur les Enjeux Sociaux (UMR 8156 CNRS - 997 INSERM - EHESS - UP13), Campus Condorcet, Aubervilliers, France, ${ }^{4}$ Université Paris Est Créteil / INSPE, Créteil, France

Introduction: The use sequencing technology becomes common practice in pediatric oncology to better understand the tumor process and to adapt treatments. This exam can eventually reveal constitutional information of interest for family members linked to the child cancer or incidental findings. This highlights some ethical, legal and societal issues related to the information that should be delivered to patients that are minors and to their relatives, the right to know or not about this data and overall the consent to these somatic genetic tests.

Materials and Methods: We surveyed the current and coming French bioethics legislation and the one on health applied to minors, the relevant international texts and recommendations formulated by legal bodies and professional societies. In addition, different consents forms were compared. Focus groups with patients and parents were organized in several pediatric oncology units to identify the information needs and to shed light on path improvements (professionals involved and temporal organization of such proposals).

Results: Initial results show disparate medical practices and an absence of specific rules regarding the somatic genetic above all when realized on minors. However, the application of general principles of health care law offers precisions and some solutions have been planned in the French draft bill on bioethics.

Conclusions: The need to regulate this practice and the communication of these data remains essential to respect the right of information of patients, their ability to consent and to improve the care of minors. This study was realized as part of the project INCa-SHS n²018-127.

S. Le Tirant: None. E. Rial-Sebbag: None. S. Julia: None. L. Hervouet: None. S. de Montgolfier: None.

P23.40.A

Intellectual functioning of adults with Silver-Russell syndrome due to IGF2/H19 hypomethylation in the 11 15 region

M. Burgevin ${ }^{1}$, A. Lacroix ${ }^{l}$, G. Brown ${ }^{1}$, M. Mikaty ${ }^{2}$, A.

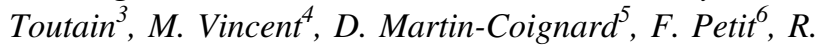


Coutant $^{7}$, C. Thauvin-Robinet ${ }^{8}$, B. Donadille ${ }^{9}$, I. Netchine ${ }^{10}$, S. Odent $t^{2,11}$

${ }^{1}$ Univ Rennes, LP3C (Laboratoire de Psychologie: Cognition, Comportement, Communication), EA 1285, Rennes, France, ${ }^{2}$ Service de Génétique Clinique, Centre de Référence Maladies Rares CLAD-Ouest, CHU de Rennes, ENR ITHACA, Rennes, France, ${ }^{3}$ Service de Génétique, CHU de Tours, Hôpital Bretonneau, UMR 1253, iBrain, Université de Tours, Inserm, Tours, France, ${ }^{4}$ Service de Génétique Médicale, CHU de Nantes, Nantes, France, ${ }^{5}$ Service de Génétique, CH du Mans, Le Mans, France, ${ }^{6}$ Clinique de Génétique, Centre de référence CLAD NordOuest, CHU Lille, Lille, France, ${ }^{7}$ Service d'endocrinologie et diabétologie pédiatrique, CHU Angers, Angers, France, ${ }^{8}$ Service de Génétique Médicale, Centre de Référence Maladies Rares "Déficiences intellectuelles de causes rares », CHU Dijon Bourgogne, Dijon, France, ${ }^{9}$ Service d'endocrinologie, Hôpital Saint Antoine, Paris, France, ${ }^{10}$ Sorbonne Universités, Inserm, UMR S 938, Centre de Recherche Saint Antoine, Hôpital Armand Trousseau, Explorations Fonctionnelles Endocriniennes, Paris, France, ${ }^{11}$ Univ Rennes, CNRS, IGDR (Institut de génétique et développement de Rennes) - UMR 6290,, Rennes, France

Introduction: Silver-Russell syndrome (SRS, OMIM\#180860, ORPHA\#813) is characterized by severe intrauterine and postnatal growth retardation, relative macrocephaly at birth, body asymmetry, prominent forehead and feeding difficulties during childhood. Two main molecular mechanisms have been identified: a maternal uniparental disomy of chromosome 7 (5-10\%), and methylation abnormalities of the 11 p15.5 region (40-60\%). To date, few studies have focused on the intellectual and cognitive profile of individuals with SRS. However, these are important concerns for families. The few existing studies are mainly focused on pediatric cohorts, but it is important to evaluate the intellectual profile and cognitive abilities of patients with SSR across lifespan.

Materials and Methods: Ten adults aged 18-39 years completed the Wechsler scale for adults. All of the patients had SRS due to epimutation in the 11 p15 region. Measures of interest included patients' intelligence quotient (IQ) and four cognitive domains: verbal comprehension, perceptual reasoning, working memory, and processing speed. Clinical and medical information were also collected from interviews and questionnaires.

Results: The mean IQ score was in the average range ( $M$ $=95.40, S D=18.55)$. Patients had better performance in verbal comprehension compared to other domains. Frequent daily difficulties were reported by patients: learning disabilities and low self-esteem were perceived in $60 \%$ of adults.

Conclusions: This study is one of the first to document the intellectual functioning of adults with SRS. It shows that early intervention and multidisciplinary care from childhood to adulthood appear to be important in this syndrome for care potential medical, cognitive and psychosocial problems.

M. Burgevin: None. A. Lacroix: None. G. Brown: None. M. Mikaty: None. A. Toutain: None. M. Vincent: None. D. Martin-Coignard: None. F. Petit: None. R. Coutant: None. C. Thauvin-Robinet: None. B. Donadille: None. I. Netchine: None. S. Odent: None.

P23.42.C

Reporting and receiving uncertain prenatal whole exome sequencing results: How might healthcare providers and patients handle uncertainty?

\section{J. E. Klapwijk ${ }^{1,2}$, H. T. Brüggenwirth ${ }^{l}$, K. E. M. Diderich ${ }^{l}$,} K. Dijkstra ${ }^{2}$, S. R. Riedijk ${ }^{1}$

${ }^{1}$ Department of Clinical Genetics, Erasmus Medical Centre, Rotterdam, Netherlands, ${ }^{2}$ Department of Psychology, Education, and Child Studies, Erasmus University Rotterdam, Rotterdam, Netherlands

Introduction: Application of prenatal whole exome sequencing (WES) offers a much broader scope of results, but can also generate more uncertainty. We aimed to investigate how uncertain prenatal WES results might be handled by healthcare professionals (HCP's) and patients and which individual characteristics may play a role. Exploring the impact of uncertainty in decision-making is instrumental in optimizing counselling in a prenatal setting.

Materials and Methods: Two experiments were conducted among 51 medical students (HCP's) and 76 psychology students (in the role of patients) respectively. Vignettes covering several types of uncertain prenatal WES results were ranked on perceived uncertainty. Medical students answered whether they would report each result to the couple (experiment 1). Psychology students indicated whether they would want to receive the result (experiment 2). In both experiments participants indicated how certain they were about their choice.

Results: In experiment 1, highly uncertain results were reported less often $(59 \%)$ than results low in uncertainty (98\%), $p<.001$. Furthermore, medical students reported more uncertainty with their choice when vignettes were high and moderate versus low in uncertainty, $p<.001$. In experiment 2, psychology students chose less often to receive highly uncertain results $(71 \%)$ than results low in uncertainty $(87 \%), p=.012$. They reported more 
uncertainty about their choice when results were moderately and highly uncertain versus low in uncertainty, $p<.001$.

Conclusions: In this study we validated the widespread hypothesis that the willingness both to convey and receive uncertain prenatal WES results decreased as uncertainty increased.

J.E. Klapwijk: None. H.T. Brüggenwirth: None. K.E. M. Diderich: None. K. Dijkstra: None. S.R. Riedijk: None.

\section{P23.44.B}

Ensuring ethical and legal compliance of cutting-edge DNA sequencing technologies: practical considerations from the $\mathrm{H} 2020$ project EASI-Genomics

\section{E. Gennet, M. Coubault-Azzouz, G. Chassang, E. Rial- Sebbag}

Inserm UMR1027, TOULOUSE, France

EASI-Genomics ('European Advanced infraStructure for Innovative Genomics) is a European Infrastructure unifying the major European genomic centers providing external access to their facilities for performing genome sequencing (human, animal, vegetal) for research purposes. The mission of EASI-Genomics is to facilitate access to cutting-edge DNA sequencing technologies to researchers from both academia and industry, within or outside of Europe.

Partners of the project have set up a process in order to ensure compliance with the relevant European ethical and legal framework at stake in human, animal or plant sequencing: a memorandum of understanding, a standard material transfer agreement and an interdisciplinary ethics check of each selected researcher before being allowed to access the sequencing services.

After a year of experience and two calls for projects, some conclusions can be drawn on the main obstacles, limitations and learning curves of such a process in guaranteeing compliance to the European ethical and legal framework. But most of all, this experience permitted to identify some recurring issues that may need particular attention and efforts to ensure ELSI compliance. This is for instance the question of sequencing of ancient DNA in compliance with access and benefit sharing principles, or of the necessary safeguards regarding CRISPR-CAS9 engineered biomaterials.

EASI-Genomics has received funding from the European Union's Horizon 2020 research and innovation program under grant agreement No 824110.

E. Gennet: None. M. Coubault-Azzouz: None. G. Chassang: None. E. Rial-Sebbag: None. 